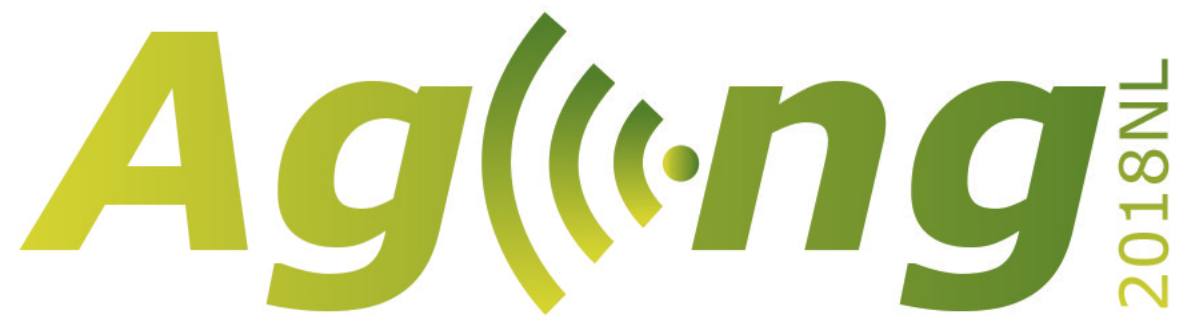

\title{
Proceedings of the European Conference on Agricultural Engineering AgEng2018
}

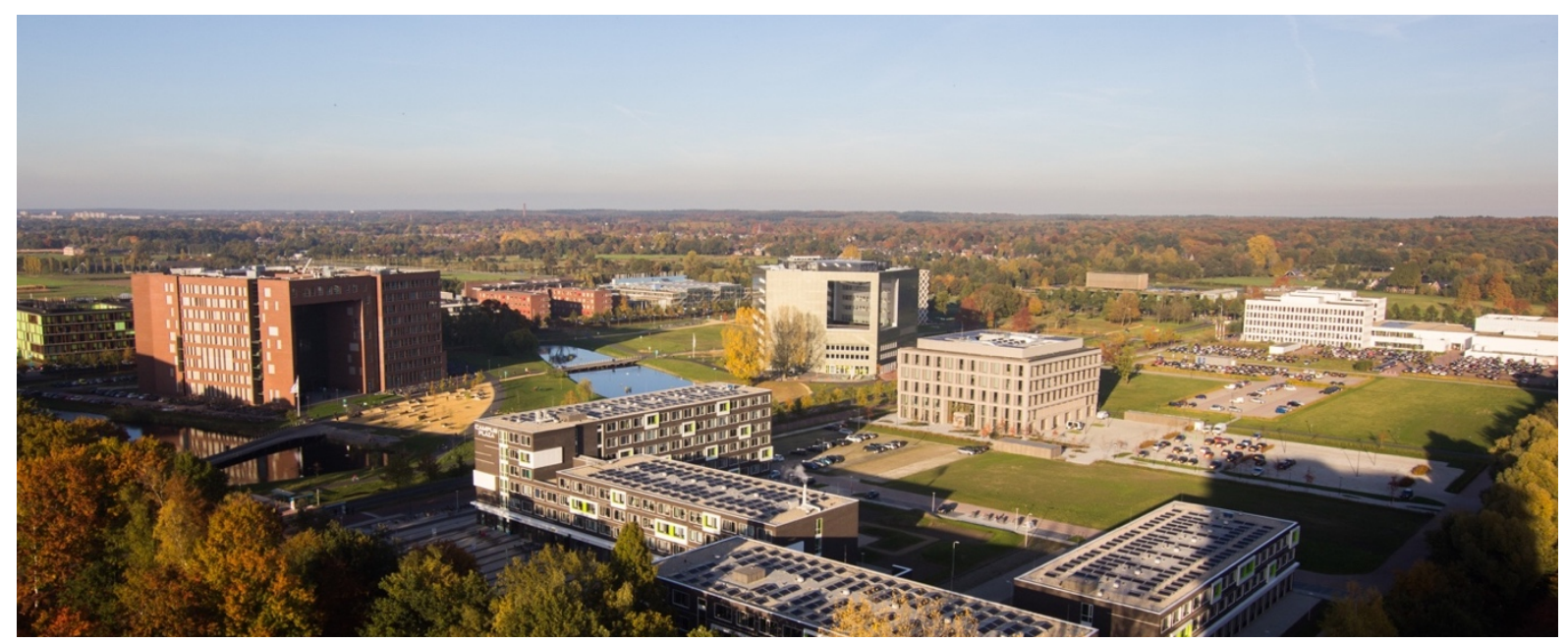

8-12 July, 2018

Wageningen, The Netherlands

Organised and published by:

Wageningen University and Research

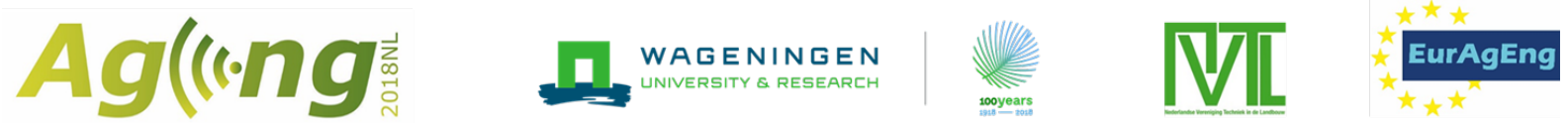




\title{
Proceedings of the European Conference on Agricultural Engineering AgEng2018
}

\author{
Edited by \\ Prof. Dr. P.W.G. (Peter) Groot Koerkamp \\ Dr. C. (Kees) Lokhorst \\ Ir. A.H. (Bert) Ipema \\ Dr. C. (Corné) Kempenaar \\ Dr. C.M. (Karin) Groenestein \\ C.G. (Casper) Van Oostrum \\ N.J. (Nardy) Ros
}

Cite as:

Groot Koerkamp, P.W.G., Lokhorst, C., Ipema, A.H., Kempenaar, C., Groenestein, C.M., van Oostrum, C.G., and Ros, N.J., 2018. Proceedings of the European Agricultural Conference, 8-12 July, Wageningen, the Netherlands. Wageningen: Wageningen University and Research.

Published by:

Wageningen University \& Research

This version is distributed under a Creative Commons Attribution (CC-BY) user license, which permits use, distribution, and repro-duction in any medium, provided the original work is properly cited. 
Papers from the bi-annual European conference on agricultural engineering held at Wageningen Campus as official event of the European Society of Agricultural Engineering (EurAgEng), Wageningen, the Netherlands, 8-12 July 2018. Abstracts of this conference can be found in a separate publication on the website http://www.eurageng.eu/

Copyright 2018, Wageningen University \& Research

PO Box 16, NL-6700 AA, Wageningen, The Netherlands

https://doi.org/10.18174/471679

Also available from www.eurageng.eu

Copyright and Reprint Permission: All rights reserved. No part of this publication may be reproduced, stored in a retrieval system or transmitted in any form or by any means without written permission from the copyright holders.

This proceeding were reproduced from manuscripts supplied by authors. Whilst every effort is made by the publisher to see that no inaccurate or misleading data, opinion or statement appears in this publication, they which to make it clear that the data and opinions appearing in the articles herein are the sole responsibility of the contributor concerned. Accordingly, the publisher, editors and their employers, officers and agents accept no responsibility or liability whatsoever for the consequences of any such inaccurate or misleading data, opinion or statement.

Not printed 


\section{Preface}

This proceedings book results from the AgEng2018 Agricultural Engineering Conference under auspices of the European Society of Agricultural Engineers, in Wageningen, the Netherlands, from 8-12 July 2018. This book contains the full papers of a selection of abstracts that were the base for the oral presentations and posters presented at the conference. We like to thank all participants, sponsors and the scientific committee of the conference for their indispensable contribution to this event. A special word of thanks goes to the municipality of Wageningen for organising a welcome reception as host city, and the many students and colleagues of chair groups and research institutes of Wageningen University \& Research that helped in so many ways to make the conference a success.

On behalf of the organising committee Peter Groot Koerkamp

\section{National organising committee}

Prof. Dr. P.W.G. (Peter) Groot Koerkamp

Dr. C.M. (Karin) Groenestein

M. (Maarten) Vrolijk

Ir. A.H. (Bert) Ipema

Dr. C. (Kees) Lokhorst

Dr. C. (Corné) Kempenaar

Wageningen University and Resesarch

Wageningen Livestock Research

Wageningen Livestock Research / Vrolijk adviseurs

Wageningen Livestock Research / retired

Wageningen Livestock Research

Wageningen Plant Research

\section{Supported by}

Oane de Hoop

OANEvents, Lelystad 


\section{Acknowledgements}

We are grateful to the following organisations and companies for their support:

NVTL, Nederlandse Vereniging Techniek in de Landbouw

Wageningen University \& Research

OANEvents

New Holland / CNH industrial, Zedelgem, Belgium

Lely, Maassluis, the Netherlands

Agrifac, Steenwijk, the Netherlands

PSI, Drasov, Czech Republic

WIWAM, Belgium

QING, Arnhem, the Netherlands

CLAAS, Harsewinkel, Germany

Kuhn, Saverne, France

Vencomatic, Eersel, the Netherlands

Big Dutchman, Vechta, Germany

Fendt, Marktoberdorf, Germany

Priva, De Lier, the Netherlands

Steketee, Stad aan 't Haringvliet, the Netherlands 


\section{Scientific committee with reviewers of abstracts}

\begin{tabular}{|c|c|}
\hline Name (in alphabetical order) & Institute/university/company, place and country \\
\hline Dr. Andre Aarnink & Wageningen University \& Research, Wageningen, the Netherlands \\
\hline Dr. Thomas Anken & Agroscope, Ettenhausen, Switzerland \\
\hline Prof. Francisco Ayuga & Universidad Politecnica de Madrid, Madrid, Spain \\
\hline Dr. Wouter Bac & TechNature, Waddinxveen, the Netherlands \\
\hline MSc Jos Balendonck & Wageningen University \& Research, Wageningen, the Netherlands \\
\hline Prof. Paolo Balsari & University of Turin, Grugliasco, Italy \\
\hline Prof. Pilar Barreiro & Technical University of Madrid, Madrid, Spain \\
\hline Dr. Thomas Bartzanas & Center for Research and Technology-HELLAS, Volos, Greece \\
\hline Prof. Nils Bjugstad & Norwegian University of Life Sciences, Ås, Norway \\
\hline Prof. Stefan Böttinger & University of Hohenheim, Stuttgart, Germany \\
\hline Prof. Demetres Briassoulis & Agricultural University of Athens, Athens, Greece \\
\hline Prof. Wolfgang Büscher & University Bonn, Bonn, Germany \\
\hline Dr. Jean-Pierre Chanet & Irstea, Aubière, France \\
\hline Dr. Bill Day & IAgrE, Hexham, United Kingdom \\
\hline Prof. Josse De Baerdemaeker & KU Leuven, Heverlee-Leuven, Belgium \\
\hline Dr. Sytze de Bruin & Wageningen University, Wageningen, the Netherlands \\
\hline Dr. Ingrid de Jong & Wageningen Livestock Research, Wageningen, the Netherlands \\
\hline Dr. Rudi de Mol & Wageningen Livestock Research, Wageningen, the Netherlands \\
\hline Dr. Jerke De Vries & Van Hall Larenstein, University of Applied Sciences, Velp, the Netherlands \\
\hline Dr. Markus Demmel & Bavarian State Research Center for Agriculture, Freising, Germany \\
\hline Dr. Marjolein Derks & Wageningen University \& Research, Wageningen, the Netherlands \\
\hline Dr. Adam Dubowski & PIMR-Industrial Institute of Agricultural Engineering, Poznan, Poland \\
\hline Prof. Pierluigi Febo & University of Palermo, Palermo, Italy \\
\hline Msc. Josi Flaba & Spw-dgarne, Awans, Belgium \\
\hline Prof. Ana I. Garcia & Universidad Politecnica de Madrid, Madrid, Spain \\
\hline Prof. Paolo Gay & Università degli Studi di Torino, Grugliasco, Italy \\
\hline Prof. Gilbert Grenier & Bordeaux Sciences Agro, Gradignan, France \\
\hline Dr. Karin Groenestein & Wageningen Livestock Research, Wageningen, the Netherlands \\
\hline Prof. Peter Groot Koerkamp & Wageningen University \& Research, Wageningen, the Netherlands \\
\hline Dr. Jan Harms & Bavarian State Research Center for Agriculture, Poing-Grub, Germany \\
\hline Dr. Jochen Hemming & Wageningen University \& Research, Wageningen, the Netherlands \\
\hline Dr. Silke Hemming & Wageningen University \& Research, Wageningen, the Netherlands \\
\hline Prof. Henk Hogeveen & Wageningen University, Wageningen, the Netherlands \\
\hline Mr. Pieter Hogewerf & Wageningen Livestock Research, Wageningen, the Netherlands \\
\hline Dr. Willem Hoogmoed & Wageningen University \& Research, Wageningen, the Netherlands \\
\hline Msc. Emmanuel Hugo & Irstea, Aubière Cedex, France \\
\hline Msc. Bert Ipema & Wageningen Livestock Research, Wageningen, the Netherlands \\
\hline Dr. Allan Leck Jensen & Aarhus University, Tjele, Denmark \\
\hline Prof. Florentino Juste & Universidad Politecnica de Valencia, Valencia, Spain \\
\hline Msc. Jan Kamp & Wageningen Plant Research, Lelystad, the Netherlands \\
\hline Dr. Claudia Kamphuis & Wageningen Livestock Research, Wageningen, the Netherlands \\
\hline Prof. Nikolaos Katsoulas & University of Thessaly, Volos, Greece \\
\hline Msc. Robert Kaufmann & Agroscope, Gossau, Switzerland \\
\hline Dr. Corné Kempenaar & Wageningen Plant Research, Wageningen, the Netherlands \\
\hline Dr. Lammert Kooistra & Wageningen University, Wageningen, the Netherlands \\
\hline Dr. Gert Kootstra & Wageningen University \& Research, Wageningen, the Netherlands \\
\hline Dr. Martin Kremmer & Deere \& Co. World Headquarters, Moline, United States \\
\hline Prof. Roland Lenain & Irstea, Aubière, France \\
\hline Prof. Kees Lokhorst & WLR/Van Hall Larenstein, Wageningen/Leeuwarden, the Netherlands \\
\hline Dr. Bastiaan Meerburg & Dutch Pest \& Wildlife Expertise Center (KAD), Wageningen, the Netherlands \\
\hline Dr. Antonis Mistriotis & Agricultural University of Athens, ATHENS, Greece \\
\hline Dr. Gert-Jan Monteny & Monteny Milieu Advies, Renkum, the Netherlands \\
\hline Dr. Julio Mosquera Losada & Wageningen Livestock Research, Wageningen, the Netherlands \\
\hline Dr. Stefan Neser & Bavarian State Research Center for Agriculture, Freising, Germany \\
\hline Prof. Filip Nicolae & Technical University of Cluj Napoca, Cluj Napoca, Romania \\
\hline Dr. Ard Nieuwenhuizen & Precision Makers, Montfoort, the Netherlands \\
\hline Msc. Michael Nørremark & Aarhus University, Tjele, Denmark \\
\hline
\end{tabular}




\begin{tabular}{|c|c|}
\hline Dr. Nico Ogink & Wageningen Livestock Research, Wageningen, the Netherlands \\
\hline Dr. Timo Oksanen & Aalto University, Espoo, Finland \\
\hline Prof. Panagiotis Panagakis & Agricultural University of Athens, Athens, Greece \\
\hline Msc. Antti Peltola & Häme University of Applied Sciences, Hämeenlinna, Finland \\
\hline Dr. Gerrit Polder & Wageningen Plant Research, Wageningen, the Netherlands \\
\hline Dr. Daniel Puente Rodríguez & Wageningen Livestock Research, Wageningen, the Netherlands \\
\hline Prof. Francisco Rovira-Mas & Universitat Politecnica de Valencia, Valencia, Spain \\
\hline Prof. Giacomo Scarascia-Mugnozza & Università di Bari, Bari, Italy \\
\hline Prof. Evelia Schettini & University of Bari, Bari, Italy \\
\hline Prof. Peter Schulze Lammers & University of Bonn, Bonn, Germany \\
\hline Dr. Dennis Snoek & Agrifirm, Apeldoorn, the Netherlands \\
\hline Prof. Bart Sonck & Institute of Agricultural and Fisheries Research, Melle, Belgium \\
\hline Dr. Claus Sörensen & Aarhus University, Aarhus, Denmark \\
\hline Dr. Kornél Szalay & National Research and Innovation Center, Gödöllö, Hungary \\
\hline Prof. Patrizia Tassinari & University of Bologna, Bologna, Italy \\
\hline Msc. David Tinker & EurAgEng Society, Bedford, United Kingdom \\
\hline Msc. Jan van de Zande & Wageningen University \& Research, Wageningen, the Netherlands \\
\hline Dr. Rik van der Tol & Wageningen University, Wageningen, the Netherlands \\
\hline Dr. Frits K. Van Evert & Wageningen University \& Research, Wageningen, the Netherlands \\
\hline Prof. Eldert van Henten & Wageningen University \& Research, Wageningen, the Netherlands \\
\hline Dr. Simon van Mourik & wageningen University, Wageningen, the Netherlands \\
\hline Prof. Gerrit van Straten & Wageningen University / AgrodynamicsSupport, Renkum, the Netherlands \\
\hline Dr. Victor van Wagenberg & Vencomatic Group, Eersel, the Netherlands \\
\hline Prof. Diego Luis Varela & University of Almería, Almería, Spain \\
\hline Dr. Cor Verdouw & Wageningen University \& Research, Wageningen, the Netherlands \\
\hline Dr. Bastiaan Vroegindeweij & Livestock Robotics, Ochten, the Netherlands \\
\hline Mr. Maarten Vrolijk & Vrolijk adviseurs, Dronten, the Netherlands \\
\hline Dr. Albert Winkel & Wageningen Livestock Research, Wageningen, the Netherlands \\
\hline Dr. Sjaak Wolfert & Wageningen University \& Research, Wageningen, the Netherlands \\
\hline Msc. Rick van de Zedde & Wageningen University \& Research, Wageningen, the Netherlands \\
\hline Dr. Guoqiang Zhang & Aarhus University, Aarhus, Denmark \\
\hline
\end{tabular}




\section{Table of contents}

PREFACE

ACKNOWLEDGEMENTS VI

SCIENTIFC COMMITTEE WITH REVIEWERS OF ABSTRACTS VII

TOPIC 1: DESIGN METHODS FOR INTEGRATED AND SUSTAINABLE FARMING SYSTEMS (FS) 1

Predictive Systems Model Simulating The Development Of Beehives And Honeybee Population

(Apis Mellifera) In Professional Apicultures

Jochen Georg Wiecha, Sascha Wörz, Heinz Bernhardt

A Model to Assess Environmental Efficiency of Pig Production Systems

Luis Lassaletta, Fernando Estellés, Arthur Beusen, Lex Bowman, Salvador Calvet, Hans van Grinsven, Jonathan Doelman, Elke Stehfest, Aimable Uwizeye, Henk Westhoeck

TOPIC 2: NEW FARMING MANAGEMENT SYSTEMS (FM) ,

Application of Drone-Based Maps in Planning Farm Operations for Sward Management in Silage Production Jussi Nikander, Markku Koistinen, Mikko Laajalahti, Oiva Niemeläinen, Jere Kaivosoja

A Territorial Services Platform for the Sustainable Precision Agriculture Applied to the Viticulture and Olive Growing Companies

Daniele Sarri, Simone Priori, Marco Antoni, Sara Gallucci, Stefania Lombardo, Marco Vieri

Application of a Modified McKendrick-von Foerster Equation to Predict Beetle Population Dynamics

(Xyleborus affinis) under Artificial Medium in Growth Chambers

Fernando Rojano, Luis Arturo Ibarra-Juarez, Andres Lira-Noriega, Federico Escobar-Sarria, Daniel González-

Tokman, Porfirio Toledo-Hernández, Brenda Tapia-Santos, Carlos A. Hernández-Linares

Performance Evaluation of Passive Radio Frequency Identification Technology in Silage Bale

Application

Katariina Penttilä, Antti Suokannas, Ilpo Pölönen

Enhanced Vineyard Descriptors Combining UAV 2D and 3D Models

Lorenzo Comba, Alessandro Biglia, Davide Ricauda Aimonino, Paolo Barge, Cristina Tortia, Paolo Gay

Internet-based Harvest Fleet Logistic Optimisation

Andrés Villa-Henriksen, Nick Skou-Nielsen, Claus A. Grøn Sørensen, Ole Green, Gareth Edwards

Connectivity of Agricultural Equipment and Applications - Strategical Approaches and Technical Standards

Matthias Rothmund

Analysis of Vine Vibration Conditions and Grape Harvest Quality in Spain under Actual Mechanized

Harvest Conditions Wilson Da Costa Neto, Pilar Barreiro, Belén Diezma

Animal Welfare Adjustment Real Time Environmental Condition of Housing (AWARTECH Project) Living Lab Setup Vasco Fitas da Cruz, Fatima Batispta, José Carlos Rico, David Botas

Low Cost integral system for irrigation remote management based on a communication nodes network with sensors, weighing lysimetry and supervision of the vegetative state for hortícultural crops Antonio Ruiz-Canales, José Miguel Molina-Martínez, Martin John Oates, Juan José Pérez Solano, Amparo Melián Navarro, Jesús Chazarra Zapata, Francisco López Peñalver, José Manuel González Esquiva

Design and Performance of an Unmanned Aerial System for Precision Pest Management Jorge Martínez-Guanter, Pablo Agüera, Juan Agüera, Manuel Perez-Ruiz

Farms, Fogs and Clouds: Data open-architecture for optimal crop growth control for loF2020 project

Manuel Muñoz-Rodríguez, Jorge A. Sanchez-Molina, Francisco Rodríguez, Manuel Berenguel, Cynthia Giagnocavo

TOPIC 3: CO-PRODUCTION AND IMPLEMENTATION OF TECHNOLOGY IN FOOD PRODUCTION SYSTEMS (FP)

An Innovation System Supporting the Adoption of Precision Agriculture in Sweden: The Case of Yara N-Sensor 
Christina Lundström, Jessica Lindblom, Magnus Ljung

Promotion of Circular Economy in Alentejo's Agri-food Sector

Vasco Manuel Fitas da Cruz, Bruno Magalhães, Cristina Ascenço, Fátima Baptista

Skills Needs for a Sustainable Farmer in the Framework of the Sagri Project

Luis L. Silva, Fátima Baptista, Vasco Cruz, José Rafael Marques-da-Silva

Adoption of automatic feeding systems in bovine livestock farms: an on-farm survey on Italian situation

Carlo Bisaglia, Massimo Brambilla, Maurizio Cutini, Alberto Assirelli

Spatial Distribution of Mechanization Levels in Italian Greenhouses

Elio Romano, Massimo Brambilla, Maurizio Cutini, Pietro Toscano, Felicetta Carillo, Carlo Bisaglia

SPARKLE Project: e-Learning Solutions to Create New Digital Agronomist Profiles on European

Students

Jorge Martínez-Guanter, Daniele Sarri, Marco Vieri, Stefania Lombardo, Manuel Pérez-Ruiz

Management System of Small Farm Machinery Hiring Business for Rice Farming Operations in

Kampar Region, Indonesia Ujang Paman, Khairizal and Hajry Arief Wahyudy

Long-Term Tracking and Automated Analysis of Tractor Behaviour with JDLINK P. Barreiro, I. Rueda, A. Rabasco, M. Garrido-Izard

\section{TOPIC 4: ROBOTICS AND SENSOR TECHNOLOGY (RM)}

Vibration Analysis and Canopy Adaptation of Citrus Tree for an Efficient Harvesting with Canopy

Shaker Technology

Sergio Castro-Garcia, Rafael R. Sola-Guirado, Gregorio L. Blanco-Roldán, Antonio J. Serrano, Juan José Garcés Iniesta, Emilio Soria Olivas, Jesus A. Gil-Ribes

PC Based Data Acquisition, Sensor Fusion and Data Syncronisation in Agriculture Based on an

Example of Measurement of Soil Surface Roughness with a 2D LIDAR Zoltan Gobor, Christian Rossow, Robert Weinfurtner, Georg Fröhlich

Robust Online Soil Roughness Measurement Using Stereo Vision Peter Riegler-Nurscher, Heinrich Prankl, Gerhard Moitzi, Helmut Wagentristl

Assessing the Spatio-Temporal Alfalfa yield variability Using Landsat-8 and Yield Monitor Data at field scale Ahmed Kayad, Khalid Al-Gaadi, ElKamil Tola, Rangaswamy Madugundu, Marco Sozzi, Francesco Marinello

Structured Design of a Novel End-effector for a Bush Trimming Robot Bart van Tuijl, Toon Tielen, Angelo Mencarelli, Jochen Hemming

Development Of Autonomous Robotic Platforms For Sugar Beet Crop Phenotyping Using Artificial Vision

Bernard Benet, Camille Dubos, Fabienne Maupas, Ghislain Malatesta, Roland Lenain

Comparison Between Ultrasonic Sensors And 2D LIDAR As Perception Systems For Autonomous

Vineyard Robots Andrés Cuenca, Francisco Rovira-Más, Verónica Saiz-Rubio

Identification of Reflected Spectral Related to Plant Circadian Rhythm Using Maximal Information

Coefficient

Shogo Nagano, Akito Sammyo, Hirokazu Fukuda

Design Of Simplified Optical Devices For The Agrofood Sector: Case Studies From Pre-Harvest To Post-Harvest Roberto Beghi, Valentina Giovenzana, Alessio Tugnolo, Riccardo Guidetti

Reliability of Autonomous Guided Systems for Vineyard Monitoring Dionisio Andujar, Jose Bengochea-Guevara, Angela Ribeiro

Evaluation Of The Potential For Automatic Herbicide In Wheat Crops Mihai Gidea, Catalin Mircea Constantinescu, Dragos Manea, Alexandru Ciorita, Laura Mihaela losub

Field Robot to Detect Plants Infected by Candidatus Liberibacter solanacearum in Horticultural Using Multispectral Computer Vision

Sergio Cubero, Santiago Lopez-Alaman, Ester Marco-Noales, Susana Sanjuan, Vicente Alegre, Silvia Barbe, Enrique Aguilar, Inmaculada Navarro, Nuria Aleixos, Jose Blasco 
Luis Alcino Conceição, José Rafael da Silva, Rute Santos, Noémia Farinha, Benvindo Maças

The Electronic Smell of the Orchard Fruit

João Valente, Rodrigo Almeida, Lammert Kooistra

Soil Organic Carbon estimation using proximal and remote sensing techniques

Theodora Angelopoulou, Athanasios Thoma Balafoutis, Dionysis Bochtis, George Zalidis

Preliminary Approach for the Detection of Olive Trees Infected by Xylella fastidiosa Using a Field

Robot and Proximal Sensing

Santiago Lopez, Sergio Cubero, Nuria Aleixos, Vicente Alegre, Beatriz Rey, Enrique Aguilar, Jose Blasco

Non-destructive Firmness Assessment of Avocado Fruit Using a Pneumatic Low Impact Device 275 Coral Ortiz, Carlos Blanes, Martin Mellado

Current potentials and challenges using Sentinel-1 for broadacre field remote sensing Martin Peter Christiansen, Morten Stigaard Laursen, Birgitte Feld Mikkelsen, Rasmus Nyholm Jørgensen, Nima Teimouri, Claus Aage Grøn Sørensen

Benchmark of Satellites Image Service For Precision Agricultural Use Marco Sozzi, Francesco Marinello, Andrea Pezzuolo, Luigi Sartori

Comparison between Different Spatial Resolution Satellites Images for Viticulture Marco Sozzi, Ahmed Galal Kayad, Andrea Pezzuolo, Luigi Sartori, Marco Dubbini, Francesco Marinello

Cruising Robot for Collecting Floor Eggs in Poultry Houses Weichao Zheng, Dongxu Zhang, Changle Pang, Guanghui Teng, Chaoyuan Wang, Baoming Li

Novel Systems for Pasture Characterization Using RGB-D Cameras and UAV-Imagery Victor Rueda-Ayala, José Manuel Peña, José María Bengochea Guevara, Mats Höglind, Christian Rueda-Ayala, Dionisio Andújar

Design Space Exploration in the Development of Agricultural Robots Frederik F. Foldager, Ole Balling, Carl Gamble, Peter Gorm Larsen, Martin Boel, Ole Green

Fast Method for the Weeds' Impact Evaluation on a Field Crop Using Smartphones

Elio Romano, Carlo Bisaglia, Massimo Palumbo, Nino Virzì, Fabiola Sciacca, Sergio Saia, Ivano Pecorella, Pasquale De Vita

Evaluation of Pasture Productivity and Quality in the Montado Ecosystem Using Proximal Sensors 329 João Serrano, Shakib Shahidian, José Marques da Silva, Francisco Moral

Lameness Recognition of Pigs Based on Skeleton Analysis and Gait Energy Image Weixing Zhu, Kaizhong Zhang, Jianxuan Qian, Xincheng Li

Estimating Pasture Nitrogen Content using Thermal Images Majeed Safa, Kirsty H Martin, Tom Maxwell, Birendra KC

UAV and Machine Learning Techniques Applied to Yield Estimation of Orange Trees Orly. Enrique Apolo-Apolo, Jorge Martínez-Guanter, Gregorio Egea, Manuel Perez-Ruiz

Data Acquisition Platform for Collecting High-Quality Images of Cultivated Weeds Simon Leminen Madsen, Mads Dyrmann, Morten Stigaard Laursen, Solvejg Kopp Mathiassen, Rasmus Nyholm Jørgensen

A 3D Low-Cost Photogrammetry Method for Weed Plants Modelling Dionisio Andujar, Mikel Calle, César Fernández-Quintanilla, Ángela Ribeiro, Jose Dorado

Challenges in modern automated Feeding Systems: Navigation Technologies Matthias Reger, Johannes Friedrich, Sascha Wörz, Jörn Stumpenhausen, Anton Sieghart, Heinz Bernhardt

SPAD and Hyperspectral Images for Sensing Chlorosis Affection in Peach Trees María Victoria Lafuente, Lourdes Lleó, Jesús Val, Jean Michel Roger, Pilar Barreiro

Integrated Study of a Picking Robot for Greenhouse Grown Sweet Pepper Yi-Chich Chiu, Li-Wen Chung, Gang-Jhy Wu

Orchardmapper: Application for Creating Tree Scale Maps FromfHigh Resolution Orthomosaics Dvoralai Wulfsohn, Oded Cohen, Ines Zamora Lagos

Development of an Autonomous Agricultural Vehicle

Pieter-Jan Note, Jan Bienstman, Chris Ganseman

\section{TOPIC 5: ENERGY AND GREENHOUSE GAS EMISSIONS (EG)}

Assessing Water Supply Sources Using Life Cycle Assessment: Study Case of Lemon Crop in South-Eastern Spain 
Bernardo Martin-Gorriz, Jose Francisco Maestre-Valero, VIctoriano Martinez-Alvarez

Sustainability Issues Related to Woody Biomass Supply From Fruit Tree Plantation Removal Alberto Assirelli, Massimo Brambilla, Giancarlo Roccuzzo, Carlo Bisaglia

Performance Study of a Standalone Direct Pumping Photovoltaic System Used for Drip Irrigation Harkani Assia, El Aissaoui Abdellah, Fihri Fassi Hichame, El Kacimi Ousama

Modeling in Matlab of a Standalone Photovoltaic Pumping System for Drip Irrigation Use and

Performance Analysis

El Aissaoui Abdellah, El Kacimi Ousama, Harkani Assia, Mansouri Mohamed

430

In-situ Performance Evaluation of PV Pumping System and Method for Yielding Pumping Capacity

for Drip Irrigation Use

EL Aissaoui Abdellah, Oudadda Mohamed, Harkani Assia

Economic Comparison and Carbon Emissions of Payload Optimized Milk Logistic Chains Michael Schmid, Sascha Wörz, Heinz Bernhardt

Milk Transport Costs and Carbon Emissions of Various Triggered Milk Logistic Chains Michael Schmid, Sascha Wörz, Heinz Bernhardt

Preliminary Study On Traction Efficiency of an Agricultural Tractor Equipped With an Extendable

Ballast Holder Massimiliano Varani, Michele Mattetti, Giovanni Molari

Biomass Heating System with Kerosene Boiler for Green House Heating Yoshimichi Yamashita, Kazuhiro Takekura, Genta Kanai, Kenichi Yakushidou

Technology for Efficient Application of Liquid Manure: A Comprehensive Modelling Approach on

Greenhouse Gas Emissions Bianca Zerhusen, Fabian Lichti, Stefan Neser

Groundwater Utilization in a Farm Heat Network Genta Kanai, Yoshimichi Yamashita

Sustainability through Digital Agriculture - Analysis of Available Greenhouse Gas Emission Indicators in Farm Management Systems Heinz Bernhardt, Leonie Spiller, Omar Hijazi

Development of In-House Lattice-Boltzmann Simulator of Anaerobic Bioreactors for Vinasse

Treatment: Preliminary Dimensionless Modelling and Sensitivity Analysis Jose Antonio Rabi, Fernando de Lima Caneppele, Rogers Ribeiro, Gustavo César Dacanal

Agricultural Tire Energy Efficiency Test Method and Dedicated Equipment to Compare and Improve

Fuel Consumption and Traction of Agricultural Tires under Real Field Conditions Kornel Szalay, Laszlo Kovacs, Gabor Bercesi, Istvan Oldal, Emmanuel Piron, Julien Charlat, Thierry Joly, Clement Poncet, Florence Tran

Fuel Consumption Analysis of Real-World Measurements Michele Mattetti, Riccardo Molinari, Eugenio Sereni, Giovanni Molari

Leftover Silage as Biogas Substrate: Processing Machinery and Costs Carina Gunnarsson, Johanna Lund,, Erik Fischer

Analysis of the Indoor Climate and Energy Use in two Animal Houses for Fattening Pigs Andrea Costantino, Lorenzo Comba, Andrea Rolfo, Paolo Cornale, Luca Battaglini, Enrico Fabrizio

Tractor Driveline Efficiency Evaluation Taking into Account Power Lost in Slippage. Maurizio Cutini, Massimo Brambilla, Carlo Bisaglia

Simulation Model of Using the Electricity and Exhaust Gas Heat Generated from Poultry Litter

Waste in their House Mohamed Nabih Omar, Abdellateif Abdelwahab Samak, Mahmoud Hassan Keshek

\section{TOPIC 6: SOIL, LAND AND WATER ENGINEERING (SW)}

Evaluation of Compaction in No-Till Vertisol Field Using Methods of Cone Index and Pedotransfer Function - Case of a Moroccan Semi-Arid Context Al Masmoudi Yassine,, El Aissaoui Abdellah, Ibno Namr Khalid

Impacts of Artificial and Natural soil Conditioners on Water holding capacity and Hydraulic Conductivity of Sandy soils Faisal Zeineldin, Khalid Biro, Mohammed Refdan Al-Hajhoj 
Alternative Underdrain Designs for Reducing Pressure Drop across Sand Media Filters

T. Pujol, G. Arbat, J. Bové, J. Puig-Bargués, M. Duran-Ros, J. Velayos, F. Ramírez de Cartagena

Effect of Sand Size and Inlet Pressure on Sand Media Filter Performance using Reclaimed Effluents

Miquel Duran-Ros, Jaume Puig-Bargués, Gerard Arbat, Joan Pujol, Francisco Ramírez de Cartagena

Development of New Version of the Spiked Shear Vane for Measuring of the Peatland Top Roots

Layer Strength Adam Piotr Dubowski, Krzysztof Zembrowski

Differential Interferometry as a Tool for Localized Study of Runoff Erosion Francisco A. Sánchez-Crespo, Eutiquio Gallego, José M. Fuentes, Francisco Ayuga, Ana I. García

Soil Conductivity: Detecting Slurry Injection Depth with Minimal Effort Jochen Georg Wiecha, Sascha Wörz, Heinz Bernhardt

Delivery Rates during Retention and Transport of Encapsulated Fertilisers In Porous Medium Fernando Rojano, Felipe Barrera-Mendez, Maria A. Luna-Velasco, Abraham Rojano

Territorial Analysis and Hydrological Modelling of the Canale D'Aiedda Basin

Francesco Gentile, Ersilia D'Ambrosio, Marinella Spanò, Ossama M.M. Abdelwahab, Antonello Lonigro, Giovanni Francesco Ricci, Giovanni Romano, Angelantonio Calabrese, Giuseppe Pappagallo, Anna Maria De Girolamo

Landscape Fragmentation in the European Context: Outcomes from a Comparative Approach Andrea De Montis, Vittorio Serra, Antonio Ledda

Effect of Irrigation Systems and Water Quantities on Corn Production under Partial Rootzone Drying

Conditions

Abdellateif Abdelwahab Samak, Mohamed Nabih Omar, Mahmoud Hassan Keshek

$\mathrm{NH}_{3}$ Emissions from Treated Buffalo Manure Application in Mediterranean Climate and Comparison

to ALFAM Model

Ester Scotto di Perta, Nunzio Fiorentino, Salvatore Faugno, Elena Cervelli, Stefania Pindozzi

Novel in Situ System for Monitoring Soil Organic Carbon by Using Mobile Vis-Nir Spectroscopy

and Machine Learning Techniques

Nikolaos Tziolas, Nikolaos Tsakiridis, Eyal Ben-Dor, Eleni Kalopesa, George Galanis, George Zalidi

\section{TOPIC 7: ANIMAL PRODUCTION TECHNOLOGIES (AP)}

Evaluation of Novel Farrowing Systems with Possibility for the Sow to Move - Effects on Working

Time Requirement

Franz Handler, Emil Blumauer, Birgit Heidinger

Long Term Investigations to Compare Different Renewable Energy Sources for Heating and

Cooling Pig Houses

Wolfgang Büscher, Manuel Krommweh, Hannah Licharz, Christian Maack

Recent Advances of Modelling and Experimental Verification of Ammonia and Greenhouse Gas

Emissions from Dairy Housing

Christopher Choi

Climate Conditions - a Status Quo at Dairy Farms in North - East Germany Jennifer Löbel, Sandra Rose-Meierhöfer, Paul Gütschow, Clemens Fuchs,

Measuring the Teat Skin Elasticity with the Help of the Suction Method Susanne Demba, Christian Ammon, Marie Neumerkel, Reiner Brunsch, Sandra Rose-Meierhöfer

Greenhouse Gas Emissions from Dairy Open Lots in China Luyu Ding, Chaoyuan Wang, Guoqiang Zhang, Zhengxiang Shi, Baoming Li, Weichao Zheng, Ligen Yu, Qifeng Li

Test of UHF Ear Tags for Piglets and Growing-Finishing Pigs on an Optimized Test Bench. Adrian Foerschner, Felix Adrion, Eva Gallmann

Differences in Light Concepts of Animal Welfare at Dairy Farms in North-East Germany Jennifer Löbel, Sandra Rose-Meierhöfer, Paul Gütschow, Clemens Fuchs

Evaluating Pig Ear Skin Temperature: Intercontinental Transport vs Housing Conditions Miguel Garrido-Izard, José M. Requejo, Eva C. Correa, Belén Diezma

The Effect of Liner Type on the Teat Load Caused by a Collapsing Liner Using a PressureIndicating Film 
Susanne Demba, Victoria Paul, Christian Ammon, Sandra Rose-Meierhöfer

Effects of the Accumulation of Heat Load Duration on the Activity Behavior of Lactating Dairy Cows

Julia Heinicke, Stephanie Ibscher, Vitaly Belik, Thomas Amon

Animal Welfare Indicators in Growing and Fattening Pigs with Different Environmental Conditions 737 Vasco Fitas da Cruz, Rui Charneca, Teresa Morgado, Catarina Martins

Development of a Test Stand for the Testing of Alternative Manure Removal Methods in Slurry

Channels

Bastian Kolb, Eva Gallmann

Ammonia Emission Assessment After Buffalo Manure And Digestate Application Ester Scotto di Perta, Yann Collas, Nunzio Fiorentino, Elena Cervelli, Salvatore Faugno, Stefania Pindozzi

Influence of Increased Light Intensity on the Lying and Excretory Behavior of Fattening Pigs Svenja Opderbeck, Barbara Kessler, Eva Gallmann

Influence of Wind Direction and Sampling Strategy on the Estimation of Ammonia Emissions in

Naturally Ventilated Barns

David Janke, Dilya Willink, Sabrina Hempel, Christian Ammona, Barbara Amon, Thomas Amon

Low Emission Slat Design for Swine Buildings

Stéphane Godbout, Francy Vanegas, Joahnn H. Palacios, Matthieu Girar, Ariane Lévesque, Alain Rousseau

Effect of Sidewall Openings on Flow Pattern and Gas Emissions of a Naturally Ventilated Dairy

Barn - A Wind Tunnel Study

Qianying Yi, Guoqiang Zhang, Marcel König, David Janke, Sabrina Hempel, Barbara Amon, Thomas Amon

Simulation of Nitrous Oxide Emission from Dairy Manure Stockpile using Dynamic Chamber

Technique

Bin Hu, Chaoyuan Wang, Haoxiang Zhao, Yejian Wang, Zhengxiang Shi, Baoming Li, Weichao Zheng

Optimal Ambient Temperature with Regard to Feed Efficiency and Daily Gain of Finisher Pigs Rikke Koch Hansen, Bjarne Bjerg

Influence of Evaporative Cooling on Respiration Rate of Lactating Cows Under Hot Climate Conditions

Severino Pinto, Harel Levit, Theresa Siemens, Gundula Hoffmann, Christian Ammon, Ilan Halachmi, Heuwieser, Thomas Amon

Modelling of Environmental Time Series in Livestock Facilities by Hybrid Model of ARIMA with

Wavelet Transform

Roberto Besteiro Doval, Tamara Arango López, M. Dolores Fernández Rodríguez, M. Ramiro Rodríguez Rodríguez

Analysis of Factors Affecting Ammonia and Methane Emissions from Pig Slurries: Slurry

Composition and Dietary Factors

Walter Antezana, Alba Cerisuelo, Fernando Estellés, Salvador Calvet

TOPIC 8: PLANT PRODUCTION TECHNOLOGIES (PP)

Mechanical Pruning of Lemon Trees

Bernardo Martin-Gorriz, Antonio Torregrosa, Carlos Martinez Barba

Management of Chemical Residual Volume Relative to Use of Stationary Spraying System in

Moroccan Greenhouse Crop Production

El Aissaoui Abdella, Khnizrou Larbi, Houmy Karim, Bouzrari Benaissa

Effect of Nozzle Type, Pressure and Height on Spray Distribution Pattern and Droplet

Characteristic

Arun Kumar, Bhabani Dash

Hyperspectral Remote Sensing Applications in Raspberry Plantation

Boglarka Keller, Laszlo Kovacs, Renata Rak, Andras Jung, Fanni Sillinger, Nora Peterfalvi, Kornel Szalay

Analysis of Different Mechanical Pruning Strategies on the Production of Clemenules Mandarin and its Costs

Guillermo Mateu Navarrete, Antonio Torregrosa Mira, Patricia Chueca Adell

Field Performance Evaluation Method for a Machine Vision Based Intra Row Hoeing Machine Jochen Hemming, Hermen de Jong, Lauwrens Struik, Jasper van Meer, Eldert J. van Henten

'Clemenrubi' Mandarin Size Development after Manual and Mechanical Thinning 
Antonio Torregrosa, Enrique Ortí, Emilio Soria, Sebastià Balasch, Coral Ortiz

Managing Weeds in Wheat and Pulses by Optimising Plant Spatial Arrangement and Sowing Time 857 Elio Romano, Carlo Bisaglia, Antonio lannuzzi, Sergio Saia, Ivano Pecorella, Pasquale De Vita

Design and Development of Machinery to Plant Daffodil Bulbs in Upland Pasture and Harvest the

Above Ground Biomass.

David Richard White, lan James Loynes, Simon Edward Cooper, Kevin Stephens, Stephen Head,

Mariecia D Fraser, Hannah Vallin, John R T Davies

Prognosis of Leaf Chlorosis in Catherina Peaches Based on RGB Images and on the Mineral

Composition of Flowers

Victoria de la Fuente, Pilar Barreiro, Lourdes Lleó, Jesús Val

TOPIC 9: GREENHOUSE PRODUCTION TECHNOLOGIES (GP)

Effect of Bench Heating on Growing Medium Temperature and Heat Loss From a Greenhouse in

Wintertime

Massimo Brambilla, Elio Romano, Maurizio Cutini, Marco Fedrizzi, Mauro Pagano, Gianluca Burchi,

Sonia Cacini, Daniele Massa, Chiara Terrosi, Carlo Bisaglia

Sensitivity Analysis of a Modified Equation for Greenhouse Energy Demand Assessment. Massimo Brambilla, Elio Romano, Maurizio Cutini, Marco Fedrizzi, Mauro Pagano, Gianluca Burchi, Sonia Cacini, Daniele Massa, Chiara Terrosi, Carlo Bisaglia

Wind Pressures on Arched Roof Greenhouses Anastasios Giannoulis, Demetres Briassoulis, Antonis Mistriotis

Effects of Infrared Radiation on Growth Parameters and Physiological Characteristics of Eggplant

Cultivation Angeliki Kavga, Vasileia Sinanoglou, Panagiotis Zoumpoulakis, Vasileios Kappatos

Effect of Dimethyl Ether (DME) Combustion on Lettuce and Chinese Cabbage Growth in Greenhouse

Waqas Qasim, Jayanta Kumar Basak, Frank Gyan Okyere, Fawad Khan, Yong Jin Lee, Hyeon Tae Kim

TOPIC 10: POST-HARVEST TECHNOLOGIES (PH)

Wall Pressures on Oblique Hoppers of Steel Silos

Eutiquio Gallego Vázquez, Adam Sadowski, Jose María Fuentes, Francisco Ayuga

Hyperspectral Imaging for Foal Meat Classification Based on Ageing Time

Ainara Lopez-Maestresalas, Kizkitza Insausti, Carlos Lopez-Mollina, Claudia Perez-Roncal, Maria Victoria Sarries, Carmen Jaren, Silvia Arazuri

Mandarin Mold Inactivation of Storage at Ambient Temperature by Non-thermal Plasma Treatment 924 Youngwook Seo, Hoeman Park, Jong Ryul Park

Detection of Astringent and Deastringent Persimmon Fruit Using Hyperspectral Imaging Technology

Sandra Munera, Nuria Aleixos, Juan Gómez-Sanchís, Cristina Besada, Sergio Cubero, Pau Talens,

Alejandra Salvador, José Blasco

Monitoring Watercress Shelf-life with VNIR Hyperspectral Image through Packaging Film Miguel Ángel Lara, Lourdes Lleó, Jean Michel Roger, Belén Diezma

Design of an Innovative Plant for Fast Freezing of Potato Dumplings Alessandro Biglia, Lorenzo Comba, Paolo Barge, Enrico Fabrizio, Davide Ricauda Aimonino, Cristina Tortia, Paolo Gay

Simultaneous Production of Functional Lipids and Natural Antioxidants From Passion Fruits Seeds 948 Carolina Cruzeiro Reis, Alexandra Mara G. Nunes Mamede, Nina Katia Silva James, Antonio Gomes Soares, Suely Pereira Freitas

Microencapsulated Functional Lipids by Association of Pomegranate Seed and Soybean Oils Nina Katia da Silva James, Meire J. A. Ferreira, Regina Isabel Nogueira, Luzimar Silva Mattos Nascimento, Suely Pereira Freitas

Development of a Machine for the Production of Liquid Wine Yeast to Induce Grape Juice

Fermentations

Lorenzo Guerrini, Alessandro Parenti, Giulia Angeloni, Piernicola Masella, Damiano Barbato, Eleonora Mari, Yuri Romboli, Giacomo Buscioni, Silvia Mangani, Simona Guerrini, Lisa Granchi

Influence of Physical and Chemicals Characteristics from Pumpkin for its Mechanized Processing 
Erich Dietmar Rössel-Kipping, Hipolito Ortiz-Laurel, Alejandro Amante-Orozco, Hector Martin Duran-Garcia, Araceli Lopez-Martinez

A New Method to Calibrate Discrete Element Models of Fibrous Agricultural Materials Adam Kovacs, Gyorgy Kerenyi

Evaluation of a Citrus Mobile Platform Using a Wireless Impact Recording Device

Gyomar Gonzalez-Gonzalez, Patricia Chueca, Coral Ortiz

TOPIC 11: NUTRIENT MANAGEMENT AND WASTE TREATMENT (NM)

Recycling Nutrients in a Circular Economy

Piet Derikx, Pieter De Wolf, Oene Oenema, Krijn Poppe, Theun Vellinga, Koos Verloop, Jan Weijma, Wyno Zwanenburg

Slurry Application Efficiency Evaluation

Christian Toft Madsen, Nick Skou-Nielsen, Thorkild Qvist Frandsen, Claus Grøn Sørensen, Gareth Edwards, Ole Green

Fertigation Equipment and Control Techniques: A Review

Stefan Vidal, Tatiana Pinho, José Boaventura-Cunha, Josenalde Oliveira, Paulo Moura Oliveira

GO EFLUENTES - A New Approach for Animal Waste Management in Portugal Vasco Manuel Fitas da Cruz, Olga Conde Moreira, Elizabeth Duarte, Henrique Trindade

Development of an Empirical Model to Prepare Nutrient Solutions with the Use of Controlled Release Fertilizers Maria Zografou, Christos Lykas

Determination of Ca2+ Absorption Rate of Young Rose Plants Grown in Hydroponic System Christos Lykas, Apostolos Zantzos, Maria Zografou

Influence Of Forced Aeration Rates On Maturation Of Composting Poultry Manure And Wood Shavings In Closed Reactor System

Waqas Qasim, Byeong Eun Moon, Frank Gyan Okyere, Fawad Khan, Jayanta Kumar Basak, Yong jin Lee, Hyeon Tae Kim 


\section{Topic 1: Design methods for integrated and sustainable farming systems}

(FS)

Theoretical concepts and methodological approaches to design novel concepts of farming systems (crops and greenhouses, animals, insects, algae, seaweed etc. and combinations thereof) that improve the performance on multiple aspects of sustainability. Examples are conservation agriculture, integrated pest management, tests and integrated assessment of concepts of farming systems, supply chain and market aspects.

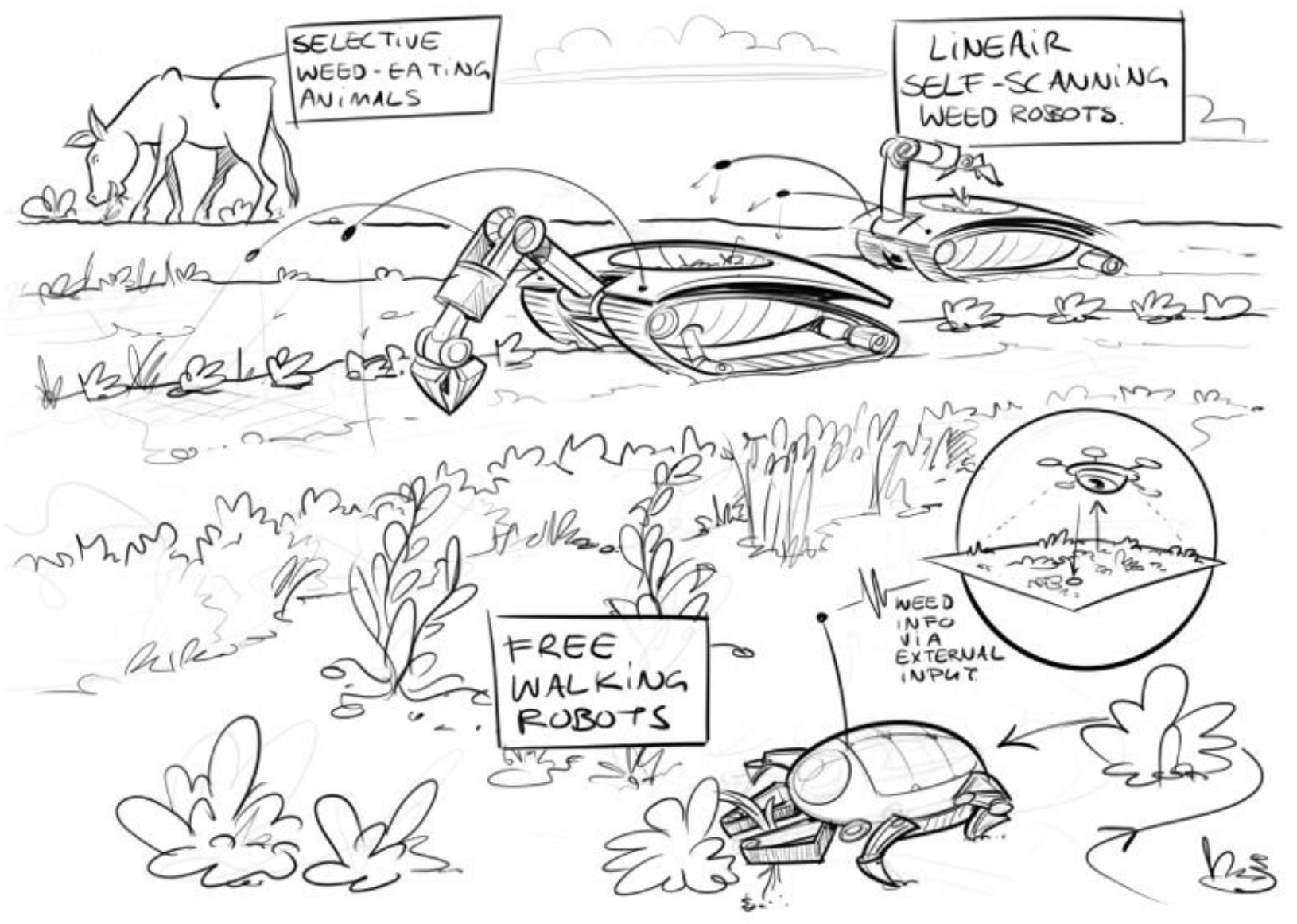




\title{
Predictive Systems Model Simulating The Development of Beehives and Honeybee Population (Apis mellifera) in Professional Apicultures
}

\author{
Jochen Georg Wiecha*, Sascha Wörz, Heinz Bernhardt \\ Chair of Agricultural Systems Engineering, Technical University of Munich, 85354 Freising, Germany \\ * Corresponding author. Email: wiecha@wzw.tum.de
}

\begin{abstract}
Worldwide, beekeepers are fighting against the parasitism through Varroa mites infesting their colonies and start mite reproduction in sealed brood combs. In order to ensure pollination maintaining healthy honeybee colonies is crucial. Long-term predictions for rearing development of a single honeybee colony can be simulated by several software-based models with the purpose to investigate the effects of pesticides on the bee colony or the general biological development of honeybees in their beehive and the surrounding environmental. Previous published models do not consider the beekeeping practice sufficiently. Ethological factors on beekeeping measures are neglected. Unfortunately, an interaction between the animals and the beekeeper cannot be studied. However, robbery of nectar among bee colonies and reinvasion of the Varroa mites must be attributed to disturbed predator-prey interaction and require the urgent intervention of the beekeeper. These models also do not consider that epigenetic switches adapt to the environmental conditions of honeybees. After swarming, several honeybee glands can develop again. All this is still missing in current prediction models. In this work, a new model for population development of honeybee colonies is presented. While using published probabilities from scholarly literature, the model covers beekeeping measures and involves for the first time the effects "robbery", "invasion of the Varroa mites", "age of queens" and "effects of professional breeding". The apicultural system is fully determined and is the fundamental for the calculations in annual frequency. With the proposed model, beekeepers are able to plan their decisions with regard to a long-term apiculture system and to observe the effects of intensified breeding or beekeeping measures on the honeybee colony. Thus, the introduced model can also be used for decision-making in beekeeping.
\end{abstract}

Keywords: Apiculture, honeybee, varroa mite, integrated pest management, beekeeping systems

\section{Introduction}

Frequently, the development of honeybee populations is in focus of reports. In addition to direct research on harmful factors and diseases, in particular, the fight against Varroa mite, researchers are also trying to predict the development of a beehive colony through models and calculation rules (Khoury et al., 2011). Several models have been developed in the past, which also take into account the corresponding brood rhythm of honeybees. However, the control of brood rhythm is due to the prevailing climatic conditions and the genetic predisposition. This complex process is thus a high variance inherent.

An exemplary representative of such a model for predicting the development of a bee colony is the "BEEHAVE" model (Becher et al., 2014). It contains a number of factors that influence the development of bees. The compilation of these factors, used in the BEEHAVE model, is based on sources and papers from numerous international publications. The use of such bee models is also attractive for the prediction of harmful effects on a bee colony by pesticides (Rumkee et al., 2015). One would like to abstain from targeted animal experiments and carry out initial tests in the model itself to do an estimate of the extent of damage.

Critical votes were given on the BEEHAVE model from an official EFSA report (European Food Safety Authority) (EFSA, 2015). EFSA evaluated the BEEHAVE model in terms of its suitability for the prediction of bee development in terms of toxic impact. The result of this investigation were complaints about numerous points in the BEEHAVE model. In addition to practical assumptions, the lack of influencing factors was also criticized (EFSA, 2015).

This work presents a model for professional beekeepers. It includes new and previously unused factors in the development of bee colonies. The period of observation is based on the development of bee populations in one region or one country for the next season. The new factors are the age of the queen, the probability of invasion of the Varroa mite and the first implementation of the beekeeper training level. Grades within the European Union are already implemented here.

\section{Materials and Methods}

Calculation concept

If you go through the models like "BEEHAVE" (Becher et al., 2014) in their degrees of freedom on a large scale, results are little realistic. Processes and results are under discussion by using a hypothetic process of brood 
rearing, because of an excel sheet with an egg laying rate for every single day of a year. For the assumptions made so far, the prediction always concerns the next day of the model and, as a result, the development of the days over the years of model runtime. However, if the prediction cannot be true for the next day, it is questionable how big the probability might be that the model will ever produce a reliable result. After all, a bee colony plans not for the next day, but for the successful wintering and reaching the next season, to further increase itself and to pass on the genes to the next generation.

The result of the equation is the bee stock of the next year in change to the current stock with the single influencing factor "normal mortality". This factor is based on observation of dying beehives without any detectable reason. The equation is therefore as in (1).

$$
H_{\text {now }} * M_{\text {natmort }}=H_{\text {next year }}
$$

$H_{\text {now }}=$ number of beehives in actual season

$H_{\text {next year }}=$ number of beehives in next season

$M_{\text {natmort }}=$ natural mortality of beehives

From the point of view of a professional beekeeper, the result on the right side of the equation is the rate at which the beekeeper has to multiply his bee colonies annually to protect himself from colony losses. Additionally, many influencing factors can damage honeybee health. Taking these into consideration yields equation 2.

$$
\begin{aligned}
& H_{\text {now }} * \sum F * M_{\text {natmort }}=B_{\text {arificial new colonies }} * H_{\text {next year }} \\
& \sum F=\text { sum of all possible factors }
\end{aligned}
$$

Separating the influence factors

For a more detailed analysis, we must separate the influencing factors and describe them individually. In the following, Figure 1 shows the factors influencing bee health and bees survivability. These factors are all on the left side of the equation in the model.

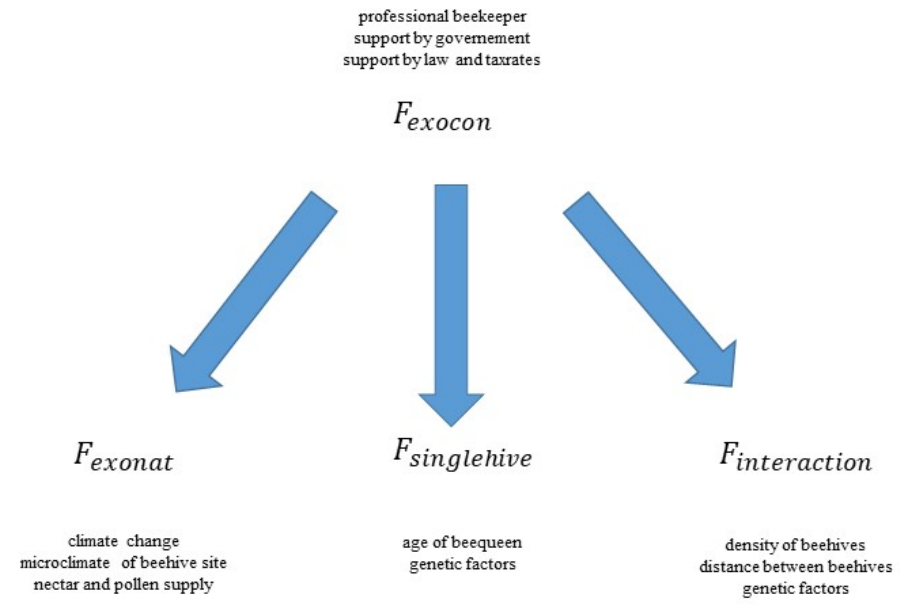

Figure 1. Flowchart of influence factors within the equation concept on the left side.

The new four groups of factors are $F_{\text {exocon }}, F_{\text {exonat }}, F_{\text {singlehive }}, F_{\text {interaction }}$. They form the sum of all possible factors as described in (2).

The "exogenous controllable factors"

Group $F_{\text {exocon }}$ presents the level of professional beekeeper in one region or member state, the possible political support by the government and the support by law and tax rates. There might be further more factors in this group. Factors in $F_{\text {exocon }}$ can be positive or negative. We call this group "exogenous controllable factors". This group is the only one, which can take affect to the other three. Therefore, $F_{\text {exocon }}$ is a steering mechanism for beekeeping in general. Interaction between factors for this group is characterized as additive. A high level of profession could occur with best support by law and government. Contrary, this proposition is often subtractive. 


$$
F_{\text {exocon }}=\sum\left(F_{1} \pm F_{2} \pm \cdots F_{n}\right)
$$

Figure 1 presents the $F_{\text {exocon }}$ as influencing all the other factor groups. In the following, we describe the mechanisms in which way $F_{\text {exonat }}, F_{\text {singlenive }}, F_{\text {interaction }}$ are controllable by $F_{\text {exocon }}$ group.

\section{The "exogenous natural factors"}

The $F_{\text {exonat }}$ is called "exogenous natural factors". In this group, we positioned the climate change, the microclimate of a single beehive location and the general nectar and pollen supply in the region round the colony. A beekeeper can take influence on climate change (of a view of a honeybee colony) by transporting the hive to a better or suitable other location. For microclimate, we face the same, as said bevor. Additionally, beekeeper can change the hive combs to suitable ones or change the kind of hive installation. In addition, the supply of nectar, pollen and honeydew can be changed by transporting the hives in different locations. Beekeepers can separate the hives for lowering the hive density. Colonies can forage much more, if there have not to many other rivalry hives nearby. We characterize the group of "exogenous natural factors" as factors multiplied with each other. In formula, this is described as in (4).

$$
F_{\text {exonat }}=\sum\left(F_{1} \times F_{2} \times \ldots F_{n)}\right.
$$

\section{The physiological factors of a single beehive}

Now, we have the group $F_{\text {singlehive }}$ to look for. In this group, for example, we put in the factors "age of bee queen" and "genetic disposition". By artificial breeding, a beekeeper could take care of having always young bee queens in his colonies. Therefore, this factor group $F_{\text {singlenive }}$ is also influenced by $F_{\text {exocon }}$ as shown in Figure 1 . The genetic disposition seems to be not impressionable, but this is, for example, the selection of the bee race used by the beekeeper. The bee race should be suitable to some factors of group $F_{\text {exonat }}$ - this will prevent colony losses in advance. The factors in $F_{\text {singlehive }}$ are multiplied as shown in (5).

$$
F_{\text {singlehive }}=\sum\left(F_{1} \times F_{2} \times \ldots F_{n}\right)
$$

\section{The factors by interaction between two and more beehives}

Interaction between honeybee hives is always negative. There are no positive benefits by working together of two honeybee colonies. After a queen loss, worker bees start foraging for another hive next to it, but without a queen, the hive is not able to transfer its genes to the next generation. It is lost forever, so there is no interaction anymore. This group is called $F_{\text {interaction }}$ and presents factors like "robbery/robbing" or "reinvasion of varroa mites". Therefore, we present the formula in negative speaking as in (6). Additionally, there is a correlation factor needed. The reason therefore is that robbing and reinvasion can occur together. They are strongly time-dependent over the year and could follow successively. Interaction depends on genetic disposition as well. One important factor is the critical distance between beehives.

$$
F_{\text {interaction }}=F_{\text {distance }} \times \sum\left(-F_{1}-F_{2} \ldots-F_{n}\right) \times F_{\text {correllaton }}
$$

For the calculation we obtain the data of from Chauzat (et al. 2013). In a region with high bee density per $\mathrm{km}^{2}$, the potential distance is lower. We calculated this fact for $\mathrm{x}=$ beehive density, and $\mathrm{y}=$ beehive distance, as we found in:

$$
y=x^{-0,5}
$$

For reason of additional data by questionnaire survey, we took the beehive density values "colonies per $\mathrm{km}^{2}$ " of the citied paper of Chauzat (et al., 2013). Now, all four groups are described well for having a point of view in every possible effect within these groups of factors. The weight for interaction was set to very high, if the distance is below $350 \mathrm{~m}$ and set to medium, if the distance is up to $800 \mathrm{~m}$. For a distance between $800 \mathrm{~m}$ and $1,5 \mathrm{~km}$, we see a low risk of interaction, but there is still one. For greater distances, the probability for interaction might be small enough for let this risk calculate with zero. For this classification, we follow Renz and Rosenkranz (2010) and there conference paper.

\section{Results and Discussion}

Completion of predictive bee model

As a result, we could put all factor groups in one equation. Therefore, we present the complete covering 
beehive model in (8). For visual presentation, we show a flowchart of the formula concept in Figure 2. We left the start value and end value of "number of beehives" $H_{\text {now }}$ and $H_{\text {next year }}$.

$$
H_{\text {now }} * F_{\text {exocon }} * F_{\text {exonat }} * F_{\text {singlehive }} * F_{\text {interaction }} * M_{\text {natmort }}=B_{\text {arificial new colonies }} * H_{\text {next year }}
$$

$F_{\text {exocon }}=$ exogenous controllable factors

$F_{\text {exonat }}=$ exogenous natural factors

$F_{\text {singlehive }}=$ physiological factors of one single beehive

$F_{\text {interaction }}=$ factors by interaction between two and more beehives

$B_{\text {artificial new colonies }}=$ new artificial colonies done by beekeeper treatment

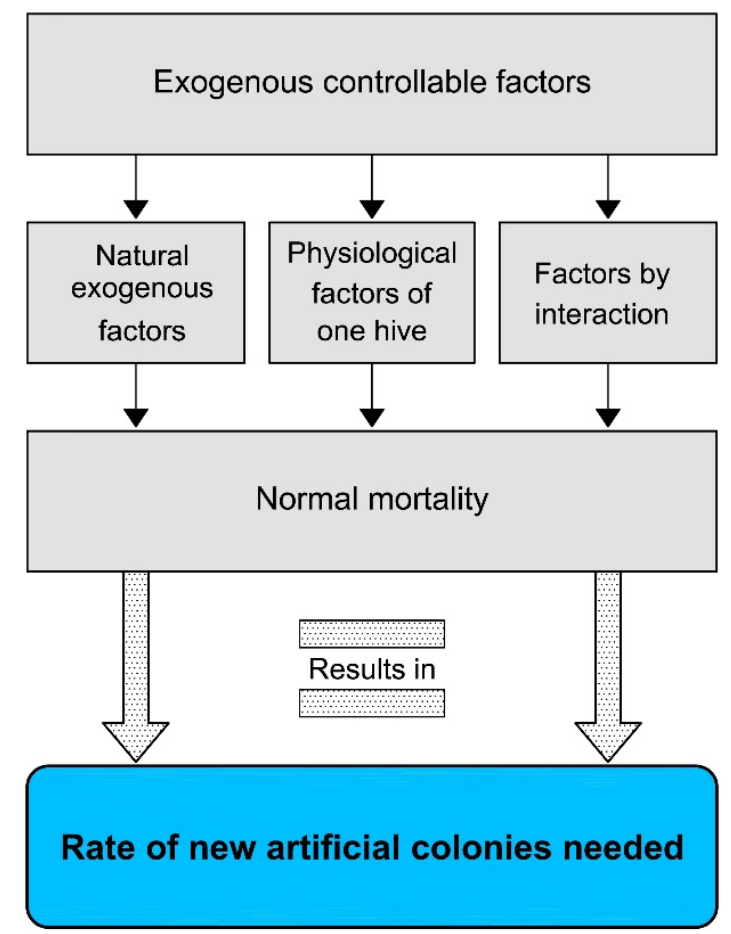

Figure 2. Flowchart shows the equivalent of equation (8).

Design of the new factors for calculation

In this paper, it is not possible to calculate all factors for being integrates in the equation. In this model the effects "robbery" and "invasion of the Varroa mites" is part of the following interaction sector. For "age of queens" and "effects of professional breeding and beekeeping" this factors are calculated in physiological factor by a single hive and as a controlled exogenous influence factor.

Factor "age of bee queen" in group "single standalone honeybee hive"

Physiological factors of a single beehive could be the age of a single honeybee queen. The queen is able to live up to five years, if she is well mated with several drones and filled up with 7.5 Mio sperm cells. Normally, this is not possible in every case. We see some of them only reaching 4 years of living. 
Table 1. Estimation or real and artificial quota of all queen ages.

\begin{tabular}{cccc}
\hline $\begin{array}{c}\text { Queen lifespan } \\
\text { in years }\end{array}$ & $\begin{array}{c}\text { Theoretical quota } \\
\text { without influence }\end{array}$ & $\begin{array}{c}\text { Estimated real quota } \\
\text { without influence }\end{array}$ & $\begin{array}{c}\text { Artificial quota by } \\
\text { professionals }\end{array}$ \\
\hline & & & $29,16 \%$ \\
2 & $20 \%$ & $22,5 \%$ & $29,16 \%$ \\
3 & $20 \%$ & $22,5 \%$ & $29,16 \%$ \\
4 & $20 \%$ & $22,5 \%$ & $12,50 \%$ \\
5 & $20 \%$ & $22,5 \%$ & $00,00 \%$ \\
$\Sigma 1,2,3$ & $20 \%$ & $10,0 \%$ & $87,5 \%$ \\
\hline
\end{tabular}

Professional beekeepers have to harvest much honey. This is only possible by strong honeybee colonies with a young and good egg-laying queen. Queens in their earliest three years are able for making big brood combs by high egg laying rates. In the theoretical model of Table 1 the sum of queens in this age is $60 \%$, where under real conditions it should be $67,5 \%$. By rising professional breeding, a beekeeper with proper knowledge will have only young queens. The artificial quota of high performance queens is $87,5 \%$ as described in Table 1 . Swarming and the quantity of nectar harvesting is highly related with the genetic disposition.

Factors "robbing" and "reinvasion of varroa mites" related to group "interaction between honeybee hives"

Interaction is a matter of distance between two hives and a matter of the health status of a colony. As mentioned in (7), we can calculate the theoretical distances between two and more beehives that we obtained from data of "beehives per $\mathrm{km}^{2}$ ". Based on the findings from Renz and Rosenkranz (2001) and the critical distances for a higher risk of interaction, we present the resulting table for EU member states in Table 2.

The probability of short distances measures the probability for interaction between two hives. However, interaction is always negative for beehives - the one might loss there honey, because of robbing, where the second hive may collect many varroa mites.

Worker bees are drifting between several colonies. Forfert (et al. 2015) found $79 \%$ drifting within short distances between hives. So the calculates values in Table 2 represent the mean of every member state. In local consideration, beehive density might by much higher or even much lower. Every beekeeper should know his surrounding well.

Density of beehives is a factor for reinvasion with mites of Varroa jacobsoni (Greatti et al. 1992). Even honeybee hives that were treated well by their beekeeper, could be reinfested in summer and autumn. For this calculated risk of mite reinvasion, the following table presents the values for every EU member state from Chauzat (et al. 2013). Additional, we compare the values of a risk by a low rate of well-educated professional beekeepers. The main problem of comparing a risk of interaction with a risk of hobby-beekeeper is the fact that every member state has its own method of collecting data form beekeeper. Table 2 can only be an estimation on the effect level of these factors. 
Table 2. Estimation of theoretical distances between two beehives.

\begin{tabular}{|c|c|c|c|}
\hline EU - member state & $\begin{array}{l}\text { theoretical distance } \\
\text { between hives in } \mathrm{km}\end{array}$ & $\begin{array}{c}\text { estimated risk of } \\
\text { interaction }\end{array}$ & $\begin{array}{c}\text { risk of low rate } \\
\text { professional beekeeper }\end{array}$ \\
\hline Austria & 0.477 & $* *$ & $\mathrm{NS}$ \\
\hline Belgium & 0.527 & $* *$ & $* * *$ \\
\hline Bulgaria & 0.426 & $* *$ & $* *$ \\
\hline Cyprus & 0.482 & $* *$ & $*$ \\
\hline Czech Republic & 0.389 & $* *$ & $* * *$ \\
\hline Denmark & 0.506 & $* *$ & $* *$ \\
\hline Estonia & 1.000 & $*$ & $* * *$ \\
\hline Finland & 3.162 & - & $*$ \\
\hline France & 0.632 & $* *$ & $*$ \\
\hline Germany & 0.725 & $* *$ & $* * *$ \\
\hline Greece & 0.296 & $* * *$ & - \\
\hline Hungary & 0.305 & $* * *$ & $*$ \\
\hline Ireland & 1.825 & $*$ & $* * *$ \\
\hline Italy & 0.520 & $* *$ & $*$ \\
\hline Kosovo & 0.392 & $* *$ & - \\
\hline Latvia & 1.000 & $*$ & $* *$ \\
\hline Lithuania & 0.745 & $* *$ & $* *$ \\
\hline Netherlands & 0.725 & $* *$ & $* * *$ \\
\hline Norway & 3.162 & - & $* * *$ \\
\hline Poland & 0.527 & $* *$ & $* * *$ \\
\hline Portugal & 0.398 & $* *$ & $* *$ \\
\hline Romania & 0.500 & $* *$ & - \\
\hline Slovakia & 0.474 & $* *$ & $* * *$ \\
\hline Slovenia & 0.360 & $* *$ & NS \\
\hline Spain & 0.451 & $* *$ & - \\
\hline Sweden & 1.825 & $*$ & $* * *$ \\
\hline United Kingdom & 0.877 & $* *$ & $* * *$ \\
\hline
\end{tabular}

Interaction level: $\quad * * *=$ high risk, $* *=$ medium risk, $*=$ low risk, "--" = no risk expected

Profession level: $\quad * * *=99 \%$ nonprofi, $* *=96 \%$ nonprofi, $*=90 \%$ nonprofi, " $\quad$ " $"=$ more than $10 \%$ professionals

By reading Table 2, some audience may question for the positive effect of having only $10 \%$ professional beekeepers. In a region with after all more than $10 \%$ professional beekeepers, transferable diseases, such as American Foul Brood might be found earlier. A better breeding by higher density of professional beekeeper, is a positive factor for all other beekeepers round the area. Therefore, there are positive or negative factors by the density of professional beekeepers that affect all other nonprofessional beekeepers in common.

Watching the development of colony population during the last view decades, we cannot confirm that there is a correlation between adding risk of interaction to the risk of low profession rate in beekeeping. This indicates that there are more factors influencing. This predictive beehive model should be continued in development. The next step is to evaluate the proportions of the four new factor classes and the correctness of the algebraic structure.

\section{Conclusions}

This study shows unequivocally the need of true and realistic honeybee model that professional beekeepers could make use of. With the presented formula and the new differentiation of the four factor groups, a new path has been taken. The presented model could not include all factors, but it is able to sort and show effects on honeybee treatment to the user.

Finally, this study could be the start in making suitable linear programming models (LP-models) for beekeeping. By inserting the factors related to the individual values for the own state, every beekeeper could predict the needs of self-making artificial new colonies every year. This is also a measure of economic efficiency. Different beekeeping practice methods could be compared and weight personally. This makes decisions in beekeeping much easier.

As EFSA mentioned on the "BEEHAVE" model, they wanted to show, how suitable this model could be for predicting the effects of pesticides on a honeybee colony. Looking in formula 8 , the uptake of pesticides is an "exogenous controlled factor" with additive characteristic taking effect with all the other factors in that group. Stopping the pesticides application, a stress factor would be leaving. In reality, we could say that this is a probability factor: Combining lethal doses for the pesticide with the theoretical mean distance to beehive, we obtain a probability for impact. Additionally, the probability for foragers visiting the location of fields spread with pesticides and having no other source of pollen and nectar, must also be calculated. 


\section{Acknowledgements}

Thanks to my adviser, Prof. Dr. Heinz Bernhardt for the support publishing papers about honeybees and Dr. Sascha Wörz for co-designing and reviewing the model concept.

\section{References}

Becher, M. A., Grimm, V., Thorbek, P. , Horn, J., Kennedy, P. J., Osborne, J. L. and Morgan, E. (2014). BEEHAVE: a systems model of honeybee colony dynamics and foraging to explore multifactorial causes of colony failure. J Appl Ecol, 51: 470-482. doi:10.1111/1365-2664.12222

Chauzat M-P, Cauquil L, Roy L, Franco S, Hendrikx P, et al. (2013). Demographics of the European Apicultural Industry. PLoS ONE 8(11): e79018. doi:10.1371/journal.pone.0079018

EFSA (European Food Safety Authority) PPR Panel (Panel on Plant Protection Products and their Residues) (2015). Statement on the suitability of the BEEHAVE model for its potential use in a regulatory context and for the risk assessment of multiple stressors in honeybees at the landscape level. EFSA Journal 2015;13(6):4125, 92 pp. doi: $10.2903 /$ j.efsa.2015.4125

Forfert N, Natsopoulou ME, Frey E, Rosenkranz P, Paxton RJ, Moritz RFA (2015). Parasites and Pathogens of the Honeybee (Apis mellifera) and Their Influence on Inter-Colonial Transmission. PLoS ONE 10(10): e0140337. doi:10.1371/journal.pone.0140337

Greatti, M. ;Milani, N. Nazzi, F. (1992). Reinfestation of an acaricide-treated apiary by Varroa jacobsoni Oud. Exp. Appl. Acarol., 16: 279-286.

Khoury DS, Myerscough MR, Barron AB (2011) A Quantitative Model of Honey Bee Colony Population Dynamics. PLoS ONE 6(4): e18491. doi:10.1371/journal.pone.0018491

Renz, M. Rosenkranz, P. (2001). Infestation dynamics and reinvasion of Varroa destructor mites in honey bee colonies kept isolated and in groups. Association of Institutes for Bee Research Report of the 48th seminar in Bad Neuenahr/Ahrweiler 27-29 March 2001, Apidologie 32 (2001) 453-520

Rumkee, J., Becher, M.A., Thorbek, P., Kennedy, P., Osborne, J. (2015). Predicting Honeybee Colony Failure: Using the BEEHAVE Model to Simulate Colony Responses to Pesticides. Environmental Science \& Technology 201549 (21), 12879-12887, DOI: 10.1021/acs.est.5b03593 


\author{
A Model to Assess Environmental Efficiency of Pig Production Systems \\ Luis Lassaletta ${ }^{a, b, c}$, Fernando Estellés ${ }^{d *}$, Arthur Beusen ${ }^{a, b}$, Lex Bouwman ${ }^{a, b}$, Salvador Calvet ${ }^{\text {, }}$, Hans \\ van Grinsven ${ }^{\text {, }}$, Jonathan Doelman ${ }^{\text {, }}$, Elke Stehfest ${ }^{\text {a }}$, Aimable Uwizeye ${ }^{\mathrm{e}}$, Henk Westhoek ${ }^{\mathrm{a}}$ \\ a PBL Netherlands Environmental Assessment Agency, PO Box 30314, 2500 GH The Hague, The Netherlands \\ ${ }^{b}$ Department of Earth Sciences_-Geochemistry, Faculty of Geosciences, Utrecht University, PO Box 80021, 3508 TA \\ Utrecht, The Netherlands \\ ${ }^{c}$ CEIGRAM/Department of Agricultural Production, Universidad Polit'ecnica de Madrid, Madrid 28040, Spain \\ ${ }^{d}$ Institute of Animal Science and Technology, Universitat Politecnica de Valencia, Camino de Vera S/n, 46022, \\ Valencia, Spain \\ ${ }^{e}$ Food and Agriculture Organization of the United Nations, Animal Production and Health Division, Viale delle Terme \\ di Caracalla, Rome 00153, Italy \\ * Corresponding author. Email: feresbar@upv.es
}

\begin{abstract}
In this work we developed a model to assess the environmental efficiency of pig production systems. Based on pork demand, production performance and dietary composition, the model predicts the global feed and land demand, nitrogen excretion and nitrogen use efficiency for pig production systems. We considered 26 regions around the world (based on IMAGE global modelling framework). For each region, productive characteristics and dietary compositions were defined from 1970 to 2050 under different scenarios. Scenarios were built following the storylines of the Shared Socio-economic Pathways. Our model outcomes suggest that pig production systems will play a significant role in the global agro-food system in 2050 including protein supply, land demand and $\mathrm{N}$ fertilization. The sustainability of future pig production systems will strongly depend on the livestock production performances and feed rations. The model suggests that limits for system efficiency will be reached in some regions. The replacement of part of the soybeans by swill and industrial by-products in the feed ration of pigs is a promising solution to increase nitrogen use efficiency as well as to reduce the land demand associated with pork production.
\end{abstract}

Keywords: nitrogen use efficiency, SSP, IMAGE, meat, swine

\title{
1. Introduction
}

The contribution monogastric production systems (pigs and poultry) to the total livestock production has increased worldwide during the last 50 years in absolute and also in relative terms. According to FAO projections (Alexandratos and Bruinsma, 2012), pig production is expected to increase $45 \%$ by 2050 . This increasing demand of pig meat is associated not only with the population rise, but also with the dietary transition in several countries towards western-type diets (Bai et al. 2018). These developments include an increasing preference for monogastric protein sources.

In terms of resource use, this transition towards a higher share of monogastric products on the total animal production has advantages and trade-offs. On the one hand pigs and chicken production systems have in general better feed conversion rates and nitrogen $(\mathrm{N})$ use efficiencies than ruminants (Gerber et al. 2014). On the other hand and unlike ruminants, monogastric animals cannot make good use of natural grasses and stovers, and their feed demand therefore does compete with demand for human food (Eisler et al. 2013). Data show that indeed the increasing production of monogastric systems is significantly rising the demand of local or imported feed grains including cereals and meals from oilcrops (Wang et al. 2018).

The production of pig meat is associated with several environmental threats along the supply chain (Winkler et al. 2016). The efficiency of the system (considered as tonnes of meat produced per tonnes of feed consumed) is key to ensure the sustainability of pig production worldwide.

Several pollutants are emitted to the air and to the water (particularly reactive $\mathrm{N}$ compounds) through the production of feed crops, during the animal production and manure management stages (Uwizeye et al. 2016). Thus, sustainability of pig production systems largely relies on the amount and type of feed consumed and as well as on the amount of nutrients excreted and the subsequent management and fate of pig manure. The composition of the feed rations is in the core issue to assess the sustainability of these systems due to: 1) the land use and emissions in the cropping systems producing feed (Uwizeye et al. 2016); 2) the competition of animal feed with human food (Schader et al. 2016) and 3) the effect of different feed rations on the amount of nitrogen excreted and in the following nitrous oxide and ammonia emitted (Sanchez-Martin et al. 2017; Sajeev et al. 2018). Thus improved feeding practices, health and herd management can reduce the environmental impact of pig production systems (Mottet et al. 2016). Improvements on production performances of intensive systems during the last decades have indeed increased the efficiency of these systems both, at the animal and at the herd level resulting in significant feed savings (e.g. Bai et al. 2014; Harchaoui and Chatzimpiros 2017). These intensive systems can 
also be very different in terms of feed demand and local impacts associated with manure management (Willems et al. 2016).

The specific objective of this work is to develop a model that allows studying how pig systems have evolved during the past decades at the global scale as a base to explore how future changes in demand for pork, pig production systems and feed rations will affect global feed demand, nitrogen use efficiency and $\mathrm{N}$ excretion in the next decades. To explore the environmental impacts and resource efficiency of alternative scenarios for demand and production of pork in 2050, we have developed a new module for the integrated assessment model IMAGE 3.0 (Stehfest et al. 2014). This module has been parametrized for the previous decades 1970-2015 and for five scenarios referred to as Shared Socioeconomic Pathways (SSPs, van Vuuren et al. 2012). These SSPs represent possible future development trajectories that result in different type of development of human societies (van Vuuren et al. 2017).

\section{Materials and Methods}

Model description

The model has been developed to be included as a module of the Integrated Model to Assess the Global Environment (IMAGE) modelling framework. IMAGE is an integrated assessment modelling framework that simulates the interactions between human activities and the environment, to explore long-term global environmental change and policy options in the areas of climate, land and sustainable development (Stehfest et al., 2014). IMAGE considers 26 regions around the world based on geographic proximity. IMAGE includes a livestock module describing livestock production. Feed demand, efficiency and nutrient excretion are calculated per year and per animal class and system from 1970. For ruminants, extensive and intensive systems are deeply described (Bouwman et al. 2005) while for monogastric animals a more simplistic approach based on excretion factors was considered.

The new pig module in IMAGE provides a more detailed representation of the pig production distinguishing three systems according to the level of intensification: backyard, intermediate and intensive. Backyard systems are partially enclosed, are devoted to subsistence or local markets and are fed with high amounts of swill. Intermediate systems are partially enclosed and they are market oriented with intermediate level of capital input. Intensive systems are fully enclosed, market oriented and are sustained on a high level of capital input (Macleod et al. 2013).

The IMAGE pig module has three sub-modules aimed to determine animal stocks and energy demand (Meat Module, Herd Module and Energy Module) per system and one sub-module aimed to calculate nitrogen intake and excretion based on animal performance and diet composition (Nitrogen Module). The model is fed by 35 input parameters ( 2 production parameters, 16 on performance and 17 feedstuffs) and 78 constants. Most of these parameters are obtained from statistical data from FAO and MacLeod et al. (2013). Some variations were considered after a comprehensive literature review.

\section{Meat Module}

The meat production module estimates the amount of meat ( $\mathrm{kg}$ of carcass) produced per system (backyard, intermediate and intensive) and IMAGE region (26) by multiplying the total Carcass Weight produced by region based in FAOSTAT figures, times the proportion of meat produced at each system and region. To obtain this fraction, we used the information of the number of heads per systems (Robinson et al. 2014) after IMAGE processing and calibration with FAO data on stocks and production.

\section{Herd Module}

The herd module estimates the number of heads by system and region and the off-take rates (OR, the fraction of the animal population that is taken out for slaughter). This model describes the functioning of the herd considering 11 different cohorts including fatteners, sows, boars and gilts at different stages 
Table 1. List of inputs and outputs of the model.

\begin{tabular}{|c|c|c|}
\hline Inputs & Description & Source \\
\hline Pig production $(\mathrm{kg})$ & $\begin{array}{l}\text { Production of carcass per IMAGE } \\
\text { region }\end{array}$ & FAOSTAT/IMAGE/MAGNET \\
\hline $\begin{array}{l}\text { Fraction of production } \\
\text { per system }(\%)\end{array}$ & $\begin{array}{l}\text { Pig production is distributed over three } \\
\text { systems (backyard, intermediate and } \\
\text { intensive), with specific intensities, } \\
\text { performances and rations }\end{array}$ & $\begin{array}{c}\text { Post-processing of } \\
\text { FAOSTAT/Robinson/Pigs module }\end{array}$ \\
\hline $\begin{array}{l}\text { Herd and performance } \\
\text { parameters }\end{array}$ & $\begin{array}{c}16 \text { parameters on herd structure, } \\
\text { mortality, fertility, replacement rates and } \\
\text { growth. }\end{array}$ & Several sources \\
\hline $\begin{array}{l}\text { Diet composition } \\
\text { (Feed rations, \%) }\end{array}$ & 17 feedstuffs human-competing or not & Several sources \\
\hline Outputs & Description & Outcome of the module \\
\hline $\begin{array}{l}\text { Meat production } \\
\text { per system }(\mathrm{kg})\end{array}$ & $\begin{array}{l}\text { Amount of CW produced per } \\
\text { system/region }\end{array}$ & Meat production module \\
\hline Off-take ratio (n.d.) & $\begin{array}{l}\text { Fraction of the animal population that is } \\
\text { taken out for slaughtered }\end{array}$ & Herd module \\
\hline Pig stock (heads) & Heads & Herd module \\
\hline $\mathrm{Kg}$ production/head & $\mathrm{CW}$ produced per head of the stock & Herd module \\
\hline $\mathrm{DM}$ input $(\mathrm{kg} / \mathrm{y})$ & Dry matter needs per system/region & Energy module \\
\hline $\begin{array}{l}\text { Feed conversion ratio (as } \mathrm{kg} \\
\text { feed } \mathrm{DM} / \mathrm{kg} \text { carcass) }\end{array}$ & $\begin{array}{l}\text { Measure of animal efficiency in } \\
\text { converting feed mass in carcass }\end{array}$ & Energy module \\
\hline Total Energy needs (ME/y) & $\begin{array}{l}\text { Metabolisable energy consumed per } \\
\text { system/region }\end{array}$ & Energy module \\
\hline $\begin{array}{l}\text { Feed conversion ratio (as kg } \\
\text { feed DM/LWout) }\end{array}$ & $\begin{array}{l}\text { Measure of animal efficiency in } \\
\text { converting feed mass in live weight of } \\
\text { slaughtered animal }\end{array}$ & Energy module \\
\hline $\mathrm{N}$ feed $(\mathrm{kgN})$ & $\mathrm{N}$ content of the feed & Nitrogen budget module \\
\hline $\mathrm{N}$ meat $(\mathrm{kgN})$ & $\mathrm{N}$ content of the CW & Nitrogen budget module \\
\hline $\mathrm{N}$ excretion $(\mathrm{kgN})$ & $\mathrm{N}$ excretion & Nitrogen budget module \\
\hline $\mathrm{N}$ lost $(\mathrm{kgN})$ & $\begin{array}{l}\mathrm{N} \text { lost in non-consumed animals and } \\
\text { other parts not included in } \\
\text { the carcass (e.g. offals) }\end{array}$ & Nitrogen budget module \\
\hline $\mathrm{N}$ use efficiency, NUE (\%) & $\begin{array}{c}\text { Measure of animal efficiency in } \\
\text { converting N input in } \mathrm{CW}(\mathrm{N}) \text { output }\end{array}$ & Nitrogen budget module \\
\hline $\mathrm{N}$ excretion $(\mathrm{kgN} / \mathrm{head} / \mathrm{y})$ & Excretion factor & Nitrogen budget module \\
\hline $\begin{array}{c}\mathrm{N} \text { excretion } \\
(\mathrm{kgN} / \mathrm{kg} \text { protein output) }\end{array}$ & Excretion factor & Nitrogen budget module \\
\hline
\end{tabular}

Note: all the results are provided per year (1970-2050), systems (3), region (26)

Carcass weight $(\mathrm{CW})$ from FAOSTAT was used to estimate the number slaughtered animals, and then to determine the number of animals needed for a given meat production at each cohort. Since CW from FAOSTAT is a weighted average of $\mathrm{CW}$ from different systems, several rules were followed to determine $\mathrm{CW}$ for different systems and regions. The number of animals per cohort was calculated according to productive and reproductive parameters. Key parameters such as litter size and the number of litters/sow/year for different regions, and productive systems were derived from a comprehensive literature review (Lañada et al., 1999; Kunavongkrit and Heard, 2000; Wabacha et al., 2004; Chimonyo et al., 2006; Kumaresan et al., 2007; Nakai, 2008; Phengsavanh et al., 2010; Hoste, 2011; Bai et al., 2014; AHDB, 2016).

\section{Energy module}

The energy module is aimed to estimate food intake. To this aim, following the IPCC (2008) approach, a metabolizable energy balance was performed. Energy needs for each category were determined according to Noblet et al.(1990), NRC (2012) and FEDNA (2013) recommendations considering needs for: maintenance, thermoregulation, growth, gestation and lactation. Total energy needs from the herd were calculated then and global food intake estimated considering energy density of diets (see section 2). Inputs needed for this module were related to animal weight, weight gain, the time animals spent in each category and housing temperature. 


\section{Nitrogen Module}

The nitrogen module aims to estimate nitrogen retention and excretion for each cohort. Since nitrogen intake is obtained from food intake and its protein content, we focused on determining nitrogen retention from animals. The amount of nitrogen retained for each cohort depended on the physiological performance of animals. We considered three main sources for Nitrogen use in pigs: growth, milk production and gestation according to NRC (2012) and FEDNA (2013).

\section{Diet parametrization}

The feed rations have been formulated considering 18 feedstuffs that includes human-competing feeds (namely temperate cereals, rice, maize, tropical cereals, pulses, roots and tubers, oil crops and other) and feed noncompeting with human food (namely brans, DDGS, forage, molasses, swill, animal source, synthetic amino acids, other residues and treated residues). Two nutritional attributes are considered for each feedstuff: 1) Energy content expressed as metabolizable energy for pigs ( $\mathrm{Mj}$ of $\mathrm{ME} / \mathrm{kg} \mathrm{DM}) ; 2$ ) crude protein content (CP\% in DM content). Feed and energy contents of each feedstuff have been obtained from Feedpedia (2018). The considered categories includes several feedstuffs (e.g. temperate cereals can be wheat, barley and oats), we weighted averaged the nutrient content based on the general allocation to feed uses provided in Faostat Food Balance Sheets for the base year (2005). Nutritional attributes are provided in Supplements. The energy and protein content of the final rations is calculated considering the feedstuffs composition and the energy and protein content of each ingredient. Total dry matter use and total nitrogen intake were calculated.

The feed rations for 2005 were defined as follows for intensive systems: Diets proposed by MacLeod et al. (2013) were considered as a baseline for all regions but for China+ where information from Bai et al. (2014) was used. We use the composition of the feed availability provided in the Food Balance Sheets, adjusted for the IMAGE regions to modify diet compositions according to the availability of each group (particularly grains) of feed for these IMAGE regions. DDGS were introduced for USA and Canada following Meisinger (2010).

Recommendations from Meisinger (2010), NRC (2012) and FEDNA (2013) were followed to keep ingredients under recommended ranges for all regions. For backyard systems, swill, residues and fibrous products were considered as the basis for pork feeding for all regions according to a literature review (Lemke et al., 2006; Phengsavanh et al., 2010; zu Ermgassen et al., 2016). FAO feed allocation information was also used to define the inclusion of other ingredients. Rations proposed by Bai et al. (2014) were used as a reference for the region China+. Rations for backyard production were considered invariable from 1970. Finally, intermediate systems rations were calculated as an average between intensive and backyard rations for each region.

\section{Scenarios description}

Five Shared Socioeconomic Pathways (SSP) have been considered based on Van Vuuren et al. (2017) and Doelman et al. (2018). SSP1 represents the "green growth" paradigm scenario with low population growth, high agricultural efficiencies (crops and livestock) and high environmental awareness. SSP2 represents the "middle of the road scenario", while SSP3 represents a fragmented world with technological stagnation and high population. SSP4 describes an unequal world with some regions evolving following different pathways, based on SSP1, SSP2 or SSP3. Finally SSP5 exemplifies a world where economic development is based on fossil fuels, technological improvements are high and environmental awareness is low. Input parameters used for future scenarios (SSP's) in intensive systems were defined based on two criteria: firstly, for each SSP, technical upper limits were considered to be achieved for all parameters and secondly, different evolution pathways for regions were defined leading to a convergence of all regions towards a high productivity scenario. Backyard systems were considered to keep unvaried and intermediate systems were expected to improve proportionally to intensive ones. It is expected that under SSP 1 and 5, productive parameters will achieve the maximum efficiency. Lower upper limits were considered for SSP2, leading to modest improvements if compared to SSP1 and 5. All parameters for SSP3 remained equal to the baseline considering no evolution (business as usual ).

\section{Results and Discussion}

Production

In all five scenarios pig meat production is expected to significantly increase for 2050 when compared to 2010 levels (107 Tg of CW, FAOSTAT) (Figure 1). The maximum increase was estimated for SSP5 (207 Tg of CW in 2050) due to a rise in the population (from 6.9 billion people in 2010 to 8.6 in 2050) and also an increase in the per capita consumption (from $16 \mathrm{~kg}$ in 2010 to $24 \mathrm{~kg} / \mathrm{cap} / \mathrm{y}$ in 2050). The lower growth in the demand is expected for SSP1 (147 Tg of CW in 2050) and it is associated with a population rise ( 8.5 billion people in 2050). The global average of per capita consumption stays relatively constant in SSP1 (17 CW/cap/y in 2050). However, in this "green growth paradigm" scenario, the per capita consumption is reduced in regions having today the socalled "Western diets" while an increase is expected in other developing regions with current low demand. 


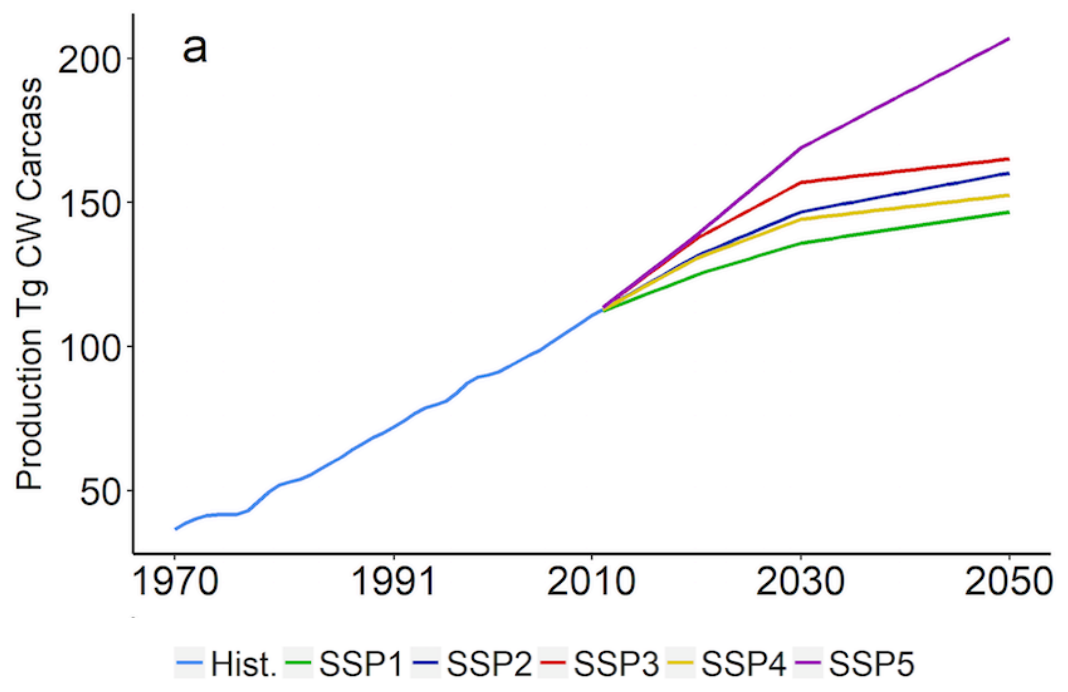

Feed use

Figure 1. Scenario-specific trajectories for pig production at global level.

The calculated feed demand expressed in dry matter (DM) has doubled during the last three decades from 226 Tg DM in 1970 to $471 \mathrm{Tg}$ DM in 2005. This demand is expected to increase in all the scenarios from $16 \%$ for SSP1 (547 Tg DM) to 34\% for SSP5 (752 Tg DM) with respect 2005 levels. In our estimation for $2005144 \mathrm{Tg}$ DM were cereal grains resulted in a share of $17 \%$ of the feed as maize and $12 \%$ as temperate cereals. The share of oil crops in the rations has increased almost $100 \%$ from 1970 and this means that the total amount used has raised from 12 to $48 \mathrm{Tg}$ DM. In SSP2, SSP3 and SSP5 the demand of oil crops is expected to increase to 63, 83 and $75 \mathrm{Tg}$ DM, respectively. Only in SSP1 is projected a significant reduction in the oil crop demand (17 Tg DM). In some regions such as Europe and USA the oil crops would be replaced by alternative protein sources such as the processed of food residues and new sources of protein together to an increase in the use of synthetic amino acids.

Feed conversion ratio

According to the model outcomes, the $\mathrm{kg}$ of feed (expressed in DM) needed to produce a $\mathrm{kg}$ of carcass (Feed Conversion Rate, FCR) have consistently decreased during the last 35 years for the intensive systems. The global weighted averaged for all systems FCR dropped from 6.3 to $4.7 \mathrm{~kg}^{\mathrm{kg}} \mathrm{kg}^{-1}$. This result indicates that in $2005,178 \mathrm{Tg}$ of DM have been saved globally due to this efficiency increase. In the backyard systems the global FCR is about $8.3 \mathrm{~kg} \cdot \mathrm{kg}^{-1}\left(7.2-18.6 \mathrm{~kg} \cdot \mathrm{kg}^{-1}\right.$ regional range in 2005) while in intermediate systems evolved from 6.9 to $4.8 \mathrm{~kg} . \mathrm{kg}^{-}$ ${ }^{1}\left(4.2-9 \mathrm{~kg} \cdot \mathrm{kg}^{-1}\right.$ regional range in 2005) and in intensive systems from 4.8 to $3.6 \mathrm{~kg}^{-\mathrm{kg}^{-1}}\left(3.5-4.4 \mathrm{~kg} \cdot \mathrm{kg}^{-1}\right.$ regional range in 2005). The total feed demand in DM was reduced $25 \%$ because of efficiency improvements during the last 35 years. The efficiency increase estimated for the historic period is predicted to be stabilized for SSP3 and increase particularly in SSP1 and SSP5 scenarios due to better performances and adjusted diets. In any case, different pathways are expected according to differential region developments (Figure 2). 

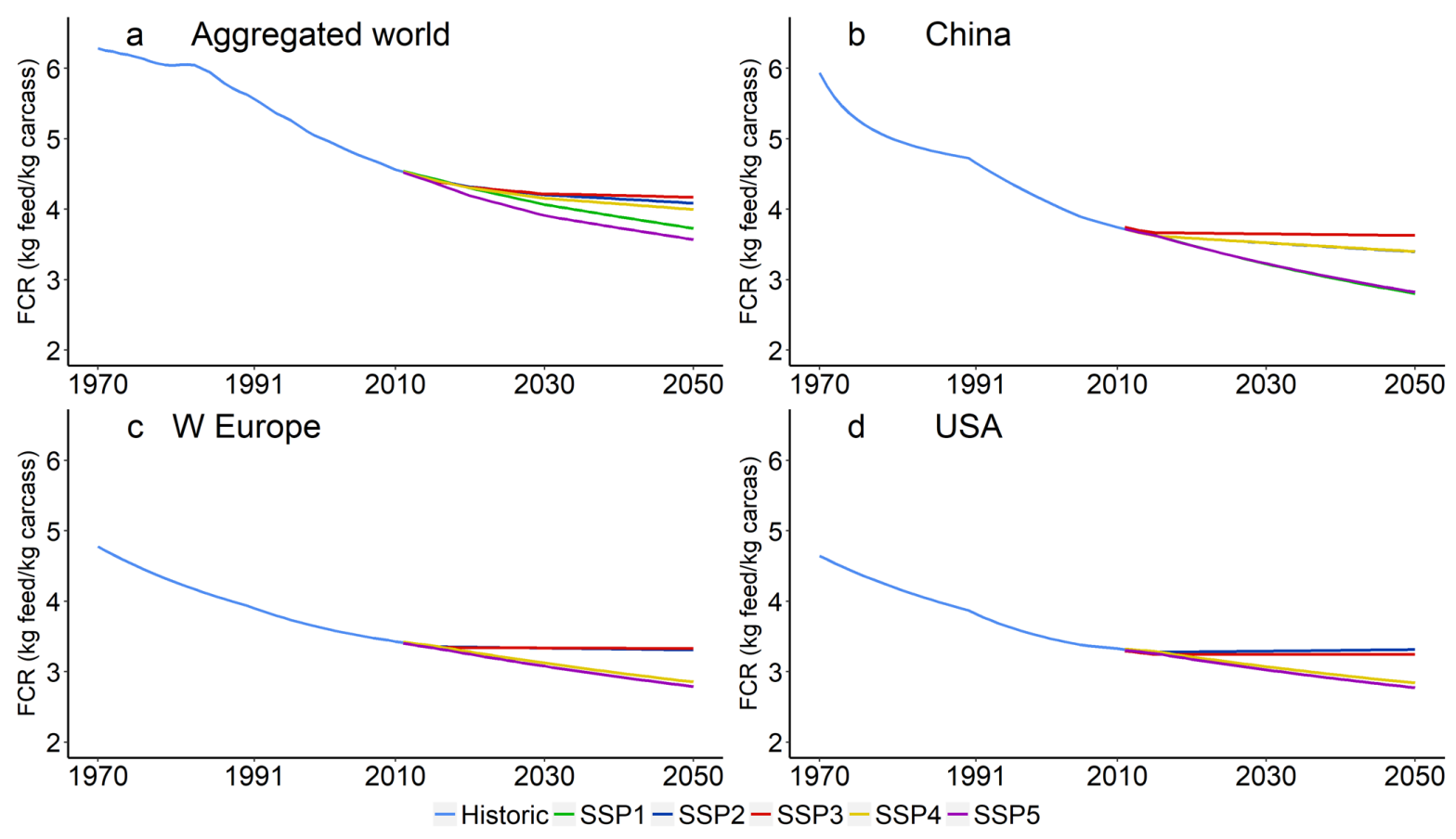

Figure 2. Scenario-specific trajectories of conversion rates (FCR) aggregated at the global and system level and for intensive systems in China, Western Europe and USA.

Nitrogen use efficiency

The weighted-average Nitrogen Use Efficiency at the herd level calculated by the model (NUE, expressed as the amount of $\mathrm{N}$ retained in the carcass per $\mathrm{N}$ input in the feed) of the world pig production systems has increased from $14.8 \%$ in 1970 to $16.5 \%$ in 2005 (Fig. 3). Even if intensive systems presented a relatively high NUE in 2005 (23\% world average) the model estimated for some regions a very moderate increase during the last 35 years associated with protein-richer diets (Fig. 3). As it has already occurred during the last decade, improved rations including lower protein content will have important effect on the NUE. This is the case of SSP1 were in 2050 all the NUEs are above $30 \%$ while in SSP5 even with a FCR similar to SSP1, a high protein content of the rations is associated with worst $\mathrm{N}$ performances.
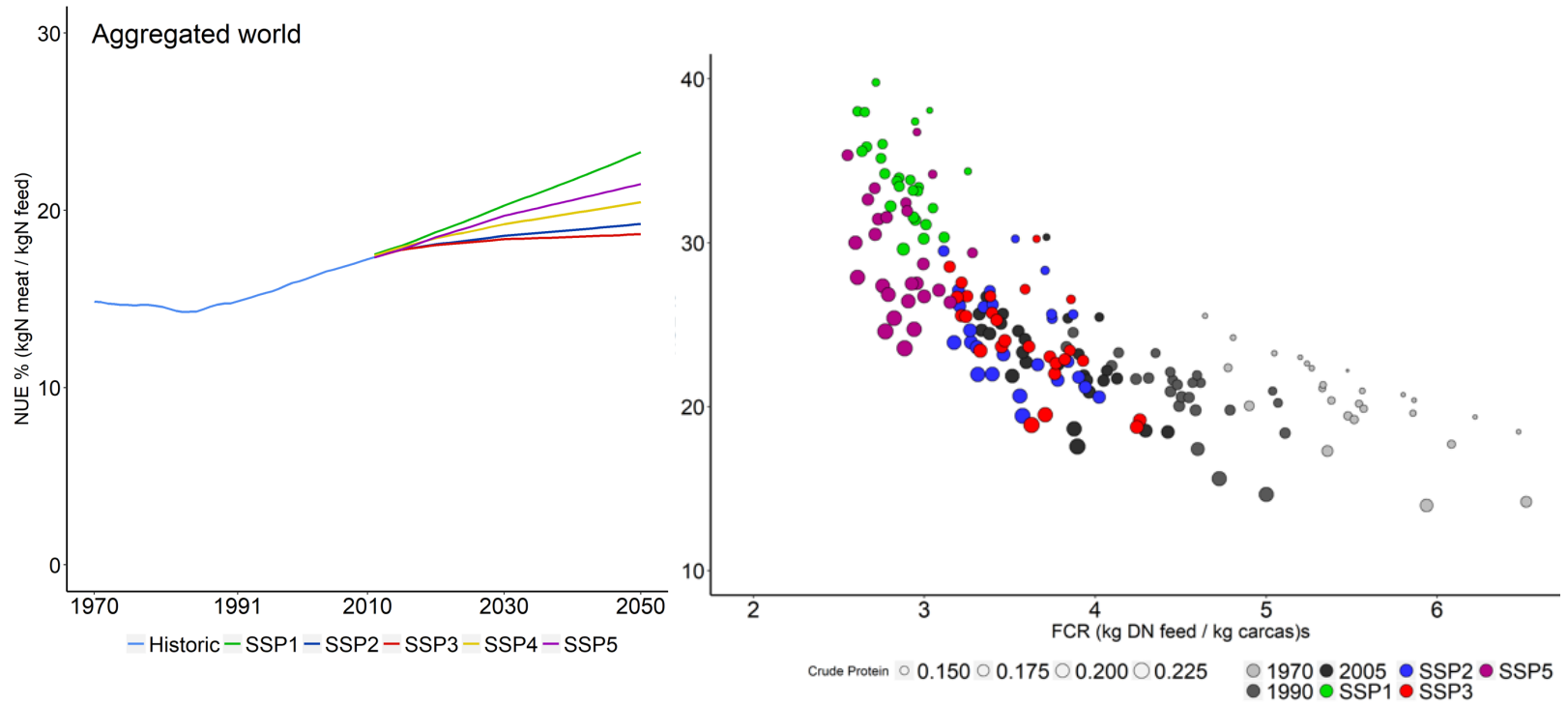

Figure 3. Left: Evolution of nitrogen use efficiency (NUE) at the herd level (calculated as the percentage of $\mathrm{N}$ retained in the carcass per $\mathrm{N}$ input in the feed). Right: Feed conversion rate (FCR) vs. nitrogen use efficiency (NUE) for the 26 IMAGE ratios different historic moments and scenarios. The size of the point represents the crude protein content of the ration. 


\section{Conclusions}

A generalized increase of efficiency occurred in the pig production systems from 1970 until 2005. It has significantly reduced feed consumption per $\mathrm{kg}$ of carcass. The significant increase in the pork demand has however produced a steady growth of the feed consumption 1970 to 2005. Only in the "green growth paradigm" scenario (SSP1) where measures at both, supply and demand side, are undertaken the total feed demand is stabilized by 2050. In SSP5, even considering efficiency progresses, the feed demand could increase due to a substantial rise of the demand. The use of alternative feeds together with moderate crude protein contents through improved feeding programs and genetic breeding are also relevant to decrease the competition with human food and to reduce the $\mathrm{N}$ excretion increasing the NUE. The relevant effect of total demand even with significant improvements at the herd level also indicates the importance of the moderation of the consumption in the human diets (SSP1). Thus the sustainability of pig production systems for the upcoming decades will rely not only on the expected efficiency improvements but also on 3 more pillars: the increase of the use of alternative feed sources, the reduction of the crude protein content in the rations and the moderation of the demand.

\section{References}

AHDB. 2016. Agriculture and Horticulture Development Board. 2014 pig cost of production in selected countries. Warwickshire, UK.

Alexandratos L, Bruinsma J. World agriculture towards 2030/2050 The 2012 Revision. In: FAO, editor. ESA Working paper No. 12-13, Roma, 2012.

Bai, Z.H., Ma, L., Qin, W., Chen, Q., Oenema, O., Zhang, F.S., 2014. Changes in Pig Production in China and Their Effects on Nitrogen and Phosphorus Use and Losses. Environmental Science \& Technology 48, $12742-$ 12749.

Bai, Z., Lee, M.R.F., Ma, L., Ledgard, S., Oenema, O., Velthof, G.L., Ma, W., Guo, M., Zhao, Z., Wei, S., Li, S., Liu, X., Havlík, P., Luo, J., Hu, C., Zhang, F., 2018. Global environmental costs of China's thirst for milk. Global Change Biology 24, 2198-2211.

Bouwman, A.F., Van der Hoek, K.W., Eickhout, B., Soenario, I., 2005. Exploring changes in world ruminant production systems. Agricultural Systems 84, 121-153.

Chimonyo, M., Dzama, K., Bhebhe, E., 2006. Genetic determination of individual birth weight, litter weight and litter size in Mukota pigs. Livestock Science 105, 69-77.

Doelman, J.C., Stehfest, E., Tabeau, A., van Meijl, H., Lassaletta, L., Gernaat, D.E.H.J., Hermans, K., Harmsen, M., Daioglou, V., Biemans, H., van der Sluis, S., van Vuuren, D.P., 2018. Exploring SSP land-use dynamics using the IMAGE model: Regional and gridded scenarios of land-use change and land-based climate change mitigation. Global Environmental Change 48, 119-135.

Eisler, M.C., Michael R. F. Lee, J.F.T., Graeme B. Martin, John Beddington, Jennifer A. J. Dungait, Henry Greathead, Jianxin Liu, Stephen Mathew, Helen Miller, Tom Misselbrook, Phil Murray, Valil K. Vinod, Robert Van Saun\& Michael Winter, 2014. Agriculture: Steps to sustainable livestock. Nature 507, 32-34.

FEDNA. 2013. Fundación Española para el Desarrollo de la Nutrición Animal. Necesidades nutricionales para ganado porcino: Normas FEDNA $\left(2^{\mathrm{a}} \mathrm{Ed}\right)$.

Feedipedia. (2018). Feedipedia: an on-line encyclopedia of animal feeds. Available at: http://www.feedipedia.org. Accessed 06/06/2018

Gerber, P.J., Uwizeye, A., Schulte, R.P.O., Opio, C.I., de Boer, I.J.M., 2014. Nutrient use efficiency: a valuable approach to benchmark the sustainability of nutrient use in global livestock production? Current Opinion in Environmental Sustainability 9-10, 122-130.

Harchaoui, S., Chatzimpiros, P., 2017. Reconstructing production efficiency, land use and trade for livestock systems in historical perspective. The case of France, 1961-2010. Land Use Policy 67, 378-386.

Hoste, R. 2011. Produktiekosten van varkens; Resultaten van InterPIG over 2009. LEI-rapport 2011-2012. Den Haag, The Netherlands.

IPCC 2008, 2006 IPCC Guidelines for National Greenhouse Gas Inventories - A primer, Prepared by the National Greenhouse Gas Inventories Programme, Eggleston H.S., Miwa K., Srivastava N. and Tanabe K. (eds). Published: IGES, Japan.

Kumaresan, A., Bujarbaruah, K.M., Pathak, K.A., Chhetri, B., Das, S.K., Das, A., Ahmed, S.K., 2007. Performance of pigs reared under traditional tribal low input production system and chemical composition of nonconventional tropical plants used as pig feed. Livestock Science 107, 294-298.

Kunavongkrit, A., Heard, T.W., 2000. Pig reproduction in South East Asia. Animal Reproduction Science 60$61,527-533$.

Lañada, E.B., Lee, J.-A.L.M., More, S.J., Taveros, A.A., Cotiw-an, B.S., 1999. The reproductive performance of sows raised by smallholder farmers in the Philippines. Preventive Veterinary Medicine 41, 171-186. 
Lemke, U., Kaufmann, B., Thuy, L.T., Emrich, K., Valle Zárate, A., 2006. Evaluation of smallholder pig production systems in North Vietnam: Pig production management and pig performances. Livestock Science 105, 229-243.

MacLeod, M., Gerber, P., Mottet, A., Tempio, G., Falcucci, A., Opio, C., Vellinga, T., Henderson, B., \& Steinfeld, H., 2013. Greenhouse gas emissions from pig and chicken supply chains - A global life cycle assessment. In: FAO (Ed.), Rome, p. 196.

Meisinger, D.J. 2010. National Swine Nutrition Guidelines. Tables on Nutrient Recommendations, Ingredient Composition, and Use Rates. U.S: Pork Center of Excellence.

Mottet, A., Henderson, B., Opio, C., Falcucci, A., Tempio, G., Silvestri, S., Chesterman, S., Gerber, P.J., 2016. Climate change mitigation and productivity gains in livestock supply chains: insights from regional case studies. Regional Environmental Change, 1-13.

Nakai, S. 2008. Reproductive performance analysis of native pig smallholders in the hillside of northern Thailand. Tropical Animal Health and Production 40, 561-566.

Noblet, J., Dourmad, J.Y and Etienne, M. 1990. Energy utilization in pregnant and lactating sows: Modeling of energy requirements. Journal of Animal Science 6, 562-572.

NRC. 2012. National Research Council. Nutrient Requirements of Swine: Eleventh Revised Edition. Washington, DC: The National Academies Press.

Phengsavanh, P., Ogle, B., Stür, W., Frankow-Lindberdg, B.E. and Lindberg, J.E. 2010. Feeding and performance of pigs in smallholder production systems in Northern Lao PDR. Tropical Animal Health and Production 42, 1627-1633.

Sajeev, E.P.M., Amon, B., Ammon, C., Zollitsch, W., Winiwarter, W., 2018. Evaluating the potential of dietary crude protein manipulation in reducing ammonia emissions from cattle and pig manure: A meta-analysis. Nutrient Cycling in Agroecosystems 110, 161-175.

Sanchez-Martín, L., Beccaccia, A., De Blas, C., Sanz-Cobena, A., García-Rebollar, P., Estellés, F., Marsden, K.A., Chadwick, D.R., Vallejo, A., 2017. Diet management to effectively abate N2O emissions from surface applied pig slurry. Agriculture, Ecosystems \& Environment 239, 1-11.

Schader, C., Muller, A., Scialabba, N.E.-H., Hecht, J., Isensee, A., Erb, K.-H., Smith, P., Makkar, H.P.S., Klocke, P., Leiber, F., Schwegler, P., Stolze, M., Niggli, U., 2015. Impacts of feeding less food-competing feedstuffs to livestock on global food system sustainability. Journal of The Royal Society Interface 12.

Stehfest, E., van Vuuren, D., Kram, T., Bouwman, L., Alkemade, R., Bakkenes, M., Biemans, H., Bouwman, A., den Elzen, M., Janse, J., Lucas, P., van Minnen, J., Müller, C. and Prins, A. 2014. Integrated Assessment of Global Environmental Change with IMAGE 3.0. Model description and policy applications, The Hague: PBL Netherlands Environmental Assessment Agency.

Uwizeye, A., Gerber, P.J., Schulte, R.P.O., de Boer, I.J.M., 2016. A comprehensive framework to assess the sustainability of nutrient use in global livestock supply chains. Journal of Cleaner Production 129, 647-658.

van Vuuren, D.P., Stehfest, E., Gernaat, D.E.H.J., Doelman, J.C., van den Berg, M., Harmsen, M., de Boer, H.S., Bouwman, L.F., Daioglou, V., Edelenbosch, O.Y., Girod, B., Kram, T., Lassaletta, L., Lucas, P.L., van Meijl, H., Müller, C., van Ruijven, B.J., van der Sluis, S., Tabeau, A., 2017. Energy, land-use and greenhouse gas emissions trajectories under a green growth paradigm. Global Environmental Change 42, 237-250.

Wabacha, J.K., Maribei, J.M., Mulei, C.M., Kyule, M.N., Zessin, K.H., Oluoch-Kosura, W., 2004. Health and production measures for smallholder pig production in Kikuyu Division, central Kenya. Preventive Veterinary Medicine 63, 197-210.

Wang, J.M., Liu, Q., Hou, Y., Qin, W., Lesschen, J.P., Zhang, F.S., Oenema, O., 2018. International trade of animal feed: its relationships with livestock density and $\mathrm{N}$ and $\mathrm{P}$ balances at country level. Nutrient Cycling in Agroecosystems 110, 197-211.

Winkler, T., Schopf, K., Aschemann, R., Winiwarter, W., 2016. From farm to fork - A life cycle assessment of fresh Austrian pork. Journal of Cleaner Production 116, 80-89.

zu Ermgassen, E.K.H.J., Phalan, B., Green, R.E., Balmford, A., 2016. Reducing the land use of EU pork production: where there's swill, there's a way. Food Policy 58, 35-48. 


\section{Topic 2: New farming management systems (FM)}

Ideas, designs of concepts and proofs of principle of new farm information and data management systems to enhance sustainability and profitability of farming. Examples are integration of multiple technologies in management and farming systems (big data, data hubs, standards), Internet of Things applications, data-based and model-based decision support.

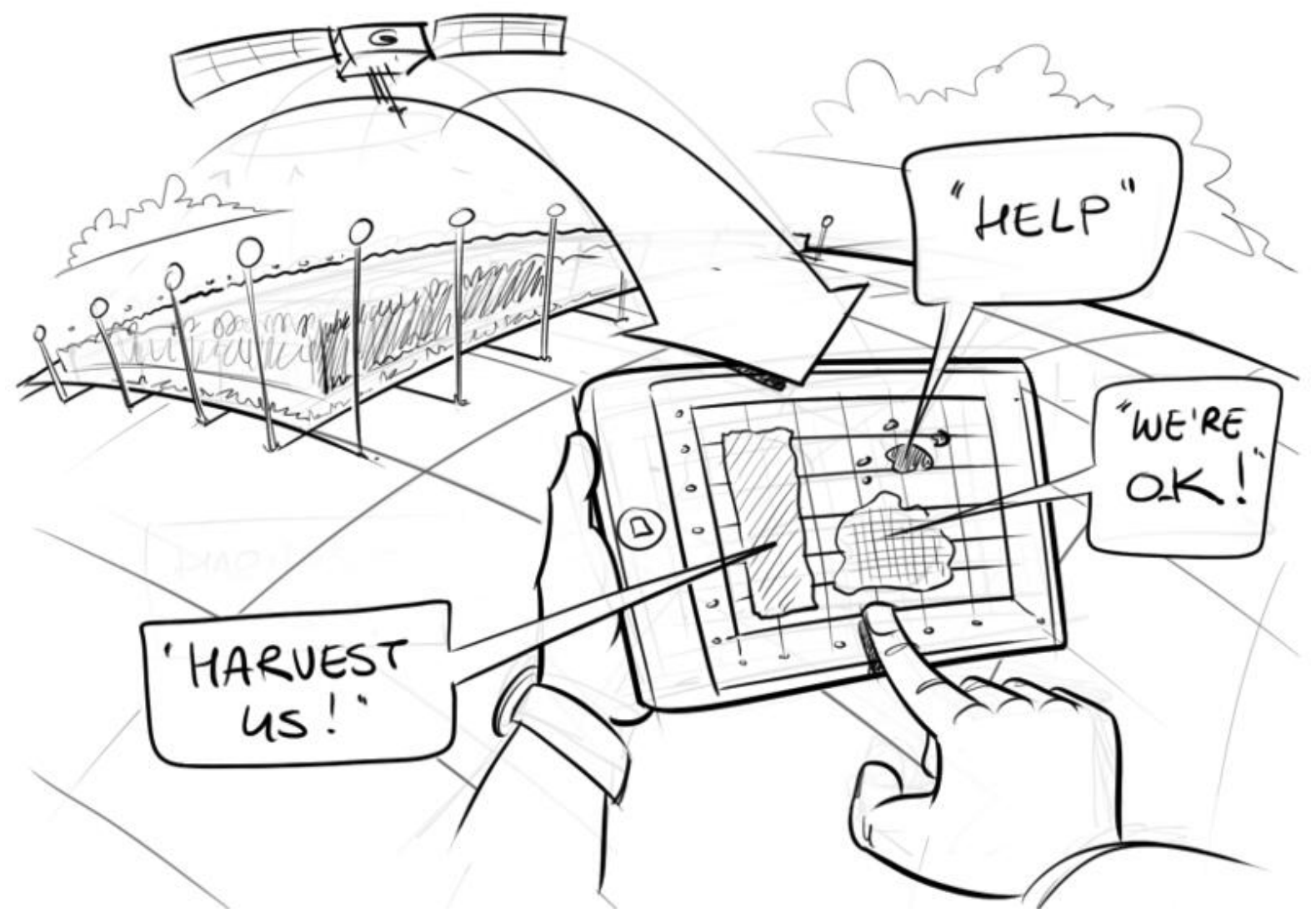




\title{
Application of Drone-Based Maps in Planning Farm Operations for Sward Management in Silage Production
}

\author{
Jussi Nikander ${ }^{\mathrm{a}}$, Hanna Huitua, Markku Koistinen ${ }^{\mathrm{a}}$, Mikko Laajalahtia ${ }^{\mathrm{a}}$ Oiva Niemeläinen ${ }^{\mathrm{b}}$, Jere \\ Kaivosoja ${ }^{a}$ \\ aProduction Systems Unit, Natural Resources Institute Finland, Maarintie 6, 02150 Espoo, Finland

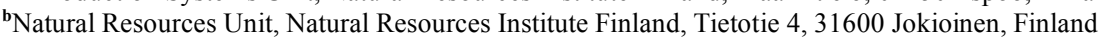

\begin{abstract}
Experience and expertise, or tacit knowledge, traditionally have a very significant role in farm management. This is especially true on smaller farms, where the scope of the farm's operations isn't necessarily overwhelming without external planning tools. However, the ongoing digitalization of farming is bringing increasing amounts of data into farm decision making. Simultaneously, the rapid decrease in the prices and significant improvements in the usability of new technology and new analysis tools are making them viable alternatives for an increasing number of farmers. Thus, in the future, traditional farm management will be increasingly supplemented - or even replaced - by management methods based on data gathered by various systems. In this work we examine how using drone-based mapping of agricultural fields can help decision making in silage production. Our work covers four important field operations: overseeding, application of both organic and mineral fertilizers, weed control, and timing of harvests. We examine how drone data can be used in planning silage farming operations, and formalize planning processes that take advantage of field maps created from the data. We will compare the drone-data utilizing process to traditional decision making to see how the drone-based data can supplement - or replace - the traditional decision making. Based on this, we will discuss how this can affect the tacit knowledge of farmers in the future. The work will both demonstrate the effect of new technology on farm management processes, as well as contribute to research on knowledge management in agriculture.
\end{abstract}

Keywords: Remoted Piloted Aircraft Systems, Grass management, farm management, knowledge management, field operation planning

\section{Introduction}

As farm sizes increase, the management of farm operations becomes increasingly time-consuming. Therefore there's an increasing need for management systems. At the same time the price of new technologies are rapidly decreasing, and therefore management systems become a viable tool also for smaller farms. Management systems, especially on large farms, change the role of the farmer towards that of a manager, with less time used in the field, and with less time to focus on each field parcel. Management systems also provide the farmer with access to detailed data about each field while on-site, and thus complement the farmer's local expertise.

These changes in farm work processes will change the experience and expertise farmers have and require. Currently, tacit knowledge of the farm, including detailed hands-on knowledge about each field, is an important part of a farmer's professional expertise. In the future, farmers are likely to spend less time on the fields and more time in the office working with data, but have access to more data while on the field.

These changes in the approach to farming will change the experience-based knowledge the farmers have. This, in turn, can have important consequences to how farming processes are planned and executed in the future. Experience-based knowledge will be less based on hands-on work in the fields and more on working with the management software and the data. Also, the software and data can complement tacit knowledge. For all farmers, the role of different decision support systems (DSS) is likely to increase, which will also affect farmer decision making. All in all, the role of data and analysis will increase and change the role of hands-on experience and local knowledge of the fields.

These changes in how farmers work can have wide-reaching consequences on the business of farming. In this work we examine the potential effects of new technology, including decision support systems and data gained from remotely piloted aircraft systems (RPAS, Drones), to the farm management processes. As our case study, we examine the management of grass sward for silage production in the Nordic context.

In the Nordic countries, the grass silage is an important part of cattle diet, especially during the winter, and grass is grown for silage in large amounts. Therefore, especially on large farms, the management of the grass silage farming is important. Sufficient amount of silage must be produced during the relatively short and cool summer to feed the livestock for the winter season. Grass is a perennial crop. However, as the annual yield decreases as the swards get older, grass fields are often plowed and re-established every 3-4 years in the Nordic countries. When operations related to the establishment and ending of the field are not taken into account, there are four principal field operations used in silage farming: overseeding, fertilization, weed control, and harvest.

Overseeding is an operation in perennial sward, at which new seeds are sown in those parts of the existing grass field where the stand is not dense enough for optimal production e.g. due to winter damage or poor establishment. The operation does not typically cover whole field area. Fertilization application is carried out for 
each harvest - in spring for the first harvest, and after the first and second harvest and for the second and third harvest, respectively. Animal manure is typically used for as large share of the fertilization need as possible on livestock farms. Mineral fertilizers are also used in addition to organic manure. Furthermore, environmental regulations restrict the application of nutrients. This has to be taken into account when fertilizer is applied. Weed control is usually carried out by chemical treatments at the early establishment phase of the grass sward - usually in the spring of the first grass year - before the perennial grass stand covers the ground. Weed control may be required also at old stands when weeds have invaded into the thinned grass stand. Silage is harvested two or three times per growing season and the first harvest is considered the most important one. First harvest is also most challenging as both the quality and quantity of yield changes rapidly at the first harvest. The yield increases and the quality for feeding decreases rapidly in the spring growth. Timing of the first cut is important to facilitate harvest of high yield at adequately high quality.

The main contribution of this work is how DSS could be used in the planning of these four operations. Aerial imagery provided by RPASs is an important data source in all operations. For each operation, we present input data required, including the RPAS data, and present the planning process for the operation that takes advantage of data and tools. Finally, we will discuss how such approach might change farming processes and what kind of influence this could have on farmer expertise and tacit knowledge.

\section{Materials and Methods}

Grass-based silage is principal feed for ruminants in the Nordic countries. Factors effecting the amount and quality of the grass yield have been intensively studied (e.g. by Niemeläinen et al. 2001a and 2012, Pulli 1980 and 1992, Virkajärvi 2004). Best solutions for practical management situations depend on e.g. geographical location, soil type, production method (conventional or organic) and type of animal the feed is produced for. In intensive dairy production the quality of the grass silage has to be high to facilitate cows to eat a high amount of silage (Rinne et al 1997a and 1997b, Kuoppala et al 2008). Environmental restrictions for various operations need to be taken into account when guidelines for management are produced. Advisory agencies as well as companies providing machinery and inputs for silage production produce general guidelines and bulletins for silage production (e.g. Toukoluoto 2018, Peltonen et al 2010)). Farmers will use their tacit knowledge of their own fields to apply the general guidelines in their conditions. Services have been established to help scheduling the first harvest, such as the Karpe-project and Mtech Digital Solutions Ltd company service links (In Finnish) http://www.karpe.fi/darvoennuste.php and https://www.mtech.fi/fi/D-arvoennuste.

Variation in yield within the field parcel has been studied relatively little so far. Niemeläinen et al (2001b) assessed the variation in grass yield, and Nykänen et al (2008) studied variation within a red clove - grass mixture stands. In the study of Nykänen et al variation in the yield was at the smallest half and at the highest twice compared to the mean yield of the field each year. Nykänen et al (2008) concluded that samples to assess the yield of a yield parcel should be taken at maximum of $100 \mathrm{~m}$ interval and for assessing the red clover content at maximum $60 \mathrm{~m}$ interval.

Potential of remotely piloted aircraft systems (RPAS) in precision agriculture has been vastly explored in recent decade (Possoch et al. 2016, Viljanen et al. 2018, Zhang 2012 for review) and the requirements for agricultural applications have been evaluated (Candiago 2015, Torres-Sánchez 2013). In the context of grassland management, the studies exploiting RPAS most often use drones equipped with different passive optical sensors, capturing reflectance from the crop canopy at the selected bands of the electromagnetic spectrum. The systems carry out processing and geo-referencing of the image mosaic together with radiometric corrections, and deliver very high resolution, geo-referenced information (Honkavaara 2013, Torres-Sánchez 2013). This information can be translated into features of interest using different parametric or non-parametric regression methods, or using physically based or hybrid approaches (see Verrelst 2015 for classification). The result may be for instance a map representing relevant grassland features, or a canopy height model (CHM) for the field. These results can be further processed to classifications and spatial delineations that can constitute the basis for grassland management decisions (Wachendorf 2018). Information sources such as in situ data, satellite imagery, or crop growth models may be used in the process.

In this work the planning processes for the four sward management field operations in silage production were modeled. The models were based on the assumption that new data sources, including RPAS imagery can provide relevant new information for the farmer, and thus enable new ways of managing silage production operations. Using these process models, the required input data was extracted, and changes to traditional silage farming planning processes were analyzed. Based on this information, possible changes in farming practices and farmer tacit knowledge were hypothesized. In this paper, the farming processes and data are discussed in Section 3, Results, and the possible influence this can have in farmer tacit knowledge in Section 4, Discussion. 


\section{Results}

In this work the Altova UModel software was used to draw the planning process models. In the model the processes are divided into tasks, depicted as green rectangles. Some of the tasks are grouped together, as they represent different parts or cases of the same subprocess. Many analyses depicted in the models are further divided into three separate steps: algorithm parameterization, calculation done by an algorithm, and review and manual adjustment of results. This basic interaction loop in exploratory data analysis is repeated in many different kinds of processes (Nikander 2012).

Yellow rectangles are used to depict collapsed subprocesses, where the tasks in the process are not explicitly shown in the Figure. These subprocesses may either be parts of the overall process that are considered out of scope of this work, or sufficiently similar to earlier parts of the same overall process that they do not need elaboration. For each field operation, a Figure depicting the planning process model, as well as the input data and the external knowledge used in the process are provided.

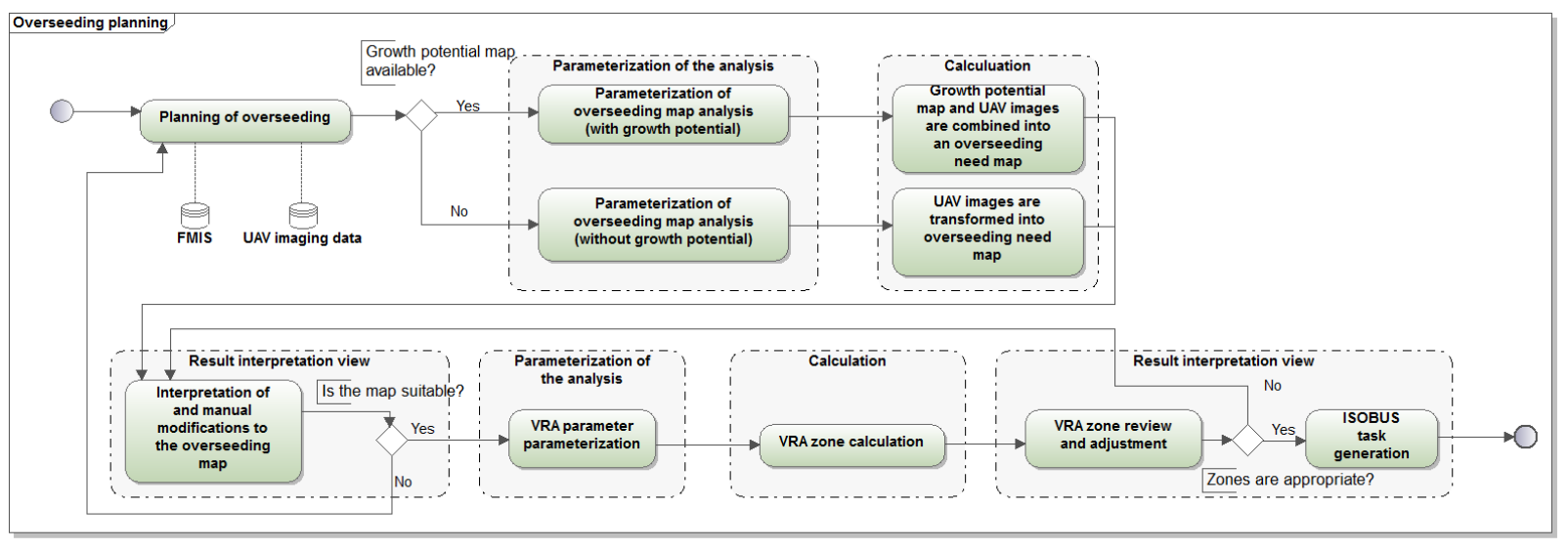

Figure 1 . The overseeding planning process.

Overseeding

Figure 1 depicts the overseeding planning process. The primary input data for the depicted process are RPAS imaging results for the fields. The orthomosaic maps created from the RPAS images used in the planning process represent the amount of biomass at the field, and low amounts of biomass correspond to locations where the grass stand is not sufficiently dense. If it is possible to sow several different types of seeds from separate containers at the same time, it would be useful to have separate maps for different species, such as one for grass and another for clover. Then it would be possible to adjust the sowing amount for each species separately.

In addition to imaging data, the farming history can be used to further refine the overseeding operation, if there is sufficient data to construct a yield potential map for each field. By combining RPAS imaging with yield potential maps, the overseeding plan can be further refined e.g. to include how dense the stand can potentially be in each location.

The planning process itself can be divided into two phases. In the first phase the RPAS images, and optionally yield potential data, is used to create an overseeding map that shows for each location the amount of additional seeds required for optimal growth. In the second phase the overseeding map is turned into Variable Rate Application (VRA) treatment zones, where each zone has a specific amount of inputs/ha that will be applied. Finally, the treatment zone map is turned into an ISOBUS task that can be executed in the field.

The user's external knowledge is used in refining both the overseeding map and the VRA treatment map. These adjustments are done based on the user's tacit knowledge and other information (e.g. field notes written on paper) that have not been encoded in the system. 


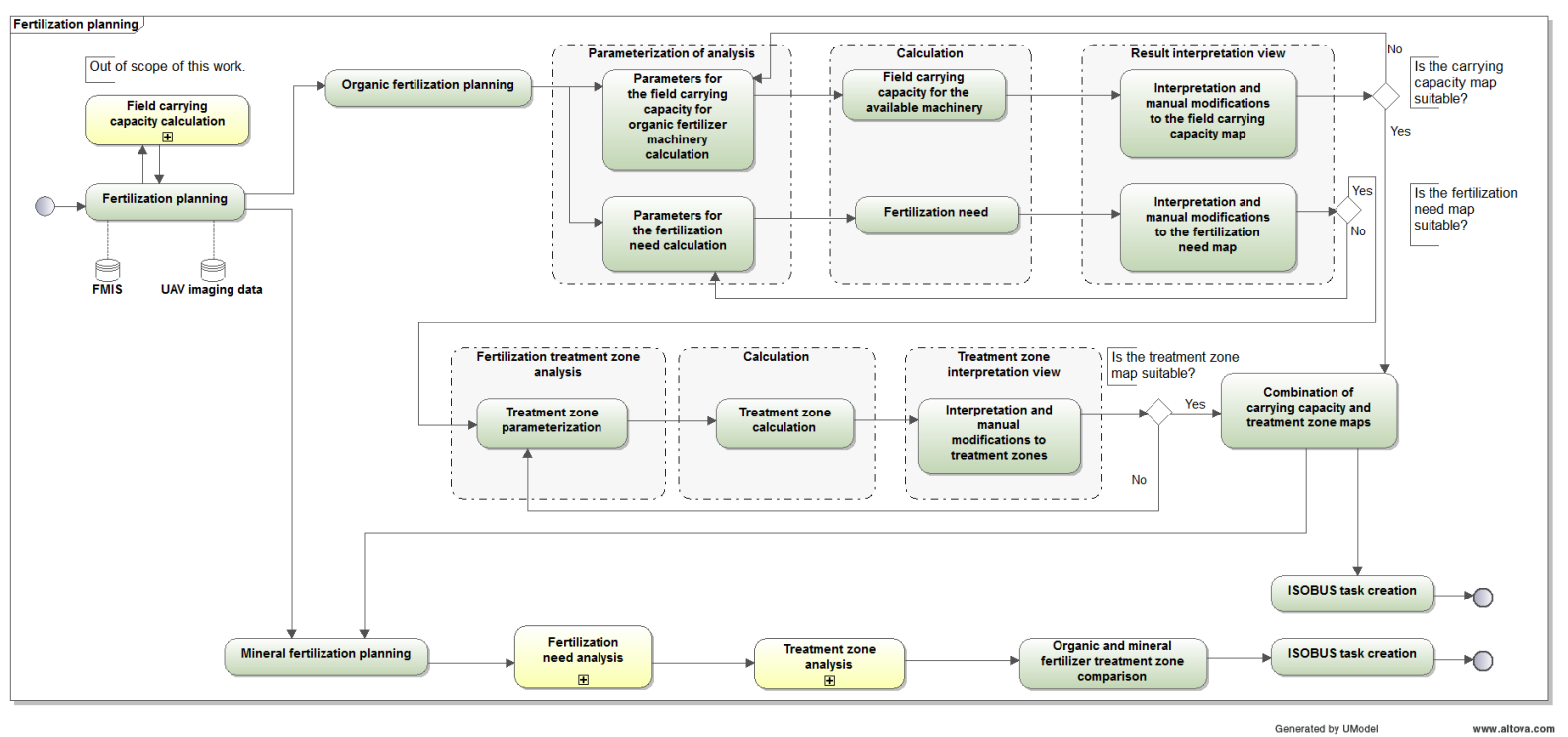

Figure 2. The fertilization planning process.

Fertilization

Figure 2 depicts the fertilization planning process. In livestock farming two types of fertilizer are used: organic fertilizer in the form of animal manure, and mineral fertilizer. In Nordic countries the preference is to use organic fertilizer where possible, and mineral fertilizers in addition. However, especially at the time of the first fertilization in the spring, the ground can still be wet and there might be parts of the fields where carrying capacity is not sufficient to support a heavy slurry injector implement or the use would cause serious soil compaction. This, in turn, necessitates the use of mineral fertilizers.

Fertilization planning is therefore divided into two separate cases: organic and mineral fertilization planning. The focus of Figure 2 is on the organic fertilization planning, since it is the preferred case and is more complex due to using heavier field machinery. The primary input data for the process are the same RPAS imaging results as for the overseeding process, field data for soil type and moisture, details of the farm machinery available, and yield potential, if available.

Soil type and moisture can be used to calculate a carrying capacity map for the field, which depicts the mount of ground pressure each location on the field can support. The carrying capacity map and details of the available machinery can be used to calculate the carrying capacity for the tractor - slurry injector combination. Meanwhile the orthomosaic from the RPAS images and the yield potential map (if available) can be used to calculate the fertilization need at each location. After the fertilization need map has been created, it needs to be turned into VRA treatment zone map, which can then be combined with the field carrying capacity map to show which locations in the field can be fertilized with organic fertilizer, and what is the fertilization need in all locations. Mineral fertilizer can then be applied to the parts of the field requiring it. The calculation of the mineral fertilizer treatment map is similar to the organic case, and thus the details are omitted from the Figure.

There are three steps in the organic fertilization process where the user can input insert their knowledge in the process. The carrying capacity map, the fertilization need map, and the VRA treatment zone map can all benefit from direct user input. Similarly, in the mineral fertilization process the fertilization need map and the VRA treatment map can be modified by the user, so there is a total of five steps where external user knowledge can be applied, if required.

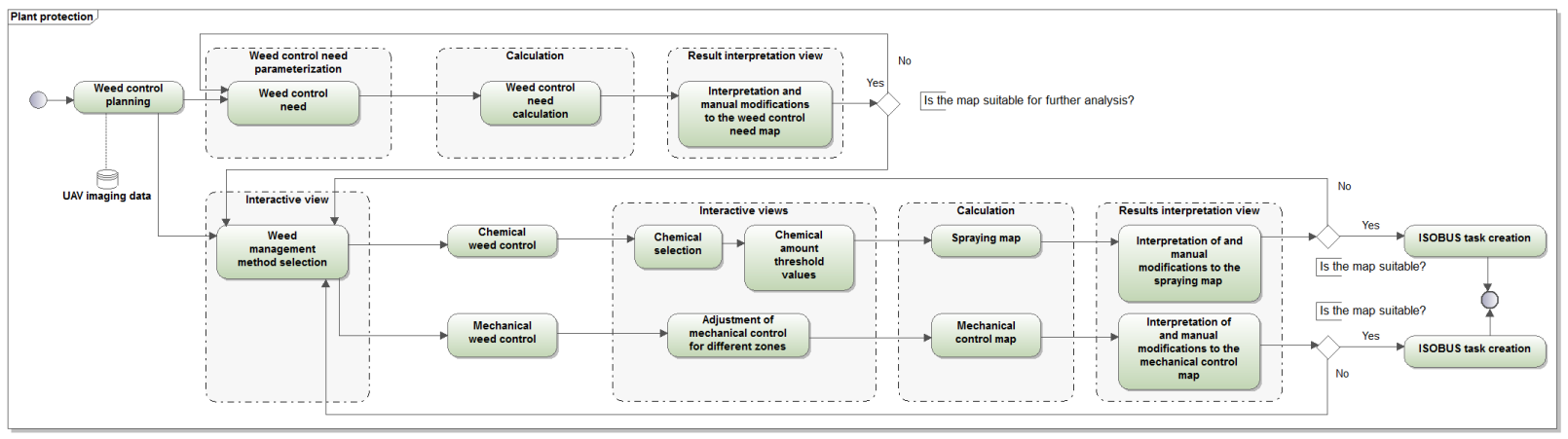

Figure 3. Weed control operation planning process. 


\section{Weed control}

Weed control in silage production can use both chemical and mechanical means of control, depending on the situation. Especially in chemical weed control, there is potential in using VRA treatment, where more detailed planning is required. For variable rate weed control, the ability to establish weed control need is crucial, and nontrivial. In this work we assume that RPAS imaging is capable of distinguishing between grass and clover and other plants growing in the grass stand. The assumption is that the other plants are weeds that need to be controlled. However, farmer observation would be needed to specify the weed species as the control agents has to be chosen according to the species to be controlled.

Figure 3 depicts the weed control operation planning process. The input data for the process are the RPAS imaging results which depict a orthomosaic map of the field with amount of weed biomass at each location. This data can be used to make the weed control need map which, in turn, can be used to plan both chemical and mechanical weed control using VRA treatment. For chemical weed control, the planning process also includes the selection of the chemical(s) to be used and the chemical amounts in different zones. The user's tacit knowledge can be inserted into the process both during the analysis of the weed control need, during the selection of the chemicals used against weed species identified in the fields, and during the development of the VRA treatment zone maps.

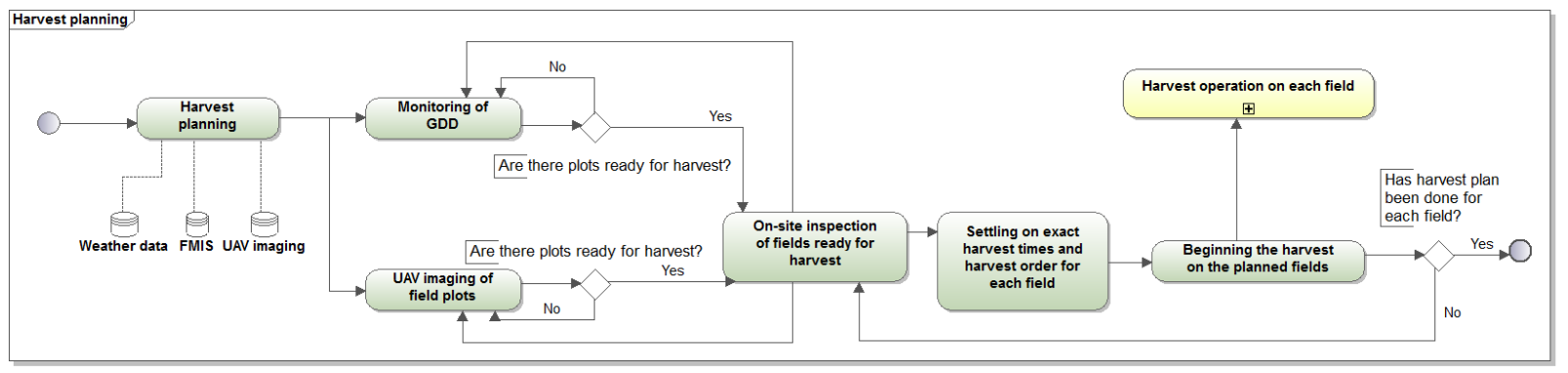

Figure 4: The harvest planning process

Harvest

The harvest planning process depicted in Figure 4 is significantly different from the other three processes included in this work. In silage farming, the most important part of harvest planning is the timing, in order to maximize the yield quantity at appropriate quality of the silage. The silage quality and yield are negatively correlated, and therefore the optimal harvest time is when the two variables are in balance to the needs at each feed production situation. Thus the focus of this process is not in the creation of a VRA field operation that can be executed, but at choosing the best possible time to harvest each field, and optimizing the order in which fields are harvested when there are several fields ready at the same time.

The input data for the process are weather data either from a meteorological institute, from weather stations, or both, including both history data and weather forecasts, if available; farming data; and RPAS imaging results depicting the amount of biomass and its quality on the fields. The optimal harvest time can either be calculated from the Growing Degree Days (GDD) at the farm, or by appropriate RPAS imaging. Imaging results also have the advantage of estimating the amount of biomass on the field and thus giving the farmer information on the amount of possible harvest before the field operation. Furthermore, the within the field variation in the quality as well as in the quantity of yield can be substantial (e.g. Niemeläinen et al. 2001). This information can also be captured in RPAS imaging. When either the GDD modeling or the images show that the fields are ready for harvest, the farmer can use their tacit knowledge to ascertain the actual situation at the field. Since grass is a perennial crop, it is common for the fields of a farm being ready for harvest around the same time. Thus, the farmer needs to optimize the order in which they handle the different fields. Furthermore, grass harvest consists of first cutting the sward, which then the grass is left for the field to dry for a short while before being harvested and stored. Thus the process of selecting the order in which the harvest operations are executed on the different fields can be a complex operation, as the farmer needs to make several operations on each field.

Table 1 contains a short overview of the data, output, and external knowledge required in each of the four processes modeled in this paper. As can be seen from the Table, the new planning processes are enabled mostly by RPAS imaging data capable for distinguishing between different types of plants; in this case grass, clover, and other plants. Furthermore, the fertilization case would require data about the soil type and amount of moisture in the fields in order to provide data about where it is possible to drive with a tractor - slurry injector -combination on the fields. In absence of sufficiently detailed data, the farmer would need to use their tacit knowledge to estimate where the fields are capable of carrying the slurry wagon. 
With the exception of the harvest operation, the ultimate output of all the planning operations is a variable rate application treatment zone map for the field or fields in question. Such a map can easily be converted into an ISOBUS task file and executed in the field using an ISOBUS-compatible tractor-implement combination. For harvest, the output of the planning process is the timing of the harvest on each field and a plan for appropriate capacity needed (machinery (tractors and wagons for silage transportation, silos for storage), labour (drivers for various machinery), materials (conservation agent) for carrying out the harvesting process smoothly, timely and efficiently.

In all processes it is possible to manually insert knowledge not encompassed in the input data into the process. The assumption is that this knowledge is mostly the farmer's tacit knowledge about the details of each of their fields. For example, subsurface drainage pipelines in the fields might not be included in a yield potential map, and can affect how the locations in their immediate vicinity react to various environmental factors. There are plenty of phenomena that can cause similar local changes in field characteristics.

Table 2. Overview of required input data, results and external knowledge required in each of the processes.

\begin{tabular}{|c|c|c|c|}
\hline $\begin{array}{l}\text { Planning } \\
\text { process }\end{array}$ & $\begin{array}{l}\text { Input data (optional data } \\
\text { in parentheses) }\end{array}$ & Output & External knowledge \\
\hline Overseeding & $\begin{array}{l}\text { (Yield potential map) } \\
\text { RPAS imaging results for } \\
\text { grass biomass in the field } \\
\text { (RPAS imaging results } \\
\text { for clover biomass in the } \\
\text { field) }\end{array}$ & $\begin{array}{l}\text { Overseeding need map } \\
\text { VRA treatment zones for } \\
\text { seeding }\end{array}$ & $\begin{array}{l}\text { Farmer knowledge of field } \\
\text { details not captured in the data. }\end{array}$ \\
\hline Fertilization & $\begin{array}{l}\text { (Yield potential map) } \\
\text { Soil type map for the } \\
\text { field } \\
\text { Moisture map for the } \\
\text { field } \\
\text { RPAS imaging results for } \\
\text { grass biomass in the field } \\
\text { (RPAS imaging results } \\
\text { for clover biomass in the } \\
\text { field) }\end{array}$ & $\begin{array}{l}\text { Fertilization need map } \\
\text { field carrying capacity map } \\
\text { for specific machinery } \\
\text { VRA treatment zones for } \\
\text { organic fertilizer } \\
\text { VRA treatment zones for } \\
\text { mineral fertilizer }\end{array}$ & $\begin{array}{l}\text { Farmer knowledge of field } \\
\text { details not captured in the data. }\end{array}$ \\
\hline Weed Control & $\begin{array}{l}\text { RPAS imaging results for } \\
\text { weed cover in the field }\end{array}$ & $\begin{array}{l}\text { Weed control need map } \\
\text { VRA treatment zones for } \\
\text { weed control }\end{array}$ & $\begin{array}{l}\text { Farmer knowledge on details } \\
\text { not captured in the data e.g. } \\
\text { identification of weed species }\end{array}$ \\
\hline Harvest & $\begin{array}{l}\text { Weather history } \\
\text { (Weather forecast) } \\
\text { RPAS imaging results for } \\
\text { amount and quality of } \\
\text { biomass in the fields }\end{array}$ & $\begin{array}{l}\text { Optimal harvest time for } \\
\text { each field } \\
\text { Capacity and time needed } \\
\text { for harvest operation and for } \\
\text { storage space }\end{array}$ & $\begin{array}{l}\text { Planning of the details of the } \\
\text { harvest operations is not } \\
\text { included in the model and may } \\
\text { require significant farmer } \\
\text { knowledge but the drone data of } \\
\text { the yield quantity per ha will } \\
\text { provide valuable information }\end{array}$ \\
\hline
\end{tabular}

\section{Discussion and Conclusions}

Traditionally, the overseeding, fertilization, weed control, and harvest operations in the Nordic countries are planned without the help from a DSS. This is especially true on small and medium farms, where the number of field plots is low, and the total cultivated area is tens of hectares. As farm sizes increase, the farm managers have less time to spend on each field, and more details and information to remember. This makes the role of farm management systems more important, as these systems can store the knowledge and provide it in a suitable form.

In general, the increased farm size, as well as the availability of management software and other technological solutions, make the adoption of various technological solutions to farm management more attractive. This, in turn, changes the farmer's job so that they spend more time in the office working with these tools, and consequently have less time to work on the fields. This decrease in time spent on the fields can then affect the farmer's tacit knowledge of each field as less time is spent to refine the knowledge on-site. RPAS imaging can supplement - or even replace to some degree - the knowledge gained from on-site work. The farmer can view their fields from the imaging results, and therefore do not necessarily need to go on-site in order to review the field.

RPAS imagery and other remote sensing data can provide a lot of information for a farmer. However, examining fields using this data is different from being on-site, which can also affect the formulation of tacit knowledge. Remote sensing may yield an excellent overview of the situation on a field, but cannot go into details in the same way. For example, aerial imagery can detect areas of poor crop growth on the field, but cannot be used to explore the details or causes at similar level of detail as being on-site. Thus increased reliance on such tools is likely to decrease the detailed knowledge of the farmer, which can affect their decision making in cases 
where very detailed knowledge of the specific field would be needed. On the other hand, mobile user interfaces to farm management software can provide the farmer on-site access to the data they work with in the office, and thus provide means to combine on-site observations with DSS results and other data stored in farm management software.

Furthermore, farmers' attitude towards farming software in Finland is that they do not want to rely too much on software. Trust on the results of DSS can be low, and a bad analysis result can easily lead to further loss of trust.

Thus there's a possibility for a clear dichotomy: increased farm sizes makes the use of management software, including DSS, and attractive prospect. However, low trust the farmers have on these systems can make them unattractive. Furthermore, the more time the farmer spends in the office using different software, the less time they have on the fields, and therefore less chance to refine their on-site knowledge of each field. In the future, we need to take these effects into account when designing how farm management and field operation processes should be supported using DSS tools.

In addition, it should be noted that farmers are not the only users groups for sward management DSS. Many farmers use agricultural contractors for many of the field operations. Many of these contractors are independent entrepreneurs working with a number of different farms. ). Silage harvesting entrepreneurs with high capacity and expensive - field machinery combinations that also require large amount of labor to run the operation smoothly, could be one group benefiting from DSS. Especially contractors working in the harvest process can have complex operations environment. E.g. ha-yield quantity would have a strong effect how many hectares the harvest chain could harvest in one day. This information (provided e.g. by drone data) would give valuable information for scheduling the harvesting.

Finally, it should be noted that this research is still very much a work in process. For example, the planning processes shown in Figures 1-4 do not take the possibility of farmers using agricultural contractors into account. It is likely that involving contractors would change the farmer's planning process sufficiently that this should be taken into account, especially if the farmer gives the contractor significant role in how the field operations are executed. There are also other aspects of the farming process that are not explicitly taken into account in this work. For example, the fertilization operation is repeated two or three times per growing season on each field. Thus the results of the previous fertilizations and harvests have an effect on the fertilization need for the subsequent operations. Thus a nitrogen uptake map for the previous harvest would be suitable input data for subsequent fertilization planning processes.

\section{Acknowledgements}

This work has been done as part of the DroneKnowledge project funded by the Challenge Finland funding by Business Finland. The goal of DroneKnowledge is to develop new applications and uses for drones and hyperspectral imaging in agriculture. DroneKnowledge is a joint project between Finnish Geospatial Research Institute, Natural Resources Institute Finland, VTT Technical Research Centre of Finland, University of Jyväskylä, and a number of Finnish companies.

\section{References}

Candiago, S., Remondino, F., De Giglio, M., Dubbini, M. and Gattelli, M., 2015. Evaluating multispectral images and vegetation indices for precision farming applications from UAV images. Remote Sensing, 7(4), pp.4026-4047.

Honkavaara, E., Saari, H., Kaivosoja, J., Pölönen, I., Hakala, T., Litkey, P., Mäkynen, J. and Pesonen, L., 2013. Processing and assessment of spectrometric, stereoscopic imagery collected using a lightweight UAV spectral camera for precision agriculture. Remote Sensing, 5(10), pp.5006-5039.

Kuoppala, K., Rinne, M., Nousiainen, J. and Huhtanen, P., 2008. The effect of cutting time of grass silage in primary growth and regrowth and the interactions between silage quality and concentrate level on milk production of dairy cows. Livestock Science, 116(1), pp.171-182.

Niemeläinen, O., Jauhiainen, L. and Miettinen, E., 2001. Yield profile of tall fescue (Festuca arundinacea) in comparison with meadow fescue (F. pratensis) in Finland. Grass and Forage science, 56(3), pp.249-258.

Niemeläinen, O., Miettinen, E., Virkajärvi, P., Alakukku, L. and Hakala, K., 2001. Variability in herbage mass and chemical composition within a timothy sward. In Proceedings of the XIX International Grassland Congress: Grassland ecosystems: an outlook into the 21st century, 11-21 February 2001, São Pedro, São Paulo, Brazil/JA Gomide (ed.), WRS Mattos (ed.), SC da Silva (ed.. Fundacão de Estudos Agrários Luiz de Queiroz. p. 122-125.

Niemeläinen, O., Niskanen, M. and Jauhiainen, L., 2012. Productivity and quality of meadow fescue, tall fescue and festulolium in silage cutting regime in Finland. In Proc. XVI International Silage Conference (pp. 174175).

Nikander, J., 2012. Interaction and visualization methods in teaching spatial algorithms and analyzing spatial data. PhD Thesis. Aalto University, Finland. 
Peltonen S, Puurunen T, Harmoinen, T., 2010. Nurmirehujen tuotanto ja käyttö (Production and use of grass silage). ProAgria Keskusten liitto. 100p.

Nykänen, A., Jauhiainen, L. and KEMPPAINEN, L., 2008. Field-scale spatial variation in yields and nitrogen fixation of clover-grass leys and in soil nutrients. Agricultural and food science, 17(4), pp.376-393.

Possoch, M., Bieker, S., Hoffmeister, D., Bolten, A., Schellberg, J. and Bareth, G., 2016. Multi-temporal crop surface models combined with the RGB vegetation index from UAV-based images for forage monitoring in grassland. The International Archives of Photogrammetry, Remote Sensing and Spatial Information Sciences, 41, p.991.

Pulli, S. 1980. Growth factors and management technique used in relatios to the developmental rhythm and yield formation pattern of a pure grass stand. Journal of the Scientific Agricultural Society of Finland. 52, pp. 281-330.

Pulli, S. 1992. An overview of grassland production in Finland. Proceedings of the $14^{\text {th }}$ general meeting of the European Grassland Federation. 8-11.6.1992. Lahti, Finland, p. XVIII-XXVIII.

Rinne, M., Jaakkola, S. and Huhtanen, P., 1997a. Grass maturity effects on cattle fed silage-based diets. 1. Organic matter digestion, rumen fermentation and nitrogen utilization. Animal Feed Science and Technology, 67(1), pp.1-17.

Rinne, M., Huhtanen, P. and Jaakkola, S., 1997b. Grass maturity effects on cattle fed silage-based diets. 2. Cell wall digestibility, digestion and passage kinetics. Animal Feed Science and Technology, 67(1), pp.19-35.

Viljanen, N., Honkavaara, E., Näsi, R., Hakala, T., Niemeläinen, O. and Kaivosoja, J., 2018. A Novel Machine Learning Method for Estimating Biomass of Grass Swards Using a Photogrammetric Canopy Height Model, Images and Vegetation Indices Captured by a Drone. Agriculture, 8(5), p.70.

Virkajärvi, P., 2004. Growth and utilization of timothy-meadow fescue pastures. Academic dissertation. University of Helsinki. Department of Applied Biology. Section of Crop Huspandry. Publication 19. $56 \mathrm{p}$.

Torres-Sánchez, J., López-Granados, F., De Castro, A.I. and Peña-Barragán, J.M., 2013. Configuration and specifications of an unmanned aerial vehicle (UAV) for early site specific weed management. PloS one, 8(3), p.e58210.

Toukoluoto N. (ed), 2018. Maatalouskalenteri 2018 (agricultural calendar 2018). ProAgria keskusten liitto. $245 \mathrm{p}$.

Verrelst, J., Camps-Valls, G., Muñoz-Marí, J., Rivera, J.P., Veroustraete, F., Clevers, J.G. and Moreno, J., 2015. Optical remote sensing and the retrieval of terrestrial vegetation bio-geophysical properties-A review. ISPRS Journal of Photogrammetry and Remote Sensing, 108, pp.273-290.

Wachendorf, M., Fricke, T. and Möckel, T., 2018. Remote sensing as a tool to assess botanical composition, structure, quantity and quality of temperate grasslands. Grass and Forage Science, 73(1), pp.1-14.

Zhang, C. and Kovacs, J.M., 2012. The application of small unmanned aerial systems for precision agriculture: a review. Precision agriculture, 13(6), pp.693-712. 


\title{
A Territorial Services Platform for the Sustainable Precision Agriculture Applied to the Viticulture and Olive Growing Companies
}

\author{
Daniele Sarri ${ }^{a^{*}}$, Simone Priori ${ }^{\mathrm{c}}$, Marco Antoni ${ }^{\mathrm{b}}$, Sara Gallucci ${ }^{\mathrm{b}}$, Stefania Lombardo ${ }^{\mathrm{a}}$, Marco Vieri ${ }^{\mathrm{a}}$ \\ ${ }^{a}$ GESAAF Agricultural, Food and Forestry System - Biosystem Engineering Division, University of Florence, Firenze, \\ 50144, Italy \\ ${ }^{\mathrm{b}}$ Copernico S.r.1., Montalcino, 53024, Italy \\ ${ }^{c}$ CREA, Research centre for agriculture and environment, Cascine del Riccio, Firenze, 50125, Italy \\ * Corresponding author. Email: daniele.sarri@unifi.it
}

\begin{abstract}
The improvement of environmental and food quality and safety, simultaneously with the pursuit of common and economic activities based on knowledge and innovation supported by an environmentally sustainable development, represent the new community guidelines (Horizon 2020). In this new approach toward the smart farming the aim of the research was the development of a territorial services platform for the sustainable precision agriculture applied to the viticulture and olive growing companies of Montalcino district (OENOSMART project). The study involved eleven companies for a total area of 330 hectares. The data were acquired both remotely by airplane RGB, multi and hyperspectral images and gamma rays and with proximal measurements on the ground and canopy. The design of services platform, based on Geoserver with db PostGIS, was conducted in four phases: structuring of the Web platform, agro-environmental parameters and aerial images data collection, data processing, organizing of layers on the platform and subsequent creation of decision supports. Collected data were processed by geostatistical analysis, interpolated between them to make thematic outputs like prescription maps shape files as decision support systems and for the implementation of variable rate management. Moreover, the cadastral data referred to each cultivation were included for intercommunication with the regional delivering body in charge of the decoupled payments by common agricultural policy (CAP). The developed platform constitutes a first Italian national case on large area of sustainable precision farming for the agricultural companies of a territory. Further, the system provides suggestions for a profitable management of big data that nowadays represents a mandatory medium-term target for the smart management of agriculture sector required by the EU community policies.
\end{abstract}

Keywords: sustainability, mapping, WebGIS competitiveness, decision support system, precision viticulture

\section{Introduction}

Health, welfare of the population, transition to sustainable and competitive social and territorial development, resistant to climate change are some of the priorities pursued by EU policies. Other objectives, promoted by the EU Cork's declaration (Cork 2.0 Conference on Rural Development) entitled "Better Life in Rural Areas" were oriented toward to the fulfilment of innovative, integrated and inclusive agricultural rural policy in the European Union. These have to be inspired by ten policy guidelines, which account on the emphasis subject of strengthening the rural value chains, rural environment and stimulate knowledge and innovation through rural communities that have to participate in the knowledge economy. The declaration stated that all type and sizes farms must have access to appropriate technologies, advanced connection to find solutions and new management tools to generate business, social and environmental benefits (EC, 2016).

Among the innovative farmers devoted to wine and olive oil production, these objectives represent some of the new challenges pursued in the productive processes by introducing precision farming technologies. This approach requires a deep and constant knowledge of data, thus information, which might sustain effective actuations strategies on farms. The data knowledge represent one of the compulsory requirements to consider the issues related to the production area where the farmer produce (land complexity, orography, climate, facilities, services etc.) and the sector production structures.

However, contextualizing such opportunities on Italian agriculture, the fragmented structure of farms characterized by the $70 \%$ of the total number of wine farm with a surface extension less than 5 hectares and an average size of just 1.3 hectares per company for the olive growing on 825.000 farms (Istat, 2013) not allows the introduction of precision farming technologies in such a way as to achieve scale economies. In this agricultural scenario for a competitive actuation of productive processes appears almost the needing data knowledge and its sharing even through service companies. The innovative precision technologies and their rapid diffusion in the agricultural sector, are being emerging solution which has a wide potential for the farms but at the same times takes some risk if not properly introduced and supported. Bramley, forerunner of precision viticulture, clearly stated in its researches the needing of proper use and introduction of new technologies for the site-specific optimization (Bramley et al.,2003). Every agricultural scenario, characterized by its diversity, has its own technological level opportunities and it is required their identification and their modality of introduction 
(Lombardo, 2018).

Along together the development of precision farming technologies there is now growing evidence the necessity of geospatial information and geodata (De Filippis et al., 2010, Terribile et al.,2017). The knowledge of soil and vegetative spatial variability at high detail is basic for most of the precision farming techniques and for sitespecific management of the crop.

Recently, the use of proximal sensors to map soil and canopy vigour spatial variability at high detail has been increasing, even in professional consultations. These sensors allow to obtain maps of certain parameters strongly related to soil physical (stoniness, texture) (Priori et al., 2014), hydrological (available water capacity, drainage) (Ortuani et al., 2016) and chemical (salinity) (Doolittle et al., 2001). At the same time, there are proximal sensors and photogrammetry techniques, which provide maps on vegetation indexing (Sarri et.al, 2015) which can be related with both soil variation and more generally, with quality and quantity responses.

These undertakings make sense since the actuation of precision farming techniques brings the production of relevant amount of data that if not properly integrated in a general management approach ends in themselves without providing economic, environmental and social benefits. The information and communication technologies (ICT) and the geographical information system (GIS) are the rising systems that may ensuring their sustainable exploitation while providing support for the actuation of sito-specific practices. The quantity and quality of available information on crop, management process and their impact on the economic turnover are key factors for the planning of activities and the production targets (i.e. quality or quantity). Therefore, it is essential to design systems, which allow the handling of huge amount of data collected by precision farming technologies and produce decision making supports. In the viticulture and olive growing some still unresolved challenges are the (a) needing to monitor crops and weather condition to differentiate production in order to improve the quality and the efficiency on input usage, (b) the knowledge needing of the resources involved in the processes for the administrative management, (c) the need for simplification of communications with the public administrations.

In the aforementioned framework the study was primarily addressed to the development of a territorial WebGIS services platform for the sustainable precision agriculture applied to the viticulture and olive growing companies. The research activities were aimed to create an innovative territorial system and promote a multiservice collaborative platform for the effective, profitable and inclusive application of precision agriculture oriented to sustainability, quality and competitiveness. As a secondary objective, the research activities aim to start a process for the development of a new entrepreneurship aimed at the widespread use of precision farming technologies and the training of new professionals (technicians and companies) able to support farms.

\section{Materials and Methods}

\section{Study Area and extent}

The study was carried out in the Montalcino district, which is located in the south-central part of Tuscany Region near Siena city, Italy (Figure 1). Specifically the study area lies between North latitude $43^{\circ} 03^{\prime} 18.7^{\prime \prime}$ and $11^{\circ} 29^{\prime} 20.6^{\prime \prime}$ East longitudes. The production area of Montalcino coincides with the historical border of the municipality of Montalcino, a district of 24,000 hectares, of which $15 \%$ covered by vineyards. The area has an almost square shape, whose sides are bordered by the Ombrone, Asso and Orcia rivers. This agricultural area is known around the world for the production on premium wine is also characterized for a huge production of olive oil and cereals. The study involved eleven companies for a total area of 250 hectares (vineyards) and 80 hectares (olive growing) respectively.
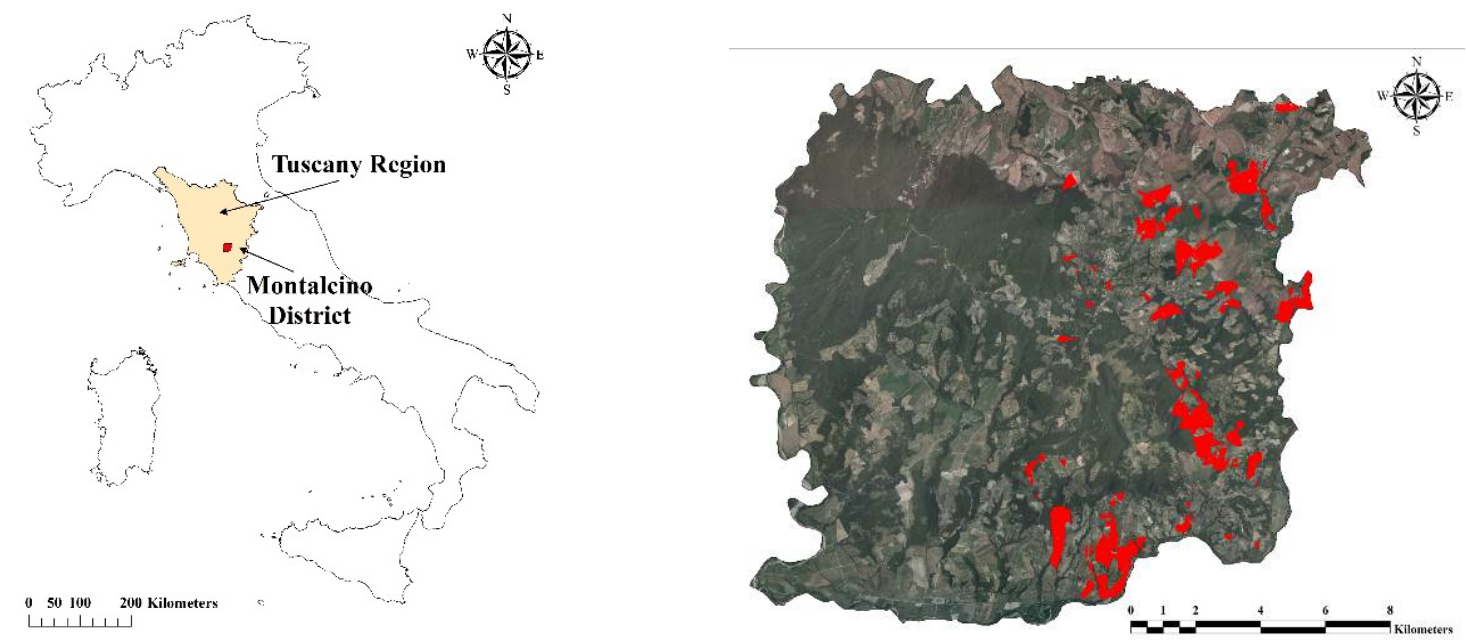

Figure 1. Overview of the study site located in Montalcino municipality (Central Italy, Tuscany region). 


\section{WebGIS Architechture}

The WebGIS platform architecture was implemented with a spatial database realized with PostGIS. This constitute the spatial database extender for PostgreSQL objects relational database in order to localize queries, which were run in SQL. The code is written in java, using the Geotools library. For server side open source Geoserver was used. This allows the management of different type of layers divided between rasters and vectors. It acts as a webserver providing, on the client side, map services following OGC standards such as WMS, WFS, WCS for the management and display of maps. Webix Jet was used like client-side JavaScript micro-framework in order to simplify the creation process of applications with complex structure and the management of big datasets by exploiting the Webix libraries. The Open Layers library was used to represent and process geographical data on the web. In particular, this library was implemented on the client side for displaying layers, raster maps, vector data prescription maps.

\section{Data mining}

Dataset connected to the WebGIS platform tool includes geo-referenced data and metadata acquired from different sources. In order to develop a decision support platform with information which can be queried by the user concerning the administrative aspects and agronomic management, cadastral data were integrated from the production units and proximal and remote surveys were carried out.

The optimization of farm management steps is an issue very much felt by farmers. Many of the processes carried out in small and medium-sized companies are not analytically monitored during the whole process with the end result of a lack of knowledge of the needing factors for the products production and their related costs. This also negatively affects the intervention ability of the farmer on the most critical phases. In this sense, the platform has been developed to store land registry information about the owners of individual plots, geometries on the single plot, cultivation plan. A specific section was dedicated to the working stages. These functionalities allow the tracking of all activities on a plot since the design phase to its ordinary management, managing costs, workmanship, staff hours. An additional design parameter considered in the development of the platform concerned the implementation of functionalities that allow the official communications with the public administration responsible for decoupled payments (Tuscany Regional Agency for Payments A.R.T.E.A.) of the European Community. In this sense, the Italian farms with surfaces devoted to the wine production since the last first January 2017 in line with the provisions of the D.M. 162 of 2015, with L.R. 68/2012 and with the deliberation G.R. November $29^{\text {th }} 2016$, n. 1192, which implements the provisions of Reg. (UE) 1308/2013 and Reg. UE 809/14 art. 17, have to communicate their vineyard surfaces. To accomplish this a computer-aided design tools (CAD) that works on high-resolution ortophoto was implemented in the platform. Moreover a communication protocol recognised by the public administration was done.

Concerning agronomic management, the activities were focused on (a) field surveys to monitor vegetative and soil characteristics by proximal sensing measurements and (b) aerial image acquisition. Moreover, to monitor and share meteorological data between farmers (c) eleven weather stations were installed. The proximal sensors used for the monitoring of plant vigour was a reflectance sensor model OptRx (Ag Leader Technology, Io., USA) which provide both NDVI and NDRE indexes. Surveys were performed in two times one before the first green pruning stage (BBCH-75) and the second at veraison (BBCH - 81/83). The aerial image acquisition was peformed with a RGB High resolution camera $\left(0,04 \mathrm{~m} \mathrm{pixel}^{-1}\right)$. For the soil analisys the sensors employed were the following: i) EM38-Mk2 electromagnetic induction sensor (Geonics Ltd., Ontario, Canada) and ii) "The Mole", gamma-ray spectroradiometer (Soil Company, The Nederlands). The datapoints measured by soil proximal sensors were interpolated within the whole field by ordinary kriging, using SAGA-Gis software. Two or three sites within each surveyed vineyard and olive groove were selected on the basis of stratified sampling strategies, using the maps obtained by the soil proximal sensors. In each selected site, a soil profile or augering was described following the FAO guidelines (Jahn et al., 2006). Soil horizons were sampled for standard laboratory analysis, such as texture, $\mathrm{pH}$, electroconductivity, organic carbon, total nitrogen, total calcium carbonates, cation exchange capacity and exchangeable bases.

The collected data by proximal canopy measurements and aerial were processed by geostatistical techniques, interpolated between them to make thematic and prescription shape files maps divided into two-three macro subplots as decision supports (e.g. selective harvesting, spreading of nutrients) but also for the implementation of variable rate managements techniques (Figure 2). Furthermore, specific measurements were carried out for the analysis of physiological parameters useful for the characterization of the identified sub plots. In particular, surveys were performed to measure the water stress of the vineyards with portable pressure chamber, chlorophyll content by means of a SPAD-502 Leaf Chlorophyll Meters, (Minolta Camera Co., Lts, Osaka, Japan) and measurements of gaseous exchanges using the CIRAS 3 portable infrared gas analyzer (PP Sistems UK). All data were georeferenced.

Finally, in the vineyards, grape samplings in the macrozones identified were carried out to make laboratory 
analyzes with the final aim of qualitatively characterizing the grapes. All the meteorological data coming from the weather stations were interpolated with ordinary kriging geostatistical method and high-resolution weather maps were made on the border of the Montalcino municipality. The weather thematisms were shared between farmers.
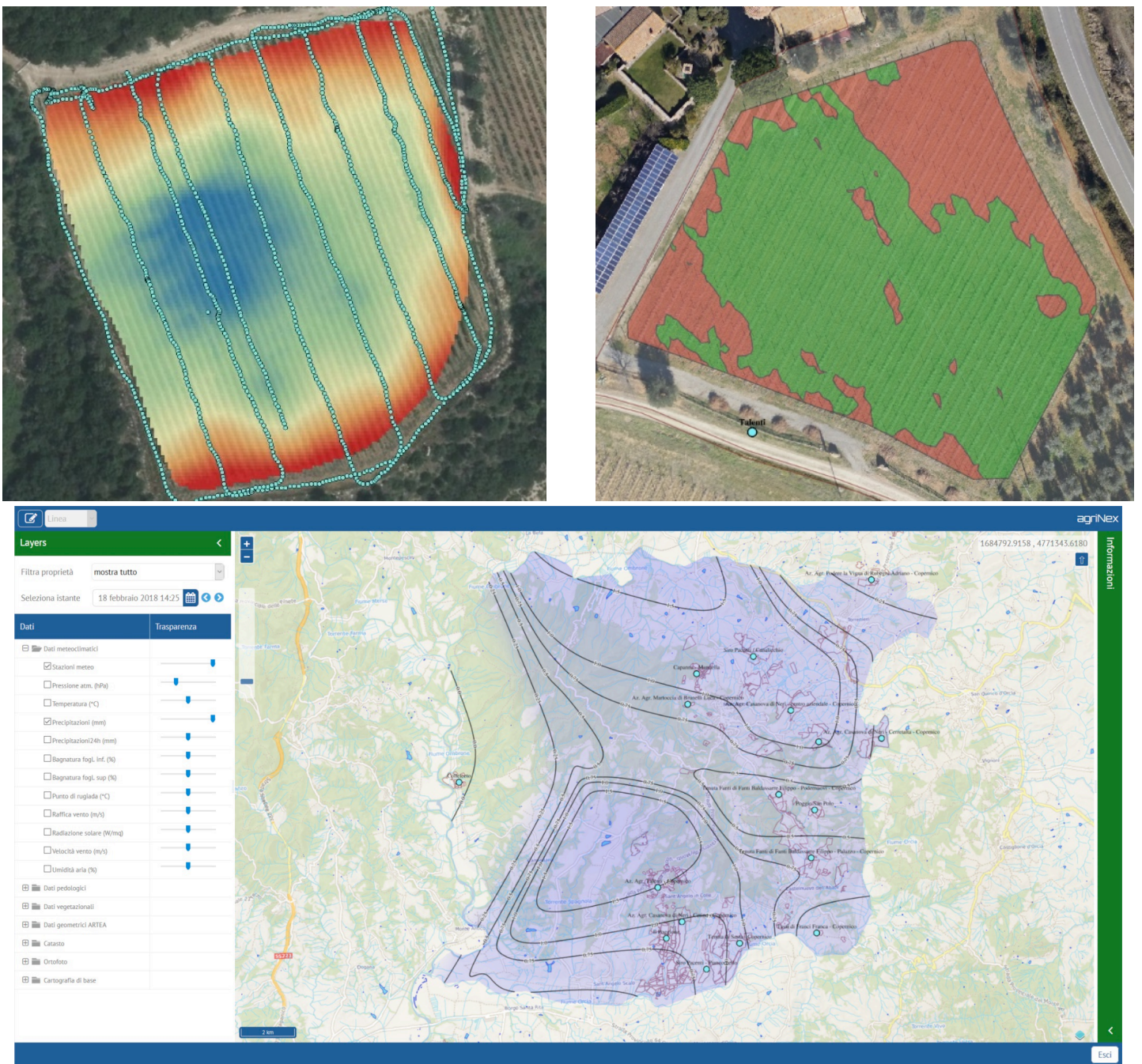

Figure 2. Top Left: Example of shapefile of the datapoints measured by EM38-MK2 proximal sensing and interpolated map of ECa2 $(0-150 \mathrm{~cm})$, obtained by ordinary kriging. Top Right: Prescription map based on proximal NDRE measurements. Central down: general overview of WebGIS platform with highlighted kriging weather map related to Montalcino district.

\section{Results and Discussion}

The aim of the ongoing study trough OENOSMART project is to develop an open platform that will be instantiated into decision support systems dedicated to sustainable, smart agriculture. The WebGIS platform collects and interpolate in multilayers agronomic, environmental and meteorological information which provie decision making support for the technical and economical management optimization of farms. As general main result, the platform provide to farmers a powerful but simple customized dashboard where they can effectively access all the available functions and information in a profiled way which appears essential to actuate optimized management strategies. The oenosmart platform includes graphical tools, procedures to view and combine spatial data (analysis and visualization), the production of tables and maps. Specifically, a Web viewer allows the visualization and querying of seven main sections which enable the end-user to navigate through spatial data layers divided into: meteorological, pedologic, vegetation, CAP communication, cadastral, ortophoto, base cartography data (Figure 3). The meteorological section provide kriging spatialized maps which show the realtime visual assessment of the trends of the major climatic parameters by means of on the fly real time processing model through the web. This information are useful for the individuation of climatic conditions that could induce the beginning of pest disease such as to assess the degree of downy mildew, as wel as insects attacks. Data on soils are provide by interactive soil maps which can be specifically queried to highlight in detail the soil type, physical features such as texture, soil apparent electrical conductivity (ECa). The layers on vegetation parameters were reported in vigour thematic maps and punctual physiological parameters measurements. The vigour maps 
were bsed on indexes as Normalized Difference Vegetative index (NDVI) or Normalized Difference Red Edge (NDRE) to take account even that part of wavelengths spectrum of near infrared part where there is a very rapid change in its reflectance between 680 and $730 \mathrm{~nm}$. The creation of vigour maps, specifically for the vineyards, is justified insofar as the high correlations have on the relationship in quality and yield. (Arnò et al.,2009, Dry, 2000; Haselgrave, 2000, Petrie, 2000, Santesteban et al.,2013, Tisseyre, 1999, ). In particular, three overall vegetation shapefiles of the study areas have been created. The first was organized into 10 classes to highlight in detail the variation in vegetative vigor. The second was organized in 2 classes using a bi-chromatic scale with the use of red identifying the sub-macro zone with low vigor and the green for the high-vigor ones in order to identify two areas with significantly different qualitative and quantitative responses. The third, of a point vector type, showed the measurements relating to the parameters of water stress, chlorophyll content and gaseous exchanges.

The layers were produced both in raster and vector format in order to allow the widest comparability and usability for any further comparison analysis with other indices, but also the uploading on machine terminals to perform variable rate treatments. Both soil and vigour maps, in conjunction with physiological measurements, may be used from the farmers to realize variable rate treatments, differentiate agronomic interventions and to diversifying the productions on quality levels.

The high-resolution images acquired by airplane flights together with the cadastral data of the plots under investigation constituted the basic tools both the management of the company registry and for the communications with the regional body used for the decoupled payments of the European Community. This was made possible by the platform thanks to the implementation of a drawing / selection tool of the area of interest (AOI). This tool allows to delineate in detail the boundaries of the single plot and to communicate and update farm data through the protocol of public administration in a such way more accurate, quickly, timely and less heavy for the farm administration. Finally, the platform archive all the activities timings and related raw materials used for carrying out cultivation steps and during the growing season and indirectly to make a simplified financial report. Indirectly this functionality provide automatic tracking and certification of products, that can be used by Regional Governments to obtain reports and data on the usage of resources and the state of the environment.

At the current stage of development, the platform is open and shared among the Oenosmart project partners farms' for the data relating to meteorological information, soils measurements and those relating to vegetative vigor obtained by proximal and aerial sensing and also to the physiological parameters of plants and ripening of the grapes. The information relating to sensible data such as the company areas, their quantification as well as the breakdown by type of crops together with those related to the management stages and the technical means and raw materials used are accessible only by the end-users. All of these information may be used from growers for the purpose of an economic valorization of the product through appropriate marketing strategies that exalt the typicality and terroir of the production areas.

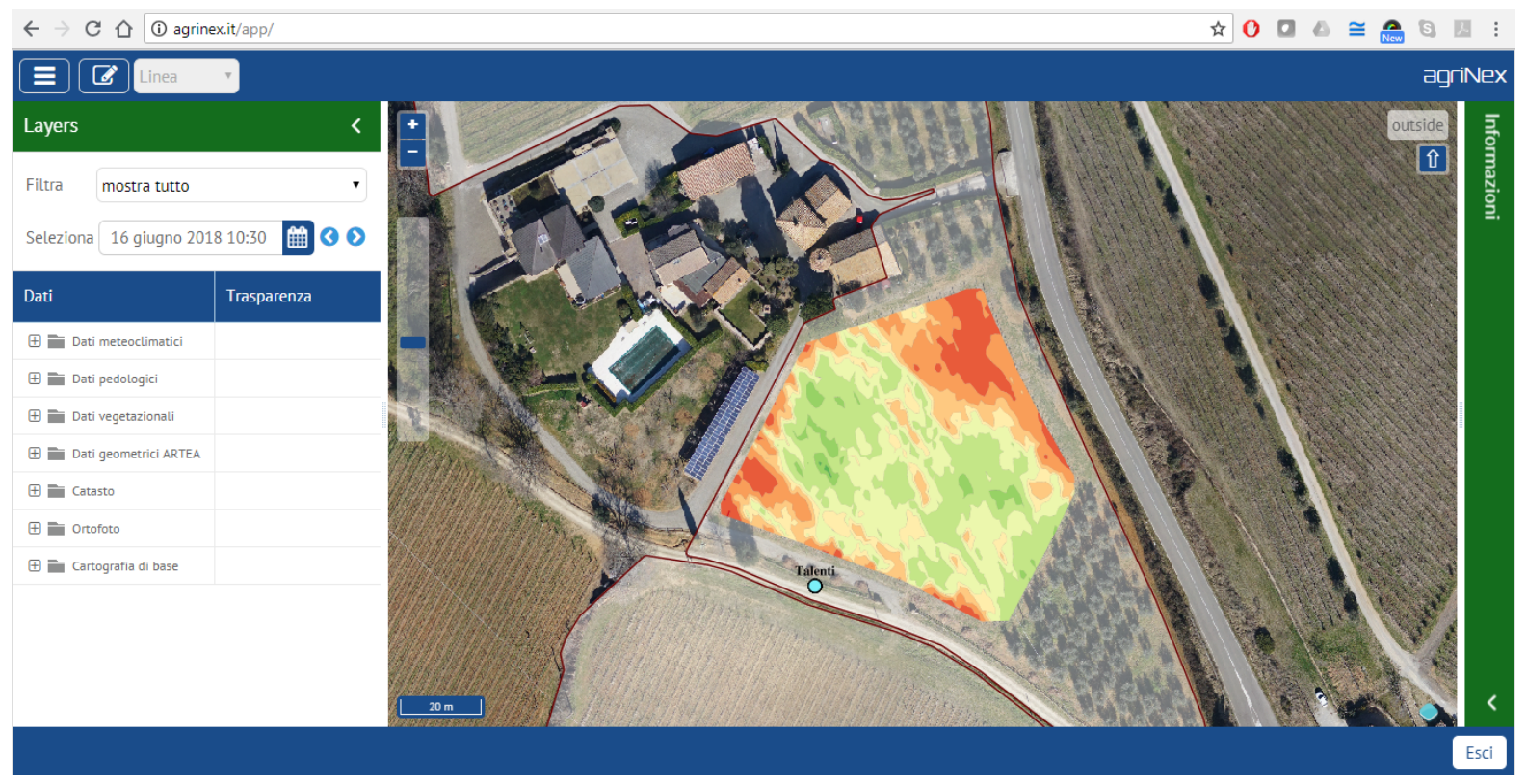

Figure 3. General outline of the dashboard developed for the Oenosmart WebGIS platform.

\section{Conclusions}

This preliminary study showed the gross potential of WebGIS platforms for the enhancement of agricultural activities leading a great opportunity to farmers in the perspective of a European Community request for the 
implementation of an increasingly precise and efficient agriculture. The developed platform offers a first wide set of information both in a proprietary and shared form, which, if properly integrated into the ordinary management of the companies, can increase the competitiveness of the farmers. The ability to access remotely via a Web viewer to thematic layers and obtain an overall view of the evolution of agronomic and meteorological variables in real time is without a doubt an essential tool for implementing the best farms strategies. This combined with the possibility to share data sets between farms falling within a territory, offers an additional support decisions tool for the farmers. Furthermore, it is increasingly necessary the need for solutions that simplify administrative management for communications between farms and public administration. The Oenosmart WebGIS platform implements an interoperability function between geographic standard formats, database which can be easily transfer. All these peculiarities, combined with the possibility of remote access even through the use of smartphones, without the need to resort to the most articulate and powerful GIS tools, make them the supporting and integrating tools of precision farming technologies in agricultural farm. Future developments will be aimed on the interested people and technologies. For the first target, the development and dissemination of WebGIS technologies as well as those of precision farming, however, requires a process of creating and updating knowledge and skills for a wide people audience ranging from end-users (farming companies) to research institutions and SMEs. To achieve this a new professional workers will be trained on the new skills necessary for the management of digital farms (Lombardo et al., 2017). For the second one, the develops will be devoted at improving and extending the digital communications with public administrations at regional and national level, the use of Web open source data, the integration of modules and algorithms for server-side processing of open source satellite data and the implementation of telemetry systems for fleet control.

\section{Acknowledgements}

This work was supported by OENOSMART project funded by the Regione Toscana, Italy (PSR 2014-2020). The authors wish to thank all the farmers, which provided technical assistance and data. Moreover, the authors want to thanks Lorenzo D'Avino, Giovanni L'Abate (CREA-AA, Firenze, Italy) and Filippo Benedetti (University of Ghent, Belgium) for help in soil proximal sensing and soil profiles description and sampling.

\section{References}

Arnó, J., Martínez-Casasnovas, J. A., Ribes-Dasi, M. and Rosell, J. R. 2009. Review. Precision Viticulture. Research topics, challenges and opportunities in site-specific vineyard management. Spanish Journal of Agricultural Research 7 (4) 779-790.

Bramley R, Pearse B, Chamberlain P., 2003. Being profitable precisely - a case study of precision viticulture from Margaret River. Australian and New Zealand Grapegrower and Winemaker, 473a:84-87

De Filippis, T., Rocchi, L., Fiorillo, E., \& Genesio, L. (2010). A WebGIS application for precision viticulture: From research to operative practices. Paper presented at the International Archives of the Photogrammetry, Remote Sensing and Spatial Information Sciences - ISPRS Archives, , 38(4W13)

Doolittle J., Petersen M., Wheeler T. (2001). Comparison of two electromagnetic induction tools in salinity appraisals. Journal of Soil and Water Conservation, 56(3), 257-262.

EC, 2016. Cork 2.0 Declaration "A Better Life in Rural Areas", Luxembourg: Publications Office of the European Union, 2016. doi:10.2762/370418 KF-01-16-997-EN-N. http://enrd.ec.europa.eu/sites/enrd/files/cork declaration en.pdf

Accessed May 132017

Istat 2013, (2013), "La struttura delle aziende agricole", https:/www.istat.it/it/archivio/167401,

Accessed June 1, 2018.

Jahn, R., Blume, H.P., Asio, V.B., Spaargaren, O., Schad, P., 2006. Guidelines for soil description. FAO, Rome (Italy).

Lombardo, S., Sarri, D., Vieri, M., \& Baracco, G. (2018). Proposal for spaces of agrotechnology co-generation in marginal areas. Atti Della Societa Toscana Di Scienze Naturali, Memorie Serie B, 125, 19-24. doi:10.2424/ASTSN.M.2018.3

Lombardo, S., Sarri, D., Vieri, M., \& Baracco, G. (2018). Proposal for spaces of agrotechnology co-generation in marginal areas. Atti Della Societa Toscana Di Scienze Naturali, Memorie Serie B, 125, 19-24. doi:10.2424/ASTSN.M.2018.3

McNEIL J. D. 1990. Geonics EM38 ground conductivity meter: EM38 operating manual. Geonics Limited, Ontario, Canada.

Ortuani B., Chiaradia E. A., Priori S., L'abate G., Canone D., Comunian A., Giudici M., Mele M., Facchi, A. (2016). Mapping Soil Water Capacity Through EMI Survey to Delineate Site-Specific Management Units Within an Irrigated Field. Soil Science, 181(6), 252-263.

Priori S., Bianconi N., Costantini E. A. (2014). Can $\gamma$-radiometrics predict soil textural data and stoniness in different parent materials? A comparison of two machine-learning methods. Geoderma, 226, 354-364. 
Sarri, D., Lisci, R., Rimediotti, M., Vieri, M., \& Storchi, P. (2015). Applications of the precision viticulture techniques in the chianti district. Paper presented at the 1st Conference on Proximal Sensing Supporting Precision Agriculture - Held at Near Surface Geoscience 2015

Santesteban, L.G., Guillaume, S., Royo, J.B. and Tisseyre, B. (2013). Are precision agriculture tools and methods relevant at the whole-vineyard scale? Precision Agriculture 14 2-17.

Terribile, F., Bonfante, A., D'Antonio, A., De Mascellis, R., De Michele, C., Langella, G., . . Basile, A. (2017). A geospatial decision support system for supporting quality viticulture at the landscape scale. Computers and Electronics in Agriculture, 140, 88-102. doi:10.1016/j.compag.2017.05.028 


\title{
Application of a Modified McKendrick-von Foerster Equation to Predict Beetle Population Dynamics (Xyleborus affinis) under Artificial Medium in Growth Chambers
}

\author{
Fernando Rojano ${ }^{\text {a*}}$, Luis A. Ibarra-Juárez ${ }^{\mathrm{b}}$, Andrés Lira-Noriega ${ }^{\mathrm{b}}$, Federico Escobar-Sarria ${ }^{\mathrm{c}}$, Daniel \\ González-Tokman $^{b}$, Porfirio Toledo-Hernández ${ }^{d}$, Brenda Tapia-Santos ${ }^{d}$, Carlos A. Hernández-Linares ${ }^{d}$ \\ a Red de Estudios Moleculares Avanzados, Instituto de Ecología, A. C. El Haya, Camino Antiguo a Coatepec 351, \\ Xalapa, 91070, México. \\ ${ }^{\mathrm{b}}$ Catedrático CONACYT, Red de Estudios Moleculares Avanzados, Instituto de Ecología, A.C., El Haya, Camino \\ Antiguo a Coatepec 351, Xalapa, 91070, México. \\ c Red de Ecoetología, Instituto de Ecología, A. C. El Haya, Camino Antiguo a Coatepec 351, Xalapa, 91070, México. \\ ${ }^{\mathrm{d}}$ Facultad de Matemáticas, Universidad Veracruzana, Circuito Gonzalo Aguirre Beltrán, Zona Universitaria, Xalapa, \\ Ver. 91090, México. \\ * Corresponding author: fernando.rojano@inecol.mx
}

\begin{abstract}
Augmenting accuracy and reliability of predictions in the woodboring beetle population dynamics is worthy information to anticipate potential plagues or extinctions, ensure sustainable ecosystems and estimate impact at new environmental scenarios due to climate change. This study focuses in the genus Xyleborus by rearing the beetle $X$. affinis, a species that can be found in United States and Mexico, such ambrosial beetle has potential to bore and disseminate fungus in trees of the Lauraceae family. In specific, it was investigated population growth rates for the $X$. affinis in four life stages (egg, larvae, pupae, adult) under three temperatures $\left(20^{\circ} \mathrm{C}, 26^{\circ} \mathrm{C}\right.$ and 32 ${ }^{\circ} \mathrm{C}$ ). The growth of the population was identified through sampling counts conducted every four days. Sampling counts of the population served to estimate the average time of development for egg life stage from 6.4 days, to 5.7 days, and to 5.6 days for $20^{\circ} \mathrm{C}, 26^{\circ} \mathrm{C}$ and $32{ }^{\circ} \mathrm{C}$, respectively; whereas for larva life stage was found from 8.3 days, to 6.2 days, and to 5.2 days for $20^{\circ} \mathrm{C}, 26{ }^{\circ} \mathrm{C}$ and $32{ }^{\circ} \mathrm{C}$, respectively. Also, estimation of the average survival rates from eggs to larva were $37.1 \%, 56.9 \%$ and $4.5 \%$ for $20{ }^{\circ} \mathrm{C}, 26^{\circ} \mathrm{C}$ and $32{ }^{\circ} \mathrm{C}$, respectively; whereas from larva to pupa were $6.7 \%, 32.9 \%$ and $0 \%$ for $20^{\circ} \mathrm{C}, 26^{\circ} \mathrm{C}$ and $32{ }^{\circ} \mathrm{C}$, respectively. Finally, the time of development was subjected to variability, which was deduced from the experimental data at each treatment, and was used to predict the expected population density using the modified McKendrick-von Foerster equation in order to recommend the periodic sampling counts accordingly to the change of life-stage, when X. affinis is subjected to a constant temperature.
\end{abstract}

Keywords: beetle rearing, insect infestation, phenology, rate of development, variability in development

\section{Introduction}

In general, woodboring beetle population density and its dynamics will be driven by availability of food, environment conditions and survival resistance of the beetle to adverse living conditions. The study of woodboring beetles has gained attention due to evidence of impact in equilibrium of ecosystems. For instance, the increment of Xyleborus population has been considered a study case in southwest of United States (Fraedrich, Johnson, and Menard 2015), because had caused a significant change in the native flora and infesting forest and farming zones. According to recent findings, Xyleborus has been attacking sassafras, avocado and redbay trees, all representative of the Lauraceae family (Koch and Smith 2008). Since its introduction of this species has continued with a prevailing growth of its population density in south-eastern United States, and progressively expanding to the west of the country. Up to now, better picture of the Xyleborus glabratus has been gained by mapping the eventual expansion (Koch and Smith 2008; Fraedrich, Johnson, and Menard 2015), and also by conducting studies in laboratory (Brar et al. 2013). Indeed, impact of Xyleborus beetles, to be considered menacing some tropical ecosystems, come from boring trees where dissemination and transport of fungal pathogen (Raffaelea lauricola) is happening. Such fungal pathogen is basic food for beetles, and causing severe damage to trees. Additionally, it has been found capabilities of horizontal transfer of ambrosial fungus dissemination among several species within the genus Xyleborus.

Under these conditions, beetles of the genus Xyleborus are not limited to the south of United States, they could also be expanding their geographical distribution to other regions that could reach Mexico. Furthermore, southern of Mexico might be fitting to the optimal biological development of the beetle due to climate and type of forest, and also an extensive region of avocado commercial plantations (e.g. Michoacan, Mexico). Additionally, such concern has raised in Mexico since there is a diversity of beetles of the same genus; that could drive with the same or more severe damage than the one observed with beetle Xyleborus glabratus in the United States.

The present investigation proposes the study of the Xyleborus affinis, considered a native beetle in Mexico, and also a threat if the fungal pathogen Raffaelea lauricola is eventually transferred to this woodboring beetle. Until now, there are not antecedents to the study of $X$. affinis under controlled conditions, and information about 
the rate of development, survival rates and the range and optimal temperature can serve to know what regions in Mexico are susceptible, and also potential regions due to climate change. Then, this research is conducted setting well-defined rearing conditions taking into account nutritional requirements, control of the micro-environment and homogeneous characteristics of the beetle (i.e. age and size) to establish population growth rates by means of indirect sampling counts and under the effect of different temperatures.

Adequate artificial medium has evolved from similar studies providing information about rearing beetles for the same genus in artificial medium (Brar et al. 2013; Biedermann, Klepzig, and Taborsky 2009) that aided in repeatability of the experiment, and collection of data with minimum interference of exogenous factors such as temperature oscillation, high density of beetles, or scarcity of food. Then, beetles were reared under the impact of three temperatures: $20{ }^{\circ} \mathrm{C}, 26{ }^{\circ} \mathrm{C}$ and $32{ }^{\circ} \mathrm{C}$, that were chosen based on previous experiments identifying an adequate range for survival of the beetles in at least the egg stage. As several other insects, beetles will adjust their metabolic rates accordingly to temperature; consequently, development and reproduction rates will be different and reaching maximum population growth at different times. Accordingly, this investigation identifies rate of development and its variability of each life-stage, taking into account differences in population abundance among a finite population set during a period of 32 days.

Rate of development to any insect has been determined through several equations which are summarised in the works of (Damos and Savopoulou-Soultani 2012; Briere et al. 1999; Logan et al. 1976). All those equations have been proposed as a function of temperature in conjunction with a threshold temperature experimentally and/or theoretically found. Alternatively, this study estimates rate of development and its variability from experimental findings at each life stage and temperature using the cumulative frequency curves, such as several studies (Règniére 1984; Bentz, Logan, and Vandygriff 2001; Falzoi et al. 2014). Such rate of development and its variability can be used to estimate population density in mathematical models such as the modified McKendrickvon Foerster equation, introduced by (Gilbert et al. 2004). Then, this work, firstly, determines the rates of development and variability for each life-stage and temperature. Secondly, there is an estimation of the survival rates. And thirdly, there is a use of the modified McKendrick-von Foerster equation to interpret population density dynamics based on the age, life-stage and temperature; making it appropriate for the experimental data obtained.

\section{Materials and Methods}

Experimental stage

\section{Living conditions of insects: protocol to build up the artificial medium}

An artificial medium was prepared to provide living conditions that could mimic the real one of the beetle. That medium was focused on the essential nutritional requirements together with physical characteristics that favour digging of galleries. Artificial medium was prepared following the methodology of (Biedermann, Klepzig, and Taborsky 2009) with minor modifications. It was used $45 \mathrm{~g}$ of sawdust of Platanus mexicana, $12 \mathrm{~g}$ of granulated agar, $6 \mathrm{~g}$ of casein, $3 \mathrm{~g}$ of starch, $3 \mathrm{~g}$ of yeast, $0.6 \mathrm{~g}$ of Wesson salt, $0.21 \mathrm{~g}$ of streptomycin, $1.5 \mathrm{~mL}$ of wheat germ, $1.5 \mathrm{~mL}$ of ethanol at $95 \%$ and $400 \mathrm{~mL}$ of distilled water. All those ingredients were mechanically mixed, and then autoclaved to $120^{\circ} \mathrm{C}$ by 120 minutes. After autoclaved process, $15 \mathrm{~mL}$ of still hot artificial medium was poured in $50 \mathrm{~mL}$ Falcon ${ }^{\circledR}$ tube and let dry inside of laminar flow cabinet overnight.

The Falcon ${ }^{\circledR}$ tube had a circular hole in its lid of approximately $8 \mathrm{~mm}^{2}$ which was covered with a mesh, allowing exchange of gases and to avoid exit of the beetle. Then, inoculation happened with one beetle was placed in the Falcon ${ }^{\circledR}$ tube, which was considered the start of the experiment.

Chambers: characteristics of the rearing system

Chambers were used for the experimental stage that had a system for temperature control. Within each chamber an Elitech URC-4 data-logger was placed to verify stability of temperature, which also logged relative humidity. A treatment was considered in each chamber that had constant temperature of $20{ }^{\circ} \mathrm{C}, 26{ }^{\circ} \mathrm{C}$ and $32{ }^{\circ} \mathrm{C}$, respectively. Also, in each chamber was placed a set of 80 Falcon ${ }^{\circledR}$ tubes as the beginning of the experiment. Chambers were closed all time and only opened for few seconds every 4 days only to get a sample of 10 Falcon ${ }^{\circledR}$ tubes, during a period of 32 days, and counting the population of eggs, larva, pupae and adults. Chambers were maintained in $20 \pm 0.25{ }^{\circ} \mathrm{C}, 26 \pm 0.31{ }^{\circ} \mathrm{C}$ and $32 \pm 0.31^{\circ} \mathrm{C}$. Given that humidity was not controlled, the natural response at each temperature of the relative humidity was as follows: $81.2 \pm 5.3 \%, 68.8 \pm 3.3 \%$ and $61.6 \pm 4.2 \%$ for the $20^{\circ} \mathrm{C}, 26^{\circ} \mathrm{C}$ and $32{ }^{\circ} \mathrm{C}$, respectively. Other gases were not recorded; nonetheless, the coherence in survival of adults until the end of the period could prove that any other gas had negligible effect.

\section{Methodology for data collection}

Beetle population can be adequately studied by means of periodic counts at each life-stage. This requirement became challenging due to beetle size (approximately $2 \mathrm{~mm}$ in adult stage), and after initiation of the experiments beetles were located at the interior of galleries. Then, accurate data was obtained by means of indirect census (i.e. 
sampling). Every 4 days the sampling was randomly choosing 10 Falcon® tubes, which had to be thoroughly dissected to identify the number of eggs, larva, pupa and adults. Such census destroyed the habitat of the beetle within the Falcon ${ }^{\circledR}$ tubes that was impossible to enumerate direct progeny. This procedure was made until to cover all life-stages ( 8 counts) and conditioned the life-cycle allowed by the artificial medium, since it was degraded and would have restrained stable survival of beetles during long periods.

\section{Modelling stage}

\section{Rate of development and its variability}

Beetles have been investigated based on the rate of development as a function of temperature by means of several empirical equations (Régnière et al. 2012); some of them have followed the Arrhenius equation to propose an universal temperature dependence. Such approach has been operational within a range of temperatures; however, out of that range of temperatures, insects could have unimodal response. Furthermore, insects could have nonlinear thermal responses, given that impact of temperature could be affecting differently at each life stage.

Even though homogenous living and feeding conditions can be provided when rearing beetles in a growth chamber, there will be an intrinsic variability in developmental rates. Thermal responses can be benchmarked in terms of distribution, and such distributions will be often found as asymmetrical (Powell and Bentz 2014). That response can then be mathematically analysed and generate models which can be useful to predict, based on specific rearing conditions, emphasising the effect of temperature by means of rate of development and its variability at each life stage.

Rate of development is computed as the inverse of development time, $t(T)$, which corresponds, for instance, to the time between oviposition and hatch or time taken between larval instars. Thus, rate of development, $r(T)$ is defined as the proportion of the total stage duration per unit of time:

$$
r(T)=\frac{1}{t(T)}
$$

This definition does not involve its variability, $v(T)$, since insects can have a deviation with respect to the mean of the rate of development. Variability can be obtained from the set of population analysed and it can be derived from the standard deviation, $\sigma$, as it is suggested in (Gilbert et al. 2004):

$$
v(T)=\frac{\sigma^{2}}{2}
$$

In this way, rate of development is better described, because using both terms it is possible to illustrate how the variability impact in the population growth rate by using the modified McKendrick-von Foerster equation.

\section{A modified McKendrick-von Foerster equation}

Original formulation of the McKendrick-von Foerster equation has been focused to various applications of mathematical biology such as cell division. Other applications have been successfully conducted to describe different biological and ecological processes subjected to age-structure (Keyfitz and Keyfitz 1997; Moenickes et al. 2012; Powell and Bentz 2014). Beetles, such as the ones considered in this investigation, have the age-structure characteristic that allows the use of the following equation:

$$
\frac{\partial p(a, t)}{\partial t}+\frac{\partial p(a, t)}{\partial a}=g(a, t, p(a, t))
$$

where $p(a, t)$ is population, $\mathrm{t}$ is time, a is age. And $g(a, t, p(a, t))$ is the total gain or loss of individuals per age per time. Also $g(a, t, p(a, t))$ can be interpreted as the mortality rate $-k p(a, t)$. Initial population must be defined as:

$$
p(a, 0)=f(a)
$$

and the boundary conditions should be set as:

$$
p(0, t)=\int_{0}^{\infty} b(a) p(a, t) d a
$$

where $b(a)$ corresponds to the birth rate.

A modification of the equation described above has been proposed by (Gilbert et al. 2004) and evaluated with enhanced performance when predicting beetle population dynamics at different temperatures:

$$
\frac{\partial p(a, t)}{\partial t}+r(T) \frac{\partial p(a, t)}{\partial a}=v(T) \frac{\partial^{2} p(a, t)}{\partial a^{2}}
$$

with $r(T)$ and $v(T)$ are the developmental rate and variability in development, respectively. Both terms are written in function of temperature since such coefficients are associated to the effect of ambient temperature. On the other hand, the term $g(a, t, p(a, t))$ or $-k p(a, t))$ is replaced by the diffusion term; meaning that either natality or mortality can be reduced to variability in development, a concept analogic to diffusivity. 


\section{Results and Discussion}

Egg abundance was notoriously successful for the temperature of $20{ }^{\circ} \mathrm{C}$ (Figure 1a). However, transition to the consecutive life stages were significantly reduced that made impossible to get adults. Additionally, the temperature slowed down the metabolic rate of them, making hard for these set of experiments to conclude if the oviposition could extend for few days more, also if consecutive life-stages could be accomplished by some individuals.

Given that this study explored the population dynamics for a beetle that has not been reported yet, a replicate of the temperature of $26^{\circ} \mathrm{C}$ was conducted to verify consistency of the results (Figure 1b). Deviation between both experiments depicted the nature of variability of beetles, and also that variability is remarked by the standard deviation found at each of the 8 counts. The consistency between the two experiments was verified through the coefficient of variation, which was found with values of 1.64 and 1.76 for experiment 1 and 2, respectively.

Temperature of $32{ }^{\circ} \mathrm{C}$ was not favourable for oviposition (Figure 1a) and it was found only during a short period (less than 24 days) that made difficult to move to consecutive life stages. Results in Figure 1 represent the average fecundity per female. Those female adults inoculated at day 0 could survive until the end of the period, but progeny was significantly depleted for temperature of $32{ }^{\circ} \mathrm{C}$; consequently, experimental data did not aid for further analysis.
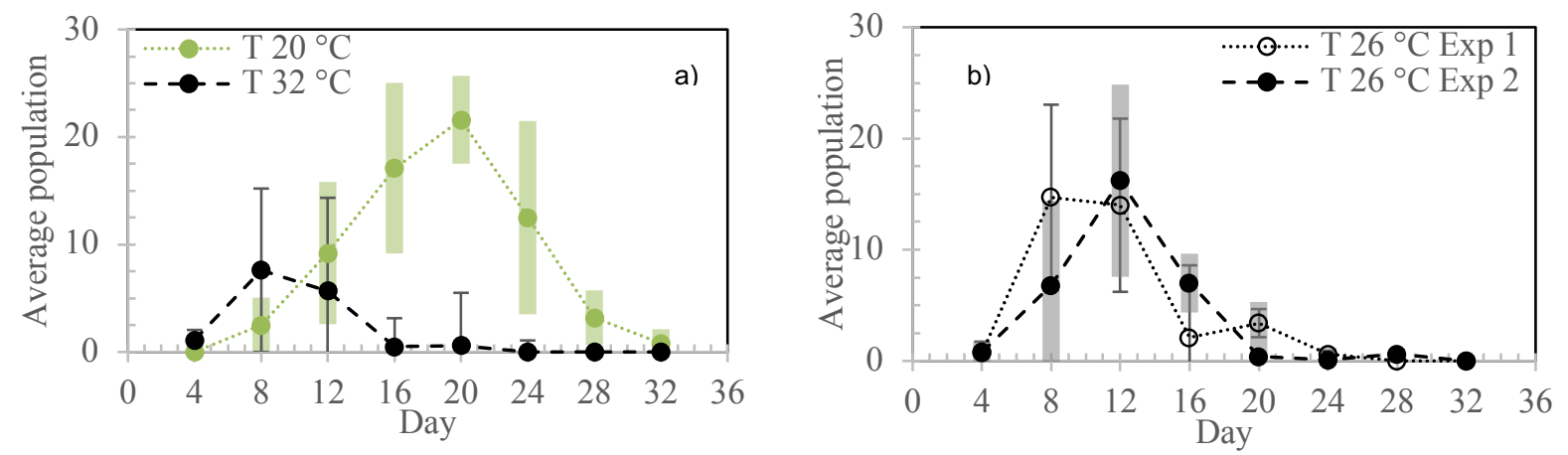

Figure 1. Abundance of eggs during a period of 32 days for $X$. affinis, each value represents an average value of 10 counts and the bars correspond to the standard deviation for temperature of $20{ }^{\circ} \mathrm{C} \mathrm{(a),} 26^{\circ} \mathrm{C}$ with a repetition (b) and $32{ }^{\circ} \mathrm{C}$ (a). Results of the consecutive life stages will be introduced in the following sections.

Rate of survival and development

The way this experiment was conducted increased accuracy in measurements due to null interference in progeny; however, such approach did not aid in a direct census of descendants. This constrain weakens estimations and/or predictions; but also this condition holds consistency of results of a population growing along time by only sampling. Then, the fraction of population surviving from one life stage to the next one is rather induced, and computed according to the curves of cumulative frequency, such as the ones presented in Figure 2. Those curves made with 8 measurements obtained from averaging 10 counts (standard deviation is indicated in Figure 1) are basically comparing consecutive life stages from eggs to larva and to pupa. Those graphs assume that each value is representative for the corresponding life stage subjected to a constant temperature.
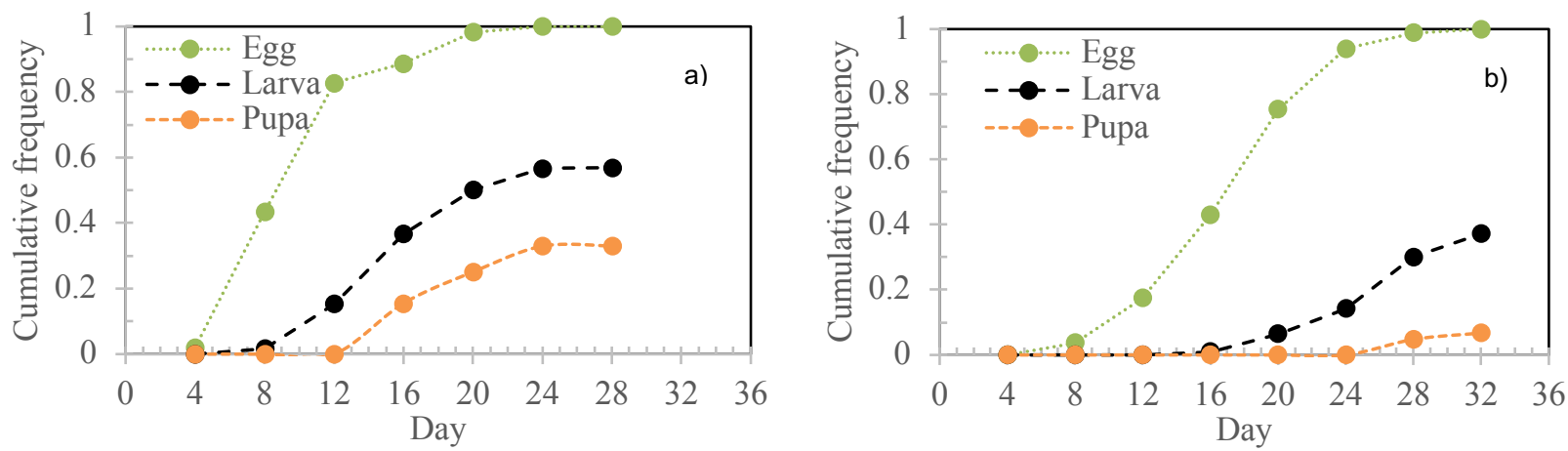

Figure 2. Cumulative frequency for egg and larva (includes averages values of Instar $1,2 \& 3$ ) at temperature of $20{ }^{\circ} \mathrm{C}$ (a) and $26^{\circ} \mathrm{C}$ (b) for $X$. affinis,; maximum cumulative frequency corresponded to the number of eggs, and that number was used to get the maximum cumulative frequency of larva and pupa. 
The procedure to determine survival rates was as follows, the cumulative frequency for eggs was taken as baseline for the consecutive life stages. Then, it was assumed that those eggs moved to the next life stage after a period of 4 days. It means that larva cumulative frequency in time $t$ (i.e. day $8,12,16, \ldots, 32$ ) was consequence of eggs cumulative frequency at time t-4 (i.e. $4,8,12, \ldots, 28$ ); schematic of the quantification is described in Figure 3. Finding survival rates with this criterion, we had an average of $37.1 \%, 56.9 \%$ and $4.5 \%$ for temperature of $20{ }^{\circ} \mathrm{C}, 26{ }^{\circ} \mathrm{C}$ and $32{ }^{\circ} \mathrm{C}$, respectively. These results were based on the abundance of population that might hatch, die or simply be present during two consecutive counts. Of course, in this investigation sampling procedure led to a different set of 10 tubes each time t.

Regarding to transition from larva to pupa was found with a survival rate of $6.7 \%, 32.9 \%$ and $0 \%$ for temperature of $20{ }^{\circ} \mathrm{C}, 26{ }^{\circ} \mathrm{C}$ and $32{ }^{\circ} \mathrm{C}$, respectively. And adult beetle population reached, after 32 days, an average value in the last count of $0.7,2.5$ and 0.3 for $20^{\circ} \mathrm{C}, 26^{\circ} \mathrm{C}$ and $32{ }^{\circ} \mathrm{C}$, respectively.

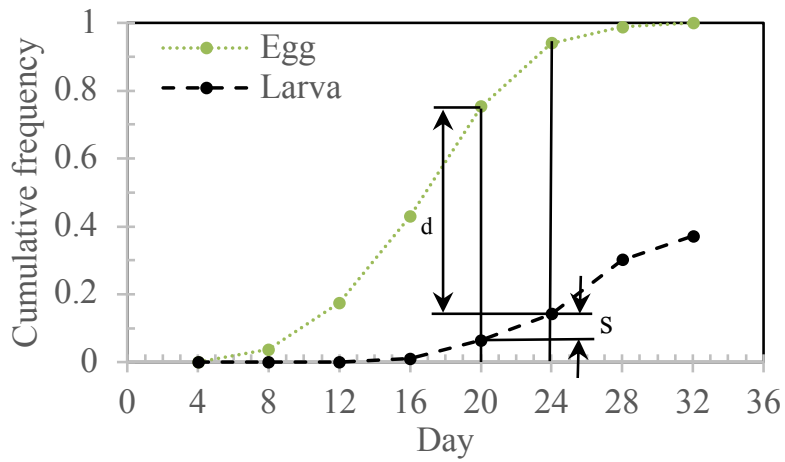

Figure 3. Schematic of the survival (s) and dead (d) proportions of X. affinis, from time t to time $\mathrm{t}+4$, between the egg and larva life stages.

On the other hand, to estimate rate of development an approach has been suggested by Regniere, 1984; such method can be accomplished by taking as reference the cumulative frequency. That work proposes to adjust the cumulative frequency to a logistic equation, and from there deduce the mean rate of development. In this study is proposed the cumulative normal distribution that can analogously represent the sigmoid curve of cumulative frequency. Then, the mean and standard deviation of the cumulative normal distribution characterise the rate of development and consequently the time of development (equation in section 2.3), within the interval of 4 days (i.e. it is expected every 4 days a change of life stage). This calculation based on the 8 mean values obtained from 10 sampling counts.

Results of the adjustment at each life stage was conducted to each of the three temperatures (Table 1). Some sampling cases were found an absence of individuals, then the number of mean values were reduced, the last column indicates the number of data considered to determine rate of development. In general, it can be understood that metabolic rate of the beetles was accelerated when temperature increased from $20{ }^{\circ} \mathrm{C}$ to $32{ }^{\circ} \mathrm{C}$; providing a shorter time of development. However, it was experimentally identified that temperature of $32{ }^{\circ} \mathrm{C}$ was not feasible to reach pupal stage, since that temperature exceeded the limiting constraint for surviving.

Table 3. Parameters of the cumulative normal distribution for each life stage of $X$. affinis.

\begin{tabular}{|c|c|c|c|c|}
\hline Temperature & $\begin{array}{l}\text { Life } \\
\text { stage }\end{array}$ & $\begin{array}{c}\text { Rate of development, } r(T) \\
\text { (1/day) }\end{array}$ & $\begin{array}{l}\text { Time of development } \\
\text { (days) }\end{array}$ & $\begin{array}{l}\text { Number } \\
\text { of data }\end{array}$ \\
\hline \multirow[t]{3}{*}{$20^{\circ} \mathrm{C}$} & Eggs & 0.1544 & 6.47 & 7 \\
\hline & Larva & 0.1195 & 8.37 & 5 \\
\hline & Pupa & 0.2139 & 4.68 & 2 \\
\hline \multirow[t]{3}{*}{$26{ }^{\circ} \mathrm{C}$} & Eggs & 0.1730 & 5.78 & 6 \\
\hline & Larva & 0.1592 & 6.28 & 6 \\
\hline & Pupa & 0.1859 & 5.38 & 3 \\
\hline \multirow[t]{3}{*}{$32{ }^{\circ} \mathrm{C}$} & Eggs & 0.1761 & 5.68 & 5 \\
\hline & Larva & 0.1913 & 5.23 & 5 \\
\hline & Pupa & $*$ & $*$ & $*$ \\
\hline
\end{tabular}

The rate of development had a standard deviation based on the number of data available, such standard deviation can be related to variability of the rate of development as specified in section 2.3 . Thus, Table 2 indicates variability that was kept within the same order of magnitude at any temperature and life stage, and it was minimised at the pupal stage at any of the three temperatures. In other words, variability does not depend on the temperature, rather could be the nature of the development of the life stage. 
Table 2. Time of development and variability for each life stage of development of $X$. affinis.

\begin{tabular}{cccc}
\hline Temperature & Life stage & Standard deviation, $\sigma(T)$ & Variability, $v(T)$ \\
\hline $20{ }^{\circ} \mathrm{C}$ & Eggs & 0.1007 & 0.0051 \\
& Larva & 0.1038 & 0.0054 \\
$26{ }^{\circ} \mathrm{C}$ & Pupa & 0.0511 & 0.0013 \\
& Eggs & 0.0971 & 0.0047 \\
& Larva & 0.1015 & 0.0052 \\
$32{ }^{\circ} \mathrm{C}$ & Pupa & 0.0663 & 0.0022 \\
& Eggs & 0.0989 & 0.0049 \\
& Larva & 0.0953 & 0.0045 \\
& Pupa & $*$ & $*$ \\
\hline
\end{tabular}

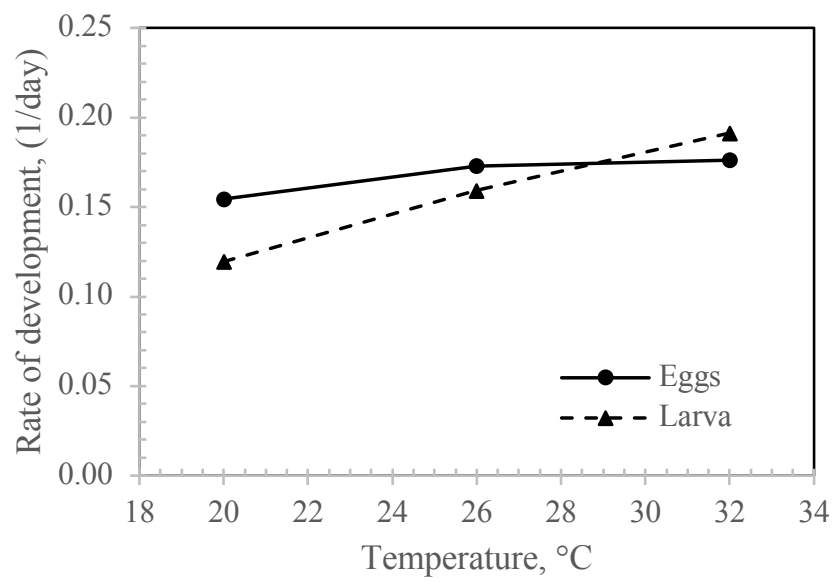

Figure 4. Rate of development for eggs and larva beetle (X. affinis) reared in artificial medium.

Since beetles will have a reproduction rate depending on temperature, the summary of the three temperatures are presented in Figure 4 for eggs and larva stages; pupa was excluded because no data was obtained at temperature of $32^{\circ} \mathrm{C}$. Given that the slope of the curve for larva (0.006) was higher than the slope of curve for eggs $(0.0018)$, it can be said that larva was more influenced by temperature than eggs; however, eggs population density was notoriously contrasting at temperature of $20^{\circ} \mathrm{C}$ and $26^{\circ} \mathrm{C}$. Upper and lower temperature limit were not determined in this study; though, further research is required.

Beetles of the genus Xyleborus had a growing population mostly with oviposition distributed along a period of time, and the condition that most of the individuals could change to the next stage of life after 4 days makes the periodic counting acceptable for deducing the population gain, and consecutively the rate of development and its variability. Still with that dynamics, data experimentally found (Table 1 and 2) can be used to depict the advance of the population based on the expected age. So, to accomplish that, the use of the modified McKendrick von Foerster partial differential equation can be used. Then, the parameters rate of development $r(T)$ and its variability $v(T)$ become the primary parameters governing the partial differential equation. Given that there is no reproduction along the age within a life stage, the boundary condition $p(0, t)=0$. Results for larva at $20{ }^{\circ} \mathrm{C}, 26$ ${ }^{\circ} \mathrm{C}$ and $32{ }^{\circ} \mathrm{C}$ are presented in Figure 5, where it is observed that maximum population density is happening at the mean time of development, and then variability governs the rate of decay before and after such mean. Those results also illustrate that adequate periodic sampling may not be similar when dealing with different temperatures, and the effect of large variability in the rate of development could give conditions to get average values of the population at any time (i.e. periodic counting) such as the case of temperature of $20^{\circ} \mathrm{C}$; whereas small variability could demand for exact and synchronous sampling according to the larva development regime. 


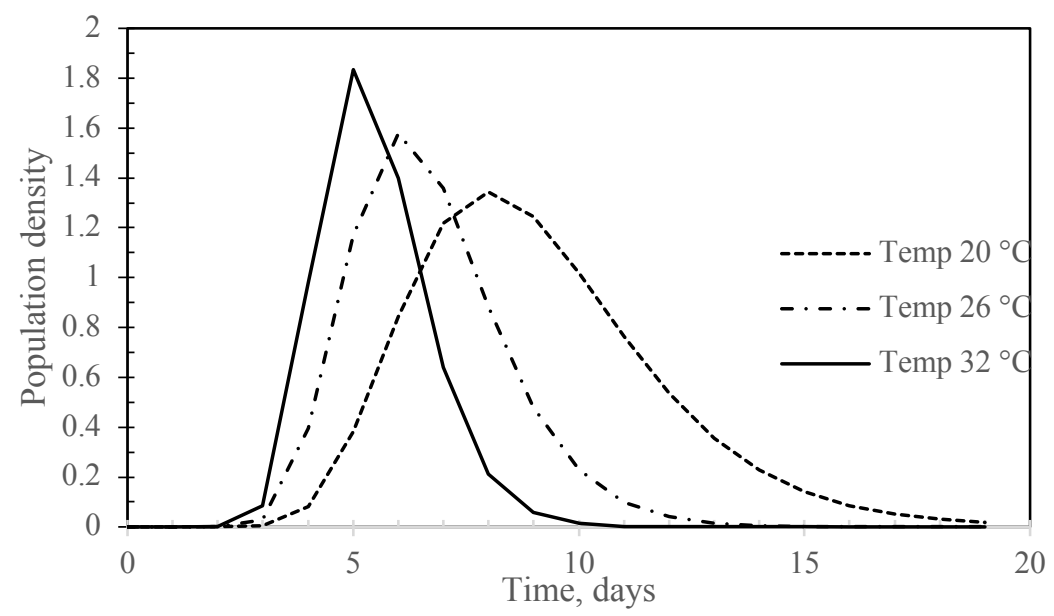

Figure 5. Estimated population density using the modified McKendrick von Foerster equation, applied at the end of the larva life stage $(a=1)$ for temperatures of $20^{\circ} \mathrm{C}, 26^{\circ} \mathrm{C}$ and $32{ }^{\circ} \mathrm{C}$.

\section{Conclusions}

This study conducted the analysis of the population growth rate applied to the beetle $X$. affinis with the following findings:

1. Experimental identification of the rate of development at the egg stage from $0.154( \pm 0.100) 1 /$ day, to $0.173( \pm 0.097) 1$ day, and to $0.176( \pm 0.098) 1$ /day for the $20^{\circ} \mathrm{C}, 26^{\circ} \mathrm{C}$ and $32{ }^{\circ} \mathrm{C}$, respectively; whereas at the larva stage from $0.119( \pm 0.103) 1 /$ day, to $0.159( \pm 0.101) 1 /$ day, and to $0.191( \pm 0.095) 1 /$ day for the $20^{\circ} \mathrm{C}, 26^{\circ} \mathrm{C}$ and $32{ }^{\circ} \mathrm{C}$, respectively.

2. Estimation of the survival rates in function of temperature from eggs to larva were $37.1 \%, 56.9 \%$ and $4.5 \%$ for $20^{\circ} \mathrm{C}, 26^{\circ} \mathrm{C}$ and $32{ }^{\circ} \mathrm{C}$, respectively; whereas from larva to pupa were $6.7 \%, 32.9 \%$ and $0 \%$ for $20^{\circ} \mathrm{C}, 26^{\circ} \mathrm{C}$ and $32^{\circ} \mathrm{C}$, respectively.

3. Use of the modified McKendrick von Foerster equation to predict the population density and its variability by using the coefficients rate of development $r(T)$ and variability $v(T)$ to aid in further investigations that define the adequate number of sampling counts, its periodicity and the number of individuals involved.

\section{Acknowledgements}

This work has been accomplished under joined efforts of the Red de Ecoetologia and Estudios Moleculares Avanzados at the Instituto de Ecologia A C, Xalapa, México. Also, it has been funded by: "Fondo Institucional de Fomento Regional para el Desarrollo Científico y Tecnológico de Conacyt-México, Proyecto 292399"

\section{References}

Bentz, Barbara J, Jesse A Logan, and JC Vandygriff. 2001. "Latitudinal Variation in Dendroctonus Ponderosae (Coleoptera: Scolytidae) Development Time and Adult Size." The Canadian Entomologist 133:375-87. http://journals.cambridge.org/abstract_S0008347X0000657X.

Biedermann, Peter H. W., Kier D. Klepzig, and Michael Taborsky. 2009. "Fungus Cultivation by Ambrosia Beetles: Behavior and Laboratory Breeding Success in Three Xyleborine Species." Environmental Entomology 38 (4):1096-1105. https://doi.org/10.1603/022.038.0417.

Brar, Gurpreet S., John L. Capinera, Paul E. Kendra, Stephen McLean, and Jorge E. Peña. 2013. "Life Cycle, Development, and Culture of Xyleborus Glabratus (Coleoptera: Curculionidae: Scolytinae)." Florida Entomologist 96 (3):1158-67. https://doi.org/10.1653/024.096.0357.

Briere, Jean Francois, Pascale Pracros, Alain Yves Le Roux, and Jean Sebastien Pierre. 1999. "A Novel Rate Model of Temperature-Dependent Development for Arthropods." Environmental Entomology 28 (1):22-29. https://doi.org/10.1093/ee/28.1.22.

Damos, Petros, and Matilda Savopoulou-Soultani. 2012. "Temperature-Driven Models for Insect Development and Vital Thermal Requirements." Psyche 2012. https://doi.org/10.1155/2012/123405.

Falzoi, Simone, Federico Lessio, Federico Spanna, and Alberto Alma. 2014. "Influence of Temperature on the Embryonic and Post-Embryonic Development of Scaphoideus Titanus (Hemiptera: Cicadellidae), Vector of Grapevine Flavescence Dorée." International Journal of Pest Management 60 (4). Taylor \& Francis:246-57. https://doi.org/10.1080/09670874.2014.966170. 
Fraedrich, SW, CW Wood Johnson, and RD Menard. 2015. "First Report of Xyleborus Glabratus (Coleoptera: Curculionidae: Scolytinae) and Laurel Wilt in Louisiana, USA: The Disease Continues Westward on Sassafras.” Florida ... 98 (4):1266-68. https://doi.org/10.1653/024.098.0445.

Gilbert, Estella, James A Powell, Jesse A Logan, and Barbara J Bentz. 2004. "Comparison of Three Models Predicting Developmental Milestones Given Environmental and Individual Variation." Bulletin of Mathematical Biology 66:1821-50. https://doi.org/10.1016/j.bulm.2004.04.003.

Keyfitz, B. L., and N. Keyfitz. 1997. "The McKendrick Partial Differential Equation and Its Uses in Epidemiology and Population Study." Mathematical and Computer Modelling 26 (6):1-9. https://doi.org/10.1016/S0895-7177(97)00165-9.

Koch, F H, and W D Smith. 2008. "Spatio-Temporal Analysis of Xyleborus Glabratus (Coleoptera: Curculionidae [Corrected] Scolytinae) Invasion in Eastern U.S. Forests.” Environmental Entomology 37 (2):44252. https://doi.org/10.1603/0046-225X(2008)37[442:SAOXGC]2.0.CO;2.

Logan, James G, D. J. Wollkind, S. C. Hoyt, and L. K. Tanigoshi. 1976. “An Analytic Model for Description of Temperatrue Dependent Rate Phenomena in Arthropods." Environmental Entomology 5:1133-40. https://doi.org/doi.org/10.1093/ee/5.6.1133.

Moenickes, Sylvia, Marieke Frassl, Jeanette Schlief, Moritz Kupisch, Michael Mutz, Frank Suhling, and Otto Richter. 2012. "Temporal Patterns of Populations in a Warming World: A Modelling Framework." Marine Biology 159 (11):2605-20. https://doi.org/10.1007/s00227-012-1996-4.

Powell, James A., and Barbara J. Bentz. 2014. "Phenology and Density-Dependent Dispersal Predict Patterns of Mountain Pine Beetle (Dendroctonus Ponderosae) Impact.” Ecological Modelling 273. Elsevier B.V.:173-85. https://doi.org/10.1016/j.ecolmodel.2013.10.034.

Règniére, Jacques. 1984. "A Method of Describing and Using Variability in Development Rates for the Simulation of Insect Phenology." The Canadian Entomologist 116 (10):1367-76. https://doi.org/10.4039/Ent1161367-10.

Régnière, Jacques, James Powell, Barbara Bentz, and Vincent Nealis. 2012. "Effects of Temperature on Development, Survival and Reproduction of Insects: Experimental Design, Data Analysis and Modeling." Journal of Insect Physiology 58 (5). Elsevier Ltd:634 47. https://doi.org/10.1016/j.jinsphys.2012.01.010. 


\title{
Performance Evaluation of Passive Radio Frequency Identification Technology in Silage Bale Application
}

\author{
Katariina Penttilä a,*, Antti Suokannas ${ }^{\text {b }}$, Ilpo Pölönen ${ }^{c}$ \\ ${ }^{a}$ Energy Efficiency Research Group, Sheet Metal Centre, School of Technology, Häme University of Applied Sciences, Lotilantie 16, \\ 37630 Valkeakoski, Finland \\ b Production Systems, Natural Resources Institute Finland, Maarintie 6, 02150 Espoo, Finland \\ c Bioeconomy Research Unit, Häme University of Applied Sciences, PL 230, 13101 Hämeenlinna, Finland \\ * Corresponding author. Email: katariina.penttila@hamk.fi
}

\begin{abstract}
Digitalization in farming sector has raised interest nowadays. By increasing knowledge and enabling unique identification of harvested crop, farmers gain relevant business profit. At present, most of silage bales are stored in stacks on the edge of field or near cowshed without any special tag identification. In some rare cases spray paint are used for identification, but real-time and unique tracking is impossible. The need for weatherproof and automated identification, combined with microclimate and geographic information offer valuable information to farmers. Research work presented in this paper presents the first steps to achieve unique bale identification and related data management. This research is focused on the utilization of passive radio frequency identification (RFID) for silage bales in stationary conditions. Passive RFID is wireless, non-line of sight identification technology, with free-air identification distances over $10 \mathrm{~m}$. When installed to application, distances decrease based on material's electromagnetic properties. As conservation of silage is based on lactic acid fermentation, with $\mathrm{pH}$ around four and dry matter variation between 20 and $45 \%$, the challenge to achieve sufficient distance raises. RFID operation principle is based on reader command and transponders' passive backscattering, where transponders harvest all their operational energy from reader's communication signal. As maximum communication signal strength is limited by telecommunication standard regulations (e.g. ETSI), transponder type plays a crucial role to guarantee adequate signal strengths to achieve sufficient communication distances. In this research, the applicability of RFID technology is measured in a silage bale of $160 \mathrm{~cm}$ in diameter, covering 360 degrees around the bale. Measurements are conducted as identification distance [meters], and transponder population [number of tags]. Based on the results, the most suitable tag for the application is chosen, and the applicability is proven. The project next steps will be on-site testing, integrating RFID reader to baler and evaluating the performance in action.
\end{abstract}

Keywords: Automated identification of silage bales, Bale Data Management System, Digitalization, Measuring Measurements, Radio Frequency Identification, Unique identification of Harvested Crop

\section{Introduction}

The revolution of digitalization has gained interest across all commercial sectors. Industry 4.0 and Internet of Things (IoT) has promised new business opportunities for several years now. Many potential applications have been introduced, but commercial side has too often forgotten. The research work presented in this paper is a part of Digibale concept, which has its origin in business needs. Radio frequency identification has been chosen as a potential identification methodology due to its wireless and non-line of sight operation principle. By integrating existing technologies and an application software in a new manner, a new concept and implementation of automated unique identification and tracking of silage bales, bale bookkeeping and open marketplace are created to modernize bale farming sector. This paper introduces the identification procedure and performance evaluation of passive radio frequency identification (RFID) technology in silage bale application.

This paper is divided to following sections: section 1.1. details the application challenges in the silage bale, section 1.2. Introduces the Digibale concept, and section 1.3. passive RFID and its challenges in silage bale application. Section 2 introduced materials and methodology used to conduct this research. In section 3, the relevant results and their analysis is presented, and section 4 concludes the research and presents the next steps in Digibale concept development and implementation.

\section{Silage bales}

In Finland, silage bales are conserved from fall until up to springtime. They are wrapped with bale net and further multiple plastic layers. Most of silage bales are stored in stacks on the edge of field or near cowshed without any special tag identification. In few cases spray paint may be used for identify the bales. These markings may be elapsed during the storage period; by making it impossible to track and identify bales, and combine them to typically used manual bookkeeping.

Bales are subjected to variable weather conditions as warm sunshine, cold, rain, snow and frost. Further, birds or rodents can cause holes to plastic layers of silage bales. Therefore, the bale identifiers, now RFID transponders 
must be placed inside the bale wrappings. However, as bale conservation is based on lactic acid fermentation, with $\mathrm{pH}$ around four and dry matter variation between 20 and 45\% (McDonald et all., 1991), the challenge of radio wave propagation in such environment arises. This challenge is further described in section 1.3.

Typical silage bale sizes varies between $1300 \mathrm{~mm}$ and $1600 \mathrm{~mm}$ in diameter. They are handled with pallet trucks in random orientation. Threfore, the ultimate target would be in achieving identification coverage of 360 degrees around the bale. This paper introduces the first steps to achieve this, by reviewing and analyzing passive RFID tag measurements in the silage bale application.

Digibale concept

Digibale concept is a concept enabling unique bale identification, collecting microclimate information, analyzing bale related data, managing bale storages, and also selling and buying silage bales in open application platform. Figure 1 presents the work packages (WPs) of Digibale project, launched for implementing the concept (Digibale project 2018). The following describes WPs shortly:

WP1: Background data collection, benchmarking and foundations:

- to collect information about the life-cycle management of bales' lifecycle

- seek information on which parameters are important for the target audience

- hardware and network connections: microclimate measurements, RFID reader and tags, database connection, supply in baler machine

- initial acceptance testing

WP 2: Building database and compatibility

- develop the unique marking of bales

- collect and export the status and location data to the database

- field testing with baler machine

WP3: Collection and use of data

- collect, analyze and combine binary identification, location and measurement data for internal and external data sources

- user interfaces for different purposes

WP 4: Business and Service Design

- Web store

- own data for farmers

- big data for researchers

This paper focuses on WP1, primarily RFID performance measurements. 


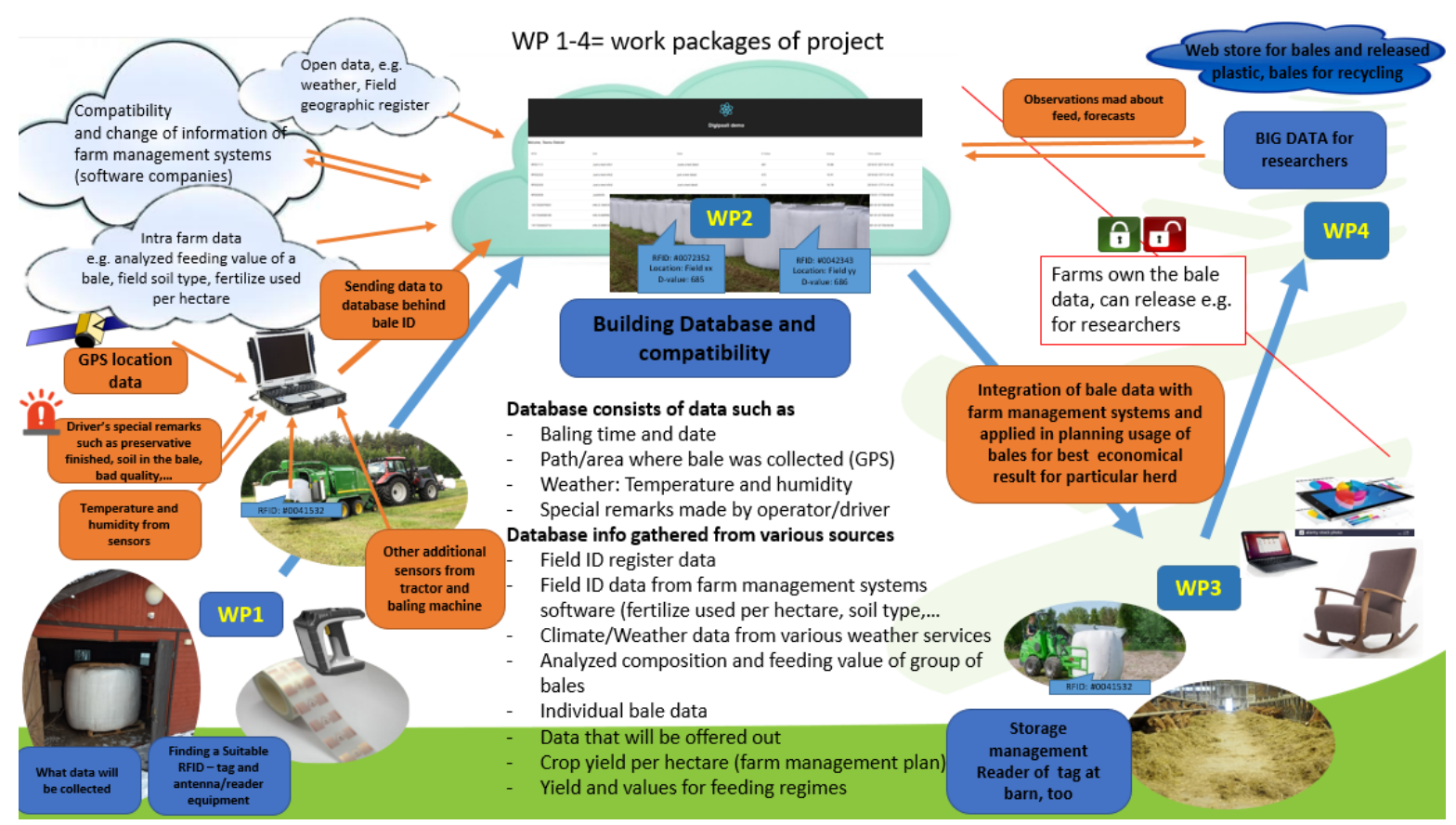

Figure 1. Schema of Digibale concept (Digibale project 2018).

Principle of RFID technology

RFID technology is an identification technology that uses radio waves for communication. Typical operational frequencies are 865-868 MHz in Europe and 13,56 MHz globally (ETSI Technologies and Glusters, 2018). The first one is based on electromagnetic waves, enabling long-range identification, while the latter is based on magnetic coupling, and operates only in near field. Some commonly known RFID applications are key cards, passports, library anti-theft systems, near field payment and metro tickets.

The operation principle of passive RFID is based on backscattering technology (Bolomey et all., 2010; Pouzin et all., 2009; She et all., 2010). Passive technology means that the transponders do not have any kind of internal power source, but it harvests its operational energy from the reader's transmission signal (Finkenzeller, 2003; Keskilammi et all., 2003). RFID reader, with external or integrated antenna, is connected to backend system and operates as master towards transponders. Based on application needs, reader send identifier polling request to its surroundings. Regional institutes, such as ETSI (European Telecommunication Standards Institute) limits the maximum allowed transmitted power level (ETSI Technical Specification, 2013). Depending on the radiation pattern of the antenna, the reader's request will spread either a narrower or a wider area, while the total amount of radiated power shall be under specified limit. If any transponder exist inside the range, the reader transmission signal will first wake the tag up, then give the identifier reading command and finally send some unmodulated signal, which the transponder will modulate and reflect backwards (Hansen, 1989; Pozar, 2004; Sydänheimo et all., 2008). The transponder modulation, which is fully passive, is based on switch connection inside transponder microchip. The modulation is on-off type amplitude modulation (Finkenzeller, 2003). Communication principle is introduced in Figure 2.

While magnetic coupling enables identification ranges up to around $1 \mathrm{~m}$, ultra-high frequency (UHF) RFID at around 866,5 MHz enables identification distances up to around $10 \mathrm{~m}$ (Keskilammi, 2004; Rao et all., 1999). These distances are maximum free air distances, meaning, that between the reader antenna and the transponder, there is only free air, without any obstacles. All absorbing materials, such as concrete wall structures, attenuate the radio signal and diminish the identification distance. In addition, the material, where the transponder is attached to, influences to the achievable identification distance. All absorbing or reflecting base materials will attenuate the communicating signal, and therefore diminish the identification distance. Absorption means that the material itself absorbs radio waves causing diminishing the maximum peak intensity (Fuschini et all., 2008; Vizmuller, 1995). Reflecting occurs when the radio wave is reflected from the base surface, and then eloped away (Appel-Hansen, 1988; Nikitin and Rao, 2006; Reitz et all., 1962; Ukkonen et all., 2004). As in the application at hand, the transponder base has strong humidity level, and large humidity variation, being between $20-45 \%$ (McDonald et all., 1991), the wrapped silage bale becomes conductive, sharing both absorbing and reflecting properties. Large humidity value leads to high conductivity (Fuschini et all., 2008; Vizmuller, 1995), further causing strong wave propagation in the bale, which diminish the amount of radio wave reaching the transponder, and therefore diminishing the identification distance. The radio wave propagated inside the bale will quickly reach the edge of the bale, and then will partially reflect back to the bale, and partially refract to the plastic wrapping 
and further outside air. Figure 3 presents reflection and refraction phenomena in silage bale application. To conclude, radio frequency identification in silage bale application requires that transponder has strong ability to harvest and backscatter radio waves in challenging environment (Byun et all., 2012; Cho et all., 2009; Lee 2008; Tang et all., 2009;).

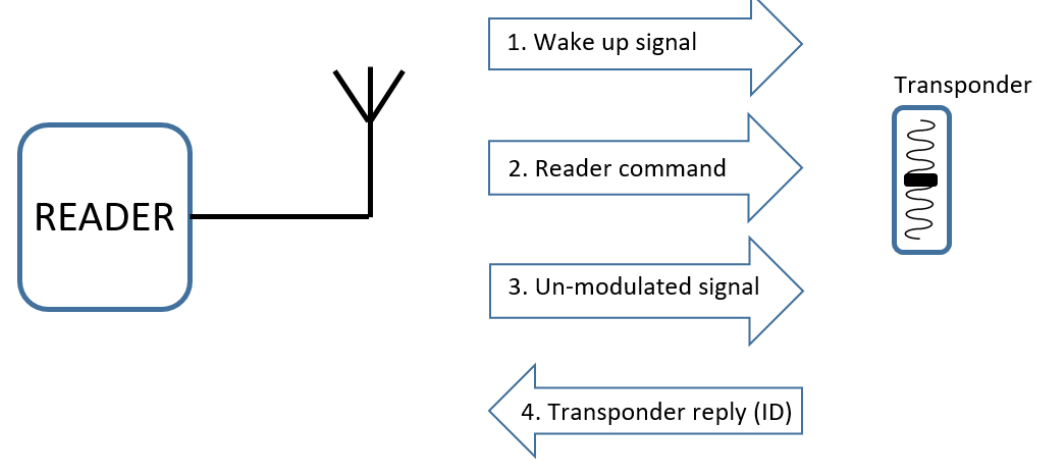

Figure 2. RFID reader - transponder communication principle

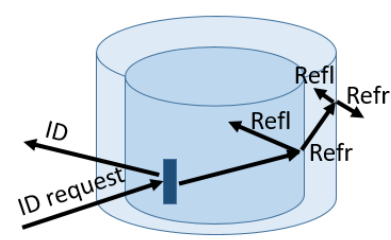

Silage

Plastic wrapping

Transponder between

silage and wrapping

$\uparrow$ Radio wave

Refl $=$ reflection

Refr $=$ refraction

Figure 3. Illustration of reflection and refraction principles in silage bale application.

\section{Materials and Methods}

This paper examines the performance of passive RFID in silage bale application. In order to conduct required measurements, this chapter introduces and argues chosen technologies and methodology.

Initial tests and test results

Initial test were taken to find out the best performing passive transponder. Totally four potential transponder types with different microchips were tested and measured in terms of free air reading distance. Tests were taken in laboratory conditions. Then the best performing transponder were chosen for application measurements. Figure 4 introduces the pictures of each tested transponder types. The choice of these transponders were based on manufacturer and retailer's recommendations.

The initial transponder test results are presented in Table 1. Results show, that the Dogbone 425-1 shows best performance, and therefore it was selected for application measurements.
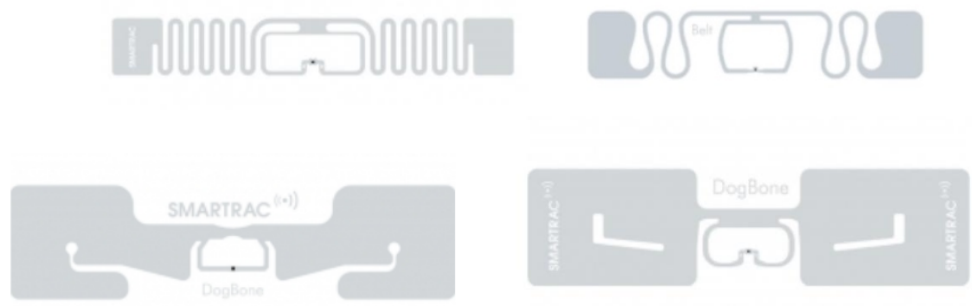

Figure 4. Top left: Belt 387-1, top right: Belt 285-3, bottom left: Dogbone 425-1 and bottom right: Dogbone 391-2. 
Table 4. Transponder free air test results.

\begin{tabular}{ccccc}
\hline $\begin{array}{c}\text { Transponder, } \\
\text { Type }\end{array}$ & $\begin{array}{c}\text { Free air reading } \\
\text { distance in laboratory } \\
\text { conditions in 0 degrees } \\
\text { angle towards the reader } \\
\text { antenna, } \mathrm{m}\end{array}$ & $\begin{array}{c}\text { Free air reading } \\
\text { distance in laboratory } \\
\text { conditions in 90 degrees } \\
\text { angle towards the reader } \\
\text { antenna, } \mathrm{m}\end{array}$ & $\begin{array}{c}\text { Microchip, } \\
\text { Type }\end{array}$ & $\begin{array}{c}\text { Transponder size, } \\
\text { mm } \times \text { mm }\end{array}$ \\
\hline Belt 387-1 & 2,6 & 3,0 & G2IL & $70 \times 10$ \\
Belt 285-3 & 2,5 & 2,6 & Monza 6 & $70 \times 14$ \\
Dogbone 425-1 & 6,8 & 7,2 & Monza R6 & $94 \times 24$ \\
Dogbone 391-2 & 6,4 & 4,6 & Ucode7 & $94 \times 24$ \\
\hline
\end{tabular}

\section{RFID components}

Radio frequency identification components were chosen based on performance criteria and integrability to the baler application. Based on initial tests presented in section 2.1., Dogbone 425-1 transponder were chosen for first bale measurements. This transponder is presented in bottom left, in Figure 4. In final application, three different readers are chosen to enable various different identification positions in the Digibale application concept. For both initial test and the first bale measurements, Impinj Speedway UHF RFID reader was used consistently.

\section{Methodology}

Application measurements were taken outside, with real silage bale, as shown in Figure 5a. Small holes were cut on the bale wrapping, and the chosen transponders were placed under the plastic wrapping. Before measurements, the cuttings were sealed with high quality tape to confirm that microclimate inside the bale wrapping remains as close as possible to the original. Cutting the bale is introduced in Figure 5b. Totally eight transponders, shown in Figure 6, were placed inside bale wrapping with even steps, to fulfilling the 360 degrees around the bale. Figure 7 shows the measurement arrangement, and directions of wave propagation between transponder and reader antenna. The positions 4, 5 and 6 in Figure 7 (numbered positions in the figure) are located in a way that the radio wave will propagate through the bale, which causes multiple reflection and refraction points presented in Figure 3. (Alonso and Finn, 1967; Kraus, 1950; Nikitin and Rao, 2008)

The identification distance was measured by moving the reader further from the bale, until the transponder inside the wrapping was no longer backscattering its identification code to the reader.
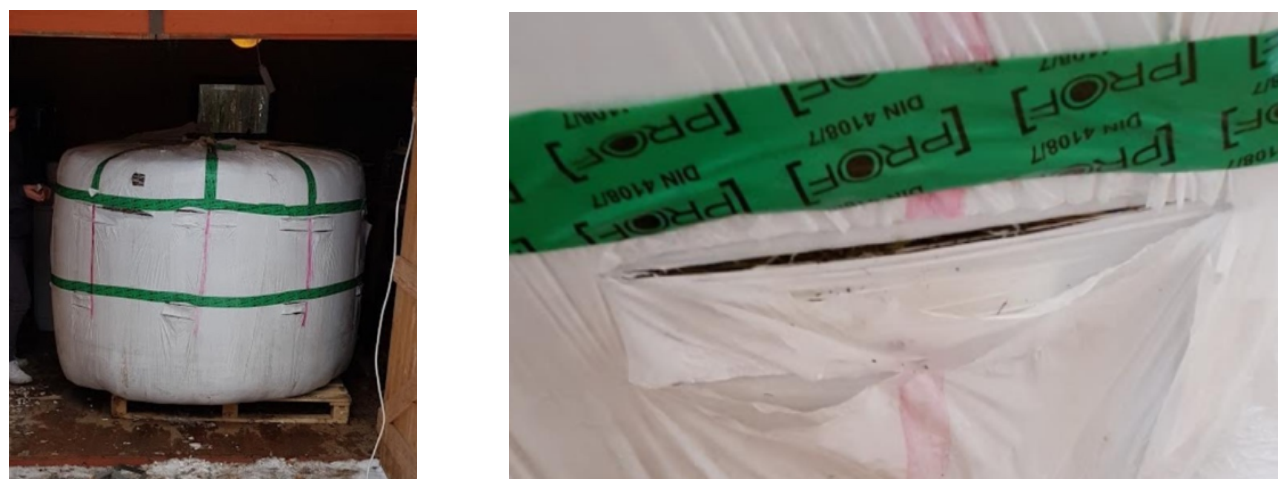

Figure 5. Silage bale. a) Test bale (left), b) bale cut (right).

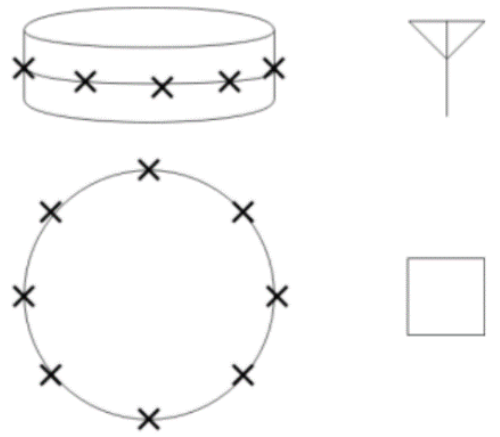

Figure 6. Schematic view of transponder placements in bale: top: side view, bottom: top view. 


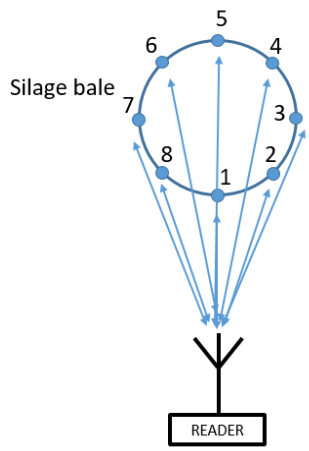

Figure 7. Measurement arrangement, top view.

\section{Results and Discussion}

The resutls for Dogbone transponder measurements are presented in Table 2. When the reader is facing the transponder (position 1 in Figure 7), or being in small angle against it (positions 2 and 8), the transponders are identified with sufficient distance. On the other hand, at opsitions 3 and 7, the transponder edge is facing the reader antenna, and the transponder antenna is not facing the transmitted wave. Therefore, transponder identification is not achieved with any distance. In positions 4, 5 and 6, the transmitted wave should propagate through the bale, which causes too much reflections and refractions, so that the electromagnetic wave elopes to its surroundings, and further attenuates in a way that the harvested wave does not give sufficient amount of energy to the transponder microchip to wake up and recognize the reader request.

Table 2. Measurement results in bale.

\begin{tabular}{ccc}
\hline $\begin{array}{c}\text { Transponder position in bale } \\
\text { according to Figure }\end{array}$ & $\begin{array}{c}\text { Simultaneous identification } \\
\text { of multiple transponders, } \\
\text { OK/NOK }\end{array}$ & $\begin{array}{c}\text { Maximum transponder identification distance, } \\
\text { mm }\end{array}$ \\
\hline 1 & OK & 3800 \\
2 & OK & 1700 \\
3 & NOK & 0 \\
4 & NOK & 0 \\
5 & NOK & 0 \\
6 & NOK & 0 \\
7 & NOK & 0 \\
8 & OK & 3600 \\
\hline
\end{tabular}

\section{Conclusions}

The results presented in this paper indicates, that passive RFID technology is suitable for unique silage bale identification. Application measurements, where the transponders were attached to a real bale, between the silage and plastic wrapping, showed sufficient performance over 90 degrees angle, or $1 / 4$ of bale, at 1,7 m distance from bale edge. In addition, point-to-point identification, in 0 degree angle, gave maximum of 3,8 $\mathrm{m}$ identification distance.These results give good starting point to upcoming field tests. The next steps for the Digibale concept develompent and piloting are field tests with Dogbone transponder to analyze the performance in real application environment. Passive RFID transponders for challenging applications have beed studied for many years, though based on author's best knowledge, silage bale transponder has not yet published or commercialized.

\section{Acknowledgements}

Authors would like to express their gratitude to Finnish Countryside Innovation Group (EIP) and Finnish Ministry of Agriculture and Forestry due to funding this project. In addition, authors are thankful BSc Khoa Dang and Mr. Bao Tran from HAMK for conducting the basic measurements according to instructions.

\section{References}

Alonso M, Finn E., 1967. Fundamental University Physics. vol II: fields and waves. Addison-Wesley Publishing Company. Reading, MA USA. 556p.

Appel-Hansen J., 1988. Accurate determination of gain and radiation patterns by radar cross-section measurements. IEEE Transactions on Antennas and Propagation. 27(5):640-646

Bolomey J.C., Capdevila S., Jofre L., Romeu J., 2010. Electromagnetic Modeling of RFID-Modulated Scattering Mechanism. Application to Tag Performance Evaluation. Proceedings of the IEEE, vol. 98, pp. 15551569. 
Byun J.-K., Choi N.-S., Kim D.-H., 2012. Optimal Design of a RFID Tag Antenna Based on Plane-Wave Incidence. IEEE Transactions on Magnetics, vol. 48, pp. 795-798. ISSN 0018-9464.

Cho C., Park I., Choo H., 2009. Design of a Circularly Polarized Tag Antenna for Increased Reading Range. IEEE Transactions on Antennas and Propagation, vol. 57, pp. 3418-3422. ISSN 0018-926X.

Digibale project. Digibale - Efficiency in life cycle control of forage bales. Luke, Natural Resources Institute Finland. https://www.luke.fi/en/projects/digipaali/ Assessed May 21, 2018

ETSI Technical Specification ETSI TS 102902 v1.2.3 (2013-04). Electromagnetic compatibility and Radio spectrum matters (ERM); Methods, parameters and test procedures for cognitive interference mitigation towards ER-GSM for use by UHF RFID using Detect-And-Avoid (DAA) or other similar techniques. http://www.etsi.org/deliver/etsi_ts/102900_102999/102902/01.02.01_60/ts_102902v010201p.pdf. Accessed: May 18, 2018

ETSI Technologies and Glusters: RFID. http://www.etsi.org/technologies-clusters/technologies/radio/rfid. Accessed May 18, 2018

Finkenzeller K., 2003. RFID handbook 2nd ed. John Wiley \& Sons Inc. West Sussex, UK 427p.

Fuschini F., Piersanti C., Paolazzi F., Falciasecca G., 2008. Analytical Approach to the Backscattering from UHF RFID Transponder. Antennas and Wireless Propagation Letters IEEE, vol. 7, pp. 33-35. ISSN 1536-1225.

Hansen R., 1989. Relationships between antennas as scatterers and as radiators. Proceedings of IEEE. 77(5):659-662

Keskilammi M, Sydänheimo L, Kivikoski M, 2003. Radio frequency technology for automated manufacturing and logistics control. Part 1: passive RFID systems and the effects of antenna parameters on operational distance. International Journal on Advanced Manufacturing Technology 21 (10-11):769-774

Keskilammi M., 2004. Importance of application specific antennas on passive long-range RFID system performance. PhD Dissertation, Tampere University of Technology Publications 516, TTY-Paino, Tampere, Finland

Kraus J., 1950. Antennas. McGraw-Hill International editions. New York, USA. 873p.

Lee B., 2008. Review of RFID tag antenna issues at UHF band. Microwave Conference 2008. APMC 2008. Asia-Pacific, pp. 1-4.

McDonald P., Henderson A.R., Heron S.J.E., 1991. The Biochemistry of Silage. Second Edition. Chalcombe Publications, Aberystwyth. p.340.

Nikitin P.V., Rao K.V.S., 2006. Theory and measurement of backscattering from RFID tags. IEEE Antennas and Propagation Magazine, vol. 48, pp. 212-218, 2006, ISSN 1045-9243.

Nikitin P.V., Rao K.V.S., 2008. Antennas and Propagation in UHF RFID Systems. IEEE International Conference on RFID. pp. 277-288.

Pozar D., 2004. Scattered and absorbed powers in receiving antennas. IEEE Antennas Propagation Magazine 46(1):144-145 12.

Pouzin A., Vuong T.P., Tedjini S., Pouyet M., Perdereau J., Dreux L., 2009. Determination of measurement uncertainties applied to the RCS and the differential RCS of UHF passive RFID tags. Antennas and Propagation Society International Symposium, APSURSI '09. IEEE, pp. 1-4, ISSN 1522-3965.

Rao K, Duan D-W, Heinrich H (1999) On the read zone analysis of radio frequency identification systems with transponders oriented in arbitrary directions. Proceedings of Microwave Conference, Asia Pacific 3:758-761

Reitz J., Milford F., Christy R., 1962. Foundations of electromagnetic Theory. Addison-Wesley, Boston Addison-Wesley Publishing Company Inc. New York, USA 390p

She K., He Y., Li B., Hou Z., Zhu Y., Zuo L., 2010. Theory and Measurement of Delta RCS for RFID Tag on Various Materials. 6th International Conference on Wireless Communications Networking and Mobile Computing, WiCOM. pp. 1-4.

Sydänheimo L., Nummela J., Ukkonen L., McVay J., Hoorfar A., Kivikoski M. 2008. Characterization of Passive UHF RFID Tag Performance. IEE Antennas and Propagation Magazine, vol. 50, pp. 207-212, 2008, ISSN 1045-9243.

Tang Z., He Y., Hou Z., Li B., 2009. The Effects of Antenna Properties on Read Distance in Passive Backscatter RFID Systems. International Conference on Networks Security Wireless Communications and Trusted Computing, NSWCTC '09., vol. 1, pp. 120-123.

Ukkonen L., Soini M., Engels D., Sydänheimo L., Kivikoski M., 2004. Effect of conductive material in objects on identification with passive RFID technology: a case study of cigarette cartoons. International Conference on Machine Automation. 24-26 Nov. 2004 Osaka, Japan, pp 569-572 20.

Vizmuller P., 1995. RF design guide: systems, circuits and equations. Artech House, Boston, USA. 281p. 


\author{
Enhanced Vineyard Descriptors Combining UAV 2D and 3D Crop Models \\ Lorenzo Comba ${ }^{b, *}$, Alessandro Biglia ${ }^{a}$, Davide Ricauda Aimonino ${ }^{a}$, Paolo Barge ${ }^{a}$, Cristina Tortia ${ }^{a}$, \\ Paolo Gay ${ }^{\text {a }}$ \\ ${ }^{a}$ DiSAFA - Università degli Studi di Torino, Largo Paolo Braccini 2, 10095 Grugliasco (TO), Italy \\ ${ }^{\mathrm{b}}$ DENERG - Politecnico di Torino, Corso Duca degli Abruzzi 24, 10129 Torino, Italy \\ *Corresponding author. Email: lorenzo.comba@polito.it
}

\begin{abstract}
Precision viticulture has been assumed as an essential approach to optimise crop-managing practices and to improve the quality of food products. To deploy proper site-specific management, addressing the intrinsic variability within a vineyard or a parcel, reliable methods for features extraction and mapping of crops must be developed. The introduction in agriculture of Unmanned Aerial Vehicle (UAV), equipped with sensors able to acquire fields planar images at several wavelengths, makes available huge amount of data with high-resolution, in terms of both spatial and temporal dimension. Recently, in addition to well-known 2D mosaicked images, innovative features leaded by modern photogrammetry allowed accurate three-dimensional models of crops (ex. $3 \mathrm{D}$ point cloud datasets) to be generated. The approach presented in this work is aimed at defining enhanced crop descriptors by exploiting information provided by both 2D and 3D crop models. Crucial phases of the procedure are the proper management of data provided by several sources, in order to achieve high consistency of the obtained huge dataset. In addition, the detection of vine rows, discriminating them from all the other elements of rural areas, plays a crucial role. The proposed methodology does not require the straightness of vine rows and it can be profitably applied to models of vineyards with curvilinear rows, also on steep terrains. Specific computing optimisation have been defined in order to reduce big data complexity. A set of 24 portion of vine rows, each made by 4 plants, has been used to validate the effectiveness of the evaluated crop canopy descriptors. The 2D maps and 3D point-cloud models have been generated by using aerial images acquired during UAV flights at 35 meters high, in a study vineyard located in Serralunga d'Alba (Piedmont, Northwest of Italy). The integration of 2D-3D information allowed to obtain good performance also in the presence of dense inter-row grassing which, usually, slightly differs from vine canopies in terms of reflectance.
\end{abstract}

Keywords: crop canopy 3D models, point-cloud processing method, crop descriptors, remote sensing, precision viticulture.

\title{
1. Introduction
}

Precision viticulture has been assumed as an essential approach to optimise crop-managing practices and to improve the quality of food products, reducing in the meanwhile the environmental impact, such as the waste of fertilisers, pesticides, fresh water and energy (Zhang et al., 2002; Gimenez et al., 2015; Lee and Ehsani, 2015; Srbinovska et al., 2015; Reina et al., 2017).

A reliable crop monitoring procedure is the base for an effective management of precision viticulture processes. For this task, remote sensing represents a powerful technology providing huge amount of data, from which valuable information can be derived. Indeed, several interesting works discussed the evaluation of plant vigour level, radiometric indices, water stresses, grapevine size and missing plants (Meggio et al., 2010; Primicerio et al., 2015; García-Tejero et al., 2016; Tang et al., 2016; Khanal et al., 2017; Primicerio et al., 2017; Terribile et al., 2017).

A fundamental requirement for providing useful remote sensing products in agriculture is the capacity to combine high spatial resolution and quick turnaround times. In this context, an additional push in agricultural remote sensing arise from the introduction of light Unmanned Aerial Vehicle (UAV), equipped with sensors able to acquire fields planar images at several wavelengths with high and very-high resolution, in terms of both spatial and temporal dimension.

Recently, in addition to well-known 2D mosaicked images (Comba et al., 2015), innovative features leaded by modern photogrammetry allowed accurate three-dimensional models of crops (e.g. 3D point cloud datasets) to be generated. A point-cloud is a large dataset of points, referred to a geodetic reference frame, representing spots of the external surface of visible objects, where light is reflected. Data for 3D crop modelling can be directly provided by laser scanner (such as light detection and ranging systems - LiDAR) (Koening et al., 2015; Bietresato et al., 2016; Mack et al., 2017) or derived from multispectral and thermal imagery (Zarco-Tejada et al., 2014; Herrero-Huerta et al., 2015; Weiss and Baret, 2017) by photogrammetry and computer vision algorithms, such as Structure from Motion (SfM).

Several published researches proved the information potentiality of this new type of crops models for monitoring and tasks assessment in biosciences, by developing reliable algorithms to exploit 3D data in agricultural (Bendig et al., 2015; Chang et al., 2017; Malambo et al, 2018), livestock (Mortensen et al., 2016; Guo 
et al., 2017) and food applications (Sture et al., 2016; Su et al., 2017).

In this work, an enhanced 2D map and 3D point-cloud processing method for reliably describe the canopy status in row-layout vineyard is presented.

\section{Materials and Methods}

The effectiveness of the proposed approach in defining proper canopy descriptors has been evaluated on a set of 24 test areas, which are sections of vine rows made by about four plants each. The whole region where the study have been conducted covers a surface of about 1 hectares located in Diano d'Alba (Piedmont, Northwest of Italy). The region, which includes one entire vineyard parcel and part of other plots, whose latitude and longitude positions in the World Geodetic System 1984 (WGS84) range between [44.6238 $44.6242^{\circ}$ ] and $\left[7.9988^{\circ}\right.$ $8.0003^{\circ}$ ] respectively (Figure 1), is characterised by a sloped land conformation, with an elevation ranging from 340 to 380 meters above sea level and a predominantly southwest orientation.

The flights paths have been planned to maintain the UAV height close to 35 meters with respect to the terrain, obtaining aerial images with a ground sample distance (GSD) of $5 \mathrm{~cm} \cdot$ pixel $^{-1}$. The acquisition campaign has been performed close to the noon on 2017, June the $29^{\text {th }}$, using an airborne 4-bands multispectral camera (Parrot Sequoia $\mathbb{R}$ ). In order to georeference the point-clouds in a geodetic coordinates frame, a set of 3 ground markers has been placed on selected vine trellis poles within the monitored area and their position has been accurately determined with a differential GNSS system. This methodology presented in this study uses both 2D highresolution multispectral map and 3D point-cloud model of the crops.

\section{Canopy descriptor from 3D point-cloud models}

The vineyard 3D point-cloud model has been generated processing a set of about 400 aerial multispectral images, using Agisoft PhotoScan ${ }^{\circledR}$ software. The obtained raw 3D point-cloud model is characterised by a mean density of about 1,450 units per meter square of map surface (Figure 1).

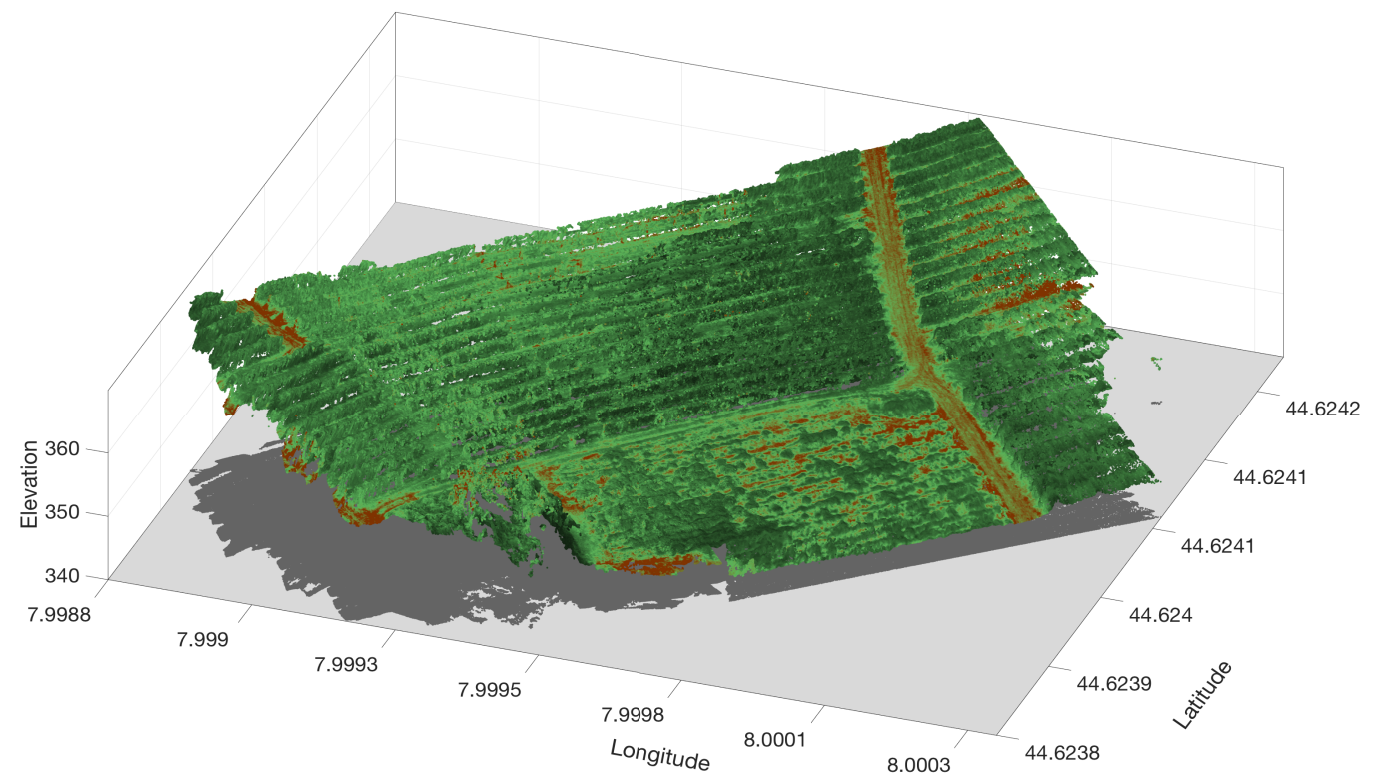

Figure 1. Point-cloud map $S^{\{\text {WGS84 }}$ of the monitored vineyard plot (June 29 $9^{\text {th }} 2017$ ).

A point-cloud map is here formally defined as a set $S^{\{\mathrm{WGS} 84\}}$ of points represented by array $p_{\mathrm{i}}=\left[\varphi_{\mathrm{i}}, \lambda_{\mathrm{i}}, e_{\mathrm{i}}\right]^{\mathrm{T}}$, with $\mathrm{i}=1, \ldots, \operatorname{card}\left(S^{\{\mathrm{WGS} 84\}}\right)$, where $\varphi_{\mathrm{i}}, \lambda_{\mathrm{i}}$ and $e_{\mathrm{i}}$ are the WGS84 latitude, longitude and elevation coordinates of point $\mathrm{p}_{\mathrm{i}}$ respectively. Each considered section of vine row $R_{j} \subset S$, with $\mathrm{j}=1, \ldots, 24$, has been represented in a local Cartesian reference frame $\{\mathrm{Loc}\}_{j}$ in order to facilitate the subsequent processing phases, as

$$
R_{j}^{\left\{\text {Loc }_{j}\right.}=\left\{p_{\mathrm{i}}=\left[x_{\mathrm{i}}, \quad y_{\mathrm{i}^{\prime}} \quad z_{\mathrm{i}}\right]^{\mathrm{T}} \in \mathbb{R}^{3} ; \mathrm{i}=1, \ldots, \operatorname{card}\left(R_{j}\right)\right\}
$$

where $x_{\mathrm{i}}, y_{\mathrm{i}}$ and $z_{\mathrm{i}}$ are the spatial coordinates of each point of the cloud map in meters (Figure 2a). The 
reference frame $\{\text { Loc }\}_{j}$ has been defined with the $\mathrm{x}$-axis aligned with the local tangent to the vine row, the $\mathrm{z}$-axis with the vertical and the $y$-axis accordingly to complete the frame. These local vine row information and the terrain model parameters, essential to obtain the relative height of each point of the model with respect to the local terrain surface, have been derived by the method described in Comba et. al. (2018). The origin $O$ of $\{\text { Loc }\}_{j}$ has been located at the soil level, aligned with a vineyard trellis close to the considered group of four vines $R_{j}$.
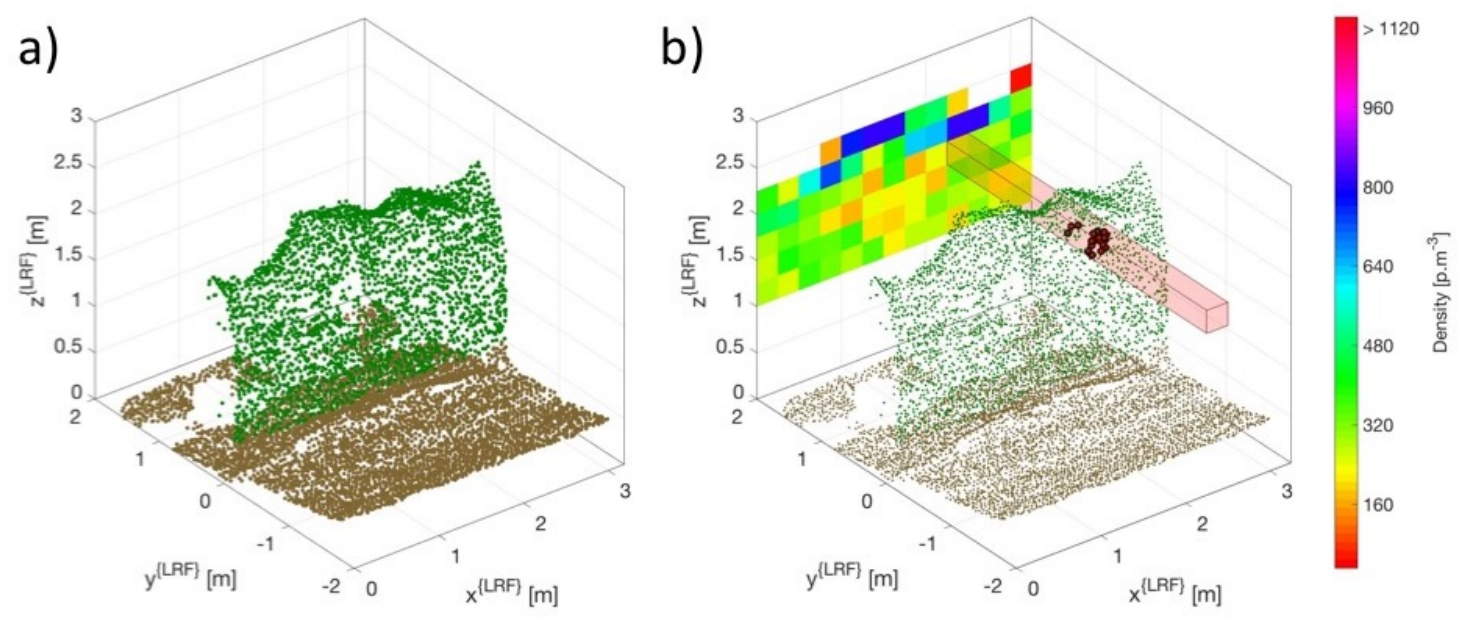

Figure 2. Portion of point-cloud $R_{13}^{\{\text {Loc }\}_{13}}$ (green dots). Points belonging to subset $T_{10,4}(d)$, with $d=0.25$ meters, are highlighted in red. Values of matrix $M_{13}$ are graphically represented in the background (images on the x-z plane), properly aligned with $\mathrm{x}$ and $\mathrm{z}$ coordinates ranges used to compute $m_{r, s}$ values.

The 3D model of the crop canopy is here processed in order to obtain a reliable descriptor, able to represent with a single number the complex spatial distribution of the leaves along the canopy wall. Considering the pointcloud section $R_{j}$, points coordinates of which are within the ranges $0 \leq x \leq x_{\max }, 0 \leq y \leq y_{\max }$ and $0 \leq z \leq$ $z_{\max }$, an analysis of the point density variability is conducted defining a subset $T_{\mathrm{r}, \mathrm{s}}$ of points within a parallelepiped with high and width equal to $d$, as

$$
T_{\mathrm{r}, \mathrm{s}}(d)=\left\{p_{i} \in \mathrm{R}_{\mathrm{j}} \mid(r-1) \cdot d \leq x_{i}<r \cdot d,(s-1) \cdot d \leq z_{i}<s \cdot d\right\}
$$

with $r \in\left\{1,2, \ldots,\left[\frac{x_{\max }}{d}\right]\right\}$ and $s \in\left\{1,2, \ldots,\left[\frac{z_{\max }}{d}\right]\right\}$.

In Figure $2 \mathrm{~b}$, a sample subset $T_{r, s}$ is highlighted in red, obtained processing section $R_{22}$ with $d$ equal to 0.1 meters. Indeed, considering the amount of point constituting the subset $T_{\mathrm{r}, \mathrm{s}}(d)$, a two-dimensional map $M_{j}$ can be derived as

$$
M_{j}=\left\{m_{r, s}=\operatorname{card}\left(T_{r, s}\right) \vee r \in\left\{1,2, \ldots,\left\lceil\frac{x_{\max }}{d}\right\rceil\right\}, s \in\left\{1,2, \ldots,\left\lceil\frac{Z_{\max }}{d}\right\rceil\right\}\right\}
$$

which represent the density distribution of the points of section $R_{j}$ along the plane x-z. As an example, the matrix $M_{13}$ obtained processing section $R_{13}$ with $d$ equal to 0.25 meters and $r \in\{1,2, \ldots, 13\}$ and $s \in\{1,2, \ldots, 6\}$ is graphically represented in Figure $2 \mathrm{~b}$. The obtained matrix $M_{j}$ is deeply affected by the parameter $d$ value, which is related to the specific degree of detail of the 2D canopy density distribution map. Indeed, small values of $d$ can lead to matrix $M_{j}$ better describing the canopy inhomogeneities. However, a too small value of $d$ generates a matrix $M_{j}$ with a lot of empty elements, drastically limiting the canopy wall map effectiveness. Several metrics and statistic can be defined, aimed at properly describing values of matrix $M_{j}$ : in this preliminary investigation, the descriptor $D_{1}$ has been defined as the product between the average value of $M_{j}$ elements and the canopy high related value $\left\lceil\frac{z_{\max }}{d}\right\rceil$, as 


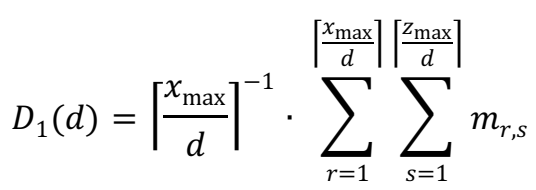

Canopy descriptor from 2D map and 3D model integration

In this section, the definition of a new crop descriptor derived from the fusion of $2 \mathrm{D}$ and $3 \mathrm{D}$ information of the canopy, considering both the spatial and the spectral content, is presented. The 2D multispectral map of the region has been generated with Pix $4 \mathrm{D}^{\circledR}$ software, with Near Infrared, Red Edge, Red and Green spectral bands. In Figure 3a, the portion of 2D map, named $I_{13}$, representing the test area 13 has been plotted in the plane $\mathrm{x}-\mathrm{y}$ using false colours (NIR, R, G bands), together with the section of point-clouds $R_{13}$. From digital number $d_{\mathrm{a}, \mathrm{b}}$ of the test portion $I_{\mathrm{j}}$ of the map, with $\mathrm{j}=1, \ldots, 24$, defined as $d_{\mathrm{a}, \mathrm{b}}=\left[d_{N I R} d_{R E} d_{R} d_{G}\right]_{a, b}$, the well know NDVI index has been computed as $n_{\mathrm{a}, \mathrm{b}}=\left[\left(d_{N I R}-d_{R}\right) \cdot\left(d_{N I R}+d_{R}\right)^{-1}\right]_{a, b}$. Indexes $a$ and $b$ point the digital number position in the image matrix, with $a \in\{1,2, \ldots, A\}, b \in\{1,2, \ldots, B\}$ and $A$ and $B$ that are the number of rows and columns of $I_{\mathrm{j}}$. For portion $I_{13}, a$ and $b$ range from 1 to 80 and from 1 to 64 respectively.
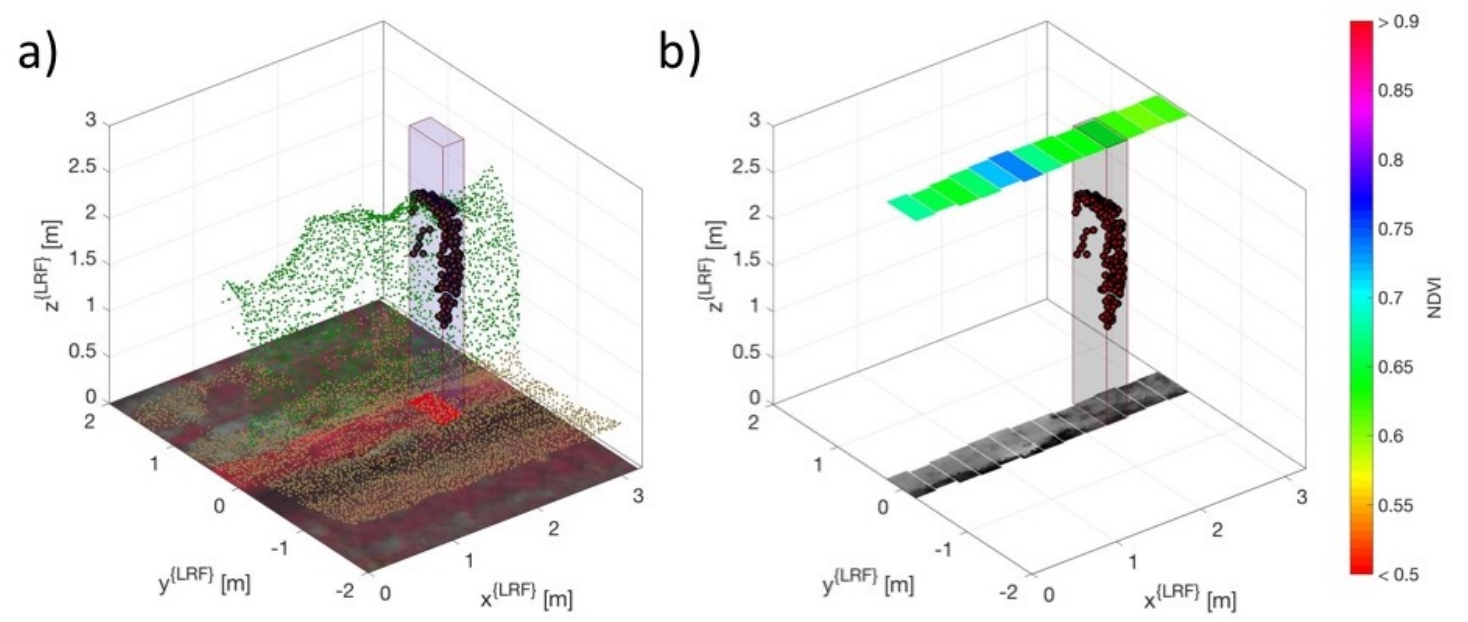

Figure 3. Portion $I_{13}$ of the 2D multispectral map (plane $x-y$ ) of the vineyard, representing the sample area 13, with false colours (NIR, R, G bands), together with the section of point-clouds $R_{13}(\mathrm{a})$. Points belonging to subset $Q_{10}(d)$, with $d=0.25$ meters, are highlighted in red. Selected set of NDVI values $H_{\mathrm{r}}$ representing vines canopy, with $r \in\{1,2, \ldots, 13\}$, are plotted in the lower x-y plane (b), while values of array $L_{13}$ are graphically represented in the upper the $\mathrm{x}-\mathrm{y}$ plane (b).

The canopy structure and variability are evaluated selecting only pixels representing the canopy from $I_{\mathrm{j}}$, by defining a subset

$$
Q_{\mathrm{r}}(d)=\left\{p_{i} \in \mathrm{R}_{\mathrm{j}} \mid(r-1) \cdot d \leq x_{i}<r \cdot d, \quad 0.5 \leq z_{i}<z_{\max }\right\}
$$

In Figure 3a, points belonging to $Q_{10}$, with $d=0.25$ meters, are highlighted in dark red, while their projection to the plane $\mathrm{x}-\mathrm{y}$ are light red coloured. The region of the soil (x-y plane) occupied by the canopy is here approximated with the rectangular polygon containing the projection of all points $p_{i} \in Q_{\mathrm{r}}$, which is defined by $x_{\mathrm{r}, \min } \leq x_{i}<x_{\mathrm{r}, \max }$ and $y_{\mathrm{r}, \text { min }} \leq y_{i}<y_{\mathrm{r}, \text { max }}$, with $x_{\mathrm{r}, \text { min }}=\min \left(\left\{x_{i}:\left[x_{\mathrm{i}}, y_{\mathrm{i}}, z_{\mathrm{i}}\right]^{\mathrm{T}} \in Q_{\mathrm{r}}(d)\right\}\right), \quad x_{\mathrm{r}, \text { max }}=$ $\max \left(\left\{x_{i}:\left[x_{\mathrm{i}}, y_{\mathrm{i}}, z_{\mathrm{i}}\right]^{\mathrm{T}} \in Q_{\mathrm{r}}(d)\right\}\right), y_{\mathrm{r}, \min }=\min \left(\left\{y_{i}:\left[\begin{array}{ll}x_{\mathrm{i}}, & y_{\mathrm{i}}, z_{\mathrm{i}}\end{array}\right]^{\mathrm{T}} \in Q_{\mathrm{r}}(d)\right\}\right)$ and $y_{\mathrm{r}, \max }=\max \left(\left\{y_{i}:\left[x_{\mathrm{i}}\right.\right.\right.$, $\left.\left.\left.\begin{array}{ll}y_{\mathrm{i}}, & z_{\mathrm{i}}\end{array}\right]^{\mathrm{T}} \in Q_{\mathrm{r}}(d)\right\}\right)$. The limits of this region, in the map $I_{\mathrm{j}}$, are located in pixel with indexes $a_{\mathrm{r}, \mathrm{min}}=$ $\left\lfloor y_{\mathrm{r}, \text { min }} \cdot w^{-1}\right\rfloor, a_{\mathrm{r}, \text { max }}=\left\lceil y_{\mathrm{r}, \text { max }} \cdot w^{-1}\right\rceil, b_{\mathrm{r}, \text { min }}=\left\lfloor x_{\mathrm{r}, \text { min }} \cdot w^{-1}\right\rfloor$ and $b_{\mathrm{r}, \text { max }}=\left\lceil x_{\mathrm{r}, \text { max }} \cdot w^{-1}\right\rceil$, where $w$ is the 
pixel spatial resolution of the 2D map. Set of pixels $n_{\mathrm{a}, \mathrm{b}}$, defined as

$$
H_{\mathrm{r}}=\left\{n_{\mathrm{a}, \mathrm{b}} \in I_{\mathrm{j}} \mid a \in\left\{a_{\mathrm{min}}, a_{\mathrm{min}}+1, \ldots, a_{\mathrm{max}}\right\}_{\mathrm{r}}, \quad b \in\left\{b_{\min }, b_{\min }+1, \ldots, b_{\max }\right\}_{\mathrm{r}}\right\}
$$

are thus selected and the average NDVI value

$$
N_{\mathrm{r}}=\left|a_{\mathrm{r}, \text { max }}-a_{\mathrm{r}, \text { min }}\right|^{-1} \cdot\left|b_{\mathrm{r}, \max }-b_{\mathrm{r}, \min }\right|^{-1} \cdot \sum_{a=a_{\mathrm{r}, \min }}^{a_{\mathrm{r}, \max }} \sum_{b=b_{\mathrm{r}, \min }}^{b_{\mathrm{r}, \max }} n_{\mathrm{a}, \mathrm{b}}
$$

computed. All values of $N_{\mathrm{r}}$, with $\mathrm{r} \in\left\{1,2, \ldots,\left\lceil\frac{x_{\max }}{d}\right\rceil\right\}$, for the section $I_{\mathrm{j}}$ are then organised in the array $L_{j}$. In Figure $3 \mathrm{~b}$, the selected group of pixels $H_{r}$, with $r=1, \ldots, 13$, and the graphical representation of array $L_{j}$ are plotted, as an example. Several metrics and statistic can be also defined for the output of this second approach: in this preliminary investigation, the descriptor $D_{2}$ has been defined as

$$
D_{2}(d)=\left\lceil\frac{x_{\max }}{d}\right\rceil^{-1} \cdot \sum_{r=1}^{\left\lceil\frac{x_{\max }}{d} \mid\right.} N_{\mathrm{r}} \cdot\left|y_{\mathrm{r}, \max }-y_{\mathrm{r}, \min }\right|
$$

where $\left|y_{\max }-y_{\min }\right|_{r}$ is the local canopy width.

\section{Results and Discussion}

The goodness of the two vine canopy descriptors $D_{1}(d)$ and $D_{2}(d)$ from 2D aerial map and 3D point-cloud model, described in section 2 , have been evaluated on 24 test areas. The set of samples have been divided in three classes on the base of the vigour and canopy density, from 'A' (lower vigour) to ' $\mathrm{C}$ ' (higher vigour), by expert infield visual assessment. During the manual classification, the presence of weak plants and portion of canopy wall

\begin{tabular}{|c|c|c|c|c|c|c|}
\hline & Source & $\begin{array}{l}\text { Sum of } \\
\text { squares }\end{array}$ & $\begin{array}{c}\text { Degrees of } \\
\text { freedom }\end{array}$ & $\begin{array}{c}\text { Mean } \\
\text { squared error }\end{array}$ & F-statistic & $\begin{array}{c}p \text {-value } \\
\text { (Prob }>\text { F) }\end{array}$ \\
\hline \multirow{3}{*}{$\begin{array}{l}\text { 우 } \\
\text { II } \\
\text { II }\end{array}$} & Groups & 1022,05151 & 2 & 511,0258 & 11,8743 & 0,00035492 \\
\hline & Error & 903,764478 & 21 & 43,0364 & & \\
\hline & Total & 1925,81598 & 23 & & & \\
\hline \multirow{3}{*}{$\begin{array}{l}\text { 엄 } \\
\text { O1 } \\
\text { II }\end{array}$} & Groups & 3634,25501 & 2 & 1817,1275 & 10,1404 & 0,00082785 \\
\hline & Error & 3763,14287 & 21 & 179,1973 & & \\
\hline & Total & 7397,39787 & 23 & & & \\
\hline \multirow{3}{*}{$\begin{array}{l}\text { 号 } \\
\text { II } \\
\text { II }\end{array}$} & Groups & 6387,88586 & 2 & 3193,9429 & 14,1629 & 0,00012765 \\
\hline & Error & 4735,81761 & 21 & 225,5151 & & \\
\hline & Total & 11123,7035 & 23 & & & \\
\hline \multirow{3}{*}{$\begin{array}{l}\text { กิ } \\
\text { II } \\
\text { II }\end{array}$} & Groups & 13119,9772 & 2 & 6559,9886 & 10,0490 & 0,00086734 \\
\hline & Error & 13708,8268 & 21 & 652,8013 & & \\
\hline & Total & 26828,8039 & 23 & & & \\
\hline \multirow{3}{*}{$\begin{array}{l}\stackrel{\text { N }}{N} \\
\text { II } \\
\text { II }\end{array}$} & Groups & 19375,3739 & 2 & 9687,6869 & 9,0992 & 0,00142558 \\
\hline & Error & 22358,2314 & 21 & 1064,6777 & & \\
\hline & Total & 41733,6053 & 23 & & & \\
\hline
\end{tabular}
with low leaf density and/or holes has been properly considered.

Table 1. Results of ANOVA analysis of canopy descriptor $D_{1}(d)$, with several values of $d$ parameter

Descriptors $D_{1}(d)$, with $d \in\{0.05,0.10,0.15,0.20,0.25\}$, being derived with the same approach, are not independent and their ability in describing the canopy variability has been investigated individually by ANOVA analysis. Results of the investigation are summarised in Table 1. All descriptors $D_{1}(d)$ prove to achieve good results, with significant differences between classes means. Descriptor $D_{1}(0.15)$ results to be the most performing one, obtaining the lowest $p$-value. For the sake of completeness, boxplots of the 24 samples of $D_{1}(0.15)$, divided in classes 'A','B' and 'C', are shown in Figure 4a. 
a)

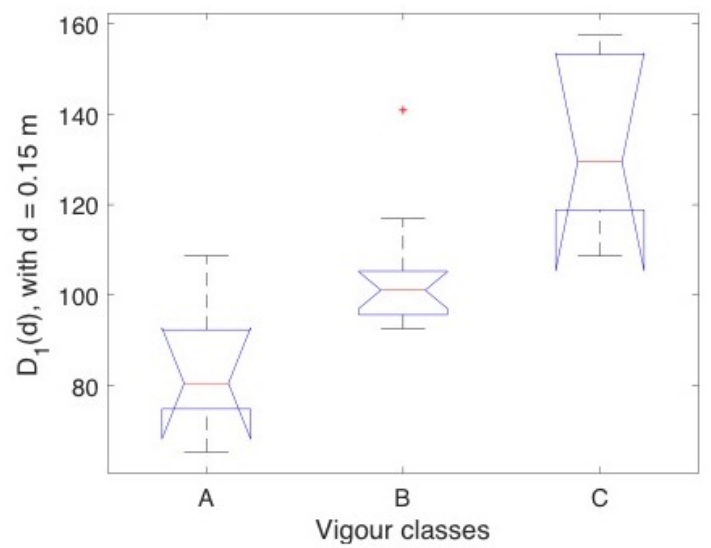

b)

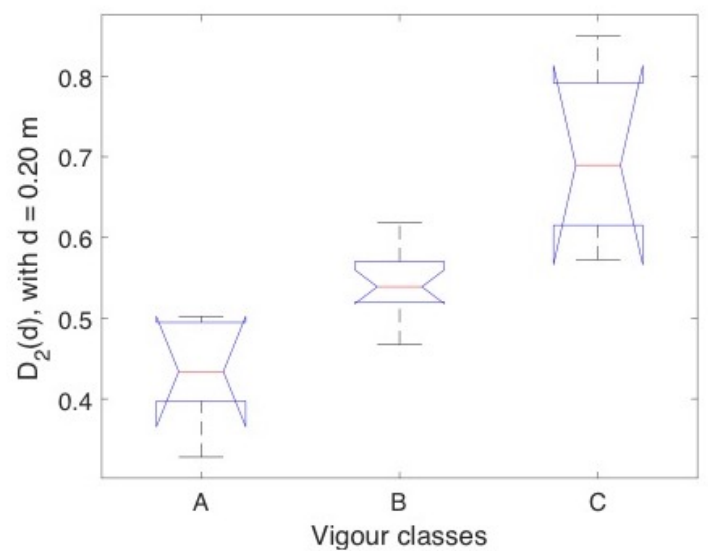

Figure 4. Boxplots of descriptor $D_{1}(d)$ (a), with $\mathrm{d}=0.15$ meters, and of descriptor $D_{2}(d)$ (b), with $\mathrm{d}=0.20$ meters.

The same analysis has been conducted for descriptors $D_{2}(d)$, with $d \in\{0.05,0.10,0.15,0.20,0.25\}$. Results of this second investigation are summarised in Table 2. All descriptors $D_{2}(d)$ prove to achieve good results, with significant differences between classes means. In this case, the best descriptor has been found to be $D_{2}(0.20)$, as shown by boxplots in Figure 4b, with the 24 samples of $D_{2}(0.20)$ grouped in classes 'A','B' and 'C'.

Table 2. Results of ANOVA analysis of canopy descriptor $D_{2}(d)$, with several values of $d$ parameter

\begin{tabular}{|c|c|c|c|c|c|c|}
\hline & Source & $\begin{array}{l}\text { Sum of } \\
\text { squares }\end{array}$ & $\begin{array}{c}\text { Degrees of } \\
\text { freedom }\end{array}$ & $\begin{array}{c}\text { Mean } \\
\text { squared error }\end{array}$ & F-statistic & $\begin{array}{l}p \text {-value } \\
\text { (Prob }>\text { F) }\end{array}$ \\
\hline \multirow{3}{*}{$\begin{array}{l}\text { In } \\
\text { Oீ } \\
\text { II }\end{array}$} & Groups & 0,2052 & 2 & 0,1026 & 9,5531 & 0,0011 \\
\hline & Error & 0,2256 & 21 & 0,0107 & & \\
\hline & Total & 0,4308 & 23 & & & \\
\hline \multirow{3}{*}{$\begin{array}{l}0 \\
\text {-1} \\
0 \\
11 \\
0\end{array}$} & Groups & 0,1796 & 2 & 0,0898 & 8,2779 & 0,0022 \\
\hline & Error & 0,2279 & 21 & 0,0109 & & \\
\hline & Total & 0,4075 & 23 & & & \\
\hline \multirow{3}{*}{$\begin{array}{l}\text { In } \\
\text { - } \\
\text { II } \\
0\end{array}$} & Groups & 0,1630 & 2 & 0,0815 & 7,8570 & 0,0028 \\
\hline & Error & 0,2179 & 21 & 0,0104 & & \\
\hline & Total & 0,3809 & 23 & & & \\
\hline \multirow{3}{*}{$\begin{array}{l}\text { O̦ } \\
0 \\
\text { II }\end{array}$} & Groups & 0,1817 & 2 & 0,0909 & 20,7711 & 0,00001 \\
\hline & Error & 0,0919 & 21 & 0,0044 & & \\
\hline & Total & 0,2736 & 23 & & & \\
\hline \multirow{3}{*}{$\begin{array}{l}\stackrel{1}{N} \\
0 \\
\text { II } \\
0\end{array}$} & Groups & 0,1557 & 2 & 0,0778 & 7,0401 & 0,0046 \\
\hline & Error & 0,2321 & 21 & 0,0111 & & \\
\hline & Total & 0,3878 & 23 & & & \\
\hline
\end{tabular}

A preliminary study of the combined effectiveness in using descriptors $D_{1}(0.15)$ and $D_{2}(0.20)$ to classify the canopy wall vigour has been performed implementing a binary decision tree algorithm. For this task, a crossvalidation approach has been adopted, using the leave-one-out cross-validation methodology. The fraction of misclassified data resulted equal to 0.12. An improvement in classification performance can be achieved increasing the number of samples. Estimates of predictor importance have been also computed for the trained decision tree model, which confirmed the need to use both the predictor $D_{1}(0.15)$ and $D_{2}(0.20)$. 


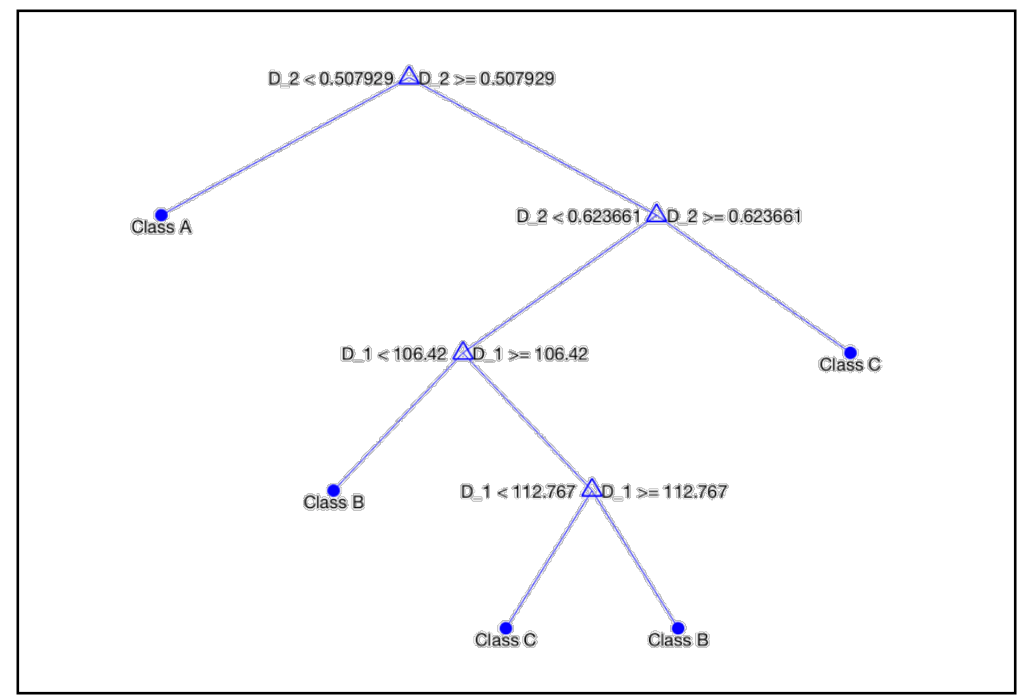

Figure 5. A decision tree diagram trained with descriptor $D_{1}(0.15)$ and $D_{2}(0.20)$.

\section{Conclusions}

In this work, a new methodology to process and merge data provided by vineyard 2D multispectral map and 3D point-cloud model has been presented, aimed at reliably describing the canopy status variability within rowlayout vineyards. The proposed approach exploits both spatial and spectral information, with particular attention to the canopy wall analysis, in term of leaves density distribution and irregularities. Two families of crop canopy descriptor have been discussed in this study, as a function of a spatial computing parameter, in order to evaluate their effectiveness in describing vines canopy degree of growth. Analysis of variance has been used to select the more preforming ones, using a sample set made by 24 portion of vine rows. A preliminary evaluation of their classification potential has been tested training a binary decision tree model, using the leave-one-out crossvalidation methodology. Obtained results are promising, although a limited number of descriptors has been still considered.

The presented data processing approach, new and with high potential, will be extended to define addition families of descriptors, evaluating the effect of several parameters. Indeed, additional crop descriptors can be used to train enhanced ensemble classifier, the effectiveness of which is strictly related to the numerosity of adopted independent variable.

\section{Acknowledgements}

This research was partially funded by project VITIFUTURE (POR FESR 2014-2020, Polo Innovazione AgriFood). The authors would like to acknowledge Germano Ettore, owner of the winery, for hosting the experimental campaign and Iway S.r.l. for supporting UAV flights.

\section{References}

Bending, J., Yu, K., Aasen, H., Bolten, A., Bennertz, S., Broscheit, J., et al., 2015. Combining UAV-based plant height from crop surface models, visible, and near infrared vegetation indices for biomass monitoring in barley. Int J Appl Earth Obs 39, 79 - 87.

Bietresato, M., Carabin, G., Vidoni, R., Gasparetto, A., Mazzetto, F., 2016. Evaluation of a LiDAR-based 3Dstereoscopic vision system for crop-monitoring applications. Comput Electron Agr 124, 1 - 13.

Chang, A., Jung, J., Maeda, M.M., Landivar, J., 2017. Crop height monitoring with digital imagery from Unmanned Aerial System (UAS). Comput Electron Agr 141, 232 - 237.

Comba, L., Gay, P., Primicerio, J., Ricauda Aimonino, D., 2015. Vineyard detection from unmanned aerial systems images. Comput Electron Agr 114, 78 - 87.

Comba, L., Biglia, A., Ricauda Aimonino, D., Gay, P., 2018. Unsupervised detection of vineyards by 3D pointcloud UAV photogrammetry for precision agriculture. Submitted to Comput Electron Agr.

García-Tejero, I.F., Costa, J.M., Egipto, R., Durán-Zuazo, V.H., Lima, R.S.N., Lopes, C.M. et al., 2016. Thermal data to monitor crop-water status in irrigated Mediterranean viticulture. Agr Water Manage 176, 80 - 90.

Gimenez, J., Herrera, D., Tosetti, S., Carelli, R., 2015. Optimization methodology to fruit grove mapping in precision agriculture. Comput Electron Agr 116, 88 - 100. 
Guo, H., Ma, X., Ma, Q., Wang, K., Su, W., Zhu, D., 2017. LSSA_CAU: An interactive 3d point clouds analysis software for body measurement of livestock with similar forms of cows or pigs. Comput Electron Agr $138,60-68$.

Herrero-Huerta, M., González-Aguilera, D., Rodriguez-Gonzalvez, P., Hernández-López, D., 2015. Vineyard yield estimation by automatic 3D bunch modelling in field conditions. Comput Electron Agr 110, 17 - 26.

Khanal, S., Fulton, J., Shearer, S., 2017. An overview of current and potential applications of thermal remote sensing in precision agriculture. Comput Electron Agr 139, 22 - 32.

Koening, K., Höfle, B., Hämmerle, M., Jarmer, T., Siegmann, B., Lilienthal, H., 2015. Comparative classification analysis of post-harvest growth detection from terrestrial LiDAR point clouds in precision agriculture. ISPRS J Photogramm 104, 112 - 125.

Lee, W.S., Ehsani, R., 2015. Sensing systems for precision agriculture in Florida. Comput Electron Agr 112, $2-9$.

Mack, J., Lenz, C., Teutrine, J., Steinhage, V., 2017. High-precision 3D detection and reconstruction of grapes from laser range data for efficient phenotyping based on supervised learning. Comput Electron Agr 135, 300 311.

Meggio, F., Zarco-Tejada, P.J., Núñez, L.C., Sepulcre-Cantó, G., González, M.R., Martín, P., 2010. Grape quality assessment in vineyards affected by iron deficiency chlorosis using narrow-band physiological remote sensing indices. Remote Sens Environ 114, 1968 - 1986.

Mortensen, A.K., Lisouski, P., Ahrendt, P., 2016. Weight prediction of broiler chickens using 3D computer vision. Comput Electron Agr 123, 319 - 326.

Primicerio, J., Caruso, G., Comba, L., Crisci, A., Gay, P., Guidoni, S., et al., 2017. Individual plant definition and missing plant characterization in vineyards from high-resolution UAV imagery. Eur J Remote Sens 50, 179 186.

Primicerio, J., Gay, P., Ricauda Aimonino, D., Comba, L., Matese, A., Di Gennaro, S. F., 2015. NDVI based vigour maps production using automatic detection of vine rows in ultra-high resolution aerial images. European Conference (10th) on Precision Agriculture, 465 - 470.

Reina, G., Milella, A., Galati, R., 2017. Terrain assessment for precision agriculture using vehicle dynamic modelling. Biosyst Eng 162, 124 - 139.

Sture, Ø., Øye, E.R., Skavhaug, A., Mathiassen, J.R., 2016. A 3D machine vision system for quality grading of Atlantic salmon. Comput Electron Agr 123, 142 - 148.

Srbinovska, M., Gavrovski, C., Dimcev, V., Krkoleva, A., Borozan, V., 2015. Environmental parameters monitoring in precision agriculture using wireless sensor networks. J Clean Prod 88, 297 - 307.

Su, Q., Kondo, N., Li, M., Sun, H., Al Riza, D.F., 2017. Potato feature prediction based on machine vision and 3D model rebuilding. Comput Electron Agr 137, 41 - 51.

Tang, J., Woods, M., Cossell, S., Liu, S., Whitty, M., 2016. Non-Productive Vine Canopy Estimation through Proximal and Remote Sensing. IFAC-PapersOnLine 49, 398 - 403.

Terribile, F., Bonfante, A., D’Antonio, A., De Mascellis, R., De Michele, C., Langella, G. et al., 2017. A geospatial decision support system for supporting quality viticulture at the landscape scale. Comput Electron Agr $140,88-102$

Weiss, M., Baret, F., 2017. Using 3D Point Clouds Derived from UAV RGB Imagery to Describe Vineyard 3D Macro-Structure. Remote Sens 9, 111.

Zarco-Tejada, P.J., Diaz-Varela, R., Angileri, V., Loudjani, P., 2014. Tree height quantification using very high resolution imagery acquired from an unmanned aerial vehicle (UAV) and automatic 3D photo-reconstruction methods. Eur J Agron 55, 89 - 99.

Zhang, N., Wang, M., Wang, N., 2002. Precision agriculture - a worldwide overview. Comput Electron Agr $36,113-132$ 


\title{
Internet-Based Harvest Fleet Logistic Optimisation
}

\author{
Andrés Villa-Henriksen ${ }^{\text {ab, }}$, Nick Skou-Nielsen ${ }^{\text {b }}$, Claus A.G. Sørensen ${ }^{\text {a }}$, Ole Green ${ }^{\text {bc }}$, Gareth T.C. \\ Edwards ${ }^{\text {b }}$ \\ a Department of Engineering, Aarhus University, Aarhus, Denmark \\ b Agro Intelligence ApS, Aarhus, Denmark \\ ${ }^{c}$ Department of Agroecology, Aarhus University, Tjele, Denmark \\ * Corresponding author. Email: avh@agrointelli.com
}

\begin{abstract}
Harvesting operations of cereal crops in a modern farming context often involves multiple vehicles, which can lead to inefficiencies and increase operational costs if they are not coordinated and used appropriately. Large distances from depot to the field, pronounced field topographies or visual barriers, e.g. hedges, can limit the operator's decision capabilities in terms of when and where an unload is taking place, and consequently make the operation less efficient. Moreover, the operation manager, who may be located at the farm office, does not have a clear overview of where the machines are at any given moment, or how far progressed the operation is. Therefore, cereal harvesting is an obvious case for utilising the potential of an internet-based harvest fleet logistic optimisation system - an application that assists the operators and manager in optimising the operation. The system created gives the user a live overview of the operation and vehicles involved, it assists the operator on where and when to unload, and optimises the path in the field to reduce the operational time. The concept system is described with focus on its architecture, its data flow and communication technologies used. The architecture is divided in three layers: sensor layer, communication layer, and application layer. The sensor layer consisting of a yield monitor, that measures the grain mass flow, and a GNSS receiver. The communication layer comprising the gateway. And the application layer covering the database, the data analysis and the interfaces. The system is based on Bluetooth communication between sensors and gateway and $3 \mathrm{G} / 4 \mathrm{G}$ communication between the gateway and the cloud. Android-based mobile devices (tablets) act at the same time as gateways and interface. The system is manufacturer independent and allows any machine to be connected, so it supports the interoperability that many farmers are seeking today.
\end{abstract}

Keywords: harvest logistics, optimised route planning, Internet of Things, agriculture, operation monitoring, Android.

\section{Introduction}

Reduced time windows of field readiness, i.e. trafficability and workability, force many farmers to perform operations hastily and timely non-optimal (Edwards et al., 2016). In addition, the competitiveness of the market pressures farmers to sell their harvest at low price levels that can endanger their business. There is therefore the need of reducing production costs and optimising operation execution times as regard trafficability and workability. In terms of the latter, farmers are compelled to increase the number of vehicles involved in operations such as harvesting to increase capacity. When multiple vehicles take part in harvesting operations of cereal and other grains, it can easily lead to inefficiencies as well as increase operational costs, if they are not coordinated appropriately. However, management of such tasks can be very difficult as the manager does not have a clear realtime overview of the location of the vehicles and when and where on- and off-loadings are happening. Furthermore, the decisions of the operators are also challenged by factors such as large distances from depot to the field, pronounced field topographies or visual barriers, e.g. hedges, especially regarding precise time and location of unloading points. Hence, an internet-based harvest fleet logistic optimisation system can increase the efficiency of harvesting operations, as well as create documentation of yield measurements and operations. Optimising the route plan of a single machine can reduce operating distance and consequently time (Edwards et al., 2017), and these effects increase proportionally the larger the number of vehicles involved in the operation (Seyyedhasani and Dvorak, 2017).

The fast growth of Internet of Things (IoT) technologies in agriculture (Tzounis et al., 2017; Verdouw, 2016), is allowing the automatic collection, storage and sharing of data, which creates new possibilities for machine monitoring and optimisation. Wireless tracking of cotton harvesting operations has been performed using RFID tags (Sjolander et al., 2011), however, the system was offline and did not transfer the data to the internet, nor did it interpret or process the data for posterior yield mapping. Live machine monitoring and performance evaluation has been achieved connecting mobile devices by Wi-Fi communication (Pfeiffer and Blank, 2015), and even though the system performs analytics on the Controller Area Network (CAN) bus data retrieved, which is shared with the operator in the cabin, the system does not optimise the operation and relies on the decisions of the operator. In a similar manner, yield CAN bus and Global Navigation Satellite System (GNSS) data from a combine harvester has been monitored live using $3 \mathrm{G}$ mobile network and OPC Unified Architecture protocols (Oksanen et 
al., 2016), but no fleet logistics optimisation was done.

A novel application that assists operators and managers and optimises harvesting operations is presented. The harvest fleet logistic optimisation system created gives the user a live overview of the operation and vehicles involved, it assists the operator in predicting time and location of future unloads, optimises the path in the field to reduce the operational time, and documents operation performance, e.g. batches, for further actions and analysis. The system employs Android mobile devices for data processing, for communicating the data to the cloud using it as the gateway, and for assisting the operator during the harvest employing it as the graphical user interface (GUI). Moreover, the system includes a web service for live monitoring of the operation, as well as for visualising documented operations from the database, including batches information.

\section{Materials and Methods}

System description

The overall architecture of the system is represented by three layers: sensor layer, communication layer, and application layer (Figure 5), following the common IoT architecture employed in agriculture (Verdouw, 2016). The sensor layer includes the yield monitoring system from the combine harvester and the GNSS receiver. The communication layer comprises a Bluetooth CAN bus adaptor that transfers the data to the Android device, and the Android device is used as the gateway for transferring the information via $3 \mathrm{G}$ and $4 \mathrm{G}$ networks. Finally, the application layer is represented by the server storing the data and the Android mobile device, which computes the data and acts as a GUI, and the web service, which also provides a GUI.

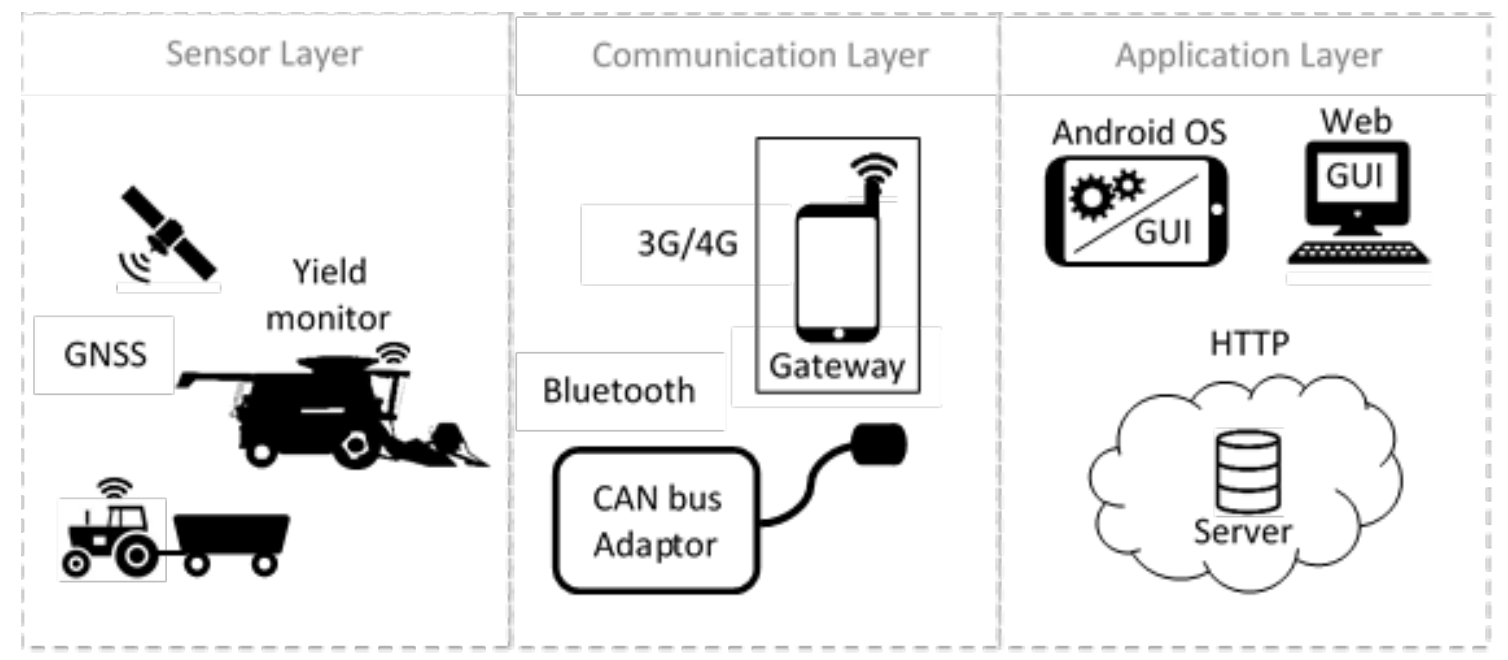

Figure 5. IoT architecture of the harvest fleet logistics optimisation system.

The five elements composing the system are: a Bluetooth CAN adaptor, a harvester mobile application, a service mobile application, a web manager service and a server (Figure 6). The Bluetooth CAN adaptor is a singleboard computer connected to the bus plug, which reads CAN bus messages and transmits them via Bluetooth with a rate of 1 message per second. The CAN adaptor retrieves CAN bus data coming from the following sensors: a mass flow sensor, i.e. an impact plate attached to a load cell; a grain moisture sensor, that measures the capacitance of the grain by passing it through two electrically conductive plates; and a GNSS sensor, e.g. the Real-Time Kinematics Global Navigation System (RTK-GPS) of the harvester. The harvester mobile application receives the CAN bus data via Bluetooth, performs the computations for optimising the route and loading points, and guides the operator via the GUI. The calculations optimise the route according to the field boundaries, working width, number of headlands, the behaviour of the harvester and the service units, i.e. tractors with grain carts, as well as the yield variations measured, so that the optimisation is an on-going process that adapts to any dynamic change in parameters. The field boundaries can be drawn in the web manager and retrieved by the harvester application in json format, or they can be recorded while harvesting the first headland track around the field, which are then stored in the database for any future further use. The service mobile application can receive GNSS data via Bluetooth from a receiver or use the inbuilt GPS of the mobile device. It also computes the route to follow according to the time and location of the unloading points defined by the harvester application, which is then communicated to the operator through the GUI. The communication between harvester and service applications elapses through the internet using $3 \mathrm{G} / 4 \mathrm{G}$ mobile networks using HTTP requests. The web manager retrieves the position of the vehicles, which is displayed live, and the batch information of the loads, i.e. its original location in the field, collected time, its weight and moisture. The final component is the server, which stores the data in an 
SQL-based database (MySQL), which can be retrieved from the web service, as well as it handles the message communications between harvester and service mobile applications and the web service. The combination of CAN adaptor and Android device adapts the combine harvester into a "thing" in an IoT context, expanding operational capabilities.

An important challenge encountered by most IoT based systems is interoperability, not only syntactical due to the great diversity of data formats (Tzounis et al., 2017; Brewster et al., 2017; Martínez et al., 2016), e.g. standardised (ISOXML, agroXML, geojson, etc.), non-standardised (XML, JSON, CSV or other types of TXT), binary (shapefile) or proprietary; but also technical interoperability due to the considerable amount of different wireless communication technologies and protocols (Tzounis et al., 2017; Ray, 2017; Oksanen et al., 2016). In the case presented here, the CAN adaptor shares the data in a proprietary text file, similar to CSV file format, and the GPS data is shared and stored in the server following NMEA 0183 standards, which are both relatively easy to handle formats. Regarding technical interoperability, the use of Android smart devices has been reported to ease some of these issues, as it can easily be programmed through applications development, it can be implemented as IoT gateways for $3 \mathrm{G}$ and $4 \mathrm{G}$ communication, it can include other wireless communication technologies such as Bluetooth, WiFi or Near Field Communication, it complies with standards and protocols that ease communication, and it can also have in-built GNSS geolocation (Hernandez-Rojas et al., 2018; Gao and Yao, 2016; Balmos et al., 2016). In addition, they can have a considerable computing capacity allowing the computations to be performed on the edge, i.e. on the devices employed, in contrast to cloud computing. The popularity of this Linux-based operating system, developed by Google, makes it a relatively cheap solution for easily implementing IoT technologies in agriculture, and are therefore an obvious choice for the harvest fleet optimisation system presented here. The android application was programmed in Java programming language.

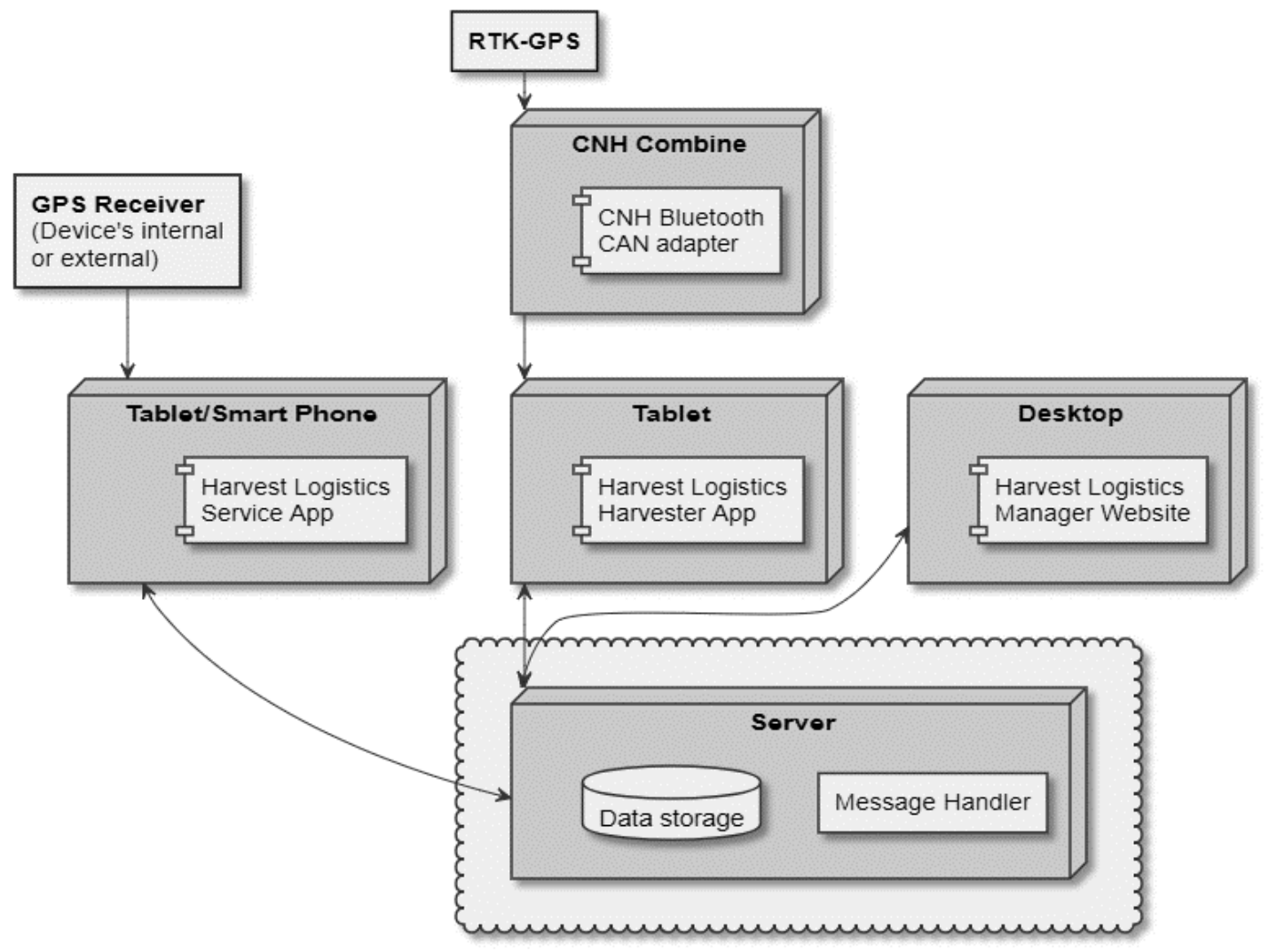

Figure 6. Deployment diagram (generated with PlantUML in Confluence).

Implementation

For testing the functionality and communication of the system, a farm in Havndal, in Jutland (Denmark) was used during their harvesting operations throughout August of 2017 (Figure 7 and Figure 8). For the operations, a New Holland CR10.90 combine harvester was serviced by two tractors with different sized grain carts with 16 and 18 tonnes of capacity, respectively. The harvester was equipped with a Samsung Galaxy S2 tablet running the harvester application, obtaining the yield and GNSS data via the CAN bus adaptor. The tractors servicing the harvester were each equipped with a Huawei Media Pad tablet running the service application, obtaining their position from a QStarz 818XT GPS receiver connected to the tablet via Bluetooth. The service 
application can also run with the internal GPS of the Android device; however, it was chosen to use an external GPS for higher position accuracy. All Android devices were plugged in to the power supply of the vehicle via a USB cable connected through a $12 \mathrm{~V}$ adaptor.

In total, the system was tested in 9 different fields harvesting diverse crops, i.e. rapeseed, rye, wheat and grass seed, each of the crops having different operational characteristics, e.g. different working speeds and yield volumes.

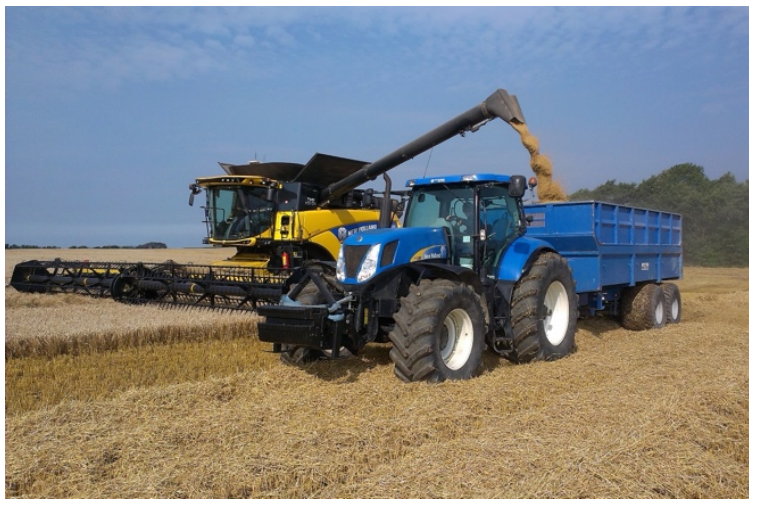

Figure 7. Transferring a load while harvesting to a grain cart.

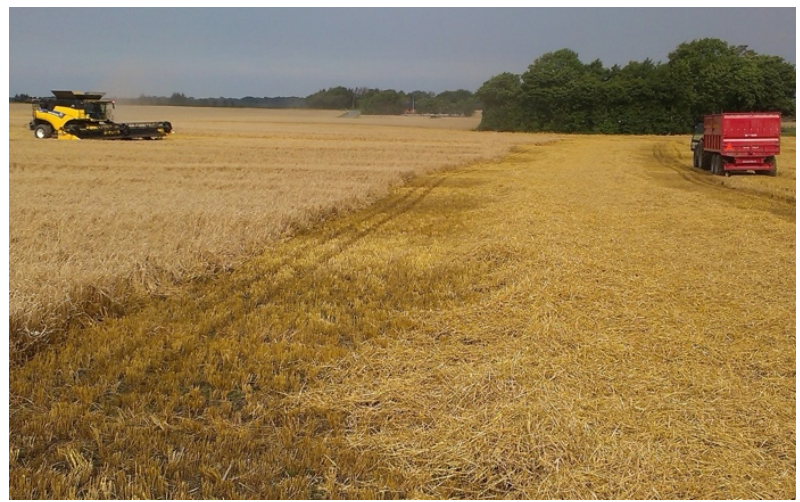

Figure 8. Second grain cart waiting for a load during the operation.

\section{Results and discussion}

The system was able to retrieve position data from the external GPS in the tractors, as well as from the RTKGPS from the harvester through the Bluetooth CAN adaptor, once the pairing and connection was established. The Bluetooth CAN adaptor was also able to transfer CAN bus data from the harvester to the harvester app without bigger issues than the yield sensor calibration, which not being properly calibrated affected the calculations of the unloading points. Some of the crops harvested had more calibration issues than others. Even if a proper calibration of the yield sensors is imperative and would be the optimal (Griffin et al., 2008; Lyle et al., 2014), many farmers do often not engage in such a task; in consequence, since most operators weigh their loads before unloading at the storage this measurement could be used as an input for auto-calibrating the harvest fleet logistics optimisation system.

The Android devices gateway functionality performed a correct communication through the message handler in the "cloud", as long as there was access to the internet via the $3 \mathrm{G}$ and $4 \mathrm{G}$ wireless communication technologies. Even though no internet connection problems were experienced, many rural areas fail to have a decent mobile network (Nakutis et al., 2016), which can limit the functionality of the system. In order to deal with internet connection problems, the system stores the last messages and computes according to them until internet connection is re-established. However, if the connection is not restored in due time, it will start affecting the optimisation, as it cannot update position and yield data. A solution could be to use the Bluetooth connection to actualise the data, when two or more vehicles are in its communication range, which is of a few meters (Jawad et al., 2017). Furthermore, it could also be possible to enhance the system with low power wide area wireless technologies that have kilometric ranges, e.g. LoRa or SigFox (Sinha et al., 2017).

The large amounts of data to be communicated in IoT contexts can become a major limitation, create latency problems and even occasionally imply high expenses of mobile data usage (Jawad et al., 2017; Jayaraman et al., 2016; Tzounis et al., 2017). However, frequently large amounts of the data transmitted to the cloud remain underutilised (Wolfert et al., 2017), meaning that the data transmitted could be limited. Edge computing can ease this challenge as it considerably reduces the amount of data transferred, along with easing the storage capabilities of the server (Ferrández-Pastor et al., 2016). In addition, the computations can be performed in near real-time, when done at the edge. For all these reasons mentioned, the harvest fleet logistics optimisation system successfully employs edge computing, being able to adapt rapidly to yield variations in the field, changes in operation speed, changes in the transport vehicles position or deviations from the optimised route proposed. The data traffic of the mobile application is sending approximately $2.9 \mathrm{~KB} \mathrm{~s}^{-1}$ and receiving $0.9-1.6 \mathrm{~KB} \mathrm{~s}^{-1}$, after the data processing is performed. If there was no processing in the tablet and the full message strings where to be sent, the mobile application would be sending approximately $6.8 \mathrm{~KB} \mathrm{~s}^{-1}$ and receiving $2.4 \mathrm{~KB} \mathrm{~s}^{-1}$, after office testing was made. A reduction of $57.35 \%$ of sent data was achieved. Furthermore, computing at device level not only reduces the amount of data transferred, but also considerably reduces the lag-time if the computations where made in the cloud, achieving near real-time optimisation. The downstream data to be retrieved from the server is minimised to the minimum for the system to function, i.e. the messages include uniquely information of the current status of the vehicle, with a rate of one message per second when the machine is moving and one message every five 
seconds when the machine is still. The stored data in the server was accessible during the operation, and after the operation was finalised, having as well available batch information of the different crops harvested.

Power consumption of the sensors and devices employed is often an important challenge when implementing IoT in agriculture (Ray, 2017; Tzounis et al., 2017; Verdouw, 2016), due to their reduced battery life. Nevertheless, in the case presented here, power consumption is not problematic as sensors and Android devices are plugged to the power supply of the vehicle.

A final but still very relevant issue to be mentioned is privacy and security of the data stored and transferred. The use of authentication protocols, signature and encryption schemes are necessary for ensuring data privacy and security (Tuna et al., 2017; Ranjan and Hussain, 2016; Oksanen et al., 2016; Tzounis et al., 2017). The system includes a username/password authentication procedure in the web service, and it relies on the inherent security of the tablets. Password encryption is used to reduce potential misuse as well as undesired interferences of third parties. Further work on protecting and securing data in the devices, the storage and communication of the system needs to be applied.

\section{Conclusions}

A novel application that assists and optimises harvesting operations was presented. The implemented harvest fleet logistic optimisation system provides a live monitoring of the operation and vehicles involved, and assists the operators with information about unloading time and location, as well as optimising the route, and documenting the operation. The system employs Android mobile devices due to their flexibility and scalability for overcoming challenges such as interoperability. The Android devices fulfilled the following functions: data retrieving, processing and data transferring, as well as assisting the operator through a GUI. Moreover, the system includes a web service for live monitoring of the operation, as well as for visualising documented operations from the database.

The harvest fleet logistics optimisation system could easily access the data from the CAN bus through the Bluetooth CAN adaptor, including yield monitor and GNSS data, as well as communicate the position information of the transport units assisting the combine harvester. However, the adequate calibration of the yield monitor is essential, as it affects the prediction of the load transferring points. The use of $3 \mathrm{G}$ and $4 \mathrm{G}$ mobile networks for communicating the data worked adequately but can present a major impediment in rural areas without a stable mobile network. Besides, the server stored the harvest operation data in the database, which could be monitored live or retrieved later from the database. The amount of data communicated through the internet was minimal to reduce latency problems and ensure the proper functionality of the system.

\section{Acknowledgements}

The authors gratefully acknowledge the Danish project Future Cropping, which has partially financed the development and testing of the system. The authors also gratefully acknowledge the support of the manager and operators of Lisbjerggaard, who allowed us to perform the testing during their farm operations.

\section{References}

Balmos, A. D., Layton, A. W., Ault, A., Krogmeier, J. V and Buckmaster, D. R. (2016) 'Investigation of Bluetooth Communications for Low-Power Embedded Sensor Networks in Agriculture', Transactions of the $A S A B E, 59(5)$, pp. 1021-1029. doi: 10.13031/trans.59.11173.

Brewster, C., Roussaki, I., Kalatzis, N., Doolin, K. and Ellis, K. (2017) 'IoT in Agriculture: Designing a Europe-Wide Large-Scale Pilot', IEEE Communications Magazine, 55(9), pp. 26-33. doi: 10.1109/MCOM.2017.1600528.

Edwards, G. T. C., Dybro, N., Munkholm, L. J. and Sørensen, C. G. (2016) 'Assessing the actions of the farm managers to execute field operations at opportune times', Biosystems Engineering. Elsevier Ltd, 144, pp. 38-51. doi: 10.1016/j.biosystemseng.2016.01.011.

Edwards, G. T. C., Hinge, J., Skou-Nielsen, N., Villa-Henriksen, A., Sørensen, C. A. G. and Green, O. (2017) 'Route Planning Evaluation of a Prototype Optimised Infield Route Planner for Neutral Material Flow Agricultural Operations', Biosystems Engineering, 153, pp. 149-157. doi: 10.1016/j.biosystemseng.2016.10.007.

Ferrández-Pastor, F., García-Chamizo, J., Nieto-Hidalgo, M., Mora-Pascual, J. and Mora-Martínez, J. (2016) 'Developing Ubiquitous Sensor Network Platform Using Internet of Things: Application in Precision Agriculture', Sensors, 16(8), p. 1141. doi: 10.3390/s16071141.

Gao, C. and Yao, K. (2016) 'The Design and Implementation of Portable Agricultural Microclimate Data Acquisition System Based on Android Platform', Proceedings - 2015 8th International Symposium on Computational Intelligence and Design, ISCID 2015, 1, pp. 210-213. doi: 10.1109/ISCID.2015.275. 
Griffin, T. W., Dobbins, C. L., Vyn, T. J., Florax, R. J. G. M. and Lowenberg-Deboer, J. M. (2008) 'Spatial analysis of yield monitor data: Case studies of on-farm trials and farm management decision making', Precision Agriculture, 9(5), pp. 269-283. doi: 10.1007/s11119-008-9072-2.

Hernandez-Rojas, D., Mazon-Olivo, B., Novillo-Vicuña, J. and Belduma-Vacacela, G. (2018) 'IoT Android Gateway for Monitoring and Control a WSN', in Botto-Tobar, M., Esparza-Cruz, N., León-Acurio, J., CrespoTorres, N., and Beltrán-Mora, M. (eds) CITT 2017: Technology Trends. Communications in Computer and Information Science. Springer, Cham, pp. 18-32. doi: 10.1007/978-3-319-72727-1_2.

Jawad, H., Nordin, R., Gharghan, S., Jawad, A. and Ismail, M. (2017) 'Energy-Efficient Wireless Sensor Networks for Precision Agriculture: A Review', Sensors, 17(8), p. 1781. doi: 10.3390/s17081781.

Jayaraman, P. P., Yavari, A., Georgakopoulos, D., Morshed, A. and Zaslavsky, A. (2016) 'Internet of things platform for smart farming: Experiences and lessons learnt', Sensors (Switzerland), 16(11), pp. 1-17. doi: $10.3390 / \mathrm{s} 16111884$.

Lyle, G., Bryan, B. A. and Ostendorf, B. (2014) 'Post-processing methods to eliminate erroneous grain yield measurements: Review and directions for future development', Precision Agriculture, 15(4), pp. 377-402. doi: 10.1007/s11119-013-9336-3.

Martínez, R., Pastor, J. Á., Álvarez, B. and Iborra, A. (2016) 'A testbed to evaluate the fiware-based iot platform in the domain of precision agriculture', Sensors (Switzerland), 16(11). doi: 10.3390/s16111979.

Nakutis, Z., Deksnys, V., Jaruevicius, I., Marcinkevicius, E., Ronkainen, A., Soumi, P., Nikander, J., Blaszczyk, T. and Andersen, B. (2016) 'Remote Agriculture Automation Using Wireless Link and IoT Gateway Infrastructure', Proceedings - International Workshop on Database and Expert Systems Applications, DEXA, 2016-Febru, pp. 99-103. doi: 10.1109/DEXA.2015.37.

Oksanen, T., Linkolehto, R. and Seilonen, I. (2016) 'Adapting an industrial automation protocol to remote monitoring of mobile agricultural machinery: a combine harvester with IoT', in IFAC-PapersOnLine 49-16. Elsevier, pp. 127-131.

Pfeiffer, D. and Blank, S. (2015) 'Real-time Operator Performance Analysis in Agricultural Equipment. Understanding Unused Potential and Ways to Improve from Day to Day', in 73rd International Conference on Agricultural Engineering, LANDTECHNIK AgEng 2015 Proceedings - Innovations in Agricultural Engineering for Efficient Farming. Hannover, Germany. Available at: https://www.researchgate.net/profile/Sebastian_Blank2/publication/283643214_Realtime_Operator_Performance_Analysis_in_Agricultural_Equipment/links/564c $7 \mathrm{~d} 8 \mathrm{e} 08 \mathrm{aeab} 8 \mathrm{ed} 5 \mathrm{e} 9 \mathrm{dcf} 4 /$ Realtime-Operator-Performance-Ānalysis-in-Agricultural-Equipment.pdf.

Ranjan, A. K. and Hussain, M. (2016) 'Terminal Authentication in M2M Communications in the Context of Internet of Things', Procedia Computer Science. The Author(s), 89, pp. 34-42. doi: 10.1016/j.procs.2016.06.006.

Ray, P. P. (2017) 'Internet of things for smart agriculture: Technologies, practices and future direction', Journal of Ambient Intelligence and Smart Environments, 9(4), pp. 395-420. doi: 10.3233/AIS-170440.

Seyyedhasani, H. and Dvorak, J. S. (2017) 'Using the Vehicle Routing Problem to reduce field completion times with multiple machines', Computers and Electronics in Agriculture. Elsevier B.V., 134, pp. 142-150. doi: 10.1016/j.compag.2016.11.010.

Sinha, R. S., Wei, Y. and Hwang, S. H. (2017) 'A survey on LPWA technology: LoRa and NB-IoT', ICT Express. Elsevier B.V., 3(1), pp. 14-21. doi: 10.1016/j.icte.2017.03.004.

Sjolander, A. J., Thomasson, J. A., Sui, R. and Ge, Y. (2011) 'Wireless tracking of cotton modules. Part 2: Automatic machine identification and system testing', Computers and Electronics in Agriculture, 75(1), pp. 3443. doi: 10.1016/j.compag.2010.09.015.

Tuna, G., Kogias, D. G., Gungor, V. C., Gezer, C., Taşkın, E. and Ayday, E. (2017) 'A survey on information security threats and solutions for Machine to Machine (M2M) communications', Journal of Parallel and Distributed Computing. Elsevier Inc., 109, pp. 142-154. doi: 10.1016/j.jpdc.2017.05.021.

Tzounis, A., Katsoulas, N., Bartzanas, T. and Kittas, C. (2017) 'Internet of Things in agriculture, recent advances and future challenges', Biosystems Engineering. Elsevier Ltd, 164, pp. 31-48. doi: 10.1016/j.biosystemseng.2017.09.007.

Verdouw, C. (2016) 'Internet of Things in agriculture.', CAB Reviews: Perspectives in Agriculture, Veterinary Science, Nutrition and Natural Resources, 11(035). doi: 10.1079/PAVSNNR201611035.

Wolfert, S., Ge, L., Verdouw, C. and Bogaardt, M. J. (2017) 'Big Data in Smart Farming - A review', Agricultural Systems. The Authors, 153, pp. 69-80. doi: 10.1016/j.agsy.2017.01.023. 


\title{
Connectivity of Agricultural Equipment and Applications - Strategical Approaches and Technical Standards
}

\author{
Matthias Rothmund ${ }^{\text {a,* }}$ \\ ${ }^{a}$ Competence Center Off-highway Control Systems, OSB AG, Munich, Germany \\ * Corresponding author. Email: matthias@isobus-experts.com
}

\begin{abstract}
Machine to cloud communication becomes an important part of agricultural work processes. For the connectivity of farm equipment to farm management applications, different approaches can be found in the market. Data platforms can be provided by machine manufacturers or farm software providers. In both cases, the 'habitat' in which a farmer works is mainly made out of one 'color', either that machines are all from same brand or farm software applications are mainly coming from the same provider. An open and multi-brand habitat could be realized if all devices in the field an also all cloud applications would follow the same communication standard. The industry is working on standardization, but even then the configuration of data flows, compatibility and reliability is still an issue for the farmer due to many endpoint to endpoint connections. By the approach of a brand-independent data-routing platform, which connects endpoints (machines, communication devices, software applications), this can be covered. All different approaches will play a role in the market due to different customer needs and preferences. The implementation of standards is urgently required to allow interoperability also between different platforms and other entities.
\end{abstract}

Keywords: Connectivity, Platform, Data exchange, Machine to cloud communication, Standardization.

\section{Introduction}

Connectivity plays an emerging role in farming systems. It includes machine to machine $(\mathrm{m} 2 \mathrm{~m})$, machine to business (m2b) and business to business (b2b) communication in which business applications like farm management tools and also on-machinery applications are increasingly cloud-based.

Connectivity in a habitat of cross-brand machinery fleets and a heterogeneous tool landscape is challenging. After having solved this problem for wire-based machinery communication by defining the ISOBUS, we are now facing the challenge to let data flow between machines in the fields and all kinds of applications. The goal is to allow farmers to freely decide for equipment and applications based on their functionality and quality and not based on their brand while being able to easily connect everything.

\section{Materials and Methods}

Cloud-based integration of machines and services

Farm Management Information Systems

Farm Management Information Systems (FMIS) have a long history since the 1970's and a couple of years ago they still were mainly living as local installations on office PCs possibly using internet-based downloads for updating information on materials like seeds, fertilizer, plant protection. Also geographic information on field boundaries etc. was used from connected services already and depending on the country or region, also the data exchange with agricultural administration entities was already possible.

Within the recent years, we have seen a massive shifting to web-based FMIS solutions due to two main advantages: (1) availability independent from determined devices and also on mobile devices and (2) no data or file management or backup concerns for the end user.

As soon as FMISs where accessible in the internet, more and more other service where connected to offer additional services for farmers and farmers now start to combine different specific features from different providers to one user-specific modular system according to their farm-specific requirements and affinities.

\section{Machine process data}

With the availability of ISOBUS systems, tractor and implement process data became available as a by-product of work processes and can be used for farm management applications and precision farming systems. Since the late 1990's, the technical realisation and also the opportunities of using that data for administrative work and field processes were investigated at Technical University Munich.

A summary of the first five years of research on using automated process data acquisition and its use for practical applications was presented in 2004 on Beijing CIGR Conference (Auernhammer et. al., 2004). It could be also shown that a web-based approach is in favour of a PC software solution in terms of data handling, flexibility and availability (Rothmund et. al, 2004). Consequently, the process data acquisition and its usage was 
designed 'as a service' as part of an agricultural service architecture (Steinberger et. al., 2008).

Today, there are more and more telemetry-based devices and services available to receive as-applied and other process data from mobile machinery and also to send prescription maps and other task data to the field equipment to support field work. The purpose of data logging is not only the usage for post-processing anymore. It is also more and more used for real-time applications like fleet-management and quality control or work process optimizing. A further goal is to feed processed information back into the precision farming data circle like for prescriptive application maps for seeding, fertilizing, spraying, tillage. Many ideas on that are more than 30 years old, but with ISOBUS on the one hand and connected machines on the other, it now becomes more easy to realize this in practical farming.

Now, FMIS providers have the challenge to integrate these telemetry based data flows from many various systems into their cloud-based FMIS services. From a physical standpoint this is easy because all entities are available in the internet through a URL or IP address. But interfaces for data content are a pre-condition for functional integration.

\section{Scenarios of connectivity 'habitats'}

Habitat in this context means a communication environment in which a farmer's equipment and farm management applications are connected to each other and also to applications or equipment of their business partners.

\section{Intra-brand habitat}

One product approach for connectivity is to create an intra-brand habitat like some of the big Ag machinery manufacturers do. The first priority of such platforms is to promote the own company's products - as far as available - all along the production chain. To close gaps in the product range for their customers, those companies invite some other players - called partners - to connect their applications or equipment or some retrofitting devices. These kind of platforms do not guarantee discrimination-free access to all providers. The marketing strategies towards the farmers emphasize the seamless easy to use data flow and interfaces. Prominent examples for such platforms are myJohnDeere.com (John Deere, 2018) or Fendt VarioDoc (AGCO, 2018).

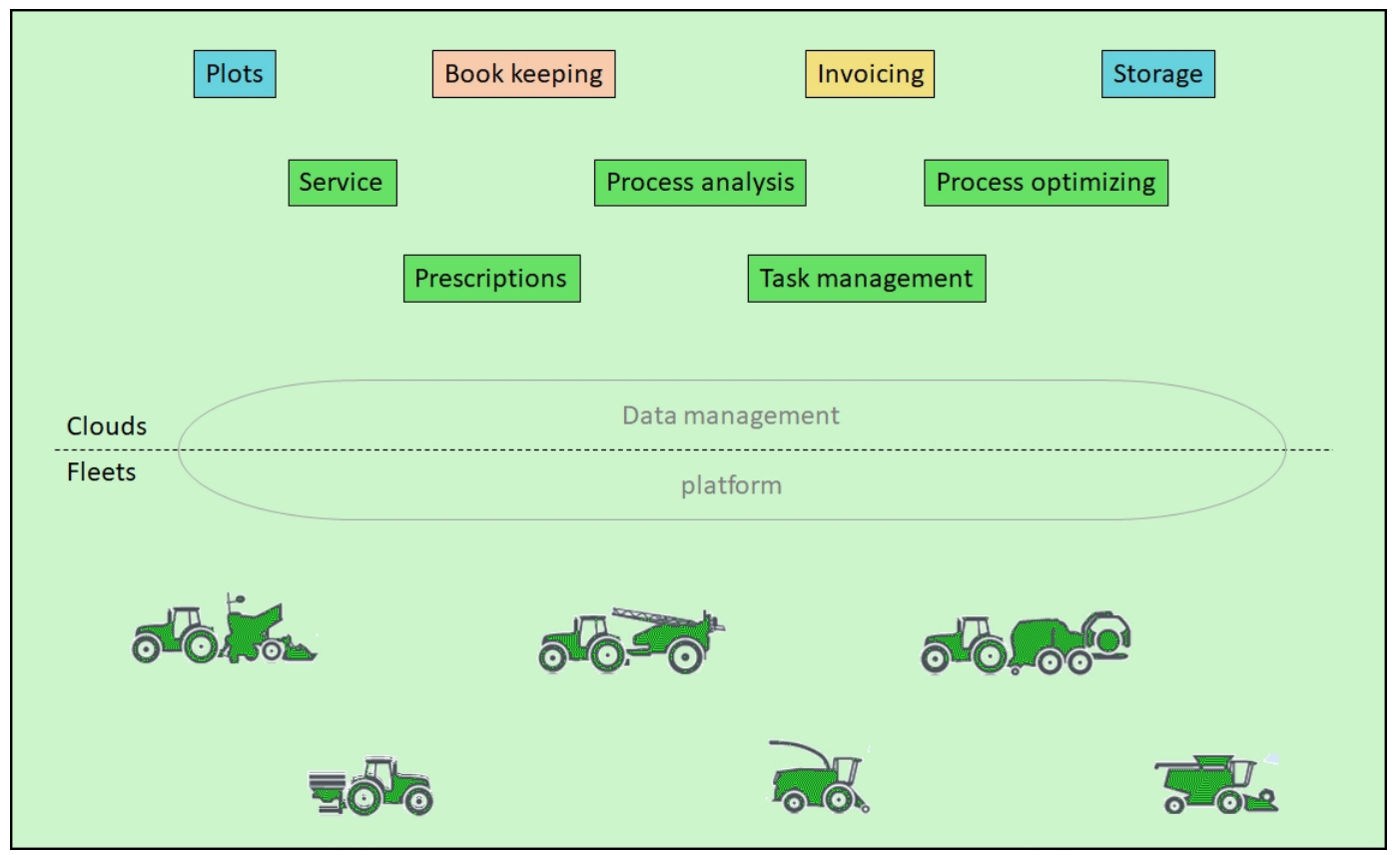

Figure 9. Equipment manufacturer-initiated intra-brand habitat for machine connectivity.

Figure 1 schematically shows such an intra-brand connectivity environment from the farmer's perspective. The whole system is made of products from "one color" except for some of the application modules on the farm management information side. External modules are connected based on the system's proprietary data interfaces. 


\section{Provider-based habitat}

Another connectivity approach is to create a platform for applications independent from tractor or equipment brands like some of the farm software companies do. Thus, different machinery brands can be connected using third-party communication devices or data-exchange standards.

Also in this approach, the main goal of the providing company has to be to promote its own applications and services as far as possible. Only gaps regarding the service product portfolio will be closed by inviting other partners and interfaces will be made available for the steps before and after the product range related to the whole product chain.

In this case, the possibilities of choosing from different products to consider the specific needs of the customer's farm are also limited for the farmer. A Current platform product in the market is for instance 365FarmNet (365FarmNet, 2018).

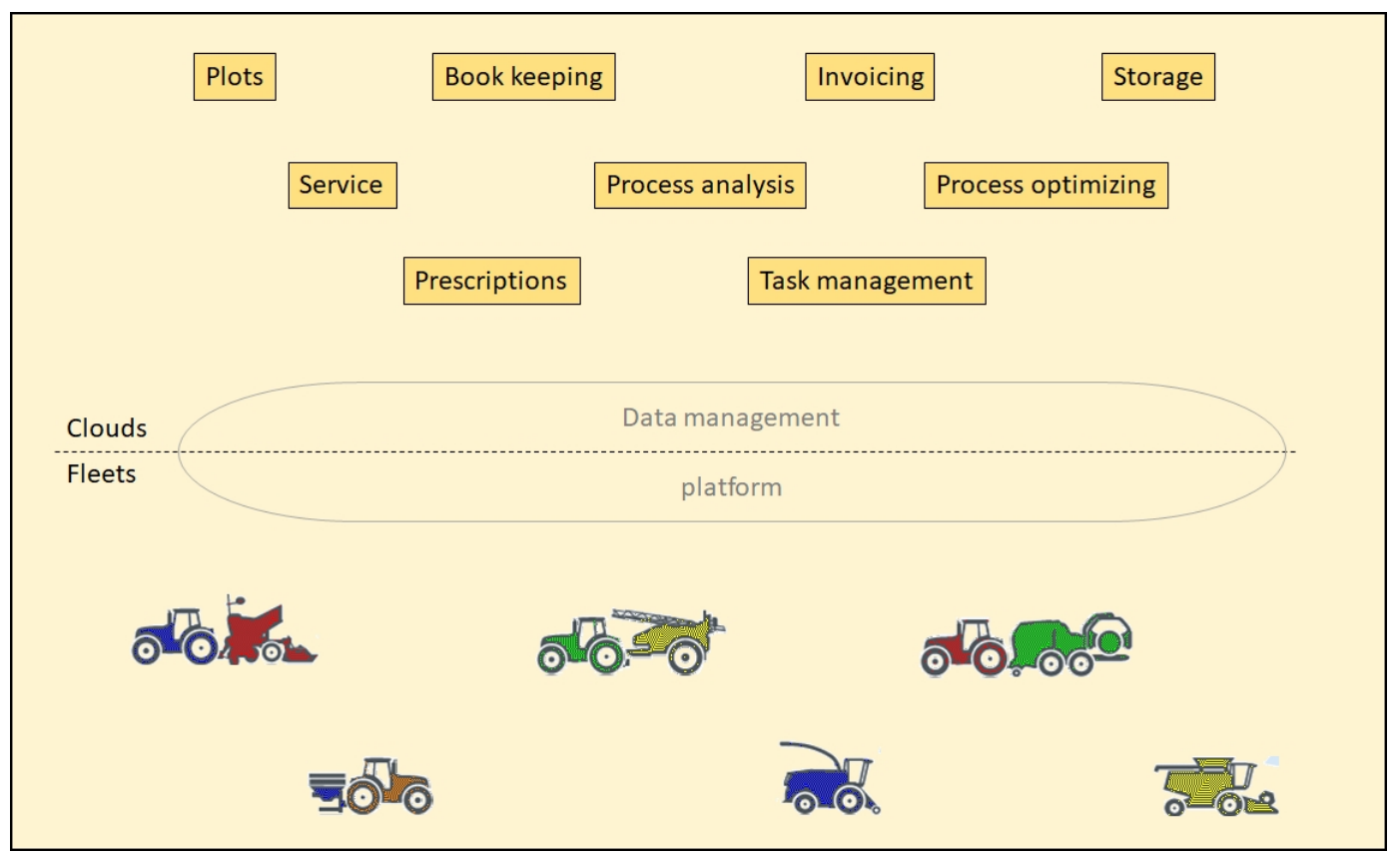

Figure 10. FMIS provider-initiated intra-brand habitat for machine connectivity.

The idea in figure 2 is similar two figure 1, but this time, the whole "color" of the system is the one of the company that provides cloud based farm management software. While different machine brands are connected, the FMIS modules are mainly coming from the platform provider. Potentially, external modules could be connected based on the system's proprietary data interfaces also in this case.

\section{Open individual habitat}

Utilizing cloud-communication standards and M2C data exchange standards, customers could build their own habitat containing many different applications from different providers and being connected to their mixed fleet of different brands of tractors and implements. A pre-condition is that all products work with standardized interfaces relying on a few norms or standards. This is currently not the case. 


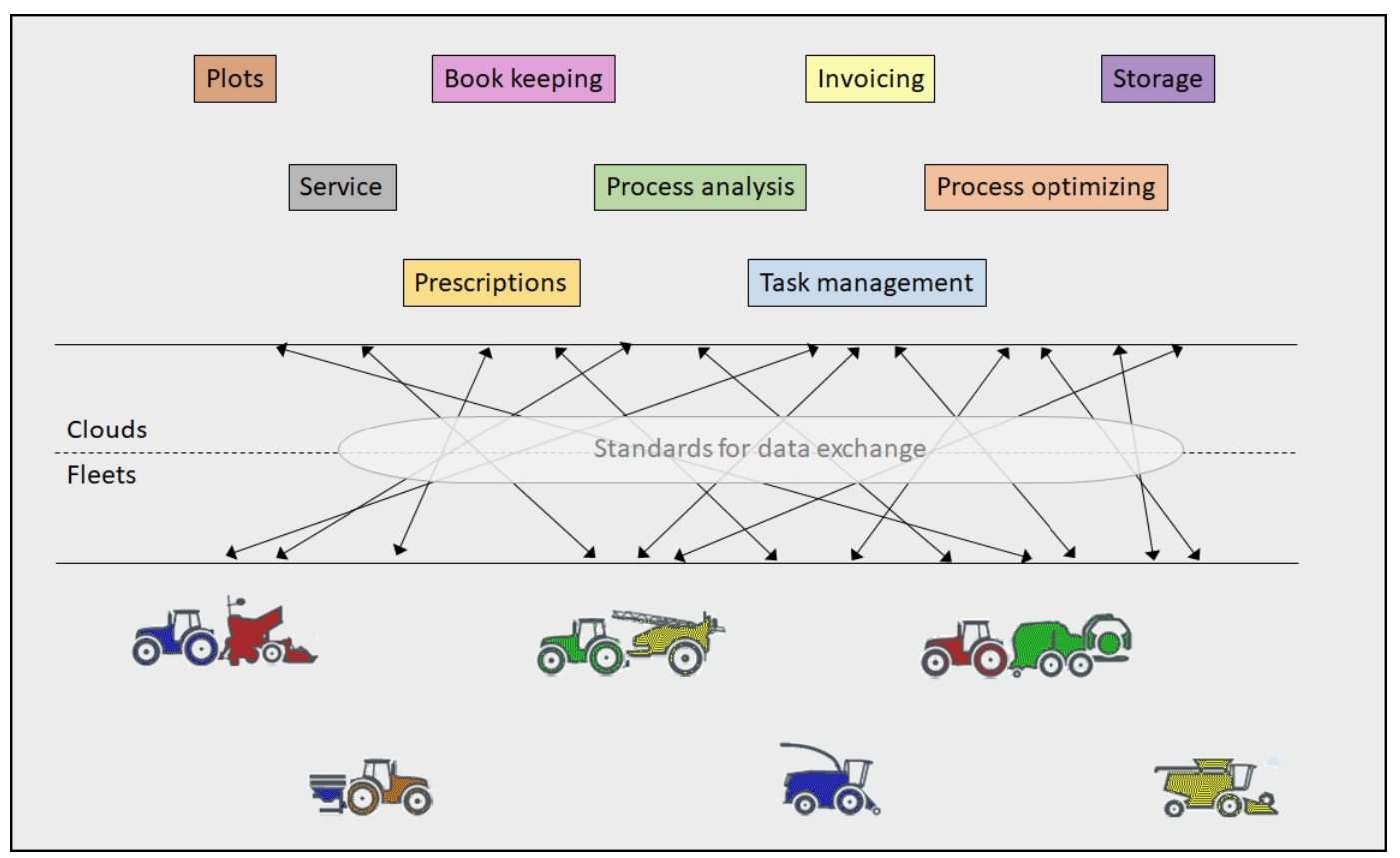

Figure 11. Open habitat for machine connectivity based on standardized data interfaces.

Like shown in figure 3, generally each farmer could freely choose the best product for his purpose for each single application, if each machine or data communication device and also each farm management software module would support the same standardized data interface.

\section{Results and discussion}

Standardization

To allow farmers and contractors to work in an open space like sketched in figure 3, the industry is working on an corresponding standardization project within its organisation called Agricultural industry Electronics Foundation (AEF, 2018).

Today, a standardized data exchange format called ISOXML exists already as part of the ISOBUS standard in ISO 11783-10. This is designed for transfer task data, prescription maps, documentation between farm office software and ISOBUS compatible field equipment. The data content is exchanged in XML and referring binary files. Some FMIS systems and ISOBUS terminals provide means to exchange the files via internet services, but most of the times, USB sticks are used.

The current ISOXML definition is not fitting future requirements of wireless and continuous data exchange and therefore in the AEF project, an Extended FMIS Data Interface (EFDI) is defined. This format will be capable of transferring the existing ISOXML data content as well as additional contents in terms of continuous process data transfer and incremental task information updates.

Whereas, it is necessary to add authentication and data description information to make the interface work throughout an open system with a number of non-distinct endpoints. Furthermore, an efficient data coding and serialization has to be chosen to reduce protocol overhead and thus allow a reliable data transfer via internet even with smaller bandwidths.

Proposed technologies besides the - compared to ISOXML - extended data model are Google Protocol Buffers (Google, 2018) for highly efficient data serialization and MQTT (MQTT, 2018) as transfer protocol in which also other protocols are still discussed and could be allowed in parallel.

Agrirouter as one example of a non-discriminating connectivity platform

leaves A standard as described under chapter 4 still lets a lot of responsibility at manufacturers and providers to make single components and apps working at the data interface. It also still keeps it complex for the single farmer to create his own system that really works seamlessly and reliable.

As an idea to cover that issue, figure 4 shows a scenario based on the same variables as the other scenarios before, but with a data-routing platform to rout data between connected endpoints. 


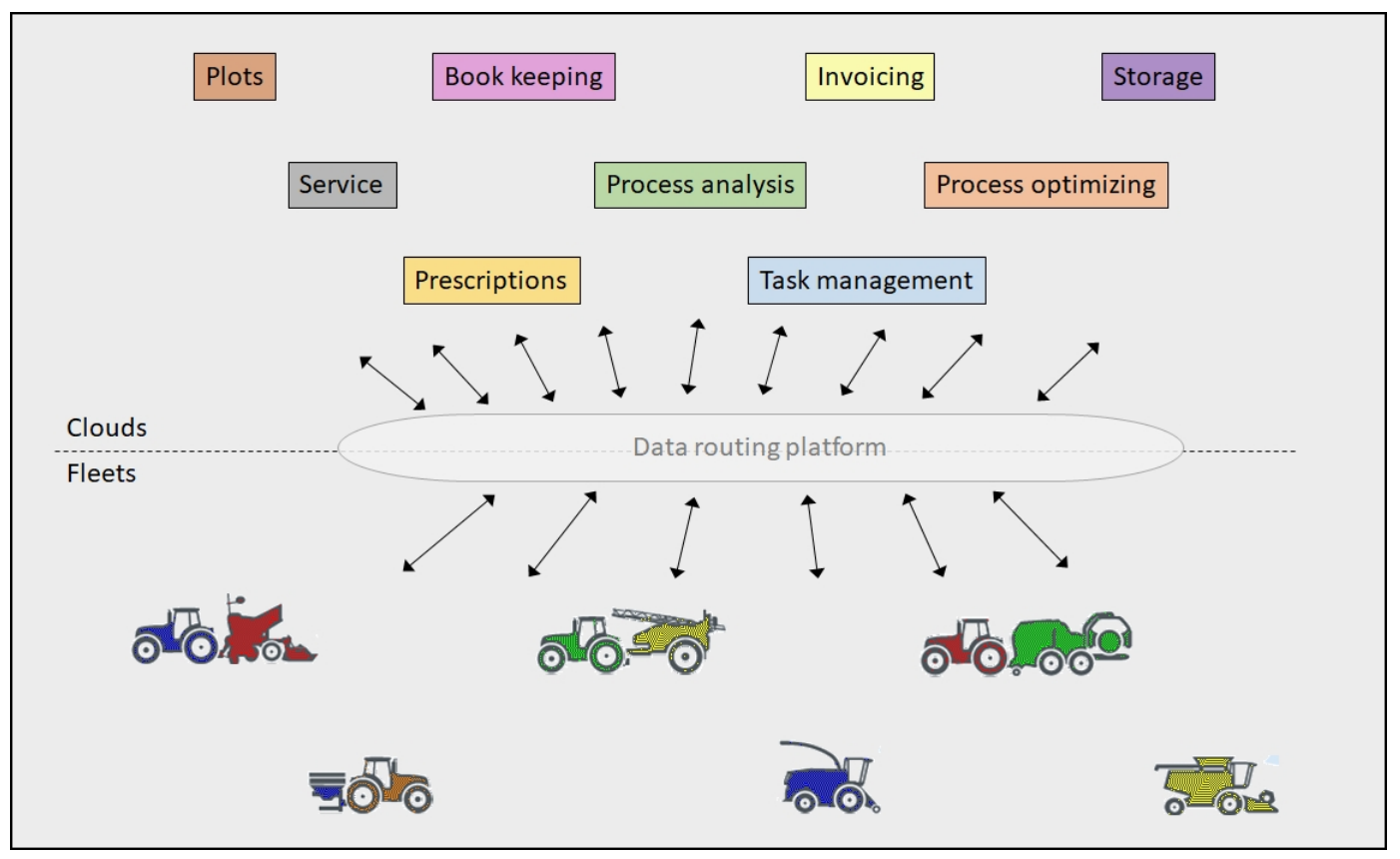

Figure 12. Open habitat for machine connectivity based on a data-routing platform.

A number of tractor and implement manufacturers founded a company called DKE-Data GmbH to create a common, brand-independent, discrimination-free connectivity platform as a product. The idea of this product, which is called agrirouter, is to create a configurable data-routing and temporary buffering for each farmer in his own cosmos. Each farmer has his own agrirouter where he configures which data are allowed to flow from one to another entity (machine or cloud application). This way, he cannot only allow data flow within his own fleet and apps but also from or to his contractors or suppliers (DKE-Data, 2018).

With such an data-routing approach, the farmer can still freely choose his machines, devices, farm management modules like in figure 3 , but at the same time it reduces complexity for the farmers and for manufacturers and app providers. Of course, the freedom of choice is limited to the apps that provide a connection to agrirouter, but anyone is invited to realize that.

One benefit is the expected improvement of stability respectively the reduced expected compatibility issues in data flows of this "endpoint to router" model compared to the "endpoint to endpoint" model as the number of the connections to be tested and verified between entities is remarkably reduced. Each device or app that has once passed the on-boarding process to the agrirouter, is automatically compatible and available to all other onboarded devices or apps. At the same time, each farmer keeps full control of each single data endpoint to endpoint data connection through his user-defined data flow configuration.

By using the EFDI standard for the ISOBUS-related data flows, the agrirouter platform additionally facilitates the on-boarding of devices and apps for the manufacturers. The use of EFDI is of course also recommended for other platforms whereby the interoperability between platforms will be enabled.

Some of the tractor manufacturers will not directly connect their machines to the platform as sketched in figure 4 , but will route the data first through their own data servers to the agrirouter which does not affect the functionality of the overall system.

\section{Conclusions}

Connectivity is one of the big trends in agricultural engineering next to progressing automatization. Besides the technological challenges and the potential benefits for farming, in this case also the possible risks are very intensely discussed. Data security on the technical side as well as trust in customer relations and legal regulations on the non-technical side are very important keywords for connected systems. In this context, different strategical approaches for connectivity platforms are subject of now and then emotional disputes among farmers or between farmers and manufacturers.

The approach of some big manufacturers and farm software providers, to collect all machine-related and fieldrelated data on company-owned data bases and connect their own or third party applications on that basis, makes customers feeling scared while others see the advantages of guaranteed compatibility. To realize a complete open space where everything can be connected just based on communication standard currently fails due to not finished standardization. It is assumable that inherent complexity and expectable compatibility issues in this approach will 
also prevent many users from using many different specific connected applications.

The open data-routing approach, which is independent from manufacturers' interests and potentially connects many different devices and apps seems to be a good compromise to benefit from both a big choice of apps and a reliable data connectivity.

However, there will be a number of different platforms and single entities which still should be able to exchange data between each other. For this purpose, the standardization, which was recently kicked-off in AEF, will play a very important role. Certified implementations of well-defined standardized interfaces will be the basis for an increasing acceptance in the market, what gives farmers the opportunity to benefit from the potentials of connectivity.

\section{Acknowledgements}

Not officially published contents, experiences, knowledge of AEF and DKE-Data were partly the basis for this paper. They were generated with the author's contribution but also with collaboration of many other people of the member companies of both organisations.

\section{References}

365FarmNet, 2018. 365FarmNet Demo. https://demo.365farmnet.com/365FarmNet/dist/index.html\#/dashboard Accessed, June 8, 2018

AEF, 2018. The AEF - Ag Industry's initiative in electronic standards implementation. https://www.aefonline.org/fileadmin/user_upload/Content/pdfs/AEF_Keynote_EN.pdf Accessed, June 8, 2018

AGCO, 2018. VarioDoc and TaskDoc ${ }^{\circ}$. https://www.agcotechnologies.com/products/detail/variodoctaskdoc/ VarioDoc Support and Training. https://www.agcotechnologies.com/support-and-training/variodoc/ Accessed, June 8, 2018

Auernhammer, A., M. Rothmund, 2004. Automated Process Data Acquisition within standardized Communication Systems and its Practical Application. In: Olympics of Agricultural Engineering, CIGR International Conference, Beijing, 2004; Vol. 2, p III-93.

DKE-Data, 2018. DKE-agrirouter, My-agrirouter. https://my-agrirouter.com/ agrirouter Produktdemo. https://my-agrirouter.com/fileadmin/additionals/demo/index-mouse-links/index.html. Accessed, June 8, 2018

Google, 2018. Protocol Buffers. https://developers.google.com/protocol-buffers/. Accessed, June 8, 2018

John Deere, 2018. Discover MyJohnDeere. https://discoveroperationscenter.com/en. Accessed, June 8, 2018.

MQTT, 2018. MQTT. http://mqtt.org/ Accessed, June 8, 2018

Rothmund, M., A. Auernhammer, 2004. A web based information system for process data designed with open source tools. In: AGENG - Engineering the future, Leuven, 2004.

Steinberger, G., M. Rothmund, H. Auernhammer, 2009. Mobile farm equipment as a data source in an agricultural service architecture. Computers and Electronics in Agriculture. 65 (2), 238-246. https://www.sciencedirect.com/science/article/pii/S0168169908002226. 


\title{
Analysis of Vine Vibration Conditions and Grape Harvest Quality in Spain under Actual Mechanized Harvest Conditions
}

\author{
Wilson Da Costa Neto ${ }^{\text {a, b }}$, Pilar Barreiro ${ }^{\text {b,* }}$, Belén Diezma ${ }^{b}$ \\ ${ }^{a}$ Campus Dom Pedrito. UNIPAMAPA. \\ ${ }^{\mathrm{b}}$ Laboratorio de Propiedades Físicas y Técnicas Avanzadas en Agroalimentación. ETSIAAB, Universidad Politécnica de \\ Madrid, Madrid, 28040, Spain \\ * Corresponding author. Email: pilar.barreiro@upm.es
}

\begin{abstract}
Grape harvest mechanization is taking place worldwide since the 90s of last century. However, little effort has been made in the systematic definition of Key Performance Identifiers (KPIs) for the characterization of the quality of mechanized tasks. Moreover, standardized automated procedures are required for the comparison of mechanized tasks irrespective machine manufacturers, field conditions, operators or many other local conditions. This study aims at providing a KPI for the characterization of vibration conditions (by means of accelerometers) as well as the quantification of grain and must losses, in this last case by means of making visible those losses that are invisible to the naked eye. To this end georeferenced data from 19 plots (42.3 ha) have been gathered in order to characterize mechanized work performance, together with corresponding grain and must losses. On the other hand, vine vibrations have been analysed by placing 4 accelerometers ( 6 channels) in dedicated positions of the vine, and computing the time and frequency response, as well as the power spectra normalized in terms of vine productivity $(\mathrm{kg})$. The results indicate that outliers found in grape losses (must and grain) can be explained in terms of the power spectra information, pointing to deficient regulation of the machines under daily circumstances.
\end{abstract}

Keywords: fleet management, harvest quality, FFT, mechatronics, sensor, automated control

\section{Introduction}

The quality of grape harvest mechanization depends on the interaction between the beater of the harvester and the structure of the plant. The main operating parameters of the mechanism that can be adjusted in the harvesters to optimize the results of their performance are beating amplitude and frequency (Chengsong, et al. 2015; Caprara and Pezzi, 2011). The transmission of the vibration through the vine is a complex phenomenon affecting the losses of grain and must and that can be monitored by accelerometers (Caprari and Pezzi, 2009; Du, X. et al. 2012). To establish relationships between the vine vibrations response and losses during harvest could help in selecting the best operating condition of the harvesters.

Moreover, a recent study (Da Costa Neto et al. 2018b) provides a calibration equation regarding the conversion between the percentage of must covering the leaves of vines with regard to the apparent must losses either in terms of 1/vine or 1/ha, as to allow comparisons between our methodology (Da Costa Neto et al. 2018a) and that presented by other researches such as Pezziet al. 2009 and 2015.

The LPF_TAGRALIA has been focused on the process of grape harvest mechanization and precision viticulture for over a decade now (Barreiro et al, 2009, Baguena et al., 2009 \& 2011) facing on-the-go yield sensing, and vine yield non-destructive estimation (Herrero et al., 2010; Diago et al., 2012; Correa et al. 2012), as well as using such information under a several year analysis. However lately, it is the evaluation of actual mechanized conditions and aggressively which has captured our interest given that South America is starting the process (da Costa Neto et al. 2017)

In this context, the objectives of this work have been a) to identify relationships between the grape harvest quality and the regulations of harvesters under actual mechanised conditions in Spain, b) to define standardized automated procedures for the comparison of mechanized tasks.

\section{Materials and Methods}

Characterization of grape harvest at experimental plots

Table 1 provides some detail on the grape harvesters used: $\mathrm{CNH}$ and Pellenc, together with their field performance in terms of effective and accessory time (TE -s- and TA -s-), and actual work capacity (Wca, ha h1) and processing ability ( $\mathrm{kg} \mathrm{h}-1)$ in the field experiments.

Grape losses due to mechanical harvest were assessed by counting and weighing the grapes on the soil (once grapes on the soil before harvest had been removed), while those grapes remaining on the vines after harvesting were expressed as percentages of vine productivity $(\mathrm{kg})$. The former is related to machine head losses, while the latter is an indicator of insufficient vibration. On the other hand, must release during mechanical harvest can be evaluated by means of water sensitive papers during harvest with varying harvester settings (shaking frequency and amplitude) and grape types (white or red) as reported by Da Costa Neto et al. (2018a); must release was 
evaluated by means of water sensitive papers during harvest with varying harvester settings (shaking frequency and amplitude) and grape type (white or red) and variety. Water sensitive papers were at several places in the vines as described at da Costa et al, 2018a, while image analysis was performed according to Costa et al. (2017) providing the percentage surface in blue (reacting to moisture). For assessing average must losses per vine in terms of total canopy and soil coverage (\%), a standard surface was considered per vine: $0.96 \mathrm{~m} 2$ at soil level (A and $\mathrm{B}$ ), and $2.88 \mathrm{~m} 2$ for the canopy (C to F). Must release was then normalized regarding vine productivity as percentage of canopy coverage per vine yield $(\% \mathrm{ckg}-1)$ as to compensate for grape type.

Table 1. Details on the grape harvesters used: $\mathrm{CNH}$ and Pellenc, together with field performance in terms of effective and accessory time (TE -s- and TA -s-), and actual work capacity (Wact, ha h-1) and processing ability (C_PR act, $\mathrm{kg} \mathrm{h}^{-1}$ ).

\begin{tabular}{|c|c|c|c|c|c|c|c|}
\hline Model & VARIETY & TYPE & $\begin{array}{c}\text { GEO- } \\
\text { METRY }\end{array}$ & TA & TE & $\begin{array}{c}\text { Wact } \\
\left(\text { ha h }^{-1}\right)\end{array}$ & $\begin{array}{c}\text { C_PR_act } \\
\left(\mathrm{kg} \mathrm{h}^{-1}\right)\end{array}$ \\
\hline $9090 X$ & AIREN & White & SQR & 16584 & 21024 & 0.66 & 15197 \\
\hline $9090 X$ & MERLOT & Red & STD & 3394 & 5310 & 0.60 & 6642 \\
\hline $9090 X$ & MERLOT & Red & R41 & 5696 & 9016 & 0.64 & 7076 \\
\hline $9090 X$ & MERLOT & Red & R41 & 2370 & 11380 & 1.05 & 11562 \\
\hline $9090 X$ & MACABEO & White & $\mathrm{R} 21$ & 2180 & 2576 & 0.65 & 7169 \\
\hline $9090 X$ & TEMPRANILLO & Red & R41 & 130 & 590 & 1.16 & 8218 \\
\hline $9090 X$ & SYRAH & Red & R41 & 2444 & 3616 & 0.76 & 3386 \\
\hline $9090 X$ & TEMPRANILLO & Red & R21 & 2228 & 2462 & 0.73 & 5182 \\
\hline $9090 X$ & SYRAH & Red & $\mathrm{R} 21$ & 862 & 1726 & 0.97 & 4334 \\
\hline $9090 \mathrm{X}$ & SYRAH & Red & R41 & 958 & 1504 & 0.92 & 3434 \\
\hline $9090 X$ & TEMPRA & Red & $\mathrm{R} 21$ & 2970 & 4626 & 0.85 & 6039 \\
\hline $9090 X$ & TEMPRA & Red & $\mathrm{R} 21$ & 1968 & 3188 & 0.92 & 6510 \\
\hline $9090 X$ & TEMPRA & Red & $\mathrm{R} 41$ & 990 & 1144 & 0.79 & 5599 \\
\hline 8015 & AIREN & White & R41 & 4194 & 12892 & 0.57 & 4407 \\
\hline 8015 & AIREN & White & STD & 8308 & 20534 & 0.64 & 4943 \\
\hline SB65 & $\mathrm{CS}$ & Red & STD & 3670 & 8889 & 0.56 & 4550 \\
\hline SB65 & $\mathrm{CS}$ & Red & & & & & \\
\hline SB65 & AIREN & White & BPL & 4235 & 9831 & 0.65 & 14883 \\
\hline
\end{tabular}

Vibration analysis

The vibration responses of the vines can be analysed trough the time domain signal, quantifying shaking time or characteristic values of acceleration, or through the frequency domain signal, obtained applying the Fast Fourier Transform algorithm.

Four accelerometers: one triaxial (B0, B1 and B2) and three uniaxials (A0, A1 and A2) were respectively attached to the trunk and brunches of a vine located inside the vineyard far from the borders of the plots as indicated in Figure 1, while the excitation for the measurement of the acceleration was based on the direct effect of the grape harvester.

From the frequency domain signal and the corresponding normalized power spectra $\left(\mathrm{W} \mathrm{h} \mathrm{kg}^{-1}\right)$, both resonant frequency $(\mathrm{Hz})$ and its amplitude were extracted as referred by Pezzi et al. 2013; Caprara and Pezzi, 2011.

\section{Results and Discussion}

Performance of machines with different grape varieties

The grape varieties harvested amount to 4 plots of Airén (harvested with 3 grape harvesters), 3 plots of Cabernet Sauvignon (SB6560), 3 plots of Merlot (9090X), 1 plot of Macabeo, 3 plots of Syrah and 5 plots of Tempranillo (9090X) to which the median of losses in the soil and plant are below $1.7 \%(0.45 \%$ in white and $1.7 \%$ red varieties) under different productivity conditions. 


\section{9090 SYRAH 480}
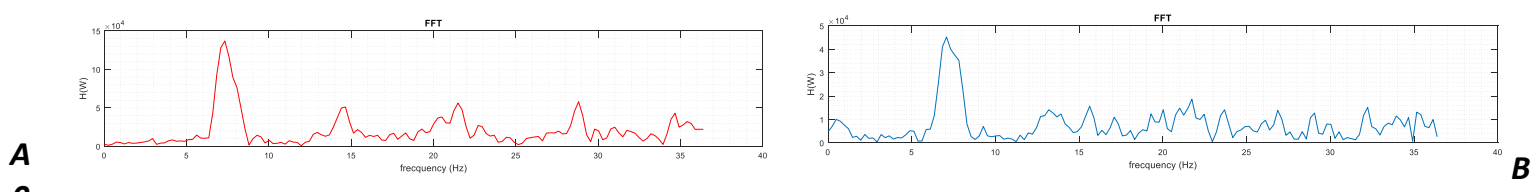

0
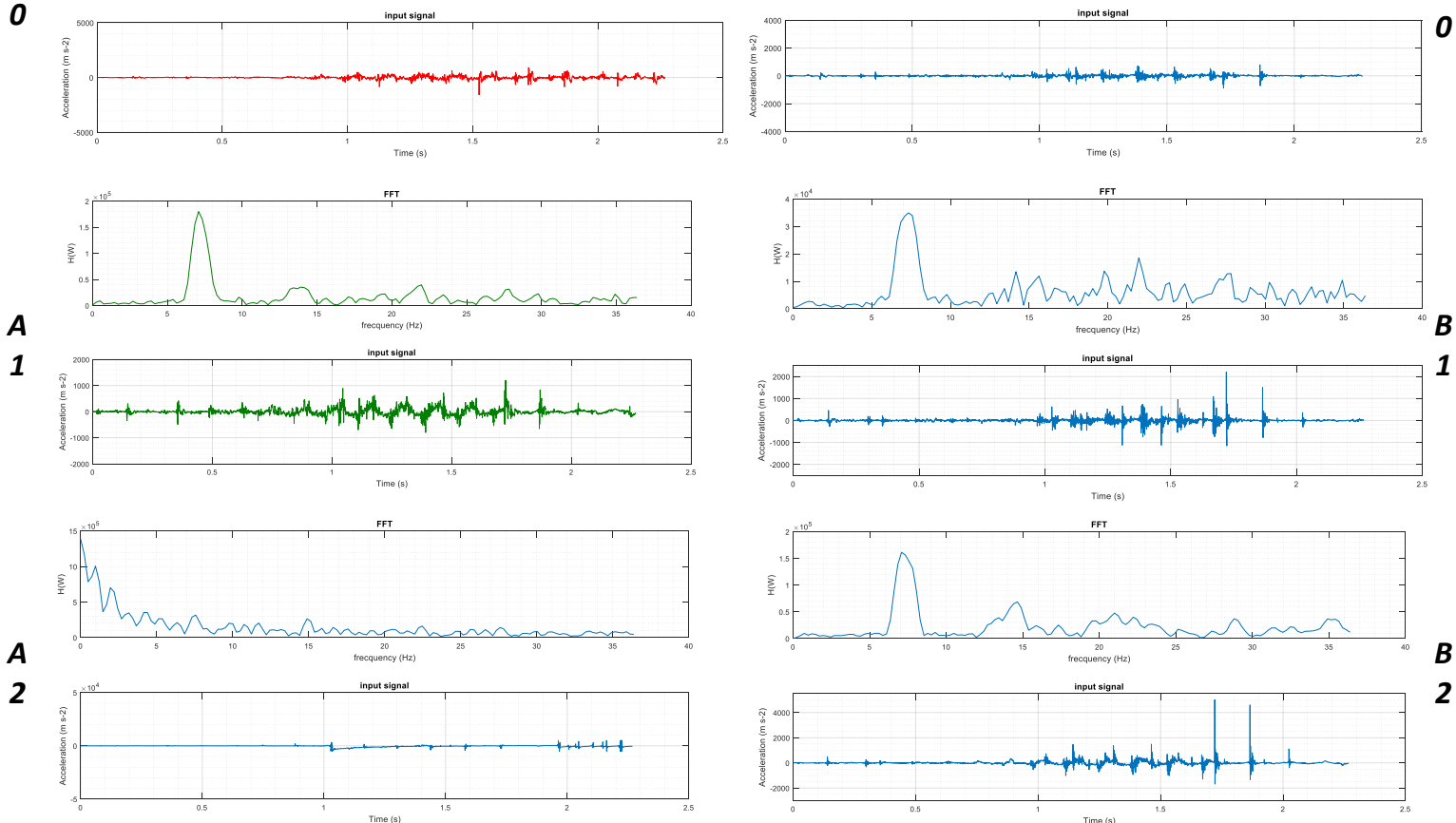

Variedad: Syrah

$N^{\circ}$ varillas: 14

BPM teórico: 480

BPM real: 425

Area de avaluación de perdidas de hojas: $0.325 \mathrm{~m}^{2}(0,25 \times 1.3 \mathrm{~m})$

Perdida de hollas: 20 (80-60)

C_Proc. $=3386 \mathrm{~kg} / \mathrm{h}$

Perdidas uvas $(S+P)=2.83 \%$

Recobrimiento de mosto $=0.052 \% / \mathrm{kg}$
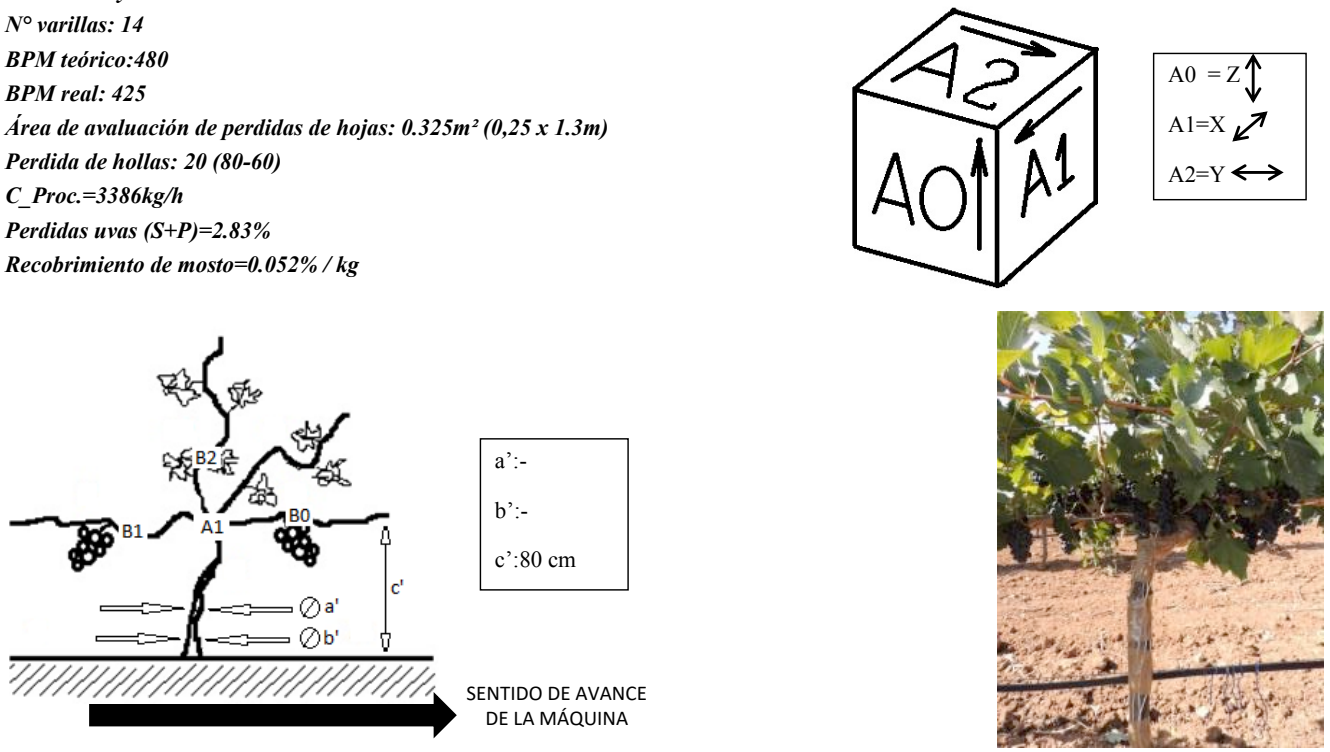

Figure 1. Field experiment and data processing process for variety Syrah, and machine 9090 with $480 \mathrm{rev} \mathrm{min}^{-1}$ setting.

Figure 2 (up) shows the results of the grape losses (\%) with respect to actual process capacity $\left(\mathrm{kg} \mathrm{ha}^{-1}\right)$ in the 18 plots evaluated (42.3 ha in total); two trends can be found in Figure 2 (left), regarding the relationship between the process capacity of the machine (kg h-1) and the hidden losses as stated by must release, that correspond to red and white varieties respectively. Only one outlier is found that corresponds to Cabernet Sauvignon in the plot with machine vibration conditions presented in Figure 3. 

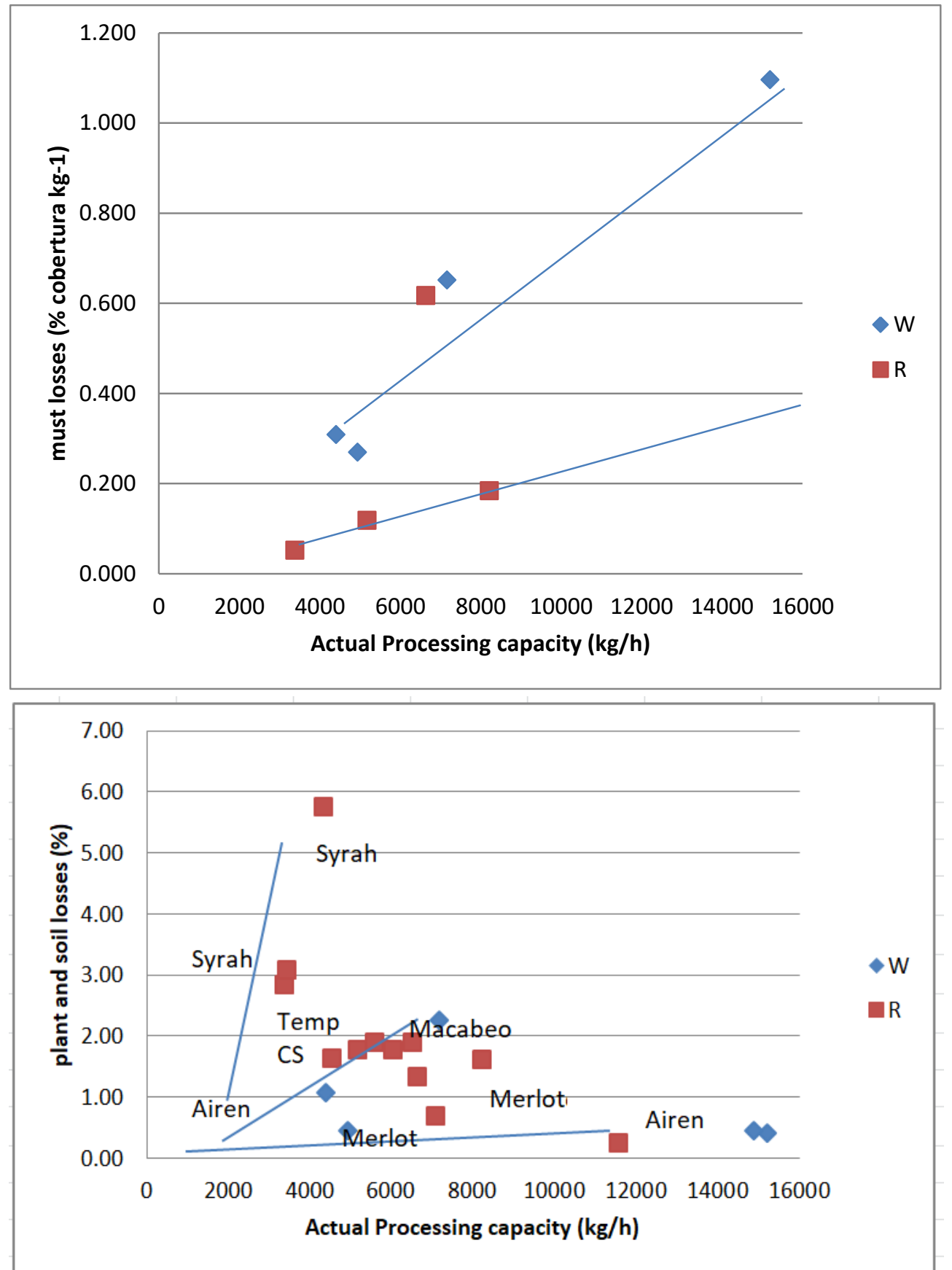

Figure 2. Grape losses with different varieties (top), and hidden losses (must release \%, top) with respect to Actual capacity of processing $\left(\mathrm{kg} \mathrm{h}^{-1}\right)$.

In Figure 2 (down), three trend lines can be observed that gather all results: losses below 1\% for Merlot and Airén (NH 9090X and Pellenc 8015) with respect to a process capacity of almost 5000 to $15000 \mathrm{~kg} \mathrm{ha}^{-1}$; losses lower than $2 \%$ in the varieties corresponding to Airén, Merlot, Cabernet Sauvignon, Tempranillo and Macabeo and the lowest values of process capacity (4000 to $\left.6000 \mathrm{~kg} \mathrm{ha}^{-1}\right)$, suggesting that this is the normal value or acceptable for losses of grapes; only the Syrah (NH9090X) presented losses close to or above 3\%, in the three plots and similar CP_act (3800 to $4300 \mathrm{~kg} \mathrm{ha}^{-1}$ ). However, most of the results are considerably low compared to (Caprara, 2009; Troncoso et al., 2002) who found an average of 3.1\% in 20 plots, of which 12 were Cabernet Sauvignon, 2 Merlot and other grape varieties not considered in this study. Caprara and Pezzzi, 2009 also found an average of total grape losses lower than 5\% and maximum $8.7 \%$ for Lambrusco Grasparossa, with grapes losses on the soil amounting to 2.0 to $2.8 \%$ which were influenced by the frequency of vibration ( 380 to $460 \mathrm{bpm}$ ). These values are higher than those found in our study when only soil losses are considered: $0.63 \%$ and $0.73 \%$ for white and red varieties). 
Analysis of hidden loses

When the hidden losses are analysed in relation to the actual process capacity of the machine comparing red and white grapes, it is found that hidden losses for white grapes tend to be higher than for red varieties under similar actual process capacity (Figure 2, up). As stated, some outliers are found in Figure 2 down corresponding to Cabernet Sauvignon which may be attributed to specific field conditions, such as excessive amount of leaves, higher yield, inadequate centring of the machine with regard to the vine line, and/or the potentially inadequate frequency range of the shafts in relation to the grape variety and its ripening stage.

Still, Figure 2 up shows two trend lines for white $\left(r^{2}=0.94\right)$ and red $\left(r^{2}=0.97\right)$ grapes that enable the estimation of the hidden losses under mechanical harvest. These values are comparable to those of L.Pari, 2013 with field losses of $23.6 \%$ for trailed harvester and $9.6 \%$ for self-propelled during the harvest of the variety Trebbiano Romagnolo white using frequencies between 450 to $700 \mathrm{rev} \mathrm{min}^{-1}$. In our research the vibrations used in white were between 420 and $480 \mathrm{bpm}$ and the must release $\left(\mathrm{m}^{2}\right.$ of surface) for the white varieties resulted in $3.8 \%$ to $12.4 \%$, and the must losses from the trailed harvester were slightly above the values disclosed by these authors through a self-propelled harvester.

\section{CSB65CST420}

A
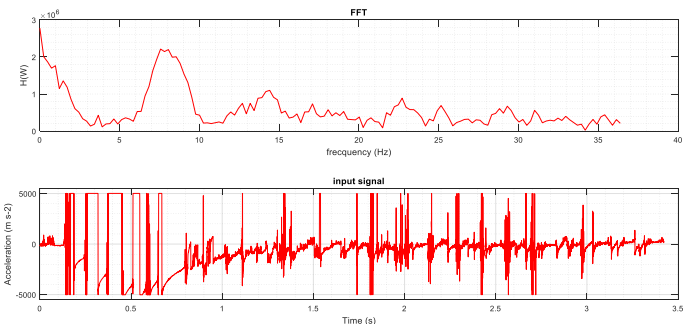

A

1
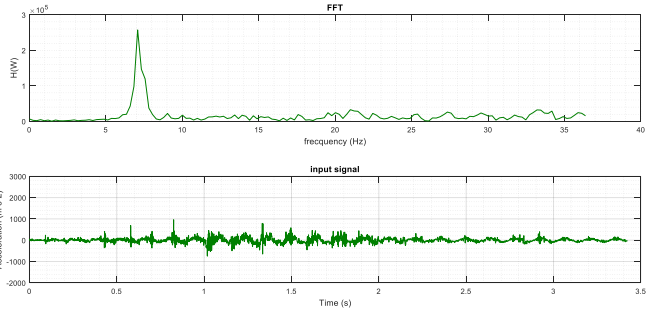

$\boldsymbol{A}$
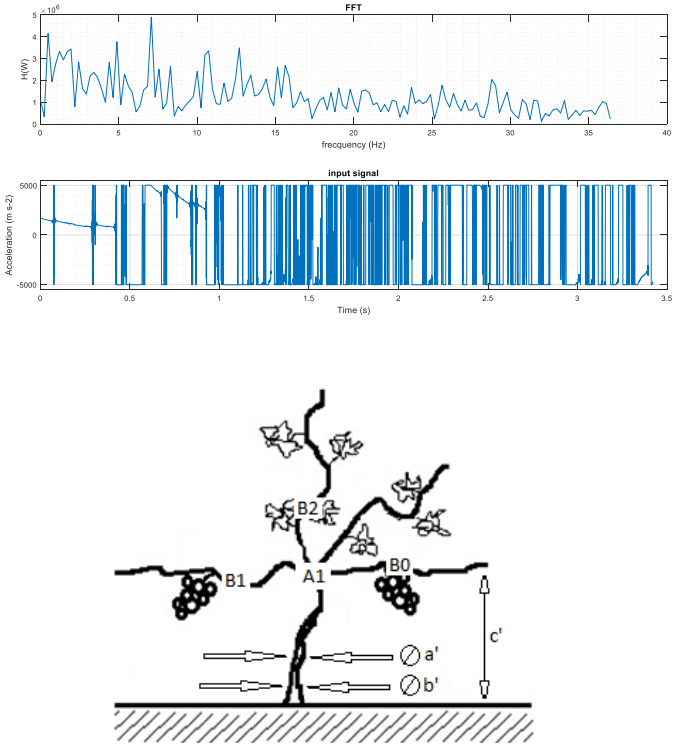
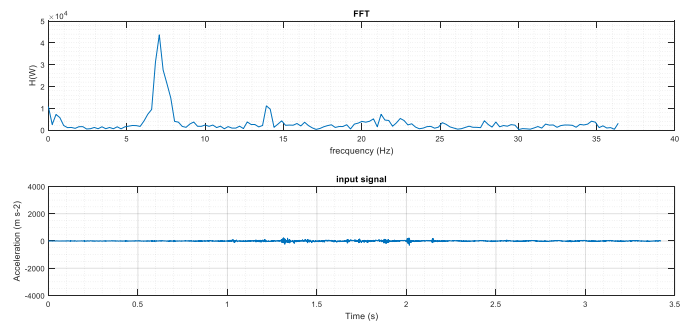

$B$

0

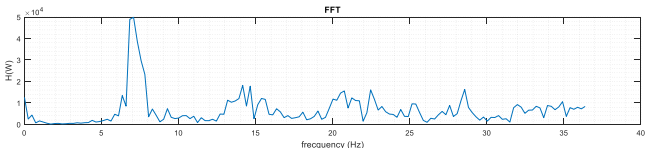

$B$

1
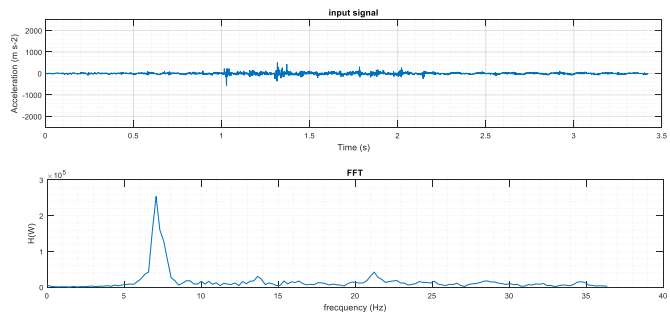

B

Figure 3. Field experiment and data processing process for variety Cabernet Sauvignon CNH SB65 with $420 \mathrm{rev} \mathrm{min}^{-1}$ setting.

Analysis variance for vibration parameters according to grape type

In this paragraph a summary is presented regarding the analysis of variance concerning the effect of grape type and machine setting on vibration conditions (Table 2). Figure 4 compares the actual vibration frequency as derived from fft and the value configure by the operator. Among several parameters, only the difference between measured and configured vibration frequencies (see Figue 5) appears to be signicative ( $\mathrm{F}=48.1$ ). 
Table 2. Analysis of variance ( $\mathrm{F}$ value and significance level) between grape types regarding accelerometer position, frequency setting, difference between frequency set and measured, and specific power of the vibration.

\begin{tabular}{|c|c|c|c|c|c|c|c|c|}
\hline \multicolumn{3}{|c|}{$\begin{array}{l}\text { Accelerometer } \\
\text { location }\end{array}$} & \multicolumn{2}{|c|}{$\begin{array}{l}\text { Actual versus set } \\
\text { vibration frequency } \\
\left(\text { rev } \text { min }^{-1}\right)\end{array}$} & \multicolumn{2}{|c|}{$\begin{array}{c}\text { Vibration frequency } \\
\text { set } \\
\left(\text { rev } \min ^{-1)}\right.\end{array}$} & \multicolumn{2}{|c|}{$\begin{array}{l}\text { Specific Power per grape type } \\
\qquad(\mathrm{W} \mathrm{h} / \mathrm{kg})\end{array}$} \\
\hline A & 0.26 & ns & 48.1 & $* *$ & 0.92 & ns & 0.35 & ns \\
\hline B & 0.64 & ns & 1.3 & $\mathrm{~ns}$ & 0.11 & ns & 0.13 & ns \\
\hline
\end{tabular}

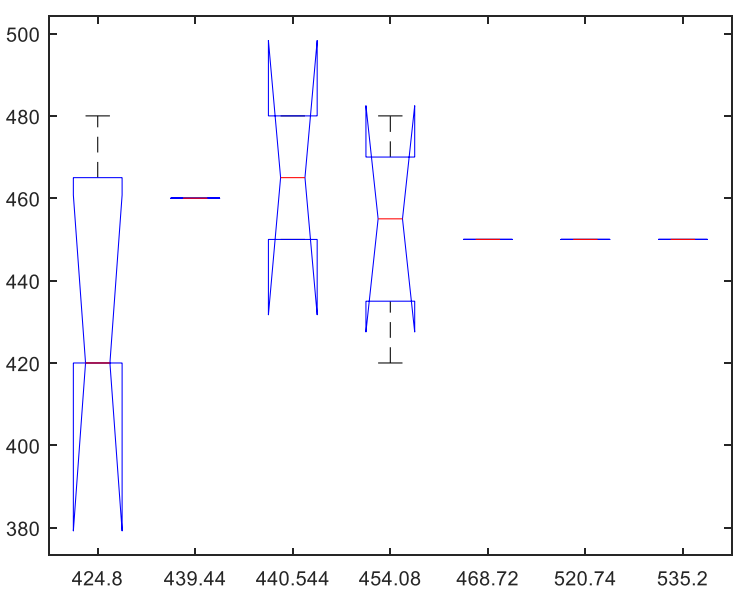

Figure 4. Measured ( $\mathrm{x}$ value) versus configured (y value) vibration frequencies $\left(\right.$ ver $\mathrm{min}^{-1}$ ) machine as derived from vibration analysis and FFT, and operator setting; accelerometers A0, A1 and A2 located on the truck.

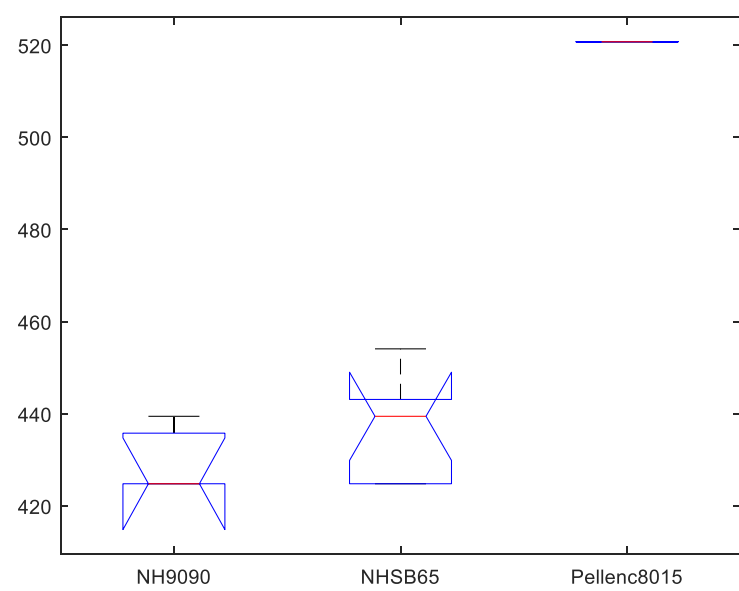

Figure 5. Vibration frequency per machine as derived from vibration analysis and $\mathrm{FFT}, \mathrm{F}=88.0 * *$; accelerometers $\mathrm{B} 0, \mathrm{~B} 1$ and $\mathrm{B} 2$ located branches.

Analysis variance for vibration parameters according to machine type and setting

In this paragraph, a summary is presented regarding the analysis of variance concerning the effect of individual harvest machines (addressed as manufacturer and model) and the settings for the vibration conditions (Table 3). The largest difference is found for the vibration frequency of Pellenc machines versus $\mathrm{CNH}$ ( $\mathrm{F}=88.0$ ), in spite of it, the specific power $(\mathrm{W} \mathrm{h} / \mathrm{kg})$ applied in either of the cases is similar $(\mathrm{F}=\mathrm{ns})$ as stated in Figures 6 and 7 . The outliers in Figure 7 correspond to the vibration conditions shown in Figure 3.

Table 3. Analysis of variance (F value and significance level) between machine type regarding accelerometer position, frequency setting, difference between frequency set and measured, and specific power of the vibration.

\begin{tabular}{ccccc}
\hline & $\begin{array}{c}\text { n setting among manufacturers } \\
\left(\text { rev } \text { min }^{-1}\right)\end{array}$ & \multicolumn{2}{c}{$\begin{array}{c}\text { Specific Power per } \\
\text { manufacturer }(\mathrm{W} \mathrm{h} / \mathrm{kg})\end{array}$} \\
\hline $\mathrm{A}$ & 0.35 & $\mathrm{~ns}$ & 0.44 & $\mathrm{~ns}$ \\
$\mathrm{~B}$ & 80.0 & $* *$ & 1.82 & $\mathrm{~ns}$ \\
\hline
\end{tabular}




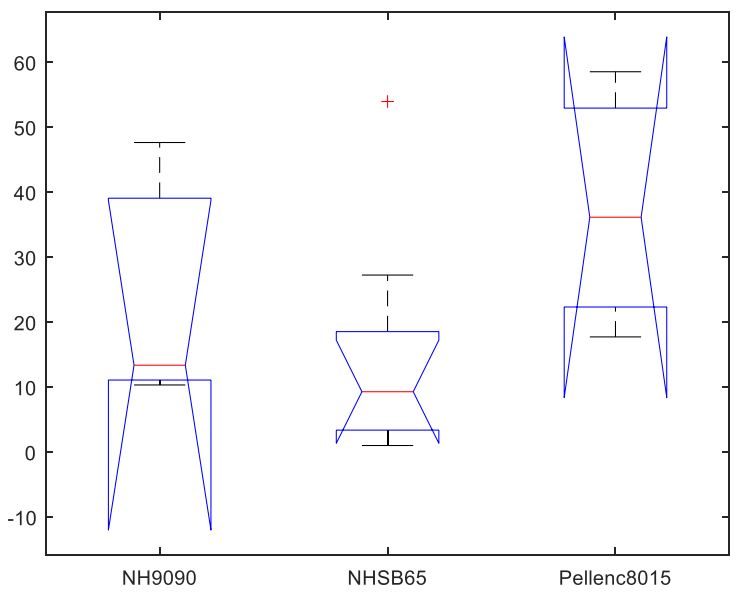

Figure 6. Specific Power( $\mathrm{W} \mathrm{h} / \mathrm{kg}$ ) per machine type as derived from vibration analysis and $\mathrm{FFT}, \mathrm{F}=\mathrm{ns}$; accelerometers A0, A1 and A2 located on the truck.

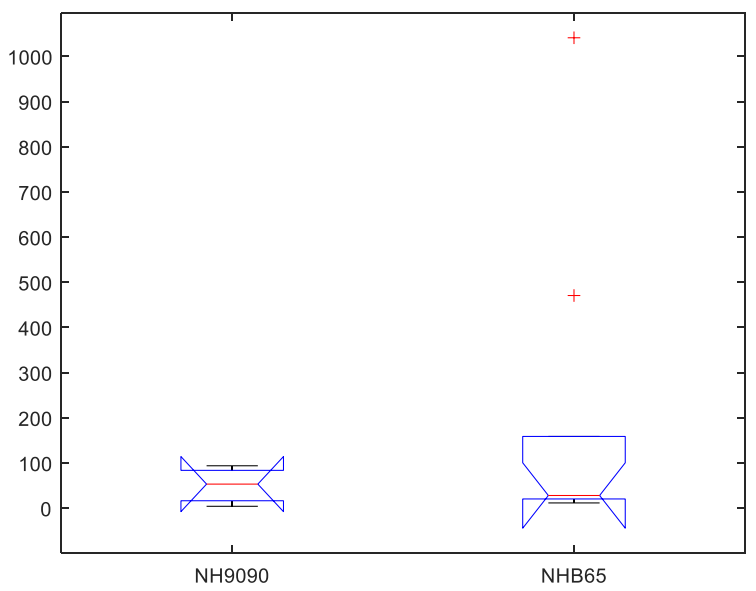

Figure 7. Specific Power(W h/kg) per machine type as derived from vibration analysis and $\mathrm{FFT}, \mathrm{F}=\mathrm{ns}$; accelerometers B0, B1 and B2 located branches. The outlier corresponds to vibrations in Figure 3.

\section{Conclusions}

The main outcome of this research can be summarized as follows:

- The adaption of actual settings of grape harvest machines according to yield $(\mathrm{kg} / \mathrm{ha})$, processing capacity (as function of ground speed) and grape type (red or white) is still limited at current stage in the Spanish context, even for machine dealers of large expertise

- Vibration analysis provides relevant information (vibration frequency and specific power) regarding the quality of the adaption of settings to local conditions

- Given the fact that many varieties are grown nowadays in the same growing area, there is need for providing some automated tools for the proper adjustment of the machine

- Occasionally, vibration conditions ten times harder in terms of specific power $(\mathrm{W} \mathrm{h} / \mathrm{kg})$ are found, which may harm the vines. In this study, such corresponded to a grape variety (Cabernet Sauvignon) with large differences in plant configuration, leaf development and yield compared to local varieties such as Tempranillo.

\section{Acknowledgements}

The authors would like to thank Case New Holland-Villarobledo (AFITOVI) for their availability in the field experiments, and Wilson Da Costa Neto gratefully acknowledges the financial support of CNPq..

\section{References}

Baguena Isiegas, E., Barreiro Elorza, P., Valero Ubierna, C., Sort, X., Torres, M., \& Ubalde, J. M. (2009). On-the-go yield and sugar sensing in grape harvester.

Baguena Isiegas, E., Barreiro Elorza, P., Valero Ubierna, C., Missotten, B., Verhoeven, J., Baerdemaeker, J. D., \& Saeys, W. (2011). Analysis of load displacement in grape harvesters and corresponding effect on dynamic weighing system under laboratory conditions.

Barreiro Elorza, P. (2009). De la vendimia mecanizada a la mecanización total del viñedo. Vida Rural, (297), 66-72.

da Costa Neto, W. V., Garrido Izard, M., Valero Ubierna, C., \& Barreiro Elorza, P. (2017). Verification of the quality of dgps signal in Rio Grande do Sul-Brazil, compared to Villarrobledo (Spain).

Caprara, C., Pezzi. C. 2009. Mechanical grape harvesting: Investigation of the transmission of vibration. Biosystem Engineering 103, 281-286.

Caprara, Caprara, C., Pezzi. C. 2011. Measuring the stresses transmitted during mechanical grape harvesting. Biosystem Engineering 110, 97-105.

Chengsong, L., Zhenjiang, G., Za, K., Lihong, W., Panpan, Y., Zhe, W. 2015. Experiment of fruit-pedicle vibration separation of wine grape. Transaction of the Chinese Society os Agricultural Engineering 31, 39-44.

Correa Farias, C., Valero Ubierna, C., \& Barreiro Elorza, P. (2012). Characterization of vineyard's canopy through fuzzy clustering and SVM over color images. 
da Costa Neto, W. V., Barreiro Elorza, P. (2018) Development of a standardized methodology for the quantification of must released during mechanical grape harvest. X Congreso de estudiantes de Ciencia, Tecnología e Ingeniería Agronómica.

da Costa Neto, Wilson Valente, et al. "First steps in the grape mechanization process in Brazil: quantitative features." Agricultural Engineering International: CIGR Journal 19.2 (2017): 110-119.

Diago, M. P., Correa, C., Millán, B., Barreiro, P., Valero, C., \& Tardaguila, J. (2012). Grapevine yield and leaf area estimation using supervised classification methodology on RGB images taken under field conditions. Sensors, 12(12), 16988-17006.

Du, X., Chen, D., Zhang, Q., Scharf, P.A., Whiting, M.D. 2012. Dynamic responses of sweet cherry trees under vibratory excitations. Biosystem Engineering 111, 305-314.

Herrero Langreo, A., Barreiro Elorza, P., Diago Santamaria, M. P., Baluja, J., Ochagavia, H., \& Tardaguila Laso, J. (2010). Pixel classification through mahalanobis distance for identification of grapevine canopy elements on rgb images.

Pezzi, F., Balducci, G., Pari, L. 2013. The comparison between the grape harvesting machines with horizontal and vertical shaking systems. Acta Horticulture 978, 257-262.

Pezzi, F., Caprara, C., and Bordini, F. 2008. Transmission of impacts during mechanical grape harvesting and transportation. Journal of Agricultural Engineering 3, 43-48.

Troncoso, C., Riquelme, J., Laurie, G., and Abarca, G. 2002. Evaluación de las ventajas relativas de la vendimia mecanizada en Chile central. Agricultura Técnica 62, 310-320. 


\title{
Animal Welfare Adjustment Real Time Environmental Condition of Housing (AWARTECH Project) - Living Lab Setup
}

\author{
Vasco Fitas da Cruz ${ }^{1}$, Fatima Batispta ${ }^{2}$, José Carlos Rico ${ }^{3}$, David Botas ${ }^{4}$ \\ ${ }^{1}$ ICAAM, Department of Rural Engineering, University of Évora. vfc@uevora.pt \\ ${ }^{2}$ ICAAM, Department of Rural Engineering, University of Évora. fb@uevora.pt \\ ${ }^{3}$ Department of Rural Engineering, University of Évora. m37275@alunos.uevora.pt \\ ${ }^{4}$ Equiporave Ibérica. david.c.botas@gmail.com
}

\begin{abstract}
AWARTECH aims to create and develop a precision animal husbandry tool that supports the sustainability of the swine value chain. The intention is to create a platform for the collection and organization of data, that allows the evaluation of a set of environmental and physiological variables and to act in real time on environmental conditions. The first test to validate the equipment and methodology for collecting and monitoring information, related to animal welfare indicators, occurred between October 2017 and January 2018. The environmental data were collected by sensors of temperature, relative humidity, air velocity and gas concentration, which are integrated in a environmental control system (Webisense) and a platform (Nidus). Webisense controlled the ventilation system, the cooling system and the heating system. Physiological data (rectal temperature, body surface temperature, $\alpha$-amylase and cortisol concentrations) were collected manually and analysed. In order to monitor the behaviour of the animals, video cameras and microphones were installed. The component of analysis of video and audio is in development, through the video and audio captures already made. This analysis aims to detect abnormal behaviours such as crowding/spacing of animals and vocalizations above certain frequencies. An individual feeding machine equipped with a scale has been installed. That allows, through an RFID system, individual monitoring and control of the quantity of food supplied and ingested; number and duration of visits; and the weight of the animals. This information allows the analysis of productive data of animals. The development of AWARTECH platform results from the integration of physiological data manually supplied and real-time data provided by Webisense, Nidus, feeding machine, video and sound analytics, that allow the control of housing environmental conditions in order to promote the animal welfare.
\end{abstract}

\section{Introduction}

The new global requirements of agricultural production, within the ethical process, are increasingly turning to the concepts of good production practices, considering animals and workers welfare, food safety and respect for the environment (Campos, 2009, cited by Baêta e Souza, 2010).

Welfare programs are based on elements that contribute to the quality of animals' life, namely the constituents of the "five freedoms" proposed by the FAWC (Farm Animal Welfare Council, 1992).

Facilities and their equipment should therefore protect animals from adverse conditions, as well as provide adequate comfort and well being, with appropriate levels of ventilation, temperature and humidity (Baêta and Souza, 2010). In this sense, intensive pig farms are in a phase of change, requiring development and reorganization.

The environmental conditions have a great impact on the productivity of different animal species, especially in poultry and swine industries, that usually work under intensive systems (Cruz and Baptista, 2006).

In intensive systems, animal comfort and welfare suffer directly with the environment, this causes difficulties in maintaining thermic balance inside the facilities, in the expression of natural behaviours and affects the productive and reproductive performance of the swine (Pandorfi, 2006 ).

The pig is considered a modifying agent of the environment where it lives, whether by the production of heat, steam, urine, or the by development of pathogens. In swine facilities, the animal is in an environment with high noise levels, steam, gases and dust that often exceed the required limits to maintain well being and health (Pandorfi, 2012).

In order for the intended environmental conditions to be met, the livestock facilities must have a monitoring and control system. However there is a need for modern facilities not to monitor only the environmental conditions, but also the behaviour and health of the animals (Banhazi and Black, 2009; Koenders et al., 2015).

In this sense, the concept of precision livestock farming is develop and it can be defined as the application of technological principles and engineering processes to the farm management (Wathes et al., 2008). This tool has the potential to improve animal welfare and increase productive outcomes (eg. weight gain) (Koenders et al., 2015), as well as to detect, in real time, certain behaviours of the animal that could indicate its level of well being. Early detection, possible through real-time monitoring, can minimize production costs by reducing the incidence of diseases and mortality (Nasirahmadi et al., 2017). 
AWARTECH Project emerges with the goal of developing a new tool that responds in real time to the environmental needs of animals through physiological, behavioural and productive indicators.

Indoor environmental parameters

Climatic (or environmental) factors, such as temperature, relative humidity and air velocity have a high influence on animals, impacting on their behavioral, physiological and immunological status (Cruz, 1997).

The pig in the phase of growth and fattening presents requirements, along with its nutritional, health, etc. necessities, so that its productivity is not affected, having engineering and environment control the goal to accomplish those conditions (Cruz, 1997; Babot and Revuelta, 2009).

\begin{tabular}{cl}
\hline PARAMETER & DESCRIPTION \\
\hline & $\begin{array}{l}\text { According to Cruz and Baptista (2006), animals are a constant source of sensible and } \\
\text { latent heat, contributing to a change in the value of indoor temperature. The indoor } \\
\text { temperature of a livestock installation is essentially the result of outdoor climatic } \\
\text { conditions, the animal species housed and the characteristics of the building. The } \\
\text { environmental temperature has direct effects on pig behaviour (Nasirahmadi, 2015) and has } \\
\text { a negative effect in their productivity (Vieira et al., 2010). The optimal temperature for } \\
\text { growing and fattening pigs has different meanings, depending on whether it is linked to } \\
\text { food intake, growth rate, food utilization efficiency or retained energy on the form of fat } \\
\text { and protein (Cruz, 1997). }\end{array}$ \\
$\begin{array}{l}\text { This is an important climatic parameter, since the concentration of humidity at high } \\
\text { levels is detrimental to the health and comfort of animals housed, as well as those involved } \\
\text { in the production process and who are in regular contact with the premises. In general, the } \\
\text { moisture content should be kept below the critical limits. However, the first difficulty arises } \\
\text { in defining this value, since it depends on some factors such as animal species, their } \\
\text { production phase and the ambient temperature. }\end{array}$ \\
$\begin{array}{l}\text { The air velocity is an important parameter, especially during winter. One of the goals } \\
\text { is to avoid cold air coming from the outside to directly reach the animals, since the inherent } \\
\text { risks of the occurrence of pneumonia may increase. On the other hand, during the summer, } \\
\text { the main objective is that the air hits directly to animals, increasing heat exchanges by } \\
\text { convection, which translates into a sensation of freshness (Cruz and Baptista, 2006). }\end{array}$ \\
$\begin{array}{l}\text { Adequate lighting should allow all tasks to be carried out within the facilites, so a } \\
\text { minimum light intensity of 40-50 lux is recommended (below 20 lux the pig has difficulties } \\
\text { in finding food and water). Although not much is known about the interaction animal- } \\
\text { luminosity, it can be concluded that some physiological processes related to reproduction, } \\
\text { growth and behaviour can be affected by light intensity of light and time length. }\end{array}$ \\
\hline Lighting
\end{tabular}

\section{Behavioral Parameters}

Behavioural diversity is an important indicator of an animal's ability to cope with the environment (Baptista et al., 2011 cited by Da Costa, 2014).

Changes in pig behaviour may be the result of various challenges, such as the ability to express behaviour according to the environment of the farm, disease or injury. Such changes are in direct conflict with the five freedoms and do not adhere to the new model of five domains (Mellor, 2016, cited by Matthews et al., 2016), and should aim to improve animal welfare and improve the sustainability of the system.

Behavioural changes in health and welfare may have an evolutionary basis (Hart, 1990; Kyriazakis et al., 1998, cited by Matthews et al., 2016), or be an inevitable consequence of the challenge. For example, exposure to pathogens is associated with changes in feeding, which may be beneficial (Kyriazakis et al., 1998, cited by Matthews et al., 2016), considering that agglomeration that can be seen as a risk factor for tail biting (Edwards, 2006; cited by Matthews et al., 2016). 


\begin{tabular}{|c|c|}
\hline BEHAVIOUR & DESCRIPTION \\
\hline Food behaviour & $\begin{array}{l}\text { Pigs are omnivorous animals, and, in intensive production systems, they focus their activity period } \\
\text { in the feeding time (Bastos, 2012). At the onset of the stress factor, the feeding level decreases, as } \\
\text { well as the number of visits to the feeder. }\end{array}$ \\
\hline Drinking behaviour & $\begin{array}{l}\text { Social factors, diet composition and texture, and environmental temperature are factors that } \\
\text { strongly influence drinking behaviour (Deen, 2010). }\end{array}$ \\
\hline Excretion behavior & Swine's usually defecate in an area far from where they eat (Deen, 2010) \\
\hline \multicolumn{2}{|l|}{ Social behaviour } \\
\hline Hierarchy & $\begin{array}{l}\text { In all phases of production, the formation of a linear social hierarchy is detected (Meese, Ewbank, } \\
\text { 1973, cited by Massari, et al., 2015). This is essential for the coexistence of the group, avoiding the } \\
\text { occurrence of aggressions and injuries (Messe and Ewbank, 1973, Lindberg, 2001, Mendl and Held, } \\
\text { 2001, cited by Massari, 2014). }\end{array}$ \\
\hline Agonistic & $\begin{array}{l}\text { In groups whose hierarchical positions are already established, aggressions (threatening, biting, } \\
\text { chewing, or jerking) (Held and Mendl, 2000, cited by Massari, 2014) may occur during the mixing of } \\
\text { unknown animals until a new dominance order is established, and in the competition for space, food } \\
\text { and resources (Fraser, 1984, Otten et al., 1997, De Jong et al., 1999, cited by Massari, 2014). It may } \\
\text { be related to a number of stress factors, related to facilities and inadequate handle (Sobestansky, } \\
\text { Zanella, 2007; cited by Baptista et al., 2011). }\end{array}$ \\
\hline Destructive & $\begin{array}{l}\text { They are those that cause injuries in animals (bites and sucking defects of the tail, ear, flank and } \\
\text { vulva, aggressive behaviors) (Zanella 1995, Sobestiansky, Zanella 2007, Baptista et al. The main } \\
\text { causes are batch mixing and overcrowding (Radostits et al., 2002, cited by Baptista et al., 2011). }\end{array}$ \\
\hline Vocalization & $\begin{array}{l}\text { It is an objective and non-invasive tool that can evidence the compromise of animal welfare based } \\
\text { on different vocal patterns (Nääs et al., 2008). According to the UK's Department of Environment, } \\
\text { Food and Rural Affairs (DEFRA) recommendation for Welfare in Pig Breeding, noise pressure values } \\
\text { above } 85 \mathrm{~dB} \text { should be avoided. According to Tolon et al. (2010), cited by Baptista et al. (2011), at } \\
\text { this level the animal may be unable to develop its natural behaviour. }\end{array}$ \\
\hline $\begin{array}{l}\text { Thermoregulatory } \\
\text { behaviors }\end{array}$ & $\begin{array}{l}\text { The appearance and detection of tremors in pigs (cold) or panting in pigs (heat) are associated } \\
\text { with extreme temperatures (WQ, 2009, cited by Da Costa, 2014). According to Da Costa (2014), it } \\
\text { was concluded that the detection of piled animals in fattening pigs allowed to determine the degree of } \\
\text { thermal comfort at temperatures slightly below the optimum temperatures recommended for the } \\
\text { species and stage of production. In the case of elevated temperatures, it is known that these, besides } \\
\text { influencing the behaviour of lying down, still impair the feeding of the animals, since food } \\
\text { consumption decreases (Jones and Manteca, 2009; cited by Da Costa, 2014). The animals are panting, } \\
\text { with a quick, shallow breath through the mouth. In addition, it is also very common to observe the } \\
\text { swirling pigs, in the water or even in the mud. This occurs in the attempt of the animals to cool } \\
\text { themselves, since pigs have very few sweat glands and are almost unable to pant because of the rigidity } \\
\text { of their trunk (Bastos, 2012). }\end{array}$ \\
\hline $\begin{array}{l}\text { Behaviors associated } \\
\text { with posture and } \\
\text { locomotion }\end{array}$ & $\begin{array}{l}\text { Posture and locomotion of pigs can be influenced by skeletal and visceral problems (Radostits et } \\
\text { al., 2007c; Matthews et al., 2016). Claudication is a major cause of slaughter of breeding pigs (Tarrés } \\
\text { et al., 2006, cited by Matthews et al., 2016), and can be caused by infection, degeneration or trauma } \\
\text { of one or more limbs. Health impairment may also be a result of changes in posture, for example, } \\
\text { easel posture (Radostits et al., 2007d, cited by Matthews et al., 2016). }\end{array}$ \\
\hline Stereotyped behaviors & $\begin{array}{l}\text { When pigs are confined in stalls or fastened for a while, some individuals exhibit stereotyped } \\
\text { behaviors such as tongue twisting, false mastication or chewing of part of the premises (Poletto et al. } \\
\text { 2010); while others become extremely inactive and non-responsive, especially when the environment } \\
\text { is not stimulating and the animal finds it difficult to cope with the environment, eg: during thermal } \\
\text { stress (Kiefer et al., 2009, cited by Baptista et al. 2011). }\end{array}$ \\
\hline Exploratory behavior & $\begin{array}{l}\text { When there is an incompatibility between the pig and the habitat, behavioral problems occur. In } \\
\text { intensive production systems, under conditions of confinement, these usually make the expression of } \\
\text { their vast behavioral repertoire impossible, since the environment is practically sterile and without } \\
\text { stimulus, directing their investigative behavior to explore the environment of confinement and / or } \\
\text { other animals present in the bay (Machado Filho and Hötzel, 2000, cited by Foppa et al., 2014). }\end{array}$ \\
\hline
\end{tabular}




\section{Physiological Parameters}

The physiological indicators of animal welfare are related to the physiological response to stress that is described by the metabolic, immunological and neuroendocrine mechanisms (Moberg, 2000).

As can be seen in the following table, metabolic mechanisms are guided by changes in rectal temperature, heart rate and respiratory rate; the neuroendocrine, which are based on changes in the endocrine levels of cortisol, endogenous opioids and acute phase proteins; and the immunological mechanisms described by immunoglobulin changes responsible for the animal's defence.

\begin{tabular}{|c|c|}
\hline MECHANISM & DESCRIPTION \\
\hline \multicolumn{2}{|l|}{ Metabolic } \\
\hline Rectal Temperature & $\begin{array}{l}\text { In a study by OMTVEDT et al. (1971), cited by Baptista et al. (2011), the } \\
\text { rectal temperature of animals exposed to stress was significantly higher than } \\
\text { those in comfort. }\end{array}$ \\
\hline Surface Temperature & $\begin{array}{l}\text { In a study, described by Baptista et al. (2011), it was concluded that the } \\
\text { animals under thermal stress presented values of superficial temperatures (nape, } \\
\text { palette and leg) approximately } 9,5 \% \text { higher than the animals submitted to thermal } \\
\text { comfort. }\end{array}$ \\
\hline - $\quad$ Heart Rate & $\begin{array}{l}\text { This adjusts to meet the needs of each situation. The high levels are produced } \\
\text { during excessive physical activity, defense and alarm reactions (Cunningham, } \\
\text { 2003, cited by Baptista et al., 2011) or the increase in body temperature (Guytin } \\
\text { \& Hall, 2006; cited by Baptista et al. 2011). }\end{array}$ \\
\hline $\begin{array}{l}\cdot \quad \text { Respiratory } \\
\text { frequency }\end{array}$ & $\begin{array}{l}\text { One of the mechanisms of thermoregulation of pigs can happen through the } \\
\text { airways. According to Baptista et al., 2011, respiratory rate can be increased by } \\
\text { thermal stress as a function of respiration to be an efficient physiological } \\
\text { mechanism that acts in maintaining thermoregulation. (Manno et al., 2006; } \\
\text { quoted by Baptista et al., 2011). }\end{array}$ \\
\hline \multicolumn{2}{|l|}{ Neuroendocrine } \\
\hline Cortisol & $\begin{array}{l}\text { It can be monitored through plasma, saliva or urine. Faced with an adverse } \\
\text { situation, cortisol levels increase, as can be confirmed in the study by Dalla Costa } \\
\text { et al. (2008), cited by Baptista et al., } 2011 \text {, when transporting adult sows, in } \\
\text { which cortisol concentration increased }(0.714 \mu \mathrm{g} \mathrm{dL}-1) \text { during transport of the } \\
\text { animals, with a subsequent decrease }(0.510 \mu \mathrm{g} \mathrm{dL}-1) \text { in the rest period. }\end{array}$ \\
\hline $\begin{array}{ll} & \text { Endogenous } \\
\text { opioids } & \end{array}$ & $\begin{array}{l}\text { The organism, in response to adverse conditions, releases the endogenous } \\
\text { opioids (beta-endorphin, enkephalin and dynorphin), which aid in coping with } \\
\text { the difficulties (Baptista et al., 2011) }\end{array}$ \\
\hline $\begin{array}{l}\text { - Acute phase } \\
\text { proteins }\end{array}$ & $\begin{array}{l}\text { The effect of stress on the serum concentration of PFAs is controversial, since } \\
\text { it is difficult to differentiate it from the effect of trauma or subclinical infection } \\
\text { (Petersen et al., 2004), however it is known that when these proteins are } \\
\text { increased, they indicate a compromise of the BEA (Piñeiro et al., 2007, cited by } \\
\text { Baptista et al., 2011). }\end{array}$ \\
\hline \multicolumn{2}{|r|}{ P } \\
\hline Immunoglobulins & $\begin{array}{l}\text { Taking into account the study of Tuchscherer et al. (2002), cited by Baptista } \\
\text { et al., 2011, stress is responsible for the decrease of these, which significantly } \\
\text { increases morbidity and mortality. }\end{array}$ \\
\hline
\end{tabular}

\section{Material and methods}

There are several technological applications (combined thermometers, capacitive sensors, anemometers, cameras, automatic weighing systems, etc.) that are applied by the precision zoo technical score since the beginning of the 21 st century. They tend to demonstrate the possibilities (current and future) of animals and environmental feedback.

The decisions of management, based on indices of thermic comfort, to a precise environmental control require appropriate materials and exact monitoring for all the parameters. The animals respond to their environment, physiological and behavioral.

In this test, different environmental, behavioral and physiological indicators were measured using different precision animal husbandry tools. All data collected was stored in real time. Next, the different measured parameters are listed, as well as the equipment used. 
Environmental Measurements

Environmental parameters generally measured and related to animal comfort include temperature, humidity, solar radiation and air velocity. They characterize, approximately, the animal environment and are measured in the area where the animals are confined (Fournel et al., 2017).

In the present moment, the measurements are made on the local and not based on meteorological states (Eigenberg et al., 2009), thus characterizing the environment where the animals are located (Fournel et al., 2017). The information obtained by the producers is available so more accurate decision can be made. (Eigenberg et al., 2009).

\section{Ambient temperature}

The devices that measure temperature depend on the alteration of some physical properties (ASHRAE, 2013, Doebelin, 1990, OMEGA, 2016 quoted by Fournel et al., 2017). Environmental temperatures of livestock facilities can be successfully measured through thermocouples or thermistors. Thermocouples offer some advantages such as their durability, relatively low costs and versatility. Thermistors have the advantage of being much more sensitive and tolerant to large temperature differences than thermocouples; however their construction makes them more fragile (CIGR, 1999, Frost et al., 1997 cited by Fournel et al. 2017, Eigenberg et al., 2009). In this assay, temperature measurements were performed through 4 "..." distributed uniformly throughout the controlled room environment.

Humidity

Investigations have shown that in harsh environments such as livestock facilities, thermal conductivity methods can be used successfully in determining the water vapor content present in the facility. The accuracy of the method decreases with lower temperatures; however, the sensors can work with high temperatures, corrosive gases and dust. A combined thermistor $\left(40-60^{\circ} \mathrm{C}\right.$ ) and a capacitive sensor ( 0 and $100 \%$ relative humidity), protected by a sintered stainless steel filter, are usually installed in livestock facilities (Banhazi, 2009, Fournel et al., 2012, Haeussermann et al., 2005, Phillips et al., 1998 cited by Fournel et al., 2017). In this test, relative humidity measurements were measured through a capacitive sensor.

Air velocity

The air velocity is measured in the vicinity of the animal to capture the heat and mass changes of the animal (Eigenberg et al., 2009). Air velocity can be measured by anemometers in a variety of ways, based on mechanical methods, pressure relationships, thermal principles and the Doppler effect. These devices are very sensitive instruments and are easily affected by traces of dust (Eigenberg et al., 2009).

In animal production applications, two types of anemometers are common, depending on the type of air flow being measured: hot wire anemometers and propeller anemometers. A hot wire anemometer is the instrument of choice for low speed air applications. Air moving at less than $0.25 \mathrm{~m} / \mathrm{s}$ is still considered air, and this condition exists in many livestock facilities (Kelly et al., 1971, Wheeler, 2012 cited by Fournel et al., 2017). In this test, the air velocity was measured through a hot wire anemometer.

\section{Physiological measurements}

The commonly measured physiological parameters related to animal comfort include body temperature, respiration rate, heart rate and body weight. There may be some difficulties associated with this type of measurements, since the intrusions to observe the animals may influence and lead to changes in the final results (Fournel et al., 2017)

\section{Body temperature}

Remote body temperature measurement isn't simple. There may be some difficulties associated depending on the location of the measurement, since the physiological function of the body part in question or the level of invasiveness of the device may cause changes in the temperature of the animal. There are mainly two categories of temperature sensors: those that are implanted surgically and those that aren't (Sellier et al., 2014 cited by Fournel et al., 2017).

In general, all devices for monitoring body temperature have their advantages and disadvantages. In this eye, given the complexity of a continuous in a real-time measurement of body temperature, a thermal chamber was used which recorded the surface temperatures of the animals. Additionally, and at weekly intervals, the rectal temperatures were measured by means of a mercury thermometer.

\section{Body weight}

The weight of an animal is an important indicator of its welfare. Any unusual change in animal weight may indicate an early warning of health problems or simply problems with feeding or ventilation equipment's (Frost et al., 1997 cited by Fournel et al., 2017).

To minimize the stress caused by traditional weighing machines, self-assessment scales were developed, such as electronic weighing platforms placed in front of a single feeder, or measurement scales based on locomotion of pigs (Gates et al., 1995, Ramaekers et al., 1995, Williams et al., 1996 cited by Fournel et al., 2017). 
The device consists of a platform that measures the voltage. The instrument is monitored through a computer that determines the weight between each record, then stores each reading and provides a distribution of the weight of the group of animals to the operator (Fournel et al., 2017). In this test, an individual automatic feeder was used, equipped with a scale that allowed, through an RFID system, individual monitoring and control of the weight of the animals per visit and day.

\section{Behavioural measurements}

The monitoring of animal behaviour, particularly in relation to its disposition in the place and its activity, has been carried out successfully through some electronic devices (Eigenberg et al., 2009). The commonly measured behavioural parameters related to animal comfort include feeding and water consumption and animal presence, activity or behaviour. These indicators may be useful for determining the health status of the animals as well as the physiological state.

Feeding

Food intake and feeding behaviour have become useful indicators of the health status of animals (Banhazi et al., 2007, Brown-Brandl et al., 2013, Kashiha et al., 2013 cited by Fournel et al., 2017). Feed consumption and changes in dietary behaviour may be related to thermal conditions (Cruz, 1997). Systems that measure feed intake (total and intake rate), along with feeding behaviour (duration, interval and number of meals per day and total time consumed) have been tested in pigs (Andree et al. Huegle, 2001, Banhazi et al., 2009, Brown-Brandl et al., 2013, Chapinal et al., 2008 cited by Fournel et al., 2017).

Some systems are equipped with an antenna that senses through a radio frequency transponder, located in the ear tag of the animal, is approach to the feeder. For each approach, a computer records the data sent by the antenna, which includes the animal number, number of the machine, time in the machine and weight, then calculates the duration of the visit and the intake. Other systems have electronic feed stations, a weighing system during feeding or with flow sensors (Fournel et al., 2017).

In this study, we used an individual automatic feed machine. This machine, through an RFID system, allowed to monitor and control individually the quantity of food supplied and ingested; and the number of visits and duration of visits per day. Through this tool it is possible to analyse the productive data of the animals through the calculation of productions indicators.

\section{Presence, activity or animal behaviour}

Automatic recording of activity (lying, stationary and moving) can be obtained using a variety of sensor systems: ultrasonic proximity sensors (Brown-Brand et al., 2000, Hillman et al., 2000 cited by Fournel et al., 2004), mercury switches (Champion et al., 1997, Ouellet et al., 2016, O'Driscoll et al., 2008 cited by Fournel et al., 2017), pedometers (Brehme et al., 2004, Walker (2006), and Muller and Schrader (2003), and Ouellet et al., 2006, Robert et al., 2009), and accelerometers (Darr and Epperson, 2009, Diosdado et al. cited by Fournel et al., 2017). However, animal behaviour is much more complex than simply recording its activity in a given area. The ability to electronically recognize specific animal behaviour (eating, fighting, biting) requires the development of a computer vision system.

Information can be collected using scanned images, defining key components of them and using numerical equations. The images are produced by a variety of physical devices, including cameras and video cameras, Xray machines, electron microscopes, radars and ultrasound. The system is calibrated by assigning behaviours based on visual observation and determining the parameter values from the numerical equations. In subsequent videos, behaviours can be identified based only on parameter values (Kristensen et al., 2006, Leroy et al., 2006, Nääs et al., 2006, Viazzi et al., 2014 cited by Fournel et al., 2017).

In this test, video cameras and microphones were used to monitor the behaviour of the animals. Currently, this system is still under development and will aim to detect abnormal behaviours such as crowding / removal of animals and vocalizations above certain frequencies.

\section{Results and discussion}

In these days, environmental controllers use set points of environmental variables, which are supposed to be optimal for an animal. These set points were derived from a combination of small laboratory experiments. Despite the application of modern techniques, this approach does not always result in the expected process, because it greatly simplifies the complex interactions between an animal and its environment (Pandorfi, 2012). The dependence of new ways of information acquisition on livestock farms, through precision animal husbandry systems, should have the capacity to activate animal management practices that are more sensitive to market signals (Fournel et al., 2017). 
Thus, precision animal husbandry technologies include:

(i) Methods for electronically measuring the critical components of production systems related to the efficiency of resource use;

(ii) Software tools intended to interpret the information collected;

(iii) Control of processes to ensure optimal animal productivity (Fournel et al., 2017).

These real-time monitoring and control systems enable the production efficiency of livestock enterprises to be radically improved (Banhazi and Black, 2009; Kuczynski et al., 2011 cited by Fournel et al., 2017).

However, it is necessary to accurately characterize, with precision, environmental conditions and predict their impact on animal performance and welfare, so over the past two decades a number of new technologies have become available for both ventilation and heating / cooling of livestock facilities, and advances were made, albeit limited, in the development of control algorithms. To obtain greater benefit from the new technologies available, it is necessary to integrate more knowledge about the interaction between animal responses and control actions in the algorithms applied (Banhazi et al., 2009 cited by Fournel et al., 2017).

About the sensors used, there is a wide variety of sensors and biosensors that can be used to measure environmental, physiological and behavioural variables. However, it is still necessary to develop low-cost equipment to measure, store and analyse variables such as body temperature, humidity and behaviour that works in aggressive, non-invasive environments and capable of functioning automatically and continuously (Wathes et al., 2008).

\section{Conclusion}

Until now, environmental control systems use set points of environmental variables derived from laboratory experiences in the past. Although there are currently a number of modern technological devices that make systems more accurate, this approach is not always efficient since it over-simplifies the complex interactions between animals and their environment (Pandorfi, 2012).

In this sense, there was a need to develop a technological and innovative strategy for thermic control of the animal environment that includes:

(i) Continuous detection (at an adequate frequency) of environmental parameters (ambient temperature and humidity, air velocity, solar radiation, air quality, etc.) and behavioural responses (growth rate, temperature body, heart and respiratory rate, movement, degree of agglomeration among animals, food and water intake, etc.), depending on the complexity of the system;

(ii) Data storage;

(iii) Data interpretation using biological response simulation models, such as animal comfort indexes that predict in real time the dynamic result of each variable, as environmental conditions vary (Fournel et al., 2017);

(iv) An online control system that automatically modifies the animal microenvironment when critical limits are breached, under the establishment of predetermined criteria (Wathes et al., 2008).

In this way, "Awartech Smart Sensing" the platform of precision livestock farming assumes itself as a pioneering system of advanced control, based on automatic and with appropriate frequency monitoring, not only of environmental variables but also of physiological and behavioral ones.

We can conclude, that AWARTECH Project appears based on the existing tools and technologies for precision livestock, positioning itself as technologically and innovative more advanced, as it encompasses and integrates all these concepts in real time and always taking into account animal welfare.

\section{References}

Babot, D. and Revuelta, M. (2009). Necessidades Funcionales. In Ganado Porcino: Diseño de alojamentos e instalaciones. Ed. Grupo Asis Biomedia, S.L., ch:2, 38-69. Zaragoza: Servet editorial.

Baêta F.C. and C.F. Souza. 2010. Ambiência em Edificações Rurais: Conforto Animal. Viçosa, Mg; Brasil: UFV.

Banhazi, T. M. and Black, J. L. (2009). Livestock Farming: A suite of electronic systems to ensure the application of best practice management on livestock farms. Australian Journal of Multi-Disciplinary Engineering, 7(1).

Bastos, F., 2012. Comportamento e bem-estar animal. (Acedido em 24-05-2017) https://www.passeidireto.com/arquivo/6083671/comportamento_e_bem-estar_animal

Cruz, V. F. (1997). Influência da estação do ano sobre as performances do porco em fase de crescimento e acabamento. Sua relação com a densidade de alojamento e a concentração energética da dieta. Dissertação de Doutoramento. Universidade de Évora, Évora. 
Cruz. V.F. and Baptista, F. J. (2006). Conceitos básicos sobre ventilação no alojamento de suínos. Revista Suinicultura 72: 40-48.

Da Costa M.R. 2014. Avaliação do bem-estar em porcos de engorda na exploração e no matadouro. Dissertação de mestrado integrado em Medicina Veterinária. Universidade de Lisboa: Faculdade de Medicina Veterinária. Lisboa

Eigenberg, R. A., Bucklin, R. A. and Brown-brandl, T. M. (2009). Chapter 6: : Instrumentation for Research and Management in Animal Agriculture. In Livestock Energetics and Thermal Environmental Management. Ed. J. A. DeShazer, ch:6, 131-149. Moscow, Idaho, USA: ASABE

Foppa, L., Caldara, F. R., Machado, S. P., Moura, R., Santos, R., Naas, I. A., and Garcia, R. G. (2014). Enriquecimento ambiental e comportamento em suínos: revisão. Brazilian Journal of Biosystems Engineering, $8(1), 1-7$.

Fournel S., Rousseau, A. N. and Laberge, B. (2017). Rethinking environment control strategy of confined animal housing systems through precision livestock farming. Biosystems Engineering 155: 96-123.

Koenders, E., Rooijakkers, L., Hertem, T. Van, Vranken, E., Berckmans, D. and Berckmans, D. (2015). Towards the development of a practical visualisation tool for farmers and other stakeholders.

Massari, J. M., Curi, T., Moura, D. J., Medeiros, B. B. L., and Salgado, D. D. (2015). Características comportamentais de suínos em crescimento e terminação em sistema " wean to finish ." Journal of the Brazilian Association of Agricultural Engineering, 4430, 646-656.

Matthews, S. G., Miller, A. L., Clapp, J., Plötz, T., and Kyriazakis, I. (2016). Early detection of health and welfare compromises through automated detection of behavioural changes in pigs. Veterinary Journal, 217, 4351.

Moberg, G.P. (2000). Biological Response to Stress: Implications for Animal Welfare. The biology of animal stress: Basic Principles and Implications for Animal Welfare. UK: CABI Publishing

Nääs, I. D. A., Campos, L. S. L., Baracho, M. D. S., and Tolon, Y. B. (2008). Uso de redes neurais artificiais na identificação de vocalização de suínos. Engenharia Agrícola, 28, 204-216.

Nasirahmadi, A., Richter, U., Hensel, O., Edwards, S. and Sturm, B. (2015). Using machine vision for investigation of changes in pig group lying patterns. Computers and Electronics in Agriculture 119, 184-190.

Nasirahmadi, A., Edwards, S. A., and Sturm, B. (2017). Implementation of machine vision for detecting behaviour of cattle and pigs. Livestock Science, 202, 25-38.

Pandorfi, H., Silva, I. D., Carvalho, J. D., and Piedade, S. M. S. (2006). Estudo do comportamento bioclimático de matrizes suínas alojadas em baias individuais e coletivas, com ênfase no bem-estar animal na fase de gestação. Engenharia Rural, 17(1), 1-10.

Pandorfi, H., Almeida, G. L. P., and Guiselini, C. (2012). Zootecnia de precisão: princípios básicos e atualidades na suinocultura. Revista Brasileira de Saúde e Produção Animal, 13(2), 558-568.

Poletto, R. (2010). Série Especial: Bem-Estar Animal. Available online: http://www.suino.com.br/Noticia/serie-especial-bem-estar-animal-por-rosangela- poletto-134530

Wathes, C. M., Kristensen, H. H., Aerts, J. and Berckmans, D. (2008). Is precision livestock farming an engineer's daydream or nightmare, an animal's friend or foe, and a farmer's panacea or pitfall ?, 64, 2-10.

Vieira, R. de F. N., Silva, K. O., Mello, S. P., and Martins, J. R. (2010). Índices de conforto na avaliação do bem estar animal de matrizes suínas em diferentes sistemas de criação. Nucleus Animalium, 2(1), 1-8. 


\title{
Low Cost Integral System for Irrigation Remote Management Based on a Communication Nodes Network with Sensors, Weighing Lysimetry and Supervision of the Vegetative State for Hortícultural Crops
}

\author{
Antonio Ruiz-Canales ${ }^{a, *}$, José Miguel Molina-Martínez ${ }^{\text {, }}$, Martin John Oates a, Juan José Pérez \\ Solano c, Amparo Melián Navarro a, Jesús Chazarra Zapata a , Francisco López Peñalver a, José Manuel \\ González Esquiva ${ }^{b}$ \\ a Escuela Politécnica Superior de Orihuela. Miguel Hernández University of Elche, Orihuela, Alicante 03312, Spain \\ ${ }^{\mathrm{b}}$ Technical University of Cartagena, Cartagena, Murcia 30202, Spain \\ ${ }^{\mathrm{c}}$ Universitat de València, Valencia 46010, Spain \\ * Corresponding author. Email: acanales@umh.es
}

\begin{abstract}
The purpose of the research project combines two main parts. In a first phase is to design and develop a hardware of communication systems and nodes of low cost and on the other hand a corresponding software meshed networks to complement commercial solutions that exist in the market. Field trials will be conducted based on the current SIGFOX and WASPMOTE technologies, to establish the weaknesses and strengths of each potential solution. Once the deficiencies are identified, the project will allow establishing trials at different geographic scales and variable conditions of the Valencian Community (Spain). A wide range of applications would be sought, from data collection for irrigation programming to precision remote control and real-time management. In parallel, a low cost system will be developed and validated, for commercial use in horticultural farms, which measure and manage irrigation accurately, in real time, according to the water balance obtained through varied sensors and compact weighing lysimetry, remotely monitoring the vegetative state of the crop through the use of digital cameras. Data and images will be sent wirelessly to a compact controller in the irrigation head, which will house irrigation management algorithms and process information in real time, and will give irrigation control orders according to the real needs of the crop. The information is sent by an ESP8266 wifi system to an external hosting where it is stored and displayed in an orderly manner, with a friendly user interface, accessible from any web browser or specific mobile applications, providing the services associated with any SCADA (historical management, warnings and alarms, changes of slogans, crop supervision, among others). The system will be linked to the hardware and software developed in the first phase and will allow obtaining specific agronomic data for large-scale applications.
\end{abstract}

\section{Introduction}

The complete management from the technological perspective of an agricultural installation must be based on a model of representation, which allows accumulating data from all existing sources of information and all the actors involved. There is currently no technological system that allows obtaining a real-time vision with a historical perspective of what happens or has happened in it. Some tasks such as monitoring variables for the calculation of indicators of all kinds (environmental, ecological, energy, production performance, etc.) are fundamental for the intelligent management of resources (Delamo et al., 2015). Currently within an agrarian installation there are elements that work in isolation, and that do not allow to obtain global, shared and connected visions of the global state. The creation of a model of representation and transmission of information corresponding to heterogeneous sources, which allows complete interoperability between different systems and technological services, is fundamental for modern management. Therefore, it is necessary to investigate a form of inter-connection of all the elements that allow an efficient access to the information and even allow ubiquitous access to the data.

To develop an open network of nodes, at the scale of a large agrarian installation, it is necessary to solve a large set of novel technical problems of various kinds derived, among other reasons, from the high non-seasonality of the environment, and limited resources of some devices (for example, in the available power, in the storage capacity and in the computing elements themselves). It is necessary to develop new concepts, algorithms and network architectures, which will have to be adapted to an environment in which there are no boundaries between physical devices, communications, computing and control.

It will also be necessary a new paradigm of processing and distributed communications that replaces the current schemes of communication networks, characterized by having rigid and centralized infrastructures, by total architectures or mostly decentralized and self-organizational. (Liao et al., 2013) In these new architectures, the different tasks of management, constitution of the network and routing of data have to be done in a distributed and cooperative way, minimizing the use of resources to the maximum (Shen et al., 2016). The fact of changing more traditional techniques towards a more decentralized paradigm has given rise to the Wireless Sensor Networks and the Internet of Things concept, which allow the creation of monitoring networks with the ability to 
publish global information through the Internet.

Admitting that the structure of the communications network will be hierarchical, it is possible to identify the different parts, their functions and the most relevant technologies.

1. At the top level of the network you have Internet that will give connectivity to data collectors and network management elements.

2. At the level immediately below would be what we call access network, which will be formed in the first instance by an operator network with 4G / 3G / GPRS radio interfaces, can be combined with Wimax (IEEE 802.16) (Andrews et al., 2007). This network will ensure coverage at any point in the area to be covered.

3. Finally, at the lowest level is the catchment network, responsible for connecting the sensor to the network. This collection network will aim to facilitate the deployment and low consumption of the sensor nodes, using selforganization techniques that will provide this network with autonomy and great adaptation to possible failures and the incorporation of new sensors. The distribution and reception networks will allow communications between neighboring nodes of the same level, so that it is possible to use distributed process techniques in the different functionalities of these networks (multi-interface routing algorithms, load balancing, QoS mechanisms, distributed signal processing, etc.). Some technologies within this section are: IEEE 802.11 (WiFi), IEEE 802.15.1 (Bluetooth), IEEE 802.15.4 (ZigBee), LoRaWAN (Schroder et al., 2016).

Once the architecture is identified, we proceed to describe the state of the art of the different technologies that sustain the research on the network, starting with the catchment network in which the wireless sensor networks formed on a meshed network or in a network would be framed. wide-area low power.

\section{Meshed networks as a catchment network}

Although sensor networks support different types of architectures, mesh networks are currently the most advisable, since they offer great ease of deployment and autonomy. On the other hand, this type of networks present scalability problems (even more considering dozens of nodes) and therefore, hierarchical solutions around clusters will be the most suitable to guarantee the necessary size (Liao et al., 2013). A meshed network is a wireless network in which not all nodes are in direct view of the rest of the nodes, so to communicate with each other they need to establish a multi-hop path. In addition, the network operates in a distributed manner and has a selfconfiguration capability. The advantages of this type of network are: a) an extension of the network coverage without the need to increase the transmission power (with the consequent improvement of the battery life time) or the sensitivity of the receiver, b) greater reliability thanks to the existence of path redundancy and c) simple network configuration.

This technology is being rapidly accepted in areas with strict requirements (metropolitan networks, company networks, automation of buildings and factories, etc.). The technology that best adapts to the characteristics of meshed networks due to its reduced power consumption and low cost are networks based on the IEEE 802.15.4 (ZigBee) standard, although communications based on IEEE 802.11 (WiFi) can also be applied and IEEE 802.15.1 (Bluetooth).

\section{Low-power wide area networks as a catchment network}

Low-power wide area networks (LPWAN) (Centenaro et al., 2013) have been designed to work with nodes powered by batteries and constitute a serious alternative to meshed networks for the constitution of the catchment network. They can be organized as a network at a regional, national or global scale and allow a secure two-way communication, with links reaching distances of several kilometers. The main difference with meshed networks is their structure, since in this case there is no multi-hop communication, and communication is established directly between a sensor node and a base station that acts as a gateway. The LPWANs are designed to provide interoperability and interconnection in the deployment of networks based on the concept of the Internet of Things, whose main applications are M2M (machine to machine) communications and smart meters. Some examples of technologies within this group are SigFox and LoRaWAN.

For the interoperability of the various levels that make up the network, the integration of protocols that allow a direct transfer of information is fundamental. In this scenario the IP protocol stack, on which the operation of the Internet is based, provides an ideal environment to be able to have versions adapted to the characteristics of meshed networks with the 6Lowpan standard (IPv6 on Low power Wireless Personal Area Networks) (Ramos et al., 2013). This project aims to provide a global solution adapted to the intrinsic requirements of agricultural systems that have strong restrictions in terms of power consumption, accessibility to the nodes and reliability of the network.

\section{Determination of water needs of crops}

In agricultural science, the determination of the water needs of a crop over time is based on measures of water 
balance in the soil, plant measures and evaporative demand. The use of soil moisture measurements has been adopted as an adequate strategy for estimating water balance in the soil and many methods have been developed to measure it (Albaugh et al., 2014). The ultimate goal of these techniques is to provide farmers with information on the most appropriate irrigation volumes to apply in each phenological period of the crop, depending on the desired levels of yield and other parameters. For the determination of soil moisture, a wide range of sensors is used (Vienken et al., 2013). There is a wide range of techniques for measuring soil moisture based on electricity and they have been applied in geophysical prospecting (Linck and Fassbinder, 2014) and agronomy (Fatas et al., 2014), among others. These measurement techniques include resistivity methods such as the Wenner method (Jiao-Jun et al., 2014), Schlumberger matrices (Mosuro et al., 2012), capacitive methods such as frequency domain reflectrometry (FDR) and time domain reflectrometry (TDR) as well as radiation-based techniques such as the neutron probe (Kodikara et al., 2014). The most simple are resistivity techniques, which are affected by a great variety of soil conditions such as composition (Kibria and Hossain, 2014), texture, variation of pH, salinity and temperature (Newill et al., 2014) can have a high effectiveness in detecting relative changes in soil moisture levels. In particular, soil temperature can affect the electrochemical properties of the soil sampled. To complicate matters further, soil temperature can vary significantly by a few centimeters deep in areas where there are significant changes in surface between day and night (Brocca et al., 2014). This is reflected as a temporary delay as depth increases (Huang et al., 2013).

In Mediterranean agriculture and particularly in the Spanish southeast, the availability of water in the soil is one of the main limitations for the practice of economically sustainable agriculture. For this reason, proper management of irrigation is the main determinant of the quality and quantity of the harvests obtained and involves determining the water needs of the crops and an optimal irrigation schedule.

Irrigation programming must answer two basic questions: a) when should it be irrigated? b) with what amount of water? The first case (when?) Requires that the irrigation period or time elapsed between two irrigations be determined. The second (how much?), Requires that the dose (or volume, or height, or application) of water be defined. To correctly program the irrigation, a certain number of basic information and knowledge (sometimes formalized in "models") are required, which are presented in Figure 1. With these elements, and the decision rules that the farmer will use ( or the irrigation computer with its control algorithms), the dose and / or irrigation frequency will be determined.

Irrigation programming methods

Mainly, three methods of irrigation programming are distinguished

1- Programming based on climate data.

An estimation of the climatic demand and the water needs of the crops (empirical formulas, evapotranspiration models, etc.) and the establishment of the water balance of the soil are carried out. This method, frequently used and recommended by FAO, requires baseline data relating to soil and crop components and climate data in real time, or referred to one-year "average" climate statistics. "And the probability of precipitation. Its main advantages are that it does not need measurements of the water status of the soil and / or the plant, and that it allows an average estimate, valid at the scale of the plot.

Reference evapotranspiration

The standardization by FAO of the estimation method of ETo, from the Penman-Monteith formula has been widely adopted by different users, and implemented as a basic tool in many applications for programming and automation of irrigation. Many authors recommend the use of this method (in the following, ETo FAO-56) because it provides consistent results in diverse climatic conditions.

The formula ETo FAO-56 was developed for a hypothetical crop of well watered grass, with an assumed height of $0.12 \mathrm{~m}$, with a fixed surface resistance $70 \mathrm{~s} \mathrm{~m}^{-1}$ and an albedo of 0.23 . In addition to air temperature and solar radiation, the FAO-56 requires knowledge of air velocity and vapor pressure deficit, which are generally obtained from nearby climate stations.

Crop coefficient

To obtain the standard crop evapotranspiration (ETc), a cultivation coefficient is applied, Kc, which takes into account the development phase of the cultivated species, the type of irrigation (sprinkling, localized ...) as well as the cultivation techniques (density of plantation, pruning ...). FAO proposes Kc values for a large number of species under standard crop conditions. An adjustment of this coefficient is needed when the conditions differ from the standard conditions in which it was obtained (Kato and Kamichika, 2006).

\section{2- Programming based on soil water status.}

The programming is based on measurements of the characteristics of the water status of the soil (water content, water potential, water balance, etc.). There is currently a long range of moisture sensors and soil water potential, with analog or numerical outputs usable for soil programming purposes (Thompson et al., 2007). It is a 
programming method widely used by farmers, due to its simplicity, but it has the disadvantage of giving specific measures, which may sometimes be not representative of the plot.

\section{3- Programming based on the water status of the crop.}

The programming is based on measurements of the characteristics of the physiological state of the crop (plant temperature, variation of diameter of organs, stem water potential, flow of sap ...). It is a relatively recent and promising method, based on the evaluation of the stress level of the crop (stress index), but which still presents some difficulties for practical applications. One of the biggest problems inherent to these sensors (or stress indicators), besides the cost of the sensors, is the interpretation of the data and the criteria to be used in irrigation programming. These methodological problems, nowadays, are not totally solved and follow the object of numerous studies on the part of the scientific community (McCarthy et al., 2014).

All of the above suggests that, for reasons of robustness, reliability and cost, it is advisable to combine the climatic method with measurements of soil water status. In response to this need, in 2008, a coordinated research project was requested from the Seneca Foundation to develop a commercial system that would help to program irrigation based on the combination of the two methods, entitled "Development and Evaluation of a Wireless Horticultural Crop Monitoring System. Application to Irrigation Programming ". The system was constituted using an agroclimatic station managed by a compact CompactRIO controller, which collected and processed the information from the climatic sensors and the wireless network of humidity and soil salinity sensors, located in the different textural zones of the plot. monitor. The algorithms of irrigation programming implemented, served as a guide to the technician of the farm to manage the available water resources adapting them to the real needs of the crops. Its application allowed to maintain the same production and quality, with a substantial saving of water and consequently of energy, with values of economic savings that even reached $40 \%$ depending on the type and period of cultivation (Fernández-Pacheco, 2014).

Despite the great advantages of this system, a series of drawbacks were observed that limited its commercial applicability by: i) requiring the in-situ determination of the Kc, for each crop, area and time of year, and ii) the numerous calibration, installation and management failures with soil moisture sensors. On the other hand it was found that access to the SCADA irrigation management system embedded in the compact controller via the $3 \mathrm{G}$ connection, required a large data consumption, so if there is no Wi-Fi network available on the farm, it is It is advisable to host the SCADA in an external Hosting or in your own server.

\section{In-situ determination of the $\mathrm{Kc}$}

The determination of Kc, requires knowing: i) the actual evapotranpiration of the crop, ETc, and ii) the reference evapotranspiration, ETo. It is recommended to use weighing lysimeters to measure ETc because they are the most accurate, but due to its high cost it is used in many occasions to estimate it through Bowen or Eddy Covariance stations. The determination of ETo, should be made from the Penman-Monteith formula.

After obtaining the values of $\mathrm{Kc}$ in the different test plots, it was observed that the values proposed by FAO were not adapted to the actual cultivation conditions. In an attempt to obtain more adequate values of Kc, the vegetation cover was measured using digital photography techniques for its estimation. Despite the fact that Kc values were closer to reality in certain phases of the crop, they were completely different from those measured in other phases, so a new methodology based on digital photography was developed to obtain the equations for their local employment, with excellent results, but with the disadvantage that the equations had to be adjusted for each zone, cycle and type of crop (Escarabajal et al., 2015), making it extremely difficult to implement an automatic irrigation programming system. In spite of these limitations, the interest for having a remote visual supervision of the state of the crop, in continuous, that allowed to study the evolution of the growth of the crop and to help in the adequate management of the same, was revealed.

\section{Calibration, installation and irrigation management with soil moisture sensors.}

Before measuring soil moisture, it is necessary to know the characteristics of the soil and the precision that is required to choose the sensor to be used appropriately. Once selected, its calibration curves should be studied for the different types of soils that were used in the manufacturer's tests. The usual thing is that the floor where they are installed is not exactly the same as those provided by the manufacturers, so it will require a new calibration. The most appropriate and simple way to carry out this process, would be using weighing lysimeters that incorporate the ground where the sensors will be used, but due to the associated cost, it recurs to carry out soil surveys with varying degrees of humidity for its Subsequent analysis in the laboratory and adjustment of humidity curves. This necessary step is ignored by most users, considering the values indicated by the manufacturer by default, with consequent measurement errors (Dimitrov et al., 2014).

The installation is also key when using this type of sensors, since the scanned volume is limited to a very small area where the sensor is located. Any error in the installation will cause incorrect readings of soil moisture and, 
therefore, poor irrigation management. So that this does not happen, there can be no stones, no air gaps, and it has to be perfectly joined to the surrounding terrain. Consequently, in most cases the terrain is adapted to the needs of the sensor so that it can make the measurement, obviating the true nature of the land where it is intended to grow (Zhao et al., 2014).

All of the above suggests that, to carry out the automatic programming of irrigation adapted to the reality of the soil, the climatic zone, the type of crop, the vegetative state and the cultivation techniques used, without the need to determine in-situ the values of Kc, the use of a network of soil-plant atmosphere sensors is required, but at a cost and size adapted to the needs of the horticultural farms, which also allow knowing different parameters to adjust the irrigation schedule and that incorporate wireless cameras for remote monitoring of crop status.

Given the drawbacks of the methods that until now have been used in precision irrigation programming, this research group developed low cost sensors within a precise, easily implemented and low cost system, complementing the measurements obtained from a lysimeter. These sensors were developed thanks to the national research project "Balances of water and carbon in vine: Effects of the variety, soil and climatic conditions and cultivation techniques" AGL2011-30408-C04-04, financed by MINECO (Spanish Ministry of Economy and Competitiveness), within this same call, between 2011 and 2014. The project included five work packages or subprojects. Specifically, the results obtained in package No. 4 "Evaluation of the effect of certain agricultural practices on the water and carbon balance and the quality of production and cultivation" over the three years, served as the basis for developing this proposal. In this subproject, researchers from the Valencian Institute of Agrarian Research (IVIA), the Miguel Hernández University of Elche (UMH) and the Polytechnic University of Cartagena (UPCT) jointly worked. Within this subproject, and with the purpose of establishing the bases for the determination of the influence of the inclination of the trellis on the water consumption and the vegetative response of the vineyard variety Bobal cultivated in a pot, the specific objective was to develop a device that allows to determine with accuracy and, in real time, the value of the evapotranspiration and of all the components that intervene in the equation of the water balance. The UMH researchers were responsible for the agronomic trials and the monitoring of the developed technology, as well as the development of low-cost sensors (Oates et al., 2015). Until reaching the specific objective, several developments were carried out. The first development consisted of the design and evaluation of a system to determine the water balance of an experimental plantation consisting of four rows of eight vines (Vitis vinifera L. cv Bobal) from the water balance obtained by measuring flows, method similar to volumetric lysimeters. Due to the limitations mentioned to be able to carry out an irrigation control with the proposed system, the research group designed and manufactured a weighing lysimeter for low cost pot crops, which was patented and is in operation. The electronics were developed to constitute a compact weighing lysimetry station through a wired modbus network connected to an agroclimatic station.

Along with these developments, several models of low-cost soil moisture sensors were advanced. The sensors were based on the Wenner resistivity method (Figure 2) and FDR and worked properly. These sensors replaced the commercial models that had been initially designed to control the humidity of the vineyard (Vitis vinifera $\mathrm{L}$ cv Bobal in pattern 110R) in a pot with localized irrigation. The sensor was powered by three $1.5 \mathrm{~V}$ batteries and used a 64kB PIC18F26K22 of memory with limited data storage and an expandable CMOS Flash chip for data transmission. The ARDUINO software was used for programming. In the same way, a brightness measurement sensor was developed from doped LEDs.

The data obtained from the irrigation management with low cost sensors showed that the risks were carried out according to the algorithms programmed in the system and that both the frequency and the duration of the same in the lysimetric mode were consistent with the rates of transpiration registered. However, the system had limitations to be used in commercial horticultural farms.

\section{Materials and Methods}

All the exposed previously have been reflected in diverse publications and patents, obtaining a considerable scientific production and technological development.

The system developed for the automatic control of irrigation by soil moisture sensors presented the following limitations for its implementation in commercial horticultural farms:

- Parameters such as soil conductivity were not taken into account in order to automatically determine the leaching ratio for each irrigation.

- Impossibility of remote access by several users with different permissions depending on the level of execution.

- The controller sent the data to the server in the cloud as files in CSV format, which made it difficult to manage them as a database for the creation of records, reports, specific data search on specific dates, etc.

- The system did not have the capacity to manage, within an agricultural holding, different plots and even within these plots several sectors, and the data read are not stored taking into account the agricultural campaign.

- Access to the program screens, that is, the SCADA display, could only be done through the use of remote 
panels offered by the controller that was used during the previous development. This implied the need to have a specific runtime of National Instruments installed on the computer from which the compact controller is to be accessed and the obligation to use Internet Explorer for visualization.

- In a horticultural farm, the distance between the sensors and the irrigation head can be greater than $1200 \mathrm{~m}$. limit of the Modbus / RS485 network used.

To correct these deficiencies, a redesign of the sensors and their connections is necessary, as well as improvements in the automatic irrigation control system to adapt it to commercial operations and an adaptation of the control algorithms for the integral irrigation management. The use of BDA techniques allows improving the current algorithms and models in order to obtain a robust system based in the obtained database. The new irrigation management system, for use in horticultural crops, must meet the following requirements:

- Portable, therefore it should be possible to change its location. For this the system will be formed by components of reduced dimensions and low cost.

- Precise: In order to accurately determine the amount of water supplied in the irrigation system, two low flow and cost flow meters will be installed.

It should have a compact controller in the irrigation head, which will receive the data from the sensors and will house the algorithms for automatic irrigation control. On the other hand, it will incorporate the possibility of receiving data from agroclimatic stations and soil moisture sensors, to give the possibility of automatic irrigation control, using traditional methods, in plots where sensors are not yet available. The obtained and analysed metadata will generate new models of growth behavior; provide more efficient irrigation and fertilization schedules; spot developing and emerging trends / correlations; and predict yield, pest likelihood, and disease spread.

The wired network will be replaced by a wireless network, which will have four nodes with the following functionalities:

- Irrigation head control node: it will be formed by a CompactRio platform that will be the sink of all the data read by the different nodes and will send this data to the cloud.

- Meteorological station node: It will have the mission of reading the data of the connected meteorological station and sending them to the control node.

- Repeater nodes: They will be installed in points where the coverage of the link does not allow the communication of distant nodes with the control node.

- Node of sensor, agricultural and image data: It will have the function of reading the data of the weighing lysimeters, of the ground sensors that are installed and of a camera that will collect the image of the plant and that will allow to establish the vegetative state Of the same.

The proposed architecture for the automatic irrigation scheduling system is shown in Figure 1.Provide sufficient details in the materials and methods section to allow your work to be reproduced.

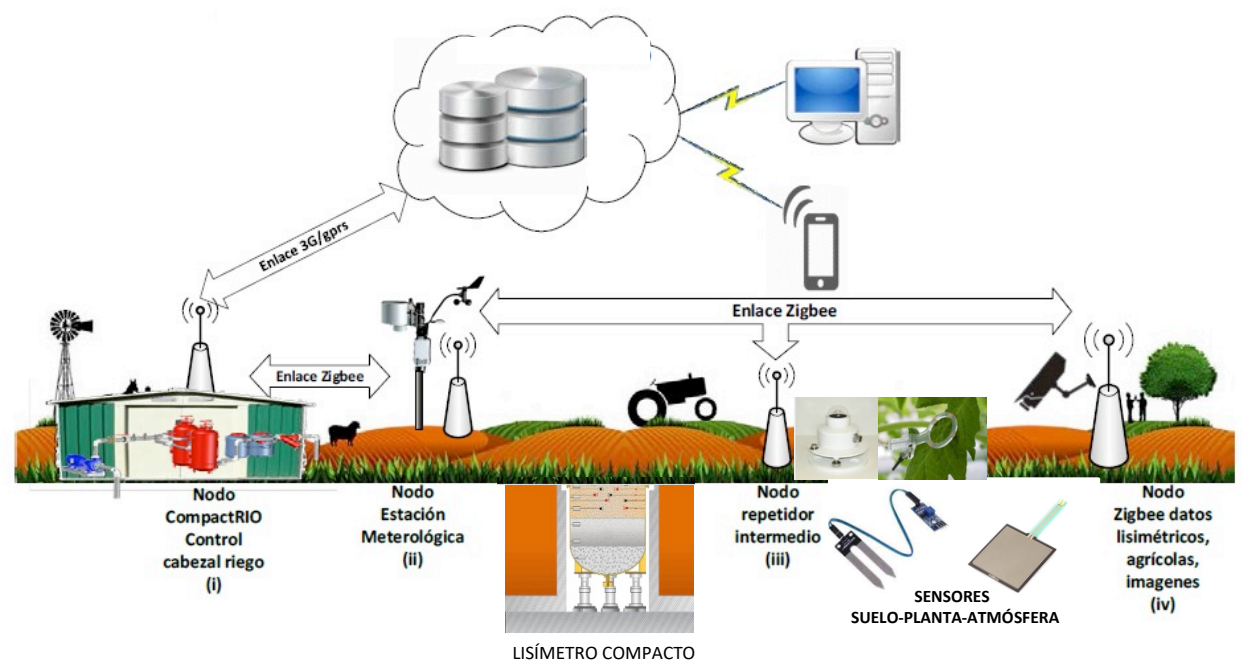

Figure 13. Architecture of the new system of automatic irrigation programming in commercial horticultural farms.

Although the compact controller will manage the irrigation independently, the data will be sent to a hosting, through Wifi or GPRS / 3G link, where a database will be managed and allow access to the SCADA from any computer or mobile device through specific APPs, able to interact with the compact controller, change slogans, view historical data, view data in real time, etc. 
Therefore, in this project, it is intended to develop a system that allows automatic programming of irrigation from soil-plant-atmosphere sensor data developed for farms with horticultural crops, whose information is sent wirelessly to a compact controller located in the irrigation head, housing the irrigation management algorithms responsible for processing said information, in real time, and giving the irrigation control orders according to the real needs of the crop. All the information will be sent via Wifi / GPRS / 3G / 4G to an external Hosting where all the information is stored and displayed in an orderly fashion through a friendly user interface, accessible from any web browser or through mobile applications designed for that purpose. providing the services associated with any SCADA such as historical management, warnings and alarms, changes of instructions, etc., and adding the monitoring of the status of the crop by wireless cameras (González-Esquiva et al., 2017).

This innovative system, which has no known precedents, will allow irrigation to be programmed efficiently, with precise control of water inputs, minimizing water and energy consumption, and maximizing the production, quality and profitability of horticultural farms. It will also connect to the network designed in parallel in order to obtain data in real time and manage the irrigation network at great distances and in areas with low coverage.

\section{Results and Discussion}

The code used to use 9-bit serial / USART communication must be strongly programmed to ensure robust and reliable communication so that it does not cause problems for long periods of time.

One problem that arose was how to get the microcontroller to address a data or address received by the serial port while it was in operating mode. There is the option of "Interruptions". In this case the microcontroller interrupts the usual execution of the program when an event occurs, performs a previously programmed code and returns to where it stayed before. By modifying the software, a code is included that manages the information received and classifies it according to whether it is an address or a data / function.

When the microcontroller has detected the information input it can differentiate if it is an address or a data, if it has received its own address and then a data is in fact the master node that has called it and orders it to perform a function. This allows us to make as many different cases as necessary to carry out certain processes.

\section{Conclusions}

The use of this network of low-cost devices for the monitoring of environmental parameters related to the management of water and energy in horticultural irrigation allows the integration of varied and large-volume information in agrarian environments, to constitute a scalable and open network. It is based on IP protocols, which allows to free applications from the need to know the configuration of communications and network management.

With this network, an open, scalable and modular network architecture is achieved that will allow access to information and system management from any device connected to the Internet.

This network will allow the integration of a low cost system, for commercial use in horticultural farms, that measure and manage irrigation accurately, in real time. The system allows obtaining specific agronomic data for large-scale applications.

Another of the improvements achieved by this system is the saving of water and energy for irrigation.

Through this technology advances are made in systems to optimally program the irrigation of horticultural crops through soil-plant-atmosphere sensors.

It is also possible to reduce the environmental impact of irrigation: the optimization of irrigation and, therefore, the reduction of fertilizers provided during irrigation, will prevent the contamination of aquifers and help solve the problem of marine eutrophication by reducing nitrates percolated, and the reduction of energy consumption will mean a reduction in the emission of greenhouse gases.

The use of this network in other analogous sectors opens a wide range in its final commercialization.

\section{Acknowledgements}

Authors are grateful with the support from the Spanish Ministry of Economy and Competitiveness (MINECO) in the project Sistema De Programacion Automatica Del Riego Por Lisimetria De Pesada Y Salinidad Del Suelo, Con Supervision Remota Del Estado Vegetativo Del Cultivo, with reference AGL2015-66938-C2-1-R.

\section{References}

Albaugh, J.M., Domec, J.-C., Maierc, C.A., Sucre, E.B., Zakiya, H., Leggettd, Z.H., King, J.S., 2014. Gas exchange and stand-level estimates of water use and gross primary productivity in an experimental pine and switchgrass intercrop forestry system on the Lower Coastal Plain of North Carolina, U.S.A. Agric. For. Meteorol. 192-193, 27-40. 
Andrews, J. G., Ghosh, A., \& Muhamed, R. Fundamentals of WiMAX: understanding broadband wireless networking. (2007) Pearson Education.

Brocca, L., Camici, S., Melone, F., Moramarco, T., Martinez-Fernandez, J., Didon-Lescot, J.F., Morbidelli, R., 2014. Improving the representation of soil moisture by using a semi-analytical infiltration model. Hydrol. Processes 28 (4), 2103-2115.

Centenaro, M., Vangelista, L., Zanella, A., \& Zorzi, M. Long-range communications in unlicensed bands: The rising stars in the IoT and smart city scenarios. IEEE Wireless Communications, 23(5), (2016). Pages 6067.

Delamo, M., Santiago Felici-Castell, Juan J. Pérez-Solano, Andrew Foster, Designing an open source maintenance-free Environmental Monitoring Application for Wireless Sensor Networks, Journal of Systems and Software (Elsevier), Volume 103, May 2015, Pages 238-247, ISSN 0164-1212.

Dimitrov, M., Vanderborght, J., Kostov, K.G., Jadoon, K.Z., Weihermüller, L., Jackson, T.J., Bindlish, R., Pachepsky, Y., Schwank, M., Vereecken, H., 2014. Soil Hydraulic Parameters and Surface Soil Moisture of a Tilled Bare Soil Plot Inversely Derived from L-Band Brightness Temperatures. Vadose Zone J. 13, 0. doi:10.2136/vzj2013.04.0075

Evans, R.G, y Buchleiter, G. W. 2000. "Controls for Precision Irrigation with Self-Propelled Systems."

Fatas, E., Vicente, J., Latorre, B., Lera, F., Vinals, V., Lopez, M.V., Blanco, N., Pena, C., GonzalezCebollada, C., Moret-Fernandez, D., 2014. TDR-LAB 2.0 Improved TDR Software for soil water content and electrical conductivity measurements. Procedia Environ. Sci. 19, 474-483 (FOUR DECADES OF PROGRESS IN MONITORING AND MODELING OF PROCESSES IN THE SOIL-PLANT-ATMOSPHERE SYSTEM: APPLICATIONS AND CHALLENGES).

Fernandez-Pacheco, DG; Molina-Martinez, JM; Jimenez, M; Pagan, FJ; Ruiz-Canales, A. 2014. SCADA Platform for Regulated Deficit Irrigation Management of Almond Trees. JOURNAL OF IRRIGATION AND DRAINAGE ENGINEERING, 140 (5). 04014008. DOI: 10.1061/(ASCE)IR.1943-4774.0000718

Goëau, H., Bonnet, P., Joly, A., Bakic' , V., Barbe, J., Yahiaoui, I., Selmi, S., Carré, J., Barthélémy, D., Boujemaa, N., Molino, J.-F., Duché, G., Perronet, A. 2013. Pl@ntnet mobile app. In: Proceedings of the 21st ACM International Conference on Multimedia, pp. 423-424.

González-Esquiva, J.M., Oates, M.J., Garcia-Mateos, G., Moros-Valle, B., Molina-Martinez, J.M., RuizCanales, A. 2017. Development of a visual monitoring system for water balance estimation of horticultural crops using low cost cameras. Computers and Electronics in Agriculture, 141: 15-26. DOI: 10.1016/j.compag.2017.07.001.

Huang, T.M., Pang, Z.H., Edmunds, W.M., 2013. Soil profile evolution following land-use change: implications for groundwater quantity and quality. Hydrol. Processes 27 (8), 1238-1252.

Jiao-Jun, Z., Hong-Zhang, K., Gonda, Y., 2014. Application of Wenner configuration to estimate soil water content in pine plantations on sandy land. Pedosphere 17 (6), 801-812.

Jiménez-Buendía, M., L. Ruiz-Peñalver, J. A. Vera-Repullo, D. S. Intrigliolo-Molina, and J. M. MolinaMartínez. 2015. "Development and Assessment of a Network of Water Meters and Rain Gauges for Determining the Water Balance. New SCADA Monitoring Software." Agricultural Water Management, New proposals in the automation and remote control of water management in agriculture: agromotic systems, 151 (March): 93-102. doi:10.1016/j.agwat.2014.11.013.

Kato, T; Kamichika, M. 2006. Determination of a crop coefficient for evapotranspiration in a sparse sorghum field. IRRIGATION AND DRAINAGE, 55 (2): 165-175. DOI: 10.1002/ird.225

Kibria, G., Hossain, M.S., 2014. Investigation of geotechnical parameters affecting electrical resistivity of compacted clays. J. Geotech. Geoenviron. Eng. 138 (12), 1520-1529.

Kodikara, J., Rajeev, P., Chan, D., Gallage, C., 2014. Soil moisture monitoring at the field scale using neutron probe. Can. Geotech. J. 51 (3), 332-345.

Liao, Y., Qi, H., \& Li, W.. Load-balanced clustering algorithm with distributed self-organization for wireless sensor networks. IEEE sensors journal, 13(5) (2013), Pages 1498-1506.

Linck, R., Fassbinder, J.W.E., 2014. Determination of the influence of soil parameters and sample density on ground-penetrating radar: a case study of a Roman picket in Lower Bavaria. Archaeol. Anthropol. Sci. 6 (1), $93-$ 106.

López-Urrea, R., A. Montoro, and T. J. Trout. 2014. "Consumptive Water Use and Crop Coefficients of Irrigated Sunflower.” Irrigation Science 32 (2): 99-109. doi:10.1007/s00271-013-0418-9.

Marsal, J., Johnson, S., Casadesus, J., Lopez, G., Girona, J., Stöckle, C., 2014. Fraction of canopy intercepted radiation relates differently with crop coefficient depending on the season and the fruit tree species. Agric. For. Meteorol. 184, 1-11. doi:10.1016/j.agrformet.2013.08.008

McCarthy, MJ; Csetenyi, LJ; Sachdeva, A; Dhir, RK. 2014. Engineering and durability properties of fly ash treated lime-stabilised sulphate-bearing soils. ENGINEERING GEOLOGY, 174: 139-148. DOI: 10.1016/j.enggeo.2014.03.001 
Mosuro, G.O., Bayewu, O.O., Oloruntola, M.O., 2012. Application of vertical electric soundings for foundation investigation in a basement complex terrain: a case study of Ijebu Igbo, Southwestern Nigeria. In: Near-Surface Geophysics and Environment Protection, pp. 29-34.

Newill, P., Karadaglic, D., Podd, F., Grieve, B.D., York, T.A., 2014. Electrical impedance imaging of water distribution in the root zone. Meas. Sci. Technol. 25 (5), http://dx.doi.org/10.1088/0957-0233/25/5/055110 (Number of article: 055110).Oates et al, 2015

M.J. Oates, A.L. Vázquez de León, D.S. Intrigliolo, J.M. Molina Martínez, A. Ruiz-Canales. 2015.

Evaluation of an experimental system of soil moisture registration for irrigation management in potted vineyard (Vitis vinifera L. CV Bobal) of multi-depth temperature compensation based in resistivity measurements. Agricultural Water Management 151 (2015) 126-135.

Panda, R.K., Patra, S., Halder, D., 2014. Low cost PVC hydraulic weighing lysimeter for measurement of crop evapotranspiration. Acta Hortic.

Ramos, M. D., Foster, A. D., Felici-Castell, S., Fos, V. G., \& Solano, J. J. P. Gatherer: an environmental monitoring application based on IPv6 using wireless sensor networks. International Journal of Ad Hoc and Ubiquitous Computing, 13(3-4), (2013). Pages 209-217.

Ruiz-Peñalver, L., J. A. Vera-Repullo, M. Jiménez-Buendía, I. Guzmán, and J. M. Molina-Martínez. 2015. "Development of an Innovative Low Cost Weighing Lysimeter for Potted Plants: Application in Lysimetric Stations." Agricultural Water Management. doi:10.1016/j.agwat.2014.09.020.

Schroder Filho, H. G., Pissolato Filho, J., \& Moreli, V. L. The adequacy of LoRaWAN on smart grids: A comparison with RF mesh technology. 2016 IEEE International Smart Cities Conference (ISC2), (2016, September). (pp. 1-6).

Shen, H., \& Bai, G.. Routing in wireless multimedia sensor networks: A survey and challenges ahead. Journal of Network and Computer Applications, 71, (2016) Pages 30-49.

Thompson, R. B., M. Gallardo, L. C. Valdez, and M. D. Fernández. 2007. "Using Plant Water Status to Define Threshold Values for Irrigation Management of Vegetable Crops Using Soil Moisture Sensors." Agricultural Water Management 88 (1-3): 147-58. doi:10.1016/j.agwat.2006.10.007.

Vera-Repullo, J. A., L. Ruiz-Peñalver, M. Jiménez-Buendía, J. J. Rosillo, and J. M. Molina-Martínez. 2015. "Software for the Automatic Control of Irrigation Using Weighing-Drainage Lysimeters." Agricultural Water Management, New proposals in the automation and remote control of water management in agriculture: agromotic systems, 151 (March): 4-12. doi:10.1016/j.agwat.2014.10.021.

Vienken, T., Reboulet, E., Leven, C., Kreck, M., Zschornack, L., Dietrich, P., 2013. Field comparison of selected methods for vertical soil water content profiling. J. Hydrol. 501, 205-2012.

Zhao, Z., Zhang, H., Li, C., Zhao, Q., Cao, L., 2014. Quantifying nitrogen loading from a paddy field in Shanghai, China with modified DNDC model. Agric. Ecosyst. Environ. 197, 212-221. doi:10.1016/j.agee.2014.08.002. 


\title{
Design and Performance of an Unmanned Aerial System for Precision Pest Management
}

\author{
Jorge Martínez-Guanter a , Pablo Agüera ${ }^{\text {b }}$, Alberto Sassu ${ }^{\text {b }}$, Juan Agüera Vega ${ }^{\text {c }}$, Manuel Pérez-Ruiz ${ }^{\text {a }}$ \\ a Aerospace Engineering and Fluid Mechanics Department, University of Seville, 41013 Seville, Spain \\ ${ }^{\mathrm{b}}$ Dronsap, a division of Agrosap, 41927 Mairena del Aljarafe, Spain \\ ${ }^{c}$ Dept. of Rural Engineering, University of Córdoba, Rabanales Campus 14014 Córdoba, Spain \\ * Corresponding author. Email: manuelperez@us.es
}

\begin{abstract}
The use of small-unmanned aerial vehicle retrofitted with spraying systems allows precision aerial applications on smaller targets.. These precision applications can result in significant savings if agrochemicals are applied only in affected areas and can contribute to avoid soil compaction caused by machinery or risks such as the exposure of operators to pesticides during treatments. In this context, this work aims at the design and development of a small application system capable of being mounted on an unmanned aerial vehicle for agrochemical spraying tasks and the subsequent analysis of the quality of the application and economic costs compared with a conventional treatment Once the equipment was developed and verified its response during flights, field trials on super-high-density olive orchards were performed to evaluate spray deposition at different spray heights, flow rates and nozzle settings. Spray deposition efficiency, droplet diameter and population and drift caused by the spraying system were analysed using water-sensitive papers at different positions on the tree structure. For the comparison with a conventional spraying equipment tasks, field tests took into account parameters such as the applied volume rate, the time performance, equipment costs and depreciation, etc., The first results obtained indicate that there is no great difference in the application costs between the one realized by the aerial vehicle and the conventional equipment. It is hoped that the conclusions of this work will serve as a basis for a debate about the existing legislation, in which this type of aerial works, which can be beneficial in specific cases, can be carried out under safe and legal conditions.
\end{abstract}

Keywords: Agrochemical application, UAV, sprayer, economic analysis, precision agriculture.

\section{Introduction}

Prevention of insects and other disease vectors plays a crucial role in current pest management. While there is widespread concern about the negative environmental consequences that may result from the application of pesticides, herbicides and other phytosanitary products in liquid form (Panneton et al., 2005), fumigation remains the most common form of application due to its low cost and efficiency (Giles et al., 2008). The implements and technologies used for this type of application have remained relatively unchanged over the last few years. This has motivated research in precision spraying, looking for a change in the way these plant protection products are applied. Thus, despite the fact that the use of hydraulic and hydro-pneumatic sprayers as implements alongside tractors is undeniably widespread in conventional herbicide and pesticide applications, the UAV technology in recent years has proven its usefulness in agriculture and vector control not only for observation and detection, but also for handling payloads, including the application of agrochemicals (Giles et al., 2015). The applying tasks by means of drones still present a considerable technical challenge, in which the payment load has a different behaviour and the power demand is significantly higher when compared to the usual tasks of capturing remote sensing information by means of sensors or cameras. In this type of aerial application, multi-rotor drones have a number of advantages over fixed-wing drones or spray drones, such as their flexibility and manoeuvrability in complex flight patterns, the non-requirement of a large landing/take-off area and the possibility of stationary flights over specific points on the ground (Zhang et al., 2016). In addition to these facilities in flight performance, the reduced exposure to chemicals and the ability to apply according to spatial and temporal variability in a plot make UAVs applications attractive from the point of view of precision agriculture. Previous developments with this type of unmanned systems for agricultural tasks such as the one carried out by Huang et al. (2009) were focused on the use of specific implements to be mounted on drones, such as the rotary electrostatic atomizer by $\mathrm{Ru}$ et al. (2011) or the study on the possibilities of coordinating a fleet of small multi-rotor vessels to carry out this type of task by Wang et al. (2013). Most of them showed a relevant aspect that has been taken into account in this work: the use of drones in spraying applications makes sense when they are used with ultra-low volume doses. These types of applications are considered key to precision plant protection, as they have achieved a higher percentage of the chemical applied being collected by the target surface than by conventional spraying (Bals, 1970). Despite this, they present technical challenges on the application, such as the relatively large size of the drops in the commercial nozzles, the high price of these or the difficulty in calculating the dose of active matter to be applied (Wen et al., 2016). Based on these considerations, this work has been developed with the aim of developing a low-cost modular spraying system, capable of being adapted to a commercial aerial vehicle and of 
carrying out ultra-low volume applications. In addition, a comparative analysis has been made of the economic costs of making an application with this vehicle as opposed to a conventional sprayer application, using as an example the treatment with a commercial insecticide that acts as bait for the olive fruit fly (Bactrocera oleae). For the rest of the document the system developed will be named ATHOS (Aerial Treatment for High Orchard System).

\section{Materials and Methods}

\section{UAV platform}

To implement the spraying system capable of applying the insecticide treatment tested, different platforms were evaluated at an initial stage based on the criteria of autonomy, load capacity, etc., selecting the DJI s1000+ commercial drone model (DJI, Shenzhen, China) from among them. This model of octocopter allowed vertical Take-Off and Landing (VTOL) and had an empty mass of about $6 \mathrm{~kg}$. This UAV can be flown remotely via a radio frequency transmitter, or autonomously, using its GNSS (Global Navigation Satellite System) receiver and its navigation and route generation system based on predefined waypoints. An ad-hoc telemetry system consisting of a radio modem mounted on board the drone and a ground receiving station provides real-time information on flight parameters (height above ground, horizontal and vertical velocities, position of the drone with respect to its 6 degrees of freedom, etc.), while an analogy video system allows real-time images to be obtained from a firstperson view and displayed on a mobile device. The maximum payload was $5 \mathrm{~kg}$, while in any case it is not possible to exceed $11 \mathrm{~kg}$ of total mass on take-off. The autonomy of the equipment by mounting the sprayer system described below has been measured on several flights with the maximum load, establishing an average flight autonomy of 14 minutes.

\section{Development of the spraying system}

The system was designed and built using low-cost materials and taking into account the limitations in the payload (Figure 1). Five elements compose the sprayer unit: the support structure, the tank to house the plant protection product, the nozzle arm or bar, the set of pipes and pump that make up the hydraulic system and the electrical spray control system. The tank, located at the bottom of the UAV, has a capacity of 5 ; the modular nozzle bar was designed to allow 2 spraying configurations: one with 4 working-nozzles with a $25 \mathrm{~cm}$ spacing between them and another with a single central anti-drift nozzle. Both the assembly and coupling frame structures with the UAV were designed and manufactured using 3D printed parts. Transparent PVC pipes with an inner diameter of $6 \mathrm{~mm}$ were selected for the pipes, while a small, independently supplied electric pump with a voltage of $12 \mathrm{~V}$ was used to drive the pipes into the circuit at a maximum operating pressure of 2.5 bar.
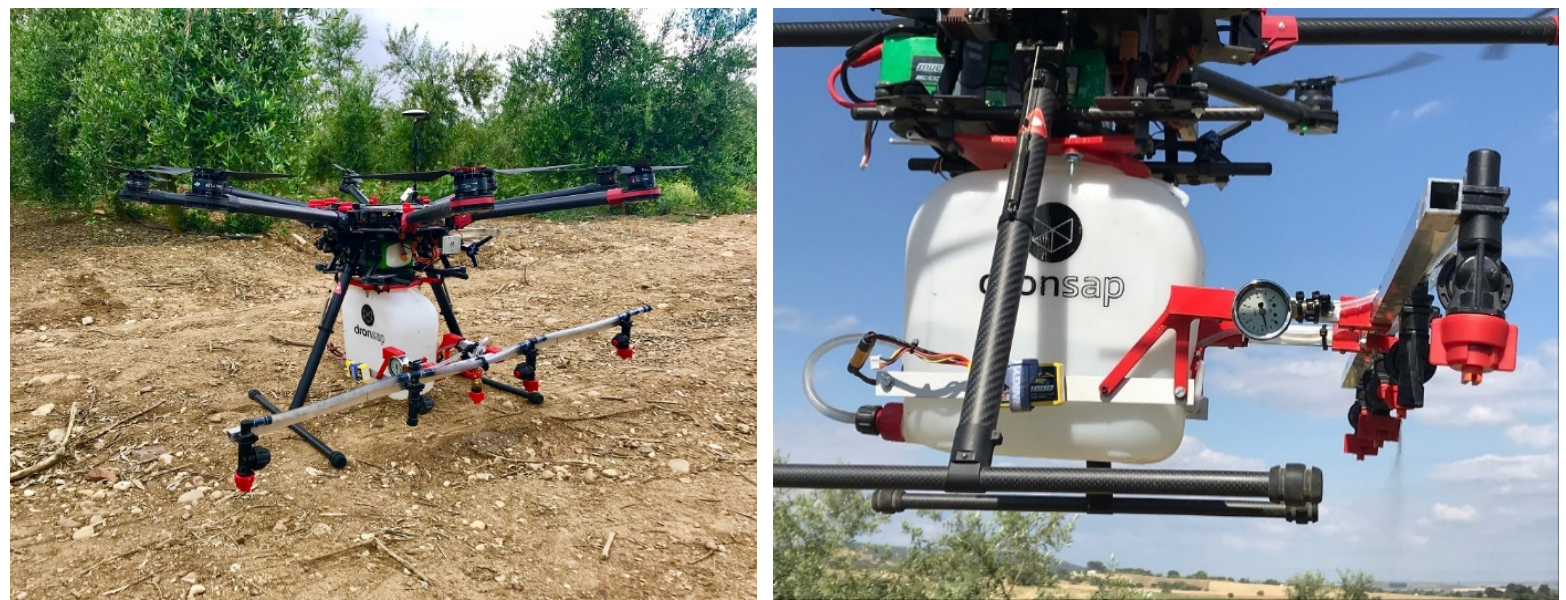

Figure 1. (left) Application equipment mounted on board the UAV (right) Detail on the spray system developed.

The spray control system allows the pump to be driven and its speed to be varied remotely from the UAV remote control station, as well as enabling autonomous application in specific areas using pre-established coordinates with the electronic system and GNSS. For the complete characterization of the equipment, laboratory tests were carried out in which the average flow rate of each nozzle in both configurations was determined at different pump regimes, measuring the liquid quantities with graduated laboratory test tubes. In addition, tests were carried out to determine the battery discharge curves with respect to weight and time, which allowed to characterize the autonomy of the equipment with different levels of payload, based on automatic recording of the telemetry received. 


\section{Field experiments}

In order to evaluate the efficiency of the application system, field tests were performed simulating the conditions of a real application. The field tests took place in an experimental exploitation of super-intensive olive trees in the facilities of the University of Córdoba. Four lines of olive trees were selected, over which flights were made with different pre-programmed routes (straight line, flights following a rectangular pattern, etc.), different application patterns and different forward speeds, with treatments being tested at 2,3 and $4 \mathrm{~m} / \mathrm{s}$ of horizontal forward speed at a height of 0.5, 1 and 2 meters above the tree canopy (Figure 2).

In these first application trials, a commercial formulation was chosen as the ingredient to be applied, which acts as bait for the olive fruit fly (Bactrocera oleae) from DOW AgroSciences. For this insecticide, whose active matter and composition is Spinosad at $0.024 \%$, an application rate of between $0.25-0.51 /$ ha is recommended to be applied in bands covering $25 \%$ of the total surface, and being diluted at a ratio of $1: 10$, which allows a treatment for 1 ha to be carried out with a deposit of the spraying system developed.

In the trees of two non-consecutive rows, two levels of height (upper and middle crown height) were established, which were covered with water-sensitive papers to evaluate the ability to deposit the spray on the tree. In addition, water-sensitive papers were placed in the rows adjacent to those treated, with the aim of capturing the possible drift formed by the application system in wind conditions. Self-developed software based on Python's OpenCV computer vision module was employed for the automatic analysis of water-sensitive papers. The droplet population, mean diameter and area covered were measured using this methodology.

\section{Economic feasibility}

Taking into account parameters such as the amount of product applied, the hourly output of the spraying equipment, the cost and payback period of the equipment, etc., a comparative study has been carried out for the same type of task if the aerial platform or conventional application equipment is used. With respect to the economic evaluation of the application using conventional equipment for localized application of fly bait, the calculations have been based on the technical considerations proposed by ASABE and MAGRAMA, collected by Boto et al. (2004). In these guidelines, the costs of agricultural operations are analysed by including: (i) fixed depreciation costs, interest, machinery accommodation, insurance and taxes; (ii) variable costs, including fuel, lubricants, tyre wear, overall repair and maintenance costs, and tractor labour; and (iii) the total hourly cost of operation, plus tractor and implement costs. For the economic evaluation of the application, a theoretical working scenario was established on an intensive olive grove plot similar to that described in Section 2.3, with a planting frame of $1.5 \times 3 \mathrm{~m}$, with a number of trees per hectare of approximately 2220 . In it, an ultra-low volume treatment is proposed, in which both equipment (both the tractor-implement and the drone) must cover the $50 \%$ of the field area (see Figure 2).
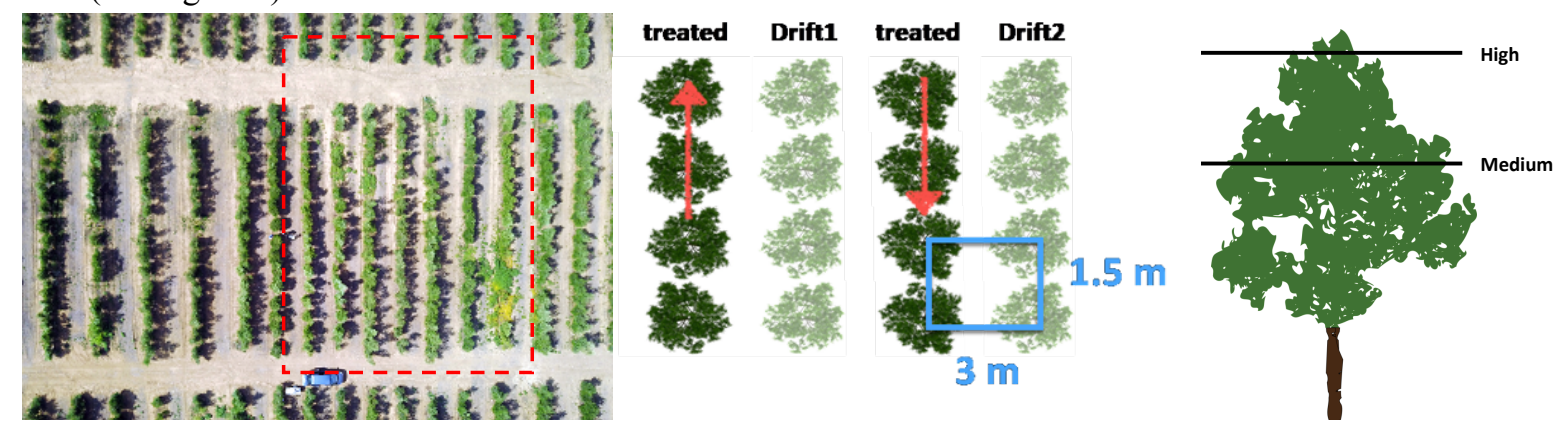

Figure 2. (Left) Aerial image of the field test area; (Centre) Diagram of the flight pattern over the olive trees and; (Right) Height measurement levels where water-sensitive papers were located on all the trees.

For the calculation of the application costs, the comparative economic study is carried out on the basis of a tractor with the characteristics listed in table 1 below and the ATHOS system described above.

Table 1. Characteristics of the tractor used in the economic assessment.

\begin{tabular}{cc}
\hline Rated Power & $78 \mathrm{~kW}$ \\
Max Power PTR & $70 \mathrm{~kW}$ \\
Acquisition Value & $30000 €$ \\
Annual working hours & $1000 \mathrm{~h} /$ year \\
Lifetime Period & 12 years \\
Working hours during lifetime period & $12000 \mathrm{~h}$ \\
\hline
\end{tabular}


Based on the preliminary results of the field trials and knowing the design and development costs, the economic assessment of the ATHOS system was carried out based on empirical data and a comparison was made with the previous analysis, since no relevant studies or bibliography have yet been found.

\section{Results and Discussion}

This section presents the preliminary results obtained with respect to the development of the application system and the comparative economic feasibility of the costs carried out between the ATHOS system and a conventional application.

\section{UAV-borne spraying system}

Once the components of the application system were integrated, the greatest complexity in the development of the platform was the need to achieve a stable and uniform flight. A number of adjustments had to be made to the centre of gravity situation and weight compensation, taking into account factors such as the possible "swell" of the liquid inside the tank during flight and the progressive decrease in the weight of the assembly as the operation is performed. It was noted that the first of these factors limits the maximum forward speed and manoeuvrability of the assembly, while the second affects the discharge curve of the battery and the power generated by the engines. In order to avoid both, the variations of the system's gravity centre during the flights were calculated and the tank support was built so that it was as close as possible to the structure, thus avoiding swaying in the air that could cause alterations in flight.

Regarding the application system, the flow rates of each of the nozzles were tested in the laboratory, collecting the samples in graduated laboratory probes and obtaining an average flow rate of $0.22 \mathrm{l} / \mathrm{min}$ for each of them at maximum pump speed. The results of the battery discharge tests and the evolution of the weight over time are shown in figure 3 below.
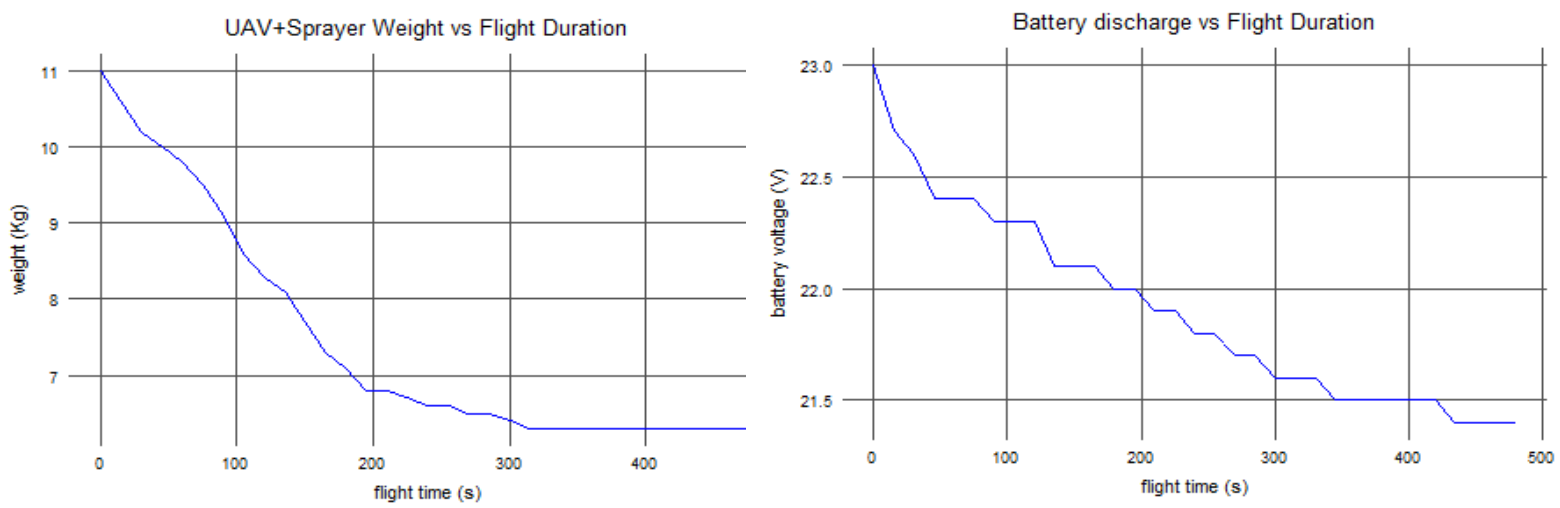

Figure 3. Curves of the evolution of the ATHOS equipment's weight (left) and the battery discharge (right) with respect to time.

From the two previous time evolutions it can be seen that the moment of maximum mass (corresponding to take-off and the first few seconds of flight) corresponds to the maximum power demand of the engines and therefore to the most marked decrease in the battery charge voltage. In addition, both graphs show that once all the phytosanitary product has been consumed (about 5 minutes or 300 seconds), the total weight of the set is stabilized at about $6 \mathrm{~kg}$, while the voltage of the barrel is still $21.8 \mathrm{~V}$. This battery charge still allows up to 10 more minutes of flight time (software warnings have been created for when the battery voltage reaches this moment), so that if the equipment had been moved a long way from the operator, there would still be time for safe return and landing.

\section{Spraying assessment using ATHOS-UAV}

In the field tests carried out to verify the correct performance of the developed aerial application system, different combinations of forward speeds and flight heights were evaluated. Final test conditions of $3 \mathrm{~m} / \mathrm{s}$ of horizontal speed and $1 \mathrm{~m}$ height above the canopy of were established. After each flight, samples of watersensitive papers were collected and analysed in the laboratory. A total of 100 papers were analysed automatically. The software developed takes as input each of the images, detects the edges corresponding to the perimeter of the hydrosensitive paper and then generates a binary image based on the threshold calculation of the values of the drops and the background. It then generates the contours of each of the elements detected in that binary image and calculates the corresponding outputs (Figure 4). One of the aspects to be evaluated was the ability of the team to effectively apply the product to the crop and to generate relatively uniform coverage. The distribution of droplet populations, the area covered by the droplets per $\mathrm{cm}^{2}$ and the average diameter of the droplets were calculated automatically, obtaining the results shown in table 2 . 
Table 2. Results of the image analysis of 100 water-sensitive papers after the ATHOS spraying application.

\begin{tabular}{cccc}
\hline $\mathrm{N}=100$ & Population $\left(\mathrm{drops} / \mathrm{cm}^{2}\right)$ & Coverage $\%$ & Avg. Drop diameter $(\mu \mathrm{m})$ \\
\hline min & 0.050 & 0 & 105 \\
median & 10.602 & 1 & 132 \\
mean & 17.508 & 2.69 & 133 \\
$\max$ & 76.973 & 15 & 206 \\
\hline
\end{tabular}
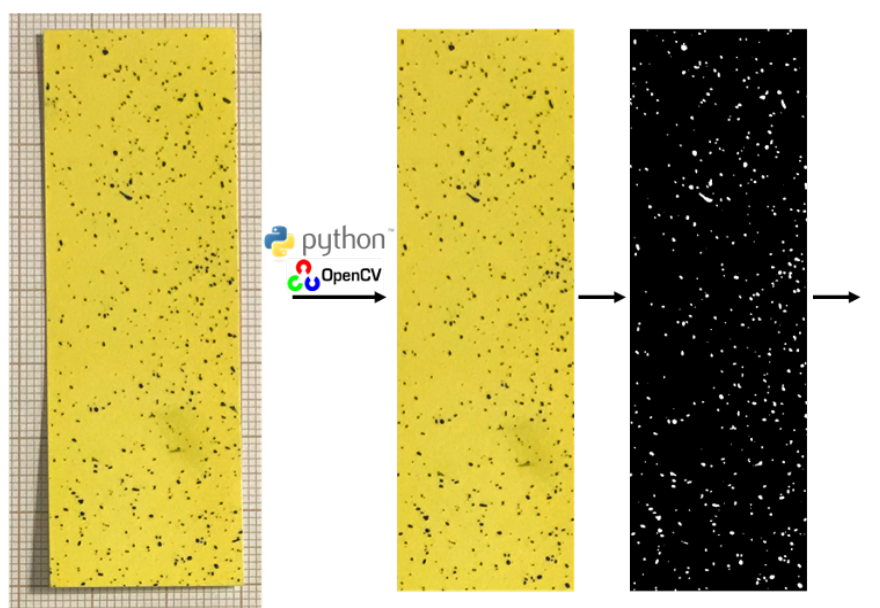

\begin{tabular}{|r|r|}
\hline & output_opencv \\
\hline Population_(drops/cm2) & 25.0506 \\
\hline Coverage \% & 2.654 \\
\hline Avg_diameter $(\mu \mathrm{m})$ & 119 \\
\hline Tree_Position & HIGH \\
\hline
\end{tabular}

Figure 4. Image Processing of water sensitive papers. RAW image is first cropped and binarized. After this, software detects the contours of the elements in the image (droplets) and count the contours. Final output shows the resulting information in a table form.

Figure 5 shows how these droplet populations and their mean diameter are distributed over the different measurement areas, showing that even under test conditions where there was a $3 \mathrm{~m} / \mathrm{s}$ wind, the drift was very low.
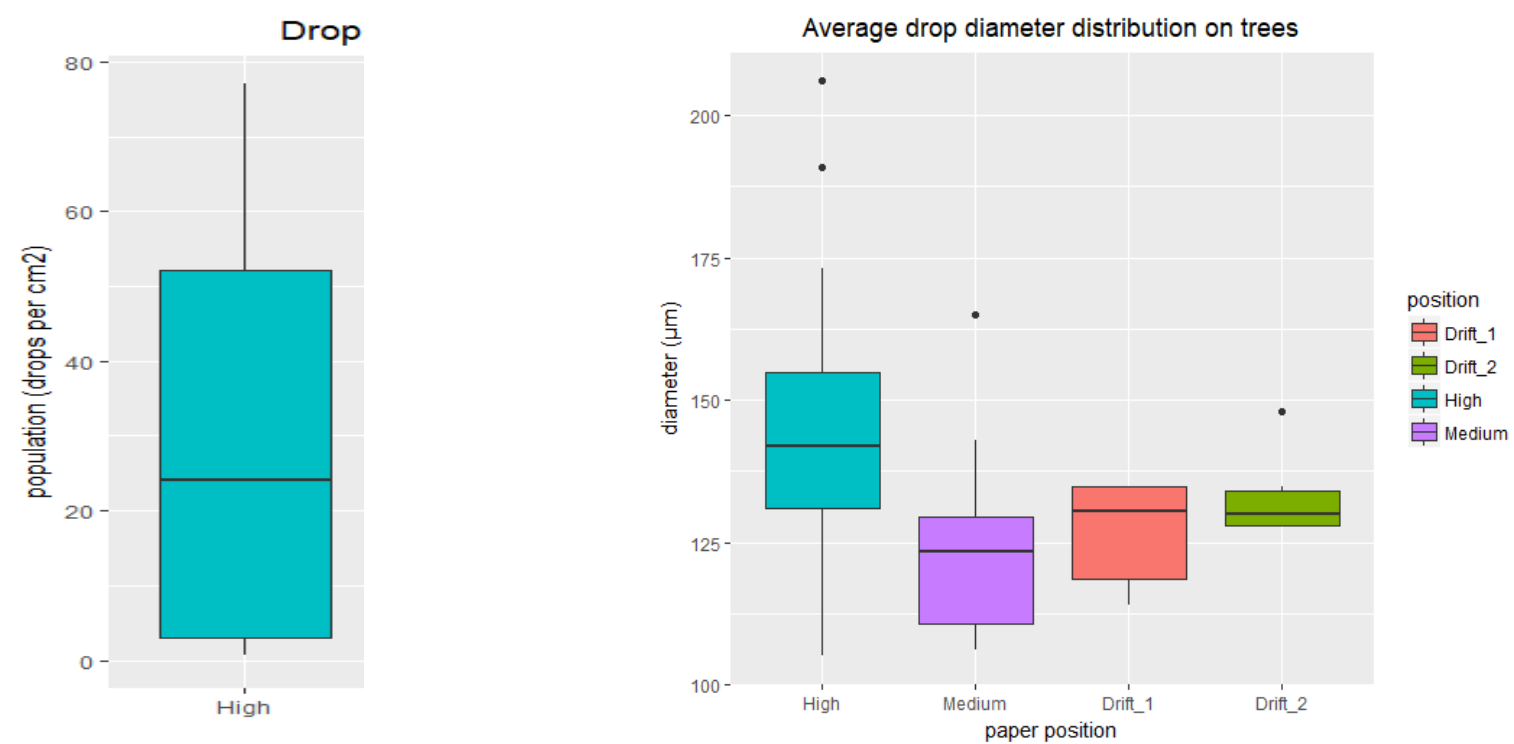

Figure 5. (left) Drop population pattern regarding the different measurement levels on olive trees. (right) Droplet diameter distribution on different measurement levels stablished.

\section{Economic assessment}

Once the machines that carry out the different operations and their capacities had been defined, the forecast of operating costs was discussed. First, the economic evaluation of the tractor and implement combination was carried out, using the following criteria: fixed costs (FC), including depreciation (using a 'combined' procedure which considers obsolescence and machine wear at the same time), interest, the cost of accommodation and insurance and taxes applicable to the combination are to be assessed. On the other hand, variable costs (VC) are calculated, including fuel and lubricant consumption, tyres wear on pneumatic tyres, overall repair and 
maintenance costs (RMC), and operator and auxiliary labour costs where applicable. Finally, the total hourly costs of the operation will be calculated, resulting from the sum of the hourly cost of the tractor and the hourly cost of the implement.

With respect to fixed costs, the evaluated items of equipment amortization, interest, housing and taxes are:

- In respect of depreciation or amortisation of the tractor, (1) is used for the calculation of the net value at the end of the operating period; (2) is used for the value of the application equipment at the end of its operating period.

$$
\begin{aligned}
& V_{N}=V_{a} \cdot 0.68 \cdot 0.92^{N} \\
& V_{N}=V_{a} \cdot 0.56 \cdot 0.885^{N}
\end{aligned}
$$

Once the net values have been calculated for both the tractor and the implement, the depreciation cost is calculated using the expression (3), obtaining an annual depreciation value of $1874.97 € /$ year.

$$
C A_{c}=\frac{V_{0}-V_{N}}{N}=\frac{30000-7500.39}{12}=1874.97 \frac{€}{\text { year }} \text { (tractor) }
$$

In the same way, the depreciation cost for the implement is calculated, obtaining an annual depreciation of $632.66 € /$ year.

- The interest cost (interest on the investment) represents the opportunity cost applied to the value of the machine. For its calculation, the accounted capital of the machine is valued, calculating half of the sum of the initial value of the machine and the net value at the end of the operating period, obtaining an accounted capital value of $18750 € /$ year. To obtain the net interest rate, the difference between commercial interest (0.06) and inflation (0.03) is calculated, obtaining 0.03 or $3 \%$ net interest. The total interest cost obtained from the product of the capital accounted for and the interest is $562.51 € /$ year.

- For the annual shedding costs, it is estimated that this is a percentage of the purchase cost of the tractor or the actual annual cost, which could be the renting of a garage or cabin for the tractor. In this case it has been set at $0.75 \%$ of the purchase price, the cost of accommodation being $225 € /$ year.

- In the same way, the annual cost in insurance and other taxes is calculated as a percentage of the acquisition value of $0.75 \%$, obtaining a cost of $225 € /$ year.

The total annual fixed costs, understood as the sum of all the above, is $3520.14 € /$ year. As it had been set at about 1000 working hours per year, it is $3.52 € /$ hour.

The Variable Costs are disaggregated as follows:

- The fuel consumption is calculated using the hourly consumption product $(\mathrm{L} / \mathrm{h})$ and the price of diesel $(€ / L)$. For the calculation of the hourly consumption, the average working power $(60 \%$ of the maximum power) and the specific consumption are estimated. Taking a fuel price of $0.80 € / \mathrm{L}$, the consumption results in a cost of $10.29 € / \mathrm{h}$.

- The lubricant consumption is taken from the tractor's technical data sheet and is valued in this case at $0.21 € / \mathrm{h}$. The oil level of the hydraulic system is set at $0.30 € / \mathrm{h}$.

- The cost of wear of the wheels and tyres is calculated considering that the tractor has 4-wheel drive, and that they have to be replaced after 4000 hours, at a cost of $600 € /$ piece. It results a cost of $0.6 € / \mathrm{h}$ from this calculation.

- For repair and maintenance costs, the average annual cost (RMC average) is taken, calculating it with respect to the acquisition value, for each year. In this way, we obtain that the total RMC in the 12 years of useful life represents $43.2 \%$ of the initial purchase value, obtaining an average annual RMC of $1080 € /$ year or $1.08 € / \mathrm{h}$.

With regard to the labour cost of the tractor driver, the estimated cost is $10 € / \mathrm{h}$, including salary and national insurance. Table 3 below summarises the total breakdown of costs as set out above. 
Table 3. Summary of Tractor + Sprayer Costs.

\begin{tabular}{ccc}
\hline Fixed Costs Tractor + Sprayer & Depreciation cost tractor & $1874.97 € /$ year \\
& Depreciation cost sprayer & $632.66 € /$ year \\
& Cost of Interest & $562.51 € /$ year \\
& Shedding costs & $225 € /$ year \\
& Insurance, taxes costs & $225 € /$ year \\
& Total fixed costs per year & $3520.14 € /$ year \\
& Total fixed costs per hour & $3.52 € / \mathrm{h}$ \\
\hline Variable costs & Fuel consumption & $10.29 € / \mathrm{h}$ \\
& Lubricant Consumption & $0.21 € / \mathrm{h}$ \\
& Hydraulic Oil Consumption & $0.30 € / \mathrm{h}$ \\
& Tyres Wear & $0.6 € / \mathrm{h}$ \\
& Repair/Maintenance & $1.08 € / \mathrm{h}$ \\
& Total variable costs per hour & $12.48 € / \mathrm{h}$ \\
\hline Labour force & Tractor driver $($ salary + national & $10 € / \mathrm{h}$ \\
\hline Total cost of conventional tractor application & insurance $)$ & $26 € / \mathrm{h}$ \\
\hline
\end{tabular}

The total costs of the conventional application using tractor and implement and adding the Fixed Costs, the Variable Costs and the operating costs is approximately $26 € / \mathrm{h}$. With regard to the ATHOS system, a useful life period of 5 years is established, with a number of flight hours of 100 hours per year. The Fixed Costs of this type of system are listed in Table 4 below.

Table 4. Fixed costs of the developed ATHOS spraying system.

\begin{tabular}{lc}
\hline Legal Declaration Operator Company/Autonomous & $300 €$ \\
Theoretical/practical/medical pilot license & $900 €$ \\
UAV insurance & $200 € /$ year \\
UAV equipment + remote control & $4000 €$ \\
Developed spraying system & $2500 €$ \\
4 Batteries & $700 €$ \\
Total fixed costs per hour & $12.3 € / \mathrm{h}$ \\
\hline
\end{tabular}

It should be noted that accommodation costs are not included in the fixed costs, due to the smaller size of the equipment, although in the event that a fleet of airborne equipment is developed, the authors consider that they should be included. The variable costs of the ATHOS system include the repair and maintenance costs, with a value of $150 € /$ year $(1.50 € / \mathrm{h})$ and the cost of the pilot's salary set at $20 € / \mathrm{h}$. The average total cost per hour of ATHOS will therefore be $33.80 € / \mathrm{h}$. The area treated per hour or hourly output is estimated using the product of the working width (in metres) by the forward speed (in kilometres/hour), divided by 10 . In the scenario described above, the working width will be 6 meters ( 3 meters of separation between rows, treatment in $50 \%$ of them) and we set the forward speed for both equipment's at $10 \mathrm{~km} / \mathrm{h}$, which gives a theoretical hourly output of $6 \mathrm{ha} / \mathrm{h}$. In the case of the tractor, the actual or real yield can be estimated by multiplying the theoretical yield by a factor of 0.8 , obtaining a value of $4.8 \mathrm{ha} / \mathrm{h}$, which implies that the application work in one hectare can be done in $12.5 \mathrm{~min}$. On the other hand, for the ATHOS system, the real or effective performance factor can be increased to 0.9 , as turns and manoeuvrability in the field are much faster and allow less time to be wasted on them. In this way, a real yield of $5.4 \mathrm{ha} / \mathrm{h}$, or $11.1 \mathrm{~min} / \mathrm{ha}$, would be obtained. It should be borne in mind that the speed of $10 \mathrm{~km} / \mathrm{h}$ set for the study could be increased in the case of ATHOS by almost twice as much, thus reducing the estimated time needed to apply one hectare.

\section{Conclusions}

The design and construction of a low-cost hydraulic sprayer model (ATHOS), capable of being mounted on board an aerial platform and of performing specific ultra-low volume applications, has been successfully completed. This equipment mounts a tank with a maximum load of 5 litres and has been field-tested for its performance in flight and feasibility to perform this type of applications, using a commercial bait product for the olive fruit fly. Droplet population, distribution on the trees, leaf area covered and droplet diameter were measured. In addition, a first comparative analysis of the application costs was carried out using conventional equipment (tractor+sprayer) and the ATHOS system developed. The following conclusions can be drawn from the present work:

- The first results obtained from the aerial application with the developed system show that a quality application can be made, with a uniform droplet population and an acceptable percentage of coverage. In addition, the average diameter of the drops has also been uniform, being the one indicated for an application of bait against olive fruit flies. Possible drift formation in windy conditions is attenuated by 
the airflow generated by the UAV rotors, which allows the product spray to be directed to the target vegetation, reducing drift. These promising results should be tested with other plant protection products and other crops in the future.

- The first results obtained after the analysis indicate that there is not much difference in the application costs between the costs of the aerial platform and the conventional equipment $(33.80 € /$ ha compared to $26 € /$ ha respectively). The analysis of the costs of the equipment developed has been carried out on the basis of estimates based on the team's own experience, since there are not yet any economic studies of this type of application carried out by other authors. It is expected that with the greater adoption of this type of technology in the near future, a greater number of studies will be conducted in this regard.

- It has been calculated that the capacity in hectares per hour of equipment such as ATHOS would be greater than that of conventional equipment, due to the fact that treatments can be carried out at a higher rate of advance and with greater manoeuvrability. The research group aims to evaluate the quality and effectiveness of this type of application with this and other active substances in different crops.

- The use of equipment such as ATHOS can represent an alternative to the traditional application, and specific applications can be made in specific coordinates (weed patches, disease foci, etc.) without the operator coming into contact with active substances that could be toxic and avoiding soil compaction due to the passage of tractors.

- This new paradigm in localised aerial applications, which can be beneficial in specific cases, must ensure that they can be carried out in conditions of operational and legal security. This should lead to a revision of the current legislation, which currently equates them to applications with much larger flight equipment (light aircraft) that can generate much more serious environmental problems than small drones.

\section{Acknowledgements}

The authors would like to thank DRONSAP, the drone division of AGROSAP, for their participation in the design of the equipment and in the trials. Also to thank the "World Olive Germplasm Bank" of the University of Cordoba for allowing us the use its facilities.

\section{References}

Bals E. J., 1970 The principles of and new developments in ultra-low volume spraying. Proceedings of the Fifth British Insecticide and Fungicide Conference, 17th to 20th November, 1969, the Hotel Metropole, Brighton, England, Vol. 1, pp. 189-193.

Boto, J.A.; Pastrana, P.; Suárez de Cepeda, M. Consumos energéticos en las operaciones agrícolas en España. Ministerio de Agricultura, Pesca y Alimentación. Madrid 2004. ISBN 84-

491-0635-4

Giles, D.K., Akesson, N.B., Yates, W.E., 2008. Pesticide application technology: research and development and the growth of the industry. Trans. ASABE 51, 397-403.

Giles D., Billing R., 2015, Deployment and performance of a UAV for crop spraying, Chemical Engineering Transactions, 44, 307-312 DOI: 10.3303/CET1544052

Huang Y., Hoffmann, W.C., Lan, Y., Wu, W., Fritz, B.K., 2009, Development of a spray system for an unmanned aerial vehicle platform. Appl. Eng. in Ag., 25, 803-809.

Panneton, B., Lacasse, B., Piché, M., 2005. Effect of air-jet configuration on spray coverage in vineyards. Biosyst. Eng. 90, 173-184.

Ru Y., Zhou, H., Fan, Q., Wu, X., 2011, Design and investigation of ultra-low volume centrifugal spraying system on aerial plant protection. Paper No. 11-10663, American Society of Agricultural and Biological Engineers. Presented at Louisville, KY USA.

Wang Z, Lan, Y., Hoffmann, W.C., Wang, Y., Zheng, Y., 2013, Low altitude and multiple helicopter formation in precision agriculture. Paper No. 13-1618681, American Society of Agricultural and Biological Engineers. Presented at Kansas City, MO, USA.

Wen, Sheng; Lan, Yubin; Zhang, Jiantao*; Li, Shenghua; Zhang, Haiyan; Xing, Hang. Analysis and experiment on atomization characteristics of ultra-low-volume swirl nozzle for agricultural unmanned aviation vehicle. Transactions of the Chinese Society of Agricultural Engineering, Volume 32, Number 20, 1 October 2016, pp. 85-93(9)

Zhang P, Deng L, Lyu Q, He S L, Yi S L, Liu Y D, et al. Effects of citrus tree-shape and spraying height of small unmanned aerial vehicle on droplet distribution. Int J Agric \& Biol Eng, 2016; 9(4): 45-52. 


\title{
Farms, Fogs and Clouds: Data Open-Architecture for Optimal Crop Growth Control for IoF2020 Project
}

Manuel Muñoz-Rodríguez ${ }^{1 *}$, Jorge A. Sanchez-Molina ${ }^{1}$, Francisco Rodríguez ${ }^{1}$ Manuel Berenguel ${ }^{1}$, Cynthia Giagnocavo²

CIESOL-ceiA3, Department of Informatics, University of Almeria, 04120, Almeria, Spain. * Corresponding author mmr411@ual.es

${ }^{2}$ Cátedra (Chair) Coexphal-UAL of Horticulture, Cooperative Studies and Sustainable Development, University of Almeria, Spain

\begin{abstract}
In this work, a traceability and decision support system (DSS) based on IoT and applicable to greenhouse tomato production based on FIWARE Cloud technology has been developed. FIWARE Context-Broker is used for processing in real time all the acquired information from the system for processing in real time all the acquired information from the system and for the persistence of the data, the notifications offered by the Context-Broker are used to notify a change in an entity to the REST API method in charge of persisting the data in MONGODB . The system consists of two parts: (I) Back-End: the part known as the data access layer, where a REST API based on the NODEJS language has been developed and will be responsible for making all the connections to the database, giving answers to the client's requests. (II) Front-End or presentation layer. The reason for the separation of layers is to ensure the security of the application by means of a token based on JWT, which is generated as response to client requests to the Back-End, to provide access to the data after verification. Another reason is to reduce computational load at the clients' side, in such a way that the Back-End will be in charge of managing the computationally heavy tasks of the application. The IoT platform integrates heterogeneous data sources in different time scales from: sensors; models; information about diseases; customer feedback; forecasts; decisions of the producers; the public agricultural and environmental authorities; etc. The data exchange will include farmers, technical engineers, production planners, handling and transport industry, that allow to know the quality and sustainability of the products. Benefits and improvements to the different actors and activities along the supply chain will be delivered in terms of: improved use of resources, better access to data and exchange, synchronization, storage reduction and cost.
\end{abstract}

Keywords: IoT, FIWARE, Agri-Data, control, Tomato

\section{Introduction}

Due to the growth of the world population, the agricultural sector faces new challenges. The FAO (Food and Agriculture Organization of the United Nations) foresees that the agricultural production will have to increase around $60 \%$ from 2015 to feed the planet in 2050, with the associated problems, related to the limitation of arable land, the increase in consumption of fresh water and climate change, which can cause a change in the current ways of production and the appearance of new pests and diseases.

Agriculture must prepare itself to face these challenges [1], the use of technology is one of the possible ways. Smart Farming [2] represents the integration of new technologies in the field of agriculture, facilitating the socalled "third green revolution" [3]. This revolution is based on the combination of tools such as the Internet of Things (IoT) [4], data processing in Big Data [5], cloud computing [6], artificial intelligence and Deep Learning, etc. The use of these technologies seeks to achieve an efficient and sustainable use of the resources available to boost production, including the optimization of water and energy use or prevention against pests and diseases. Modern agriculture is based on a series of tools to help in the improvement of decision making, crop monitoring activities and tools to improve the quality, production and quantity of food. It is a system in constant evolution.

To help the implementation of smart farming, a series of sensors, actuators and devices capable of collecting the context information that is generated in the greenhouse, are needed to perform a subsequent treatment of these data [7]. Several agronomic data management platforms using IoT technologies are being developed, often driven by initiatives of the European Union (FIWARE - https://www.fiware.org/, IoT-EPI - https://iot-epi.eu/, OPENIoT - www.openiot.eu, IoT-A- https://cordis.europa.eu/project/rcn/95713 es.html, universAAL http://www.universaal.info/, and many more). Some of these platforms, when used in the agriculture framework, area natural evolution of the so-called Farm Management Systems to make them compatible with cloud computing.

The unique Almería (Spain) agricultural model consists of 15,000 family farmers in peri-urban areas, with an average landholding of 2 ha, dedicated to protected F\&V (Fruits and Vegetables) greenhouse cultivation, and predominantly organized in cooperatives. Its organizational and production structure allows it to provide food not only for local and national markets, but also to satisfy a large part of Europe's demand for fresh F\&V. It contributes $30 \%$ of Andalucía's agricultural value and supplies 45,000 agricultural jobs to people from 150 different nationalities. However, the question of environmental and economic sustainability, or "greening cities" is also 
important in tracing the value chain of this agricultural model. Data sharing, and corresponding benefits and improvements, will include all types of stakeholders in the supply/value chain, allowing awareness of quality, origin, sustainability and traceability issues [5]. Moreover, the IoT system augmented with decision system support will contribute to achieve: a) the standardization and interoperability of information b) technological and scientific added value through innovative techniques; c) supply chain, quality and safety management, through the recording of agricultural processes, d) economic added value through improved products and processes, and e) social and environmental added value, through the improvement of air, soil and water quality and overall socialeconomic-environmental sustainability.

\section{Material and Methods}

The data used to develop the first version of the platform were acquired from the Cajamar Foundation's Experimental Station greenhouses in El Ejido, Almería Province, Spain (2 $2^{\circ} 43^{\prime} 00^{\prime}$ ' W, 36 48' 00' N, and 151 $\mathrm{m}$ a.s.1.). The tomato crops grew in a multispan "Parral-type" greenhouse, with a surface of $877 \mathrm{~m}^{2}(37.8 \times 23.2$ $\mathrm{m}$ ) and has a polyethylene cover, automated ventilation with windows in the north and south walls, a roof-flap window in each span, $20 \times 10$ threads $\mathrm{cm}^{-1}$ mesh "bionet" anti-insect screens. The greenhouse orientation is east to west, whilst crop rows are aligned north to south. The growing conditions and crop management are very similar to those in commercial tomato greenhouses. The climate parameters inside the greenhouse were monitored continuously each 30 seconds. Outside the greenhouse, a weather station measured air temperature and relative humidity with a ventilated sensor, solar radiation, photosynthetic active radiation with a silicon sensor, rain detector, $\mathrm{CO}_{2}$ concentration, wind direction, and wind speed. The cover temperature sensors were located on the east and west sides (two sensors each). During the experiments, the indoor climate variables were also taken, especially air temperature, relative humidity, solar radiation with a pyranometer, photosynthetic active radiation, soil and cover temperature, water and electricity consumption, an irrigation demand tray, water content, electrical conductivity (EC) and temperature of substrate, and $\mathrm{CO}_{2}$ concentration. As actuators, biomass heater of $150 \mathrm{~kW}$, four LEDs lights of $300 \mathrm{~W}$ each, $\mathrm{CO}_{2}$ enrichment, humidification of high pressure, dehumification of $980 \mathrm{~L} \mathrm{day}^{-}$ ${ }^{1}$ and a $95 \mathrm{~kW}$ hot-air heater. The data acquisition system is made up of three Compact Fieldpoints and one CompactRIO from National Instruments connected by Ethernet protocol to a wireless router that sends all the information to the data acquisition computer, where a Supervisory Control and Data Acquisition (SCADA) was designed. Moreover, data from lab analysis and field notebook, as well as weather prediction and third party cloud services were used.

\section{Results and Discussion}

For the integration of the heterogeneous data taken from the different sensors, lab analysis, field notebook, prediction and third party cloud services, the UC 4.2 of the Internet of Food and Farm project (iof202.eu) has designed FIWARE-based IoT platform (Figure 1) to relate all our infrastructures.

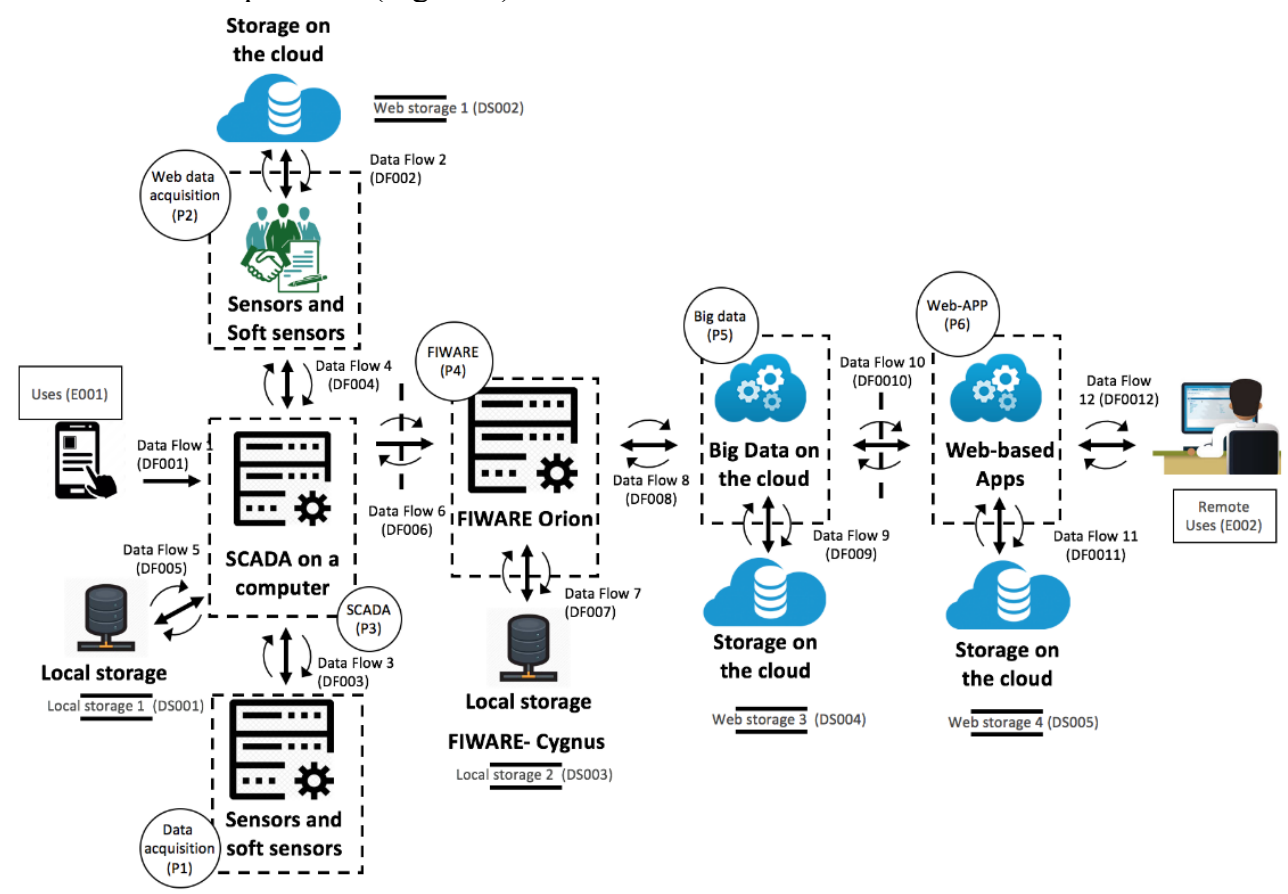

Figure 1. Scheme of the IoT platform for decision support in tomato crops in greenhouses. 
We are using the FIWARE standard NGSI-V10 [8], proposed to solve the incompatibility problem that exists in the IoT world between communication protocols and data models. This model is based on entities, attributes, values and metadata (Figure 2).

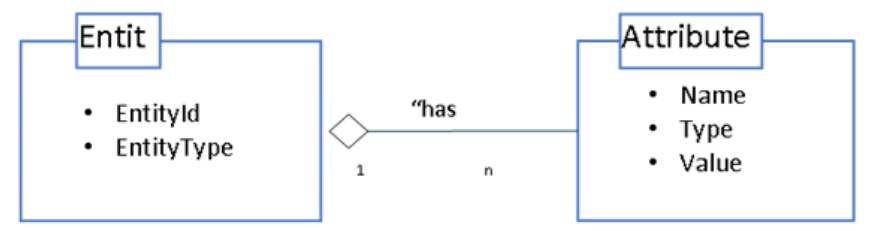

Figure 2. Scheme of the data model based on NGSI-V10.

The architecture on which this project is based is the following (Figure 3):

- $\quad$ FIWARE and communication: for communication between the sensors, actuators and the cloud, a supervisory control and data acquisition system (SCADA) is used in the experimental station, which is the responsible for collecting the information from the sensors and actuators for later sending to the cloud.

The available FIWARE enabler called ContextBroker [10] is used to manage the data, which is responsible for managing the context information using the NGSI information model, based on entities and attributes. It is therefore responsible for managing the context information from the sensors and actuators of the greenhouse, for subsequent treatment in real time. For the persistence of data, it is necessary to make subscriptions to the ContextBroker so that it notifies about a change in the previously created entities, in which case it will provide the notification to the Back-End in charge of managing the persistence in MongoDB [11].

- Back-End: is the part known as the data access layer. A REST API based on the NODEJS language [12] has been developed. This is in charge of making all the connections to the database, thus responding to the client's requests. The main reason of why the decision has been made to separate the presentation layer (Front-End) of the data access layer (Back-End) [13] is to guarantee the security of the application by means of a token based on JWT, which is generated and requests the part of the client to the Back-End and until not verified, it will not have access to the data. Another reason is to reduce the computational load of the client, in such a way that the Back-End is in charge of managing the heavy tasks of the application. In addition to the aforementioned, since a REST API is available, an integration can be made with any other Front-End that may be developed in the future.

- Front-End: this is the part known as the presentation layer, through which the end user can perform the necessary queries for later viewing. As Front-End we have opted to use a framework such as Angular4 and Bootstrap [14], based on JavaScript and html, CSS, JQuery. The advantage of using a Front-End separated from the data access layer is the security of the application, since it does not have any data related to the access to the database and also encrypted passwords can be used, plus a token, which is generated and checked when requesting data from the Back-End, so that if it is not correct, it does not return a response. 


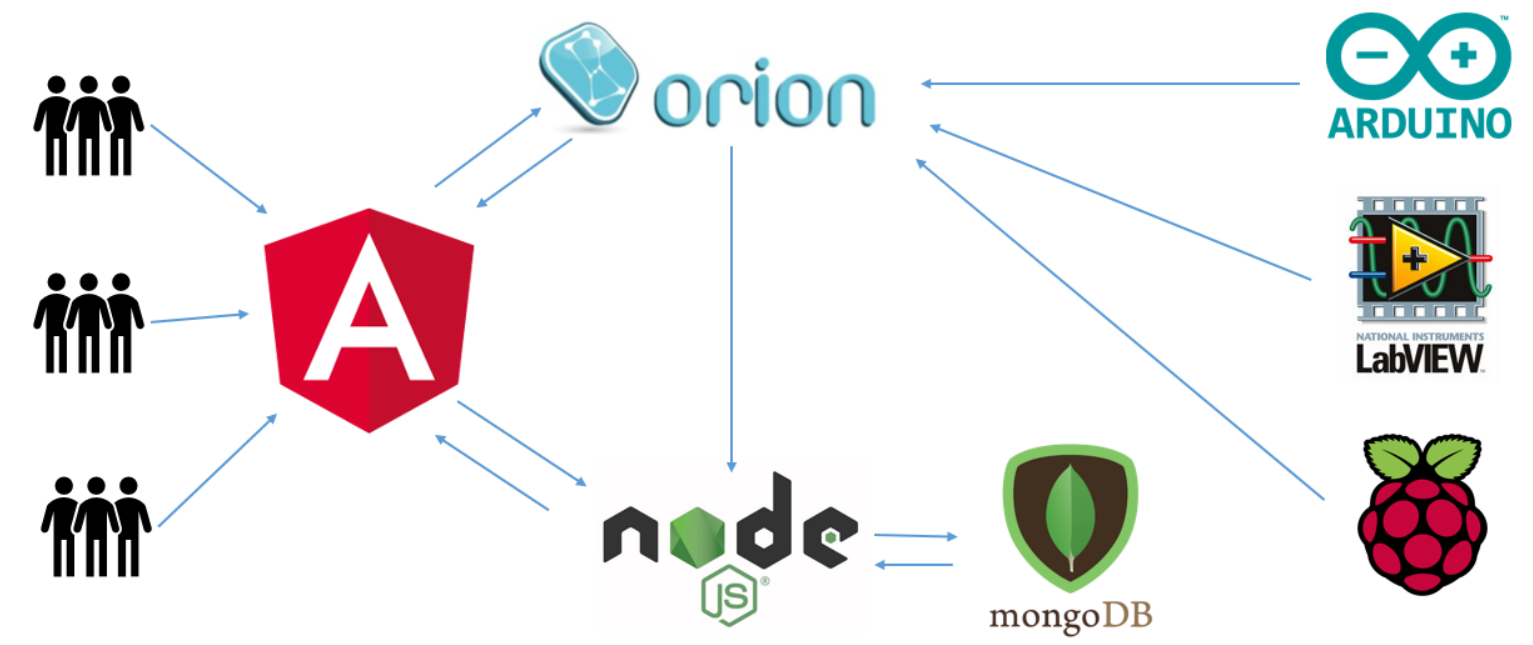

Figure 3. Scheme of the structure of the proposed IoT platform.

\section{Conclusions}

An Internet-based data exchange system for greenhouse crops has been designed. This IoT web-based traceability and decision support system (DSS) is focused on efficiency in the use of water, fertilizers and of energy, production planning, safety, transparency and quality standards.

The IoT platform (developed in FIWARE) is the core of our project, it has been integrated to obtain a standard in data modeling and obtain information on management, plant quality parameters, security and the application of quality standards. The FIWARE technology helps us to manage the context information of all the data generated in this production process from sensors, field laptops, laboratory analysis and models, notifying the change of some entity in order to perform the persistence of the data, thus having a data warehouse for further treatment with Big Data or Artificial Intelligence.

This is the first step with the final aim of having information on production and management in the whole value chain available to end users to make them aware of quality, origin, sustainability and traceability (Figure 4).

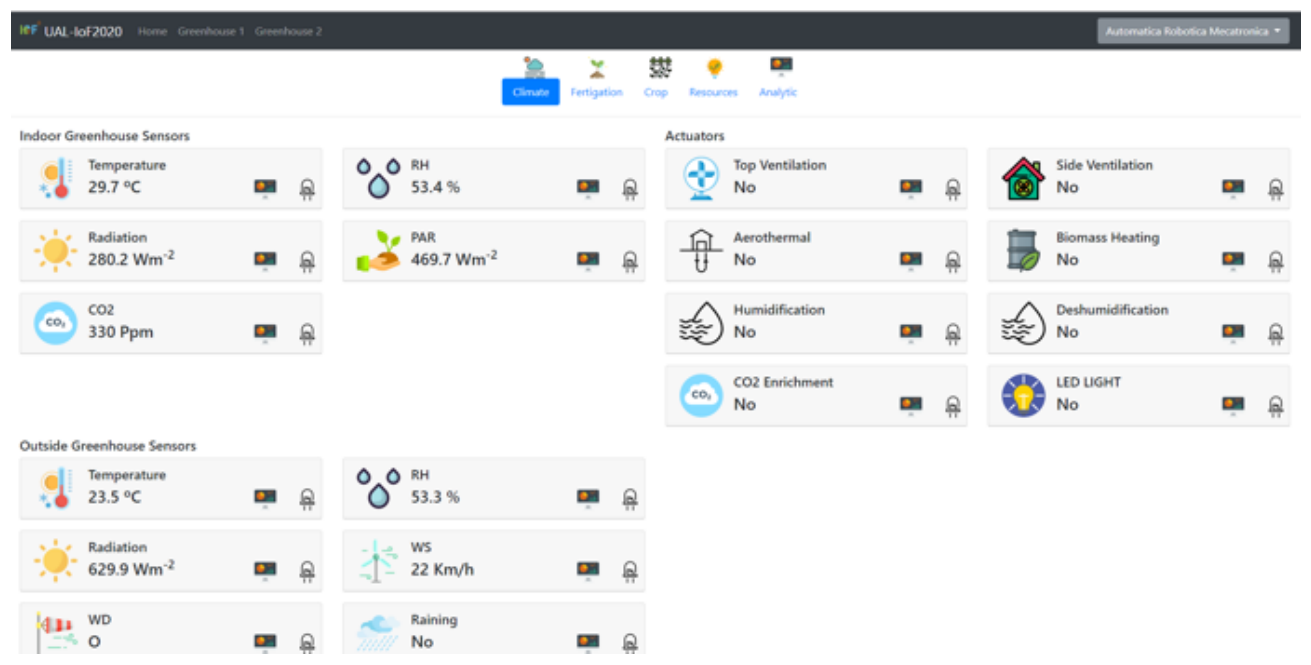

Figure 4. Greenhouse climate data.

\section{Acknowledgement}

This work has been developed within the framework of the Project IoF2020-Internet of Food and Farm 2020, funded by the Horizon 2020 Framework Programme of the European Union, Grant Agreement no. 731884 and by the National R+D+i Plan Project DPI2014-56364-C2-1-R of the Spanish Ministry of Economy and Competitiveness and ERDF funds. 


\section{References}

[1] R. R. Shamshiri et al., "Advances in greenhouse automation and controlled environment agriculture: A transition to plant factories and urban agriculture," Int. J. Agric. Biol. Eng., vol. 11, no. 1, pp. 1-22, 2018.

[2] J. Liu and J. P. Tao, "Research and application of agricultural greenhouse intelligence platform based on IoT (Internet of Things) and cloud computing," Int. J. Simul. - Syst., Science \& Tech., vol. 17, no. 5, p. 8.1-8.5, 2016.

[3] G. Gomes and A. Pérez García, "El proceso de modernización de la agricultura latinoamericana," Rev. CEPAL, 8, 57-77, 1979.

[4] J. Gubbi, R. Buyya, S. Marusic, and M. Palaniswami, "Internet of Things (IoT): A vision, architectural elements, and future directions," Futur. Gener. Comput. Syst., vol. 29, no. 7, pp. 1645-1660, Sep. 2013.

[5] A. Kamilaris, A. Kartakoullis, and F. X. Prenafeta-Boldú, "A Review on the Practice of Big Data Analysis in Agriculture." Comput. Electron. Agric., 143, 23-37, 2017.

[6] K. R. Bidua and C. N. Patel, "International Journal of Innovative and Emerging Research in Engineering Internet of Things and Cloud Computing for Agriculture in India," Int. J. Innov. Emerg. Res. Eng., vol. 2, no. 12, 2015 .

[7] V. I. Adamchuk et al., "On-the-go soil sensors for precision agriculture," Comput. Electron. Agric., vol. 44, pp. 71-91, 2004.

[8] M. Fazio, A. Celesti, F. G. Marquez, A. Glikson, and M. Villari, "Exploiting the FIWARE cloud platform to develop a remote patient monitoring system," in 2015 IEEE Symp. on Computers and Communication (ISCC), 2015, pp. 264-270.

[9] P. Rajalakshmi and S. Devi Mahalakshmi, "IOT based crop-field monitoring and irrigation automation," in 2016 10th Int. Conf. on Intelligent Systems and Control (ISCO), 2016, pp. 1-6.

[10] B. Moltchanov and O. R. Rocha, "A context broker to enable future IoT applications and services," in 2014 6th Int. Congress on Ultra Modern Telecommunications and Control Systems and Workshops (ICUMT), 2014, pp. 263-268.

[11] Y.-S. Kang, I.-H. Park, J. Rhee, and Y.-H. Lee, "MongoDB-Based Repository Design for IoT-Generated RFID/Sensor Big Data,” IEEE Sens. J., vol. 16, no. 2, pp. 485-497, Jan. 2016.

[12] S. Tilkov and S. Vinoski, "Node.js: Using JavaScript to Build High-Performance Network Programs," IEEE Internet Comput., vol. 14, no. 6, pp. 80-83, Nov. 2010.

[13] J. L. Tacilla Ludeña, J. L. Tacilla Ludeña, and J. L. Tacilla Ludeña, "Sistema informático web de gestión de incidencias usando el FRAMEWORK angulares y NODEEJS para la empresa REDTEAM SOFTWARE LLC," Univ. Priv. Antenor Orrego - UPAO, 2016.

[14] J. RAMOS MARTÍNEZ, "Desarrollo de una aplicación Web CRM usando un framework libre," Universidad Politècnica de València, Sep. 2017. 


\section{Topic 3: Co-production and implementation of technology in food production systems (FP)}

Experiences and research into systems addressing the human aspects for farmers, workers, consumers and society involved in the development, implementation and use of new technology and systems in food production and processing. Examples include acceptance/rejection, safety and risk and management thereof, responsible development and democratization.

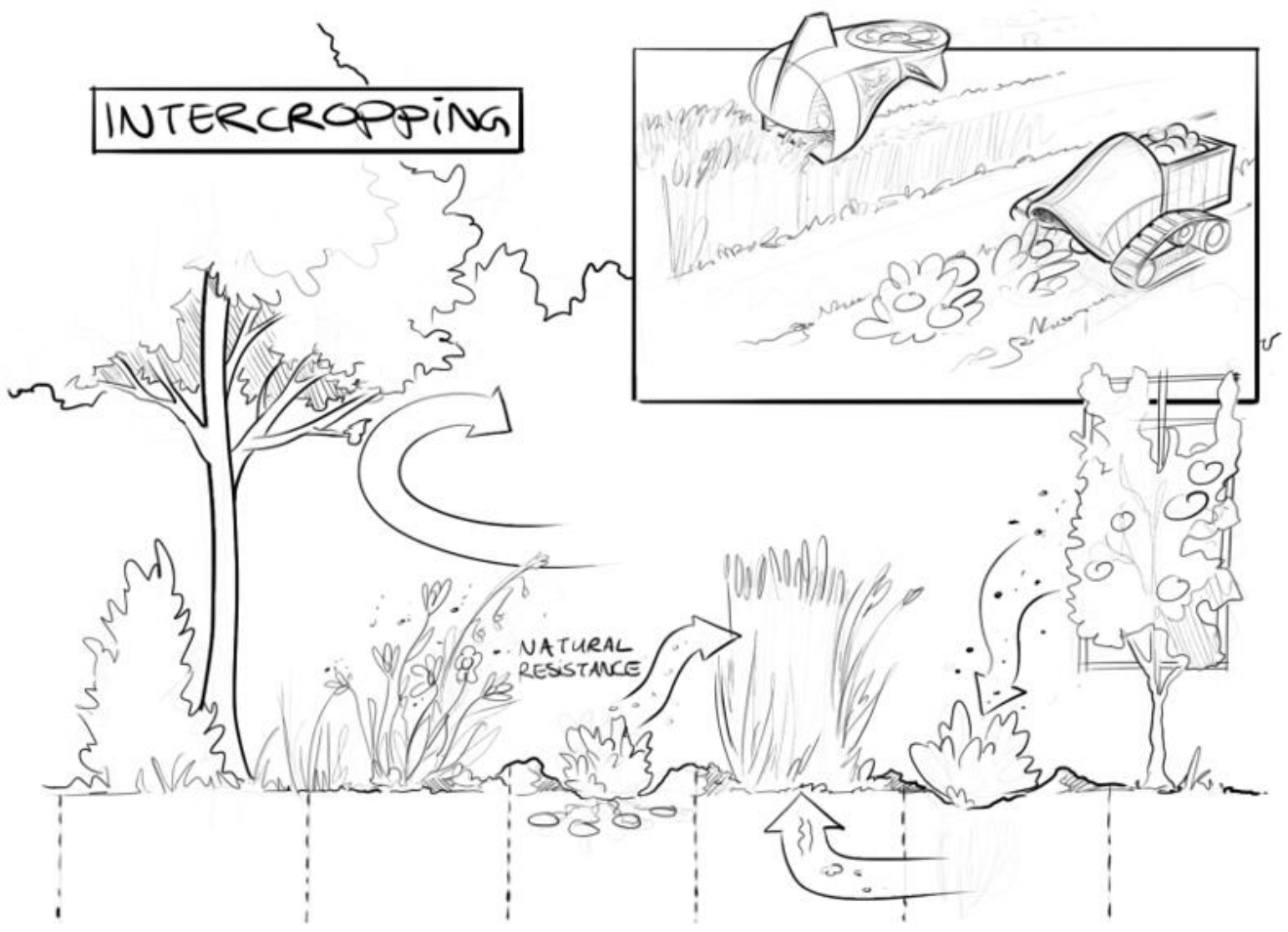




\title{
An Innovation System Supporting the Adoption of Precision Agriculture in Sweden: the Case of Yara N-sensor
}

\author{
Christina Lundström ${ }^{\mathrm{a}^{*}}$, Jessica Lindblom ${ }^{\mathrm{b}}$, Magnus Ljung ${ }^{\text {a }}$

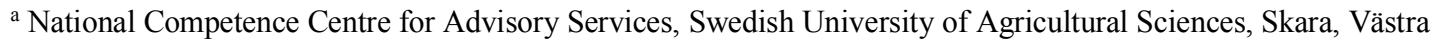 \\ Götaland, Sweden \\ ${ }^{\mathrm{b}}$ School of Informatics, Interaction lab, University of Skövde, Skövde, Västra Götaland, Sweden \\ * Corresponding author. Email: christina.lundstrom@slu.se
}

\begin{abstract}
This paper aims to investigate and analyse the underlying innovation system concerning the Yara N-sensor adoption in Sweden from 2000 to 2017, in order to identify potentials, challenges and barriers to more general precision agriculture development in Sweden. The study addresses the following two research questions: 1) How can the innovation system concerning Yara N-sensor be described? 2) What are the main implications for the regime shift from considering fields as homogenous entities to a heterogeneous ditto? To answer the research questions a qualitative inquiry using an explorative approach was conducted in 2017. The first author performed interviews with different actors within the Swedish agricultural knowledge and innovation system. The data concerning the innovation system was analysed by using seven functions from innovation system literature. The findings revealed: 1) public discourse concerning limited fertilisation possibilities. 2) New requisites concerning possibilities to develop precision agriculture technology. 3) An innovation system, driven by a group of entrepreneurial people employed at a small number of companies in close contact with a regionally funded network of precision agriculture supported by the Swedish University of Agricultural Sciences, advisory services, an institute, companies and public actors. One the one hand, there would probably not have been any precision agriculture network, without the entrepreneurs who laid their eyes on the first Yara N-sensor in Germany 1997. On the other hand, there may not have been 250 Yara N-sensors in Sweden 2018 if there has not been a precision agriculture network back in the days.
\end{abstract}

Keywords: Decision support system, within field variation, advisory services,

\section{Introduction}

There is a huge interest for digitisation and the development is fast considering precision agriculture (PA) and new technology in agriculture. In parallel, the environmental impact from agriculture has been an important issue for a long time. In the 1990s there were intense discussions on nutrient leaching and the need for better adaptation of fertilisation to crop need. In Sweden the strategic decision was to implement a public funded advisory project - Focus on Nutrients (http://www.greppa.nu/om-greppa/om-projektet/in-english.html) - that started in 2000. The project focused on fertilisation strategies focusing on manure, and then fields were viewed as homogenous entities. Simultaneously the development of PA started. In 1995, the GPS signal was made accessible and it became interesting to use it in farming situations. Gradually, it became possible for farmers to treat their fields heterogeneously. To succeed, farmers need credible and usable agricultural decision support systems (AgriDSS). However, uptake and acceptance of complex AgriDSS has generally been slow in Sweden as in other parts of the world (Aubert, 2012; Lindblom et al., 2017). The European Parliament (2016) points out that: "the full potential of precision agriculture is not yet harvested. We only see a first series of precision farming practices implemented on small number of farms. These precision farming are making farming more easy rather than giving crop plants and animals the optimal treatment at the right time and lowest scale possible. For the latter, the adoption rate is still very low". In times were society are focusing on innovation in order to handle a broad range of challenges this lack of PA technology is considered a problem and a change concerning support of innovation processes are discussed (EU SCAR, 2015). EU SCAR (2015) suggests that information and communications technology (ICT) will change agriculture in an essential way due to strategies considering operation, management and farm structure in a way comparable with the introduction of tractors and pesticides. In 2000, the Yara N-sensor (YNS) was introduced to the Swedish market. It has had an important symbolic value in the national discussion and development of PA technology. Nevertheless, it took until 2016 before a Yara representative claimed that "the plug has gone out" considering YNS use and selling, meaning that finally a broader group of farmers seem to accept, trust and use the YNS.

The knowledge needed to deal with complex situations as fertilisation is diverse and must be locally adapted. Thus, the farmers' experience and knowledge from their farms is central as well as the advisors' knowledge based on science and experience (Hoffman et al., 2007). In addition, PA technology can provide an improved decision base by handling a huge amount of data from different kinds of measurements. PA technology can supplement and facilitate farm management, i.e. technology is essential for handling large data samples, measuring properties that cannot be detected by the human vision system, and providing valuable, credible representations of complex 
situations that clarify and support actions without losing the complexity (Lindblom et al., 2017; Lundström \& Lindblom, 2018). Consequently, PA technology support, but does not replace decision-makers. The adoption of new technology or knowledge is a learning process that involves 1) the collection, integration and evaluation of new information and 2) the adaptation of the innovation to the user's situation (Pannel et al., 2006). Thus, relevant knowledge must be provided both from the inside (probably the farmer) and the outside (possibly the advisor) (Leeuwis, 2004). The inside perspective could be tacit knowledge and the outside perspective scientific knowledge. The solution is to use both and combine them to a locally fitting solution.

The traditional view of diffusion of innovation (Rogers, 2003) offered a linear model concerning innovators, early adopters, where the late majority and laggards have shown to be limited (Busse et al., 2014). The systemic view on innovation processes is consistent, but widened in relation to the traditional view of the Agricultural Knowledge and Innovation system (AKIS) (EU SCAR, 2015). AKIS traditionally embraced a system of education, research and extension, but it needs to involve many more actors beyond the traditional ones in the food chain who has impact on farmers' decision-making (EU SCAR, 2015). In line with this remark, technology change could be viewed in a broader sense following Heckert et al (2007. p. 414) who define it as "the development of technology in interaction with the system in which the technology is embedded". This innovation system is defined by its hardware, software and orgware, where the orgware consists of the different parts of the innovation system. Marelba (2002), among others, described the parts in an innovation system model as 1) Products; 2) Agents and organizations; 3) Mechanisms of interactions; 4) Knowledge and learning processes; 5) Institutions; 6) Basic technologies inputs, demand and related links and complementarities; and 7) Processes of competition and selection. However, parts and structure are not enough to fully understand, how an innovation system works. The dynamics between actors within the structure need to be further understood (Busse et al, 2014; Heckert et al, 2007). The importance of understanding the underlying dynamics is because they create functions that support or prohibit adaptation of a possible innovation. Heckert et al. (2007) discuss functions of innovation systems as a number of processes crucial for well-functioning innovation systems. The identified functions are: 1) Entrepreneurial activities, 2) Knowledge development, 3) Knowledge diffusion through networks, 4) Guidance of the search, 5) Market formation, 6) Resources mobilization; and 7) Creation of legitimacy/counteract resistance to change.

Using the perspective of functions instead of structures and actors, may provide an assessment of the whole system including visualise barriers and sub-optimal aspects (Eastwood et al., 2017). Heckert et al. (2007) claimed that analysis of traditional innovation system mainly studies social structures, missing the dynamic perspective. Heckert et al. (2007) used functions for systematisation of empirical work and for mapping system dynamics. Additionally, the traditional analysis focuses mainly on the macro level (institutions) and less on the micro level (entrepreneurs), which is a tentative risk since innovation is both an individual and collective act (Edquist, 2001), especially when the individual perspective in innovation is fundamental (Heckert et al. 2007). This kind of systemic approach for investigating innovation systems often explains why innovation is slow and hard to influence. Often it is not two competing technology systems, rather two competing innovation systems (new or already established), that are struggling. Kemp (1994) claimed that the prevailing technology, including its design and function, has already benefited from different evolutionary improvements, such as costs and performance characteristics, better user understanding and the adaptation of the socio-economic environment in terms of accumulated knowledge, capital outlays, infrastructure, available skills, production routines, social norms, regulations and lifestyles.

This paper aims to investigate and analyse the underlying innovation system concerning the YNS adoption in Sweden from 2000 to 2017, in order to identify potentials, challenges and barriers to more general PA development in Sweden. The study addresses the following two research questions: 1) How can the innovation system concerning YNS be described? 2) What are the main implications for the regime shift from considering fields as homogenous entities to a heterogeneous ditto?

\section{Materials and Methods}

The project authors decided to use an explorative approach using qualitative methods (Patton, 2002). This qualitative inquiry was conducted in 2017, when the first author performed interviews with different actors within the Swedish AKIS. Twelve farmers were interviewed by phone, ten who have an YNS and two who have not. The author contacted the farmers at a conference and the two who did not use YNS were identified through the other farmers. Earlier projects had resulted in complementary information concerning farmers who do not use PAtechnology. This prior information influenced this project. In addition, interviews were conducted with representatives from advisor services, researchers, PA companies, Yara, an agricultural institute, companies selling machine services to farmers and participants within the public funded project Focus on Nutrients. The advisor interviews were mainly performed by telephone, while the rest were performed in real life meetings. The interviews took between 20 minutes up to two hours. When making interviews by phone, notes were taken. Face to face interviews were recorded and transcribed. The collected data was analysed, using the earlier presented 
framework of functions of innovation systems (Heckert et al., 2007), using the seven functions 1) entrepreneurial activities , 2) knowledge development, 3) knowledge diffusion through networks, 4) guidance of the search, 5) market formation, 6) resources mobilization and 7) creation of legitimacy/counteract resistance to change.

\section{Setting the scene}

Yara N-sensor is the result of a doctoral work in Hanninghof, Germany 1997 (Reusch, 1997). In Sweden at that time there was an ongoing discussion about agricultural development, which was connected to environmental issues and technology. A couple of individuals had a big interest and the opportunity to work with issues connected to PA, which was a rather new concept at the time. Most companies selling requisites to farmers were regionally connected at that time and some had funds as well as people working with development. One such company was Odal (from 2000 denoted Lantmännen) (http://www.lantmannenlantbruk.se/en/). Odal, for example, worked with soil maps, GPS and crop sensors. In 1996 a precision agriculture network, Precision agriculture Sweden (POS), was established in the western part of Sweden due to a Odal initiative in cooperation with the Swedish University of Agriculture and Agroväst, a regional funded Agribusiness, which has a kind of broker role concerning R\&D in the western part of Sweden. The POS network started as a regional network, but became national in 2002. This network is still active, funded regionally and open for all interested at universities, companies, authorities and organisations (http://www.precisionsskolan.se/). The first written report from the POS network described a visit to Germany and Hanninghof in 1997, where ten people from the POS network witnessed the first developed YNS used in practice. The year after, the first Swedish YNS was demonstrated at a farm in the western part of Sweden. In 1999, the YNS was used in practice at 1000 hectares and field trials were performed in wheat. In 2000, 15 entrepreneurs were highly engaged and started to sell fertilisation services on the request from Odal and its counterparts in other regions. Yara (Hydro Agri at that time) introduced a hand-held YNS for field trial measurements. The public funded project Focus on Nutrients started, and the YNS was mentioned for the first time in the yearly report on fertilisation recommendations published by the Swedish Board of Agriculture. Hence, Yara (former Hydro Agri) has a unique role in Swedish agriculture. It is the dominating company for fertilisers at the Swedish market, at least partly due to the product potassium nitrate and a prevailing long history in Sweden. Selling fertilisers is Yara's main issue, but to still keep their exceptional position they claim that they need to be trustworthy, both in agricultural and environmental discussions. Consequently, Yara cooperates with other actors and one example is the sharing of costs for Swedish fertilisation field trials with the public funded Focus on Nutrients and The Rural Economy and Agricultural Societies (one third each).

The interest concerning YNS was big, when it was introduced. YNS received rewards, it was shown at exhibitions and it was mentioned in farmers' newspapers. In 2004, Hydro Agri changed its name to Yara and in a survey to its customers about $95 \%$ of the farmers had heard about YNS. In 2005, it became possible for anybody to buy an YNS. In 2006, there was almost $40 \mathrm{~N}$-sensors in Sweden and in 2010, the numbers had increased to 80, and the sale was steady but slow. However, since the Swedish introduction the interest among farm advisors were rather low. The POS network had precision agricultural courses for farmers, students and advisors, but with very limited success among advisors. In 2009, the hand-held YNS was further developed and slowly Yara began to lend hand-held YNS to advisory services in order to make measurements in field trials. All collected data was, and still is, published on a public website, managed by the Swedish University of Agricultural Sciences (SLU), and it is under open access for anybody, except for the algorithms calculating the $\mathrm{N}$ amount in the YNS, which is still a secret. The interest among advisory services slowly increased during the years. One important reason was that Yara let advisory services borrow a hand-held YNS if they shared their experience and the collected data.

In 2010, POS developed an advisory module regarding PA at the request from the Focus on Nutrients. In this module, one option was to make a cost-benefit analysis on an YNS investment. During 2013-2014, POS developed an alternative to the YNS, CropSAT (https://cropsat.se/). CropSAT uses satellite images, allowing the users to set levels of Nitrogen (N) and then calculates variable rate application files. Since 2015, the Focus on Nutrients has funded CropSAT and today all farmers in the major agricultural regions of Sweden have access to it on a free-to use website. In 2015, advisory services started to sell a measurement service to farmers that used a hand-held YNS to collect data from not fertilised field spots in order to evaluate $\mathrm{N}$ mineralisation. By the use of the handheld YNS, advisory services increased their engagement concerning questions about $\mathrm{N}$ mineralisation and variation both between fields and within fields. In the same year, the company Lantmännen (the national version of Odal) introduced a flour from wheat, produced by contractors with a term to use a $\mathrm{N}$ field sensor for fertilisation. Finally, this year (2015) had very special whether conditions in Sweden, resulting in high yields, high N optimum and low protein content in many crops and many farmers lost money due to high yield but with too low protein content. However, many farmers who had used the YNS were successful in both yield and protein content and the plug for YNS had finally gone out! 


\section{Results and discussion}

The findings revealed an innovation system, driven by a group of entrepreneurial people employed at a small number of companies (the main owner of the YNS, the others selling the product) in close contact with a regionally funded network of PA supported by the SLU, advisory services, an institute and public actors.

Applying the seven functions suggested by Heckert et al (2007) in the analysis of the collected data clarifies and describes the innovation system and the adoption process of the YNS in the following way.

Entrepreneurial activities

Initially, a group of people, interested in agricultural technology worked together in the western part of Sweden. They tested GPS, satellite image technology, yield mapping etc, and thanks to regional funding, they started POS and were able to continuing working together via their own organisations. When one of these persons were asked what made it happen, he answered: "There were no complicated features at that time ... if you just come up with good ideas, it was possible to get it funded. So a lot of good teamwork and a little bit of stubbornness".

When asked about how well-developed the YNS was when it came to Sweden, he said: "sufficiently finished for us to bet on it ... if you want to be first, you cannot wait until it's completely finished ... but then it's important to hang on ... we did not matter very much about it".

This reveals that they were engaged doers with a bit of freedom within their own organisations. The POS network became a community of practice, which in turn became an important R\&D environment for the development of YNS. One person in the Yara staff said: "We have worked closely with Hanninghof, which I do not think all countries have done... not such a great interest ... and I think that may be due to POS. We have been challenged a lot from POS ... and eh ... we have also had the opportunity to get help and use POS as sparring partners".

The person responsible for YNS in Sweden, has been tightly connected to POS, by the functional role as technology expert. However, he also has a close contact with the farmers using YNS. Many farmers highlighted the crucial contact with him and that he was a very important person for them when they decided to adopt the technology. Farmers describe him as a person who struggle to be supportive concerning any question or problem that turned up. At least in the beginning people were interested in testing the YNS and then forming good relations to the representatives from Yara and Odal were of major importance. One farmer said: "When you get a wild and crazy idea, there must be someone saying: Well, that will work"'and these representatives from Yara and Odal did provide that kind of support.

Knowledge development

Learning is central to all innovation processes. A farmer has to learn how to use a new tool, generalised knowledge must be adapted to local conditions. During the years, Yara has developed a kind of $\mathrm{N}$ fertilisation strategy that is based on four incremental levels. The first level is to follow recommendations due to an average yield level, the second level is to reflect on the considerable variations between years and fields following weekly information from field trials during spring seasons, delivered by Yara, Focus on Nutrients, and/or advisory services. At the third level, the farmers use a spadmeter (https://www.konicaminolta.eu/en/measuringinstruments/products

/colour-measurement/chlorophyll-meter/spad-502plus/introduction.html) to measure in their own fields and compare the obtained measurements with field trails done during the spring. Finally, at the last level the farmers are well aware of within field variation and use an YNS to adapt the N amount to it. In parallel, the Focus on Nutrients which has worked mainly on questions concerning manure and adaptations on an average level, still cooperating with POS in developing an advisory module for PA, accomplishment of advisory seminars, exhibitions, and funding CropSAT.

The analysis reveals that the YNS innovation system has some limitations in utilisation of farmers' experience. At the yearly conference for YNS farmers, the main issue is how to accomplish the knowledge transfer to the farmers. There is not many elements of knowledge and experience exchanges, rather an expectation from farmers on Yara to present new knowledge to the users. The focus has mainly been on learning by searching and analysis of collected data from field trials and research, and not so much form the more first-hand experiences of learning by doing by farmers using the tool in practice. However, some farmers follow Yara's recommendations concerning YNS use very strictly, while other farmers use it in additional crops and at different times than recommended. Additionally, farmers adjust the sensor when fertilising, believing in the technology, but still being aware of its limitations. Still, the obtained findings do not tell anything concrete about to what extent farmers may be important to YNS development or user strategies. However, the close contact between the Yara representative and the farmers has certainly have big influence, even though it is rather difficult to evaluate this aspect afterwards. 
Knowledge diffusion through networks

As already mentioned POS has been a crucial network for YNS development and adoption in Sweden. It has been an arena for learning by interacting, since POS involve actors from companies, organisations, authorities, and universities. Another important network is the YNS network that consists of farmers who meet during two days every year. The analysed data from the interviews mirror that it may be the case, or at least have been, a bit special to be an YNS farmer.

Guidance of the search

This activity refers to activities that have positive effect on visibility and clarity among technology users. In the 1990s the discussion concerning environmental impact from agriculture was intense. In 1991, EU introduced the nitrate directive and every member country formulated and introduced a good agricultural practice concerning N fertilisation. Sweden joined EU in 1995, and in Denmark a strict account for fertilisation was introduced in 1998. Discussions about doing the same were held in Sweden. Finally, Sweden decided to choose the learning track instead of the Danish forcing ditto, and Focus on Nutrients was the outcome. In parallel, new technology was developed. In 1995, the GPS signal was released and made PA on an interesting accuracy possible. PA and YNS have two main goals. 1) To increase farmers profitability by an increase of yield and quality in crops, and 2) to increase adaptation of fertilisation to crop need and thus result in a decrease in environmental impact. The discussion concerning nutrient leaching and environmental impact from agriculture has increased the interest and awareness among many actors for PA in Sweden. For Yara, the YNS was a strategic venture to be trustworthy in selling fertilisers.

\section{Market formation}

In the late 1990s there was a big confidence in PA. However, after some years of testing the technology it became clear that the field system was more complex than expected and that the $\mathrm{N}$ mineralisation and yield was difficult to predict. The complexity of the field, the difficulty to actually test and see what happens and the lack of clear cost-benefit connections to the local field made the interest somehow reduced. The PA technology seemed at first rather easy to use, but it could be quite demanding for the individual farmer. In spite of some problems to make YNS to communicate with other technologies in the tractor, it was rather easy to use. To make PA and YNS interesting for the farmers, the within field variation must be very easy to grasp. Everybody knows that there is a variation, but the amount does on the one hand variate a lot between different fields and on the other hand between different years. It is harder to change a behaviour than to do as one always has done. Farmers buying advisory services did not seem to have much support concerning YNS, because the majority of advisors did not support it. Many of the early adopters did not seem to buy any advisory services. Lately, advisors interest have increased significantly. However, for some reasons it seems more difficult to estimate an average amount of $\mathrm{N}$ and tell the YNS, than doing it in the traditional way and set an average amount of $\mathrm{N}$ for the whole field. One advisor said: "Yes ... it will be even more sharp in a way". It is easier to set an average that everybody know is more or less wrong. Some advisors claim that farmers, not the early adopters though, buy an YNS and then expect the advisor to set the average. Or, in some cases they even think that the YNS can decide for itself, being a kind of expert system. Some advisors were critical to how Yara described the YNS, as some kind of automatic fertiliser. But, some advisors realise that: "Here we have a device that can help you make decisions, but in the end of the day nobody knows. You can never get a better result then what you tell the device yourself".

An YNS need a rather new fertiliser spreader to function correctly. If the farmer had an older fertiliser spreader it seemed to be more expensive to hire someone else to make the fertilisation, since cost-benefit analysis for variable spreading is blurred. However, many farmers speculate that they are buying an YNS when they need a new fertiliser spreader, but not until then. In Sweden, regional county administrative boards have introduced investment subsidies for environmental friendly technology and YNS are entitled to those. That is an important signal to the farmers and advisors.

\section{Resources mobilization}

Yara has worked on a long-term basis, even though one employee said that the YNS has been questioned a couple of times and if the company in hindsight had known how long time it would have taken to make it accepted on the Swedish market they may have hesitated to put so much effort in the product. However, YNS has been important for Yara's trustworthiness, its uniqueness on the Swedish market and have fitted well with their image as an environmental friendly company. The work in Sweden, with close cooperation concerning field trials, open data, lending hand-held sensors to advisory services and authorities is exceptional. The same has not happened in any other country, due to a Yara representative. 
Creation of legitimacy/counteract resistance to change

As stated earlier, one important actor representing legitimacy of the YNS is POS. The network has been important for its entrance to university as well as college education. Since YNS has been a good practical example on PA, and POS has used it in education of farmers, students and advisors. Nowadays, YNS has been on the Swedish market for so long that at least one individual who has grown up on a farm that used YNS, now works as an advisor. For him, using the YNS was a normal activity and his starting point was considering a field as a heterogeneous entity. The introduction of CropSAT in 2015 increased farmers and advisors consciousness of within field variation. Suddenly it was possible for anybody to evaluate the variation in biomass before fertilisation. Nevertheless, rather few advisors used the CropSAT tool. Some of them hesitated to decide the N levels, whereas others said that their farmers did not have "that kind of farm", because the farmers were not interested or "not so technically advanced" and "when nobody asks the question, nothing will happen". This group of advisors waited for the farmers to react and said that: "the customer pushes the development by demand". Still some advisors used CropSAT maps together with their customers to get another image from the field. One advisor said that: "CropSAT is part of my concept". In some cases they used CropSAT to decide how to calibrate the YNS (Lundström \& Lindblom, 2018).

The Swedish Board of Agriculture has signalled through its yearly report on recommendation for fertilisation and liming. In these reports, very little is written about within field variation. YNS was mentioned for the first time in 2000, and 15 years later almost the same formulation was still there. The starting point is to adapt the average amount of $\mathrm{N}$ to the field. However, in the last five years or so the public project Focus on Nutrients has borrowed hand-held YNS from Yara to make N mineralisation measurements in not fertilised spots during spring. Thus, since a couple of years the Focus of Nutrients, Advisory services and Yara communicate variation in $\mathrm{N}$ mineralisation between fields and years. But, still in 2018 representatives from the Focus on Nutrients, present average figures of $\mathrm{N}$ mineralisation from different fields instead of introducing a discussion of the need for within field variation. It is hard to change the starting point and consider a field as an entity that may have within field variation that may be worth to consider. One important reason for this way of acting is that normally there is only one spot in the field without fertilisation intended for $\mathrm{N}$ mineralisation evaluation.

However, considering within field variation of nutrients, is probably not a common starting-point for the ordinary Swedish farmer and advisor in 2018. Although, farmers have used the YNS technology in Sweden since 2000 , advisor organisations sell a service to farmers based on the technology and CropSAT has been accessible for anybody since 2015, but no organisation (as far as we know) have decided to really include the technology and change their common advisory strategies considering fertilisation.

Now 20 years have passed since Yara (then Hydro Agri) introduced the first YNS in Sweden and so far, 250 YNS have been sold. Much work has been done but still much remains to be done in order to change people's starting point from regarding a field as a homogenous entity to seriously consider it as a heterogeneous ditto. These findings show a complex innovation system, where many of the functions do work, even though it has taken some years. Yara has been patient and achieved a lot. Probably, the public discussion on environmental impact on the hand and the threat about initiation of a fertilisation account as in Denmark, on the other hand has been important, but there is also a genuine interest for Swedish agriculture. It is important to remember that the Focus on Nutrients had manure management highest on their priority list when it started. However, more clarity considering within field variation in the recommendations for liming and fertilisation from the Swedish Board of Agriculture could have had a positive impact, since many advisors consider and follow their recommendations.

In the beginning of the PA era, there was big expectations of the use of yield mapping. After some years it became clear that the yield map just was a receipt, it was impossible to use it as many people first had expected. Many farmers have told us about their beautiful coloured yield maps. Nowadays, the majority of farmers do not even print or look at the map, since they do not know what to do with them. However, when using an YNS or other PA technology, it is interesting to be able to evaluate the obtained results. One advisor said: "Many [farmers] have yield mapping technology, but nobody looks at it... that is how you want to apply the YNS to see the differences and learn the results of YNS in comparison with the yield map". For the credibility of YNS it would be interesting to be able to contrast and compare the YNS maps with the yield maps, not in order to validate the YNS, rather to use them for learning and to get an approximate cost-benefit analysis on the local field. By reconsidering PA technology from a tool used for decision making to a tool that supports learning, i.e. changing perspective from goal-oriented thinking to thinking in terms of learning could increase both usability and credibility. This kind of change would also help some advisors, who back off when the complexity increases, instead of using the tool that creates the complexity together with the farmer in order to learn together and reach a better result. However, in order for this kind of change to occur in practice, many advisors would need to have support from colleagues and back-office. Currently, it does not seem to be a joint strategy for how to handle new PA and other technology among many advisory services, instead it depends on the individual advisor. Farmers who use the YNS address the possibility of learning while using the YNS. One farmer said: "It (YNS) triggers you to reflect... which the fertiliser spreader does not do... If you think that I spread 40 kilograms per hectare 
last year, and I will do the same this year... and then you experience a variation in $N$ demand, while driving the YNS. What is the reason for this variation? How do I manage such a variation? Should I increase or decrease the amount of N? It triggers me to walk in the field and reflect more." Many farmers express that the use of YNS makes the crop production more interesting and when something is interesting, it motivates you to learn more about the issue. In a questionnaire 2017 to YNS farmers, $95 \%$ marked I agree on the statement: "I feel curious and have a lot to learn in crop production", though the majority of these farmers probably are among the most competent farmers in Sweden.

At the end of the day, the profitability is the most important issue for the individual farmer. Thus, without being able to evaluate cost-benefit on your own fields, the interest to make an investment and increase complexity in practice without being able to evaluate the obtained result, probably make many farmers hesitate. Complex technology as YNS is difficult to evaluate properly (Pannel et al, 2006). Good results in research and field trials do not guarantee the same result at the local fields. That is what PA is all about. One of the informants said concerning the YNS: "You could have got faster results. But, agriculture still is a fairly conservative industry or an industry where you... You can say there is no room for doing too many bad investments and then it requires good evidence that it will pay off... It requires interest and a technical knowledge. And at the same time it's not absolutely crucial either ... so while some earn money, those who have not invested, have not lost so terrible". However, in 2015 when exceptional weather conditions resulted in record high yields, the $\mathrm{N}$ fertilisation did not let both ends meet, resulting in too low protein content. Farmers who had used the YNS did reach better result and the interest from others eventually increased. Finally, the plug had gone out!

\section{Conclusions}

YNS has come to stay in Sweden, even though it took some time. However, the resistance from mainly the advisors, has triggered Yara and POS to continue work on this issue. In this era of digitisation and fast development of new technology, there is an urgent need for competent advisors who can combine agricultural issues and technology in order to support farmers. Today, most advisors have their main competence in crop production not in technology. Advisory services must consider this issue, because many farmers say they do not use advisory services due to low competence among advisors, but if there were competence, they would pay for it. It is also remarkable that there is no education for engineers or IT people, with the emphasis on agriculture in Sweden.

Finally, it is striking to stress as well as acknowledge the importance of creating open networks where different actors can exchange ideas, experiences and knowledge and how important the culture and community of practices of such networks are in order to function for what they initially are aimed for. On the one hand, there would probably not have been any POS network, without the entrepreneurs who laid their eyes on the first YNS in Germany 1997. On the other hand, there may not have been 250 YNS in Sweden 2018 if there has not been a POS network back in the days.

\section{Acknowledgements}

We would like to thank all persons who have participated in the data collection and thus has made this publication possible. We also want to thank the Stiftelsen Lantbruksforskning who has financially supported the project.

\section{References}

Aubert, B. A., A. Schroeder, J. Grimaudo, 2012. IT as enabler of sustainable farming. Decision Support Systems. 54 (1), 510-520. DOI:https://doi.org/10.1016/j.dss.2012.07.002

Busse, M., A. Doernberg, R. Siebert, A. Kuntosch, W. Schwerdtner, B. König, et al., 2014. Innovation mechanisms in German precision farming. Precision Agriculture. 15 (4), 403-426. http://dx.doi.org/10.1007/s11119-013-9337-2.

Eastwood, C., L. Klerkx, R. Nettle, 2017. Dynamics and distribution of public and private research and extension roles for technological innovation and diffusion: Case studies of the implementation and adaptation of precision farming technologies. Journal of Rural Studies. 49, 1-12. https://doi.org/10.1016/j.jrurstud.2016.11.008

Edquist, C., 2001. Innovation Systems and Innovation Policy: the state of the art. Invited Paper for DRUID's Nelson-Winter Conference. 2001. Aalborg, Denmark.

EU Scar, 2015. Agricultural knowledge and Innovation Systems towards the future. A foresight paper.

Standing Committee on Agricultural Research (SCAR). Strategic Working Group AKIS-3 Report. EUR 27692 EN. https://publications.europa.eu/en/publication-detail/-/publication/4d9a1811-c3fb-11e5-8d0801aa75ed71a1/language-en Accessed May 5, 2018. 
European Parliament, 2016. Precision agriculture and the future of farming in Europe Scientific Foresight Study. EPRS European Parliamentary Research Service. Scientific Foresight Unit (STOA) PE 581.892. http://www.veteffect.eu/news/precision-agriculture-and-future-farming-europe . Accessed June 6, 2018.

Hekkert, M.P., R.A.A. Suurs, S.O. Negro, S. Kuhlmann, R.E.H.M. Smits, 2007. Functions of innovation systems: A new approach for analysing technological change. Technological Forecasting \& Social Change. 74, 413-432. https://doi.org/10.1016/j.techfore.2006.03.002

Hoffmann, V., K. Probst, A. Christinck. 2007. Farmers and researchers: How can collaborative advantages be created in participatory research and technology development? Agriculture and Human Values. 24 (3), 355-368. DOI: $10.1007 / \mathrm{s} 10460-007-9072-2$

Kemp, R., 1994. Technology and the transition to environmental sustainability-the problem of technological regime shifts. Futures. 26 (10), 1023-1046. https://doi.org/10.1016/0016-3287(94)90071-X

Leeuwis, C., 2004. Communication for rural innovation. Rethinking agricultural extension. Oxford UK: Blackwell Science.

Lindblom, J., C. Lundström, M. Ljung, A. Jonsson, 2017. Promoting Sustainable Intensification in Precision Agriculture: Review of Decision Support Systems Development and Strategies. Precision Agriculture. 18 (3). 309-331. DOI: 10.1007/s11119-016-9491-4

Lundström, C., J. Lindblom, 2018. Considering Farmers' Situated Knowledge of Using Agricultural Decision Support Systems (AgriDSS) to Foster Sustainable Farming Practices: The Case of CropSAT. Agricultural Systems. 159, 9-20. DOI: 10.1016/j.agsy.2017.10.004

Malerba, F., 2002. Sectoral systems of innovation and production. Research Policy. 31 (2), $247-264$. https://doi.org/10.1016/S0048-7333(01)00139-1

Pannel, D. J., G. R. Marshall, N. Barr, A. Curtis, F. Vanclay, R. Wilkinson, 2006. Understanding and promoting adoption of conservation practices by rural landholders. Australian Journal of Experimental Agriculture. 46 (11), 1407-1424. DOI: https://doi.org/10.1071/EA05037

Patton, M. Q., 2002. Qualitative research and evaluation methods. (3 ${ }^{\text {rd }}$ Ed.) London: Sage.

Reusch, S., 1997. Entwicklung eines reflexionsoptischen Sensors zur Erfassung der Stickstoffversorgung landwirtschaftlicher Kulturpflanzen. Forschungsbericht Agrartechnik des Arbeitskreises Forschung und Lehre der Max-Eyth-Gesellschaft Agrartechnik im VDI (VDI-MEG) 303, Kiel, Germany.

Rogers, E. 2003. Diffusion of innovation. Fifth edition. NY: Free press. 


\title{
Promotion of Circular Economy in Alentejo's Agri-Food Sector \\ Vasco Fitas da Cruz ${ }^{1}$, Bruno Magalhães ${ }^{2}$, Cristina Ascenço ${ }^{2}$, Fatima Batispta ${ }^{1}$ \\ ${ }^{1}$ ICAAM, Department of Rural Engineering, University of Évora. vfc@uevora.pt \\ ${ }^{2}$ ISQ - Instituto de Soldadura e Qualidade, Lisbon,
}

\begin{abstract}
In a world of increased lack of resources, it is imperative to promote the transition to a more circular economy, seen as an alternative business model to promote competitiveness, reducing simultaneously the consumption and dependency of raw materials and energy. The main goal of "Alentejo Circular" project was to raise awareness and mobilize Alentejo' s agrifood sectors to adopt the circular economy model, aiming to create value for enterprises, and consequently for the region, by transferring knowledge on the best practices and technologies for efficient resource use and waste valorisation. The work was focused on the wine, olive oil and pig farming industries, due to their current and growing importance for the regional economy. Through national and international technical site visits and bibliographic research, a current diagnosis of circularity in the addressed regional industries was as carried out, as well the assessment of circular economy best practices, at national and international level. The resources and wastes were characterized and quantified improving the knowledge of recovery cycles with a list of best practices being gathered as priority for actions to be taken by the regional stakeholders, in order to support the transition towards a circular economic model. As a result of this study, it was possible to conclude that the best available practices in these sectors, such as, smart farming and the use of renewable energy are already in use across the region, however these practices are only implemented by a limited number of the regional companies. By means of several public sessions across the territory and a web 2.0 based platform, the interaction between stakeholders, including companies, public authorities and the scientific community increased, resulting in shared experience and knowledge on alternative use of resources, thus settling the conditions for the development of future synergies.
\end{abstract}

\section{Introduction}

In a world of increased lack of resources, it is imperative to promote the transition to a more circular economy, seen as an alternative business model to promote competitiveness, reducing simultaneously the consumption and dependency of raw materials and energy.

The Alentejo, like other regions of Portugal and the planet, faces major economic and environmental challenges today. This region has very specific characteristics associated with its geographic location, unique culture and economic situation in constant change. Through an analysis that combines the characteristics of the region and the economic sectors under study, a step forward can be taken in the transition from the linear economic model, based on a logic of extraction - production - consumption - elimination, thus dependent on the intensive use of natural resources for a circular economic model based on the preservation of natural capital and the optimization of resource efficiency through the circulation of products, components and materials with the greatest possible use in technological and biological cycles. This transition is inevitable, also for the Alentejo, which is now beginning to experience the consequences of climate change and scarce resources.

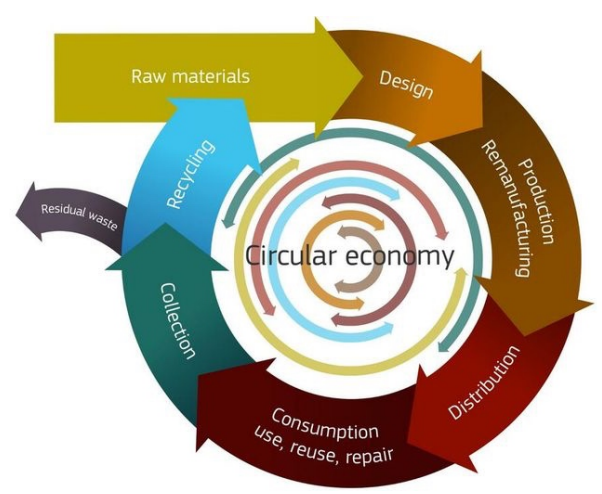

Figure 1. Circular Economy Model (Source: Ellen MacArthur Foundation).

In this context, the ISQ - Institute of Welding and Quality and the University of Évora created the project Alentejo Circular The main goal of "Alentejo Circular" project was to raise awareness and mobilize Alentejo' s agrifood sectors to adopt the circular economy model, aiming to create value for enterprises, and consequently for 
the region, by transferring knowledge on the best practices and technologies for efficient resource use and waste valorisation.

\section{Material and methods}

The work was focused on the wine, olive oil and pig farming industries, due to their current and growing importance for the regional economy. In the first stage, an analyse of the current legislation was made followed for interviews with national and regional administrative and technical authorities related with the 3 industries identified as relevant for the project.

The diagnosis of the regional situation and the identification of the main factors responsible for the waste production was made through national and international technical site visits. Through the analysis of the data collected through the completion of a survey with economic agents, and through bibliographic research, it was possible to perform a characterization and positioning of the Alentejo at the level of circular economy practices. A current diagnosis of circularity in the addressed regional industries of different dimension was carried out, as well the assessment of circular economy best practices, at national and international level. Also, the identification of barriers and opportunities was done. The resources and wastes were characterized and quantified improving the knowledge of recovery cycles with a list of best practices being gathered as priority for actions to be taken by the regional stakeholders, in order to support the transition towards a circular economic model.

The final stage of the project was the information, awareness and audition actions made by means of several public sessions (seminars, reflection sessions and networking sessions) across the territory and a web 2.0 based platform that's include a list of the companies that are register, a map of supply and demand of by-products and their localization and a discussion forum.

\section{Results and discussion}

A total of 20 field visits were realized (table 1). 11 in the wine sector, 11 in the olive oil sector and 7 in the pig farming sector.

Table 1. Number of field visits by sector.

\begin{tabular}{|c|c|c|c|}
\hline \multirow{2}{*}{$\begin{array}{c}\text { No EMPRESAS } \\
\text { VISITADAS }\end{array}$} & Vinho & Azeite & Suinicultura \\
\cline { 2 - 4 } & 11 & 11 & 7 \\
\hline 20 & &
\end{tabular}

The visits were realized in all Alentejo territory (figure2) and over all the kinds of companies (big, medium and low dimension)

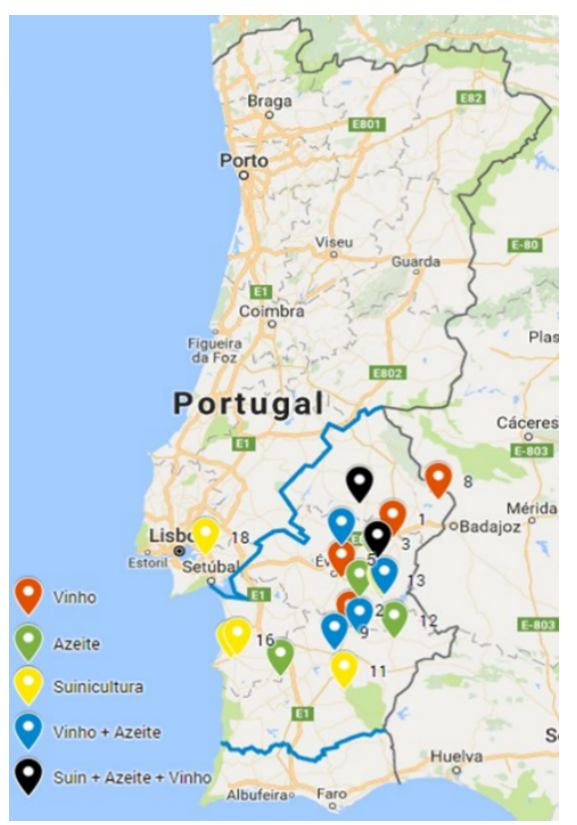

Figure 2. Field visits map. 
A total of 14 national and international benchmarking actions ( 3 in the win sector 7 in the olive oil sector and 4 in the pig farming sector, table 3 ) have been done through field visits followed by technical discussions.

Table 2. National and international benchmarking visits

\begin{tabular}{|c|c|c|c|}
\hline \multirow{2}{*}{ № VISITAS } & \multicolumn{3}{|c|}{ Fileira } \\
\cline { 2 - 4 } & Vinho & Azeite & Suinicultura \\
\hline
\end{tabular}

Good practices identified in the recovery of waste and by-products from wine and olive oil were as follows:

- Agronomic valorization of organic compost: stings, oilcake, pruning, leaves, sludge from WWTP

-Tartarates: Use of tartaric acid salts in the wine stabilization process

-Production of wine spirits in distilleries from oilcake and lees of wine

-Energy valorization of biomass: Olive seeds, pruning

-Animal feed: Mice, oilcake (wine); leaves and pruning (olive oil)

In pig farming those good practices, in the production activities are the application of slurry as fertilizer organic compound (agronomic valorization) by direct application in soil or integrated in composting process. In pig meat transformation sector, the follow good practices were identified:

- $\quad$ Bones - Flour (animal feed)

- Blood - Plasma Extraction

- $\quad$ Fat - Lard and animal feedstuffs

- Guts - Food industry

In table 3 is it possible to see the information, awareness and networking events organized in the frame of the project.

Table 3. Alentejo Circular Events.

\begin{tabular}{|c|c|c|c|}
\hline TIPOLOGIA EVENTO & $\begin{array}{c}\text { PÚBLICO } \\
\text { ALVO }\end{array}$ & DATA & LOCAL \\
\hline 10 evento de apresentação e 1 a jornada de reflexão & Multisetorial & 07 de fevereiro & Évora \\
\hline 1a sessão de sensibilização e 2 a jornada de reflexão & Multisetorial & 14 de março & Santarém \\
\hline 2a sessão de sensibilização & Suinicultura & 13 de abril & Alvalade de Sado \\
\hline 3ạ sessão de sensibilização & Vinho & 18 de abril & $\begin{array}{c}\text { Reguengos de } \\
\text { Monsaraz }\end{array}$ \\
\hline 4⿳亠口冋 sessão de sensibilização & Azeite & 11 de maio & Moura \\
\hline 5a sessão de sensibilização e 3 a jornada de reflexão & Multisetorial & 7 de junho & Elvas \\
\hline 6ạ sessão de sensibilização e 1a sessão de networking & Multisetorial & Setembro & Évora \\
\hline 7ạ sessão de sensibilização e $2^{a}$ a sessão de networking & Multisetorial & Setembro & Beja \\
\hline 8aㅗ sessão de sensibilização e $3^{a}$ a sessão de networking & Multisetorial & Setembro & Portalegre \\
\hline Evento de encerramento e $4 \mathbf{a}$ jornada de reflexão & Multisetorial & 25 de Outubro & Évora \\
\hline
\end{tabular}

The data collected indicate: that:

- Large majority of producers of wine and olive oil are under integrated production and protection regime. Many are in conversion to organic production.

-Industrial symbiosis was limited to the sharing of resources and optimization of processes by industries of the same group, and the supply of organic matter (leaflets, leaves) by cooperatives to their producers

- There are punctual economic agents that receive organic matter from other ranks for the production of compost. However, there is interest of several economic agents in receiving MO for enrichment of their agricultural soils 


\section{Conclusions}

In order to support the transition to a circular economy, a survey was made of the practices used in the wine, olive oil and pork industry in the Alentejo region. In this geographic area of Portugal, the efficient use of resources is of great relevance due to different factors such as the scarce availability of water and the need to preserve natural capital, seeking a sustainable balance between environmental and economic issues.

As a result of this study, it was possible to conclude that the best available practices in these sectors, such as, smart farming and the use of renewable energy are already in use across the region, however these practices are only implemented by a limited number of the regional companies.

The interaction between stakeholders, including companies, public authorities and the scientific community increased, resulting in shared experience and knowledge on alternative use of resources, thus settling the conditions for the development of future synergies.

\section{References}

Amaro, S., Ribeiro, L., Paralta, E., \& Pinto, F. C. (n.d.). Aplicação de Efluentes de suiniculturas como fertilizantes na agricultura: Impacto na qualidade dos meios hídricos. Associação Portuguesa Dos Recursos Hídricos, 1-9.

Brunori, E., Farina, R., Biasi, R., 2016. Sustainable viticulture: The carbon-sink function of the vineyard agroecosystem. Agriculture, Ecosystems and Environment 223: 10-21.

Ellen Macarthur Foundation - www.ellenmacarthurfoundation.org

Di Giovacchino, L., N. Constantini, M. L. Ferrante, e A. Serraiocco. Influence of malaxation time of olive paste on oil extraction yields and chemical and organoleptic characteristics of virgin olive oil obtained by a centrifugal decanter at water saving. Grasas y Aceites, 2002: 179-186.

Marçal, C. (2014). Gestão integrada de resíduos do setor vinícola e análise do ciclo de vida do produto. Instituto Superor de Agronomia - Universidade de Lisboa, Lisboa. 


\title{
Skills Needs for a Sustainable Farmer in the Framework of the SAGRI Project
}

\author{
Luis L. Silva*, Fátima Baptista, Vasco F. Cruz, José R. Marques-da-Silva
}

Departamento de Engenharia Rural and ICAAM - Instituto de Ciências Agrárias e Ambientais Mediterrânicas, Escola de Ciências e Tecnologia, Universidade de Évora, Núcleo da Mitra, Ap. 94, 7002-554 Évora, Portugal

* Corresponding author. Email: 1lsilva@uevora.pt

\begin{abstract}
In the EU almost $50 \%$ of the territory is covered by farmland (both arable and permanent grassland), which means that agriculture plays a key role in land management and has a huge responsibility in the preservation of natural resources. In order to practice a sustainable agriculture, farmers responsible for the management of farmland must adopt correct and environmental friendly practices, using appropriate technology and complying with EU regulations for a sustainable agriculture. Recent developments in science and technology, that could be an added value for farmers' crop and land management, are still unutilized in many situations because farmers have not been introduced to them or have not been trained to use them. The SAGRI project (http://www.sagriproject.eu/) is an ERASMUS+ Project which main goal is to advance the skills of European farmers and agricultural extension staff through the development of new curricula and teaching programmes that integrate in a practical way the latest developments in agricultural applied research. The purpose is to provide farmers and agricultural stakeholders with knowledge, skills and competencies in the field of agro-environmental technology for a sustainable agriculture. There were identified six major areas, where significant technological developments occurred and that can help farmers for a more sustainable agriculture: 1) Precision agriculture; 2) Integrated pest management; 3) Agricultural reuse of organic residuals; 4) Drip irrigation and water-conserving technologies; 5) Renewable energy; and 6) Bioenergy and energy crops. For each of these topics there were identified job-specific skills with a highlight in the awareness for all agricultural sustainability aspects and in the introduction to major technological developments in the specific areas. These skills will be the basis for the developing of new innovative curricula integrating the latest advancements of the "agri-tech" sector, and training courses for agricultural workers.
\end{abstract}

Keywords: ERASMUS+, Sustainable agriculture, Training, Skills, Competencies.

\section{Introduction}

In the EU almost $50 \%$ of the territory is covered by farmland (both arable and permanent grassland), which means that agriculture plays a key role in land management and has a huge responsibility in the preservation of natural resources. The desired relationship between agriculture and the environment can be captured by the term "sustainable agriculture". In order to practice a sustainable agriculture, farmers responsible for the management of farmland must adopt correct and environmental friendly practices, using appropriate technology and complying with EU regulations for a sustainable agriculture.

To do so, farmers must be educated in agreement with the "sustainable agriculture" concept, which was defined in the US law in 1990 (Gold, 2015) as an integrated system of plant and animal production practices having a sitespecific application that will over the long-term:

- $\quad$ Satisfy human food and fiber needs;

- Enhance environmental quality and the natural resource base upon which the agriculture economy depends;

- Make the most efficient use of non-renewable resources and on-farm resources and integrate, where appropriate, natural biological cycles and controls;

- $\quad$ Sustain the economic viability of farm operations;

- $\quad$ Enhance the quality of life to farmers and society as a whole.

A critical issue in the 21 st century for increasing the sustainability of agricultural production are the changes and adaptations required in agricultural education in order to make it more effectively, contributing to the improvement of sustainable agricultural production and rural development (Van Crowder et al., 1998). Recent developments in science and technology, that could be an added value for farmers' crop and land management, are still unutilized in many situations because farmers have not been introduced to them or have not been trained to use them. According to the EU Agricultural and Farm Economics Brief n. 9 (European Commission, 2015a) even in 2013, the vast majority of EU farmers have learned their profession only through practical experience. This was particularly the case for the oldest farmers, among which more than $80 \%$ never had any agricultural training. And close to one-third of all farmers were above the normal retirement age of 65 . In 2013, the majority of EU farmers (i.e., the persons responsible for the day-to-day management of a farm) were older than 55 years and only $6 \%$ were younger than 35 years. But the lack of agricultural training was not only a problem among the oldest farmers, in 2013 it also affected about $60 \%$ of the youngest farmers, who will need advanced knowledge if they want to stay in business. As technological developments transform the agriculture and forestry sectors highly 
qualified people will be much more needed (an estimated increase by one-third, or almost 300 thousands of new jobs). Simultaneously, employment of low qualified workers will decline significantly - by more than 1.1 million of jobs in 2015-2025 (Cefedop, 2016).

The "Skills Alliance for Sustainable Agriculture - SAGRI" project (http://www.sagriproject.eu/) is an ERASMUS+ Project which main goal is to advance the skills of European farmers and agricultural extension staff through the development of new curricula and teaching programmes that integrate in a practical way the latest developments in agricultural applied research. The purpose is to provide farmers and agricultural stakeholders with knowledge, skills and competencies to understand and analyse agro-environmental systems as natural ecosystems modified by human activity, with an emphasis on agro-environmental technologies that can be applied to achieve crop sustainable production by means of improved systems' management.

In this paper, we tried to identify the skills needs in the field of agricultural technology (agri-tech), required for a farmer or agricultural worker to be able to practice a "sustainable agriculture", with a particular focus on "green skills" and "digital skills".

Skilled agricultural workers are generally defined as those responsible for growing, managing and harvesting crops; rearing livestock and managing forests (Cedefop, 2016). According to the EU Skills Panorama (2014), changes in technology, work organisation and available tools have and are changing the skills requirements of agricultural workers, concerning:

a) Green skills. Skilled agricultural workers increasingly need to have a holistic awareness of sustainability. This may relate to understanding Climate changing, the need for carbon emission reduction, renewable energy, biofuels, water resources and ecosystems management, and to be updated with new regulations and legislation linked to the sustainability agenda.

b) Digital or technological skills. Skilled agricultural workers will need to be able to understand and apply new technologies related to: primary production for both food and non-food uses, soil science, crop and livestock genetics, agri-chemicals and general purpose technologies such as remote sensors, satellites and robotics.

According to Cedefop's European skills and jobs survey (ESJS), the key 5 skills for skilled agricultural, forestry and fishery workers are teamwork, problem solving, learning, planning and job-specific skills (Cefedop, 2016). In this paper we will focus mainly in the analysis of job-specific skills.

\section{Materials and Methods}

When defining job-specific skills we must be aware that not all agricultural workers or farmers have sufficient knowledge to understand all the new developments in agriculture applied research, since some of them require a minimum education level. Therefore, prior to identifying the skills it is mandatory to define the agricultural worker profile to whom they are destined. In the SAGRI project we decided to analyse the skills needs of an agricultural worker or farmer considering that he or she would have a minimum education level of high school and a basic knowledge and experience in agriculture, at a practical level. Six major areas were identified, where significant technological developments occurred and that can help farmers for a more sustainable agriculture: 1) Precision agriculture; 2) Integrated pest management; 3) Agricultural reuse of organic residuals; 4) Drip irrigation and water-conserving technologies; 5) Renewable energy; and 6) Bioenergy and energy crops.

Within these six areas there where defined different skills that will be the basis for the developing of new innovative curricula integrating the latest advancements of the "agri-tech" sector, and training courses for agricultural workers. The training courses will be composed by six modules (corresponding to the six above mentioned thematic areas) each one with a total of 50 hours of student learning effort, that includes in class lectures, self-study, the use of an Open Educational Resources (OER) platform developed in the framework of the project, field visits and evaluation.

\section{Results and Discussion}

Transversal skills

Although this paper is mainly about the job-specific skills for agricultural workers, there are some generic and transversal skills that agricultural workers need to have in order to adapt to changing production processes, and to other sector-specific changes and challenges (Cedefop, 2016).

- Information and Communication Technology (ICT) skills. Nowadays the information available in the World Wide Web about agriculture (production systems, technology, results from research, new machinery, equipment and products for agriculture) is enormous. For the farmer to benefit from this information he (or she) has to know how to find it in the internet. Free agricultural software or on-line platforms that can interact with the farmer and give him specific information and e-tools useful for its activity are also available for those that can use a computer, a smartphone or a tablet. Communication technology gives farmers greater control over their access and exposure to information. It enables them to take initiative as information seekers, rather than adopting a passive role as information recipients (Meera et al., 2004). Text messaging is one of the most widely used mobile data service 
worldwide, and many services or equipment can use this tool to interact with the farmer providing him with real time information. This can be very useful for farm management.

- Desktop software skills. The use of common word processors and spreadsheets is essential for data management and are becoming an important skill in farming practice, allowing workers to process information collected from different sensors and mapping systems (European Commission, 2014). Software can also store digital evidence to be presented to national and EU agricultural regulators on the fulfilment of subsidy conditions.

- An up-to-date understanding of evolving EU and national regulations for the agricultural sector and awareness of sustainable practices are requirements to improve the efficient use of resources (European Commission, 2014).

- Climate change awareness. Climate change and environmental degradation increase farmers' responsibilities on conservation and environmental management. Farmers need to maintain the productivity of their land while facing extreme weather events, potential water shortages etc. There is a growing need for skilled agricultural workers to understand how environmental sustainability is integral and applicable to their everyday practice (i.e. managing pesticide and other chemical use, reducing carbon dioxide emissions, using renewable energy, and managing water resources) (European Commission, 2015b).

\section{Specific skills}

The following presented skills are job-specific skills that have been identified in the framework of the SAGRI project for each of the proposed training modules. They are job-specific skills with a highlight in the awareness for all agricultural sustainability aspects and in the introduction to major technological developments in the specific areas.

\section{Precision agriculture}

Precision Agriculture (PA) is "a holistic and environmentally friendly strategy in which farmers can vary input use and cultivation methods - including application of seeds, fertilizers, pesticides, and water, variety selection, planting, tillage, harvesting - to match varying soil and crop conditions across a field" (Srinivasan, 2006). The adoption of PA may be represented as a five-step cyclical process including data collection, diagnostics, data analysis, precision field operations and evaluation. It uses information technology, satellite positioning (GNSS) data, remote sensing and proximal data gathering (European Commission, 2014).

The implementation of PA has become possible thanks to the development of sensor technologies combined with procedures to link mapped variables to appropriate farming practices such as tillage, seeding, fertilization, herbicide and pesticide application, harvesting and animal husbandry (European Commission, 2014). With PA technologies the farmer can increase yields and/or profitability in a sustainable and environmentally friendly way. Several studies have demonstrated the economic and ecological benefits of PA tools over conventional techniques (e.g. Stafford, 2006; Silva et al., 2007; Takacs-Gyorgy, 2008). It is a complex process requiring different types of knowledge and expertises, usually available through consulting, advisory and training services. Nevertheless, the farmer or agricultural worker is a key factor for the implementation of PA. He must be aware of the benefits of PA, and must have some skills regarding the use of Precision technology. According to Pierpaolia et al. (2013) one of the main reasons for farmers not to adopt PA technologies are insufficient skills and competences to manage PA tools.

The agricultural workers required skills in Precision technology are:

a) Notions on the concept and principles of Precision Agriculture and the potential benefits from its use. The implementation of PA schemes enables farmers to: i) be more economically competitive; ii) improve farm sustainability; iii) improve crop quality and yield; iv) to attain more homogeneous products; v) secure traceability of products; and vi) be better adapted to comply with regulations regarding the use of nutrients and chemicals (Bakhtiari and Hematian, 2013).

b) Notions on the criteria for PA adoption and implementation. It is important for the farmer to be aware that the implementation of PA requires clear evidence of significant spatial and temporal variability in soil and crops conditions within a field or within fields in a region. The fields with the greatest opportunities for PA are those which reveal a high degree of yield variation. A high degree of variation will mean higher variable rate application of inputs and, therefore, greater economic and environmental benefits in comparison with uniform management. It is also important to analyse aspects as farm size, expected reduction in costs, higher revenues to provide a suitable cost/benefit ratio, the total income, land tenure, the farmer's level of computing skills, access to information and location, which are also important factors influencing the adoption of PA (Pierpaolia et al., 2013).

c) Notions on the better techniques and technologies to evaluate field and crop variability. The farmer may not be able to use these techniques, due to the required expertize to operate different sensors, equipment and computer software, but he must be aware of the best techniques that can be used to evaluate soil and crop variability in order to be able to acquire the correct consulting services for his own situation. Over the last few decades, many new technologies have been developed for, or adopted to, agricultural use, such as: yield monitors, sensors developed 
to quantify the physiological status of crops (e.g. Nitrogen sensors, crop vegetation index sensors), geophysical sensors to measure soil properties, such as apparent electrical conductivity (ECa) sensors, and low-cost remote sensing techniques. Topographic, crop and soil data can be attained from aerial photographs, photographs from unmanned aerial vehicles (UAV), satellite images and proximity sensors. These data can then be incorporated in Geographical Information Systems to attain diverse thematic maps, such as elevation maps, soil electric conductivity maps, $\mathrm{pH}$ maps, yield maps, vegetation index maps and others. Notions about the characteristics and accuracy of the referred data sources, such as, spatial resolution, frame size and equivalent ground area, are also important to identify the best data source according to the assessment goals.

d) Skills for implementation and/or use of precision technologies. Precision technologies can be used in several agricultural activities: i) Controlled Traffic Farming (CTF) and auto-guiding systems are the most successful applications on arable land showing clear benefits in many cases; ii) Variable Rate Application (VRA) methods, for fertilizer or pesticide application, for irrigation, etc, can be used with different success depending to the specific factors of the application. Its use allows precise seeding, optimization on planting density and improved application rate efficiency of herbicides, pesticides and nutrients, resulting in cost reduction and reducing environmental impact. There is some evidence from research which shows that environmental degradation is reduced when PA methods are applied, including increased fuel use efficiency resulting in lowering carbon footprints. PA has been identified as a way to meet EU directives in Member States to reduce agro-chemicals (Zhang et al., 2002); iii) Precision Livestock Farming (PLF) that relies on automatic monitoring of individual animals and it is used for meat, milk and egg production and for monitoring animal behaviour, welfare and productivity and also their physical environment (European Commission, 2014). There are a lot of monitoring equipment that the farmer can use to collect data after a proper training; iv) Precision irrigation. In high-value crops, precise irrigation methods are developing rapidly in order to save water while improving yields and fruit quality. A simple division of a field in different individual controlled irrigation sectors, can allow to apply different irrigation depths according to specific field and crop variations.

The farmer must be aware of the main available precision technologies, including different sensors required to collect field data for crop status evaluation, and what is necessary to use them.

\section{Integrated pest management}

"Integrated pest management means careful consideration of all available plant protection methods and subsequent integration of appropriate measures that discourage the development of populations of harmful organisms and keep the use of plant protection products and other forms of intervention to levels that are economically and ecologically justified and reduce or minimise risks to human health and the environment. 'Integrated pest management' emphasises the growth of a healthy crop with the least possible disruption to agroecosystems and encourages natural pest control mechanisms" (European Parliament and Council Directive 2009/128/EC).

For a farmer to adopt an integrated pest management strategy he must have the following skills:

a) Notions on general goals and principles of integrated pest management (Directive 2009/128/EC Annex III);

b) To know relevant national legislation and regulations for the adoption of integrated pest management.

c) Notions on integrated pest management strategies and techniques, including:

i. Notions on risk assessment, economic threshold levels and pest control methods (agronomic, biological, genetic, biotechnical and chemical);

ii. Information on the general principles and crop or sector-specific guidelines for integrated pest management;

iii. Harmful organisms monitoring methods. Harmful organisms must be monitored by adequate methods and tools, where available. Such adequate tools should include observations in the field as well as scientifically sound warning, forecasting and early diagnosis systems, where feasible, as well as the use of advice from professionally qualified advisors.

d) Decision making. Based on the results of the monitoring the professional user has to decide whether and when to apply plant protection measures. For harmful organisms, threshold levels defined for the region, specific areas, crops and particular climatic conditions must be taken into account before treatments, where feasible. Sustainable biological, physical and other non-chemical methods must be preferred to chemical methods if they provide satisfactory pest control (Directive 2009/128/EC Annex III).

e) Notions on the standards for a sustainable use of plant protection products, including:

i. Definition, classification, toxicity and eco-toxicity, and authorized plant protection products in integrated pest management strategies.

ii. Application methods of plant protection products, including equipment characteristics, selection and maintenance needs.

iii. Procedures for preparing pesticide application equipment for work, including its calibration, and for its operation with minimum risks to the user, other humans, non-target animal and plant species, biodiversity and the 
environment, including water resources.

iv. Measures to minimise risks to humans, non-target organisms and the environment: safe working practices for storing, handling and mixing pesticides, and decontamination and disposal of empty packaging, other contaminated materials and surplus pesticides (including tank mixes), whether in concentrate or dilute form; recommended ways to control operator exposure (personal protection equipment).

\section{Agricultural reuse of organic residuals}

The term "organic residuals" includes several different waste categories. Among them are the organic fraction of municipal solid waste, animal wastes or effluents, agro-industrial co-products, by-products and effluents, biosolids (organic solids or sludge remaining after sewage treatment) and forestry and agricultural crops byproducts and wastes. There is considerable potential for beneficial use of organic residuals through land applications. These beneficial uses can contribute to sustainable agricultural production and support a triple bottom line with positive economic, social and environmental outcomes. However, land application of organic residuals has some risks and costs, and these must be carefully evaluated and managed (King et al., 2011).

In order to reuse organic residues in agriculture in a sustainable way, farmers must have the following skills:

a) Notions on available organic residuals and their potential uses. Types, characteristics, availability and agricultural potential uses of major organic residuals.

b) To know the legislation regarding the use of organic residuals. National regulations, limits for contaminants level, pre-treatment regulations, pathogen regulations, limits on direct discharge into water bodies, etc.

c) Notions on environmental and economic aspects of using organic residuals. Crop residues, manure or compost can be used in agriculture reducing the use of fertilizers, offsetting the environmental impacts of its use (the energy consumption for its production, or gas emissions and leaching), the use of pesticides or irrigation water (European Commission, 2010). This may also bring economic benefits for farmers. However, there can be some biological and chemical risks (from direct exposure or from contamination of food and water), depending on the source of the organic residuals or its previous treatment. Treating material to reduce or eliminate pathogens, for example, will affect costs associated with beneficial uses. To optimize economic benefits, the viability of all end use options must be factored into decision making processes. Land application of organic residuals may only be feasible if certain economic incentives are instituted. These incentives may evolve as a result of a fuller understanding of the benefits of reusing organic residuals (King et al., 2011).

d) Notions on transport, storage and treatment requirements for different organic residuals. Previous to its use, organic residues may have to be transported to the application site, and in some cases stored or transformed prior to its application. In many cases there are specific rules (best management practices) and regulations that must be followed.

e) Notions on organic residuals management and treatment techniques that could be performed in the agricultural farm. There are several treatment techniques that the farmer can apply in the management of organic residuals. Composting is one of the most ecological technologies for the management of the bio-waste, allowing its material valorisation (Scotti et al., 2016). On-farm composting could be an efficient, cost-effective and environmentally safe biological process for the recycling of residual agricultural biomasses (Maniadakis et al., 2004). Application of organic amendments, such as compost, has been successfully proposed in many cases to improve soil structure and fertility, as well as to suppress soil-borne pathogens (Scotti et al., 2016).

\section{Drip irrigation and water-conserving technologies}

Climate changing and the increase of the competition for water use from other activity sectors is increasing water scarceness for agriculture. Therefore it is very important to provide agricultural workers with skills to improve the use of available water, increasing water use efficiency and farmers profitability. Drip irrigation has the potential to achieve the highest uniformity and efficiency values of all irrigation methods. Yet, it is possible to observe drip irrigation systems with poor uniformity and application efficiency resulting from various causes, such as inadequate maintenance, low inlet pressure or pressure fluctuations, emitter clogging and inadequate system design (Hsiao et al., 2007). A correct use of these systems is very important not only to preserve water or the environment, but also because water efficiency will be an increasingly important factor for competitiveness (CEC, 2008).

The agricultural worker required skills for a better use of drip irrigation systems are:

a) Manage irrigation following an irrigation scheduling. Irrigation scheduling is the farmers' decision process related to 'when' to irrigate and 'how much' water to apply to a crop (Pereira, 1999). It requires knowledge on crop water requirements and yield responses to water, the constraints of the irrigation system ("how" can the system apply the desired irrigation water), the availability of the water that supplies the irrigation system, and the knowledge of soil properties, such as soil water holding capacity, field capacity, wilting point, etc, that can affect irrigation scheduling. The agricultural worker must be aware of the importance to manage irrigation according to 
an established irrigation scheduling, either based on crop water evapotranspiration and/or soil water availability. He also must understand the effect of meteorological parameters (air temperature, wind, solar radiation, etc) over the crop evapotranspiration to choose the right time to irrigate. For example, scheduling irrigation during night will allow a reduction in evapotranspiration, thus improving irrigation efficiency and saving water. The reduction of applied irrigation water, due to the improvement of irrigation efficiency, will also allow an energy saving due to less pumping hours. It is recognised that the adoption of appropriate irrigation scheduling practices could lead to increased yields and greater profit for farmers, significant water savings, reduced environmental impacts of irrigation and improved sustainability of irrigated agriculture (Smith et al., 1996).

b) Definition of different irrigation goals. Although the primary irrigation goal is to satisfy full crop water requirements, to achieve maximum yield, there can be other objectives associated with crop quality, the scarceness of water resources, economic return, etc. The agricultural worker must understand the concepts of full irrigation, supplemental irrigation, deficit irrigation (including regulated deficit irrigation (RDI) and partial root drying (PRD)), water use efficiency (WUE) or water productivity (WP), and be able to adapt the irrigation scheduling to different irrigation goals. Different irrigation strategies have been proven to successfully increase WUE reducing water use. For example, the successful use of RDI in fruit trees and vines demonstrated not only increases in water productivity, but also in farmers' profits (Fereres and Soriano, 2007).

c) Irrigation system evaluation. The agricultural worker must know all the irrigation system components, from the pump to the dripper, to be able to evaluate, at any time, their operating status and to perform changes in its operating mode that can improve its performance. For this reason, it is essential that the farmer knows and is able to use the equipment required to measure the major operating parameters, such as manometers to measure the system operating pressure, flowmeters or other flow measuring techniques to ensure a proper dripper discharge, etc.

d) Maintenance of irrigation systems. The irrigation system maintenance is also another very important issue. The agricultural worker must know how to maintain the irrigation system to ensure his lifespan. Drip irrigation systems, more than other irrigation systems, require a good maintenance, including annual operations to clean filters, pipes and drippers. Only with a good maintenance it is possible to guarantee its proper operation, which is essential to enhance water use efficiency.

e) Irrigation evaluation and monitoring. The agricultural worker must understand the concepts of irrigation efficiency and uniformity, and know how to perform a field irrigation evaluation determining its efficiency and uniformity. Only based on this information will he/she be able to improve the irrigation performance, saving water and increasing water use efficiency or water productivity. Excessive irrigation stimulates run-off, leach-outs of fertilisers and pesticides and soil erosion. The determination of performance indicators requires specific equipment, including soil moisture sensors, which he must know how to operate. Soil moisture sensors are also essential for irrigation monitoring. Irrigation performance and actual meteorological data can produce variations in soil water availability different from those expected, demanding real time corrections on the irrigation scheduling. Therefore, a continuous monitoring of soil water content to improve water use efficiency is essential.

f) Notions on the use of low quality irrigation water. There are many examples of soil salinization and yield reductions due to the use of irrigation waters with high salinity content. The farmer must be aware of the effects of using low quality irrigation water on soils and crops. Irrigation scheduling may need to be adapt (irrigation frequency, irrigation depths) when the farmer uses low quality irrigation waters. Additional irrigation, using good quality water, may also be needed to promote salts leaching. Excessive and inappropriate fertilizers used in fertigation systems can also contribute for soil salinization. It is important to know how to select fertilizers in order to minimize their salinization effect.

\section{Renewable energy}

Climate change and the global agenda to reduce $\mathrm{CO}_{2}$ emissions are among the most pressing international challenges of the present day. Together they form the single most significant factor driving a preference for renewable energy over energy from fossil fuels (ILO, 2011). The renewable energy sector has the potential to deliver substantial reductions in energy related emissions of greenhouse gases and other pollutants. Renewable energies offer the full range of energy services - heat, light, electricity and mechanical energy (IPCC, 2011). The main renewable energy technologies are wind, solar, geothermal, hydropower and bioenergy. Some of them can be more easily used in a farm, like solar or geothermal energy. Solar energy, for example, can be used to produce electricity and heat. Photovoltaic panels can be used to power different farm operations, pumping water, lighting and electric fences. Solar heat collectors can be used for house, livestock buildings and greenhouses heating, in drying systems and to provide hot water for dairy operations, cleaning and sanitary uses. Geothermal energy can be used to generate heat for farm buildings heating. Bioenergy due to its importance will be addressed in more detail in another training module.

The Agricultural workers' required skills regarding renewable energy are:

a) Environmental awareness. Farmers must be aware of the environmental advantages of using renewable energies. 
b) Notions on all possible renewable energy sources. It is important for farmers to know what types of renewable energy sources and technologies they can use and the environmental, social and economic benefits of using them. The farmer should be able to identify renewable energy sources that are most appropriate for their own situation, from a technical and economical aspect. Off-grid renewable energy solutions (meaning in areas not connected to a central grid) can often make economic sense even without subsidies or policy support (ILO, 2011).

c) To know national legislation and regulations promoting the use of renewable energies. Many governments have favoured the connection of smaller renewable energy capacity generating facilities to electricity grids. This can be a business opportunity for farmers, allowing to increase profit and farm sustainability. Many governments have also promoted the use of renewable energies from subsidizing the installation of wood chip fired heating systems to subsidizing bioethanol or biodiesel. A good knowledge of national subsidy policies regarding the use of renewable energy can be an incentive to its use.

d) Notions on safety measures for using different renewable energy equipment. All equipment has specific rules for its installation and safe use that must be known and complied with. Its compliance will allow better performance of the equipment, increasing its lifespan, and the decrease of potential risks to people interacting with them.

\section{Bioenergy and energy crops}

The term bioenergy refers to energy derived from any organic matter that is available on a renewable basis. It may use any of a wide range of inputs, including forest and mill residues, agricultural crops residues, as well as processing residues, wood and wood waste, animal production wastes, aquatic plants, fast-growing trees and herbaceous crops, municipal and industrial wastes, among other sources (ILO, 2011).

Bioenergy outputs can include electricity, fuels such as bioethanol and biodiesel, and heat, with more than one of these sometimes coming from the same process. They can also include chemicals and other materials with potential for further processing.

Bioenergy uses a number of different types of process (ILO, 2011). The biomass may be burned directly to produce heat and/or for the generation of electricity. Other possibilities are: i) a process to produce a liquid fuel such as biodiesel or bioethanol; ii) gasification process to produce gases which can be stored and used to produce electricity, or iii) a process of anaerobic digestion to produce methane, which may then be used to generate electricity or thermal energy.

Biomass production requires substantial numbers of agricultural or forestry workers to plant, manage and harvest biomass crops for as long as the bioenergy facility is in operation. Efficient production also relies on skills in agricultural science.

For the purpose of this report we will define agricultural worker skills in the bioenergy sector considering mainly, the subsectors of: i) anaerobic digestion; ii) dry agricultural residues (poultry litter, straw and spent mushroom compost); iii) energy crops; iv) liquid biofuels and v) wood. Although, some of these subsectors are also included in the "agricultural reuse of organic residues" module.

The agricultural workers' required skills regarding bioenergy and energy crops include:

a) Environmental awareness. Farmers must be aware of the environmental benefits and impacts of bioenergy.

b) Notions on the range of bioenergy resources, conversion technologies and markets. It is important for farmers to know what types of bioenergy resources and technologies they can use and the environmental, social and economic benefits of using them. They must be introduced to the technical, business, environmental, policy and legal aspects of: i) anaerobic digestion; ii) Dry agricultural residues (poultry litter, straw and spent mushroom compost); iii) Energy crops; iv) Liquid biofuels and v) Wood.

c) Notions on handling, transport and storage of biomass, bioenergy products and by-products. To a proper use and production of bioenergy resources the farmer must have a basic knowledge on how to handle, transport and storage biomass, bioenergy products and by-products.

d) To identify which bioenergy solutions are most appropriate for their own situation, technically and financially.

e) Notions on how to evaluate energy crops as a farm business opportunity. Characteristics (including yields) and requirements of energy crops. Comparison of different energy crops, including financial evaluation. Agricultural practices for establishment and production of energy crops. Cutting cycles and harvesting options, including baling, bundling or chipping as appropriate. Logistics of supply following harvesting, including immediate use or drying and storage. Possibilities for transport and preliminary processing of biomass.

\section{Conclusions}

The above mention skills are those that have been identified as the necessary and possible to transmit to agricultural workers in the frame of the SAGRI project. Main skills will be focused in: 
i) Gaining awareness about principles and objectives of different agricultural practices that are friendly to the environment and at the same time economically viable;

ii) Gaining awareness of different legislation and regulations regarding the adoption and use of these different agricultural practices;

iii) Be able to identify the data, equipment and techniques to acquire field data, that the farmer must have or hire in order to adopt the above mentioned agricultural practices; and,

iv) Be able to recognize the benefits and to evaluate the technical and economic feasibility to adopt any of these agricultural practices.

In some modules it may also be possible to give to the agricultural workers (farmers) the information and/or training that can enable them to use some of the equipment required for implementation of the proposed agricultural practices. It will be also desirable to present to farmers actual examples of the application of different technologies explaining its benefits and constrains. If possible this can be complemented with field demonstrations.

It is our belief that the acquisition of these skills is an important step to achieve a more technologically advanced and social, economic and environmentally sustainable agriculture. Agricultural workers that conclude successfully the training courses, will be awarded with the certification of "sustainable farmers" according to the standards of ISO 17024.

\section{Acknowledgements}

The project "Skills Alliance for Sustainable Agriculture - SAGRI" was financed by the European Union Programme ERASMUS+ - Key Action 2: Sectors skills alliances (Contract no. OI 174011).

\section{References}

Bakhtiari, A.A., A. Hematian, 2013. Precision Farming Technology, Opportunities and Difficulty. International Journal for Science and Emerging Technologies with Latest Trends: 5(1), 1-14. http://www.ijsett.com/images/Paper(1)1-14.pdf

Commission of the European Communities (CEC), 2008. Follow up Communication on Water Scarcity and Droughts in the European Union COM (2007) 414 final, [SEC(2008) 3069], Brussels.

Cedefop, 2016. Analytical Highligts. Skilled agricultural, forestry and fishery workers: skills opportunities and challenges. (http://skillspanorama.cedefop.europa.eu/en/analytical highligths/skilled-agricultural-forestryand-fishery-workers-skills-opportunities-and) accessed in January, 2017.

European Commission, 2010. Accompanying the Communication from the Commission On future steps in bio-waste management in the European Union. Assessment of the management of bio-waste. Commission staff working document. Brussels, SEC(2010) 577 final.

European Commission, 2014. Precision agriculture: an opportunity for EU farmers: potential support with the CAP 2014-2020. Study.

European Commission, 2015a. EU farms and farmers in 2013: an update. EU Agricultural and Farm Economics Briefs n. 9. (https://ec.europa.eu/agriculture/sites/agriculture/files/rural-areaeconomics/briefs/pdf/009 en.pdf)

European Commission, 2015b. Towards a long-term strategy for European agricultural research and innovation by 2020 and beyond. Background paper, EU pavilion at Expo Milan.

European Parliament and Council, 2009. Directive 2009/128/EC. Establishing a framework for Community action to achieve the sustainable use of pesticides. Official Journal of the European Union. L 309/71-86.

EU Skills Panorama, 2014. Skilled agricultural workers analytical highlight (http://skillspanorama.cedefop.europa.eu/en/analytical highlights/prospects-skilled-agricultural-workers)

Fereres, E., A. Soriano, 2007. Deficit irrigation for reducing agricultural water use, Journal of Experimental Botany, 58 (2): 147-159. https://doi.org/10.1093/jxb/erl165

Gold, M.V. (2015) Sustainable Agriculture: Information Access Tools. United States Department of Agriculture. https://www.nal.usda.gov/afsic/sustainable-agriculture-information-access-tools\#define, accessed in May, 2018.

Hsiao, T.C., P. Steduto, E. Fereres, 2007. A systematic and quantitative approach to improve water use efficiency in agriculture. Irrigation Science, 25, 209-231. https://doi.org/10.1007/s00271-007-0063-2

ILO, 2011. Study of occupational and skill needs in renewable energy: final report / International labour office, ILO skills and employability Department (EMP/SKILLS), European Commission. Geneva.

International panel on climate change (IPCC), 2011. Special report on renewable energy sources and climate change mitigation, prepared by the Working Group III of the Intergovernmental panel on climate change. Geneva. 
King, G.M., J.P. Brooks, S. Brown, C. Gerba, G.A. O'Connor, I.L. Pepper, 2011. Land application of organic residuals: Public health threat or environmental benefit?. American Society for Microbiology. (https://www.asm.org/index.php/education?id=7489:biorep8-2011)

Maniadakis, K., K. Lasaridi, Y. Manios, M. Kyriacou, T. Manios, 2004. Integrated waste management through producers and consumers education: composting of vegetable crop residues for reuse in cultivation. Journal of Environmental Science Health B 39, 169-183. https://doi.org/10.1081/PFC-120027447

Meera, S., A. Jhamtani, D. Rao, 2004. Information and Communication Technology in Agricultural Development: A Comparative Analysis of Three Projects from India. Agricultural Research and Extension Network Paper No. 135. Washington DC: World Bank.

Pereira, L.S., 1999. Higher performance through combined improvements in irrigation methods and scheduling: a discussion. Agricultural Water Management, 40, 153-169. https://doi.org/10.1016/S03783774(98)00118-8

Pierpaolia E., G. Carlia, E. Pignattia, M. Canavari, 2013. Drivers of Precision Agriculture Technologies Adoption: A Literature Review, Procedia Technology 8, 61 - 69. https://doi.org/10.1016/j.protcy.2013.11.010

Scotti, R., C. Pane, R. Spaccini, A.M. Palese, A. Piccolo, G. Celano, M. Zaccardelli, 2016. On-farm compost: a useful tool to improve soil quality under intensive farming systems. Applied Soil Ecology, 107: 13-23. https://doi.org/10.1016/j.apsoil.2016.05.004

Silva, C.B., S.M.L.R. Do Vale, F.A.C. Pinto, C.A.S. Muller, A.D. Moura, 2007. The economic feasibility of precision agriculture in Mato Grosso do Sul State, Brazil: A case study. Precision Agriculture, 8(6), 255-265. https://doi.org/10.1007/s11119-007-9040-2

Smith, M., L.S. Pereira, J. Beregena, B. Itier, J. Goussard, R. Ragab, L. Tollefson, P. Van Hoffwegen (Eds.), 1996. Irrigation Scheduling: From Theory to Practice. FAO Water Report 8, ICID and FAO, Rome.

Srinivasan, A. (Ed.), 2006. Handbook of Precision Agriculture. Principles and Applications. Food Products Press. The Haworth Press, Inc. NY.

Stafford, J.V., 2006. The role of technology in the emergence and current status of precision agriculture, In Srinivasan, A. (Ed.), Handbook of Precision Agriculture. Principles and Applications. Food Products Press, The Haworth Press, Inc. NY., Chapter 2, 19-56.

Takacs-Gyorgy, K. 2008. Economic aspects of chemical reduction on farming: role of precision farmingWill the production structure change?. Cereal Research Communications, 36: 19-22. http://www.jstor.org/stable/90002630

Van Crowder, L., W.L. Lindley, Th.H. Bruening, N. Doron, 1998. Agricultural education for sustainable rural development: Challenges for developing countries in the 21 st century. The Journal of Agricultural Education and Extension, 5(2): 71-84. https://doi.org/10.1080/13892249885300201

Zhang, N., M. Wang, N. Wang, 2002. Precision agriculture-a worldwide overview, Computers and Electronics in Agriculture, 36: 113-132. https://doi.org/10.1016/S0168-1699(02)00096-0 


\title{
Adoption of Automatic Feeding Systems in Bovine Livestock Farms: an On-Farm Survey on Italian Situation
}

\author{
Carlo Bisaglia a, ", Massimo Brambilla a , Maurizio Cutini ${ }^{\text {a }}$, Alberto Assirelli ${ }^{\text {b }}$ \\ ${ }^{a}$ CREA Centro di ricerca Ingegneria e Trasformazioni agroalimentari, via Milano 43, 24047 Treviglio (BG), Italy \\ ${ }^{\mathrm{b}}$ CREA Centro di ricerca Ingegneria e Trasformazioni agroalimentari, via della Pascolare 16, 00015 Monterotondo \\ (Roma), Italy \\ * Corresponding author. Email: carlo.bisaglia@crea.gov.it
}

\begin{abstract}
Although the automatic feeding system (AFS) is not the sole method to achieve precision feeding in dairy cows, its adoption allows a novel way to manage the daily feeding distribution that includes impacts on feed intake rhythm, leftover, animal behaviour, labour share and barn design. Moreover, the possible interactions with other automatic systems in the barn enable to plan a higher level of automation and precision in livestock farms. Italy has been adopting AFS in dairy farms for very short time, albeit with a relative delay compared to Northern European Countries that started the adoption of AFS in the early 2000. Since 2013, more than 60 Italian farms have introduced AFS both in junction with other automation and not. To know the motivation and, in the case, the constraints to further development of these technologies, a survey was carried out among these farms selected asking directly at all the manufacturers - or their dealers - present in Italy. The survey has considered: (a) the farming system, (b) grouping and housing, (c) scheduling and work organisation, (d) engineering aspects of the AFS, and (e) costs. The investigated farms have adopted AFSs provided by five manufacturers. The farming systems cover all the systems present in Italy for bovine livestock (milk production for dairy, milk production for long ripening cheese, beef production) and all the possible geographic areas (mountain, dry hill, irrigated plans). Herd dimension ranges from less than 20 to more than 300 dairy cow and 700 to 1.000 fattening bulls according to the various contexts the farms operate in and includes 5 Italian Regions (Lombardia, Piemonte, Veneto, Trentino-Alto Adige and Emilia Romagna) with distinctive dairy or beef specifications. The workload is diminished for most the farmers and installation costs range from 400 to $1200 € \mathrm{cow}^{-1}$ depending on the complexity of the project. Even if with a delay concerning other Countries, Italy has begun to adopt AFS in dairy farms. A further increase in the number of these systems is also expected for specialised fattening bulls farms, mainly located in Piemonte, Veneto and Lombardia Regions.
\end{abstract}

Keywords: Total Mixed Ration, automation, bovine livestock.

\section{Introduction}

The EU-28 dairy sector, in 2016, produced approximately 168.3 million tonnes of milk, the $96 \%$ of which is from cows (Eurostat, 2017). The same source (Eurostat, 2015) indicates, in 2014, an estimated meat production from bovine animals (beef and veal) of 7.4 million tonnes, the $87 \%$ of which is from beef. The intense technological development the European livestock is being got through over the past 40 years has allowed the improvement of livestock production and quality of farming activities.

Mechanisation, in particular, replacing part of the labour force, has eliminated or somehow decreased the extent of strenuous and dangerous tasks conducted by men, with benefits regarding the health and safety of agricultural workers and higher profits arising following the increased labour productivity. Moreover, the powerful informatisation of production processes has resulted in the development of specific software allowing for both the control of machines, farm plants and the information processing of farm (production, animal health and welfare, economic) data.

Nevertheless, the increasing use of machines and production systems has led to a gradual increase in the energy consumption of livestock farming, and this is a negative aspect, especially in the current situation, with the general economic crisis, the rising trend in the cost of global energy and the environmental constraints.

Automation could be an answer to these problems in particular for agriculture sector that results to be one of the five work-sectors with the highest potential for automation, especially for physical works (McKinsey Global Institute, 2017).

However, concerning dairy and beef cattle feeding, the automation of feed preparation and distribution operations is a very important and difficult task because of the physiology of these animals. Being them ruminants, they need a special diet featuring not only nutritional elements, but also the physical aspect related, in particular, to the content and the size of the solid particles (mainly fiber). To date, the reference diet form for ruminants is the Total Mixed Ration or TMR (Barmore, 2002), consequently the automation system design has to be able to perform TMR diets. Moreover, animal nutrition is the most important item in the production costs of milk and meat, both because of the costs for fodder, feed and supplements purchase and production and the cost of labour and the machinery needed for on-farm feed preparation and distribution operations, so the automation has to be 
economically feasible as well. In addition, for the meat sector, beef production has a relatively long production cycle, in particular if compared with pig-meat production, and its feed efficiency is generally lower if compared with other animals. Moreover, the diversity of bovines produced for slaughter (feeding mode, age, dairy or meat breeds, etc.) makes the production systems more complex.

All these factors have led to tight margins and low profitability; the purchasing power of consumers is also a key determinant of the level of animal products consumption.

In recent years (Figure 1), several automatic feeding systems (AFS) have been designed and introduced into the dairy livestock (Pompe, 2004) and bull fattening livestock (Serap et al., 2017) providing new suggestions to managing and designing livestock activities (Da Borso et al., 2017); in fact, the automated preparation and distribution of the food, along with the milking, are the operations research and experimentation activities focus the most on.

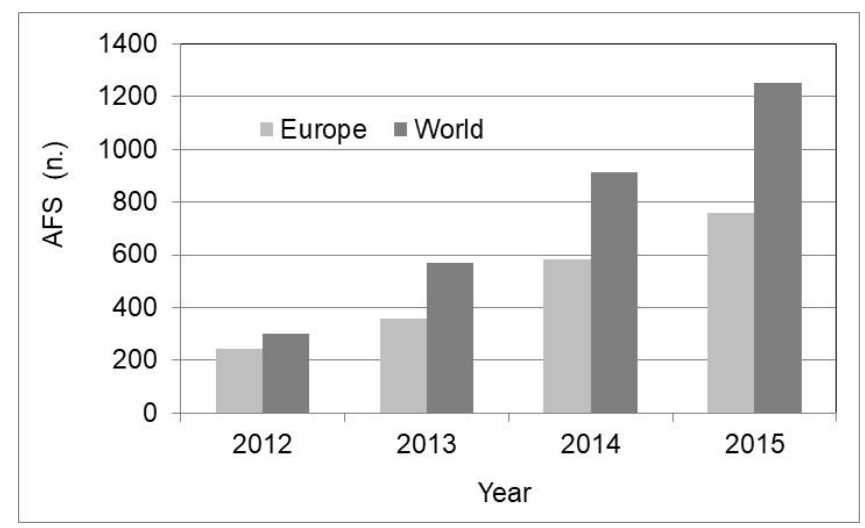

Figure 1. The diffusion of AFS in Europe and in the World (eight manufacturers, from Oberschätzl et al., 2015, modified).

Today there are more than twenty manufacturers that make the reference market for AFS, and that produce also other automatic systems for animal husbandry (i.e. milking and cleaning systems) so that the interaction between the various robotised components becomes increasingly desirable.

Considering the potential of these systems to solve some of the problems listed before, this work aims to present an overview of the current status of AFS in Italy, highlighting the current elements of strength and criticality of this type of technology following the users' opinions and perceptions.

\section{Materials and Methods}

To deep the knowledge of AFS diffusion and appreciation in Italy, the information provided by five manufacturers, who started installations in our country from 2013, have been used. Nevertheless, the market provides farmers with a higher number of manufacturers to meet the growing interest they have about these technical solutions.

A survey on the diffusion of AFS in Italy was carried out asking commercial offices of the manufacturers producing such plants the location of the installed ones. These allowed to get in touch with farmers that agreed to answer a questionnaire aimed at pointing out the structural aspects of the farming activity (size of the herd, energetic consumptions, characteristic elements of barn construction) and the propensity of the farmer to innovation and the motivations related to the purchase of an AFS.

The study consisted of two parts: firstly, the manufacturers involved in the Italian market were detected, contacted by phone and asked for the total number of farmers using their AFS, the address, the type of production (milk or beef), the dimension of the herds and the year of installation/start of use.

After completing this work, a second part of the survey was conducted directly into selected farms, including a range of producers from the earliest to the newest thus providing a scale of how the transition progression has changed, if any, over time.

Considering the rapid evolution of the situation and/or the dependence, in some cases, of the farmers' decision from public subsidies (mainly from Regional Development Plans), the "intention to buy" of some farmers or the farmers that asked a quotation to the manufacturer but that not have completed the installation at the moment of the survey were not included in the study; in any case, these aspects gave an impression of the potential and growing interest for the AFSs from the Italian livestock sector. 


\section{Results and Discussion}

Overall, the AFS today regard almost sixty farms that purchased them from five manufacturers. Although the situation is under continuous evolution, as always with new technologies, a first map provides interesting odds (Figure 2). AFS are currently distributed in five Italian Regions (Lombardia, Piemonte, Veneto, Trentino-Alto Adige and Emilia Romagna) with distinctive dairy/beef specifications (ISTAT, 2018).

The observed size of the herds ranged from 18 to 150 dairy cows, but consistencies of up to 300 dairy cows were also pointed out. The main breed was Holstein Frisian with also Brown and Jersey in some cases. Following the heterogeneity of the rearing conditions, milking production was very wide and ranged between 8,500 and $10,500 \mathrm{~kg}_{\text {milk }} \mathrm{y}^{-1} \mathrm{cow}^{-1}$. However, following the installation of AFS, almost all the breeders reported a gain of their milk production (1-4 $1 \mathrm{cow}^{-1}$ day $\left.^{-1}\right)$ compared to that related to the previous mode of animal feeding.

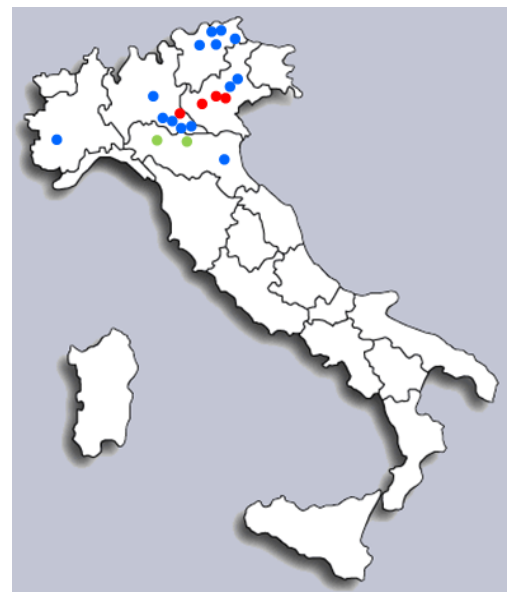

Figure 2. Distribution of the surveyed AFS in Italy (five manufacturers): blue dot=dairy farms, green dot=dairy farm for Protected Designation of Origin (PDO) production, red dot=beef production (in some cases one dot represents more than one farm).

With reference to meat production livestock, the size herd ranged from 700 to 1000 beefs with mainly French breeds (Charolaise, Limousine, Aubrac). The animals were generally bought at 350-400 kg weight and sloughtered at 600-700 kg, according to the breed they belong to. Also in this case, an average gain of the daily weight was recorded (130-150 $\mathrm{g} \mathrm{beef}^{-1}$ day $\left.^{-1}\right)$ compared to that related to the previous mode of animal feeding.

Concerning farmers interest in approaching AFS installation, the $60 \%$ of the analysed farms also had an automatic milking system (AMS) installed. According to some farmers, the priority they had when considering the purchase of an AFS, was to achieve a good level of automation of the feed ration (preparation and distribution) and only afterwards their interest was focused on AMS too. The installation costs ranged from 400 to 1200 $€ /$ animal depending on the complexity of the project and in light of the structure the machine should have been installed.

Data processing and the AFS description the farmers provided allowed the depiction of the following results. The constant improvement AFS distinctive elements undergo (e.g. the so-called kitchen, functional accessories, controlling devices), together with the considerable competition among manufacturers, has gradually brought to the market more and more evolved generations of AFS. Following farmers and manufacturers descriptions, a brief report of AFS distinctive elements found in the surveyed farms follows.

\section{The kitchen}

The kitchen is a permanently covered place that stores all the ingredients of the ration, even for two-threes days, and where the distributing wagon is reloaded automatically according to frequency intervals that the farmer establishes. The kitchen can be refurbished daily or can have the autonomy of more than one day, depending on the shelf life of the ingredients (i.e. shorter for the silages, longer for hay and strow). However, it is usually manageable with great flexibility by the operator.

When the wagon operates only ration distribution, the kitchens have fixed mixers that undergo the same management of standard TMR mixers.

In case the AFS foresees the adoption of more complex mixing-distributing wagons, the kitchen may have higher variability following the design of the manufacturers. It may consist of self-unloading containers supported by automated vertical silos for feed. These containers, thanks to conveyor belts, cutting devices and augers, are capable of measuring all the ingredients to be introduced into the wagon. Kitchen equipment also fits customer 
needs as for the farms producing milk for Parmigiano Reggiano cheese. They must follow the ParmigianoReggiano production regulation to sell cheese with the Protected Designation of Origin (PDO) label, and this means that using additives is not permitted (European Commission, 2006 and 2011). In this case, AFS's kitchens have specific devices separating the soil particles - vector of clostridia - from the hay, to guarantee the quality of the affined ingredients and reduce problems during cheese making and ageing.

Another typical design for the kitchen foresees a covered reinforced floor to store the dry ingredients together with vertical automated silos for feed: self-loading cranes provide for the dosing of the ingredients into the mixingdistributing wagon. When milk is for the typical production of long-aged cheeses, rations are based on hay but do not foresee the use of silages: in this case, water dispensers are added to kitchen equipment to guarantee the TMR diet with the proper humidity.

The AFS surveyed have demontrated to well manage the great variability of diets and feeding strategies present in Italy. Only as an example, a syntesis of the different diets and situation found was elaborated and presented in Table 1.

Table 1. Average characteristics of three main different TMR groups managed by the AFS in Italian condition. (The data are indicative: a very wide range of variants is foreseen inside each group following local feed availability, animal production and age/breed requirements).

\begin{tabular}{cccc}
\hline $\begin{array}{l}\text { Ingredient } \\
\text { (in order of preferred loading) }\end{array}$ & $\begin{array}{c}\text { Dairy milk } \\
\text { production }\end{array}$ & $\begin{array}{c}\text { PDO milk } \\
\text { production }\end{array}$ & $\begin{array}{c}\text { Beef } \\
\text { production }\end{array}$ \\
\hline Hay (weight ratio on wet basis, \%) & $10-15$ & 42 & - \\
Straw (weight ratio on wet basis, \%) & - & - & 7 \\
Silage (weight ratio on wet basis, \%) & $79-84$ & - & 38 \\
Concentrates (weight ratio on wet basis, \%) & 5.5 & 20.5 & 54.5 \\
Minerals (weight ratio on wet basis, \%) & 0.5 & 0.5 & 0.5 \\
Water (weight ratio on wet basis, \%) & - & 37 & - \\
Physical properties & & $210 \pm 10$ & $300 \pm 5$ \\
Bulk density (kg m ${ }^{-3}$, on wet basis) & $270 \pm 7$ & 28 & 18 \\
\hline Indicative daily quantity of feed (kg animal ${ }^{-1}$, on wet basis) & 30 & &
\end{tabular}

As it can be seen, following the milk destination (dairy production or PDO production) the ration changes dramatically being the silages not permitted in the second case thus affecting the bulk density of the diet (that affects the complete mixer volume exploitation) and accentuating the need for chopping hay. For beef production the main aspect reported by farmers is related to the number of boxes to serve and the number of diets to perform (starter, fattening and finishing).

The wagon

The wagon very frequently has the volume of 2-4 $\mathrm{m}^{3}$ (but customizable solutions are possible) and operates in two ways: it distributes a ration that has undergone mixing in the kitchen, or it mixes and distributes the ration after the loading with all the ingredients in the kitchen. In the first case, the distributing wagon is fed by medium and large volume mixers $\left(6-50 \mathrm{~m}^{3}\right)$, driven by electric motors, and whose number is equal to that of groups of animals to be fed with different rations. The operator fills the fixed mixers exactly as it would do for feeding a normal mixing wagon, but the difference is the distribution, whose schedule is automatic according to the flexible times established by the operator. In the second case, the working procedure foresees operators first fill the mixingdistributing wagons with the individual ingredients of the expected rations. Subsequently, the mechanical parts inside the wagon operate mixing and further shredding of the ingredients to obtain the designed TMR that is automatically distributed in the barn to the target group.

Both these wagon typologies also put the ration close again to the feeding alley to contrast the continuous sorting action of the cows looking for the most palatable ingredients. The operator chooses to operate it in a distributing or putting close mode running it at full load or empty.

If, on the one hand, the operating mode of the wagon acts on the structure and the distinctive elements of the kitchen, on the other, the way the wagon moves and operates within the animal houses affects the structure of the building. In fact, wagons surveyed can move according to three principles: suspended, guided and self-propelled.

The suspended wagon follows a rail connected to the building structure: it has the advantage that it is possible to overcome any unevenness or difference in the height of the barn surface. Nevertheless, the need to have a barn with adequate supporting structures is a fundamental prerequisite as all the weight of the wagon at full load is charged on it. The guided wagon system foresees the presence of a rail whose only task is to drive the wagon along the path it has to follow, but not to bear it, since it has wheels that also ensure traction. The self-propelled 
wagon is the most recent solution: it moves autonomously based on the information received from boarded sensors such as GPS and ultrasound, as well as from metal guides immersed in the paving. The advantage of these systems is that it is independent of the loading characteristics of the structure, but requires a sufficiently levelled moving surface with an adequate coefficient of friction.

All these solutions have the advantage of reduced dimensions of the wagon: in this way, both in case of restructuring and new building construction, animals are provided with more space allowance. Wagon propulsion is electric, based on a dedicated line or rechargeable batteries reducing from 30 to $40 \%$ the energy requirements compared with previous mechanized feeding system following farmer's declarations.

\section{Feeding strategies}

The questionnaire pointed out that AFS allow for a reduction of feed wastes and improve the animal intake of the ration. The reared animals showed to adapt easily to the new feeding system, and the simple transit of the wagon in the feeding alley (regardless the distributing or putting close mode) encourages them to get close to the alley resulting in substantial increase in feed intake. Moreover, in addition to the fixed scheduling of the distributing times (which the farmer sets and leaves more or less unchanged during the 24 hours or the year), other dynamic types of management are available as well, taking into account the effective consumption of the TMR animals operate and/or seasonal variability (i.e. winter/summer) in feeding rhythm. The wagon constantly records animal food consumption. The control system acquires this information to act on the frequency the daily distributions occurs as well as on their interval to satisfy the needs of the animals. In this way, any variation (diurnal, seasonal, behavioural) possibly affecting the feed intake can be promptly managed.

Thanks to such a usage flexibility AFS contribute to the improvement of the working conditions of the operator, in particular concerning the schedules for feeding operations, which results in a better, more rational and frequent administration of the ration during the day. As a matter of fact, in the first hours after the distribution, the ration is generally subjected to an initial voracious intake by the animals that, only afterwards, make a thorough selection in its most desirable parts. What remains in the manger (the most coarse and less desirable fractions) is equal to $2-4 \%$ of the distributed dose. According to some interviewed, increasing the frequency of distribution up to 8 daily supplies (on average) interspersed every 3 hours (a wide range of adjustments is possible as well), resulted in lowering the competition among the animals, providing interesting indications about the level of animal welfare these systems would allow reaching and reducing leftover to an average of $1.5 \%$ of the daily distributed dose.

However, although Italy is one of the Countries that has actively studied and introduced the adoption of the TMR diet, besides being one of the first producers in the world of feeder-mixer wagons (both trailed and selfpropelled), some critical factors caused this technology not to be of immediate interest for Italian breeders. Among these:

- the concern that in some circumstances the silage could undergo degradation during intermediate conservation phases following the increase of its temperature, the restart of the lactic fermentation or anomalous ones;

- the potential difficulty of the management of hay amounts (significantly higher than those of the North European Countries and particularly high in PDO milk production), which has low apparent bulk density that could cause a failure of the shredding-loading operations.

The results and the farmers perception recorded in this preliminary study, seems to show a growing interest towards this technology and a great adaptability of the proposed systems to the large variability of Italian situations (barns design and architecture, animal production, local availability or limitation of feedstuffs) providing interesting development prospects.

\section{Conclusions}

Since 2013, in Italy about sixty farms have, currently, adopted AFSs provided by five manufacturers. The farming systems cover all the systems present in Italy for bovine livestock (milk production for dairy, milk production for long ripening cheese, beef production). The herd dimension ranges widely: from less than 20 to more than 300 animals, concerning dairy farms, and 700 to 1,000 concerning beef on the base of the various contexts the farms operate in (mountain, dry hills or plan irrigated areas). Five are the Regions were AFS operate (Lombardia, Piemonte, Veneto, Trentino-Alto Adige and Emilia Romagna) with distinctive dairy/beef specifications.

AFS installation costs ranged from 400 to $1200 € \mathrm{cow}^{-1}$ depending on the complexity of the project and following their installation. The workload diminished for most of the farmers but, overall, it becomes more flexible (generally no more fixed time are needed). A general gain in milk and meat production (respectively 1-4 $1 \mathrm{cow}^{-1}$ day $^{-1}$ and 130-150 $\mathrm{g} \mathrm{beef}^{-1}$ day $^{-1}$ ) is reported by most the farmers. This is attributable to the higher frequency of 
distribution (ranging from 5 to 10 times day ${ }^{-1}$ ) compared with the previous feeding system. Managing different rations following the different group/breed performances and energy aspects are the main advantages reported by the farmer (30-40\% of energy requirements reduction) together with a better flexibility in daily workload. Investment and long stemmed forages management are, on the other hands, the main fears farmers had before the purchase. The development of these systems could lead to a deeper adoption of precision livestock farming in particular when coupled with other automatic systems installed into the barn.

\section{Acknowledgements}

This work was supported by the Italian Ministry of Agriculture (MiPAAF) under the AGROENER project (D.D. n. 26329, 1 April 2016) - http://agroener.crea.gov.it/.

The Authors are grateful to the AFS manufacturers and to the farmers adopting these systems for their help and cooperation.

\section{References}

Barmore J.A. 2002. Fine-tuning the ration mixing and feeding of high production herds. In: Proc. of Tri-state dairy nutrition conference, Fort Wayne, Grand Wayne Center, Indiana, USA, 16-17 April.

Da Borso F., Chiumenti A., Sigura M., Pezzuolo A. 2017. Influence of automatic feeding systems on design and management of dairy farms. Journal of Agricultural Engineering 2017; XLVIII(s1):642, p 48-52. DOI:10.4081/jae.2017.642

EC-European Commission. 2009. Council Regulation 8EC) No. 510/2006 "Parmigiano Reggiano". Official Journal of the European Union, 16.4.2009, pp: 16-19.

EC-European Commission. 2009. Commission regulation (EU) No 794/2011 of 8 August 2011 approving amendments to the specification for a name entered in the register of protected designations of origin and protected geographical indications (Parmigiano Reggiano (PDO)). Official Journal of the European Union, 09.08.2011, pp: 19-20.

Eurostat. 2015. Meat production statistics - 2015 Edition. [http://ec.europa.eu/eurostat/statisticsexplained/index.php?title=Meat production statistics\#Beef and veal .28bovine meat.29] accessed June, 2018.

Eurostat. 2017. Agriculture, forestry an fishery statistics - 2017 edition. [http://ec.europa.eu/eurostat/web/products-statistical-books/-/KS-FK-17-001] accessed April, 2018.

ISTAT - The National Institute for Statistics. 2018. Italian statistical yearbook, Chap. 13: agriculture [in Italian].

McKinsey Global Institute. 2017. A future that works: automation, employment and productivity. McKinsey\&Company, January 2017. [https:/issuu.com/fredzimny/docs/mgi-a-future-that-works-fullreport 937f4a8b6a24f6/17] accessed June, 2018.

Oberschätzl R., Haidn B., Neiber J., Neser S. 2015c. Energy consumption of automatic feeding systems in farms under practical conditions. In: Proceedings of the $12^{\text {th }}$ Conference Construction, Engineering and Environment in Livestock Farming. Freising, Germany, 8-10 September 2015. Editor: Kuratorium für Technik und Bauwesen in der Landwirtschaft e.V. (KTBL).

Pompe J.C.A.M., Nieuwenhuizen A.T., Lokhorst C., Kettenis D.L. 2004. Discrete-event simulation model for automatic precision feeding of dairy cows. AgEng Leuven 2004 - Engineering the Future, Technologisch Instituut vzw, Leuven, pp. 574-575 (Extended abstract in hard copy of proceedings, Vol. 572; full paper on CD ROM).

Serap G., Özgül A., Gökhan G. 2017. Futuristic applications for profitable beef production systems. Journal of Environmental Science and Engineering A 6 (2017), 625-634. DOI:10.17265/2162-5298/2017.12.005. 


\title{
Spatial Distribution of Mechanization Levels in Italian Greenhouses
}

\author{
Elio Romano, Massimo Brambilla, Maurizio Cutini, Pietro Toscano, Carlo Bisaglia* \\ ${ }^{\text {a }}$ CREA Research Centre for Engineering and Agro-Food Processing, via Milano 43, 24047 Treviglio (BG), Italy \\ *Corresponding author. Email: carlo.bisaglia@crea.gov.it
}

\begin{abstract}
Greenhouse cultivation in Italy represents an important production sector within the horticultural sector, with strong economic importance. Protected crops in the Mediterranean basin cover more than 40,000 hectares (ISMEA2016). Regarding volume, the regions that play a predominant role in Italian greenhouse cultivation are Campania (25.74\%), Sicily (23.54\%) and Lazio (19.53\%). Then follow Veneto (9.45\%), Sardinia (6.80\%) and Lombardy $(6.21 \%)$. Greenhouse distribution in Italy covers different latitudes and the cultivations conducted provide different management with very different technologies and materials. Also, the mechanisation of the production structures is very varied and foresees different degrees of use. The mechanisation in greenhouses can provide for the various operations and are conditioned, as well as by the characteristics of the species in cultivation, vegetables or flowers, the type of greenhouse used and the type of cultivation adopted. The present study has the main objective to observe the distribution of current mechanisation levels in protected production companies and to verify any influences from income or the surface and structure. Therefore the data of the Italian agricultural accounting information network (R.I.C.A. - Rete di Informazione Contabile Agricola that belongs to the Farm Accountancy Data Network - FADN), which is an annual sample survey established by the European Economic Commission in 1965 with the Regulation 79/56 and updated with the EC Reg. 1217/2009, were studied. The FADN sample allows an average national coverage of $95 \%$ of the utilised agricultural area (UAA), $97 \%$ of the value of the Standard Production, $92 \%$ of the Work Units.
\end{abstract}

Keywords: Agriculture, Protected Crops, Process Optimization.

\section{Introduction}

The impacts that agriculture has in our lives are multiple (Tilman et al., 2002): since its origin, it has constantly increased its efficiency, and nowadays it ensures the production of both food and energy, materials and services. Sustainability has become the paradigm for all this, in particular when land is managed with multiple objectives (Klapwijk et al., 2014). Currently, to assess which factors (or combination of them) can drive agricultural change towards sustainability or not a scenario analysis using an integrated agricultural landscape model is required (Carmichael et al., 2004). In this way, the use of indicators could point out which are the potential causes of modifications (as well as combinations of them) to the characteristics of farming systems to foresee and check the changes in the degree of the achieved sustainability (Florin et al., 2013).

There are some different definitions of spatial autocorrelation: among these, Upton and Fingleton (1985) defined spatial autocorrelation as a property that mapped data have whenever they exhibit any organised pattern. These authors say that spatial autocorrelation exists whenever there is systematic spatial variation in values across a map or patterns in the values recorded at locations with the locations given. Positive autocorrelation is when high values at one locality are associated with high values at neighbouring localities, while negative autocorrelation arises when high values and low values alternate between adjacent localities (e.g. like tiles on a checkerboard). According to Griffith (1991) and Goodchild (1987), spatial autocorrelation deals simultaneously with both location and attribute information: an index of comparison points out the way values deviate from a random pattern. Most published measures of spatial autocorrelation can be recast as a (normalized) cross-product statistic indexing the degree of relation between the corresponding entries from two matrices. One that specifies the spatial connections among a set of $n$ locations, and another that provides for a very explicit definition of similarity between the set of values on some $x$ variable realized over $n$ locations (Hubert and Arabie, 1991).

As Tille et al. (2018) have pointed out, the measure of the degree of spatial spreading of a sample can have great interest provided that the used index come with clear bounds and its scale of measurement allows to assess the level of spatial spreading in absolute terms. Within this framework, the Moran index of spatial autocorrelation can effectively indicate the level of spatial concentration of a given sample of georeferenced observations.

The aim of the work is to evaluate i) any possible connection between the mechanisation level of the farms (mechanical power - $\mathrm{kW}$ ) and their geographic distribution, ii) the impact of the proximity between similar farms by economic orientation (that is an indirect indicator of their possible aggregation in consortia and cooperatives) on farm profitability and its achieved mechanization level. 


\section{Materials and Methods}

Dataset description

The processed dataset belongs to the FADN (Farm Accountancy Data Network) that is an instrument for evaluating the income of agricultural holdings and the impacts of the Common Agricultural Policy). The observations included in the dataset are all the agricultural holdings in the European Union having at least 1 hectare. Those with less than 1 hectare of utilised agricultural area (UAA) are included provided that they produce more than a specified amount of output.

Within this statistical universe, the used dataset refers to the 2012-2014 three-year time span. Overall, it includes 779 horticultural-floricultural farms, producing in greenhouses: these belonged to four production classes (horticultural, floricultural, mixed and plant nursery). Each observation included the following variables:

i. $\quad$ specific net income $(€ / \mathrm{ha})$;

ii. agricultural area (ha);

iii. the number of working units;

iv. $\quad$ gross saleable product $(€)$;

v. type of farming (by the four classes mentioned above);

vi. the mechanisation level (expressed as the sum of all the machinery powers available in the farm $-\mathrm{kW}$ )

vii. Geographic coordinates (Longitude and Latitude).

Methodology and data processing

Anselin (1991) defined spatial autocorrelation as a territorial cluster including objects with similar features in their parameters. The spatial autocorrelation of the dataset was studied using the Moran index that is defined in $\mathrm{Eq}$ (1). It tests for global spatial autocorrelation for continuous data basing on the cross-products of the deviations from the mean and is calculated for $n$ observations on a variable $x$ at locations $i, j$ as:

$$
I=\frac{n}{\sum_{i=1}^{i=n} \sum_{j=1}^{j=n} W_{i, j}} \cdot \frac{\sum_{i=1}^{i=n} \sum_{j=1}^{j=n} W_{i, j}\left(x_{i}-\bar{x}\right)\left(x_{j}-\bar{x}\right)}{\sum_{i=1}^{I=n}\left(x_{i}-\bar{x}\right)^{2}}
$$

here, is the mean of the $x$ variable; $W_{i j}$ is the binary weight matrix of the general cross-product statistic (by convention, it equals 1 in case two different observations are adjacent; it is 0 when they are not adjacent).

Just like the Pearson correlation coefficient, the values of Moran index range from +1 in case of strong positive spatial autocorrelation (meaning that there is significant clustering), to -1 if there is strong negative spatial autocorrelation that means perfect dispersion. Moran index zero value refers to perfect randomness of the observations. This measures how much an object is similar to others surrounding it: if objects are somehow "attracted" (or repelled) by each other, it means that the observations are not independent.

Data processing foresaw the use of the "R" statistical software (R Development Core Team, 2008) with the "rgdal", "sp" and "spdep" packages. The "rgdal" package converted the geodetic data into WGS 84 Coordinate System. Afterwards, the "sp" package processed the data pointing out the proximity effects resulting from the vicinity farms have each other. This considered as indicator the number of production sites included within $30 \mathrm{~km}$ of distance from each site. Eventually, the "spdep" package calculated the Moran index to study the spatial dependance of the data.

Moreover, to increase the knowledge attained from the considered variables, all data, underwent standardisation and multivariate processing using Principal Components Analysis (PCA) to find out the existence of groups or classes, not a priori defined, and whose existence is not accidental (Todeschini, 1998).

The graphic elaboration of the company distribution has been developed thanks to the "Surfer" software (Surfer, 2008), basing the statistical interpolation on the kriging algorithm.

\section{Results and Discussion}

The Moran index provides information on spatial autocorrelation in 2-dimensional space considering at the same time both feature locations and feature values. Spatial autocorrelation plays an important role in the geographical analysis; however, applying the calculus to the considered dataset did not point out any significant correlation between the mechanisation level $(\mathrm{kW})$ and the location of the sites. The Moran scatterplot (Figure 1) reports this information: in it, the $\mathrm{x}$ - and the $\mathrm{y}$-axis refer to the considered normalised variable and to its normalised spatial delay: the Moran index is the angular coefficient of the regression line between these two values. The dispersion of the dots representing the observations among the four classes results in a substantial absence of correlation ( $\left.I_{\text {statistic }}=0.12\right)$ meaning that the mechanisation level $(\mathrm{kW})$ can't be considered somehow dependent from the geolocation of the farms. 


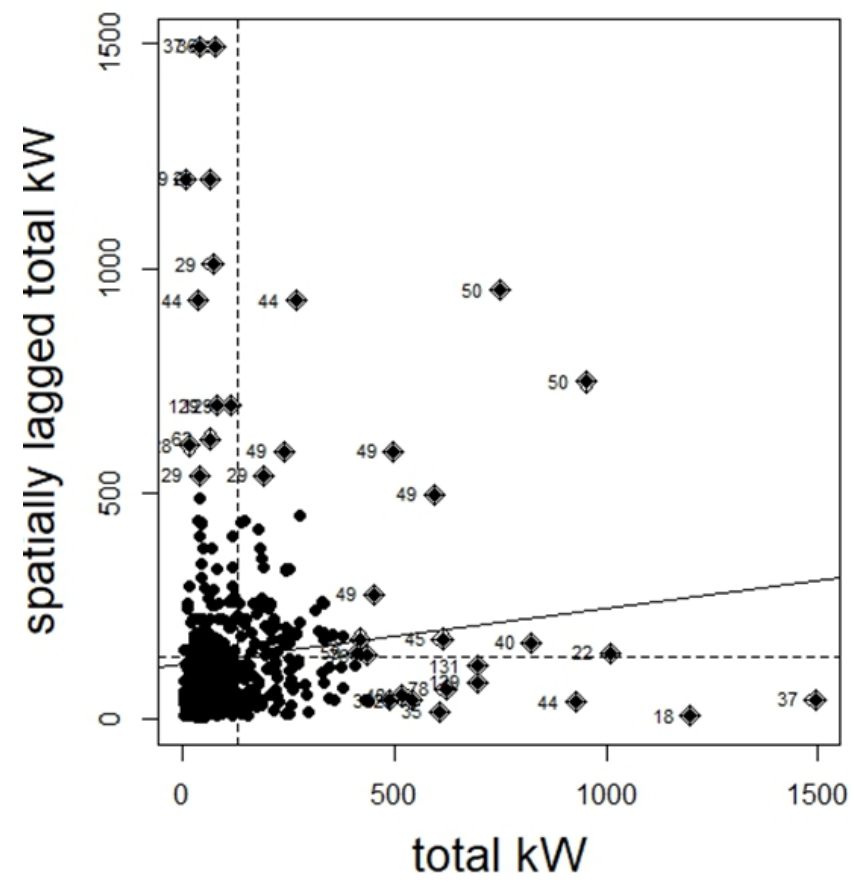

Figure 14. Moran scatter plot related to the equipment of company machines expressed in $\mathrm{kW}$.

The spatial distribution of the agricultural holdings of the dataset underwent data processing also with Surfer statistical mapping software (Surfer, 2008): the output is the contour plot Figure 2. In it, the geographical interpolation of the georeferenced data has been extended to the whole national territory based on Kriging method. It appears clear that, within the radius of $30 \mathrm{~km}$ from each site, the highest concentrations of production sites can be retrieved in the North West part of the country (Liguria) and in the western part of Tuscany (more in detail the evidenced region corresponds to the districts of Pistoia and Lucca). A similar analysis performed with " $\mathrm{R}$ " " $s p$ " package) resulted in a map pointing out the sites with higher concentration of farms (Figure 2, on the right): here different colours identify a number of classes ranging from different minimums and maximums (e.g. from 2 to 69 sites; from 69 to 136 sites and so on). The former zone is that of horto-floricultural production that takes place in Liguria: here a large number of farms are operating in a very restricted area because of the peculiar geological assessment. As a matter of fact, the region is dominated by mountainous territory and greenhouses mainly operate in valleys and in terraced fields producing mainly aromatic herbs (basil) and flowers. The latter is the district of nursery greenhouses that is highly developed in the hilly area in the West of Prato up to the province of Lucca.
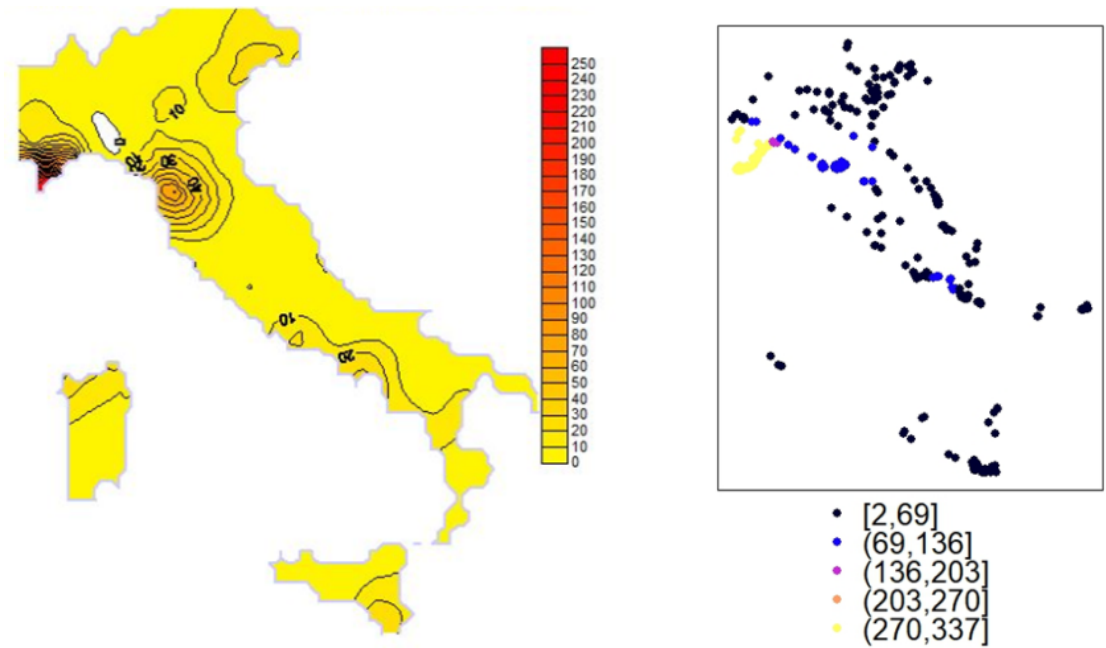

Figure 15. Contour map representing the number of horticultural companies within a radius of $30 \mathrm{~km}$ from each site (on the left) and a representation of the proximity effect resulting from.

Other interesting zones appear to be those related to the districts in the plan sited in the province of Latina that 
at the morphological level is characterized by two distinct parts. On one side there is the vast plain of the Agro Pontino and, on the other, the hilly and mountainous area of the Monti Lepini, Aurunci and Ausoni, inside which there are the two small planes of Fondi and Minturno. Here greenhouse production is mainly devoted to grow tomatoes, zucchini and watermelon. Since 1980 the number of farms operating in the nursery sector has being growing as well (Regione Lazio, 2010)

The other zone that has been pointed out is the district of Ragusa where, in the coastal zone, many greenhouse production sites have started their activity that is mainly devoted to the production of tomatoes, peppers, aubergines, cucumbers and watermelons. As a matter of fact, the Sicilian greenhouse production accounts for about $27 \%$ of the national greenhouse production (ISTAT, 2004): it is mainly developed in the southern coastal area where greenhouses without heating system (usually simple structures made of wood and with plastic covers) can produce in any season (OSEAAS, 2006).

The Northern East districts of Udine, Gorizia and Monfalcone (Friuli Venezia Giulia) where nurseries for flower production prevail (Cisilino et al., 2016).

A further elaboration of the data with the "rgdal" package resulted in a map that highlights the potential network between the observed farms (Figure 3). The shown network derives from the Delaunay triangulation that defines the relationships between the neighbouring farms whose Euclidean distance is considered as a Sphere of Influence that, to be defined, needs the weakest networks to be removed (Avis and Horton, 1985).

Here, the aforementioned zones appear to expand their influence in the surrounding territory: again the districts of Albenga and Sanremo (Liguria) together with those of the provinces of Lucca appear to be those with the wider spheres of interest while the districts of Latina and Vittoria, despite the high density of connection, appear to more isolated. What's worth noticing is the number of greenhouses in Lombardy, Friuli and the coastal line of the Marche regions that, despite their sparse distribution on the territory show a high degree of connection/influence among themselves and with other production sites.
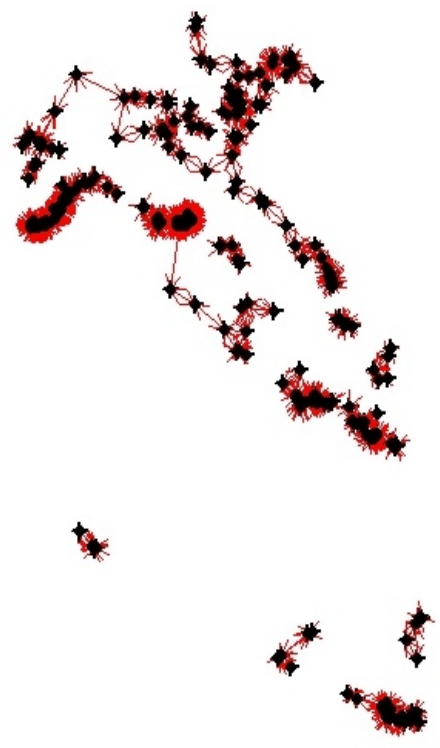

Figure 16. Representation of the connection network elaborated according to distances with "Distance-Based Neighbours" of the "rgdal" R's package.

Multivariate analysis by means of Principal Component Analysis (PCA), considering all the variables, achieved an overall variance explanation percentage of $76.4 \%$ (57.4\% on the firs principal component and $19.1 \%$ on the second). In the biplot (Figure 4), concerning the first principal component, the variables that affect it the most are the agricultural area, the achieved mechanization level (expressed as the overall power of the machinery $-\mathrm{kW}$ ) and the number of the working units. The second PC was affected mainly by the proximity effect that farms have each other. These variables contribute to the overall description of the dynamics behind this production sector. In particular, being the vector of the mechanization level (total $\mathrm{kW}$ ) opposite to that of the proximity effects, it comes out a negative relation between these two variables that means that more isolated farms tend to have higher power machinery. 


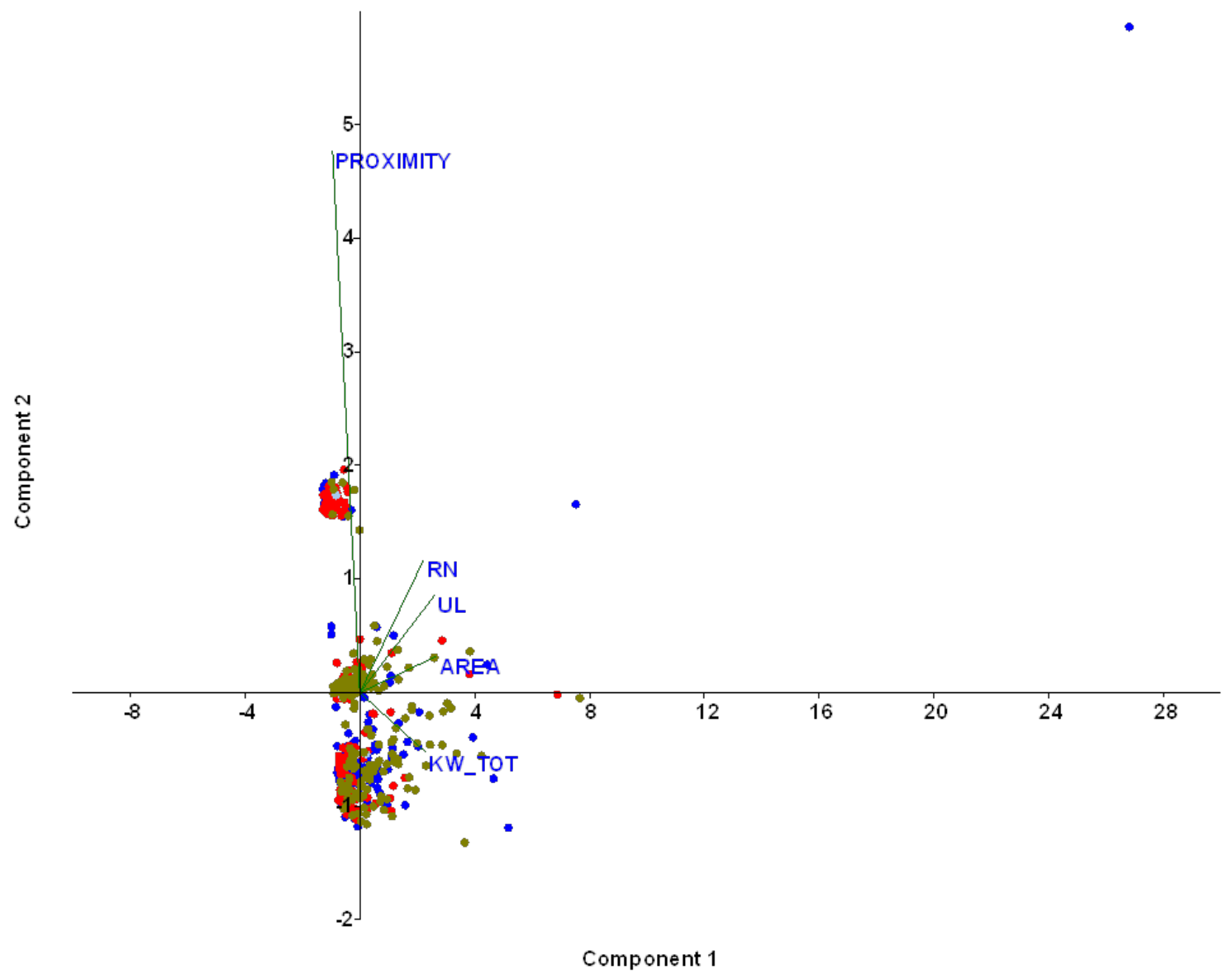

Figure 17. PCA scatter plot showing the weights the studied variable have on the dataset.

\section{Conclusions}

In our study a dataset representing the Italian agricultural production sites using greenhouses underwent statistical analysis to point out if there is an interaction between farm localization, and the achieved mechanization level to check the possible existence of clustering effect.

The Moran index gave no indication of spatial autocorrelation meaning that, if any clustering effect should be pointed out, the reason is not connected to the spatial greenhouses have.

At the same time, further processing pointed out that a clustering effectively exists, nevertheless it seems more related to the interaction farms have more than on their geographical distribution: probably, this can be the consequence of the presence of specific infrastructures(e.g. highways, irrigation consortia, harbours and suitable markets).

On the base of this processing, spatial autocorrelation can play an important role in geographical analysis.

However, there is still room for improvement of this method as the Moran index itself turned out not to be sufficient to point out any connection between the mechanisation level horticultural farmers achieve and the localization of the farms.

Further processing using the multivariate approach (and including the Moran index in the dataset) needs to be carried out in order to define specific indexes and solutions that take into account the peculiarities of agricultural production.

\section{Acknowledgements}

This work was supported by the Italian Ministry of Agriculture (MiPAAF) under the AGROENER project (D.D. n. 26329, $1^{\text {st }}$ April 2016) - http://agroener.crea.gov.it/ 


\section{References}

Anselin, L. and Rey S. 1991. Properties of tests for spatial dependence in linear regression models. Geographical Analysis, 23. 112-131. https://doi.org/10.1111/j.1538-4632.1991.tb00228.x

Avis, D. and Horton, J. 1985. Remarks on the sphere of influence graph. Discrete Geometry and Convexity. New York Academy of Sciences, In Goodman, J. E., editor, New York, pp 323-327.

Bivand, R.S.; Pebesma, E.; Gómez-Rubio V. 2008. Applied Spatial Data Analysis with R, Springer-Verlag, New York pp. 239-251

Carmichael, J., Tansey, J., Robinson, J., 2004. An integrated assessment modeling tool (Review). Global Environmental Change, 14(2), 171-183. http://dx.doi.org/10.1016/j.gloenvcha.2003.12.002

Cisilino F., Zannutig G., Zilli G., 2016. Il sistema agricolo del Friuli Venezia Giulia. http://www.crea.gov.it/wp-content/uploads/2017/05/quadrotto FVG2016 DEFweb.pdf Accessed June 2018.

FAOSTAT-Food and Agriculture Organization of the United Nations, FAOSTAT Database, 2008. http://faostat.fao.org/site/362/DesktopDefault.aspx?PageID=362

Accessed May 15, 2018

Florin M.J., Van Ittersum M.K., Van de Ven G.W.J. 2013. Family farmers and biodiesel production: systems thinking and multi-level decisions in Northern Minas Gerais, Brazil. Agricultural Systems, 121, 81-95. http://dx.doi.org/10.1016/j.agsy.2013.07.002

Goodchild, M.F. 1987. Spatial Autocorrelation. CATMOG 47.

Griffith, D.A. 1987. Spatial Autocorrelation: A primer. Association of American Geographers, Resource Publications in Geography.

Klapwijk C.J., van Wijk M.T., Rosenstock T.S., Van Asten P.J.A., Thornton P.K., Giller K.E. 2014. Analysis of trade-offs in agricultural systems: current status and way forward. Current Opinion in Environmental Sustainability, 6, 110-115. http://dx.doi.org/10.1016/j.cosust.2013.11.012

Moran P. A. P.. 1950. Notes on continuous stochastic phenomena. Biometrika, 37 (1/2), 17-23. https://doi.org/10.1093/biomet/37.1-2.17

OSEAAS - Osservatorio sull'Economia del Sistema AgroAlimentare della Sicilia. 2006. La filiera ortofrutticola in Sicilia. http://www.coreras.it/news/volumi/2017/05/22/la-filiera-ortofrutticola-in-sicilia-60/ Accessed June 2018.

Regione Lazio. 2010. L'agricoltura del Lazio: un'analisi dei dati del Censimento 2010. http://www.regione.lazio.it/binary/prtl statistica/statistica normativa/RapportoDatiDefinitivi6CensimentoAgric olturaRegioneLazio.pdf Accessed June 2018.

R Development Core Team. 2008. R: a language and environment for statistical computing. R Foundation for Statistical Computing, Vienna (2008).

Surfer ${ }^{\circledR}$ from Golden Software, LLC (www.goldensoftware.com)"

Tillé Y., Dickson M.M., Espa G., Giuliani D. 2018. Measuring the spatial balance of a sample: A new measure based on Moran's I index. Spatial Statistics, 23: 182-192. https://doi.org/10.1016/j.spasta.2018.02.001

Tilman D., Cassman K.G., Matson P.A., Naylor R., Polasky S. 2002. Agricultural sustainability and intensive production practices. Nature, 418, 671-677. http://dx.doi.org/10.1038/nature01014

Todeschini, R., 1998, Introduzione alla Chemiometria. Naples, Italy: EdiSES.

Upton, G.J., and Fingleton, B. 1985. Spatial data analysis by example, volume 1: Point pattern and quantitative data. Wiley, Toronto Singapore, Brisbane, New York, Chichester. 


\title{
SPARKLE Project: e-Learning Solutions to Create New Digital Agronomist Profiles on European Students
}

\author{
Jorge Martínez-Guanter a , Daniele Sarri ${ }^{\text {b }}$, Marco Vieri ${ }^{\text {b }}$, Stefania Lombardo ${ }^{\text {b }}$, Manuel Pérez-Ruiz ${ }^{\text {a }}$ \\ ${ }^{a}$ Aerospace Engineering and Fluid Mechanics Department, University of Seville, 41013 Seville, Spain \\ ${ }^{\mathrm{b}}$ Dipartimento di Gestione dei Sistemi Agrari, Alimentari e Forestali, University of Firenze, Piazzale delle Cascine, 15, \\ Firenze, Italy \\ * Corresponding author. Email: manuelperez@us.es
}

\begin{abstract}
Recent advances in new technologies applied to agriculture are creating a gap in the employability of agronomy students. In the short term, future-oriented farmers, motivated by the increasing market competitiveness, will demand the existence of small and medium-sized enterprises (SMEs) capable of applying innovative solutions to agriculture. These new companies should have business models capable of providing a "digital accompaniment" to these farmers, also involving research institutions. Nowadays, at European level, students are well prepared in agronomic terms but lack knowledge about generating new business models based on the effective application of new technologies for Sustainable Precision Agriculture (SPA). The aim of the project is to disseminate knowledge and innovation, linking universities, farmers and students by providing a learning opportunity to students, but also by enabling universities to validate real solutions to specific problems in applying SPA in field conditions with the help of the farmer's experience. The SPARKLE project will contribute to build a methodological a theoretical framework for future-oriented agripreneurs, coupled with the development of e-learning based educational solutions, in order to facilitate achieving a new awareness and maturity levels among SME, students and educators on technologies, business and innovation in agriculture. The expected outputs of the project will be to acquire knowledge and skills for universities by training more students in SPA technologies and business. With this in mind, it will be possible for students to have the opportunity to manage a real experience with companies specializing in SPA. Therefore, companies are given the opportunity to get directly involved in training and the opportunity to interact with students and future technicians. In the long term, the expected results will be to create impact through growing synergies between the educational offer of the University and the companies, extending the higher education offer through the crossing between new technologies in agriculture and new business models.
\end{abstract}

Keywords: sustainable precision agriculture, digital agricultural business models, education, agripreneurs,

\section{Introduction}

European farm sector is currently at a crossroad: embracing the future or staying stuck in old practices. The future is represented by the so-called "Precision Agriculture" (PA), that is defined as "an integrated informationand production-based farming system that is designed to increase long term, site-specific and whole farm production efficiency, productivity and profitability while minimizing unintended impacts on wildlife and the environment" (CEMA, 2016). PA is not a technology, but an approach and a network of different technologies (digitalization, metadata, Internet of Things, IT, etc.) that can be adopted in agriculture.

The future of agriculture are the so-called "agripreneurs 4.0": an entrepreneur who works in agriculture and adopts in its business PA technologies. This means that the agripreneur needs a mix of competencies: a full set of entrepreneurial skills (to allow her/him to start and run efficiently a business) and, at the same time, a full set of PA related knowledge (in order to being able to take advantage of PA technologies), along with agricultural skills (related to "traditional" farming activity). In addition to this, PA should also consider the environmental impact: this is why in this project we will talk about Sustainable Precision Agriculture (SPA), as that farming system which respects environmental issues.

Regarding the actual agricultural University education, it is clear that the third set of competencies (in agriculture production) is available in all the institutions, while the other (entrepreneurial skills and PA knowledge) are, in many cases, missing. There is a need for improvement of the university education in agricultural sector, to train students on the relevant skills for becoming agripreneurs 4.0 (entrepreneurship + SPA knowledge), which is currently barely absent.

For being able to offer the students a new training program, it is fundamental for the Universities to establish more direct contacts with farmers (both traditional and PA), researchers and students, for better understanding the level of knowledge on PA and identifying the entrepreneurial needs for starting new SPA businesses.

At the same time, it is difficult for Universities to being able to seize the whole SPA dimension, as this is complex and composed by different technologies which change and develop in short time, and thus transferring competencies to both traditional farmers and SPA farmers. It becomes fundamental for teachers and researchers to develop new collaborations with other Universities, but also companies working in the sector (providers of PA 
services) and PA farmers, in order to have an overall vision on the field and being able to foresight technologic and market changes.

The needs identified above can be summarized as follows: (i) Lack of innovation potentiality self-awareness of farm companies; (ii) lack of knowledge transfer of SPA; (iii) lack of SPA knowledge and entrepreneurial skills of agricultural science students and (iv) lack of SPA and entrepreneurial educational offer of Universities.

Based on these needs, the main aim of the SPARKLE project is to build the necessary expertise in "agripreneurship" for more effective entrepreneurial education and innovative business practice. Accordingly, the project specific objectives include:

i. $\quad$ To increase "agripreneurship" among students, entrepreneurs and academics.

ii. To provide needs targeted entrepreneurial educational offer for Universities.

iii. To transform the agricultural sector into a SPA-oriented system.

The specific objectives will be achieved through actions, which are described in detail in the materials and methods section.

\section{Materials and Methods}

To guarantee that the project's results are appropriate independently of geographical location, a consortium of 11 European participants has been formed, involving leading companies, universities, research centers and reference farmers from Italy, Greece, Portugal and Spain, to manage action for achieving positive changes in education and business ecosystem.

Three different sectors (Arable crops, Orchards and Viticulture) will be part of the scope of the project, in an approach that guarantees a scientific robust and consistent research activity while ensuring that the outputs will be based on the best knowledge available for training. This section describes the methodologies and working groups in which the SPARKLE project is organized in order to achieve the objectives described above.

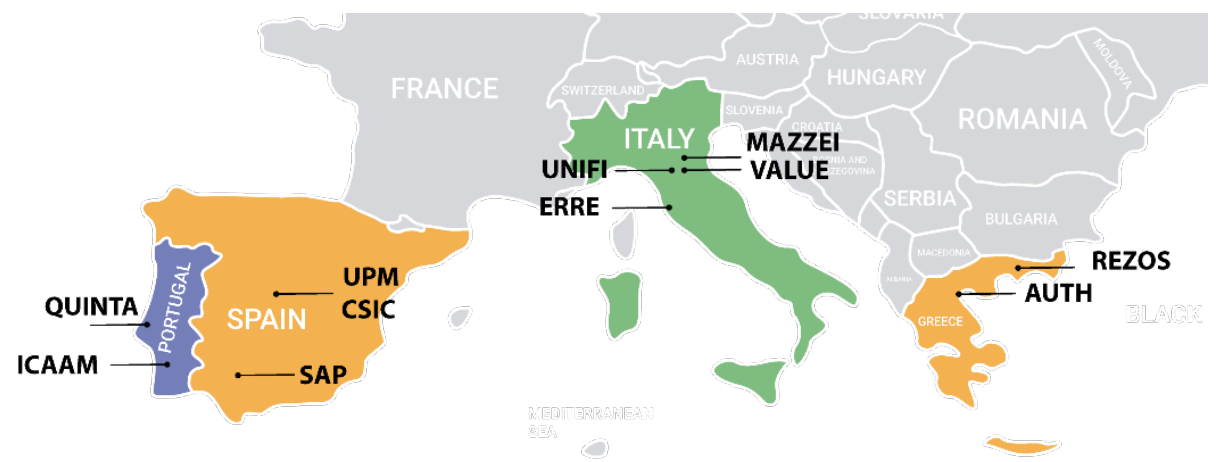

Figure 18. Map of the European members of the SPARKLE consortium.

SPARKLE project methodology

The SPARKLE project intends to transfer to agricultural science students the entrepreneurial skills and Precision Agriculture knowledge, in order to create the so-called "Agripreneur 4.0", and consists of two main phases:

i) The Preparation Phase, devoted to collect and analyse information in detail about educational needs and skills required for the new digital agronomist profiles, and to design and test the e-learning courses and educational resources that form the core of the SPARKLE project. This phase has three main steps:

a) Step 1: will result in specifying educational needs and creating innovative reports on same fundamental topics, for developing the educational packages:

- Preparing and executing deep in details surveys to analyse educational needs among three most relevant target groups (PA researchers, students and farms already adopting PA technologies);

- Conducting a detailed analysis on technological trends in PA sector and analysing the future trends of the adoption of robotics in agriculture;

- Collecting the business cases of successful farms adopting PA technologies;

- Producing methodological recommendations concerning pedagogical strategies, didactic approaches and the content of the educational offer.

b) Step 2: will be focused on the production of educational offer by: 
- Developing the technical characteristics of the e-learning courses;

- Developing the core e-learning course.

c) Step 3: will aim to make the educational offer fully operational by:

- Producing a unified methodology of testing and validating the e-learning offer,

- Testing and validating the e-learning offer in Italy, Portugal, Greece and Spain

- Producing and testing some new coaching methods, involving academic support and intrapreneurship experience, for developing advanced business models for traditional farms willing to adopt PA technologies

ii) The Implementation Phase, devoted to train a significant group of European students to become "agripreneurs 4.0) in their respective countries. To this end, the project in one of its working groups will support a competition between the students, where the partners will launch an open "call for challenges" addressed to traditional farmers. The companies will also be invited to present their activities and needs. Reference PA-adopting farms will spread the call within their network. Each University will select a students' team that will have the chance to reinforce its training within one of the traditional farms that participated in the call for challenges. The selection will be done by a panel of University and Company representatives, based on some common criteria, inspired by the following principles: i) originality of the idea/project, ii) business idea innovative potential and iii) feasibility of the idea.

The students will be invited to work on business plans for the farms participating in the call and the best team will be awarded with a coaching support for developing the BP and a week of intrapreneurship experience within the farm which launched the challenge.

\section{SPARKLE project organisation}

The consortium designed the project to guarantee the achievement of Aims and Objectives presented in the Introduction section through planned WPs, tasks and milestones (table 1), as well as to ensure the quality of processes, results and their evaluation (WP 7-8). Th process below will be followed to achieve this (figure 2).

Table 1. SPARKLE milestones and quality indicators.

\begin{tabular}{|c|c|c|c|}
\hline Milestones & & Indicators & \\
\hline $\begin{array}{l}\text { ML1: Training needs } \\
\text { assessment for students in } \\
\text { Agricultural Sciences }\end{array}$ & $\begin{array}{l}\text { Participants from the different } \\
\text { target groups selected basing } \\
\text { on clear criteria }\end{array}$ & $\begin{array}{l}\text { The three target groups have } \\
\text { been reached with an acceptable } \\
\text { dimension of samples for in- } \\
\text { depth analyses }\end{array}$ & $\begin{array}{l}\text { Data collection through } \\
\text { surveys and their analysis } \\
\text { have been accomplished }\end{array}$ \\
\hline $\begin{array}{l}\text { ML2: Precision Agriculture } \\
\text { Technological Foresight }\end{array}$ & $\begin{array}{l}\text { Technology foresight } \mathrm{n} \text { two } \\
\text { agricultural lines has been } \\
\text { performed producing relevant } \\
\text { information for the training } \\
\text { course }\end{array}$ & $\begin{array}{l}\text { Significant number of best } \\
\text { practices has been identified } \\
\text { and analysed }\end{array}$ & \\
\hline $\begin{array}{l}\text { ML3: Precision Agriculture } \\
\text { Farms: business models for } \\
\text { training and application }\end{array}$ & $\begin{array}{l}\text { A significant number of } \\
\text { business models has been } \\
\text { identified and analysed }\end{array}$ & $\begin{array}{l}\text { Business models collected are a } \\
\text { relevant support for students to } \\
\text { develop innovative business } \\
\text { models for conventional farms } \\
\text { wiling to adopt PA technologies }\end{array}$ & \\
\hline $\begin{array}{l}\text { ML4: State-of-the-art and } \\
\text { future applications of } \\
\text { robotics in agriculture }\end{array}$ & $\begin{array}{l}\text { Consistent information on the } \\
\text { state of the art in robotics and } \\
\text { agriculture }\end{array}$ & $\begin{array}{l}\text { Significant number of robotic } \\
\text { applications has been identified } \\
\text { and analyzed }\end{array}$ & \\
\hline $\begin{array}{l}\text { ML5: Fully prototyped e- } \\
\text { learning modules }\end{array}$ & $\begin{array}{l}\text { Pedagogical and technical } \\
\text { criteria for online courses } \\
\text { defined and respected }\end{array}$ & $\begin{array}{l}\text { All agreed topics for core e- } \\
\text { learning course covered and } \\
\text { target groups' need addressed }\end{array}$ & \\
\hline $\begin{array}{l}\text { ML6: Accomplished Pilot } \\
\text { Actions -Educational } \\
\text { packages tested and } \\
\text { evaluated by students }\end{array}$ & $\begin{array}{l}\text { Target groups' needs } \\
\text { validated through } \\
\text { questionnaires }\end{array}$ & $\begin{array}{l}\text { Target groups reached with } \\
\text { expected quantity in the pilots }\end{array}$ & $\begin{array}{l}\text { Pilot Actions fully } \\
\text { delivered in accordance } \\
\text { with quality assurance } \\
\text { criteria }\end{array}$ \\
\hline $\begin{array}{l}\text { ML7: Report on } \\
\text { effectiveness of } \\
\text { intrapreneurship experience }\end{array}$ & $\begin{array}{l}\text { Results analysis has helped } \\
\text { the last improvements }\end{array}$ & $\begin{array}{l}\text { Specific needs and requirements } \\
\text { are effectively addressed }\end{array}$ & $\begin{array}{l}\text { Results and analysis has } \\
\text { helped the last } \\
\text { improvements }\end{array}$ \\
\hline $\begin{array}{l}\text { ML8: e-Learning courses } \\
\text { final version }\end{array}$ & $\begin{array}{l}\text { Rules for adaptation and } \\
\text { further mainstreaming agreed }\end{array}$ & $\begin{array}{c}\text { Educational packages } \\
\text { disseminated by associated } \\
\text { partners' network }\end{array}$ & \\
\hline
\end{tabular}




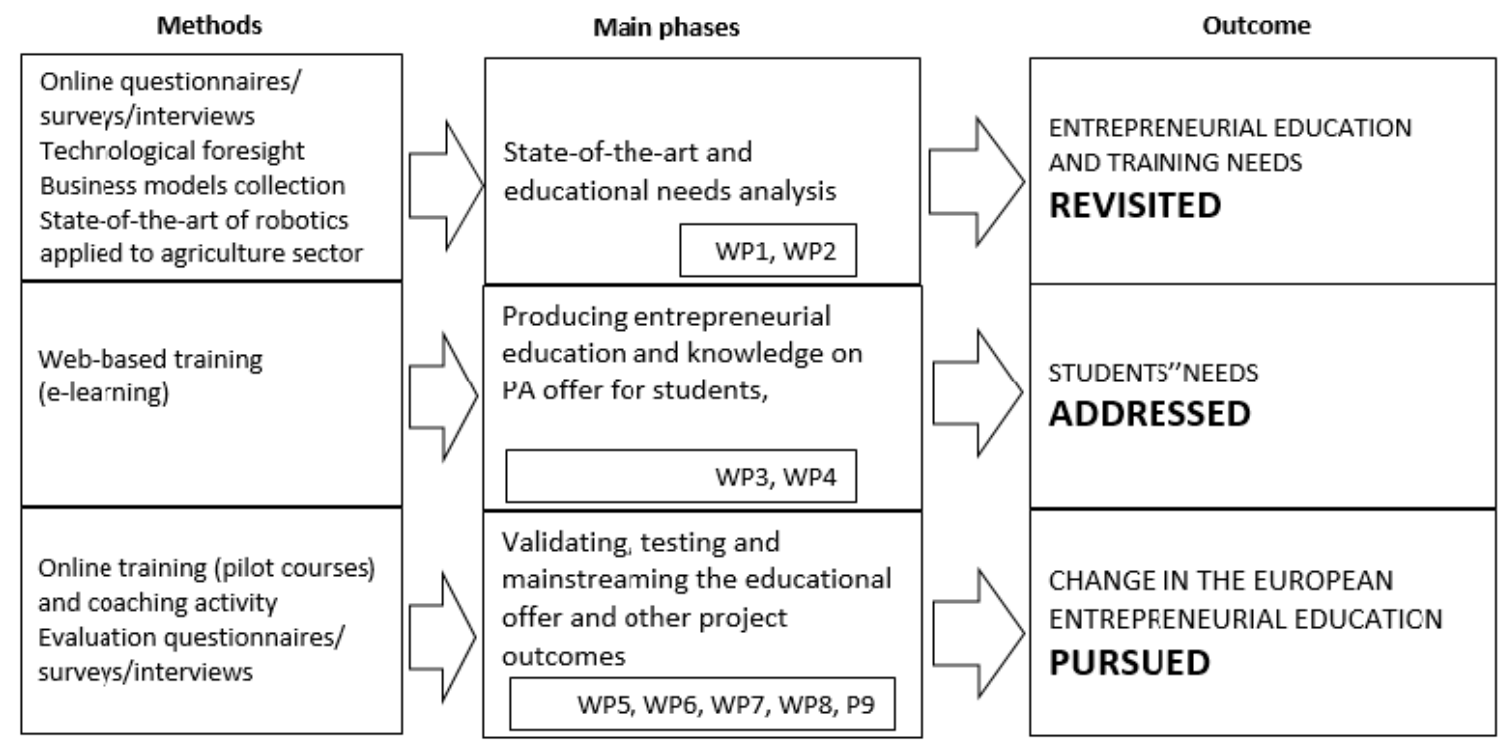

Figure 2. Schedule of activities by work package.

The framework consists of the following WPs (Figure 4):

- WP1 is aimed at establishing a detailed training needs assessment by conducting surveys, in order to have actual information for the following development of the e-learning courses.

- WP2 is aimed to create a consistent scientific background for the educational materials and supporting the teaching activity to be performed in WP4

- WP3 will identify a coherent architecture for the educational packages and will elaborate the common materials.

- WP4 will test the educational packages, developed and assembled in WP3 (the training will embed e-learning and activities in class - mainly at the beginning and the end of the training) and to improve the packages, based on piloting feedback. The partnership will then release the ultimate version of the materials.

- WP5 is aimed to plan and implement a business model competition, in order to allow the attendees to work on real business cases and learning to develop innovative solutions for traditional farmers.

- WP6 (Mgmt.): will devoted to manage the overall project's activity and to organize and participate in virtual and in-person meetings, monitor the deadlines, take care of periodic reports and support each WP leader in the WP management

- WP7 (QA): Quality assurance plan by conducting in-deep Quarterly Reports in order to formalize all the activities performed.

- WP8 (Eva.): Partners will be responsible for the internal evaluation of each WP.

- WP9 (Diss.): All the partners will be involved in a strong dissemination and exploitation activities in order to present the results and exploit the materials, also adopting mainly the EIP-AGRI platform as cooperative platform. $3)$.

The project will last 36 months and the work will be distributed according to the following schedule (Figure

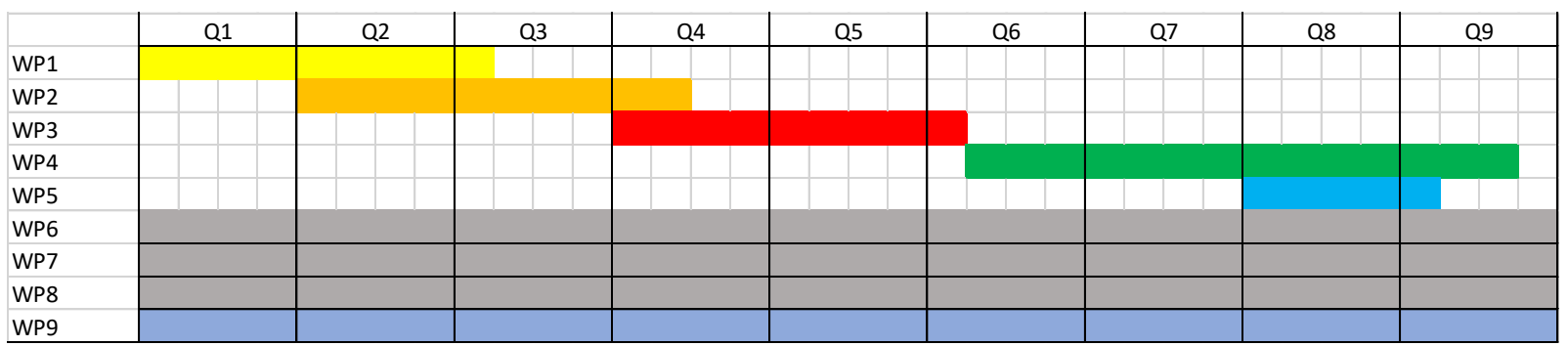

Figure 3. Schedule of activities by work package. 


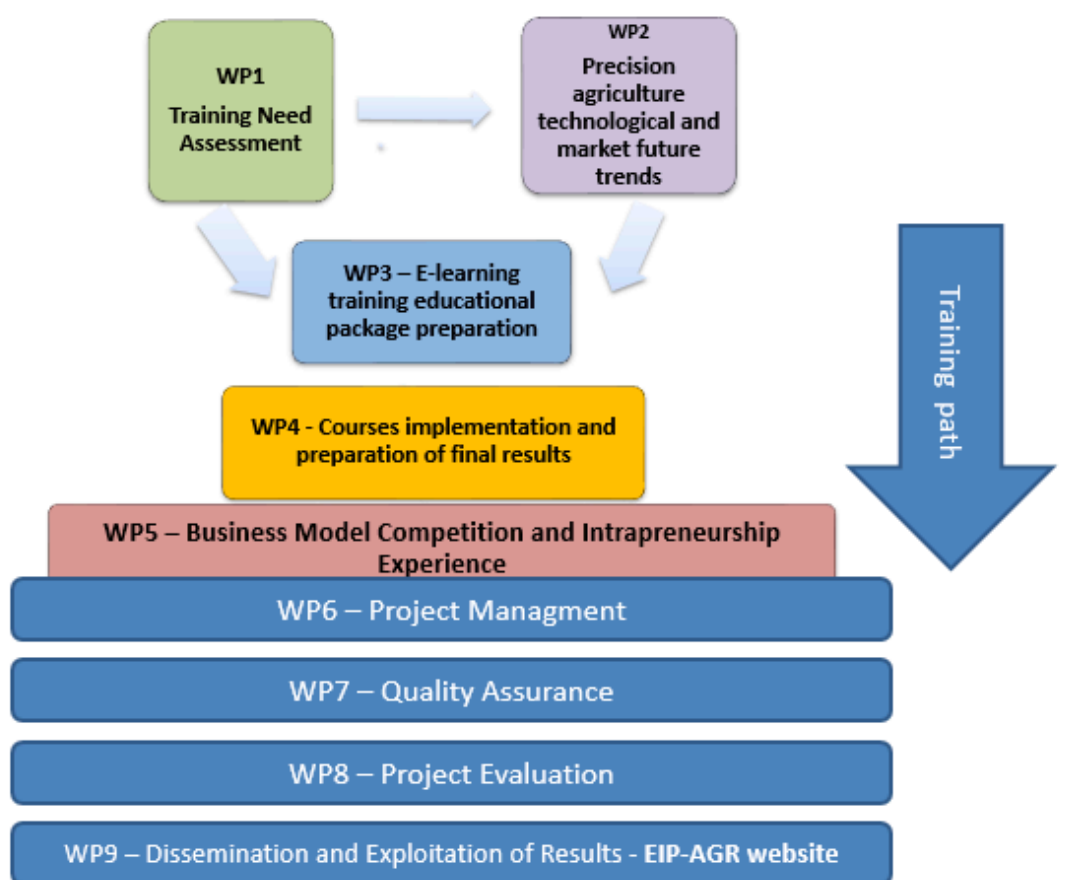

Figure 4. Relation between the different work packages during SPARKLE project.

\section{Results and Discussion}

Due to the recent development of the project, no results have yet been obtained. However, these statements will guide the project consortium and will be the subject of empirical verification and theoretical confirmation in the course of the proposed project (Figure 5):

1. Knowledge transfer in sustainable precision agriculture and business models in this sector among academics, students and entrepreneurs is limited in Europe;

2. Current entrepreneurship and precision agriculture educational offer does not meet the labour market needs related to agribusiness and new farms,

3. Employing sustainable precision agriculture and agribusiness models into entrepreneurial education and business practice will enable individuals and organisations to better address/manage professional challenges in this sector.

SPARKLE refers to the study "Precision agriculture and the future of farming in Europe" made by the Science and Technology Options Assessment (STOA) that warn on the necessity of skilled workforces in precision agriculture and report some data about training in Europe. This study makes the state of art about precision farming in Europe and tell that most farmers are not formally trained, $70 \%$ has only practical experience.

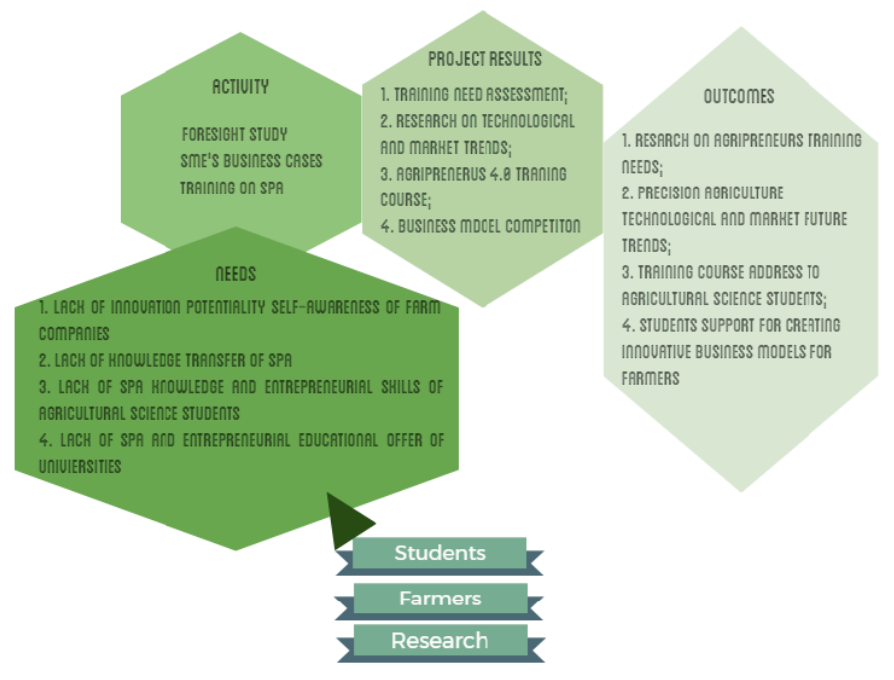

Figure 5. Expected results and project outcomes from SPARKLE activities. 
As expected results, the SPARKLE project aims to develop and implement a comprehensive training course for agricultural science students, addressing the different skills and entrepreneurial education and SPA knowledge that will be demanded in the future. In addition, the challenges that the techniques and technologies applied in the SPA will present, such as robotics skills, programming and data analysis, will be addressed from the perspective of their applicability from a practical point of view in advanced business models.

To reach this objective, the partnership will conduct and extensive training need assessment (Result 1) and will collect advanced contributions from some partners (Result 2) for creating an innovation educational package (e-learning courses + course book). To complete the training path and giving the students the possibility of realizing what they studied for, Universities and farms will launch a business model competition (result 4) for helping traditional farms to develop their business adopting PA technologies.

\section{Conclusions}

Fostering innovation in higher education in agricultural field, it is necessary to make young people prepared with the right blend of both job-specific and cross-cutting core skill to be able to access the labour market. In addition to this new technology and existing business models will have to be combined to make a technology successful but changes in technology and business models are both possible.

While Precision Agriculture techniques are now at the point of becoming commercially feasible and the market for PA is expected to grow every year with an average 12 per cent through 2020, the farm sector (especially small medium farmers) is not yet fully ready to embrace this change. In fact, companies identify that the main costs associated there are learning costs for the farmers to develop management schemes and calibrate the machinery. If the farmers of the future, currently students, could learn these lessons and if they could learn how to adopt the technological revolution that would be for them an inestimable asset for their businesses. At the same time, transferring this knowledge to already established companies would give a boost to their business activity. In this framework, SPARKLE Erasmus + project is the core of a pioneering initiative in agriculture. In fact, to achieve and answer to all needs found and to train Agripreuners 4.0 is necessary to connect sectors and target groups through collaboration. Furthermore, for speeding up innovation, technology and business knowledge shall be mix in a cross-over approach between farmers, students and research having as result brand-new SPA business model, people trained and SPA diffusion in small and medium enterprises (SME). The mix between different field and different knowledges can realize awareness on SPA, realizing the Agripreuner 4.0 value chain.

\section{References}

http://cema-agri.org/sites/default/files/EPRS_STU\%282016\%29581892_EN.pdf

\section{Acknowledgements}

The authors would like to thank all the members of the consortium for their contribution to the project and their contribution to the creation of the new digital agronomist profiles. The members of the consortium are:

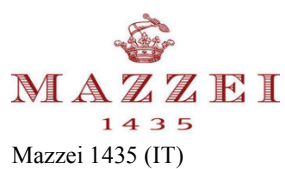

Mazzei 1435 (IT)

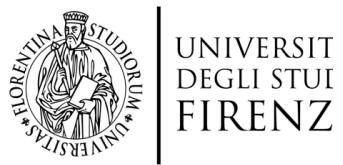

Università di Firenze (IT)

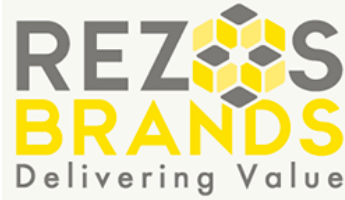

Rezos Brand (GR)

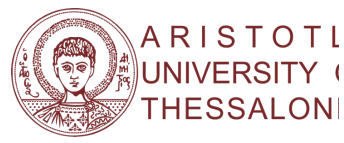

Aristotle University of Thessaloniki (GR)

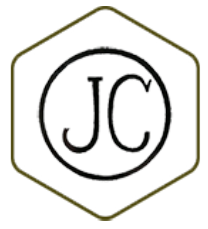

Quinta da Cholda SA (PT)

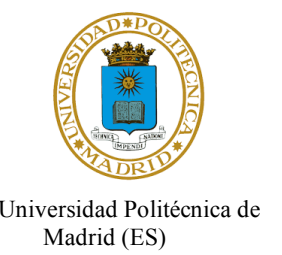

University of Évora (PT)

Madrid (ES) 


\title{
Management System of Small Farm Machinery Hiring Business for Rice Farming Operations in Kampar Region, Indonesia Ujang Paman*, Khairizal and Hajry Arief Wahyudy
}

\author{
Department of Agribusiness, Faculty of Agriculture, Riau Islamic University \\ Jl. Kaharudin Nasution No. 113 P. Marpoyan, Pekanbaru 28284, Riau, Indonesia \\ *Corresponding author: pamanu@agr.uir.ac.id
}

\begin{abstract}
Development of using farm machines for agricultural operations has created business opportunities for smallholder farmers in rural areas. Custom hire offering machinery services for farmers is one of business models at the farm level which has been adopted widely in many developing countries with different management system and economic benefit depending farming practices. This paper attempts to examine the management system of small farm machinery hire businesses for rice farming operations in Kampar Region. We have surveyed and purposively selected as 20 groups of hire service providers of small farm machinery in the region. Group managers and machine operators were personally interviewed using questionnaires to collect primary data during September - October 2017. The results showed that the small farm machinery hire services were small businesses managed by farmer groups and operated within village area. They managed 2 - 4 kind of farm machines and offered hiring services for their group members according to machine owned with a lower charge rate. The businesses became sources of increasing and diversifying family incomes for smallholder farmers in the region. Moreover, the availability of the farm machinery hire businesses was helpful stallholder farmers to access farm machinery for being mechanized their rice farming operations.
\end{abstract}

Keywords: Management system, Machinery hiring business, Small-scale rice farming, Kampar region.

\section{Introduction}

Agricultural mechanization today has widely expanded over the world. However, the application of mechanization in farming practices still differs significantly between developed and developing countries. The present level of the mechanization in the developing countries is still relatively low and differs significantly across the countries (Kienzle et al., 2013) and even from region to region within the country (Singh and Zhao, 2016). It is because many developing counties face various constraints and continue to go on today. Main constrains to develop mechanization are low purchasing power of farmers who are mostly smallholders farmers, lack of welltrained operators and mechanics for making machinery repairs and maintenance, and inadequate maintenance and repair facilities for supporting machinery management system. Twenty years ago, Rijk (1998) confirmed that agricultural sector in developing countries is dominated by small-scale farming, many of which are less than two hectares.

During the last few decades, many developing countries have strived to accelerate the technological transformation of mechanization through introducing small farm machinery for smallholder farmers. The utilization of mechanical power instead of human and animal power offers a potential for increasing agricultural efficiency and productivity primarily food crops. The sustainability of agriculture in the world today cannot be distinguished from the development of farm mechanization at the farm level. Many developing countries have succeeded to significantly increase rice production and achieve self-sufficiency, and even exported rice to other countries.

The agricultural machinery use has been adopted through custom hiring system that helped to increase the utilization of the agricultural machinery in many countries (Abdullah, 2016). Although the operation model may not be compatible with the conditions in every country (Chancellor, 1971), the custom hiring system has been adopted in many developing countries in order to provide farm machinery to smallholder farmers (Koike, 2009; Paman et al., 2010; Paman et al., 2014; Paman et al., 2016; Singh, et al., 2013; Rahman, et al., 2013; Chahall, et al., 2014). The machinery hire services could be provided by governmental agencies as well as private entrepreneurs/co-operatives (Singh, 2013).

In Indonesia, farm machinery hire services have developed and widely expanded over the country and have been small businesses managed by farmer groups. Based on data from Agricultural Department of Indonesia (2011), number of farm machinery hire services groups in Indonesia in 2010 accounted to 12,612 and about 707 (5.6\%) were located in the Riau Province. Currently, most of small farmers are increasingly depending on custom hiring of farm machinery to perform farm works, particularly for labour intensive operations such as tillage, threshing, and milling operations. Successful farm machinery business depends greatly on management system adopted by the business. The purpose of this paper is to examine the management system of small farm machinery hire businesses for rice farming operations in Kampar Region. 


\section{Materials and Methods}

This research was carried out using survey method which located in Kampar Region, Riau Province, Indonesia. A total of 20 hire business groups were selected from 15 districts in the region. The districts are rice production areas in Kampar Region and have a high level of mechanization adoption on farm. Most farmers use farm machinery for their farming operations provided by hire business groups. The survey area of the research is showed in Fig. 1.

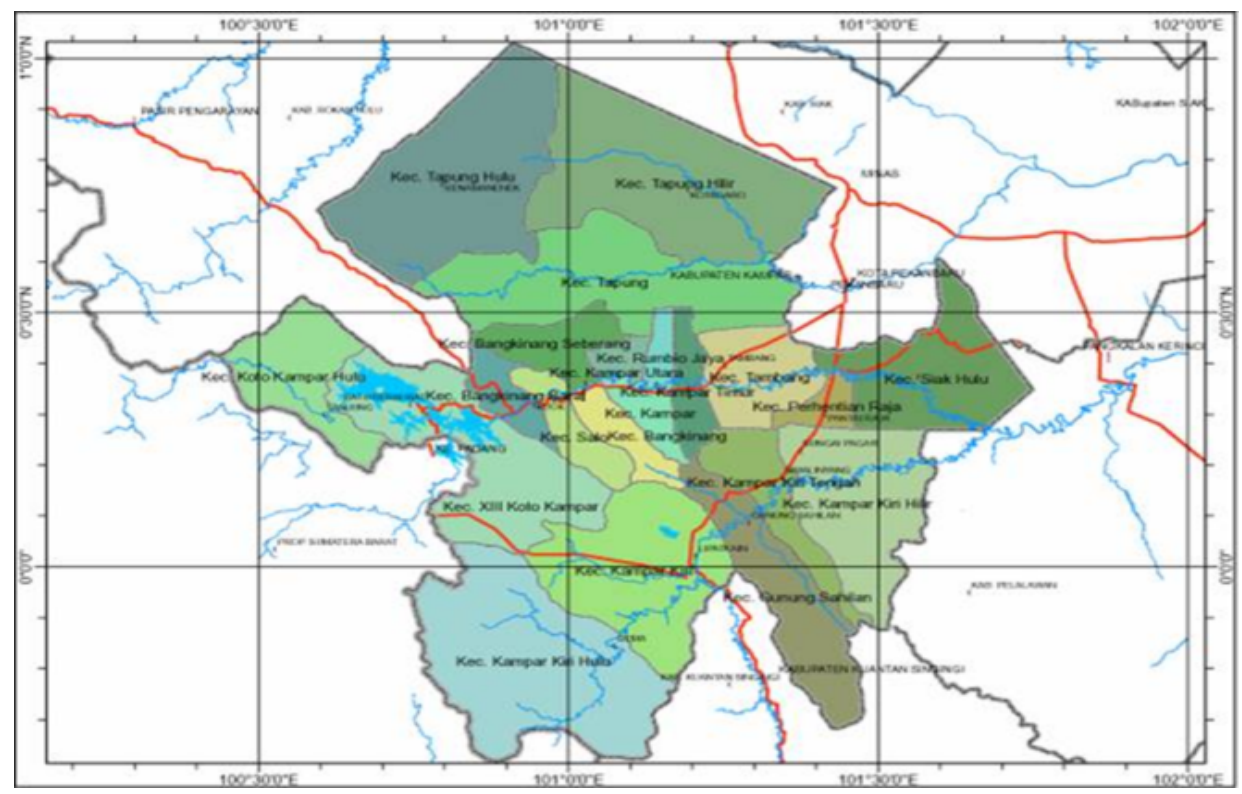

Figure 1. Map of Kampar Region showing the research location.

Data required in this research consisted of primary and secondary data. Primary data obtained through personal interviews with managers of hire business groups, machinery operators, and farmer's group leaders by using questionnaires. The collected data included number and type of owned machines, rates of service charge and payment procedures, number farmer groups and their members, and other information corresponding to this research. While, secondary data were collected from Food Crops Service of Kampar Region, Statistical Office of Kampar region and District Offices. The secondary data included type and number of farm machinery in the region, ownership system, and other related data. The collected data were tabulated and then analyzed by using descriptive-quantitative approaches.

\section{Results and Discussion}

Types and number and of farm machinery on rice farming

Farm machinery in Kampar Region has widely expanded during the last ten years over the region. Most of the farm machinery is still concentrated on the rice production areas. Types and number of farm machines available in the region are still limited (Table 1). The major types of farm machinery on farm today are four-wheel tractors, two-wheel tractors (power tillers), irrigation pump, power threshers, dryers, and rice milling units (RMUs). Therefore, farm operations which can be performed by using farm machines depend on the machine types available, such as land preparation, water pumping, threshing, harvesting, and milling. Paman et al (2018) reported that the high level of mechanization was found on land preparation and milling operations. 
Table 1. Type and number of farm machines in Kampar Region in 2016.

\begin{tabular}{clrrr}
\hline No. & Type of machines & Good condition & Breakdown & Total \\
\hline 1 & Two-wheel tractor & 195 & 26 & 221 \\
2 & Four-wheel tractor & 7 & 2 & 9 \\
3 & Irrigation pump & 257 & 53 & 310 \\
4 & Power thresher & 333 & 60 & 393 \\
5 & Dryer & 17 & 8 & 25 \\
6 & Rice milling unit (RMU) & 70 & 8 & 78 \\
\hline
\end{tabular}

Source: Food Crops and Horticulture Services of Kampar Region. 2017

Table 1 shows that the largest number of farm machines is power thresher (393 units) and the second one is irrigation pump ( 310 units). Two-wheel tractor is found to be 221 units and this tractor type is very popular among farmers for tillage operation. Four- wheel tractor is smallest number (9 units) and this tractor is not suitable for any small-scale farm. Furthermore, the machines are not all in good condition. There are some machines in breakdown condition and the machines are difficult to be repair due to lack of spare parts and mechanics.

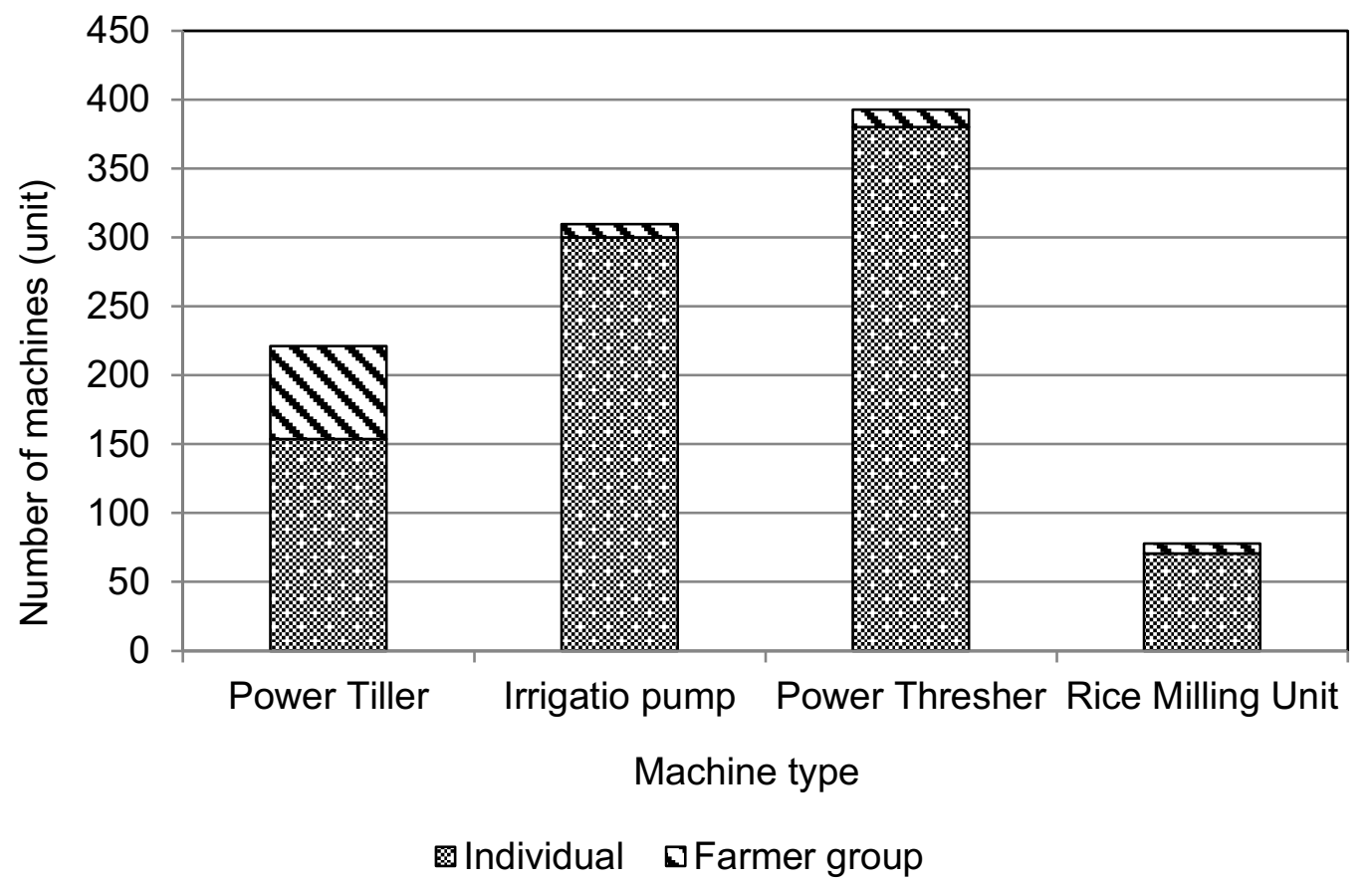

Figure 2. Farm machinery ownership in Kampar region.

Farm machinery in survey areas is owned by individual and hire business groups. Table 2 shows that most of farm machinery is owned by individual. The machines are purchased by farmers themselves and mainly used to work their farms. They also provide hiring services for other farmers to fully use machine capacity and receive money from the services. They usually offer hire services to neighbor farmers who require farm machines to work their farm operations.

Organizational and membership structures of hire business groups

Machinery hire businesses in Kampar Region are managed by farmer groups and individual farmer. The hire service groups have been established in villages which have paddy field areas and produce rice every year. The farm machines managed by the group came from government aid through mechanization development program. There is only one machinery hire business available in each village. The establishment of the groups is to make easy for distributing farm machinery to be managed effective and efficient and then the machines can be accessed easily by smallholder farmers.

Farm machinery hire service is a small business group and involves only by few persons in its operation management. Therefore, form of organizational structure of the business group is very simple and has only few job positions (Fig. 3). It is because of types of job only provide hire services for perform farm operations to group 
members. The organization of hiring business group is managed by a manager and assisted by a vice manager, a secretary, and a treasurer. The organizational structure is equipped with three persons as operator, mechanic and group leader. While, village chief and extension officer have a position as advisers in the organizational structure.

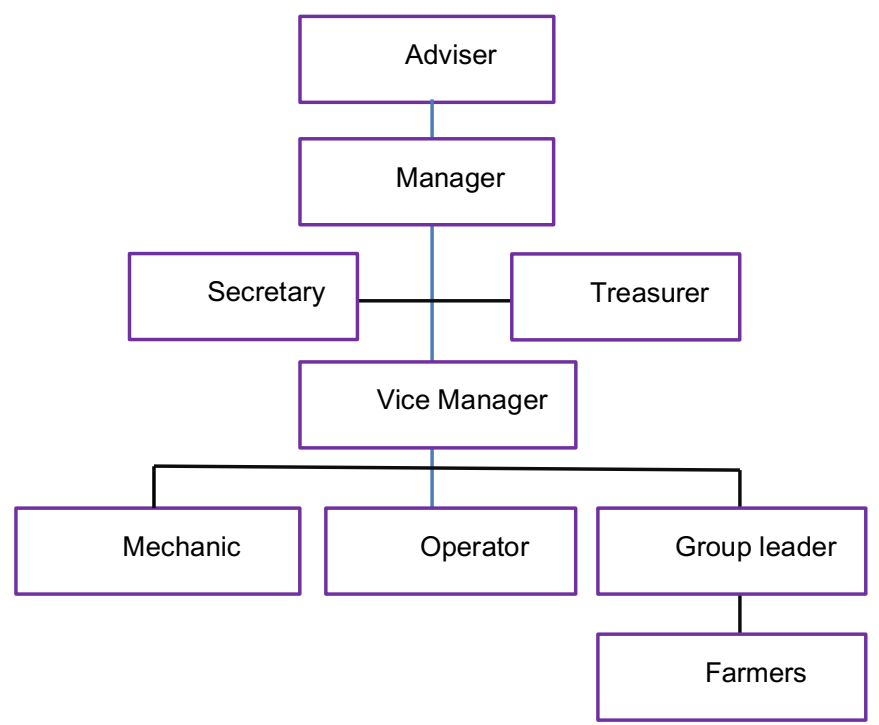

Figure 3. Organizational structure of hire business groups.

There is not monthly salary for manager and other management staffs. They receive wage at the end of season, exception operators. The operator wage is paid in installments during season. The operator wage is calculated as much as $50 \%$ from profit obtained from job contract. The rest will be divided for advisers, manager, vice manager, secretary, treasurer, and group leader, but there is no wage from hire business group for mechanic. The mechanic will receive wage from repair and maintenance services. The amount of wage depends on number of job contracts and their position in the organizational structure.

Member of the hire business groups consisted of the whole farmer groups available in a village. The member of the groups is farmers who cultivate rice in the village. Therefore, the number of the group member depends on number of farmer groups and/or rice farmers in the village area, so the number of hire business group member differs across groups. The village that has wide paddy field areas and many farmers has more group or more farmer members. The structural member of hire business groups is presented in Fig. 4.

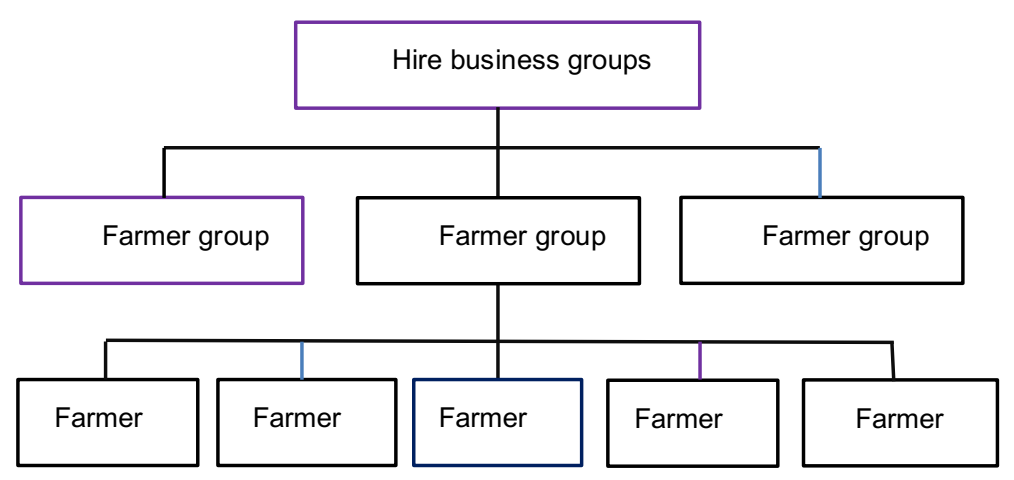

Figure 4. Membership structure of hire business groups.

The hire business groups provide services primarily for farmers who were registered as group members. The procedure to get job order is begun from farmers. At the beginning of growing season, farmers can propose any jobs to group leader to be continued to vice manager in order to make a decision for the job contract between farmers and hire business group. After contract is agreed between farmers and manager, the job can be begun. Farmers must also pay $50 \%$ of total payment of the contract as down payment. This payment is used to purchase fuel and oil. The rest will be paid after job completely finish. In few cases, farmers failed to pay off the second 
payment due to economic reason. They will pay it after harvesting rice, but there is no interest imposed for delaying the second payment. Such cases have caused financial problems when hire business groups will pay operator wage and repair costs.

\section{Rates of Service Charge}

Rates of service charge for various types of farm machinery offered by hire business groups are established based on agreement between village chief, group manager, farmer group leader, and extension officer. The rates are made to include costs of operational machines such as fuel, oil, operator wage, repair and maintenance, and profit for hire business group. Therefore, the rates of service charge differed across hire business group and type machines or operations. The rates offered to group members were lower than rates of private (individual) hire business available in the vicinity. It is because the hire business groups have purpose not only to get profit but also to help small farmers to get farm machinery service to mechanize their farming operations. Hence, small farmers who have not buy or owned machines yet can work their farm using the machines without purchasing them.

Rates of service charge are established under different basis. Tillage operations are calculated in meter square $\left(\mathrm{m}^{2}\right)$ and the rates included for irrigation pump. The rates for harvesting, threshing, and milling operations are determined based on kilogram $(\mathrm{kg})$. The rates of charge can differed across groups with the same type of operations. The rates of tillage operations, for example, varied among machine types (moldboard flow, rotary tillers, or hydro tillers), hire service groups, and field conditions (such as weed conditions and distance from the machine centre to the location of farm area). The charge for moldboard flow is the highest rate of tillage operations because the operations are performed twice including primary and secondary tillage. The machine is usually used when paddy field is lack of water supply primarily on dry season. Operators usually use water pump to supply water into the paddy field. The charge included for the water pump operation.

Table 2. Rates of service charge for various types of farm machinery.

\begin{tabular}{lccc}
\hline Machine types & Operation Types & Calculation basis & Rates (IDR) \\
\hline Moldboard Flow & Tillage preparations & $\mathrm{M}^{2}$ & $200(\$ 0.015)$ \\
Rotary Tillers & & & $180(\$ 0.014)$ \\
Hydro Tillers & & & $150(\$ 0.012)$ \\
Power Thresher & Threshing & KG & $300(\$ 0.023)$ \\
Rice Milling Unit & Milling & & $500(\$ 0.038)$ \\
\hline
\end{tabular}

Note: US\$ 1 is equivalent to about IDR 13,000 based on the average exchange rate in 2017.

Revenue and Profit

The total revenue and profit derived by hire business groups each operational season is presented in Table 3. The total revenue was obtained as US $\$ 3,792.7$ and the largest revenue derived from rotary tiller operation. Total cost was required as US $\$ 2,690.5$ and the highest cost was for rotary tiller operation. With operating five types of machines, hire business groups received profit as US \$1,102.2 each operational season. This profit was mostly derived from rotary tiller operation, accounting to US \$338. The results showed that hire business groups are viable business model in village areas in Kampar Region. The profit has become an additional income for farmers involved in the business.

Table 3. Revenue and profit derived each machine type managed by hire business groups (US\$).

\begin{tabular}{lcrc}
\hline Type of machine & \multicolumn{1}{c}{ Cost } & Revenue & \multicolumn{1}{c}{ Profit } \\
\hline Moldboard Flow & 691.6 & 970.5 & 278.9 \\
Rotary Tillers & 806.7 & $1,144.7$ & 338.0 \\
Hydro Tillers & 592.1 & 904.1 & 312.0 \\
Power Thresher & 166.2 & 201.3 & 35.1 \\
Rice Milling Unit & 433.9 & 572.1 & 138.2 \\
Total & $2,690.5$ & $3,792.7$ & $1,102.2$ \\
\hline
\end{tabular}




\section{Conclusions}

The small farm machinery hire services were small businesses managed by farmer groups and operated within village area. They managed $2-4$ kind of farm machines and offered hiring services mainly for their group members with a lower rate of charge. The machinery hire businesses became sources of increasing and diversifying family incomes for smallholder farmers in the region.

\section{References}

Abdullah, K. 2016. Present Status and Future Prospects of Agricultural Machinery Industry in Indonesia. Agricultural Mechanization in Asia, Africa, and Latin America, 47(2): 71-74.

Agricultural Department of Indonesia. 2011. Annual Report. Jakarta.

Chancellor, W. J. 1971. Mechanization of small farms in Thailand and Malaysia by tractor hire services. Trans. ASAE, 14(6): 847-854, 859.

Chahal, S. S., P. Kataria, S. Abbott, and B. S. Gill. 2014. Role of Cooperatives in Institutionalization of Custom Hiring Services in Punjab. Agricultural Economics Research Review, 27: 103-110.

Food Crop and Horticulture Services of Kampar Region. 2017. Agricultural Statistics 2016. Bangkinang.

Kienzle, J., Ashburner, J. E. and Sims, B. G. 2013. Mechanization for Rural Development: A Review of Patterns and Progress from Around the World. Food and Agriculture Organization of the United Nations. Rome.

Koike, M. 2009. Custom Hire Systems for Agricultural Machines in Southeast Asia - In a Rural Community in Thailand. Engineering in Agriculture, Environment and Food, 2(4): 144-149.

Mada, D. A. and S. Mahai. 2013. The Role of Agricultural Mechanization in the Economic Development for Small Scale Farms In Adamawa State. The International Journal of Engineering and Science, 2(11): 91-96.

Paman, U., S. Uchida, and S. Inaba. 2010. Economic Potential of Tractor Hire Business in Riau Province, Indonesia: A case study of small tractors for small rice farms. Agricultural Engineering International: the CIGR Journal, 12(1): 135-142.

Paman, U., Inaba, S. and Uchida, S. 2014. Farm Machinery Hire Services for Small Farms in Kampar Regency, Riau Province, Indonesia. Applied Engineering in Agriculture, 30(5): 699-705.

Paman, U., Inaba, S. and Uchida, S. 2016. Economic Aspects of Machinery Hire Services Managed by Farmer Groups in Kampar Regency, Indonesia. Applied Engineering in Agriculture, 32(2): 169-179.

Paman, U., H. A. Wahyudhy, and Khairizal. 2018. Effect of Farm Machinery Development on Using Women Labour in Kampar Region, Indonesia. Research Report, Research and Community Services Institution of Riau Islamic University, Pekanbaru.

Rahman, A., M. Latifunnahar, and M. M. Alam. 2013. Financial Management for Custom Hire Service of Trar in Bangladesh. Agricultural Engineering International: CIGR Journal, 6(3): 28 - 33.

Singh, G., and B. Zhao. 2016. Agricultural Mechanization Situation in Asia and the Pacific Region. Agricultural Mechanization in Asia, Africa and Latin America. 47(2): 15-25.

Singh, R. S. 2013. Custom Hiring and Scope of Entrepreneurship Development in Farm Machinery. Agricultural Mechanization in Asia, Africa and Latin America, 44(2): 26-32.

Singh, S., H. S. Kingra, and Sangeet. 2013. Custom Hiring Services of Farm Machinery in Punjab: Impact and Policies. Indian Research Journal Extension Education, 13(2): 45-50. 


\title{
Long-Term Tracking and Automated Analysis of Tractor Behaviour with JDlink \\ Barreiro, $\mathbf{P}^{\mathbf{1}}$; Rueda, $\mathbf{I}^{\mathbf{2}}$; Rabasco, $\mathbf{A}^{\mathbf{1}}$; Garrido-Izard, $\mathbf{M}^{\mathbf{1}}$ \\ ${ }^{1}$ LPF_TAGRALIA. Technical University of Madrid. (UPM) CEI Moncloa. UP4 \\ ${ }^{2}$ John Deere Ibérica, Madrid, Spain \\ * Corresponding author. Email: pilar.barreiro@upm.es
}

\begin{abstract}
JDLINK is an application for farm management under a precision agriculture strategy (variable rate application of inputs with geolocated data and maintenance alerts among others. It allows accessing to isolated or groups of machines, with the possibility of downloading CAN BUS data in periods established by use (engine hours) or by date. JDLINK can be considered a SCADA (System Control - SC- and Data Acquisition -DA-) where the visualization of data is indispensable to the manager of the fleet of machines. Our approach in this research is clear: What practical recommendations in terms of energy efficiency, work performance and improvement of use (maintenance) can be made based on the automate analysis of the information contained in JDLINK? For this prospective study, two tractors were used: one $8345 \mathrm{R}$ of $380 \mathrm{HP}$ (1000 hours) belonging to a cooperative in Navarra (Spain), and another one 7230R of 230HP (500 hours) belonging to a private farmer in Ciudad Real (Spain); we chose 42 variables: 17 motor, 10 on maintenance and 17 derived from the tasks performed. Then we programed and tested an automated multivariate analysis for the recognition of agricultural activity patterns which consists of a Principal Component Analysis (PCA), an unsupervised cluster (Cluster) and a multiple analysis of variance or MANOVA. The use of the automated procedure has led to the isolation of several patterns as identifiable tasks four in the case of $8345 \mathrm{R}$ and six different patterns for the 7230R tractor. As a consequence, a multivariate pattern became available and thus the identification of machine performance and fuel consumption pattern $\left(1 \mathrm{ha}^{-1}\right)$. Moreover, clear conclusions were derived upon the misuse of tractor ballast, low level and excessive variability of slippage (below $4 \%$ on average basis. At present this procedure is available for dealers to provide highly valuable agricultural consultancy for farmers.
\end{abstract}

Keywords: Multivariate pattern, chemometrics, CAN BUS data, machine performance, advisory service, ISOBUS

\section{Introduction}

By the end of s. XX, advanced agricultural mechanization based on auto-guidance, electronic control, M2M communication and ISOBUS (the standard for information interchange between tractor and machinery) opened a whole set of possibilities for the optimization of in-field routes and yield management zones, inputs efficiency analysis and the use of fleets of vehicles: this is now called Agriculture 2.0. The onset of Geographic information Systems together with agent based computing opened a new step in the development of agriculture knowledge and capabilities which in some areas is labelled as Agriculture 3.0: not necessarily happening after A2.0 since small plot do not required variable rate technology. Nowadays with the emergence of remote data warehouse, and cloud computing, we face possibilities that could not be witnessed before, all gathered under the term Agriculture 4.0; the EU has set the goal for 2020 of ubiquitous internet access in the rural areas with 30Mbytes s-1 speed (Barreiro et al., 2017).

Digital Agriculture is part of terrestrial digital technology and aims to create an open information network at the service of economic and social development, environmental protection, research and the dissemination of knowledge, through the development of software and hardware (Yong et al., 2002). In the event that there is sufficient bandwidth in farms in the future, it is reasonable to assume that all agricultural systems would eventually become Internet-based services hosted on cloud servers, instead of using software applications users installed on a personal computer (Schuster et al., 2011).

Telemetry is today the cornerstone of Formula1; its use in fleet management and optimization of intermodal transport logistics of goods and services is also known, with a real-time location of vehicles from a control centre, as well as remote monitoring of the quality of the cargo. In agricultural machinery also, telemetry allows the extension of the connection from manufacturing centres to local dealers, and end-users (Lenz 2007). John Deere has displayed a great effort in fleet management by stablishing MyJohnDeere as remote platform. In this way the maintenance requirements and needs are transmitted remotely, wirelessly, from the end-user to the dealer, while successive versions of the tractors software and firmware can in turn be updated (and adapted to local needs).

Recently, iGreen, a public-private research project with 23 participants, has analysed and verified the procedures for hosting and sharing data based on cloud services from several agricultural machinery manufacturers. With two basic components: machine-level connectors (MC), and in-line (OB) boxes (Blank et al., 2013). In such work, the M2M communication data shared within a week showed that the real-time information transmitted via WiFi (IEEE 802.11g) was delayed between 0.1 to $3 \mathrm{~s}$ with success higher than $90 \%$; 
when it is carried out via $3 \mathrm{G}$ it has a delay of 1 to 10 s with success above $65 \%$, however when documentation information is to be transmitted (it does not require real time) the delay was found between 5 to 85 minutes with $100 \%$ success.

The iGreen concept was also presented by Gansemer et al. in 2013, with the participation of CLAAS. It starts from the premise that the agricultural sector is characterized by heterogeneous machines, with a large number of entities participating in the processes, and with a high cost of operation. The inefficiency in this context is mainly due to the lack of optimization of the location of the machines in synchronized operations such as harvesting, the existence of avoidable downtime (such as the need to travel to update the firmware of the machines), and the incompatibilities of communication between machines. To eliminate the last point, it proposes the use of open M2M standards in what it calls the M2M value chain. It offers clear and detailed flow charts and information control between the different agents: machinery manufacturers, farmers, production managers (for example cooperatives), and machinery service companies that could benefit from this value chain. This article addresses two scenarios: 1) the synchronization of machine chains of various manufacturers (clearly the case under iGreen), and 2) the remote update of firmware and software. In the first, not only the initial programming of the machines is contemplated, but the modification of the dynamic schedule according to work contingencies; in the latter, there are more than 30 elements / actions that are automatically modified or activated in the process of software update.

In this research we approach fleet management with the use of JDLINK, under the following target: What practical recommendations in terms of energy efficiency, performance and improvement of use (maintenance) can be made based on the automate analysis of the information contained in JDLINK?

Table 5. Variables downloaded from JDLINK.

\begin{tabular}{|c|c|c|c|c|}
\hline Category & Subcategory & Acronym & Description & PCA DATA \\
\hline \multirow{17}{*}{ Motor } & \multirow{6}{*}{ Work } & RW & Regime (rev min-1) & 2 \\
\hline & & LW & Load $(\%)$ & 5 \\
\hline & & $\mathrm{cW}$ & Hourly consumption $\left(1 \mathrm{~h}^{-1}\right)$ & 8 \\
\hline & & $\mathrm{CW}$ & Total consumption (1) & 11 \\
\hline & & $\mathrm{T}_{-} \mathrm{TBJO}$ & Assigned time (\%) & 26 \\
\hline & & $\mathrm{v}$ TBJO & Speed $\left(\mathrm{km} \mathrm{h}^{-1}\right)$ & 37 \\
\hline & \multirow{6}{*}{ Transport } & RT & Regime (rev min-1) & 3 \\
\hline & & LT & Load $(\%)$ & 6 \\
\hline & & $\mathrm{cT}$ & Hourly consumption $\left(1 \mathrm{~h}^{-1}\right)$ & 9 \\
\hline & & $\mathrm{CT}$ & Total consumption (1) & 12 \\
\hline & & T_TPTE & Assigned time (\%) & 27 \\
\hline & & $\mathrm{v}$ TPTE & Speed $\left(\mathrm{km} \mathrm{h}^{-1}\right)$ & 38 \\
\hline & \multirow{4}{*}{ Iddle } & $\mathrm{RR}$ & Regime (rev min-1) & 1 \\
\hline & & LR & Load $(\%)$ & 4 \\
\hline & & $\mathrm{cR}$ & Hourly consumption $\left(1 \mathrm{~h}^{-1}\right)$ & 7 \\
\hline & & $\mathrm{CR}$ & Total consumption (1) & 10 \\
\hline & Management & T_IPM & Intelligent management time (\%) & 13 \\
\hline \multirow{9}{*}{ Tasks } & \multirow{3}{*}{ Traction and drive } & Slip P1 a p9 & Ranges in figure (\%) & $28-36$ \\
\hline & & T_TDF & PTO time $(\%)$ & 24 \\
\hline & & $\mathrm{T}$ IDM & Time front-wheel drive $(\%)$ & 25 \\
\hline & \multirow{6}{*}{ Auxiliaries } & H1 & Flow ves 1 & 39 \\
\hline & & $\mathrm{H} 2$ & Flowves 2 & 40 \\
\hline & & H3 & Flow ves 3 & 41 \\
\hline & & $\mathrm{H} 4$ & Flow ves 4 & 42 \\
\hline & & H5 & Flow ves 5 & 43 \\
\hline & & Elevator & Position & 44 \\
\hline \multirow{10}{*}{ Maintenance } & & TmR & $\mathrm{T}^{\mathrm{a}}$ average temperature $\left({ }^{\circ} \mathrm{C}\right)$ & 14 \\
\hline & & TMT & $\mathrm{T}^{\mathrm{a}}$ max transmission $\left({ }^{\circ} \mathrm{C}\right)$ & 15 \\
\hline & & $\mathrm{TMH}$ & $\mathrm{T}^{\mathrm{a}}$ max hydraulic $\left({ }^{\circ} \mathrm{C}\right)$ & 16 \\
\hline & & $\mathrm{TmT}$ & $\mathrm{T}^{\mathrm{a}}$ average transmission $\left({ }^{\circ} \mathrm{C}\right)$ & 17 \\
\hline & & TMR & $\mathrm{T}^{\mathrm{a}}$ max cooling $\left({ }^{\circ} \mathrm{C}\right)$ & 18 \\
\hline & & $\mathrm{TmH}$ & $\mathrm{T}^{\mathrm{a}}$ average hydraulic $\left({ }^{\circ} \mathrm{C}\right)$ & 19 \\
\hline & & $\mathrm{V}$ & Average voltage (V) & 20 \\
\hline & & N1 & $\begin{array}{c}\text { Number of interrupted cleanings } \\
\text { of exhaust filter }\end{array}$ & 21 \\
\hline & & $\mathrm{N} 2$ & $\begin{array}{l}\text { Number of exhaust filter } \\
\text { cleanings completed }\end{array}$ & 22 \\
\hline & & $\mathrm{NN}$ & Fuel level $(\%)$ & 23 \\
\hline
\end{tabular}




\section{Materials and Methods}

JDLINK is one of the modules of MyJohnDeere among which the Operations Centre also stands out. If the former is an application for farm management under a precision agriculture strategy (variable rate application of inputs with geo-located data, maintenance alerts, geo-fences, job management, documentation), the second, JDLINK, allows access to individual or groups of machines, with the possibility of downloading CAN BUS data (more than fifty variables) in periods established by use (engine hours) or by date. JDLINK can be considered a SCADA (System Control - SC- and Data Acquisition -DA-) where the visualization of data to the manager is mandatory for fleet management. For this prospective study, two tractors were used: one 8345R of $380 \mathrm{HP}$ (1000 hours) belonging to a cooperative in Navarra (Spain), and another one 7230R of 230HP (500 hours) belonging to a private farmer in Ciudad Real (Spain)(.

The first step in the automated analysis of agricultural machinery is to establish the frequency and the amount and format of data to be downloaded (by date or hours of use). For each period, JDLINK provides more than 40 series of data that can be categorized by types of use: idle, work and transport based on speed (see Table 6); Assignment performed internally, using small Matlab routines (MATLAB R2015b, MathWorks, Inc., Natick, MA, USA) that read the files, collates the variables and aggregate the data into consistent matrices. In our case, we chose 42 variables: 17 motor, 10 on maintenance and 17 derived from the tasks performed (see Table 5).

Table 6. Categorization of use in agricultural machinery according to JDLINK.

\begin{tabular}{ccc}
\hline & Mowers & Tractors \\
\hline Iddle & Engine on and 0 ground speed & Engine on and 0 ground speed \\
Work & Feeder and engine on & Ground speed between 0 and $20 \mathrm{~km} \mathrm{~h}^{-1}$, or else 0 ground speed and PTO \\
Transport & Feeder and engine on & on. \\
\hline
\end{tabular}

The second process is the programming and realization of an automated multivariate analysis for the recognition of agricultural activity patterns and their corresponding characterization of the machines performance, which consists of a Principal Component Analysis (PCA), an unsupervised cluster (K-means Cluster) and a multiple analysis of variance or MANOVA.

\section{Results and Discussion}

Figure 19a (left) shows that the use of the automated procedure has led in the 8345R tractor to the isolation of four different patterns as identifiable tasks: heavy and light traction, PTO activated task and hauling; six different patterns (Figure 19) have been found for the 7230R tractor: heavy and light traction activities, heavy and light PTO tasks, and heavy and light hauling. With this information and a slight feedback to the farmer (equipment list in use and actual width) it was possible to make a unique match between equipment and task.

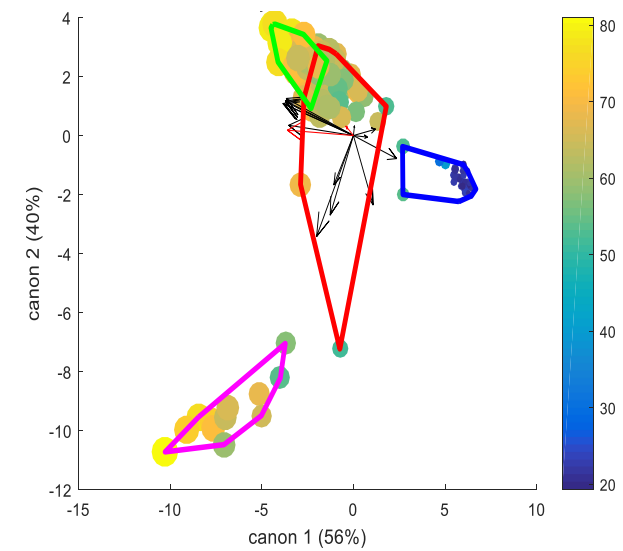

$8345 R$

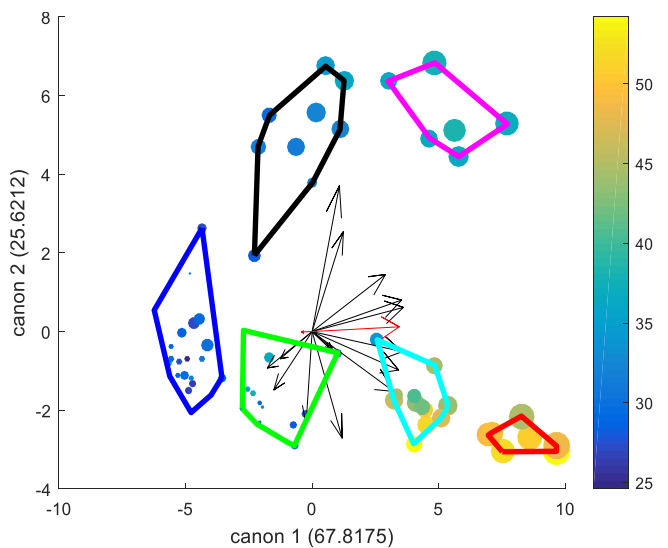

$7230 \mathrm{R}$

Figure 19. Results from the k-means clusters: marker color refers to the engine load under working conditions; marker size indicates fuel consumption ( $\mathrm{h}-1)$ and; line color identify the tasks segregated with the unsupervised procedure: a(left) $8345 \mathrm{R}$ and $\mathrm{b}$ (right) $7230 \mathrm{R}$. 
Table 7 and Table 8 provide some quantitative characteristic features for each task considering tractor $8345 \mathrm{R}$ and 7230R, such as engine load (\%) and speed (rev min-1)during work, together with fuel consumption (1 h-1), the percentage of time with front traction active, PTO speed $\left(\mathrm{rev} \mathrm{min}^{-1}\right)$ and several slippage ranges $(\%)$.

Table 7. Characteristic features for the four tasks identified in tractor 8345R.

\begin{tabular}{|c|c|c|c|c|}
\hline & $\begin{array}{l}\text { Heavy hauling } \\
\text { (green) }\end{array}$ & $\begin{array}{l}\text { Light hauling } \\
\text { (red) }\end{array}$ & Transport (blue) & $\begin{array}{c}\text { PTO activated } \\
\text { (magenta) }\end{array}$ \\
\hline $\mathrm{RW}\left(\right.$ rev $\min ^{-1}$ ) & 1656 & 1580 & 1359 & 1731 \\
\hline LW (\%) & 70.21 & 62.96 & 23.95 & 67.58 \\
\hline $\operatorname{cW}\left(I h^{-1}\right)$ & 45.76 & 39.48 & 12.60 & 45.30 \\
\hline CW (I) & 211.42 & 161.17 & 19.25 & 203.96 \\
\hline TW (\%) & 92.45 & 84.55 & 31.29 & 91.39 \\
\hline IPM (\%) & 0.09 & 0.33 & 2.14 & 18.21 \\
\hline TDM (\%) & 93.60 & 83.35 & 16.47 & 87.76 \\
\hline TDF (\%) & 0.00 & 1.89 & 0.53 & 79.38 \\
\hline $\mathrm{RT}\left(\operatorname{rev} \mathrm{min}^{-1}\right)$ & 1439 & 1404 & 1590 & 1424 \\
\hline LT (\%) & 39.13 & 38.52 & 38.52 & 36.83 \\
\hline cT $\left(\mathrm{I} \mathrm{h}^{-1}\right)$ & 22.39 & 21.35 & 23.90 & 21.13 \\
\hline
\end{tabular}

Table 8. Characteristic features for the six tasks identified in tractor 7230R

\begin{tabular}{|c|c|c|c|c|c|c|}
\hline & $\begin{array}{c}\text { Transport } \\
\text { at harvest } 1 \\
\text { (red) }\end{array}$ & $\begin{array}{l}\text { Light hauling } \\
\text { with semi- } \\
\text { chisel (blue) }\end{array}$ & $\begin{array}{c}\text { Mower } 1 \\
\text { (magenta) }\end{array}$ & $\begin{array}{l}\text { heavy hauling } \\
\text { with semi- } \\
\text { chisel (green) }\end{array}$ & $\begin{array}{c}\text { Mower } 2 \\
\text { (black) }\end{array}$ & $\begin{array}{c}\text { Transport } \\
\text { at harvest } 2 \\
\text { (cyan) }\end{array}$ \\
\hline $\mathrm{RW}\left(\mathrm{rev} \min ^{-1}\right)$ & 1786,11 & 1252,91 & 1695,17 & 1338,10 & 1560,28 & 1736,77 \\
\hline LW (\%) & 49,79 & 28,79 & 37,00 & 31,63 & 31,94 & 45,19 \\
\hline $\operatorname{cW}\left(I h^{-1}\right)$ & 21,36 & 9,08 & 15,35 & 10,87 & 12,47 & 19,02 \\
\hline CW (I) & 196,23 & 17,54 & 140,38 & 12,57 & 86,68 & 88,11 \\
\hline TW (\%) & 87,88 & 39,52 & 91,39 & 25,10 & 77,19 & 81,66 \\
\hline IPM (\%) & 0,68 & 4,21 & 0,53 & 8,37 & 1,40 & 0,33 \\
\hline TDM (\%) & 91,70 & 6,56 & 68,10 & 63,23 & 13,28 & 92,86 \\
\hline РTO (\%) & 0,00 & 1,12 & 86,00 & 1,09 & 60,21 & 2,22 \\
\hline $\mathrm{RT}\left(\right.$ rev $\left.\min ^{-1}\right)$ & 1773,94 & 1681,32 & 1677,10 & 1734,47 & 1719,11 & 1690,17 \\
\hline LT $(\%)$ & 52,29 & 46,55 & 48,27 & 50,77 & 49,14 & 48,03 \\
\hline cT $\left(I^{-1}\right)$ & 23,99 & 20,38 & 20,88 & 22,71 & 21,94 & 21,12 \\
\hline
\end{tabular}

Using the feedback from the farmer, only in the case of the $8345 \mathrm{R}$ was it necessary to segregate between mouldboard and chisel (both distributed among heavy and light traction depending on plots and soil conditions), which was done by means of a refined subset of data (Figure 20), see calibration and validation sets. 


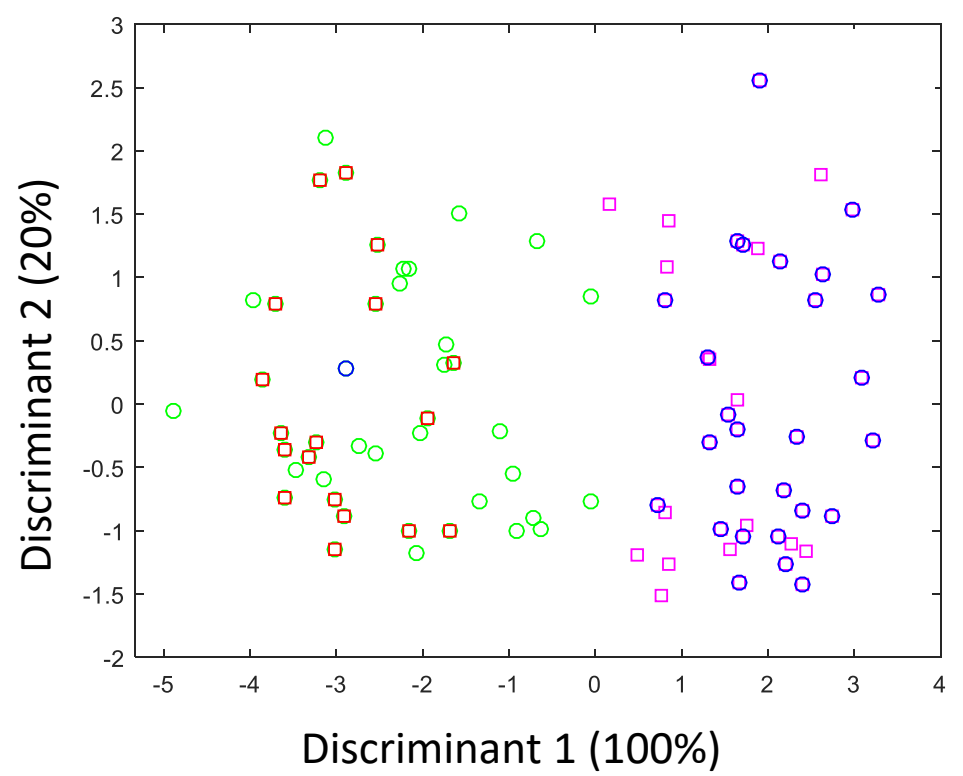

Figure 20. Supervised procedure use to segregate between mouldboard and chisel in tractor 8345R: calibration and validation sets (red and green for mouldboard, and blue and magenta for chisel implements respectively).

Figure 21 provides as an example the distribution along the season of the daily duration of use in tractor 7320 , together with the type of task assigned each day according to the previous indicated procedure.
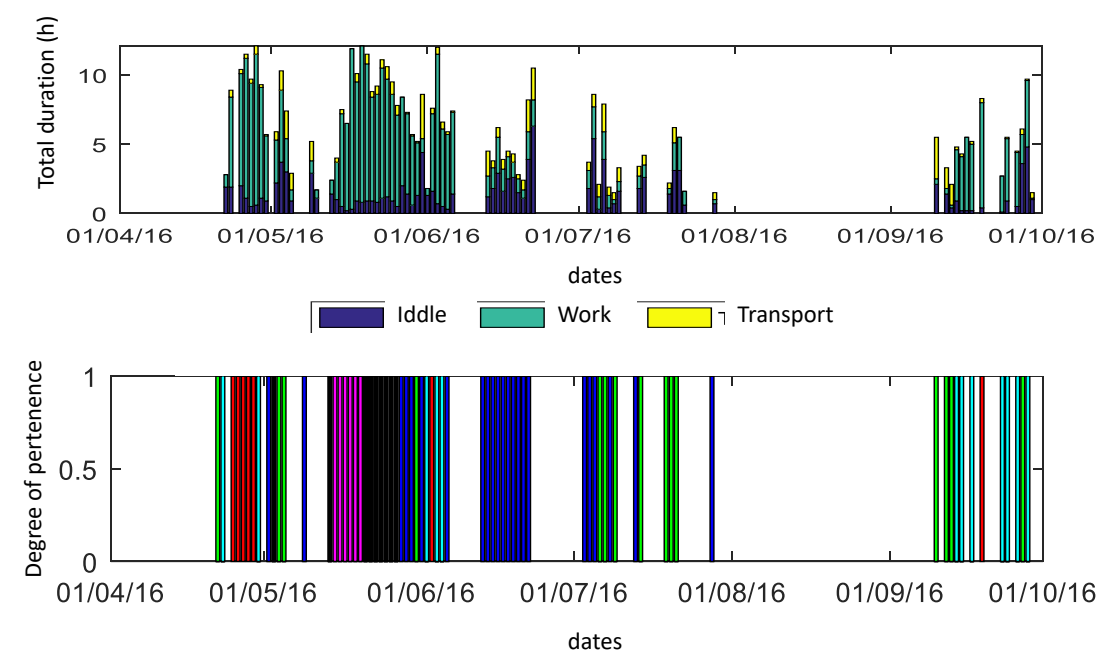

\begin{tabular}{|c|c|c|c|c|c|}
\hline cluster 1 & cluster 2 & ] cluster 3 & cluster 4 & cluster 5 & cluster 6 \\
\hline $\begin{array}{c}\text { Transport at } \\
\text { harvest } 1\end{array}$ & $\begin{array}{l}\text { Light hauling } \\
\text { with semi- } \\
\text { chisel }\end{array}$ & Mower_1 & $\begin{array}{l}\text { heavy hauling } \\
\text { with semi- } \\
\text { chisel }\end{array}$ & Mower_2 2 & $\begin{array}{c}\text { Transport at } \\
\text { harvest } 2\end{array}$ \\
\hline
\end{tabular}

Figure 21. Example of task distribution along the season, as well as daily tractor use duration for 7230 R.

As a consequence of the above mentioned procedure for both $8345 \mathrm{R}$ and $7230 \mathrm{R}$ tractors together with the actual widths of the implements provided by the farmers, it was possible to quantify machine performance (ha h1) as well as fuel consumption pattern (1 ha-1). Moreover, a clear misuse of the tractors was found concerning the selection of counterweights, as well as a poor and excessively variable slippage (generally below 4\%). Also an improper employment of the differential blockage was found for mouldboard tasks. Fuel consumption in the case of tractor $8345 \mathrm{R}$ was validated with the direct data of the farmer as stated in Table 9. 
Figure 22 shows the variability in fuel consumption for each period of 5 hours versus ground speed in the case of tractor $8345 \mathrm{R}$. A main feature that arises from this representation is the large differences obtained for the same task in different plots. Therefore there is a large knowledge to be gained when systematically aplying the unsupervised identification of tasks that makes use of of multivariate cloud-contained tractor data.

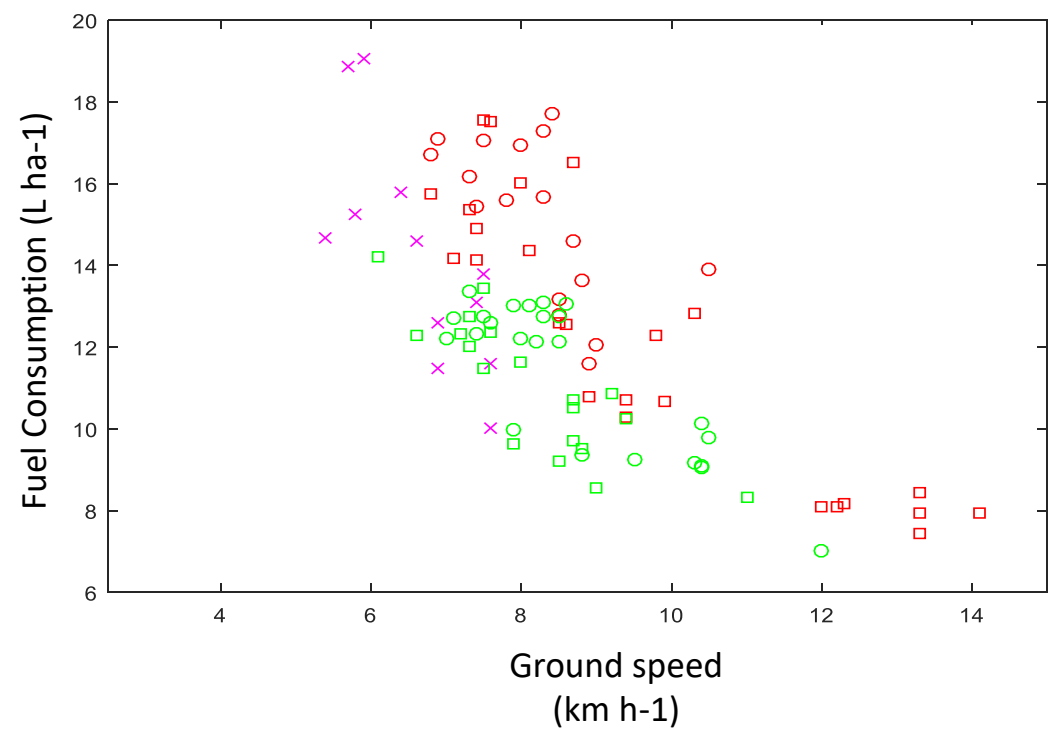

Figure 22. Fuel consumption for each period of 5 hours versus ground speed in the case of tractor 8345R.

Table 9. Tractor task performance $\left(\mathrm{ha} \mathrm{h}^{-1}\right)$, and fuel consumption $\left(1 \mathrm{~h}^{-1}\right.$ and $\left.1 \mathrm{ha}^{-1}\right)$ for $8345 \mathrm{R}$. Total fuel consumption along the season $(485 \mathrm{~h})$ was 215881.

\begin{tabular}{|c|c|c|c|c|c|}
\hline & PTO activated & $\begin{array}{c}\text { Chisel } \\
\text { Light hauling }\end{array}$ & $\begin{array}{c}\text { Chisel } \\
\text { Heavy hauling } \\
\end{array}$ & $\begin{array}{l}\text { Mouldboard } \\
\text { Light hauling }\end{array}$ & $\begin{array}{c}\text { Mouldboard } \\
\text { Heavy hauling }\end{array}$ \\
\hline $\begin{array}{l}\text { Task performance } \\
\left(\mathrm{ha} \mathrm{h}^{-1}\right)\end{array}$ & 3.32 & 4.82 & 4,11 & 2,94 & 3,12 \\
\hline $\begin{array}{l}\text { Fuel consumption } \\
\text { under work }\left(1 \mathrm{~h}^{-1}\right)\end{array}$ & 45.3 & 38.3 & 43,48 & 41,04 & 47,38 \\
\hline $\begin{array}{l}\text { Fuel consumption } \\
\text { under work }\left(1 \mathrm{ha}^{-1}\right)\end{array}$ & 13.64 & 7.95 & 10,58 & 13,97 & 15,16 \\
\hline $\begin{array}{l}\text { Total fuel } \\
\text { consumption under } \\
\text { work }\left(\mathrm{l} \mathrm{h}^{-1}\right)\end{array}$ & 46.49 & 40.03 & 44,4 & 44,32 & 48,43 \\
\hline $\begin{array}{l}\text { Total fuel } \\
\text { consumption }\left(1 \mathrm{ha}^{-1}\right)\end{array}$ & 14.00 & 8.31 & 10,80 & 15,09 & 15,50 \\
\hline Work duration (h) & 60 & 125 & 85 & 95 & 120 \\
\hline $\begin{array}{l}\text { Total fuel (l) } \\
\text { consumption (l) }\end{array}$ & 2789 & 5003 & 3774 & 4210 & 5812 \\
\hline Aprox. surface (ha) & 204 & 630 & 357 & 301 & 383 \\
\hline $\begin{array}{l}\text { Field validated fuel } \\
\text { consumption under } \\
\text { work }\left(1 \mathrm{ha}^{-1}\right)\end{array}$ & 18.63 & & & & \\
\hline
\end{tabular}




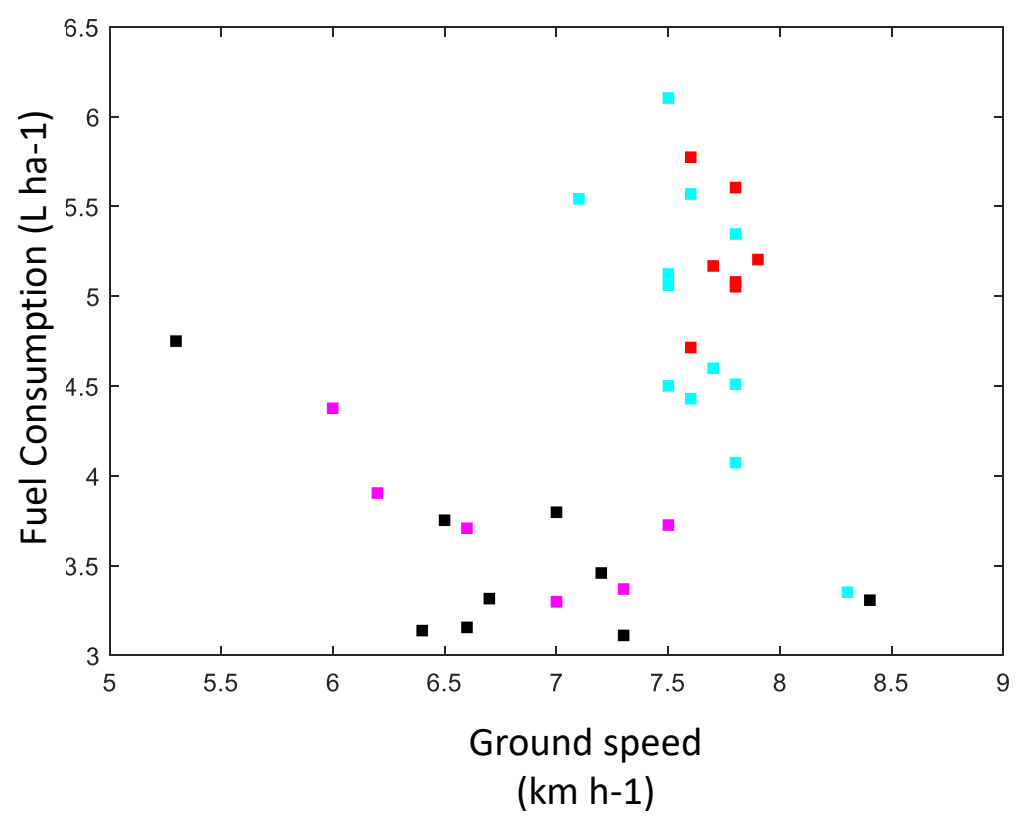

Figure 5. Fuel consumption for each period of 5 hours versus ground speed in the case of tractor 8345 R.

Table 10 and Table 11 present the percentage of time the tractor worked under different slippage conditions for tractor $8345 \mathrm{R}$ and $7230 \mathrm{R}$. The bold and red number indicates the median slippage value for each task.

Table 10. Percentage of time in which tractor $8345 \mathrm{R}$ worked under increasing slippage conditions.

\begin{tabular}{cccccc}
\hline & $\begin{array}{c}\text { PTO } \\
\text { activated }\end{array}$ & $\begin{array}{c}\text { Chisel } \\
\text { Light hauling }\end{array}$ & $\begin{array}{c}\text { Chisel } \\
\text { Heavy } \\
\text { hauling }\end{array}$ & $\begin{array}{c}\text { Mouldboard } \\
\text { Light hauling }\end{array}$ & $\begin{array}{c}\text { Mouldboard } \\
\text { Heavy hauling }\end{array}$ \\
\hline P1: $0-2 \%$ & 50.12 & 24.59 & 17.36 & 7.45 & 9.53 \\
P2: $2-4 \%$ & 24.10 & 28.70 & 24.26 & 9.86 & 10.25 \\
P3: $4-6 \%$ & 11.17 & 19.98 & 14.08 & 11.83 & 11.74 \\
P4: $6-8 \%$ & 7.18 & 10.92 & 9.71 & 12.54 & 13.01 \\
P5: $8-10 \%$ & 2.12 & 5.39 & 7.51 & 11.95 & 13.18 \\
P6: $10-12 \%$ & 0.51 & 2.95 & 6.19 & 11.10 & 10.30 \\
P7: $12-14 \%$ & 0.51 & 1.66 & 4.34 & 8.65 & 4.92 \\
P8: $14-16 \%$ & 0.10 & 0.74 & 3.12 & 6.42 & 3.84 \\
P9: $16-18 \%$ & 0.10 & 0.19 & 2.08 & 4.21 & \\
\hline
\end{tabular}

Table 11. Percentage of time in which tractor 7230R worked under increasing slippage conditions.

\begin{tabular}{ccccccc}
\hline & semi-chisel & Transport 1 & Mower 1 1 & Transport 2 & Mower 2 & semi-chisel \\
\hline $0-2 \%$ & 48.7 & 41.1 & 78.7 & 51.7 & 69.9 & 53.2 \\
$2-4 \%$ & 28.9 & 16.7 & 9.6 & 20.9 & 10.8 & 26.3 \\
$4-6 \%$ & 11.7 & 9.9 & 2.9 & 8.4 & 3.8 & 9.5 \\
$6-8 \%$ & 3.8 & 7.4 & 1.8 & 5.3 & 2.7 & 4.1 \\
$8-10 \%$ & 1.6 & 4.3 & 1.3 & 1.3 & 1.5 & 1.2 \\
$10-12 \%$ & 0.8 & 2.3 & 1.1 & 1.0 & 1.3 & 0.8 \\
$12-14 \%$ & 0.3 & 1.1 & 0.3 & 0.6 & 1.1 & 0.3 \\
$14-16 \%$ & 0.3 & 0.7 & 0.0 & 0.0 & 0.9 & 0.1 \\
$16-18 \%$ & 0.1 & 0.4 & 0.0 & 0.3 & 0.4 & 0.1 \\
$>18 \%$ & 3.7 & 16.1 & 4.3 & 10.4 & 7.6 & 4.3 \\
\hline
\end{tabular}




\section{Conclusions}

In accordance with the previous results, an action plan has been established in 4 phases for each tractor. For tractor $8345 \mathrm{R}$ it is proposed: 1) Reducing the idling time in hauling tasks to half the current value (50\%), that is to say, 200 haul hours, $100 \mathrm{~h}$ are idling and reduced to $50 \mathrm{~h}$ (given that the average consumption at idle is $3.84 \mathrm{l}$ $\mathrm{h}^{-1}$, in 50 hours you would save about 2001 of diesel fuel). 2) To teach the farmer how to properly use the electronic engine management during task, which according to the available technical studies could lead to a $20 \%$ reduction in fuel consumption at work; in this case amounting to $81 \mathrm{~h}-1$ (for 10\% reduction) in 400 hours of mouldboard and chisel, that is, 32001 of fuel that could be saved for tractor $8345 \mathrm{R}$ during a season. 3) Improving the traction conditions so that the median slippage ( $50 \%$ of the time) is between 6 and $8 \%$ and its variability is reduced (this means studying the counterbalance with the concession and learning how to use the differential lock reasonably).4) To carry out a second round of indirect estimates, such as areas worked to see if they fit with the farm's data as a validation test for the proposed procedure.

In the case of tractor 7230R, the recommendations can be summarised as: 1) the improvement in terms of consumption does not seem very relevant (on average it is around $51 \mathrm{ha}^{-1}$ ) since the tractor is being used at lowmedium power levels: always $<55 \%$ load and $<1800 \mathrm{rev}$ min-1). 2) It is necessary to reconsider the ballasting procedures and the use of the front traction due to inadequate slippage values. 3) Mowing without front traction has shown the least consumption compared to the activated front traction: $2.51 \mathrm{ha}^{-1}$ compared to $3.51 \mathrm{ha}^{-1}$. 3) May and June are the months of maximum activity ( $>10 \mathrm{~h}$ daily), therefore the use of optimization procedures for task sequence seems a promising tool to improve efficiency.

\section{Acknowledgements}

The authors would like to thank John Deere Ibérica in the persons of Miguel Langens and Belén Luque, and JD Dealers: Agritrasa (Sara Hervas), and Víctor Perez Agrícola S.L. (Xabier de Miguel) for making this research feasible.

\section{References}

Barreiro, P. 2014. Qué se puede esperar de la agricultura digital. VIDA RURAL 388, suplemento MAQ pp:5056

Yong, Liang, et al. "The main content, technical support and enforcement strategy of digital agriculture." Geospatial Information Science 5.1 (2002): 68-73.

Blank, Sebastian, et al. "iGreen: A ubiquitous dynamic network to enable manufacturer independent data exchange in future precision farming." Computers and electronics in agriculture 98 (2013): 109-116.

Gansemer, Sebastian, et al. "Machine-to-machine communication for optimization of information chain in agricultural business." Intelligent Data Acquisition and Advanced Computing Systems (IDAACS), 2013 IEEE 7th International Conference on. Vol. 1. IEEE, 2013.

J. Lenz, R. Landman, and A. Mishra. "Customized Software in Distributed Embedded Systems: ISOBUS and the Coming Revolution in Agriculture". Agricultural Engineering International: the CIGR Ejournal. Manuscript ATOE 07 007. Vol. IX. July, 2007

Schuster, Edmund W., et al. "Machine-to-machine communication for agricultural systems: An XML-based auxiliary language to enhance semantic interoperability." Computers and electronics in agriculture 78.2 (2011): 150-161. 


\section{Topic 4: Robotics and sensor technology (RM)}

Research and development of mechatronic and (semi-autonomous) robotic systems and machine vision for agricultural applications, from breeding and seeding to monitoring in primary production and post-harvest processing. Contributions may include sensing technology such as 3D vision, hyperspectral imaging, spectroscopy, acoustics, use of satellites and drones, classification techniques including use of artificial intelligence for e.g. phenotyping and 3D reconstruction of plants, animals and environment.

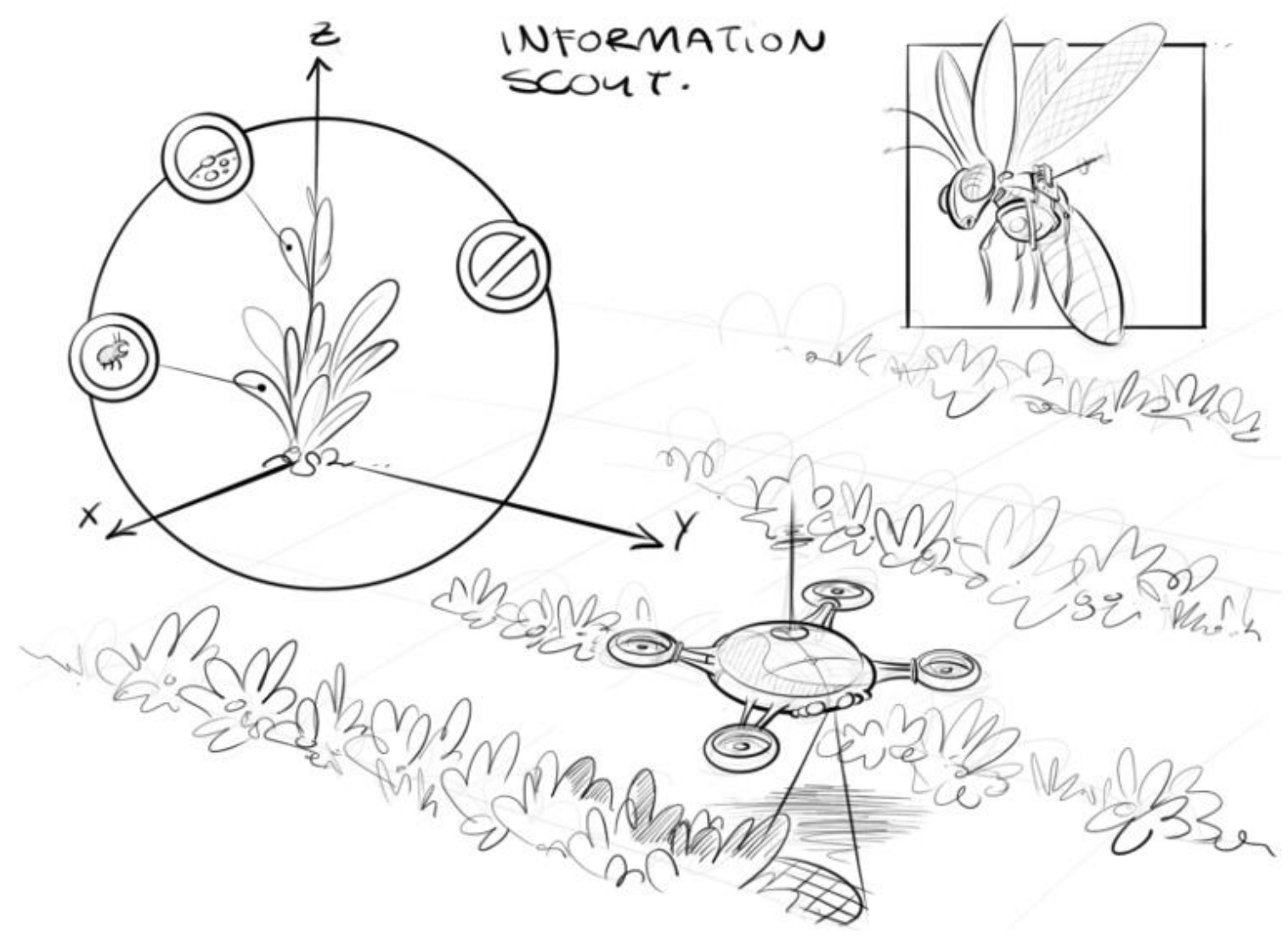




\title{
Vibration Analysis and Canopy Adaptation of Citrus Tree for an Efficient Harvesting with Canopy Shaker Technology
}

\author{
Sergio Castro-Garciaa,", Rafael R. Sola-Guirado ${ }^{a}$, Gregorio L. Blanco-Roldán ${ }^{a}$, Antonio J. Serrano ${ }^{b}$, \\ Juan José Garcés Iniesta ${ }^{b}$, Emilio Soria Olivas ${ }^{b}$, Jesús A. Gil-Ribes ${ }^{a}$ \\ ${ }^{\text {a }}$ Department of Rural Engineering, University of Cordoba, Cordoba, 14071, Spain \\ b IDAL, Intelligent Data Analysis Laboratory, Universidad de Valencia, Spain \\ * Corresponding author. Email: scastro@uco.es
}

\begin{abstract}
The mechanical harvesting of juice oranges can contribute to enhance profitability of farms and solve the problem of labour availability. The growing demand of the juice market generates the need for more efficient harvesting system. The canopy shaker systems are a current technology used to detach the fruit from the tree by means of the interaction of machine rods with the tree canopy. Since the most of citrus production is hand harvested, a mutual adaptation between machine and tree is requires for an efficient result. The objective of this work is to establish recommendations to reach high values of fruit detachment efficacy able to eliminate a sequent hand harvesting process. Field tests were carry out with a lateral tractor-drawn canopy shaker in four commercial plots of sweet orange in south of Spain. The canopy vibration during the harvesting process was measured with a set of triaxial accelerometer sensors with a datalogger placed on bearing branches in 90 trees. The vibration process, fruit production and branch properties were analysed. Vibration parameters showed a large variability in different points into the tree canopy. The harvesting efficiency values were reduced from an average value of $85 \%$ in zones of canopy in contact with rods to a mean value of $27 \%$ in zones without direct contact. The improvement of fruit detachment efficiency is possible if both hedge and machinery were mutually adjusted. The hedge should be training to facilitate the rods access and placing the fruit production on the outside of the canopy. The machine, working at mean frequency value of $4.1 \mathrm{~Hz}$, must to produce a high level of resultant acceleration $\left(100-200 \mathrm{~ms}^{-2}\right)$ in the branches during a period of time higher than $0.921 \mathrm{~s}$ to reach a $100 \%$ fruit detachment.
\end{abstract}

Keywords: Citrus sinensis (L.) Osbeck, mechanical harvesting, acceleration, vibration time, OneR

\section{Introduction}

The Spanish citrus production represents over $50 \%$ of the cultivated area in the European Union, being the seventh producer country worldwide with 3.2 million tons (FAOSTAT, 2016). Citrus orchards are primarily oriented for the fresh market, although fruit production for industrial transformation increase interest when fruit price is low, and famer's profitability is compromised due to high production costs. In Spain, $80 \%$ of citrus orchards have less than 5 ha (INE, 2016) and is challenging with high operating costs. The mechanical harvesting plays an important role since it is one of the most important expenses for citrus farmers (Brotons-Martínez et al. 2018). For example, in Andalusia during the 2014-15 season, the manual harvesting represented $26 \%$ of the total average cost in orange and 34\% in tangerine (Junta de Andalucía, 2015).

The application of the mechanical harvesting of citrus for juice could provide a reduction of the costs by $50 \%$ whilst increasing the labour productivity by 10X under Florida conditions (Roka and Hyman 2012). However, its implantation in the citrus sector is missing yet, because adapted orchards and efficient machines are required. Thus, there are limited number of available commercial machines for mechanical harvesting of citrus, therefore orchards are not adapted to the conditions of those machines. This need of adaptation gives rise to a lack of orientation of the Spanish citrus sector to the industrial transformation since the manual harvesting provides low or no profitability.

Canopy shaker systems are a harvesting technology that has been successfully developed for juice fruits for last three decades, mainly in Florida (Sanders, 2005). The canopy vibration is generated by the contact of the rods of the machine with the tree. These rods have an alternating or rotational movement that penetrate and compress the tree canopy, producing impulse excitations on branches and fruits. The forced vibration of the tree canopy causes the fruit detachment. Nowadays, the main criteria for the design and improvement of canopy shaker systems are minimum tree (Pu et al. 2018) and fruit damage (Ortiz et al., 2011) with maximum fruit removal efficiency (Savary et al. 2010). Both machine regulations and tree hedge training must be studied and adapted to improve the mechanization of citrus plantations.

The objective of this study was to analyse and predict the vibration process of fruit bearing branches under field conditions with canopy shaker systems in order to increase the fruit detachment process. Both machine interaction with tree canopy and canopy hedge were analysed to enhance a vibration process to harvest citrus for juice. 


\section{Materials and Methods}

The mechanical harvesting tests were carried out in Cordoba (Spain) in May 2017 during the sweet orange harvest season (Citrus sinensis (L.) Osbeck cv. 'Valencia') for juice production, when flowering was ending and before the natural immature fruitlets fell in June. Trees were planted into wide hedge, over $0.4 \mathrm{~m}$ ridges and had wide row distances to allow machine manoeuvrability and the use of canopy shaker harvesters (Figure 1). Table 1 shows the characteristics of the four harvesting plots mechanically harvested.

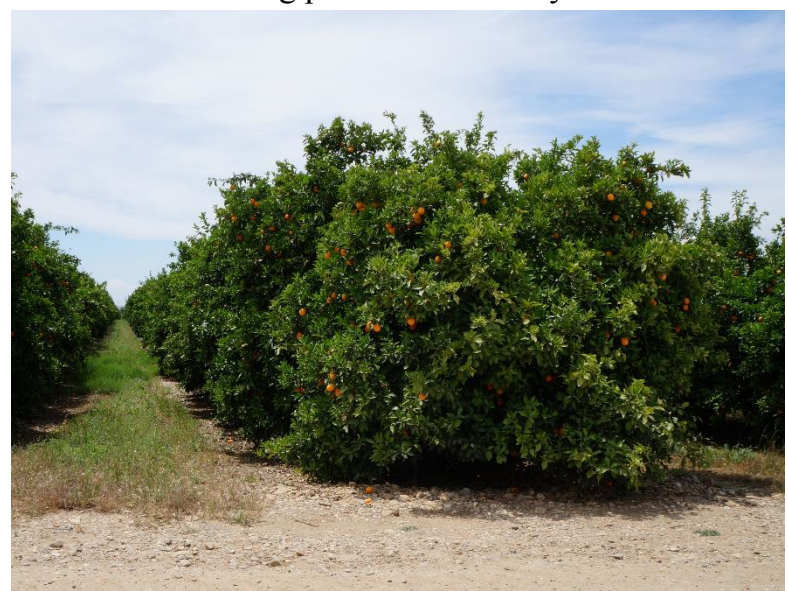

Figure 1. Sample of a wide hedge of sweet orange tree used to mechanical harvesting.

Table 1. Characteristics of citrus orchards mechanically harvested with canopy shaker system.

\begin{tabular}{ccccc}
\hline & Plot 1 & Plot 2 & Plot 3 & Plot 4 \\
\hline Date planted & 2006 & 2005 & 2007 & 2005 \\
Trees per ha & 440 & 330 & 440 & 330 \\
Tree distance (m) & $7 \times 3$ & $7 \times 4$ & $7 \times 3$ & $7 \times 4$ \\
Hedge height (m) & 4.0 & 4.0 & 4.3 & 4.4 \\
Hedge width (m) & 3.9 & 4.1 & 4.5 & 4.6 \\
\hline
\end{tabular}

The mechanical harvesting was carried out with a tractor-drawn continuous canopy shaker system (Oxbo 3210, Byron, New York) working under regular conditions featuring a ground speed range between 1 and $1.5 \mathrm{~km} \mathrm{~h}-1$, a vibration frequency close to $4.5 \mathrm{~Hz}$ and removing the fruit into the ground. The harvesting tests were carried out ensuring the contact of machine shaker system with tree canopy (Figure 2).

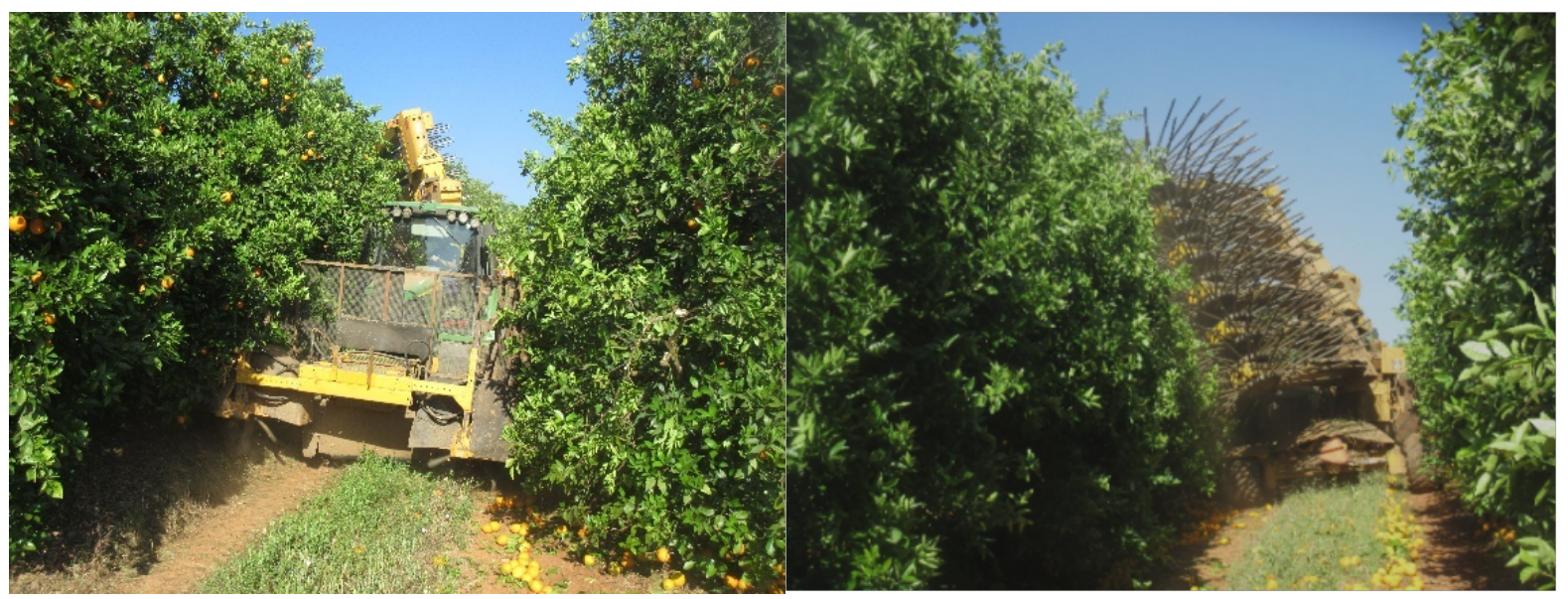

Figure 2. Tractor-drawn continuous canopy shaker system (Oxbo, 3210) used in citrus harvesting tests.

A total of 90 fruit-bearing branches with mature fruits, distributed in different positions of the tree canopy, were selected. The branches were located between 0.75 and $2.15 \mathrm{~m}$ high from the ground and between 0.1 to 2.5 $\mathrm{m}$ from the line of the trunks to the outside of the canopy, to ensure that it was a representative harvestable part of the hedge. In each branch, a measurement point was determined close to the fruits, where the diameter of the branch, its position into the canopy, as well as the number of fruits before and after harvesting. Fruit detachment 
was determined according to the number of fruits removed in each branch before and after harvesting. Fruit detachment was calculated as a percentage or as a categorical variable, with special interest where $100 \%$ of the fruits of the branch were removed.

The branch vibration measurement was recorded with a set of triaxial MEMS accelerometer sensors (Gulf Coast Data Concepts LLC X200-4, Waveland, MS) with a measurement range of $\pm 200 \mathrm{~g}, 16$-bit resolution, a sensitivity of $0.06 \mathrm{~m} \mathrm{~s} \_2$ and a sampling frequency of $400 \mathrm{~Hz}$. Figure 3 shows the placement of the sensor in the branch.

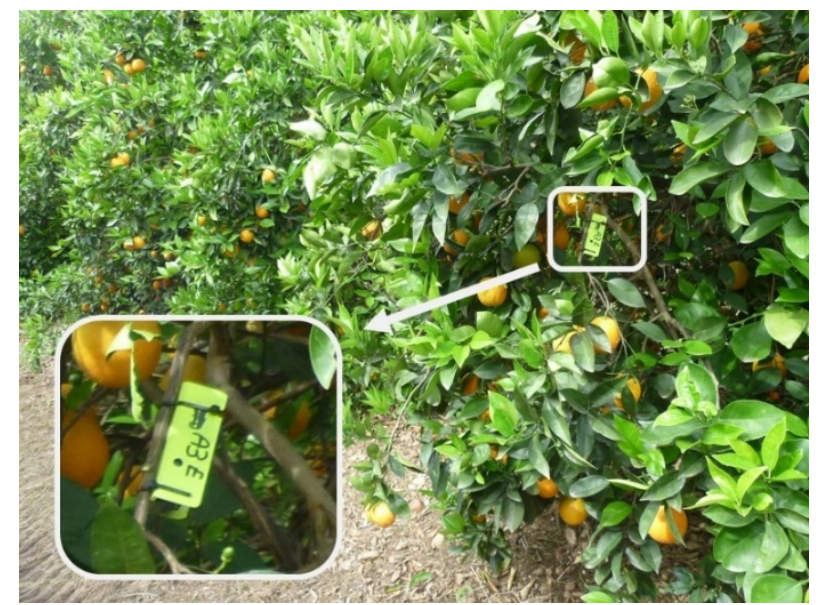

Figure 3. Placement of the acceleration sensor on the fruit-bearing branch on the outside of the hedge.

The analysis of the acceleration signals was performed in time domain using $\mathrm{R}$ open software (R Core Team, 2016) and in time domain using NVGate v8.0 software, with a Fast Fourier Transformation with 401 lines in a frequency range of $0-156.2 \mathrm{~Hz}$ with a $0.3905 \mathrm{~Hz}$ resolution. In the time domain, the resultant acceleration value $\left(\mathrm{A}_{\mathrm{r}}\right)$, that is, the vector sum of the three measurement axes $\left(\mathrm{a}_{\mathrm{x}}, \mathrm{a}_{\mathrm{y}}, \mathrm{a}_{\mathrm{z}}\right)$ on each accelerometer was performed using Eq. (1).

$$
A_{r}=\sqrt{a_{x}^{2}+a_{y}^{2}+a_{z}^{2}}
$$

Figure 4 shows a sample of resultant acceleration measured in branch. In the time domain, the vibration variables were:

- Vibration time ( $\mathrm{T}_{\mathrm{vib}}$ ): elapsing time between the first and last event with an $\mathrm{A}_{\mathrm{r}}$ value from $20 \mathrm{~ms}^{-2}$ to values up to $600 \mathrm{~ms}^{-2}\left(\mathrm{~T}_{\text {vib_20 }}, \mathrm{T}_{\text {vib_30 }}, \mathrm{T}_{\text {vib_40 }}, \mathrm{T}_{\text {vib_50 }}, \mathrm{T}_{\text {vib_100, }} \mathrm{T}_{\text {vib_200, }} \mathrm{T}_{\text {vib_300, }} \mathrm{T}_{\text {vib_400, }} \mathrm{T}_{\text {vib_500, }} \mathrm{T}_{\text {vib_>600 }}\right.$ ).

- Vibration time in a range of $A_{r}\left(T_{A r}\right)$ : duration of exposure of branch to a range of $A_{r}$ values $\left(T_{A r} 20-30\right.$, $\mathrm{T}_{\mathrm{Ar} \_30-40}, \mathrm{~T}_{\mathrm{Ar} \_40-50}, \mathrm{~T}_{\mathrm{Ar} \_} 50-100, \mathrm{~T}_{\mathrm{Ar} \_100-200}, \mathrm{~T}_{\mathrm{Ar} \_200-300,} \mathrm{~T}_{\mathrm{Ar} \_}$300-400, $\left.\mathrm{T}_{\mathrm{Ar} \_400-500}, \mathrm{~T}_{\mathrm{Ar}}>600\right)$.

- Mean peak acceleration $\left(\mathrm{A}_{\mathrm{pk}}\right)$ : the mean value of the first 10 peak values of $\mathrm{A}_{\mathrm{r}}$.

In the frequency domain, the vibration variables were:

- Frequency: number of cycles per second (Hz) of the rod movement into the canopy.

- RMS acceleration (ARMS): root mean square value (RMS) of acceleration value for each accelerometer axis for the frequency of vibration. 


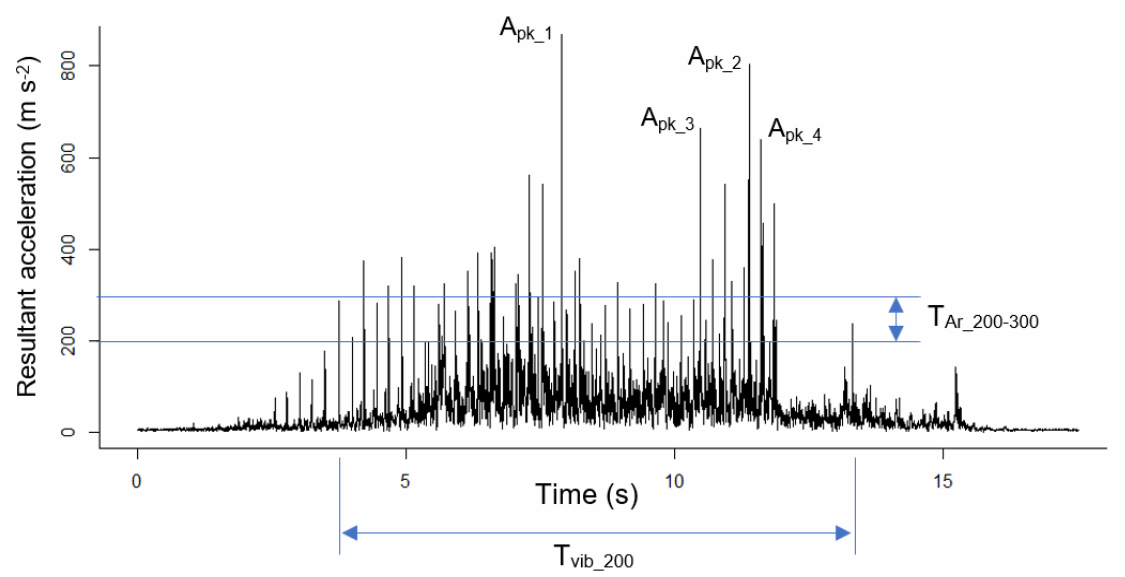

Figure 4. Example of resultant acceleration $\left(A_{r}\right)$ in the time domain measured in a fruit-bearing branch. $T_{\text {vib_200: elapsing }}$ time between the first and last event with an $A_{r}$ value of $200 \mathrm{~ms}^{-2} . T_{A r} 200-300$ : Vibration time in a range of $A_{r}$ between 200 and $300 \mathrm{~ms}^{-2}$. $A_{\mathrm{pk} \_n}$ : $n$ peak value of $A_{r}$.

The statistical analysis was focused on predict the fruit detachment according to vibration variables and branch measurements as predicted parameters. It was due to the importance of fruit detachment to propose suggestions both for machine design and regulation and for tree training to enhance the mechanical harvesting process.

\section{Results and Discussion}

The branch measurements were classified into 20 geometric sectors into the canopy. Each sector is within an interval of $0.5 \mathrm{~m}$ from the trunk line and height from the ground. The distance to the trunk was ranged from 0 to $2.5 \mathrm{~m}$ and the distance to the ground from 0.5 to $2.5 \mathrm{~m}$. The tested branches had a mean diameter of $7.9 \pm 2.4 \mathrm{~mm}$ (mean $\pm \mathrm{sd}$ ) and fruit detachment efficiency was $69.1 \pm 40.7 \%$. Figure 5 shows the distribution of vibration parameters, fruit detachment and branch diameter in the tree canopy. As it was expected, these variables have their highest values in the contact zone of the rod with the tree canopy. In this way, Whitney (1999) recommended to limit the hedge size so the rods enter the entire canopy width and height to reach high fruit detachment efficiency values. In this study, the fruit detachment efficiency values were reduced from an average value of $85 \%$ in zones of canopy in contact with rods to a mean value of $27 \%$ in zones without direct contact.

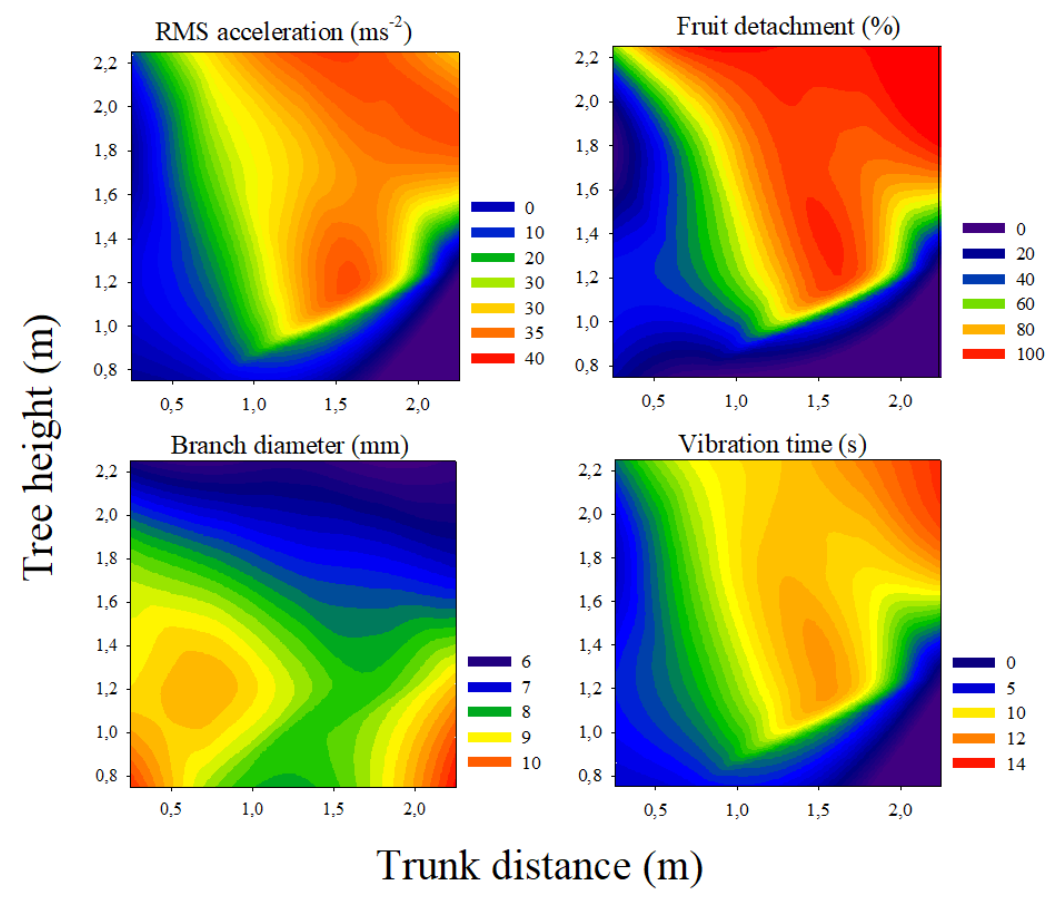

Figure 5. Distribution of acceleration, fruit detachment, branch diameter and vibration time in the tree canopy under mechanical harvesting with canopy shaker. 
Previous studies reported the location of the remain fruit after the harvesting fruit in the tree canopy (Whitney, 1999). The branch diameters showed were reduced from the top of the canopy to the ground. The branch vibration was characterized by a frequency value of $4.1 \pm 0.5 \mathrm{~Hz}$ and with low variation, in the range recommended for the work of these machines (Castro-Garcia et al., 2017). However, the distribution of ARMs, vibration time and fruit detachment variables showed a canopy distribution similar to interaction of the rod machine with the canopy. Similar ARMS values were reported by Savary et al. (2010) where highest resultant acceleration values where located at the end of the branches. The vibration time was identified as an import parameter to detach fruit with canopy shaker. Field tests with three canopy shaker, Castro-Garcia et al 2018 recorded the required time to detach fruit ranged from 1.45 to $5.75 \mathrm{~s}$ which could involve limitation to machine ground speed. In fact, Shamshiri et al. (2013) reported a usual ground speed of $1.8 \mathrm{~km} \mathrm{~h}^{-1}$ for canopy shakers. Burns et al. (2005) showed that mechanical harvesting at a ground speed of $3.2 \mathrm{~km} \mathrm{~h}^{-1}$ reduced fruit removal efficiency compared with 2.4 and $1.6 \mathrm{~km} \mathrm{~h}^{-1}$.

The variable distribution in the canopy showed that branches were subjected to different vibration levels and, therefore, different fruit detachment values were obtained. In order to achieve an efficient harvesting process, the objective of the study was to predict the vibrations requirement to get a fruit detachment efficiency with a value of $100 \%$, where a subsequent hand harvesting was not necessary. However, the results generated showed a high linear correlation between variables (Figure 6). The high correlation between variables indicates that fruit detachment prediction could not have a single solution.

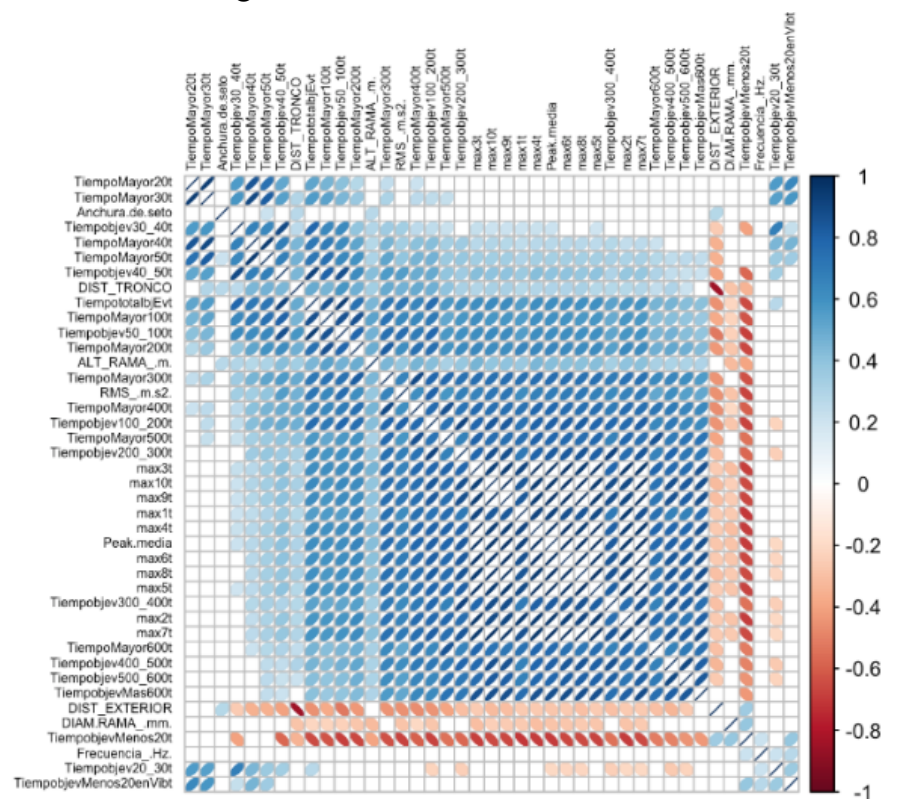

Figure 6. Graphical display of a correlation matrix.

The analysis of the data was focused on discretization of quantitative variables that could discriminate if there was a fruit detachment with value $100 \%$ and with a reasonable success. Due to its simplicity and efficiency, the OneR (one rule) algorithm was used (Holte, 1993). OneR algorithm uses a supervised partition discretization method with equal size intervals, using the information gain property. It is a classification algorithm that searches for a rule for each predictor variable and chooses as the only rule the one that has the lowest total error, that is, the one that best contributes to the predictability of the model, based on the frequency tables. With this analysis, two rules were proposed: a) one to determine if there was any value of fruit detachment and b) another rule to determine if fruit detachment was $100 \%$. Figure 7 shows the combination of two OneR rules with four outcomes possibilities.

a) The optimal rule to determine fruit detachment (hit score of 91.01\%) was the vibration time (s) in a range 40 to $50 \mathrm{~ms}^{-2}\left(\mathrm{~T}_{\text {Ar_ }} 40-50\right)$ :

- If $\mathrm{T}_{\mathrm{Ar} \_40-50}=(0.0228,0.718]$ then fruit detachment $=$ No fruit detachment

- If $\mathrm{T}_{\mathrm{Ar}} 40-50=(0.718,2.24]$ then fruit detachment $=$ Any fruit detachment value

b) The optimal rule to determine $100 \%$ fruit detachment value (hit score of $91.01 \%$ ) was the vibration time (s) in a range 100 to $200 \mathrm{~ms}^{-2}\left(\mathrm{~T}_{\mathrm{Ar}} 100-200\right)$ :

- If $\mathrm{T}_{\mathrm{Ar} \_100-200}=(-0.00327,0.921]$ then fruit detachment $=$ No $100 \%$ fruit detachment 
- If $\mathrm{T}_{\mathrm{Ar} \_} 100-200=(0.921,3.27]$ then fruit detachment $=100 \%$ fruit detachment

The branch vibration process showed four possibilities according to vibration time and resultant acceleration values:

- No fruit detachment: TAr_40-50 $<0.718 \mathrm{~s}$ and TAr_100-200 $<0.921 \mathrm{~s}$.

- Some fruit detachment: TAr_40-50 $>0.718 \mathrm{~s}$ and TAr_100-200<0.921 s.

- $100 \%$ fruit detachment: TAr_40-50 $>0.718 \mathrm{~s}$ and TAr_100-200 $>0.921 \mathrm{~s}$.

- No records for TAr_40-50<0.718 s and TAr_100-200 $>0.921 \mathrm{~s}$.

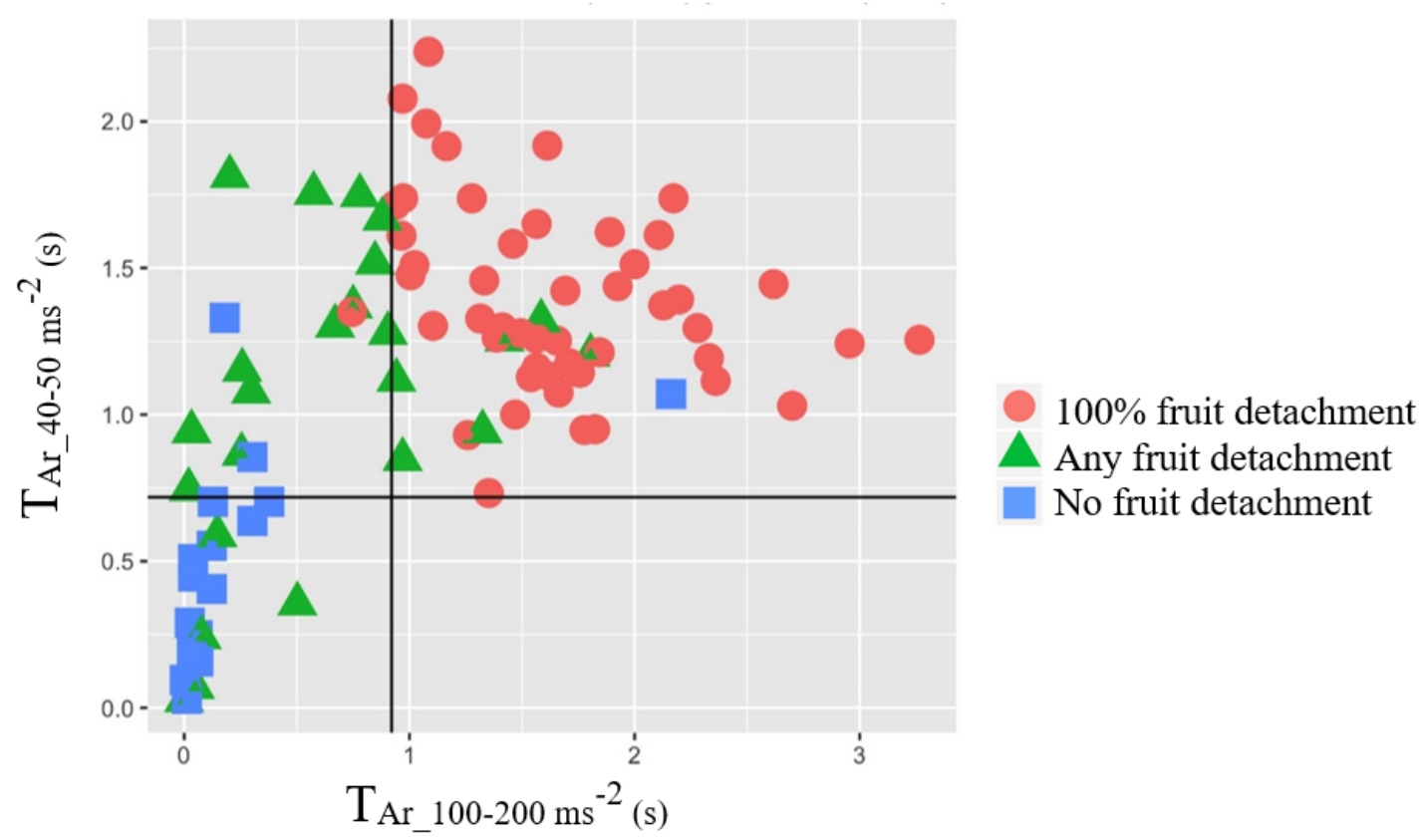

Figure 7. Distribution of fruit detachment values according to vibration time (s) in a range 40 to $50 \mathrm{~ms}^{-2}\left(\mathrm{~T}_{\mathrm{Ar}} 40-50\right)$ and vibration time (s) in a range 100 to $200 \mathrm{~ms}^{-2}\left(\mathrm{~T}_{\mathrm{Ar} \_40-50}\right)$.

\section{Conclusions}

The citrus mechanical harvesting with canopy shaker reported a mean fruit detachment efficiency of $85 \%$ in the direct contact of the rods with the canopy and a mean value of $27 \%$ in areas without direct contact. The improvement of fruit detachment efficiency is possible if both hedge and machinery were mutually adjusted. The hedge should be training to facilitate the rods access and placing the fruit production on the outside of the canopy. The machine, working at mean frequency value of $4.1 \mathrm{~Hz}$, must to produce a high level of resultant acceleration (100-200 ms-2) in the branches during a period of time higher than $0.921 \mathrm{~s}$ to reach a $100 \%$ fruit detachment.

\section{Acknowledgements}

This work has been supported by research projects RTA2014-00025-C05-03 and RTA2014-00025-C05-05 from the National Institute for Agricultural and Food Research and Technology (INIA, Spain) financed by FEDER funds.

\section{References}

Brotons-Martínez, J.M., B. Martin-Gorriz, A. Torregrosa, I. Porras, 2018. Economic evaluation of mechanical harvesting of lemons. Outlook on Agriculture. 47, 44-50.

Burns, J.K., Iii. R.S. Buker, F.M. Roka, 2005. Mechanical harvesting capacity in sweet orange is increased with an abscission agent. HortTechnology. 15(4), $758 \mathrm{e} 765$.

Castro-Garcia, S., G.L. Blanco-Roldán, L. Ferguson, E.J. González-Sánchez, J.A. Gil-Ribes, 2017. Frequency response of late-season 'Valencia' orange to selective harvesting by vibration for juice industry. Biosystems Engineering. 155, 77-83.

Castro-Garcia, S., R.R. Sola-Guirado, J.A. Gil-Ribes, 2018. Vibration analysis of the fruit detachment process in late-season 'Valencia' orange with canopy shaker technology. Biosystems Engineering. 170. 130-137. 
FAOSTAT, 2016. Food and agriculture data. http://www.fao.org/faostat/en/\#data/QC Accessed April 25, 2018.

Holte, R.C. 1993. Machine Learning 11. 63.

INE, 2016. Instituto Nacional de Estadística. Encuesta sobre la estructura de las explotaciones agrícolas. http://www.ine.es Accessed April 25, 2018.

Junta de Andalucia, 2014. Observatorio de precios y mercados. Costes medios de producción. Campaña 20142015. http://www.juntadeandalucia.es/agriculturaypesca/observatorio Accessed April 25, 2018.

Ortiz, C., J. Blasco, S. Balasch, A. Torregrosa, 2011. Shock absorbing surfaces for collecting fruit during the mechanical harvesting of citrus. Biosystems Engineering. 110: 2-9.

$\mathrm{Pu}, \mathrm{Y}$, A. Toudeshki, R. Ehsani, F. Yang, J. Abdulridha, 2018. Selection and experimental evaluation of shaking rods of canopy shaker to reduce tree damage for citrus mechanical harvesting. International Journal of Agricultural and Biological Engineering. 11, 48-54.

R Core Team, 2016. R: A language and environment for statistical computing. R Foundation for Statistical Computing, Vienna, Austria. https://www.R-project.org/.

Roka, F.M., B.R. Hyman, 2012. Mechanical harvesting of sweet oranges for juice processing. Acta Horticulturae, 965. 241-244.

Sanders, K., 2005. Selective picking head for citrus harvester. Biosystems Engineering. 90(3). 279-287.

Savary, S.K.J.U., R. Ehsani, J.K. Schueller, B.P. Rajaraman, 2010. Simulation study of citrus tree canopy motion during harvesting using a canopy shaker. Transactions of the ASABE. 53. 1373-1381.

Shamshiri, R., R. Ehsani, J.M. Maja, F.M. Roka, 2013. Determining machine efficiency parameters for a citrus canopy shaker using yield monitor data. Applied Engineering in Agriculture, 29(1), 33e41.

Whitney, J.D., 1999. Field test results with mechanical harvesting equipment in Florida oranges. Applied Engineering in Agriculture. 15(3). 205-210. 


\title{
PC Based Data Acquisition, Sensor Fusion and Data Synchronisation in Agriculture Based on an Example of Measurement of the Roughness of the Soil Surface using 2D LiDAR
}

\author{
Zoltan Gobor*, Robert Weinfurtner, Christian Rossow and Georg Fröhlich \\ Bavarian State Research Center for Agriculture, Institute for Agricultural Engineering and Animal Husbandry, Am \\ Staudengarten 3, 85354 \\ * Corresponding author. Email: zoltan.gobor@lfl.bayern.de
}

\begin{abstract}
By the developing of a device for fully automated attaching of the supporting wires in hop gardens, a dependency between the area capacity, the efficiency and the roughness of the soil surface was analysed using a simplified 3D model. Since the hop gardens have not been designed for the attaching of the supporting wires by a machine, often prevailed suboptimal conditions during the testing and consequently, the number of outages increased. The malfunctioning was caused by the suboptimal inertial forces of the machine components, as well as due the inadequate sensor technology and suboptimal environmental parameters. In order to be able to assure further improvements, the working conditions were analysed and a hypothesis stated that the driving paths and the related soil roughness affects the area capacity and the quality of the attached wires. The paper highlights the design of a method in which a 2D LiDAR will be used for measuring the configuration of the terrain (fixed lanes) immediately before the application of the device for wire attaching. The problems considering the optimal position of the LiDAR and the data correction based on the measured roll and pitch angles of the LiDAR, as well as the measured accelerations were analysed. The data fusion and synchronisation considering two PC-based data acquisition sources with different sampling rates have been described and the advantages and the disadvantages of such an approach discussed. It is expected that the appropriately acquired sensor data with an adequate resolution on one hand, combined with the information about the outages on the other hand, should provide more detailed insights for defining constrains which will assure achieving optimal area capacities and accordingly support the decision making to choose the optimal strategy concerning the further development of the device.
\end{abstract}

Keywords: LiDAR, PC-based data acquisition, sensor fusion, data synchronisation

\section{Introduction}

State of the art

A prototype of a device for automated attaching of the supporting wires for hops has been developed within the innovation project (Gobor et al., 2012). During the development and the testing of the prototype, introduction of a redundant mobile data acquisition system for further data analysis was recognised as affordable (Gobor 2013, 2015). The idea was oriented toward a solution providing supplementary information considering the system performance, as well as the information according to failures and their causes. In the following project we were involved in the development of a zero-series device for the automated attaching of the supporting wires, as well as in the development of an online diagnostic system implemented on the embedded real-time controller. The results gained from the innovation project as well as the insights gained during the testing of the prototype under field conditions served as backgrounds for the new developments.

As well the farmer as the operator would like to achieve working under optimal conditions while minimizing the mistakes, failures or outages. Such an approach comprises carrying out the task with an optimal speed close to the maximally permitted one. Thus, high area capacity can be reached. Unfortunately, not only the machine and the operator, but the environmental parameters, such as the roughness of the soil surface, the atmospheric conditions, the quality of the wire, dirt, vibration and shock affects the quality of the work. Such type of noise introduced in the automatic control cycle can move the system beyond the steady-state range and cause irregularities. Such irregularities cannot be simply predicted, their observation under real condition is monotonous and time-consuming without any guarantee that the expected failure will occur. Hence, we tried to decompose the problem to measurable elements, in order to find parameters which initiate the irregularities and are responsible for outage of the machine. Based on the experiences from previous project, as well as on the carried out field experiments, the roughness of the soil surface and accordingly the driving condition in the hop garden were defined as the factors which should be investigated.

Simulation using a simplified 3D model of the machine

When using the device under unsuitable environmental conditions (in this case we consider uneven soil surface with positive and negative hindrances), the inertia of individual components installed in the machine, affects the appropriate reaction to the disturbing influences (see Figure 1). In order to be able to analyse the problems in a 
controlled manner simulations of the behaviour of the simplified 3D CAD model on a terrain with positive and negative hindrances using Autodesk Inventor were carried out. Using the simulation model, the behaviour of the machine was checked by specifying defined motion patterns. To make the simulation efficient, logical and user friendly the constraints, simplifications and relationship between input and output conditions needs to be approved. Oscillatory displacements are introduced on the front axle as well as on the rear right wheel of the model (see figure 1) and the results showed that the displacements of the wire reception in the $x$-direction and in the $y$-direction are connected via a linear equation with the displacements on the right rear wheel. The displacement of the $x$-axis has the same sign as the causative displacement when the right rear wheel is displaced. Similarly, several scenarios were carried out approving that there are amplified linear dependencies between the absolute position of the end effector (head for knotting) of the machine and the positive/negative hindrances on the path. Furthermore, important outcomes were the required speeds of the hydraulic cylinders safeguarding the position of the head in the appropriate position in order to assure the wire attaching without outage while moving on the ground with hindrances. For sake of validation of the results and accordingly to confirm the appropriate dimensioning of the hydraulic components, a realistic input speeds still have to be determined so that the simulations can reflect the reality. For this reason, the results must be validated by practical experiments.
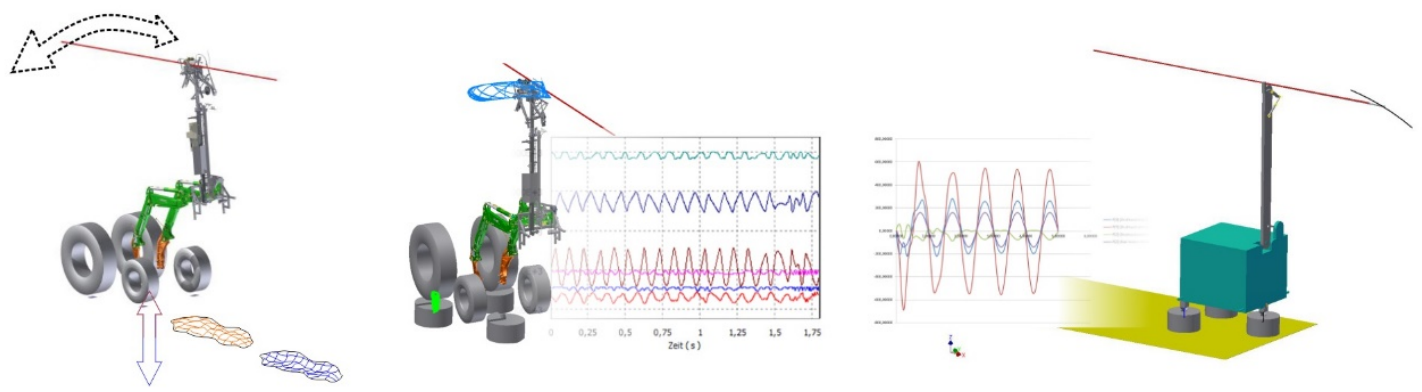

Figure 23. Simulation of the machine behaviour on a terrain with positive and negative hindrances using 3D Model (left: idea; middle: simulation model nr.1; right: simulation model 2).

The analysis of the field trial results (see figure 2) showed a possible dependency of the type and the number of failures on the driving conditions and on the properties of the soil surface (e.g. by driving along fixed paths; frozen vs. muddy paths; soil texture -relation of the sandy, silty, clay components; etc.).

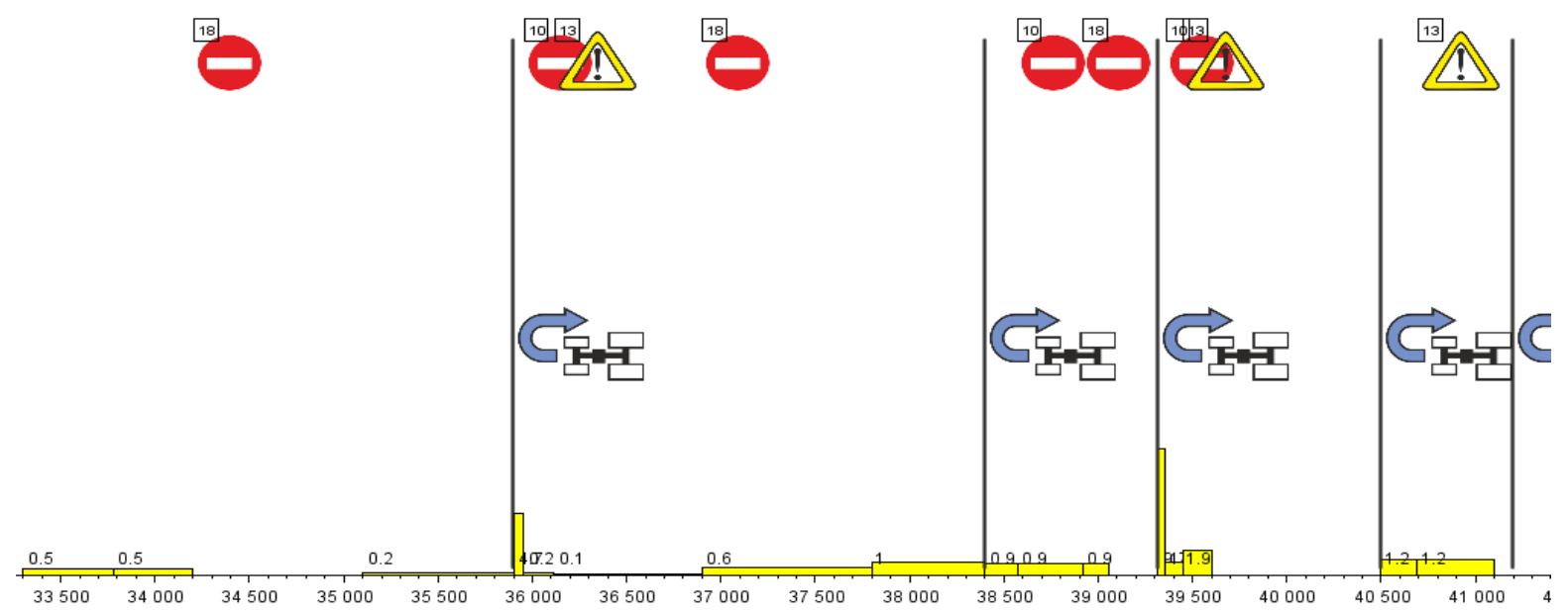

Figure 24. An example of the automated analysis of a field test events and area capacity: manually acquired, saved in excel, analysed in Scilab (field test carried out on $19^{\text {th }}$ January 2017).

By comparing the simulation results with the protocols obtained from field trials it becomes obvious that a deeper analysis of the driving path with exact information about the position and the type of the irregularities could provide aspects for further optimisation.

Application of LiDAR sensor for measurements in agriculture

There are many examples of the application of LiDAR for measurements not only in agriculture in which the 
sensor is used for inspection, object and surface modelling (Van der Linden et al., 2008) or (Ball, 2015). JimenezBerni et al. (2018) used LiDAR for measuring the canopy height as well as ground coverage. For determining the canopy height, the difference between the measured values above the canopy and a point representing the surface or a measurement representing the average can be used. Another LiDAR usage is described by Garrido, et al. (2015) in which measurement at different stage were used to determine the plant development. Synchronous usage of several LiDAR sensors allocated on different positions and thus covering a different field of view (FoV) provide data for accurate object reconstruction, after the data fusion. Seifert et al. (2016) analysed the suitability of a 2D laser scanner for the localisation of the maintenance spots on a pasture. As main advantage the relatively high ground resolution, the non-destructive measurement and the ability to distinguish between the leftovers and the area without vegetation within the sward were recognised. The output of a $2 \mathrm{D}$ laser scanner comprise the distance between the rotating laser head and the surface from which the beam is reflected and the characteristic energy received from the reflected beam, depending on the surface properties. For 3D data analysis the third dimension is provided through a translatory motion of the LiDAR in the longitudinal direction. For the analysis it is important to distinguesh between a 3D point cloud and triangulated data.

\section{Objectives}

A truly multidisciplinary approach to system design requires not only simulation capability within domains of mechanical, control, electrical engineering and embedded computing but also synergistically combines these disciplines (MacCleery and Kassas, 2008). The functionality of the modern machines relies on an ever greater share of the control software. Because of the increasing complexity the control software must be tested at an early stage of development to avoid risk of errors in the field. According to Benet (2017) the LiDAR is not sensitive to lighting variation. Furthermore S. Van der Linden et al. (2008) showed that the sensor depth measurement was not affected by the soil texture and soil moisture content. In order to be able to carry out the validation of our sensor constellation, we used these hypotheses and in the first instance carried our measurement on the chosen test field under different environmental conditions. The fusion information from acceleration and inclination sensor are important while according to Benet (2017), definition or rather calculation of the exact position is required in order to achieve the desired accuracy. Benet (2017) furthermore addressed the problems of vibrations which can under certain circumstances cause misdetection. Consequently, the information about the acceleration of the sensor head in such situation could be very valuable.

\section{Materials and Methods}

Sensors, hardware and configuration

The idea was to use a 2D laser scanner for the analysis of the soil profile in dynamical measurements. For this purpose an available 2D laser scanner (Pepperl+Fuchs R2000) with a TCP/IP as communication protocol was used. Additionally, with a NI cDAQ 1972 (National instruments, 2017) a specially adapted PC based data acquisition system was built, comprising of an inclination (Pepperl+Fuchs INY360D) and an acceleration sensor (Pepperl+Fuchs ACY04), to provide additional data about the angular position, velocity and acceleration of the laser scanner. Thus, correction of the data measured with the laser scanner can be calculated using the inverse kinematic approach and accurate measurement can be assured while the carrier vehicle continuously moves along the path containing hindrances causing positive and negative changes in pitch and roll angles (see Figure 3). As carrier we use a tractor John Deere 544, with following characterisitcs: $14.9 \mathrm{~kW} / 20$ PS; length $1905 \mathrm{~mm}$; width $1110-1167 \mathrm{~mm}$; wheelbase $1280 \mathrm{~mm} ; 18 \times 8.50-8$ tyres at the front axle and $26 \times 12.00-12$ tyres for the rear axle. The data acquisition of the inclination and the acceleration sensors was based on the odometry, triggered by a rotary encoder (Hengstler RI36) mounted trough adequate transmission to the rear left wheel of the carrier vehicle. Main characteristics of the sensors are listed in the table 1. 
Table 12. Main characteristics of the sensors.

2D LiDAR sensor - Pepperl + Fuchs R2000

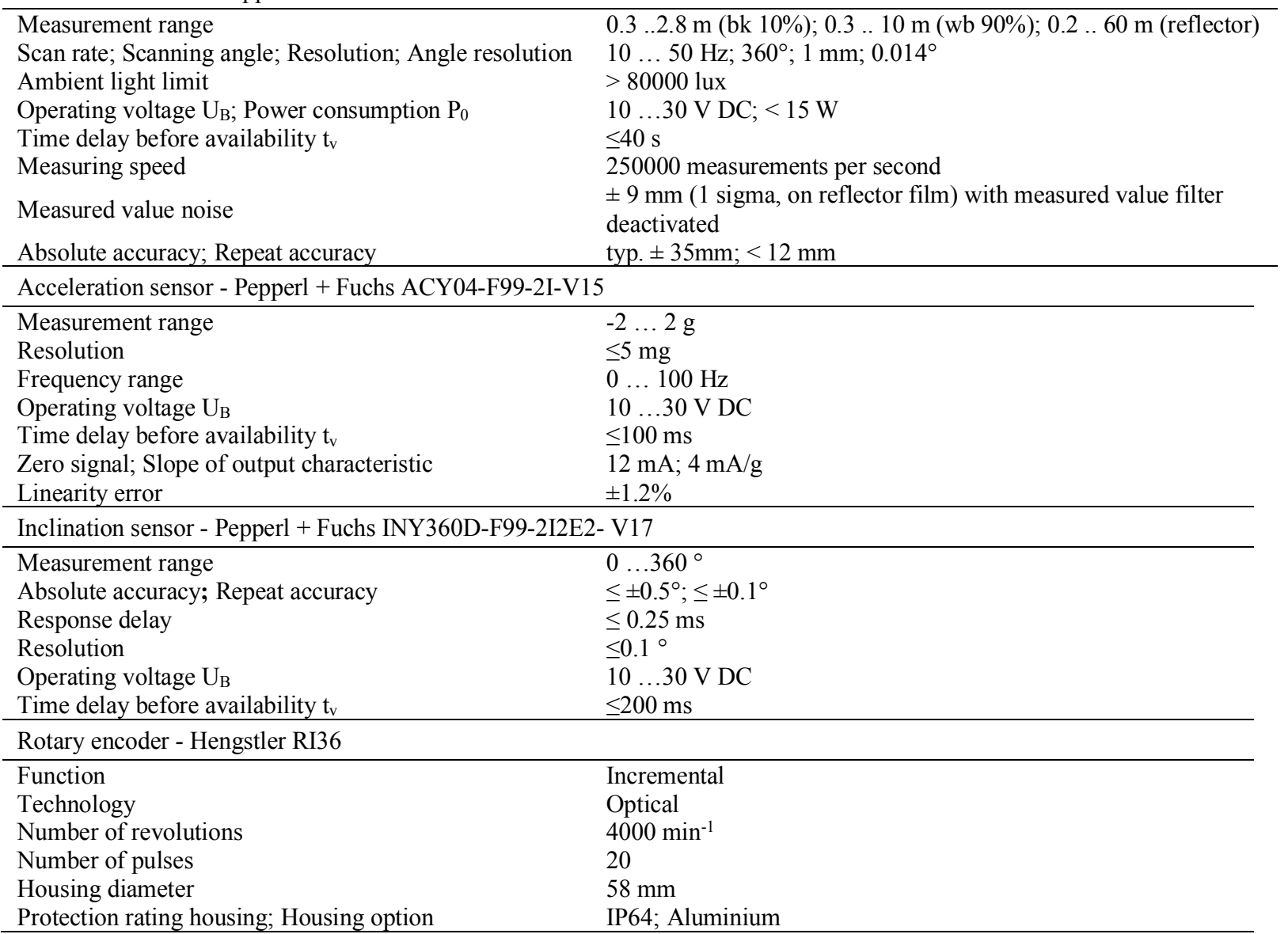
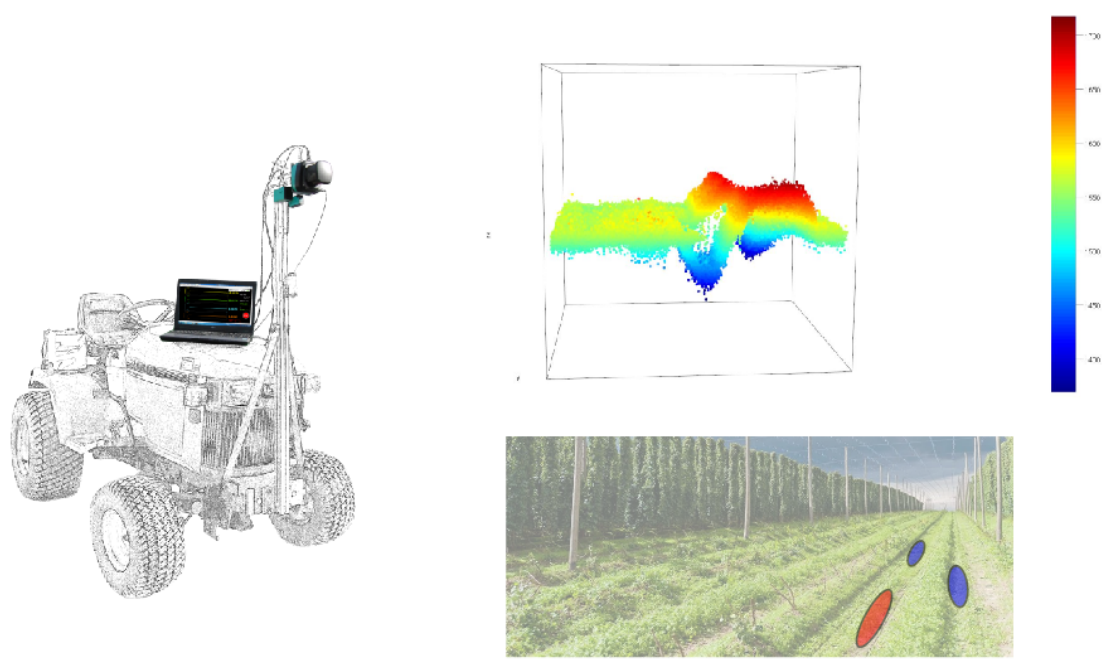

Figure 25. Carrier vehicle with sensors (left); first interpretation of the measured data (up right); example of a description of the terrain with positive and negative hindrances (bottom right).

For the communication and data acquisition with laser scanner TCP/IP protocol over Ethernet and for data acquisition from the rotary encoder, acceleration and inclination sensor a USB based NI cDAQ 9172 and a standard- Windows 7 notebook (i5-Quadcore 3.6 GHz processor, 4 GB RAM, SSD) were used.

In order to gain initial experience under the laboratory conditions, a test bench was built on which the laser 
scanner is mounted stationary and the work table can be moved with the test object at a defined forward speed (see Figure 3).
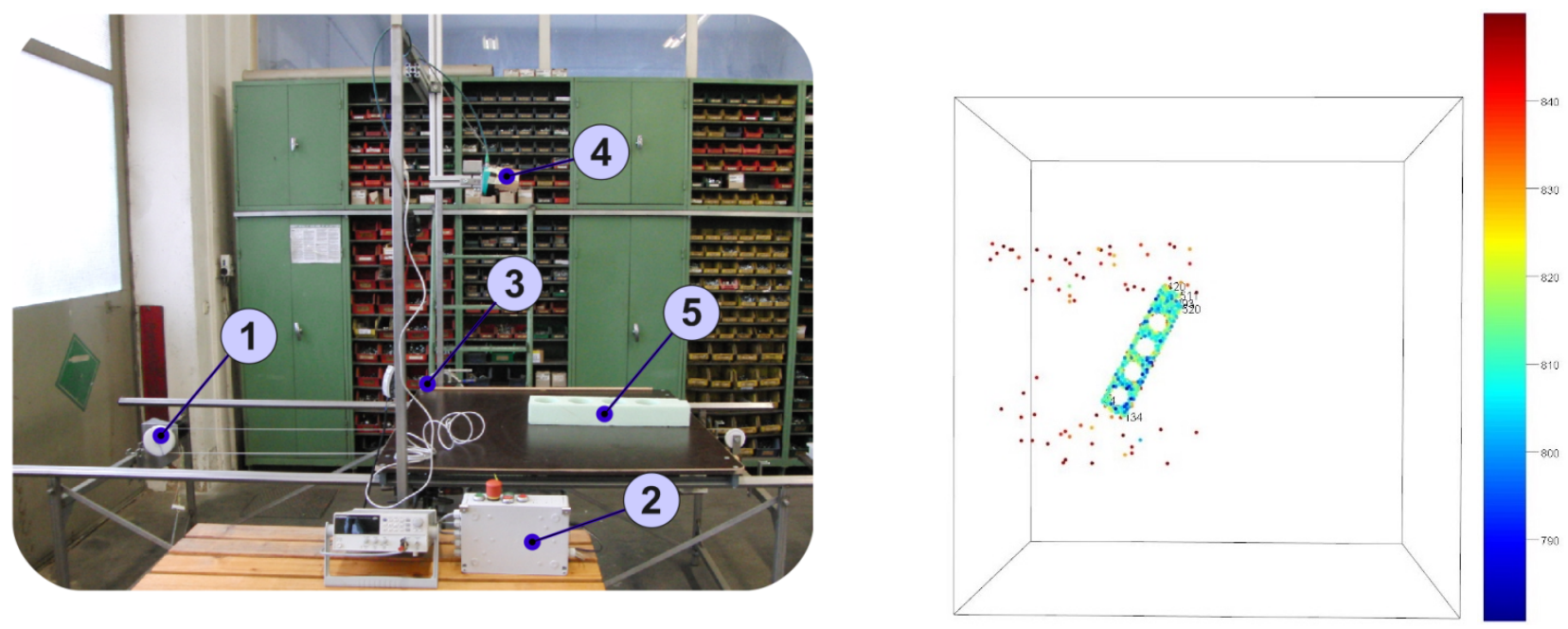

Figure 26. Test bench for the laser scanner under laboratory conditions: (1) - stepper motor for workbench positioning; (2) stepper controller; (3) - rotary encoder for acquiring the workbench position; (4) - 2D laser sensor (LiDAR); (5) - test sample [left]; results of the measurement of a test sample with a special pattern presented in R [right].

\section{Data acquisition}

As the cost of computing decrease and the amount of the data increase, the likelihood that applications will fail due to numerical errors accumulate. Hence, a not fully deterministic testing software (model), running under Windows, was created. The aim was to replicate the operating modes and use simulated signals in order to allow demonstration of the operating sequences to the user and to test the software prior to its actual commissioning. Two software solutions have been developed: for the acquisition of the data from the laser scanner, a solution in C\# and for the position-based acquisition of the inclination and acceleration of the laser head during the forward motion, a solution in LabVIEW (see Figure 6). The Software "Programm R2000_C Demo.exe" was developed in $\mathrm{C \#}$ based on the template, we become with courtesy of the R\&D of the Pepperl+Fuchs, using Visual Studio 2010 IDE. The communication between the sensor and the PC was acquired using the Ethernet interface and html requests. The measurement data are saved in CSV file format. All data are time-stamped, according to the computer system time and each measurement is characterised by the settings like the frequency of the sensor head rotation and the sampling rate saved in the file name. The program sets or gets parameter trough http request in single steps using temporary port (TCP/IP) for communicating with the laser scanner. The scanner measures at a predefined constant frequency and based on the combination of the frequency and the sampling rate delivers approximately same sized data packages through the UDP. One complete measurement usually consists from multiple data packages. Each package includes a header and the actual scan data. The information in the header are built from: the number of the delivered data points, the scan frequency, the number of the scanned data points and the number of the points within the data package. After completing the delivery of one data package, angular position and the time stamp (system time - start time of the measurement) are assigned as supplementary information and the so prepared dataset moved to a buffer variable from which the data are saved into a CSV file every 2000 milliseconds.

For the data acquisition from the rotary encoder, acceleration and inclination sensor using a compact modular data logging system CompactDAQ (National Instruments) another software solution was designed in LabVIEW (National Instruments, 2017). In order to test different approaches in this software, the sampling from the sensors was not time, but position based. The data from the acceleration and the inclination sensors were acquired after a trigger from the rotary encoder and combined in one dataset with the time stamp from the PC and the absolute position, calculated from the encoder position. In a preliminary measurement it is necessary to define a calibration coefficient which defines the resolution of the encoder impulses in relation to the travelled distance. By changing the mechanical transmission between the encoder and the element building physical connection with the surface (tractor tires, size of the wheel on the test bench etc.) it is possible to realise different measurement resolution by means of replacing the interfacing component and not the sensor or the carrier vehicles. In principle, similar results can be achieved with software filtering, but in such case a sensor (encoder) with much higher resolution need to be implemented. The software allows visualization of the measured data as well as data streaming. The screenshots of the front panel of the data acquisition software is presented in figure 5. The TDMS file format is used for saving the data without any signal conditioning and analysis, only the position of the rotary encoder is transformed to 
represent the travelled distance in longitudinal direction in relation to the start point defined by the first impulse of the encoder after the software measurement have been started. TDMS is the recommended data format because of the higher file writing speed and the smaller disk footprint in relation to the ASCII files format. The number of the acquired channels in the software as well as within the TDMS file can be configured. The software were developed with the aim to intelligently respond to a stimulus such as sensor signals or HMI, using an approach based on the state machines which is one of the most powerful and typical approaches (Bitter et al. 2007). Thus the software can be easily extended with new functionalities.
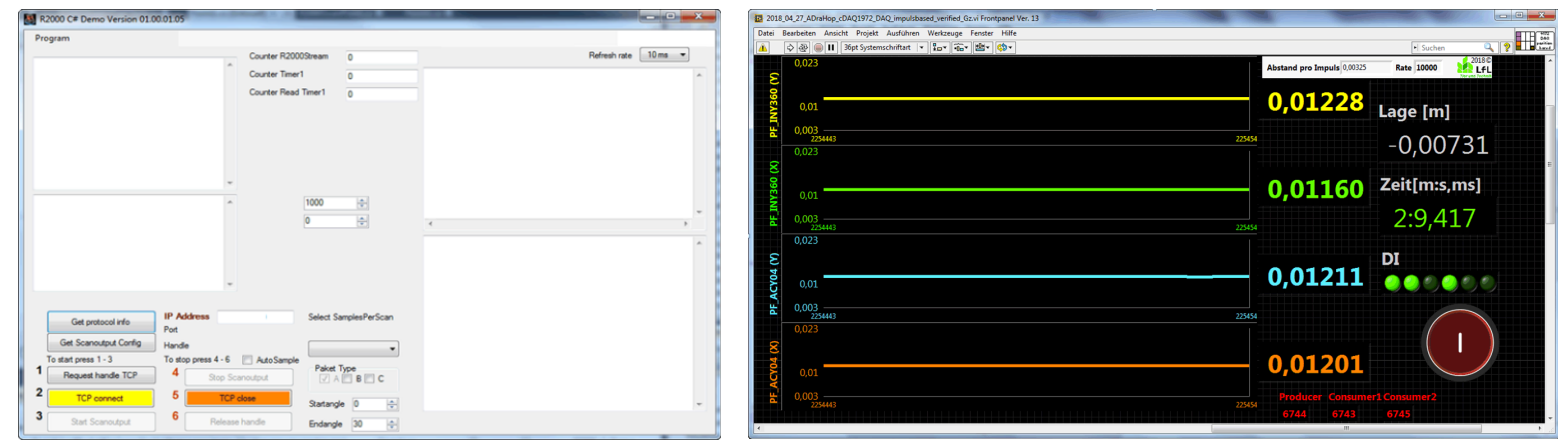

Figure 27. Front panel of the in C\# developed software for the data acquisition with the laser scanner (left); front Panel of the in the LabVIEW created software for the position-based acquisition of the inclination and the acceleration (forward motion).

Data processing and correction based on the measured roll and pitch angles of the LiDAR

Statistical software R and Scilab were compared and used for analysis and data visualisation by the data evaluation. The data synchronisation between the sensors and thus, the fusion has been developed based on the methods described in Garrido et al. 2015. The measured data are combined based on a timestamp, because the PC system time was written in both software solutions as a timestamp. The measurement were carried out with an assumption that both software solutions, if executed in parallel, do not use the same resources, do not cause interrupts in one another, or are not blocked with background processes and have the same priority.

Testing under controlled environmental conditions

The sensors were comprehensively tested on the workbench and thereafter a special carrier was designed allowing rigid and flexible connection (depending on the adjustment intensifies the vibrations generated on a carrier vehicle). While mounted on the carrier vehicle the sensors were tested while driving over known positive obstacles under different environmental conditions. A test field with markers, as well as positive and negative obstacles were built. Measurements with and without direct exposure to sunlight, wet and dry conditions of the soil surface and with and without effects of intensified vibrations of the carrier vehicle on the LiDAR were carried out as given in table 2 and figure 6 . The developed test system should provide an adequate supporting environment for development and testing of the hypothesis under laboratory and field conditions. With the on such way developed system the reliable and repeatable introduction of different type of phenomena (above described irregularities) within control cycle is possible.

Table 13. Design of the experiments for testing the 2D laser scanner under different environmental conditions.

\begin{tabular}{cccc}
\hline Number of repetitions & Soil surface characteristics & Illumination & $\begin{array}{c}\text { Intensified } \\
\text { vibrations }\end{array}$ \\
\hline 10 & dry & shadow & yes \\
10 & dry & shadow & no \\
10 & dry & direct & no \\
10 & dry & direct & yes \\
10 & wet & shadow & no \\
10 & wet & direct & yes \\
10 & wet & direct & no \\
\hline
\end{tabular}

\section{Results and Discussion}

The developed system for testing the LiDAR in order to provide appropriate analysis of the surface and the objects on which the carrier vehicle moves allows different type of testing. Not only the surface, but also the vegetation as an example for agricultural application can be analysed, if the sensor data are adequately interpreted. Considering the reliability of the system it needs to be mentioned that during the advancements along the path on 
which the hindrances are present, as mentioned by Benet (2017), the results can be easily misinterpreted, especially if the system begins to oscillate. The measurement confirmed, that there is no significant difference considering the measurements carried out under different conditions, such as soil moisture or direct or indirect illumination, if only the measured distance as output parameter from the LiDAR sensor is observed. Nevertheless, the parallel observation of the measured distance and the intensity of the reflected energy indicated, that there could be a dependency between the moister contain and the level of the reflected energy. Due to the fact that our preliminary intent was to provide information for further optimisation of device for automated attaching of the supporting wires for hops and our finding gone beyond the simple data interpretation, we need to carry out additional experiments and define additional algorithm for the data fusion especially having in mind the vibrations, which can under certain circumstance lead to misinterpretation. For this purpose the data from the acceleration sensor can be of great importance. The first results of a measurement under field conditions are presented in figure 7.
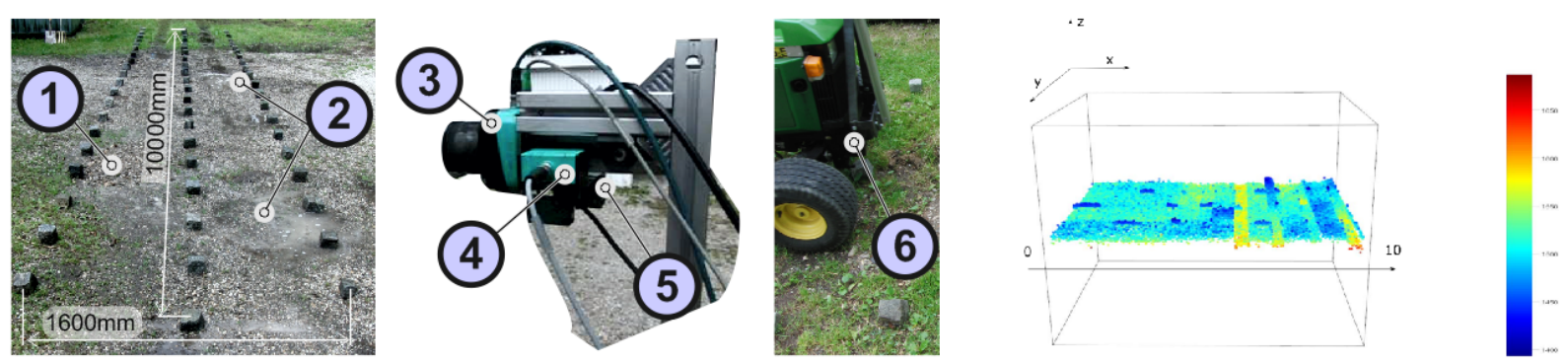

Figure 28. Test field with markers (1) - positive obstacle, (2) - negative obstacles (left); position of the sensors on the carrier (3) - 2D laser scanner, (4) acceleration sensor, (5) - inclination sensor (left middle); sensor carrier allowing intensification of the tractor vibrations (6) - adapter joint with springs; example of the measurement results with intensified vibrations of the tractor without the compensation based on the inclination and acceleration - possibly the oscillations can be visualised (right).
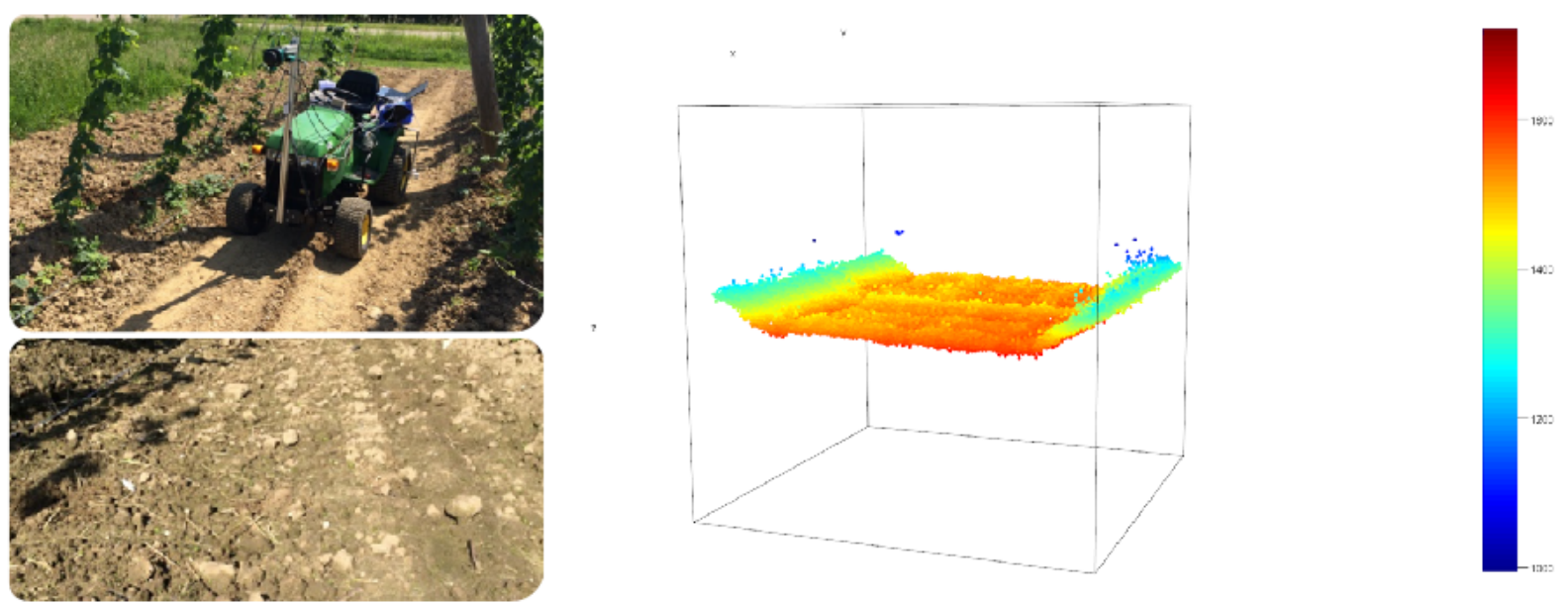

Figure 29. Preliminary results of a measurement under field conditions: carrier vehicle with sensors (upper left position); example of the soil surface characteristics (bottom left position) results of the data analysis (right).

\section{Conclusions}

Based on the preliminary results gained during the measurements under laboratory and simulated filed conditions, the application of the LiDAR in combination with odometry, as well as accurate measurement of the LiDAR angular position and acceleration can be positively evaluated. While developing the software for the data acquisition from LiDAR as well as the software for the event based data acquisition comprising the from the rotary encoder, the inclination sensor and acceleration sensor two approaches were comprehensively tested and evaluated. The project contributes to improvement of the design process of mechatronic systems in agriculture and the achieved results can be implemented in further projects. 


\section{Acknowledgements}

The project "Entwicklung eines Gerätes zur voll-automatischen Drahtaufhängung im Hopfenbau” was funded by the German Federal Agency for Agriculture and Food (BLE) as a part of the innovation support program of the German Federal Ministry of Food, Agriculture, and Consumer Protection (BMELV). The project "Weiterentwicklung des Geräts zur vollautomatischen Aufhängung von Aufleitdrähten in Hochgerüstanlagen im Hopfen: Optimierung, Erprobung und Fertigung der Nullserie" is funded by the German Federal Agency for Food and Agriculture (BLE) and Rentenbank within the DIP (Agricultural Innovation Partnership) programme. The authors thank to all involved employees of the Institute for Agricultural Engineering and Animal Husbandry of the Bavarian State Research Center for Agriculture and the private limited company Soller (Geisenfeld, Germany) for successful cooperation.

\section{References}

Benet B., 2017. Multi-sensor fusion method for crop row tracking and traversability operations. AXEMAEurAgEng Paris (SIMA) https://www.eurageng.eu/axema-eurageng-paris-2017-(sima) Accessed Jun 12, 2018.

Bitter R., Mohiuddin T., Nawrocki M., 2007. LabVIEW:Advanced Programming Techniques, Second Edition, Boca Raton London New York: CRC Press. P.520

Fraser, I. (general editor)., 2010. Guide to application of the Machinery Directive 2006/42/EC. 2nd ed. https://ec.europa.eu/docsroom/documents/9483/attachments/1/translations/en/renditions/pdf. Accessed 12 June 2018.

Garrido, M., D.S. Paraforos, D. Reiser, M. Vazquez Arellano, H.W. Griepentrog, and C. Valero, 2015. 3D maize plant reconstruction based on georeferenced overlapping LIDAR point clouds. Remote Sensing 7 (12), 17077-17096. https://doi.org/10.3390/rs71215870

Gobor, Z., G. Fröhlich, H. Soller and J. Portner., 2012. First operating experiences with a prototype for automated attaching of the supporting strings that the hop vines grow on in high-trellis hop gardens.

International conference of agricultural engineering, CIGR-AgEng2012, Valencia 8-12 July, Papers book, ISBN 978-84-615-9928-8 p.6

Gobor, Z., 2013. Data acquisition and automated data analysis in prototype development - Testing of the prototype for automated attachment of the supporting wires in hop gardens. In $1^{\text {st }}$ international Mobile Machine Control (MMC) conference, Nuremberg (Germany), Jun 12-13, CAN in Automation GmbH, pp. 03-15 - 03-21

Gobor, Z., 2015. Development of a diagnostic tool for performance analysis during the testing of agricultural implements. SCILABTEC 2015 7th International scilab user conference. Paris May 21-22.

Jimenez-Berni, J.A., D.M. Deery, P. Rozas-Larraondo, A.G. Condon, G.J. Rebetzke, R.A. James, W.D. Bovill, R.T. Furbank and X.R.R. Sirault, 2018. High Throughput Determination of Plant Height, Ground Cover, and Above-Ground Biomass in Wheat with LiDAR. Frontiers in Plant Science 9.

https://doi.org/10.3389/fpls.2018.00237

MacCleery, B. and Z.M. Kassas, 2008. New mechatronics development techniques for FPGA-based control and simulation of electromechanical systems. Proceedings of the 17th IFAC World Congress; Seoul, Korea, 6-11 July

National instruments, 2017. cDAQ-9172 Specifications. http://www.ni.com/documentation/en/compactdaqchassis/latest/cdaq-9172/specs/ Accessed Jun 1, 2018.

Schmidt, D., 2010. An Introduction to Floating-Point Behavior in LabVIEW. National Instruments Corporation. $\mathrm{ftp} / / / \mathrm{ftp}$. ni.com/pub/devzone/tut/floating_point_in_labview.pdf Accessed Jun 12, 2018.

Seiferth, B., Z. Gobor, and S. Thurner, 2016. Localization of pasture maintenance spots by a 2D laser scanner. Proceedings of the international Conference on Agricultural Engineering CIGR - AgEng 2016 Jun. 2629, Aarhus, Denmark, p.10 http://conferences.au.dk Accessed Jun 12, 2018.

Van der Linden, S., A.M. Mouazen,J. Anthonis, H. Ramon and W. Saeys, 2008. Infrared laser sensor for depth measurement to improve depth control in intra-row mechanical weeding. Biosystems Engineering. 100 (3), pp 309-320 https://doi.org/10.1016/j.biosystemseng.2008.03.010 


\author{
Robust Online Soil Roughness Measurement Using Stereo Vision \\ Peter Riegler-Nurscher ${ }^{\text {a, }}$, Heinrich Prankl ${ }^{\text {a }}$, Gerhard Moitzi ${ }^{\text {b }}$, Helmut Wagentristl ${ }^{\text {b }}$ \\ a Josephinum Research, Rottenhauserstraße 1, Wieselburg, Austria \\ ${ }^{\mathrm{b}}$ University of Natural Resources and Life Sciences, Vienna, Austria \\ * Corresponding author. Email: p.riegler-nurscher@josephinum.at
}

\begin{abstract}
Soil Roughness is an important agronomic soil parameter. It influences plant emergence as well as plant growth and plays an important role in soil erosion. The main goal at point of seeding is to get a good settled seedbed which is not to rough crumbled for good plant emergence and not to fine crumbled to avoid soil erosion and silting. These agronomic properties at seeding can be determined by soil roughness. Thus, a robust method for soil roughness measurement is needed for mapping the soil roughness on fields and to control machines according to the site specific roughness. Tillage machines might be controlled online during field work to get more homogeneous and more efficient working results. Furthermore, roughness maps, generated with overlapping GNSS data, might be used for later precision farming applications, for instance site specific fertilizing. Our proposed method is based on cost-effective stereo cameras and mobile processors. The method can be implemented in real time on a moving machine. Challenging environmental influences, like dust, lighting and vibrations, have been taken into account. The resulting roughness indices consider directional roughness, like stripe patterns. These constant soil patterns are generated by the directed movement of the tillage machine. To evaluate the method, the roughness measurements and aggregate size of the soil were compared in different field tests. The robustness of the method was investigated in various tests under different soil conditions. Also machine parameters which influence the resulting roughness, like driving speed or PTO speed, were evaluated. Finally, the influence of soil roughness on the plant emergence was evaluated.
\end{abstract}

Keywords: stereo vision, tillage, seeding, soil roughness

\title{
1. Introduction
}

The working intensity of a tillage machine influences different agronomic aspects like plant emergence and soil erosion and has a direct impact on economic efficiency. For regulation of the tillage operation it is required to measure the tillage results, especially soil roughness, accurately in motion. Traditional methods like a pin meter or the chain method (see Allmaras et al. 1966) are contact based which cannot be used in mobile applications. There is a need for a contactless method which does not wear out on the soil surface. In recent years many machine vision based methods have been published. All these methods share the reconstruction of the soil surface into a 3D point cloud. Methods using a laser scanner (see Turner et al. and Zhixiong et al.) are very accurate at this task, but also very expensive due to the hardware needed. Laser scanners are also very sensitive to dust and mechanical vibrations. Investigations on the measurement uncertainty of laser scanners are published in Boehler et al. (2003). Active 3D camera sensors, like the Microsoft Kinect ${ }^{\mathrm{TM}}$ sensor used in Marinello et al. (2015), allow fast generation of the 3D point cloud due to the strong hardware integration. However, these sensors do not work under strong sunlight, what makes them impractical for online outdoor applications. Photogrammetric approaches, like proposed in Gilliot et al. (2017), are very accurate. These methods rely on a reference pattern, like a frame, and multiple images taken from different poses, so they are impractical for online use. Stereo camera systems on the other hand are passive, in principle motion insensitive and relatively cheap. The results on stereo camera based soil roughness measurements published in Riegler et al. (2014) and Taconet et al. (2007) are very promising. A rich amount of information is delivered through camera based systems and therefore open a wider range of future applications. The work of Jester and Klik (2005) compare mechanical methods like the chain method and pin meter and optical methods like laser scanner and stereo cameras for estimation of soil roughness. Their work focuses on data capturing and an assessment of computational cost.

The proposed method is based on a stereo camera to reconstruct the soil surface. The focus of the method is on a robust detection which copes with various environmental influences, like dust, residues and light changes. We show the environmental influences on the camera system and our countermeasures to cope with those. For method evaluation we compare the measurements with the weighted mean clod diameter, the plant emergence and the tillage intensity.

\section{Materials and Methods}

The proposed method is based on utilization of stereo camera images. To get accurate measurements the cameras have to be calibrated once at the beginning. The calibration step estimates the intrinsic camera parameters, like focal length and focal point, and extrinsic parameters which define the geometric location of the two cameras 
to each other. One key aspect of robust and durable measurements is a stable mounting of the cameras to avoid changes in the extrinsic parameters.

The real-time estimation of the roughness is done on a single board computer based on ARM architecture. The computation of the roughness value should be as efficient and fast as possible to get a high frame rate for online measurements. This aspect was given special attention in method development.

The estimation pipeline

An overview of the estimation pipeline is shown in Figure 30. The input for the estimation of the roughness are two images (Figure $31 \mathrm{a}$ and b) from a stereo camera. The baseline between the camera centers is $50 \mathrm{~mm}$, to achieve the required depth resolution. The initial step is to rectify the images and afterwards to find corresponding points in the left and right image. The proposed method builds on the block matching algorithm provided in OpenCV (OpenCV library, 2018) by K. Konolige for finding these corresponding points. The block matching algorithm is dependent on rich surface texture to work well. Soil provides these rich texture, and therefore the soil surface can be reconstructed efficiently and accurately. The block matching algorithm delivers a semi dense disparity map (see Figure $31 \mathrm{c}$ ) which can be converted into a point cloud (see Figure $31 \mathrm{~d}$ ) by using the camera calibration parameters. The density of the point cloud is mainly dependent on the image quality. To quantify the image quality $\mathrm{q}$ the ratio of found correspondences to image size in pixels is used. The value of q ranges from 0 , which implies that no correspondences were found, to 1 which indicates that all possible correspondences are found.

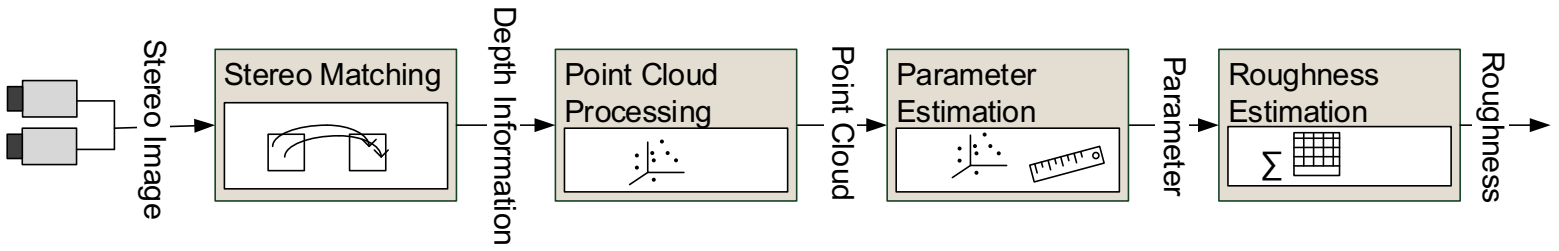

Figure 30. Pipeline of the roughness estimation algorithm.

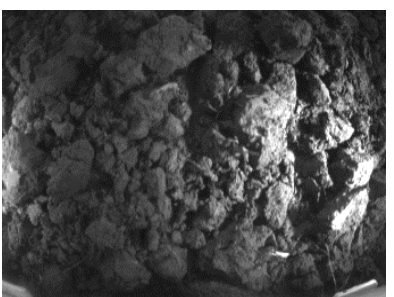

a)

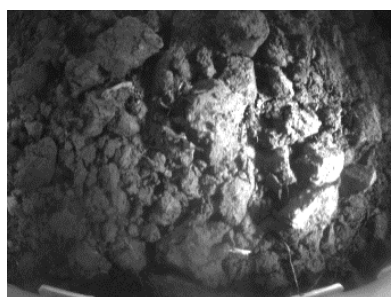

b)

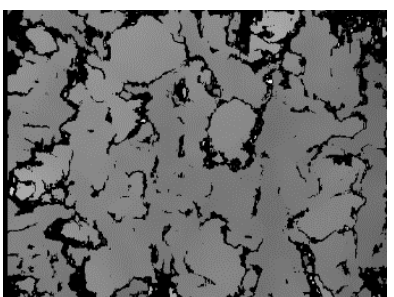

c)

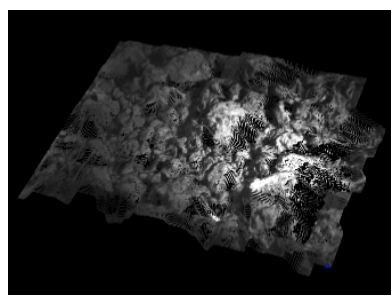

d)

Figure 31. a) left camera image; b) right camera image; c) disparity map; d) reconstructed point cloud (Digital Elevation Model, DEM).

The 3D point cloud is further processed to get parameters like height measurements or surface normal. These parameters are the basis for the final estimation of the roughness. We evaluated different roughness indices, and focused on the most promising roughness indices RC, see Equation (1), as presented in Taconet and Ciarletti (2007) and AoN (average angle of surface normals) as implemented by Riegler T. et al. (2014). The RC index calculates the statistical variance of the height $z$ for each point in the point cloud. The variants of the RC index can be implemented very efficiently and are suitable for use in real-time applications.

$$
R C=\sqrt{\left\langle\left(z(x, y)-\langle z(x, y)\rangle_{X, Y}\right)^{2}\right\rangle_{X, Y}}
$$

Most tillage machinery produce directed roughness patterns, like furrows. To eliminate these directed patterns, the roughness is estimated in two different directions with $\mathrm{RCx}$, see Equation (2), and RCy. This results in a higher roughness transversal to the driving direction $\mathrm{RCy}$ compared to the roughness along the driving direction $\mathrm{RCx}$. The roughness is normalized over lines in $\mathrm{x}$ direction for the $\mathrm{RCx}$ and not in both directions, as for the RC.

$$
R C_{X}=\left|\sqrt{\left\langle\left(z(x, y)-\langle z(x, y)\rangle_{X}\right)^{2}\right\rangle_{X}}\right\rangle_{Y}
$$

The AoN roughness index depends on the calculation of surface normals, which is more time consuming. Our method uses the normal estimation algorithm, based on integral images, implemented in the PCL library (Holzer et al., 2012). The parameter smoothing_size of the algorithm defines how many points are used for the calculation 
of the surface normals. The modification of this parameter is necessary to cancel noise in the point cloud. All angles in $\mathrm{z}$ direction of the surface normals, shown in Figure 32 are combined into the AoN index. Following equation shows the calculation of the AoN roughness index. The average angle to the $x-y$ plane is calculated over all N surface normals $S_{i}$.

$$
A o N=\frac{1}{N} \sum_{\mathrm{i}=0}^{\mathrm{N}} \operatorname{acos}\left(\frac{S_{i, Z}}{\left\|S_{i}\right\|}\right)
$$

The captured area per sample is about $60 \mathrm{~cm} \mathrm{x} 40 \mathrm{~cm}$ at a mounting height of $30 \mathrm{~cm}$ above the ground. To increase the captured area we calculate a weighted sliding mean window over several sample points, which in addition leads to a smoothing of the results.

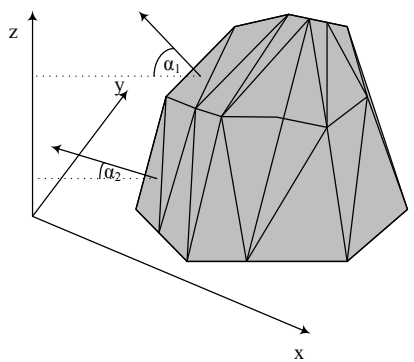

a)

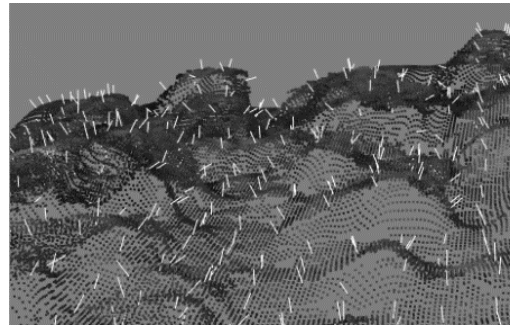

b)

Figure 32. a) Angle $\alpha$ in $z$ direction of surface normal; b) surface normals on soil surface.

Handling residues

At tillage operations residues often occur. One challenge of the system is to detect residues to avoid them at the roughness estimation. We tried two different approaches to cope with residues, a geometric filtering on the point cloud and a pixel-wise segmentation based on the "SoilCover" algorithm proposed in Riegler-Nurscher et al. (2018). Details and related work to different soil cover detection methods can be found in Riegler-Nurscher et al. (2018). These filtering approaches work till a certain amount of residue cover, where still enough soil is visible.

The filtering, based on the 3D point cloud, detects unexpected spikes within the depth map and marks them. This simple approach filters spikes with bigger height in proportion to its cross-section, as caused by straws. The $3 \mathrm{D}$ point cloud filtering can be implemented very efficiently and is suitable for real-time applications.

The second approach based on the SoilCover algorithm works only with RGB stereo cameras. One color image of the stereo camera (see Figure 33 a) is classified pixel-wise into the categories soil, living biomass and dead biomass (see Figure $33 \mathrm{~b}$ ). The classification result is projected into the disparity map and pixel which are not classified as soil are removed from it (see Figure $33 \mathrm{c}$ ). This approach works more robust but on the other hand is more computationally intense and must be optimized for online application.

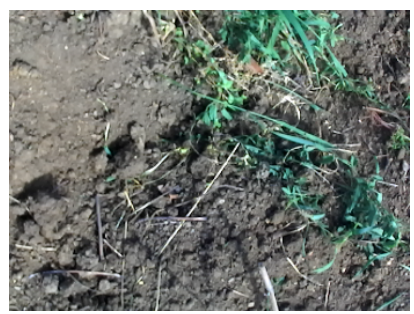

a)

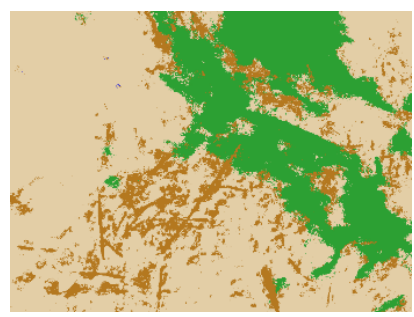

b)

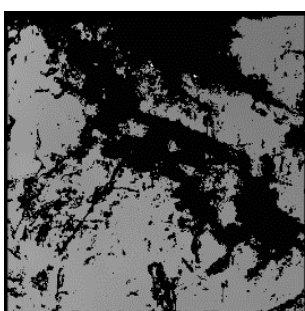

c)

Figure 33. a) left RGB image of the stereo camera; b) classification result of the SoilCover algorithm; c) filtered disparity map.

Other environmental influences

One mayor issue for camera systems is dust, especially in rough agricultural environments. We distinguish between two types of dust. Dust and dirt on the glass cover of the camera and dust clouds in the atmosphere between camera and soil surface. Dirt on the camera glass cover can be detected by comparing the left and right camera image. This approach works till a certain size of dirt particles, after a certain glass cover, the overall stereo correspondence detection quality sinks noticeable. Very strong dust between soil and camera also reduces the detection quality. In both cases a notification is sent to the user.

The basis for good stereo matching is enough light. The light captured by the image sensors can be increased by increasing the shutter time, which in contrast decreases the feasible moving speed of the camera due to motion 
blur. The proposed method utilizes a velocity value to calculate the slowest shutter time while still avoiding blurring. The velocity value can come from a tractor CAN bus or can be calculated from GPS readings. Additional to these countermeasures, LED-headlights mounted on the camera housing are used in bad light conditions. These headlights allow use of the system under all light conditions, even at night time.

\section{Results and Discussion}

Following chapter shows the results of roughness measurements compared to the mean clod diameter, to the working intensity and to the plant emergence. All roughness measurement result in this chapter use the filtering approach based on the $3 \mathrm{D}$ point cloud.

Comparison to the mean clod diameter

We compared roughness measurements of the stereo camera to a sieve analysis. Soil samples of a size of about $25 \times 25 \mathrm{~cm}$ and $5-7 \mathrm{~cm}$ in depth where sieved and weighted. The sieve grid sizes were 40, 20, 10, 5, 2.5 and 1.25 $\mathrm{mm}$. The measured masses were combined to a mean weighted diameter (MWD, equation see Kirchmeier et al., 2005) and compared to the measured roughness at the sample position.

Table 14. Correlation between measured soil roughness index and weighted mean clod diameter.

\begin{tabular}{ccc}
\hline & $\begin{array}{c}\text { Location 1: } \\
\text { Groß Enzersdorf, AT }\end{array}$ & $\begin{array}{c}\text { Location 2: } \\
\text { Krummnussbaum, AT }\end{array}$ \\
\hline $\begin{array}{c}\text { Pearson correlation between mean clod diameter and soil } \\
\text { roughness index RC }\end{array}$ & 0.550 & 0.657 \\
Statistical significance two-tailed & 0.00002 & 0.00008 \\
\hline $\begin{array}{c}\text { Pearson correlation between mean clod diameter and soil } \\
\text { roughness index AoN }\end{array}$ & 0.455 & 0.449 \\
Statistical significance two-tailed & 0.00001 & 0.0130 \\
\hline $\begin{array}{c}\text { N } \\
\text { Soil: \% sand; \% silt, \% clay } \\
\text { Date }\end{array}$ & 52 & 30 \\
\hline
\end{tabular}

The correlation between the results of the sieve analysis and the roughness measurements are shown in Table 1. The lower correlation at location 1 can be described by a lower range of roughness values. At location 1 the sample position was determined by GPS. This increases the uncertainty of the measurement due to the GPS-Error. At location 2 the roughness was estimated at the exact position where the sieve analysis sample was taken.

\section{Example Images}

The following Figure 34 should give an impression of the range of roughness on a field. It shows images of different clod sizes and the corresponding roughness values $\mathrm{RC}$ and AoN. These images where taken after a rotary harrow/seed drill combination on a test site in Schollach, Austria. The camera was mounted at the very back of the seed drill facing downwards. The driving speed was constant at about $6 \mathrm{kph}$. 


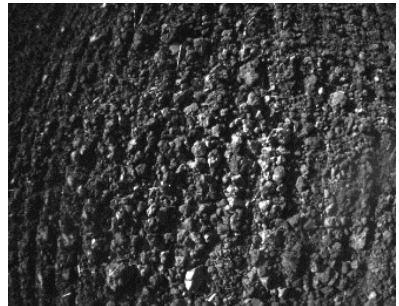

RC 4.3 / AoN 1.0

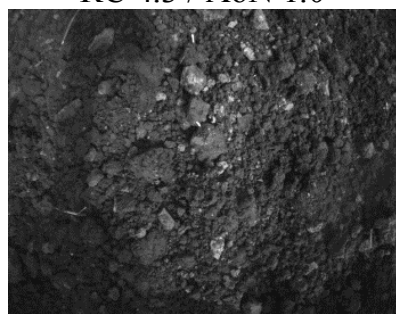

$\mathrm{RC} 8.0 / \mathrm{AoN} 25.1$

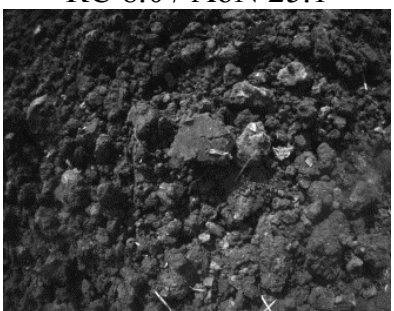

RC 14.0 / AoN 30.5

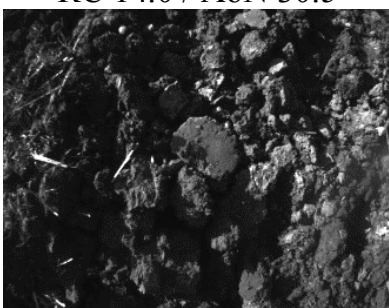

RC 18.0 / AoN 29.6

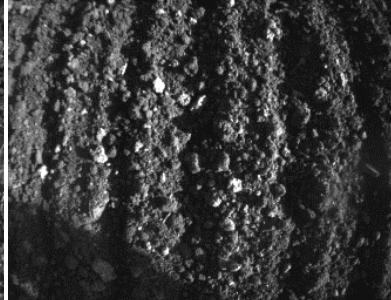

RC 5.0 / AoN 18.4

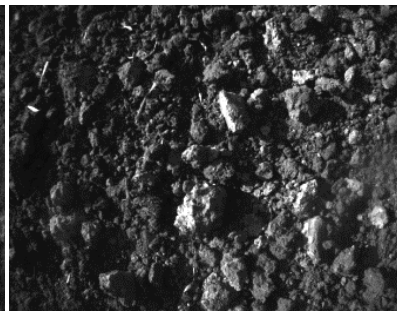

$\mathrm{RC} 9.0$ / $\mathrm{AoN} 23.8$

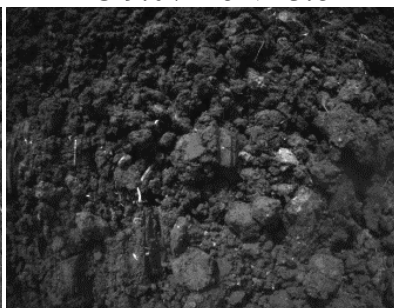

RC 15.0 / AoN 28.7

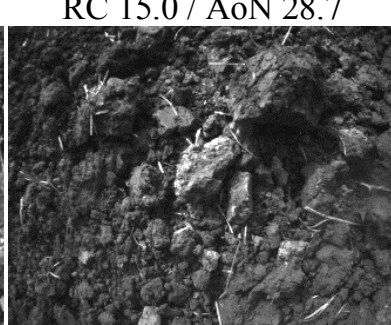

RC 20.0 / AoN 30.8

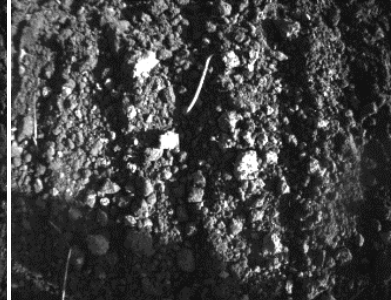

RC 6.0 / AoN 21.0

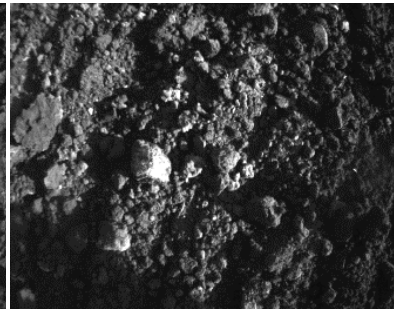

RC 11.0 / AoN 24.3

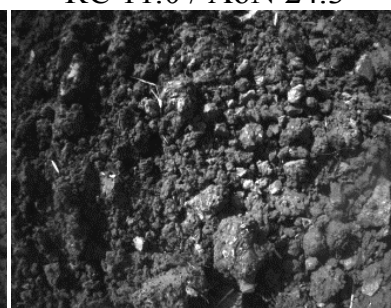

RC 16.0 / AoN 28.1

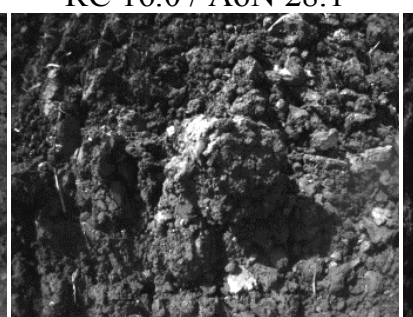

RC 24.0 / AoN 29.3

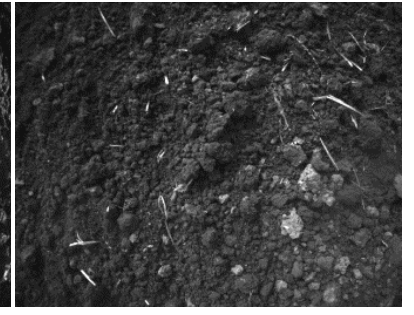

RC 7.0 / AoN 22.8

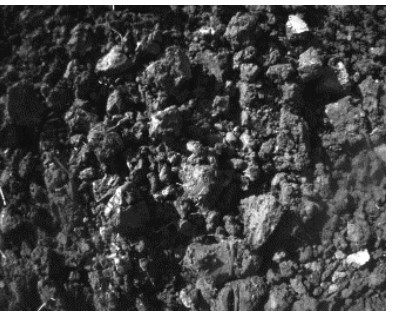

RC 13.0 / AoN 29.4

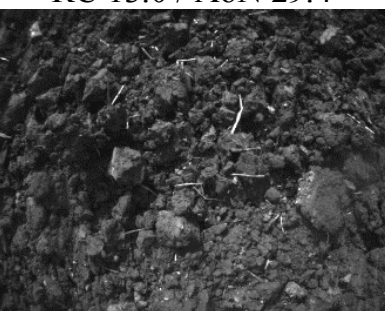

RC 17.0 / AoN 30.0

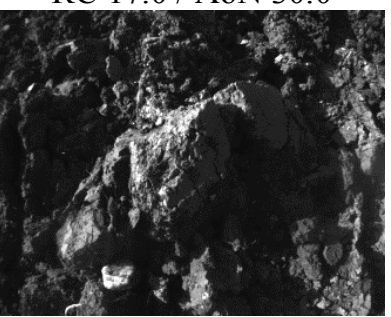

RC 30.2 / AoN 32.6

Figure 34. Example images with corresponding $\mathrm{RC}$ values in ascending order.

Comparison between soil roughness and working intensity

As a basis for controlling a tillage machine with the measured roughness, we tested the correlation between the soil roughness $\mathrm{RC}$ and the tillage intensity of a rotary harrow. The tillage intensity of a rotary harrow is mostly influenced by the rotation speed of the tines and the overall driving speed. The driving speed was estimated via GPS. Figure 35 shows the measured roughness and the tine loops per metre in a diagram. The value of the $\mathrm{x}$ axis equals single-tine rotational speed divided by driving speed $(\mathrm{rpm} /(\mathrm{m} / \mathrm{s}))$, representing tine loops per metre. As expected, the roughness decreases with increased intensity of the working process. 

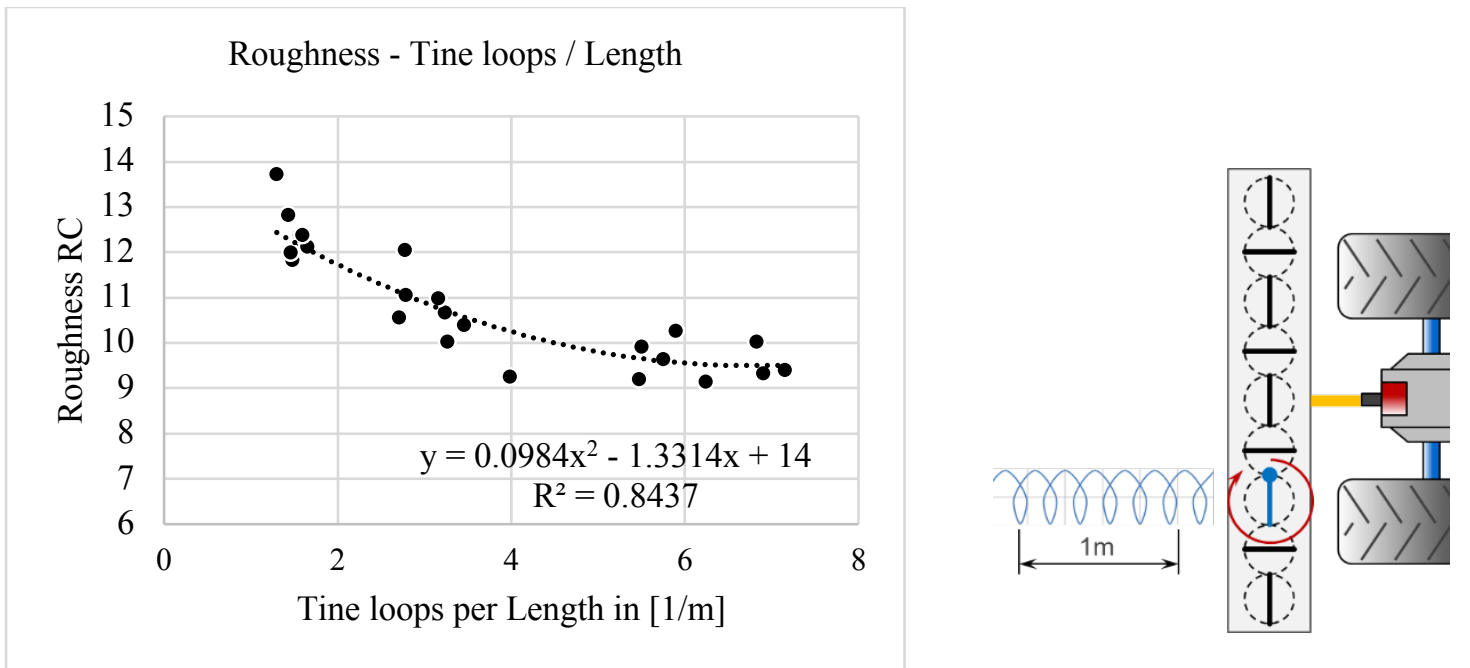

Figure 35. Comparison of soil roughness to rotary harrow tine speed. Each point equals the mean value of one track. $\mathrm{N}=$ 24. The left image shows the tine loops per metre soil.

Mapping of soil roughness and field emergence

In combination with a GPS the method can be used to map the soil roughness on a field. Figure 36 a) shows mapping results on a field in Wieselburg, Austria. In addition, we measured the plant emergence of winter wheat on the field via the SoilCover method (Riegler-Nurscher et al., 2018). The mapping of the plant cover is shown in Figure $36 \mathrm{~b}$ ). The correspondences between soil roughness and plant cover measurement points were estimated by mean values within voronoi polygons. The pearson correlation over all these correspondences was $-0,622$ between plants and soil roughness RC and $-0,557$ for the correlation between plants and AoN. This means more roughness leads to lower plant emergence, as expected.

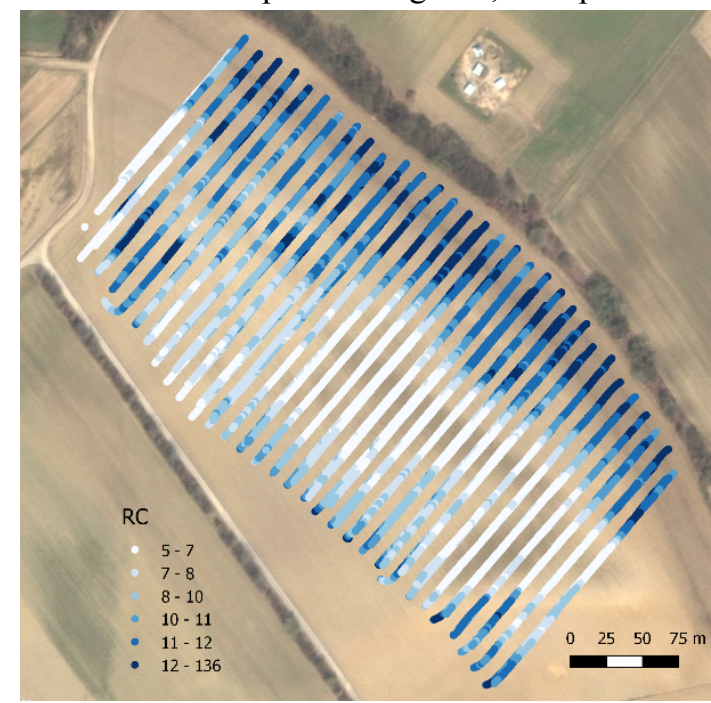

a)

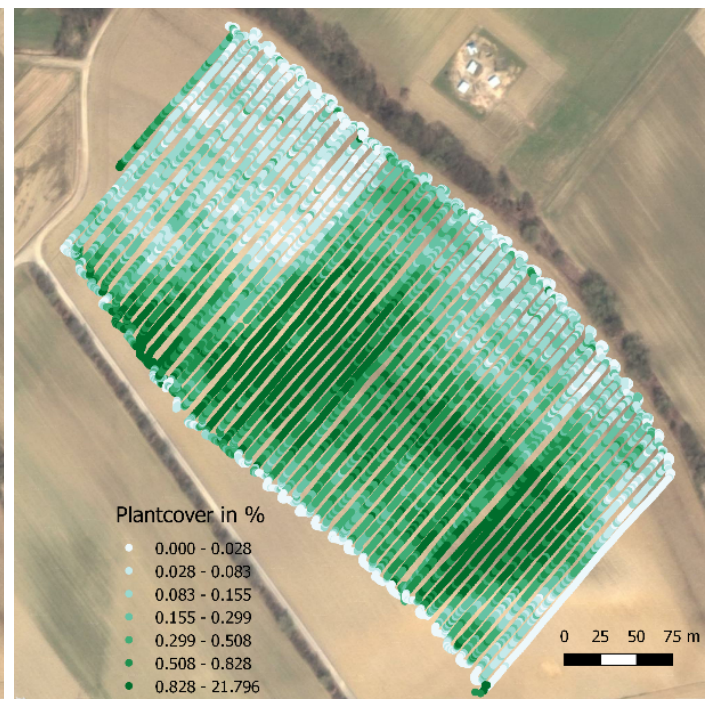

b)

Figure 36. Roughness measured after seeding on $26^{\text {th }}$ October 2017 in Wieselburg, Austria on the left a); and plant emergence (2D plant coverage) estimated by the SoilCover method (Riegler-Nurscher et al., 2018) on the right b).

\section{Conclusions}

Soil roughness is an important factor for plant emergence and soil erosion. Controlling tillage machines in real-time is dependent on a robust method for soil roughness measurement. The proposed method based on stereo vision is able to reconstruct the soil surface and to calculate soil roughness indices. The algorithm is able to detect and cope with environmental influences like dust, residues and different light conditions. Comparison to the mean weighted diameter, to the working intensity and to the plant emergence show promising results. 


\section{Acknowledgements}

The Project is funded by the Austrian Federal Ministry of Transport, Innovation and Technology (BMVIT) under the program "Bridge 1" between November 2015 and December 2017.

\section{References}

Allmaras, R. R.; Burwell, R. E.; Larson, W. E.; Holt, R. F.; Nelson, W. W., 1966. Total Porosity and random roughness of the interrow zone. U.S. Department of Agriculture, Conservation Research Report No. 7, pp. 1-22

Boehler, W., Vicent, M. B., Marbs, A., 2003. Investigating laser scanner accuracy. The International Archives of Photogrammetry, Remote Sensing and Spatial Information Sciences 34 (Part 5), XIX. CIPA Symposium, 30.9.4.10.2003, Antalya, Turkey

Gilliot, J. M., Vaudour, E., \& Michelin, J., 2017. Soil surface roughness measurement: A new fully automatic photogrammetric approach applied to agricultural bare fields. Computers and Electronics in Agriculture, 134, 6378.

Holzer, S., Rusu, R. B., Dixon, M., Gedikli, S., Navab, N., 2012. Adaptive Neighborhood Selection for RealTime Surface Normal Estimation from Organized Point Cloud Data Using Integral Images, IROS 2012

Jester, W., Klik, A., 2005. Soil surface roughness measurement - methods, applicability, and surface representation. Catena 64, pp. 174-192

Kirchmeier, H, Geischeder, R. Demmel, M., 2005. Bearbeitungseffekt und Leistungsbedarf von Kreiseleggen mit unterschiedlicher Kreiselgeometrien. Landtechnik 60 (4), pp.196-197

Marinello, F., Pezzuolo, A., Gasparini, F., Arvidsson, J., \& Sartori, L., 2015. Application of the Kinect sensor for dynamic soil surface characterization. Precision Agriculture 16, 601-612.

OpenCV library, 2018. http://opencv.org/, accessed 5 June 2018

Riegler, T., Rechberger, C., Handler, F., \& Prankl, H., 2014. Image processing system for evaluation of tillage quality. Landtechnik.

Riegler-Nurscher, P., Prankl, J., Bauer, T., Strauss, P., Prankl, H., 2018. A machine learning approach for pixel wise classification of residue and vegetation cover under field conditions, Biosystems Engineering, 169, 188-198

Taconet, O.; Ciarletti, V., 2007. Estimating soil roughness indices on a ridge-and-furrow surface using stereo photogrammetry. Soil and Tillage Research 93, pp. 64-76

Turner, R., Panciera, R., Tanase, M. A., Lowell, K., Hacker, J. M., \& Walker, J. P., 2014. Estimation of soil surface roughness of agricultural soils using airborne LiDAR. Remote Sensing of Environment, 140, 107-117

Zhixiong, L., Nan, C., Perdok, U. D., \& Hoogmoed, W. B., 2005. Characterisation of soil profile roughness. Biosystems Engineering, 91(3), 369-377 


\title{
Assessing the Spatio-Temporal Alfalfa Yield Variability Using Landsat-8 and Yield Monitor Data at Field Scale
}

\author{
Ahmed Kayad ${ }^{\text {a,b,c*, }}$ Khalid Al-Gaadi ${ }^{\text {b,d }}$, El-Kamil Tola ${ }^{\text {b }}$, Rangaswamy Madugundu ${ }^{\text {b }}$ \\ Marco Sozzi $^{\text {a }}$, Francesco Marinello ${ }^{\text {a }}$ \\ a Department of Land, Environment, Agriculture and Forestry, University of Padova, Legnaro (PD), 35020, Italy. \\ ${ }^{\mathrm{b}}$ Precision Agriculture Research Chair (PARC), King Saud University, Riyadh, 11451, Saudi Arabia. \\ ${ }^{c}$ Agricultural Engineering Research Institute (AEnRI), Agricultural Research Centre, Giza, 12619, Egypt. \\ ${ }^{d}$ Department of Agricultural Engineering, King Saud University, Riyadh, 11451, Saudi Arabia. \\ * Corresponding author. Email: agkayad@gmail.com
}

\begin{abstract}
Yield mapping is considered as the first logical step in Precision Agriculture (PA). In this study, a hay yield monitoring system was mounted on a large rectangular baler to assess alfalfa yield during baling process. The first step was to calibrate the system under different baler working conditions (baler chamber pressure and forward speed). The system provided an average $\mathrm{R}^{2}$ value of 0.87 . Secondly, the calibrated system was used to investigate the spatial yield variations in an alfalfa cultivated area of 23.5 ha under a center pivot irrigation system located in the eastern province of Saudi Arabia. The system was used to monitor the harvesting operation during five alfalfa cuts, dated 20 Oct, 5 Dec, 16 Feb, 2 Apr and 6 May in 2013-14 growing season. Yield monitor data sets were analysed using the ArcGIS software program and classified into five zones $(>2,2.01-3,3.01-4,4.01-5$, and $<5$ ton/ha). Additionally, the maps of the five cuts were combined in one composite map to estimate the seasonal impact on different yields. Geospatial analysis of yield maps showed significant spatial variations in the study area despite the fact that all agricultural practices (irrigation, planting, etc.) were kept constant. During the study period, 18 landsat- 8 images were analysed and compared to yield monitor data. Results revealed significant correlations between yield and NIR reflectance, SAVI and NDVI with $\mathrm{R}^{2}$ values of $0.69,0.68$ and 0.63 , respectively. On the other hand, results showed that the yield was, on the average, temporally varying among the different cuts with systematic spatial distribution trend for all cuts within the detected zones. Significant yield seasonal variations, as large as $17 \mathrm{t} / \mathrm{ha}$ (almost $300 \%$ of the minimum), necessitates the development of a yield forecast model to support a proper agronomic input optimization.
\end{abstract}

Keywords: Yield Monitoring, Precision Agriculture, Hay Baler, Alfalfa, Remote Sensing.

\section{Introduction}

Alfalfa (Medicago sativa) is one of the most important forage crops, which contains high nutrition value and digestibility. It is cultivated in more than 80 countries in an area exceeding 35 million ha. The most common use of alfalfa is in hay bales after a drying process (Radovic et al., 2009).

The spatial and temporal variations on crops and pasture yield may be related to environmental factors or management decisions (Trotter et al., 2008). Mapping yield variability is a useful tool for farm managers to understand the variability within their fields, and subsequently, support their management decisions (Maughan, 2012).

Currently, a wide range of satellite remote sensing applications has been adopted for different purposes, such as for environmental monitoring, meteorology and agriculture. Considering the commercial large farms with large areas, the conventional yield monitoring methods can be inefficient. Therefore, the remote sensing application in estimating crop yield has recently gained importance and popularity due to the improvements in the spatial and spectral resolution and the high temporal revisit of used satellites.

Most of research studies targeting yield prediction though remotely sensed (RS) data have two main strategies. One strategy is to involve the RS data with plant physiological and meteorological models to predict crop development and yield. The second strategy; however, relies on predicting yield directly from RS data, which works only if there is a direct relation between total leaf or biomass and harvest index, such as grain yield (Ferencz et al., 2004).

Trotter et al. (2008) investigated the relation between different vegetation indices and the biomass of triticale, native pasture, sorghum and alfalfa. Results showed that SAVI provided an $\mathrm{R}^{2}$ value that ranged between 0.87 to 0.69 for triticale and sorghum biomass, respectively. While alfalfa showed a poor relationship with $\mathrm{R}^{2}$ value of less than 0.1 , which was attributed to NDVI saturation.

\section{Materials and Methods}

This study was conducted on a commercial farm (Todhia Arable Farm) located at the eastern province of Saudi 
Arabia. One of the farm fields cultivated with alfalfa under a center pivot irrigation system was designated as the study field.

A large rectangular baler (CLAAS model: Quadrant 3200) equipped with a hay yield monitor (Model: Harvest Tec 500) was, after subjected to intensive calibration, used to bale and monitor alfalfa yield. The calibration of the yield monitor provided an $\mathrm{R}^{2}$ value of 0.87 (Kayad et al., 2015). This system was utilized to monitor the alfalfa yield for 5 cuts dated 20 Oct, 5 Dec, 16 Feb, 2 Apr and 6 May for 2013-2014 season.

Eighteen Landsat 8 satellite images were downloaded from the Earth Explorer service located at USGS/EROS, Sioux Falls, SD, USA (http://lpdaac.usgs.gov). These images were radiometrically corrected and subset to the region of interest using the ENVI software program. The vegetation indices were calculated using the ArcGIS software program (V. 10, ESRI, Redlands, CA, USA). The indices, including VI, EVI, NDVI, SAVI, GRVI and GNDVI, were calculated using equations 1-6 (Panda et al., 2010).

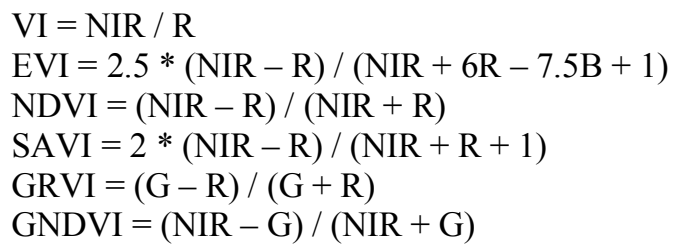

Where: R, G, B and NIR are the red, green, blue and infrared bands of Landsat- 8 images.

The calculated vegetation indices from Landsat- 8 data were overlaid with the actual yield data from the yield monitor. These data layers, for each cut, were subjected to statistical analysis.

\section{Results and Discussion}

The five maps of alfalfa yield from the study area showed a huge variation in field productivity. The spatial distribution of the yield was great enough to produce three distinctive zones with high, medium and low yield. This distribution was similar for the five cuts; however, the average yield generally decreased in cool periods. In order to investigate the seasonal impact on field productivity, the cumulative yield was calculated by combining the five cuts in one map. Results of this combination showed that the low productivity zone started from 6.14 ton/ha, while the high productivity zone reached a little more than 23 ton/ha. The five cut yield maps and the cumulative yield map are shown in figure (1).

Vegetation indices at different crop ages derived from Landsat-8 satellite images, such as NIR, VI, EVI, NDVI, SAVI, GRVI and GNDVI, have been correlated to alfalfa yield for the four alfalfa cuts dated 5 Dec, 16 Feb, 2 Apr and 6 May in 2013-14 growing season. Investigation of the relationships between the vegetation indices and the alfalfa hay yield revealed that the best hay yield indicative measures were observed to be NIR, SAVI and NDVI vegetation indices. Based on $\mathrm{R}^{2}$ values, these relationships were found to be stronger for data related to the 16 Feb, 2014 cut compared to the other three cuts (Table 1). For cut dated 16-Feb 2014, NIR, SAVI and NDVI vegetation indices provided the highest $\mathrm{R}^{2}$ values of $0.54,0.55$ and 0.53 , respectively, at a crop age of 16 days. The NIR correlation with estimated yield is shown in Figure 2. Also, the cumulative vegetation indices were calculated for each cut and correlated with the alfalfa yield. The highest correlations were observed for cumulative NIR, SAVI and NDVI with $R^{2}$ values of $0.69,0.68$ and 0.63 , respectively (Kayad et al., 2016).

Feb, 2014 alfalfa cut was during the winter season with a rainy weather, where a cooler weather existed and low irrigation practices were performed compared to the remaining cuts. During this period, crop produced an average low hay yield of $2.45 \mathrm{t} / \mathrm{ha}$ after a long growth period of 73 days. This could be attributed to the cold period from 5 December, 2013 to 16 February 2014, where grasses required a period of cool temperatures for flowering, and only reproductive stems produced during that period. A previous description for this cut conditions explained the different reasons for the relative high relationship between yield and indices from this cut in particular. The highest $\mathrm{R}^{2}$ value for this cut was observed from landsat-8 image at a crop age of 16 days. After this age, the NIR reflectance was saturated due to high leaf area index (Hanna et al., 1999), which explained the weak relationship.

It was observed, in Feb., 2014 cut, that in a crop age of 71 days, there were significant relations between alfalfa yield and all the VIs investigated in this study. Only in this cut, the growing period was 73 days, which was a long period compared to the other cuts (46, 45 and 35 days). This long period enabled the crop to enter into another physiological stage (flowering) that may have affected the light reflectance from this crop, and subsequently, the VIs.

For other cuts, the poor relation between alfalfa yield and vegetation indices could be due to high plant population and density with high level of green material and leaf area index, which may have saturated the NIR reflectance. These findings are in agreement with the results reported by Trotter et al. (2008) and Hanna et al. (1999). 
Alfalfa hay yield prediction from satellite images requires more research using different bands of light wavelengths, more VIs and to be investigated in different seasons. Even if it is not easy to estimate the yield from passive VIs, there is a specific stage after growing and before NIR saturation, between any two cuts according to seasons, which can provide the best period for alfalfa yield prediction (approximately the age between 15 to 23 days). Yield variation in the study area was huge; suggesting another study to understand the reasons for this variability, which could be due to topography or soil properties.
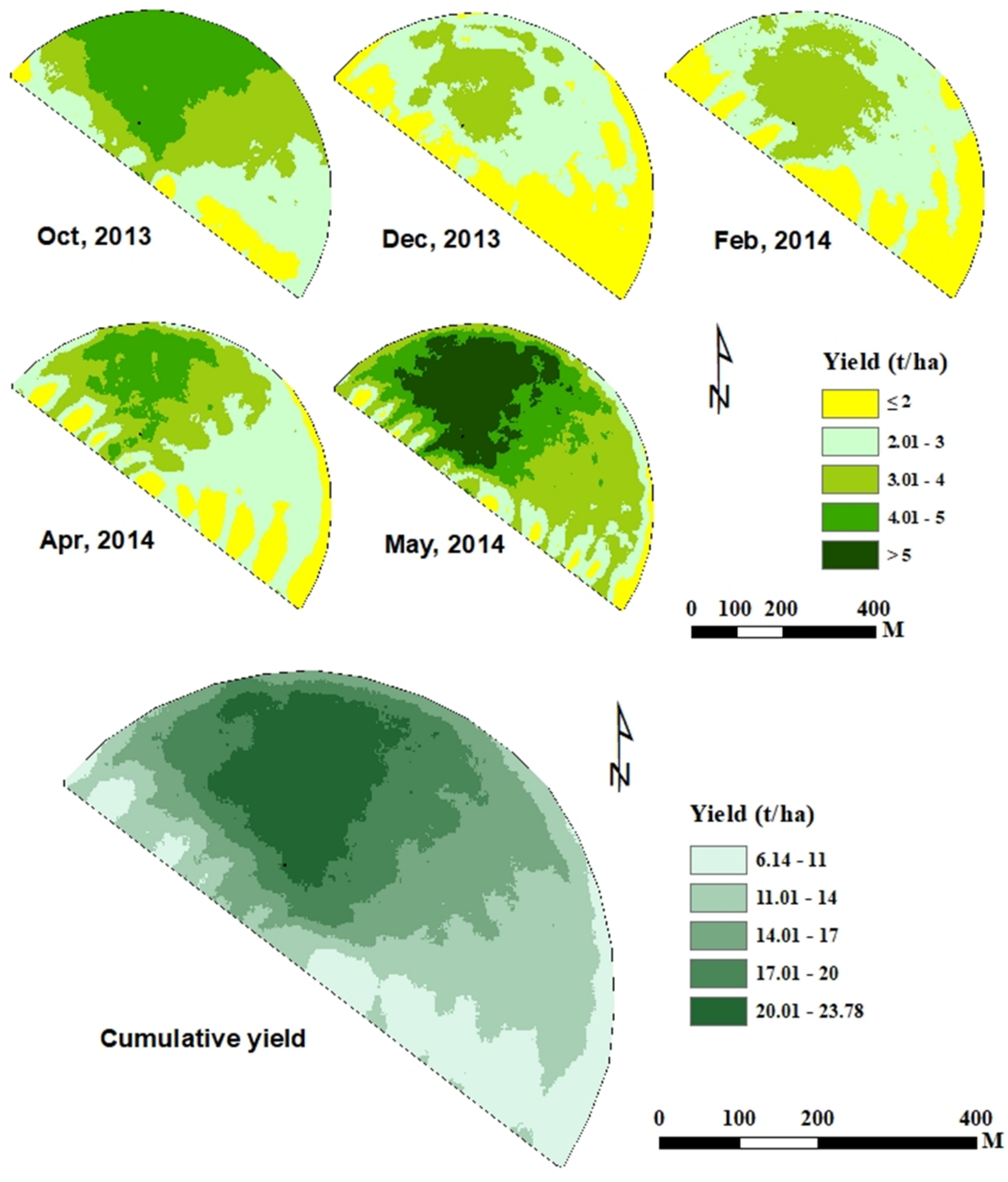

Figure 1. Five alfalfa yield maps from Oct, 2013 to May, 2014 and the cumulative yield map. 
Table 1. Correlation $\left(\mathrm{R}^{2}\right)$ between NIR, SAVI, NDVI with alfalfa yield at different ages.

\begin{tabular}{ccccc}
\hline Date & Crop Age & NIR & SAVI & NDVI \\
\hline 03-Nov-2013 & 14 & 0.195 & 0.192 & 0.174 \\
10-Nov-2013 & 21 & 0.034 & 0.017 & 0.011 \\
26-Nov-2013 & 37 & 0.012 & $>0.001$ & 0.013 \\
05-Dec-2013 & 46 & 0.057 & 0.016 & 0.004 \\
05-Dec-2013 & 46 & & & \\
\hline 21-Dec-2013 & 16 & 0.539 & 0.555 & 0.528 \\
28-Dec-2013 & 23 & 0.466 & 0.391 & 0.159 \\
13-Jan-2014 & 39 & 0.324 & 0.120 & 0.008 \\
22-Jan-2014 & 48 & 0.465 & 0.388 & 0.269 \\
29-Jan-2014 & 55 & 0.224 & 0.110 & 0.014 \\
14-Feb-2014 & 71 & 0.376 & 0.458 & 0.561 \\
16-Feb-2014 & 73 & & & \\
\hline 02-Mar-2014 & 14 & 0.137 & 0.040 & 0.004 \\
11-Mar-2014 & 23 & 0.248 & 0.154 & 0.018 \\
18-Mar-2014 & 30 & 0.146 & 0.061 & 0.070 \\
27-Mar-2014 & 39 & 0.203 & 0.060 & 0.018 \\
02-Apr-2014 & 45 & & & \\
\hline 12-Apr-2014 & 10 & 0.105 & 0.018 & 0.004 \\
19-Apr-2014 & 17 & 0.067 & 0.002 & 0.010 \\
28-Apr-2014 & 26 & 0.060 & 0.026 & 0.013 \\
05-May-2014 & 34 & 0.096 & 0.040 & 0.017 \\
06-May-2014 & 34 & & & \\
\hline
\end{tabular}

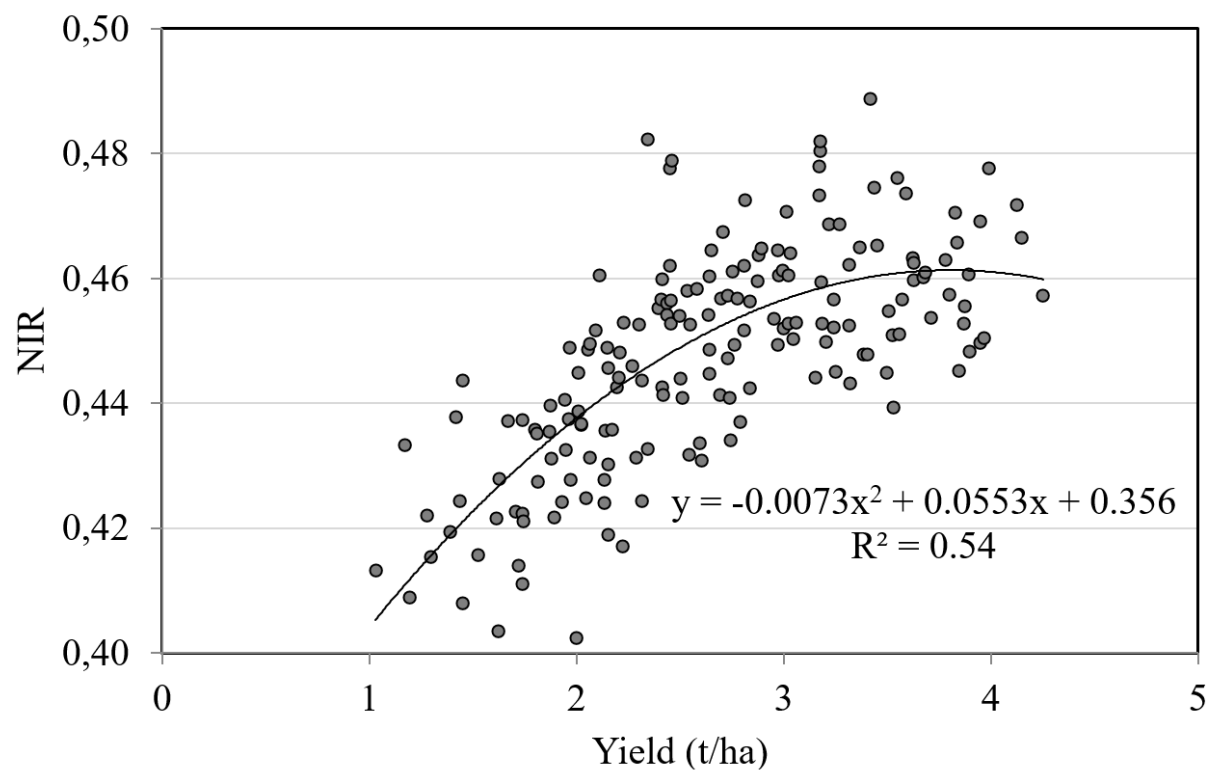

Figure 2. Estimated yield versus NIR of December 21, 2013.

\section{Conclusions}

NIR, SAVI and NDVI were found to be the best vegetation indices to predict alfalfa hay yield. These indices were found to be more correlated to yield during the cool season with an average $\mathrm{R}^{2}$ value of 0.56 at a crop age of 16 days. Also, it was observed that the correlation decreased as the crop age exceeded 20 days.

\section{Acknowledgements}

This study was financially supported by King Saud University, Vice Deanship of Research Chairs. The unlimited cooperation and support extended by Todhia Arable Farm (TAF) in carrying out the research work are gratefully acknowledged. 


\section{References}

Ferencz, C., P. Bognar, J. Lichtenberger, D. Hamar and G. Tarscai. 2004. Crop yield estimation by satellite remote sensing. International Journal of Remote Sensing, 25: 4113-4149.

Hanna, M.M., D.A. Steyn-Ross and M. Sten-Ross. 1999. Estimating biomass for New Zealand pasture using optical remote sensing techniques. Geocarto International, 14: 89-94.

Kayad A, Al-Gaadi K, Tola E, Madugundu R, Zeyada A. Performance evaluation of hay yield monitoring system in large rectangular baler. American-Eurasian Journal of Agricultural \& Environmental Sciences. 2015; 15: $1025-1032$.

Kayad, A, Al-Gaadi, K, Tola, E, Madugundu, R, Zeyada, A, Kalaitzidis, C. Assessing the spatial variability of alfalfa yield using satellite imagery and ground-based data. PLoS ONE 2016,11, e0157166.

Maughan, J.D., S. K. Mathanker, T.E. Grift and A.C. Hansen. 2012. Yield Monitoring and Mapping Systems for Hay and Forage Harvesting: A Review. ASABE Annual International Meeting, Hilton Anatole, Dallas, Texas, July 29 - August 1, 2012.

Panda SS, Ames DP, Panigrahi S. Application of Vegetation Indices for Agricultural Crop Yield Prediction Using Neural Network Techniques. Remote Sensing. 2010; 2: 673-696.

Radovic, J., D. Sokolovic and J. Markovic. 2009. Alfalfa-most important perennial forage legume in animal husbandry. Biotechnology in Animal Husbandry, 25: 465-475.

Trotter, T.F., P.S. Fraizer, M.G. Trotter and D.W. Lamp. 2008. Objective biomass assessment using an active plant sensor (crop circleTM) - preliminary experiences on a variety of agricultural landscapes. 9th International Conference on Precision Agriculture (ICPA). Denver, Colorado, USA. July 20 - 23, 2008. 


\author{
Structured Design of a Novel End-Effector for a Bush Trimming Robot \\ B.A.J. van Tuijl, A.P.M. Tielen, A. Mencarelli, J. Hemming \\ Wageningen University \& Research, Greenhouse Horticulture, P.O. Box 644, 6700 AP, Wageningen, The Netherlands \\ * Corresponding author. Email: bart.vantuijl@wur.nl
}

\begin{abstract}
The European TrimBot2020 project researches the robotics and vision technologies to prototype the first autonomous outdoor garden trimming robot. The robot navigates over different terrains, approaches boxwood plants and trims them to a desired shape. The robot platform is based on a modified Bosch robot lawnmower, which navigates autonomously using 3D-based vision scene analysis. During trimming a robotic arm is controlled by visual servo in order to trim the bush. A novel end-effector had to be designed to guarantee flexibility of the manipulator, precision of trimming and smoothness of the trimmed bush surface. This paper describes the structured design of this bush trimmer. When faced with a design problem with many interconnecting system elements, structured design is a tool to be used to iteratively and step by step guide the designers in making the right design choices at the right moment during the different design phases. First, preliminary research is done to analyse the problem and to assess the goals of the end-effector. Second, the functions are determined and working principles are found and put into a coherent structure. Finally, this leads to a composition of several preliminary designs of which the most promising one is determined. This design is built as a working prototype. Next to this, 3D-Computer Aided Design (CAD) tools and rapid prototyping is used to test ideas along the design process. The final design, based on contra-rotating blades, was discussed in terms of how and to what extent it has met the requirements, objectives and functions found during the structured design process. Moreover, the results of lab and field tests have shown the first functional results and points of improvements have been identified. A novel trimming method, by contra-rotating blades, has been found using structured design which meets the demands and limitations of other system components of the robot.
\end{abstract}

Keywords: robot, end-effector, structured design, bush trimming, mobile robot.

\title{
1. Introduction
}

In agriculture, robots and autonomous systems are nowadays utilized in more and more areas in for example grafting, packaging and harvesting of fruits, flowers and vegetables (Bac et al., 2014). Recently, for instance, the company Cerescon introduced an asparagus harvesting robot in the Netherlands (https://www.cerescon.com/NL/home) and the EU project Sweeper nears its completion of the sweet-pepper harvesting robot (http://www.sweeper-robot.eu/).

Nowadays, many people own a robotic lawnmower and thus automatic gardening has also raised the interest of companies and researchers on robotics, computer vision and artificial intelligence. In this paper, we present the development of an end-effector using structured design for a gardening robot that will allow the robot to trim bushes. It is currently under development in the EU project "Trimbot2020" (http://trimbot2020.webhosting.rug.nl/).

The TrimBot2020 project researches the robotics and vision technologies to prototype the first outdoor garden trimming robot. The robot will navigate over varying terrain, approach bushes and hedges to trim them to an ideal shape. The following European partners develop the vision soft- and hardware and control algorithms; University of Groningen (The Netherlands), University of Amsterdam (The Netherlands), University of Edinburgh (Scotland), Alberts-Ludwigs Unversitaet Freiburg (Germany) and the Eidgenoessische Technische Hochschule Zürich (Switzerland). The robot hardware; the robot carriage, manipulator, end-effectors and the control will be developed by: Robert Bosch GmbH (Germany) and Wageningen University \& Research (The Netherlands). With that many partners, the broad scope of interests and varying requirements it is of key importance to structure the design process. In this paper the design methods are addressed to guide the process of designing the Trimbot endeffector. Secondly, the outcome will be presented with which the first laboratory and field tests were made. Third, the results are discuss and reflect back on the design of this first version of the end-effector.

\section{Materials and Methods}

Design methods

Systematic design, assists researchers in design choices, structures the design process and stimulates creativity. Systematic design methods include either process-based design methods or systems engineering methods. Examples of process-based methods are structured design (Siers, 2004) or engineering design (Cross, 2000). The use of these two methods turned out to be effective in previous research (Van Henten et al., 2006; Bakker, 2010; Nieuwenhuizen, 2009). Examples of systems engineering in agriculture is the determination of number of robot arms, multiple arm configuration, degrees-of-freedom, and horticulture workspace design (Edan, Miles, 1994). 
Systematic design is the process of gradually developing the hardware and software components of a (robotic) system using information regarding needs of stakeholders, project objectives, requirements, functions, and working principles. Systematic design assures that a decision for a certain design is not just based on technical trade-offs, but also matches project objectives, project requirements, and needs of stakeholders. Definition of the requirements is a critical step in systematic design. In horticulture an end-effector must deal with varying shapes and sizes which might be taken care of using a dexterous or anthropomorphic hand. But on the other side, due to the hostile environment, the frequency of operation and the low market potential for robots in horticulture which drives the need for cheap solutions this dexterity must not be solved using a complex mechanism with a lot of moving parts, (Bac, 2014). The challenge is to find a low-cost, robust, simple and above all elegant design that ensures dexterity with a minimum of moving parts. The end-effector design problem must be thorough analysed to come up with the simplest solution. According to (Edan, 2000) for horticultural end-effectors, "the feasibility of the design concept can only be evaluated in field conditions under actual operating conditions".

\section{Structured design}

The design method used in this paper is based on structured design (Siers, 2004) and the first phase (out of three in total) of Reflexive Interactive Design (RIO in Dutch), (Bos, 2010). The first steps in RIO include a thorough analysis of the problem by listing the key challenges, actors, literature research and establishing the brief of requirements (BOR) (Figure 37, left). The second phase is based on structured design from which step three to five are used (Figure 37, right). Step one and two of structured design more or less coincide with step one, two and three of RIO. The structured design method is detailed in (Figure 38). This method is divided in five steps, starting with a preliminary research, problem definition, assessment of working principles, design, shaping and realization.

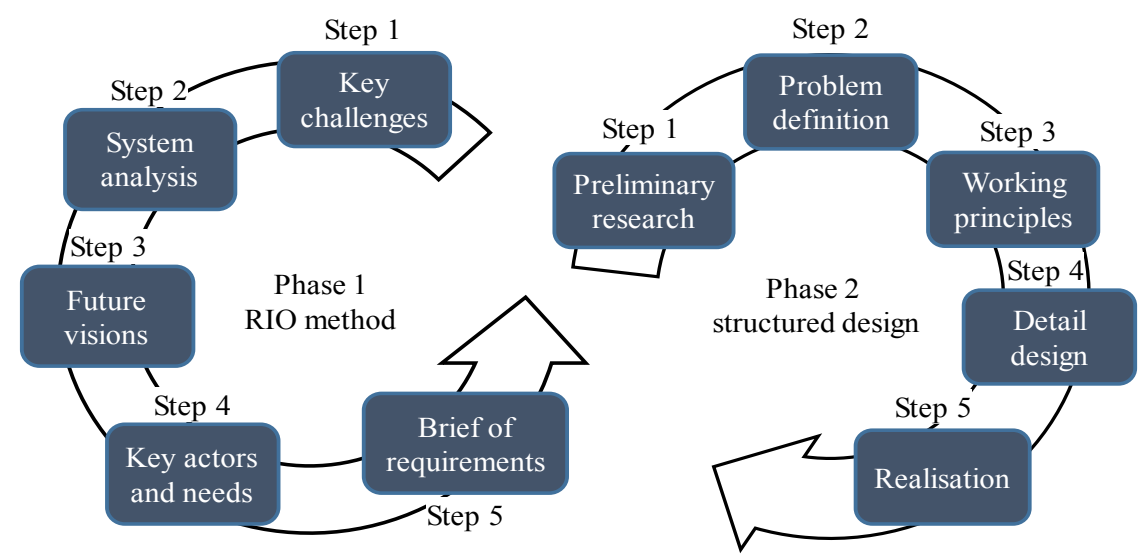

Figure 37. Graphical overview of the first phase of the RIO method. Hereafter, in phase 2, the methodical design method is used. Each phase consists of different steps (Bos, 2010).

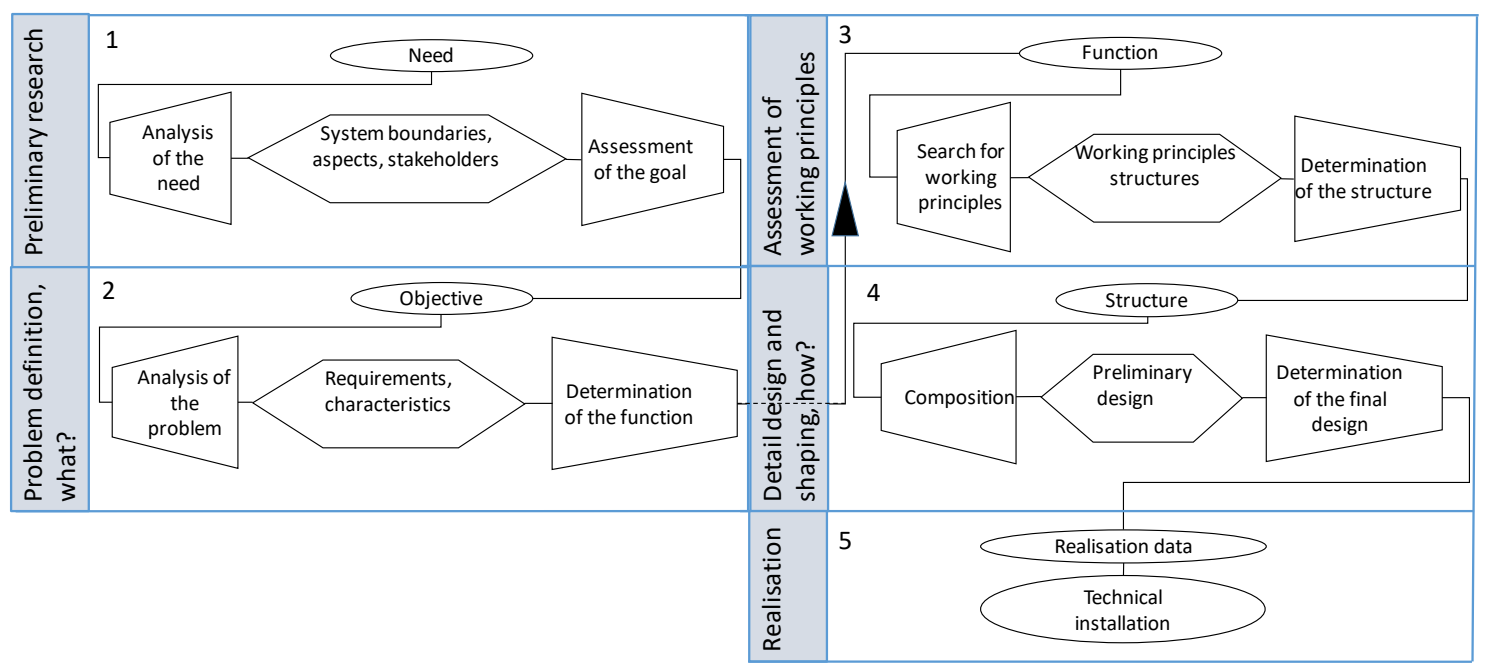

Figure 38. Overview of the five steps in structured design of Van den Kroonenberg. Adapted from (Siers, 2004). 
Each step in both design techniques consists of the same structure. First, it starts with an input followed by a diverging stage then an arrangement stage and finally a converging stage from which we draw conclusions, Figure 38. The result of one step is the input of the next step. Furthermore, the results of each step are not definitive and the development is an iterative method. For example, if a key function is missing during the development of concepts, this step will be adapted to make sure the solution space remains open for alterations. Due to the compactness of the article the description of all steps is not possible. We will discuss the most important findings, conclusions and discussions.

\section{Results and Discussion}

Determining needs, functions and requirements of the Trimbot end-effector

\section{Determining the Key challenges}

The first step of the RIO method is to determine the key challenges in the design of the Trimbot end-effector. The following drawing shows which system elements contribute to this process, Figure 39 . The key challenges describe what the end-effector must do in order to have a successful demonstration at the end of the Trimbot project. The description of the key challenges is used to derive the functions of the end-effector and consequently their attributes described in terms of requirements (fixed or variable), demands and wishes.

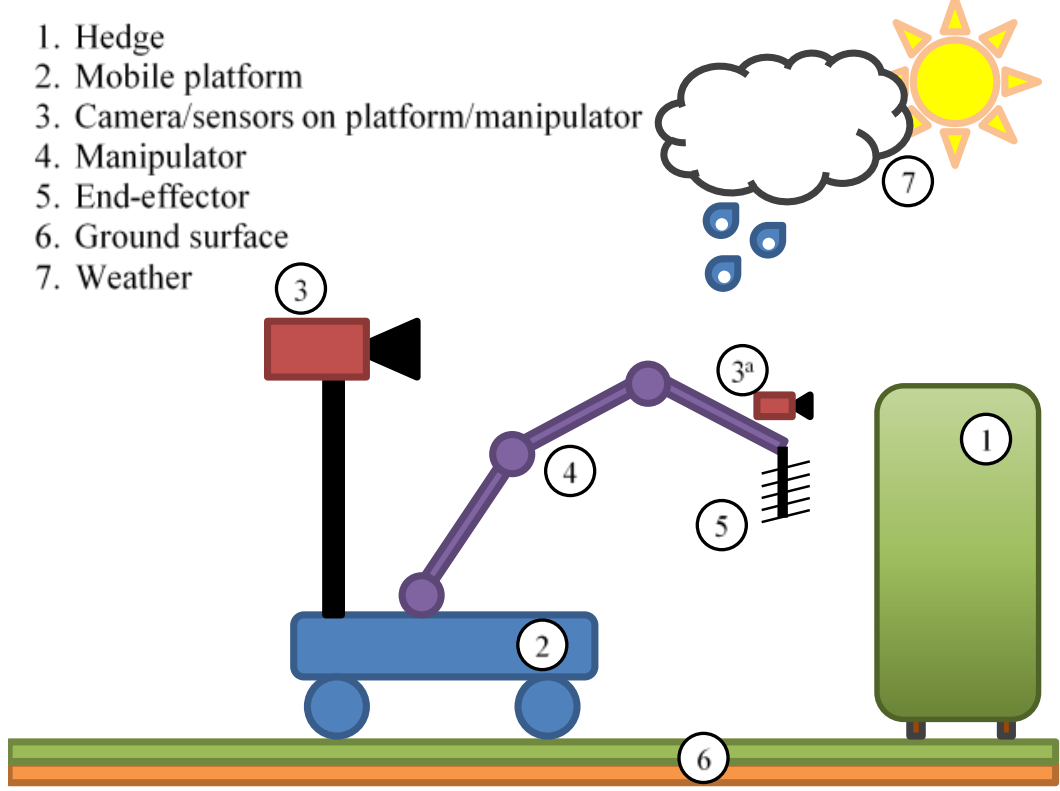

Figure 39. System elements of Trimbot which might contribute in terms of key challenges to design the end-effector.

The given system elements are used to derive the key challenges by analysing the following:

- What is the influence of element X on the Trimbot end-effector.

- How does this relate to the functions, behaviour and needs of the Trimbot end-effector.

Before we enter into this process the following demarks the design of the Trimbot end-effector and determines at a basic level what will be excluded from the design process:

- The aim is to design and build the Trimbot end-effector up to Technology Readiness Level (TRL) 5-6. Meaning, to build a prototype ready for testing in its relevant environment.

- Safety for the human end-user is not incorporated in the design of the Trimbot end-effector.

- The focus is on the design of the trimming tool. All other elements are outside the scope of our project.

- Engineering for cost-effective manufacturing is also outside the scope of the project.

Overall Key challenge and challenges related to system elements

The following key challenge was found analysing the description of work of the Trimbot 2020 EU proposal. "To design a trimming tool for an outdoor hedge and bush trimming robot for demonstration purposes, which can carried by a six degree of freedom manipulator to trim hedges and bushes to their, to be specified, shape by converting information given by the sensor system mounted on the end-effector into a desired action of the endeffector". Below some challenges of the end-effector that are based on the system elements are listed:

- Weather conditions: design an end-effector which is able to withstand different weather conditions and is able to perform well in different outdoor weather conditions (Sun, rain, wind, etc.). 
- Sensor system \& plant model: within the "limited" set of representations (line, surface) of the garden elements given by the sensor system, the trimming tool must be able to be orientated and positioned by the manipulator and to follow this set of representations.

- To design the right combination of end-effector and robotic arm (mechanism, size, accuracy) given the properties of the sensor system (accuracy, detail level).

- Given a defined quality from the plant model, controlled/measured by the sensor system, the manipulator will position and orientate the end-effector to a predefined point with a certain accuracy and then follows a succession of connected paths along a given line. The end-effector must perform its task along that path within the desired quality and within the reach of the robotic arm.

- During garden travel, the end-effector must not destabilize the platform and prevent branches from getting stuck.

- The end-effector should be able to trim with a predefined quality and accuracy based on predefined specifications, (see Table 16).

With these key challenges and the system elements in mind we are now able to conduct a system analysis.

\section{System analysis}

In order to visualize and understand the system, the Trimbot system is analysed from the description of work EU document. This gives insight in the problems/challenges and which functions needs to be performed in order to trim a bush autonomously and have a successful demonstration at the end of the project. Below a short overview of the different functions are given (Table 15) and one level is drawn in function blocks in Figure 40. In the end a complete function block diagram was made for the total system which at a later stage helped to design the control software for the Trimbot.

Table 15. Partial overview of functions and their sub-functions of the Trimbot system.

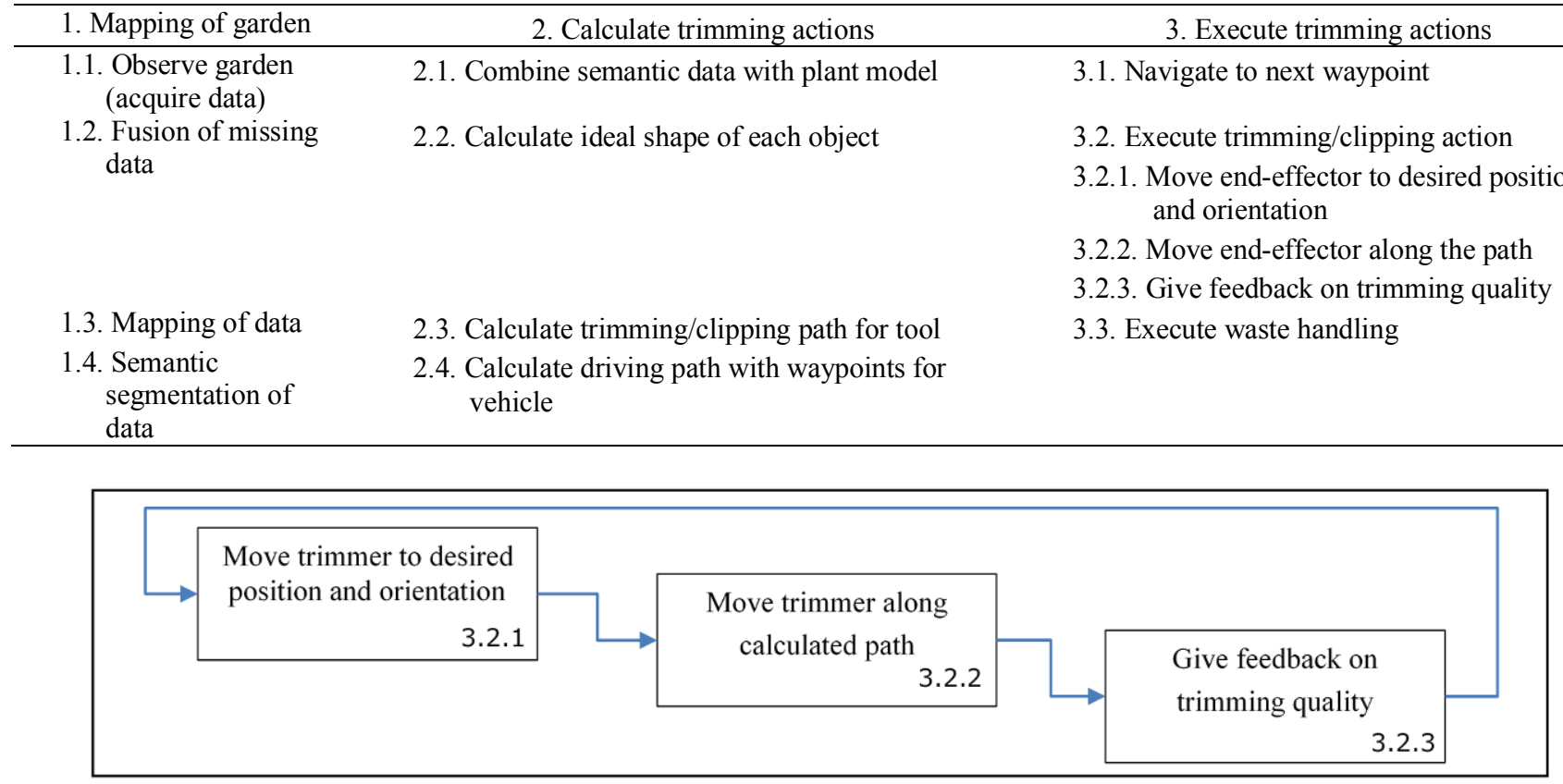

Figure 40. State machine function block of level 3.2 (execute trimming actions level 3).

Key Actors \& Needs

The purpose of this design step is to define the actors and their needs which play a role in the problem. This prevents the product of being based on only one actor. After identifying the actors, their viewpoint on the problem and their basic needs are being investigated. This ensures no viewpoints are missed during the design.

- Potential manufacturer

- Attractive product for customers.

- Explore and utilize new markets.

- Low-cost and safe tool to trim bushes.

- European Union

○ Induce technical development.

- To stimulate cooperation between universities and private companies. 
- A successful demonstration of the system resulting in a positive exploitation of the end results.

- Research groups vision

- A stable mechanism to mount camera's, sensors and lighting systems.

- An unimpeded view from the camera's, sensors mounted on the end-effector towards the bush.

- Research groups modelling and control

- Stable and predictable sensor information from the camera's and sensors mounted on the endeffector.

- To easily convert that information into trimming actions which are executed as predicted.

- Research groups trimming operations

- Use information about location and ideal trimming path to execute the trimming action.

- Development of a computer controlled end-effector.

- Development of a robot arm with visual servoing to enable the end-effector to perform its task.

- Visitors, demonstration and dissemination

○ Properly working machine.

- High technological level.

\section{Brief of Requirements}

Based on the key challenges and the needs of the key actors, a Brief Of Requirements (BOR) is constructed. In Table 16, for each component a set of requirements is stated. This gives the total of the design constraints in which solutions should be found. The fixed requirements should always be met and the variable requirements have a certain range. During the later design step, different technical solutions will be reviewed using this BOR.

Table 16. Brief of requirements (BOR) related to end-effector design (this is not the complete BOR).

\begin{tabular}{|c|c|c|c|c|c|c|c|c|c|}
\hline nr. & Component & Requirements & $\begin{array}{c}\text { fixe } \\
\mathrm{d} \\
\end{array}$ & $\begin{array}{c}\text { var- } \\
\text { iabl } \\
\mathrm{e} \\
\end{array}$ & $\begin{array}{c}\text { desi- } \\
\text { rabl } \\
\mathrm{e}\end{array}$ & target & $\begin{array}{c}\text { min. } \\
\text { value }\end{array}$ & $\begin{array}{l}\max . \\
\text { value }\end{array}$ & unit \\
\hline 1 & Common requirements & The system should be able to operate autonomously. & $\mathrm{x}$ & & & & & & \\
\hline 2 & & The system should be able to handle a relative humidity of $90 \%$. & & $\mathrm{x}$ & & 95 & 90 & 100 & $\mathrm{RH} \%$ \\
\hline 3 & & The system should be able to operate during sun-radiation of $1000 \mathrm{~W} / \mathrm{m} 2$ & & $\mathrm{x}$ & & 1200 & 1000 & 1200 & $\mathrm{~W} / \mathrm{m} 2$ \\
\hline 4 & & The system should be able to handle at least $5 \mathrm{~mm} / \mathrm{h}$ rain & & & $\mathrm{x}$ & 15 & 5 & - & $\mathrm{mm} / \mathrm{h}$ \\
\hline 5 & Robotic arm & The robotic arm should move without damaging the plant while reaching its desired position. & $\mathrm{x}$ & & & & & & \\
\hline 6 & & The robotic arm should be able to position and to orientate the trimmer as required & $\mathrm{x}$ & & & & & & \\
\hline 7 & & The robotic arm should be able to withstand the reaction forces during cutting. & & $\mathrm{x}$ & & 25 & 20 & 50 & $\mathrm{~N}$ \\
\hline 8 & & The robotic arm should be able to bring the tool to a height of at least 0.6 meter. & & $\mathrm{x}$ & & 0.8 & 0.6 & - & $\mathrm{m}$ \\
\hline 9 & & The robotic arm should be able to bring the tool 0.05 meter from the ground surface & & $\mathrm{x}$ & & 0 & 0 & 0.05 & $\mathrm{~m}$ \\
\hline 10 & & The robotic arm should have a accuracy of at least $5 \mathrm{~mm}$ at the desired point. & & $\mathrm{x}$ & & 0 & 0 & 5 & $\mathrm{~mm}$ \\
\hline 11 & & The robotic arm should have accuracy of at least $5 \mathrm{~mm}$ while following a path. & & $\mathrm{x}$ & & 0 & 0 & 5 & $\mathrm{~mm}$ \\
\hline 12 & & The robotic arm should have a payload of at least $2 \mathrm{~kg}$ & & $\mathrm{x}$ & & 3 & 2 & 10 & $\mathrm{~kg}$ \\
\hline 13 & & The robotic arm should be able to operate on a low voltage DC power supply & & & $\mathrm{x}$ & 12 & 6 & 24 & V DC \\
\hline 14 & Hedge trimming tool & The trimming tool should not harm other branches (touching is ok). & $\mathrm{x}$ & & & & & & \\
\hline 15 & & The trimming tool should be able to cut hedges, spheres, cubes, cones, pyramids and variations thereof. & $\mathrm{x}$ & & & & & & \\
\hline 16 & & The trimming tool should be able to cut surfaces with an accuracy lower than $5 \mathrm{~mm}$. & & $\mathrm{x}$ & & 2 & 0 & 5 & $\mathrm{~mm}$ \\
\hline 17 & & The trimming tool should be able to cut hedges with branches of at least a diameter of $4 \mathrm{~mm}$. & & $\mathrm{x}$ & & 4 & 4 & - & $\mathrm{mm}$ \\
\hline 18 & & The trimming tool should be lighter than $2 \mathrm{~kg}$. & & $\mathrm{x}$ & & 0.25 & 0 & 1 & $\mathrm{~kg}$ \\
\hline 19 & & The trimming tool should be able to cut at least $2 \mathrm{~m} 2$ per hour. & & $\mathrm{x}$ & & 5 & 2 & 10 & $\mathrm{~m} 2 /$ hour \\
\hline 20 & & The trimming tool should be autonomous interchangeable within 3 minutes . & & & $\mathrm{x}$ & 1.5 & 0 & 3 & $\min$ \\
\hline 21 & & The trimming tool should be able to measure the surface orientation and position (according to the plant model) & & & $\mathrm{x}$ & & & & \\
\hline 22 & Both robotic arm \& tool & The combination of robotic arm and tool hould be able to cut the desired hedges and roses. & $\mathrm{x}$ & & & & & & \\
\hline 23 & & The system should be able to cut hedges with a height of 0.5 meter. & & $\mathrm{x}$ & & 0.9 & 0.5 & - & $\mathrm{m}$ \\
\hline 24 & & The system should be able to cut hedges with a depth of 0.5 meter. & & $\mathrm{x}$ & & 0.9 & 0.5 & - & $\mathrm{m}$ \\
\hline
\end{tabular}

Preliminary research, evaluation of State-of-the-art in consumer and professional hedge trimmers

A comprehensive research is done in current state-of-the-art on bush trimming tools. Based on that, different designs are evaluated. In Figure 41 left, examples are shown of a motorized and a manual trimming tool. Motorized trimmers usually consist out of two blades of which one is usually stationary where a second moves over the stationary blade. Manual trimmers have a scissor type of configuration in which the blade length varies (Figure 41, second picture). The blades of professional trimmers, Figure 41 right, move both in opposite directions to provide a higher cut capacity. Most professional trimming tools are not easy adjustable for different plant shapes. Adaptability or flexibility is an important requirement for Trimbot. 


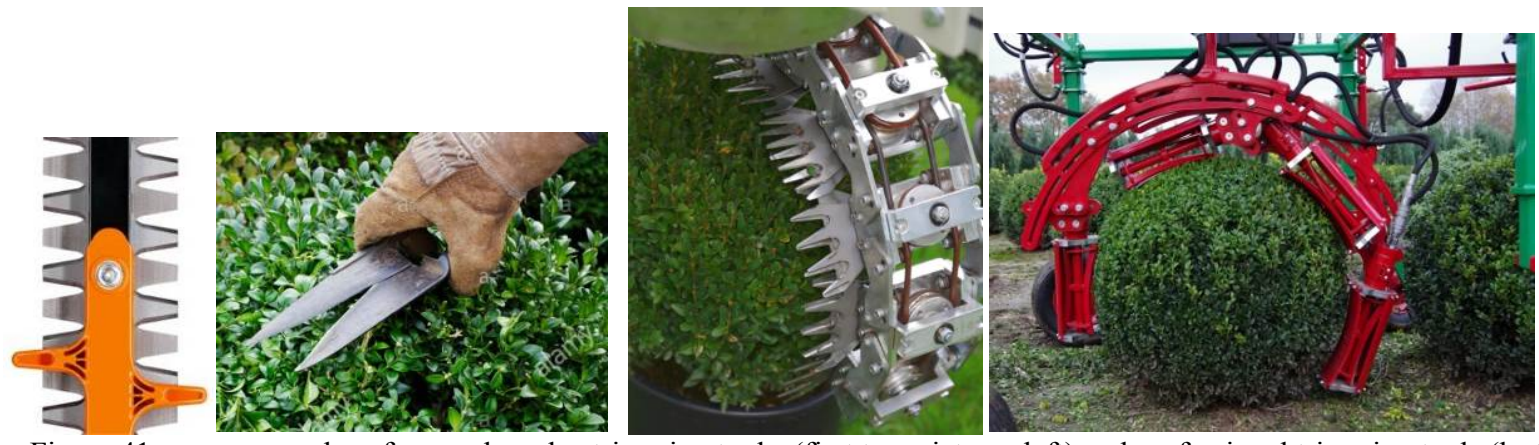

Figure 41. some examples of manual garden trimming tools, (first two pictures left) and professional trimming tools (last two pictures right).

Problem definition, evaluation of hedge trimmers in regards to the system element "manipulator"

The way the manipulator is intend to be controlled has an impact on the configuration of the end-effector and vice versa. From early on, the actors of the research group "modelling and control" expressed their need that the end-effector should ideally be used as a milling type cutter. That means that the last axis of the manipulator should coincide with the rotation axis of the hedge trimmer, Figure 42 left. In that way, the joint control of the manipulator resembles computer numerical control $(\mathrm{CNC})$ as is used in 6 axis $\mathrm{CNC}$ milling machines.
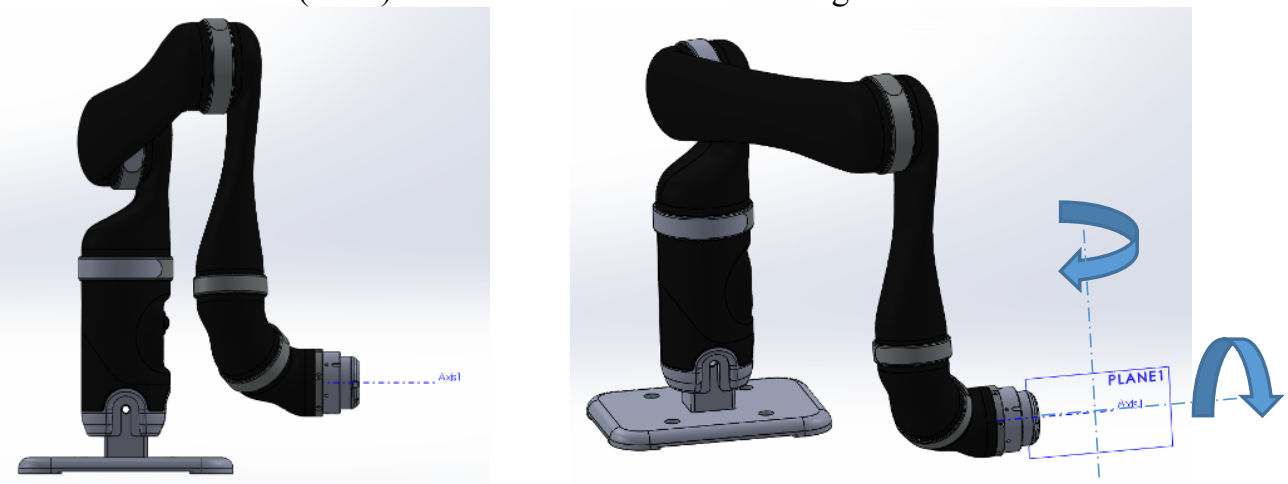

Figure 42. Left; last axis of the manipulator (Kinova Jaco 2 manipulator 6 degrees of freedom, Kinova, Boisbriand, Canada), coincides with the trimming tool. Right; a planar tool would add two degrees of freedom to control.

Using a planar like tool configuration, as shown in Figure 42 right, would add two more degrees of freedom to control by the manipulator. In that sense, coinciding axes offer benefits to solve more easily the joint control in mathematical terms. From that follows, using the idea of coinciding axes a circular knife would be a logical choice.

\section{Working principles and structures}

As shown in Figure 43, a morphologic chart has been made in order to evaluate the solution space for the design for the end-effector. For each function needed to fulfil the trimming task, different solutions (called concepts) have been explored. Also concepts from other fields (not agricultural) are evaluated, like cutting with heat, laser or high pressured water jets. The following examples of functions are evaluated (not a complete overview):

Method of separating a branch: concepts to separate the unwanted part of the branch

Configuration of sets of knives: if multiple knifes are used, ideas are found of how they are moved relative to each other. If a single knife is used, single is chosen.

Configuration to the branch: methods of how the knife(s) is/are applied relative to the branch.

Movement of knife: if a double blade is chosen, how the cutting blade moves relative to the contra-blade.

Energy supply: method of applying the force needed to perform the cutting task.

After all concepts were inserted in the chart, multiple design lines were drawn, by choosing one concept for each function. The performance of those design lines have been discussed with the key actors and some iterations to the concepts choices have been made to come to the final design solution. This final design line for the endeffector related to the method of cutting (in red) is drawn in Figure 43. 


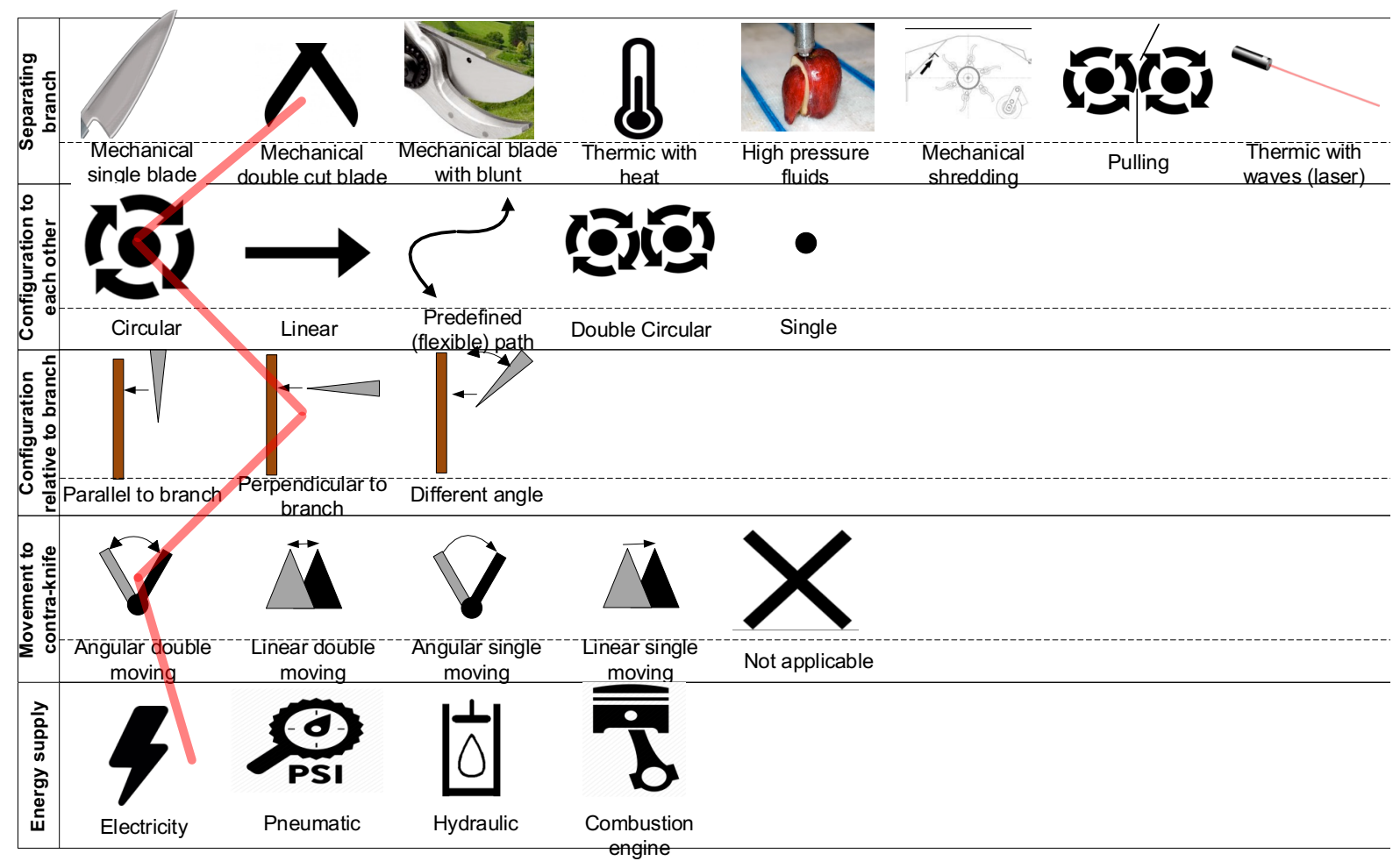

Figure 43. Morphological chart of working principles related to functions of the end-effector.

Detailed design and first realisation of the Trimbot end-effector

Summarizing the design steps of former paragraphs led to the most promising idea to have a circular knife configuration which axis coincides with the last axis of the manipulator. Because professional hedge trimmers, with linear knifes moving both in opposites directions, are successful in cutting branches in one movement, that particular mechanical configuration will be copied. This idea results in two circular counter rotating sets of knifes. One of these will have sharp edges whereas the other will act as an anvil with blunt edges. To enable the counter rotating movement of the end-effector blades we choose to buy an existing power tool (made by Powerplus tools, Lier, Belgium) that had that feature build in. This machine was stripped down so that the gearbox could be used in the design as presented in Figure 44. Furthermore, the original $230 \mathrm{~V}$ motor is replaced by a $24 \mathrm{~V}$ DC servo motor and control. This enables us to monitor the motor current and speed and provides the Trimbot platform to start and stop the end-effector using simple IO commands. The total weight is 2.1 kilograms and the motor and gear is protected by a 3D printed housing with embedded rubber seals to protect it from dust and rain and to give it an aesthetic look. Flat surfaces on the side and on top of the gear housing will provide space to mount camera's, sensors and or lighting systems (during this point in the project it was still under discussion what camera or sensor systems to use). The end-effector is mounted on the manipulator using 6 screws providing a quick way to swap the tool if needed. The first design looks as follows, Figure 44. 

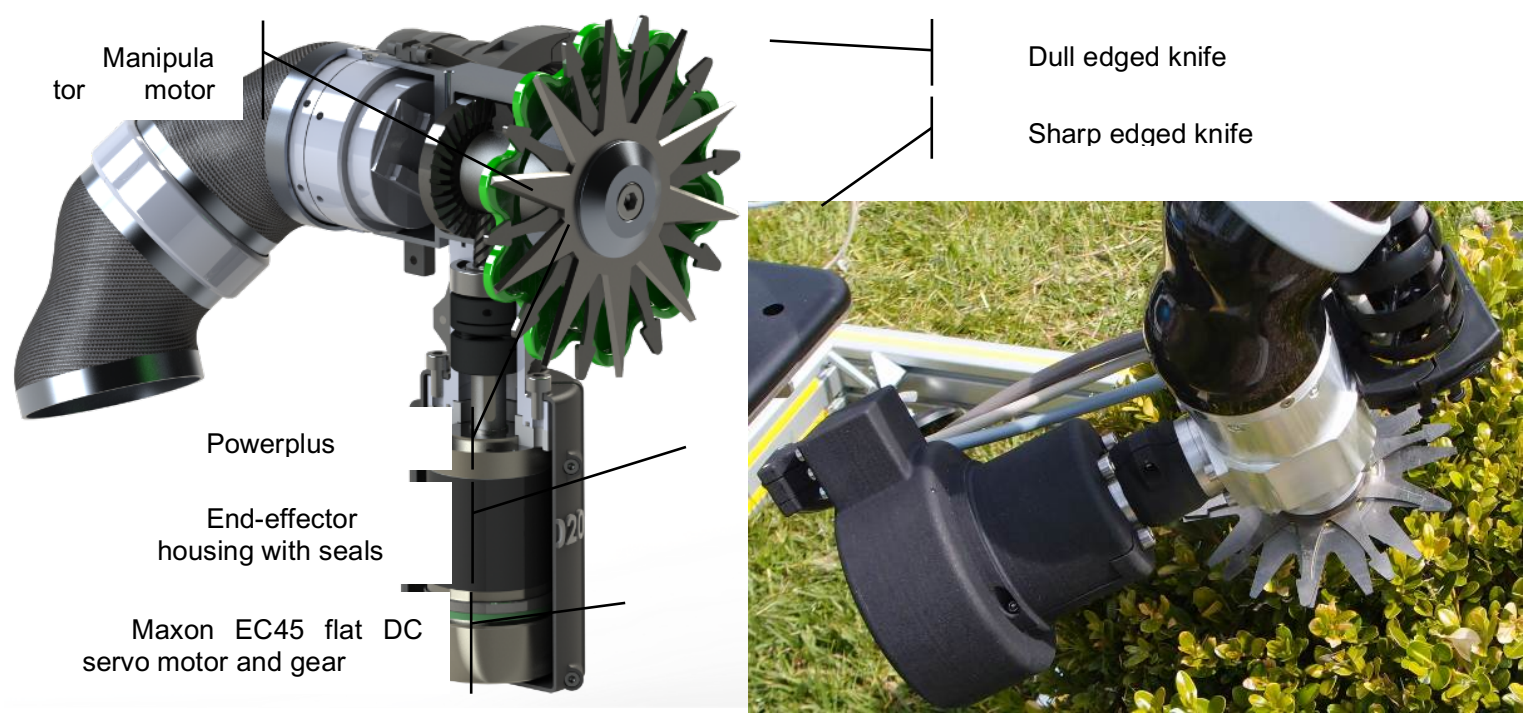

Figure 44. Cross section view (left) and the end-effector mounted on the manipulator during a first field test (right).

Final results

The manipulator with end-effector was mounted on a rig with a bush in front of it placed on a turn table to provide full access to the bush from all sides, Figure 45 left. Cameras were mounted on top of the end-effector to provide feedback of the initial position of the manipulator. The rotational speed of the blades was chosen by reviewing the resulting quality of the cut which turned out to operate best at $180 \mathrm{rpm}$. Next to that, the lab trail proved that the end-effector could easily cut through all the branches, Figure 45, right.
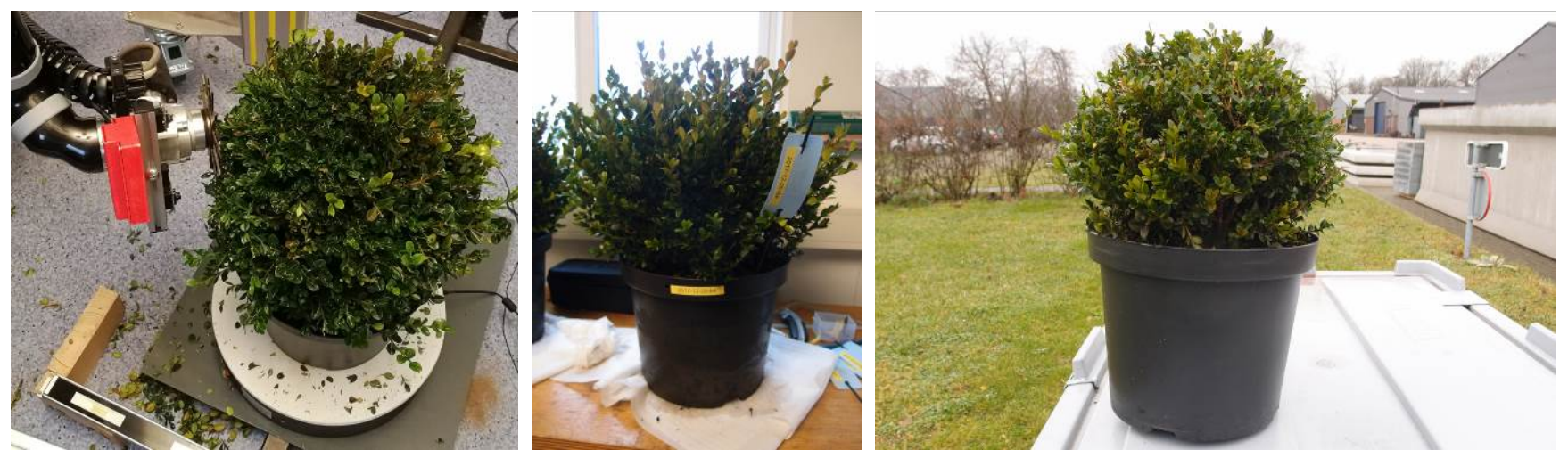

Figure 45. left; end-effector mounted on the manipulator during a lab test. The bush is set on a rotation table to provide access from all sides. Middle; bush before trimming and after trimming; right.

\section{Discussion}

The total system should be able, according to the BOR (Table 16), to trim the surface within $+/-5 \mathrm{~mm}$. Currently, the first evaluation (not presented in this paper) revealed that the system is not able to achieve this goal. Although many factors beside the end-effector design contribute to this, four are related to the end-effector design. First, the weight of the cables to power and control the DC servo motor of the end-effector were not taken into account during the design process. This ads 2 more kilograms to the configuration. The manipulator itself is designed to aid people with a disability and is not rigid enough to keep a high positioning accuracy with this extra weight. It therefore deforms and cannot follow the path within the desired limits given by the manipulator controller. Second, the motion planning strategy assumed an isotropic outgrowth of the braches of the bush (from the centre of the bush growing outwards) while in reality the braches grow anisotropic (from the base of the plant upwards towards the light). The design team currently thinks that the performance could improve if we take that into account into the motion planning of the manipulator. How to address this is still under discussion, a first idea is to plan the motion beginning at the base of the plant and then to move upwards to the top and to trim the bush in the direction of the branches. Third, the motor mounted directly underneath the tool obstructs the manipulator from getting at the lower parts of the plants. We have advised the key actor from the motion control group to add 
a constraint in the cost function of the last manipulator joint to turn the motor upwards while cutting near the ground surface. And finally, the camera housing and the position should be revised. Its current design, which was quickly added to the system at the last moment, is mechanically not sufficiently rigid. In the next few months leading to the field test during the summer of 2018 these problems will be addressed. The mass will be lowered by providing extra mounting points of the cables along the manipulator to distribute the weight, mount the motor at one of the joints of the manipulator and drive the blades using a flexible shaft. And finally, an integrated camera/sensor housing will be added to the end-effector that better protects the cameras and prevents the cameras from changing their mounting pose due to mechanical impact as requested by the vision group.

\section{Acknowledgements}

This project received funding from the European Union's Horizon 2020 research and innovation program under grant No. 688007 (TrimBot2020) and from the Dutch Topsector Tuinbouw and Uitgangsmaterialen.1

\section{References}

A.P. Bos, 2010. Reflexive Interactive Design (RIO), Wageningen UR Livestock Research, report 344

Cross, N., 2000. Engineering design methods: strategies for product design. Wiley. 212 p.

C.W. Bac, E.J. van Henten, J. Hemming, Y. Edan, 2014. Harvesting robots for high-value crops: state-of-theart review and challenges ahead. Journal of Field Robotics (2014), Vol. 31(6), p. 888-911

Nieuwenhuizen, A.T., 2009. Automated detection and control of volunteer potato plants. PhD thesis Wageningen University, Wageningen, The Netherlands (2009) Ch. 1.

Siers, F.J., 2004. Methodisch ontwerpen. Noordhoff Uitgevers B.V. 223 p

T. Bakker, K. Asselt, J. Bontsema, J. Müller, G. van Straten, 2010. Systematic design of an autonomous platform for robotic weeding. Journal of Terramechanics. 47. 63-73. 10.1016/j.jterra.2009.06.002.

Trimbot 2020 description of work: A gardening robot for hedge and topiary trimming. Proposal acronym: TrimBot2020 Work programme topic: ICT-24-2015/Robotics - advance key technologies relevant for industrial and service robotics

Van Henten, E. J. (2006). Greenhouse Mechanization: State of the Art and Future Perspective. Acta Horticulturae (710), p. 55-69.

Y. Edan, D. Rogozin, T. Flash, G.E. Miles, 2000. Robotic melon harvesting. IEEE Transactions on Robotics and Automation, 16(6), 831-835.

Y. Edan, G.E. Miles, 1994. Systems engineering of agricultural robot design. IEEE Transactions on Systems, Man and Cybernetics, 24(8), 1259-1265. 


\title{
Development of Autonomous Robotic Platforms for Sugar Beet Crop Phenotyping using Artificial Vision \\ Bernard Benet ${ }^{\text {a, }}$, Camille Dubos ${ }^{\text {a }}$, Fabienne Maupas ${ }^{\text {b }}$, Ghislain Malatesta ${ }^{\text {b }}$, Roland Lenain ${ }^{\text {a }}$ \\ ${ }^{\mathrm{a}}$ IRSTEA, UR TSCF, 9 avenue Blaise Pascal, 63178 Aubière, France \\ ${ }^{\mathrm{b}}$ ITB, 45 rue de Naples, 75008 Paris, France \\ * Corresponding author. Email: bernard.benet@irstea.fr
}

\begin{abstract}
Phenotyping is a major challenge in international agronomic competition. In a perspective of modern and sustainable agriculture, understanding the relationship between genotype and phenotype according to the environment is one of the major projects of agronomic research. Artificial vision devices embedded on robotic platforms, working in visible or hyperspectral color fields permit to carry out many geometric and colorimetric measurements on crops. From this information, operations like crop varieties comparisons and disease detection are realized. For sugar beet crops, phenotyping operations are made from a two small leaf stage up to the final stage just before harvesting task. Two robotic devices were used to make colorimetric and geometrical measurements on sugar beet plants. An autonomous mobile robot navigating in crop lines for little growth stages, embedded two cameras. A first one, with an oblique orientation permitted to realize autonomous crop raw tracking and the second one in a vertical position was used to record cartographic images and make detailed measurements on sugar beet plants. The second robotic platform was a manipulator arm with 6 degrees of freedom, fixed on a mobile linear axis to make measurements for advanced growing stages. Active perception operations realized with the embedded camera fixed at its extremity, consisted in locating by artificial vision the plant leaves in 3D environment and from this information, the camera was automatically positioned at various desired heights and orientations for each detected leaf, for carrying out, with accuracy, image acquisitions and measurements. Experimentations realized with both robotic platforms, for various sugar beet growing stages, shown the interest of these devices for following and analyzing in detail the geometric and colorimetric evolution of sugar beet plants in the fields, in order to carry out some phenotyping measurements and particularly for detecting some diseases.
\end{abstract}

Keywords: Mobile robot, vision, active perception, manipulator arm, phenotyping

\section{Introduction}

In recent years, the use of new technologies has become widespread in agriculture, through precision farming, with the aim of improving agricultural operations. The evolution of increasingly sophisticated perception sensors has enabled the development of high performance autonomous navigation systems, which can in particular perform agricultural tasks of crop monitoring, and measurements for different types of plants, while limiting human intervention, relatively restrictive. An important point of growing interest for the agricultural community is the protection of crops against a variety of factors that lead to reduced yields, such as diseases, that can affect plants during the growth process. Agriculture has become much more than just a way to feed ever-growing populations. Indeed, plants have become an important source of energy, and are a fundamental piece in the puzzle to solve the problem of global warming. There are several diseases that affect plants with the potential to cause devastating economic, social and ecological losses. In this context, diagnosing diseases in an accurate and quick way is of utmost importance. There are several ways to detect plant pathologies. Vision devices are currently used to detect some diseases, which can be seen in the visible or invisible lighting spectrum. So, it is necessary to work with RGB cameras and also with hyperspectral devices to develop image processing tools for disease detection. A large amount of information on the subject can be found in the papers by (Bock et al., 2010), (Mahlein et al., 2012) and (Sankaran et al., 2010). Fungal diseases have recently led to losses in world production, especially for sugar beet, wheat or maize. Also, in (Sharr et al., 2016), we find a comparison of leaf detection algorithms presented at the Leaf Segmentation Challenge in 2014. Article in (Barbedo et al, 2013) contains a state of the art of disease detection from different types of sensors (RGB, thermal, hyperspectral ...). The improvement of disease detection by automatic objective tools, which could replace tedious and imperfect human visual detection, has therefore become a major concern for agricultural producers. Reliable disease detection methods at the microscopic scale are also used (Henson et al., 1993) and (Hobbs et al., 1987), but are destructive because they require samples of plants. There is therefore a need for new innovative techniques to detect diseases as quickly as possible in crops, to prevent the spread of these diseases over large areas. The implementation of sensors technologies and algorithms for automatic phenotyping are of increasing importance. Sensors such as 3D Timeof-Flight cameras can be used for phenotyping under outdoor conditions (Kloze et al, 2011) and (Paulus et al, 2014). The influences of direct light, speed, humidity and dust on the measurements of the characteristics of the cameras and color dependence, noise level and depth resolution were analyzed by the application of phenotyping.

The benefit of analyzing the information is the ability to develop strategies to optimize the quality of their processing, e.g. next to spectral information on these important phenotyping methods is the exact measurement 
of the plant's morphological characteristics, for example, leaf size, leaf configuration, angle of the tree, plant height or stem thickness. In this paper, we will focus on the development of phenotyping tools and techniques to realize automatically by artificial vision, geometric and colorimetric measurements on sugar beet plants. In this application, we will be interested in two phenotyping platforms, to work on different stages of plant growth. On the one hand, a mobile robot carrying two vision systems to navigate crops for low lift stages and perform different measurements, and on the other hand, a mobile manipulator was carrying a camera for high growth stages. With regard to this second platform, the objective is to develop an autonomous robotic system allowing the camera to be placed at the desired location with respect to the plants, by means of active perception techniques. Its goal is to realize precise image acquisitions, in order to detect and identify leaf diseases, which usually appear when the plants have reached an advanced stage of maturity, and to quantify the diseased surfaces. During the first year of this project, a feasibility study, related to the development of a robotic system intended to perform measurements by artificial vision for the detection of diseases on beet leaves and the study of their propagation in crops, was carried out. This study led to the choice of the robotic system named BettyBot chosen for phenotyping operations and particularly for disease detection on sugar beet plants.

\section{Materials and Methods}

Two robotic platforms were used for phenotyping operations: a light mobile robot for low level stages and a big one composed of a linear axis and a manipulator arm, for high level stages.

\section{Mobile robot}

For little vegetation stages, a light mobile robot (weight: $30 \mathrm{~kg}$, length: $50 \mathrm{~cm}$, width: $30 \mathrm{~cm}$ ) was used (Figure 1). This one embedded two cameras to carry out two operations: a Webcam and a Gigaethernet device. The first one, in an oblique position, permits to achieve a crop row tracking by visualizing three beet lines and the second one realizes image acquisitions on the central line to obtain a cartographic image and make measures for phenotyping operations. Both operations were achieved in parallel and in real time. Image processing algorithms were developed in order to autonomously follow the crop lines, realize measurements and record images of the central line. At the end of the navigation, the 2D cartography of the central line was obtained. From this one, geometric and colorimetric information were obtained (beet counting, spacing between plants, leaf area, level of red, green and medium blue, presence of black areas in the leaves, ...).
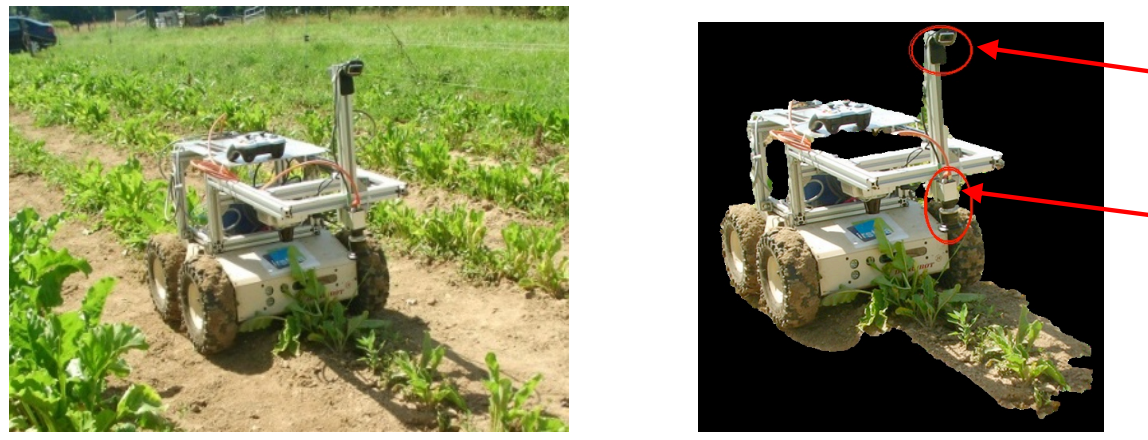

Webcam camera

Resolution: $1280 \times 720$

GigaEthernet camera

Resolution: 2044 x 2044

Figure 1. Mobile robot with both embedded cameras.

Various robotic platforms carrying vision systems to perform various agricultural operations have been developed, for phenotyping operations, as example (Subramanian et al., 2012) and (Gustavo et al., 2012). (Åstrand and Baerveldt, 2012) have developed a robotic platform, similar to our platform, with the use of two vision devices for weed control on sugar beet crop lines, one for crop raw tracking and the other for detection and measurements on crops. Different methods are used for vision tracking by detecting the vanishing point (Ding et al., 2014 ) or (Kong et al., 2013). The intuitive idea is to make an assumption about the relative position between the vehicle and the crop lines that must be corrected by observing the image. With the development of precision agriculture, a lot of work has been done in order to be able to use assistance robots (assistance to the driving of agricultural machines) or even autonomous ones. For this purpose, it is necessary to be able to follow cultures in a precise way. Concerning the use of a camera for those crop raw tracking, different approaches are used. Hough method is often applied on images to find crop lines, after applying thresholding and morphological operations, such as (Rovira-Mas et al., 2005) or (Montalvo et al., 2012). An interesting way of determining the position and orientation of crop lines in the image is outlined in (Gee et al., 2008). The author starts from a wide view of the rows (at least 6 visible) that segment from the function of excess of green, and applied two successive Hough methods to finally obtain the vanishing point and the width of the rows. One way to detect crop lines when many 
weeds are present is also exposed in the literature. To that end, two thresholding operations are used. The first one permit to isolate plants and the second one is applied to distinguish the plants from the weeds.

Finally, to find the culture lines, a linear regression is made around the average position of the rows. This method is certainly very powerful, but the model used imposes that the variations of position of the lines in the image is weak. Given the requirements of the specifications and a priori knowledge of the environment, a bottomup approach seems to be preferred. Indeed, there are two general principles for a procedural approach: the socalled top-down approach and the bottom-up approach. In the first case, we start from a large amount of data that must be broken down and sorted in order to obtain a result. In the second case, we try to confirm a hypothesis thanks to the data of the sensors (which requires information on the environment in which the system evolves).

In our application, for crop raw tracking with mobile robot, a geometric model of the robot has been defined, taking into account the position of the Webcam on the robot as well as the geometry of the ground and the spacing between the beet lines (Figure 2), to achieve a line tracking task with the Webcam, which was positioned so that it could visualize the vanishing point of the crop lines on the images.

\section{Geometric parameters of the robot model}

- X0 lateral deviation of the robot from the central line

- -Y0 the distance from the robot's center to the camera following the direction of robot moving

- Z0 the height of the camera relative to the center of the robot

- $\alpha$ yaw angle

- $\beta$ pitch angle

- $\gamma$ angle of the camera relative to the plane of robot moving
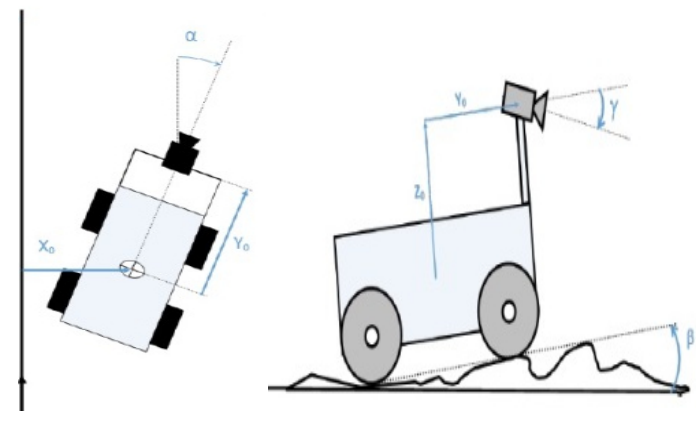

Figure 2. Mobile robot geometric model for crop row tracking.

The technique used in this application is to define restricted search areas, from the knowledge of the environment, in which the objects to be detected (beet leaves) must be located. One of the major interests of these methods is to reduce the computation time by only looking at interesting parts of the image while ensuring a result consistent with reality. To be able to find the lines of culture, one estimates at first (from the knowledge a priori) the state of the mobile robot, in real time. Then, the lines around their estimated position are searched for the current state of the system. To carry out these operations, a model of the robot with the camera, in the given environment, must be developed. The complete image processing method is the following: first, the original image is thresholded and binarized, using image processing algorithms such as color classification and mathematical morphology operations (OpenCV functions), to detect beet leaves. Then, the image is divided into different horizontal and vertical zones ( 3 columns corresponding to three crop lines, in which the gravity centers of each leaf are searched). A linear regression operation allows obtaining sugar beet rows for the three lines. From this information, the application of a Kalman filtering makes it possible to automatically define and limit the search zones of the three beet lines, during the successive searches of beet leaves on the images acquired by the camera (Figure 3). Finally, visual control operations are applied for the crop row autonomous tracking task.

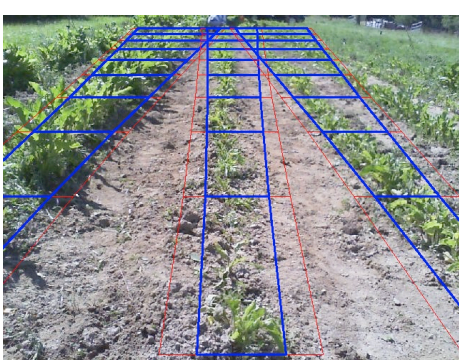

Line detection $1^{\text {st }}$ step

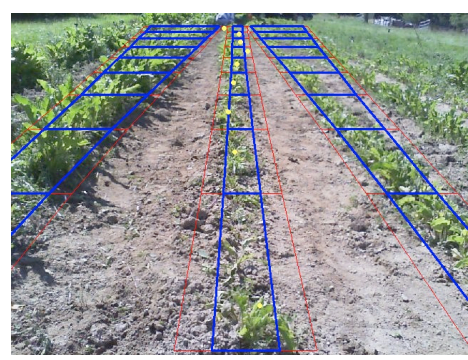

Line detection Medium step

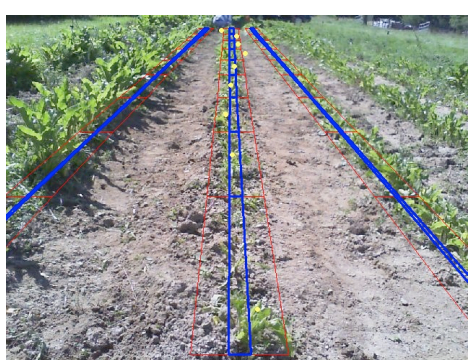

Line detection final step

Figure 3. Detection of crop lines using Kalman filtering.

\section{Bettybot Robot}

Figure 4 below shows Bettybot robot in a laboratory with a camera at its extremity, which will be used for phenotyping measurements for advanced level stages. It included a motorized linear axis, on which a manipulator arm with six degrees of freedom is mounted/fixed (UR5 Universal Robot model). At the end of this arm, a vision sensor such as a color camera or a hyperspectral camera was embedded to collect/take images on sugar beet crops, 
with the possibility to acquire images with various desired heights and orientations. Figure 5 presents this robot embedded on a tractor for the application in the fields.

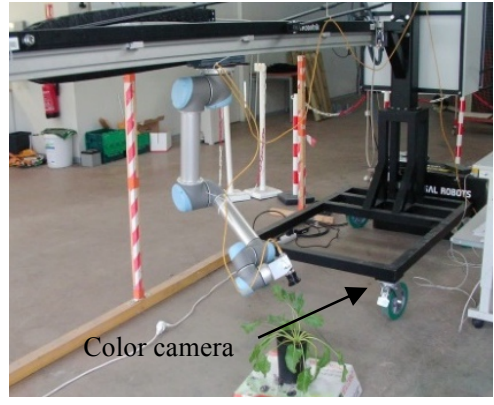

Figure 4. Bettybot robot with GigaEthernet camera.

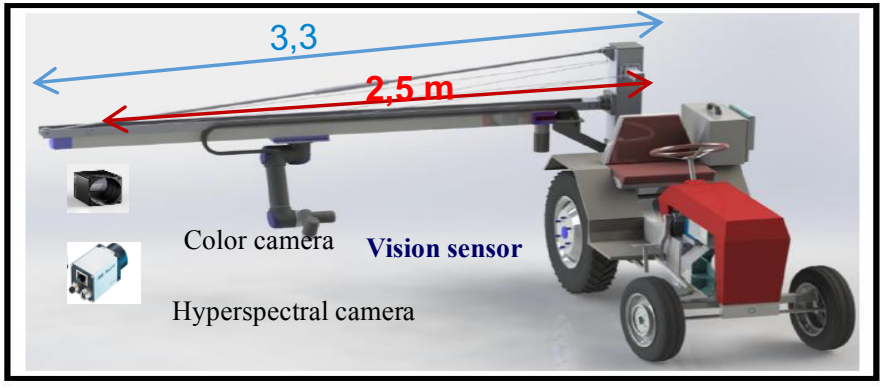

Figure 5. Bettybot embedded on a tractor.

The general application with this robotic platform is shown in Figure 6. The tractor navigates outside the crop lines and stops when the linear axis is aligned with a crop line. Then, the mobility of linear axis and manipulator arm permit to acquire and compute images on lengths of beet line of about $3 \mathrm{~m}$.

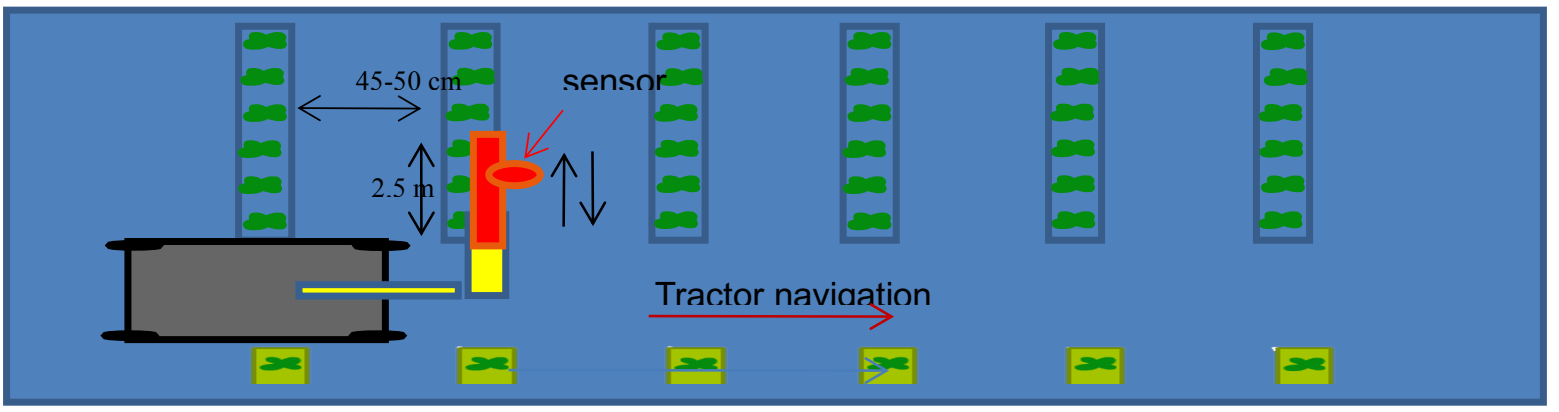

Figure 6. General operations in sugar beet crop lines.

The use of such a system makes it possible to observe the beet leaves according to different points of view, thanks to the complementarity between the manipulator arm and the linear axis, which enables the sensor to reach a large number of positions and orientations in relation to the leaves and to be able to work on different plants. However this association causes difficulties in the control. Indeed, this system is redundant, that is to say it has more control variables (degrees of freedom) than sensor position data. Here, our system has seven joints, so seven variables of commands on which one can act, to place the sensor to a pose (position and orientation) defined by 6 values ( 3 for the position and 3 for the orientation). There are infinite configurations of the system to place the sensor in a desired pose. Control/command algorithms have been developed for this operation, by acting on the six degrees of freedom of the UR5 arm and taking into account the problems of redundancy. The interest of the phenotyping platform is that its behavior adapts according to the perception of the state of the vegetation. Thus, the movements of the system must be enslaved from observation algorithms of beet plants, to ensure the most accurate detection as possible. It is also necessary to take into account the specificities related to the evolution in natural environments in these controls, such as the variation of the brightness, to adapt the behavior of the robot. The main task is to detect the beet leaves and then observe them using the sensor located at the end of the manipulator arm, in order to detect diseases. This task can be broken down into different functions: the first will be to detect the different leaves to be observed, then to place the sensor close enough above them, and finally to move around to determine the angle of view allowing obtaining the maximum relevant information for the detection of diseases. The work done so far is focused on two areas: perception, through crop detection, and control, including, among other things, the management of system redundancy.

Sugar beet leaf detection

A pixel color classification in RGB color space, using SVM method, was made, in order to discriminate both pixel types (sugar beet leaf and ground pixel) (Figure 7), considering different ground colors and beet leaves corresponding to various level stages (various green colors). 

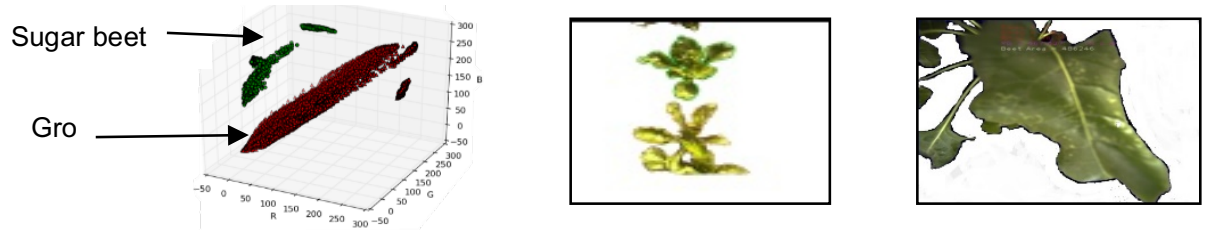

Figure 7. Color discrimination between sugar beet leaf / ground points using SVM classification.

\section{Results and Discussion}

Crop row tracking result

Figure 8 shows an example of a result obtained from monitoring beet line detection, with Webcam camera with the three steps of the developed algorithm: in (a) thresholding operations with the application of SVM classification and morphological operations to detect sugar beet crops and remove noise, in (b) the gravity centers of leaves in each defined rectangular area are obtained, and in (c) an example of final result obtained is presented, with the use of a Kalman filtering operation for improving the crop line detection. From this information and taking into account the position and orientation data of the camera embedded on the robot, the lateral deviation between the robot and the central line was obtained. Then a referenced vision command was applied for autonomous robot navigation.

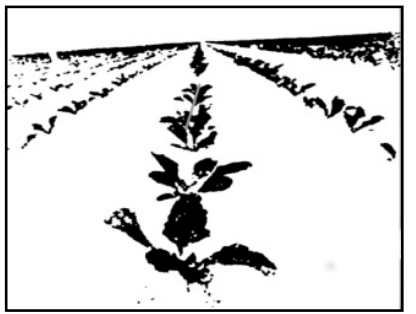

(a)

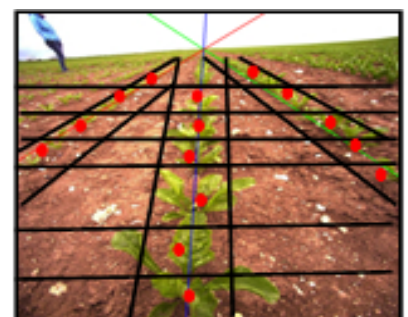

(b)

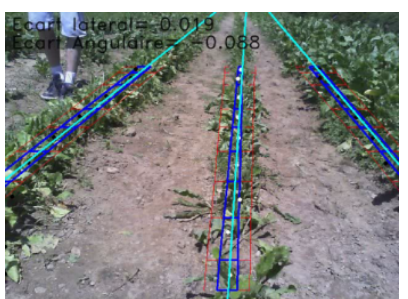

(c)

Figure 8 . Sugar beet line detection with Webcam camera.

2D mapping of beet crop lines

With the GigaEthernet camera, the objective was to acquire images on the central line, to obtain, after image processing, a cartographic image. From this cartographic image, geometric measurements can be made, such as the counting of plants and leaves, the spacing between the plants, the leaf area, but also colorimetric measurements, allowing to analyze in detail the colorimetric variations. on the different leaves (Figure 9).

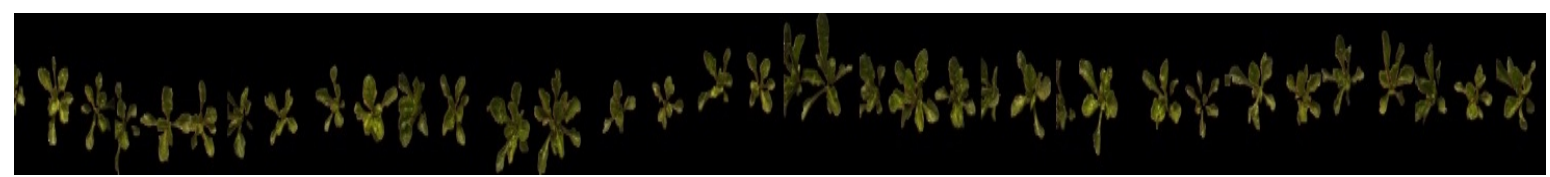

Figure 9. Cartographic image of a sugar beet line obtained with GigaEthernet camera.

From this 2D mapping, geometric and colorimetric measurements were carried out, such as beet plant counting, leaf area of each plant, distance between plant, mean color in RGB or in HSV color space, in order to analyze the color difference between leaves, to detect some disease and to see the growing level of the culture. Also, data obtained with these cartographic images, at different time during the growing evolution, permit to make comparisons between crop varieties and also between fields. 
Detection and localization of beet leaves with Bettybot robot

An image processing job has been performed. The objective was to detect the beet leaves of a potted plant in a global manner and to achieve measures on this set of pixels belonging to the beet leaves, such as the position of the gravity center and total leaf area (Figure 10). From these image data, control/command operations of UR5 manipulator arm was performed. The goal was to use the image data to position the robot extremity above a plant.
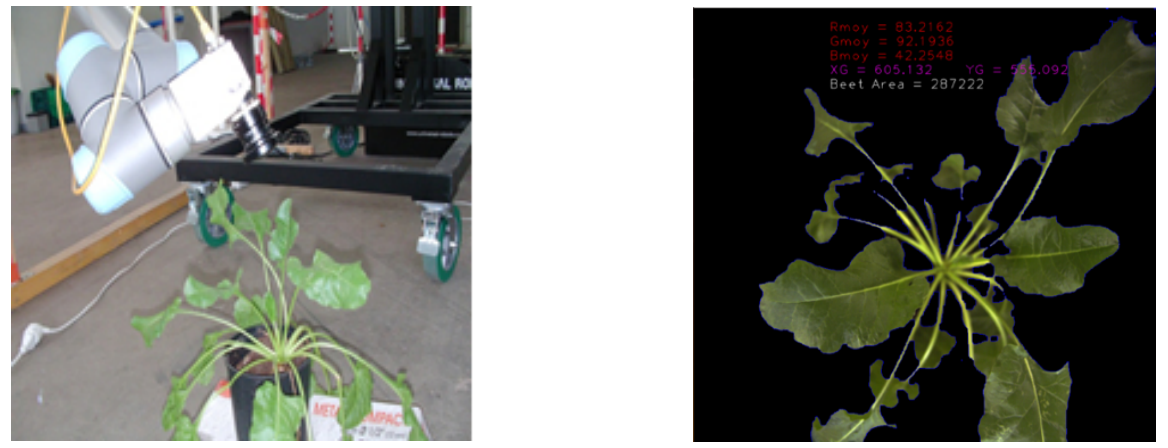

Figure 10. Detection of sugar beet leaves by image processing.

The desired pose of the sensor is estimated according to the sensor data. First, an image processing algorithm extracts the green areas of the observed image (by thresholding and morphology) then retrieves the different information that is the position of the barycenter of this area in the image and its size in pixels, as shown in the previous figure. The goal is to superimpose the center of gravity of this area with the center of the image. For that, one calculates the displacement to be carried out in the camera frame, for the moment simply by displacing the sensor in a plane parallel to the ground, then one expresses this displacement in the global coordinate system. The presented works, in this paper are based directly on the works of (Spong et al., 2001) and (Craig, 2005). The objective of this project is to detect beet leaves and then observe them using the sensor located at the end of the manipulator arm in order to detect diseases. This objective can be broken down into different tasks: the first will be to detect the different leaves to be observed, then to position themselves at the top close enough, then to move around to determine the angle of view allowing obtaining the maximum of relevant information for the detection of diseases. At first, the work done so far has been to move the camera attached to the manipulator arm above a plant. The position of the desired effector is then obtained. A performance criterion must be defined to reduce the possibilities. There are articles on trajectory generation and control mobile manipulators based on the value of manipulability. This criterion was defined in (Yoshikawa, 1984) and represents the ability of a manipulator robot to modify the pose of its effector in all directions. It is used in (Yamamoto and Yun, 1992) to determine the joint coordinates of the manipulator arm corresponding to maximum manipulability, these coordinates being subsequently fixed and only the mobile platform moves to follow the trajectory. In (Bayle et al., 2003), the theory developed in (Yamamoto and Yun, 1992) has been extended to all mobile manipulators. Thus, this criterion defines the whole system, and makes it possible to generate, from kinematic models, coordinated movements of the whole system. More recently, this criterion has been optimized for manipulator arms with six degrees of freedom (Zang et al., 2016). There are also different ways to formulate the problem of control of mobile manipulators. In (Soetanto et al., 2003), three formulations are listed: the achievement of a desired pose by the terminal organ, path tracking or trajectory tracking. Finally, a second aspect that may be interesting to dig is the definition of an image quality metric. Indeed, the purpose of the system is to enable detection of diseases, which implies obtaining images of sufficiently good quality. The criteria for image resolution and sharpness are to be taken into account. Moreover, as the system must be used outdoors, it will be subject to variations in brightness, but also to glare due to the sun or the movement of leaves.

\section{Mobility control of Bettybot robot}

Once the installation of the desired effector is estimated, the system is set in motion according to a control law which has been developed to manage the redundancy of the system. To control the robot, we then base ourselves on optimizing the working space of the manipulator arm. The ultimate goal is to come to observe leaves according to different points of view, it is necessary that the orientation of the camera can be modified. Therefore, the working space of the manipulator arm should be as large as possible, when the camera is centered over a plant, to allow this change of orientation. To do this, we base ourselves on the distance between the desired position of the camera and the limits of the working space of the manipulator arm (Figure 11). If this position is inside the working space of the manipulator arm, only the latter is set in motion. On the other hand, if the position to be reached is too far away, the support translate to center the working space of the manipulator arm above the plant to be observed, ie in order to center the base of the manipulator arm to the maximum above the plant. In practice, a coefficient $\lambda$ is defined as a function of the distance between the desired position of the sensor and the limits of 
the working space of the manipulator arm, along an axis parallel to that of the linear axis. Its values are in the range $[-1 ; 1]$ so that when the desired pose of the sensor is close to the center of the working space, $\lambda$ is zero and, in this case, only the manipulator arm is set in motion. On the other hand, if the desired pose is outside the working space, or close to the limit, then $\lambda= \pm 1$ (the sign differs according to the direction of the displacement to be performed), and the Linear axis only moves in order to center the base of the manipulator arm above the plant. If the plant to be observed is between the two previous cases, then the manipulator arm and the linear axis move in a coordinated way. Once this coefficient $\lambda$ is determined, two commands are sent: one for the linear axis which is proportional to $\lambda$, and one second for the manipulator arm joints, which is inversely proportional to $\lambda$.

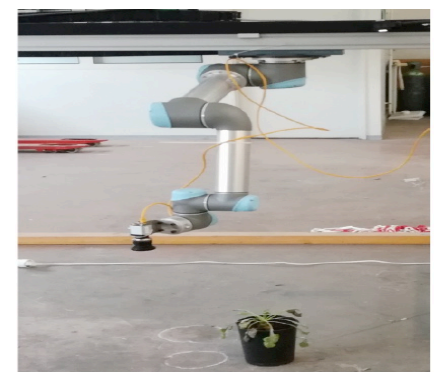

a) Plant inside the working space of the arm

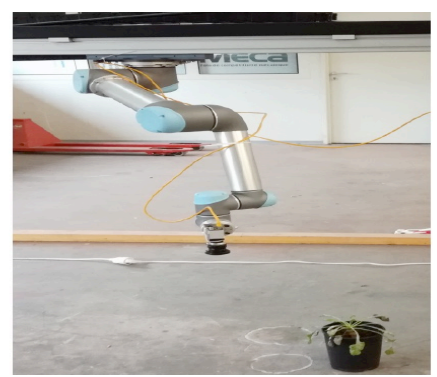

b) Plant outside the working space of the arm

Figure 11. Influence of the space between the desired position for the camera and the working space of manipulator arm.

In Figure (a), it may be noted that the plant is located almost under the base of the manipulator arm, fixed on the support in translation. In this case, the arm alone can be positioned above the plant, while keeping sufficient freedom of movement to allow the change of orientation of the camera. In contrast, in Figure (b), the plant being too far from the base of the manipulator arm, the translation of the support is then necessary to allow a change of orientation once the camera centered above the plant. The previously discussed command approach has been tested on the actual system. The following curves in (figure 12) represent the evolution of three elements: the lambda coefficient, the linear axis speed and the respective speeds of each of manipulator arm joints as a time function. The left column of each element shows the behavior of the system in the case where the plant to be observed is in the working space of the manipulator arm (Figure 11, case a), while the right one represents the case where the plant is located at outside the workspace (Figure 11, case b).
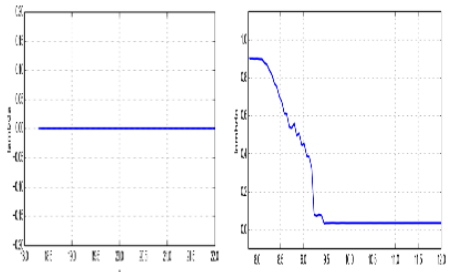

(a) Lambda evolution
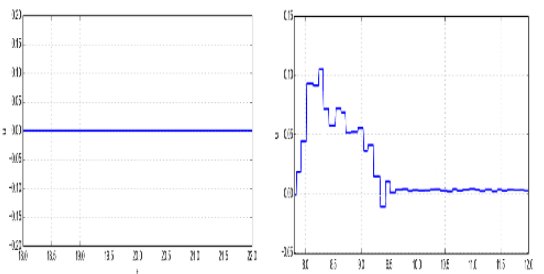
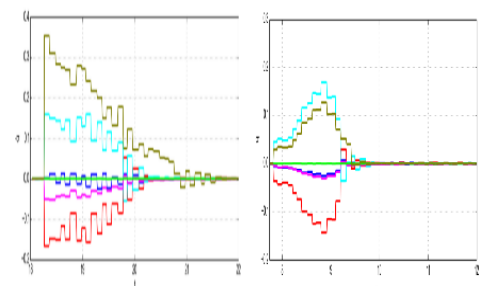

(c) Evolution of manipulator arm joints speed

(b) Evolution of linear axis speed Figure 12. Experimental results with Bettybot Robot.

In the first case, as the desired pose of the sensor is located in the working space, the coefficient $\lambda$ is zero and only the manipulator arm is moving. Indeed, the speed of the linear axis is zero in this case, while the articular velocities of the manipulator arm evolve as a function of time, until becoming zero when the sensor is centered above the plant. In the second case, the desired pose of the sensor is located at the limit of the workspace. The value of $\lambda$ then almost equal to 1 and the speed on the linear axis is therefore non-zero. It is also observed that the evolution of the speed of the axis follows the evolution of $\lambda$, it increases initially until reaching the maximum speed, then decreases at the same time as $\lambda$, which corresponds to the relationship of proportionality between the two sizes. During this time, the articular velocities are rather weak at the beginning, as $\lambda$ is large, then increase as $\lambda$ decreases, and become null when the sensor is above the plant. The developed algorithm for mobility and control of Bettybot robot, in order to move the camera at a desired position from a sugar beet crop position is satisfying. It makes it possible to weight the displacement of the linear axis and the manipulator arm as a function of the working space of the manipulator arm and permit to optimize this space in order to execute observation trajectories around the leaves. In addition, the calculation of the coefficient between the two commands can be parameterized according to the desired behavior: it is possible to give more weight to the movement of the manipulator arm, or, on the contrary, to promote the displacement of the linear axis. 


\section{Conclusions}

In 2017, the robotic platform Bettybot was taken in hand, by looking at different aspects in the field of robotics, to meet the needs of the Phénaufol project: mobility of the linear axis and the arm UR5, perception with the using of a color camera, attached to the end of the manipulator arm, control / command operations, working on the one hand in open loop, by sending commands on the 7 degrees of freedom of Bettybot, to position the color camera at the desired locations to make acquisitions and image processing, and secondly in closed loop, to automatically control the 7 degrees of freedom, from the image data obtained, in real time, by image processing. Acquisition and image processing work was also carried out for early growth stages, with the Jaguar mobile robot, in order to obtain map images of beetroot lines and to perform geometric and colorimetric measurements.

Next experimentations realized in laboratory and in the fields, will consist to apply and optimize the developed methods about perception by vision and control/command of Bettybot robot, working on several aligned sugar beet crops, at high growing stage, taking into account the redundancies of the seven degrees of freedom for robot control, and the lighting variations in the fields which can affect the sugar beet leaves detection. Concerning artificial vision, one important work will consist in the detection of each plant independently, using new image processing methods and for each plant, the aim will be to detect its leaves and to obtain some geometric data such as gravity center and the position of the central vein. Therefore, this will allow coming to observe the plant leaves one by one, and to test different observation trajectories. It will also be possible to integrate the calculation of an important image quality characteristic, such as brightness, for example, in order to determine the best point of view concerning this parameter. This approach could be extended to other parameters, depending on the characteristics of the image necessary for good detection. Also, this robot could be also used to achieve measurements on other crops, such as maize, wheat or sunflower, for various precision agriculture operations.

\section{References}

Åstrand, Björn and Baerveldt, Albert-Jan. A mobile robot for mechanical weed control. Halmstad University, School of Information Science, Computer and Electrical Engineering (IDE), 2003. International Sugar Journal, ISSN 0020-8841, Vol. 105, no 1250, p. 89-95

Barbedo J.G.A.. Digital image processing techniques for detecting, quantifying and classifying plant diseases. SpringerPlus, 2(1) :1, 2013.

Bayle B., Fourquet J.Y., and Renaud M.. Manipulability of wheeled mobile manipulators : Application to motion generation. The International Journal of Robotics Research, 22(7-8) :565-581, 2003.

Bock, C. H., Poole, G. H., Parker, P. E., and Gottwald, T. R. 2010. Plant disease severity estimated visually, by digital photography and image analysis, and by hyperspectral imaging. Crit. Rev. Plant Sci. 29:59-107, USA.

Craig J.J.. Introduction to robotics : mechanics and control. Pearson education international. Pearson, Prentice Hall, Upper Saddle River, NJ, 3. ed., international ed edition, 2005. OCLC : 249488662.

Ding W., Li Y., Wang W., and Zou Y.. Vanishing point detection algorithm for urban road image based on the envelope of perpendicular and parallel lines. 34, 10. 2014.

Gée Ch., Bossu J., Jones G., and Truchetet J.. Crop/weed discrimination in perspective agronomic images. Computers and Electronics in Agriculture, 60(1) :49-59, January 2008.

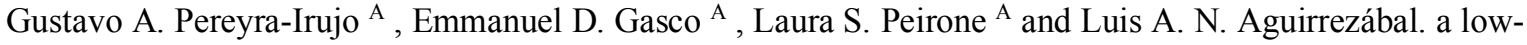
cost platform for phenotyping plant growth and water use. Functional Plant Biology 39(11) 905-913, 2012

Henson J.M. and French R.C.. The Polymerase Chain Reaction and plant disease diagnosis. Papers in Plant Pathology, $31: 81-109,1993$.

Hobbs H.A., Reddy D.V.R., Rajeshwari R., and Reddy A.S.. Use of Direct Antigen Coating and Protein A Coating ELISA Procedures for Detection of Three Peanuts Viruses. Plant Disease, 71 :747-749, 1987.

Klose R., Penlington J., Ruckelshausen A.. Usability study of 3D Time-of-Flight cameras for automatic plant phenotyping. University of Applied Sciences Osnabrück/ Faculty of Engineering and Computer Science, Albrechtstr. 30 ; 49076 Osnabrück; Germany

Kong H., Sarma S.E., and Tang F.. Generalizing Laplacian of Gaussian Filters for Vanishing-Point Detection. IEEE Transactions on Intelligent Transportation Systems, 14(1) :408-418, March 2013.

Mahlein A.K. , Steiner U., Hillnhütter C., Dehne H.W. and Oerke E.C.. Hyperspectral imaging for small-scale analysis of symptoms caused by different sugar beet diseases. Plant Methods2012 8:3, Germany

Montalvo M., G. Pajares, J. M. Guerrero, J. Romeo, M. Guijarro, A. Ribeiro, J. J.Ruz, and J. M. Cruz. Automatic detection of crop rows in maize fields with high weeds pressure. Expert Systems with Applications, 39(15) :11889-11897, November 2012Paulus S., Behmann J., Mahlein A.K., Plumer L. and Kuhlmann H. LowCost 3D Systems: Suitable Tools for Plant Phenotyping. Institute of Geodesy and Geoinformation (IGG)Geodesy, University of Bonn, Nussallee 17, Bonn 53115, 2014. Germany 
- Rovira-Más F., Zhang Q., Reid J.F., and Will J.D.. Hough-transform-based vision algorithm for crop row detection of an automated agricultural vehicle. Proceedings of the Institution of Mechanical Engineers, Part D : Journal of Automobile Engineering, 219(8) :999-1010, August 2005.

-Sankaran S., Mishra A., Ehsani R., Davi C.. A review of advanced techniques for detecting plant diseases. Computers and Electronics in Agriculture72 (2010) 1-13, USA

-Scharr H., Minervini M., French A.P., Klukas C., Kramer D.M., Liu X., Luengo I., Pape,G J.M.,. Polder, D. Vukadinovic, X. Yin, and S. A. Tsaftaris. Leaf segmentation in plant phenotyping : a collation study. Machine Vision and Applications, 27(4) :585-606, May 2016.

- Soetanto D., Lapierre L., and Pascoal A.. Adaptive, non-singular path-following control of dynamic wheeled robots. In Decision and Control, 2003. Proceedings. 42nd IEEE Conference on, volume 2, pages 1765-1770. IEEE, 2003.

- Spong M.W., Hutchinson S., and Vidyasagar M.. Robot dynamics and control. Wiley, New York, second edition edition, 2004.

-Subramanian R., Spalding E.P., Ferrier N.J. A high throughput robot system for machine vision based plant phenotype studies. 2012 at Springerlink.com.

- Yamamoto Y. and Yun X.. Coordinating Locomotion and Manipulation of a Mobile Manipulator. 1992.

- Yoshikawa T. Analysis and Control of Robot Manipulators with Redundancy. Robotics research : the first international symposium, pages 735-747, 1984.

- Zhang Y., Yan X., Chen D., Guo D., and Li W.. QP-based refined manipulability-maximizing scheme for coordinated motion planning and control of physically constrained wheeled mobile redundant manipulators. Nonlinear Dynamics, 85(1) :245-261, July 2016 


\title{
Comparison between Ultrasonic Sensors and 2D Lidar as Perception Systems for Autonomous Vineyard Robots
}

\author{
Andrés Cuenca a, *, Francisco Rovira-Más a, Verónica Saiz-Rubio a \\ a Agricultural Robotics Laboratory (ARL), Universitat Politècnica de Valencia, Camino de Vera s/n, Valencia 46022, \\ Spain \\ * Corresponding author. Email: ancuecu1@upv.es
}

\begin{abstract}
The VineScout project aims to demonstrate, and eventually take to market, an innovative expert field monitoring system (decision support system) embedded in a small-medium size and cost-efficient vineyard robot. Autonomous robots need agile perception systems to execute navigation algorithms, and handle emergency stops in critical situations. This study focuses on the performance of auto-guidance and obstacle detection routines for the robot. In a first approximation to this problem, ultrasonic sensors were used for navigation and safeguarding. The suitability of the sonar network depended on sound cone dimension and interference crosstalk errors. Due to echo interference, ultrasonic sensors had to be separated $90 \mathrm{~cm}$, producing a perception gap in the central area ahead of the robot, which is crucial for safety issues. As an alternative, a $2 \mathrm{D}$ non-rotational lidar sensor was used to provide eleven measurements simultaneously. Both perceptive solutions have been compared to determine which one is the most appropriate as a safety system. Both types of sensors were mounted in the same support assembly, measuring the range to several targets in static and dynamic tests. For the trials, the sensing frame was attached to a robot prototype, which traveled the field in real working conditions. Three type of targets were considered in the trials, being target $A$ a cylindrical element, target $B$ a rectangular box, and target $C$ a person. The results showed that the 2D lidar covers well the dead zone of the central area, and it provides an increased safety zone for the robot when combined with the ultrasonic technology. Furthermore, the lidar-based range measurements were more reliable than the ultrasonic-based distances, regardless of surface texture and position. Further research is in progress to find out if the forward-looking lateral sonars are useful to increase the perceptive capabilities of the robot or, on the contrary, if they may be omitted to reduce complexity.
\end{abstract}

Keywords: ultrasonic sensor, lidar, safety systems, autonomous robot, agricultural robots, vineyard automation

\section{Introduction}

In agriculture in general, and in viticulture in particular, product quality is considered a key element to make the crop profitable. The use of intelligent robots (Kamarolzaman et al., 2017) and autonomous operations (Ksanen, 2013 ) is gradually increasing in the field. For this reason, it is important to have robust safety and control systems to avoid potential safety hazards and navigation accidents. The European VineScout project aims to deliver a robot to monitor vineyards, and assist in irrigation and harvesting decision-making. The current robot prototype is self-guided, and its ultimate goal is to make automatic water stress maps of the vineyard to determine the optimal time for grape harvesting. This study focuses on the detection of objects (including people) in front of the robot when moving in the forward direction. Ultrasonic sensors have been extensively used in Precision Agriculture operations for canopy profiling (Gil and Escolà, 2007; Gil et al., 2013), as well as rotating lidar sensors (Rosell et al., 2009). The first perceptive engine of VineScout implemented ultrasonic sensors for safety and navigation. The suitability of ultrasonic sensor networks depends on the dimensions of the sonic cone and the interference between sensors (Zaman et al., 2007). To avoid interferences, two frontal ultrasonic sensors had to be separated $90 \mathrm{~cm}$, resulting in a blind zone in the central area in front of the robot. As an alternative, a non-rotational lidar rangefinder was added to the robot to increase robustness in ranging measurements. Both sensing technologies have been compared for different applications (Llorens et al., 2011; Tumbo et al., 2002; Whitney et al., 2002), and this paper adds to previous work the specific fusion of short-range sonar with non-rotational lidar for the safety system of autonomous robots when traveling along vineyard rows.

\section{Materials and Methods}

The schematic depicted in Figure 1 shows the hardware architecture of the system that was developed for conducting the series of field tests. The 2D lidar sensor used was the Multi-Ray LED Scanner OMD8000-R2100R2-2V1 (Pepperl + Fuchs, Mannheim, Germany), which gives 11 measures at a time as a result of 11 emitters, covering an 88-degree scanning zone. The two ultrasonic sensors mounted in the testing frame of Figures 1 and 3 are the MB7139 XL-TrashSonar-WR (MaxBotix Inc., Fort Mill, SC, USA), which had to be separated $80 \mathrm{~cm}$ (center to center) to avoid wave interferences between them. The GNSS receiver used in the system to record the robot motion was a SX Blue L1/L2 GPS (Geneq Inc., Montreal, Quebec, Canada). The computer used for data acquisition was the central computer mounted inside the robot, a modular computer following the PC-104 
architecture (Sundance multiprocessor technology Ltd., Chesham, UK), that also managed the sonar, lidar, and GNSS sensors through an Arduino Mega 2560 board (Arduino LLC, Ivrea, Italy) to acquire data.

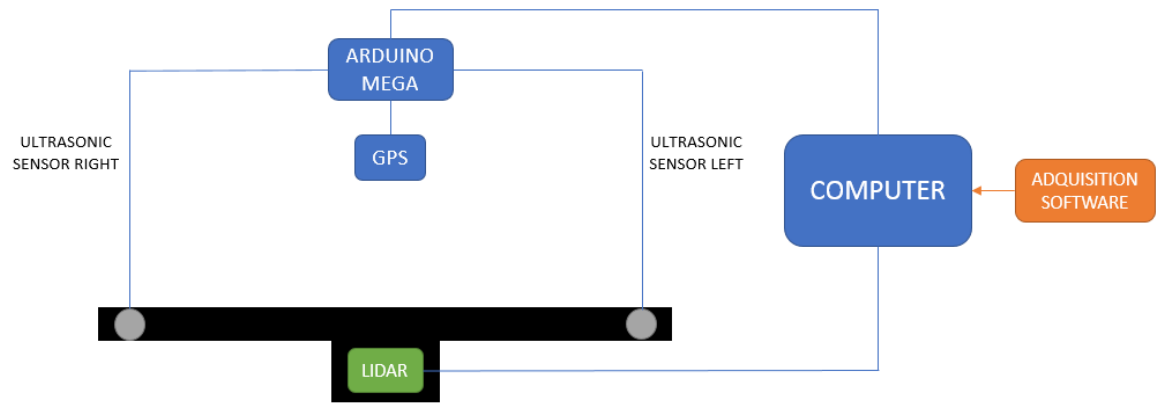

Figure 1. Perception engine: system architecture.

Data acquisition was carried out through a customized application written in Visual Basic, which included data-logging functions and the graphic interface of Figure 2. In the developed software, data streaming can be individually observed from each particular sensor. The sampling frequency for all the tests was $1 \mathrm{~Hz}$.

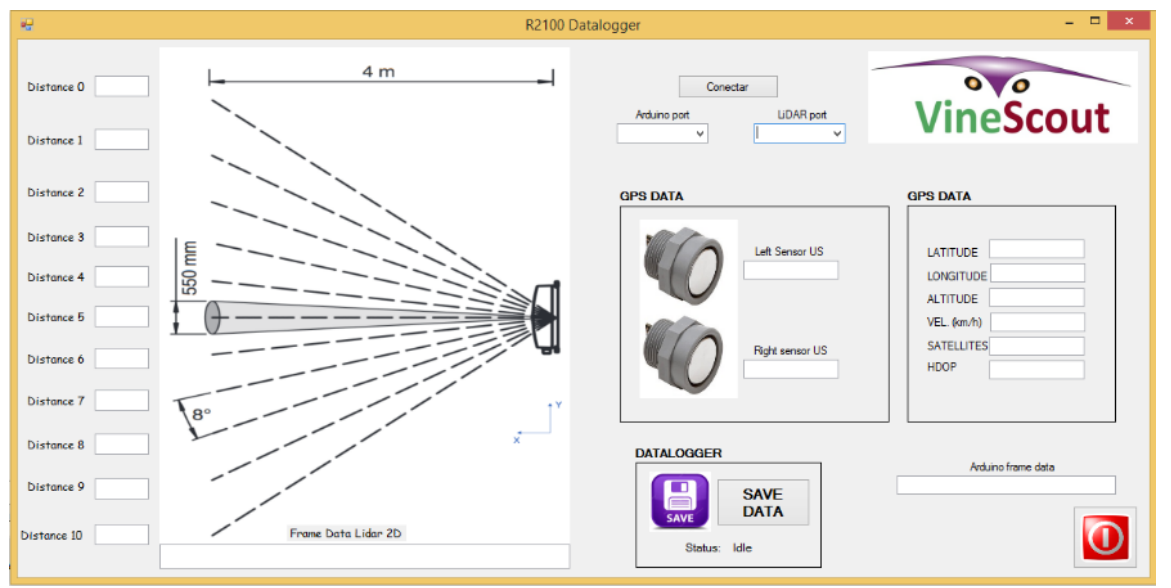

Figure 2. Data-logging user interface.

All the tests were carried out in the experimental field of the Universitat Politècnica de Valencia (Valencia, Spain), which is located in the campus surroundings. The field includes three rows of different grape varieties, with 20 meters in length and a row spacing of 3 meters. As shown in Fig. 3, the robot was positioned in the geometrical center of the row, surrounded by vegetation on both sides to simulate typical working conditions. The supporting structure for the sensors was located at an approximate high of $0.9 \mathrm{~m}$ with respect to the ground. Field tests were carried out in both static and dynamic conditions, trying to simulate real working situations with human obstacles as targets. The set of tests can be divided into three different cases T1, T2, and T3.

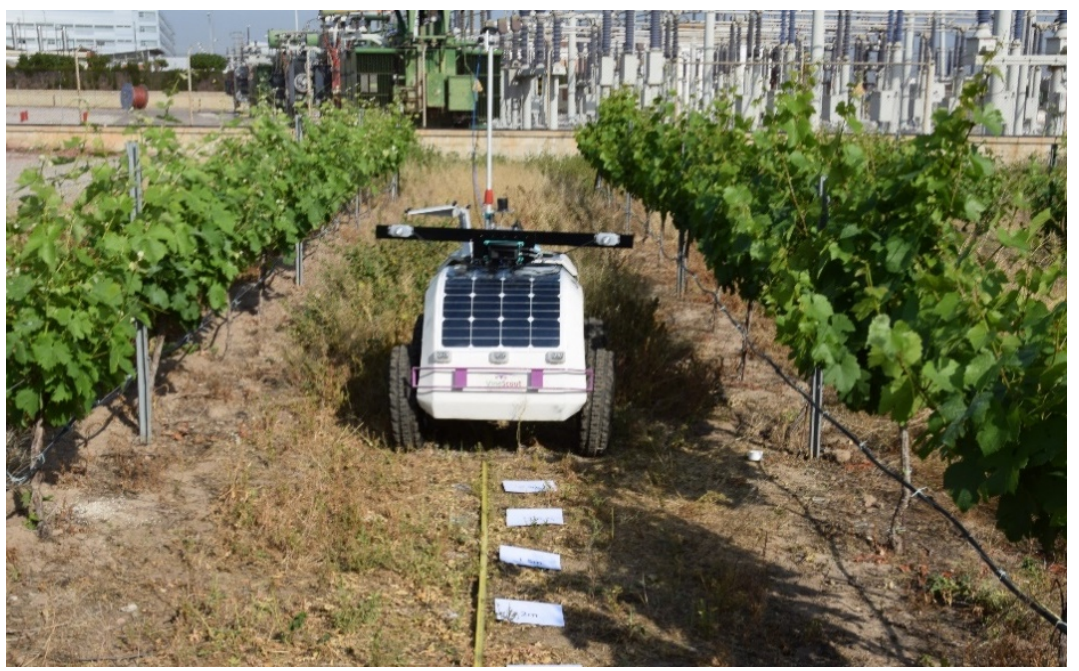

Figure 3. Robot preparation for the field tests. 
Static field tests (T1)

In this first set of tests, static measurements were made after varying the target distance from the robot between $3.5 \mathrm{~m}$ and $0.5 \mathrm{~m}$, at intervals of $0.5 \mathrm{~m}$ (Figure 4). For each of the seven positions, 10 sets of measurements were taken. Each set collected eleven distance measurements provided by the 2D lidar, and range measurements for each ultrasonic sensor. T1 tests included three independent repetitions.
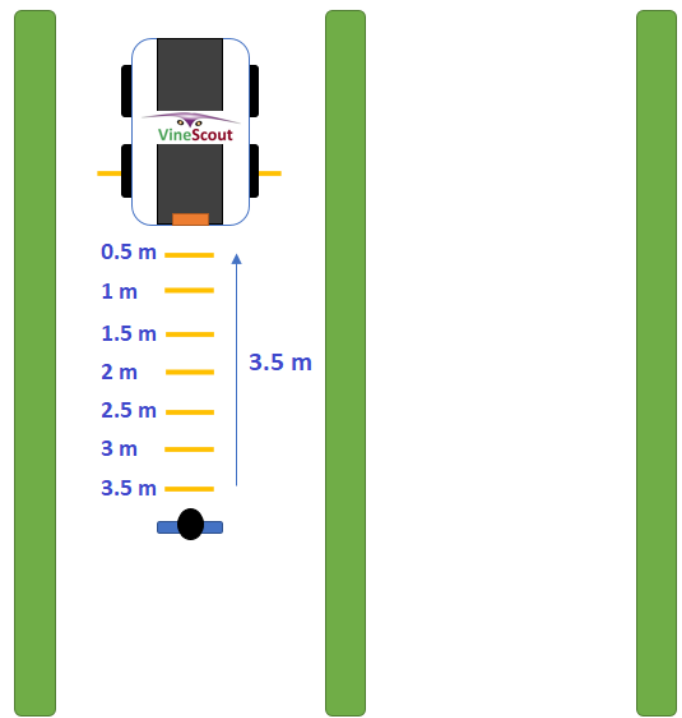

Figure 4. Diagram test for T1.

Dynamic field test 1 (T2)

For this set of tests, the target person remained static at $3.5 \mathrm{~m}$ from the robot front. The acquisition of range data began when the robot initiated its motion, moving forward towards the target at a constant speed of approximately $0.5 \mathrm{~m} / \mathrm{s}$, and acquiring data at a sampling frequency of $1 \mathrm{~Hz}$ (Figure 5). Three repetitions of T2 tests were performed.

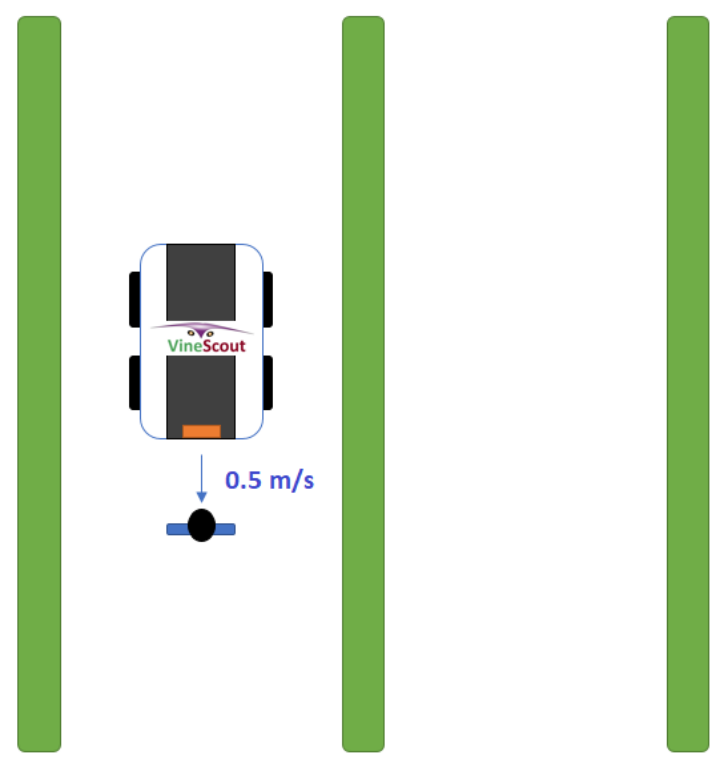

Figure 5. Diagram test T2. 
Dynamic field test 2 (T3)

In these field tests, the robot remained static at 3.5 meters from the target, and the person advanced towards the robot following a $\mathrm{Z}$ track (Figure 6). As in the previous tests, data acquisition was performed at a sampling frequency of $1 \mathrm{~Hz}$, repeating the experiment three times.
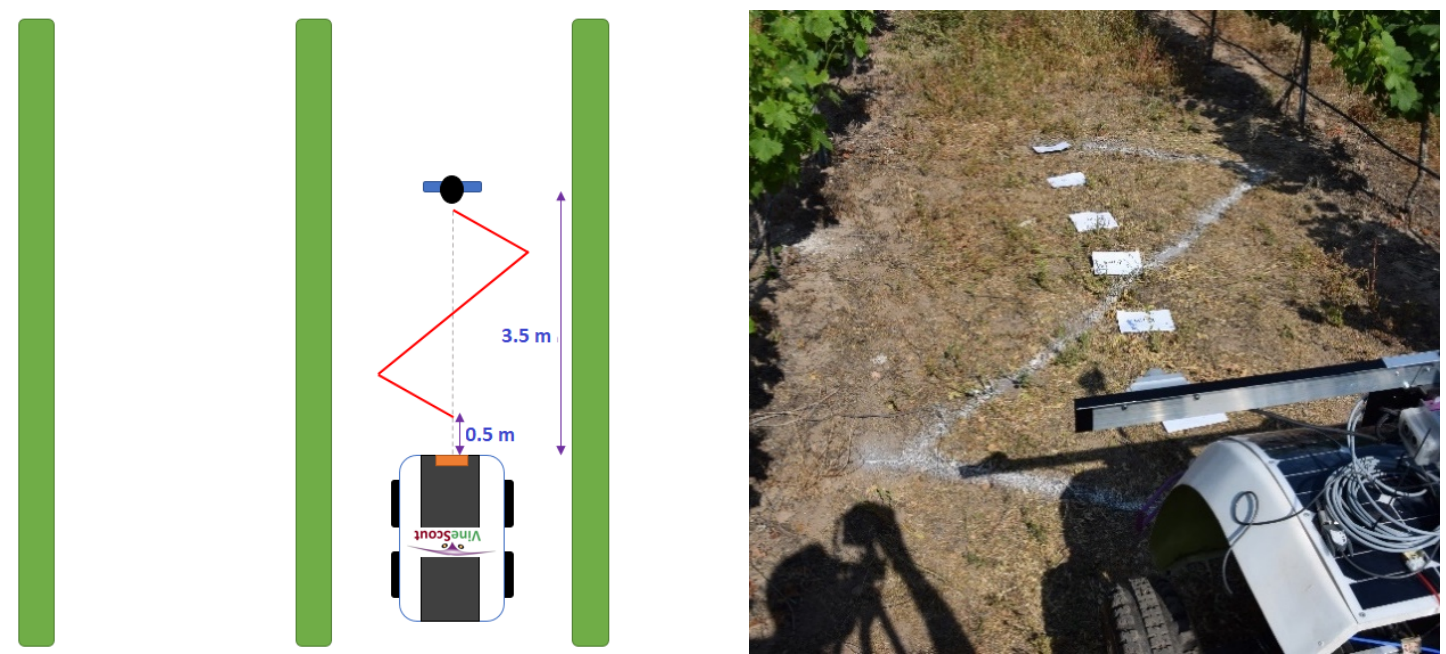

Figure 6. Diagram (left) and photo (right) for test T3.

\section{Results and Discussion}

Static field test (T1)

The origin of coordinates $(0,0)$ in Fig. 7 indicates the position of the robot. The Y-coordinate axis represents the distance (range) from the sensors to the target object in centimeters, tracking the point where the signal impacted the person. Figure 7 represents the specific case where the obstacle (person) was located $2 \mathrm{~m}$ ahead of the robot. The plot superimposes the three repetitions of the tests. In this case, it shows that lidar beams 0 to 4 and 8 to 10 (from right to left) detected the vines, and the three central beams $(5,6$, and 7 ) hit the target in the middle of the row.

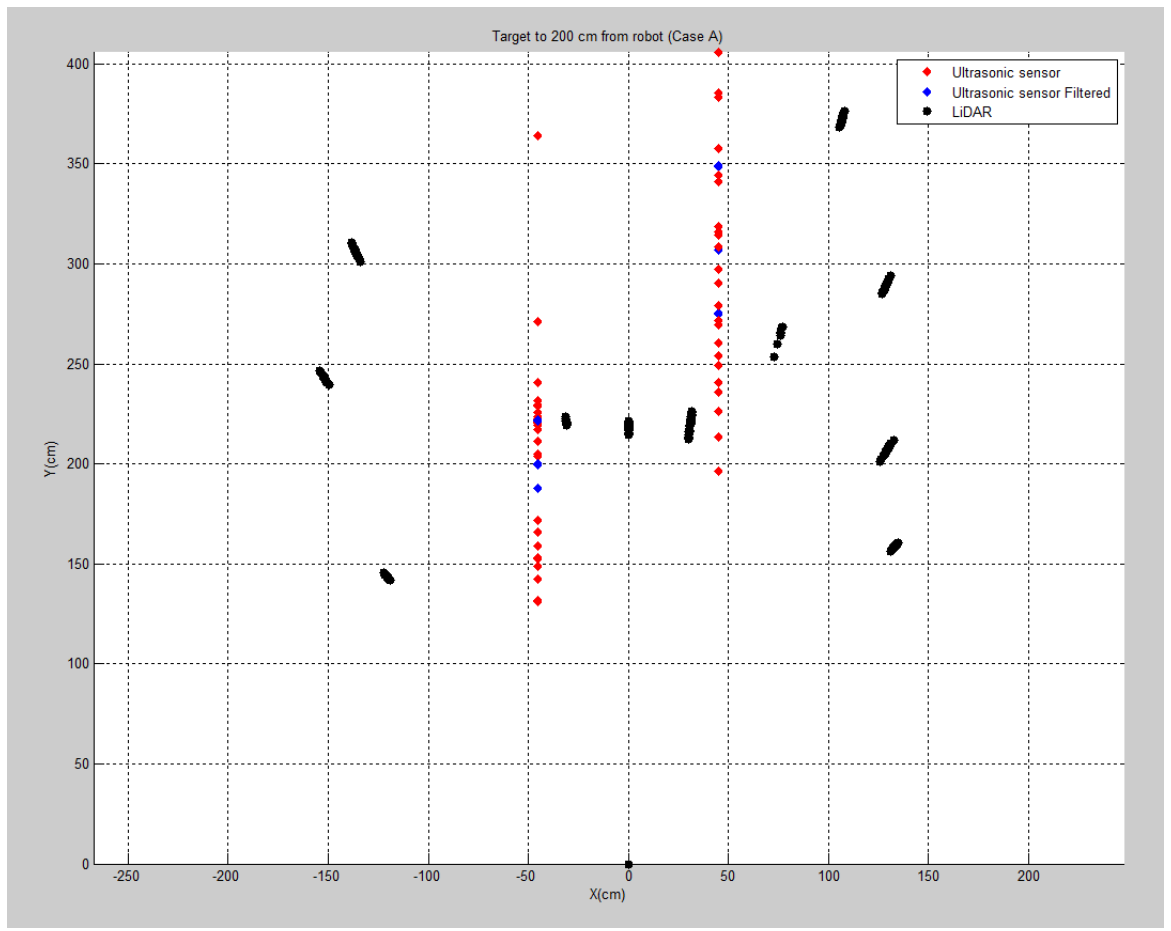

Figure 7. Test T1: Target at $2 \mathrm{~m}$ from the robot. 
In the case of the ranges measured by the two ultrasonic sensors (red dots), a great dispersion in the data was observed. For comparison purposes, sonar data was filtered by applying a moving average after removing extreme values. In particular, out of the ten values that composed each series, the highest and the lowest value from each repetition was discarded, averaging the remaining eight values. The six blue points in Figure 7 indicate the average values of each repetition (three points per sonar). The left sonar yielded similar ranges to the lidar; however, the ultrasonic sensor on the right side gave too imprecise values to be used for safeguarding. The reason behind the unlike behavior for the left and right ultrasonic sensors was probably due to the fact that the target was mostly inside the perceptive cone of the left sensor, but only near the edge of the sonic cone of the right sensor. According to the results of the analysis performed for test $\mathrm{T} 1$, the $2 \mathrm{D}$ lidar was clearly more reliable than the ultrasonic sensors for detecting a static person in front of an autonomous agricultural robot.

Dynamic field test 1 (T2)

In the first dynamic test (T2), a person moved straight towards a static robot. Figure 8 shows the results of the tests, where the abscissa axis represents the ranges measured by each sensor in $\mathrm{cm}$, and the $\mathrm{Y}$-axis provides the time elapsed ( $s$ ) since the beginning of the test. Only the central beams $\left(\mathrm{d}_{4}, \mathrm{~d}_{5}\right.$, and $\left.\mathrm{d}_{6}\right)$ of the $2 \mathrm{D}$ lidar were represented in Fig. 8, as the other eight beams only perceived the surrounding vineyard canopies rather than the target. The plot evidences that there exists a great dispersion for the values provided by the ultrasonic sensors (red dots). Sonar estimates oscillate with values over 2 meters off for the same measuring instant. This problem occurred because the target entered the blind zone that exists between the two sonic cones. In this case, it was not possible to filter out the signal since each value was obtained dynamically when the robot was moving, and therefore it was not practical to apply a moving average. In the case of the 2D lidar, when the target was at 3.5 meters from the robot, just at the beginning of the run, there were two beams $\left(\mathrm{d}_{5}\right.$ and $\mathrm{d}_{6}$ in Figure 8$)$ that detected the target. After passing the proximity line of $2 \mathrm{~m}$ (until $0.5 \mathrm{~m}$ from the robot front), a third beam ( $\left.\mathrm{d}_{4}\right)$ began to provide measurements for the target. The three repetitions verified that at least two beams were always detecting the target. This test confirmed the results of T1, proving that the lidar is more reliable than the ultrasonic sensors for detecting targets in open fields.

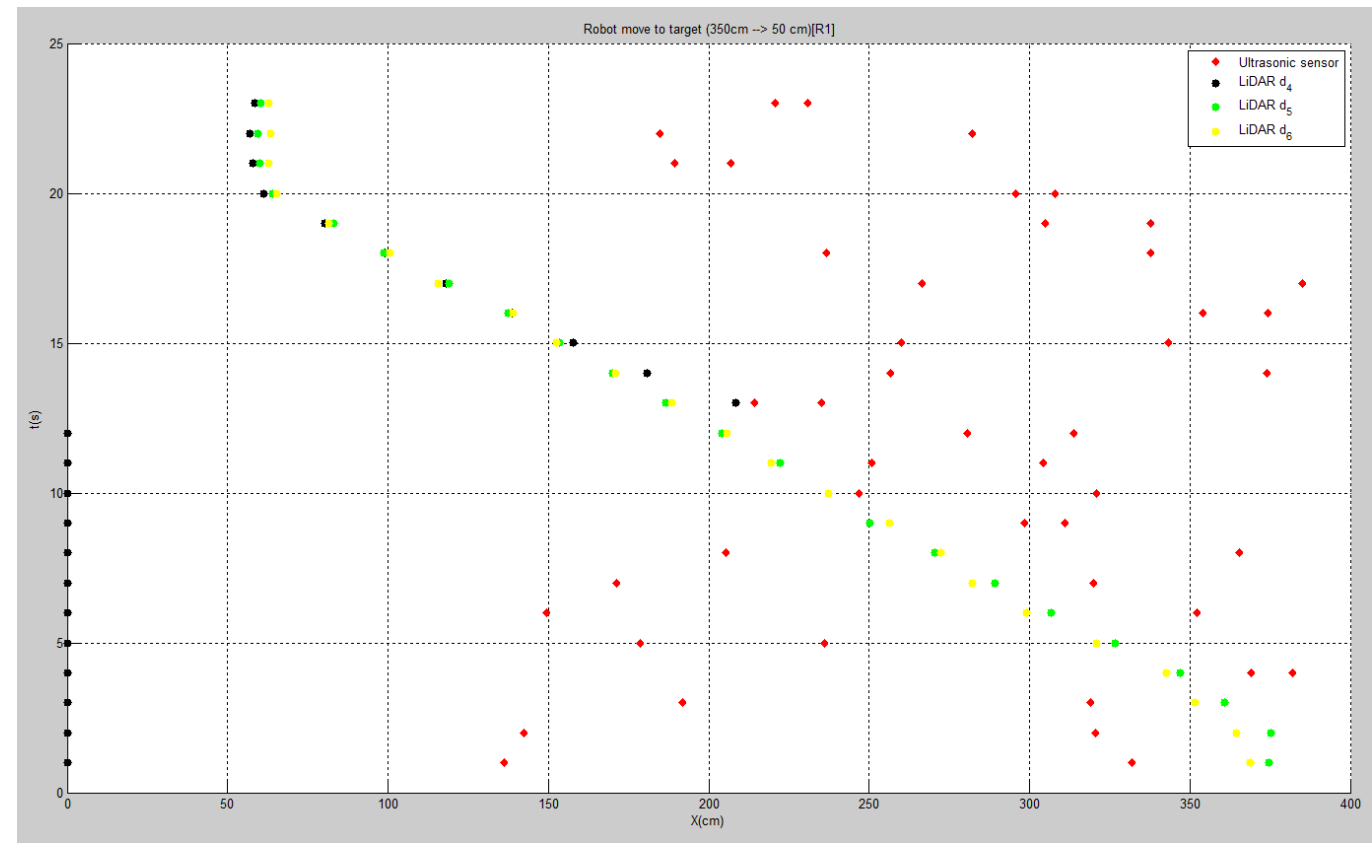

Figure 8. Test T2: robot moving towards the target.

Dynamic field test 2 (T3)

A zig-zag trajectory towards the robot requires separating the measurements obtained by each sonar. Figure 9 represents distance versus time for the second dynamic test $\mathrm{T} 3$ in a similar way to Figure 8, but making a distinction between both sonar rangefinders. The diagonal trajectory of the target was well tracked by the central beams of the 2D lidar, detecting the approaching target when the person interfered with their signal. Specifically, the central beam ( $\mathrm{d}_{5}$, in green) detected the target at the beginning of the test (with the target at around 3.5 meters), 
and again at around 2 meters, and finally at about 0.5 meters. The beams $\mathrm{d}_{4}$ (black) and $\mathrm{d}_{6}$ (yellow), which are not totally centered, detected the target when it traversed the beam signals near the canopy of the plants. The ultrasonic sensors, as observed in previous experiments, produced data with high dispersion, although some observations can be drawn from the range plot. The response of the sonar significantly varied with the detection zone; between $2 \mathrm{~m}$ and $3.5 \mathrm{~m}$, sonar data could be used if a filter is implemented. However, for ranges below $2 \mathrm{~m}$, the estimates were highly unstable, and therefore it is not recommended to use ultrasonic sensors for these close distances, which are typically important for safeguarding in autonomous vehicles.

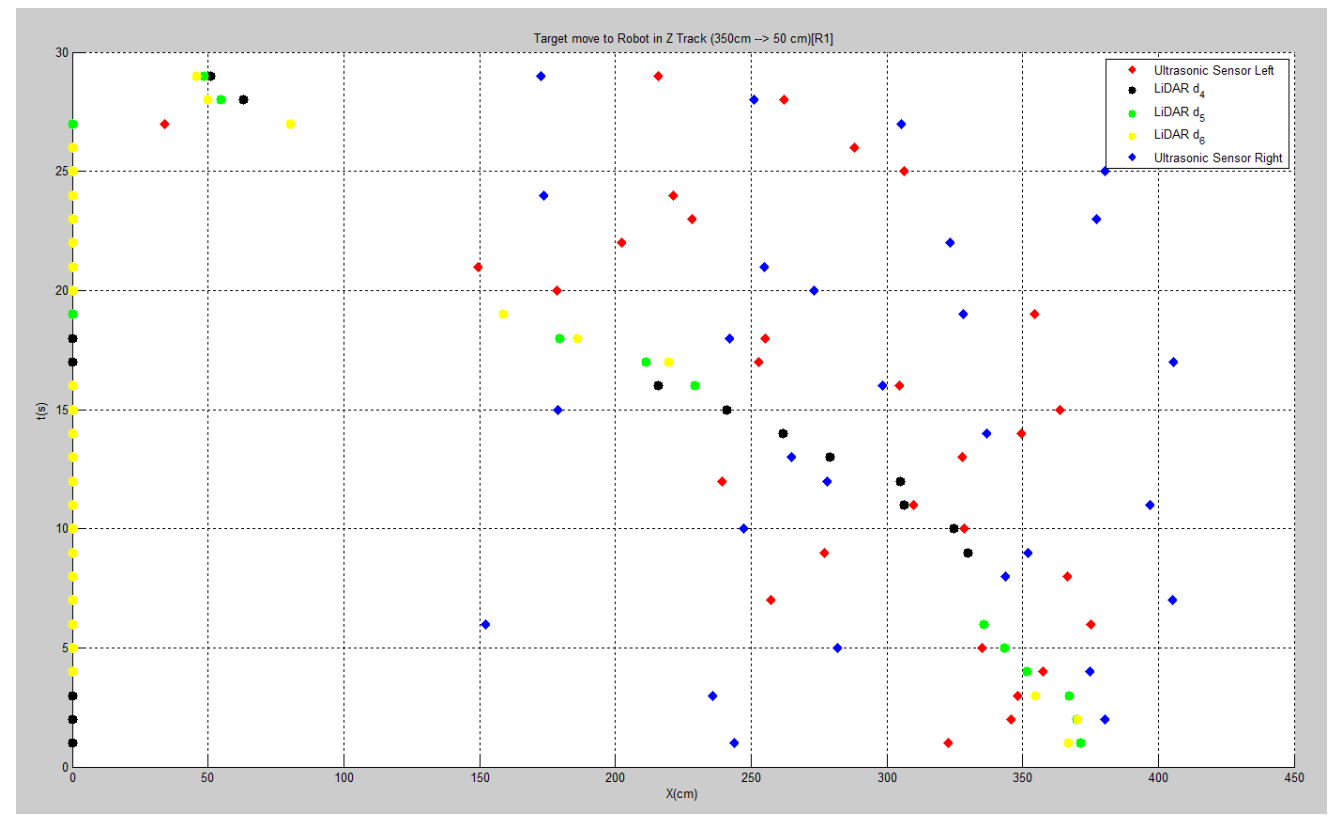

Figure 9. Test T3: target moving towards the robot in zig-zag.

\section{Conclusions}

The work described in this paper establishes a comparison between two ranging technologies for safety in autonomous robots: ultrasonic sensors and a non-rotational 11-beam 2D lidar. All the experiments were conducted in real environments inside a vineyard field. Test results demonstrated that the lidar rangefinder was always superior to the sonar in both static and dynamic situations. This superiority was based on accuracy, repeatability, and stability. Although the sonar sensors provided correct estimates in some circumstances, stability could never be assured for long periods of time, even in the short duration of the dynamic tests $(25 \mathrm{~s}-30 \mathrm{~s})$. The only clear advantage for the sonar was in the purchasing cost $(100 €$ versus $1,000 €)$, but the fact that cone amplitude requires a separation between sensors of at least $80 \mathrm{~cm}$, makes this technology inconvenient for many agricultural applications, where dead zones may lead to serious accidents. The lidar rangefinder was tested against the sun and in the shade of the vines, with the purpose of detecting potential interferences of sunlight with the beams, but the outputs were always stable and robust. Surface texture did not affect the behavior of the sensor either. Future steps in the VineScout project will integrate the lidar sensor as a key navigation aid for autonomous mapping of vineyards.

\section{Acknowledgements}

This research article is part of a project that has received funding from the European Union's Horizon 2020 research and innovation programme under grant agreement $\mathrm{N}^{\circ} 737669$. The opinions expressed reflect only the authors' view. Neither the European Commission, nor the funding agency, nor its services are responsible for any use that may be made of the information this publication contains. The authors want to express their gratitude to Montano Pérez Teruel and Juan José Peña Suárez for their assistance in the mechanical adjustments of the robot, as well as Mostafa Al-Shamari for his help in the field trials.

\section{References}

Gil, E.; Escolà, A. Variable Rate Application of Plant Protection Products in Vineyard using Ultrasonic Sensors. In 9th Workshop on Sustainable Plant Protection Techniques in Fruit Growing (SuProFruit), Alnarp, Sweden, 11-14 September 2007; pp. 61-62. 
Gil, E.; Llorens, J.; Llop, J.; Fàbregas, X.; Gallart, M. Use of a terrestrial LIDAR sensor for drift detection in vineyard spraying. Sensors 2013, 13, 516-534.

Kamarolzaman N.A.F.M., Yeong C.F., Ming Su E.L., Chik S.F., Duan F., Tan J.T.C., Tan P.H., Chin P.J.H. 2017. Smart Advertising Robot with Data Analytics Using Machine Vision. Elektrika. VOL. 16, NO. 3, 2017, 1116.

Ksanen, T., 2013. Accuracy and performance experiences of four wheel steered autonomous agricultural tractor in sowing operation. 9th International Conference on Field and Service Robotics, 9-11 December.pp. 114.

Llorens, J.; Gil, E.; Llop, J.; Escolà, A. Ultrasonic and LIDAR sensors for electronic canopy characterization in vineyards: Advances to improve pesticide application methods. Sensors 2011, 11, 2177-2194.

Rosell, J.R.; Llorens, J.; Sanz, R.; Arnó, J.; Ribes-Dasi, M.; Masip, J.; Escolà, A.; Camp, F.; Solanelles, F.; Gràcia, F. et al. Obtaining the Three-Dimensional Structure of Tree Orchards from Remote 2D Terrestrial LIDAR Scanning. Agric. For. Meteorol. 2009, 149, 1505-1515.

Tumbo, S.D.; Salyani, M.; Whitney, J.D.; Wheaton, T.A.; Miller, W.M. Investigation of Laser and Ultrasonic Ranging Sensors for Measurements of Citrus Canopy Volume. Appl. Eng. Agric. 2002, 18, 367-372.

Whitney, J.; Tumbo, S.; Miller, W.; Wheaton, T.A. Comparison between ultrasonic and manual measurements of citrus tree canopies. In Proceedings of the ASAE Annual International Meeting, St. Joseph, MI, USA, 28-31 July 2002.

Zaman, Q.U.; Schumann, A.W.; Hostler, H.K. Quantifying Sources of Error in Ultrasonic Measurements of Citrus Orchards. Appl. Eng. Agric. 2007, 23, 449-453. 


\title{
Identification of Reflected Spectral Related to Plant Circadian Rhythm Using Maximal Information Coefficient
}

\author{
Shogo Nagano a, b, Akito Sammyo a , Hirokazu Fukuda a, c, * \\ ${ }^{a}$ Graduate School of Engineering, Osaka Prefecture University \\ ${ }^{\mathrm{b}}$ Research Fellow of the Japan Society for the Promotion of Science \\ ${ }^{c}$ Japan Science and Technology Agency, Precursory Research for Embryonic Science and Technology \\ * Corresponding author. Email: fukuda@me.osakafu-u.ac.jp
}

\begin{abstract}
Plant factories have attracted attention as a breakthrough to solve the food production crisis. In the plant factory, there is an advantage that the light-dark cycle can be flexibly controlled, so that the circadian rhythm in plants can be regulated by light conditions and plant growth could be maximized by matching the circadian rhythm with the light-dark cycle. However, the circadian rhythm is difficult to measure without destruction of plants, e.g. analyse of gene expression. In this research, we focused on a hyperspectral camera (HS camera) to measure the circadian rhythm non-destructively. An HS camera is an instrument that analyses the reflected light from an object as high-precision wavelength information. In a previous study, we have found that the internal time of the circadian clock (circadian phase) can be estimated by combining an HS camera and machine learning, but the effective wavelengths for the estimation have not been clarified. Here, we identified wavelengths as indicators of circadian phase by analysing all combinations of two different wavelengths. Green perilla (Perilla frutescens) was grown and measured every 2 hours for 2 days. Maximal information coefficient (MIC) was used for all combinations of $525 \mathrm{~nm}$ to $900 \mathrm{~nm}(25 \mathrm{~nm}$ interval) to evaluate the association between two wavelengths. Furthermore, we constructed time-series data of MIC (time-series-MIC) and figured out that the correlation between time-series-MIC of the 1st and 2nd days is maximized when two wavelengths were chosen from the photosynthetically active radiation region $(400 \mathrm{~nm}$ to $700 \mathrm{~nm})$ or the near infrared region $(700 \mathrm{~nm}$ to $900 \mathrm{~nm})$. This study suggests that circadian phase can be measured by two different wavelengths of light reflectance of leaves.
\end{abstract}

Keywords: circadian clock, hyperspectral camera, maximal information coefficient, nondestructive analysis, Perilla frutescens

\section{Introduction}

Plant factories that enable cultivation of plants in closed spaces have attracted attention in recent years as a breakthrough measure for the food crisis and cultivation techniques in areas unsuitable for agriculture (Morimoto et al., 1995). As one of the features of this plant factory, there is the point that the light cycle can be freely adjusted. For that purpose, we focused on the "circadian clock", which is closely related to light. Circadian clocks have a rhythm with a period of about 24 hours, and it is known that growth is maximized by matching this cycle with the light-dark cycle of light (Dodd et al., 2005). Therefore, it is important to adjust the light-dark cycle to measure the date and time.

As a measurement method of the circadian clock, there is the "molecular timetable method", which measures the circadian clock using enormous genetic information, and it is applied to various kinds of plants, such as lettuce and tomato (Ueda et al., 2004, Higashi et al., 2016). However, since this method is costly and requires about 3 months to acquire data, the human cost and monetary cost are a problem, as well as that measurement cannot be done unless the plant body is destroyed. Therefore, this is reflected in the measurement results for the circadian clock in a light environment that are not suitable for adjusting the light-dark cycle of light. Also, the luciferase luminescence measurement method is a non-destructive measurement method of the circadian clock. In this method, it is possible to measure the rhythm of the clock gene CCA1 by measuring luminescence of Arabidopsis thaliana using the firefly derived recombinant CCA1::LUC (Nakamichi et al., 2004). However, this method requires the use of genetically modified organisms, and it is difficult to apply it to plant production as with the molecular timetable method.

Therefore, in this research, we focused on the spectroscopic measurement method, which is a non-destructive and noncontact measurement method. Spectroscopic measurement methods include near infrared spectroscopy, a chlorophyll-meter, multispectral imaging (MSI), hyperspectral imaging (HSI), and the like. Studies on nondestructive measurement of tomato soluble solid content have been conducted as an example using a near infrared light content light method (Kfuriyati et al., 2004). Further, there is a device for non-destructive measurement of chlorophyll concentration using a chlorophyll meter SPAD-502 (Konica Minolta Inc., Tokyo, Japan). MSI and HSI spectroscopically measure reflected light, and the name changes depending on the number of measurement bands. These spectroscopic measurement methods are applied to various fields such as detection of tumors and hematomas, remote sensing from a satellite, defect inspection of industrial products, and the like (Duan et al., 
2014, Lee et al., 2012, Panasyuk et al., 2007). In addition, as a research example relating to the circadian rhythm, there is an example in which time change of the amount of chlorophyll was measured using MSI (Pan et al., 2015), and in the previous study of this research group, using HSI and machine learning, the circadian clock rhythm was estimated successfully. On the other hand, because machine learning was used, the problem is that the effective wavelength for time measurement is unknown.

In this research, we focused on a hyper-spectral camera (hereinafter called "HS camera") in order to measure circadian rhythm at low cost and instantaneously. An HS camera is a measuring instrument that measures reflected light of an object to be photographed as high-precision spectroscopic information, and is also useful when the target spectral distribution is unknown. We attempted to measure circadian rhythm from two specific wavelengths by using a statistical method for spectral data acquired with an HS camera.

\section{Materials and Methods}

Cultivation methods

Perilla (Perilla frutescence var. crispa f. viridis) was used as the type of test plants. The seeds were cultivated at $25^{\circ} \mathrm{C}$ for 12 days with a red LED (PPFD: $80 \mu \mathrm{mol} \mathrm{m}{ }^{-2} \cdot \mathrm{s}^{-1}$ ) at $12 \mathrm{~h}$ light period, $12 \mathrm{~h}$ dark period (12 L:12 D). Next, in a sponge containing moisture, seedlings were transplanted. The tray with sponge corners $(200 \mathrm{~mm} \times 250$ $\mathrm{mm} \times 560 \mathrm{~mm})$ was covered by a film. The seeds in this tray were irradiated with fluorescent light $(130 \mu \mathrm{mol}$ $\mathrm{m}^{-2} \cdot \mathrm{s}^{-1}$, TBL-14, manufactured by SANYO Electric Co., Ltd., Osaka, Japan) using a growth chamber (MLR-351 HNB; / 5N; OHM ELECTRIC INC., Saitama, Japan). There, the trial plants were transplanted to another hydroponic culture system $(1200 \mathrm{~mm} \times 650 \mathrm{~mm} \times 2000 \mathrm{~mm}$; Mansei Corporation, Osaka, Japan), and under fluorescent lighting under long-day conditions for 34 days in an environment of $23 \pm 1{ }^{\circ} \mathrm{C}, 164 \mu \mathrm{mol} \mathrm{m}{ }^{-2} \cdot \mathrm{s}^{-1}, 16$ $\mathrm{L}: 8 \mathrm{D}$. For cultivation, we use fertilizer (N:P2O5:K2O:CaO:MgO= 18.5:5.1:7.6:8.2:3.7:0; Otsuka A formulation, Otsuka Chemical Co., Ltd., Osaka, Japan) diluted with water.

Sampling and analysis methods

After cultivating for 34 days under long-day conditions, we switched to the constant condition (LL condition). A total of 250 samples were sampled every 2 hours, 25 points (hereinafter called $T=0 \sim T=46$ ), 10 samples per time from 24 hours after switching to the LL condition. For sampling at each time, multiple individuals were randomly selected (supplementary materials are being prepared). Sampled leaves were imaged immediately using the hyperspectral camera GS-OP (EBA Japan Co., Tokyo, Japan). This camera has a pixel resolution of 8-bit VGA $(640 \times 480$ pixels $)$ and it is possible to measure 141 consecutive wavelengths at $350 \mathrm{~nm}-1050 \mathrm{~nm}(5 \mathrm{~nm}$ interval $)$. In this study, the imaging area was set to be about $250 \times 188 \mathrm{~nm}$. For spatial resolution, the number of pixels in the vertical and horizontal directions is 65 pixels per inch.

The light source was installed on the side of the object to be measured with halogen light (CHP 250, 250 W; Caster Lighting Japan Co., Ltd., Osaka, Japan), and the object to be measured was a standard reflector plate (KHG 3421-WB, X-Rite Inc, Tokyo, Japan). Before shooting the measurement target, ten samples were taken after acquiring the whiteboard data. All steps were carried out between sampling times \pm 5 minutes. The measured data were saved as a spectral cube of $640 \times 480 \times 141$ (width $\times$ length $\times$ wavelength). 
Preprocessing of hyperspectral data

A

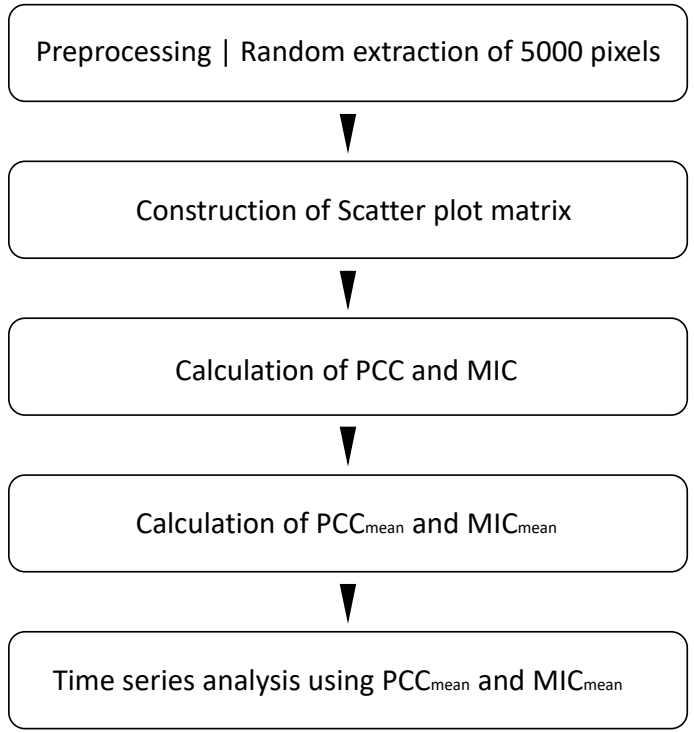

B
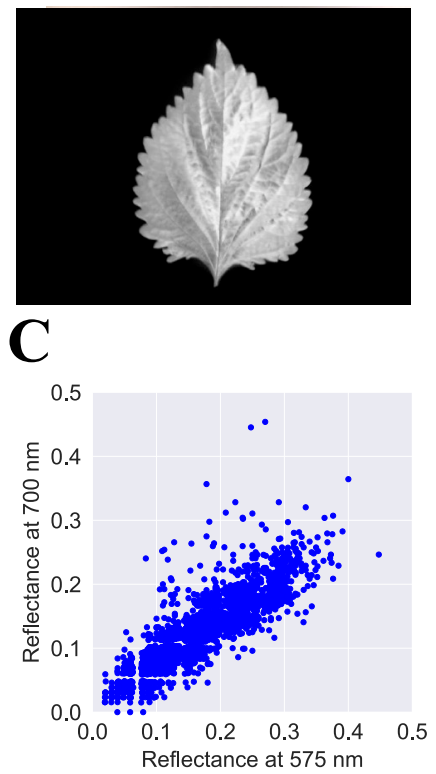

Figure 1. Outline of the preprocessing and data analysis. Flow chart of the analysis (A). The sample image of the objective (B). The sample scatter matrix of the relationship between two wavelengths (C).

Figure 1 shows an outline of the analysis contents in this research. Figure 1A shows a flow chart of the analysis, and Figure 1B shows an example of the HS data actually used. Figure 1C shows an example of a scatter plot created using data selected in pre-processing.

First, reflectance data were acquired from the acquired HS data based on the following equation.

$$
\text { Reflectance rate }=\frac{\text { Raw data }}{\text { White data }}
$$

NDVI (Normalized Difference Vegetation Index) was used for the extraction of leaf area (ROI, Region of Interest) as shown below. NDVI is an index showing the distribution state and activity of vegetation.

$$
\mathrm{NDVI}=\frac{(\mathrm{IR}-\mathrm{R})}{(\mathrm{IR}+\mathrm{R})}
$$

Here, IR represents the reflectance in the red region, and $\mathrm{R}$ represents the reflectance in the near infrared region. In this study, average of $695 \mathrm{~nm}$ to $740 \mathrm{~nm}$ was used as IR data and $630 \mathrm{~nm}$ to $655 \mathrm{~nm}$ was used as R data. Each pixel was calculated using NDVI, and the area with NDVI $>0.5$ was defined as the leaf area. We randomly extracted 5000 pixels from the leaf area defined using Python. This series of processing was performed for all the leaves, and finally spectral data ( $525 \mathrm{~nm}$ to $900 \mathrm{~nm}, 25 \mathrm{~nm}$ interval) of 5000 pixels were selected for each of 250 leaves.

Time-series analysis using statistical methods

The selected 5000 pixels were analyzed using a statistical method. First, a scatter plot matrix ( $25 \mathrm{~nm}$ interval, $15 \times 15$ ) was created for all 250 leaves using PCC evaluating linear relevance. Next, the average of scatter plot matrix of 10 leaves sampled at the same time was calculated and defined as $\mathrm{PCC}_{\text {mean. }}$. Finally, the time series change of $\mathrm{PCC}_{\text {mean }}$ was calculated.

A series of analyzes carried out with PCC was also carried out with MIC as well. MIC is a statistical method used in evaluating nonlinear relevance, and PCC may obtain nonlinear correlation also for a distribution determined to have no correlation (Reshef et al., 2011, Kinney and Atwal., 2014). 


\section{Results}

Evaluation of association between two wavelengths

Figure 2 shows the analysis result of PCC for a single leaf at $T=0$. Figure $2 \mathrm{~A}$ shows a scatter plot matrix. The results show a linear association, non-linear association and no correlation related to the combination of two wavelengths. For example, Figure 2B is the correlation between $800 \mathrm{~nm}$ and $825 \mathrm{~nm}$ and the calculation result of PCC was 0.88 , which represents the linear correlations. On the other hand, Figure $2 \mathrm{C}$ is the correlation between $700 \mathrm{~nm}$ and $750 \mathrm{~nm}$ and PCC is 0.68 , which shows lower correlations compared to Figure 2B, but a non-linear correlation can be found. From these results, only linear correlations can be found by a scatter plot matrix, so we analysed the non-linear associations by using MIC analysis.
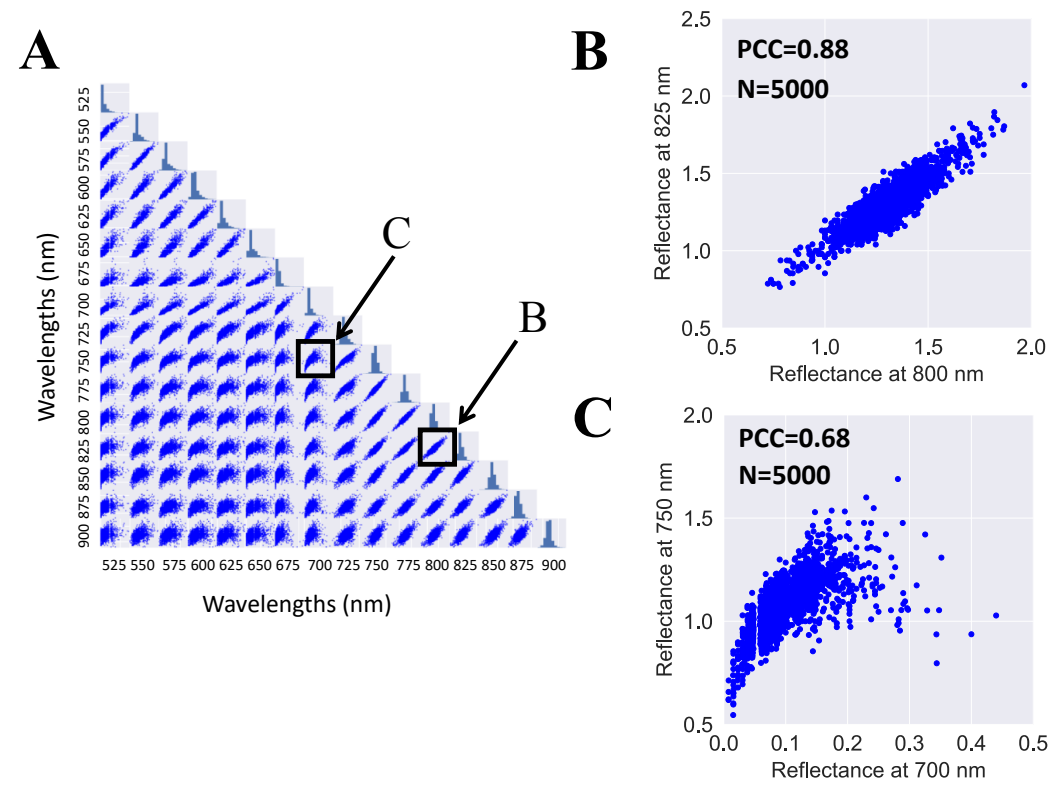

Figure 2. Result of scatter plot matrix analysis. Scatter plot matrix of single leaf at $T=0$ (A). Sample of scatter plot with linear correlation (B).

Calculation of $\mathrm{PCC}_{\text {mean }}$ and $\mathrm{MIC}_{\text {mean }}$

Figure 3 shows the $\mathrm{PIC}_{\text {mean }}$ and $\mathrm{MIC}_{\text {mean }}$ analysis results for $T=0$. Figure $3 \mathrm{~A}$ shows the results of $\mathrm{PCC}_{\text {mean. }}$ On the other hand, the MIC mean results in Figure 3B are MIC 0.53 and 0.37, close amounts. This means the MIC mean extracts both linear and non-linear results.

A

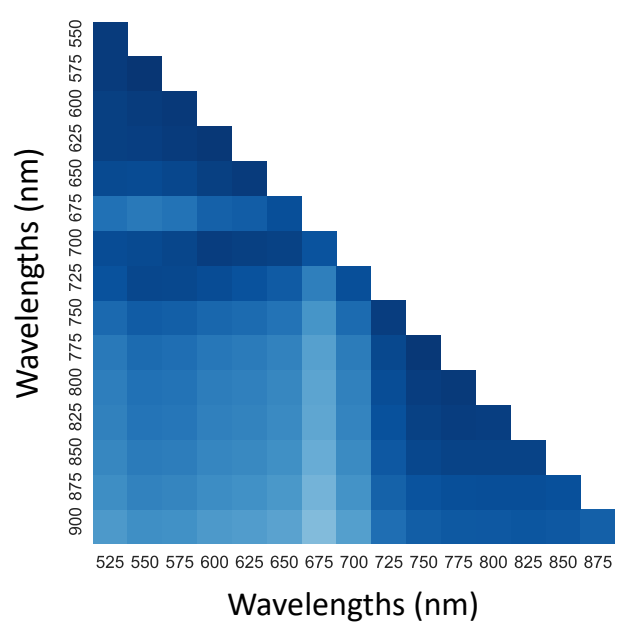

B

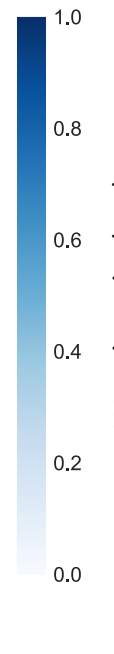

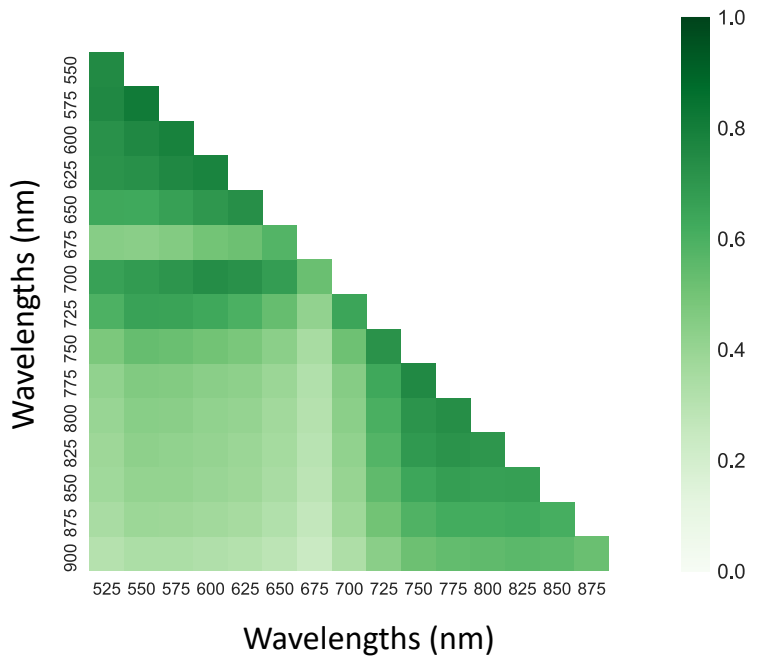

Figure 3. Result of $\mathrm{PCC}_{\text {mean }}$ and $\mathrm{MIC}_{\text {mean }}$ at $T=0$. Result of $\mathrm{PCC}_{\text {mean }}$ at $T=0$ (A). Result of $\mathrm{MIC}_{\text {mean }}$ at $T=0$ (B). 
Time-series analysis of PCC and MIC

Time series analysis for each combination of scatter plot matrix is done (120 patterns). Figure 4 shows the three different $\mathrm{PCC}_{\text {mean }}$ and $\mathrm{MIC}_{\text {mean }}$ results $(600 \mathrm{~nm}$ and $650 \mathrm{~nm}, 725 \mathrm{~nm}$ and $750 \mathrm{~nm}, 575 \mathrm{~nm}$ and $850 \mathrm{~nm})$ in which periodicity was found. Each figure shows $T=0-22 \mathrm{~h}$ (Day 1 ) and $T=24-46 \mathrm{~h}$ (Day 2 ). Each figure shows that the first period and the second period show close patterns.

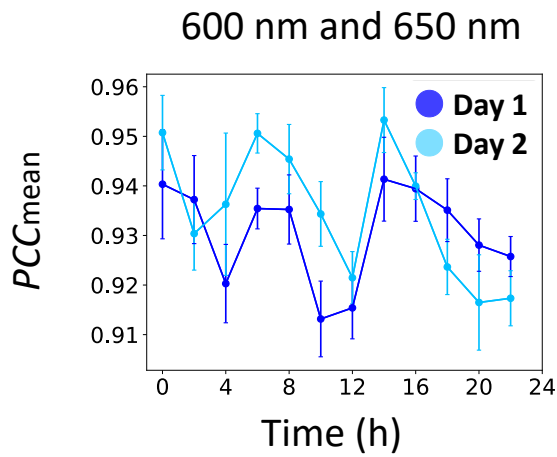

$725 \mathrm{~nm}$ and $750 \mathrm{~nm}$

$575 \mathrm{~nm}$ and $850 \mathrm{~nm}$
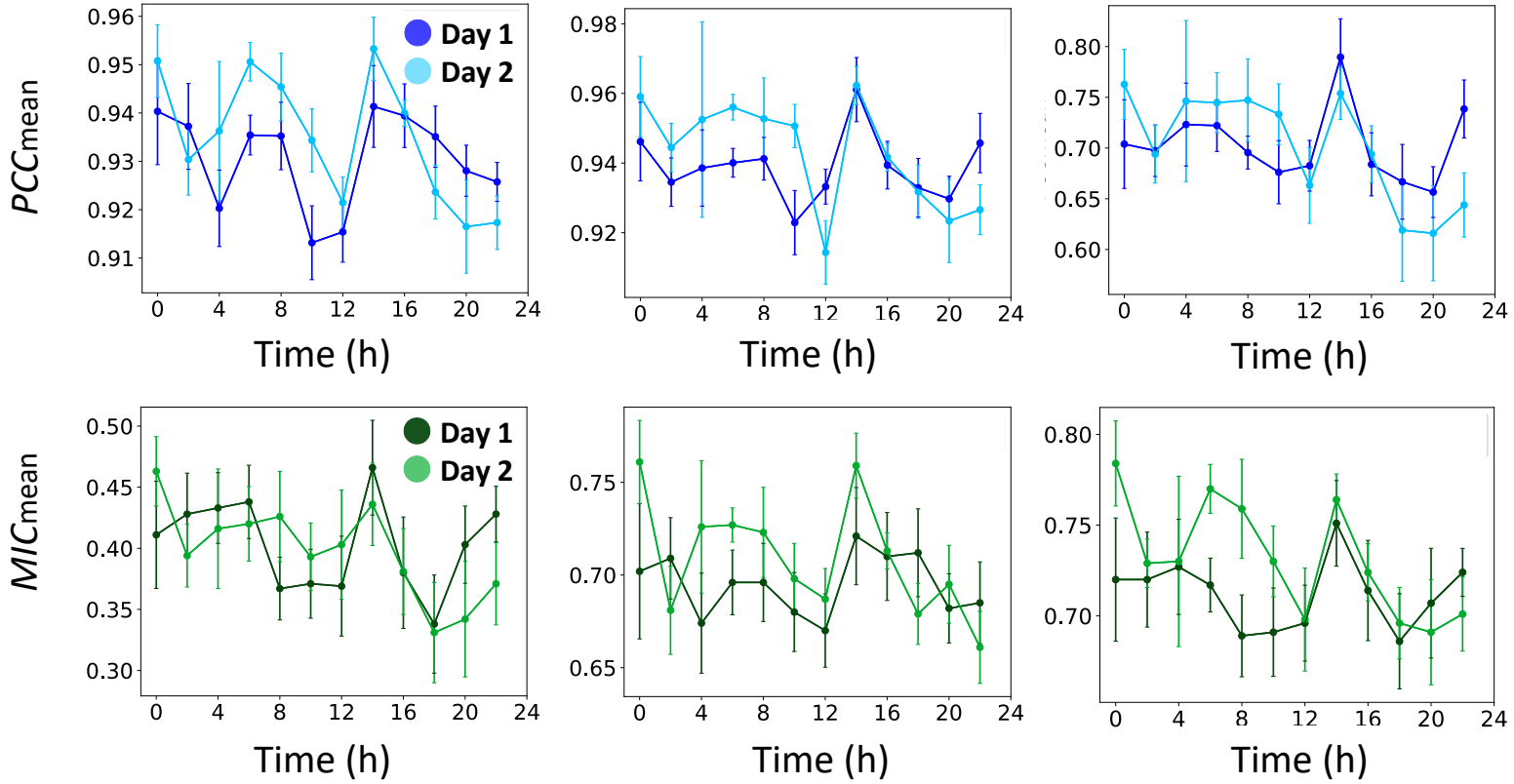

Figure 4. Time-series analysis result of scatter plot matrix of $\mathrm{PCC}_{\text {mean }}$ and $\mathrm{MIC}_{\text {mean }}$.

\section{Discussion}

In this research, we analysed periodic relationship by using two different wavelengths. In the many combinations, the first period and second period show a close relationship. Especially, by using one wavelength from $400 \mathrm{~nm}$ to $700 \mathrm{~nm}$ and other wavelength from $700 \mathrm{~nm}$ to $2500 \mathrm{~nm}$, the rhythm was found clearly. This is due to the photosynthetic activity wavelengths $(400 \mathrm{~nm}-700 \mathrm{~nm})$ and moisture content wavelengths $(700 \mathrm{~nm}$ $2500 \mathrm{~nm}$ ) (Okuzaki et al., 2017, McCree, 1972, Inada, 1976). Figure 5A is the scatter plot matrix of the first period and second period, and Figure 5B shows the histogram related to the combination of wavelengths. The same analyses were done for Figure 5C and 5D. From these results, it was suggested that the combination of photosynthetic activity and moisture content results in the high correlation. Also, Figure 4 shows approximately 12 hour period rhythms, whereas the circadian rhythms show approximately 24 hour rhythms. It is suggested that this result is due to the data compression from the scatter plot to the correlation coefficient. 

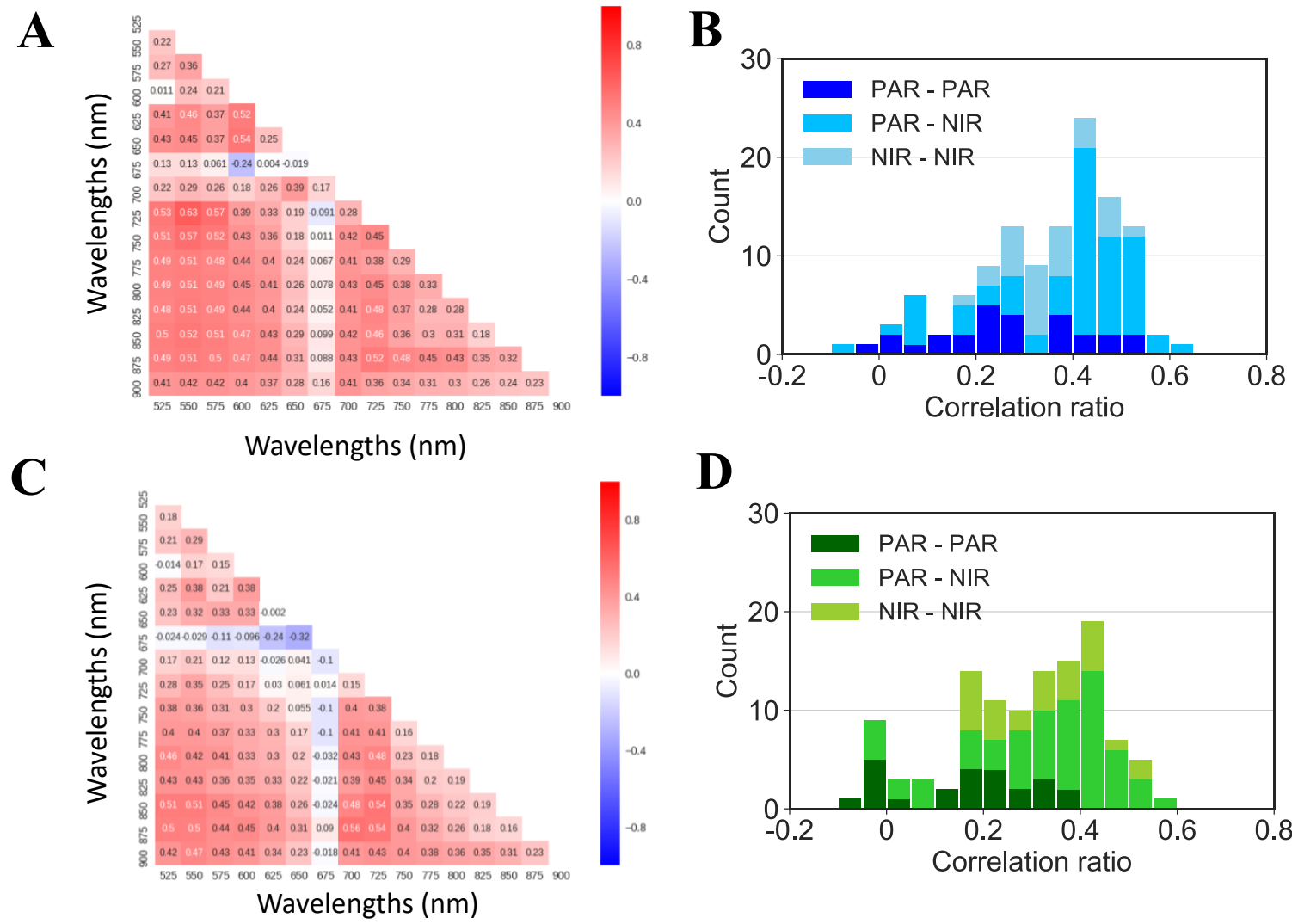

D

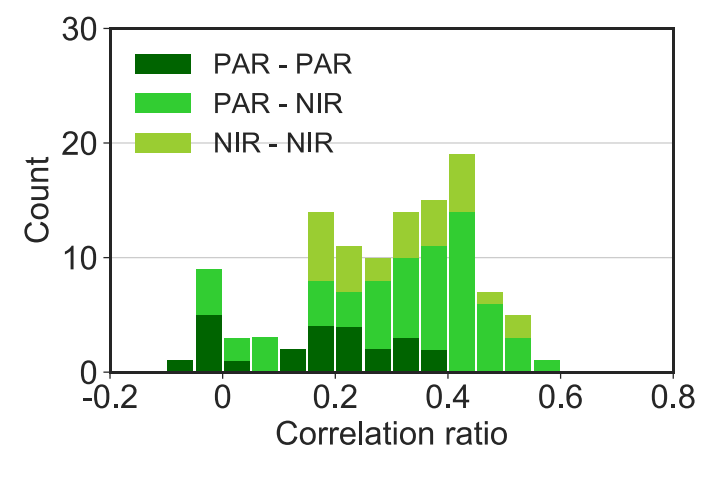

Figure 5. The difference of correlation ratio between the combinations of two wavelengths. Scatter plot matrix of correlation ratio between PCC of first day and second day (A). Histogram of correlation ratio of PCC related to two different wavelengths (B). Scatter plot matrix of correlation ratio between MIC of first day and second day (C). Histogram of correlation ratio of MIC related to two different wavelengths (D).

\section{Conclusions}

In this research, we observed a circadian clock derived periodic rhythm non-destructive and non-invasively. Furthermore, this research suggested that the circadian clock derived periodicity could be observed by using a statistical method applied to two different wavelengths. This research analysed circadian rhythms derived from periodic rhythms by using only two different wavelengths, which will lead to a circadian rhythm measurement method applicable to the industry.

\section{Acknowledgements}

This study was supported, in part, by Grants-in-Aid for Scientific Research (16H05011) and Precursory Research for Embryonic Science and Technology (no. JPMJPR15O4) of the Japan Science and Technology Agency (to H.F.).

\section{References}

Dodd, A. N. et al., 2005. Plant Circadian Clocks Increase Photosynthesis, Growth, Survival, and Competitive Advantage. Science. 309(5734), 630-633.

Duan, S.B. et al., 2014. Inversion of the PROSAIL model to estimate leaf area index of maize, potato, and sunflower fields from unmanned aerial vehicle hyperspectral data. International Journal of Applied Earth Observation and Geoinformation Geoinf. 26, 12-20.

Higashi, T. et al. 2016. Detection of diurnal variation of tomato transcriptome through the molecular timetable method in a sunlight-type plant factory. Frontiers in plant science. 7,87

Inada, K., 1976. Action spectra for photosynthesis in higher plants. Plant and Cell Physiology. 17(2), 355-365.

Khuriyat, N. et at., 2004. Precise near infrared spectral acquisition of intact tomatoes in interactance mode. Journal of near infrared spectroscopy. 12(6), 391-395.

Kinney, J. B. and Atwal, G. S., 2014. Equitability, mutual information, and the maximal information coefficient. Proceedings of the National Academy of Sciences of the United States of America. 111(9), 33543359 . 
Lee, J. D. et al., 2012. Analysis of Concrete Reflectance Characteristics Using Spectrometer and VNIR Hyperspectral Camera. International Archives of the Photogrammetry, Remote Sensing and Spatial Information Science. 39, B7

McCree, K. J., 1971. The action spectrum, absorptance and quantum yield of photosynthesis in crop plants. Agricultural Meteorology. 9, 191-216.

Morimoto, T. et al., 1995. Optimal control of physiological processes of plants in a green plant factory. Control Engineering Practice. 3 (4), 505-511.

Nakamichi, N. et al., 2004. Characterization of Plant Circadian Rhythms by Employing Arabidopsis Cultured Cells with Bioluminescence Reporters. Plant and Cell Physiology. 45(1), 57-67.

Okuzaki, Y. et al., 2017. Spectrometry for ecologists. Japanese Journal of Ecology. 67, 2017.

Pan, W. J., et al. 2015. Nondestructive and intuitive determination of circadian chlorophyll rhythms in soybean leaves using multispectral imaging. Scientific reports. 5, 11108

Panasyuk, S. V. et al., 2007. Medical hyperspectral imaging to facilitate residual tumor identification during surgery. Cancer biology \& therapy. 6(3), 439-446.

Reshef, D. N. et al., 2011. Detecting novel associations in large data sets, Science. 334(6062), 1518-1524.

Ueda, H. R. et al., 2004. Molecular-timetable methods for detection of body time and rhythm disorders from single-time-point genome-wide expression profiles. Proceedings of the National Academy of Sciences of the United States of America. 101(31), 11227-11232. 


\title{
Design of Simplified Optical Devices for the Agrofood Sector: Case Studies from Pre-
}

\section{Harvest to Post-Harvest}

\author{
Roberto Beghi**, Valentina Giovenzana $^{\text {a }}$, Alessio Tugnolo ${ }^{a}$, Riccardo Guidettia \\ ${ }^{a}$ Department of Agricultural and Environmental Sciences - Production, Landscape, Agroenergy (DiSAA), Università \\ degli Studi di Milano, via Celoria 2, Milano 20133, Italy \\ * Corresponding author. Email: roberto.beghi@unimi.it
}

\begin{abstract}
The Italian agri-food system is mostly characterized by small and medium-sized enterprises (SME). Due to their size and the small possibility of significant investments in analytical instrumentation, they are characterized by the lack of crucial information for the management and control of their processes. The main parameters related to important information to better lead the decision-making stages of the production process (e.g. decide the harvest time, monitoring fermentations, monitoring shelf life and post-harvest life, etc.) need to be measured in a simple, quick and objective way. From this point of view, the optical techniques based on visible near-infrared spectroscopy (vis/NIR) are an established, simple and rapid application for the analysis of many productions quality related parameters. A number of vis/NIR and NIR optical systems are available on the market. These devices potentially represent a valid support to the various supply chains. Nevertheless, the available optical systems are complex and expensive devices and their real use in SME is still very limited. The aim of the research was to design, build and test concepts and prototypes of low-costs and user-friendly devices useful for different applications from pre-harvest to post-harvest. Tests for the selection of specific wavelengths for the analysis of grapevine leaf water status, for grape and blueberry ripening evaluation, and for senescence monitoring of freshcut baby leaf salad during shelf life were conducted. In particular, for the grape ripeness analysis a Light Emitting Diode (LED) based prototype equipped with a customized optical fiber for the measurement of the spectral reflectance at four specific wavelengths $(630,690,750$ and $850 \mathrm{~nm})$ was proposed and tested. The use of these devices could be helpful for a more rational and objective monitoring of the products quality, leading to an optimization of the supply chains to provide a better product to the final consumer.
\end{abstract}

Keywords: vis/NIR and NIR spectroscopy; fruit and vegetable; quality; ripeness; water stress; chemometrics.

\section{Introduction}

The Italian agri-food system is mostly characterized by small and medium-sized enterprises (SME). Due to their size and the small possibility of significant investments in analytical instrumentation, they are characterized by the lack of crucial information for the management and control of their processes. The main parameters related to important information to better lead the decision-making stages of the production process (e.g. decide the harvest time, monitoring fermentations, monitoring shelf life and post-harvest life, etc.) need to be measured in a simple, quick and objective way.

The water deficit for vine is linked to phenological stages, duration of water deficit and its intensity level. Irrigation increases photosynthesis and grape yield by 1.5 to 4 times. This behaviour depends on the irrigation timing, the amount of water applied, the environmental conditions, and the differential phenological sensitivity of berry quality between different varieties and other cultural practices. This results in a need of rapid water status monitoring systems in order to better manage crop and irrigation scheduling for a high water use efficiency.

At present, ripening stage and harvest time are estimated mainly by visual assessment based on growers' experience, which can result in unsatisfactory homogeneity of the fruit lots and large variability from farm to farm. Operators need simple and portable devices capable to provide fast ripeness assessment of a large set of samples in the field to help them to determine the best harvest time.

Consumers' interest in berry fruits has recently increased together with the growing demand for healthproducing foods, considering the berries' bioactive and health-protecting properties. Due to these aspects, blueberries, blackberries, and raspberries are a rich source of antioxidant compounds, particularly of flavonoids and anthocyanin (López et al., 2010). Moreover, the growing demand for these fruits may represent an important income generating opportunity for marginal rural areas, because their cultivation is particularly suited for small farms and mountain regions.

The non-destructive techniques, and in particular the optical analysis in the region of near-infrared (NIR) and visible-near infrared (vis/NIR), have been developed considerably over the last 20 years (Guidetti et al. 2012; Nicolai et al. 2007). NIR and vis/NIR spectroscopy are based on the study of the interaction of electromagnetic radiation with the structure of the food product. Molecular bonds like $\mathrm{OH}, \mathrm{CH}, \mathrm{CO}$ and $\mathrm{NH}$ are subject to vibrational energy changes when irradiated by the radiation. The energy absorption of organic molecules in vis/NIR region occurs when molecules vibrate and this is translated into an absorption spectrum (Cen and He, 
2007). Many studies reported in the literature have evaluated the applicability of vis/NIR and NIR spectroscopy to obtain a effective system to perform a wide check on fruits. For example, several applications to estimate the ripeness parameters of different fruit species can be found, especially on grapes (Bellincontro et al., 2009, Cozzolino, 2006). These technologies are extensively used also in postharvest. Ready-to-eat products are characterized by a shelf-life shorter than that of the original unprocessed raw material. The sequence of operations necessary to produce a fresh-cut product (i.e. washing, trimming, peeling and/or cutting) promotes the biochemical and microbial instability of the product itself. These foods are often subjected to rapid loss of colour, organic acids, vitamins and other compounds that determine flavour and nutritional value. Monitoring the quality decay of fresh-cut products is necessary to control the freshness level during the entire production chain and to ensure quality product for the consumer. Hence, also fresh-cut fruit and vegetable sector could be greatly helped by new analytical methods that are accurate, rapid and could be integrated into the production chain to better managing the shelf life of minimally processed products and to meet consumer demand

These optical approaches, however, are always related to the analysis of wide spectra (thousands of wavelengths or variables) and, therefore, require multivariate techniques for data processing to build predictive models. In order to explain the chemical information encoded in the spectral data, chemometric analysis is required (Cogdill and Anderson, 2005). Moreover, the limitations to NIRS adoption by the pre- and postharvest sector could be attributed to cost, technical limitations, grower resistance and supply chain weakness (Magwaza et al., 2012). Hence, to avoid these limitations, simplified handheld devices are desirable. For a simplification and greater diffusion of these non-destructive techniques, in recent years, interest has shifted towards the development of portable systems that could be used in pre- and post-harvest (Zude et al., 2006; Temma et al., 2002; Walsh et al., 2000).

Portable vis/NIR instruments have been tested in controlled laboratory conditions or directly in the field. Concerning laboratory applications, Puangsombut et al. (2012) investigated the feasibility of using vis/NIR spectroscopic devices in transmission mode to predict the soluble solids content (SSC) and total acidity of freshcut products. Regarding applications in uncontrolled field conditions, Larrain et al. (2008) developed a portable NIR instrument (640-1300 nm) for determining ripeness in wine grapes, Guidetti et al. (2008) tested a vis/NIR device working in reflectance configuration to predict blueberry ripeness, Guidetti et al. (2010) evaluated grape quality parameters with a portable vis/NIR system, finally, Beghi et al. (2012) assessed the nutraceutical properties of apples through a visible and near-infrared portable system. All these approaches, nevertheless, rely always on wide spectral ranges (thousands of wavelengths) and thus require multivariate techniques for data processing to build calibration and prediction models. Complex mathematical techniques (chemometrics) are required to explain chemical information encoded in spectral data.

Chemometrics can be also used for the selection of a small number of relevant variables, which represent the most useful information contained in the full spectra (Xiaobo et al., 2010). In this way the spectral noise and the variables containing redundant information can be eliminated. Moreover, a reduced cost for potential miniaturized devices, realized to work at only these selected wavelengths, can be foreseen.

Few examples of commercial non-destructive devices based on a small number of wavelengths are already available on the market. These applications are mainly dedicated to fruits. For example, the University of Bologna (Costa et al., 2011) patented innovative and simplified NIRs equipment, namely DA-Meter for apple and KiwiMeter for kiwi. These systems are used for the analysis of the ripeness level of the fruit through indices based on differences in absorbance between specific wavelengths. This type of instrument, simple and portable, can be used directly on the fruit on the trees and can help growers in taking decision regarding the best cultural management practices (such as pruning, thinning, nutrition, etc.). In this way the heterogeneity of the product can be reduced and, therefore, can be simplified the management of lots product during post-harvest.

The aim of this group of researches was to design, build and test concepts and prototypes of low-costs and user-friendly devices useful for different case-study applications from pre-harvest to post-harvest. The authors conducted tests for the selection of specific wavelengths for the analysis of grapevine leaf water status (Giovenzana et al., 2018), for grape (Civelli et al., 2015; Giovenzana et al., 2015; Giovenzana et al., 2014) and blueberry (Beghi et al., 2013) ripening evaluation, and for senescence monitoring of fresh-cut baby leaf salad during shelf life (Beghi et al., 2014).

\section{Materials and Methods}

Different experimental setups were carried out for the different matrices considered.

Samplings

Grapevine leaf water status

Spectral measurements were performed on 440 leaves (cv. Biancolella) in the vis/NIR (400-1000 nm) and NIR $(1100-2000 \mathrm{~nm})$ range for water status monitoring directly in field using two portable optical devices.

Spectral measurements were acquired directly in the field on the leaf under natural water stress conditions. Air 
temperature during experimental acquisitions ranged between $20^{\circ} \mathrm{C}$ and $26^{\circ} \mathrm{C}$. Field datasets of spectra were assumed to randomly embed possible environment influencing factors in a representative way.

As references, a Scholander pressure chamber was used to measure the water potential $(\Psi, \mathrm{MPa})$ equipped with a manometer with a $0.05 \mathrm{MPa}$ scale division and 0-2.5 $\mathrm{MPa}$ pressure range. Since the goal of the work is the development of a useful device for evaluating the $\Psi$ mainly in drought periods where the grapevine is exposed to the risk of water stress, measurements were made directly in the field during the night in pre-dawn conditions (pre-dawn leaf water potential, $\Psi$ plwp) (3.00 to 5.00 hours, solar time; sunrise in September about 6.30 hour, in July about 6.00 hour) and leaf water potential ( $\Psi$ lwp) was also measured during the day (7.00 to 10.00 hours, solar time), on fully expanded and undamaged leaves for each plant chosen from the mid-upper part of the canopy.

\section{Grape ripeness}

The experimental plan monitored the grape ripening process in the Valtellina viticultural area (Sondrio, northern Italy, approx. $46.2^{\circ} \mathrm{N}, 9.9^{\circ} \mathrm{E}$ ) during the last period of ripening just before harvest. The experimentation was carried out on red grape Nebbiolo cultivar (ecotype Chiavennasca), one of the most important varieties in Italy. Samples were drawn from 17 different zones, throughout the entire wine area of the valley, in order to represent environmental variability and monitor the entire production region of DOC (controlled denomination of origin) and DOCG (controlled and guaranteed denomination of origin) wines. A total of 68 samples of red grapes were collected on four sampling dates. For every date, non-destructive analyses were carried out on each sample using a portable commercial vis/NIR spectrophotometer and then destructive chemical analyses were performed.

The total soluble solids (TSS) content was measured using a digital pocket refractometer while grape titratable acidity ( $\mathrm{g}$ tartaric acid $\mathrm{dm}^{-3}$ ) was measured using an automatic titrator. The Glories method (Glories, 1984) was used to estimate the grape phenolic content. According to this method, potential (PA) and extractable anthocyanins (EA), extracted at $\mathrm{pH} 1$ and $\mathrm{pH} 3.2$, respectively $\left(\mathrm{mg}\right.$ anthocyanins $\left.\mathrm{dm}^{-3}\right)$, and total polyphenols (TP) were evaluated. The phenolic compound quantification was based on the optical density (OD) measurement at $520 \mathrm{~nm}$ and $280 \mathrm{~nm}$ for anthocyanins and polyphenols, respectively, using a UV/vis spectrophotometer.

\section{Blueberries ripeness}

In this case a total of $942 \mathrm{Vis} / \mathrm{NIR}$ fruit spectra in the range of 445-970 nm were acquired for Vaccinium corymbosum ("Brigitta" cultivar) during two different growing seasons, cultivated in different farms in the area of Valtellina, Italy. Spectra measurements were taken in the field on individual berries along their equator region after artificial illumination with a probe tip.

Field spectra acquisitions of berries were carried out at weekly intervals. Just after measurement of the plants, fruits samples were picked and divided into four ripeness classes according to the criteria used by expert growers (mainly relying on size and surface color distribution). According to commercial classification, blueberry fruits were graded as follows (figure 1):

- $\quad$ Ripeness grade 1 (R1 = totally unripe): green, small-sized berries, needing 3-5 weeks to commercial harvest;

- $\quad$ Ripeness grade 2 (R2 = unripe): green skin over more than half of the surface, medium-sized berries, needing 1.5-3 weeks to commercial harvest;

- $\quad$ Ripeness grade 3 (R3 = close to ripeness): almost completely blue-pigmented (except the stem's end), nearly full or full-sized berries, needing 1 week or less to commercial harvest;

- $\quad$ Ripeness grade 4 (R4 = fully ripe): completely blue-pigmented (included stem's end), full-sized berries, ready to be harvested.
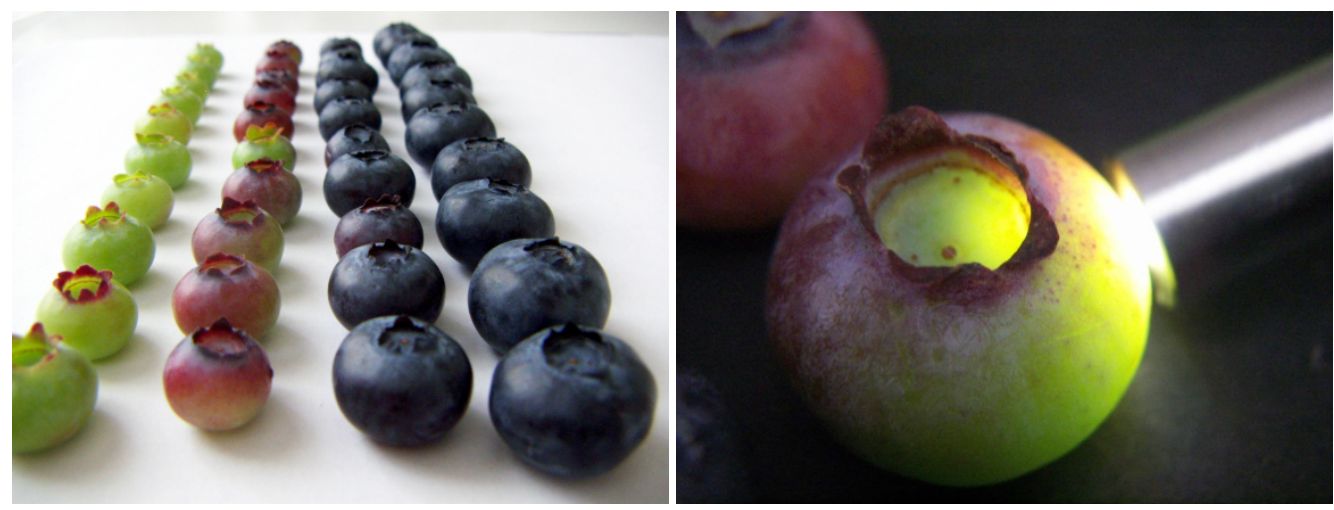

Figure 1. Left: blueberries grouped into four ripeness grades according to commercial classification. Right: illustrative example of spectral measurement on a freshly detached berry; in this study, the acquisition of berries' spectra was done with undetached fruits on the plant (Beghi et al., 2013). 
Senescence monitoring of fresh-cut baby leaf salad

Three storage/shelf-life temperatures were investigated: $4.0 \pm 0.5^{\circ} \mathrm{C}, 10.0 \pm 0.5^{\circ} \mathrm{C}$ and $20.0 \pm 0.5^{\circ} \mathrm{C}$.

The duration of the experimentation was different for the different storage temperature. The Valerianella packages preserved at $4{ }^{\circ} \mathrm{C}$ and $10{ }^{\circ} \mathrm{C}$ were sampled for 16 days, while samples stored at $20^{\circ} \mathrm{C}$ were analyzed only for 7 days, due to the rapid degradation of the lettuce at this temperature. The numbers of sampling points during storage monitoring for the fresh-cut leaves were therefore 10,11 and 6 for $4{ }^{\circ} \mathrm{C}, 10{ }^{\circ} \mathrm{C}$ and $20{ }^{\circ} \mathrm{C}$, respectively.

The quality decay of samples was evaluated by chemical parameters ( $\mathrm{pH}$, moisture and total polyphenols content) and by a non-destructive optical device (vis/NIR spectroscopy).

\section{Vis/NIR spectroscopy measurements}

Spectral acquisitions were performed on samples using a vis/NIR portable system operating in the wavelength range of 400-1000 nm (Jaz, OceanOptics, USA), and an NIR (only on grapevine leaves) working in the range 1100-2000 nm (TG-cooled NIR II, Hamamatsu Photonics, Japan). Both the optical equipment sets consisted of five components: 1) a lighting system (halogen lamp), 2) a fiber-optic probe for reflection measurement, 3) a spectrophotometer, 4) hardware for data acquisition and instrument control, and 5) the power supply. Spectra were acquired in reflectance mode: light radiation was guided from the light source to the surface of the sample through a Y-shaped, bidirectional fiber-optic probe (OceanOptics, USA). Samples were illuminated by the radiation produced by the lighting system, and the backscattered component as guided by an optical fiber to be measured by the spectrophotometer.

Spectral resolutions were $0.3 \mathrm{~nm}$ and $8.0 \mathrm{~nm}$ for vis/NIR and NIR systems, respectively.

\section{Data analysis}

Chemometric analyses were performed using The Unscrambler ${ }^{\circledR} 9.8$ software package (CAMO ASA, Norway) in order to extract the maximum usable information from the spectral data and to select the most significant wavelengths. The correlation between the spectral data matrix and the reference parameters were carried out using the PLS regression algorithm. Depending on the dimension of the available datasets, leave-moreout cross-validation with different cancellation groups was used to validate the PLS regression models. To evaluate model accuracy, the statistical parameters used were the coefficient of determination in calibration $\left(\mathrm{R}^{2}\right.$ cal $)$, the coefficient of determination in cross-validation $\left(\mathrm{R}^{2} \mathrm{cv}\right)$, the root mean square error of calibration (RMSEC) and the root mean square error of cross-validation (RMSECV). The best calibrations were selected based on minimizing the RMSECV.

RCA was carried out for relevant variable selection, representing the most useful information of the full vis/NIR spectral region (Xiaobo et al., 2010, Chong and Jun, 2005).

Standardized regression coefficients of the PLS model were used for the elaboration. The standardization considered both the standard deviation of reflectance and the standard deviation of the reference data. Therefore, the selected wavelengths were employed for the elaboration of MLR models. MLR is a regression method that, compared with PLS, allows to develop models using only few important variables, to predict the outcome of a response. This method is also well-suited when the number of variables is less than the number of samples and is not affected by collinearity. Verifying the prediction ability of the MLR models was performed to study the efficiency of the selected wavelengths.

\section{Results and Discussion}

Results for grapevine leaf water status monitoring

Standardized regression coefficients for the NIR region showed a larger number of candidate wavelengths compared to bands highlighted for the vis/NIR range. The statistics of the MLR models (table 1) were equal to $\mathrm{R}^{2}$ val about 0.7 for MLR models arising from vis/NIR spectra. Similar results were obtained using both crossvalidation and test set validation. Regarding the NIR range, acceptable models were obtained only using the selection of fourteen EVs, with analogues $\mathrm{R}^{2}$ values to those obtained for the vis/NIR region. In this range the use of only five EV not allowed to calibrate a good regression model due to a lack of sufficient useful information contained in them.

A comparison between PLS derived from the full vis/NIR spectra (2048 variables) and MLR arising only from the five EVs was carried out. The overall calibration and validation results of the MLR models for $\Psi$ prediction were satisfactory, although the performance of the MLR models were worse than the good PLS models. Overall results were satisfactory considering that are predictive models based on indirect correlations between spectra and a physical parameter as water potential, not only directly related to specific overtones absorption of $\mathrm{OH}$ bond, but 
also to modifications concerning the firmness and turgidity of tissues with effects on scattering of the first layer of the leaf tissue.

Regarding the vis/NIR range, $\mathrm{R}^{2}$ value for $\Psi$ prediction decreased in cross-validation from 0.77 for PLS to 0.69 for MLR, while in test set validation similar results were obtained $\left(\mathrm{R}^{2}=0.68\right.$ and 0.67 , respectively).

Greater differences were highlighted in the comparison between PLS and MLR models for the NIR range. In this case, the loss of performance by using a small number of EV was evident; using the whole available spectral range excellent results were obtained, instead fair MLR models were calculated using fourteen EVs, with both internal and test set validation. Overall, a reasonable loss of information was noticeable between the PLS models calculated using the total wavelengths ranges and the MLR models employing only the selected EV.

Table 1. Statistics related to the MLR models obtained by vis/NIR and NIR spectroscopy (Giovenzana et al., 2018).

\begin{tabular}{|c|c|c|c|c|c|c|c|c|c|}
\hline & & & & \multicolumn{3}{|c|}{ Calibration } & \multicolumn{3}{|c|}{ Validation } \\
\hline $\begin{array}{c}\text { Spectral } \\
\text { region }\end{array}$ & Pretreatment & $\begin{array}{c}\text { Validation } \\
\text { method }\end{array}$ & $\mathrm{N}^{\circ}$ of $\mathrm{EV}$ & Samples & $\mathrm{R}_{\text {cal }}^{2}$ & $\mathrm{RMSE}_{\mathrm{cal}}$ & Samples & $\mathrm{R}_{\text {val }}^{2}$ & $\mathrm{RMSE}_{\mathrm{val}}$ \\
\hline \multirow[t]{2}{*}{ Vis-NIR } & \multirow[t]{2}{*}{ Smoothing } & $\begin{array}{c}\text { Cross- } \\
\text { validation }\end{array}$ & \multirow[t]{2}{*}{5} & 407 & 0.70 & 0.30 & 407 & 0.69 & 0.30 \\
\hline & & Test set & & 279 & 0.65 & 0.33 & 150 & 0.68 & 0.32 \\
\hline \multirow{4}{*}{ NIR } & \multirow{4}{*}{$\begin{array}{l}\text { Smoothing + } \\
\text { der2 }\end{array}$} & $\begin{array}{c}\text { Cross- } \\
\text { validation }\end{array}$ & \multirow[t]{2}{*}{5} & 405 & 0.32 & 0.39 & 405 & 0.30 & 0.40 \\
\hline & & Test set & & 279 & 0.56 & 0.43 & 150 & 0.45 & 0.51 \\
\hline & & $\begin{array}{c}\text { Cross- } \\
\text { validation }\end{array}$ & \multirow[t]{2}{*}{14} & 407 & 0.71 & 0.27 & 407 & 0.69 & 0.28 \\
\hline & & Test set & & 279 & 0.63 & 0.32 & 150 & 0.63 & 0.33 \\
\hline
\end{tabular}

Results for grape ripeness evaluation

Correlations between the spectral data matrix and technological (total soluble solids, TSS) and phenolic (polyphenols) parameters were carried out using partial least square (PLS) regression. Standardized regression coefficients of the PLS model were used to select the relevant variables, representing the most useful information of the full spectral region. To support the variable selection, a qualitative evaluation of the average spectra and loadings plot, derived from principal component analysis (PCA), were considered.

The three selected wavelengths were $670 \mathrm{~nm}$, corresponding to the chlorophyll absorption peak, $730 \mathrm{~nm}$, equal to the maximum reflectance peak, and $780 \mathrm{~nm}$, representing the third overtone of $\mathrm{OH}$ stretching. PCA and multiple linear regression (MLR) were applied on the three selected wavelengths in order to verify their effectiveness. Simple equations for TSS and polyphenols prediction were calculated. A comparison between PLS derived from the full vis/NIR spectra and MLR arising only from the three wavelengths was carried out on TSS and TP. The overall calibration and prediction results of the MLR models, both for TSS and TP prediction, were satisfactory, although the performance of the MLR models (table 2) were slightly worse than the good PLS models. The obtained RMSECV values were similar for the PLS and MLR models. RPD value for TSS decreased from 2.26 for PLS to 2.13 for MLR and, regarding TP, RPD showed a decrement from 1.98 to 1.76 , respectively. PLS and MLR models for both the quality parameters showed very low bias values and almost the same slope (around 0.8 ).

Table 2. Statistics of the MLR models, based on the three selected wavelengths $(670,730,780 \mathrm{~nm})$ to predict the ripening parameters of the Nebbiolo grape (Giovenzana et al., 2014). TSS=total soluble solid content; TP=total polyphenols; $\mathrm{OD}=$ optical density.

\begin{tabular}{|c|c|c|c|c|c|c|c|c|c|}
\hline \multirow{2}{*}{$\begin{array}{l}\text { Ripening } \\
\text { Parameters }\end{array}$} & \multirow{2}{*}{ Units } & \multicolumn{2}{|c|}{ Calibration } & \multicolumn{2}{|c|}{ Cross-validation } & \multirow[b]{2}{*}{ Slope } & \multirow[b]{2}{*}{ RMSECV } & \multirow[b]{2}{*}{ RMSECV\% } & \multirow[b]{2}{*}{ RPD } \\
\hline & & $\mathrm{R}_{\text {cal }}^{2}$ & RMSEC & $\mathrm{R}_{\mathrm{cv}}^{2}$ & Bias & & & & \\
\hline TSS & ${ }^{\circ}$ Brix & 0.75 & 0.77 & 0.71 & -0.006 & 0.74 & 0.83 & $3.9 \%$ & 2.13 \\
\hline $\mathrm{TP}$ & $\mathrm{OD}_{280} \mathrm{~nm}$ & 0.74 & 3.95 & 0.70 & -0.034 & 0.73 & 4.30 & $11.9 \%$ & 1.79 \\
\hline
\end{tabular}

Results showed that only a small loss of information was noticeable between the PLS model calculated using 2048 wavelengths and the MLR models employing only three effective variables. The results demonstrate the feasibility of a simplified, low-cost handheld device for ripeness assessment in the field and a possible functional scheme and a prototype was proposed (figure 2). 


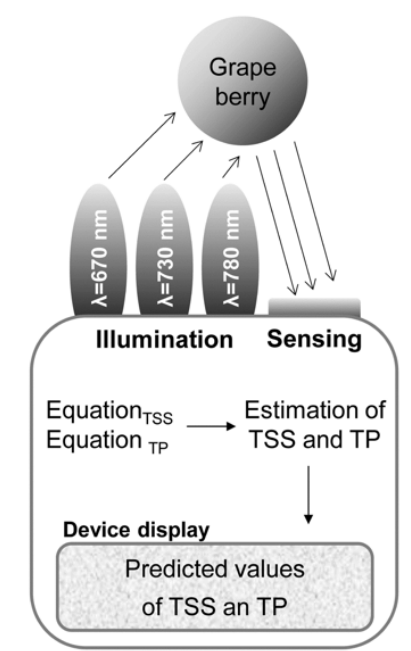

Figure 2. Functional scheme of a simple, handheld optical device implementing measurements of the signal intensity at the selected wavebands and equations to estimate total soluble solids (TSS) and total polyphenols (TP) for supporting grower's decisions on optimal harvest date.

Results for blueberry ripeness evaluation

A principal component analysis of fruits spectra highlighted that two main wavebands $(680 \mathrm{~nm}$ and $740 \mathrm{~nm})$ can maximize the differences between fully ripe samples and those close to ripeness or unripe. Hence, spectral values at 680,740 , and $850 \mathrm{~nm}$ (the latter being an additional normalization waveband) were used to create a blueberry ripeness index (BRI) as a linear combination of two spectral ratios: $\mathrm{S} 1=\log (\mathrm{I} 680 / \mathrm{I850})$ and $\mathrm{S} 2=$ (I740/I850).

The definition of specific ripeness thresholds for the BRI according to more or less selective criteria was tested, and the ripeness classification capability was then assessed on a separate validation set of 471 berries. When applying a less selective threshold approach, the BRI correctly classified as ripe $85 \%$ of manually graded fully ripe berries, whereas $13 \%$ of close but not yet ripe validation samples were misclassified as fully ripe and ready to harvest. Comparatively, when a more demanding ripeness threshold was applied, the amount of nearly ripe berries misclassified as ripe decreased to $8 \%$, but, the amount of fully ripe berries not identified as ripe rose to $25 \%$. In both cases, none of the unripe validation samples was erroneously classified as a ripe fruit.

These results, which were obtained with a BRI defined by spectral measurements at just three discrete wavelengths, point to the feasibility of a simple, microcontroller-based, handheld optical device able to implement the BRI to quickly assess the ripeness of sets of berries during the last and most delicate stages of the ripening process.

Results for senescence monitoring of fresh-cut baby leaf salad

Through PLS-RCA technique, standardized regression coefficients of PLS models were used to select the relevant variables, representing the most useful information of full spectral region. In this case study, the four selected wavelengths were $520 \mathrm{~nm}, 680 \mathrm{~nm}, 710 \mathrm{~nm}$ and $720 \mathrm{~nm}$. For pH, moisture and TP prediction, the statistics of the MLR models were equal to $\mathrm{R}^{2} \mathrm{cv}=0.70,0.75,0.80$ and $\mathrm{RPD}=1.83,2.08,2.48$, respectively. A comparison between PLS derived from the full vis/NIR spectra (400-1000 nm) and MLR arising only from the four wavelengths showed that the overall calibration and prediction results of the MLR models, for all the parameters, were satisfactory, although the performance of the MLR models was slightly worse than the good PLS models, as expected. RPD value for $\mathrm{pH}$ decreased from 2.54 for PLS to 1.83 for MLR, regarding the MC, RPD showed a slight decrement from 2.25 to 2.08, and for TP from 3.19 to 2.48 for PLS and MLR respectively (table 3). 
Table 3. Statistics of the MLR models, based on the four selected wavelengths $(520,680,710,720 \mathrm{~nm})$ to predict the freshness level of Valerianella leaf samples, and of the PLS models (cross-validation) (Beghi et al., 2014). LV=latent variables.

\begin{tabular}{|c|c|c|c|c|c|c|c|c|c|c|c|c|}
\hline \multirow[b]{2}{*}{ Quality parameters } & \multirow[b]{2}{*}{$\mathrm{N}^{\circ}$} & \multirow[b]{2}{*}{ Mean } & \multicolumn{4}{|c|}{ Calibration MLR } & \multicolumn{3}{|c|}{ Cross-validation MLR } & \multicolumn{3}{|c|}{ Cross-validation PLS } \\
\hline & & & $\mathrm{SD}$ & $\mathrm{R}^{2}$ & RMSEC & RPD & $\mathrm{R}^{2}$ & RMSECV & RPD & $\mathrm{R}^{2}$ & RMSECV & RPD \\
\hline $\mathrm{pH}$ & 24 & 6.45 & 0.33 & 0.82 & 0.13 & 2.54 & 0.70 & 0.18 & 1.83 & 0.86 & 0.13 & 2.54 \\
\hline $\mathrm{TP}\left(\mathrm{mg} / 100 \mathrm{~g}_{\text {eq gallic acid }}\right)$ & 16 & 267 & 40.3 & 0.88 & 12.38 & 3.26 & 0.80 & 16.28 & 2.48 & 0.89 & 12.64 & 3.19 \\
\hline $\mathrm{MC}(\%)$ & 24 & 93.38 & 0.27 & 0.87 & 0.09 & 3 & 0.75 & 0.13 & 2.08 & 0.84 & 0.12 & 2.25 \\
\hline
\end{tabular}

\section{Conclusions}

Different feasibility studies were performed on several pre- and post-harvest matrices. Correlations between the optical data and reference parameters were carried out using partial least square (PLS) regression for spectra and using multiple linear regression (MLR) for data from the effective wavelengths selected for the different applications.

The overall calibration and prediction results of the MLR models were satisfactory, although the performance of the MLR models was generally slightly worse than the PLS models, a small loss of information using only the selected wavelengths is noticeable. Consequently, the applicability of LED lightning system and inexpensive hardware could be envisaged to build simplified optical systems.

Moreover, a first prototype of simplified optical device was realized for grape ripeness analysis, paying attention to versatility and modularity. The possibility to adjust light sources with a specific choice of wavelengths for LEDs, makes it possible a future use of the same simplified optical device for many different applications (i.e. ripeness evaluation, chemicals and physical properties prediction or shelf life analysis) and for different food matrix.

An evolution and an engineering of these systems will be desirable in order to obtain compact, pocket-size, inexpensive and easy to use devices.

\section{References}

Beghi R., Giovenzana V., Civelli R., Malegori C., Buratti S., Guidetti R., 2014. Setting-up of a simplified handheld optical device for decay detection in fresh-cut Valerianella locusta L. Journal of Food Engineering 127: $10-15$.

Beghi R., Giovenzana V., Spinardi A., Bodria L., Guidetti R. and Oberti R., 2013. Derivation of a blueberry ripeness index with a view to a low-cost, handheld optical sensing device for supporting harvest decisions. Transactions of the ASABE, 56(4): 1551-1559.

Beghi, R., Spinardi, A., Bodria, L., Mignani, I., \& Guidetti, R. 2012. Apples nutraceutic properties evaluation through a visible and near-infrared portable system. Food and Bioprocess Technology, DOI 10.1007/s11947-0120824-7.

Bellincontro, A., I. Nicoletti, M. Valentini, A. Tomas, D. De Santis, D. Corradini, and F. Mencarelli. 2009. Integration of Nondestructive Techniques with Destructive Analyses to Study Postharvest Water Stress of Winegrapes. Am. J. Enol. Vitic. March 2009 60:57-65.

Cen, H. \& He, Y. 2007. Theory and application of near infrared reflectance spectroscopy in determination of food quality. Trends in Food Science \& Technology, 18, 72-83.

Chong, I.G., and C.H. Jun. 2005. Performance of some variable selection methods when multicollinearity is present. Chemom. Intell. Lab. Syst., 78, 103-112.

Civelli, R., Giovenzana, V., Beghi, R., Naldi, E., Guidetti, R., \& Oberti, R. 2015. A Simplified, Light Emitting Diode (LED) Based, Modular System to be Used for the Rapid Evaluation of Fruit and Vegetable Quality: Development and Validation on Dye Solutions. Sensors, 15(9), 22705-22723.

Cogdill, R.P., and C.A. Anderson. 2005. Efficient spectroscopic calibration using net analyte signal and pure component projection methods. J. Near Infrared Spectrosc. 13(3), 119-132.

Costa, G., Bonora, E., Fiori, G., \& Noferini, M. 2011. Innovative non-destructive device for fruit quality assessment. Acta Horticulturae, 913, 575-581.

Cozzolino, D., R.G. Dambergs, L. Janik, W.U. Cynkar, and M. Gishen. 2006. Analysis of grapes and wine by near infrared spectroscopy. Journal of Near Infrared Spectroscopy. Chichester, UK. 14, 279-289.

Giovenzana V., Beghi R., Malegori C., Civelli R., Guidetti R., 2014. Wavelength selection with a view to a simplified handheld optical system to estimate grape ripeness. Am. J. Enol. Vitic. 65(1): 117-123. 
Giovenzana, V., Beghi, R., Parisi, S., Brancadoro, L., \& Guidetti, R. 2018. Potential effectiveness of visible and near infrared spectroscopy coupled with wavelength selection for real time grapevine leaf water status measurement. Journal of the Science of Food and Agriculture, 98(5), 1935-1943.

Giovenzana, V., Civelli, R., Beghi, R., Oberti, R., \& Guidetti, R. 2015. Testing of a simplified LED based vis/NIR system for rapid ripeness evaluation of white grape (Vitis vinifera L.) for Franciacorta wine. Talanta, 144, 584-591.

Glories Y., 1984. The colour of red wines. Connaissance de la Vigne et Du Vin, 18, 195-217.

Guidetti R., Beghi R. \& Giovenzana V. 2012. Chemometrics in Food Technology. In: InTech (Eds.), Chemometrics in Practical Applications, ISBN: 978-953-51-0438-4.

Guidetti, R., Beghi, R., \& Bodria, L. 2010. Evaluation of Grape Quality Parameters by a Simple Vis/NIR System. Transaction of the ASABE, 53(2), 477-484.

Guidetti, R., Beghi, R., Bodria, L., Spinardi, A., Mignani, I., \& Folini, L. 2008. Prediction of blueberry (Vaccinium corymbosum) ripeness by a portable Vis-NIR device. Acta Horticulturae 310, 877-885.

Larrain, M., A.R. Guesalaga, E. Agosin. 2008. A Multipurpose Portable Instrument for Determining Ripeness in Wine Grapes Using NIR Spectroscopy. Instrumentation and Measurement, IEEE Transactions 57(2), $294-302$.

López J., Uribe E., Vega-Gálvez A., Miranda M., Vergara J., Gonzalez E., Di Scala K. 2010. Effect of Air Temperature on Drying Kinetics, Vitamin C, Antioxidant Activity, Total Phenolic Content, Non-enzymatic Browning and Firmness of Blueberries Variety O'Neil. Food And Bioprocess Technology, 3(5), $772-777$.

Magwaza, L. S., Opara, U. L., Nieuwoudt, H., Cronje, P. J., Saeys, W., \& Nicolaï, B. 2012. NIR spectroscopy applications for internal and external quality analysis of citrus fruit - a review. Food and Bioprocess Technology, 5(2), 425-444.

Nicolai, B.M., Beullens, K., Bobelyn, E., Peirs, A., Saeys, W., Theron, K.I., \& Lammertyna, J. 2007. Nondestructive measurement of fruit and vegetable quality by means of NIR spectroscopy: A review. Postharvest Biology and Technology, 46, 99-118.

Puangsombut A., S. Pathaveerat, A. Terdwongworakul, and K. Puangsombut. 2012. Evaluation of internal quality of fresh-cut pomelo using vis/NIR trasmittance. Journal of texture studies, 43, 445-452.

Temma, T., Hanamatsu, K., \& Shinoki, F. 2002. Development of a portable near infrared sugar-measuring instrument. J. Near Infrared Spectrosc., 10, 77-83.

Walsh, K.B., Guthrie, J.A., \& Burney, J.W. 2000. Application of commercially available, low-cost, miniaturised NIR spectrometers to the assessment of the sugar content of intact fruit. Aust. J. Plant Physiol., 27, 1175-1186.

Xiaobo, Z., Z. Jiewen, M.J.W. Povey, M. Holmes, and M. Hanpin. 2010. Variables selection methods in nearinfrared spectroscopy. Analytica Chimica Acta 667, 14-32.

Zude, M., Herold, B., Roger, J.-M., Bellon-Maurel, V., \& Landahl, S. 2006. Non-destructive tests on the prediction of apple fruit flesh firmness and soluble solids content on tree and in shelf life. J. Food Eng., 77, 254260. 


\title{
Reliability of Autonomous Guided Systems for Vineyard Monitoring
}

\author{
Andújar D a,*, Jose Maria Bengochea-Guevara a, Angela Ribeiro a \\ ${ }^{a}$ Centre for Automation and Robotics, CSIC, Arganda del Rey, Madrid 28500, Spain \\ * Corresponding author. Email: d.andujar@csic.es
}

\begin{abstract}
Homogeneous use of pesticides in the majority of the cropping areas is the most common method for pest control. Intra-plant variation is not considered which leads to the use of a non-adjusted dose. These facts demonstrate that the dose of sprayed products should be adapted to the growing state at each location. Information about crop status is of high value for agricultural management. The use of plant models allows site specific management and yield estimations. The models can be analyzed to obtain information such as height of tree-rowvolume, which is used to determine the amount of agrochemicals, nutrients or grow regulator to apply at each location. The use of new technologies is of high impact in agriculture, and viticulture is the agricultural sector with more technological advances. The creation of spatial variability maps for site-specific management is still a challenge. The use of 3D structural maps can adapt the application of inputs within the fields. This work assess the reliability of 3D models using large maps of vineyards for site-specific management and explore the economic profitability of its usage. A mobile platform, based on a commercial electric vehicle, was developed and equipped with different on-board sensors for crop monitoring. The platform based on RTK-GNSS receiver and IMU information allowed operating in autonomous mode for vineyard monitoring. A low-cost RGB-D sensor, Microsoft Kinect v2 sensor was installed on-board the platform for construction of 3D crop maps. The reconstruction of the field objects was fully automatic based on ICP algorithms for 3D reconstructions of large areas, such as a complete row crop. The experiment showed that the use of robotic platforms would reduce the operational cost and it would increase economic savings.
\end{abstract}

\section{Introduction}

The homogeneous use of pesticides in the majority of the cropping areas is the most common method for pest control (Peteinatos et al., 2013). However, growing period starts with no leaves and the canopy increase gradually. Thus, plant protection plans should focus efforts in adapting application rates and not in the use of a fixed amount of active ingredient per hectare, which can over-dose or under-dose the needed treatment. In addition, intra-plant variation is not considered which leads to the use of a non-adjusted dose. Information about crop status is of high value for agricultural management. Productivity and efficiency can be improved by including spatial information in field work processes.

The use of plant models allows site specific management. When individual plants are modelled is also of high value for research purposes. Models show information about structural parameters such as tree height and tree volume (Andújar et al. 2017). Plant height derived from models allows the use of differential plant protection product application. Muharam (2013) showed the relation of plant height and nitrogen nutrition status, allowing the use of a site-specific application of products. Similarly, plant height is used for site specific weed management. Different weed species could be discriminated based on height models at real time for patch treatment (Andujar et al., 2012). The use of distance measurements is also used for tree characterization. The distance from a measurement point to the tree is used to adapt a variable rate of plant protection products in relation to the variability of crop width. Although, discrete measurement show a good usage for variable rate, the use of continuous models based on the third dimension show better resolutions and more precise applications.

Plant digital models are used not only in phenotyping processes. Structural and geometric parameters have been usually calculated through high cost and time consuming methods, which are related to destructive manual processes. The use of electronic devices rapidly characterizes crops by non-destructive, accurate and repeatable methods. Several methods have been tested on-field from fluorescence sensors or spectral reflectance sensors able to separate bare ground from plants to visible imaging systems (Burgos-Artizzu et al., 2010). The use of 3D model is showing the great possibilities of crop modelling for agricultural management. Thus, emerging techniques such as RGB-D cameras creates models from which extracted information allows to take the better decision. The models can be analyzed to obtain information such as height of tree-row-volume which is used to determine the amount of agrochemicals,

New technologies is of high impact in agriculture, and viticulture is the agricultural sector with more technological advances (Arnó et al., 2009). The creation of spatial variability maps for site-specific management is still a challenge. The use of 3D structural maps can adapt the application of inputs within the fields. The objective of this study was to assess provide information about vineyard crop status using 3D structural maps trough the design of a mobile platform, based on a commercial electric vehicle, equipped with different on-board sensors for crop monitoring and the devolopment of ICP algorithms for 3D reconstructions of large areas. 


\section{Materials and Methods}

On-field inspection vehicle

The Kinect v2 RGB-D sensor (Microsoft, Redmond, WA, USA), operates at 30 fps, supplies RGB images with a resolution of $1920 \times 1080$ pixels together with depth information with a resolution of $512 \times 424$ pixels. The system calculates the distance from the speed of light in air by estimating the received light phase at each pixel with knowledge of the modulation frequency with a range of the sensor is $0.5-4.5 \mathrm{~m}$. A Kinect v2 sensor was placed in a field platform (Figure 1) using an aluminium support structure. The field platform is based on a Twizy Urban 80 model (Renault, Valladolid, Spain), which has a $13 \mathrm{~kW}$ electric motor and can travel up to $80 \mathrm{~km} / \mathrm{h}$. The vehicle is ultra-compact, and unladen weight of $450 \mathrm{~kg}$. The electric motor of the vehicle allows negligible vibration at speeds below $3 \mathrm{~km} / \mathrm{h}$, which is convenient for high-quality information acquisition.

The sensor was connected to the on-board computer, which was equipped with an Intel Core i7-4771@3.5GHz processor, 16 GB of RAM, and an NVIDIA GeForce GTX 660 graphic card. The platform is also equipped with a RTK-GNSS receiver, an R220 receiver (Hemisphere, Scottsdale, AZ, USA), which provides location data at a $20 \mathrm{~Hz}$ sample rate with an error below $2 \mathrm{~cm}$.

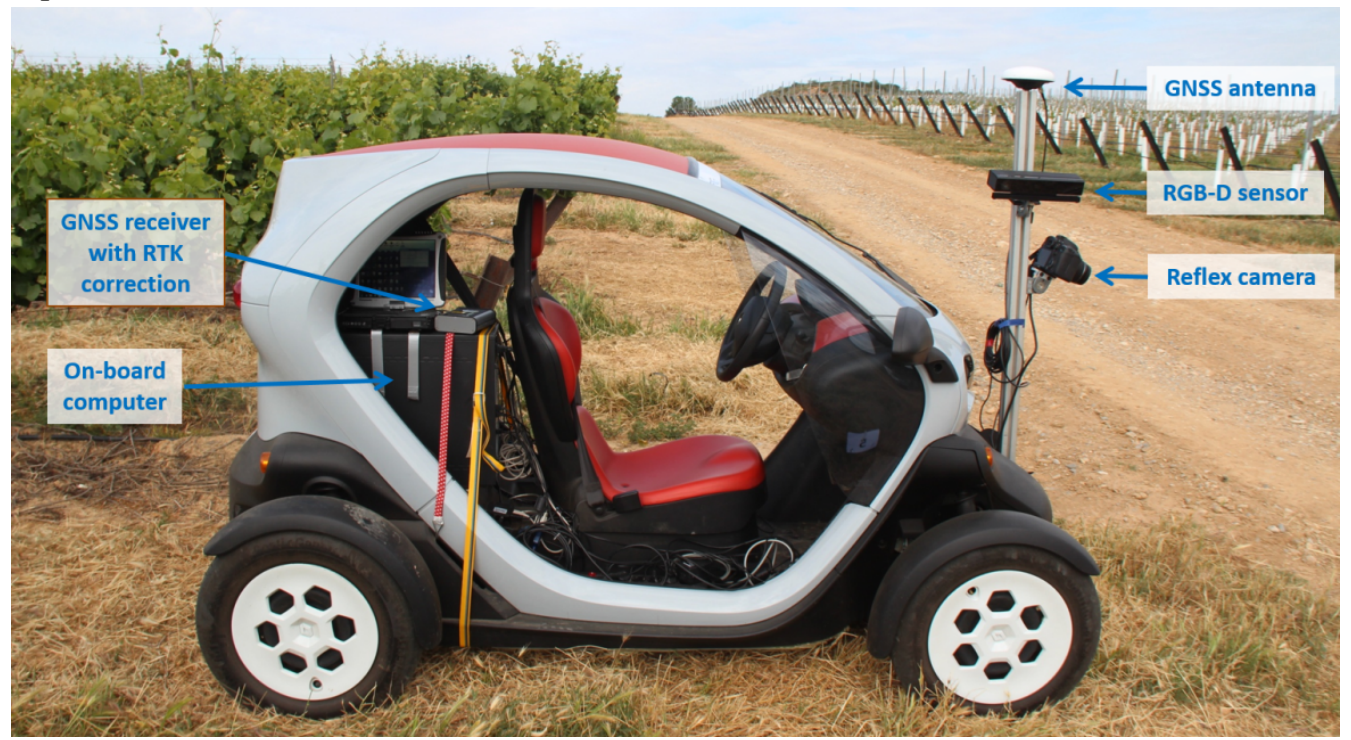

Figure 46. Field platform and on-board equipment.

Point Cloud generation method

The algorithm reconstruct large regions using the fusion of different overlapped depth images (Curless \& Levoy, 1996), storing information only on the voxels closest to the detected object and accessing to the stored information by using a hash table. Given a new input depth image and known camera position, the ray-casting technique (Roth, 1982) is used to project a ray from the camera focus for each pixel of the input depth image to determine the voxels in the 3D world that cross each ray. Thus, the voxels related to the depth information are determined. Once the surface of the scene has been extracted using the ray-casting technique, this information is used to estimate the position and orientation of the camera with 6 degrees of freedom when a new input image arrives. The last estimation is conducted with a variant of the iterative closest point (ICP) algorithm (Chen \& Mendioni, 1992) and provides a point cloud as the output, which in this case is a woody row that does not appear to be as straight as the original row, showing detectable drift (Figure 2).

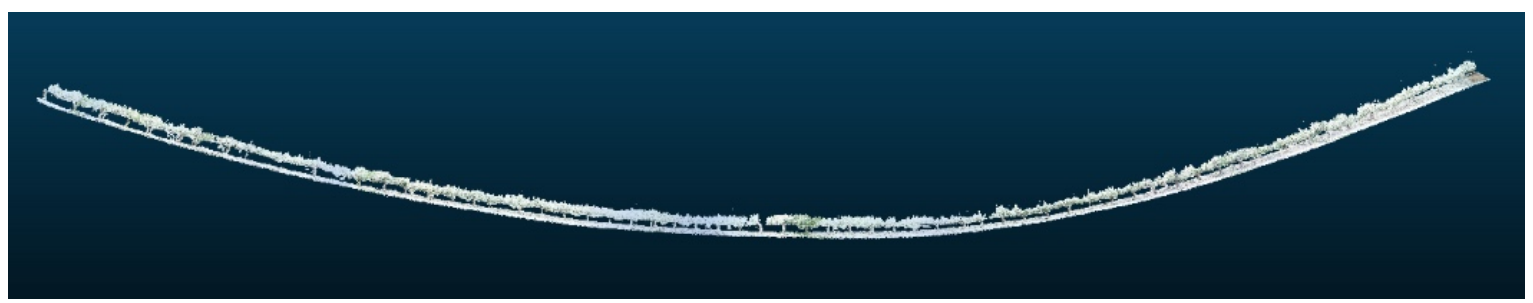

Figure 2. 3D reconstruction of a vineyard crop row with a drift. 
Finally, a method was designed and developed to accommodate this drift using the minimal scene information that can be acquired from the field platform during its travel, such as the geographical location of the starting and ending points of each sampling row and the fact that trees are typically planted in straight lines.

\section{Filtering}

Firstly isolated points in the point cloud are eliminated. A point is considered an outlier if the average distance to its 64 nearest neighbours is greater than the standard deviation of the distance to the neighbours of all the points (Figure 3).

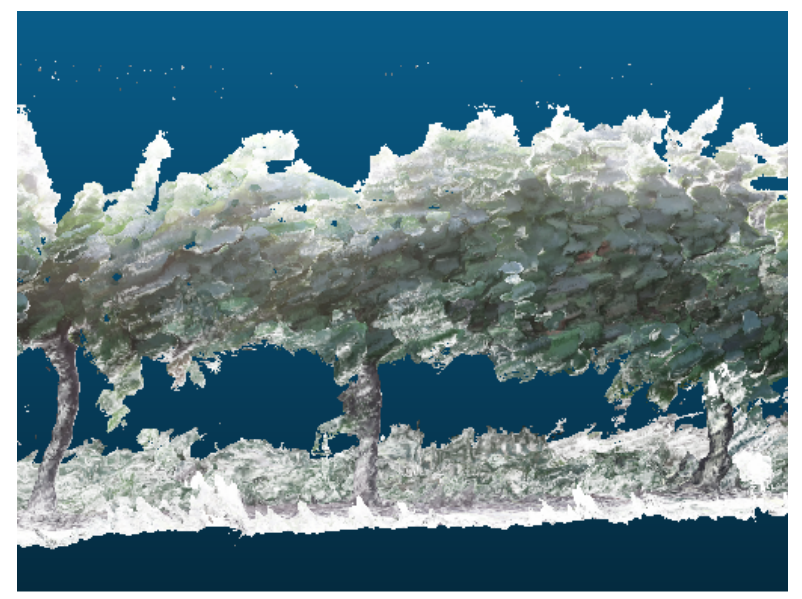

(a)

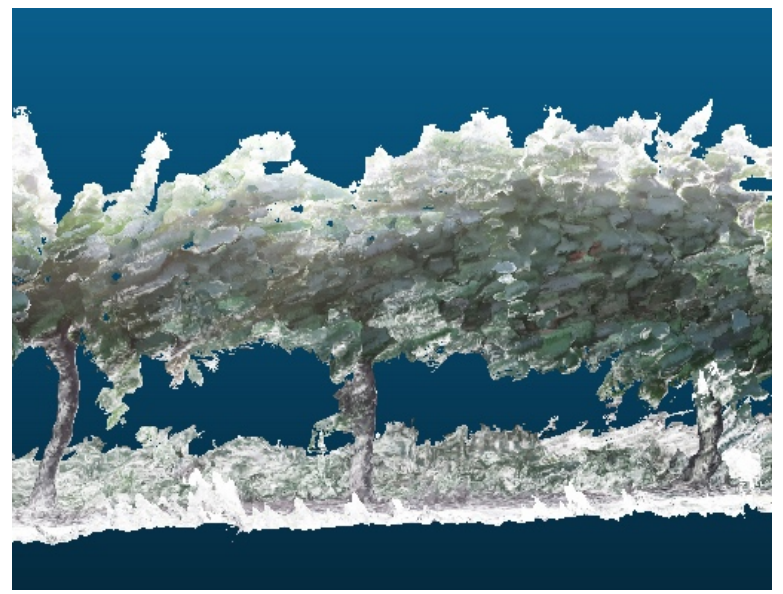

(b)

Figure 3. 3D reconstruction of a vineyard crop row with a drift.

Estimation of a model line

Following is required to find a line that longitudinally models the $3 \mathrm{D}$ reconstruction of the tree row previously generated. This model line should be adjusted as much as possible to the path following the tree row and will be used in the next steps to segment the row in sections of the same length. The point cloud is divided into smaller groups of equal numbers of points and the centroids of the points in each group is estimated. The number of groups is estimated from the total length of the tree row and the known geographical position of the starting and ending point of the row. In general, it is sufficient to divide the row into groups of approximately $5 \mathrm{~m}$ since it has been experimentally shown that the effect of the drift of $5 \mathrm{~m}$ is essentially negligible. thus, the points that define the model line are not at the same distance, as shown in Figure 4.

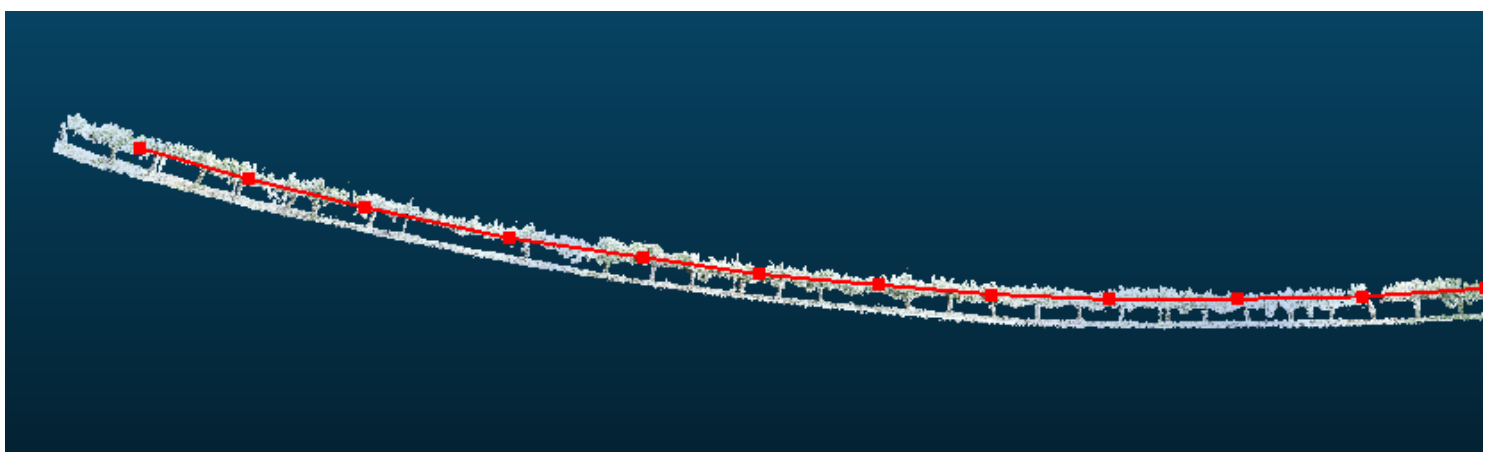

Figure 4. Part of the model line of the 3D reconstruction of Figure 2.

By determining the endpoints of the row to incorporate these to the points that form the model line, the points of the first group are projected onto the extension of the line connecting the first and second centroids. The projected point most distant to the first centroid is chosen as the starting point of the model line since, in a frontal view, all other points of the tree row are to the right of the centroid. The procedure to be followed is similar in the case of the ending point estimation. All points of the last group are projected on the extension of the model line that connects the penultimate and last centroids, choosing as the ending point of the model line the point projected as more distant to the last centroid since all the points of the tree row are to the left of this centroid (Figure 5). 


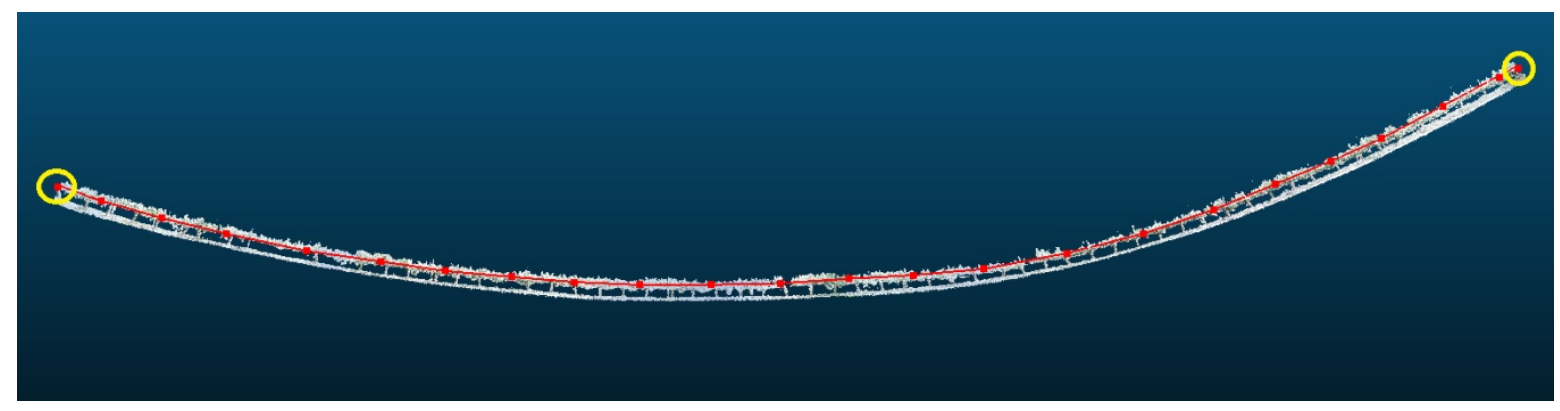

Figure 5. Yellow circles represent the endpoints of the tree row added to the model line of Figure 4.

Following the model is splitting into sections of the same length .Once an accurate model line of the crop is obtained, the line is divided into segments of the same length. The point cloud is divided into sections, each containing a fragment of the $3 \mathrm{D}$ reconstruction.

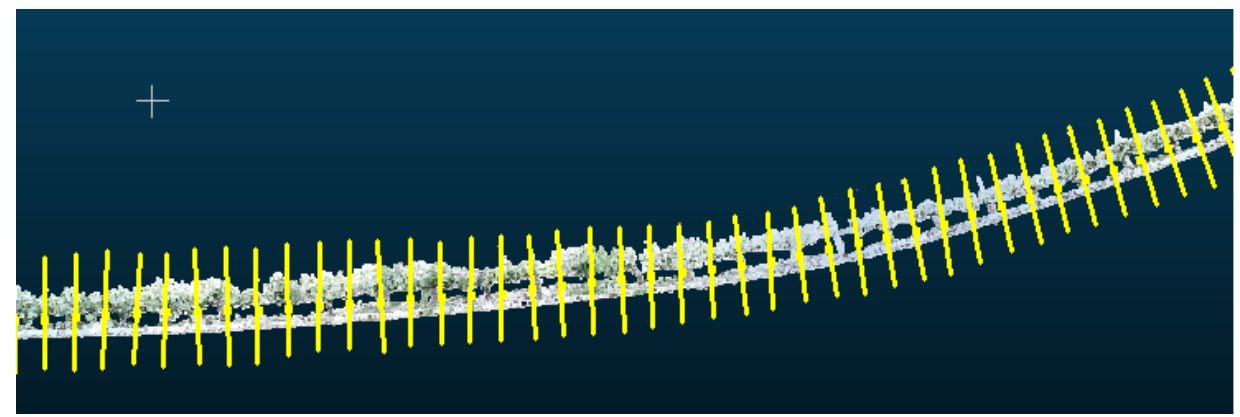

Figure 6. Part of the 3D reconstruction shown in Figure 2 split into sections.

Section correction

The coordinate system is changed. The applied transformation matrix places the origin in the initial position of the crop, centred in the soil, with the X-axis in the direction of the crop row, the Z-axis in the direction of tree growth, and the $\mathrm{Y}$-axis in the direction of the crop depth (Figure 7). The vector that indicates the direction of the crop row in each section can be calculated using the starting and ending points of the model line segment contained in the section, drawn as a red line in Figure 8. However, this the direction of this vector does not coincides with the direction of the actual tree row appearing in the $3 \mathrm{D}$ reconstruction. To eliminate the drift, each section must be corrected in such a manner that the section must be rotated to align the vector that indicates the direction of the model line in the reconstructed section (Xsection) with the direction of the $\mathrm{X}$-axis of the actual section (Xreference).

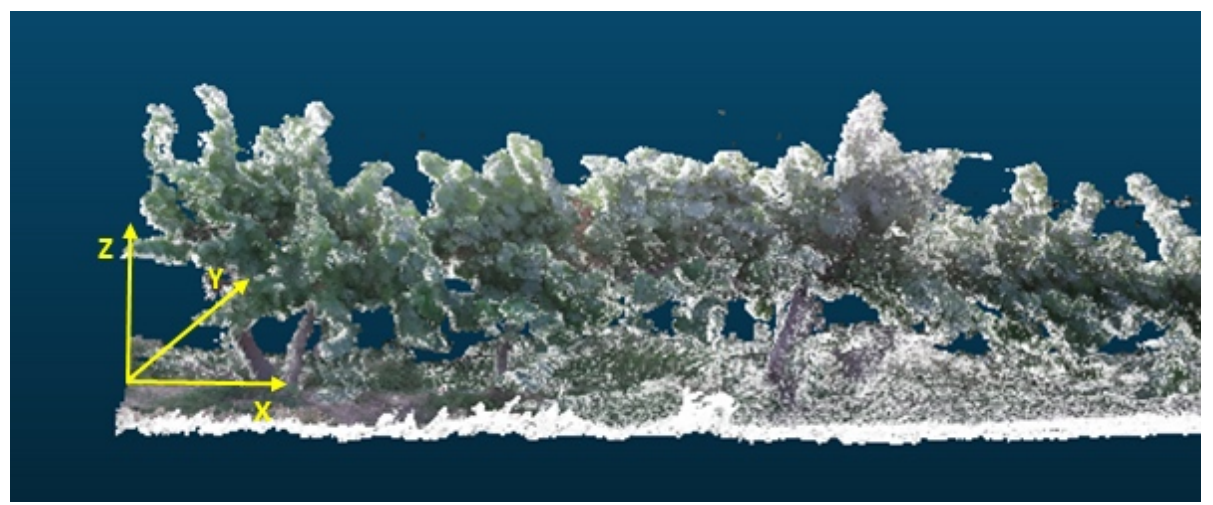

Figure 7.The coordinate system defined in yellow. 


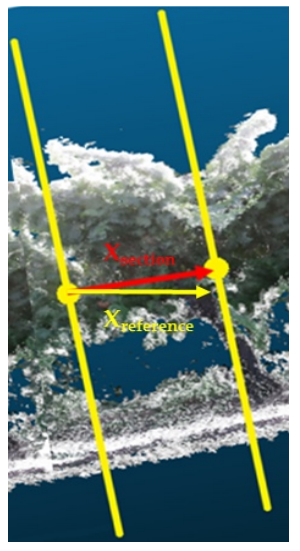

Figure 8. The X-axis of the section (Xsection) is represented in red, and the X-axis of the reference (Xreference) is represented in yellow.

Once the drift of each section has been corrected, the sections are aligned; thus, the drift produced during 3D reconstruction is corrected. Figure 9 shows a reconstruction of a vineyard row with drift and the result obtained after applying the developed procedure.

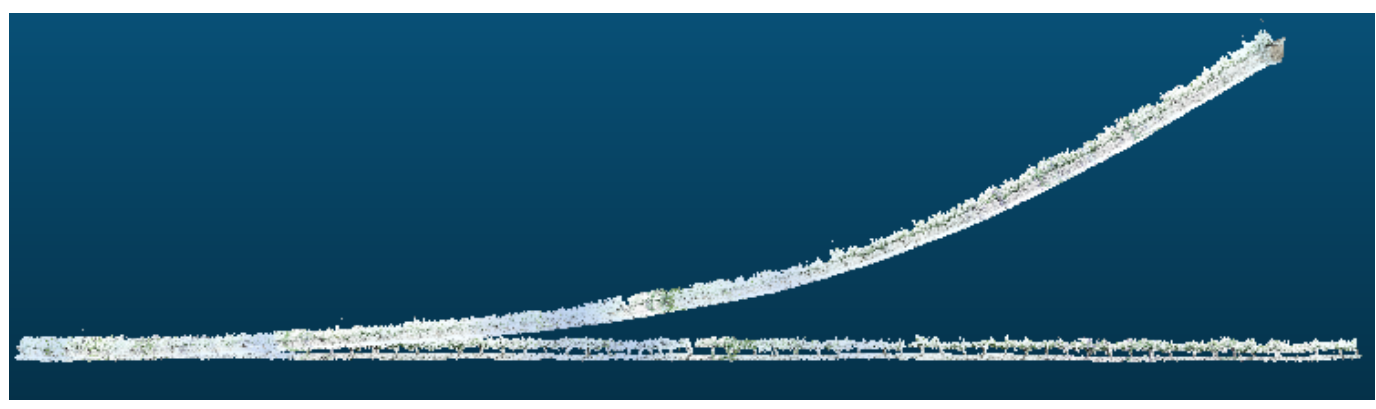

Figure 9. 3D reconstruction of a tree row with drift (up) and the result after the drift correction procedure is applied (down).

\section{Results and Discussion}

The systes was tested at by Codorniu S.A. vineyards (Raimat, Lleida, Spain) during spring 2016. The aforementioned inspection platform moving at $3 \mathrm{~km} / \mathrm{h}$ was used to collect the information. The sensor Kinect v2 was oriented to the crop rows at $1 \mathrm{~m}$ from the crop row. From each row inspected, the starting and ending geographical positions of the row supplied by the RTK-GPS receiver from the vehicle were stored. With the information supplied by the Kinect v2 sensor and stored in the on-board computer of the vehicle, the 3D reconstruction of the sampled rows of vines was performed using a computer equipped with an Intel Core i76900K@3.2GHz processor, 64 GB of RAM, and an NVIDIA GeForce GTX Titan X graphics card to create a a triangular mesh (Figure 13) obtained from the point cloud using the marching cubes algorithm.

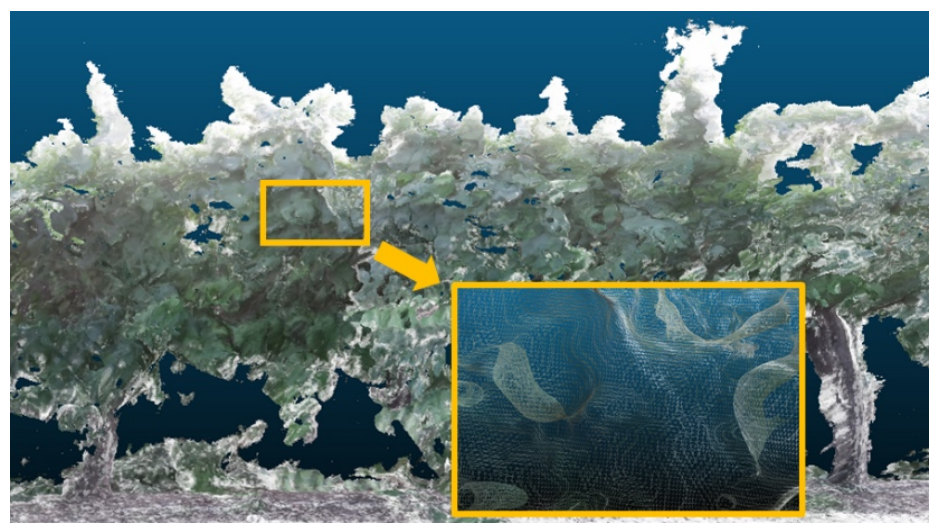

Figure 10. Details of a 3D reconstruction of a vine that shows the triangular mesh obtained from a point cloud. 
During the several experiments conducted, although 3D reconstructions of long crop rows were properly performed, drift appears in all the tests conducted throughout the year. Using the described method it was possible to correct the drift. The performance of the proposed approach was analysed through a 3D reconstruction of two rows of the vineyard with lengths of $85 \mathrm{~m}$ and $105 \mathrm{~m}$. Smaller number of leaves in the vineyards is correlated with a smaller deformation in the 3D reconstructions. This behaviour arises because the ICP algorithm, which estimates the position and orientation of the Kinect sensor, is not as effective when the same structure is repeated. It seems that, when the length of the row to be reconstructed is greater, the deformation that appears in the 3D reconstruction is greater because of the error accumulated along the row.

Table 17. Results of the drift correction methodology for two row lengths.

\begin{tabular}{cccccc}
\hline Sampling & $\mathrm{N}^{\circ}$ of Points & Mean Angle $\left(^{\circ}\right)$ & Std. Dev. Angle $\left(^{\circ}\right)$ & Maximum Angle $\left(^{\circ}\right)$ & Length $(\mathrm{m})$ \\
\hline Short rows & 8118581 & 4,19 & 2,86 & 10,64 & 89,93 \\
Long rows & 9839601 & 3,83 & 2,36 & 10,74 & 111,67 \\
\hline
\end{tabular}

The use of autonomous vehicles would also reduce significantly the cost (Bengochea-Guevara, et al 2016). An autonomous mobile robot to inspect a crop field with full coverage would reduce the human labour which is related to the highest cost of mapping. The use of autonomous platforms would also have significant advantages in terms of flexibility, adaptability and environmental benefits compared to traditional agricultural machinery and can be adapted to many of the advances in machine vision, decision support and application (Pedersen et al,. 2017).

\section{Conclusions}

A low-cost crop monitoring using a medium-size platform equipped with various on-board sensors tp create accurate $3 \mathrm{D}$ models of woody crop rows was developed.. It also incorporates software specifically developed to accurately generate 3D maps of the crops. The implemented algorithm provides good results for the automatic 3D reconstruction of large areas under uncontrolled lighting conditions. The drift that usually appears in the 3D reconstruction of long rows, those greater than $25 \mathrm{~m}$, can be properly handled with the proposed algorithm.

\section{Acknowledgements}

This research was partly funded by the AGL2014-52465-C4 and AGL2017-83325-C4 projects (Spanish Ministry of Economy and Competition) and by the RYC-2016-20355 agreement.

\section{References}

Andújar, D., J. Dorado, J.M. Bengochea-Guevara, J. Conesa-Muñoz, C. Fernández-Quintanilla, Á. Ribeiro, 2017. Influence of Wind Speed on RGB-D Images in Tree Plantations. Sensors 17(4), E914.

Andújar D, M. Weis, R. Gerhards, 2012. An Ultrasonic System for Weed Detection in Cereal Crops. Sensors. 12(12), 17343-17357.

Arnó, J., J.M. Vallès, J. Llorens, R. Blanco, J. Palacín, R. Sanz, J. Masip, M. Ribes-Dasi, J.R. Rosell, 2006. Ground Laser Scanner Data Analysis for LAI Prediction in Orchards and Vineyards. In: Proceedings of International Conference on Agricultural Engineering 2006, Bonn, Germany, 3-6 September 2006.

Burgos-Artizzu X.P., A. Ribeiro A. Tellaeche, G. Pajares, C. Fernández-Quintanilla,2010. Analysis of natural images processing for the extraction of agricultural elements. Image and Vision Computing 28:138-149. doi: 10.1016/j.imavis.2009.05.009.

Chen, Y., G. Medioni, 1992. Object modelling by registration of multiple range images. Image and Vision Computing, 10, 145-155.

Curless, B., M. A, Levoy, 1996. Volumetric method for building complex models from range images. In Proceedings of the 23rd annual conference on Computer graphics and interactive techniques. ACM. $303-312$.

Muharam, F.M., K.F., Bronson, S.J., Maas, G.L., Ritchie, 2014. Inter-relationships of cotton plant height, canopy width, ground cover and plant nitrogen status indicators. Field Crop Research. 169, 58-69.

Pedersen S.M., S., Fountas, C.G., Sørensen, F.K., Van Evert, B.S., Blackmore, 2017. Robotic Seeding: Economic Perspectives. In: Precision Agriculture: Technology and Economic Perspectives. Progress in Precision Agriculture Pedersen S., Lind K. (eds). Springer, Cham. 167-179.

Peteinatos, G. G., Weis, M., Andújar, D., Rueda Ayala, V. and Gerhards, R. (2014), Potential use of groundbased sensor technologies for weed detection. Pest. Manag. Sci., 70: 190-199. doi: 10.1002/ps.3677

Roth, S. D. Ray casting for modeling solids. Computer graphics and image processing1982, 18, 109-144. 


\title{
Evaluation of the Potential for Automatic Herbicide in Wheat Crops
}

\author{
Gidea, Mihai a $^{\text {* }}$, Constantinescu ${ }^{\text {b }}$, Catalin Mircea ${ }^{\text {b, }}$ Manea, Dragos ${ }^{\text {c }}$, Ciorita, Alexandru ${ }^{\text {a }}$ \\ ${ }^{a}$ University of Agronomic Sciences and Veterinary Medicine of Bucharest, Romania \\ ${ }^{\mathrm{b}}$ University of Craiova, Romania \\ ${ }^{\mathrm{c}}$ National Institute of Research - Development for Machines and Installations Designed to Agriculture and Food Industry - \\ INMA, Romania \\ * Corresponding author. Email: gideam@yahoo.com
}

\begin{abstract}
Due to its importance in human nutrition, the wheat crop is cultivated on an area of $220 \mathrm{mil}$ ha. Weed control in wheat crops is one of the most important technological components, the share of weed control expenditure in the production cost structure is $15-20 \%$, and their non-application results in production losses which, depending on the weednes level, can go up to compromising the crop. In conventional farming, weed control is done by uniform application of the herbicidal mixture over the entire surface. Although the herbicide is applied uniformly over the entire surface, the weedess area is less than half. In this context, the research was based on the adaptation of herbicidal equipment for precision herbicide, only there where weeds are. In order to meet these requirements, each nozzle on the spray boom was fitted with a quick solenoid valve (normally closed type), that will be open only where the weeds are. Each solenoid valve is individually controlled by the control system as a result of processing the images taken with a video camera. The principle of precision spraying consists in identifying areas covered by weeds by extracting green areas, through segmentation of the acquired image, and then removing the area occupied by wheat plants from the total green surface. Following the adaptation of the herbicide equipment, field tests consisted of determining the spectrum of weednes, participation and constance of weedness before and after using the equipment. In order to test the efficacy of the adapted herbiciding equipment, the experimental field was split in half, one half was herbicidated uniformlly across the surface, and the other half with precision herbicide equipment started. The degree of weed combat in wheat crops determined two weeks after application of the herbicide ranged from $98 \%$ to uniform application of the herbicide and $83 \%$ in the case of precision herbicide compared to the non-herbicidal control.At the harvesting time the yield don't show a significance diference between uniformely spray and precision spray. From an economical point of view, the production costs has recorded a decrease of about $10 \%$.
\end{abstract}

Keywords: weed, weedness spectrum, weed detection, image procesing.

\section{Introduction}

On the global scale, wheat is one of the most important cultures, with ever increasing cultivated surfaces, reaching $220 \mathrm{mil}$ ha in 2014 [20]. In response to the continuous growth of the world population, cultivation methods must be improved, so that the yields obtained will allow for all needs to be met, while keeping the cultivated area constant.

The weedness of crops is a problem with negative impact on the production of all crops. In the wheat crops, the production losses exceed $48 \%$ [1], mainly due to competition of the crop plants for light vegetation, water, nutrients, but also by alelophatic effects on the rhizosphere [2]. The size of the losses caused by the weeds are determined by the type of weeds and their density, the time of infestation and the duration of the infestation [3].

The level of losses caused by the weedness is maximal when the resources are limited and the weedness occurs simultaneously with the growth of the culture [4]. The level of production decreases with the increase of the degree of weedness, there is a correlation between the duration of the competition and the competitive pressure on the culture [5]. Local pedoclimatic conditions influence the feed spectrum, so each area has certain characteristics of the weeds, so for each area there are specific weed control strategies [6]. Under the conditions of the minimum tillage system compared to the classic system, the degree of weedness infestation increases by $22-78 \%$ [7].

Research has shown that weeds appear in patches of varying sizes and densities, in a field alternating with areas without weeds or highly weedness areas [8]. The SSWM concept for site-specific weed management is to identify, analyze and manage the spatial and / or temporary variability of weed populations to optimize economic yields, sustainability of cultivation systems and environmental protection [9]. Many research has been done on the use of SSWM technologies to increase the efficiency of herbicide use in agricultural systems. In the crop, the surfaces covered with weed is range from 40 to $50 \%$ of the total area, and significant herbicide consumption reductions can be achieved through precision herbicides[10].

As of now, efforts to reduce the number of herbicides are concentrated on the automatic application of herbicides having as a basis the weed / crop discrimination. In the case of maize crops, the issue of discrimination during the crop processing process has been resolved by the use of two subsystems: a fast, real-time image 
processing (FIP) system and a more precise and accurate Row Detection (RCRD) is used together with the first systemto make corrections[11].

Several methods are used to discriminate weeds / cultures: multispectral analysis, by using a spectrograph $(660-1060 \mathrm{~nm}$ with a resolution of $5 \mathrm{~nm})$ in potato culture, 3 weed species with a $89 \%$ grading rate could be identified [ 12], by combining the analysis at different wavelengths, bands can obtain characteristic spectral signatures for each weed species [13]; analysis of the difference in weight between species and crops by using Lidar [14], form analysis, after filtration of the image, the formulas containing chlorophyll are selected, and then on the basis of an algorithm the segmentation and the classification of the identified forms are performed[16, 15].

Provide an adequate background here, but avoid a detailed literature survey or a summary of the results. State also the objectives of the work in this section.

\section{Materials and Methods}

Research on the adaptation of herbicide equipment for the automatic accuracy of herbicide administration on weed areas began from the finding that in a chain the weed distribution is not uniform, with the areas fed alternating with weed-free areas. The objective of the research is the comparative evaluation of the herbicide equipment adapted to the classical method.

The research on the determination of the degree of weedness, spectrum and distribution of nuruien was carried out in the spring before the first internode, $\mathrm{BBCH}$ 30. The research was carried out in the South of Romania in the Romanian field, in the experimental field of Probstdorfer Saatzucht Romania SRL, Modelu , Calarasi N 44.2557 / E 27.5152 on the Chernozem cambic. Cultivated wheat variety Hubert, 400 germinable seeds / m2, sown on 3.Oct.2016.

Due to the specific climatic conditions, low-temperature winters, there are no monocotyledonous species in this wheat field. The presence of monocotyledonous species is sporadic and does not require methods of control.

The classical method of weed mapping has determined the speckling spectrum characterized by the average level of impregnation, participation and constancy. He used the metric frame to determine the specter.

The mean value $\mathrm{a}=\mathrm{S} / \mathrm{N}$, where $\mathrm{S}$ represents the total number of plants of a particular weed species found at all determinations and $\mathrm{N}$ the number of points of determination, hence the average number of weeds of a given type per square meter.

Participation $\mathrm{P} \%=\mathrm{m} * 100 / \mathrm{M}$, where $\mathrm{M}=$ represents the average number of weeds per square meter, the sum of all the averages of the identified species.

Constance, $\mathrm{K} \%=\mathrm{n} * 100 / \mathrm{N}$, where $\mathrm{n}$ is the number of points in which a particular weed species was present.

The area occupied by the species $\mathrm{Sk}=\pi * \mathrm{r} 2$, where $\mathrm{r}$ is the average radius of the ground projection of the $\mathrm{k}$ species of the weeds.

The total area occupied by weeds ST =, where $\mathrm{k}$ is the number of species identified, $\mathrm{S}$ is the average area of weed projection $\mathrm{k}$.

Equipment adapted for automatic precision herbicide management

To achieve the objective of the research was taken a trailed sprayer machine with the length of $12 \mathrm{~m}$ lances, with the capacity of $1800 \mathrm{l}$ tank, without any automation. Adaptation of the equipment started with the realization of a system of automatic adjustment of flow and pressure in the pumping system correlated with the speed of displacement of the aggregate. This was done by mounting an inductive sensor on the wheel, a pair of pressure sensors and a flowmeter that sends the information to a central unit and a motorized solenoid valve which controls dynamic pressure from the

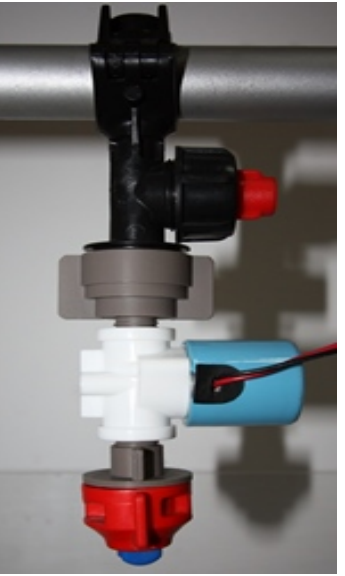

Figure 1. Detail solenoid valve - nozzle. herbicide ramp.

The second stage of the adaptation consisted of replacing the spray nozzles with a set of adapters and a quick valve solenoid with $12 \mathrm{~V}$ supply, normally closed. The opening and closing of the solenoid valve is controlled by an individual control module based on the processing of images purchased with a Raspberry Pi NoIR - Infraredsensitive camera for each individual nozzle.

Vegetation identification is done by real-time processing of images taken with the camera. Virtually every purchased frame is processed and analyzed. For the processing of catches, OpenCV specific functions were used. Thus, for green detection, the image capture (cam.read function, camcorder type defined by the VideoCapture function) is captured. For faster processing, the image can be resized using the OpenCV resize function after which the image is fit with the inRange applied to the image and with the color thresholds as arguments. This is basically a masking of the image based on green content. For better contrast and deeper color difference, the gamma curve and also the brightness of the image have changed. The green clusters were then identified and 
marked with red rectangles. The algorithm can be applied in real-time to captured images through the camera.

Selection of a particular area is necessary to select as the weed detection area the space between the crop rows. To get better filtering / masking, one filter can be used for each elemental color. Evaluating the effectiveness of the applied treatment. For the comparative evaluation of the functionality of the adapted sprayer equipment, half of the nozzles on the ramp were automatically controlled on the basis of the processed images, and the other half with all open valves for a uniform application of the treatment. In order to accurately quantify the amount of solution used, each half of the ramp was fed through a flowmeter.

Two weeks after application of the herbicide treatment, its efficacy was assessed by determining the degree of weed control. At harvest, the crops were quantised separately for the two variants. Rival Superstar 75 GD was used with Rival Superstar 75 GD with 37.5\% tribenuron-methyl active substances and 37.5\% clorsulfuron at a dose of $20 \mathrm{~g} /$ ha with a herbicide blending standard of 300 liters / ha. The area of the experimental plot was 1 ha.

Statistical analysis was performed using XLSTAT and Anova running under Excel.

\section{Results and Discussion}

The results of the determination of the coverage spectrum and the area occupied by each species of weed in the two studied locations are centralized in Table 1. From the data on the average number of weeds we can see that the average number of weeds $/ \mathrm{m}^{2}$ ranged from 1.1 to Convolvulus arvensis and 39.8 to Veronica hederifolia.

Analyzing the participation of weed species in the specter spectrum, it is found that annual species have a much larger share compared to perennial species, so that annual species of dicotyledonous species have a participation of $88.5 \%$ and the perennial ones of $11.5 \%$. It is found that the main weed species enriching the wheat culture are Veronica hederifolia, Stelaria media and Galium aparine.

The constant presence of weed species varied considerably between $68.7 \%$ in Veronica hederifolia, early spring germination ephemeric species, and $11.3 \%$ in Galinsoga parviflora species with late spring germination.

The diameter of the soil weed projection varied between $2.1 \mathrm{~cm}$ in the species Solanum nigrum which starts in vegetation later and $13.8 \mathrm{~cm}$ in the average Stelaria weeds, which germinate at lower temperatures.

Table 1 Determination of the weedness spectrum of the experimental plots and the area occupied by the weeds $\left(\mathrm{m}^{2}\right)$.

\begin{tabular}{lrrrrrr}
\hline Species & $\mathrm{M}$ & $\mathrm{P}$ & $\mathrm{K}$ & $\mathrm{d}$ & $\mathrm{s}$ & $\mathrm{S}$ \\
\hline Veronica hederifolia & 39,8 & 21,0 & 68,7 & 12,6 & 124,6 & 716,1 \\
Solanum nigrum & 4,8 & 2,5 & 23,1 & 2,1 & 3,5 & 0,1 \\
Poligonum convolvulus & 8,8 & 4,6 & 46,7 & 5,6 & 24,6 & 4,7 \\
Chenopodium album & 12,2 & 6,4 & 34,9 & 6,2 & 30,2 & 8,3 \\
Papaver roheas & 24,4 & 12,9 & 56,2 & 10,2 & 81,7 & 144,3 \\
Stelaria media & 35,1 & 18,5 & 52,1 & 13,8 & 149,5 & 506,6 \\
Sinapis arvensis & 2,8 & 9,3 & 22,9 & 4,1 & 13,2 & 0,8 \\
Galinsoga parviflora & 3,6 & 1,9 & 11,2 & 2,2 & 3,8 & 0,03 \\
Galium aparine & 36,2 & 19,1 & 68,6 & 11,6 & 105,6 & 501,4 \\
Annual dicotyledons & 167,7 & 96,4 & & & & 1882,2 \\
& & & & & & \\
Convolvulus arvense & 1,1 & 0,6 & 20,6 & 5,4 & 22,9 & 0,0 \\
Cirsium arvense & 6,4 & 3,4 & 46,7 & 10,8 & 91,6 & 9,2 \\
Sonchus arvense & 5,8 & 3,1 & 48,2 & 9,2 & 66,4 & 81,7 \\
Capsela bursa pastoris & 8,4 & 4,4 & 48,6 & 10,2 & 11,7 & 14,8 \\
Perennial dicotyledons & 21,7 & 11,5 & & 12,2 & 116,8 & 29,8 \\
Total & 189,4 & 107,8 & & & & 1912,0 \\
\hline
\end{tabular}

Prior to post-emergence treatments, there were no significant differences in weedness spectrum, average weed $/ \mathrm{m}^{2}$, participation and constancy on the surface of the sole tested. The area occupied by the weeds was 1912 $\mathrm{cm}^{2}$, so the weed's soil coverage was $19.12 \%$.

Table 2 summarizes the data on the comparative evaluation of the results obtained using the two methods of uniform and precision treatment. Two weeks after the application of the treatments, the degree of weedness was determined and it was found to be $96.2 \%$ in the case of application of uniform herbicide treatment on the whole surface and $91.8 \%$ in the automatic application of herbicides only where there are weeds . 
Table 2 Results of comparative determinations after application of herbicide treatments.

\begin{tabular}{lccc}
\hline & $\begin{array}{c}\text { Herbicide treatment applied } \\
\text { uniformly over the entire } \\
\text { surface }\end{array}$ & $\begin{array}{c}\text { Automate herbicide treatment with } \\
\text { herbicide only where weeds have been } \\
\text { identified }\end{array}$ & Diference \\
\hline Weed control level & $96,2 \%$ & $91,8 \%$ & 4,4 \\
The amount of solution used & $2961 / \mathrm{ha}$ & $182 \mathrm{l} / \mathrm{ha}$ & 114 \\
Equivalent herbicide dose & $98,7 \%$ & $61,3 \%$ & 37,4 \\
Obtained yield production $(\mathrm{kg} / \mathrm{ha})$ & 5620 & 5380 & 240 \\
\hline
\end{tabular}

Analyzing the data on the amount of solution used for the herbicide in the two variants there is a decrease in the quantity of the solution used from 296 to $1821 /$ ha, so a solution saving of $1141 /$ ha. Although the area actually covered with weeds was $19.12 \%$ the area herbicidated according to the amount of herbicide consumed was $61.3 \%$. This difference is explained by the fact that the nozzles have a fixed surface area to be treated, the area not covered $100 \%$ by weeds. Practically, non-herbicidal surfaces were the areas where no weeds were identified at the time of application.

By applying the herbicidal method using the adapted equipment, the amount of herbicide was reduced by $37.4 \%$, thus reducing the specific weed control expenditure by $37 \%$.

The obtained yields were $5620 \mathrm{~kg} /$ ha in the case of applying uniform treatments on the whole surface and $5380 \mathrm{~kg} / \mathrm{ha}$ in the case of precision herbicides with the adapted equipment. The difference of $240 \mathrm{~kg} / \mathrm{ha}$ between the two tested variants is not statistically assured.

\section{Conclusions}

1. In order to adapt the equipment for automatic precision application of the herbicide treatments only in the furbished areas were used independent modules - controller - solenoid valves

2. By using the adapted equipment, only the weed areas have been herbicized, thus significantly reducing the herbicidal surface.

3. The degree of weed control, although smaller than the uniform application of herbicides on the entire surface, but did not significantly affect the level of production.

4. The use of tailored equipment has led to reduced weed control specific costs

5. Using this method, the level of harvested yields did not show a statistically significant decrease.Type your research findings here in the Conclusions section. Do not replicate information from the Materials and Methods section, instead focus on answering the research questions and the goal of the research.

\section{Acknowledgements}

The research was founded by Executive Agency for Higher Education, Research, Development and Innovative Funding (UEFISCDI), by contract number 33BG2016_PN-III-P2-2.1-BG-2016-0472.

\section{References}

[1] Khan, M., Haq, N.Wheat crop yield loss assessment due to weeds(2002) Sarhad Journal of Agriculture, 18, pp. 449-453

[2] A. Khaliq, S. Hussain, A. Matloob, A. Wahid, F. AslamAqeous swine cress (Coronopus didymus) extracts inhibit wheat germination and early seedling growth, Int. J. Agric. Biol., 15 (2013), pp. 743-748

[3] Estorninos Jr., L.E., Gealy, D.R., Gbur, E.E., Talbert, R.E., McClelland, M.R.Rice and red rice interference. II. Rice response to population densities of three red rice (Oryza sativa) ecotypes(2005) Weed Science, 53 (5), pp. 683-689.

[4] R.L. Zimdahl, Fundamentals of Weed Science, (third ed.), Elsevier Inc, U.S.A. (2007), pp. 151-156

[5] S. Ciuberkis, S. Bernotas, S.R.J. FelixEffect of weed emergence time and intervals of weed and crop competition on potato yield, Weed Technol., 21 (2007), pp. 213-218;

[6] Bogdan Ileana, T.Rusu, Stefania Gadea, I. Ivan, Paula Moraru, A. Pop, 2011, Effective weed control in maize. Buletin USAMV, Agricultură, 68 (1)/2011, ISSN 1454 - 2382 .Ed. AcademicPres Cluj-Napoca. p. 53-59, http://journals.usamvcj.ro/agriculture/article/download/6419/5709

[7] Rusu, T., Petru Guş and Ileana Bogdan, 2006, The influence of minimum soil tillage systems on weed density, frequency of phytopatogenous agents and crop yields of soybean, wheat, potato, rape and corn. Journal of Food, Agriculture \& Environment, vol. 4(1/2006), p. 225-227, ISSN: 1459-0255, http://www.isfae.org/scientficjournal/2006/issue1.php, Section Agriculture, ISSN: 1459-0263.

[8] Clay SA, Lems GJ, Clay DE, Forcella F, Ellsbury ME, Carlson CG, 1999, Sampling weed spatial variability on a field wise scale, Weed Science 47, 674-681 
[9] Shaw, D. R. (2005), Remote sensing and site-specific weed management. Frontiers in Ecology and the Environment, 3: 526-532. doi:10.1890/1540-9295(2005)003[0526:RSASWM]2.0.CO;2 198 ;

[10] Marshall, E.J.P. Field-scale estimates of grass weed populations in arable land. Weed Res. 1988, 28, 191-

[11] Burgos-Artizzu X, Ribeiro A, Guijarro M, Pajares G, Real-time image processing for crop/weed discrimination in maize fields, Computers and electronics in agriculture, volume 75, issue 2, february 2011, pages 337-346;

[12] Borregaard, T., Nielsen, H., Norgaard, L., Have, H., 2002. Crop-weed discrimination by line imaging spectroscopy. Journal of Agricultural Engineering Research 75,389-400

[13] Piron A, Leemans, V Kleynen, O, Lebeau, F, Destain, M.-F., 2008a. Selection of the most efficientwavelength bands for discriminatingweeds fromcrop.Computers and Electronics in Agriculture 62, 141148

[14] Li Hanzhe, Zhai C, Weckler P, Wang N, Yang S, Zhang B. A Canopy Density Model for Planar Orchard Target Detection Based on Ultrasonic Sensors. Sinha DN, Pantea C, eds. Sensors (Basel, Switzerland). 2017;17(1):31. doi:10.3390/s17010031;

[15] Pedro Javier Herrera P J, Dorado J, Ribeiro A, A Novel Approach for Weed Type Classification Based on Shape Descriptors and a Fuzzy Decision-Making Method, Sensors 2014, 14, 15304-15324, doi:10.3390/s 140815304, ISSN 1424-8220, http://www.mdpi.com/1424-8220/14/8/15304/pdf.

[16] P Milberg, E Hallgren, Yield loss due to weeds in cereals and its large-scale variability in Sweden,Field Crops Research,Volume 86, Issues 2-3, 2004, Pages 199-209, ISSN 0378-4290,

[17] Christensen SE, Nordbo E, Heisel T, and Walter AM (1998). Overview of developments in precision weed management, issues of interest and future directions being considered in Europe. Proceedings of Precision weed management of crops and pastures workshop. Charles Sturt University, May 6-7th, 1998. pp. 3-13. Ed. RW Medd and JE Pratley. CRC for Weed Management Systems.

[18] Gerhards R (2007). Managing weeds with respect to their spatial and temporal heterogeneity. Proceedings (abstracts) of the 2nd Conference on Precision Crop Protection. Bonn, Germany, October 10-12th, 2007.

[19] Lutman PJW and Miller PCH (2007). Spatially variable herbicide application technology; opportunities for herbicide minimisation and protection of beneficial weeds. Research Review No. 62 (Project No. RD-20063298), HGCA, UK.

[20] http://www.fao.org/faostat/en/\#data/QC 


\author{
Field Robot to Detect Plants Infected by Candidatus Liberibacter solanacearum in \\ Horticultural Crops using Multispectral Computer Vision \\ Sergio Cubero ${ }^{1}$, Santiago López-Alamán ${ }^{1}$, Ester Marco-Noales ${ }^{2}$, Susana Sanjuan ${ }^{3}$, Vicente Alegre ${ }^{4}$, \\ Silvia Barbé ${ }^{4}$, Enrique Aguilar ${ }^{4}$, Inmaculada Navarro ${ }^{4}$, Nuria Aleixos ${ }^{5}$, José Blasco ${ }^{1^{*}}$ \\ 1) Centro de Agroingeniería. Instituto Valenciano de Investigaciones Agrarias (IVIA). CV-315, km 10,7 - 46113 \\ Moncada (Valencia), Spain \\ ${ }^{2)}$ Centro de Protección Vegetal y Biotecnología . Instituto Valenciano de Investigaciones Agrarias (IVIA). CV-315, km \\ 10,7 - 46113 Moncada (Valencia), Spain \\ 3) Agrícola Villena S. Coop. Carretera del Puerto, S/N, 03400 Villena (Alicante), Spain \\ 4) TRAGSA. CV-315, km 10,7 - 46113 Moncada (Valencia), Spain \\ ${ }^{5)}$ Departamento de Ingeniería Gráfica. Universitat Politècnica de València (UPV). Camino de Vera, s/n, 46022 Valencia, \\ Spain \\ * Corresponding author email: blasco_josiva@gva.es
}

\begin{abstract}
A low cost field robot has been built capable of transporting a series of sensors through horticultural fields, with the aim of early detecting problems in the crop by means of proximal sensing techniques. The robot is operated by remote control and is driven by two electric motors coupled to the wheels and powered by batteries. The sensors include different thermal, colour and multispectral cameras in the visible and the near-infrared range that are synchronised with the advance of the robot by means of an encoder coupled to the axis of the motors. The position of each image is geolocated using a GPS. An industrial computer receives the encoder pulses and triggers the cameras, also receiving and storing the images and GPS information for further processing. The inspection area is located beneath the robot with the cameras focusing downwards (to the crop). To avoid the negative influence of direct sunlight, the area had been covered with a canvas and illuminated artificially with four-spot halogen lights. A telescopic extension system between 100 and $200 \mathrm{~cm}$ allows the robot to adapt to crops with different row widths. The first trials were carried out in a carrot test field located in Villena (Spain) to detect plants infected with Candidatus Liberibacter solanacearum. The crop was inspected every month from sow to harvest. Labels were placed on 100 plants to guarantee their individual identification in the images. During the harvest, these plants were collected separately, identified and analysed in the laboratory using molecular techniques in order to determine whereas they were infected or not. Several maps of the field have been created using spectral indexes at a very high resolutions between $0.5 \mathrm{~mm} / \mathrm{pixel}$ and $2.5 \mathrm{~mm} /$ pixel depending on the camera.
\end{abstract}

Keywords: Field robot, computer vision, multispectral imaging, proximal sensing, asymptomatic detection

\title{
1. Introduction
}

Candidatus Liberibacter solanacearum (CaLsol) is a bacterium that affects the phloem of plants. The transmission of the disease occurs mainly through insect vectors, although it can also occur by grafting or seeds (Antolinez et al., 2017). In Europe it is causing damage mainly in carrots. In Spain, the bacteria are distributed in several areas, especially affecting carrots and have already been detected in some potato plantations, although the bacteria can also affect plants such as celery, parsnips, parsley or fennel. Another haplotype also affects tomatoes, peppers, aubergines, tobacco, good grass or mint.

The most prominent symptoms in these plants are the wrinkling of the leaves, generalized chlorosis, purplish discoloration and atrophied growth of shoots and roots. The disease in the aerial part of these crops causes growth retardation, erection of the new foliage, generalized chlorosis, purpurea coloration of the foliage with basal cupping of the leaves and interruption of fruit formation. However, symptoms may be confused by those caused by other pathogens (i.e. phytoplasma, Spiroplasma citri) and, in other cases, while the fruits are affected the aerial pat remain asymptomatic which makes difficult their visual detection. Hence, it is necessary to carry out frequent sampling and laboratory analysis to determine the presence of the disease in the plants, which is difficult and expensive to do it on a large area of the crop.

The use of remote sensing in precision farming can help to study a large spatial extent and with high resolution to detect plant diseases (Baylis, 2017). These techniques have evolved over the last three decades and can be performed at different scales, depending on the area to be monitored as well as the spatial and spectral resolution required (Martinelli et al., 2015). At leaf level, spectral information can be collected at a high spatio-temporal resolution, and can be achieved using hand sensors or mounted on agricultural vehicles. On the other hand, the reduction of prices and miniaturization make unmanned aerial systems (UAS) increasingly popular for rapid monitoring at crop level (Vicent and Blasco, 2017). This work aims to help in the early detection of crops infected by the CaLsol at plant and crop level, using high resolution proximal sensing sensors mounted on a electrical platform designed and developed for this purpose. 


\section{Materials and Methods}

Field robot and experimental crop

For this purpose, an electric terrestrial vehicle (Fig. 1a) remotely operated has been created to incorporate on board a full set of sensors for monitoring entire fields. A telescopic system allows adapting the width of the robot to the needs of the field in a range between 100 and $200 \mathrm{~cm}$ because the geometry of the crop allows the wheels to circulate only by a few lines separated by a certain distance that can vary from one crop to another. On the other hand, this system allows to reduce its size to be transported in a van. The robot and all the sensors are powered by a battery while an electric generator is used to illuminate the scene using four light halogen lamps, each of one located in the four corners of the scene. These are used to capture the images under controlled conditions, preventing the negative effect of natural changing conditions and also the direct sun lighting. Aligned at the topmiddle of the chamber are the sensors to capture all the inspection area.

To capture the images at determined distances avoiding gaps and overlapping, a programmable system was developed to synchronise the trigger of the cameras with the advance of the vehicle using two optical encoders coupled to each drive wheels. The signal from optical encoders was also used to assist in the guidance of the vehicle through a proportional-integral-derivative controller (PID). In addition, an application was developed to run in the industrial computer and control the triggering and image acquisition of all cameras during the field tests. In addition, a GNSS receiver (Hiper SR, TOPCON Corp. Japan) with RTK correction was installed in the vehicle, allowing geolocation with an accuracy of around $1 \mathrm{~cm}$ to identify each of the crop plants in the images.

The experiments have been carried out in a carrot crop (Figure 1b) located in Villena, Alicante (Spain) for two years. Red lines delimit the monitored area containing 27 cultivated rows. Inside this area, some patches were treated with different products to prevent the infections in the area delimited by the yellow lines in Figure $1 \mathrm{~b}$. Each of these 27 cultivated rows was composed of three lines of plants with a total width of $1.5 \mathrm{~m}$ approximately that were inspected at the same time. In each of the two seasons tested, six inspections of the whole area were carried out, one every month during the crop cycle from the sowing to the harvesting (June-October) to observe the evolution of the plants during their growth and try to detect the infection as early possible. During the last survey of the field, 100 plants were marked using red discs to be later identified in the images. These plants were collected separately and taken to the laboratory to undergo a spectral analysis with a spectrometer (200 to 1800 $\mathrm{nm}$ ) and with a hyperspectral camera (400 to1000 nm), and also using an ultraviolet induced fluorescence imaging system. Finally, and a molecular analysis using specific real-time polymerase chain reaction (q-PCR) was performed to determine the presence or absence of infection in the leaves and use these plants for reference in the further creation of statistical models.

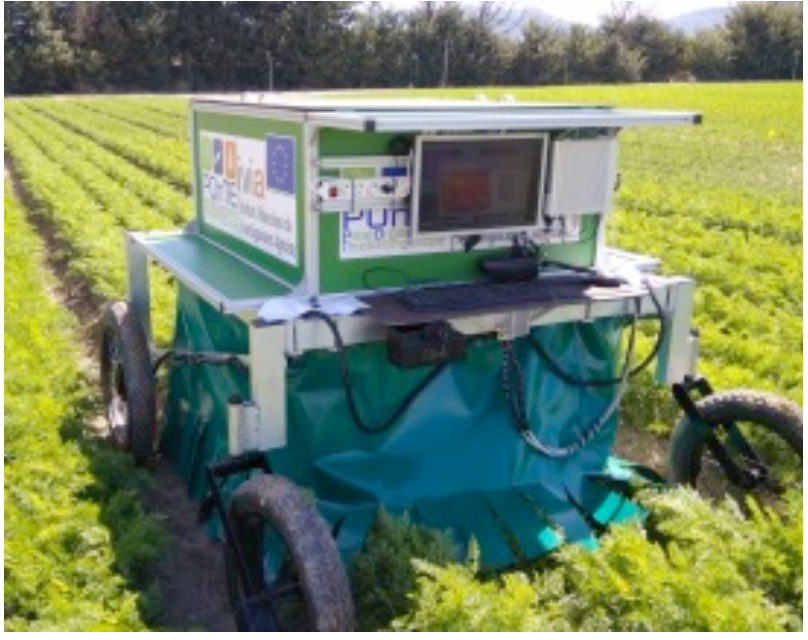

a)

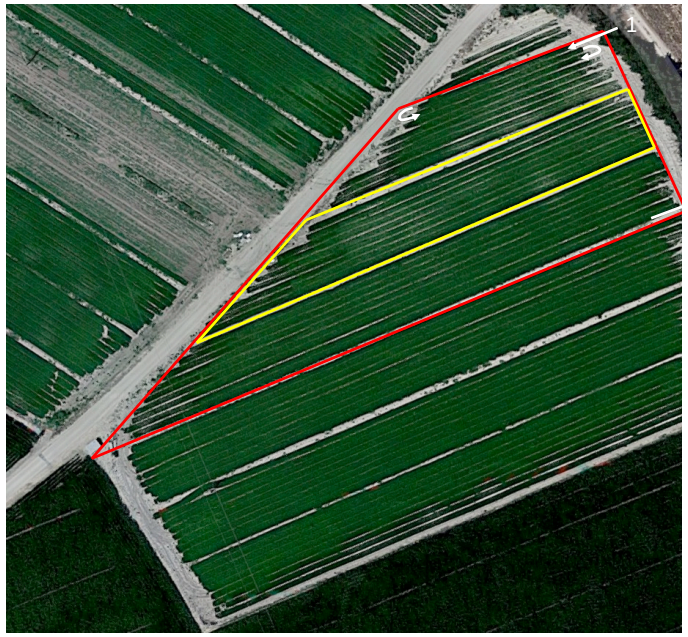

b)

Figure 1. a) Remote-guided vehicle; and b) carrot crop in Villena, Alicante (Spain).

Sensors

The sensors mounted in the terrestrial vehicle (Fig. 2) include three DSLR (Digital Single Lens Reflex) cameras (EOS 600D, Canon Inc, Japan), two of them modified to capture images in near infrared (NIR) from 700 to $1000 \mathrm{~nm}$, and blue NDVI (normalized difference vegetation index), a thermal camera (A320, FLIR Systems, USA) and a multispectral camera (CMS-V, Silios Technologies, France) capable of capturing eight monochromatic images in 558, 589, 623, 656, 699, 732, 769 and $801 \mathrm{~nm}$. The DSLR cameras allow to capture images of the crop with a resolution of $0.5 \mathrm{~mm} / \mathrm{pixel}$ and the thermal and the multispectral camera could obtain 
images with a spatial resolution of $2.5 \mathrm{~mm} /$ pixel. All the cameras were configured to capture images synchronised with the advance of the vehicle about one image per meter with integration times less than $4 \mathrm{~ms}$ to avoid moving or blurry images.

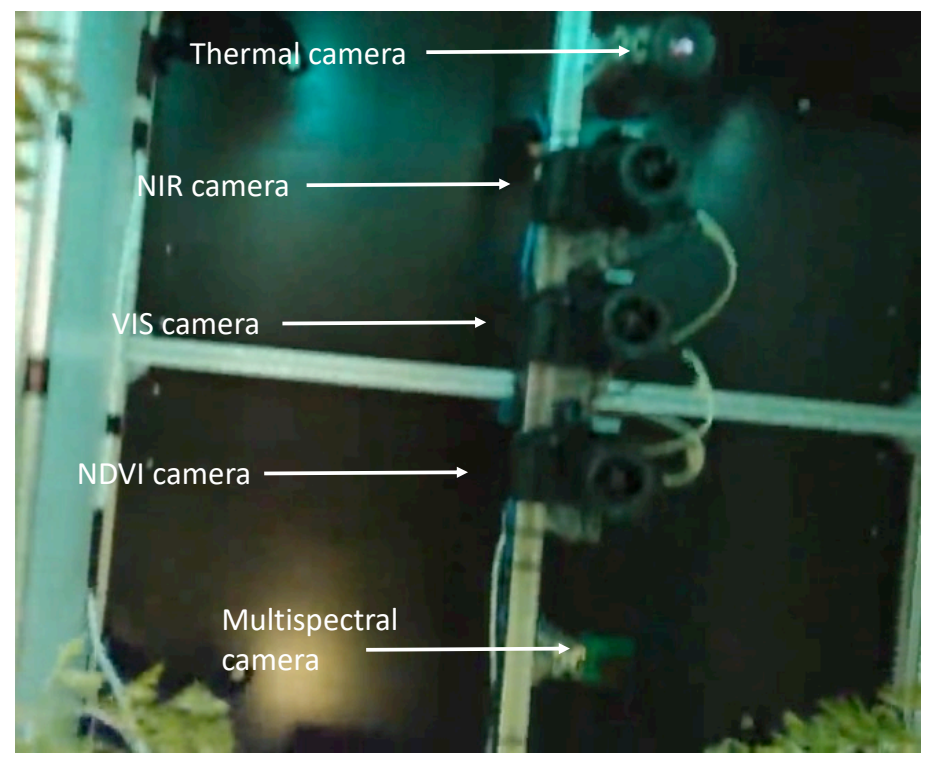

Figure 2. Image captured from the crop side, showing the sensors on board from the ground.

\section{Results and Discussion}

The main result at this stage is the creation of a robotic solution, remotely operated that can transport different proximal sensing equipment to inspect horticultural crops, illuminating the scene to prevent direct solar radiation. The robot advances smoothly at a very accurate speed which allows the creation of precise high resolution maps of the crops. Starting from the images collected by each camera of the whole crop, images in different bands of each of the rows cultivated are made. The images are being analysed to create maps showing different spectral indexes (Zarco-Tejada, 2012) from the images captured in different spectral bands (Fig. 3).

The results of the field analyses in these two years of trials show no clear signs of CaLsol in the spectral information acquired during the monitoring period. However, no information was obtained from negative samples to be able to compare in the images since the great majority of the samples presented positive results in the detection of the CaLsol bacteria. The results of the laboratory analysis (both, spectral and molecular) together with the analysis of these specific plants captured in the field, will serve to create maps of crop with different vegetative indexes showing potential infections that will be contrasted with the information collected by the Bacteriology Unit of IVIA and visual inspection provided by the technicians of the cooperative Agricola Villena.

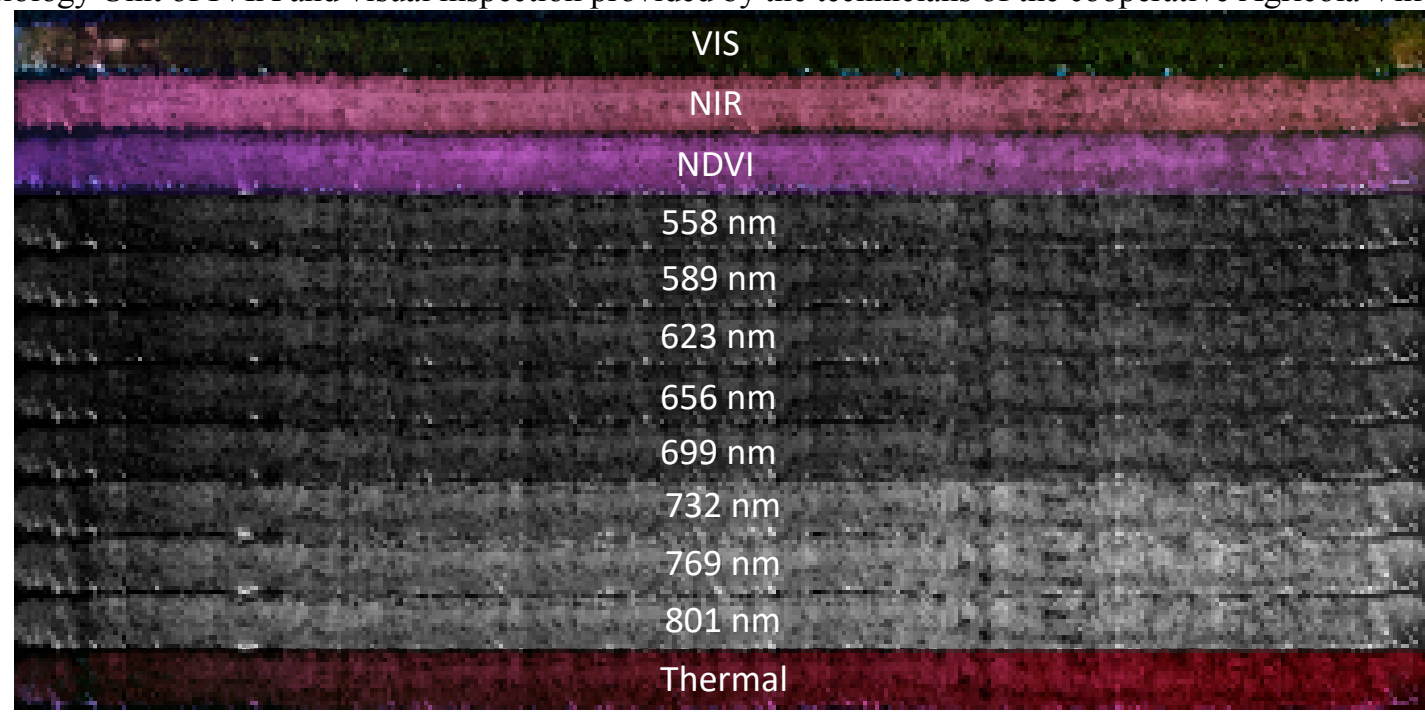

Figure 3. Image of one of the rows of the crop captured in different spectral bands. 


\section{Acknowledgements}

This work was partially supported by funding from the European Union's Horizon 2020 research and innovation programme under grant agreement No 635646 POnTE (Pest Organisms Threatening Europe).

\section{References}

Antolinez CA, Fereres A, Moreno A. (2017). Risk assessment of 'Candidatus Liberibacter solanacearum' transmission by the psyllids Bactericera trigonica and B. tremblayi from Apiaceae crops to potato. Scientific Reports 7, Article number: 45534

Baylis A (2017) Advances in precision farming technologies for crop protection. Outlooks on Pest Management, 28: 158-161

Martinelli F, Scalenghe R, Davino S, Panno S, Scuderi G, Ruisi P, Villa P, Stroppiana D, Boschetti M, Goulart LR, Davis CE, Dandekar AM. 2015. Advanced methods of plant disease detection. A review. Agronomy for Sustainable Development 35: 1-25.

Vicent A, Blasco J. (2017) When prevention fails. Towards more efficient strategies for plant disease eradication. New Phytologist, 214: 905-908.

Zarco-Tejada PJ, González-Dugo V, Berni JAJ. (2012) Fluorescence, temperature and narrow-band indices acquired from a UAV platform for water stress detection using a micro-hyperspectral imager and a thermal camera. Remote Sensing of Environment, 117: 322-337 


\title{
Sustainable Management and Yield Monitoring of an Annual Mixture Forage
}

\author{
Luís A. Conceição ${ }^{\text {a, }}$, José Rafael da Silva ${ }^{\text {b }}$, Rute Santos a, Noémia Farinha ${ }^{\text {c }}$, Benvindo Maças ${ }^{\text {d }}$ \\ ${ }^{a}$ IPP Polytechnic Institute of Portalegre, Portugal. and ICAAM - Instituto de Ciências Agrárias e Ambientais \\ Mediterrânicas, Instituto de Investigação e Formação Avançada, Universidade de Évora, Núcleo da Mitra, 7000 Évora, \\ Portugal \\ ${ }^{\mathrm{b}}$ Departamento Engenharia Rural, Escola de Ciências e Tecnologia, ICAAM - Instituto de Ciências Agrárias e \\ Ambientais Mediterrânicas, Instituto de Investigação e Formação Avançada, Universidade de Évora, Núcleo da Mitra, \\ Agroinsider (www.agroinsider.com), 7000 Évora, Portugal. \\ ${ }^{c}$ IPP Polytechnic Institute of Portalegre, Praça do Município 11, 7300-110 Portalegre, Portugal \\ ${ }^{\mathrm{d}}$ Research Unit of Biotechnology and Genetic Resources (INIAV), 7300 Elvas, Portugal \\ * Corresponding author. Email: luis_conceicao@ipportalegre.pt
}

\begin{abstract}
The need for sustainable intensification of fodder production to support extensive animal production systems in the Mediterranean regions requires new forms of mixtures, new forms of mechanization and the adoption of georeferenced sensory technologies that allow spatial and temporal analysis to mapping field and crop variations. The objective of the present study was to delineate site-specific management zones and cost effective crop by means of soil intelligent surveys, map yield and chlorophyll indices, measured by satellite, of a mixture forage under no-till based on annual ryegrass and annual leguminous plants. The experiment took place in an area of 31 ha under a circular pivot in the Alentejo region, Portugal at the coordinate's $38^{\circ} 53^{\prime} 39^{\prime}$ 'N $7^{\circ} 03^{\prime} 03^{\prime}$ ' $\mathrm{W}$. The field was mapped for soil apparent electrical conductivity with a Veris 2000 XA sensor combined with the use of a Global Navigation Satellite System, soil and crop samples were georeferenced in 16 different locations and normalized difference vegetation index (NDVI) evaluated by remote sensing using Sentinel -2. Data were processed by linear model and geospatial ArcGIS software. Statistical analysis resulted in significant correlation coefficient values between fresh matter yield, dry matter yield and plant families' composition and, spatial auto correlation to NDVI along the crop cycle was found. The survey carried out with the Veris sensor showed that there was spatial auto correlation for soil apparent electrical conductivity $\left(\mathrm{EC}_{\mathrm{a}}\right)$ and topography and two different areas of high and low $\mathrm{EC}_{\mathrm{a}}$ were identified. Although there was not spatial auto correlation for biomass suggesting that crop yield could be affected by other factors than soil, plant families composition concerning legume percentage was different $(\mathrm{p}=0.0039)$ between two areas of $\mathrm{EC}_{\mathrm{a}}$, even though no statistical differences were found regarding grasses and other families.
\end{abstract}

Keywords: no till, on-the-go sensors, forages, remote sensing

\section{Introduction}

Taking into account the natural climate conditions of the hot and dry summer and cold and wet winter of the Mediterranean regions, pasture in extensive systems is not sufficient to feed livestock herds. So farmers are forced to supplement cattle during wintertime and end of summer with preserved annual forages namely those sown in the previous autumn/ early winter.

Even though these forages were less cost effective, when compared to those sown in spring in the last two years, forages price is hitting livestock sector, mainly because of the irregularity of temperatures and rainfall patterns. Unfortunately, in the Alentejo region, shallow and degraded soils through erosion and loss of nutrients due to many decades of intensive cereal monoculture (Serrano et al., 2014) also limits, in many cases, forage production (Freixial \& Carvalho, 2013).

Therefore, it is of most importance in these regions to improve new sustainable soil and crop management practices based on new forms of mechanization, cultivars and crop management. Increasing the share of bio diverse forages supported on different combinations of grass-legume cultivars, adapted to rainfed farming, result in a lower price protein, when compared to soybeans or rapeseed meal, and help to reduce the mineral nitrogen fertilization due to legume content.

Furthermore, conservation agriculture systems improve soil properties by means of better infiltration, reducing runoff and soil erosion and can sequester large amounts of $\mathrm{CO}_{2}$ equivalents into soil organic matter (Kertész \& Madarász, 2014). They also benefit wildlife, reduce soil compaction and save fuel energy, when compared to other traditional soil practices such as conventional tillage (Govaerts et al., 2009; Soane et al., 2012). Nevertheless, the advantages of sustainable practices are not always appealing to farmers, because of the limited formalised interest in soil conservation, amongst the conventional farming community and industry in general. Thus, monitoring the ecosystem using on the go sensors will contribute to the understanding of the value of adaptive management to forage crops under natural conditions, identifying research needs, determining sitespecific management and improving cost effectiveness (Stafford, 2000, Basso, 2003). 
Currently, the use of Global Navigation Satellite Systems (GNSS) and Geographical Information Systems (GIS) combined with geospatial measurements of soil apparent electrical conductivity $\left(\mathrm{EC}_{\mathrm{a}}\right)$ and remote sensing, is one of the most reliable techniques to characterize the spatial and temporal pattern of soil and crops properties within fields (Sousa et al., 2017). Europe's Global Monitoring for Environment and Security programme, while providing systematic global acquisitions of high resolution multispectral imagery, also became a friendly option to online study the canopy chlorophyll content and leaf chlorophyll concentration indicating plant health and potential gross primary productivity (Frampton et al., 2013; Gitelson et al., 2006).

This trial presents the findings of the first year of a three years study to delineate site-specific management zones by means of soil intelligent surveys, map yield and chlorophyll indices, measured by satellite, of a mixture forage under no-till based on annual ryegrass and annual leguminous plants.

\section{Materials and Methods}

Site Characteristics and initial conditions of the trial

The study was conducted in Southern Portugal, on a 31 ha irrigated field, by means of a center pivot system located at the Comenda farm, owned by the National Institute of Agricultural and Veterinary Research (NIAVR) at the coordinate's $38^{\circ} 53^{\prime} 39^{\prime}$ ' $\mathrm{N} 7^{\circ} 03^{\prime} 03^{\prime}$ ' $\mathrm{W}$. The field is located in a plane area with light slopes. Climate, according to Köppen-Geiger is Csa. Figure 1 shows the Ombrothermic diagram from monthly mean temperatures and rainfalls data collected by the NIAVR meteorological station in the 2016/17 campaign.

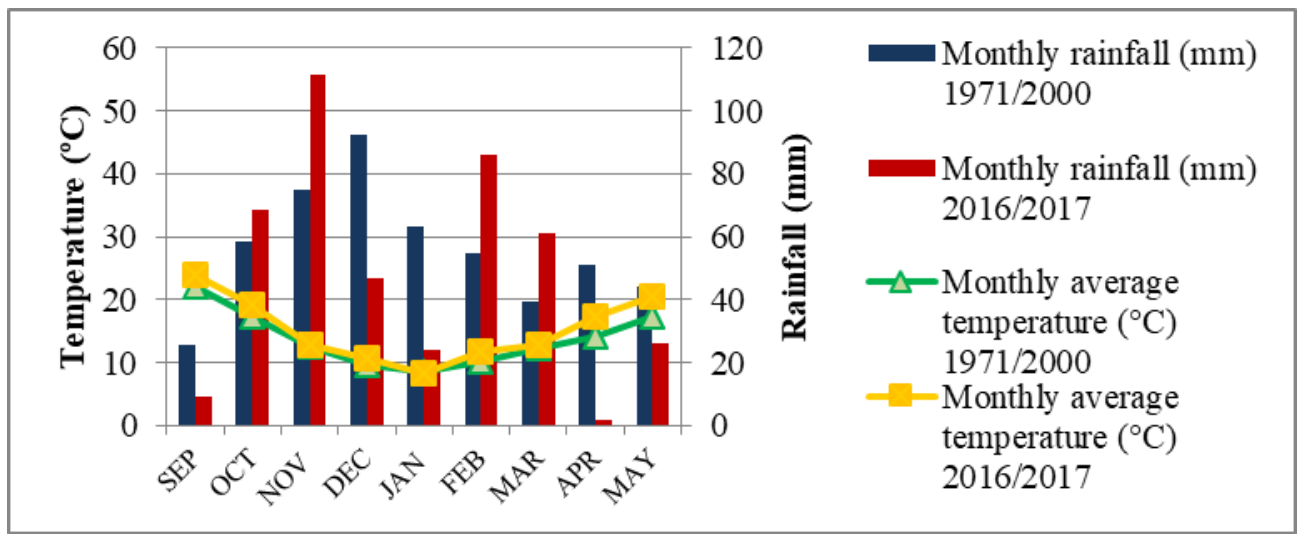

Figure 1. Ombrothermic diagram in 2016/2017 campaign.

The predominant soil of the field is, according to the FAO classification, a Luvisol matching to a Pag and $\mathrm{Sr}$ Mediterranean soil types (Cardoso, 1974). Pag is characterized by brown to brownish or light grey colour, sandy or loamy sandy sometimes with coarse elements of quartz throughout its profile and a structure without aggregates or weak fine granules and $\mathrm{Sr}$ of brown or reddish brown colour, usually with many coarse elements throughout its profile, of moderate or weak fine granular structure. The experimental area is under no-till for the second year.

\section{Soil Assessments}

In a preliminary analysis, the apparent soil electrical conductivity $\left(\mathrm{EC}_{\mathrm{a}}\right)$ was mapped with a Veris $2000 \mathrm{XA}$ contact-type sensor (Veris Technologies, Salina, KS, USA) in the $0-0.5 \mathrm{~m}$ depth equipped with a global positioning system (GPS) antenna pulled to a conventional tractor. $\mathrm{EC}_{\mathrm{a}}$ survey was conducted at an average speed of $8 \mathrm{~km} \mathrm{~h}^{-1}$ along 50 parallel transects spaced $12 \mathrm{~m}$ apart registering one measurement per second (Figure 2). To improve the accuracy of swat passages and forward speed, tractor was equipped with a light bar guidance system Trimble EZ-Guide 250 model+Ez steer (Trimble Navigation Limited, Sunnyvale-California, USA). 


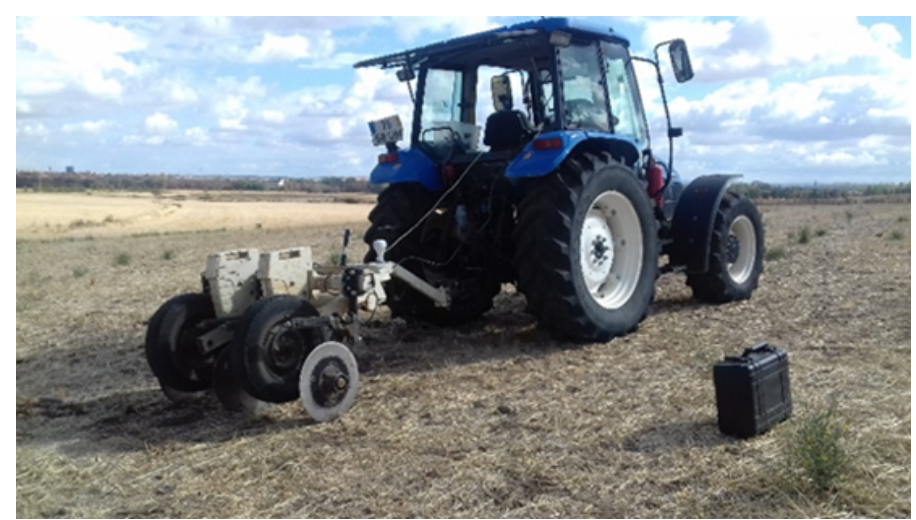

Figure 2. Veris 2000 XA contact-type sensor pulled to a conventional New Holland TL100.

Soil intelligent survey considered the $\mathrm{EC}_{\mathrm{a}}$ map classes and the field was sampled in 16 georeferenced locations obtained with ArcGIS (Ver. 2010) software. A GPS-receiver Magellan Mobile Mapper CX was used to track the pre-determined location of the sample points in the field to collect soil and forage samples. For each of the sampled points a standard soil characterization of soil gravimetric moisture, texture, bulk density, $\mathrm{pH}$, organic matter content, phosphorus, and potassium has been made at the sowing date.

Sowing operation and crop management

Sowing of a mix forage crop (Table 1) under no-till took place in mid-October after irrigation and weed control of a glyphosate based herbicide on a prescription map of the areas previously identified by a low altitude remote sensing survey. Images were obtained with a Canon Powershot A490 camera (Tokyo, Japan) modified to capture Near Infrared (NIR) light $(750-950 \mathrm{~nm})$ assembled to a hexacopter Unmanned Aerial Vehicle (UAV). The UAV followed a flight plan across 30 waypoints covering the entire field at an altitude of $120 \mathrm{~m}$ above ground level at a speed of $5.2 \mathrm{~ms}^{-1}$.

Table 1. Mix forage composition and seed rates.

\begin{tabular}{lc}
\hline Grass/Legume Cultivar & Seed rate $\left(\mathrm{kg} \mathrm{ha}^{-1}\right)$ \\
\hline Lolium multiflorum var. westerwoldicum & 14 \\
Lolium multiflorum var. Italicum & 10 \\
Trifolium isthmocarpum & 1 \\
Trifolium suaveolens & 7 \\
Trifolium michelianum & 2 \\
\hline
\end{tabular}

At sowing operation, a basal dressing of $150 \mathrm{~kg} \mathrm{ha}^{-1} \mathrm{NPK} 7-21-21$ fertilizer was applied. Along the crop cycle, two more nitrogen applications at a rate of $100 \mathrm{~kg} \mathrm{ha}^{-1}$ took place, the first one in the late January at crop tillering and, the second one in March immediately after a first cut for bailage. In the end of May, a second cut of the crop for hay took place.

\section{Crop Sample Analysis and Remote Sensing Survey}

Forage samples of $1 \mathrm{~m}^{2}$ areas were harvested $3 \mathrm{~cm}$ height in tillering, stem elongation (first cut) and flowering (second cut) matching stages 4, 8 and 10 of the Feekes growth stage (Lancashire et al. 1991).

The collected samples were weighted to determine the Fresh Matter (FM) production per hectare, and subsamples in small paper bags placed in $65^{\circ} \mathrm{C}$ until constant weight to determine forage moisture content and Dry Matter (DM) content. Samples were separated in legumes, grasses and other families and the relative percentage of each was determined.

The Sentinel 2, NDVI (Eq.1) index was calculated according to Rouse et al. (1974) and the following equation (NIR-Near infrared band; Red - Red infrared band).

$$
N D V I=(N I R-R e d) /(N I R+R e d)
$$

\section{Statistical analysis}

Topographic and $\mathrm{EC}_{\mathrm{a}}$ spatial variability maps were created using ordinary kriging interpolation with ArcView software, version 10.2 (Redlands, USA). Statistical analysis of the collected data consisted in descriptive statistics (means and standard deviations), Student t-tests for identification of significant differences between a two level factor (use or no use of herbicide), Pearson's correlation coefficients for assessment of association between 
variables, and a general linear model that considered fresh and dry matter yield, dry matter content and botanical composition (\% grasses, \% legumes and \% other families) as dependent variables; and herbicide use, sampling moment and $\mathrm{EC}_{\mathrm{a}}$ class (high or low) as independent factors, followed by a Tukey's test for post hoc results. Statistical calculations were performed using Statistica software, version 12.0 (StatSoft ${ }^{2}$, Tulsa, USA).

\section{Results and Discussion}

The survey carried out with the Veris sensor showed that there was spatial auto correlation for $\mathrm{EC}_{\mathrm{a}}$ and topography and two different areas of high and low $\mathrm{EC}_{\mathrm{a}}$ were identified. Figure 3 show the $\mathrm{EC}_{\mathrm{a}}$ and topographic map of the field trial.

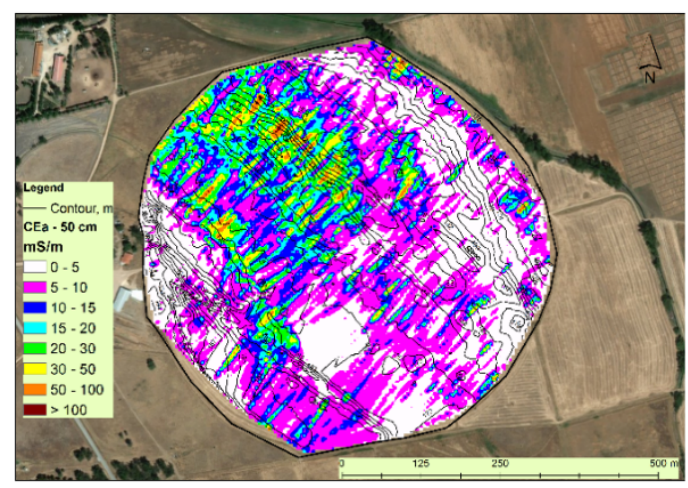

Figure 3. $\mathrm{EC}_{\mathrm{a}}$ and topographic map of the field trial.

Experimental variogram (points) and theoretical exponential variogram (lines) for soil apparent electrical conductivity and topography are shown in Figure 4
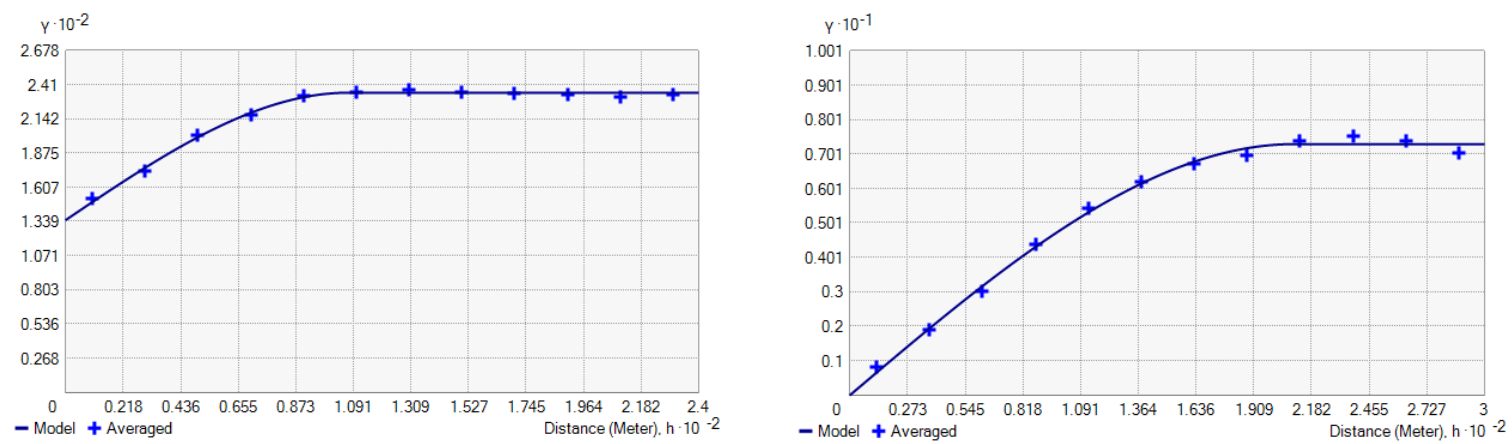

Figure 4. (Left) Experimental and theoretical exponential variograms for soil apparent electrical conductivity and topography (right).

The $\mathrm{EC}_{\mathrm{a}}$ soil data structural analysis showed that the theoretical spherical model provided the best fit for $E_{a}$ evaluations with a nugget value of 134.8 partial sill of 99.6 and range of $107 \mathrm{~m}$. Field topography data structural analysis showed that the theoretical spherical model provided the best fit for topography evaluations with a nugget value of 0.0 partial sill of 7.3 and range of $210 \mathrm{~m}$. These values according to Cambardella et al. (1994) by the ratio of nugget to sill denote a strong spatial dependence of topography but weak of soil $\mathrm{EC}_{\mathrm{a}}$ probably due to the heterogeneous of soil components.

The average values of soil analysis of the experimental field considering two major classes of EC $\mathrm{a}_{\mathrm{a}}, 0-30 \mathrm{mS} / \mathrm{m}$ and $30-100 \mathrm{mS} / \mathrm{m}$ are shown in Table 2.

Table 2. Soil analysis results of experimental field at sowing date.

\begin{tabular}{ccccccccccc}
\hline $\begin{array}{c}\mathrm{EC}_{\mathrm{a}} \\
\text { Class } \\
(\mathrm{mS} / \mathrm{m})\end{array}$ & $\begin{array}{c}\text { Soil } \\
\text { Texture }\end{array}$ & $\begin{array}{c}\text { Sand } \\
(\%)\end{array}$ & $\begin{array}{c}\text { Silt } \\
(\%)\end{array}$ & $\begin{array}{c}\text { Clay } \\
(\%)\end{array}$ & $\begin{array}{c}\text { Available } \\
\text { Phosphorus } \\
\left(\mathrm{mg} \mathrm{kg}^{-1}\right)\end{array}$ & $\begin{array}{c}\text { Available } \\
\text { Phosphorus } \\
\left(\mathrm{mg} \mathrm{kg}^{-1}\right)\end{array}$ & $\begin{array}{c}\mathrm{pH} \\
\text { Organic } \\
\text { Mater } \\
(\%)\end{array}$ & $\begin{array}{c}\text { Gravimetric } \\
\text { moisture } \\
(\%)\end{array}$ & $\begin{array}{c}\text { Bulk } \\
\text { Density }\end{array}$ \\
\hline $0-30$ & $\begin{array}{c}\text { Sandy } \\
\text { loam }\end{array}$ & 71.5 & 13.4 & 15 & 168.2 & 99.3 & 6.8 & 1.5 & 7.5 & 1.3 \\
$30-100$ & Loam & 61.2 & 18.7 & 21.5 & 162 & 79 & 6.9 & 1.14 & 9 & 1.4 \\
\hline
\end{tabular}


These results suggest the presence of two main areas with different soil $\mathrm{EC}_{\mathrm{a}}$ classes and textures. A loam texture in the area with high values of $\mathrm{EC}_{\mathrm{a}}$ and soil gravimetric moisture and a coarser sandy loam texture in the area with low $\mathrm{EC}_{\mathrm{a}}$ values and lower soil gravimetric moisture content. Therefore, these results agree with Lund et al. (1999) and Doolittle, J.A \& Brevik, C. (2014) where under non-saline conditions EC a $_{\mathrm{a}}$ is positively correlated with clay content.

Total yields, considering the addition of the sampling at the tillering phase (approx. 19\% DM), the 1st cut (baleage, approx. 32\% DM) and the $2^{\text {nd }}$ cut (hay, approx. 41\% DM), averaged $25.44 \mathrm{ton} /$ ha of FM and 6.53 ton/ha of DM. According to the seed manufacturer's technical catalogue, expected DM yield for this seed mixture in conventional tillage conditions should vary between 6 and 14 ton/ha of DM. Our result corresponds to the lower values in this range, but is comparable to the results of Bueno et al. (2007) on Italian ryegrass under no tillage systems. Crespo (2015) and Chastain et al. (2017) found that tillage system had an influence on yield parameters in annual ryegrass, with more noticeable differences being found in environmentally less favourable years, with no tillage systems showing inferior yields. In our trial, despite being located in an area under pivot, irrigation was not possible before mid-April for lack of water. The decrease values in temperature and reduced rainfall in January may have affected the tillering phase of the crop and determined the observed values.

Figure 5 show the NIR image of the experimental field were blue-grey colour corresponds to the area for weed control in 19 ha from the total 31 ha.

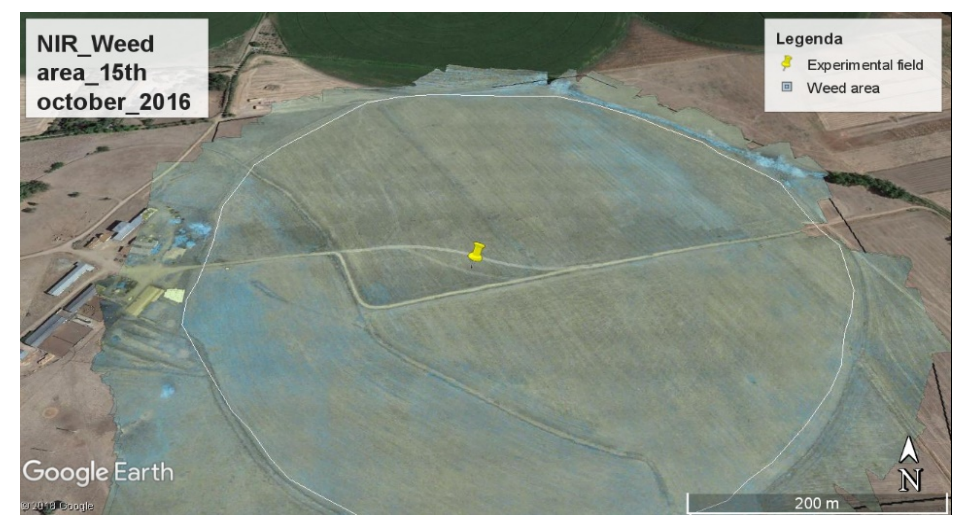

Figure 5. NIR image of the experimental field before sowing to weed control in the blue-grey areas.

There were apparent differences between the area where herbicide was used and the area where no herbicide was applied. We compared mean fresh and dry matter yields, as well as botanical composition (expressed in \% DM of grasses, legumes and other families) at the tillering phase, the $1^{\text {st }}$ and $2^{\text {nd }}$ cuts, using Student's t test (Table 3).

Table 3. Differences between yield results and botanical composition of the areas with and without herbicide application at tillering phase, $1^{\text {st }}$ and $2^{\text {nd }}$ cuts (Student's t test).

\begin{tabular}{|c|c|c|c|}
\hline & \multicolumn{3}{|c|}{ Tillering $(\mu \pm$ Std.Dev. $)$} \\
\hline & Herbicide $(n=10)$ & No herbicide $(\mathrm{n}=6)$ & $\mathrm{p}$ \\
\hline FM (kg/ha) & $7861.37 \pm 4082.81$ & $9508.89 \pm 7545.92$ & 0.330 \\
\hline DM (kg/ha) & $2136.02 \pm 1046.86$ & $2240.52 \pm 1017.26$ & 0.737 \\
\hline$\%$ Grasses & $88.33 \pm 12.46$ & $40.56 \pm 31.19$ & $0.000 * * *$ \\
\hline$\%$ Legumes & $3.70 \pm 5.53$ & $2.00 \pm 5.13$ & 0.295 \\
\hline \multirow[t]{3}{*}{$\%$ Other } & $7.97 \pm 12.14$ & $51.94 \pm 34.42$ & $0.000 * * *$ \\
\hline & \multicolumn{3}{|c|}{$I^{s t}$ cut (baleage) $(\mu \pm$ Std.Dev.) } \\
\hline & Herbicide $(n=10)$ & No herbicide $(n=6)$ & $\mathrm{p}$ \\
\hline FM (kg/ha) & $9981.00 \pm 2864.24$ & $6250.00 \pm 2238.13$ & $0.017 * *$ \\
\hline DM (kg/ha) & $3089.70 \pm 1039.49$ & $2004.33 \pm 846.60$ & $0.049^{*}$ \\
\hline$\%$ Grasses & $91.80 \pm 5.31$ & $34.00 \pm 24.27$ & $0.000 * * *$ \\
\hline$\%$ Legumes & $1.20 \pm 1.62$ & $0.00 \pm 0.00$ & 0.095 \\
\hline \multirow[t]{3}{*}{$\%$ Other } & $7.00 \pm 6.09$ & $66.00 \pm 24.27$ & $0.000 * * *$ \\
\hline & \multicolumn{3}{|c|}{$2^{\text {nd }}$ cut (hay) $(\mu \pm$ Std.Dev. $)$} \\
\hline & Herbicide $(n=10)$ & No herbicide $(n=6)$ & $\mathrm{p}$ \\
\hline FM (kg/ha) & $3548.00 \pm 1676.27$ & $4193.33 \pm 2823.04$ & 0.572 \\
\hline DM (kg/ha) & $1446.18 \pm 758.74$ & $1737.83 \pm 1229.24$ & 0.563 \\
\hline$\%$ Grasses & $90.80 \pm 8.69$ & $71.50 \pm 20.97$ & $0.021 *$ \\
\hline$\%$ Legumes & $7.00 \pm 8.18$ & $5.33 \pm 8.26$ & 0.700 \\
\hline$\%$ Other & $2.20 \pm 2.86$ & $23.17 \pm 24.84$ & $0.000 * * *$ \\
\hline
\end{tabular}

Significance using Student's t test: * significative $(\mathrm{P}<0.05)$; ${ }^{*}$ very significative $(\mathrm{P}<0.01) ; * *$ highly significative $(\mathrm{P}<0.001)$ 
Herbicide application significantly increased both fresh and dry matter yields at the $1^{\text {st }}$ cut, but not at the $2^{\text {nd }}$. Relative amounts of grasses were significantly higher and relative amounts of other families were significantly lower in the area where herbicide was used, both in the $1^{\text {st }}$ and $2^{\text {nd }}$ cut results. Student's t test was also used to compare total fresh and dry matter yields in both areas; results show a significantly higher fresh matter total yield $(p=0.030)$ in the area where herbicide was used, but no significant differences were found in dry matter yields. Results show a noticeable heterogeneity for the analysed values, patent in the high values of standard deviations. Pearson's correlation coefficients ( $r$ ) between yield results, botanical composition, normalized difference vegetation index (NDVI) and apparent electrical conductivity $\left(\mathrm{EC}_{\mathrm{a}}\right)$, in the three sampling moments, are presented in Table 4. As expected, $r$-values between fresh and dry matter yields were highly significant $(p<0.001)$ in all three sampling moments. We also found a strong negative correlation between percentage of grasses and percentage of other families in all three sampling moments, which probably mirrors the differences found between the area where herbicide was applied and the area without herbicide.

Table 4. Pearson's correlation coefficients between yield results, botanical composition, NDVI and $\mathrm{EC}_{\mathrm{a}}$, considering the 16 sampling points (with and without herbicide application) over 3 sampling moments.

\begin{tabular}{|c|c|c|c|c|c|c|c|c|}
\hline & $\begin{array}{c}\text { FM } \\
(\mathrm{kg} / \mathrm{ha})\end{array}$ & $\begin{array}{c}\mathrm{DM} \\
(\mathrm{kg} / \mathrm{ha})\end{array}$ & $\mathrm{DM}(\%)$ & $\begin{array}{l}\text { Grasses } \\
(\% \mathrm{DM})\end{array}$ & $\begin{array}{l}\text { Legumes } \\
\text { (\%DM) }\end{array}$ & $\begin{array}{l}\text { Other } \\
(\% \mathrm{DM})\end{array}$ & NDVI & $\begin{array}{c}\mathrm{EC}_{\mathrm{a}} \\
(\mathrm{mS} / \mathrm{m})\end{array}$ \\
\hline \multicolumn{9}{|l|}{ Tillering } \\
\hline FM (kg/ha) & 1.00 & $0.88 * * *$ & $-0.62 * * *$ & $-0.64 * *$ & -0.28 & 0.38 & $0.84 * * *$ & 0.11 \\
\hline $\mathrm{DM}(\mathrm{kg} / \mathrm{ha})$ & & 1.00 & -0.22 & $-0.77 * * *$ & -0.30 & 0.49 & $0.73 * * *$ & 0.10 \\
\hline DM (\%) & & & 1.00 & 0.03 & 0.01 & 0.08 & $-0.68 * *$ & 0.01 \\
\hline Grasses $(\% \mathrm{DM})$ & & & & 1.00 & 0.35 & $-0.78 * * *$ & -0.40 & -0.34 \\
\hline Legumes (\%DM) & & & & & 1.00 & -0.30 & -0.01 & $-0.51 *$ \\
\hline Other $(\% \mathrm{DM})$ & & & & & & 1.00 & 0.18 & 0.15 \\
\hline NDVI & & & & & & & 1.00 & -0.14 \\
\hline $\mathrm{EC}_{\mathrm{a}}(\mathrm{mS} / \mathrm{m})$ & & & & & & & & 1.00 \\
\hline \multicolumn{9}{|l|}{ 1st cut (baleage) } \\
\hline $\mathrm{FM}(\mathrm{kg} / \mathrm{ha})$ & 1.00 & $0.86^{* * *}$ & -0.17 & $0.53 *$ & 0.18 & $-0.53 *$ & $0.62 * *$ & -0.32 \\
\hline $\mathrm{DM}(\mathrm{kg} / \mathrm{ha})$ & & 1.00 & 0.33 & 0.43 & 0.19 & -0.43 & 0.40 & -0.18 \\
\hline $\mathrm{DM}(\%)$ & & & 1.00 & -0.14 & 0.04 & 0.13 & -0.36 & 0.32 \\
\hline Grasses $(\% \mathrm{DM})$ & & & & 1.00 & 0.43 & $-1.00 * * *$ & 0.39 & -0.43 \\
\hline Legumes (\%DM) & & & & & 1.00 & -0.46 & 0.25 & -0.11 \\
\hline Other (\%DM) & & & & & & 1.00 & -0.40 & 0.42 \\
\hline NDVI & & & & & & & 1.00 & -0.06 \\
\hline $\mathrm{EC}_{\mathrm{a}}(\mathrm{mS} / \mathrm{m})$ & & & & & & & & 1.00 \\
\hline \multicolumn{9}{|l|}{ 2nd cut (hay) } \\
\hline FM (kg/ha) & 1.00 & $0.94 * * *$ & 0.06 & -0.21 & 0.06 & 0.17 & -0.15 & 0.33 \\
\hline $\mathrm{DM}(\mathrm{kg} / \mathrm{ha})$ & & 1.00 & 0.38 & -0.34 & 0.10 & 0.27 & -0.15 & 0.29 \\
\hline DM (\%) & & & 1.00 & -0.32 & 0.19 & 0.22 & -0.04 & -0.18 \\
\hline Grasses $(\% \mathrm{DM})$ & & & & 1.00 & -0.10 & $-0.90 * * *$ & 0.20 & -0.30 \\
\hline Legumes (\%DM) & & & & & 1.00 & -0.35 & -0.20 & $-0.51 *$ \\
\hline Other (\%DM) & & & & & & 1.00 & -0.10 & $0.51^{*}$ \\
\hline NDVI & & & & & & & 1.00 & $-0.60 * *$ \\
\hline $\mathrm{EC}_{\mathrm{a}}(\mathrm{mS} / \mathrm{m})$ & & & & & & & & 1.00 \\
\hline
\end{tabular}

NOTE: Significant $r$ values: $*-p<0.05 ; * *-p<0.01 ; * * *-p<0.001$

The relative content of grasses was negatively correlated with fresh and dry matter yields at the tillering phase; at the $1^{\text {st }}$ cut, fresh matter yield was positively correlated with percentage of grasses, and at the $2^{\text {nd }}$ cut, there was no correlation between both variables. Field observation suggests that at tillering phase, fresh and dry matter yields were noticeably influenced by other families content (mainly Diplotaxis catholica), because of their earlier growth; at the $1^{\text {st }}$ cut, grasses were already dominant in the area where herbicide was used, and represented around $34 \%$ of dry matter in the area with no herbicide application; finally, at the $2^{\text {nd }}$ cut, grasses were already prevalent in both areas, hence explaining the loss of association. 
A strong positive correlation was found between NDVI and fresh matter yield at tillering phase; r-value was lower at the $1^{\text {st }}$ cut, and correlation was lost at the $2^{\text {nd }}$ cut. NDVI is highly correlated with plant productivity ("greenness"), and therefore, is a good estimator of changes in aboveground biomass. Because NDVI measures photosynthetic activity, it also correlates positively with chlorophyll content, and in turn, nitrogen levels in plants (Hogrefe et al., 2017). Our results seem to show a good level of association between NDVI and aboveground biomass in earlier phases of plant development, that is then lost when dry matter content rises to $40 \%$. When Pearson's correlation coefficients are calculated considering all observations (16 sampling points over three sampling moments, $\mathrm{n}=48)$, r-values are highly significant $(\mathrm{p}<0.001)$ for fresh and dry matter yields (positive correlations) and dry matter content (negative correlation) (Figure 6).
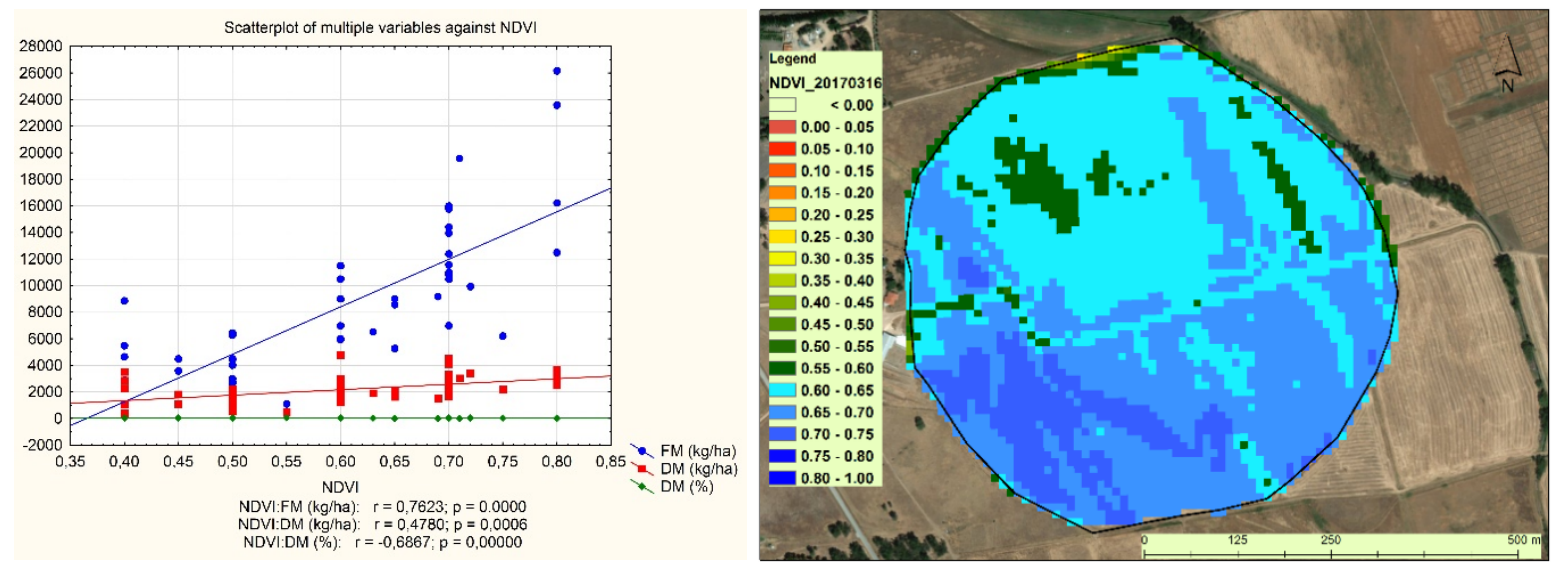

Figure 6. Scatterplot of fresh and dry matter yields and dry matter content against NDVI (Pearson's $r$ and $p$ values are presented below) (on the left) and spatial distribution of crop NDVI in the $1^{\text {st }}$ cut (on the right).

Additionally, there was a strong negative correlation between NDVI and $\mathrm{EC}_{\mathrm{a}}$ in the $2^{\text {nd }}$ cut (Figure 7). As mentioned earlier, association between aboveground biomass and NDVI was not significant when dry matter content reached $40 \%$ (less "greenness"). Considering that relative content of other families in this sampling moment was the lowest and relative content of legumes was the highest, we can assume an influence of nitrogen content in aboveground biomass in this correlation.
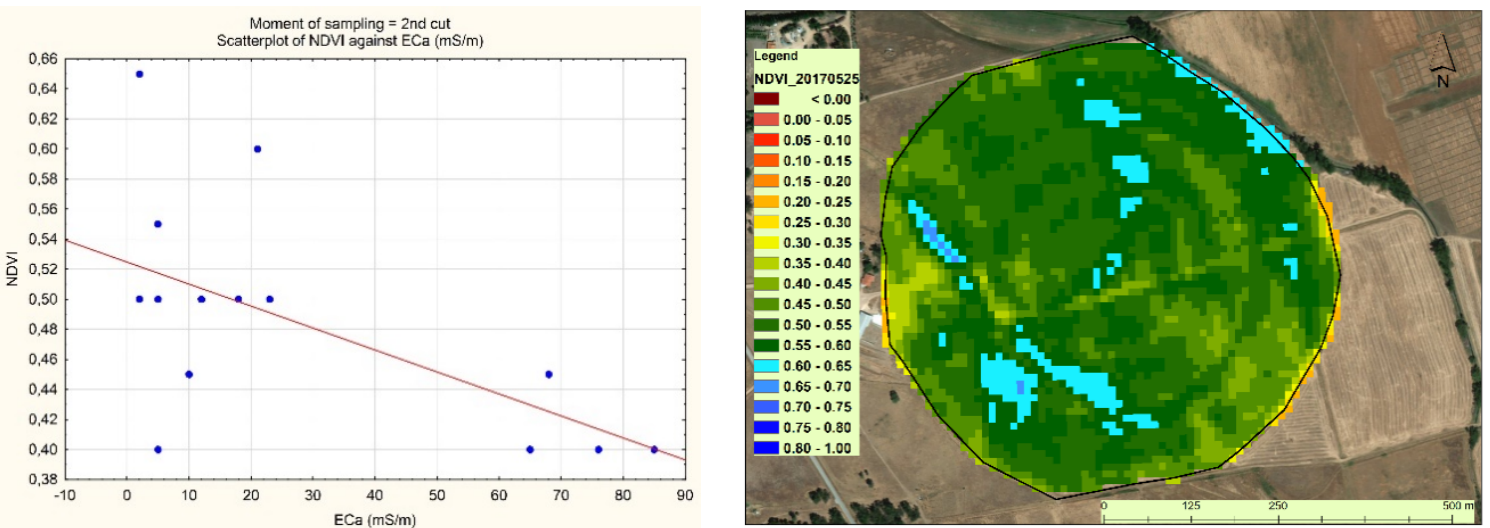

Figure 7. Scatterplot of NDVI against $\mathrm{EC}_{\mathrm{a}}$ at the $2^{\text {nd }}$ cut (on the left) and spatial distribution of crop NDVI in the $2^{\text {nd }}$ cut (on the right).

In recent years, several studies have been conducted in different grass and legume crops grown under different climatic conditions, with and without irrigation, attempting to find relationships between patterns in soil characteristics and crop growth using $\mathrm{EC}_{\mathrm{a}}$ measurements. Even though $\mathrm{EC}_{\mathrm{a}}$ was correlated with crop yield at harvest, a constant statistical linear relationship could only be verified in a few studies, while the majority of studies found varying relationships between $\mathrm{EC}_{\mathrm{a}}$ and crop yield, and different statistical approaches did not reveal a steady relationship between $\mathrm{EC}_{\mathrm{a}}$ and crop yield (Stadler et al. 2015). According to Marques Da Silva et al. (2008) based on the annual weather conditions, dry years and fine soil textures are those with the highest NDVI and $\mathrm{EC}_{\mathrm{a}}$ indices; on the other hand, flooding impact of wet conditions limiting crop development limits NDVI values.

In this study, there were significant correlations of $\mathrm{EC}_{\mathrm{a}}$ with relative content in legumes at the tillering phase and at the $2^{\text {nd }}$ cut (moderate negative correlation, $r=-0.51$ ), and with relative content in other families at the $2^{\text {nd }}$ 
cut (moderate positive correlation, $r=0.51$ ). A scatterplot of the 48 observations ( 16 sampling points at 3 sampling moments) for each of the botanical composition variables according to $\mathrm{EC}_{\mathrm{a}}$ (Figure 8) clearly shows that $\mathrm{EC}_{\mathrm{a}}$ in the study area varied in two separate ranges: $\mathrm{EC}_{\mathrm{a}}$ lower than $25 \mathrm{mS} / \mathrm{m}$ ("low" $\mathrm{EC}_{\mathrm{a}}$ ), and $\mathrm{EC}_{\mathrm{a}}$ higher than $65 \mathrm{mS} / \mathrm{m}$ ("high" ECa). Although Pearson's r-values were only statistically significant for relative content in legumes, regression lines seem to point to lower relative contents in grasses and legumes, and higher content in other families, in the areas where $\mathrm{EC}_{\mathrm{a}}$ was higher than $65 \mathrm{mS} / \mathrm{m}$. This may also support the correlation between NDVI and $\mathrm{EC}_{\mathrm{a}}$ at the $2^{\text {nd }}$ cut, as has been previously discussed.

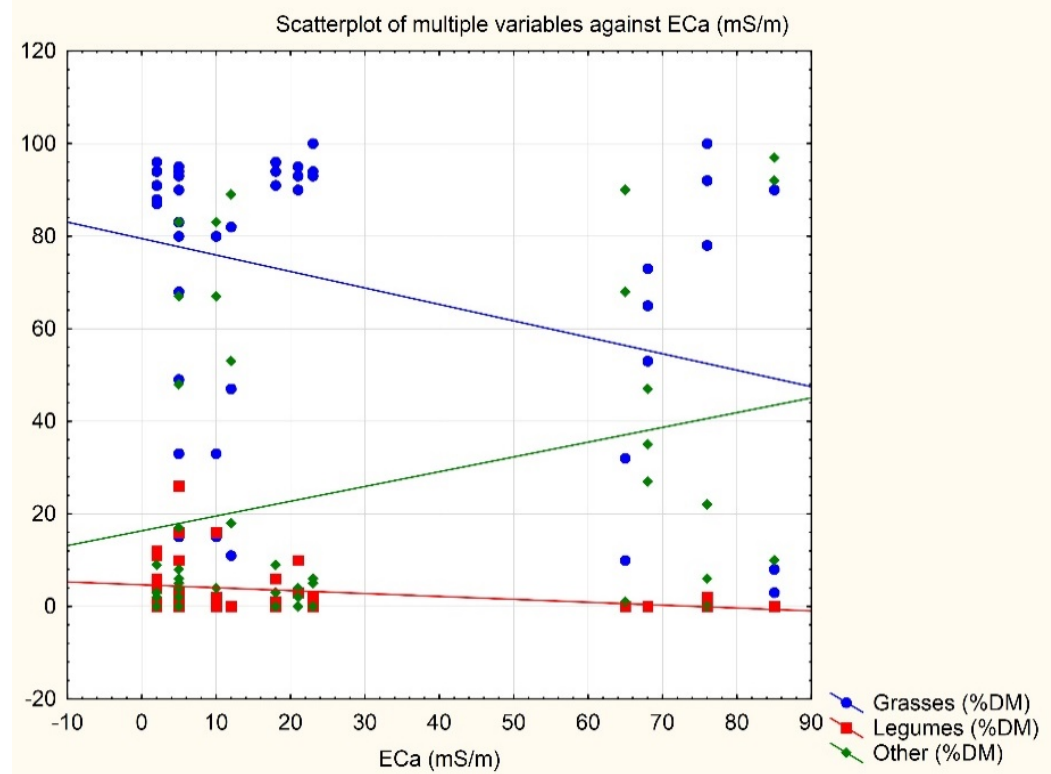

Figure 8. Scatterplot of botanical composition variables against $\mathrm{EC}_{\mathrm{a}}$ considering the 3 sampling moments and 16 sampling points.

Considering all these results, a multivariate model was used to assess the influence of factors: sampling moment, herbicide use and $\mathrm{EC}_{\mathrm{a}}$ class (high or low) over fresh and dry matter yields, dry matter content, botanical composition variables and NDVI. The multivariate tests of significance showed significant influence of sampling moment and herbicide use $(\mathrm{p}<0.001)$, whereas $\mathrm{EC}_{\mathrm{a}}$ Class showed no significant effect in the model.

Univariate results showed a significant influence of sampling moment over fresh and dry matter yield, dry matter content and botanical composition variables, a significant influence of herbicide use over the relative contents in grasses and other families, and a significant influence of $\mathrm{EC}_{\mathrm{a}}$ over relative content in legumes and NDVI. Post hoc results for significant influence of the three factors are presented on Table 5.

Table 5. Mean values and post hoc results (Tukey's test) for the different factors in the linear model.

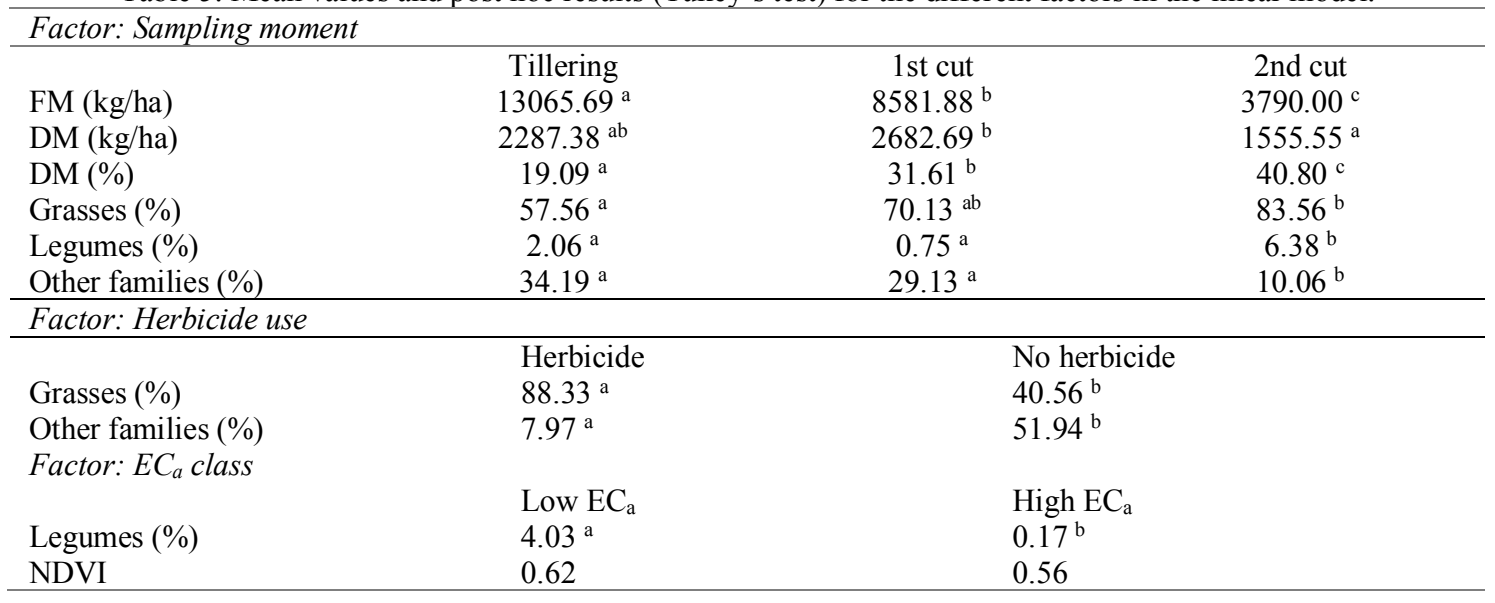

NOTE: Different letters mean significantly different means $(\mathrm{p}<0.05)$. 
The influence of sampling moment on fresh and dry matter yields is expected, as well as on dry matter content, as it reflects the plants' development cycles. The botanical composition differences along the three sampling moments can be justified by different growth rates and regrowth capacity of seeded plants, when compared to those of weeds (other families). Herbicide use had a noticeable influence on relative content of weeds in the crop, hence conditioning grasses content, by families' competition. Relative overall content in legumes was unexpectedly low, and even more so in areas with high $\mathrm{EC}_{\mathrm{a}}$, pointing to an important influence of soil physical and chemical properties on legumes development. Plant families composition concerning legume percentage was different $(\mathrm{p}=0.0039)$ between these two areas, even though no statistical differences were found regarding grasses and other families. Despite the model's univariate results showing a significant influence of $\mathrm{EC}_{\mathrm{a}}$ class on NDVI, mean NDVI values for low and high $\mathrm{EC}_{\mathrm{a}}$ areas were not significantly different, according to post hoc Tukey's test.

\section{Conclusions}

In this study, data from a soil sensor for electrical conductivity and remote sensing were used to delineate site-specific management and crop monitoring. Two different areas were identified considering soil $\mathrm{EC}_{\mathrm{a}}$ and soil textures as well as the need of herbicide at the sowing moment. Total DM yield was probably affected by the lack of an appropriate water management at crop's tillering stage. Taking into account crop sample moments and families composition, there were significant correlations of $\mathrm{EC}_{\mathrm{a}}$ with relative content in legumes at the tillering phase and at crop $2^{\text {nd }}$ cut as well as with the relative content in other families at crop's $2^{\text {nd }}$ cut. Along the crop cycle, a strong positive correlation was found between NDVI and fresh matter yield at tillering phase, becoming lower at the $1^{\text {st }}$ cut and lost at the $2^{\text {nd }}$ cut.

In order to ensure this management approach, it is of most importance to confirm these preliminary results in the next trials.

\section{Acknowledgements}

This work was funded by IPP - Polytechnic Institute of Portalegre and ICAAM - Institute of Agrarian and Environmental Sciences of the University of Évora. The authors would like to express their gratitude to Willem Carp for UAV remote sensing service, Ana Barradas, Ana Lucia Rodrigues, Nuno Henriques and José Pais for their support in crop management.

\section{References}

Basso, B., 2003. Perspectives of precision agriculture in conservation agriculture. In Garcia Torres, L, Benites, J., Martinez-Vilela, A., Holgado-Cabrera, A. (Eds), Conservation agriculture: Environment, farmers experiences, innovations, Socio economy, Policy. Springer Netherlands, 281-288.

Bueno, J., Amiama, C., Hernanz, J.L., 2007. No-tillage drilling of Italian ryegrass (Lolium multiflorum L.): Crop residue effects, yields and economic benefits. Soil and Tillage Research, Volume 95, Issues 1-2: 61-68, doi: 10.1016/j.still.2006.11.002.

Cambardella, C.A., Moorman, T.B., Novak, J.M., Parkin, T.B., Karlen, D.L., Turco, R.F., Konopka, A.E., 1994. Field-scale variability of soil properties in Central Iowa soils. Soil Sci. Soc. Am. J. 58, $1501-1511$.

Cardoso, J.C., 1974. A classificação dos solos de Portugal. Portuguese soil classification. Nova versão Boletim de solos do S.R.O.A, 17:14-46.

Chastain, T.G., Garbacik, C.J., Young, W.C., 2017. Tillage and establishment system effects on annual ryegrass seed crops. Field Crops Research, Volume 209: 144-150, doi: 10.1016/j.fcr.2017.04.017.

Crespo, D., 2015. Portugal: Um País de Solos diversos mas pobres - Papel das Pastagens biodiversas na sua recuperação e uso Sustentável. Portugal: a country of diverse but poor soils - The role of biodiversed pastures in their recovery and sustainable use. Colóquio "Biodiversidade dos Solos". INIAV. Oeiras. 34pp

Doolittle, J.A., Brevik, E.C., 2014. The use of electromagnetic induction techniques in soils studies. Geoderma, 223-225, 33-45.

Frampton, W.J., Dash, J., Watmough, G., Milton, E.J., 2013. Evaluating the capabilities of Sentinel-2 for quantitative estimation of biophysical variables in vegetation. ISPRS Journal of Photogrammetry and Remote Sensing 82 (2013) 83-92. https://doi.org/10.1016/j.isprsjprs.2013.04.007

Freixial, R., Carvalho, M., 2013. A agricultura de conservação e a sementeira directa em pastagens. (Conservation agriculture and no-till in pastures). Revista Vida Rural, Abril, 2013, 32-34.

Gitelson, A.A., Viña, A., Verma, S.B., Runquist, D.C., Arkebauer, T.J.,Keydan, G., Leavitt, B.,Ciganda, V., Burba, G.G., Suyker A.E., 2006. Relationship between gross primary production and chlorophyll content in crops: Implications for the synoptic monitoring of vegetation productivity. J. Geophys. Res., 111: D08S11

Govaerts, B., Sayre, K.D., Goudeseune, B., De Corte, P., Lichter, K., 2009. Conservation agriculture as a sustainable option for the central Mexican highlands. Soil \& Tillage Research, 103, 222-230. 
Hogrefe, K.R., Patil, V.P., Ruthrauff, D.R., Meixell, B.W., Budde, M.E., Hupp, J.W., Ward, D.H., 2017. Normalized Difference Vegetation Index as an Estimator for Abundance and Quality of Avian Herbivore Forage in Arctic Alaska. Remote Sensing, 9, 1234; doi: 10.3390/rs9121234.

Kertész, A., Madarász, B., 2014. Conservation Agriculture in Europe. International Soil and Water Conservation Research, Vol. 2, No. 1, 2014, 91-96.

Lancashire, P.D., Bleiholder, H., Langeluddecke, P., Stauss, R., van den Boom, T., Weber, E., Witzen-Berger, A., 1991. A uniform decimal code for growth stages of crops and weeds. Ann. Appl. Biol. 119 (3): 561601. doi:10.1111/j.1744-7348.1991.tb04895.x

Lund, E.D., Colin, P.E., Christy, C., Drummond, P.E., 1999. Applying soil electrical conductivity technology to precision agriculture. In: International Conference on Precision Agriculture, 4, 1998, Madison. Proceedings Madison: ASA-CSSA-SSSA: 1089-1100.

Marques da Silva, J.R., Peça, J.O., Serrano, J.M., Carvalho, M., Palma, P., 2008. Evaluation of spatial and temporal variability of pasture based on topography and the quality of the rainy season. Precision Agric (2008) 9: 209. https://doi.org/10.1007/s11119-008-9066-0

Rouse, J.W., Haas, R.H., Schell, J.A., Deering, D.W., 1974. Monitoring vegetation systems in the Great Plains with ERTS, In: S.C. Freden, E.P. Mercanti, and M. Becker (eds) Third Earth Resources Technology Satellite-1 Syposium. Volume I: Technical Presentations, NASA SP-351, NASA, Washington, D.C., pp. 309-317.

Serrano, J., Shaidian, S., Da Silva, J.M., 2014. Spatial and Temporal Patterns of Apparent Electrical Conductivity: DUALEM vs. Veris Sensors for Monitoring Soil Properties. Sensors, 14 (6), 10024-10041; doi: 10.3390/s140610024

Soane, B.D., Ball, B.C., Arvidsson, J., Basch, G., Moreno, F., Roger-Estrade, J., 2012. No-till in northern, western and south-western Europe: A review of problems and opportunities for crop production and the environment. Soil \& Tillage Research, 118, $66-87$.

Sousa, A.M.O., Gonçalves, A.C., Da Silva, J.R.,2017. Above-Ground Biomass Estimation with High Spatial Resolution Satellite Images. In Biomass Volume Estimation and Valorization for Energy: Intech Eds. 47-70, doi: $10.5772 / 62678$

Stadler, A., Rudolph, S., Kupischa, M., Langensiepena, M., van der Krukb, J., Ewert, F., 2015. Quantifying the effects of soil variability on crop growth using apparent soil electrical conductivity measurements. Europ. J. Agronomy 64 (2015) 8-20, doi: 10.1016/j.eja.2014.12.004

Stafford, J.V., 2000. Implementing precision agriculture in the 21st century. Journal of Agricultural Engineering Research, 76, 267-275. 


\title{
The Electronic Smell of the Orchard Fruit
}

\author{
João Valente*, Rodrigo Almeida, and Lammert Kooistra \\ Laboratory of Geo-Information Science and Remote Sensing, Wageningen University, Wageningen 6708 PB, Netherlands \\ * Corresponding author. Email: joao.valente@wur.nl
}

\begin{abstract}
Assessing fruit maturity at the end of the season is a hard task for fruit growers. This task is either made by visual inspection - which is tedious and time consuming - or using destructive procedures for measuring biophysical properties of the fruits, such as the sugar content. An alternative to measure the ripeness of fruits is measuring the volatile organic compounds emitted by the fruits. An important compound produced by the fruits in this stage is ethylene $\left(\mathrm{C}_{2} \mathrm{H}_{4}\right)$. The recent advances in electrochemical semiconductors have enabled the rapid growth of electronic noses technologies and applications. Nevertheless, the research reported where its characteristics and limitations are explored only addresses experiments in controlled and indoor settings. Therefore, many questions remain regarding the electronic noses applicability in outdoor environments. This work presents preliminary evidences that there are good chances that ethylene can be detected outdoors via an electronic nose placed within an orchard field. The results presented are measurements acquired in a Conference pear (Pyrus communis) orchard in September 2017. The measurements where acquired on several points within the field, and the maximum ethylene detection shows an increase of $10 \%$ over 400 seconds. These results were contrasted with a theoretical study where gas dispersion patterns can be appreciated when subject to the wind speeds recorded in the field. The simulation results indicated a good correlation between the practical and the theoretical simulation results. To the best of our knowledge this work is the first to report results from measurements using electronic noses in a non-controlled environment, and detecting spatial-temporal variability of natural gas sources.
\end{abstract}

Keywords: Electronic nose, Fruit orchard management, Fruit maturity, Ethylene detection, Uncontrolled setups

\section{Introduction}

The worldwide population is increasing so fast that we will not be able to produce food to everyone. This unbalance chain has triggered the alarms from industrials and academics. Many collaborations between the two parties have been carried out over the last years where the aim is to find agrotechnologic solutions that aim to increase food production and preserve its natural quality.

For farmers, to determine the best time for harvesting is key. This time must take into consideration some marketing flexibility, i.e. shelf-life, and reaching a sufficient quality for the consumer. While harvesting immature fruits results in poor quality when ripe and higher susceptibility to mechanical damage, harvesting overripe fruits results in soft and flavourless product, with a very short shelf-life (Kader 1997).

To develop an effective marketing strategy and to increase the efficiency of the use of labour and resources, maturity indices exist to support farmer's decision making. These indices should in theory be simple enough to be performed by farmers, fruit handlers and quality control personnel. Moreover, the samples should be easy to acquire in the field and in inspection points, be objective, i.e., a measurement instead of a subjective evaluation, and be non-destructive.

As fruit matures and ripens, several Volatile Organic Compounds (VOC) are synthesized and emitted by fruits which yield the characteristic aroma of the fruit. In Baietto and Wilson (2015) several fruit aroma characteristic VOC are described. The aroma, as perceived by the human nose is a very simple maturity assessment but as with visual inspection, is a very subjective method. The VOC can also be detected using digital sensors, electronic noses or gas sensors, that can measure the concentration of a certain VOC or a mixture of different VOC and with that infer on the maturity of the fruit.

The first non-destructive attempt for monitoring fruit ripeness using electronic noses is reported in the work from Brezmes et al. (2000). The authors proposed an alternative method in a controlled setup that skip the conventional fruit decomposition. In the following years, several other attempts using similar approaches have been reported (e.g., Gomez et al. 2006, Esser et al. 2012, Ma et al. 2016). Those studies demonstrate that it's possible to classify fruit maturity at several levels, e.g., unripe, half-ripe, ripe, and overripe., using electronic noses, but in a controlled setup through a chamber. To the best of our knowledge, there is no evidence of any attempt for sensing ethylene concentrations within an uncontrolled facility like a fruit orchard field.

The overall goal of this works is to investigate the potential use of electronic noses for inferring the fruit maturity of fruit orchards: 1) Design a low cost and easily replicable ethylene sensor system; 2) Define an experimental setup for testing it within an uncontrolled environment; 3) Substantiate the practical results with a theoretical model; and 4) Discuss the experimental setup re-design. 


\section{Materials and Methods}

Experimental setup

The first measurements performed with the sensor prototype were conducted in Houten in a commercial producer farm, applying a ground sampling methodology. The goal here was to check whether the sensor was capturing some signal and where hand held measurements in the ground, at breast height and in the line, were suitable for detecting ethylene concentrations.

The experiments were carried out in the very morning of the day that the pears were found ready to be harvested by experts and so where there was more probability to detect ethylene. A set of nine points were defined. Seven points where place equidistantly (each 5 meters) in the same row and two other points where placed equidistantly in adjacent rows on west. The measurements where obtained following the points and positions within the points order, i.e., 1-Ground, 2-Breast height (at 1.65 meters above the ground), 3-In line (in the branches). The experimental field and sample locations are displayed in Figure 1.
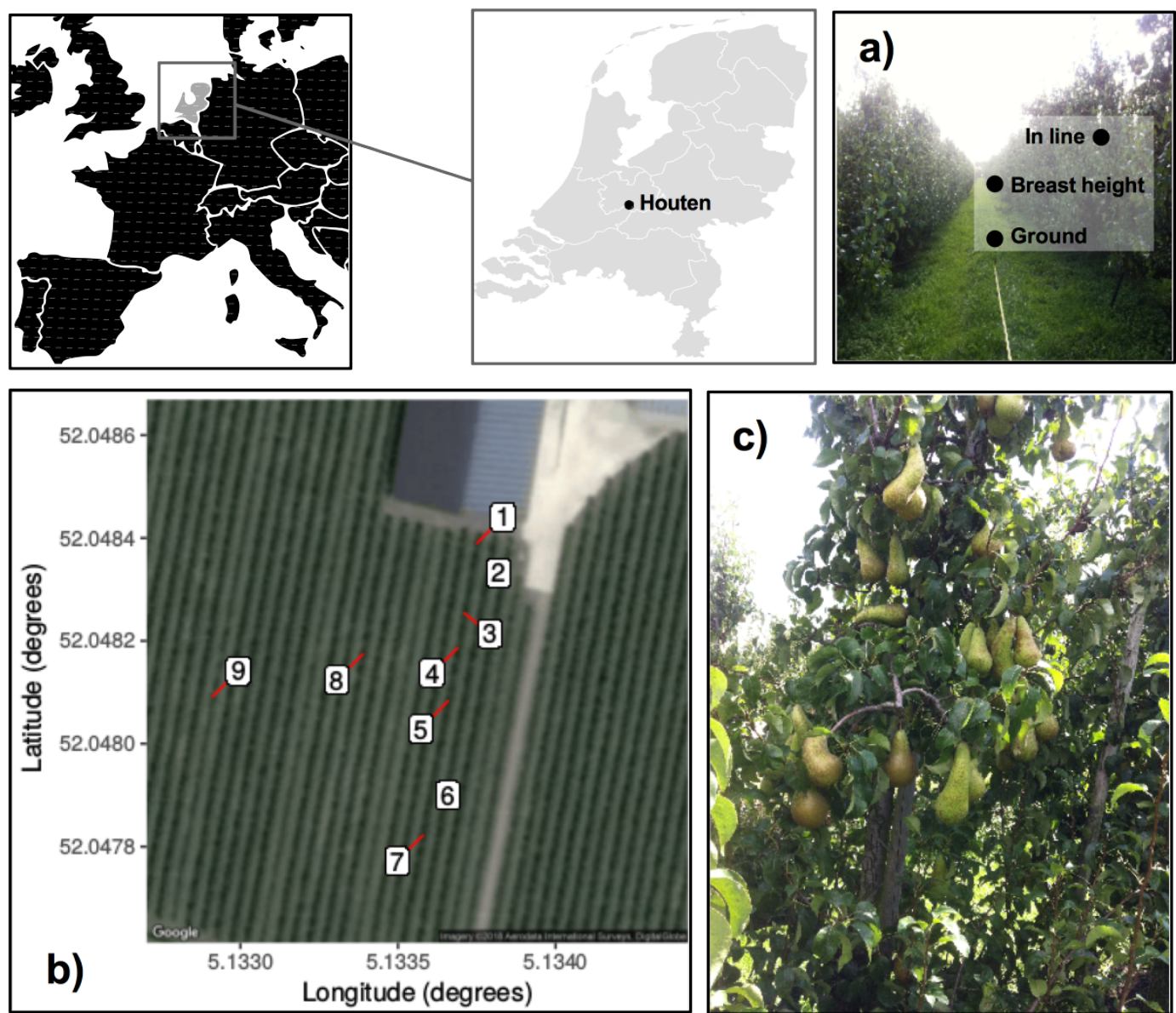

Figure 1. Experiment carrying out on September $12^{\text {th }}, 2017$ on the harvest day: a) Field measurement heights in each point; b) Geo-reference position from the field points; and c) Conference pear at the harvest time.

Low-cost ethylene sensing system

A prototype ethylene sensor has been developed based in an electrochemical gas sensors, also referred as a Taguchi gas sensors (TGS) and more often electronic nose (e-nose). The prototype, as illustrated in Figure 2 has four main components: 1) Arduino UNO; 2) Adafruit Assembled Data Logging shield for Arduino; 3) Winsen ME4-C2H4 electrochemical gas sensor; and 4) Power source.

The electronic nose sensor specification sheet reports a sensing range of 0 to $100 \mathrm{ppm}$ of $\mathrm{C} 2 \mathrm{H} 4$ and a response (amount of time it takes the sensor to detect the presence of ethylene) and recovery time of 100s. The sensor prototype is equipped with a memory card when the device is on, with a configurable measurement frequency, measurements are recorded with the respective time-stamp and output signal from the sensor. In these experiments one measurement per second $(1 \mathrm{~Hz})$ was set as the measurement frequency.

The overall prototype working current is less than $100 \mathrm{~mA}$. The autonomy using a 9 volts' battery is less than 4 hours, while with a $1400 \mathrm{mAh}$ LiPo battery we can increase autonomy up to 14 hours. 

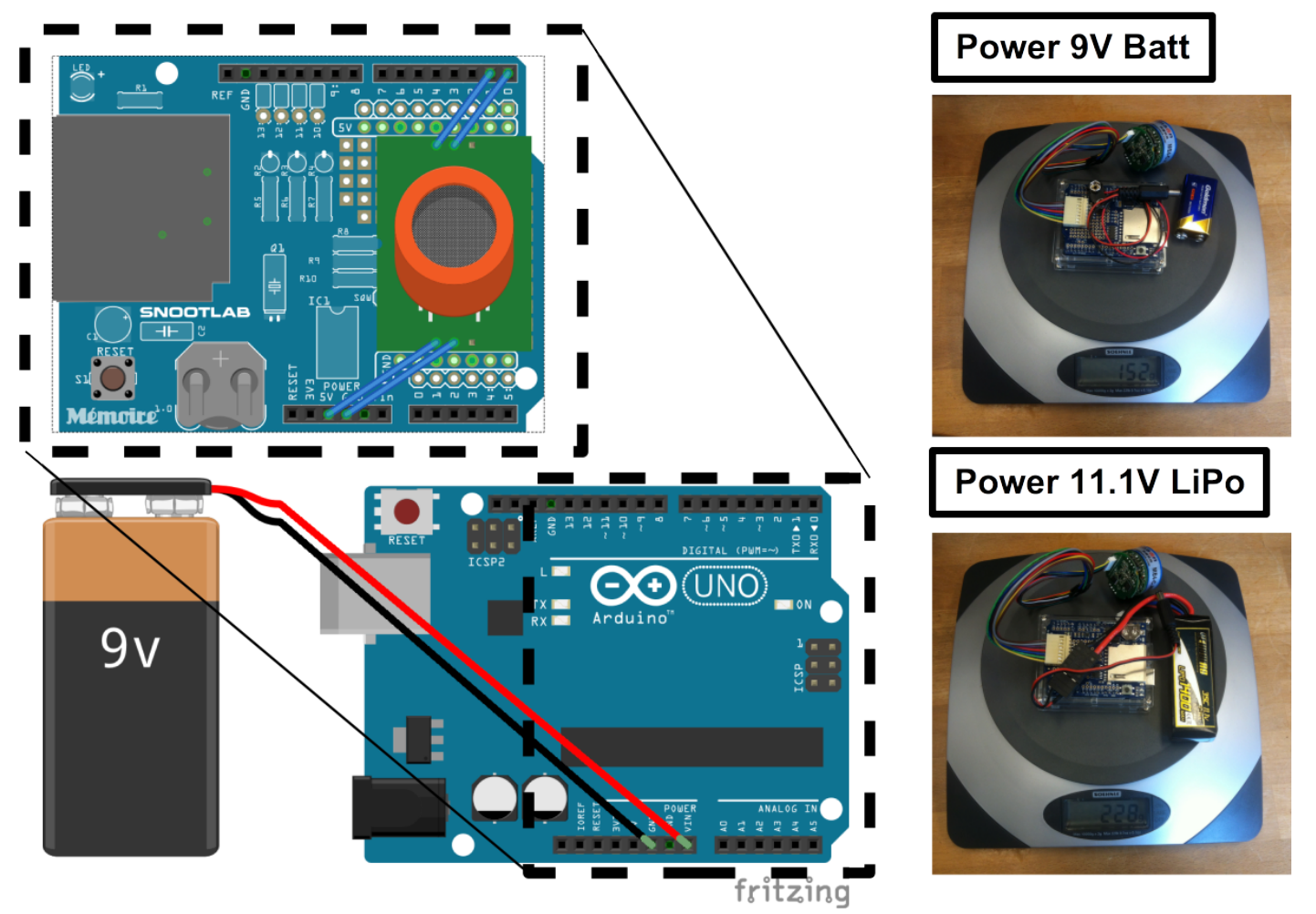

Figure 2. First prototype of the low-cost sensor prototype for detecting ethylene: The assemblage schematic using Arduino-based electronic boards; and final prototype with different power sources (rechargeable and non-rechargeable).

\section{Results and discussion}

Measured ethylene detections

The average wind speed during those measurements was of about $1.3 \mathrm{~ms}^{-1}$ (west), measured with a hand-held anemometer. The ambient temperature was around the $19^{\circ} \mathrm{C}$.

Before starting the measurements, the system was turned on half an hour before to ensure the sensor is at the steady state during the measurements. The sampling time in each point was from 4-15 minutes, where the goals was to sample each position from 4-5 minutes.

The data acquired with the electronic nose sensing system is shown in Figure 3. This result seemed to show promise since at the time of the measurements, the harvest was under way and the measurements obtained suggest the absence of fruits in a fraction of the field. The picking started in the early morning going from the most east tree row until the latest row on the west. In the first measurements - point 1 to point 6 - some ethylene is detected, especially in the first point, where the sensor output reaches the maximum in this measurement set. In the vicinity of Point 6 to 9, which confirm the hypothesis that there was no signal because no fruits were on the tree since that area was already harvested.

The measurements from the three first points from the last row show stronger detection signals and special in point 1 , where the signal shows an increase of $10 \%$ over 400 seconds. The difference of magnitudes between the sensors might explain because the first point was more protected from the wind because of the shed that was close to the row, or due to the shed itself since is a fruit storage facility.

Furthermore, a brief qualitative evaluation was performed considering three characteristics that were important to analyse: Settling time; detection time; and variance. The settled time is defined as the amount of time that the signal is settled (continuous); the detection time is defined as how long it takes for the first detection; and the variance regards to the peaks frequency in the signal. The results suggest that the ethylene concentrations are higher on the ground, and also at the breast height. 

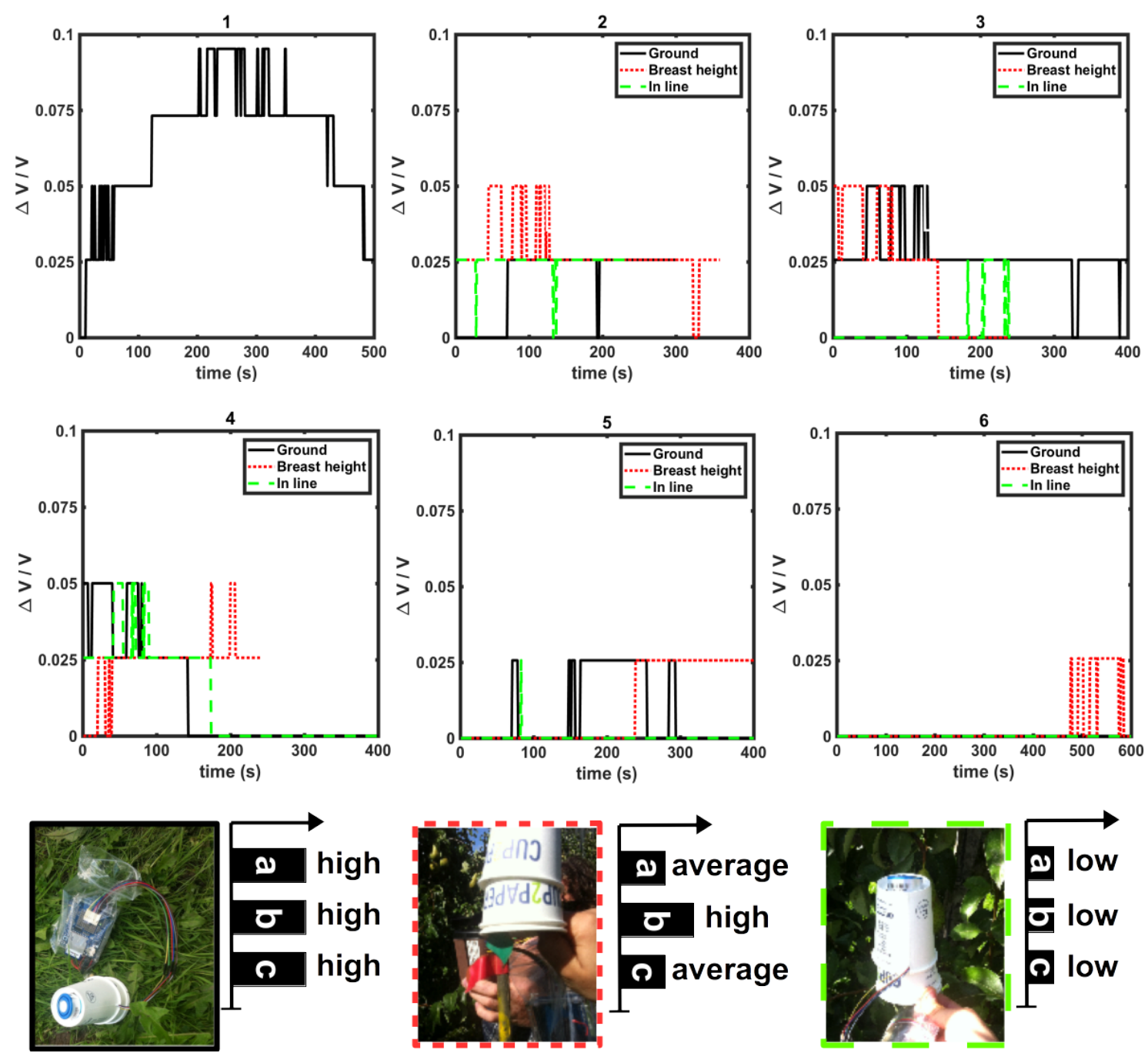

Figure 3. Ethylene measurements from point 1 to 6 at the different positions within the field. The qualitative assessment of the performance o: a-Settling time; b-detection time; and c) c-Peaks frequency.

Simulated ethylene emissions subject to wind speed

The experiments shown in last section shown that the sensor can detect the presence of fruit, the ethylene emission source, although it's hard to draw conclusion about the amount of ethylene concentration measured. The detection and no detection of ethylene are not enough to infer on the pears maturity.

To obtain a model that could provide an estimation for the optimal harvest time we need a more descriptive signature from the ethylene concentrations on the field. There is a hypothesis that the environment changes like the wind during the measurements could had affected the sensor sensibility. Although the average during the measurements was about $1.3 \mathrm{~ms}^{-1}$, a maximum wind speed from $3.3 \mathrm{~ms}^{-1}$ was registered.

The dispersion of the ethylene was studied when subject to wind through a modelling and simulation approach using the as parameters the physical properties from the orchard and from the fruits. For that purpose, the GADEN gas dispersion simulation framework was employed. GADEN is a gas dispersion simulation framework that not only emulates the gas dispersion on a three-dimensional space but also considers obstacles and air flow dynamics (Monroy et al., 2017).

In the orchard model four different zones (volumes) have been defined: Environment (it compresses all the simulated area); Main volume (bounding area around the overall modelled field); In rows (bounding volume corresponding to the orchard rows); and In-between rows (bounding volume corresponding to the orchard inbetween rows).

The impact of wind speed in the average ethylene concentration is summed up in Figure 4. The results indicate clearly that the ethylene concentration is higher when not subject to wind speeds higher than $1 \mathrm{~ms}^{-1}$ and that concentration values drops significantly between $1-2 \mathrm{~ms}^{-1}$. For an increase of $1 \mathrm{~ms}^{-1}$ we get between $67 \%-92 \%$ decrease in the average ethylene detection. 


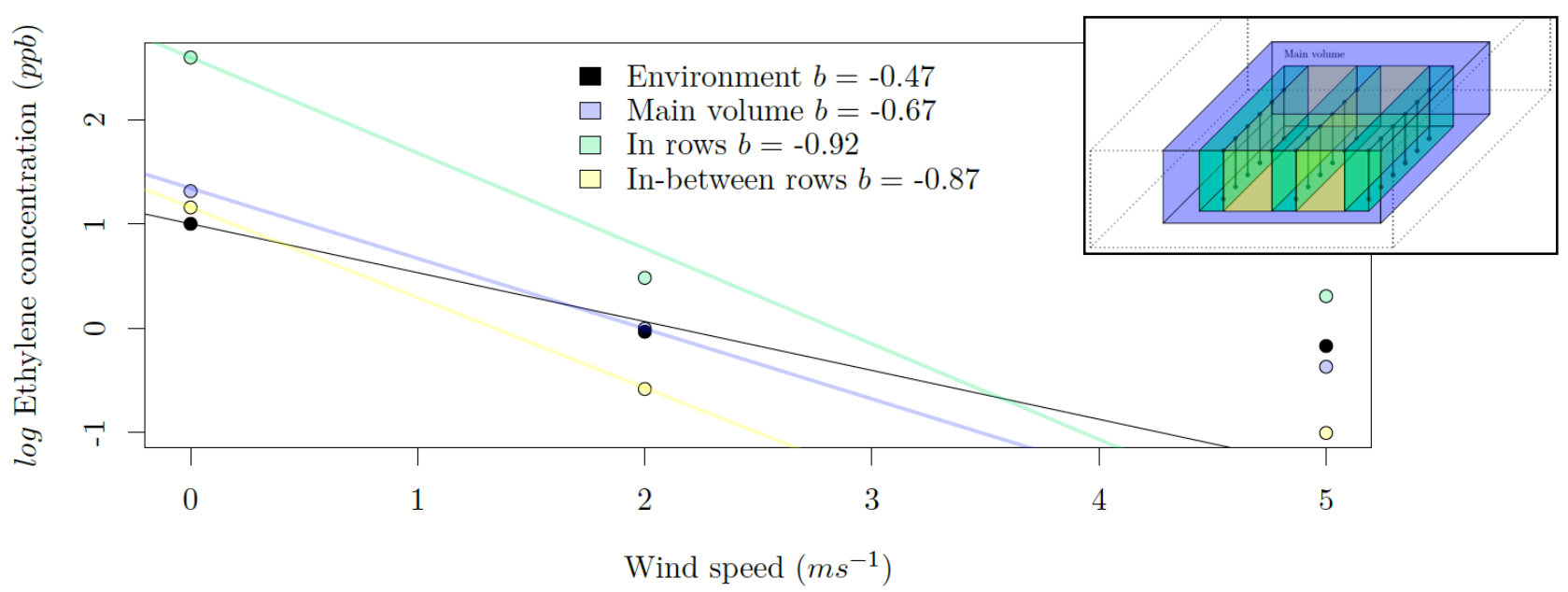

Figure 4. Relation between wind speed and average ethylene concentration in the 4 different zones within the model orchard. The coloured lines represent the trend line for each zone, as given by the equation $y=a+b x$ where $b$ is the decrease in $\log (\mathrm{ppb})$ per additional unit of wind speed $\left(\mathrm{ms}^{-1}\right)$.

The results reported in the previous section are a starting point to carry out further experiments that can yield more meaningful conclusions. What is clear from the field measurements performed is that detecting and measuring ethylene in an uncontrolled environment is a difficult challenge. Furthermore, several lessons were learned regarding to the instrumentation used and the environment conditions:

Sensitivity: In these experiments only one type of sensors was used. It's important to provide from other ethylene gas sensors to exclude all the hypothesis of mal functioning of the current sensors system and have additional ground truth. Look for more sensible sensors if available. Introducing the sensor in an air inletoutlet camera following the hypothesis that might help to increase the sensor sensibility.

Sampling: The sampling scheme must be upgraded. It is important to have system that is able to increase effective sampling area and the sampling frequency. The sampling time should also be increase to the order of hours. The time series must take longer to increase the probability of detection and to get more informative signals.

Robustness: The sensor system must be robust enough to record data in reliable way. The developing of a new prototype weather proofed should be considered. Important to protect and increase the lifetime of the system. Additionally, the design should be oriented to portability and to be easy to be use in the field.

Environment condition: Wind conditions in the orchard are an important variable in the ethylene concentration dynamics within the orchard, but other variable such like humidity and temperature most probably increase the uncertainty from the measurements. Therefore, in next experiment is important to monitor those variable during the experiment and have it into account in each sample.

Calibration: The raw data outputted from the sensor is not quantifiable until a proper calibration is done. It's important to define a zero-measurement reference in order to understand more about the magnitude from the measurements. This should be achieved in next trial in a controlled environment with an ethylene gas source.

\section{Conclusions}

This paper report what might be the first tentative to measure ethylene emissions in a ripped fruit orchard by an electrochemical sensor. Although, the concentrations registered were not enough to infer in the fruit maturity, there is a good indication that ripe and not ripe fruit areas are detectable within the field.

Moreover, a theoretical model from the orchard was provided to understand how the wind speed could affect the ethylene concentrations and thus the detection. The simulations show that to carry out further research and ensure reliable detection signatures it's important to avoid wind in the target environment.

Finally, a set of major challenges in measuring ethylene in an uncontrolled environment are identified to continue with this study. 


\section{Acknowledgements}

This work was supported by the SPECTORS project (143081) which is funded by the European cooperation program INTERREG Deutschland-Nederland. The authors would also like to thank to Fruitbedrijf G.H.J. Jongerius BV for providing the field test and for the fruitful discussions about pears orchards.

\section{References}

Baietto, M. and Wilson, A., 2015. Electronic-nose applications for fruit identification, ripeness and quality grading. Sensors 15(1):899-931.

Brezmes, J., Llobet, E., Vilanova, X., Saiz, g., and Correig, X., 2000. Fruit ripeness monitoring using an electronic nose. Sensors and Actuators B: Chemical, 69(3):223 - 229.

Esser, B., Schnorr, M., and Swager, T. M., 2012. Selective detection of ethylene gas using carbon nanotubesbased devices: Utility in determination of fruit ripeness. Angewandte Chemie International Edition, 51(23):57525756.

Gómez, A. H., Hu, G., Wang, J., and Pereira A, G., 2006. Evaluation of tomato maturity by electronic nose. Computers and Electronics in Agriculture, 54(1):44-52.

Ma, L., Wang, L., Chen, R., Chang, K., Wang, S., Hu, X., Sun, X., Lu, Z., Sun, H., Guo, Q., et al., 2016. A low cost compact measurement system constructed using a smart electrochemical sensor for the real-time discrimination of fruit ripening. Sensors, 16(4):501.

Monroy, J., Hernandez-Bennets, V., Fan, H., Lilienthal, A., and Gonzalez-Jimenez, J. (2017). Gaden: A 3d gas dispersion simulator for mobile robot olfaction in realistic environments. Sensors, 17(7):1479.

Kader, A. A., 1997. Fruit maturity, ripening, and quality relationships. In: International Symposium Effect of Pre-\& Postharvest factors in Fruit Storage 485:203-208. 


\title{
Soil Organic Carbon Estimation using Proximal and Remote Sensing Techniques
}

\author{
Theodora Angelopoulou ${ }^{\text {a, b, * }}$, Athanasios T. Balafoutis ${ }^{\text {a }}$, Dionysios Bochtis ${ }^{\text {a }}$, George Zalidis ${ }^{\text {b,3 }}$ \\ ${ }^{a}$ Centre of Research and Technology - Hellas (CERTH), Institute for Bio - economy and Agri - Technology (iBO), \\ Thessaloniki, Thermi 57001, Greece \\ ${ }^{\mathrm{b}}$ Laboratory of Remote Sensing, Spectroscopy, and GIS, Department of Agriculture, Aristotle University of \\ Thessaloniki, Thessaloniki, 54124, Greece \\ ${ }^{\mathrm{c}}$ Interbalkan Environment Center (i-BEC), 18 Loutron Str., Lagadas 57200, Greece \\ * Corresponding author. Email: d.angelopoulou@certh.gr
}

\begin{abstract}
Soil Organic Carbon (SOC) is considered as one of the most important factors characterizing soils' quality and health and consequently affecting agricultural productivity. Additionally, soil is the second largest $\mathrm{C}$ pool with an estimated storage capacity of $1500 \mathrm{Pg} \mathrm{C}$. Since soil is not a static reservoir, SOC estimation is of great use to ensure adequate agricultural production combined with sustainable soil management practices for climate change adaptation and mitigation. However, even if laboratory analytical methods for SOC content determination are considered reliable in individual field level, such methods cannot be used for large area scale measurements. To that end, more efficient, rapid, non-destructive and cost-effective estimations need to be developed and assessed. The challenges to measure and monitor SOC led to the need of (i) revised methodologies, (ii) updated remote and proximal sensing methods and (iii) simplified field information that could be used for SOC estimation, since there is still lack of a common measuring protocol with mutual acceptable results. The availability of hyperspectral data has led the scientific community to make a turn towards remote and proximal sensing techniques for estimating soil parameters. Hence, the aim of this paper is to review the studies that used remote and proximal sensing techniques for SOC estimation, highlighting the advantages and limitations of each approach. The focus is on hyperspectral sensors i.e. satellite, airborne and UAV based, and proximal sensing techniques in the visible and near infrared (vis - NIR) regions in laboratory and in situ conditions.
\end{abstract}

Keywords: soil reflectance spectroscopy, satellites, airborne, in-situ, laboratory, vis-NIR

\section{Introduction}

Factors like climate change, land degradation and biodiversity loss have led soils to become one of the world's most vulnerable resources (FAO, 2017). Soil Organic Matter (SOM) is of great importance as it affects the physical, chemical and biological properties of soil, ensuring its proper function by improving its structure and increasing water and nutrient retention (Ontl and Schulte, 2012). SOC is part of the C cycle and stores about 1500 Pg C, being the second largest C pool (Jobbágy and Jackson, 2000). It plays an important role in climate change mitigation and therefore needs to be estimated in large scales (Crowther et al. 2016). Soil is a complex mixture with large variability from site to site or even within the same field thus, identifying its components is a laborious but essential procedure. Precision agriculture aims at sensing such variability for more efficient and site-specific applications of fertilizers, water, manure and other inputs (Mulla, 2013).

Monitoring of SOC stock in a systematic basis requires the use of a rapid and cost-effective method that can be applied in different soil types. Consequently, analysing SOC by a single method that can be applied in diverse circumstances remains a great challenge since SOC is not evenly distributed over large areas, depths, soil types and landscape positions (Gehl et al., 2007). To that end, a variety of methods to measure and assess SOC dynamics have been developed, but up to date, there is no standardized approach to measure total soil carbon concentration (Davis et al., 2017). Remote and proximal sensing techniques are now widely used and evaluated to that purpose. The main principal of these techniques lay on energy-matter interactions. The electromagnetic radiation radiated on a specific material can be either reflected or absorbed in specific wavelengths, consequently the reflected radiation of a material is a function of wavelength, surface material absorption, emissivity and reflectance characteristics (Stuart, 2004) Vis-NIR spectroscopy is based on characteristic vibrations of chemical bonds in molecules. Specifically, in the Vis (400-700nm) region electronic transitions occur, while in the NIR (700$2500 \mathrm{~nm}$ ) weak overtones and combinations of these vibrations occur due to stretching and bending of the $\mathrm{N}-\mathrm{H}$, O-H, and C-H bonds (R.A. Viscarra Rossel et al., 2006). Hence, energy matter interactions in the vis - NIR regions could be used for qualitative and quantitative analysis of soil properties. Stark et al. (1986) introduced Near Infrared Analysis (NIRA) in an attempt to make this technique more known to researchers. Ben-Dor and Banin (1995) studied laboratory NIRA to evaluate its potential for predicting soil properties and found that at the regions 1400-1900 nm the $\mathrm{OH}$ groups have strong absorption features mainly due to water content in the soil. SOC generally affects spectral reflectance in the visible region, mainly at 550-700nm due to change in soil colour, as the concentration increases the soil appears darker (Ladoni et al., 2010) and narrow peaks between 2100-2300nm (Castaldi et al., 2018). 
The aim of this study was to review the recent advances (last decade) in proximal and remote sensing technologies using the visible and near infrared region and highlight the problems and needs towards SOC estimations.

\section{Material and methods}

\section{Sensor Types}

Different types of sensors can be mounted on satellites, aircrafts and Unmanned Aerial Vehicles (UAVs) i.e. remote sensing, or on tractors/vehicles and handheld i.e. proximal sensing. The main two types of sensors are: i) passive sensors that are instruments designed to receive and measure natural emissions produced by Earth's surface and atmosphere and ii) active sensors which are radar instruments used for measuring signals transmitted by the sensor that were reflected, refracted or scattered by the Earth's surface or its atmosphere (Grunwald et al., 2015). Sensors are also characterized by their spatial, spectral, temporal and radiometric resolution. Spatial resolution specifies the pixel size of the acquired images covering the earth surface (Atkinson and Aplin, 2004). High spatial resolution could be from $0.31-4.0 \mathrm{~m}$ i.e. WorldView-4, Pleiades-1B, to few centimeters for UAVs and ground proximal sensors. Low spatial resolution ie Hyperion $(30 \mathrm{~m})$ refers to large pixel size that could eventually lead to mixed pixels i.e. bare soils mixed with vegetation. The temporal resolution refers to the frequency a sensor can make a measurement in the same area to assess temporal patterns (Turner et al., 2003). For satellites ranges between high temporal resolution ( 24 hours -3 days) to low temporal resolution of 16 days, while for airborne, UAV and ground proximal applications is user dependable. Spectral resolution is the number of spectral bands in which the sensor can collect the reflected radiance (Goetz et al., 1985). An important information is the position of bands in the electromagnetic spectrum (microwave, infrared, visible, ultraviolet, Xray). For agricultural application and specifically for SOC estimations, it was found that the vis-NIR and mid infrared region (2500-20000nm) provide accurate predictions (England et al., 2018) Radiometric resolution describes the sensitivity of a sensor to detect small differences in reflected or emitted energy (Tucker, 1980). According to the number of spectral bands and their width there are three sensor categories: a) panchromatic band that has a wide bandwidth of a couple of hundred nanometres with high signal to noise ratio and spatial resolution, b) multispectral that has tenths of bands with narrower bandwidth (40-100nm), and c) hyperspectral that comprises of hundreds of bands hence, making them more capable of capturing more detail in spectral and spatial resolution (Shaw and Burke 2003).

\section{Proximal Sensing}

\section{Laboratory}

Scientific community for years focused on using soil reflectance spectroscopy in laboratory environment that provide more controlled conditions (Dalal and Henry, 1986; Cécillon et al., 2009). Laboratory measurements require sample preparation (i.e. drying and sieving) and after the spectroscopic measurement the use of data preprocessing techniques, model calibration and validation. To solve the calibration problem of spectral data analysis, scientists use mathematical and statistical methods also known as chemometrics (Geladi, 2003), such as. Principal Component Analysis (PCA), Partial Least Square Regression (PLSR) and more (Islam et al., 2003; Viscarra Rossel et al., 2006). The most common method used is the PLSR which is a useful approach to model linear relationships between reflectance spectra and soil attributes. However, soils reflectance behaviour suggests that PLSR may be proven insufficient for non-linear relationships (Wold et al., 2001). To that end, different calibration techniques start to be evaluated. Towards this scope, Artificial Neural Networks (ANN) (Viscarra Rossel and Behrens, 2010, Goldshleger et al., 2012), Support Vector Machines (SVM) (Wetterlind et al., 2013; Gholizadeh et al., 2015) and other data mining techniques are frequently used. Considering the selection of the calibration technique is one of the main factors influencing the prediction models Nawar et al. (2016) compared three different techniques i.e. PLSR, support vector regression (SVR), and multivariate adaptive regression splines (MARS) for clay and SOM content in salt affected soils. The best calibration technique was found to be MARS with the use of Continuum Removal as pre-processing technique that gave coefficient of determination $\mathrm{R}^{2}=0.81$, root mean square error $\mathrm{RMSE}=0.22 \%$ and residual prediction deviation $\mathrm{RPD}=2.27$. The other two techniques showed less accuracy, while PLSR gave better prediction models for SOM than SVR. The study also showed high correlation of OM and raw spectra between the 550-680nm regions. In the same context and to improve the performance of the SVMR model, Peng et al. (2014) used an appropriate wavelength selection with a successive projection algorithm (SPA). Twenty-eight wavelengths were selected by the application of the SPA algorithm being mainly in the near infrared region, probably because the stretching and bending of $\mathrm{NH}, \mathrm{OH}$ and $\mathrm{CH}$ bonds are concentrated in this region depicting high correlation with SOC content. The SPA-SVMR provided the best predictive models $\left(\mathrm{R}^{2}=0.73\right)$ in comparison with SVMR and SPA-PLSR $\left(\mathrm{R}^{2}=0.62\right)$.

Although, studies have shown the accurate estimation of SOC in the laboratory with the use of soil reflectance spectroscopy in the Vis-NIR region (Brown et al., 2006; Viscarra Rossel et al., 2006; Vohland et al., 2014), most 
reliable predictions are achieved for soil samples of the same field and soil type depicting the local character of these predictions (St. Luce et al., 2014).

However, there are several factors affecting soil spectra like soil moisture, thus, many authors tried to create prediction models of SOC for different levels of moisture (Rienzi et al., 2014; Wijewardane et al., 2016). Nocita et al. (2013) proposed a methodology by using the normalized soil moisture index (NMSI) and divided the artificially moistened samples into four classes according to that index. The PLSR modelling showed that when dry calibration models were used to predict wet samples low predictions were given (RMSE of $30.21 \mathrm{~g} \mathrm{C} \mathrm{kg}^{-1}$ ), while calibration models developed from moist samples had better accuracy. Specifically, for the higher moisture content they resulted in RMSE of $3.58 \mathrm{~g} \mathrm{C} \mathrm{kg}^{-1}$. Hence, towards the development and exploitation of national and global spectral libraries of dry soil samples there is a need to remove the effect of soil moisture when moist samples are to be measured. Direct standardization (DS) and external parameter orthogonalization (EPO) are methods that could be used to correct this effect. Roudier et al. (2017) found that the aforementioned methods gave similar results when a large number of rewetted samples were used to calibrate these corrections, while for a smaller sample size EPO outperformed DS. This indicated that spectra from soil moist samples require a correction prior to SOC modelling from Soil Spectral Libraries (SSLs). To minimize the local character of the predictions using soil reflectance spectroscopy, Stevens et al. (2013) used the LUCAS soil database (20.000 samples) that has samples from 23 member states of the European Union. By that mean the soil heterogeneity could be potentially covered as these samples represent most of soil types and SOC content in a variety of land uses. The models' performance depended on the calibration size and the variance of SOC values with the best generated models created for croplands, grasslands and mineral soil where the RMSEP ranged from $4-8.9 \mathrm{~g} \mathrm{C}$ $\mathrm{kg}^{-1}$ respectively. It was also observed that sand content affects the error of prediction, mainly in low SOC concentrations. Yet, the results showed that the use of large scale SSLs for accurate SOC predictions in local scale still needs further investigation. In order to address this problem, Lobsey et al. (2017) developed a new method for local calibration using existing SSLs called ReSampling-Local (RS-LOCAL). The aim was to minimize the collected samples or even not collect any samples for site specific calibrations. The concept of the developed algorithm was to evaluate and remove irrelevant data from existing SSLs in order to hold the most appropriate information for site specific calibrations. As a data driven method it does not consider the different soil types, analytical methods and standards resulting in better local models than those of site-specific approach using a great amount of samples. The study was conducted in two areas and the results with the use of only 20 site-specific samples were $\mathrm{R}^{2}=0.78$ and 0.84 respectively.

\section{In situ}

Advancements in precision agriculture have led to the development of in situ soil sensors for rapid, cost effective and real-time data acquisition site specific management. These technologies concern either on-the-go sensors mounted on tractors or hand-held instruments. Sinfield et al. (2010) outlined the specifications for any onthe-go sensor to be successful in five key points: (i) readiness for future measurements free from residuals of former measurements, (ii) permit soil contact either periodically or in continuation, (iii) successfully derive soil samples, (iv) present an adequate volume/mass of soil in a sufficient range of the sensor and (v) perform an accurate measurement. Still utilizing soil reflectance spectroscopy in the field requires the application of methods to eliminate the effect of water, soil texture and more. Soil sensors for in situ measurements could be divided into different categories ranging from the soil depth of the measurement, whether they are mobile or static, mounted on a tractor/vehicle and their spectral resolution. To exploit the potential of diffuse reflectance spectroscopy (DRS), Kodaira and Shibusawa, (2013) upgraded a prototype real-time soil sensor (RTSS) that was subsequently mounted on a tractor. The unit of the sensor comprised of a computer, a halogen lamp, a micro CCD (couplecharged device) camera, two spectrophotometers and a differential global positioning system. The sensor was designed to acquire spectra at soil depths from $0.05-0.35 \mathrm{~m}$. After each measurement by the RTSS, two soil samples were taken from the same point the measurement was made and twelve soil properties were estimated. The speed of the tractor was that of $0.56 \mathrm{~m} \mathrm{~s}^{-1}$ similar to the speed needed for some field operations. The results gave an accuracy of $\mathrm{R}^{2}=0.93$ for SOM providing valuable information for variable rate applications. Veris Technologies developed an optical soil sensor (OpticMapper ${ }^{\mathrm{TM}}$ ) with two wavelengths $(660$ and $940 \mathrm{~nm})$ for SOM and six coulter electrodes for cation exchange capacity (CEC) estimations. The sensor was tested through an experiment held in 15 fields with different soil types and SOM variations. At a 4-cm depth and with a speed of $10-15 \mathrm{~km} / \mathrm{h}$, the collected data points were approximately $150-200$ per field. For model calibration the Multiple Linear Regression (MLR) was used as it was assumed that relationship between SOM and the two selected wavelengths was linear at SOM levels below 5\%. It was observed that same SOM concentrations at different fields showed different spectral response probably due to variations on soil moisture levels hence, a soil moisture sensor could be simultaneously used. (Kweon et al., 2013).

Aiming to estimate the effect of soil moisture and texture on the predictions of SOC and total nitrogen (TN), Kuang and Mouazen, (2013) conducted an experiment in five different fields with an on-line sensor developed by 
Mouazen and Ramon, (2006) mounted on a tractor. The sensor penetrates soil at a certain depth and smoothens the surface were the spectral measurement is acquired. The model calibration and validation was made for processed soils, fresh soils and on-line measurements. The RMSEP for on-line measured OC, ranged from 1.29$1.90 \mathrm{~g} \mathrm{C} \mathrm{kg}^{-1}$. They concluded that soil moisture content, sand and silt fractions have a negative effect in the models' accuracy, while clay content increases accuracy in dried samples. Furthermore, they proposed that field measurements are to be done in dry soil conditions especially when the clay content is relative high. Knadel et al., (2015) also used a multi-sensor platform (MSP) from Veris Technologies in two different fields. The difference from the previous study was that in this study they compared the performance of a single sensor, to sensor data fusion for predicting SOC concentrations, sand, clay and silt. Specifically, SOC and clay of the one test site was better predicted using a fusion of sensor data $\left(\mathrm{R}^{2}=0.94\right)$, while for sand, silt and clay better prediction was obtained using only spectral data.

To minimize external weather conditions in the field, Rodionov et al. (2015) developed a closed dark chamber mounted on a tractor with a spectrometer inside of with a spectral range of 350-2500 nm for on-line measurements. The spectra were acquired in two different sampling conditions, at a stop and go mode and in continuation. The latter showed spectral discontinuities due to different integration times, though this effect could be eliminated by reducing the number of spectra per scan. SOC was predicted after selecting an already developed appropriate model for different soil moisture content. The stop and go mode gave $\mathrm{R}^{2}=0.63$, while the same samples with conventional laboratory spectral measurements gave $\mathrm{R}^{2}=0.94$. Though this study was held in bare soil conditions, there are factors affecting in situ vis-NIR spectroscopic measurements, such as green vegetation and straw cover leading to an overestimation of SOC. Rodionov et al. (2016) in a combined field and laboratory study aimed to distinguish green vegetation and straws from SOC using the same spectral data and the same devise with Rodionov et al. (2015). In addition to the spectral measurements, soil colour measurements were also performed, but the results were proven to be unsatisfactory, thus no further testing was made. Firstly, the experiment was conducted in laboratory conditions where soil samples with different green plant and straw coverage were measured. To characterize straw cover, the Cellulose Absorbance Index (CAI) was used and for green vegetation several known indices were used such as the Normalized Difference Vegetation Index (NDVI) etc. CAI and NDVI were estimated and then subtracted to prevent SOC overestimation. In conclusion, the spectral response of straw was not very characteristic as to be discriminated from other materials hence both the use of CAI and NDVI is needed. Although this study showed the overestimation of SOC content with the presence of green plant and straw coverage, the proposed method gave average predictions with $\mathrm{R}^{2}=0.58-0.63$.

Regarding the simultaneous estimation of several soil properties the use of a single sensor is not adequate (Ge et al., 2011). To that end, Viscarra Rossel et al. (2017) developed the Soil Condition Analysis System (SCANS) that integrates a soil core sensing system. The system includes four sensors combined with analytic statistics and modelling and can estimate approximately thirteen physical, biochemical and mineralogical soil properties. It takes about 30 minutes for 50 measurements along the soil core. To achieve the transferability of the calibrated models with models from spectral libraries of dry soil, the EPO algorithm was used to eliminate the effect of water in the soil spectra. For modelling, the RS-LOCAL algorithm was used providing a more efficient exploitation of the local spectral libraries (Lobsey et al., 2017). The results from spectroscopic modelling were promising with SOC showing the greatest accuracy $\left(\mathrm{R}^{2}=0.83\right)$ for a repeated 10 -fold cross validation and $\mathrm{R}^{2}=0.81$ with independent validation. The advantage of SCANS is that it provides accurate, fast and cost-efficient predictions in high spatial resolution at various depths that the user defines. Accordingly, Franceschini et al. (2018) used an on-the-go vis - NIR Veris Spectrometer and aimed to remove effects other than soil moisture, mainly those related to sensor movement during spectral acquisition. To that end, they estimated the performance of three treatments: the EPO, DS and orthogonal signal correction (OSC). The overall aim was to reduce differences occurring when the measurements were performed under different conditions (i.e. in situ and laboratory). Although the application of different spectral treatments improved the prediction models still the results were inferior, specifically for SOM $\left(\mathrm{R}^{2}<0.10\right)$, to model generated in laboratory conditions, probably due to positioning inconsistencies of the factors affecting on-the-go proximal sensing.

There are portable instruments able to be used for in situ proximal soil sensing equipped with a handheld contact probe for direct measurements. Gras et al. (2014) evaluated seven different practices for estimating topsoil properties in the field using an ASDLabSpec 5000 spectrometer. These procedures were either directly to the soil surface, in cores extracted with an auger or clods from the core of the site. It was observed that different spectral acquisition procedures gave different results especially for SOM estimations providing an increase in RPD values by 0.7 . A portable spectrophotometer LabSpec 2500 was also used by Cambou et al. (2016) for in field measurements of SOC. The spectra were acquired with a contact probe from the outer core of samples collected with a manual auger. The analytical method though for SOC and SOC stock estimations was not performed in the same samples resulting in poor predictions with $\mathrm{R}^{2}=0.75$ and $\mathrm{R}^{2}=0.70$ respectively. 


\section{Remote Sensing}

\section{Satellites}

Imaging spectroscopy with wide spatial resolution can be achieved by sensors onboard satellites. These data have the advantage of providing a synoptic view and high temporal resolution compared to ground measurements (Cécillon et al., 2009). The limitation in their use derives from the atmospheric correction of the data needed along with ground observations, therefore there are limited studies using satellite data for SOC estimation (Croft et al., 2012). In an Australian case study, Gomez et al. (2008) compared the predictions of SOC using field vis-NIR and remote sensing data from the Hyperion sensor on board the EO-1 satellite $(400-2500 \mathrm{~nm})$. To do so, the field spectra were resampled to cover the Hyperion data. They found that it was not possible to determine the SOC content when the amount of SOC dropped below $1 \%$, irrespective of the spectral resolution and the number of soil samples. In situ Vis - NIR measurements compared to Hyperion data gave values of $\mathrm{R}^{2}=0.66$ and 0.51 respectively hence, the use of Hyperion hyperspectral data should be as useful as the use of field vis-NIR data. Satellite remote sensing could also be used as auxiliary data for SOM mapping. Recently, Mirzaee et al. (2016) used ordinary and hybrid geostatistical methods to predict SOM content. It was concluded that information from Landsat 7 ETM+ imagery were potential auxiliary variables to improve spatial prediction, monitoring and development of high quality SOM maps, while the coefficient of variation of SOM content reached to $63.3 \%$ with the use of artificial neural network-simple kriging (ANNSK). In the same context, Mondal et al. (2017) used a hybrid interpolation method (regression kriging) combined with eight selected variables derived from remote sensing platforms to estimate SOC distribution. The use of these variables provided auxiliary information for SOC distribution regarding its spatial interpretation, resulting in more accurate interpolation results than using an ordinary kriging method. Wang et al. (2018) investigated how the use of environmental covariates obtained from remote sensing, i.e. seasonal fractional cover, could improve the digital mapping of SOC. For modelling purposes three different machine learning techniques were used: i) random forest, ii) boosted regression trees and iii) support vector machines with and without the use of the environmental variables. The overall results showed that seasonal fractional cover improved the model's accuracy by about $2.8-12.7 \%$ and could aid local scale digital mapping in areas lacking data. In the scope of forthcoming new satellite platforms, Castaldi et al. (2016) evaluated their prospective to estimate soil texture and SOC compared to four known multispectral and hyperspectral imagers. In this study, they utilized soil spectral libraries and resampled spectral data to simulate each imagers specifications as well as their declared or actual noise. The results indicated that satellite sensors are of limited use for quantitative estimations of SOC with an accuracy between $\mathrm{R}^{2}=0.49-0.67$. The conclusion was though, that the forthcoming imagers, EnMap satellite $\left(\mathrm{R}^{2}=0.67\right)$, could marginally improve mapping and monitoring of $\mathrm{SOC}$ in comparison to current imagers.

\section{Airborne}

Recent advances in remote sensing technology have reduced the cost and increased the viability of these approaches for several precision agricultural applications. Airborne multi- and hyperspectral imaging provide a large amount of spectral information and thus could be of great use for soil properties estimation (Liaghat and Balasundram, 2010). Since the application of remote sensing techniques can have many constraints (Ben-Dor et al., 2008), bare soil studies provide more controllable conditions and are more preferable for reflectance data acquired by airborne mounted sensors (Denis et al., 2014). Stevens et al. (2010b) were the first to use Penalizedspline Signal Regression (PSR) and least square Support Vector Machine Regression (SVMR) calibration techniques to estimate SOC with the Airborne Hyperspectral Sensor 160 (AHS) (Caravan International Corporation, USA). They concluded that sub setting the data according to different parameters such as soil type, improved the prediction models with the use of PSR for local calibrations presenting the best results, while the SVMR performed better for global datasets. The reason could be attributed to the non-linear relationship of SOC with reflectance spectra concerning global calibrations.

Nevertheless, airborne data still need atmospheric correction and favorable weather conditions, while difficulties arise from large pixel size and varying quality of the sensor's stability and sensitivity (Brook and Dor, 2011). Another aspect of prohibition in the use of airborne sensors is the low signal to noise ratio mainly in the region between 1900-2500nm, probably due to electro-optics during flight and atmospheric absorption. Stevens et al. (2008) compared three different approaches for SOC estimations: the AHS 160 sensor, laboratory and in situ spectral measurements. Results showed that accuracy was decreasing from laboratory to airborne sensing techniques with RPD values from 1.47 to 2.11 respectively. The local character of the prediction models was noted, explaining that they depend on the soil type and SOC ranging values. Although airborne hyperspectral imaging gave poor predictions, its potential is encouraging. Controlling external parameters could increase the performance of a model. Hbirkou et al. (2012) attempted to minimize the effects of soil moisture by conducting the flight of the airborne hyperspectral sensor HyMap (450-2500 nm) (Integrated Spectronics, Sydney, Australia) after a period of dry weather. The more controllable the conditions, the better models were created ranging from $\mathrm{R}^{2}=0.73$ for dry homogenous with no vegetation cover sites to 0.51 for the most unfavorable conditions 
respectively. Soil moisture though, is not the only soil surface parameter that affects spectral responses i.e. surface roughness and green vegetation or straw residues (Lagacherie et al., 2008). The assessment of bare soil fraction in the contrast of photosynthetic and non-photosynthetic active vegetation fractions was studied by Franceschini et al. (2015). They proposed and tested a methodology for assessing the soil spectral mixture based on bare soil fractional cover. The predictions for organic matter content with the use of the ProSpecTIR V-S sensor (SpecTIR LLC), equipped with two imaging sensors to capture the spectral range from $400-2500 \mathrm{~nm}$, were found substantially less accurate $\left(\mathrm{R}^{2}=0.33\right)$ than those in laboratory conditions $\left(\mathrm{R}^{2}=0.70\right)$. This result was mainly affected by soil spectral albedo near the region 700-1200 nm and wavelengths greater than $2040 \mathrm{~nm}$. Bartholomeus et al.(2011) worked in maize fields with varying fractional coverage and tried to improve the quantitative estimates of soil properties by introducing a spectral unmixing approach to remove vegetation influence that limits the accuracy of soil properties estimation. The procedure was named Residual Spectral Unmixing (RSU). They found that a PLSR based prediction of SOC is very sensitive to vegetation influence and is over or underestimated depending on the spectral pre-processing technique used. Overall, the RSU gave predictions similar to studies with bare soils, showing the within field SOC variation.

Combining airborne hyperspectral data with synchronous field spectra measurements could be proven beneficial, but still needs further research since different strategies for sampling spectral data give different calibration performances (Vaudour et al., 2016). Land use changes require a specific land management for SOC preservation. Imaging spectroscopy could facilitate these practices with appropriate techniques that involve spectral unmixing for soil - vegetation deconvolution, atmospheric and geometric corrections (Bayer et al., 2016). Remote sensed data were used for the spatial assessment of multiple ecosystem services in subalpine grasslands. Homolová et al. (2014) suggested a more integrated approach combining remote sensed images, land use and Digital Elevation Model (DEM) maps with field observations. The study showed that data acquired by the AISA Dual system (Specim, Finland) $(400-2450 \mathrm{~nm})$ provided better results for $\mathrm{SOC}\left(\mathrm{R}^{2}=0.73\right)$ compared to results of the plant trait-based model $\left(\mathrm{R}^{2}=0.31\right)$ for ecosystem properties (green biomass, litter mass, crude protein content, species diversity and soil carbon content). Another study aimed to create multi-temporal composites of the Airborne Prism Experiment (APEX) in support of digital mapping for bare soils of heterogeneous agricultural areas (Diek et al., 2016). By correcting the spectral variations that occur over time due to weather and soil conditions, and management practices bare soil identification was possible. However, for SOM estimations the $\mathrm{R}^{2}$ was $0.39 \pm 0.04$. The same airborne imaging spectrometer was used by Castaldi et al. (2018) combined with data from the LUCAS topsoil database (Tóth et al., 2013). A bottom-up approach was used to create SOC maps of bare cropland fields. This approach does not require analytical laboratory measurements, but instead uses laboratory spectral data for different soil variables estimation. The PLSR models were tested on a completely independent validation dataset giving similar RMSE of $4.3 \mathrm{~g} \mathrm{C} \mathrm{kg}^{-1}$ to the traditional approaches RMSE $=3.6 \mathrm{~g}$ $\mathrm{C} \mathrm{kg}{ }^{-1}$.

While it is common to use the full spectral range to model soil properties, there has been an attempt for specific spectral variable selection to remove spectra providing no information with a possibility to improve the predictive ability for independent validation samples. Vohland et al. (2017) used the HyMap sensor for spectral acquisition and for modelling purposes they combined PLSR with three different spectral variables selection methods, namely (i) competitive adaptive reweighted sampling (CARS), (ii) a method that "iteratively retains informative variables" (IRIV) and (iii) a genetic algorithm (GA). It was observed that these combinations could be a valuable tool for improving soil properties estimation accuracies. Specifically, for SOC estimations the application of GA generated $\mathrm{R}^{2}=0.94$ for laboratory measurements and $\mathrm{R}^{2}=0.85$ for airborne measurements.

The AHS was used by (Peón et al., 2017) to estimate SOC content in slightly vegetated burned mountain areas. Simple and multiple linear regression models were used, and a comparison was made to evaluate their accuracy against the PLSR models. The estimation of $\mathrm{R}^{2}=0.62$ showed the greater absolute correlation in the wavelengths of 610 and $679-681 \mathrm{~nm}$. The best prediction models were applied to each pixel of the image to generate Total Organic Carbon maps. The results were in agreement with several other research work (H. M. Bartholomeus et al., 2008; Mouazen, Maleki, De Baerdemaeker, \& Ramon, 2007; Stevens et al., 2010).

\section{Unmanned Aerial Systems}

In the last few years the improvements in the resolution, capabilities and cost of the new sensors has led to the wider use of small Unmanned Aerial Systems (UAS) (Whitehead and Hugenholtz, 2014). The use of (UAS) has become more frequent in the agricultural sector mainly due to their capability for large areas cover at high spatial and temporal resolution (d'Oleire-Oltmanns et al., 2012). Despite the advantages of this method there is still limited research in the field of soil properties predictions and few for environmental monitoring. To our knowledge only one study has been done for SOC estimation. Aldana-Jague et al. (2016) aimed to develop a method for high resolution multispectral information acquisition using a UAS platform and test its efficiency for SOC predictions. To that end, the Rothamsted Long-term Experiments (LTEs) were used. A multispectral camera $(480-1000 \mathrm{~nm})$ was mounted on an octocopter that detects six spectral bands $(480-550-670-780-880-1000 \mathrm{~nm} \pm 10 \mathrm{~nm})$ that 
capture significant parameters were chosen. The flight campaign was held in cloudless sky with low vegetation cover and dry conditions to minimize the effects of soil moisture. The images were corrected for random and nonsystematic noise. The proposed methodology showed great potential for SOC monitoring with a mean coefficient of determination of $95 \%$ and a RMSE of $0.21 \%$ in cross validation. However, it was observed an overestimation for low SOC concentrations and an underestimation of the high SOC values.

\section{Results and discussion}

Remote and proximal soil sensing are techniques that differ in terms of spatial and spectral resolution, thus are used for different purposes according to the need of each research or as complimentary data. The evolution of remote imagers from panchromatic to multispectral and hyperspectral could potentially improve SOC accounting, though mostly for bare soils. However, higher spectral resolution could lead to greater signal to noise ratio, while sensors are also affected by other parameters i.e. soil moisture, roughness, green vegetation and atmospheric conditions. (Ge et al., 2011). On the other hand, proximal soil sensors in the vis-NIR region could provide valuable information for soil properties estimation, but lack in spatial variability. To address this problem mobile sensors are more frequently used and developed, but need further improvements. Nowadays, new emerging technologies are becoming available and have led to the development of smaller and cheaper and micro-electromechanical systems (MEMs) in the vis-NIR region.

Although acquiring the spectroscopic data is a rather feasible and rapid procedure, information is still hidden in the data. To extract this information, multivariate statistic technics known as chemometrics to relate spectral data to the conventionally measured soil properties coupled with pre-processing techniques are used. To that end, apart from the most commonly used PLSR method, which describes linear relationships between the variables (the dependent and the independent data), machine learning techniques such as AAN, boosted regression Trees, Support Vector Machines are also evaluated.

Many countries have started initiatives to create national Soil Spectral Libraries (Australia, European Union, Brazil, China). Though building these large databases is more cost effective than using conventional analytical methods for soil properties determination, there are some drawbacks in their use when soil properties of small areas are to be estimated. To increase the accuracy of such predictions several methods are now being used like spiking and RS-LOCAL that benefit from an already created calibration set. Furthermore, there are some challenges due to the great variation in soil sampling methods, the instrumentation used, the model calibration and pre-processing techniques that affects not only the measurements but the comparability between the results. As SOC and consequently SOC stocks is a property of great interest for soil fertility and climate change, the agreement on a common protocol is very important for date harmonization.

Detrimental effects that affect SOC spectral measurements need to be addressed and solved. These factors are soil moisture, soil roughness, vegetation cover etc. To that end, the use of various algorithms and calibration models are evaluated and tested for model transferability from laboratory to the field where conditions differ.

\section{Conclusions}

The present review aimed to highlight the progress that has been done in the last decade using remote and proximal soil sensing in vis - NIR region for SOC estimations. Satellites are proven to be an efficient auxiliary data source for SOC estimation, while airborne sensors could provide more accurate models. In addition, unmanned aerial vehicles are still of limited use for this purpose, but there is an increasing interest towards their application due to their low cost and spatial coverage. Laboratory measurements are now a well-established method to estimate soil properties, but still need a lot of sample preparation. To that end, mobile soil sensors that estimate SOC in real time are extensively evaluated simultaneously with other sensors for a broader range of soil properties estimation. To efficiently use vis-NIR spectra, SSLs are now developed, however with a non-agreed protocol. Overall, the results are promising for SOC estimations and more research could be focused on selecting the proper instrumentation, calibration techniques and pre-processing methods.

\section{Acknowledgements}

The work was supported by the project "Research Synergy to address major challenges in the nexus: energyenvironment-agricultural production (Food, Water, Materials)" - NEXUS, funded by the Greek Secretariat for Research and Technology (GSRT) - Pr. No. MIS 5002496.

\section{References}

Aldana-Jague, E., G. Heckrath, A. Macdonald, B. van Wesemael, and K. Van Oost,. 2016. UAS-based soil carbon mapping using VIS-NIR (480-1000 nm) multi-spectral imaging: Potential and limitations. Geoderma. 275 55-66. http://doi.org/10.1016/j.geoderma.2016.04.012. 
Atkinson, P. M., and P. Aplin,. 2004. Spatial variation in land cover and choice of spatial resolution for remote sensing. International Journal of Remote Sensing. $25 \quad$ (18),3687-3702. http://doi.org/10.1080/01431160310001654383.

Bartholomeus, H., L. Kooistra, A. Stevens, M. van Leeuwen, B. van Wesemael, E. Ben-Dor, and B. Tychon,. 2011. SoAldana-Jague, E., G. Heckrath, A. Macdonald, B. van Wesemael, and K. Van Oost,. 2016. UAS-based soil carbon mapping using VIS-NIR (480-1000 nm) multi-spectral imaging: Potential and limitations. Geoderma. 275 55-66. http://doi.org/10.1016/j.geoderma.2016.04.012.

Bartholomeus, H., L. Kooistra, A. Stevens, M. van Leeuwen, B. van Wesemael, E. Ben-Dor, and B. Tychon,. 2011. Soil Organic Carbon mapping of partially vegetated agricultural fields with imaging spectroscopy. International Journal of Applied Earth Observation and Geoinformation. 13 (1),81-88. http://doi.org/10.1016/J.JAG.2010.06.009.

Bartholomeus, H. M., M. E. Schaepman, L. Kooistra, A. Stevens, W. B. Hoogmoed, and O. S. P. Spaargaren,. 2008. Spectral reflectance based indices for soil organic carbon quantification. Geoderma. 145 (1-2),28-36. http://doi.org/10.1016/J.GEODERMA.2008.01.010.

Bayer, A. D., M. Bachmann, D. Rogge, M. Andreas, and H. Kaufmann,. 2016. Combining Field and Imaging Spectroscopy to Map Soil Organic Carbon in a Semiarid Environment. IEEE Journal of Selected Topics in Applied Earth Observation and Remote Sensing. 9 (9),3997-4010. http://doi.org/10.1109/JSTARS.2016.2585674.

Ben-Dor, E., and A. Banin,. 1995. Near-Infrared Analysis as a Rapid Method to Simultaneously Evaluate Several Soil Properties. Soil Science Society of America Journal. 59 (2),364-372. http://doi.org/10.2136/sssaj1995.03615995005900020014x.

Ben-Dor, E., R. G. Taylor, J. Hill, J. A. M. Demattê, M. L. Whiting, S. Chabrillat, and S. Sommer, 2008. Imaging Spectrometry for Soil Applications. Advances in Agronomy. 97 321-392. http://doi.org/10.1016/S00652113(07)00008-9.

Brook, A., and E. Ben Dor,. 2011. Supervised vicarious calibration (SVC) of hyperspectral remote-sensing data. Remote Sensing of Environment. 115 (6),1543-1555. http://doi.org/10.1016/J.RSE.2011.02.013.

Brown, D. J., K. D. Shepherd, M. G. Walsh, M. Dewayne Mays, and T. G. Reinsch,. 2006. Global soil characterization with VNIR diffuse reflectance spectroscopy. Geoderma. 132 (3),273-290. http://doi.org/10.1016/j.geoderma.2005.04.025.

Cambou, A., R. Cardinael, E. Kouakoua, M. Villeneuve, C. Durand, and B. G. Barthès,. 2016. Prediction of soil organic carbon stock using visible and near infrared reflectance spectroscopy (VNIRS) in the field. Geoderma. 261 151-159. http://doi.org/10.1016/j.geoderma.2015.07.007.

Castaldi, F., S. Chabrillat, A. Jones, K. Vreys, B. Bomans, and B. van Wesemael,. 2018. Soil Organic Carbon Estimation in Croplands by Hyperspectral Remote APEX Data Using the LUCAS Topsoil Database. Remote Sensing. 10 (2),153. http://doi.org/10.3390/rs10020153.

Castaldi, F., A. Palombo, F. Santini, S. Pascucci, S. Pignatti, and R. Casa,. 2016. Evaluation of the potential of the current and forthcoming multispectral and hyperspectral imagers to estimate soil texture and organic carbon. Remote Sensing of Environment. 179 54-65. http://doi.org/10.1016/j.rse.2016.03.025.

Cécillon, L., B. Es, C. Gomez, D. Ertlen, V. Génot, M. Hedde, A. Stevens, and J.-J. Brun,. 2009. Assessment and monitoring of soil quality using near infrared reflectance spectroscopy (NIRS). European Journal of Soil Science. 60 770-784. http://doi.org/10.1111/j.1365-2389.2009.01178.x>.

Croft, H., N. J. Kuhn, and K. Anderson,. 2012. On the use of remote sensing techniques for monitoring spatiotemporal soil organic carbon dynamics in agricultural systems. CATENA. 94 64-74. http://doi.org/10.1016/J.CATENA.2012.01.001.

d'Oleire-Oltmanns, S., I. Marzolff, K. Peter, and J. Ries,. 2012. Unmanned Aerial Vehicle (UAV) for Monitoring Soil Erosion in Morocco. Remote Sensing. 4 (11),3390-3416. http://doi.org/10.3390/rs4113390.

Dalal, R. C., and R. J. Henry,. 1986. Simultaneous Determination of Moisture, Organic Carbon, and Total Nitrogen by Near Infrared Reflectance Spectrophotometry1. Soil Science Society of America Journal. 50 (1), 120. http://doi.org/10.2136/sssaj1986.03615995005000010023x.

Davis, M., B. Alves, D. Karlen, K. Kline, M. Galdos, and D. Abulebdeh,. 2017. Review of Soil Organic Carbon Measurement Protocols: A US and Brazil Comparison and Recommendation. Sustainability. 10 (2),53. http://doi.org/10.3390/su10010053.

Denis, A., A. Stevens, B. van Wesemael, T. Udelhoven, and B. Tychon,. 2014. Soil organic carbon assessment by field and airborne spectrometry in bare croplands: accounting for soil surface roughness. Geoderma. 226-227 94-102. http://doi.org/10.1016/J.GEODERMA.2014.02.015.

Diek, S., M. Schaepman, and R. de Jong,. 2016. Creating Multi-Temporal Composites of Airborne Imaging Spectroscopy Data in Support of Digital Soil Mapping. Remote Sensing. 8 (11),906. http://doi.org/10.3390/rs8110906.

England, J. R., R. A. Viscarra Rossel, R. Armando, and V. Rossel,. 2018. Proximal sensing for soil carbon accounting 45194 101-122. http://doi.org/10.5194/soil-4-101-2018. 
FAO,. 2017. Soil Organic Carbon the Hidden Potential. Page Food and Agriculture Organization of the United Nations Rome, Italy.

Franceschini, M. H. D., J. A. M. Demattê, L. Kooistra, H. Bartholomeus, R. Rizzo, C. T. Fongaro, and J. P. Molin,. 2018. Effects of external factors on soil reflectance measured on-the-go and assessment of potential spectral correction through orthogonalisation and standardisation procedures. Soil and Tillage Research. 177 1936. http://doi.org/10.1016/j.still.2017.10.004.

Franceschini, M. H. D., J. A. M. Demattê, F. da Silva Terra, L. E. Vicente, H. Bartholomeus, and C. R. de Souza Filho,. 2015. Prediction of soil properties using imaging spectroscopy: Considering fractional vegetation cover to improve accuracy. International Journal of Applied Earth Observation and Geoinformation. 38 358-370. http://doi.org/10.1016/J.JAG.2015.01.019.

Ge, Y., J. A. Thomasson, and R. Sui,. 2011. Remote sensing of soil properties in precision agriculture: A review. Frontiers of Earth Science. 5 (3),229-238. http://doi.org/10.1007/s11707-011-0175-0.

Gehl, R. J., C. W. Rice, R. J. Gehl, and C. W. Rice,. 2007. Emerging technologies for in situ measurement of soil carbon. Climatic Change. 80 43-54. http://doi.org/10.1007/s10584-006-9150-2.

Geladi, P.,. 2003. Chemometrics in spectroscopy. Part 1. Classical chemometrics. Spectrochimica Acta Part B: Atomic Spectroscopy. 58 (5),767-782. http://doi.org/10.1016/S0584-8547(03)00037-5.

Gholizadeh, A., L. Borùvka, R. Vašát, M. Saberioon, A. Klement, J. Kratina, V. Tejnecký, and O. Drábek,. 2015. Estimation of potentially toxic elements contamination in anthropogenic soils on a brown coal mining dumpsite by reflectance spectroscopy: A case study. PLoS ONE. 10 (2),1-14. http://doi.org/10.1371/journal.pone.0117457.

Goetz, A. F. H., G. Vane, J. E. Solomon, and B. N. Rock,. 1985. Imaging spectrometry for earth remote sensing. Science. 228 (4704),1147-1153. http://doi.org/10.1126/science.228.4704.1147.

Goldshleger, N., A. Chudnovsky, E. Ben-Dor, N. Goldshleger, A. Chudnovsky, and E. Ben-Dor,. 2012. Using Reflectance Spectroscopy and Artificial Neural Network to Assess Water Infiltration Rate into the Soil Profile. Applied and Environmental Soil Science. 2012 1-9. http://doi.org/10.1155/2012/439567.

Gomez, C., R. A. Viscarra, and A. B. Mcbratney,. 2008. Soil organic carbon prediction by hyperspectral remote sensing and fi eld vis-NIR spectroscopy: An Australian case study. Geoderma. $146403-411$. http://doi.org/10.1016/j.geoderma.2008.06.011.

Gras, J. P., B. G. Barthès, B. Mahaut, and S. Trupin,. 2014. Best practices for obtaining and processing field visible and near infrared (VNIR) spectra of topsoils. Geoderma. 214-215 http://doi.org/10.1016/j.geoderma.2013.09.021.

Grunwald, S., G. M. Vasques, and R. G. Rivero,. 2015. Fusion of Soil and Remote Sensing Data to Model Soil Properties. Advances in Agronomy. 131 1-109. http://doi.org/10.1016/BS.AGRON.2014.12.004.

Hbirkou, C., S. Pätzold, A. K. Mahlein, and G. Welp,. 2012. Airborne hyperspectral imaging of spatial soil organic carbon heterogeneity at the field-scale. Geoderma. 175-176 21-28. http://doi.org/10.1016/j.geoderma.2012.01.017.

Homolová, L., M. E. Schaepman, P. Lamarque, J. G. P. W. Clevers, F. de Bello, W. Thuiller, and S. Lavorel,. 2014. Comparison of remote sensing and plant trait-based modelling to predict ecosystem services in subalpine grasslands. Ecosphere. 5 (8), http://doi.org/art100.10.1890/ES13-00393.1.

Islam, K., B. Singh, and A. Mcbratney,. 2003. Simultaneous estimation of several soil properties by ultraviolet, visible, and near-infrared reflectance spectroscopy. Australian Journal of Soil Research. 41 1101-1114. http://doi.org/10.1071/SR02137.

Jobbágy, E. G., and R. B. Jackson,. 2000. The vertical distribution of soil organic carbon and its relation to climate and vegetation. Ecological Applications. 10 (2),423-436. http://doi.org/10.1890/10510761(2000)010[0423:TVDOSO]2.0.CO;2.

Knadel, M., A. Thomsen, K. Schelde, and M. H. Greve,. 2015. Soil organic carbon and particle sizes mapping using vis-NIR, EC and temperature mobile sensor platform. Computers and Electronics in Agriculture. 114134 144. http://doi.org/10.1016/J.COMPAG.2015.03.013.

Kodaira, M., and S. Shibusawa,. 2013. Using a mobile real-time soil visible-near infrared sensor for high resolution soil property mapping. Geoderma. 199 64-79. http://doi.org/10.1016/J.GEODERMA.2012.09.007.

Kuang, B., and A. M. Mouazen,. 2013. Non-biased prediction of soil organic carbon and total nitrogen with vis-NIR spectroscopy, as affected by soil moisture content and texture. Biosystems Engineering. 114 (3),249258. http://doi.org/10.1016/J.BIOSYSTEMSENG.2013.01.005.

Kweon, G., E. Lund, and C. Maxton,. 2013. Soil organic matter and cation-exchange capacity sensing with on-the-go electrical conductivity and optical sensors. Geoderma. 199 80-89. http://doi.org/10.1016/J.GEODERMA.2012.11.001.

Ladoni, M., H. A. Bahrami, S. K. Alavipanah, and A. A. Norouzi,. 2010. Estimating soil organic carbon from soil reflectance: a review. Precision Agriculture. 11 (1),82-99. http://doi.org/10.1007/s11119-009-9123-3. 
Lagacherie, P., F. Baret, J.-B. Feret, J. Madeira Netto, and J. M. Robbez-Masson,. 2008. Estimation of soil clay and calcium carbonate using laboratory, field and airborne hyperspectral measurements. Remote Sensing of Environment. 112 (3),825-835. http://doi.org/10.1016/J.RSE.2007.06.014.

Liaghat, S., and S. K. Balasundram,. 2010. A Review: The Role of Remote Sensing in Precision Agriculture. American Journal of Agricultural and Biological Sciences. 5 (1),50-55.

Lobsey, C. R., R. A. Viscarra Rossel, P. Roudier, and C. B. Hedley,. 2017. Rs-Local Data-Mines Information From Spectral Libraries To Improve Local Calibrations. European Journal of Soil Science. 68 (6),840-852. http://doi.org/10.1111/ejss.12490.

St. Luce, M., N. Ziadi, B. J. Zebarth, C. A. Grant, G. F. Tremblay, and E. G. Gregorich,. 2014. Rapid determination of soil organic matter quality indicators using visible near infrared reflectance spectroscopy. Geoderma. 232-234 449-458. http://doi.org/10.1016/J.GEODERMA.2014.05.023.

Mirzaee, S., S. Ghorbani-Dashtaki, J. Mohammadi, H. Asadi, and F. Asadzadeh,. 2016. Spatial variability of soil organic matter using remote sensing data. CATENA. 145 118-127. http://doi.org/10.1016/J.CATENA.2016.05.023.

Mondal, A., D. Khare, S. Kundu, S. Mondal, S. Mukherjee, and A. Mukhopadhyay,. 2017. Spatial soil organic carbon (SOC) prediction by regression kriging using remote sensing data. The Egyptian Journal of Remote Sensing and Space Science. 20 (1),61-70. http://doi.org/10.1016/J.EJRS.2016.06.004.

Mouazen, A. M., M. R. Maleki, J. De Baerdemaeker, and H. Ramon,. 2007. On-line measurement of some selected soil properties using a VIS-NIR sensor. Soil and Tillage Research. 93 (1),13-27. http://doi.org/10.1016/J.STILL.2006.03.009.

Mouazen, A. M., and H. Ramon,. 2006. Development of on-line measurement system of bulk density based on on-line measured draught, depth and soil moisture content. Soil and Tillage Research. 86 (2),218-229. http://doi.org/10.1016/j.still.2005.02.026.

Mulla, D. J.,. 2013. Twenty five years of remote sensing in precision agriculture: Key advances and remaining knowledge gaps. Biosystems Engineering. 114 (4),358-371. http://doi.org/10.1016/j.biosystemseng.2012.08.009.

Nawar, S., H. Buddenbaum, J. Hill, J. Kozak, and A. M. Mouazen,. 2016. Estimating the soil clay content and organic matter by means of different calibration methods of vis-NIR diffuse reflectance spectroscopy. Soil and Tillage Research. 155 510-522. http://doi.org/10.1016/J.STILL.2015.07.021.

Nocita, M., A. Stevens, C. Noon, and B. van Wesemael,. 2013. Prediction of soil organic carbon for different levels of soil moisture using Vis-NIR spectroscopy. Geoderma. $199 \quad 37-42$. http://doi.org/10.1016/J.GEODERMA.2012.07.020.

Ontl, T., and L. Schulte,. 2012. Soil Carbon Storage. Nature Education Knowledge. 3 (10),35-45.

Peng, X., T. Shi, A. Song, Y. Chen, and W. Gao,. 2014. Estimating Soil Organic Carbon Using VIS/NIR Spectroscopy with SVMR and SPA Methods. Remote Sensing. 6 (4),2699-2717. http://doi.org/10.3390/rs6042699.

Peón, J., C. Recondo, S. Fernández, J. F. Calleja, E. De Miguel, and L. Carretero,. 2017. Prediction of topsoil organic carbon using airborne and satellite hyperspectral imagery. Remote Sensing. 9 (12),1211. http://doi.org/10.3390/rs9121211.

Rienzi, E. A., B. Mijatovic, T. G. Mueller, C. J. Matocha, F. J. Sikora, and A. Castrignanò,. 2014. Prediction of Soil Organic Carbon under Varying Moisture Levels Using Reflectance Spectroscopy. Soil Science Society of America Journal. 78 (3),958. http://doi.org/10.2136/sssaj2013.09.0408.

Rodionov, A., S. Pätzold, G. Welp, R. Pude, and W. Amelung,. 2016. Proximal field Vis-NIR spectroscopy of soil organic carbon: A solution to clear obstacles related to vegetation and straw cover. Soil and Tillage Research. 163 89-98. http://doi.org/10.1016/j.still.2016.05.008.

Rodionov, A., G. Welp, L. Damerow, T. Berg, W. Amelung, and S. Pätzold,. 2015. Towards on-the-go field assessment of soil organic carbon using Vis-NIR diffuse reflectance spectroscopy: Developing and testing a novel tractor-driven measuring chamber. Soil and Tillage Research. 145 93-102. http://doi.org/10.1016/J.STILL.2014.08.007.

Roudier, P., C. B. Hedley, C. R. Lobsey, R. A. Viscarra Rossel, and C. Leroux,. 2017. Evaluation of two methods to eliminate the effect of water from soil vis-NIR spectra for predictions of organic carbon. Geoderma. 296 98-107. http://doi.org/10.1016/J.GEODERMA.2017.02.014.

Sinfield, J. V., D. Fagerman, and O. Colic,. 2010, January 1. Evaluation of sensing technologies for on-the-go detection of macro-nutrients in cultivated soils. Elsevier.

Stark, E., K. Luchter, and M. Margoshes,. 1986. Near-Infrared Analysis (NIRA): A Technology for Quantitative and Qualitative Analysis. Applied Spectroscopy Reviews. 22 (4),335-399. http://doi.org/10.1080/05704928608060440.

Stevens, A., M. Nocita, G. Tóth, L. Montanarella, and B. van Wesemael,. 2013. Prediction of Soil Organic Carbon at the European Scale by Visible and Near InfraRed Reflectance Spectroscopy. PLoS ONE. 8 (6), http://doi.org/10.1371/journal.pone.0066409. 
Stevens, A., T. Udelhoven, A. Denis, B. Tychon, R. Lioy, L. Hoffmann, and B. van Wesemael,. 2010a. Measuring soil organic carbon in croplands at regional scale using airborne imaging spectroscopy. Geoderma. 158 (1-2),32-45. http://doi.org/10.1016/j.geoderma.2009.11.032.

Stevens, A., T. Udelhoven, A. Denis, B. Tychon, R. Lioy, L. Hoffmann, and B. van Wesemael,. $2010 \mathrm{~b}$. Measuring soil organic carbon in croplands at regional scale using airborne imaging spectroscopy. Geoderma. 158 (1-2),32-45. http://doi.org/10.1016/J.GEODERMA.2009.11.032.

Stevens, A., B. van Wesemael, H. Bartholomeus, D. Rosillon, B. Tychon, and E. Ben-Dor,. 2008. Laboratory, field and airborne spectroscopy for monitoring organic carbon content in agricultural soils. Geoderma. 144 (12),395-404. http://doi.org/10.1016/J.GEODERMA.2007.12.009.

Stuart, B. H.,. 2004. Infrared Spectroscopy: Fundamentals and Applications. Methods.

Tóth, G., A. Jones, and L. Montanarella,. 2013. The LUCAS topsoil database and derived information on the regional variability of cropland topsoil properties in the European Union. Environmental Monitoring and Assessment. 185 (9),7409-7425. http://doi.org/10.1007/s10661-013-3109-3.

Tucker, C. J.,. 1980. Radiometric resolution for monitoring vegetation: How many bits are needed? International Journal of Remote Sensing. 1 (3),241-254. http://doi.org/10.1080/01431168008948235.

Turner, W., S. Spector, N. Gardiner, M. Fladeland, E. Sterling, and M. Steininger,. 2003. Remote sensing for biodiversity science and conservation. Trends in Ecology \& Evolution. 18 (6),306-314. http://doi.org/10.1016/S0169-5347(03)00070-3.

Vaudour, E., J. M. Gilliot, L. Bel, J. Lefevre, and K. Chehdi,. 2016. Regional prediction of soil organic carbon content over temperate croplands using visible near-infrared airborne hyperspectral imagery and synchronous field spectra. International Journal of Applied Earth Observation and Geoinformation. 49 24-38. http://doi.org/10.1016/j.jag.2016.01.005.

Viscarra Rossel, R. A., and T. Behrens,. 2010. Using data mining to model and interpret soil diffuse reflectance spectra. Geoderma. 158 46-54. http://doi.org/10.1016/j.geoderma.2009.12.025.

Viscarra Rossel, R. A., C. R. Lobsey, C. Sharman, P. Flick, and G. McLachlan,. 2017. Novel Proximal Sensing for Monitoring Soil Organic C Stocks and Condition. Environmental Science and Technology. 51 (10),5630-5641. http://doi.org/10.1021/acs.est.7b00889.

Viscarra Rossel, R. A., D. J. J. Walvoort, A. B. McBratney, L. J. Janik, and J. O. Skjemstad,. 2006. Visible, near infrared, mid infrared or combined diffuse reflectance spectroscopy for simultaneous assessment of various soil properties. Geoderma. 131 (1),59-75. http://doi.org/10.1016/j.geoderma.2005.03.007.

Vohland, M., M. Ludwig, S. Thiele-Bruhn, and B. Ludwig,. 2014. Determination of soil properties with visible to near- and mid-infrared spectroscopy: Effects of spectral variable selection. Geoderma. 223-225 (1), http://doi.org/10.1016/j.geoderma.2014.01.013.

Vohland, M., M. Ludwig, S. Thiele-Bruhn, and B. Ludwig,. 2017. Quantification of Soil Properties with Hyperspectral Data: Selecting Spectral Variables with Different Methods to Improve Accuracies and Analyze Prediction Mechanisms. Remote Sensing. 9 (11),1103. http://doi.org/10.3390/rs9111103.

Wang, B., C. Waters, S. Orgill, J. Gray, A. Cowie, A. Clark, and D. L. Liu,. 2018. High resolution mapping of soil organic carbon stocks using remote sensing variables in the semi-arid rangelands of eastern Australia. Science of The Total Environment. 630 367-378. http://doi.org/10.1016/J.SCITOTENV.2018.02.204.

Wetterlind, J., B. Stenberg, and R. A. V. Rossel,. 2013. Soil analysis using visible and near infrared spectroscopy. Methods in molecular biology (Clifton, N.J.). 953 95-107. http://doi.org/10.1007/978-1-62703$152-36$.

Whitehead, K., and C. H. Hugenholtz,. 2014. Remote sensing of the environment with small unmanned aircraft systems (UASs), part 1: a review of progress and challenges 1. Journal of Unmanned Vehicle Systems. 02 (03),6985. http://doi.org/10.1139/juvs-2014-0006.

Wijewardane, N. K., Y. Ge, and C. L. S. Morgan,. 2016. Prediction of soil organic and inorganic carbon at different moisture contents with dry ground VNIR: a comparative study of different approaches. European Journal of Soil Science. 67 (5),605-615. http://doi.org/10.1111/ejss.12362.

Wold, S., M. Sjöström, and L. Eriksson,. 2001. PLS-regression: a basic tool of chemometrics. Chemometrics and Intelligent Laboratory Systems. 58 (2), 109-130. http://doi.org/10.1016/S0169-7439(01)00155-1. 


\author{
Preliminary Approach for the Detection of Olive Trees Infected by \\ Xylella fastidiosa using a Field Robot and Proximal Sensing \\ Santiago López ${ }^{1}$, Sergio Cubero ${ }^{1}$, Nuria Aleixos ${ }^{2}$, Vicente Alegre ${ }^{3}$, Beatriz Rey ${ }^{2}$, Enrique Aguilar ${ }^{3}$, \\ José Blasco $^{1^{*}}$ \\ ${ }^{1)}$ Centro de Agroingeniería. Instituto Valenciano de Investigaciones Agrarias (IVIA). CV-315, km 10,7 - 46113 \\ Moncada (Valencia), Spain \\ ${ }^{2)}$ Departamento de Ingeniería Gráfica. Universitat Politècnica de València (UPV). Camino de Vera, s/n, 46022 Valencia, \\ Spain \\ ${ }^{3)}$ TRAGSA. CV-315, km 10,7 - 46113 Moncada (Valencia), Spain. \\ * Corresponding author email: blasco_josiva@gva.es
}

\begin{abstract}
A small field robot was designed and built within the framework of the $\mathrm{H} 2020$ project Xylella fastidiosa Active Containment Through a Multidisciplinary-Oriented Research Strategy (XF-ACTORS). The robot is remotely driven and provided with different proximal sensing equipment for the early detection of $\mathrm{Xf}$ in olive groves, including thermal, colour and multispectral cameras, and a 2D laser scanner (LiDAR) to obtain the 3D structure of the crop. The equipment is completed by a GPS to geolocate the data obtained and an IMU (inertial measurement unit) to correct the data captured by the LiDAR. An industrial computer triggers the sensors and controls the data acquisition, which is synchronised with the advance of the robot by means of a pulse encoder coupled to the axis of the motor. Then, crop maps can be created off-line after the analysis of the collected data to show graphically potential $X f$ infection in the trees. Owing to the height of the olive trees inspected, the cameras were placed on a platform that can be elevated up to $200 \mathrm{~cm}$. Two batteries power the electric motors attached to the wheels, thereby allowing a continuous inspection for approximately six hours (a field of about 4 ha). A series of tests have been carried out in an olive orchard showing slight symptoms of $X f$ infection in the region of Apulia, southern Italy. During the first tests, the robot inspected each row in both directions with the cameras pointing to one side, so as to inspect all sides of the trees. The tests were mainly focused on the development of the mechanics, navigation systems, sensors and data acquisition. Synchronised and geolocated images of the whole crop were also captured with the cameras in different climatic conditions, as well as with the laser scanner for later comparison to the in-situ observations.
\end{abstract}

Keywords: Robotics, computer vision, multispectral imaging, Lidar, asymptomatic detection

\title{
1. Introduction
}

Xylella fastidiosa (Xf) was first detected in Italy (Apulia region, southern Italy) in 2013 (Martelli et al., 2016) and it has expanded rapidly throughout the region causing severe damage to olive crops. The detection throughout Corsica (France) and in southeastern France and the more recent detections in Mallorca (2016), Alicante (2017) and Madrid (2018) in Spain, has greatly increased the awareness of the threat that this pathogen poses to European agriculture and environment (Purcell, 1997). As a consequence of the $X f$ outbreak in Italy affecting mostly olive trees, almost the entire province of Lecce (Apulia Region) has been demarcated as "infected area" and only containment measures are enforced. The project XF-ACTORS (EU H2020 GA N ${ }^{\circ} 727987$ ) faces the problems from a multidisciplinary research approach. Among the solutions proposed, it is included the setting up of proximal and remote-sensing tools for early diagnostic and surveillance programs.

The representativeness of diagnostics relies strongly on proper sampling design, both at the field and the individual plant level but large spatial extent and fine resolution can only be achieved through extensive testing at relatively high cost (Vicent and Blasco, 2017). Then, the use of remote sensing to map the distribution of plant diseases has evolved considerably during the last three decades and can be performed at different scales, depending on the area to be monitored as well as the spatial and spectral resolution required (Martinelli et al., 2015). While manned aircraft remains the only alternative to obtain maps at larger scales with optimum spatial and spectral resolution for the early detection of disease outbreaks (Calderón et al., 2015), at the plant level the spectral information can be gathered at high spatio-temporal resolution using sensors mounted on regular agricultural vehicles.

This work presents a small field robot built for the early detection of $X f$ in olive crops. The robot is remotely driven and provided with different proximal sensing equipment including thermal, colour, multispectral and hyperspectral cameras, and a 2D Laser Imaging Detection and Ranging (LiDAR) to obtain the 3D structure of the crop. The equipment is completed by a Global Positioning System (GPS) to geolocate the data obtained and an Inertial Measurement Unit (IMU) to minimise the negative influence of moving on uneven ground. 


\section{Materials and Methods}

Terrestrial vehicle

The small robot has been designed and constructed to carry on board the sensing equipment and inspect a field with olive trees from 5 to 6 meters high. A required restriction of the robot is a relatively small size, so that it can be easily transported in a van, thereby its maximum size is $100 \times 60 \mathrm{~cm}$. Apart from the sensing equipment, it mounts two easily replaceable gel batteries that power two electrical motors, an industrial computer, a folding screen, and all the electronics required to operate the robot and capture synchronised and georeferenced data. The batteries last $>6 \mathrm{~h}$ when fully charged. It is driven wireless with a remote control. A platform containing the cameras can be elevated up to $200 \mathrm{~cm}$ for better measurement of the highest trees. A programmable system has been developed to synchronise the cameras with the advance of the vehicle, so that images can be acquired at predetermined distances while the robot advances. An IMU unit allows later corrections of the inaccuracies due the irregularity of the ground. The sensors mounted on the robot included two DSLR cameras (EOS 600D, Canon Inc, Japan) one of them modified to obtain NDVI (Normalized Difference Vegetation Index) images, a multispectral camera (CMS-V, Silios Technologies, France) that can obtain simultaneous images in eight different wavelengths $(558,589,623,656,699,732,769$ and $801 \mathrm{~nm})$, a low cost hyperspectral system (spectrograph Imspector V10, Specim Spectral Imaging Ltd., Finland + camera uEye 5220CP, iDS Imaging Development Systems GmbH, Germany) and a thermal camera (A320, FLIR Systems, USA). All cameras were configured to capture one image per meter synchronised with the advance of the robot.

In addition, the entire grove was scanned using a LiDAR (LMS111, Sick AG, Germany) with an effective sweep angle of $270^{\circ}$ and resolution of $0.5^{\circ}$. All data were geolocated using a GPS (Hiper SR, TOPCON Corp. Japan) and corrected trough the data captured by a 9-axis absolute orientation sensor (BNO055, Adafruit learning system, USA). The LiDAR, the GPS and the orientation sensor units were configured to operate in free range, with the highest resolution of each sensor. Thereby, the synchronisation between data from three units was done by means of the time stamp (in ms) provided in the data streams captured by every sensor.

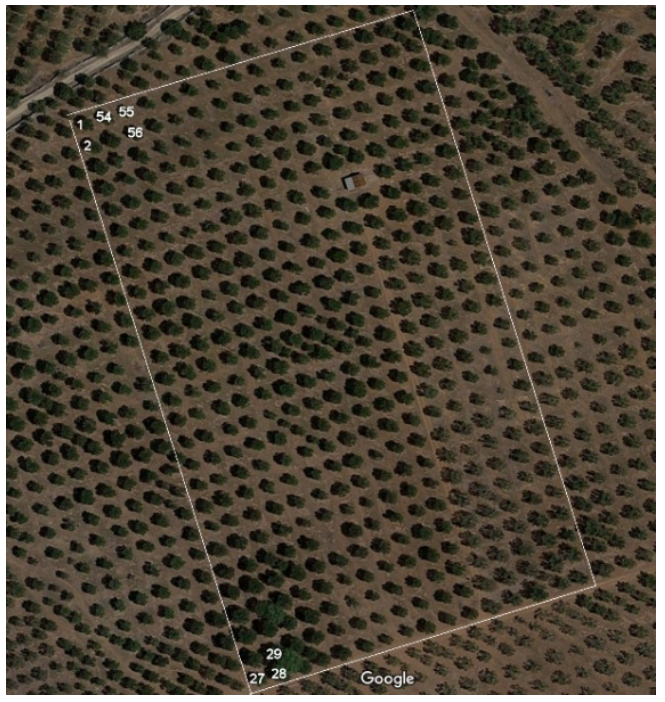

a)

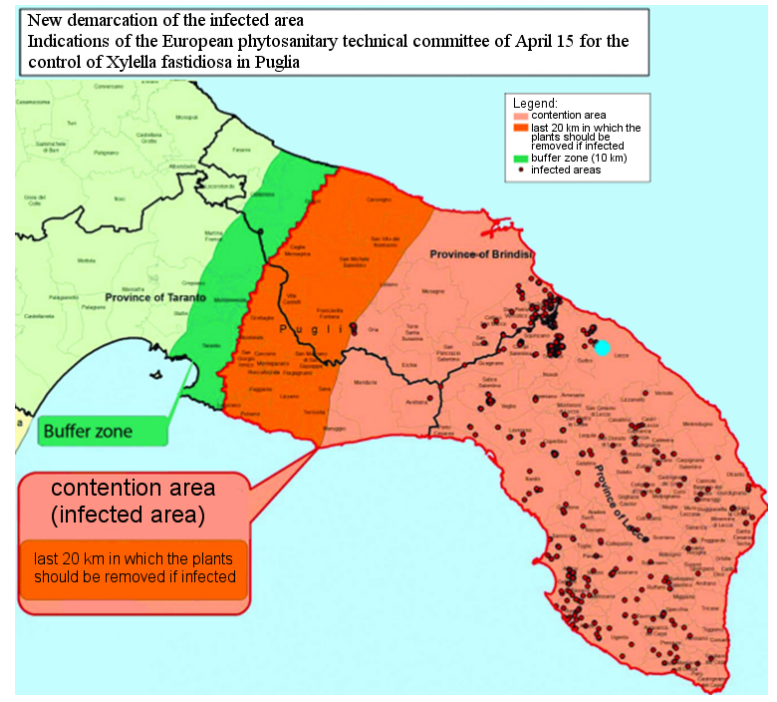

b)

Figure 1. a) Experimental olive grove located in the Lecce Province; and b) Demarcations of the infected area in the Apulia Region (Italy) including the indications of the European phytosanitary technical committee on Xylella fastidiosa.

Field tests

Two experiments were carried out in the Lecce province in September 2017 and June 2018, in an olive grove known as C20 (Fig. 1a) with a size of $3 \mathrm{Ha}$. This field is located in the contention area demarcated by the UE (Fig. 1b). The robot was deployed in the field. The robot advanced in one row acquiring the images of the trees on one side and turn back in the same row acquiring the images of the trees in the other side. During the tests, the vehicle crossed the entire field firstly in one direction (vertical) and later in the other direction (horizontal), obtaining images and data of four sides of each tree with all the sensors. On the drive up, the vehicle imaged one row of trees, imaging an adjacent row on its way back. The first tests (September 2017) were used to fine-tune the robot, electronics, setting up and programming all sensors, collecting preliminary data and improve autonomy and ease of handling, while the latest tests (June 2018) while the second tests were already carried out to collect data on 
the orchard structure and try to detect the presence of the infection in the trees. Before, during and after the inspection, images of a standardised colour checker (ColorChecker SG Chart, X-Rite Inc, USA) and a white reference target (Spectralon $99 \%$, Labsphere, Inc, NH, USA) where acquired for further correction of the images. The sensors worked correctly and the images that were obtained from the entire field are awaiting analysis.

Moreover, a visual inspection of each tree was done based on the severity of its symptoms. Each of the four sides of the tree that were then monitored by the robot were measured on a scale of 1 (no symptoms) to 5 (dead tree). However, as the field is currently active, the farmer uses to remove the branches in poor condition so the visible symptoms do not always correspond to the actual degree of infection. This is a critical problem to obtain reference data on early detection of asymptomatic trees infected but does not affect the detection of the symptoms when present. Apart from measuring the visual symptoms, some trees were analysed using techniques of molecular biology to accurately detect the presence of the infection.

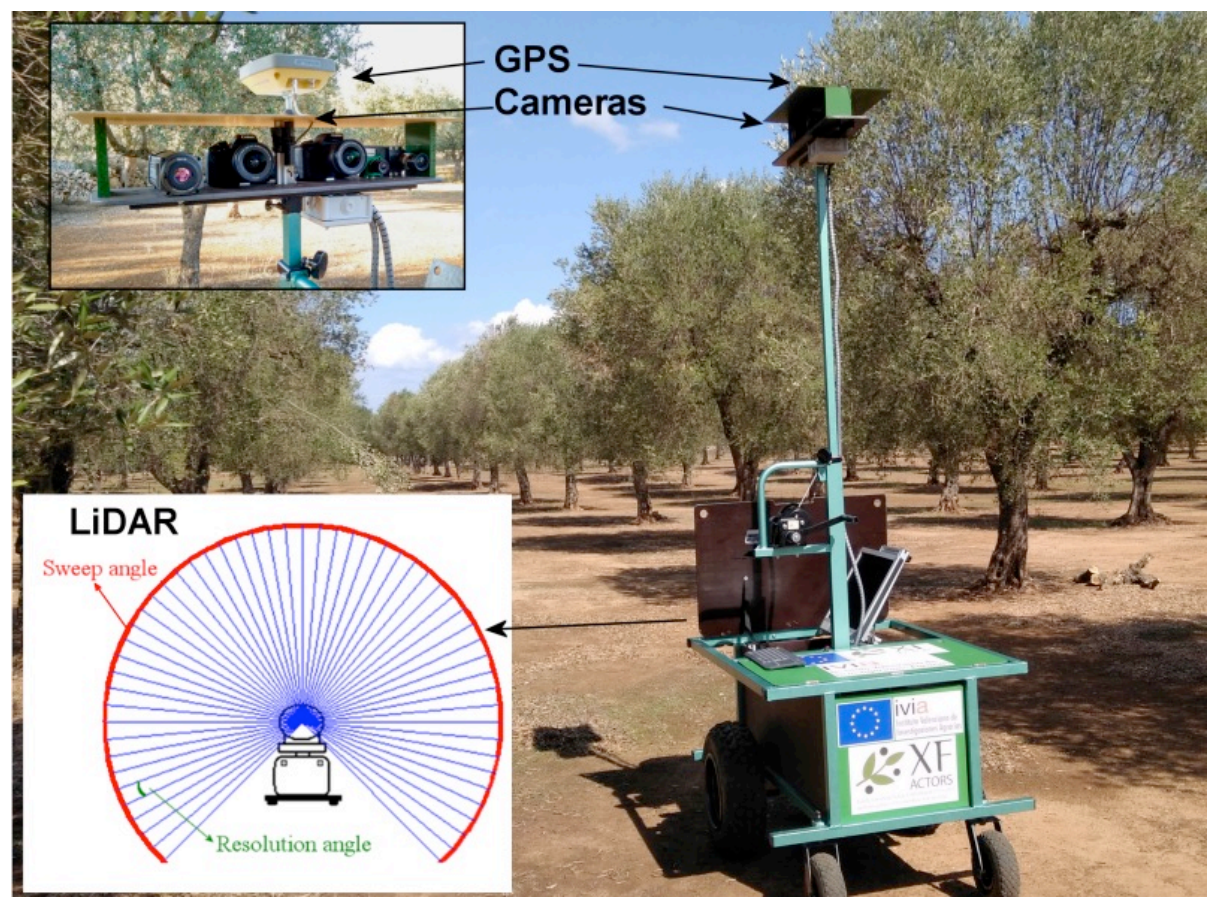

Figure 2. a) Remotely driven robot with details of the sensors mounted.

\section{Results and Discussion}

The robot work properly during the tests, being capable of continuously inspect the whole field and was able to inspect the entire field without interruptions capturing more than 77000 images. All sensors worked well capturing data synchronised with the advance of the robot. The trees were labelled following a matrix strategy, that is, in rows and columns corresponding with the navigation path of the robot, and the images captured associated to their corresponding tree. At this time, all the images captured are still under analysis. The data from LiDAR, the GPS and the IMU sensors, were stored in ASCII format, recording one file per row/column scanned. The LiDAR coordinates, originally in millimetres in a cylindrical coordinate system, were converted to meters in a Cartesian coordinate system and subsequently geolocated with the GPS reference using the WGS 84 / UTM coordinates system in the 34T zone. Later, these coordinates were corrected by means of the IMU unit using the pitch, yaw and roll angles to minimise the impact of the irregularities of the ground on the movement of the robot. Finally, Point clouds describing tree rows from opposite viewing angles were then merged to generate the full $360^{\circ}$ rendering of the trees as shown in Fig. 3. The synchronisation of the both LiDAR sensor and GPS and IMU units was done by means of the time stamp in the data streams captured with a time resolution of one millisecond. All the software to extract and process this information has been developed in MATLAB (8.5 R2015aSP1, The MathWorks Inc., USA). 

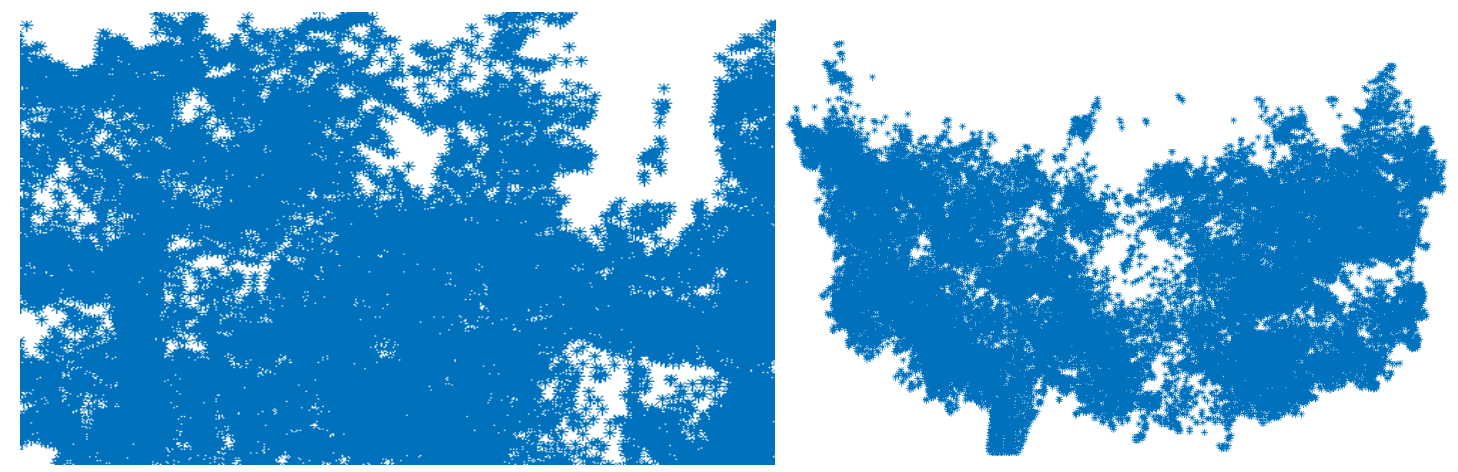

Figure 3. Three-dimensional points in UTM coordinates both representing two different views of one of the olive trees in the scanned field.

After the 3D rendering of the trees, all the points of each tree in the field were projected into the corresponding UTM zone as a top view of the field, and the contour (profile) of the top view of every tree, calculated as the convex hull, has been extracted. This profile is intended to be used to calculate the leaf area density (LAD) (1), the leaf area index (LAI or cumulative LAD) (2) and the normalised difference vegetation index (NDVI) (3). Next, the LiDAR-based renderings of the trees will be combined with spectral indices retrieved from the passive cameras.

Figure 4 shows the projection of several trees (top views) in the UTM zone superimposed on a Google satellite view of the field. The red colour has been chosen in order to highlight the dots projection from the satellite picture.

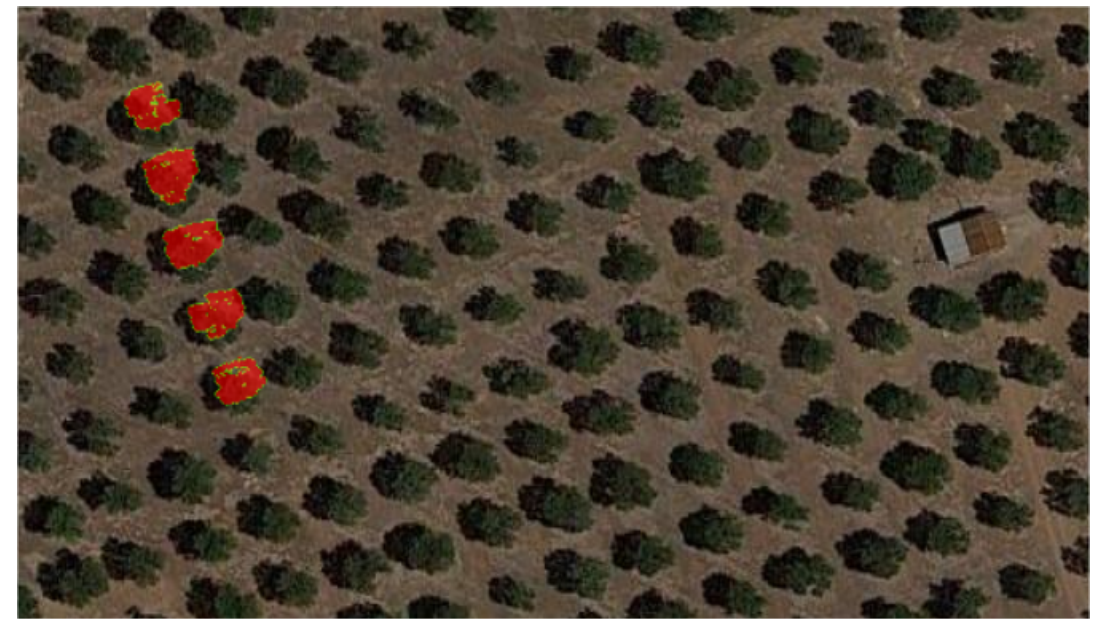

Figure 4. Projection of the 3D scanned points superimposed on a Google satellite view of the field.

$$
\begin{aligned}
& L A D=u(z) \\
& L A I(z)=\int_{z=0}^{h} L A D(z) d z ; \quad \begin{array}{ll}
\operatorname{In~}^{2} \mathrm{~m}^{-2} \text { units, where } h \text { goes from } 0 \\
\operatorname{LAD}(\mathrm{z})=\text { surface density coefficient; }
\end{array} \\
& N D V I=\frac{N I R-V I S}{N I R+V I S} \quad \begin{array}{l}
\mathrm{NIR}=\text { near infrared reflectance; } \\
\mathrm{VIS}=\text { visible reflectance; }
\end{array}
\end{aligned}
$$

\section{Acknowledgements}

This work was partially supported by funding from the European Union's Horizon 2020 research and innovation programme under grant agreement No 727987 Xylella Fastidiosa Active Containment Through a multidisciplinary-Oriented Research Strategy (XF-ACTORS)

\section{References}

Calderón R, Navas-Cortés JA, Zarco-Tejada PJ. 2015. Early detection and quantification of Verticillium wilt in olive using hyperspectral and thermal imagery over large areas. Remote Sensing 7: 5584-5610. 
Martinelli F, Scalenghe R, Davino S, Panno S, Scuderi G, Ruisi P, Villa P, Stroppiana D, Boschetti M, Goulart LR, Davis CE, Dandekar AM. 2015. Advanced methods of plant disease detection. A review. Agronomy for Sustainable Development 35: 1-25.

Martelli GP, Boscia D, Porcelli F, Saponari M. 2016. The olive quick decline syndrome in south-east Italy: A threatening phytosanitary emergency. European Journal of Plant Pathology 144: 235-243.

Purcell AH. 1997. Xylella fastidiosa, a regional problem or global threat? J Plant Pathol. 79: 99-105.

Vicent A, Blasco J. (2017) When prevention fails. Towards more efficient strategies for plant disease eradication. New Phytologist, 214: 905-908. 


\title{
Non-Destructive Firmness Assessment of Avocado Fruit Using a Pneumatic Low Impact Device
}

\author{
Coral Ortiz $^{a^{*}}$, Carlos Blanes ${ }^{b}$, Martín Mellado ${ }^{b}$ \\ a Rural and Agri-food Engineering Department, Universitat Politècnica de València, Valencia 46022, Spain \\ ${ }^{\mathrm{b}}$ Instituto Universitario de Automática e Informática Industrial, Universitat Politècnica de València, Valencia 46022, \\ Spain \\ * Corresponding author. Email: cortizz@dmta.upv.es
}

\begin{abstract}
The decrease of fruit firmness is a physiological process that occurs during fruit ripening. Avocado fruit is one of the most rapidly ripening fruits. Different methods have been developed and used for firmness measurement. The objective of this research study was to test a developed laboratory prototype designed for the non-destructive assessment of avocado firmness. A set of 150 'Hass' variety avocados, size 16, were selected, divided in 5 sets of 25 avocados and stored in the laboratory under room conditions. In order to assess firmness decrease, in every measuring session during 5 days, a set of 25 avocados was measured, destructively and non-destructively. The non-destructive firmness measuring prototype was an impact of a pad actuated with a pneumatic low friction cylinder. The pad and the cradle have a vacuum jamming system capable to be adapted to the irregular fruit shape. An accelerometer, located at the base of the pad, registered the deceleration of the rod. Signal were processed for getting three non-destructive parameters. After the non-destructive test, the fruits were destructively measured by a compression test. The designed prototype is capable to register data related to the avocado firmness without damaging the fruit. Non-destructive parameters registered from the prototype were highly correlated to the destructive firmness. A multiple regression model explained destructive firmness according to the non-destructive parameters from the prototype $(\mathrm{R} 2=77.4 \%)$. Further research should be done in order to improve the adjustment of the prototype to assess avocado firmness.
\end{abstract}

\section{Introduction}

The avocado (Persea americana Mill.) is a climacteric fruit. This fruit has an increase in ethylene production at the beginning of ripening (Jeong et al., 2002). This climacteric increase in ethylene production is associated with faster ripening speed. Avocado is one of the most rapidly ripening fruits, often finishing ripening 5 to 7 days after harvest (Seymour and Tucker, 1993). The decrease of fruit firmness is a physiological process that occurs during fruit ripening.

Different methods have been developed and used for firmness measurement. Traditional measuring methods, like Effegi-tester and conical probes, are based on destructive procedures (Mazhar et al., 2014). Some nondestructive devices has been developed as the Sinclair iQ Firmness Tester, the Electronic Firmometer and other based on low mass impact (Chen and Tjan, 1998; Valero et al., 2007). However, the used of this devices for avocado firmness analysis is not extensively applied.

The objective of this research study was to test a developed laboratory prototype designed for the nondestructive assessment of avocado firmness.

\section{Materials and Methods}

Materials

A set of 150 'Hass' variety avocados, size 16, were selected and divided in 5 sets of 25 avocados, Figure 1. Fruits were stored in the laboratory under room conditions $\left(22.9 \pm 2.8^{\circ} \mathrm{C}\right.$ and $64.8 \pm 3.0 \%$ relative humidity). In order to assess firmness decrease, in every measuring session (Monday-1, Tuesday, Thursday, Friday and Monday-2) a set of 25 avocados was measured, destructively and non-destructively.

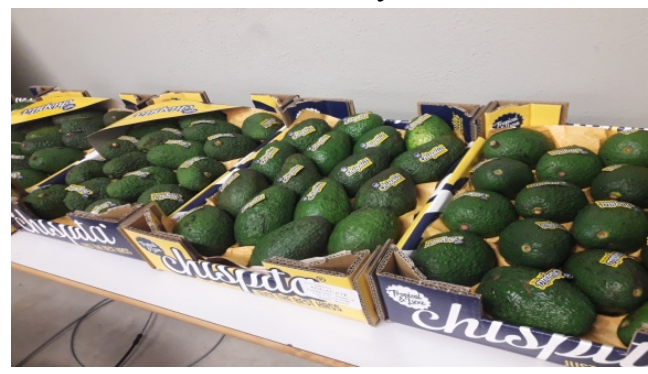

Figure 1. 'Hass' avocado sets. 
The non-destructive firmness measuring was provided by the analysis of the impacts of a pad, moved by a low friction pneumatic cylinder (SMC MQQTB16-50D), against every avocado. Figure 2. The pad located at the pneumatic cylinder end of rod (A) and the pad located at the cradle $(C)$ has a vacuum jamming system (A) capable to be adapted to the irregularities of fruit shape. A mono-axial accelerometer ADXL278, attached to the pad (A), registered impacts decelerations. Signal was transformed according to a Labview program.

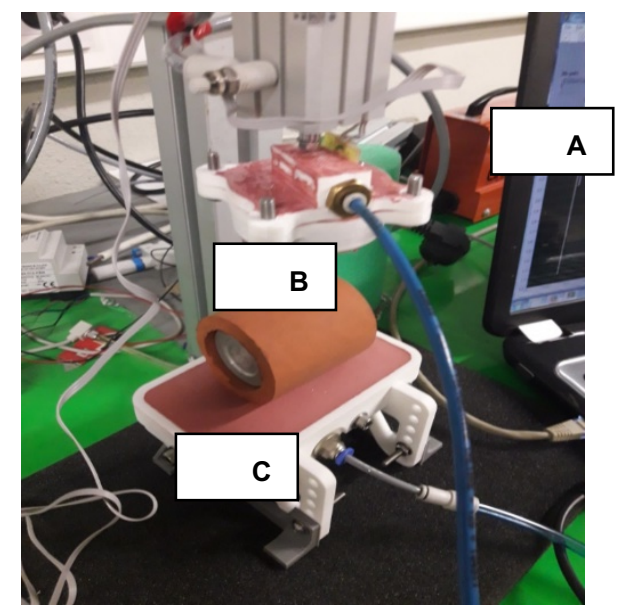

Figure 2. Prototype device used for assessment of avocados firmness. (A) Pneumatic cylinder end of rod, (B) rubber cylinder for calibration, (C) cradle.

Methodology

All the fruits were non-destructively measured at two points, one at the middle of the outer equatorial surface skin side (A) and at the opposite side (B). Five repetitions were done per side.

Weight and diameter of all the avocados was also measured before the destructive test.

After being measured by the non-destructive prototype, the fruits were destructively measured by a compression test ( $5 \mathrm{~mm}$ deformation, $10 \mathrm{~mm}$ rod diameter and $0.001 \mathrm{~m} / \mathrm{s} \mathrm{speed}$ ) using a universal test machine (Ibertest, www.ibertestint.com). Three destructive firmness measurements were done per fruit, one at the same point and two other more at the equatorial surface.

Every measuring session, the prototype was calibrated with a rubber cylinder (Figure 2, B) with a steel centre (63 $\mathrm{mm}$ length, $52 \mathrm{~mm}$ diameter, $7.8 \mathrm{~mm}$ width and $62.2 \mathrm{~N}$ compression resistance $(5 \mathrm{~mm}$ deformation, $10 \mathrm{~mm}$ rod diameter and $0.001 \mathrm{~m} / \mathrm{s}$ speed).

Impact device operation

Jamming transition of granular material provide the possibility to control the hardness of the pads A and C (Jaeger 2014).

Avocados were placed over the cradle B. During 0.03 seconds A and C pads are blown ensuring soft behavior during the first impact. After that, negative pressure was applied inside the pads. Pads changed from soft to hard. This process ensures that the pads have the shape of every avocado analyzed. After that five impacts per side were done to every avocado and its signals were recorded and processed in a computer.

Three non-destructive parameters were obtained from the processing of the accelerometer registered data. Only the deceleration of the impact time was analysed and processed to obtain the three parameters: VE (energy of the impact), AvSlpA (average of the deceleration slope at the first contact) and MaxA (maximum values).

\section{Results and Discussion}

Destructive firmness, measurement by destructive methodology, decrease along time as it was expected (Figure 3). 


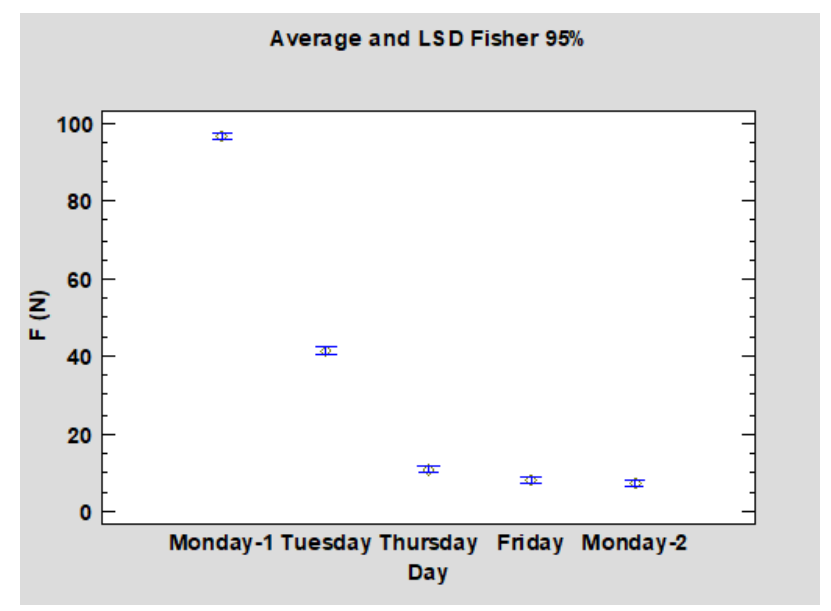

Figure 3. Destructive firmness (F, N) along days.

Analysing the development of the non-destructive parameters extracted from the prototype along days, it was confirmed a very similar decrease, Figure 4.

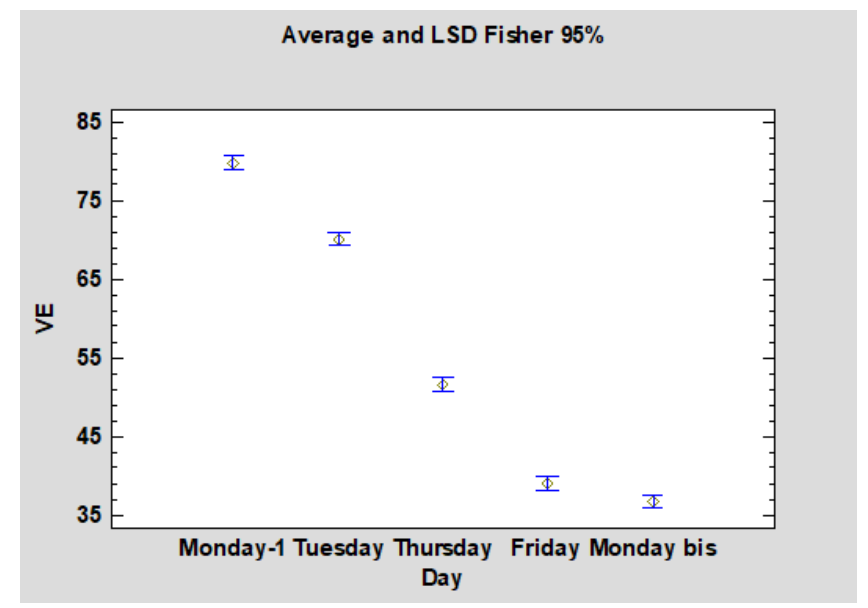

Figure 4. Non-destructive measurement (VE) along days.

According to the Duncan's multiple range test, the non-destructive parameters obtained VE, AvSlpA or MaxA, was significantly different every day.

The three non-destructive parameters obtained from the prototype were highly correlated to the destructive firmness (correlation coefficient higher than 0.8 in all the cases).

A multiple way ANOVA analysis was developed in order to assess the effect of the different factors studied. The side of the avocado or the repetition did not significantly affect the non-destructive parameters, Table1. The measurements at both sides and the five repetitions at each side were similar. This fact confirmed the possibility of only testing one of the sides and carrying out one repetition per fruit.

Table 18. Multiple ways ANOVA analysis of the effect of the factors day, side and repetition on the parameters AvSlpA

\begin{tabular}{cccccc}
\multicolumn{7}{c}{ extracted from the prototype. } \\
\hline Source & Sum of squares & $\mathrm{df}$ & Mean squareo & F-ratio & $\mathrm{p}$-value \\
\hline A:Day & 86,2602 & 4 & 21,5651 & 588,65 & 0,0000 \\
B:Side & 0,0019805 & 1 & 0,0019805 & 0,05 & 0,8161 \\
C:Repetition & 0,0596683 & 4 & 0,0149171 & 0,41 & 0,8036 \\
& & & & & 1,79 \\
AB & 0,262783 & 4 & 0,0656958 & 0,1278 \\
AC & 0,0846703 & 16 & 0,00529189 & 0,14 & 1,0000 \\
BC & 0,00135052 & 4 & 0,000337629 & 0,01 & 0,9998 \\
Residuals & 44,5112 & 1215 & 0,0366347 & & \\
Total & 131,182 & 1248 & & & \\
\hline
\end{tabular}


Finally, a multiple regression model was carried out in order to relate destructive firmness to the nondestructive parameters, Figure 3 . A $77.4 \%$ of the variability was explained by the model (1).

$\mathrm{F}(\mathrm{N})=-129.35+0,75 *$ weight $+1,99 * \mathrm{MAX}-253,10 * \mathrm{AV}$

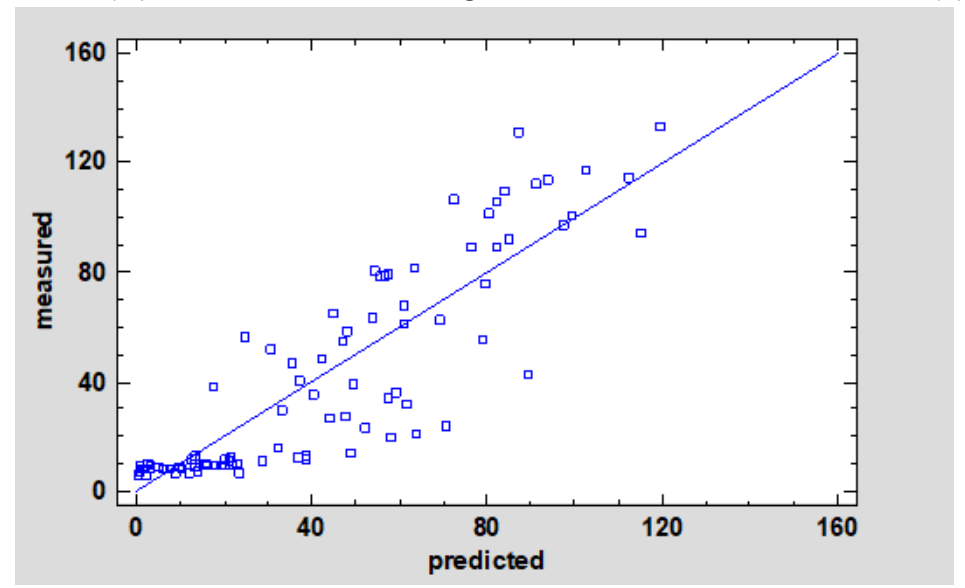

Figure 5. Observed and predicted data from the multiple regression to explain destructive firmness with the non-destructive parameters from the prototype.

\section{Conclusions}

The designed prototype is capable to register data related to the avocado firmness without damaging the fruit. Non-destructive parameters registered from the prototype were highly correlated to the destructive firmness. A multiple regression model explained destructive firmness according to the non-destructive parameters from the prototype $(\mathrm{R} 2=77.4 \%)$. Further research should be done in order to improve the adjustment of the prototype to assess avocado firmness.

\section{References}

Howarth, M.S., I. Shmulevich, C. Raithatha, Y. Ioannides. 2003. Online non-destructive avocado firmness assessment based on low-mass impact technique. Proceedings V World Avocado Congress, 679-685

Mazhar, M., D. Joyce, A. Lisle, R. Collins, P. Hofman. 2014. Comparison of firmness meters for measuring 'Hass' avocado fruit firmness. ISHS Acta Horticulturae 1119: XXIX International Horticultural Congress on Horticulture (DOI: 10.17660/ActaHortic.2016.1119.22)

Seymour, G.B., J. Tayler, G.A. Tucker. 1993. Biochemistry of Fruit Ripening, Chapman and Hall, London (1993), pp. 53-81

Jeong, J., D.J. Huber, S.A. Sargent. 2002. Influence of 1-methylcyclopropene (1-MCP) on ripening and cellwall matrix polysaccharides of avocado (Persea americana) fruit. Postharvest Biology and Technology 25, 241256

Chen, P., Y. Tjan. 1998. A real-time impact sensing system for online firmness sensing. Proceedings of the Ag. Eng. Conference, Oslo (1998). Paper 98-F-006

Valero, C., C.H. Crisosto, D. Slaughter. 2007. Relationship between nondestructive firmness measurements and commercially important ripening fruit stages for peaches, nectarines and plums, Postharvest Biology and Technology, 44 (3), 248-253, ISSN 0925-5214,

(DOI: $10.1016 /$ j.postharvbio.2006.12.014).

Jaeger, H., 2014. Celebrating Soft Matter's 10th Anniversary: Toward jamming by design. Soft matter, 11(1), pp.12-27. 


\title{
Current Potentials and Challenges using Sentinel-1 for Broadacre Field Remote Sensing
}

\author{
Martin Peter Christiansen a, ${ }^{\text {a }}$, Morten Stigaard Laursen ${ }^{a}$, Birgitte Feld Mikkelsen ${ }^{\mathrm{b}}$, Nima Teimouria ${ }^{\mathrm{a}}$, \\ Rasmus Nyholm Jørgensen ${ }^{\mathrm{a}}$, Claus Aage Grøn Sørensen ${ }^{\mathrm{a}}$ \\ a Department of Engineering, Aarhus University, Finlandsgade 22, 8200 Aarhus N, Denmark \\ ${ }^{b}$ AgroTech, Danish Technological Institute, Agro Food Park 15, 8200 Aarhus N, Denmark \\ ${ }^{c}$ SDU UAS Center, University of Southern Denmark, Campusvej 55, 5230 Odense M, Denmark \\ * Corresponding author. Email: mapc@mmmi.sdu.dk
}

\begin{abstract}
ESA operates the Sentinel-1 satellites, which provides Synthetic Aperture Radar (SAR) data of Earth. Recorded Sentinel-1 data have shown a potential for remotely observing and monitoring local conditions on broad acre fields. Remote sensing using Sentinel-1 have the potential to provide daily updates on the current conditions in the individual fields and at the same time give an overview of the agricultural areas in the region. Research depends on the ability of independent validation of the presented results. In the case of the Sentinel-1 satellites, every researcher has access to the same base dataset, and therefore independent validation is possible. Well documented research performed with Sentinel-1 allow other research the ability to redo the experiments and either validate or falsify presented findings. Based on current state-of-art research we have chosen to provide a service for researchers in the agricultural domain. The service allows researchers the ability to monitor local conditions by using the Sentinel-1 information combined with a priori knowledge from broad acre fields. Correlating processed Sentinel-1 to the actual conditions is still a task the individual researchers must perform to benefit from the service. In this paper, we presented our methodology in translating sentinel-1 data to a level that is more accessible to researchers in the agricultural field. The goal here was to make the data more easily available, so the primary focus can be on correlating and comparing to measurements collected in the broadacre fields. We illustrate the value of the service with three examples of the possible application areas. The presented application examples are all based on Denmark, where we have processed all sentinel-1 scan from since 2016.
\end{abstract}

Keywords: synthetic aperture radar, crop monitoring, backscatter analysis, crop growth stages, winter wheat

\section{Introduction}

Free Big data have become available in recent years from different remote sensing sources. Both optical and synthetic aperture radar (SAR) remote sensing solutions are possible such as the Sentinel-1 (Torres et al. 2012), and Sentinel-2 (Drusch et al. 2012) satellites (ESA, Paris, France) within the European Copernicus program and the Landsat-7 (Roy et al. 2016) and Landsat-8 (Roy et al. 2014) provided in cooperation between the National Aeronautics and Space Administration and U.S. Geological Survey. Data from these different remote sensing satellite solutions are freely available and have the potential to open up a broad spectrum of monitoring and prediction applications for the agricultural domain (Dalla Mura et al. 2015), (Kussul et al. 2017).

The Sentinel-1 satellite data have already been used in many articles related to the agricultural domain with interesting results (Erten et al. 2016), (Zhou, Li, and Pan 2018), (Jin et al. 2018), (Kumar et al. 2017). The Sentinel1 data over agricultural fields have been used to estimate parameters such as crop type, plant height, biomass, Green Area Index (GAI) and vegetation water content.

Scientific research depends on the ability to perform an independent validation of the presented results (Sagan 2011). In the case of the Sentinel-1 satellites, every researcher have access to the same base data set and therefore independent validation is possible. Well documented research performed with Sentinel-1 remote sensing data will allow other research the ability to redo the experiments and either validate or falsify findings.

To estimate different crop parameters, one requires reference data from actual fields or a priori created knowledge from literature and research projects. The demands from agricultural to Sentinel-1 and remote sensing satellites in general means reference data is highly relevant for the development process of estimation methods. Public available reference data provides a way to ensure researchers in the same field have the chance to redo the processing and validate the results.

Another challenge is the level of understanding one needs to have in pre-processing sentinel-1 data (Pierdicca, Pulvirenti, and Pace 2014), before an estimation and correlation in an agricultural context can be performed. The needed level of understanding for Sentinel-1 pre-processing creates a need to provide well-documented steps for agricultural researchers to follow. Well documented pre-processing step ensures data are readily available for experts outside the SAR research field and also allows reproduction on the same experiments to either falsify or validate previously created results. The challenge is the number of details one needs to document of these satellitebased systems, to ensure other researchers can reproduce the results. 
Based on current research and evaluated results, we currently provide a service for researchers in the agricultural domain. The service allows researchers in the agricultural domain the ability to monitor local conditions by using the recorded sentinel-1 information combined with a priori knowledge from broad acre fields. Correlating the possessed sentinel- 1 to actual conditions is still a task the individual researchers must perform to utilize the service.

\section{Materials}

Satellite data sources

Different free data sources are available for remote sensing with an agricultural use case (Roy et al. 2014), (Li, Jiang, and Feng 2014), (Bosch et al. 2016), (Berger et al. 2012). Currently, only the ESA's Sentinel satellite missions continually provide free sensory data for both multispectral and SAR images via the Copernicus data hub. From the Copernicus open access data hub (Müller et al. 2016), (Klein et al. 2017), one can request and download Sentinel- 1, 2 and 3 products recorded over a specific region. The Sentinel data products need to be processed using software such as SNAP before one can utilize it for a particular purpose.

\section{Sentinel-1}

Two products created from Sentinel-1 recordings has been analyzed in the agricultural domain, which is Single Look Complex (SLC), and Ground Range Detected (GRD) SAR data (Torres et al. 2012). Significant parts of the current research for the agricultural domain using Sentinel-1 data have been focusing on GRD since it comes more preprocessed and has four to five times smaller package size compared SLC. The GRD standard products come as either $\mathrm{VV}+\mathrm{VH}$ or $\mathrm{HH}+\mathrm{HV}$, with a swath of $250 \mathrm{~km}$ (Torres et al. 2012). On a global scale where broadacre crops are grown, Sentinel-1 mainly records data for the VV or VV+VH polarizations. For the growth season 2016/17 in Denmark, Sentinel GRD VV+VH data (dual polarisation) are available, from the two sentinel-1 satellites, with one to three days in between. The GRD products come in both ascending and descending recording mode and from different satellite tracks.

\section{External services to combine with the Sentinel data}

In agriculture, Sentinel-1 satellite data generally needs to be combined with a prior knowledge about SAR measurements to land and vegetation, or with models created by correlating local observations from broad acre fields with the SAR measurements. In this section, we present the different external data sources relevant when analysing Sentinel-1 data.

\section{Land-parcel identification system}

The Land-parcel identification system (LPIS) can be used retrieve information about active fields for each season and what the individual applicants are currently growing. The applicant is the farmer which has requested funding from the European Agricultural Guarantee Fund (EAGF) for a specific field. The field boundaries are drawn manually, by the individual applicant, on orthophotos recorded with planes over Denmark. The farmers are expected to exclude unfarmed land and ineligible features from the recorded field boundaries (Phil Wynn Owen et al. 2016). According to the EAGF, the estimated error level in LPIS was $2.9 \%$ in 2014, and close to half was related to the marking of the correct area. An error level $2.9 \%$, makes LPIS data an interesting reference source and annotated dataset for analysing Sentinel data from individual fields. Combining Sentinel-1 time series and LPIS data could be a future means to track and predict errors in the data supplied by the individual farmer (Giasat s.r.o 2017).

\section{Nordic field trial system}

Nordic Field Trial System (NFTS) is a repository provided by SEGES and Danish Technological Institute to design experiments plans and store recorded data from field trials (Hansen et al. 2004). Experiments from Denmark, Norway, and Sweden have been recorded using NFTS since 2006. The NFTS repository is a source of reference datasets with fields spread out all over Denmark and the other Nordic countries. NFTS is an open access repository that provides the public with the ability to retrieve broadacre field experiment plans and results via their homepage. Growth stage, crop treatment, sensor recordings, yield estimates for different crop types are some of the relevant parameters that can be extracted from the NFTS repository. Globally alternative data sources exist such as the AgTrials repository that attempts to use a standardized definition from the Crop Ontology for all measurements (Hyman et al. 2017). 


\section{Fieldbabel service}

The Fieldbabel service is intended to allow agricultural researchers the ability to rapidly compare and correlate data collected in the field with remote sensing measurement from satellites. The fieldbabel service is built on open-source software to handle preprocessing of the request from the users and export the results as QGIS projects (Shelestov et al. 2013). Commercial alternatives exist to QGIS, but in this case, we are attempting to keep the source code and functionality open to researchers without the need to pay additional fees for using the service.

Sentinel Application Platform (SNAP) is the standard software tool provided by ESA (Zuhlke et al. 2015), jointly developed by Brockmann Consult, Array Systems Computing and C-S, to process data from the Sentinel satellites. The Fieldbabel service is based upon the SNAP API and tools from ESA to process and extract the relevant area.

Python 2.7 running on an Ubuntu 16.04 server is used for both the frontend and backend parts of the presented service. The frontend is based on the python Flask API (Grinberg 2018) which is a micro web framework and template development. Shapefiles added to the Qgis project are handled using python Shapefile Library (Butler 2005), which can be used to process, store and exchange GIS information.

Methods

\section{Sentinel-1 GRD preprocessing pipeline}

In this section, we present the processing steps that are used on the field babel service for preprocessing the different sentinel satellite data sources. The preprocessing pipeline performs the procedure illustrated in the SNAP graph in Figure 47.

Read $\rightarrow$ Calibration $\rightarrow$ Speckle-Filter $\rightarrow$ Terrain-Correction $\rightarrow$ Write

Figure 47. The preprocessing stages used on the fieldbabel server. The raw GRD are read, processed in the SNAP toll and the result saved on the filedbabel server.

The GRD product is loaded with the SNAP tool and calibrated to the sigma nought area on the ground (Sabel et al. 2012), providing a layer in which the values are directly related to the radar backscatter (Sabel et al. 2012). To remove noise, a Lee sigma speckle filter (Lee et al. 2009) with a single look; a window size of 7x7; a sigma value of 0.9 and a target window size of $3 \times 3$ is used. The terrain correction operation is intended to compensate for distortions so the geometry of the layer will be as close as possible to the actual world. For terrain correction, the pipeline utilizes the Range doppler terrain correction (Schubert et al. 2015) with the digital elevation model parameter set to "SRTM 1Sec. HGT" and the pixel spacing of $10 \mathrm{~m}$.

\section{Fieldbabel processing of requests}

The Fieldbabel server follows the processing flow illustrated in Figure 48. The processes are split up into two stages; one is the web-service that handles the user requests directly, and the second is the actual processing and creation of the Qgis project.
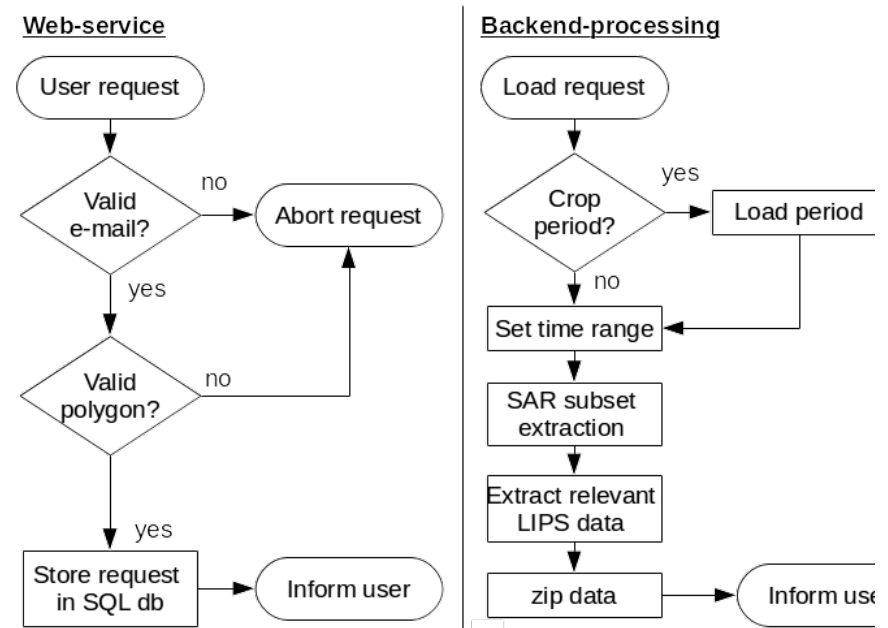

Figure 48. Flowchart of the fieldbabel server processing of requests. 
Web-service

The web-service is designed to handle user-requests for their selected areas of Denmark. A Geojson polygon and an e-mail are submitted and evaluated via the web interface. If the e-mail address is valid and the geojson contains a single polygon with an area no larger than a single degree latitude/longitude, the request is stored in the SQL database on the server and set for processing.

\section{Backend-processing}

The processing is performed using a background process running on the Linux server. The server loads the relevant period for the selected area. The server fetches the relevant preprocessed sentinel data for the selected period and area and exports the layers as GeoTIFF's. The backend processing utilises the layers created with the preprocessed pipeline, by extracting a subset of the layer and converting the VV and VH values into dB scale. Based on the Sentinel-1 output from the preprocessing pipelines, the GeoTIFF values for the red, green and blue bands are represented by $\mathrm{VV}(\mathrm{dB})$ and $\mathrm{VH}(\mathrm{dB})$ and $\mathrm{VV}(\mathrm{dB}) / \mathrm{VH}(\mathrm{dB})$ respectively.

\section{Qgis project creation}

The GeoTIFF files are packaged as a Qgis project for the request with preset color ranges for each band. The visual color range for the GeoTIFF in Qgis is set by having analyzed the value spectrum of all layers over a whole year, in a specific track of Sentinel-1 scans from Denmark. The relevant LPIS data for the same period and area is added to the Qgis project as a final step before being compressed into a zip file. When the Qgis project have been packed into a zip file, the user is informed with a link for the project to download.

\section{Crop selection for analysis}

Information about the start and end of a crops regular growing season can be used to track the temporal behavior in the recorded Sentinel-1 data. Local weather and geographic specific conditions impact the growing season for any given area, making it impossible to provide exact dates. Different crops are seeded and harvested at particular periods of the year, for Nordic countries such as Denmark, since they are seasonally dependent. Information about crop seasons is highly relevant for researchers outside the agricultural domain, such as engineers with images and signal processing backgrounds. The temporal information about the crop ensures researchers the ability to analyse the behavior when they are active. By researching the normal seeded and harvesting for different crop types, this information has been added to the web-service. The field babel service can extract the relevant Sentinel-1 scans for the chosen period, based on the selected crop type.

The primary source for determining crop seeding and harvesting time is the homepage https://dyrkplant.dlbr.dk/, which is a guide for Danish farmers. Since local wheat and other external factors still impact the growing season, the time-period has been extended with a month in each end. The current stored crop period is documented in Table 19, where $\mathrm{xx}$ in the date representing the year. A plus or minus year value indicates if the crops season is part of multiple years.

Table 19. Table with chosen crop type, seasonal start and end date, when extracting data from the pre-processed Sentinel-1 layers on the Fieldbabel server.

\begin{tabular}{cccc}
\hline $\begin{array}{c}\text { Danish name } \\
\text { (LPIS entry) }\end{array}$ & $\begin{array}{c}\text { English name } \\
\text { (Fieldbabel) }\end{array}$ & start date & end date \\
\hline & All & $01-01-20 \mathrm{xx}$ & $31-12-20 \mathrm{xx}$ \\
Majs & Corn & $15-03-20 \mathrm{xx}$ & $15-11-20 \mathrm{xx}$ \\
Vårbyg & Spring barley & $01-03-20 \mathrm{xx}$ & $01-09-20 \mathrm{xx}$ \\
Sukkerroer & Sugar beat & $01-04-20 \mathrm{xx}$ & $01-02-20 \mathrm{xx}(+1)$ \\
Vårraps & Spring rape & $01-03-20 \mathrm{xx}$ & $01-10-20 \mathrm{xx}$ \\
Vinterraps & Winter rapeseed & $01-07-20 \mathrm{xx}(-1)$ & $01-08-20 \mathrm{xx}$ \\
Vinterhvede & Winter wheat & $15-08-20 \mathrm{xx}(-1)$ & $01-10-20 \mathrm{xx}$ \\
\hline
\end{tabular}

Case studies

\section{Crop-type distinction}

Classification and mapping of crop-types have been a common focus for remote sensing (Lopez-Sanchez and Ballester-Berman 2009) for the last decade. The sensitivity of C band SAR data to crop type different features have been validated in (Picard, Le Toan, and Mattia 2003). A number of articles have been published, which indicate that sentinel-1 data can be used to distinguish crop types, by training based on known reference data 
(Skriver et al. 2011).

Data from the LPIS database can be a means to validate the Qgis projects created with Fieldbabel, by determining if different crop types are visually distinguable. For this paper an area with an bounding box of (WGS84: latitude $\min _{\text {nin }} 55.2025$, longitude $\min 10.3275$ and latitude $\max _{\max }$ 55.0700, longitude $\max _{\text {ax }}$ 10.5839), was chosen from the 1 st of june 2017 where the significant crop types in Denmark are in their late stages of development with the exception of maize and beets (Olesen et al. 2012).

\section{Sampling of field data}

Collecting reference data from broadacre fields to correlate Sentinel-1 measurements against can be a timeconsuming and labor-intensive task. The goal in collecting reference data is not to cover each field meter by meter, but to acquire samples from larger areas where $\mathrm{VV}$ or $\mathrm{VH}$ is reporting variation compared to the rest of the field. One way to perform this automatically would be to download the newest Sentinel-1 datasets and cluster areas with high and low values for either VV or VH.

In this example, a field analysed by shrinking the LPIS field boundary with $30 \mathrm{~m}$ using erosion. The intention with the erosion step is only to allow crop values to be part of the time-temporal series, by shrinking the inner area. The $30 \mathrm{~m}$ value is chosen based on three factors; an assumption that each processed layer have maximum alignment error of $\pm 10 \mathrm{~m}$; the headland of the field is the least consistently seeded; since the farmers manually draw the LPIS field, erosion of the boundaries ensures the processing avoids input from minor manual drawing inaccuracies.

To determine where a sample should be collected, a K-mean clustering algorithm for grids is used to evaluate the distribution of measurements. The K-mean algorithm is set to search with three cluster bins, based on an approach that combines hill-climbing (Wu et al. 2008) and iterative minimum distance (Forgey 1965). The number of cluster bins is set to three to evaluate the homogeneity of the field and cluster the measurements into the main areas of variation. For this paper, fields with only a few meters of variation in elevation is select and analysed, using the k-mean clustering method.

\section{Phenological stages}

The web-service allows researchers the ability to monitor the full crop growth by obtaining timely information about the crop condition regarding sentinel-1 data. According to literature (Veloso et al. 2017) the VV/VH profile are in good agreement with the GAI for winter wheat. GAI is measured as the ratio of green material in the ground area it covers. The GAI index is known to follow the growth stages of the crop. According to (Gerry Boyle et al. 2016 ) the GAI will peak between growth stage $39-59$ o for winter wheat. This make is interesting to attempt to compare growth stage and the $\mathrm{VV}(\mathrm{dB}) / \mathrm{VH}(\mathrm{dB})$ ration as a time series, to validate if the same peak scenario is present. If $\mathrm{VV}(\mathrm{dB}) / \mathrm{VH}(\mathrm{dB})$ ratio follows the $\mathrm{GAI}$ it could indicate sentinel-1 data can be used to provide estimates of the current growth stage of winter wheat.

For trial 010811616 in the NFTS database, the progress of winter wheat growth stage has been noted throughout the growing season. In trial 010811616, there exist five fields scattered throughout Denmark, where the same experiment was performed. Other trails in the NFTS have also logged the growth stages of winter wheat and other crops, but trial 010111616 was the only one found where notation was done consistently for all fields.

\section{Results and Discussion}

The result resection focuses on a number of example cases to illustrate what the service can be used for in the agricultural domain. The results are based on a combination of literature study and experiments using the collected data.

\section{Crop type distinction based on Qgis data}

The different crop-field types in the Sentinel-1 image processed with the per-processing pipeline can be seen in Figure 49, which was recorded on the first of june 2017. Fields with maize, spring barley and winter rape are directly distinguishable in Figure 49, since the color patterns differ significantly. Winter barley and wheat seems to have identical structures in the produced Qgis image. The similarity between winter barley and wheat is expected since they would look identical in the $10 \mathrm{~m}$ pixel level this time of year. One should note the arrow in Figure 49, where a field seems to contain both winter rape and winter wheat. The marked area might indicate an error made by the farmer when drawing the field boundary. 


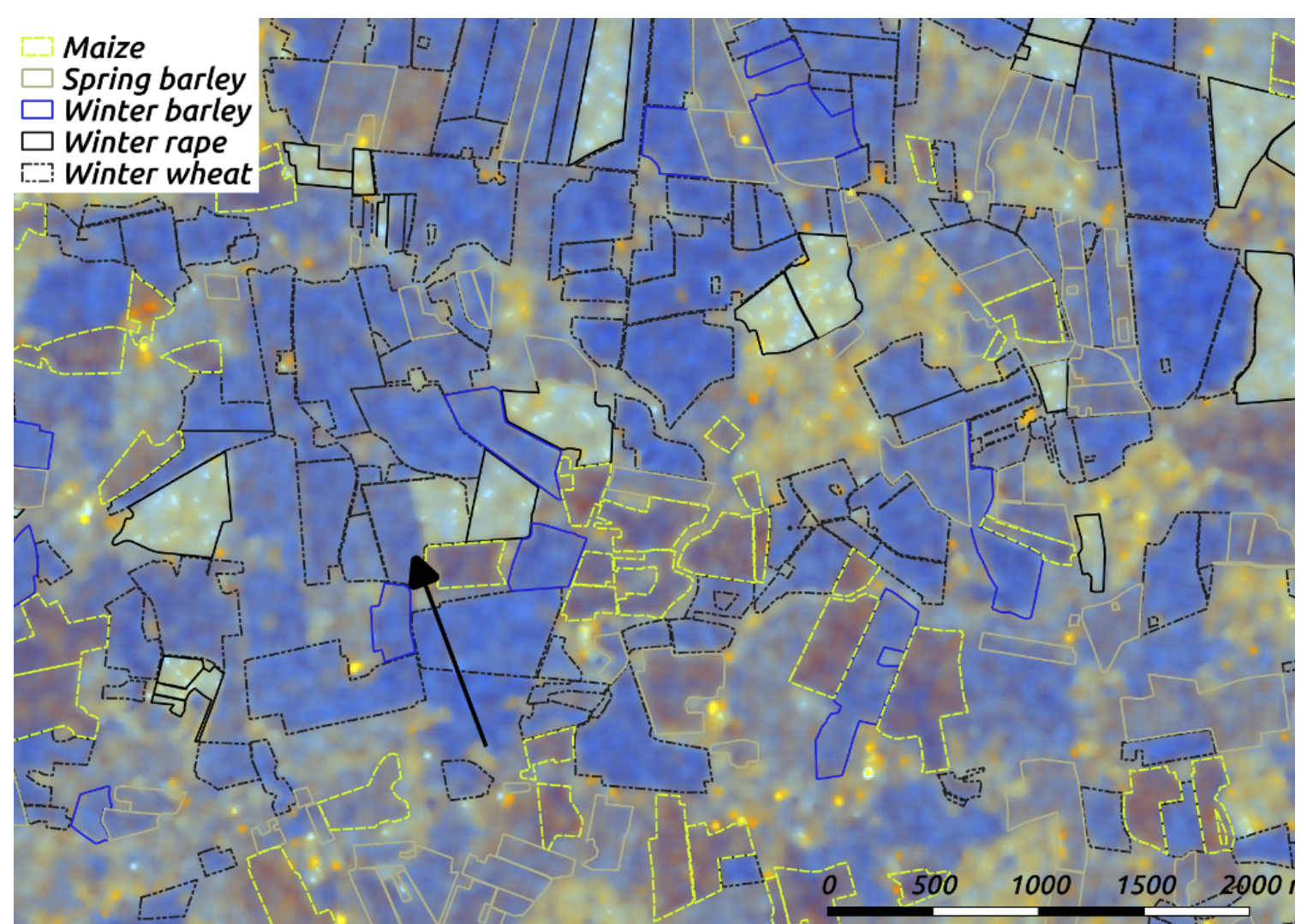

Figure 49. Processed SAR image from the 1st of june 2017 with marking of the five crop types: Maize, Spring barley, Winter barley, Winter rape, Winter wheat.

The achieved result indicates the processed sentinel-1 images provided by the fieldbabel service can be used to identify different crop types, with the exception of winter barley and wheat. By automatically tracking the different crop types in the Sentinel-1 images, since the start of the year, we would expect one could also distinguish winter barley and wheat.

Sampling in the field based on current Sentinel-1 data

The results of the k-means clustering can be seen in Figure 50. Since the crop field is somewhat flat, the clustering should not be significantly affected by the elevation. 


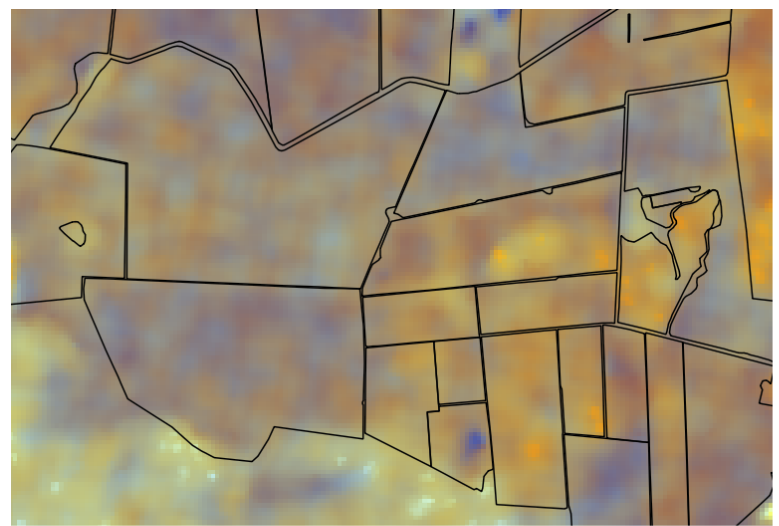

(a)

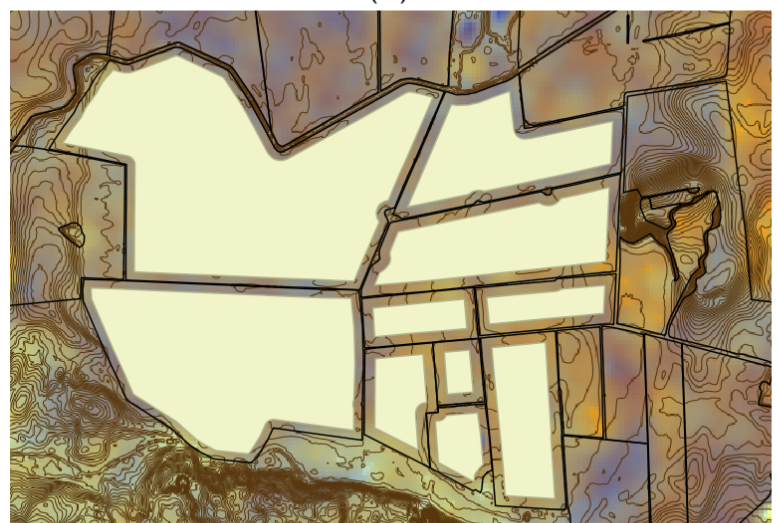

(c)

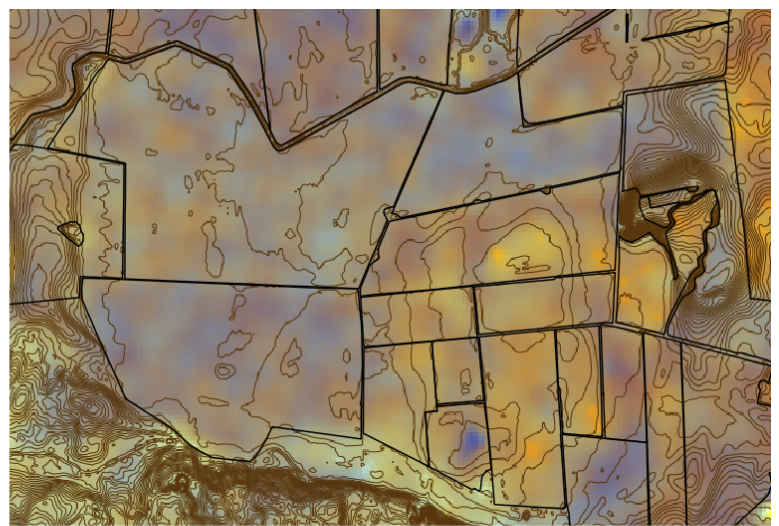

(b)

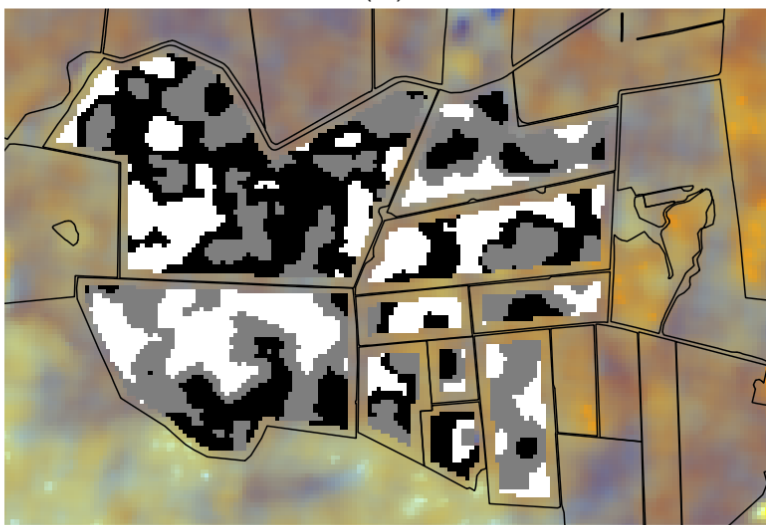

(d)

Figure 50. The processing of individual fields using k-means clustering, to create maps of where to collect samples. The red lines in subfigure (b) represents elevation differences of more than 0.5 meters. (c) Erosion is used to shrink the field boundary.(d) Each field is analysed separately using k-means clustering.

From the above-mentioned figure on can see that the clusters do not seem to follow the evaluation on the area. This approach could be used to plan sampling reference data from a field for both manual collection and more automated systems such as a drone.

Time series analysis: Phenological stages

After processing trough farm report manager, the time-temporal plot results for the $\mathrm{VV}(\mathrm{dB}) / \mathrm{VH}(\mathrm{dB})$ ratio can be seen in Figure 51 for two of the five fields.

Other trials in the Nordic field trial data have also logged the growth stages of winter wheat and other crops, but trial 010111616 was the only one found where notation was done consistently for all fields. From the growth stages compared to the ratio of $\mathrm{VV}(\mathrm{dB}) / \mathrm{VH}(\mathrm{dB})$ it can be seen, that the curve starts to rise around stage 24-25 and peaks around growth stage 39 . The peaking spot on $\mathrm{VV}(\mathrm{dB}) / \mathrm{VH}(\mathrm{dB})$ curve show a tendency similar to GAI for winter wheat, which we would be expected based on earlier studies (Veloso et al. 2017). The dataset indicates a tendency, we would expect generally to be present for winter wheat fields where the correct Sentinel-1 data products are recorded. The example with winter wheat growth stages and Sentinel-1 data also match the previous findings published by other researchers. 


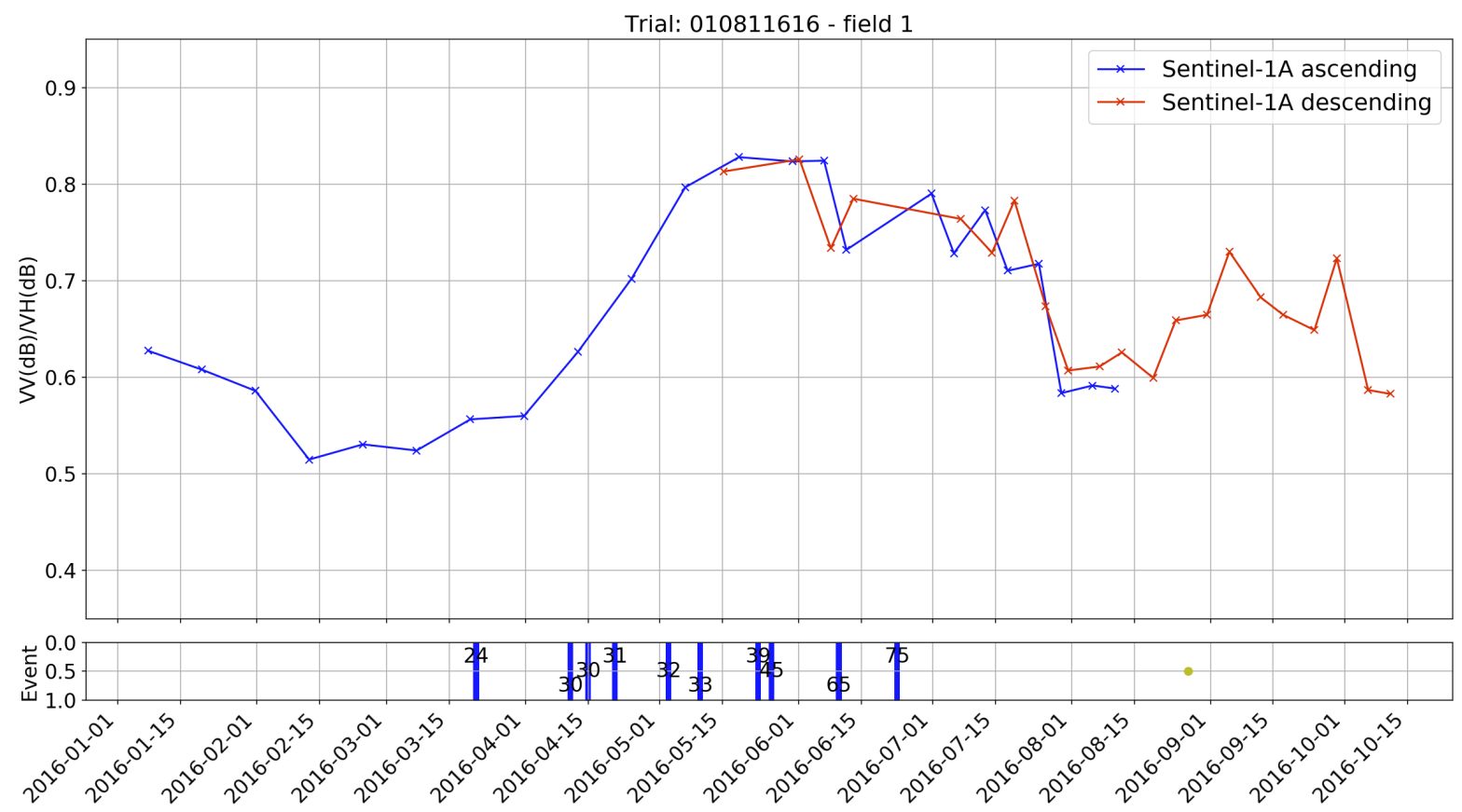

(a)

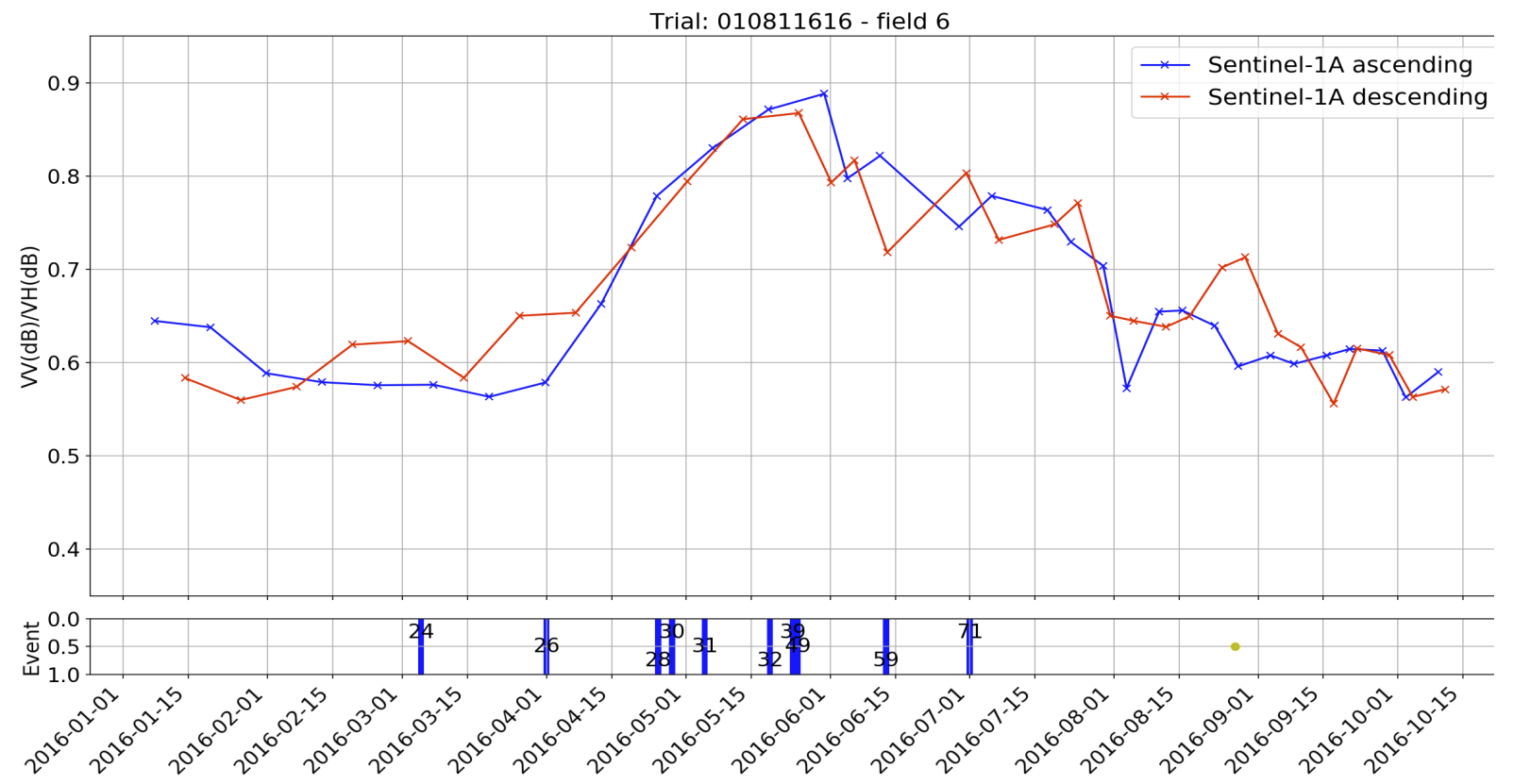

(b)

Figure 51. Plot of the mean VV/VH value for two specific winter wheat field against time (upper). Below is the reported growth stage of these winter wheat in NFTS.

From a big data perspective automatically determining growth stages of winter winter could be a means to automatically plan field operations such as fertilizing, spraying and harvesting. Automated prediction of winter wheat growth stages could be evaluated on upcoming seasons and will also be explored in the future. 


\section{Conclusions}

In this paper, we have presented our Fieldbabel system for pre-processing Sentinel-1 recording for the agricultural domain. The system is intended for non-SAR processing experts, so focus can be on correlation with field samples. Three different application examples have been presented to illustrate the possible potential of the Fieldbabel service. By providing both application examples and the service, we hope it the will provide more agricultural researchers with the ability to discover new uses for Sentinel-1 data.

\section{Acknowledgements}

The work presented here is supported by the FutureCropping and SqMFarm projects funded by Innovation Fund Denmark and GUPD, The Danish AgriFish Agency, respectively. Data From the National Field Trials, from SEGES L\&F are used for ground truth.

\section{References}

Berger, Michael, Jose Moreno, Johnny A. Johannessen, Pieternel F. Levelt, and Ramon F. Hanssen. 2012. "ESA's Sentinel Missions in Support of Earth System Science. Remote Sensing of Environment 120: 84-90. https://doi.org/10.1016/j.rse.2011.07.023.

Bosch, Marc, Zachary Kurtz, Shea Hagstrom, and Myron Brown. 2016. “A Multiple View Stereo Benchmark for Satellite Imagery. In Applied Imagery Pattern Recognition Workshop (AIPR), 2016 IEEE, 1-9. IEEE.

Butler, Howard. 2005. "A Guide to the Python Universe for ESRI Users. ArcUser Mag. April-June, 34-37.

Dalla Mura, Mauro, Saurabh Prasad, Fabio Pacifici, Paulo Gamba, Jocelyn Chanussot, and Jón Atli Benediktsson. 2015. "Challenges and Opportunities of Multimodality and Data Fusion in Remote Sensing. Proceedings of the IEEE 103 (9): 1585-1601.

Drusch, M, U Del Bello, S Carlier, O Colin, V Fernandez, F Gascon, B Hoersch, et al. 2012. "Sentinel-2: ESA's Optical High-Resolution Mission for GMES Operational Services. Remote Sensing of Environment 120: 25-36.

Erten, Esra, Juan M Lopez-Sanchez, Onur Yuzugullu, and Irena Hajnsek. 2016. "Retrieval of Agricultural Crop Height from Space: A Comparison of SAR Techniques. Remote Sensing of Environment 187: 130-144.

Forgey, Edward. 1965. "Cluster Analysis of Multivariate Data: Efficiency vs. Interpretability of Classification. Biometrics 21 (3): 768-769.

Gerry Boyle, Joseph Lynch, Richie Hackett, Steven Kildea, David Wall, John Spink, Deirdre Doyle, Shay Phelan, and Dermot Forristal. 2016. "The Winter Wheat Guide. Teagasc.

Giasat s.r.o. 2017. "Sentinel-2 Agriculture - Czechagri. Final Report. Gisat.

Grinberg, Miguel. 2018. Flask Web Development: Developing Web Applications with Python. O’Reilly Media, Inc.

Hansen, Ole K., Ulrik Bräuner Nielsen, Øyvind M. Edvardsen 1, Brynjar Skúlason, and Jan-ole Skage. 2004.

"Nordic Provenance Trials with Abies Lasiocarpa and Abies Lasiocarpa Var. Arizonica: Three-Year Results. Scandinavian Journal of Forest Research 19 (2): 112-26. https://doi.org/10.1080/02827580410024098.

Hyman, Glenn, Herlin Espinosa, Paola Camargo, David Abreu, Medha Devare, Elizabeth Arnaud, Cheryl

Porter, Leroy Mwanzia, Kai Sonder, and Sibiry Traore. 2017. "Improving Agricultural Knowledge Management: The AgTrials Experience. F1000Research 6.

Jin, Xiuliang, Lalit Kumar, Zhenhai Li, Haikuan Feng, Xingang Xu, Guijun Yang, and Jihua Wang. 2018. “A Review of Data Assimilation of Remote Sensing and Crop Models. European Journal of Agronomy 92: 141-152.

Klein, Thomas, Mats Nilsson, Anders Persson, and Bertil Håkansson. 2017. "From Open Data to Open Analyses-New Opportunities for Environmental Applications? Environments 4 (2). https://doi.org/10.3390/environments4020032.

Kumar, P, R Prasad, DK Gupta, VN Mishra, AK Vishwakarma, VP Yadav, R Bala, A Choudhary, and R Avtar. 2017. "Estimation of Winter Wheat Crop Growth Parameters Using Time Series Sentinel-1A SAR Data. Geocarto International, 1-15.

Kussul, Nataliia, Mykola Lavreniuk, Sergii Skakun, and Andrii Shelestov. 2017. "Deep Learning Classification of Land Cover and Crop Types Using Remote Sensing Data. IEEE Geoscience and Remote Sensing Letters 14 (5): 778-782.

Lee, J. S., J. H. Wen, T. L. Ainsworth, K. S. Chen, and A. J. Chen. 2009. “Improved Sigma Filter for Speckle Filtering of SAR Imagery. IEEE Transactions on Geoscience and Remote Sensing 47 (1): $202-13$. https://doi.org/10.1109/TGRS.2008.2002881.

Li, Peng, Luguang Jiang, and Zhiming Feng. 2014. "Cross-Comparison of Vegetation Indices Derived from Landsat-7 Enhanced Thematic Mapper Plus (ETM+) and Landsat-8 Operational Land Imager (OLI) Sensors. Remote Sensing 6 (1): 310-329. https://doi.org/10.3390/rs6010310. 
Lopez-Sanchez, Juan M, and J David Ballester-Berman. 2009. "Potentials of Polarimetric SAR Interferometry for Agriculture Monitoring. Radio Science 44 (2).

Müller, Andreas, Thomas Esch, Tobias Storch, Hubert Asamer, Christoph Reck, Gunter Schreier, Erhard Diedrich, and Eberhard Mikusch. 2016. "An Operational Data Handling and Processing Environment for Copernicus Sentinel 1, 2 and 3 Data.

Olesen, Jørgen E, Christen Duus Børgesen, Lars Elsgaard, Taru Palosuo, RP Rötter, AO Skjelvla ag, Pirjo Peltonen-Sainio, et al. 2012. "Changes in Time of Sowing, Flowering and Maturity of Cereals in Europe under Climate Change. Food Additives \& Contaminants: Part A 29 (10): 1527-1542.

Phil Wynn Owen, Nikolaos Milionis, Ioulia Papatheodorou, Kristian Sniter, Helder Faria Viegas, Jan Huth, Ramona Bortnowschi, et al. 2016. "The Land Parcel Identification System: A Useful Tool to Determine the Eligibility of Agricultural Land - but Its Management Could Be Further Improved. Special Report 25. EUROPEAN COURT OF AUDITORS.

Picard, Ghislain, Thuy Le Toan, and Francesco Mattia. 2003. "Understanding C-Band Radar Backscatter from Wheat Canopy Using a Multiple-Scattering Coherent Model. IEEE Transactions on Geoscience and Remote Sensing 41 (7): 1583-1591.

Pierdicca, Nazzareno, Luca Pulvirenti, and Gaetano Pace. 2014. "A Prototype Software Package to Retrieve Soil Moisture from Sentinel-1 Data by Using a Bayesian Multitemporal Algorithm. IEEE Journal of Selected Topics in Applied Earth Observations and Remote Sensing 7 (1): 153-166.

Roy, David P, V Kovalskyy, HK Zhang, Eric F Vermote, L Yan, SS Kumar, and A Egorov. 2016. "Characterization of Landsat-7 to Landsat-8 Reflective Wavelength and Normalized Difference Vegetation Index Continuity. Remote Sensing of Environment 185: 57-70.

Roy, David P, MA Wulder, Thomas R Loveland, CE Woodcock, RG Allen, MC Anderson, D Helder, et al. 2014. "Landsat-8: Science and Product Vision for Terrestrial Global Change Research. Remote Sensing of Environment 145: 154-172.

Sabel, Daniel, Zoltan Bartalis, Wolfgang Wagner, Marcela Doubkova, and Jean-Pierre Klein. 2012. "Development of a Global Backscatter Model in Support to the Sentinel-1 Mission Design. Remote Sensing of Environment 120: 102-12. https://doi.org/10.1016/j.rse.2011.09.028.

Sagan, Carl. 2011. The Demon-Haunted World: Science as a Candle in the Dark. Ballantine Books.

Schubert, Adrian, David Small, Nuno Miranda, Dirk Geudtner, and Erich Meier. 2015. "Sentinel-1A Product Geolocation Accuracy: Commissioning Phase Results. Remote Sensing 7 (7): 9431-9449. https://doi.org/10.3390/rs70709431.

Shelestov, A. Yu., A. N. Kravchenko, S. V. Skakun, S. V. Voloshin, and N. N. Kussul. 2013. "Geospatial Information System for Agricultural Monitoring. Cybernetics and Systems Analysis 49 (1): 124-132. https://doi.org/10.1007/s10559-013-9492-5.

Skriver, Henning, Francesco Mattia, Giuseppe Satalino, Anna Balenzano, Valentijn RN Pauwels, Niko EC Verhoest, and Malcolm Davidson. 2011. "Crop Classification Using Short-Revisit Multitemporal SAR Data. IEEE Journal of Selected Topics in Applied Earth Observations and Remote Sensing 4 (2): 423-431.

Torres, Ramon, Paul Snoeij, Dirk Geudtner, David Bibby, Malcolm Davidson, Evert Attema, Pierre Potin, et al. 2012. "GMES Sentinel-1 Mission. Remote Sensing of Environment 120: 9-24.

Veloso, Amanda, Stéphane Mermoz, Alexandre Bouvet, Thuy Le Toan, Milena Planells, Jean-François Dejoux, and Eric Ceschia. 2017. "Understanding the Temporal Behavior of Crops Using Sentinel-1 and Sentinel2-like Data for Agricultural Applications. Remote Sensing of Environment 199: 415-426.

Wu, Xindong, Vipin Kumar, J Ross Quinlan, Joydeep Ghosh, Qiang Yang, Hiroshi Motoda, Geoffrey J McLachlan, et al. 2008. "Top 10 Algorithms in Data Mining. Knowledge and Information Systems 14 (1): 1-37.

Zhou, Tao, Zhaofu Li, and Jianjun Pan. 2018. "Multi-Feature Classification of Multi-Sensor Satellite Imagery

Based on Dual-Polarimetric Sentinel-1A, Landsat-8 OLI, and Hyperion Images for Urban Land-Cover Classification. Sensors 18 (2): 373.

Zuhlke, M., N. Fomferra, C. Brockmann, M. Peters, L. Veci, J. Malik, and P. Regner. 2015. "SNAP (Sentinel Application Platform) and the ESA Sentinel 3 Toolbox. In Sentinel-3 for Science Workshop, 734:21. ESA Special Publication. 


\author{
Benchmark of Satellites Image Services for Precision Agricultural Use \\ Marco Sozzi a,", Francesco Marinello a, Andrea Pezzuolo a, Luigi Sartori ${ }^{\text {a }}$ \\ a TeSAF, Department of Land, Environment, Agriculture and Forestry, University of Padova, Viale dell'Università 16, \\ Legnaro, Italy \\ * Corresponding author. Email: marco.sozzi@phd.unipd.it
}

\begin{abstract}
Over the last years, the attention of satellites images providers on precision agriculture has increased, leading to an enhancement of features resolution. At present, several satellites images services exist, which provide very different products for different demands. Specifically, constellations of nanosatellites are now able to produce daily images all over the world with a spatial resolution as low as $3 \mathrm{~m}$ in the case of Planet Scope. Besides, Sentinel-2 constellation is now completely positioned, and it provides data free of charge with a revisit time of 5 days and spatial resolution of $10 \mathrm{~m}$. The present work is aimed at comparing spatial, temporal, spectral and radiometric resolution taking into account the farmer's requirements to benchmark the major satellites images providers. Concurrently, the study analyses the computational power needs per unit of area, for each single downloadable product. Finally, the costs of single images related to the minimum purchased areas are considered. The integration of satellite services with digital agriculture technologies could be profitable for both small and large farms. Small farmers require low-cost services, but with enough resolution to manage modest plots of land. On the other hands, big farmers, despite the possibility they have to make significant investments, can use lowresolution images, due to the relevant dimensions of the fields; additionally, big farms are more frequently equipped with variable rate technologies, and they can more easily take advantage of variability detection by satellites images.
\end{abstract}

Keywords: Benchmark, Nanosatellites, Digital Agriculture, Sentinel-2 MSI, Planet Labs

\title{
1. Introduction
}

Digital agriculture takes advantages of precision agriculture techniques and information technologies to help make the best decisions in business and agricultural processes (Shen et al. 2010). In digital agriculture, sensors are integrated into a more complex system, which includes data analysis and decision support systems. In addition, digital agriculture aims to increase the automatization of decision-making process using big data to improve the productivity of farmer and companies (Kamilaris et al. 2017). Thus, digital agriculture benefits from the high amount of information provided by networks of sensors and satellites (Brown 2015).

Over the years, numerous sensing systems were developed to explore the sub-fields variability, including sensors for yield mapping, vegetation status, and soil variability (Dubbini et al. 2017). On the other hands, several approaches were performed to identify agricultural fields variability using optical and radar satellites images. A wide range of sensors is nowadays carried by satellites. Indeed, the high cost of satellites technologies forced companies to set up on satellites more sensors, to increase the number of potential users. Thanks to the significant number of sensors available through satellites, different useful agricultural features can be investigated through remote sensing. Continuous improvement in remote sensing technologies leads to a better data available for precision agriculture (Mulla 2013).

According to the spectral resolution of sensors, satellites allow to detect and monitor several characteristics of crops and soil, during the whole production process. Sensors able to detect radio waves (RADAR) are used to estimate soil moisture and biomass production, by exploiting backscatter effects (Martínez-Fernández et al. 2016). The thermal region of the electromagnetic spectrum has application in soil and vegetation water content evaluation, irrigation scheduling, drought monitoring, mapping of residues, and crop maturity detection (Khanal et al. 2017). Indexes obtained by sensors focused on short wave infrared (SWIR) are able to detect, on a large scale, several soil properties as mineralogy, texture, organic carbon, and salinity (Mulder et al. 2011).

Remote sensing in near-infrared (NIR) and visible (VIS) region of the electromagnetic spectrum for agriculture was deep investigated over the years. Several vegetation indexes were developed, mostly based on the difference between reflectance in near-infrared and red by exploiting the healthy vegetation spectral signature (Barati et al. 2011; Huete 1997). Many studies have already demonstrated that vegetation indexes like NDVI and SAVI can successfully identify sub-fields variability to perform precision agriculture practices (Ortega-Blu and MolinaRoco 2016; Pezzuolo et al. 2016). Besides, vegetation indexes can be used to define biophysical features of vegetation like LAI or fAPAR (Herrmann et al. 2011). To exploit results in precision agriculture, vegetation indexes evolution should be monitored during the crop cycle. Good monitoring of crops requires at least one sensing per month, during the whole growing season. In addition, a collection of historical archive of satellites visible images is available, which can be used by farmers to delineate temporally stable zones (Crestey et al. 2017). 
Nowadays satellites images providers make available many products suitable for precision agriculture and the sub-fields variability investigation. Spatial resolution ranges from dozens of centimetres to kilometres with revisit time ranging between daily up to weeks. Similarly, the highest spectral resolution available is some nanometres (hyperspectral satellites), but the most common sensors have spectral bands with a band-width of about a hundred nanometers and can collect radiations from ultraviolet to infrared wavelengths. Significant advances in satellites technologies are always limited by engineering and economic compromises related to optics, bandwidth, and orbital configuration which limits ideal resolution features (Houborg and McCabe 2016). To overcome such limitations, constellations of nanosatellites was proposed, characterised by a large number of small satellites with compact sensors (Esper et al. 2000). Besides, intergovernmental organisations such as European Space Agency (ESA) and National Aeronautics and Space Administration (NASA) have made available constellations of satellites with multispectral sensors for free.

Due to the number of sensors available for precision agriculture, a comparative analysis is needed, to assist the selection of the best satellites sensors for farmers. According to the precision agriculture needs, the following features for ideal satellites images can be defined:

- Spatial resolution in accordance with the average agriculture fields size (typically $\leq 30 \mathrm{~m}$ );

- Revisit time which allows at least one sensing per month ( $\leq 30$ days);

- Spectral resolution of bands focused on the region of the spectral bands used for vegetation indexes calculation (from $450 \mathrm{~nm}$ to $900 \mathrm{~nm}$ );

- Low computational power needs for data processing;

Based on the previous points, a cost-effectiveness evaluation of the most common satellites constellations was performed, considering sensors characteristics, price per hectare, and the computation demand, compared with the advantages provided by precision agriculture.

\section{Materials and Methods}

An analysis based on surveys and prices list of numerous satellites images providers was performed, to determine the satellites at one's disposal for precision agriculture. Characteristics, price per unit and costeffectiveness are proposed in the following paragraphs.

Satellites sensors

The present section gives a summary of the main satellites available and relevant for precision agriculture.

- Deimos-2: $\quad$ A full private satellite developed by Elecnor company (Spain). It is the second sensor of Deimos missions. Deimos-2 is a minisatellite $(\sim 310 \mathrm{~kg})$, which can provide $0.75 \mathrm{~m}$ panchromatic and $4 \mathrm{~m}$ multispectral images in 4 channels VIS/NIR. The average global revisit time $\left( \pm 45^{\circ}\right)$ is 2 days, with a radiometric resolution of 10 bits/pixel. Spectral resolution ranges from $466 \mathrm{~nm}$ to $892 \mathrm{~nm}$. An agreement exists between Deimos Imaging and SI Imaging Services.

- Dove (Planet): $\quad$ A constellation of nanosatellites provides by Planet Labs Inc (US). At present Doves constellation consist of more than 175 satellites, which are able to provide several daily images all over the world. Spatial resolution is $3 \mathrm{~m}$ for multispectral images in 4 channels VIS/NIR, with a radiometric resolution of 16 bits/pixel. Spectral resolution ranges from 420 to $900 \mathrm{~nm}$.

- GeoEye-1: $\quad$ Provides $0.46 \mathrm{~m}$ panchromatic and $1.84 \mathrm{~m}$ multispectral images in 4 channels VIS/NIR. Spectral resolution ranges from $450 \mathrm{~nm}$ to $920 \mathrm{~nm}$ with a temporal resolution of 3 days. Radiometric resolution of the GeoEye- 1 sensor is 11 bits/pixel. It is provided by Digital Globe (US).

- Kompsat-2/3/3A: Kompsat missions were developed by KARI (Korea Aerospace Research Institute). The images are provided by SI Imaging Services. All Kompsat sensors have a spectral resolution in the range $450-900 \mathrm{~nm}$ in 4 channels VIS/NIR and a radiometric resolution of 14 bits/pixel. Spatial resolution is different for each sensor: Kompsat-2 is $1 \mathrm{~m}$, Kompsat3 is $0.7 \mathrm{~m}$, and Kompsat-3A is $0.55 \mathrm{~m}$. Similarly, the revisit time for Kompsat-2 is 6 days, and for Kompsat-3 and $3 \mathrm{~A}$ is 3 days. An agreement exists between Deimos Imaging and SI Imaging Services.

- Landsat-7/8: $\quad$ Landsat-7/8 ware developed as a collaboration between NASA and the U.S. Geological Survey (USGS). Spatial resolution ranges from $15 \mathrm{~m}$ in panchromatic, $30 \mathrm{~m}$ in multispectral to $60 / 100 \mathrm{~m}$ in thermal bands. Landsat-7/8 have a radiometric resolution of $8-12$ bits/pixel, with a revisit time of 8 days. Spectral resolution in VIS/NIR ranges from 450 to $900 \mathrm{~nm}$ in 4 channels, but sensor provides up to 11 bands. 
- Pleiades-1A/1B:

- Rapideye:

- Sentinel-2:

- Spot-6/7:

- WorldView-2/3/4:
The Pleiades is a constellation of two satellites built by Airbus Defence and Space (France). They provide daily images, with a spatial resolution of $0.5 \mathrm{~m}$ panchromatic and $2 \mathrm{~m}$ multispectral in 4 channels VIS/NIR. Radiometric resolution is $12 \mathrm{bits} / \mathrm{pixel}$ and spectral resolution range from 450 to $915 \mathrm{~nm}$.

A constellation of 5 satellites owned by BlackBridge, a Planet Labs Inc company (US). Spectral resolution ranges from 440 to $850 \mathrm{~nm}$ in 5 channels VIS/NIR, with a radiometric resolution of 12 bits/pixel. It provides images with 5.5 days (at nadir) interval. Spatial resolution is $5 \mathrm{~m}$ for all bands-

Sentinel-2 is a constellation of two satellites (2A-2B) developed by European Space Agency (ESA). Spatial resolution ranges from $10 \mathrm{~m}$ in VIS/NIR, $20 \mathrm{~m}$ in red edge bands, up to $60 \mathrm{~m}$ in cirrus and UV bands. Spectral resolution in VIS/NIR ranges from 458 to 900 $\mathrm{nm}$ in 4 channels, but sensors provide at least 12 bands. Sentinel-2 have a radiometric resolution of $16 \mathrm{bits} /$ pixel, with a revisit time of 5 days.

Spot-6/7 are satellites built by Airbus Defence and Space (France). Spatial resolution is $1.5 \mathrm{~m}$ panchromatic and $6 \mathrm{~m}$ multispectral in 4 channels VIS/NIR. Revisit time is one day, with a spatial resolution of $0.5 \mathrm{~m}$ panchromatic and $2 \mathrm{~m}$ multispectral in 4 channels VIS/NIR. Radiometric resolution is $12 \mathrm{bits} /$ pixel and spectral resolution range from 455 to $890 \mathrm{~nm}$.

Worldview satellites were developed by Digital Globe (US). WorldView-2 has a spatial resolution of $0.5 \mathrm{~m}$ panchromatic and 1.85 in multispectral, with a spectral resolution from 450 to 1040 in VIS/NIR. It is a multispectral sensor with up to 8 channels. The spatial resolution of WorldView-3 is $0.3 \mathrm{~m}$ panchromatic and $1.24 \mathrm{~m}$ in multispectral. Compared to WorldView-2, it provides additional 8 bands in the shortwave infrared and 12 bands for desert, aerosol and water. WorldView-4 has a spatial resolution of $0.3 \mathrm{~m}$ panchromatic and 1.24 multispectral in 4 channels VIS/NIR

Table 20 Summary table of sensors features.

\begin{tabular}{|c|c|c|c|c|}
\hline & $\begin{array}{c}\text { Spatial resolution } \\
m\end{array}$ & $\begin{array}{c}\text { Spectral resolution VIS/NIR } \\
n m\end{array}$ & $\begin{array}{c}\text { Radiometric resolution } \\
\text { bits pixel }{ }^{-1}\end{array}$ & $\begin{array}{c}\text { Revisit time } \\
d d\end{array}$ \\
\hline Deimos-2 & 0.75 & $466-697 / 770-892$ & 10 & 2 \\
\hline Dove (Planet) & 3 & $420-700 / 770-900$ & 16 & 1 \\
\hline GeoEye-1 & 0.5 & $450-690 / 780-920$ & 11 & 3 \\
\hline Kompsat-2 & 1 & $450-690 / 760-900$ & 14 & 6 \\
\hline Kompsat-3 & 0.7 & $450-690 / 760-900$ & 14 & 3 \\
\hline Kompsat-3A & 0.55 & $450-690 / 760-900$ & 14 & 3 \\
\hline Landsat- $7 / 8$ & 15 & $450-690 / 770-900$ & $8-12$ & 8 \\
\hline Pleiades-1A/1B & 0.5 & $430-720 / 750-950$ & 12 & 1 \\
\hline RapidEye & 5 & $440-685 / 690-850$ & 12 & 5.5 \\
\hline Sentinel-2 & 10 & $458-680 / 785-900$ & 16 & 5 \\
\hline Spot-6/7 & 1.5 & $455-695 / 760-890$ & 12 & 1 \\
\hline WorldView-2/3/4 & $0.3-0.5$ & $450-690 / 770-1040$ & 11 & 1 \\
\hline
\end{tabular}

Costs and benefits assessment

According to the performed analysis, price per area unit, data processing and download time are the primary cost items of satellites images application for precision agriculture.

Taking advantage from price list and quotation requested to several satellites images providers, a database of the minimum price per hectare was developed. The analysis was based on the minimum price between recent acquisition and fresh archive not older than 90 days (in the most case was not older than 60 days). Fresh images are required in precision agriculture both for definition of prescription maps and monitoring. Spatial resolution was evaluated as panchromatic images, according to the possibility to perform a pan-sharpening process to enhance the spatial resolution of multispectral images. Spectral resolution was main focused on visible and nearinfrared bands, in accordance with the vegetation indexes calculation needs.

Data processing of satellites images is not trivial. It is a time-consuming task, especially if atmospheric correction is needed. On the other hand, vegetation indexes calculation requires only a band math operation and its processing time is proportional to the amount of area analysed. A survey conducted between professional users of remote sensing and GIS estimates the time consuming of images download, rectification, masking, indices calculation, legend-making, and producing output in one hour per image downloaded. According to the labour cost per hour, data processing cost is fixed at $100 \$$ per images (without image interpretation and advising). 
Table 21 Cost features for satellites sensors.

\begin{tabular}{|c|c|c|c|c|}
\hline & $\begin{array}{l}\text { Minimum order area } \\
\qquad h a\end{array}$ & $\begin{array}{l}\text { Price per } \\
\text { unit } \\
\$ h a^{-1}\end{array}$ & $\begin{array}{l}\text { Minimum area } \\
\text { price* } \\
\$\end{array}$ & $\begin{array}{l}\text { Computational } \\
\text { demand } \\
K B h a^{-1}\end{array}$ \\
\hline Deimos-2 & 10000 & 0.060 & 700 & 50 \\
\hline Dove & 10000 & 0.012 & 218 & 8 \\
\hline GeoEye-1 & 10000 & 0.275 & 2850 & 100 \\
\hline Kompsat-2 & 2500 & 0.055 & 237.5 & 20 \\
\hline Kompsat-3 & 2500 & 0.110 & 375 & 50 \\
\hline Kompsat-3A & 2500 & 0.160 & 500 & 100 \\
\hline Landsat-7/8 & 3700000 (one scene) & 0 & 100 & 0.5 \\
\hline Pleiades-1A/1B & 10000 & 0.213 & 2225 & 100 \\
\hline Rapideye & 10000 & 0.012 & 218 & 4 \\
\hline Sentinel-2 & 1200000 (one scene) & 0 & 100 & 0.63 \\
\hline Spot-6/7 & 10000 & 0.045 & 550 & 8 \\
\hline $\begin{array}{l}\text { WorldView- } \\
2 / 3 / 4\end{array}$ & 10000 & 0.275 & 2850 & 130 \\
\hline
\end{tabular}

*minimum area price is obtained by minimum order area times price per unit plus data processing cost

Based on first half 2018 report of Rewheel/research, in OECD countries the median fully allocated gigabyte price was 1.3\$ GB-1 (April 2018). The Rewheel report analysed 4G mobile broadband or stationary (fixedwireless) plans of 41 countries all over the world. The download cost per area unit was calculated considering the number of KB ha-1. The analysis considered five images per year, according to precision agriculture monitoring needs. The average economic advantages due to precision farming were fixed at $30 \$ \mathrm{ha}^{-1}$ as proposed by Colaço and Bramley (2018).

\section{Results and Discussion}

Data analysis of all satellites sensors was performed considering the parameters showed in the previous chapter.

Despite a limited cost of images per hectare, the minimum purchasable area affects the final cost of satellites images predominantly. All satellites services with a spatial resolution lower than $10 \mathrm{~m}$, provide images with a minimum order area, after which the price increase proportionally with the purchased area. For agriculture use, the threshold of minimum area is excessively high (the lowest threshold is 2500 hectares), and the proportional price can be reached only buy few farms or consociated companies.

The minimum order area affects even the download cost. Considering one scene as the minimum downloadable area, Landsat 7-8 and Sentinel-2 have a download cost comparable with Worldview 2-3-4 and Pleiades 1A/B, which have a computational demand (KB/ha) hundreds of times larger.

All satellites sensors viewed provide multispectral images with a spectral resolution between 450 and $900 \mathrm{~nm}$, by ensuring an effective vegetation indices calculation. Spatial resolution is the main features that affect the subfields variability detection. The first approach of sensors choice must consider spatial resolution as the primary factor, evaluating that with the minimum area price. In Fig. 1 is shown how the minimum area price evolves according to the spatial resolution (pixel size). Even in this case, the values are affected by the minimum purchasable area. Worldview 2-3-4, Geoeye-1, and Pleiades 1A/B provide images with a sub-meter resolution and have the highest value of minimum area price. Sensors with a spatial resolution of 10-15 meters, like Sentinel2 and Landsat 7-8 have the lowest value of minimum price area, which is influenced only by download cost. An exponentially inverse correlation exists between price and spatial resolution, confirmed by an $\mathrm{R}^{2}$ of 0.9474 . Thus, the pixel size is the main parameters constituent the price of satellites images.

The radiometric resolution does not have a significant variation between sensors evaluated. Values range from $10 \mathrm{bits} / \mathrm{pixel}$ in case of Deimos-2, up to $16 \mathrm{bits} / \mathrm{pixel}$ in case of Dove and Sentinel-2. The radiometric resolution does not affect vegetation indices measurement considerably for agricultural needs, but it may be considered to detect variability with a value of NDVI close to the saturation (Gu et al. 2013). 


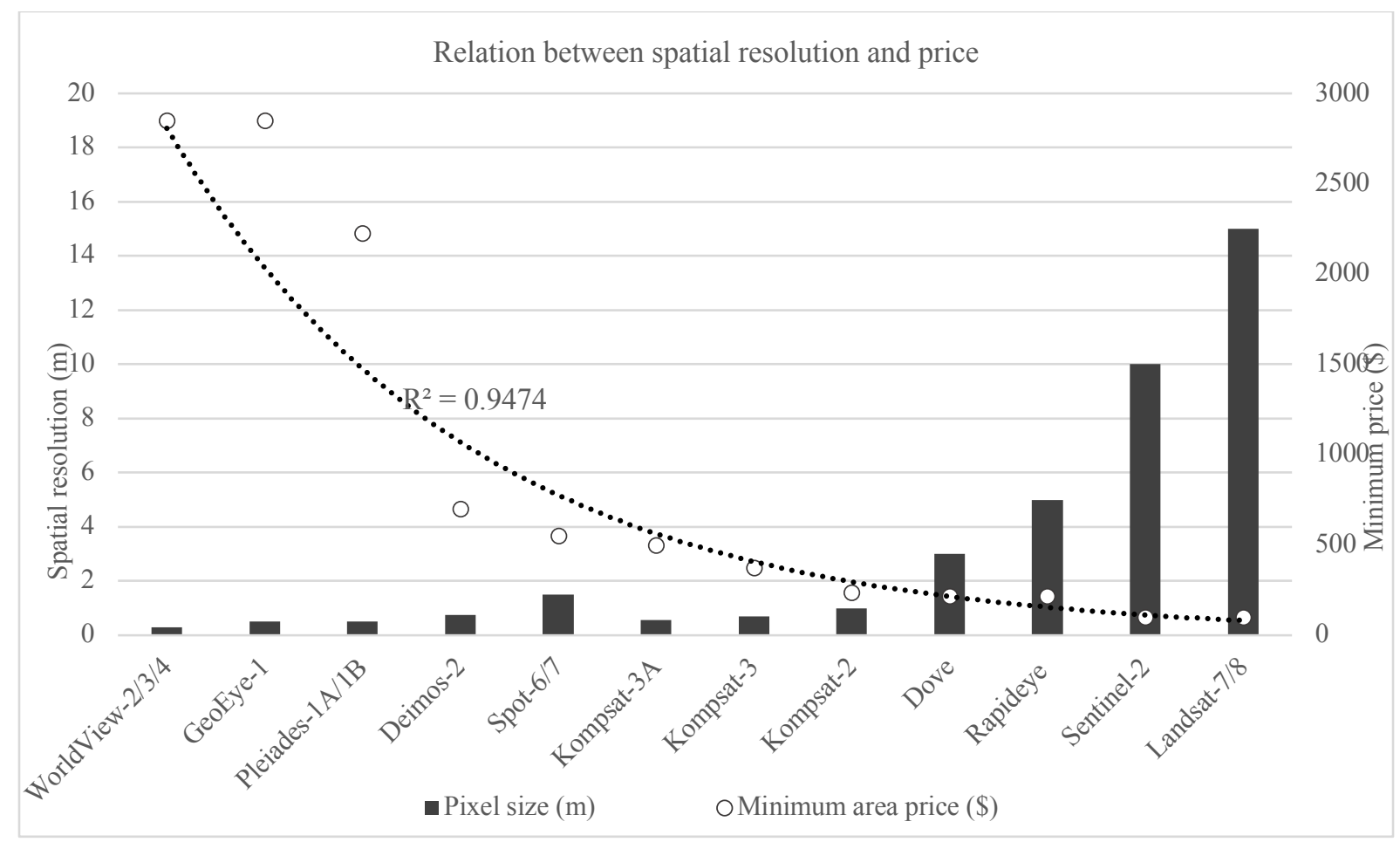

Figure 52 Comparison between price and spatial resolution.

Based on the minimum price area and the estimate precision agriculture advantages $\left(30 \$ \mathrm{ha}^{-1}\right)$, a calculation of the break-even point for all the sensors was performed. The application of precision agriculture using free of charge satellites is convenient at approximately 17 hectares. On the other hands, the use of satellites with a submeter spatial resolution is justified only with 370 or 470 hectares, Pleaides 1A/B and Worldview-2/3/4-GeoEye1 respectively. Differently, Deimos- 2 sensor provides images with a sub-meter spatial resolution, but the breakeven point is reached at 115 hectares, showing a reverse trend.

Cost effectiveness of satellites images

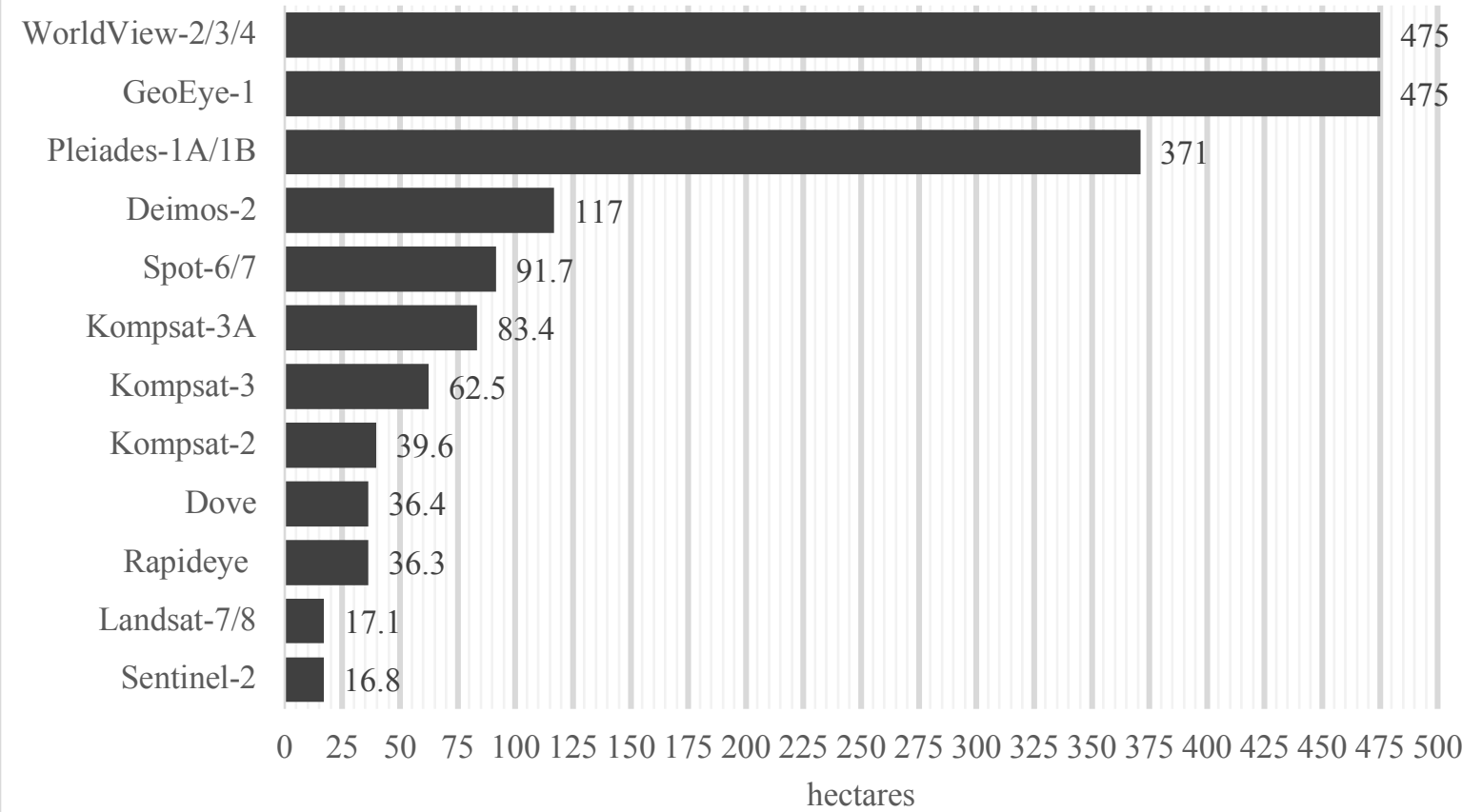

Figure 53. Break Even point of satellites images, in hectares. 
Farms with an extension of 40 hectares can profitably exploit both Devos/Plane Lab and RapidEye images. In this case, Planet satellites have the advantage of a better spatial resolution, and a more frequent revisit time. Besides, RapidEye users benefit from and additional bans close to the Red Edge. A kind of attention is needed to Kompsat satellites. Due to the low minimum area required (2500 ha), the break-even point is reached from Kompsat 2, Kompsat-3 and Kompsat-4 around 40, 65 and 85 hectares respectively. Therefore, Kompsat satellites give the possibility to use high-resolution images ( $\sim 1$ meter) also to farms around $60-80$ hectares.

\section{Conclusions}

This work was aimed at analysing satellites image providers to evaluate the features of satellites sensors according to precision agriculture needs. The analysis of satellites characteristics such as resolution, revisit time, and minim cost showed that profitability of satellites images for precision agricultural application starts at 17 hectares. In this case, the fields shape should be considered, to reduce the border effect noise, which is particulate acute with a spatial resolution of 10-15 m (Sentinel-2 and Landsat7/8 sensors). Besides, sensors with a spatial resolution of 3-5 m (Doves-Planet and Rapideye) are profitable starting from 40 hectares. Finally, sub-meters resolution images are sustainable with more than 85 hectares, thanks to the lower minimum purchasable area of Kompsat satellites.

With a focus on the European situation, the average utilised agricultural area per holdings is 16.1 hectares, ranging from 1.2 hectares per holding of Malta, up to 133 hectares per holdings of Czech Republic (EU-28 Eurostat, 2013). In this situation the satellites image use should mainly regards free of charge satellites. An enlargement of the average size of farms could increase the interest of farmer to more accurate satellites images.

\section{Acknowledgements}

This study was performed thanks to data provided by e-GEOS, LAND INFO Worldwide Mapping, Geocento, Satellite Imaging Corporation, Sysdeco Italia s.r.l., Harris geospatial, and Planet Labs Inc (Planet Team (2017). Planet Application Program Interface: In Space for Life on Earth. San Francisco, CA. https://api.planet.com).

\section{References}

Barati, S., Rayegani, B., Saati, M., Sharifi, A., \& Nasri, M. (2011). Comparison the accuracies of different spectral indices for estimation of vegetation cover fraction in sparse vegetated areas The Egyptian Journal of Remote Sensing and Space Sciences. The Egyptian Journal of Remote Sensing and Space Sciences, 14, 49-56. doi:10.1016/j.ejrs.2011.06.001

Brown, M. E. (2015). Satellite Remote Sensing in Agriculture and Food Security Assessment. Procedia Environmental Sciences, 29, 307. doi:10.1016/J.PROENV.2015.07.278

Colaço, A. F., \& Bramley, R. G. V. (2018). Do crop sensors promote improved nitrogen management in grain crops? Field Crops Research, 218, 126-140. doi:10.1016/J.FCR.2018.01.007

Crestey, T., Pichon, L., \& Tisseyre, B. (2017). Potential of freely available remote sensing visible images to support growers in delineating within field zones. Advances in Animal Biosciences, 8(02), 372-376. doi:10.1017/S2040470017000437

Dubbini, M., Pezzuolo, A., De Giglio, M., Gattelli, M., Curzio, L., Covi, D., et al. (2017). Last generation instrument for agriculture multispectral data collection. Agricultural Engineering International: CIGR Journal, 19(1), 87-93.

Esper, J., Panetta, P. V., Ryschkewitsch, M., Wiscombe, W., \& Neeck, S. (2000). NASA-GSFC nano-satellite technology for Earth science missions. Acta Astronautica, 46(2-6), 287-296. doi:10.1016/S0094-5765(99)002143

Gu, Y., Wylie, B. K., Howard, D. M., Phuyal, K. P., \& Ji, L. (2013). NDVI saturation adjustment: A new approach for improving cropland performance estimates in the Greater Platte River Basin, USA. Ecological Indicators, 30, 1-6. doi:10.1016/J.ECOLIND.2013.01.041

Herrmann, I., Pimstein, A., Karnieli, A., Cohen, Y., Alchanatis, V., \& Bonfil, D. J. (2011). LAI assessment of wheat and potato crops by VEN $\mu \mathrm{S}$ and Sentinel-2 bands. Remote Sensing of Environment, 115(8), 2141-2151. doi:10.1016/j.rse.2011.04.018

Houborg, R., \& McCabe, M. (2016). High-Resolution NDVI from Planet's Constellation of Earth Observing Nano-Satellites: A New Data Source for Precision Agriculture. Remote Sensing, 8(12), 768. doi: $10.3390 /$ rs 8090768

Huete, A. (1997). A comparison of vegetation indices over a global set of TM images for EOS-MODIS. Remote Sensing of Environment, 59(3), 440-451. doi:10.1016/S0034-4257(96)00112-5

Kamilaris, A., Kartakoullis, A., \& Prenafeta-Boldú, F. X. (2017, December 1). A review on the practice of big 
data analysis in agriculture. Computers and Electronics in Agriculture. Elsevier. doi:10.1016/j.compag.2017.09.037

Khanal, S., Fulton, J., \& Shearer, S. (2017). An overview of current and potential applications of thermal remote sensing in precision agriculture. Computers and Electronics in Agriculture, 139, $22-32$. doi:10.1016/J.COMPAG.2017.05.001

Martínez-Fernández, J., González-Zamora, A., Sánchez, N., Gumuzzio, A., \& Herrero-Jiménez, C. M. (2016). Satellite soil moisture for agricultural drought monitoring: Assessment of the SMOS derived Soil Water Deficit Index. Remote Sensing of Environment, 177, 277-286. doi:10.1016/J.RSE.2016.02.064

Mulder, V. L., de Bruin, S., Schaepman, M. E., \& Mayr, T. R. (2011). The use of remote sensing in soil and terrain mapping — A review. Geoderma, 162(1-2), 1-19. doi:10.1016/j.geoderma.2010.12.018

Mulla, D. J. (2013). Twenty five years of remote sensing in precision agriculture: Key advances and remaining knowledge gaps. Biosystems Engineering, 114(4), 358-371. doi:10.1016/j.biosystemseng.2012.08.009

Ortega-Blu, R., \& Molina-Roco, M. (2016). Evaluation of vegetation indices and apparent soil electrical conductivity for site-specific vineyard management in Chile. Precision Agriculture, 17(4), 434-450. doi:10.1007/s11119-016-9429-x

Pezzuolo, A., Cillis, D., Marinello, F., \& Sartori, L. (2016). Relationship Between Satellite-Derived Ndvi and Soil Electrical Resistivity: a Case Study. In Proceeding of 6Th International Conference on Trends in Agricultural Engineering 2016 (pp. 484-489).

Scudiero, E., Teatini, P., Corwin, D. L., Deiana, R., Berti, A., \& Morari, F. (2013). Delineation of site-specific management units in a saline region at the Venice Lagoon margin, Italy, using soil reflectance and apparent electrical conductivity. Computers and Electronics in Agriculture, 99, 54-64. doi:10.1016/j.compag.2013.08.023

Shen, S., Basist, A., \& Howard, A. (2010). Structure of a digital agriculture system and agricultural risks due to climate changes. Agriculture and Agricultural Science Procedia, 1, $42-51$. doi:10.1016/J.AASPRO.2010.09.006 


\author{
Comparison between Different Spatial Resolution Satellites Images for Viticulture \\ Marco Sozzi $^{\text {**, Ahmed Galal Kayad }}{ }^{\mathrm{a}}$, Andrea Pezzuolo ${ }^{\mathrm{a}}$, Luigi Sartori ${ }^{\mathrm{a}, \mathrm{b}}$, Marco Dubbini ${ }^{\mathrm{c}, \mathrm{d}}$, Francesco \\ Marinello $^{\mathbf{a}, \mathbf{b}}$ \\ a TeSAF, Department of Land, Environment, Agriculture and Forestry, University of Padova, Viale dell'Università 16, \\ Legnaro, Italy \\ ${ }^{b}$ NEOS srl, Spin off of the University of Padova, Piazzetta Modin, 12, 35129 Padova (PD), Italy \\ ${ }^{\mathrm{c}}$ Department of History and Cultures, University of Bologna, Bologna, Italy \\ d SAL Engineering srl, 48026 Russi (RA), Italy \\ * Corresponding author. Email: marco.sozzi@phd.unipd.it
}

\begin{abstract}
Knowledge of spatial variation of vegetative vigour is essential for site-specific management and variable rate application. Specifically, precision viticulture requires continuous monitoring of the vigour due to the mutable condition during the year and to frequent treatments (10 to 15) during the growing season. Over the last few years, several solutions for vegetation variability surveying have been proposed, mainly based on optical remote and proximal sensing. In precision viticulture, images from remote sensing are influenced by inter-row spacing, which can somehow mask or distort field variability. Analysis of high-resolution satellites or UAV imagery are not trivial, due to the computing power needs, however inter-row spacing can be in these cases recognized, segmented and eventually removed. On the other hand, medium resolution satellites imagery does not allow identification of single rows, while they can easily provide general averaged information. The spatial resolution of the images plays a preeminent role in vineyard variability sensing and understanding. In the present work, vineyard variability is evaluated through vegetation indices, which were estimated taking advantage of multispectral images at different resolutions, from Planet Scope, RapidEye and Sentinel 2 satellites respectively providing 3, 5 and 10 meters pixel sizes. The experimentation took place in a 5 hectares vineyard located in Northern part of Italy. Data were compared with UAV images, opportunely re-sampled in order to fit the spatial resolution of different considered satellites. The comparison was carried out considering references statistical parameters calculated on the basis of spectral images. Fast Fourier transform, and root mean square was been considered to exploit differences and relation between sources images.
\end{abstract}

Keywords: precision viticulture, Sentinel-2 MSI, RapidEye, Planet Labs, Unmanned Aerial Vehicle (UAV) imagery

\title{
1. Introduction
}

Expert's consciousness of advantages and benefits in precision agriculture is raising, powered by challenge for more sustainable agriculture and the food security issue (Far and Rezaei-Moghaddam 2018). Indeed, precision agriculture maintains and improves the environment while it enhances the sustainability of food supply, taking advantages from sensors, information systems, and enhanced machinery (Gebbers and Adamchuk 2010). Sensors provide information related to crops, soil, and nutrients availability. Specialized machineries implemented with variable rate technologies allow to perform site-specific management. A target input distribution reduces losses from excessive applications and nutrients imbalances (Bongiovanni and Lowenberg-Deboer 2004). Moreover, precision agriculture improves energy efficiency of food production process (Cillis et al. 2016).

In viticulture, site-specific management is spreading rapidly, due to the high standard quality needs and the continuous development of this sector. Precision viticulture allows to enhance the oenological features of grapevine thanks to quality variability management and differential harvesting. Several variable rate technologies were proposed in recent years in viticulture, in order to improve agrochemicals spreading (Llorens et al. 2010), fertilization and pruning. In viticulture, management zones defined by cluster analysis of sensors data can be assessed in terms of yield, considering quantity and quality parameters. Spatial variation of yield is mainly influenced by soil properties, topography, and nutritional status of vines (R. G. V. Bramley and Hamilton 2007). Variable rate technologies in viticulture allow a significant reduction of chemicals spreading, even in uniform vineyards, due to the differences observed in canopy volume along the line (Llorens et al. 2010).

In modern viticulture, manual sampling and chemicals analysis at high spatial resolution are prohibitive in terms of cost and time. Consequently, the use of sensors for quick and easy sampling is necessary (Arnó et al. 2009). Over the last years, several sensors have been proposed to estimate variability at sub-fields scale. Optical sensors, both proximal and remote (De Benedetto et al. 2013), are widely used in variability detection for precision agriculture. Vegetation indices take advantages from the different reflectance of plants along the electromagnetic spectrum, focusing especially in near-infrared and red (Huete 1988). Several vegetation indices have been developed, not only for land monitoring but also for agriculture use. In precision viticulture, specific vegetations indices have been also proposed, such as Plant Cell Density (G. V Bramley et al. n.d.) and Photosynthetic Vigor Ratio. At the same time most common vegetation indices such as Normalized Difference Vegetation Index 
(NDVI) are well investigated (Johnson et al. 2003). All these indices exploit high reflectance in near-infrared and low reflectance in red bands of healthy vegetation, in order to detect stressed vegetation. In addition, these indices are correlated with structural and physiological characteristics of the vine (Leaf Area Index -LAI- and Fraction of Absorbed Photosynthetically Active Radiation -FAPAR-). Leaf reflectance and vegetation indices are correlated to water content, biochemical substances and photosynthetic pigments (Matese et al. 2015). Moreover, vegetation indices and satellite images can be used to optimize the harvest of high-quality grapes (Crestey et al. 2017).

Due to the tree structure and inter-rows spacing, viticulture needs, more than other crops, high-resolution data in order to properly quantify vine status. High-resolution images can be used for vine signal extraction, through thresholding of the histogram in a grayscale image, to isolate reflectance due to the inter-row spacing (Smit et al. 2010). High-resolution images can be provided by satellites or by unmanned aerial vehicles (UAVs). Satellites, as Worldview-3/4 or Quickbird, can provide images with a spatial resolution of $0.30 \mathrm{~m}$, in spectral bands from visible to near infrared. High-resolution images allow to define a threshold to eliminate inter-row noise, but the minimum cost can reach $2000 €$ for 25 square kilometres. On the other hands, UAVs are becoming widespread for precision farming use, and through photogrammetry algorithms, they can provide fields reconstruction with a spatial resolution of less than $0.10 \mathrm{~m}$ even in multispectral bands (Turner et al. 2010). Concurrently, a UAV survey is still expensive, and is not always justified. Besides, satellites images, which are commonly used in precision agriculture, are widespread and affordable for some $€ /$ ha or even free of charge.

Sub-fields variability of vineyards can be investigated through UAVs and satellites. In this work, NDVI variability was evaluated, detected by three mid-resolution satellites (Planet Labs, RapidEye and Sentinel 2) correlated and analyzed in comparison with NDVI data generated after UAV monitoring operations. UAV data were collected in the same fields and at the same time of satellite data, with the aim to understand advantages and limits of indexes estimations from satellites themselves.

\section{Materials and Methods}

Several contiguous vineyards were selected as study area in the North of Italy. Vineyards were characterized by a white grape variety with a grasses inter-row of $3 \mathrm{~m}$ not cultivated. Vines were trained by spurred cordon on sandy soil, with a plant density of 3700 plants hectare. On these areas were collected multispectral images from UAV and three different satellites (Planet, RapidEye, and Sentinel-2).

The UAV survey was carried out between 8 and 9 August 2016, on a sunny day, with no clouds noise. UAV was equipped with MAIA sensor (EOPTIS SRL / SAL ENGINEERING SRL). MAIA is a multispectral camera with an array of nine sensors, composed by one RGB colour and eight monochromes (Dubbini et al. 2017). Such optics allow to collect data in the VIS-NIR spectrum from 390 to $950 \mathrm{~nm}$. All optics have a focal length of 7.5 $\mathrm{mm}$ and a display field of view of $43^{\circ}$. Images collected were analysed with Agisoft PhotoScan Professional software through photogrammetry algorithm, to obtain multispectral orthophotos of all vineyards. Multispectral images obtained were characterized by $0.02 \mathrm{~m}$ spatial resolution. According to MAIA spectral bands, NDVI calculation was performed using band 5 (red) and band 8 (near-infrared).

Planets Labs Inc provides satellites images collected by a constellation of nanosatellites (Doves). This constellation is currently composed of more than 175 satellites equipped with multispectral sensors VIS/NIR (420$900 \mathrm{~nm}$ ). All these nanosatellites provide daily images all over the world with a spatial resolution of $3 \mathrm{~m}$. An image sensed on the same day of the UAV flight was chosen and downloaded from Planet website. The product was already geometrically and radiometrically corrected. Red and near-infrared bands were used for NDVI calculation.

RapidEye missions is a constellation of 5 satellites owned by BlackBridge, a Planet Labs Inc company (US). Spatial resolution is $5 \mathrm{~m}$, with a revisit time of 5.5 days. Spectral resolution ranges from 440 to $850 \mathrm{~nm}$ in 5 channels VIS/NIR.

Radiance and then reflectance correction was carried out through by applying scale radiometric factor and exo-atmospheric coefficients to the digital number, in order to obtain top of the atmosphere reflectance. According to the spectral resolution of RapidEye, NDVI calculation was performed using band 3 (red) and band 5 (nearinfrared).

Sentinel-2 is a constellation of two sensors (2A-2B) developed by European Space Agency (ESA). Sentinel-2 constellation provides images with $10 \mathrm{~m}$ spatial resolution with a spectral resolution ranging from 440 to 2280 $\mathrm{nm}$. The average global revisit time is 5 days. An image with the same level of correction (top of the atmosphere) was chosen and downloaded from Copernicus Open Access Hub. Ten meters resolution NDVI was calculated using band 4 (red) and band 8 (near infrared).

A comparison between MAIA, Planet, RapidEye and Sentinel-2 spectral bands is showed in Fig.1 (where $\rho$ is reflectance, $\lambda$ wavelength, and CWL is central wavelength).

Images obtained by MAIA, Planet, RapidEye and Sentinel-2 were analysed with GIS software both from reflectance correction and NDVI calculation. Five random plots of one hectare were selected in the study area, 
avoiding agricultural roads and boundaries. UAV, Planet, RapidEye and Sentinel-2 images were clipped inside the five random plots and exported as a tagged image file. All of the images were analysed with Scanning Probe Image Processor (SPIPTM developed by Image Metrology A/S) software in order to understand how the variability is detected by different sensors. Statistical analysis was aimed at recognizing the periodic pattern, number and magnitude of summits and variability. Variability parameters were studied considering highest resolution images (0.02 m UAV, $3 \mathrm{~m}$ Planet, and $5 \mathrm{~m}$ RapidEye) and after down sampling to the lowest $10 \mathrm{~m}$ resolution.

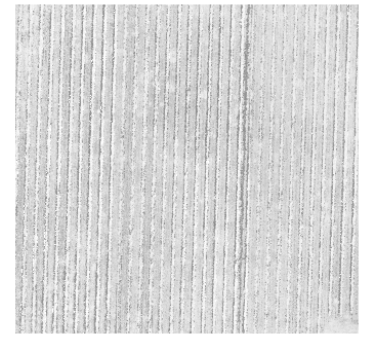

UAV installed sensor

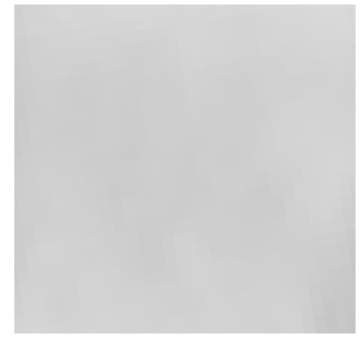

Planet satellite

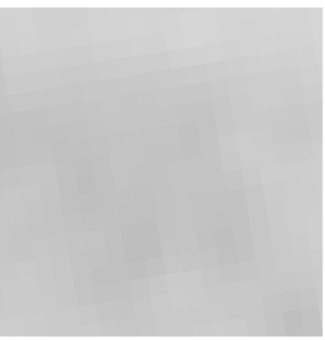

RapidEye satellite

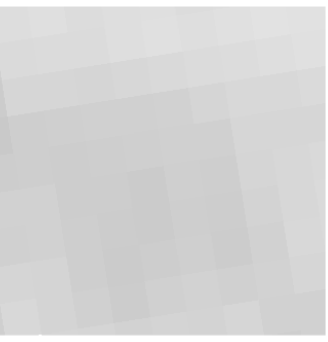

Sentinel-2 satellite

Figure 1. Comparison between images.

\section{Results and Discussion}

The first evaluated parameter was the square root of the mean square (Root Mean Square -RMS). This parameter was chosen as quantification of the variability detected by sensors. Root mean square was assessed through the following formula:

$$
R M S=\sqrt{\frac{1}{M N} \sum_{k=0}^{M-1} \sum_{i=0}^{N-1}\left[z\left(x_{k}, y_{i}\right)\right]^{2}}
$$

As amplitude parameter, Root Mean Square describes values deviations from the mean pixels value of the image. As shown in Fig.2 images sensed by MAIA can detect significantly more variability than satellites, primarily due to the better spatial resolution. Sentinel-2 and RapidEye images do not show statistical differences in variability detection, even if spatial resolution of RapidEye is half of Sentinel-2. Despite a spatial resolution of $3 \mathrm{~m}$, Planet images showed the lowest value of Root Mean Square.

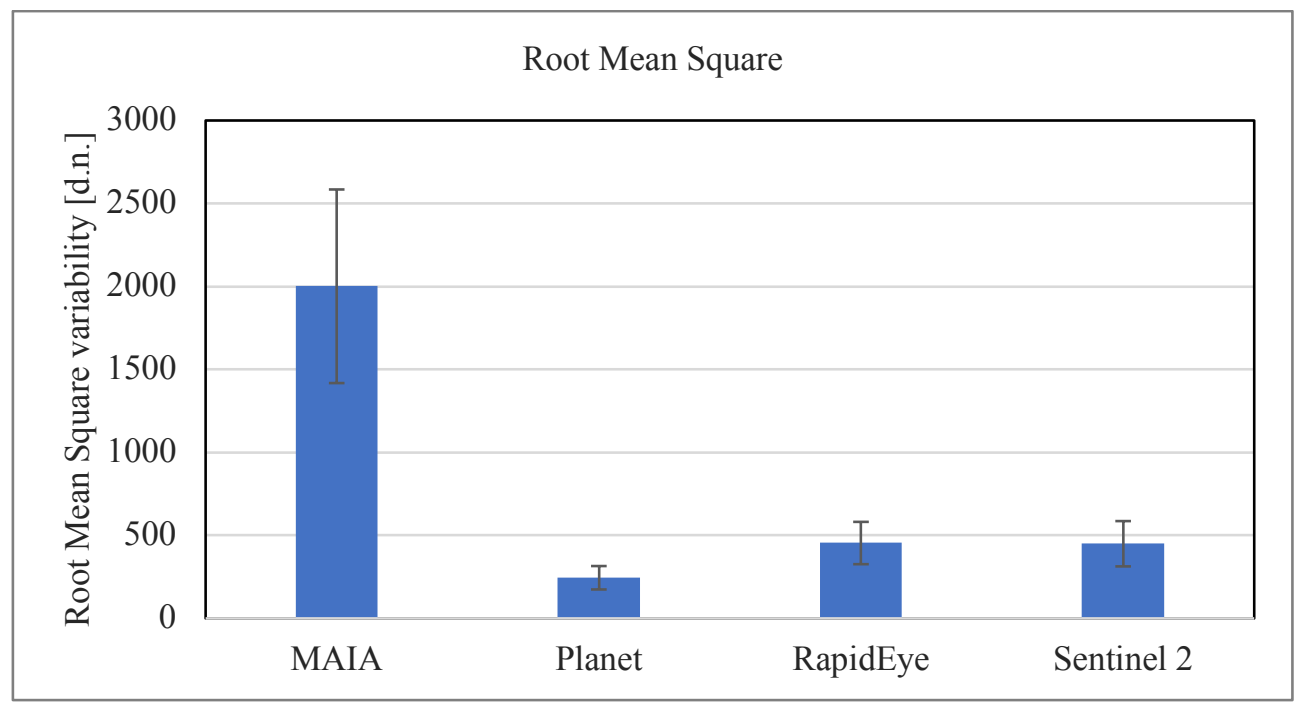

Figure 2. Variability detected by sensors.

The Reduced Peak Height is a measure of summits height above the low closest value. This is a parameter defined by ISO 13565-2 Standard. Reduces Peak Height quantifies summits variability, estimating the sensors capacity to detect a sudden change of NDVI peaks values. Reduced Peak Height is reported in Fig.3. As for RMS, the UAV mounted sensor MAIA showed high sensed variability. Planet, RapidEye, and Sentinel-2 do not have significant differences in Reduced Peak Height, but Planet tends to exhibit lower values. 


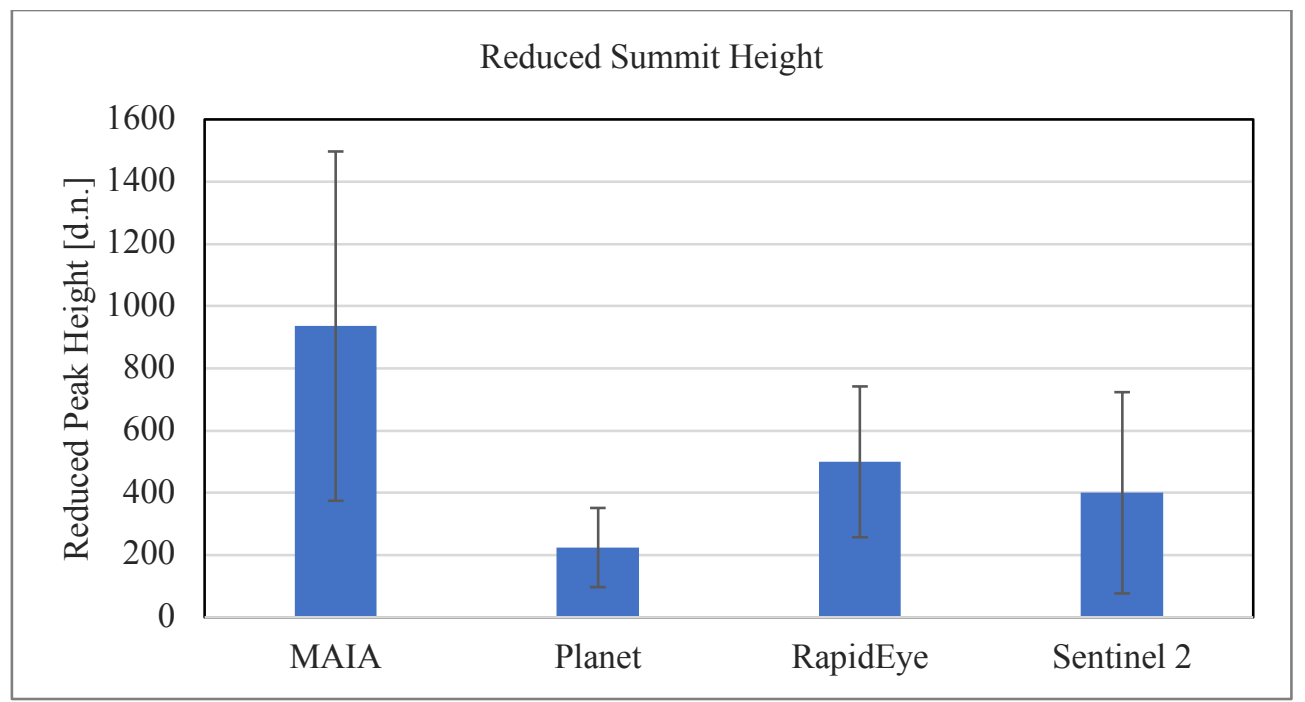

Figure 3. Variability on a small scale.

In resampled images some spatial parameters were calculated as for instance Density of summits and Texture direction index. The density of summits estimates the number of summits per area (Fig.4), decreasing in an inverse proportion with spatial resolution. Density of summits was assessed through the following equation:

$$
\text { Density of summits }=\frac{\text { Numbers of local maximum }}{(M-1)(N-1) \delta x \delta y}
$$

All satellites data exhibit the same trend, and a similar number of summits per area is reached at $5 \mathrm{~m}$ and 10 m spatial resolution.

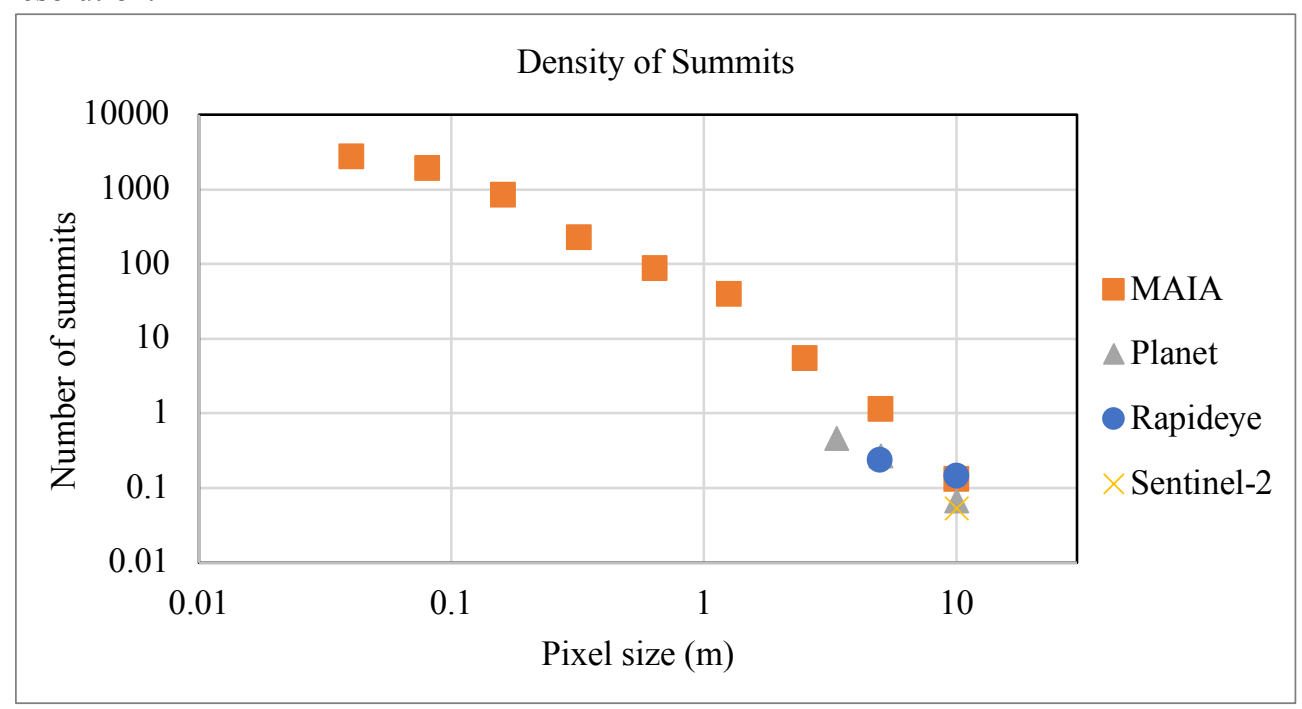

Figure 4. Number of summits per area.

Texture direction index measures how dominant the dominating direction is. It is defined as the average amplitude sum divided by the amplitude sum of the dominating direction. This function takes advantages of Fast Fourier Transform, and is calculated according to the following equation:

$$
\text { Texture direction index }=\frac{\sum_{i=0}^{M-1} A\left(\frac{i x}{M}\right)}{M A_{\max }}
$$

As reported in Fig. 5, a dominant direction pattern exists up to $1.25 \mathrm{~m}$ spatial resolution. Starting from this point a dominant direction is not recognisable. The main pattern which affects the direction in vineyards is row direction. According to vineyards features, a direction pattern is not sensed once spatial resolution gets over the inter-row spacing $(3 \mathrm{~m})$. 


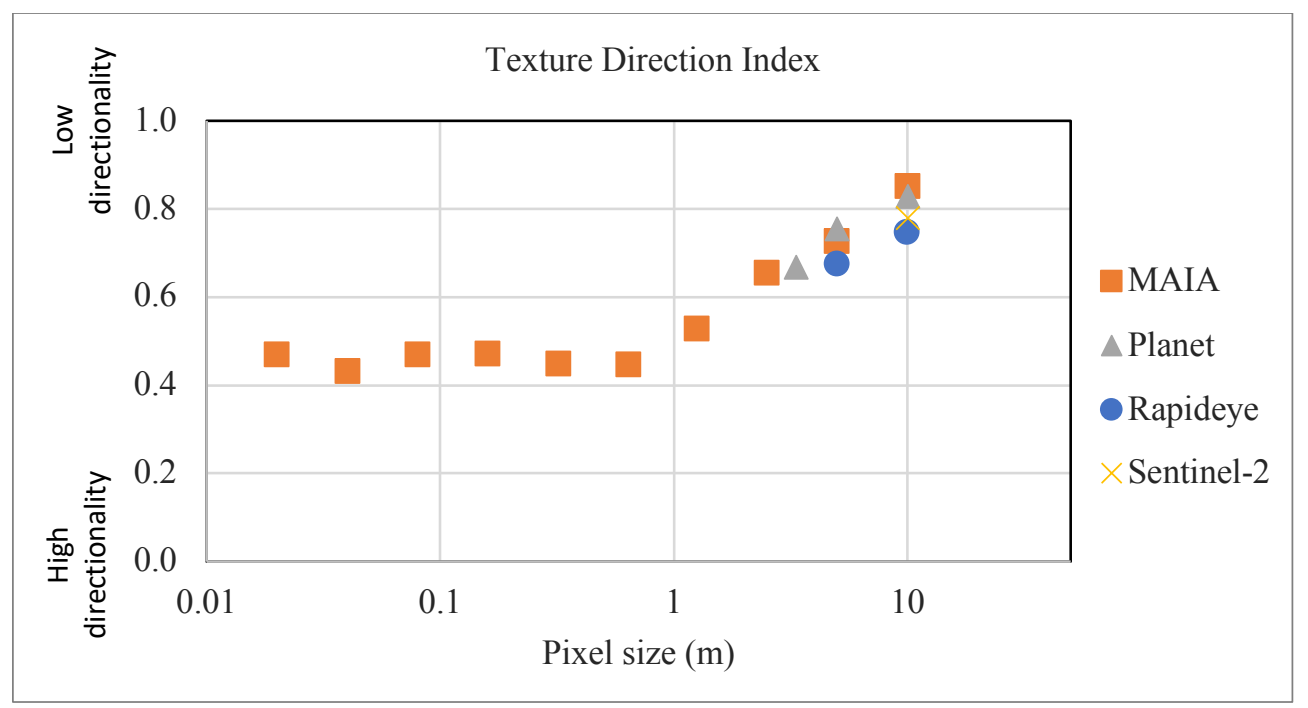

Figure 5. Amplitude of the dominating direction.

\section{Conclusions}

The present work was aimed at evaluating the capacity to assess vineyards variability through different sensors. A statistical analysis of images pattern was performed on images sensed with multispectral camera (MAIA), and three different spatial resolution satellites (Planet, RapidEye, and Sentinel-2). The analysis showed that vineyards row-interrow spacing significantly affect the capacity to detect variability by sensors. Satellites images did not show statistical differences each other, suggesting a performance equivalence between private and free of charge satellites services. Additional research may include high-resolution satellites service, which are able to provide images with a spatial resolution lower than inter-row spacing.

\section{Acknowledgements}

This study was performed thanks to data provided by Planet Team (2017). Planet Application Program Interface: In Space for Life on Earth. San Francisco, CA. https://api.planet.com

\section{References}

Arnó, J., Martínez Casasnovas, J. A., Ribes Dasi, M., \& Rosell, J. R. (2009). Review. Precision viticulture. Research topics, challenges and opportunities in site-specific vineyard management. Spanish Journal of Agricultural Research, 7(4), 779. doi:10.5424/sjar/2009074-1092

Bongiovanni, R., \& Lowenberg-Deboer, J. (2004). Precision Agriculture and Sustainability. Precision Agriculture, 5(4), 359-387. doi:10.1023/B:PRAG.0000040806.39604.aa

Bramley, R. G. V., \& Hamilton, R. P. (2007). Terroir and precision viticulture: Are they compatible? Journal International des Sciences de la Vigne et du Vin, 41(1), 1-8. doi:10.20870/oeno-one.2007.41.1.855

Bramley, G. V, Trought, M. C. T., \& Praat, J.-P. (n.d.). Vineyard variability in Marlborough, New Zealand: Characterising variation in vineyard 1 performance and options for the implementation of Precision Viticulture. 234 R. https://publications.csiro.au/rpr/download?pid=csiro:EP10416\&dsid=DS1. Accessed 3 June 2018

Cillis, D., Marinello, F., Pezzuolo, A., Basso, B., \& Sartori, L. (2016). Precision in conservation agriculture : first results of an experimental study. In Environment, Sustainable Agriculture and Forest Management.

Crestey, T., Pichon, L., \& Tisseyre, B. (2017). Potential of freely available remote sensing visible images to support growers in delineating within field zones. Advances in Animal Biosciences, 8(02), 372-376. doi: $10.1017 /$ S2040470017000437

De Benedetto, D., Castrignano, A., Diacono, M., Rinaldi, M., Ruggieri, S., \& Tamborrino, R. (2013). Field partition by proximal and remote sensing data fusion. Biosystems Engineering, 114(4), 372-383. doi:10.1016/J.BIOSYSTEMSENG.2012.12.001

Dubbini, M., Pezzuolo, A., De Giglio, M., Gattelli, M., Curzio, L., Covi, D., et al. (2017). Last generation instrument for agriculture multispectral data collection. Agricultural Engineering International: CIGR Journal, 19(1), 87-93.

Far, S. T., \& Rezaei-Moghaddam, K. (2018). Impacts of the precision agricultural technologies in Iran: An analysis experts' perception \&amp; their determinants. Information Processing in Agriculture, 5(1), $173-184$. doi:10.1016/J.INPA.2017.09.001

Gebbers, R., \& Adamchuk, V. I. (2010). Precision Agriculture and Food Security. Science, 327(5967), 828- 
831. doi:10.1126/science. 1183899

Huete, A. . (1988). A soil-adjusted vegetation index (SAVI). Remote Sensing of Environment, 25(3), 295-309. doi:10.1016/0034-4257(88)90106-X

Johnson, L. F., Roczen, D. E., Youkhana, S. K., Nemani, R. R., \& Bosch, D. F. (2003). Mapping vineyard leaf area with multispectral satellite imagery. Computers and Electronics in Agriculture, 38(1), 33-44. doi:10.1016/S0168-1699(02)00106-0

Llorens, J., Gil, E., Llop, J., \& Escolà, A. (2010). Variable rate dosing in precision viticulture: Use of electronic devices to improve application efficiency. Crop Protection, 29(3), 239-248. doi:10.1016/j.cropro.2009.12.022

Matese, A., Filippo, S., \& Gennaro, D. (2015). Technology in precision viticulture : a state of the art review. International Journal of Wine Research, 7, 69-81. doi:10.2147/IJWR.S69405

Smit, J. L., Sithole, G., \& Strever, A. E. (2010). Vine Signal Extraction - an Application of Remote Sensing in Precision Viticulture. American Journal of Enology and Viticulture, 31(2). http://jsaa.ac.za/index.php/sajev/article/viewFile/1402/615. Accessed 9 October 2017

Turner, D., Lucieer, A., \& Watson, C. (2010). Development of an Unmanned Aerial Vehicle (UAV) for hyper resolution vineyard mapping based on visible, multispectral, and thermal imagery. Proceedings of 34th International Symposium on Remote Sensing of Environment, 4. https://pdfs.semanticscholar.org/e783/bb830fc89c832cbbbbdc037acc9fcf16155d.pdf 


\author{
Cruising Robot for Collecting Floor Eggs in Poultry Houses \\ Weichao Zheng a , Dongxu Zhang ${ }^{\text {a }}$, Changle Pang ${ }^{\text {a }}$, Guanghui Teng ${ }^{\text {a }}$, Chaoyuan Wang ${ }^{\text {a }}$, Baoming Li ${ }^{\mathrm{a}}$ \\ ${ }^{a}$ College of Water Resources and Civil Engineering, China Agricultural University, Key Laboratory of Agricultural \\ Engineering in Structure and Environment, Ministry of Agriculture, Beijing 100083, China \\ * Corresponding author: E-mail address: pancl@cau.edu.cn (C. Pang).
}

\begin{abstract}
Current conveyor-type systems for poultry breeders and cage-free systems for laying hens can automatically collect eggs from the nests. However, collecting the floor eggs is a great challenge for the management and mostly require the labours to walk around and collect by hand daily. Facing the global stacked cage system is rapidly changing to cage-free systems, the challenge becomes event great. In this paper, an automatic cruising robot for picking up floor eggs was developed. It was comprised of egg grasping system, placement, driving mechanism, and UWB positing system. The detailed parameters were designed and perfected in different situations. The egg grasping system was used as the flexible material-paddle wheel, which can greatly reduce the broken egg rate. The placement mechanism was based on the vertical cross bars design, and the precision and completion rate were improved by a stepping motor. The UWB positing system can provide the position data, therefore, the automatic cruising robot would execute the stationary program. The surrounding information was precisely positioned by ultrasonic and infrared sensors, so the cruising robot can complete obstacle avoidance, collecting egg and placing them on the egg tray functions during the non-dead angle cruise.
\end{abstract}

Keywords: Autonomous robot, picking up, obstacle avoidance, route planning

\title{
1. Introduction
}

Current conveyor-type systems for poultry breeders and cage-free systems for laying hens can automatically collect eggs from the nests. However, eggs are regularly found on the floor. These floor eggs mostly require the farm labours to walk around and collect by hand daily. Once they are left on the floor, more hens are likely to lay eggs around it. Floor egg which even are collected by hand can also cause the reduction of egg quality. Therefore, collecting the floor eggs is a great challenge for the management in conveyor-type systems for poultry breeders and cage-free system for laying hens, resulting in the increasing labour cost and greater risk in cross-contamination between the facilities. Facing the global stacked cage system is rapidly changing to cage-free systems, the floor egg challenge becomes event great. Due to the high labour cost, an automatic collecting device is desired for these farms. An egg collecting device for floor eggs in loose housing systems for laying hens was developed, but showed a low success rate and high broken rate during collection (Vroegindeweij, et al., 2014). Using rapid advances in Computer Vision and Machine Learning, researchers developed an autonomous robotic system for picking up floor eggs (Joffe and Usher, 2017). Our approach is designed to be a cost-effective cruising robot for collecting floor eggs by using more reliable mechanical designing.

\section{Materials and Methods}

Hardware description

And the main components are as follows (Figure 1):

-Egg grasping system, mainly including the paddle reel to "picking up" eggs and the collecting track.

-Placement mechanism, mainly including the vertical cross bars and conveyor belt mechanism.

-UWB positioning system, mainly include 4 based stations setting on the wall and 1 label setting on the robot.

-Driving mechanism, mainly include the body and the tracked wheels. 

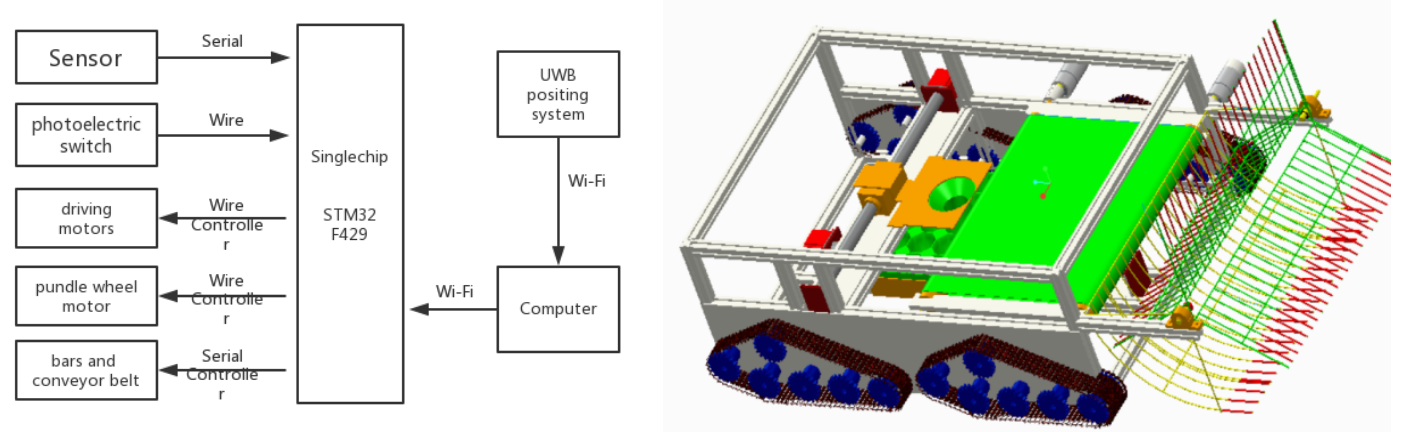

Figure 54. Robot system overview.

Software description

The robot is controlled by micro control system STM32F429 Series. The computer can get the location of robot depending on the UWB positioning system. The MCU communicates with the computer by the router and then control the motors to adjust its position or rotate the wheel to pick up eggs on the basis of whether there is a deviation between the current location and desired location.

Egg picking and placement

The egg picking progress of the robot starts when the photoelectric switch is blocked by eggs. The singlechip control the motors to drive wheel rotating to collect the eggs while the collecting track cooperating with it to restrict the eggs. To reduce the breakage of this progress, flexible materials is used to the paddle wheel and collecting track. The paddle wheel is also designed as little curved to touch eggs gently.

The placement system mainly includes the vertical cross bars and conveyor belt mechanism. When the conveyor belt mechanism transfer eggs to the cloth funnel, vertical cross bars was driven to the regularly changed position to lay eggs down to the tray. The funnel is short but the friction between the egg and the funnel is strong enough to reduce the declining speed of the egg to avoid for broken eggs.

\section{Locating and cruising}

It is very convenient to install Orbital equipment because the modern poultry house was spacious, but it is not assure for the cruising robot accuracy locating. We develop a convenient positioning system-UWB Positioning System (It includes 4 based stations setting on the wall and 1 label setting on the robot) and the software to calculate the destination. And the theory is easy as follows:

Firstly, we measure the realistic position of the based stations (the Coordinate origin can be set arbitrarily but the station A best) and then put into our software.

Secondly, the clock of MCU is synchronous, so we can get the distance of the label to every based station easily. And then the location data is transport to the software on the computer to build the three-dimensional model and calculate the coordinate of the label according to the designed algorithm.

Finally, the computer will send the coordinate to the singlechip, which will get further adjustment rely on a deviation between the current location and desired location.

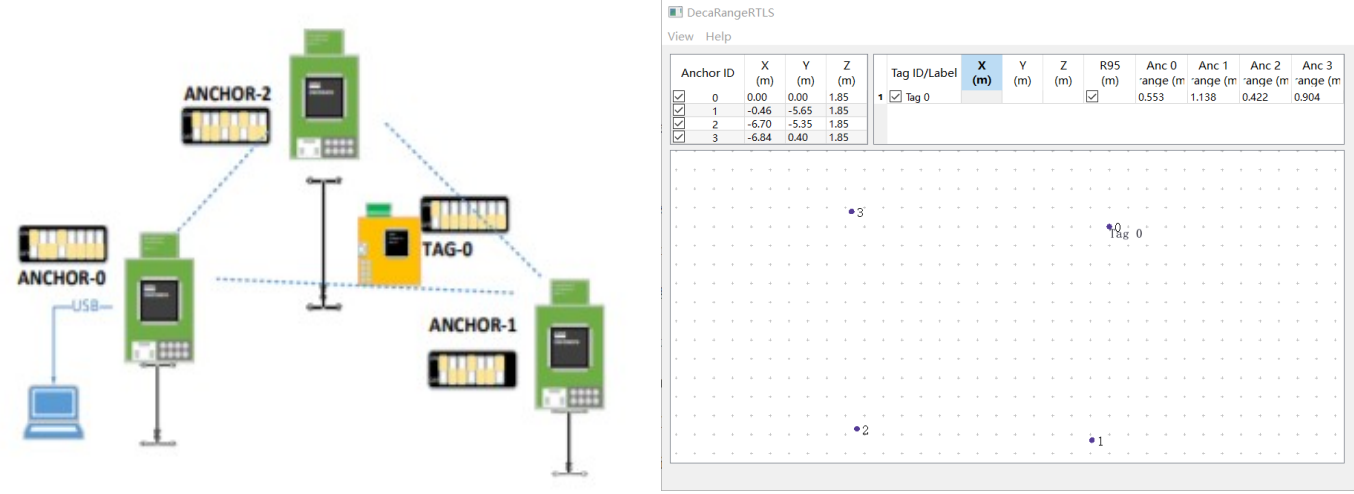

Figure 2. UWB positing system and software.

The error of above-mentioned positioning system can be limited to $10 \mathrm{~cm}$ and it is enough to design the 
cruising path. The main principles to design cruising path is to cover every inch of the house as much as possible. Because of the limited accuracy and the blocking of the equipment in the house, it is impossible for the robot to cruise without any repeated land. So the robot will do some invalid scanning works.

Another challenge is, however, to recognize and avoid the obstacles such as the equipment and the wall. Just using UWB is not enough, so ultrasonic sensors and infrared sensors is introduced to recognize them. There are four group sensors setting on the robot and the almost situation as the picture will be written into the program. And then the robot can cruise in the poultry house smoothly.

Future work

The study is ongoing at the time of writing. The success rate of the system needed to be tested. Consummating the algorithm of UWB positing system will make the robot more effective and smart to execute the moving command and improve the accuracy of the system. Four based stations may be inadequate, more stations or change their position might be needed. Another planned upgrading is using new hardware, especially changing the singlechip to a more powerful one. Another non-negligible improvement is the material, to mitigate the impact force between eggs and robot.

\section{Conclusion}

The robotic platform demonstrates the feasibility of complex behaviours like egg picking and cruising in a lab test. The results shows that the accuracy of the positing system is a little inadequate and there is some instability during the signal transportation between robot and computer. Egg picking still requires thorough evaluation and improvement to produce a robust solution suitable for commercial production. However, this platform shows the reliability of using affordable and easy components and UWB positing system can produce a realize way to optimize cruise path for the robot.

\section{References}

Joffe, B. P., \& Usher, C. T. (2017). Autonomous robotic system for picking up floor eggs in poultry houses. In 2017 ASABE Annual International Meeting (p. 1). American Society of Agricultural and Biological Engineers.

Vroegindeweij, B. A., Kortlever, J. W., Wais, E., \& van Henten, E. J. (2014). Development and test of an egg collecting device for floor eggs in loose housing systems for laying hens. 


\title{
Novel Systems for Pasture Characterization Using RGB-D Cameras and UAV- imagery
}

\author{
Victor Rueda-Ayala ${ }^{a, *}$, José M. Peña ${ }^{\text {}}$, José Bencochea-Guevara ${ }^{c}$, Mats Höglind ${ }^{\text {a }}$, Christian Rueda- \\ Ayala $^{c}$, Dionisio Andújar ${ }^{c}$

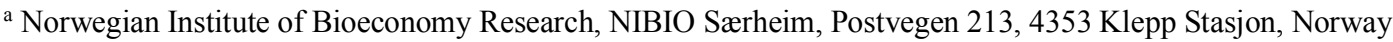 \\ ${ }^{\mathrm{b}}$ Centre for Automation and Robotics, CSIC, Arganda (Madrid), Spain \\ ${ }^{\mathrm{c}}$ Institute of Agricultural Sciences, CSIC, Serrano 115b, 28006 Madrid, Spain \\ * Corresponding author. Email: patovicnsf@gmail.com
}

\begin{abstract}
Reliable assessments of grassland growth and development enable accurate and timely management decision taking and making (e.g. application of slurry or grass cutting). In Norway, these assessments are commonly done by farmers, based on their experience, which is subjective and possibly erroneous. Current machine vision and geospatial technologies enable objective, time flexible and cost-effective plant monitoring, although, little implementation has been achieved for grasslands. Therefore, we evaluated the capability of two procedures to study and model pasture architecture in 3D, aiming at computation of grass height and biomass. Using the Microsoft Kinect ${ }^{\circledR}$ v2 sensor, on-ground depth images were acquired on selected $1 \times 1 \mathrm{~m}$ plots and reconstructed 3D point clouds via Kinect Fusion algorithms. Paralelly, a low-cost unmanned aerial vehicle (UAV) captured alongside a set of aerial Red-Green-Blue (RGB) images with $90 \%$ field overlap and the Structure from motion (SfM) technique composed the mosaic image, including also a 3D model of the study plots. Image data and 3D models were processed with object-based image analysis for estimation of grass heights and other parameters, to be compared with ground-truth observations (rising plate-meter measurements, manual cuttings and determination of dry weight biomass). Twenty sampling plots were measured in two experimental fields (10 plots per field) at the NIBIO Særheim Research Station, containing grass mixtures of importance for local farmers and of varying sward densities and plant heights. A higher accuracy of the Kinect-based procedure was reflected on the results to model grass architecture and compute plant height. However, the UAV-based procedure required significantly less time for data acquisition and elaboration of 3D models. We point out some advantages and limitations of each procedure. The use of UAVs facilitated operability and coverage of large surfaces, compared with on-ground systems. However, the on-ground sensing captured fine details of plants, producing more accurate measurements.
\end{abstract}

Keywords: 3D crop modeling, remote sensing, on-ground sensor, depth images.

\section{Introduction}

Pasture characterization is crucial for a good management and planning of the optimal cutting time. Sensing technologies contribute enormously to model vegetation and aid management in current agriculture. Models can show environmental stress problems, diseases, or the necessity of applying an agricultural task, at the right timing. Sensor data provide information to process based modelling throughout the complete life cycle, increasing knowledge and providing optimal solutions (Heege and Thiessen, 2013). Including information from vegetation models and spatial information in decision-making processes contributes to improve efficiency of crop management. Spatial information adds up value to decision-support models, allowing a site-specific management within the field. Pastures require consecutive supervision along their life cycle, because as in most perennial crops, a great potential of biomass production is available (Hopkins, 2000). A continuous supervision of a spatial model could lead to better decision making for pasture management, including operations such as determining the optimal cutting time, fertilization and need for reseeding.

The use of 3D models allows characterizing automatically plant morphology, which for breeding programs and genetic management is needed to accurately monitor agronomic processes and apply decision-making programs (Chaivivatrakul et al., 2014). Capture of the third dimension in the form of a 3D point cloud with X-Y$\mathrm{Z}$ coordinates can be created through different processes. The most common system uses a LiDAR (Light Detection and Ranging) in combination with a displacement of the sensor to acquire de Z coordinate (Rosell-Polo et al., 2015). This method is fast and accurate, although, it needs of calibration and displacement. The associated cost also increases as the resolution increases (Yandún Narváez et al., 2016). These drawbacks can be compensated with a new generation of sensing devices based on Time-of-Flight cameras such as RGB-Depth cameras, which combine depth information with the color scene in single shoots. The combination of frames allows reconstructing big crop surface areas (Bengochea-Guevara et al., 2018). Such sensors have been used for several agricultural purposes, being Microsoft Kinect ${ }^{\circledR}$ v2 the most frequently used. Wang and Li (2014) calculated onion bulb volume and concluded that the accuracy of these systems is satisfactory. Foliar density of threes was estimated for autonomous spraying (Correa et al., 2013). Andújar et al. (2016) using a dual methodology separating crops and weeds from soil in maize crops under field conditions. The methodology 
included height selection and RGB segmentation using a unique model for plant discrimination.

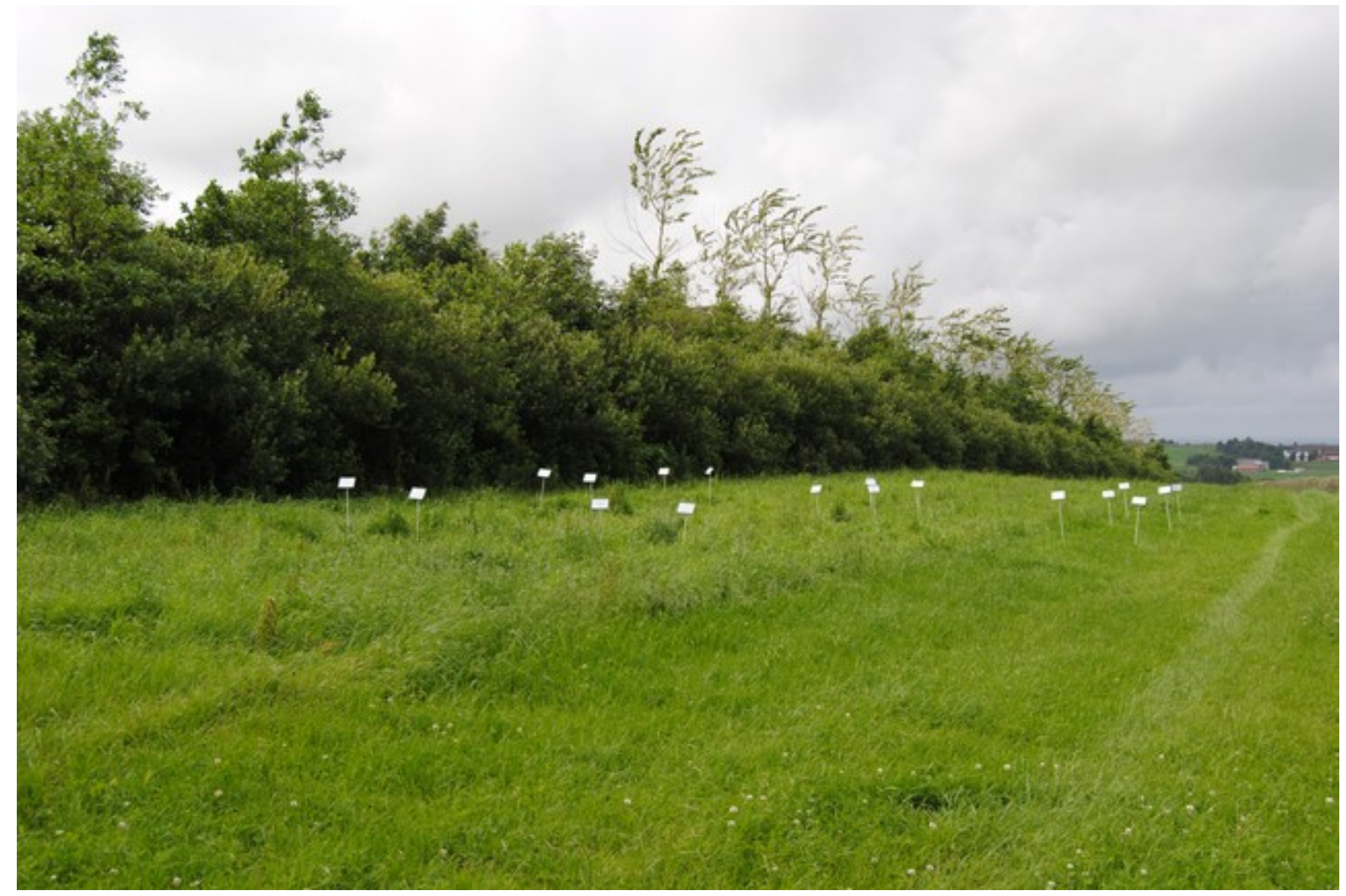

Figure 1. Field test conducted at NIBIO Særheim (Klepp stasjon).

Even though on-ground scanning has proved to be feasible for crop characterization, other methods based on aerial platforms such as UAVs can also be used to construct high-resolution models using different sensors. Such platforms can cover large areas and operate independently of soil conditions (Sankey et al., 2017). In addition, automatic aerial vegetation characterization systems allow more flexibility than ground-based systems, at reduced operational time and costs. The use of photogrammetry from UAVs has proved high functionality in different studies. It can be applied to create high spatial resolution images when flying at low altitudes, with large overlapping between images. The data can be processed through Structure from Motion reconstruction for building the 3D model. The method has been tested in olive trees to calculate canopy area, tree height and crown volume by generation of digital surface models and OBIA algorithm (Torres-Sánchez et al., 2015). New challenges in agriculture require sensing devices and technological advances to help improve crop quality and yield. As for other crops, grassland farmmers are in need of increasing productivity, to which advanced technological support could contribute. Digital models could assist in the optimization of inputs, leading to better distribution or reduction of fertilizers, pesticides or seeds, e.g., by site-specific reseeding of pastures. In addition, a more environmentally friendly management would be achieved, because chemical contamination and residues will be avoided in the forage, if pesticies use was reduced. Thus, the objective of this study was to evaluate the possibilities of using on-ground and aerial methods to characterize pastures of different species mixtures by calculating biomass, volume and plant height values.

\section{Materials and Methods}

Aerial and on-ground characterization systems were tested on a field located at the NIBIO Særheim station (Klepp stasjon, Norway). The field of 0.5 ha was mapped by UAV and 10 subsamples of $1 \mathrm{~m}^{2}$ were placed for ground-truth validation (Figure 1). The grass mixture was composed of $85 \%$ timothy (Phleum pratense L.), $10 \%$ perennial ryegrass (Lolium perenne L.), and 5\% annual ryegrass (Lolium multiflorum L.). The field was sown in 2015 and yearly fertilized with 10, 8 and 6 tons of liquid manure at early spring (March-April), after first cut (June-July) and after second cut (August-September), respectively, corresponding to ca. $260 \mathrm{~kg} \mathrm{ha}^{-1}$ year ${ }^{-1}$. Additions of $\mathrm{P}$ and $\mathrm{K}$ fertilizer were done according to the expected yield and soil nutrient status, with application at early spring of 40 and $140 \mathrm{~kg} \mathrm{ha}^{-1}$ of $\mathrm{P}$ and $\mathrm{K}$, respectively. Field assessments were conducted simultaneously using two sensing systems, an on-ground and an aerial, during July-August 2017 when pasture was fully developed, at anthesis stage. 
The on-ground method was based on a RGB-D system. The RGB-D sensor Kinect ${ }^{\circledR}$ v2 (Microsoft, Redmond, WA, USA) was used; it operates at $30 \mathrm{fps}$, supplies RGB images with a resolution of $1920 \times 1080$ pixels together with depth information with a resolution of $512 \times 424$ pixels. The device is equipped with a standard RGB camera of $1080 \mathrm{p}$, a depth camera, an infrared camera and an array of microphones. The distance is calculated for every pixel in the scene by the method of Time-of-Flight method by phase detection, i.e., the distance is calculated based on the time that a pulse of light takes to travel from the light source to the impacted plant and back to the sensor. The RGB camera can adapt automatically the exposure time of the RGB to obtain brighter images at limiting light conditions. The IR camera can take a clear view into the darkness with a resolution of $512 \times 424$ pixels. The opening field of view (FOV) is different for every camera. The IR camera has a FOV of 70 degrees horizontally and depth perception is limited to 60 degrees vertically. The range this camera works in depth goes from 0.5 to $4.5 \mathrm{~m}$ of distance from the sensor, although in outdoors conditions, the maximum range decreases. Studies conducted outdoors under different daytime illumination conditions showed that the sensor provides valid depth measurements up to $1.9 \mathrm{~m}$ during sunny days, while the distance increases up to $2.8 \mathrm{~m}$ under the diffuse illumination of an overcast day (Andújar et al., 2016). The needed overlap to fuse the acquired images is reached by a frame rate than can be set up to $30 \mathrm{fps}$ while data acquisition.

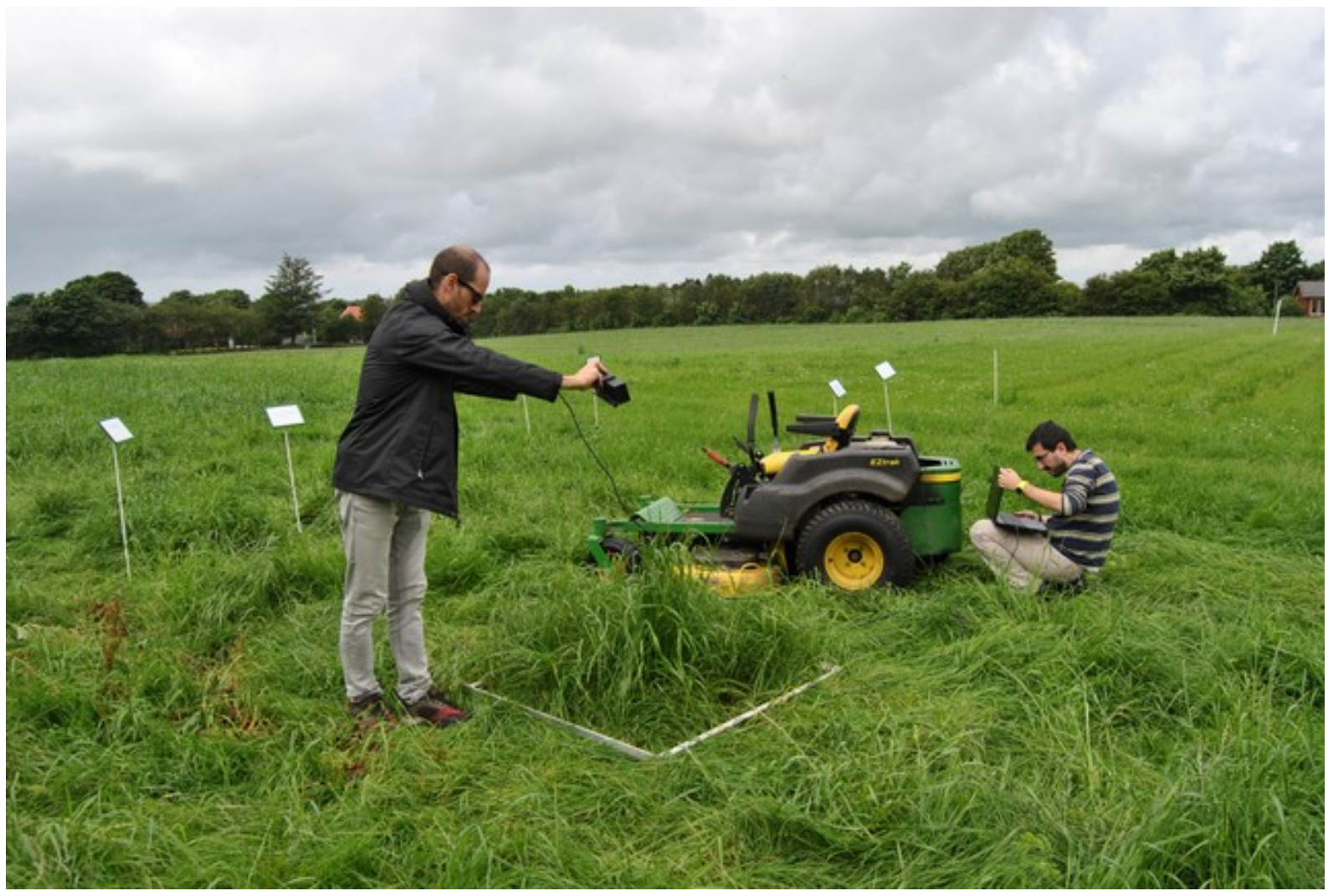

Figure 2. RGB-D adquisition system while working at the sample plots.

An Intel laptop computer with Windows 8 supported by Kinect SDK was used for data collection. The SDK (software development kid) helps to acquire data by classes, functions and structures by providing the necessary drivers for the sensor, and some sample functions that were implemented for the measurements combined with some OpenCV functions (OpenCV, 2018). The sensor was hand held pointing out the field samples from top view (Figure 2). The method developed for point cloud generation reconstructs large regions using the fusion of different overlapped depth images based on a previous development (Curless and Levoy, 1996), storing information only on the voxels closest to the detected object and accessing the stored information by using a hash table. After that, for every new input depth image and knowing camera position, the ray-casting technique (Roth, 1982) is used to project a ray from the camera focus for each pixel of the input depth image, to determine the voxels in the 3D world that cross each ray. Then, the voxels related to the depth information are determined. The next step is conducting variation of the iterative closest point (ICP) algorithm, which produces a point cloud as output. The modified algorithm creates a point cloud by detecting the overlapping areas in sequential frames by assessing the relative position of the Kinect sensor for each frame to create a 3D model and removing outliers from the mesh (Bengochea-Guevara et al., 2018). Outliers may appear isolated in the point cloud. A point was considered an outlier when the average distance to its 64 nearest neighbour was greater than the standard deviation of the distance to the neighbours of all points (Figure $\underline{3}$ ). 

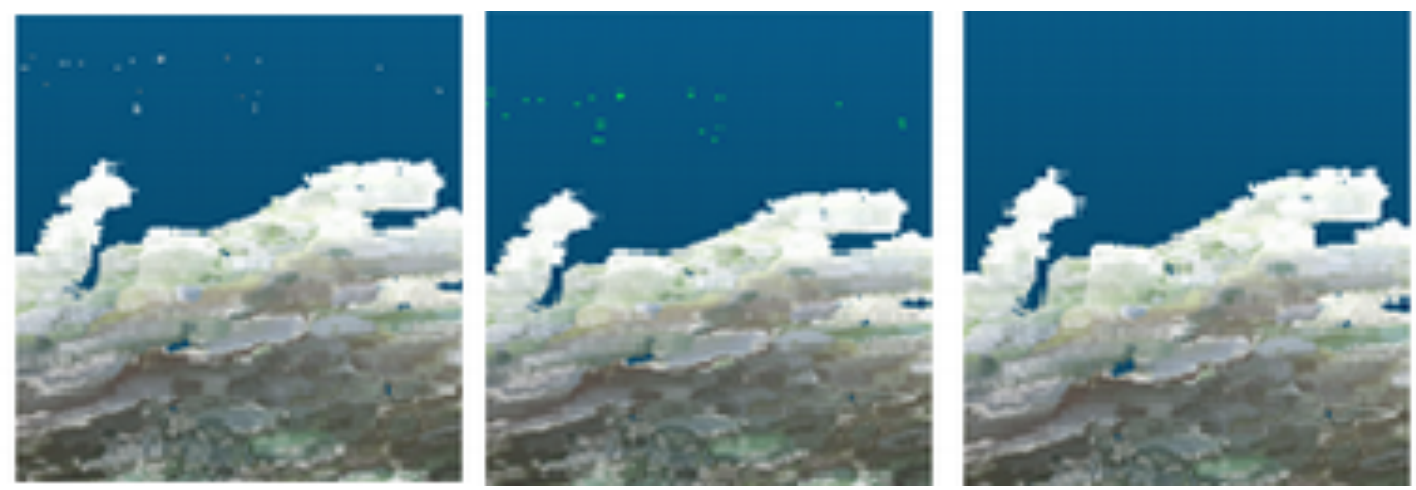

Figure 3. Section of the 3D reconstruction: before filtering (left); removed points are marked in fluorescent (middle) and after filtering (right).

Once the 3D representation of the whole pasture sample was available (Figure 4), we could estimate volumes. Firstly, an alpha shape (Edelsbrunner and Mücke, 1994) or volume that envelops the set of 3D points had to be obtained. The alpha parameter specifies how tight the body fits the points. There are many software libraries to address this issue. In the developed system, the R 'alphashape3d' package based on the original Edelsbrunner and Mucke's work (Edelsbrunner and Mücke, 1994) was used. Figure $\underline{5}$ shows different alpha shapes according to the alpha value selected for the same point cloud. Higher values showed very loose shapes, whereas lower values generated tight bodies. Finally, the same function library that allowed calculation of the alpha shapes, allowed also volume estimation.

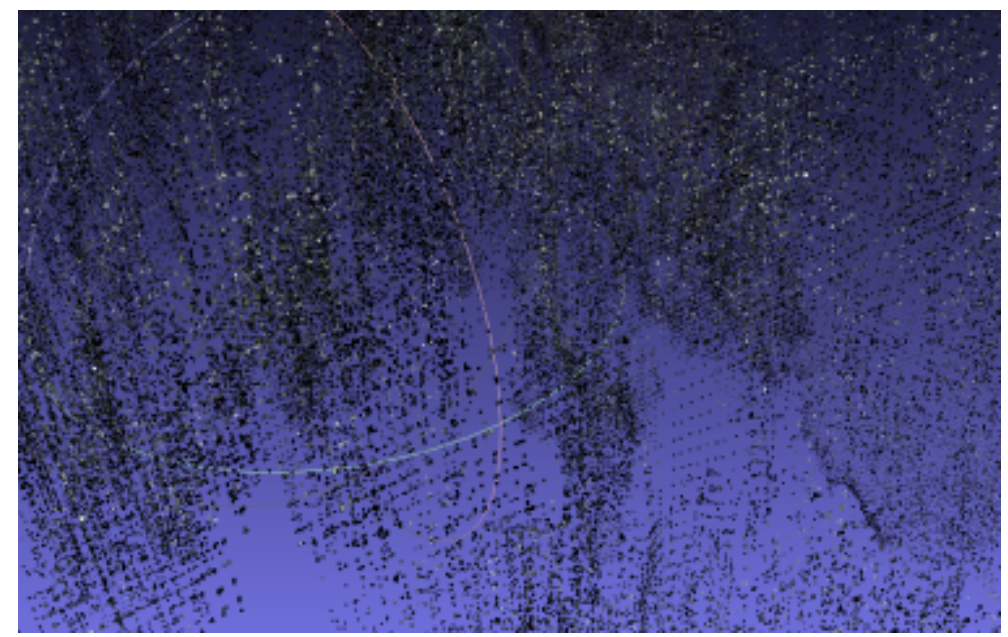

Figure 4. Point clouds created by RGB-D Microsoft Kinect ${ }^{\circledR}$ v2 system.

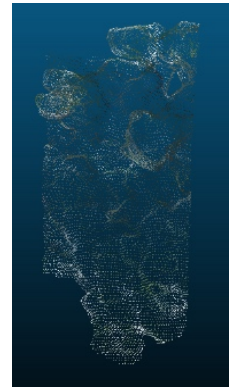

(a)

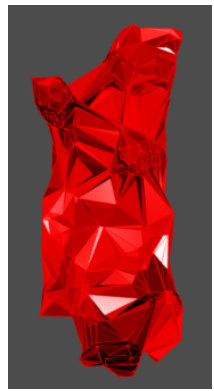

(b)

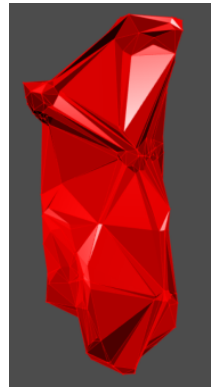

(c)

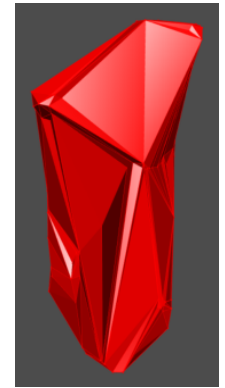

(d)

Figure 5. Alpha shapes for the same point cloud. (a) and (b) alpha $=0.1$, (c) alpha $=0.2$ y (d) con alpha $=0.4$.

A UAV-based on a quadcopter DJI Mavic Pro combining a 4K digital camera and location information, was used for aerial imaging. The camera mounted on the UAV had a $28 \mathrm{~mm}$ lens with a Field of Fiew (FOV) of 78.8 
degrees and a resolution of 4,000 $\times 3,000$, capable of shooting 12.35 megapixel photos; the camera was 3-axis stabilized by its drone's gimbal (https://www.dji.com). The acquired aerial imagery was tested and compared with the RGB-D on-ground system (Figure 6). The UAV flew autonomously following the programed route by an internal GPS receiver using Litchi APP. The images were used to reconstruct the Digital Surface Model by photogrammetry process.

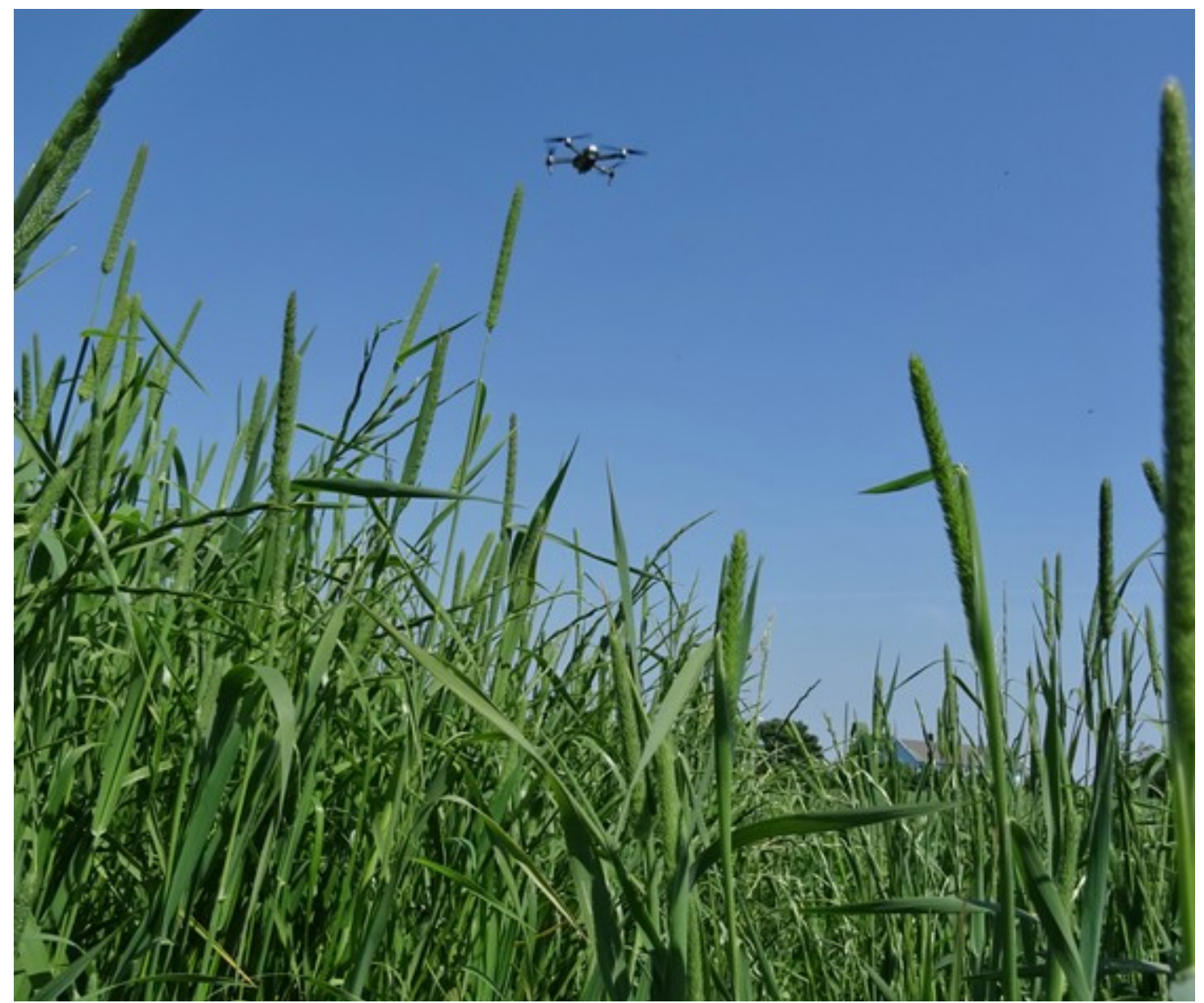

Figure 6. Detail of the aerial sampling in a pasture field using the UAV platform.

The UAV flight mission was set up to take continuously photos every two seconds. The software Agisoft PhotoScan Professional Edition (Agisoft LLC, St. Petersburg, Russia) version 1.0.4 was used for 3D model generation. This software allows a fully automatic process for aligning images, building field geometry, and orthophoto generation (Figure $7 \mathrm{a}$ ). The modelling process was set up in three main stepts, (1) aligning images, (2) building field geometry, and (3) dense point cloud generation. The software searches for common points in the images to create a dense 3D point cloud to create the final model is created of a 3D polygon mesh. The DSM and the ortho-mosaics were then joined to create a 4-band multi-layer file of the RGB bands and the DSM. 3D features, such as volume were calculated by integrating the volume of the individual pixels below the top of the crop (de Castro et al., 2018) (Figure 7b). A desktop computer equipped with an Intel Core i7-4771@3.5 GHz processor, 16 GB of RAM, and NVIDIA GeForce GTX 660 graphic card was used for image processing and 3D modelling. 


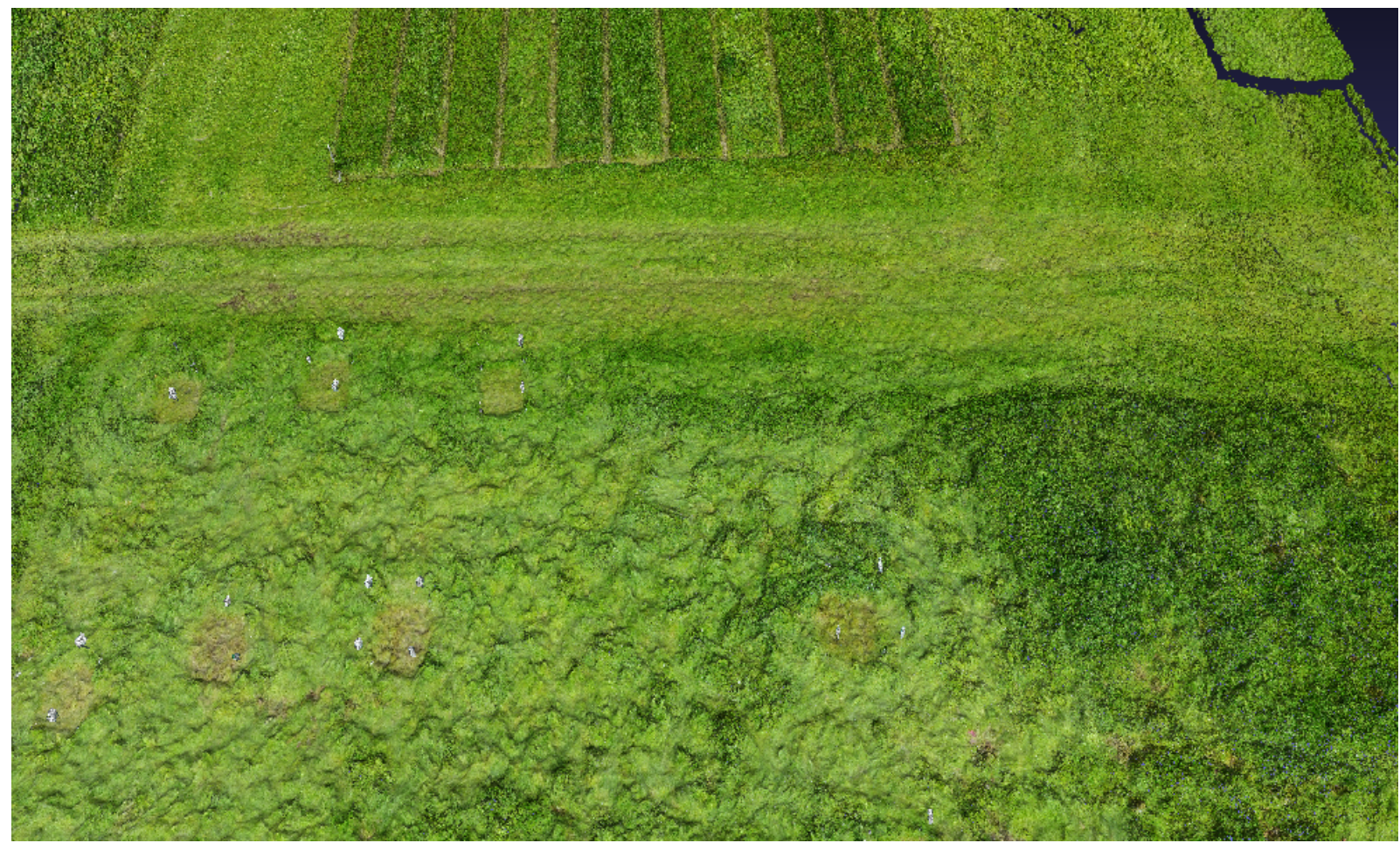

(a)

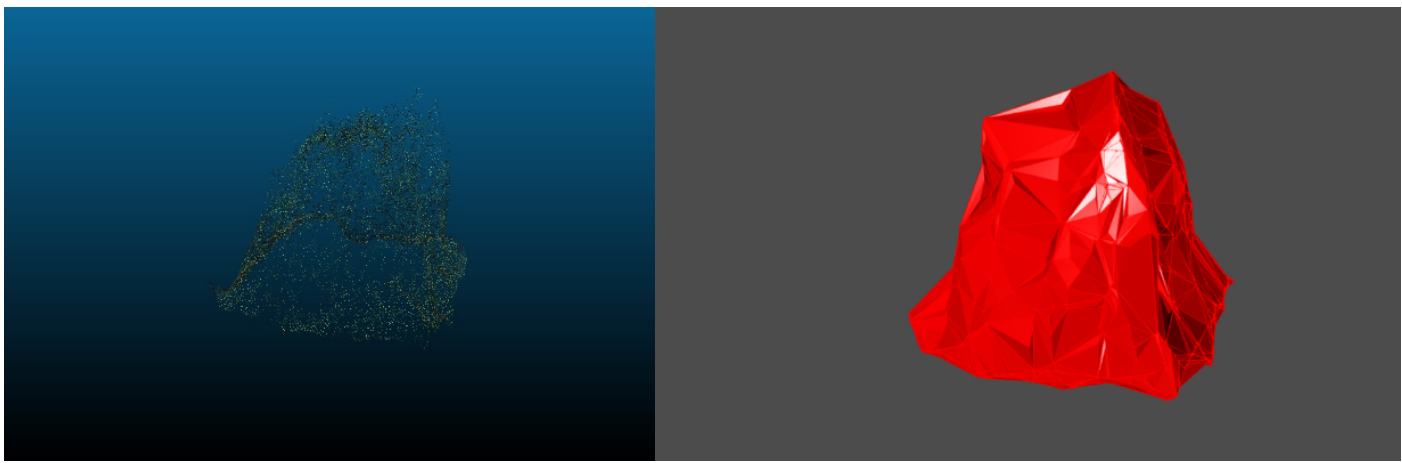

(b)

(c)

Figure 7. Model constructed by photogrammetry methods and processes of point cloud of DSM model and point cloud and solid generation.

After acquiring the information with both systems, the canopy height was determined on five random locations per plot (Figure $\underline{8}$ a). Additionally, the compressed sward height was determined using a rising plate meter (Figure $\underline{8 b}$ ). The compressed height of pastures is of high importance since it is an indicator of the growth rate and herbage yield. Thereafter, all grass plants of each sampling plot were cut at ground level and taken to the laboratory for dry biomass determination. Biomass dry weight was determined after oven-drying the samples at $80^{\circ} \mathrm{C}$ during $48 \mathrm{~h}$.

\section{Results and Discussion}

The analyzed systems in the present study showed differences in data resolution, therefore data analysis indicated differences in plant height and volume determination capability by each system. It is difficult to determine pasture volume and plant height accurately, since they vary highly across the field. In spite of this difficulty, there was a reasonably good agreement between the UAV-based and Kinect-based model estimates with the manual measurements. Kinect assessments showed a good correlation between the plate-meter measured and model-estimated pasture volume. End-details of leaves in the field were difficult to reconstruct with either system, thus reducing the height estimation capability by the developed models. However, the plate-meter minimizes such end-details, as it bends down the largest leaves during measurement (Figure $\underline{8} \mathrm{~b}$ ). A significant relationship between measured plant height $(\mathrm{cm})$ and Kinect volume $\left(\mathrm{m}^{3}\right)$ estimations was identified at $P<0.01$ with $R^{2}=0.87$ (Figure 9a). Pasture volume estimated with Kinect was also positively correlated to the measured biomass per unit area, with a $R^{2}=0.65$ (Figure $\underline{9}$ b). A slightly poorer correlation was found between pasture 
volume estimated by the UAV-based system and the actual sward dry biomass, with a $R^{2}=0.54$ (Figure $9 \mathrm{c}$ ). As expected, biomass was highly correlated with actual plant height (Figure $9 \mathrm{~d}$ ).

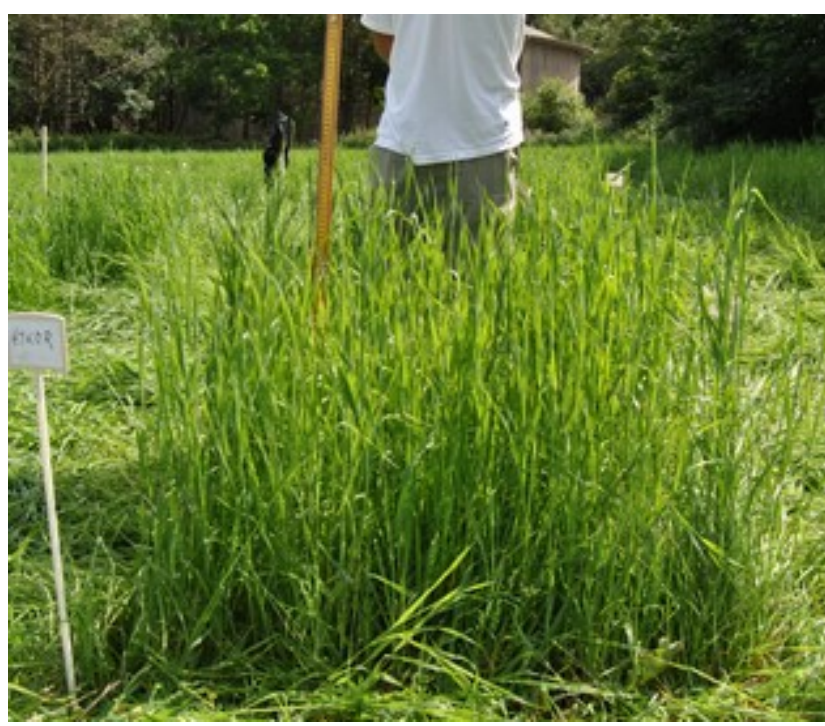

(a)

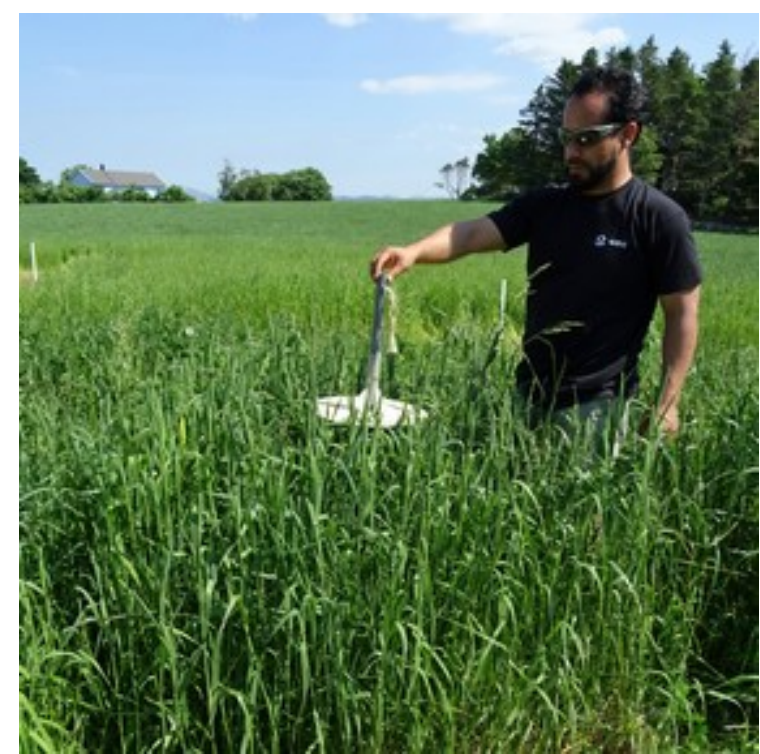

(b)

Figure 8. Height measurements with a ruler meter (a) and a rising plate-meter (b).

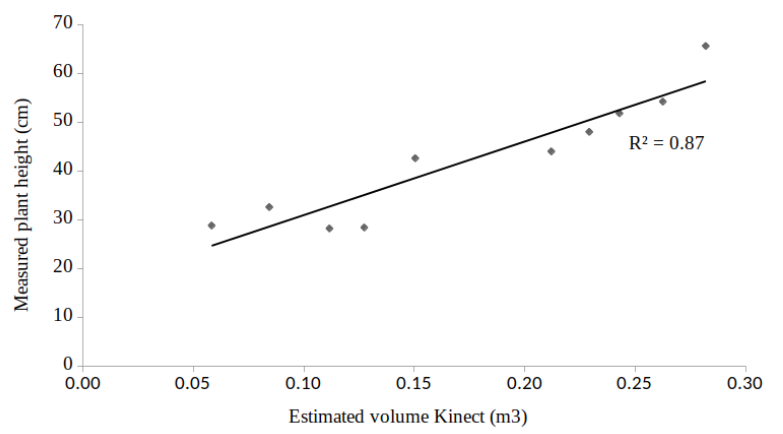

(a)

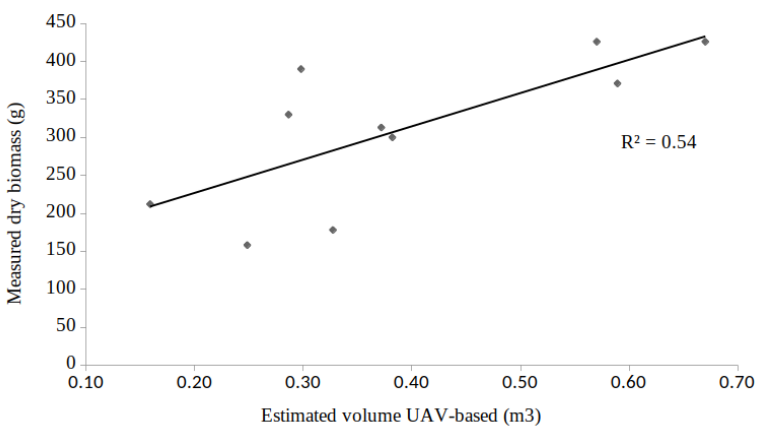

(c)

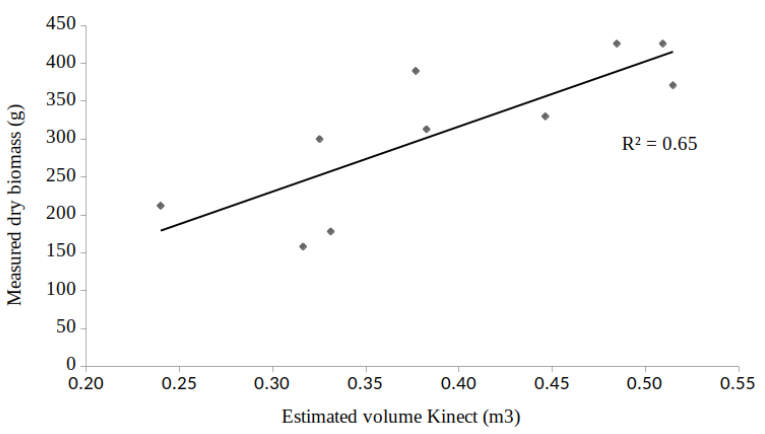

(b)

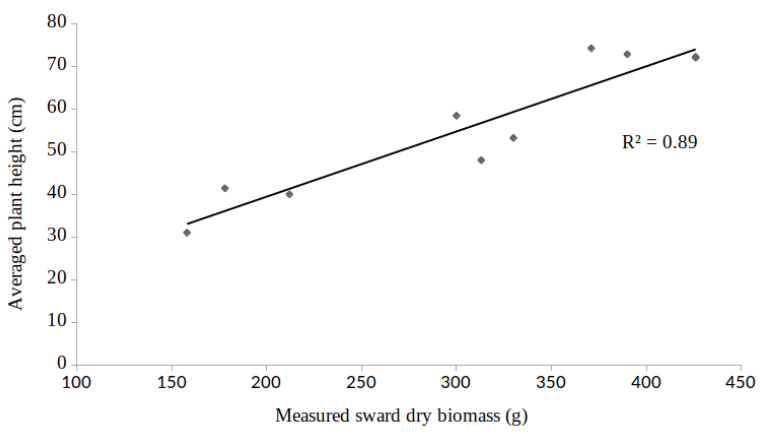

(d)

Figure 9. Regression analysis comparing a) pasture volume using Kinect 3D modeling vs. actual plant height; b) pasture volume using Kinect 3D modeling vs. dry biomass; c) pasture volume using UAV modeling vs. dry biomass ; d) dry biomass vs. actual plant height.

In general, the sward height measured with both studied sensing systems was slightly lower than the manually and plate-meter height. Although, the reconstruction with aerial images was similar to the reality, some details of the plants (e.g., tips of leaves) tended to be sometimes missed by the camera or the sensor-based models. 
Comparing UAV-based with Kinect-based systems, the costs differ between systems. It has been argued that cost of aerial imaging is lower and can cover bigger areas (Anderson et al., 2008). The advantage of using UAV-based sampling was notorious, with whole-field coverage in less than 12 minutes. Contrarily, the Kinect-based system needed considerably more time for all sampling plots. Further development of the ground-based systems would contribute to a more cost-effective sampling, which will increase the incentive for using automatic characterization systems for decision making in grassland production. The on-ground vegetation characterization with Kinect system may require more investment, due to the need of an additional ground vehicle. However, the current state of development, especially of auto-guided ground vehicles, would allow increased monitoring capacity. In the end, which system will be most cost efficient will depend on the technical development of the two systems including the vehicles carrying the sensors.

\section{Conclusions}

The use of UAV- and Kinect-based systems showed their feasibility for a reasonably good determination of plant height and volume of pastures, compared with manual measurements, even though manual determination of these parameters is highly difficult for such plant species. UAVs facilitated operability and coverage of large surfaces, while the Kinect system captured fine details of plants, producing more accurate models and estimations. Any of the tested systems offer already a huge advantage for pasture architecture characterization, which would be of immense help for carrying out assessments on grassland research and especially for decision-making in forage production. In this matter, it is important to recall that pasture measurements rely mostly on manual sampling (e.g., with a plate-meter and destructive biomass sampling) of heights and biomass, which are tedious and highly time-consuming tasks. Resolution of UAV acquired imagery is extremely affected by other conditions external to the camera sensor, such as sunlight, clouds, wind speed and climate, which affect the models for parameter estimation. Furthermore, a high investment needs to be done for data analysis software, if a reliable and accurate model is to be obtained. In our study, it could be observed a slight advantage of using the Kinect-based, regarding the height and volume estimation efficiency. The Kinect system (RGB plus depth perception camera) produced data of higher fidelity for the development of vegetation architecture models. Still, operation costs are a challenge to overcome. Increasing speeds for on-ground platforms would improve the performance of these systems, to monitor more area. On-ground RGB-D data can be effectively analysed with open source software, as it was done in this study, which may compensate and challenge the expenses.

\section{Acknowledgements}

This research was funded by the projects AGL2017-83325-C4-1-R and AGL2017-83325-C4-3-R (Spanish Ministry of Economy and Competition) and by the RYC-2016-20355 agreement, Spain, and by the Department of Grassland and Livestock, NIBIO.

\section{References}

Anderson, J.E., Plourde, L.C., Martin, M.E., Braswell, B.H., Smith, M.L., Dubayah, R.O., Hofton, M.A., Blair, J.B., 2008. Integrating waveform lidar with hyperspectral imagery for inventory of a northern temperate forest. Remote Sensing of Environment 112, 1856-1870. doi: 10.1016/j.rse.2007.09.009.

Andújar, D., Dorado, J., Fernández-Quintanilla, C., Ribeiro, A., 2016. An approach to the use of depth cameras for weed volume estimation. Sensors 16, 972. doi:10.3390/ s16070972.

Bengochea-Guevara, J.M., Andújar, D., Sánchez-Sardana, F.L., Cantuña, K., Ribeiro, A., 2018. A low-cost approach to automatically obtain accurate $3 d$ models of woody crops. Sensors 18. doi:10.3390/s18010030.

Chaivivatrakul, S., Tang, L., Dailey, M.N., Nakarmi, A.D., 2014. Automatic morphological trait characterization for corn plants via $3 \mathrm{~d}$ holographic reconstruction. Computers and Electronics in Agriculture 109, 109-123. doi:10.1016/j.compag.2014. 09.005.

Correa, C., Valero, C., Barreiro, P., Ortiz-Cañavate, J., Gil, J., 2013. Usando kinect como sensor para pulverización inteligente, in: VII Congreso Ibérico de Agroingeniería y Ciencias Hortícolas; UPM: Madrid, Spain, pp. 1-6.

Curless, B., Levoy, M., 1996. A volumetric method for building complex models from range images, in: Proceedings of the 23rd Annual Conference on Computer Graphics and Interactive Techniques, ACM, New York, NY, USA. pp. 303-312. doi:10.1145/237170. 237269.

de Castro, A.I., Torres-Sánchez, J., Peña, J.M., Jiménez-Brenes, F.M., Csillik, O., López-Granados, F., 2018. An automatic random forest-obia algorithm for early weed mapping between and within crop rows using uav imagery. Remote Sensing 10. doi:10.3390/rs10020285.

Edelsbrunner, H., Mücke, E.P., 1994. Three-dimensional alpha shapes. ACM Trans. Graph. 13, 43-72. doi:10.1145/174462.156635. 
Heege, H.J., Thiessen, E., 2013. Sensing of crop properties, in: Precision in Crop Farming: Site Specific Concepts and Sensing Methods: Applications and Results. Springer Netherlands, Dordrecht, pp. 103-141. doi:10.1007/978-94-007-6760-7_6.

Hopkins, A., 2000. Grass: its production and utilization. British Grassland Society. 11.

OpenCV, 2018. The open source computer vision. URL: https://www.opencv.org/. last accessed on 2018-04-

Rosell-Polo, J.R., Auat-Cheein, F., Gregorio, E., Andújar, D., Puigdomènech, L., Masip, J., Escolà, A., 2015. Chapter three - advances in structured light sensors applications in precision agriculture and livestock farming, Academic Press. volume 133 of Advances in Agronomy, pp. 71-112. doi:10.1016/bs.agron.2015.05.002.

Roth, S.D., 1982. Ray casting for modeling solids. Computer Graphics and Image Processing 18, $109-144$. URL: http://www.sciencedirect.com/science/article/pii/0146664X82901691, doi:https://doi.org/10.1016/0146$664 X(82) 90169-1$.

Sankey, T., Donager, J., McVay, J., Sankey, J.B., 2017. Uav lidar and hyperspectral fusion for forest monitoring in the southwestern usa. Remote Sensing of Environment 195, 30-43. doi:10.1016/j.rse.2017.04.007.

Torres-Sánchez, J., López-Granados, F., Serrano, N., Arquero, O., Peña, J.M., 2015. High-throughput 3-d monitoring of agricultural-tree plantations with unmanned aerial vehicle (uav) technology. PLOS ONE 10, 1-20. URL: 10.1371/journal.pone.0130479, doi:10.1371/journal.pone.0130479.

Wang, W., Li, C., 2014. Size estimation of sweet onions using consumer-grade rgb-depth sensor. Journal of Food Engineering 142, 153-162. doi:10.1016/j.jfoodeng.2014.06. 019.

3 Yandún Narváez, F.J., del Pedregal, J.S., Prieto, P.A., Torres-Torriti, M., Cheein, F.A.A., 2016. Lidar and thermal images fusion for ground-based 3d characterisation of fruit trees. Biosystems Engineering 151, 479-494. doi:10.1016/j.biosystemseng. 2016.10.012. 


\author{
Design Space Exploration in the Development of Agricultural Robots \\ Frederik F. Foldager ${ }^{1,2, *}$, Ole Balling ${ }^{1}$, Carl Gamble ${ }^{4}$, Peter Gorm Larsen ${ }^{1}$, Martin Boel ${ }^{2}$ and Ole \\ Green $^{2,3}$ \\ ${ }^{1}$ Department of Engineering, Aarhus University, Aarhus, Denmark \\ ${ }^{2}$ Agro Intelligence Aps, Aarhus, Denmark \\ ${ }^{3}$ Department of Agroecology, Aarhus University, Tjele, Denmark \\ ${ }^{4}$ Department of Computing, Newcastle University, United Kingdom \\ *Corresponding author. Email: ffo@eng.au.dk
}

\begin{abstract}
Within the last decade, an increased focus in the agricultural industry is drawn towards autonomously driving field robots. The general tendency is that autonomous field robots are designed to accommodate a wide range of field operations which increases the requirement to the field robots in terms of velocity, traction force, etc. When removing the human driver from the loop, the engineers have to investigate and evaluate the performance of the robot in numerous configurations and what-if scenarios during the development of the robot. To address the issue of developing a robot with a high number of possible configurations and load cases, it is proposed to apply a model-based approach. The method presented here relies on the use of co-simulation using the Functional Mockup Interface (FMI). Valuable insight into the performance is obtained by modelling and co-simulating models of the robot dynamics, steering controller and tools. Each model is developed in a suitable format and exported as a Functional Mock-up Unit (FMU). To accommodate an increasing number of different tools to be driven autonomously, Design Space Exploration (DSE) is applied to perform a number of co-simulations. It is described how the FMUs of the robot dynamics and tools are established. Two examples are presented to show the use and advantage of the FMI. The models shown are based on either a mathematical description or based on soil-bin test data that is included in an FMU and hereby applicable for co-simulation.
\end{abstract}

Keywords: Co-simulation, Robotics, Vehicle Dynamics, Design Space Exploration

\title{
1. Introduction
}

The research and development of autonomous field robots for arable farming are gaining traction in both the agricultural industry and academia (Bechar and Vigneault, 2016; Christiansen et al., 2018; Foldager et al., 2018). This work presents a method for supporting the development of the autonomous field robot, Robotti ${ }^{I}$ as shown in Figure 1. The robot is a tool-carrier, meaning that the robot is designed for accommodating a wide span of field operations. This causes certain requirements to the design of the robot and the engineers behind. By combining the robot with several tools, a large number of load cases emerge. By removing the human driver from the field operation, an initial evaluation has to be conducted during the product development. To maintain a short time to market and reduce the number of hours spent testing different configurations with several tools in the fields, a structured simulation-based approach is needed to address the increasing number of possible configurations.

This work presents a method for applying the Functional Mock-up Interface ${ }^{2}$ (FMI) to support the research and development of the robot and the applicable tools. This method is applied to investigate the physical characteristics of the robot when changing the tool. The robot, the steering controller and the tools are modelled separately and joined in a co-simulation using the co-simulation orchestration engine (Thule et al. 2018; Lausdahl et al. 2017). By utilising the co-simulation techniques of the INTO-CPS project (Fitzgerald et al. 2016, 2015) it is possible to perform a number of simulations of the robot in the different configurations using the Design Space Exploration (DSE) feature (Gamble 2017). Using co-simulation allows for testing tools and robot configurations effectively in the cyber-physical domain before entering the fields (Christiansen et al., 2015).

This work is initiated by developing a basis DSE of the robot and steering controller which performs the simulations of the robot in the different configurations of possible widths, masses and position of the centre of gravity. This DSE is then the applied in three scenarios - scenario 0 describes the motion and load distribution of the robot without any external load. In scenarios one and two, the tool model is integrated into the co-simulations consisting of models describing respectively a mechanical weeder and a sprayer. The mechanical weeder is characterised by being soil engaging, while the sprayer is characterised by having no soil contact but a high weight. As more tools become relevant in the development of the robotic platform, the number of tool FMUs will increase.

\footnotetext{
${ }^{1} \mathrm{http}: / /$ agrointelli.com/robotti-diesel.html\#rob.diesel

${ }^{2}$ http://fmi-standard.org
} 


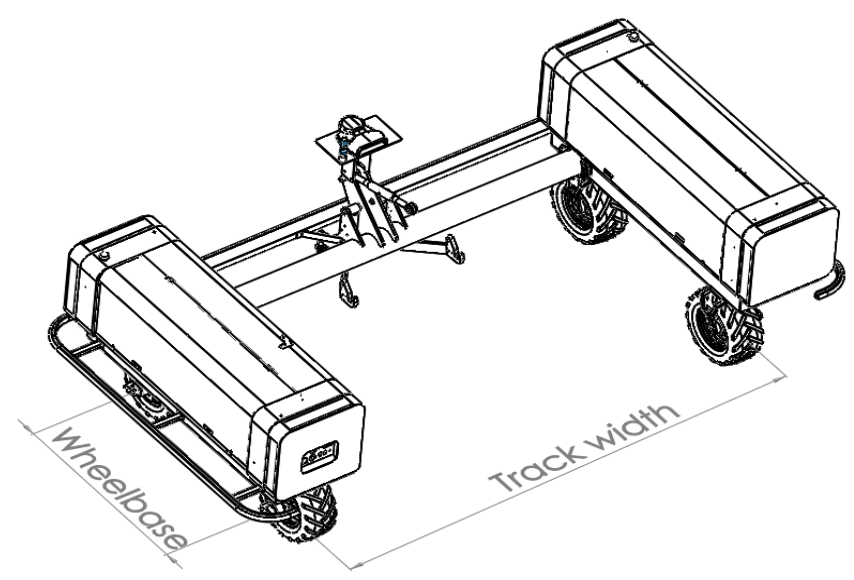

Figure 55. Agricultural tool carrier, Robotti.

This paper is structured as follows, section 2 presents the materials and methods including the underlying mechanism of performing a co-simulation using the INTO-CPS technology and the concept of DSE. Furthermore, a Continuous Time (CT)-model of the robot dynamics, CT-models of the tools and a Discrete Event (DE)-model of the steering controller are presented. Section 3 presents results of two co-simulations reflecting scenarios one and two. The first DSE experiment investigates the distribution of normal loads as a function of the kinematic constraints of the robot, loaded and unloaded. Secondly, the results of a co-simulation of the robot, a tool and the steering controller are shown to indicate the possibilities of using FMUs in the research and development of such industrial applications. Finally, section 4 provides a number of concluding remarks, discussion and points to directions for future work.

\section{Materials and Methods}

The scope of this work is to obtain a generic model-based virtual test setup of the robot that allows for investigating a number of load cases of the robot in a virtual setting before entering the fields. A series of virtual tests predicts, among other parameters, the normal loads on the wheels and the draught forces acting on the robot as a consequence of mounting a given tool. This is important in the assessment of the already existing robot, as well as applicable in the research and development of future versions and configurations of the robot.

A versatile modelling and simulation setup is obtained using FMI, which gives the possibility of easily interchanging models of the robot, steering controller and tools to suit a given scenario. The components are modelled individually and exported as FMUs. The models are based on the physical properties of the robot in the specific configurations. This section covers the general introduction to the INTO-CPS Technology, the robot and the models applied in the co-simulations.

\section{FMI and the INTO-CPS Technology}

The "Integrated Tool Chain for Model-based Design of Cyber-Physical Systems" (INTO-CPS) project supplies the majority of the technology used in this study (Larsen et al., 2016). The underlying standard is the FMI version 2.0 standard for co-simulation (Blochwitz et al. 2011). It was decided to use FMI as the interface for the different simulation and testing tools since it is a mature standard created in the MODELISAR project (ITEA Office Association 2015) with an active community. The core FMI compliant tool of the INTO-CPS tool chain is a Co-simulation Orchestration Engine called Maestro supporting both fixed and variable step size simulations (Thule et al. 2018).

The tools used in this work include the SysML tool Modelio (Bagnato et al. 2017) the Overture VDM tool used for DE modelling (Larsen et al. 2010) and the 20-sim tool used for CT modelling (Kleijn 2006). In addition, the INTO-CPS Application has been used to launch Maestro for co-simulation and multiple co-simulations are invoked via a DSE feature.

\section{Design Space Exploration}

When building the FMUs for a multi-model, the engineer has the option to define parts of those models as being parameters, where parameters may have their default value changed at the start of a simulation during the initialisation phase. These parameters can represent physical properties of the model such as mass or width of a device, or perhaps the traction between the tyres and the ground. Equally, parameters may also describe cyber 
properties of the controller hardware and software, such as the cycle time for the controller or parameters of the controller algorithm. Since the engineer may wish to compare many designs to find the best, DSE requires the engineer to define objective scripts, these process the raw simulation results produced by a simulation and output values that characterise the performance of the system being modelled in a way that supports comparison of different designs (Gamble 2017). Designs often have multiple objectives to optimise and so the INTO-CPS support includes both exhaustive and genetic (Deb 2012) search algorithms to automatically search the design space and to return a Pareto front representing the best designs found in that design space (Fitzgerald et al. 2017).

\section{The Robot and Simulation Setup}

The robot is a four-wheeled self-propelled autonomous field robot. The mechanical setup of the robot consists of two modules connected by a rod, see Figure 2 . The rod is constrained such that it is fixed to the left module and rotates freely relative to the right module. The type of constraint is chosen to ensure continuous surface contact. Due to a number of possible modifications of the robot, the left to right weight proportion can vary between configurations. The robot is designed such that a tool can be mounted on the robot through a standard three-point linkage. The robot steers on the two front wheels using an Ackermann description (Gillespie 1996). This means that the relative orientation of the two front wheels is calculated separately, $\delta_{o}$ (outer wheel) and $\delta_{i}$ (inner wheel) according to the direction of the turn:

$$
\delta_{i}=\tan ^{-1} \frac{L}{R-\frac{T}{2}} \text { and } \delta_{o}=\tan ^{-1} \frac{L}{R+\frac{T}{2}}
$$

where $L$ is the wheelbase, $T$ is the track width and $R$ is the desired turning radius.

The track width of the robot can be configured with three possible values. Furthermore, the position of the centre of gravity can vary as the consequence of the mass of components in each module. The values applied for tests in the basis DSE are shown in Table 1. The parameters are included in a configuration file that initiates the DSE. The purpose of the basis DSE is to capture the possible physical configurations of the robot in a single series of co-simulations. In this study an exhaustive search algorithm is chosen to sweep over all parameters.

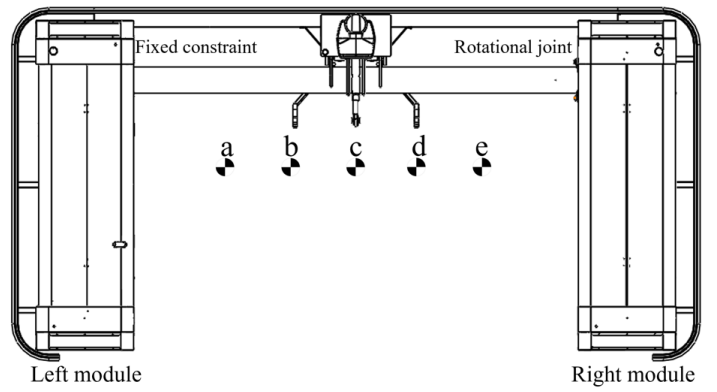

Figure 56. Sketch of the robot.
Table 1. Inputs for basis DSE configuration file.

\begin{tabular}{ll}
\hline Mass, $m_{r}:$ & $800 \mathrm{~kg}, 900 \mathrm{~kg}, 1000 \mathrm{~kg}$ \\
Track width, $T:$ & $0.9 \mathrm{~m}, 2.2 \mathrm{~m}, 3.2 \mathrm{~m}$ \\
Wheelbase, $L:$ & $1.5 \mathrm{~m}$ \\
$\begin{array}{l}\text { Left to right proportion, } l r \\
\{\mathrm{a}, \mathrm{b}, \ldots, \mathrm{e}\}:\end{array}$ & $0.3,0.4,0.5,0.6,0.7$ \\
$\begin{array}{l}\text { Front to rear weight } \\
\text { proportion, } f r:\end{array}$ & 0.4 \\
Velocity, $v:$ & $(60 \%$ front, $40 \%$ rear $)$ \\
\hline
\end{tabular}

Robot Dynamics

To obtain a feasible representation of the robot, the underlying physics of the machine-dynamics is the foundation of the model. In this study, the governing forces acting in the system is considered by representing the normal loads and tire forces. Furthermore, the effects of mounting a given tool are modelled by considering the weight, the position, and the traction force exerted from the tool.

The orientation of the robot is obtained by numerically integrating the rotational acceleration, $\ddot{\phi}$, eq. 2 , twice as a function of time (Balling, O. and Bernard 2011).

$$
\ddot{\phi}=\frac{1}{I_{z z}}\left(\sum F y_{f} \cdot a-\sum F y_{r} \cdot b\right),
$$

where $a$ and $b$ is the distance from the front and rear wheel axle to centre of gravity in the longitudinal direction. $\sum F y_{f}$ and $\sum F y_{r}$ is the sum of lateral tire forces on the front wheels and the rear wheels, $a=f r$. $L$, and $b=(1-f r) \cdot L, I_{z z}$ is the estimated yaw inertia of the robot.

Similar, the lateral velocity component $\dot{y}$ of the robot is obtained by integrating the lateral acceleration:

$$
\ddot{y}=\frac{1}{m}\left(\sum F y_{f}+\sum F y_{r}\right)-v \cdot \dot{\phi},
$$

where $v$ is the forward velocity and $m$ is the total mass of the robot and tool.

Due to the constraint of the modules and rod, the normal loads on the wheels differ. The normal loads are estimated from the static equivalence, corresponding to an assumption of the robot driving under quasistatic conditions on a plane surface. The normal loads are calculated for each wheel. This is crucial when estimating the 
maximum traction force on each wheel.

It is assumed that the vertical load of the lift mounted tools is shared equally on the left and right module. The kinematic constraints dictate that the moment exerted from the lift mounted tool to the machine can only be transmitted through the fixed constraint in the left side, whereas the rotational joint only transmits vertical forces. The normal loads on each wheel are calculated as follows:

$$
\begin{aligned}
& \text { Normal load on the front left wheel: } \\
& \text { Normal load on the front right wheel: } \\
& \text { Normal load on the rear left wheel: } \\
& \text { Normal load on the rear right wheel: }
\end{aligned}
$$

$$
\begin{gathered}
N l f_{L}=\frac{1}{L} \cdot\left(m_{r L} \cdot g \cdot b-\frac{F_{v}}{2} \cdot\left(L-l_{\text {ext }}\right)\right) \\
N l f_{R}=m_{r R} \cdot g \cdot \frac{b}{L}-\frac{F_{v}}{2}, \\
N l r_{L}=\frac{1}{L} \cdot\left(a \cdot m_{r L} \cdot g-M_{\text {ext }}\right), \\
N r_{R}=m_{r R} \cdot g \cdot a / L,
\end{gathered}
$$

where $M_{\text {ext }}=\left(F_{v} / 2 \cdot l_{\text {ext }}-h \cdot F_{l}\right), g$ is gravity, $F_{v}$ and $F_{l}$ is the vertical and longitudinal force exerted from the tools, $l_{\text {ext }}$ is the distance from the front wheels to the centre of gravity of the tool. It is assumed that the point of action of the force is located in the centre of the robot. $m_{r L, R}$ is calculated based on the left to right ratio of the mass of the robot. Furthermore, it assumed that the robot drives on a flat surface, only characterized by a friction coefficient between the tire and the surface. The model including equations (2)-(7) is implemented in 20-sim. From 20-sim the model is exported as an FMU.

\section{Steering Controller}

A model of the steering controller is implemented in VDM-RT using the Overture tool, from where an FMU is exported. The purpose of the controller is to navigate the virtual robot on a predefined path. The control signal is defined as a function of the angular misalignment between the heading of the robot and the orientation towards the next virtual GPS point. Based on the angular misalignment, the controller transmits the steering signal to the front wheels, $\delta_{f}$.

\section{External Tool models}

The tool FMU is a placeholder for external forces acting on the robot which is highly dependent on the type of tool. Two methods are proposed for including external forces in an FMU. First, a simple model of a sprayer is included in the co-simulation by modelling the decay in the mass as a function of time. Second, test results have been interpolated and integrated into a CT-model that is exported as an FMU to fit in the modelling and simulation framework.

\section{Sprayer FMU}

A lift mounted sprayer is modelled by including an initial mass of the sprayer and a mass decay rate corresponding to the rate by which the tank is emptied. The sprayer is modelled simply by including an additional point load in a specific position, $l_{\text {ext }}$ on the robot.

The model is implemented in 20-sim and exported as an FMU. The model is shown in Listing 1, $\mathrm{m}_{-} 0$ is the mass of the sprayer with an empty tank. The decay_rate is the rate at which the sprayer is emptied, and m_init is the initial total mass of the sprayer.

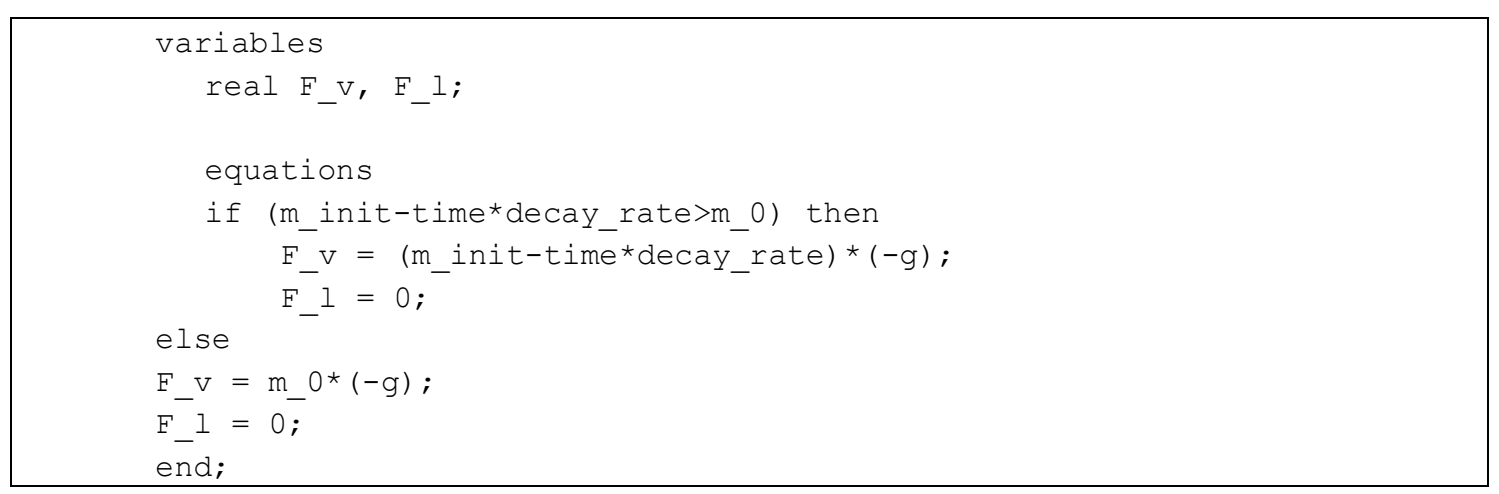

Listing 1. Sprayer FMU.

In this example, the sprayer exerts only a vertical force in a distance $l_{\text {ext }}$ from the front wheels. Since there are no draught forces, the longitudinal force, $F_{-} 1$, is zero. 


\section{Mechanical weeder FMU}

The reaction forces of a mechanical weeder are included in the co-simulations. In this case, soil-bin test data is included in the simulations by interpolating the data and exported as an FMU. The test is performed by Znova et al., the test is explained in details in (Znova et al. 2018). The test was performed to correlate the reaction forces and soil deformations of an $L$-shear designed for mechanical weeding. The forces are measured on a single share in three directions. The reaction forces were measured with different working depths, $d$ and forward velocities, $v$. In this study, only the longitudinal and vertical forces acting on the shear are included. It is assumed that the sum of lateral forces is zero due to symmetry, and therefore negligible.

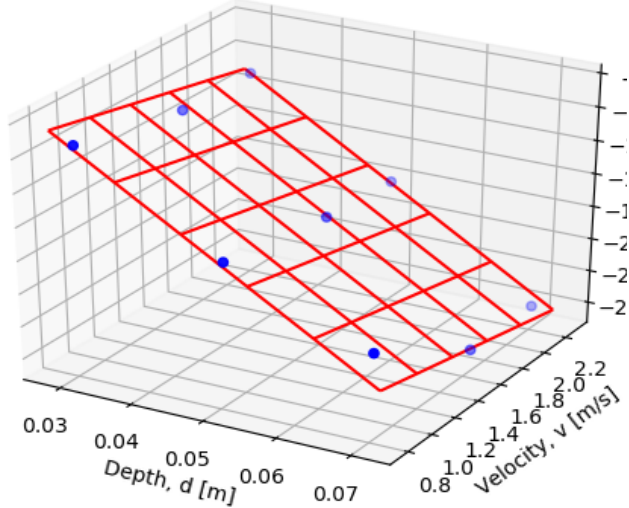

(a) Forces in the longitudinal direction, $F_{l}$

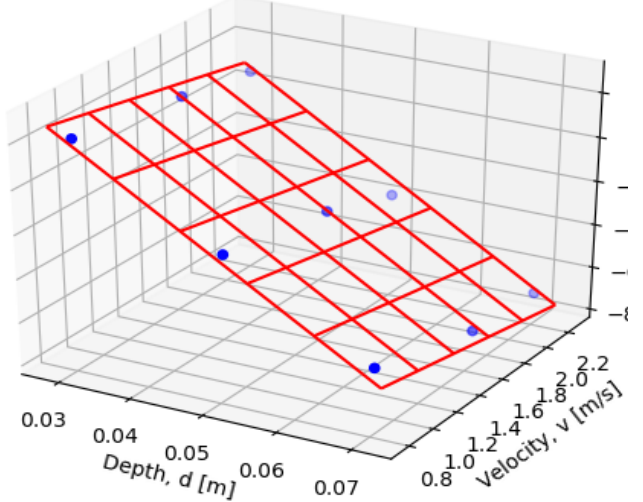

(b) Forces in the vertical direction, $F_{v}$

Figure 57. Reaction force of one L-shear, the dots represents the data-points measured in steady state. The red surface represents the interpolated function. is

The test data is interpolated into two linear functions of two variables. The measured and the interpolated data

shown in Figure 3. The establishment of the two functions, $F_{l}(d, v)$ and $F_{v}(d, v)$ are computed using the least square

method. Both functions of depth $(d)$ and forward velocity $(v)$. The raw data is measured in steady state, likewise, it is assumed that the shear operates under quasistatic and steady state conditions. Furthermore, linearity within the domain of test is assumed.

$$
F=\left\{\begin{array}{l}
F_{l}(d, v) \\
F_{v}(d, v)
\end{array}\right\}=\left[\begin{array}{lll}
-3183.3 & -20.9 & 23.8 \\
-1932.0 & -11.6 & 89.4
\end{array}\right]\left\{\begin{array}{l}
d \\
v \\
1
\end{array}\right\}
$$

The inputs for this FMU are respectively, velocity, weeding depth, and position of the implement. The corresponding output of the FMU is the force components in longitudinal and vertical direction.

The implementation of the interpolated data is shown in Listing 2, where $A_{-} i j$ is the coefficient in eq. (8). The depth is a global value that is defined when initializing the simulation. The velocity component is passed from the steering controller FMU. In a similar fashion as the sprayer model, the mechanical weeder FMU is implemented in 20-sim and exported as an FMU.

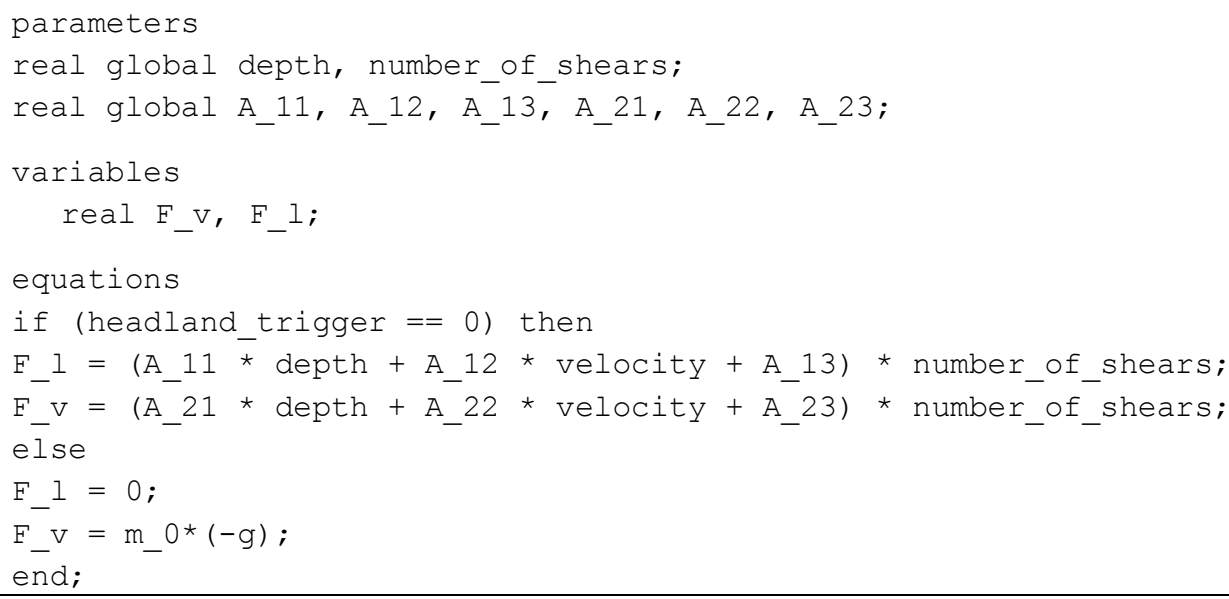

Listing 2. Mechanical Weeder FMU. 


\section{Results and Discussion}

The results of the DSEs are presented in the following section. Three DSEs are performed using the basis DSE configuration. The scope of the first DSE is to estimate the normal loads on the wheels without any tools attached. The normal loads of the unloaded robot are shown in Figure 4a. The initial verification is done by comparing the sum of normal forces with the total mass of the robot.

A similar static analysis is conducted using the sprayer tool FMU, section 2.6.1. In this DSE, the decay rate is set to zero, and the initial mass is set to $300 \mathrm{~kg}$. In this example, it is assumed that the vertical force acts in a position centred $1.0 \mathrm{~m}$ behind the front wheels.

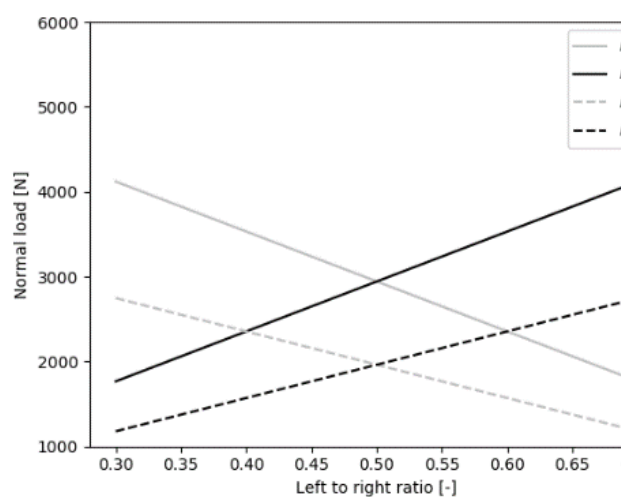

(a) Normal loads, unloaded

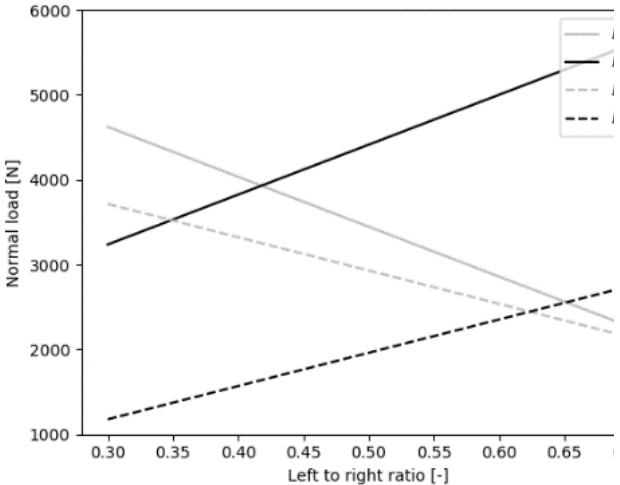

(b) Normal loads, loaded by $300 \mathrm{~kg}$

Figure 4. Results of the DSE.

In Figure $4 \mathrm{a}$ it is seen, that the position where the minimum ratio between the maximum and minimum normal loads are, as expected, exactly at 0.5 which is preferable from a vehicle dynamics and stability point of view. Though, as it is shown in Figure 4b, by loading the robot through the three-point linkage, the characteristics of the weight distribution changes. In Figure $4 \mathrm{~b}$, it is seen, that the point of where the minimum ratio exist between the maximum and the minimum normal load is found at 0.6 , meaning that $60 \%$ of the load should be located on the right module due to the constraint.

FMI provides the standard for simulating interacting, time-dependent systems. This is demonstrated by performing a DSE including a time-dependent mass reduction, correspondingly to emptying the tank of a sprayer. The result is shown in Figure 5, where the normal loads in the different configurations are shown as a function of time. In this example, the rate at which the tank is drained is set arbitrarily high to highlight the evolvement in the model. This illustrates one result that can be extracted directly from the co-simulations.
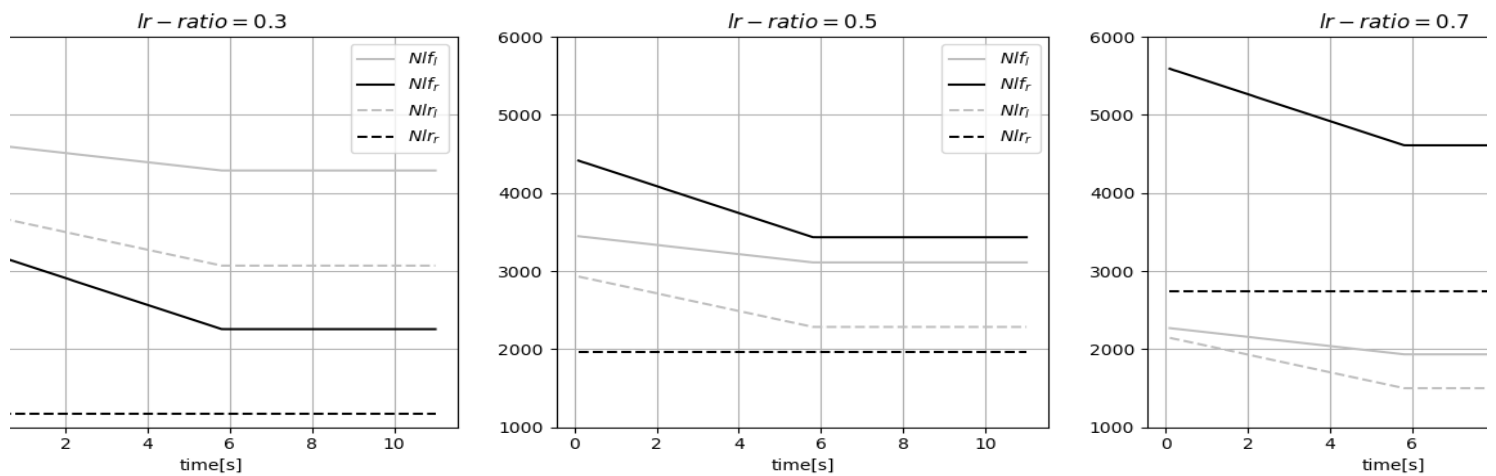

Figure 5. Normal loads as function of time.

Finally, a co-simulation is performed using the mechanical weeder tool FMU. Here a headland trigger is applied to change the load case from dragging the mechanical weeder through the soil to lifting the tool when driving from mainland to headland. In this example, it is assumed that five shears are soil-engaging at a depth of $0.05 \mathrm{~m}$ with a velocity of $1 \mathrm{~ms}^{-1}$. The mechanical weeder has an estimated mass of $50 \mathrm{~kg}$ when lifted from the soil. Furthermore, it is assumed that the vertical force acting on the robot is exerted only from the shears within in the working area of the field. The simulated trajectory is shown in Figure 6, and the forces acting between the robot and the tool are illustrated in Figure 7 as a function of time. The vertical load is calculated positive in the upwards direction. 


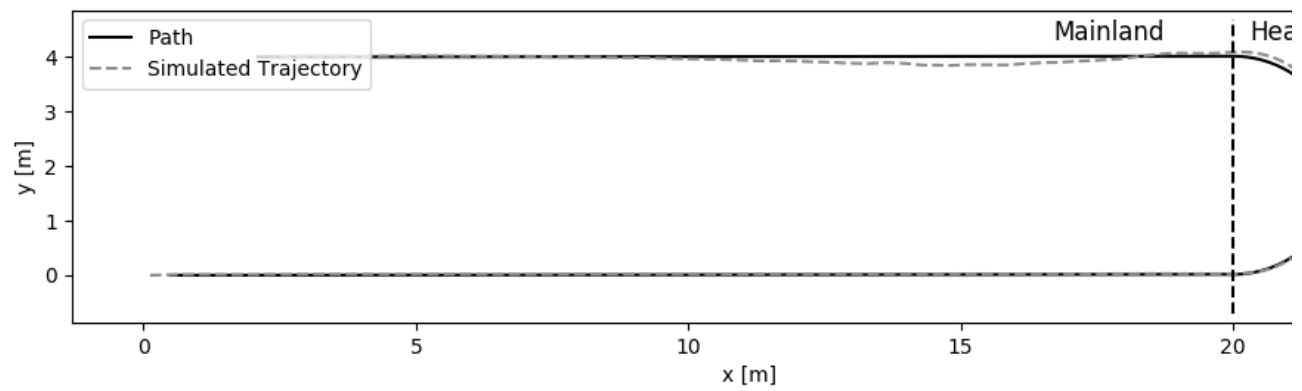

Figure 6. Visualisation of the simulated trajectory and desired path of the robot.

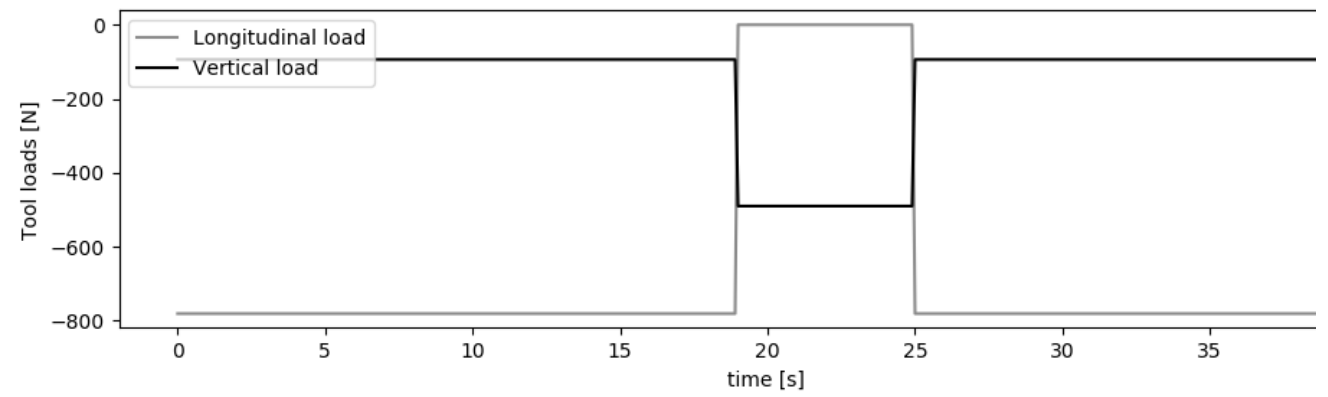

Figure 7. Normal loads and traction forces in the weeder DSE.

The initial conditions of the simulation are $\left\{x_{0}, y_{0}, \phi_{0}, \dot{x}_{0}, \dot{y}_{0}, \dot{\phi}_{0}\right\}=\left\{0,0,0,1.0 \mathrm{~ms}^{-1}, 0,0\right\}$, the simulations are performed with a fixed time-step of 0.1 seconds.

In Figure 6 it is seen that the robot slightly overshoots the path, this can be eliminated by further optimising the steering controller of the robot. This is not in the scope of this work but a possible application of the DSE setup presented in this paper. The two examples presented here illustrate only a limited number of the possible results that can be obtained through the proposed modelling and simulation approach.

\section{Conclusion}

In this study, a method for applying a model-based approach for supporting the development of Robotti, is shown. By utilizing the INTO-CPS Tool Chain, multiple co-simulations of the robot, steering controller, and an external tool model using DSE from which the optimal load distribution have been estimated.

The versatility of using FMUs is shown by developing a simulation setup that allows for virtually investigate the loads and performance of the robot and easily change the FMUs of the external tool correspondingly to changing the tool on the robot in the physical domain. Two approaches of describing external tools are presented, by modelling and by including measured data in an FMU. The method applied here will be the foundation for including a wide range of tools in future studies of the robot by continuously developing a model-library of external tool FMUs that can be applied in performing various virtual tests. Furthermore, this allows for including models or data from external collaborators or stakeholders which have great potential. The results of this work will be a part of designing and evaluating future versions of the robot.

To increase the confidence in the models, validation studies have to be performed. Likewise, the models of the robot dynamics and the steering controller can be investigated further in terms of including models of more components such as the hydraulic systems, electronic systems, sensors, etc. Furthermore, the inclusion of topology data through the FMI is a point for future research. This will be beneficial in the development of any field robot or off-road vehicle. This will allow for deeper investigation into the path tracking performance when driving on slopes. A topology-FMU could be included in the same manner as presented here for the external tool FMUs. Finally, modelling and simulation of soil-tool interaction as well as soil-tire interaction is a topic for future research. Simulations of soil-tire interaction will lead to valuable insights in the robot performance under changing soil conditions, e.g. simulating the manoeuvrability of the robot driving on clay soil or in sandy soil. Likewise, the inclusion of high-fidelity soil-tool simulations will be a topic of future research. To realistically estimate reaction forces and soil displacements in a high-fidelity model will enhance the capabilities of model-based engineering within the agricultural domain. 


\section{Acknowledgements}

The VDM-RT steering-controller applied in this study is implemented based on an existing controller setup developed during the INTO-CPS project (Ouy et al. 2016, 2017). Of this, we would like to acknowledge Martin Peter Christensen.

\section{References}

Bagnato, A, E Brosse, I Quadri, and A Sadovykh. 2017. "The INTO-CPS Cyber-Physical System Profile.” In DeCPS Workshop on Challenges and New Approaches for Dependable and Cyber-Physical System Engineering Focus on Transportation of the Future. Vienna, Austria.

Balling, O. and Bernard, J. 2011. "Course Notes on Vehicle Dynamics, Aarhus University."

Bechar, Avital, and Clément Vigneault. 2016. "Agricultural Robots for Field Operations: Concepts and Components." Biosystems Engineering 149: 94-111. doi:10.1016/j.biosystemseng.2016.06.014.

Blochwitz, T, M Otter, M Arnold, C Bausch, C Clauß, H Elmqvist, A Junghanns, et al. 2011. "The Functional Mockup Interface for Tool Independent Exchange of Simulation Models." In 8th International Modelica Conference, 105-14. Munich, Germany.

Christiansen, Martin, Peter Larsen, and Rasmus Jørgensen. 2015. "Robotic Design Choice Overview Using CoSimulation and Design Space Exploration.” Robotics 4 (4): 398-421. doi:10.3390/robotics4040398.

Christiansen, Martin Peter, Morten Stiggaard Laursen, Rasmus Jørgensen, and Ibrahim Hameed. 2018. "Collaborative Model Based Design of Automated and Robotic Agricultural Vehicles in the Crescendo Tool."

Deb, Kalyanmoy. 2012. Optimization for Engineering Design: Algorithms and Examples. PHI Learning Pvt. Ltd.

Fitzgerald, John, Carl Gamble, Peter Gorm Larsen, Kenneth Pierce, and Jim Woodcock. 2015. "Cyber-Physical Systems Design: Formal Foundations, Methods and Integrated Tool Chains." In FormaliSE: FME Workshop on Formal Methods in Software Engineering. Florence, Italy.

Fitzgerald, John, Carl Gamble, Richard Payne, and Benjamin Lam. 2017. "Exploring the Cyber-Physical Design Space.” In Proc. INCOSE Intl. Symp. on Systems Engineering, 27:371-85. Adelaide, Australia. doi:10.1002/j.23345837.2017.00366.x.

Fitzgerald, John, Carl Gamble, Richard Payne, Peter Gorm Larsen, Stylianos Basagiannis, and Alie El-Din Mady. 2016. "Collaborative Model-Based Systems Engineering for Cyber-Physical Systems -- a Case Study in Building Automation." In Proc. INCOSE Intl. Symp. on Systems Engineering. Edinburgh, Scotland.

Foldager, F.F., P.G. Larsen, and O. Green. 2018. Development of a Driverless Lawn Mower Using Co-Simulation. Lecture Notes in Computer Science (Including Subseries Lecture Notes in Artificial Intelligence and Lecture Notes in Bioinformatics). Vol. 10729 LNCS. doi:10.1007/978-3-319-74781-1_23.

Gamble, Carl. 2017. "Comprehensive DSE Support."

Gillespie, T. D. 1996. Fundamentals of Vehicle Dynamics. SAE International.

ITEA Office Association. 2015. "ITEA 3 Project, 07006 MODELISAR."

Kleijn, Christian. 2006. "Modelling and Simulation of Fluid Power Systems with 20-Sim." Intl. Journal of Fluid Power 7 (3).

Larsen, Peter Gorm, Nick Battle, Miguel Ferreira, John Fitzgerald, Kenneth Lausdahl, and Marcel Verhoef. 2010.

“The Overture Initiative -- Integrating Tools for VDM." SIGSOFT Softw. Eng. Notes 35 (1). New York, NY, USA: ACM: 1-6. doi:10.1145/1668862.1668864.

Larsen, Peter Gorm, John Fitzgerald, Jim Woodcock, Peter Fritzson, Jörg Brauer, Christian Kleijn, Thierry Lecomte, et al. 2016. "Integrated Tool Chain for Model-Based Design of Cyber-Physical Systems: The INTO-CPS Project." In CPS Data Workshop. Vienna, Austria.

Lausdahl, Kenneth Guldbrandt, Casper Thule, Peter Gorm Larsen, J Höll, A Klueber, M Pfeil, and V Fässler. 2017. “The INTO-CPS Co-Simulation Orchestration Engine - Experiences with FMI 2.0 and Proposed Extensions." In .

Ouy, Julien, Thierry Lecomte, Martin Peter Christiansen, Andres Vill Henriksen, Ole Green, Stefan Hallerstede, Peter Gorm Larsen, et al. 2016. "Case Studies 2, Public Version." http://into-cps.org/fileadmin/intocps.org/Filer/D1.2a_Case_Studies.pdf.

Ouy, Julien, Thierry Lecomte, Frederik Forchhammer Foldager, Andres Villa Henriksen, Ole Green, Stefan Hallerstede, Peter Gorm Larsen, et al. 2017. "Case Studies 3, Public Version." http://into-cps.org/fileadmin/intocps.org/Filer/D1.3a_Case_Studies.pdf.

Thule, Casper, Kenneth Lausdahl, Peter Gorm Larsen, and Gerd Meisl. 2018. "Maestro: The INTO-CPS CoSimulation Orchestration Engine." Submitted to Simulation Modelling Practice and Theory.

Znova, Liubava, Bo Melander, Aleksander Lisowski, Jacek Klonowski, Jaroslaw Chlebowski, Gareth T.C. Edwards, Søren Kirkegaard Nielsen, and Ole Green. 2018. "A New Hoe Share Design for Weed Control: Measurements of Soil Movement and Draught Forces during Operation.” Acta Agriculturae Scandinavica Section B: Soil and Plant Science 68 (2): 139-48. doi:10.1080/09064710.2017.1367837. 


\title{
Fast Method for the Weeds' Impact Evaluation on a Field Crop Using Smartphones
}

\author{
Elio Romano $^{\text {a, }}$, Carlo Bisaglia ${ }^{a}$, Massimo Palumbo ${ }^{b}$, Nino Virzi ${ }^{b}$, Fabiola Sciacca ${ }^{b}$, Sergio Saia ${ }^{c, d}$, \\ Ivano Pecorella ${ }^{c}$, Pasquale De Vita ${ }^{c}$ \\ ${ }^{\text {a }}$ Consiglio per la ricerca in agricoltura e l'analisi dell'economia agraria (CREA), Centro di ricerca Ingegneria e \\ Trasformazioni Agroalimentari (CREA-IT), Sede di Treviglio. Via Milano, 43 - 24047 Treviglio (BG), Italy \\ ${ }^{\mathrm{b}}$ Consiglio per la ricerca in agricoltura e l'analisi dell'economia agraria (CREA), Centro di ricerca Cerealicoltura e \\ Colture Industriali (CREA-CI), Lab. di Acireale. Corso Savoia 190 - 95024 Acireale (CT), Italy \\ c Consiglio per la ricerca in agricoltura e l'analisi dell'economia agraria (CREA), Centro di ricerca Cerealicoltura e \\ Colture Industriali (CREA-CI), Sede di Foggia. S.S. 16 km $675-71122$, Foggia, Italy \\ ${ }^{\mathrm{d}}$ Consiglio per la ricerca in agricoltura e l'analisi dell'economia agraria (CREA), Centro di ricerca Cerealicoltura e \\ Colture Industriali (CREA-CI), CREA-CI, Sede di Vercelli. S.S. 11 per Torino, Km 2,5 - 13100, Vercelli, Italy \\ * Corresponding author. Email: elio.romano@crea.gov.it
}

\begin{abstract}
The amount of weeds and the composition of its population can influence wheat grain yield and its quality, especially in organic farming systems, and have implication for the management of the system in space and time. Thus, knowing the weed flora traits with precise and on time reading are important factors for agronomic management both in conventional and organic farming and can play a role in decision-making at wide scale. The information technology available today can allow for cognitive investigations on various approaches. Promising studies are being conducted for the education of machine learning procedures such as neuronal networks or classification and regression trees for the recognition of the species. However, farmers presently lack of equipment for automatic weed identification and location. This frequently led to a large use active ingredients, or other mechanical means of weed containment, irrespective of their certain need, with high environmental impact and great economic commitment. The goal of this work was to develop an assessment methodology for unwanted grasses from images taken by a smartphone to be applied in field crops. The methodology is based on the analysis of the RGB and localization information available on most of the smartphones available on the market. An image reading algorithm was set-up with the $\mathrm{R}$ statistical processing program (The $\mathrm{R}$ Foundation for Statistical Computing) through which the R, G and B matrices were studied, and the weed percentages were estimated. Sampling was carried out in two locations and in two successive moments; at the same time of the collection of pictures, weed biomass and individuals number were measured. The surveys were repeated on the same positions thanks to the smartphone geolocation. The present work reports the results related to the processing of the pictures collected in the geo-localized sampling points and shows the correspondence with the on-the-spot evaluation, in order to indicate the reliability of this fast system to indicate the presence of weeds on the crop.
\end{abstract}

Keywords: sensing technology, cereals, environment, sustainability.

\section{Introduction}

Weeds are one of the most challenging problems for farmers, threatening their ability to produce good-quality food cost-effectively (Oerke, 2006). Weed control is decisive for a successful crop in terms of grain yield, quality and profitability and fallouts in the rotation (Frenda et al. 2013).

Weed management is extremely important and consists in carrying out the control strategies in a targeted and timely manner. In particular, the rational of weed management does not include the complete eradication of the weed flora, but rather that of containing it under certain 'intervention threshold', below of which the cost of intervention is higher than the drawbacks on profitability.

Weed management strategies based on the use of active ingredients (a.i., commonly referred as 'chemicals') has a marked effect on the presence of weeds, but imply a high economic pressure on farmers. In addition, it also has negative impacts on the environment and can induce weeds resistance to the a.i. group (Owen, 2016, Ramsden et al., 2017; Strassemeyer et al., 2017).

For such reasons, decreasing the dependence on the use of a.i. has become a distinct objective within the EU with the EU directive 2009/128/EC, concurrently with an overall ambition to maintain or increase productivity at the one time.

However, transition to a fully integrated weed management will require a considerable change in agricultural practices as weed control in most arable crops still relies heavily on herbicides (Deytieux et al., 2012; Hillocks and Cooper, 2012). In particular, the acquisition of the information of the actual and mean weed pressure on the crop and weed composition can allow for the choice of the most suitable strategy of control.

Among the likely strategies, a first step could be to evaluate whether the rate of each a.i. could be reduced without reducing yields. Reduced doses of a.i. can control weeds sufficiently when the selection and dose of a.i. are carefully adapted to the flora. Indeed, label recommendations are set to be efficient under many different 
conditions and commonly the doses are therefore higher than necessary for very specific weed compositions under optimal spraying conditions (Kudsk, 2008). Such strategies were applied to reduce the chance of selection for weeds tolerant to the a.i.

Also studies on the use of decision-making support for weed control require the integration of information on the impact of pre-existing weed biomass, to facilitate efficient and sustainable management of the sector (Kitchen 2008, Franco et al., 2017).

Images, taken by unmanned aerial vehicles (UAV, e.g. drones) or by hand-held cameras, can bear a wealth of information on the conditions of the field and can be easy and cheap to take up. However, from a computational perspective, such imagery holds pixel-data strongly needing further elaboration in efficient and reliable ways for extracting the desired information. Based on such knowledge, it is possible to develop sound and useful decision support for farmers and decision makers in information intensive practices in agriculture (Fountas et al. 2006; Sorensen et al. 2010).

UAV have a high potential to give information on the weed positioning in the field, the weed flora composition and describe the weed' impact, however their actual cost and limits in their use are not sufficiently encouraging their application.

Studies on more complex equipment based on hand-driven drones, have however demonstrated the need, especially for crops with narrower canopy structure and sown in rows, such as wheat, to use information coming from the ground (Gonzalez-de-Santos et al. 2016). In the last decade, various methods of reading images caught by cameras have been set up in order to evaluate the density of the crop and the presence of the weed biomass.

Researchers found reading algorithms based on computer image simplification, but intercepted methods had problems when the weed species to be discriminated showed a canopy structure similar to the crop and could thus be scarcely discriminated (Xuewen et al. 2018).

So, the present study is a preliminary assessment of an algorithm implemented in an application for smartphones, which is based on pictures taken by the smartphone and provides for the quantification of the percentage on total pixels representing the soil to ponder the presence of weed biomass against the crop. The implemented app is a simple application that requires the operator, after taking the picture, to draw on the same pictures lines representing the cultivation rows.

Therefore, this research has the main objective of verifying if the algorithm for extrapolation of the percentage of weed biomass corresponds to the quantity actually present and, consequently, if the application that uses it can be a reliable tool for the choices of use and the evaluation of the possible reduction of phytosanitary to be used to support the cultivation in production in terms of quantity and quality.

\section{Materials and Methods}

The tests were conducted in two locations, one in northern Italy (Treviglio, BG, $45^{\circ} 31^{\prime} 10 \mathrm{~N}, 9^{\circ} 33^{\prime} 50 \mathrm{E}$ ) and one in southern Italy (Foggia, FG, $41^{\circ} 28^{\prime} 00 \mathrm{~N}, 15^{\circ} 30^{\prime} 0 \mathrm{E}$ ). The test fields were chosen for the optimal soil characteristics for cereal cultivation and for their uniform orography. Therefore, two hectares were planted one with durum wheat (Triticum durum) in Foggia and one with (Triticum aestivum) in Treviglio.

Sowing density was $200 \mathrm{~kg}$ seed ha ${ }^{-1}$. Durum wheat cultivar sown in Foggia was Cappelli, soft wheat cultivar sown in Treviglio was Azzorre. Both were sown with a $15 \mathrm{~cm}$ inter-row width. Weeds were not controlled.

On each experimental field, at the beginning of the stage of preparation, pictures were taken from 50 random coordinates in the field, with a smartphone with an ability to return images of 12 Mpixels. Each picture was taken with a known reference measurement of the area, placing a rigid square of $0.16 \mathrm{~m}^{2}$ on the ground (Fig. 1).

The square was placed so as to have the sides parallel and perpendicular to the rows. The operator placed the smartphone at a height of about 1 meter, in a position as parallel as possible to the ground. After the shot, the geographical coordinates were recorded, through the indications of the location of the smartphone. The photos were repeated after one month, at the end of the phenological stage of preparation, in the same geographical coordinates (with the approximation of the location of the smartphone). 


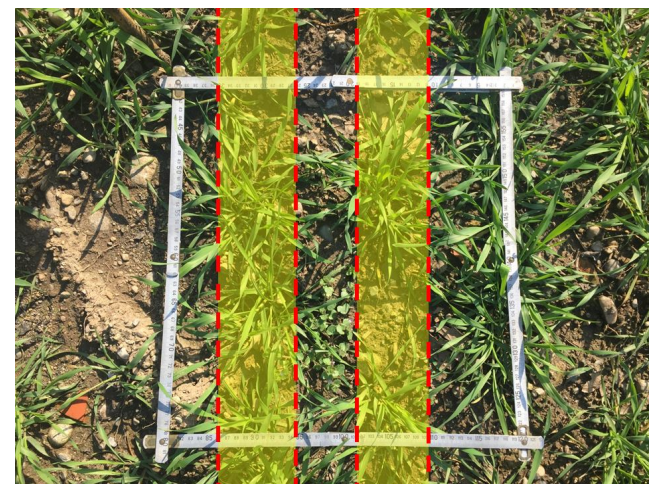

Figure 1. A picture with the lines indicating of the rows (yellowed bad with red hatched lines).

The images, altogether 200, were elaborated through the statistical program "R" statistical software (R Development Core Team, 2008), using the EBImage package. This R package provides general purpose functionality for image processing and analysis. It was adopted in the context of (high-throughput) microscopybased cellular assays, EBImage offers tools to segment cells and extract quantitative cellular descriptors (Olès et al., 2018; Pau et al., 2010; Pennekamp et Schtickzelle, 2013; Fabian et al., 2018). This allows the automation of such tasks using the R programming language and facilitates the use of other tools in the R environment for signal processing, statistical modelling, machine learning and visualization with image data.

Since the application requires the operator to draw lines corresponding to crop rows on the picture, in each image the rows were identified and, with parallel lines, the thickness of each row, so as to highlight the inter-row without the crop in progress. The thickness of the row varied in the two phases studied and was defined image by image. In this way, the algorithm proceeded by clipping the inter-row, for the extraction of the pixels related to the presence of the weeds. The images were made up mainly of three elements: the crop (wheat), the earth and weeds (mostly present in the inter-row). The discriminating ability of the transformation processes is very reliable, when the plant species must be distinguished from the ground, whereas it was particularly hard to distinguish weeds from the green background of the crop. Afterwards, the weed biomass has been characterized in its RGB components, through samples of pixels from the image parts representing weeds. Therefore, unlike Xuewen (2018), the intensity of gray has not been calculated, but the thresholds of the RGB components of the weed biomass was characterized, through ten samples for each pixel. Likewise, with the same number of samples, the RGB thresholds characterizing the bare soil and the crop were also produced.

In that way, it was possible to request, from the processing software, the extraction, from the sampling area (the inter-row) only pixels that corresponded to the characteristics of the RGB thresholds of the biomass found (Fig. 2). Furthermore, before the evaluation of the percentage density, a filter available in the EBImage package was applied, and executed by the medianFilter function, which replaces every pixel with the median of its next pixels, in order to have a wider reading margin with respect to the biomass edges (Fig. 3). Also the watershed function of the same EBImage package was applied (Fig. 4).

The watershed function identifies and separates objects that stand out of the background (zero). It inverts the image and as it uses water, fills the resulting valleys (pixels with high intensity in the source image) until another object or background is met. The deepest valleys become indexed first, starting from 1.

In case of a binary image, its distance map can serve as the input heightmap. The distance map, which contains for each pixel the distance to the nearest background pixel, can be obtained by distmap function (Oleś et al., 2018).

The following phase was then to count the pixels and calculate the ratio between the pixels counted and the total number of pixels in the test area, for the quantification of the percentage of weed biomass.

For the evaluation of the efficiency of the algorithm used, the correct detection rates and the false detection rate were considered. The evaluation of the algorithm's efficiency was based on the correct detection rate of weeds and false detection rate, which are two common and generic indicators in assessing the performance of the detection algorithms in several topics (Xuewen et al., 2011). From each image, the density of presence of the weed biomass was calculated, using the described algorithm. The evaluation of the real density of the percentage of weed biomass was instead obtained in the field, from the manual count of the weed plants in the inter-rows inside the square used for the shot.

The dataset was subjected to the test of Shapiro-Wilk to verify the assumption of normality of variance and the Levene test for the homogeneity of the variances. Then, data were subjected to analysis of the variance (ANOVA) to verify the repeatability of the obtained values and the eventual significance of the differences between the treatments and between the actual biomass values present and those obtained after the image analysis. 


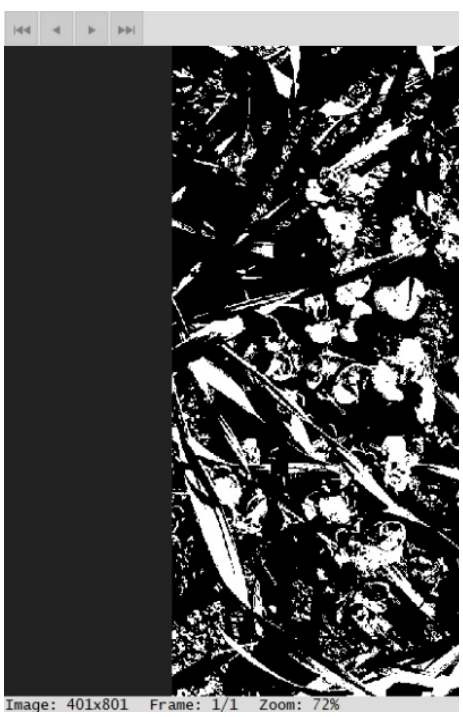

Figure 2. Image after thresholding method processed.

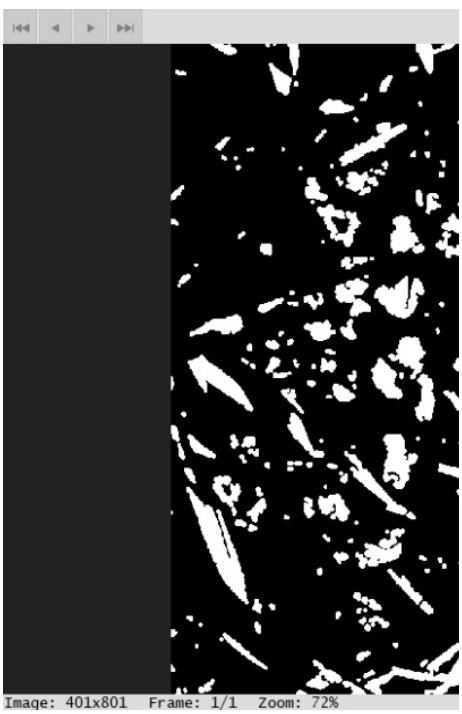

Figure 3. Image after medianFilter function processed.

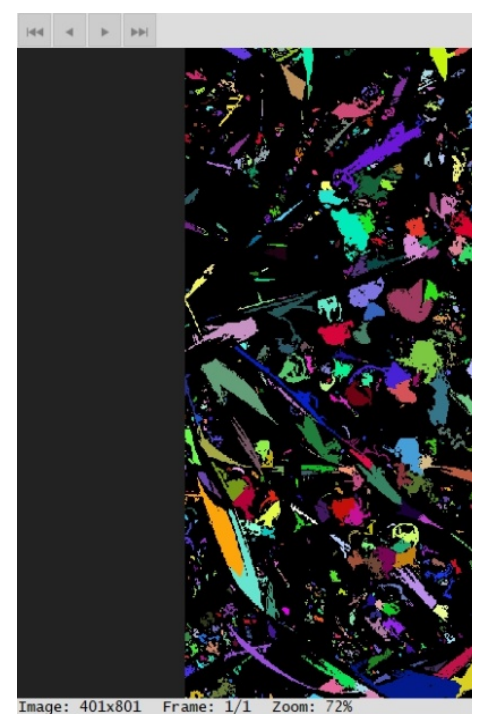

Figure 4. Image after watershed function processed.

\section{Results and Discussion}

If considering that no weed control was applied, a low percentage of weed biomass was found, which resulted on average $13.59 \%$ in Foggia and $11.55 \%$ in Treviglio. Such low percentages were likely due in both sites to a dry and cold winter compared to the long-term climatic patterns, which did not encourage the emergence, winter survival and growth of weeds. Sampling for the characterization of the weed biomass was from $0.46 \pm 0.028$ (mean \pm standard deviation) to $0.74 \pm 0.04$ for $\mathrm{R}$ channel, from $0.60 \pm 0.12$ to $0.98 \pm 0.01$ for $\mathrm{G}$ channel and from $0.33 \pm 0.11$ to $0.72 \pm 0.09$ for B channel.

The lowest percentage recorded was $7.12 \%$ in the first phase in Treviglio, measured by the analysis of the image as $6.99 \%$, then with a delta lower than the percentage point. The highest percentage recorded was $16.98 \%$ observed in the second phase Treviglio, measured by the analysis of the image as 17.98, with a delta of one percentage point. Overall, the differences between the actual percentage values of weed biomass and those extracted from the image processing process was $0.83 \%$, with exact values and a highest error of $1.44 \%$.

Table 1. Weed's percent measured by image analysis and observed and measured in the two studied fields (1 = Foggia, 2 = Treviglio).

\begin{tabular}{|c|c|c|c|c|c|c|c|c|}
\hline Condition & \multicolumn{4}{|c|}{ Actual \% weed } & \multicolumn{4}{|c|}{ Estimated \% Weed } \\
\hline & Mean & Min & Max & $\mathrm{SD}$ & Mean & Min & Max & $\mathrm{SD}$ \\
\hline Field 1 & 13.59 & 10.01 & 16.98 & 2.30 & 12.76 & 9.02 & 17.98 & 1.89 \\
\hline Phase 1 & 11.43 & 10.01 & 12.98 & 0.79 & 11.19 & 9.02 & 14.10 & 1.10 \\
\hline Phase 2 & 15.76 & 13.06 & 16.98 & 0.72 & 14.32 & 13.02 & 17.98 & 1.00 \\
\hline Field 2 & 11.55 & 7.12 & 15.00 & 2.40 & 10.72 & 6.99 & 16.00 & 1.89 \\
\hline Phase 1 & 9.57 & 7.12 & 11.56 & 1.16 & 9.25 & 6.99 & 12.10 & 1.23 \\
\hline Phase 2 & 13.52 & 11.00 & 15.00 & 1.52 & 12.20 & 11.00 & 16.00 & 1.11 \\
\hline & 12.57 & 7.12 & 16.98 & 2.56 & 11.74 & 6.99 & 17.98 & 2.14 \\
\hline
\end{tabular}

Percentage of weeds in the second phase was higher than in the first (Fig. 5) (p-value $<0.05$ ). However, a difference ( $p$-value $<0.01$ ) was also observed between the two methods of computing the percentage of biomass, with lower values for image analysis than direct sampling. Similarly, an interaction between the method and phase of observation occurred ( $p$-value $<0.05$ ), suggesting that the method of extraction of values based on the proposed algorithm has responses that are affected by the percentage actually present and that probably underestimates the actual values at low percentages or overestimate at increasing amounts of weeds. 


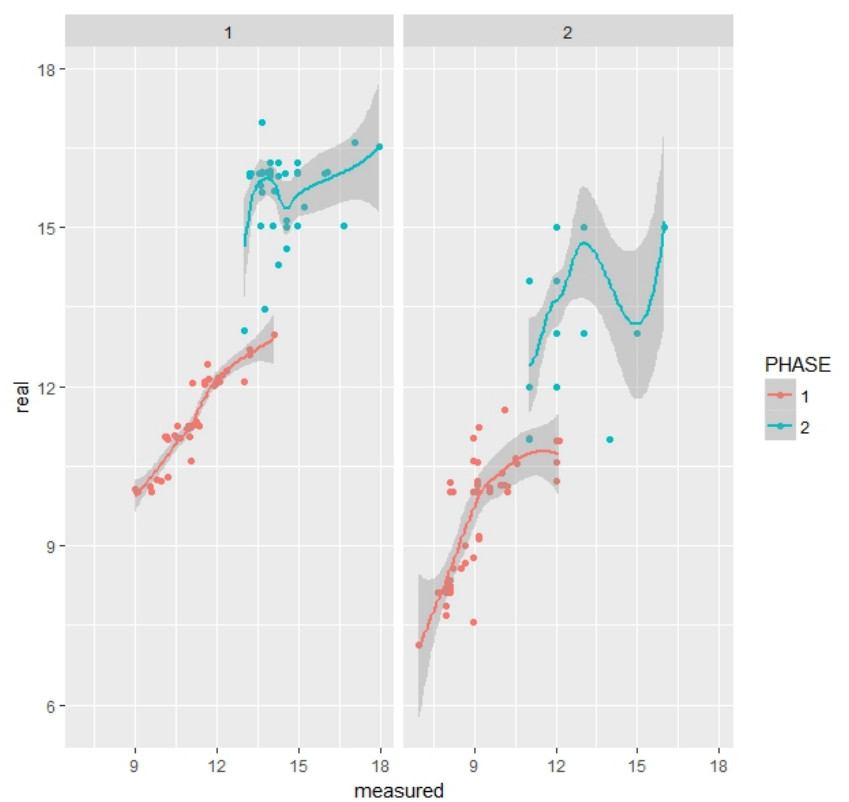

Figure 5. Percentage values of measured and actual weed biomass in the two fields (at the top of the panel, $1=$ Foggia, $2=$ Treviglio) in the 2 consecutive observed phases (in legend). Shaded areas are confidence (95\%) intervals.

The correlation coefficient between the real dataset and the measured dataset was 0.93 for the data coming from Foggia, and 0.97 from the data coming from Treviglio.

The study of the ratio of the gap between the real value and that measured with respect to the real value showed that the estimation capacity of the proposed algorithm was, on average, over $80 \%$ of matching fitness. Transition from growth phase 1 to phase 2 resulted in a lower correspondence and greater response variability (Fig. 6), which was mostly characterized by a mean reduction of the ratio and an increase of the values in the upper quantiles of the ratio.

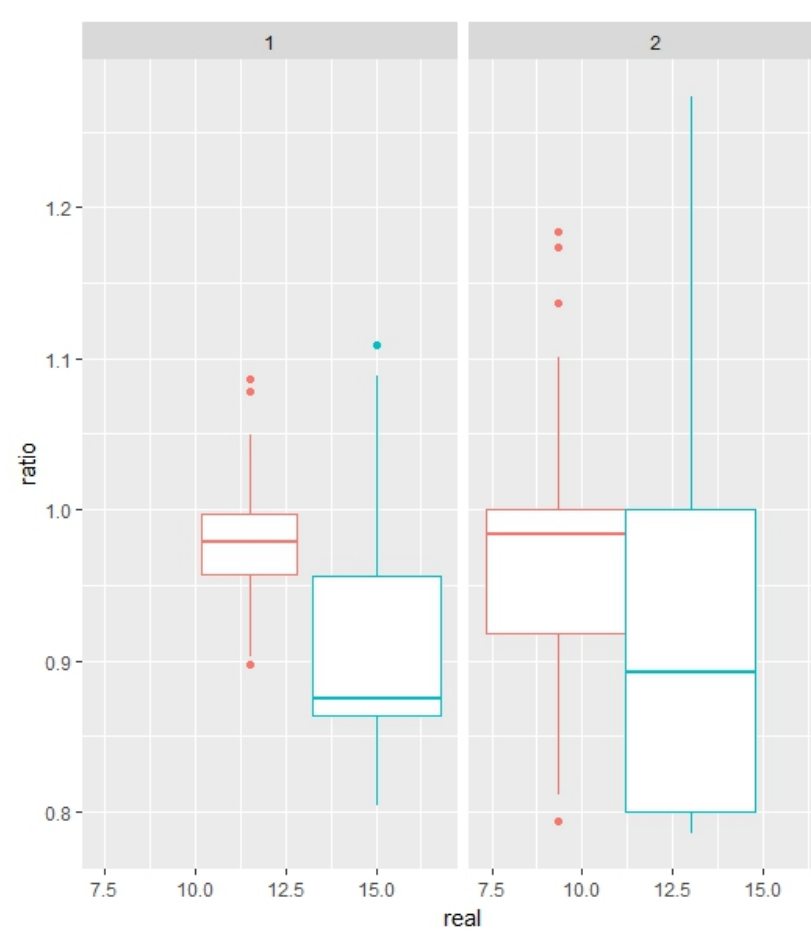

Figure 6. Box and whiskers plot of the ratio of correspondence real/measured in the two fields (at the top of the panel, 1 = Foggia, $2=$ Treviglio) in the 2 consecutive observed phases (in legend). Shaded areas are confidence $(95 \%)$ intervals.

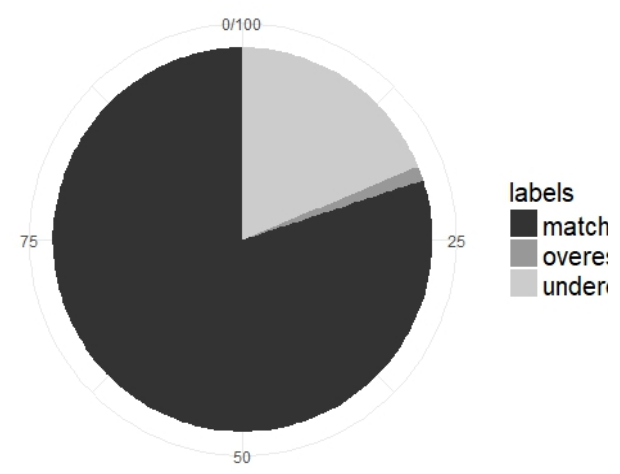

Figure 7. Percentage matching rates (correct matching $=80 \%$, overestimated $=1.2 \%$, underestimated $=18.8 \%$ ). 
The samples correctly interpreted by the image analysis system, those overestimated and those underestimated were counted. The values exactly corresponding to the actual value were considered as correctly interpreted with a confidence interval of $2 \sigma$ of the values of the same test condition. So, the overestimation and underestimation was indicated when the actual value were higher or lower than the measured value by more than $2 \sigma$.

According to this categorization, the samples indicated as matching were $80 \%$, those overvalued $1.2 \%$ and those underestimated $18.8 \%$ (Fig. 7).

The accuracy test revealed that the proposed algorithm had a sensitivity of $127 /(127+32)=0.80$ and a specificity of $9 /(9+2)=0.82$ (Table 2 ). These values indicated that the algorithm in the study correctly classified $80 \%$ of the pixels defined as weeds (i.e. true positives) and $82 \%$ of the samples defined as non-weedy (i.e. true negatives) compared to the actual estimate. Specificity was $2 \%$ higher than sensitivity, which can imply that this algorithm had a slight tendency to classify the samples as non-weedy even if the trained operators classified them as weeds (i.e., false positive, type I error). Overall, this digital measurement method accurately classified $68 \%$ of the pixels (i.e., $[127+9] / 200)$.

Table 2. Comparison of image classification by the algorithm and operators using a total of 200 samples.

\begin{tabular}{cccc}
\hline Reference condition & Measured Weed & Measured Non-Weed & Percent correct \\
\hline Real Weed & 127 (true positives) & 32 (type II error) & $\%$ \\
Real not-weed $^{*}$ & 2 (type I error) & 9 (true negatives) & $0.80^{*}$ \\
Correctly classified images $^{*}$ & & & $0.82^{* *}$ \\
& & & 0.68
\end{tabular}

\footnotetext{
${ }^{*}$ Sensitivity

** Specificity
}

\section{Conclusions}

The image analysis proposed in this research involved the treatment of pictures from two experimental fields of wheat cultivation with the aim of providing an effective method to be included in an application for the smartphone or other proximal sensing devices useful for the defining the presence of weed.

In the process of discrimination of weeds, the information protocol uses the characteristics of position indicated by the same user, thus concentrating in inter-spaces. The algorithm has implemented methodologies present in the literature about the same problem of weeds, but has integrated innovative software processes and packages currently used in areas of cell discernment.

The procedure resulted highly sensitive and showed a high specificity towards the weed biomass. Overall, the proposed processing system showed $80 \%$ of results corresponding to the actual percentage of biomass present, with errors in only $18 \%$ of cases and an underestimation never higher than $2 \%$. The picture shots repeated over time on the same sampling site showed that the deviation between the values measured with the proposed algorithm and the actual ones had a trend related to the growth of both the crop and the weed biomass. The interference of the protruding foliage of the main crop gave the discrimination system in difficulty and generates less reliable results, which could be harder to apply to systems with reduced row distance efficient in controlling weeds (De Vita et al. 2017).

Nonetheless, it is very likely that the proposed process will have greater precision and more correspondence to the actual values when crop and weed share less chances of intermingling, such as in crops with larger interrows, including corn, soybean, sunflower or sorghum.

The implementation of the algorithm in the application for the smartphone or automated systems is therefore possible, which will allow expressing in real time, soon following the picture shots, the value of present weed biomass, and thus facilitate the operator in the choices of intervention, in the best economic optimization of resources and towards the sustainability of agricultural management.

\section{Acknowledgements}

This work was supported by the Italian Ministry of Agriculture (MiPAAF) under the BIODURUM project (D.D. n. 5424, 17 may 2013). The authors acknowledge the work of Mr. Elia Premoli for the valuable help provided.

\section{References}

De Vita, P., Pecorella, I., Colecchia, S.A., Saia, S., 2017. Reduced inter-row distance improves yield and competition against weeds in a semi-dwarf durum wheat variety. Eur. J. Agron. 86:69-77 Doi: 
10.1016/j.eja.2017.02.003

Deytieux, V., Nemecek, T., Knuchel, R.F., Gaillard, G., Munier-Jolain, N.M., 2012. Is integrated Weed management efficient for reducing environmental impacts of cropping systems? A case study based on life cycle assessment. Eur. J. Agron. 36, 55e65.

Fabian E., Kamieniczna M., Kurpisz M., Stachowska E. (2018) Human Sperm Morphology Analysis using a Digital Holographic Microscope. In: Gzik M., Tkacz E., Paszenda Z., Piętka E. (eds) Innovations in Biomedical Engineering. IBE 2017. Advances in Intelligent Systems and Computing, vol 623. Springer, Cham. DOI: https://doi.org/10.1007/978-3-319-70063-2_7

Fountas, S., Wulfsohn, D., Blackmore, B. S., Jacobsen, H. L., \& Pedersen, S. M. (2006). A model of decisionmaking and information flows for information-intensive agriculture. Agricultural Systems, 87, 192-210.

Franco, C., Pedersen, M. S., Papaharalampos, H., Ørum, J.E. 2017. The value of precision for image-based decision support in weed management. Precision Agric (2017) 18:366-382. DOI 10.1007/s11119-017-9520-y .

Frenda, A.S., Ruisi, P., Saia, S., Frangipane, B., Di Miceli, G., Amato, G., Giambalvo, D., 2013. The Critical Period of Weed Control in Faba Bean and Chickpea in Mediterranean Areas. Weed Science, 61(3):452-459. doi: http://dx.doi.org/10.1614/WS-D-12-00137.1

Gonzalez-de-Santos, P., Ribeiro, A., Fernandez-Quintanilla, C., Lopez-Granados, F., Brandstoetter, M., Tomic, S., Pedrazzi, S., Peruzzi, A., Pajares, G., Kaplanis, G., Perez-Ruiz, M., Valero, C., Del Cerro, J., Vieri, M., Rabatel, G., Debilde, B., 2016. Fleets of robots for environmentally-safe pest control in agriculture. Precision Agric DOI 10.1007/s11119-016-9476-3.

Hillocks, R.J., Cooper, J.E., 2012. Integrated pest management - can it contribute to sustainable food production in Europe with less reliance on conventional pesticides? Outlook Agric. 41, $237 \mathrm{e} 242$.

Kitchen, N. R. (2008). Emerging technologies for real-time and integrated agriculture decisions. Computers and Electronics in Agriculture, 61, 1-3.

Kudsk, P., 2008. Optimising herbicide dose: a straightforward approach to reduce the risk of side effects of herbicides. Environmentalist 28, 49e55.

Oerke, E.-C., 2006. Crop losses to pests. J. Agric. Sci. 144, 31-43.

Oleś, A., Pau, G., Sklyar, O., Huber, W. 2018. Image processing and analysis toolbox for R. URL https://github.com/aoles/EBImage

Owen, M.D.K., 2016. Diverse approaches to herbicide-resistant weed management. Weed Sci. 64, 570-584.

Pau, G. Fuchs, F., Sklyar, O., Boutros, M., Huber, W. 2010. EBImage—an R package for image processing with applications to cellular phenotypes Bioinformatics, Volume 26, Issue 7, 1 April 2010, Pages 979-981, https://doi.org/10.1093/bioinformatics/btq046

Pennekamp F., Schtickzelle N. 2013. Implementing image analysis in laboratory-basedexperimental systems for ecology and evolution:a hands-on guide. Methods in Ecology and Evolution 2013, 4, 483-492 doi: 10.1111/2041-210X.12036

Ramsden, M.W., Kendall, S.L., Ellis, S.A., Berry, P.M., 2017. A review of economic thresholds for invertebrate pests in UK arable crops. Crop Prot. 96, 30-43.

Sorensen, C. G., Pesonen, L., Fountas, S., Suomi, P., Bochtis, D., Bildsøe, P., et al. (2010). A user-centric approach for information modelling in arable farming. Computers and Electronics in Agriculture, 73, 44-55.

Strassemeyer, J., Daehmlow, D., Dominic, A., Lorenz, S., Golla, B., 2017. SYNOPS-WEB, an online tool for environmental risk assessment to evaluate pesticide strategies on field level. Crop Prot.

Wu, X., Xu, W., Song, Y., Cai, M. 2011. A detection Method of Weed in Wheat Field on Machine Vision. Procedia Engineering, 15, 1998-2003. 


\title{
Evaluation of Pasture Productivity and Quality in the Montado Ecosystem Using Proximal Sensors
}

\author{
João Serrano ${ }^{\text {a,*, }}$, Shakib Shahidian ${ }^{\text {a }}$, José Marques da Silva a ${ }^{\text {, Francisco Moral }}{ }^{\text {b }}$ \\ ${ }^{a}$ Departamento de Engenharia Rural, ICAAM- Instituto de Ciências Agrárias e Ambientais Mediterrânicas, Escola de \\ Ciências e Tecnologia, Universidade de Évora, Apartado 94, 7006-554 Évora, Portugal \\ ${ }^{\mathrm{b}}$ University of Extremadura, Departamento de Expresión Gráfica, Avenida de Elvas s/n, 06006 Badajoz, Spain \\ * Corresponding author. Email: jmrs@uevora.pt
}

\begin{abstract}
Montado is a silvo-pastoral system characterized by open canopy woodlands of mainly Quercus suber and Quercus rotundifolia, mingled in some areas with other Mediterranean tree species, with natural or cultivated grassland in the undercover and grazing animals. A decline of these ecosystems has been reported since the end of the nineteenth century in southern Portugal. In order to ensure a sustainable management, there is an urgent need to produce knowledge on the resilience thresholds of these systems. Monitoring of indicators for a timely evaluation of pasture variability in Montado is particularly complex and an important challenge due to the existence of different strata (soil, pasture, trees and animals). The conventional methods for determining key components of pasture productivity and quality are time consuming and expensive, hence the interest in evaluating faster and cost effective tools. Monitoring pasture productivity and quality over time is critical for defining the nutritional value of pastures and designing balanced diets for grazing animals. The aims of this study were to evaluate two proximal sensors with potential to monitor relevant variables in the Montado ecosystem and demonstrate their application in a case study: a capacitance probe and an active optical sensor (AOS). This work uses data from a dryland biodiverse Mediterranean pasture, collected during spring of 2016 and 2017. The significant and strong correlations between capacitance and pasture biomass and between normalized difference vegetation index (NDVI) measured by AOS and pasture crude protein (CP) and pasture fibre (NDF, neutral detergent fibre) can make an important contribution to determination of key components of pasture productivity and quality and to support decisions in the implementation of site-specific pasture and grazing management. This correlation can be used for the management of animal grazing intensity and calculation of feed supplementation needs throughout the vegetative cycle of the pasture.
\end{abstract}

Keywords: Pasture monitoring, optical sensors, Grassmaster, normalized difference vegetation index, biomass, crude protein.

\section{Introduction}

Montado is a silvo-pastoral system characterized by open canopy woodlands of mainly Quercus suber and Quercus rotundifolia, mingled in some areas with other tree species, with natural or cultivated grassland in the undercover and grazing animals (Serrano et al., 2018). This ecosystem is at risk, evidencing a strong reduction of area in the last decades (Pinto-Correia and Godinho, 2013), it is therefore important to improve knowledge about this ecosystem and its potential and limitations. This is located in areas with a Mediterranean climate, with a strong seasonality and high variability (David et al., 2013), with direct influence on the vegetative cycle of dryland pastures.

The existing knowledge on the distribution and characteristics of pasture productivity and quality in the montado is still insufficient to develop conservation strategies (Seddaiu et al., 2013). This difficulty results from the characteristic variability of this ecosystem, accentuated by the presence of trees (David et al., 2013) and by the dynamics of animal grazing (Schellberg et al., 2008). The information about the spatial and temporal variability of pastures constitutes the basis to estimate available feed, a fundamental decision support tool for the farm manager to formulate the most appropriate management strategy for grazing rotations, nutrient management, and yield prediction (Bernardi et al., 2016). Monitoring of pasture quality through the evaluation of protein and fibre content over time is critical to define the nutritional value of pastures and design balanced diets for grazing animals (Demanet et al., 2015). The impact of related grazing management decisions, such as pasture allocation, paddock residence time and supplementary feeding, could have consequences on grazing pressure and on overall sustainability of the system (Sales-Baptista et al., 2016). As such, to maintain the important socio-economic role and the high nature value of these systems in the long term it is essential to understand the components of the system and to understand the interactions between them (David et al., 2013; Peco et al., 2006).

Intensive grid-sampling is generally regarded as one of the most accurate means of evaluating spatial variability and mapping a field in detail (Brevik et al., 2006), but traditional on-field pasture clipping and dryweighing sample quadrats of known area (Harmoney et al., 1997) and the necessary laboratory analysis are timeconsuming, labour intensive and cost prohibitive, not viable to implement as routine at farm level (Schaefer and Lamb, 2016), leading to increased interest in automated monitoring methods (Handcock et al., 2016). 
The application of sensing techniques to grazed production systems is difficult due to the complexity of these environments. Grassland systems are highly variable in composition, structure, and age and are continually changing in response to a range of drivers including grazing, pest and weed ingress, fertility, and moisture status (Pullanagari et al., 2013). However, grassland can benefit from technological developments that have been made for arable crops over the past decades (Schellberg et al., 2008). Active optical sensors (AOS) or electronic capacitance probes are some examples of these technological developments, normally combined with the use of global navigation satellite systems (GNSS) and geographical information systems (GIS). The "GrassMaster II" capacitance meter (Novel Ways Electronic, Hamilton, New Zealand) has been used previously (Serrano et al., 2011) in Mediterranean pastures, throughout its vegetative cycle, with variations of floristic composition, phenological stage or pasture moisture content. The significant relationship found between pasture productivity and corrected meter reading (CMR) of this electronic capacitance probe could justify the interest in performing calibration tests. AOS also was used previously in Mediterranean pastures (Serrano et al., 2016), demonstrating the potential, when mounted on mobile platforms, to capture rapid changes in the proportion of photosynthetically active vegetation and to provide the basis for a more responsive management of pasture in livestock ecosystems (Handcock et al., 2016). At the same time, several indexes, including the normalized difference vegetation index (NDVI), derived from high-resolution satellite imagery, begin to be used as a promising non-destructive tool to quantitatively predict and map the biomass of annual pastures (Cicore et al., 2016). It is, therefore, expected that in the coming years new technologies will emerge in response to this challenge of crop monitoring, taking into consideration the possibilities offered by remote sensing.

In this study, the intrinsic features of high spatial-temporal variability of Mediterranean grazed pastures were assessed with the objective of evaluating the suitability of two proximal sensing techniques (an active optical sensor and a capacitance probe) for easily monitoring seasonal variability of pasture productivity and quality and support to decision making in the implementation of site-specific pasture and grazing management (Figure 1).

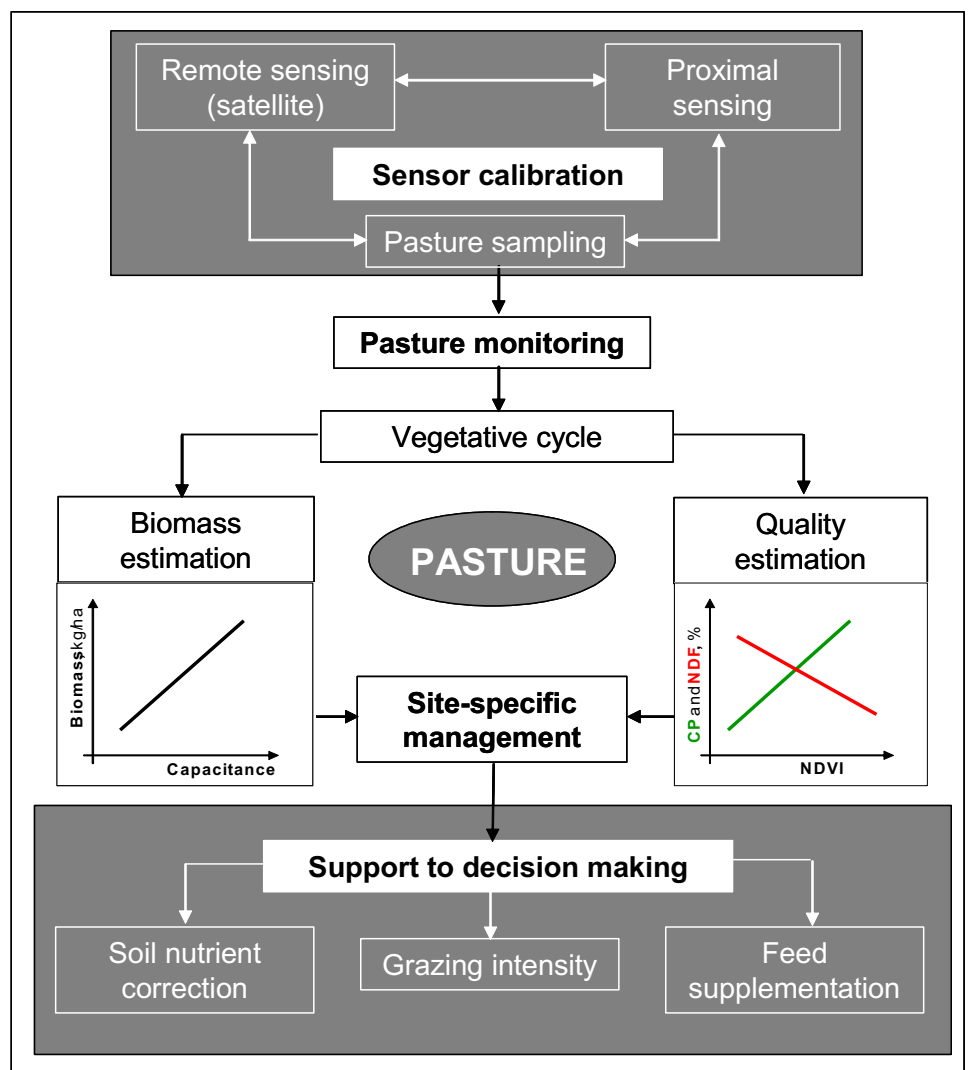

Figure 1. Calibration of proximal sensors for pasture biomass and quality monitoring: tools for site-specific management of Mediterranean pastures in the montado ecosystem.

\section{Materials and Methods}

Study area

The studied field, with an area of $2.3 \mathrm{ha}$, is located at the Mitra farm (coordinates $38^{\circ} 32.2^{\prime} \mathrm{N} ; 8^{\circ} 01.1^{\prime} \mathrm{W}$ ), at Évora University, Southern Portugal. This field of oak trees (Q. ilex ssp. rotundifolia Lam.), with a relatively reduced tree density (approximately 10 trees $\mathrm{ha}^{-1}$ ), has had an understory of natural pasture for the past 30 years and is grazed by sheep in a rotational system. A permanent biodiverse pasture (legumes and grasses) was planted 
in October 2013 at the same time that $150 \mathrm{~kg} \mathrm{ha}^{-1}$ of phosphate fertilizer (super phosphate 18\%) was applied. Since March 2014 the pasture has been grazed permanently by 15 adult Black Merino sheep. The predominant soil of this field is classified as a Cambisol derived from granite (FAO, 2006). Acid Cambisols are not very fertile and are mainly used for mixed arable farming and as grazing and forest.

Characterization of the climate

The Mediterranean climate can be considered a transition between temperate and dry subtropical climates. It is characterized by summer drought, variable rainfall, and mild or moderately cold winters. The monthly average temperature is between $8^{\circ} \mathrm{C}$ and $24^{\circ} \mathrm{C}$; minimum temperatures are close to $0^{\circ} \mathrm{C}$ between December and February. The annual rainfall in the region is between 400 and $600 \mathrm{~mm}$; rainfall occurs mainly between October and March and is practically non-existent during the summer.

Figure 2 illustrates the average thermo-pluviometric diagrams of the Évora meteorological station between 1981 and 2010 and the monthly rainfall between September 2015 and August 2016 and between September 2016 and August 2017.

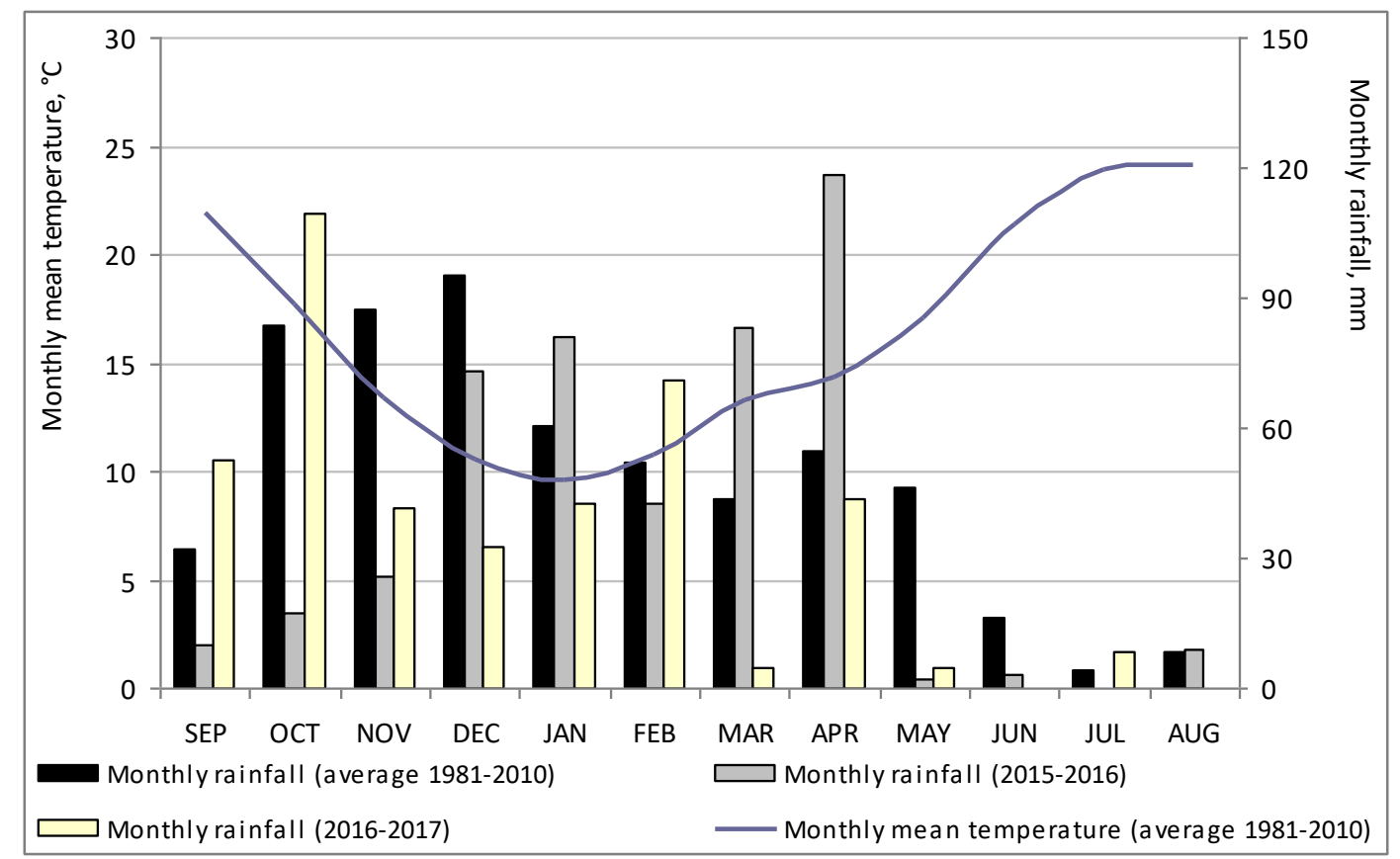

Figure 2. Average thermo-pluviometric diagrams of the Évora meteorological station between 1981 and 2010 and the monthly rainfall between September 2015 and August 2016 and between September 2016 and August 2017.

Figure 2 shows very significant difference between the agricultural years of 2015/2016, 2016/2017 and the average historical values in terms of average monthly precipitation, with direct influence on the vegetative cycle of the dryland pastures in Alentejo. In terms of annual accumulated rainfall, the average historical value is of $585.3 \mathrm{~m}$, while in 2015/2016 was of $465.8 \mathrm{~mm}$ and in 2016/2017 was $411.7 \mathrm{~mm}$. The concentration of rainfall in the months of April and May is an important factor for maintaining the growth of the pasture and lengthening its vegetative cycle. In June, the combined influence of smaller rainfall and higher temperatures naturally impacts the productivity and quality of the pasture. In regards to the accumulated rainfall between March and June, the average historical value is $161.9 \mathrm{~mm}$, while in 2016 and 2017 the rainfall was, respectively, 206.5 and $53.1 \mathrm{~mm}$. This difference is aggravated when we observe the distribution of the monthly rainfall. Thus, while the average historic values of accumulated rainfall are 43.9, 55.0, 46.5, and $16.5 \mathrm{~mm}$, respectively, in March, April, May, and June, for the same period the following values were observed: 82.9, 118.2, 2.1, and $3.3 \mathrm{~mm}$ in 2016 and 5.0, 43.5, 4.6, and $0.0 \mathrm{~mm}$ in 2017 .

\section{Pasture monitoring}

The evolution of the pasture was recorded in 24 sampling geo-referenced points, in grazing exclusion cages, at each monitoring date: monthly during spring 2016 (March, April, May, and June) at the end of winter (February) and then monthly during spring 2017 (March, April, May, and June). Multispectral and capacitance measurements were carried out prior to pasture cut. 
Vegetation multispectral measurements

The multispectral bands were measured with a commercial OptRx ${ }^{\circledR}$ AOS (Figure 3(a)), constructed by Ag Leader (2202 South River Side Drive Ames, Iowa 50010, USA), associated with a Trimble GNSS GeoExplorer 6000 series model 88951 with sub-meter precision (Trimble: GmbH, Am Prime Parc 11, 65479 Raunheim, Germany) and its power source (small portable battery).

The sensor, placed on a platform standing $0.7 \mathrm{~m}$ above the ground surface (about $0.5 \mathrm{~m}$ above the pasture, considering an average pasture height of $0.2 \mathrm{~m}$ ), measures simultaneously three visible and infrared bands: (1) RED (670 nm with a bandwidth of $20 \mathrm{~nm})$; (2) RED EDGE (728 nm with a bandwidth of $16 \mathrm{~nm})$; and (3) NIR (775 nm with basically everything under $750 \mathrm{~nm}$ being filtered). With two of the previous spectral bands, NDVI vegetation index was calculated considering the following expression (Equation (1)):

$$
\mathrm{NDVI}=\frac{\mathrm{NIR}-\mathrm{RED}}{\mathrm{NIR}+\mathrm{RED}}
$$

The operator stood still at the area of each geo-referenced point and performed measurements for a 2 min period. The values of NDVI were organized in a spreadsheet and associated with the coordinates of the respective sampling points to calculate the mean and standard deviation (SD) of NDVI from about 120 measurements taken at each point.

\section{Capacitance measurements}

Each measurement of capacitance with the Grassmaster probe (Figure 3(b)) was preceded by an air humidity-level correction. The capacitance readings (CMR) were registered after the instrument had been positioned vertically over the vegetation, some $0.2-0.3 \mathrm{~m}$ away from the operator's body. In each measuring area, 10 readings were carried out with the probe and averaged. Greater detail on the operation of the probe can be found in Serrano et al. (2011) and Serrano et al. (2016).
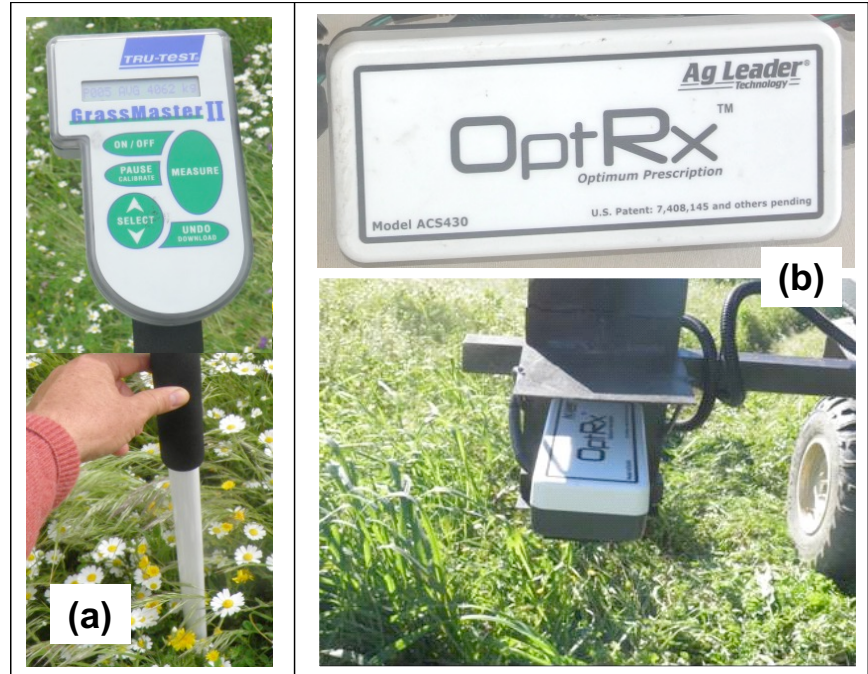

Figure 3. Capacitance probe (“GrassMaster II"; (a)) and active optical sensor (“OptRx®”; (b)) used in field trials.

Pasture sample collection and analysis

After sensor data acquisition, the operator placed a metallic rim with a $0.1 \mathrm{~m}^{2}$ area on pasture. Inside each sampling point, pasture was harvested with a portable electric grass shears at $1-2 \mathrm{~cm}$ above ground level and stored in marked plastic bags. The collected pasture samples were then taken to the Pasture and Forage Technology Laboratory of the University of Évora, where they were weighed to establish pasture productivity in terms of green matter $\left(\mathrm{kg} \mathrm{GM} \mathrm{ha}^{-1}\right)$. The dehydrated samples were analysed in order to determine the key component of pasture quality (crude protein, $\mathrm{CP}$, and neutral detergent fibre, NDF, in \%) using conventional method of wet chemistry according to the Association of Official Analytical Chemists (AOAC, 2005).

Statistical analysis of the data

Descriptive statistics analysis, including mean and standard deviation (SD) were performed for each data set of pasture and sensors parameters. Statistical procedures were performed using 'MSTAT-C' software with a significance level of $95 \%(\mathrm{p}<0.05)$. The statistical treatment of the results consisted of simple linear regression. 
Linear regression analysis was used to study the relationships between sensor and pasture variables. The Pearson correlation coefficients ' $r$ ' for the statistically significant $(\mathrm{p}<0.05)$ regression relations were then presented.

\section{Results and Discussion}

Pasture and sensors parameters variability patterns

Table 1 presents the mean $\pm \mathrm{SD}$ of the pasture and sensor parameters in the set of 24 sampling points of the studied field for each sampling date, in spring of 2016 and 2017. Figure 4 shows the patterns of evolution of pasture biomass productivity (green matter) in spring of 2016 and 2017.

Table 1. Descriptive statistics of pasture and sensor parameters in the set of 24 sampling points of the studied field for each sampling date.

\begin{tabular}{lcc}
\hline Parameter & 2016 & 2017 \\
\hline$G M, k g h a^{-1}$ & - & $6588 \pm 3161$ \\
February & $11,426 \pm 6328$ & $11,050 \pm 5375$ \\
March & $16,903 \pm 8264$ & $12,771 \pm 7106$ \\
April & $24,148 \pm 13,584$ & $5242 \pm 2151$ \\
May & $11,003 \pm 6483$ & $1604 \pm 744$ \\
June & - & \\
CP, \% & $13.8 \pm 2.8$ & $16.9 \pm 4.9$ \\
February & $11.9 \pm 3.5$ & $15.4 \pm 3.3$ \\
March & $10.3 \pm 3.0$ & $9.2 \pm 2.6$ \\
April & $7.6 \pm 2.5$ & $9.1 \pm 2.0$ \\
May & - & $6.7 \pm 1.7$ \\
June & $-5.1 \pm 5.3$ & \\
NDF, \% & $52.9 \pm 5.9$ & $50.5 \pm 6.4$ \\
February & $64.0 \pm 4.4$ & $60.6 \pm 4.2$ \\
March & $66.8 \pm 6.1$ & $70.7 \pm 3.5$ \\
April & - & $76.0 \pm 3.7$ \\
May & & \\
June & & $0.779 \pm 0.092$ \\
FDVI & $0.780 \pm 0.063$ & $0.807 \pm 0.061$ \\
February & $0.732 \pm 0.065$ & $0.583 \pm 0.100$ \\
March & $0.644 \pm 0.063$ & $0.342 \pm 0.078$ \\
April & $0.345 \pm 0.073$ & $0.197 \pm 0.025$ \\
May & - & $4489 \pm 631$ \\
June & $6992 \pm 1879$ & $4941 \pm 905$ \\
CMR & $8564 \pm 2101$ & $3804 \pm 545$ \\
February & $7497 \pm 2160$ & - \\
March & $5735 \pm 1953$ & \\
April & & \\
May & & \\
June & & \\
\hline
\end{tabular}

GM- Green matter; CP- Crude protein; NDF- Neutral detergent fibre; NDVI- Normalized difference vegetation index; CMR- Capacitance.

These results show the typical evolution of productivity and quality parameters of dryland Mediterranean pastures throughout their vegetative cycle: an increase in the yield (GM and DM) from winter to late spring, and an inverse pattern of pasture quality (reduction of nutritional value, $\mathrm{CP}$, and a continuous increase of NDF with the approach of summer). The peak of production (May 2016 and April 2017, Figure 4) and the quality decline occur sooner or later in the Spring, depending on the distribution of precipitation in the months of March to June. According to Murray et al. (2007), the concentration of rainfall between March and June is one of the major factors governing annual pasture production, mainly due to its influence on maintaining the growth of the pasture and extending its vegetative cycle.

In regards to parameters measured by sensors, CMR anticipate the productivity curve: in 2016 increases from March to April and decreases in May and June; in 2017, increases from February to March and decreases in April and May. On the other hand, on average, the tendency of NDVI was to decrease throughout the vegetative cycle of the pasture, from March to June, which reflects the corresponding decrease in the vegetative vigour of the pasture (Gitelson, 2004).

The variability measured by $\mathrm{CV}$ is high, especially in pasture productivity (Biomass, $40-60 \%$ ) and capacitance (CMR, 15-35\%), being lower in the case of pasture quality (CP, 20-30\%, and NDF, 5-20\%) and NDVI (8-20\%). 


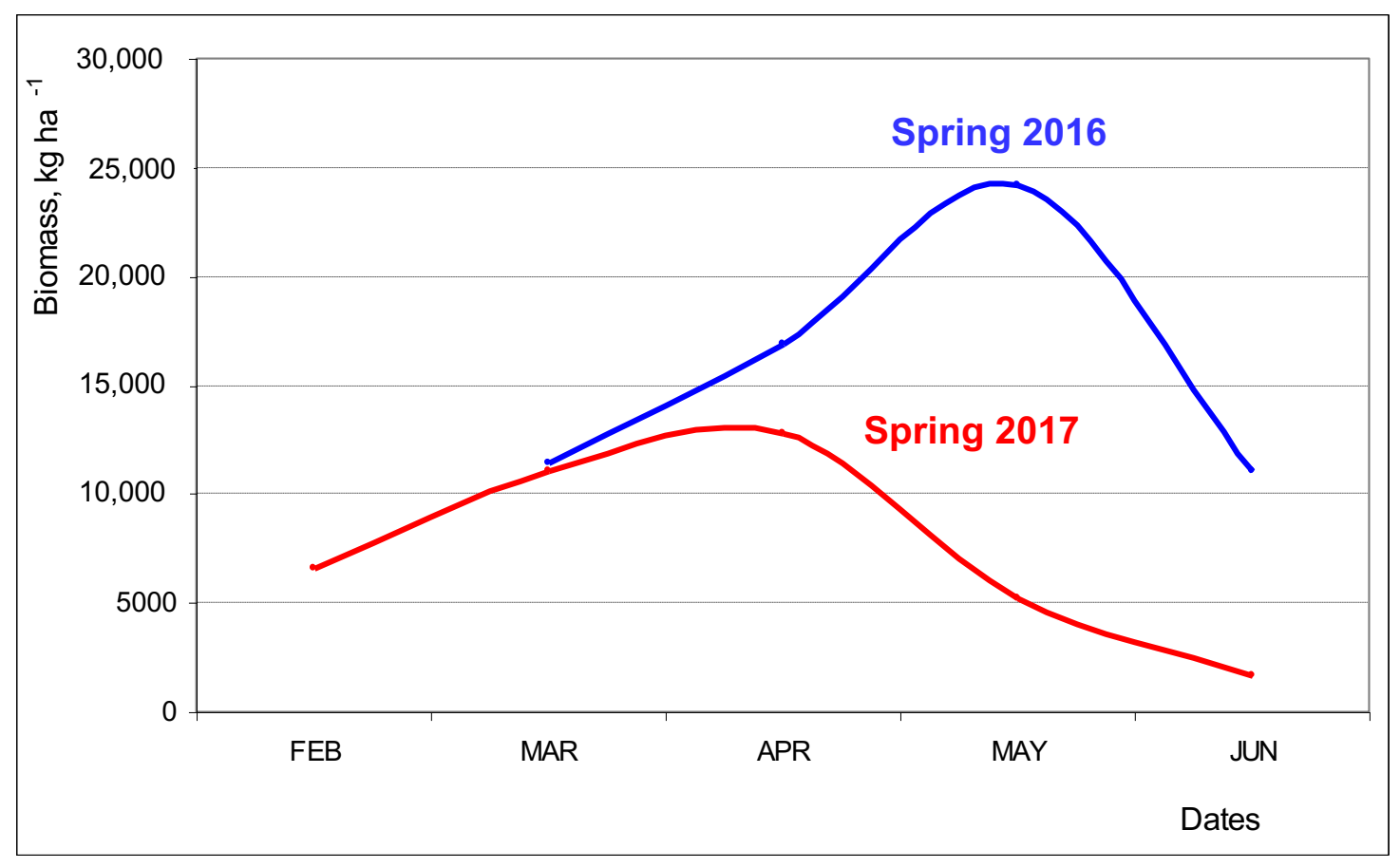

Figure 4. Pasture biomass (green matter) evolution in spring 2016 and 2017.

Correlation between pasture and sensor parameters

Table 2 presents the correlation coefficients between parameters obtained by sensors and pasture parameters using the set of 24 sampling points in each sampling data.

Table 2. Correlation coefficients (r) between biomass and capacitance (CMR), between crude protein (CP) and NDVI and between neutral detergent fibre (NDF) and NDVI, in 2016 and 2017.

\begin{tabular}{|c|c|c|c|c|c|}
\hline Correlation & February & March & April & May & June \\
\hline GM vs CMR (2016) & - & $0.729 * *$ & $0.818 * *$ & $0.609 * *$ & $0.658 * *$ \\
\hline GM vs CMR (2017) & $0.590 * *$ & $0.749 * *$ & $0.686 * *$ & $0.397 *$ & - \\
\hline CP vs NDVI (2016) & - & $0.507 * *$ & $0.527 * *$ & $0.587 * *$ & ns \\
\hline CP vs NDVI (2017) & $0.803 * *$ & $0.661 * *$ & $0.470 *$ & $0.491 *$ & $0.773 * *$ \\
\hline NDF vs NDVI (2016) & - & ns & ns & ns & ns \\
\hline NDF vs NDVI (2017) & $-0.678 * *$ & ns & ns & $-0.486 * *$ & $-0.631 * *$ \\
\hline
\end{tabular}

GM- Green matter; CMR- Capacitance; CP- Crude protein; NDVI- Normalized difference vegetation index; NDF- Neutral detergent fibre; ns-Correlation not significant; *-Correlation significant at the 0.05 level; ***-Correlation significant at the 0.01 level.

The correlations between GM and CMR were positive and significant at all moments of evaluation, being stronger in April $2016(\mathrm{r}=0.818 ; \mathrm{p}<0.01)$ and in March $2017(\mathrm{r}=0.719 ; \mathrm{p}<0.01)$, that is, when the CMR is maximum (8564 \pm 2101 and $7082 \pm 1761$, respectively), in each year in the month prior to the maximum pasture productivity (May and April, respectively).

The correlation between CP and NDVI was also positive and significant in all sampling months, being stronger in May $2016(\mathrm{r}=0.587 ; \mathrm{p}<0.01)$ and February $2017(\mathrm{r}=0.803 ; \mathrm{p}<0.01)$. These correlations may be justified by the operational principle of the active optical sensor: it detects vegetation with higher levels of chlorophyll (photosynthetically active vegetation, abundant in green vegetation), which can be attributed to absorbance of visible radiance by chlorophyll, and this will be correlated with CP levels (Pullanagari et al., 2013).

The correlation between NDF and NDVI was negative and significant only in 2017, with the highest value occurring in February.

These results reinforce the inter-annual variability associated with the effect of rainfall on pasture development and the differentiated response of the sensors under these conditions.

The correlations between the same parameters but involving the overall sampling points (24) and dates (9) (n $=216$ points) are presented in Figure 5 . 


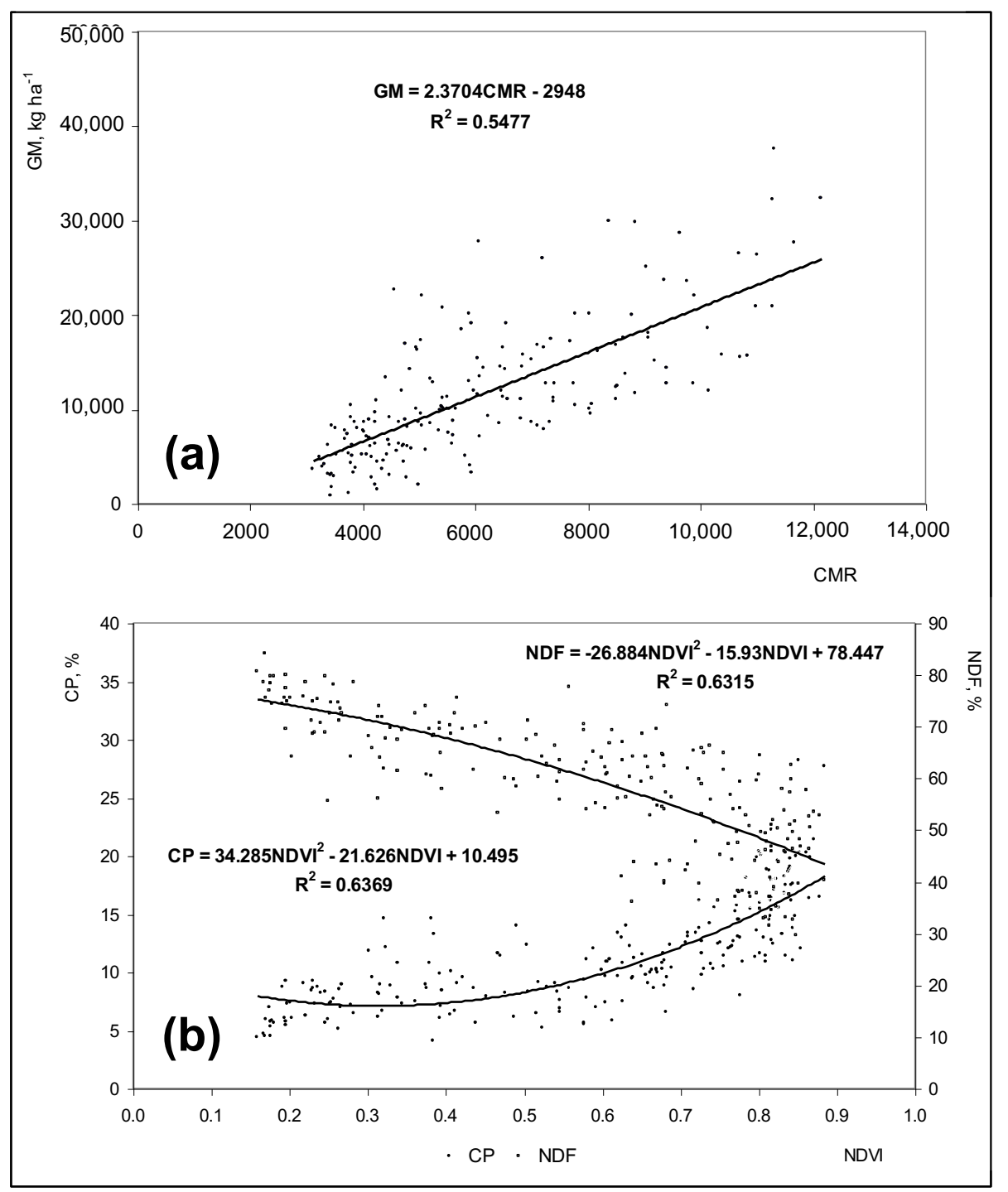

Figure 5. Correlations between: (a) pasture biomass ( $\mathrm{GM}$, in $\left.\mathrm{kg} \mathrm{ha}^{-1}\right)$ and capacitance (CMR); (b) pasture quality (CP and $\mathrm{NDF}$, in $\%$ ) and NDVI, using the set of 216 sampled points (24 points $\times 9$ dates).

These results confirm the practical interest of the Grassmaster II probe as a fast method of estimating the productivity of the Mediterranean pastures in Southern Portugal and are in line with the studies of Serrano et al. (2011) and Serrano et al. (2016). These results also show the accuracy of the estimation of pasture quality based on NDVI measured by proximal AOS. However, pastures have high variability as a result of diversity of species, morphology and interactions with animal grazing, natural environmental conditions and management practices (Pullanagari et al., 2013), whereby, changes in species composition in space and time can affect these relationships, requiring further research in different sites and types of biodiverse pastures.

\section{Conclusions}

Estimation of pasture productivity and quality is an important step for the farmer in terms of planning animal stocking, organizing animal lots, and determining supplementary feeding needs throughout the year. In the present work, the correlation between pasture and proximal sensor parameters was consistent between capacitance and pasture productivity, between NDVI and pasture quality. Natural changes in species composition in space and time can affect these relationships, requiring further research under these conditions, in different sites and types of biodiverse pastures.

These results highlight the importance of developing an ideal pasture monitoring system that combines large scale satellite remote sensing images coupled with fine-scale information provided through high-resolution proximal sensing data. An integrated pasture monitoring system will provide a much better understanding and sustainability of the livestock production system, serving as the basis for the most appropriate management strategies, such as soil nutrient correction, yield prediction, animal grazing intensity or feed supplementation. 


\section{Acknowledgements}

This work is funded by National Funds through FCT - Foundation for Science and Technology under the Project UID/AGR/00115/2013.

\section{References}

AOAC, 2005. Official Methods of Analysis of AOAC International, 18th ed.; AOAC International: Arlington, VA, USA.

Bernardi, A. C. C., Bettiol, G. M., Ferreira, R. P., Santos, K. E. L., Rabello, L. M., Inamasu, Y., 2016. Spatial Variability of Soil Properties and Yield of a Grazed Alfalfa Pasture in Brazil. Precision Agriculture, 17(6), 737752.

Brevik, E., Fenton, T., Lazari, A., 2006. Soil electrical conductivity as a function of soil water content and implications for soil mapping. Precision Agriculture, 7, 393-404.

Cicore, P., Serrano, J., Shahidian, S., Sousa, A., Costa, J.L., Marques da Silva, J. 2016. Assessment of the spatial variability in tall wheatgrass forage using LANDSAT 8 satellite imagery to delineate potential management zones. Environmental Monitoring and Assessement, 188,1-11.

David, T.S., Pinto, C.A., Nadezhdina, N., Kurz-Besson, C., Henriques, M.O., Quilhó, T., Cermak, J., Chaves, M.M., Pereira, J.S., David, J.S., 2013. Root functioning, tree water use and hydraulic redistribution in Quercus suber trees: a modeling approach based on root sap flow. Forest Ecology and Management, 307, 36-146.

Demanet, R., Mora, M.L., Herrera, M.A., Miranda, H., Barea, J.M., 2015. Seasonal variation of the productivity and quality of permanent pastures in Adisols of temperate regions. Journal of Soil Science and Plant Nutrition, 15(1), 111-128.

FAO, 2006. World Reference Base for Soil Resources. World Soil Resources Reports No. 103. Food and Agriculture Organization of the United Nations, Rome, Italy.

Gitelson, A. A., 2004. Wide dynamic range vegetation index for remote quantification of biophysical characteristics of vegetation. Journal of Plant Physiology, 161(2), 165-173.

Handcock, R.N., Gobbett, D.L., González, L.A., Bishop-Hurley, G.J., McGavin, S.L. 2016. A pilot project combining multispectral proximal sensors and digital cameras for monitoring tropical pastures. Biogeosciences, 13, 4673-4695.

Harmoney, K.R., Moore, K.J., George, J.R., Brummer, E.C., Russell, J.R., 1997. Determination of pasture biomass using four indirect methods. Agronomy Journal, 89(4), 665-672.

Murray, R. I., Yule, I. J., Gillingham, A. G., 2007. Developing variable rate application technology: modelling annual pasture production on Hill Country. New Zealand Journal of Agricultural Research, 50, 41-52.

Sales-Baptista, E., d'Abreu, M.C., Oliveira, M.I.F. 2016. Overgrazing in the Montado? The need for monitoring grazing pressure at paddock scale. Agroforestry System, 90(1), 57-68.

Schellberg, J., Hill, M.J., Gerhards, R., Rothmund, M., Braun, M., 2008. Precision agriculture on grassland: applications, perspectives and constraints. European Journal of Agronomy, 29, 59-71.

Seddaiu, G., Porcua, G., Luigi, L., Roggero, P., Agnelli, A., Cortic, G., 2013. Soil organic matter content and composition as influenced by soil management in a semi-arid Mediterranean agro-silvo-pastoral system. Agriculture, Ecosystems and Environment, 167, 1-11.

Peco, B., Sánchez, A.M., Azcárate, F.M., 2006. Abandonment in grazing systems: consequences for vegetation and soil.

Agricultural Ecosystems and Environment, 113(1), 284-294.

Pinto-Correia, T., Godinho, S., 2013. Chapter 4 Changing agriculture changing landscapes: what is going on in the high

valued Montado. In: Agriculture in Mediterranean Europe: between old and new paradigms. Emerald Group Publishing Limited, p. 75-90.

Pullanagari, R.R., Yule, I.J., Tuohy, M.P., Hedley, M.J., Dynes, R.A., King, W.M., 2013. Proximal sensing of the seasonal variability of pasture nutritive value using multispectral radiometry. Grassland Forage Science, $68,110-119$.

Serrano, J., Peça, J., Silva, J.M., Shahidian, S. 2011. Calibration of a capacitance probe for measurement and mapping of dry matter yield in Mediterranean pastures. Precision Agriculture, 12, 860-875.

Serrano, J., Shahidian, S., Silva, J.M. 2016, Monitoring pasture variability: optical OptRx_crop sensor versus Grassmaster II capacitance probe. Environmental Monitoring and Assessment, 188(2), 1-17.

Serrano, J., Shahidian, S., Marques da Silva, J., Carvalho, M., 2018. A Holistic Approach to the Evaluation of the Montado Ecosystem Using Proximal Sensors. Sensors, 18, 570. 


\title{
Lameness Recognition in Pigs Based on Skeleton Analysis and Gait Energy Image \\ Weixing Zhu ${ }^{\text {a, *, Kaizhong Zhang a }}$, Jianxuan Qian ${ }^{\text {a }}$, Xincheng $\mathbf{L i}^{\text {a }}$ \\ ${ }^{a}$ School of Electrical and Information Engineering, Jiangsu University, Zhenjiang, Jiangsu, China \\ * Corresponding author. Email: zwxbest@126.com
}

\begin{abstract}
Animal gait can reflect its status of health. In order to identify the normal walking and lame walking of pigs, a method based on skeleton analysis and gait energy image was proposed in this paper. Firstly, the gait cycle of pigs was detected by the method of skeleton analysis. Then the gait features of pigs were characterized using the gait energy image (GEI). Two-dimensional principal component analysis (2DPCA) method was used to reduce the dimension of gait energy image of pigs. Finally, the nearest neighbor classifier was used to recognize the normal walking and lame walking of pigs. The samples in the pig's gait database were tested using the above method. Experimental results show that the recognition rate is $93.25 \%$. This study provides a new idea for identifying pigs with abnormal gait and lameness using computer vision technology.
\end{abstract}

Keywords: Skeleton analysis, Gait energy image, Two-dimensional principal component analysis, Pigs, Lameness recognition.

\section{Introduction}

Animal gait reflects its status of health, motion function and mental activity (Dawkins, 2004). The study found that the pig lame behavior generally indicates the occurrence of some diseases, such as pig foot and mouth disease and pig swollen disease. An experienced breeder can usually recognize lame behavior of pigs by observing their ways of walking. If we design a system that can automatically detect lameness in pigs, it would be very useful in preventing the spread of swine diseases in time and reducing economic losses.

As a new kind of biometrics, gait recognition has become a hot research topic in the field of computer vision. Relevant researches mainly focus on gait-based individual recognition and abnormal gait recognition in the elderly. There are relatively a few studies on animal gait analysis. Zhu established pig's skeleton model based on anatomy knowledge. Combined with the change of angle of the forelimb joint and the key silhouette wavelet moment, SVM classifier was used to recognize normal and abnormal gait of pigs. The recognition rate is $85 \%$. However, the paper does not detect the gait cycle of pigs and the recognition rate is not high (Zhu, Zhang, 2010). Bahr analyzed the relationship between lame behavior and some parameters in the image, and proved that there is a strong correlation between trackway overlap, hoof time, back arch and the level of lame (Bahr et al,.2009). Wang proposed a gait recognition method based on the back curvature of the pigs, using the angle feature to detect the gait cycle of the pigs, extracting the back curvature features and using the nearest neighbor classifier for recognition. However, the features extracted by this method are more single, and the back curvature feature needs to be extracted frame by frame, which is not efficient (Wang, 2015). Han proposed a method based on the gait energy image (GEI) to characterize the gait characteristics of human beings. The principal component analysis and multiple discriminant analysis were used to realize the identification of human beings (Han, Bhanu, 2005). Zhou proposed a gait impairment detection method for the elderly based on the patch-GEI. By segmenting the gait energy image, LDA method was used to detect gait impairment of the elderly in each patch-GEI (Zhou et al., 2015).

This paper proposed a method for identifying lameness in pigs based on skeleton analysis and gait energy image (GEI). Firstly, the binary silhouette image of pigs was extracted by image preprocessing, and the gait cycle of pigs is detected by skeleton analysis. The gait energy image of pigs was calculated in one gait cycle. Then, the characteristics of gait energy image were extracted, and 2DPCA method was used to reduce the dimension of gait energy image of pigs. Finally, according to the feature after dimension reduction, nearest neighbor classifier is used to identify the normal walking and lame walking of pigs.

\section{Materials and Methods}

Image acquisition

The video material used in this experiment was taken on a scale farm of Zhenjiang Xima Animal Husbandry Co., Ltd. The images of normal pigs and lame pigs were collected using Microsoft Kinect camera with an image resolution of $640 \times 480$ and a frame rate of $30 \mathrm{fps}$. The methods involved in the experiment were run on a computer with hardware: intel CORE i5 2.5GHZ, 4G memory. 
Image sequence preprocessing

In this paper, the target pig foreground image was extracted by background subtraction, and the mean filtering method was used to reduce the effect of noise. Then, the target pig foreground image was binarized by OTSU method. Finally, target pig binary image was obtained through morphology processing. The preprocessing process was shown in Figure 1.

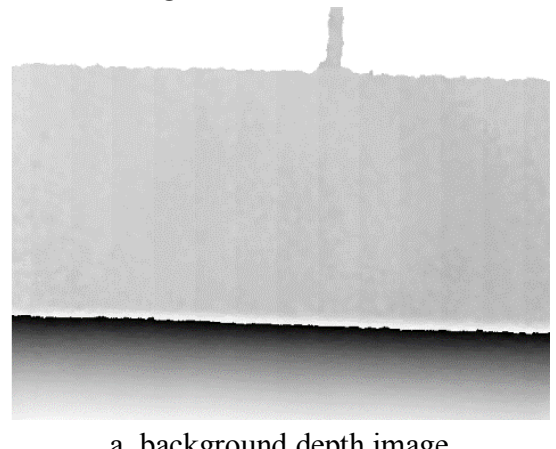

a. background depth image

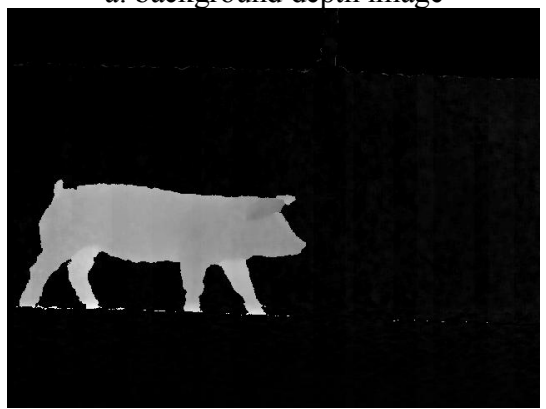

c. target pig foreground image

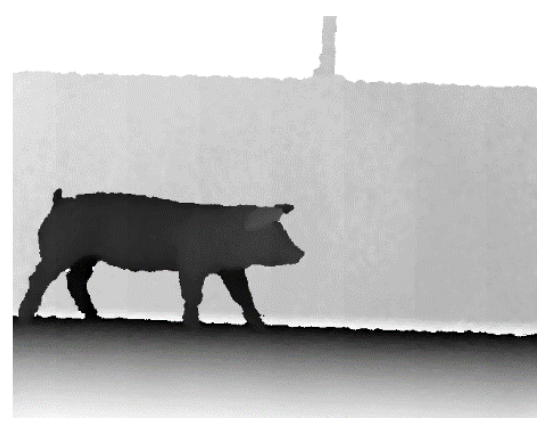

b. target depth image

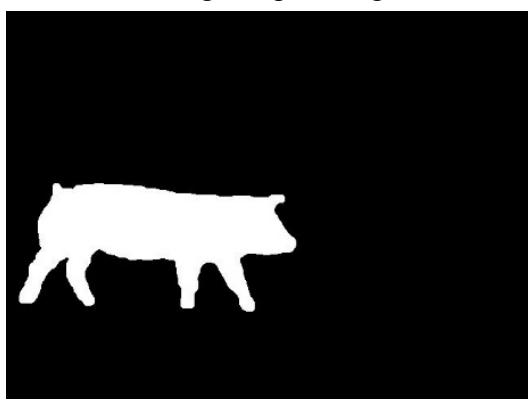

d. target pig binary image

Figure 1. The preprocessing process.

Gait cycle detection of pigs

Skeleton and endpoints matching

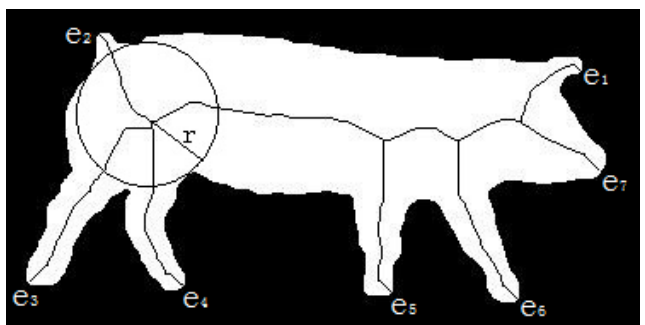

Figure 2. Standard skeleton of pigs.

Skeleton of pigs was constituted by the collection of largest inscribed circle center points inside contour constitute. All pixels on the skeleton are called skeleton points, and the pixel points on the skeleton that have only one adjacent skeleton point are called the skeleton endpoints (Liu et al., 2014). Morphology method was used to extract the skeleton of pigs, and the corrosion and reconstruction algorithm (Yi, 2009) was used to prune the skeleton. The pruned skeleton of pigs is shown in Figure 2, which is called standard skeleton of pigs. The standard skeleton of pigs contains seven skeleton endpoints from $e_{1}$ to $e_{7}$, corresponding to the ear endpoint, the tail endpoint, the two hindlimb endpoints, the two forelimb endpoints and the mouth endpoint respectively. Due to the non-rigid body movement of pigs, the limbs or head may deform relative to the main trunk, sometimes resulting in missing endpoints of the skeleton. Therefore, determining the endpoints of the skeleton is the key to the detection of the gait cycle (to determine the specific parts of the pig, such as forelimbs, ear, etc.).

In Figure 2, starting from the top-right ear endpoint, the endpoints of the skeleton are ordered in the counterclockwise direction ( $\left.e_{1}, e_{2}, e_{3}, e_{4}, e_{5}, e_{6}, e_{7}\right)$, and the path between two adjacent skeleton endpoints is called the adjacent point skeleton path. Seven adjacent point skeleton paths $\left(e_{1} \rightarrow e_{2}, e_{2} \rightarrow e_{3}, e_{3} \rightarrow e_{4}, e_{4} \rightarrow e_{5}\right.$, 
$\left.e_{5} \rightarrow e_{6}, e_{6} \rightarrow e_{7}, e_{7} \rightarrow e_{1}\right)$ can be found on the standard skeleton of pigs, denoted as $P_{i}(\mathrm{i}=1,2 \ldots 7)$. The adjacent point skeleton path was used to characterize the endpoints of the skeleton, so as to construct the feature vector of the skeleton endpoints. For example, the feature of the endpoint $e_{1}$ was obtained by the skeleton path $e_{1} \rightarrow e_{2}$.

According to the pixel distance, the adjacent point skeleton path $P_{i}$ is divided by M-1 equally. M maximum inscribed circle radiuses is taken as the first M-dimension of the feature vector of the skeleton endpoint, and then increase the length of $P_{i}$, which constitutes the $(\mathrm{M}+1)$-dimension feature vector. Since the feature vector only has the invariance of translation and rotation, considering that the target scale of the pig will change when walking, in order to have scale invariance, the normalization was done in this paper, as shown in Formula (1) (2) (3).

$$
\begin{aligned}
& R_{i t}=\frac{r_{i t}}{r_{\max }} \quad(t=1,2 \ldots M) \\
& L_{i}=\frac{l_{i}}{l_{\max }} \quad i=(1,2 \ldots n) \\
& V\left(e_{i}\right)=\left(R_{i 1}, R_{i 2}, \ldots, R_{i M}, L_{i}\right)
\end{aligned}
$$

In Formula (1) (2) (3), $r_{\max }$ is the maximum of the $r_{i t} . l_{\max }$ is the maximum of the $l_{i}$. i represents the ith endpoint, and $t$ represents the th point.

The normalized feature vectors have scale, translation and rotation invariance. Therefore, the similarity between endpoint feature vector of the skeleton to be measured and that of the standard skeleton can be used to determine the ownership of the endpoints on the skeleton to be measured. The similarity measure formula $d\left(V, V^{\prime}\right)$ can be used to calculate by Formula (4).

$$
d\left(V, V^{\prime}\right)=\sum_{i=1, j=1}^{M} \frac{\left(R_{i}-R_{j}^{\prime}\right)^{2}}{R_{i}+R_{j}^{\prime}}+\alpha \frac{\left(L-L^{\prime}\right)^{2}}{L+L^{\prime}}
$$

where $\alpha$ is the weight. $V$ is the endpoint feature vector of the skeleton to be measured $\left(R_{1}, R_{2}, \ldots, R_{M}, L\right)$, and $V^{\prime}$ is the endpoint feature vector of the known standard skeleton $\left(R_{1}^{\prime}, R_{2}^{\prime}, \ldots, R_{M}^{\prime}, L^{\prime}\right)$.

When doing the skeleton endpoints matching experiments, the selected parameter $\mathrm{M}=40, \alpha=30$. Experiments show that this method is very robust. The method can get the correct matching result even when the posture of the pig body is deformed. For example, the forelimb overlaps in Figure 3, and the endpoints matching diagram is shown in Figure 4.
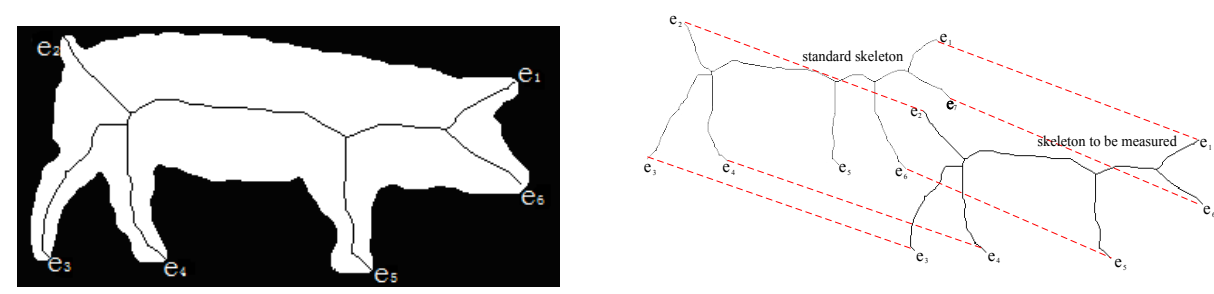

Figure 3. Skeleton of pig when forelimb overlaps. Figure 4. Endpoints matching diagram.

\section{Detection of gait cycle}

Using the above skeleton endpoints matching method to determine the pig's two forelimb endpoints and extract their coordinates. If the determined forelimb endpoint was only 1, so, the two forelimbs overlap. In order to distinguish two different forelimb endpoints, setting from the tail of the pig to head direction, the left is left forelimb endpoint, and the right is right forelimb endpoint. Since the value of each pixel in the depth image reflects the distance information of the target from the depth camera, the left and right forelimb endpoints can be determined by using the depth value. In order to reduce the error caused by noise, this paper selects the average of the depth values of the forelimb endpoint and its 8 neighborhood pixels as the depth value of forelimb endpoint. The left and right forelimb endpoints were determined by comparing the depth values of the two endpoints.

Pig is a four-footed-hoofed animal. Its walking process involves the alternating movement of forelimbs and hindlimbs. In this paper, the gait cycle is detected by the movement of forelimbs. So, the difference between the abscissa value of the left forelimb endpoint and the abscissa value of the right forelimb endpoint can be used as 
the gait feature of the pigs, which called the relative distance between forelimb endpoints.

$$
d_{t}=x_{1 t}-x_{2 t}
$$

where $X_{1 t}$ and $X_{2 t}$ represent the abscissa values of the left and right forelimb endpoints in th frame respectively.

In general, if the left forelimb of pigs takes the largest distance when ignoring the influence of noise, $d_{t}$ reaches the maximum (positive). When the two forelimbs of the pigs overlap, $d_{t}$ is 0 . When the right forelimb of the pigs takes the largest distance, $d_{t}$ reaches the minimum (negative). Apply the formula (5) to calculate $d_{t}$ for each frame and plot the curve of $d_{t}$ over time. Fig. 5 is a curve of $d_{t}$ over time for a normal pig walking without stress. The horizontal axis represents the number of frames and the vertical axis represents $d_{t}$ (pixels). As can be seen from Figure $5, d_{t}$ showed quasi-periodic changes. Therefore, the number of frames between adjacent maxima on the curve can be used as the gait cycle $\mathrm{T}$ value of the pigs.

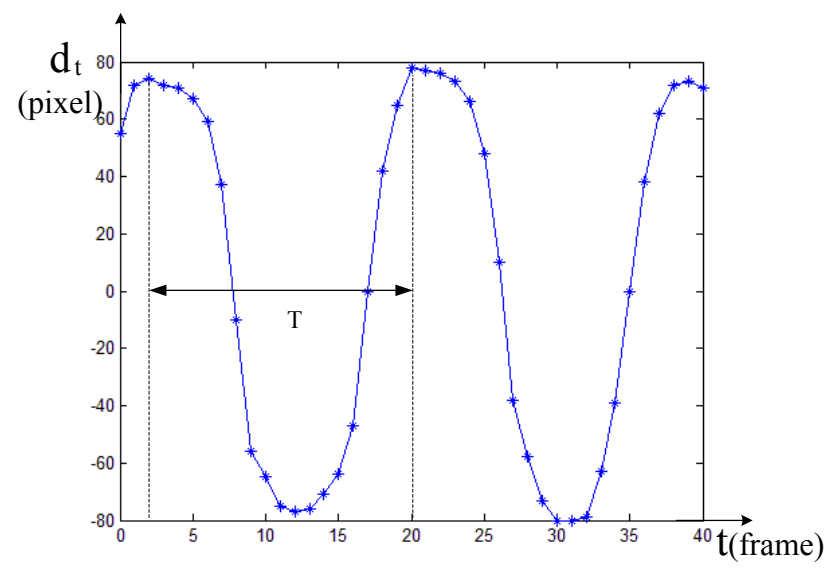

Figure 5. The relative distance change carve between forelimb endpoints.

Gait energy image and features dimension reduction

Gait energy image

Gait Energy Image (GEI) is an effective representation of spatio-temporal gait features. Unlike other gait features, Gait Energy Image presents a series of gait sequence images into a two-dimensional image, while retaining the shape, frequency and phase of gait information (Liu, 2009). The gait energy image $G(x, y)$ is defined as:

$$
G(x, y)=\frac{1}{T} \sum_{t=1}^{T} B_{t}(x, y)
$$

where $\mathrm{T}$ is the gait cycle, and $B_{t}(x, y)$ represents the pixel value ( 0 or 1$)$ of the th frame binary silhouette image at the coordinates $(x, y)$.

Before the gait energy image of pigs is calculated, the binary silhouette image of pigs needs to be normalized. So, in this paper, a target template of pigs with width of 180 and height of 90 was selected.

The gait energy image not only includes all the characteristics of pig walking process, but also because it is the average of gait frame images, it can reduce the sensitivity of image noise to a certain extent. In Figure 6, the left are binary silhouette images in one gait cycle of pigs, and the rightmost one is the gait energy image of the pig calculated by using the formula (6). It can be seen from Figure 6 that the gait energy image (right) reflects the main morphology of the binary silhouette image of the pigs (left) and its changing process in one gait cycle. 


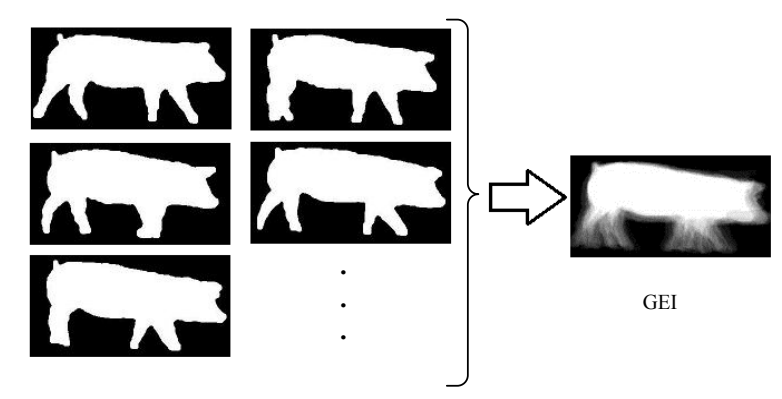

Figure 6. gait energy image synthesis of pigs.

In the gait energy image of pigs, the pixel with the highest gray value (white) corresponds to parts of the pig body whose relative position changes little in a complete gait cycle, such as the head and trunk. Pixels with gray values between the lowest and the highest (gray) correspond to parts of pig body whose relative position changes more frequently in a gait cycle, such as the forelimbs and hindlimbs of pigs. The former (white) can be called the static region, which mainly contains the posture information of pigs. The latter can be called the dynamic region, which mainly reflects the body movement of the pig during walking. The black area in gait energy image is the background.

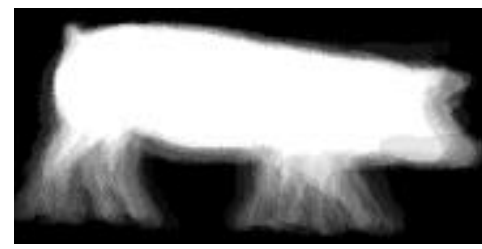

Figure 7. GEI of normal pig.

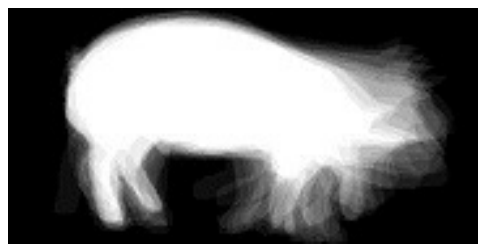

Figure 8. GEI of lame pig.

Normal pig walking is a stable process. A healthy pig body motion system will produce a stable, continuous and symmetrical gait, and body shaking is less. While the lame pig walked, the back showed an arched posture, and the back showed a certain degree of shaking. Especially, the head and neck of the pig swung to a very large extent. The gait was disorderly, discontinuous and asymmetrical. Figure 7 is a GEI of a normal pig when walking without stress and Figure 8 is a GEI of a lame pig with one forelimb injured when walking without stress. By comparing the two gait energy images, we can see that the head dynamic region of the normal pigs is smaller and more symmetrical, while that of the lame pig is relatively large and irregular. The gray distribution of the forelimb dynamic region in normal pigs was uniform, while the gray distribution of forelimb dynamic region in lame pigs was uneven. Because of the large differences in the grayscale distribution between the gait energy image of normal pigs and lame pigs, we selected the gait energy image to characterize the gait features, so as to identify the normal walking and lame walking of pigs.

Features dimension reduction

Because dimension of the gait energy image is too high $(90 \times 180)$, Two-dimensional principal component analysis (2DPCA) method (Yang et al., 2004) was used to reduce the dimension of GEI, and the gait energy characteristics after dimension reduction were obtained. The basic idea of dimension reduction in 2DPCA is as follows: Firstly, the input sample image matrix is normalized. Then the covariance matrix of the normalized samples is calculated and its eigenvalues and eigenvectors are calculated. Finally, the eigenvectors corresponding to the larger eigenvalues are selected to construct the projection matrix, and the original sample image matrix is projected to this direction to reduce the dimension.

Considering a image matrix $\mathrm{A}(m \times n)$, its projection matrix is $X \in R^{n \times d}(n \geq d)$. The image matrix $\mathrm{A}$ is projected to the $\mathrm{X}$ direction, and the matrix $\mathrm{Y}(m \times d)$ is obtained.

$$
Y=A X
$$

In formula (7), $\mathrm{Y}$ is the eigenvector generated by projection of the image matrix A onto $\mathrm{X}$. According to the distribution of feature vector $\mathrm{Y}$ to determine the best projection matrix, therefore, a criterion function was needed to measure the pros and cons of the projection matrix X. In the 2DPCA, the total dispersion of the projected feature vector $\mathrm{Y}$ is used as a criterion function.

$$
J(X)=\operatorname{tr}\left\{S_{Y}\right\}
$$


In formula (8), $S_{Y}$ is the covariance matrix of $\mathrm{Y} . \operatorname{tr}\left\{S_{Y}\right\}$ is the trace of $S_{Y}$. When the maximum of the criterion function $J(X)$ is obtained, it indicates that the total dispersion of the projected feature vector Y reaches the maximum, and $\mathrm{X}$ is the best projection matrix at this time. The trace of the covariance matrix of $\mathrm{Y}$ can be written as:

$$
\begin{aligned}
\operatorname{tr}\left\{S_{Y}\right\} & =\operatorname{tr}\left\{E\left[(Y-E Y)(Y-E Y)^{T}\right]\right\} \\
& =\operatorname{tr}\left\{E\left[(A X-E(A X))(A X-E(A X))^{T}\right]\right\} \\
& =\operatorname{tr}\left\{X^{T} E\left[(A-E A)^{T}(A-E A)\right] X\right\}
\end{aligned}
$$

The covariance matrix of the image matrix is defined as:

$$
G=E\left[(A-E A)^{T}(A-E A)\right]
$$

From formula (10), $\mathrm{G}$ is a nonnegative positive definite matrix, which can be obtained from the sample. Assuming there are $\mathrm{N}$ sample images with the same size $(m \times n)$, which is represented by $A_{k}(k=1,2, \ldots, N)$. The average image matrix is $\bar{A}=\frac{1}{N} \sum_{k=1}^{N} A_{k}$. So G can be expressed as:

$$
G=\frac{1}{N} \sum_{k=1}^{N}\left(\left(A_{k}-\bar{A}\right)^{T}\left(A_{k}-\bar{A}\right)\right)
$$

Formula (8) can be written as:

$$
J(X)=\operatorname{tr}\left(X^{T} G X\right)
$$

The formula (12) is also called generalized total distribution criterion, where $\mathrm{X}$ is the best projection matrix with each column orthogonal, and $\mathrm{X}$ is the projection axis for maximizing. Its physical meaning is: the total discrete degree of the eigenvectors obtained by the projection of the sample image matrix on this axis reaches maximum. Generally, the optimal projection axis is the eigenvector corresponding to the largest eigenvalue of the G. However, it is not feasible to select only one optimal projection axis. Usually, a series of normalized eigenvectors (d) corresponding to the largest eigenvalue of the $\mathrm{G}$ is selected, which constitute projection matrix X.

$$
\left\{\begin{array}{c}
\left\{X_{1}, X_{2}, \ldots X_{d}\right\}=\arg \max (J(X)) \\
X_{i}^{T} X_{j}=0, i \neq j, i, j=1,2, \ldots d
\end{array}\right.
$$

In formula (13), $\arg \max (J(X))$ represents the set of eigenvectors $\left\{X_{1}, X_{2}, \ldots X_{d}\right\}$ when $J(X)$ was maximum.

After obtaining the projection matrix $X=\left(X_{1}, X_{2}, \ldots X_{d}\right)$, the dimension of GEI of pigs was reduced. Suppose a GEI of pigs is A, then

$$
Y_{k}=A X_{k} \quad k=1,2, \ldots d
$$

Since the 2DPCA method selects d principal component vectors, the projected feature vectors consists of $Y=\left(Y_{1}, Y_{2}, \ldots Y_{d}\right)$. Therefore, the size of GEI $(m \times n)$ is reduced to $(m \times d)$, and $\mathrm{Y}$ is called GEI characteristic matrix.

\section{Results and Discussion}

In order to validate the feasibility of the proposed algorithm, the first step is to build gait database of pigs. The gait database used in this paper contains 400 gait cycle sequences, which are obtained from 200 normal pigs walking gait cycle sequences And 200 lame pigs walking gait cycle sequence, and then tested in this gait database. 


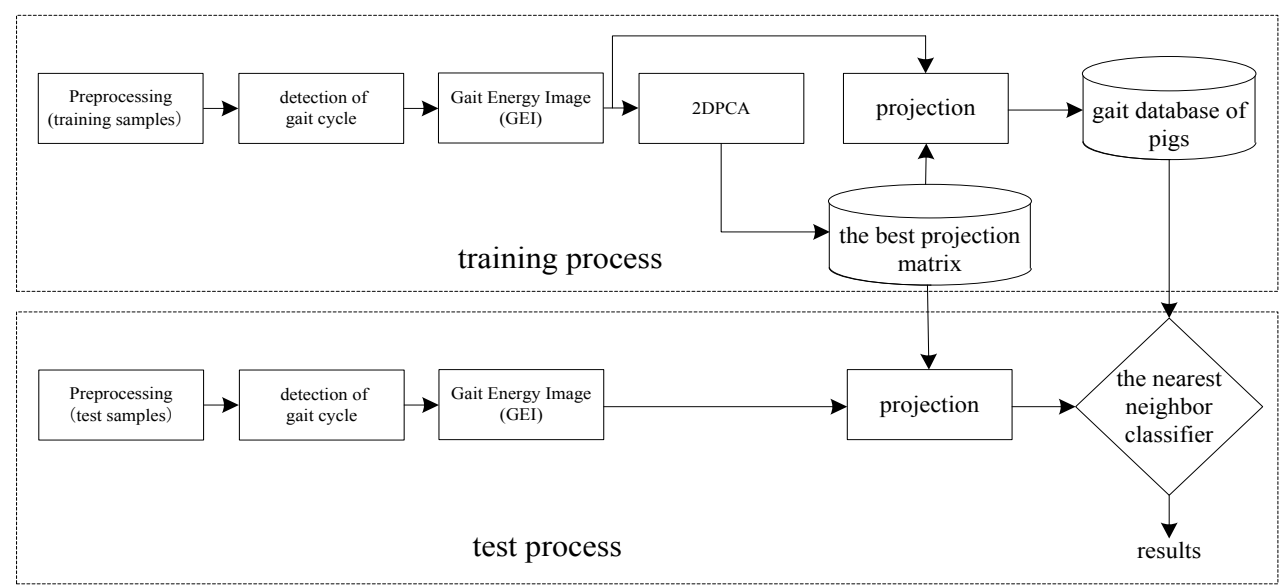

Figure 9. The experimental framework.

The experimental framework is shown in Figure 9. During the training process, binary silhouette images of pigs were obtained by preprocessing. The gait cycle of pigs was detected by skeleton analysis, and then the gait energy image of pigs was calculated. The GEI of normal pigs and lame pigs were taken as training sample images respectively and the best projection matrix was obtained by 2DPCA. Finally, the gait energy image feature matrix of normal pigs and lame pigs are obtained through the feature reduction of the training GEI samples.

In the test process, each test sample is subjected to the same preprocessing process, gait cycle detection and GEI calculation as the training process. Then, the testing GEI sample is projected onto the best projection matrix obtained from the training process, so as to obtain the gait energy image feature matrix of the test sample. Finally, the nearest neighbor classifier is used to identify the normal walking and lame walking of the pigs.

Using nearest neighbor classifier and Euclidean distance for classification, distance measurement $D(B, A)$ used Euclidean formula (15) to calculate:

$$
D(B, A)=\sum_{i=1}^{d}\left\|B_{i}-A_{i}\right\|_{2}
$$

where $B_{i}$ and $A_{i}$ represents the ith column of the matrix B and A respectively.

Because the number of gait database established in this paper is not too large, the K-fold cross validation method is used to verify the experiment. The specific steps are as follows: (1) Divide the sample set $\mathrm{S}$ into $\mathrm{K}$ (K $=8$ ) disjoint subsets $\left\{S_{1}, S_{2}, \ldots S_{8}\right\}$, and each subset contains 50 samples. (2) Select one subset as the test sample in turn, and the remaining 7 subset is used as the training samples. (3) Train the best projection matrix by training samples. (4) The test sample is projected onto the best projection matrix, using the nearest neighbor classifier to get the recognition rate. (5) The average of the recognition rate obtained from $\mathrm{K}(\mathrm{K}=8)$ experiments is used as the final recognition rate of the model. According to this method, the final recognition rate is $93.25 \%$.

Table 1. Recognition of the algorithm in this paper.

\begin{tabular}{lccc}
\hline & number of GEI & correct recognition number & recognition rate \\
\hline normal pigs & 200 & 182 & $91.0 \%$ \\
lame pigs & 200 & 191 & $95.5 \%$ \\
total & 400 & 373 & $93.25 \%$ \\
\hline
\end{tabular}

The experimental results in Table 1 show that the correct recognition rate of normal pigs is $91.0 \%$ and that of lame pigs is $95.5 \%$. Therefore, the algorithm can easily miscalculate normal walking of normal pigs as lame walking of lame pigs. The main reason for the above misjudgement is that by observing GEI of pigs, some normal pigs may appear to walk and stop in some cases. In addition, the head sometimes appear certain amplitude of shaking up and down.

In the 2DPCA experiment, each eigenvalue corresponds to a eigenvector, and the number of eigenvalues selected will have a great influence on the final experimental result. In order to select the optimal number of principal components, the experiment was done about the effect of different values of $\mathrm{d}$ on the correct recognition rate, as shown in Figure 10. 


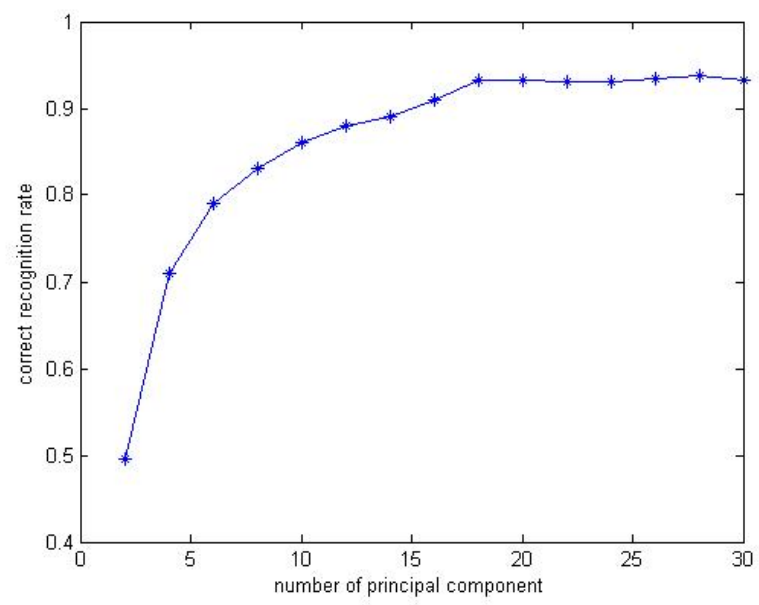

Figure 10. The correct recognition rate under different number of principal component.

As can be seen from Figure 10, when the number of selected principal components $\mathrm{d}$ is small, the correct recognition rate increases as $d$ increases. When $d$ is 18 , the correct recognition rate reaches $93.25 \%$. If continue to increase the $\mathrm{d}$, the correct recognition rate no significant change. According to the above analysis, this paper chooses the number of principal components as 18. Compared with the traditional PCA, 2DPCA reduces the computational complexity and preserves the two-dimensional structural features of GEI, so the recognition is better.

\section{Conclusions}

(1) In this paper, a skeleton analysis method is used to detect the gait cycle of pigs. Through the skeleton and skeleton endpoint feature description of pigs, the similarity between feature vectors of the skeleton endpoints was used to determine ownership of the endpoints on the skeleton to be measured, and then the pig gait cycle is detected according to the relative distance between forelimb endpoints. The experimental results show that the method of skeleton analysis can effectively detect the gait cycle of pigs.

(2) This paper proposes a gait energy image based method to characterize the gait features of pigs. According to the difference of gray distribution on the GEI of normal pigs and lame pigs, 2DPCA was used to reduce the dimension of gait energy image of pigs. Finally, the nearest neighbor classifier is used for classification and recognition, the recognition rate reaches $93.25 \%$. The experimental results show that it is effective to use the GEI to characterize the gait features of pigs, and it is feasible to apply it to lameness behavior recognition of pigs.

\section{Acknowledgments}

This project was supported by the National Natural Science Foundation of China (Grant No. 31172243) and a project funded by the Priority Academic Program Development of Jiangsu Higher Education Institutions.

\section{References}

Bahr C, Leroy T, Song X Y, et al, 2009. Automatic detection of lameness in dairy cattle - analyzing image parameters related to lameness. Livestock Environment VIII. Iguassu Falls, Brazil, 31 August - 4 September 2008.

Dawkins M, 2004. Using behaviour to assess animal welfare. Animal Welfare, 13(1):3-7.

Han J, Bhanu B, 2005. Individual recognition using gait energy image. IEEE Transactions on Pattern Analysis \& Machine Intelligence, 28(2), 316-322.

Liu B, Zhu W, Yang J, et al, 2014. Extracting of pig gait frequency feature based on depth image and pig skeleton endpoints analysis. Transactions of the Chinese Society of Agricultural Engineering, 30(10), 131-137.

Liu L, 2009. Gait based human identification research for safety-critical environments. MS Thesis. College of automation, Harbin Engineering University, Harbin, China.

Wang J, 2015. Gait recognition of pigs based on curvature of the back. MS Thesis, School of Electrical and Information Engineering, Jiangsu University, Zhenjiang, China.

Yang J, Zhang D, Frangi A F, et al, 2004. Two-dimensional PCA: a new approach to appearance-based face representation and recognition. IEEE Trans Pattern Anal Mach Intell, 26(1), 131-137.

Yi C, 2009. Identity Recognition based on Human Motion Analysis using Skeleton and Periodic 
Characteristics. MS Thesis. School of Electronic Information and Communication, Huazhong University of Science and Technology, Wuhan, China.

Zhou C, Mitsugami I, Yagi Y, 2015. Detection of gait impairment in the elderly using patch - GEI. Ieej Transactions on Electrical \& Electronic Engineering, 10(S1), S69-S76.

Zhu W, Zhang J, 2010. Identification of abnormal gait of pigs based on video analysis. In 2010 3rd International Symposium on Knowledge Acquisition and Modeling, KAM 2010, 394-397. 


\author{
Estimating Pasture Nitrogen Content using Thermal Images \\ Majeed Safa $^{a^{*}}$, Kirsty E. Martin ${ }^{\mathrm{b}}$, Thomas M.R. Maxwell ${ }^{\mathrm{b}}$, Birendra KC \\ a Department of Land Management and Systems, Lincoln University, New Zealand \\ b Department of Agricultural Sciences, Lincoln University, New Zealand \\ ${ }^{c}$ Aqualinc Research Limited, Christchurch, New Zealand \\ * Corresponding author. Email: Majeed.Safa@lincoln.ac.nz
}

\begin{abstract}
This study explored the possibility of estimating nitrogen content of a pasture grass using thermal images and multiple linear regression (MLR), based on the premise that plant herbage with a higher $\mathrm{N}$ content would be absorbing more light energy for active photosynthesis, therefore be emitting less energy as heat. This is the first reported study to use thermal infrared images and MLR to estimate pasture nitrogen content under different conditions of nitrogen $(\mathrm{N})$ fertiliser. The research was conducted in a controlled-climate environment to isolate the effect of a key environmental parameter, available soil N, on pasture grass herbage temperatures. The project was the first step towards developing a smart fertiliser spreader to manage $\mathrm{N}$ applications based on plant temperature. A field experiment was conducted to determine the degree of the correlation between leaf $\mathrm{N}$ content and the surface temperatures of perennial ryegrass (Lolium perenne) herbage. Using a thermal imaging camera, periodic measurements of the herbage surface temperatures were made in conjunction with herbage cuts and analysis of grass dry matter for $\% \mathrm{~N}$ content. At the same time, other environmental factors, such as air temperature and humidity, were also measured. As data constituted the core of the study, the database should be flexible, accessible and simple, for both data entry and data analysis. Subsequently, a MLR model was developed to predict $\mathrm{N}$ content based on herbage temperatures and the other factors measured. The final MLR model was developed based on three input variables: plant temperature, soil moisture, and illuminance (Lux). Comparing actual and predicted data showed that the MLR model could be fitted to pasture nitrogen content and accounted for around $81 \%$ and $85 \%$ of the variance in the training and validation data, respectively. The outcome of this study will aid the development of technology for estimating nitrogen content of perennial ryegrass under field conditions which is seen as critical in the design of an advanced fertiliser spreader to manage nitrogen application on farms.
\end{abstract}

Keywords: Modelling, Pasture, Thermal images, Nitrogen, Multiple Linear Regression

\title{
1. Introduction
}

Remote sensing is a branch of thermal remote sensing that deals with the acquisition, processing and interpretation of data obtained in the thermal infrared (TIR) region of the electromagnetic (EM) spectrum (Prakash, 2000). Thermal remote sensing measures emitted radiation from the surface of a targeted object (Sabins, 1996). In thermal remote sensing, the invisible radiation patterns of objects are converted into visible images, which are called thermal images (Ishimwe, Abutaleb, \& Ahmed, 2014). Thermal images are used for analysing different properties related to growth, yield and adaptation to biotic (disease, insects) or abiotic (drought and salinity) stress.

Portable, hand-held or thermal sensors that are mounted on an airplane or satellite or vehicle are used to acquire thermal images of any object of interest (Li, Zhang, \& Huang, 2014). As infrared instruments can be portable and/or hand-held, the thermal infrared technology can be applied directly by the user on the ground. A thermal camera, a signal processing unit and an image acquisition system, forms the basis of infrared thermal imaging systems (Fitzgerald et al., 2006; Ishimwe et al., 2014).

Thermal remote sensing is based on the principle that everything above absolute zero $\left(0 \mathrm{~K}\right.$ or $-273.15^{\circ} \mathrm{C}$ or $-459^{\circ} \mathrm{F}$ ) emits radiation in the infrared range of the electromagnetic spectrum (Prakash, 2000). Approximately $80 \%$ of the energy thermal sensors receive is emitted by land surfaces thus, it is easiest to analyse surface temperature from the thermal infrared signal (Quattrochi \& Luvall, 2004). In addition, the simplicity of operating thermal imaging systems has seen it being applied in various fields such as agriculture, civil engineering, industrial maintenance, aerospace, medicine, pharmacy and veterinary science (Vadivambal \& Jayas, 2011). Different fields where thermal imaging has been used as a research tool is described in da Luz and Crowley (2010). Thermal imaging techniques have also been used also in forestry ecosystem studies (da Luz \& Crowley, 2010; Kokaly, Asner, Ollinger, Martin, \& Wessman, 2009) food industries (Manickavasagan, Jayas, White, \& Paliwal, 2005) and it is presently refined for integration into precision farming (Liu et al., 2000).

In fact, thermal imaging systems are applicable for analyzing the geometry and morphology of any material and process that emits or absorbs heat (Hellebrand et al., 2006). Use of thermal imaging in agriculture includes nursery and greenhouse monitoring, irrigation scheduling, plant disease detection, estimating fruit yield, 
evaluating fruit maturity (Ishimwe et al., 2014; Tilling et al., 2007). Various researchers used thermal imaging for monitoring water stress of different crops such as wine-grape (Möller et al., 2007) and wheat (Tilling et al., 2007). If irrigation supply is sufficient to meet crop water requirement, leaves recieve enough water to satisfy transpiration demand that help to maintain cool canopy temperature. However, if irrigation supply is limited then transpiration and associated evaporative cooling are reduced resulting in higher canopy temperatures. Thermal imaging thus provides relevant information for real time irrigation scheduling and $\mathrm{N}$ concentration measurement (Fitzgerald et al., 2006).

Tilling et al. (2007) and da Luz and Crowley (2010) have described the principle behind thermal imaging. A plant leaf contains a certain amount of water per unit area which varies with nutrients and water deficiency (Fitzgerald et al., 2006). The concentration of $\mathrm{N}$ and water in leaves greatly influence the light reflected by a plant (Haboudane, Miller, Tremblay, Zarco-Tejada, \& Dextraze, 2002). Well-hydrated and healthy plants strongly reflect infrared wavelengths (Gates, Keegan, Schleter, \& Weidner, 1965). As plant tissues dehydrate, the reflectance decreases (Pinter et al., 2003). Therefore, by analysing internal leaf structures through thermal imaging, it is possible to judge the turgidity of different leaves and the nutrient content of various crops (AlAbbas, Barr, Hall, Crane, \& Baumgardner, 1974; Hu, Du, \& He, 2011; Schepers, Blackmer, Wilhelm, \& Resende, 1996; Thomas \& Gausman, 1977).

Blackmer, Schepers, and Varvel (1994), Gao (1995), Osborne, Schepers, Francis, and Schlemmer (2002) and Penuelas, Filella, Biel, Serrano, and Save (1993) have described the use of reflected light to measure nitrogen (N) and water status in plants. A crop's response to $\mathrm{N}$ fertiliser applications is mostly governed by water status in the plant, with the less water stressed plants showing greater $\mathrm{N}$ response (Fitzgerald et al., 2006). Therefore, regular monitoring of plant available water and crop $\mathrm{N}$ status is essential to match $\mathrm{N}$ supply with spatial and temporal soil water availability (Tilling et al., 2007). Regular monitoring of plant available water and crop N status would guide targeted $\mathrm{N}$ applications only to those areas where crop is facing $\mathrm{N}$ stress (deficiency) but where plant available water is sufficient. Thus, modern thermal imaging techniques with high resolution provides relevant information for real time irrigation scheduling and the measurement of $\mathrm{N}$ concentrations (Fitzgerald et al., 2006).

Some studies of $\mathrm{N}$ content in different plants using various spectral imaging technology include CabreraBosquet, Sánchez, Rosales, Palacios-Rojas, and Araus (2010), Nguyen and Lee (2006) and Tilling et al. (2007). Cabrera-Bosquet et al. (2010) used near infrared reflectance spectroscopy (NIRS) to estimate the N content in maize grown under different water applications. The genotypic differences in N content using NIRS technology varied according to the variation in water applications, confirming that NIRS technique is capable of predicting $\mathrm{N}$ level in maize. The result indicated that the NIRS technology can be used to improve nitrogen and water use efficiency. Nguyen and Lee (2006) used hyperspectral canopy reflectance (300-1100 nm) to assess N status in rice at various growth stages. The results revealed that hyperspectral canopy reflectance data can be used to develop an acceptable and accurate model to predict $\mathrm{N}$ concentration and $\mathrm{N}$ density in rice. Tilling et al. (2007) used thermal imaging to estimate the $\mathrm{N}$ status of wheat farmed under irrigated and rainfed conditions, by applying different amounts of $\mathrm{N}$. The result indicated that the mean temperature of rainfed wheat was $6.5^{\circ} \mathrm{C}$ and $2.7^{\circ} \mathrm{C}$ warmer than irrigated wheat at full plant cover status, during years 2004 and 2005, respectively. The plot temperature was affected by irrigation supply, plant cover and $\mathrm{N}$ treatment, with the higher the irrigation supply, plant cover and $\mathrm{N}$ application, the lower was the plot temperature (Tilling et al., 2007). Rainfed plots were consistently more stressed than irrigated plots. Safa and Maxwell (2015) developed an artificial neural networks (ANN) model to predict pasture nitrogen content in small glasshouse pot experiment. The final ANN model was developed based on three input variables: plant temperature, number of days after planting, and number of days after the last nitrogen application

The concentration of leaf $\mathrm{N}$ largely determines the level of chlorophyll present in the leaves which greatly influences the light reflected by a plant (Haboudane et al., 2002). Measurement of light reflected from crop leaves and canopies can be used to determine chlorophyll content and thus leaf $\mathrm{N}$ concentration. However, the reflection of light from a plant leaf is also affected by other characteristics, such as plant cover, plant size, plant age, and leaf angle. (Shimshi, 1967) found that even if soil moisture content is a non-limiting, a N deficiency would induce stomatal closure in various crop species, such as beans, wheat, sugar beets, maize, groundnuts.

Since thermal imaging generates different coloured images based on leaf $\mathrm{N}$ content, analysing thermal images can determine nitrogen deficiency. In fluorescence images, a higher blue-green, higher Chl-F at $690 \mathrm{~nm}$ (Corp et al., 2003; Heisel, Sowinska, Miehé, Lang, \& Lichtenthaler, 1996) and reduced chlorophyll concentration implies a nitrogen deficiency (Liu et al., 2000). The response to different levels of fertilizer use varies with crop type (Costa, Grant, \& Chaves, 2013). For example, thermal imaging of spring barley showed a higher canopy temperature from barley plants with no $\mathrm{N}$ fertiliser applied compared to barley plants fertilised with $165 \mathrm{~kg} \mathrm{~N} / \mathrm{ha}$ (Chaerle, Hagenbeek, Vanrobaeys, \& Van Der Straeten, 2007). However, the opposite result was observed for wheat plants (Tilling et al., 2007). According to Maxwell and Johnson (2000), light energy absorbed by chlorophyll molecules are utilized for three processes: 1) some proportion of energy is utilised to drive 
photosynthesis; 2) some proportion of light energy is dissipated as heat; and 3) some proportion is re-emitted as light-chlorophyll fluorescence.

The thermal imaging technique offers great potential to study plant $\mathrm{N}$ content, allowing deeper investigation of specific phenomena such as the nature of stomatal closure and the impact of different management strategies on crop water status (Costa et al., 2013). However, more research is needed to ascertain the degree of the link between plant leaf temperature and leaf $\mathrm{N}$ content. This study estimated $\mathrm{N}$ content in a pasture grass using thermal images and multiple linear regression (MLR). It was hypothesized that perennial ryegrass herbage with a higher $\mathrm{N}$ content would absorb more light energy for active photosynthesis, and therefore, emits less light energy as heat.

\section{Material and Methods}

An on-farm experiment was conducted to determine the degree of the correlation between leaf $\mathrm{N}$ content and the surface temperature of perennial ryegrass (Lolium perenne) herbage. The field study was developed to monitor different environmental factors and measure their sensitivity in the final model as well. Using a thermal imaging camera, periodic measurements of herbage surface temperatures were made in conjunction with herbage cuts and herbage dry matter analysis for $\% \mathrm{~N}$ content. At the same time, other environmental factors such as humidity, soil temperature, light intensity and air temperature were measured. In this study, a Flir E30 thermal camera was used to measure pasture herbage temperatures with a range between 7.5 and $13 \mu \mathrm{m}$.

The experiment was conducted at the Lincoln University Research Dairy Farm, Canterbury, New Zealand (436'ㅅ, 172 $\left.46^{\prime} \mathrm{E}\right)$. The soil type was a free-draining Templeton fine sandy loam (Immature Pallic soil; Hewitt 2010). The N-fertiliser rates were $0,45,90,180,315$ and $450 \mathrm{~kg} \mathrm{~N} / \mathrm{ha} /$ year. Main plots areas were $3 \mathrm{~m} \mathrm{x}$ $12.6 \mathrm{~m}$, and subplots $3 \mathrm{~m}$ x $2.1 \mathrm{~m}$ (Martin et al., 2017). Perennial ryegrass 'One 50' was sown as pure swards. Periodic measurements of the surface temperature of the herbage were made using a mobile platform from which infrared measurements of the herbage temperature were made from $1 \mathrm{~m}$ above the plots. The emissivity value of thermal images was selected as $95 \%$. Five photos from sides and centre of the plots were taken in each sampling and soil moisture, air temperature, illuminance (Lux), air velocity, and soil temperature of each spot were measures simultaneously.

For data analysis, the thermal images were analysed using Flir software to find the average temperature of the leaves (Figure 1). The soil surface temperature would change the accuracy of the data measurement, therefore, only the average temperature of the areas covered by plants were measured. As data constituted the core of the study, the database should be flexible, accessible and simple for both data entry and data analysis. Subsequently, after five monthly herbage cuts (spring to summer; September, October, November, December and January) a MLR model was developed to predict leaf $\mathrm{N}$ content based on herbage temperatures and the other factors measured.

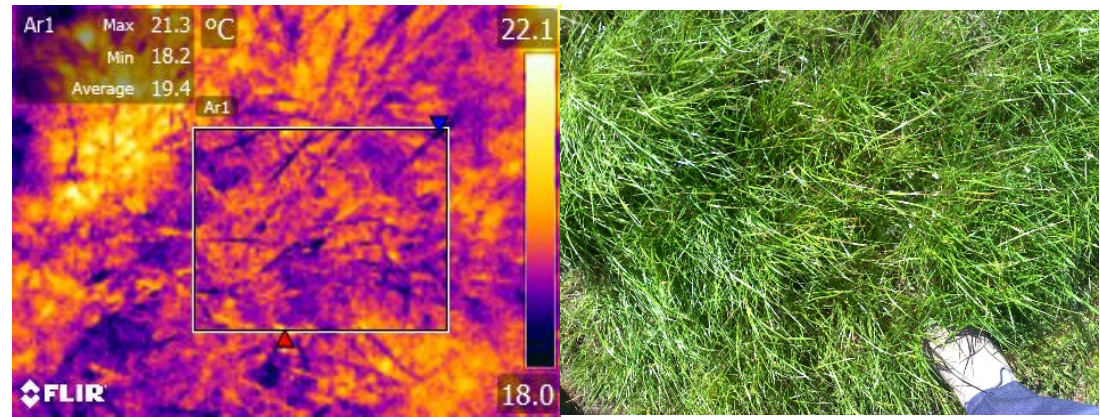

Figure1. Thermal and digital photos of a spot.

The multiple linear regression model was selected and developed for predicting pasture nitrogen content. Compared with nonlinear models, linear regression models are easier and more practical for solving the different problems (Catalina, Iordache, \& Caracaleanu, 2013).

For use in the model, it was necessary to select a limited number of variables without any selective bias (M. Safa \& Samarasinghe, 2011). A simple model with the highest $\mathrm{r}^{2}$ was designed through a combination of forward, backward and stepwise regression adjustments. Terms were maintained in the final model if they were significant at $\mathrm{p}=0.05$ (Alvarez, 2009). In the first step, the relationship between energy consumption and each input variable was tested with a simple linear regression using the $\mathrm{r}^{2}$ as the decision criterion. Then, a multiple linear regression model was developed for predicting the energy consumption as: 
where $a_{0}-a_{n}$ are the regression coefficients and $V_{0}-V_{n}$ are the independent variables. The model was in a linear form to represent the linear relationships of the dependent variable with the independent variable. After running the model, predictions on the validation data were estimated. In this study, the model was developed with a minimum number of variables to capture energy consumption in as simple a form as possible. After investigating several variables, plant temperature, soil moisture, and illuminance (Lux)were selected as the independent variables with which to develop the models.

Several methods of error estimation were proposed. The mean square error (MSE) over all training patterns (Eq. 2) was the most commonly used error indicator. MSE was very useful to compare different models; it showed a network's ability to predict the correct output. The MSE can be written as:

$$
M S E=\frac{1}{2 N} \sum_{i}^{N}\left(t_{i}-z_{i}\right)^{2}
$$

where $t_{i}$ and $z_{i}$ are the actual and predicted outputs for the $i^{\text {th }}$ training pattern, and $\mathrm{N}$ is the total number of training patterns (Catalina et al., 2013; Samarasinghe, 2007). The root mean square error (RMSE) is another error estimation method, which shows the error in the units of actual and predicted data.

\section{Results}

The results of several models were investigated to find the model with the minimum margin of error. Three independent variables was selected including soil moisture, illuminance (Lux), and plant temperature (C) to predict N\% content in the herbage samples. The actual RMSE of the final MLR model was estimated to be $0.21 \%$ $\mathrm{N}$ and $0.12 \% \mathrm{~N}$ for the training and validation data, respectively. This was the lowest RMSE between several MLR models examined in this study. As shown in Figures 2 and 3, the $\% \mathrm{~N}$ estimated by the MLR accounted for $81 \%$ and $85 \%$ of the actual variability in $\% \mathrm{~N}$ in training and validation data, respectively.

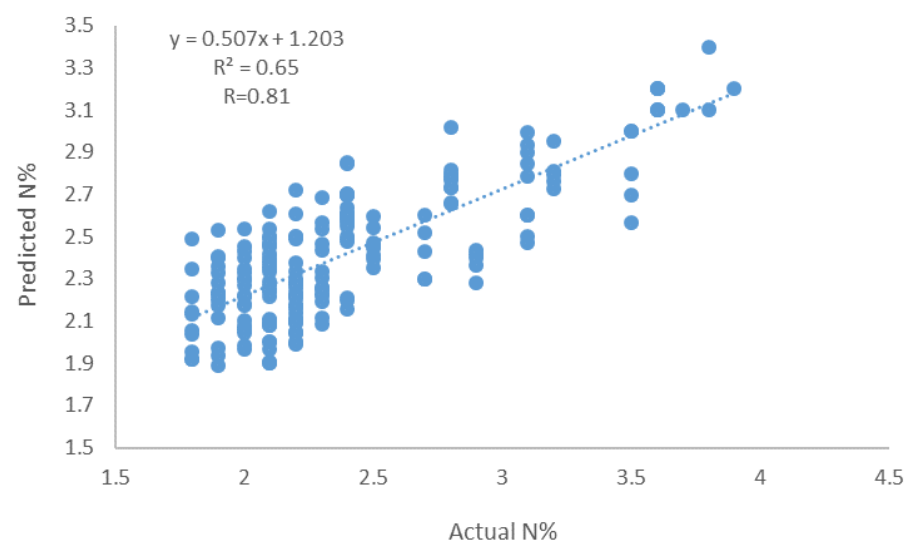

Figure 2. Relationships between observed and predicted pasture nitrogen content (Training) using the MLR model.

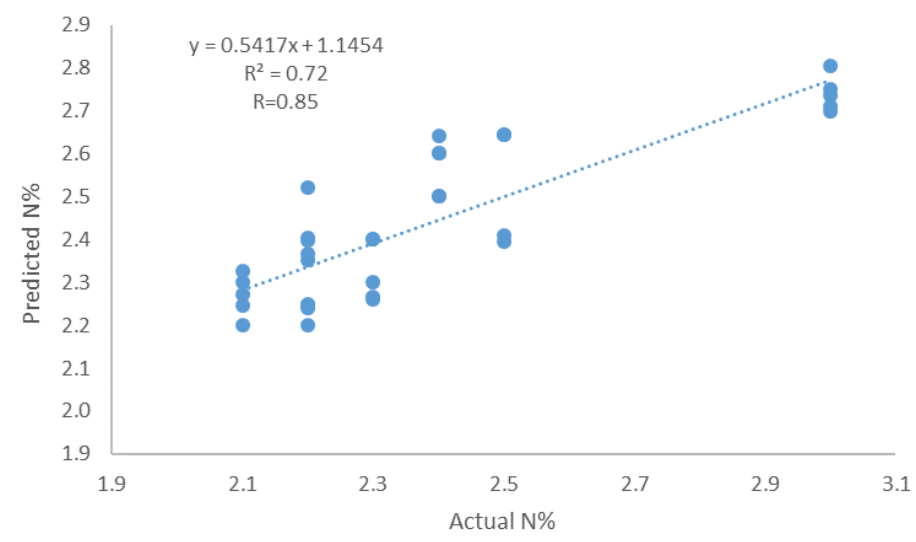

Figure 3. Relationships between observed and predicted pasture nitrogen (Validation) using the artificial neural networks model. 
As shown in Figure $2 \& 3$, the final model predicted pasture nitrogen content with a accaptable ? margin of error. Comparing the training and validation data showed that the correlation between predicted and actual $\% \mathrm{~N}$ in both training and validation data were similar. It is recommended other input variables should be examined to improve the model under different conditions. There were several factors which could influence the final results. However, the result of this study was very encouraging and the final model was able to predict pasture nitrogen content with an acceptably low error.

\section{Conclusions}

This study has shown it was possible to estimate nitrogen content of perennial ryegrass (Lolium perenne) herbage under different soil $\mathrm{N}$ fertiliser applications under field conditions. The results showed that a MLR model with basic input variables was capable of predicting pasture nitrogen content within acceptable errors. The accuracy of the data was a critical factor in developing a prediction model with a minimum margin of error. The final model predicted pasture nitrogen content $(\% \mathrm{~N})$ based on plant temperature, soil moisture, and illuminance (Lux). The main challenge of this method is collecting accurate data under farming conditions in the field.

It is possible to use the hypothesis of the current project in an advanced precision farming project to reduce nitrogen consumption by using thermal images, artificial neural networks, and machine vision to improve fertiliser use efficiency. Using other modelling techniques such as artificial neural networks would improve the accuracy of the model. The study has great potential to reduce nitrogen consumption, farmers' costs and environmental impacts on New Zealand dairy farms. The method used in this study can be developed for other plants and agricultural products. Future studies to compare other modelling methods and other input variables are strongly recommended.

\section{References}

Al-Abbas, A., Barr, R., Hall, J., Crane, F., \& Baumgardner, M. (1974). Spectra of normal and nutrientdeficient maize leaves. Agronomy Journal, 66(1), 16-20.

Alvarez, R. (2009). Predicting average regional yield and production of wheat in the Argentine Pampas by an artificial neural network approach. European Journal of Agronomy, 30(2), 70-77.

Blackmer, T. M., Schepers, J. S., \& Varvel, G. E. (1994). Light reflectance compared with other nitrogen stress measurements in corn leaves. Agronomy Journal, 86(6), 934-938.

Cabrera-Bosquet, L., Sánchez, C., Rosales, A., Palacios-Rojas, N., \& Araus, J. L. (2010). Near-Infrared Reflectance Spectroscopy (NIRS) Assessment of $\delta 180$ and nitrogen and ash contents for improved yield potential and drought adaptation in maize. Journal of agricultural and food chemistry, 59(2), 467-474.

Catalina, T., Iordache, V., \& Caracaleanu, B. (2013). Multiple regression model for fast prediction of the heating energy demand. Energy and Buildings, 57(0), 302-312.

doi:http://dx.doi.org/10.1016/j.enbuild.2012.11.010

Chaerle, L., Hagenbeek, D., Vanrobaeys, X., \& Van Der Straeten, D. (2007). Early detection of nutrient and biotic stress in Phaseolus vulgaris. International Journal of Remote Sensing, 28(16), 3479-3492.

Corp, L. A., McMurtrey, J. E., Middleton, E. M., Mulchi, C. L., Chappelle, E. W., \& Daughtry, C. S. (2003). Fluorescence sensing systems: In vivo detection of biophysical variations in field corn due to nitrogen supply. Remote sensing of environment, 86(4), 470-479.

Costa, J. M., Grant, O. M., \& Chaves, M. M. (2013). Thermography to explore plant-environment interactions. Journal of experimental botany, 64(13), 3937-3949.

da Luz, B. R., \& Crowley, J. K. (2010). Identification of plant species by using high spatial and spectral resolution thermal infrared (8.0-13.5 $\mu \mathrm{m})$ imagery. Remote Sensing of Environment, 114(2), 404-413.

Fitzgerald, G., Rodriguez, D., Christensen, L., Belford, R., Sadras, V. O., \& Clarke, T. (2006). Spectral and thermal sensing for nitrogen and water status in rainfed and irrigated wheat environments. Precision Agriculture, 7(4), 233-248.

Gao, B.-C. (1995). Normalized difference water index for remote sensing of vegetation liquid water from space. Paper presented at the SPIE's 1995 Symposium on OE/Aerospace Sensing and Dual Use Photonics.

Gates, D. M., Keegan, H. J., Schleter, J. C., \& Weidner, V. R. (1965). Spectral properties of plants. Applied optics, 4(1), 11-20.

Haboudane, D., Miller, J. R., Tremblay, N., Zarco-Tejada, P. J., \& Dextraze, L. (2002). Integrated narrowband vegetation indices for prediction of crop chlorophyll content for application to precision agriculture. Remote sensing of environment, 81(2), 416-426.

Heisel, F., Sowinska, M., Miehé, J. A., Lang, M., \& Lichtenthaler, H. K. (1996). Detection of nutrient deficiencies of maize by laser induced fluorescence imaging. Journal of plant physiology, 148(5), 622-631.

Hellebrand, H., Herppich, W., Beuche, H., Dammer, K., Linke, M., \& Flath, K. (2006). Investigations of plant infections by thermal vision and NIR imaging. International agrophysics, $20(1), 1$. 
$\mathrm{Hu}, \mathrm{Z}$., Du, W., \& He, X. (2011). Application of infrared thermography technology for irrigation scheduling of winter wheat. Paper presented at the Multimedia Technology (ICMT), 2011 International Conference on.

Ishimwe, R., Abutaleb, K., \& Ahmed, F. (2014). Applications of Thermal Imaging in Agriculture-A Review. Advances in Remote Sensing, 3(03), 128.

Kokaly, R. F., Asner, G. P., Ollinger, S. V., Martin, M. E., \& Wessman, C. A. (2009). Characterizing canopy biochemistry from imaging spectroscopy and its application to ecosystem studies. Remote Sensing of Environment, 113, S78-S91.

Li, L., Zhang, Q., \& Huang, D. (2014). A Review of Imaging Techniques for Plant Phenotyping. Sensors, 14(11), 20078-20111.

Liu, Q., Gu, X., Li, X., Jacob, F., Hanocq, J., Friedl, M., . . Tian, G. (2000). Study on thermal infrared emission directionality over crop canopies with TIR camera imagery. Science in China Series E: Technological Sciences, 43(1), 95-103.

Manickavasagan, A., Jayas, D. S., White, N. D., \& Paliwal, J. (2005). Applications of Thermal Imaging in Agriculture-A Review. Canadian Society for Engineering in Agricultultural, Food and Biological Systems, $05-$ 002.

Maxwell, K., \& Johnson, G. N. (2000). Chlorophyll fluorescence-a practical guide. Journal of Experimental Botany, 51(345), 659-668.

Möller, M., Alchanatis, V., Cohen, Y., Meron, M., Tsipris, J., Naor, A., . . Cohen, S. (2007). Use of thermal and visible imagery for estimating crop water status of irrigated grapevine. Journal of Experimental Botany, 58(4), 827-838.

Nguyen, H. T., \& Lee, B.-W. (2006). Assessment of rice leaf growth and nitrogen status by hyperspectral canopy reflectance and partial least square regression. European Journal of Agronomy, 24(4), 349-356.

Osborne, S., Schepers, J. S., Francis, D., \& Schlemmer, M. R. (2002). Detection of phosphorus and nitrogen deficiencies in corn using spectral radiance measurements. Agronomy Journal, 94(6), 1215-1221.

Penuelas, J., Filella, I., Biel, C., Serrano, L., \& Save, R. (1993). The reflectance at the 950-970 nm region as an indicator of plant water status. International journal of remote sensing, 14(10), 1887-1905.

Pinter, J., Paul J, Hatfield, J. L., Schepers, J. S., Barnes, E. M., Moran, M. S., Daughtry, C. S., \& Upchurch, D. R. (2003). Remote sensing for crop management. Photogrammetric Engineering \& Remote Sensing, 69(6), 647-664.

Prakash, A. (2000). Thermal remote sensing: concepts, issues and applications. International Archives of Photogrammetry and Remote Sensing, 33(B1; PART 1), 239-243.

Quattrochi, D. A., \& Luvall, J. C. (2004). Thermal remote sensing in land surface processing, CRC Press, Boca Raton, USA.

Sabins, F. F. (1996). Remote sensing: principles and applications, 3rd edition, William H. Freeman and Company, New York, USA.

Safa, M., \& Maxwell, T. (2015). Predicting Pasture Nitrogen Content using ANN Models and Thermal Images Paper presented at the 21st International Congress on Modelling and Simulation, Gold Coast, Australia.

Safa, M., \& Samarasinghe, S. (2011). Determination and modelling of energy consumption in wheat production using neural networks: "A case study in Canterbury province, New Zealand". Energy, 36(8), 51405147. doi:http://dx.doi.org/10.1016/j.energy.2011.06.016

Samarasinghe, S. (2007). Neural networks for applied sciences and engineering : from fundamentals to complex pattern recognition. Boca Raton, FL: Auerbach.

Schepers, J. S., Blackmer, T., Wilhelm, W., \& Resende, M. (1996). Transmittance and Reflectance Measurements of CornLeaves from Plants with Different Nitrogen and Water Supply. Journal of Plant Physiology, 148(5), 523-529. 461 .

Shimshi, D. (1967). LEAF CHLOROSIS AND STOMATAL APERTURE*. New Phytologist, 66(3), 455-

Thomas, J., \& Gausman, H. (1977). Leaf reflectance vs. leaf chlorophyll and carotenoid concentrations for eight crops. Agronomy journal, 69(5), 799-802.

Tilling, A. K., O’Leary, G. J., Ferwerda, J. G., Jones, S. D., Fitzgerald, G. J., Rodriguez, D., \& Belford, R. (2007). Remote sensing of nitrogen and water stress in wheat. Field Crops Research, 104(1), 77-85.

Vadivambal, R., \& Jayas, D. S. (2011). Applications of thermal imaging in agriculture and food industry-a review. Food and Bioprocess Technology, 4(2), 186-199. 


\title{
UAV and Machine Learning Techniques Applied to Yield Estimation of Orange Trees
}

\author{
Orly. E. Apolo-Apolo, Jorge Martínez-Guanter, Gregorio Egea, Manuel Pérez-Ruiz \\ Aerospace Engineering and Fluid Mechanics Department, University of Seville, 41013 Seville, Spain \\ * Corresponding author. Email: manuelperez@us.es
}

\begin{abstract}
An accurate and early estimation of citrus yield is important for allowing both farmers and agricultural cooperatives to sell their products. Farmers require accurate yield estimates since such information is key for predicting the volume of stock necessary at the supermarkets and for organizing harvesting operations. In many cases, visual estimates of yield are done but these are not accurate. The main goal of this study was to develop an image processing method to detect and count the number and size of citrus fruits on individual trees using a machine learning algorithm and neural network. A total of 40 images (canopy left and right sides) were acquired from a commercial citrus orchard with an Unmanned Aerial Vehicle (UAV). The algorithm and neural network were tested on 20 citrus trees. The citrus yield estimations and actual mass of the fruit per tree were compared. The errors showed very promising values, and therefore, the method shows great potential for providing yield estimations for citrus and other types of fruits.
\end{abstract}

Keywords: fruit detection, machine learning, citrus, neural networks, harvest.

\section{Introduction}

Citriculture is an activity that has relevance in Spanish agribusiness (Castel et al., 1987). Currently, the total area devoted to citriculture in Spain is 297,000 ha, with an annual production of approximately $7 \times 10^{6} \mathrm{t}$. Most of this production is located in the regions of Andalusia and Valencia, with 82,000 and 161,000 ha, respectively (Gironés-Vilaplana et al., 2014). In this context, Spain has become the largest producer and exporter of sweet orange fruit in Europe despite the strong market competitiveness of countries outside the EU (García-Tejero, 2010; Cardeñosa et al., 2015).

Harvesting is usually the most critical operation in any crop since it involves enormous complexity in terms of contracting labour and selling the product. In the case of orange fruits, fruit harvesting usually accounts for $25 \%$ of the total production costs and requires nearly $50 \%$ of the labour of the crop since mechanized harvesting is not yet widespread in this crop (Agustí Fonfría, 2012; Palacios, 2005). Choosing the optimum harvest time depends on the maturity index, fruit colouring, juice content and size, among other factors (Reig-Martínez and Picazo-Tadeo, 2004). However, although these parameters are important, it is crucial for both farmers and cooperatives to accurately estimate crop production since doing so is key for predicting the volume of stock necessary at the supermarkets and for organizing harvesting operations.

Throughout history, several precision farming applications have been tested in citrus fields to estimate yield (Whittaker et al., 1987; Slaughter and Harrell, 1989; Whitney et al., 1999). These methodologies have been based on the agronomic conditions of the crop, historical crop yield data and visual observation of the crop (Srivastava et al., 2007). In Spanish orange orchards, visual techniques are often used to estimate yield; however, these methods are highly subjective, as they depend on technician knowledge and experience. An error between the actual yield and yield prediction of 15 to $25 \%$ can occur (Pérez-Ruiz et al., 2017). Hence, developing a new tool to estimate yield in orange trees with high accuracy or at least with an error less than that of visual methods may help farmers by supporting their economic profitability and reducing costs.

In the last ten years, thanks to the advances in computers, artificial intelligence (AI) and digital cameras (Sonka et al., 2014), many researchers have sought to develop methods for fruit yield estimation using image segmentation techniques (Haralick et al., 1973; Teixidó et al., 2012). For instance, some earlier studies of fruit recognition were conducted for apple (Stajnko et al., 2005; Linker et al., 2012), citrus (Hannan et al., 2009; Kurtulmus et al., 2011) and tomatoes (Aggelopoulou et al., 2011; Yamamoto et al., 2014). However, many of these methods use only the spectral response between pixels as a unique feature to detect and count fruits (Burnett and Blaschke, 2003). Currently, although many of these methods continue using the same rules, the development of new softwares programming languages and algorithms have opened the possibility to carry out object-based image analysis (OBIA). OBIA allows to work with groups of pixels, edges, shapes and even textures. One of these programs is Python $^{\mathrm{TM}}$, an open-source software that allows the development of algorithms capable of detecting colour on images with great precision (Prateek, 2015). Furthermore, artificial Neural Networks (ANNs), which are computation systems that attempt to mimic (or at least, are inspired by) the neural connections in our nervous system, have improved noticeably over the last decade thanks to the advances in computer systems (Rosebrock, 2017). ANNs have advanced due to Google Keras, OpenCV and TensorFlow, which are open-source software libraries for numerical computation using neural networks that promote a simple and intuitive syntax based on 
Python $^{\mathrm{TM}}$ (Rosebrock, 2016; Collet, 2017). All these tools are part of what experts define as machine learning, which is the ability machines (computers) have to automatically learn and is also known as AI. Scientists hope that these technologies can replace the knowledge and intuition that farmers have always had (Curioni, 2018).

Finally, despite the many algorithms and ANNs that have been developed to estimate yields of different types of crops, most of these methods use images taken at ground level, such as the method presented by Yamamoto et al. (2014). Although these methods yield accurate results some of them are time consuming, laborious, costly and unviable for orange orchards due the size of the cultivated area (Whitehead et al., 2014). Unmanned aerial vehicle (UAVs), which are being successfully used in precision farming for different crops and tasks, provide an alternative that is fast and inexpensive for farmers (González et al., 2018). The main advantages of these vehicles are their ability to be transported, their price and the results that they obtain (Costa et al., 2012; Martínez et al., 2016).

The main goal of this project was to develop and test a fruit detection system using RGB images obtained flying an UAV in combination with ANNs using Keras and TensorFlow. Moreover, a script based on the OpenCV library to obtain the size of orange fruit was tested.

\section{Materials and Methods}

Field description and UAV imagery acquisition

The research was carried out in 2017 and took place over a 4-ha area of a commercial citrus orchard (Citrus sinensis L. cv. Navelina) near Seville, southwestern Spain (Latitude: 37.512574; Longitude: -5.956659). Sampling was conducted on 20 individual trees randomly selected from a total of 1,654 trees. Forty images were acquired under natural lighting conditions using a Phantom 3 (SZ DJI Technology Co. Ltd.). This device is a commercial quadcopter fitted with a camera sensor, which is an RGB Sony Exmor 1/2.3" CMOS with a lens FOV of 94², a focal length of $20 \mathrm{~mm}$, a focal ratio $\mathrm{f} / 2.8 \mathrm{f}$, and focus to infinity. The image resolution of the camera is $4,000 \times$ 3,000 pixels. The flight altitude was between 5 and $6 \mathrm{~m}$ according to previous experience, and the manual flight option was used. The workflow of imagery acquisition followed the same pattern for each tree. Two pictures from the left and right canopy sides were taken (Figure 1). Finally, a short wood ruler calibrated in centimetres $(\mathrm{cm})$ was used during picture taking to determine the average fruit size.

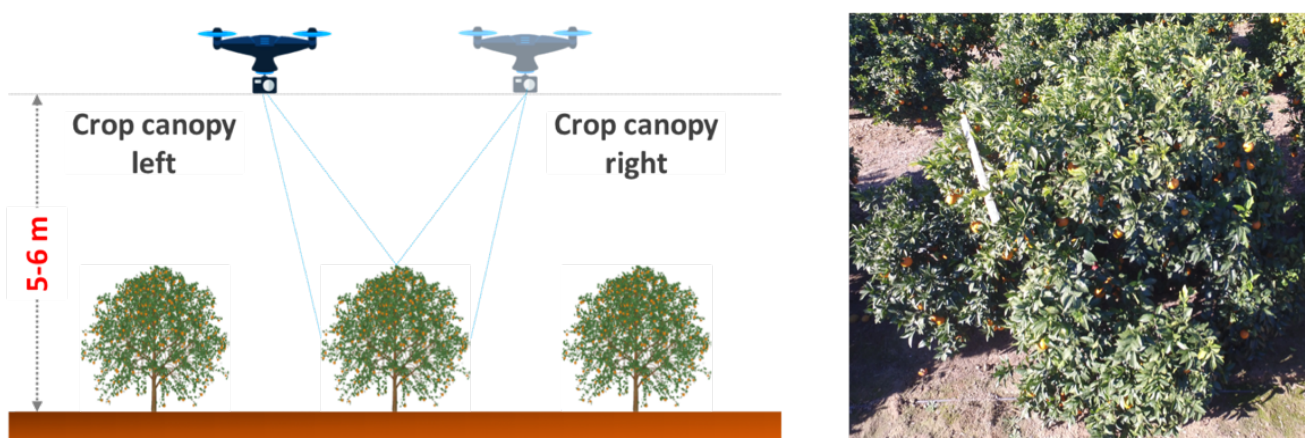

Figure 58. Illustration of the process using UAVs (left). An original citrus tree canopy image (right).

\section{Harvesting}

The citrus trees were harvested manually, and a representative sample of 10 randomly selected fruits/tree was taken to estimate the average fruit weight in grams and size in millimetres. The fruit was picked and placed into picking sacks. Then, the fruit was weighed and dumped from the picking sacks into boxes ( $20 \mathrm{~kg}$ per box $)$, which were manually dumped into a field transport vehicle. A gauge calibrated in millimetres and balance with an accuracy in grams were used to measure the fruit size and weights, respectively.

\section{Computer equipment and software used}

The capacity of a computer (memory RAM, processor, and graphics card, among others) to store and manipulate large amounts of data is one of the greatest challenges in machine learning (Rosebrock, 2017). In this project, the hardware used for all the processes was a MacBook Pro (MacOs High Sierra 10.13.4) with a 2.5 GHz Intel Core 17 processor with 16 GB of RAM and a Graphics AMD Radeon R9 M370X 2048 MB Intel Iris Pro 1536 MB. The Open Source Computer Vision (OpenCV) library (http://opencv.org/), which includes several hundred computer vision algorithms, was used to process images (Rosebrock, 2016). The development of an ANN has traditionally been a complex process; however, in the last few years, new tools, such as Keras and TensorFlow, have emerged to facilitate this process. Keras (https://keras.io/) is a Python library that abstracts away much of the complexity of building a deep neural network, leaving a very simple, nice, and easy-to-use interface to rapidly 
build, test, and deploy deep learning architectures (Murata et al., 2017). TensorFlow is also an open-source software library for numerical computation using data flow graphs (Mayer et al., 2017). TensorFlow is extremely flexible, allowing network computation to be deployed to multiple CPUs, GPUs, servers, or even mobile systems without having to change a single line of code (Rosebrock, 2017). This ability makes TensorFlow an excellent choice for training distributed deep learning networks in an architecture- neutral manner. A combination of both tools was used in this project.

\section{Delimiting the zone of interest to detect fruit}

The images taken show other trees that are not the target; thus, the zone of interest must be delimited using a mask, an extremely powerful and useful technique in computer vision and image processing (Rosebrock, 2016). The first step was to compute the centre of the image by dividing the width $(4,000 \mathrm{px})$ and height $(3,000 \mathrm{px})$ by two; hence, the origin of coordinates for each image was $(2,000,1,500 \mathrm{px})$. Then, a white rectangle $(2,000,1,600$ px) was drawn to focus only on the target tree. The remaining pixels were turned off, shown as a black colour on each image (Figure 2).
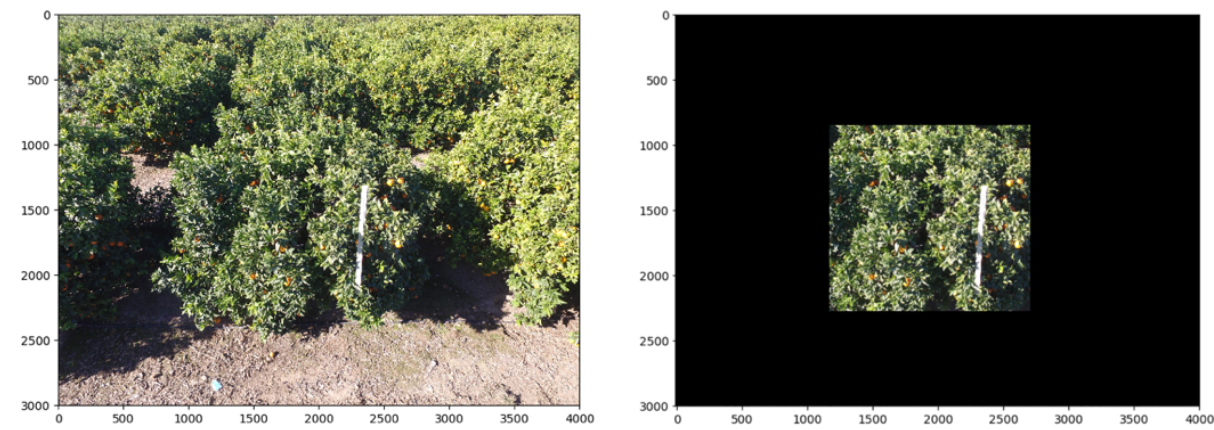

Figure 2. Original image of the orange tree (left) and a mask image (right) where the mask pixels with values greater than zero are shown.

Imagery processing to calculate the fruit size

Measuring the size of fruits in an image is similar to computing the distance from a camera to an object; in both cases, a ratio that measures the number of pixels per a given metric must be defined (Rosebrock et al., 2016). In this project, to achieve this goal a "calibration" using a reference object (wood ruler) was performed. The reference object has two important properties: (1) the dimensions (in terms of width and height) in a measurable unit $(\mathrm{cm})$ are known and (2) the reference is easy to find and identifiable either in terms of the location of the object or in its appearance.

The wood ruler used as a reference object has a known width $(5 \mathrm{~cm})$ and height $(100 \mathrm{~cm})$, with a total area of $500 \mathrm{~cm} 2$. This object was measured in pixels on each image using a Python script. A width of $50.50 \pm 1.43$ pixels and a height of $747.06 \pm 3.81$ pixels were obtained based on its associated bounding box (Figure 3 ). In this manner, there are approximately $37,753.8$ pixels per every $500 \mathrm{~cm}^{2}$ in each image. As is generally known, a fruit is more or less a circular object, and its total area may be calculated by knowing its diameter (D) by the expression $A=\frac{\pi \cdot D^{2}}{4}$. Hence, considering the diameter of a circle, the fruit size $\left(\mathrm{F}_{\mathrm{z}}\right)$ can be approximated using Eq. (1).

$$
F_{z}=\sqrt{\frac{4 A}{\pi}}
$$

Due to the detection of fruits with a very small size in terms of the number of pixels, a minimum value of 32 px for both width and height was defined. Using this ratio and a Python script in combination with OpenCV, the size of each fruit detected in an image was computed (Figure 3). 
a)

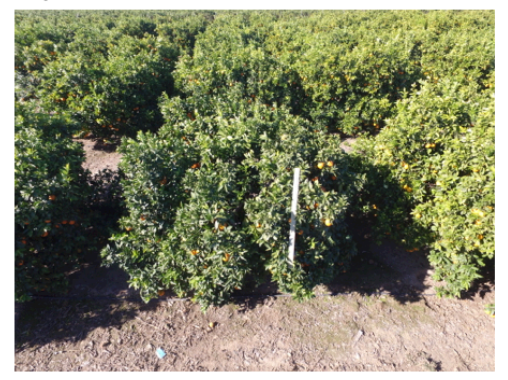

b)

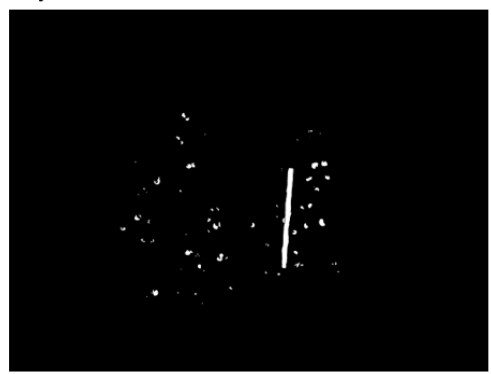

c)

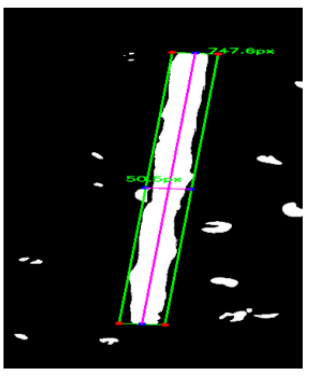

Figure 3. Flowchart of the theoretical image processing steps in Python, where the colour of the original RGB image (b) is first transformed into a binary image with black and white colours (b). Then, taking the wood ruler as a reference (c), the fruit size is computed, yielding the number of pixels for both width and height, respectively. Finally, the fruit size (Fz) is calculated based on the total number of pixels.

Building a deep learning image dataset

To train an ANN, a large number of representative images, also known as a dataset, must be available (Rosebrock, 2017). Deep learning and machine learning models without sufficient training data cannot learn the underlying discriminative patterns required to make robust classifications on testing data (Sonka, et al. 2014). This set of images must be a sample of the problem being solved. In this case, the features of oranges on tree canopies can differ dramatically (e.g., green fruits, fruits of different sizes and shapes, fruits covered by branches and leaves). Thus, although the size of the dataset is not very important, the variations in the samples is crucial since data augmentation increases the accuracy and generalization of an ANN model (Arefi, 2013).

In this project, four datasets with 100, 200,300 and 400 pictures were developed using Google Images, as suggested by Rosebrock (2017) (Figure 4). The first step was to use the query term "orange tree" and a script of JavaScript to gather, download and save the images, URLs using Python. Then, since not every image downloaded was relevant, a manual pruning selection was performed.

a)

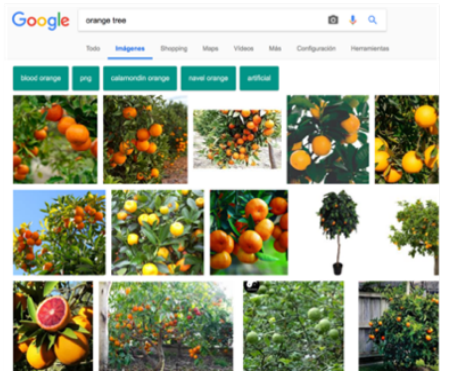

b)

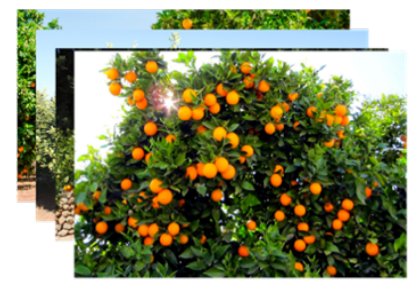

c)

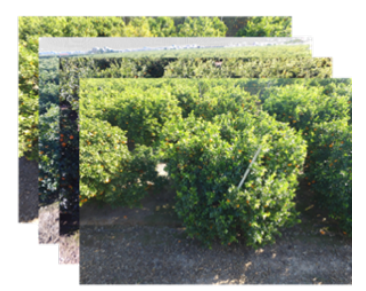

Figure 4. Entering the query "orange tree" and letting the pictures load in the browser (a), set of training images (b) and set of testing images (c).

Training and use of an artificial neural network to count the number of fruits

ANNs are a class of machine learning algorithms inspired by the structure and function of the brain that learn from data and specialize in pattern recognition (Rosebrock, 2017). To understand how neural networks function, knowledge of the different parts of a network and how it works is useful. Any ANN has an input neuron, which represents an input based on the information being classified. Each number of the input neurons is given a weight at each synapse. Then, for each neuron in the next layer, the outputs of all synapses coming to that neuron along with a bias are added and applied to an activation function. Finally, the output of that function will be treated as the input for the next synapse layer, repeating the process until the output is reached.

In this project, due to the variety of different kinds of fruits in the images, a labelling process showing where the fruits are located in each image from the training datasets is necessary (Figure 5). The tool used for this process was labelImg (https://github.com/tzutalin/labelImg), an effective open-source image labelling tool. Once all fruits are labelled, an XML file is generated with label data and the coordinates of the bounding rectangles for each fruit in the image. Then, an object detection model provided by TensorFlow must be chosen (https://github.com/tensorflow/models). These kinds of models are pre-trained classifiers that have a specific neural network architecture. Choosing a correct model is very important since some models have a high speed but lower accuracy and vice versa. The faster $\mathrm{CNN}$ inception v2 model was used in this project. 


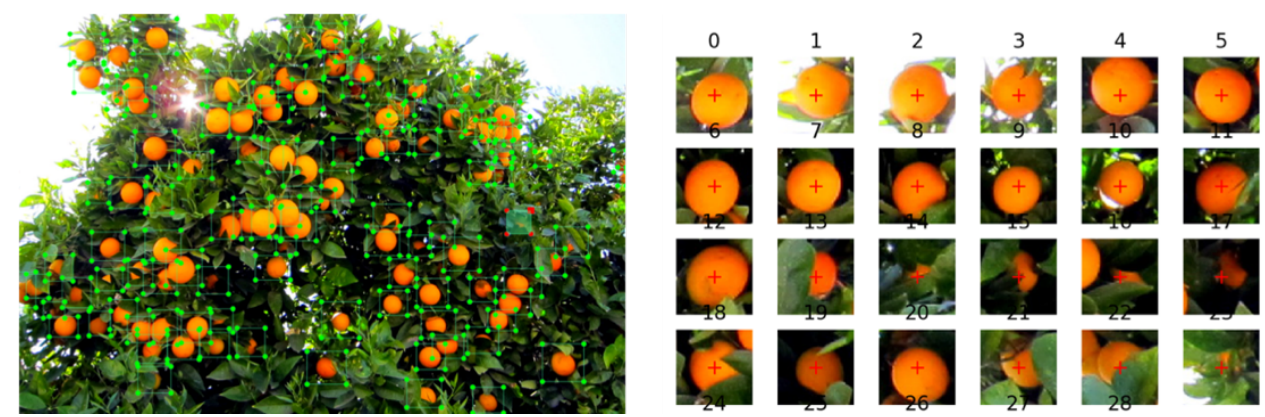

Figure 5. Labelling process in the testing images (left) and different kinds of fruits labelled (right).

On the basis of foregoing the set of training images and other additional steps, all files necessary to train ANN models are generated, and the training process can be initialized. Due to the four datasets being tested in this project, a selection process to determine the best training dataset was necessary. The classification accuracies of the training datasets were $92.85 \%, 93.51 \%, 95.13 \%$ and $98.67 \%$. The classification accuracy is defined by a loss function whose values imply how well or poorly a certain model behaves after each interaction of optimization. Then, the goal when training a machine learning model is to minimize the loss function by changing the weight vector values through different optimization methods, such as increasing the number of images, thereby increasing our classification accuracy. Moreover, another parameter under consideration is the time required to train each model since 6, 12, 18 and 24 hours were taken to train each dataset. With this in mind, the dataset with 400 images and a classification accuracy of $98.67 \%$ was selected to detect fruits in all images.

Once the model was trained, it was used to detect orange fruits in the images taken with the UAV (Figure 6). Each orange fruit is labelled with a green box showing the accuracy (as a percentage) that the algorithm has in recognizing a fruit. In other words, a higher percentage indicates a higher level of accuracy.

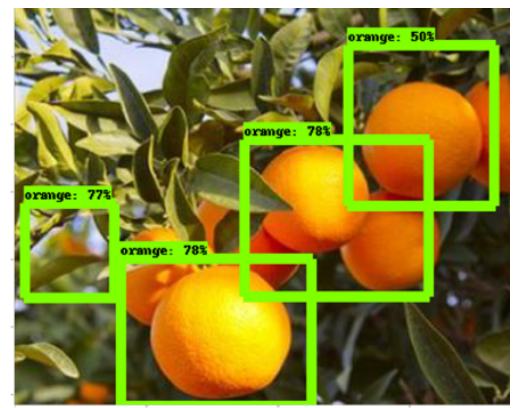

Figure 6. Process of fruit detection with green box labeling.

Statistical analysis

In each experiment, fruit size and number of fruits were analysed separately, each tree representing an experimental unit. Analysis of the statistical error and variance was performed with the statistical package Statgraphics (Statgraphics Centurion XV), and some graphics were developed using Python ${ }^{\mathrm{TM}}$ and the Matplotlib library. Images from each tree (left and right canopy sides) were grouped together, and the number of fruits from each tree was compared with visual counts of fruits using linear regression analyses. To compare the actual yield with the estimated yield, the canopy of an orange tree was assumed to have four sides with a number of fruits similar to that estimated in the analysed canopy sides. Hence, the average number of fruits detected in each tree side was multiplied by four. Finally, the total yields obtained from the yield estimation using computer vision and the visual estimation made by a professional technician during the harvester season were compared to actual yield data.

\section{Results and Discussion}

Fruit size

The fruit size estimated with the model was very similar to the measured fruit size (Table 1). As mentioned before, only fruits with a pixel number higher than $32 \mathrm{px}$ were used. Furthermore, for each tree, an average number of pixels for all fruits detected is shown. Despite the approximate nature of this approach, the model accuracy was high explaining $87 \%$ of the observed variability in fruit size (not shown), so it could be applied to orange fruit management by optimizing the resources required to harvest, transport, store and, if needed, manufacture of the orange fruit product. 
Table 1. Comparison of measured and estimated fruit sizes. Wpixeles and Hpixels denote the number of pixels of fruits in the Width and Height dimensions.

\begin{tabular}{lcccc}
\hline Tree & Measured fruit size $(\mathrm{mm})$ & Wpixels & Hpixels & Estimated fruit size $(\mathrm{mm})$ \\
\hline 1 & $75.34 \pm 2.67$ & 68.00 & 54.00 & $78.69 \pm 1.65$ \\
2 & $71.22 \pm 1.88$ & 58.00 & 46.00 & $67.07 \pm 0.56$ \\
3 & $72.26 \pm 3.18$ & 56.00 & 49.00 & $68.02 \pm 1.23$ \\
4 & $72.60 \pm 1.15$ & 58.00 & 45.00 & $66.34 \pm 2.30$ \\
5 & $76.44 \pm 3.06$ & 61.00 & 50.00 & $71.71 \pm 1.20$ \\
6 & $75.40 \pm 1.67$ & 63.00 & 53.00 & $75.04 \pm 0.60$ \\
7 & $72.12 \pm 1.60$ & 58.00 & 51.00 & $70.63 \pm 0.34$ \\
8 & $67.74 \pm 2.23$ & 53.00 & 45.00 & $63.42 \pm 2.04$ \\
9 & $71.88 \pm 2.70$ & 56.00 & 49.00 & $68.02 \pm 1.03$ \\
10 & $74.40 \pm 1.80$ & 59.00 & 52.00 & $71.93 \pm 1.11$ \\
11 & $82.72 \pm 3.10$ & 65.00 & 58.00 & $79.73 \pm 2.12$ \\
12 & $79.6 \pm 2.89$ & 64.00 & 56.00 & $77.74 \pm 1.22$ \\
13 & $73.82 \pm 2.89$ & 48.00 & 65.00 & $72.53 \pm 1.43$ \\
14 & $86.80 \pm 2.83$ & 68.00 & 56.00 & $80.13 \pm 3.32$ \\
15 & $83.16 \pm 2.62$ & 69.00 & 59.00 & $82.85 \pm 2.34$ \\
16 & $84.32 \pm 2.45$ & 65.00 & 67.00 & $85.69 \pm 2.11$ \\
17 & $84.40 \pm 3.21$ & 68.00 & 70.00 & $89.59 \pm 1.21$ \\
18 & $91.04 \pm 2.41$ & 74.00 & 70.00 & $93.46 \pm 1.34$ \\
19 & $79.96 \pm 1.84$ & 68.00 & 56.00 & $80.13 \pm 0.54$ \\
20 & $77.5 \pm 3.21$ & 64.00 & 56.00 & $77.74 \pm 1.23$ \\
\hline
\end{tabular}

Fruit yield estimation

Finally, the number of fruits obtained with the best dataset model for each crop canopy was compared with the number of fruits counted by visual methods in each image for each crop canopy. An $\mathrm{R}^{2}$ with a value close to 1 indicates that the trained model has good accuracy in terms of the detected and counted fruits (Figure 7).
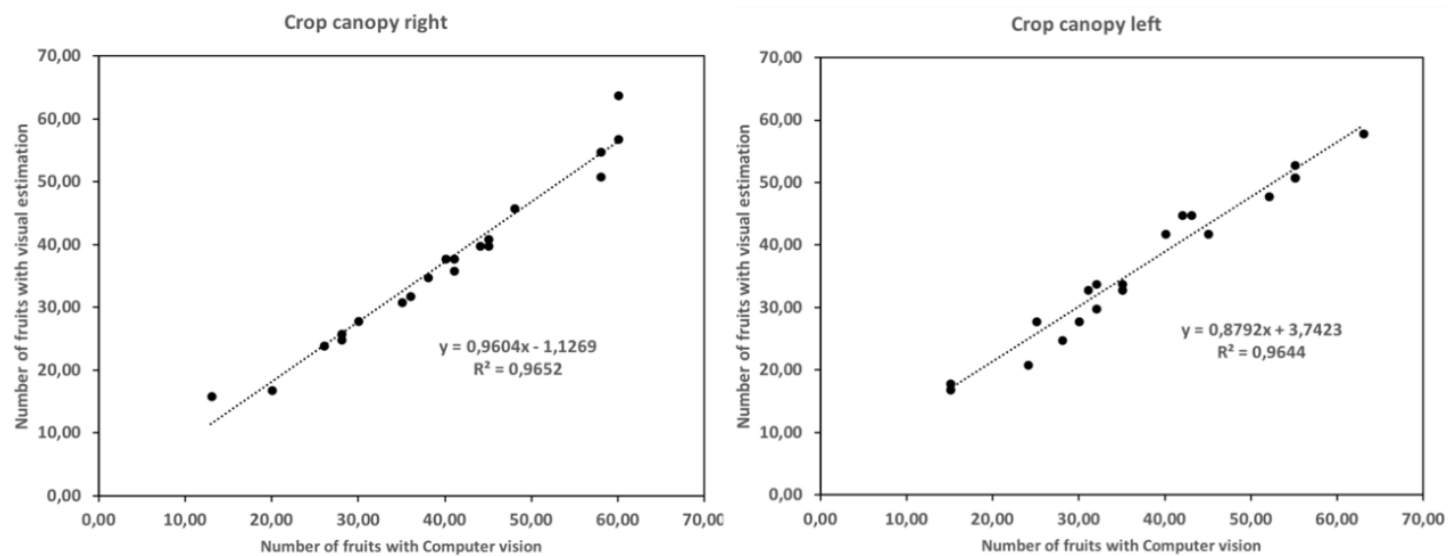

Figure 7. Number of fruits detected in the left and right canopy sides compared with the number of fruits visually counted in each image.

The actual orchard yield was $217,000 \mathrm{~kg}$ and the yield estimated by the professional technician was approximately $200,000 \mathrm{~kg}$. Table 2 shows the number of fruits detected by the model ( Nofcl and Nfrc) and the yield estimated per tree (Yest). The estimated yield can be calculated considering the number of trees in the field $(1,654)$ and the average yield of a tree $(132.96 \mathrm{~kg})$, resulting in an estimated yield of $219,909 \mathrm{~kg}$. Comparing the estimated yields with the actual yield indicates that an error of $1.54 \%$ was made with the model versus the error of $7.83 \%$ made by the professional technician. 
Table 2. Weight per tree harvested, average weight of fruit and number of fruit estimated with the model.

\begin{tabular}{lccccl}
\hline Tree & Yobs $(\mathrm{kg} /$ tree $)$ & Wofh $(\mathrm{kg} /$ fruit $)$ & Nofcl & Nfrc & Yest $(\mathrm{kg} /$ tree $)$ \\
\hline 1 & 187.00 & 0.55 & 45.00 & 42.00 & 154.80 \\
2 & 145.80 & 0.45 & 40.00 & 42.00 & 144.48 \\
3 & 199.10 & 0.45 & 52.00 & 48.00 & 172.00 \\
4 & 120.00 & 0.43 & 32.00 & 34.00 & 123.84 \\
5 & 139.10 & 0.49 & 32.00 & 30.00 & 120.40 \\
6 & 162.80 & 0.27 & 63.00 & 58.00 & 208.12 \\
7 & 122.10 & 0.24 & 55.00 & 53.00 & 197.80 \\
8 & 123.20 & 0.25 & 55.00 & 51.00 & 197.80 \\
9 & 133.50 & 0.45 & 35.00 & 34.00 & 130.72 \\
10 & 139.30 & 0.50 & 31.00 & 33.00 & 123.84 \\
11 & 116.30 & 0.64 & 25.00 & 28.00 & 77.40 \\
12 & 137.40 & 0.49 & 43.00 & 45.00 & 122.12 \\
13 & 126.30 & 0.48 & 35.00 & 33.00 & 108.36 \\
14 & 132.40 & 0.30 & 55.00 & 51.00 & 194.36 \\
15 & 80.60 & 0.29 & 30.00 & 28.00 & 111.80 \\
16 & 112.80 & 0.58 & 24.00 & 21.00 & 86.00 \\
17 & 146.40 & 0.58 & 28.00 & 25.00 & 110.08 \\
18 & 85.70 & 0.72 & 15.00 & 17.00 & 48.16 \\
19 & 145.20 & 0.28 & 42.00 & 45.00 & 149.64 \\
20 & 110.10 & 0.27 & 15.00 & 18.00 & 77.40 \\
Average & 133.26 & 0.43 & 37.60 & 39.70 & 132.96 \\
\hline
\end{tabular}

Yobs: Measured fruit mass per tree; Wofh: Measured mass of individual fruits (average of 10 measured fruits); Nofcl: Estimated number of fruits (left canopy side); Nfrc: Estimated number of fruits (right canopy side); Yest: Estimated tree yield according to the expression Yest $=(\mathrm{Nofcl}+\mathrm{Nfrc}) \cdot 0,43 \cdot 4$.

More accurate estimates of the fruit size and number of fruits can be obtained with images taken at lower altitude since the resolution of each piece of fruit increases. Moreover, the accuracy of the model will increase considerably if the ANN is trained using a dataset with images from a UAV instead of different kinds of images from the Internet.

\section{Conclusions}

The results of this project, both for in terms of the number of fruits and fruit size, were promising. However, one of the primary limitations is the time and capacity of the equipment to train the models.

The fruit size data show that an average fruit diameter can be estimated with relatively high precision; however, this approach is generic in nature since all fruits detected cannot be computed due to some of them being covered by leaves, branches and other elements of the orange tree canopy. Nevertheless, these results allow us to affirm that the model developed is feasible for estimating the fruit size in an orange orchard.

The model yield estimation was closer to the actual yield than the visual estimates made by a professional technician. These results are promising and make us optimistic about the possibility to develop a more accurate model to estimate yields of orange orchards.

Future work involves the integration of the proposed algorithm with a Raspberry Pi with a camera mounted on a UAV to detect the yield of an orange field in real-time. Moreover, a new dataset with more images could be built to achieve more accurate performance.

\section{Acknowledgements}

The authors would like to thank Pablo Agüera, a UAV pilot from Soluciones Agrícolas de Precisión, S.L. (AGROSAP), for help and support during the images acquisition. Finally, the authors want to thank the Predoctoral Research Fellowship for the development of the University of Seville R\&D\&I program (IV.3 2017).

\section{References}

Aggelopoulou, A.D.; Bochtis, D.; Fountas, S.; Swain, K.C.; Gemtos, T.A.; Nanos, G.D., 2012. Yield prediction napple orchards based on image processing. Precis. Agric. 12, 448-456. https://doi.org/10.1007/s11119-0109187-0.

Arefi, A.; Motlagh, A., 2013. Development of an expert system based on wavelet transform and artificial neural networks for the ripe tomato harvesting robot. Aust. J. Crop Sci. 7(5), 699-705.

Collet, F., 2017. Deep Learning with Python. Manning Publications. 384 p. 
González, F., Mcfadyen, A., Puig, E., 2018. Advances in Unmanned Aerial Systems and Payload Technologies for Precision Agriculture. Boca Raton: CRC Press. 488 p.

Kurtulmus, F.; Lee, W.S.; Vardar, A. Green citrus detection using "eigenfruit”, color and circular Gabor texture features under natural outdoor conditions. Comput. Electron. 78 (2), 140-149. https://doi.org/10.1016/j.compag.2011.07.001.

Hannan, M.W.; Burks, T.F.; Bulanon, D.M., 2009. A Machine Vision Algorithm Combining Adaptive Segmentation and Shape Analysis for Orange Fruit Detection. Agric. Eng Int. CIGR J., XI, 1281.

Haralick, R.M.; Shanmugam, K.; Dinstein, I. Textural Features for Image Classification. IEEE Trans. Syst. Man. Cybern. 1973, 3, 610-621. http://dx.doi.org/10.1109/TSMC.1973.4309314.

Mayer, S., Le, H. V., Henze, N., 2017. Machine learning for intelligent mobile user interfaces using TensorFlow. MobileHCI '17 Proceedings of the 19th International Conference on Human-Computer Interaction with Mobile Devices and Services

Article No. 62. http://dx.doi.org/10.1145/3098279.3119915.

Murata, T., Onuki, Y., Nukui, S., Inagi, S., Qiu, X., Watanabe, M., Okamoto, H., 2017. Predicting Relations Between RDF Entities by Deep Neural Network. The Semantic Web: ESWC 2017 Satellite Events. https://doi.org/10.1007/978-3-319-70407-4_43.

Pérez-Ruiz, M., Martinez Guanter, J., Appolo-Apolo, O. E., 2017. Estimación de la producción de fruta en cítricos a través de tecnologías basadas en drones y visión artificial. Comunicación en congreso. IX Congreso Ibérico de AgroIngeniería 2017. Bragança (Portugal). 2017.

Ruiz-Ruiz, G.; Gómez-Gil, J.; Navas-Gracia, L.M., 2009. Testing different color spaces based on hue for the environmentally adaptive segmentation algorithm (EASA). Comput. Electron. Agric.68 (1), 88-96. https://doi.org/10.1016/j.compag.2009.04.009.

Slaughter, D. C., Harrell, R., 1987. Color vision in robotic fruit harvesting. Transactions of the ASAE 30(4): 1144-1148. http://dx.doi.org/10.13031/2013.30534.

Stajnko, D.; Čmelik, Z., 2005. Modelling of Apple Fruit Growth by Application of Image Analysis. Agric. Conspec. Sci. 70, 59-64.

Rosebrock, A., 2016. Practical Python and OpenCV+Case Studies (Starter Bundle). Pyimagesearch.com.

Rosebrock, A., 2017. Deep Learning for Computer Vision with Python, Practitioner Bundle, 1st Edition (1.2.2), Pyimagesearch.com.

Sonka, M., Hlavac, V., Boyle, R., 2014. Image Processing, Analysis, and Machine Vision. Springer US. https://doi.org/10.1007/978-1-4899-3216-7.

Teixidó, M., Font, D., Pallejà, T., Tresanchez, M., Nogués, M., Palacín, J., 2012. Definition of linear color models in the RGB vector color space to detect red peaches in orchard images taken under natural illumination. Sensors, 12, 7701-7718. https://doi.org/10.3390/s120607701.

Triboni, H. de R., Barbosa, J. C., 2011. Estimativa do número de frutos por amostragem de parte da copa em laranjeiras. Rev. Bras. Frutic. 26 (3), 454-458. http://dx.doi.org/10.1590/S0100-29452004000300020.

Wang, Q.; Nuske, S.; Bergerman, M.; Singh, S., 2009 Automated Crop Yield Estimation for Apple Orchards. Proceedings of the 13th International Symposium on Experimental Robotics (ISER 2012), Quebec, QC, Canada, 18-21 June 2012; pp. 745-758. https://doi.org/10.1007/978-3-319-00065-7_50.

Whittaker, A. D., Miles, G. E., Mitchell, O. R., Gaultney, L. D., 1987. Fruit location in a partially occluded image. Transactions of the ASAE 30(3): 591-597. http://dx.doi.org/10.13031/2013.30444.

Whitney, J. D., Wheaton, T. A., Miller, W. M., Salyani, M., 1998. Site-specific yield mapping for Florida citrus. Proc. Fla. State Hortic. Soc. 111: 148-150. http://dx.doi.org/10.13031/2013.5451.

Whitney, J. D., Ling, Q.,Wheaton, T. A., Miller, W. M., 1999. A DGPS yield monitoring system for Florida citrus. Applied Engineering in Agriculture 17(2): 115-119. http://dx.doi.org/10.13031/2013.5451.

Whitney, J. D., Miller, W. M., Wheaton, T. A., Salyani, M. et al., (1999) Precision farming applications in Florida citrus. 17(2):115-119.http://dx.doi.org/10.13031/2013.5795. 


\title{
Data Acquisition Platform for Collecting High-Quality Images of Cultivated Weeds
}

\author{
Simon Leminen Madsen a,*, Mads Dyrmann a, Morten Stigaard Laursen ${ }^{\text {a }}$, \\ Solvejg Kopp Mathiassen ${ }^{\text {b }}$, Rasmus Nyholm Jørgensen ${ }^{\text {a }}$ \\ ${ }^{a}$ Department of Engineering, Aarhus University, Aarhus, Denmark \\ ${ }^{b}$ Department of Agroecology, Aarhus University, Slagelse, Denmark \\ * Corresponding author. Email: slm@eng.au.dk
}

\begin{abstract}
In modern agriculture, there is an increasing focus on a reduction in herbicide usage. As a result, machine learning algorithms are applied in several weed detection and classification applications. To make robust detection and classification, training data for such algorithms must include a wide variety of weed species and present diversity in the appearance of these. Ideally, training data should be collected in real-world fields to reflect reality most accurately. However, acquiring high quantities of in-situ images of all weed species plus annotation of these is time-consuming and prone to human errors. This paper presents a data acquisition platform providing a reliable and efficient way of collecting high-quality images of weeds, cultivated in a controlled environment. Each of the collected images covers approximately $0.28 \mathrm{~m}^{2}$ and have a ground resolution of $\sim 6.6$ pixels $/ \mathrm{mm}$. The controlled environment makes additional annotation unnecessary, thereby reducing the risk of errors. Diversity in plant appearance has been achieved by applying different growth conditions. The platform has been used to acquire images each day of 39 weed species, divided over two growth seasons. The weeds are cultivated under three different growth conditions, from emergence to the 6 to 8-leaf stage.
\end{abstract}

Keywords: Weed Management, Automated Data Collection, Proximal Sensing, Precision Agriculture, Machine Learning

\section{Introduction}

In modern agriculture, there is an increasing focus on reducing the amount of herbicides applied in the field to combat weed growth. A reduction in the amount of applied herbicides is motivated by both environmental and economic incentives (Been et al., 2010; Christensen et al., 2009, 2003; Gerhards, 2013; Jørgensen et al., 2007; Petit et al., 2015; Timmermann et al., 2003). One strategy for achieving the desired reduction is to target the weed control to the weeds present in the field. The targeted weed management involves identification of which herbicide to use and where to apply it to achieve the best treatment. However, this require that we have some knowledge of which weed species are present in the field and ideally where these are. In recent years several promising weed detection and classification algorithms have been developed (Dyrmann, 2017; Dyrmann et al., 2017; Lottes et al., 2016; Milioto et al., 2017). In order to train these kinds of data-driven algorithms, large datasets are needed. These datasets should ideally contain examples of several different weed species and include a large intraspecies variety with respect to visual appearance to make the classification algorithms as robust as possible.

Today different weed datasets exist, of which some are publicly available. These include the images used by (Søgaard, 2005) to classify shepherd's purse (Capsella bursa-pastoris), Scentless mayweed (Tripleur-ospermum inodorum) and Charlock (Sinapis arvensis). Even though the dataset consists of 34 different species, the variation within each species is limited, as there on average is less than 12 samples per species of which most are at the same growth stage. Another weed dataset is provided by (Chebrolu et al., 2017), who have published annotated RGB-Depth, GPS and lidar data from sugar-beet fields. This dataset is best suited for non-chemical weed control and not for monitoring weed populations, since the weed species are not known for each individual plant. The Plant Seedlings Dataset by (Giselsson et al., 2017) is acquired in greenhouses under controlled conditions and consists of images of $\sim 960$ plants of 12 different species. The dataset tracks the development of the plants from germination and beyond the 8 leaf-stage. Therefore, some of the plants are larger than what is expected to be observed in-field, since weed control must be applied earlier to be effective. The Plant Seedlings Dataset cover some intraspecies variation, due to the temporal tracking, but the dataset only contains a limited number of species and only applies one growth condition, which is known to greatly affect the visual appearance of plants.

Real world data acquisition is preferable since the weeds occur in the natural environment. However, after annotating more than 30000 plants from more than 200 different fields, we have seen that the variety of some common species is still not covered. Furthermore, the annotation of weeds is time-consuming and is prone to errors: A test using high-resolution images has shown that when two consultants are to annotate the same weed seedlings, they disagree on the species for about $12 \%$ of the samples (Dyrmann and Midtiby, 2016). Some weed species are difficult to distinguish, especially at early development stages, and the annotators might fail in recognizing them as different species. This issue could be due to the annotators having a bias towards a certain species they have observed earlier in their analysis or simply because they do not know the correct species and label it as the closest match they do recognize. The issue of species bias could be problematic if for instance an invasive species, which requires special treatment, is incorrectly annotated. Real world data acquisition have 
previously been conducted by manually collecting images in fields using hand-held cameras or mobile phones, but other data collection systems also utilize motorized vehicles or UAVs (Berge et al., 2012; Laursen et al., 2017; Leminen Madsen et al., 2017; Peña et al., 2013; Peteinatos et al., 2014; Torres-Sánchez et al., 2013)

Alternatively, it is possible to collect data in a controlled environment, where the specific weed species are being cultivated under controlled conditions. The annotation in such a setup comes for free, due to the deterministic nature of such experiments. Furthermore, it also enables tracking of the plants over time to observe, how different species develop from seedlings to full grown plants, which helps covering most of the variation within each species. Finally, it is possible to induce different growth conditions for the plants, such as different combinations of soil texture, moisture and nutrition, to observe the effects on the appearance.

At early growth stages, different plant species often look alike as the plants have not yet developed their true leaves, which carry most of the 'visual identity' of these. Well-illuminated images with high resolution are therefore essential to ensure that plants are easy to recognize in the images. This paper presents a data acquisition platform for collecting such high-quality images of weeds, cultivated in a controlled environment. The designed platform provides a consistent and reliable way of collecting high-quality images to be applied in further research. The platform is designed to be easy to use and require minimum technical skills and operator interaction to collect data when set up.

The data collected using this platform is expected to be used in future research for improving weed detection and classification algorithms.

\section{Materials and Methods}

The data acquisition platform was established at the semifield area at Aarhus University, Research Center Flakkebjerg. The semi-field area is an outdoor area equipped with tables designed for pot experiments. The experiment tables are constructed with a portable shield automatically covering the weed plants in case of rain or heavy wind thereby protecting them from adverse weather conditions. The tables are automatically watered by flooding from a pre-set program. The weed plants are sown in polystyrene growth boxes $(40 \times 40 \times 12 \mathrm{~cm})$ allowing watering from underneath.

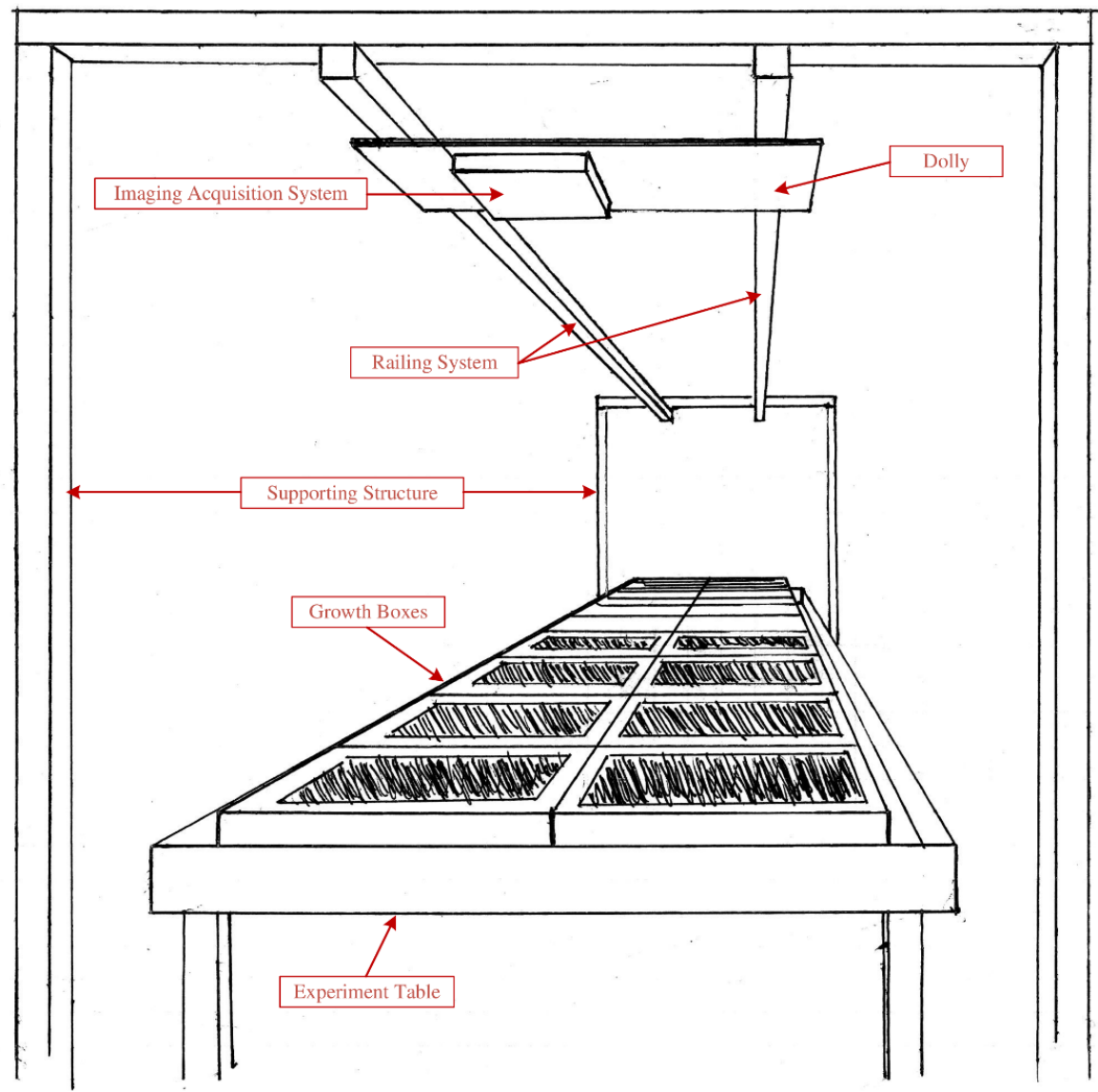

Figure 59: Sketch of the proposed data acquisition platform seen from the end of the experiment table. 
To mount a camera in a suitable height a base construction was built. The base construction consists of a set of wooden frames placed at even intervals around the experiment tables, with a railing system in the top. On the rails a camera dolly is mounted, in which an imaging acquisition system can be mounted. The railing system and the dolly allows for a smooth movement of the imaging acquisition system along the length of the experiment tables, in order to take pictures of all growth boxes. The railing system covers approximately 16 meters in length divided between two experiment tables, which gives the system a maximum capacity of 70 growth boxes per trial.

Figure 59 shows a sketch of the experimental setup, including experiment table, growth boxes, supporting structure, railing system, dolly and imaging acquisition system. The figure visualizes the expected relative placements of the different platform components. Figure 60 shows images of the final implementation of the data acquisition platform. Figure 60a shows a close-up of the imaging acquisition system mounted on one of the setup's two rail dollies. Figure $60 \mathrm{~b}$ shows an overview of the entire setup.

\section{Imaging acquisition system}

The imaging acquisition system consists of a Point Grey GS3-U3-123S6C camera (BC, Canada) mounted with a Schneider Kreuznauch Xenon-TOPAZ XN 2,0/38-0901 38mm lens (Bad Kreuznach, Germany). Scene illumination is provided by a Paul C. Buff. AlienBee ABR800 Xenon ring flash (Tennessee, America). The camera assembly is mounted in the centre of the ring flash, using a custom 3D printed mount, and the diffuser and reflector is both mounted on the ring flash. The flash is triggered through the camera $\mathrm{I} / \mathrm{O}$. The ISO/Gain of the camera is set to unity gain / base ISO and the shutter speed set to the lowest value providing an acceptable exposure. The aperture is set based on a circle of confusion of $7 \mu \mathrm{m}$, a distance-to-ground of $1.66 \mathrm{~m}$ and a desired depth of field of $8 \mathrm{~cm}$, yielding $\mathrm{F} / 4$. The height of $1.66 \mathrm{~m}$ yields a field of view of approximately $62 \times 45 \mathrm{~cm}$ and provides a ground resolution of $\sim 6.6$ pixels $/ \mathrm{mm}$.

The imaging system is controlled by an NVIDIA TX2 (California, America) equipped with a 500GB SSD. The system is equipped with a lidar-based distance sensor, Lightware SF11/C (Olifantsfontein, South Africa), which measures the absolute position along the track on which the platform is mounted. Image acquisition is controlled based on the measured distance, so when the dolly is moved in a direction away from the start position, images are recorded for every $\sim 0.15$ meters. To eliminate too much redundancy in the data, the system is only triggered when moving in one direction and not when moved back to the start position. The embedded computer runs ROS (Quigley et al., 2009) and is launched at boot, this allows for a simple workflow in which the operator only has to power up the system, wait for it to boot, drag along the dolly, which automatically causes images to be recorded, return to the start position and power down the system. Powering on and off the system is done with a single switch wired directly to the soft power button, a high intensity led has then been added to clearly show the power state even under outside directly lit conditions. In order to maintain a backup and allow for timely inspection of the images during the trial, images are synchronized to the central university storage system through the university wifi on which the embedded computer is connected at the trial site.

The imaging acquisition system is similar to that used for in-field data collection with minor alterations (Laursen et al., 2017). This provides a good basis for comparing images collected in a controlled vs. a real environment.

\section{Sowing Process and Growth Conditions}

The seeds used for the trials were supplied from the seed bank at Research Center Flakkebjerg. Seeds of Fallopia convolvulus, Polygonum lapathifolia, Plantago major, and Cirsium arvense were buried in wet sand at a temperature of $5{ }^{\circ} \mathrm{C}$ for 3 weeks before sowing to improve germination. The seeds were placed on the soil surface (approximately 50 to 100 seeds per box depending on species) and covered by $1 \mathrm{~cm}$ of the growth medium. The boxes were watered by a light shower from the top to the soil capacity before being moved to the experiment tables.

Each weed species was applied three unique growth conditions with respect to growth medium, amount of watering, and fertilizers. By applying different growth conditions, we increase the plant diversity with respect to the visual appearance in the collected images. The different growth conditions are summarized in Table 22. The

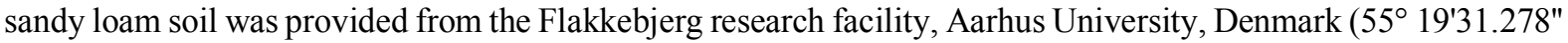

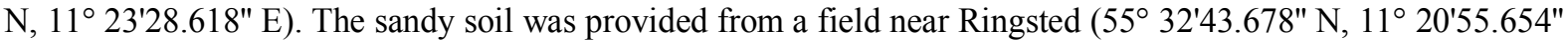
E). The potting mixture was composed of the sandy loam soil, sand, and peat $(2: 1: 1$ by weight) to give optimum growth conditions for the weeds. Growth condition G1 and G3 were watered regularly three times daily, whereas G2 was not watered after establishment. After emergence, the seedlings were thinned as required to avoid plant leaves covering each other. Each growth box is marked with a unique barcode, to identify the individual boxes and thereby, the individual species and growth condition applied to each box. 
Table 22. Summary of applied growth conditions.

\begin{tabular}{cccc}
\hline ID & Growth medium & Watering level & Fertilizer Level \\
\hline G1 & Potting mixture & Optimum & Optimum \\
G2 & Sandy loam soil & Sub-optimum & Optimum \\
G3 & Sandy soil & Optimum & Low \\
\hline
\end{tabular}

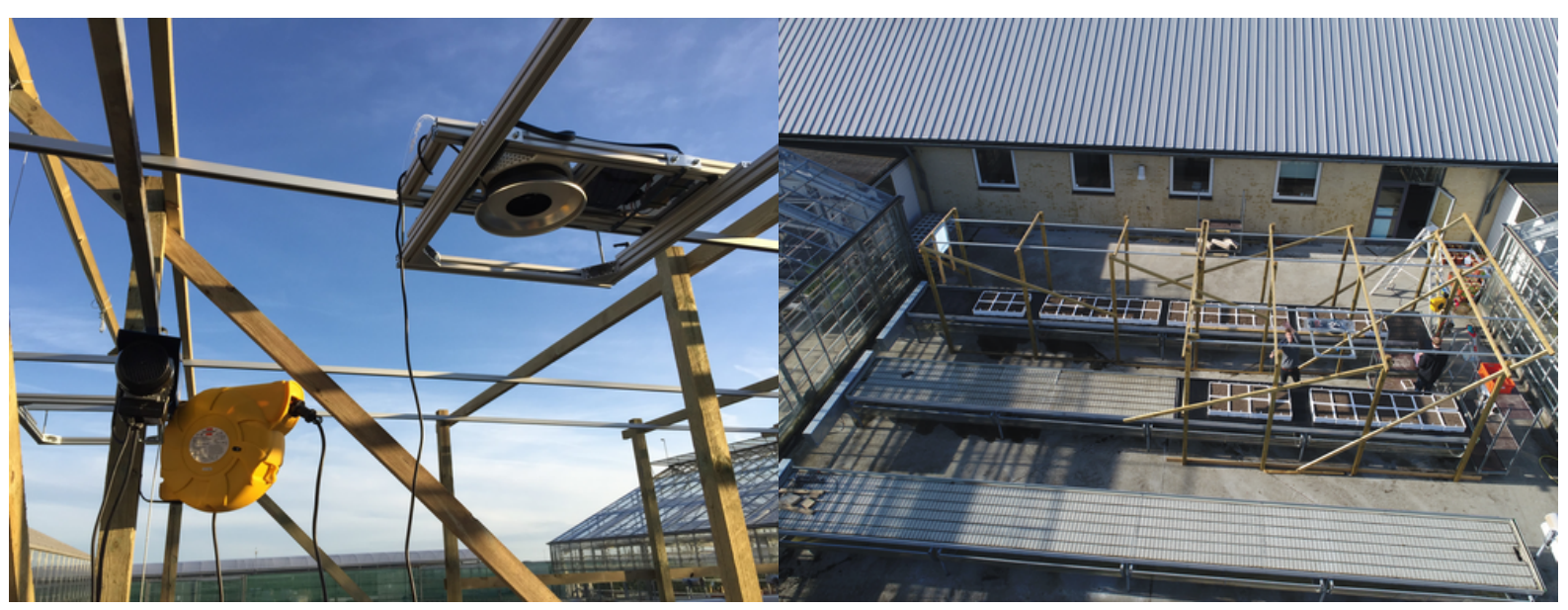

(a)

(b)

Figure 60: Final implementation of the data acquisition platform. (a) The imaging acquisition system mounted on one of the rail dollies. (b) Overview photo of the entire data acquisition setup with supporting structure and railing system. 
Table 23. List of weed species. (a) Spring growth season 2017. (b) Autumn growth season 2017

(a)

\begin{tabular}{llll}
\hline$I D$ & Name & Latin Name & EPPO Code \\
\hline 1 & Scented mayweed & Matricaria chamomilla & MATCH \\
2 & Field forget-me-not & Myosotis arvensis & MYOAR \\
3 & Common chickweed & Stellaria media & STEME \\
4 & Common lambsquarter & Chenopodium album & CHEAL \\
5 & Shepherd's purse & Capsella bursa-pastoris & CAPBP \\
6 & Scentless mayweed & Tripleurospermum inodorum & MATIN \\
7 & Cornflower & Cyanus segetum & CENCY \\
8 & Night-flowering catchfly & Silene noctiflora & MELNO \\
9 & Common nightshade & Solanum nigrum & SOLNI \\
10 & Burning nettle & Urtica urens & URTUR \\
11 & Black knotweed & Fallopia convolvulus & POLCO \\
12 & Knotted persicaria & Persicaria lapathifolia & POLLA \\
13 & Oilseed rape & Brassica napus & BRSNN \\
14 & Charlock & Sinapis arvensis & SINAR \\
15 & Field pansy & Viola arvensis & VIOAR \\
16 & Dove's-foot cranesbill & Geranium molle & GERMO \\
17 & Common poppy & Papaver rhoeas & PAPRH \\
18 & Common field speedwell & Veronica persica & VERPE \\
19 & Common field speedwell & Veronica persica & VERPE \\
$20^{* *}$ & Greater plantain & Plantago major & PLAMA \\
$21 * *$ & Red dead-nettle & Lamium purpureum & LAMPU \\
$22 * *$ & Canada thistle & Cirsium arvense & CIRAR \\
& $*$ Duplicate & &
\end{tabular}

** Did not germinate

(b)

\begin{tabular}{|c|c|c|c|}
\hline$I D$ & Name & Latin Name & EPPO Code \\
\hline 1 & Cleavers & Galium aparine & GALAP \\
\hline 2 & Eastern groundsel & Senecio vernalis & SENVE \\
\hline $3 * *$ & Common groundsel & Senecio vulgaris & SENVU \\
\hline 4 & Common mugwort & Artemisia vulgaris & ARTVU \\
\hline 5 & Red chickweed & Anagallis arvensis & ANGAR \\
\hline 6 & Common storkbill & Erodium cicutarium & EROCI \\
\hline 7 & Common hairy tare & Vicia hirsuta & VICHI \\
\hline 8 & Annual sowthistle & Sonchus oleraceus & SONOL \\
\hline 9 & Petty spurge & Euphorbia peplus & EPHPE \\
\hline 10 & Nipplewort & Lapsana communis & LAPCO \\
\hline $11 * * *$ & Corn speedwell & Veronica arvensis & VERAR \\
\hline 12 & Curled dock & Rumex crispus & RUMCR \\
\hline 13 & Annual meadowgrass & Poa annua & POAAN \\
\hline 14 & Loose silky bent & Apera spica-venti & APESV \\
\hline 15 & Italian ryegrass & Lolium multiflorum & LOLMU \\
\hline 16 & Blackgrass & Alopecurus myosuroides & ALOMY \\
\hline 17 & Barren brome & Anisantha sterilis & BROST \\
\hline 18 & Common wild oat & Avena fatua & AVEFA \\
\hline 19 & Narrow-leaved plantain & Plantago lanceolata & PLALA \\
\hline 20 & Canada thistle & Cirsium arvense & CIRAR \\
\hline $21 *$ & Corn speedwell & Veronica arvensis & VERAR \\
\hline \multirow[t]{2}{*}{22} & Redshank & Persicaria maculosa & POLPE \\
\hline & $\begin{array}{l}* \text { Duplicate } \\
* * \text { Did not germinate } \\
* * * \text { Sown later, since ori }\end{array}$ & d not germinate & \\
\hline
\end{tabular}




\section{Results}

The setup has been used throughout two growth seasons: a spring season and an autumn season. In spring the weeds were sown on May 5, 2017 and were tracked in the period from May 15, 2017, to June 20, 2017 equal to 36 days, whereas in autumn the weeds were sown on August 31, 2017, and were tracked in the period from September 4, 2017 to November 3, 2017 equal to 60 days. Due to better weather conditions throughout the spring compared to the autumn, the weeds developed faster, and images were recorded twice a day (once a day on weekends) during this period. In the autumn season images were recorded once a day. The recordings were performed at varying times of the days. Table 23 provides a summary of the cultivated weed species for the spring and autumn growth seasons 2017.

Table 24 shows examples on some of the collected images. The images show how the appearance of the weed species scentless mayweed (Tripleurospermum inodorum) change over time and under different growth conditions. The appearance of the plants changes greatly as an effect of time and the different growth conditions, which induces high intraspecies diversity into the collected data, which is desired. The soil appears darker in some images, which is a result of the watering process. Figure 61 shows a few examples of the image quality.

It should be mentioned that not all weed species were recorded throughout the entire duration of the experiment. The system was slower than expected in writing data to the storage, resulting in some data being lost when the system was powered off before the data buffer was empty. This issue was easily corrected when discovered, by giving the system enough time to save the images to the storage before powering off in between each trial.

Table 24. Development of the weed scentless mayweed, Tripleurospermum inodorum (MATIN), in the three different growth conditions. The weeds in the images were sown on May 5, 2017.

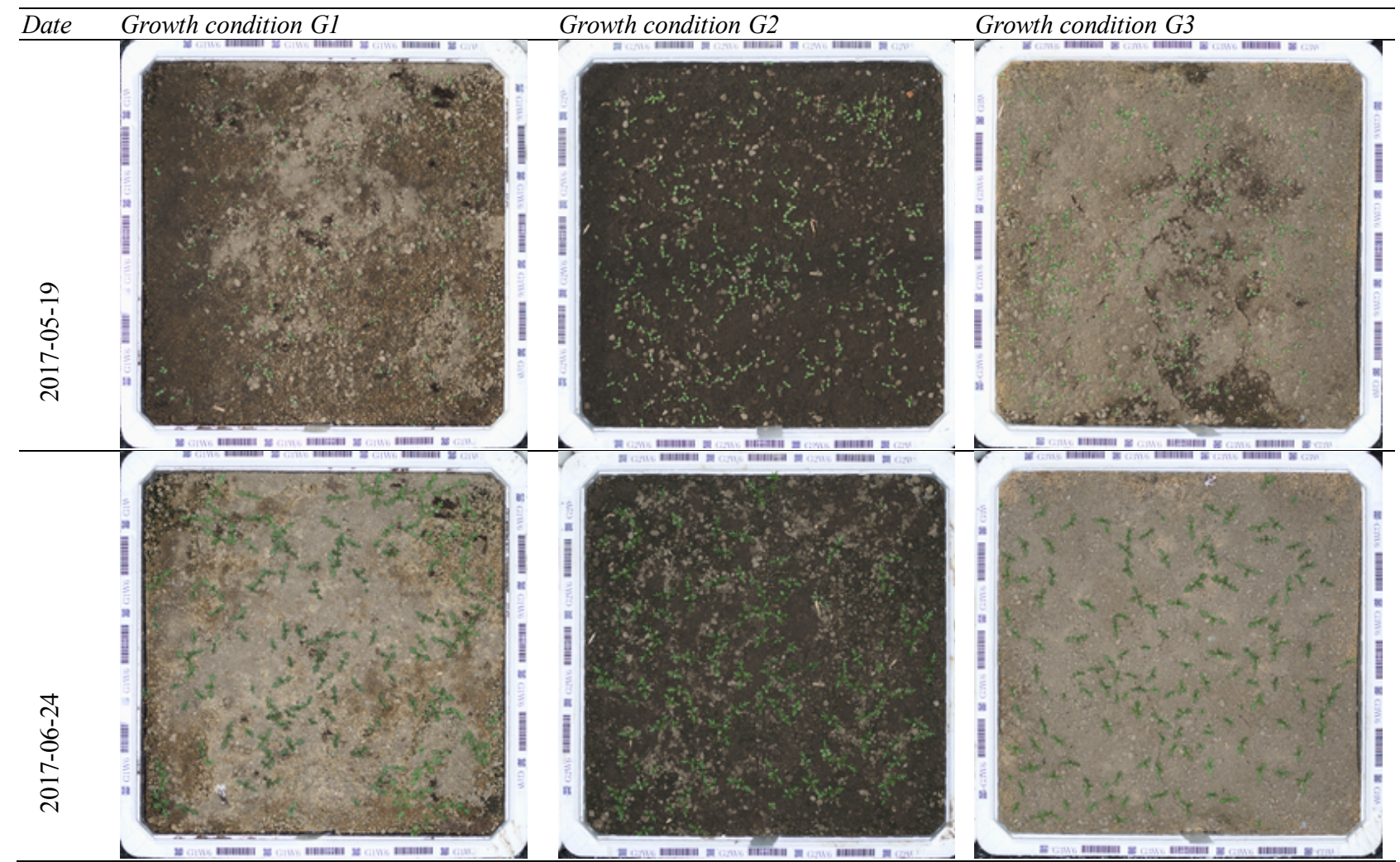

Continued on next page ... 
Table 24.Ccontinued from previous page.

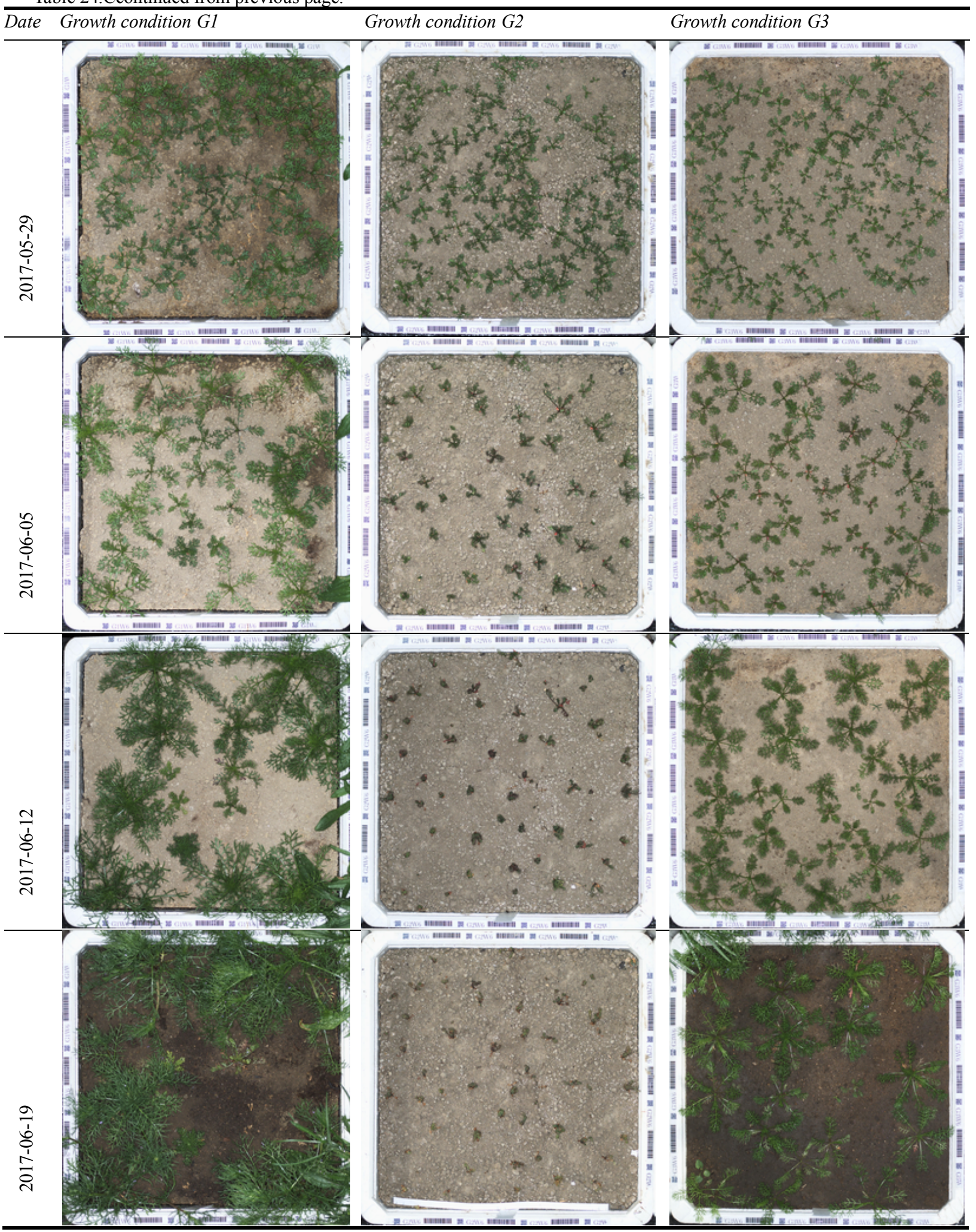






Figure 61. One of the captured images with cropped samples. Image show the weed scentless mayweed, Tripleurospermum inodorum (MATIN) with growth condition G1. The image is taken on 24 May, 2017. 


\section{Discussion \& Conclusions}

The developed platform provides a reliable and efficient way of collecting high-quality images of weeds. The images are easily annotated, due to the weeds being cultivated in a controlled environment. Annotation only requires identification of the individual growth boxes, which can easily be achieved through manual annotation or automated software. The controlled environment also allows for applying multiple combinations of growth conditions, which greatly affects the appearance of the cultivated weeds and thereby increase the intraspecies diversity. The drawback of the weeds being cultivated in a controlled environment might be that the settings are too perfect and not reflecting a natural environment sufficiently. Additionally, the controlled environment only has limited space for the plants to grow, due to the size of the growth boxes, which results in an unnatural background for the plants close to the edges, especially as the weeds becomes large.

The images collected with this data acquisition platform have a ground resolution of 6.6 pixels $/ \mathrm{mm}$, which should be sufficient for training and for evaluating weed detection and classification algorithms. The ground resolution is slightly lower compared to datasets collected using high-end consumer cameras e.g. (Giselsson et al., 2017), but this system has a significantly higher throughput in the amount of collected images versus the amount of work required for collecting images. The collected images still have a higher ground resolution compared to those collected in a real-world setup (Chebrolu et al., 2017; Laursen et al., 2017). Furthermore, the integrated ring flash setup ensures there are plenty of light and no hard shadows even in strong sunlight. However, it is observed that when the weed plants become large, the images become slightly blurred, due to the limited focal depth. This observation is not expected to have any influence on the expected application of the collected data since ideally the weeds need to be identified and treated well before they develop into the 6-8 leaf stage. When using the setup in the future, the focal depth will be increased by closing the aperture and the flash-output will be increased to compensate.

The platform is designed to be easy to use and requires a minimum of user interaction, due to the single switch design. However, it would have been beneficial to provide some sort of visual feedback to the experiment technicians to avoid issues, such as loss of data due to untimely power off of the system. The issue of loss of data due to untimely power off, was solved by simply letting the system be powered on for longer after each trial.

The collected images using this platform are expected to be used for boosting existing weed detection, segmentation and classification algorithms. With this data acquisition platform, it is possible to generate large amounts of annotated training data, that can be used for training modern machine learning algorithms. An issue with existing weed detection and classification systems is that they perform poorly on some weed species if they are rare and their diversities are not covered in the existing training sets. With this platform, it is possible to reduce this issue by simply cultivating samples of these rare species. Furthermore, the platform enables capturing the temporal development of the plant and it might be possible to include this information in the training of the algorithms to boost the performance on detecting the weeds at all growth stages.

\section{Acknowledgements}

This work is founded by Innovation Foundation Denmark as part of the RoboWeedMaPS project (J.nr. 615000027B)

\section{References}

Been, T.H., B. Consiglio, A., Evans, N., Gouache, D., Gutsche, V., E. Jensen, J., Kapsa, J., Levay, N., MunierJolain, N., Nibouche, S., Raynal, M., Rydahl, P., 2010. Review of new technologies critical to effective implementation of decision support systems and farm management systems (DSS's) and Farm Management Systems (FMS's. Crit. Policy Stud.

Berge, T.W., Goldberg, S., Kaspersen, K., Netland, J., 2012. Towards machine vision based site-specific weed management in cereals. Comput. Electron. Agric. 81, 79-86. https://doi.org/10.1016/J.COMPAG.2011.11.004

Chebrolu, N., Lottes, P., Schaefer, A., Winterhalter, W., Burgard, W., Stachniss, C., 2017. Agricultural robot dataset for plant classification, localization and mapping on sugar beet fields. Int. J. Rob. Res. https://doi.org/10.1177/0278364917720510

Christensen, S., Heisel, T., Walter, A.M., Graglia, E., 2003. A decision algorithm for patch spraying. Weed Res. 43, 276-284. https://doi.org/10.1046/j.1365-3180.2003.00344.x

Christensen, S., Søgaard, H.T., Kudsk, P., Nørremark, M., Lund, I., Nadimi, E.S., Jørgensen, R., 2009. Sitespecific weed control technologies. Weed Res. 49, 233-241.

Dyrmann, M., 2017. Automatic Detection and Classification of Weed Seedlings under Natural Light Conditions.

Dyrmann, M., Jørgensen, R.N., Midtiby, H.S., 2017. RoboWeedSupport - Detection of weed locations in leaf occluded cereal crops using a fully convolutional neural network. Adv. Anim. Biosci. 
https://doi.org/10.1017/S2040470017000206

Dyrmann, M., Midtiby, H.S., 2016. Evaluation of intra variability between annotators of weed species in color images, in: CIGR2016 AgEng Conference.

Gerhards, R., 2013. Site-Specific Weed Control, in: Heege, H.J. (Ed.), Precision in Crop Farming: Site Specific Concepts and Sensing Methods: Applications and Results. Springer Netherlands, Dordrecht, pp. $273-294$. https://doi.org/10.1007/978-94-007-6760-7_10

Giselsson, T.M., Jørgensen, R.N., Jensen, P.K., Dyrmann, M., Midtiby, H.S., 2017. A Public Image Database for Benchmark of Plant Seedling Classification Algorithms.

Jørgensen, L.N., Noe, E., Langvad, A.-M., Jensen, J.E., Ørum, J.E., Pinnschmidt, H., Qvist Bøjer, O., 2007. Vurdering af Plantevaern Onlines økonomiske og miljømaessige effekt. Bekæmpelsesmiddelforskning fra Miljøstyrelsen 115.

Laursen, M.S., Jørgensen, R.N., Dyrmann, M., Poulsen, R.N., 2017. RoboWeedSupport - Sub millimeter weed image acquisition in cereal crops with speeds up till $50 \mathrm{~km} / \mathrm{h}$. ICPA 2017 - 19th Int. Conf. Precis. Agric.

Leminen Madsen, S., Dyrmann, M., Laursen, M.S., Jørgensen, R.N., 2017. RoboWeedSupport - SemiAutomated Unmanned Aerial System for Cost Efficient High Resolution in Sub-Millimeter Scale Acquisition of Weed Images, in: ICPA 2017 - 19th International Conference on Precision Agriculture.

Lottes, P., Hoeferlin, M., Sander, S., Müter, M., Schulze, P., Stachniss, L.C., 2016. An effective classification system for separating sugar beets and weeds for precision farming applications, in: 2016 IEEE International Conference on Robotics and Automation (ICRA). pp. 5157-5163. https://doi.org/10.1109/ICRA.2016.7487720

Milioto, A., Lottes, P., Stachniss, C., 2017. REAL-TIME BLOB-WISE SUGAR BEETS VS WEEDS CLASSIFICATION for MONITORING FIELDS USING CONVOLUTIONAL NEURAL NETWORKS, in: ISPRS Annals of the Photogrammetry, Remote Sensing and Spatial Information Sciences. https://doi.org/10.5194/isprs-annals-IV-2-W3-41-2017

Peña, J.M., Torres-Sánchez, J., de Castro, A.I., Kelly, M., López-Granados, F., 2013. Weed Mapping in EarlySeason Maize Fields Using Object-Based Analysis of Unmanned Aerial Vehicle (UAV) Images. PLoS One 8, e77151. https://doi.org/10.1371/journal.pone.0077151

Peteinatos, G.G., Weis, M., Andújar, D., Rueda Ayala, V., Gerhards, R., 2014. Potential use of ground-based sensor technologies for weed detection. Pest Manag. Sci. https://doi.org/10.1002/ps.3677

Petit, S., Munier-Jolain, N., Bretagnolle, V., Bockstaller, C., Gaba, S., Cordeau, S., Lechenet, M., Mézière, D., Colbach, N., 2015. Ecological Intensification Through Pesticide Reduction: Weed Control, Weed Biodiversity and Sustainability in Arable Farming. Environ. Manage. 56, 1078-1090. https://doi.org/10.1007/s00267-0150554-5

Quigley, M., Conley, K., P Gerkey, B., Faust, J., Foote, T., Leibs, J., Wheeler, R., Y Ng, A., 2009. ROS: an open-source Robot Operating System, ICRA Workshop on Open Source Software.

Søgaard, H.T., 2005. Weed classification by active shape models. Biosyst. Eng. https://doi.org/10.1016/j.biosystemseng.2005.04.011

Timmermann, C., Gerhards, R., Kühbauch, W., 2003. The Economic Impact of Site-Specific Weed Control. Precis. Agric. 4, 249-260. https://doi.org/10.1023/A:1024988022674

Torres-Sánchez, J., López-Granados, F., De Castro, A.I., Peña-Barragán, J.M., 2013. Configuration and Specifications of an Unmanned Aerial Vehicle (UAV) for Early Site Specific Weed Management. PLoS One 8, e58210. https://doi.org/10.1371/journal.pone.0058210 


\author{
A 3D Low-Cost Photogrammetry Method for Weed Plants Modelling \\ Dionisio Andujar a,", Mikel Calle ${ }^{\mathrm{b}}$, César Fernández-Quintanilla ${ }^{\mathrm{b}}$, Ángela Ribeiro ${ }^{\mathrm{a}}$, Jose Dorado ${ }^{\mathrm{b}}$ \\ ${ }^{a}$ Centre for Automation and Robotics, CSIC, Arganda del Rey, Madrid 28500, Spain \\ ${ }^{\mathrm{b}}$ National Museum of Natural Sciences, CSIC, 28006 Madrid, Spain \\ ${ }^{\mathrm{c}}$ Institute of Agricultural Sciences, CSIC, 28006 Madrid, Spain \\ * Corresponding author. Email: d.andujar@csic.es
}

Abstract

New methods for plant phenotyping are crucial nowadays in basic and applied plant research. Plant phenotyping allows gathering information about plant architecture, which is fundamental to improve plant characterization, plant selection, treatment or agronomical management. In this sense, 3D processing algorithms and new devices for plant modeling are continuously arising and they are expanding rapidly to morphologically characterize plants. However, there are still some technical aspects to be improved, such as an accurate reconstruction of the end-details. The proposed study adapts low-cost techniques, i.e. Structure from Motion (SfM) and MultiView Stereo (MVS) for the 3D reconstruction of plant models. Different weeds with differential shape and architecture were selected for modelling. The capabilities and limitations of these methods were compared to accurately extract various parameters related to the morphology of these weeds. Plant reconstruction was made applying SfM to an input set of images covering each individual weed. Two rounds were done during the image acquisition process which guarantees a minimum overlapping between images of $90 \%$. After acquiring the images, the actual height of plants was measured. The images were processed to create a dense point cloud using MVS to obtain a 3D polygon mesh. The created models were compared with ground truth data of plant height and leaf area index. The results showed that each individual plant was reconstructed properly, with only small parts missing. Some errors at the end of the leaves and branch borders occurred and some part of the reconstruction did not show end details, mainly in the monocot weeds. The analysis of estimated and measured plant heights and LAI showed good values in every case.

Keywords: SfM, MVS, 3D model, plant phenotyping.

\title{
1. Introduction
}

Plant modelling can characterize stress from biotic or abiotic factors, biomass production, weed discrimination, fruit characterization, yield, leaf characteristics, root morphology, photosynthetic efficiency, etc. Traditionally these factors have been assessed by experts relying on visual scoring which creates differences between expert opinions and they are quite time-consuming. Thus, the goal of plant phenotyping is usually to measure those plant characteristics that lead to a common decision without different judges. It measures complex shapes which are related to every decision for plant selection, treatment or agronomical management (Walter et al., 2015). However, increasing knowledge and expertise require of technological developments from sensors to platforms. Many of the current sensing techniques are based on two dimensional characteristics such as hyperspectral or thermal imaging which are highly dependent of the angle and distance to the plant to measure. Thus, 3D modelling is a choice that allows assessing the morphological parameters of a plant. The concept is rapidly expanding and new methods are becoming more attractive. The techniques include visible images (Arvidsson et., al 2011), LiDAR (Guo et., al 2017), structured light (Nguyen et al 2015), spectroscopy (Gutierrez et al 2016), thermal images (Ludovisi et., al 2017), etc. The most common technique is based on visible imaging based on sensors sensitive to visible bands of light. They are of low cost and easy to operate. The obtained images under controlled conditions can be related with yield, nutrition stress, vigor, biomass or other related parameters. It use in a period of time can rapidly describe growing dynamics by analyzing the canopy by color selection algorithms able to segment the RGB (Peteinatos et., al 2014). This method is fast and simple. However, it only provides limited information. The challenge of leaf overlapping, leaf number or plant biomass quantification is still an issue that needs of further research. Although, it usability is limited in breeding programs, the use in agronomical task is of high importance in the development of algorithm for pest detection or crop management. Similarly, fluorescence imaging provides information about metabolic status by fluorescent light exposition prior to measure (Fahlgren et., al 2015). It shows effects of stress or diseases due to the metabolic changes occurred in the chloroplast. Thus, this technique shows the photosynthetic activity and it relates with physiological changes. Fluorescence can identify those plants more susceptible or perturbations. This method is usually used for measuring induced stress and measuring the response. Thermal imaging is generally use for water stress measuring (Humplík et., al 2015). It calculates leaf temperature which is related with evapotranspiration. It usage is extended to almost every crop from cereals to trees, showing drought stress though estimation of leaf water content. Also, imaging spectroscopy is used as rapid indicator in phenotyping processes (Gutierrez et al., 2016). Spectral reflectance from leaves quantify some vegetation indexes related to leaf area, biomass or yield.

Although every sensor provides valuable information, new sensors and techniques have arisen. They are able to create 3D models which perfectly characterize plant shape and morphology, extracting several parameters that help in breeding and agronomic programs. Far from a unique system, the caption of the third dimension can be acquired by many ways and principles. In the case of scanners they provide a dimensional line per reading. By 
displacing the sensor the third dimension is then created. Light detection and ranging (LiDAR) is the most used method. They allow scanning at high frequency and at large distances. However, cost is high when resolution increases. Similarly, RGB-D cameras such as Kinect v2 have been widely used for plant characterization in agriculture. Andújar et al. (2016) proposed the use of depth cameras for detection of weed infestation on maize field. They concluded that discrimination is possible with low-cost sensors on the same way of expensive laser scanners, which opens the door for other sensors in some of the plant phenotyping scenarios. The distance from the camera to the plant is calculated based on the disparity or shift of the plant in various images. Also, the algorithm greatly affects the accuracy of the depth model and non-controlled illumination gets worse the quality of the models. Indoors applications have shown their capability for plant reconstruction. Takizaka et., al (2005) reconstructed plants models and extracted information of plant height, leaf area and shape. Ivanov et al. (1995) characterize maize plants in field conditions from several point of view images to describe plant structure from the leaf orientation, leaf area distribution and leaf position. Combination of cameras and images from Structure from motion techniques (SfM) techniques usually produces a sparse set of points in $3 \mathrm{D}$. This method estimates the common position of the cameras and a set of points. Following, using a region growing algorithm, a dense cloud of points is created (Lhuillier et al., 2002). Similarly, Multiview stereo (MVS) is developing rapidly. It allows the creation of dense 3D models through the fusion of images, even on real time (Vogiatzis et., al 2011), with budget hardware.

This work assessed the possibilities of low-cost tools to reconstruct weed plants at treatment time. The study was conducted to assess the use of SfM and MVS techniques to characterized weeds The objectives were to explore method capabilities and limitations to extract weed parameter related with physical plant parameters.

\section{Materials and Methods}

Site Study and Field Measurements

Field measurements were conducted on a maize field located at the experimental farm "La Poveda" (Arganda del Rey, Madrid, Central Spain) during April 2017. Three weed species with contrasting shape and plant structure were considered. The selected species were the most troublesome ones in maize field in mediterranean regions. Monocots and dicots were chosen for the experiment looking for structural differences. The species were: Xanthium strumarium, Sorghum halepense, and Datura ferox. Weed assessments were made during May when maize was at stage 12 to 14 of BBCH scale (Lancashire, et al 1991) and weeds ranged from $\mathrm{BBCH} 10$ to $\mathrm{BBCH}$ 20. At sampling time, weed heights ranged from $4 \mathrm{~cm}$ to $30 \mathrm{~cm}$. The samples were chosen looking for different weed stages. A total of ten samples per specie were selected. Weeds were selected randomly within the field looking for a representative sample of the population within $95 \%$ confidence interval of mean plant height. The adjacent weeds were removed avoiding the interference into the model with the studied plant. The ground disturbance was also considered in order to keep the angle of stem and leaves. Then three $10 \mathrm{~cm}$ graphic scales were located on the ground in triangular shape around the plant, being the desired plant in the center of the triangle. The graphic scales help while model creation and rescaling the model to real measurements in post-processing.
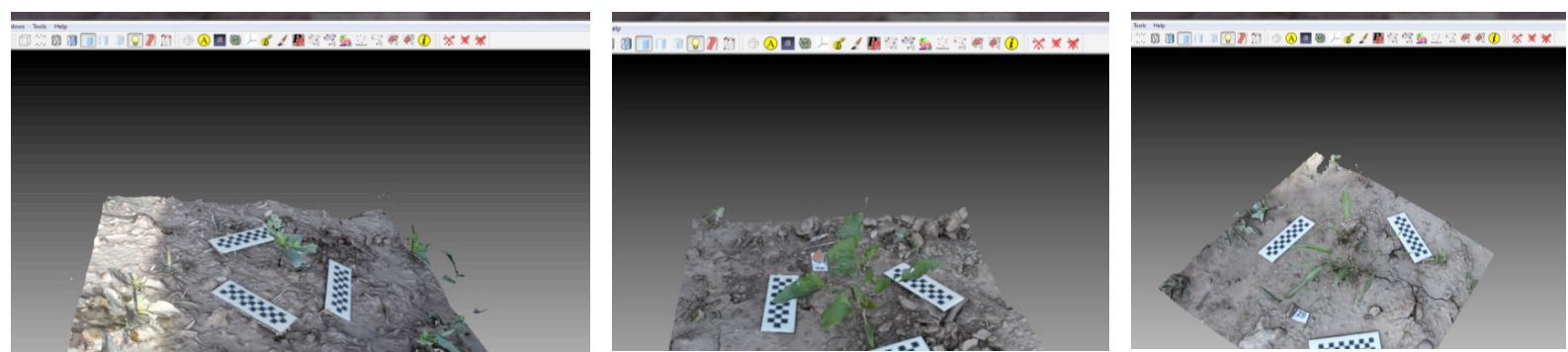

Figure 62. Examples of some different weed models.

The reconstruction was made applying structure from motion ( $\mathrm{SfM}$ ) for an input set of images covering the weed plant. The images were generated slightly moving the camera, producing baseline image pairs (Figure 1).. Images were sequentially taken following a concentric track respect to the plant axis using three different angles, from perpendicular to top view. A Nikon D5500 (Canon, Tokyo, Japan) camera was used to take the images. The distance was kept constant at $50 \mathrm{~cm}$ to the plant from the camera objective. The full covering of weed plants needed of 40 to 50 images per plant according to its size. Due to the use of SfM technique for the plant reconstruction the camera positions are not predefined due to the variations can be corrected by the algorithm. 


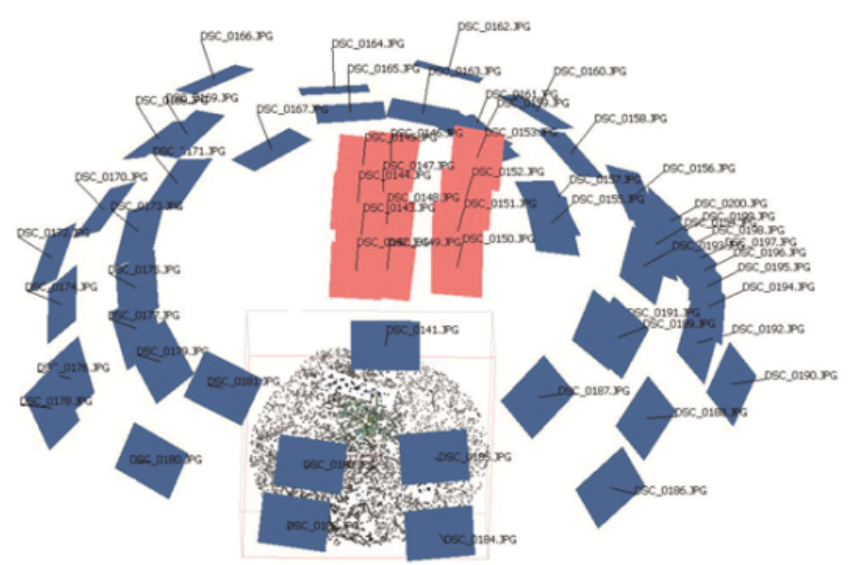

Figure 2. Set of images taken around a weed plant while image acquisition.

Following, the actual height of every weed was manually measured. Thereafter, weeds were cut for determination of biomass and Leaf Area (LA). Dry biomass weight were determined by drying the samples of the weed plant in paper bags. For LA calculation all leaves and stems were placed on a white surface and top images were acquired. A standard $100 \mathrm{~cm} 2$ black square was also placed in the image as a reference in order to calculate, by correlation, the LA of each sample (Figure 3). The camera Nikon D5500 was also used to take the images. The RGB images were transformed to binary images. A linear combination of the RGB planes with coefficients $(r=$ $-0.884, \mathrm{~g}=1.262, \mathrm{~b}=-0.311$ ) was performed. The applied coefficients were obtained by a genetic algorithm optimization process that proved to perform better than Excess Green coefficients (ExG $=2 \mathrm{G}-\mathrm{R}-\mathrm{B})$. Following, the Otsu's thresholding method separated the plant objects pixel-wise into foreground and background in a binary image. The binary image was processed to calculate the LA determined for black pixels while white ones denoted the background.
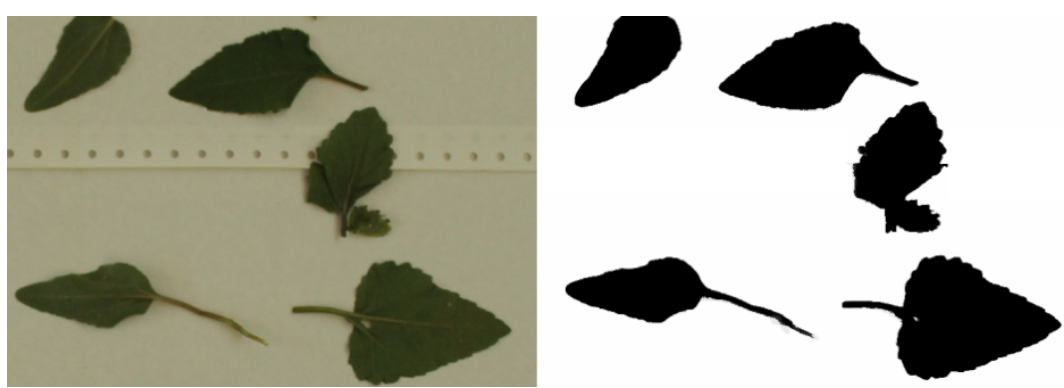

Figure 3. Example of RGB images (left side) used to quantify the leaf area, after their transformation to binary images (right side) and subsequent application of the Otsu's thresholding method.

Data Processing

Previously to build the plant surface mesh, a dense point cloud by using multiple view stereo is created. DSM generation was performed using the Agisoft PhotoScan Professional Edition software (Agisoft LLC, St. Petersburg, Russia) version 1.0.4 build 1874. The process was fully automatic. The process of model creation was formed for a sequence of phases: (1) aligning images, (2) building field geometry, and (3) point cloud generation. Point clouds were processed on an Intel laptop with Windows 7 and a 2 Gb Nvidia GeForce GTX graphic card with a Graphics Processing Unit (GPU). The final model is a 3D polygon mesh that represents the plant shape and show the geometry of every processed weed plant.

Solid 3D model were processed off line in the open source software Meshlab®. The software was used to manage and plot the stored data, creating a model with the point cloud previously created in Agisoft. The incorporated tools allow cleaning, managing and processing the $3 \mathrm{D}$ model. The meshes were processed in different steps: (1) Filter and removal of outliers and noise reduction by filters. Individual points were removed using statistical filters. Those points out of the grid more than $0.5 \mathrm{~cm}$ were automatically removed. (2) Neighbor weeds were cleaned. The target weed plant was isolated after removing every source of interference inside the bounding box. (3) The obtained meshes were processed to extract the main parameters and compared with the actual parameters. The LA of each model was calculated using Meshlab. The model height was also measured and compared with the actual weed height. The calculated LA was compared with dry biomass and actual LA. The analysis of the LA is important in determining the plant's growing stage or health status. It also is an indicator for operational tasks, such as herbicide application (Andújar et al., 2013). 
Statistical analyses

The created 3D models were validated with the actual values using regression methods. Previously to the regression analysis, a correlation analysis was conducted to make an initial examination of bivariate relationships among the variables. Pearson's correlation coefficient was used to analyze simple linear relationships between the actual ground truth values and those values measured within the model.

\section{Results and Discussion}

Every plant was properly reconstructed with only small parts missing. At the end of the leaves and branch borders triangulation some errors occurred and some part of the reconstruction did not show end details. However, very small errors were obtained in every case with some little differences between weed species. The case of dicots plants (X. strumarium and D. ferox) showed small differences with the case of monocot one (S. halepense). The measured parameters on the models showed a high correlation with the ground truth data. Indeed, a good consistency in linear correlation equations between the actual parameters of the modeled weed plants and the measured values on the digital 3D model was found. All measured parameters in the models were highly correlated with R2 lying from 0.998 to 0.833 . Level of detail and errors can be reduced by lowering the distance of the camera to the plant and increasing the number of images per sample. However, this effect would increase the acquisition and processing times.

Regarding height measurement, $X$. strumarium plants showed an average of $12 \mathrm{~cm}$ in height. The correlation with values extracted from the model was high $(R 2=0.998)$ and significant at $P<0.01$. The comparison of the $D$. ferox model and ground truth showed an average height of $7.25 \mathrm{~cm}$. Regression analysis showed a strong correlation $(\mathrm{R} 2=0.997)$ between actual values and those extracted from the model (figure 4$)$. In the case of $\mathrm{S}$. halepense estimated stem length was more complicate than in the dicot weeds. The beginning and end of the stem are difficult to identify due to the insertion of leaves and the stem curves. The obtained values indicate the potential of this budget photogrammetry methodology for small weeds reconstruction. According to the obtained results, the reconstruction of monocot weeds is more difficult than dicots. Sampling conditions such as illumination or wind were controlled to create high detailed models.

a) Xanthium strumarium

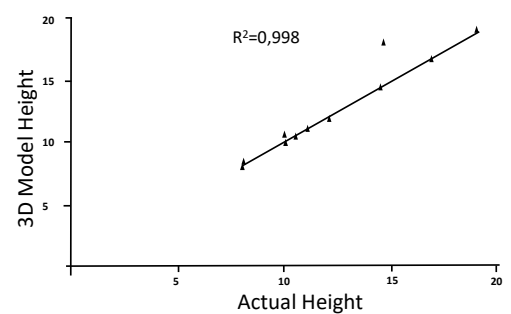

b) Datura ferox

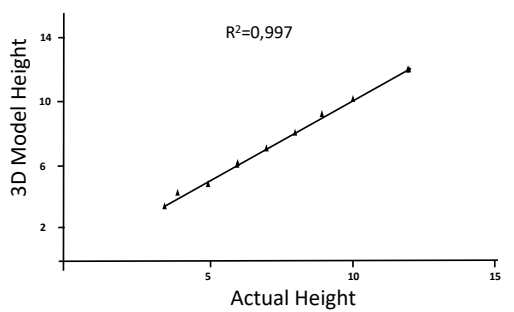

c) Sorghum halepense

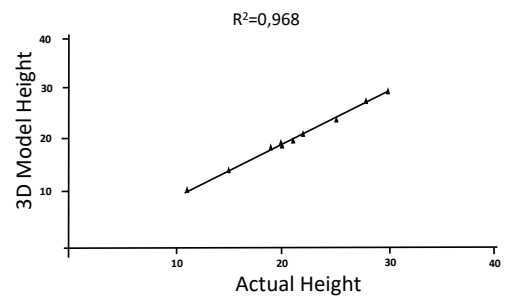

Figure 4. Regression analysis comparing actual weed height vs. measured height using 3D modelling.

The LA measurements showed similar tendencies to height estimations. Apparently, the case of dicots is more accurate than S. halepense predictions. However, the differences between species are quite low. X. strumarium plants showed a real LA average of $116.34 \mathrm{~cm} 2$ and a value of $115.91 \mathrm{~cm} 2$ in the model. The correlation with values extracted from the model was significant at $\mathrm{P}<0.01$ with $\mathrm{R} 2=0.966$ (figure 5). Regarding, regression analysis, the values had a strong correlation $(\mathrm{R} 2=0.978)$ between actual values and those extracted from the model. The same tendency that was showed on height values was found for LA in the case of S. halepense. Although, the accuracy of prediction was lower than in the dicot species, it was highly significant. Regression analysis were significant at $\mathrm{P}<0.01$ with $\mathrm{R} 2=0.841$ (figure 5). Similar tendency was observed when comparing LA with actual dry biomass weight values (Figure 6).
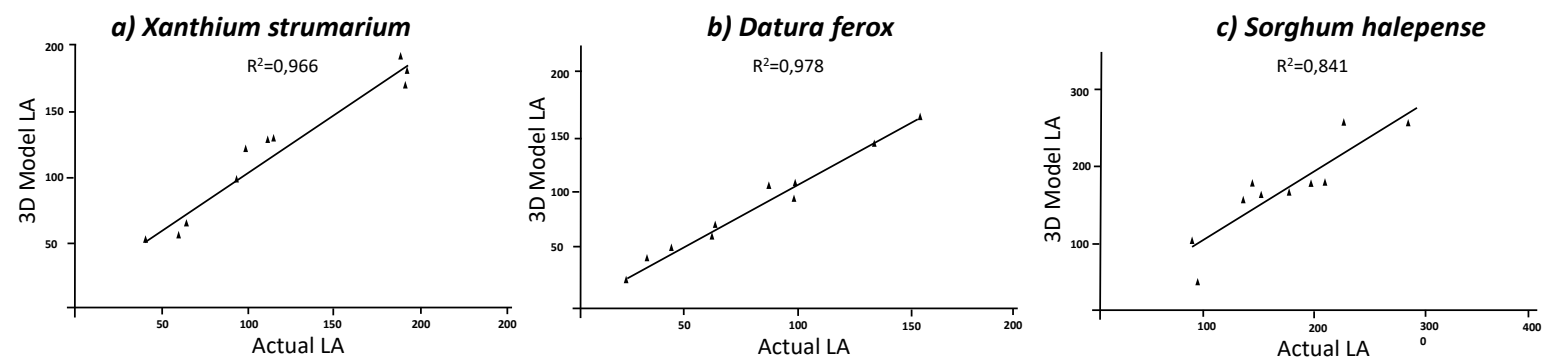

Figure 5. Regression analysis comparing actual leaf area (LA) vs. estimated leaf area using 3D modelling. 

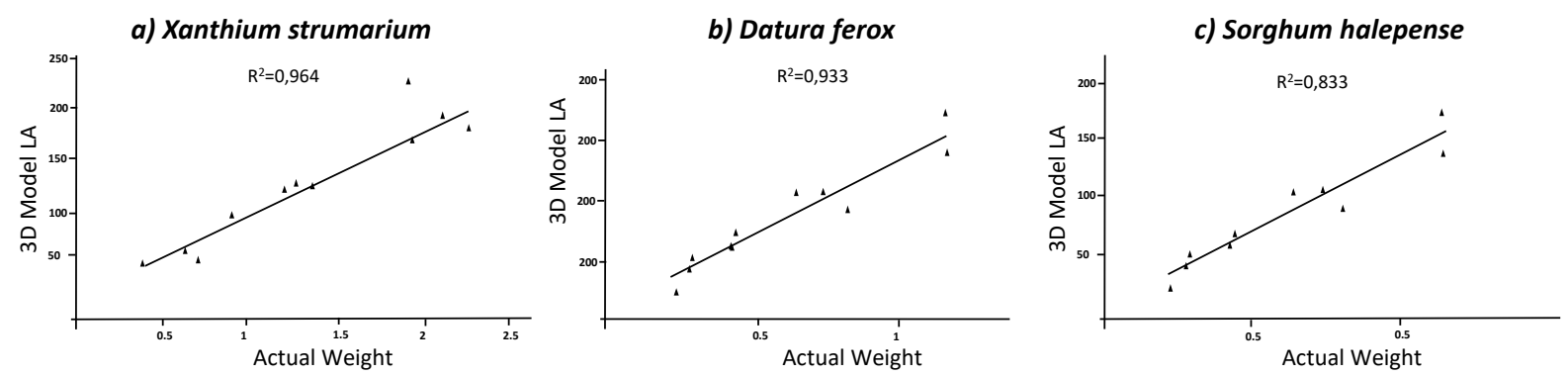

Figure 6. Regression analysis comparing estimated leaf area (LA) vs. actual dry biomass weight.

Thus, regarding differences between species, SfM technique is a proper method to analyze the actual values of height and an LA. In addition, the SfM method calculates the camera position automatically and avoid the use of scanning structures and difficult methodologies for image acquisition such as de case of stereo vision (Biskup et al., 2007). Since, the camera position does not need to be fixed, the image acquisition process is quite fast compared to other methods. Similarly, Quan et al. (2006) designed a fast semi-automatic technique for modeling plants employing multiple view stereo for plant reconstruction. As in the current research, branch details were not fully reconstructed. Thus, simple, fast, and accurate methods are need for plant reconstruction. Although, high level of detail is sometimes required in breading programs, the use of faster and simpler methods are usually more suitable for agronomical task. The use of SfM in outdoors conditions allowed the creation of detailed models with lower effort than complex methods. Also, the use of this methodology would help to take decision over plants growing on field conditions, which use to differ with those plants cultivated under greenhouse conditions. The combination of ToF cameras and imagining system such as stereo vision would improve the level of detail in the model while constructing denser depth maps (Song et al., 2011). The sensor fusion can help to increase the level of detail and reduce processing time.

\section{Conclusions}

Further efforts should be done on extracting some additional structural information such as leaf inclination and overlapping and stem diameter which can be of help for decision support systems. The time-cost and needed details for the different approaches of this technique should be studied. The end details could be necessary for breading programs or botanical classification. However, the integration of the models in on-field precision treatments would need of less details and lower processing times. The limit and implication of detectable details should be studied. The studied plants had different structures which had led to different resolutions while the image acquisition and operational processing systems were the same. The complexity and shape of the plants would need of classificators for automated decision making programs. The obtained results showed that weed plant reconstruction at high detail is possible with a budget and simple system usable in several scenarios, even for phenotyping processes.

\section{Acknowledgements}

This research was funded by the projects AGL2017-83325-C4-1-R and AGL2017-83325-C4-3-R (Spanish Ministry of Economy and Competition) and by the RYC-2016-20355 agreement.

\section{References}

Andújar, D., A. Escolà, J.R. Rosell-Polo, C. Fernández-Quintanilla, J. Dorado, 2013. Potential of a Terrestrial LiDAR-Based System to Characterise Weed Vegetation in Maize Crops. Computers and Electronics in Agriculture. 92, 11-15. https://doi.org/10.1016/j.compag.2012.12.012

Andújar, D., J. Dorado, C. Fernández-Quintanilla, A. Ribeiro, 2016. An Approach to the Use of Depth Cameras for Weed Volume Estimation. Sensors, 16, 972. https://doi.org/10.3390/s16070972

Biskup, B., H. Scharr, U. Schurr, U. Rascher, 2007. A Stereo Imaging System for Measuring Structural Parameters of Plant Canopies. Plant, Cell \& Environment. 30, 1299-1308. https://doi.org/10.1111/j.1365$\underline{\text { 3040.2007.01702.x }}$

Gutiérrez, S., J. Tardaguila, J. Fernández-Novales, M.P. Diago, 2016. Data Mining and NIR Spectroscopy in Viticulture: Applications for Plant Phenotyping under Field Conditions. Sensors, 16, 236. https://doi.org/10.3390/s16020236

Fahlgren, N., M.A. Gehan, I. Baxter, 2015. Lights, Camera, Action: High-Throughput Plant Phenotyping is Ready for a Close-Up. Current Opinion in Plant Biology. 24, 93-99. https://doi.org/10.1016/j.pbi.2015.02.006

Humplík, J.F., D. Lazár, A., Husičková, L, Spíchal, 2015. Automated Phenotyping of Plant Shoots Using Imaging Methods for Analysis of Plant Stress Responses - A Review. Plant Methods. 11, 29. https://doi.org/10.1186/s13007-015-0072-8

Ivanov, N., P. Boissard, M. Chapron, B, Andrieu, 1995. Computer Stereo Plotting for 3-D Reconstruction of a Maize Canopy. Agricultural and Forest Meteorology., 75, 85-102. https://doi.org/10.1016/0168-1923(94)02204-W 
Quan, L., P. Tan, G. Zeng, L. Yuan, J. Wang, S.B. Kang, 2006. Image-based plant modeling. ACM Transactions on Graphics (TOG). 25, 599-604. https://doi.org/10.1145/1179352.1141929

Lancashire, P.D., H. Bleiholder, T.V.D. Boom, P. Langelüddeke, R. Stauss, E. Webber, A. Witzenberger, 1991. A Uniform Decimal Code for Growth Stages of Crops and Weeds. Annals of Applied Biology. 119, 561601. https://doi.org/10.1111/j.1744-7348.1991.tb04895.x

Lhuillier, M., L. Quan, 2002. Match Propagation for Image-Based Modeling and Rendering. Pattern Analysis and Machine Intelligence. IEEE Trans. Pattern Anal. Mach. Intell. 24, 1140-1146. https://doi.org/10.1109/TPAMI.2002.1023810

Ludovisi, R., F. Tauro, R. Salvati, S. Khoury, G. Mugnozza Scarascia, A. Harfouche, 2017. UAV-Based Thermal Imaging for High-Throughput Field Phenotyping of Black Poplar Response to Drought. Frontiers in Plant Science. 8,1681. https://doi.org/10.3389/fpls.2017.01681

Nguyen, T.T., D.C. Slaughter, N. Max, J.N. Maloof, N. Sinha, 2015. Structured Light-Based 3D Reconstruction System for Plants. Sensors, 15, 18587-18612. https://doi.org/10.3390/s150818587

Peteinatos, G.G., M. Weis, D. Andújar, V. Rueda Ayala, R. Gerhards, 2014. Potential Use of Ground-Based Sensor Technologies for Weed Detection. Pest Management Science., 70, 190-199. https://doi.org/10.1002/ps.3677

Song Y., C.A. Glasbey, G.W.A.M. van der Heijden, G. Polder, J.A. Dieleman. 2011. Combining Stereo and Time-of-Flight Images with Application to Automatic Plant Phenotyping. In: Image Analysis. SCIA. Lecture Notes in Computer Science, vol 6688. Springer, Berlin, Heidelberg. 23-25 May 2011. https://doi.org/10.1007/9783-642-21227-7 44

Takizawa, H., N. Ezaki, S. Mizuno, S. Yamamoto, 2005. Plant Recognition by Integrating Color and Range Data Obtained Through Stereo Vision. Journal of Advanced Computational Intelligence and Intelligent Informatics, 9, 630-636. https://doi.org/10.20965/jaciii.2005.p0630

Vogiatzis, G., C. Hernández, 2011. Video-Based, Real-Time Multi-View Stereo. Image and Vision Computing. 29, 434-441. https://doi.org/10.1016/j.imavis.2011.01.006

Walter, A., F. Liebisch, A. Hund, 2015. Plant Phenotyping: From Bean Weighing to Image Analysis. Plant Methods. 11, 14. https://doi.org/10.1186/s13007-015-0056-8 


\author{
Challenges in modern automated Feeding Systems: Navigation Technologies

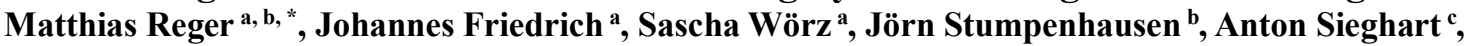 \\ Heinz Bernhardt ${ }^{a}$ \\ ${ }^{\text {a }}$ Department of Life Science Engineering, Technical University of Munich, 85354 Freising, Germany \\ ${ }^{b}$ Department of Agriculture and Food Economy, University of Applied Sciences Weihenstephan-Triesdorf, 85354 \\ Freising, Germany \\ ${ }^{c}$ SILOKING Mayer Maschinenbau GmbH, 84529 Tittmoning, Germany \\ * Corresponding author. Email: matthias.reger@tum.de
}

\begin{abstract}
Germany is a high-tech location, especially in agriculture with over 7.800 automatic milking systems (AMS) installed in Germany, with a clearly evident upward-trend [HEMMERLING et al. 2016, HARMS 2017]. Based on the steep rise of AMS a similar development in AFS (automated Feeding Systems) seems likely. The technical advances are mainly attributed to time and labour savings for the farmer and the plan to meet nutritional and behavioural aspects of the cows. Recently Strautmann presented their study "Verti-Q", a fully automated feeding system, on the Agritechnica 2017. Previously Schuitemaker, Lely, Hirl, LucasG and Siloking announced fully or partly automated feeding systems, so that self-driving, automatic feeding systems can be seen as a future solution in dairy industry. Challenging for most manufacturers is that no standards for self-propelled AFS are existing, which are said to be the state of the art. Especially the various environments on farms cause critical situations for machine operations, such as tough weather conditions. To reach the set safety level, durable sensing and a comprehensive safety concept are necessary. In preliminary tests a novel radar scanner and a laser 2D-scanner were tested. They proved to be possible solutions for navigational and safety tasks in AFS. The sensors collected data of the environment of a farm. The acquired data were used to create digitals maps of the stable and showed the applicability of these methods. Quality data for mapping algorithms have been improved steadily with a selfdesigned high-complex physical system, where already Monte Carlo filtered data has been processed with an extended Kalman filter. With further progression concerning the prototyped radar-scanner and its data-processing, radar as well as laser will provide the sensory for a self-driving AFS.
\end{abstract}

Keywords: AFS, feeding, dairy, automation, navigation

\title{
1. Introduction
}

Current automated feeding systems reduce time and labour efforts for the farmers. Furthermore they enable multiple feeding times to meet the animals' behaviour. Nevertheless farmers still have to cope with drawbacks. Whether they manually fill up a feed store every two days (maximum) or every single load, a human intervention is necessary. Most of these systems are also bound to a certain layout, because they are customized into the building and not self-propelled. To improve productivity in automated feeding the upcoming developments need to lead towards self-driving and self-loading feeding systems. To reach this goal we tested radar and laser as navigation technologies under practical conditions and improved the accuracy of laser-mapping by constructing a high-complex physical system.

\section{Materials and Methods}

Testing Environment and Experimental Setup

The experiments were conducted at the experimental station "Veitshof" of the TUM (Technical University of Munich). A self-propelled robot was equipped with a novel radar scanner and a standard Sick AG-laser scanner. The laser scanner and the radar scanner were arranged one above the other centred above the steering axle (Fig. 1). This should minimize the not negligible measuring differences that especially occur while cornering. The route led through a dairy barn and around it (Fig. 1) on changing surface. One lap was had a distance of approximately $100 \mathrm{~m}$. Beforehand the experimental driving a digital 2D-map of the track was generated with the laser scanner and a mapping algorithm. Way points were set along the route (teach-in) and formed the desired trajectory.

Technical and Software Equipment

The experiments were performed with the robot Heros 224 WST of Innok Robotics GmbH. The robot measures $966 \mathrm{~mm} \times 730 \mathrm{~mm} \times 776 \mathrm{~mm}$ (without scanners) at $105 \mathrm{~kg}$. The drive power of the two batterypowered motors was $800 \mathrm{~W}$. The robot had a 2WD differential drive and a two-wheel pendulum axle. The drive wheels were rubber pneumatic tires with tractor profile at a nominal wheel diameter of $410 \mathrm{~mm}$.

Navigation was performed with a laser scanner of the LMS 100 type by Sick AG. Its light source lies in the infrared range at a wavelength of $905 \mathrm{~nm}$. It has an opening angle of 270-degrees and a working range between $0.5 \mathrm{~m}$ and $20 \mathrm{~m}$. The used radar scanner was in the prototype stage. It generated data for further development. It is a 360-degree short-range radar with a range of about $10 \mathrm{~m}$. The range depends on the target objects and is restricted by the software. The distance resolution is about $2 \mathrm{~cm}$. The sensor is able to separate/distinguish several consecutive targets. The angular resolution depends on the update rate and is between 8 and 12 degrees. The 2D- 
updaterate is adjustable between $1 \mathrm{~Hz}-2 \mathrm{~Hz}$ and limited to $50-100$ measurements/rotation, respectively.

The Software "Cartographer" was used for digital mapping. The robot operating system "ROS" was installed on the robot. For data pre-processing and post-processing Microsoft Excel 2016 and Matlab 2016b were used.

\section{Data Fusion}

In a first step the raw data of the laser scanner were processed to remove the measurement noise and thus to increase its accuracy. Measurement accuracy was then improved further by using an Extended Kalman filter based on a high-resolution physical system with associated measuring system. It is therefore called data fusion using Kalman filtering.

Physical System, Measurement System and Extended Kalman Filter

The physical and measurement system is currently under patent review and therefore cannot be described here.

Quality Characteristics

The measurement results of the considered trails were evaluated on the basis of different quality characteristics. Accuracy, precision, measurement resolution, consistency and condition are criteria for assessing results obtained by evaluating sensor data.

\section{Definition Accuracy}

The accuracy of a measurement system is the degree of deviation of measurement data to a true value. Accuracy was estimated by determining the nearest neighbours of the travel trajectories to the target trajectory using the 1 nearest neighbour method (1NN-method) and Euclidean distance formation. (WÖRZ et al. 2017)

\section{Definition Precision}

The reproducibility of a measuring system describes to what extent samples scatter under the same conditions at several repetitions. The precision was estimated by determining the nearest neighbours of the travel trajectories among each other by $1 \mathrm{NN}$-method. (WöRZ et al. 2017)

In this work, a tolerance limit of $0.05 \mathrm{~m}$ is specified for Precision and Accuracy. This tolerance limit can be selected by the user and thus assume a different value for other scenarios.

\section{Definition Measurement resolution}

The measurement resolution of the measuring system describes the physical variable that can still be measured (DIN 1319-1). Accuracy and precision as well as consistency and measurement resolution determine the quality of the navigation.

\section{Definition Consistency} 1).

If the measurement results of an evaluated experiment are accurate and precise, they are consistent (DIN 1319-

\section{Definition Condition}

If minor changes in the input data of the measurement data after the execution of the algorithm result in minor changes in the output data, the measurement data are well conditioned. Otherwise they are badly conditioned. The condition of the algorithm influences the navigation. The condition dominates the robustness of an algorithm. Poor conditioning of the algorithm may imply false navigation. (DIN 1319-1)

\section{Experimental Procedure}

The radar scanner and the laser scanner were active and generated data continually. The current time was logged hand-written and with time stamps in the data records to be assigned exactly. From a starting position the robot followed the route automatically and stopped automatically after one lap. For the next turn it was started manually with a "free start", means after started driving manually the automatic mode takes over. The driving speed was at $0.6 \mathrm{~m} / \mathrm{s}$. Each run had 10 laps.

Run 1 was a not representative trial run. Due to problems in run 2 (robot shook out sometimes) only run 3 and 4 were considered for the evaluation of the laser scanner data. The problems of run 2 have been solved by doubling the number of saved waypoints. In total in 20 laps with about 1000 measurements/laps a dataset of about 20,000 measurements was generated. The evaluation of the radar scanner considered the generated data of runs 2,3 and 4. Laser and radar data was saved as .bag-file with ROS. The laser data was than saved as a .txt-file. Subsequently, the measurement data could be used for the evaluation in Microsoft Excel 2016 and Matlab 2016b. In addition to the trajectories of the individual rides of the passageways, the waypoints were also provided as a text file. 
Monte Carlo Filter vs. Kalman Filter

This comparison was based on the dataset of run 3. Only the data which was generated in the stable has been taken into account.

The prerequisite for this comparison was a least-squares best approximation to the corrected Y-mean values of the Monte Carlo filter by means of an absolute term. The absolute term was just the average of the corrected Y-mean values of the Monte Carlo filter. This can be assumed to be the Y-value of the true trajectory without restriction of generality. It was also defined: (FRIEDRICH 2017)

Error $1:=$ Monte Carlo filter Y-measurement data-Monte Carlo filter Y-mean of measurement data (MK1)

Error $2:=$ Kalman filter Y-measurement data-Monte Carlo filter Y-mean of measurement data $\quad$ (MK2)

After calculating the absolute "Error 1" (MK1) and "Error 2" (MK2), their difference was calculated for each measurement point $i$ generated in the stable.

\section{Laser Navigation Evaluation}

Before the calculation of the Accuracy the laser dataset was prepared in two steps. Distances were calculated using the $1 \mathrm{NN}$-method i.e. one-nearest-neighbour method. The first and last point of the dataset had to tally roughly. Furthermore, the dataset was checked for irregularities and other discrepancies, e.g. missing data. For the $1 \mathrm{NN}$-method, the trajectory was used as the reference trajectory, which hat the lower number of measured data. After calculating the distances with the $1 \mathrm{NN}$-method, the data was processed a second time. Measured data was adjusted if the measurement data of one of the trajectories had a gap at one point and another trajectory had no gap at this point. At the start and end of measurement data errors occurred because of curve driving. Different steering angles during cornering led to very different positions at the end of the turn. Therefore, these measurement data were adjusted if they were influenced by the previous curve. The goal of this two-stage pre-processing and cleansing of the dataset was to obtain the most reliable data base possible to enable robust evaluation results.

\section{Radar Data}

In general, the measurements were noisy and the generated data were not optimal. Some Sectors of the radar sensor were interfered or no data was recorded at some point. The sensors hardware was at an advanced stage of development. However, mechanics and data transfer were still preliminary and therefore limited the update rate.

The raw radar material was first subjected to threshold filtering to clean up the radar noise. All measured values under a certain threshold were cut off. The processed data was fed into a mapping algorithm for radar data. The live position data from the laser scanner were used as a reference to create the map by accumulating the sensor data.

The radar mapping algorithm is at a very early stage of development, different from laser mapping algorithms. A determination of the position by means of the radar data was not possible.

\section{Results and Discussion}

\section{Monte Carlo Filter vs. Kalman Filter}

Z38 represents the Monte Carlo filter trajectory and X38 represents the Kalman filter trajectory.

If the inequality (abs(error 1) - abs(error 2)) >0 holds for a measuring point $i, i \in N, 0 \leq i \leq|Z 38|,|X 38|$, then the Kalman filter will reconstruct better for this measurement point $i$ (FRIEDRICH 2017).

If the inequality (abs(error 1) - abs(error 2)) >0 holds for a measuring point $i, i \in N, 0 \leq i \leq|Z 38|,|X 38|$, then the Kalman filter will reconstruct better for this measurement point $i$ (FRIEDRICH 2017).

Fig shows |Error 1| - |Error 2|. The measurements improved by the Kalman filter are positive (values from 0 to $\approx 7 \cdot 10^{-4}$ ) and those improved by the Monte Carlo filter are negative (values from 0 to $\approx-3 \cdot 10^{-4}$ ). The Kalman filter reconstruction improved the Monte Carlo filter reconstruction in 231 out of 448 cases. Thus, $51.6 \%$ of the measured data is improved by the Kalman filter reconstruction. In the 217 cases where it does not improve the Monte Carlo filter reconstruction, it negligible impairs the reconstruction. Furthermore, the plot shows that the grade of improvement by the Kalman filter is higher than the grade of improvement by the Monte Carlo filter. The reason for this is that the amplitude of the positive values is greater than the amplitude of the negative value (FRIEDRICH 2017). 


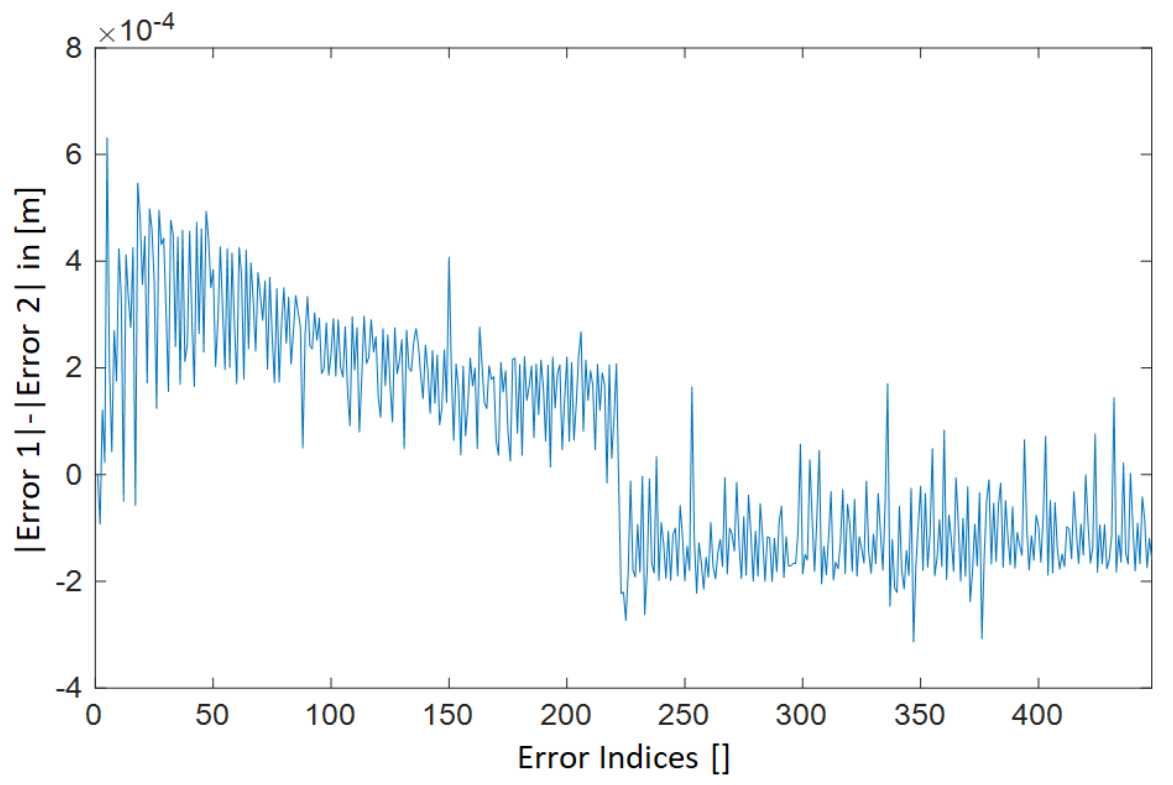

Figure 1. Plot of |Error 1|-|Error 2|.

It could be that the Monte Carlo filter has produced outliers because it is a step-like lowering. The Monte Carlo filter eventually does not work well while dealing with ill posed measurement data originating from inconsistency. Here, real-time applications are especially crucial.

With the Monte Carlo filter, a possibly error-prone motion and measurement update produces suitable reconstruction possibilities for a point to be reconstructed, which are then merged into a reconstructed point by means of distribution theory. Big disadvantage: the reconstruction depends only on the last reconstructed point.

If the measurement or the last reconstructed point is strongly error-prone, this can yield to a distorted reconstruction of the true trajectory. In addition, in case of a too low sampling rate, the disaster is perfect. The Monte Carlo filter is plainly too probability-based.

\section{Laser Navigation Evaluation}

Due to unstable software or a fully occupied operating system, measurement gaps occurred during lap 4. In the following evaluation of the laser navigation data the data of lap 4 was not considered. For future reliable operation the software must be adapted for real-time applications by using a real-time operating system.

On average, the accuracy was $0.0487 \mathrm{~m}$ and the standard deviation was $0.0286 \mathrm{~m}$. The maximum value for the accuracy was $0.1406 \mathrm{~m}$ (R3L10; Round 3 Lap 10), the lowest value was $0.0047 \mathrm{~m}$ (R3L1). The difference between the highest and the lowest value was $0.1359 \mathrm{~m}$. The measurement data for run 3 was judged to be accurate. Fig shows the Accuracy box plots of each lap of run 3. Lap 4 has been eliminated because a significant amount of measurement data was missing. (FRIEDRICH 2017)

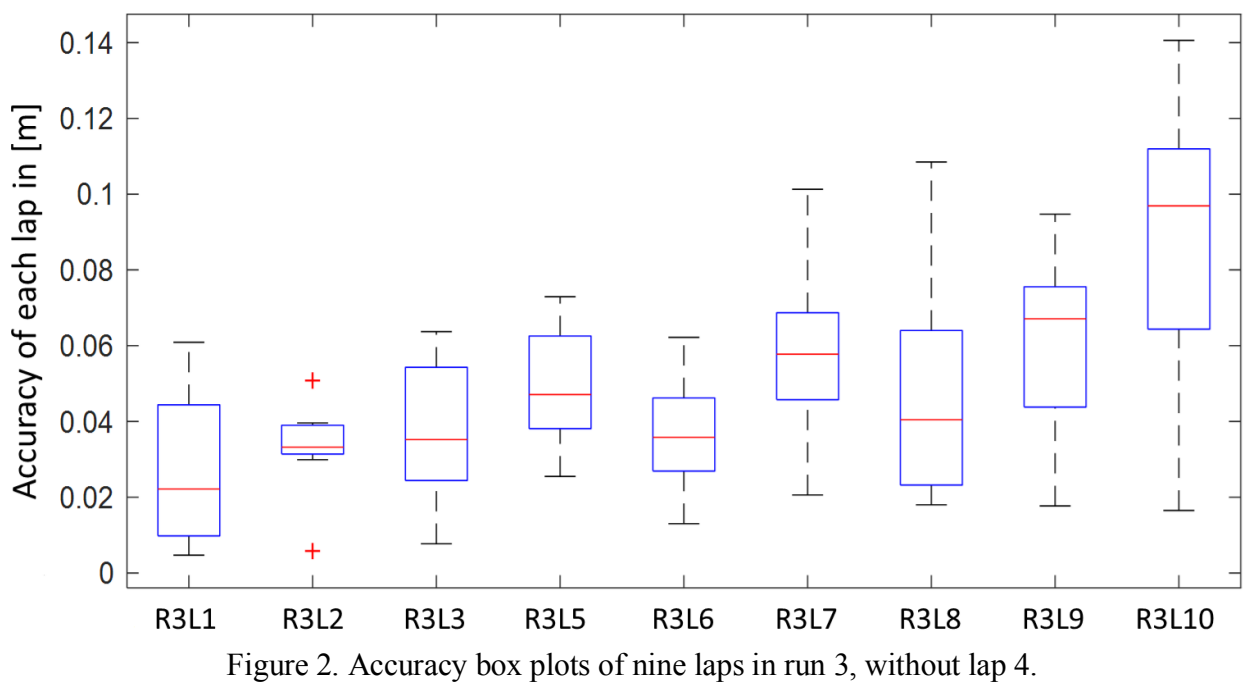


What can be derived from Fig is an upward trend from lap 1 to 10 . In comparison with Table 1 there is a strong correlation between the number of measurement data and the accuracy. Laps with a higher number of measured data can be judged as more accurate than laps were the number of measured data is lower. For lap 10 compared to the other laps, at least 70 less measured data were recorded. The number of available measurement data is related to the performance of the SLAM algorithm. A higher number of measurement data allows conclusions to be drawn about the more accurate mapping of the environment. This allows better differentiation of the body and the measurement data is more accurate and precise.

\begin{tabular}{cccccccccc}
\multicolumn{10}{c}{ Table 1. Number of measured data of each lap in run 3. } \\
\hline Run 3 & L1 & L2 & L3 & L5 & L6 & L7 & L8 & L9 & L10 \\
Data $\mid$ & 524 & 529 & 506 & 525 & 514 & 522 & 448 & 449 & 378 \\
\hline
\end{tabular}

The accuracy of the laser measurement data was sufficient enough.

For the measured data of the 9 considered trajectories (without lap 4) the nearest neighbours were calculated with respect to the Euclidean distance using the $1 \mathrm{NN}$-method. A total of 15.584 nearest neighbours were included for the trajectories. Fig simultaneously compares the precision of the trajectories in a histogram. (FRIEDRICH 2017)

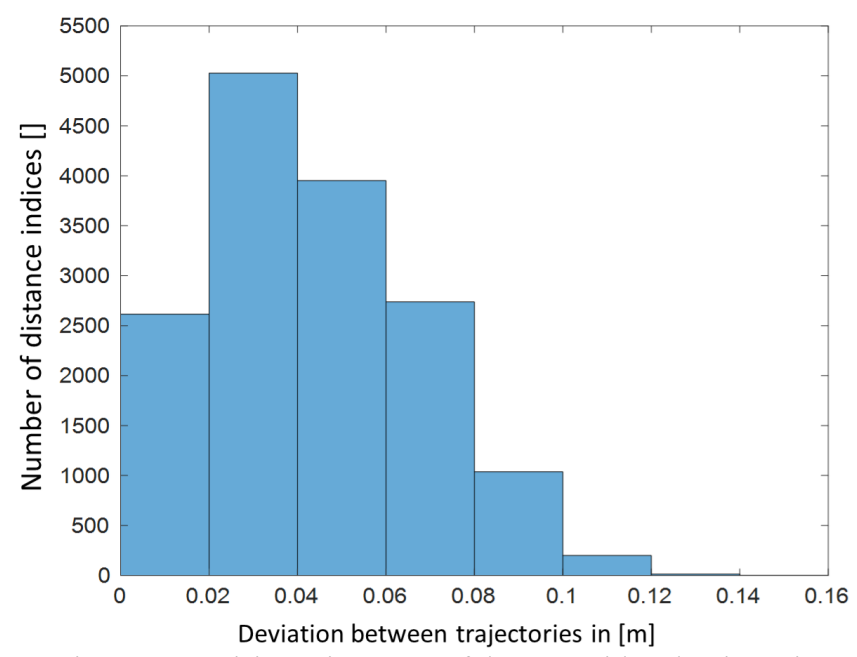

Figure 3. Precision-Histogram of the 9 considered trajectories.

$16.5 \%$ of the values were below $0.02 \mathrm{~m}, 32.3 \%$ were between $0.02 \mathrm{~m}$ and $0.04 \mathrm{~m}, 25.3 \%$ were between $0.04 \mathrm{~m}$ and $0.06 \mathrm{~m}, 17.5 \%$ were between $0.06 \mathrm{~m}$ and $0.08 \mathrm{~m}, 6.7 \%$ were between $0.08 \mathrm{~m}$ and $0.10 \mathrm{~m}, 1.3 \%$ were between $0.1 \mathrm{~m}$ and $0.12 \mathrm{~m}, 0,0008 \%$ were between $0.12 \mathrm{~m}$ and $0.14 \mathrm{~m}$ and $0.00006 \%$ of the values were between $0.14 \mathrm{~m}$ and $0.16 \mathrm{~m}$. This means that $1.4 \%$ of the values were above $0.1 \mathrm{~m}$. On average, the precision was $0.0439 \mathrm{~m}$ and the standard deviation was $0.0238 \mathrm{~m}$. The maximum value for the Precision was $0.1511 \mathrm{~m}$, the lowest value was $0.0001 \mathrm{~m}$. As a result, the difference between the maximum value and the lowest value was $0.1510 \mathrm{~m}$. Thus, the measurement data was considered precise. The statistical evaluation of the three pairs of trajectories with the largest or smallest distance value showed no significant difference between the mean values of the trajectory pairs. The null hypotheses are not necessarily correct. Nevertheless, it can be assumed in practice that there is nothing wrong with the correctness of the null hypotheses. Since a very small FDR (false discovery rate) was determined, this trial can be classified as highly accurate. (FRIEDRICH 2017)

Since the measurement data has already been assessed as accurate and precise they were therefore also consistent. A robust test evaluation always requires a consistency of the evaluation algorithms. Thus, these are extremely reliable evaluation results.

To obtain a consistency of the evaluation algorithms, of course, a corresponding measurement resolution must be present. As an example, the measurement resolution of the laser position data can be cited here, which proved to be sufficiently small for the experiments. The measurement resolution of the laser data was in the micrometre range.

The results of the Kalman-Filter for close-coupled inputs were close-coupled outputs. Consequently, it can be said that the Kalman-Filter was very well conditioned because the output data was constantly dependent on the input data.

\section{Rader Data}

The processed radar data has been overlaid over the laser scanner map (Figure 1). The data is congruently 


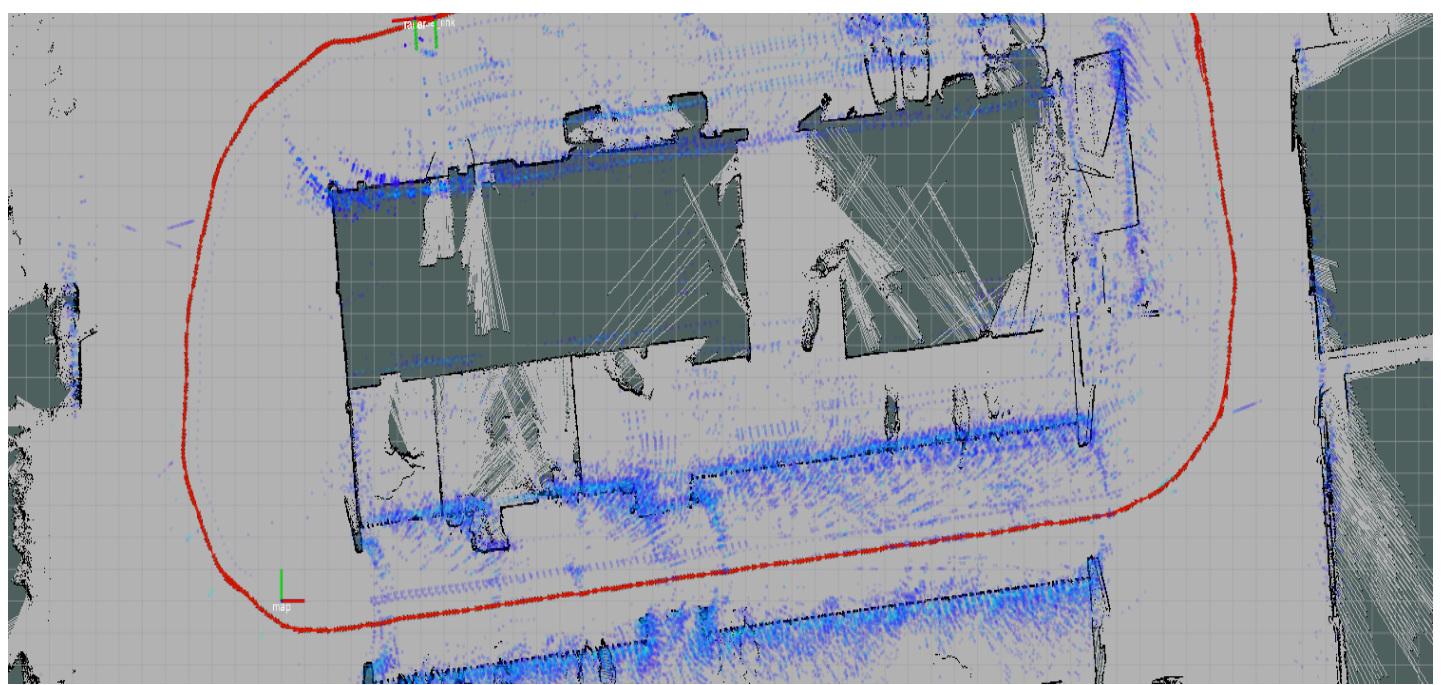

Figure 1. The processed radar data (blue dots) overlaid onto the radar scanner map with the desired trajectory (red line).

The used radar scanner is still in the prototype stage, so some software and hardware issues arose which primarily caused data transfer and recording disturbances. In the future, a new navigation or mapping algorithm will be developed on the basis of an existing algorithm. Currently the radar measurements have a resolution of about $2 \mathrm{~cm}$. With post-processing, a potential accuracy of about $1 \mathrm{~mm}$ within a resolution cell is possible. Therefore, positioning accuracies between 1 and $10 \mathrm{~cm}$, similar to the laser scanner, will be achieved in the future.

Furthermore, the experiments showed that navigation based on a radar scanner will work in the future. The radar scanner should allow more stable localization due to its characteristics. Once the software has been further developed and stabilized, the experiments must be repeated in a similar manner with the aim of obtaining more consistent results. The further experiments will initially primarily deal with the resolution, the target recognition and the range, only then the mapping will continue. Furthermore, the hardware will be improved, which in turn will lead to further attempts.

\section{Conclusions}

Both, laser and radar, are suitable technologies for future AFS. Even though the evaluated radar scanner was in the prototype stage its potential was clearly evident. Particularly the data processing with adapted software offers many possibilities for optimization. The problems that occurred relating to the hardware can easily be rectified and enhanced. Due to its characteristics it promises a more stable localization in outdoor areas. The laser scanner technology is already mature. It enables high-precision mapping of the environment and consequently precise navigation. It can also take on security duties. By a meaningful combination of both and the associated data fusion, they represent a reliable, secure and future-proof solution for automated machines.

The aim, to qualitatively improve the reconstruction of the laser raw data has been achieved. The reconstruction based on the Monte Carlo filter could be improved in more than half of the cases by the selfdesigned physical system using an Extended Kalman filter. Furthermore it was proven that the laser navigation can succeed in an agricultural environment. The physical system enables an even more detailed mapping of the environment and as a result of this a more precise navigation for automated machinery. Without mechanical periphery the system remains flexible and can automatically adapted to changing conditions, e. g. varying fill level in horizontal silos. The navigation meets the required accuracy with respect to compliance with safety distances. The physical system means a step towards fully automated AFS.

\section{Acknowledgements}

The authors thank the Bavarian State Ministry of Education and Culture, Science and Art for its support of this research project, the Mayer Maschinenbau Ltd. as industrial partner in this research project and the Innok Robotics Ltd. as partner for automation issues. 


\section{References}

Friedrich, J., 2017. Technologische Analyse der freien Navigation von mobilen AFS im Milchviehstall. Unpublished Bachelor Thesis, Technical University of Munich, Freising, 2017

Harms, J., 2017. Automatisches Melken - Erfahrungen, Tipps und Tricks. LfL-Information zum Info-Tag “Automatisches Melken”, March 15, 2017, https://www.lfl.bayern.de/ilt/lehrschau/153087/index.php, Accessed June 10, 2018

Hemmerling, U., 2016. Kapitel 2.3 - Moderne Tierhaltung. In: Situationsbericht 2016/17 Trends und Fakten zur Landwirtschaft, Deutscher Bauernverand, 12/2016, https:/www.bauernverband.de/23-moderne-tierhaltung, Accessed June 10, 2018.

Woerz, S., M., Mederle, V., Heizinger, H., Bernhardt, 2017: A novel approach to piecewise analytic agricultural machinery path reconstruction. Engineering Optimization. $60 \quad(1), \quad 1-24$. https://doi.org/10.1080/0305215X.2017.1289742. 


\author{
SPAD and hyperspectral images for sensing chlorosis affection in peach trees \\ María Victoria Lafuente ${ }^{1}$, Lourdes Lleó ${ }^{2 *}$, Jesús Val ${ }^{1}$, Jean Michel Roger ${ }^{3}$ Pilar Barreiro $^{2}$ \\ ${ }^{1}$ Estación Experimental de Aula Dei (CSIC), Zaragoza, España \\ ${ }^{2}$ LPF TAGRALIA, UPM CEI Moncloa, UP4, Madrid, Spain \\ 3 UMR ITAP Irstea - SupAgro \\ * Corresponding author. Email: lourdes.lleo@upm.es
}

\begin{abstract}
The peach tree is one of the fruit species with the highest production and area cultivated in Spain. For peach cultivation, the range of available rootstock is high, since several species, or even hybrids among them, can be used as rootstocks. Peach varieties are markedly influenced by the rootstock. In fact, many works show this influence, both in productive and physiological aspects. The nutritional diagnosis of the peach tree, usually based on the mineral analysis of leaves, is often late for the application of corrections before harvesting. On the other hand, the leaf diagnosis of ferric chlorosis is imprecise in many cases, due to the narrow range of variation in leaf iron concentrations. SPAD measurements are commonly used for chlorophyll content sensing in leaves, showing relation with chlorosis development. However, the SPAD is measured locally by transmission. Hyperspectral images could be able to sense the evolution and the distribution of ferric chlorosis on the leaf surface, to clearly study the development and the variability of such affection. Thirty-two trees, belonging to seven different rootstocks, and three leaves per tree were considered for hyperspectral measurements. SPAD measurements were acquired from their leaves and flowers. Mineral composition (Al, B, Ca, Cu among others) was also determined. The SPAD appears correlated with several minerals in leaf: $\mathrm{Ca}, \mathrm{S}, \mathrm{Fe}, \mathrm{Cu}$ and $\mathrm{Sr}$, restricted to $\mathrm{Ca}$ and $\mathrm{Sr}$ when comparing the SPAD with the mineral composition in flower. There appears to be a very large dispersion among SPAD values for the same rootstock which points the existence of isolated individuals with leave chlorosis. According to the visual identification of experts $\mathrm{SPAD}>=32$ was set to sound trees, $\mathrm{SPAD}<24$ was labelled as trees severely affected by chlorosis, and intermediate chlorosis level in-between. Comparing the average spectra of sound leaves with affected ones, three main features were observed; a) a general increase of reflectance from 500 to $600 \mathrm{~nm}$, with a peak around 550, b) a red-edge displacement to the left, and c) an overall weak decrease of reflectance at NIR range probably due to the increase of the chlorosis affection. Several authors notices that rededge moves towards left with the decreasing of chlorophyll content. Besides, a global increase in the visible range can also be caused by a decrease in chlorophyll due to nutritional stress.
\end{abstract}

Keywords: Fruit quality, spectral fingerprint, spectral features, remote sensing, NDVI ground truth, SPAD, IoT (Internet of things), precision agriculture.

\title{
1. Introduction
}

Peach varieties are markedly influenced by the rootstock. In fact, many works show this influence, both in productive and physiological aspects. The nutritional diagnosis of the peach tree, usually based on the mineral analysis of leaves, is often late for the application of corrections before harvesting. On the other hand, the leaf diagnosis of ferric chlorosis is imprecise in many cases, due to the narrow range of variation in leaf iron concentrations. SPAD measurements are commonly used for chlorophyll content sensing in leaves, showing relation with chlorosis development. However, the SPAD is measured locally by transmission. Hyperspectral images could be able to sense the evolution and the distribution of ferric chlorosis on the leaf surface, to clearly study the development and the variability of such affection.

Most of the reflectance changes observed in plants under stress conditions, at leaf scale, occur in the visible part of the spectrum, due to changes in the pigment content: loss of chlorophyll and carotenoids (Behmann et al., 2014). In fact, the loss of chlorophyll (both a and b) results in an increase in VIS reflectance in maize and sunflower with nutritive stress, iron deficiencies (Mariotti et al., 1996), with the same effect observed also in wheat and barley under Fe, S, Mg and Mn deficiencies (Masoni et al., 1996). Literature indicates that N, P and K deficiencies also promote an increase of VIS reflectance in maize (Al-Abbas et al., 1974). Carter (1993) studied the spectral response of many plants to different stress conditions, and VIS reflectance increased in all the plants independently of the type of stress; NIR reflectance, however, was less sensitive.

The leaf nutrient concentrations in chlorotic trees have been generally studied only at the standard sampling time for this area, in June-July (120 days after full bloom; DAFB), (Abadía et al, 1985; Köseoglu, 1995). At 120 $\mathrm{DAFB}, \mathrm{Fe}$ chlorosis generally increases the leaf $\mathrm{K}$ concentrations and decreases leaf $\mathrm{Ca}$ concentrations, and consequently causes marked increases in the K/Ca ratio (Abadía et al, 1985; Heras et al., 1976; Montañés et al., 1990). Other works have also shown that the $\mathrm{P} / \mathrm{Fe}$ ratio could be higher in chlorotic than in green leaves (Abadía et al., 1985; Köseoglu, 1995). Some research groups have used the $\mathrm{K} / \mathrm{Ca}$ and $\mathrm{P} / \mathrm{Fe}$ nutrient ratios to assess $\mathrm{Fe}$ chlorosis (Abadía et al, 1985; Dong, 1987; Heras et al, 1976; Ji et al., 1985; Mengel el al, 1984). Other studies have proposed the use of alternative, earlier dates in the vegetative cycle for tree leaf analysis, such as 60 DAFB (Montañés and Sanz, 1994; Montañés et al., 1990; Sanz and Montañés, 1993; Sanz et al., 1991, 1992). Therefore, alternative non-destructive methods to leaf analysis have been proposed to prognose nutrient deficiency, using hyperspectral images. 
VIS reflectance usually increases at green and yellow bands in plants under stress conditions (Milton et al., 1991). Thus, loss of chlorophyll a and b causes an increase of VIS reflectance. Bands at 550 and $680 \mathrm{~nm}$ were determined as the most sensitive to detect loss of chlorophyll due to stress conditions (Merzlyak et al., 2003). The objective of the present research is to stablish a first step for the use of hyperspectral images as a tool for chlorosis sensing affection. This work constitutes a previous study; focused on a) a detailed discussion of SPAD values variation of the leaves and trees and their relationship with mineral composition and on $b$ ) the main features of the spectra extracted from hyperspectral images and their relationship with SPAD classification.

\section{Materials and Methods}

The trial was carried out at The Experimental Station of Aula Dei (CSIC) Zaragoza, Spain. Thirty-two peach trees, belonging to seven different rootstocks, and three leaves per tree were considered. SPAD measurements $(n=288)$ were carried out on three leaves per tree (tree repetition per leaf). For hyperspectral measurements three leaves per tree were also considered. SPAD measurements were acquired from their leaves and flowers. Mineral composition ( $\mathrm{Al}, \mathrm{B}, \mathrm{Ca}, \mathrm{Cu}$ among others) was also determined considering a group of leaves for each tree, independent form the group of SPAD and hyperspectral measurements.

The hyperspectral vision system consists of a push-broom CCD camera (Andor Luca) equipped with a spectrograph Headwall Photonics HyperspecTM VNIR (spectral range: 400 to $1000 \mathrm{~nm}$ ). The spectral binning was configured to obtain 189 wavelengths (spectral resolution $3.17 \mathrm{~nm}$ ). The acquisition and the storage of the images were made through specific software (Headwall HyperespecTM). The illumination was provided by two halogen lamps with regulated and variable intensity. Each group of three leaves (of the same tree) was placed on a platform that moved under the camera (Micos-MOCO motor). The sample was scanned line by line according to the movement (push-broom system). The spatial resolution was $260 \mu \mathrm{m}$. Once the raw images were acquired, the corresponding relative reflectance hypercube was computed, containing the relative reflectance spectrum of each pixel of the image with respect to a reference (mean spectrum of a barium sulfate white reference).

\section{Results and Discussion}

SPAD measurements

According to the visual identification of experts SPAD $>=32$ was set to sound trees, $\mathrm{SPAD}<24$ was labelled as trees severely affected by chlorosis, and intermediate chlorosis level in-between. According to it, there appears to be a very large dispersion among SPAD values for the same rootstock which points the existence of isolated individuals with leave chlorosis.

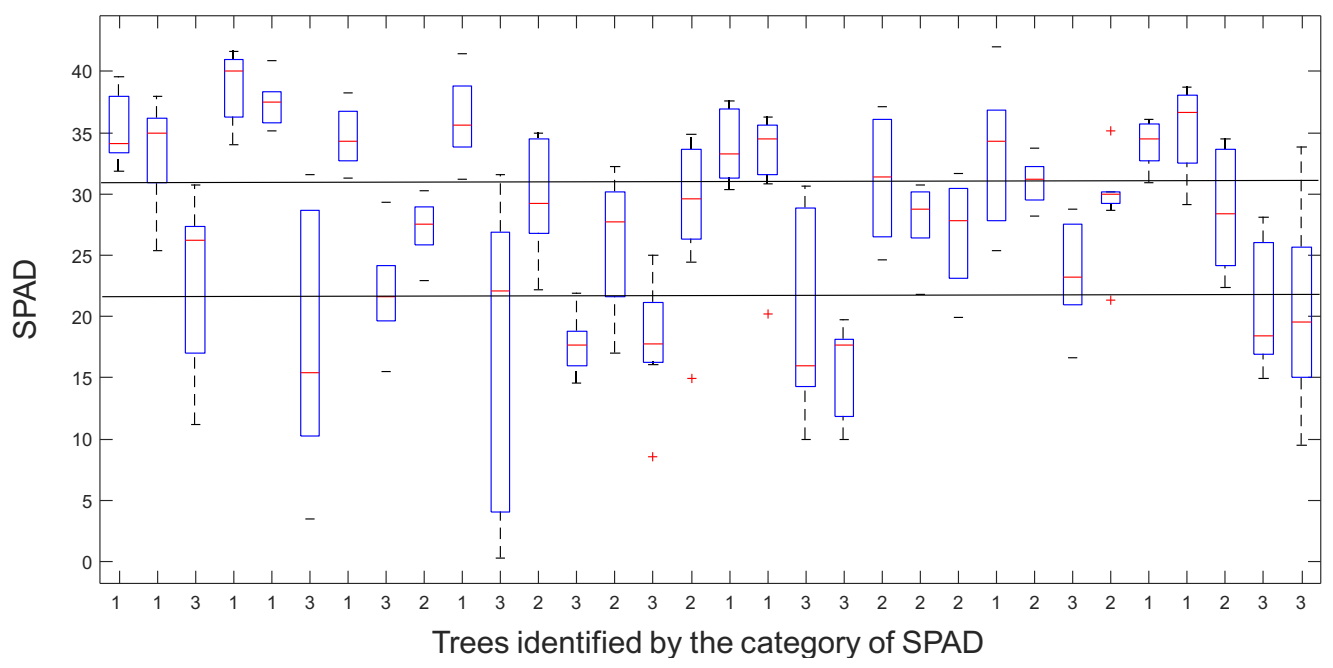

Figure 63. SPAD values ranges for each tree (nine values per tree). Horizontal red lines (median), box and whiskers: interquantile and whole range intervals.

Figure 1shows the SPAD values range (box and whiskers: interquantile and whole range intervals respectively) within each tree considering the nine values of SPAD for each tree. Category of trees (x-axis) are assigned according to their SPAD average value. It could be appreciated that there are trees presenting a huge range of variation such as: 6, 11 and 19 which also belong to SPAD category 3 (SPAD $<24$ ), while more homogeneous ones are also found: 9, 28 etc., generally with almost all their values corresponding to sound tissues (category 1, SPAD > 32). All trees classified as category 1 show values of SPAD higher than 24; so those trees never show 
leaves with large probably chlorosis affection. The range of variation of SPAD within trees of category 3 is very high showing a wide heterogeneity in the development of chlorosis within the tree.

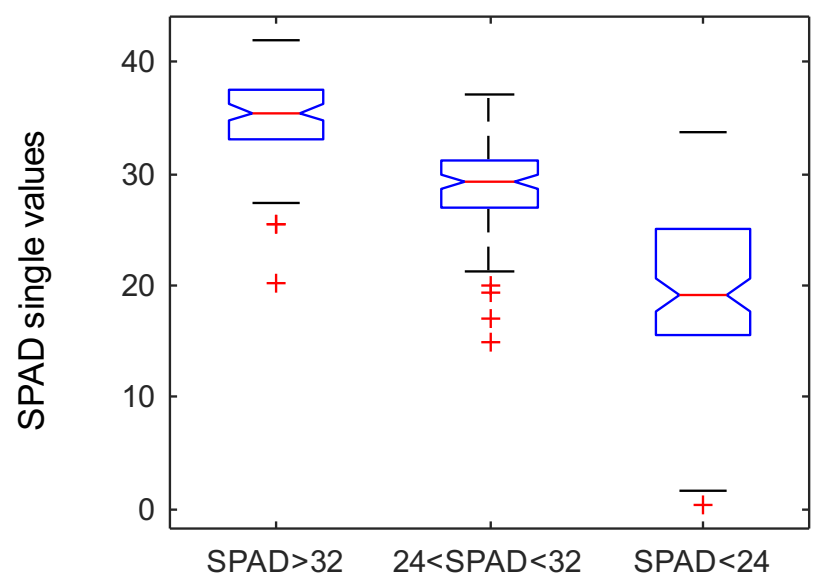

Figure 2. SPAD values ranges (288 values); for each class of SPAD considering the average of the values of the tree. Median, horizontal red lines, box edges correspond to first and third quantiles, and whisker, for the whole range excluding the outliers.

The average value of SPAD was computed for each tree category (nine values per tree, 32 trees, 288 values in total). Then, trees were classified into chlorosis affection level according to SPAD limits (class 1, SPAD $>=32$ sound; class 2, 24<SPAD $<32$ intermediate affected; class 3, SPAD $<24$ severely affected). Figure 2 presents the range of variation for each SPAD class. This graph shows that the SPAD values belonging to category 1 (sound trees) are the highest, also with the lowest variability within such category; on the contrary category 3 (trees with intense chlorosis) show the lowest values but with very high large dispersion. Trees classified as sound present almost all unique SPAD class values (all SPAD above 32), whereas class 3 trees present a high range of variation of individual values: ranging from intermediate and to severely affected individuals by chlorosis.

Table 1. Analysis of variance of SPAD values considering two factors: tree and leaf within the tree.

\begin{tabular}{|c|c|c|c|c|c|}
\hline \multicolumn{6}{|c|}{ Analysis of Variance } \\
\hline Source & Sum Sq. & $d . f$. & Mean Sq. & $\mathrm{F}$ & Prob $>F$ \\
\hline \multicolumn{6}{|c|}{ 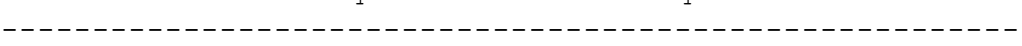 } \\
\hline tree & 12783.8 & 31 & 412.379 & 47.08 & 0 \\
\hline leaf & 16.4 & 2 & 8.199 & 0.94 & 0.394 \\
\hline tree*leaf & 5029.4 & 62 & 81.119 & 9.26 & 0 \\
\hline Error & 1681.7 & 192 & 8.759 & & \\
\hline Total & 19511.2 & 287 & & & \\
\hline
\end{tabular}

Table 1 shows that the differences of SPAD values between trees are significant and that the values of SPAD within leaves are very homogeneous in comparison with the values within trees.

\section{Relation between SPAD and mineral composition of leaves}

When comparing the SPAD with the mineral composition in flower, the SPAD values correlate with several minerals in the leaf composition such a $\mathrm{Ca}, \mathrm{S}, \mathrm{Fe}, \mathrm{Cu}$ and $\mathrm{Sr}$, restricted to $\mathrm{Ca}$ and $\mathrm{Sr}$ at flower level.

Table 2 shows the linear correlation coefficients between macro elements and SPAD at leave and flower level. The correlation coefficients between macro nutrients and SPAD are significant at leave level for Ca, S and $\mathrm{K}$, while at flower level only Ca seems related to SPAD.

Table 2. Linear correlation coefficients between macro-nutrients and SPAD at flower and leave level.

\begin{tabular}{cccccc} 
& $\mathrm{K}$ & $\mathrm{Ca}$ & $\mathrm{P}$ & $\mathrm{Mg}$ & $\mathrm{S}$ \\
\hline FLOWER & $\mathrm{ns}$ & $0.4455^{* *}$ & $\mathrm{~ns}$ & $\mathrm{~ns}$ & $\mathrm{~ns}$ \\
LEAVE & $-0.3091^{* *}$ & $0.4736^{* *}$ & $\mathrm{~ns}$ & $-0.1487^{*}$ & $0.4336^{* *}$ \\
\hline
\end{tabular}


Table 3 shows the linear correlation coefficients between micro elements and SPAD at flower and leave level. As found before, higher correlation coefficients are found at leave than at flower level: for leaves, $\mathrm{Ca}, \mathrm{S}, \mathrm{Fe}, \mathrm{Cu}$ and $\mathrm{Sr}$ appear to be relevant, while at flower level only $\mathrm{Ca}$ and $\mathrm{Sr}$ show significant correlation.

Table 3. Linear correlation coefficients between micro-nutrients and SPAD at flower and leave level.

\begin{tabular}{ccccccccc}
\hline & $\mathrm{Na}$ & $\mathrm{Al}$ & $\mathrm{Fe}$ & $\mathrm{Cu}$ & $\mathrm{Zn}$ & $\mathrm{Sr}$ & $\mathrm{Mn}$ & $\mathrm{B}$ \\
\hline FLOWER & $\mathrm{ns}$ & $0.1517^{*}$ & $0.1460^{*}$ & $0.1854^{*}$ & $\mathrm{~ns}$ & $0.4096^{* *}$ & $0.1200^{*}$ & $0.2099^{*}$ \\
& & & & & & & & - \\
LEAVE & $\mathrm{ns}$ & $\mathrm{ns}$ & $0.3345^{* *}$ & $0.3092^{* *}$ & $0.1049^{*}$ & $0.4565^{* *}$ & $0.1448^{*}$ & $0.3710^{* *}$ \\
\hline
\end{tabular}
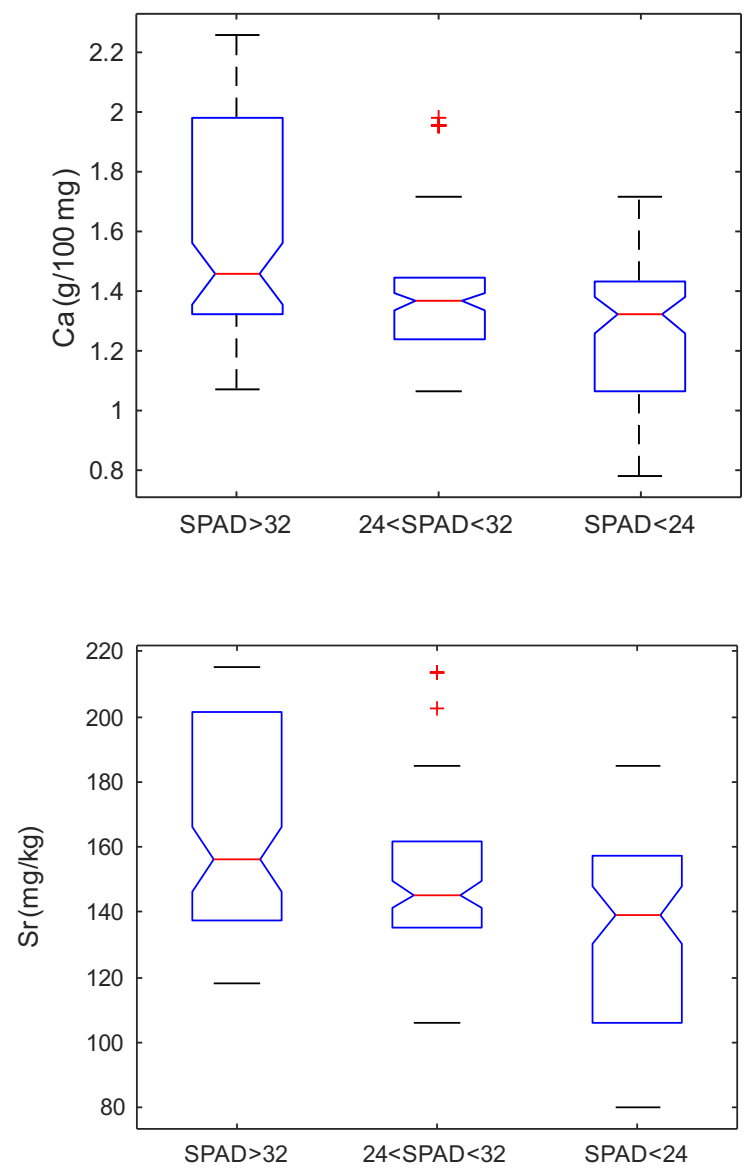

Figure 3. Mineral content of $\mathrm{Ca}$ and $\mathrm{Sr}$, considering the average SPAD values per tree and for each category of chlorosis: median, horizontal red lines, box edges corresponding to first and third quantiles, and whisker referring to whole range excluding the outliers (out of normal distribution).

Spectral features extracted from hyperspectral images

Comparing the average spectra of sound leaves with affected ones, three main features are found; a) a general increase of reflectance from 500 to $600 \mathrm{~nm}$, with a peak around 550, b) a red-edge displacement to the left, and c) an overall weak decrease of reflectance at NIR range (probably due to the degradation of leave microstructure due to the increase in chlorosis affection). Several authors have already noticed that the red-edge moves towards left for decreasing chlorophyll content. Besides, a global increase in the visible range can also be attributed to the decrease in chlorophyll under nutritional stress. 


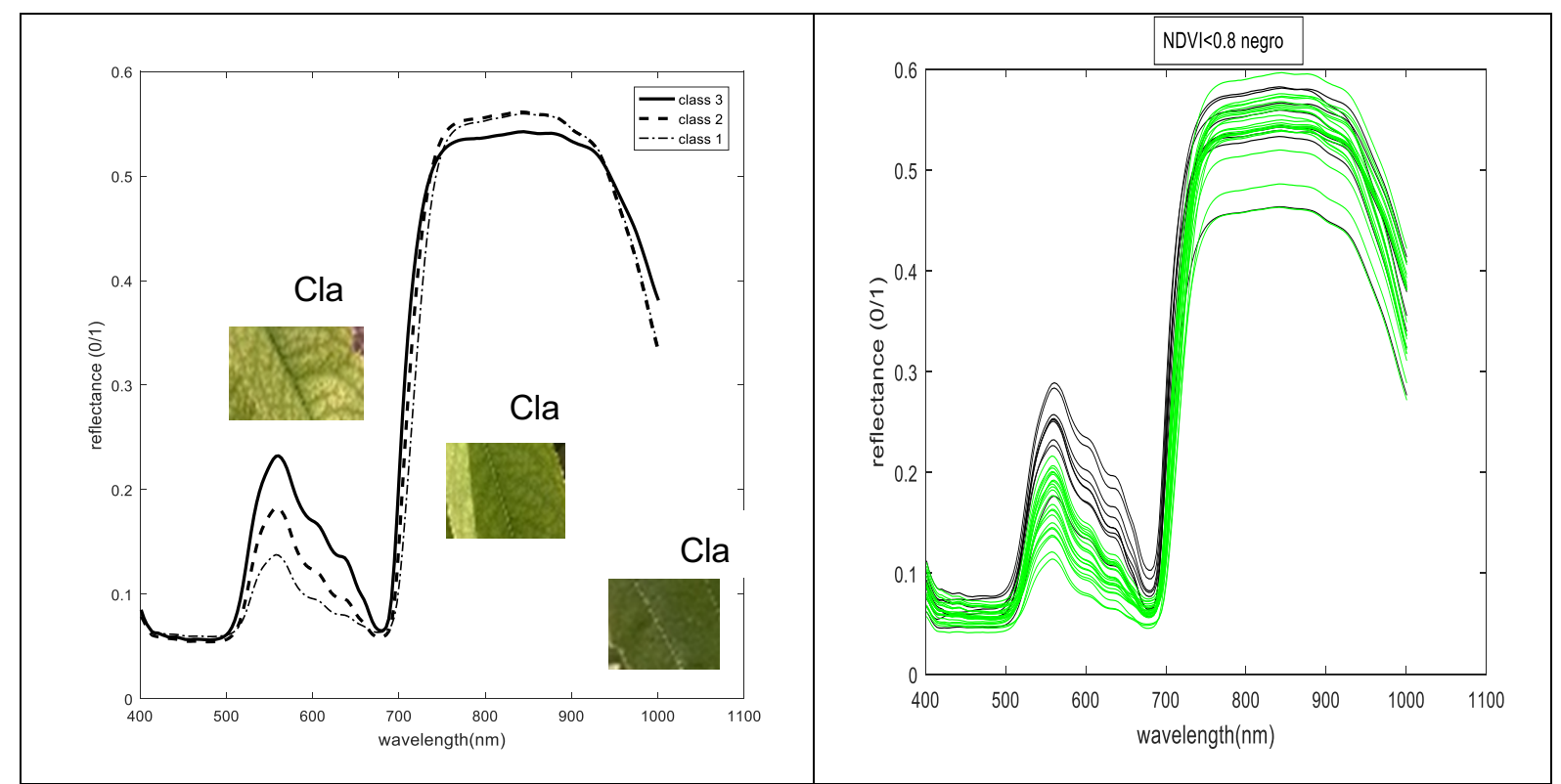

Figure 4. Left, three representative average spectra and RGB images belonging to three samples of leaves. Each spectrum corresponds to one of the types of leaf according to SPAD classes. Right, the average spectra of the 32 leaves; green and black spectra correspond to above and below 0.8 in NDVI.

Figure 4 provides examples of average leaf images and spectra for class 1 (dotted) to class 3 (bold) where a strong decrease of chlorophyll absorbance is found. HASTA AQUÍ. Comparing the average spectra of category 1 to 3, three main features are observed; a) a general increase of reflectance from 500 to $600 \mathrm{~nm}$, with a peak around $550 \mathrm{~nm} ; \mathrm{b}$ ) a red-edge displacement to the left; and c) an overall weak decrease of NIR reflectance probably due to the increase in chlorosis affection. In Figure 4 (right) the average spectra of leaves above and below 0.8 in NDVI is presented: leaves with NDVI below 0.8 (likely to have chlorosis affection according to remote sensing) show high reflectance level between 500 to $700 \mathrm{~nm}$, and a displacement of the red edge to the left. Several authors have already noticed that red-edge moves towards left with decreasing chlorophyll content (Lara et al., 2016; Mutanga and Skidmore, 2007; Van der Meer and Jong 2002). Besides, a global increase in the visible range can also be caused by a decrease in chlorophyll due to nutritional stress in accordance also with literature Ustin et al. (2009).
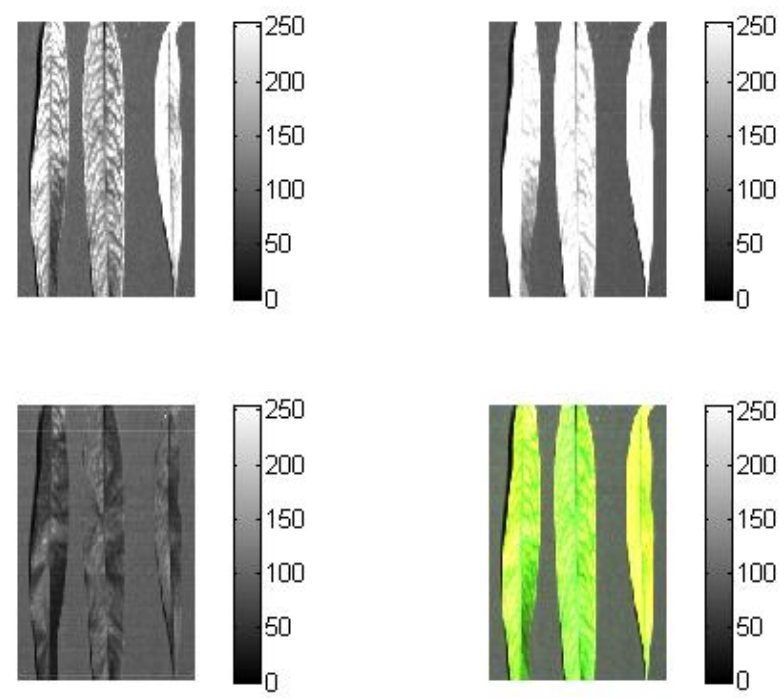

Figure 5. Three examples of images of leaves belonging to different SPAD classes: from left to right: intermediate SPAD, high value of SPAD and low value of SPAD. On the first row, Red (left) and Green (right) images; on the second row, Blue (left) and RGB images (right) resulting from the composition of the other three. 

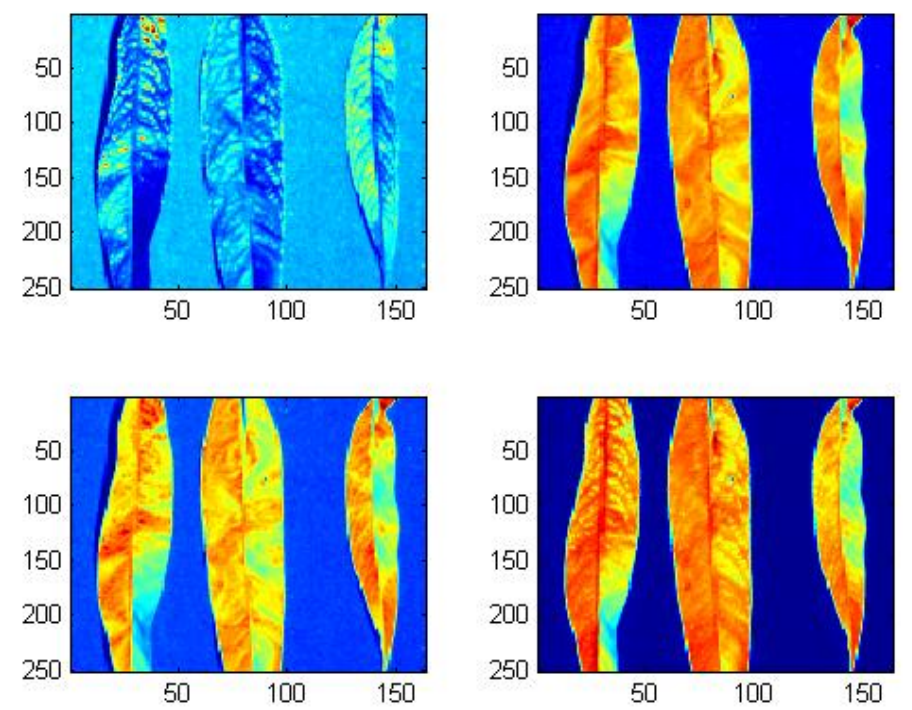

Figure 6. Three examples of images (in false color) of leaves with probably different grade of chlorosis: from left to right: intermediate SPAD, high value of SPAD and low value of SPAD. On the first row, $682 \mathrm{~nm}$ (left) and $803 \mathrm{~nm}$ (right) images; on the second row, addition image $682 \mathrm{~nm}+803 \mathrm{~nm}$ (left) and subtraction image $682 \mathrm{~nm}-803 \mathrm{~nm}$ (right).

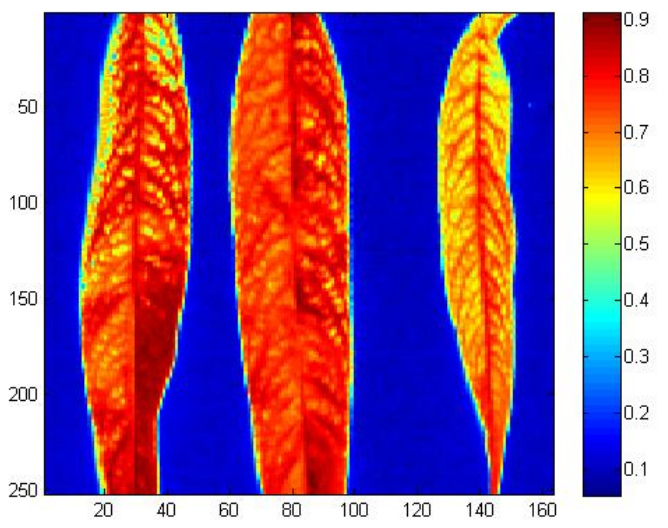

Figure 7. Three examples of NDVI images of leaves with probably different grade of chlorosis: from left to right: intermediate SPAD, high value of SPAD and low value of SPAD.

Figure 7 shows three examples of NDVI images of leaves with different grade of chlorosis. It can be observed that the leaf with lowest value of SPAD (belonging to class 3 , on the right) also shows lower values of NDVI; most of their values of their pixels are below 0.8 . It can be seen the spatial distribution of the chlorosis affection. On the contrary, the leaf (sound tissue) in the middle shows almost all the values of the pixels above 0.8 . And the leaf on the left, intermediate level of NDVI, in accordance to its intermediate class of SPAD.

\section{Conclusions}

The SPAD used as a reference for the identification of chlorosis in peach leaf appears correlated with several leaf minerals: $\mathrm{Ca}, \mathrm{S}, \mathrm{Fe}, \mathrm{Cu}$ and $\mathrm{Sr}$, restricted to $\mathrm{Ca}$ and $\mathrm{Sr}$ when $\mathrm{SPAD}$ is compared with the mineral composition in flower.

The SPAD presents a great variation in the leaves of peach trees in the cases of trees with a large and intense development of chlorosis (SPAD <24), while healthy trees (SPAD> 32) show more uniform (and high) SPAD values in the spatial distribution of the leaf. The variability of SPAD in the leaves of trees affected by chlorosis is clearly much higher than for healthy trees.

The SPAD and NDVI, which are the traditional tools to evaluate chlorosis, can be explained in terms of VISNIR overall reflectance of the leaves, and may be attributed to changes in leaf pigments (visible spectra) and the alteration of tissue microstructure (NIR reflectance). Soon, and on the basis of these same data, the spatial evolution of the areas affected by chlorosis inside the leaf will be analysed, in order to increase the knowledge about the origin and development of this physiopathology that has an important effect on the yield ( $t$ ha- 1 ) and on the quality of the peaches. 
The results of this study clearly point the interest in developing IoT-lowcost multispectral cameras that could be installed in the fields with some type of icloud storage for real time crop surveyannce and variable rate nitrogen fertilization advisory service.

\section{Acknowledgements}

The authors would like to thank the research group Alimentos de Origen Vegetal from Estación Experimental Aula Dei (CSIC) from Zaragoza, Spain for the availability of peach plots and their previous experience concerning peach quality as related to soil properties.

\section{References}

Abadía A, Sanz M, De las Rivas J and Abadía J 1989 Photosynthetic pigments and mineral composition of iron efficient pear leaves. J. Plant Nutr. 12, 827-838

Dong M 1987 The relationship between HCl-extractable iron and chlorosis in leaves. Plant Physiol. Comm. 4, 27-30.

Heras L, Sanz M and Montañés L 1976 Corrección de la clorosis férrica en melocotonero y su repercusión sobre el contenido mineral, relaciones nutritivas y rendimientos. An. Estac. Exp. Aula Dei (Zaragoza) 13, 261289. 776.

Köseoglu A T 1995 Effect of iron chlorosis on mineral composition of peach leaves. J. Plant Nutr. 18, 765-

Lara, M.A.; Diezma Iglesias, Belen; Lleó García, Lourdes; Roger, Jean-Michel; Garrido, Y.; Gil, M. I. y RuizAltisent, Margarita (2016). Hyperspectral Imaging to Evaluate the Effect of Irrigation Water Salinity in Lettuce. "Applied Sciences-Basel", v. 6 (n. 12); pp. 2-18.

Mengel K, Breininger M Th and Bubl W 1984 Bicarbonate, the most important factor inducing iron chlorosis in vine grapes on calcareous soil. Plant Soil 81, 333-344

Merzlyak, M.N., Solovchenko, A.E. and Gitelson, A.A., 2003. Reflectance spectral features and nondestructive estimation of chlorophyll, carotenoid and anthocyanin content in apple fruit. Postharvest Biology and Technology, 27(2), pp. 197-211.

Milton, N.M., Eiswerth, B.A., Aeger, C.M. 1991. Effect of phosphorus deficiency on spectral reflectance and morphology of soybean plants. Remote Sensing Environment. 36, 121-127.

Montañés L, Sanz M, Gomez V and Heras L 1990. Evolución de nutrients en hoja de melocotonero (Prunus persica L. Batsch) y producción. An. Estac. Exp. Aula Dei (Zaragoza) 20, 15-26.

Montañés L and Sanz M 1994 Prediction of reference values for early leaf analysis for peach trees. J. Plant Nutr. 17, 1647-1657.

Mutanga, O., Skidmore, A.K., 2007. Red edge shift and biochemical content in grass canopies. ISPRS Journal of Photogrammetry and Remote Sensing 62 (1), 34-42.

Sanz M, Heras L and Montañés L 1991 Foliar diagnosis in peach: reference nutrient contents throughout the season. An. Estac. Exp. Aula Dei (Zaragoza) 20, 87-94. Sanz M, Cavero J and Abadía J 1992 Iron chlorosis in the Ebro river basin, Spain. J. Plant Nutr. 15, 1971-1981.

Ustin, S.L., Gitelson, A.A., Jacquemoud, S., Schaepman, M., Asner, G.P., Gamon, J.A., Zarco-Tejada, P. 2009. Retrieval of foliar information about plant pigment systems from high resolution spectroscopy. Remote Sensing of Environment 113, S67-S77.

Van Der Meer, F.D., Jong, S.M., 2002. Imaging spectrometry: basic principles and prospective applications. Remote Sensing and Digital Image Processing 4, 113- 135 (Springer. Chapter 5). 


\title{
Integrated Study of a Picking Robot for Greenhouse Grown Sweet Pepper Yi-Chich Chiu ${ }^{a^{*}}$, Li-Wen Chung ${ }^{\text {a }}$, Gang-Jhy Wu ${ }^{\text {a }}$ \\ a Department of Biomechatronic Engineering, National ILAN University, Yilan, 26047, Taiwan \\ * Corresponding author. Email: yichiu@niu.edu.tw
}

\begin{abstract}
A robot was developed for sweet pepper harvesting in greenhouse. The robot has five major components: a six-degree-of-freedom robot manipulator, a harvesting end effector, a machine vision system, a mobile-platform which was modified from electric powered lift-platform-truck, and a control system which employs LabVIEW software package to control and monitor the harvesting processes. The machine vision system uses two color cameras to capture the image of targeted sweet pepper, and determines the position and orientation of its stalk through imagine processing techniques. The end-effector is a two-finger gripper installed with a set of cutting blade on one side of the fingers, and with sponge pads on inner side of both fingers. It was designed to grip and cut the stalk of the targeted sweet pepper, and secure the cut-off pepper between padded fingers with no damage. A primary test using 58 plastic sweet pepper models with $1500 \times 1500 \mathrm{~mm}$ simulated vertical harvesting zone and $500 \times 500 \mathrm{~mm}$ imagine shot was conducted to check the essential functions of the developed robot. The success harvest rate was $93.7 \%$. Another practical performance test which harvested 42 peppers with real sweet pepper plants in simulated greenhouse cultivating setup resulted in $81 \%$ success rate. Out of 8 failed cases, 5 was unable to accurately identify the position of the pepper stalk; 1 was cutting fail, and the other 2 dropped off when was transferred to collecting basket. The time for harvesting one pepper is about 21.8 seconds. The developed robot proved to be feasible and practical for automatic sweet pepper harvesting in greenhouse.
\end{abstract}

Keywords: Sweet pepper, Machine vision, Robot, End-effector, Greenhouse

\section{Introduction}

Protected cultivation requires large investments of human resources, but shortages in agricultural labor have continued to grow. Therefore, finding replacements for traditional manual labor using automated facilities or robots has become a necessary adaptation in agriculture. Robots have been used in numerous sectors such as the manufacturing, medicine, agriculture, disaster relief, entertainment, and space industries. Robotic arms, which can imitate human or animal movements, have been applied as replacements for human labor in dangerous, laborintensive, and repetitive tasks. Taiwan, where intrafacility harvesting is reliant on human labor, is faced with an aging agricultural population and rising labor costs. Faced with such crises, robots could be ideal candidates for application in traditionally human harvesting tasks to enhance production efficiency, improve product quality control, and lower production costs. Bell peppers, which are one of the four major crops of the family Solanaceae, are a common and valuable crop worldwide; their global cultivation area is second only to those of potatoes and tomatoes (Wang et al., 2013). China possessed the largest bell pepper production area in the world (accounting for approximately $37 \%$ of that of the world), followed by Indonesia (approximately $14 \%$ ); the total production area in Asia accounted for approximately $64 \%$ of that of the world. Bell peppers (Capsicum annuиm L.) are divided into sweet peppers and chili peppers according to whether they contain spicy flavors (Li, 2005). The primary bell pepper production zones in Taiwan are located in Chiayi, Nantou, Pingtung, Yunlin, Kaohsiung, Tainan, and Hualien. Typically, bell peppers are cultivated through basket suspension to improve product quality consistency (Li, 2006).

Kondo et al. (2010) created a gripper for harvesting tomatoes from suspended cultivation. The upper finger was designed to cut and grab; the lower finger was positioned at the bottom of the gripper and installed with a sensor to ensure that the gripper moved along the stalk; the width between the two fingers was $8 \mathrm{~mm}$. The gripper was installed on a Mitsubishi RH-6SH5520 robotic arm for harvesting. The arm had $4 \mathrm{df}$, a load capacity of $6 \mathrm{~kg}$, and a maximal action length of $550 \mathrm{~mm}$. The average time to complete a harvest was $15 \mathrm{~s}$, and 10 out of the 20 samples were successfully harvested. Wang et al. (2016) designed a tomato harvesting gripper capable of holding and cutting stems. An infrared sensor was used to detect the tomatoes within action length of the gripper, and a pneumatic drive was subsequently activated to move the gripper toward the fruits.

Chiu et al. (2013) developed a greenhouse tomato harvester using the LabVIEW 7.1 graphic control language. The harvester successfully grabbed large, medium, and small test balls on $94.83 \%, 91.83 \%$, and $89.63 \%$ of the attempts, respectively. The average time to grab each sample was $35.86 \mathrm{~s}$, equating to an average of 100.1 balls that could be successfully grabbed per hour. In the subsequent experiment harvesting real tomatoes, a gripper was designed to harvest tomatoes with diameters of 40-90 $\mathrm{mm}$. The gripper used a $15-\mathrm{mm}$ suction cup that exerted a suction force of $7.2 \mathrm{~N} / \mathrm{cm}^{2}$. The gripper, which also featured a vertical action length of $60 \mathrm{~mm}$ and was able to bend its "fingers," was able to effectively harvest the tomatoes. The successful harvest rate was $95.3 \%$, and the average time taken to harvest each fruit was $74.6 \mathrm{~s}$.

Hemming et al. (2014) established a robotic bell pepper harvester that featured a mobile vehicle, a 9-df arm, two distinct harvesting grippers, a 3D time-of-flight camera, two charge-coupled-device video cameras, and a light source. The two grippers employed for harvesting the bell peppers were the fin-ray type and lip type. Fin- 
ray types involve first grabbing a fruit before cutting it, whereas lip types involve first sucking a fruit and then severing it vertically. In a nonsimplified environment (with nearby leaves present and fruits overlapping one another), the rates of successful harvest were $6 \%$ for the fin-ray type and $2 \%$ for the lip type, and the average time taken to harvest each fruit was $94 \mathrm{~s}$.

This paper describes a robotic bell pepper harvester designed to move and harvest bell peppers automatically inside a facility. The harvester consisted of five components, namely a robotic arm, a harvesting gripper, an MV unit, a lift platform, and a control unit. The LabVIEW 2016 graphic control language developed by National Instruments (hereafter referred to as NI, TX, USA) was employed to write the human-machine interface (HMI) and the major control program. A two-finger gripper and lift platform for harvesting bell peppers were created according to suggestions provided by farmers and experts interviewed for this study. Stereoscopic 3D vision was implemented in the MV unit to calculate the spatial coordinates of bell peppers through image processing. The completed robotic harvester was capable of moving and harvesting bell peppers automatically inside a facility, thus reducing labor costs and improving harvest efficiency.

\section{Materials and Methods}

Physical Analysis of Bell Peppers

A total of 50 Bell peppers was randomly selected from the Kaoping Tomato Farm, and their stalk lengths (from the peduncle to the calyx), stalk widths (where the thickest and thinnest were separately measured), fruit heights, fruit mass, fruit lengths (the length of the long side viewed from above), stalk angles (the angle between the shoulder and the stalk), and fruit width (the length of the short side viewed from above) were measured. The measured values were recorded and analyzed as a reference when designing the gripper for the bell pepper harvester.

According to physical analysis of the bell peppers, the average thickest stalk, average length, and average distance from the top of the fruit to the thinnest part of the stalk were $11.7 \mathrm{~mm}, 30.8 \mathrm{~mm}$, and $19 \mathrm{~mm}$, respectively. Accordingly, the gripper was designed to harvest a bell pepper with a maximum stalk width of 15 mm and a mass of 79-160 g. With the robotic arm positioned on top of the fruit, a 20-mm upward and 35-mm forward movement would allow it to reach the thinnest part of the stalk.

\section{Designing the Robotic Bell Pepper Harvester}

The design of the robotic bell pepper harvester consisted of five parts: 1) A robotic arm unit, which consisted of the arm itself and its controller. 2) A gripper unit, which consisted of a two-finger double action pneumatic gripper, gripper fingers, holders, and blades. The gripper unit was installed at the front end of the arm to locate and grip the target objects. 3) An MV unit, which captured and processed images through the use of the video cameras and lenses. 4) A robotic platform unit, which was the robotic platform itself. 5) A control unit, which consisted of the arm and platform control systems. The platform was used to carry the video cameras, the platform and arm control boxes, the arm, and a fruit basket. Installed at the end of the arm was the bell pepper harvesting gripper. The LabVIEW graphic control language was used to integrate the actions, image capture, and analysis control of all the units, thereby enabling the harvester to automatically move and harvest bell peppers.

\section{Arm Unit}

The robotic arm employed in this study was a KR 6 R900 SIXX model made by KUKA (Germany). The arm was a 6-df industrial grade robotic arm that consisted of six axes, namely the base twisting (A1), base bowing (A2), forearm flapping (A3), forearm twisting (A4), wrist flapping (A5), and wrist twisting (A6) axes. A1 encompassed a rotation range of $170^{\circ}$; the upper arm (A2-A3), forearm (A3-A5), and wrist (A5 to the flange surface) featured respective lengths of 455,420 , and $80 \mathrm{~mm}$. The total action radius was $901.5 \mathrm{~mm}$; the maximum speed of the gripper was $2 \mathrm{~m} / \mathrm{s}$; the minimum movement of the arm was $1 \mathrm{~mm}$; and the maximum holding weight was $6 \mathrm{~kg}$.

The arm could be operated manually or automatically. Manual activation enabled control of each individual action. When the arm was activated automatically, its central control program transmitted commands to the arm controller using Ether Control Automation Technology (hereafter referred to as EtherCAT) to designate target coordinates.

\section{Gripper Unit}

The gripper unit was designed according to the results of the random sampling physical analysis, which indicated that the average thickest stalk width and stalk length of the bell peppers were $11.7 \mathrm{~mm}$ and $30.8 \mathrm{~mm}$, respectively. After physical analysis of bell peppers, the gripper was designed using the Solid Works 2014 computer-aided design software (Figure 1 (a)). The gripper was installed on the flange surface at the A6 axis of the robotic arm and attained its locating, grabbing, and placing capabilities from the arm. The pneumatic drive adopted to control the opening and closing of the gripper was an MPG-810N two-finger double action pneumatic gripper designed by Zimmer (Germany). The gripper, created through the use of aluminum alloy, could maintain its open state with a force of $270 \mathrm{~N}$ without air intake. It featured physical dimensions of 42.0 (width) $\times 69.0$ $($ height $) \times 33.0$ (depth) $\mathrm{mm}$ and a mass of $280 \mathrm{~g}$ and was designed as a two-finger double action gripper. A razor 
blade was installed on the gripper fingers using screws and could be replaced when worn. Both fingers were covered with foam to mitigate the damage sustained during bell pepper harvest. The thickness of each finger was $20 \mathrm{~mm}$, the length of the blade was $40 \mathrm{~mm}$, and the maximal opening range was $18 \mathrm{~mm}$ (Figure 1 (b)). When the gripper closed, it severed the stalk of the target bell pepper using the installed razor blade, completing the harvest process.

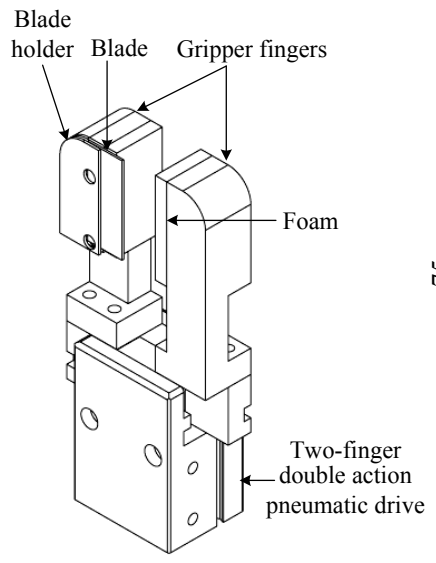

(a)

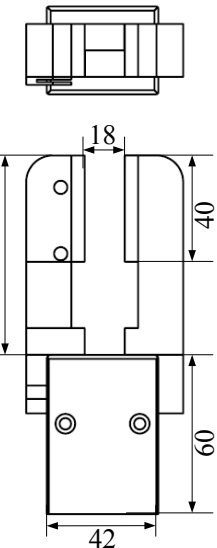

Unit : mm

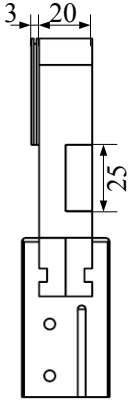

(b)

Figure 1. Schematics of the gripper: (a) anoxometric view and (b) specification.

\section{Unit}

The MV unit comprised video cameras and a lens. The cameras captured images and transmitted them to the control unit for processing. Two C-MOS color video cameras (model UI-3240CP-C-HQ, IDS, Germany) were employed to enable stereoscopic 3D vision. The 2016 LabVIEW Vision Assistant module (NI) was adopted to process the images and identify the characteristics and coordinates of the bell peppers.

(a) Stereoscopic 3D Vision

According to the dual-camera standard stereo geometry as described by Li et al. (2015), when the optical axes of two cameras are parallel to each other, the two side-by-side images will exhibit identical $y$-coordinates, where $X, Y$, and $Z$ represent the horizontal, vertical, and depth spatial coordinates, respectively; $x_{1}$ and $y_{1}$ represent the horizontal and vertical imaging positions of the object in the left image; $x_{\mathrm{r}}$ represents the horizontal imaging position of the object in the right image; $b$ represents the moving distance of the camera; and $f$ represents the focal distance of the camera. Two cameras were used to create a stereoscopic 3D vision system, and the parallax between the feature points of the two images was used to calculate the 3D spatial coordinates of the target object. The distance from the center point between the two cameras was set as $150 \mathrm{~mm}$, and the focal distance was set as $8 \mathrm{~mm}$; two images were simultaneously captured by the control unit. Once the feature point coordinates of the target object in the two images were determined, standard stereo geometry was applied to calculate the 3D spatial coordinates of the object for the gripper to locate.

(b) Image Processing and Analysis

The right camera was used as the primary camera for capturing and processing images. The cameras were activated and used to capture images through the vision acquisition command in the control unit. The images of were then processed through the LabVIEW Vision Assistant. The backgrounds and targets were separated through adjustment of the hue, saturation, and lightness. Subsequently, the boundary objects and smaller particles were removed through thresholding, and the target images were truncated and had any holes filled. A particle analysis was performed to identify the core positions, stalk positions, and boundary positions of the bell peppers, thus completing the image processing. The image signals were transmitted to LabVIEW to calculate the coordinates of the objects. Figure 2 depicts the image processing procedure. 


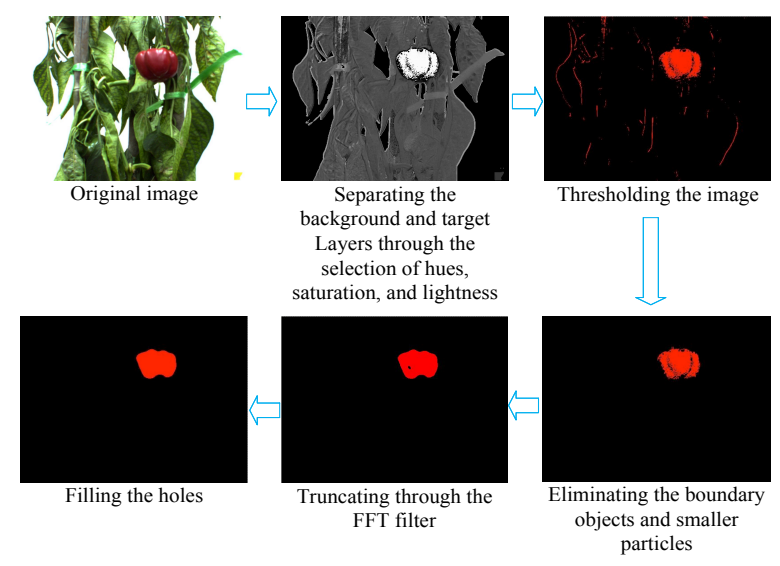

Figure 2. Processing the image of a bell pepper.

\section{Robotic Platform Unit}

The robotic platform unit was used to carry the robotic arm, the lift platform control unit, the arm control unit, the master computer system, the motor drive, and the MV unit. Field measurement revealed the width of the aisles in the laboratory greenhouse to be $850 \mathrm{~mm}$. On the basis of interviews with farmers and experts, the robotic platform must be no wider than $550 \mathrm{~mm}$ to ensure that it did not collide with fruits. Therefore, the platform was designed with specifications of 2000 (length) $\times 545$ (width) $\times 1200$ (height) $\mathrm{mm}$, and the height of the lift platform was $600 \mathrm{~mm}$. The robotic platform featured biaxial positioning control, which involved forward and backward movements (x-axis) and ascending and declining movements of the lift platform (y-axis) to improve its ability to traverse its environment and conduct the harvesting tasks.

The platform was controlled by employing a DVP-32EH00R3 programmable logic controller (PLC) developed by Delta Electronics (Taiwan). The modes of control were divided into manual and automatic modes. The manual mode involved operating each part of the robotic platform by pressing buttons on the platform itself. The automatic mode involved writing system programs, connecting them to the PLC through a serial communication card (RS-232), and controlling the PLC using the LabVIEW HMI. The PLC could drive and halt the movements of each arm axis and record the return amounts of movement through pulses of the encoder, thereby calculating actual movement distances. For the x-axis, a rotary encoder (HTR-HB-8-1800A-V, Hontko, Taiwan) was implemented to calculate the horizontal movement distance of the platform according to its pulse signals. The encoder featured a hollow axial appearance; the hollow shaft was $8 \mathrm{~mm}$ in diameter and connected directly to the shaft of the motor. The resolution of the encoder was 1800 pulses/cycle, and the diameter of the wheel was $200 \mathrm{~mm}$. On the y-axis, a wire encoder (HLS-S10-1A-V, Hontko) was adopted to calculate the vertical movement distance of the lift platform according to its pulse signals. The encoder underwent a measuring trip of $1000 \mathrm{~mm}$ and had a resolution of $1 \mathrm{~mm}$.

A 12-V direct-current (DC) 250-W brushless motor (model BL90M12R8B00144250, Trumman, Taiwan) was jointly applied with the motor driver to enable horizontal movement of the robotic platform at a 4000-rpm rotating speed. The motor decelerated the platform through its joint application with the 1:28 double-shaft reducer (model EC82M, Trumman). A 12-V DC hydraulic motor (model ELTX-800, Winner Hydraulics, Taiwan) was applied to drive the single-scissor hydraulic lifting device of the robotic platform. The hydraulic motor featured a maximal output torque of $4.1 \mathrm{Nm}$, consumed $1500 \mathrm{~W}$ of power, and rotated at $3000 \mathrm{rpm}$. The maximum movement distance of the platform was $600 \mathrm{~mm}$.

\section{Control Unit}

The movement and coordination controls for the arm, MV, and robotic platform were integrated using a control system developed with LabVIEW. EtherCAT was employed to connect the control system to the arm. The LabVIEW Vision Assistant was used to capture, process, and analyze the images of the bell peppers. Subsequently, the coordinates and information for the mature bell peppers were transmitted to the arm, which then located and harvested the identified fruits and placed them in the basket. The control unit consisted of arm control, platform control, and MV control (Figure 3). 


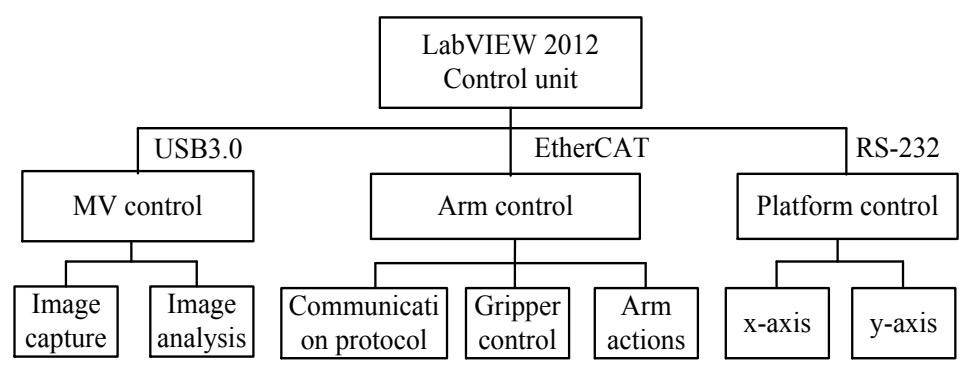

Figure 3. Structure of the robotic bell pepper harvest control system.

Moreover, in the first phase, the control system communicated with the arm and platform, and the arm returned to its initial position to complete the initialization process. In the second phase, the camera captured, processed, and analyzed images of the mature bell peppers. Once detected, the mature bell peppers were numbered and harvested in a sequential order from the upper left to the lower right of the robots field of view. In the third phase, once no mature bell peppers remained in vision, the platform moved to the next visual frame and repeated the second phase until all harvesting tasks in the area were finished.

The arm first returned to its initial position and received the coordinates for the target bell pepper from the control system. The arm then opened its gripper and moved to the front of the fruit without making contact. The gripper turned to the appropriate angle and the arm reached forward. Because the length of the fruit was $73.3 \pm$ $5.8 \mathrm{~mm}$ according to the previous physical analysis, the arm was commanded to reach forward $35 \mathrm{~mm}$. After the gripper reached the stalk, the pneumatic drive closed the gripper to sever the fruit from the plant. Subsequently, the gripper moved the fruit to the fruit basket. The arm then returned to its initial position, completing the harvest of one bell pepper.

\section{Experimental Methods \\ Analyzing the Depth Positioning Errors of the Stereoscopic 3D Vision}

The spatial depth information of the bell peppers was processed and analyzed using the stereoscopic 3D vision. As aforementioned, field measurement revealed the width of the aisles in the laboratory greenhouse to be 850 $\mathrm{mm}$. Therefore, the platform was placed 450-750 $\mathrm{mm}$ away from the plants, and the $x$-axis action length of the arm was set as $\pm 330 \mathrm{~mm}$ at its maximal reach. A random number generator was used to generate depth values between 450 and $750 \mathrm{~mm}$ and width values of $\pm 330 \mathrm{~mm}$. The bell peppers were hung in the space indicated by these values and their images were captured. A total of 30 bell peppers were used in this experiment. By using the bell pepper shoulders as the datum points, the errors were calculated and presented as absolute values. The errors between the depth information and the actual measured depths were identified through image processing.

Measuring the Time to Locate Fruits through Integrating the Robotic Arms and MV

The time required for the robotic harvester to completely capture and analyze the images and complete the harvesting tasks were investigated. A group of 25 sets of coordinates was established using a random number generator that featured depth values of 450-750 $\mathrm{mm}$ and width values of $\pm 330 \mathrm{~mm}$. The bell peppers were hung in the space indicated by these values and harvested using the robotic arm. The total time spent capturing and analyzing the images and completing the harvesting tasks was recorded.

\section{Testing the Performance of the Robotic Platform}

The robotic platform was capable of $x$-axis (forward and backward) and $y$-axis (ascending and descending) movements. The positioning accuracy of the platform was examined to assess any errors in its movement. The $x$ axis positioning test involved examining its forward movement on horizontal ground. The performance of the platform with no object loaded was tested at speeds of 20,40 , and $60 \mathrm{~mm} / \mathrm{s}$ and distances of 200,400 , and 600 $\mathrm{mm}$. Each was repeated five times. The maximal $y$-axis movement range of the platform was $600 \mathrm{~mm}$. The platform's ascending and descending movement was tested. The positioning test was carried out with a fixed speed of ascent of $125 \mathrm{~mm} / \mathrm{s}$ and a speed of descent of $40 \mathrm{~mm} / \mathrm{s}$. The platform was tested with distances of 100 , $200,300,400$, and $500 \mathrm{~mm}$. Each test was repeated five times to measure the actual arrival positions of the platform.

\section{Gripper Positioning Test Using Bell Pepper Models}

Red bell pepper models were employed to test the integrated positioning system of the robotic harvester in the laboratory. Based on the earlier physical analysis of the bell peppers (average fruit width of $65.8 \pm 5.8 \mathrm{~mm}$ and an average fruit height of $69.1 \pm 7.8 \mathrm{~mm}$ ), plastic bell pepper models with widths of $70 \mathrm{~mm}$ and heights of $80 \mathrm{~mm}$ were used as target objects. The test zone was a $1500 \times 1500 \mathrm{~mm}$ area, divided into nine $500 \times 500 \mathrm{~mm}$ visual frames. The movement of the platform in the $x$ and $y$ directions for each test was $500 \mathrm{~mm}$. The test zone was divided into a $3 \times 3$ grid designated as Frames A-I (Figure 4). Each frame was assigned 0-2 bell peppers through the random number generator. Similarly, the coordinates of each bell pepper in the frames were also randomly assigned. The bell pepper models were placed at the assigned coordinates. Each test was repeated five times. The 
robotic platform moved along the grid, and the robotic harvester automatically conducted the experiment. Beginning at the starting point, the platform moved sequentially from Frames A to I. The platform performed MV identification to confirm the number and coordinates of the models within each frame. The arm then positioned and reached for the stalk of the identified pepper model; when the point was within $10 \mathrm{~mm}$ of the center of the gripper, the positioning was considered complete. Subsequently, the arm returned to its initial position. If two pepper models were within the frame, then the arm repeated its positioning task until all the models within the frame were harvested. The platform then moved to the next frame to repeat the harvesting process until the tests in all nine frames were complete. The overall success rate was then calculated according to the test results.

Testing the Integrated Robotic Harvester System by Using Real Bell Peppers

The real bell peppers adopted in the test were Kinfujiu bell peppers from Xingang, Taiwan. The bell pepper plants had heights of 1200-1500 $\mathrm{mm}$ and the pot housing each plant had a diameter of $300 \mathrm{~mm}$. A total of three pots were employed in the test, which encompassed a $1500 \times 1500 \mathrm{~mm}$ area divided into a grid with nine $500 \times$ $500 \mathrm{~mm}$ visual ranges. The movement of the platform in the $x$ and $y$ directions in each test was $500 \mathrm{~mm}$. The platform was programmed to move through the grid harvesting the bell peppers, during which time the leaves from the nearby fruits were removed. The automated harvesting processes of the robot were controlled using LabVIEW. Beginning from the starting point, the platform moved along the grid sequentially from Frames A to I.

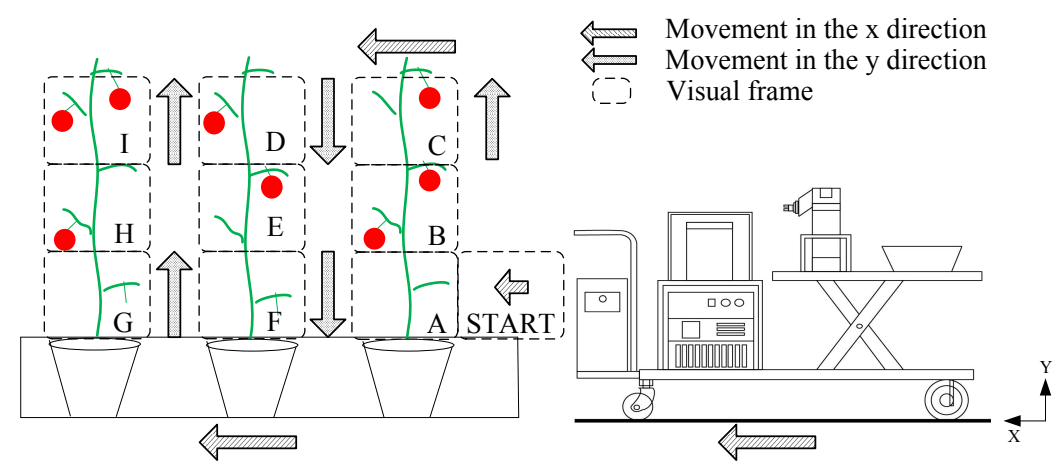

Figure 4. Schematics of the harvesting zone.

The platform performed MV identification to confirm the number and coordinates of the fruits within the frame. The arm then positioned and reached for the stalk of a fruit, closed its gripper to sever the stalk, grabbed the fruit with its fingers, placed it in the basket, and returned to its initial position. If more than one fruit was within the visual frame, then the arm repeated the harvesting procedure until all the fruits within the frame were harvested. The platform then moved to the next frame to repeat the harvesting process until the fruits in all nine frames were harvested. The overall success rate was then calculated according to the test results.

\section{Results and Discussion}

Development of the Robotic Bell Pepper Harvester System

The robotic bell pepper harvester (Figure 5) was designed to move automatically and assess whether bell peppers were present through image capture. When a bell pepper was detected, the robotic arm was used to harvest the fruit and place it into the fruit basket, completing one automated harvest. The developed robotic platform was narrow, enabling it to move along the narrow aisles inside a greenhouse.

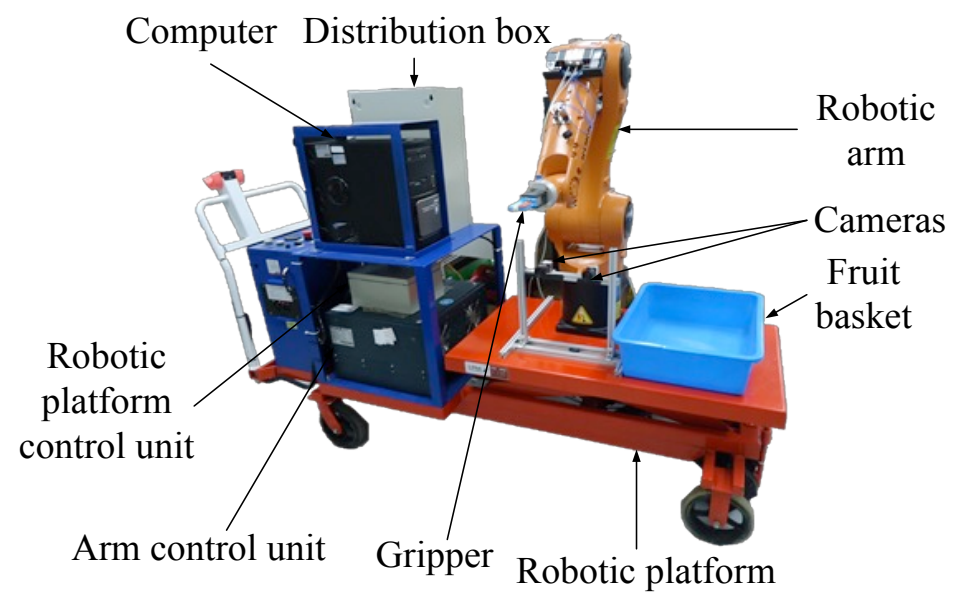

Figure 5. Intrafacility robotic bell pepper harvester. 
The robotic harvester was controlled using LabVIEW. The HMI displayed the image analysis results, the actual locations of the bell peppers, and the instant processing results of the images within the visual frames. The Internet protocols of the arm and the master program were placed in the same domain to enable data transmission between the two units. The images captured and processed through the MV were displayed in the HMI, and the actual positions of the bell peppers were calculated for the arm to identify. When multiple bell peppers were detected by the system, their positions were displayed sequentially for harvesting.

Analysis on the Depth Positioning Errors of the Stereoscopic 3D Vision

The coordinates of 30 bell peppers were randomly generated, and their depth information was calculated through image processing and analysis; subsequently, an analysis was conducted on the errors between the information and the actual depths. The results revealed the largest, smallest, and average absolute values of the depth errors to be $5.5,0.1$, and $3.4 \pm 1.7 \mathrm{~mm}$. The largest diameter of the stalk the gripper blade could sever was $40 \mathrm{~mm}$. Therefore, the center points of the gripper fingers were designated as the datum points for gripping the stalk of a bell pepper, and the largest absolute value of the depth errors had to be $\pm 20 \mathrm{~mm}$ for the stalk to fall within range of the fingers for successful severing. The largest depth positioning error of the stereoscopic 3D vision fulfilled the design requirements of the gripper fingers.

Analysis of the Time Taken to Complete the Harvesting Process

The maximum movement speed of the robotic arm was $2 \mathrm{~m} / \mathrm{s}$, but the speed was adjusted to $1.5 \mathrm{~m} / \mathrm{s}$ to prevent vibrations caused by full-speed movement from affecting its positioning accuracy in the harvesting task. The times taken to complete the tasks of capturing and analyzing images, operating the arm, and finishing the harvest were separately recorded. Figure 6 illustrates timing analysis of the harvesting procedure. According to the analysis, the time required to capture a pair of images through the left and right lenses was approximately $3.6 \mathrm{~s}$; to calculate the stalk position was approximately $1 \mathrm{~s}$; to transmit the coordinates of the fruit from the control unit to the arm required $0.7 \mathrm{~s}$; to analyze the images and calculate the coordinates of the fruit required $5.3 \mathrm{~s}$; opening the gripper required $0.5 \mathrm{~s}$; stretching the arm to the position of the fruit required $4.6 \mathrm{~s}$; and for the gripper to ascend, move forward, change its angle, descend, and close required $1 \mathrm{~s}$ each; it took $4.2 \mathrm{~s}$ for the arm to return to its initial position. Accordingly, the total time of arm operation was $14.3 \mathrm{~s}$, and the slowest process was the arm reaching the position of the target fruit. The total time to complete the entire harvest procedure was approximately $19.7 \mathrm{~s}$.

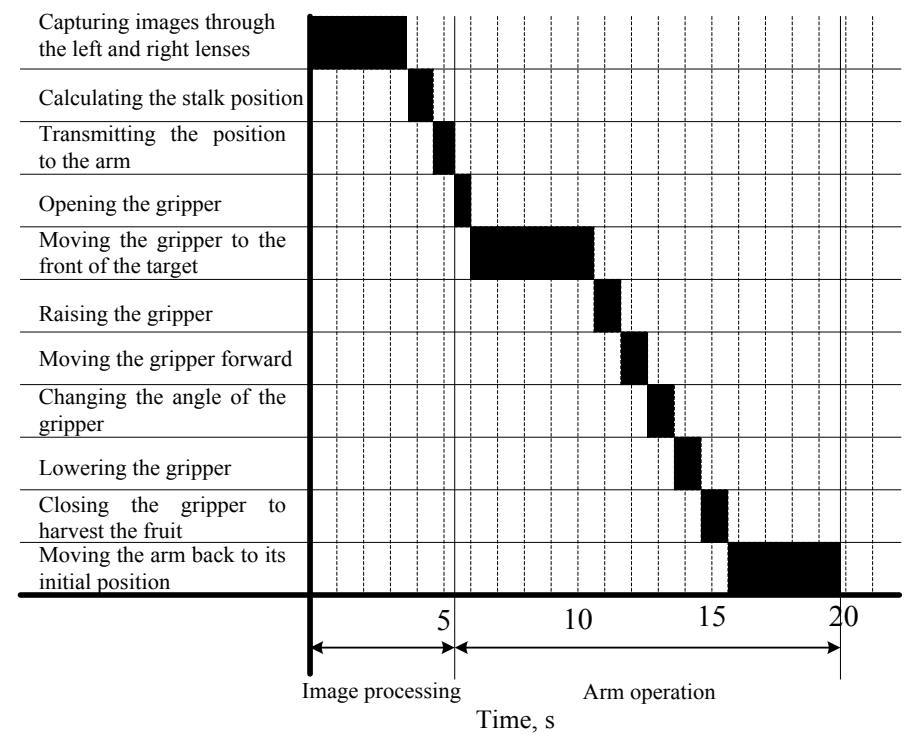

Figure 6. Timing diagram of the harvesting procedure.

Analysis on the Performance of the Robotic Platform

The robotic platform was created to carry the robotic arm, the MV, and other related hardware devices for the harvesting task. The errors in the $x$ and $y$ direction walking distances of the platform were clarified by integrating the system with that of the arm during harvest. The platform was tested at varying speeds in the $x$ and $y$ directions to determine the ideal speed for operation. Regression analysis revealed the errors between the theoretical amount of movement and the actual amount of movement, and an error correction program was written. The actual amount the platform moved was measured for set distances of 200, 400, and $600 \mathrm{~mm}$ in the $x$ direction at speeds of 20, 40 , and $60 \mathrm{~mm} / \mathrm{s}$. The results, indicated that the movement error was largest when the platform moved $200 \mathrm{~mm}$ at a speed of $60 \mathrm{~mm} / \mathrm{s}(11.5 \pm 0.53 \mathrm{~mm})$. When the platform moved at $20 \mathrm{~mm} / \mathrm{s}$, the movement errors at the distances 
of 200, 400, and $600 \mathrm{~mm}$ were $1.5 \pm 1.27,1.2 \pm 0.42$, and $0.7 \pm 0.48 \mathrm{~mm}$, respectively. This indicated that the movement error was minimal when the platform moved at the slowest speed. The loaded platform positioning test was conducted at fixed speeds of ascent and descent of 125 and $40 \mathrm{~mm} / \mathrm{s}$, respectively. The movement errors of the platform when ascending and descending were measured for movement distances of 100, 200, 300, 400, and $500 \mathrm{~mm}$. According to the results, during both ascent and descent the positioning accuracy increased over larger distances, and the movement error decreased with a decline in movement speed. In the ascent test, the movement error was smallest when the platform moved by $500 \mathrm{~mm}(1.06 \pm 0.09 \mathrm{~mm})$; in the descent test, the movement error was also smallest when the platform moved by $500 \mathrm{~mm}(1.02 \pm 0.29 \mathrm{~mm})$.

Analysis of the Integrated Robotic Harvester System Positioning Using Bell Pepper Models

The robotic harvester was tested using bell pepper models to clarify the MV unit's ability to identify targets and the gripper's positioning success rates. The area of the test was divided into a grid encompassing Frames. The harvest positioning test was repeated five times, generating success rates of $92.3 \%, 100 \%, 84.6 \%, 100 \%$, and $91.7 \%$ for an average of $93.7 \%$. The MV identification success rate was $100 \%$. In the unsuccessful instances, the gripper positioned a model successfully but prematurely touched its stalk, affecting the entire branch of models and causing all the other models to shake, thus impairing the subsequent positioning processes. In the test of an integrated robotic tomato harvester system by Lin (2009), the success rates of test balls with diameters of 60, 70, and $80 \mathrm{~mm}$ were $94.83 \%, 91.83 \%$, and $89.63 \%$, respectively. The bell pepper models employed in this study had a width of $70 \mathrm{~mm}$ and a height of $80 \mathrm{~mm}$. The success rate in this study was lower than the success rate of the test with balls with diameters of $60 \mathrm{~mm}$ by Li (2009), but it was higher than for the balls with diameters of 70 and 80 $\mathrm{mm}$ in that same study.

\section{Analysis of the Test Using Real Bell Peppers}

According to the results of the test using real bell peppers, which was conducted in a $1500 \times 1500 \mathrm{~mm}$ area, the time for the robotic platform to move $500 \mathrm{~mm}$ was $24.5 \mathrm{~s}$, the time for the lift platform to ascend $500 \mathrm{~mm}$ was $6.3 \mathrm{~s}$, and the time for the lift platform to descend $500 \mathrm{~mm}$ was $5.2 \mathrm{~s}$. When the robotic harvester detected no bell pepper within its visual frame, it stopped for $1 \mathrm{~s}$ before continuing to the next frame; when it spotted one bell pepper in its visual frame, it harvested the bell pepper in approximately $21.8 \mathrm{~s}$. In the test, which involved 42 mature bell peppers, the MV correctly identified and positioned all the mature bell peppers for a success rate of $100 \%$. However, the stems of five of the bell peppers were unsuccessfully positioned because of their inclination; consequently, the success rate of the gripper positioning tasks was $88.1 \%$. A total of 34 out of the 42 bell peppers were successfully harvested for a success rate of $81 \%$. Of the eight unsuccessful harvests, five were caused by the aforementioned unsuccessful stalk positioning; two were caused by dropping the fruits on the way to the basket - which was because of insufficient thickness of the foam on the gripper leading to an unstable grip; and one was caused by unsuccessful severing of the residual strings from the severed stalk.

\section{Conclusions}

An automated intrafacility robotic bell pepper harvester was successfully developed in this study, with red bell peppers chosen as the targets. LabVIEW 2016 was used to integrate the control programs of all the units. The two-finger gripper was designed with a maximal opening range of $18 \mathrm{~mm}$ for harvesting tasks. EtherCAT and the knowledge representation language copyrighted by KUKA were adopted for communication integration. The radius of the operation of the arm was $901.5 \mathrm{~mm}$, and the movement error was less than $1 \mathrm{~mm}$, indicating the range of operation and control accuracy were satisfactory. Two complementary metal-oxide semiconductor video cameras were applied to establish a stereoscopic $3 \mathrm{D} \mathrm{MV}$, which demonstrated a positioning accuracy of $\pm 5 \mathrm{~mm}$ and a maximal error of $5.5 \mathrm{~mm}$. The robotic platform was loaded with the robotic arm, the gripper, the MV, and the control units, and was able to move automatically within the experimental facility.

Experiments were conducted using the integrated robotic bell pepper harvester system in a $1500 \times 1500 \mathrm{~mm}$ area divided into nine $500 \times 500 \mathrm{~mm}$ visual frames. The robotic platform performed harvesting tests according to the aforementioned sequential order of the visual frames. From the results of the test conducted using 58 bell pepper models, the successful harvest rate was $93.7 \%$. In the test using 42 real bell peppers, 34 were successfully harvested for a success rate of $81 \%$; notably, the rates of successful gripper positioning and MV targeting were $88.1 \%$ and $100 \%$, respectively. Among the eight unsuccessful harvests, two were caused by dropping of the fruits on their way to the basket because insufficient thickness of the foam on the gripper led to an unstable grip; one was caused by unsuccessful severing of the pepper stalk; and five were caused by the inclined stems, which prevented effective stalk positioning. The time for the platform to move $500 \mathrm{~mm}$ was $24.5 \mathrm{~s}$ and the time for its lift platform to ascend $500 \mathrm{~mm}$ and descend $500 \mathrm{~mm}$ was 6.3 and $5.2 \mathrm{~s}$, respectively. When the robotic harvester detected no bell peppers within a visual frame, it stopped for $1 \mathrm{~s}$ and moved to the next frame; when it spotted one bell pepper within a frame, it harvested the fruit in approximately $21.8 \mathrm{~s}$. This experiment was conducted inside the laboratory; future studies may apply the harvester in actual bell pepper harvesting tasks within agricultural facilities, thereby proving the harvester's ability to conduct real-world automated intrafacility bell pepper harvests. 


\section{Acknowledgements}

We would like to express our thanks to the Ministry of Science and Technology of Taiwan for funding this project. We would like to thank Professor F. Ou-Yang for his kind suggestions. Thanks are also extended to C. F. Lin for his kind assistance.

\section{References}

Chiu, Y. C., S. Chen, and J. F. Lin, 2013. Study of an autonomous fruit picking robot system in greenhouses. Engineering in Agriculture, Environment and Food 6(3), 92-98. https://doi.org/10.1016/S1881-8366(13)80017-1.

Hemming, K. J., C. W. Bac, B. A. J. van Tuijl, R. Barth, J. Bontsema, and E. Pekkeriet, 2014. A robot for harvesting sweet-pepper in greenhouses. AgEng Paper No. 0-8. Zurich. http://www.geyseco.es/geystiona/adjs/comunicaciones/304/C01140001.pdf, 2017.

Kondo, N., K. Yata, M. Iida, T. Shiigi, M. Monta, M. Kurita, and H. Omori, 2010. Development of an endeffector for a tomato cluster harvesting robot. Engineering in Agriculture, Environment and Food 2(1), 24-30. https://doi.org/10.1016/S1881-8366(10)80007-2.

Li, A. J., 2006. Basket cultivation techniques for sweet peppers in the facility. Taoyuan Agricultural Technology Report 51, 1-4.

Li, M. J., 2005. Sweet Pepper. Taiwan's farm Highlights: Farming (2), 533-536. Taiwan, Taipei: Council of Agriculture, Executive Yuan.

Wang, G., Y. Yu, and Q. C. Feng., 2016. Design of end-effector for tomato robotic harvesting. IFACPapersOnLine 49(16), 190-193. 


\title{
Orchardmapper: Application for Creating Tree Scale Maps from High Resolution Orthomosaics \\ Dvoralai Wulfsohn ${ }^{\text {a, }}$, Oded Cohen ${ }^{\text {b }}$, Ines Zamora Lagos ${ }^{c}$ \\ ${ }^{a}$ Geco Enterprises Centro de I+D, San Vicente TT, Region de O’Higgins 2970000, Chile \\ ${ }^{b}$ Image Analysis Consultant, Kiryat Tivon, Israel \\ c Dayenu Ltda, San Fernando, Region de O’Higgins 3071116, Chile \\ * Corresponding author. Email: gecochile@gmail.com
}

\begin{abstract}
The availability of georeferenced maps of individual trees and their characteristics such as position in the orchard, canopy area and vegetation indices provide valuable information about spatial variability and crop status for plant-scale precision fruticulture and viniculture. The OrchardMapper desktop application was developed to detect trees and derive individual tree variables from 2D orthomosaics created from images obtained using unmanned aerial vehicle (UAV) platforms. The application is currently being used as part of a commercial precision agriculture and fruit yield estimation service. Applications include mapping of spatial variability of tree vigour for precision canopy management, derivation of tree sampling protocols, tree inventories and detection of missing plants. The algorithm is general enough to permit detection of trees under a wide range of crown coverage structures, ranging from closed carpet-like canopies of overhead trellis systems, to almost- or fully- discrete canopies characteristic of fruit and nut tree orchards. It also estimates portions of the canopy under shading or overexposure (situations resulting in loss of information pixels). We present a general overview of the software and algorithm and evaluated detection performance for four orchard structures. The cases are for multispectral othomosaics of a table grape vineyard, a wine grape vineyard, an apple orchard and an almond orchard with different training systems producing different conditions of canopy closure. Tree numbers ranged from 1500 to 3900 trees in the orchard blocks. Detection of rows and their direction was achieved at close to $100 \%$ detection rate. Tree detection rates (counts and positions) were $98 \%$ or better.
\end{abstract}

Keywords: Aerial imagery, Image analysis, Orchards, Plant-scale, Precision Agriculture, Software, Tree Detection, UAV, Vineyards

\section{Introduction}

The availability of georeferenced maps of individual trees and their characteristics such as position in the orchard, canopy area and vegetation indices provide valuable information about spatial variability and crop status for plant-scale precision fruticulture and viniculture.

A number of developments have been reported for automatic recognition and inventory of trees (or missing trees) planted on grid patterns at the individual plant scale from high resolution aerial or UAV images (RobbezMasson and Foltête, 2005). Typically, algorithms have been developed for specific species or canopy type (crown shape and connectivity). Koc-San et al. (2018) presented a method for automatic detection of citrus trees, their location and size from UAV multispectral images and DEMs. She et al. (2014) and Quiros and Khot (2016) developed algorithms for tree counting from images of nursery trees. Kattenborn et al. (2014) and Kalantar et al. (2017) presented algorithms for oil palm inventories using UAV derived point clouds and images, respectively. A number of investigators have used LIDAR scanners or high density point clouds generated from UAV images to estimate tree location, height and crown width from 3D models. Kattenborn et al. (2014) made use of the TreeVis software developed by Weinacker et al. (2004), which includes 3D models for trees extracted from laser scanning data. Koc-San et al. (2018) presented a review of the literature and summary of previous studies for forest and agricultural tree (location) detection and (crown outline) extraction from different remote sensing platforms.

In this paper we provide a brief overview of the OrchardMapper software, which was developed for tree and plant detection from high-resolution images and is being used in our precision farming advisory services to fruit and nut, vineyards and horticultural growers. Applications include mapping of plant characteristics for precision canopy management, derivation of tree sampling protocols for yield estimation (Ehsani et al., 2016), tree inventories, and detection of missing plants. 


\section{Materials and Methods}

General Description of Algorithm

The OrchardMapper $(\mathrm{OM})$ algorithm detects the structure of orchards (rows and plants) and the characteristics of each row (length, number of plants) and plant (including projected area, geographic coordinates, row and treein-row position, vegetation index, shaded area) from high-resolution orthomosaics, such as derived from images captured from UAV (drone) platforms. The program can analyse RGB and 3-band multspectral orthomosaics in JPEG or Tiff (with World files) or GeoTiffs.

Utilising some predefined data OM detects the characteristics of repetitive elements (the plants or trees) in the image, and fits these repetitive elements to a model that defines the geometry of the orchard and the positions of each plant in the orchard.

The assumptions are: 1) rows are close to linear, 2) the row and tree spacing is within a range predefined by the user, 3) most of the orchard may be constructed as a grid of repeated elements ("tiles" or small blocks, e.g. $3 \times 5$ of trees).

The boundaries of the block or orchard may be irregular, and the trees may have irregular forms. The user is required to delineate the borders of the blocks or orchard manually (the ROI), and prescribe limits on some variables such as plant spacing and fraction of foliage coverage.

The general steps of the OM algorithm are:

1. Create a (reflectance-) calibrated vegetation index map (depending on the camera channels this may be a map of relative NDVI, ENDVI, GNDVI, or Excess Green).

2. Detect well-structured tiles of $N$ trees by $M$ rows.

3. Analyse the tiles to characterise the properties of the repeated elements and their spacing.

4. Match an approximation of linear rows to the plants detected in the tiles.

5. Refine this approximation to compensate for irregularities in the distances between trees between and along rows.

6. Construct a KNN classifier based on Step 3 to separate artefacts from trees.

7. Using this classifier and a wavelet detection method, detect the trees along the rows predicted in Step 5.

\section{Materials and Methods}

The evaluated orthomosaics are of three plantations in central Chile and one apple orchard in Argentina, created from UAV aerial images. Images were obtained using an 8-rotor UAV (Octocopter-XL, HiSystems $\mathrm{GmbH}$, Moormerland, Germany) equipped with a gimbal-mounted modified consumer camera, a Canon SX 230HS with NIR-G-B bands (www.maxmax.com). The camera was configured for each mission with 1/2000 exposure time, custom white balance, fixed focus (infinity), and fixed ISO and f-stop. Images of two $1.2 \times 1.2 \mathrm{~m}$ reflectance panels of $38 \%$ andc $60 \%$ reflectance over the VIS-NIR range (Group Technology VIII), respectively, were captured from altitudes of between 20-30 m above the ground at the start and end of each UAV mission. Missions were typically flown at $100 \mathrm{~m}$ altitude above terrain, providing image resolutions of about $3 \mathrm{~cm} \mathrm{px}^{-1}$. A CHDK script was used to automatically trigger the camera at intervals selected to provide a minimum $62 \%$ side overlap between flightlines and $75 \%$ along flight lines. The orthomosaics were assembled using either pix4dmapper (Pix4D S.A., Lausanne, Switzerland, www.pix4d.com) or Agisoft Photoscan Professional (Agisoft LLC, St. Petersburg, Russia, www.agisoft.ru) (Wulfsohn and Zamora, 2014).

We use four cases to illustrate some of the different canopy structures that can be analysed by OM (Table 1):

a) A 37-year old table grape (Vitis vinifera 'Crimson Seedless') plantation located in Colchagua province, Chile, planted using the traditional Spanish Parron (overhead) trellis system and using angled end posts (which changes tree spacing and canopy coverage at the borders). The image was obtained in the month of December when the plants are in the stage of green berry growth and trained with three leaf layers, producing a carpet like coverage as seen from the air, with gaps at the intersections of four trees (Fig. 1a). The images were obtained with the purpose of providing the producer a map of tree-NDVI classes for precision agriculture management.

b) A 3-year old irregular shaped organic vineyard (Vitis vinifera 'Syrah') planted on steep slopes of the foothills near Placilla, Colchagua province, Chile (elevations changed from 340 to 380 m.a.s.l. over the block). The canopy is formed on a Guyot VSP (Vertical Shoot Positioned, VSP) trellis. The image was obtained in the summer month of January at berry growth stage, providing a view of clearly separated rows, with touching vines along the rows (Fig. 1b). A number of rows are discontinuous due to the shape of the block, and there are gaps due to missing trees along rows. The images were obtained with the purpose of providing the producer with an inventory of the number of trees established in the plantation, the numbers and locations of missing trees in each row for replanting.

c) A mature apple (Malus $x$ domestica) orchard in the Patagonia region of Argentina (Fig. 1c). The 
image is of clearly distinct rows and closely touching tree canopies. The main variety is Pink Lady®, with Fuji trees dispersed on regular grid, visible as larger crown projected areas. This orchard presents a high variability in crown size, and the final row is cast under shadow of wind shelter trees to the east. The purpose of the analysis was to provide a tree-scale NDVI map for the producer and for design of a sampling scheme for yield estimation.

d) An almond (Prunus Dulcis) orchard with three different varieties planted in the coastal region of Chile (Fig. 1d). The images were obtained in the early summer (November). Both rows and trees are easily separable, despite some touching of tree canopies along rows. Due to topography and elevation changes of $10 \mathrm{~m}$ over the block, some nonlinearity of rows are seen on the 2D orthomosaic, especially on the northwest part. The purpose of the mission was to provide a tree-level vigour map (tree NDVI and crown area) for the producer and to make an optimal selection of trees to sample for yield estimation.

Table 1. General description of the orchards.

\begin{tabular}{|c|c|c|c|c|c|c|c|}
\hline $\begin{array}{l}\text { Ortho- } \\
\text { mosaic }\end{array}$ & Crop & Variety & $\begin{array}{l}\text { Training } \\
\text { system }\end{array}$ & $\begin{array}{c}\text { Area } \\
\text { (ha) }\end{array}$ & $\begin{array}{l}\text { Planting } \\
\text { distance } \\
(\mathrm{m})\end{array}$ & $\begin{array}{l}\text { Number } \\
\text { of rows }\end{array}$ & $\begin{array}{l}\text { Number } \\
\text { of trees }\end{array}$ \\
\hline Image a & Tablegrape & $\begin{array}{l}\text { Crimson } \\
\text { Seedless }\end{array}$ & Spanish Parron & 2.0 & $3 \times 3$ & 60 & 2166 \\
\hline Image b & Winegrape & Syrah & Guyou & 0.86 & $2 \times 1$ & 54 & 3909 \\
\hline Image $\mathrm{c}$ & Apple & $\begin{array}{l}\text { Pink Lady } \\
\text { and Fuji }\end{array}$ & $\begin{array}{l}\text { Modified Central } \\
\text { Leader }\end{array}$ & 2.0 & $3.5 \times 2$ & 46 & 3098 \\
\hline Image $\mathrm{d}$ & Almonds & $\begin{array}{l}\text { Non Pareil, } \\
\text { Carmen, Fritz }\end{array}$ & Vase & 4.2 & $6 \times 3.8$ & 41 & 1567 \\
\hline
\end{tabular}

All analysis were conducted on a Toshiba Portege 930 laptop, Intel core i5-3320M 2.6 GHz, 1 CPU with 4 threads, 16 GB RAM, Intel ${ }^{\circledR}$ HD graphics 4000 graphics card running Windows 7 64-bit. Orthomosaics were uploaded to OrchardMapper (OM) and rescaled for analysis to $10 \mathrm{~cm} / \mathrm{px}$ resolution. Each orthomosaic image was calibrated for reflectance in $\mathrm{OM}$ from photos of the calibration panels taken during flights as described in section 2.2, using inbuilt calibration capabilities.

ROIs of the blocks for analysis were outlined manually in OM. A gallery of orchard structure types (Fig. 2a) was used to set recommended algorithm parameter values, with small adjustments as related to differences between the mosaic orchard structures versus that in the gallery. Values of row and tree spacing were updated according to Table 1. The algorithm was then run. (Further adjustments and rerunning the algorithm were done to find a satisfactory solution if the initial solution was not satisfactory. Algorithm runs for the presented cases took less than one minute each on the laptop). When a solution was accepted, the result tables and maps (vegetation index map, tree segmentation map) were exported in formats suitable for upload to a GIS. The detected trees are presented to the user in OM using octagonal polygons (Fig. 2b). The user may edit results (adding and deleting rows and trees, moving and reshaping trees, reordering row and tree labelling); however, no editing was done for this study; OM algorithm solutions were exported unchanged for purposes of performance evaluation. Tables of block, row, and tree addresses and characteristics were exported as CSV files for posterior upload to a GIS (vers. 2.18.7, QGIS Development Team, 2016) for inspection and plotting of results (Fig. 3). A tree segmentation map was also exported for each case (Fig. 3a).

Detections (tree count and position) were overlaid on the original mosaic in the GIS (Fig. 3b) and based on their positions (or absence) categorised as False Positives (objects incorrectly counted as a tree), True Positives (correctly detected trees), False Negatives (missed trees) and True Negatives (correctly detected missing trees). Detection performance was summarised using Sensitivity and Specificity,

$$
\begin{aligned}
\text { Sens } & =\frac{T P}{T P+F N} \\
\text { Spec } & =\frac{T N}{T N+F P}
\end{aligned}
$$

where $T P=$ number if true positives, $F N=$ number of false negatives (Type II error), $T N=$ number of true negatives, $F P=$ number of false positives (Type I error). 

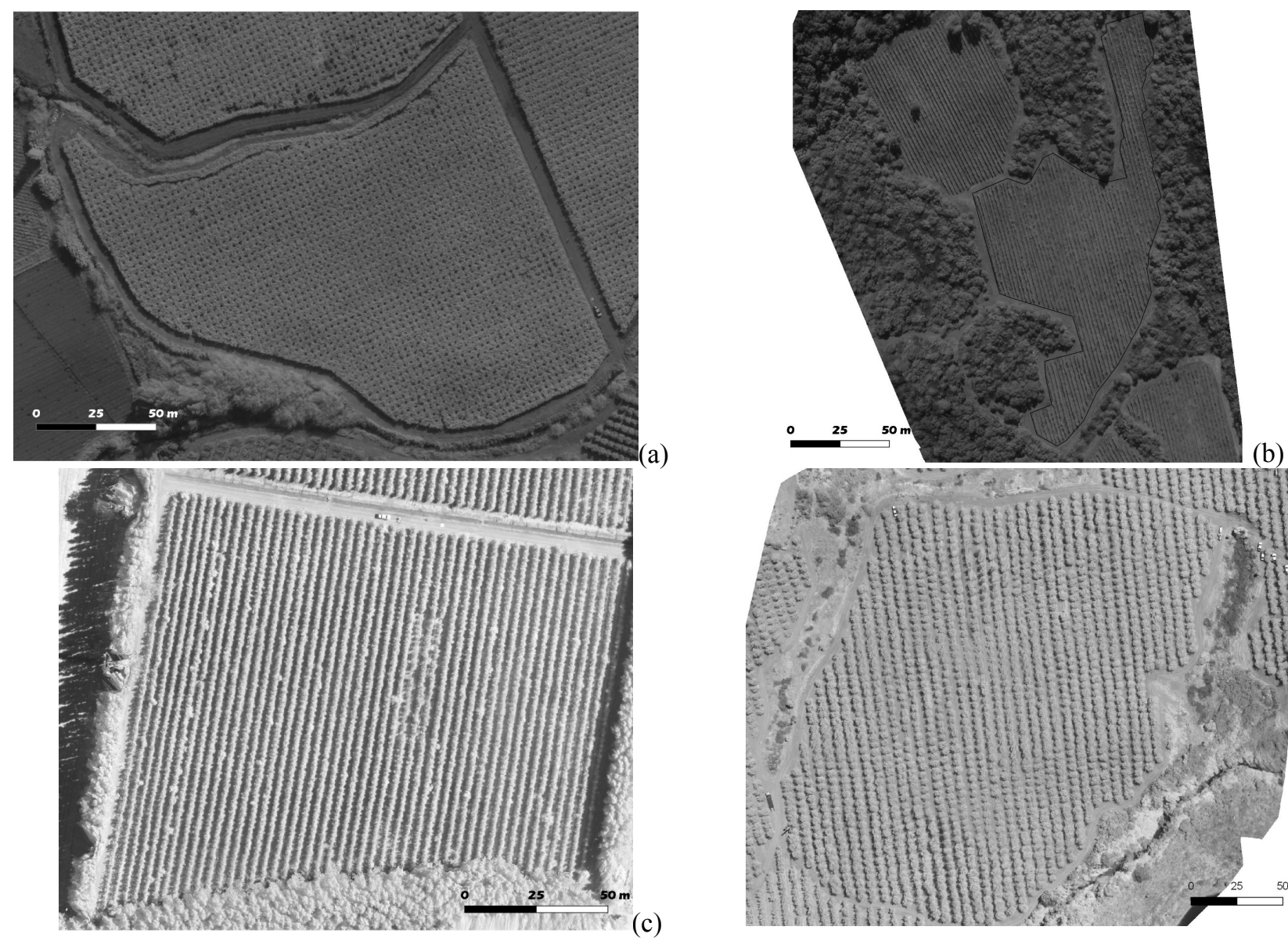

a)

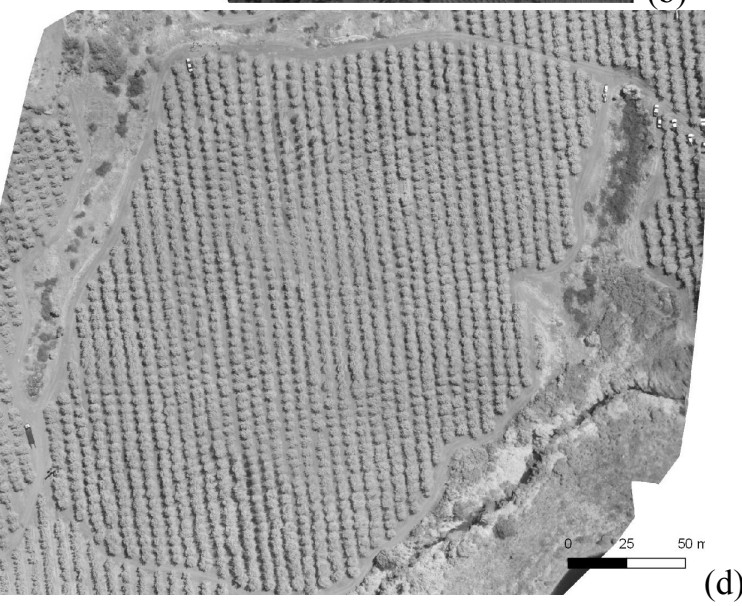

Figure 1. Multispectral mosaic images of the orchards. (a) Tablegrape (var. Crimson Seedless) vineyard (b) Hillside vineyard (var. Syrah) (c) Apple (var. Pink Lady) orchard (d) Almond orchard (four varieties). 


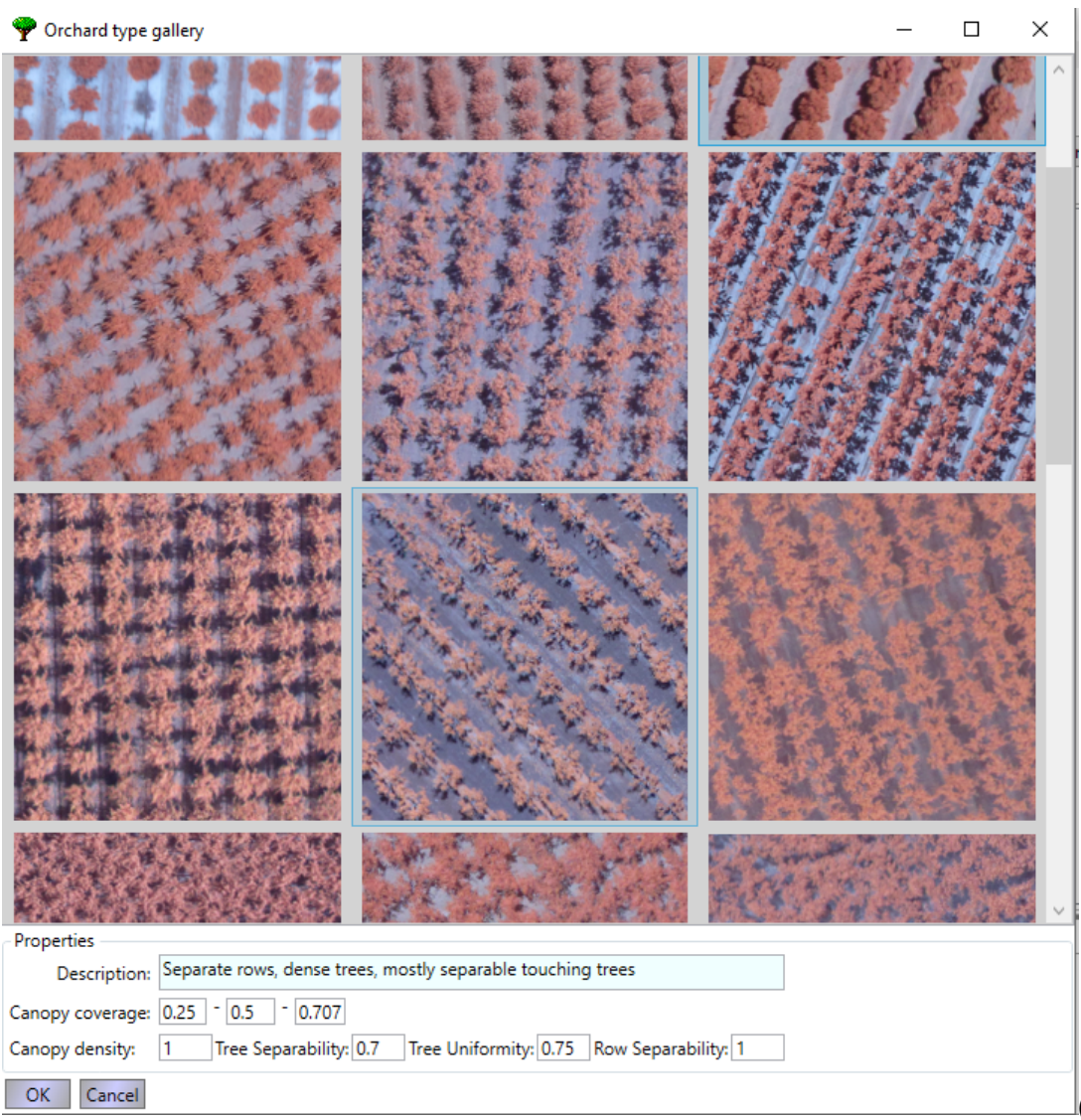

(a)

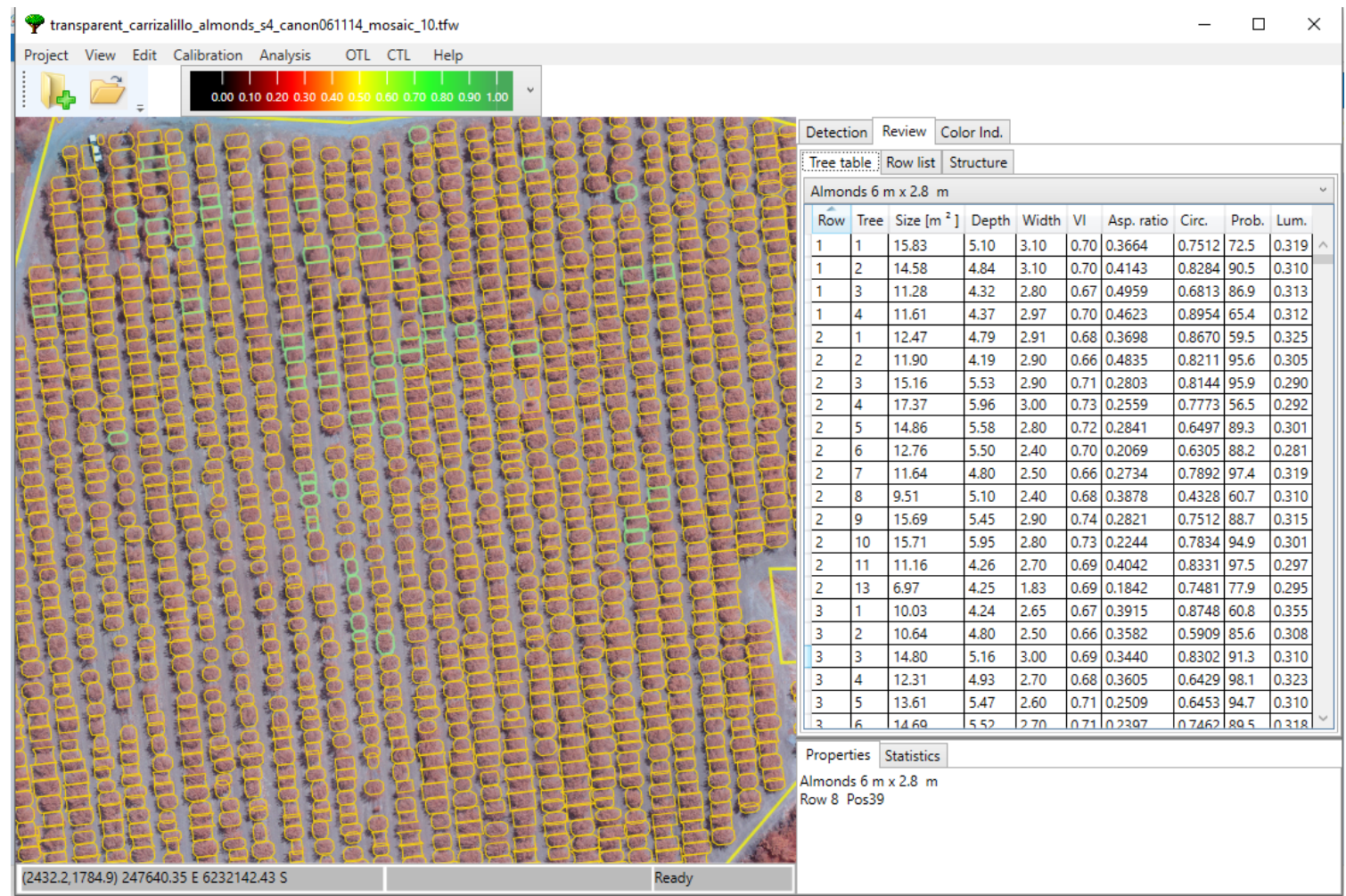

(b)

Figure 2. OrchardMapper. (a) The Orchard type gallery was used to select general algorithm parameters. (b)Tree detection for the almond orchard (image d). Detected trees are outlined using octagons. The results tables and maps are exported for mapping in other software. 


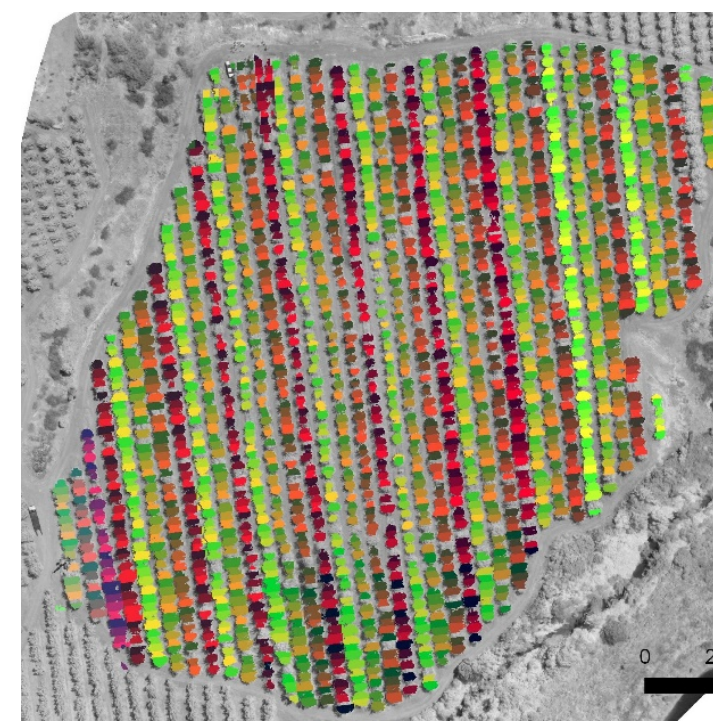

(a)

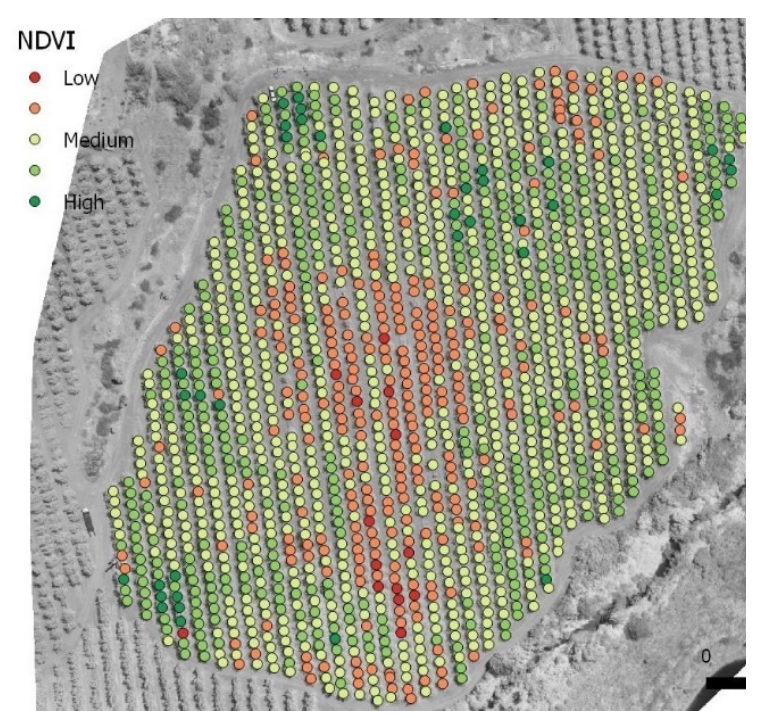

(b)

Figure 3. Maps created from OM outputs for the almond orchard. (a) Segmented tree image as a map overlay on the orthomosaic. Colours have no quantitative meaning; they are used to distinguish between trees. (b) Map of individual tree NDVI dsiplayed in a GIS from the OM Tree output file.

\section{Results and Discussion}

The canopy structures included a closed carpet-like canopy of a table grape orchard, discrete rows with touching trees and many tree gaps of a vineyard, discrete rows with touching trees of an apple orchard, and discrete trees (with minimal touching) of an almond orchard. Detection results with respect to row and tree counts are reported in Table 2. For three of the four mosaics, rows and their orientations were detected without error $(100 \%$ sensitivity on row count). Tree count sensitivity was $98 \%$ and greater for the four cases.

Table 2. OrchardMapper detection results for count of rows and trees. Percent performances are relative to the true values reported in Table 1.

\begin{tabular}{|c|c|c|c|c|c|c|c|c|c|}
\hline \multirow[t]{3}{*}{ Orto-mosaic } & \multicolumn{2}{|c|}{ Detection Result } & \multicolumn{7}{|c|}{ Performance * } \\
\hline & \multirow{2}{*}{$\begin{array}{l}\text { Number } \\
\text { of Rows }\end{array}$} & \multirow{2}{*}{$\begin{array}{l}\text { Number } \\
\text { of Trees }\end{array}$} & \multirow{2}{*}{$\begin{array}{c}\text { Rows } \\
\text { Sensitivity } \\
(\%)\end{array}$} & \multicolumn{6}{|c|}{ Trees } \\
\hline & & & & $F N$ & $T N$ & $F P$ & $T P$ & $\begin{array}{c}\text { Sensitivity } \\
(\%)\end{array}$ & $\begin{array}{c}\text { Specificity } \\
(\%)\end{array}$ \\
\hline a tablegrape & 60 & 2126 & 100 & 37 & 2 & 2 & 2124 & 98.3 & 50.0 \\
\hline b winegrape & 54 & 3886 & 100 & 32 & 41 & 9 & 3877 & 99.2 & 82.0 \\
\hline c apple & 45 & 3040 & 97.8 & 61 & 0 & 3 & 3037 & 98.0 & 0 \\
\hline $\mathrm{c}$ almond & 41 & 1571 & 100 & 0 & 5 & 4 & 1567 & 100.0 & 55.5 \\
\hline
\end{tabular}

${ }^{*} F N=$ number of false negatives, $T N=$ number of true negatives, $F P=$ number of false positives, $T P=$ true positives

For the closed-canopy table grape orchard, the great majority of misses $(F N)$ were along edges of the parron where tree spacing and size is often different due to the way the parron is supported (angled posts) and differing vine vigour and extent at the borders. Two gaps in the canopy created by missing trees were correctly detected $(T N)$. The two false positives $(F P)$ were the result of incorrectly detected foliage crossing gaps as trees.

Detection results for the vineyard were very good with all rows correctly identified, and over $99 \%$ of the trees correctly detected. The specificity was also high ( $82 \%$ ) due to the low count of false positives (low Type-I error) compared to correctly detected misses $(T N)$. Most false positives $(F P)$ were due to small bushes (native to the hills) near the irregular shaped border, aligned with parts of the rows. As an aside, in this vineyard, additional vines were also planted beneath the end-posts, at a reduced spacing than the rest of the vines (usually the end-ofrow vines were planted close to the final post). A ground-truthing revealed that not all rows had a growing vine under the end-post, and when they did, it was not possible to distinguish from a regular end-vine with foliage possibly extending to the end-posts. This ambiguity is one source of error of counts at row ends. Similarly, at the edges of blocks of continuous foliage next to gaps in rows, foliage is often of very low vigour (projected area and vegetation index value). OM generally did not detect these as new vines, which may or may not be correct, depending on the health of neighbouring vines. These are not detectable from aerial images, even of high resolution and even by the human eye, a challenge also discussed by Tang et al. (2016). We did carry out a field 
survey to record missing vines at row ends and vines planted under the end-posts, but given the impossibility of counting these latter vines on mosaics, they were not included in the count or analysis.

In the image of the apple orchard (Fig. 1(c)) the row along the eastern edge of the orchard is under shadow of the wind break trees along that edge, producing very low vegetation index which were not characterised by $\mathrm{OM}$ as foliage. Thus, the error due to missing a row is related to the low brightness and contrast of the original orthomosaic in this zone. Of the 61 false negatives reported in Table 2 for the apple orchard, 60 are for trees along the undetected row; thus, only one tree was not detected in all remaining rows. The 3 false positives were all cases where foliage from neighbouring trees crossed over the gap left by a missing tree and was classified as a tree by OM. The larger projected profile areas of the larger crowns of Fuji trees were correctly delineated in the tree segmentation map and crown area data.

The almond orchard presented the least complex orchard structure, with easily separated rows and trees. All missing trees were correctly detected, and there were no undetected trees. There were errors introduced as four false positives near row edges, especially at the northwest border where there is sufficient row curvature that end trees deviated sufficiently as to appear to line between the imaginary extension of its row and the neighbouring row, an effect arising from changes in topography as observed on a $2 \mathrm{D}$ projection.

Given the wide range of tree shapes and connectivity that can be analysed by the OrchardMapper algorithm, one might well expect that performance will not be as good as an algorithm designed for a specific species or orchard types. Nevertheless, for the above illustrated cases, OM performed very well compared to other methods reported in the literature for tree detection from images.

Koc-San et al. (2018) detected tree locations and crowns on UAV images of citrus (with 60, 196 and 96 trees per plot, respectively). Our almond orchard case presents canopy structures similar to citrus plantations. Tree detection (sensitivity) was close to $100 \%$, but one FP was generated and 2 FNs on the image containing 196 trees. Small size crowns could not be detected because of algorithm limitations imposed by the use of the circular Hough transform to detect round-shaped crown of citrus trees. Crown extraction accuracy was computed by comparing detected crown pixels with manually digitised areas, resulting in sensitivity values between $81 \%$ and $94 \%$.

Quiros and Khot (2016) developed an algorithm for counting trees in high-resolution images of in-field apple nurseries, in which there may be large variability in canopy shapes, spacing, and growth and the presence of weeds and possibly rocks. The algorithm was evaluated using individual and mosaic images captured from 10, 20, 40 and $50 \mathrm{~m}$ altitude using a multispectral imaging sensor. A counting accuracy of $95 \%$ (of an image with 485 plants) was obtained using mosaic images taken from $10 \mathrm{~m}$ altitude $\left(0.35 \mathrm{~cm} \mathrm{px}^{-1}\right)$, whereas accuracies were reduced to $88 \%$ and $75 \%$ for mosaics obtained from $40 \mathrm{~m}$ and $50 \mathrm{~m}$ altitudes, respectively. The orchard structure created by very small plants is not represented in the case studies that we present above, so a direct comparison cannot be made with $\mathrm{OM}$ in this paper.

Kalantar et al. (2017) developed an semi-automatic algorithm that combined template mapping and objectbased image analysis to count oil palm trees in UAV images of oil palm plantations. They obtained detection rates (sensitivity) of $91 \%$ of a plantation with 509 trees using the combined method. They obtained 73 false positives and 45 false negatives with the method. The plantation structure in the images presented quite a mix of zones with trees located on a gird-like pattern, and others with more deviations from the structure, and deviating rows, so that a direct comparison cannot be made with the cases presented in this paper. The approach we use for highly heterogeneous plantation is to divide the plantation into two or more zones (ROIs), in which each ROI has trees in approximately aligned rows for analysis.

The basis of the OM algorithm which is to detect statistical patterns (repeating tiles) provides a powerful approach to detecting individual trees and their structures under a broad range of orchard structures and tree canopy shapes. In general, errors in detection can be attributed to units that can be considered as extreme outliers or due to lack of detail or information and artefacts in the orthomosaic reconstruction. The outliers may arise from short-range, but large changes in row and/or tree spacing, high nonlinearities of rows, and pixel scale loss (strong shadows or specular reflection) or degradation (shaded foliage vegetation indices) in the mosaic. The challenges introduced by shadows and shade are particular to the high resolution images obtained by low altitude UAVs, and are not an issue for satellite images or airplanes. Some such effects are seen on the apple orchard mosaic, which was obtained under windy conditions. Another observation is that at small scales, separation of trees is more straightforward, but at higher scales (zooming in on the image pixels), touching pixels representing tree foliage are more difficult to separate for the segmentation step. It could be expected that higher quality multispectral images (such as obtained using true multispectral cameras) and cameras with better dynamic range than the adapted consumer cameras used, could facilitate more precise tree crown segmentation.

\section{Conclusions}

The OrchardMapper algorithm is sufficiently flexible and powerful to be able to detect trees planted in rows under a broad range of canopy coverage fractions and tree crown shapes, ranging from closed carpet-like canopy of a table grape orchard, discrete rows with touching trees and many tree gaps of a vineyard, discrete rows with touching trees of an apple orchard, and almost-discrete trees of an almond orchard. Two of the orchards were 
planted on steep slopes, which distort row linearity and tree spacing seen on the orthomosaics.

OM correctly detected all rows in the orchards, with the exception of one row under strong shadow. The software detected from $98 \%$ to $100 \%$ of trees correctly from the mosaics. Sources of errors included high nonlinearity of rows so that trees nearly aligned with the orientation of an adjacent row, foliage crossing the gap where a tree was missing, and very low vigour foliage at the edges of vineyard rows or gaps between vines.

Block, row and tree results provided data convenient for upload to a GIS for creating maps for a number of applications such as tree inventories, plant-scale vegetation index maps for precision agriculture, for preliminary crop scouting and trees selection for yield estimation and fruit quality surveys, and for quantitative summaries of block, row- and tree-scale variability of tree characteristics.

\section{Acknowledgements}

OrchardMapper was developed as part of the projects "Pronofrut: Desarrollar un Método Práctico para Estimar Cosecha en Frutales a Nivel Predial con un Rango de Error Conocido, para Entregarlo al Mercado como un Servicio de Estimación de Cosecha" (11IEI-12803) and "Ajustes y Empaquetamiento para Contar con un Producto Tecnológico Validado Comercialmente, que Permitirá Entregar un Servicio de Estimación de Cosecha de Fruta con un Error Conocido No Superior al 10 Porciento" (15ITE2-39616) with partial financing by CORFO-INNOVA CHILE (https://www.corfo.cl/sites/cpp/movil/webingles) and by Dayenu Ltda (www.dayenu.cl). We thank the producers, winery and fruit exporters for their collaboration and Victor Ahumada (Dayenu Ltda), UAV pilot.

\section{References}

Ehsani, R., D. Wulfsohn, J. Das, and I. Zamora Lagos. 2016. Yield estimation; A low-hanging fruit for application of small UAS. Resource, 23 (4), 16-18. St. Joseph, Mich.: ASABE.

QGIS Development Team. 2016. QGIS Geographic information system. Open Source Geospatial Foundation. URL http://qgis.osgeo.org

Kalantar, B., M. Oludare Idrees, S. Mansor, and A. A. Halin. 2017. Smart counting - oil palm tree inventory with UAV. Coordinates. 17-22. May 2017.

Kattenborn, T., M. Sperlich, K. Bataua, and B. Koch. 2014. Automatic single palm tree detection in plantations using UAV-based photogrammetric point clouds. The International Archives of the Photogrammetry, Remote Sensing and Spatial Information Sciences, XL-3, 139-144. doi:10.5194/isprsarchives-XL-3-139-2014

Koc-San, D., S. Selim, N. Aslan, and B.T. San. 2018. Automatic citrus tree extraction from UAV images and digital surface models using circular Hough transform. Computers and Electronics in Agriculture. 150, 289-301. doi.org/10.1016/j.compag.2018.05.001

Quiros, J. J., and L. R. Khot. 2016. Potential of low altitude multispectral imaging for in-field apple tree nursery inventory mapping. IFAC-PapersOnLine 49-16, 421-425.

Robbez-Masson, J.M., and J.C. Foltete. 2005. Localising missing plants in squared-grid patterns of discontinuous crops from remotely sensed imagery. Computers and Geosciences. 31, 900-912. doi:10.1016/j.cageo.2005.02.013

She, Y., R. Ehsani, J. Robbins, J. N. Leiva, and J. Owen. 2014. Applications of small UAV systems for tree and nursery inventory management. In Proceedings of the 12th International Conference on Precision Agriculture, Sacramento, CA, USA, June.

Tang, J., M. Woods, S. Cossell, S. Liu, and M. Whitty. 2016. None-productive vine canopy estimation through proximal and remote sensing. IFAC-PapersOnLine. 49-16, 398-403. doi 10.1016/j.ifacol.2016.10.073

Weinacker, H., B. Koch, and R. Weinacker. 2004. TreesVis-a software system for simultaneous 3D-real-time visualisation of DTM, DSM, laser raw data, multispectral data, simple tree and building models. International Archives of Photogrammetry, Remote Sensing and Spatial Information Sciences, 36, 90-95.

Wulfsohn, D., and I. Zamora Lagos, 2014. The use of a multirotor and high-resolution imaging for precision horticulture in Chile: An industry perspective. In Proceedings of the 12th International Conference on Precision Agriculture, Sacramento, CA, USA, June. 


\author{
Development of an Autonomous Agricultural Vehicle \\ Pieter-Jan Note $^{\text {a, }}$, Jan Bienstman ${ }^{\text {a }}$, Chris Ganseman ${ }^{\text {a }}$ \\ ${ }^{a}$ Flanders Make, Core Lab MotionS, Celestijnenlaan 300 / B4027, 3001 Heverlee, Belgium \\ * Corresponding author. Email: Pieter-jan.note@flandersmake.be
}

\begin{abstract}
This paper describes the developments of an autonomous agricultural vehicle at Flanders Make. Flanders Make is a Flemish research institute, aiming to bridge the gap between universities and manufacturing companies by providing top-class research and infrastructure. One of the research domains is autonomous vehicles. Over the past years, Flanders Make has invested in the development of an autonomous driving platform based on the Nvidia Drive PX2 hardware. This hardware and software platform is now being used in several projects and implemented on different vehicles ranging from an AGV to an on-highway car and bus and off-highway equipment such as an agricultural tractor. In this paper, the development of our autonomous tractor is presented. This paper covers the goal of the Off-Highway Equipment project, the equipment used, the project results and next steps.
\end{abstract}

Keywords: Flanders Make, test infrastructure, sensor fusion, perception, motion planning, control, New Holland T7 tractor, Nvidia Drive PX2, MicroAutoBoxII

\title{
1. Introduction
}

Flanders Make is a strategic research centre for the manufacturing industry. It's mission is to strengthen the international long-term competitiveness of the Flemish manufacturing industry. Flanders Make is driven by more than 400 highly specialised researchers operating from Flanders Make sites in Lommel, Leuven and Kortrijk as well as from 10 research facilities at the major Flemish universities. Flanders Make supports SME and large manufacturing companies with excellent research in two application domains:

Products: vehicles and machines

Processes: assembly

Flanders Make is organised in 4 competence clusters with both internal and external core labs.

Over the years, Flanders Make has build experience with different autonomous vehicles, ranging from indoor AGV's to On-Highway and Off-Highway vehicles. This experience has also been put into practice for industrial use cases.

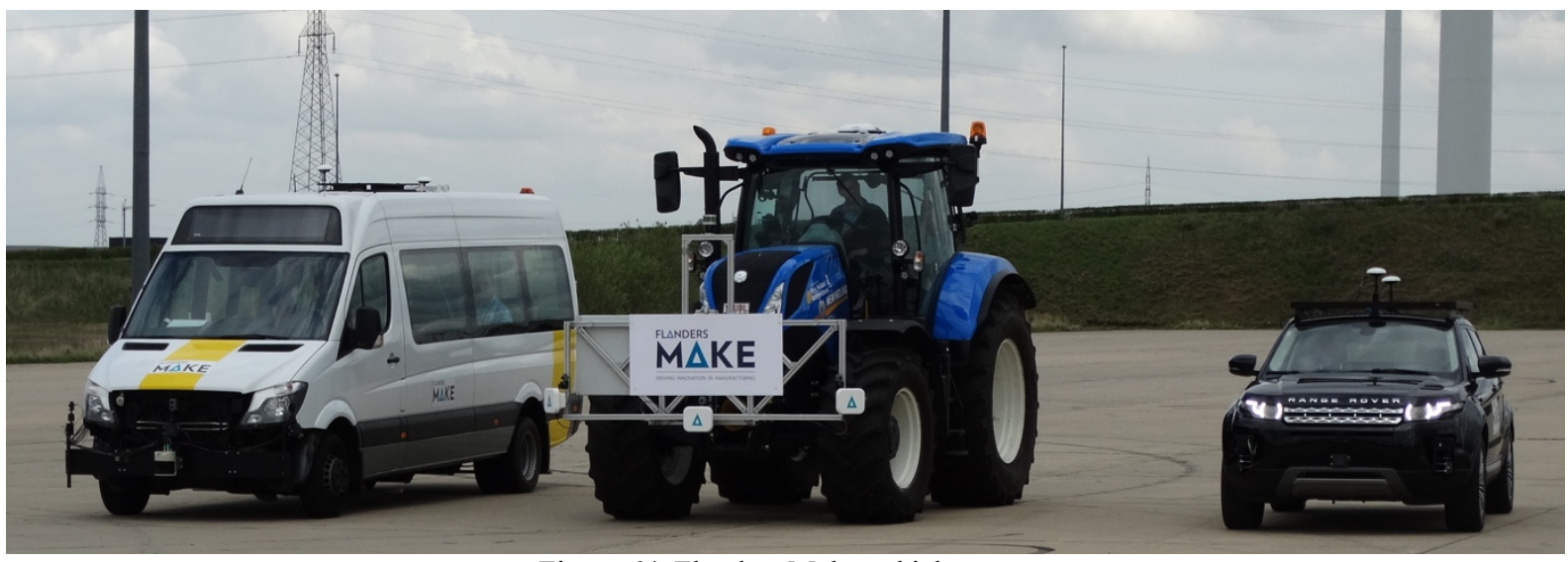

Figure 64. Flanders Make vehicles.

In the beginning of 2017, the Off-Highway Equipment project started. The goal of this project is to extend the infrastructure of Flanders Make with an autonomous Off-Highway vehicle. A New Holland T7 tractor with front loader and rear implement was chosen for this purpose. During this project, a portable platform will be developed to allow autonomous driving and operation for any Off-Highway vehicle. The second goal is the electrification of an agricultural implement.

This infrastructure will allow to test newly developed technology and components in off-highway conditions. It provides complementary infrastructure to the available On-Highway vehicle infrastructure. 


\section{Materials and Methods}

A New Holland T7.175 SideWinder II tractor was chosen and acquired by Flanders Make. This tractor was specifically chosen since it already has a high level of factory automation. The tractor is equipped with the Precision Land Management (PLM) system consisting of several subsystems such as the advanced GPS localization system with RTK correction, Intellirate, Intelliview and two way communication with Isobus III technology between tractor and implement. Additionally, two implements, a New Holland front loader 770TL and an articulated arm of Vandaele are acquired. These acquisitions allow Flanders Make to perform research on autonomous driving of the tractor and automate work

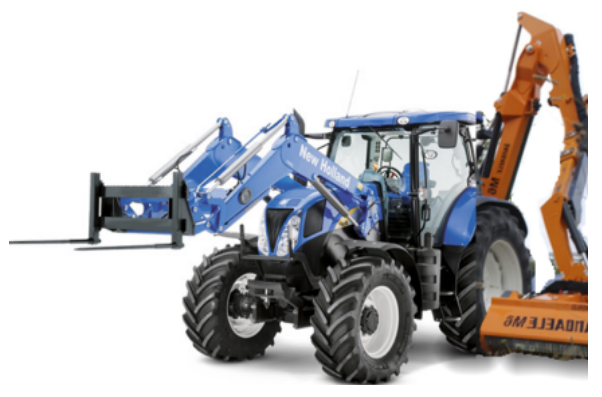
functions with the implements.

Up till now, most of the agricultural and construction actuators are hydraulic actuators since they are able to deliver high forces in a dense format. Considering the electrification trend in many industries, some manufacturers are experimenting with electric actuators ${ }^{3}$. One of the goals of the project is the electrification of the actuators of the articulated arm, which is expected to contribute to energy saving while enhancing the controllability and accuracy of the arm. Therefore, the hydraulic actuators are to be replaced with electric actuators. The high power for the electric actuators will come from a separate generator connected to the PTO.

Several modifications will thus be made to the tractor/implements to accommodate for the additional sensors, power components, computational components, electric actuators and cabling. The additional sensors required for scanning and monitoring of the environment are placed on sensor frames. There will be one sensor frame on the front of the tractor to detect objects in the path of the tractor and one sensor frame on the roof of the tractor for full surround awareness and positioning. Additional sensor frames dedicated to each implement can be added. The computational components, network and storage will be mounted in a box - our system box - on the roof of the tractor cabin. An extra box - powerbox - containing batteries, inverter/charger and energy management provides ample power for all sensors and the systembox. This allows us to have long test cycles in the field, with or even without the engine of the tractor running. A picture of the tractor with frames and boxes and an insight of the powerbox can be seen in Figure 65.

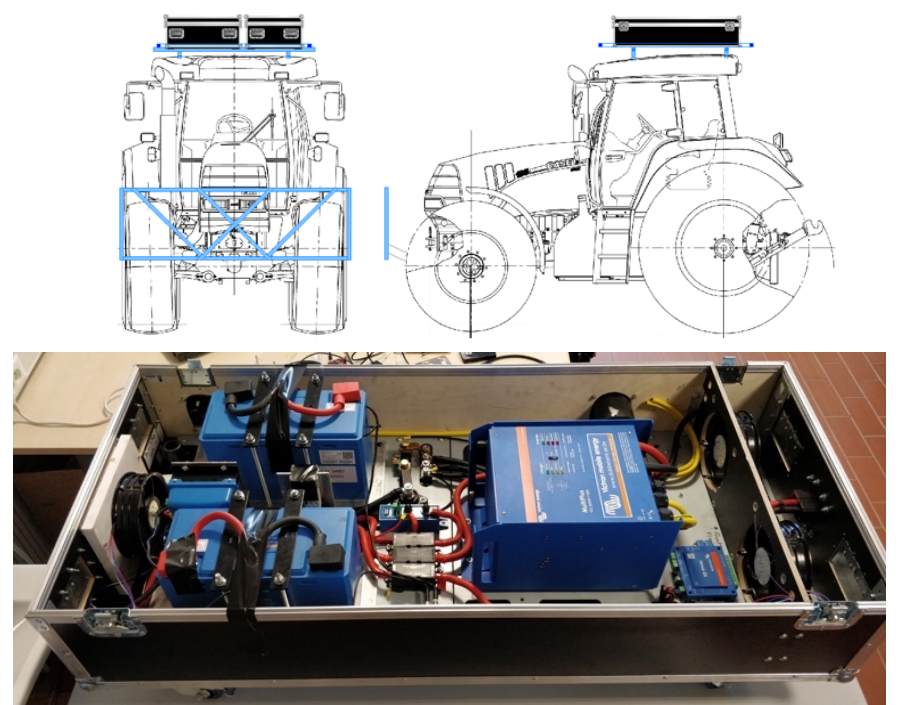

Figure 65. Left: Tractor with front and top sensor frame. Boxes are also mounted on the top frame. Right: powerbox.

The autonomous platform which will be developed for the tractor, consists of several component groups (See Figure 66):

- Sensors required to perceive the environment and detect objects. Two groups can be defined: Sensors for the global localisation and mapping of the tractor, and sensors for the local perception, localization and control of the implement in its smaller environment. For each group, safety sensors are required.

- Computational units \& Software required to merge and interpret the sensor data by sensor fusion (perception),

https://www.volvoce.com/global/en/news-and-events/news-and-press-releases/volvo-ce-unveils-100percent-electric-compact-excavator-prototype/ 
plan the actions (motion planning), control the actuators and move the implements

- Actuators required to steer, accelerate and decelerate the tractor

These component groups are further detailed in the following paragraphs.

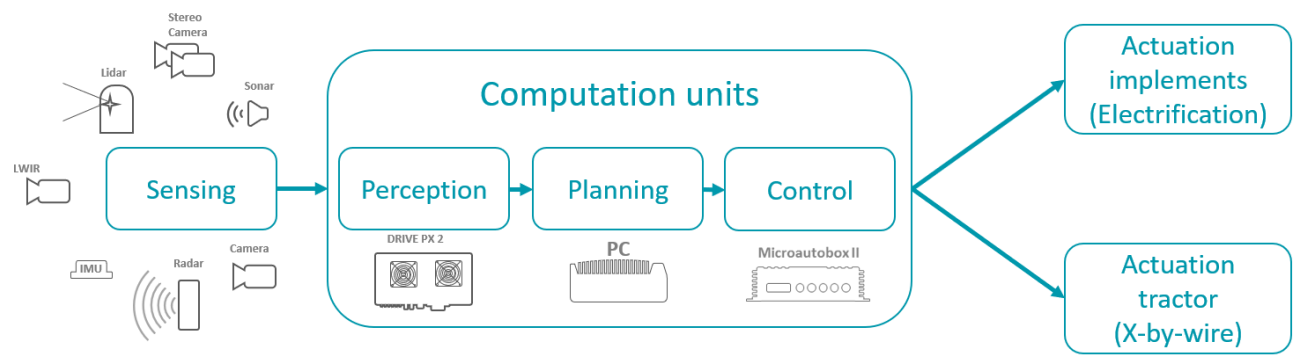

Figure 66. Schematic overview of the components of the autonomous platform.

\section{Sensing}

Different types of sensor technologies exist such camera, radar, lidar, ultrasonic technology. One type of input will never be adequate. Each sensor technology has different strengths and weaknesses. Our platform offers a multitude of sensors and allows to combine different types, experiment with it and improve fusion algorithms.

In our current setup, for front obstacle detection and planning, the data from 3 Leopard Imaging GMSL AR0231 cameras are fused with the data from 3 Continental ARS 408-21 radars. The Leopard Imaging GMSL cameras are specifically suited for its use in ADAS and autonomous driving applications. They are also supported on Nvidia's Drive PX2 platform which features 12 direct camera inputs. The Continental ARS 408-21 radars are long range radars working in the $76-77 \mathrm{GHz}$ frequency band. These radars are compact sensors which are especially suited for anti-collision systems in outdoor autonomous vehicles such as cranes, fork lifts, tractors, harvest combines. The combination of the cameras and radars is used for the detection and classification of objects. For the interpretation of the radar data, the vehicle speed and yaw rate is required. The vehicle speed is taken from the CAN bus of the tractor while an external IMU from Xsens (MTi-300 AHRS) provides inertial data (acceleration and rotation i.e. pitch, roll \& yaw rate) and absolute heading. Four extra IMU's (MTi-30 series) can be added on sensor frames for additional compensation and for movement and vibration measurements.

On top of the tractor, two GNSS-RTK systems will be used: the already factory installed GNSS-RTK system for Precision Land Management (PLM), and an AsteRx-U system of Septentrio, to which we have better access and control. The built-in system will be used for extra logging and as a reference comparison.

A Velodyne VLP 16 LiDAR is currently used as a safety sensor for scanning the immediate surroundings of the tractor. If an object is detected within the safety perimeter, the emergency brake procedure is initiated. Beside the sensors above, we have extra radars, lidars, camera's (GMSL+ FLIR GigE), ultrasonic sensors (Bosch) and infrared camera's (LWIR) available for both global and local perception and detection around the vehicle and the implement.

\section{Computation \& control}

Over the past years, Flanders Make has developed a custom, flexible software platform that can be deployed on different autonomous vehicles, ranging from indoor AGV's to On-Highway and Off-Highway vehicles. In this project, the Flanders Make middleware runs on the Drive PX2 (DPX2) platform from Nvidia, the most used AI supercomputer for self-driving cars at this moment. It forms the main heart of the perception, detection and planning processes. For rapid prototyping of perception algorithms, RTMaps, which is another middleware, is used too. It allows to quickly develop AV/ADAS algorithms to be run on the DPX2.

In order to process the amount of streaming data coming from all sensors, high computational power is required. Nvidia's Drive PX2 platform is exactly tailored for this. Because agricultural and/or street image data is typical non-deterministic (i.e. one cannot rely on or predict which and how many objects are in the field of view), a typical/classical machine vision processing is unsuited. Therefore, the Nvidia platform uses deep learning techniques for object recognition.

On the other hand, for reliable control and actuation, a real-time (deterministic) computing system, with guaranteed millisecond responsiveness is compulsory, considering one is driving a 6 ton, 150 horsepowered vehicle. For this, a dSPACE real-time system was chosen, i.e. the MicroAutoBoxII (MAB2). The MAB2 provides already plenty of general IO, interfacing and processing power. To be prepared for future needs, an extra internal FPGA and a remote RapidPro Control and Signal Conditioning unit is available.

Next to the DPX2 and the MAB2, a powerful embedded PC (Neousys Nuvo-5095GC) serves as development station. It runs Matlab/Simulink and Controldesk for Rapid Control Prototyping on the dSPACE platform and it is also the development station for RTMaps applications.

Additionally, the PC can also act as processing unit, as it has a GPU card add-on, and provides extra CAN, ethernet and USB interfaces for connecting sensors. 
Finally, a central 10Gig ethernet switch and fiber-coupled large storage NAS, together with router, firewall and remote access through Wifi/4G completes the picture.

Figure 67 shown below, summarizes the system architecture:

- Nvidia Drive PX2 (DPX2): performs functions such as sensor fusion and motion planning.

- $\quad$ MicroAutoBoxII (MAB2): to perform functions such as longitudinal and lateral control.

- Embedded PC: to develop Simulink models for the MAB2, to develop RTMaps applications for the DPX2 and to develop and deploy Flanders Make own middleware software suite.

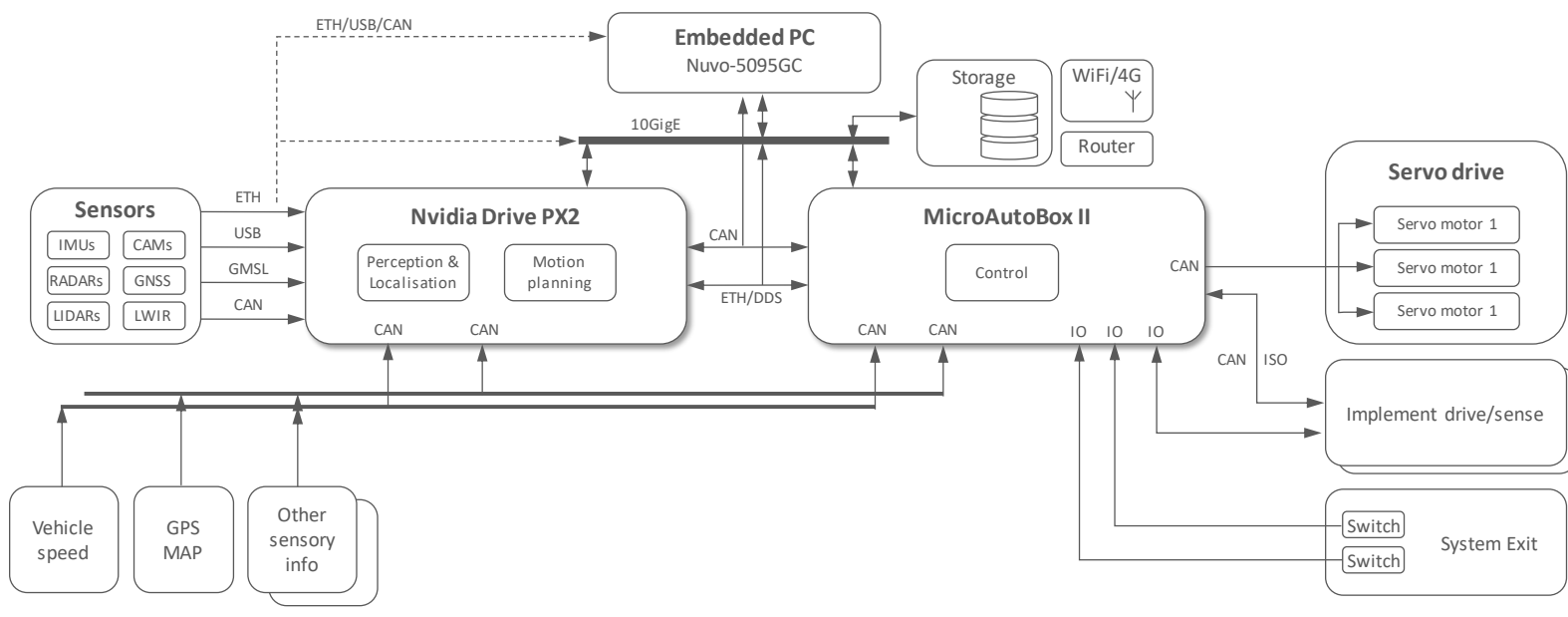

Sense Perception Application Control Actuation

Figure 67. Architecture of the Autonomous Agricultural Vehicle platform.

The MAB2 runs real time code developed using Matlab/Simulink. By way of ControlDesk, which runs on the embedded PC, users can access and interact with the MAB2. Similarly, the RTMaps applications which run on the DPX2, are developed on and controlled by the PC. The PC by itself can also run RTMaps applications. In this way, an extremely powerful and flexible environment is created, allowing engineers with different backgrounds to work on each submodule, using the most optimal or available

- $\quad$ solution or application $(\mathrm{C}++$, Python, OpenCV, Matlab, Simulink, ...)

- environment (Linux/Windows)

- hardware: DPX, MAB2, PC,

- $\quad$ hardware interfaces (CAN, USB, Ethernet, FlexRay, GMSL, ...)

- protocol: CAN, CANopen, ISOBUS, J1939, DDS, RS232/485, ...)

- $\quad$ architecture \& SDK support: x86 vs ARM based

Actuation

There are two options to actuate the tractor: the first one is directly using the drive-by-wire system of the NH T7 tractor and the second one is implementing an external drive-by-wire system. In this project, the goal is to cover both options, starting with developing a new external drive-by-wire system to physically actuate the steering wheel, gas and brake pedals of the tractor. The benefit of the development of this drive-by-wire system allows for deployment in other off-highway vehicles which do not have a drive-by-wire system implemented. To drive the tractor, the steering wheel, gas and brake pedal need to be controlled. This is done with three servomotors from ESTUN(EMJ-08ASB22) and ESTUN servodrive ETS 101010APG-CAN.

On the gas and brake pedals of the tractor, two ABB safety switches are mounted to detect when the person in the tractor wants to take manual control over the tractor. Pressing one of the pedals will trigger the autonomous system exit which will result in manual control over the tractor and retraction of the servomotor levers controlling the pedals.

\section{Results and Discussion}

Sensor fusion and perception

Figure 68 shows different footage of the tractor approaching two walking pedestrians. Even under rainy weather conditions, the system was capable of detecting and recognising the two pedestrians, even from farther away as shown in the pictures. (At larger distance, the pedestrians are visible in full resolution, but would be hardly visible in this image compilation.) The upper left image shows the scenery taken with an external camera. The middle upper image is the output of the LIDAR captured from the screen. It is a reconstructed point cloud 
projected from a virtual point above and (far) behind the tractor. The centre blob corresponds with the tractor cabin, which is just behind the LIDAR. (The LIDAR is mounted in front of the tractor, a little elevated, as can be seen in the upper left image). The insert in the upper middle image, enlarged right, shows the lidar dots projected on a normal visual camera image, where one can see the two pedestrians. The bottom right picture shows a detail of the above where the pedestrians can be distinguished.(see arrow). The centre bottom row images are a snapshot of the raw visual and radar input from left, centre and right camera/radar units. The fused output, on the bottom left picture, shows the known objects in the radar plot and labelled green rectangles around the recognised pedestrians. (more footage at https://youtu.be/PWqEj3P6mFA?t=86).
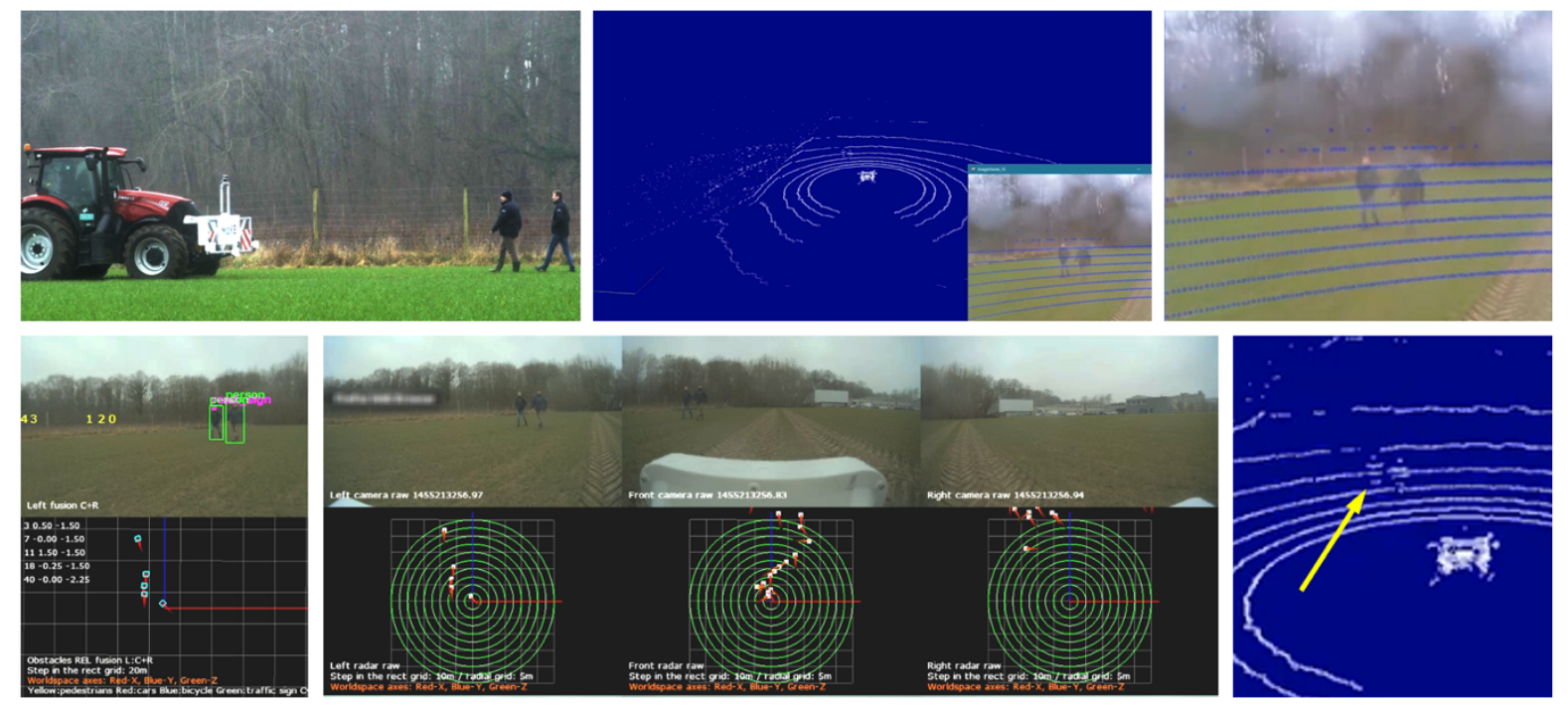

Figure 68. Example of object detection and recognition using sensor fusion. (Upper row: left: external view, middle: projected scenery from lidar, right: detail of visual image with lidar measurements superposed. Bottom row: left: fused result with object labeling, middle: raw camera and radar input, right: detail of lidar image.)

Actuation

For development purpose, a dummy bench with steering wheel and pedals has been built.(See Figure 69). On this bench, the external drive-by-wire system is mounted, which can be installed later on the tractor. This allows us to develop and test our actuation and control algorithms in lab environment before rolling them out on the real tractor. Communication between the Estun servo controller and dSPACE is done by way of CANopen and the interface using Matlab/Simulink has been implemented. Readout of all motor parameters like speed, torque, angle is realized and actuation control is chosen to be position control, with limits on torque and speed. Direct X-bywire control of the T7 is yet under development. The readout of CAN messages is generally not a problem, however, for controlling, which means sometimes overtaking, translating or mimicking other system commands, requires in depth knowledge and can impair safety systems of the tractor.

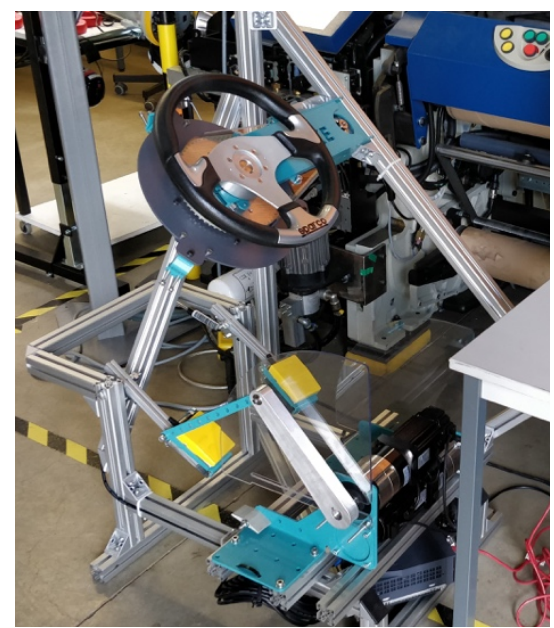

Figure 69. bench with steering wheel and pedals for testing custom drive-by-wire system. 


\section{Next steps}

The purpose of the platform described above is enabling tests of newly developed technology and components in off-highway conditions. Practically, it is in continuous progress: new applications and algorithms are developed or existing ones are improved. Further integration and rollout of equipment and hardware is ongoing. A validation test is planned where the vehicle is to drive a specified track on a test field, including turning, forward and backward driving, acceleration and deceleration and stopping for an randomly positioned obstacles. Related to electrification, recently the articulated arm without hydraulics has been delivered. This arm will be equipped with electrical linear actuators. Investigations and market study for choosing the actuators is ongoing.

\section{Conclusions}

This paper presented the developments of an autonomous agricultural vehicle at Flanders Make. A versatile and powerful infrastructure for developing and testing autonomous driving and operation of any Off-Highway vehicle has been put in place. It provides many state of the art sensors and processing power for autonomous and assisted operation of any agricultural vehicle using modern techniques like deep learning AI. Next steps are the further extension towards the work function of the tractor, as it is the work executed that matters. This encompasses the electrification of the implement, accurate control of it and equiping the implement with perceptive sensors.

\section{Acknowledgements}

This research was supported by Flanders Make vZw, the strategic research centre for the manufacturing industry.

\section{References}

Birem M., Shopovska I., Jovanonv L., Veelaert P. Philips W., Lehaen K., 2017. A Hybrid Fusion Based Frontal-Lateral Collaborative Pedestrian Detection and Tracking. IEEE 20th International Conference on Intelligent Transportation Systems. 16-19/10/2017

Flanders Make project video Platform Autonomous driving https://www.youtube.com/watch?v=PWqEj3P6mFA, Accessed September 21, 2018

Intempora RTMaps https:/intempora.com/products/rtmaps.html, Accessed September 21, 2018

dSpace MicroAutoBox II https://www.dspace.com/en/inc/home/products/hw/micautob/microautobox2.cfm, Accessed September 21, 2018

Deserve EU project http://www.deserve-project.eu/, Accessed September 21, 2018 


\section{Topic 5: Energy and greenhouse gas emissions (EG)}

Reduction of environmental impact of operations in food and biomass production and processing by reducing energy demand, energy storage technologies, and new non-fossil energy sources. Examples are new implements and tractors including smart control, integration of energy from wind, bio-refinery and wastes, and reduction of emissions.

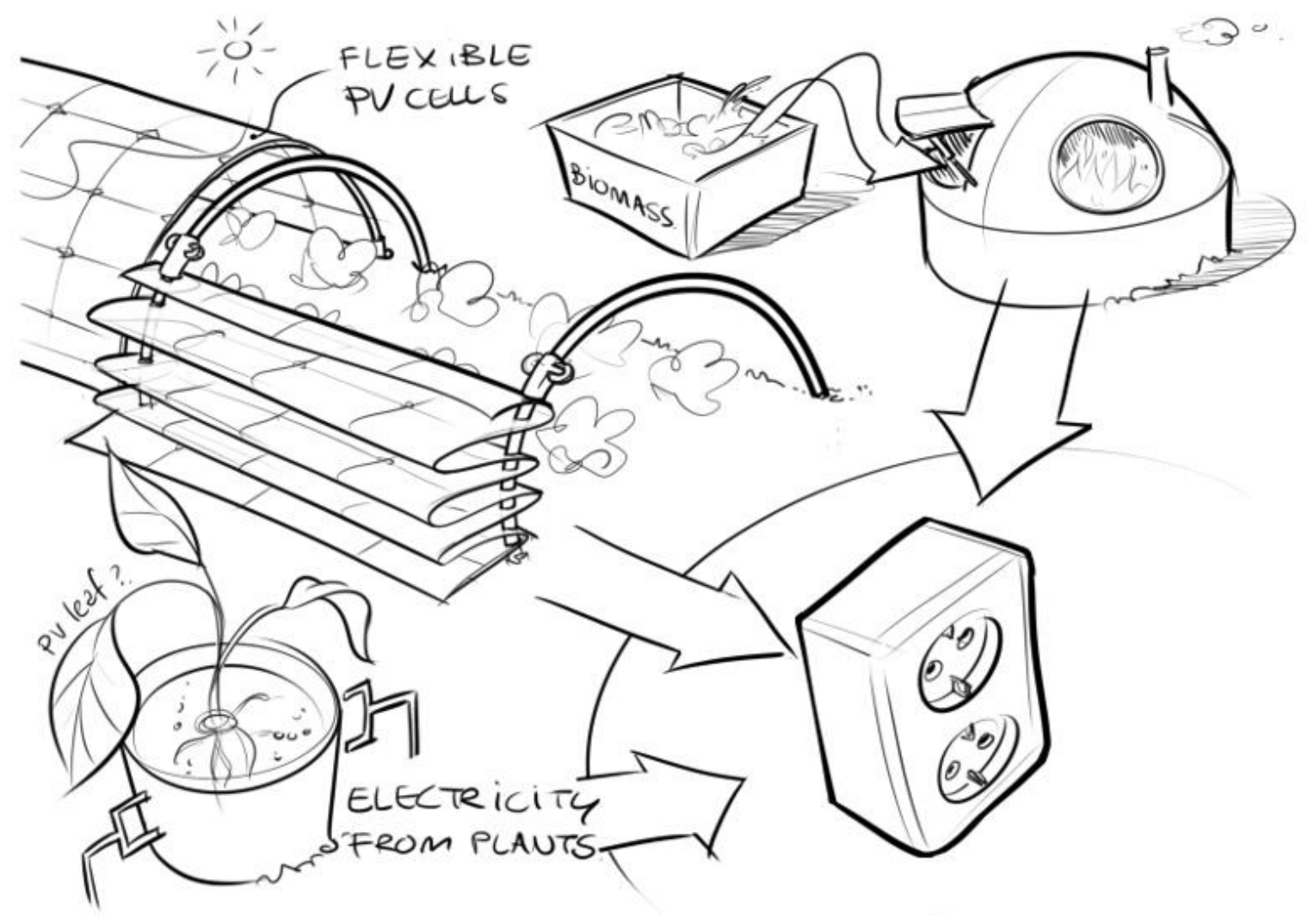




\title{
Assessing Water Supply Sources Using Life Cycle Assessment: Study Case of Lemon Crop in Southeastern Spain
}

\author{
Bernardo Martin-Gorriz *, Jose Francisco Maestre-Valero, Victoriano Martínez-Alvarez \\ Escuela Técnica Superior de Ingeniería Agronómica, Universidad Politécnica de Cartagena. Paseo Alfonso XIII, 48. \\ 30203 Cartagena, España. \\ * Corresponding author. Email: b.martin@upct.es
}

\begin{abstract}
The Segura river basin, in southeastern Spain, is one of the areas with the highest water scarcity, not only in Spain but also in Europe. As a result, in such a region, farmers can have up to six different water supply sources for irrigation (surface water, groundwater, reclaimed water, desalinated brackish groundwater, desalinated seawater and inter-basin Tagus-Segura water transfer). Moreover, water reallocation by water trading between concession holders is also possible (i.e. water markets), which in practice represents an additional water supply source. Water markets are one of the most promising instruments for reallocating water resources in mature water economies since they are capable of increasing the economic efficiency of water use and reducing the economic impact of scarcity. This paper analyses the environmental impacts of production of a representative lemon orchard irrigated with each one of the above-mentioned water supply sources. Lemon has been selected as it is the perennial crop with the greatest area in the basin. A life cycle assessment was used to quantify ten midpoint impact categories and the cumulative energy demand of the process. The system boundary was considered from raw material extraction to farm-gate based on lemon production. The results showed large variation in the indicators. In summary, fertilisers production was the key contributor to global warming potential, acidification, eutrophication, human toxicity and toxicity of air, water, and soil. On the other hand, in water scarcity circumstances irrigation with a high percentage of desalinated seawater showed the highest value of global warming potential and cumulative energy demand, which is justified by the high-energy requirements to produce desalinated seawater. In addition, the analysed environmental indicators of water markets resources with the basin were similar to those of the inter-basin Tagus-Segura water transfer.
\end{abstract}

Keywords: Water resource, Global warming potential, GWP, LCA, Irrigation, Citrus.

\section{Introduction}

Agriculture in semi-arid and arid regions is highly dependent on water for irrigation. However, water availability is already problematic in many regions, with imbalances between renewable resources and total demands jeopardising irrigated agriculture and its resilience. Moreover, global climate models predict that available water resources will diminish under arid and semi-arid climates, exacerbating water scarcity problems in the near future in many areas around the world (Faurès et al., 2013).

A clear example of this circumstance is the Segura River Basin (SRB; southeast Spain), which is a basin that is severely affected by drought problems. Nevertheless, the Segura River Basin is the European region with the highest production of open field early fruit and vegetables, despite its agricultural production being limited by a water supply that is markedly lower than the water demand. This complex paradox is putting pressure on agriculture in this basin, which cannot meet current or future demand for irrigation by relying solely on conventional water sources. Consequently, the exploration of other new agricultural water supply options such as regional transfers, desalinated seawater and reclaimed water is absolutely essential to sustain agricultural production (Martínez-Alvarez et al., 2016).

In this context, the specific aim of this study is to quantify the environmental impact of using non-traditional water sources for lemon orchards irrigation. The analyses to be conducted will be based on different scenarios of water supply associated to drought periods. Life Cycle Assessment (LCA) methodology will be used to quantify the environmental impacts of different scenarios; and finally, to identify the agricultural practices or inputs which give rise to the most significant environmental problems and to propose adaptation measures in the Segura River Basin agriculture.

\section{Materials and Methods}

Study area and water supply scenarios

The study was performed in the semi-arid Campo de Cartagena irrigation district (CCID), located in the southeast of the SRB. Such a district, with an area around 34,131 ha, has been selected for the study as it is the largest irrigation district in the SRB. A wide range of crops are grown in the CCID, with a predominance of vegetables (lettuce, broccoli, artichoke and melon) and citrus fruits (lemon, orange and mandarin trees). Within citrus, lemon trees is the irrigated perennial crop with the greatest area in the basin. In addition, the CCID manages 
water resources from a wide range of origins (CHS, 2015):

- SW (surface water): water diverted from the rivers.

- GW (groundwater): water that farmers extract from the underlying aquifer.

- EW (external water): water transferred to the SRB through the Tagus-Segura water transfer.

- DBW (desalinated brackish water): salty water from the Campo de Cartagena irrigation district drainage system that is desalinated for reuse in irrigation.

- DW (desalinated seawater): seawater desalinated following reverse osmosis processes and remineralisation post-treatments must be adapted to tailor agricultural water quality requirements.

- RW (reclaimed water): recycled sewage from several local populations.

- WM (water markets): water transfer between basins with intervention of irrigation communities for agricultural use.

The management of these sources usually follows the multiple strategy concepts, as all these sources contribute to an irrigation mix that allows increasing water availability for irrigation (Martinez-Alvarez et al., 2016). Under these conditions, farmers feel free to use the adequate irrigation mix in each period of time (month or year) according to the availability of each water source and price. In this context, and focusing on the water scarcity circumstances outlined above, the following three water supply scenarios (WS) for the CCID have been analysed (Fig. 1):

- WS1: Scenario with normal water availability. In this scenario, the water transferred from the Tagus basin in central Spain (EW) represents the most important available water resource with $60 \%$. Due to the large inter-annual variation of water transfer between the Tagus and Segura basins in the last years (Soto-García et al., 2013), the year 2011 has been selected for the WS1 scenario as it is a year with normal water supply in the CCID (Martin-Gorriz et al., 2014).

- WS2: Transition scenario between normal water availability period and drought period. In this scenario, the water transferred from central Spain decreases considerably due to an incipient drought period, as well as the use of groundwater. This hypothetical scenario considers $-50 \%$ in EW and $50 \%$ in GW. Additionally, the SW was reduced to $0 \%$. The water supply reduction in these scenarios was compensated with prospected increases in WM $(+50 \%)$ and DSW $(+50 \%)$ (Fig. 1b). WM and DSW are presented as valid adaptive measures to water scarcity.

- WS3: Drought scenario. In this water-stress scenario where there is persistent water scarcity, the traditional water sources are not available (EW, GW, DBW, WM and SW), and DSW represents an alternative for irrigated agriculture. In this context, DSW replaces traditional water sources since it provides water for irrigation independently of climate changes and drought (Fig. 1c). It should be noted that this hypothetical scenario has to date never happened in the CCID.
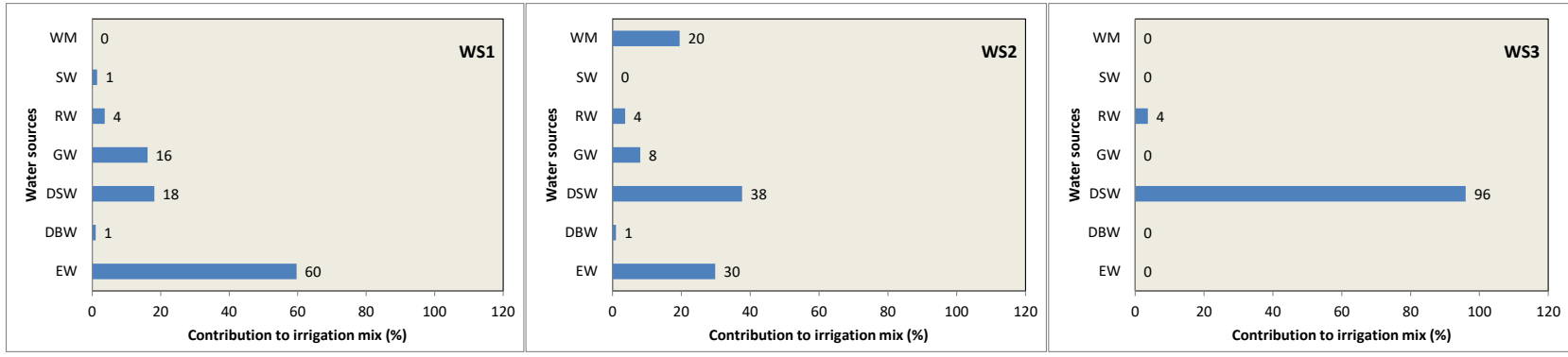

Figure 1. Irrigation mix contribution by each water source in three water supply scenarios.

Life cycle assessment

The environmental impacts of lemon production in the CCID have been assessed using the LCA methodology and following the ISO 14040-44 standards (ISO, 2006a, 2006b). The goal and scope definition are discussed in Section 2.1, whilst system boundaries are reported in Sections 2.2. The life cycle inventory (LCI) and data sources are detailed in Section 2.3; and the software and impact assessment method are discussed in Section 2.4.

\subsection{Goal and scope definition}

This analysis aims to evaluate the environmental sustainability of the three water supply scenarios in lemon cultivation, and provide in-depth understanding of the environmental impacts as well as of the processes and inputs that are implemented in order to: (a) promote more sustainable cultivation systems, in terms of their total environmental impacts; and (b) further reduce their environmental footprint, by selecting alternative renewable energy sources. 
The unit of assessment, also known as the functional unit in LCA, was $1 \mathrm{~kg}$ of lemons for fresh consumption.

\subsection{System boundaries}

In the present study, the "cradle-to-gate" approach was used, considering all the production processes involved from raw materials extraction (i.e. the cradle) to the point where the final product is made available at the gate of the field. Based on the system boundaries, the production processes under study considered both foreground and background systems (Fig. 2), and included a set of life-cycle production stages, namely, fertilisers production, agrochemicals production, nursery production, drip irrigation system, agricultural machinery and field operations on farm.

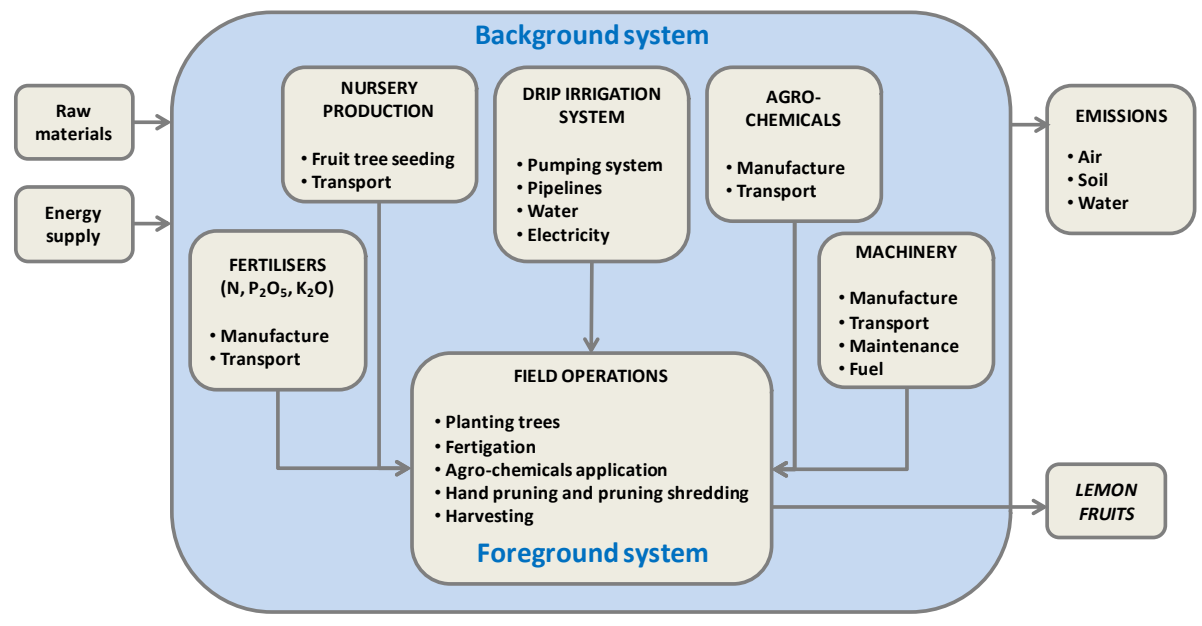

Figure 2. Flow diagram of the main stages included in the LCA study.

\subsection{Life cycle inventory (and data sources)}

LCI considers all the relevant inputs and outputs for processes that occur during the life cycle of a product. The data in Table 1 show the inputs and outputs of lemon orchards in the CCID. This data has been obtained by means of surveys to farmers (Segura et al., 2006) and may be assumed to be representative of typical mean values for agricultural practices in this area for lemon cultivation. The specific energy by WS was calculated weighted according to the product of the percentage of water used by water source used in the irrigation mix per its specific energy. It should be noted that Table 1 also includes the specific energy by water source proposed by several authors (Aparicio et al., 2017; Hospido et al., 2013; Martin-Gorriz et al., 2014; Martínez-Alvarez et al., 2017; Megarejo and Montaño, 2009; Soto-García et al., 2013) and the final specific energy by WS used in the analysis calculated according to the irrigation mix contribution by water source (Fig. 1). 
Table 1. Life-cycle inventory (LCI) per hectare of lemon cultivation.

\begin{tabular}{|c|c|c|}
\hline Inputs/Outputs & Unit & Amount \\
\hline Yield & $\mathrm{kg} / \mathrm{ha}$ & 41,400 \\
\hline Land use & ha & 1 \\
\hline $\mathrm{N}$ fertilisers: & $\mathrm{kg}-\mathrm{N} / \mathrm{ha}$ & 240 \\
\hline - Ammonium nitrate & $\mathrm{kg}-\mathrm{N} / \mathrm{ha}$ & 123 \\
\hline - Potassium nitrate & $\mathrm{kg}-\mathrm{N} / \mathrm{ha}$ & 40 \\
\hline - Calcium nitrate & $\mathrm{kg}-\mathrm{N} / \mathrm{ha}$ & 19 \\
\hline - $\quad$ Nitric acid & $\mathrm{kg}-\mathrm{N} / \mathrm{ha}$ & 44 \\
\hline - Monoammonium phosphate & $\mathrm{kg}-\mathrm{N} / \mathrm{ha}$ & 14 \\
\hline $\mathrm{P}_{2} \mathrm{O}_{5}$ fertilisers: & $\mathrm{kg}-\mathrm{P}_{2} \mathrm{O}_{5} / \mathrm{ha}$ & 70 \\
\hline - Monoammonium phosphate & $\mathrm{kg}-\mathrm{P}_{2} \mathrm{O}_{5} / \mathrm{ha}$ & 70 \\
\hline $\mathrm{K}_{2} \mathrm{O}$ fertilisers: & $\mathrm{kg}-\mathrm{K}_{2} \mathrm{O} / \mathrm{ha}$ & 140 \\
\hline - $\quad$ Potassium nitrate & $\mathrm{kg}-\mathrm{K}_{2} \mathrm{O} / \mathrm{ha}$ & 140 \\
\hline Diesel for field operations & $\mathrm{kg} / \mathrm{ha}$ & 18 \\
\hline Water for irrigation & $\mathrm{m}^{3} / \mathrm{ha}$ & 4400 \\
\hline \multicolumn{3}{|l|}{ Agro-chemicals: } \\
\hline - Pesticides & $\mathrm{kg} / \mathrm{ha}$ & 17 \\
\hline - Herbicides & $\mathrm{kg} / \mathrm{ha}$ & 41 \\
\hline \multicolumn{3}{|l|}{ Specific energy by water source: } \\
\hline - DSW & $\mathrm{kWh} / \mathrm{m}^{3}$ & 4.87 \\
\hline - $\quad$ DBW & $\mathrm{kWh} / \mathrm{m}^{3}$ & 2.5 \\
\hline - $\quad \mathrm{GW}$ & $\mathrm{kWh} / \mathrm{m}^{3}$ & 0.9 \\
\hline - $\quad \mathrm{MW}$ & $\mathrm{kWh} / \mathrm{m}^{3}$ & 1.198 \\
\hline - $\quad \mathrm{RW}$ & $\mathrm{kWh} / \mathrm{m}^{3}$ & 0.78 \\
\hline - $\quad$ SW & $\mathrm{kWh} / \mathrm{m}^{3}$ & 0.06 \\
\hline - $\quad \mathrm{EW}$ & $\mathrm{kWh} / \mathrm{m}^{3}$ & 1.21 \\
\hline \multicolumn{3}{|c|}{ Specific energy by water supply scenario: } \\
\hline - WS1 & $\mathrm{kWh} / \mathrm{m}^{3}$ & 1.80 \\
\hline - $\quad \mathrm{WS} 2$ & $\mathrm{kWh} / \mathrm{m}^{3}$ & 2.58 \\
\hline - $\quad$ WS3 & $\mathrm{kWh} / \mathrm{m}^{3}$ & 4.71 \\
\hline
\end{tabular}

2.4 The life-cycle stages are grouped as follows, to make it easier for them to be understood:

Nursery production: The stage of nursery production was considered and included in this study. Data for the nursery production phase was mainly taken from the Ecoinvent v.3.4 database. The activity starts with the production of rootstocks and stems, and the process of grafting. This activity included agricultural machinery, mineral and organic fertilisers, pesticides and ground cultivation.

Drip irrigation system: This stage included: (i) the manufacture and transport of on-farm infrastructure (pumps, a fertigation system, electrovalves, an automatic irrigation programmer and filters, PVC pipes and PE irrigation laterals) and the construction of a shed for head pumping, fertilisers tank and farm machinery storage; (ii) water consumption for irrigation; (iii) electricity required on-farm and off-farm for irrigation according to the water source (Table 1); and (iv) emissions related to infrastructure and electricity. The emission factor for electricity includes indirect emissions attributable to the extraction, production and transport of electricity as well as to the electricity lost in delivery in the network. For these calculations, it was assumed that the electricity in Spain is generated by several sources, with electricity mix factor being obtained from the Ecoinvent v.3.4 database. The lifetime of the shed and irrigation system was defined for 20 years and for a 10-ha lemon farm. This was divided annually considering the lifetime and land unit $(1 \mathrm{ha})$. Following the methodology proposed by Hospido et al. (2013), the study excluded off-farm irrigation infrastructures of LCA, i.e. channels, reservoirs, desalination plants, collective pressurised network, etc.

Agro-chemicals: Data for the production of pesticides and herbicides was obtained from processes inventoried in the Ecoinvent v.3.4 database. This stage included the required infrastructure, transport of primary and secondary materials to the plant, synthesis of the chemical components required and disposal or treatment of the generated waste. The name of the pesticide and the amount of active ingredient applied are used to model the environmental fate in the inventory. The environmental fate is assumed to be $100 \%$ to soil.

Fertilisers: This stage included manufacture $\left(\mathrm{N}-\mathrm{P}_{2} \mathrm{O}_{5}-\mathrm{K}_{2} \mathrm{O}\right.$ production), packaging, and transport to the farm. $\mathrm{N}$-fertilisers included emissions to air $\left(\mathrm{N}_{2} \mathrm{O}, \mathrm{NH}_{3}, \mathrm{NO}_{\mathbf{x}}\right)$, water $\left(\mathrm{NO}_{3}\right)$ and soil (element content); $\mathrm{P}_{2} \mathrm{O}_{5}$-fertiliser included emissions to water $\left(\mathrm{P}, \mathrm{PO}_{4}\right)$ and soil (element content), and $\mathrm{K}_{2} \mathrm{O}$-fertiliser included emissions to water $(\mathrm{K})$ and soil (element content). Data for this stage regarding emissions and energy consumption were taken from the Ecoinvent v.3.4 database.

Machinery for field operations: This stage included the manufacture, transport, maintenance, repair, as well as waste management for specify machinery to field operations, namely tractor ( $2 \mathrm{WD}, 60 \mathrm{~kW})$, air-blast sprayer 
(pesticides), sprayer tank (herbicides), chipper (pruning shredding), and trailer to transport lemon fruits harvested on-farm. Machine use has been calculated following the methodology proposed by Audsley et al. (1997) for agricultural machines. Moreover, the type of machinery, weight, lifetime and utilization have been adapted according to Spanish conditions (Marquez, 2004); the data for this stage regarding emissions and energy consumption were taken from the Ecoinvent v.3.4 database.

Field operations: This stage included the following field work processes: planting trees, fertigation (fertilisation plus irrigation), pesticides and herbicides application (3 times/year each one), hand pruning, pruning shredding and harvesting. These operations with machinery generate emissions from diesel consumption and emissions from inputs such as fertilisers, pesticides and herbicides due to their use. Planting trees was weighted in lifetime years of the orchard ( 20 years). The application of fertilizers was included in irrigation that was powered by electricity. For all inputs, consideration was given to the transport of materials in the field via tractor and trailer.

\subsection{Software and impact assessment method}

The impact assessment has been estimated according to the Center of Environmental Science (CML) of Leiden University in The Netherlands, CML-IA methodology (Guinée et al., 2002), using SimaPro 8.5 software (Pre Consultants, 2015). Ten midpoint impact categories, defined according to (Guinée et al., 2001) have been considered in the present study (Table 2). Furthermore, an energy flow indicator, cumulative energy demand (CED), which represents the energy demand in the process, has been analysed in order to assess the energetic performance of crop production. CED was focused on the energy used throughout the life cycle of a product, including the energy consumed during the extraction, manufacturing and disposal of the raw and auxiliary materials (Frischknecht et al., 2005, 2007). Finally, a water use indicator was included in our study (water supplied to the crop).

These impact categories were selected because of their relevance in agriculture and energy processes. Abiotic depletion, global warming and cumulative energy demand are important indicators related to energy consumption. Emissions related to agricultural inputs, mainly fertilisers, are major contributors to global warming and cumulative demand potential because of the energy consumption in the manufacturing process, as well as energy consumption in irrigation. Ammonia and nitrate emissions from $\mathrm{N}$-fertilisers are important contributors to acidification and eutrophication, respectively. Emissions contribution to photochemical oxidant formation may have significant consequences on human health, ecosystems and crops; and finally, human and ecotoxicity (air, water, and soil) are important indicators related to chemical products (agro-chemicals and fertilisers).

Table 2. Environmental impact categories, measurement units and main contributors in each impact category.

\begin{tabular}{|c|c|c|c|}
\hline Impact category & Acronym & Units & Main contributing substances \\
\hline $\begin{array}{l}\text { Abiotic depletion potential } \\
\text { (fossil fuels) }\end{array}$ & ADP & $\mathrm{MJ} \mathrm{FU}^{-1}$ & Fossil resources (oil, gas, coal) \\
\hline Acidification potential & AP & $\mathrm{kg} \mathrm{SO}_{2}$ eq FU ${ }^{-1}$ & $\begin{array}{l}\text { Ammonia }\left(\mathrm{NH}_{3}\right), \\
\text { Nitrogen oxides }\left(\mathrm{NO}_{\mathrm{x}}\right)\end{array}$ \\
\hline Eutrophication potential & EP & $\mathrm{kg} \mathrm{PO}^{-3}{ }_{4}$ eq $\mathrm{FU}^{-1}$ & $\begin{array}{l}\text { Ammonia }\left(\mathrm{NH}_{3}\right) \text {, Nitrogen oxides } \\
\left(\mathrm{NO}_{\mathrm{x}}\right) \text {, Nitrate }\left(\mathrm{NO}^{-3}\right)\end{array}$ \\
\hline $\begin{array}{l}\text { Fresh water ecotoxicity } \\
\text { potential }\end{array}$ & FEP & $\underset{1}{\mathrm{~kg}} \underset{1,4-\mathrm{DB}}{\text { eq } \mathrm{FU}^{-}}$ & $\begin{array}{l}\text { Chemical oxygen demand (COD), } \\
\text { Chemical products }\end{array}$ \\
\hline Global warming potential & GWP & $\mathrm{kg} \mathrm{CO}_{2}$ eq $\mathrm{FU}^{-1}$ & $\begin{array}{l}\text { Carbon dioxide }\left(\mathrm{CO}_{2}\right) \text {, Dinitrogen } \\
\text { monoxide }\left(\mathrm{N}_{2} \mathrm{O}\right)\end{array}$ \\
\hline Human toxicity potential & HTP & $\underset{1}{\mathrm{~kg}}$ 1,4-DB eq FU- & $\begin{array}{l}\text { Methane }\left(\mathrm{CH}_{4}\right) \\
\text { Emissions of toxic substances to air, } \\
\text { water and soil }\end{array}$ \\
\hline Terrestrial ecotoxicity & TE & $\mathrm{kg} \underset{1}{1,4-\mathrm{DB}}$ eq $\mathrm{FU}^{-}$ & Chemical products \\
\hline Ozone layer depletion & OLD & $\mathrm{kg} \underset{1}{1,4-\mathrm{DB}}$ eq $\mathrm{FU}^{-}$ & Chemical products \\
\hline $\begin{array}{l}\text { Photochemical oxidation } \\
\text { potential }\end{array}$ & POP & $\mathrm{kg} \mathrm{C}_{2} \mathrm{H}_{4}$ eq FU $\mathrm{FU}^{-1}$ & $\begin{array}{l}\text { Carbon monoxide }(\mathrm{CO}) \\
\text { Sulphur dioxide }\left(\mathrm{SO}_{2}\right)\end{array}$ \\
\hline Marine aquatic ecotoxicity & MAE & $\mathrm{kg} \underset{1}{1,4-\mathrm{DB}}$ eq $\mathrm{FU}^{-}$ & Chemical products \\
\hline Cumulative energy demand & CED & $\mathrm{MJ} \mathrm{FU}^{-1}$ & $\begin{array}{l}\text { Coal, natural gas, oil, uranium, energy, } \\
\text { gross calorific value }\end{array}$ \\
\hline Water use & WU & $\mathrm{LFU}^{-1}$ & Water \\
\hline
\end{tabular}

FU: Functional Unit. 


\section{Results and discussion}

Environmental impacts per output unit

\section{Analysis of contribution by impact category}

Fig. 2 summarises the relative contribution of each stage by impact category to the lemon production in a scenario with normal water availability (WS1). The main contributors to all impact categories are fertilisers production and electricity consumption for irrigation (fertigation). Indeed, fertilisers production causes $71.90 \%$ of the eutrophication effects, $58.63 \%$ of the acidification impacts, $55.76 \%$ of the global warming, $39.58 \%$ of the abiotic resource depletion, $32.99 \%$ of the ozone layer depletion, $26.80 \%$ of the photochemical oxidation, and around $99 \%$ of other impact categories (human toxicity, fresh water aquatic ecotoxicity, marine aquatic ecotoxicity, and terrestrial ecotoxicity). Within fertilisers, ammonium nitrate production is the main contributor of fertilisers to the global warming, acidification, and eutrophication impact categories, with 55\%, $24 \%$ and $21 \%$ respectively (Data not shown).

Moreover, electricity consumption for irrigation causes $64.46 \%$ of the photochemical oxidation, $53.08 \%$ of the ozone layer depletion, $50.08 \%$ of the abiotic resource depletion, $37.75 \%$ of the acidification impacts, $36.18 \%$ of the global warming, and $23.89 \%$ of the eutrophication effects.

In contrast, the contribution to all impact categories of the foreground system (field operations) was less than $5 \%$. It should be noted that electricity for irrigation (specific energy in $\mathrm{kWh} / \mathrm{m}^{3}$ ), shown in Table 1, included both off-farm and on-farm electricity consumption and the share of electricity for irrigation on-farm was $9 \%$ in WS1, $7 \%$ in WS2 and 4\% in WS3. In the CCID, the on-farm energy consumption was used for pressurising the irrigation system, which requires $0.17 \mathrm{kWh} / \mathrm{m}^{3}$ (Soto-Garcia et al., 2013).

Cumulative energy demand allows to assess the energetic performance of crop production. In this sense, electricity consumption for irrigation $(68 \%)$ and fertilisers production $(27 \%)$ were the main contributors to CED.

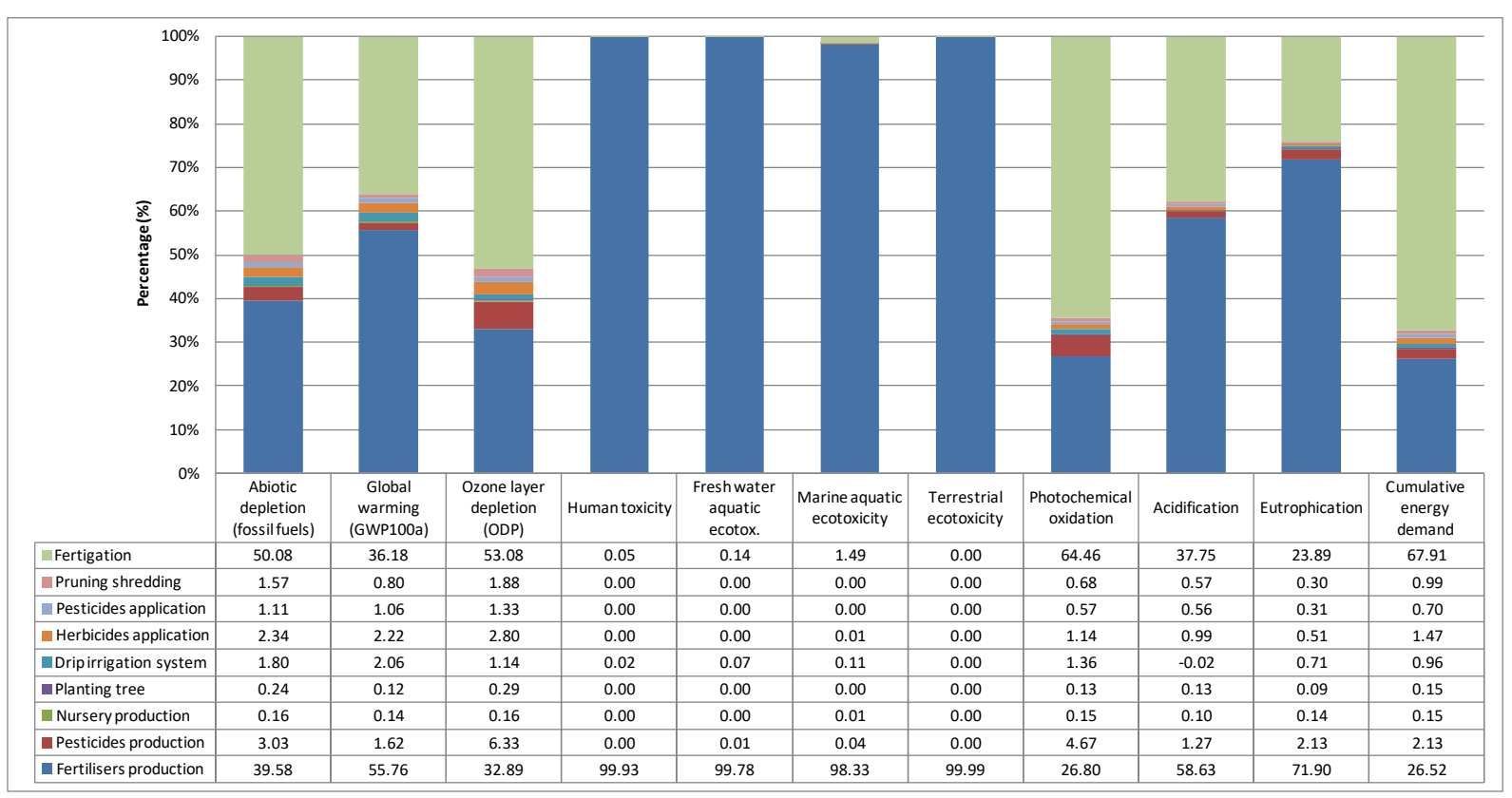

Figure 2. Analysis of the environmental impacts of lemon production in Southeastern Spain in WS1.The relative scale $(100 \%)$ shows the contribution of different stages.

\section{Comparative analysis by water scenario}

Table 4 summarises the environmental impacts of lemon production by WS. The LCA of lemon revealed how the lemon production increased its environmental impacts when replacing traditional water sources (WS1) with MW and DSW (WS2) and even more so when replacing it with only DSW (WS3). The use of water sources with high specific energy consumption was the potential contributor to global warming, ozone layer depletion, acidification, eutrophication, photo-oxidant formation and cumulative energy demand. This occurred as a result of incorporating a greater proportion of water sources with higher specific energy, mainly DSW which is the source with the most specific energy into the irrigation mix (WS2 and WS3) (Table 1). 
Table 4. Analysis of the environmental impacts of lemon production (FU) by water scenario.

\begin{tabular}{lcccc}
\hline Impact category & Unit & WS1 & WS2 & WS3 \\
\hline Abiotic depletion potential (fossil fuels) & $\mathrm{MJ}$ & 1.79 & 2.18 & 3.25 \\
Global warming potential (GWP100a) & $\mathrm{kg} \mathrm{CO}$ eq & 0.23 & 0.26 & 0.36 \\
Ozone layer depletion potential & $\mathrm{kg} \mathrm{CFC}-11 \mathrm{eq}$ & $1.74 \mathrm{E}-08$ & $2.14 \mathrm{E}-08$ & $3.23 \mathrm{E}-08$ \\
Human toxicity potential & $\mathrm{kg} \mathrm{1,4-DB} \mathrm{eq}$ & 35.45 & 35.46 & 35.48 \\
Fresh water aquatic ecotox. potential & $\mathrm{kg} \mathrm{1,4-DB} \mathrm{eq}$ & 14.12 & 14.13 & 14.16 \\
Marine aquatic ecotoxicity potential & $\mathrm{kg} \mathrm{1,4-DB} \mathrm{eq}$ & 7891.68 & 7942.74 & 8082.17 \\
Terrestrial ecotoxicity potential & $\mathrm{kg} \mathrm{1,4-DB} \mathrm{eq}$ & 2.04 & 2.03 & 2.03 \\
Photochemical oxidation potential & $\mathrm{kg} \mathrm{C} \mathrm{H}_{4}$ eq & $3.67 \mathrm{E}-05$ & $4.70 \mathrm{E}-05$ & $7.50 \mathrm{E}-05$ \\
Acidification potential & $\mathrm{kg} \mathrm{SO}_{2}$ eq & $1.65 \mathrm{E}-03$ & $1.92 \mathrm{E}-03$ & $2.66 \mathrm{E}-03$ \\
Eutrophication potential & $\mathrm{kg} \mathrm{PO}_{4}$ eq & $6.19 \mathrm{E}-04$ & $6.83 \mathrm{E}-04$ & $8.58 \mathrm{E}-04$ \\
Cumulative energy demand & $\mathrm{MJ}$ & 3.52 & 4.34 & 6.60 \\
Water use & $\mathrm{L}$ & 108 & - & - \\
\hline
\end{tabular}

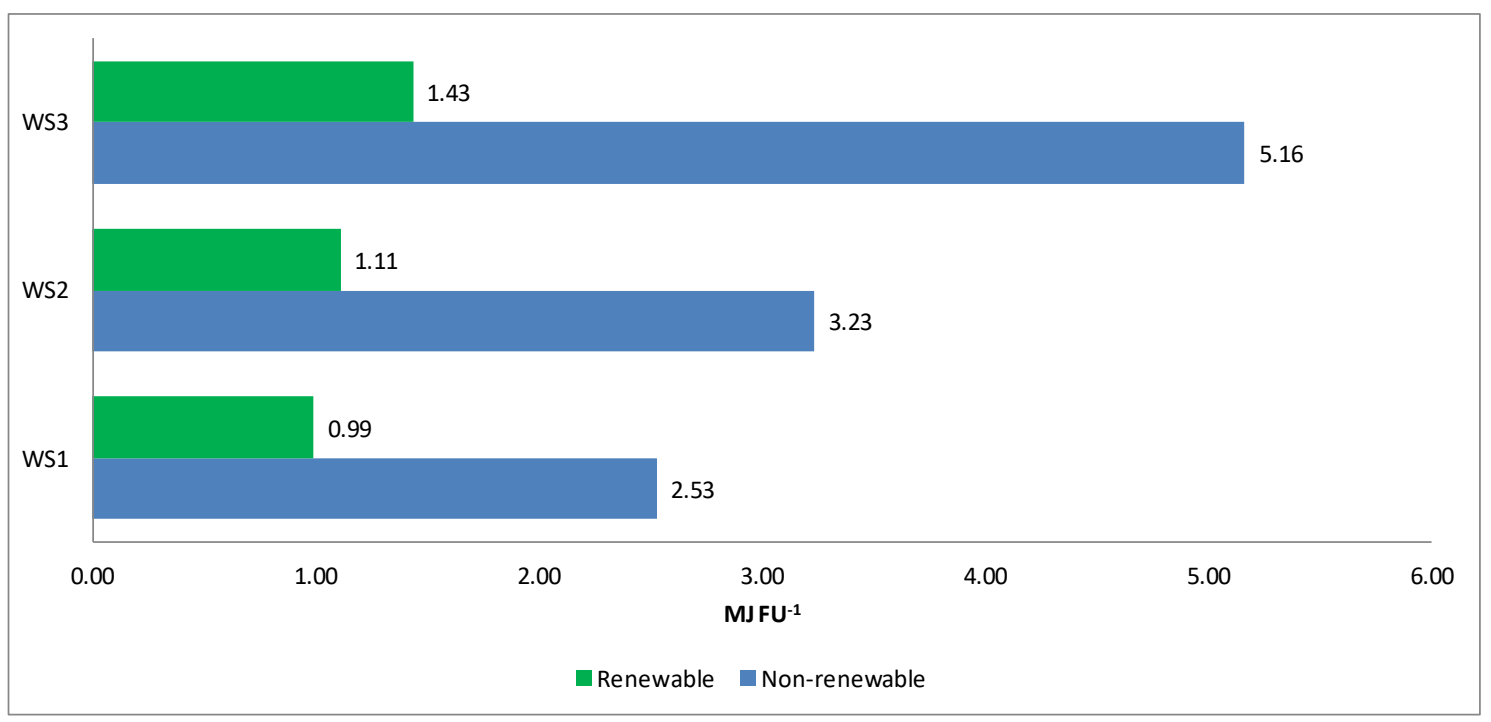

Figure 3. Share of renewable and non-renewable cumulative energy demand by water scenario.

Cumulative energy demand can be divided into renewable (biomass, wind, solar, geothermic and water) and non-renewable (fossil, nuclear and biomass) sources. Fig. 3 shows the share of renewable and non-renewable CED as a result of the increase in electricity consumption for irrigation from WS1 to WS3. In this sense, it is necessary to increase the use of renewable energies because the relative contribution from non-renewable energies to the electric generation mix is higher than renewable energies.

In the present study, the most relevant source of environmental impacts is fertilisers production. Several authors have obtained similar results in conventional citrus cultivation in Spain (Ribal et al., 2016; Sanjuan et al., 2005) and in Italy (Beccali et al., 2009; Pergola et al., 2013). In this sense, several authors have evaluated practical options to reduce the negative environmental impacts associated to mineral fertilisers. Hasler et al. (2015) studied the impact of different mineral fertiliser products and reported that choosing the right sources of mineral $\mathrm{N}$ fertiliser reduced the cradle-to-gate emissions by $20 \%$ compared with the worst alternatives.

The use of irrigation water in arid and semi-arid areas is essential for citrus growers, therefore the second key factor (electricity use for irrigation) has particular significance in the present study since conventional water sources are in clear recession as a result of climate change. In this matter, DSW offers an attractive alternative in arid coastal regions lacking clear alternative water sources (Martinez-Alvarez et al., 2016). However, it presents higher energy requirements compared to other water sources. Even though in our study the amount of water used 
per hectare was in the same range as the literature value for other drip irrigation systems in Spain (Sanjuan et al., 2005) and in Italy (Pergola et al., 2013), electricity consumption was strongly higher. The main reason for the difference is that those authors only considered on-farm electricity consumption. Sanjuan et al. (2005) considered drip irrigation with a $30 \mathrm{~m}$ pressure at the beginning of the watering station. Equally, Pergola et al. (2013) for conventional lemon production used $0.12 \mathrm{kWh} / \mathrm{m}^{3}$. Nevertheless, our study considered the specific energy associated with irrigation both off-farm as well as on-farm. It should be highlighted that in our study case the offfarm share of electricity for irrigation supposes a high percentage (91\% in WS1, 93\% in WS2 and 96\% in WS3). The main environmental impacts of electricity are ozone depletion, photochemical oxidation and global warming potential due to the electricity generation based on fossil fuels and the abiotic resource depletion due to the fossil fuels extracted. In the context of Spain, the contribution from non-renewable energy to the electricity generation mix was $61.1 \%$ in 2016 (IDAE, 2017). However, the situation should improve slightly in the coming years in all impacts categories with the increase of renewable energy sources in the Spanish electricity mix (Foidart et al., 2010). In this sense, the present use of renewable electricity generation with solar photovoltaic panels on-farm has been tested in olive orchards (Lopez-Luque et al., 2015; Moral et al., 2009) and lettuce crops (Marrou et al., 2013; Rothwell et al., 2016). In our study case, the benefit of using solar photovoltaic panels on-farm to produce electricity would be limited, as a consequence of the low percentage of electricity consumption on-farm (9\% in WS1, 7\% in WS2 and 4\% in WS3).

Finally, when focusing on water use, lemon production in the Segura River Basin shows a smaller water use (108 L/FU) compared with other authors that reported values of $162 \mathrm{~L} / \mathrm{FU}$ for lemon production in Italy (Pergola et al., 2013), $140 \mathrm{~L} / \mathrm{FU}$ for conventional lemon in Sicily (Beccali et al., 2013), and $142 \mathrm{~L} / \mathrm{kg}$ for orange in Spain (Sanjuan et al., 2005).

\section{Conclusions}

The use of LCA to compare the environmental impacts of three irrigation mix strategies in lemon cultivation brings interesting elements in the debate about the background of water source and the influence of that origin on the environmental impact assessment level. The main conclusions drawn from this work are summarised below:

- Fertilisers production was the main contributor to global warming potential, acidification, eutrophication, human toxicity and toxicity of air, water, and soil.

- Irrigation process plays a very important role in water scarcity periods, due to the high energy demand required to produce DSW in comparison with other conventional water sources.

- Lemon production in the Segura River Basin shows a smaller water use as compared with other Mediterranean citrus production areas.

- Efforts to reduce the environmental impacts of cropping lemon under intensive agriculture practices should be focused on controlling the use of fertilisers or selecting those with lower impact.

\section{Acknowledgements}

This work was supported by the Fundación Séneca-Agencia de Ciencia y Tecnología de la Región de Murcia [19280/PI/14].

\section{References}

Aparicio, J., L. Candela, O. Alfranca, J.L. García-Arostegui, 2017. Economic evaluation of small desalination plants from brackish aquifers. Application to Campo de Cartagena (SE Spain). Desalination. 411, 38-44.

Audsley, E., (coord.) S. Alber, R. Clift, S. Cowell, P. Crettaz, G. Gaillard G, J. Hausheer, O. Jolliet, R. Kleijn, B. Mortensen, D. Pearce, E. Roger, H. Teulon, B. Weidema, H. Van Zeijts, 1997. Harmonisation of Environmental Life Cycle Assessment for Agriculture. Final Report. Concerted Action AIR3-CT94-2028. European Commission. DG VI Agriculture.

Beccali, M., M. Cellura, M. Iudicello, M. Mistretta, 2009. Resource consumption and environmental impacts of the Agrofood sector: life cycle assessment of Italian citrus-based products. J. Environ. Manage. 43, 707-724.

CHS, 2015. Estudio General sobre la Demarcación Hidrográfica del Segura [General study on the River Basin District of the Segura]. Confederación Hidrográfica del Segura, Murcia.

Faurès, J.M., D. Bartley, M. Bazza, J. Burke, J. Hoogeveen, D. Soto, P. Steduto, 2013. Climate smart agriculture sourcebook. FAO, Rome, $557 \mathrm{p}$.

Foidart, F., J. Oliver-Solá, C.M. Gasol, X. Gabarrell, J. Rieradevall, 2010. How important are current energy mix choices on future sustainability? Case study: Belgium and Spain-Projections towards 2020-2030. Energy Policy. 38, 5028-5037.

Frischknecht, R., N. Jungbluth, H.J. Althaus, G. Doka, R. Dones, T. Heck, et al., 2005. The ecoinvent database: overview and methodological framework. Int J LCA. 10, 3-9. 
Frischknecht, R., N. Jungbluth, H.J. Althaus, G. Doka, R. Dones, R. Hischier, S. Hellweg, S. Humbert, M. Margni, T. Nemecek, M. Spielmann, 2007. Implementation of Life Cycle Impact Assessment Methods: Data v2.0. ecoinvent report No. 3, Swiss centre for Life Cycle Inventories, Dübendorf, Switzerland.

Guinée, J.B. M. Gorrée, R. Heijungs, G. Huppes, R. Kleijn, A. de Koning, L. van Oers, A. Wegener Sleeswijk, S. Suh, H.A. Udo de Haes, H. de Bruijn, R. van Duin, M.A.J. Huijbregts, 2002. Handbook on life cycle assessment. Operational guide to the ISO standards. Part III: Scientific background. Kluwer Academic Publishers, ISBN 14020-0228-9, Dordrecht, $692 \mathrm{p}$.

Guineé, J.B., G. Huppes, R. Heijungs, 2001. Developing an LCA guide for decision support. Environ Manage Health. 12(3), 301-311.

Hasler, K., S. Bröring, S.W.F. Omta, H.W. Olfs, 2015. Life cycle assessment (LCA) of different fertilizer product types. Eur. J. Agron. 69, 41-51. http://dx.doi.org/10.1016/ j.eja.2015.06.001.

Hospido, A., M. Núñez, A. Antón, 2013. Irrigation mix: how to include water sources when assessing freshwater consumption impacts associated to crops. Int J Life Cycle Assess. 18, 881-890.

IDAE, 2017. Retrieved from http://www.idae.es/articulos/renewable-energies-spain

ISO, 2006a. ISO 14040:2006. Environmental Management. Life cycle assessment. Principle and Framework. International Organization for Standardization, Geneva, Switzerland.

ISO, 2006b. ISO 14044:2006. Environmental Management. Life cycle assessment. Requirements and Guidelines. International Organization for Standardization, Geneva, Switzerland.

López-Luque, R., J. Reca, J. Martínez, 2015. Optimal design of a standalone direct pumping photovoltaic system for deficit irrigation of olive orchards. Applied Energy. 149, 13-23.

Márquez, L., 2004. Maquinaria agrícola. Madrid. B\&H editores S.L. 700 p.

Martin-Gorriz, B., M. Soto-García, V. Martínez-Alvarez, 2014. Energy and greenhouse-gas emissions in irrigated agriculture of SE (southeast) Spain. Effects of alternative water supply scenarios. Energy. 77, 478-488.

Martínez-Alvarez, V., J. Calatrava, D. Martinez-Granados, B. Martin-Gorriz, 2016. Analysis of water markets as an adaptive tool to water scarcity in SE Spain's agriculture. In ASABE Annual International Meeting, Orlando, Florida USA, July 17-20. St. Joseph, Mich. Paper Number: 162456070; DOI: 10.13031/aim.20162456070.

Martínez-Alvarez, V., M.J. González-Ortega, B. Martin-Gorriz, M. Soto-García, J.F. Maestre-Valero, 2017. The use of desalinated seawater for crop irrigation in the Segura River Basin (south-eastern Spain). Desalination. 422, 153-164.

Marrou, H., J. Wery, L. Dufour, C. Dupraz, 2013. Productivity and radiation use efficiency of lettuces grown in the partial shade of photovoltaic panels. Eur. J. Agron. 44, 54-66.

Melgarejo, J., B. Montaño, 2009. La eficiencia energética del trasvase Tajo-Segura. Cuaderno Interdisciplinar de Desarrollo Sostenible. 3, 173-193.

Moral, F.J., F. López-Rodríguez, F. Cuadrados, A. Ruiz Celma, 2009. Computer assisted sizing of photovoltaic systems for drip irrigation of olive orchards in semi-arid climate. Span. J. Agric. Res. 7 (3), 503-512.

Pergola, M., M. D’amico, G. Celano, A.M. Palese, A. Scuderi, G. Di Vita, G. Pappalardo, P. Inglese, 2013. Sustainability evaluation of Sicily's lemon and orange production: an energy, economic and environmental analysis. J Environ Manag. 128, 674-682.

PRe Consultants, 2014. SimaPro. The Netherlands. Retrieved from http://www.pre-sustainability. com/SimaPro

Ribal, J., C. Ramírez-Sanz, V. Estruch, G. Clemente, N. Sanjuán, 2016. Organic vs. conventional citrus. Impact assessment and variability analysis in the Comunitat Valenciana (Spain). Int J Life Cycle Assess. http://dx.doi.org/10.1007/s11367-016-1048-2

Rothwell, A., B. Ridoutt, G. Page, W. Bellotti, 2016. Environmental performance of local food: trade-offs and implications for climate resilience in a developed city. J. Clean. Prod. 114, 420-430.

Sanjuán, N., L. Úbeda, G. Clemente, F. Girona, A. Mulet, 2005. LCA of integrated orange production in the Comunidad Valenciana (Spain). Int J Agric Resour Gov Ecol. 4(2), 163-177.

Segura, P., A. García, B. Costantini, 2006. Estudio técnico-económico de los procesos de producción agrícola y de transformación (manipulación y confección) de las principales orientaciones hortofrutícolas de la Región de Murcia. Asociación Murciana de Organizaciones de Productores Agrarios (AMOPA), Murcia, 591 p (in Spanish).

Soto-García, M., B. Martin-Gorriz, P.A. García-Bastida, F. Alcón, V. Martínez-Alvarez, 2013. Energy consumption for crop irrigation in a semiarid climate (south-eastern Spain). Energy. 55, 1084-1093. 


\title{
Sustainability Issues Related to Woody Biomass Supply from Fruit Tree Plantation Removal
}

\author{
Alberto Assirelli a , Massimo Brambilla* b, Enrico Santangelo a , Giancarlo Roccuzzo ${ }^{\text {c }}$, Carlo Bisaglia ${ }^{\text {a }}$

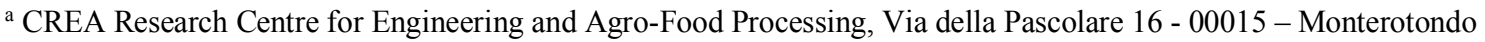 \\ (Roma), Italy \\ ${ }^{\mathrm{b}}$ CREA, Research Centre for Engineering and Agro-Food Processing, Via Milano, 43 - 24047 Treviglio (BG), Italy \\ ${ }^{\mathrm{c}}$ CREA Research Centre for Olive, Citrus and Tree fruits, Via la Canapona, 1Bis - 47100 Forlì (FC), Italy \\ * Corresponding author. Email: massimo.brambilla@,crea.gov.it
}

\begin{abstract}
In Europe, there is a large surface of land cultivated with fruit trees (i.e. vineyards, olive and fruit orchards) providing valuable amounts of woody biomass from pruning and plant removal at the end of the plantation cycle. Such biomass is generally burnt in open air or shred on topsoil. In few cases, this material is used as fuel for biomass power stations provided that harvesting technologies are adequate for costs optimization and product quality. To assess and deepen the sustainability issues related to such a supply chain, a field survey was carried out on the various phases of biomass harvesting and transporting from the uprooting of a peach tree orchard placed in the surroundings of Ravenna (Emilia-Romagna, Italy).Trees were planted in a low-density orchard, spaced at $6.0 \mathrm{~m}$ between rows and $3.5 \mathrm{~m}$ on the row, at 476 plants ha $^{-1}$ (the total area was $1.96 \mathrm{ha}$ ). The biomass collection chain foresaw the following phases: i) plant uprooting and windrowing, ii) biomass stacking, iii) lorry loading with and without compaction; iv) biomass transport to the power station. According to results, personnel represented $18.8 \%$ to $30.15 \%$ of the total costs $\left(€ \mathrm{ha}^{-1}\right)$ for phases i) and ii) while the kind of truck (articulated or not) was found to deeply affect the loading times (they doubled in case of articulated trucks) more than the compaction system that caused a $+35.7 \%$ increase in working time. The collection system was also found to affect the quality of the supplied biofuel following the cleaning of root biomass from soil residues. This study shows the importance of a specific set up of the logistic chain related to the recovery of orchards stumps that should be focused on both economic and technologic issues (e.g. reducing the topsoil pollution of the harvested underground biomass) to meet the needs of power stations.
\end{abstract}

Keywords: Uprooting operations, Cleaning units, Bioenergy chain.

\section{Introduction}

Regardless of the crop form, the termination practices in permanent crops produce significant volumes of wood biomass, which can be exploited as a renewable fuel in bioenergy plants. Many examples are available for the collection and logistics of the pruning residues (Pari et al., 2017). These are mainly focused on vineyard (Spinelli et al., 2010), but the studies also concerned peach (Pari et al., 2018), kiwifruit (Spinelli et al., 2012), olive (Acampora et al., 2012; Spinelli and Picchi, 2010), almond (Pari et al., 2016). However, the collection of the residual stump too has been investigated in forest trees (Laitila and Nuutinen, 2015; Picchio et al., 2012) and in orchard fruit (Picchio et al., 2012; Picchi et al., 2012; Pari et al., 2017). More recently, a detailed evaluation of the removal of the clear-cut biomass of infected kiwi orchards was presented by Manzone et al. (2017).

The recovery in a single step of the whole-plant of the depleted orchard can be an interesting solution, but adequate harvest and logistics operations should be defined. One of the main problem is the production of a "clean" feedstock, because the contamination by soil and dirt particles from stump can reduce the commercial value, until the rejection of the product (Picchi et al., 2012; Pari et al., 2017).

Peach (Prunus persica (L.) Batsch) represents an important permanent crop: in 2012, in Italy, it exceeded 62,000 hectares. It is the second permanent crop for importance in Italy after the orange (ISTAT, 2018). It may yield around 2.5-3 t ha ${ }^{-1}$ of wood biomass, corresponding to hundred tons of feedstock potentially available annually by the renewal cycle. The amount may be higher in the intensive plantation $\left(1,000\right.$ plant ha $\left.^{-1}\right)$ diffused $^{-1}$ in Italy (Garcìa-Galindo et al., 2016).

The extraction of the whole plant can be deemed an interesting new approach to exploit as a whole all the residues of an orchard at the end of the cycle. The objective of the present work was to carry out a preliminary analysis mainly focused on the logistic phase including i) the hauling of the biomass extracted (whole plant) or pruned (aboveground biomass), delivered compacted or loose; ii) the hauling of the comminuted biomass.

\section{Materials and Methods}

The Site

The study took place in San Bartolo, Ravenna-Italy (4421'30.77"N 12¹1'50.54" E) in Northern Italy. The plantation was a peach orchard having a layout $6 \times 3.5 \mathrm{~m}$ grown in training system "Italian delayed vase". The 
orchard was 14 years-old, at the end of turnover. The total surface area collected was 0.90 ha

The mechanization lines

The work was carried out within an integrated supply chain focused on the efficient exploitation of the residues of permanent crops. Although the chain was conceived as a whole, it may be separated into two sections (Fig. 1).

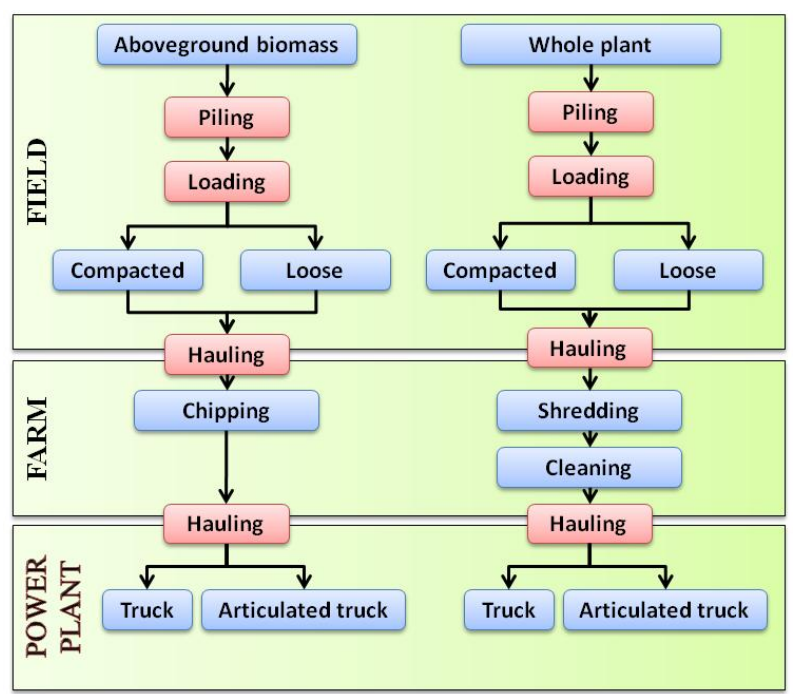

Figure 70. Schematic representation of the two supply chain sections. The red box shows the phase analysed in the present study.

The first concerned the logistics associated with the management of the aboveground biomass without the stumps (left on the ground for later handling). The second focused on the extraction and management of the whole plant (aboveground biomass plus the stump). In the field, both the types of residue were collected and piled at the border.

The biomass was transported to the farm centre. The delivering was performed using either a truck having a working volume of $55 \mathrm{~m}^{3}$ or an articulated truck with the working volume of $90 \mathrm{~m}^{3}$ : both the machines were equipped with a crane. Once loaded on the truck, the biomass was left loose or compacted. In the latter case, a trunk section weighing $450 \mathrm{~kg}$ hooked to the crane was used to partially break and compact the loaded biomass (Figure 2). The compaction was accomplished in two steps during the filling of the track/trailer (at the half and full volume), causing a partial or total breakage of the branches. Every single truck and trailer transporting the biomass (compacted or loose) was weighed before transporting using a weighbridge.

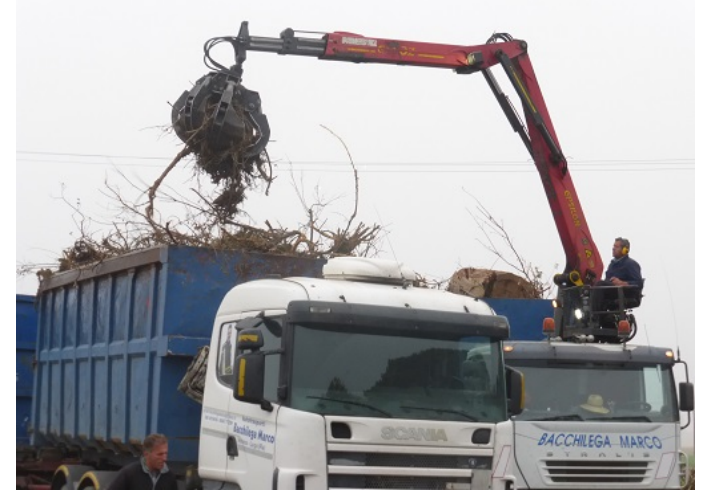

Figure 71. Compaction phase during the loading of the whole trees.

At the farm centre, the aboveground biomass was chipped, while the whole plants were pre-grounded and cleaned to remove the soil from the stump. Usually the first does not present problems for the combustion plants, while the biomass produced from the whole plant requires cleaning to reduce the soil pollution of the woody biomass below the $10 \%$. Both the biomasses (aboveground part only and whole plant with stump) were transported and unloaded by roll-off container truck and container trailer/semitrailer. Finally, the comminuted biomass (wood chips or pre-grounded material) were conferred to the power plant. All the machines used in the study are listed in Table 1. 
The extraction, piling, and loading phases were analyzed following the CIOSTA (Comité International d'Organisation Scientifique du Travail en Agriculture) methodology and the recommendations of the Italian Society of Agricultural Engineering (A.I.I.A.) 3A R1. A reference to these methods can be found in Bodria et al. (2006)

The bulk density of the pre-grounded biomass was calculated in compliance with the standard UNI EN 15103:2010 using a normalized cylinder with the capacity of $0.026 \mathrm{~m} 3$ : to do this, three samples for each truck were taken.

During the hauling, the average speed of three travels was recorded for each treatment (truck or articulated truck transporting compacted or loose feedstock). Comprehensively, the vehicles travelled on unpaved roads (39), ordinary roads (85), and passed through inhabited centers (48). The drivers declared the fuel consumptions by onboard instruments.

To assess the overall costs for transportation, given the free negotiation in compliance with the Italia law 1st March 2005, n. 32 and with the Legislative Decree 21st November 2005, n. 286, as reference we considered the last tariffs available for these kind of transport that were issued with the Ministry Decree 18th November 1982 (Ministero Italiano dei Trasporti, 1982). Following this, it was decided to use the value of $0.0051 € \mathrm{~kg}-1 \mathrm{for}$ trailer trucks and $0.0066 € \mathrm{~kg}-1$ for articulated trucks. In addition, we adopted the value of $1.71 € \mathrm{~km}-1$ that refers to the lower volume class that does not include articulated trucks, and the value of $1.79 € \mathrm{~kg}-1$ for articulated trucks. Such differences depend to the fact that transporting the whole plants requires high volume machinery given the low specific weight of the biomass. These high volume always require oversized trucks and trailers. Costs were referred to the amount of biomass transported $(\mathrm{kg})$.

Table 25. Machines used in the study.

\begin{tabular}{cccc}
\hline Work phase & Type & Make & Model \\
\hline \multirow{2}{*}{$\begin{array}{c}\text { Extraction and } \\
\text { piling }\end{array}$} & Truck excavator & Hyundai & 140 LCD7 \\
& Chipper & Pezzolato & $1000 / 1000$ \\
& Shredder & Doppstradt & DW 30/60 \\
Loading and & Rotary Sieving & Farwick & Mustang \\
hauling & Trailer truck & Scania & 420 \\
\hline
\end{tabular}

Data processing was carried out with the PAST (Hammer et al., 2001) and MINITAB 17 softwares (MINITAB, 2017): after checking for data homoscedasticity and normality data underwent analysis of variance with one-way ANOVA and GLM procedures followed by post hoc comparisons with the Tukey's HSD test.

\section{Results and Discussion}

The extraction of the whole trees and their piling stored in the field (Figure 3 ) registered a production capacity higher than 1 ha day ${ }^{-1}$. Whole tree extraction was the most time-consuming phase requiring $13.1 \mathrm{~h} \mathrm{ha}^{-1}$, while piling needed just $2.2 \mathrm{~h} \mathrm{ha}^{-1}$ (Table 3 ). As a consequence, the uprooting and windrowing accounted for the $88 \%$ of the cost per hectare. According to results, as percentage of the total cost the personnel represented $18.8 \%$ for phase 1 and $30.15 \%$ for phase 2 (Table 2). The kind of truck (articulated or not) was found to deeply affect the loading times (they doubled in case of articulated trucks) more than the compaction system that caused a $+35.7 \%$ increase in working time.

The collection system was found to affect also the quality of the supplied biofuel following the cleaning of root biomass from soil residues. Within the collection chain this phase is the most expensive and requires as lot of attention in the organization of the work and field preparation. Both biomass windrowing and piling may affect the quality of the biomass because, after uprooting, plants are pushed on soil surface. In this way the soil particles may pollute the woody biomass lowering the quality of the fuel. The compaction of the heaps allowed to increase the performance of logistic chain improving the uniform unloading of the biomass on the platform.

Table 26. Costs analysis of the whole plant management.

\begin{tabular}{lccc}
\hline Working phase & $\begin{array}{c}\text { Operative } \\
\text { capacity }\left(\mathrm{h} \mathrm{ha}^{-1}\right)\end{array}$ & $\begin{array}{c}\text { Personnel costs } \\
\left(€ \mathrm{ha}^{-1}\right)\end{array}$ & $\begin{array}{c}\text { Total costs } \\
\left(€ \mathrm{ha}^{-1}\right)\end{array}$ \\
\hline 1) Uprooting and windrowing & $13.1 \pm 1.2$ & $196.56 \pm 18.03$ & $982.82 \pm 90.13$ \\
2) Whole plant piling & $2.2 \pm 0.2$ & $32.83 \pm 3.02$ & $109.42 \pm 10.08$ \\
\hline
\end{tabular}




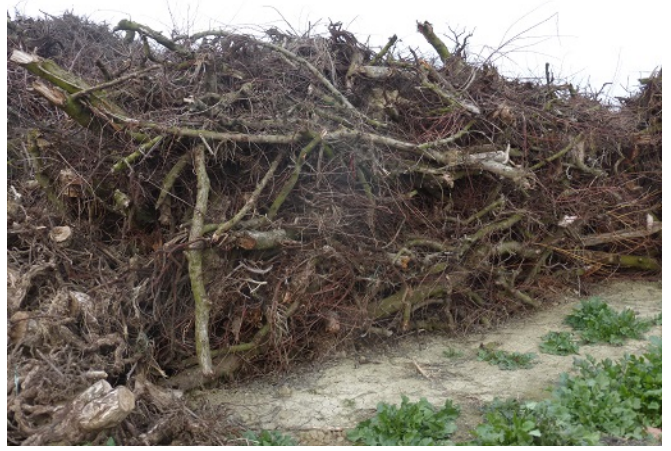

Figure 72. The compacted biomass stored in the field.

Concerning whole plant harvesting including stumps, there was the need to guarantee an adequate level of cleaning of the wood biomass from soil particles: this required an additional step of pre-grinding with a shredder followed by cleaning through a rotary sieve. During the work, some random samples were analyzed before and after the cleaning to estimate the efficiency of pre-grinding. Although not validated statistically, the system tested in the present study gave positive results regarding the amount of soil removal and, hence, the overall decrease of soil pollution. Before the cleaning, the sample had 18-20\% of impurities (mainly dust and ground), while soon after the cleaning the soil pollution decreased to 5-7\%. However, the cleaning phase showed the same productivity of the pre-grinding phase but determined an increase of operational costs caused by the use of two different machines (data not shown). When the plants were collected without the stumps, a chipper was used for the comminution of the biomass. The wood chip may be deemed ready for direct combustion in a biomass power plant.

Both the steps needed to set up a separate area inside the farm to optimize the efficiency of the machines. Transport of loose or compacted biomass could reduce the soil pollution following the partial breakage caused by the compaction of the roots. On average, the loading of the articulated truck was more time-consuming than the loading on the trailer truck. In both cases, biomass compaction resulted in significantly higher loading times: 43 $v s 32$ minutes in case of the articulated truck and 22 vs 15 minutes for the trailer truck (data not shown).

In the open field, biomass compaction resulted in having a significant influence also on the loading time (Figure 4, left). However, the higher amount of biomass transported after compaction allowed to compensate for the longer time required for loading..

For each vehicle, the loading time was longer in case of compaction, but the difference between the compaction and loose material was lower for the articulated truck $(11 \mathrm{~min})$ than for the trailer truck (13 min) (data not shown). The trailer truck showed a significant lower loading time for compaction $\left(0.36 \mathrm{vs} 0.47 \mathrm{~min} \mathrm{~m}^{-3}\right)$ and for loose material $\left(0.28\right.$ vs $\left.0.40 \mathrm{~min} \mathrm{~m}^{-3}\right)$ than the articulated truck (Figure 4 , left). The loading time of the loose material in the articulated truck was comparable with that of the compacted biomass in the trailer truck $(0.36 \mathrm{vs} 0.40 \mathrm{~min}$ $\mathrm{m}^{-3}$ ). Although in the articulated truck the vehicles to load were two, the simplified loading (loose) allowed to reduce the average time per loaded volume. Such a result must be kept into account in defining the most costeffective supply chain.

The hauling time was unaffected by biomass compaction (Figure 4, right), but it was dependent on the type of truck (articulated or not). The useful volume of trailer truck increased by $21 \%$ compared to the simple truck, exceeding $20 \mathrm{t}$ the volume of the former. However, the weight of the load and vehicle manoeuvrability affected the speed maintained during transportation. On the road network used for the travels, within the same kind of vehicle, a different type of compaction showed the same transport times and hourly costs.
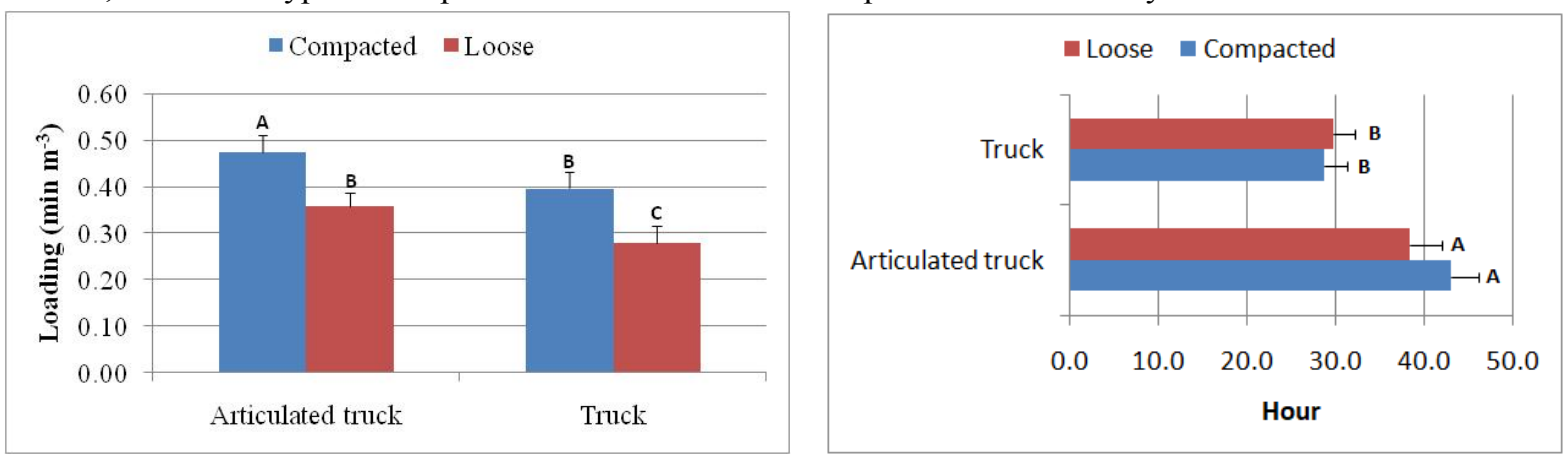

Figure 73. Loading times (on the left) and total hauling times (on the right) for truck or articulated truck from the field to the farm centre. Data are expressed as mean \pm standard deviation. Different letters indicate significant differences $(\mathrm{p}<0.0001)$ after Tukey's HSD test. 
Table 3. Productivity and main costs of extraction and piling.

\begin{tabular}{cccc}
\hline & Type & Extraction & Piling \\
\hline & Productivity $\left(\mathrm{h} \mathrm{ha}^{-1}\right)$ & $13.1 \pm 1.20$ & $2.2 \pm 0.2$ \\
Machine Cost $^{*}$ & $€ \mathrm{~h}^{-1}$ & 50.0 & 75.0 \\
& $€$ ha & 982.8 & 109.4 \\
Fuel $^{* *}$ & Consumption $\left(\mathrm{L} \mathrm{h}^{-1}\right)$ & 20.0 & 28.0 \\
& Cost $\left(€ \mathrm{ha}^{-1}\right)$ & 262.1 & 61.3 \\
Personnel cost $^{* * *}$ & $€ \mathrm{~h}^{-1}$ & 15.0 & 15.0 \\
& $€ \mathrm{ha}^{-1}$ & 196.6 & 32.8 \\
\hline
\end{tabular}

${ }^{*}$ In accordance with the local (provincial) mechanical agricultural service scale of rates.

${ }^{* *}$ Ordinary prices

${ }^{* * *}$ In compliance with the National Collective Agreement of agricultural employees

Each comminuted or untreated biomass had a different bulk density that, in turn, resulted in a variable capacity of biomass delivery (Figure 5). The complexity of the different logistic options did not allow to have a consistent number of replicates, but the data were drawn from a simulation of realistic conditions. In all cases, the biomass hauled with the articulated vehicle was higher compared to the trailer truck. When in the form of wood chips, the amount of the hauled biomass using the articulated truck was the highest $(27.4 \mathrm{t})$. On the other side, the transport of the whole plants with the trailed truck reduced the amount of biomass to $10.4 \mathrm{t}$. The pre-grinding of the plants can be deemed an interesting solution. When the whole plants were grinded with the shredder, the delivered volume increased to $18.6 \mathrm{t}$ with the trailed truck and to $25.03 \mathrm{t}$ with the articulated truck: these values are comparable with those registered for the wood chips.

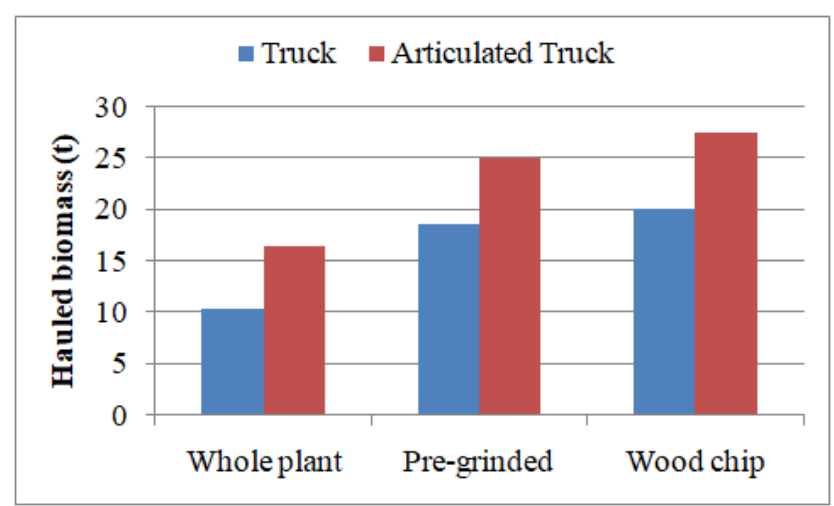

Figure 74. Amount of hauled biomass ( $t$ ) with different degree of comminution.

Overall transportation costs to move the biomass from the field to farmyard ranged from $0.0032 €$ to $0.0067 €$ for any trip (from the farm to the biomass plant and backward). Figure 6 represents the interval plots of the averages related to the considered mechanization lines (kind of truck and presence/absence of biomass compaction at loading).

The analysis of the variance pointed out that both the kind of truck and the biomass compaction significantly act on the total costs of the operation $(p<0.05)$. On average, the cheaper way to transport the biomass from the farm to the plant is to use the articulated truck both with and without biomass compaction $(0.0033 \pm 0.000047 €$ $\mathrm{kg}^{-1}$ and $0.0042 \pm 0.00012 € \mathrm{~kg}^{-1}$ ) while using trailer trucks basically results in doubling the costs that range from $0.0052 \pm 0.000074 €$ with biomass compaction and $0.0066 \pm 0.00019 € \mathrm{~kg}^{-1}$ without it.

Accordingly, the choice of the articulated truck to bring the biomass from the orchard to the farmyard may result in a significant saving of money for the farmer: during the loading, the filling of the dumper is more practical and the compaction of biomass easier and faster. As a consequence, the saving of time and money is higher. Despite this, the economic criterion is only one side of the coin. Another fundamental issue is the field accessibility. Owing to their dimension, articulated trucks have a wider turning radius (requiring the absence of tight turns), but also a higher specific load. This on the one hand may damage the surface of the farm tracks, and on the other hand (and this is even worse), when entering the field, may induce soil compaction resulting in substantial damage of soil structure. 


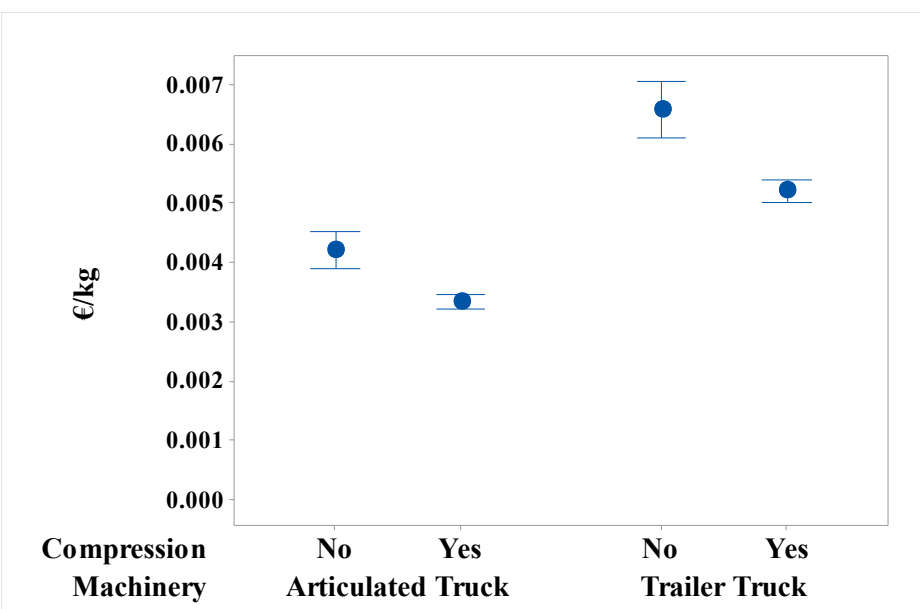

Figure 75. Interval plot representing the total costs for biomass harvesting (average $\pm 95 \%$ confidence interval).

\section{Conclusions}

The logistic approach foreseeing the collection of the whole-plant has encouraged some companies to design and implement different harvesting systems. Nevertheless, the technologies available for whole-plant harvesting are however still unsatisfactory because of the contrast between delivery performances and the low quality of the end product due to the soil particle pollution of the woody biomass.

The whole-plant harvesting appears as a promising technique able to reduce the cost of the logistic chain. The harvesting of the whole biomass in a single step allows for avoiding the double passage for the removal of trunks and stumps. The system should foresee a further step which for the whole trees is the pre-grinding/cleaning, while for the trees collected without the stumps is the direct chipping. In both cases, there is the need of a dedicated area in the farm to increase machinery efficiency.

When the chain includes the compaction of the biomass during the loading, the system may result even more convenient allowing for a further simplification of the production chain that leads to produce renewable fuel ready to use.

The choice of the proper transportation is important not only under the economic point of view, but also in view of the sustainability of the production system. To pay attention at the the mass of the machineries means to preserve the condition of soils and farm tracks, two key points in the concept of the conservative agriculture.

\section{Acknowledgements}

This work was supported by the Italian Ministry of Agriculture (MiPAAF) under the AGROENER project (D.D. n. 26329, 1 april 2016) - http://agroener.crea.gov.it/.

It shall be considered that any reference to specific makes and models is solely made to help readers to assess the study correctly, and it does not involve any endorsement of specific makes and models to the exclusion of similar machines produced by other manufacturers.

\section{References}

Acampora, A., Croce, S., Assirelli, A., Del Giudice, A., Spinelli, R., Suardi, A., Pari, L., 2013. Product contamination and harvesting losses from mechanized recovery of olive tree pruning residues for energy use. Renew. Energy 53, 350-353. doi:10.1016/j.renene.2012.12.009

Bodria, L., Pellizzi, G., Piccarolo, P., 2006. Meccanica Agraria vol II: la meccanizzazione, Edizioni Il Sole24ORE, Bologna, Italy.

Garcìa-Galindo, D., Gòmez-Palmero, M., E., P., Germer, S., Pari, L., Alfano, V., Dyjakon, A., Sagarna, J., Rivera, S., Poutrin, C., 2016. Agricultural pruning as biomass resource: generation, potentials and current fates. An approach to its state in Europe, in: 24th European Biomass Conference and Exhibition, 6-9 June 2016, Amsterdam. pp. 1579-1595.

Hammer, Ø., Harper, D.A.T., Ryan, P.D. 2001. PAST: Paleontological statistics software package for education and data analysis. Palaeontologia Electronica 4, 1. http://palaeo-electronica.org/2001_1/past/issue1_01.htm

ISTAT (Istituto Italiano di Statistica). Principali Coltivazioni Legnose Āgrarie. Available online: https://www.istat.it/it/archivio/124365 (accessed on 29 March 2018).

Laitila, J., Nuutinen, Y., 2015. Efficiency of Integrated Grinding and Screening of Stump Wood for Fuel at Roadside Landing with a Low-Speed Double-Shaft Grinder and a Star Screen. Croat. J. Foorest Eng. 36, $19-32$. 
Manzone, M., Gioelli, F., Balsari, P., 2017. Kiwi clear-cut: First evaluation of recovered biomass for energy production. Energies 10, 1837. doi:10.3390/en10111837.

Ministero Italiano dei Trasporti. 1982. Ministree Decree: Approvazione delle tariffe per i trasporti di merci su strada per conto di terzi sul territorio nazionale. Italian Republic Official Journal n. 342 of $14^{\text {th }}$ december 1982.

Pari, L., Scarfone, A., Alfano, V., Bergonzoli, S., Gallucci, F., Santangelo, E., 2017. Testing Open-Air Storage of Stumps to Provide Clean Biomass for Energy Production. Energies 10, 1725. doi:10.3390/en10111725.

Pari, L., Suardi, A., Del Giudice, A., Scarfone, A., Santangelo, E., 2018. Influence of chipping system on chipper performance and wood chip particle size obtained from peach prunings. Biomass and Bioenergy In press. doi:10.1016/j.biombioe.2018.01.002

Pari, L., Suardi, A., Santangelo, E., García-galindo, D., Scarfone, A., Alfano, V., 2017. Current and innovative technologies for pruning harvesting : A review. Biomass and Bioenergy 107, 398-410. doi:10.1016/j.biombioe.2017.09.014

Pari, L., Suardi, A., Scarfone, A., Acampora, A., Frackowiak, P., Adamczyk, F., Santangelo, E., 2016. Harvesting of almond (Prunus dulcis, Mill.) pruning in Spain using a new chipper prototype, in: 24th European Biomass Conference and Exhibition, 6-9 June 2016, Amsterdam. pp. 6-9.

Picchi, G., Pari, L., Scarfone, A., Barontini, M., Spinelli, R., 2012. Unearthing the hidden resource: biomass from rootstock recovery. Biofuels, Bioprod. Biorefining 6, 246-256. doi:10.1002/bbb

Picchio, R., Verani, S., Sperandio, G., Spina, R., Marchi, E., 2012. Stump grinding on a poplar plantation: Working time, productivity, and economic and energetic inputs. Ecol. Eng. 40, 117-120. doi:10.1016/j.ecoleng.2011.11.012.

Spinelli, R., Magagnotti, N., Nati, C., 2010. Harvesting vineyard pruning residues for energy use. Biosyst. Eng. 105, 316-322. doi:10.1016/j.biosystemseng.2009.11.011

Spinelli, R., Magagnotti, N., Nati, C., Pari, L., Vanneste, J.L., 2012. Recovering kiwifruit pruning residues for biomass production. Trans. ASABE 55, 1-8.

Spinelli, R., Picchi, G., 2010. Industrial harvesting of olive tree pruning residue for energy biomass. Bioresour. Technol. 101, 730-735. doi:10.1016/j.biortech.2009.08.039 


\author{
Performance Study of a Standalone Direct Pumping Photovoltaic System Used \\ for Drip Irrigation \\ Harkani Assia, El Aissaoui Abdellah, Fihri Fassi Hichame, El Kacimi Ousama \\ ${ }^{a}$ Laboratory of Agricultural Machinery, National Institute of Agronomic Research, Settat 26000, Morocco \\ ${ }^{\mathrm{b}}$ Laboratory Engineering, Industrial Management and Innovation, Hassan $1^{\text {st }}$ University, Faculty of Sciences and \\ Technologies, Settat 26000, Morocco \\ * Corresponding author. Email: assiaharkani91@gmail.com
}

\begin{abstract}
Renewable energy sources for irrigation have been widely used in recent decades. Performance study of a standalone photovoltaic pumping system confronted to actual behaviour of incident irradiance is investigated in this work. The system is based on a photovoltaic generator, a MPPT/ DC-DC converter, and a diaphragm DC motor pump connected to calibrated (Flow versus Pressure) orifices that simulate a small drip irrigation network, mounted in parallel scheme to vary the pump operating point according to timely varied irradiance. The result showed that daily trend of solar irradiance influenced considerably operating behaviour of the pump, consequently the performance of the standalone PV system is affected as there is inadequation between charges of the PV generator and the DC pump. The hydraulic effeciency trends showed a dispersion and varying performances according to irradiance change and to hydraulic system unstability. A method of gouverning hydraulic performance is proposed to improve system efficiency and pressure stability as requirements for water distribution uniformity in drip irrigation network under by a standalone PV pumping system.
\end{abstract}

Keywords: Standalone, Photovoltaic Pumping System, Performances, Irradiance, Efficiency, DC pump.

\title{
1. Introduction
}

Agriculture production in developing countries is mainly based on rainfall for annual crop production and small pumping systems based on groundwater for vegetable crops irrigation.

In Morocco, small scale farming is dominant (about 90\%) and need significant technological solutions for improving its sustainability. During the last decades, agricultural productions systems were most vulnerable to energy prices rising (fuel and electricity) and consequently final costs of agricultural products are undermined.

This constraint induced farmers to transform their diesel based irrigation pumping systems to butane use for propulsing gasoline based engines. Butane is a low cost energy because it is subsidized by the government. There are approximately more than 100000 ha, that are butane based irrigated using butane (MEMSD, 2014). The consumption of butane in agriculture is about $800 \mathrm{ktep}$ per year (CCCC, 2014). As alternative, the promotion of using alternative energy is becoming important according to existing average irradiance in Morocco (about 2.253 $\mathrm{kWh} /\left(\mathrm{m}^{2 *}\right.$ year) $)(\mathrm{GIZ}, 2011)$. Moroccan government has initiated a national program on photovoltaic pumping in 2013 to promote irrigation pumping for small scale farming representing a workforce of $90 \%$ and an area of 44\% (MAPM, 2013). Odeh et al. (2006) compared PV water pumping systems to diesel based systems, and showed better technical and economical performances for equivalent hydraulic energy capacities up to 8000 $\mathrm{m} 4 /$ day, $4100 \mathrm{~m} 4 /$ day and $2600 \mathrm{~m} 4 /$ day for interest rate values of $0 \%$ (subsidized project), $10 \%$ (average market rate in developing countries) and 20\% (high market rate), respectively. Reca et al. (2016) showed that drip irrigation is potentially the main method for use of $\mathrm{PV}$ pumping systems according to benefits of energy and water saving for sustainable farming systems. Performance of PV pumping systems depends mainly on its design. Sizing is considered as a first step that could lead to system reliability and cost effectiveness (Renu and al., 2016). Robust sizing leads to reliable and affordable standalone PV pumping systems for irrigation. However, knowledge on crop management, soil and irrigation systems adaptation are required (Firatoglu and al., 2004; Glasnovic and al., 2007; Gad and al., 2011).

Adaptation of PV panels to pump requirement cannot be satisfied without integration of a robust DC-AC or DC-DC energy conversion for the pumping systems performances. Many studies dealt with PVWPS performance's integrating MPPT devices (Sefriti and al., 2015). Akhirio and al., (2009) simulated PVWPS performance using two MPPT algorithms of IncCond and P \& O on Matlab/Simulink. They found that low efficiency of PVWPS can be improved by 35\% using MPPT technology and the system valorized more than $99 \%$ of the PV panel's energy to satisfy more than $87 \%$ of the water requirement. Khan and al. (2012) studied performance of standalone PVWPS based on DC-DC converter coupled to a DC pump. The system efficiency has been improved by integrating close loop control with DC-DC buck converter to increase its daily pumping output. Essam and al. (2016) developped a standalone PVWPS based on batteries energy storage to drive BLDC motorpump. They proposed an integrated control strategy based on use of MPPT, speed control and hysteresis current of the BLDC motor and batteries charging/discharging optimization to improve the overall system efficiency. 
Others references are done on hydraulic aspect to improve PVWPS efficiency using centrifugal and positive displacement pumps. Centrifugal pumps are characterized to efficiently work for low pressure (less than $100 \mathrm{~m}$ ) and high flow rate requirements. Worldwide, it represents popular pumping equipment used to deliver to $90 \%$ of water (Guiqiang and al., 2017; Levon and al., 2015).

According to affinity law, centrifugal pump efficiency depends on its rotational speed to offer low yield at low speed. The low performance of centrifugal pumps is an actual problem of standalone PVWPS to cause hydraulic instability of connected drip irrigation at low and varying insulation (Walski, 2003; Chandel, 2015). In last decades, use of DC pumps has been promoted to improve performance of PV based irrigation systems. In fact, technologies emergence of DC brushless motors and positive displacement pumps improved cost efficiency for decreasing maintenance rate and efficient use of DC pumps in PV agricultural irrigation. Compared to centrifugal pumps, positive-displacement pumps are characterized to efficiently work at high pressure (more than 100m) and low flow rate requirements. But, it cannot be appropriate to be associated directly to PV panels without adequate linking between photovoltaic energy input and irrigation network charge. Positive-displacement pumps are only used for DC low-voltage applications ( 24 to $48 \mathrm{~V}$ ) to deliver small daily flows (up to $5 \mathrm{~m}^{3} /$ day). Protogeropoulos et al., (2000) and Kashyap et al. (2013) stated that more improvements are needed for standalone PVWPS to satisfy water demand in small drip irrigation systems with control of surrounded pressure change due to influence of daily insulation variation. Hamidat and al. (2004) studied performance of PVWPS using both centrifugal and positive displacement pumps. They used a multistage centrifugal pump coupled to AC motor and three-phase inverter. The second pump is coupled to DC/DC converter actuating a BLDC motor. They showed that a positive displacement pump has an efficiency of $45 \%$ compared to centrifugal pump efficiency or only $14 \%$. In other study, Hamidat and al. (2009) compared PVWPS performances of centrifugal and positive displacement pumps simulation for different sites characterized by different atmospheric conditions (Saharan climate, Saharan climate and raining in summer). A comparison between electrical and hydraulic performances of both centrifugal and positive displacement pumps showed better efficiency, less energy losses and high water volume pumped by the positive displacement pump. Naresh and al., (2012) simulated a PVWPS using MATLAB/ Simulink. They used MPPT and Permanent Magnet motor (PMDC) to show that PMDC motors are technically and economically efficient compared to AC motors in terms of water discharge rate.

This paper aims to study the performance of a standalone PVWPS based on a DC positive displacement pump facing hydraulic instability of a drip irrigation network due to varying solar irradiance. Studying a standalone PVWPS based on a DC pump is of importance to find better adequation between pressure potential of a positive displacement pump to serve a hydraulic network (mounted of parallel nozzles to simulate drip irrigation raws). The parallel scheme simulates operating behavior of a small drip irrigation system that requires a constant operating pressure with a uniformity distribution.

\section{Materials and Methods}

A schematic of the experimental setup used for testing the direct coupled PV pumping system is presented in Fig. 1. PV panel (Monocrystal Silicon Module, SharpTM 175W), an MPPT controller (Victron Blue Solar 48/24 / 12V), a DC diaphragm Pump (FLOJET, 12V DC, 144W) and a hydraulic ramification of seven identical calibrated orifices (Albuz, 11004, $1.3 \mathrm{~L} / \mathrm{min}$ at 3 bar) mounted in parallel scheme to simulate outputs of a connected irrigation network and to evaluate flow rate with reference to pressure potential created by the pump using orifice hydraulic model $\mathrm{Q}=\mathrm{K} * \mathrm{Px}$.

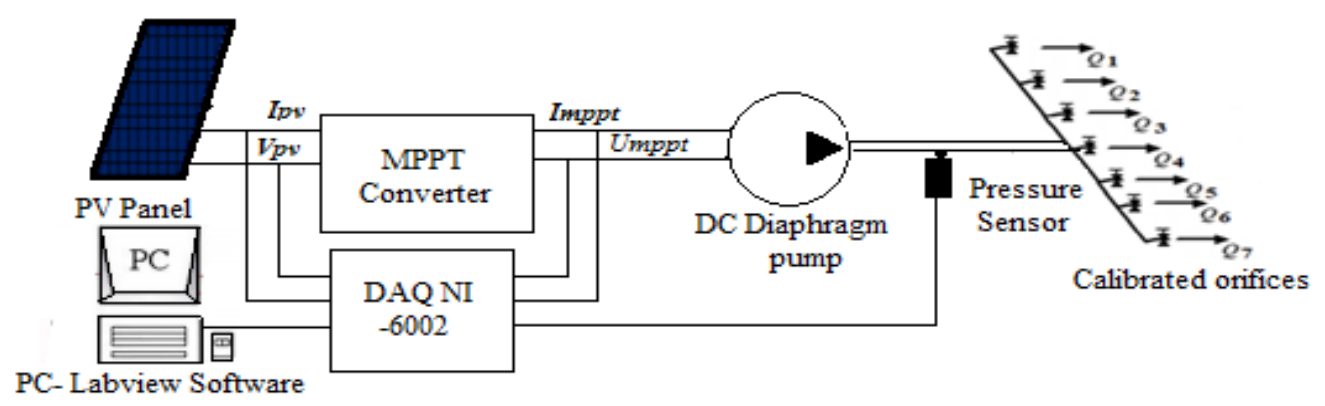

Figure 1. Test bench of a photovoltaic DC pumping system without capacitance. 
Equations

Electrical and hydraulic characteristics computation was based on the equations below,

- Voltage and current sensors (ACS712) were installed at upstream and downstream points of the MPPT converter to evaluate electrical power at the both levels using the equations 1 and 2.

$$
\begin{aligned}
& P p v=V p v^{*} I p v \\
& P m p p t=V m p p t * \operatorname{Im} p p t \\
& I p v=\left(a^{*} V_{s} 1\right)+b \\
& \operatorname{Im} p p t=\left(a^{\prime} * V_{s} 2\right)+b^{\prime}
\end{aligned}
$$

- Current sensors were calibrated using an acid lead battery source supplying a circuit of parallel mounted automotive lamps (12 VDC, $55 \mathrm{Watt})$. The calibration model is presented in equation 3.

$$
Y=14.333 x-36.149
$$

- $\quad$ Pressure of sensor is calculated based on the following calibration model:

$$
Y=0.3427 x^{2}-2.6075 x+4.918
$$

To evaluate the flow rate orifices outlet, hydraulic model (Eq.5) taken experimentally by calibration was developed:

$$
\begin{aligned}
& Q(L / \min )=K^{*} P^{x}(\text { bar }) \\
& (0.4759<x<0.5745)
\end{aligned}
$$

A hydraulic ramification of seven identical calibrated orifices characteristics were presented in Table 1.

Table 27. Orifices hydraulic parameters' (experimental test).

\begin{tabular}{ccc}
\hline Orifices number & $\mathrm{K}$ & $\mathrm{x}$ \\
\hline Orifice 1 & 1.1557 & 0.5006 \\
Orifice 2 & 1.1718 & 0.5186 \\
Orifice 3 & 0.5778 & 0.5384 \\
Orifice 4 & 0.968 & 0.5745 \\
Orifice 5 & 1.1576 & 0.5053 \\
Orifice 6 & 1.1369 & 0.5042 \\
Orifice 7 & 0,7663 & 0.4759 \\
\hline
\end{tabular}

\section{Results and Discussion}

Morocco is located on $34^{\circ} 0^{\prime} 47 \mathrm{~N} ;-6^{\circ} 49^{\prime} 57 \mathrm{O}$ on longitude and latitude with annual global solar radiation is about $2.253 \mathrm{kWh} /\left(\mathrm{m}^{2 *}\right.$ year) (GIZ, 2011). A variation in global solar radiations on a 25 May 2017 has been presented in figure 2 .

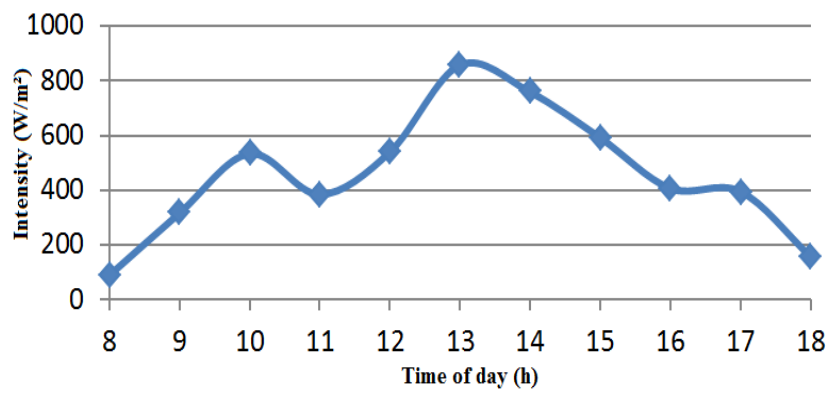

Figure 2. Radiation curve of the 25-05-2017. 
The maximum solar intensity recorded was $858 \mathrm{~W} / \mathrm{m}^{2}$. At $11 . \mathrm{am}$, the intensity decreased due to a cloudy environment, and merely affected the performance of load connected to the circuit i.e. motor-pump.

Performance of the PVWPS studied by monitoring the pump behavior according to solar radiation for the even hours of the day (at $8 \mathrm{am}, 10 \mathrm{am}, 12 \mathrm{pm}, 02 \mathrm{pm}, 04 \mathrm{pm}$ and at $6 \mathrm{pm}$ ), showed changes in pump efficiency.

Figure 3a shows the pump response relative to low irradiance (8.am) of $100 \mathrm{~W} / \mathrm{m} 2$. Seven flow behaviors are presented with reference to the number of engaged orifices to displace the pump operating point as flow rate decreasing causes an increase in pressure. This displacement showed that it is possible to operate pump not only to satisfy requirement of operating an orifice around a stable and predefined operating pressure (network requirement of a drip irrigation system) but also to satisfy condition of better performance. In fact, adaptation between the number of engaged orifices and actual irradiance is needed to operate the pump around its better performance. When a system pressure is satisfied, the performance is correlated to an engaged part of the hydraulic network (number of engaged orifices).

The characteristic curves, flow / pressure $[Q=f(P)]$ and hydraulic power / pressure $[\mathrm{Ph}=\mathrm{f}(\mathrm{P})]$ don't converge with reference curves of the pump as solar irradiance is lacking in the morning for a best performance convergence. Second graph (Figure 3a/08.am) illustrates varying behavior of hydraulic powers due to the variation of flow and pressure induced by variation of operating orifices. This make possible control of the pump operating point and prediction of its convergence for best efficiency and/or for satisfying operating pressure required for best uniformity distribution of connected drip irrigation system. Third graph (Figure 3a/08.am) shows an overall system efficiency varying from 1 to $63 \%$ (average of $20 \%$ ). This dispersion in efficiency is due to the standalone system behavior as any capacitance is set to regulate and stabilize instantaneous behavior of flow / pressure influenced by a solar irradiance change.

The results obtained at 10.am (Fig.5c) showed three hydraulic [flow vs pressure / power vs pressure] behaviors. In fact, the incident irradiance $(534 \mathrm{~W} / \mathrm{m} 2)$ impacted the pump to operate with three orifices that converge the operating point to increase efficiency to be $49 \%$. The system has shown good hydraulic performance, since the pump holds the maximum pressure ( 4.5 bar).

At noon, the system had a better hydraulic performance as the pump reached its the maximum flow rate vs its pressure characteristics ( $16 \mathrm{~L} / \mathrm{min}$ vs 4.6 bar) (Figure $3 \mathrm{e}$ ).

As the pump not performed at low level of sunshine, the flow/pressure adequacy was required to optimize pump efficiency according to adaptation of hydraulic equivalence model of the connected orifices (effect of the orifices number switched on). However, at 02.pm and at $04 \mathrm{pm}$, convergence of the performance curves is observed (Figs. g and i) as irradiance at $02 \mathrm{pm}$ and $04 \mathrm{pm}$ were important to operate the pump without decreasing the number of operating orifices. The superposition of the actual curves with the references makes it possible to locate the optimal operating point of the pump for each behavior (number of switched orifices). The solar irradiance increase influenced positively the pump efficiency to be more than $35 \%$.

The system performance at $6 \mathrm{pm}$ (Figure 3k) showed a behavior similar to the one of $8 \mathrm{am}$. This is practically due to a reduction in incident irradiance $(160 \mathrm{~W} / \mathrm{m} 2)$. The third graph (Figure $3 \mathrm{k}$ ) shows an efficiency varying from $3 \%$ and $65 \%$ with an average of $20 \%$. This difference in flow / pressure behavior was induced mainly by an alternance of sunlight/shadow. 

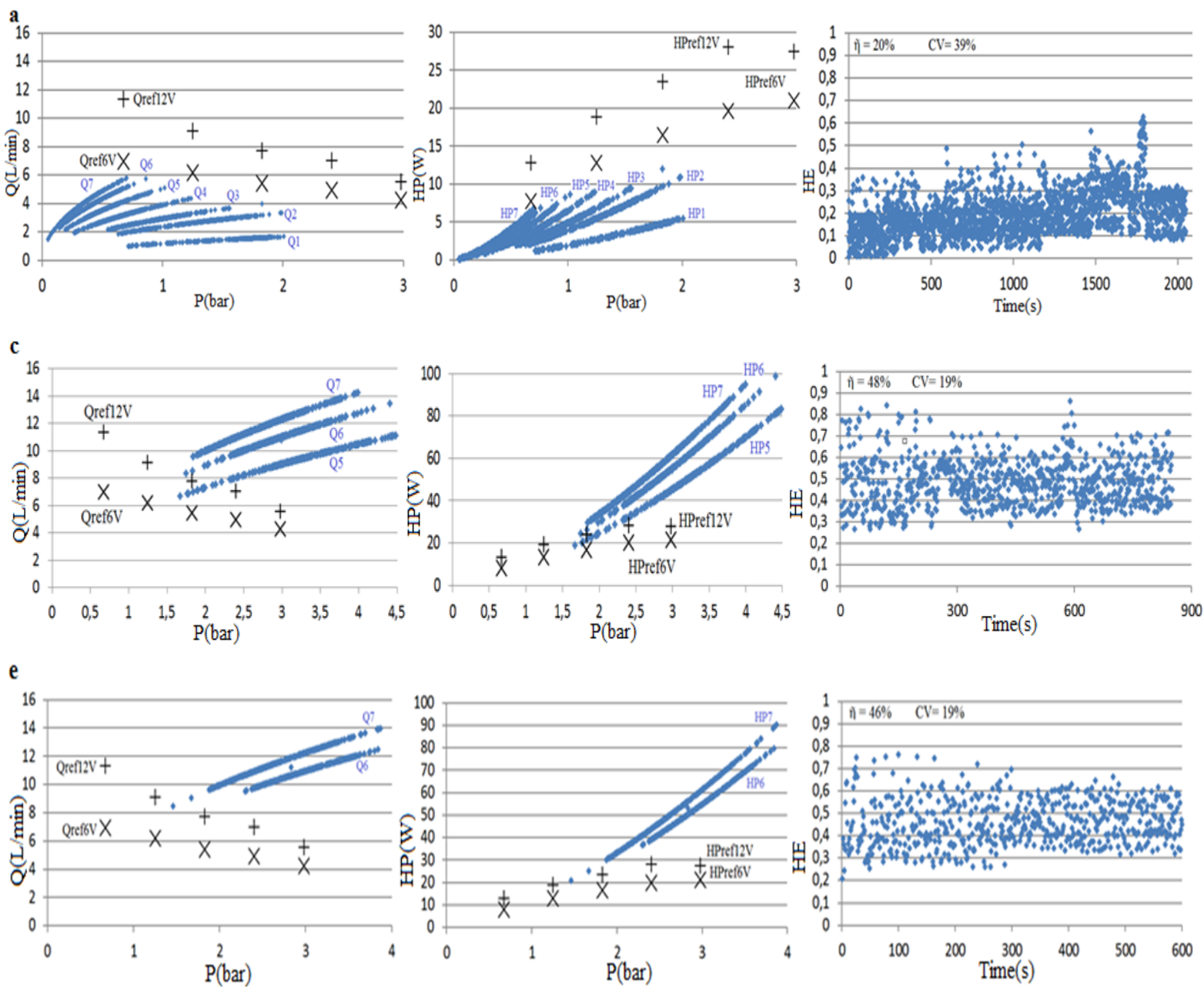

Figure 3. Performance of PVWPS at $8 \mathrm{am}, 10 \mathrm{am}$ and at $12 \mathrm{pm}$. 

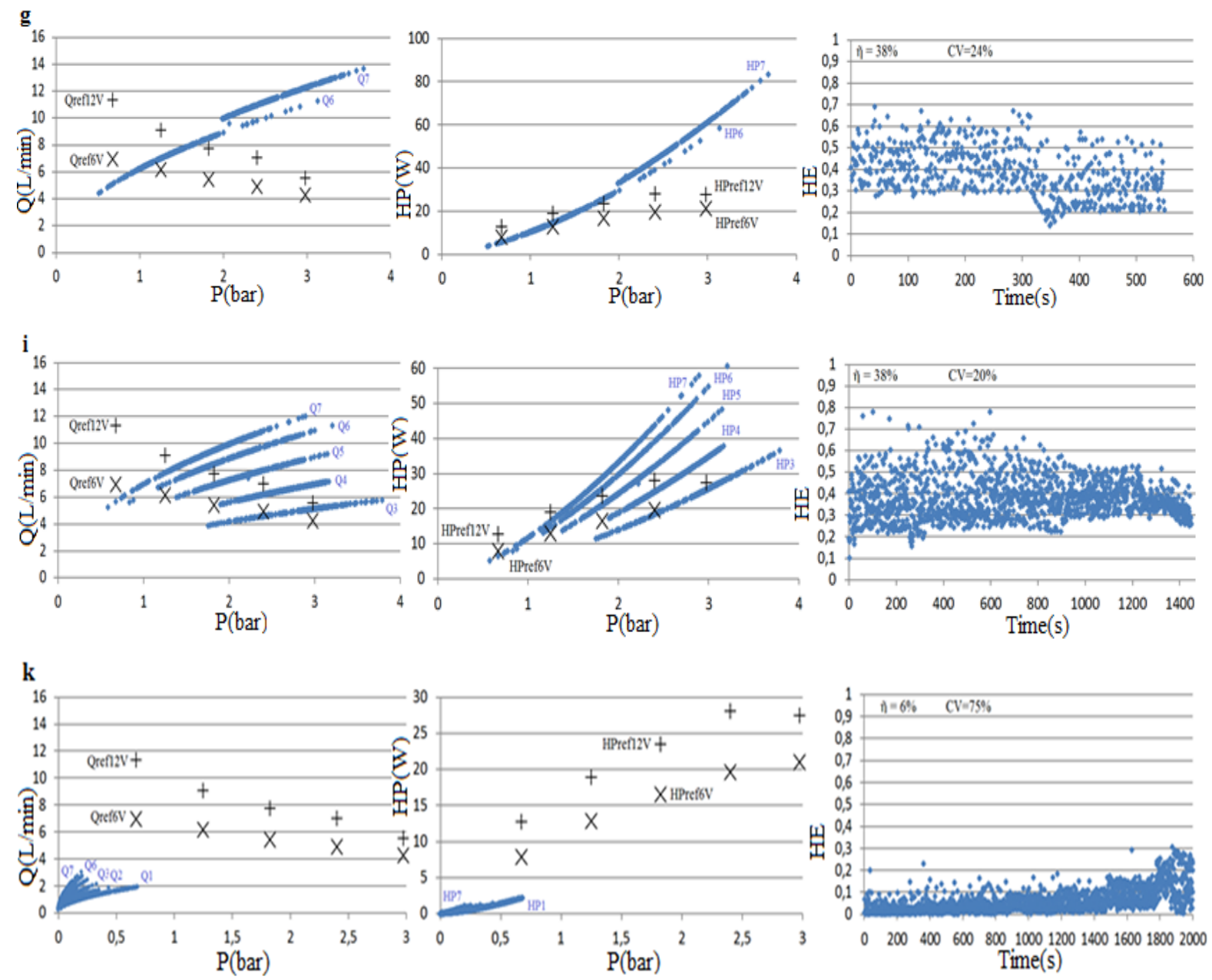

Figure 4. Performance of PVWPS at $2 \mathrm{pm}, 4 \mathrm{pm}$ and at $6 \mathrm{pm}$.

According to the pumping system response for each configuration of the number of operating orifices, a significant dispersion in terms of efficiency is shown (Table.2). There is a negative influence of change in irradiance on the pump performance for a standalone PVWPS if the pump operating point is kept static. In fact, the pump operating points need an adjustment to maintain its highly hydraulic performance and its pressure stability. The adaptation of the pump operating point using orifices switching on/off is of importance to efficiently valorize actual irradiance at upstream level to adequately fit it with a portion of a connected drip irrigation network (number of switched on orifices). 
Table 28. Daily performance of PVWPS according to irradiance change and portion of switched on orifices

\begin{tabular}{|c|c|c|c|c|c|}
\hline $\begin{array}{l}\text { Daily } \\
\text { time }\end{array}$ & $\begin{array}{c}\text { Irradiance } \\
\left(\mathrm{W} / \mathrm{m}^{2}\right)\end{array}$ & $\begin{array}{l}\text { Engaged } \\
\text { orifices }\end{array}$ & $\begin{array}{c}\text { Mean Hydraulic } \\
\text { Yield } \\
\%(\mathrm{CV} \%)\end{array}$ & $\begin{array}{c}\text { Mean } \\
\text { Pressure } \\
\text { Bar }(\mathrm{CV} \%)\end{array}$ & $\begin{array}{l}\text { Mean flow rate } \\
\text { L/min }(\mathrm{CV} \%)\end{array}$ \\
\hline \multirow{7}{*}{ 08. am } & \multirow{7}{*}{90} & 7 & $13(47)$ & $00.33(34)$ & 03.84 (19) \\
\hline & & 6 & $16(35)$ & $00.42(23)$ & $03.91(12)$ \\
\hline & & 5 & $18(16)$ & $00.52(24)$ & 03.55 (13) \\
\hline & & 4 & $19(35)$ & $00.66(24)$ & 03.07 (13) \\
\hline & & 3 & $22(30)$ & $00.93(21)$ & $02.78(11)$ \\
\hline & & 2 & $29(29)$ & $01.26(21)$ & $02.60(11)$ \\
\hline & & 1 & $22(28)$ & $01.45(21)$ & 01.38 (11) \\
\hline \multirow{3}{*}{$10 \mathrm{am}$} & \multirow{3}{*}{534} & 7 & $49(22)$ & $02.77(15)$ & $11.70(08)$ \\
\hline & & 6 & $49(16)$ & $00.34(12)$ & $10.88(06)$ \\
\hline & & 5 & $47(17)$ & $03.26(15)$ & $09.32(08)$ \\
\hline \multirow[t]{2}{*}{ 12. pm } & \multirow[t]{2}{*}{540} & 7 & $44(22)$ & $00.38(15)$ & $11.46(08)$ \\
\hline & & 6 & $47(15)$ & $02.99(10)$ & $10.09(05)$ \\
\hline \multirow[b]{2}{*}{ 02. pm } & \multirow[b]{2}{*}{759} & 7 & $44(19)$ & $02.66(12)$ & $11.47(06)$ \\
\hline & & 6 & $32(25)$ & $01.35(23)$ & $07.14(12)$ \\
\hline \multirow{5}{*}{ 04. pm } & \multirow{5}{*}{407} & 7 & $36(26)$ & $01.96(20)$ & $09.04(11)$ \\
\hline & & 6 & $39(26)$ & 01.95 (18) & $08.70(09)$ \\
\hline & & 5 & $39(19)$ & $02.18(13)$ & $07.55(07)$ \\
\hline & & 4 & $39(14)$ & $02.58(10)$ & $06.40(05)$ \\
\hline & & 3 & $36(11)$ & $02.83(12)$ & $04.95(06)$ \\
\hline \multirow{7}{*}{ 06. pm } & \multirow{7}{*}{158} & 7 & $02(76)$ & $00.05(56)$ & $01.37(31)$ \\
\hline & & 6 & $03(72)$ & $00.06(54)$ & $01.34(29)$ \\
\hline & & 5 & $03(63)$ & 00.08 (48) & $01.30(26)$ \\
\hline & & 4 & $04(52)$ & $00.12(41)$ & $01.23(22)$ \\
\hline & & 3 & $05(52)$ & $00.16(44)$ & $01.10(23)$ \\
\hline & & 2 & 09 (41) & $00.26(35)$ & 01.16 (19) \\
\hline & & 1 & $14(43)$ & $00.35(41)$ & $01.33(23)$ \\
\hline
\end{tabular}

\section{Conclusions}

The performance of a standalone PVWPS was monitored under varying solar irradiance and varying hydraulic operating point of the pump/orifices connection. The portion of hydraulic network (number of switched on orifices) can be simulated by an equivalent hydraulic model of a defined number of a calibrated orifices. An equivalent model of a given hydraulic circuit can be tested experimentally for establishing a governing diagram of the performance pump with reference to incident irradiance and to pressure required at downstream level.

\begin{tabular}{lll} 
& & \multicolumn{1}{l}{ Nomenclature } \\
BCDC & $:$ & Alternatif current. \\
CCCC & $:$ & Crushless Direct Current. \\
DC & $:$ & Direct Current. \\
HP (W) & $:$ & Hydraulic Power \\
HE & $:$ & Hydraulic Effeciency \\
Imppt & $:$ & Output Current of MPPT. \\
IncCond & $:$ & Incremental Conductance. \\
Ipv & $:$ & Photovoltaic Current. \\
Ktep/ Koe & $:$ & kilotonne d'équivalent pétrole; kilotonne of oil equivalent \\
MAPM & $:$ & Ministry of Agriculture and Fishery \\
MEMSD & $:$ & Ministry of Energy, Mines and Sustainable Development. \\
MPPT & $:$ & Maximum Power Point Tracking. \\
P (bar) & $:$ & Pressure \\
P\&O & $:$ & Perturb and Observe. \\
PMDC & $:$ & Permanent Magnet Direct Current \\
PV & $:$ & Photovoltaic \\
PVWPS & $:$ & Photovoltaic Water Pumping System \\
PWM & $:$ & Pulse Width Modulation \\
Q (L/min) & $:$ & Flow rate \\
Qn & $:$ & Flow rate curve for n orifices switched on
\end{tabular}




$\begin{array}{lll}\text { Umppt } & : & \text { Output Voltage of MPPT } \\ \text { Vpv } & : & \text { Photovoltaic Voltage }\end{array}$

\section{References}

Akihiro Oi, T., Anwari, M., Taufik, M. 2009. Modeling and simulation of photovoltaic water pumping system. In the Third Asia International Conference on Modelling \& Simulation. 978-0-7695-3648-4/09 \$25.00@ 2009 IEEE.

Arafat Khan, Md. T., Md. Rishad, A., Ibne Ahmed, S., and Islam Khan, S. 2012. Design and performance analysis of water pumping using solar PV. In the 2nd International Conference on the Developments in Renewable Energy Technology (ICDRET), Dhaka, Bangladesh, 5-7 Jan. 2012.

Chandel, S.S., Nagaraju Naik, M., Chandel, R. 2015. Review of solar photovoltaic water pumping system technology for irrigation and community drinking water supplies. Renewable and Sustainable Energy Reviews ,49 1084-1099. https://doi.org/10.1016/j.rser.2015.04.083

Essam, E. A., Zaki, A.M., El-sotouhy. M.M. 2017. Design and control of a standalone PV water pumping system. Journal of Electrical Systems and Information Technology. JESIT-96. https://doi.org/10.1016/j.jesit.2016.03.003

Firatoglu, Z. A., and Yesilata, B. 2004. New approaches on the optimization of directly coupled PV pumping systems.SolEnergy, 77:81-93. https://doi.org/10.1016/j.solener.2004.02.006

Gad, H.E., and Safya, M. 2011. Using photovoltaic array for solar water pumping in Toshka region. In the fifteenth international water technology conference. IWTC15. Egypt.

Giz, Aderee. 2011. Étude du potentiel de développement de l'énergie photovoltaïque dans les régions de Meknès-Tafilalet, Oriental et Souss-Massa-Drâa..

Glasnovic, Z., and Margeta, J. 2007. A model for optimal sizing of photovoltaic irrigation water pumping systems. Sol Energy, 81:904-16. https://doi.org/10.1016/j.solener.2006.11.003

Guiqiang, L.., Yi J., Akram, M.W., Xiao C. 2017. Research and current status of the solar photovoltaic water pumping system - A review. Renewable and Sustainable Energy Reviews, 79 (2017) 440-458. https://doi.org/ 10.1016/j.rser.2017.05.055

Hamidat, A., Benyoucef, B., and Hartani, T. 2003. Small-scale irrigation with photovoltaic water pumping system in Sahara regions. Renewable Energy 28 1081-1096. https://doi.org/10.1016/S0960-1481(02)00058-7

Hamidat, A., Benyoucef, B. 2008 .Mathematic models of photovoltaic motor-pump systems. RenewEnergy, 33:933-42. https://doi.org/10.1016/j.renene.2007.06.023

Kashyap, M., Chanana, S., Jai Singh, A. 2013. SOLAR POWERED PMDC MOTOR DRIVE. In Conference on Advances in Communication and Control Systems (CAC2S)

Kolhe, M., Joshi, J. C., and Kothari, D. P. 2004. Performance Analysis of a Directly Coupled Photovoltaic Water-Pumping System. IEEE Transactions on energy conversion, VOL. 19, NO. 3. https://doi.org/ 10.1109/TEC.2004.827032

Levon, G., Valery, V., Zoja, R. 2015.PLC-based pressure control in multi-pump applications. Electrical, Control and Communication Engineering. https://doi.org/ 10.1515/ecce-2015-0008

Naresh, B., Madhu, P., Prasad, K.R.K. 2012. Analysis of De solar water pump and generalized photovoltaic model using Matlab/Simulink. In Intl. Conf. on Advances in Computer, Electronics and Electrical Engineering, 978-981-07-1847-3

Odeh, I., Yohanis, Y.G., and Norton, B. 2006. Economic viability of photovoltaic water pumping systems. Solar Energy, 80 850-860. https://doi.org/ 10.1016/j.solener.2005.05.008

Protogeropoulos, C., and Pearce, S. 2000. Laboratory evaluation and system sizing charts for a 'second generation' direct PV-powered, low cost submersible solar pump. Solar Energy, Vol. 68, No. 5, pp. 453 _474. https://doi.org/10.1016/S0038-092X(00)00005-0

Reca, J., Torrente, C., Lopez-Luque, R., and Martínez, J. 2016. Feasibility analysis of a standalone direct pumping photovoltaic system for irrigation in Mediterranean greenhouses. Renewable Energy, 85 (2016) 1143e1154. https://doi.org/10.1016/j.renene.2015.07.056

Renu. Birinchi, B., Basudev, P., Sastry, O.S., Atul, K., and Bangar M. 2017. Optimum sizing and performance modeling of solar photovoltaic (SPV) water pumps for different climatic conditions. Solar Energy, 155 (2017) 1326-1338. https://doi.org/10.1016/j.solener.2017.07.058

Sefriti, B., and Boumhidi, I. 2015. Neural network incremental conductance MPPT algorithm for photovoltaic water pumping system. Institute of Electrical and Electronics Engineers (IEEE), 978-1-4799-7560-0/15/\$31. https://doi.org/ 10.1109/SITA.2015.7358383

Walski, T., Zimmerman, K., Dudinyak, M., and Dileepkumar, P. 2003. Some surprises in estimating the efficiency of variable-speed pumps with the pump affinity laws. In the World Water \& Environmental Resources Congress 


\title{
Modeling in Matlab/Simulink of a Standalone Photovoltaic Pumping System for Drip Irrigation Use and Performance Analysis
}

\author{
El Aissaoui Abdellah ${ }^{\text {a }}$, El Kacimi Ousama ${ }^{\text {b }}$, Harkani Assia ${ }^{\text {a }}$, Mansouri Mohamed ${ }^{\text {b }}$ \\ ${ }^{a}$ Laboratory of Agricultural Machinery, National Institute of Agronomic Research, Settat 26000, Morocco \\ b Dep. Process Engineering, ENSA-Khouribga 25000 ,Morocco \\ * Corresponding author. Email: ab elaissaoui@yahoo.fr
}

\begin{abstract}
This study dealt with simulation for optimal design of a stand-alone photovoltaic solar pumping system directly coupled to drip irrigation network in the context of arid climate of central region of Morocco. The simulation was achieved using Matlab/Simulink environment. It provides theoretical study and modeling techniques based on equivalent electrical circuits to improve PV system design for optimal energy efficiency and cost of installation. The structure of the pumping system is presented using the mathematical models of different constituents. The modeling of the optimal operating point is done using the MPPT method based on the incrementing of the conductance. The assessment of the pumping system model is done using actual sunlight data provided by the meteorological station of the INRA center of Settat. One set of data is used as input for testing the model response in sunny conditions. The simulation results showed that the system is stable and reaches its equilibrium state in a relatively short time. The model is of importance to serve as tool for designing of a standalone PV pumping system based on actual irradiance data. It allows possibility of studying influence of ambient temperature and solar radiation on the PV and hydraulic performances.
\end{abstract}

Key words: Photovoltaic pumping system, drip irrigation, MPPT, Conductance Increment (INC).

\section{Introduction}

In 2013, Moroccan government launched a program to promote the use of photovoltaic water pumping system (PVWPS) for small farms (less of $5 \mathrm{ha}$ ) representing a workforce of $90 \%$ with an area of $44 \%$ (Giz \& Aderee, 2011). Small farmers potentially use PV pumping system to satisfy irrigation requirements and animal production need. The PV systems consist of electronic, electrical and mechanical subsystems making adoption of the technologies no easy for carrying out adequate design, installation or maintenance.

Many studies focused on modeling PV systems to evaluate and improve electric and hydraulic performances. ALTAS (2007), studied a PV array using PV cells mathematical model to approach effects of solar irradiation and temperatures changes in Matlab interface. The model was evaluated using direct coupled DC load and showed that that changes in temperature and solar radiation affect voltage and current outputs of the PV array. Akhirio et al, (2009) evaluated performance of two MPPT algorithms, IncCond and P \& O., to improve system performance. The algorithms were implemented in Matlab / Simulink to show that PV water pumping efficiency was improved by $35 \%$ using MPPT. In fact, the MPPT technology valorizes up to $99 \%$ of the PV panels capacity and consequently $87 \%$ of the water could be pumped. Gad and Safya (2011) developed a Matlab code for sizing and predicting performance of a directly coupled photovoltaic pumping system. The system was designed and tested using winter climatic data at worst conditions to show performance endurance in low irradiance potential. Results showed that for a pumping head of $50 \mathrm{~m}$, the system was able to pump 5.82 and $4.83 \mathrm{~m} 3$ /day per unit array area in summer and winter operating period, respectively.

The study showed that the proposed simulation model improved efficiently and accurately the sizing, optimization and prediction of performance for different designs and operating parameters. Chokri al,. (2015) worked on dynamic modeling of PVWPS control to manage energy performance. The model was based on the system components sizing to ensure a reliable power supply and minimize PV energy losses using Matlab/Simulink interfaces. A method of regulating operating water head of the PV pumping system was developed using fuzzy logic algorithm. The algorithm was performed to read solar irradiance and water level from three tanks and delivers in real time the reference speed of the three pump motors. To prove the effectiveness of the algorithm, the proposed fuzzy control method was implemented, and interface controller was made. They concluded that the controller improved the energy gain to be $88.5 \%$ against a reference gain of $71.4 \%$ (Bakelli et al.,2011) developed a model using Matlab for improving performance of PV pumping system according to climatic conditions. Analytical method was proposed for sizing and assessing PVWPS performance using economic indicator of life cycle cost (LCC) profitability. The procedure proposed was based on water consumption profile, head tank capacity and peak power of the PV system. The model was helpful to perform optimal pumping system configurations.

This work aims to evaluate performance of a direct coupled PVWPS system using Matlab/Simulink model related to actual solar irradiation data acquired in arid region of Settat-Morocco. 


\section{Materials and Methods}

The solar cell model is represented by the equivalent circuit (Figure 1). This model is used to directly calculate model parameters (Townsend, 1989) according to its high accuracy.

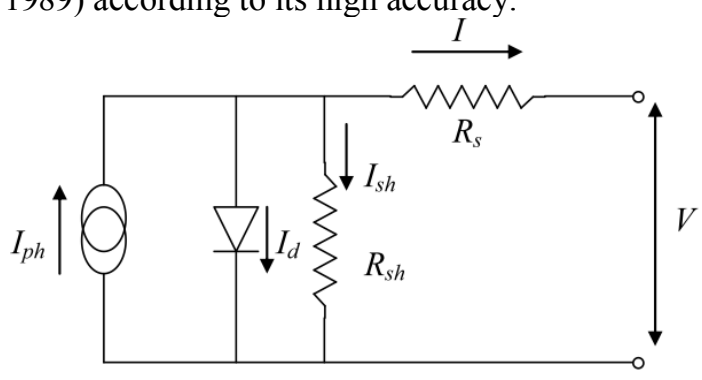

Figure 76. Equivalent circuit of PV module.

A PV array is a nonlinear power source. For a potential irradiance, the current-voltage relationship is given by:

$$
\begin{aligned}
& I p v=I p h-I s[\exp ((q *(V p v+R s * I p v)) /(n * K * T))-((V p v+R s * I p v) / R s h)] \\
& T=T a+K t . \Phi \\
& I p h=(I s c+T c o e f .(T-T o f f s)) . \Phi / 1000 \\
& I S=I s 0 . T^{3} \cdot \exp (-e . U g a p / K . T) \\
& A=e /(n . K . T)
\end{aligned}
$$

Where: Ta: Ambient temperature; Isc: short circuit current (A); Iso: Reference reverse saturation current of the diode. These parameters allow determining the current- voltage characteristics for any specific cell temperature and radiation level. Ipv, Iph, Is, q, Vpv, Rs, n, K, T and Rsh are photovoltaic panel current, photo-current depending on the intensity of irradiation, reverse saturation current of the diode, electron charge $(1.602 * 10-19 \mathrm{C})$, cell output voltage $(\mathrm{V})$, series resistance $(\mathrm{Rs})$, non-ideality factor, Boltzmann constant $\left(1.38^{*} 10-23 \mathrm{~J} /{ }^{\circ} \mathrm{K}\right)$, actual temperature of the cell $\left({ }^{\circ} \mathrm{K}\right)$ and shunt resistance $(\mathrm{Rsh})$, respectively.

Modeling hydraulic pump of PVWPS was based on hydraulic characteristics of centrifugal pump equations estimated by the "Pleider- Peterman" model (Betka A. and Attali A., 2010) as follow:

$$
H=a 0 . W^{2}+a 1 \cdot Q \cdot W \cdot Q+a 2 \cdot Q^{2}
$$

The pump hydraulic power $(\mathrm{Hp})$, torque $(\mathrm{T})$ and efficiency $(\mathrm{\eta})$ are expressed by the following relationships:

$$
\begin{aligned}
& H p=p \cdot g \cdot Q \cdot H \\
& \eta=\mathrm{Hp} / \mathrm{Ps} \\
& T=K . \Omega^{2}+T 0
\end{aligned}
$$

Where the symbols are defined as follows:

Q: Flow rate of water (m3 / s); H: Total Head (m); Hp: Hydraulic power (W); $\rho$ : Water density $(1000 \mathrm{Kg} / \mathrm{m} 3)$; Ps: Shaft power; K: Proportionality factor ( $\mathrm{Nm} / \mathrm{rads}$ ); T0: static torque; $\Omega$ : Rotation speed. a0, a1, a2: Coefficients given by the manufacturers.

To evaluate performances of the standalone PVWPS, a simulation using Matlab/Simulink was developped (Figure 3). The system based on 45 PV panel (Thin film, Mitsubishi MA100T2 Module, 100W), a MPPT controller (Incremental Conductance algorithm), an AC motor with Centrifugal Pump (Lorentz PS 4000C-SJ815) (Figure 2). 


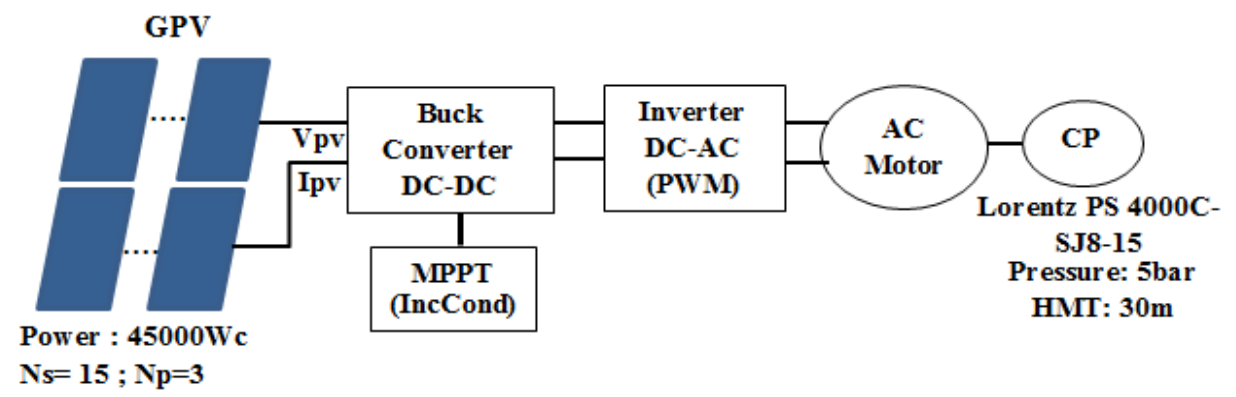

Figure 77. Diagram scheme of the photovoltaic pumping system.

The PVWPS is presented in Figure 3 by four blocks diagram: i) Photovoltaic generator; ii) Buck converter DC-DC with MPPT controller (Incremental inductance algorithm); iii) Inverter DC-AC; iv), Centrifugal pump.

Study case simulation

The simulated case is based on satisfying the highest water requirements $\left(100 \mathrm{~m}^{3} /\right.$ day $)$ for irrigating two hectares during the month of June (Table 1). The pump was set to deliver water at the potential pressure of 4 bars (Table 2) to cover downstream operating pressure and friction loss of connected hydraulic circuit.

Table 29. Input parameters (irradiance, temperature) and water requirements.

\begin{tabular}{lllllll}
\hline Months & Feb & Mar & Apr & Mai & Jun & Jul \\
\hline $\mathrm{W} / \mathrm{m}^{2}$ & 324 & 401.31 & 500.64 & 477.47 & 524.68 & 511.78 \\
Temp $\left({ }^{\circ} \mathrm{C}\right)$ & 12.42 & 11.97 & 15.64 & 18,82 & 22,55 & 26,40 \\
$\mathrm{M}^{3} / \mathrm{ha} /$ Day & 6.13 & 11.74 & 35.23 & 35.25 & 48.46 & 47.20 \\
$\mathrm{M}^{3}$ day/2ha & 12,26 & 23,48 & 70,46 & 70,5 & 96,92 & 94,4 \\
\hline
\end{tabular}

Table 30. PV and pump parameters.

\begin{tabular}{cr}
\hline Input parameters (June) & Output hydraulic parameters \\
$\mathrm{E}=524.68 \mathrm{~W} / \mathrm{m}^{2}$ & $\mathrm{Bbj}=100 \mathrm{~m}^{3} /$ day \\
$\mathrm{Tmoy}=22,55^{\circ} \mathrm{C}$ & $\mathrm{P}(\mathrm{bar})=4 \mathrm{bar}$ \\
\hline
\end{tabular}

\section{Results and Discussion}

Performance of a direct coupled PVWPS was evaluated using Simulink model (Figure 3). Results of Figure $4 \mathrm{a}$ and $4 \mathrm{~b}$ showed panels behavior at standard conditions (Temperature of $25^{\circ} \mathrm{C}$ and irradiance of $1000 \mathrm{w} / \mathrm{m}^{2}$ ). Figure 5a, represents simulated (I-V) curves for a constant temperature $\left(25^{\circ} \mathrm{C}\right)$ and a variable irradiance (from $400 \mathrm{~W} / \mathrm{m}^{2}$ to a $1000 \mathrm{~W} / \mathrm{m}^{2}$ ), while (I-V) curves for different temperature levels (from $10^{\circ} \mathrm{C}$ to $50^{\circ} \mathrm{C}$ ) and a constant irradiance $\left(1 \mathrm{Kw} / \mathrm{m}^{2}\right.$.) is represented in Figure $5 \mathrm{~b}$. Power characteristic curve showed a considerable change relative to irradiance while an insignificant variation according to temperature levels (Figure 6). Figure $7 \mathrm{~b}$ showed that the perturbation caused by irradiance variation was limited using the incremental conductance algorithm (IncCond), as a result the system had a good convergence towards the MPP in a short period time and remained stable with a low ripple rate, comparing to Figure 7a where there is no MPPT used. The simulation was tested according to actual irradiation data provided by the weather station installed at INRA-Settat (06/06/2017). Data contains irradiation and temperature measurements at each hour in day (Figure $8 \&$ Figure 9). According to analytical computations summarized in Table 2, Figures 10 and 11 both shows respectively the evolution of optimal flow and discharge pressure in relation to time. The results showed that the system begins to pump water after an hour (due to low temperature at $6 \mathrm{am}$ ) before being stabilized at a constant flow equal to $5.8 \mathrm{~m} 3 / \mathrm{hr}$ with a pressure of 4 bar. Figure 12 showed that in the end of the day, more than $100 \mathrm{~m} 3$ of water was pouned in a tank. We note according to this result that the quantity of water drawn daily by the PV pumping system satisfies the expressed needs and the system showed stabilized in short period time. 


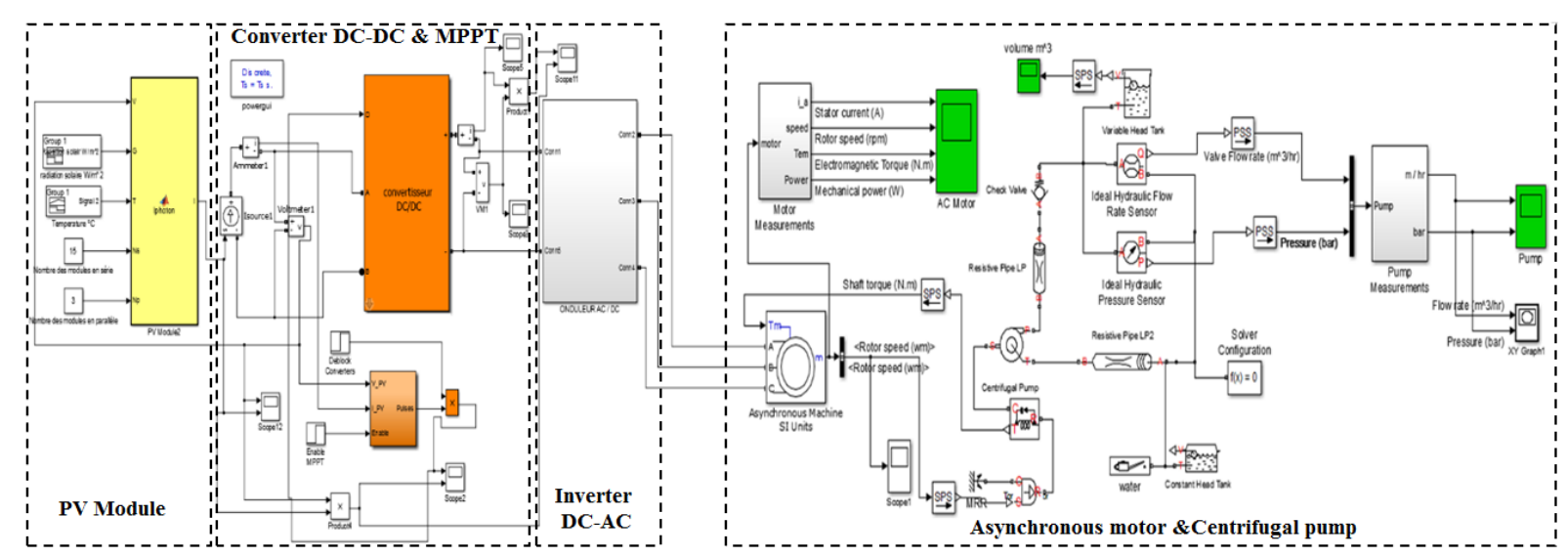

Figure 78. Photovoltaïc water pumping system model by Matlab/Simulink.
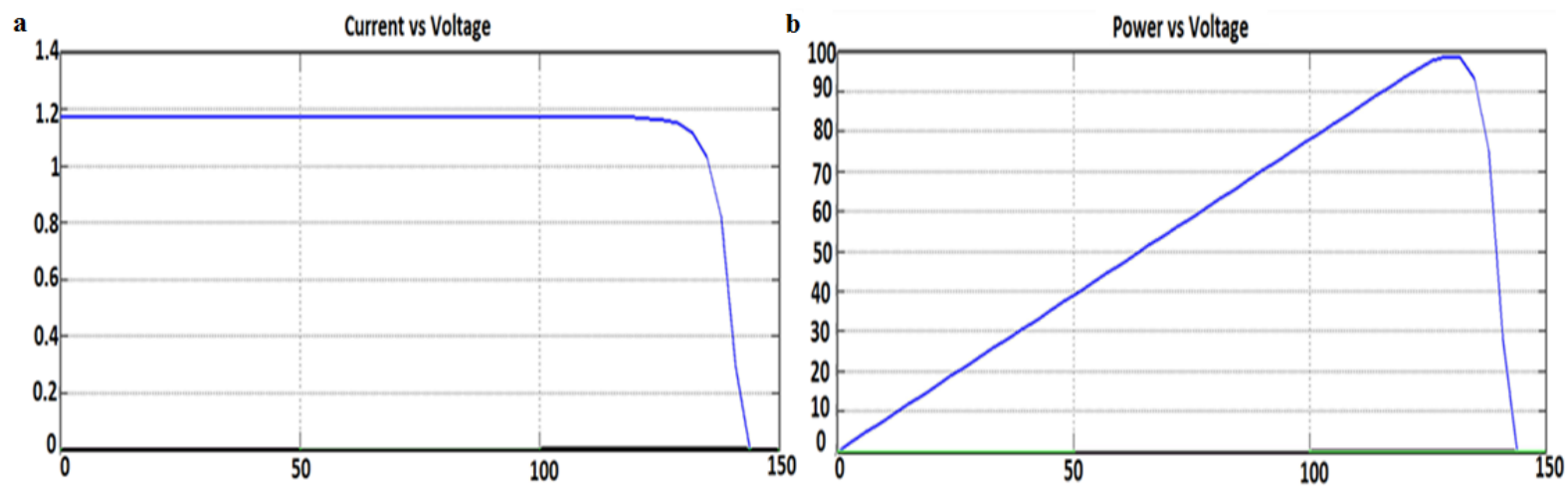

Figure 79. a) Current-voltage characteristics I (V) model; b) Power-voltage characteristics I (V) model.

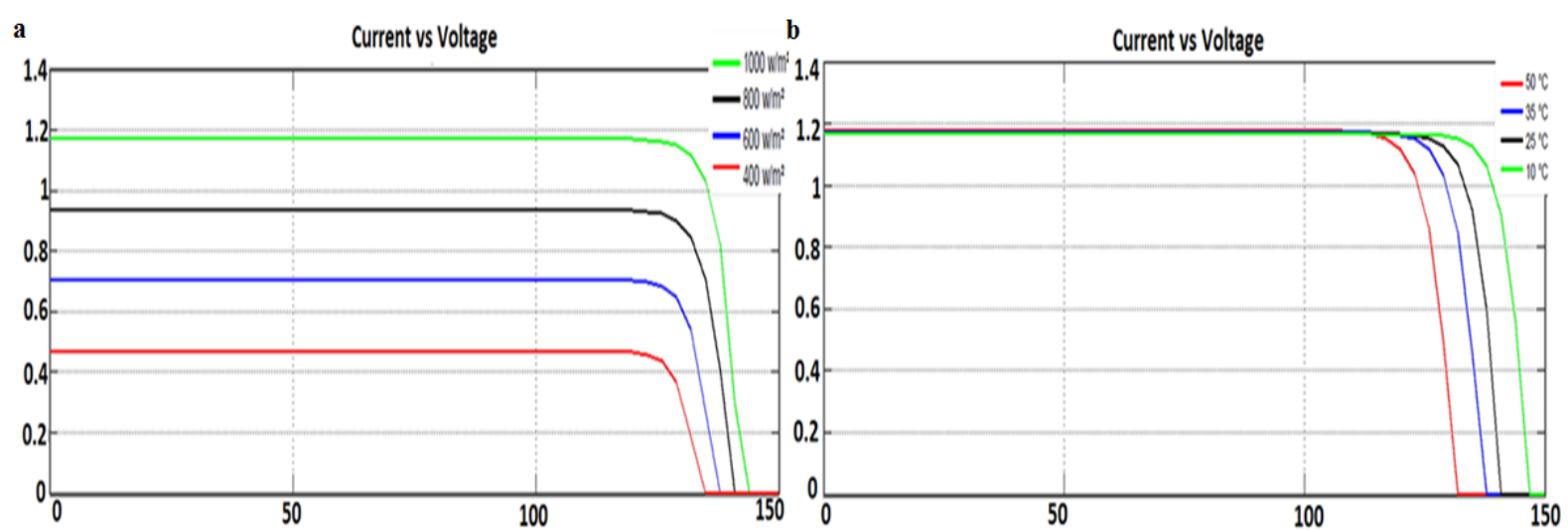

Figure 80. a) Curves (I-V) for variable irradiances and a temperature of $25^{\circ} \mathrm{C}$; b) Curves (I-V) for variable temperatures and irradiance of $1 \mathrm{Kw} / \mathrm{m}^{2}$. 


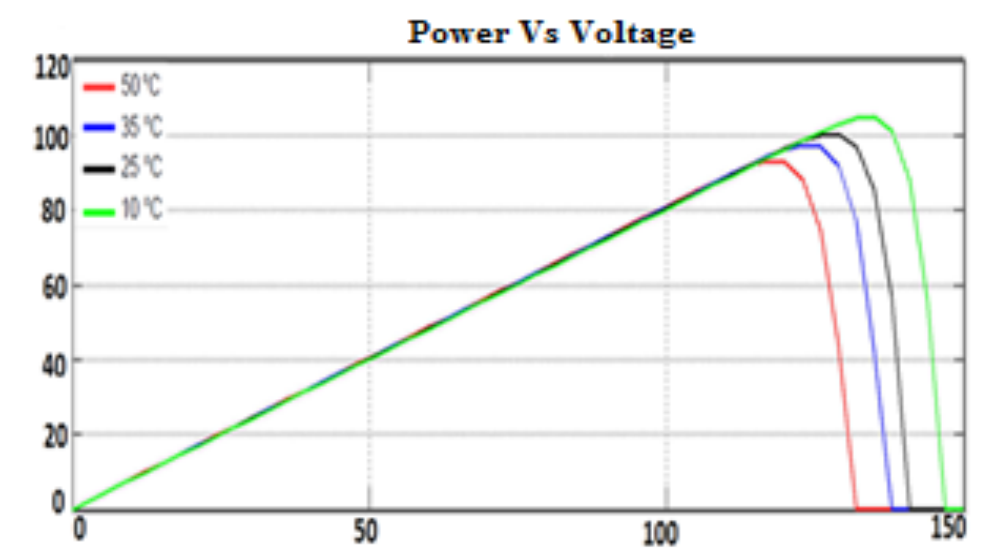

Figure 81 . Curves $(\mathrm{P}-\mathrm{V})$ for variable temperature and irradiance of $1 \mathrm{KW} / \mathrm{m}^{2}$.

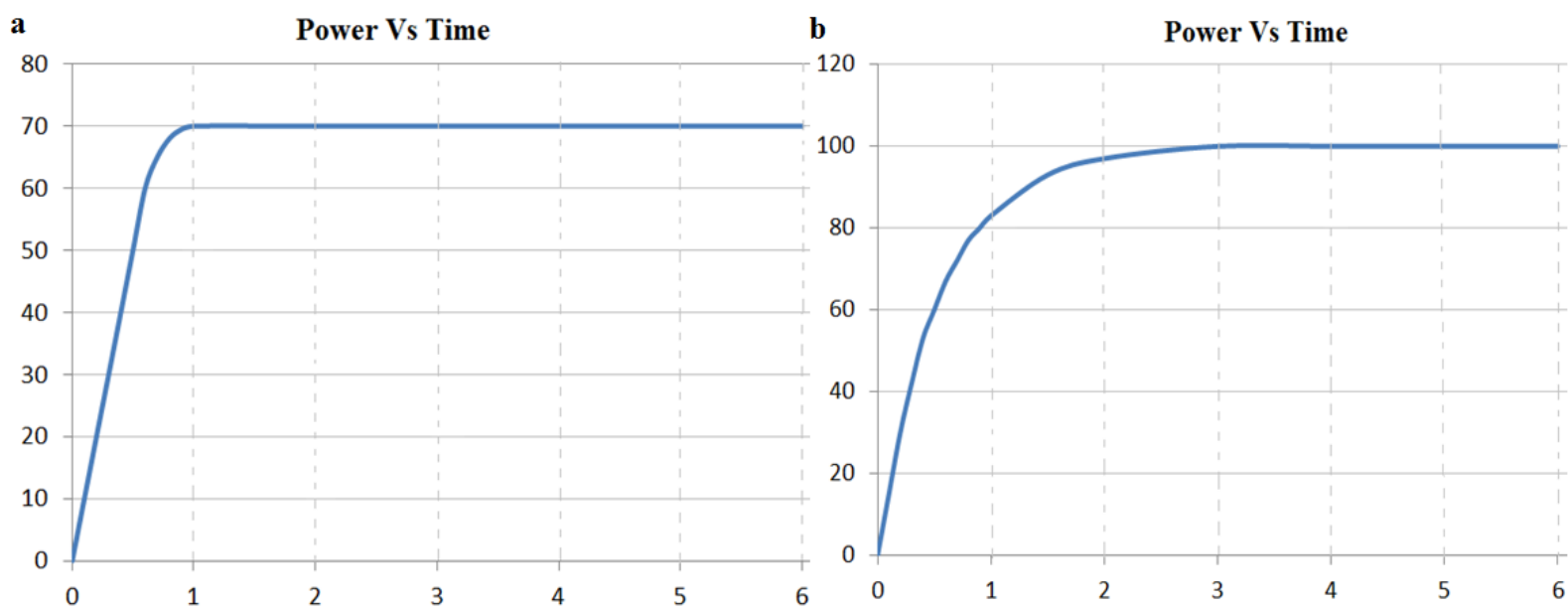

Figure 82. a) Power Module at $\mathrm{T}=25^{\circ} \mathrm{C}$ and $\mathrm{G}=1000 \mathrm{~W} / \mathrm{m}^{2}$ without MPPT control; b) Power module at $\mathrm{T}=25^{\circ} \mathrm{C}$ and $\mathrm{G}=1000 \mathrm{~W} / \mathrm{m}^{2}$ with MPPT control.

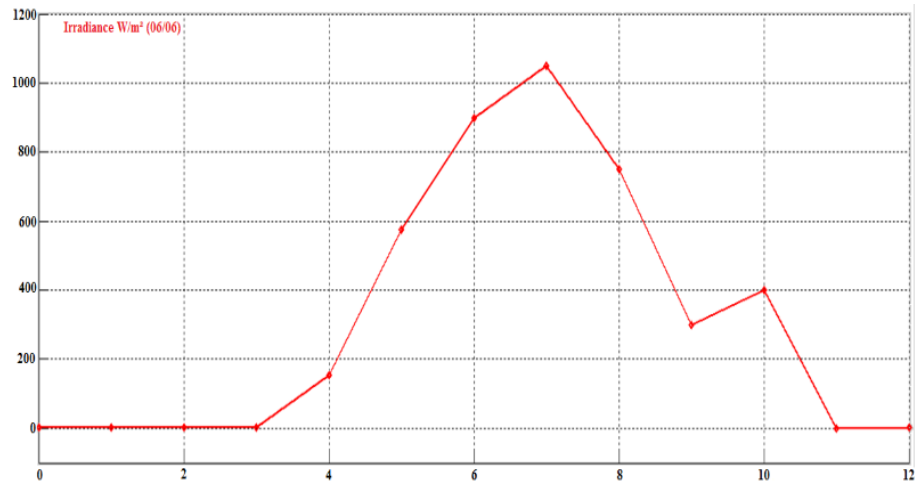

Figure 83. Irradiation values (06/06) estimated by interpolation in the MATLAB functions. 


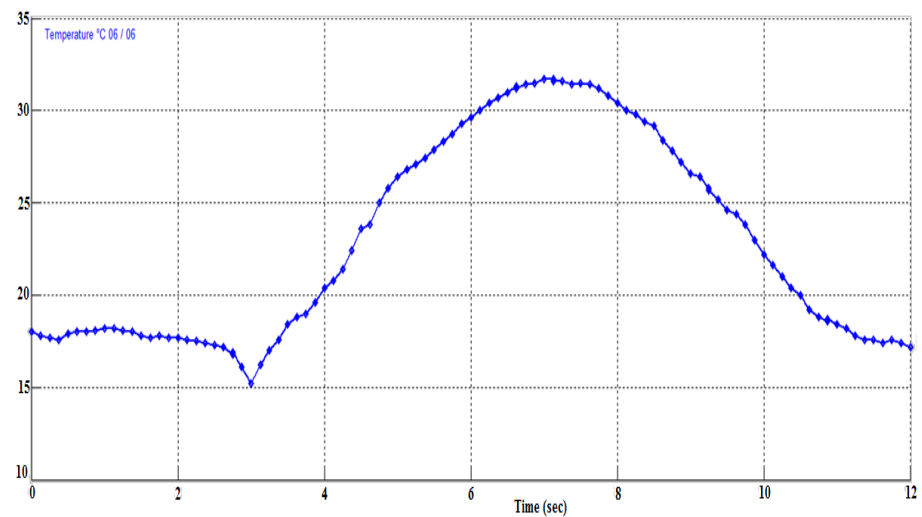

Figure 84 . Temperature values (06/06) estimated by interpolation in MATLAB functions.

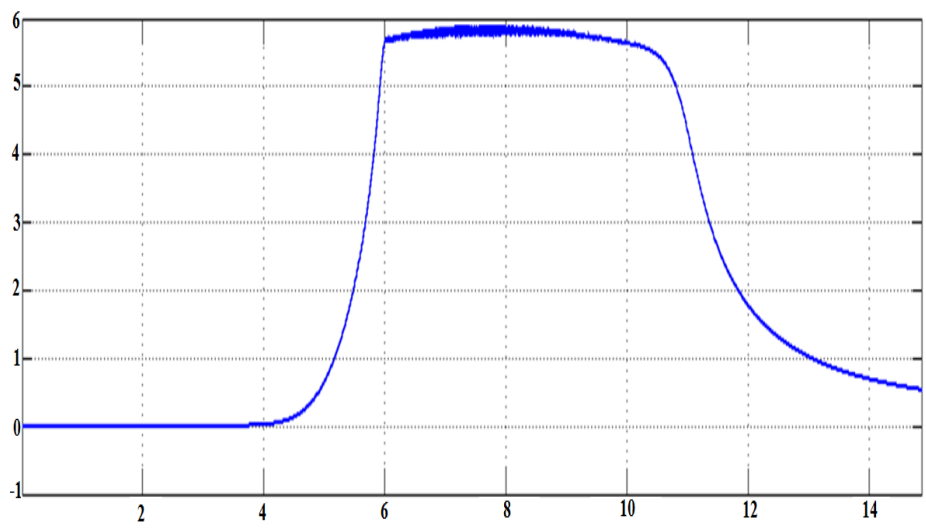

Figure 85 . The gross water requirement in $(\mathrm{m} 3 / \mathrm{h})$.

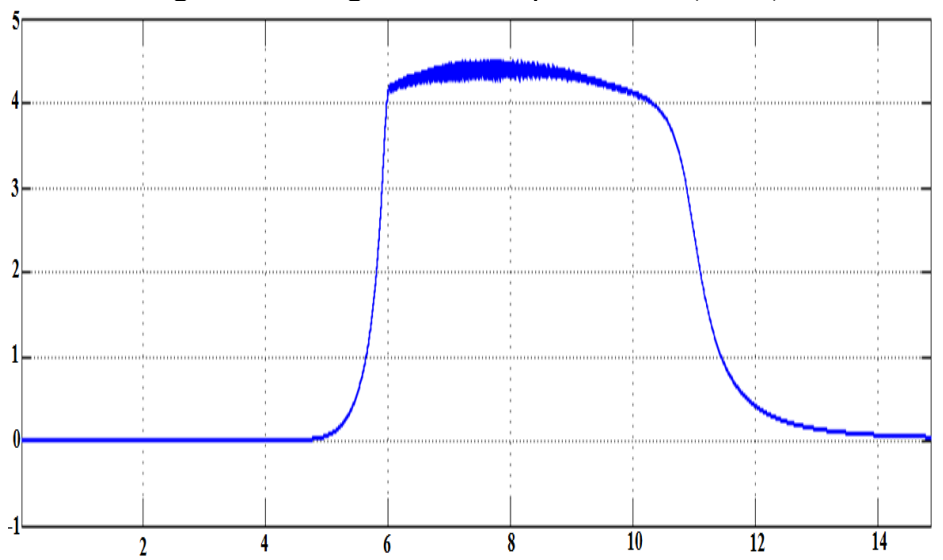

Figure 86. Discharge pressure in bar.

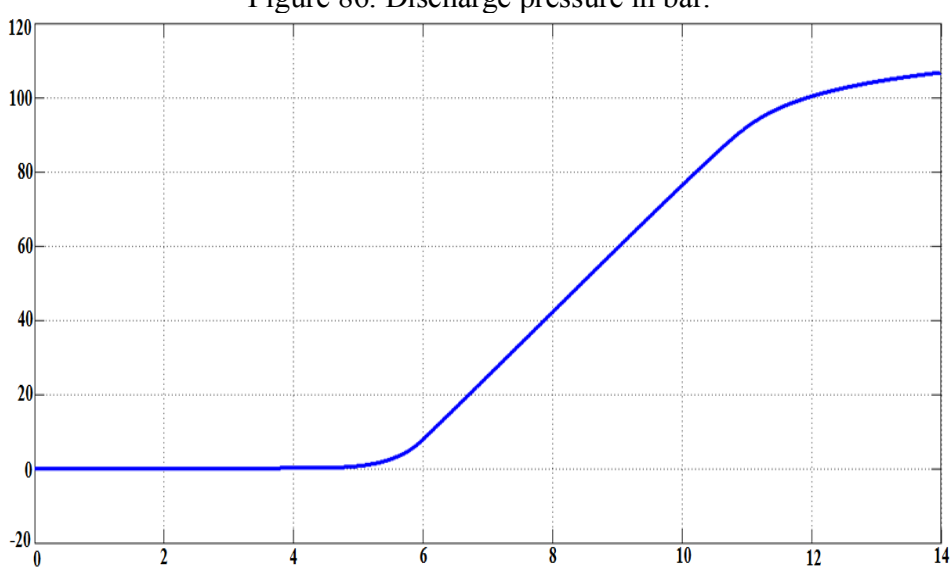

Figure 87 . Water volume $(\mathrm{m} 3)$ stored in the tank at the end of the day $(06 / 06)$. 


\section{Conclusions}

This study aims to simulate a stand-alone solar pumping system for drip irrigation in Settat-Morocco. The mathematical modeling of PVWPS using Matlab/Simulink was developed. The pumping system model was validated by implementing actual irradiance data collected from weather station installed at National Institute of Agricultural Research. The influence of ambient temperature and solar radiation on the energy and hydraulic performance of the system was studied. The simulation results showed that the system is stable and reaches its equilibrium state in a relatively short period time.

$\begin{array}{lll}\text { DC } & : & \text { Direct Current } \\ \text { PV } & : & \text { Photovoltaic } \\ \text { IncCond } & : & \text { Incremental Conductance } \\ \text { MPPT } & : & \text { Maximum Power Point Tracking } \\ \text { P\&O } & : & \text { Perturb \& Observe } \\ \text { PVWPS } & : \text { PV Water Pumping System } \\ \text { LCC } & : \text { Life Cycle Cost } \\ \text { AC } & : \text { Alternatif Current } \\ \text { Ha } & : \text { Hectares }\end{array}$

\section{References}

Akihiro Oi, T., Anwari, M., Taufik, M. 2009. Modeling and simulation of photovoltaic water pumping system. In the Third Asia International Conference on Modelling \& Simulation. 978-0-7695-3648-4/09, IEEE.

Altas I.H,. and Sharaf A.M,. 2007. A Photovoltaic Array Simulation Model for

Matlab-Simulink GUI Environment. 1-4244-0632-3/07, IEEE. http://doi.org/10.1109/ICCEP.2007.384234

Betka A. and Attali A.2010. Optimization of a photovoltaic pumping system based on the optimal control theory. Solar Energy 84 (2010) 1273-1283. http://doi.org/10.1016/j.solener.2010.04.004

Chokri Ben Salah, Mohamed Faouzi Mimouni b, Mohamed Ouali. 2015. A real-time control of photovoltaic water-pumping network. Computers and Electrical Engineering 46 (2015) 14-28.

https://doi.org/10.1016/j.compeleceng.2015.06.026

Helmy E. Gad and Safya M. El-Gayar. 2011. Using Photovoltaic Array for Solar WaterPumping in Toshka Region, Egypt. In the Fifteenth International Water Technology Conference, IWTC 2011, Alexandria, Egypt ;

Townsend, T. U. 1989. A method for estimating the long-term

performance of direct-coupled photovoltaic systems. MS thesis. Madison, Wis.: University of WisconsinMadison, Department of Mechanical Engineering.

Yahia Bakelli, Amar Hadj Arab b, Boubekeur Azoui.2011. Optimal sizing of photovoltaic pumping system with water tank storage using LPSP concept. Solar Energy 85 (2011) 288-294

https://doi.org/10.1016/j.solener.2010.11.023 


\title{
In-situ Performance Evaluation of PV Pumping System and Method for Yielding Pumping Capacity for Drip Irrigation Use
}

\author{
EL Aissaoui Abdellah ${ }^{a^{*}}$, Oudadda Mohamed ${ }^{\text {b }}$, Harkani Assia ${ }^{\text {a }}$ \\ a Agricultural Engineering Laboratory, INRA PO Box 586 Settat, Morocco. \\ ${ }^{\mathrm{b}}$ Agricultural Engineering Department, IAV Hassan II, PO Box 6202, Rabat, Morocco. \\ * Corresponding author. Email: abdellah elaissaoui@yahoo.fr
}

\begin{abstract}
Photovoltaic (PV) water pumping constitute an innovative solution for sustainable irrigated agricultural in Morocco. The present study treats technical and economic aspects related to actual use of PV pumping systems for drip irrigation in Morocco. The technical study is based on investigating 14 farms to evaluate performance of PV pumping installation according to potential irradiance. The economic study was done using LCC (Life Cycle Cost) method to compare the cost of the cubic meter taken using PV and diesel technologies mostly used by Moroccan farmers. Reconversion of existing diesel pumping systems to photovoltaic one was tested using an original method based on calculating monthly system peak power, and adoption of a set of parallel pumps instead of using a single pump for the same equivalent power. Furthermore, proposition of a management method is done to optimally operate PV pumping system for adequate synchronization between hydraulic yield and actual daily irradiance. The results of the surveys showed satisfaction of the farmers when using PV technologies, but it is of importance to use hybrid systems (PV/Diesel) to overcome deficiency of using only PV system. The technical study showed that use of small pumps in parallel improved the efficiencies and makes the pumping system running at $100 \%$ with the photovoltaic generator without compensation by use of diesel energy as done for hybrid systems. Analysis cost of water cubic meter production showed the importance of PV system compared to the cost production using diesel energy (differences ranging between $11 \%$ and $87 \%$ with an average of $61 \%$ ). The proposed method for increasing daily water pumped using PV technology showed that it is possible to design more economical PV systems as the maximum peak power demand for each month can carried out using small power pumps in parallel and adjusting switched power with actual irradiance. Using such method of yielding pumping capacity for drip irrigation is of importance to optimize a design of photovoltaic pumping systems in terms of cost and efficiency.
\end{abstract}

Key words: Water pumping system, Photovoltaic energy, Performance, Design

\section{Introduction}

Water availability and accessibility are two main factors for rural development in developing countries. Due to reduced rainfall in many arid areas, availability of surface water was affected. For satisfying water demand, there is a need to mobilize underground resources using pumping technologies. The use of water pumping systems has significantly increased to exploit deep groundwater. Moreover, low pressurized drip irrigation techniques are becoming more demanded for saving water and energy. In fact, energy requirement for agricultural water pumping increased and use of efficient pumping technologies is of importance to improve performance of pumping and irrigation systems. The energy problem is even more acute in isolated sites where the use of conventional resources is often very expensive. Indeed, several technical and economic constraints, such as fuel transportation and periodic maintenance of diesel engines, oriented farmers for searching and using alternative energy sources. Use of alternative energy is of importance to promote sustainability in irrigated agriculture system. Photovoltaic pumping technologies was considerably grown up to implement governmental strategies looking for use of proper energy in a sustainable farming system. However, design and use of photovoltaic pumping still need more R\&D actions to improve technologies performance and to satisfy cost-effectiveness for a large adoption of the PV technologies in developing countries.

PV pumping systems are widely based on using submersible centrifugal pumps for pumping up underground water. By referring to affinity law, centrifugal pump characteristics can be varied according to variable rotor speed, and as a result pump flow rate, head, and power will be changed. Several authors studied implementation of the affinity laws for optimal management of PV pumping system to maximize daily water volume output (Chandrasekaran et al., 2014; Elgendy et al., 2012; Marchi et al., 2010; Khader et al., 2013).

Muñoz et al. (2015) developed a large PV pumping systems model using SISIFO interface to predict amount of pumped water relative to PV size, type by system performance analysis. They simulated the model with $20 \mathrm{~kW}$ PVWPS demonstrator installed in Spain. The system was composed of a PV generator, a variable frequency inverter, an AC centrifugal pump and a solar array tracker for improving system efficiency (water pumped according to incident irradiance ( $\mathrm{m} 3 / \mathrm{kWh}$ )). The affinity laws curves (head vs flow rate) were used to determine operating points of the pump. The simulation model showed that the output efficiency reached $2049 \mathrm{kWh} / \mathrm{kWp}$ 
of which $1158 \mathrm{kWh} / \mathrm{kWp}$ was converted as useful hydraulic energy equivalent to $1637 \mathrm{~m} 3 / \mathrm{kWp}$ or $32740 \mathrm{~m} 3$ per year as pumped water. The system performance was only $46 \%$ and need use pump and motor having higher efficiencies for improving the overall efficiency of the simulated large system.

Moubarak et al. (2017) used affinity laws to change pump characteristics by varying its speed, and consequently flow rate, head, and power were adjusted. They adapted the affinity laws to achieve the pump hydraulic requirements while the power delivered to the pump motor remains unchanged by shifting the pump to operate around new pump curves. A Matlab/Simulink model of the PV pumping system was evaluated over a wide range of weather and loading conditions (Moubarak et al., 2017).

This work treats technical and economic aspects related to actual performance of managing PV pumping system based on centrifugal pump for supplying drip irrigation system in arid context of Morocco. A management method was proposed to improve performance of a PV pumping system case according to incident solar irradiance during June, July and August as peak months for irrigation water demand.

\section{Materials and Methods}

This work consisted on a survey study to evaluate PVWPS actual performances and on a study case to propose method for improving hydraulic performances:

Survey study: The survey concerned fourteen PVWPSs to evaluate their operating conditions with reference to energetic and hydraulic performances. The survey was based on a questioner sheet to collect PVWPS general information and on measuring technical parameters for evaluating actual electric and hydraulic performances of three existing pumping stations of small, medium and large installed power.

PVWPS case study

Performance of a large PVWPS $(22 \mathrm{~kW})$ was approached with three scenarios to propose a management method of splitting and switching pump powers for better electric and hydraulic performances according to variability of solar irradiance. The finality of proposing a management method converges with possibility of satisfying irrigation water requirements without link to thermal pumping as done mostly in the PVWPS object of the survey. Electrical and hydraulic measurements were online done to collect performance data between $10 \mathrm{am}$ and $4 \mathrm{pm}$. Actually, solar irradiance occurrence was not sufficient to continuously operate the three phase AC motor/pump at the required speed range without stopping and speed fluctuation problems. The measurements were effectively started at 11:00 am, taking into account the system boot, which was only done around 10:30 am. To find adequate exploitation of the PVWPS power according to potential irradiance, the management method proposed consists on governing energy performance of centrifugal pump for three configurations. The configurations of using one large pump power (1st variant: $22 \mathrm{~kW}$ ), two medium pump powers ( 2 nd variant: $2 * 11$ $\mathrm{kW}$ ) and small pump powers (3rd variant: $3 * 7.5 \mathrm{~kW}$ ), were compared. The pumps are mounted in parallel and managed by switching on according to supply of the installed PV array power $(22 \mathrm{kWc})$ and its potential variation with reference to daily solar irradiance occurrence.

Performance evaluation of each variant was based on comparing pumped and required water volumes.

Optimal energy management of a centrifugal pump consists on valorising the PV array potential using adequate pump power with reference to operating it at its higher rotational speed or frequency of $50 \mathrm{~Hz}$. In fact, actual flow rate provided by a pump depends on its rotational speed, consequently on the potential frequency processed by the connected inverter according to occurring solar irradiance. The flow rate of a centrifugal pump can be governed (Eq. 1) to adequately adjust its efficiency and optimally use varying occurrence of a daily solar irradiance. The characteristic curve of a pump turning at constant rotational speed is presented by the following equation:

$$
H=a Q^{2}+b Q+c
$$

Where: $\mathrm{H}, \mathrm{Q}$ and $\mathrm{a}, \mathrm{b}, \mathrm{c}$ are head, flow rate, and constants of speed and diameter for a centrifugal pump model, respectively (Paugh, 1984).

Centrifugal pumps are governed by affinity laws (Eq.2) showing impact of rotational speed change on flow rate, head and power. The affinity laws is important to manage pump rotational speed point for better energetic and hydraulic performances. The affinity law expressions are given for a fixed rotor diameter by the following equations (Wilson, 2009)

$$
\frac{Q 1}{Q 2}=\frac{N 1}{N 2} ; \frac{H 1}{H 2}=\left(\frac{N 1}{N 2}\right)^{2} ; \frac{P 1}{P 2}=\left(\frac{N 1}{N 2}\right)^{3}
$$

Where: $\mathrm{H}, \mathrm{Q}$ and $\mathrm{N}$ are head, flow rate and speed, respectively. 
The method proposed to improve performance management of PV pumping systems is based on determining optimal centrifugal pump parameters according to affinity law and available photovoltaic power supplied by a PV generator. In fact, the power produced by a photovoltaic generator and supplied to a centrifugal pump mainly depends on irradiance potential G:

$$
P=G^{*} A * \eta P V * \eta i n v
$$

Where: $\mathrm{A}, \eta \mathrm{PV}$ and $\eta$ inv are photovoltaic generator surface, photovoltaic panel output and photovoltaic inverter output, respectively.

The third affinity law (Eq. 2) can be used to find a second operating point of the pump rotational speed N2 corresponding to a power P2. Characteristic curves for a centrifugal pump can be given for two rotational speeds N1 and N2 as follow:

$$
\begin{aligned}
& \left\{H 1=a_{1} \cdot Q_{1}{ }^{2}+b_{1} \cdot Q_{1}+c_{1}(N 1)\right. \\
& \left\{H 2=a_{2} \cdot Q_{2}{ }^{2}+b_{2} \cdot Q_{2}+c_{2}(N 2)\right.
\end{aligned}
$$

By applying law affinity, parameters a2, b2 and c2 can be obtained by the following equations:

$$
a_{1}=a_{2} ; b_{1}=b_{2} \cdot \frac{N_{2}}{N_{1}} ; c_{1}=c_{2} \cdot\left(\frac{N_{2}}{N_{1}}\right)^{2}
$$

Then, for a constant head $\mathrm{H}$ corresponding to a potential of the PV pumping system, the pumped flow rate for a given irradiance $\mathrm{G}$ is as follow:

$$
Q_{2}=\frac{-b_{2}-\sqrt{b^{2}-4 \cdot a_{2} \cdot\left(c_{2}-H\right)}}{2 a_{2}}
$$

\section{Results and Discussion}

\section{Survey Results}

The survey done on fourteen PVWPS showed adoption of drip and surface irrigation systems. PV generators were used for pumping underground water from wells at depth varying between 70 and $120 \mathrm{~m}$ and also for transferring water storage tank to drip irrigation network. All farmers targeted by the survey use hybrid pumping systems (PV generators and thermal engines) to cover irrigation water requirements. An economic evaluation of PV pumping system was done using LCC method, in order to compare different power options and to determine the most cost-effective system design. Results showed that initial cost of PV pumping system (18000\$) was very high compared to thermal pumping system (3500\$). The cost of pumping water using photovoltaic energy was ranging between 0.02 and $0.085 \$ / \mathrm{m} 3$. The cost of thermal pumping ranged between 0.04 and $0.161 \$ / \mathrm{m} 3$. The cost of PV water pumping was relatively low compared to thermal (diesel) water pumping. The gain due to use of PV generator vary from $11 \%$ to $88 \%$ with an average of $63 \%$.

\section{Study case results}

Analyze of actual operating of the pumping systems showed the possibility of improving its energetic and hydraulic performances and using only photovoltaic array for covering average daily water requirement. The study case based on a $22 \mathrm{~kW}$ PVWPS showed that installation of one large pump cannot work widely at its nominal frequency $(50 \mathrm{~Hz})$ to satisfy conditions of maximum daily efficiency according to irradiance occurrence. Prospection showed that maximum operating frequency $(84 \%)$ was occurred only for a few number of hours (four to five hours per day). Difficulty of reaching nominal frequency and operating pump at its nominal speed during a long daily period is due to inefficiently operate a large pump power $(22 \mathrm{~kW})$ at low and medium irradiance conditions (from 200 to $600 \mathrm{~W} / \mathrm{m}^{2}$ ). The photovoltaic generator is adequate for optimal pump operating only at higher irradiance period $\left(>600 \mathrm{~W} / \mathrm{m}^{2}\right)$. Use of small and medium pump powers mounted in parallel was effectively improved operating conditions of the pumps for relatively lower dent irradiance, longer day period and higher efficiency. Choosing small $(2 * 11 \mathrm{~kW})$ and medium $(3 * 7.5 \mathrm{~kW})$ pump powers helped to improve PV energy use and hydraulic performance of the PVWPS for effective head of $70 \mathrm{~m}$. Indeed, the system test using both variants showed better performance to satisfy water requirements in the three peak months. (June, July and August). The 
daily water requirements were evaluated at $522.6 \mathrm{~m} 3,595.1 \mathrm{~m} 3$ and $618.2 \mathrm{~m} 3$ for June, July and August, respectively (Figs 1, 2 and 3).

\section{a. First variant}

This variant consists on evaluating performance of one large pump power $(22 \mathrm{~kW})$ as the reference case actually adopted. The pump characteristic curve relative to nominal operating speed is as follow:

$$
H=-0.0036 Q_{2}-0.4909 Q-134.05
$$

Results (Fig.1) showed limitation to satisfy water requirement as the pump cannot efficiently operate in low irradiance (less than $352 \mathrm{~W} / \mathrm{m} 2$. Even if the daily operating period was 9 hours, the daily pumped water was computed to be $455 \mathrm{~m} 3,541 \mathrm{~m} 3$ and $581 \mathrm{~m} 3$ for June, July and August, respectively. The water requirement are not satisfied in this variant as the pumped volumes were only $86 \%, 91 \%$ and $93 \%$ for June, Jully and August, respectively.

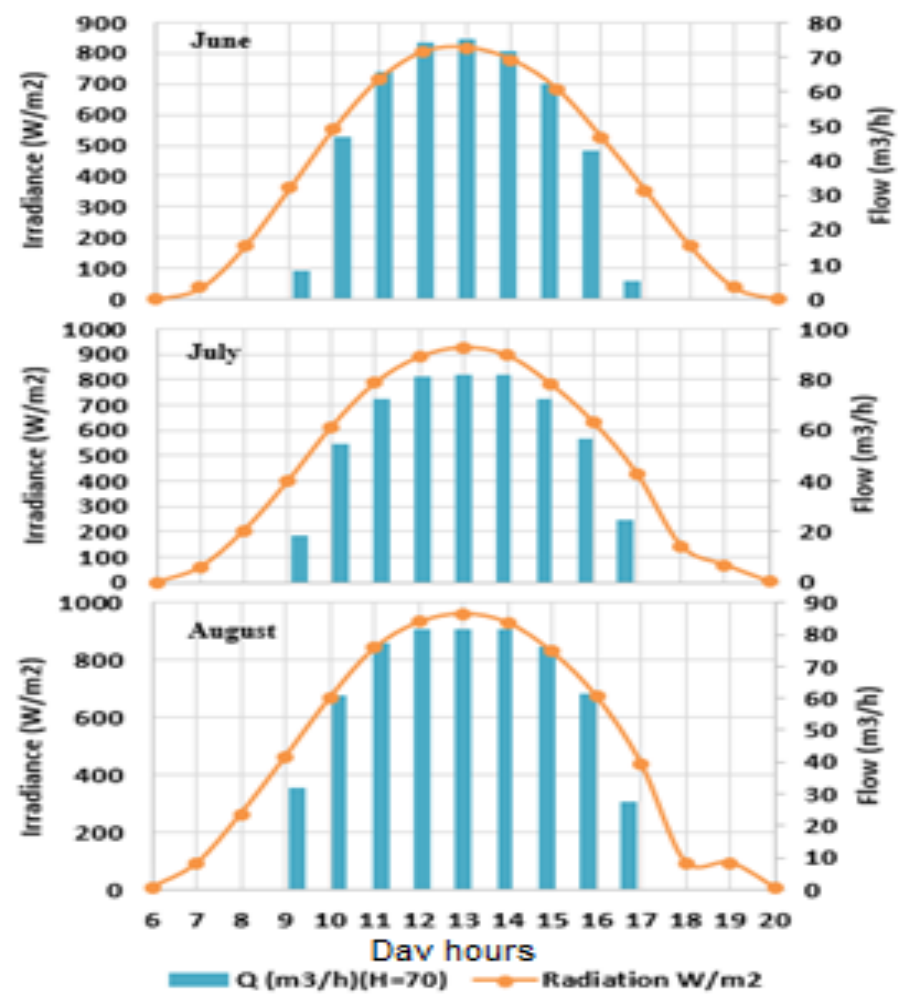

Figure 88. Average daily pumped water versus irradiance (case of using one large pump of $22 \mathrm{~kW}$ ).

\section{b. Second variant}

Implementation of this variant was based on using two similar pump powers mounted in parallel $(2 * 11 \mathrm{~kW})$. The pump characteristics curve at nominal operating speed is as follow:

$$
H=-0.0035 Q_{2}-0.0905 Q+132.97
$$

Figure 2 showed that use of two pumps increase the daily operating period to more than 10 hours. This increase is due to possibility of operating one pump at in low irradiance (less than $200 \mathrm{~W} / \mathrm{m}^{2}$ ) and engaging second pumps as irradiance became higher than $460 \mathrm{~W} / \mathrm{m}^{2}$. Consequently, the daily pumped water was increased to cover water demand by $103 \%, 102 \%$ and $103 \%$ for June, July and August, respectively. 


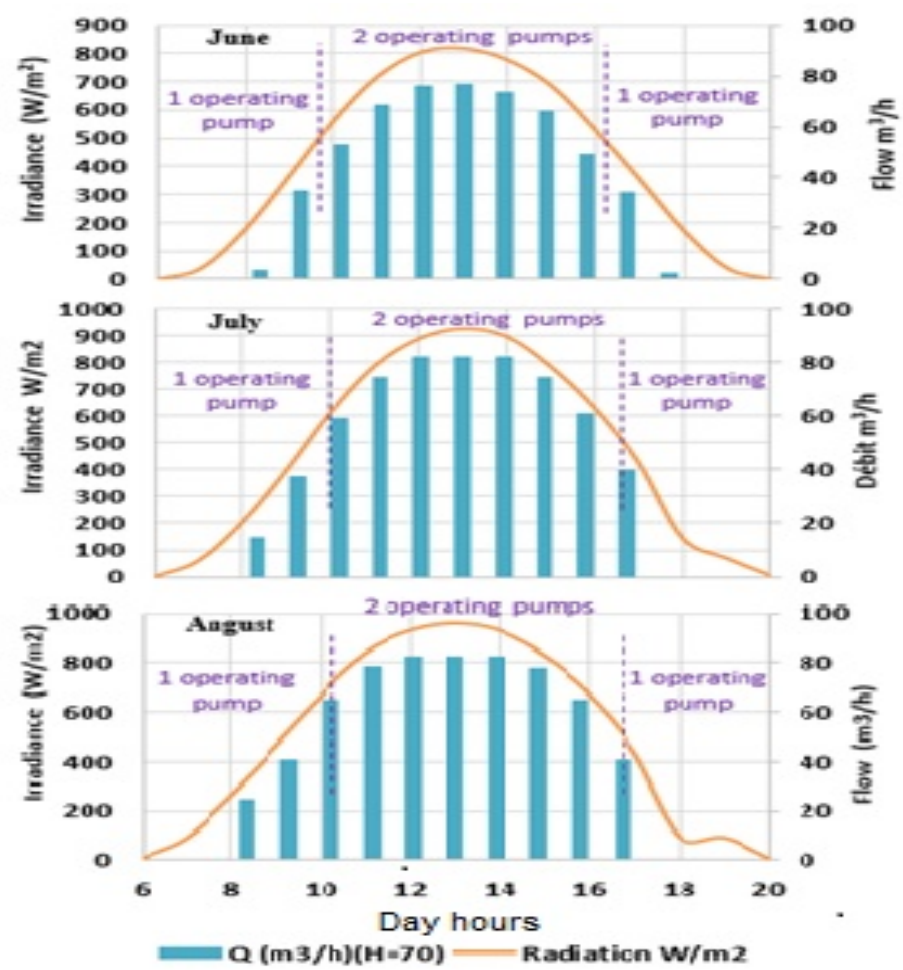

Figure 89. Average daily pumped water versus irradiance (case of using two medium pump powers of $11 \mathrm{~kW}$ ).

\section{c. Third variant}

This variant consists on using three small pumps of in parallel. The pump characteristic curve is represented as follow:

$$
H=-0.0571 Q_{2}-2.8571 Q+187.14
$$

Figure 3 showed considerable improvement of daily operating period (about 12 hours. This increase is due importance of using adapted pump power to take advantage from low irradiance occurring during sunrise and sunset periods. In fact, PV generator was optimally adapted to operate one pump at low irradiance (less than 200 $\mathrm{W} / \mathrm{m} 2$ ), two pumps as irradiance became between 200 and $450 \mathrm{~W} / \mathrm{m} 2$ and three pumps for irradiance higher than $450 \mathrm{~W} / \mathrm{m}^{2}$. Such scenario satisfied water requirement by $108 \%, 104 \%$ and $104 \%$ for June, July and August, respectively. 


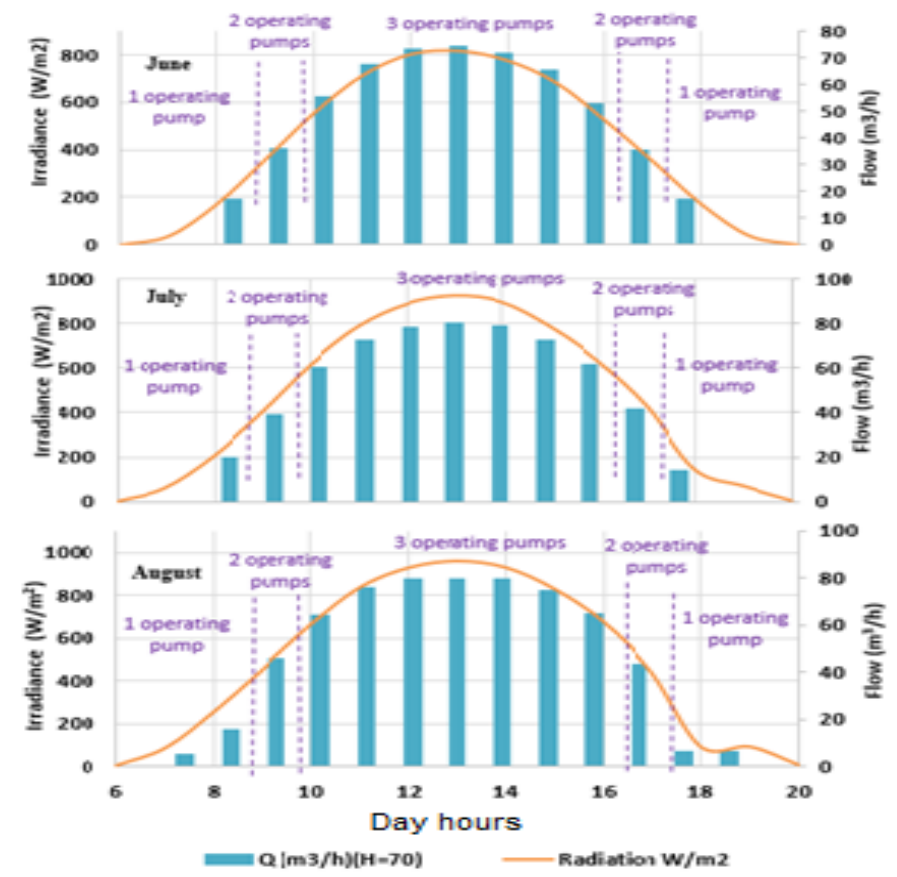

Figure 90. Average daily pumped water versus irradiance (case of using three small pumps of $7.5 \mathrm{~kW}$ ).

Water requirements are satisfied by $90 \%$ (1st variant), $103 \%$ (2nd variant) and $105 \%$ (3rd variant). It is better to adopt two or three pump mounted in parallel than choosing on pump of similar installed power.

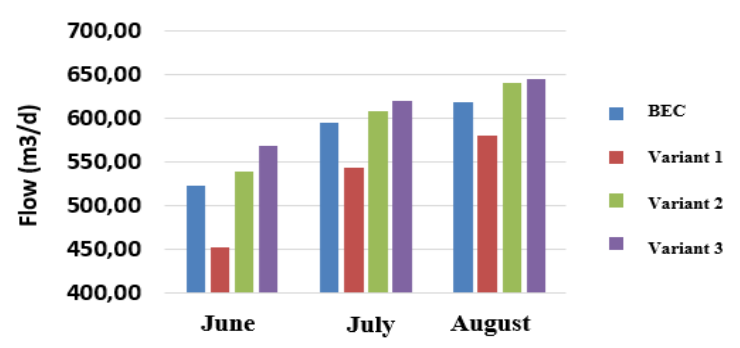

Figure 91. Average daily water requirements and pumped volumes.

Better performance of both variant 1st and 2nd) is due to adaptation of PV power potential to improve pumps operating and management satisfying higher hydraulic efficiencies. The average pump yields were $67 \%, 72.16 \%$ and $73.18 \%$ for 1 st, 2 nd and 3rd variants, respectively (Fig. 4).

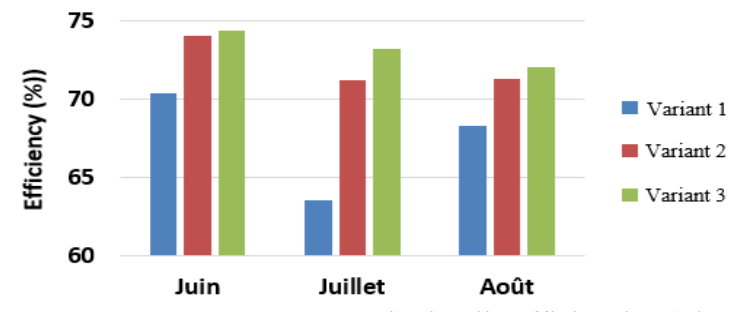

Figure 92. Pump powers management vs hydraulic efficiencies (Three variants).

Installation of PVWPS based on one large pump power (case of $22 \mathrm{~kW}$ ) is not adequate as its optimal operating is only ensured during peak solar irradiance period around midday. This study case showed that use of three small pump powers $(7.5 \mathrm{~kW})$ in parallel instead of a single large pump $(22 \mathrm{~kW})$ was effective solution to enlarge PVWPS service period and to improve their energetic and hydraulic performances.

\section{Conclusions}

This study was based on field survey to evaluate actual PVWPS performance and to propose a management hydraulic. The field survey showed that farmers disposing of PVWPS cannot complete water requirement deficit 
without using thermal engine to power their pumping system. Maximum valorization of available installed PV power depends on use of adequate hydraulic power and its timing with reference to occurrence of daily irradiance. The proposed power management method in the study case showed feasibility of using only PV energy supply to totally cover water requirements of the farming system. In fact, using a set of pump powers mounted in parallel instead of using one large pump of equivalent power can be an effective solution for enlarging operating period of PVWPS and take more advantage from occurring irradiance to carry out high hydraulic efficiencies.

\section{References}

Chandrasekaran, N. and Thyagarajah, K. (2014) Simulation and Experimental Validation of AC Motor and PMDC Motor Pumping System Fed by Photovoltaic Cell. Indian Journal of Engineering and Material Sciences, 21, 93-103.

Elgendy, M.A., Zahawi, B. and Atkinson, D.J. (2010) Comparison of Directly Connected and Constant Voltage Controlled Photovoltaic Pumping Systems. IEEE Transactions on Sustainable Energy, 1, 184-192.

Marchi, A., Simpson, A.R. and Ertugrul, N. (2012) Assessing Variable Speed Pump Efficiency in Water Distribution Systems. Drinking Water Engineering and Science, 5, 15-21.

Khader, S., and Daud, A. (2013) PV-Grid Tie System Energizing Water Pump. Smart Grid and Renewable Energy, 4, 409-418.

Moubarak, A.; El-Saady, G. and Ibrahim, E.N.A. (2017) Variable Speed Photovoltaic Water Pumping Using Affinity Laws. Journal of Power and Energy Engineering, 5, 50-71.https://doi.org/10.4236/jpee.2017. 511005.

Muñoz J., Carrillo J.M., Martínez-Moreno F., Carrasco L.M., Narvarte L. (2015), Modeling and simulation of large PV pumping systems, 31st European Photovoltaic Solar Energy Conference and Exhibition.DOI:10.4229/EUPVSEC20152015-6BV.5.42.

\section{Nomenclature}

$\begin{array}{ll}\text { PVWPS } & : \text { Photovoltaic Water Pumping System } \\ \mathrm{kW} & : \text { Kilo-Watt } \\ \mathrm{kWc} & : \text { Kilo Watt Peak } \\ \mathrm{AC} & : \text { Alternatif Current } \\ \mathrm{DC} & : \text { Direct Current } \\ \mathrm{MPPT} & : \text { Maximum Power Point Tracking }\end{array}$




\title{
Economic Comparison and Carbon Emissions of Payload Optimized Milk Logistic Chains
}

\author{
Michael Schmid ${ }^{\text {a*}}$, Sascha Wörz ${ }^{\text {a }}$, Heinz Bernhardt ${ }^{\text {a }}$ \\ ${ }^{a}$ Chair of Agricultural Systems Engineering, Technical University of Munic, Am Staudengarten 2, 85354 Freising, \\ Germany \\ * Corresponding author. Email: michael.schmid@wzw.tum.de
}

\begin{abstract}
This contribution copes with the optimization of the payload and carbon emission rates in milk logistic chains between milk producers and dairy processor by full cost accounting, where two different milk logistic chains and two different milk draw areas are examined. The use of a milk transport truck and its profitability requires a monetary and carbon emissions valuation between different triggered milk chains and its payloads. This contribution aims at determining the full costs and carbon emission rates for five different payload scenarios. Exemplarily, the various payload marginal costs are calculated for two milk logistic chains and two milk draw areas. The cost calculation of payload triggered logistic chains uses full cost accounting. The carbon emission rates are determined by the consumption-based method. Thereby, the full cost accounting and the carbon emission rates differ and increase with the decrease of the payload and increasing distances between milk producer and milk processing company. Studying payload triggered milk logistic chains and carbon emissions is beneficial for strategical consulting and decision-making when thinking of milk removers from the viewpoint of a dairy processor.
\end{abstract}

Keywords: Milk logistic chain; carbon emissions; payload optimization; milk transport costs.

\section{Introduction}

In Germany, dairy production is the most important branch of the agriculture, while the dairy industry occupies the second largest segment in the German food industry (BMEL 2017). The structural transformation in milk producers caused by technological progress and the site-specific concentration and the increase in capacity of milk processing companies require increasing distances for milk collection and milk transport, according to which the optimization of milk logistics costs and $\mathrm{CO}_{2}$ emissions becomes more important. Simultaneously, the technical progress of milk logistics in the area of payload optimization offers new possibilities for milk collection and milk transport. Thereby, the $\S 34$ "axle load and total weight" of the road traffic approval order prescribes a permissible total weight of maximum $40 \mathrm{t}$ (Bundesministerium der Justiz und für Verbraucherschutz 26.04.2012). In honour of this occasion, the determination of a payload-optimized milk collection and milk transport requires an economic and sustainable valuation of different payload triggered milk logistic chains. By adjusting the tank volume size to the possible payload, the payload can also be increased. In addition, the reduction in the weight of the driver's cab by eliminating possible equipment offers optimization potential in terms of payload increase. Quinlan et al. calculated in 2012, modeling a milk collection semitrailer for the Irish dairy industry for the periods after the milk quota full costs of $0,81-0,98 € \mathrm{Ct} / \mathrm{kg}$ milk depending on the volatility of the delivered milk quantity. At the same time $\mathrm{CO}_{2}$ emissions were analyzed, it ranged from 3,80 to 4,44 $\mathrm{g} \mathrm{CO}_{2} / \mathrm{kg}$ milk (Quinlan et al. 2012). The FAO reported for the Organization for Economic Cooperation and Development member states (USA, Australia, Spain, UK, Norway and Sweden) of CO2 emissions of milk logistics amounting to $16 \mathrm{~g}$ (FAO 2012). Instead of providing milk removers with blanket equipment advice when buying a new milk collection or milk transport vehicle, the purpose of this paper is to calculate the total payload-specific and carbon dioxide emissions in $\mathrm{kg} \mathrm{CO}_{2}$ equivalent for two differently located milk catchment areas and two process variants for milk logistics.

\section{Materials and Methods}

The simulation model used was developed by Schmid et al. and is based on the economic and sustainable evaluation of the components of the milk logistics: Outward/return run to the milk draw area, assembly driving, on-farm-routine activities, on-farm pumping, outward/return run to the trailer / semitrailer, on-trailer-routine activities, on-trailer pumping, in dairy-processor-routine activities, plant pumping and in plant cleaning (Schmid et al. 2018a).

In this article, the full costs of milk logistics are charged by means of the method of full cost calculation for the process variant of milk logistics four-axle truck solo unbroken and four-axle truck solo with milk transport semitrailer broken (Steinhauser et al. 1992). Full costs and $\mathrm{CO}_{2}$ emissions are analysed for five different payload scenarios. In the process variant four-axle truck solo, the four-axle truck with a payload Ia to Ie travels from the dairy processor into the milk draw area, collects milk from the milk producers and travels back to the dairy processor. In the process variant four-axle truck with milk transport semi-trailer broken runs the four-axle with a 
payload of Ia-If from the dairy processor to the milk draw area, fills in the center of gravity the milk transport trailer with a payload IIa to IIf by pumping and moves for reasons of cleaning at least once during the three-day triggered milk logistic to dairy processor back. For reasons of payload optimization, the milk transport trailer has no milk acceptation equipment. The modeling of the specific full costs and $\mathrm{CO}_{2}$ emissions of the milk logistics is carried out in process variant II respectively for the combination of the same payload classes of the four-axle solos and the milk transport trailer. Table one below illustrates the payload categories for the four-axle truck solo and the milk transport semitrailer.

Table 1. Payload triggered classes for the four-axle truck solo Ia-Ie (left) and the milk transport semitrailer IIa-IIe (right).

\begin{tabular}{lllc}
\hline Abbreviation & Payload & Abbreviation & Payload \\
\hline Ia & 16.600 & IIa & 27.400 \\
Ib & 16.900 & IIb & 27.700 \\
Ic & 17.200 & IIc & 28.000 \\
Id & 17.500 & IId & 28.300 \\
Ie & 17.800 & IIe & 28.600 \\
\hline
\end{tabular}

The outward and the return run to the milk draw area includes the time required and the full costs for the outward and the return run from the dairy processor to the barycenter of the milk draw area. This distance is 35 $\mathrm{km}$ in the distance scenario A and $250 \mathrm{~km}$ in the distance scenario B. The milk collection required for milk collection includes the full costs and the time required for milk collection between the 120 dairy farmers, which is $5,5 \mathrm{~km}$ on average. The average milk quantity per milk producer is $3.000 \mathrm{~kg} / \mathrm{milk}$ producer during and threeday milk collection cycle, so that during the two-day analysis period $360,000 \mathrm{~kg}$ of milk must be collected and transported to the dairy. The on-farm-routine activity per milk producer is $1,56 \mathrm{~min}$, while the on farm pumping is $615 \mathrm{~kg} / \mathrm{min}$. For each pumping process to the trailer/semitrailer, a routine work time of 1,97 min per pumping operation is estimated. The on-trailer pumping power is $1.114 \mathrm{~kg} / \mathrm{min}$. The time required to in dairy-processorroutine activities is $15,83 \mathrm{~min} /$ discharge. The plant pumping is estimated with $906 \mathrm{l} / \mathrm{min}$. The time required for cleaning is 33,66 minutes per discharge. The hourly wage of the milk collection truck driver is estimated at $20 €$ / h. At $12 € / \mathrm{h}$ the hourly wage of the milk transport trailer truck driver is lower than that of the milk collection truck driver, since the pure "milk transport" requires less expertise.

The calculation of the specific $\mathrm{CO}_{2}$ emissions of the milk logistics is realized by the weel-to-wheel method. This includes the direct $\mathrm{CO}_{2}$ emissions from diesel combustion and the indirect $\mathrm{CO}_{2}$ emissions released in fuel processing and fuel logistics (Kranke et al. 2011).

$$
\mathrm{G}_{\mathrm{T}}=\mathrm{F}^{*} \mathrm{~g}_{\mathrm{t}}
$$

In this formula $\mathrm{G}_{\mathrm{T}}$ sets the weel-to-wheel carbon emissions in $\mathrm{kg} \mathrm{CO}_{2}$-equivalent $\left(\mathrm{CO}_{2}\right)$; $\mathrm{F}$ the diesel consumption in 1 ; and $\mathrm{g}_{\mathrm{t}}$ is the tank-to-wheel carbon emission factor $\left(3,15 \mathrm{~kg} \mathrm{CO}_{2} \mathrm{e} / \mathrm{l}\right.$ fuel).

The specific full costs and $\mathrm{CO}_{2}$ emissions are calculated respectively for process variant $\mathrm{I}$ and process variant II, each grouped into five payload classes, and the two milk catchment areas A and B. Table two below summarizes the scenarios described.

Table 2. Scenarios between milk draw area A and B and process variant I and II.

\begin{tabular}{cll}
\hline & Milk draw area A $(35 \mathrm{~km})$ & Milk draw area B $(250 \mathrm{~km})$ \\
\hline Process variant I & AIa, AIb, AIc, AId, AIe & BIa, BIb, BIc, BId, BIe \\
Process variant II & AIIa, AIIb, AIIc, AIId, AIIe & BIIa, BIIb, BIIc, BIId, BIIe \\
\hline
\end{tabular}

The abbreviations shown in the scenario matrix in Table 2 are the basis of the explanations in the results section. 


\section{Results and Discussion}

The specific full costs of milk logistics for distance class A are illustrated in figure one below for process variant I in blue and for process variant II in red.

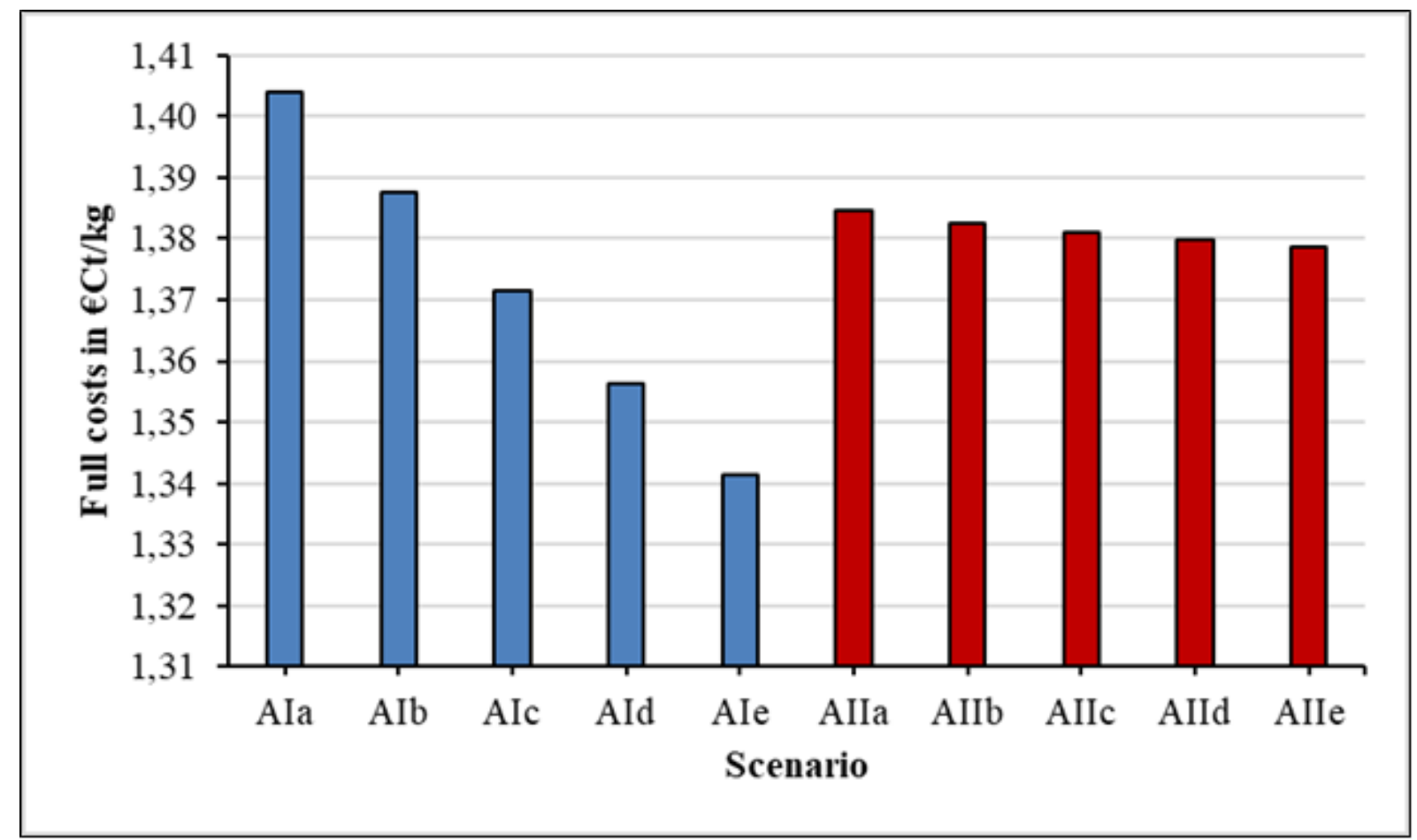

Figure 1. Specific payload triggered milk logistic chain full costs in distance class A for process variant I (blue) and process variant II (red).

The full costs of milk logistics shown in Figure 1 are between $1,34 € \mathrm{Ct} / \mathrm{kg}$ milk in scenario A for process variant $\mathrm{I}$ in scenario AIe and $1,40 € \mathrm{Ct} / \mathrm{kg}$ milk in scenario AIa. In process variant II in scenario A, the full costs of milk logistics range from $1,37 € \mathrm{Ct} / \mathrm{kg}$ in scenario AIIe up to $1,38 € \mathrm{Ct} / \mathrm{kg}$ milk in scenario AIIa. The reason given for the reduction in the full cost of milk logistics for both variants is the increase in payload, which reduces the distance covered for milk collection and milk transport. At maximum payload, the number of group trips in scenario AIe decreases by $4,3 \%$, and the number of transport trips by $0,43 \%$ in comparison to scenario AIa. In comparison of both milk logistics chains in the producer removal scenario A, process variant I from payload class $\mathrm{c}$ to payload class e has lower full costs of milk logistics than all payload classes in process variant II, because the relative savings potential in process variant I is higher than in process variant II. The economic savings potential from the point of view of a dairy between the selection of the milk logistics chain with the lowest full costs compared to the milk logistics chain with the highest full costs in the distance class A in process variant I is 225 $€ /$ milk collection cycle and $27.353 € /$ year, in process variant II $21 € /$ milk collection cycle and $2.605 € /$ year. Since in process variant II the relative payload optimization due to the use of the optimized milk transport semitrailer are lower than in the milk logistics chain I, the absolute optimization possibilities in the milk logistics chain I are higher.

The specific full cost of milk logistics for distance class B is shown in Figure 2 below. 


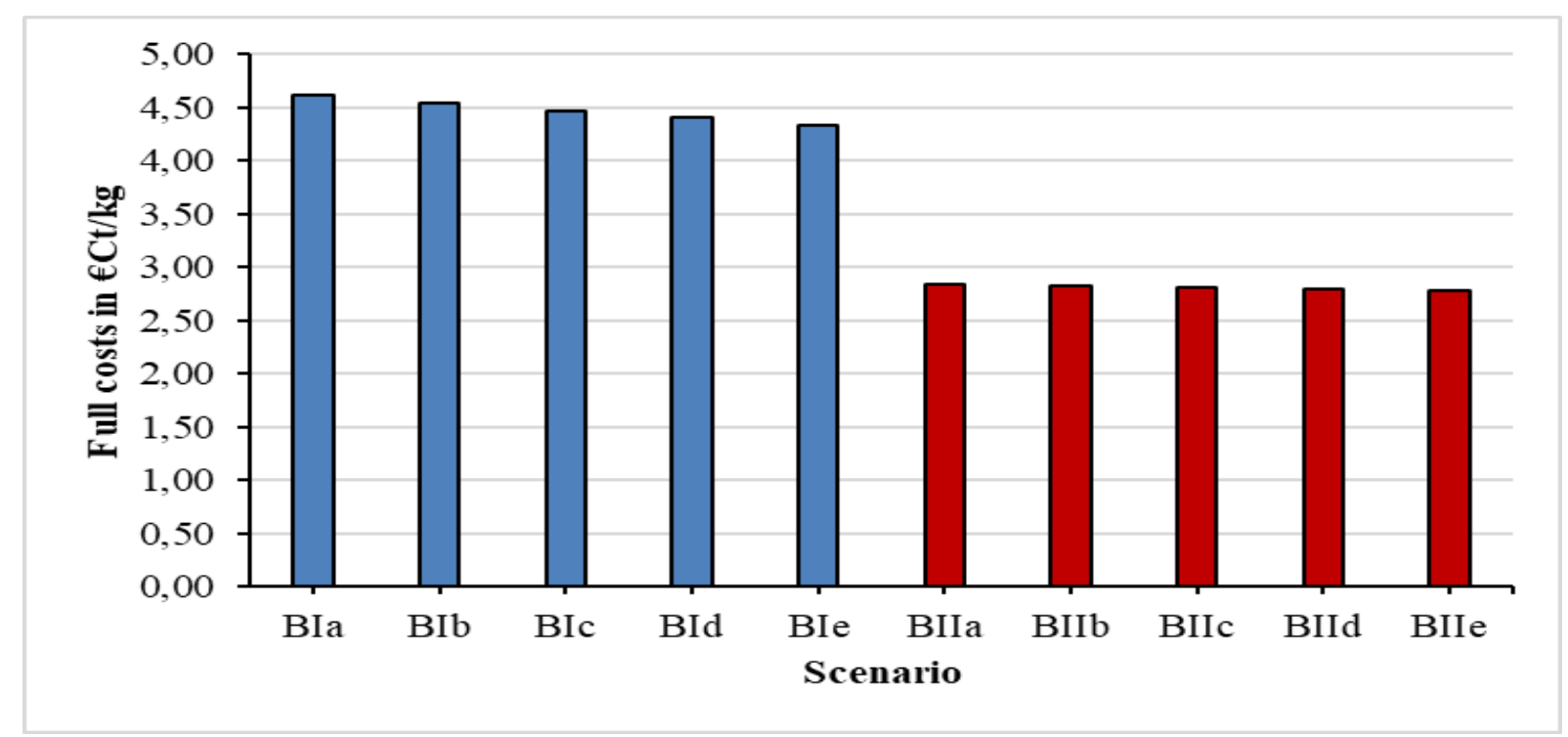

Figure 2. Specific payload triggered milk logistic chain full costs in distance class B for process variant I (blue) and process variant II (red).

In distance class $\mathrm{B}$, the full costs of milk logistics in process variant I vary between $4,34 € \mathrm{Ct} / \mathrm{kg}$ milk in scenario BIe and $4,61 € \mathrm{Ct} / \mathrm{kg}$ milk in scenario BIa. The full costs of milk logistics in process variant II are between $2,77 € \mathrm{Ct} / \mathrm{kg}$ milk in scenario BIIe and $2.84 € \mathrm{Ct} / \mathrm{kg}$ milk in scenario BIIa. In this way payload optimization in distance class $\mathrm{B}$ in process variant I can save $1.008 € / \mathrm{milk}$ collection cycle and $122.640 € /$ year from the point of view of a dairy processor. In process variant II, this economic savings potential through payload optimization amounts to $252 € /$ milk collection cycle and $30.660 € /$ year. Overall, the full costs of milk logistics in distance class $\mathrm{B}$ range from $2,77 € \mathrm{Ct} / \mathrm{kg}$ milk in scenario BIIe up to $4,61 € \mathrm{Ct} / \mathrm{kg}$ milk in scenario BIa so that through the optimized process variant $6.624 € /$ milk collection cycle and $805.920 € /$ year can be saved from the viewpoint of a dairy processor. This economic saving is justified by the reduction of transport by $44 \%$ through the use of milk transport semi-trailers, which have both a 10,8 thigher payload compared to the four-axle solo and a lower wage level of the truck driver.

The comparison of the specific process-type full cost of milk logistics in Scenario A and Scenario B has shown that with decreasing payload and increasing the distance between the milk draw area and the dairy, the full cost of milk logistics increases. This illustrates that as the distance between the milk draw area and the dairy processor grows, the relative performance of the process variant with milk transport by milk transport semitrailer increases because the number of transport journeys is reduced below the analyzed milk quantity and supplier structure. From a transport distance of $40 \mathrm{~km}$, the payload-optimized milk logistics chain four-axle truck with milk transport by semitrailer is economically justified in this model.

The specific $\mathrm{CO}_{2}$ emissions for Scenario A are illustrated in Figure 3 below. 


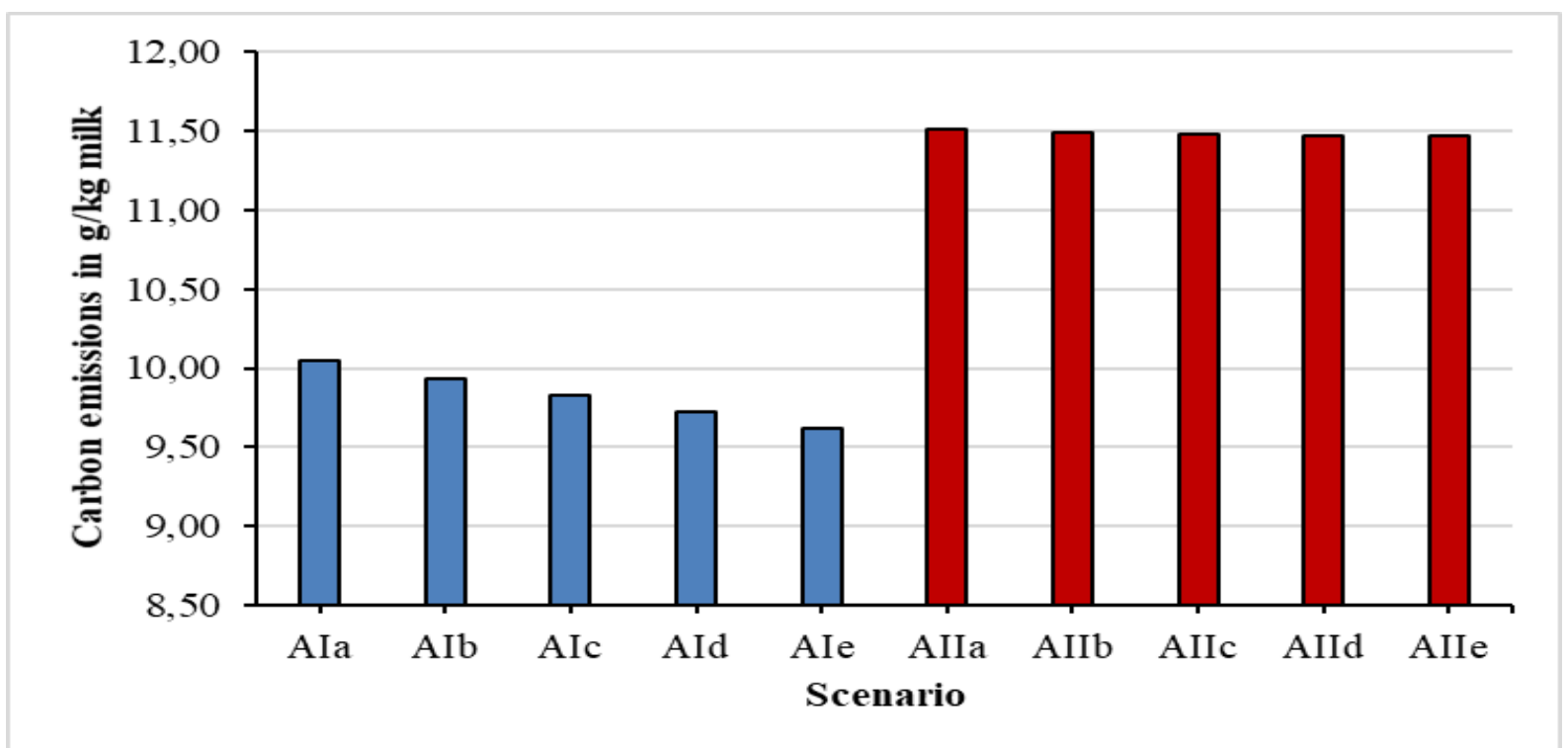

Figure 3. Specific payload triggered $\mathrm{CO}_{2}$ emissions in distance class A for process variant I (blue) and process variant II (red).

In process variant $\mathrm{I}$ in scenario $\mathrm{A}$, the $\mathrm{CO}_{2}$ emissions are between 9,62 $\mathrm{g} \mathrm{CO}_{2} / \mathrm{kg}$ milk in the milk logistics scenario AIe and 10,05 $\mathrm{g} \mathrm{CO} 2 / \mathrm{kg}$ milk in the milk logistics scenario AIa, so that the specific CO2 emissions can be reduced by $0,43 \mathrm{~g} \mathrm{CO}_{2} / \mathrm{kg}$ milk or $4.3 \%$ can be reduced. Thus, selecting the payload class with the lowest specific $\mathrm{CO}_{2}$ emissions compared to the payload class with the highest specific $\mathrm{CO}_{2}$ emissions provides a potential savings of $0,16 \mathrm{t} \mathrm{CO}_{2}$ per milk collection cycle and $28,18 \mathrm{t} \mathrm{CO}_{2}$ per year. In scenario $\mathrm{A}$ in process variant II, the $\mathrm{CO}_{2}$ emissions range from $11,46 \mathrm{~g} \mathrm{CO}_{2} / \mathrm{kg}$ milk for scenario AIIe to $11,50 \mathrm{~g} \mathrm{CO}_{2} / \mathrm{kg}$ milk for scenario AIIa. By optimizing the payload, it is possible to save $14,4 \mathrm{~kg} \mathrm{CO} / \mathrm{milk}$ collection cycle and $5,26 \mathrm{t} \mathrm{CO}_{2} /$ year. The comparison of the specific $\mathrm{CO}_{2}$ emissions of process variant I and process variant II in distance class A shows that the specific $\mathrm{CO} 2$ emissions of all payload classes in process variant I are lower than in process variant II. The omission of the components of milk logistics outward/return run to the trailer and on-trailer pumping justified the lower $\mathrm{CO}_{2}$ emissions of process variant I compared to process variant II.

Figure four below exemplifies the specific $\mathrm{CO}_{2}$ emissions of the milk logistics for the far-away distance scenario B.

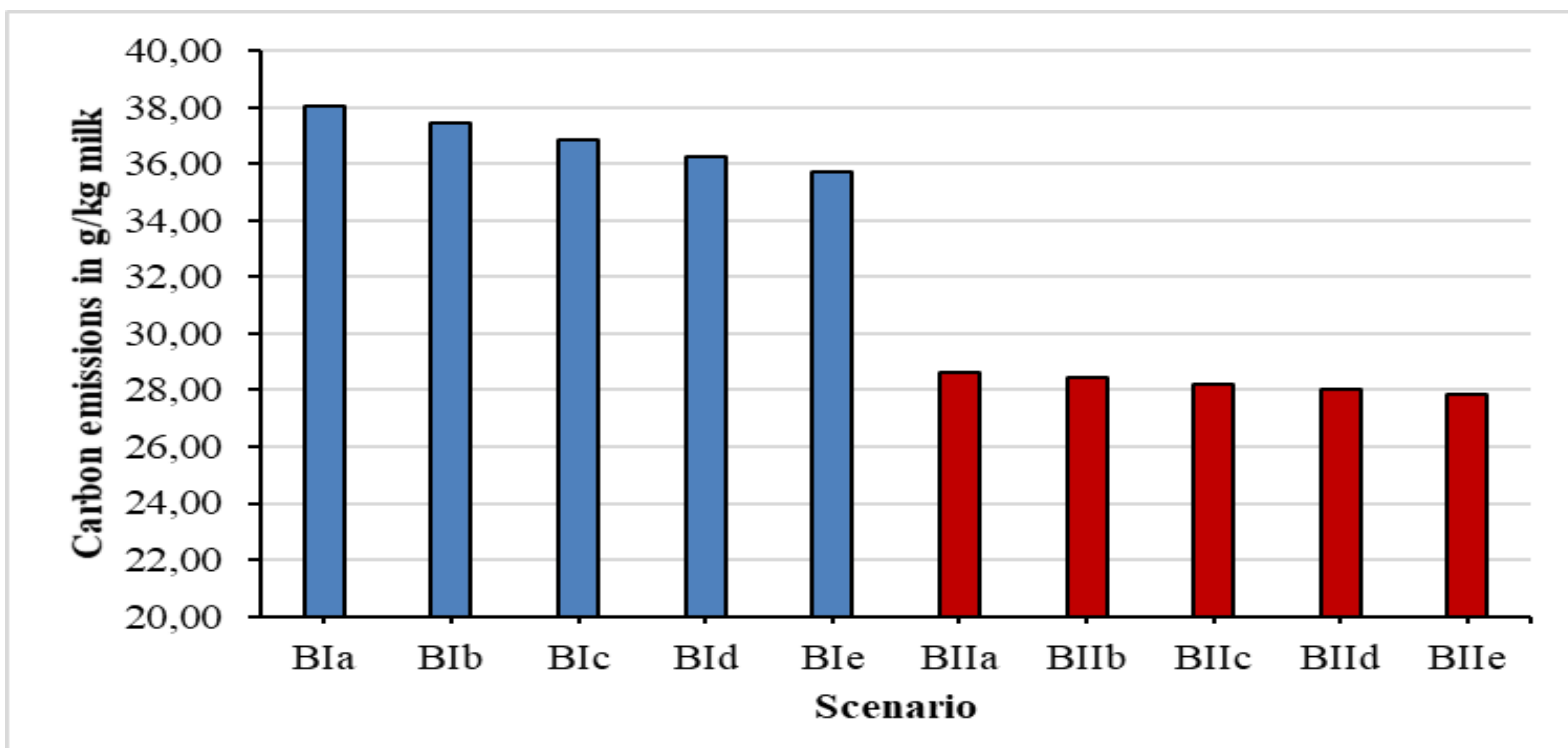

Figure 4. Specific payload triggered $\mathrm{CO}_{2}$ emissions in distance class $\mathrm{B}$ for process variant I (blue) and process variant II (red).

The specific $\mathrm{CO}_{2}$ emissions in scenario $\mathrm{B}$ in process variant I range between $35,73 \mathrm{~g} \mathrm{CO} / \mathrm{kg}$ milk for the payload class BIe and 38,04 $\mathrm{g} \mathrm{CO}_{2} / \mathrm{kg}$ milk, so that by selecting the payload-optimized process variant $\mathrm{I} 0,83 \mathrm{t}$ $\mathrm{CO}_{2} /$ milk collection cycle and $151,35 \mathrm{t} \mathrm{CO}_{2}$ year can be saved. For process variant II in distance class $\mathrm{B}$, the $\mathrm{CO}_{2}$ emissions of the milk logistics range between 27,86 $\mathrm{g} \mathrm{CO}_{2} / \mathrm{kg}$ milk for the payload class BIIe and 28,62 $\mathrm{g} \mathrm{CO}_{2} / \mathrm{kg}$ 
milk for the payload class BIIa. Therefore, by optimizing the payload in this process variant, it is possible to save $0,27 \mathrm{t} \mathrm{CO}_{2} /$ milk collection cycle and $49,80 \mathrm{tCO}_{2}$ per year. The $\mathrm{CO}_{2}$ emissions of the milk logistics chain II shown in figure 4 are lower than the specific $\mathrm{CO}_{2}$ emissions of process variant $\mathrm{I}$ in all payload classes, because the reduced number of transport journeys means that less $\mathrm{CO}_{2}$ is emitted in spite of the process steps on-trailer pumping and outward/return run to the trailer / semitrailer.

The $\mathrm{CO}_{2}$ emissions recorded for both distance classes illustrate an increase in specific $\mathrm{CO} 2$ emissions per $\mathrm{kg}$ of milk with a raise in the distance between the milk draw area and the dairy processor and a decrease in the payload of a particular process variant. At the same time, the specific full costs of milk logistics boost with a decrease in payload and an increase in the distance between the milk draw area and the dairy processor. Therefore, when purchasing new milk collection and milk transport vehicles to reduce the full costs and carbon emissions, payload optimized vehicle variants are to be purchased. In addition, the full costs and $\mathrm{CO}_{2}$ emissions of the milk logistics can be reduced by using the optimal process variant of the milk logistics depending on the distance to the dairy. At the same time, the sustainable and economic optimization potential increases with an increase in the distance between the milk catchment area and the dairy. This raise in the specific full costs of milk logistics and an increase in the potential for optimization with an increase in the distance between the milk draw area and the dairy processor were confirmed by Schmid et al (Schmid et al. 2018b). Compared to the collected specific full costs and $\mathrm{CO}_{2}$ emissions of milk logistics by Quinlan et al. the analyzed results of this contribution are higher because the distance between the considered milk draw areas and dairies is larger than the modeled distances in Ireland (Quinlan et al. 2012).

\section{Conclusions}

In this contribution, the payload triggered milk logistic chain full costs and carbon emissions are analyzed for two different distance scenarios between dairy processor and milk draw area. Thereby, the full costs and $\mathrm{CO}_{2}$ emissions accelerate with an increase in distance and a decrease in payload. The selection of payload and procedure-type full costs provides an economic savings potential of up to $27 \%$. The technical progress in the field of payload optimization and process-type-dependent milk logistics planning contributes both to cost reduction and to more sustainable milk logistics. Therefore, this article provides objective indicators for dairies processors that advise milk removers about investment decisions. As milk removers may incur higher costs or less bestbefore dates as a result of investing in payload optimized milk collection and milk transport vehicles, this marginal cost should be determined from the perspective of a milk remover as part of further research.

\section{References}

BMEL (2017): Milchbericht 2017 des Bundesministeriums für Ernährung und Landwirtschaft. Berlin.

Bundesministerium der Justiz und für Verbraucherschutz (26.04.2012): Straßenverkehrs-ZulassungsOrdnung. (StVZO).

FAO (2012): Greenhouse Gas Emissions from the Dairy Sector. A Life Cycle Assessment.

Kranke, A.; Schmied, M.; Schön, Andrea D. (2011): CO2-Berechnung in der Logistik. Datenquellen, Formeln, Standards. 1. Aufl., Stand August 2011. München: Vogel.

Quinlan, C.; Kaene, M.; Connor, D.; Shalloo, L. (2012): Milk transport costs under differing seasonality assumptions for the Irish dairy industry. In: International journal of dairy technology 2012 (65), S. 22-31.

Schmid, M.; Wörz, S.; Bernhardt, H. (2018a): Kalkulationsrechnung für ein optimales Milch Supply Chain Management. In: 21. Arbeitswissenschaftliches Kolloquium: Arbeit in der digitalen Transformation, S. 186-195.

Schmid, M.; Wörz, S.; Bernhardt, H. (2018b): Vergleich von Verfahrensvarianten einer Milchlogistikkette zwischen Milcherzeuger und Molkerei. In: Informatik in der Land-, Forst- und Ernährungswirtschaft, S. 219-222.

Steinhauser, H.; Langbehn, C.; Peters, U. (1992): Allgemeiner Teil. Produktionsgrundlagen, Produktionstheorie und Rechnungssysteme mit Planungsrechnungen; 75 Übersichten. 5., neubearb. Aufl. Stuttgart: Ulmer (UTB für Wissenschaft Uni-Taschenbücher Agrarwissenschaften, 113). 


\title{
Milk Transport Costs and Carbon Emissions of Various Triggered Milk Logistic Chains
}

\author{
Michael Schmid ${ }^{\text {a*}}$, Sascha Wörz ${ }^{\text {a }}$, Heinz Bernhardt ${ }^{\text {a }}$ \\ ${ }^{a}$ Chair of Agricultural Systems Engineering, Technical University of Munic, Am Staudengarten 2, 85354 Freising, \\ Germany \\ * Corresponding author. Email: michael.schmid@wzw.tum.de
}

\begin{abstract}
The optimization triggered milk chain and its process variant requires a monetary valuation of the milk collection cycles and different milk logistic chains. This contribution aims at determining the full costs and the carbon emission rates of a milk supply chain during a period of six days for a one-, two-, and three-day triggered milk chain. Exemplarily, the various triggered marginal costs and carbon emissions are estimated for two milk logistic chains and two milk draw areas. The cost calculation of various triggered milk logistic chains uses full cost accounting. The carbon emission rates are determined by the consumption-based method. The unique full cost accounting and carbon emission rates of the triggered milk chains and the various process variants differ. The analysis of the individual scenarios constitutes an indicator for the payment to the milk producer of a various triggered and processed milk chain from the viewpoint of a dairy processor.
\end{abstract}

Keywords: Milk logistic chain, carbon emissions, milk collection cycles, milk transport costs.

\section{Introduction}

The structural transformation among milk producers and societal and political debates about climate-relevant greenhouse gas emissions in the logistics sector pose a new challenge for dairy processors, whose industry occupies the second largest share in the German food industry (Janze et al. 2017; Strogies und Gniffke 2017). Simultaneously, the technical progress of milk logistics, milk storage and milk cooling enable the milk collection interval to be extended to up to three days. However, the determination of an optimal milk collection cycle, an optimal process variant of milk logistics and the specific carbon emissions require an evaluation of different milk collection cycles and different process variants of milk logistics.

Compared to other transport goods, milk logistics is a part-load transport of the perishable and continuously produced commodity raw milk. For the dairy processors, these properties demand milk collection in planned tours and, for the dairy farmers, a milk storage and milk cooling facility adapted to the milk quantity and collection cycle. The milk collection cycle defines the triggered milk chain at the milk producer, which influences the delivery quantity from a logistical point of view per entry procedure. As early as 2009, Weindlmaier and Betz conducted a study on the "current situation of milk collection in Germany and Austria". This confirmed the development from the one-day triggered milk chain to the two-day triggered milk chain with a share of $60 \%$ in the total milk deliveries of the year 2006. Thereby, a cost saving through the abrupt junction of a one- to two-day triggered milk chain of 0,3 to $0,4 € \mathrm{Ct} / \mathrm{kg}$ milk is proved (Betz und Weindlmaier 2006; Weindlmaier und Betz 2009). Today, the share of two-day triggered milk chain in Germany is about $90 \%$; few dairy processors collect milk in the one- or three-day day milk collection cycle.

Since the year 2007, a three-day triggered milk chain is possible in Germany with the replacement of the national milk quality regulation by the EU-regulation (EG) No. 853/2004 (Europäische Union 25.06.2004). In the three-day triggered milk chain, the Dutch dairy company Campina Friesland has been collecting milk in the Netherlands since 1985; in Germany, milk from this dairy company has been collected by around 50\% of the milk producers in the three-day triggered milk chain since 2008. The cost saving potential of this conversion is estimated at 10 to $15 \%$ compared to the two-day triggered milk chain. In return, dairy producers receive a supplement of $0,25 € \mathrm{Ct} / \mathrm{kg}$ milk for the additional tank storage capacity and the additional cooling costs for the three-day day triggered milk chain compared to the two-day triggered milk chain. The milk from the dairy company Söbbeke in Germany, too, has only been collected since 2009 in the three-day triggered milk chain. The milk producers receive a lump-sum surcharge of $0.5 € \mathrm{Ct} / \mathrm{kg}$ milk for the resulting extra effort compared to the one-day triggered milk chain. As a result, this dairy company can estimate the economic savings potential of up to $25 \%$. (Liste 2011). Because of this potential for optimization, the three-day triggered milk chain has been practiced in Ireland for a long time (Quinlan et al. 2012; Quinlan et al. 2010).

Instead of generating blanket surcharges dependent on milk collection cycles, the purpose of this paper is to quantify the milk collection cycle-dependent milk logistics full costs and carbon emissions in $\mathrm{CO}_{2}$ equivalent for two milk catchment areas and two process variants of milk logistics. 


\section{Materials and Methods}

The simulation model used was developed by Schmid et al. and is based on the economic and sustainable evaluation of the components of the milk logistics: Outward/return run to the milk draw area, assembly driving, on-farm-routine activities, on-farm pumping, outward/return run to the trailer / semitrailer, on-trailer-routine activities, on-trailer pumping, in dairy-processor-routine activities and plant pumping and in plant cleaning (Schmid et al. 2018).

In this contribution the full costs and $\mathrm{CO}_{2}$ emissions of milk logistics are estimated for the process variant three-axle truck with two-axle trailer broken and for the process variant three-axle truck with two-axle trailer and milk transport by semitrailer. In process variant three-axle truck with two-axle trailer broken drives the three-axle truck with a payload of $16.000 \mathrm{~kg}$ with a two-axle trailer with a payload of $9.000 \mathrm{~kg}$ from the dairy processor to the barycenter of the milk draw area, hangs the trailer there, collects milk from the milk producers, fills the trailer by pumping, hooks up the trailer and returns to the dairy. In process variant three-axle truck with two-axle trailer and milk transport by semitrailer drives the three-axle truck with a payload of $16,000 \mathrm{~kg}$ with a two-axle trailer with a payload of 9,000 kg from the dairy processor to the barycenter of the milk draw area, hangs the trailer there, collects milk from milk producers, fills the trailer and the semitrailer by pumping, hitches the trailer and, drives back to the dairy processor at least once during the milk collection cycle for cleaning reasons. For payload optimization reasons, the milk transport trailer has no milk intake equipment and has a payload of $28.000 \mathrm{~kg}$.

The outward and the return run to the milk draw area involves the time required and the full costs for the outward and the return run from the dairy processor to the barycenter of the milk draw area. Thereby, the distance in the present milk logistics scenario is $100 \mathrm{~km}$. Milk collection between milk producers describes the time needed and the cost of milk collection between the 100 milk producers with an average milk production of $1.500 \mathrm{~kg} / \mathrm{day}$. For this, the average distance between the milk producers in intermediate producer removal class $\mathrm{A}$ is $2,5 \mathrm{~km}$, in intermediate producer removal class B $5,0 \mathrm{~km}$. The on-farm-routine activity per milk intake includes the time required for the uptake and release for the milk intake per milk producer, which is 1,56 min per milk producer. The performance of the milk on-farm pumping is based on $615 \mathrm{~kg} / \mathrm{min}$. The routine activity per milk transfer operation includes the time required for the uptake and devise of the milk pumping device, which is 1.97 min per pumping operation. The pumping rate is estimated at $1.114 \mathrm{~kg} / \mathrm{min}$. The routine activities required to discharge the raw milk in the dairy processor to check the raw milk's workability necessitate 15,83 min per discharge. The time required for cleaning is estimated 33,66 min per discharge. The unloading capacity of the raw milk in the dairy is estimated to be $906 \mathrm{l} / \mathrm{min}$ (Schmid et al. 2018).

The calculation of the specific $\mathrm{CO}_{2}$ emissions of the milk logistics is realized by the weel-to-wheel method. This includes the direct $\mathrm{CO}_{2}$ emissions from diesel combustion and the indirect $\mathrm{CO}_{2}$ emissions released in fuel processing and fuel logistics (Kranke et al. 2011).

$$
\mathrm{G}_{\mathrm{T}}=\mathrm{F}^{*} \mathrm{~g}_{\mathrm{t}}
$$

In this formula $\mathrm{G}_{\mathrm{T}}$ sets the weel-to-wheel carbon emissions in $\mathrm{kg} \mathrm{CO}_{2}$-equivalent $\left(\mathrm{CO}_{2}\right)$; $\mathrm{F}$ the diesel consumption in 1 ; and $\mathrm{g}_{\mathrm{t}}$ is the tank-to-wheel carbon emission factor $(3,15 \mathrm{~kg} \mathrm{CO} 2 \mathrm{e} / \mathrm{l}$ fuel).

Specific milk logistics costs and $\mathrm{CO}_{2}$ emissions are determined for three different milk collection cycles, two intermediate producer removals and two different milk logistics chains for a six-day period. Thus, during the analysis period, $900.000 \mathrm{~kg}$ of milk must be collected from milk producers and transported to the dairy processor. At the one-day milk collection cycle (I), milk producers are approached daily, six times during the analysis period. Every second day, and thus three times during the analysis period, milk is collected by dairy farmers at the twoday milk collection cycle (II). The three-day milk collection cycle (III) is characterized by a milk collection every third day and thus a double milk collection during the observation period. Table one below summarizes the analyzed scenarios. 
Table 3. Scenarios between intermediate producer removal and milk collection cycle.

Intermediate producer removal A

$(2,5 \mathrm{~km})$
Intermediate producer removal B

$(5,0 \mathrm{~km})$

$\begin{array}{llc}\text { One-day milk collection cycle (I) } & \mathrm{AI}^{*}, \mathrm{AI} \# & \mathrm{BI} \text {, BI\# } \\ \text { Two-day milk collection cycle (II) } & \text { AII*, AII\# } & \text { BII*, BII\# } \\ \text { Three-day milk collection cycle (III) } & \text { AIII*, AIII\# } & \text { BIII*, BIII\# }\end{array}$

*Process variant three-axle truck with two-axle trailer broken

\# Process variant three-axle truck with two-axle trailer and semitrailer broken

The abbreviations shown in Table one serve as a basis for the descriptions in the result section.

\section{Results and Discussion}

The specific full costs of milk logistics for intermediate producer removal class A (blue) and intermediate producer removal claas B (red) are shown in Figure 1 below.

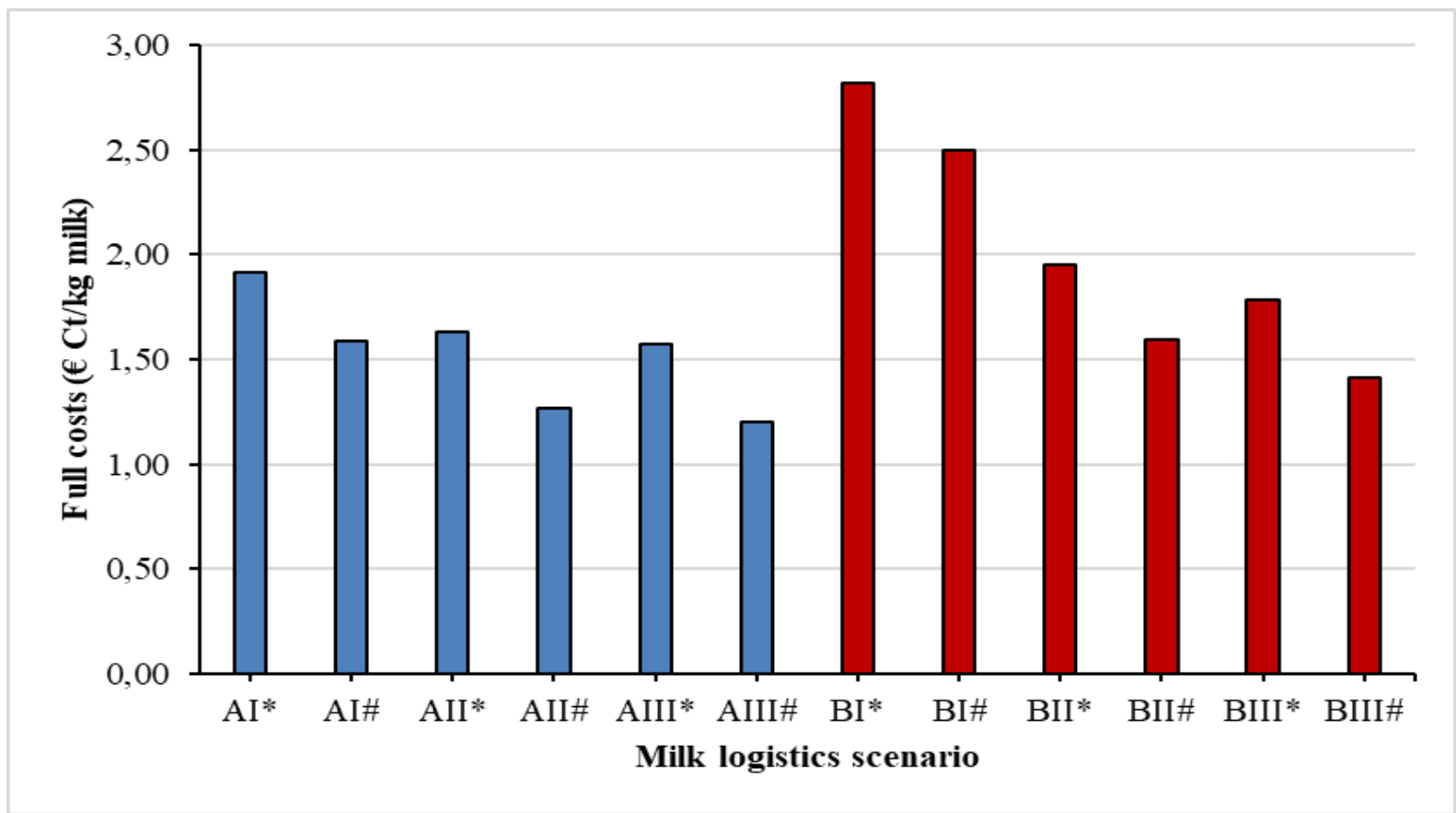

Figure 1. Milk logistics full costs for intermediate producer removal scenario class A (blue) and intermediate producer removal scenario B class (red).

The full costs of milk logistics in intermediate producer removal class A vary between 1,20 €Ct/kg milk in the milk logistics scenario AIII\# and $1,91 € \mathrm{Ct} / \mathrm{kg}$ in the milk logistics scenario AI*. In intermediate producer removal class $\mathrm{B}$, the full costs of milk logistics range from $1,41 € \mathrm{Ct} / \mathrm{kg}$ in the milk logistics scenario BIII\# to $2,82 € \mathrm{Ct} / \mathrm{kg}$ in the milk logistics scenario $\mathrm{BI}^{*}$. The $100 \%$ higher intermediate producer removal in intermediate producer removal class $\mathrm{B}$, compared to intermediate producer removal class $\mathrm{A}$, demonstrates the higher full cost of milk logistics within each milk collection cycle. The reduction of the absolute collection distance during the six-day analysis period reasons the decrease in the full cost of milk logistics with the extension of the milk collection cycle in both intermediate producer removal classes. The relative transport travel reduction of $8,3 \%$ in the process variant three-axle truck with two-axle trailer and milk transport semitrailer broken compared to the process variant three-axle truck with two-axle trailer broken justified in both intermediate producer removals within a milk collection cycle the lower full cost of milk logistics process variant three-axle truck with two-axle trailer and milk transport semitrailer broken. 
The marginal differences between the lowest and highest full cost of the milk logistics scenario, both within the intermediate producer class A and intermediate producer class B, are shown in table two below.

Table 4. Margins between lowest and highest full costs of milk logistics for intermediate producer removal class A and intermediate producer removal class B.

\begin{tabular}{lccc}
\hline Account costing & Unit & $\begin{array}{c}\text { Intermediate producer } \\
\text { Removal class A }\end{array}$ & $\begin{array}{c}\text { Intermediate producer } \\
\text { removal class B }\end{array}$ \\
\hline Lowest full costs of milk logistics & $€ C t / \mathrm{kg}$ milk & 1,20 & 1,41 \\
Lowest full costs of milk logistics & $€ /$ analysis period & $10.793,15$ & $12.701,13$ \\
Highest full costs of milk logistics & $€ C t / \mathrm{kg}$ milk & 1,91 & 2,82 \\
Highest full costs of milk logistics & $€ /$ analysis period & $17.213,77$ & $25.382,87$ \\
Margin & $€ C t / \mathrm{kg}$ milk & 0,71 & 1,41 \\
Margin & $€ /$ analysis period & $6.420,62$ & $12.681,75$ \\
\hline
\end{tabular}

By optimizing the process variant and the milk collection cycle, from the point of view of a dairy processor in intermediate producer removal class $\mathrm{A}$, it is possible to save $6.421 €$ /analysis period and in intermediate producer removal class B $12.682 €$ /analysis period. As already mentioned, in both intermediate producer removal classes, the process variant three-axle truck with two-axle trailer and milk transport semitrailer broken down in the threeday pick-up interval has the lowest full cost of milk logistics. Therefore, the following description and discussion of the milk collection cycle-dependent surcharges based on the process variant three-axle truck with two-axle trailer and milk transport semitrailer broken.

In intermediate producer removal class $\mathrm{A}$, the full costs of milk logistics will be reduced by $0,32 € \mathrm{Ct} / \mathrm{kg}$ of milk or $19,97 \%$ by extending the one to a two-day milk collection cycle. In intermediate producer removal class $\mathrm{B}$, this economic savings potential is $0,90 € \mathrm{Ct} / \mathrm{kg}$ milk or $36,23 \%$ from the point of view of a dairy processor. These margins could, from the point of view of the dairy processor, be given to milk producers as much as possible for the additional costs of increasing milk storage capacity and longer refrigeration duration resulting from the extension of the milk collection cycle. The milk producer's extra milk allowance surcharge of $0,15 € \mathrm{Ct} / \mathrm{kg}$ milk granted by many dairy processors is below to the reported marginal surcharges in both intermediate producer removal classes. The marginal surcharge imposed in intermediate producer removal scenario $\mathrm{A}$ is equal to the marginal surcharge of 0,30 - 0,40 €Ct/kg milk levied by Betz and Weindlmaier (Betz und Weindlmaier 2006). The results of both scenarios illustrate the increase in the economic savings potential by extending the collection interval with increasing of the intermediate producer removal.

Extending from a two to a three-day triggered milk chain reduces the full costs of milk logistics in the intermediate producer removal scenario A by $0,07 € \mathrm{Ct} / \mathrm{kg}$ or $5,58 \%$. This extension of the milk collection cycle in scenario B provides an economic potential saving of $0,18 € \mathrm{Ct} / \mathrm{kg}$ milk or $11,28 \%$. The marginal surcharge of $0,5 € \mathrm{Ct} / \mathrm{kg}$ for the extension of the two-day milk collection cycle to a three-day milk collection cycle, as reported by the organic processor Söbbeke, is also below the differences of $0,72 € \mathrm{Ct} / \mathrm{kg}$ milk (intermediate producer removal class $\mathrm{A}$ ) and $1,38 € \mathrm{Ct} / \mathrm{kg}$ of milk (intermediate producer removal class B). From these results, it is obvious that the economic saving potential increases by increasing of the milk collection cycles as the inter-generator removal increases because, at the same time, the absolute distance of inter-generator removal decreases within the six-day analysis period. From a practical point of view, economic advantages can be achieved by extending the milk collection interval from the point of view of a dairy processor, as long as the individual milk quantity per collection interval does not exceed the payload of the process variant.

The results of specific $\mathrm{CO}_{2}$ emissions for intermediate producer removal scenario class $\mathrm{A}$ (red) and intermediate producer removal scenario class B (blue) are shown in Figure two below. 


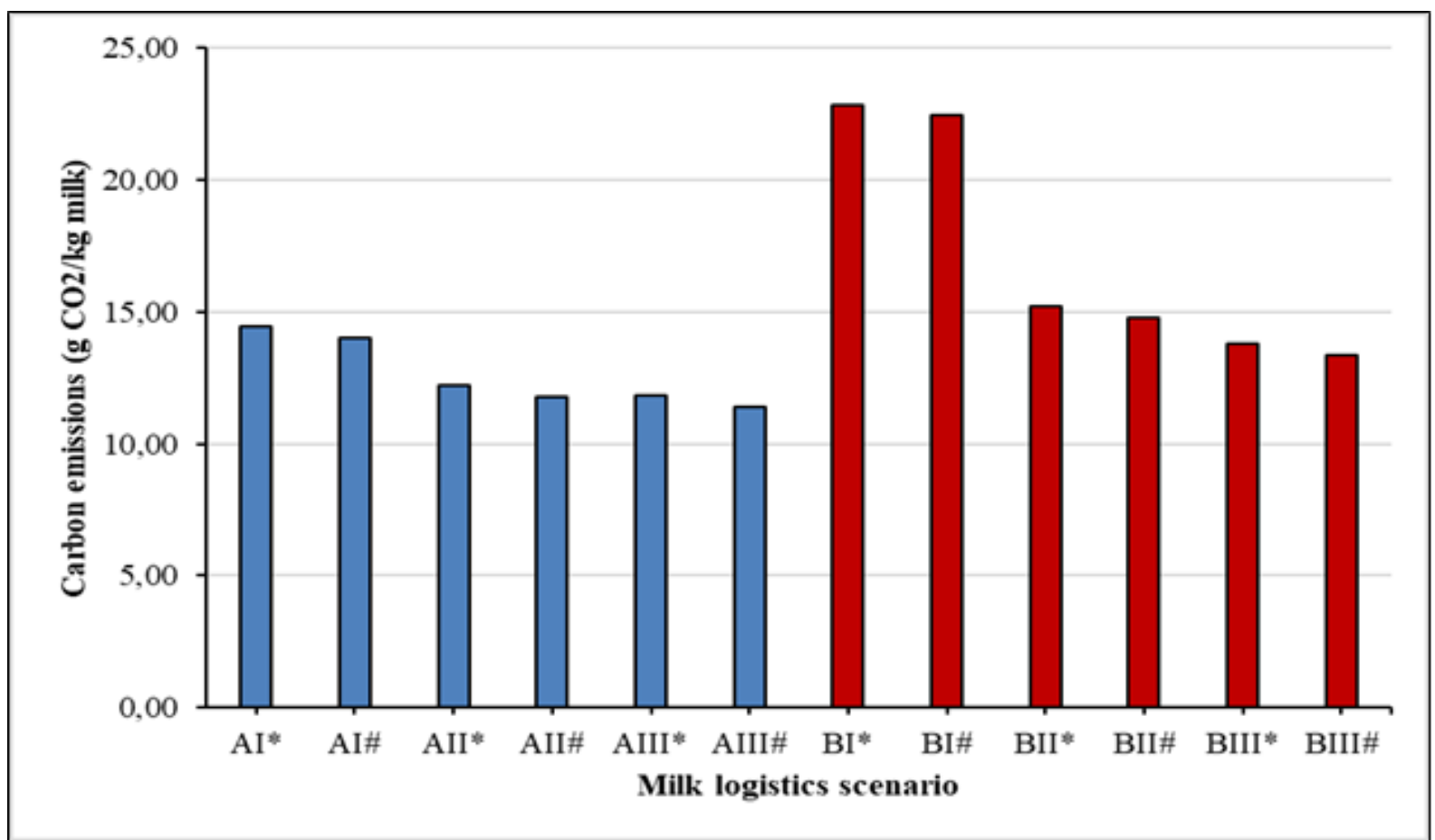

Figure 2. Milk logistics carbon emissions for intermediate producer removal class A (blue) and intermediate producer removal class B (red).

The $\mathrm{CO}_{2}$ emissions in the intermediate producer removal class A range from $11,38 \mathrm{~g} \mathrm{CO}_{2} / \mathrm{kg}$ milk in the milk logistics scenario AIII\# to $14,42 \mathrm{~g} \mathrm{CO}_{2} / \mathrm{kg}$ milk in the milk logistics scenario AI*. In intermediate producer removal scenario $\mathrm{B}$, the $\mathrm{CO}_{2}$ emissions from milk logistics differ between $13,35 \mathrm{~g} \mathrm{CO}_{2} / \mathrm{kg}$ milk in the milk logistics scenario BIII\# and 22,86 g CO$/ / \mathrm{kg}$ milk in the milk logistics scenario $\mathrm{BI}^{*}$. The $\mathrm{CO}_{2}$ emissions of intermediate producer removal class $\mathrm{B}$ have $18 \%$ to $37 \%$ higher $\mathrm{CO}_{2}$ emissions from milk logistics compared to the $\mathrm{CO}_{2}$ emissions of intermediate remover class $\mathrm{A}$ within the milk collection cycles, because the average intermediate producer removal in class B is $100 \%$ higher than in intermediate producer removal in class A. Simultaneously, extending the milk collection cycle up to three days due to milk-collection route savings will result in a reduction in $\mathrm{CO}_{2}$ emissions of up to $17 \%$ in intermediate producer removal scenario $\mathrm{A}$ and up to $40 \%$ in intermediate producer removal scenario B. As can be seen in figure two, the process variant three-axle truck with two-axle trailer and milk transport semitrailer illustrates lower specific $\mathrm{CO}_{2}$ emissions per $\mathrm{kg}$ of milk compared to the process variant three-axle truck with two-axle trailer due to the payload-optimized milk transport by milk transport semitrailer the number of total transport journeys during the analysis period is reduced by 8,3 $\%$. Table three below exemplifies the $\mathrm{CO}_{2}$ limit differences between the milk logistics chains with the lowest and highest $\mathrm{CO}_{2}$ emissions, respectively, for the two intermediate producer removal classes.

Table 3. Margins between lowest and highest carbon emissions of milk logistics for intermediate producer removal class A and intermediate producer removal class B.

\begin{tabular}{lccc}
\hline Carbon emmision calculation & Unit & $\begin{array}{c}\text { Intermediate producer } \\
\text { removals class A }\end{array}$ & $\begin{array}{c}\text { Intermediate producer } \\
\text { removals class B }\end{array}$ \\
\hline Lowest carbon emissions of milk logistics & $\mathrm{g} \mathrm{CO}_{2} / \mathrm{kg} \mathrm{milk}$ & 11,38 & 13,35 \\
Lowest carbon emissions of milk logistics & $\mathrm{kg} \mathrm{CO}_{2} /$ analysis period & $10.238,39$ & $12.013,16$ \\
Highest carbon emissions of milk logistics & $\mathrm{g} \mathrm{CO}_{2} / \mathrm{kg} \mathrm{Milch}$ & 14,42 & 22,86 \\
Highest carbon emissions of milk logistics & $\mathrm{kg} \mathrm{CO}_{2} /$ analysis period & $12.977,99$ & $20.576,76$ \\
Margin & $\mathrm{g} \mathrm{CO}_{2} / \mathrm{kg} \mathrm{Milch}$ & 3,04 & 9,52 \\
Margin & $\mathrm{kg} \mathrm{CO}_{2} /$ analysis period & $2.739,60$ & $8.563,60$ \\
\hline
\end{tabular}

The optimization potential of selecting the process variant with lowest $\mathrm{CO} 2$ Emissions compared to the process variant with the highest $\mathrm{CO}_{2}$ emissions is $2.740 \mathrm{~kg} \mathrm{CO}_{2}$ intermediate producer removals class A and $8.564 \mathrm{~kg}$ $\mathrm{CO} 2$ in intermediate producer removals class B during the six-day analysis period. Since the process variant threeaxle truck with two-axle trailer and semitrailer have lower carbon emissions than the process variant three-axle truck with two-axle trailer, their $\mathrm{CO}_{2}$-emitting savings potential is discussed below. In this process variant, the carbon emissions can be reduced by $2,23 \mathrm{~g} / \mathrm{kg}$ milk or $15,88 \%$ in the intermediate producer removal scenario A by switching from the one- to the two-day triggered milk logistic chain. Extending the one-day triggered milk 
logistic chain to the two-day triggered milk logistic chain in intermediate producer removal scenario B provides a $\mathrm{CO}_{2}$ savings potential of 7,68 $\mathrm{g} / \mathrm{kg}$ of milk, or $34,19 \%$. By extending a two-day triggered milk logistic chain to the three-day milk logistic chain, $1,43 \mathrm{~g} \mathrm{CO}_{2} / \mathrm{kg}$ milk or $9,68 \%$ can be saved in intermediate producer removal class $\mathrm{B}$. In comparison between the two distance scenarios, the $\mathrm{CO}_{2}$ emissions of the individual process variants in the milk collection cycle $\mathrm{B}$ are higher than in the milk collection cycle $\mathrm{A}$, because the distance between dairy farmers is $100 \%$ higher. Thus, the specific $\mathrm{CO}_{2}$ emissions increase with increasing distance between the dairy farmers.

A study for the entire Irish dairy industry showed $\mathrm{CO}_{2}$ emissions to milk logistics between milk producer and dairy from 3,78 to 4,45 $\mathrm{CO}_{2} \mathrm{~g} / 1$ milk (Quinlan et al. 2012). The $\mathrm{CO}_{2}$ emissions calculated for the two milk logistics scenarios in this paper are higher than those reported by Quinlan et al. (2012) analyzed $\mathrm{CO}_{2}$ emissions for the Irish dairy industry. The higher $\mathrm{CO}_{2}$ emissions recorded are due to the greater distance between the milk draw area and the dairy and between the milk producers, which reflects typical South German structures. In addition, for the in Irland used process variants semitrailer unbroken, the components of milk logistics outward/return run to the trailer/semitrailer, on-trailer-routine activities and on-trailer pumping are not necessary.

The comparison of the classified milk collection cycles and process variants show a match between the lowest full costs and the lowest $\mathrm{CO}_{2}$ emissions as well as between the highest full costs and highest $\mathrm{CO}_{2}$ emissions, because the cost block fuel accounts for about $50 \%$ of the full cost of a truck and $\mathrm{CO}_{2}$ emissions are calculated via this consumption-based method. Within the intermediate producer removal scenarios and milk collection cycles, the full costs of milk logistics are reduced more by optimizing the process variant than by $\mathrm{CO}_{2} \mathrm{emissions}$, since the lower costs of the dairy transport process variant are mainly due to the higher payload and higher hourly wages of the truck driver compared to the milk transport trailer driver. In addition, in the process variant with the milk transport semitrailer $90 \%$ more milk is pumped during the analysis period, which requires fossil energy.

\section{Conclusions}

This contribution determines the milk collection cycle-dependent and process variant-dependent full costs and $\mathrm{CO}_{2}$ emissions of the milk logistics between a dairy processor and two differently structured milk draw areas. With the increase in the distance between the milk producers, with a constant transport distance between the milk draw area and the dairy processor, the full costs and $\mathrm{CO}_{2}$ emissions of the milk logistics increase when comparing the same collection intervals. The extension of the daily milk collection cycle to a three-day milk collection cycle, and the selection of an optimal process variant of the milk logistics provide an economic saving potential of up to $50 \%$, a $\mathrm{CO}_{2}$ saving potential of up to $42 \%$. Thus, the technical progress in the field of milk logistics, milk storage and milk cooling technology contribute as well as to cost optimization as to the greenhouse gas reduction.

Since milk producers incur marginal costs for the milk tanker's higher tank capacity and longer refrigeration time compared to the one- or two-day milk collection cycle, additional costs for further milk production are considered by a dairy producer to be a fair milk collection cycle-dependent supplement from a milk producer perspective. The quantification of an optimal triggered milk logistic chain and an optimal process variant of the milk logistics with simultaneous analysis of the $\mathrm{CO}_{2}$ emissions provide first optimization possibilities of the logistics planning between milk producer and dairy processor without consideration of an overall scenario with defined milk intake and milk discharge possibilities. In addition, the results and the methods can be used for sustainable milk producer acquisition serve for practical use in dairy companies.

\section{References}

Betz, J.; Weindlmaier, H.: Kosteneinsparung durch die Umstellung auf die zweitägliche Milcherfassung. In: Milchwirtschaftliche Forschung Weihenstephan Jahresbericht 2006.

Europäische Union (25.06.2004): Verordnung (EG) Nr. 853/2004 Des Europäischen Parlaments und des Rates vom 29. April 2004 mit spezifischen Hygienevorschriften für Lebensmittel tierischen Ursprungs. Verordnung (EG) Nr. 853/2004.

Gudehus, T. (2011): Logistik. Grundlagen - Strategien - Anwendungen. 4., aktualisierte Aufl. 2010. Berlin, Heidelberg: Springer-Verlag Berlin Heidelberg.

Janze, C.; Theuvsen, L.; Schmidt, C. (2017): Konjunkturbarometer Agribusiness in Deutschland 2018.

Kranke, A.; Schmied, M.; Schön, Andrea D. (2011): CO2-Berechnung in der Logistik. Datenquellen, Formeln, Standards. 1. Aufl., Stand August 2011. München: Vogel.

Liste, P. (2011): Kommt die dreitägige Milchabholung? Zwei Molkereien in Deutschland erfassen die Milch nur noch alle drei Tage. Kann das bundesweit Schule machen? Online verfügbar unter https://www.topagrar.com/archiv/Kommt-die-dreitaegige-Milchabholung-535054.html, zuletzt aktualisiert am 01.03.2011, zuletzt geprüft am 25.03.2018.

Quinlan, C.; Keane, M.; O'Connor, D.; Shalloo, L. (2010): The transport implications for Ireland of the 
elimination of the milk quota regime in 2015. In: Proceedings of ITRN 2010, S. 1-15.

Quinlan, C.; Kaene, M.; Connor, D.; Shalloo, L. (2012): Milk transport costs under differing seasonality assumptions for the Irish dairy industry. In: International journal of dairy technology 2012 (65), S. 22-31.

Schmid, M.; Wörz, S.; Bernhardt, H. (2018): Kalkulationsrechnung für ein optimales Milch Supply Chain Management. In: 21. Arbeitswissenschaftliches Kolloquium: Arbeit in der digitalen Transformation, S. 186-195.

Steinhauser, H.; Langbehn, C.; Peters, U. (1992): Allgemeiner Teil. Produktionsgrundlagen, Produktionstheorie und Rechnungssysteme mit Planungsrechnungen; 75 Übersichten. 5., neubearb. Aufl. Stuttgart: Ulmer (UTB für Wissenschaft Uni-Taschenbücher Agrarwissenschaften, 113).

Strogies, M.; Gniffke, P. (2017): Submission under the United Nations Framework Convention on Climate Change and the Kyoto Protocol 2017.

Weindlmaier, H.; Betz, J. (2009): Zur aktuellen Situation der Milcherfassung in Deutschland und Österreich im Jahr 2007. In: Deutsche Milchwirtschaft 60, S. 12-17. 


\title{
Preliminary Study on Traction Efficiency of an Agricultural Tractor Equipped With an Extendable Ballast Holder
}

\author{
Massimiliano Varani ${ }^{\text {a, }}$, Michele Mattetti ${ }^{\text {a }}$, Giovanni Molari ${ }^{\text {a }}$ \\ ${ }^{a}$ Department of Agricultural and Food Sciences -University of Bologna, viale G. Fanin 50, 40127,Bologna Italy \\ * Corresponding author. Email: massimiliano.varani@unibo.it
}

\begin{abstract}
In the last decades, agricultural tractor optimization was the main topic of countless studies. In fact, a more efficient tractor leads to higher durability and reduced energy losses, thus saving on fuel consumption. In particular, many studies were conducted on the optimal weight distribution among tractor front and rear axles because this aspect deeply influences traction efficiency. Therefore, in recent years some agricultural machinery manufacturers tried to develop innovative ballast systems to obtain the maximum traction efficiency in every field operation and soil condition. One example of these innovative ballast systems is a special ballast holder that could be installed both on front or rear three point hitch, able to move the ballast up to $1 \mathrm{~m}$ over its original position in the longitudinal direction. This movement is granted by a mechanical linkage actuated by the tractor hydraulic system. The aim of this paper is the tractive performance comparison of a tractor equipped with this special ballast holder between its two extreme configurations, device fully closed and device fully extended. Field tests were performed on a loam soil with a 4WD tractor with a maximum engine power of $194 \mathrm{~kW}$ and a ballasted weight of $9590 \mathrm{~kg}$. With each ballast holder configuration, drawbar tests were carried out by towing another tractor with similar weight as a loading unit. Tractor operating parameters were acquired through a CAN-Bus data logger, while drawbar pull and speed were acquired respectively with a load cell and a GPS receiver. The analysis of the results shows that a higher traction efficiency was reached with the device in the fully extended configuration, especially in high slip condition. The obtained results confirm that the tractor weight distribution on the axles influence its traction efficiency.
\end{abstract}

Keywords: agricultural tractor, ballast holder, tractive efficiency, weight distribution, field tests

\section{Introduction}

The food demand growth due to the increase of the world population implies that modern agriculture has to increase its productivity and efficiency at the same time (Olesen and Bindi, 2002). One method to overcome this issue is the usage of more powerful and efficient machines, for this reason in the last decades front wheel assist (FWA) tractors gained more and more importance compared to two wheel drive (2WD) tractors. (Kutzbach, 2000). However, an improper tractor ballasting could worsen significantly efficiency and productivity of the machine. The estimation of the correct ballasting is strongly correlated to many parameters, such as soil conditions, tires type, inflation pressure and field operation.

The first attempts to predict the correct ballasting for every working condition were based mainly on field experience (Bloome et al., 1983). Especially during the 70's, these empirical models were proposed by tractor manufacturers to their customers using simple tables that considered parameters like tractor weight, maximum power, size of tires and speed of the field operation. Another method to analyse the influence of tractor load distribution on traction efficiency is through the development of analytical models, most of them based on the traction equations developed by Brixius (Brixius, 1987). Tractive performances are strongly influenced by Gross Traction Ratio (GTR) that is the ratio between the traction force and the dynamic load on the wheel. Since tractors doesn't operate always in constant working conditions, a ballast configuration that generates a GTR of 0.54 is considered a good compromise, because it permits to have good traction performances in most of the conditions (Zoz, 1987). In recent years agricultural machineries manufacturers invested in the development of devices able to modify the tractor load distribution faster and more efficiently. An example of these innovative ballast systems is the "Multiplier Counterweight" designed by ALI s.r.l. (Anghiari, AR, Italy), a special ballast holder to move the ballast up to $1 \mathrm{~m}$ over its original position in the longitudinal direction. The aim of this paper is the tractive performance comparison of a tractor equipped with an extendable ballast holder between its two extreme configurations, device fully closed and device fully extended.

\section{Materials and Methods}

The tests were carried out with a New Holland T7.260 (Table 1) fitted with a "Multiplier Counterweight" ballast holder designed by ALI s.r.l. (Figure 1 and Figure 2). 
Table 31. Tractor characteristics.

\begin{tabular}{lc}
\hline Tractor & New Holland T7.260 \\
Max engine power $[\mathrm{kW}]$ & 194 \\
Max torque @ $1500 \mathrm{rpm}[\mathrm{Nm}]$ & 1349 \\
Unballasted weight $[\mathrm{kg}]$ & 8590 \\
\hline
\end{tabular}

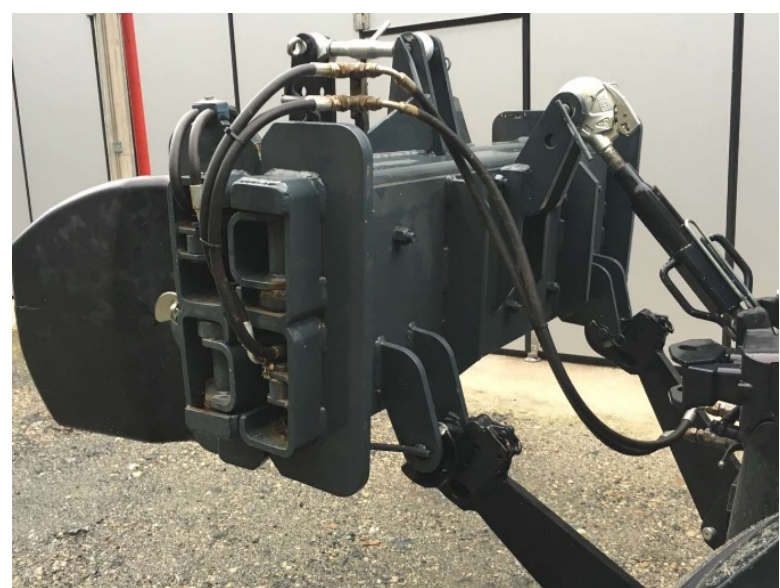

Figure 93. Ali s.r.l. "Multiplier Counterweight" in fully closed configuration fitted with a $500 \mathrm{~kg}$ ballast.

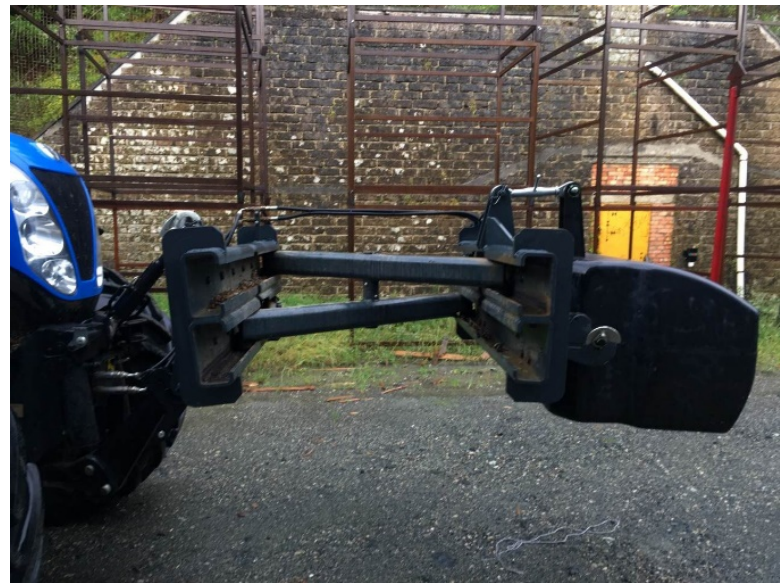

Figure 94. Ali s.r.l. "Multiplier Counterweight" in fully extended configuration fitted with a $500 \mathrm{~kg}$ ballast.

This special ballast holder has been installed on the front three point hitch of the tractor with a $500 \mathrm{~kg}$ ballast. The movement of the ballast in the longitudinal direction is granted by a mechanical linkage actuated by the tractor hydraulic remotes. The weight of the "Multiplier Counterweight" without any ballast connected is $500 \mathrm{~kg}$ and the maximum reachable extension of the device is $1 \mathrm{~m}$. The weight distribution on the tractor axles with the ballast holder fully closed and with the ballast holder fully extended (named respectively as "FC" and "FE" in the following) is shown in Table 2.

Table 32. Tractor equipped with extendable ballast holder weight distribution in static condition.

\begin{tabular}{cccc}
\hline Test condition & Total tractor weight [kg] & Weight on the front axles [\%] & Weight on the rear axles [\%] \\
\hline Ballast holder FC & 9590 & 56.31 & 43.69 \\
Ballast holder FE & 9590 & 58.76 & 41.24 \\
\hline
\end{tabular}

The New Holland tractor equipped with the ballast holder was connected to a Case IH Maxxum 115 properly ballasted in order to test the tractive performances of the first tractor. This layout permits to use the towed tractor (Case Maxxum 115) as a loading unit that gradually increases the resistance force acting on the tractor under test (Figure 3). The two tractors were joined by a steel chain and a load cell (full-scale range $=3 * 10^{5} \mathrm{~N}$ ) to measure the draft force during the tests. 


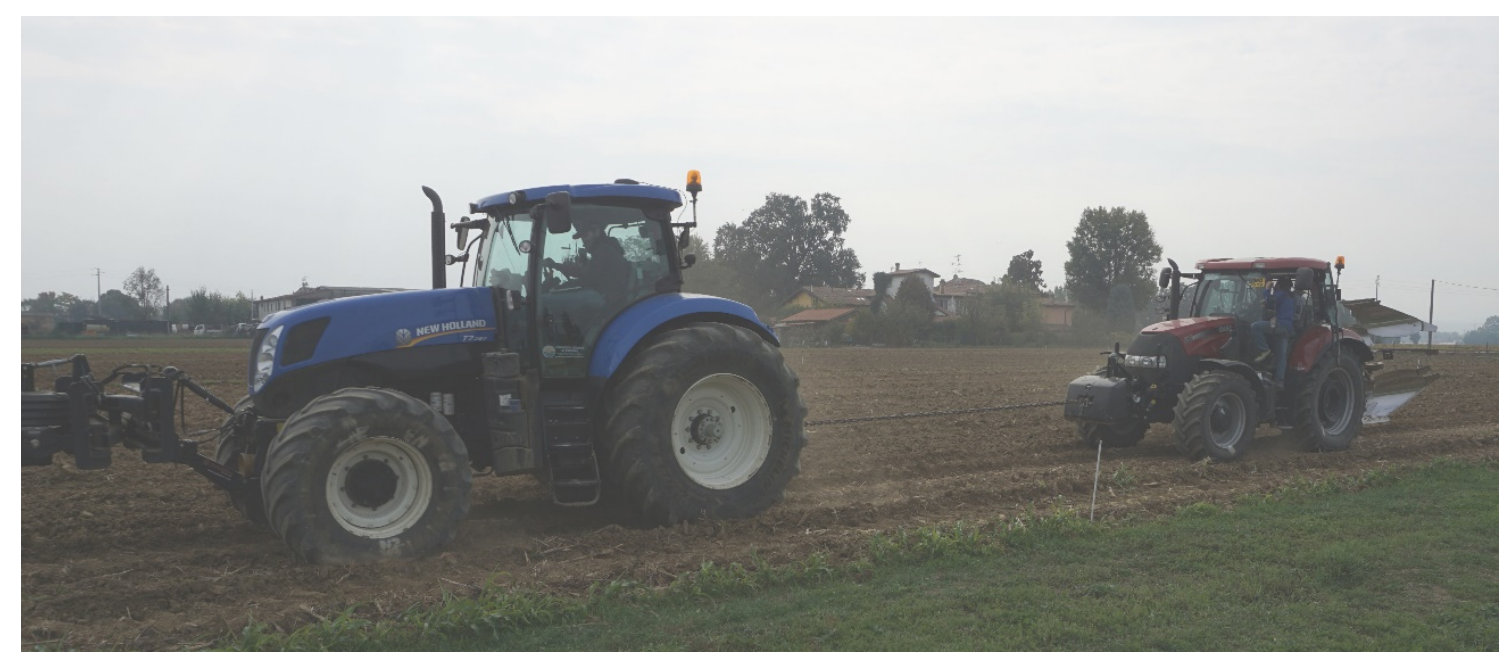

Figure 95. Tractive performance test performed with a Case IH Maxxum 115 used as a loading unit.

Moreover, on the New Holland tractor a GPS receiver (IPESpeed, IPETronik GmbH, Baden Baden, Germany) and CAN-Bus data logger (CanCase XL Log, Vector Informatik,GmbH, Struttugart, Germany) were installed, so that performance parameters such as speed, slip and engine power were acquired.

The tests were carried out in FC and FE ballast holder configurations on a loam soil as classified by the USDA Textural Soil Classification (USDA, 1987). The liquid limit (LL) and the plastic limit (PL) of the soil were $36.16 \%$ and $22.6 \%$, respectively. The mean value of moisture content was $16.87 \%$ and the standard deviation was $1.68 \%$ (ASTM, 2010).

Tractive Efficiency (TE) was calculated with Eq. 1

$$
T E=\frac{D^{*} V}{P}
$$

where $\mathrm{D}$ is the draft force, $\mathrm{V}$ is the actual speed of the tractor and $\mathrm{P}$ is the power available at the axles.

The calculated values of TE in each test condition were fitted with an exponential regression curve in relationship with the slip percentage.

\section{Results and Discussion}

The measured values of TE in relationship with the tractor slip percentage in both tested conditions are reported in Figure 4.

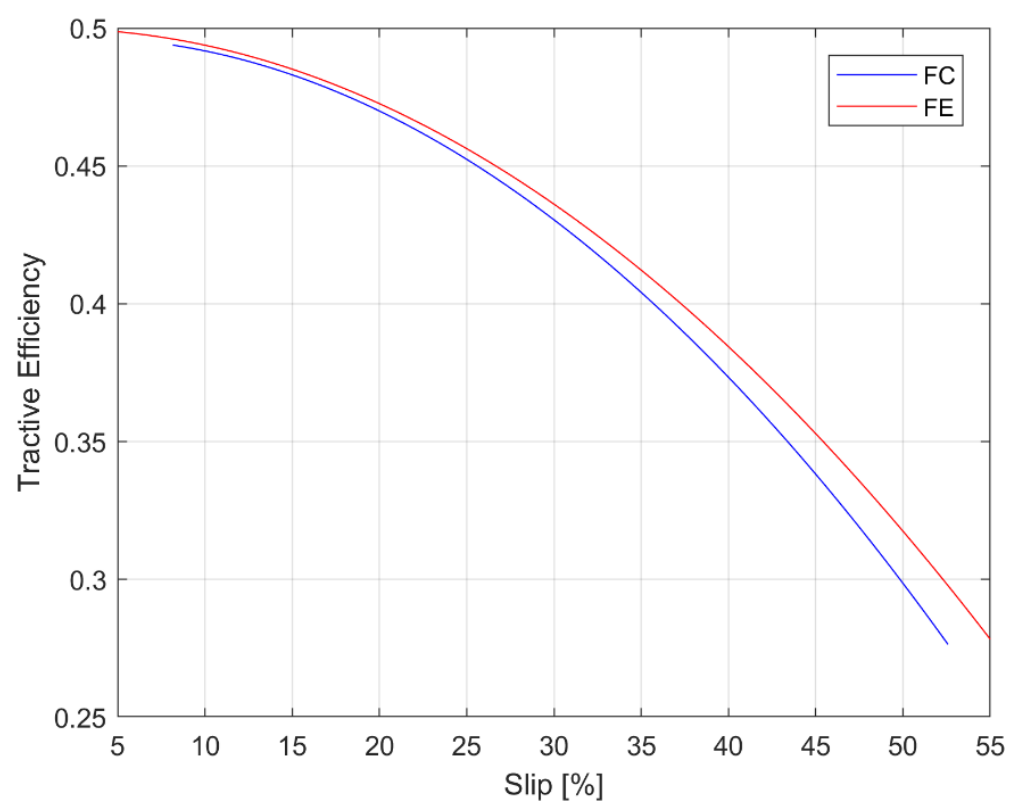

Figure 96. Tractive efficiency of the New Holland tractor equipped with the extendable ballast holder in Fully Closed(FC) and Fully Extended(FE) configuration. 
The coefficients of determination $\left(\mathrm{R}^{2}\right)$ of the exponential regression curves were 0.90 and 0.95 for the FC and the FE condition respectively. Test conditions with slip percentage under $5 \%$ were not considered due to the difficulties to obtain such conditions in a field test. One can note that at low slip values TE has the highest values and the two curves almost overlap. For example, at $10 \%$ slip percentage the difference in terms of TE values between FC and FE is less than 0.01. With higher slip percentages the two curves are no longer overlapping and the difference increase with the slip percentage. For example, at $50 \%$ slip percentage the difference in terms of TE values between FC and FE is around 0.03. The mild difference in terms of TE between FC and FE configuration is due to the fact that the weight transfer on the tractor axles produced by the ballast holder is limited (Table 2).

\section{Conclusions}

In this paper, a preliminary study has been made on the tractive efficiency of an agricultural tractor equipped with an extendable ballast holder. The analysis of the results shows that the change of weight distribution between the tractor axles due to the use of an extendable ballast holder slightly influences the tractive efficiency of the tractor, especially with high slip percentages. The results introduced in this paper will be useful for the evaluation of the benefits in terms of tractive efficiency generated by the adoption of an extendable ballast holder.

A further development of this work may be the arrangement of new field tests with a heavier ballast, in order to amplify the weight transfer effect produced by the extendable ballast holder on the tractor axles. A further future development may be the evaluation of the performances of an extendable ballast holder during real field operations.

\section{References}

ASTM, 2010. Test Methods for Liquid Limit, Plastic Limit, and Plasticity Index of Soils.ASTM International. Bloome, P.D., Summers, J.D., Khalilian, A., Batchelder, D.G., 1983. Ballasting recommendations for twowheel and four-wheel drive tractors [Tractor performance]. Microfiche Collect. USA.

Brixius, W.W., 1987. Traction prediction equations for bias ply tires. Am. Soc. Agric. Eng. Microfiche Collect.

Kutzbach, H.D., 2000. Trends in Power and Machinery. J. Agric. Eng. Res. 76, 237-247. https://doi.org/10.1006/jaer.2000.0574

Olesen, J.E., Bindi, M., 2002. Consequences of climate change for European agricultural productivity, land use and policy. Eur. J. Agron. 16, 239-262. https://doi.org/10.1016/S1161-0301(02)00004-7

USDA, 1987. Soil Mechanichs Level I

Zoz, F.M., 1987. Predicting tractor field performance (updated). Am. Soc. Agric. Eng. Microfiche Collect. USA. 


\title{
Biomass Heating System with Kerosene Boiler for Green House Heating
}

\author{
Yoshimichi Yamashita $^{\text {a,*, }}$, Kazuhiro Takekura ${ }^{b}$, Genta Kanai a, Kenichi Yakushidou ${ }^{\text {c }}$ \\ ${ }^{a}$ Tohoku Agricultural Research Center, Fukushima Research Station, National Agriculture and Food Research \\ Organization (NARO), Fukushima City, Fukushima Pref., Japan \\ ${ }^{\mathrm{b}}$ Central Region Agricultural Research Center, NARO, Tsukuba City, Ibaraki Pref., Japan \\ ${ }^{c}$ Kyushu Okinawa Agricultural Research Center, NARO, Koshi City, Kumamoto Pref., Japan \\ * Corresponding author. Email: yamashitay351@affrc.go.jp
}

\begin{abstract}
Biomass boiler system is important to substitute fossil fuel with woody biomass. Generally, the biomass boiler system has a heat storage tank filled with water in order to store hot water converted from combustion heat. With a heat storage tank, even a small burner can heat the greenhouse. However, according to the heating load, the biomass boiler is comparatively easy to control the quantity of woody biomass, but difficult to turn on and off; therefore, we developed a biomass heating system using a rotary kiln-type burner combined with kerosene boiler. The Heat storage tank of our system contained $8 \mathrm{~m}^{3}$ water, we kept the water from $30^{\circ} \mathrm{C}$ to $60{ }^{\circ} \mathrm{C}$ using Kerosene boiler. Furthermore, we made the water temperature rising to $70^{\circ} \mathrm{C}-90{ }^{\circ} \mathrm{C}$ by the biomass boiler with the rotary kiln-type burner. For heating the greenhouse, we used the radiator which was made by automobile component, and it provided warm air converted from the hot water. The heat output of the radiator was about $20 \mathrm{~kW}$ with $60{ }^{\circ} \mathrm{C}-80{ }^{\circ} \mathrm{C}$ water temperature. This system controlled switching on and off the fan of the radiator. Field tests were performed by heating a strawberry-cultivation greenhouse in Japan from December 2017 to March 2018. The greenhouse was double layer greenhouse. Outside temperature was from about $-10{ }^{\circ} \mathrm{C}$ to $10{ }^{\circ} \mathrm{C}$, and the minimum temperature was $-17^{\circ} \mathrm{C}$. The greenhouse was able to be heated to keep $10^{\circ} \mathrm{C}$ or $18^{\circ} \mathrm{C}$ at night, depending on the growing stages of strawberries. The temperature control accuracy in the greenhouse was $\pm 2^{\circ} \mathrm{C}$. When the water temperature was $70^{\circ} \mathrm{C}$ in daytime, the total amount of the heat radiation from the heat exchanger in the greenhouse was about 1,409 MJ from around 5:00 p.m. to 9:00 a.m.
\end{abstract}

Keywords: greenhouse heating, biomass boiler, woody biomass, heat storage tank, rotary kiln-type burner, radiator

\section{Introduction}

We devised a greenhouse heating system using biomass boiler to substitute fossil fuel with woody biomass during greenhouse heating for horticulture (Takekura et al., 2018). Our biomass boiler has a heat storage tank filled with water in order to store hot water converted from combustion heat. With a heat storage tank, even a small burner can heat the several greenhouses.

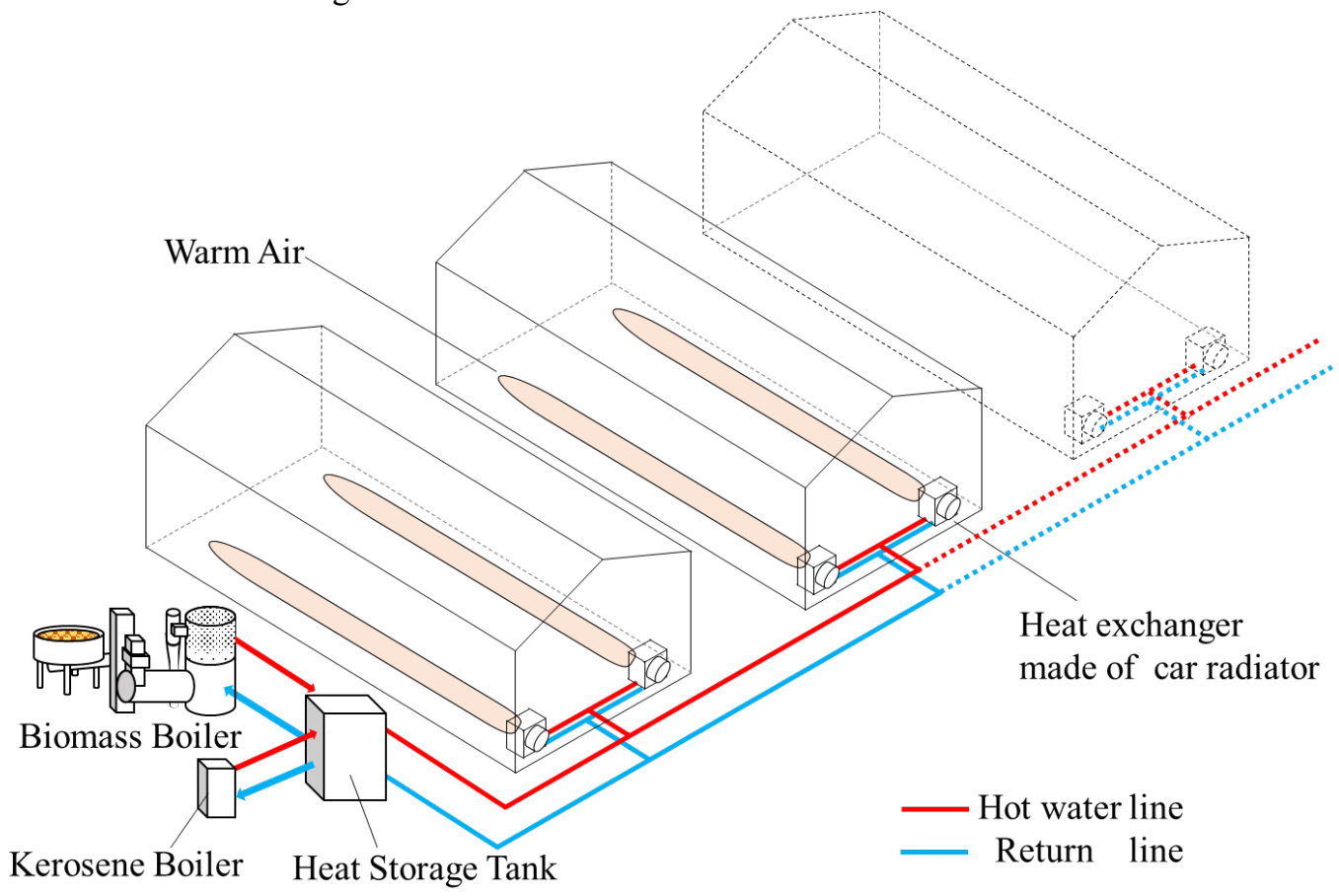

Figure 1. Concept of the Biomass Heating System with kerosene boiler. 
According to the heating load, the biomass boiler is able to control feeding the quantity of woody biomass; however, like any other biomass boiler system, turning on and off frequently during a day is difficult, makes combustion efficiency lower, and causes wasting biomass.

Generally, greenhouse which needs heating has a greenhouse heater using fossil fuel. In this case, the new biomass heating system is mainly used and the old greenhouse heater is used as a back up to ensure redundancy. On the other hand, it costs too much money to buy the both in building a new greenhouse with heater. Therefore, we improved our system, and developed a biomass heating system using a rotary kiln-type burner combined with kerosene boiler (Figure 1). Field test was conducted to examine its validity during a whole winter.

\section{Materials and Methods}

Greenhouse

We conducted a field test in the mountain area of Fukushima Pref. Japan. The greenhouse was double layer greenhouse used for high-bench strawberry culture. The size of the greenhouse is about $260 \mathrm{~m}^{3}$, and the production area was about $160 \mathrm{~m}^{2}$.

\section{Biomass Heating System with Kerosene Boiler}

The biomass heating system with kerosene boiler was located inside the greenhouse. The system consists of six component: a screw conveyor-type quantitative feeder, a rotary kiln-type burner (116 kW), a heat exchanger, a heat storage tank $\left(8 \mathrm{~m}^{3}\right)$, a kerosene boiler $(68.6 \mathrm{~kW})(\mathrm{PG}-7002$, CHOFU SEISAKUSHO Co.,Ltd), and two heat exchangers with fans for greenhouse heating (Figure 2). In this test, fuel for biomass boiler was wood pellets. Both the biomass boiler and the kerosene boiler can be used at the same time.

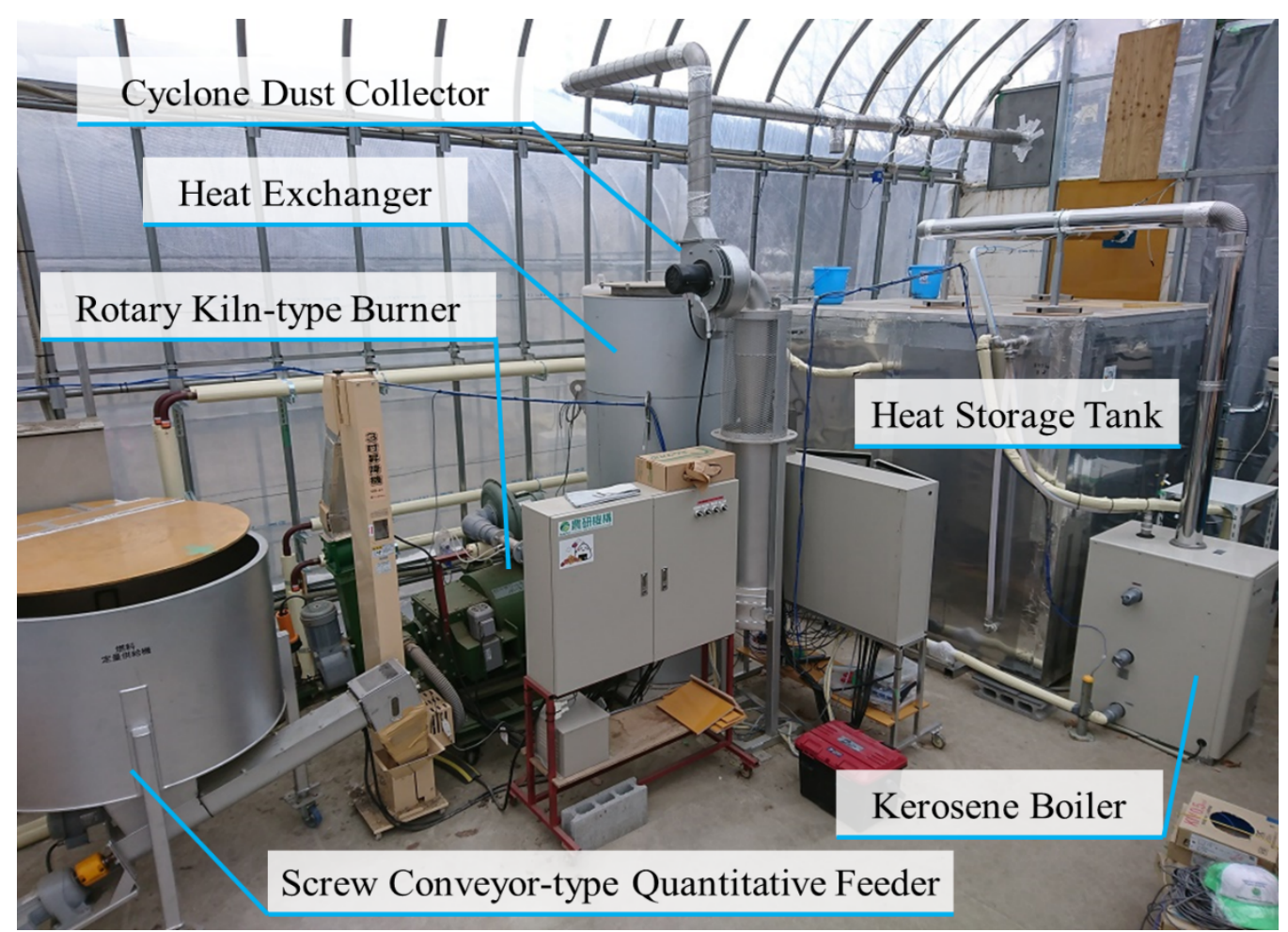

Figure 2. Biomass heating system with kerosene boiler.

Green house heating control procedure

For heating the greenhouse, we used two heat exchangers with fans made of the car radiator, and they provided warm air converted from the hot water. This system controlled switching on and off the fan of the radiator.

We used a low-cost force ventilated radiation shield NIAES-09S located at $1.5 \mathrm{~m}$ from the bottom in the centre of production area (Fukuoka et al., 2011). In the shield we put a temperature sensor (K-type thermocouple) and connected the temperature controller (E5CB, OMRON Corporation), which sent control output to the programmable relays (FL1F Smart Relay, IDEC Corporation) in order to control the temperature in the greenhouse. Also, we put another temperature sensor (U23-002, Onset Computer Corporation.) inside the shield, 
because the K-type thermocouple is used for control of the temperature controller. According to the growing stages of strawberries, the set temperature in the production area changed (Table 1).

Table 1. Set Temperature of Greenhouse heating.

\begin{tabular}{|c|c|c|c|}
\hline & Date & Daytime $\left[{ }^{\circ} \mathrm{C}\right]$ & Night $\left[{ }^{\circ} \mathrm{C}\right]$ \\
\hline First period & Dec.12-Dec.20 & 22 & 16 \\
\hline Second period & Dec.21-Feb. 8 & 22 & 18 \\
\hline Third period & Feb.9-Mar.31 & 22 & 10 \\
\hline
\end{tabular}

*Day time was 9:30 - 16:00 *Night was 16:00-9:30

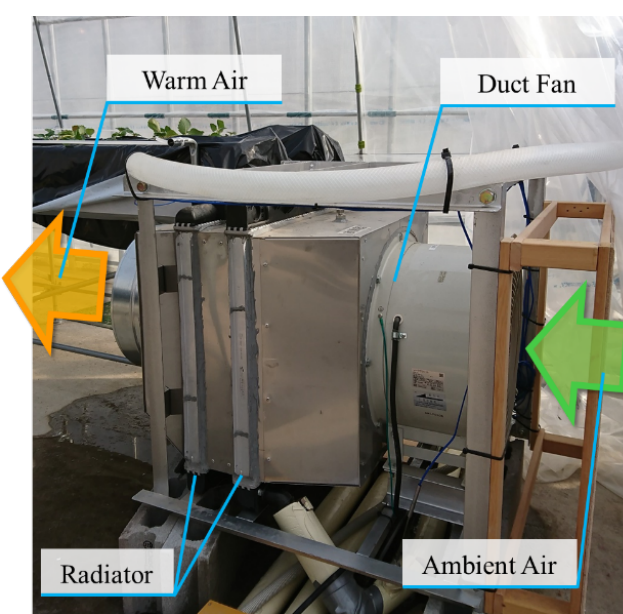

(a) Picture of the heat exchanger in a greenhouse

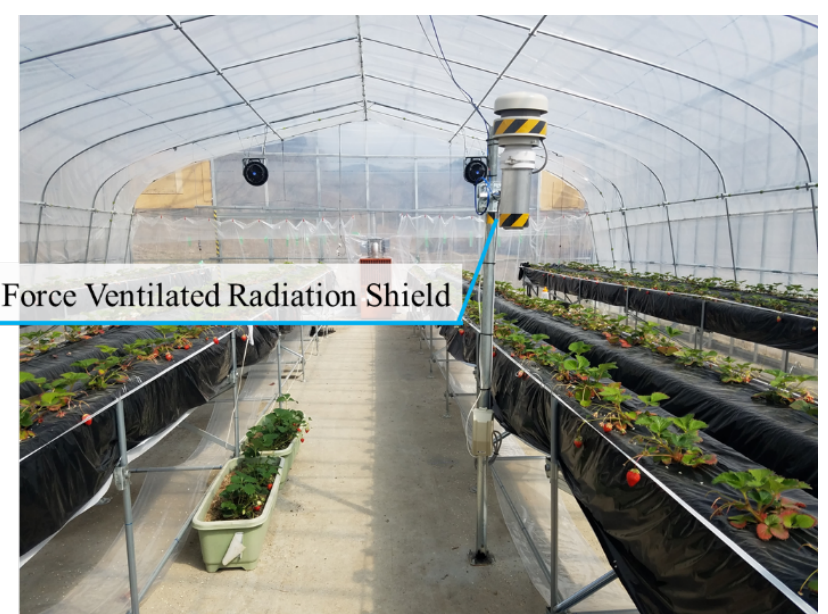

(b) Picture of the production area

Figure 3. Greenhouse heating apparatus.

\section{Results and Discussion}

Test of the biomass heating system without kerosene boiler

The trial of biomass heating system without kerosene boiler was held and a result was shown in Figure 4 and Figure5. The trial of combustion test for making hot water was held from around 9:00 - 17:00 on December 20th, 2017.

As the result of this test, the water temperature of the heat storage tank rose up to about $70{ }^{\circ} \mathrm{C}$. The heat radiation from the heat exchanger was calculated based on the difference between the input and output temperature and flow ratio of water. When the water temperature was $70^{\circ} \mathrm{C}$ in daytime, the total amount of the heat radiation from the heat exchanger in the greenhouse was about 1,409 MJ from around 17:00 to 9:00.

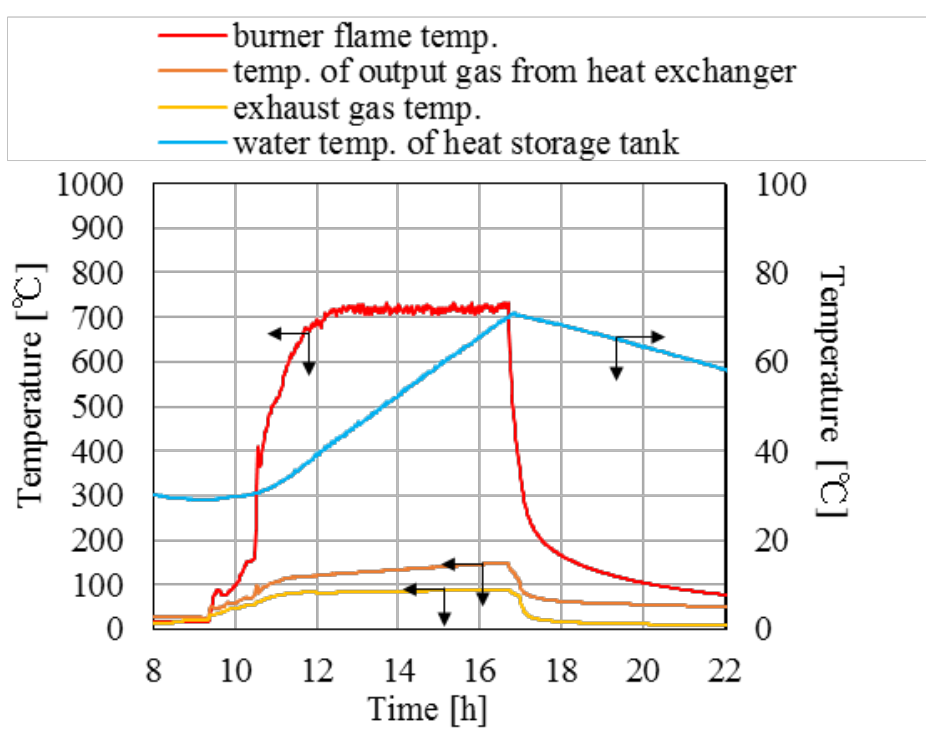

Figure 4. Temperature changes in the biomass heating system. 

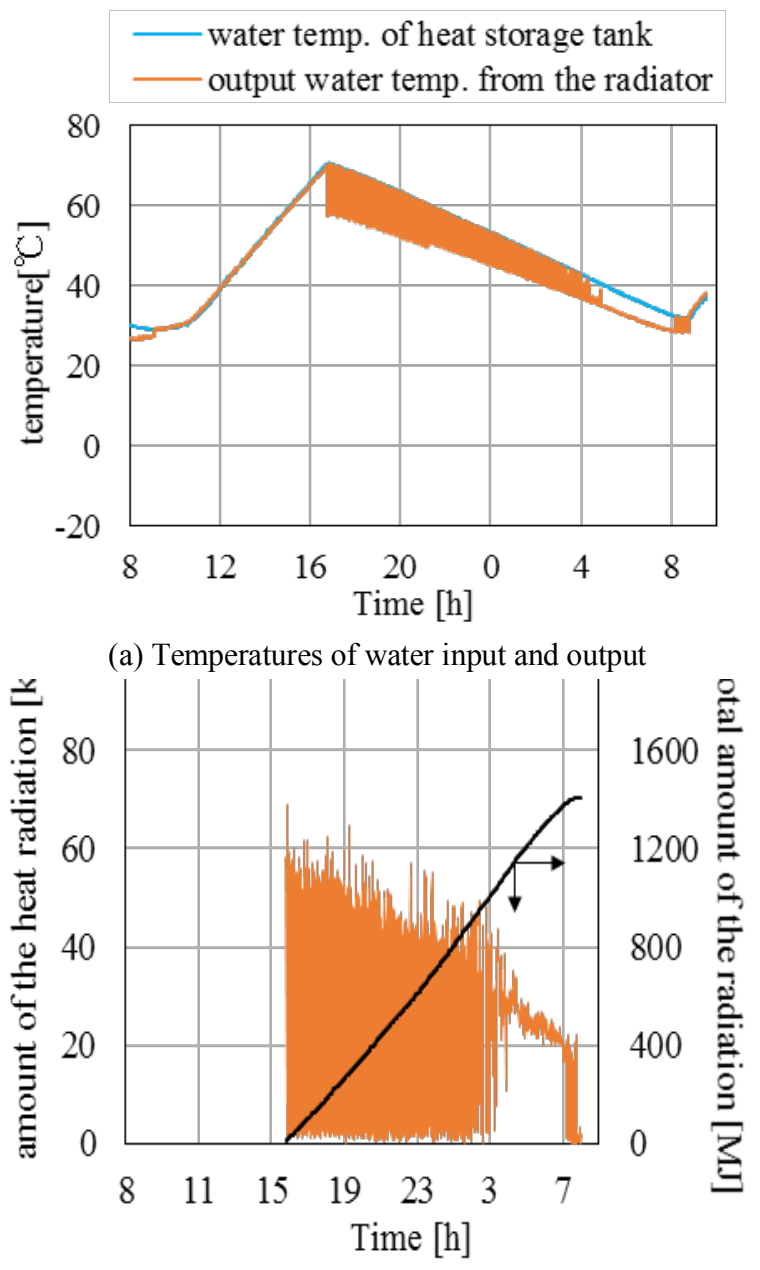

(b) Heat radiation from the radiator in the greenhouse

Figure 5. Heat radiation from the radiator-type heat exchanger.

Test of the biomass heating system with kerosene boiler

The tests of the biomass heating system with kerosene boiler were held and the result was Figure 6 and Figure 7. Field tests were performed from December 2017 to March 2018. The greenhouse was double layer: a small greenhouse was built inside a big greenhouse, therefore we measured the three temperatures of outside, inside greenhouse, and production area. In order to grow strawberries well, we have to keep the greenhouse the set temperature from $10^{\circ} \mathrm{C}$ to $18{ }^{\circ} \mathrm{C}$. In other words, it is important that minimum temperature of the production area bellow the set temperature. Water temperature of the heat storage tank was controlled by kerosene boiler (Table 2).

As the result of the tests, the temperature control accuracy in the greenhouse was $\pm 2{ }^{\circ} \mathrm{C}$ during the first period, because no greenhouse circulation fans were in the production area. After the first period, the circulation fans were installed. Therefore, even at -17 ambient temperature, the temperature control accuracy in the greenhouse was $\pm 1{ }^{\circ} \mathrm{C}$ during the other periods. Drastic temperature changed between the periods when trials of combustion test for making hot water were conducted.

Table 2. Water temperature of the heat storage tank.

\begin{tabular}{lc}
\hline \multicolumn{1}{c}{ Date } & Water temperature of the Heat Storage Tank $\left[{ }^{\circ} \mathrm{C}\right]$ \\
\hline Dec. 20 - Feb. & $50-60$ \\
Feb. 2 - Feb. 5 & $30-40$ \\
Feb.5 - Feb.7 & $40-50$ \\
Feb.7 - Feb.13 & $30-40$ \\
Feb. 13 - Feb. 19 & $40-50$ \\
Feb. 19 - & $30-40$ \\
\hline
\end{tabular}




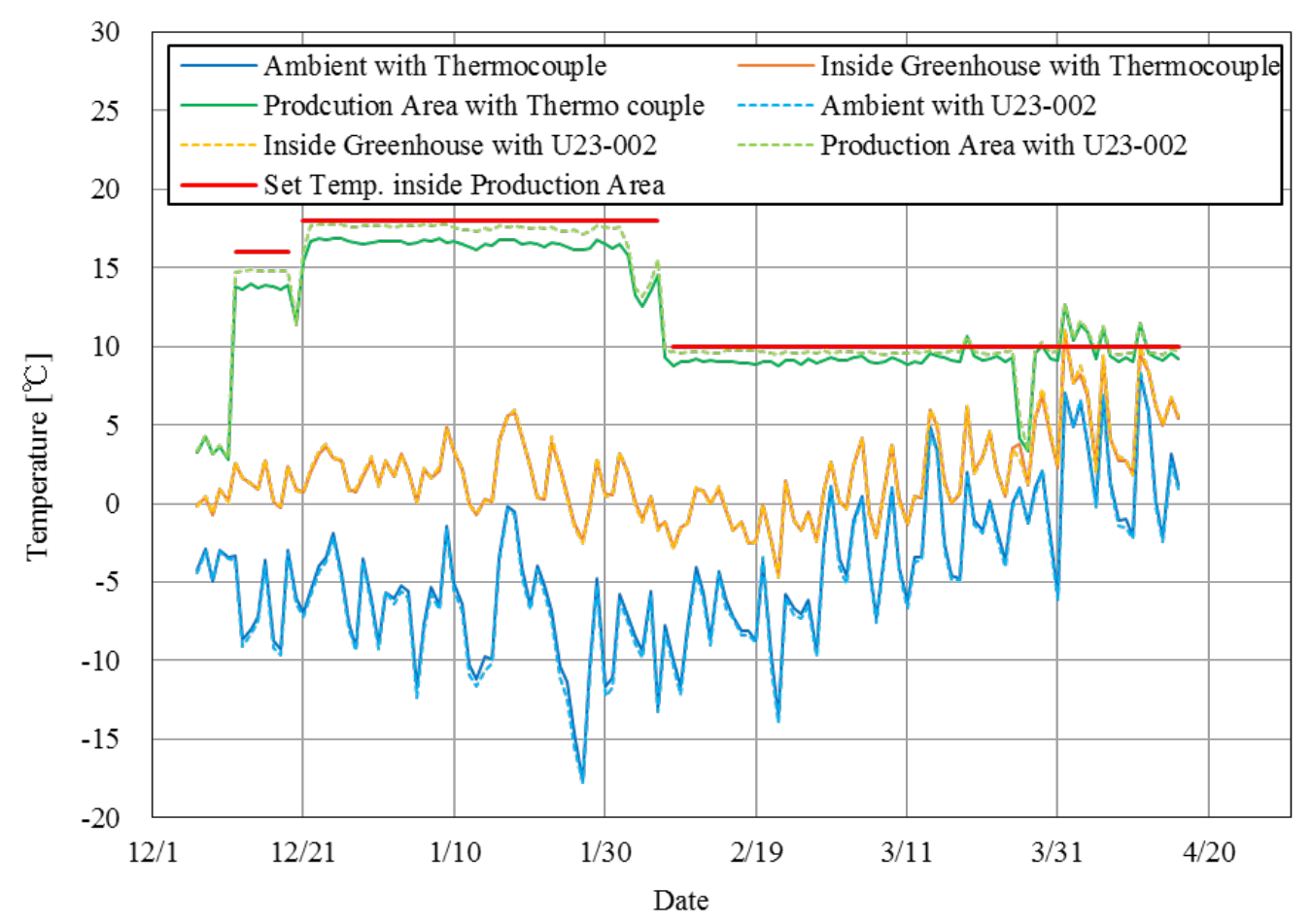

Figure 6. Minimum temperature changes during this field tests.

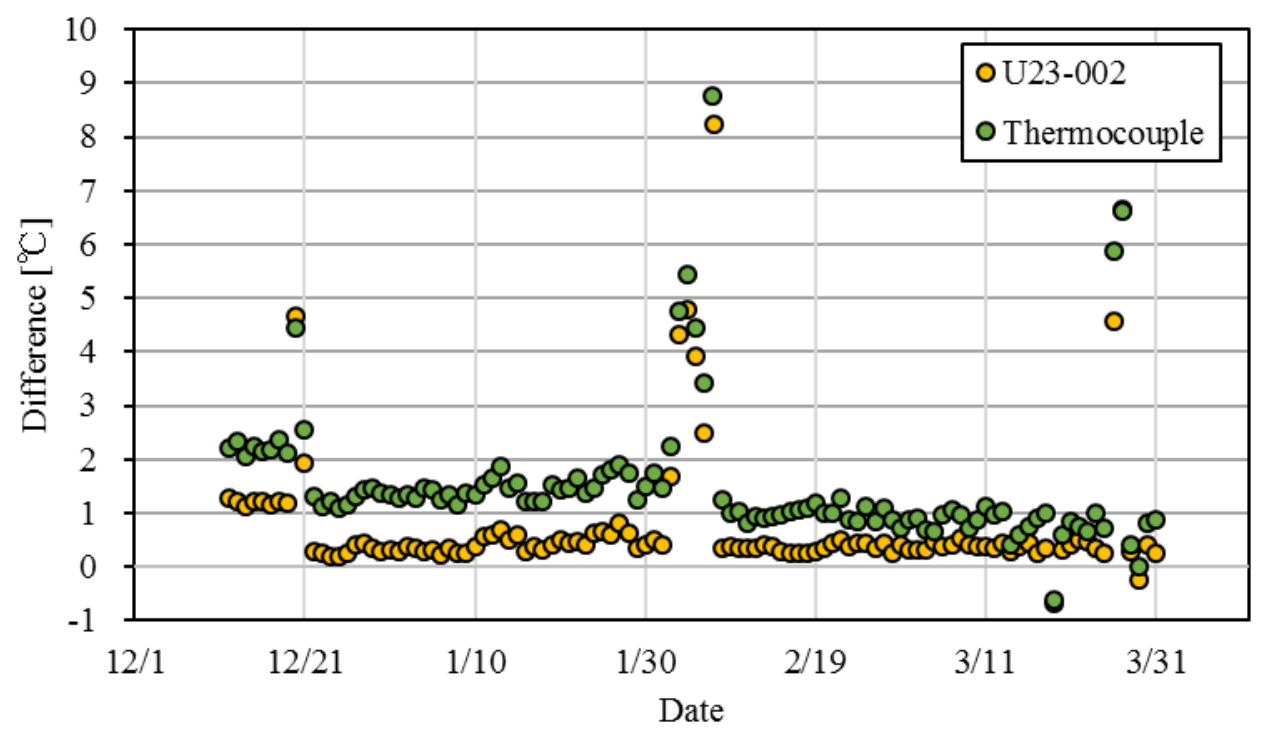

Figure 7. Differences between set temperature and measured temperature.

\section{Conclusions}

We combined heat storage tank with biomass boiler and kerosene boiler and devised a greenhouse heating system. With a heat storage tank, even a small kerosene boiler was able to keep the greenhouse from $10^{\circ} \mathrm{C}$ to 18 ${ }^{\circ} \mathrm{C}$ at night and the temperature control accuracy in the greenhouse was $\pm 1{ }^{\circ} \mathrm{C}$. In the next step, we conduct field tests in which a few greenhouses are heated, and we develop an efficiency operation method of this system.

\section{Acknowledgements}

This work was conducted under "A Scheme to Revitalize Agriculture and Fisheries in Disaster Area through Deploying Highly Advanced Technology" by the Ministry of Agriculture, Forestry and Fisheries, Japan. 


\section{References}

Fukuoka, M., Kuwagata, T., Mayumi, Y., Yamada, Y. 2011. A guide for building your own "NIAES-09"-A low-cost force-ventilated radiation shield utilizing construction materials. The Society of Agricultural Meteorology of Japan. 11, A10-A16

Takekura, K., Yamashita, Y., Kanai, G., Yakushido, K., Ahn, D., 2018. Development of Greenhouse Heating System using Biomass Boiler. The Society of Agricultural Structures. 49 (1), 24-29. 


\title{
Technology for Efficient Application of Liquid Manure: a Comprehensive Modelling Approach on Greenhouse Gas Emissions
}

\author{
Bianca Zerhusen", Fabian Lichti, Stefan Neser \\ Institute of Agricultural Engineering and Animal Husbandry, Bavarian Research Center of Agriculture, Freising 85354, \\ Germany \\ * Corresponding author. Email: Bianca.Zerhusen@1fl.bayern.de
}

\begin{abstract}
The goal of this study is to describe all effects of liquid manure application technologies on greenhouse gas (ghg) emissions at farm level. A literature review showed, that despite the existence of a big number of research done in this field, there is still a lack of understanding overall ghg emission dynamics. As there is a big uncertainty in predicting nitrous oxide emissions and so far none of the field studies included the full set of relevant pathways of nitrogen loss and all influencing factors (e.g. location factors), the current work aims to analyse and describe coherences and the range of possible manifestations rather than derive absolute numbers for ghg emission reductions through liquid manure application technology. A modelling approach was developed to show selfenforcing and counteractive influences using values of diverse studies. The results highlight different forces that need to be considered to achieve a ghg emission reduction. Furthermore, results show which framework conditions promote or hinder the implementation of liquid manure application technology at farm level. The liquid manure application technology provides a big potential to reduce ghg emissions. The efficiency of liquid fertilizers due to application close to the soil and roots can be expressed by the mineral fertilizer equivalent. Such high efficiencies can be accounted for in ghg modelling by savings of manure that are available for fertilization elsewhere and replace mineral fertilizers there. Our findings emphasise, that to ensure a ghg emission reduction through the use of liquid manure application technology, it should go hand in hand with a reduction of manure input, being the biggest control variable in ghg modelling. From the found results the conclusion can be drawn, that ghg modelling needs to address manifold effects to develop functional ghg mitigation strategies and not to mislead by monocausal findings.
\end{abstract}

Keywords: ammonia emissions, mineral fertilizer equivalent, $\mathrm{CO}_{2}$ equivalent, broadcast application, injection, trailing shoe, trailing hose

\section{Introduction}

Nutrient losses to the environment are an important concern of intensive animal farming systems, threatening air and water quality (Chardon et al., 2012). International policy aims to reduce different pollutants to water and air. To achieve this, laws and agreements on mitigation goals are established in Europe. There are the Kyoto Protocol focusing on greenhouse gas (ghg) emissions, the Gothenburg Protocol referring to substances threatening air quality like ammonia $\left(\mathrm{NH}_{3}\right)$ but also leading to eutrophication of water bodies and acidification of soils and there is the nitrate directive for the protection of aquatic biospheres. In Germany recently a new regulation on fertilization has come into force (DüV) and a novel national emission ceiling is at discussion. $95 \%$ of ammonia emissions in Germany result from agriculture (UBA, 2018). Manure spreading accounts for the biggest share with $41 \%$ of national ammonia emission in 1990 , while today (2016) it makes up $31 \%$ and $40 \%$ when excluding or including digested residues of energy crops respectively (Haenel et al., 2018). Agriculture, including land use and land use change, accounts for $12.5 \%$ of ghg emissions in Germany (NIR 2017). Thereby, nitrous oxide $\left(\mathrm{N}_{2} \mathrm{O}\right)$ emissions make up $31 \%$ of agricultural emissions and are an important ghg within farming systems besides carbon dioxide $\left(\mathrm{CO}_{2}\right)$ and methane $\left(\mathrm{CH}_{4}\right)$. Single contribution sources to the $\mathrm{N}_{2} \mathrm{O}$ emissions are: $3.8 \%$ manure management in housing and storage, $18.7 \%$ fertilizer induced emissions, harvest residues and pasture, $2.8 \%$ peatland under agricultural use and 6\% mineral soils under agricultural use (Osterburg, 2017). In the German national climate protection plan (Klimaschutzplan 2050) a reduction of ammonia is mentioned as a goal to simultaneously reduce greenhouse gas (ghg) emissions.

Mitigation strategies for either $\mathrm{NH}_{3}$ or ghg emissions often emphasize the synergies between the two impact categories. However, there is confusion in the debate about mitigation measures and whether they are proposed to mitigate ghg emissions or $\mathrm{NH}_{3}$ emissions and air pollution. More precisely, there is a big uncertainty in discussions about ammonia emissions and their special role in carbon footprint assessments in regard of their multiple indirect effects to global warming. Ghg balances do often account for indirect $\mathrm{N}_{2} \mathrm{O}$ emissions after nitrogen deposition occurring in consequence of ammonia emissions (e.g. German emission inventory by Haenel et al., 2018 or ghg balance approaches on farm level KTBL, 2016). However, other effects like direct $\mathrm{N}_{2} \mathrm{O}$ emissions might be affected by manure application technology. While general approximation of emission shares is possible using the methodology provided by the IPCC, this methodology seems superficial (Chadwick et al., 2011 ) and insufficient to describe bio-physical coherences in organic manure management on farms. 
We face "challenges of understanding and facilitating management action in complex, uncertain and changing situations" (Patterson et al., 2013). Choosing the right mitigation tools for environmental protection is a very complex task as there are multiple pollutants, drivers, actors and environmental outcomes (Collins et al., 2018; Patterson et al., 2013; Gunningham \& Sinclair, 2005). However, integrated approaches including coherences, indirect effects and long-term effects are rare (Brink \& Grinsven, 2011; Oenema et al., 2015).

Actual European efforts to describe "best available technology" (BAT) in intense livestock farming systems, in terms of cost-effective and environmental friendly methods, aim to provide information on available measures, mitigation potentials and threats like to animal health or pollution leakage effects (Santonja et al., 2017; Lyon et al., 2016). A leakage effect can be e.g. the adaption of the feeding strategy, which might influence product amounts or quality, feed utilization or manure storage emissions.

The biggest potential of ammonia emission reduction is seen in the use of special manure application technology, with manure management being the biggest contributor and the most cost-effective sector (Loyon et al., 2016; Webb et al. 2015). The use of application techniques can lower emissions, save fertilizer and increase $\mathrm{N}$ use efficiency (NUE) (Oenema et al., 2015). Manure can be spread precisely; odour and $\mathrm{NH}_{3}$ emissions can be reduced, as well as crop contamination (Thorman, 2011). Farms with low N input and inefficient N-management might even profit from precise application technology with higher yields. In some countries, slurry injection is the most recommended technique, though showing some limitations of implementation (Fangueiro et al., 2015). Wulf et al., 2001 state, that wrong conclusions might be drawn concerning effective ghg mitigation options, when only considering single trace gases and that further studies are needed, which integrate additional aspects like fuel consumption or applicability.

The goal of the study is to generate a better understanding of coherences in ghg modelling, thereby outlining different viewpoints on appearing effects and implementing the findings into a broader system perspective. In our modelling approach, we focus on the application of liquid manure from dairy cattle in the process of winter wheat production in Germany as an exemplary study-subject to simultaneously analyse possible occurring effects on ghg balances: trace gas emissions, NUE and fuel use.

\section{Materials and Methods}

Technology for liquid manure application

Among the BAT the following application technologies are described (Santonja et al., 2017; EUC, 2017):

- Low pressure broadcast spreader + Incorporation (with plough, tine cultivator or disc harrow)

- band application technology (also referred to in literature as trailing hose)

- trailing shoe

- Injector, open slot

- Injector, closed slot

- High pressure injector

- Irrigation (in combination with slurry water delusion)

- Slurry acidification (in the slurry tanker)

Most studies focus on the first five technologies, while evidence on high pressure injectors and irrigation is missing. They have been excluded from the current study as their distribution is rare, which might be attributed to comparably high costs. Slurry acidification has also been excluded as $\mathrm{NH}_{3}$ mitigation potential and long term effects on the soil are scientifically discussed and need further prove.

In the reviewed papers, a clear description of influencing factors and of technology use (e.g. open or closed slot injection, or only cutting a line or a v-shape) could not always be found. In this context, we want to emphasise the importance of those experimental parameters to derive adequate models.

Review on effects on ghg emission modelling due to different application technology and determination of modelling assumptions

Firstly, a literature review has been done on possible effects due to different application techniques with regard to ghg balances. All relevant nitrogen losses are discussed as well as effects on yield and N-uptake. Furthermore, framework conditions that promote or hinder the implementation of liquid manure application technology at farm level were summarized.

Secondly, ghg emissions where calculated using common modelling algorithms and comparing modelled data with findings from different field studies. To analyse ghg balances, it is assumed that yields and therefore cultivation area are equal over all scenarios of manure application. Thus, one ha of agricultural land and the wheat 
grain dry matter (DM) yield are used as functional units for ghg balances, describing the manifold impacts in the process of manure application in the wheat production chain.

The global warming potential (GWP) translates the effect of different trace gases to global warming due to retention time in the atmosphere and reflexion characteristics into carbon dioxide equivalents $\left(\mathrm{CO}_{2} \mathrm{e}\right)$ as a common basis for calculations. The GWP was assumed to be $28 \mathrm{kgCO}_{2} \mathrm{e} \mathrm{kg}^{-1}$ for $\mathrm{CH}_{4}$ and $265 \mathrm{kgCO}_{2} \mathrm{e} \mathrm{kg}^{-1}$ for $\mathrm{N}_{2} \mathrm{O}$ according to a 100 year time frame (IPCC, 2013). All literature values using other GWP values were translated into present figures.

\section{State of knowledge}

\section{$\mathrm{NH}_{3}$ emissions}

Many studies prove the effect of ammonia reduction due to special application technology placing liquid manure near to the ground or beneath the soil surface into slots or incorporating liquid manure directly after application (Tamm et al., 2016; Wulf, 2003; Webb et al. 2009, Hou et al., 2015). The highest $\mathrm{NH}_{3}$ emissions appear within 24 hours. Avoiding $\mathrm{NH}_{3}$ emissions trough burying liquid manure into the soil and rooting zone can result in higher NUE but also in a higher $\mathrm{N}$ pool in the soil, which is source for further emissions like $\mathrm{N}_{2} \mathrm{O}$ (Thorman, 2011; Chadwick et al., 2011). Additionally, deposition of nitrogen trough aerosol particles on plants and soil surfaces is building a source of so called indirect $\mathrm{N}_{2} \mathrm{O}$ emissions that are relevant for ghg modelling. The ALFAM database (Hafner et al., 2015) and model (Søgaard et al., 2002) describe coherences in $\mathrm{NH}_{3}$-modelling. Besides application technologies, air temperature, wind speed and water content are the main determinants (Huijsmans et al., 2018; Søgaard et al., 2002). $\mathrm{NH}_{3}$ emissions are depending on surface area and air exposure, therefore an impact of soil types could be explained by infiltration capacity, being lowest in clay soils, moderate in peat soils and high in sandy soils (Huijsmans et al., 2018). In literature volatile ammonia emissions are mainly calculated on basis of total ammoniacal nitrogen (TAN), its chemical precursor.

\section{$N$-uptake, yield and NUE}

Different studies state, that reduced $\mathrm{NH}_{3}$ application techniques also increase crop N-uptake or yield and therefore add value to manures (Webb et al., 2010; Oenema et al., 2015). This is true, if the farms had insufficient amounts of organic manures before. On the other hand, farms with high amounts of organic fertilizer can actually reduce mineral fertilizer amounts due to higher NUE. Findings on N-uptake and yield are not always statistically significant and vary between observation years and being subject to uncertainty of open air field trials (Webb et al., 2010). Nevertheless, there is evidence that precise application of liquid manure into the rooting zone, prevention of emissions and conservation of plant available nutrients can lead to higher NUE. Lichti, 2013 found trailing shoe application with a higher N-uptake of plants and also higher dry matter (DM) yields than trailing hose application for different crops, but no statistical differences could be found in winter wheat between broadcast spreading and trailing hose application of digested residues. In maize, however, incorporation showed even higher $\mathrm{N}$ uptake rates than with trailing hose.

Organic fertiliser efficiencies of scientific studies are only comparable if the calculation method is clear and necessary background information for conversion (e.g. amounts and properties of fertilizers like DM, $\mathrm{pH}, \mathrm{N}-$ content etc.; yields and properties of harvest goods) is given. Besides $\mathrm{NH}_{3}$-loss, leaching and runoff as well as immobilization of $\mathrm{N}$ should be taken into account to explain $\mathrm{N}$-uptake and yield formation. Measurement times for $\mathrm{N}$-loss and plant $\mathrm{N}$-uptake over 12 months are still rare and study numbers rather small so that further investigation in field studies, especially in crop rotations, are needed.

\section{$\mathrm{N}_{2} \mathrm{O}$ emissions}

$\mathrm{N}_{2} \mathrm{O}$ is formed during microbial degradation processes in the soil, namely nitrification and denitrification. Nitrification is the conversion of ammonia to nitrate via oxidation. Denitrification is the stepwise conversion of nitrate to $\mathrm{N}_{2} \mathrm{O}$ and $\mathrm{N}_{2}$ under mainly facultative anaerobic conditions. Both processes can occur timely and locally depending on oxygen availability and shortage and they do depend on each other. The processes are described in more detail by Petersen, 2011.

As $\mathrm{NH}_{3}$ is a volatile gas and is formed immediately with liquid manure spreading, it might be subtracted from the readily available nitrogen $\left(\mathrm{NH}_{4}-\mathrm{N}, \mathrm{NO}_{3}^{-}-\mathrm{N}\right.$ and uric acid-N) to calculate $\mathrm{N}_{2} \mathrm{O}$ emissions, as those occur over a longer period depending on biochemical transformation in soils. However, increased $\mathrm{N}_{2} \mathrm{O}$ emissions do not only depend on the mineral N-Pool in the soil but also on organic carbon content, temperature, moisture and oxygen, manure constitution and $\mathrm{pH}$ (Chadwick et al., 2011, Thorman, 2011; Petersen, 2011). Besides, clogging of soil pores (Chadwick et al., 2011) and soil compaction (Webb et al., 2010) due to heavy machinery and therefore infiltration rate or runoff of slurry are affecting the soil N-Pool. There might be an accumulation of water in compacted zones after rain events triggering denitrification and $\mathrm{N}_{2} \mathrm{O}$-formation. Organic matter and water holding capacity (especially to be found in clay soils) are positively correlated with $\mathrm{N}_{2} \mathrm{O}$ emissions (Wulf et al., 2001). 
Rainfall and soil water content are important determinants for $\mathrm{N}_{2} \mathrm{O}$ formation (Sistani et al. 2010). Moreover, with rainfall other N-losses might occur (e.g. leaching).

$\mathrm{N}_{2} \mathrm{O}$ emissions vary broadly among different studies, showing no effect or an increase in $\mathrm{N}_{2} \mathrm{O}$-emissions after slurry application (Webb et al., 2010). In their review Chadwick et al., 2011 found field experiment results which indicate, that $\mathrm{N}_{2} \mathrm{O}$ emissions might increase, when liquid manure is injected into the soil (Rodhe et al., 2006, Thomsen et al., 2010, Sistani et al., 2010). The studies were conducted in grassland fertilized with cattle slurry, in cereal crops and corn each fertilized with pig slurry. Similar findings are described by Wulf, 2003. However, effects could not be statistically secured between different years (Sistani et al., 2010 und Thomsen et al., 2010). A significant increase in $\mathrm{N}_{2} \mathrm{O}$-emissions for manure incorporation and injection were found by Hou et al., 2015, taking into account 75 observations from 69 field experiments. The variation was between +26 to $+199 \%$. In scenario analysis basing on three different pig farm systems to assess $\mathrm{NH}_{3}$ and ghg mitigation measures along the manure management chain, they found ghg emissions to increase by $16 \%$ with manure injection due to increased $\mathrm{N}_{2} \mathrm{O}$ emissions. It has to be pointed out here, that indirect $\mathrm{N}_{2} \mathrm{O}$ emissions and plant nutrition effects have been neglected in the study.

\section{$\mathrm{CH}_{4}$ emissions}

Wulf et al., 2001 found $\mathrm{CH}_{4}$-emissions from application of digested residues and their contribution to $\mathrm{CO}_{2} \mathrm{e}$ emissions to be rather low, what is in line with other reports on liquid manure application (Loyon et al., 2016). Soils can even be $\mathrm{CH}_{4}$ sinks (Rodhe et al., 2006). Sistani et al., 2010 found $\mathrm{CH}_{4}$ emissions to be increased in injection compared with surface application and presumed this to result from methanogenic activity in soils after incorporation.

\section{Nitrate leaching}

Nitrate leaching is an important control variable in N-cycles and strongly depending on soil characteristics and rainfall. Application technology could reduce nitrate leaching if precise manure application into the rooting zone and splitting of fertilization reduce $\mathrm{NO}_{3}{ }^{-}$content in the soil and increase NUE trough the plant uptake. Then again, reduced $\mathrm{NH}_{3}$ application technology might lead to higher leaching rates when slurry is buried or placed into soil slots, which might be a problem especially on sandy soils. Application of liquid manure in spring instead of fall is a preferred method to reduce $\mathrm{NO}_{3}{ }^{-}$leaching (Thorman, 2011) and may reduce direct and indirect $\mathrm{N}_{2} \mathrm{O}$ emissions if there are favourable conditions (low temperatures - resulting in less $\mathrm{N}_{2} \mathrm{O}$ and $\mathrm{NH}_{3}$ emissions, good soil conditions and no heavy rainfall to provide good infiltration, crop growth and $\mathrm{N}$ uptake). Even so, those conditions vary strongly between different years. Other strategies to reduce leaching refer e.g. to crop residue and cover crop management or manure planning (Collins et al., 2018).

\section{Fuel use and $\mathrm{CO}_{2}$ e emission}

Fuel consumption is depending on many factors like production systems, crop production management and technical factors e.g. width and weight of equipment, power of the tractor (Pelletier et al., 2014). Soil workability and field trips are also influencing fuel use. $\mathrm{CO}_{2}$ e emissions result from combustion of fuel in the engine but also from upstream activities for its provision.

\section{Technology implementation in farms - Chances and limits}

Obviously, mitigation potential and verification are important criteria for implementation of mitigation measures. But also feasibility is an important aspect, which describes the configuration of measures for specific farms and considers the decision-making framework. In terms of application technology, feasibility is determined by field-settings (size, slope, stones and heaviness of soil, locally different $\mathrm{N}$ needs), farm predisposition (empiric experience, management targets, organic fertilizer capacity, amortisation of existing stocks, leasing/ machinery contractors, Know-How, stakeholders), effort (time, investment, expenditure of establishment) and legal framework (water pollution control, fertilization regulations, air pollution control, subsidies). Patterson et al., 2013 describe important factors for implementation of measures to mitigate water pollution and thereof find farm history and contingency; institutional arrangements; collaboration; engagement; vision and strategy; knowledge building and brokerage; resourcing and entrepreneurship.

Furthermore, different impacts on sustainability need consideration. Prompt incorporation of slurry within one process step means a reduction in working time and might have positive effects on workload peaks (labour, management). Contractors or machine leasing can assure the best available technology (Webb et al., 2015). However, machine capacity and availability might be a challenge when all farms use weather forecasts to reduce $\mathrm{NH}_{3}$-emissions (Huijsmans et al., 2018). Effects on acidification and eutrophication, soil compaction due to heavy machinery need to be addressed and might again lead to $\mathrm{N}_{2} \mathrm{O}$ emissions and yield effects. Broadcasting is cheap and fast (Webb et al. 2015), incorporation and precise placement is avoiding $\mathrm{NH}_{3}$-emissions but might increase 
work, energy use and costs. Uneven distribution due to wind, a contamination of plants or increased spreading surface with harvest residues may increase $\mathrm{NH}_{3}$ emissions. Plant-canopy size and height determine applicability and protection against solar radiation and wind. Damages on plants trough cutting by application technology should be reduced (harmonizing tool settings and crop growth) and application on freshly harvested grass might lead to inefficient $\mathrm{NH}_{3}$ mitigation (Webb et al. 2015). Application shortly before harvest might lead to contamination and conservation problems. Slurry application in growing crops enables better timely distribution to crop growth and wides working time frames (field trafficability, machine availability). Better local distribution of manure to the rooting zone might increase NUE of organic fertilizers and might have special benefits for dry soils. Additionally, slurry viscosity and possible dilution determine technology applicability. Stony, slopy fields, wet soils and injection depth are influencing the applicability and efficiency of $\mathrm{NH}_{3}$ mitigation (Webb et al. 2015; Tamm et al., 2016).

\section{Modelling ghg emissions}

The basis for modelling ghg emissions is provided by a study of Wulf, 2003, who compared different manure application techniques in terms of trace gas emissions $\left(\mathrm{N}_{2} \mathrm{O}, \mathrm{CH}_{4}\right.$ and $\left.\mathrm{NH}_{3}\right)$. A more detailed description of the same trail is provided by Wulf et al., 2001. In the experiments $30 \mathrm{~m}^{3}$ of digested residue were applied manually, simulating different application technologies. Broadcast spreading, trailing hose, trailing shoe and injection (not specified) were analysed. The trial was performed in Western Germany, on a well-drained Luvisol (loamy soil) and the observation year was rather warm and dry. Wulf et al., 2001 calculated $\mathrm{CO}_{2}$ e emissions resulting from $\mathrm{N}_{2} \mathrm{O}$ on basis of measured values subtracted by control plot emissions (background concentration emitted by soils). In contrast, in the IPCC methodology to assess $\mathrm{N}_{2} \mathrm{O}$ from mineral soils, the emissions from soil and fertilization are not disaggregated. Therefore, background emissions were added for the consideration of trace gas emissions from a field study with our modelling results of further influencing factors. Information on the cultivated crop is missing. Further modelling steps are based on assumptions and we determined to consider winter wheat production. Additionally, it was assumed, that a maximum of $170 \mathrm{kgN}$ of organic fertilizer from dairy production was applied, equalling an amount of $44 \mathrm{~m}^{3}$ of slurry (applicable deductions for field loss and standard values for manure properties were used according to Wendland et al., 2018).

Depending on the research question, there are different ways to calculate the NUE of organic fertilization (Lichti, 2013). The yield equivalent to mineral fertilizer (YEM) compares fertilizer amounts, which lead to the same yield and is especially useful for efficiency calculations with focus on economically relevant DM yields.

$\mathrm{YEM}=(($ application rate organic fertilizer $) /($ application rate mineral fertilizer $)) * 100$

The mineral fertilizer equivalent (MFE) compares the N-uptake from mineral and organic fertilized plots at the same fertilization rate and is especially useful to compare different fertilizers.

$\mathrm{MFE}=((\mathrm{N} \text { Uptake organic }-\mathrm{N} \text { Uptake control plot }) /(\mathrm{N} \text { Uptake mineral-N Uptake control plot }))^{*} 100$

Nitrogen uptake or application rate can either be calculated as total nitrogen $\left(\mathrm{N}_{t}\right)$ or ammoniacal nitrogen (TAN). YEM and also MFE seem appropriate indicators for fertilizer use efficiency under the premise of constant DM yields, assuming the difference between NUE (due to soil or climate) may not lead to a variation in absolute fertilization or yield level and with the objective to compare MFE of different application technologies.

Lichti, 2013 analysed yield and N-uptake for shallow injection with a trailing shoe, trailing hose and broadcast spreading (with a metal plate for downward direction of the manure stream). In the three years experiment with biogas residues applied to winter wheat in Bavaria, Lichti, 2013 found the MFE to be 30\% on a silty loam soil and $32 \%$ on a loamy sand soil referring to $\mathrm{N}_{\mathrm{t}}$. The YEM was $34 \%$ on average for digested biogas residue applied with trailing hose. In our modelling approach we assumed the manure efficiency for trailing hose to be $35 \%$, defined in accordance with these findings. Assumed efficiencies of $40 \%$ for trailing shoe and $55 \%$ of injection are somewhat higher. NUE of $30 \%$ and $35 \%$ were assumed for broadcasting and trailing hose application respectively. Due to higher fertilizer efficiencies, the effective $\mathrm{N}$ in organic fertilizer varies between manure application technologies (see Tab. 1). The difference in effective fertilizer $\mathrm{N}$ can be interpreted as the amount of mineral fertilizer saved in conventional farms due to more efficient $\mathrm{N}$ use through more precise application techniques. 
Table 33. NUE assumed for our modelling approach and resulting fertilizer savings (derived from Lichti, 2013).

\begin{tabular}{cccc}
\hline & $\begin{array}{c}\text { mineral fertilizer equivalent } \\
{[\%]}\end{array}$ & $\begin{array}{c}\text { effective fertilizer N } \\
{\left[\mathrm{kg} \mathrm{N}^{*} \mathrm{ha}^{-1}\right]}\end{array}$ & $\begin{array}{c}\text { mineral fertilizer savings } \\
{\left[\mathrm{kg} \mathrm{N}^{*} \mathrm{ha}^{-1}\right]}\end{array}$ \\
\hline broadcast & 30 & 51.0 & 0.0 \\
trailing hose & 35 & 59.5 & 8.5 \\
trailing shoe & 40 & 68.0 & 17.0 \\
injection & 55 & 93.5 & 42.5 \\
\hline
\end{tabular}

$\mathrm{CO}_{2} \mathrm{e}$ credits were calculated for mineral fertilizer savings assuming the use of calcium ammonium nitrate (CAN). Emissions for upstream activity (production of mineral fertilizers) were $9.46 \mathrm{kgCO}_{2} \mathrm{e} \mathrm{kgN}^{-1}$ (Weidema et al., 2013) and direct and indirect $\mathrm{N}_{2} \mathrm{O}$ emissions were calculated according to KTBL, 2016.

Fuel use was assumed to be $41 \mathrm{ha}^{-1}$ for broadcast application, $51 \mathrm{ha}^{-1}$ for trailing hose, $71 \mathrm{ha}^{-1}$ for trailing shoe and $121 \mathrm{ha}^{-1}$ for injection and was derived from practical energy efficiency consulting (Schmidt, 2014). Fuel use for heavy machines was discussed (Webb et al., 2015) to be between 18 and $25 \mathrm{~h}^{-1}$ for broadcasting and $\mathrm{NH}_{3}$ reduced application, respectively. Assuming a working time of $16-18 \mathrm{~min} \mathrm{ha}^{-1}$ the values correspond to our modelling assumptions. Emission of diesel fuel production $\left(0.49 \mathrm{~kg} \mathrm{CO}_{2} \mathrm{e} \mathrm{l}^{-1}\right)$ and combustion $\left(2.53 \mathrm{kgCO}_{2} \mathrm{e}^{-1}\right)$ where derived from Weidema et al., 2013.

To create an approach that is more related to practice and includes mineral fertilization, further assumptions were made. Yield was assumed to be $91 \mathrm{dt}$ FM, representing the three year average value (2014-2016) of a "A quality" winter wheat crop in Bavaria (LfL, 2017). Fertilization was modelled according to the German fertilizer ordinance (DüV) using the guideline for fertilization of arable land and grassland (Wendland et al., 2018). The standard nitrogen need was assumed to be $241 \mathrm{kgN} \mathrm{ha}^{-1}$ and deductions were made for a typical mineral nitrogen content in the soil (-54 $\mathrm{kgN}$ for the county Oberbayern according to LfL, 2018) and continuous organic fertilization $(-17 \mathrm{kgN})$. Permissible gaseous ammonia losses $(17.6 \%)$ were subtracted and the minimum effectiveness of fertilizer $\mathrm{N}$ was accounted for with $50 \%$.

In the data derived from experiments (Wulf, 2003), $\mathrm{N}_{2} \mathrm{O}$ emission factors can be calculated referring to the amount of organic fertilizer supplied. Assuming application amounts of $120-170 \mathrm{kgN}$ (corresponding to estimated $\mathrm{N}$ application and wheat fertilizer needs) values range from 0.0085 to $0.132 \%$ and are therefore more than 10 -fold smaller than the assumed ghg emission factor of 1\% by IPCC, 2006. Values for mineral fertilizer savings and calculated mineral fertilization amounts were adapted to analyse resulting implications.

As there is a high variability in $\mathrm{N}_{2} \mathrm{O}$ emissions between different locations, an assessment of effects due to this uncertainty was done. Actual IPCC methodology accounts for $\mathrm{N}_{2} \mathrm{O}$ emissions with $1 \%$ of total $\mathrm{N}$ applied to the soil, giving an interval of $0.3-3 \%$ (IPCC, 2006). Besides, a different approach of modelling $\mathrm{NH}_{3}$ emissions, NUE and fertilization was made to investigate the actual compilation of emission factors as used in emission inventories (Haenel et al., 2018; KTBL, 2016). Emission factors used for modelling in this study and comparable values from meta-analyses by Hou et al., 2015 and by Webb et al., 2010 are presented in Tab. 2. Differences between studies might be explained by criteria like manure amounts spread, crop type, N-use, yield, vegetation and following incorporation processes. Depth and volume of injection slots also have effects on ammonia emissions (Rhode et al., 2006; Webb et al., 2010). Hence, different findings on $\mathrm{NH}_{3}$ emission reduction can be contributed to a missing differentiation between technological aspects, different field trial settings but also $\mathrm{NH}_{3}$ measurement methodology (Webb et al., 2010). It was assumed that $\mathrm{NH}_{3}$-loss corresponds to a loss of $100 \%$ of plant available nitrogen. The resulting scenarios comprise additional consideration of $\mathrm{N}_{2} \mathrm{O}$ emissions from leaching and were used as basis for the $\mathrm{N}_{2} \mathrm{O}$-uncertainty assessment.

Table 34. Emission factors for $\mathrm{NH}_{3}$ emissions referring to total ammoniacal nitrogen (TAN) and resulting $\mathrm{NH}_{3}$ reduction due to precise placement of liquid dairy cattle manure, which were used for modelling ([1] Haenel et al., 2018; [2] KTBL, 2016) and further literature values ([3] Hou et al., 2015; [4] Webb et al., 2010).

\begin{tabular}{|c|c|c|c|c|c|c|}
\hline Application technology & $\begin{array}{c}\mathrm{NH}_{3}-\mathrm{N} \\
\operatorname{kg~kgTAN}^{-1}\end{array}$ & $\begin{array}{c}\mathrm{NH}_{3}-\mathrm{N} \\
\text { kg kgTAN }^{-1}\end{array}$ & $\begin{array}{l}\text { Reduction of } \\
\mathrm{NH}_{3} \%\end{array}$ & $\begin{array}{c}\text { Reduction of } \\
\mathrm{NH}_{3} \%\end{array}$ & $\begin{array}{c}\text { Reduction of } \\
\mathrm{NH}_{3} \%\end{array}$ & $\begin{array}{c}\text { Observations/ } \\
\text { studies }\end{array}$ \\
\hline $\begin{array}{l}\text { Brroadcasting, } \\
\text { without incorporation }\end{array}$ & 0.5 & $\begin{array}{c}0.48 \\
(0.3-0.68)\end{array}$ & - & & - & $229 / 20$ \\
\hline $\begin{array}{l}\text { Trailing hose, } \\
\text { underneath vegetation }\end{array}$ & 0.35 & $\begin{array}{c}0.21 \\
(0.14-0.32)\end{array}$ & 0.3 & $\begin{array}{c}0.35 \\
(0-0.75)\end{array}$ & $\begin{array}{c}0.55 \\
(0.37-0.67)\end{array}$ & $100 / 11$ \\
\hline trailing shoe & 0.36 & $\begin{array}{c}0.21 \\
(0.8-0.36)^{*}\end{array}$ & 0.28 & $\begin{array}{c}0.65 \\
(0.38-0.74)\end{array}$ & $\begin{array}{c}0.7 \\
(0.5-0.82)^{*}\end{array}$ & $39 / 5^{*}$ \\
\hline $\begin{array}{l}\text { Incorporation } \\
\text { and injection }\end{array}$ & 0.05 & $\begin{array}{c}0.11 \\
(0.5-0.21) \\
\end{array}$ & 0.9 & $\begin{array}{c}0.7-0.8 \\
(0.23-0.99)^{* *} \\
\end{array}$ & $\begin{array}{c}0.80 \\
(0.72-0.86) \\
\end{array}$ & $106 / 11$ \\
\hline Source & {$[1] \&[2]$} & [3] & {$[1] \&[2]$} & [4] & [3] & [3] \\
\hline
\end{tabular}


Furthermore, we wanted to verify and compare fuel use assumptions with the help of the fuel use calculator (focus on manure spreading at the field level) and with the calculation tool for standard crop production chains (KTBL, 2018a\&b) (derive standard process values for the whole winter wheat production chain). Further values for the whole production chain were assessed according to earlier work (Maze et al., 2017) corresponding with assumptions hereafter chosen for modelling ghg emissions of the single process step of liquid manure application. Field-farm distance and field size was varied starting with the lower values of $2 \mathrm{~km}$ and 2 ha, which represent rather small farm structures of Bavaria. The field size upper values where chosen with 5 ha and $14 \mathrm{~km}$ to show the dependency of the fuel use to these factors. Mechanisation was chosen with a $67 \mathrm{~kW}$. As the supposed amount of liquid manure spread to the field is quite high, it could be assumed that the fertilization is splitted (e.g. $25 \mathrm{~m}^{3}$ fertilized in early spring and $19 \mathrm{~m}^{3}$ applied to the tillering stage $\mathrm{BBCH} 30-32$ ). A maximum slurry amount of 30 $\mathrm{m}^{3} \mathrm{ha}^{-1}$ could be chosen within the fuel use calculator. A minimum of $5 \mathrm{~m}^{3}$ was chosen as bottom line.

\section{Results and discussion}

Results of ghg modelling

Wulf, 2003 found no statistical difference between different application technologies when considering trace gas emissions. As fuel use is increasing with the incorporation into soils, heavy machinery or smaller transport volumes the fuel use is greatest for injection. Still, the importance of fuel use (expressed as $\mathrm{CO}_{2} \mathrm{e}$ ) is rather small and does not lead to big changes in the ghg balance (Fig. 1). Accounting for increasing NUE on the other hand shows big relevance in ghg balance results. Considering additional mineral fertilization, total emissions were 1,646 $\mathrm{kg} \mathrm{CO}_{2} \mathrm{e} \mathrm{ha}^{-1}$ for broadcasting, 1,508 $\mathrm{kg} \mathrm{CO}_{2} \mathrm{e} \mathrm{ha}{ }^{-1}$ for trailing hose, 1,381 $\mathrm{kg} \mathrm{CO}_{2} \mathrm{e} \mathrm{ha}^{-1}$ for trailing shoe and $1,021 \mathrm{~kg} \mathrm{CO}_{2} \mathrm{e} \mathrm{ha}^{-1}$ for injection.

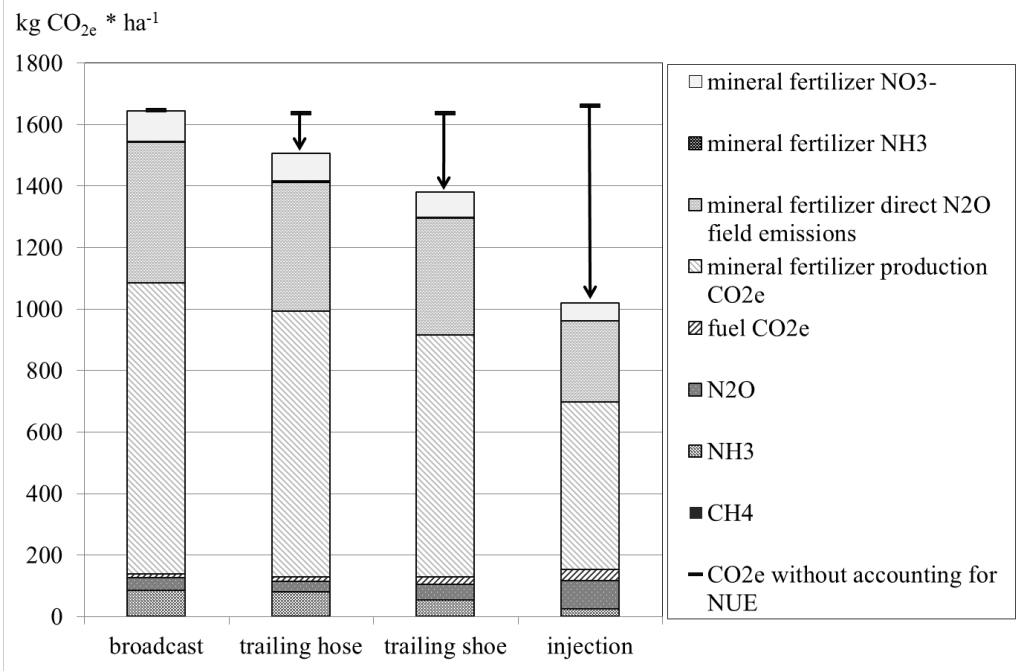

Figure 97. Ghg emissions of different application techniques for liquid manure according to measurements of Wulf, 2003 (including $\mathrm{N}_{2} \mathrm{O}$ background emissions and adapted GWP); supplemented by assumptions for fuel use and mineral $\mathrm{N}$ fertilization under consideration of different NUE and with the premise of a constant yield level. Reduced emissions due to mineral fertilizer savings are indicated with an arrow.

Adaptions of $\mathrm{N}_{2} \mathrm{O}$ emissions (10-fold smaller than the standard IPCC factor) showed no relevant change in the direction of coherences and hence, did not change the general predication (not presented). Uncertainty of $\mathrm{N}_{2} \mathrm{O}$ emission is high and influences from different location factors are not yet well-known. Effects resulting from this uncertainty are presented in Fig. 2. Emissions range from 1,407 to 5,267 $\mathrm{kgCO}_{2} \mathrm{e} \mathrm{ha}^{-1}$; credits for mineral fertilizer savings range from 0 (149) to 881. Absolute amounts and also relative importance of fertilizer savings change due to different $\mathrm{N}_{2} \mathrm{O}$ emission factors. However, in all cases NUE has a visible effect on ghg balance results.

Thorman, 2011 quote studies with 7 or up to $14 \%$ and calculated $\mathrm{N}_{2} \mathrm{O}$ emissions from findings of Wulf, 2003 were even lower than the lower boundary of $0.3 \%$. Web et al., 2010 show with a model calculation that reducing $\mathrm{NH}_{3}$ emissions with slurry injection and therefore indirect $\mathrm{N}_{2} \mathrm{O}$ emissions after deposition of $\mathrm{N}$ was in the same range with an increase of direct $\mathrm{N}_{2} \mathrm{O}$ emissions by $35 \%$, which exceeds their findings. In contrast, data from Wulf et al., 2001 showed significant increases in direct $\mathrm{N}_{2} \mathrm{O}$ emissions that were even greater. 


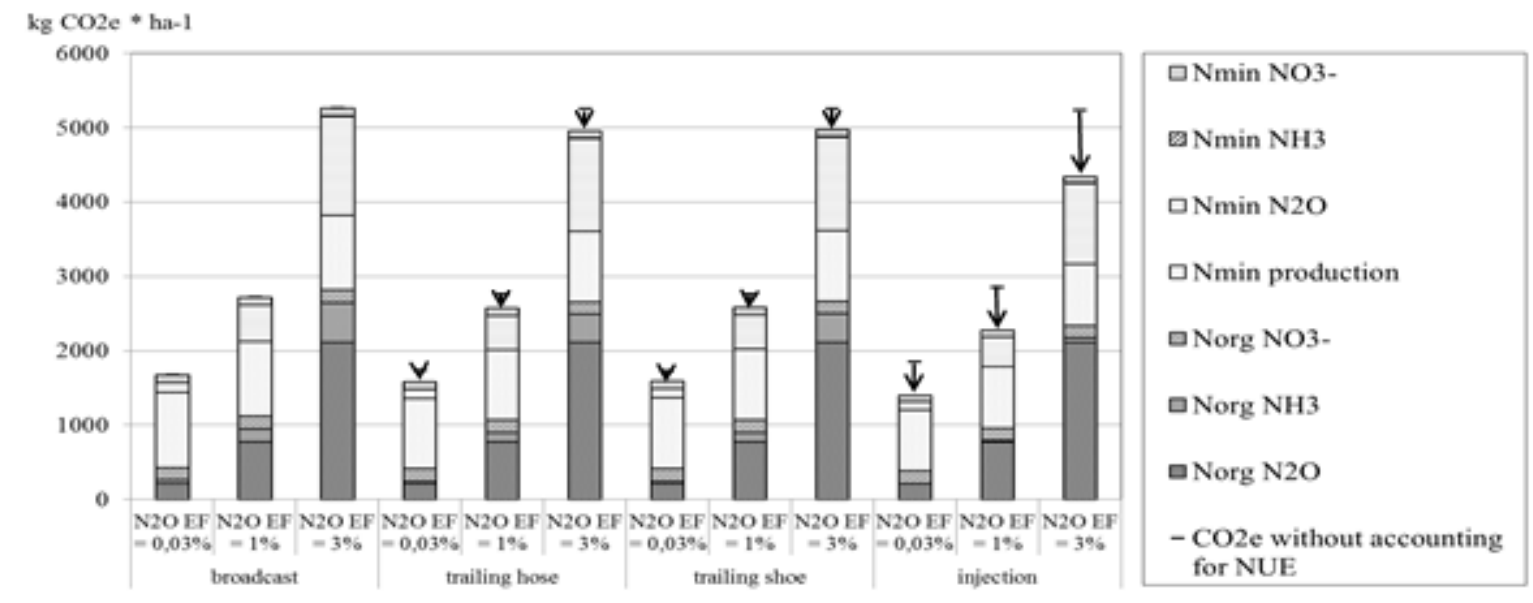

Figure 98. Uncertainty assessment of $\mathrm{N}_{2} \mathrm{O}$-emissions using the IPCC methodology for national ghg inventories as calculation basis, including modelled mineral fertilization under consideration of NUE of different application technologies.

The final proof on $\mathrm{N}_{2} \mathrm{O}$ emissions can't be made as there are only a few studies and long-time assessments are missing. Looking at the ghg emissions for mineral and organic fertilization, the share of upstream emissions of mineral fertilizer makes up a big part of the ghg balance results, especially where low $\mathrm{N}_{2} \mathrm{O}$ emission factors were used.

Lichti, 2013 found $6 \%$ and $2 \%$ higher wheat grain yields with trailing shoe application at the beginning of the vegetation period or to stem elongation stage and $\mathrm{N}$ uptake differences were $9 \%$ and $5 \%\left(100-116 \mathrm{kgN} \mathrm{ha}^{-1}\right)$ compared to trailing hose. From another perspective, to achieve the same DM yields of winter wheat, the trailing hose application would need to apply $16 \%$ more $\mathrm{N}_{\mathrm{t}}$ or $10.9 \mathrm{~kg} \mathrm{~N}$ in absolute numbers. In comparison, our calculations resulted in $9 \mathrm{~kg} \mathrm{~N}$ or $10 \%$ more mineral nitrogen. The relative difference is small. Yield level in the study of Lichti, 2013 was with 72-76 DM dt ha-1 slightly lower than $78 \mathrm{dt}$ DM assumed in our modelling approach.

The magnitude of overall fuel consumption of the winter wheat process chain ranged from 70 to $1001 \mathrm{ha}^{-1}$. However, the organic fertilization amounts as assumed in the KTBL-standard scenario were much lower than our assumptions ( 10 to $15 \mathrm{~m}^{3} \mathrm{ha}^{-1}$ applied in a single field operation compared to $44 \mathrm{~m}^{3}$ ).

Pelletier et al. 2014 found in their study in cooperation with Canadian farmers fuel consumption from soil preparation made up between $32 \%$ and $50 \%$ of fuel consumption over the whole production cycle of winter wheat with values of $39.61 \mathrm{ha}^{-1}$, $55.61 \mathrm{ha}^{-1} 76.01 \mathrm{ha}^{-1}$ for no tillage, reduced tillage and conventional ploughing respectively. Similar values are described by the standard values from KTBL for fuel consumption in wheat cultivation with 35,78 and $841 \mathrm{ha}^{-1}$ for mineral fertilized fields. Pelletier et al., 2014 find values of $1.81 \mathrm{ha}^{-1}$ for fertilization with ammonium nitrate, $14.71 \mathrm{ha}^{-1}$ for cattle slurry and $7.71 \mathrm{ha}^{-1}$ for pig slurry. Additional stirring and loading result in $31.1 \mathrm{l} \mathrm{ha}^{-1}$ and $12.51 \mathrm{ha}^{-1}$ for cattle and pig slurry respectively. Emissions of fuel use for spreading manure on the field are presented in Tab. 3. Our assumptions are included in the table and seem to cover rather small amounts of slurry application or small farm structures but are in line with other findings.

Table 35. Ghg emissions in $\mathrm{kg} \mathrm{CO}_{2}$-equivalent per ha according to model-scenarios.

\begin{tabular}{|c|c|c|c|c|c|c|}
\hline \multirow{2}{*}{$\begin{array}{l}\text { slurry amount; } \\
\text { average field size; } \\
\text { field-farm distance }\end{array}$} & \multirow[t]{2}{*}{ broadcast } & \multirow{2}{*}{$\begin{array}{c}\text { trailing } \\
\text { hose }\end{array}$} & \multicolumn{3}{|c|}{ trailing shoe } & \multirow[t]{2}{*}{ Injection } \\
\hline & & & light & medium & heavy & \\
\hline $5 \mathrm{~m}^{3} ; 2 \mathrm{ha} ; 2 \mathrm{~km}$ & 7 & 11 & 20 & 21 & 21 & 26 \\
\hline $5 \mathrm{~m}^{3} ; 5 \mathrm{ha} ; 14 \mathrm{~km}$ & 15 & 16 & 25 & 26 & 27 & 32 \\
\hline $30 \mathrm{~m}^{3} ; 2 \mathrm{ha} ; 2 \mathrm{~km}$ & 20 & 20 & 28 & 29 & 30 & 36 \\
\hline $30 \mathrm{~m}^{3} ; 5 \mathrm{ha} ; 14 \mathrm{~km}$ & 73 & 59 & 66 & 66 & 67 & 79 \\
\hline scenario assumptions & 12 & 15 & 21 & & & 36 \\
\hline
\end{tabular}

As the presented figures were created using a lot of assumptions and single case studies, they are of a more qualitative nature for understanding coherences. For a more in-depth understanding we want to point to the literature review. 


\section{Conclusion}

Ghg emissions from application of manure are influenced by factors like constitution of manure, technology, soil parameters and weather (Loyon et al., 2016). From what we found in literature, description of technology and information on fertilizers, soils and weather conditions are vital to improve actual knowledge in the field of ghg emissions. The assessment of higher $\mathrm{N}_{2} \mathrm{O}$ emissions due to injection is not yet included in farm and process models to calculate ghg emission balances and there is still a lack of knowledge on ghg emission dynamics. Choosing the precise amount of manure to serve $\mathrm{N}$ needs of plants and achieve profitable yields and qualities is a challenging task for farmers and requires good knowledge of manure efficiencies, timely application effects, soil properties and weather conditions. These effects need to be analysed in more detail in field trials because they are crucial for modelling and to determine $\mathrm{NH}_{3}$ - and $\mathrm{N}_{2} \mathrm{O}$ emissions, $\mathrm{N}$-leaching, or NUE in management systems.

Our findings show, that $\mathrm{NH}_{3}$ mitigation via efficient liquid manure application in combination with reduced mineral fertilizer amounts offer a great ghg mitigation potential and application technology can be an important control variable. In our approach we showed, that the assessment of NUE of organic fertilizers in ghg modelling has a great impact on ghg balance results. Therefore, this approach can advance comprehensive ghg modelling for farms and single processes. The consideration of NUE of organic manures could be used in future studies as a simple alternative to more complex dynamic $\mathrm{N}$ models.

However, the assessment of N-cycle dynamics is still of great importance due to coherences and positive effects of organic fertilization on yield, on soil organisms, stability against erosion and leaching. Effects of application technology on soil structure and constitution (compaction, runoff, denitrification zones, long-term fertility), residue management and crop rotation should be assessed in periods under consideration, which are longer than one year or a single crop vegetation period. To develop efficient and feasible ghg mitigation methods, interaction in the combination with other measures need to be addressed (e.g. choice of fertilizer type, additives like nitrification inhibitors, acidification of manure, crop rotation, intercropping, fertilizer application time and splitting, feeding strategies etc.). Hou et al., 2015 propose the combination of different mitigation options along the manure chain can avoid pollution swapping and can lead to maximum emission reduction. Still, there is research needed in the field of side effects and interactions of mitigation methods. When assessing mitigation measures, also the actual development status, trade-offs and long term effects of those measures need to be considered. For example, the implementation of acidification of manure is hindered as it may lead to a modification of soil properties in $\mathrm{pH}$ and metal concentration and there is still research to be done to assess long term effects (Fangueiro et al., 2015).

Further economic assessment and assessment of effects on yield and quality are needed. As actual fertilization levels are high, a yield effect with a small reduction of fertilizer application is not expected. However, quality might be affected. Mineral fertilizer savings due to higher NUE of organic manure can lead to economic savings. On the other hand, investment costs for new technologies are high and there might be additional effort by applying manure close to the ground and adapted to plant growth (e.g. fuel). For a successful use of precise liquid manure application technology, fertilizer amounts need to be reduced with increasing NUE to avoid losses to other environmental compartments and to go easy on resources (in case of mineral fertilizers). Knowledge on $\mathrm{N}$ use efficiency like fertilizer needs and plant available nitrogen are the key to environmental protection (ghg emissions, $\mathrm{NH}_{3}$ emissions) and cost effectiveness of liquid manure application technology.

\section{Acknowledgement}

The conference contribution was elaborated within a project on ghg mitigation in agriculture (FKZ: $\mathrm{KL} / 16 / 03$ ) funded by the Bavarian state ministry for Food, Agriculture and Forestry (BayStMELF).

\section{References}

Brink, C.; Grinsven, H. (2011): Costs and benefits of nitrogen in the environment. 513-540pp IN: The European Nitrogen Assessment, ed. Mark A. Sutton, Clare M. Howard, Jan Willem Erisman, Gilles Billen, Albert Bleeker, Peringe Grennfelt, Hans van Grinsven and Bruna Grizzetti. Published by Cambridge University Press

Chardon, X.; Rigolot, C.; Baratte, C.; Espagnol, S.; Raison, C.; Martin-Clouaire, R.; Rellier, J.P.; Le Gall, A.; Dourmad, J. Y.;Piquemal, B.; Leterme, P.; Paillat, J.M.; Delaby, L.; Garcia, F.; Peyraud, J.C.; Morvan, T.; Faverdin, P. (2012): MELODIE: a whole-farm model to study the dynamics of nutrient in dairy and pig farms with crops. Animal 6-10, p. 1711-1721. DOI: 10.1017/S1751731112000687

Chadwick, D.; Sommer, S.; Thorman, R.; Fangueiro, D.; Cardenas, L.; Amon, B.; Misslebrook, T. (2011): Manure management: Implications for greenhouse gas emissions. Animal Feed Science and Technology 166-167, p. $514-531$ 
Collins, A.L.; Price, J.P.; Zhang, Y.; Gooday, R.; Naden, P.S.; Skirvin, D. (2018): Assessing the potential impacts of a revised set of on-farm nutrient and sediment "basic" control measures for reducing agricultural diffuse pollution across England. Science of the Total Environment 621, p.1409-1511

EUC (European Commission), (2017): Commission implementing decision (EU) 2017/302 of 15 February 2017 establishing best available techniques (BAT) conclusions, under Directive 2010/75/EU of the European Parliament and of the Council, for the intensive rearing of poultry or pigs (notified under document C(2017) 688). L43/231 Official Journal of the European Union

Fangueiro, D.; Surgy, S; Fraga, I.; Cabral, F.; Coutinho (2015): Band application of treated cattle slurry as an alternative to slurry injection: Implications for gaseous emissions, soil quality and plant groth. Agriculture, Ecosystems and Environment 211, p.102-111

Gunningham, N.; Sinclair, D. (2005): Policy instrument choice and diffuse source pollution. Journal of Environmental Law 17-1, p. 51-81, DOI: 10.1093/envlaw/eqi003

Haenel, H.-D.; Rösemann, C.; Dämmgen, U.; Döring,U.; Wulf, S.; Eurich-Menden, B.; Freibauer, A.; Döhler,H.; Schreiner, C.; Osterburg, B. (2018): Calculations of gaseous and particulate emissions from German agriculture 1990 - 2016 Report on methods and data (RMD) Submission 2018. Thünen Report 57. Braunschweig, Germany ISBN 978-3-86576-181-1

Hafner, S. D.; Pedersen, S. V.; Pacholski, A. (2015): The ALFAM2 project - Predicting ammonia loss from field-applied manure. RAMIRAN 2015 (Recycling of Agricultural, Municipal and Industrial Residues in Agriculture Network) - 16th International Conference Rural-Urban Symbiosis, 8th - 10th September 2015, Hamburg, Germany

Hou, Y.; G. L. Velthof; O. Oenema (2015): Mitigation of ammonia, nitrous oxide and methane emissions from manure management chains: a meta analysis ands integrated assessment. Global change biology 21, p. 1239-1312 DOI: $10.1111 / \mathrm{gcb} .12767$

Huijsmans, J. F. M.; Vermeulen, G. D.; Hol, J. M. G.; Goedhart, P. W. (2018): A model for estimationg seasonal trends of ammonia emission from cattle manure applied to grassland in the Neatherlands. Atmospheric Environment 173, p. 231-238

IPCC (2006): IPCC guidelines for national greenhouse gas inventories. Volume 4 Agriculture, Forestry and Other Land Use. Hayama: Institute for Global Environmental Strategies (IGES).

IPCC (2013): Climate Change 2013: The Physical Science Basis. Contribution of Working Group I to the Fifth Assessment Report of the Intergovernmental Panel on Climate Change [Stocker, T.F., D. Qin, G.-K. Plattner, M. Tignor, S.K. Allen, J. Boschung, A. Nauels, Y. Xia, V. Bex and P.M. Midgley (eds.)]. Cambridge University Press, Cambridge, UK/New York, NY, USA, 1535 pp.

KTBL (2016): Berechnungsstandard für einzelbetriebliche Klimabilanzen (BEK) in der Landwirtschaft. AG-

BEK - Handbuch. (In German)

https://www.ktbl.de/fileadmin/user upload/Allgemeines/Download/BEK/Handbuch.pdf Accessed: 08.06.2018

KTBL, (2018a): Verfahrensrechner Pflanze. Web-Application (In German) Kuratorium für Technik und Bauwesen in der Landwirtschaft e.V., Darmstadt, Germany https://daten.ktbl.de/vrpflanze/home.action;jsessionid=2ACC224FC82CA111DDE4C72F37AF6549 Accessed: 11.06 .2018

KTBL, (2018b): Dieselbedarfsrechner. Web-Application (In German) Kuratorium für Technik und Bauwesen in der Landwirtschaft e.V., Darmstadt, Germany https://daten.ktbl.de/dieselbedarf/main.html Accessed: 11.06.2018

LfL, (2017): Deckungsbeitragsrechner. Bayerische Landesanstalt für Landwirtschaft (LfL) https://www.stmelf.bayern.de/idb/default.html Accessed:11.06.2018

LfL, (2018): Nmin für Wintergetreide und Winterraps. Bayerisches landwirtschaftlichen Wochenblatt 208 9/2018, p. 40 Bayerische Landesanstalt für Landwirtschaft, Freising. http://www.lfl.bayern.de/iab/duengung/028149/index.php Accessed: 01.06.2018

Lichti, F. H. (2013): Bewertung und Optimierung der Nährstoff und Umweltwirkung von Gärrückständen aus der Biogasgewinnung. (In German) Dissertation. Technical University of Munich, Chair of Plant Nutrition. Germany

Loyon, L.; Burton, C.H.; Misselbrook, T.; Webb, J.; Philippe, F.X.; Aguilar, M.; Doreau, M.; Hassouna, M.; Veldkamp, T.; Dourmad, J. Y.; Bonmati, A.; Grimm, E.; Sommer, S. G. (2016): Best available technology for European livestock farms: Availability, effectiveness and uptake. Journal of Environmental Management 166, p. 1-11

Maze, M.; Zerhusen, B.; Effenberger M. (2017): A database approach for simulating the produced greenhouse gas emissions from biogas plants, Chemical Engineering Transactions 58, p. 595-600 DOI: 10.3303/CET175810

Oenema, O.; Gyldenkaerne, S.; Oenema, J. (2015): Economic costs of nitrogen management in Agriculture. In: S. Reis et al. Costs of ammonia abatement and the climate Co-benefits. Chapter 2. Springer Science \& Bussiness Media Dordrecht. DOI 10.1007/978-94-017-9722-1_2 
Osterburg, 2017: Die Rolle der Landwirtschaft in der Klimaschutzpolitik. (In German) Agrarwissenschaftliches Symposium. Herausforderung Klimawandel. 21.09.2017 Hans Eisemann-Zentrum, Freising.

http://www.hez.wZw.tum.de/fileadmin/Agrarwissenschaftliches Symposium/AgrarSymp 2017/Praesentationen/ Osterburg Landwirtschaft und Klimaschutzpolitik 92017 v1.pdf Accessed: 01.06.2018

Patterson, J. J.; Smith, C; Bellami, J. (2013): Understanding enabling capacities for managing the 'wicked problem' of nonpoint source water pollution in catchments: A conceptual framework. Journal of Environmental Management 128, p. 441-452

Petersen, S. O. (2011): Methane and Nitrous oxide emissions from livestock manure: The scientific basis. Emissionen in der Tierhaltung - Treibhausgase, Umweltbewertung, Stand der Technik. KTBL Tagung 06.08.12.2011, Kloster Banz, Bad Staffelstein KTBL Schrift 491 ISBN 978-3-941583-59-7

Pelletier, F.; Godbout, S.; Belzile, L.; Li, J. (2014): Modelling fuel use for specific farm machinery and operations of wheat production IN: Schenck, R., Huizenga, D. (Eds.), 2014. Proceedings of the 9th International Conference on Life Cycle Assessment in the Agri-Food Sector (LCA Food 2014), 8-10 October 2014, San Francisco, USA

Rodhe, L.; Pell, M.; Yamulki, S. (2006): Nitrous oxide, methane and ammonia emissions following slurry spreading on grassland. Soil Use Management 22, p. 229-237 DOI: 10.1111/j.1475-2743.2006.00043.x

Santonja, G. G.;Georgitzikis, K; Scalet, B.; Montobbio, P.; Roudier, S; Sancho, L. D. (2017): Best Available Techniques (BAT) Reference Document for the Intensive Rearing of Poultry or Pigs - Industrial Emissions Directive 2010/75/EU (Integrated Pollution Prevention and Control) ISBN 978-92-79-70214-3

Schmidt, J. (2014): Potentiale der Energieeinsparung im Ackerbau und Grünland. (In German) Land-EnergieTag im Landkreis Starnberg. Landtechnik- und Energieberater FZ 3.11 AELF Ingolstadt

Sistani, K. R.; Warren, J. G.; Lovanh, N.; Higgins, S.; Shearer, S. (2010): Greenhouse gas emissions from swine effluent applied to soil by different methods. Soil Science Society of America Journal 74 - 2, p. $429-435$

Søgaard, H.T.; Sommer, S.G.; Hutchings, N.J.; Huijsmans, J.F.M.; Bussink, D.W.; Nicolsol, F. (2002): Ammonia volatilization from field-applied animal slurry - the ALFAM model. Atmospheric Environment 36, p. 3309-3319

Tamm, K.; Vettik, R.; Viil, P.; Võsa, T. (2016): Comparative survey of manure spreading technologies. Estonian Crop Research Institute Jānis Kažotnieks Latvian Rural Advisory and Training Centre. The survey was commissioned by the Estonian Chamber of Agriculture and Commerce and NGO Latvian Farmers Parliament

Thorman, R. E. (2011): Nitrous oxide emissions following the application of livestock manure - an integrated approach. Emissionen in der Tierhaltung - Treibhausgase, Umweltbewertung, Stand der Technik. KTBL Tagung 06.-08.12.2011, Kloster Banz, Bad Staffelstein KTBL Schrift 491 ISBN 978-3-941583-59-7

Thomsen, I. K.; Pedersen, A. R.; Nyord, T.; Petersen, S. O. (2010): Effects of slurry pre-treatment and application technique on short-term $\mathrm{N}_{2} \mathrm{O}$ emissions as determined by a new non-linear approach. Agriculture Ecosystem and Environment 136, p. 227-235

UBA (2018): Emissionen ausgewählter Luftschadstoffe nach Quellkategorie. (In German). https://www.umweltbundesamt.de/sites/default/files/medien/384/bilder/3 tab_emi-ausgew-luftschadst_2017-0612 0.png Accessed: 06.02.2018

Webb J, Eurich-Menden B, Dämmgen U, Agostini F (2009): Review of published studies estimating the abatement efficacy of reduced-emission slurry spreading techniques. In: Sutton MA, Reis S, Baker S (Hrsg.): Atmospheric Ammonia: detecting emission changes and environmental impacts; results of an Expert Workshop under the Convention on Long-range Transboundary Air Pollution. Springer, Dordrecht, p. 195-202.

Webb, J.; Pain, B.; Bittman, S.; Morgan, J. (2010): The impacts of manure application methods on emissions of ammonia, nitrous oxide and on crop response-A review. Agriculture, Ecosystems and Environment 137, p. 39-46 DOI:10.1016/j.agee.2010.01.001

Webb J., Morgan J., Pain B. (2015) Cost of Ammonia Emission Abatement from Manure Spreading and Fertilizer Application. In: Reis S., Howard C., Sutton M. (eds) Costs of Ammonia Abatement and the Climate CoBenefits. Springer, Dordrecht. DOI https://doi.org/10.1007/978-94-017-9722-1_6

Weidema, B. P. et al. (2013): The ecoinvent database: Overview and methodology, Data quality guideline for the ecoinvent database version 3, www.ecoinvent.org Accessed: 01.06.2018

Wendland, M.; Diepolder, M.; Offenberger, K.; Raschbacher, S. (2018): Leitfaden für die Düngung von Ackerund Grünland Gelbes Heft Stand: 2018 (In German). LfL-Information. Bayerische Landesanstalt für Landwirtschaft (LfL). Freising, Januar 2018.

Wulf, S.; Maeting, M.; Bergmann, S.; Clemens, J. (2001): Simultaneous Measurement of NH3, N2O and CH4 to Assess Efficiency of Trace Gas Emission Abatement After Slurry Application. Phyton 41 (Austria) Special Issue: Nitrogen emissions, p. 131-142

Wulf, S. (2003): Untersuchung der Emission direkt und indirekt klimawirksamer Spurengase (NH3, N2O und $\mathrm{CH} 4$ ) während der Lagerung und nach der Ausbringung von Kofermentationsrückständen sowie Entwicklung von Verminderungsstrategien (In German). Project Report- Deutsche Bundesstiftung Umwelt. DBU-AZ 08912. 


\title{
Groundwater Utilization in a Farm Heat Network
}

\author{
Genta Kanai a,", Yoshimichi Yamashita ${ }^{\text {a }}$ \\ a Tohoku Agricultural Research Center, Fukushima Research Station, National Agricultural Research Organization \\ (NARO), Fukushima City, Fukushima Pref., Japan. \\ * Corresponding author. Email: kanaigen@affrc.go.jp
}

\begin{abstract}
There are various demands for heating and cooling in a farm. The dryer of grain, raising seedling and heating of greenhouse and farm office or working space in winter have demands of heating. And refrigerators for products and cooling of greenhouse in summer have demands of cooling. Our purpose is to reduce the energy consumption in a farm by building the Farm Heat Network (FHN) that is consisted of networks of hot and cold water and heaters and coolers. Hot water will be supplied to the network from biomass boiler, solar heater and other heat sources. Cold water will be supplied to the other network from heat pump or other cold sources. As a part of that system groundwater can be utilized for both heat and cold sources. Because the temperature of groundwater keeps almost the same temperature throughout the year it can be utilized for cooling in summer and heating in winter. We built a system to heat and cool green house for flower production utilizing groundwater. The capacity for cooling and heating of the system was examined as a part of FHN. A result of winter night trial of our system was as follows, the average temperature of input air to the heat exchanger was $0.4{ }^{\circ} \mathrm{C}$, the output air $8.7^{\circ} \mathrm{C}$, the input groundwater temperature to the heat exchanger $10.1^{\circ} \mathrm{C}$, the output $4.6^{\circ} \mathrm{C}$ and the average heating capacity $5.1 \mathrm{~kW}$ with the groundwater flow rate $13.5 \mathrm{~L} / \mathrm{min}$. And a result of summer night trial of our system was as follows, the average temperature of input air to the heat exchanger was $20.4{ }^{\circ} \mathrm{C}$, the output air $17.2{ }^{\circ} \mathrm{C}$, the input groundwater temperature to the heat exchanger $14.9^{\circ} \mathrm{C}$, the output $19.6^{\circ} \mathrm{C}$ and the average cooling capacity 4.2 $\mathrm{kW}$ with the groundwater flow rate13.1 L/min.
\end{abstract}

Keywords: groundwater, heating, cooling, green house, heat efficiency

\section{Introduction}

Our purpose is to reduce the energy consumption in a farm by building the Farm Heat Network (FHN) that is consisted of networks of hot and cold water and heaters and coolers. Hot water will be supplied to the network from biomass boiler, solar heater and other heat sources. Cold water will be supplied to the other network from heat pump or other cold sources. But those hot or cold water must be produced artificially heated or cooled.

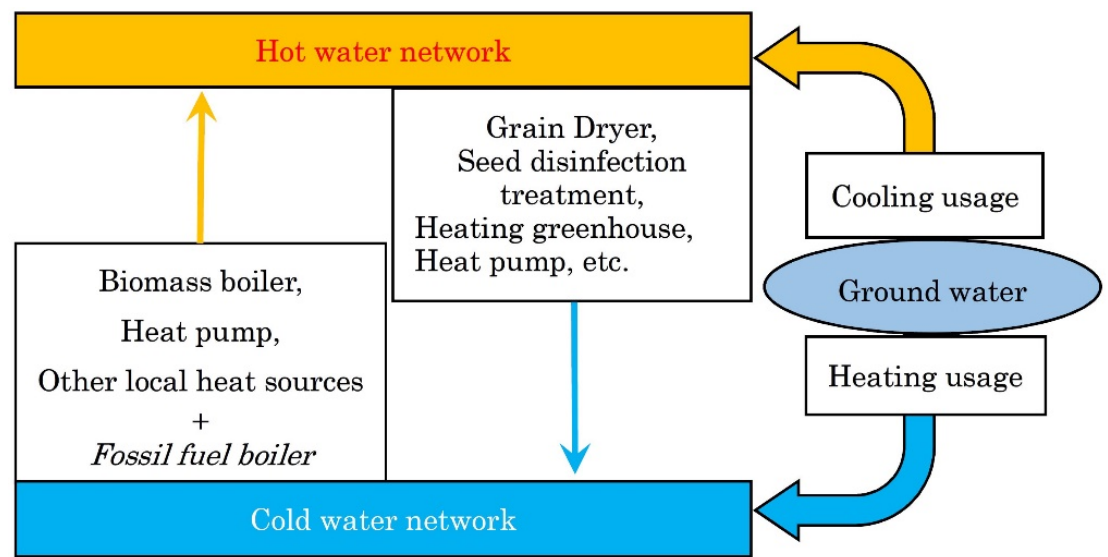

Figure 1. Concept of Farm Heat Network (FHN) utilizing ground water.

On the other hand, the ground water keeps stable temperature through a year. And many farms located in mountain side have their rich source of ground water, spring or well. The temperature of the ground water is almost same temperature as the average ambient temperature of the place. That means it can be used as heat source in winter and cold source in summer. Output water of cooling usage will be supplied to hot water network of FHN because it has absorbed heat through cooling usage. And output water of heating usage will be supplied to the cold water network of FHN because it has been cooled through heating usage.

As a part of the system we examined the heating and cooling system for greenhouse utilizing groundwater. The greenhouse has almost $400 \mathrm{~m} 2$ located on the mountain area of Fukushima Pref. Japan. Our system does not have enough capacity for the size of the greenhouse. The system was examined especially on its basic validity and capability. 


\section{Materials and Methods}

Greenhouse

The greenhouse is used for flower production from June to September without heater or cooler. The production area is almost $400 \mathrm{~m}^{2}$. and located on the mountain area of Fukushima Pref. Japan.

\section{Groundwater}

The farm has a well and the groundwater is utilized for spraying the flowers of its greenhouses. But as a water source it can afford for other usage. And a heat exchanger was connected to the well.

\section{Heat Exchanger}

The heat exchanger from groundwater to the air of the greenhouse was made with 3 radiators. Those are originally parts of car with $3 \mathrm{~L}$ diesel engine. The three radiators were connected serially

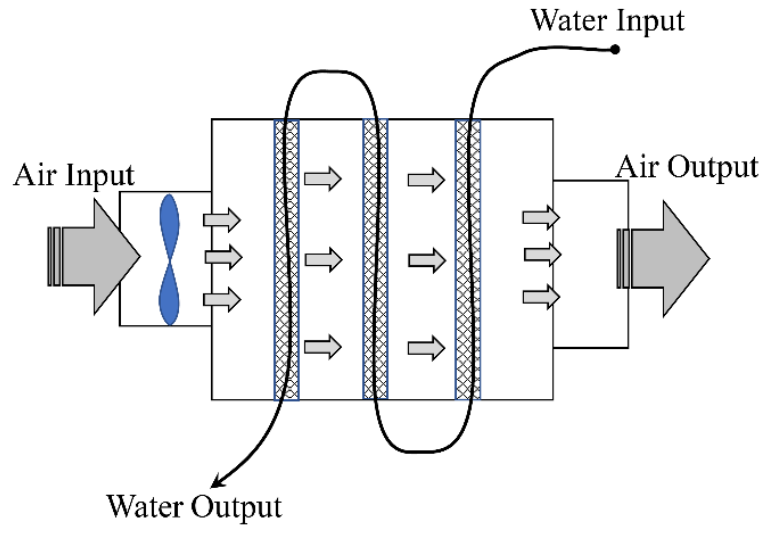

(a) Schematic of the heat exchanger.

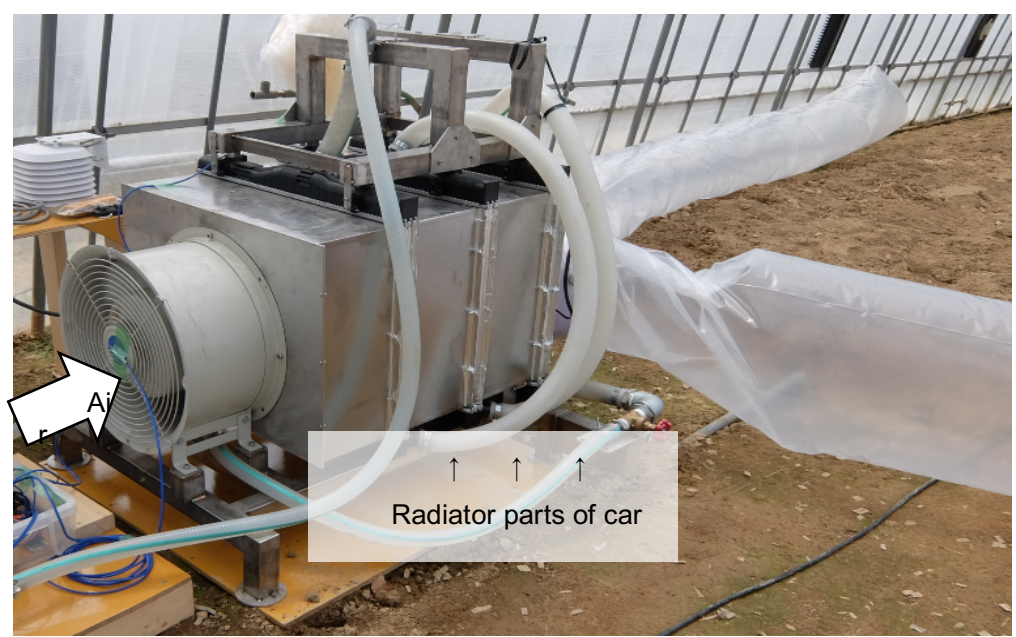

(b) Picture of the heat exchanger in a greenhouse

Figure 2. Heat exchanger used in our trial.

Data Logging

Temperatures of water input and output, air input and output and ambient air of inside and outside of the greenhouse were measured and logged by every second with a data logger (GL240, Graphtec.)

\section{Results and Discussion}

Heating test

The trial of greenhouse heating was held and a result was shown in Fig.3 and Fig.4. The trial was held at a 
winter night, 18:00-6:00. The groundwater was pumped up and stored in a tank and supplied to the heat exchanger by another pump.

The temperature of the input water to the heat exchanger seems stable through the night and the average was $10.1^{\circ} \mathrm{C}$. The temperature of the output water shows fluctuated and the average was $4.6^{\circ} \mathrm{C}$. The fluctuation seems as the result of fluctuation of flow ratio of water. The average flow ratio was $13.5 \mathrm{~L} / \mathrm{min}$. and the fluctuation is caused by the pump control logic to keep the certain pressure in the bottom of the water tank.

The average temperature of input air to the heat exchanger, the inside air temperature of the greenhouse, was $0.4^{\circ} \mathrm{C}$. The input air temperature changed around $0^{\circ} \mathrm{C}$, the small changes seems as the result of the effect of outside temperature and wind of the greenhouse. The average temperature of output air from the heat exchanger, the heating air, was $8.7^{\circ} \mathrm{C}$, higher than the input by $8.3^{\circ} \mathrm{C}$.

As the result of heating test the average heating capacity was $5.1 \mathrm{~kW}$. The heat output was calculated based on the difference of the input and output temperature and the flow ratio of water. The heating capacity was too small for the greenhouse size and it is difficult to examine the effect of our system to the greenhouse temperature.

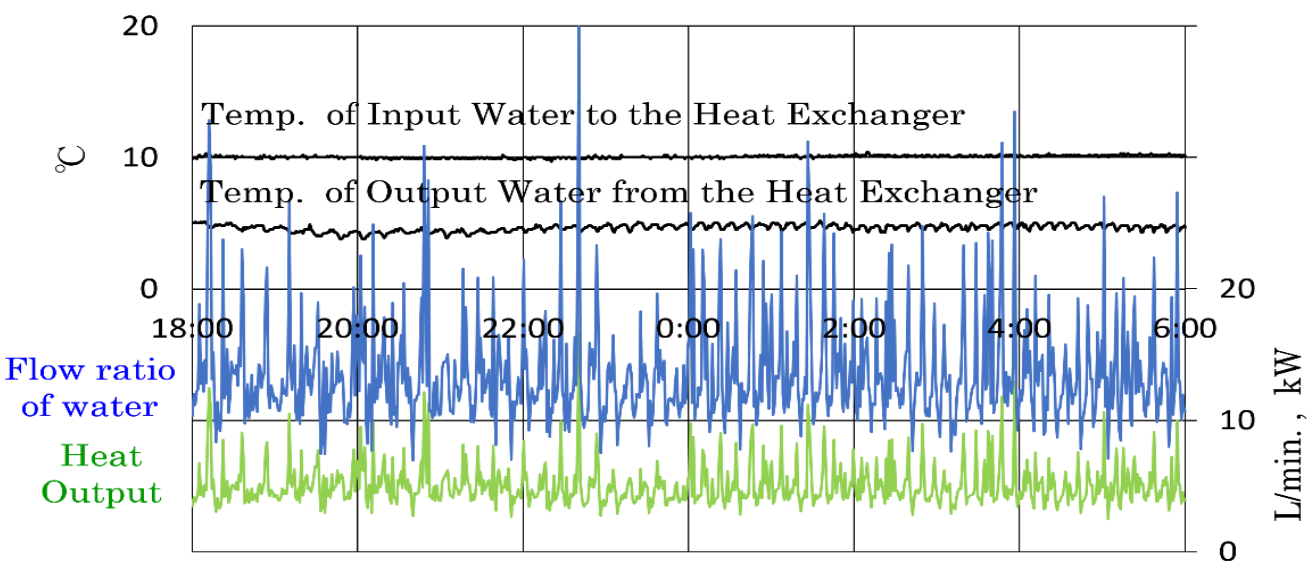

Figure 3. Heat output and temperature changes of water input and output of the heat exchanger in a heating test.

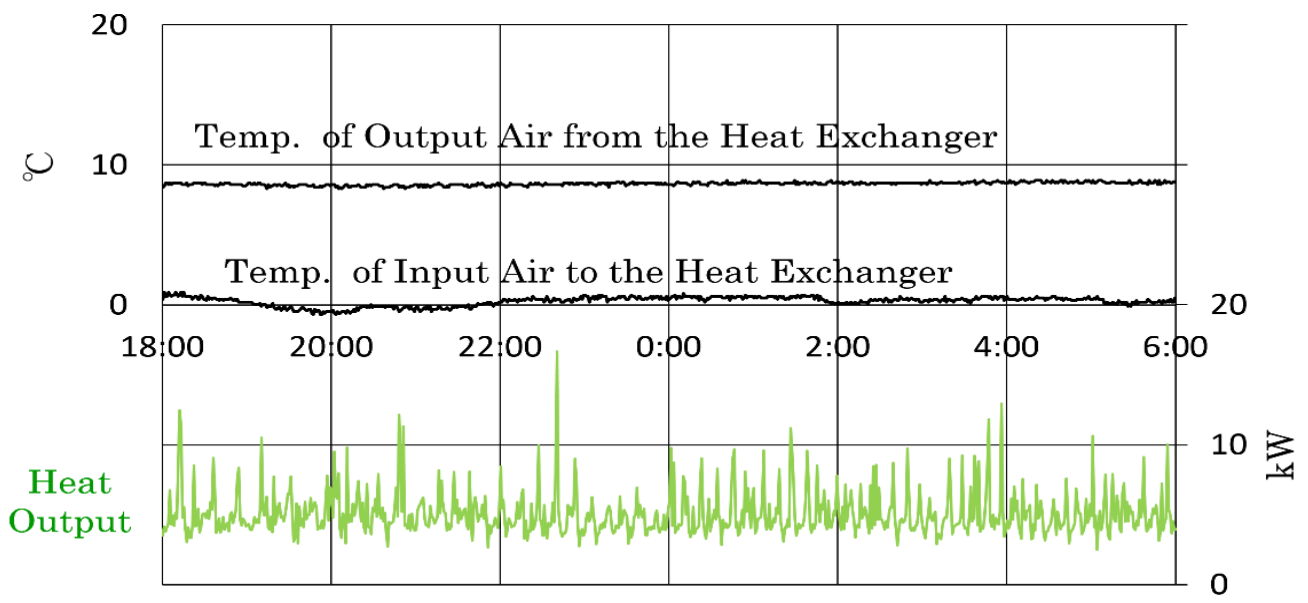

Figure 4. Heat output and temperature changes of air input and output of the heat exchanger in a heating test.

\section{Cooling test}

The trial of greenhouse cooling was held and the result was shown in Fig.5 and Fig.6. The trial was held at a summer night, 18:00-6:00. The groundwater was pumped up and stored in a tank and supplied to the heat exchanger by another pump, those are same conditions as the heating test.

The temperature of the input water to the heat exchanger seems stable through the night and the average was $14.9^{\circ} \mathrm{C}$. The temperature of the output water was almost $22^{\circ} \mathrm{C}$ at $18: 00$ and $18^{\circ} \mathrm{C}$ at $3: 00$ the average was $19.6^{\circ} \mathrm{C}$. The temperature changing seems effected by the changes of the input air temperature. The average flow ratio of water was $13.1 \mathrm{~L} / \mathrm{min}$. and the fluctuation is caused by the pump control logic, the same phenomena as the heating test.

The average temperature of input air to the heat exchanger, the inside air temperature of the greenhouse, was $20.4^{\circ} \mathrm{C}$. The input air temperature showed decreasing from $23^{\circ} \mathrm{C}$ at $18: 00$ to $19^{\circ} \mathrm{C}$ at 3:00. That can be explained 
as a part of daily temperature changing of the ambient air. The average temperature of output air, the cooling air temperature, was $17.2^{\circ} \mathrm{C}$, lower than the input by $3.2^{\circ} \mathrm{C}$

As the result of cooling test, the average of cooling capacity was $4.2 \mathrm{~kW}$. The heat output was calculated based on the difference of the input and output temperature and the flow ratio of water. The capacity was too small for the greenhouse size and it is difficult to examine the effect of our system to the greenhouse temperature.

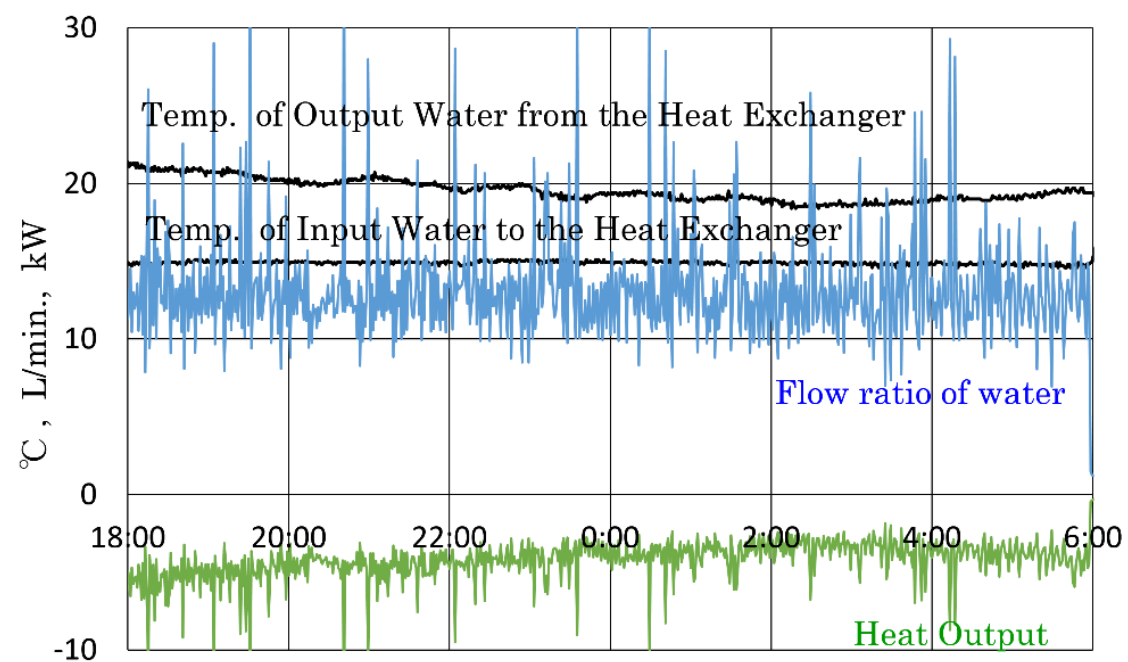

Figure 5. Heat output and temperature changes of water input and output of the heat exchanger in a cooling test.

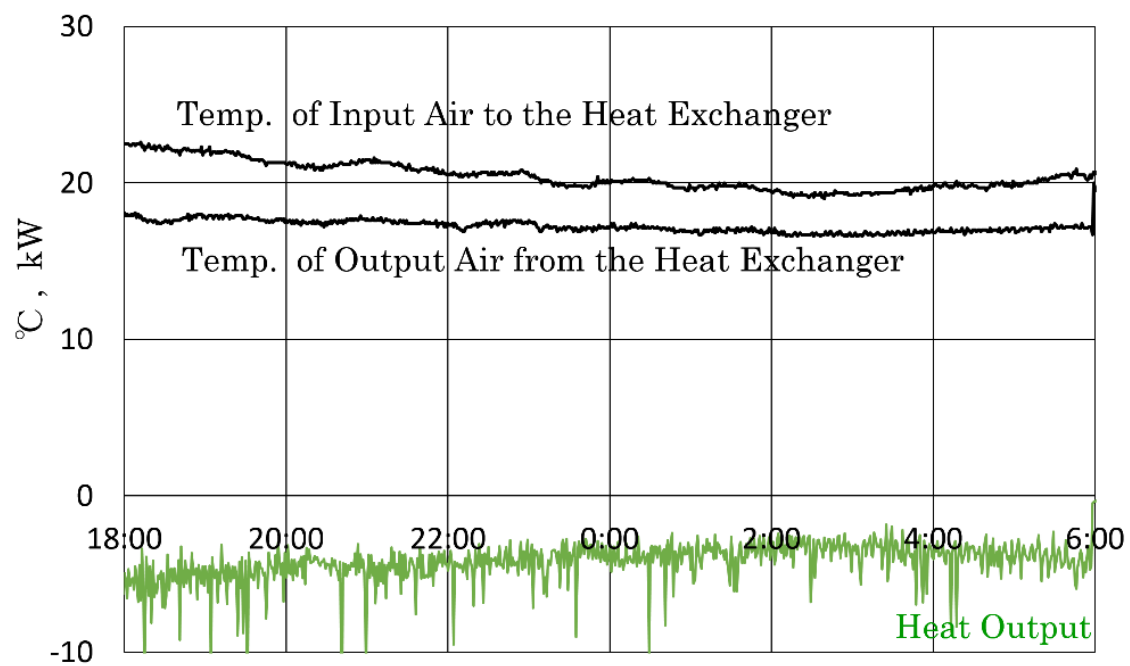

Figure 6. Heat output and temperature changes of air input and output of the heat exchanger in a cooling test.

\section{Conclusions}

Here the same system was used for heating and cooling. And for both usage a certain capacity was demonstrated as a first step. The heating capacity was $5.1 \mathrm{~kW}$, the cooling $4.7 \mathrm{~kW}$. The reason of the difference of capacities seems caused by the difference of temperatures of the input water and the input air. In the heating test the difference was $9.7^{\circ} \mathrm{C}$, the water was $10.1^{\circ} \mathrm{C}$ and the air $0.4^{\circ} \mathrm{C}$. In the cooling test the difference was $5.5^{\circ} \mathrm{C}$, the water was $14.9^{\circ} \mathrm{C}$ and the air $20.4^{\circ} \mathrm{C}$. In next step the appropriate capacity heating and cooling system to the greenhouse will be introduced and examined. And the final purpose of the research is to construct the FHN system containing the groundwater utilizing system.

\section{References}

Kanai,G. and Kowata, H. 2016. Wood Boiler as a Multi Solid Fuel Burner in a Farm Heat Network. http://conferences.au.dk/uploads/tx powermail/2016cigr ageng full paper template kanai.pdf, Accessed June $7,2018$. 


\title{
Sustainability through Digital Agriculture - Analysis of Available Greenhouse Gas Emission Indicators in Farm Management Systems
}

\author{
Heinz Bernhardt a ${ }^{\text {,* }}$, Leonie Spiller ${ }^{\text {a }}$, Omar Hijazi ${ }^{\text {a }}$ \\ a Agricultural System Engineering, Technical University of Munich, 85354 Freising, Germany \\ * Corresponding author. Email: heinz.bernhardt@wzw.tum.de
}

\begin{abstract}
Considering all sources and influencing factors of greenhouse gas (GHG) emissions in agriculture, the individual and accurate calculation of GHG emissions is a challenge. As farmers must report several farming activities, a growing number of farmers are using farm management information systems (FMIS) to do so. Additionally, agricultural equipment is becoming increasingly digital; therefore automating GHG calculation of farms seems possible. This thesis examined whether calculating agricultural GHG emissions with the help of data from FMIS, geo-information systems (GIS) and ISOBUS is possible. For this purpose, all required input data was aligned with available data from automated sources. For all emissions related to the topic of soil and plants, 40 different data inputs are needed: 21 can be directly transferred from sources such as ISOBUS or FMIS, four of them have to be calculated out of several available data and 15 of them are not available from digital sources. Emissions from agricultural machinery require 15 different data inputs: nine of them are directly available from digital sources, one of them has to be indirectly calculated and five of them are not available via automated sources. The calculation of GHG emissions from cattle and dairy requires 43 data inputs: 26 of them are available through automated sources, two are indirectly available and 15 of them are not available. The reference value for the required accuracy grade was set to 1.00: the average accuracy of available data in the area soil and plants was 1.01 , for agricultural machinery 1.03 and for animals 0.97 . The examination points out that it is indeed possible to calculate GHG emissions in agriculture with the help of digital sources. Yet, for some of the calculations, literature values are still needed. For further steps regarding the research topic, this thesis recommends to evaluate farmers' perspective on automated GHG calculations and to compare automated GHG calculations with average GHG values in agriculture.
\end{abstract}

Keywords: Digital farming, Sustainability, Carbon Footprint, Smart Farming

\section{Introduction}

The debate on sustainability is playing a more and more important role in European agriculture. The greenhouse gas emissions induced by production processes are a decisive factor in this process. On the one hand, they are a decisive factor in the commitment of individual states to reduce greenhouse gas emissions. On the other hand, there is an increasing demand for information about emissions by food retailers as these are a marketing instrument.

A recent description of sustainable development from 1987 is by definition a development that does not only satisfy the needs of our generation, but also does not neglect the needs of future generations (Brundtland, 1987). In addition, other levels have been added to the term sustainability, which are equally important. The so-called triple-bottom-line addresses the three dimensions of sustainability: social, environmental and economic aspects. Considering them together is difficult, especially because there is no common unit of measurement (Slaper \& Hall, 2011).

Sustainability in agriculture has additional dimensions and a special relevance. This is based on the complexity of agriculture and its numerous positive and negative effects on the environment: on the air, the soil, fauna and flora and the people involved. These result in many aspects which build a sustainable agriculture: higher productivity, more efficient use of resources, education, improved water quality, clean fuel production and food safety. The relevance of these factors varies regionally, especially between developing and industrialized countries (Lal, 2008).

Above all, sustainable agriculture takes into account long-term goals and can look very different from one region to another, which is influenced by the various climatic and environmental conditions. It is also described as dynamic in regards to respond to changes in the environmental, social or economic environment, for example through innovation. Sustainable agriculture also strives for a holistic perspective in which all aspects of the system are considered (Horrgian, Lawrance \& Walker, 2002). Simply put, agriculture is only sustainable if the output of all components is in balance with the input (Lal, 2008)

\section{Materials and Methods}

In general, farmers have to document a wide range of activities, so more and more farmers switch to farm management systems. It will be used to calculate agricultural greenhouse gas emissions with the help of automated 
data from Farm Management Systems.

Based on the IPCC system and the following balancing models (IPCC, 2013; Hülsbergen, 2002; Zehetmeier, Zickgraf, Effenberger \& Zerhusen, 2017; Denef, Paustian, Archibeque, Biggar \& Pape, 2012; EPA, 2005) in agriculture, the required input data for the calculation of GHG emissions are extracted. Table 1 can be seen as an example of this treatment, which also contains information about the unit and purpose.

Table 36. Input data required for direct engine emissions.

\begin{tabular}{ccc}
\hline Required input data & unit & purpose \\
\hline fuel consumption $*$ & 1 & emissions \\
number of machines of one type & - & fuel consumption farm level \\
annual operating hours & $\mathrm{h}$ & fuel consumption farm level \\
futed power & $\mathrm{kW}(\mathrm{IPCC})$ & fuel consumption farm level \\
utilization & $(0-1)$ & EF \\
vehicle type & - & EF \\
fuel type & - & fuel consumption area level \\
working width & $\mathrm{m}$ & fuel consumption area level \\
working depth & $\mathrm{cm}$ & fuel consumption area level \\
mass & $\mathrm{kg}$ & fuel consumption area level \\
ha & &
\end{tabular}

${ }^{*}$ If fuel consumption exists

To review the research questions, these are compared with the automated data sources. Automated data sources are defined as sources of input data that can be automatically read from other GHG emission calculation software systems. These are for example DDI's from ISOBUS, data from FMIS and GIS. In addition, their accuracy level is checked by matching the required unit size with the available unit size. If a smaller / more accurate unit is available than needed, a higher level of accuracy is assumed. If the available data source does not completely correspond to the required input data but is only a part of it, a literature value is often necessary. This does not imply any digital data availability, so they have to be offset with literature values before use in order to match the required input data. This is indicated in the result tables by the source value "literature value".

The focus of this work is on agriculture, livestock (only dairy cattle and cattle) and agricultural machinery. Not included in the analysis and literature searches are the areas of biogas / fermentation, land use change, combustion of organic material, special crop and rice cultivation and the keeping of other farm animals (except dairy cattle and cattle). The unobserved areas were not considered due to their complexity or low distribution / application in Germany and not because of their relevance to your GHG potential.

\section{Results and Discussion}

The results are subdivided into the areas soil / plant, machinery and animals. The required data for the sustainability analysis is compared with the available data from automated sources. If the available values are available in a more accurate unit and can be read out directly, these results in an accuracy value of 1.1. If the value is available in the same unit, this results in an accuracy value of 1 . With indirect calculation or a more inaccurate unit, this results in an accuracy value of 0.9 and thus more inaccurate than the required level. Literature values are not classified in their type of use as non-automated, since these results are only intended to represent which data can be read individually. In practice, however, it would then be possible to store the required values from the literature as a database.

\section{Soil / plant}

The results of the Soil and Plants category match required input data with available data in the areas of nitrogen emissions, $\mathrm{CO} 2$ emissions and mineral fertilizers. For this purpose, DDIs (via ISOBUS), FMIS and GIS will be used.

In total 40 different emission indicators are needed to calculate emissions from soil and plants. 21 of the required data are directly readable, for example from FMIS or ISOBUS. Four are available via indirect calculation, which means that they can be calculated indirectly through several readable factors. 15 required indicators are at least partially not automatically readable, so these data would usually have to be determined by literature values and thus not individually. Nevertheless, one could automate this data to the extent that required literature values are stored as databases.

In the overall average of the soil and plant data, the accuracy level is 1.01 and thus 0.01 just above the required accuracy level of 1 . 


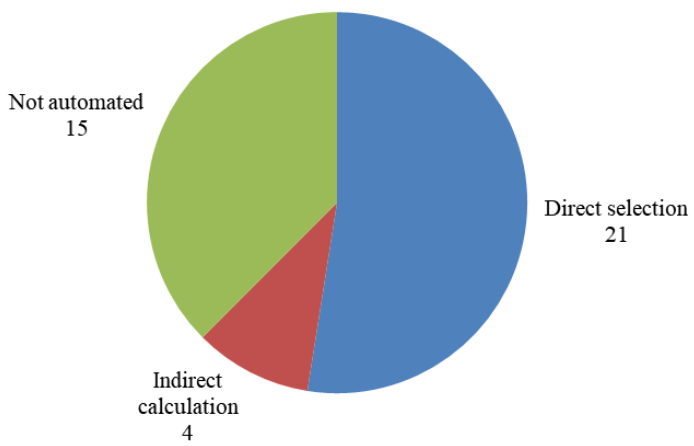

Figure 1. Data availability soil and plant.

Machinery

The chapter "Results Machine Emissions and Data" compares available data for direct and indirect emissions with required input data. For this purpose, DDIs (via ISOBUS) and FMIS will be used.

In total, 15 required input data are identified. Nine are directly readable and thus automatically available. A required value has to be calculated indirectly over several automated values and five values are not available or at least partially not automated - this is especially true for the range of stationary machines. On average, the accuracy level at the scale described at the beginning of Chapter 4 is 1.03 , which is 0.03 above the required accuracy level of 1 .

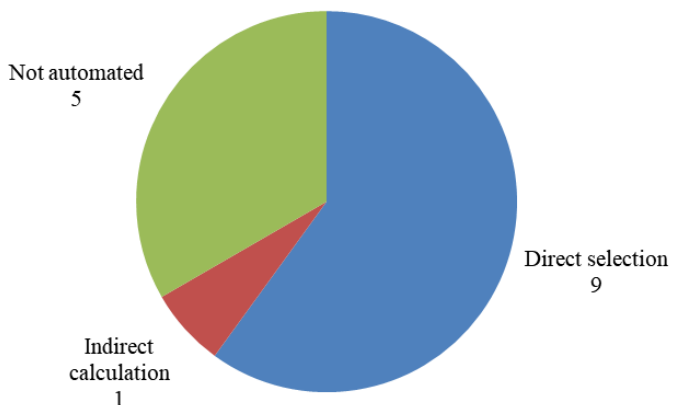

Figure 2. Data availability machinery.

Animals

The chapter "Animal Emissions and Data" results from the comparison of required input data and available automated data in the areas of direct emissions, slurry systems, organic fertilizers and weight / gain. For this purpose, FMIS are used.

A total of 43 different input data were identified to determine GHG in livestock with a focus on cattle and dairy cattle. 26 of the required data are directly readable and thus automatically available. Two of the required input data can be automatically calculated using several available data. At least some of the data are not automatically available, so literature values have to be used here. The accuracy according to the scale described at the beginning of chapter 4 is 0.97 in total and thus on average 0.03 below the required accuracy level of 1 . This is due to the numerous literature values required, all of them were rated with an accuracy level of 0.9 .

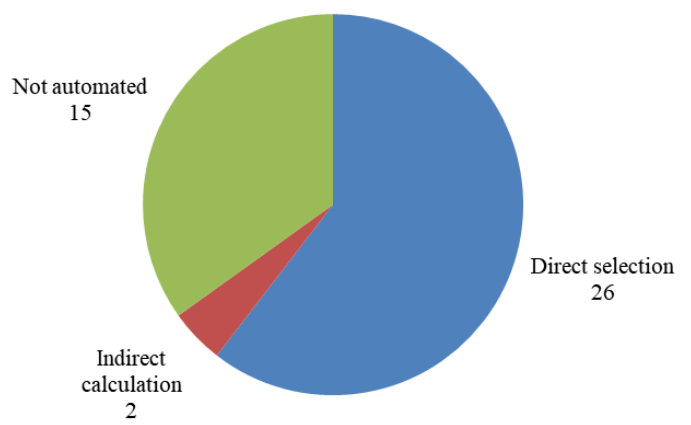

Figure 3. Data availability animals. 


\section{Conclusions}

The aim of this work was to check whether input data required for the calculation of GHGs in agriculture can be drawn from automated sources such as FMIS or ISBUS. In view of the fact that in all three categories (soil / plant, machinery and animals) at least half of all required data are directly readable, it is clear that partial automation is possible from this perspective. The available accuracy levels are, as far as the scale used permits, at an acceptable level (maximum deviation of 0.03 according to a defined scale). Thus, from the results of this work, one can conclude that digitization in agriculture favours a simplified and more accurate GHG calculation, paving the way to more sustainable agriculture. This supports the thesis of Sørensen (2010) that the digitization of agriculture enables farmers to meet external demands for sustainability and efficiency. Automated calculation could also be performed by Tubiello et al. (2014) solve the problem of required data volumes and inaccuracies. Another advantage of the individual and automated THG calculation is the reference level. Due to the high accuracy of the available input data in many areas, GHG emissions can be calculated not only at farm level but also at the product, machine or time level. This enables accurate reference results, which in turn are the basis for research on THG avoidance strategies. Hülsbergen (2002) has also stated that this accuracy is necessary in order to carry out consultations on GHG prevention.

From the perspective of farmers, the biggest advantage of automated GHG calculation is certainly the time savings in the documentation.

In the future, if compensation for greenhouse gas reductions is paid in agriculture, the implementation of avoidance strategies could be more easily verified by automated transfer.

\section{References}

Brundtland, G., 1987. Report of the World Comission on Environment and Development: Our common future. United Nations - General Assembly, p. 54.

Denef, K., K. Paustian, S. Archibeque, S. Biggar, D. Pape, 2012. Report of Greenhouse Gas Accounting Tools for Agriculture and Forestry Sectors. Interim report to USDA under Contract No. GS23F8182H.

EPA, 2005. User's Guide for the Final NONROAD2005 Model - Technical Report. EPA - United States Environmental Protection Agency .

Horrgian, L., R.S. Lawrance, P. Walker, 2002. How Sustainable Agriculture Can Address the Environmental and Humand Health Harms of Industrial Agriculture. Research Review - Environmental Health Perspectives, p 445-456.

Hülsbergen, K.-J., 2002. Entwicklung und Anwendung eines Bilanzierungsmodells zur Bewertung der Nachhaltigkeit landwirtschaftlicher Systeme. Halle/Saale: Shaker Verlag.

IPCC, 2006. 2006 IPCC Guidelines for National Greenhouse Gas Inventories - Volume 2 Energy. Accessed 02. november 2017, http://www.ipcc-nggip.iges.or.jp/public/2006gl/

Lal, R., 2008. Soils and sustainable agriculture. A review. Agronomy for sustainable development, p 57-64.

Slaper, T. F., T. J. Hall, 2011. The Triple Bottom Line: What is it and how does it work? IBR - Indiana Business Review, p. 4.

Sørensen, C., S. Fountas, E. Nash, L. Personen, D. Bochtis, S. M. Pedersen, S. B. Blackmore, 2010. Conceptual model of a future farm management information system. Computers and Electronics in Agriculture 72(1), S. 3747. doi:10.1016/j.compag.2010.02.003

Tubiello, F. N., M. Salvatore, R. D. Golec, A. Ferrara, S. Rossi, R. Biancalani, A. Flammini, 2014. Agriculture, Forestry and Other Land Use Emissions by Sources and Removals by Sinks - 1990-2011 Analysis. FAO Statistics Division.

Umweltbundesamt, 2010. Aktualisierung des Modells TREMOD - Mobile Machinery (TREMOD-MM).

Zehetmeier, M., W. Zickgraf, M. Effenberger, B. Zerhusen, 2017. Treibhausgas-Emissionen in bayerischen landwirtschaftlichen Betrieben. Bayrische Landesanstalt für Landwirtschaft. 


\title{
Development of In-House Lattice-Boltzmann Simulator of Anaerobic Bioreactors for Vinasse Treatment: Preliminary Dimensionless Modelling and Sensitivity Analysis
}

\author{
José A. Rabi ${ }^{\text {a, }}$, Fernando L. Caneppele ${ }^{\text {a }}$, Rogers Ribeiro ${ }^{\text {b }}$, Gustavo C. Dacanal ${ }^{\text {b }}$ \\ ${ }^{a}$ Department of Biosystems Engineering, University of São Paulo - Pirassununga campus, Pirassununga, SP 13635-900, \\ Brazil \\ ${ }^{\mathrm{b}}$ Department of Food Engineering, University of São Paulo - Pirassununga campus, Pirassununga, SP 13635-900, Brazil \\ * Corresponding author. Email: jrabi@usp.br
}

\begin{abstract}
Sugarcane vinasse is a by-product from the ethanol industry whose large-scale exploitation has long pointed to ferti-irrigation in sugarcane crops after anaerobic treatment. Anaerobic packed bed reactor (APBR) arises as an attractive treatment system so as to preserve vinasse quality as bio-fertiliser while allowing biogas recovery, thus mitigating both greenhouse gas emission and groundwater contamination. Bearing in mind the computational modelling of APBR for cost-effective and sustainable operation, an in-house numerical simulator has been developed via lattice Boltzmann method (LBM). LBM has the ability of numerically simulating transport phenomena, fluid flow and moving boundaries without solving Navier-Stokes equations. Inspired by Anaerobic Digestion Model No 1, such in-house LBM simulator has initially relied on a time-dependent one-dimensional model in primitive variables. In view of supporting (while simplifying) prospective scale-up, the present work casts this initial model and LBM simulator in dimensionless form. Original model parameters (e.g. interstitial fluid velocity, species diffusivity, kinetic coefficients, and bioreactor length) were successfully lumped into fewer dimensionless parameters (e.g. mass-transfer Péclet and Damköhler numbers). Computationally implemented in D1Q2 lattice, the LBM simulator remained fully operational in dimensionless form. A sensitivity analysis showed that gradients of dimensionless species concentrations were smoothed not only with respect to axial (i.e. spatial) variation but also in terms of time variation in diffusion-dominant APBR operation.
\end{abstract}

Keywords: sugarcane vinasse, anaerobic treatment, biogas, computational modelling, lattice Boltzmann method.

\section{Introduction}

Sugarcane vinasse is a waste from ethanol industry whose large-scale use has pointed to ferti-irrigation in sugarcane crops (Robertiello, 1982). As high levels of organic matter, salt and nutrients are found in sugarcane vinasse, its direct deposition onto soil may lead to serious environmental impact in the long term (Madejón et al., 2001). Anaerobic treatment has been pointed as proper procedure prior to ferti-irrigation (Vlissidis and Zouboulis, 1993) so as to preserve fertilising qualities of vinasse (Moraes et al., 2014) while recovering biogas (Grisi et al., 2012). Hence, anaerobic treatment of vinasse can mitigate both ground-water contamination and emission of greenhouse effect gases (España-Gamboa et al., 2011). Due to relatively simpler design and operation, low cost and process stability, anaerobic packed bed reactor (APBR) arises as attractive system for vinasse treatment (Ferraz Jr. et al., 2014).

As the comprehensive knowledge of bioprocesses invokes equations requiring numerical methods (Gluszcz et al., 2011; Naessens et al., 2012), computational modelling has indeed supported bioreactor engineering (Parco et al., 2007; Brannock et al., 2010). As phenomenological models have broader application scope than data-driven ones (de Souza-Santos, 2010), the shift from black-box to white-box simulation is vital for process virtualisation (Sam Saguy, 2016).

While simulation models of wastewater treatment have been developed (Jeppsson, 2017), new numerical methods have been envisaged for bioprocesses such as lattice Boltzmann method (LBM) (van der Sman, 2007). As LBM does not directly solve Navier-Stokes equations (Succi, 2001), it renders simpler codes (Mohamad, 2011) to simulate fluid flow, transport phenomena and multiphase-multicomponent systems (Sukop and Thorne Jr., 2006). By relying on a time-dependent (i.e. dynamic) one-dimensional (1-D) model, an in-house LBM simulator of continuous-flow APBR for vinasse treatment has been developed, with equations initially expressed in primitive variables (Fortunato et al., 2018).

Together with Buckingham's $\Pi$ theorem, dimensional analysis has long supported scale-up (Bird et al., 1960; Lim et al., 2007). Accordingly, this work casts aforesaid model (Fortunato et al., 2018) in dimensionless form, thus lumping influencing parameters into fewer dimensionless numbers. The corresponding in-house LBM simulator was properly adapted to a dimensionless version in line with (Rabi, 2017). 


\section{Continuous-Flow APBR for Vinasse Treatment}

Experimental APBR set-up

The present work numerically simulates an existing laboratory-scale APBR for sugarcane vinasse treatment (Ferraz Jr. et al., 2014), comprising feeding module, bed section, effluent collection module, and biogas collection module, as sketched in Figure 99(a). LBM simulations herein performed refer to dimensionless concentrations of chemical species within the bed section. As the present work is mainly focused on computational modelling, one may refer to (Ferraz Jr. et al., 2014) for more details concerning the corresponding experimental study.

Mathematical model in primitive variables

The dynamic 1-D model for continuous-flow APBR for vinasse treatment is inspired by Anaerobic Digestion Model No 1 - ADM1 (Batstone et al., 2002). APBR bed section is then modelled as stratified porous medium where species concentrations are functions of axial coordinate $z$ and time $t$. Porosity $\varepsilon$ is assumed uniform within the solution domain as well as constant throughout the process. By further assuming constant volumetric flow of vinasse, interstitial fluid velocity $v$ results uniform and constant. Bearing in mind the fluid phase as sketched in Figure $99(b)$, chemical species concentrations $y_{i}=y_{i}(z, t)$ are ruled by the following partial differential equation:

$$
\frac{\partial y_{i}}{\partial t}+v \frac{\partial y_{i}}{\partial z}=D_{i} \frac{\partial^{2} y_{i}}{\partial z^{2}}+\dot{r}_{i}
$$

where $D_{i}$ is species diffusivity in vinasse and $\dot{r}_{i}$ refers to species generation/consumption rates via chemical reactions.

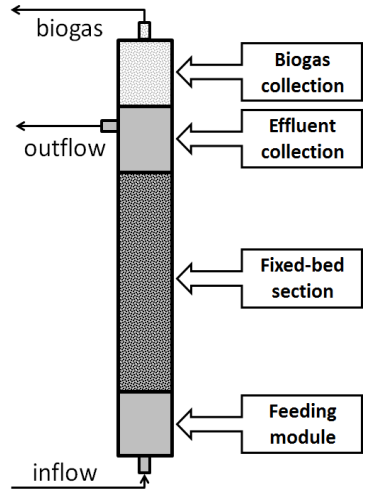

(a)

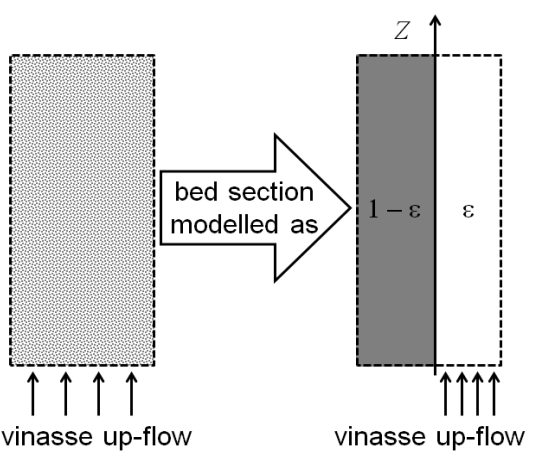

(b)

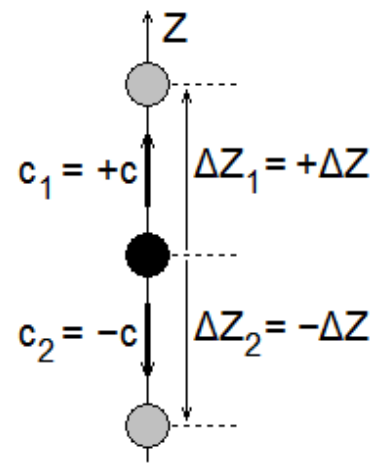

(c)

Figure 99. (a) Sketch of laboratory-scale APBR for sugarcane vinasse treatment, adapted from (Fortunato et al., 2018); (b) one-dimensional (1-D) approach for the bed section; (c) linear structure of D1Q2 lattice for 1-D LBM simulations.

At its initial stage (Fortunato et al., 2018), the LBM simulator solves Eq. (1) for selected species listed in Table 37 (to be extended towards more species in future developments). By relying on a common auxiliary parameter $a_{1,7}=a_{1,7}(z, t)$, Table 37 also shows rates $\dot{r}_{i}$ for Eq. (1). By introducing specific Monod maximum uptake rate $k_{\mathrm{m}, \mathrm{su}}$ and Monod half saturation constant $K_{\mathrm{S} \text {,su }}$ (Batstone et al., 2002) for sugar degraders, aforesaid common parameter $a_{1,7}$ is defined as:

$$
a_{1,7}(z, t)=k_{\mathrm{m}, \mathrm{su}} \frac{y_{1}(z, t)}{y_{1}(z, t)+K_{\mathrm{S}, \mathrm{su}}} y_{7}(z, t)
$$

Table 37. Continuous-flow APBR for vinasse treatment: species concentrations and related generation/consumption rates thus far considered in the preliminary version of the dynamic 1-D model and LBM simulator.

\begin{tabular}{ccc}
\hline Symbol & Concentration (in kg-COD m ${ }^{-3}$ ) of: & Generation/consumption rate $\dot{r}_{i}$ \\
\hline$y_{1}$ & chemical oxygen demand (COD) & $-a_{1,7}$ \\
$y_{2}$ & acetic (ethanoic) acid & $\left(1-Y_{\mathrm{su}}\right) f_{\mathrm{C} 2, \mathrm{su}} a_{1,7}$ \\
$y_{3}$ & propionic (propanoic) acid & $\left(1-Y_{\mathrm{su}}\right) f_{\mathrm{C} 3, \mathrm{su}} a_{1,7}$ \\
$y_{4}$ & butyric (butanoic) acid & $\left(1-Y_{\mathrm{su}}\right) f_{\mathrm{C} 4, \mathrm{su}} a_{1,7}$ \\
$y_{5}$ & dissolved hydrogen gas $\left(\mathrm{H}_{2}\right)$ & $\left(1-Y_{\mathrm{su}}\right) f_{\mathrm{H} 2, \mathrm{su}} a_{1,7}$ \\
$y_{7}$ & biomass (for degradation) & $Y_{\mathrm{su}} a_{1,7}-k_{\mathrm{d}} \cdot y_{7}$ \\
\hline
\end{tabular}


As Table 37 shows, further parameters to assess rate $\dot{r}_{i}$ comprise the biomass yield $Y_{\text {su }}$ and the catabolismonly yield $f_{i, \text { su }}(i=\mathrm{C} 2, \mathrm{C} 3, \mathrm{C} 4$ and $\mathrm{H} 2$ ), with dissolved sugar being the substrate for both yields (Batstone et al., 2002). One may split rate $\dot{r}_{7}$ into generation rate $\left(Y_{\mathrm{su}} \cdot a_{1,7}\right)$ and endogenous decay rate $\left(-k_{\mathrm{d}} \cdot y_{7}\right)$, where $k_{\mathrm{d}}$ is the kinetic coefficient. All rates are functions of position $z$ and time $t$, i.e. $\dot{r}_{i}=\dot{r}_{i}(z, t)$, as they ultimately depend on either $y_{1}=y_{1}(z, t)$ or $y_{7}=y_{7}(z, t)$.

Initial conditions (at $t=0$ ) for Eq. (1) are imposed as:

$$
y_{i}(z, 0)=y_{i, 0}
$$

Boundary conditions at APBR inlet can be imposed by relying on known concentrations $y_{i \text {,feed }}$ in the feeding sugarcane vinasse. Accordingly, at $z=0$ one may impose either Dirichlet condition:

$$
y_{i}(0, t)=y_{i, \text { feed }}
$$

or Danckwerts condition (which simplifies to Dirichlet condition for $D_{i}=0$ ):

$$
v y_{i, \text { feed }}=v y_{i}(0, t)-\left.D_{i} \frac{\partial y_{i}}{\partial z}\right|_{z=0} \Leftrightarrow y_{i}(0, t)=y_{i, \text { feed }}+\left.\frac{D_{i}}{v} \frac{\partial y_{i}}{\partial z}\right|_{z=0}
$$

At APBR exit ( $z=L)$ null Neumann condition (for developed concentration profile) has been imposed as:

$$
\left.\frac{\partial y_{i}}{\partial z}\right|_{z=L}=0
$$

Mathematical model in dimensionless variables

By following the 1-D approach sketched in Figure 99(b), the present work casts the dynamic 1-D APBR model into dimensionless form. Accordingly, the following dimensionless variables are put forward:

$$
Z=\frac{z}{L} \quad, \quad \tau=\frac{v t}{L} \quad, \quad Y_{i}=\frac{y_{i}}{y_{1, \text { feed }}}
$$

Bed length $(L)$, theoretical residence time $(L / v \neq 0)$ and COD concentration in feeding vinasse $\left(y_{1, \text { feed }} \neq 0\right)$ are assigned as non-null reference values respectively for dimensionless axial position, process time, and species concentrations.

With the help of dimensionless variables in Eq. (7) and generation/consumption rates $\dot{r}_{i}$ in Table 37, one can cast Eq. (1) into the following dimensionless form for species $i=1$ to 5 :

$$
\frac{\partial Y_{i}}{\partial \tau}+\frac{\partial Y_{i}}{\partial Z}=\frac{1}{\mathrm{Pe}_{\mathrm{m}, i}} \frac{\partial^{2} Y_{i}}{\partial Z^{2}}+\mathrm{Da}_{\mathrm{su}} f_{i} A_{1,7}
$$

whereas the dimensionless form of Eq. (1) for species $i=7$ becomes:

$$
\frac{\partial Y_{7}}{\partial \tau}+\frac{\partial Y_{7}}{\partial Z}=\frac{1}{\mathrm{Pe}_{\mathrm{m}, 7}} \frac{\partial^{2} Y_{7}}{\partial Z^{2}}+\mathrm{Da}_{\mathrm{su}} f_{7} A_{1,7}-\mathrm{Da}_{\mathrm{d}} Y_{7}
$$

Besides a dimensionless auxiliary parameter $A_{1,7}$ in Eqs. (8) and (9), the following dimensionless numbers also arise:

$$
\mathrm{Pe}_{\mathrm{m}, i}=\frac{L v}{D_{i}} \quad, \quad \mathrm{Da}_{\mathrm{su}}=\frac{k_{\mathrm{m}, \mathrm{su}} L}{v} \quad, \quad \mathrm{Da}_{\mathrm{d}}=\frac{k_{\mathrm{d}} L}{v} \quad, \quad A_{1,7}=\frac{Y_{1}}{Y_{1}+K_{\mathrm{S}, \mathrm{su}} / y_{1, \text { feed }}} Y_{7}
$$

where $\mathrm{Pe}_{\mathrm{m}, i}$ is Péclet number for mass transfer, $\mathrm{Da}_{\mathrm{su}}$ and $\mathrm{Da}_{\mathrm{d}}$ are Damköhler numbers respectively for species generation and consumption (Lim et al., 2007). The dimensionless factors $f_{i}$ in Eqs. (8) and (9) are defined in Table 38 .

Table 38. Dimensionless factors $f_{i}$ related to generation/consumption rates of chemical species.

\begin{tabular}{ccccccc}
\hline Species & $i=1$ & $i=2$ & $i=3$ & $i=4$ & $i=5$ & $i=7$ \\
\hline Factor $f_{i}$ & -1 & $\left(1-Y_{\mathrm{su}}\right) f_{\mathrm{C} 2, \mathrm{su}}$ & $\left(1-Y_{\mathrm{su}}\right) f_{\mathrm{C} 3, \mathrm{su}}$ & $\left(1-Y_{\mathrm{su}}\right) f_{\mathrm{C} 4, \mathrm{su}}$ & $\left(1-Y_{\mathrm{su}}\right) f_{\mathrm{H} 2, \mathrm{su}}$ & $Y_{\mathrm{su}}$ \\
\hline
\end{tabular}

Initial and boundary conditions, Eqs. (3)-(6), are cast in dimensionless form as:

$$
Y_{i}(Z, 0)=Y_{i, 0} \quad, \quad Y_{i}(0, \tau)=Y_{i, \text { feed }} \quad \text { or } \quad Y_{i}(0, \tau)=Y_{i, \text { feed }}+\left.\frac{1}{\mathrm{Pe}_{\mathrm{m}, i}} \frac{\partial Y_{i}}{\partial Z}\right|_{Z=0} \quad,\left.\quad \frac{\partial Y_{i}}{\partial Z}\right|_{Z=1}=0
$$


where:

$$
Y_{i, 0}=y_{i, 0} / y_{1, \text { feed }} \quad \text { and } \quad Y_{i, \text { feed }}=y_{i, \text { feed }} / y_{1, \text { feed }}
$$

Numerical Solution via Lattice Boltzmann Method (LBM)

LBM treats any medium (whether fluid or solid) as constituted by fictitious particles moving and colliding with each other in a fictitious lattice structure. LBM mathematically relies on distribution functions $\phi$ of those particles, whose moments provide macroscopic medium properties (van der Sman, 2007). Particle distribution functions $\phi$ are ruled by Boltzmann transport equation, which is numerically solved in LBM in terms of the fictitious lattice assigned to the true medium (Succi, 2001; Sukop and Thorne Jr., 2006; van der Sman, 2007; Mohamad, 2011).

As a dynamic 1-D model is proposed, so-called D1Q2 lattice is lined up with APBR vertical axis $Z$. The repetitive linear structure of D1Q2 comprises a central lattice site linked to two adjacent sites, one on each side as sketched in Figure 99(c). Let $\Delta Z$ and $\Delta \tau$ be the dimensionless uniform distance between two adjacent sites and the dimensionless advancing time step, respectively. D1Q2 allows two streaming velocities to particles, namely $\vec{c}_{1}=+\Delta Z / \Delta \tau \hat{z}$ (forward) and $\vec{c}_{2}=-\Delta Z / \Delta \tau \hat{z}$ (backward), being $\hat{z}$ the unit vector in upward direction. Particle distribution functions $\phi_{i, k}$ are assigned to each dimensionless concentration $Y_{i}$ and for each streaming link $k(=1$ or 2). At any dimensionless position $Z$ and time $\tau$, one can retrieve $Y_{i}$ simply as:

$$
Y_{i}(Z, \tau)=\sum_{k} \phi_{i, k}(Z, \tau)=\phi_{i, 1}(Z, \tau)+\phi_{i, 2}(Z, \tau)
$$

In order to obtain $\phi_{i, k}$ one writes Boltzmann transport equation for each lattice link $k$, thus yielding (dimensionless) lattice-Boltzmann equation in 1-D (Succi, 2001; Sukop and Thorne Jr., 2006; van der Sman, 2007; Mohamad, 2011):

$$
\frac{\partial \phi_{i, k}(Z, \tau)}{\partial \tau}+c_{k} \frac{\partial \phi_{i, k}(Z, \tau)}{\partial Z}=\frac{\phi_{i, k}^{\mathrm{eq}}(Z, \tau)-\phi_{i, k}(Z, \tau)}{\Delta \tau_{\mathrm{relax}, i}}
$$

where $\Delta \tau_{\text {relax }, i}$ and $\phi_{i, k}^{\mathrm{eq}}$ are respectively relaxation time and equilibrium distribution function (in dimensionless form).

Based on dimensionless $\Delta Z$ and $\Delta \tau$, space-time discretization of Eq. (14) then yields an algebraic equation whose numerical evolution is carried out in two iterative steps. During the collision step, functions $\phi_{i, k}$ are updated from instant $\tau$ to $\tau+\Delta \tau$ for each link $k$ and at each lattice site as:

$$
\phi_{i, k}(Z, \tau+\Delta \tau)=\left(1-\omega_{i}\right) \phi_{i, k}(Z, \tau)+\omega_{i} \phi_{i, k}^{\mathrm{eq}}(Z, \tau)+w_{k} \dot{S}_{i} \Delta \tau
$$

where $\omega_{i}=\Delta \tau / \Delta \tau_{\text {relax }, i}$ is known as relaxation parameter whereas weighting factors $w_{k}$ are $w_{1}=w_{2}=1 / 2$ in D1Q2. As source/sink terms $\dot{S}_{i}$ are introduced at this LBM step, Eqs. (8) and (9) then require:

$$
\dot{S}_{i}= \begin{cases}\mathrm{Da}_{\mathrm{su}} f_{i} A_{1,7} & , i=1 \text { to } 5 \\ \mathrm{Da}_{\mathrm{su}} f_{7} A_{1,7}-\mathrm{Da}_{\mathrm{d}} A_{1,7} & , \quad i=7\end{cases}
$$

In the streaming step, collision results are transported to adjacent lattice sites as:

$$
\phi_{i, k}\left(Z+\Delta Z_{k}, \tau+\Delta \tau\right)=\phi_{k}(Z, \tau+\Delta \tau)
$$

with $\Delta Z_{1}=+\Delta Z$ and $\Delta Z_{2}=-\Delta Z$ respectively for forward and backward streaming. LBM simulation consists of iterating Eqs. (15) and (17) throughout the solution domain $(0 \leq Z \leq 1)$ until final instant is reached ( $\tau=\tau_{\text {final }}=$ $v \cdot t_{\text {final }} / L$ ).

In addition to Eq. (13), true macroscopic medium is linked to LBM simulation through relaxation parameters $\omega_{i}$ and particle distribution functions at equilibrium $\phi_{i, k}^{\mathrm{eq}}$. Bearing in mind the definition of mass-transfer Péclet number $\mathrm{Pe}_{\mathrm{m}, i}$ in Eq. (10), the following expression holds in dimensionless variables (Rabi, 2017):

$$
\frac{1}{\omega_{i}}=\frac{D_{i} \Delta t}{(\Delta z)^{2}}+\frac{1}{2} \Rightarrow \frac{1}{\omega_{i}}=N_{Z} \frac{\mathrm{Ma}}{\mathrm{Pe}_{\mathrm{m}, i}}+\frac{1}{2}
$$

where $\mathrm{Ma}=\Delta \tau / \Delta Z$ is lattice-based Mach number and $N_{Z}=1 / \Delta Z$ (so that $N_{Z}+1$ is the total number of lattice sites). As Eqs. (8) and (9) consider diffusive-convective transport, dimensionless equilibrium functions are (Rabi, 2017): 


$$
\phi_{i, k}^{\mathrm{eq}}(Z, \tau)=w_{k} Y_{i}(Z, \tau)\left(1+v \Delta t / \Delta z_{k}\right) \Rightarrow \phi_{i, k}^{\mathrm{eq}}(Z, \tau)=w_{k} Y_{i}(Z, \tau)(1 \pm \mathrm{Ma})
$$

where the sign before Ma is positive $(+)$ for forward streaming $(k=1)$ and negative $(-)$ for backward streaming $(k=2)$.

In view of Eq.

(11), initial conditions (at $\tau=0)$ are imposed as:

$$
\phi_{i, k}(Z, 0)=w_{k} Y_{i}(Z, 0)
$$

At inlet $(Z=0), \phi_{i, 2}(0, \tau)$ is obtained through backward streaming from the adjacent site, i.e. $\phi_{i, 2}(0, \tau)=$ $\phi_{i, 2}(\Delta Z, \tau)$, while $\phi_{i, 1}(0, \tau)$ is obtained by imposing either Dirichlet condition or Danckwerts condition together with Eq. (13):

$$
\phi_{i, 1}(0, \tau)=Y_{i, \text { feed }}(0, \tau)-\phi_{i, 2}(0, \tau) \quad \text { or } \quad \phi_{i, 1}(0, \tau)=\frac{\phi_{i, 1}(\Delta Z, \tau)+\phi_{i, 2}(\Delta Z, \tau)+\mathrm{Pe}_{\mathrm{m}, i} \Delta Z Y_{i, \text { feed }}}{1+\operatorname{Pe}_{\mathrm{m}, i} \Delta Z}-\phi_{i, 2}(0, \tau)
$$

At exit $(Z=1), \phi_{i, 1}(1, \tau)$ is obtained via forward streaming from the adjacent site, i.e. $\phi_{i, 1}(1, \tau)=\phi_{i, 1}(1-\Delta Z, \tau)$, whereas $\phi_{\mathrm{i}, 2}(1, \tau)$ is obtained by imposing null Neumann condition together with Eq. (13):

$$
\phi_{i, 2}(1, \tau)=\phi_{i, 2}(1-\Delta Z, \tau)
$$

In Eq. (21), it is worth noting that Danckwerts inlet condition reduces to Dirichlet condition as $\mathrm{Pe}_{\mathrm{m}, i} \rightarrow \infty$.

\section{Results and Discussion}

Input data to perform LBM simulations of APBR were obtained from (Fortunato et al., 2018) in primitive form, as shown in Table 39, Table 40 and Table 41, along with dimensionless counterparts assessed via Eqs. (10) and (12) as well as Table 38. As Table 41 evidences, the number of influencing ADM1-APBR parameters is reduced in dimensionless form. In addition, dimensionless LBM parameters were adopted as $\Delta Z=0.01$ and $\Delta \tau=$ 0.001 so as to render relatively small lattice-based Mach number (in this case, $\mathrm{Ma}=\Delta \tau / \Delta Z=0.1$ ) for numerical stability purposes (Mohamad, 2011). In all LBM simulations, only Danckwerts condition, Eq. (21), was imposed

\begin{tabular}{|c|c|}
\hline Primitive values & Dimensionless values \\
\hline$y_{1,0}=y_{2,0}=y_{3,0}=y_{4,0}=y_{5,0}=0$ & $Y_{1,0}=Y_{2,0}=Y_{3,0}=Y_{4,0}=Y_{5,0}=0$ \\
\hline$y_{7,0}=y_{7, \text { feed }}=0.1 \mathrm{~kg}-\mathrm{COD} \mathrm{m}^{-3}$ & $Y_{7,0}=Y_{7, \mathrm{feed}}=0.00405$ \\
\hline$y_{1, \text { feed }}=24.7 \mathrm{~kg}-\mathrm{COD} \mathrm{m}^{-3}$ & $Y_{1, \text { feed }}=1$ \\
\hline$y_{2, \text { feed }}=0.5013 \mathrm{~kg}-\mathrm{COD} \mathrm{m}^{-3}$ & $Y_{2, \text { feed }}=0.02030$ \\
\hline$y_{3, \text { feed }}=0.0801{\mathrm{~kg}-C O D ~ \mathrm{~m}^{-3}}^{-}$ & $Y_{3, \text { feed }}=0.00324$ \\
\hline$y_{4, \text { feed }}=0.5266 \mathrm{~kg}-\mathrm{COD} \mathrm{m}^{-3}$ & $Y_{4, \text { feed }}=0.02132$ \\
\hline$y_{5, \text { feed }}=0.0001 \mathrm{~kg}-\mathrm{COD} \mathrm{m}^{-3}$ & $Y_{5, \mathrm{feed}}=4.049 \times 10^{-6}$ \\
\hline
\end{tabular}
at APBR inlet for the sake of conciseness as well as because it is more physically consistent than Dirichlet counterpart (Rabi, 2017).

Table 39. Input data for LBM simulations of APBR for vinasse treatment: initial and feeding concentrations.

Table 40. Input data for LBM simulations of APBR for vinasse treatment: species generation/consumption parameters.

\begin{tabular}{cc}
\hline Primitive values & Dimensionless values ${ }^{*}$ \\
\hline$a_{1,7}$ assessed from $y_{1}, y_{7}, K_{\mathrm{S}, \mathrm{su}}$ and $k_{\mathrm{m}, \mathrm{su}}$ & $A_{1,7}$ assessed from $Y_{1}, Y_{7}, K_{\mathrm{S}, \mathrm{su}} / y_{1, \text { feed }}$ \\
$Y_{\mathrm{su}}=0.10$ & $f_{7}=0.10$ \\
$f_{\mathrm{C} 2, \mathrm{su}}=0.41$ & $f_{2}=0.369$ \\
$f_{\mathrm{C} 3 \mathrm{su}}=0.27$ & $f_{3}=0.243$ \\
$f_{\mathrm{C} 4, \mathrm{su}}=0.13$ & $f_{4}=0.117$ \\
$f_{\mathrm{H} 2, \mathrm{su}}=0.19$ & $f_{5}=0.171$ \\
\hline
\end{tabular}

* Dimensionless factor $f_{1}=-1$ is already defined in Table 38.

Table 41. Input data for LBM simulations of APBR for vinasse treatment: ADM1 and APBR parameters.

\begin{tabular}{cc}
\hline Primitive values & Dimensionless values \\
\hline$L=1 \mathrm{~m}$ & $\mathrm{Pe}_{\mathrm{m}, i}=100$ \\
$v=2 \mathrm{~m} \mathrm{~d}^{-1}$ & \\
$D_{i}=0.02 \mathrm{~m}^{2} \mathrm{~d}^{-1}$ & $\mathrm{Da}_{\mathrm{su}}=15$ \\
$k_{\mathrm{m}, \mathrm{su}}=30 \mathrm{~d}^{-1}$ & \\
$K_{\mathrm{S}, \mathrm{su}}=0.5 \mathrm{~kg}-\mathrm{COD} \mathrm{m} \mathrm{Ca}_{\mathrm{d}}=0.01$ \\
$k_{\mathrm{d}}=0.02 \mathrm{~d}^{-1}$
\end{tabular}


LBM simulations in (Fortunato et al., 2018) were performed by assuming the same diffusivity $D_{i}=0.02 \mathrm{~m}^{2}$ $\mathrm{d}^{-1}$ for all species $\left(i=1\right.$ to 5 and 7). While aforesaid $D_{i}$ renders $P_{\mathrm{m}, i}=100$ (which is a realistic value shown in Table 41), the present work performs additional LBM simulations by adopting mass-transfer Péclet numbers one order of magnitude above (i.e. $\mathrm{Pe}_{\mathrm{m}, i}=1000$ ) as well as below (i.e. $\mathrm{Pe}_{\mathrm{m}, i}=10$ ). The idea is to verify how species concentrations are affected as APBR operation changes from diffusion-dominant (low $\mathrm{Pe}_{\mathrm{m}, i}$ ) to convectivedominant (high $\mathrm{Pe}_{\mathrm{m}, i}$ ) scenario.

At two instants $(\tau=1$ and $\tau=2)$, Figure 100 shows how dimensionless concentrations $Y_{i}(Z, \tau)$ vary within the solution domain $(0 \leq Z \leq 1)$ as simulated for $\mathrm{Pe}_{\mathrm{m}, i}=10,100$ and 1000 . It is worth mentioning that measurements of species concentration as a function of APBR position are extremely awkward (if not unfeasible). As expected, concentration gradients are smoothed as APBR operation becomes more diffusive (i.e. for relatively lower $\mathrm{Pe}_{\mathrm{m}, i}$ ), which is inherent in transport phenomena modelled by $2^{\text {nd }}$-order derivatives with respect to spatial coordinates, Eqs. (1) and (8). As Figure 100(f) evidences, significant differences can arise in concentration profiles depending on the operation scenario.

Still, one may come across models neglecting diffusive transport. If differential equations lack diffusive terms, they remain (at most) parabolic with respect to the spatial dependence (if convective terms are kept). Consequently, a second boundary condition is dismissed (usually at downstream exit) thus enabling the use of marching numerical methods (e.g. Runge-Kutta). Conversely, the present work does consider diffusive terms in model equations, Eqs. (1) and (8), towards comprehensiveness as well as scale-up. While convective transport is strictly one-directional (i.e. along with fluid flow), diffusive transport occurs both forward and backward. In view of that, diffusion plays indeed a major role in process where fluid flow is relatively feeble and such is the case of wastewater treatment in APBR.

Despite gradient smoothing due to diffusive transport is of spatial nature, time effects are equally noted in species concentrations. Figure 13 shows how dimensionless species concentrations $Y_{i}(Z, \tau)$ vary over process time $\tau$ at APBR exit $(Z=1)$ as LBM-simulated for $\mathrm{Pe}_{\mathrm{m}, i}=10,100$ and 1000. Again, species concentration gradients become smoother for diffusive-dominant scenarios (i.e. for relatively lower $\mathrm{Pe}_{\mathrm{m}, i}$ ). 


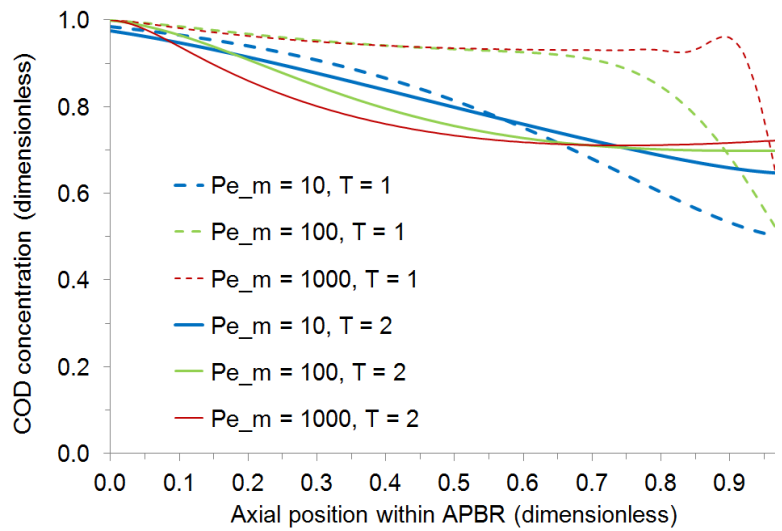

(a)

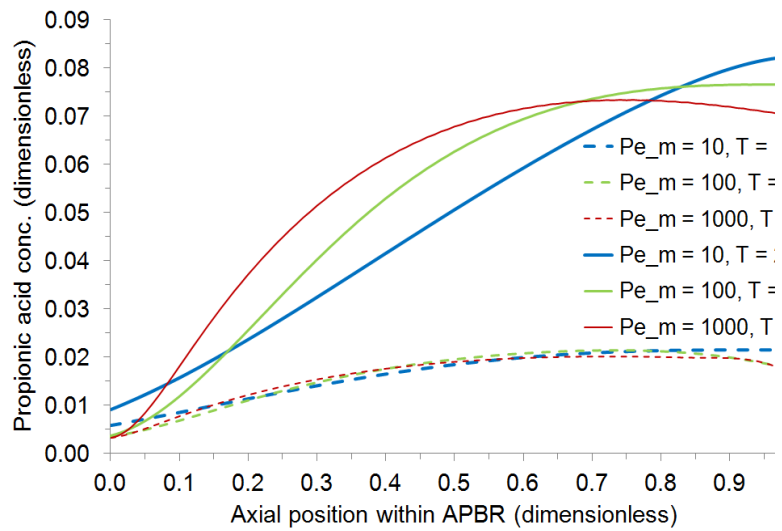

(c)

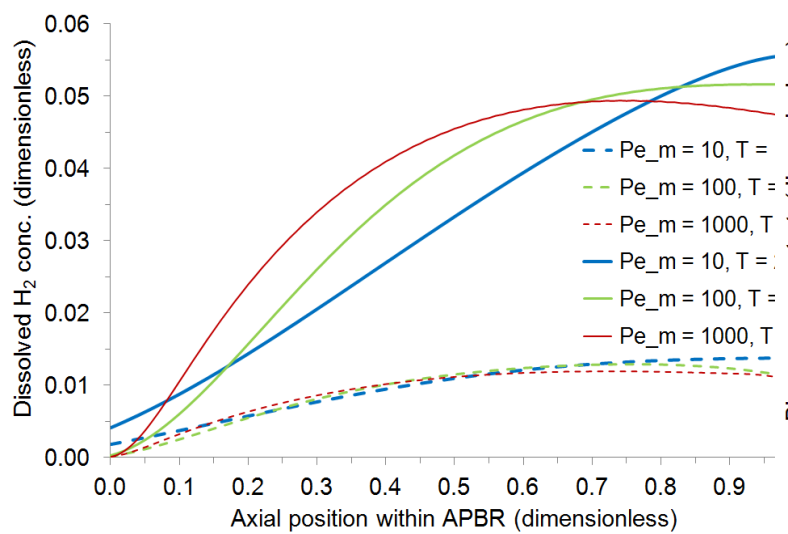

(e)

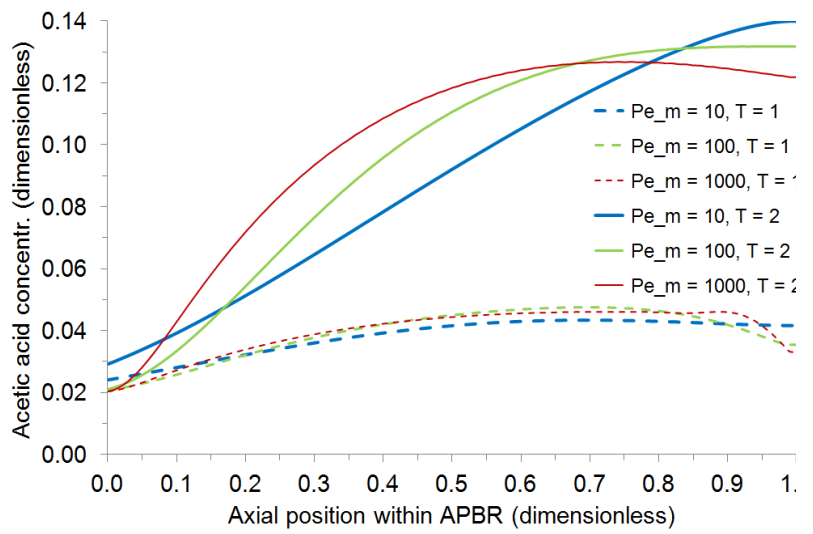

(b)

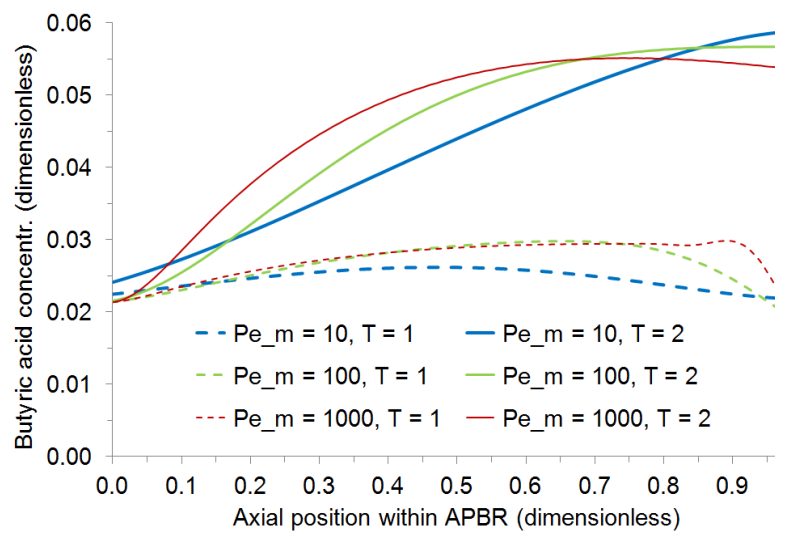

(d)

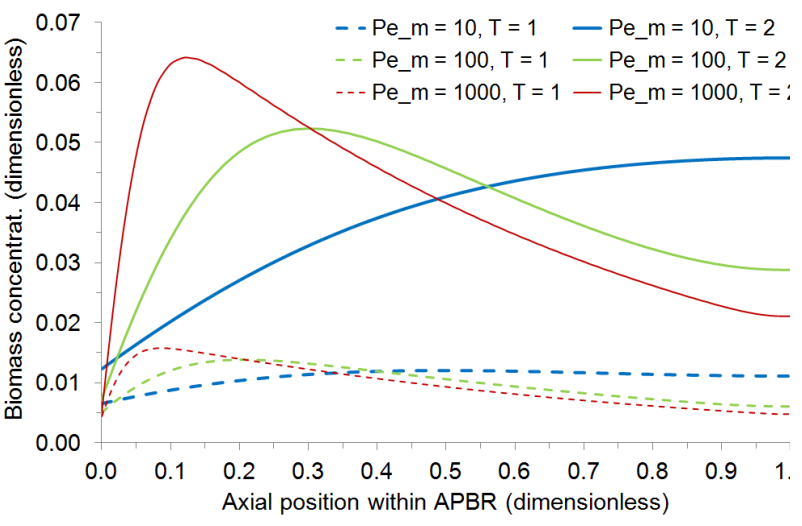

$(f)$

Figure 100. LBM simulations of 1-D variation of species concentrations at $\tau=1$ (dashed line) and $\tau=2$ (solid line), for $\mathrm{Pe}_{\mathrm{m}, i}=10,100$ and 1000: $(a) \mathrm{COD},(b)$ acetic acid, $(c)$ propionic acid, $(d)$ butyric acid, $(e)$ dissolved $\mathrm{H}_{2},(f)$ biomass.

It is noteworthy that species concentrations at the exit $(Z=1)$ depend not only on process time $\tau$ but also on mass-transfer Péclet number $\mathrm{Pe}_{\mathrm{m}, i}$ (and ultimately on species diffusivity $D_{i}$, provided that both bed length $L$ and interstitial velocity $v$ are known parameters). Bearing particularly in mind the model framework in primitive variables, one may exploit this dependence to fine-tune species diffusivity against experimental data (e.g. species concentration measured at APBR exit throughout process time).

Inasmuch as the model framework will be extended towards additional chemical species (other than those in Table 37) while multi-parameters optimisation routines will be coupled into the LBM simulator, aforesaid finetuning task is left for future work. Yet and for the sake of preliminary comparison, just after 1 day (i.e. at $\tau=2$ ) COD concentration at APBR exit $(Z=1)$ was measured as $26.1 \mathrm{~kg}-\mathrm{COD} \mathrm{m}^{-3}$ ( $Y_{1} \cong 1.057$ ) (Ferraz Jr. et al., 2014), which is slightly higher but at the same order of magnitude of LBM simulations in Figure 13(a). 


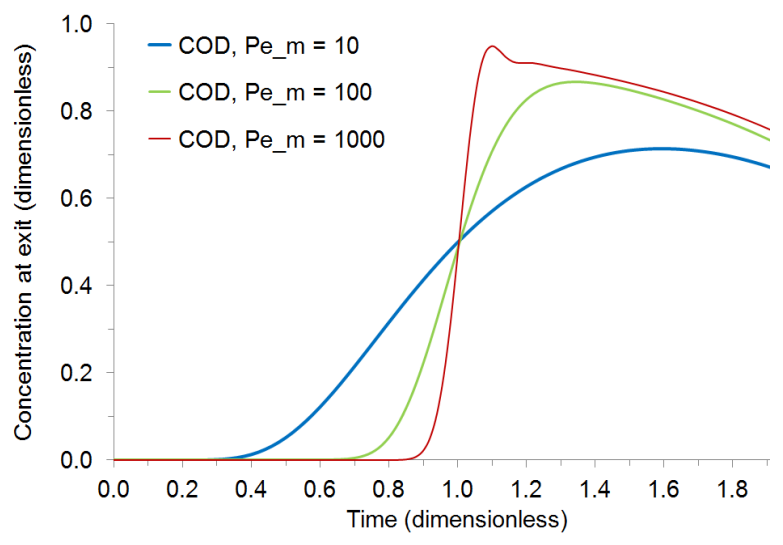

(a)

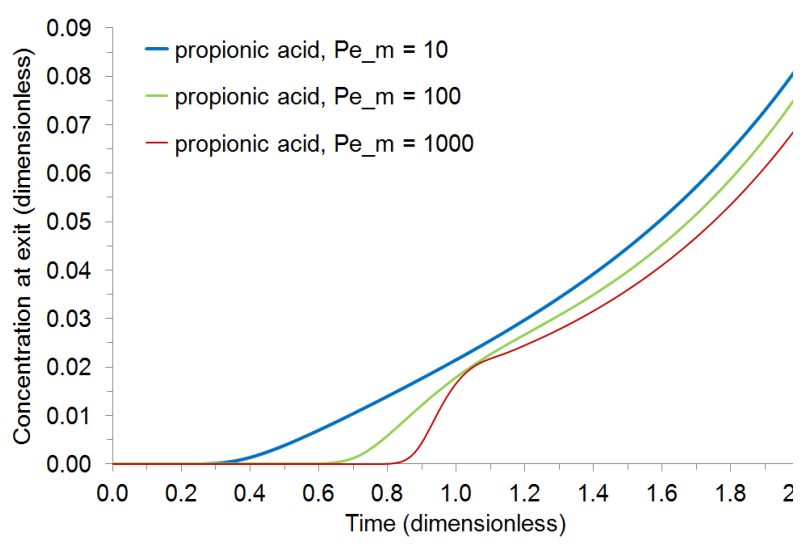

(c)

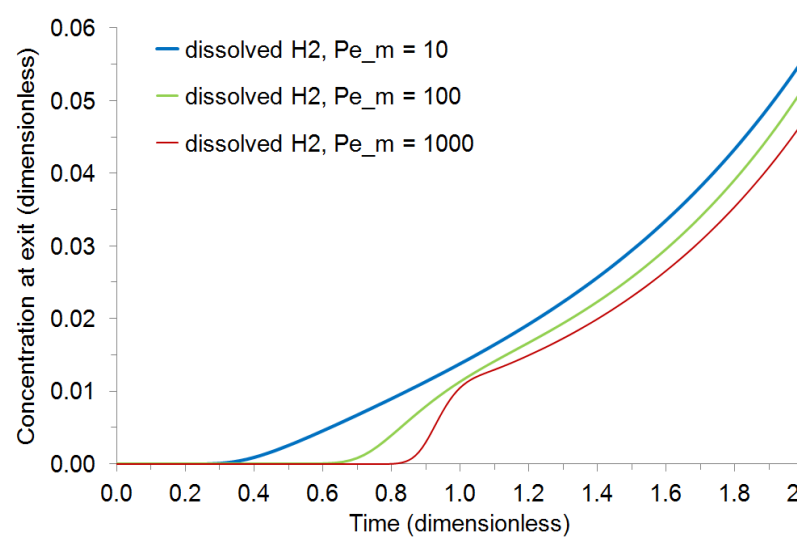

(e)

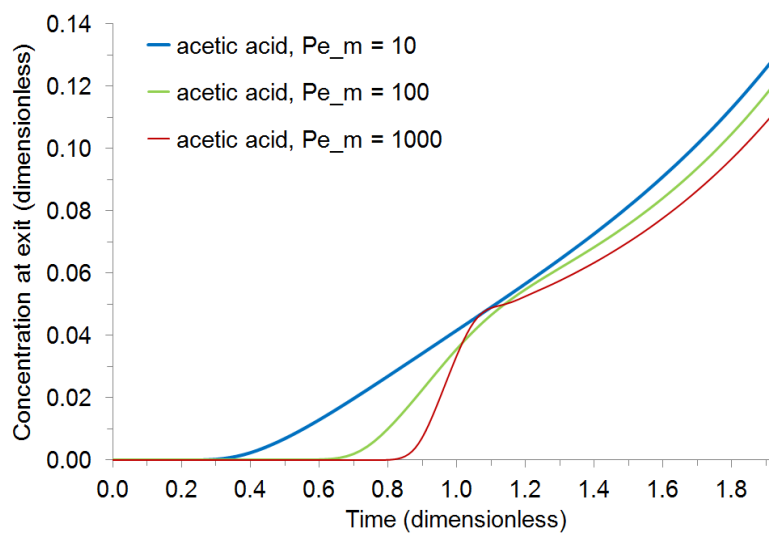

(b)

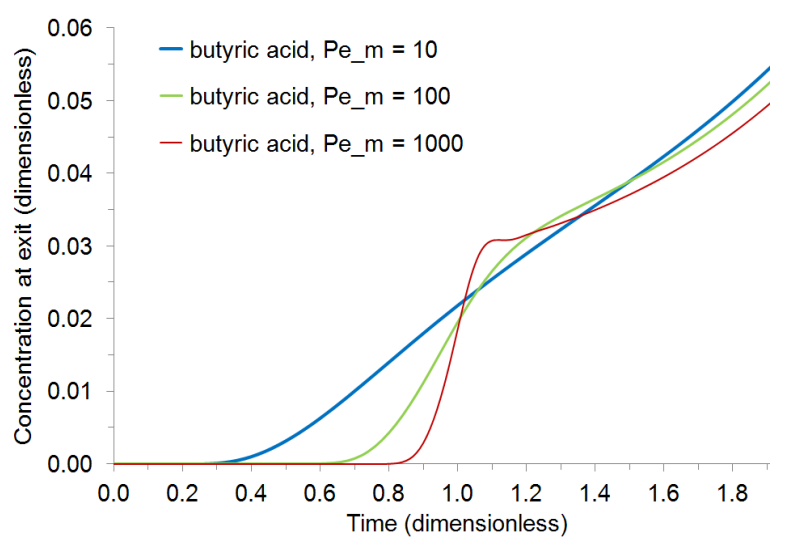

(d)

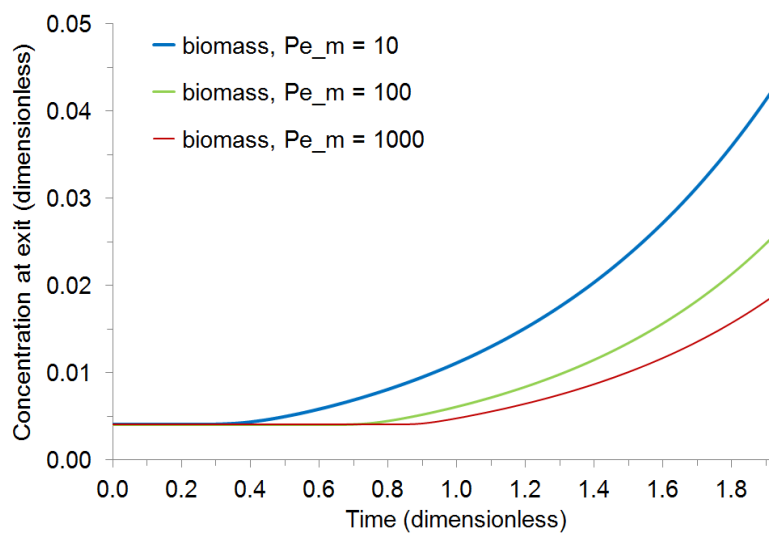

$(f)$

Figure 101. LBM simulations of time variation of species concentrations at APBR exit $(Z=1)$, for $\mathrm{Pe}_{\mathrm{m}, i}=10,100$ and 1000: (a) COD, (b) acetic acid, (c) propionic acid, $(d)$ butyric acid, $(e)$ dissolved $\mathrm{H}_{2},(f)$ biomass.

\section{Conclusions}

Bearing in mind prospective scale-up steps, the LBM simulator remained fully operational in dimensionless form as primitive influencing parameters $\left(L, v, D_{i}\right.$ and $\left.k_{i}\right)$ were successfully lumped into fewer dimensionless numbers $\left(\mathrm{Pe}_{\mathrm{m}, i}\right.$ and $\left.\mathrm{Da} i\right)$. Sensitivity analyses of dimensionless species concentrations $Y_{i}(Z, \tau)$ were performed through LBM simulations. In diffusion-dominant scenarios (i.e. as $\mathrm{Pe}_{\mathrm{m}, i} \rightarrow 0$ ), dimensionless concentration gradients were visibly smoothed not only with respect to spatial variation but also in terms of their time variation. Future developments comprise the extension of the APBR model framework towards additional chemical species and the coupling of multi-parameters optimisation (i.e. best-fitting) routines into the LBM simulator. 


\section{Acknowledgements}

The present work is part of ongoing research aiming at conceiving and developing in-house LBM simulators of agroindustrial processes. Authors are grateful to $\mathrm{CNPq}$ - Brazilian National Council for Scientific and Technological Development for their financial support (process 420293-2016-7).

\section{References}

Batstone, D.J., J. Keller, I. Angelidaki, S.V. Kalyuzhnyi, S.G. Pavlostathis, A. Rozzi, W.T. Sanders, H. Siegrist, and V.A. Vavilin, 2002. The IWA Anaerobic Digestion Model No 1 (ADM1). Water Science and Technology. 45 (10), 65-73. https://www.ncbi.nlm.nih.gov/pubmed/12188579 $780 \mathrm{p}$.

Bird, R.B., W.E. Stewart, and E.N. Lightfoot, 1960. Transport Phenomena. New York: John Wiley \& Sons.

Brannock, M., G. Leslie, Y. Wang, and S. Buetehorn, 2010. Optimising mixing and nutrient removal in membrane bioreactors: CFD modelling and experimental validation. Desalination. 250 (2), 815-818. http://dx.doi.org/10.1016/j.desal.2008.11.048

de Souza-Santos, M.L., 2010. Solid Fuels Combustion and Gasification: Modeling, Simulation, and Equipment Operations. Boca Raton: CRC Press. 508 p.

España-Gamboa, E., J. Mijangos-Cortes, L. Barahona-Perez, J. Dominguez-Maldonado, G. Hernández-Zarate, and L. Alzate-Gaviria, 2011. Vinasses: characterization and treatments. Waste Management \& Research. 29 (12), 1235-1250. https://doi.org/10.1177/0734242X10387313

Ferraz Jr., A.D.N., J. Wenzel, C. Etchebehere, and M. Zaiat, 2014. Effect of organic loading rate on hydrogen production from sugarcane vinasse in thermophilic acidogenic packed bed reactors. International Journal of Hydrogen Energy. 39 (30), 16852-16862. https://dx.doi.org/10.1016/j.ijhydene.2014.08.017

Fortunato, V.A., F.L. Caneppele, R. Ribeiro, and J.A. Rabi, 2018. Development of in-house lattice-Boltzmann simulator of bioreactors for wastewater treatment: basic concepts and initial results. Water Science and Technology. 77 (3), 838-847. https://doi.org/10.2166/wst.2017.597

Głuszcz, P., J. Petera, and S. Ledakowicz, 2011. Mathematical modeling of the integrated process of mercury bioremediation in the industrial bioreactor. Bioprocess and Biosystems Engineering. 34 (3), 275-285. https://doi.org/10.1007/s00449-010-0469-8

Grisi, E.F., J.M. Yusta, and R. Dufo-López, 2012. Opportunity costs for bioelectricity sales in Brazilian sucroenergetic industries. Applied Energy. 92, 860-867. https://doi.org/10.1016/j.apenergy.2011.08.045

Jeppsson, U., 2017. The Benchmark Simulation Modelling Platform - Areas of Recent Development and Extension. In: Frontiers in Wastewater Treatment and Modelling - Lecture Notes in Civil Engineering 4. Palermo: Springer. Ed., G. Mannina. 81-91.

Lim, L.C., S.S. Sablani, and A.S. Mujumdar, 2007. Dimensional Analysis. In: Handbook of Food and Bioprocess Modeling Techniques. Boca Raton: CRC Press. Eds., S.S. Sablani, M.S. Rahman, A.K. Datta, and A.R. Mujumdar. 533561.

Madejón, E., R. López, J.M. Murillo, and F. Cabrera, 2001. Agricultural use of three (sugarbeet) vinasse composts: effect on crops and chemical properties of a Cambisol soil in the Guadalquivir river valley (SW Spain). Agriculture, Ecosystems and Environment. 84 (1), 55-65. http://dx.doi.org/10.1016/S0167-8809(00)00191-2

Mohamad, A.A., 2011. Lattice Boltzmann Method: Fundamentals and Engineering Applications with Computer Codes. London: Springer-Verlag. 178 p.

Moraes, B.S., T. Junqueira, L.G. Pavanello, O. Cavalett, P.E. Mantelatto, A. Bonomi, and M. Zaiat, 2014. Anaerobic digestion of vinasse from sugarcane biorefineries in Brazil from energy, environmental, and economic perspectives: Profit or expense? Applied Energy. 113, 825-835. https://doi.org/10.1016/j.apenergy.2013.07.018

Naessens, W., T. Maere, N.R. Ratkovich, S. Vedantam, and I. Nopens, 2012. Critical review of membrane bioreactor models: Part 2: hydrodynamic and integrated models. Bioresource Technology. 122, 107-118. https://doi.org/10.1016/j.biortech.2012.05.071

Parco, V., G. Du Toit, M. Wentzel, and G. Ekama, 2007. Biological nutrient removal in membrane bioreactors: denitrification and phosphorus removal kinetics. Water Science and Technology. 56 (6), 125-134. https://doi.org/10.2166/wst.2007.642

Rabi, J.A., 2017. Comparing Dirichlet and Danckwerts Inlet Condition for Bioaffinity Chromatography via Lattice Boltzmann Simulations. In: IMACS Series in Computational and Applied Mathematics 20. Rome: IMACS. Eds., D. Mansutti, and R.M. Spitaleri. 131-140.

Robertiello, A., 1982. Upgrading of agricultural and agro-industrial wastes: The treatment of distillery effluents (vinasses) in Italy. Agricultural Wastes. 4 (5), 387-395. https://doi.org/10.1016/0141-4607(82)90033-6 
Sam Saguy, I., 2016. Challenges and opportunities in food engineering: modeling, virtualization, open innovation and social responsibility. Journal of Food Engineering. 176, 2-8. https://doi.org/10.1016/j.jfoodeng.2015.07.012

Succi, S., 2001. The Lattice Boltzmann Equation for Fluid Dynamics and Beyond. Oxford: Clarendon Press. $288 \mathrm{p}$.

Sukop, M.C., and D.T. Thorne Jr., 2006. Lattice Boltzmann Modeling - An Introduction for Geoscientists and Engineers. Berlin Heidelberg: Springer-Verlag. 172 p.

van der Sman, R.G.M., 2007. Lattice Boltzmann Simulation of Microstructures. In: Handbook of Food and Bioprocess Modeling Techniques. Boca Raton: CRC Press. Eds., S.S. Sablani, M.S. Rahman, A.K. Datta, and A.R. Mujumdar. 15-39.

Vlissidis, A., and A.I. Zouboulis, 1993. Themophilic anaerobic digestion of alcohol distillery wastewaters. Bioresource Technology. 43 (2), 131-140. https://doi.org/10.1016/0960-8524(93)90172-8 


\title{
Agricultural Tire Energy Efficiency Test Method and Dedicated Equipment to Measure the Fuel Consumption and Traction of Agricultural Tires Under Real Field Conditions
}

\author{
Szalay K. , Kovács L. a, Bércesi G. a, Oldal I. a , Piron E. ${ }^{\text {, }}$, Charlat J. ${ }^{\text {c }}$, Joly T. ${ }^{\text {c, Poncet C. }}$, Tran F. \\ a NARIC Institute of Agricultural Engineering, 2100 Gödöllő Tessedik Sámuel w. 4., Hungary \\ ${ }^{\mathrm{b}}$ Research Institute of Science and Technology for Environment and Agriculture, Clermont-Ferrand 9 avenue Blaise \\ Pascal CS 2008563178 Aubière France \\ ${ }^{c}$ Manufacture Francaise Des Pneumatiques Michelin, Ladoux 63118 Cébazat, France \\ * Corresponding author. Email: szalay.kornel@mgi.naik.hu
}

\begin{abstract}
Sustainable way of agriculture is one of the fundamental pieces of our viable future. Most policy makers, stakeholders, production companies, farmers have realized the importance of energy efficiency. The economic impact of energy waste is unambiguous. Fewer people understand or care its environmental aspects. The ENTAM as the European Network of Testing stations of Agricultural Machines and equipment does. It has settled up a new ENTAM Technical Working Group in order to draft a common methodology to test the agricultural tire energy efficiency, in close cooperation with Michelin. The aim was to find an easy still a representative measurement method with reproducible records on real agricultural fields, in standardized soil conditions. A novel dedicated equipment has been built which uniquely provides the possibility to measure dimensionless tire performance indices in a standardized way. The prototype covers a wide range of tire dimensions and is capable of applying large vertical loads and drive torques. In the practice it will play a key role to determine optimal tire settings and can decisively contribute to the sustainable way of agricultural practice.
\end{abstract}

\section{Introduction}

Global background

Sustainability, sustainable development are well-known terms which have many, sometimes rather different definitions. The very basic topics and related challenges are common. They all agree that climate change, energy and fossil fuel scarcity have high importance in this regard. Reduction of greenhouse gas emission and fuel consumption are two of those key elements. Agricultural production plays decisive role in the sustainable future so energy efficiency of agricultural machines is getting more and more important.

Mobile machinery and the pneumatic tires in particular play a very significant role in this context. For a tractor, one third of the mechanical energy delivered by transmissions to the wheels is lost. Some energy is lost in soil compaction, by tire slippage, and by rubber viscoelasticity. The remaining two-thirds are used for pulling, carrying and traction.

Studies have been published evaluating the specific contribution of the engine, the transmission, or the tires on the fuel consumption performance. The role of a tire to the fuel consumption in the field is significantly determined by its tractive efficiency as defined in ASABE, D497 (2009).

The tractive efficiency can be evaluated, based on physical variables that can be measured directly at the wheel centre, namely the drive torque $(\mathrm{T})$, the rotation speed of the wheel $(\omega)$, the net traction force (NT), the actual travel speed (V), the vertical load in the contact patch area (W). Some analytical vehicles and laboratory equipment conceptually composed of a chassis and a tested wheel were developed in the past, in order to record "on-the-go" measurements of these variables (Kassai and Szente, 2005; Andreas and Hans-Joachim, 2015). Although, no standard test exists so far, so as to evaluate the performance of the tires under real working conditions, on the field. In 2011 according to this new requirement the 9th ENTAM Technical Working Group has been established in order to draft a common methodology to test the "Agricultural Tire Energy Efficiency".

The working concept of a novel dedicated test equipment had been laid down to uniquely provide possibility to measure dimensionless tire performance indices in a standardized way and to form the basis of a tire-only energy performance. The equipment and the standardized test method have been jointly and gradually developed by the NARIC Hungarian Institute of Agricultural Engineering (MGI) and the Michelin Tire Company, in cooperation with the IRSTEA National Research Institute of Science and Technology for Environment and Agriculture. The name of the equipment had been created as an acronym based on the possible application fields of the unique single wheel tester: FAST $^{2}$. In this paper the authors wish to present a review of the technical capacities of the latest prototype of the new equipment, designed to be large enough to test the biggest agricultural tires of today and the coming years. 
Stakes and aims

Key economical stakes for farmers are productivity and fuel consumption. The tires contribute decisively to these stakes through traction and energetic efficiency. Tire deformation, soil deformation and tire slippage have a large impact on tire energy loss mechanisms. It is therefore fundamental to reproduce pertinently tire/soil interaction. The aims of the FAST2 machine project are to provide a tool to be able to evaluate traction and energetic efficiency of tire alone in the field, independently of the vehicle and in real soil conditions. The technical challenges for the project team are to achieve both good measurement accuracy and machine sturdiness, to be able to perform tests in field conditions.

\section{Materials and Methods}

ENTAM test method

A common methodology for testing agricultural tire energy efficiency has been set up by the ENTAM network, as ASABE; EP542 (R2009). This test method allows coming up with a dimensionless tire-only energy performance index called PLI (Pull Loss Index) (Fancello et al., 2013; Fancello et al., 2015; Szalay et al., 2016). Formula for calculating PLI is shown in Figure 1.

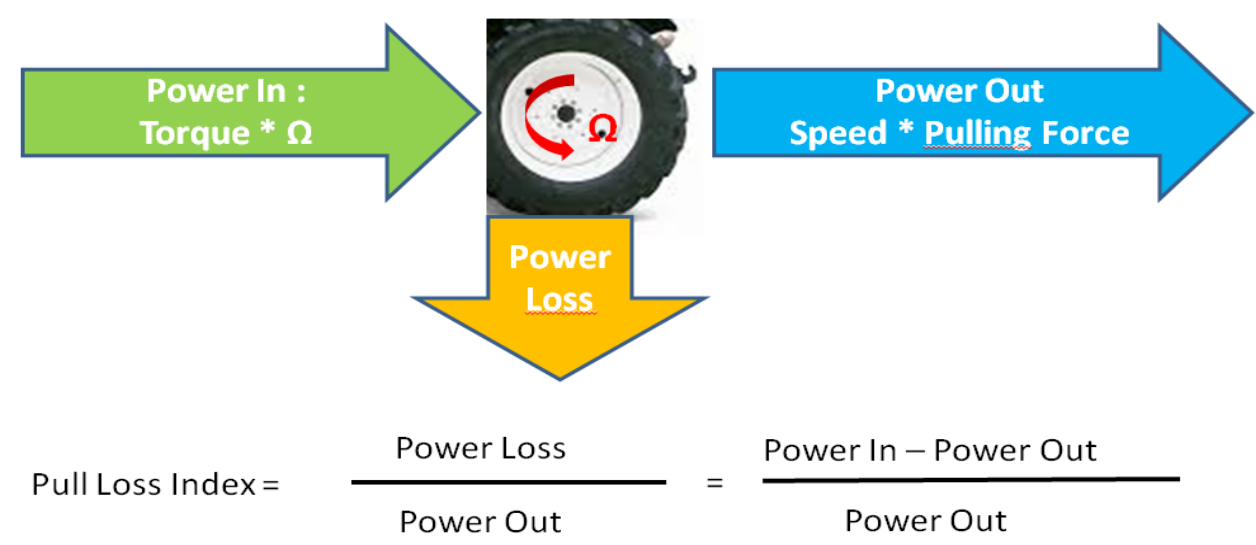

Figure 1. Formula for calculation of PLI.

For example, having a PLI value of $50 \%$ for a given tire means: if the power requirement at the drawbar is $100 \mathrm{~kW}$, then $150 \mathrm{~kW}$ of power is needed at the wheel axle.

Results presented in section ASABE; S313 were obtained following ENTAM test protocol, namely by respecting - among others - 3 criteria on the soil:

- texture: clay, silt, sand resp. between 5 and $15 \%, 10$ and $30 \%, 55$ and $75 \%$,

- moisture content (water mass / dry mass) between 10 and 14\%,

- cone index value (according to ASABE EP542 and ASABE S313 (Dessèvre, 2010; Forissier, 2009)

between 0.5 and $0.9 \mathrm{MPa}$.

Example of ENTAM test method results: comparison of 4 dimensions in 2016

Following previous studies performed in the years before ASABE; EP542 (2009), many experiments were conducted in 2016 at IRSTEA Montoldre place, by use of IRSTEA single wheel tester shown in Figure 2.

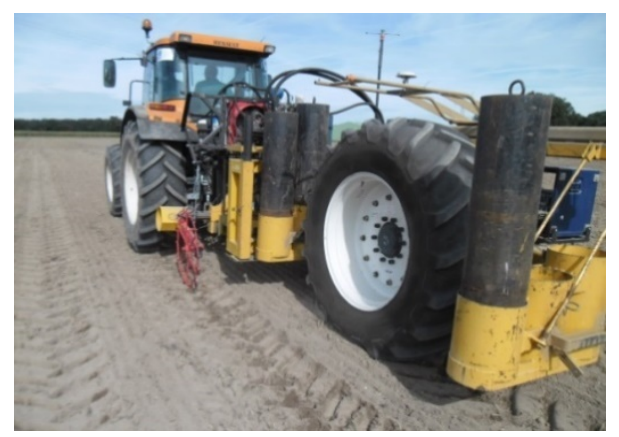

Figure 2. IRSTEA single wheel tester performing a tire efficiency test in a field. 
The ability to sort tires using the ENTAM test method has been specifically addressed during the study and test plan included repeatability, field characteristics and field preparation influences. Four different tires, from Tire 1 to Tire 4 on the graph, were evaluated using each manufacturer recommendations for inflation pressure, regarding the applied load. For each tire and test configuration, 4 different measurements were carried out successively using increasing traction coefficients (defined as the ratio pull force over vertical force) from 0.1 to 0.4 by step of 0.1 , and following ENTAM test recommendation (data collection on 4 successive zones during an up-down round trip in the field). Each tire has been tested 2 times on random places in the same field, and tire 2 has also been tested in another field, and in the same place as both first evaluations, but after a new soil preparation (Figure 3.).

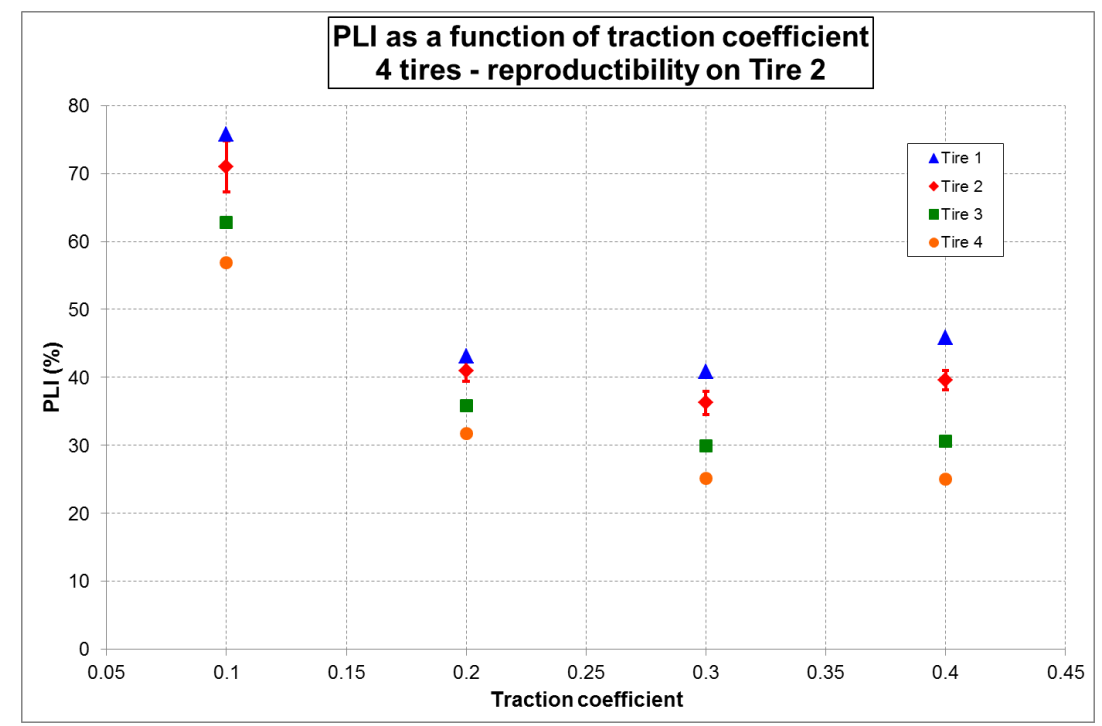

Figure 3. Results using 4 different tires, 4 different traction coefficients for each tire. Reproducibility for tire 2 is presented using error bars.

Results obtained show the robustness of the method and its ability to sort different tires. Whatever the traction coefficient is on the global range, the global sort stays the same. Reproducibility results presented for tire 2 are based on a Student test. The magnitude of the error bar is smaller than the difference between tires. As a consequence, the difference between tires is significant since the error bar takes into account field effect, in-the field test position and successive soil preparations.

\section{Results}

Specifications of the $\mathrm{FAST}^{2}$ machine

The main specifications of FAST $^{2}$ machine are:

- Tire diameter : 1460 to $2500 \mathrm{~mm}$

- Tire width : 311 to $973 \mathrm{~mm}$

- $\quad$ Tire seat : 30 to 50 inches

- Net traction : - 1000 to $7200 \mathrm{daN}$

- Vertical load : 2000 to $12000 \mathrm{daN}$

- $\quad$ Torque : -1250 to 9000 daN.m

- Travel speed in field : up to $15 \mathrm{~m} / \mathrm{h}$

- Wheel rotation speed : up to $9 \mathrm{rad} / \mathrm{s}$

- Tire slip ratio : up to $25 \%$

The FAST ${ }^{2}$ machine enables to get 4 types of results:

- PLI and CLI indexes with ENTAM test method

- Traction efficiency curve

- Moving resistance 
Global description of the machine

The $\mathrm{FAST}^{2}$ machine is a self-propelling vehicle with a measurement post to test tire only. The following 3D model illustrates the complete structure of $\mathrm{FAST}^{2}$ with the measurement system situated in the middle (Figure 4).

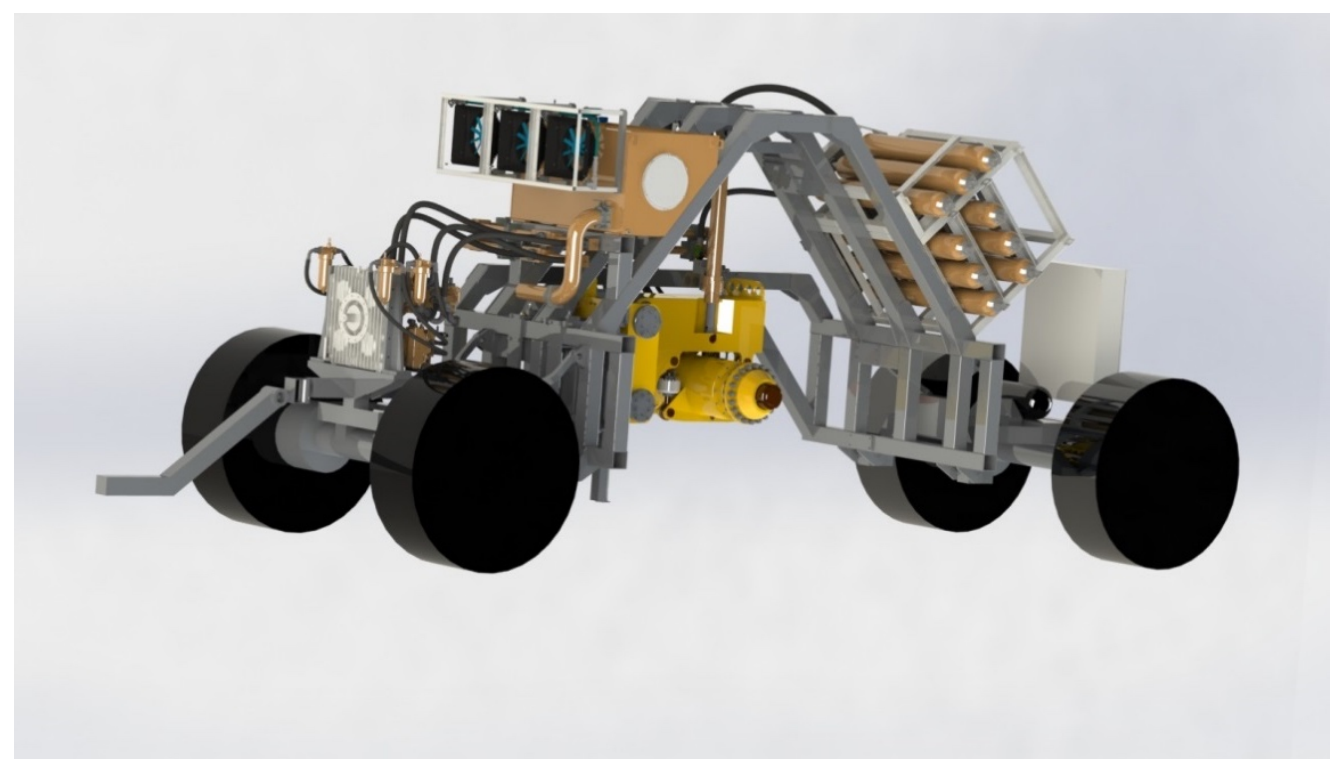

Figure 4. The 3D model of the equipment.

Principle of measurement of $\mathrm{FAST}^{2}$ machine

In order to have the ability to sort tires based on the ENTAM test method accurate and repeatable measurements are needed of drive torque $(T)$, the rotation speed of the wheel $(\omega)$, the net traction force $(\mathrm{NT})$, the actual travel speed (V), the vertical load in the contact patch area (W). A new isostatic measurement principle has been designed (Figure 5). Key elements of the configuration are high accuracy 1 direction load cells. Traction force (NT) is measured by load cell 1 (LC1). Vertical load is measured by LC2, LC3, LC4. The calculation of NT and $\mathrm{W}$ are done with the following simple formulas:

$$
\mathrm{NT}=\mathrm{LC} 1 \quad \mathrm{~W}=\mathrm{LC} 2+\mathrm{LC} 3+\mathrm{LC} 4
$$

In addition to the $1 \mathrm{D}$ load cells a torque measuring flange is built into the spindle to accurately measure the drive torque (T). Traction force (NT) and vertical load (W) are measured by load cells. In addition to the 1D load cells a torque measuring flange is built into the spindle to accurately measure the drive torque (T).

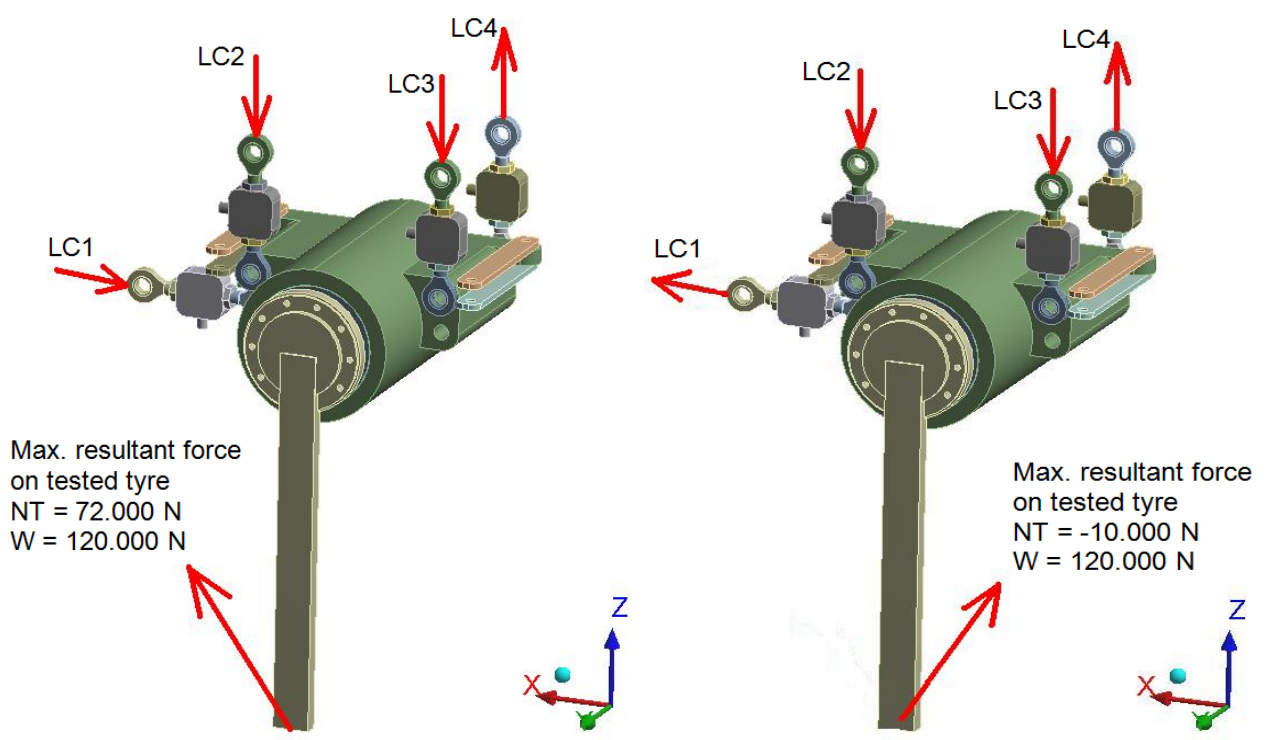

Figure 5. Isostatic measurement system: Forces in case of drive (left) and brake modes (right). 
Principle of kinematic (Szente et al., 2010) of FAST ${ }^{2}$ machine

The FAST ${ }^{2}$ can be used for tests in field in "self propelled double axle" configuration (Figure 6). The whole mass of FAST ${ }^{2}$ lies on the trailer's front and rear axles. The tested wheel is situated between the front and rear shaft of the trailer. In general, the tractor controls the trajectory (direction) of the equipment. The front and rear axles are steerable. Through the PTO the tractor drives four hydraulic pumps which provide hydrostatic drive for the tested wheel in the middle, the four peripheral wheels through gearboxes and also feed the two hydraulic cylinders. One is to set the measuring position other is to express the vertical load on the tested wheel. Testing wheel is centred to the tractor's axle. The driving force delivered by the tested wheel is counteracted by the peripheral wheels based on the desired test mode. In case the measured wheel is driven (drive mode) the four peripheral wheels are broke. In case the measured wheel is broke (brake mode) the four peripheral wheels are driven. The drive of the measured wheel and the peripheral wheels are both hydrostatic, driven by two separate hydraulic circles but pumps are mechanically connected. With adequate control the extra traction force awaken on the tested wheel (NT) is available to drive the peripheral wheels - it saves the energy. A part of the energy used for braking is used for driving (patent pending). The efficiency of energy reuse depends mostly on the slip ratio of the wheels (tested wheel and peripheral wheels). The travel speed of FAST ${ }^{2}$ is controlled through the regulation of the four peripheral wheels speed.

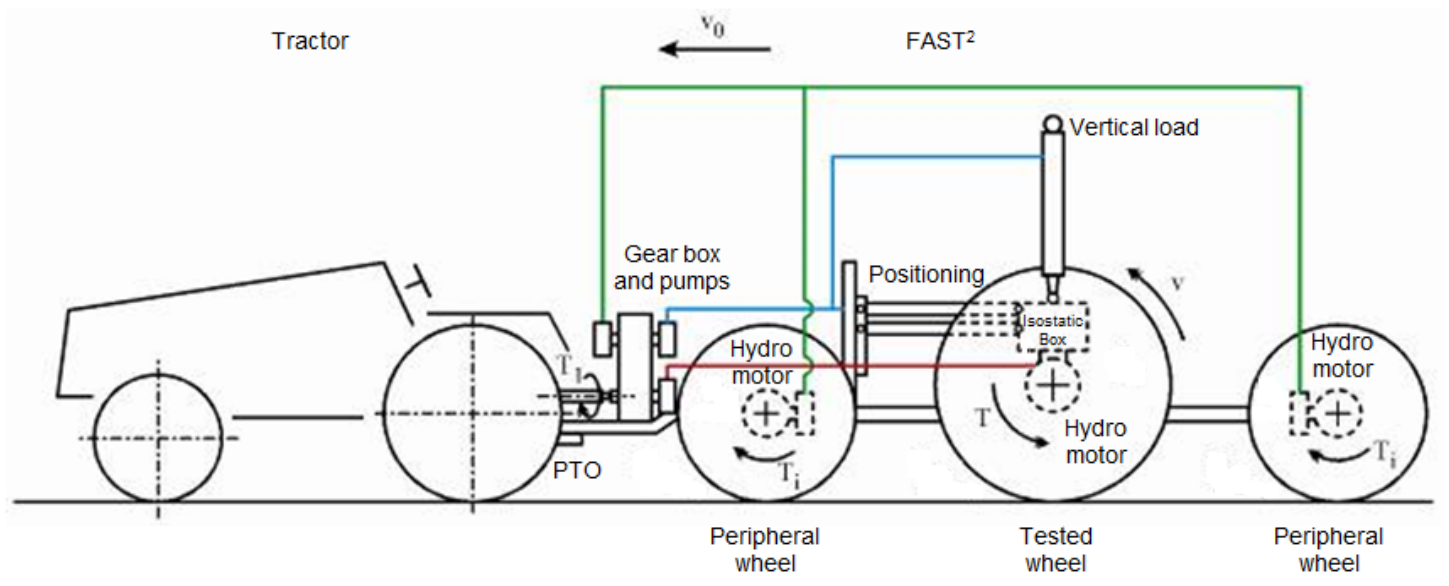

Figure 6. "self-propelled double axle" configuration (drive mode).

Green and red lines illustrate the active hydrostatic drivelines. Blue line represents the oil flow to feed the hydraulic cylinders to set the desirable measuring position and to provide vertical loads on the tested wheel.

\section{Conclusion}

The first measurement results showed high potential in performing tire-only measurements under field conditions. In order to assure the desired accuracy and quality of the collected dataset a new measurement principle has been designed. The equipment's new design has been finished and currently the manufacturing phase is under finalization period. With the fully operational prototype the first field tests will be carried out in 2018. The unique features of $\mathrm{FAST}^{2}$ will make possible to test the biggest agricultural tires (tires with diameters up to $2.5 \mathrm{~m}$ and width up to $0.90 \mathrm{~m}$; vertical loads up to $120000 \mathrm{~N}$, traction force from $-10000 \mathrm{~N}$ to $+72000 \mathrm{~N}$ ) even under extreme usage conditions (speeds up to $15 \mathrm{~km} \cdot \mathrm{h}^{-1}$ up to $40 \%$ slip ratio in the field) or on the road, driving or braking torque of the tested tire up to $90000 \mathrm{Nm}$ ). In the coming years, this test equipment will be used to strengthen the understanding of the basics of the tire performance, depending on the tire design (tire construction; tire tread), the various types of soft soils, and the usage conditions (vertical load; inflation pressure; applied driving torque and resulting slip rate). FAST $^{2}$ can be a useful test equipment to enforce the standard test methods for performances that are key factors in minimizing the environmental impact of mechanized agriculture. FAST $^{2}$ can contribute to a standard test for fuel consumption in the field. Following the $1^{\text {st }}$ field tests for reception, the FAST ${ }^{2}$ machine will be available to perform test the tests described in this paper (PLI/CLI, traction efficiency curve, moving resistance) for external customers, under operation of trained ENTAM Network institutes.

\section{Acknowledgement}

Authors would like to express their gratitude to all colleagues from NARIC MGI, IRSTEA and Michelin, who have contributed in the project. Special thanks go to:

- $\quad$ From NARIC MGI: Márk Szente, Bakk János, István Keppler 
- From Michelin: Patrick Vervaet, Matthieu Vandemoortele

- From IRSTEA: Denis Miclet

Their theoretical and practical knowledge, experience and support provided during all these years are strongly appreciated.

\section{References}

ASABE; EP542 (R2009).: "Procedures for Using and Reporting Data Obtained with the Soil Cone Penetrometer"

ASABE; S313; "Soil Cone Penetrometer"

ASABE, D497 "Agricultural Machinery Management Data" 2009.

Dessèvre, D. 2010. A review of the main effects of the tires and their usage, on the fuel consumption of agricultural tractors. EurAgEng 2010.

Forissier, J.F. 2009. Methodological precautions for tractor fuel consumption's measurement Land Technik AgEng 2009.

Fancello G., Szente M, Szalay K., Kocsis L., Piron E., Marionneau A. 2013. New experimental method for measuring the energy efficiency of tires in real condition on tractors. VDI AgEng 2013.

Fancello G., Szente M., Kovács L., Kocsis L., Szalay K., Piron E., Miclet D. Héritier P. 2015. Agricultural Tire Energy Efficiency test method link with specific fuel consumption for measuring the efficiency of agricultural tires under real conditions on tractors, VDI AgEng 2015.

Kassai, Zs. and Szente, M 2005. Complex comparison of tractor transmission systems between Continuously Variable Transmission and Powershift Transmission. VDI-MEG 2005.

Szalay K., Szente M., Kovács L., Kocsis L., Bablena A., Bércesi G., Piron E., Miclet D., Héritier P., Fancello G. 2016. Agricultural tire energy efficiency tests: a commitment toward a sustainable agricultural production, AgEng 2016.

Szente, M.; Kriston, S.; Forissier, J. F. 2010. A New Single Wheel Tester - Global Traction and Motion Resistance Measuring System, EurAgEng 2010.

Andreas A., Hans-Joachim T. 2015. DLG powermix chassis dynamometer - the field on a test bench, VDI AgEng 2015 


\title{
Fuel Consumption Analysis of Real-World Measurements
}

\author{
Michele Mattetti ${ }^{a}, *$, Riccardo Molinari ${ }^{\mathrm{a}}$, Eugenio Sereni ${ }^{\mathrm{b}}$, Giovanni Molari ${ }^{\mathrm{a}}$ \\ ${ }^{a}$ Department of Agricultural and Food Sciences - University of Bologna, viale G. Fanin, 50, 40127, Italy \\ ${ }^{\mathrm{b}} \mathrm{CNH}$ Industrial S.p.A., viale delle Nazioni 55, Modena, Italy \\ * Corresponding author. Email: michele.mattetti@unibo.it
}

\begin{abstract}
The trend in agriculture is toward more sustainable and efficient crop productions. The efficiency of a crop production can be evaluated as a ratio between energy costs and crop yield. The highest energy cost is that of mechanization fuel consumption. This information is usually estimated with empirical formulas. The most commonly adopted formula was established by the ASAE and it was derived from the Nebraska Tractor Test reports. The procedure that developed those reports are based on constant load tests, but tractors, under real field activities, operate with variable loads and therefore, concerns can be made in using this formula. Nowadays, due to the widespread integration of CAN-BUS, much information is easily available from any modern tractor and they can be used for an exact estimation of tractor fuel consumption. The aim of this paper was the collection and the analysis of fuel consumption data acquired under real operating conditions. A fleet of tractors of the same family were used in different farms for cultivation. Tractors operated with different cultivators (i.e. different working width and depth, tool type, etc) and worked on different soils and land profiles. On each tractor, a CANBus data logger was installed and the following operating parameters were acquired: engine torque and speed; vehicle speed; and fuel consumption. For each tractor, data were acquired for an hour. From the acquired signals, power requirements and frequency plots were calculated for each acquisition and all the data were statistically analysed. The data analysis revealed that in-field average engine power is limited to the $75 \%$ of the maximum engine power due to the fact the implement draft is highly variable. Moreover, the in-field fuel consumption varies from tractor to tractor due to the fact each tractor was used with different power demand by the driver.
\end{abstract}

Keywords: agricultural tractors, fuel consumption prediction, field tests, sustainable agriculture.

\section{Introduction}

Agriculture is a high energy demanding activity, which contributes to about $20 \%$ of the greenhouse emissions (Rosenzweig \& Hillel, 2000). An important contributor of these emissions is the fuel combustion during agricultural operations (Meisterling et al. 2009). Fuel consumed in agricultural productions depends on various factors, such as technology, operational conditions, farm conditions and management. The prediction of fuel consumption for each condition is crucial for understanding how to use more responsibly the input energy.

Fuel consumption caused by agricultural mechanisation can be measured or obtained scientific literature. Measurements are reliable but also expensive to carry out. A well-known approach for estimating the fuel consumption of agricultural field activities is the ASAE method (ASAE, 2006, 2011). This method permits to calculate the draft requirement of each field activity and machine setting (with a range of variability of $+/-50 \%$ ), which can be used to calculate the engine load in terms of ratio of equivalent Power Take-Off (PTO) power to the rated PTO power of the tractor under study. From this ratio, fuel consumption can be predicted with a formula based on PTO power test results from a large sample of Nebraska Tractor Test Lab (NTTL) reports of the 70s. A more refined approach is the Grisso's formula for specific tractors model (GF), where the fuel consumption formula is based on NTTL report of the specific tractors model under study. This formula demonstrated to be $6 \%$ more precise than the ASAE fuel consumption formula (Grisso et al. 2008). NTTL test procedure is based on a steady-state test run at five different engine operating points. However, engine during real-world operations is run under different states (idling, headland turns, working state passes) and under highly variable load (from the 30\% up to $80 \%$ of the maximum power) (Mattetti et al. 2018), therefore those formulas can lead to erroneous results.

The widespread integration of CAN-Bus into modern agricultural tractors permits to easily acquire engine operating parameters in many machines (Perozzi et al. 2016). Previous studies reported a maximum error of $6.22 \%$ between the physical fuel rate measurement and the CAN-Bus fuel rate measurement (Marx, 2015). Therefore, CAN-Bus is an attractive solution for obtaining fuel consumption data and measurements under real operating conditions were carried out for few operations (Pitla et al. 2016). However, no study reporting fuel consumption data coming from a large fleet of tractors under real operating conditions has been conducted so far. This will be beneficial to verify and update GF. The aim of this paper was the collection and the analysis of fuel consumption data acquired from a large fleet of tractors under real operating conditions and compare the results with those predicted from GF. 


\section{Materials and Methods}

The comparison between predicted and measured fuel consumption was carried out on a sample of 54 tractors with nominal power ranging from 257 to $441 \mathrm{~kW}$ (figure 1). Data were mainly collected during ploughing, cultivating and seeding operations, performed by different drivers among the European and the Northern America areas.

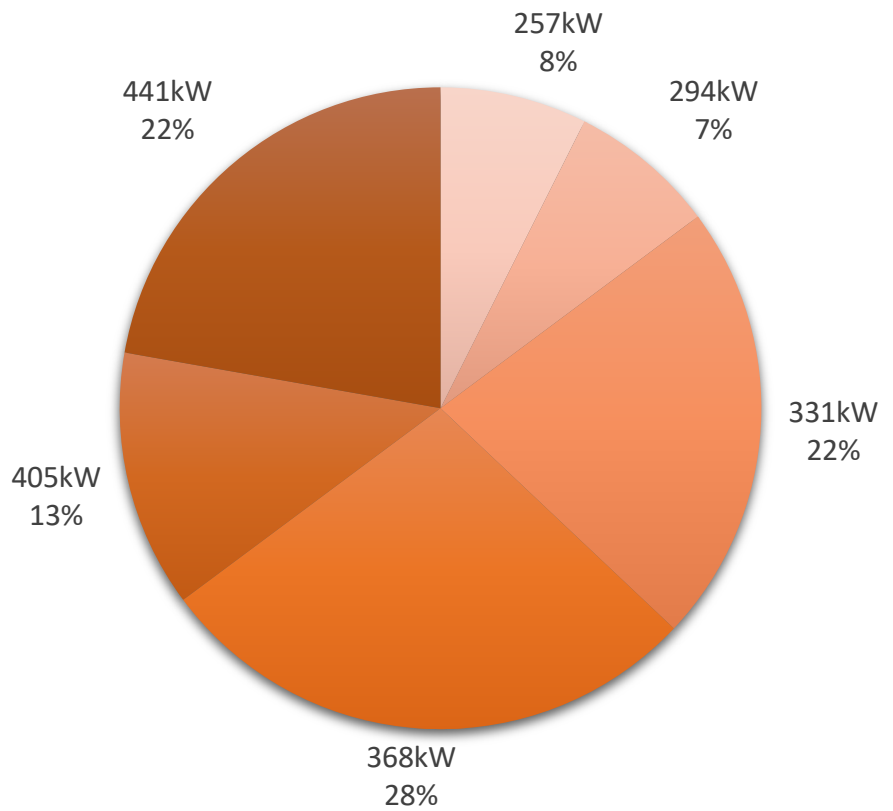

Figure 102. Pie chart of the tractor population according to their nominal power.

On every vehicle, a CAN-Bus data logger was installed to record the following signals (sampled at $100 \mathrm{~Hz}$ ):

Engine speed in $\mathrm{rpm}\left(R P M_{F E S}\right)$

Engine torque $\left(T_{E}\right)$

Fuel rate $\left(Q_{m}\right)$

The relative frequency of $Q_{m}$ was calculated and in function of the maximum frequency, datasets were classified into two categories called in text narrow band (NB) and broad band (BB). The threshold values of the maximum function to be classified as NB was $25 \%$, otherwise the dataset was classified as BB (Figure 103).
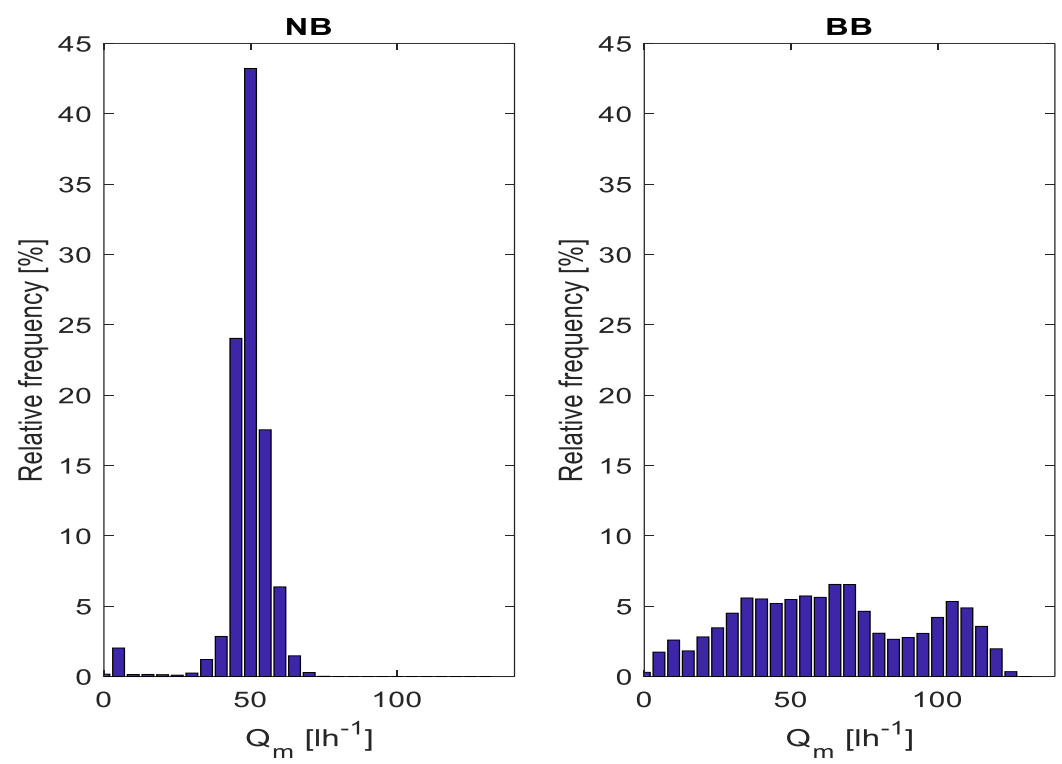

Figure 103. Sample of a dataset classified as NB (on left) and as BB (on right). 
Then, the delivered engine power $(P)$ was then calculated as follows:

$$
P=T_{E} R P M_{\text {fes }} \frac{2 \pi}{60}
$$

The predicted fuel consumption $(Q)$ was calculated using GF, where its parameters were extracted from NTTL test reports for each different tractor (figure 4).

$$
Q=(a X+b) *[1-(N-1) *(c X-d)] * P_{P T O}
$$

with:

$$
\begin{aligned}
& X=P / P_{P T O} \\
& N=\frac{R P M_{\text {fes }}}{R P M_{\text {rated }} @ T_{E}}
\end{aligned}
$$

Being $a, b, c$ and $d$ parameters (Grisso, Vaughan, \& Roberson, 2008) and RPM rated $@ T_{E}$ was derived from interpolation of the "Varying Power and Fuel Consumption" section parameters in NTTL test reports. For each dataset, the Instant Percentage Error (IPE) was calculated as follows:

$$
I P E=\frac{Q-Q_{m}}{Q_{m}} 100
$$

To smooth IPE from spikes caused by signal inconsistencies, IPE was filtered (using a low-pass filter with a cutoff frequency of $15 \mathrm{~Hz}$ ). To provide a global view of GF's effectiveness in different operating conditions, the Mean Percentage Error (MPE) was calculated as follows:

$$
M P E=\left(\sum_{i}^{N} I P E_{i}\right) / N
$$

Being $\mathrm{N}$ the number of samples of IPE. To verify the goodness of the smoothing process, $M P E$ s of the raw and filtered $I P E$ s were compared and for all the datasets, the maximum deviation was limited to about $0.15 \%$. Since $M P E$ covered a wide range of values throughout the datasets, for each tractor, IPE was plot together with $X$ to investigate their correlation. Finally, a comparison of MPE in NB and BB datasets was conducted, and the mean MPE of both groups were calculated.

\section{Results and Discussion}

In Figure 104, the trend of IPE with respect to $X$ of a dataset is reported. IPE decreases with $X$ passing from 92 to -19 , that means, GF overestimates the fuel consumption for low engine loads (lower than 0.58) but underestimate the fuel consumption for high engine loads. IPE is zero when $X$ ranges from 0.58 to 0.68 . Moreover, most of the data lie on a boundary which can be described as hyperbolic.

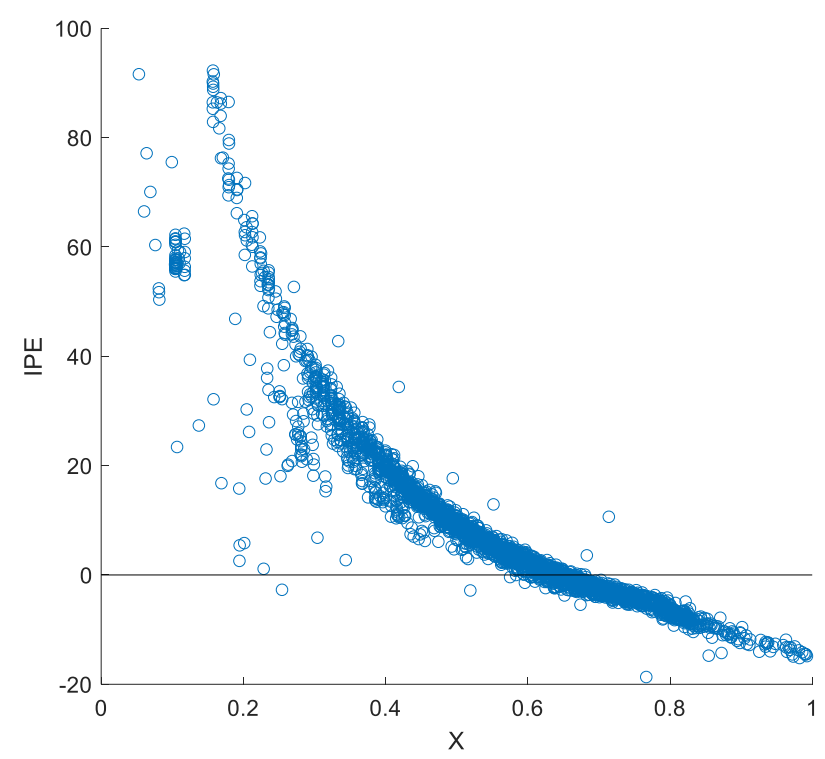

Figure 104. Trend of IPE with respect to $X$ for a sample dataset. 
In Figure 105, $M P E$ values for $\mathrm{BB}$ and $\mathrm{NB}$ groups are reported. $M P E$ of $\mathrm{BB}$ group is significantly higher than $\mathrm{NB}$, indeed for BB group, $M P E$ ranges from -8.4 to 54.7; while for NB $M P E$ ranges from -9.3 to 10.9. The mean value of $M P E$ of $\mathrm{BB}$ and $\mathrm{NB}$ groups is 8.6 and 0.7 , respectively.
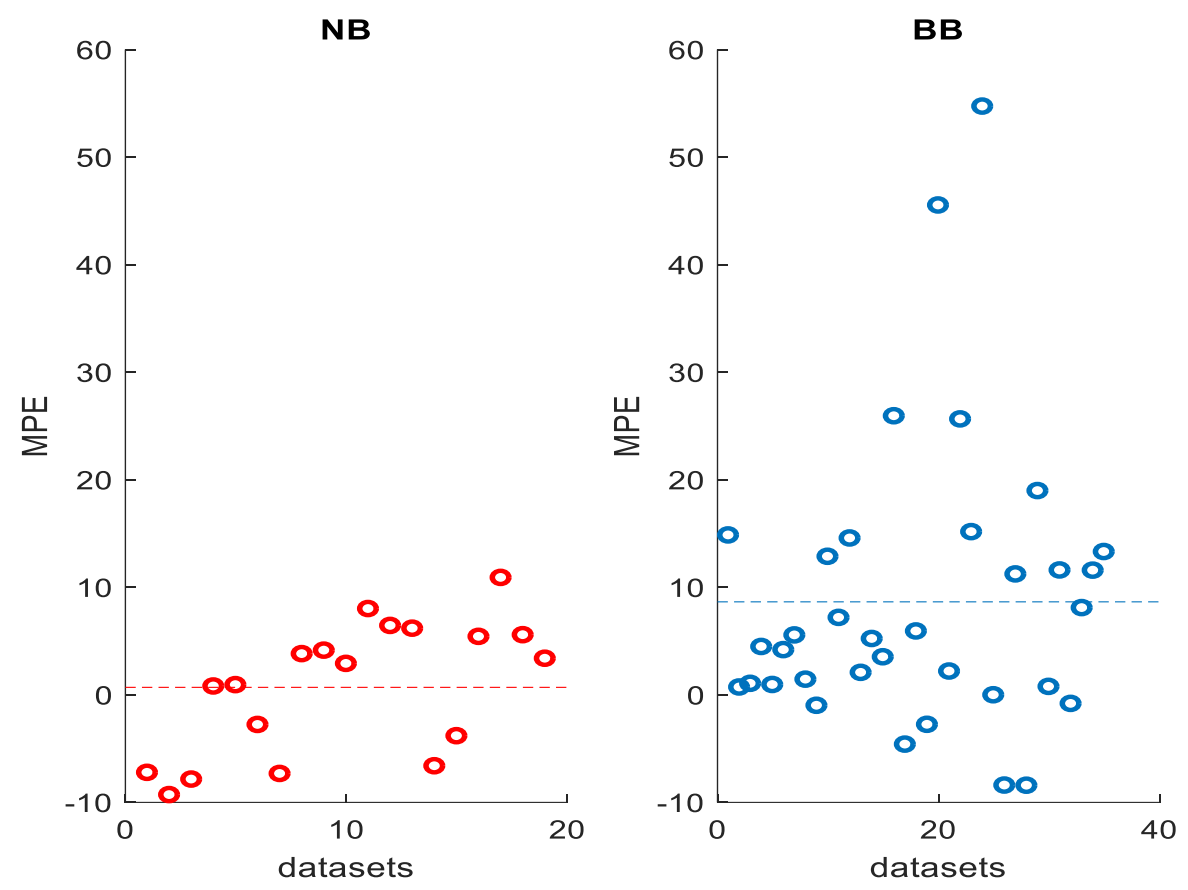

Figure 105. MPEs for the different datasets of NB (on left) and BB (on right). The dashed lines on each plot represents the average value of the group.

NB so represents more stationary datasets than BB, the decrease of MPE in NB could be due to a loss of accuracy in transitional intervals that are more often observed in BB. This can be explained by the fact that GF was derived from NTTL reports, where fuel consumption is measured at five different steady operating points ( $100 \%$ of the maximum pull and $50 \%, 75 \%$ at maximum and reduced throttle). Thus, GF contains no information about transitional states, which could differ from one condition to another.

\section{Conclusions}

In this paper, fuel consumption data from real-world measurements carried out on a large sample of tractors was analysed. These data were compared with those predicted from GF. Due to the different engine load states which may affect the fuel consumption, the comparison was carried using the actual values of the time series, rather than their average values. This permitted to evaluate the conditions where the formula effectiveness was the best. Indeed, the average value comparisons could work well only on steady-state conditions, which are far from real-world conditions. GF predicts very well the fuel consumption for engine loads between 58 and $68 \%$ of the maximum engine power. As a future activity, the analysis of a much wider sample of datasets would be of a great interest to search for other parameters influencing MPE, so that correction factors could be added to the formula, so that the prediction of fuel consumption can be improved.

\section{References}

ASAE, A. 2006. ASAE EP496.3 - Agricultural Machinery Management. ASAE. ASAE, A. 2011. ASAE D497.7 - Agricultural Machinery Management Data. ASAE.

Frye, W. W. 1984. Energy Requirement in No-Tillage. In No-Tillage Agriculture (pp. 127-151). Springer, Boston, MA. https://doi.org/10.1007/978-1-4684-1467-7_6

Grisso, R. D., Vaughan, D. H., \& Roberson, G. T. 2008. Fuel Prediction for Specific Tractor Models. Applied Engineering in Agriculture, 24(4), 423-428. https://doi.org/10.13031/2013.25139 
Heichel, G. H. 1976. Agricultural Production and Energy Resources: Current farming practices depend on large expenditures of fossil fuels. How efficiently is this energy used, and will we be able to improve the return on investment in the future? American Scientist, 64(1), 64-72.

Marx, S. E. 2015. Controller Area Network (CAN) Bus J1939 Data Acquisition Methods and Parameter Accuracy Assessment Using Nebraska Tractor Test Laboratory Data.

Mattetti, M., Maraldi, M., Sedoni, E., \& Molari, G. 2018. Optimal criteria for durability test of stepped transmissions of agricultural tractors. Biosystems Engineering, Submitted.

Meisterling, K., Samaras, C., \& Schweizer, V. 2009. Decisions to reduce greenhouse gases from agriculture and product transport: LCA case study of organic and conventional wheat. Journal of Cleaner Production, 17(2), 222-230. https://doi.org/10.1016/j.jclepro.2008.04.009

Perozzi, D., Mattetti, M., Molari, G., \& Sereni, E. 2016. Methodology to analyse farm tractor idling time. Biosystems Engineering, 148, 81-89. https://doi.org/10.1016/j.biosystemseng.2016.05.007

Pitla, S. K., Luck, J. D., Werner, J., Lin, N., \& Shearer, S. A. 2016. In-field fuel use and load states of agricultural field machinery. Computers and Electronics in Agriculture, 121, 290-300. https://doi.org/10.1016/j.compag.2015.12.023

Rosenzweig, C., \& Hillel, D. 2000. Soils and global climate change: Challenges and opportunities. Soil Science, 165(1), 47-56. https://doi.org/10.1097/00010694-200001000-00007

Wold, M., Kocher, M., Hoy, R., Lammers, A., \& Blankenship, E. 2011. Development of a Calibration Procedure for Diesel Fuel Flow Measurement with a Coriolis Effect Meter (1-15). American Society of Agricultural and Biological Engineers. https://doi.org/10.13031/2013.37797 


\title{
Leftover Silage as Biogas Substrate: Processing Machinery and Costs \\ Carina Gunnarsson a,", Johanna Lund ${ }^{\text {a, }}$, Erik Fischer ${ }^{\text {b }}$ \\ ${ }^{a}$ RISE Agrifood and Bioscience, RISE Research Institute of Sweden, Uppsala, Sweden \\ ${ }^{b}$ DBFZ Deutsches Biomasseforschungszentrum gemeinnützige GmbH, Leipzig, Germany \\ * Corresponding author. Email: carina.gunnarsson@ri.se
}

\begin{abstract}
Large amounts of leftover silage are available in agriculture and in municipalities harvesting meadows and grassland. At the same time, biogas plants using crop-based substrate are looking for alternative feedstocks in order to adapt to EU RED and to increase profitability. Silage that is not needed or not suitable for feeding animals can be an excellent biogas substrate, but the material is often coarse and pre-treatment is necessary. This paper describes a case study seeking to replace $20 \%$ of the crop-based substrate used today (maize, whole-crop silage and grain) at the Jordberga biogas plant in southern Sweden with leftover silage bales. Three different mobile machines for chopping silage bales were tested in practical trials, to evaluate which was most effective at reducing particle length and damaging the structure of the grass, to improve biodigestion. Two of the machines used a hammermill technique for chopping and the third used knife rotors. The resulting particle length, energy consumption and capacity were measured. The costs of silage bale handling system, from the farm or storage site to the processing unit for chopping and then to the digester, were also calculated. All three machines managed to chop silage with a dry matter (DM) content of $40-70 \%$. The chopping effect was more complete with respect to particle length for silage with lower DM content than for silage with higher DM content. Based on the test results the cost of chopping, which included machine and tractor, diesel, work, maintenance and use of a wheel loader for handling bales and unwrapping the plastic, ranged between 14.6 and $25.6 € / \mathrm{t}$ wet weight depending on lifetime of the machines. Purchase of the silage bales and transport by tractor and trailer to the biogas plant were the main costs in the system.
\end{abstract}

Keywords: silage bales, biogas, pre-treatment, chopping, machinery, costs.

\section{Introduction}

In order to reduce greenhouse gas emissions and limit their contribution to global climate warming, the European Union Renewal Energy Directive (EU RED) requires at least 10\% of transport fuels used in member states to come from renewable sources by 2020 . Today, biogas accounts for $2 \%$ of fuel sales, which is equivalent to $1.7 \mathrm{TWh}$. However, to achieve the Swedish government target of a fossil-independent vehicle fleet by 2030, $12 \mathrm{TWh}$ of biogas will be required for the transport sector (Energigas, 2018). Achieving this goal in a sustainable way will be a challenge.

There are large amounts of leftover silage in agriculture and in municipalities that harvest meadows and grassland. This biomass is currently a disposal problem and a cost. At the same time, biogas plants are experiencing increasing demand for substrates that do not compete with production of animal feed and food.

Leftover silage can be an excellent biogas substrate, provided it is effectively pre-treated. This study examined $20 \%$ replacement of today's crop-based substrates with leftover silage from agriculture and municipalities. The Jordberga biogas plant in Scania (southern Sweden) was used as a case study, but the results are applicable to other regions in Sweden.

The objective of the study was to identify appropriate technologies and equipment for pre-treatment of various types of leftover silage for biogas generation. This was done through practical tests and by calculating the resulting costs for processing silage bales, including the chopping procedure and work and energy use in handling the bales on the site before and after chopping.

The study was conducted by RISE Agrifood and Bioscience in collaboration with: the German Biomass Research Center (Deutsches Biomasseforschungszentrum, DBFZ); Gasum, the owner of the biogas plant in Jordberga; the County Administration Board of Scania; and Fogda Farm. The result of the study is also presented in Swedish in Lund et al. (2018).

\section{Materials and Methods}

Machine tests

Based on previous studies and experiences by the research team, an initial inventory was conducted on suitable machines for processing silage bales, including machines currently used in Germany for chopping silage. Mobility of the machine was considered an advantage, since it allowed it to be used on different sites, ensuring high annual use and lowering the capital costs.

Three machines were selected for practical tests: Roto Grind, RS CutMaster and I-GRIND. These machines 
represent different chopping techniques; Roto Grind and I-GRIND use a hammermill technique, whereas RS CutMaster uses knife rotors. Roto Grind has been on the market for a long time, while the other two machines are new. All three machines are mobile and tractor-powered. The practical tests were conducted on farms in Sweden (Roto Grind), Germany (RS CutMaster) and Denmark (I-GRIND).

All machines were tested with bale silage harvested on two different meadows in Scania, CAB 1 and CAB 2, which was provided by the County Administration Board (CAB) for Scania. These meadows were cut only once during the season, in the middle of September, resulting in long, fibrous silage. The bales were transported to the three farms where the tests were carried out. In addition, one or two different types of silage available locally on each farm (called feed silage in Table 1) were included in the tests. Round bales were tested in all cases except one, where square bales of silage (IG-3) were tested with I-GRIND.

Table 1. Information about the test runs performed on each machine; type of silage/machine adjustment used, bale code and number of bales in each run.

\begin{tabular}{cccc}
\hline Machine & Type of silage/machine adjustment & Bale code & Number of bales \\
\hline Roto Grind (RG) & CAB 1 & RG-1 & 4 \\
& CAB 2 & RG-2 & 4 \\
& Feed silage & RG-3 & 4 \\
RS CutMaster (CM) & RG-4 silage & CM-1 & 4 \\
& CAB 1, with counterknifes & CM-1a & 0,5 \\
& CAB 1, without counterknifes & CM-2 & 0,5 \\
& CAB 2, with counterknifes & CM-3 & 1 \\
I-GRIND (IG) & Feed silage, with counterknifes & CM-3a & 0,5 \\
& Feed silage, without counterknifes & IG-1 & 0,5 \\
& CAB 1, without sieve & IG-2 & 4 \\
& CAB 2, without sieve & IG-3 & 4 \\
\hline
\end{tabular}

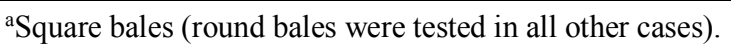

Diesel consumption and bale chopping capacity were measured during the tests and the function of the machines in terms of chopping effect was observed. Furthermore, samples were taken for analysis of particle length and dry matter (DM) content. A standardised test procedure was used, where the bales were weighed, plastic wrapping and netting was removed and the bales were fed into the chopping machine. For the Roto Grind and RS CutMaster tests, a device measuring fuel consumption was connected to the CAN-bus system of the tractor. For I-GRIND, the diesel tank was filled up before and after each test run, and consumption was recorded as the difference.

\section{Analysis of particle length}

To evaluate the ability of the selected machines to chop the silage, samples of the chopped material were run in a sorting machine at the RISE facilities in Uppsala. This machine sorts material by length into eight different fractions: $<4.5,4.5-8,8-12,12-20,20-33,33-54,54-90$ and $90-150 \mathrm{~mm}$. If the sample contains material over 150 $\mathrm{mm}$, two additional fractions are sorted by hand; $150-250 \mathrm{~mm}$ and $>250 \mathrm{~mm}$. Thus, sorting of a sample can result in at most 10 fractions (see example in Figure 1). The sorting machine and its function are described in detail by Gale \& O’Dogherty (1982).

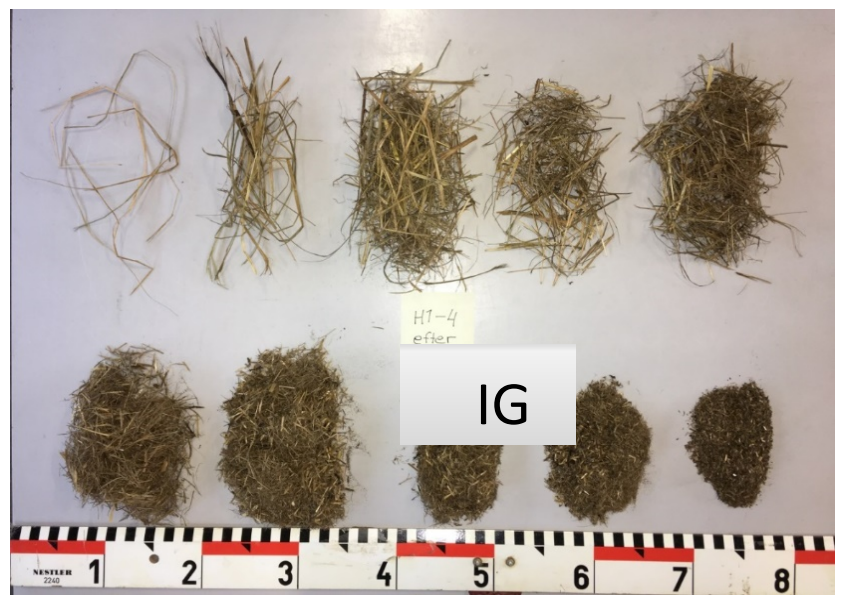

Figure 1. Set of 10 fractions obtained after sorting a sample of silage IG-1 from County Administration Board (CAB 1), chopped with I-GRIND without sieve. 
The weights of the different fractions were entered into a computer programme that calculates the median chop length of the sample (i.e. the length that divides the sample into two equal parts by weight), based on the assumption that the particle length distribution follows a log-normal distribution. Using the curve obtained, the particle length distribution was calculated for the part of the sample exceeding $50 \mathrm{~mm}$ and the part of the sample less than $10 \mathrm{~mm}$. Based on experiences from Gasum, the limits were chosen in light of the fact that particle length shorter than $10 \mathrm{~mm}$ is desired for silage for biogas production and that particle length greater than about $50 \mathrm{~mm}$ may cause handling problems in the biogas process.

\section{Cost evaluation}

Costs were calculated based on the capacity and diesel consumption measured in the tests, based on replacing $20 \%$ of the methane produced from dedicated energy crops at Jordberga biogas plant, corresponding to $3268 \mathrm{Nm}^{3}$ methane/day, with methane produced from silage bales. Silage bales were assumed to weigh $800 \mathrm{~kg}$ wet weight (ww), with a DM content of $42 \%$.

The total chopping costs included the cost of the chopping machine and its operation, and also the costs of handling the bale before and after chopping. The cost calculations were done in SEK and the rate of exchange was set to 1 Euro $=10 \mathrm{SEK}$

\section{Machine costs}

The depreciation cost for buying the machine was included. The purchase price of Roto Grind was set at $€ 20,500$, that for RS CutMaster at $€ 90,000$ and that for I-GRIND at $€ 97,000$. It was assumed that the machines were used for their entire lifetime and that they were replaced when the maximum operating time was reached. This means that variable depreciation cost was calculated, by dividing the purchase price by the total lifetime (in hours) of the machine.

The lifetime of a machine, i.e. the number of running hours, can be difficult to estimate. Both I-GRIND and RS CutMaster are newly developed machines, so long-term data on their operating life are lacking. The life expectancy also depends on how well the machine is maintained and on the quality of the bales. The calculations were therefore performed for three lifetimes; 2000, 4000 and $8000 \mathrm{~h}$.

An interest cost, calculated as an annual cost based on the average purchase cost and the residual value at $2 \%$ interest, was added to the cost of the machine.

\section{Tractor costs for powering the machine}

The costs for the tractor needed to power the machine were based on the size of tractor used in the practical tests carried out on farms and were calculated as a fixed cost per hour, according to hourly rate data taken from Maskinkalkylgruppen (2017). A $140 \mathrm{~kW}$ tractor was used for Roto Grind, while a $220 \mathrm{~kW}$ tractor was used for both Cut Master and I-GRIND.

\section{Diesel consumption for chopping}

All three machines tested were tractor-driven and the cost of diesel for their operation was calculated based on fuel measurements made during chopping tests on some the bales from the County Administration Board (bales RG-1 and RG-2 in Table 1). The price of diesel was set to $0.9 € / \mathrm{L}$, according to Maskinkalkylgruppen (2017).

\section{Work}

It was assumed that one person can carry out all the work involved in bale chopping; removing the plastic, feeding the material into the chopping machine, operating the machine and removing the silage after chopping. The labour costs were set at $28 € / \mathrm{h}$ according to Maskinkalkylgruppen (2017).

\section{Maintenance}

There was no good basis for calculating maintenance costs, since two of the machines are new and practical experience of their long-term operation is lacking. Contacts with manufacturers and users of the machines indicated that the maintenance costs are highly dependent on the type and condition of the silage chopped.

We used a method whereby total repair and maintenance costs are related to the machine's purchase price (I) and to annual use (H), see Gunnarsson \& Hansson (2004). The repair and maintenance factor (RM) was set at $80 \%$ of the purchase price for all three machines, according to ASABE (2006). Maintenance cost per hour $(\mathrm{Ku})$ was calculated as:

$$
K u=(I \times R M) \div H
$$

\section{Wheel loader and equipment for removing plastic from bales}

The costs for use of a wheel loader to handle bales and to remove the plastic was calculated as a fixed cost per hour during the chopping operation, using hourly rates from Maskinkalkylgruppen (2017).

The depreciation cost (A) for a device mounted on the front loader of the wheel loader to remove plastic and netting from the bales was calculated as:

$$
A=a f(\operatorname{Inv}-R v)
$$


where af is the annuity factor, Inv is the purchase price (here $€ 5,500)$ and $\mathrm{Rv}$ is the residual value (here set to $0)$. The annuity factor was calculated as:

$$
\text { af }=p /\left(1-(1+p)^{-t}\right)
$$

where $\mathrm{p}$ is the interest (set to $2 \%$ ) and $\mathrm{t}$ is depreciation time (set to $5 \mathrm{yr}$ ), which gives an annuity factor of 0.2122 .

\section{Cost of purchasing and transporting bales from farm or storage to biogas plant}

In the calculations, the purchase price of the bales was set at $16 € /$ bale or $20 € / \mathrm{t} \mathrm{ww}$, corresponding to the price of a silage bale of feed quality. However, the price depends very much on quality, but also on supply and demand, which means that variations between years can be high.

The cost of transporting bales from farm to biogas plant was calculated for a tractor and a trailer with space for 28 bales. The time for loading and unloading was set to one minute per bale. The distance between farm and plant was set to $30 \mathrm{~km}$ and the transport speed was set at $30 \mathrm{~km} / \mathrm{h}$ (Amon et al., 2007). The hourly rate for the tractor and trailer, including driver and fuel, was taken from Maskinkalkylgruppen (2017).

\section{Results and Discussion}

Technical evaluation

\section{Analysis of particle length distribution}

Particle length analysis revealed that shorter median chop length resulted in a large proportion of material with length $<10 \mathrm{~mm}$ (Table 2). Correspondingly, longer median shop length resulted in a large part of material with length $>50 \mathrm{~mm}$. Four of the samples (CM-1, CM-1a, CM-2, IG-2) contained relatively large amounts of clumps of chopped material. For these, it was concluded that particle length analysis in the sorting machine would not give a fair result, as in principle the machine sorting must be done separately for each individual particle. Therefore it is unfortunately not possible to compare the result of chopping with the different machines for the same type of silage.

Before the tests, it was expected that there would be problems chopping bales with a high water content. In the tests with Roto Grind, the resulting particle length was longest for silage RG-4, which also had the highest DM content of the bales tested. However, since silage of different origins was tested, factors other than DM content could have influenced the results. Due to the clumps in some of the samples of chopped silage produced by RS CutMaster and I-GRIND, corresponding analysis could not be conducted for these machines.

Table 2. Median chop length and weight- $\%$ of fractions $>50 \mathrm{~mm}$ and $<10 \mathrm{~mm}$ obtained from the test silage bales using Roto Grind (RG), RS Cut Master (CM) and I-GRIND (IG).

\begin{tabular}{cccc}
\hline Test silage & $\begin{array}{c}\text { Median chop length, } \\
\text { mm }\end{array}$ & $\begin{array}{c}\text { Particles }>50 \text { mm, } \\
\text { weight-\% }\end{array}$ & $\begin{array}{c}\text { Particles }<10 \text { mm, } \\
\text { weight-\% } \%\end{array}$ \\
\hline RG-1 & 13 & 9 & 39 \\
RG-2 & 11 & 7 & 46 \\
RG-3 & 12 & 5 & 42 \\
RG-4 & 22 & 21 & 21 \\
CM-3 & 20 & 14 & 14 \\
CM-3a & 29 & 30 & 37 \\
IG-1 & 16 & 19 & 17 \\
IG-3 & 29 & 31 & \\
\hline
\end{tabular}

\section{Diesel consumption and capacity}

Diesel consumption during the tests, the capacity of the machines to chop the test bales (based on operating time and the weight of the bales) and DM content in samples of the chopped silage are shown in Table 3. 
Table 3. Measured diesel consumption per hour and per ton wet weight (ww) and per ton dry matter (DM), calculated machine capacity (per ton ww and per ton DM) and measured DM content of the chopped material in the tests performed with Roto Grind, RS CutMaster and I-GRIND.

\begin{tabular}{|c|c|c|c|c|c|c|c|}
\hline \multirow[t]{2}{*}{ Machine } & \multirow[t]{2}{*}{ Test silage } & \multicolumn{3}{|c|}{ Diesel consumption } & \multicolumn{2}{|c|}{ Capacity } & \multirow{2}{*}{$\begin{array}{r}\mathrm{DM} \\
\text { content } \\
\%\end{array}$} \\
\hline & & $\mathrm{L} / \mathrm{h}$ & $\mathrm{L} / \mathrm{t} \mathrm{wW}$ & $1 / \mathrm{t} \mathrm{DM}$ & t ww/h & t DM/h & \\
\hline \multirow{3}{*}{$\mathrm{RG}$} & RG-2 & 31 & 3.3 & 7.4 & 9.3 & 4.2 & 45 \\
\hline & RG-3 & 34 & 2.8 & 7.0 & 12.1 & 4.8 & 40 \\
\hline & RG-4 & 21 & 6.4 & 8.2 & 3.3 & 2.5 & 78 \\
\hline \multirow{4}{*}{$\mathrm{CM}$} & CM-1a & 41 & 4.7 & 9.6 & 7.9 & 3.9 & 46 \\
\hline & CM-2 & $39-43$ & 4.6 & 8.6 & 8.7 & 4.6 & 53 \\
\hline & CM-3 & 34 & - & - & - & - & 70 \\
\hline & CM-3a & $27-38$ & - & - & - & - & 70 \\
\hline IG & IG-1 & 61 & 4.5 & 8.8 & 13.4 & 6.8 & 51 \\
\hline
\end{tabular}

\section{Chopping costs}

Replacement of $20 \%$ of crop-based biogas production at Jordberga biogas plant with biogas production based on silage bales of feed quality would require 12 ton DM/day. The costs of chopping the corresponding amount of silage bales with the different machines are shown in Figure 2. The calculations were carried out for different values of capacity and lifetime of the machines (Table 4). In practical operation, the capacity can vary widely depending on e.g. the quality of the silage and the experience of the driver handling the machine. The lifetime of the machine can also vary widely, depending on the quality of the silage and e.g. maintenance. In addition, the maintenance costs were difficult to determine in this case, because two of the machines are new and there are no long-term operating data available. It is therefore relevant to consider capacity and lifetime as variables, and the values in Table 4 can be used as a basis for estimating costs depending on what is considered relevant in different cases.

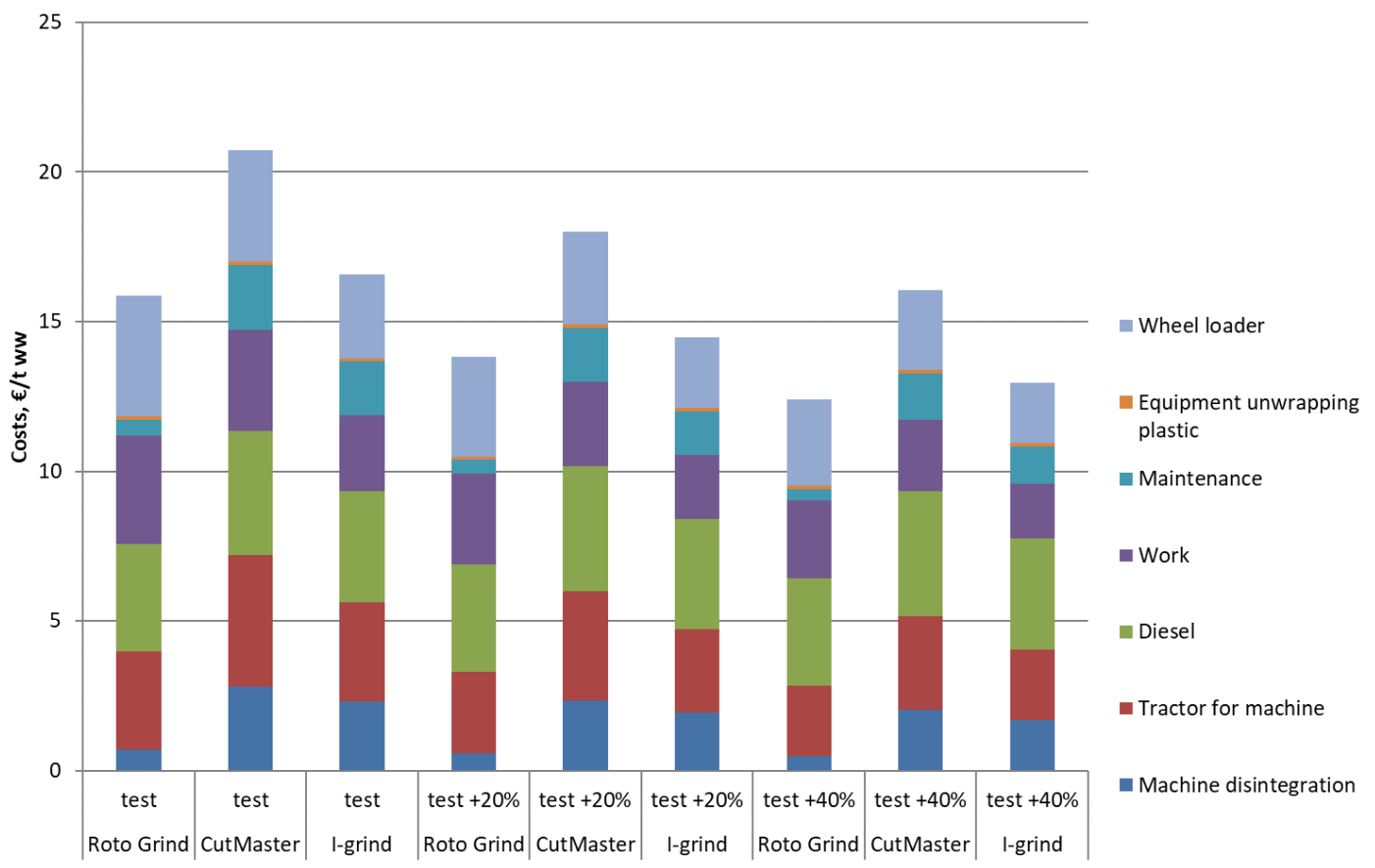

Figure 2. Costs per ton wet weight (€/t ww) for chopping silage bales with the Roto Grind, RS CutMaster and I-GRIND machines, assuming a lifetime of $4000 \mathrm{~h}$. 
Table 4. Summary of costs in $€ / t$ wet weight (ww) for chopping silage bales with the Roto Grind (RS), RS CutMaster (CM) and I-GRIND (IG) machines, operated at varying capacity (measured capacity $+0 \%,+20 \%$ and $+40 \%$, and capacity stated by the manufacturer) for $2000 \mathrm{~h}, 4000 \mathrm{~h}$ and $8000 \mathrm{~h}$ total lifetime of the machine.

\begin{tabular}{|c|c|c|c|c|c|c|c|c|c|}
\hline \multirow{3}{*}{ Cost, $€ / \mathrm{t} w \mathrm{w}$} & \multicolumn{9}{|c|}{ Lifetime, $\mathrm{h}$} \\
\hline & \multicolumn{3}{|c|}{2000} & \multicolumn{3}{|c|}{4000} & \multicolumn{3}{|c|}{8000} \\
\hline & $\mathrm{RG}$ & $\mathrm{CM}$ & IG & $\mathrm{RG}$ & $\mathrm{CM}$ & IG & RG & $\mathrm{CM}$ & IG \\
\hline Capacity measured in test ${ }^{*}$ & 17.1 & 25.6 & 20.6 & 15.9 & 20.7 & 16.6 & 15.3 & 18.3 & 14.6 \\
\hline $\begin{array}{l}\text { Capacity measured in test } \\
\quad+20 \%\end{array}$ & 14.8 & 22.1 & 17.8 & 13.8 & 18.0 & 14.5 & 13.3 & 16.0 & 12.8 \\
\hline $\begin{array}{l}\text { Capacity measured in test } \\
\quad+40 \%\end{array}$ & 13.2 & 19.5 & 15.8 & 12.4 & 16.1 & 13.0 & 12.0 & 14.3 & 11.5 \\
\hline $\begin{array}{l}\text { Capacity stated by } \\
\text { manufacturer** }\end{array}$ & 10.6 & 10.2 & 16.1 & 10.0 & 8.9 & 13.2 & 9.6 & 8.2 & 11.7 \\
\hline
\end{tabular}

Based on the test results, the costs of chopping varied between 14.6 and $25.6 € / t \mathrm{ww}$ depending of lifetime, see Table 4, for the three machines. Higher chopping capacity decreased cost whereas lower capacity increased costs. These costs included machine and tractor, diesel, work, maintenance and use of a wheel loader for handling bales and unwrapping the plastic. The average chopping costs were lowest for Roto Grind and slightly higher for I-GRIND and RS CutMaster in that order (see Figure 2 and Table 4). The higher costs for RS CutMaster and IGRIND were due to higher depreciation costs, as these machines had higher purchase cost and thus higher maintenance costs, which were linked to machine purchase costs in this study. The slightly lower costs for IGRIND than for RS CutMaster are explained by the higher capacity measured in the I-GRIND tests. At $40 \%$ higher capacity than measured in our tests, the RS CutMaster would have about the same costs as Roto Grind and I-GRIND (Table 4). This $40 \%$ higher capacity corresponds to approximately 12 tons $\mathrm{ww} / \mathrm{h}$, which is well below the average capacity specified for the RS CutMaster by the manufacturer (approximately 30 tons ww/h).

The chopping capacity of the machines can be expected to increase if larger numbers of bales are chopped per handling occasion than the number used in the practical tests, because the capacity was not maximised at the beginning and the end of the test sessions and the amount of silage fed to the cutting unit was small. For example, for Roto Grind and I-GRIND the capacity was measured for four bales chopped in succession, while for RS CutMaster the capacity was measured during chopping of only one bale. The capacity achieved in the tests may also increase with increasing practical experience by the person operating the machine. During the tests on Roto Grind, the machine was operated by the person who normally uses the machine on the farm, but chopping silage bales with the machine was new to him, as the machine is usually used for chopping straw. Both RS CutMaster and I-GRIND were operated by farm staff with experience of the machines, but their experience of handling round bale silage was limited.

As machine capacity increases, the time required to chop the desired number of bales decreases, reducing the running costs of the chopping machine and also other costs that depend on capacity and time, such as the cost of the wheel loader and labour costs. Maintenance costs are also linked to annual machine usage and decrease with increased capacity.

The lifetime of a machine depends on e.g. service and maintenance, as well as the type of biomass that has to be chopped and how contaminated it is with soil, stones, metal pieces etc. Since two of the machines tested here (RS CutMaster and I-GRIND) are new, data on long-term operation are lacking. With increasing life length, the purchase cost of a machine can be divided across more hours, which reduces the capital cost. As Figure 2 shows, the estimated maintenance costs were higher for RS CutMaster and I-GRIND than for Roto Grind. This is because they were calculated in relation to the purchase cost, which is much lower for Roto Grind. However, since RS CutMaster and I-GRIND are new and long-term data are lacking, the maintenance costs for these machines are uncertain.

\section{Purchase and transport costs for silage bales}

The purchase cost of silage bales was set to $20 € / \mathrm{tww}$, but the price varies greatly from case to case. In some cases, biogas plants have to buy bales and pay for transport to the biogas plant, while in other cases the bales are delivered without cost to the gate of the biogas plant. How much biogas plants can and will pay for bales depends on the quality of the bales and the amount of biogas that can be obtained on digesting the bales. In general, the methane yield is dependent on e.g. species composition, harvest time and decomposition rate. In some situations, 
for example when meadow grass is harvested by municipalities for recreational reasons, the municipality may pay a reception fee, which means that the biogas plant is paid to accept the bales.

In the present case, transport of bales was estimated to cost $6.5 € / \mathrm{tww}$, at $30 \mathrm{~km}$ of transport distance from farm to biogas plant by tractor and trailer.

These bale purchase and transport costs are large compared with the costs of chopping shown in Figure 2 and Table 4 and can therefore be a determining factor in the use of silage bales by biogas plants.

\section{Conclusions}

- All three machines tested managed to chop silage with DM content varying between 40 and $70 \%$. The chopping effect with respect to particle length was better on silage with lower DM content than on silage with higher DM content.

- Based on the test results, the cost of chopping silage bales varied between 14.6 and $25.6 € / \mathrm{t}$ ww for the different machines. The costs included machine and tractor, diesel, work, maintenance and use of a wheel loader for handling bales and removing plastic wrapping.

- Based on the test results, RS CutMaster had higher total chopping costs than Roto Grind and I-GRIND. The main reasons for this were lower measured capacity of RS CutMaster and higher purchase and maintenance costs of RS CutMaster (and I-GRIND). In order to reach the same cost level, the capacity of RS CutMaster would have to be $12 \mathrm{tww} / \mathrm{h}$, i.e. about $40 \%$ higher capacity than measured in the tests.

- The cost of purchasing the chopping machine accounted for only a small part of the total costs of chopping. Other costs were for the tractor powering the machine, diesel, labour and use of a wheel loader to handle the bales.

- In-depth studies are needed to determine the relationships between machine settings, capacity, energy consumption and effect on biogas exchange for different types of silage. Two of the machines tested here are new (RS CutMaster, I-GRIND) and long-term operating data are lacking, resulting in some uncertainty in the calculations and assessments for those machines.

\section{Acknowledgements}

The project was funded by the Swedish Energy Agency, Gasum, County Administration Board for Scania and Fogda Farm. Many thanks to the farmers and machinery companies involved in the tests Mr Marx in Schillingen and Annaburger, Germany, Grønning Smede \& Maskinforretning and Skinnerups Maskinstation, Denmark and Hans Cederlöf, Salbohed, Sweden.

\section{References}

Amon, T., Hopfner-Sixt, K., Amon, B. \& Bauer, A. 2007. Handbuch. Optimierung der Beschaffungs- und Distributionslogistik bei grossen Biogasanlagen, Universität fûr Bodenkultur Wien, Department für Nachhaltige Agrarsysteme, Institut für Landtechnik.

ASABE. 2006. Agricultural Machinery Management Data. ASAE D497.5. American Society of Agricultural and Biological Engineers, St. Joseph, MI, USA.

Energigas. 2018. Energigas Sverige. www.energigas.se

Gale, G.E. \& O'Dogherty, M.J.O. 1982. An apparatus for the assessment of the length distribution of chopped forage. Journal of Agricultural Engineering Research 27, 35-43.

Gunnarsson, C. \& Hansson, P-A. 2004. Optimisation of field machinery for an arable farm converting to organic farming. Agricultural Systems 80, 85-103.

Lund, J., Gunnarsson, C., Fischer, F., Sundberg, M., Tersmeden, M. 2018. Outnyttjat ensilage till förnybar energi [Unutilized silage for renewable energy]. RISE Rapport 2018:28, RISE, Borås Sweden.

Maskinkalkylgruppen. 2017. Maskinkostnader- underlag och kalkylexempel för lantbruksmaskiner. Maskinkalkylgruppen \& HIR Skåne, Bjärred, Sweden. 


\author{
Analysis of the Indoor Climate and Energy Use in Two Pig Houses \\ Andrea Costantino ${ }^{\text {a,* }}$, Lorenzo Comba ${ }^{\text {a }}$, Andrea Rolfo ${ }^{\text {a }}$, Paolo Cornale ${ }^{\text {b }}$, Luca Battaglini ${ }^{\text {b }}$, Enrico \\ Fabrizio $^{\text {a }}$ \\ ${ }^{a}$ Department of Energy, TEBE Research Group, Politecnico di Torino, Torino, Torino 10129, Italy \\ ${ }^{b}$ Department of Agricultural, Forest and Food Sciences, University of Torino, Grugliasco, Torino 10095, Italy \\ * Corresponding author. Email: andrea.costantino@polito.it
}

\begin{abstract}
Data collection in the agricultural sector is becoming easier due to the improvement of different technologies (e.g. wireless transmission and sensor technologies). Collected data are used to obtain information with the final aim of increasing the production, both in terms of quality and quantity. The data collection process is particularly relevant in the livestock subsector due to its fundamental role in feeding the future world population and because it is characterized by high energy consumption. The use of energy in livestock housing systems is strongly dependent on climate control, especially for some animal productions, such as pigs and poultry. For this reason, data about indoor climate conditions and energy consumption for climate control can be useful to increase the farm productivity and sustainability while improving the optimal animal welfare. In this work, a subset of data (31 days) coming from a long-term monitoring campaign (about one year) is analysed. The data were acquired through monitoring systems for the measurement of indoor air temperature, relative humidity and total electrical loads of two growing-finishing pig houses. The analysis of these data aims at evaluating the environmental conditions inside the houses and at quantifying the electrical energy consumption that is attributable to the different energy uses. The analysis showed that both pig houses have similar indoor climate conditions and the indoor air temperatures and relative humidity values are quite uniform inside both the enclosures. The total electrical loads were analysed using a specifically developed algorithm that is able to identify the partial electrical loads at each time step, due to activities inside the pig houses. The algorithm estimates that ventilation, with an overall energy consumption of $1087 \mathrm{kWh}$, is the predominant component of the electrical energy consumption, reaching a share that represents $87.1 \%$ of the total electrical energy consumption.
\end{abstract}

Keywords: monitoring systems, livestock houses, climate control, animal welfare, energy consumption, data analysis.

\title{
1. Introduction
}

In the last years, the fast improvement of sensor technologies, smart mobile devices, wireless transmission technologies and cloud data sharing has facilitated the collection of a great amount of data (Koseleva and Ropaite, 2017) that can provide new valuable information through specific analysis. In the agricultural sector, the use of data, especially big data, is concurring to the digitalization of the farms, a process that is considered the third revolution in agriculture (Pham and Stack, 2018). This revolution is leading to the "smart farm" (Tyagi, 2016), a notion that refers to a new approach to agriculture management, where the production processes are supported by digital technology and biotechnology (Kamilaris et al., 2017). Smart farming plays an important role in tackling the challenges of feeding the future world population that United Nations (2015) estimates to reach 9.7 billion of people before 2050 . This population growth will entail a rise in food demand by about $70 \%$ more than the current amount (FAO, 2009).

Livestock subsector will be crucial in smart farming, because livestock products provide $33 \%$ of proteins and $17 \%$ of kilocalories that are currently consumed worldwide (Thornton, 2010). Furthermore, livestock production is estimated to increase too and according to FAO investigations, meat production will increase by about $70 \%$ and dairy product approximatively by 58\% (FAO, 2012). Most of the worldwide livestock production is obtained from animals that are reared under intensive conditions (FAO, 2011), therefore, the collection and analysis of data from intensive livestock systems can help to increase the sustainability of the farm sector. The collected data, in fact, can provide information to farmers, animal scientists and engineers for developing integrated strategies to decrease the needed resources, to increase the production and the revenues, to improve the farm management and to contribute in ameliorating animal welfare.

One of the key point of intensive livestock production is climate control due to its direct relation with both energy consumption and animal welfare. To maintain adequate indoor climate conditions inside the livestock houses, in fact, different mechanical systems are adopted. These systems entail an energy consumption that, depending on the reared species, can represent a considerable share of the total on-farm direct energy consumption (Costantino et al., 2016). Climate control also affects the on-farm indirect energy consumption (Beckman et al., 2013), a share of energy that is connected to the production of feed for the reared animals. When indoor climate conditions are not adequate to the animals, they suffer from thermal stress and adopt phenotypic responses (e.g. variation of feed intake) that affect the feed conversion ratio (Fregley and Blatteis, 1996; Nardone et al., 2010), 
with negative consequences on the indirect energy consumption, production and farm profit (St-Pierre et al., 2003).

In literature, indoor environmental parameters and energy consumption of various building typologies (e.g. public buildings and offices) have been studied (Zhao et al., 2013; Erkoreka et al., 2016), but few of them are focused on livestock houses. Part of the studies that are focused on livestock houses adopts a numerical approach aimed at developing simulation models to have the needed datasets (Bustamante et al., 2017; Costantino et al., 2018). The other part adopts an experimental approach by the development and installation of monitoring systems inside the livestock houses (Shen et al., 2016; Gray et al., 2017), but very few of them are based on long-term monitoring campaign that are focused on indoor environmental conditions and energy consumption.

Aim of the work

Aim of the present work is to evaluate the indoor climate conditions and the electrical energy consumption of two growing-finishing pig houses that are located in North West of Italy. The analysis could provide useful information to the farmer for the improvement of the production from a point of view of both quantity (increasing the feed conversion ratio) and quality (improving animal welfare). Furthermore, the presented data analysis aims to quantify the electrical energy consumption that is attributable to the various electrical loads that are used during the production cycle. To obtain this data, a specific algorithm was developed and implemented in Matlab ${ }^{\circledR}$ environment.

Analysis of intensive housing system for pigs from an energy point of view is particularly relevant because this livestock production is characterized by high on-farm energy consumption of both direct and indirect energy. For example, a great share of the direct on-farm electrical energy consumption is due to ventilation, an energy use that is estimated to be around $35 \mathrm{kWh} \cdot \mathrm{m}^{-2} \cdot \mathrm{year}^{-1}$ (Costantino et al., 2016), representing roughly the half of the total electrical energy consumption of the entire pig production.

Pig houses are chosen as target of the present work due to the importance of this livestock production in the geographical area where the analysed pig houses are located. Furthermore, pork is the greatest European meat production, with more than 23.4 million of tons produced in 2016 in EU28 (Eurostat, 2017).

\section{Materials and Methods}

Description of the case studies

The analysed case studies are two growing-finishing intensive pig houses that are located in the province of Cuneo (North West of Italy). The two pig houses are quite similar. Both the buildings are equipped with fully slatted floor and have gable roof and reinforced concrete structure (pillars, beams and pit walls). The walls of the buildings are made of concrete piled hollow blocks and the rooves are made of prefabricated sandwich panels with a double layer of pre-painted steel sheets with an interposed layer of high-density spread polyurethane. The air inlets of both the buildings are made of polycarbonate hollow sheets and they are in the north and south walls. In pig house $\mathrm{B}$, some air inlets are also in the roof.

Building A has a gross floor area of about $285 \mathrm{~m}^{2}$ and a gross volume of roughly $1400 \mathrm{~m}^{3}$. Gross floor area of building B is about $400 \mathrm{~m}^{2}$ and the gross volume is roughly $2400 \mathrm{~m}^{3}$. In pig house B, two thermal zones are considered because a bearing wall divides the enclosure in two equal parts. Both the thermal zones are equipped with an independent climate control system.

In pig house $\mathrm{A}$, about 150 pigs were present during the monitoring campaign, while in pig house $\mathrm{B}$ the pigs were approximately 250 . Therefore, the stocking densities in house A and B were similar, being 0.52 and 0.63 pigs $\cdot \mathrm{m}^{-2}$, respectively.

The adequate indoor climate conditions are guaranteed by air heaters (for heating) and pit exhaust fans (for indoor air quality control and cooling).

Description of the monitoring systems

The monitoring systems that were installed inside the analysed pig houses were similar and they monitored both the indoor environmental conditions (air temperature and relative humidity) and the total electrical loads of the houses.

The indoor environmental data were obtained using the following sensors (accuracy noted in brackets):

- a thermistor sensor for the measurement of air temperature $\left( \pm 0.21^{\circ} \mathrm{C}\right)$ and relative humidity $( \pm 2.5 \%)$ embedded in a portable logger;

- a resistance temperature detector PT1000 sensor $\left( \pm 0.15^{\circ} \mathrm{C}\right)$ connected to an analog logger.

The data loggers aimed at monitoring the environmental parameters were set with a logging time step of 10 minutes.

The environmental sensors were evenly distributed inside both the analysed houses at about $2 \mathrm{~m}$ of height from the floor level. In both pig houses, a PT1000 sensor was arranged on the grid of the exhaust fans, to measure 
the exhaust air temperature. In pig house A, 5 temperature sensors and 4 relative humidity sensors were installed, while in pig house B there were 5 temperature sensors and 3 relative humidity sensors that were placed in one of the two enclosures inside the house. The outdoor environmental parameters were monitored using a data logger arranged outside the houses. A PT1000 sensor was placed near the air inlets of each house to monitor the temperature of outdoor air entering inside the house.

The monitoring of the total electrical loads was done using kilowatt transducers (accuracy: $\pm 1 \%$ ) for 3-phase systems connected to analogue loggers set with a logging time step of 10 seconds. A kilowatt transducer connected to a data logger was placed in the electrical panel of each house.

The monitoring systems logged values approximately during one year, acquiring data during two different pig production cycle that were carried out during the warm and cold seasons.

Identification of the partial electrical loads

The kilowatt transducer sensors monitored the total electrical load $(L)$ of each pig house. To have detailed information about the set of partial electrical loads $\left(l_{\mathrm{i}}\right)$ that were present at each time step, a new methodology for loads identification and unbundle has been developed.

The total monitored electrical power $L(\tau)$, at the time instant $\tau$, is here defined as

$$
L(\tau)=\vec{a}(\tau)^{\mathrm{t}} \cdot \vec{l}+e\left(\|\vec{a}(\tau)\|_{1}\right)[\mathrm{kW}]
$$

where $\vec{a}(\tau)$ is a column vector which elements are Boolean variables $\pi_{i} \in\{0,1\}$, the values of which is related to the presence at time $\tau$ of the load $l_{i}$, with $i=1, \ldots, n_{l}$, which are organized in array $\vec{l}$. Parameter $n_{l}$ is the total quantity of known partial electrical loads. These values of partial electrical loads were obtained through specific test measurements that were carried out at the beginning of the monitoring campaign. For the pig house A, the set of seven partial electrical loads that were identified during the test measurements are shown in Table 42.

Addend $e$ of Eq. (1) represents the total error of the measurement at each time step. This error, related to the accuracy of the sensor, is related to both the measurement of $L(\tau)$ and of every partial load $l_{i}$, and thus, it is function of the number of detected active loads. Furthermore, $e$ includes also the presence of partial electrical loads that were not expected and, therefore, that were not measured with the specific tests that were carried out at the beginning of the monitoring campaign.

For pig house A, vectors $\vec{a}(\tau)$ and $\vec{l}$ can be expressed as follows

$$
\vec{a}(\tau)=\left[\begin{array}{c}
\pi_{\text {Feed }} \\
\pi_{\text {Light }, 1} \\
\pi_{\text {Light, } 2} \\
\pi_{\text {Fan, } 1} \\
\pi_{\text {Fan, } 2} \\
\pi_{\text {Fan,3 }} \\
\pi_{\text {Fan, } 4}
\end{array}\right] \quad \vec{l}=\left[\begin{array}{c}
l_{\text {Feed }} \\
l_{\text {Light }, 1} \\
l_{\text {Light }, 2} \\
l_{\text {Fan, } 1} \\
l_{\text {Fan, } 2} \\
l_{\text {Fan, } 3} \\
l_{\text {Fan }, 4}
\end{array}\right]
$$

To understand which partial loads are present and the value of $e$ at each time step, a specific new algorithm was developed and implemented in Matlab ${ }^{\circledR}$ environment. The algorithm is based on the following optimization function

$$
\widehat{\vec{a}}(\tau)=\min _{\vec{a}(\tau)}(e)
$$

where $\widehat{\vec{a}}(\tau)$ is the $\vec{a}(\tau)$ vector that minimizes the $e$ function obtained from Eq. (1) as

$$
e=\vec{a}^{t} \cdot \vec{l}-L[k W]
$$

Constraints of the minimization procedure are

and

$$
\pi_{i} \in\{0,1\}
$$

$$
\sum_{n=4}^{7} \pi_{i} \leq 1
$$

The condition expressed by Eq. (6) has to be fulfilled because $l_{\mathrm{Fan}, 1}, l_{\mathrm{Fan}, 2,} l_{\mathrm{Fan}, 3}$ and $l_{\mathrm{Fan}, 4}$ represent different velocities of the same fans and, thus, cannot exist at the same time step $\tau$. 
Table 42. Main partial electrical loads that were monitored in pig house A.

\begin{tabular}{ccc}
\hline Load & Elements & $\begin{array}{c}\text { Power } \\
{[\mathrm{kW}]}\end{array}$ \\
\hline Feed distribution & $l_{\text {Feed }}$ & 0.861 \\
Light 1 & $l_{\text {Light, } 1}$ & 0.224 \\
Light 2 & $l_{\text {Light, },}$ & 0.126 \\
Fans (speed 1) & $l_{\text {Fan, },}$ & 1.022 \\
Fans (speed 2) & $l_{\text {Fan, }, 2}$ & 1.436 \\
Fans (speed 3) & $l_{\text {Fan, } 3}$ & 1.534 \\
Fans (speed 4) & $l_{\text {Fan, } 4}$ & 1.839 \\
\hline
\end{tabular}

\section{Results and Discussion}

Presentation of the dataset

The long-term monitoring campaign lasted almost a year and, during that period, data concerning environmental parameters and the total electrical loads were acquired. In this work, a subset of 31 days (during the warm season) of the entire dataset was considered for the data analysis.

\section{Subset of environmental parameters}

The subset of the environmental parameters contains data that were acquired with a time step of 10 minutes, therefore 4464 measurements were logged by each sensor during the considered 31 days. The averages values of indoor air temperature and relative humidity on an hourly basis for pig house $\mathrm{A}\left(\overline{\mathrm{air}}_{\mathrm{ir}, \mathrm{A}}\right.$ and $\left.\bar{\varphi}_{\mathrm{air}, \mathrm{A}}\right)$ and pig house $\mathrm{B}$ $\left(\bar{t}_{\text {air,B }}\right.$ and $\left.\bar{\varphi}_{\text {air,B }}\right)$ are calculated as the arithmetical mean between all the measurements and their trends are shown in Figure 106.

Trends of average indoor air temperatures ( $\bar{t}_{\mathrm{air}, \mathrm{A}}$ and $\left.\bar{t}_{\mathrm{air}, \mathrm{B}}\right)$ and relative humidity values $\left(\bar{\varphi}_{\mathrm{air}, \mathrm{A}}\right.$ and $\left.\bar{\varphi}_{\mathrm{air}, \mathrm{B}}\right)$ appear similar with $\bar{t}_{\mathrm{air}, \mathrm{A}}$ slightly lower than $\bar{t}_{\mathrm{air}, \mathrm{B}}$. This difference could be due to the slightly lower animal density of pig house A or due to the solar shadings (e.g. roof overhangs) that decrease the incidence of solar radiation on the building envelope. The maximum difference between $\bar{t}_{\mathrm{air}, \mathrm{A}}$ and $\bar{t}_{\mathrm{air}, \mathrm{B}}$ is calculated to be $2.19^{\circ} \mathrm{C}$.

The trends of indoor air relative humidity $\bar{\varphi}_{\text {air,A }}$ and $\bar{\varphi}_{\text {arr, }}$ shown in Figure 106 look similar, with $\bar{\varphi}_{\text {air,B }}$ slightly lower than $\bar{\varphi}_{\text {air,A }}$. The maximum difference that was monitored between $\bar{\varphi}_{\text {air,A } \mathrm{A}}$ and $\bar{\varphi}_{\text {air,B }}$ was $10.3 \%$. This trend is similar to the air temperature one, being relative humidity strongly dependant from air temperature.

During the monitoring period, the maximum value of $\bar{t}_{\mathrm{air}, \mathrm{A}}$ was $33.2^{\circ} \mathrm{C}$, while the maximum value of $\bar{\varphi}_{\text {air,A }}$ was $80.2 \%$. The same values in pig house B were $34.4^{\circ} \mathrm{C}$ for $\bar{t}_{\text {arr, B }}$ and $80.3 \%$ for $\bar{\varphi}_{\text {air,b. Similar values results higher }}$ than the ones recommended by literature (Rossi et al., 2004). In particular, during the monitoring period, all the values of $\bar{t}_{\mathrm{air}, \mathrm{A}}$ and $\bar{t}_{\mathrm{air}, \mathrm{B}}$ were higher than $18{ }^{\circ} \mathrm{C}$ (considered as the optimal set point value). Even though the indoor air temperatures were not the ones recommended by literature, the growth performance of pigs can be considered satisfactory, because the animals grew of about $0.9 \mathrm{~kg} \cdot \mathrm{head}^{-1} \cdot \mathrm{day}^{-1}$ (values were averaged on the entire productive cycle).

To evaluate the uniformity of air temperature and relative humidity inside the analysed pig houses, the Root Mean Square Error (RMSE) was calculated for each considered sensor $k$ as

$$
\operatorname{RMSE}_{\mathrm{k}}=\sqrt{\frac{\sum_{\mathrm{i}=1}^{N_{\mathrm{i}}}\left(x_{\mathrm{k}, \mathrm{i}}-\bar{x}_{\mathrm{i}}\right)^{2}}{N_{\mathrm{i}}-1}}
$$

where $x_{\mathrm{k}, \mathrm{i}}$ is the value measured by sensor $k, \bar{x}_{\mathrm{i}}$ is the mean value and $N_{\mathrm{i}}$ is the considered number of measurements.

The maximum value of RMSE for indoor air temperature in pig house $A$ was $1.62^{\circ} \mathrm{C}$. This value was measured by the PT 1000 sensor placed on the exhaust fan. If the values measured by this sensor are not taken into account, the RMSE values for indoor air temperature have a range between $0.37^{\circ} \mathrm{C}$ and $0.55^{\circ} \mathrm{C}$. A similar calculation was carried out for the relative humidity and the RMSE values are within the range $1.29 \%-1.35 \%$. 


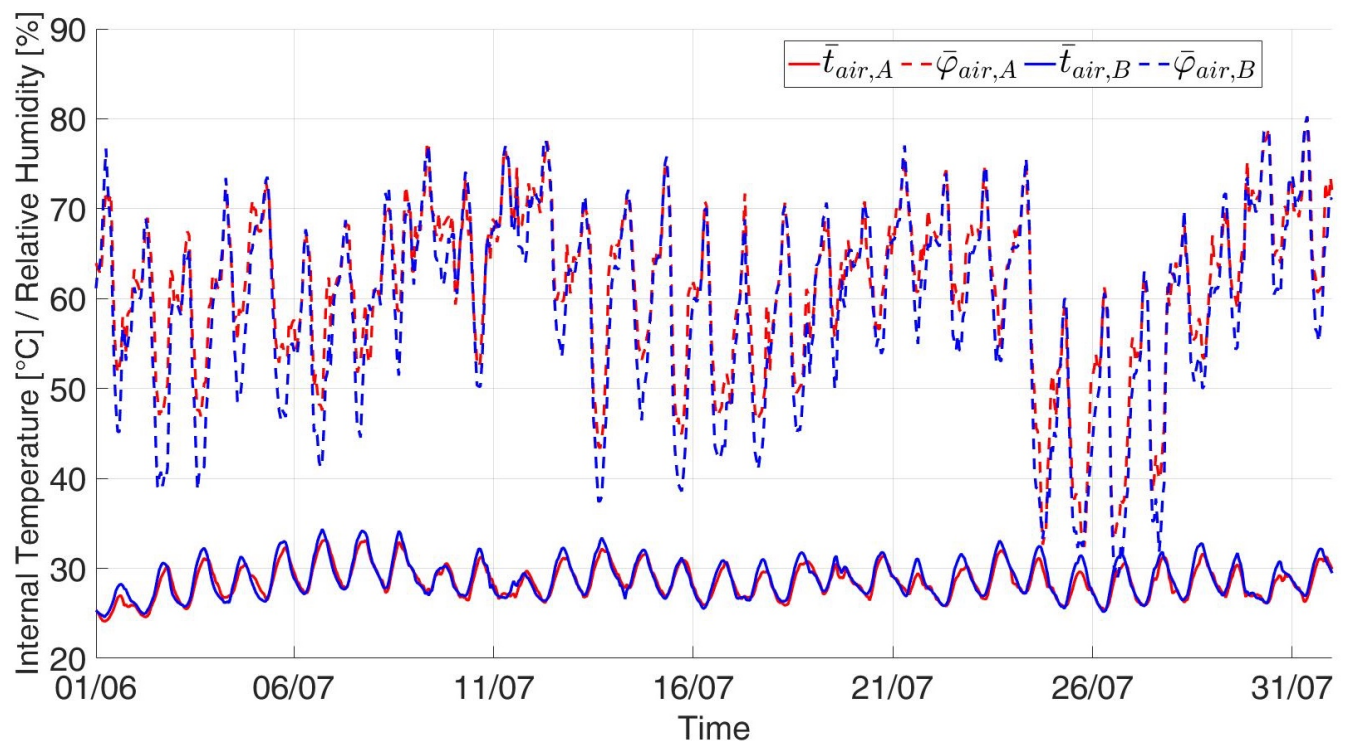

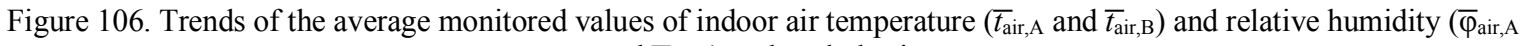
and $\bar{\varphi}_{\text {air,B }}$ ) on hourly basis.

In pig house $\mathrm{B}$ the maximum value of RMSE calculated for indoor air temperature is $0.88^{\circ} \mathrm{C}$. This value refers to the temperature sensor that was placed on the exhaust fan. If this sensor is not considered, the RMSE values are between $0.37^{\circ} \mathrm{C}-0.69^{\circ} \mathrm{C}$. RMSE values concerning relative humidity are between $0.66 \%$ and $2.00 \%$. This analysis shows that the indoor air temperature and relative humidity inside the pig houses are uniform and differences are only found in the exhaust air temperatures. In pig house A, the exhaust air temperature (monitored on a north-facing fan) is lower than $\overline{\bar{a}_{\mathrm{air}, \mathrm{A}}}$ during most of the monitored period. This difference can be explained by the adiabatic cooling process that takes place when indoor air crosses the pit before being exhausted. In pig house $\mathrm{B}$, the exhaust air temperature is higher than $\overline{\overline{t a i r}_{\mathrm{B}} \mathrm{B}}$ during most of the monitored period. This difference could be due to the fact that the PT1000 sensor is placed on a south-facing fan and solar radiation influences the measure. Solar radiation, in fact, increases the temperature of the fan grid at which the sensor was fixed.

Subset of electrical loads

The subset includes 267,840 total electrical loads that were acquired with a time step of 10 seconds. In Figure 107 , the trend of the total monitored electrical loads in pig house A is shown on a 10 seconds basis. The chart shows that electrical loads were always present in pig house A during the entire analysed period. From July the $1^{\text {st }}$ to the $31^{\text {st }}$, the monitored total electrical load was constantly around $1.8 \mathrm{~kW}$ with some peaks around $2.6 \mathrm{~kW}$.

In Figure 108, the monitored total electrical loads that are shown in Figure 107 were grouped in classes $(50 \mathrm{~W}$ of range) and their frequencies are shown in logarithmic scale. From the chart, some clusters of total electrical loads that are characterized by high absolute frequency stand out. Their identification is possible referring to the values shown in Table 42. The highest frequency in Figure 108 is related to a total electrical load of about $1.8 \mathrm{~kW}$ and can be attributable to the activation of fans at speed 4 . The second highest frequency is related to a total electrical load of about $1.4 \mathrm{~kW}$ and could be due to the activation of fans at speed 2 . The other more relevant frequencies are around $2.2 \mathrm{~kW}$ and $2.6 \mathrm{~kW}$ and they are found respectively when fans at speed $2(1.436 \mathrm{~kW})$ and $4(1.839 \mathrm{~kW})$ are activated at the same time of feed distribution $(0.861 \mathrm{~kW})$. The feed distribution itself has a not negligible absolute frequency (around $0.8 \mathrm{~kW}$ ) and can be appreciated in the first part of the chart. 


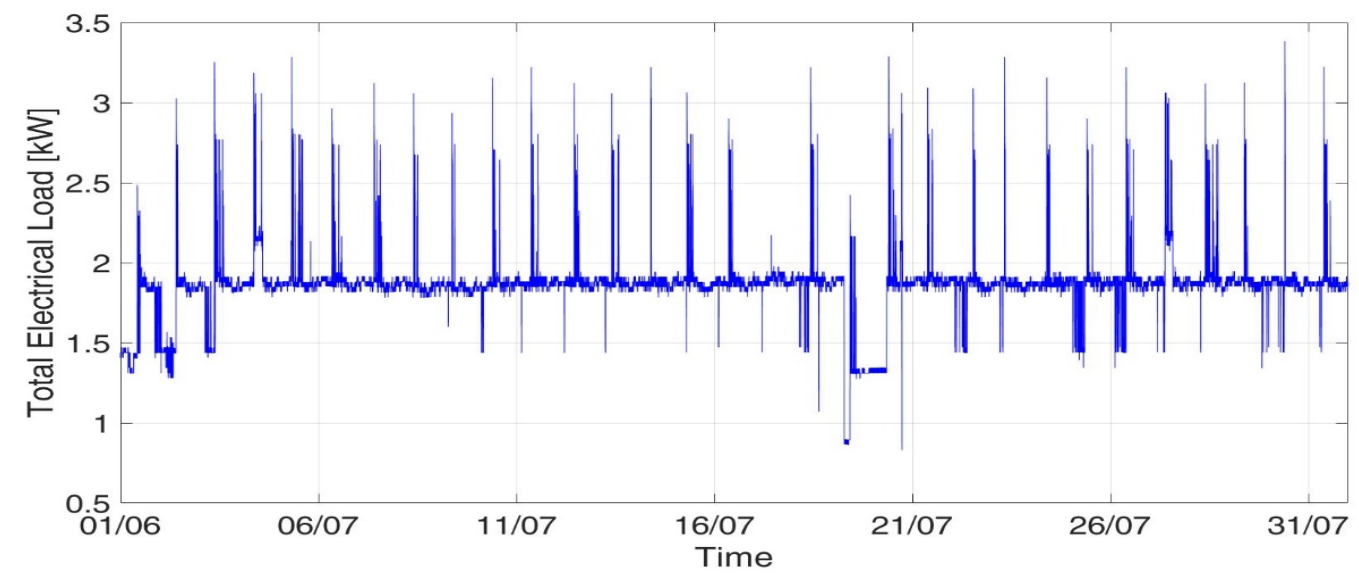

Figure 107. Trend of the total electrical loads that were monitored in pig house A.

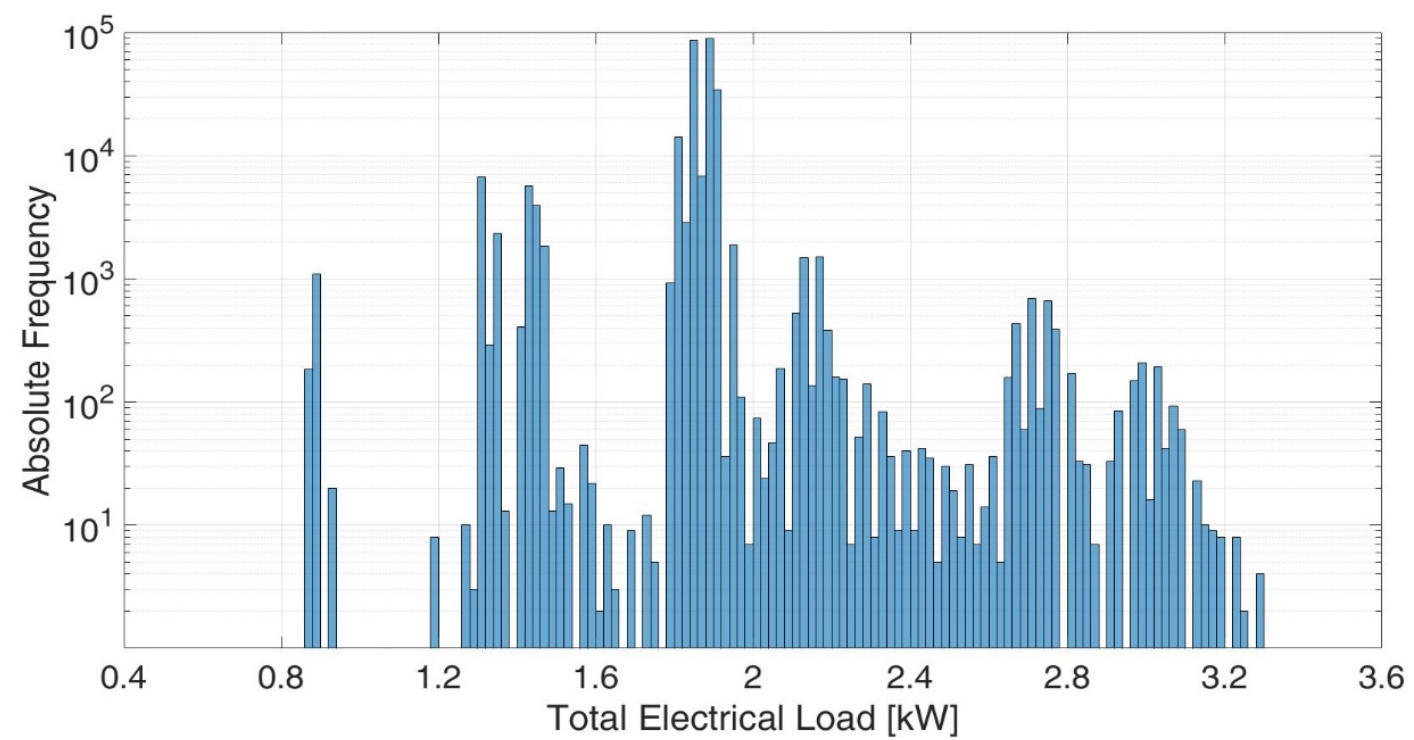

Figure 108. Frequencies of the classes (width: $50 \mathrm{~W}$ ) of total electrical loads that were measured in pig house A. Frequencies are reported using logarithmic scale.

Analysis of the total electrical energy consumption

In section 3.1.2 the subset of the total electrical loads that were monitored by the data loggers are presented. To obtain the shares of electrical energy consumption due to the various electrical loads, the methodology described in section 2.3 was applied and the obtained partial electrical loads were integrated over the monitored period. In Table 43, the absolute and relative values of electrical energy consumption of pig house A during the analysed period are shown. For the sake of clarity, error defined in Eq. (4) has been omitted. During the monitored period, the electrical energy consumption of pig house A was $1245.8 \mathrm{kWh}$. Most of this energy consumption was due to the fans $(87.1 \%)$, while feed distribution and light represented a minor energy electrical consumption share $(12.9 \%)$. From this analysis, the importance of ventilation for cooling the animals during warm periods stands out. In fact, the monitored period was characterized by high outdoor air temperatures and, for this reason, the fans were always activated. In particular, they worked for most of the time (64.8\% of the working hours) at speed 2 to decrease the indoor air temperature using an outdoor air flow.

To evaluate the precision of the algorithm in the evaluation of the error $e$, the mean error $\bar{e}$ was calculated as

$$
\bar{e}=\frac{\sum_{\mathrm{j}=1}^{N_{\mathrm{m}}}\left|e_{\mathrm{j}}\right|}{N_{\mathrm{m}}}[\mathrm{kW}]
$$

where $e_{\mathrm{j}}$ is the error measured in the time step $\tau_{\mathrm{j}}$ and $N_{\mathrm{m}}$ is the total number of measurement that are considered $(267,840)$. For pig house $\mathrm{A} \bar{e}$ is equal to $126 \mathrm{~W}$.

In Figure 109 the daily electrical energy consumption of pig house A is shown according to the considered different energy uses. During the monitored period, the daily energy consumption is almost always around $45 \mathrm{kWh}$ and ventilation represents the main energy consumption share. Lower values are estimated on July the $19^{\text {th }}$ and $20^{\text {th }}$, where the electrical energy consumption decreases at about $33 \mathrm{kWh}$ (July the $19^{\text {th }}$ ) and $40 \mathrm{kWh}$ (July the $20^{\text {th }}$ ). 
In Figure 109, also the $e$ term of Eq. (1) was taken into account. The error overestimates the daily electrical energy consumption of about $4 \mathrm{kWh}$ each day.

Table 43. Absolute and relative values of energy consumption for energy use in pig house A. The table shows also the working hours of each appliance load.

\begin{tabular}{cccc}
\hline Load & $\begin{array}{c}\text { Electrical consumption } \\
{[\mathrm{kWh}]}\end{array}$ & $\begin{array}{c}\text { Share on the total energy } \\
\text { consumption }\end{array}$ & $\begin{array}{c}\text { Working hours } \\
{[\mathrm{h}]}\end{array}$ \\
\hline Feed distribution & 12.2 & $1.0 \%$ & 14 \\
Light 1 & 142.1 & $11.5 \%$ & 634 \\
Light 2 & 4.9 & $0.4 \%$ & 39 \\
Fans (Speed 1) & 60.4 & $4.8 \%$ & 59 \\
Fans (Speed 2) & 807.5 & $64.8 \%$ & 562 \\
Fans (Speed 3) & 0.8 & $0.0 \%$ & 1 \\
Fans (Speed 4) & 218.0 & $17.5 \%$ & 119 \\
\hline Total & 1245.9 & $100 \%$ & - \\
\hline whereof for fans & 1086.7 & $87.1 \%$ & 741 \\
\hline
\end{tabular}

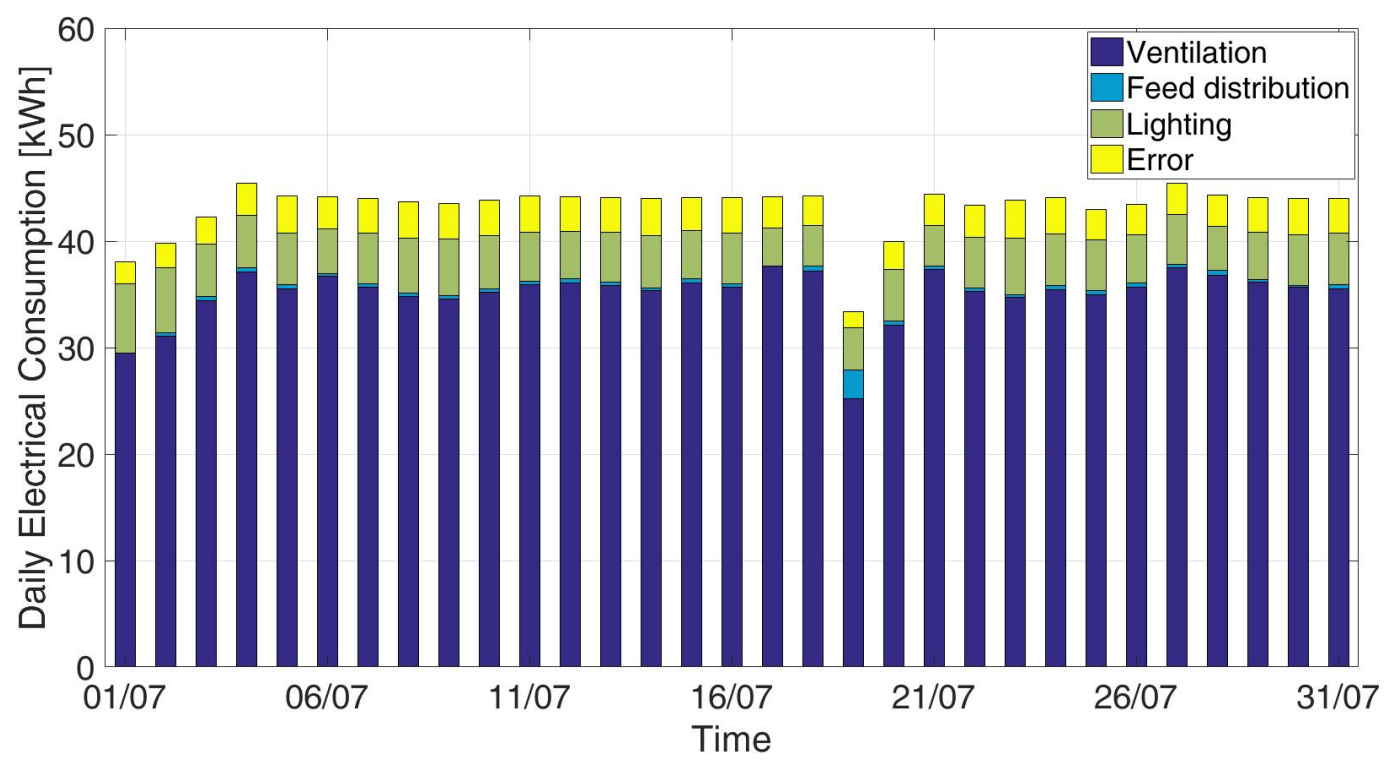

Figure 109. Daily electrical energy consumption for the different energy uses in pig house A.

According to this analysis, the energy consumption due to ventilation during the analysed period is about $233 \mathrm{Wh} \cdot$ head $^{-1} \cdot$ day $^{-1}$, while lighting entails an electrical energy consumption of $32 \mathrm{Wh} \cdot$ head $^{-1} \cdot$ day $^{-1}$. Electrical energy consumption for feed distribution is estimated to be $3 \mathrm{Wh} \cdot \mathrm{head}^{-1} \cdot \mathrm{day}^{-1}$. The total electrical energy consumption without considering the error is estimated to be $268 \mathrm{~W} \cdot \mathrm{head}^{-1} \cdot \mathrm{day}^{-1}$.

From these values, some considerations can be done. First, the electrical energy consumption for lighting during a year can be estimated. Considering that the pig farm usually carries out two production cycles of about 160 days during a year, the electrical energy consumption for lighting can be estimated to be about $1536 \mathrm{kWh} \cdot \mathrm{year}^{-}$ ${ }^{1}$, a value that is equal to $5.4 \mathrm{kWh} \cdot \mathrm{m}^{-2} \cdot \mathrm{year}^{-1}$. This value may underestimates the real consumption, because the sanitary empty periods are not considered in the estimation and because during the cold season lights are expected to work more hours than in warm season because the number of hour of natural light is lower.

Another consideration concerns the mean electrical power that is needed for the lighting. Starting from the energy consumption of $32 \mathrm{Wh} \cdot \mathrm{head}^{-1}$ day $^{-1}$ a mean electrical power of $0.7 \mathrm{~W} \mathrm{~m}^{-2}$ can be estimated. This value is considerably lower than the sensible heat of the pigs, therefore it can be omitted in the development of energy simulation models.

The last consideration concerns the energy use for feed distribution. In this case, an estimation of the yearly energy consumption due to feeding distribution cannot be done, because pigs vary considerably the intake feed according to age and climate conditions. Furthermore, $3 \mathrm{Wh} \cdot \mathrm{head}^{-1} \cdot \mathrm{day}^{-1}$ seems a low value for this energy use. More analysis have to be carried out to prove the reliability of the developed algorithm and to understand if a time step of 10 second is enough short to correctly monitor the trend of this electrical load. 
Concerning the electrical energy consumption due to ventilation an estimation for the entire year cannot be carried out because it is strictly dependant by many factors, such as the outdoor climate conditions and the set point regulation according to the ages of the pigs.

Those energy consumptions (for feeding and ventilation) can be estimated through specifically developed energy simulation models that can consider all the needed variables.

\section{Conclusions}

In this work, data coming from monitoring systems inside two pig houses for growers and finishers were analysed. The analysed data concern both environmental parameters (indoor air temperature and relative humidity) and energy consumption values (total electrical loads).

The analysis showed that the monitored values of indoor air temperature and relative humidity that were monitored by several sensors are similar between them. This proves that the considered indoor environmental parameters are uniform inside the pig houses. Furthermore, the mean indoor climate conditions are also quite similar between the two pig houses.

The developed algorithm for the partial electrical loads identification was reliable and showed that most of the electrical energy consumption were due to ventilation, making feed distribution and lighting shares negligible. The use of the algorithm also showed that fans were always activated during the monitored period usually working at speed 2. In particular, the estimated energy consumption due to fans during the considered period was about $1087 \mathrm{kWh}$, a value equal to $268 \mathrm{~W} \cdot \mathrm{head}^{-1} \cdot \mathrm{day}^{-1}$.

Considering the partial electrical load due to fans together with the trends of indoor air temperature, it stands out that, even though the ventilation flow rate was high during almost all the monitored period, indoor air temperatures in both pig houses were considerably higher than the values recommended by literature. According to the average feed conversion ratio, the high indoor air temperatures of the enclosure do not seem to affect the growth performance of the pigs. Nevertheless, the installation of more effective cooling systems (e.g. evaporative pads) is recommended for increasing the animal welfare and for further improving the performance of the pigs.

The present work shall be improved through the implementation of the air velocity measurements in the monitoring systems. Indeed, air velocity represents a fundamental aspect for the animal welfare, both in warm and cold periods. If the air speed in various spots inside the houses is known, it may be possible to regulate the fan speed to have adequate air velocities inside the house and to evaluate, for example, which pens can be used as hospital pens for sick and injured pigs.

Room for improvement lies also in the developed algorithm. Currently the algorithm analyses the total electrical load considering the single time step. To have more reliable results, a machine-learning algorithm can be developed for the partial loads identification which can also consider processing results obtained in the previous time step, with a specific a time window.

\section{Acknowledgements}

This work was carried out in the framework of the "EPAnHaus - Energy Performance of livestock houses" project that has been supported by financial funding of SIR (Scientific Independence of young Researchers) 2014, grant number RBSI141A3A, funded by MIUR (Italian Ministry of Education, Universities and Research).

\section{References}

Beckman, J., A. Borchers, and C.A. Jones, 2013. Agriculture's supply and demand for energy and energy products. EIB-112. U.S. Department of Agriculture, Economic Research Service, Washington D.C., USA.

Bustamante E., S. Calvet, F. Estellés, A.G. Torres, A. Hospitaler, 2017. Measurement and numerical simulation of single-sided mechanical ventilation in broiler houses. Biosystems Engineering. 160, 55-68. https://doi.org/10.1016/j.biosystemseng.2017.05.009.

Costantino, A., E. Fabrizio, A. Biglia, P. Cornale, L. Battaglini, 2016. Energy use for climate control of animal houses: the state of the art in Europe. Energy Procedia. 101, 184-191. https://doi.org/10.1016/j.egypro.2016.11.024.

Costantino, A., E. Fabrizio, A. Ghiggini, M. Bariani, 2018. Climate control in broiler houses: A thermal model for the calculation of the energy use and indoor environmental conditions. Energy and Buildings. 169, 110-126. https://doi.org/10.1016/j.enbuild.2018.03.056.

Erkoreka, A., E. Garcia, K. Martin, J. Teres-Zubiaga, L. Del Portillo, 2016. In-use office building energy characterization through basic monitoring and modelling. Energy and Buildings. 119, 256-266. https://doi.org/10.1016/j.enbuild.2016.03.030.

EUROSTAT, 2017. Agriculture, forestry and fishery statistics - 2017 edition. Statistical books. European Union, Luxembourg. 
FAO (Food and Agriculture Organization of the United Nations), 2009. Global agriculture towards 2050. High Level Expert Forum Issues Paper. FAO, Rome, Italy.

FAO (Food and Agriculture Organization of the United Nations), 2011. Energy-smart food for people and climate. Issue Paper. FAO, Rome, Italy.

FAO (Food and Agriculture Organization of the United Nations), 2012. Energy-Smart Food at FAO: an Overview. FAO, Rome, Italy.

Fregley, M.J., C.M. Blatteis (Eds.), 1996. Handbook of physiology. Oxford: Oxford University Press.

Gray, J., T.M. Banhazi, A.A. Kist, 2017. Wireless data management system for environmental monitoring in livestock buildings. Information Processing in Agriculture. 4, 1-17. http://dx.doi.org/10.1016/j.inpa.2016.12.001.

Kamilaris, A., A. Kartakoullis, F.X. Prenafeta-Boldù, 2017. A review on the practice of big data analysis in agriculture. Computers and Electronics in Agriculture. 143, 23-37. http://dx.doi.org/10.1016/j.compag.2017.09.037.

Koseleva, N., G. Ropaite, 2017. Big data in building energy efficiency: understanding of big data and main challenges. Procedia Engineering. 172, 544-549. https://doi.org/10.1016/j.proeng.2017.02.064.

Nardone, A., B. Ronchi, N. Lacetera, M.S. Ranieri, U. Bernabucci, 2010. Effects of climate changes on animal production and sustainability of livestock systems. Livestock Science. 130 (1-3), 57-69. https://doi.org/10.1016/j.livsci.2010.02.011.

Pham, X., M. Stack, 2018. How data analytics is transforming agriculture. Business Horizons. 61, 125-133. https://doi.org/10.1016/j.bushor.2017.09.011.

Rossi, P., A. Gastaldo, P. Ferrari, 2004. Ricoveri, attrezzature e impianti per l'allevamento dei suini (in Italian). Verona (Italy): Edizioni L'Informatore Agrario. 315 p.

Shen, W., G. Liu, Z. Su, R. Su, Y. Zhang, 2016. Design and implementation of livestock house environmental perception system based on wireless sensor networks. International Journal of Smart Home. 10 (5), 69-78. http://dx.doi.org/10.14257/ijsh.2016.10.5.08.

St-Pierre, N.R., B. Cobanov, G. Schnitkey, 2003. Economic losses from heat stress by U.S. livestock industries. Journal of Dairy Science. 86 (Supplement), E52-E57. https://doi.org/10.3168/jds.S0022-0302(03)74040-5.

Thornton, P.K., 2010. Livestock production: recent trends, future prospects. Philosophical Transactions of the Royal Society B (Biological Sciences). 365, 2853-2867. https://doi.org/10.1098/rstb.2010.0134.

Tyagi, A.C., 2016. Towards a second green revolution. Irrigation and Drainage. 65, 388-389. https://doi.org/10.1002/ird.2076.

United Nations, 2015. World Population Prospects: The 2015 Revision, Key Findings and Advance Tables. Working Paper No. ESA/P/WP.241. Department of Economic and Social Affairs, Population Division, New York, USA.

Zhao, L., J. Zhang, R. Liang, 2013. Development of an energy monitoring system for large public buildings. Energy and Buildings. 66, 41-48. https://doi.org/10.1016/j.enbuild.2013.07.007. 


\title{
Tractor Drive Line Efficiency Evaluation Taking into Account Power Lost in Slippage
}

\author{
Maurizio Cutini*, Massimo Brambilla, Carlo Bisaglia \\ Consiglio per la ricerca in agricoltura e l'analisi dell'economia agraria (CREA), Centro di ricerca Ingegneria e \\ Trasformazioni agroindustriali (CREA-IT), Via Milano, 43 -24047 Treviglio (BG), Italy \\ *Corresponding author. Email: maurizio.cutini@crea.gov.it
}

\begin{abstract}
The primary purpose of agricultural tractors is to perform drawbar work that is mathematically defined by the pull force and forward speed of the machine. An ideal tractor converts all the fuel energy into useful work at the drawbar. This implies the maximization of both engine fuel and drive train mechanical efficiencies allowing the optimization of tractive advantage by selecting an optimum travel speed for any given operation. Optimizing tractor efficiency is still actual as research activity, in particular in terms of emissions and energetic points of view. Tractors' testing has followed this technical development and a wide range of methodologies, both standard or following internal protocols, have been developed. In this frame, the OECD Tractor Codes could be considered a reference for many experimental procedures. The adoption of OECD Code 2 standard for drawbar power and fuel consumption assessment allows obtaining comparable data (measurements are carried out at maximum engine power), but opens the issue that tractors maximum drawbar power changes with gear or speed. To deepen such knowledge, the data of 100 tractors of different engine power and weight were analysed with the aim of evaluating the specific driveline efficiency (the driveline power net to losses due to slippage). The whole dataset underwent Linear Regression Analysis (LRA) aimed at pointing out the relations between the power at the drawbar and the losses due to slippage. Then the power lost as slippage was calculated with the hypothesis that the power at the drawbar is function of: i) the maximum power at the power take off, ii) the power used for the displacement of the vehicle, iii) the power lost for slip. The model fitted with $\mathrm{R}^{2}>99 \%$ allowing to calculate the driveline efficiency.
\end{abstract}

\section{Introduction}

Agricultural tractors are machines designed for pulling. Following their technical evolution, they added such a wide range of different tasks to their working capability that they are now considered as mobile central power units.

The born concept of a wheeled tractor was developed at the end of the nineteenth century. It can be also associated to the first internal combustion engine available for ploughing that substituted the steam engines that, however, had few applications and very low little use in agriculture.

Tractor testing has followed the technical developments and a wide range of methodologies, standards or internal protocols aimed to cover all the parts that occur to optimize the traction performance.

The studies were focused on the improvement of engine, driveline, tires efficiencies together with optimal tractor settings (Molari and Sedoni, 2008; Harris and Rethmel, 2011; Smerda and Cupera, 2010; Janulevicius et al., 2014; Monteiro et al., 2013). Investigations were also carried out on the effect of different fuel types on traction and on emissions (Da Cunha et al., 2013).

The main aims concerned the optimization of the traction performance not only as in terms of material engineering but above all concerning labour and energy efficiency (Gil-Sierra et al., 2007; Turker et al., 2012; Grisso and Kocher, 2004), also by using models (Wong and Huang, 2006; Zoz and Grisso, 2003) verified by experimental results or bench-test data (Sabbioni et al., 2011; Lacour et al., 2014; Tiwari et al., 2010). Furthermore, in-depth investigations on soil properties have been deeply investigated as well to determine their influence on traction efficiency and performance (Lyasko, 2010; Filho et al., 2010).

An experimental drawbar test is required in order to investigate so many different factors affecting the traction. This consists in the application of an horizontal force at the agricultural tractor drawbar while it is being run on a test track or on a field, and measuring the main tractor vehicle out-puts (i.e.: force, forward speed, wheel slip, fuel consumption).

Besides, the requirement of measuring the drawbar force has led to the development of specific sensors (Hvasi et al., 2012) and, in particular, purpose-designed loading devices able to generate an horizontal force at the drawbar of the tested tractor while it is moving forward (Cutini and Bisaglia, 2016). This mobile loading device is defined as a "dynamometric vehicle" (DV) or "dynamometer" following the statement by the PAMI - Prairie Agricultural Machinery Institute (PAMI, 1996).

The ideal tractor converts all the fuel energy into useful work at the drawbar, this implies the maximization of both engine and drive train mechanical efficiencies, allowing the optimization of the tractive advantage by selecting an optimum travel speed for any given operation. 
The drawbar power is mathematically defined by the pull force $(\mathrm{kN})$ and forward speed $\left(\mathrm{m} \mathrm{s}^{-1}\right)$ of the machine. The OECD Code 2 standard (OECD, 2018) for drawbar power and fuel consumption assessment allows obtaining comparable data (measurements are carried out at maximum engine power), but opens the issue that tractors maximum drawbar power changes with gear or speed, in case of continuously variable transmission.

To deepen such knowledge, the data of 100 tractors of different engine power and weight, obtained following the OECD Code 2 standard, were analysed with the aim of evaluating the specific driveline efficiency (the driveline power net to losses due to slippage). The whole dataset underwent Linear Regression Analysis (LRA) aimed at pointing out the relations between the power at the drawbar and the losses due to slippage.

\section{Materials and Methods}

Investigations into traction performances have to solicit drawbar power or traction effort. The data of 100 tractors of different engine power and weight, obtained following the OECD Code 2 standard (fig. 1), were analysed with the aim of evaluating the specific driveline efficiency (the driveline power net to losses due to slippage).

\begin{tabular}{|c|c|c|c|}
\hline \multicolumn{4}{|c|}{ Engine, Transmission, Power take-off Specifications } \\
\hline Make, Model & New Holland & \multicolumn{2}{|r|}{ Iveco $8035.05 R$} \\
\hline Type, Supercharging & irect injection & \multirow{2}{*}{\multicolumn{2}{|c|}{$\begin{array}{r}\text { None } \\
\text { vertical in line }\end{array}$}} \\
\hline Cylinders, Disposition & & & \\
\hline Capacin, Cooling & $2930 \mathrm{~cm}^{3}$ & \multirow{2}{*}{\multicolumn{2}{|c|}{$\begin{array}{r}\text { By water } \\
\text { Mechanical }\end{array}$}} \\
\hline Gear box & & & \\
\hline Number of fonward and reverse speeds & & \multicolumn{2}{|l|}{8} \\
\hline Speed at rated engine speed & & from 2.37 & to $30.12 \mathrm{~km} / \mathrm{h}$ \\
\hline Standarc & & $540 \mathrm{~min}^{-1}$ & $1000 \min ^{-1}$ \\
\hline Power take-off speed at rated engine speed & & $565 \min ^{-1}$ & $1072 \min ^{-1}$ \\
\hline Diamet & & $35 \mathrm{~mm}$ & $35 \mathrm{~mm}$ \\
\hline Number of splin & & 6 & \\
\hline \multicolumn{4}{|l|}{ Power take-off Test } \\
\hline $\begin{array}{l}\text { Power, Engine and Power take-off speed } \\
\text { Hourly and specific consumption }\end{array}$ & $39.4 \mathrm{~kW}$ & $\begin{array}{r}2300 \min ^{-1} \\
12.501 \mathrm{~h}\end{array}$ & $\begin{array}{r}565 \min ^{-1} \\
267 \mathrm{~g} / \mathrm{kWh}\end{array}$ \\
\hline \multicolumn{4}{|l|}{ Test at maximum power at rated engine speed } \\
\hline $\begin{array}{l}\text { Power, Engine and Power take-off speed } \\
\text { Hourly and speciffe consumption } \\
\text { Test at standard Power take-off speed }\end{array}$ & $39.4 \mathrm{~kW}$ & $\begin{array}{r}2300 \min ^{-1} \\
12.501 \mathrm{~h}\end{array}$ & $\begin{array}{r}565 \mathrm{~min}^{-1} \\
267 \mathrm{gkWh}\end{array}$ \\
\hline $\begin{array}{l}\text { Power, Engine and Power take-off speed } \\
\text { Hourly and specific consumption }\end{array}$ & $39.1 \mathrm{~kW}$ & $\begin{array}{r}2202 \mathrm{~min}^{-1} \\
12.21 \mathrm{th}\end{array}$ & $\begin{array}{r}541 \mathrm{~min}^{-1} \\
262 \mathrm{~g} / \mathrm{kWh}\end{array}$ \\
\hline Torque rige & & & $26.0 \%$ \\
\hline Maximum torque. Engine speed corresponding & & $205.9 \mathrm{Nm}$ & $1205 \mathrm{~min}^{-1}$ \\
\hline \multicolumn{4}{|l|}{ Drawbar Test } \\
\hline Rear tyres & & $7.5-16$ & $16.9-30$ \\
\hline Test 1 & & inbal & isted \\
\hline Total Mass & & $2560 \mathrm{~kg}$ & $3060 \mathrm{~kg}$ \\
\hline Maximum drawbar pull & & $23.1 \mathrm{kN}$ & $23.5 \mathrm{kN}$ \\
\hline & & $4.69 \mathrm{~km} / \mathrm{h}$ & $3.17 \mathrm{~km} / \mathrm{h}$ \\
\hline power & & $30.6 \mathrm{~kW}$ & $31.4 \mathrm{~kW}$ \\
\hline at speed of & & $9.14 \mathrm{~km} / \mathrm{h}$ & $8.96 \mathrm{~km} / \mathrm{h}$ \\
\hline \multicolumn{4}{|l|}{$\begin{array}{l}\text { Power Lift and Hydraulic Performance Test } \\
\text { Hydraulic system }\end{array}$} \\
\hline $\begin{array}{l}\text { Maximum lifting force at hitch points, at frame } \\
\text { At maximum hydraulic power }\end{array}$ & & $19.6 \mathrm{kN}$ & $18.0 \mathrm{kN}$ \\
\hline Flow rate, Pressure, Power & $42.8 \mathrm{1} / \mathrm{min}$ & $17.5 \mathrm{MPa}$ & $12.5 \mathrm{~kW}$ \\
\hline
\end{tabular}

Figure 1. Example of a summary of the OECD code 2 results.

This standard code indicates how to measure the tractor's performance in terms of power and fuel consumption. The main assessments are the power take off (PTO) test and the drawbar power test. It indicates the methodology for measuring the maximum PTO and drawbar power of an agricultural tractor. The drawbar test is carried out with the engine at full throttle and in different selected gears, or speeds if the tractor has continuously variable transmission. There is the need of a device generating the horizontal force at the drawbar of the tested tractor (Figure 2), up to a maximum wheel slip of 15\% (Zoz and Brixius, 1979) or the maximum drawbar power, in each gear or speed. 


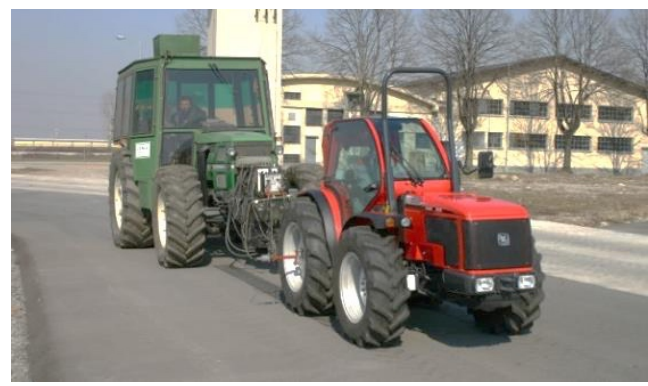

Figure 2. An example of the layout of a drawbar power test.

It is necessary to measure the force and the forward speed in order to calculate the power at the drawbar, as shown by the following Eq (1).

$$
P_{d b}=F_{d b} \cdot s
$$

where $P_{d b}$ is the power at the drawbar, $\mathrm{kW} ; F_{d b}$ is the maximum force measured at the drawbar in different gears or speeds, $\mathrm{kN}, s$ is the forward speed of the test tractor, $\mathrm{m} \mathrm{s}^{-1}$.

The test has to be carried out on a level surface, usually made of concrete or asphalt. As a result, it is also possible to obtain the fuel consumption values. The dataset takes into account the maximum power and fuel consumption at the PTO, these data are measured and taken as reference as ideal behaviour of the tractor.

The other considered performance is the maximum drawbar power at different speed, where the slippage value must be lower than $15 \%$. The other considered variables are tractor mass $(\mathrm{kg})$ and drawbar pull $(\mathrm{kN})$.

Following this protocol resulted in one maximum drawbar power for each tested gear or speed for any of the 100 tested tractors so that the final dataset contained up to 584 observations.

First of all it was verified a correlation between slippage and drawbar pull following the hypothesis reported in the $\mathrm{Eq}(2)$.

$$
\text { Slip }=f(D, W)
$$

where Slip is the per cent slippage, dimensionless; $D$ is the drawbar pull, daN; $W$ is the static weight of the tractor, $\mathrm{kg}$.

The calculation of the efficiency of the drive line was carried out following the hypothesis that the power measured at the power take off was the maximum ideally available on the test track. The power losses taken into account were:

- efficiency of the drive-line;

- power used for the displacement of the vehicle;

- power lost for slip

With this aim the "expected" results would have had to follow Eq.3:

$$
P_{d b}=\alpha \cdot P_{P T O}-\beta \cdot P_{v d}-P_{s}
$$

Where $P_{d b}$ is the power at the drawbar (measured), $\mathrm{kW}$; Р РТО is the maximum power at the PTO (measured), $\mathrm{kW}$; Pvd is the power used for the displacement of the vehicle (calculated as weight and speed), $\mathrm{kW} ; P_{s}$ is the power lost for slip, $\mathrm{kW} ; \alpha$ is the efficiency of the drive train, dimensionless; $\beta$ is a rolling resistance coefficient, dimensionless.

Concerning $\alpha$ an indication of its value was already given in the standard ASAE D497.7: it refers to the ratio between drawbar power and power take off power only and its value for a $4 \mathrm{WD}$ tractor driven on a concrete surface is 0.88 . Also the rolling resistance coefficient $(\beta)$ was previously assessed by the same ASAE standard and its value is expected to be around 0.04 .

The whole dataset underwent Linear Regression Analysis (LRA) aimed at pointing out the relations between the power at the drawbar and the losses due to slippage. The software used was Minitab 17 statistical software.

The variables taken into account were:

- maximum power at the PTO;

- tractor's mass;

- drawbar power;

- drawbar pull;

- forward speed;

- slip;

- maximum drawbar pull;

- surface friction (obtained as maximum drawbar pull/tractor's weight). 
Interactions between variables were carried out till the third order. In order to manage the choice of predictive variables stepwise regression was adopted to fit regression models. All candidate variables were involved, the deletion of each variable using a chosen model fit criterion was tested by backward elimination, deleting the variable whose loss gives the most statistically insignificant deterioration of the model fit.

At this point the dataset was divided into two equally numerous datasets representing the training set and the test set. The former was used to determine the model relating the power at the drawbar with the power at the PTO and the power lost due to the slip of the wheels; the latter was used afterward to validate the obtained relation.

The quantitative results were expressed as: the Pearson's correlation coefficient $r$, the coefficient of determination $\mathrm{R}^{2}$, the root mean square error (RMSE), the percent bias of prediction (PBIAS) and the dimensionless index Nash-Sutcliffe Efficiency (NSE), (Moriasi et al., 2007). Ideally, RMSE and PBIAS values are 0 , and low values indicate an accurate equation prediction; NSE values range between $-\infty$ and 1, value between 0 and 1 are generally considered as a measure of satisfactory prediction level for an equation.

\section{Results}

The first verification of a correlation between slippage, drawbar pull and tractor's weight was carried out following the hypothesis reported in the Eq (2). The outcomes of the regression procedure were two interesting and similar equations. The former is $\mathrm{Eq}(4)$.

$$
\text { Slip }=-0.000634 \cdot D+13.634 \cdot D / W
$$

that presented R-sq=86.99 and R-sq $(\operatorname{adj})=86.94$, and with both $\mathrm{D}$ and $\mathrm{D} / \mathrm{W}$ estimations having $\mathrm{p}$-value $=0$.

The second regression outcome was obtained simplifying the model of Eq (2). to check if the ratio D/W only could adequately represent the slip of the wheels. This relation is hereafter given in Eq (5).

$$
\text { Slip }=9.285 \cdot D / W
$$

that presented R-sq=82.51 and R-sq $(\operatorname{adj})=82.48$, and with $\mathrm{D} / \mathrm{W}$ coefficient having a $\mathrm{p}$-value $=0$.

The high level of correlation found between slip and drawbar pull allowed to suppose that the power lost for slippage is correlated to the tractor's pull.

As reported in "Methods", the power loss as slippage was calculated indirectly. The power at the drawbar is measured so it has been chosen as dependant. The other variables were considered independent and were all involved in the regression analysis. The deletion of each variable using a chosen model fit criterion was tested by backward elimination, deleting the variable whose loss gives the most statistically insignificant deterioration of the model fit. With this aim the "expected" model would have to follow Eq.3.

$$
P_{d b}=\alpha \cdot P_{P T O}-\beta \cdot P_{v d}-P_{s}
$$

A first regression equation was developed on 50 tractors randomly chosen that defined the so called "training set" to be used for the equation coefficients assessment. The obtained equation (model) was afterwards tested on the remaining 50 tractors not included in the training set (the so called test set).

The regression equation resulting from the training set was:

$$
P_{d b}=0.9395 \cdot P_{P T O}-0.04579 \cdot P_{v d}-0.02124 \cdot P_{P T O} \cdot \operatorname{Slip}+0.00938 \cdot P_{v d} \cdot \text { Slip }
$$

it had both R-sq and R-sq (adj) equal to 99.78, with all the coefficient resulting significant $(\mathrm{p}<0.05)$.

During the validation phase, the correlation coefficient and the $\mathrm{R}^{2}$ turned out to be 0.99 , the RMSE achieved the value of $5.25 \mathrm{~kW}$ and the PBIAS was $1.5 \%$. This means that a quite good prediction level was achieved with a little underestimation occurring in high power tractors (Figure 3). The NSE index for this set of data was found to be 1.0 and this support the goodness of the obtained equation. 


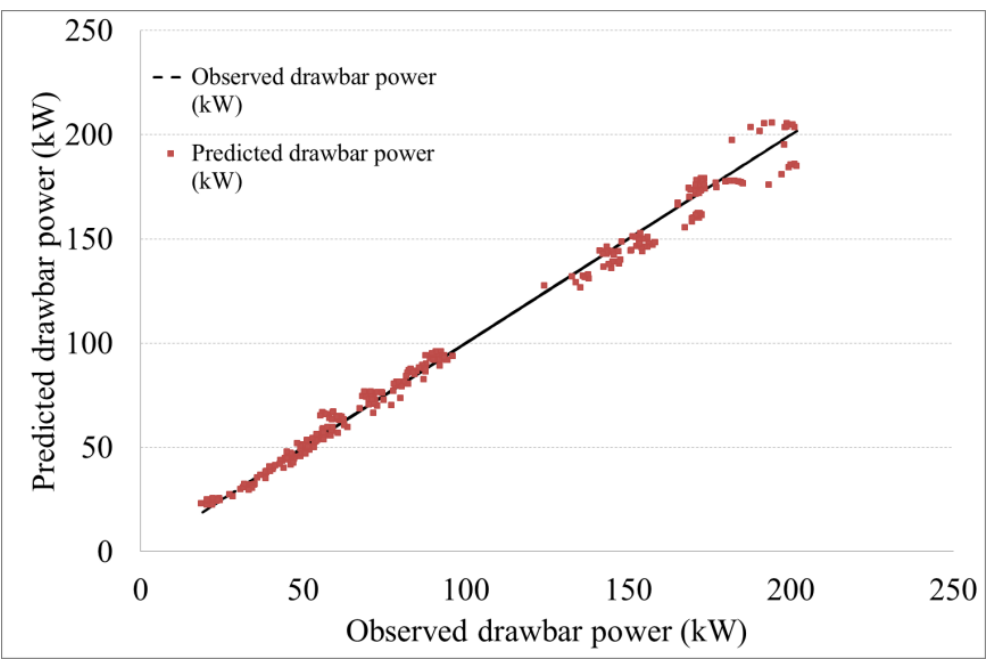

Figure 3. Scatterplot representing the predicted drawbar powers calculated through Equation 6 versus the measured values reported in the test set. The full line is the $1: 1$ reference.

The equation shows also the effect that the drawbar power increases with the increasing of speed till reaching a maximum and then starting to decrease. In this way is able to consider the effect of the power used for the displacement of the vehicle, that increases with speed and is considered negative, and the effect of the power lost for slip, that decreases with the increasing of the speed.

This consideration is useful to calculate the correct setting of the tractor for reducing slippage or rolling resistance at varying of the task to be performed.

\section{Discussion}

The results of equation 6 show the possibility of evaluating the efficiency of tractors not only as specific fuel consumption at the power take off that remain obviously one of the main indicator characterizing engine efficiency, but also the possibility of evaluating the driveline contribution with the capability of considering rolling resistance and the power loss for slippage.

The high value of correspondence of model output to the dataset $\left(\mathrm{R}^{2}=0.99\right)$ if on the one hand it is partially due to the relation that is known to exist between the power at the power take off and that at the drawbar, on the other, opens an interesting issue. In fact, it is worth noticing the it highlights the obtained values of $\alpha$ and $\beta$ $(0.9395$ and 0.04579$)$ are really close to those that can be retrieved from the ASAE standard meaning that these two losses of power can be taken into account in a distinct way.

Moreover, the development of Eq (6) has made possible to verify the relation between slip and power loss that is commonly considered straight (each per cent point of slippage correspond to $1 \%$ of power loss). Effectively, the relation between $\mathrm{Eq}$ (6) results and the measured slippage results in a correlation coefficient of 0.94 confirming, even in this case, what has been previously reported in literature.

\section{Conclusions}

The data of 100 tractors of different engine power and weight were analysed with the aim of evaluating the specific driveline efficiency (the driveline power net to losses due to slippage). The whole dataset underwent Linear Regression Analysis (LRA) aimed at pointing out the relations between the power at the drawbar and the losses due to slippage.

Then the power lost as slippage was calculated with the hypothesis that the power at the drawbar was function of the maximum power at the power take off, of the power used for the displacement of the vehicle and the power lost for slip.

The model fitted with $\mathrm{R}^{2}>99 \%$ allowing to calculate the driveline efficiency.

The results show the possibility of evaluating the efficiency of tractors not only as specific fuel consumption at the power take off that remain obviously one of the main indicator characterizing engine efficiency, but also the possibility of evaluating the driveline contribution with the capability of considering rolling resistance and the power loss for slippage.

The developed algorithm has been approached without any classification of tractor as type, mass, capacity, power, geometry, etc. but the results could be extended to attempts of clustering that could allow to find the parameters that could contribute to improve the efficiency of tractor. 
Results could be extended as methodology also to test on field. Also in this case ASAE presents interesting models that could provide useful indications for the development of a model representing the studied relation when tractors are run on soil.

\section{Acknowledgements}

This work was supported by the Italian Ministry of Agriculture (MiPAAF) under the AGROENER project (D.D. n. 26329, 1 April 2016) - http://agroener.crea.gov.it/

\section{References}

ASAE D497.7, Mar 2011. Agricultural Machinery Management Data.

Cutini M., Bisaglia C., 2016. Development of a dynamometric vehicle to assess the drawbar performances of high-powered agricultural tractors. J. of Terramechanics $65 \quad$ (2016) 73-84, http://dx.doi.org/10.1016/j.jterra.2016.03.005

Da Cunha Siqueira W.; Fernandes H.C.; Teixeira M.M.; Santos N.T.; Abraho S.A., Performance on the traction of an agricultural tractor drive tires fed with mixtures of petroleum diesel and raused soybean oil. Revista Ceres, 2013, 60 (6), pp.793-801.

Filho A.G.; Lancas K.P.; Leite F.; Acosta J.J.B.; Jesuino P.R., Performance of agricultural tractor on three different soil surfaces and four forward speeds. Revista Brasileira de Engenharia Agricola e Ambiental, 2010,14 (3), pp. 333-339.

Gil-Sierra J.; Ortiz-Canavate J.; Gil-Quiros V.; Casanova-Kindelan J., Energy efficiency in agricultural tractors: A methodology for their classification. Applied Engineering in Agriculture, 2007, 23 (2), pp. 145-150.

Grisso R.D.; Kocher M.F.; Vaughan D.H., Predicting tractor fuel consumption. Applied Engineering in Agriculture, 2004, 20 (5), pp. 553-561.

Harris, B.J.; Rethmel, B.R., Comparison of IF and standard marked metric radial ply tires; American Society of Agricultural and Biological Engineers Annual International Meeting 2011, ASABE 2011, 7, pp. 5461-5472

Hvasi M. R.; Shiekhdavoodi M. J.; Shini N. A.; Sami M. Design, Development and Evaluation of a Digital Tractor Dynamometer. Asian Journal of Agricultural Sciences 4(2): 145-148, 2012.

Janulevicius A.; Pupinis G.; Kurkauskas V., How driving wheels of front loader tractor interact with the terrain depending on tire pressures. Journal of Terramechanics, 2014, 53 (1), pp. 83-92.

Lacour S.; Burgun C.; Perilhon C.; Descombes G.; Doyen V., A model to assess tractor operational efficiency from bench test data. Journal of Terramechanics, 2014, 54, pp. 1-18.

Lyasko M.I., How to calculate the effect of soil conditions on tractive performance. Journal of Terramechanics, 2010, 47 (6), pp. 423-445.

Minitab (2010). Minitab 17 statistical software. State college, PA: Minitab.

Molari G.; Sedoni E., Experimental evaluation of power losses in a power-shift agricultural tractor transmission. Biosystem Engineering, 2008, 100 (2), pp. 177-183.

Monteiro L.A.; Albiero D.; de Souza F.H.; Melo R.P.; Cordeiro I.M., Tractor efficiency at different weight and power ratios. Revista Ciencie Agronomica, 2013, 44 (1), pp.70-75.

Moriasi, D.N., Arnold, J.G., Van Liew, M.W., Bingner, R.L., Harmel, R.D., \& Veith T.L. 2007. Model evaluation guidelines for systematic quantification of accuracy of watershed simulations. Trans. ASABE, 50(3): 885-900. http://dx.doi.org/10.13031/2013.23153

OECD Code 2, Feb. 2015 OECD Standard Codes for the official testing of agricultural and forestry tractors performance.

Prairie Agricultural Machinery Institute (PAMI), Research Update, Standardized Tractor Performance Testing. What it is - and isn't; July, 1996.

Sabbioni E.; Negrini S.; Braghin F.; Melzi Lng S., A 2D model for tractor tire-soil interaction: Evaluation of the maximum traction force and comparison with experimental results. SAE Technical Papers, 2011.

Smerda T.; Cupera J., Tire inflation and its influence on drawbar characteristics and performance - Energetic indicators of a tractor set. Journal of Terramechanics, 2010, 47 (6),pp. 395-400.

Tiwari V.K.; Pandey K.P., Pranav P.K., A review on traction prediction equations. Journal of Terramechanics, 2010, 47 (3), pp.191-199.

Turker U.; Ergul I.; Eroglu M.C., Energy efficiency classification of agricultural tractors in Turkey based on OECD tests. Energy Education Science and Tecnology Part A: Energy Science and Research, 2012, 28 (2), pp. 917-924.

Wong J.Y.; Huang W., "Wheels vs. tracks" - A fundamental evaluation from the traction perspective. Journal of Terramechanics, 2006, 43 (1), pp. 27-42.

Zoz F. M.; Grisso D. R. Tractor and Traction 596 Performance. ASAE Publication Number 913C0403. 2003.

Zoz F. M., Brixius W. W. Traction Prediction for Agricultural Tires on Concrete. Summer Meeting of ASAE and CSAE, ASAE paper n ${ }^{\circ}$ 79-1046; 1979. 


\title{
Simulation Model of Using the Electricity and Exhaust Gas Heat Generated from Poultry Litter Waste in their House
}

\author{
Mohamed Nabih Omar ${ }^{a, *}$, Abdellateif Abdelwahab Samak ${ }^{a}$, Mahmoud Hassan Keshek ${ }^{a}$ \\ a Agricultural Engineering Department, Faculty of Agriculture, Menoufia University, 32511 Shebin EL-Kom, Egypt \\ * Corresponding author. Email: mohamedomar0007@yahoo.com
}

\begin{abstract}
The poultry industry is an important industry in Egypt and consumes a large amount of energy. This study was done to estimate the renewable energy generated from the broiler waste and reused it in the broiler farm. A mathematical model consisting of algebraic equations was developed for broiler house heating by exhaust gas from generator engine integrated with a heater exchanger. The equations were activated by MATLAB program in four stages. The first one, calculate the required broiler house heating energy according to the recommended inside air, outside air temperature and heat transfer through the broiler house structure. The second stage, estimate the broiler litter waste produced from broiler house and calculated its bio-gas production in $\mathrm{m}^{3} / \mathrm{day}$. The third stage, calculate the electricity generated and heat energy of exhaust gas from the generator. Finally, calculate the energy saved by balancing energy produced from wastes to the energy required for the broiler house. The results of the developed model show that, about $19.85 \mathrm{kWh}_{\text {day }}{ }^{-1}$ of electricity can be generated from the bio-gas produced from the broiler waste and $32.28 \mathrm{kWh} \mathrm{day}^{-1}$ of waste heat energy recovered from generator exhaust gas. This adaptation would be a valuable addition of renewable energy in country existing energy system. The saved energy from broiler litter wastes was ranged about 45 to $53 \%$ of the total required energy. The values of thermal loss during the broiler cycle production from Transmission and ventilation were about 48.78 and $44.19 \%$ of total required energy. The minimum correlation coefficients between predicted and measurements values were 0.940 , 0.9478 and 0.936 for inside temperature, biogas generation, electricity generation. Using the electricity and heat energy from exhaust gas which, generated from waste as an input for broiler house operations is a new approach for reducing operating costs and considering as a new application of renewable energy.
\end{abstract}

Keywords: Exhaust Gas Heat, Poultry Waste, Renewable Energy, Electricity

\section{Introduction}

Owing to the relatively high cost and uncertain availability of fossil fuels, considerable attention has been given to the new and renewable energy sources as an alternative means of the heating. We must develop and looking for new and efficient energy sources. Accordingly, new sources of energy are being developed to conserve available energy sources Oeztuerk and Baescetin-celik (2003). As the new energy source, the waste heat is used to supplement the heating needs of a greenhouse tomato crop in the cooled season omar (2011). Energy conservation is unquestionably of great importance to all countries, but for developing countries which meet most of their energy needs through imports, it comes into prominence. Energy consumption has rapidly increased. Due to industrialization, climate change, growth of population and rising standards of living Erdem and Cemek (2018). Livestock houses for intensive animal farming are characterized by high energy consumption due to climate control that is needed to guarantee animals' welfare and to maximize their production. Considering that the price of non-renewable energy sources is predicted to rise, the energy topic in the livestock sector becomes crucial. For this reason, knowledge about energy performance and energy use in all the branches of this field is fundamental to achieve a considerable energy saving and a higher level of sustainability Andrea et al.,(2018). The role of livestock breeding for intensive animal husbandry is particularly important because it can provide many opportunities to increase energy performance. In fact, these buildings are generally characterized by high energy consumption for different uses, and climate monitoring can represent a significant share of energy consumption of the breeding farm. But it provides a large amount of energy consumed Rossi et al., (2013). Also, space cooling and heating in farm building consumption more than one to third of the total energy consumed Mangan et al., (2016). Hence, to decrease the energy demand in Farm buildings is to improve their thermal insulation. Installing insulation not only increases a building's energy performance, but enhances indoor environmental quality as well Anastaselos et al., (2017). Poultry are sensitive and greatly affected by temperature and air exchange fluctuations during all rearing phases Song and King (2015). Failure to provide the ideal environment in broiler house results in reduced profitability because of slow growth rates, reduced feeding, poor feed conversion, and increased disease and mortality rates Daghir (2008). Providing the optimal environment in a broiler house is of paramount importance. It is well known that heating and cooling costs are among the highest expenses for poultry producers. Therefore, energy conservation is critical in poultry production due to its potential to save money, conserve resources, and reduce air pollution. Optimum environmental control to increase the productivity of livestock farming has become more important. Environmental factors including, humidity, air temperature ventilation rate 
and contaminants are generally controlled by the airflow which depends on ventilation in the broiler house Seo et al., (2009). Conditions of breeding strongly depend on the formation of ventilation as well as the isolation of buildings during the cold season. Therefore, it is necessary to create ventilation design for both optimal environmental control and energy saving Seo at al., (2009).In winter, where external temperatures are low, low ventilation rates is preferred. These rates are slow around 0.25 to 1 cubic feet per minute). The air exchange rate of $0.1\left(\mathrm{~min}^{-1}\right)$ was used to adjust the input velocity of each ventilation type assuming that each case had an identical ventilation rate Bustamante et al.,(2017). In recent years with increasing prices of fossil fuels in world, the biogas has become an alternative of renewable energy, and each day more countries are creating renewable energy laws that encourage farmers to use anaerobic digesters Pao and Fu HC (2013), Salomon and Silva Lora (2009). Poultry farms are used to produce poultry, but there are residues from this process. These residues are of great importance as they are used in the process of fertilization because they contain a high percentage of nitrogen. But this waste can be used in the biogas production as a new application for the production of renewable energy. And the use of wastes in energy production does not affect the use of waste in fertilization. The use of waste inside the ferment produces organic fertilizer of high quality, which does not affect the economic importance of waste. Biogas is mainly composed of $\mathrm{CO}_{2}$ and $\mathrm{CH}_{4}$ and finds application in heat, electricity generation and as a vehicular fuel Shen et al.,(2015). Dunstan and Probert (2001) reported that the electrical generation efficiency of the biogas engine is $25-35 \%$, and Thermal to-electrical power outputs is varied from 1:1 to 2:1, and the overall efficiency when waste heat usefully employed is $70-78 \%$. The utilization of exhaust waste heat is very important source of the energy. The exhaust gases from such installations represent a significant amount of thermal energy that traditionally has been used for combined heat and power applications. In addition, another major part of energy input is lost in the form of heat via the cooling system. So a substantial improvement in fuel economy would result Willits et al., (2003). About of 35\% of the fuel energy is generated electrical power, heat losses are about $65 \%$ and the portion of heat that can be utilized is $55 \%$. This $55 \%$ of the heat is available at various temperatures, which also depend on the engine producer according to Zupancic et al., (2003). Biogas was used in engine, which has been modified to operate under dual-fuel condition to generate electricity. Use biogas fuel in engine the thermal efficiency of approximately $23 \%$ may be expected for electricity generation. Fuel consumption is on average, about 550 and $450 \mathrm{~g} \mathrm{~kW}^{-1} \mathrm{~h}^{-1}$ for dual-fuel and normal diesel operations, respectively Tippayawong et al., (2007). Phan and Wattanavichien (2007) presents an experimental investigation of a small IDI biogas premixed charge diesel dual fueled CI engine used in agricultural applications. The exhaust gas temperature at dual-fuel can reach to $\left(270^{\circ} \mathrm{C}\right)$. And the cooling water temperature at dual-fuel, it is $\left(97-105^{\circ} \mathrm{C}\right)$ at different engine speed. According to Houdkov et al. (2008) the balanced system show that the daily amount of mixed raw sludge is $111 \mathrm{t}$ of dry matter was evaluated. During anaerobic digestion, biogas is produced and burnt in the cogeneration units and the amount of $88.1 \mathrm{MWh}$ of power and 141.8MWh of heat is produced. Majority of heat is used for heating sludge in digesters $(76.9 \mathrm{MWh})$.the electrical generation efficiency $=29 \%$ and thermal efficiency $=47 \%$. The quality of the biogas was around $68 \%$ of methane, resulting in a specific consumption of the generator group of $0.94 \mathrm{~m}^{3} / \mathrm{kWh}$ and a global efficiency of $17 \%$ Felipe et al.,(2018). $50 \mathrm{Kg}$ of poultry waste produced $3.0 \mathrm{~m}^{3}$ biogas day $^{-1}$ and every $1 \mathrm{~m}^{3}$ of biogas is equivalent to $20 \mathrm{MJ}$ or $5.56 \mathrm{kWh}$ of thermal energy Shen et al.,(2015). This could be an additional benefit for poultry industries. The poultry waste producing capacity of the farm of $93,075 \mathrm{~kg} \mathrm{year}^{-1}$ the biogas generation could be estimated to $6,648 \mathrm{~m}^{3}$. This could generate $36.96 \mathrm{MWh}$ of thermal energy. The main objective of the study is to Simulated and validation of a mathematical model for Optimization of Using the electricity and heat energy of exhaust gas which, generated from broiler litter waste as an input for broiler house operations, is a new approach for reducing operating costs and considering as a new application of renewable energy.

\section{Materials and Methods}

Mathematical models

The model was designed for evaluation performance of the broiler house that heating with the exhaust gas from the engine. This study was done to estimate the renewable energy generated from the broiler waste and reused it in the broiler farm and reused heat energy loses from exhaust gas. A mathematical model consisting of algebraic equations was developed for broiler house heating by exhaust gas from generator engine integrated with a heater exchanger. The equations were used by MATLAB program in four stages. The first one, to calculate the broiler house heating required according to recommended inside air temperature, outside air temperature and heat loss through structure and ventilation. The second stage, to estimate the broiler litter waste produced and calculated its biogas production $\mathrm{m}^{3} / \mathrm{day}$. The third stage, calculate the electricity generated from biogas and recover heat of exhaust gas from the generator. Finally, to calculate the energy saved by energy production from wastes to the energy required of the broiler house.

Heating required for the broiler house

To determine the heating required for the broiler farm, a thermal equilibrium system must be established between both the lost heat and the added heat to maintain temperature stability bird's age, which will depend on 
the stage of age of the broilers and external weather conditions. In this study the heat added will be obtained from the heat of the exhaust gases from electricity generation, additional electric heaters and the heat generated by the birds themselves. The heat loss includes heat transfer through structural, latent and sensible heat lost with ventilation.

\section{The heat loss from broiler house}

As mentioned previously, total heat loss is a function of two components: Transmission heat loss through house structure, and Ventilation. To evaluate transmission loss, the first step is to calculate the surface area of the structure. This surface area should be subdivided into the various materials employed. After determining the total surface area (A) of the various construction materials, this value is then combined with a design temperature difference $(\Delta \mathrm{T})$ and a heat loss factor $(\mathrm{U})$ for each component. For broiler house, ventilation is generally analyzed via the air change method. This method is based upon the number of times per hour $(\mathrm{ACH})$ that the air in the broiler house was replaced by cold outside air. The number of air changes which occur is a function of indoor wind speed, relative humidity and bird mass

\section{Heat energy losses by heat transfer through the structural (Transmission)}

The heat losses by the broiler farm structural depended on the difference between recommended temperatures for the broilers and outdoor air temperature and Physical properties of the structural materials.

Heating degree-days required

Degree-hour temperature (HDh) is considered as one of the simplest methods of estimation of heating required energy for buildings. It can be obtained by summation the average difference between outdoor air temperature and recommended temperatures at a certain period interval Erdem and Cemek 2018

The number of heating degree hour (HDh) in broiler house production at a certain period was determined using Equation (1) according to De Rosa et al., 2014.

$$
H D h=\sum_{i=1}^{i=n}\left(T_{\text {recom }}-T_{o}\right)
$$

Where, $\mathrm{n}$ is the total number of hours in the day 24 hours, $\mathrm{T}_{\text {recom }}$ is the recommended temperature for broilers and $\mathrm{T}_{\mathrm{o}}$ is the mean outside air temperature.

Table 1. Average weekly recommended and outside temperature at certain period from 14 January to 24 February.

\begin{tabular}{lcccccc}
\hline Week & 1 & 2 & 3 & 4 & 5 & 6 \\
\hline Recommended temperature, ${ }^{\circ} \mathrm{C}$ & 32 & 29 & 26 & 24 & 22 & 21 \\
Average weekly outside temperature ${ }^{\circ} \mathrm{C}$ & 15.96 & 15.15 & 14.93 & 21.47 & 17.09 & 18.73 \\
\hline
\end{tabular}

The walls, roof and windows structure

In this study, the walls is made of $12 \mathrm{~cm}$ bricks, covered with $2 \mathrm{~cm}$ inner plaster, $3 \mathrm{~cm}$ outer plaster and $3 \mathrm{~cm}$ inside insulation layer as shown (Figure 1). The single insulation material Extruded polystyrene foam boards are used. Thermal properties of the material used in the wall, roof, insulation, window and removed wall are given in table 2.

Table 2. Physical properties for elements of wall, roof and window according to Erdem 2018; Omar 2011and Taha 2003.

\begin{tabular}{|c|c|c|c|c|c|c|}
\hline & structure & $\begin{array}{c}\text { Thicknes, } \\
\text { m }\end{array}$ & $\begin{array}{c}\mathrm{K}, \\
\mathrm{W} / \mathrm{mK} \\
\end{array}$ & $\begin{array}{c}\alpha_{\mathrm{i}} \\
\mathrm{W} / \mathrm{m}^{2} \mathrm{~K} \\
\end{array}$ & $\begin{array}{c}\alpha_{o}, \\
W / m^{2} K \\
\end{array}$ & $\begin{array}{c}\mathrm{R}, \\
\mathrm{m}^{2} \mathrm{~K} / \mathrm{W}\end{array}$ \\
\hline \multirow{4}{*}{ Wall } & Internal plaster (cement -based) & 0.02 & 1.400 & \multirow{4}{*}{7} & \multirow{4}{*}{25} & 0.014 \\
\hline & Insulation material & 0.03 & 0.03 & & & 1 \\
\hline & Brick & 0.12 & 0.486 & & & 0.25 \\
\hline & External plaster (cement-based) & 0.03 & 1.400 & & & 0.021 \\
\hline Windows & Polyethylene (PE) & 0.0002 & 0.030 & 4 & 20 & \multirow{3}{*}{0.16} \\
\hline Roof & Concrete & 0.20 & 1.255 & 7 & 25 & \\
\hline Moved wall & Polyethylene (PE) & 0.0002 & 0.030 & 4 & 9 & \\
\hline
\end{tabular}




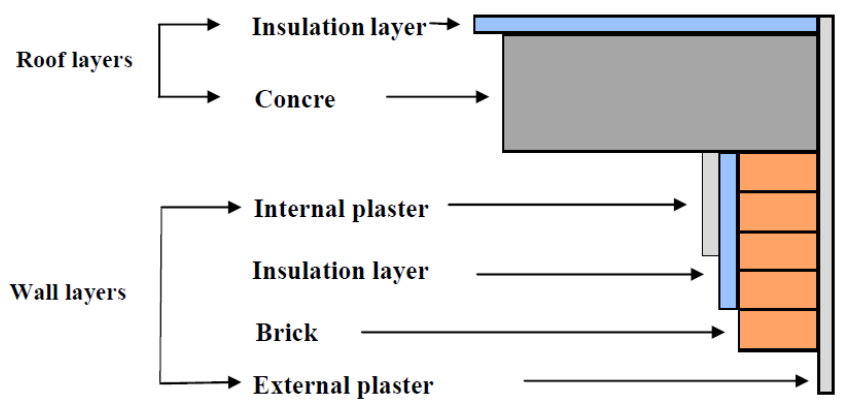

Figure 1. section of the wall and roof layers structure.

To calculate the heat loss through a unit's surface can be obtained by following equation:

$$
q_{w, r, w i n d o, m w}=U_{w, r, d, m w} \cdot A_{w, r, w i n d o, m w} \cdot\left(T_{\text {recom }}-T_{o}\right)
$$

Where $\mathrm{q}_{w, r, \text { windo,mw }}$ is heat loss through a unit surface of walls, roof, side windows moving wall and the $\mathrm{U}$ $w, r$, windo, $m w$ is the total heat transfer coefficient of walls, roof, side windows moving wall, $\mathrm{T}_{\text {recom }}$ is base temperature, and $T_{0}$ is mean outside air temperature. The daily heat losses from the farm calculated by following equation:

$$
q_{\text {losses }}=24 \times U A\left(T_{\text {recom }}-T_{o}\right)
$$

The total heat transfer coefficient for the structures can be computed as:

$$
\begin{aligned}
& U_{w}=\frac{1}{\left(\frac{1}{\alpha_{i}}+R_{w}+R_{p}+R_{i n s}+\frac{1}{\alpha_{o}}\right)} \\
& U_{r}=\frac{1}{\left(\frac{1}{\alpha_{i}}+R_{r}+R_{i n s}+\frac{1}{\alpha_{o}}\right)} \\
& U_{\text {windo }}=\frac{1}{\frac{1}{\alpha_{i o}}+\frac{1}{\alpha_{o w}}} \\
& U_{m w}=\frac{1}{\frac{1}{\alpha_{i o}}+\frac{1}{\alpha_{o w}}}
\end{aligned}
$$

Where $\alpha_{i}$ and $\alpha_{o}$ are the inside and outside convection heat transfer coefficients, respectively, $R_{w}, R_{\text {ins }}$ and $R_{p}$ are the heat resistance of the wall, the insulation layer and the plaster layer, which is

$$
R_{w}=\frac{X_{w}}{K_{w}}, R_{\text {ins }}=\frac{X_{\text {ins }}}{K_{\text {ins }}}, R_{P}=\frac{X_{p}}{K_{p}}
$$

Where, $\mathrm{x}_{\mathrm{w}}, \mathrm{X}_{\text {ins }}, \mathrm{X}_{\mathrm{p}}$ and $\mathrm{k}_{\mathrm{w}}, \mathrm{k}_{\text {ins }}, \mathrm{k}_{\mathrm{p}}$ are the thickness and thermal conductivity of the Brick, Internal and External plaster and insulation material, respectively.

Heating losses with ventilation of a broiler house

The ventilation is one of the most important tools for controlling broiler house climate and which also can greatly influence on the environmental conditions such as temperature, humidity and carbon dioxide concentration that all effect on the production inside the environmental controlled rooms.

The energy removed by ventilation flux $q_{v e n t}$ from inside to outside due to natural ventilation through a ventilation opening can be calculated according to Harmanto (2006) as following:

$$
q_{\text {vent }}=n \rho m c_{p}\left(T_{\text {recom }}-T_{o}\right)
$$

Where, qvent, thermal heat loss from broiler house air due to ventilation, $\mathrm{kWh}, n$, air exchange number, $\mathrm{h}^{-1}, m$, is bird mass, $\mathrm{kg}, \rho$, density of the air, $\mathrm{kgm}^{-3}, \mathrm{C}_{\mathrm{p}}$, specific heat of the air, $1.047, \mathrm{kJkg}^{-1} \mathrm{~K}^{-1}, \mathrm{~T}_{\text {reco }}$, inside temperature, $\mathrm{K}, \mathrm{T}_{\mathrm{o}}$, outside temperature, $\mathrm{K}$.

The typical ventilation rate during the cold season was calculated using Eq. (10) Seo et al., (2009)

Minimum recommended ventilation rates

$$
n=0.8 \mathrm{~m}^{3} \text { hour }^{-1} \mathrm{Kg}_{\text {liveweight }}^{-1}
$$


Heat and water vapour Produced by broiler

The heat and water vapour emitted by the animals were estimated according to Rojano et al., (2014), which recommend a basal heat metabolism empirically related to the weight of the animal. Eqs. (11), (14) compute the total heat, sensible and latent heat, respectively. Total heat was calculated by applying Eq. (11):

$$
A H=10.62 m^{0.75} \times\left(1+\frac{20\left(20-T_{i n}\right)}{1000}\right)
$$

Where, $\mathrm{AH}$ is the total heat generated from the bird, $\mathrm{m}$ is bird mass (live weight), $\mathrm{T}_{\text {in }}$ is average indoor temperature. Bird mass was estimated based on a curve identified in the work of Rojano et al., (2014):

$$
m=0.000346 d^{2}+0.0236706 d-0.024548
$$

The sensible heat $\mathrm{AH}$ s was estimated with Eq. (13) and latent heat deduced from Eq. (14):

$$
\begin{aligned}
& A H s=0.61 \times A H-\left(\frac{0.228}{1000}\right) \times\left(T_{i n}\right)^{2} \\
& A H l=A H-A H s
\end{aligned}
$$

The required energy for lighting

Lighting programs are a key factor for good broiler performance and flock welfare. Suitable lighting program was used. The intensity and distribution of light alters broiler activity. Uniform distribution of light throughout the house is essential to the success of any lighting program. The energy for lighting calculating from equation (15) Management Guide

$$
q_{\text {lighting }}=5 \cdot A\left[W^{-2}\right]
$$

The daily energy requirement for heating is computed as: $q_{\text {suply }}=q_{\text {los }}+q_{\text {vent }}-q_{\text {produce }}$

Estimating the amount of poultry waste produced

The average amount of the waste produced from the house during the cycle was found about, $125 \mathrm{~g} \mathrm{day}^{-1}$ bird $^{-}$ 1. This amount was variations depended on conversion rate of the broiler, according to Management Guide.

Mathematical model for biogas

A comprehensive, fundamentally-based mathematical model that predicts energy requirements to operate a digester at a specified temperature and the resulting gas production was developed. Gebremedhin et al., (2004).

Biogas production

Biogas production per day is calculated as:

$$
B G=\frac{0.5}{H R T}\left[C_{o}-C_{t}\left({ }^{\chi}, t\right)\right] V \frac{T_{a}}{T_{d}} \rho_{b i o}
$$

Where, $B G$, is biogas production , $m^{3} d a y^{-1}, H R T$, is hydraulic retention time, days, $C_{0}$, is the total volatile solids inflow, $\mathrm{kg} \mathrm{m}^{-3}, T S$, is the ratio of total solids in the inflow, $\%$. $\rho$, is the density of the inflow, $\mathrm{kg} \mathrm{m}^{-3}, \rho_{b i o}$ , is the density of the biogas, $=1.1 \mathrm{kgm}^{-3}, C_{t}(x, t)$, is total substrate degradation in the digester, $\mathrm{kgm}^{-3}, V$, is digester volume, $V=H R T \cdot v$, , $\mathrm{m}^{3}, T_{a}$, is $273.2^{\circ} \mathrm{K}$, and, $T_{d}$, is temperature inside digester $=311.2(=273.2+38){ }^{\circ} \mathrm{K}$.

The total substrate degradation $\left(C_{t}(x, t)\right)$ can be determined from $\mathrm{kgm}^{-3}$

$$
\begin{aligned}
& \left.C_{T}=\frac{C_{o} k e^{\left(-\frac{1}{k}\right)}}{u_{m}^{2} V \cdot H R T}\left\{\left(V u_{m}-2 v k\right)\left[1-e^{\left(-u_{m} \cdot H R T / k\right.}\right)\right]+H R T \cdot v \cdot u_{m}\left[1+e^{\left(-u_{m} \cdot H R T / k\right)}\right]\right\} \\
& u_{m}=0.013 T_{o}-0.129
\end{aligned}
$$


$C_{o}=0.863 \cdot T S \cdot \rho$

Where, $k$ is constant $=1.26, u_{m}$ is bacterial growth rate, day, $v$ is flow rate of manure slurry into the digester, $m^{3} d a y^{-1}$.

Table 3. The different parameters used in the equations of model for biogas production.

\begin{tabular}{lllllllll}
\hline Item & HRT & $\mathrm{C}_{\mathrm{o}}$ & $v$ & $\mathrm{~T}_{\mathrm{a}}$ & $\mathrm{T}_{\mathrm{o}}$ & $\mathrm{k}$ & $\rho$ & $\mathrm{TS}$ \\
\hline Value & 25 & 83.71 & 0.375 & 273.2 & 311.2 & 1.26 & 970 & $9 \%$ \\
\hline
\end{tabular}

Energy requirement for biogas system

For the digester energy balance, model was derived, which comprises the major forms of energy produced and consumed. Consumption of energy results mainly due to stirring and substrate heating. Energy requirement for biogas production per day is calculated as following equations according to Lubken et al., 2007.

$$
E_{\text {stir }}=V_{\text {liq }} \cdot S \cdot t_{s}
$$

Where, $E_{\text {stie, }}$ is the energy for stirring, $\mathrm{kWhd}^{-1}, V_{\text {liq, }}$, is the liquid volume, $\mathrm{m}^{3}, \mathrm{~S}$, is the specific power of the stirrer $=0.005, \mathrm{kWm}^{-3}$ and $\mathrm{t}_{\mathrm{s}}$, is the time for stirring use every half hour for $10 \mathrm{~min}, 6 \mathrm{hd}^{-1}$

$$
E_{\text {sub-heat }}=v \cdot c\left(T_{d}-T_{\text {substrate }}\right)
$$

Where, $E_{\text {sub-heat }}$, is the energy for heating substrate, $\mathrm{kWhd}^{-1}, c$ is the heat capacity of the substrate, $4.1 \mathrm{~kJ} \mathrm{~kg}$ ${ }^{1} \mathrm{~K}^{-1}, T_{\text {substrate }}$ is the temperature of the substrate $=$ as the weather of area, about $293 \mathrm{~K}$

Energy production form generation engine when used biogas as a fuel

Electricity generation and Thermal energy production

The calculation uses an IC engine fired with biogas as a fuel to generate electricity and thermal energy with a nominal power output of $3.7 \mathrm{~kW}$.

$$
\begin{aligned}
& E_{\text {elect }}=Q_{\text {bio }} \cdot H_{\text {bio }} \cdot \mathrm{\eta}_{\text {elect }} \\
& E_{\text {therm }}=Q_{\text {bio }} \cdot H_{\text {bio }} \cdot \mathrm{\eta}_{\text {thermal }}
\end{aligned}
$$

Where, $E_{\text {elect }}, E_{\text {therm }}$ is the electricity and thermal energy generation, $\mathrm{kWhd}^{-1}, Q_{b i o}$, is the biogas fuel consumption), $\mathrm{m}^{3} \mathrm{~d}^{-1}, H_{b i o}$, is the heating value of biogas, $21 \mathrm{MJm}^{-3}, \eta_{\text {elect }}$ and $\eta_{\text {thermal }}$ is the electrical and thermal degree of efficiency, $25 \%$ and $40 \%$.

Net energy production

Calculated the net energy from electricity generated and heat energy of exhaust gas from the generator by the following equation.

$$
E_{\text {net }}=\left(E_{\text {elect }}-E_{\text {stir }}\right)+\left(E_{\text {therm }}-E_{\text {sub-heat }}\right)
$$

Heat energy recovery from exhaust gas

The energy balance in the engine includes that the input energy and output energy. Energy is supplied to the engine as a chemical energy of the fuel and leaves as energy in the cooling water, exhaust, brake work and extraneous heat transfer, which is often termed 'heat loss'. The waste heat from exhaust gas is used to supplement the heating needs of a broiler house. Thermal energy recovered by passing exhaust gas through heat exchanger units. The exhaust gas temperature was measured at the input and output end to calculate the energy added by the engine or removed by broiler house. The following equations used to determine the maximum heat that can be recovered from the exhaust gas, Omar 2011.

$$
\begin{gathered}
q_{\text {recover }}=m_{\text {exh }} \cdot c p_{\text {exh }}\left(T_{\text {exh }}-T_{o}\right) \\
m_{\text {exh }}=v_{\text {exh }} \cdot A_{\text {tub }} \cdot \rho_{\text {exh }}
\end{gathered}
$$


Where, $\mathrm{m}_{\text {exh }}$, mass flow rate of the exhaust gas, $\mathrm{kg} \mathrm{s}^{-1}, v_{\text {exh }}$, exhaust gas velocity, $\mathrm{m} \mathrm{s}^{-1} \mathrm{~A}_{\text {tub}}$, cross section area of the exhaust gas tube, $\mathrm{m}^{2}$, $\rho_{\text {exh }}$, density of exhaust gas, $\mathrm{kg} \mathrm{m}^{-3}$, cpexh, specific heat of the exhaust, $\mathrm{J} \mathrm{kg}^{-1}{ }^{\circ} \mathrm{C}^{-1}$, $\mathrm{T}_{\mathrm{exh}}$, inlet temperature of the hot exhaust gas, ${ }^{\circ} \mathrm{C}$

\section{Heater exchanger efficiency}

As a result of used the heat exchanger, there is some loss in the heat of the fluid available for use in the actual heating equipment. This heat loss depends upon the type of used heat exchanger. Therefore the heater efficiency will calculated to evaluation this heater for heating transfer.

$$
\eta_{\text {heater }}=\frac{Q_{\text {input }(\text { heater })-} Q_{\text {output }(\text { heater })}}{Q_{\text {input }}(\text { heater })} \times 100
$$

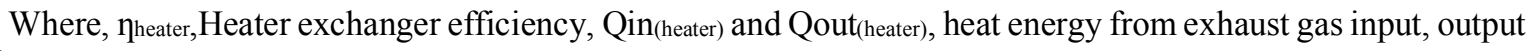
to heater

Differential equation

To calculate the temperature of the indoor air of broiler the following differential equation was used Taha (2003)

$$
T=\frac{1}{\rho V_{\text {air }} c_{p}} \int_{t=0}^{t=n}\left(q_{\text {sup }}-q_{\text {loe }}\right) d t
$$

Experimental setup

The experimental setups used in the work are shown in the schematic diagram (figure 2).

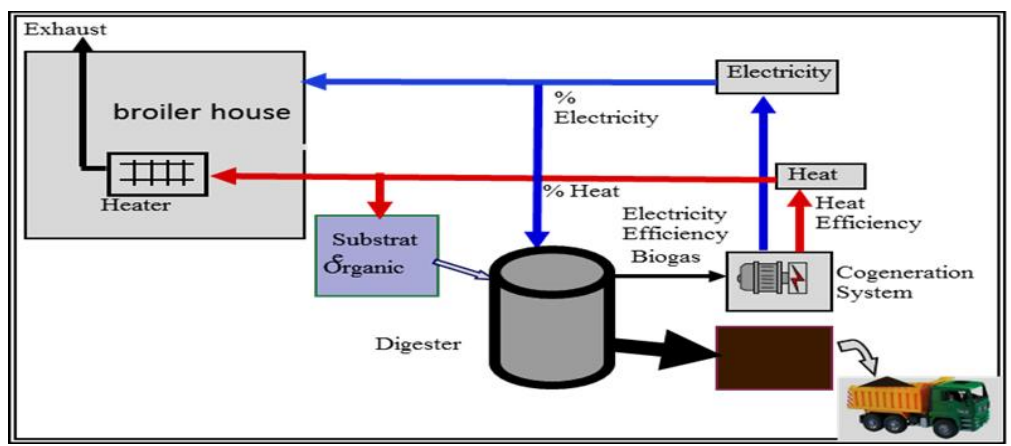

Figure 2. Schematic diagram of the experimental setup used in this work.

Broiler house construction and its materials

To achieve the objective of the study, A cycle of production (6 weeks) in a broiler house was selected for this study, that was in Menoufia Governarate, egypt (Geographically, the chosen place's latitude angle is $30^{\circ} 54^{\prime}$ degrees). Heat exchanger unit constructed were carried out during winter season 2017/2018. The broiler house had a direction of east-west design to take the maximum benefit from the sun during winter season and high level of ventilation rate of the summer season. The total area under the broiler house was $150 \mathrm{~m}^{2}$ with the dimensions as $8 \times 18.75 \mathrm{~m}$ and height of $3 \mathrm{~m}$ with a naturally ventilation during winter season. The steractural of the house , the Brick was the main in all walls and had an inner layer of plaster ( sand and cement) with thickness of $3 \mathrm{~cm}, 3$ $\mathrm{cm}$ inside layer of the insulation and $2 \mathrm{~cm}$ of outside plaster. The roof was made of $20 \mathrm{~cm}$ from concrete and 3 $\mathrm{cm}$ layer of insulation was added to the exterior of the roof. Polyethylene was the main material used in all window with $0.003 \mathrm{~cm}$ of thkness. By determining the thermal requirements of birds, it was found that they needed a high temperatures and a small area (based on the recommended poultry breeding) during the first period. The energy needs begin to decrease over time and the required of area increases. From this important observation, a moving head wall was built inside the farm, made of metal frame and covered with a layer of polyethylene. This wall will move freely on the farm to reduces space according to required area. The production cycle was divided into three periods each one two-week and the farm area was divided into three equal parts, each part equal to $8 \times 6.25$ meters. It represents the area of the farm during the first two weeks is very suitable for birds in the first age of birds. During the next two weeks, the wall is moved to increase the area of the farm to the second stage with a length of 12.5 meters. In the last two weeks, the area of the farm is the total area of 18.75 meters. This wall plays a very 
important role in the energy consumed within the farm. Which reduces the size of the farm during the period of high thermal requirements and thus reduce the added energy to meet the thermal needs of the farm. As well as reduce the electricity energy consumed and also reduce the energy lost during the transmission of heat from the broiler house. The physical properties of these materials are indicated in Table 1. Two heaters located in the house were uniformly distributed along the length, and each had a heating capacity of $5 \mathrm{~kW}$. The bird density was 10 birds $\mathrm{m}^{-2}$ according to the recommendation from broiler management guide.

\section{Heat Exchanger}

The heat exchanger is designed to remove heat from the exhaust gas and transfer it to the broiler house without mixing the exhaust gas with the inside air. It is the most equipment commonly used to heat the broiler house.

\section{The experimental Engine and generator}

The engine was used in this study to move the generator was a $3.7 \mathrm{~kW}$ Water Cooled Diesel Engine. The compression ratio was 17:1. Four-stroke, naturally aspirated, water-cooled, compression ignition. Biogas was supplied through a plastic pipe and mixed with inlet air from the engine filter air.

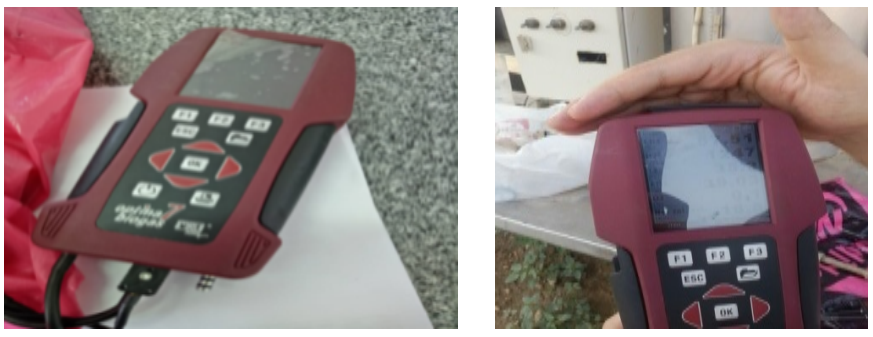

Figure 3. The OPTIMA 7 Biogas.

Measurements

Biogas generation and the specification of produced biogas was measured by OPTIMA 7 Biogas (figure 3) it is suitable for biogas as well as flue gas and cogeneration engine measurements. Simultaneous measurements biogas components: $\mathrm{O}_{2}, \mathrm{H}_{2} \mathrm{~S}, \mathrm{CH}_{4}$ and $\mathrm{CO}_{2}$ and temperature. Temperatures inside, outdoor air of the broiler house and in three points (from the engine, before and after the heater exchanger) temperature were measured using IC (LM35) sensors. The data collection and recording frequency were recorded every 1 minute and 1 hour average of each measurement by using a LabJacks data logger.

\section{Results and Discussion}

The results of the model validation were discussed under the following heads:

The results of energy loss obtained from the solution of equations

Discussion of the results obtained from the solution of equations for calculate the poultry house heating required according to recommended inside house temperature, outside air temperature and heat loss.

Table 4. Calculate the heat loss of the steady- state condition for the conventional broiler house at total cycle time.

\begin{tabular}{|c|c|c|c|c|c|c|c|c|c|}
\hline \multirow[t]{2}{*}{ Element } & & \multicolumn{3}{|c|}{ Area, $\mathrm{m}^{2}$} & \multirow[t]{2}{*}{$\begin{array}{l}\text { Heat resistance } \\
\mathrm{m}^{2} \mathrm{KW}^{-1}\end{array}$} & \multirow{2}{*}{\multicolumn{2}{|c|}{$\begin{array}{l}\text { Energy loss, } \\
\text { kW.h }\end{array}$}} & \multirow{2}{*}{\multicolumn{2}{|c|}{$\begin{array}{c}\text { Energy loss, } \\
\%\end{array}$}} \\
\hline & & First stage & second stage & third stage & & & & & \\
\hline \multirow[t]{11}{*}{ structure } & Roof & 50 & & & 1.34 & 187.09 & 515.67 & 4.18 & 11.52 \\
\hline & & & 100 & & & 181.91 & & 4.06 & \\
\hline & & & & 150 & & 146.66 & & 3.28 & \\
\hline & Wall & 41.25 & & & 1.47 & 141.32 & 351.38 & 3.16 & 7.85 \\
\hline & & & 65.25 & & & 108.68 & & 2.43 & \\
\hline & & & & 113.25 & & 101.38 & & 2.26 & \\
\hline & Window & 20.25 & & & 0.3 & 338.92 & 820.17 & 7.57 & 18.32 \\
\hline & & & 33.75 & & & 274.61 & & 6.14 & \\
\hline & & & & 47.25 & & 206.64 & & 4.62 & \\
\hline & Removed & 24 & & & 0.36 & 333.7 & 495.94 & 7.46 & 11.08 \\
\hline & wall & & 24 & & & 162.24 & & 3.62 & \\
\hline \multirow{3}{*}{\multicolumn{2}{|c|}{ ventilation }} & & & & & 745.99 & 1977.83 & 16.67 & 44.19 \\
\hline & & & & & & 699.06 & & 15.62 & \\
\hline & & & & & & 532.78 & & 11.90 & \\
\hline \multirow{3}{*}{\multicolumn{2}{|c|}{ lighting }} & & & & & 52.5 & 315 & 1.17 & 7.04 \\
\hline & & & & & & 105 & & 2.35 & \\
\hline & & & & & & 157.5 & & 3.52 & \\
\hline \multirow{3}{*}{\multicolumn{2}{|c|}{ Total }} & 159 & & & & 1799.52 & 4475.98 & 40.20 & 100 \\
\hline & & & 274 & & & 1531.50 & & 34.22 & \\
\hline & & & & 389 & & 1144.96 & & 25.58 & \\
\hline
\end{tabular}


It is clear from the table 4 that there is a significant impact of the inside insulation moved wall used on the farm. Where the results showed that, the largest value of thermal loss was in the first stages compared to the following two stages, despite the small size of the farm at this stage. This means that if the total size of the farm was used at this stage, the energy consumption will increase by $200 \%$. The same result was in the second stage, which is to reduce the energy consumption in this stage by $100 \%$. The table show that the total energy required to add to the farm was $4475.98 \mathrm{kWh}$ during the cycle. The largest energy lost value was $40.2 \%$ and $18.32 \%$ from the first stage and windows and the lowest energy lost was $7.85 \%$ for the walls. We must increase the layer thickness of the insulation to reduce the energy losses and used double layers polyethylene in the windows.

Predicted energy generated from biogas's mathematical model and measured value

The results of mathematical model predicted the energy of operate a digester at a specified temperature $38^{\circ} \mathrm{C}$ and energy generated by used biogas production as a fuel in the $3.7 \mathrm{~kW}$ engine. The daily flow rate of substrate was $0.375 \mathrm{~m}^{3} \mathrm{day}^{-1}$ it production $13.2 \mathrm{~m}^{3} \mathrm{day}^{-1}$ biogas. The results (figure 4 ) shows that, the electricity, thermal energy production, energy requirement for biogas production presses and the net energy production were 847.36, $1355.78,811.86$ and $1,391.28 \mathrm{kWh}$ for total period of broiler cycle production.

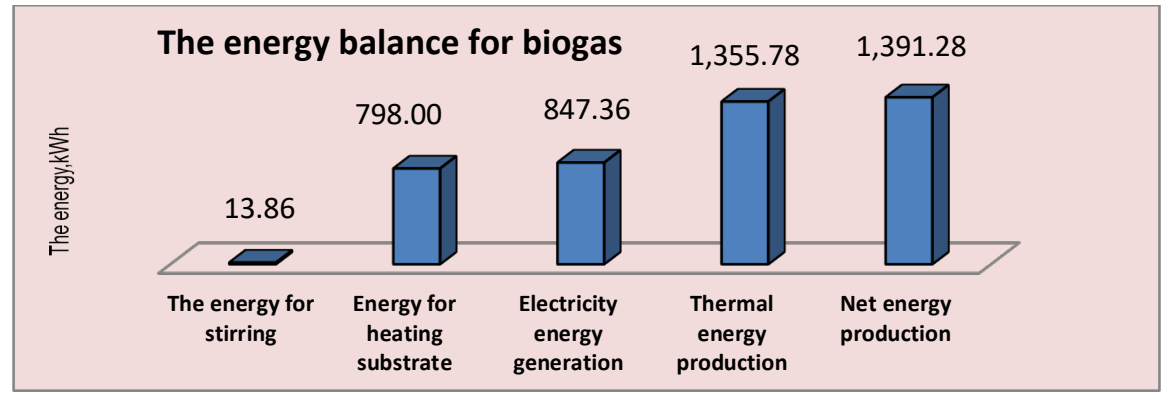

Figure 4. Total energy required for the broiler house.

Table 5. The biogas production specification

\begin{tabular}{lcccccc}
\hline & $\begin{array}{c}\text { Bio gas, } \\
\mathrm{m}^{3} \text { day }\end{array}$ & $\begin{array}{c}\mathrm{HLV}, \\
\mathrm{MJm}^{-3}\end{array}$ & $\begin{array}{c}\mathrm{CH}_{4}, \\
\%\end{array}$ & $\begin{array}{c}\mathrm{CO}_{2}, \\
\%\end{array}$ & $\begin{array}{c}\mathrm{H}_{2} \mathrm{~S}, \\
\mathrm{ppm}\end{array}$ & $\begin{array}{c}\mathrm{O}_{2}, \\
\%\end{array}$ \\
\hline $\begin{array}{l}\text { Predicted value from model } \\
\text { Measured value }\end{array}$ & 13.2 & 21 & -- & -- & -- & -- \\
-- & 19.9 & 50.8 & 44.38 & 901 & 0.1 \\
\hline
\end{tabular}

Average values of biogas yield, biogas yield rate and composition during the experimental cycles are presented in Table 6. It was observed that the Predicted from model and measured value was 13.2 and 12.65 $\mathrm{m}^{3}$ day $^{-1}$. A good agreement between the predicted and measured values was obtained during the entire modelling period. The average biogas heating value was 21 and $19.9 \mathrm{MJm}^{-3}$ for the use from references and measured value with measured specific methane at $50.8 \% \mathrm{CH}_{4}$ concentration in the biogas. The results obtained from this experiment are high compared to some result from literature at mesophilic conditions but are in line with other.

\section{Efficiency of heater exchanger, electrical and thermal degree}

The results in the (figure 5) showed that the efficiency of the heater exchanger designed and used for heating broiler house from references and measured was 90 and $81.3 \%$. That means that $18.7 \%$ from the engine exhaust gas was lost but this efficiency was agreement. The measured efficiency of electrical and thermal degree of the generation by using bio gas as a fuel was found 23.4 and $34.7 \%$. Although the values were taken from references and that used in the model were 25 and $40 \%$. The difference was due to the change in the true value for the heating value of biogas fuel and that used in the model and generator case and its efficiency.

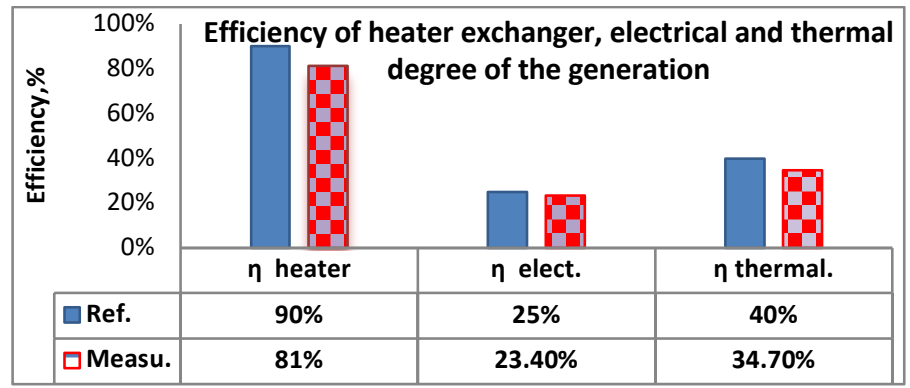

Figure 5: Total energy required for the broiler house. 
The energy balance of the broiler house

The figure 6 shows that the total lost energy as well as the electrical energy required for lighting to maintain the environmental factors (temperature, ventilation and intensity of light) on the farm at the required levels. It was discerned from the (figure 6) that the total energy lost from transmission and ventilation was 49 and $44 \%$. The energy required to lighting was $7 \%$ of the total energy wanted to supplement.

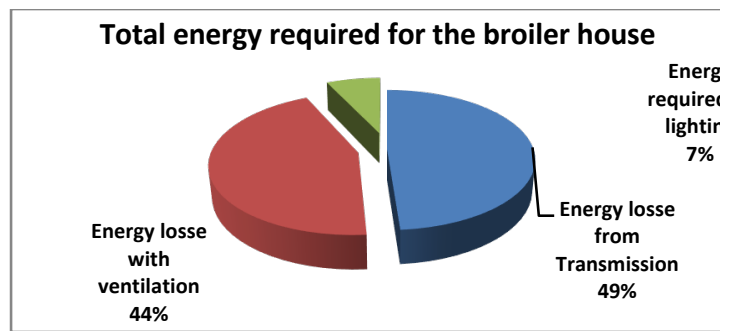

Figure 6. Total energy required and supplement for the broiler house.

The total energy required to supplement to the broiler house as well as the share of energy generated from the waste broiler in covering this energy shows in (Figure 6). Only $2 \%$ of the energy needed for the energy balance came from the poultry itself through the breathing process. The electricity and heat energy of exhaust gas which, generated from broiler litter waste as an input for broiler house operations portion by $49 \%$ of the total energy required. This is a great value that needs to be taken care of; the rest of the energy needed to add through the heaters was $49 \%$.

The indoor air of broiler temperature

To view the results of the model validation, three periods were chosen for each period representing two consecutive days. The first period is the seventh and eighth day of the cycle, the second period is represented by the twenty-one and the twenty-tow and the third period were represented on the thirty-five and thirty-six of the cycle. The predicted inside air temperatures of broiler house using the computer model along with arithmetic average of measured values at different time of the day are shown in (figure 7).

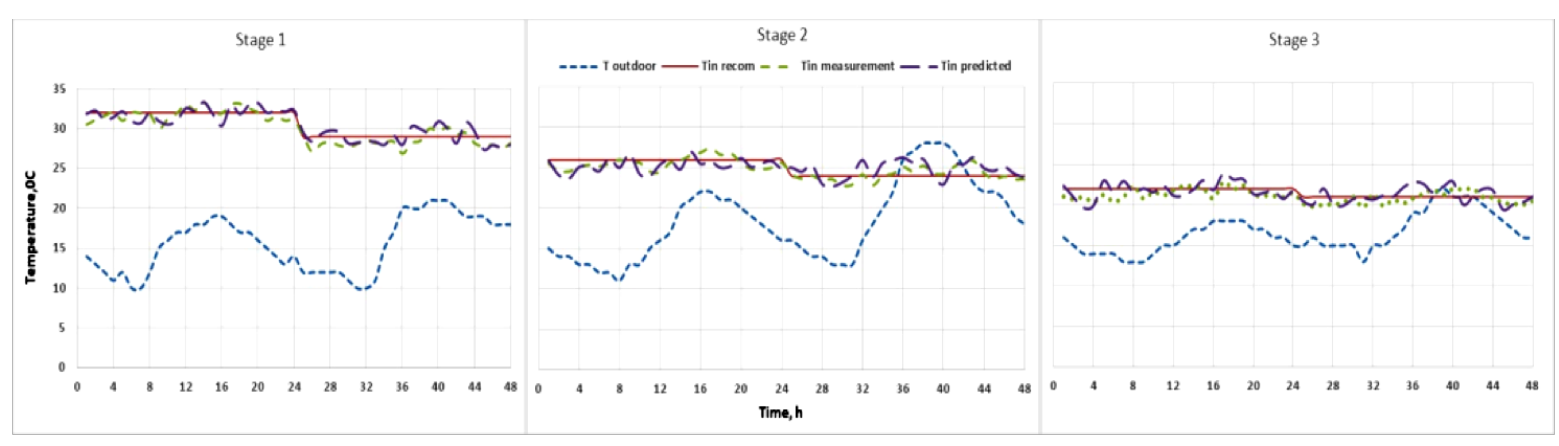

Figure 7. Comparison between measured and predicted of the inside air temperature.

The air temperatures outdoor fluctuate with the daily amplitude becoming larger at the noon. It reached the peak at noon and decline in late afternoon and early morning as shown in the (figure 7). The air temperature inside house designed by the recommendation as show in table 1 it appeared in the (figure7) as a fixed value (horizontal line). The maximum values of the measured and predicted temperature were $(33.2,33.37),(27.37,26.7),(22.83$, $23.86)^{\circ} \mathrm{C}$ in first stage, second and third stage. The minimum value were $(27,27.4),(22.8,22.7),(19.7,19.38)^{\circ} \mathrm{C}$ in first period, second and third respectively. The result indicated that, the high different between measured or predicted and recommended temperature was $1.86^{\circ} \mathrm{C}$ and this value was found in the third stage while, the lower different between measured or predicted and recommended temperature was $2{ }^{\circ} \mathrm{C}$ found in the first stage. That is a big rapprochement between the measured or predicted and recommended temperature, which is means the model is respected and Correspondence between the simulated and the measured air temperatures inside the broiler house.

It can be observed from (figure 8) that, the maximum difference between the measured and predicted temperature was $2.1^{\circ} \mathrm{C}$, the maximum difference between the measured and recommendation temperature was 2 ${ }^{\circ} \mathrm{C}$ and the maximum difference between the predicted and recommendation temperature was $2.3{ }^{\circ} \mathrm{C}$. The correlation coefficient between values was $\mathrm{R}^{2}=0.9447$ (figure 8 ). That is means a correspondence between the 
simulated and the measured air temperatures inside the broiler house. The air temperature inside the house is influenced by ventilation, air temperature outside the house, heat production from the broiler and heating system.
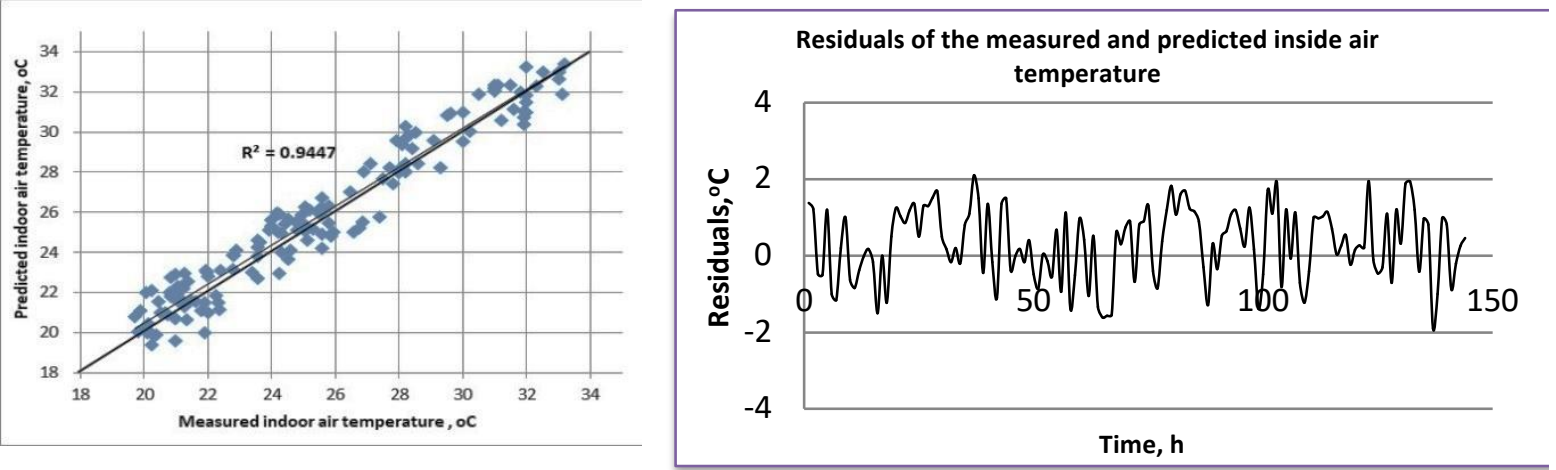

Figure 8. The correlation coefficient and residuals of the measurement and predicted inside air temperature, ${ }^{\circ} \mathrm{C}$.

The difference between calculated and measured temperatures can be explained by several different causes. This may be due to the change in the speed of the outside air, which would cause a change in the convective heat transfer coefficient as well as the external temperature and thus increase the amount of heat lost by heat transfer or ventilation. The values of convective heat transfer coefficients and the conductivity used in the model may vary based on the values found in the previous research, in a small fraction of their true values, leading to small differences between measured and calculated values. The number of opening and closing of the farm door was not taken into account in the model. The stability of the amount of added energy from the heat of the exhaust or electric heaters and the thermal needs less or more may lead to the same difference.

\section{Conclusions}

The electricity, thermal energy production, energy requirement for biogas production presses and the net energy production were $847.36,1355.78,811.86$ and $1,391.28 \mathrm{kWh}$ for total period of broiler cycle production. The daily flow rate of substrate was $0.375 \mathrm{~m}^{3}$ day $^{-1}$ it production $12.65 \mathrm{~m}^{3} \mathrm{day}^{-1}$ biogas. The Efficiency measured of electrical and thermal degree of the generation by using bio gas as a fuel was found 23.4 and $34.7 \%$. The maximum difference between the measured and predicted temperature was $2.1^{\circ} \mathrm{C}$, the maximum difference between the measured and recommendation temperature was $2{ }^{\circ} \mathrm{C}$ and the maximum difference between the predicted and recommendation temperature was $2.3{ }^{\circ} \mathrm{C}$. The correlation coefficient between values was $\mathrm{R}^{2}=0.9447$. As a whole, a good agreement between the predicted and measured values was obtained during the entire modelling period. This means that the model can be used to predict a thermal performance of the broiler house in a wide range of solar radiation, variable rate of ventilation, change of building structural materials and temperatures. A large amount of energy was lost during the ventilation process. Ventilation must therefore be carried out within a heat exchanger where heat is exchanged between the indoor heated air and cold air. Using waste heat as an input for broiler house operations is a new mechanism for reducing operating costs. Found that the electricity and heat energy of exhaust gas which, generated from broiler litter waste as an input for broiler house operations portion by $49 \%$ of the total energy required.

\section{References}

Anastaselos D, S. Oxizidis, A.M. Papadopoulos. 2017. Suitable thermal insulation solutions for Mediterranean climatic conditions: a case study for four Greek cities. Energy Efficiency.10 (5) 1081- 1098. https://doi.org/10.1007/s12053-016-9504-1.

Andrea, C., E. Fabrizio, A. Ghiggini, M. Bariani. 2018. Climate control in broiler houses: A thermal model for the calculation of the energy use and indoor environmental conditions. Energy \& Buildings 169 (2018) 110-126 https://doi.org/10.1016/j.enbuild.2018.03.056

Bustamante,E., S. Calvet, F.J. García-Diego, A.G. Torres, A. Hospitaler, 2017. Measurement and numerical simulation of single sided mechanical ventilation in broiler houses. Sustainability. 7(2):2066-2085

Daghir N.G. 2008. Poultry Production in hot Climatic, Broiler management in hot climates. 2008:60-227.

De Rosa, M., V. Bianco , F. Scarpa, L.A. Tagliafico. 2014. Heating and cooling building energy demand evaluation; a simplified model and a modified degree days approach. Applied Energy. 128. (2014) 217: 229. https://doi.org/10.1016/j.apenergy.2014.04.067

Dunstan, D. and D. Probert. 2001. Increasing electricity generation per unit of fossil fuel so expended in diesel engines Applied Energy 70 (3) 267-280. https://doi.org/10.1016/S0306-2619(01)00030-7 
Erdem K., B. Cemek , 2018. A study on environmental impact of insulation thickness of poultry building walls. Energy 150 (2018) 583-590. https://doi.org/10.1016/j.energy.2018.02.153

Felipe,P.S., S. N., Melegari de Souza, D. S. Kitamura, C. E. C. Nogueira, 2018. Brazil Energy efficiency of a micro-generation unit of electricity from biogas of swine manure. Renewable and Sustainable Energy Reviews.82 (3) 3900-3906https://doi.org/10.1016/j.rser.2017.10.083

Gebremedhin, K., U. Wu, C.Gooch, and P. Wright. 2004. Simulation of Heat Transfer for Biogas Production 2004 ASAE/CSAE Annual International Meeting Sponsored by ASAE/CSAE 1 - 4 August 2004

Harmanto, H.J. Tantau, and V.M. Salokhe. 2006. Microclimate and Air Exchange Rates in Greenhouses covered with Different Nets in the Humid Tropics Biosystems Engineering (2006) 94 (2), 239-253 doi:10.1016/j.biosystemseng.2006.02.016

Houdkova, L., J. Boran, V. Ucekaj, T. Elsaser, and P.Stehlik, 2008.Thermal processing of sewage sludge - II, Appl. Therm. Eng. 28 (16) 2083:2088. https://doi.org/10.1016/j.applthermaleng.2008.04.005

Lubkena,M., M. Wicherna, M. Schlattmannb, A. Gronauerb, and H. Horna. 2007. Modelling the energy balance of an anaerobic digester fed with cattle manure and renewable energy crops. Water research 41 (2007) 4085 - 4096. https://dx.doi:10.1016/j.watres.2007.05.061

Mangan S.D., G.K.,Oral .2016 . Energy and cost analyses of solar photovoltaic (PV) microgeneration systems for different climate zones of Turkey. Energy Power Eng. 8(03):117-129.

Oeztuerk, H.H. and A. Baescetincelik, A, 2003. Energy and Exergy Efficiency of a Packed-bed Heat Storage Unit for Greenhouse Heating. Biosystems Engineering. 86 (2), 231-245. https://doi.org/10.1016/S1537-5110(03)134$\underline{X}$

Omar, M.N. 2011. Environmental control of some vegetable crops inside greenhouse Ph.D. Thesis, ,Faculty of Agriculture, Minoufiya University, Minoufiya, Egypt .

Pao H.T., H.C.Fu.2013. Renewable energy, non-renewable energy and economic growth in Brazil. Renew Sustain Energy Rev 2013;25:381-92. http://dx.doi.org/10.1016/j. rser.2013.05.004.

Phan, M.D. and K.Wattanavichien, K. 2007.Study on biogas premixed charge diesel dual fuelled engine Energy Conversion and Management 48 (8) 2286-2308. https://doi.org/10.1016/j.enconman.2007.03.020

Rojano, F., P.E. Bournet, P. Robin, M. Hassouna, C.Y. Choi and M. Kacira. 2014. Test of two different schemes through CFD to include heat and mass transfer induced by animals inside a broiler house. In International conference of agricultural engineering (Zurich, Switzerland, July 6-10).

Rossi, P. , A. Gastaldo , G. Riva , C. de Carolis, Progetto Re Sole . 2013. (Final Draft), Centro Ricerche Produzioni Animali (CRPA), Reggio Emilia, Italy.

Salomon K.R., L.E. Silva Lora .2009. Estimate of the electric energy generating potential for different sources of biogas in Brazil. Biomass and Bioenergy .33 (9):1101-1107. http://dx. doi.org/10.1016/j.biombioe.2009.03.001.

Seo, I.-H., I.B. Lee, O.K. Moon, H.T. Kim, H.S. Hwang, S.W. Honga, J.P. Bitog, J.I. Yoo, K.S. Kwon, Y.H. Kim, J.W. Han, 2009. Improvement of the ventilation system of a naturally ventilated broiler house in the cold season. Biosystems Engineering. 104.(1) 106-117.https://doi.org/10.1016/j.biosystemseng.2009.05.007

Shen, Y., J.L.Linville, M.Urgun-Demirtas, M.M.Mintz, S.W.Snyder. 2015. An overview of biogas production and utilization at full-scale wastewater treatment plants (WWTPs) in the United States: challenges and opportunities towards energy-neutral WWTPs. Renewable and Sustainable Energy Reviews. 50, 346-362. https://doi.org/10.1016/j.rser.2015.04.129

Song D.J., A.J.King . 2015. Effects of heat stress on broiler meat quality. World's Poultry Science Journal 71(04):701-709

Taha, A.T. 2003. Simulation Model of Energy Fluxes in Passive Solar Greenhouses with a Concrete NorthWall. Ph.D. Thesis, University of Hannover, Germany.

Tippayawong N., A. Promwungkwa, and P. Rerkkriangkrai. 2007. Long-term operation of a small biogas/diesel dual-fuel engine for on-farm electricity generation biosystems engineering 98 ( 2007 ) 26 - 32

Willits, D.H., J.M. Marbis, . and J.Cheng. 2003. Waste Heat Utilization in a Greenhouse Used for the Removal of Nutrients from a Swine Waste Stream

Zupancic, G.D. and M. Ros. 2003. Heat and energy requirements in thermophilic anaerobic sludge digestion ; Renewable Energy 28 (14) 2255-2267. https://doi.org/10.1016/S0960-1481(03)00134-4. 


\section{Topic 6: Soil, land and water engineering (SW)}

Technology and systems for management of water and nutrients in soil, soil quality and rural planning. Contributions can address irrigation and drainage, logistics of equipment in the field, losses and emissions, soil compaction and carbon sequestration.

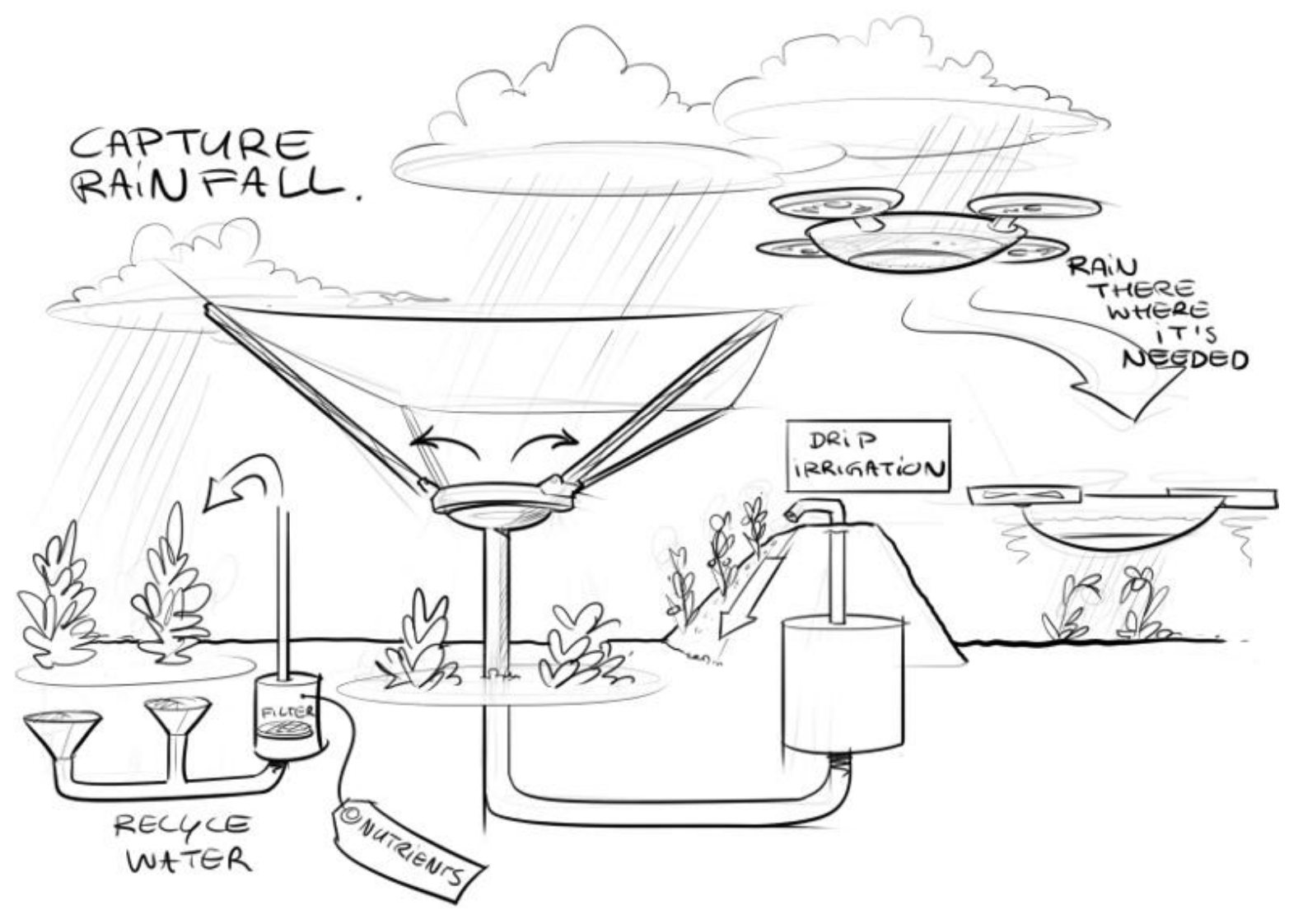




\title{
Evaluation of Compaction in No-till Vertisol Field Using Methods of Cone Index and Pedotransfer Function - Case of a Moroccan Semi-arid Context
}

\author{
Al Masmoudi Yassine ${ }^{\text {a,b*, }}$ El Aissaoui Abdellah ${ }^{\text {a }}$, Ibno Namr Khalid ${ }^{\text {b }}$ \\ ${ }^{a}$ Laboratory of Agricultural Machinery, National Institute of Agronomic Research, Settat, 26000, Morocco \\ b Laboratory of Geosciences and Environment Technics, Chouaib Doukkali University, Faculty of Science, El-Jadida, \\ Morocco \\ * Corresponding author. Email: $\underline{\text { Yassine.almasmoudi@gmail.com }}$
}

\begin{abstract}
This study aims to evaluate compaction of a fifteen years old no-till vertisol field crop (40.91\% clay $44.16 \%$ loam $14.93 \%$ sand) to find spatially potential correlation between compacted clusters and relative physicochemical soil properties. Measurements are done on soil strength (using vertical penetrometer), bulk density, organic matter, total limestone, and Atterberg limits to evaluate soil compaction and its pedotransfer function. Experimental results showed a range of the bulk density of [ 1.30 to $1.80 \mathrm{~g} / \mathrm{cm} 3]$ in the boundaries and another in [1.01 to $1.40 \mathrm{~g} / \mathrm{cm} 3]$ in the center of the field crop. The matter organic content averages were $2.23 \%$ and $2.91 \%$ for the profiles of $0-10 \mathrm{~cm}$ and $10-20 \mathrm{~cm}$, respectively. Analysis of total limestone content showed a spatial decreasing gradient situated between the ranges of [3.5-14 \%] and [0.5-3\%] for the soil profile of [0-10 $\mathrm{cm}]$. The total limestone gradient decreased in the soil profile of $[10-20 \mathrm{~cm}]$ within the range $[4.5-21 \%]$ and $[1-$ $4 \%$ ]. Soil strength (Ss) behaviour showed relatively a decreasing gradient between the ranges of [11.5-19 MPa] and [0.1-11 MPa]. Atterberg limits showed that is important to respect intervention delays to avoid soil compaction due to soil water content. The results showed an important compaction level in the extremities of the field due to importance of machines/tools traffic impact and intensity of tractor turning and operation number with no respect of soil plasticity during the cropping season. Compaction was founded to be related to limestone gradient of the field. The limestone content affected soil strength and its susceptibility to compaction. Based on evaluated parameters, the estimation of vertisol sensitivity to compaction was achieved by using a pedotransfer function an R2 of 0.9039 .
\end{abstract}

Keywords: Compaction, Vertisol, Strength, Atterberg Limits, Limestone, pedotransfer function.

\section{Introduction}

Soil compaction is becoming a critical problem facing modern agriculture that impacts the environment and food production with its intensity and extent (Durán et al., 2008). It is also a main raison of the degraded 33 million ha in Europe (Akker et al., 2001), and about 4 million ha in western Australia (Carder and Grasby, 1986). This phenomenon is prevalent in almost every continent: Azerbaijan, Japan, Russia, France, China, Ethiopia, New Zealand and Australia (Aliev, 2001; Ohtomo and tan, 2001; Bondarev and Kuznetsova, 1999; Tardieu, 1994; Suhayda and al, 1997; Mwendera and Saleem, 1997; Russell and al, 2001; Hamza and Anderson, 2003).

Heavy machine traffic and tillage tools are the main sources of this constraint. Severity of compaction depends on soil texture and structure, bulk density (BD), organic matter content (OMC), water content and total limestone (TL) (Panayiotopoulos et al., 1994; Raper and al., 1998; Mosaddeghi and al., 2000). The dynamic of compaction is mainly influenced by soil moisture content (Soane and Van Ouwerkerk, 1995; Hamza and Anderson, 2005). Vertisols are among the most sensitive soils to compaction; they have high yield potential and are used intensively for crops production in many countries. Their high clay and water contents during agricultural operations make them essentially sensitive to compaction, this latter may occur during a cropping cycle and may negatively affect crop performance (Radford, 2000). this type of soil can respond to compaction in different ways depending on soil structure and type of dominant clay (So and cull 1984). Some crop field losses were due to road traffic and tillage practices (Alakukku et al., 1996; Radford et al., 2007) and its effects on yield loss of different crops (Honsson and Reeder, 1994; Filipovic et al., 2006). Most of Moroccan soils are vertisols and the degree of soil compaction is an important soil structural attribute that needs characterization, such as bulk density, which gives immediately comparable values (Hakansson and Lipiec, 2000). In general bulk density is equal to 1.37 and 1.66 $\mathrm{g} / \mathrm{cm} 3$ for non-compacted and compacted soil respectively in the $(0-30 \mathrm{~cm})$ profile (Herbauts et al. 1996). It is the frequently used to evaluate the degree of soil compaction (Panayiotopoulos et al., 1994). The soil strength parameter is also used as an indicator of soil compaction, as it reflects soil resistance to root penetration (Taylor, 1971; Mason et al., 1988; Panayiotopoulos et al., 1994; Hamza and Anderson, 2001, 2003). Sinnet et al (2008) showed that $90.7 \%$ of roots are observed in penetration resistance class less than $3 \mathrm{Mpa}$. Maintaining an adequate organic matter content in the soil stabilizes the soil structure and makes it more resistant to compaction (Cochrane and Aylmore, 1994; Thomas et al., 1996), and decreases its bulk density and soil strength (Sparovek et al., 1999; Carter, 2002). Since soil moisture content strongly influences compaction sensitivity, variation of the soil moisture 
makes it possible to identify three levels: liquid limit, plastic limit and solid limit, called Atterberg limits that are defined by differences in soil behavior according to moisture content.

The study of Atterberg allows to conclude deducing that is important to respect intervention delay to avoid creation of compaction creation due to soil water content effect (Destain, 2013).

Spatial analysis of compaction intensity at plot scale was based on computing precompression stress (Pc) using pedotransfer functions. Horn and Fleige (2003) and Horn et al (2005) defined the parameter as an indicator of soil resistance to compaction at different scales from farm to regional levels. They found a correlation between precompression stress and soil physical parameters formulated as pedotransfer function. Texture (clay, silt and sand content), structure and moisture content are the most soil physical properties studied for developing these functions (Gupta and Larson, 1982; Imhoff et al., 2004; Lebert and Horn, 1991; McBride, 1989; Smith et al., 1997). Several authors characterized soil structure by density or others measurable variables such as permeability (Alexandrou and Earl, 1998; Canarache et al., 2000; Imhoff et al., 2004; Lebert and Horn, 1991; McBride, 1989; Rücknagel et al., 2007; Salire et al., 1994). In fact, the Pc computation requires knowledge of pedological (texture, organic matter), mechanical (bulk density, cohesion, internal friction angle) and hydraulics (water content available, water content not available, air capacity, hydraulic conductivity) parameters.

$\mathrm{Pc}$ values were classified into 6 clusters from very low $(\mathrm{Pc}<30)$ to extremely high $(\mathrm{Pc}>150)$, and vertisol sensitivity to compaction can be evaluated according to these precited classes (Dimitri D'or, 2014).

This study aims to evaluate compaction of a no-till vertisol field crop and to find spatially potential correlation between compacted clusters and relative physicochemical properties to develop a pedotransfer function related to soil strength and its precompression stress $(\mathrm{Pc})$.

\section{Materials and Methods}

The experiment was done in a vertisol field crop of an area of 1 ha and an historic of fifteen years no-till practice. This field is situated in the Chaouia region of Morocco (X: -7.6222, Y: 32.9556). Soil physical parameters (organic matter content (OMC), Bulk density (BD) and total limestone (TL)), soil strength, plasticity (Atterberg limit) were evaluated using systematic sampling for each cluster of $10^{*} 10 \mathrm{~m}^{2}$.

The OMC was measured by Walkley and Black method (ref or standard) based on the measurement of total organic carbon (eq.1).

$$
O M C(\%)=\operatorname{TOC}(\%) * 1.724
$$

Where TOC is the total organic carbon

Measurement of the total limestone (TL) was evaluated by Bernard Calcimeter Method (ref or standard) and quantified by equation 2 .

$$
\operatorname{CaCO3}(\%)=\left[\Delta V *(P T) * 10^{-4}\right] / M
$$

Where: $\Delta \mathrm{V}, \mathrm{PT}$ and $\mathrm{M}$ are difference of volume, intersection pressure-temperature and mass of soil sample, respectively.

The soil strength was measured by a $\mathrm{V}$ shaped cone penetrometer $\left(30^{\circ}\right)$. This penetrometer was equipped with an electronic pressure sensor. Data were acquired (Sampling frequency of $2 \mathrm{~Hz}$ ) in Volt using an oscilloscope/Logger (Agilent U1604B) and transferred to bar based on the curve calibration (Fig.2). The penetrometer was mounted on a tractor framework and actioned by a $12 \mathrm{~V}$ DC electric motor with constant speed of $2.5 \mathrm{~cm} / \mathrm{s}$. 


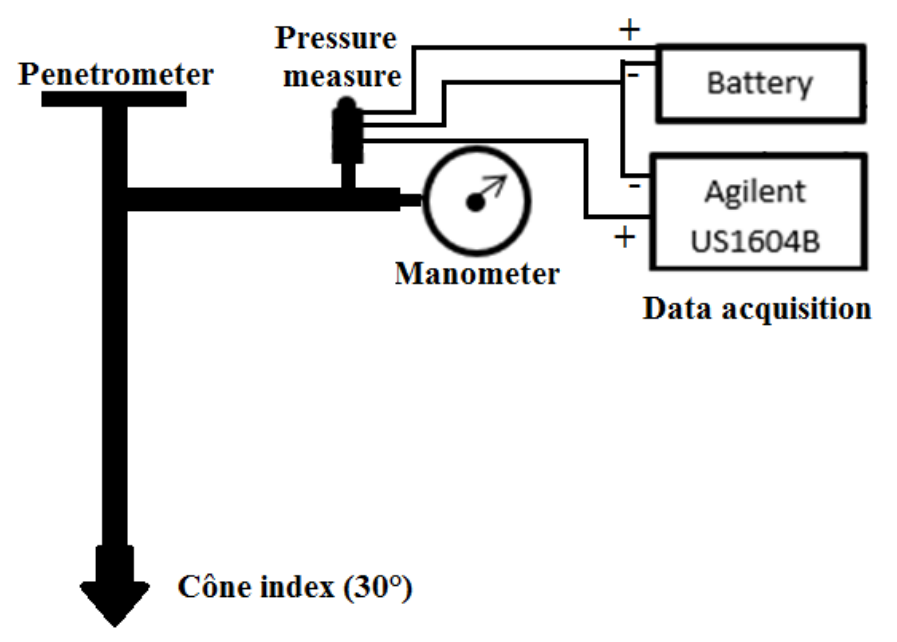

Figure 1. Experimental protocol for soil strength measurement.

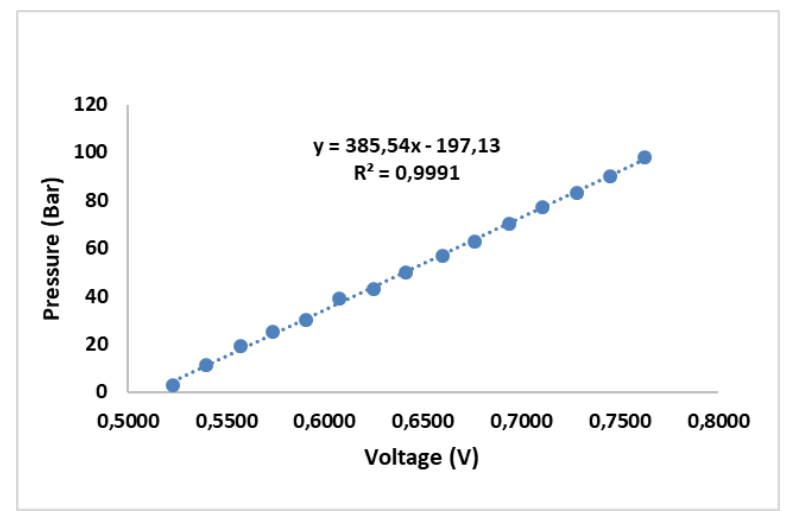

Figure 2. Soil strength calibration curve (pressure vs voltage).

Vertisol plasticity was evaluated by searching Atterberg limits using a hydraulic method press (Kirchhof, 2000). Figure 3 shows a prototype of a press developed in our laboratory and used for plastic limits evaluation within to ASTMD 4318-17 standard. The plasticity limits of vertisol looked for the limit mostly soil susceptible to compaction (Sridharan, 2004). Evaluation was based on twenty-eight soil sampling ( 7 treatment and four repetitions). Each soil sample $\left(269.26 \mathrm{~cm}^{3}\right.$ of granules having less than $2 \mathrm{~mm}$ ) was poured in a press cylinder and humidified by $150 \mathrm{ml}$ of tap water. The compression (up to 6 bar) and soil strength measurements were noted at the end of 1st, 2nd; 3rd; 4th; 5th, 10th and 15th days to find soil response to compression (strength as a function of moisture content) in order to approach effect of moisture content on soil compaction for an eventual prediction of optimal delay intervention in the field and avoid compaction created by machines and tools in occurring soil plasticity period.

Soil data evaluation was done using a spatial interpolation method (Inverse Distance to Power or IDP) and Surfer Software. The IDP is considered as a robust method for data interpolation and map realization (Irmak. 2010)

Correlation analysis of Pc and soil strength was done using JMP SAS software.
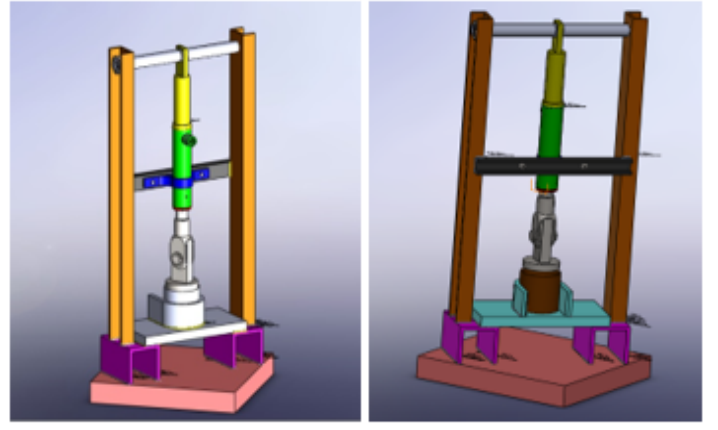

Figure 3. Scheme of hydraulic press used for soil samples compression to assess their response to compaction at varying moisture contents. 


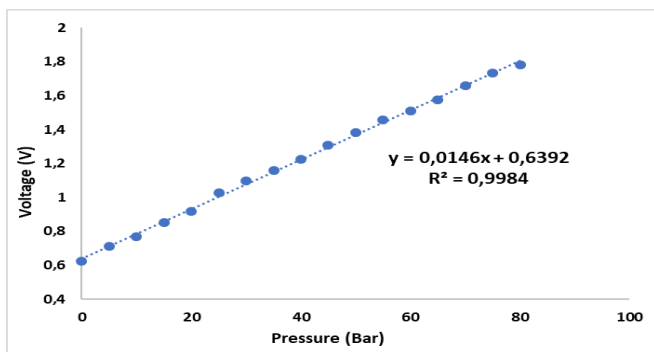

Figure 4. Hydraulic press calibration (pressure vs voltage).

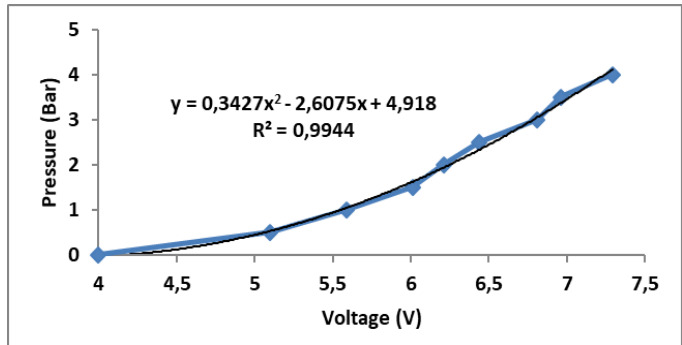

Figure 5. Soil strength calibration curve (soil strength vs voltage).

\section{Results and Discussion}

Soil strength assessment

Soil strength spatial distribution (Fig. 6) showed a significant compaction level in the boundaries limits of the field matrix. It is due to traffic importance and tractor turns. Results showed also an important compaction gradient (from simple to quadruple) between upstream and the downstream sides of the field. The compaction gradient was related to importance of limestone distribution. In fact, comparison between soil strength and limestone spatial distributions (Fig. 5 and 6) showed that both parameters where proportionally related. There is a potential effect of limestones proportion on compaction severity according to presence of limestone slab in the studied soil matrix. Referring to Laamal (1982), susceptibility to compaction is relatively more important in loamy soil than in clayey soil.

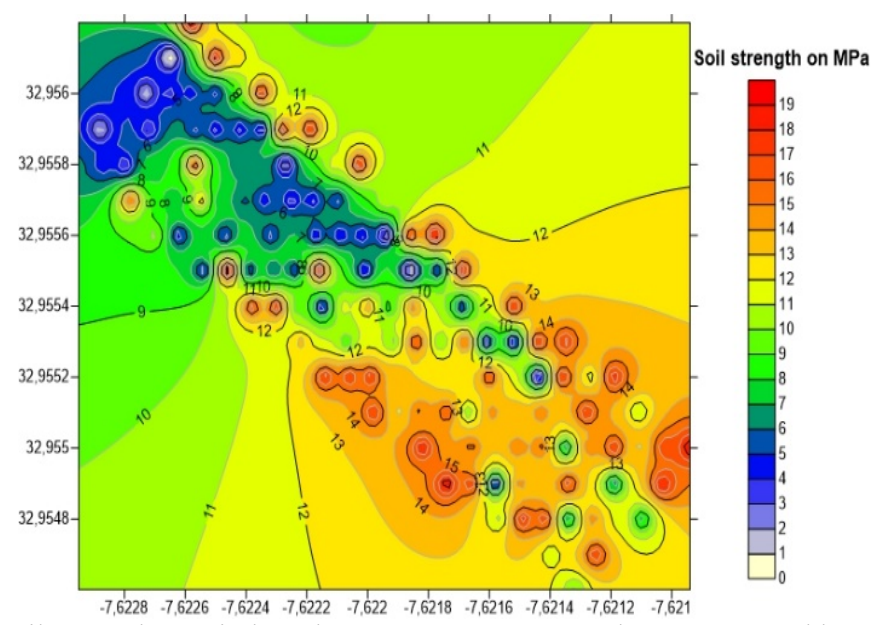

Figure 6 . Soil strength matrix based on cone penetrometer data represented by IDP method. 


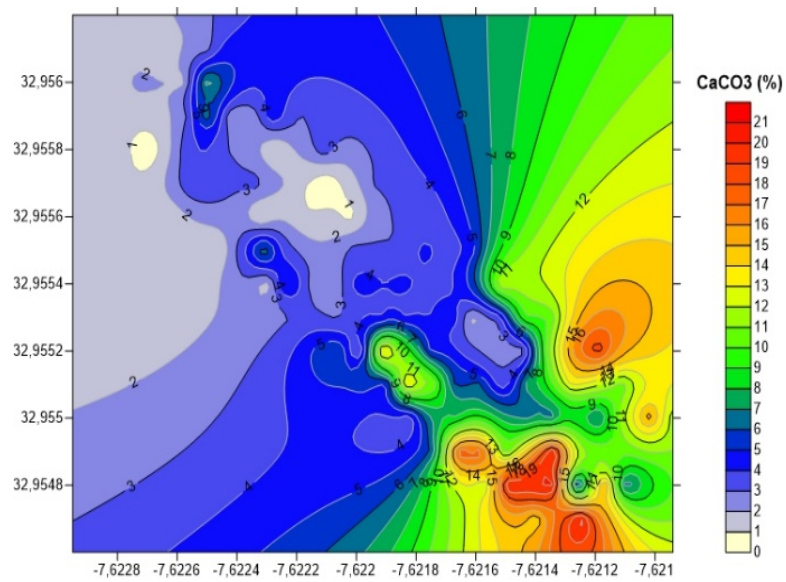

Figure 7. Total limestone matrix based on analysis data represented by "Distance to Power IDP" method.
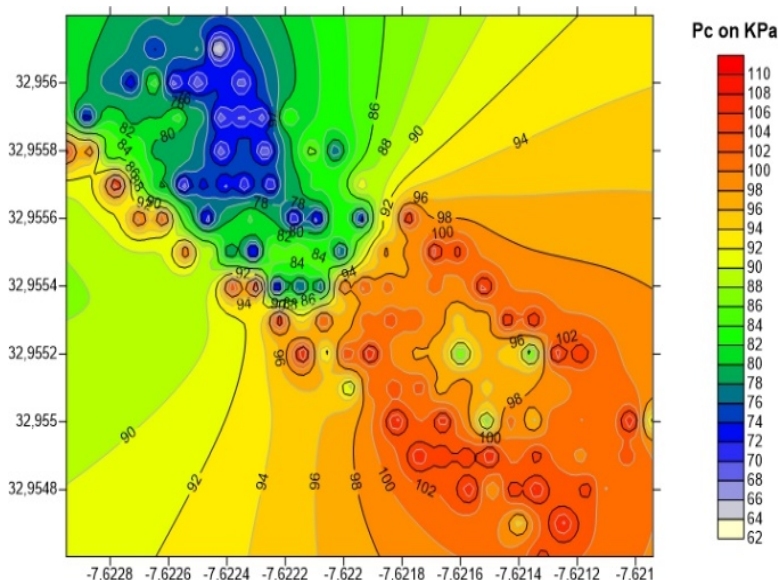

Figure 8. Pc matrix based on pedotransfer data represented by "Distance to Power IDP" method.

Atterberg limits showed that soil water content dynamic has an influence on its sensitivity to compaction within plasticity limits (Fig.7). It is of importance to avoid machines traffic during soil plasticity period to overcome compaction problem. Furthermore, monitoring plasticity to manage timing of introducing machines in susceptible soil to compaction can served as a decision tool based on temporal soil water content dynamic.

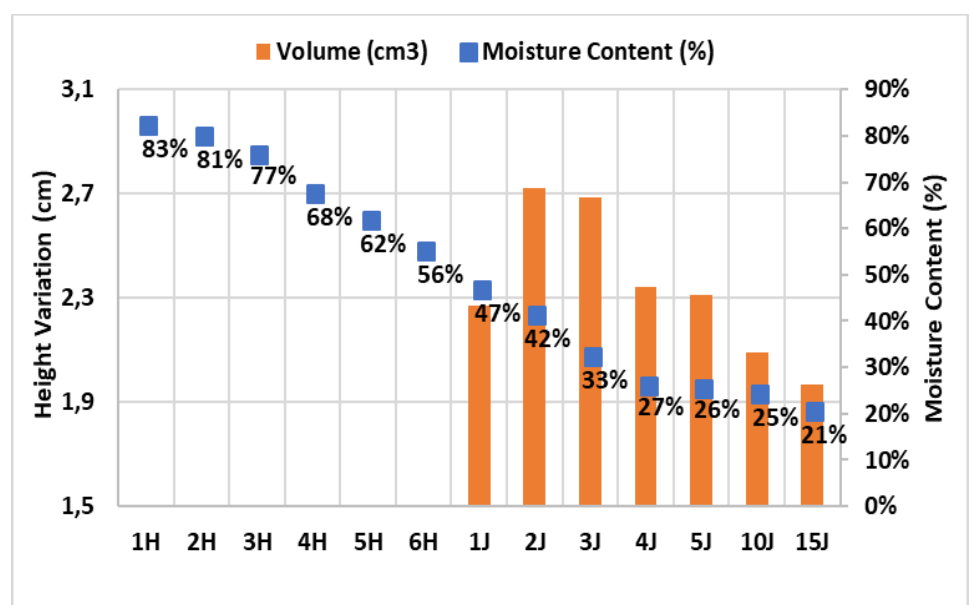

Figure 9. Soil sensitivity to compaction as a function of water content and Atterberg limits.

Results of the effect of plasticity on the samples compacted by the press was done using the cone penetrometer. Resistance to penetration are represented as a function of the penetration depth and moisture content for each sample. Figure 9 showed three ranges of low, high and medium resistance for depths of 0-3, 3-5 and 5-7 cm, respectively. 
According to the response of samples to penetration resistance, two ranges of 0.2-0.5 and 0.5-1 MPa relative to pressing delays from 1 to 15 days. Plasticity state occurs to show different s-shaped curves showing a dynamic of increasing soil penetration resistance as the soil moisture content decrease.

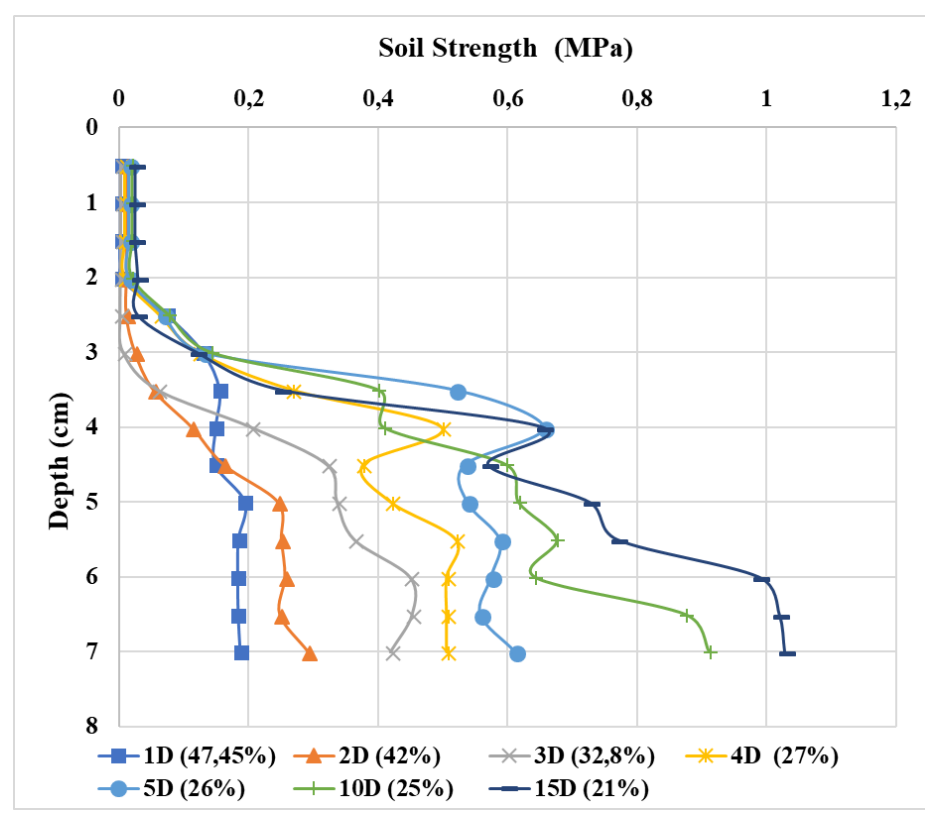

Figure 10. Soil strength response (MPa) vs Depth $(\mathrm{cm})$ and moisture content (during 15 days period).

Precompression stress computation

Pc computation was done to evaluate indirectly susceptibility of soil to compaction (Dimitri et al, 2014). Computation of Pc using physical parameters of the soil matrix showed three distinct classes of compaction (low, medium high) (Fig. 8).

Precompression stress Vs Soil strength correlation (Pedotransfer function)

Precompression stress and soil strength were investigated by a pedotransfer function to evaluate field crop susceptibility to compaction. Figures 10,11,12,13 showed that precompression stress and soil strength are highly correlated $\left(\mathrm{R}^{2}\right.$ around $\left.90 \%\right)$ at the boundaries limits at the middle of the field, this relationship provided means to assess response of soil to compaction using Pc as indirect method as done before by Dimitri et al. (2014) for both field and regional scales. The water content state constitutes a determinant parameter to optimally manage timing for introducing machines in the field crop and avoiding traffic when soil is in plastic state. The Pc pedotransfer function takes into account soil water state represented by field capacity and potential welting points.

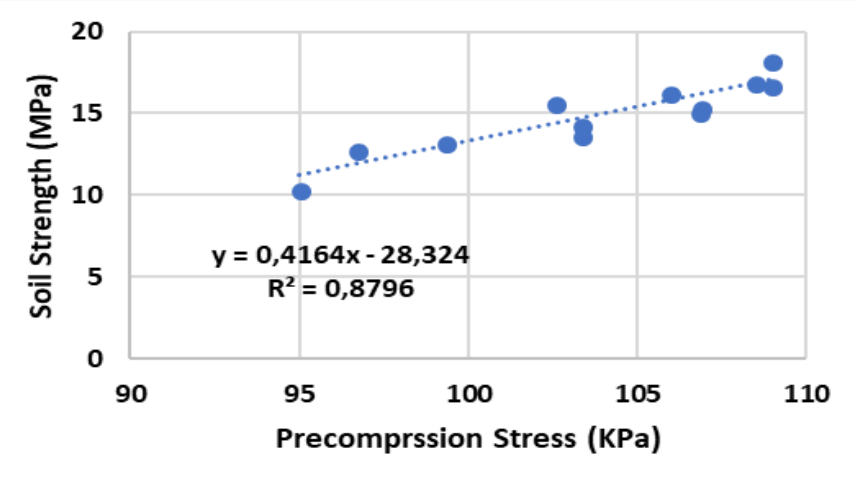

Figure 11. Pc vs soil strength (filed crop center I). 


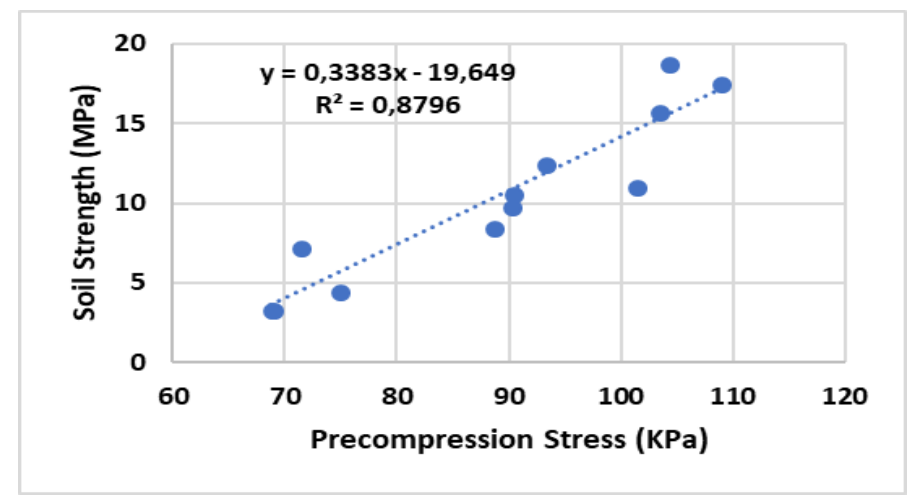

Figure 12: Correlation between Pc and soil strength in the second part (center) of field crop.

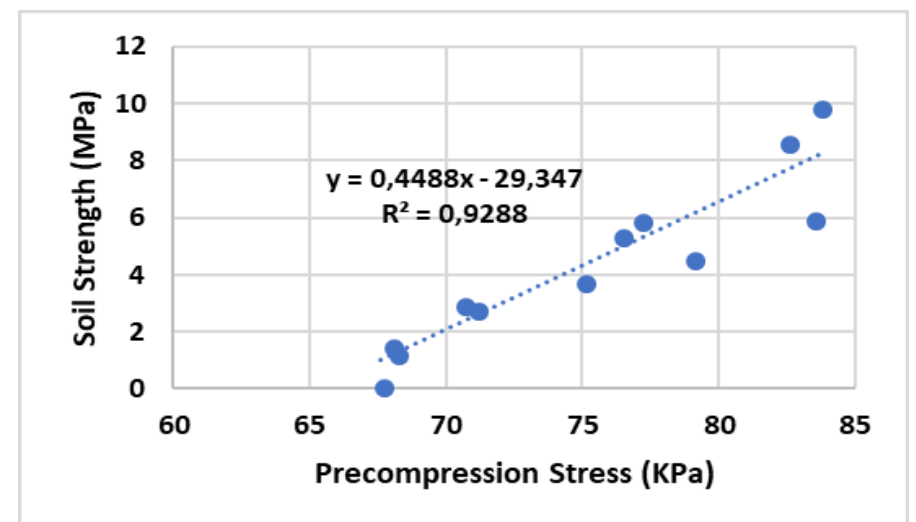

Figure 13: Correlation between Pc and soil strength in the third part (center) of field crop.

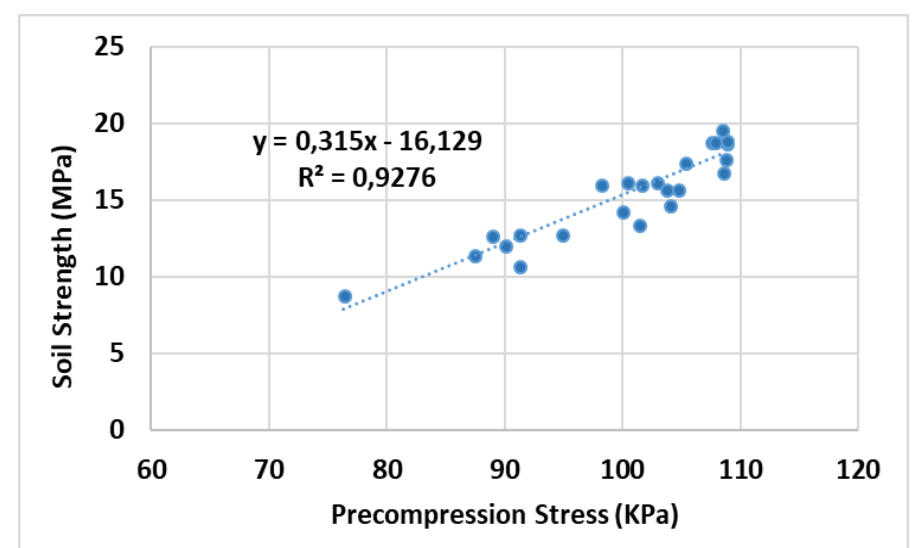

Figure 14: Correlation between Pc and soil strength in the boundaries part of field crop.

\section{Conclusions}

The correlation between several parameters (bulk density, organic matter, total limestone, soil strength and Precompression stress) can detect the factors influencing the intensity of soil compaction. Indeed, the total limestone is one of these parameters which can increase soil susceptibility to compaction. Vertisol sensitivity to compaction is high as its moisture content coincides within the plasticity state according to evaluation of the soil strength test. According to the precompression computation, soil susceptibility to compaction was adequately 
evaluated. The pedotransfer function requires knowledge of texture, organic content, bulk density, cohesion, internal friction angle, moisture content and saturated hydraulic conductivity.

Future Work

The study showed that is possible to assess the response of soil to compaction using Pc as indirect method and soil strength as direct method and it is important to upscale it to a regional level.

\title{
Nomenclature:
}

\author{
BD: Bulk Density \\ OMC: Organic Matter Content \\ TOC: Total Organic Content \\ TL: Total Limestone \\ Pc: Precompression Stress \\ ATL: Atterberg Limits \\ IDP: Inverse Distance to a Power
}

\section{References}

Akker, J.J.H., Canarache, A., 2001. Two European concerted actions on subsoil compaction. Landnutzung und Landentwicklung 42, 15-22.

Alakukku, L., 1996. Persistence of soil compaction due to high axle load traffic I. Short-term effect on the properties of clay and organic soils. Soil Tillage Res. 37, 211-222. https://doi.org/10.1016/01671987(96)01016-1

Alexandrou, A. and Earl, R., 1998. The relationship among the pre-compaction stress, volumetric water content and initial bulk density of soil. Journal of Agricultural Engineering Research, 71: 75-80. https://doi.org/10.1006/jaer.1998.0300

Aliev, K., 2001. Current problems with regard to mechanization and greening of farming in Azerbaijan/Mezhdunarodnyi Sel'skokhozyaistvennyi Zhurnal 5, 57-61.

Ayse Irmak and David R Maidment Geostatistical Analyst

Bondarev, A.G., Kuznetsova, I.V., 1999. The degradation of physical properties of soils in Russia and ways to minimize it. Pochvovedeni 9, 1126-1131.

Canarache, A., Horn, R. and Colibas, I., 2000. Compressibility of soils in a long-term field experiment with intensive deep ripping in Romania. Soil and Tillage Research, 56: 185-196. https://doi.org/10.1016/S01671987(00)00143-4

Carder, J., Grasby, J., 1986. A framework for regional soil conservation treatments in the medium and low rainfall agricultural district. Department of Agriculture,Western Australia, Research Report 1/86, pp. 120.

Carter, M.R., 2002. Soil quality for sustainable land management: organic matter and aggregation interactions that maintain soil functions. Agronomy J. 94, 38-47. https://doi.org/10.2134/agronj2002.3800

Cochrane, H.R., Aylmore, L.A.G., 1994. The effects of plant roots on soil structure. In the 3rd Triennial Conference "Soils 94". pp. 207-212.

Destain, 2013. La compaction des sols de wallonie, departement de sciences et technologies de l'environnement ULG (Gemloux Agro-Bio Tech), 2 passages des des departs 5030 gembloux

Dimitri D'or, and M-F-Destain 2014 Toward a tool aimed to quantify soil compaction risks at a regional scale : application to wallonia (belguim) Ephesia consult, tienne de mont, 10-5140 (Belguim).

Durán Zuazo VH, Rodríguez Pleguezuelo CR, 2008. Soil-erosion and runoff prevention by plant covers. A review. Agron Sustain Dev 28:65-86. https://doi.org/10.1051/agro:2007062

Filipovic, D.; Husnjak, S.; Kosutic, S. and Gospodaric, Z. 2006. Effects of tillage systems on compaction and crop yield of Albic Luvisol in Croatia. J. Terramechanics. 43:177-189. https://doi.org/10.1016/j.jterra.2005.04.002

Gupta, S.C. and Larson, W.E., 1982. Predicting soil mechanical behaviour during tillage, In: Predicting Tillage Effects on Soil Physical Properties and Processes, American Society of Agronomy, pp. 151-178 Special Publication 44, Chapter 10).

Håkansson I, Lipiec J 2000. A review of the usefulness of relative bulk density values in studies of soil structure and compaction. Soil Till Res 53:71-85. https://doi.org/10.1016/S0167-1987(99)00095-1

Hamza, M.A., Anderson, W.K., 2003. Responses of soil properties and grain yields to deep ripping and gypsum application in a compacted loamy sand soil contrasted with a sandy clay loam soil inWestern Australia. Aust. J. Agric. Res. 54, 273-282. https://doi.org/10.1071/AR02102

Hamza, M.A., Anderson,W.K., 2001. Effect of competition between gypsum, potassium, iron and sulphur on lupin. In the 2nd Australia- New Zealand Conference on Environmental Geotechnics. 28-30 November, 2001. Newcastle, Australia, pp. 95-97. 
Herbauts J, El Bayad J, Gruber W, 1996. Influence of logging traffic on the hydromorphic degradation of acid forest soils developed on loessic loam in middle Belgium. Forest Ecol Manag 87:193-207 https://doi.org/10.1016/S0378-1127(96)03826-1

Honsson, I. and Reeder, R. C. 1994. Subsoil compaction by vehicles with high axle load-extent, persistence and crop response. Soil Tillage Res. 29(2-3):277-304.

Horn, R. et al., 2005. SIDASS project Part 5: Prediction of mechanical strength of arable soils and its effects on physical properties at various map scales. Soil and Tillage Research, 82: 47-56. https://doi.org/ https://doi.org/10.1016/j.still.2005.01.007

Horn, R., Fleige, H. 2003. A method for assessing the impact of load on mechanical stability and on physical properties of soils. Soil and Tillage Research 73, 89-99. https://doi.org/10.1016/S0167-1987(03)00102-8

Imhoff, S., Da Saliva, A.P. and Fallow, D., 2004. Susceptibility to compaction, load support capacity and soil compressibility of Hapludox. Soil Sci. Soc. Am. J., 68: 17-24. https://doi.org/10.2136/sssaj2004.0017

Jin Li and Andrew D. Heap 2008 A Review of Spatial Interpolation Methods for Environmental Scientists GeoCat \# 68229

kirchhof G. et H.B.SO 2008. La Compaction du vertisol, université de Queensland departement de l'agriculture brisbane. Qld 4074, Australie

Lebert, M. and Horn, R., 1991. A method to predict the mechanical strength of agricultural soils. Soil and Tillage Research, 19: 275-286. https://doi.org/10.1016/0167-1987(91)90095-F

M.A Hamza and W.K. Anderson, 2005 soil compaction in cropping systems. A review of the nature, causes and possible solutions department of agriculture, westerne Austraia, Dryland Research Institute, merredin, WA 6415, Australia, Soil and tillage 82(2005) 121-145 https://doi.org/10.1016/i.still.2004.08.009

Mason, E.G., Cullen, A.W.J., Rijkse, W.C., 1988. Growth of two Pinus radiata stock types on ripped and ripped/bedded plots at Karioi forest. N. Zeal. J. Forestry Sci. 18, 287-296.

McBride, R.A., 1989. Estimation of density-moisture-stress functions from uniaxial compression of unsaturated, structurated soils. Soil and Tillage Research, 13: 383-397. https://doi.org/10.1016/01671987(89)90045-7

Mosaddeghi, M.R., Hajabbasi, M.A., Hemmat, A., Afyuni, M., 2000. Soil compactibility as affected by soil moisture content and farmyard manure in central Iran. Soil Tillage Res. 55, 87-97. https://doi.org/10.1016/S0167-1987(00)00102-1

Mwendera, E.J., Saleem, M.A.M., 1997. Hydrologic response to cattle grazing in the Ethiopian highlands. Agric. Ecosyst. Environ. 64, 33-41.

Ohtomo, K., Tan, C.C.A., 2001. Direct measurement of soil deformation using the bead-grid method. J. Agric. Eng. Res. 78, 325-332.

Panayiotopoulos, K.P., Papadopoulou, C.P., Hatjiioannidou, A., 1994. Compaction and penetration resistance of an Alfisol and Entisol and their influence on root growth of maize seedlings. Soil Tillage Res. 31, 323-337. https://doi.org/10.1016/0167-1987(94)90039-6

Radford BJ, Yule DF, McGarry D and Playford C (2007). Amelioration of soil compaction can take 5 years on a vertisol under no till in the semi-arid subtropics, Soil and tillage Research 97 249-55. http://dx.doi.org/10.1016/i.still.2006.01.005

Radford, B.J., Bridge, B.J., Davis, R.J., McGarry, D., Pillai, U.P., Rickman, J.F., Walsh, P.A., Yule, D.F., 2000. Changes in the properties of Vertisol and responses of wheat after compaction with harvester traffic. Soil Tillage Res. 54, 155-170. http://doi.org/10.1016/S0167-1987(00)00091-X

Raper, R.L., Reeves, D.W., Burt, E.C., 1998. Using in-row subsoiling to minimize soil compaction caused by traffic. J. Cotton Sci. 2, 130-135.

Rücknagel, J., Hofmann, B., Paul, R., Christen, O. and Hülsbergen, K.-J., 2007. Estimating precompression stress of structured soils on the basis of aggregate density and dry bulk density. Soil and Tillage Research, 92: 213-220. https://doi.org/10.1016/j.still.2006.03.004

Russell, J.R., Betteridge, K., Costall, D.A., Mackay, A.D., 2001. Cattle treading effects on sediment loss and water infiltration. J. Range Manage. 54, 184-190. https://doi.org/10.2458/azu jirm v54i2 russell

Salire, E.V., Hammel, J.E. and Hardcastle, J.H., 1994. Compression of intact subsoils under short-duration loading. Soil and Tillage Research, 31: 235-248. https://doi.org/10.1016/0167-1987(94)90083-3

Sinnett D, Morgan G, Williams M, Hutchings T 2008. Soil penetration resistance and tree root development. Soil Use Manage 24:273-280 https://doi.org/10.1111/j.1475-2743.2008.00164.x

Smith, C.W., Johnston, M.A. and Lorentz, S., 1997. Assessing the compaction susceptibility of South African forestry soils. II, Soil properties affecting compactibility and compressibility. Soil and Tillage Research, 43: 335354. https://doi.org/10.1016/S0167-1987(97)00023-8

$\mathrm{So} \mathrm{Hb}$ and Cull $\mathrm{P}$, 1984. effect on soil compaction on cotton growth in the emerald irrigation area. The Australian cotton grower. May-July 52-57.

Soane B, Van Ouwerkerk C (1995) Implications of soil compaction in crop production for the quality of the environment. Soil Till Res 35:5-22 https://doi.org/10.1016/0167-1987(95)00475-8 
Sparovek, G., Lambais, M.R., Silva, A.P., Tormena, C.A., 1999. Earthworm (Pontoscolex corethrurus) and organic matter effects on the reclamation of an eroded Oxisol. Pedobiologia 43, 698- 704.

Suhayda, C.G., Yin LiJuang, Redmann, R.E., Li Jiandong, 1997. Gypsum amendment improves native grass establishment on saline-alkali soils in Northeast China. Soil Use and Management 13, 43-47

Tardieu, F., 1994. Growth and functioning of roots and of root systems subjected to soil compaction. Towards a system with multiple signalling. Soil Tillage Res. 30, 217-243. https://doi.org/10.1016/01671987(94)90006-X

Taylor, H.M., 1971. Effect of Ss on seedling emergence, root growth and crop yield. Compaction of Agricultural Soils, American Society of Agricultural Engineering, pp. 292-305.

Thomas, G.W., Haszler, G.R., Blevins, R.I., 1996. The effect of organic matter and tillage on maximum compactibility of soils using the proctor test. Soil Sci. 161, 502-508. 


\title{
Impacts of Artificial and Natural soil Conditioners on Water Holding Capacity and Hydraulic Conductivity of Sandy soils
}

\author{
Faisal Zeineldin $^{a, *}$, Khalid Biro ${ }^{\mathrm{b}}$ and Mohammed Refdan Al-Hajhoj ${ }^{\mathrm{c}}$ \\ a a,b, Water Studies Centre, King Faisal University, Al-Hofuf, Al-Hassa 31982, Saudi Arabia \\ c, Faculty of Agricultural Sciences and Food Science, Al-Hofuf, Al-Hassa 31982, Saudi Arabia \\ * fzeineldin@kfu.edu.sa
}

\begin{abstract}
In arid and semi-arid regions like the Kingdom of Saudi Arabia, efficient use of irrigation water requires adoption of appropriate agricultural practices of water conservation. Improving hydraulic properties of sandy soil to be as if that of loamy soils enhances soil available water and minimizes deep percolation. Addition of natural and artificial soil conditioners to sandy soils attested to be effective in rising water holding capacity and reducing saturated hydraulic conductivity. This study was to investigate impacts of addition of super-absorbent polymer (K- crossed-linked polyacrylamide), as an artificial conditioner on hydraulic properties of sandy soil, along with three natural soil conditioners; dry cattle manure, rich clay soil (silt clay loam soil), and date palm biochar. Water holding capacity of amended and un-amended sandy soils evaluated under selected low soil matric tensions (-0.2, $-0.4,-0.6,-0.8$ and -1 bars), while their saturated hydraulic conductivity assessed with TOP-Bench high precise digital apparatus. The results showed all conditioners enhanced the retention ability of sandy soil, but they had different impacts on saturated hydraulic conductivity. Water-holding capacity of amended sandy soils increased by about $379 \%, 200 \%, 74 \%$ and $32 \%$ with respect to effect of addition of $10 \%$ date palm biochar, $0.4 \% \mathrm{~K}-\mathrm{HCP}$ polymer, $10 \%$ silt-clay-loam soil and 10\% cattle manure respectively. On other hand, the date palm biochar, siltclay-loam, and cattle manure decreased saturated hydraulic conductivity of sandy soil by about $74 \%, 59 \%$ and $47 \%$ respectively, while the K-HCP polymer increased it by about $31 \%$. The date palm biochar has the highest potential to improve hydraulic properties of sandy soil; therefore, it has the potential to save irrigation water and reduce deep percolation water losses.
\end{abstract}

Keywords: Sandy soil, K- highly crossed-linked polyacrylamide polymer, dry cattle manure, biochar and richclay soil

\section{Introduction}

Sandy soils found in most of the agricultural regions of the Kingdom of Saudi Arabia. A sandy soil consist of $70-90 \%$ sand, $0-30 \%$ silt, and 0-15\% clay, and characterized by low water holding capacity (WHC) due to small specific surface of the sand particles (7 and 9) and characterized by high saturated hydraulic conductivity $\left(K_{S}\right)$. Sandy soils reach saturation point in shorter time as compared to clay soils. The higher percentage of silt and clay sized particles in a soil, the higher $W H C$ and lower $K_{\text {s. }}$. Water-holding capacity of a soil is limiting factor to availability of soil water and nutrients to plants (4). Texture of sandy soils varies from very fine to course, depending on soil particles distribution and accordingly the $W H C$ varies from high to low while the $K_{s}$ from low to high. Agricultural land of Al-Hassa oasis in eastern region of the Kingdom covered mostly by sandy soils, which enhanced formation of two vast evaporation lakes (Aleyoon and Alsfer) down its slope to the east towards the Arabian gulf. The combined effects of low $W H C$ and high $K_{\mathrm{s}}$ of sandy soils, along with over irrigation lead to loss of irrigation water as deep percolation water below the root zone (3). In fact, the presence of the two lakes, under such hot arid region, for decades until now requires continuous huge supply of water to meet the evaporation losses. Therefore, the oasis' agricultural drainage water, which keep flowing throughout the year via drainage canals (D1 and D2) into the lakes, assumed as one of the main sources to maintain the water of the lakes. Although recently more modern irrigation systems implemented on the oasis farms to reduce deep percolation water, but still significant amount of the irrigation water, i.e. $50 \%$, drains into the two lakes.

Biochar is a carbon-rich natural soil conditioner, which produced from thermal decomposition of different organic material at temporaries less than $700^{\circ} \mathrm{C}$ in the absence of the oxygen $(6,7$, and 9). The positive potential impacts of the biochar soil conditioner well known, which included carbon sequestration, improved crop yield and enhanced water retention. Loamy sand's water holding capacity increased by $233 \%$ and $133 \%$ when mixed by rate of $10 \%$ switchblade grass biochar and hemlock biochar respectively (12). Water holding capacity of sandy loam sand, $16 \%$, doubled when amended by $9 \%$ mass of woody biochar, yellow pine from pyrolysis at $400{ }^{\circ} \mathrm{C}$ (13).

Application of cattle manure to sandy soils has potentials benefits such as improving soil organic carbon, increasing soil water retention and soil nutrient availability and reducing compatibility. Previous studies showed that manure application increased soil retention and plant available water $(5,14$ and 15). In Nebraska research station, a study indicated amended cattle manured fine sandy loam soil retained $18 \%$ more soil water at -0.33 bars and $20 \%$ more at -15 bars potential, which in turn resulted into $16 \%$ more plant available water compared to 
unlamented fine sandy loam soil (5). Besides that, attraction of a soil for water increases with the increase of the organic matter content.

Super-absorbent hydrogel polymers are highly cross-linked polyacrylamide polymers, made up of waterinsoluble acrylamide and potassium acrylate, [-CH2- $\mathrm{CH}(\mathrm{COOK})-] \mathrm{n}$. They used in agriculture to enhance waterholding capacity of sandy soils. Leaching polymer amended sandy soil with tab water, initially caused decrease in $K_{\mathrm{s}}$ of the soil followed by steady increase; however, leaching with distilled water cause decrease of the $K_{\mathrm{s}}(3)$. Number of studies indicated superabsorbent polymers are capable of improving the physic-chemical properties of sandy soils $(1,2,10$, and 11). Superabsorbent polymer found to enhance absorbing and retaining abilities of sandy soils and enabling gradual release of soil water and nutrients to plants uptake. Application 4 gram of two superabsorbent polymers, Super AB A200 and Herbosorb, to a kilogram of sandy soil increased soil available water by $350 \%$ and $320 \%$ respectively (4). Highly crossed-linked polyacrylamide polymer (Stockosorb $₫$ K 400) when added to a sandy soil at varying concentrations $(0.04 \%, 0.08 \%, 0.12 \%, 0.20 \%$ and $0.4 \%$ weight by weight), the highest concentration, $0.4 \%$, changed the water holding capacity from typical sand to loam or silty clay (6). The objective of the study is to evaluate impacts of three natural conditioners (dry cattle manure, rich-clay soil, and biochar) and an artificial conditioner (K- Highly Crossed-linked Polyacrylamide Polymer, K-HCPP) on water holding capacity and saturated hydraulic conductivity of sandy soils.

\section{Materials and Methods}

Soils and natural soil conditioners

Soils (sandy and clay) and dry cattle manure collected from the surface of $15 \mathrm{~cm}$ fields on King Faisal Agricultural and Veterinary Research, Al-Hassa, KSA. They were air dried, grounded and sieved by $2 \mathrm{~mm}$ mesh screen, at the Water Study Center lab. Biochar made from date palm leaves by a small-scale pyrolysis system at the Center of Excellence for Dates palm. Then crushed by hand and sieved to collect particles sized $<2 \mathrm{~mm}$, as recommended for agricultural application. A soil mechanical analysis method used to determine soil particles distribution of the sandy soil as shown in Table 1. Based on the USDA classification, the sandy soil was classified as medium sand soil (52\% medium sand), which served as control in laboratory experimental studies. Obtained clay soil was texturally classified as silt clay loam soil ( $49.2 \%$ silt, $37.3 \%$ clay, $13.5 \%$ sand), which served as a natural conditioner beside the dry cattle manure and the biochar that made from leaves of date palm trees (Khalas Cultivar).

Table 1. Sandy soil particles distribution.

\begin{tabular}{l|ccc}
\hline Soil Separate & $\begin{array}{c}\text { Diameter ranger } \\
\text { (millimeters) }\end{array}$ & $\begin{array}{c}\text { Sandy soil particles } \\
\text { percent distribution }\end{array}$ & $\begin{array}{c}\text { Cumulative } \\
\text { percent }\end{array}$ \\
\hline Gravel & 2.0 and larger & 0.00 & 0.00 \\
Very Coarse Sand & $2.0-1.0$ & 1.25 & 1.25 \\
Coarse Sand & $1.0-0.5$ & 18.66 & 19.91 \\
Medium Sand & $0.5-0.25$ & 52.03 & 71.94 \\
Fine Sand & $0.25-0.1$ & 21.21 & 93.15 \\
Very Fine sand & $0.1-0.05$ & 6.10 & 99.25 \\
Fines & $<0.05$ & 0.75 & 100 \\
\hline
\end{tabular}

\section{K-HCP-Polymer}

Pre-hydrated hydrogel form of the K-HCP polymer was prepared using 12.5 grams dry powder of the polymer (Stockosorb® 500 micro) and 1-liter of tab water $(1.3 \mathrm{dS} / \mathrm{m})$. Initially, the powder of the K-HCPP left into the water to absorb water and to swell for almost 30 minutes, and then stirred several times until no more absorption or swelling take place. Retention and release abilities of the hydrogel of K-HCPP to water examined against selected soil matric tensions $(-0.1,-0.3,-0.5,-0.7,-0.9,-1,-3,-5,-10$, and -15 bars), as shown in Table 2 , using pressure plate apparatus. Besides measuring the electrical conductivity $(\mathrm{ECw})$ of released water from the hydrogel. Moreover, the dehydration and rehydration abilities of the K-HCPP investigated under heat of the sun in the summer. A dish filled with pre-hydrated K-HCPP placed outdoor under sun heat for period of thirty days. As shown in Figure 1, about $90 \%$ of its water weight evaporated, and when rehydrated again, its rehydration ability observed to be much slower as compared to the initial hydration ability, i.e. approximately about $60 \%$. 
Table 2. Retention and release abilities of K-HCPP

\begin{tabular}{cccc}
\hline Soil matric Suction & Released Water & Retained Water & $E C_{w}$ of released Water \\
\hline bars & $\%$ & $\%$ & $\mathrm{mS} / \mathrm{cm}$ \\
-0.1 & 28.4 & 71.6 & 4.2 \\
-0.3 & 37.4 & 62.6 & 4.1 \\
-0.5 & 46.0 & 54.0 & 4.3 \\
-0.7 & 51.0 & 49.0 & 4.2 \\
-0.9 & 52.2 & 47.8 & 3.8 \\
-1 & 55.4 & 44.6 & 3.7 \\
-3 & 68.4 & 31.6 & 4.9 \\
-5 & 71.0 & 29.0 & 3.7 \\
-10 & 76.1 & 23.9 & 3.2 \\
-15 & 82.9 & 17.1 & 4.4 \\
\hline
\end{tabular}

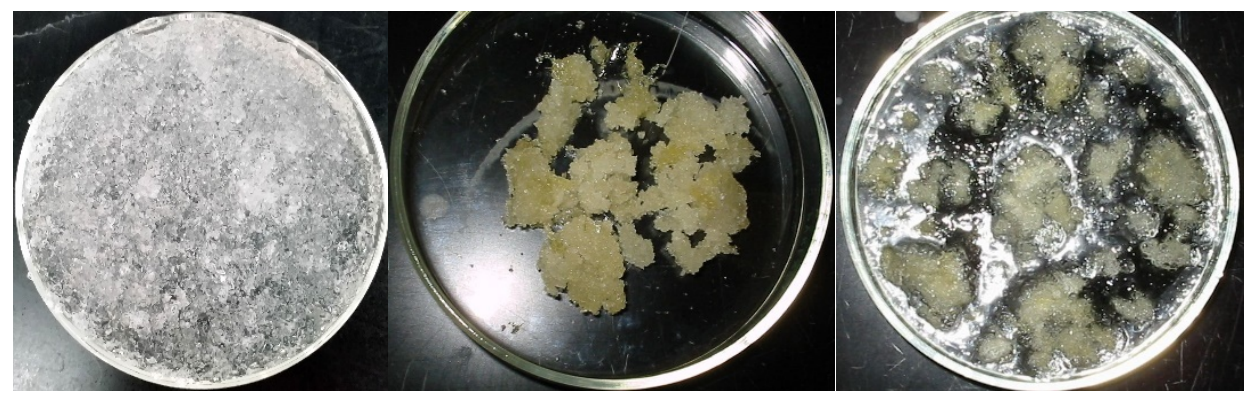

Hydrated Polymer Dehydration ability (90\%) Rehydration ability (60\%)

Figure 1. Dehydration and rehydration of the K-HCP polymer.

\section{Amendment of sandy soil}

On mass basis, sandy soil amended separately by addition of $10 \%$ dry cattle manure, $10 \%$ silt-clay-loam soil, $0.4 \% \mathrm{~K}-\mathrm{HCPP}$, and by mixture of the three conditioners together. In addition, the sandy soil amended by addition of $5 \%$ biochar and $10 \%$ biochar of date palm leaves. Then water-holding capacity, at -0.33 bar, and wilting point, at -15 bar, and the total available water (TAW) of these amended sandy soils determined with a pressure plate apparatus in the laboratory. The formula of American Society for Testing and Materials (8) used to determine the WHC on mass basis, was written as follows:

$$
W H C=\frac{\text { mass }_{\text {Wet }}-\text { mass }_{\text {dry }}}{\text { mass }_{\text {dry }}} \times 100 \%
$$

In addition to that, soil moisture content of the sandy soil and the amended sandy soils evaluated against selected low tensions of soil matric $(-0.0,-0.2,-0.4,-0.6,-0.8$, and -1 bars). On the other hand, impacts of the additions of the soil conditioners on saturated hydraulic conductivity of sandy soils measured with Bench-Top $K_{s}$ smart device, which connected to a computer via USB to manage running of the measurement of $K_{s}$ with help of interactive program, see Figure 2. In fact, the device measures the $K_{s}$ based on principle of Darcy's law. Initially, soil packed into device's sampler $\left(250 \mathrm{~cm}^{3}\right)$ by a process of maneuvering and tapping up to a specific density based on soil type. Since no volume changes occur upon saturation of sandy soil and silty clay loam soil amended sandy soil samples, their density set to be $1.56 \mathrm{~g} / \mathrm{cm}^{3}\left(390 \mathrm{~g} / 250 \mathrm{~cm}^{3}\right)$, typical to soil's density of the cultivated areas in the Kingdom. However, densities for the polymer amended sandy soil, manure amended sandy soil and conditioners mixture (silty clay loam soil + K-HCPP and dry cattle manure) amended sandy soil were 1.27, 1.33 and $1.2 \mathrm{~g} / \mathrm{cm}^{3}$ respectively. This is because their volume changed upon saturation. Initially all soil samples were saturated overnight from bottom to top with degassed tap water $(1.3 \mathrm{dS} / \mathrm{m})$.

The pressure sensor of the device is to detect the hydraulic gradient, the driving force for volumetric water flux density, $Q$, which passes through the soil sample column. Falling head option, resumes from $5 \mathrm{~cm}$ head, was chosen for all the $K_{\mathrm{s}}$ measurements of the experiment. The coefficient of the saturated hydraulic conductivity, $K_{s}$, that is shown in equation 3 derived from Darcy's relations of equation 2. Equation 3 is embedded into the interactive program in the computer to estimate the $K_{s}$, where the water flux density, $Q$, multiplied by the soil sample, $L$, divided by soil sample area $A$ and time t, and hydraulic difference $\Delta H$ along the flow direction.

$$
q=\frac{Q}{A t}=K_{s} \frac{\Delta H}{L}
$$




$$
K_{S}=\frac{Q L}{A t \Delta H}
$$

Where $\frac{Q}{A t}=$ water flux density and $\frac{\Delta H}{L}=$ hydraulic gradient

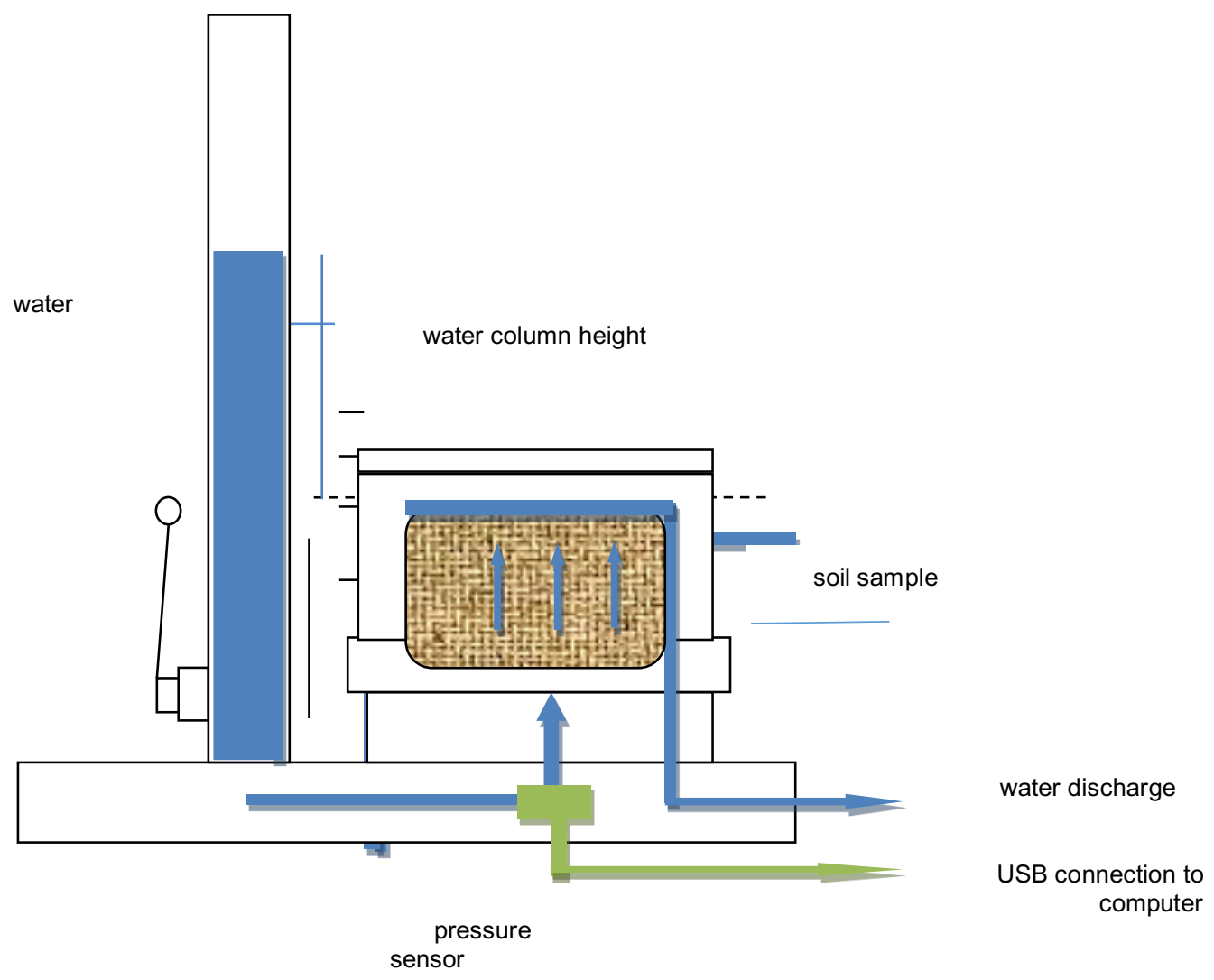

Figure 2. Bench-Top $K_{s}$ smart device.

\section{Results and Discussion}

\section{K-HCP polymer}

The K-HCP is superabsorbent hydro-gel polymer and it is an insoluble organic compound. According to laboratory assessment, the K-HCP polymer is capable of swelling and absorbing about $80 \%$ of its dry weight. Obtained data from laboratory experiment as shown previously in Table2, indicated the K-HCP polymer is capable of releasing up to about $83 \%$ of its absorbed water at wilting point tension. The average electrical conductivity of the released water during the range of tensions ( -0.1 to -15 bars) was $4.1 \mathrm{mS} / \mathrm{cm}$, which can be diluted when be mixed with sandy soil. The percentage retention of the $\mathrm{K}-\mathrm{HCP}$ polymer at field capacity, $\mathrm{FC}=-0.33$ bars, and wilting point, $\mathrm{WP}=-15$ bars, the $\mathrm{K}-\mathrm{HCPP}$ were found $62.6 \%$ and $17.1 \%$ respectively. This indicates the $\mathrm{K}-\mathrm{HCP}$ polymer has the potential to enhance irrigation water conservation within vicinity of the root zone and at same time decrease amount deep percolation losses by gravitational forces. Therefore, the results point out that K-HCP polymer has high TAW (FC-WP), about $45.5 \%$, which is nine folds of the TAW of sandy soil.

Impacts on WHC of amended sandy soil

Influences of the K-HCP polymer and soil the natural conditioners on water holding capacity of sandy soil presented in Table 2 and Figure 3. Low soil matric suctions $(-0.1,-0.2,-0.3,-0.4,-0.5,-0.6,-0.7,-0.8,-0.9$ and 1 bars) were investigated because most of the TAW of sandy soils is readily available for plant uptake within a range between 0.0 to- 1 bars, beside it is importance for plant irrigation scheduling. As shown in Figure 3 , the results imply addition of the K-HCP-Polymer has a potential to increase water-holding capacity of sandy soil by 2 to 3 folds, while the natural conditioners (silt-clay-loam soil and the manure) by $1 / 2$ to 1 folds. However, below -0.4 bar suction; the mixture of all three conditioners (polymer + silt-clay-loam soil + dry-cattle-manure) has higher impacts on water holding capacity of sandy soil than the K-HCP polymer. 


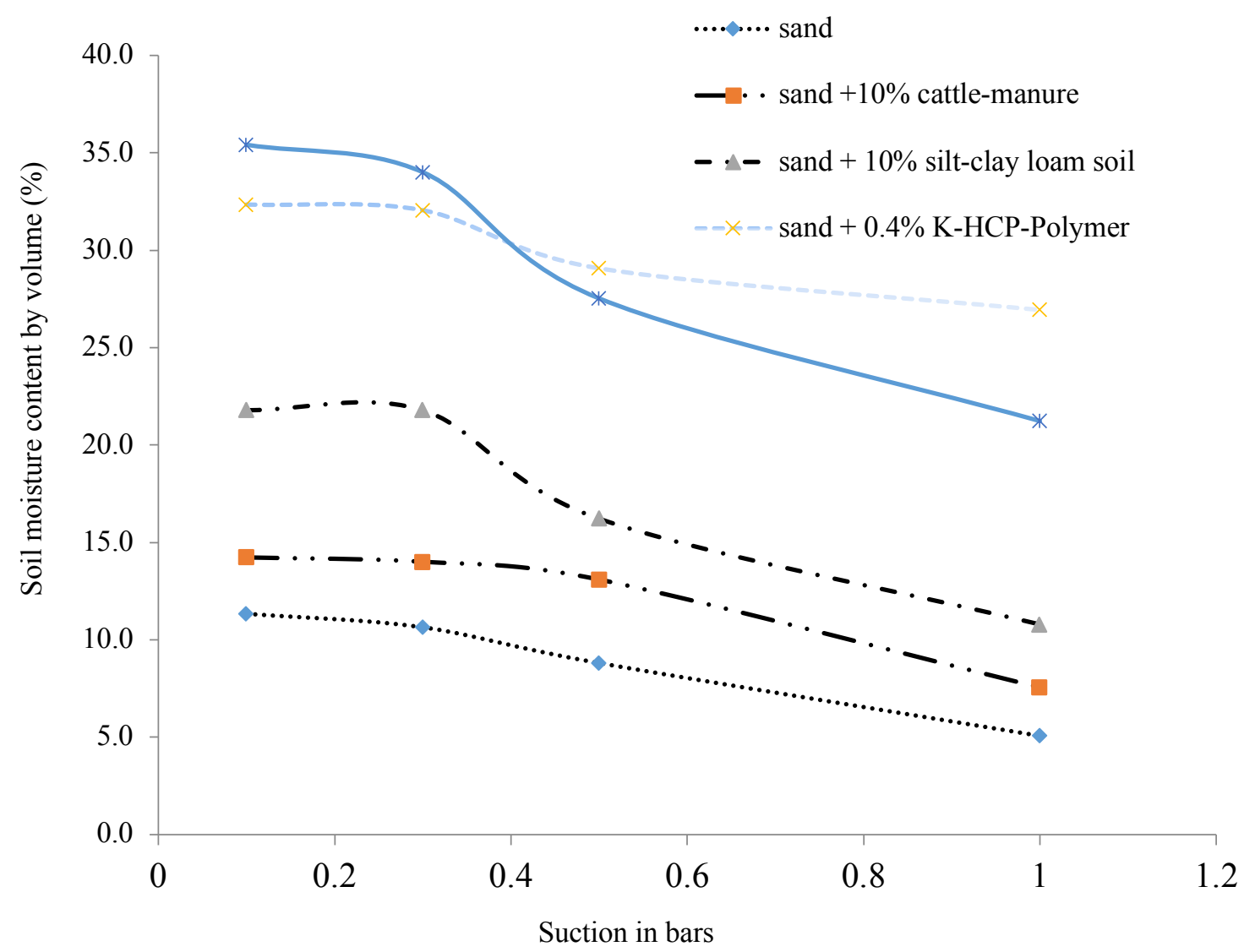

Figure 3. Water holding capacity of amended sandy soil.

As shown in Table 3, the investigations revealed that the $W H C$ of sandy soil increased by $379 \%, 200 \%, 74 \%$ and $32 \%$ due to addition of $10 \%$ date palm biochar, $0.4 \% \mathrm{~K}-\mathrm{HCP}$ polymer, $10 \%$ silt-clay-loam soil, and $10 \%$ cattle manure, on mass basis respectively.

Table 3. Percent change in water holding capacity of amended sandy soils.

\begin{tabular}{lccc}
\hline Soil & $\begin{array}{c}\text { WHC means } \\
\left(\mathrm{w}_{\text {wet }} / \mathrm{w}_{\mathrm{dry}} * 100\right)\end{array}$ & STDEV & $\begin{array}{c}\text { Impact on } W H C \text { as } \\
\text { percent change }\end{array}$ \\
\hline Sand (control) & 6.82 & \pm 0.54 & 0 \\
Sand + 0.4\% K-HCP-Polymer & 20.6 & \pm 2.96 & +200.1 \\
Sand + 10\% cattle-manure & 8.98 & \pm 0.65 & +31.6 \\
Sand + 10\% silt-clay loam soil & 11.86 & \pm 1.2 & +73.9 \\
Sand+0.4 K-HCP-Polymer + 10\% cattle- & 21.8 & \pm 4.42 & +219.5 \\
$\quad$ manure +10\% silt-clay loam soil & & & +379 \\
Sand +10\% biochar & 32.7 & \pm 2.1 & +114.1 \\
Sand +5\% biochar & 14.6 & \pm 1.9 & \\
\hline
\end{tabular}

Impact on $\mathrm{K}_{\mathrm{s}}$ of amended sandy soil

Impact of the hydrogel K-HCP polymer and the soil natural conditioners on $K_{s}$ of sandy soils, revealed in Table 4. Addition of $0.4 \% \mathrm{~K}-\mathrm{HCP}$ polymer, on mass basis, increased saturated hydraulic conductivity of sandy soil in range of $27-36 \%$, with average of $30.5 \%$ increase as negative impact. While addition of $10 \%$ date palm biochar, $10 \%$ rich clay soil (silt clay loam), and $10 \%$ cattle to sandy soil, on mass basis, reduced the $K_{s}$ by more than $74 \%$, $59 \%, 47 \%$ respectively as positive impact. The impacts of the addition of mixture of $0.4 \% \mathrm{~K}-\mathrm{HCP}-\mathrm{Polymer}$ with $10 \%$ cattle-manure and $10 \%$ silt-clay loam soil on $K_{\mathrm{s}}$ of the amended sandy soil dominated by the effect of the polymer, which increased it by $26 \%$ as compared to the control. Although the silt-clay loam soil and the cattle manure had postive impacts on $K_{\mathrm{s}}$, but the negative impact of the polymer dominated in the mixture impact. Addition of mixture of artificial and natural conditioners (K-HCP polymer, silt-clay-loam soil and cattle manure) to sandy soil enhanced water-holding capacity and increased $K_{\mathrm{s}}$ relatively less than the impact of K-HCP polymer alone, 25.9\%. as shown in Table 4. In Figure 4, on site application of the mixture of the conditioners to sandy soil showed good growth of grasses as compared to growth of grasses in bare sandy soil. This indicated the amended 
sandy soil with this mixture of soil conditioners provided enough soil water and organic nutrient fertilizers for the grass growth.

Table 4. Percent change of saturated hydraulic conductivity $\left(K_{\mathrm{s}}\right)$.

\begin{tabular}{lccc}
\hline Soil & $\begin{array}{c}K_{s} \text { average } \\
\mu \mathrm{m} / \mathrm{s}\end{array}$ & STDEV & $\begin{array}{c}\text { Impact on } K_{s} \text { as } \\
\text { percent change }\end{array}$ \\
\hline Sand (control) & 98.1 & \pm 6.7 & 0 \\
Sand + 0.4\% K-HCP-Polymer & 128 & \pm 4.3 & +30.5 \\
Sand + 10\% cattle-manure & 52.4 & 3 & -46.6 \\
Sand + 10\% silt-clay loam soil & 40.7 & \pm 2.1 & -58.5 \\
Sand + 0.4\% K-HCP-Polymer + 10\% & 123.5 & \pm 9.8 & +25.9 \\
$\quad$ cattle-manure + 10\% silt-clay loam soil & & & -73.9 \\
Sand +10\% biochar & 25.6 & \pm 10.1 & -41.08 \\
Sand +5\% biochar & 57.8 & \pm 4 & \\
\hline
\end{tabular}

Figure 4. Impact of the mixture of soil conditioners ( $0.4 \%$ HCPP, $10 \%$ cattle manure and $10 \%$ silty clay loam soil) of grasses grown on sandy soil.
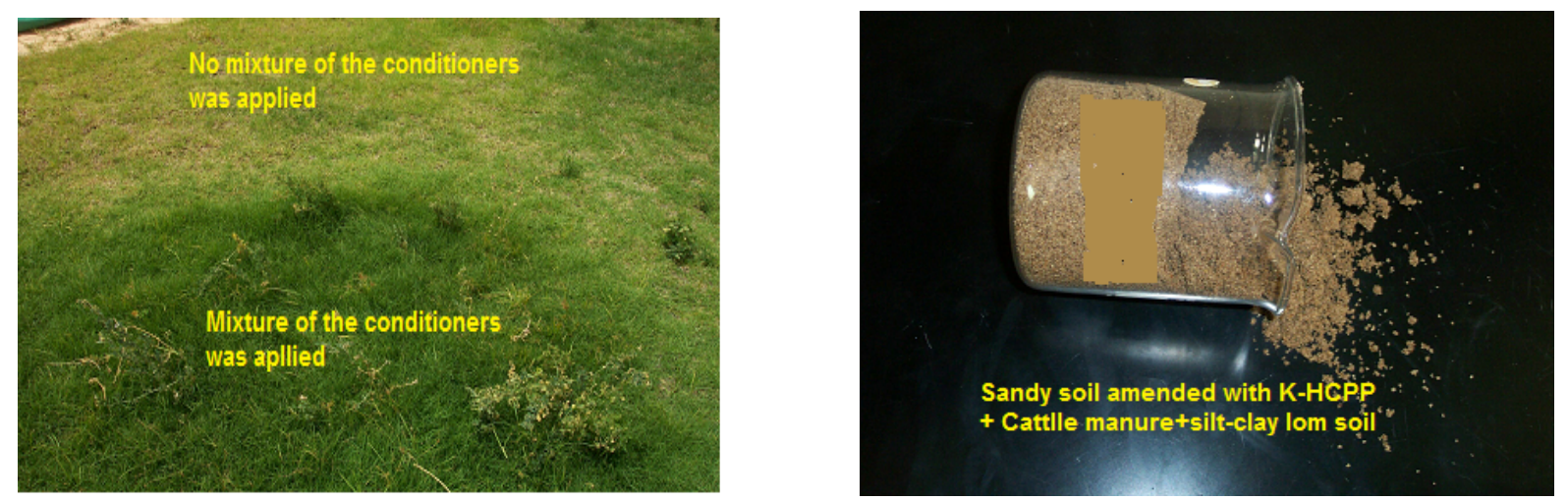

Impact of date palm biochar on $\mathrm{pH}$ and $\mathrm{EC}_{\mathrm{e}}$

Although impacts of the addition of $10 \%$ date palm biochar on water holding capacity and $K_{\mathrm{s}}$ of sandy soils were interesting and promising, but the electrical conductivity $\left(\mathrm{EC}_{\mathrm{e}}\right)$ and $\mathrm{pH}$ of the soil water extract of the amended sandy soil were relatively high, i.e. $14 \mathrm{mS} / \mathrm{cm}$ and 9.9 respectively. The soil water extract obtained using standard saturation paste method for obtaining soil water extract. As matter of fact, the ideal soil water condition for growing crops that the electrical conductivity $\left(\mathrm{EC}_{\mathrm{e}}\right)$ of the soil water extract should not exceed $4 \mathrm{mS} / \mathrm{cm}$ and its $\mathrm{pH}$ should be within 7.0. Although, low rates of mixing of date palm biochar, i.e. $5 \%$ and $2 \%$, with sandy soil were tested but still the outcome of the $\mathrm{EC}_{\mathrm{e}}$ and the $\mathrm{pH}$ were relatively high, so further investigations are needed. 


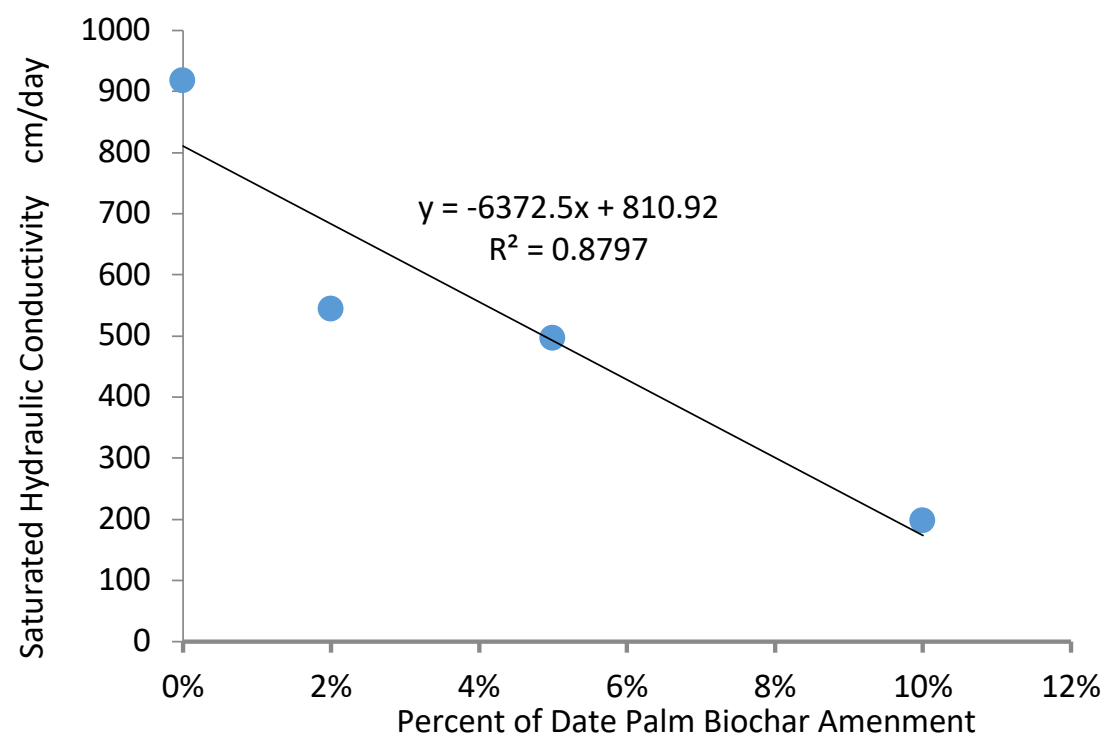

Figure 5. Saturated hydraulic conductivity of the amended sandy soil at different mixture rates (0\%-10\%).

\section{Conclusions}

All of the natural soil conditioners (date palm biochar, silt-clay-loam soil and cattle manure) and the artificial soil conditioner (K-HCP polymer improved water-holding capacity of sandy soils. The impacts of the soil conditioners (natural and artificial) on saturated hydraulic conductivity of sandy soils vary from positive to negative. The date palm biochar found to have the highest potential in enhancing water-holding capacity and reducing statured hydraulic conductivity of sandy soil. However, it high rates of addition (5\%-10\%) resulted into high $\mathrm{EC}_{\mathrm{e}}$ and $\mathrm{pH}$ values, which affect suitability of the amended sandy soil for crop growth. Despite, the K-HCP polymer, its amendment impact came second to date palm biochar in improving water-holding capacity of sandy soil, but it has the highest negative impact by increasing saturated hydraulic conductivity of amended sandy soil. Amended sandy soil by mixture of artificial and natural conditioners (K-HCP polymer, silt-clay-loam soil and cattle manure) enhanced water-holding capacity and suitability for grass growth.

\section{References}

Abd El-Rehim H.A., El-Sayed A. Hegazy, H. L. Abd El-Mohdy. 2004. Radiation Synthesis of Hydrogels to Enhance Sandy, Soils Water Retention and Increase Plant Performance. Journal of applied Polymer science. Voulme 93, issue 3.

Abd El-Rehim H.A., El-Sayed A. Hegazy, H. L. Abd El-Mohdy. 2006. Effect of various environmental conditions on the swelling property of PAAm/PAAcK superabsorbent hydrogel prepared by ionizing radiation. Journal of applied Polymer science. Voulme 101, issue 6.

Bhardway, A.K; Shainberg, I; Goldstein, D; Warrington, D N; Levy G J. 2007. Water Retention and Hydraulic Conductivity of Cross-linked Polyacrylamides in Sandy Soils. Soil Science Society of American Journal, 71,2; Proquest Pg.406

Heyder A.K., Narges Z. 2013, The Effect of Superabsorbent Polymers on the Water Holding Capacity and Water Potential of Karkhe Noor Sandy soils. International Journal of Scientific Research in Knowledge (IJSRK), 1(9).pp.317-324.

Humberto Blanco-Canqui, Gary W, Hergert and Rex A. Neilsen. 2015. Cattle manure application reduces compatibility and increases water retention. Soil Sci. Soc. Am. J. 79:212-223

Huttermann A., M. Zommorodi and K. Reise. 1999. Addition of hydrogels to soil for prolonging the survival of Pinus halepensis seedlings subjected to drought. Soil and Tillage Research. Volume 50, Issues 3-4. 295-304.

Lehmann, J., \& Joseph, S. (2009). Biochar for environmental management: science and technology, London, GB: Earthscan.

Maraseni, T.N. 2010. Maximizing the benefits, International Journal of Environmental Studies, 67, 319-327. 
Potter, S.R., Andrews, S., Atwood, J.D., Kellogg, R.L., Lemunyon, J., Norfleet, L., \& Oman, D. (2006). Model Simulation of Soil Loss, Nutrient Loss, and Change in Soil Organic Carbon Associated with Crop Production. U.S. Department of Agriculture, Natural Resources Conservation Service, Conservation Effects Assessment Project Report

Silberbush M., E. Adar and Y. De Malach. 1993. Use of an hydrophilic polymer to improve water storage and availability to crops grown in sand dunes II. Cabbage irrigated by sprinkling with different salinities. Agricultural water management. Vol.23, Issue 4. 315-327.

Sivapalan S. 2001. Effect of polymer on soil water holding capacity and plant water use efficiency. Proceedings of the 10 th Australian Agronomy Conference, Australian Society of Agronomy

Yu, O.Y., M. Harper, M. Hoepfl, and D. Domemuth. 2017. Characterization of Boichar and Its Effects on Water Holding Capacity of Loamy Sand Soil: Comparison Of Hemlock Biochar and Switchblade Grass Biochar Characteristics. Environmental Progress and Sustainable Energy, Vol.36, No.5, pp 1474-1479.

Yu O-K-Youn, Brian Raichled and Sam Sink. 2013. Impact of biochar on water holding capacity of loamy sand soil. International Journal of Energy and Environmental Engineering. http://www.jounal.ijeee.com/content/4/1/44

Walia, M.K., S.S. Walia, and S.S. Dhaliwal. 2010. Long-term effect of integrated nutrient management of properties of Typic Ustochrept after 23 cycles of an irrigated rice (Oryza sativa L.)-wheat (Triticum aestivum L.) system. J. Sustain. Agric. 34:724-743. doi:10.1080/10440046.2010.507519

Zhang, S., X. Yang, M. Wiss, H. Grip, and L. Lovdahl. 2006. Changes in physical properties of a loess soil in China following two long-term fertilization regimes. Geoderma 136:579-587. doi:10.1016/j.geoderma.2006.04.015 


\title{
Alternative Underdrain Designs for Reducing Pressure Drop across Sand Media filters
}

\author{
T. Pujol ${ }^{a}$, G. Arbat ${ }^{b^{*}}$, J. Bovéb ${ }^{b}$ J. Puig-Bargués ${ }^{b}$, M. Duran-Ros ${ }^{b}$, J. Velayos ${ }^{a}$, F. Ramírez de \\ Cartagena $^{b}$ \\ ${ }^{a}$ Department of Mechanical Engineering and Industrial Construction, University of Girona, C. de Maria Aurèlia \\ Capmany, 61, 17003 Girona, Catalonia, Spain \\ ${ }^{\mathrm{b}}$ Department of Chemical and Agricultural Engineering and Technology, University of Girona, C. de Maria Aurèlia \\ Capmany, 61, 17003 Girona, Catalonia, Spain \\ * Corresponding author. Email: gerard.arbat@udg.edu
}

\begin{abstract}
Reduction of pressure drop across sand media filters - which are broadly used for preventing emitter clogging - should help reducing energy consumption in microrrigation systems. Previous research has shown that most of the pressure drop is located at sand filter underdrain. In this work, the effect of the nozzle geometry on the pressure drop of a sand filter was experimentally studied. Four nozzles were analysed: one commercially produced with a conical shape and three alternative cylindrical underdrains that were built for the experiment and that differed in the location and the number of slots. Experiments in both filtration mode and backwashing conditions for a wide range of superficial velocities were carried out at the laboratory. The results reported that a reduction of the filter energy consumption greater than $20 \%$ could be achieved by simply modifying the position of the slots above the surface of the underdrain element. The effects of the nozzle were further investigated by means of an analytical model that predicted the pressure drop of the water flow through the filter. The model confirmed that the distribution of the slots in the underdrain was a critical factor for determining the length of the region with a nonuniform flow within the sand. When using the commercial nozzle at flow rates $>0.85 \mathrm{l} / \mathrm{s}$, this region produced the major contribution to energy losses in the filter due to increases in the tortuosity of the water path within the porous medium. From these results, it is suggested that an affordable way to increase the energy efficiency of already existing installations would be to replace the current underdrain elements with new improved designs that have $40 \%$ more slots in its cylindrical surface.
\end{abstract}

Keywords: Drip irrigation, nozzle, analytical model, pressure drop, energy consumption.

\section{Introduction}

Microirrigation systems allow for highly efficient water use. They are also the most suitable irrigation systems for applying reclaimed wastewater for irrigation (Capra and Scicolone, 2007). Both reasons explain why microirrigation is extensively used in many countries such as India, China, Spain and USA, with microirrigated areas in each of these countries over 1.5 Mha (ICID, 2011). In the case of Spain, microirrigation covers up to the $49 \%$ of the irrigated land (MAGRAMA, 2015) and Israel, the country with the highest percentage of microirrigated surface area, this system accounts $73 \%$ of the total irrigated surface area.

In general, the adoption of more efficient irrigation systems, as microirrigation, has led to an increase in the use of energy (Tarjuelo et al., 2015). The modernisation of the irrigation systems in Spain has reduced the consumption of water per hectare by $21 \%$ between 1950 and 2007, but the corresponding energy demand has increased by $657 \%$ (Corominas, 2010). In fact, Spain, with values above $774 \mathrm{GWh}$, is the Mediterranean country with the highest energy demand for irrigation (Daccache et al., 2014). To cope with this problem, several studies have been carried out at the irrigation network level in Spain which have suggest solutions to reduce energy consumption in pressurised systems (Carrillo Cobo et al., 2014; Jiménez-Bello et al., 2015; Moreno et al., 2010), and the pumping equipment (Fernández García et al., 2014; Mora et al., 2013; Pérez-Urrestarazu and Burt, 2012), but there is a lack of studies aimed at analysing the filtration system.

In a microirrigation system, the energy needs are mainly due to providing the required pressure in the filters; a pressure which is well above that required for the drip emitters. Although the trend is to operate with emitters at lower pressures, the pressure requirements of filters have not been substantially changed. Burt (2010) already identified that a reduction of the pressure required for filtration could significantly reduce pressure requirements in the microirrigation systems. It is noticeable that pressure drop produced by the auxiliary elements of a porous medium filter is quantitatively more important than that occurring in the porous medium itself (Burt, 2010; Mesquita et al., 2012),

Arbat et al. (2011) used computational fluid dynamics (CFD) techniques to predict pressure losses produced by the different elements of a microirrigation sand filter and pointed out the importance of the underdrain design in the filter's hydraulic behavior and therefore in the resulting pressure drop produced by the entire filter. Mesquita et al. (2012) studied the pressure drop produced in three different commercial filters, which had the three different 
underdrain configurations most commonly used by manufacturers: manifold, disc and nozzle types. According to their results, the nozzle underdrain-type filter was the one that produced less pressure drop, independent of the media size and bed depths tested in their experiments. Bové et al. (2015b) presented a new underdrain design that modified the geometry of a commercial nozzle by (1) increasing the cross-sectional area at the outlet of the nozzle and (2) placing some of the slots at the top of the nozzle whereby a more direct exit of the streamlines was favoured and therefore the pressure drop was reduced. Based on the computational fluid dynamics (CFD) simulations, the new underdrain designed by Bové et al. (2015b) reduced the pressure drop in the complete filter by $35 \%$ compared with the similar commercial nozzle.

The objectives of the present paper are: (1) to construct three different new underdrains following the suggestions of previous CFD studies by Bové et al., (2015b) and the analytical study of Arbat et al., (2013) who focused on improving the efficiency of sand media filters; (2) to test under laboratory conditions the three new underdrain designs and to compare the resulting pressure drop with those produced by a commercial nozzle; and (3) to use a modified version of the analytical model of Arbat et al. (2013) modified by Pujol et al. (2016) in order to predict and explain the effect of the different underdrain configurations, the granular medium, and their interactions.

\section{Materials and Methods}

Experimental setup

The laboratory sand filter was a scaled version of a commercial filter (Arbat et al., 2013). The main dimensions of the laboratory filter were an inner diameter (DF) of $200 \mathrm{~mm}$ and a total length (LT) of $750 \mathrm{~mm}$ (Fig. 1). An inner plate with an orifice in the middle was welded $133 \mathrm{~mm}$ above the bottom of the filter and was used as a location for fitting the underdrain element (nozzle) and, also, for supporting the sand media. Inlet and outlet filter pipes were of $50.8 \mathrm{~mm}$ inner diameter that included a digital manometer Leo 2 (Keller, Winterthur, Switzerland; $\pm 0.07 \%$ accuracy) for recording the pressure.

Tap water was pumped in a closed-circuit system with a centrifugal pump Prisma 20/4 M (ESPA, Banyoles, Spain). The flow rate was measured with a magnetic flowmeter MP400-CP PTFE DN20 (Comaquinsa, Llinars del Vallès, Spain). A screen filter (Regaber, Parets del Vallès, Spain) was added in the pipe line to prevent particles entering the sand filter and retaining the sand during backwashing conditions. It had an inner diameter of $165 \mathrm{~mm}$ and a $130 \mu \mathrm{m}$ filtration mesh.

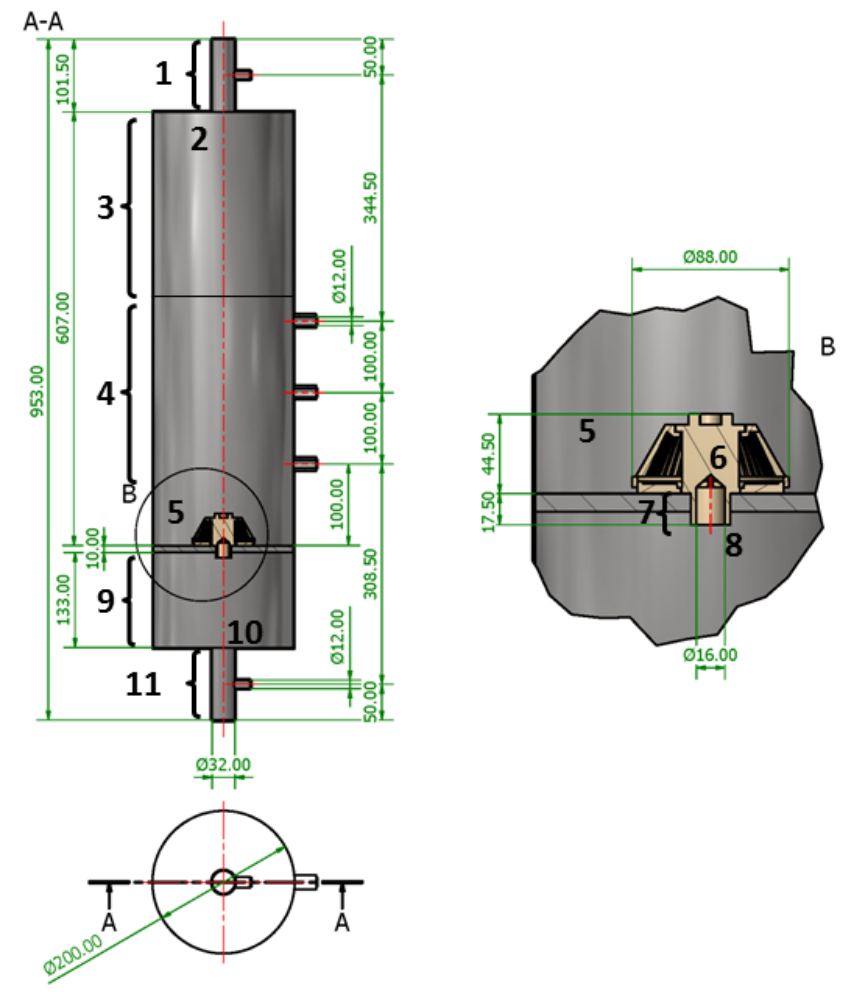

Figure 1. General dimensions of the laboratory filter (units in $\mathrm{mm}$ ). 


\section{Nozzle types}

The consequences of using four different types of nozzles were investigated. The commercial conical nozzle made by Regaber (Parets del Vallès, Spain) used 45 rectangular slots of dimensions $0.45 \mathrm{~mm}$ x $30 \mathrm{~mm}$ distributed as shown in Fig. 2a. Data obtained with this commercial element were used as reference values for comparison. A cylindrical stainless steel nozzle with exactly the same number of slots and dimensions as the commercial one (see Fig. 2b) was designed. Besides its external geometry (cylindrical instead of conical) the main difference in the new nozzle was the lack of inner structural elements and a slightly reduced size of the diameter of the outlet orifice. Two additional cylindrical stainless steel nozzles were designed with the same external and internal geometry. The first had the same open area as the commercial nozzle, and the previous cylindrical nozzle, but it had 12 slots located at the top of the nozzle (see Fig. 2c). Thus, only 33 slots of $0.45 \mathrm{~mm}$ x $30 \mathrm{~mm}$ were placed along the cylindrical surface. Lastly, a cylindrical stainless steel nozzle with 63 slots of $0.45 \mathrm{~mm}$ x $30 \mathrm{~mm}$ located at the cylindrical surface (see Fig. 2d) was designed. The filtration surface of the nozzle was increased by $40 \%$ with respect to the previous filters.

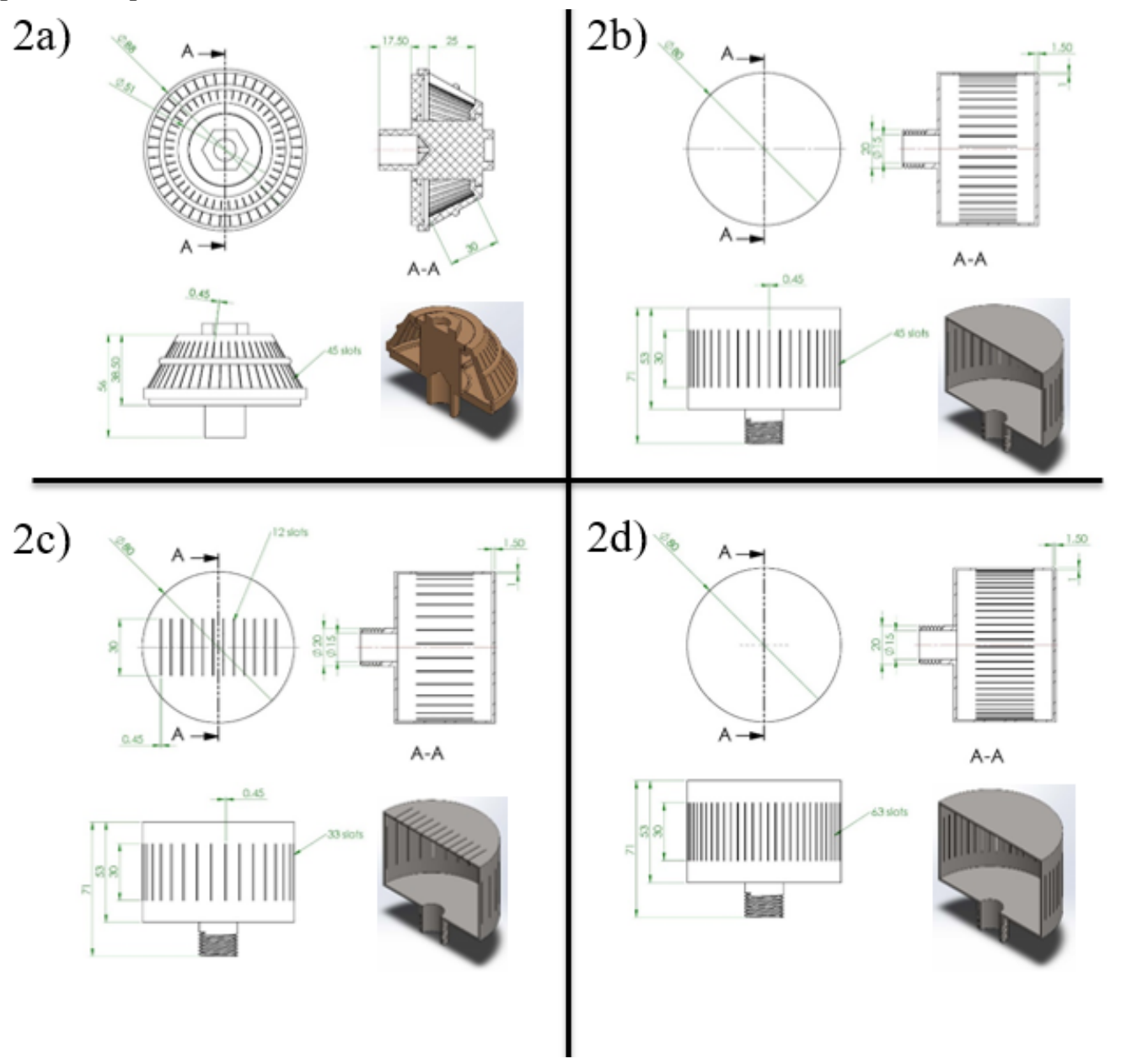

Figure 2. Nozzles analysed: a) commercial, b) stainless steel one with the same number of slots than case a located at the cylindrical surface, c) as in case b except with slots also at the top and d) as in case b except with $+40 \%$ slots at the cylindrical surface. Units in $\mathrm{mm}$.

Experimental procedure

The characteristic curve of the sand filter (i.e., the relationship between the superficial velocity $v_{s}=4 Q /\left(\pi D_{F}^{2}\right)$ with $\mathrm{Q}$ the volumetric flow rate, and the pressure difference between the filter inlet and the filter outlet $\mathrm{D} p=p_{\text {in }}$ $\left.p_{o u t}\right)$ was obtained for both filtration and backwashing regimes and for different filter configurations. The same procedure was followed in each individual experiment. Initially, the valve at the filter outlet was closed until the inlet pressure reached approximately $400 \mathrm{kPa}$. With this position of the valve, the mean flow-rate obtained during $120 \mathrm{~s}$ was calculated from the readings of the flowmeter and the minimum and maximum pressures at the filter inlet and outlet (pin and pout) for the same period of time were registered with the digital manometer. After that, the procedure was repeated by opening the outlet valve until the outlet pressure was reduced approximately 20 $\mathrm{kPa}$. The procedure was repeated until the outlet valve was completely opened, and thus the sand filter characteristic curve could be obtained. Each experiment was repeated 3 times to ensure repeatability of the data. 
At the end of each experiment the filter was opened in order to examine the porous medium. The sand particles remained disaggregated, no biofilm formation was observed and the sand top surface was flat. Therefore, it was assumed that no preferential channels were formed.

\section{Analytical model}

The analytical model developed by Arbat et al. (2013) for predicting the pressure loss in sand filters was slightly modified according to Pujol et al. (2016). The changes consisted of 1) neglecting a term related to the decrease in the number of available channels as the flow approaches the nozzle and whose contribution to the total energy losses was minor (Eq. (A.22) in Arbat et al., 2013), and 2) changing the expression for determining the energy losses at the outlet tube of the nozzle (Eq. (A.6) in Arbat et al., 2013) by simplifying it in terms of a pressure drop in series configuration (minor loss at the tube inlet, major loss through the tube length and further minor loss at the tube outlet).

The total pressure loss in the filter was defined as $\Delta p_{12}(\mathrm{~Pa})$, and was obtained by adding the pressure loss through the sand medium $\Delta_{p s}(\mathrm{~Pa})$ and the pressure loss through water only regions $\Delta_{p w}(\mathrm{~Pa})$,

$$
\Delta p_{12}=\Delta p_{s}+\Delta_{p w} \text {. }
$$

Pressure losses in water only regions were divided into primary and secondary losses. Primary losses $\Delta_{\text {pwp }}(\mathrm{Pa})$ in water only regions of the filter with diameter $\mathrm{D}(\mathrm{m})$ and length $\mathrm{L}(\mathrm{m})$ followed the Darcy-Weisbach equation (White, 2011):

$$
\Delta p_{w p}=f \frac{L}{D} \frac{\rho}{2} v^{2}=f \frac{L}{D^{5}} \frac{8 \rho}{\pi^{2}} Q^{2}
$$

with $v$ the mean flow velocity $(\mathrm{m} / \mathrm{s}), Q$ the volumetric flow rate $\left(\mathrm{m}^{3} \mathrm{~s}^{-1}\right), \rho$ the water density $\left(\mathrm{kg} \mathrm{m}^{-3}\right)$ and $f$ the friction factor (dimensionless). For the range of flow rates analysed in this study, the Reynolds number $\operatorname{Re}=$ $v D \rho / \mu$, with $\mu$ the fluid viscosity ( $\mathrm{Pa} \mathrm{s}$ ), was within the range $4000<\mathrm{Re}<10^{5}$. Therefore, the friction factor could be expressed using the Blasius formula $f=0.316 \mathrm{Re}^{-1 / 4}$.

The secondary loss contribution $\Delta p_{w m}(\mathrm{~Pa})$ followed the expression (White, 2011):

$$
\Delta p_{w m}=K \frac{\rho}{2} v^{2}=K \frac{8 \rho}{\pi^{2} D^{4}} Q^{2}
$$

with $K$ the dimensionless minor loss coefficient. In a sudden expansion from a region of diameter $d$ to a region of diameter $D(>d)$, the value of the minor loss coefficient was $K=\left(1-d^{2} / D^{2}\right)^{2}$ (White, 2011). In a sudden contraction, from diameter $D$ to diameter $d(<D)$, the secondary loss coefficient was $K=0.42\left(1-d^{2} / D^{2}\right)$ (White, 2011).

Pressure losses through the sand bed $\Delta p_{s}(\mathrm{~Pa})$ were also divided into primary $\Delta p_{s p}(\mathrm{~Pa})$ and secondary $\Delta p_{s m}$ (Pa) ones. Primary losses $\Delta p_{s p}$ were expressed as:

$$
\Delta p_{s p}=72 \lambda \frac{\mu}{\phi^{2} D_{p}{ }^{2}} \frac{(1-\varepsilon)^{2}}{\varepsilon^{3}} \frac{4 Q}{\pi D_{F}^{2}} L
$$

where $\lambda$ was the tortuosity that accounted for the fact that channels were not totally straight (dimensionless), $\varepsilon$ was the sand porosity (dimensionless), $D_{p}$ was the mean particle diameter $(\mathrm{m}), \phi$ was the sphericity factor (dimensionless with $\phi D_{p}$ the particle effective diameter), $D_{F}$ was the filter diameter $(\mathrm{m})$ and $L$ was the length of the sand column $(\mathrm{m})$.

Secondary losses $\Delta p_{s m}(\mathrm{~Pa})$ followed the expression (Arbat et al., 2013):

$$
\Delta p_{s m}=K_{s} \frac{L}{\varepsilon^{2} \phi D_{p}} \frac{\rho}{2} \frac{16 Q^{2}}{\pi^{2} D_{F}^{4}}
$$

with $K_{s}$ the dimensionless minor loss coefficient for the sand medium.

The sand medium was divided into two regions: region I with uniform flow not affected by the nozzle and region II close to the nozzle and with non-uniform flow.

In region I, values $\lambda=150 / 72$ in Eq. (4) and $K_{s}=3.5(1-\varepsilon) / \varepsilon$ in Eq. (5) were used for a total sand column length $L$ of $L_{u}$ (length of sand with uniform flow). Thus, Eqs. (4) and (5) became the Ergun equation (Macdonald et al., 1979 ) that has been successfully adopted for determining the pressure losses in uniform porous media (Hicks, 1970; McCabe et al., 2001; du Plessis and Woudberg, 2008; Bové et al., 2015a).

In region II, a column length $L$ of $L_{n u}$ (length of sand with non-uniform flow) was used, the value of which is discussed in the next section. The parameter for the primary loss in Eq. (4) was $\lambda=150 x / 72$, being $x=D_{F 0} / D_{F 1}$, the ratio for the equivalent filter diameters at the uniform region $\left(D_{F 0}=D_{F}\right)$ and at the nozzle $\left(D_{F 1}=(4 S / \pi)^{1 / 2}=\right.$ 
$0.028 \mathrm{~m}$ with $S=607.5 \times 10^{-6} \mathrm{~m}^{2}, S$ being the filtration surface area of the nozzle). In the region II, the minor loss expression Eq. (5) used $K_{s}=K_{x} x\left(x^{2}+x+1\right) / 3$ with $x=D_{F 0} / D_{F 1}$ and $K_{x}=1.42$, being the expected minor loss coefficient for a sudden contraction plus expansion across the water channels found in the porous medium (Arbat et al., 2013).

The total pressure loss in the filter was calculated as a series of pressure drops along the different contributions found in the filter. The total pressure drop was calculated with Eq. (2) (primary losses in water only regions) in zones 1, 3, 7, 9 and 11 of Fig. 1, Eq. (3) (minor losses with coefficients for a sudden expansion) in zones 2 and 8 of Fig. 1, Eq. (3) (minor loss with coefficients for a sudden contraction) in zone 10 of Fig. 1, and Eqs. (4) and (5) in region I (zone 4 of Fig. 1) and in region II (zone 5 of Fig. 1). At the entrance of the outlet tube of the nozzle, zone 6 in Fig. 1, Eq. (3) (secondary losses) was assumed with $K=0.8$ for the commercial nozzle (corresponding to a re-entrant inlet) and with $K=0.2$ for the cylindrical nozzles here designed (corresponding to a slightly rounded inlet) in agreement with their inner geometries that differed from sudden contraction conditions (White, 2011).

\section{Results and Discussion}

Filtration

Figure 3 shows the experimental pressure drop across the filter for different nozzles with a sand height equal to $\mathrm{H}=340 \mathrm{~mm}$ (Fig. 3a) and $\mathrm{H}=140 \mathrm{~mm}$ (Fig. 3b). The cylindrical nozzle with the same open area as the commercial nozzle led to a pressure drop slightly reduced for the same superficial water velocity (vs). The distribution of the slots in a vertical position along the cylindrical surface may tend to increase the pressure drop in comparison with the commercial nozzle where the slots occupied an inclined position more suitable for the water trajectories through the porous medium. However, the absence of inner elements in the stainless steel nozzle (see Fig. 2) may be the reason for a lower pressure drop across this element with respect to the commercial element.

In Fig. 3a-b it can be observed that the cylindrical nozzle, with exactly the same open area as both the commercial and the cylindrical underdrains but with 12 slots at the top of the nozzle, substantially decreased the pressure drop. The values of the pressure change are similar than those produced with a higher open area $(+40 \%$ slots) but located along the cylindrical surface instead of at the top. This indicates that the position of the slots strongly modified the flow trajectories through the sand. Reducing the tortuosity of the water channels within the porous medium obtained by, for example, locating the slots at the top of the nozzle (i.e., in a perpendicular plane with respect to the mean water trajectory) decreased the filter pressure drop a $20 \%$ value compared with the cylindrical configuration and by $25 \%$ when compared with the commercial nozzle.

For the $\mathrm{H}=340 \mathrm{~mm}$ case, Fig. 4 shows the variation in the pressure drop (in \%) for the whole filter when substituting the nozzle with slots at the cylindrical surface by (1) the nozzle with the same passing surface area but with slots distributed at both the cylindrical and top circular faces and (2) the nozzle with a $40 \%$ increase in open area with slots distributed in the cylindrical face. These results were obtained after fitting a second order polynomial curve to the data shown in Fig. 3a with a correlation coefficient $r>0.990$ for all cases. At low flow rates, the change in the distribution of slots in the nozzle may lead to a $20 \%$ reduction in the pressure drop across the entire filter. This showed how the energy savings of a filtration system might substantially increase simply by using underdrain elements with an optimised distribution of the slots. For example, in the scaled filter, with a pump working at $601 \mathrm{~min}^{-1}$ at $25 \%$ net efficiency, the calculated power required for pumping water through the filter was $240 \mathrm{~W}$ with the commercial nozzle whereas it reached $183 \mathrm{~W}$ only $(23 \%$ energy saving) with the cylindrical nozzle with the same open area and 12 slots at the top. 

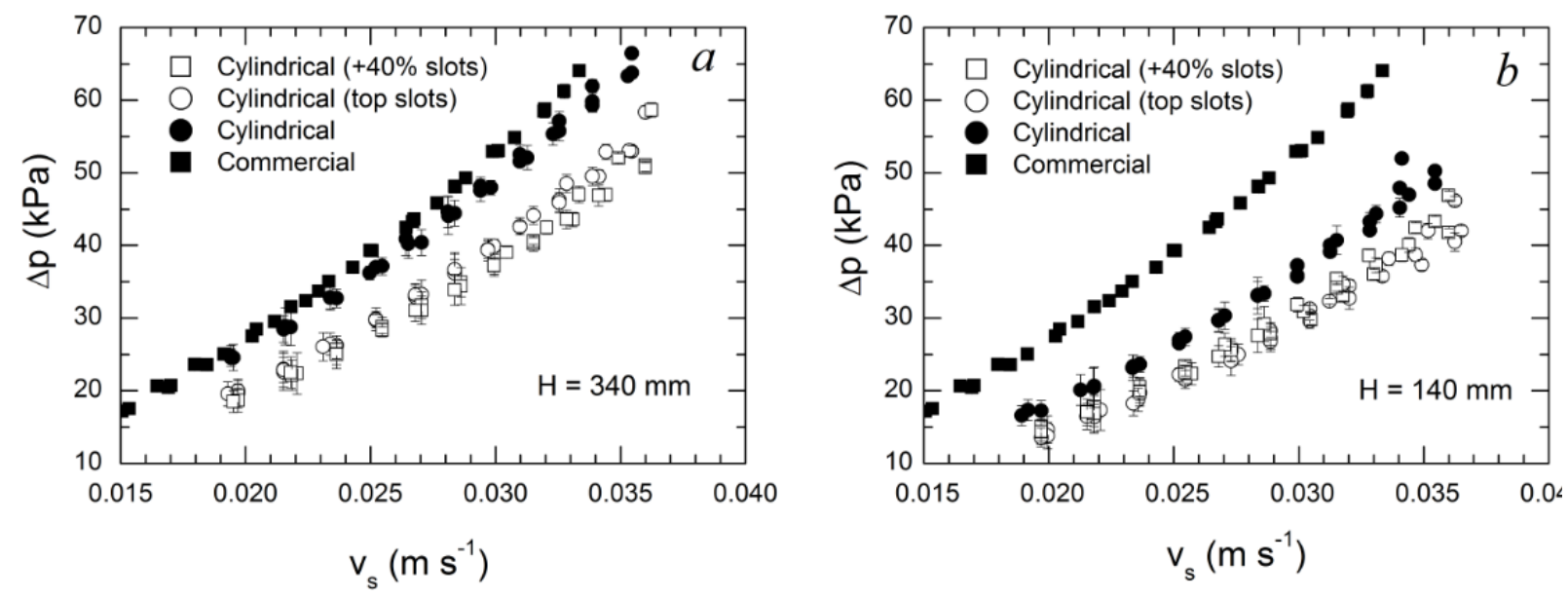

Figure 3. Results under filtration regime with a sand height $\mathrm{H}=340 \mathrm{~mm}$ (a) and $\mathrm{H}=140 \mathrm{~mm}$ (b).

The results obtained above were predicted by the analytical model, as it is shown in Fig. 5 for the $\mathrm{H}=340 \mathrm{~mm}$ case. The dashed line refers to the results obtained with the analytical model for the whole filter (water and porous medium) but assuming uniform flow throughout the sand (i.e., rejecting the existence of region II and with $\mathrm{L}=\mathrm{H}$ in region I). Since the commercial nozzle had a complex inner structure in the underdrain element, the analytical secondary losses were larger than those corresponding to the empty stainless steel nozzles. This decrease in the pressure drop regarding the commercial nozzle was also observed in the data and explained above when analysing Fig. 3.

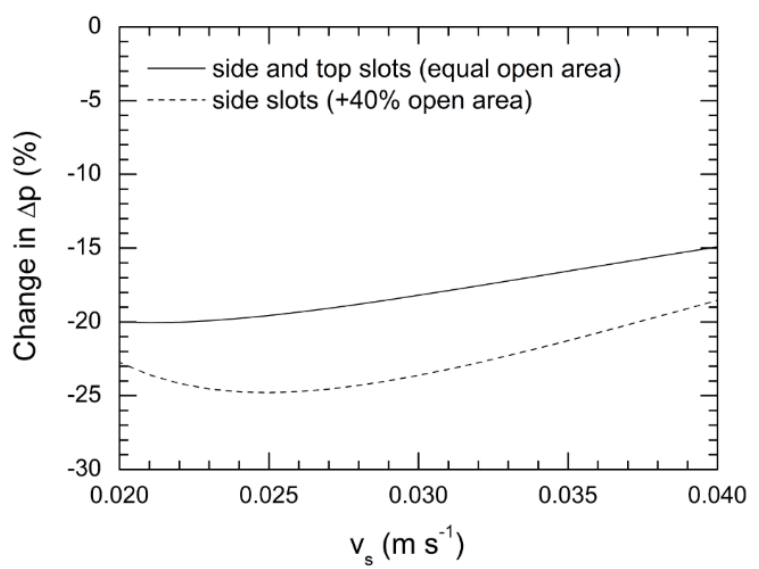

Figure 4. Changes of the pressure drop when using the nozzle shown in Fig. $2 b$ in comparison with that of Fig. 2c (solid line) and of Fig. $2 \mathrm{~d}$ (dashed line) for a sand height $\mathrm{H}=340 \mathrm{~mm}$ under the filtration regime. 


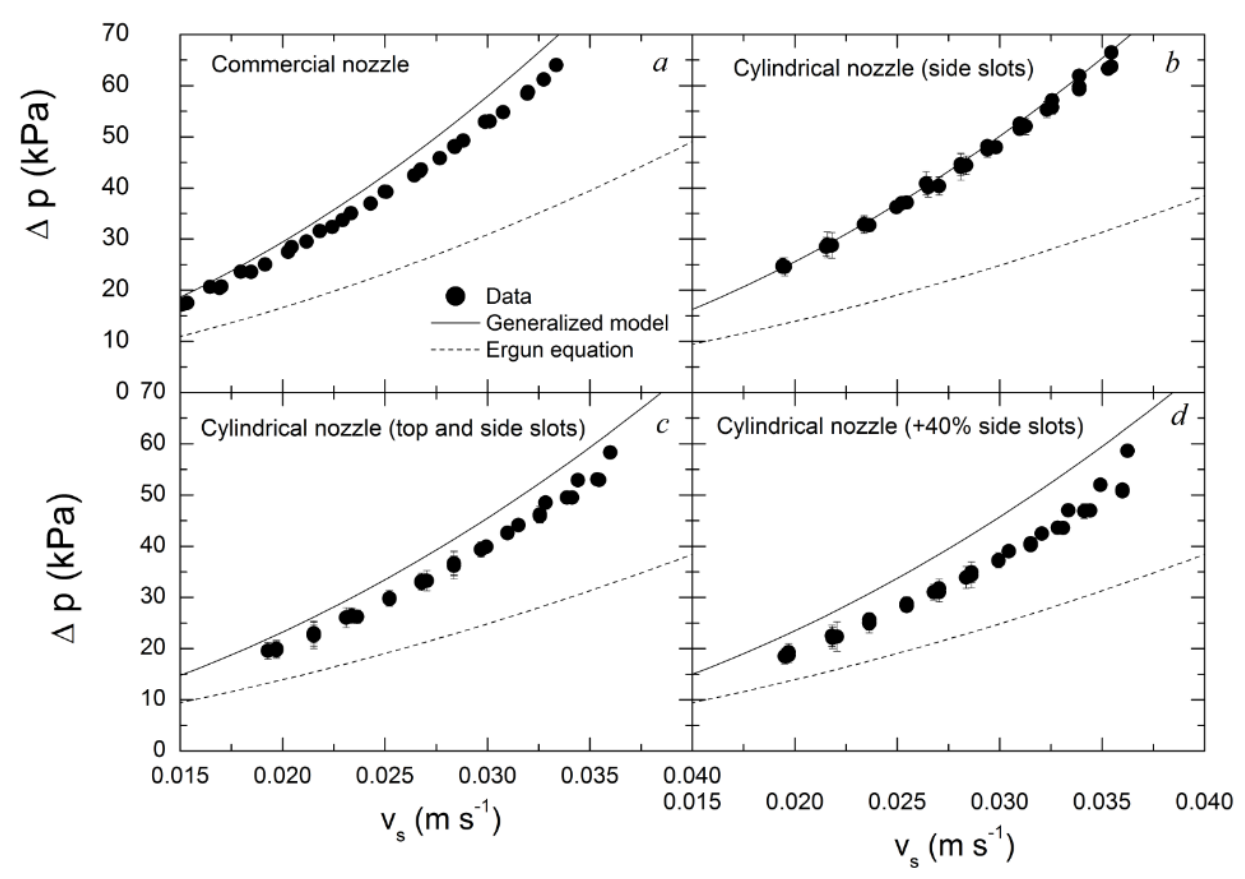

Figure 5. Results under filtration regime with a sand height $\mathrm{H}=340 \mathrm{~mm}$ (dots) in comparison with the analytical model. Solid line: model with non-uniform flow within the sand. Dashed line: model neglecting the existence of a non-uniform flow region within the sand.

The model agreed well with the measurements as long as the non-uniform water flow zone (region II) was included within the porous medium (see the solid line in Fig. 5). The length of the region with non-uniform flow $L_{n u}$ was estimated using a very simple criterion (see Fig. 6). It was assumed that, very close to the nozzle, the position of the water front within the sand at a given time took a shape similar to the nozzle element but with higher dimensions. As the water front approached the nozzle, the surface of the water front shrunk until it finally matched the nozzle open area. Thus, the value of $L_{n u}$ was calculated as the distance between the nozzle surface and the virtual surface with a value equal to the filter cross-sectional area $3.14 \times 10^{-2} \mathrm{~m}^{2}\left(=\pi D_{F}^{2} / 4\right)$ with a shape equal than the nozzle but with higher dimensions (see the scaled geometries in Fig. 6). Following this criterion, the value of $L_{n u}$ may be understood as the length of the water path within the sand region where the water front reduced its surface area. In this region, a progressive decrease in the number of water channels was assumed and, therefore, a non-zero contribution to the secondary loss term.

By using this criterion, the non-uniform distance for the commercial nozzle $\left(L_{n u}\right)$ was $18 \mathrm{~mm}$ (commercial case; Fig. $6 \mathrm{a}$ ), the distance $\left(L_{n u}\right)$ for the cylindrical nozzle including the slots at the top circular area was $9 \mathrm{~mm}$ (Fig. 6c), and the distance $(L m u)$ for the nozzle with slots at the cylindrical surface only was $14 \mathrm{~mm}$ (Fig. $6 \mathrm{~b}$ ) since a scaled virtual geometry with the same height was employed in the latter case due to the expected path of the water (almost horizontal in case of only having slots in the side area). Note in Fig. 5 that the simple analytical model agreed well with the experimental behaviour and correctly reproduced the effect of using nozzles with different geometries. The pressure drop differences between the solid and the dashed lines in Fig. 5 corresponded to the effects of the non-uniform flow region within the sand whose contribution greatly varied as a function of the nozzle shape. 

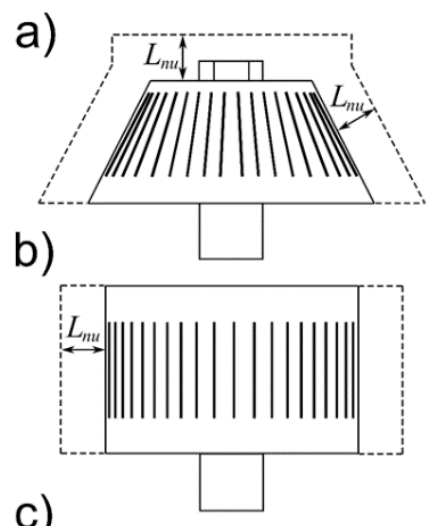

c)

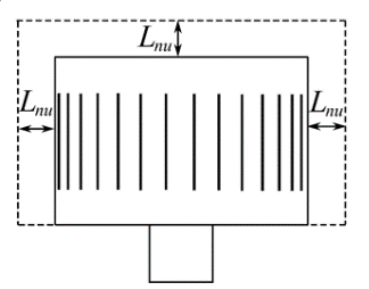

Figure 6. Criterion for determining the non-uniform flow length $L_{n u}$. The dashed line is a virtual geometry with the same surface area than that of the uniform region $\left(\pi D_{F}^{2} / 4\right)$. Case a) non-uniform flow length for the commercial nozzle. Case b) non-uniform flow length for cylindrical nozzles with slots at the cylindrical surface only. Case c) non-uniform flow length for the cylindrical nozzle with slots at both the top and the cylindrical surfaces (lengths not to scale).

\section{Backwashing}

The results obtained under backwashing conditions are shown in Fig. 7. Pressure drop differences were almost the same when using different nozzle types. This indicated that the flow acquired the minimum fluidization velocity reaching an expanded bed. In this case, the differences in the pressure drop were only a function of the energy losses generated when the water flowed through the nozzle (in reverse direction). At the same superficial velocity, the energy required for cleaning the porous medium during the backwashing process was similar to that needed for filtration. For example, at $v_{\mathrm{s}}=0.02 \mathrm{~m} \mathrm{~s}^{-1}, \Delta p=26 \mathrm{kPa}$ in backwashing configuration and $\Delta p=27 \mathrm{kPa}$ and $\Delta p=19 \mathrm{kPa}$ in filtration mode (commercial nozzle and cylindrical nozzle with top slots, respectively). Thus, the backwashing mode must be carefully taking into account when analysing the economic cost of the irrigation system, as already suggested by Burt (2010).

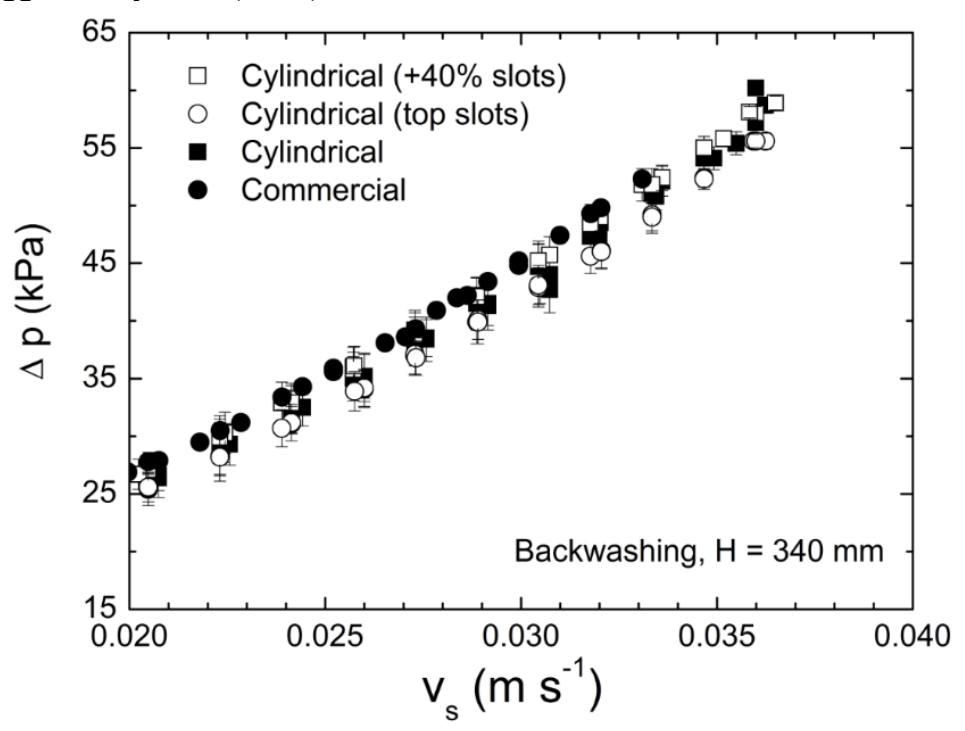

Figure 7. Results under backwashing conditions with a sand height $H=340 \mathrm{~mm}$. 


\section{Conclusions}

According to the first objective of this work, three underdrains with geometrical configurations based on improvements suggested by previous theoretical studies were constructed. These underdrains were then tested in laboratory and the effects of using different underdrain geometries in a sand filter led to the conclusion that the nozzle shape highly influenced the overall pressure drop. The key effect of the underdrain was the modification of the water path within the porous medium, which changed the tortuosity of the water channels within the sand and substantially varied the energy losses. It was found that with the same open area, a proper location of the slots, with some at the top of the cylindrical nozzle, a reduction of $25 \%$ in the energy required for filtration may be achieved. This significant increase in the filter efficiency was also reached when substituting the classical nozzle with an underdrain using $40 \%$ more slots in its cylindrical surface. By contrast, all nozzles produced the same pressure drop when working in a backwashing regime since all reached fluidised bed conditions.

The modification of the analytical model of Arbat et al. (2013), which was our third objective, agreed well with the experimental data and clearly showed that the main contribution to the pressure drop corresponded to the region close to the nozzle with non-uniform water flow within the sand. This non-uniform flow region was highly dependent on the nozzle geometry. Therefore, a very affordable way to improve the energy efficiency of new and, especially, installed filtration systems may consist of substituting the underdrain elements with optimised designs. This modification may be scheduled to coincide with the task of replacing the sand in accordance with the standard maintenance requirements of the filter. Values of more than a $20 \%$ reduction of the filter energy consumption during filtration mode conditions are expected.

\section{Acknowledgements}

The authors would like to express their gratitude to the Spanish Ministry of Economy, Industry and Competitiveness for their financial support for this experiment though grant AGL2015-63750-R. P. Sala, S. Saus and J. Vicens provided very helpful technical assistance.

\section{References}

Arbat, G., Pujol, T., Puig-Bargués, J., Duran-Ros, M., Barragán, J., Montoro, L., Ramírez de Cartagena, F. (2011). Using computational fluid dynamics to predict head losses in the auxiliary elements of a microirrigation sand filter. Transactions of the ASABE, 54(4), 1367-1376.

Arbat, G., Pujol, T., Puig-Bargués, J., Duran-Ros, M., Montoro, L., Barragán, J., Ramírez de Cartagena, F. (2013). An experimental and analytical study to analyze hydraulic behavior of nozzle-type underdrains in porous media filters. Agricultural Water Management, 126, 64-74.

Bové, J., Arbat, G., Duran-Ros, M., Pujol, T., Velayos, J., Ramírez de Cartagena, F., Puig-Bargués, J. (2015). Pressure drop across sand and recycled glass media used in micro irrigation filters. Biosystems Engineering, 137, 55-63.

Bové, J., Arbat, G., Pujol, T., Duran-Ros, M., Ramírez de Cartagena, F., Velayos, J., Puig-Bargués, J. (2015). Reducing energy requirements for sand filtration in microirrigation: Improving the underdrain and packing. Biosystems Engineering, 140, 67-78. doi: 10.1016/j.biosystemseng.2015.09.008.

Burt, C. M. (2010). Hydraulics of commercial sand media filter tanks used for agricultural drip irrigation. ITRC Report No. R 10001. San Luis Obispo, California: Irrigation Training and Research Center.

Capra, A., and Scicolone, B. (2007). Recycling of poor quality urban wastewater by drip irrigation systems. Journal of Cleaner Production, 15(16), 1529-1534. doi: http://dx.doi.org/10.1016/j.jclepro.2006.07.032.

Carrillo Cobo, M. T., Camacho Poyato, E., Montesinos, P., Rodríguez Díaz, J. A. (2014). New model for sustainable management of pressurized irrigation networks. Application to Bembézar MD irrigation district (Spain). Science of the Total Environment, 473-474, 1-8. doi: 10.1016/j.scitotenv.2013.11.093.

Corominas, J. (2010). Agua y energía en el riego, en la época de la sostenibilidad [Water and Energy in irrigation, at the age of sustainability]. Ingeniería del agua, 17(3).

Daccache, A., Ciurana, J. S., Rodriguez Diaz, J. A., Knox, J. W. (2014). Water and energy footprint of irrigated agriculture in the Mediterranean region. Environmental Research Letters, 9(12). doi: 10.1088/17489326/9/12/124014

du Plessis, J. P., Woudberg, S. (2008). Pore-scale derivation of the Ergun equation to enhance its adaptability and generalization. Chemical Engineering Science, 63(9), 2576-2586. doi: http://dx.doi.org/10.1016/j.ces.2008.02.017.

Fernández García, I., Moreno, M. A., Rodríguez Díaz, J. A. (2014). Optimum pumping station management for irrigation networks sectoring: Case of Bembezar MI (Spain). Agricultural Water Management, 144, 150-158. doi: 10.1016/j.agwat.2014.06.006.

Hicks, R. (1970). Pressure drop in packed beds of spheres. Industrial \& engineering chemistry fundamentals, 9(3), 500-502. 
ICID. (2011). Sprinkler and micro irrigated area, retrieved December 1, 2015, from http://www.icid.org/sprin_micro_11.pdf.

Jiménez-Bello, M. A., Royue-̄a, A., Manzano, J., Prats, A. G., Martínez-Alzamora, F. (2015). Methodology to improve water and energy use by proper irrigation scheduling in pressurised networks. Agricultural Water Management, 149, 91-101. doi: 10.1016/j.agwat.2014.10.026.

Macdonald, I. F., El-Sayed, M. S., Mow, K., Dulllen, F. A. L. (1979). Flow through porous media - The Ergun equation revisited. Industrial and Engineering Chemistry Fundamentals, 18(3), 199-208.

MAGRAMA. (2015). Encuesta de Superficies y Rendimientos de Cultivos (ESYRCE), retrieved January 1, 2015, from http://www.magrama.gob.es/es/estadistica/temas/estadisticas-agrarias/regadios2015_tcm7404816.pdf..

McCabe, W. L., Smith, J. C., Harriott, P. (2001). Unit Operations of Chemical Engineering (Sixth edition). New York: McGraw-Hill.

Mesquita, M., Testezlaf, R., Ramirez, J. (2012). The effect of media bed characteristics and internal auxiliary elements on sand filter head loss. Agricultural Water Management, 115, 178-185.

Mora, M., Vera, J., Rocamora, C., Abadia, R. (2013). Energy Efficiency and Maintenance Costs of Pumping Systems for Groundwater Extraction. Water Resources Management, 27(12), 4395-4408. doi: 10.1007/s11269013-0423-z.

Moreno, M. A., Córcoles, J. I., Tarjuelo, J. M., Ortega, J. F. (2010). Energy efficiency of pressurised irrigation networks managed on-demand and under a rotation schedule. Biosystems Engineering, 107(4), 349-363. doi: 10.1016/j.biosystemseng.2010.09.009.

Pujol, T., Arbat, G., Bové, J., Puig-Bargués, J., Duran-Ros, M., Velayos, J., Ramírez de Cartagena, F. (2016). Effects of the underdrain design on the pressure drop in sand filters. Biosystems Engineering, 150. 1-9. doi: 10.1016/j.biosystemseng.2016.07.005.

Tarjuelo, J. M., Rodriguez-Diaz, J. A., Abadía, R., Camacho, E., Rocamora, C., Moreno, M. A. (2015). Efficient water and energy use in irrigation modernization: Lessons from Spanish case studies. Agricultural Water Management, 162, 67-77. doi: http://dx.doi.org/10.1016/j.agwat.2015.08.009.

Pérez-Urrestarazu, L., Burt, C. M. (2012). Characterization of pumps for irrigation in central California: Potential energy savings. Journal of Irrigation and Drainage Engineering, 138(9), 815-822. doi: 10.1061/(ASCE)IR.1943-4774.0000463.

White, F. M. (2011). Introduction to Fluid Mechanics (Seventh edition.). New York: McGraw-Hill. 


\title{
Effect of Sand Size and Inlet Pressure on Sand Media Filter Performance using Reclaimed Effluents
}

\author{
Miquel Duran-Ros, Jaume Puig-Bargués, Gerard Arbat *, Joan Pujol, Francisco Ramírez de \\ Cartagena \\ Department of Chemical and Agricultural Engineering and Technology, University of Girona, Girona, Catalonia, 17003, \\ Spain \\ * Corresponding author. Email: gerard.arbat@udg.edu
}

\begin{abstract}
Sand media filters are specially recommended for preventing emitter clogging when irrigation water with high pollutant load is used in micro irrigation. The objective of this work was to assess those operational conditions related to media size and filter inlet pressure that could yield to a better emitter protection when wastewaters are used. So, an experiment was carried out in a micro irrigation system that had a sand media filter for filtering a reclaimed effluent. Two sand effective diameters $(0.41$ and $0.63 \mathrm{~mm})$ and three filter inlet pressures $(200,300$ and $400 \mathrm{kPa}$ ) were tested. Filtered volume, pressure loss across the filter, inlet $\mathrm{pH}$, temperature, turbidity and electrical conductivity and outlet turbidity data were collected every minute in a supervisory control and data acquisition system. Volume used for filter backwashing was also recorded after each filtration cycle. As the reclaimed effluent was supplied by a wastewater treatment plant, there were some variations in its quality across the experiment. Greater sand size significantly $(\mathrm{P}<0.05)$ reduced filtered volume and turbidity removal. On the other hand, 300 $\mathrm{kPa}$ filter inlet pressure yielded the greatest filtered volume and the lowest backwashing water consumption. Turbidity removal was significantly higher $(\mathrm{P}<0.05)$ for both 300 and $400 \mathrm{kPa}$ than for $200 \mathrm{kPa}$. Results suggest that sand media filter performance is optimised using sand media with an effective size of $0.41 \mathrm{~mm}$ and an inlet pressure of $300 \mathrm{kPa}$.
\end{abstract}

Keywords: Filtration, Micro irrigation, Drip irrigation, Clogging, Turbidity.

\section{Introduction}

Micro irrigation is the irrigation system used in $6.21 \%$ of worldwide-irrigated area, being $66.89 \%$ of micro irrigation irrigated area located in emerging and developing countries (ICID, 2017). Micro irrigation is being adopted in more farms because it increases both land and water productivity, although its energy consumption is higher (Tarjuelo et al., 2015). In addition, micro irrigation has more crop, environmental and sanitary advantages when reclaimed effluents are used for agricultural irrigation (Trooien and Hills, 2007), which is an increasing practice in areas with limited water resources (WWAP, 2017).

Filtering the effluent properly and effectively is needed for preventing emitter clogging to keep the micro irrigation systems operating as designed (Trooien and Hills, 2007). Screen, disc, media and hydro cyclonic filters are the most common filter types used in micro irrigation systems. However, sand media filters are considered the standard for filtration protection of micro irrigation systems, especially when effluents are used because, due to their higher particle, salt, nutrient and biological concentrations, they present increased clogging hazards (Trooien and Hills, 2007). Ravina et al. (1997), Capra and Scicolone (2004) and Duran-Ros et al. (2009a), among others, experimentally verified that sand media filters decreased emitter clogging compared with screen and disc filters when effluents were used.

Pressurized high flow sand media filters are the most popular in micro irrigation systems (Nakayama et al., 2007). Flowrates, sand type, height and size as well as operating pressures are the common parameters that filter manufacturers' recommend for a proper sand media filter operation that should yield a removal of emitter clogging particles that can be measured by parameters such as total suspended solids (TSS), turbidity or particle count.

Several authors (Puig-Bargués et al., 2005; Duran-Ros et al., 2009a; Gómez et al., 2010; Elbana et al., 2012; Tripathi et al., 2014; Wen-Yong et al., 2015) have analysed the effect of sand media filters on effluents characteristics under different operational conditions. Although removal efficiencies were dependent on effluent characteristics, the previous experiments found that greater removals were achieved with smaller sand sizes. Nakayama et al. (2007) pointed out that finer filtration occurs at lower velocities although de Deus et al. (2015) found just the opposite for particles greater than $60 \mu \mathrm{m}$. However, neither the effect of filter inlet pressure nor the effect on filtration velocity have been assessed for pressurized sand media filters using reclaimed effluents. Thus, the objective of this paper was to analyse the performance of a sand media filter for micro irrigation systems working under different sand sizes, inlet pressures and filtration velocities. 


\section{Materials and Methods}

Experimental set-up

A filtration platform with a sand filtration system was used to carry out the filtration experiments with the reclaimed effluents produced at the wastewater treatment plant (WWTP) of Celrà (Girona, Catalonia, Spain), which treats urban and industrial wastewater using a sludge process. The hydraulic scheme of the filtration unit can be observed at Figure 1.

The sand filtration system consisted of two sand filters in parallel (Regaber, Parets del Vallès, Spain), both with an internal diameter of $0.51 \mathrm{~m}$ and a filtration surface of $0.20 \mathrm{~m}^{2}$. The sand filters were filled with $175 \mathrm{~kg}$ of silica sand as a single filtration layer. The sand filters had an automatic backwashing system triggered by a preset pressure difference of $50 \mathrm{kPa}$, as suggested by Ravina et al. (1997), which was measured by a pressure transmitter with flush diaphragm. Backwashings were carried out for more than $2 \mathrm{~min}$.

The flowrate at the filter outlet was measured by an electromagnetic flow-metre. A turbine volume-metre with impulse emitter was used to determine backwashing water volume.

The effluent provided by the WWTP was taken using a $4 \mathrm{~kW}$ pump that had a frequency converter that allowed operating under different filter inlet flowrates and pressures.

In order to obtain water quality data and their variation, $\mathrm{pH}$, temperature, dissolved oxygen and turbidity sensors were installed. These devices were connected to a supervisory control and data acquisition (SCADA) system previously developed (Duran-Ros et al., 2008) and adapted to this experiment. The SCADA system both allowed the control of filter performance and the collection of filter data performance. This required a computer and two programmable logic controllers (PLC).

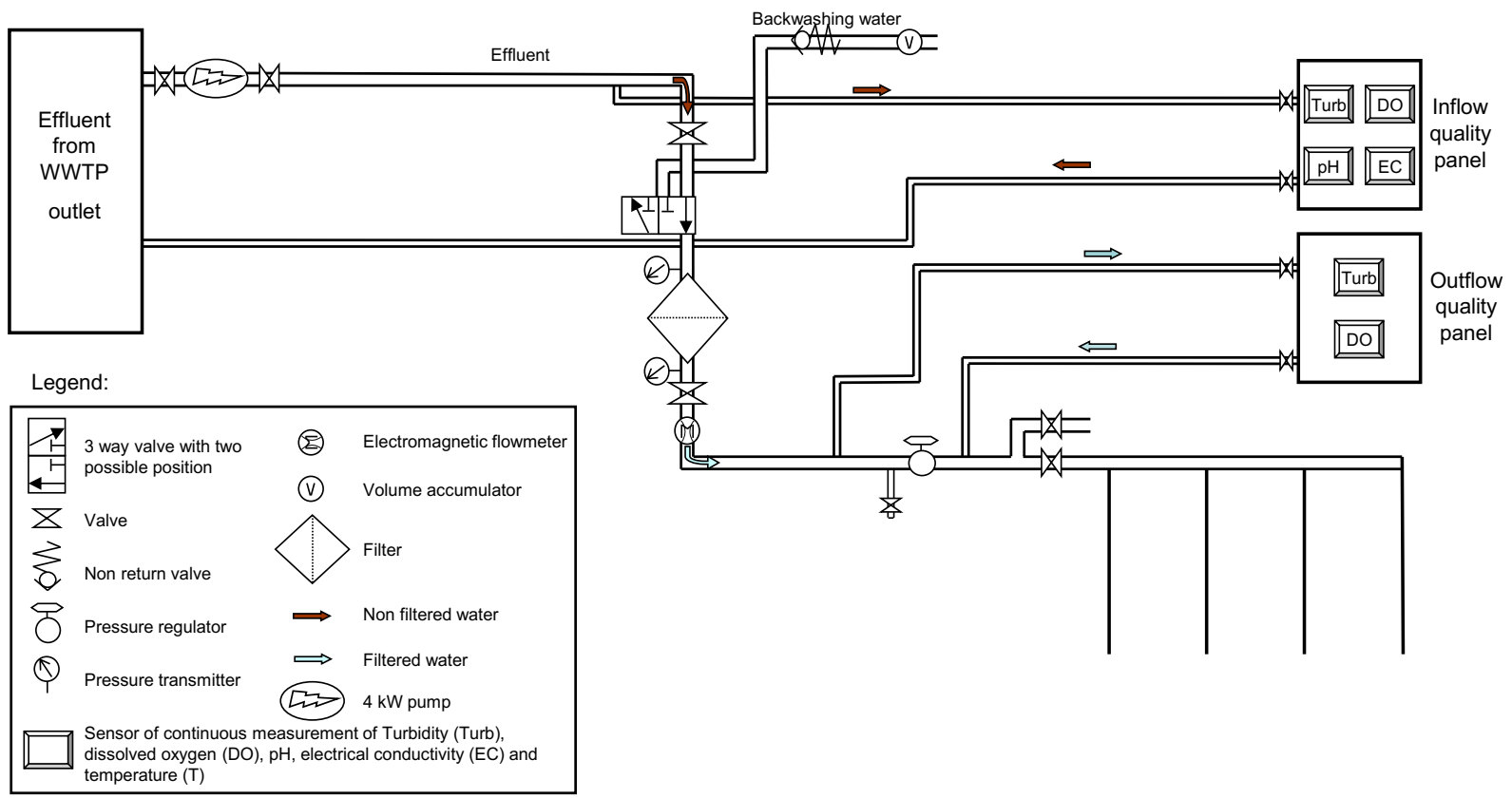

Figure 110. Hydraulic scheme of the sand filtration unit and location of monitoring and control equipment.

Sand media

Two silica sand media with different sizes were used. Both were supplied by Sibelco Hispania (Bilbao, Basque Country, Spain) and they were obtained from a quarry, and then they were washed and dried. Sand media were characterised in the laboratory by determining their sand effective diameter $\left(\mathrm{d}_{\mathrm{e}}\right)$ and sand uniformity coefficient $(\mathrm{UC})$. Sand effective diameter is the size of the screen opening which allows $10 \%$ of the sand mass to pass, while sand uniformity coefficient is the ratio between the screen pores that let pass 60 and $10 \%$ of the sand. Both parameters were determined for each sand media through three independent, homogeneous and representative sand samples, which were dried and sieved through twelve stainless steel screens ranging from 0.06 to $2.00 \mathrm{~mm}$ pores. Then, each screen was weighed to determine the retained sand and the particle size distribution curve, which allowed the computation of $\mathrm{d}_{\mathrm{e}}$ and $\mathrm{UC}_{\mathrm{s}}$. According to the results, the first sand media had a $\mathrm{d}_{\mathrm{e}}=0.41 \mathrm{~mm}$

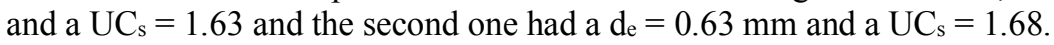


Operational procedure

Performance of the sand filtration unit was studied under three different filter inlet target pressures: $300 \mathrm{kPa}$, $400 \mathrm{kPa}(33 \%$ higher than $300 \mathrm{kPa}$ ) and $200 \mathrm{kPa}$ ( $33 \%$ lower pressure). Filter inlet pressures were set programming the target values in the pump frequency converter. This procedure caused that filtration velocity across the filter had some differences between experimental conditions.

The experiment was carried out from August 2014 to October 2015 during four irrigation periods. There were different breaks due mainly to lack of effluent supply, operational problems, electrical breakdowns and winter season. Overall, filters operated $1562 \mathrm{~h}$, on an average $8 \mathrm{~h}$ daily basis. Table 1 summarises the experimental conditions and the number of filtration cycles that are included in this study.

Irrigation time and filtration cycles under each experimental conditions were slightly different due to variations on effluent characteristics and filter performance. By using this procedure, there were differences in inlet pressure values that were kept within $\pm 20 \%$ of target inlet pressures, except for $\mathrm{d}_{\mathrm{e}}=0.63 \mathrm{~mm}$ and $200 \mathrm{kPa}$, when maximum pressure was $23.5 \%$ above. These differences in inlet pressure caused also differences in filter inlet flowrate and filtration velocities, as it can be seen in Table 1 .

Table 44. Experimental conditions during the experiment.

\begin{tabular}{|c|c|c|c|c|c|c|c|}
\hline $\begin{array}{r}\text { Sand } \\
\text { effective } \\
\text { size } \mathrm{d}_{\mathrm{e}} \\
(\mathrm{mm})\end{array}$ & $\begin{array}{r}\text { Sand } \\
\text { uniformity } \\
\text { coefficient } \\
\mathrm{UC}_{\mathrm{s}}(-) \\
\end{array}$ & $\begin{array}{c}\text { Target } \\
\text { filter inlet } \\
\text { pressure } \\
(\mathrm{kPa}) \\
\end{array}$ & $\begin{array}{l}\text { Average (and } \\
\text { range) filter inlet } \\
\text { pressure } \\
(\mathrm{kPa})\end{array}$ & $\begin{array}{c}\text { Average (and } \\
\text { range) filtration } \\
\text { velocity } \\
(\mathrm{m} / \mathrm{h}) \\
\end{array}$ & $\begin{array}{l}\text { Irrigation } \\
\text { time } \\
\text { (h) }\end{array}$ & $\begin{array}{c}\text { Number } \\
\text { of filtration } \\
\text { cycles } \\
(-)\end{array}$ & Dates \\
\hline \multirow[t]{3}{*}{0.41} & 1.63 & 200 & $\begin{array}{c}228 \\
(216-235)\end{array}$ & $\begin{array}{c}15.0 \\
(14.4-16.2)\end{array}$ & 220 & 15 & $\begin{array}{r}28 / 07 / 2015 \\
-09 / 09 / 2015\end{array}$ \\
\hline & & 300 & $\begin{array}{c}318 \\
(294-337)\end{array}$ & $\begin{array}{c}16.8 \\
(13.8-19.2)\end{array}$ & 314 & 16 & $\begin{array}{r}09 / 10 / 2015 \\
-29 / 10 / 2015\end{array}$ \\
\hline & & 400 & $\begin{array}{c}375 \\
(350-403) \\
\end{array}$ & $\begin{array}{c}17.4 \\
(16.2-19.6)\end{array}$ & 244 & 20 & $\begin{array}{r}09 / 09 / 2015 \\
-08 / 10 / 2015 \\
\end{array}$ \\
\hline \multirow[t]{3}{*}{0.63} & 1.68 & 200 & $\begin{array}{c}241 \\
(225-247)\end{array}$ & $\begin{array}{c}23.3 \\
(20.9-25.7)\end{array}$ & 295 & 36 & $\begin{array}{r}26 / 08 / 2014 \\
-20 / 10 / 2014\end{array}$ \\
\hline & & 300 & $\begin{array}{c}320 \\
(314-325)\end{array}$ & $\begin{array}{c}27.2 \\
(26.2-28.0)\end{array}$ & 247 & 29 & $\begin{array}{r}06 / 08 / 2014 \\
-25 / 08 / 2014\end{array}$ \\
\hline & & 400 & $\begin{array}{c}398 \\
(381-425) \\
\end{array}$ & $\begin{array}{c}18.4 \\
(14.2-20.7) \\
\end{array}$ & 242 & 23 & $\begin{array}{r}20 / 10 / 2014 \\
-19 / 05 / 2015 \\
\end{array}$ \\
\hline
\end{tabular}

Effluent characteristics are shown in Table 2. Following the risk classification proposed by Nakayama and Bucks (1991), the effluent had a low clogging risk for the turbidity (highly related with suspended solids) and a medium clogging risk for the $\mathrm{pH}$. Unfortunately, data from dissolved oxygen sensor located of the outflow quality panel was missed from most of the experiment because the instrumentation was not working properly.

Table 2. Mean \pm standard deviation of the effluent $\mathrm{pH}$, temperature, turbidity and dissolved oxygen at filter inlet. Different letters mean that inlet values of a parameter were significantly different $(\mathrm{P}<0.05)$.

\begin{tabular}{cccccc}
\hline $\begin{array}{c}\text { Sand } \\
\text { effective size } \mathrm{d}_{\mathrm{e}} \\
(\mathrm{mm})\end{array}$ & $\begin{array}{c}\text { Target filter } \\
\text { inlet pressure } \\
(\mathrm{kPa})\end{array}$ & $\begin{array}{c}\mathrm{pH} \\
(-)\end{array}$ & $\begin{array}{c}\text { Temperature } \\
\left({ }^{\circ} \mathrm{C}\right)\end{array}$ & $\begin{array}{c}\text { Turbidity } \\
(\mathrm{FTU})\end{array}$ & $\begin{array}{c}\text { Dissolved oxygen } \\
\left(\mathrm{mg} \mathrm{l}^{-1}\right)\end{array}$ \\
\hline 0.41 & 200 & $7.26 \pm 0.22 \mathrm{~b}$ & $26.02 \pm 1.86 \mathrm{~d}$ & $11.90 \pm 4.43 \mathrm{bc}$ & $2.43 \pm 0.44 \mathrm{ab}$ \\
& 300 & $7.07 \pm 0.11 \mathrm{a}$ & $21.96 \pm 0.81 \mathrm{a}$ & $17.11 \pm 6.45 \mathrm{~d}$ & $3.61 \pm 0.49 \mathrm{c}$ \\
& 400 & $7.02 \pm 0.08 \mathrm{a}$ & $24.61 \pm 1.24 \mathrm{bc}$ & $15.44 \pm 6.87 \mathrm{dc}$ & $2.71 \pm 0.61 \mathrm{a}$ \\
\hline 0.63 & 200 & $7.84 \pm 0.11 \mathrm{c}$ & $26.37 \pm 1.22 \mathrm{~d}$ & $8.62 \pm 3.35 \mathrm{~b}$ & $2.14 \pm 0.30 \mathrm{ab}$ \\
& 300 & $7.97 \pm 0.10 \mathrm{~d}$ & $25.87 \pm 1.84 \mathrm{~cd}$ & $3.79 \pm 0.66 \mathrm{a}$ & $3.38 \pm 0.78 \mathrm{c}$ \\
& 400 & $7.96 \pm 0.20 \mathrm{~d}$ & $23.73 \pm 1.38 \mathrm{~b}$ & $14.60 \pm 6.74 \mathrm{dc}$ & $1.98 \pm 0.25 \mathrm{~b}$ \\
\hline
\end{tabular}


Data treatment and statistical analysis

Filter run time, filtered flowrate, inlet and outlet filter pressure, inlet and outlet effluent parameters, number of automatic back-washings and back-washing water volume were recorded every minute by the SCADA system.

Removal turbidity efficiencies were calculated using Eq. (1):

$$
R E_{\text {Turbidity }}=\frac{\text { Turbidity }_{i}-\text { Turbidity }_{o}}{\text { Turbidity }_{i}}
$$

where $R E_{\text {Turbididy }}$ is filter removal efficiency for turbidity, dimensionless; Turbidity $i$ is filter inlet turbidity, FTU; and Turbidityo is filter outlet turbidity, FTU.

Backwashing water consumption was computed with Eq. (2):

$$
B W C=\frac{B W V}{F W V+B W V} \cdot 100
$$

where $B W C$ is the percentage of backwashing water consumption, dimensionless; $B W V$ is backwashing water volume, $\mathrm{m}^{3}$; and FWV is filtration water volume, $\mathrm{m}^{3}$.

In order to assess the effect of the different independent variables (sand size and inlet pressure) on filtered volume, filter removal efficiency for turbidity and BWC, an analysis of variance (ANOVA) was carried out using the IBM SPSS Statistical program (IBM, Armonk, New York, USA). As filtration velocity varied for each target inlet pressure, as it was discussed in Section 2.3, it was initially included in the statistical analysis as a co-variable. However, if filtration velocity had not a significant effect, which happened for most of the parameters, the ANOVA was carried out again without including it as co-variable. Tukey's pairwise comparison test was used to identify if means were different. The significance level used in the statistical analyses was set at 0.05 .

\section{Results and Discussion}

Filtered volume

According to the analysis of variance, filtered volume was significantly dependent on sand media size and filter inlet pressure $(\mathrm{P}<0.05)$. As the interaction between both factors was not significant $(\mathrm{P}>0.05)$, the effect of sand media size and filter inlet pressure will be analysed separately (Figures 2 and 3).

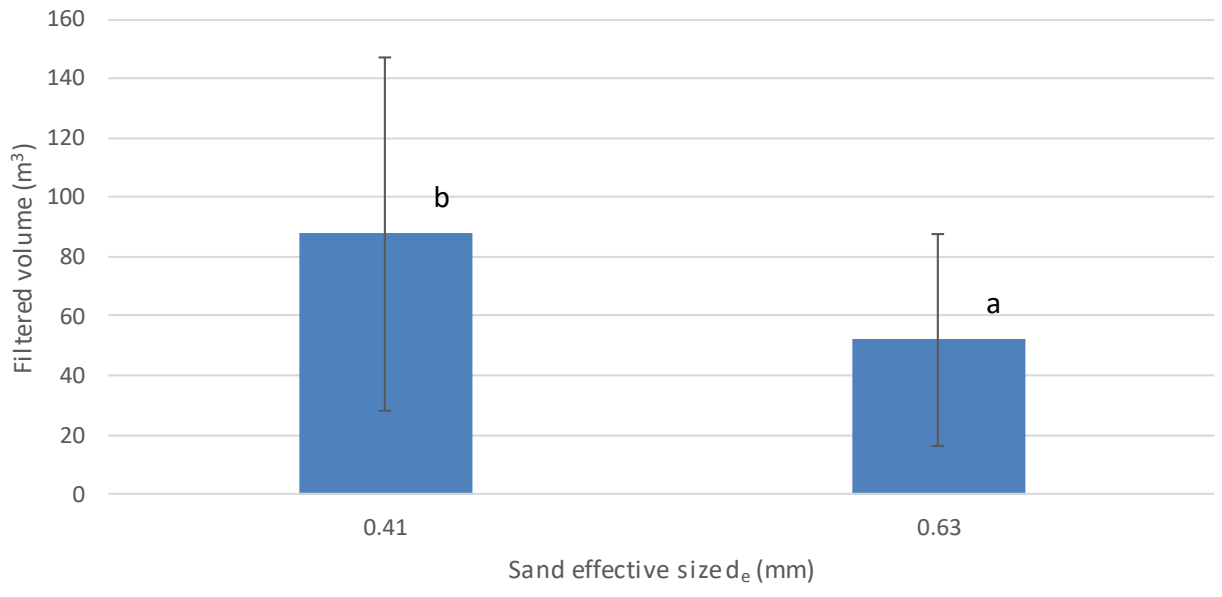

Figure 2. Effect of sand media size on filtered volume. Different letter means significant differences $(\mathrm{P}<0.05)$. 


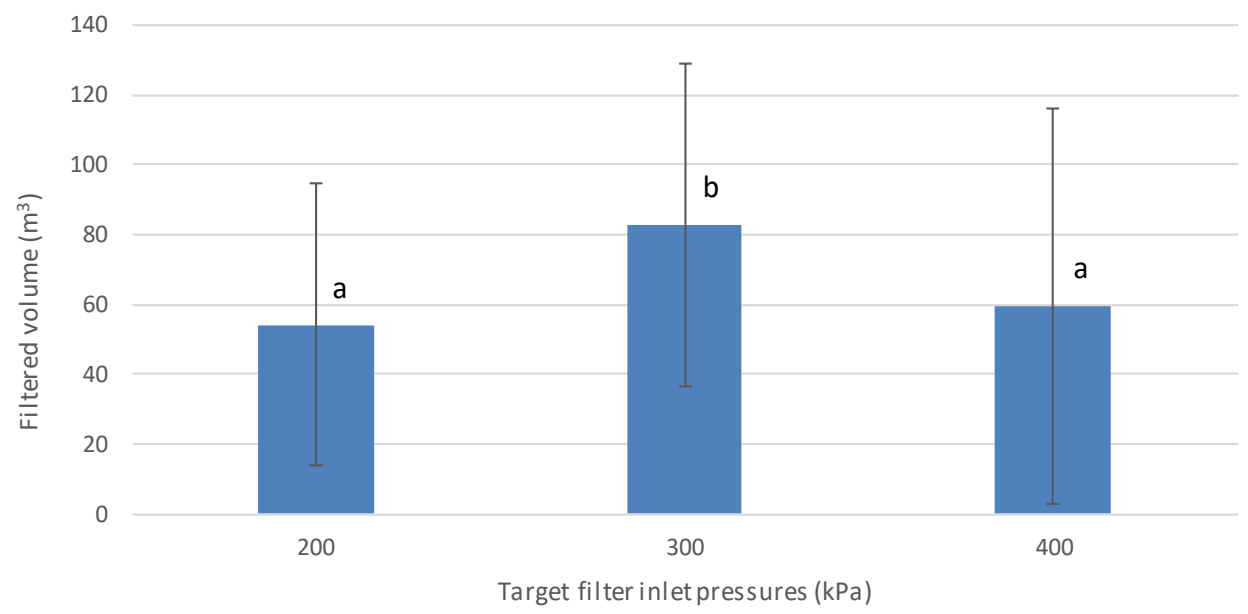

Figure 3. Effect of filter inlet pressure on filtered volume. Different letter means significant differences $(\mathrm{P}<0.05)$.

According to Figure 2, filtered volume was higher when a sand effective size of $0.41 \mathrm{~mm}$ was used. This does not agree with Duran-Ros et al. (2009a), who found that filtration cycles were larger with greater sand sizes due to its lower pressure loss. When greater sand size sand was used effluent turbidity was clearly smaller (9.00 FTU on average) regarding the effluent supplied when smaller sand was in the filters (that had a mean value of 14.82 FTU), as it has been shown in Table 2. However, $\mathrm{pH}$ was more basic during the experiments with $\mathrm{d}_{\mathrm{e}}=0.63 \mathrm{~mm}$. Average temperature $\left(25.32^{\circ} \mathrm{C}\right.$ versus $\left.24.20^{\circ} \mathrm{C}\right)$ was also higher in these experiments, meaning that biological growth could be promoted causing more clogging and faster pressure loss across sand media filter. Lower values of dissolved oxygen for the reclaimed effluent when sand with $\mathrm{d}_{\mathrm{e}}=0.63 \mathrm{~mm}$ was used can be explained by this higher temperature, as warm water holds less oxygen than cold water, or by an increase in microbiological activity. Thus, it might be that the reclaimed effluent that the WWTP supplied when coarser sand was used, had more biological pollutant load and, consequently, filtration cycles were shorter. However, the increase in the ratio of pressure loss across the filter and filtered volume (Table 3) show no significant differences between filtration carried out with different sand sizes and inlet pressures, with averages of 1.21 and $1.18 \mathrm{kPa} \mathrm{m}^{-3}$ for 0.41 and 0.63 $\mathrm{mm}$ sand size, respectively.

Table 3. Mean \pm standard deviation of the increase of pressure loss regarding filtered volume. No significant differences were found $(\mathrm{P}>0.05)$.

\begin{tabular}{ccc}
\hline $\begin{array}{c}\text { Sand effective size } \mathrm{d}_{\mathrm{e}} \\
(\mathrm{mm})\end{array}$ & $\begin{array}{c}\text { Target filter inlet } \\
\text { pressure }(\mathrm{kPa})\end{array}$ & $\begin{array}{c}\text { Pressure loss/filtered volume } \\
\left(\mathrm{kPa} \mathrm{m}^{-3}\right)\end{array}$ \\
\hline 0.41 & 200 & $1.20 \pm 1.22$ \\
& 300 & $0.47 \pm 0.18$ \\
& 400 & $1.96 \pm 1.65$ \\
\hline 0.63 & 200 & $1.22 \pm 0.68$ \\
& 300 & $0.90 \pm 0.82$ \\
& 400 & $1.41 \pm 0.75$ \\
\hline
\end{tabular}

Turbidity removal

Turbidity removal was significantly dependent $(\mathrm{P}<0.05)$ on sand media size and filter inlet pressure, being the interaction between these factors not significant. So, the effect of sand size and filter inlet pressure on turbidity removal is analysed independently in Figures 4 and 5, respectively. 


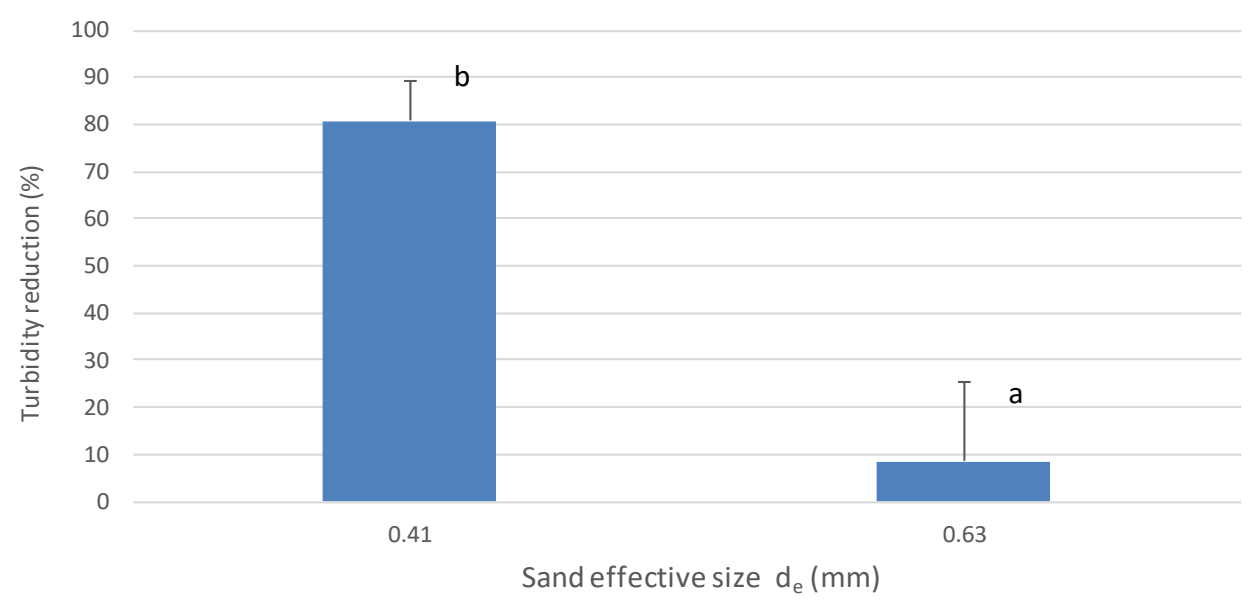

Figure 4. Effect of filter sand effective size on turbidity removal. Different letter means significant differences $(\mathrm{P}<0.05)$.

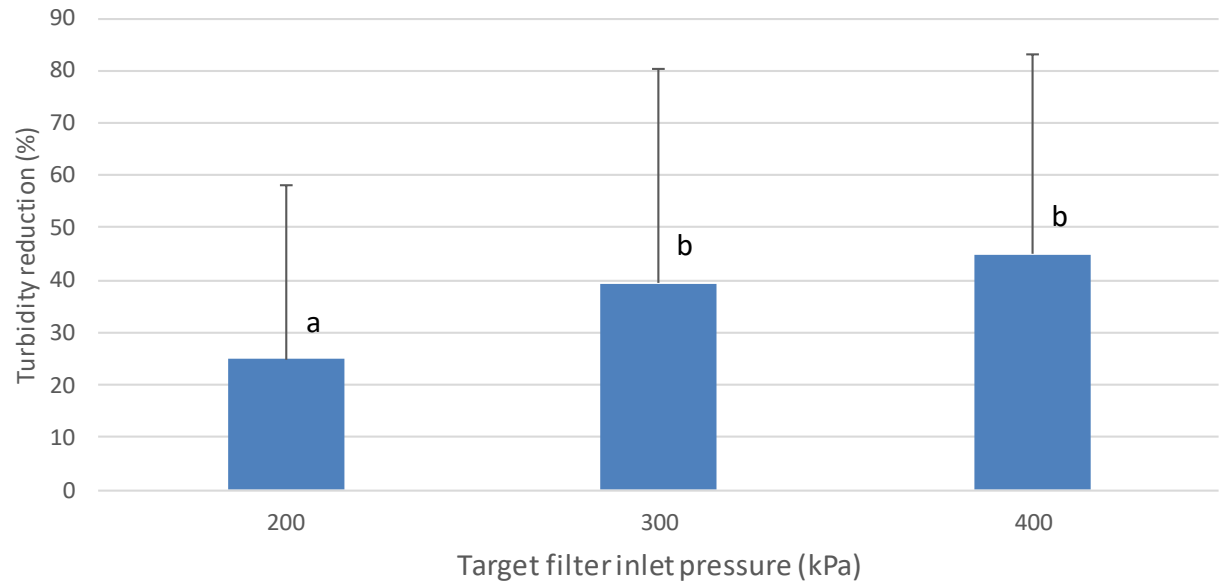

Figure 5. Effect of filter inlet pressure on turbidity removal. Different letter means significant differences $(\mathrm{P}<0.05)$.

Turbidity removal was $80.88 \%$ with $\mathrm{de}_{\mathrm{e}}=0.41 \mathrm{~mm}$ sand size but drop down to $8.43 \%$ with sand with a $\mathrm{de}_{\mathrm{e}}=0.63$ $\mathrm{mm}$. Removals achieved were higher and lower for smaller and larger sand effective size, respectively, that removals observed by Duran-Ros et al. (2009a) and Elbana et al. (2012) working with similar effluents. The average $8.43 \%$ turbidity removal observed by $0.63 \mathrm{~mm}$ effective size was also clearly below other reported turbidity removals using other effluents (Puig-Bargués et al., 2005; Gómez et al., 2010; Tripahti et al., 2014). Higher inlet turbidity values (Table 2) do not explain this different performance in turbidity removal.

Average turbidity removal was $45.00 \%$ for a filter inlet pressure of $400 \mathrm{kPa}$. However, this turbidity removal efficiency was not significantly different than removal achieved working at $300 \mathrm{kPa}$, which was of $39.37 \%$. When the target inlet pressure was reduced to $200 \mathrm{kPa}$, turbidity removal $(24.89 \%)$ was significantly smaller $(\mathrm{P}<0.05)$ than that observed at both 300 and $400 \mathrm{kPa}$ pressures. Better filter performance at higher pressures was also observed by Duran-Ros et al. (2009b) in disc and screen filters. Although an inlet pressure of $400 \mathrm{kPa}$ yielded the best turbidity removal, the increase in energy consumption needed for reaching this pressure should be taken into account for analysing if is interesting to work at this pressure or, it is better to reduce energy costs diminishing the filter inlet pressure to $300 \mathrm{kPa}$.

Backwashing water consumption

The volume of water used for filter backwashing was measured using a turbine volume-metre (Figure 1) and recorded in the SCADA system. The percentage of backwashing water consumption was computed using Eq. (2) and it is shown in Table 4. 
Table 4. Mean \pm standard deviation of backwashing water consumption (\%).

\begin{tabular}{ccc}
\hline $\begin{array}{c}\text { Sand effective size } \mathrm{d}_{\mathrm{e}} \\
(\mathrm{mm})\end{array}$ & $\begin{array}{c}\text { Target filter inlet } \\
\text { pressure }(\mathrm{kPa})\end{array}$ & $\begin{array}{c}\text { Backwashing water consumption } \\
(\%)\end{array}$ \\
\hline 0.41 & 200 & $0.73 \pm 0.69 \mathrm{ab}$ \\
& 300 & $0.29 \pm 0.11 \mathrm{a}$ \\
& 400 & $1.03 \pm 0.84 \mathrm{~b}$ \\
\hline 0.63 & 200 & $1.06 \pm 0.61 \mathrm{~b}$ \\
& 300 & $0.35 \pm 0.28 \mathrm{a}$ \\
& 400 & $1.27 \pm 1.08 \mathrm{~b}$ \\
\hline
\end{tabular}

Only filter inlet pressure had a significant effect $(\mathrm{P}<0.05)$ on backwashing water consumption, but not sand effective size and the interaction between sand size and pressure. Results of mean separation of backwashing water consumption regarding pressure are shown in Figure 5. Backwashings carried out when filter inlet pressure was $300 \mathrm{kPa}$ significantly consumed less backwashing water $(0.32 \% \mathrm{BWC}$ on average) than those consumed for inlet pressures of 200 and $400 \mathrm{kPa}(0.89 \%$ and $1.15 \%$ BWC, respectively). These values were obtained with a similar number of filtration cycles, that ranged from 43 to 51 for inlet pressures of 400 and $200 \mathrm{kPa}$, respectively.

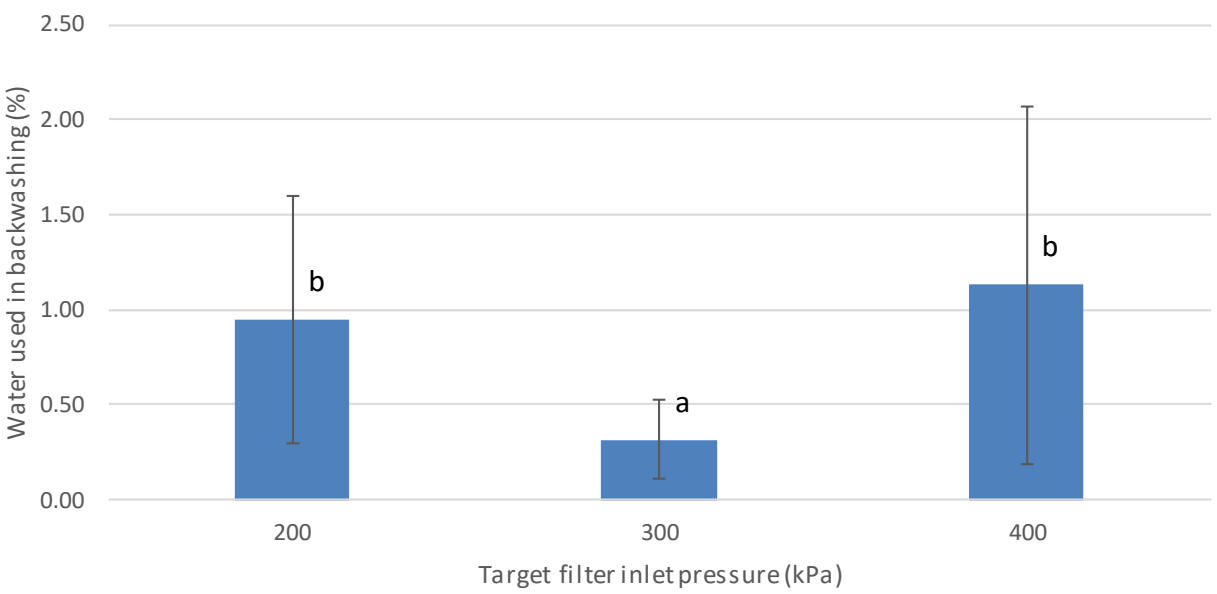

Figure 6. Effect of filter inlet pressure on the percentage of water used in backwashings. Different letter means significant differences $(\mathrm{P}<0.05)$.

Average backwashing water consumption percentages have been below $1.15 \%$, which are similar to those found by Duran-Ros et al. (2009a) (1.1\% BWC with sand with $\left.\mathrm{d}_{\mathrm{e}}=0.40 \mathrm{~mm}\right)$, Ravina et al. (1997) (0.5-1.5\% with $\mathrm{de}=1.0 \mathrm{~mm}$ ) but smaller than the $3.0 \%$ observed by Tajrishy et al. (1994) with $\mathrm{d}_{\mathrm{e}}=0.45 \mathrm{~mm}$.

\section{Conclusions}

The present work aimed to determine the performance in removing turbidity, and therefore reducing emitter clogging risk, when reclaimed effluent are used as irrigation water, of a pressurised sand media filter commonly used in micro irrigation systems. Two different effective sand sizes and three different filter inlet pressures were tested in this experiment. Despite that experiment was carried out at different filtration velocities for the different inlet pressures, the effect of filtration velocities was not statistically significant on results. Turbidity removal was significantly increased with a sand effective size of $0.41 \mathrm{~mm}$ and both filter inlet pressures of 300 and $400 \mathrm{kPa}$. Smaller turbidity reductions were observed with a sand effective size of $0.63 \mathrm{~mm}$ and filter inlet pressure of 200 $\mathrm{kPa}$.

Backwashing water consumption only was significantly dependent on filter inlet pressure, being the minimum average $(0.32 \%)$ reached with an inlet filter pressure of $300 \mathrm{kPa}$.

According to the results, the best sand filter performance is achieved using sand with an effective size of 0.41 $\mathrm{mm}$ and operating under a filter inlet pressure of $300 \mathrm{kPa}$, as these conditions maximize turbidity removal and minimize backwashing water consumption.

\section{Acknowledgements}

The authors would like to express their gratitude to the Spanish Ministry of Economy, Industry and Competitiveness for their financial support for this experiment through grant AGL2015-63750-R. 


\section{References}

Capra, A., Scicolone, B., 2004. Emitter and filter tests for wastewater reuse by drip irrigation. Agricultural Water Management. 68 (2), 135-149. https://doi.org/10.1016/j.agwat.2004.03.005.

de Deus, F.P., Testezlaf, R., Mesquita, M., 2015. Eficiência de filtros de areia pressurizados na remoção de diferentes tamanhos de partículas da água de irrigação. Pesquisa Agropecuária Brasileira. 50 (10), 939-948. https://doi.org/10.1590/S0100-204X2015001000010.

Duran-Ros, M., Puig-Bargués, J., Arbat, G., Barragán, J., Ramírez de Cartagena, F., 2008. Definition of a SCADA system for a microirrigation network with effluents. Computers and Electronics in Agriculture. 64, (2), 338-342. https://doi.org/10.1016/j.compag.2008.05.023.

Duran-Ros, M., Puig-Bargués, J., Arbat, G., Barragán, J., Ramírez de Cartagena, F., 2009a. Effect of filter, emitter and location on clogging when using effluents. Agricultural Water Management. 96 (1), 67-79. https://doi.org/10.1016/j.agwat.2008.06.005.

Duran-Ros, M., Puig-Bargués, J., Arbat, G., Barragán, J., Ramírez de Cartagena, F., 2009b. Performance and backwashing efficiency of disc and screen filters in microirrigation systems. Biosystems Engineering. 103 (1), 35-42. https://doi.org/10.1016/j.biosystemseng.2009.01.017.

Elbana, M., Ramírez de Cartagena, F., Puig-Bargués, J., 2012. Effectiveness of sand media filters for removing turbidity and recovering dissolved oxygen from a reclaimed effluent used for micro-irrigation. Agricultural Water Management. 111, 27-33. https://doi.org/10.1016/j.agwat.2012.04.010.

Gómez, M., Plaza, F., Garralón, G., Pérez, J., Gómez, M.A., 2010. Comparative analysis of macrofiltration processes used as pre-treatment for municipal wastewater reuse. Desalination. 255, 72-77. https://doi.org/10.1016/j.desal.2010.01.014.

ICID, 2017. Sprinkler and micro irrigated area. URL http://www.icid.org/sprinklerandmircro.pdf. Accessed April 12, 2018.

Nakayama, F.S., Boman, B.J., Pitts, D.J., 2007. Maintenance, in: Lamm, F.R., Ayars, J.E., Nakayama, F.S. (Eds.), Microirrigation for Crop Production. Elsevier, Amsterdam, pp. 389-430.ç

Nakayama, F.S., Bucks, D.A., 1991. Water quality in drip/trickle irrigation: A review. Irrigation Science. 12 (4), 187-192. https://doi.org/10.1007/BF00190522.

Puig-Bargués, J., Barragán, J., Ramírez de Cartagena, F., 2005. Filtration of effluents for microirrigation systems. Transactions of the ASAE. 48 (3), 969-978. https://doi.org/10.13031/2013.18509.

Ravina, I., Paz, E., Sofer, Z., Marcu, A., Schischa, A., Sagi, G., Yechialy, Z., Lev, Y., 1997. Control of clogging in drip irrigation with stored treated municipal sewage effluent. Agricultural Water Management. 33 (2-3), 127-137. https://doi.org/10.1016/S0378-3774(96)01286-3.

Tajrishy, M.A., Hills, D.J., Tchobanoglous, G., 1994. Pretreatment of secondary effluent for drip irrigation. Journal of Irrigation Drainage Engineering. 120 (4), 716-731. https://doi.org/10.1061/(ASCE)07339437(1994)120:4(716).

Tarjuelo, J.M., Rodriguez-Díaz, J.A., Abadía, R., Camacho, E., Rocamora, C., Moreno, M.A., 2015. Efficient water and energy use in irrigation modernization: Lessons from Spanish case studies. Agricultural Water Management. 162, 67-77. https://doi.org/10.1016/j.agwat.2015.08.009.

Tripathi, V.K., Rajput, T.B.S., Patel, N., 2014. Performance of different filter combinations with surface and subsurface drip irrigation systems for utilizing municipal wastewater. Irrigation Science. 32 (5), 379-391. https://doi.org/10.1007/s00271-014-0436-2.

Trooien, T.P., Hills, D.J., 2007. Application of biological effluent, in: Lamm, F.R., Ayars, J.E., Nakayama, F.S. (Eds.), Microirrigation for Crop Production. Elsevier, Amsterdam, pp. 329-356.

Wen-Yong, W., Yan, H., Hong-Lu, L., Shi-Yang, Y., Yong, N., 2015. Reclaimed water filtration efficiency and drip irrigation emitter performance with different combinations of sand and disc Filters. Irrigation and Drainaige. 64 (3), 362-369. https://doi.org/10.1002/ird.1909.

WWAP, 2017. The United Nations World Water Development Report 2017. Wastewater: The Untapped Resource. UNESCO, Paris. 


\title{
Development of New Version of the Spiked Shear Vane for Measuring of the Peatland Top Roots Layer Strength
}

\author{
Adam P. Dubowski ${ }^{\text {a,* }}$, Krzysztof Zembrowski ${ }^{\text {a }}$ \\ a PIMR - Industrial Institute of Agricultural Engineering, 60-963 Poznan, Poland \\ * Corresponding author. Email: ad@man.poznan.pl, ad@pimr.eu
}

\begin{abstract}
Many wetlands in Poland require special care to restore them as breeding areas for endangered bird species. Using tracked snow groomers, tracked trailers and farm tractors is not eco-friendly practice and can conflict with conservation goals. To improve protection of these sites Industrial Institute of Agricultural Engineering (PIMR) developed tracked vehicles and technologies that will be more useful in formal paludiculture. Spiked shear vane is manually rotated using a commercial torque wrench equipped with gyroscopic sensor. Tool is good but its measurement only describes strength of peat in small area. Increased diameter of the plate should yield a truer estimation of the peat top layer strength. After preliminary field tests new version of the plate was build. Radius of plate increased from $0.1 \mathrm{~m}$ to $0.194 \mathrm{~m}$, spikes length (0.062) is over twice shorter comparing with first one $(0.163 \mathrm{~m})$. Number of spikes was preliminary increased from 13 to 25 (two axis of symmetry), in next step increased to 45 spikes (four axis of symmetry) and in newest version of the plate reduced again to 25 spikes on four axis of symmetry. The length of spikes corresponds to the operating zone of track's bars of vehicles that were built in PIMR as well bars that are used in snow grooming vehicles in Poland. The volume of the sample rotated by the spikes - corresponds to $20,6 \%$ of the volume of the top roots layer which is between the bars of one track of the PIMR's vehicle. Spiked tool is equipped with removable wheels for easier transport handling in the field. New version of tool will facilitate relatively fast manual measurement of peat top roots layer strength and should be helpful in estimating mobility on wetlands of different types of tracked vehicles with total mass up to $7 \mathrm{t}$.
\end{abstract}

Keywords: spiked plate, track, vehicle, environment, protection

\section{Introduction}

Many wetlands in Poland, as well as in Europe, require special care to restore them as breeding areas for endangered bird species. Using tracked snow groomers, tracked trailers and farm tractors is not eco-friendly practice and can conflict with conservation goals, mainly, through disturbance of the ground surface (Banaszuk et al., 2016; Dubowski et al., 2013, 2014; Zembrowski et al., 2014a, 2016, 2017; Pawlowski et al., 2014). Environment problems with use of snow groomers were also noticed in Germany (Schroeder et al., 2015; Wichtmann et al., 2016).

To improve protection of these sites Industrial Institute of Agricultural Engineering (PIMR) developed tracked vehicles and technologies that will be more useful in formal paludiculture (Pawlowski et al., 2014; Zembrowski et al., 2014b, 2015, 2016, 2017). Problem of proper peatland strength is very important for safety operations and work of the different types of vehicles especially these that were designed and tested by PIMR. The mobility of the vehicles unit within the boggy terrains usually depends on the strength of the plants and their roots in the upper layer of the peat.

\section{Materials and Methods}

In PIMR a penetrometer and a moisture meter were used to measure the strength of the upper root layer but it turned out that Penetrologger (EIJKELKAMP) with conical head was not very useful and reliable for measuring mechanical properties of the peat layer in the earlier field tests (Dubowski et al., 2012). In PIMR, the Penetrolloger tool has been modified, that is: the cone head, dia. $25.4 \mathrm{~mm}$, with $30^{\circ}$ - top angle was replaced with the plate head with the same base area $\left(5 \mathrm{~cm}^{2}\right)$ - it was adapted to measure modulus of elasticity (Zembrowski et al., 2016).

The analysis of results obtained by Uusitalo and Ala-Ilomäki (2013), during field trials led to similar conclusions: in peaty soils the penetrometer gives poor estimations on the properties of the topsoil. Instead of using penetrometer they designed manually operated plate loading device for measuring the modulus of elasticity, the diameter of the plate applied was $0.07 \mathrm{~m}$.

For measuring the shear modulus of the top vegetation layer - the manually operated spiked shear vane (spiked plate) was built. It is based on the idea of Ala-Ilomaki (2013) from the METLA - Finish Forest Research Institute. In Finland, spiked plate was found to be significant predictor for quantifying rut depth of harvesters and forwarders (net mass 7-12t). During the logging operation, the average rut depth of the harvester varied from 7 to $29 \mathrm{~cm}$ and rut depth after forwarding from 9 to $32 \mathrm{~cm}$. 
PIMR's version of spiked plate with digital torque wrench

PIMR's version of the spiked plate, similar to the original vane head designed by Ala-Ilomaki (2013) has two rows of steel spikes attached perpendicular to a circular steel base plate. Diameter of plate is $0.22 \mathrm{~m}$, its thickness $10 \mathrm{~mm}$, spikes with length $0.163 \mathrm{~m}$ and a diameter $12 \mathrm{~mm}$ (Figure 1). The distance of spike hole centres from the plate centre were $0 \mathrm{~mm}, 31.33 \mathrm{~mm} 62.66 \mathrm{~mm}$ and $94.00 \mathrm{~mm}$. To the plate's upper surface is attached a handle steel tube (outer dia. $40 \mathrm{~mm}$, thickness of $3 \mathrm{~mm}$ with square hole (Figure 2) for the tip of the digital torque wrench. To insert the spikes of the plate into the top root layer, is hit perpendicularly against the root mat to facilitate the compression of any loose material (Figure 3). Handle design is adequate to omit its torsion angle during measuring operation. The tool is manually rotated by $5-15^{0}$ using digital torque wrench. Mass of the spiked tool is $7.9 \mathrm{~kg}$.

In PIMR we use the FACOM torque wrench model: E.316-340D equipped with gyroscopic measurement of angular rotation and strain gauge measurement of torque. Mass of torque wrench is $2.5 \mathrm{~kg}$.

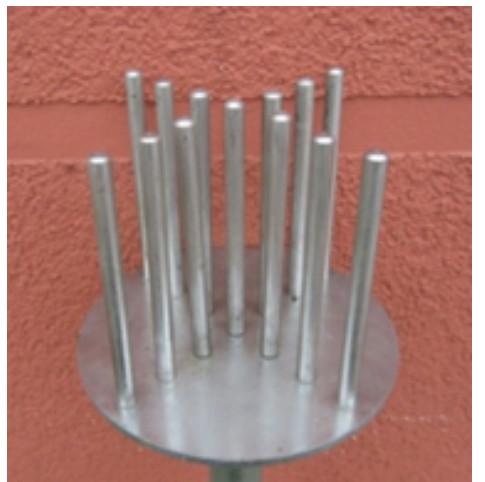

Figure 111. PIMR's version of the spiked plate (spiked shear vane) (archive PIMR-BE).

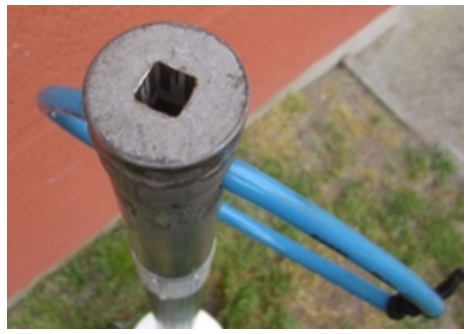

Figure 2. Square hole for the tip of the torque wrench (archive PIMR-BE).

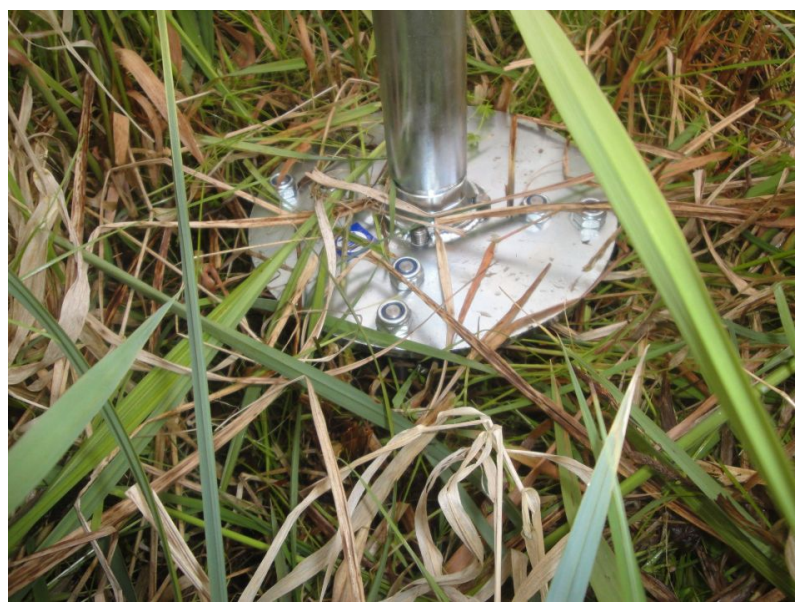

Figure 3. Spiked plate (build in PIMR) during field tests (archive PIMR-BE).

The advantage of this method of measuring shear modulus is the simplicity and speed of measurement, the practical lack of damage to the root system of plants and the small nuisance of measurements when measured moments of forces do not exceed $100 \mathrm{Nm}$. However, some difficulties with this method are the necessity of manual transport of the spiked plate instrument and torque wrench and a considerable effort related to the measurement of the moment of forces in the range up to $340 \mathrm{Nm}$. While conducting research on a more concise ground, the need to perform several dozen measurements of shear modulus is becoming a very exhaustive task.

In order to facilitate measurements with a torque wrench and to reduce the effort of people who measure shear modulus on the torque wrench handle, an additional pipe was assumed which increased the lever arm length by about $0.5 \mathrm{~m}$ (total wrench mass with the tube is $2.7 \mathrm{~kg}$ ) (Figure. 4). 


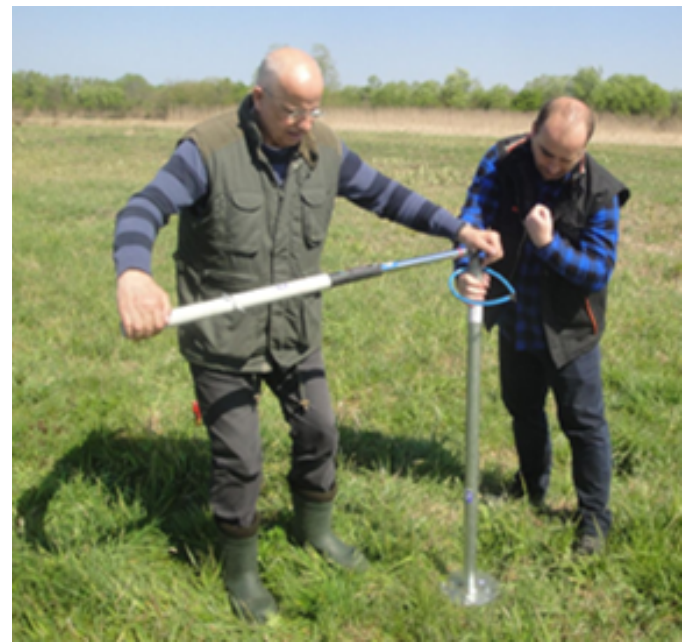

Figure 4. Torque wrench handle with a pipe as an additional arm (archive PIMR-BE).

Spiked plate is good but output of 13 spikes shear vane measurement only describes strength prevailing in very small spots. After preliminary field tests we took decision to increase diameter of the plate, this should yield a truer estimation of the top layer strength of the peat.

New models of the spiked plate

First model of the plate (dia. 0,42 m) was based on the design of the 13 spikes plate it is on the two axis of symmetry were placed 25 spikes. The distance between each pair of spikes' hole centres from the plate centre was $31.33 \mathrm{~mm}$. Length of the. spike was reduced to $62 \mathrm{~mm}$ (Figure 5).

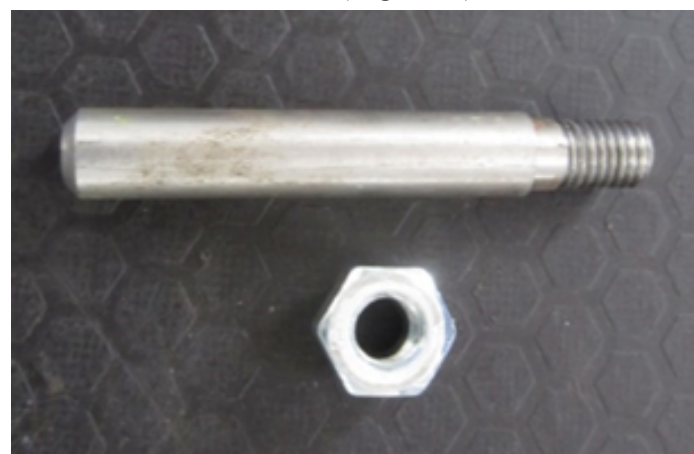

Figure 5. Short spike (length $62 \mathrm{~mm}$, dia. $12 \mathrm{~mm}$ ), with self-locking nut (archive PIMR-BE).

Second model of the plate had four axis of symmetry with total number of the spikes 45 (Figure $6 \& 7$ ), other parameters of spikes placement and theirs dimensions were as in first model. Increasing up to four the number of axis of symmetry, in which the spikes are mounted, allows for a more even transfer of the moment of strength from the torque wrench to a cylindrical sample of peat soil that rotated by an angle of $5-15^{0}$.

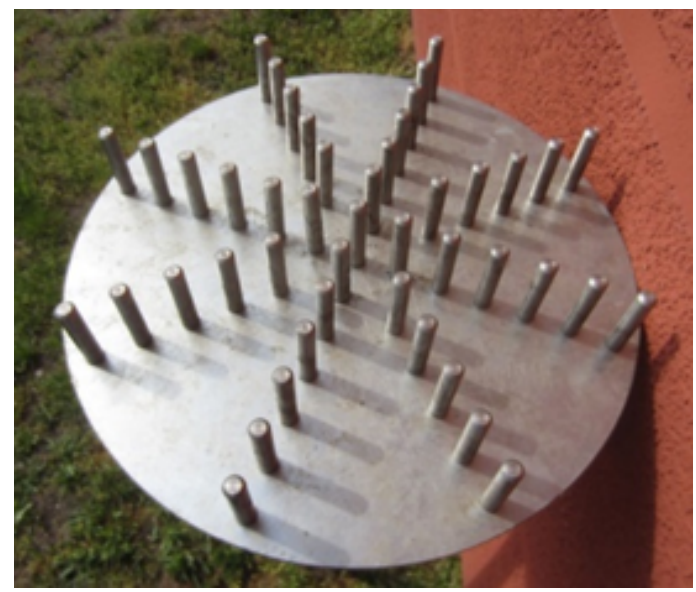

Figure 6. Spiked plate with 45 spikes (archive PIMR BE). 


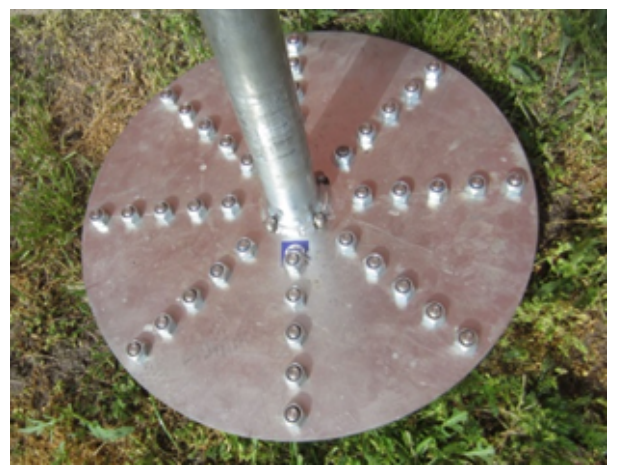

Figure 7. The spikes were mostly bolted using self-locking nuts (archive PIMR BE).

In the next short field tests, second model of the plate was working fine, but too short distance between spikes caused that plate was not easy to clean from the residues of the peat, but much easier than in the case of spiked plate with 13 spikes (Figure 8).

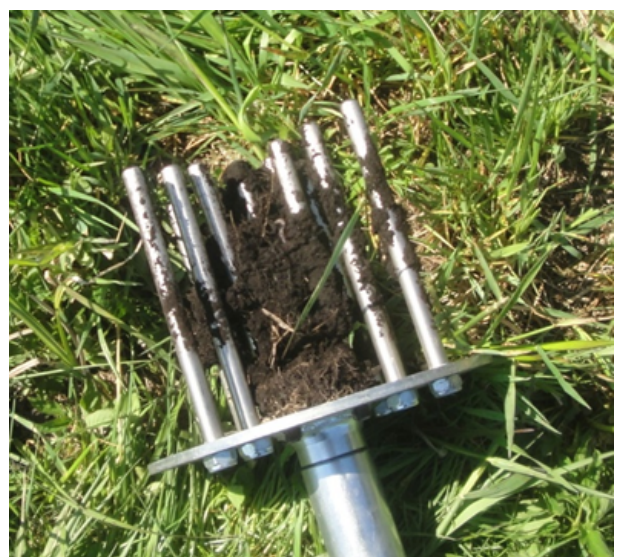

Figure 8. 13 Spikes plate with peat residues (archive PIMR-BE).

Manual transfer of the plate device was so cumbersome that it was decided to transport it on an additionally mounted axis with wheels (Figure 9). In a later phase of field work, a three-person PROWLER tracked off-road vehicle was used for transporting spiked plate (Figure 10).

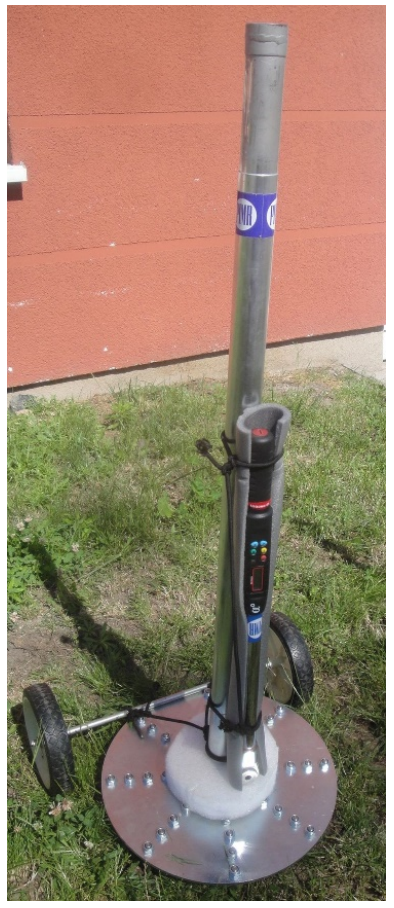

Figure 9. Spiked plate with torque wrench ready for transport on wheels (archive PIMR-BE). 


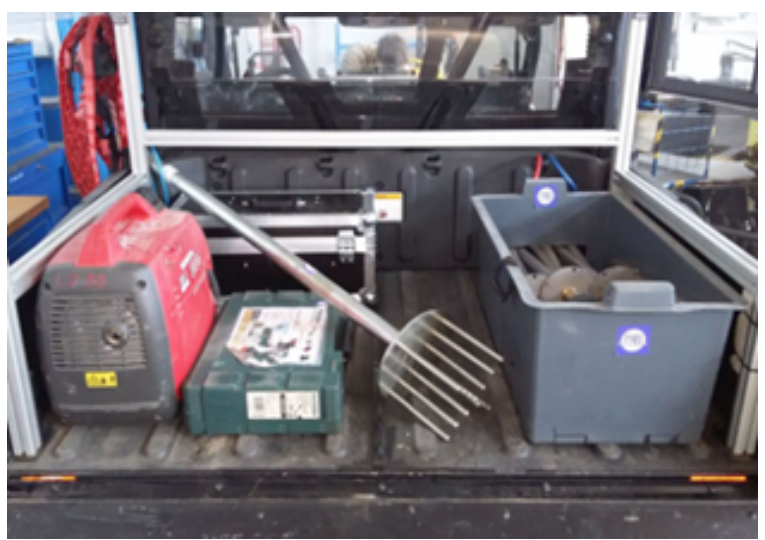

Figure 10. PROWLER - serves also for transporting spiked plate (Zembrowski et al., 2016).

New version of the spiked plate with 25 spikes

The spiked plate was modified again and spikes were spaced apart on twice longer distance $(66.66 \mathrm{~mm})$ as result the new version of the spiked plate with 25 spikes was build (Figure 11). The holes, after the removed spikes, have not been blinded to facilitate the evacuation of air and water from underneath the plate that is rapidly immersed in the boggy peat surface.

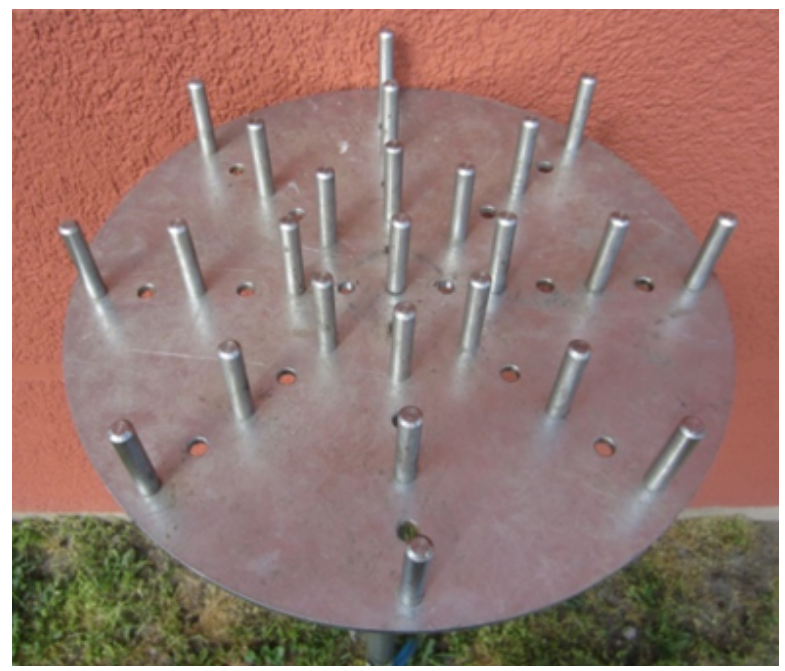

Figure 11. View of the new version of the plate with 25 spikes (archive PIMR-BE).

\section{Results and Discussion}

The new version of the spiked plate, with 25 spikes, is designed to determine the strength of the top peat roots layer in the working zone of tracks of the tracked vehicles developed and built in PIMR (Figure 12). The weight of the tracked tractor is about $5 \mathrm{t}$, the pressure during the penetration of bars into the ground is $\sim 0.037 \mathrm{MPa}$, and under the support rubbers it decreases by up to $0.011 \mathrm{MPa}$. In the course of the research, tracks of PIMR's vehicles would not be observed to sink deeper than the height of the bars $(41 \mathrm{~mm})$. The length $(62 \mathrm{~mm})$ of the spikes of the newer version of the plate also corresponds approximately to the height of metal bars mounted in snow grooming vehicles adapted in Poland for work on wetlands (Dubowski et al., 2013; Pawlowski et al., 2014).

Only one model of the delta type track is used in PIMR's tracked vehicles (Figure 13, Pawlowski et al., 2014; Zembrowski et al., 2014, 2017)). Each bar of the track has length $0.786 \mathrm{~m}$, height $41 \mathrm{~mm}$ and width $43 \mathrm{~mm}$. When moving on wetlands, merely 12 steel bars of the each track penetrates into the ground, it is on the length $(1.3 \mathrm{~m})$ of the track which is under its 5 support wheels. Estimated volume of the soil between 12 bars of the track is approximately $0.034 \mathrm{~m}^{3}$.

PIMR tracked vehicles practically do not devastate the ground, they do not cause its levelling as it has in the case of ratracs (snow grooming vehicles adapted to work on wetlands) and not making deep ruts like it is in the case of much heavier harvesters and forwarders.

Data on the strength (shear modulus) of the upper root layer of the peat, collected using spiked plates should be helpful in determining the areas on which workers as well as tracked vehicles can safely move on. 


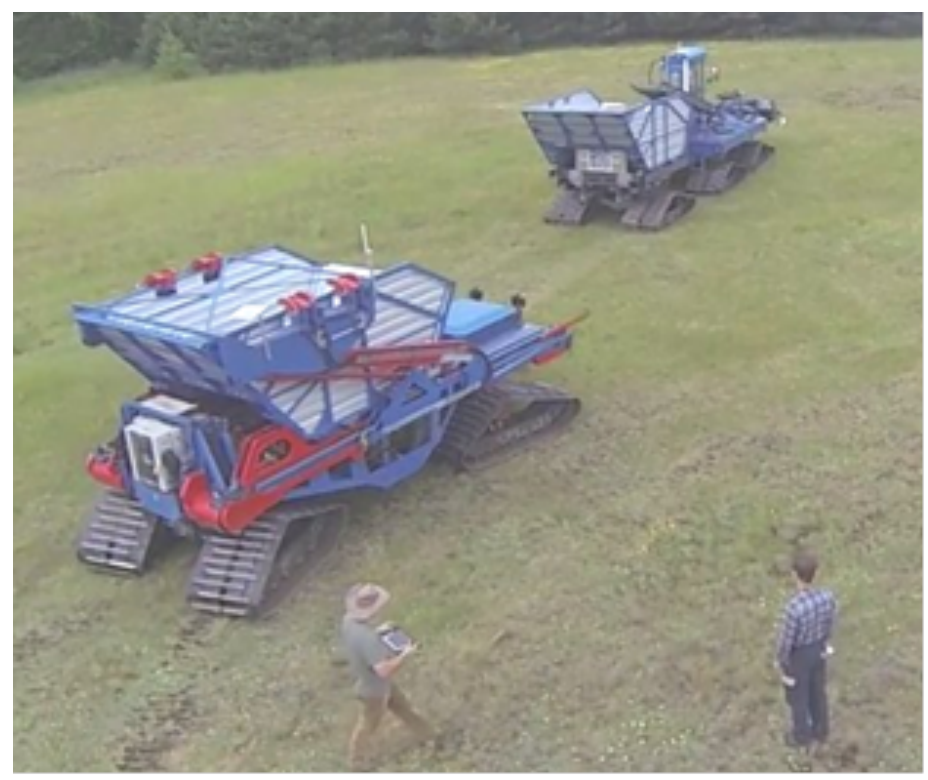

Figure 12. Preliminary field tests with autonomous tracked vehicle and tracked unit near Trzcianka, Poland. In the pilot tests the tracked vehicle was steered by remote control panel (Zembrowski et al., 2017).
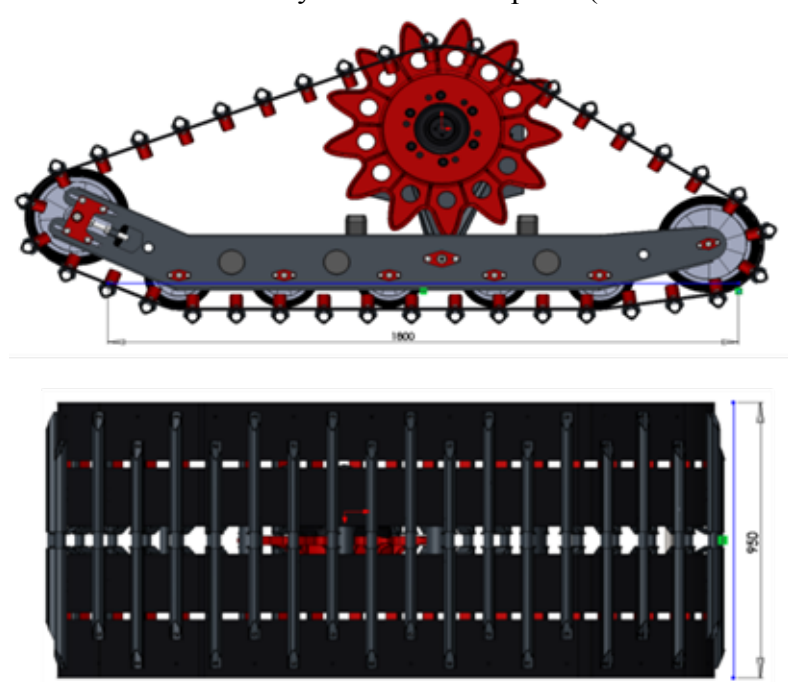

Figure 13. Delta type track designed by PIMR. Usually, only 12 bars of the track penetrate into the ground (archive PIMR$\mathrm{BE})$.

Comparison of surface and volume of both spiked plates (SP) is shown in the table 1.

Table 1.

\begin{tabular}{cccc}
\hline Parameter / Version of SP & $25 \mathrm{SP}$ & $13 \mathrm{SP}$ & Ratio of the 25/13 SP \\
\hline Surface of the soil sample $\left[\mathrm{m}^{2}\right]$ & 0.1182 & 0.0314 & 3.76 \\
Volume of the soil sample $\left[\mathrm{m}^{3}\right]$ & 0.007 & 0.005 & 1.4 \\
\hline
\end{tabular}

In the newer version of spiked plate with 25 spikes the sample surface is 3.76 times bigger than in the original version, in the analogical comparison, its volume is 1.4 times bigger.

Volume of soil sample rotated by spiked plate with 25 spikes is $20.6 \%$ of volume of peat layer between 12 bars of one track of the PIMR's vehicle. 


\section{Conclusions}

When compared to the original spiked shear vane (13 spikes), the new version of the spiked plate with 25 spikes seems be better and more appropriate for measuring the strength of the peat top roots layer, especially from point of view of work of the tracked vehicles with total mass up to $7 \mathrm{t}$, which are designed and used by PIMR.

The results that will be obtained during measurements, with use of new spiked plate, should be helpful in estimating mobility of different types of tracked vehicles on wetlands, this is important for safety of theirs work as well as for better environment protection.

We think that method of measuring strength of top root layer should be mechanized and spiked plate should be included as a part of the research stand, which should ready to be mounted on different type of vehicles.

\section{Acknowledgements}

The work related to the construction of the PIMR's tracked vehicles and the construction or adaptation of instruments for testing the strength of the peat top layer were carried out with funds from the following research projects: PBS2/B8/11/2013, UOD-DEM-1-145/00, WND-POIG.01.03.01-00-164/09 .

\section{References}

Ala-Ilomaki Jari , 2013. Spiked shear vane - a new tool for measuring peatland top layer strength. Suoseura Finish Peatland Society. ISSN 0039-5471, Suo 64 (2-3):113-118.

Banaszuk P., Kamocki A.K., Zarzecki R. , 2016. Mowing with invasive machinery can affect chemistry and trophic state of rheophilous mire. Eco. Eng. 86 (2016), 31-38.

Zembrowski K. , Dubowski A.P., Stobnicki P., 2014a. Vehicles for biomass harvesting and transportation on protected wetlands - an assessment of their work and the impact on the environment. Logistyka 4/2014, 15261535.

Zembrowski K., Dubowski A. P., Rakowicz A., Weymann S., 2014b. Development of tracks constructions for specialized vehicle. Logistyka 6/2014, pp. 11750-11758.

Zembrowski K., Dubowski A. P., Vicente N., Rakowicz A., Danielak M., Siczynski L., Stobnicki P., Weymann S., 2015. New version of the turn system of the tracks in PIMR's specialized vehicle. Logistyka 3/2015, 50695075.

Wendelin Wichtmann, Christian Schroeder \& Hans Joosten (eds.) (2016) Paludiculture - productive use of wet peatlands. Schweizerbart Science Publishers. Stuttgart.

Dubowski A. P., Zembrowski K., Weymann S., Karbowski R., Rakowicz A., Potrykowska A., Wojniłowicz L., 2012. New method for biomass bales coupling and rolling them on boggy terrain, especially in National Parks and Natura 2000 protected areas. International Conference of Agricultural Engineering CIGR AGENG2012, Valencia, Spain, July 8-12, 2012.

Dubowski A. P., Zembrowski K., Wojnilowicz L., Rakowicz A., Weymann S., Stobnicki P., Mac J., 2014. The Tracked Vehicles Unit - for stopping unwanted vegetation successions on wetlands. International Conference of Agricultural Engineering AGENG2014, Zurich, Switzerland, July 6-10 2014. http://www.geyseco.es/geystiona/adjs/comunicaciones/304/C04100001.pdf . Accessed May 17,2018.

Pawlowski T., Zembrowski K., Dubowski A. P., 2014. Integrated technology and multimodule tracked vehicles unit for wetlands protection. Scientific Proceedings XXII International Scientific Technical Conference “trans \& MOTOAUTO'14", Year XXII, Volume 2, 96-99, 2014. file://C:/Users/user/Documents/AGENG_2018/26.INTEGRATED\%20TECHNOLOGY\%20AND\%20MULTI MODULE\%20TRACKED\%20VEHICLES\%20UNIT\%20FOR\%20WETLANDS\%20PROTECTION.pdf . Accessed May 17, 2018 .

Dubowski A. P., Zembrowski K., Rakowicz A., Pawlowski T., Weymann S. , Wojnilowicz L., 2013. Developing new-generation machinery for vegetation management on protected wetlands in Poland, Mires and Peat. http://mires-and-peat.net/pages/volumes/map13/map1311.php . Accessed May 17, 2018,

Schroeder C.; Dahms T.; Paulitz J., Wichtmann W., and Wichmann S. (2015). Towards large-scale paludiculture: addressing the challenges of biomass harvesting in wet and rewetted peatlands. Mires and Peat. http://mires-and-peat.net/media/map16/map_16_13.pdf. Accessed May 17, 2018.

Uusitalo J., Ala-Ilomäki J., 2013. The significance of above-ground biomass, moisture content and mechanical properties of peat layer on the bearing capacity of ditched pine bogs. Silva Fennica vol. 47 no. 3 article id 993. https://doi.org/10.14214/sf.993. Accessed May 17, 2018.

Zembrowski K., Siczynski L., Vicente N., Stobnicki P., Pawlowski T.; Danielak M., Sobocki S., Dubowski A.P., 2016. Rebuilding of the wheeled all-terrain vehicle into the tracked mini-tractor of the biomass train. The 4th CIGR AGENG International conference „Agricultural and Biosystems Engineering - Automation, 
Environment and Food Safety” $\quad$ Aarhus, Denmark, 2016.

http://conferences.au.dk/uploads/tx_powermail/2016cigr_ageng_paper_pimr_pl.pdf. Accessed May 17, 2018.

Zembrowski K., Dubowski A. P., Polasik M., 2017. Three phase technology of harvesting and transportation of biomass from wetlands to nearby warehouses. International Conference RRR2017, Greifswald, Germany. https://www.lensescape.org/mov/moorwissen/rrr2017/presentationen/Dubowski_RRR2017_Session_2.pdf . Accessed May 17, 2018.

EIJKELKAMP. https://en.eijkelkamp.com/products/field-measurement-equipment/penetrologger-set-a.html . Accessed May 21, 2018.

FACOM - digital torque wrench. http://facom.com.pl/en/6821-e316-340d-tw-elec-torque-angle-340.html . Accessed May 16, 2018.

METLA. http://www.metla.fi/index-en.html . Accessed May 22, 2018.

PROWLER, Arctic Cat. https://intl.www.arcticcat.com/ . Accessed May 21, 2018. 


\title{
Differential Interferometry as a Tool for Localized Study of Runoff Erosion
}

\author{
Francisco A. Sánchez-Crespo*a, Eutiquio Gallego ${ }^{a}$, José M. Fuentes ${ }^{\mathrm{a}}$, Francisco Ayuga a , Ana I. García ${ }^{a}$ \\ a BIPREE Research Group, Universidad Politécnica de Madrid, Madrid, Madrid 28040, Spain \\ a* Corresponding author. Email: fasanchezcrespo@gmail.com
}

\begin{abstract}
New space missions like Sentinel-1 (European Commission and European Space Agency Copernicus Programme partnership) are able to obtain SAR (Synthetic Aperture Radar) images with high frequency, resolution, range and, above all, availability, which allows the use of techniques like differential interferometry in new fields. Interferometry technique is based in the accurate measurement of the go and back travel of electromagnetic radiation between a SAR Radar and the earth surface, registering intensity and phase of returning radiation into SAR images. Comparing two images of the same earth surface area it is possible to make an interferogram, in which the information of phase difference is highly related to terrain topography, and its deformations can be graphically represented through maps. The aim of this study is to evaluate the possibilities of using differential interferometry technique for localized monitoring of runoff erosion studies, evaluating calculation parameters, use limits and results meanings. Qualitative studies in a monitored basin in Daganzo de Arriba (Madrid) has offered good results: erosion proportional to the intensity of rain, use and land cover of each plot, and the results showed independence from crops activities as harvesting. Quantitative studies in a pilot plot in El Molar (Madrid) was made comparing interferometry results with the deformation patterns obtained with direct measurements taken with a LIDAR and processed with $\mathrm{M} 3 \mathrm{C} 2$ algorithm of CloudCompare software. Although results of both methods share the same order of magnitude and, in most cases, differences are within the RMS error, some of the measurements were not conclusive, probably due to the lack of horizontal accuracy needed to make a cell to cell comparison.
\end{abstract}

Keywords: SAR images, interferogram, LIDAR, runoff erosion

\section{Introduction}

Although differential interferometry over SAR images is not a new technique (European Space Agency ERS missions go back to 1991), the resolution, coverage, frequency and availability of images offered by Sentinel-1 mission of the Copernicus Project (joint venture of the European Space Agency and European Commission) allows new uses and applications (European Space Agency, 2003; Smith et al., 2000)

Sentinel-1 Mission:

The Sentinel-1 mission consists of two satellites equipped with synthetic aperture radars (SAR) that work in the $\mathrm{C}$ band of microwaves, which enables images to be taken both day and night, and independently of cloud conditions.

The Sentinel-1 satellites have a quasi-polar orbit with a phase shift of $180^{\circ}$ in the same plane, so each satellite visits a certain area of the Earth's surface at least once every 12 days, and each area of the Earth's surface is visited by one of the two satellites at least once every 6 days (European Space Agency, 2003).

The equipped sensors have 4 acquisition modes: Strip Map, Interferometric Wide Swath, Wave and Extra Wide Swath.

The Interferometric Wide Swath (IW) mode has a medium-high resolution ( $5 \times 20$ meters), very good coverage (250 km band) and, above all, high availability, with the images ready for downloading few hours after its acquisition. These characteristics make it the most appropriate mode for the present study.

The IW mode captures the images in three sub-strips using the Earth Observation technique with Progressive SAR Scans (TOPSAR - Terrain Observation with Progressive Scans SAR). Each of these sub-bands consists of several bursts.

The TOPSAR mode presents a series of improvements over the previous ScanSAR mode, related to the electronic control of the orientation of the bursts and synchronization between passes that manage to improve the SNR (Signal to Noise Ratio) and DTAR (Distributed Target Ambiguity Ratio), achieving images with less noise and deformations.

\section{Differential Interferometry}

Interferometry in synthetic aperture radars (InSAR) is equipped in these satellites, and it allows accurate measurement of the go and back travel of radiation, recording the intensity and phase of the electromagnetic signal in a SAR image. A SAR image is composed of a two-dimensional mosaic of elements called pixels, each referenced to a small area of the earth's surface or cell. Each pixel contains the intensity and phase information of the bounced radiation in its corresponding cell or land surface (Rucci et al. 2012). 


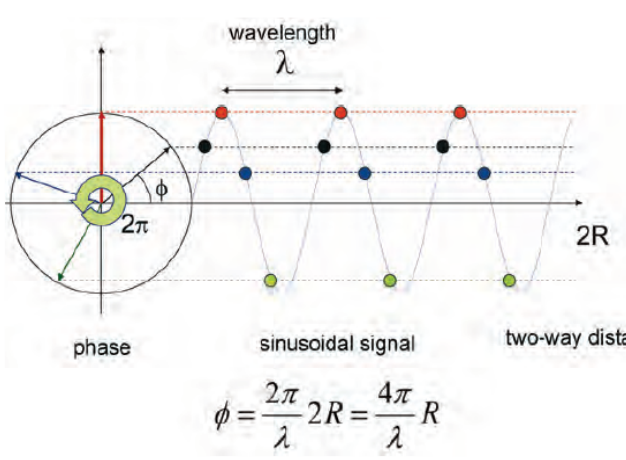

Figure 1. Phase shift (ESA-TM19 InSAR Principles)
Figure 2. Differential Interferometry satellites geometry. Source ESA-TOPS Interferometry Tutorial.

SAR interferometry takes advantage of the phase difference between two SAR observations from the same area of the earth's surface, taken from slightly different sensor positions, to extract the information about the distance to the ground.

After a corregistration at pixel level is made and by combining the phase differences of the two observations, an interferogram can be generated where the information of the phase difference is highly related to the terrain topography and their deformations can be mapped.

The phase difference can be due to 5 factors:

$$
\Delta \varphi=\Delta \varphi_{\text {fat }}+\Delta \varphi_{\text {elearion }}+\Delta \varphi_{\text {displacement }}+\Delta \varphi_{\text {ammosphier }}+\Delta \varphi_{\text {mose }}
$$

Where:

$\Delta \varphi_{\text {flat: }}$ Earth's curvature.

$\Delta \varphi$ elevation: Topography.

$\Delta \varphi$ displacement: Deformations of the land between both acquisitions.

$\Delta \varphi$ atmosphere: Due to differences in humidity, pressure and temperature between acquisitions.

$\Delta \varphi_{\text {noise: }}$ Temporal changes in reflectors, volume scattering and acquisition angles.

The interferometric analysis tries to eliminate the sources of error (flat, atmosphere and noise) in order to remain only with the contributions of interest: elevation (to generate digital terrain models) and displacement (to analyze changes on the terrain) (Veci, 2016).

If the topography contribution is subtracted from the interferogram, the resulting phase difference would correspond to the deformation patterns between the two acquisition dates.

Simultaneously to the interferogram, a coherence band is generated, showing how similar both images are at the pixel level on a scale from 0 (low) to 1 (high).

The loss of coherence between a pair of images can be due to several factors:

$$
\gamma=\gamma_{T} \cdot \gamma_{G} \cdot \gamma_{V} \cdot \gamma_{P}
$$

Where:

$\gamma_{\mathrm{T}}$ Temporal: Cannot be avoided, it is due to differences in the terrain between both shots, and precisely one of the objectives of differential interferometry.

$\gamma_{\mathrm{G}}$ Geometric: Due to satellites orbits errors, it can be partially eliminated.

$\gamma_{\mathrm{V}}$ Volumetric: Due to the existing vegetation, it cannot be avoided.

$\gamma_{P}$ Processing: Due to calculation errors. It must be avoided.

The technique of differential interferometry has been used successfully in the analysis of subsidence due to civil works, landslides, earthquakes and volcanic eruptions (ESA-EO College, 2017). 


\section{Materials and Methods}

Two types of tests have been carried out:

Quantitative verification in a pilot plot

Qualitative verification in a small monitored basin

Quantitative verification

A quantitative verification of the erosion values obtained by TOPSAR differential interferometry was carried out.

A small plot was used for verification measures. It had to be easily monitored to identify possible elements present during the passage of satellites that could disturb the measurements (people and dogs walking, cows, cars, etc.), with reference elements nearby to identify horizontal inaccuracy and with possible control points nearby (areas with zero deformation).

Simultaneously, a LIDAR device was used to get measurements in the plot on dates matching with the passage of the satellites, to compare the values obtained by means of LIDAR and differential interferometry. (figure 3 )
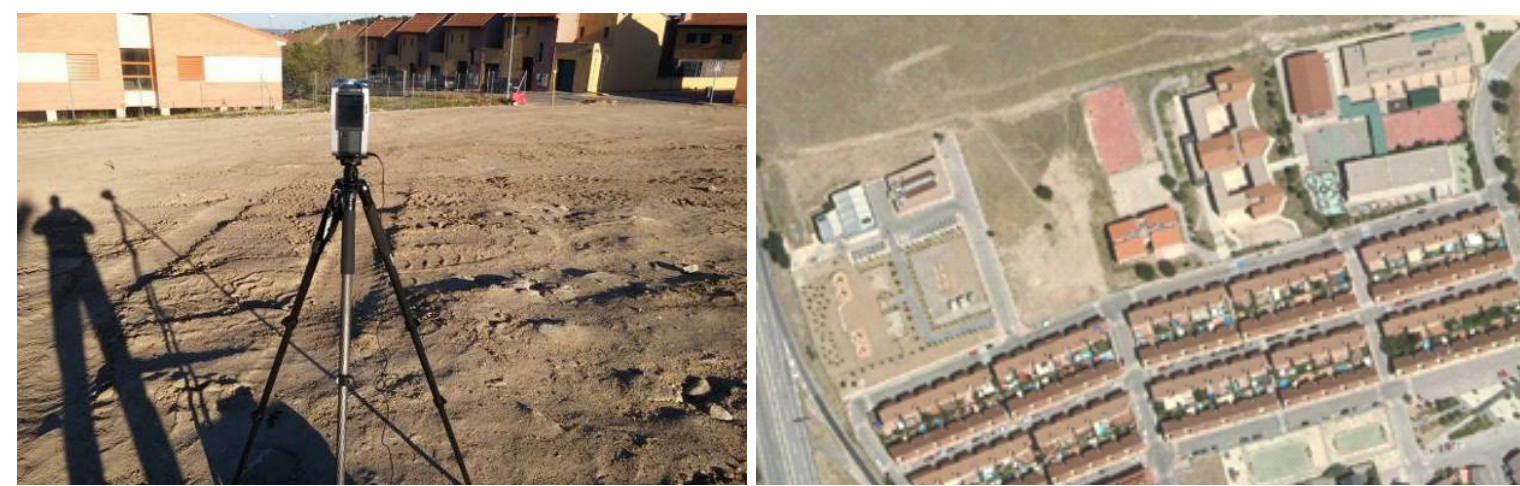

Figure 3. Plot localization, between buildings and sports courts that can be used as reference and control points. The plot has no vegetation coverage, with a lot of wheelprints to favor runoff erosion.

Every 6 days observations were made in three campaigns:

Campaign 1: 23/03/2017 to 10/04/2017 (1 points cloud/model, 4 spheres)

Campaign 2: 28/04/2017 to 09/07/2017 (2 points clouds /model, 4 spheres)

Campaign 3: 30/12/2017 to 23/01/2018 (2 points clouds /model, 6 spheres)

A LIDAR point clouds model of the plot surface was made for every satellite visiting day of the campaign with a FARO 3D LASER. To avoid and minimize the radial error, each model consists in two points cloud taken from two points inside the plot and separated by approximately 14 meters (Sánchez-Crespo et al., 2017).

The processing of the models and its point clouds has been made with CloudCompare open source software (CloudCompare opensource documentation, 2015)

Point clouds and models were assembled using four reference spheres.

Before making comparisons between models, each model is processed with a texture identification algorithm (CANUPO) to eliminate the vegetation and left only the bare soil (Brodu \& Lague, 2012)

A Multiscale Model to Model Cloud Comparison (M3C2) algorithm is used to measure differences between models (figure 4). (Lague et al., 2013)

Two sources of errors have been detected in LIDAR measures:

Turning mechanism of the FARO 3D LASER.

Assembling point clouds to form models and assembling models to make comparisons.

Errors from the same source are combined using Root Mean Square (RMS) technique:

$$
E_{T}=\sqrt[2]{E_{1}^{2}+E_{2}^{2}+\ldots+E_{n}^{2}}
$$

Errors from different sources are combined using Root Square Sum (RSS) technique:

$$
E_{T}=\sqrt[2]{E_{1}^{2}+E_{2}^{2}+\ldots+E_{n}^{2}}
$$


The results are normalized and exported to raster using a $10 \mathrm{~cm}$ cell dimension.

QGIS open source software was used to calculate mean deformation values of the $10 \mathrm{~cm}$ raster into a $13,93 \mathrm{~m}$ cell raster corresponding to the raster result of the differential interferometry analysis (Quantum GIS opensource documentation, 2018)

TopSAR Differential Interferometry analysis was made using ESA SNAP software (Veci, 2016)

The VV polarization of ascending orbits IW images was used and pixel to pixel interpolation has been made with an adaptive algorithm with 21 interpolation points. Interferometric pairs have been corregistered with the spectral enhance technique (SNAP - ESA, 2018)

A SRTM 1sec HGT DEM was used to remove the topographic phase.

Results were exported to an ETRS89 raster with a $13.93 \mathrm{~m}$ cell size.

Qualitative verification

Surface deformation patterns of Arroyo del Monte basin (Madrid, Spain) has been monitored using differential interferometry over TOPSAR images (Martinez et al., 2002). At the same time this basin has been visually monitored to register surface conditions, land coverage and crops changes, erosion gullies and flowed areas, etc. Then, results of the differential interferometry have been analysed using the information collected over the field.

The TOPSAR images have been obtained from European Space Agency Scientist Data Hub. The characteristics of the images are:

Satellites Sentinel S-1A and S-1B / IW (Interferometric Wide Swath)/ SLC (Single Look Capture)/ VV polarization/

Ascending orbit

The Sentinel Application Platform SNAP Desktop tool from the European Space Agency is used (Veci, 2016). The differential interferometry analysis of TOPSAR satellite images with this tool allows detecting small variations (of the order of millimetres) in the elevation of the earth surface, although the following restrictions must be considered:

The water surfaces and areas with abundant vegetation show little coherence in the results, while the bare, paved floors and buildings show a high coherence in the results.

The moisture of the soil can affect the penetration of the $\mathrm{C}$ Band microwaves, so different moisture conditions could induce to small variations detection (figure 5).

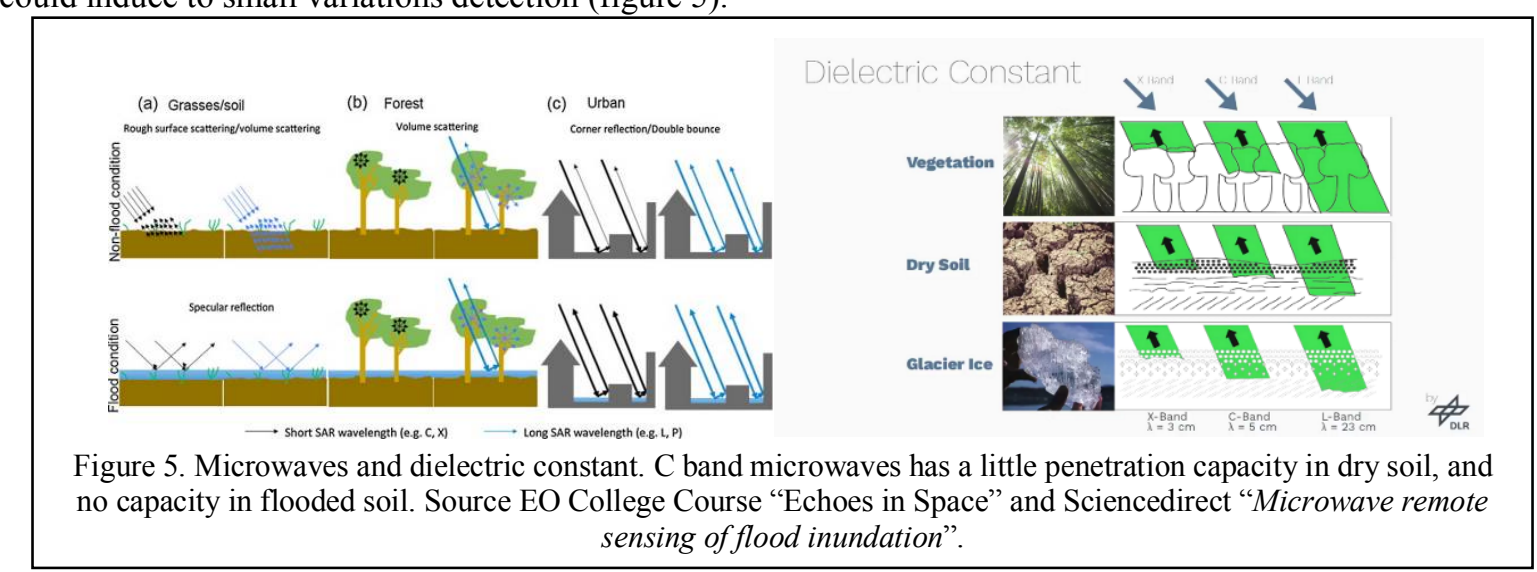

Variations in height of the land between one intake and another may be due to different factors other than erosion processes:

- Vegetation growth, tillage and cultivation practices and new or changing elements.

- Other geological processes (clay expansion, land subsidence, earthquakes, hillsides sliding, limestone dissolution, etc.).

Vertical correction adjustment has been done using control point (zero deformation). The control points have been selected due to their high coherence (the $5 \%$ quantile with higher coherence, usually with a coherence above 0.94), and the correspondent adjustment has been made using a raster obtained from the interpolation of the values of displacement of the control points

The interpolation methods analysed include Inverse distance Weighting (IDW) and Krigging, with the results of IDW with an exponent value of 2 to be a good candidate to interpolate the adjustment from the first analyzes made (ESRI, 2016).

\section{Results and Discussion}

Quantitative verification

The goal of this analysis is to get differences between LIDAR and Differential Interferometry lower than the LIDAR error. 
The visual results of LIDAR measures (with the equivalent TOPSAR grid), and TOPSAR differential Interferometry (coherence and deformation) were recorded. An example of the kind of analysis can be seen in figure 7:

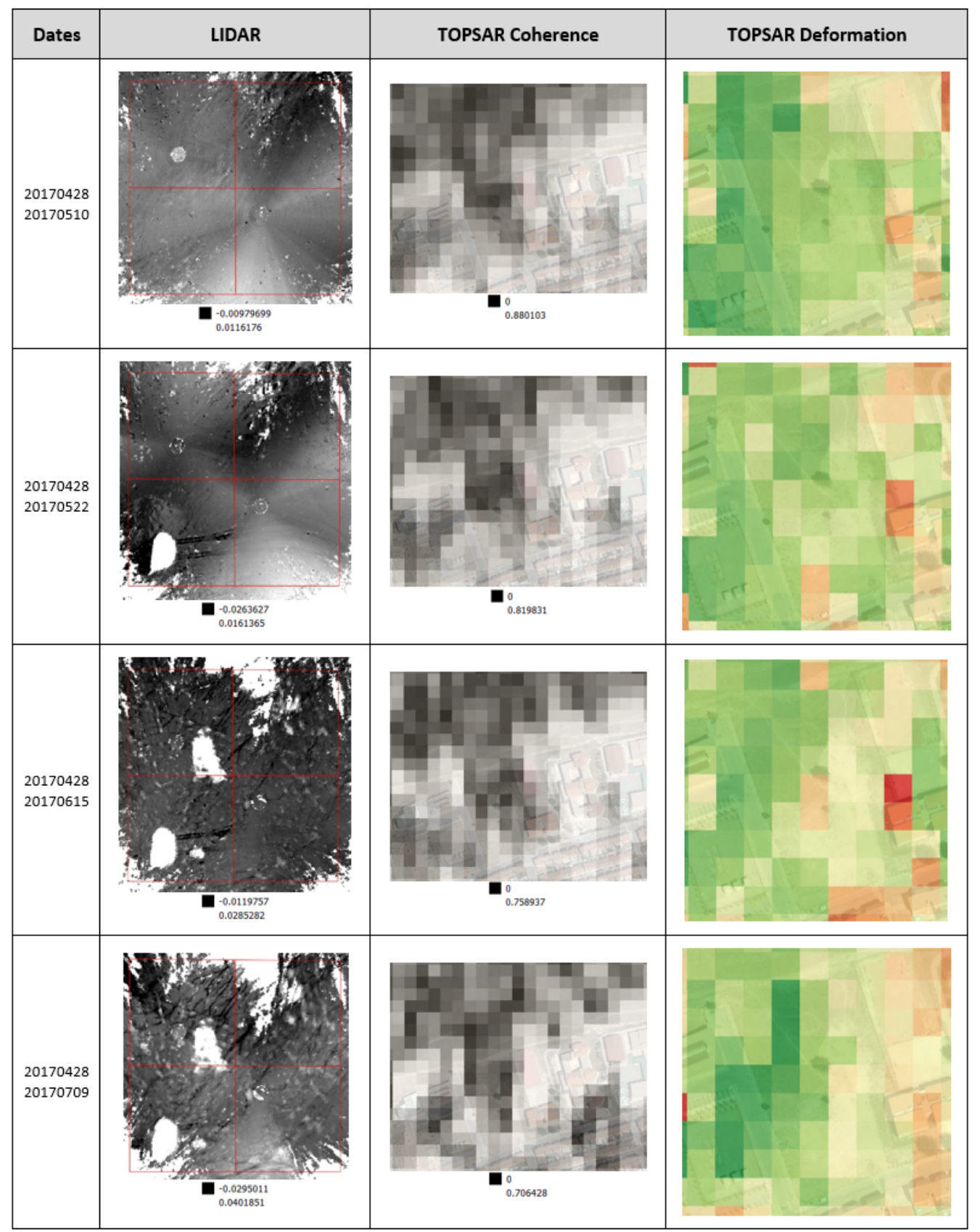

Figure 6. LIDAR and TOPSAR comparison. 
Table 45. Numerical comparisons between LIDAR and TOPSAR.

\begin{tabular}{|c|c|c|c|c|c|c|c|c|c|c|c|c|}
\hline \multicolumn{13}{|c|}{ INSAR TOPSAR MEASURES } \\
\hline Reference & 20170504 & 20170428 & 20170428 & 20170428 & 20170428 & 20170428 & 20170510 & 20170522 & 20170603 & 20170615 & 20170627 & 20171230 \\
\hline Aligned & 20170516 & 20170510 & 20170522 & 20170615 & 20170709 & 20170510 & 20170522 & 20170603 & 20170615 & 20170627 & 20170709 & 20180111 \\
\hline Horizontal unaccuracy & $1 \mathrm{~N}$ & $2 \mathrm{~N}$ & $2 \mathrm{~N}$ & $2 \mathrm{~N}$ & $2 \mathrm{~N}$ & $2 \mathrm{~N}$ & $2 \mathrm{~N}$ & $2 \mathrm{~N}$ & $1 \mathrm{~N}+1 \mathrm{E}$ & $2 \mathrm{~N}$ & $2 \mathrm{~N}(3 \mathrm{~N})$ & $2 \mathrm{~N}(3 \mathrm{~N})$ \\
\hline Vertical error adjustment & 0.0006 & -0.0234 & -0.0609 & -0.0669 & -0.1607 & -0.0234 & -0.0302 & 0.0006 & -0.0346 & 0.0312 & -0.0514 & -0.0035 \\
\hline \multicolumn{13}{|l|}{ Original Measure } \\
\hline A & 0.0019 & -0.0238 & -0.0575 & -0.0676 & -0.1617 & -0.0238 & -0.0358 & 0.0019 & -0.0311 & 0.0327 & -0.0520 & -0.0049 \\
\hline B & 0.0010 & -0.0235 & -0.0585 & -0.0672 & -0.1645 & -0.0235 & -0.0358 & 0.0010 & -0.0348 & 0.0311 & -0.0522 & -0.0024 \\
\hline c & 0.0015 & -0.0233 & -0.0583 & -0.0676 & -0.1612 & -0.0233 & -0.0349 & 0.0015 & -0.0357 & 0.0326 & -0.0507 & -0.0043 \\
\hline D & 0.0007 & -0.0236 & -0.0581 & -0.0669 & -0.1639 & -0.0236 & -0.0352 & 0.0007 & -0.0340 & 0.0321 & -0.0519 & -0.0030 \\
\hline \multirow{2}{*}{\multicolumn{13}{|c|}{ Corrected Measure }} \\
\hline & & & & & & & & & & & & \\
\hline $\mathrm{A}^{\prime}$ & 0.0013 & -0.0004 & 0.0035 & -0.0008 & -0.0009 & -0.0004 & -0.0056 & 0.0013 & 0.0035 & 0.0015 & -0.0006 & -0.0014 \\
\hline B' & 0.0003 & 0.0000 & 0.0024 & -0.0003 & -0.0038 & 0.0000 & -0.0056 & 0.0003 & -0.0002 & 0.0000 & -0.0008 & 0.0011 \\
\hline$C^{\prime}$ & 0.0009 & 0.0001 & 0.0026 & -0.0007 & -0.0005 & 0.0001 & -0.0047 & 0.0009 & -0.0011 & 0.0014 & 0.0007 & -0.0008 \\
\hline$D^{\prime}$ & 0.0001 & -0.0001 & 0.0028 & -0.0001 & -0.0031 & -0.0001 & -0.0049 & 0.0001 & 0.0006 & 0.0009 & -0.0006 & 0.0005 \\
\hline Global $A^{\prime} B^{\prime} C^{\prime} D^{\prime}$ & 0.0007 & -0.0001 & 0.0028 & -0.0005 & -0.0021 & -0.0001 & -0.0052 & 0.0007 & 0.0007 & 0.0010 & -0.0003 & -0.0001 \\
\hline & & & & & & & & & & & & \\
\hline \multicolumn{13}{|c|}{ LIDAR MEASURES } \\
\hline Reference & 20170504 & 20170428 & 20170428 & 20170428 & 20170428 & 20170428 & 20170510 & 20170522 & 20170603 & 20170615 & & 20171230 \\
\hline Aligned & 20170516 & 20170510 & 20170522 & 20170615 & 20170709 & 20170510 & 20170522 & 20170603 & 20170615 & 20170627 & 20170709 & 20180111 \\
\hline Error Position R1 & 0.0028 & 0.0021 & 0.0021 & 0.0021 & 0.0021 & 0.0021 & 0.0022 & 0.0022 & 0.0041 & 0.0019 & 0.0021 & 0.0025 \\
\hline Error Position R2 & 0.0027 & 0.0039 & 0.0039 & 0.0039 & 0.0039 & 0.0039 & 0.0015 & 0.0486 & 0.0019 & 0.0018 & 0.0021 & 0.0021 \\
\hline EP Reference & 0.0027 & 0.0031 & 0.0031 & 0.0031 & 0.0031 & 0.0031 & 0.0019 & 0.0344 & 0.0032 & 0.0018 & 0.0021 & 0.0023 \\
\hline Error Position A1 & 0.0047 & 0.0024 & 0.0022 & 0.0019 & 0.0044 & 0.0022 & 0.0022 & 0.0041 & 0.0019 & 0.0021 & 0.0044 & 0.0020 \\
\hline Error Position A4 & 0.0022 & 0.0022 & 0.0486 & 0.0018 & 0.0022 & 0.0027 & 0.0486 & 0.0019 & 0.0018 & 0.0021 & 0.0022 & 0.0020 \\
\hline EP Aligned & 0.0037 & 0.0023 & 0.0344 & 0.0018 & 0.0035 & 0.0024 & 0.0344 & 0.0032 & 0.0018 & 0.0021 & 0.0035 & 0.0020 \\
\hline EP Total & 0.0032 & 0.0027 & 0.0244 & 0.0025 & 0.0033 & 0.0028 & 0.0244 & 0.0244 & 0.0026 & 0.0020 & 0.0029 & 0.0021 \\
\hline RMS CC Reference & 0.0051 & 0.0068 & 0.0068 & 0.0068 & 0.0068 & 0.0068 & 0.0073 & 0.0125 & 0.0090 & 0.0088 & 0.0109 & 0.0101 \\
\hline RMS CC Aligned & 0.0050 & 0.0073 & 0.0125 & 0.0088 & 0.0083 & 0.0073 & 0.0125 & 0.0090 & 0.0088 & 0.0109 & 0.0124 & 0.0053 \\
\hline RMS CC Compare & 0.0120 & 0.0110 & 0.0093 & 0.0083 & 0.0117 & 0.0110 & 0.0098 & 0.0102 & 0.0083 & 0.0083 & 0.0111 & 0.0061 \\
\hline RMS CC Total & 0.0081 & 0.0085 & 0.0098 & 0.0080 & 0.0091 & 0.0085 & 0.0101 & 0.0107 & 0.0087 & 0.0094 & 0.0115 & 0.0075 \\
\hline Max. Possible Error & 0.0087 & 0.0090 & 0.0263 & 0.0084 & 0.0097 & 0.0090 & 0.0264 & 0.0267 & 0.0091 & 0.0096 & 0.0118 & 0.0078 \\
\hline & & & & & & & & & & & & \\
\hline A & -0.0077 & -0.0003 & -0.0126 & 0.0037 & -0.0009 & -0.0003 & -0.0122 & 0.0205 & -0.0038 & -0.0013 & -0.0039 & -0.0015 \\
\hline B & -0.0059 & -0.0030 & -0.0141 & -0.0031 & -0.0073 & -0.0030 & -0.0116 & 0.0156 & -0.0037 & -0.0007 & -0.0035 & 0.0017 \\
\hline c & 0.0065 & 0.0017 & -0.0026 & 0.0143 & 0.0115 & 0.0017 & -0.0041 & 0.0164 & 0.0011 & -0.0015 & -0.0017 & 0.0027 \\
\hline D & -0.0024 & 0.0012 & -0.0022 & -0.0013 & 0.0018 & 0.0012 & -0.0033 & -0.0004 & 0.0013 & -0.0011 & 0.0042 & -0.0015 \\
\hline Global A B C D & -0.0024 & -0.0001 & -0.0079 & 0.0034 & 0.0013 & -0.0001 & -0.0078 & 0.0130 & -0.0012 & -0.0011 & -0.0012 & 0.0004 \\
\hline \multicolumn{13}{|c|}{ INSAR TOPSAR MEASURES VS LIDAR MEASURES } \\
\hline & & & & & & & & & & & & \\
\hline Permissible range & 0.0087 & 0.0090 & 0.0263 & 0.0084 & 0.0097 & 0.0090 & 0.0264 & 0.0267 & 0.0091 & 0.0096 & 0.0118 & 0.0078 \\
\hline \multicolumn{13}{|l|}{ Diferencia } \\
\hline A & 0.0090 & 0.0001 & 0.0161 & 0.0045 & 0.0001 & 0.0001 & 0.0067 & 0.0192 & 0.0074 & 0.0028 & 0.0033 & 0.0001 \\
\hline B & 0.0062 & 0.0029 & 0.0165 & 0.0028 & 0.0035 & 0.0029 & 0.0060 & 0.0152 & 0.0034 & 0.0007 & 0.0027 & 0.0007 \\
\hline c & 0.0057 & 0.0016 & 0.0052 & 0.0150 & 0.0120 & 0.0016 & 0.0006 & 0.0156 & 0.0023 & 0.0029 & 0.0024 & 0.0035 \\
\hline D & 0.0026 & 0.0013 & 0.0050 & 0.0013 & 0.0049 & 0.0013 & 0.0017 & 0.0006 & 0.0007 & 0.0020 & 0.0047 & 0.0019 \\
\hline Global A B C D & 0.0030 & 0.0000 & 0.0107 & 0.0038 & 0.0034 & 0.0000 & 0.0026 & 0.0123 & 0.0019 & 0.0021 & 0.0009 & 0.0005 \\
\hline
\end{tabular}

As can be seen in the visual and numerical results:

1.- Results obtained by both study methods (LIDAR and differential interferometry) not only have the same order of magnitude, but in most cases difference between values obtained by both methods are within the margin of error of the LIDAR readings, so differential interferometry is an acceptable method to measure surface changes over the time. Note that at 22/05/2017 occurred an unexpected error of the movement mechanism of the LIDAR in the second position, so error and differences are greater than in the rest of the days.

2.- Cells with high coherence (whiter ones) should coincide spatially with buildings or sports tracks, but there is a lack of horizontal precision and a displacement that must be evaluated in each interferometric pair. Therefore, differential interferometry technique may not be suitable for an accuracy evaluation of very small areas.

Qualitative verification

We have selected two observations, one after raining and other in a dry period, to analyse the differential interferometry results: 


\section{$28 / 02 / 2018 \rightarrow 06 / 03 / 2018$}

Precipitation: $105 \mathrm{~mm}$

No agricultural activities.

Deformation range $>8 \mathrm{~cm}$

Red and orange areas: Areas with erosion (up to $4 \mathrm{~cm}$ ). Fallow lands without vegetative coverage.

Yellow areas: No erosion. Cereal crops.

Soft green areas: This area needs of interpretation. There are extensive areas of soft green color that cannot correspond to sedimentation areas.

Likely recorded elevation is due to clay expansion phenomena or changes in the dielectric constant of the soil that cause a more superficial rebound of the c-band microwaves rather than erosion phenomena (it was the first rain after a dry period).

Deep green areas: Visual inspection of the basin showed that, in this event, green areas corresponded to sediment and flooded areas.
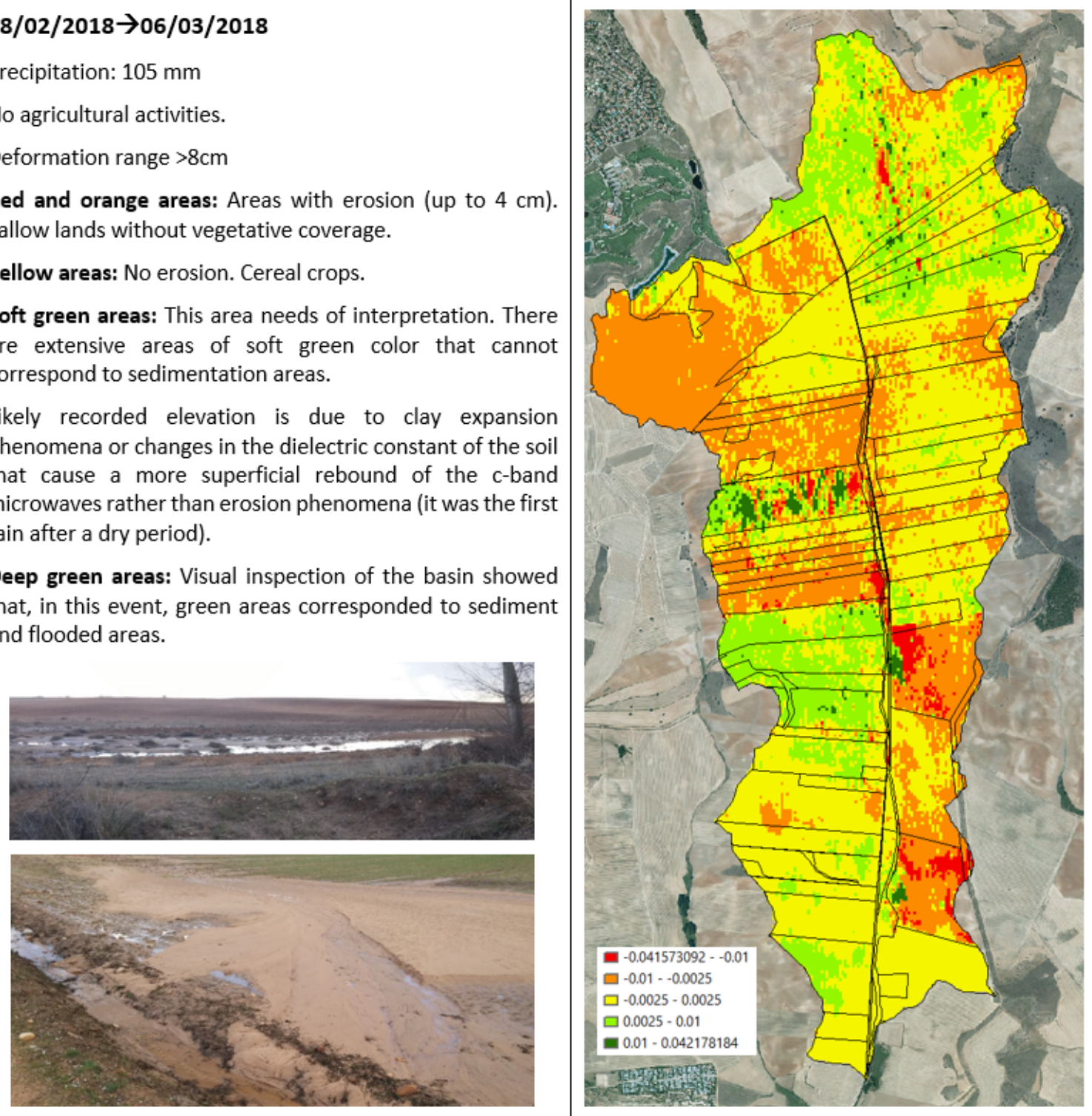


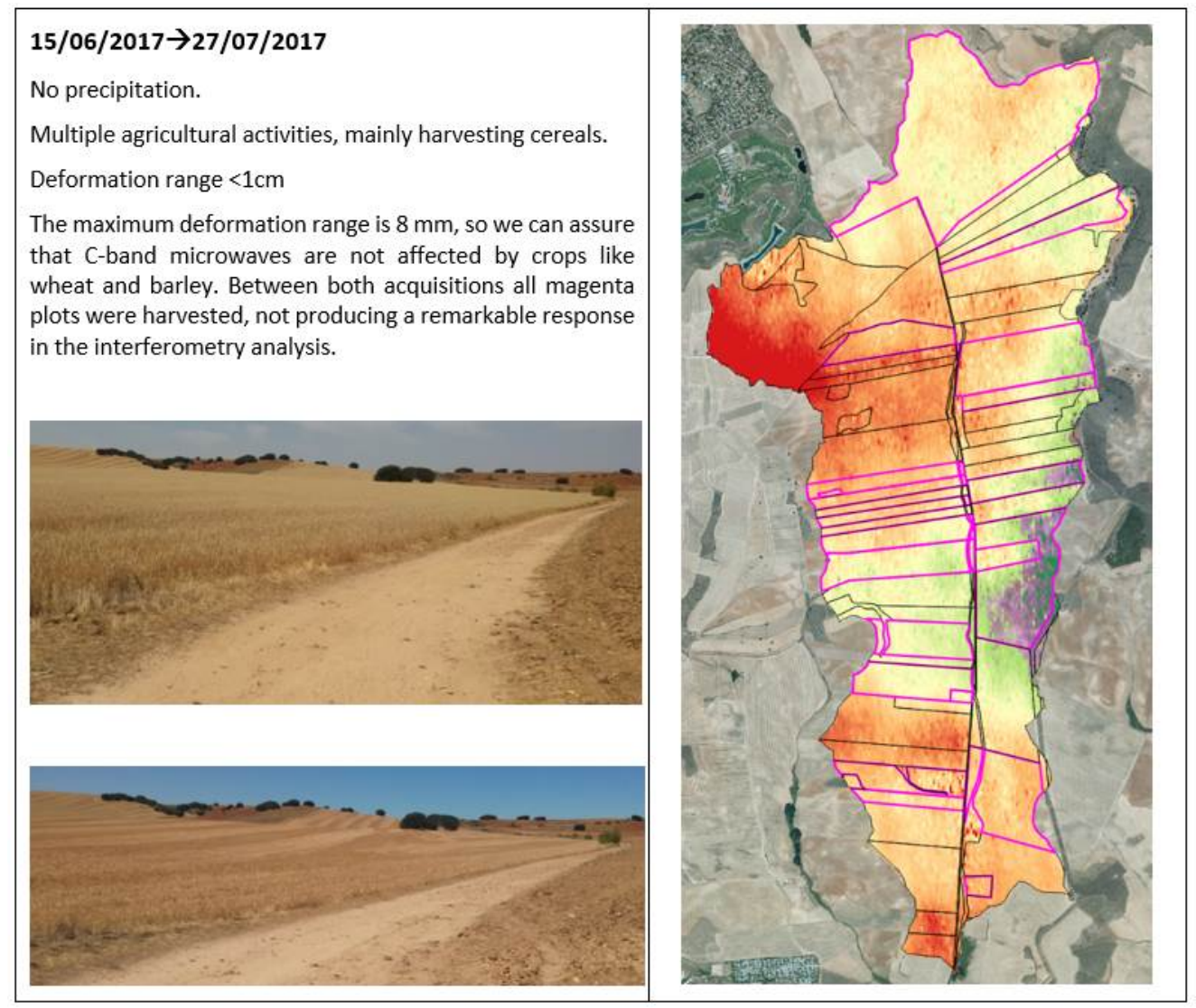

\section{Conclusions}

Differential interferometry technique over TOPSAR images is useful to study runoff erosion:

Qualitative values of runoff erosion are consistent with the field observations.

Quantitative values of runoff soil erosion are correct but depending on the possibility of having control points to zero and the way to distribute the correction.

The procedure is adequate for large areas.

It is not significantly affected by crops.

The loss of coherence favours its use for short periods of time.

In all cases, it is mandatory to check that no other parallel phenomena have occurred (earthquakes, subsidence, landslides, expansion of clays, etc.).

\section{Acknowledgements}

The authors want to thank the Institution "Cátedra Rafael Dal-Ré/TRAGSA" for supporting the Ph. D. thesis of Francisco A. Sánchez-Crespo

\section{References}

Brodu, N., \& Lague, D. 2012. 3D terrestrial LIDAR data classification of complex natural scenes using a multi-scale dimensionality criterion: Applications in geomorphology. ISPRS Journal of Photogrammetry and Remote Sensing, 68, 121-134.

ESA-EO College. 2017. Course "Echoes in Space"

European Space Agency-European Comission. 2013. Sentinel-1 User Handbook.

Lague, D., Brodu, N., \& Leroux, J. 2013. Accurate 3D comparison of complex topography with terrestrial laser scanner: Application to the Rangitikei canyon (NZ). ISPRS Journal of Photogrammetry and Remote Sensing, $82,10-26$.

Martinez, V., Garcia, A. I., \& Ayuga, F. 2002. Distributed routing techniques developed on GIS for generating synthetic unit hydrographs. Transactions of the ASAE, 45(6), 1825.

Rucci, A., Ferretti, A., Guarnieri, A. M., \& Rocca, F. 2012. Sentinel 1 SAR interferometry applications: The outlook for sub millimeter measurements. Remote Sensing of Environment, 120, 156-163. 
Sánchez-Crespo, F. A., Ayuga, F., García, A. I. 2017. Interferometry as a tool for localized study of runoff erosion, IX Iberian Congress on Agro-engineering - Bragança, Portugal.

Smith, L. C., Alsdorf, D. E., Magilligan, F. J., Gomez, B., Mertes, L. A., Smith, N. D., \& Garvin, J. B. (2000). Estimation of erosion, deposition, and net volumetric change caused by the 1996 Skeiðarársandur jökulhlaup, Iceland, from synthetic aperture radar interferometry. Water Resources Research, 36(6), 1583-1594.

Veci, L.. 2016. Sentinel-1 Toolbox-SAR Basic Tutorial / Interferometry Tutorial / TOPS Interferometry Tutorial. European Space Agency

Internet resources:

CloudCompare opensource documentation. 2015. http://www.cloudcompare.org/doc/ Last visit June 2018

ESRI, 2016. Comparing Interpolation Methods. http://desktop.arcgis.com/en/ Last visit June 2018

Quantum GIS opensource documentation. 2018. http://www.qgis.org. Last visit June 2018

SNAP - ESA Sentinel Application Platform. http://step.esa.int Last visit June 2018. 


\title{
Soil conductivity: Detecting Slurry Injection Depth with Minimal Effort \\ Jochen Georg Wiecha*, Sascha Wörz, Heinz Bernhardt \\ Chair of Agricultural Systems Engineering, Technical University of Munich, 85354 Freising, Germany \\ * Corresponding author. Email: wiecha@wzw.tum.de
}

\begin{abstract}
Semi-liquid manure is a valuable fertilizer. Worldwide, pig or cattle slurry ensures efficient supply of plant nutrients in many crops. Current laws and future regulations demand to apply semi-liquid manure on soil surface or to inject the slurry immediately into the soil during application. The results are emission reductions and reduction of nutrient loss. Correct slurry application depth is a significant factor in each crop. If the roots reach the injected slurry at the optimal plant growth, we will observe a maximum consumption of nutrients and a suitable intake. In field trials and research facilities, the potentially successful slurry deposition depth after injection is checked by digging with spade. Depending on the structure of the soil and the presence of larger particles and stones slurry injection depth may vary. By a large number of samples, the variance depending on soil conditions, moisture and nutrient supply can be determined. This requires lots of work and much time. For quick and reliable checking of the correct deposition depth of the semi-liquid manure, we present a measurement system based on conductivity. The system delimits the processed soil areas from the slurry, measures the vertical extent of the fertilizer applied and can detect the zone of mixed loosened soil and semi-liquid manure in deposition depth. This results in time-consuming field trials for the scientific staff. Fast data collection makes the measurement results comparably, since the environmental influences during data acquisition can change less strongly than over a long trial period.
\end{abstract}

Keywords: Soil, conductivity, slurry, semi-liquid manure

\section{Introduction}

Advanced requirements for high quality crop production and livestock feeding are sophisticated. A central point in management of agricultural crops is the correct supply of nutrients to the plant root. Fertilizer is an important steering mechanism for triggering and planning plant growth. This ensures the farmer to have a valuable control element staring with the root development (Paungfoo-Lonhienne et al., 2012). Additionally, this is a prevention for plant health.

In agricultural engineering so-called strip-till devices, liquid manure cultivators and liquid manure injectors were developed for rapid slurry application into the soil (Warner and Godwin, 1988). All these systems aim to reliably deposit the fertilizer at a pre-defined depth into soil and avoid ammonia volatilization from slurry (Vandré et al., 1997). In order to check the deposition depth, it is customary to open the soil point-like by spade and to assess the cut after the excavated manure peel. This is very time-consuming, when dealing with a large sample size.

Aim of this study is the detection of the exact depth of the semi-liquid manure after deposition into soil. The work presented here introduces the "slurry meter" - an easy and quick to use option that can replace the extensive work with the spade on the field. It is described by the circuit diagram and the components used. Therefore, it is easy to replicate the "slurry meter".

\section{Materials and Methods}

The difference, between soil in its current state and a soil well supplied with fertilizer, is the large increase in nutrients, achieved by fertilization. This corresponds to a big source of minerals, which results in a change of conductivity in the deposit horizon. The conductivity of livestock slurry from cattle and swine used Juriček (1990) during his work. Juriček was able to obtain conclusions of dry matter by conductivity. This makes use of the application from the presented work.

There are already existing measurement systems for the detection of conductivity (Sophocleous and Atkinson, 2015). They work at soil samples, or should be able to give general information about the nutrient supply (Provolo and Martínez-Suller, 2006) and the density of slurry (Jäger, 1957). A depth probe, locating the slurry strand after the deposition of an application unit on the field, does not exist yet.

Slurry meter concept based on 1. Kirchhoff's law

The following description is basing on the first Kichhoff's rule. Kichhoff first formulated in 1845 that in a node of an electrical network, the sum of the inflowing currents is equal to the sum of the outflowing currents (Küpfmüller und Kohn, 2013). Therefore, we can set up the governing equations: 


$$
\begin{aligned}
& \qquad \frac{U_{p}}{U_{\text {sig }}}=\frac{R_{g e s}}{R_{\text {ref }}} \\
& U_{p}=\text { test voltage } 9 \mathrm{~V}, 12 \mathrm{~V} \text { or } 15 \mathrm{~V} \\
& U_{s i g}=\text { voltage measured on slurry meter } \\
& R_{g e s}=\text { sum of resistance } \\
& R_{\text {ref }}=\text { reference resistance } \\
& R_{\text {soil }}=\text { soil resistance }=R_{g e s}-R_{\text {ref }} \\
& \qquad R_{g e s}=R_{\text {soil }}+R_{\text {ref }}
\end{aligned}
$$

The equations below derive by algebraic rearrangements.

$$
\begin{aligned}
& \frac{U_{p}}{U_{\text {sig }}}=\frac{R_{\text {soil }}+R_{\text {ref }}}{R_{\text {ref }}} \\
& \frac{U_{p}}{U_{\text {sig }}} * R_{\text {ref }}=R_{\text {soil }}+R_{\text {ref }} \\
& \frac{U_{p}}{U_{\text {sig }}} * R_{r e f}-R_{\text {ref }}=R_{\text {soil }} \\
& R_{\text {soil }}=R_{\text {ref }} *\left(\frac{U_{p}}{U_{\text {sig }}}-1\right)
\end{aligned}
$$

The soil as a resistor is the result of the reference resistor multiplied with a difference of the test voltage $U_{p}$ divided by the voltage measured on slurry meter spike minus 1 .

Suitable probe for field trials into the soil

For the construction of the measurement probe, we used the finished puncture unit "AM-50 Messsonde" from Pronova Umweltmesstechnik GmbH without the commonly connected devices. After removing the supplied plug, we adapted the two included conductions to suitable new connectors.

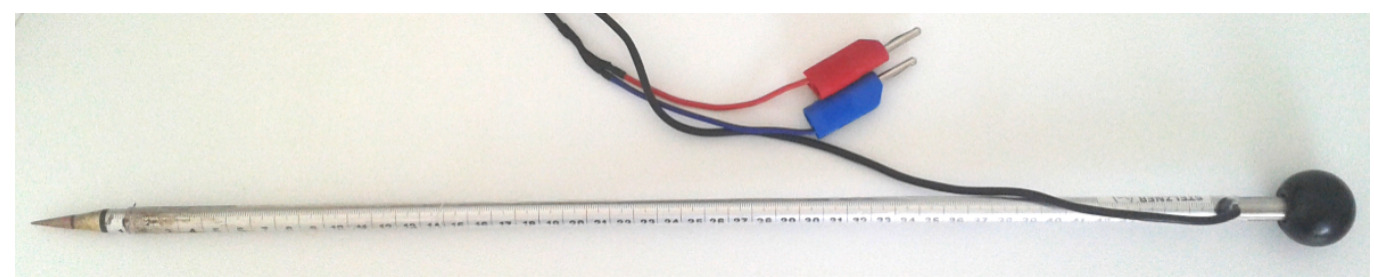

Figure 1. Slurry meter with new conectors.

There are two electrically conductive poles on the spike side of the probe, separated by a plastic element. We connect both poles to the conductor cables, which emerge at the upper end of the probe. The main part of the probe is made of stainless steel and has an overall length of $0.5 \mathrm{~m}$. At the upper end there is a knob made of plastic. This enables powerful punctures by the probe vertically into the ground.

For visual depth measurement, we glued a self-adhesive measuring tape to the probe. The starting point of the count on the measurement tape begins directly above the two conductive poles. In order to protect the probe and the self-adhesive measuring tape for a long time from liquid manure, we attached a shrinkable tubing.

\section{Circuit diagram}

In Figure 2, the wiring diagram is provided. The circled position on the symbolic slurry meter results in soil resistance. We generate a reference voltage of $9 \mathrm{~V} \mathrm{DC}, 12 \mathrm{~V} \mathrm{DC}$ and $15 \mathrm{~V}$ DC. During field trials, we change these voltages by a switcher. We are logging the reference voltage and the test voltage during the centimeteraccurate penetration into the soil. Reference resistor was set to $1 \mathrm{k} \Omega$ in all experiments in this study. 


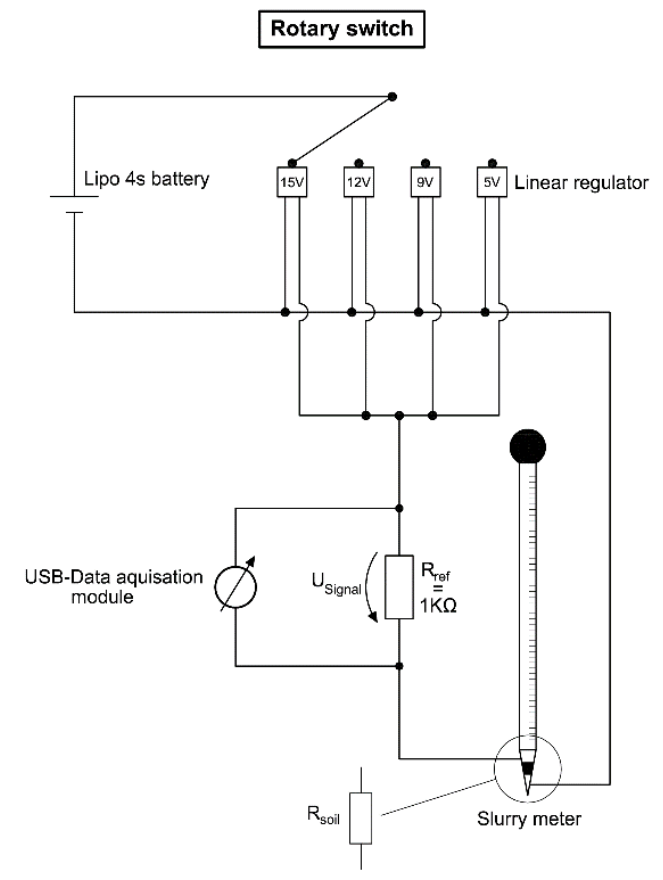

Figure 2. "Slurry meter" circuit diagram.

Figure 2 shows the circuit diagram of the slurry measurement system. The electrical circuit models a series of connection of two ohmic resistors. The first resistor is a reference resistor above which a certain reference voltage declines. In the soil, the manure probe meets the second resistance, the so-called soil or manure resistance. We determine it using Kirchhoff's rules (1. Kirchhoff's law) and the definition of ohmic resistance.

Field trials design and experimental setup

In general, we divide the trials into four sections:

- Experiment 1 - Puncture only in unloosened soil and only in mixed manure. The aim of the experiment is to show, whether the soil and manure differ sufficiently in their conductivity that they can be approved for subsequent experiments. We strive for a sufficiently large difference in conductivity between both media.

- Experiment 2 - This is a field test to show the potential applications of the slurry meter. This second field trial consists of three groups divided into three simulated deposition depths of $10 \mathrm{~cm}, 15 \mathrm{~cm}$ and $20 \mathrm{~cm}$. In the following, all combinations of reference voltages $9 \mathrm{~V} \mathrm{DC}, 12 \mathrm{~V} \mathrm{DC}$ and $15 \mathrm{~V}$ DC and simulated deposition depth just listed bevor, are covered. The measurement protocol indicates the deviation of the resistance from the reference resistor.

The manure measurement probe is tested with $n=25$ trials in each group of different voltages. We perform this in all three groups of deposition depths. For each tested depth, we planted a row on farmland for experimental purposes to prevent influence of a working device. We measured the generated deep gutter in cm and logged. We observe the penetration of slurry meter visually and protocols it by hand activating "enter" on laptop for each centimeter. LabView Software does a time shot every second with all data - the current depth, the current reverence voltage and the current voltage measured on slurry meter spike. Cattle manure is used, which was been mixed before the experiment.

After applying the liquid manure into the prepared rows, these are refilled with the excavated soil material and reconsolidated. We start the measurement without waiting a certain time since we injected the manure. For the use of field trials, we designed the manure measurement probe. We would promptly measure during the test of fieldwork equipment for manure application.

- Experiment 3 - This is a test for refinement of data as collected in experiment 2. For a new single deposition depth, the experiment is done with $n=30$ trials of punctures. Therefore, we excavate the soil circularly to a depth of $20 \mathrm{~cm}$. The refilled sequence has the following structure: Horizon 1 - refilled soil from the soil surface to 12 $\mathrm{cm}$ depth. Horizon 2 - manure horizon of $4 \mathrm{~cm}$ height. Horizon 3 - refilled soil below the manure horizon to the first excavated depth of $20 \mathrm{~cm}$.

We slow down the puncture speed in experiment 3 in order to observe the visual detection of the penetration depth of the manure measurement probe more accurately. The speed of data acquisition via the LabView software is set to 100 values per second for experiment 3. Observation of the penetration depth of slurry meter is visual, as 
described before.

The aim of experiment 3 is to show, whether the movements of slurry meter spike during data acquisition is responsible for the mean variance and whether we could fix it by significantly fine logging. Therefore, we do not need the laptop with complete equipment on the field anymore. We will see the manure depth practically in Rdata or in the excel sheet. In relation to the deposition depth, we measure slurry "start value" and "end value". In addition, you can detect the jump speed (suddenly occurring ascent or descent) during puncture. This indicates a better manure mixing with loosened soil.

- Experiment 4 -We have to investigate, whether s single probe with slurry meter is possible to detect correct deposition depth only by watching data in $\mathrm{R}$. The aim of this experiment is to cover the whole phase of puncturing well, without losing any important information. From the dataset obtained by experiment 3 , we cut out a sequence for own trail and analyze it be R.

Software for data collection and data analysis

We conduct the field trials with portable laptop Lenovo R400 connected via USB to a NI-USB - 6002 from National Instrument Corporation and the build circuit presented in Figure 3. Data collection software is LabView version 2016.

Statistical data analysis is performed with R x64 3.4.2 (R Core Team, 2017) and Lattice (Sarkar, 2008). In the first step of field trial (results of 3.1), logging speed is set to one value per second. The trial for refinement, logging frequency was one hundred values per second.

\section{Results and Discussion}

\section{Result of experiment 1}

Bevor starting the main field trial 2, we applied measurement on unloosened soil and on the livestock slurry from cattle separately. Observed conductivity should differ. Figure 3 shows the result presented as soil resistance. We did this experiment with $12 \mathrm{~V}$ DC reference voltage only.

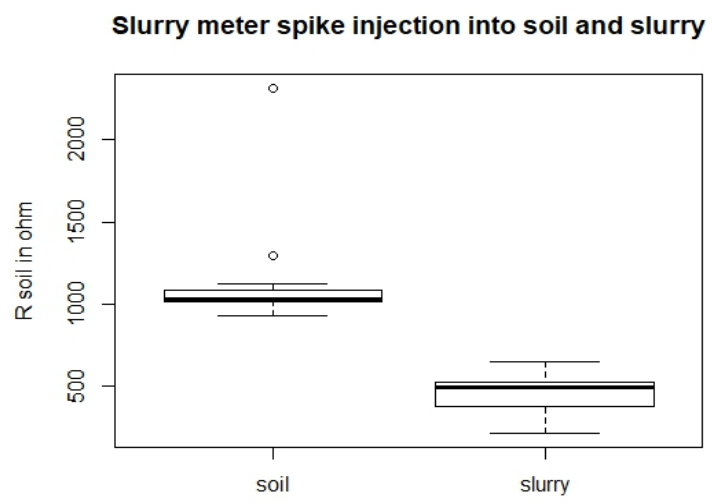

Figure 3. Test with 12 volt for probe penetration in standalone soil and standalone slurry.

In Figure 3 slurry meter spike punctures into soil and slurry separately. The injection depth was $15 \mathrm{~cm}$. During this trial, the slurry meter do not move up or down in soil. The mean of measured test voltage $\mathrm{U}_{\text {sig }}$ was $5,7 \mathrm{~V}$ DC for the soil and 8,3 V DC for the slurry. Soil resistance falls down to a range of $200 \Omega$ till $600 \Omega$ during slurry contact. In field trial area, soil is merged well over the years and plowsole is deeper, than the tested depth of 15 $\mathrm{cm}$. Therefore, it is permissible to do experiment 1 not in a deeper region of more than $20 \mathrm{~cm}$ of soil.

The test was done in unloosened soil, because of the higher contact pressure. In loosened soil, trapped air will rise the soil resistance additionally. Differences between soil and slurry have to be found even under perfect contact conditions. Therefore, the soil was not tilled previously.

In the next step, we put the slurry meter spike in mixed slurry from livestock cattle. Doing so, slurry covers the whole spike with the two conductors. The bigger variance during measurement in slurry is the result of the liquid and semiliquid phase of the slurry. Conductivity even of unloosened soil and slurry are significant different. Therefore, they are suitable for the test design of the following field trials.

\section{Results of experiment 2}

In the next step, we will have to evaluate, whether the slurry meter could provide good values for all artificial deposition depths. The trial was performed with $9 \mathrm{~V} \mathrm{DC}, 12 \mathrm{~V} \mathrm{DC}, 15 \mathrm{~V}$ DC and a slurry depth of $10 \mathrm{~cm}, 15 \mathrm{~cm}$ and $20 \mathrm{~cm}$ under the surface. Figure 4 shows the exemplary result for measurement with $12 \mathrm{~V}$ reference voltage. 


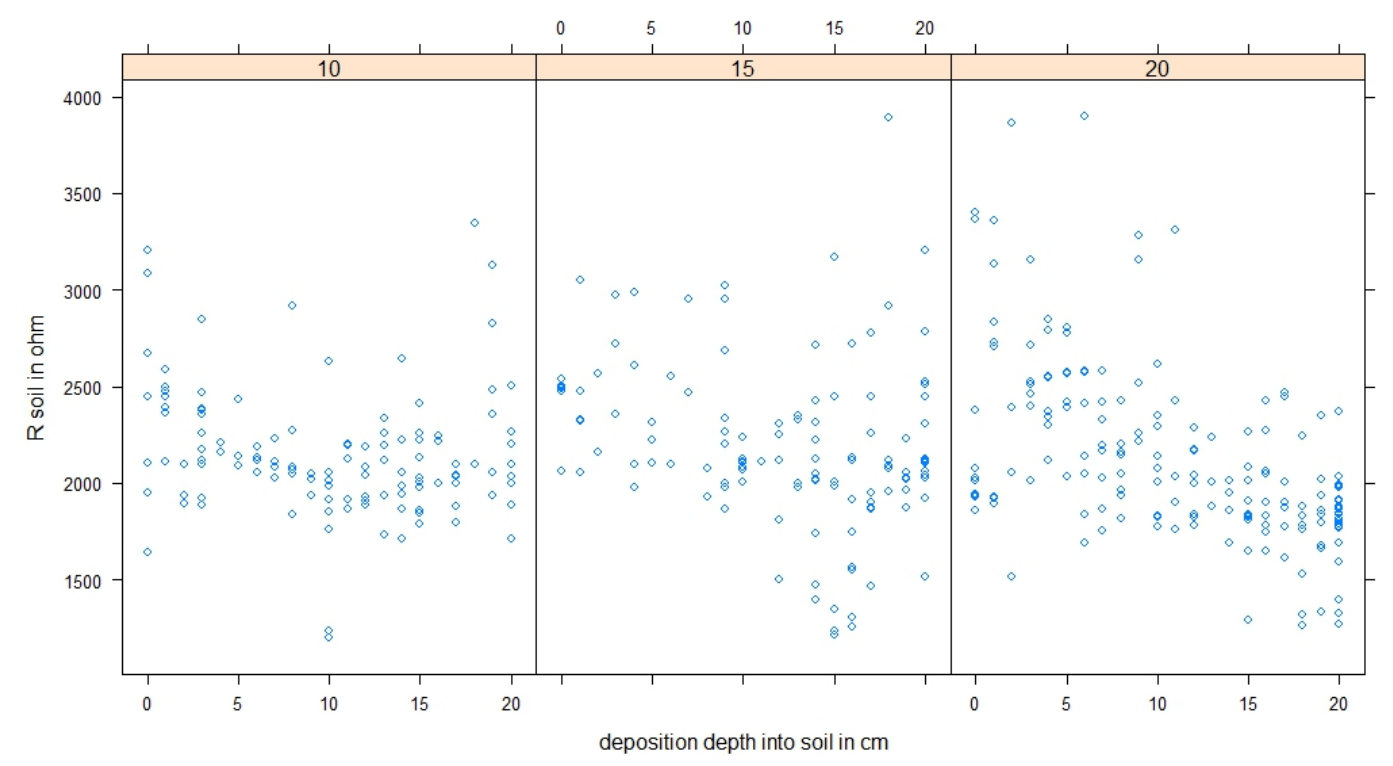

Figure 4. Result of field trail with $12 \mathrm{~V}$ measuring.

We used the reference voltage of $12 \mathrm{~V}$ DC in the diagram of Figure 4. Voltage of $9 \mathrm{~V}$ showed insufficient values and $15 \mathrm{~V}$ measurement was not significant better. For presenting reliable data, we choose to keep the results with $12 \mathrm{~V}$ reference voltage in this study. Values of soil resistance above $4 \mathrm{k} \Omega$ were cut off in Figure 4.

$\mathrm{X}$-axis shows the soil depth during the test. The $\mathrm{y}$-axis is the soil resistance on the spike of the slurry meter. In the upper line, we grouped the slurry depth during field trail in $10 \mathrm{~cm}$ (left part of Figure 4), $15 \mathrm{~cm}$ (middle) and $20 \mathrm{~cm}$ (on the right side). For each group $\mathrm{n}=25$ trials were performed. The logging frequency is the reason for having a couple of values during injection trial.

First, we have to notice that there is a discrepancy in the R_soil of Figure 4 and Figure 3. This might be due to the different tillage before the experiments - no tillage in experiment 1 , soil opening and resealing in experiment 2. The trapped air and an incomplete reconsolidation are the reasons for a higher soil resistance, observed in experiment 2.

On every expected point for the artificial deposition depth, we observe a lower soil resistance in the range of applied slurry. However, we have to admit a wide range of fault values. In the left part of Figure 4, slurry might be mixed with loosened soil during reconsolidation near the surface. In the middle part, Figure 4 presents low soil resistance in the range of $15 \mathrm{~cm}$ deposition depth as expected. Additionally, there are many fault values as well. On the right side, we obtain lowest soil resistance near $20 \mathrm{~cm}$ deposition depth. The large scatter indicates slurry mixing again with loosened soil near the surface. Due to the plowsole below $20 \mathrm{~cm}$ depth, the manure cannot mix down further more in the right part of Figure 4 . The resulting deposit of liquid manure remains stable. Therefore, we measured values for $20 \mathrm{~cm}$ depth more accurately.

To do a refinement of measurement and earn exact data, we have two options:

- First, we do much more test points on the field with a high expansion of time and work.

- Second, we refine the framerate during the spike injections to become means of each centimetre spike going down into soil. Because of the aim of this study and the willingness to present a system with less effort, we decided to do second and generate a refinement of software and data collection.

\section{Results of experiment 3}

Figure 5 presents the results of experiment 3 with new single slurry depth. Out of 30 trials, $n=23$ could be evaluated. The logging speed was set to $100 \mathrm{~Hz}$ resulting in a large dataset with $\mathrm{n}=99,591$ values taken. Values above $2 \mathrm{k} \Omega$ where cut off. As described in section 2.4 for experiment 3, we see a wide range of weak values in the upper "experiment horizon 1" again, reaching from one until nine-centimeter soil depth. Because of the big data pool, the mean in every single boxplot shows correct values as presented in section 3.1 for conductivity in soil bevor reaching the slurry horizon 2 . The quantum of fault values is related to the slurry meter pulling back out of the soil for doing the next trial. Slurry can hold contact and remains on the spike of the slurry meter. Soil depth 10 and 11 shows the spike reaching the slurry horizon.

For the next centimeters, the probe reaches the "slurry horizon 2 " between $12 \mathrm{~cm}$ and $17 \mathrm{~cm}$ as presented in Figure 5. The conductivity rises and the soil resistance falls down between $200 \Omega$ and $600 \Omega$. The fault values 
become less and the moisture of the slurry let rise conductivity additionally. Now slurry meter spike exits the slurry horizon 2 and test voltage is falling back again.

Now, the slurry meter reaches the "experiment horizon 3 ". Pulling up the slurry meter out of the soil is the reason for rising fault values on soil depth 19 and 20.

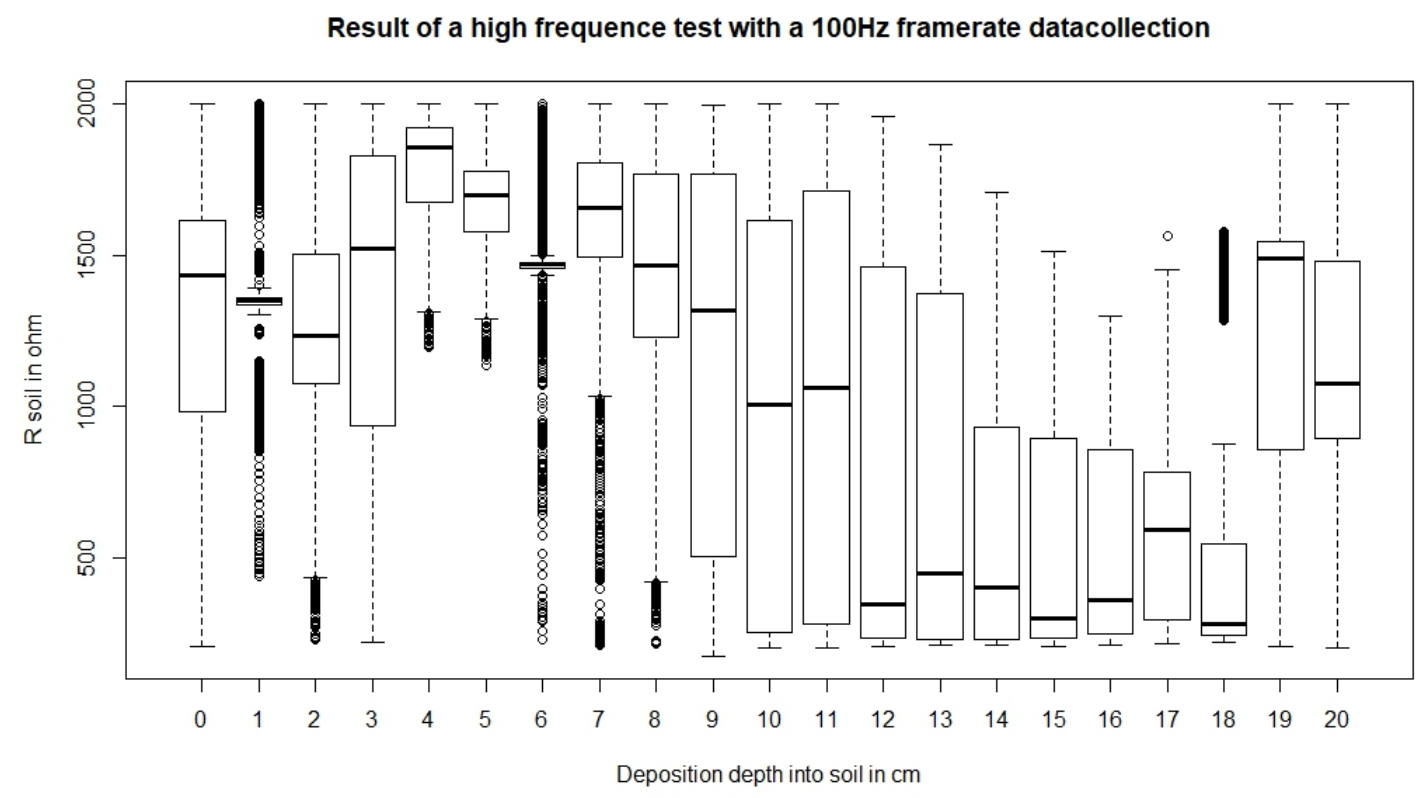

Figure 5. Result of the second field trial with $12 \mathrm{~V}$ testvoltage and with a single new deposition depth.

The manure measuring system "slurry meter" is a moving device. At any point in time, the conductivity can change because of trapped air in the soil after tillage. If the soil is no longer cohesively at the spike of the slurry meter, the conductivity is immediately significant worse. The inaccuracy between $1 \mathrm{~cm}$ and $7 \mathrm{~cm}$ might occur duo to the spike dirtying. Spike, touching the soil surface, have minimal slurry on the electrical conductors left. In the first $3 \mathrm{~cm}$ presented in Figure 5, this is the reason for better conductivity. Accordingly, now spike is cleaned by the soil reaching the next few centimeters. This results in a higher soil resistance as observed in experiment 2. Cleaning the slurry meter spike before every single trail might prevent getting fault values as presented in Figure 5 for the soil depth of $1 \mathrm{~cm}$ and $7 \mathrm{~cm}$.

Smallest grains and particles of sand or clay can influence the conductivity. Only with a very large sample size and high data density, we recognize the true values by the mean of every centimeter.

Results of experiment 4 with a view on a single puncture

For evaluating, if it is possible to obtain the $100 \mathrm{~Hz}$ data without the scientific equipment of laptops, we performed a test on a single puncture. The aim is not to lose any information during puncturing.

In Figure 6 shows the result for one spike injection with $100 \mathrm{~Hz}$ logging frequency. We took the small picture on the test laptop screen during field trial. It shows the precision of the measurement in comparison to the later evaluation with statistical software. For the reason that refinement is suitable, the calculated data done with $\mathrm{R}$ software is similar to the screenshot. A bigger variance is due to the effect of a fast increase or decrease of the conductivity. 


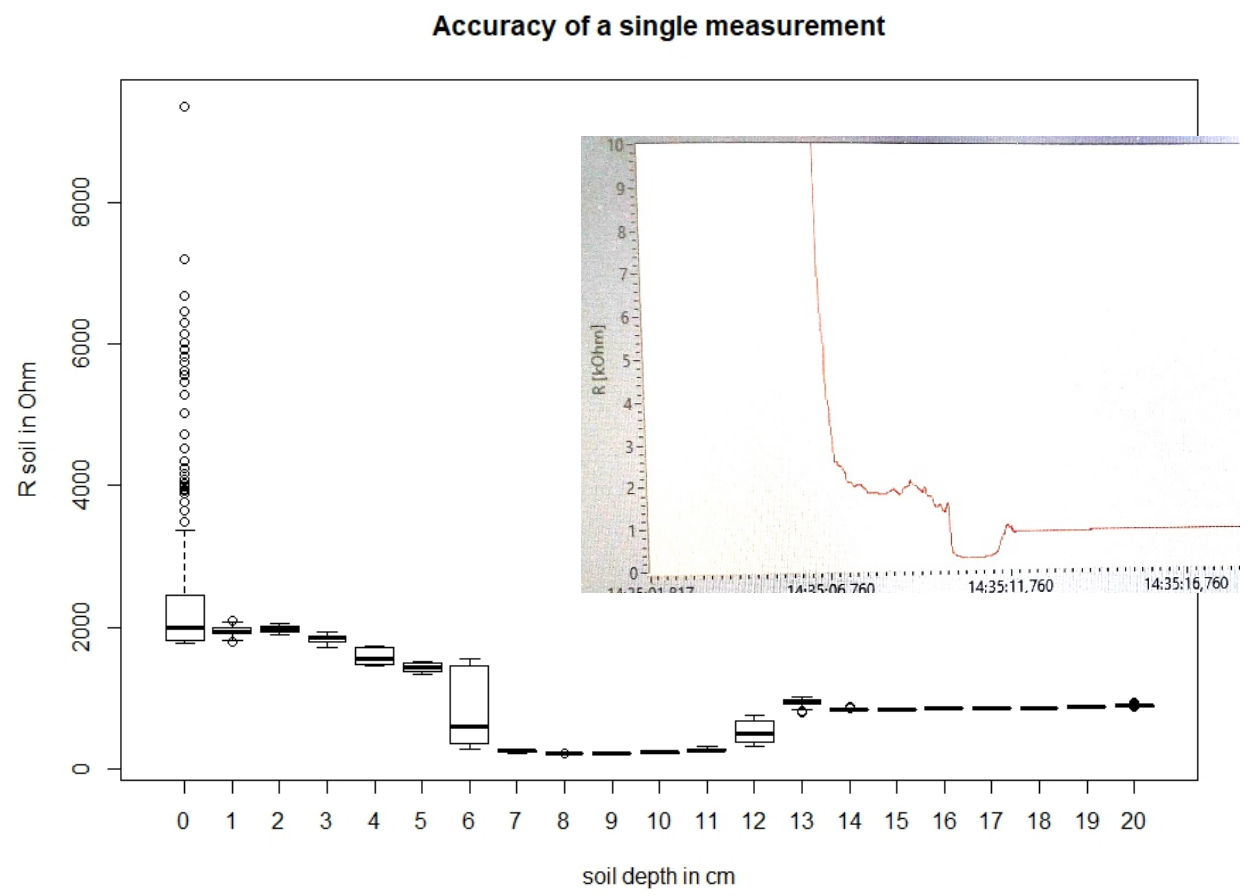

Figure 6. Diagramm is the result of $\mathrm{R}$ data analysis. The picture on the right is the equivalent screenshot.

Figure 6 is the result of $n=1953$ values taken between 19 seconds observant time for only one spike injection in soil. A resistance of $200 \Omega$ presents the presence of slurry in this single trial during measurement. After exiting the slurry horizon, resistance is not reaching the value as bevor. This is due to the fact of minimal slurry remaining on the spike that moves deeper into soil. The effect persists, until we pull slurry meter backwards out of the soil for doing the next trial. As mentioned in section 3.3, cleaning the slurry meter spike bevor the next trail could reduce fault values.

\section{Conclusions}

The presented slurry meter is suitable to detecting the slurry deposition depth. Without the need for digging holes into soil surface, slurry is detectable by punctures with the presented probe. It is very cheap to buy the equipment needed. With the information given in this paper, a quick replica is easy to finish.

For very low slurry deposition depth of only $5 \mathrm{~cm}$ and a deposition depth greater $20 \mathrm{~cm}$, this slurry meter should not be applied. In the upper soil horizon, slurry is so close to the surface. The first contact of the slurry meter spike is too early for a reliable measurement. For a measurement in depth of more than $20 \mathrm{~cm}$, the risk of touching the plowsole is higher. More pressure on top of the slurry meter could result in a weak measurement.

The aim of this study was to make tests with the slurry meter in a tilled soil on top of the plowsole. If the data collection rate is set to $100 \mathrm{~Hz}$, we will detect the deposition depth well. Therefore, we have to slow down the puncture speed during spike injection into soil

For this reason, a new kind of soil sensor is now available. The slurry meter signals every passing horizon. It might be even possible to detect more than one slurry horizon. Therefore, the use of "slurry meter" could affect future research.

\section{Acknowledgements}

My thanks go to the entire team of the Chair of Agricultural Systems Engineering at the Technical University of Munich, who helped to implement the idea. Special thanks are due to the coauthors and the support by Ms. Kathrin Ziegler being involved in the field trials. Mr. Stefan Huber did the programming with the LabView software. Thanks for that. 


\section{References}

Jäger, H. (1957). Elektrische Messung von Bodenverdichtungen unter landwirtschaftlichen Fahrzeugen. Landtechnische Forschung 1957, H. 6

Juriček, J. (1990). Einfluß des Trockenmassegehalts auf die Veränderung der Dichte und der spezifischen elektrischen Leitfähigkeit der Schweinegülle. Agrartechnik Berlin 40 (4), Forschungsinstitut für Landtechnik Rovinka (ČSSR)

Küpfmüller, K., Kohn, G. (1993). Theoretische Elektrotechnik und Elektronik. 14. Auflage. Springer Verlag, 1993

Paungfoo-Lonhienne, C., Visser, J., Lonhienne, T.G.A. et al. Plant Soil (2012). Past, present and future of organic nutrients. 359: 1. https://doi-org.eaccess.ub.tum.de/10.1007/s11104-012-1357-6

Provolo, G., Martínez-Suller, L. (2006). In situ determination of slurry nutrient content by electrical conductivity. Bioresource Technology 98 (2007) 3235-3242

R Core Team (2017). R: A language and environment for statistical computing. R Foundation for Statistical Computing, Vienna, Austria. URL https://www.R-project.org/.

Sarkar, Deepayan (2008). Lattice: Multivariate Data Visualization with R. Springer, New York. ISBN 978-0387-75968-5

Sophocleous, M., Atkinson, J. K. (2015). A novel thick-film electrical conductivity sensor suitable for liquid and soil conductivity measurements. Sensors \& Actuators: B. Chemical, 5 July 2015, Vol.213, pp.417-422

Vandré, R., Clemens, J., Goldbach, H., Kaupenjohann, M. (1997). NH, and N,O Emissions after Landspreading of Slurry as Influenced by Application Technique and Dry Matter-Reduction. I. NH, Emissions. Z. Pflanzenernährung und Bodenkunde, 160. 303-307 (1997)

Warner, N. L., Godwin, R.J. (1988). An Experimental Investigation into Factors influencing the Soil Injection of Sewage Sludge. Journal of agric. Engng Res. (1988) 39, 287-300 


\title{
Delivery Rates during Retention and Transport of Encapsulated Fertilisers in Porous Medium
}

\author{
Fernando Rojano $^{\text {a,* }}$, Felipe Barrera-Méndez ${ }^{\text {a,b }}$, Maria A. Luna-Velasco ${ }^{c}$, Abraham Rojano ${ }^{d}$ \\ a Red de Estudios Moleculares Avanzados, Instituto de Ecología, A C. Carretera Antigua a Coatepec 351, El Haya, \\ Xalapa, 91070, México. \\ ${ }^{\mathrm{b}}$ Cátedra CONACYT en el Instituto de Ecología AC, Xalapa, Veracruz, México \\ ${ }^{c}$ Medio Ambiente y Energia, Centro de Investigacion en Materiales Avanzados. Miguel de Cervantes 120, Complejo \\ Industrial Chihuahua, 31136, México. \\ ${ }^{\mathrm{d}}$ Universidad Autónoma Chapingo, Carretera México-Texcoco Km 38.5 Chapingo, 56230, México \\ * Corresponding author. Email: fernando.rojano@inecol.mx
}

\begin{abstract}
Fertilisers applied to soils commonly undergo a quick removal by leaching that greatly diminish nutritional benefits to the plant. To solve such problem, this investigation proposes augmenting effectiveness by means of a controlled release of the fertiliser. The present investigation suggests a process of synthesising particles that contain fertiliser and having an average size between $1 \mu \mathrm{m}$ and $2 \mu \mathrm{m}$. For this study, a fertiliser $\left(\mathrm{KH}_{2} \mathrm{PO}_{4}\right)$ was immobilised with chitosan, and later was evaluated with the aim to identify in first place efficiency of synthesis of micro-particles, and in second place delivery rates along time. The evaluation compared results between $\mathrm{KH}_{2} \mathrm{PO}_{4}$ and $\mathrm{KH}_{2} \mathrm{PO}_{4}$ immobilised with chitosan in order to know the capacity of retention and transport within the soil. To conduct experiments, soil was represented by a column length of $0.1 \mathrm{~m}$ with porous medium, which was constituted of silica gel with average size particle of $51 \mu \mathrm{m}$. Milli- $\mathrm{Q}^{\mathrm{TM}}$ water was used for a series of experiments that assessed the micro-particles through induced leaching. It was found that $17 \%$ of the $\mathrm{KH}_{2} \mathrm{PO}_{4}$ was delivered during the first induced leaching, and corresponded to the $\mathrm{KH}_{2} \mathrm{PO}_{4}$ that was not immobilised with chitosan. Additionally, every $48 \mathrm{~h}$ there was an induced leaching that was conducted 4 times, and delivered $69 \%$ of the $\mathrm{KH}_{2} \mathrm{PO}_{4}$. Then, the experimental rig was also modelled by means of the general rate model. The model included axial transport, convection, and accumulation in the mobile and stationary phase to get unidimensional predictions along the column. In this way, it was possible to predict the concentrations after water moved down through the column, as an indicator of delivery rates. Thus, presenting in this investigation a technological process to build up micro-particles made of $\mathrm{KH}_{2} \mathrm{PO}_{4}$ with chitosan, which can be considered as a feasible technique to increase the lifetime of fertiliser in soils; and an experimental and modelling assessment of the induced leaching happening in a column under specific operating conditions.
\end{abstract}

Keywords: fluids in columns, tracers in soils, axial dispersion, lixiviation

\section{Introduction}

Application of agrochemical compounds to the soil has been intensively used in the last century with the aim to augment availability of nutrients, fungal control and also to enhance potential of the microbiota processing nutrients to the plant. Those agrochemicals have been firstly developed and tested in lab; then, assessment on the field is necessary because adversity of local weather, mainly due to precipitation, could greatly diminish their effectiveness. Once an agrochemical is deposited in soil, investigation about its transport and fate should be known. For that, it has been paid attention to the rates of transport and dispersion, since it is crucial to keep those agrochemicals as much as possible close to the target (i.e. roots). This investigation is motivated due to great concerns about leaching that have recently arisen (Broschat 1995), as is the transportation away from the target of those agrochemicals, carrying them to groundwater reserves, and becoming the main limitation to effectively use them.

Alternatives have been proposed to reduce the problem of leaching, such as application at the right time and amount, adequate management of irrigation, use of the peripheral technology (climate forecasting, soil analysis) to identify the exact amount and time to apply those agrochemicals. However, such alternatives can be subjected to the need of soil and local climate dataset analyses, increment of labour to execute several applications, and handle uncertainty at forecasting local weather and bias measurements when analysing soils.

Additionally to the options aforementioned, it is possible to immobilise agrochemicals to delay its delivery accordingly to the needs of the plant. In particular, this study has been focused in the feasibility of incrementing the effectiveness of fertilisers. For that, several choices have been explored as it is summarised in (Shaviv 2001; Han, Chen, and Hu 2009; Kashyap, Xiang, and Heiden 2015). The immobilisation can increase effectiveness when fertilisers are deposited in soil, and also the same technological process can be feasible for many other valued-added compounds of interest in agriculture. As it has been proved in previous studies (Han, Chen, and $\mathrm{Hu}$ 2009; Han, Chen, and Hu 2009; Tomaszewska and Jarosiewicz 2006; Yang et al. 2016), immobilisation can serve to augment the retention time and also provide the controlled delivery rates needed by the plant. 
For immobilising fertilisers several materials could be used, among the more important materials are: rosin coating, carboxyme-thylcellulose/hydroxyethylcellulose, polystyrene foam, super absorbent formula, polydopamin, and chitosan (Trinh, Kushaari, and Basit 2015). From all these options, we chose chitosan because it has been identified with minimum impact that could alter the regular physical, chemical or biological processes observed in the soil. Additionally, chitosan is qualified as a promising option for micro-formulations due to its higher surface area, induction of systemic activity due to smaller particle size and higher mobility (Kashyap, Xiang, and Heiden 2015). Furthermore, it is biocompatible, biodegradable, highly permeable, low cost, no toxic and with excellent film forming ability.

Therefore, the interest in this investigation is the development of a technological process to produce suitable micro-particles that can be applied on the field, it proposes the synthesis of the micro-particle by integrating chitosan with the fertiliser through a physical-chemical process. Later, this investigation is focused on the assessment in laboratory conditions of the micro-particles by means of a column with porous medium. This section proves the effectiveness of immobilising potassium phosphate $\left(\mathrm{KH}_{2} \mathrm{PO}_{4}\right)$ with chitosan and also the retention that can be achieved. Consecutively, experimental data is used to support the modelling stage through the use of fundamentals of mass transfer.

In summary this work is oriented to cover the following objectives:

1. Synthesise micro-particles of $\mathrm{KH}_{2} \mathrm{PO}_{4}$ with chitosan.

2. Determine characteristics and operating conditions of the column with porous medium to evaluate retention and leaching of $\mathrm{KH}_{2} \mathrm{PO}_{4}$ immobilised with chitosan.

3. Use of the experimental results for validating a model that includes axial transport, convection and accumulation of micro-particles.

\section{Materials and Methods}

Physical and chemical properties of the fertiliser and chitosan

The $\mathrm{KH}_{2} \mathrm{PO}_{4}$ is a salt freely soluble in water and insoluble in ethanol with molecular weight of 136.09. The $\mathrm{pH}$ has been found within the range of 4.3 to 4.7. This fertiliser was provided by the company J. T. Baker with a purity of $99.4 \%$ and it contains $0.005 \%$ of insoluble matter. On the other hand, chitosan is a polymer soluble acidic to neutral solutions, with low molecular weight between $50 \mathrm{kDa}$ and $190 \mathrm{KDa}$ based on viscosity. Chitosan was provided by the company Sigma Aldrich ${ }^{\mathrm{TM}}$.

Synthesis of the micro-particles

A $10 \mathrm{mg} \mathrm{mL}^{-1}$ stock solution of $\mathrm{KH}_{2} \mathrm{PO}_{4}$ was prepared dissolving the fertiliser in Milli- $\mathrm{Q}^{\mathrm{TM}}$ water. In a similar way, a $10 \mathrm{mg} \mathrm{mL}^{-1}$ stock solution of chitosan was prepared dissolving $2 \mathrm{~g}$ of the carrier in $1 \%$ acetic acid solution and taking it to a volume of $200 \mathrm{~mL}$. The solution was steered overnight and filtered out to remove insoluble impurities. For each $100 \mathrm{~mL}$ of the final formulation with a 2.5:1 ratio of $\mathrm{KH}_{2} \mathrm{PO}_{4}$ and chitosan, the following quantities were used: $25 \mathrm{~mL}$ of the carrier solution, $10 \mathrm{~mL}$ of the fertiliser solution, and $65 \mathrm{~mL}$ of Milli-Q ${ }^{\mathrm{TM}} \mathrm{water}$ to reduce the viscosity.

To obtain the micro-particles, the solution was then dried by the spray drying technique, using the device YAMATO-ADL311S. The operating conditions were: temperature of $120{ }^{\circ} \mathrm{C}$, air pressure of $0.3 \mathrm{MPa}$, vapour extraction rate of $6 \mathrm{~m}^{3} \mathrm{~min}^{-1}$, and feeding flow rate of $3.6 \mathrm{~mL} \mathrm{~min}{ }^{-1}$ to obtain a particle in a range from $1 \mu \mathrm{m}$ to 2 $\mu \mathrm{m}$. Additionally, microparticles of pure chitosan were obtained by drying, at the same operational conditions, 25 $\mathrm{mL}$ of the carrier solution with $75 \mathrm{~mL}$ of Milli- $\mathrm{Q}^{\mathrm{TM}}$ water.

General specifications of the column of percolation

A column with porous medium was used to assess the micro-particles obtained through the process indicated in section 2.2. In contrast to evaluation of tracers in soil columns that usually have a length between $0.3 \mathrm{~m}$ and 2 $\mathrm{m}$, a diameter from $0.1 \mathrm{~m}$ to $0.5 \mathrm{~m}$, and having gravity and capillarity as the driving forces governing the process of leaching (Naka et al. 2016), this research proposes a column with a length of $0.1 \mathrm{~m}$ and diameter of $0.01 \mathrm{~m}$ that can be pumped to quickly evaluate retention and leaching. The dimensions of the column demands for a porous medium made of micro-particles. An option for this configuration is silica gel with average diameter of the particle of $51 \mu \mathrm{m}$ and pore size $\left(60 \mathrm{~A}^{\circ}\right)$ quantified in a ratio of volume and weight of $0.7-0.85 \mathrm{~cm}^{3} \mathrm{~g}^{-1}$, and surface area $\geq 4800 \mathrm{~m}^{2} \mathrm{~g}^{-1}$; this material is commonly used for columns of chromatography. Also, silica gel provided by SigmaAldrich ${ }^{\mathrm{TM}}$ was chosen because avoided perturbations that regularly are observed in soils such as acidity/basicity, activity of microorganisms, salts among others. In summary, the experimental rig specifications and equipment are illustrated in Figure 1. 


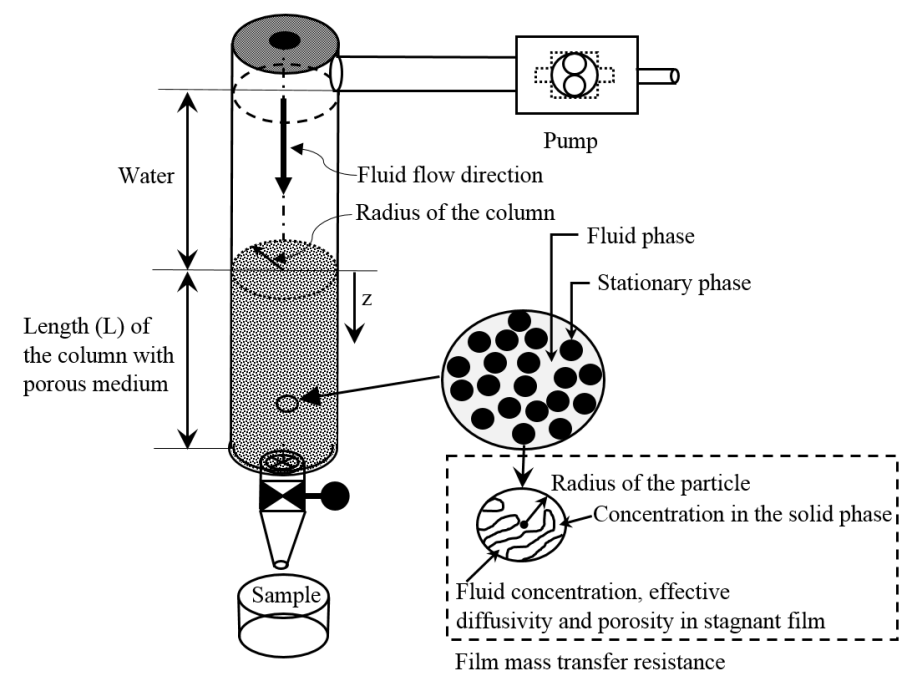

Figure 1. Column and porous medium specifications and equipment.

\section{General rate model}

The transport and fate of fertilisers can adequately be analysed by using the fundamentals of mass transport happening in porous medium. The corresponding analysis can be studied by only focusing in axial motion of the fluid flow; then, the phenomena involved can be simplified in a one-dimensional approach, and the model still have enough accuracy of the estimation ( $\mathrm{Lu}$ et al. 2016). With this simplification, the main elements of the experiment can be useful for the modelling; which can be classified in three phases: the mobile phase (fluid concentration) flowing between particles and porous medium, the stagnant film (static fluid immobilised in the macroporos of the porous medium), and the stationary phase (the fine sand particles where adsorption may occur).

Additionally, modelling can be accomplished when assuming some conditions that could reduce the level of complexity. For instance, regarding the mobile phase, it is considered that the fluid is not compressible, and such fluid flow is at constant velocity. Once the fluid is passing through the porous medium there will be no change in its volume and temperature. Also, the fluid will not be absorbed (fluid at the interior of the particles of the porous medium). Since the fluid will move in axial direction, its gradient will be significantly larger with respect to the radial direction gradient.

In the stationary phase (particles of the porous medium) will be assumed that particles are spherical and uniform in diameter. And the stagnant film will assume that fluid flow will be static when reaching the macroporos, and there will be instantaneous local equilibrium between the surface of the macroporos and the static fluid at the interior of the macroporo's particle.

Fluid flow characteristics must be conditioned to a constant diffusion, which will be independent of the effects at mixing water and the fertiliser. In addition, all the mechanisms contributing to axial mixing are grouped together in one coefficient known as dispersion. For instance, the value of the dispersion coefficient may change when fluid motion is induced due to influence of tortuosity by packing the porous medium, which is reducing the axial molecular diffusion, the anastomosis problem created by the small channels throughout the porous medium after running a fluid, and "dead volumes".

\section{Governing equations}

The general rate model is proposed to analyse axial, convective and accumulation of mass in the fluid flow. The equation below states from left to right: the dispersion, advection, change over time and accumulation.

$$
-D_{f} \frac{\partial^{2} C_{f}}{\partial z^{2}}+v \frac{\partial C_{f}}{\partial z}+\frac{\partial C_{f}}{\partial t}+\frac{\left(1-\varepsilon_{b}\right)}{\varepsilon_{b}} \frac{\partial q}{\partial t}=0
$$

where $D_{f}$ is coefficient of axial dispersion, $\mathrm{m}^{2} \mathrm{~s}^{-1} . C_{f}$ is fluid concentration, $\mathrm{kmol} \mathrm{m}^{-3} . v$ is interstitial velocity, $\mathrm{m} \mathrm{s}^{-1} . z$ is axial coordinate, $\mathrm{m} . t$ is time, s. $\varepsilon_{b}$ is void fraction volume of the porous medium, Adim. $q$ is concentration of the fluid adsorbed by the porous medium.

Interfacial fluid flow (between the mobile phase and the porous medium) is:

$$
\frac{\partial q}{\partial t}=\frac{3 k}{R_{p}}\left(C_{f}-C \underset{R=R_{p}}{p}\right)
$$

where $R_{p}$ is radius of particle of the porous medium. $C_{p, R=R_{p}}$ is concentration of the static fluid located within the macroporos of the porous medium particles, as a reference value to be assigned at the level of surface of the 
particle radius. $k$ is coefficient of mass transfer of the film around the particles of the porous medium

On the other hand, analysing the interaction of the macroporos with fluid flow can be estimated by means of the equation below that computes with terms from left to right: the accumulation in the microporous and macroporos of the stationary phase, change over time of the fluid concentration in the solid phase, and the radial diffusion inside the particle of the porous medium:

$$
\left(1-\varepsilon_{p}\right) \frac{\partial C_{p}{ }^{*}}{\partial t}+\varepsilon_{p} \frac{\partial C_{p}}{\partial t}+\varepsilon_{p} D_{p}\left[\frac{1}{R^{2}} \frac{\partial}{\partial R}\left(R^{2} \frac{\partial C_{p}}{\partial R}\right)\right]
$$

where $\varepsilon_{p}$ is porosity. $C_{p}{ }^{*}$ is concentration of the fluid in the solid phase (porous medium) of the particle. $D_{p}$ is effective diffusivity driven by the porosity of the column. And concentration $C_{p}{ }^{*}$ is directly linked with the isotherms of Langmuir.

Initial and boundary conditions

The governing equation for axial transport considers that initial concentrations (i.e. $t=0$ ) will be as follows:

$$
\begin{aligned}
& C_{f}=C_{f}(t, z) \\
& C_{p}=C_{f}(t, R, z)
\end{aligned}
$$

where the column has axial $(z)$ and radial $(R)$ coordinates to indicate the location of the concentration in the fluid $\left(C_{f}\right)$ and in the particle $\left(C_{p}\right)$.

Then, boundary conditions will assume that fluid concentration at the top surface (i.e. $z=0$ ) will be:

$$
\frac{\partial C_{f}}{\partial z}=\frac{v}{D_{f}}\left(C_{f}-C_{f}(t)\right)
$$

whereas the fluid concentration at the end of the column ( $L=$ Length of the column) can be stated as:

$$
\frac{\partial C_{f}}{\partial z}=0
$$

And at the level of the surface $\left(R=R_{P}\right)$ of the particle of the porous medium we will have a concentration:

$$
\frac{\partial C_{p}}{\partial R}=\frac{k}{\varepsilon_{p} D_{p}}\left(C_{f}-C_{p, R=R_{p}}\right)
$$

\section{Results and Discussion}

The synthesis of the micro-particles was conducted to obtain chitosan and chitosan- $\mathrm{KH}_{2} \mathrm{PO}_{4}$ particles, in order to compare theirs rates of transport through the porous medium. The ratio of chitosan and $\mathrm{KH}_{2} \mathrm{PO}_{4}$ was $2.5: 1$ to provide the more homogeneous and spherical particles. Then, $\mathrm{KH}_{2} \mathrm{PO}_{4}$ immobilised with chitosan were used for the scanning electron microscope (SEM) image (Figure 2).

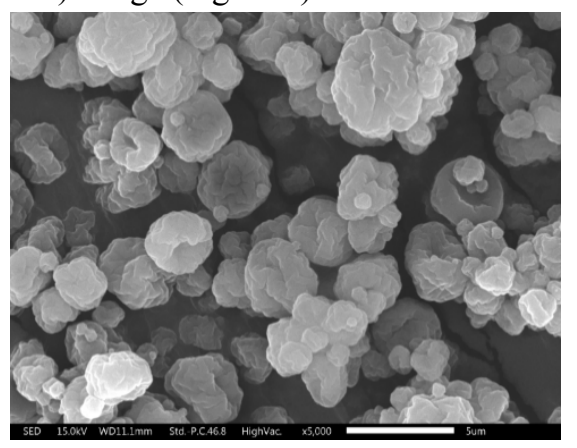

Figure 2. SEM image of micro-particles of fertiliser $\mathrm{KH}_{2} \mathrm{PO}_{4}$ immobilised with chitosan.

The porous medium of the column was constituted of silica gel that was washed with Milli- $\mathrm{Q}^{\mathrm{TM}}$ water, to get the minimum electro-conductivity (EC) equivalent to values less than $12 \mu \mathrm{S} \mathrm{cm}^{-1}$. The procedure to assess transport of micro-particles was conducted by comparing three samples: 1) fertiliser, 2) chitosan, and 3) fertiliser immobilised with chitosan. Each sample was pumped down at a constant velocity through the porous medium with a length of $0.1 \mathrm{~m}$. The sample was placed in the top of the column and mixed with silica gel located within the first $0.5 \mathrm{~cm}$; then, $12.5 \mathrm{~mL}$ of Milli-Q ${ }^{\mathrm{TM}}$ water with temperature of $24( \pm 0.5)^{\circ} \mathrm{C}$ was used for induced leaching, those experiments were conducted at room temperature of $25^{\circ} \mathrm{C}$. Fluid average interstitial velocity was $v=$ $4 Q /\left(\pi d^{2} \epsilon_{b}\right)=0.00054 \mathrm{~m} \mathrm{~s}^{-1}$, where $Q$ is the flow rate in $\mathrm{m}^{3} \mathrm{~s}^{-1}, d$ is the particle diameter, $\varepsilon_{b}=0.4$ is void fraction of the silica gel particles in porous medium. The time $t$ required to pass a volume of water equivalent to the volume of the porous medium was equal to $440.5 \mathrm{~s}$. 
Firstly, the evaluation of the fertiliser was conducted. Three runs were covered, and then sampling of the induced leaching was each $0.5 \mathrm{~mL}$. After those samples were collected, then EC was measured. A calibration curve was obtained for the fertiliser to convert from EC units to $\mathrm{mg} \mathrm{mL}^{-1}$, finding that $C_{f}=0.0014 *$ $E C\left(\right.$ in $\left.\mu \mathrm{S} \mathrm{cm}^{-1}\right)+0.0004$. Experimental results considered time equal to 1 when it reached the $440.5 \mathrm{~s}$, and fluid concentration was equal to 1 , when it was equal to the amount of fertiliser initially placed at the top of the column. To make sure validity of the induced leaching, the sum of all sample fluid concentrations had to be near the initial amount of fertiliser placed in the column; our experiments found in average more than $95 \%$ of the fertiliser lixiviated. The three runs and its average value are presented in Figure 3.

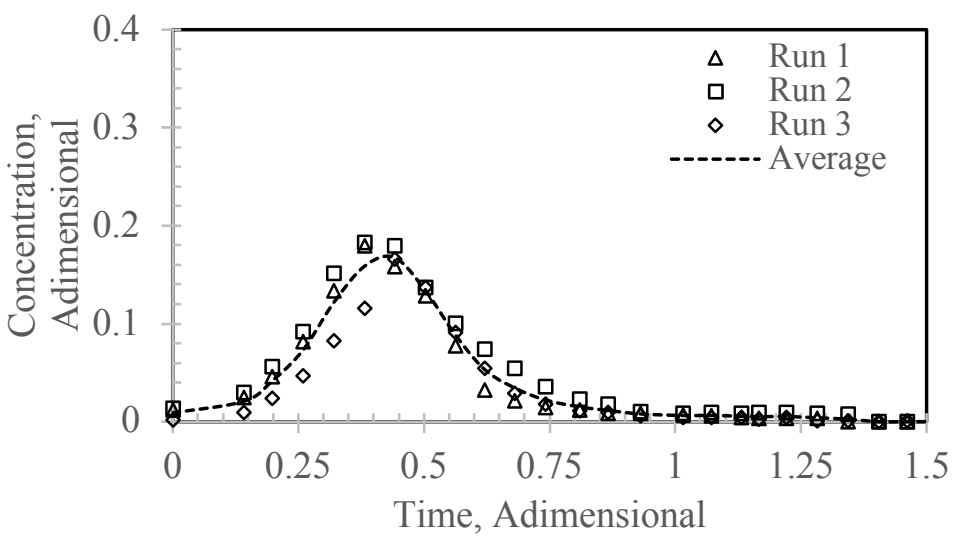

Figure 3. Experimental data of $\mathrm{KH}_{2} \mathrm{PO}_{4}$ concentrations in a column of $10 \mathrm{~cm}$-length with porous medium.

The evaluation of micro-particles of chitosan and fertiliser immobilised with chitosan was conducted with the same operating conditions aforementioned (i.e. the induced leaching of the fertiliser). In this case, the induced leaching of fertiliser immobilised with chitosan was firstly covered and EC values were found. Then, induced leaching of the chitosan was conducted. In this way, EC values of the chitosan were subtracted to the EC values of the fertiliser immobilised with chitosan, to know the EC values corresponding to the fertiliser. After that, EC values were converted to fluid concentration. Experimental results of the fertiliser and fertiliser immobilised with chitosan are presented in Figure 4, where average concentration for only fertiliser introduced in Figure 3 was presented again. It was found that immobilising the fertiliser with chitosan can significantly reduce the losses by leaching, given that only $17 \%$ of the fertiliser was delivered.

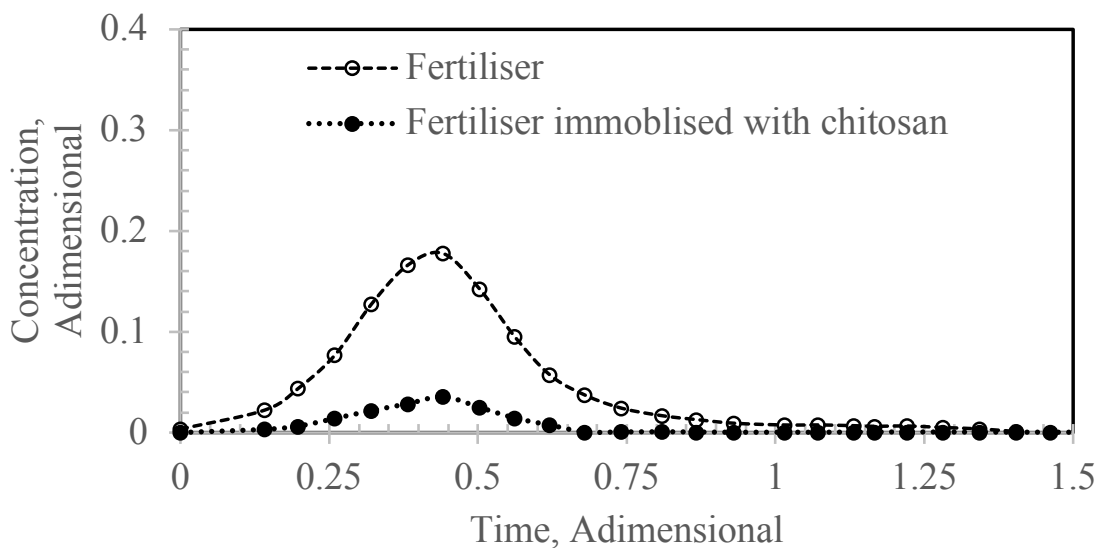

Figure 4. Concentrations for fertiliser, chitosan and immobilised fertiliser $\mathrm{KH}_{2} \mathrm{PO}_{4}$ with chitosan.

Since the fertiliser immobilised with chitosan had capability of retention, it was necessary to conduct every 48 $\mathrm{h}$ an induced leaching during 4 times under same operating conditions explained in section corresponding to experimental results presented in Figure 3. Those outcomes served to know the fertiliser rates that may be delivered. The assessment considered the column with chitosan and the one with fertiliser immobilised with chitosan. After each induced leaching both columns were kept at room temperature of $25{ }^{\circ} \mathrm{C}$, and the porous medium wet as a consequence from the previous induced leaching. It was found that after 5 repetitions of the induced leaching, the total fertiliser delivered was approximately $86 \%$ of the total. The cumulative delivery rates at each induced leaching were estimated and presented in Figure 5. Maximum fertiliser released was equal to 1 when the total amount of fertiliser immobilised in chitosan was delivered. 


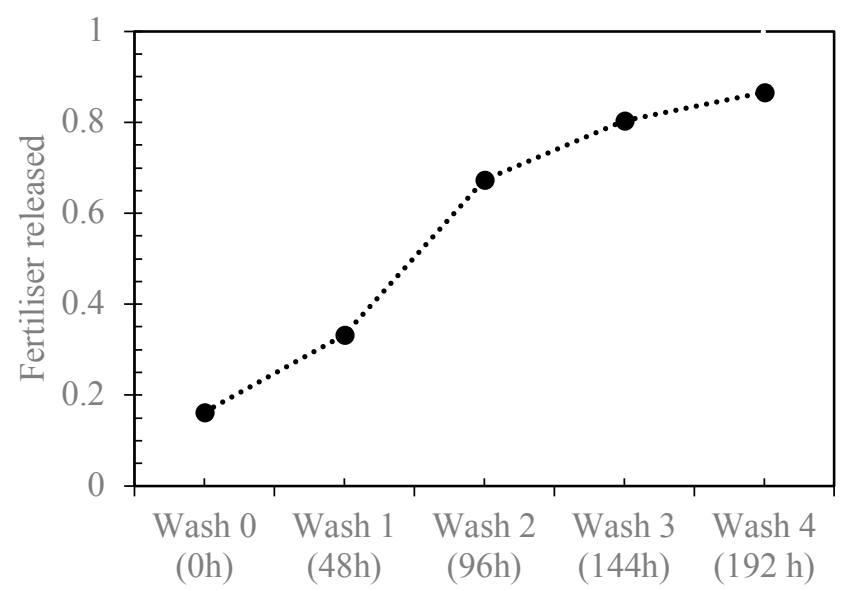

Figure 5. Cumulative delivery of the immobilised fertiliser $\mathrm{KH}_{2} \mathrm{PO}_{4}$ with chitosan.

The modelling stage is assisted by the general rate model, which is commonly used in chromatography for separation of molecules based on its size. In this case, such equations are used for only predicting the axial dispersion based on the experimental data presented before. The equations and initial and boundary conditions were transformed in dimensionless way based on Table 1, to get the governing equations as:

$$
\begin{aligned}
& -\frac{1}{P e} \frac{\partial^{2} C_{f}}{\partial z^{2}}+\frac{\partial C_{f}}{\partial z}+\frac{\partial C_{f}}{\partial \tau}+\xi\left(C_{f}-C_{p, r=1}\right)=0 \\
& \frac{\partial}{\partial \tau}\left[\left(1-\varepsilon_{p}\right) C_{p}^{*}+\varepsilon_{p} C_{p}\right]-\eta\left[\frac{1}{r^{2}} \frac{\partial}{\partial r}\left(r^{2} \frac{\partial C_{p}}{\partial r}\right)\right]=0
\end{aligned}
$$

At the initial conditions $(\tau=0)$ we have:

$$
\begin{aligned}
& C_{f}=C_{f}(0, z) \\
& C_{p}=C_{f}(0, r, z)
\end{aligned}
$$

At the boundary $(z=0)$ conditions we have:

$$
\frac{\partial C_{f}}{\partial z}=P e\left(C_{f}-\frac{C_{f}(t)}{C_{f, \max }}\right)
$$

Table 1. Terms conversion to estimate for predictions of fluid concentrations in dimensionless approach.

$$
\begin{aligned}
& C_{f}=\frac{C_{f}}{C_{f, \max }} \quad C_{p}=\frac{C_{p}}{C_{f, \max }} \quad C_{p}^{*}=\frac{C_{p}^{*}}{C_{f, \max }} \quad \tau=\frac{\vartheta * t}{L} \quad r=\frac{R}{R_{p}} \\
& Z=\frac{Z}{L} \quad P e=\frac{\vartheta * L}{D_{f}} \quad B i=\frac{k * R_{p}}{\epsilon_{p} * D_{p}} \quad \eta=\frac{\epsilon_{p} * D_{p} * L}{2 * v * R_{p}} \quad \begin{array}{l}
\xi * B i * \eta *\left(1-\epsilon_{b}\right) \\
\epsilon_{b}
\end{array}
\end{aligned}
$$

The estimation of the concentration $C^{*}{ }_{p}$ at the solid phase of the particles was linked directly with the Langmuir isotherm, detailed implementation is provided in the work of (Lu et al. 2016). Then, predictions were focused in the effluent of the column in a way to estimate fluid concentrations as the ones presented in Figure 4. The solution of the general rate model is accomplished numerically since the non-linearity caused by the Langmuir isotherm. The numerical solution had to use the finite element formulation for predicting the fluid concentration. The estimation of concentrations in the particle phase needed of the orthogonal collocation formulation, where the Langmuir isotherms were treated. Then, the partial differential equations can become a set of ordinary differential equations solved by means of the software Matlab.

The estimation of the fluid concentration by means of the general rate model required values of porosity $\varepsilon_{p}=$ 0.4, an effective diffusion constant (Saripalli et al. 2002), which was estimated by means of $D_{p}=$ $D_{m} \times \epsilon_{b} \times \Phi \times \tau^{-1}$, where $D_{m}$ is the free diffusion of water, $\delta$ is the constrictivity value $(0.43)$, $\varepsilon_{b}$ is the porosity (0.4), and $\tau$ is the tortuosity (1.448) derived from a porous medium made of spheres (Ghanbarian et al. 2013). Then, finding that $D_{p}=1.1495 \times 10^{-10} \mathrm{~m}^{2} \mathrm{~s}^{-1}$. An estimated mass transfer film rate of $k=1.7 \times 10^{-3} \mathrm{~m} \mathrm{~s}^{-1}$, and an average particle radius of $R_{p}=25.5 \mu \mathrm{m}$. High solubility of the fertiliser aided in the assumption that it was diluted with an exponential decay, and it was possible to assume a step function equal to 1 minus the cumulative normal distribution with mean and standard deviation equal to 0.18 , during the first $215 \mathrm{~s}$ as the initial 
concentration. Then, predictions of the general rate model were adjusted to experimental data in order to estimate the coefficient of dispersion $D_{f}$, which was approximately to $6.32 \mathrm{~mm}^{2} \mathrm{~s}^{-1}$.

Results of the fluid concentrations in Figure 6 correspond to the ability of the general rate model to be adjusted based on the identified coefficient of dispersion. It should be noted that values assumed to other variables such as the mass transfer film, porosity and void fraction may lead to some bias estimations; nonetheless, differences between the experimental and modelled data created a RMSE of 0.009 and 0.002 for the fertiliser and fertiliser immobilised with chitosan, respectively. These simulations were focused for the first induced leaching, as a way to identify and compute the level of dispersion. The approach to find out the level of dispersion was by means of least squares that compared the curve of concentrations identified experimentally with the one obtained from simulations.

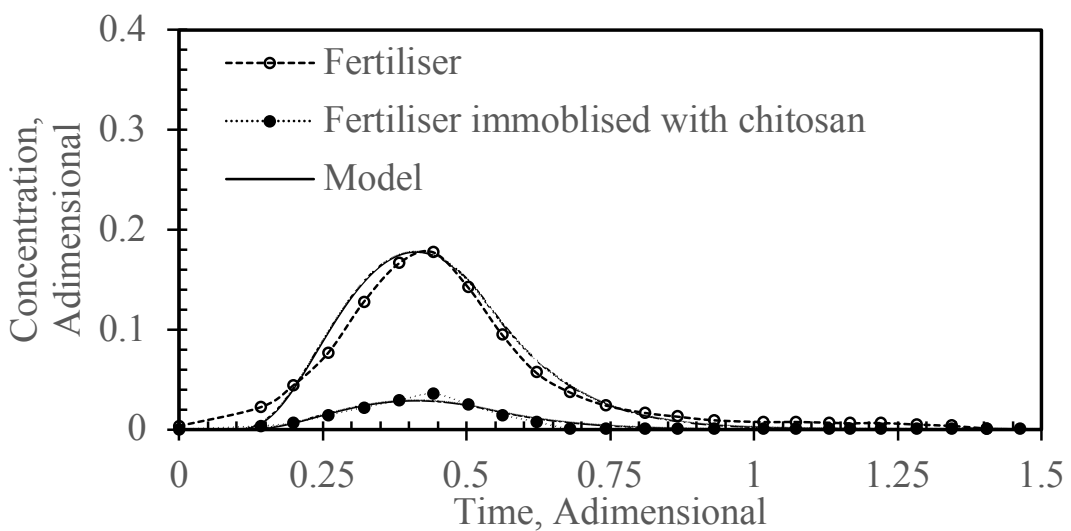

Figure 6. Concentration predictions of the fertiliser with and without chitosan by the general rate model.

\section{Conclusions}

The present work aimed to present a technological process to build up and evaluate micro-particles that can delay the delivery of a fertiliser. Firstly, it was presented the synthesis of micro-particles that were obtained with a size between $1 \mu \mathrm{m}$ and $2 \mu \mathrm{m}$. The synthesis mainly immobilised the fertiliser by means of chitosan. Then, benchmarking of those micro-particles were conducted based on the experimental results of induced leaching obtained from a column with homogeneous physical properties of the porous medium. The induced leaching process in the column by means of a pump accelerated the process, and it was achieved in approximately 12 minutes. The transport and fate of fertilisers immobilised with chitosan were compared with the ones obtained with only fertiliser in order to demonstrate differences of leaching; these experiments found that fertiliser immobilised with chitosan could deliver $17 \%$ of the total during the first induced leaching. Consecutively, every 48 hours there was an induced leaching during 4 times, delivering up to $86 \%$ of the fertiliser. Finally, the concentrations during the first induced leaching of both, only the fertiliser and the fertiliser immobilised with chitosan, were predicted by means of the general rate model, and its predictions were in agreement with experimental results. Then, this model can be used for additional predictions and reduce the time required in the experimental stage.

\section{Acknowledgements}

This investigation has been funded by: "Fondo Institucional de Fomento Regional para el Desarrollo Científico y Tecnológico de Conacyt-México, Proj. 292399".

\section{References}

Broschat, T. K. 1995. "Nitrate, Phosphate, and Potassium Leaching from Container-Grown Plants Fertilized by Several Methods." HortScience 30 (1):74-77.

Ghanbarian, Behzad, Allen G. Hunt, Robert P. Ewing, and Muhammad Sahimi. 2013. "Tortuosity in Porous Media: A Critical Review." Soil Science Society of America Journal 77 (5):1461. https://doi.org/10.2136/sssaj2012.0435.

Han, Xiaozhao, Sensen Chen, and Xianguo Hu. 2009. "Controlled-Release Fertilizer Encapsulated by Starch/polyvinyl Alcohol Coating.” Desalination 240 (1-3):21-26. https://doi.org/10.1016/j.desal.2008.01.047.

Kashyap, Prem Lal, Xu Xiang, and Patricia Heiden. 2015. "Chitosan Nanoparticle Based Delivery Systems for Sustainable Agriculture." International Journal of Biological Macromolecules 77. Elsevier B.V.:36-51. https://doi.org/10.1016/j.ijbiomac.2015.02.039.

$\mathrm{Lu}$, Yangcheng, Jing He, Longwen Wu, and Guangsheng Luo. 2016. "Relationship between Breakthrough 
Curve and Adsorption Isotherm of Ca(II) Imprinted Chitosan Microspheres for Metal Adsorption." Chinese Journal of Chemical Engineering 24 (2). Elsevier B.V.:323-29. https://doi.org/10.1016/j.cjche.2015.10.002.

Naka, Angelica, Tetsuo Yasutaka, Hirofumi Sakanakura, Ute Kalbe, Yasutaka Watanabe, Seiji Inoba, Miyuki Takeo, et al. 2016. "Column Percolation Test for Contaminated Soils: Key Factors for Standardization." Journal of Hazardous Materials 320. Elsevier B.V.:326-40. https://doi.org/10.1016/j.jhazmat.2016.08.046.

Saripalli, K. Prasad, R. Jeffery Serne, Philip D. Meyer, and B. Peter McGrail. 2002. "Prediction of Diffusion Coefficients in Porous Media Using Tortuosity Factors Based on Interfacial Areas." Ground Water. https://doi.org/10.1111/j.1745-6584.2002.tb02512.x.

Shaviv, Avi. 2001. "Advances in Controlled-Release Fertilizers" 71:1-49. https://doi.org/10.1016/S00652113(01)71011-5.

Tomaszewska, Maria, and Anna Jarosiewicz. 2006. "Encapsulation of Mineral Fertilizer by Polysulfone Using a Spraying Method.” Desalination 198 (1-3):346-52. https://doi.org/10.1016/j.desal.2006.01.032.

Trinh, Thanh H., Kuzilati Kushaari, and Abdul Basit. 2015. "Modeling the Release of Nitrogen from Controlled-Release Fertilizer with Imperfect Coating in Soils and Water." Industrial and Engineering Chemistry Research 54 (26):6724-33. https://doi.org/10.1021/acs.iecr.5b01281.

Yang, Xiuyi, Jibiao Geng, Chengliang Li, Min Zhang, and Xiaofei Tian. 2016. "Cumulative Release Characteristics of Controlled-Release Nitrogen and Potassium Fertilizers and Their Effects on Soil Fertility, and Cotton Growth." Scientific Reports 6 (December). Nature Publishing Group. https://doi.org/10.1038/srep39030. 


\title{
Territorial Analysis and Hydrological Modeling of the Canale d'Aiedda Basin \\ Francesco Gentile ${ }^{a^{*}}$, Ersilia D'Ambrosio ${ }^{a}$, Marinella Spanò ${ }^{a}$, Ossama M.M. Abdelwahab ${ }^{b}$, Antonio Lonigro ${ }^{a}$, Giovanni Francesco Riccia ${ }^{a}$, Giovanni Romano ${ }^{a}$, Angelantonio Calabrese ${ }^{c}$, Giuseppe Pappagallo ${ }^{c}$, Anna Maria De Girolamo \\ ${ }^{a}$ Department of Agricultural and Environmental Science, University of Bari Aldo Moro, Bari, Italy \\ ${ }^{b}$ Department of Agricultural Engineering, Faculty of Agriculture, Cairo University, 12613 Giza, Egypt \\ ${ }^{c}$ Water Research Institute, National Research Council, Bari, Italy \\ * Corresponding author. Email: francesco.gentile@uniba.it
}

\begin{abstract}
The Canale d'Aiedda basin, together with the other basins of the Jonico-Tarantino territory, is part of the environmental area of Taranto. This site is recognized by the Italian State as an area that requires remediation of soil, subsoil, surface water and groundwater, in order to avoid environmental and health damages. The Canale d'Aiedda has the largest extension among the basins in the area and flows into the Mar Piccolo. The objective of the present work is a territorial analysis at supporting hydrological and water quality models in the Canale d'Aiedda basin. The results of this analysis defined in detail the geological, morphological and hydrological features of the basin, as well as the land use and main environmental characteristics. The database includes information concerning agricultural practices, climatic data, soil parameters, groundwater data, and surface water quality parameters. The information produced by the data analysis and the surveys carried out on the territory were archived in a GIS environment. The results of the field and laboratory activities are related to two specific objectives: i) monitoring of streamflow and water quality; ii) model calibration and validation. Data set up was carried out aimed at the implementation of two hydrological distributed models, SWAT and AnnAGNPS, for the estimation of water discharge, sediment yield and nutrient loads deriving from point and non-point pollution sources.
\end{abstract}

Keywords: Mediterranean catchment, temporary river, territorial analysis, stream water monitoring, agricultural pollution, Geographic information systems (GIS).

\section{Introduction}

In recent decades, hydrological and water quality models have been largely used in environmental studies (Abdelwahab et al., 2014; Abouabdillah et al., 2014, De Girolamo et al., 2015; De Girolamo et al., 2017a, Ricci et al., 2018). Ecohydrological models, such as Soil and Water Assessment Tool (SWAT) (Arnold et al., 1998) and Annualized Agricultural Non-Point Source (AnnAGNPS) (Theurer and Cronshey, 1998), are able to simulate the water cycle and constitute useful tools for river basin management and for identifying source areas of pollutants. In addition, models have a great potential in developing scenarios in order to test environmental measures to reduce pollution (Abdelwahab et al., 2016) or to mitigate climate change impacts on water resources (De Girolamo et al., 2017b; Spanò et al., 2017).

The model implementation requires parameters and environmental data for the set-up, the calibration and validation. Depending on the complexity of the model, data requirement can be very large (De Girolamo and Lo Porto, 2012; Bisantino et al., 2015), this may constitute a limit in the model applicability (Oueslati et al., 2015). Official data used by modellers may derive from different sources (i.e., Regional Environmental Agency, Municipalities, River basin Authority, Civil Protection Service, etc.) and generally are not uniform in terms of time scale and spatial resolution. Moreover, it is not rare the case of time series that include several lacks. Hence, before applying a model, it is necessary to collect and accurately cross and check available data, which should be integrated with field campaigns, farmers' and citizens' interviews to clarify inconsistency in data or to update datasets. In an area where streamflow and water quality data are not available, it could be also necessary to design a monitoring plan for water quality sampling and to install a number of gauging stations for streamflow measurements. The above-mentioned activities are time-consuming and may require considerable economic resources. Although fundamental for a model application, they generally receive little attention in the publications.

In the present work a territorial analysis of the Canale d'Aiedda basin was developed. A Geographic Information System (GIS) and a database were implemented including a large number of data coming from different sources and from monitoring activities specifically designed. The results, on the one hand, increase the knowledge on the study area and its actual environmental status and, on the other hand, constitute a methodology aimed at supporting hydrological model implementation. 


\section{Materials and methods}

Study area

The investigated basin is the Canale d'Aiedda, which together with the other basins of the Jonico-Tarantino territory is part of the environmental area of Taranto, in Southern Italy (Figure 112). This catchment, which covers a drainage area of approximately $408 \mathrm{~km}^{2}$, is representative of Mediterranean regions, in terms of climate, land cover, management practices and data availability. The Canale d'Aiedda basin flows into the second inlet of the Mar Piccolo (literally "Small Sea"), which is a sea inner, semi-enclosed basin. The Mar Piccolo has lagoon features and it is divided into two inlets: the first one of $8.1 \mathrm{~km}^{2}$ and with a maximum depth of $13 \mathrm{~m}$ and the second one of $12.6 \mathrm{~km}^{2}$ and with a maximum depth of $8 \mathrm{~m}$. The Mar Piccolo is connected with the open sea, Mar Grande (literally "Big Sea"), through two channels. The surrounding zone represents sensitive environmental area due mostly to the considerable concentration of industrial and port sites including a very large steel company, a refinery and other industrial activities. Due to the extent of the environmental impact in terms of health and ecological risk, and the detriment to the cultural and environmental heritage, the Ministry of the Environment, with the National Law 426/98, declared Taranto "Contaminated Site of National Interest - SIN" (i.e., "Sito di Interesse Nazionale"). More specifically, the Taranto SIN consists of the SIN Mare (i.e., Sea SIN, $70 \mathrm{~km}^{2}$ ) and SIN Terra (i.e., Land Sea, $43 \mathrm{~km}^{2}$ ).

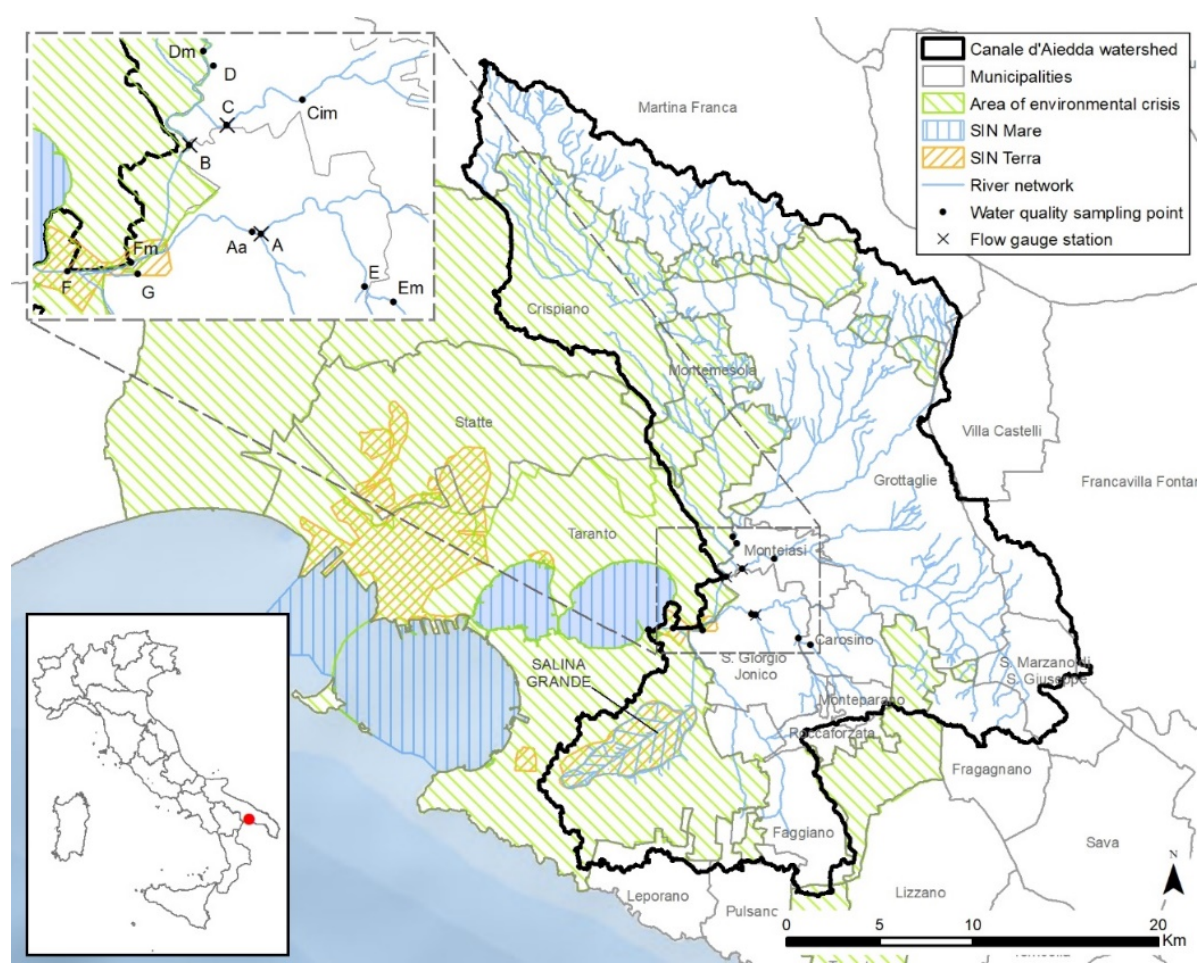

Figure 112. Study area.

The basin includes ten medium-sized urban agglomerations and five urban wastewater treatment plants (WWTPs), which discharge into the river system of the Canale d'Aiedda. The river, through an artificially channelled terminal trunk, flows in the second inlet of Mar Piccolo in correspondence of the wet zone "Regional Nature Reserve Swamp la Vela". This protected area (116 ha) is included in the Site of Community Importance (SCI) "Mar Piccolo" (IT9130004) and is characterized by typical Mediterranean natural vegetation (i.e., Mediterranean maquis) able to offer shelter and food to many species of resident and migratory birds.

The study area is characterized by a flat territory, with a maximum elevation of $520 \mathrm{~m}$ a.s.1., and by the presence of zones morphologically depressed like the Salina Grande (literally "Great Saline") of ca. $9 \mathrm{~km}^{2}$, which through the Canale Maestro enters in the Canale d'Aiedda. In the watershed, the extensive agriculture is the predominant land use, which is characterized by many fruit tree species, mostly vineyards and olive groves, and by different types of vegetables. There is also a significant presence of simple arable crops like durum wheat, uniformly distributed throughout the basin. The presence of zootechnical activities is low and mostly localized in the northern zone. 
Surface water monitoring

The Canale d'Aiedda watershed has been monitored since August 2017. Three flow gauge stations within the catchment (A, B, C - Figure 112) register water levels and temperature through a pressure sensor with internal data logger (Figure 113). These sensors measure water level with different frequencies. A semi-hourly time scale is considered if the water level is lower than $0.30 \mathrm{~m}$, while during floods (water level higher than $0.30 \mathrm{~m}$ ), the time interval is $15 \mathrm{~min}$ and it remains the same until the water height is higher than $0.25 \mathrm{~m}$ (flood recession). The choice of the location the instruments were installed was done taking into account the accessibility and visibility of the sites, in order to avoid vandalism acts. Stable river banks was another factor considered in the choice (Gentile et al., 2010).

Water velocity measurements were performed considering different flow conditions, by means of a flow module (ISCO 750 Area-Velocity Flow Module) or by means of a float measurement (Turnipseed and Sauer, 2010), when it was not possible to use the flow module, since the water height was lower than $0.04 \mathrm{~m}$.

Surface water samples are taken at fortnightly or monthly time intervals in 12 sections $\left(\mathrm{A}, \mathrm{A}_{\mathrm{a}}, \mathrm{B}, \mathrm{C}, \mathrm{Cim}, \mathrm{D}\right.$, $\mathrm{D}_{\mathrm{m}}, \mathrm{E}, \mathrm{E}_{\mathrm{m}}, \mathrm{F}, \mathrm{F}_{\mathrm{m}}, \mathrm{G}$ - Figure 112). The choice of the monitoring sections was done considering, on the one hand, the hydrological model implementation and calibration requirements and, on the other hand, the characteristics of the environment. Thus, samples from sections $A, B, C$ are taken, as well as from identified point sources $\left(A_{a}\right.$, $\mathrm{Cim})$ and spring $(\mathrm{G})$. Moreover, samplings were done to analyse water quality upstream $\left(\mathrm{D}_{\mathrm{m}}, \mathrm{E}_{\mathrm{m}}\right)$ and downstream (D, E) from the discharge of the two WWTPs of Monteiasi (ca. $2 \mathrm{~km}$ upstream from B) and San Giorgio Jonico (ca. $3 \mathrm{~km}$ upstream from A). Monitoring activities are also performed at the watershed closing section (F), as well as in a river section $(\mathrm{Fm})$, which is $250 \mathrm{~m}$ upstream from $\mathrm{F}$, and is not subject to Mar Piccolo saltwater intrusion.

The concentrations of Total Suspended Solids (TSS), ammonia (N-NH4), nitrate (N-NO3), nitrite (N-NO2), total nitrogen (TN), mineral phosphorus (P-PO4), and total phosphorus (TP) are determined in the Department of Agricultural and Environmental Science and Water Research Institute laboratories according to the 2090-B APAT-IRSA/CNR analytical standard method.
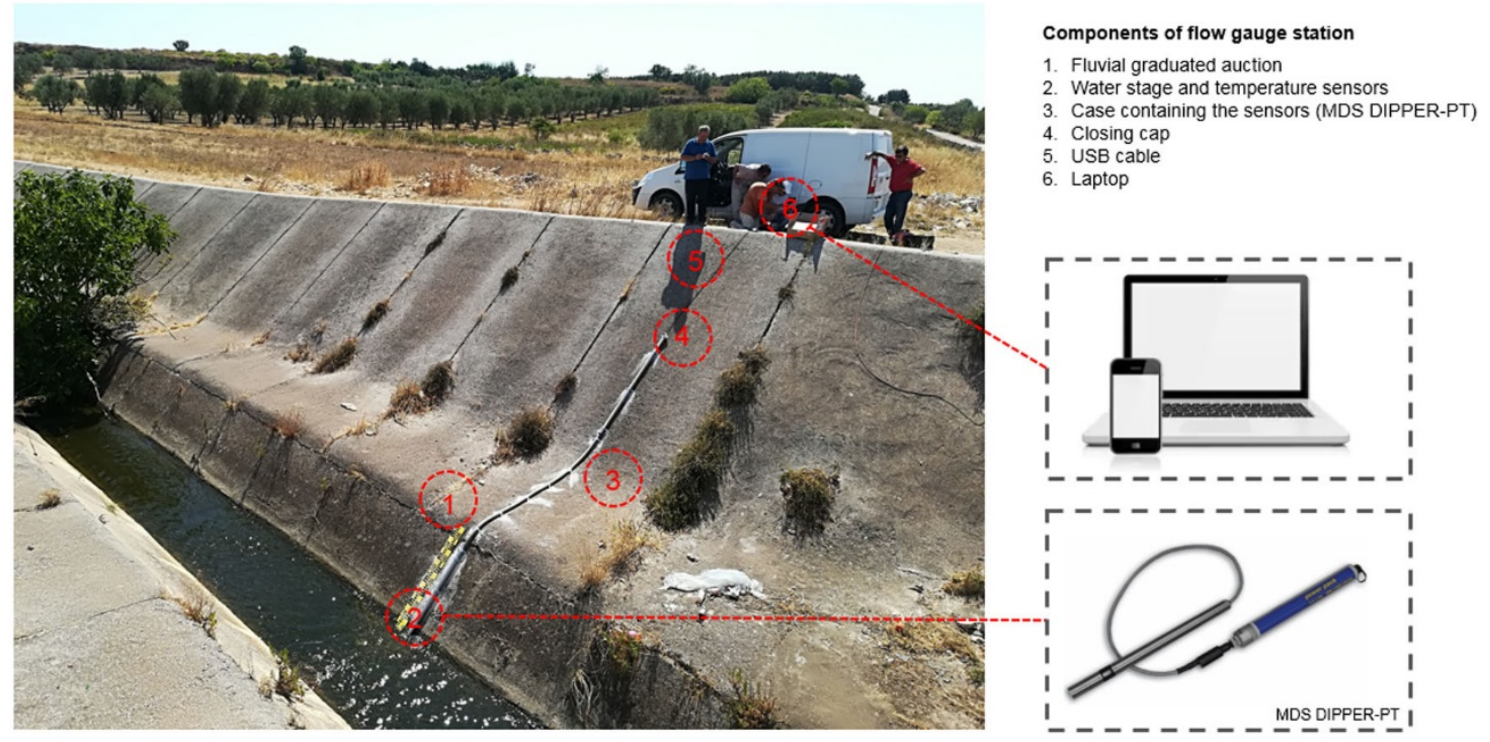

Figure 113. Flow gauge station (station B).

\section{Territorial analysis}

Data were derived from different sources. Table 1 shows data, their source, resolution, and format. Data were not uniform in terms of time scale and spatial resolution. In the last decades, several studies were conducted in the study area by Regional Environmental Agency, Municipalities, River basin Authority, Civil Protection Service. Unfortunately, the results and data of the previous studies were not archived in a unique database.

Having in mind the final aim of the present study, which was to create a GIS and a database for supporting hydrological and water quality models, all these studies were collected as well as the available data, which were crossed and integrated with farmers' and citizens' interviews, field activities and literature research. Therefore, detailed land use and soil maps, as well as information on soil characteristics, river network conformation, agricultural practices, climate, and point sources were obtained.

The software Soil Water Characteristics, implemented by the USDA Agricultural Research Service (nrcs.usda.gov), was used in order to calculate soil hydraulic properties (i.e., hydraulic conductivity, available 
water content, bulk density) based on soil texture (clay and sand content of soil layers). Interviews with farmers and local dealers were performed in order to collect the agronomic data needed by the hydrological models. The required information includes type, timing and amount of fertilizers used for each crop, annual crop yields, tillage operations, irrigation supply and grazing. Fertilizers were distinguished between synthetic and animal manure. Nutrients from animal manure and grazing were quantified, multiplying the live weight of each animal type by the animal-specific TN excretion rates (Perelli and Pimpini, 2003). Livestock densities were collected from the National Agricultural Census on a municipal scale (ISTAT, 2010). A distinction between indoor and outdoor farming was made. TN loss during manure handling and storage (27.5\%) was considered for manure produced by indoor farming (Fulhage et al., 2002). Several farmers and/or local dealers were selected in order to gain information covering the whole catchment (De Girolamo et al., 2017c). Field surveys in collaboration with the Reclamation Consortium of Stornara and Tara were carried out, in order to obtain a real river network representation, and understand the hydraulic operation of the Salina Grande.

Table 46. Territorial database.

\begin{tabular}{|c|c|c|c|}
\hline Name & Source & Information & Format \\
\hline $\begin{array}{l}\text { Digital Terrain Model } \\
\text { (DTM) }\end{array}$ & Puglia Region & $\begin{array}{c}\text { DTM has a spatial resolution of } 8 \\
\text { meters }\end{array}$ & asc \\
\hline Administrative limit & Istat & Delimitation of municipalities & $\operatorname{shp}$ \\
\hline Land Use map & Puglia Region & $\begin{array}{l}\text { Specifications of the CORINE Land } \\
\text { Cover project (IV level) }\end{array}$ & $\operatorname{shp}$ \\
\hline $\begin{array}{l}\text { Soil map and soil } \\
\text { characteristics }\end{array}$ & $\begin{array}{c}\text { Puglia Region } \\
\text { Joint Research Centre - ESDAC }\end{array}$ & Soil map and soil data & $\begin{array}{l}\text { shp } \\
\text { rst }\end{array}$ \\
\hline $\begin{array}{c}\text { Hydro- } \\
\text { geomorphological map }\end{array}$ & Puglia Region & $\begin{array}{l}\text { River network, caves, springs, } \\
\text { sinkholes }\end{array}$ & $\operatorname{shp}$ \\
\hline $\begin{array}{l}\text { Regional Technical } \\
\text { map (CTR) }\end{array}$ & Puglia Region & $\begin{array}{l}\text { Topography (e.g., river network, } \\
\text { channel, contours) }\end{array}$ & $\operatorname{shp}$ \\
\hline $\begin{array}{l}\text { Regional Territorial } \\
\text { Landscape Plan }\end{array}$ & Puglia Region & Landscape elements to be protected & $\operatorname{shp}$ \\
\hline $\begin{array}{l}\text { Cadastre of caves and } \\
\text { natural cavities }\end{array}$ & Puglia Region & $\begin{array}{c}\text { Location of karst elements (caves, } \\
\text { sinkholes) }\end{array}$ & $\operatorname{shp}$ \\
\hline $\begin{array}{l}\text { Groundwater } \\
\text { database }\end{array}$ & Puglia Region & $\begin{array}{l}\text { Groundwater quality between } 2006- \\
2016\end{array}$ & $\begin{array}{l}\text { shp } \\
\text { xls }\end{array}$ \\
\hline $\begin{array}{l}\text { Surface water } \\
\text { database }\end{array}$ & $\begin{array}{l}\text { Puglia Region } \\
\text { Arpa Puglia }\end{array}$ & Water quality between 2011-2018 & $\begin{array}{l}\text { shp } \\
\text { xls }\end{array}$ \\
\hline WWTPs database & Arpa Puglia & $\begin{array}{l}\text { Treated wastewater quality between } \\
2012-2017\end{array}$ & xls \\
\hline $\begin{array}{l}\text { WWTPs discharged } \\
\text { volumes }\end{array}$ & Acquedotto Pugliese & $\begin{array}{l}\text { Mean annual water volumes discharged } \\
\text { into the river system by the WWTPs }\end{array}$ & xls \\
\hline Meteorological data & $\begin{array}{c}\text { Civil Protection Puglia Region } \\
\text { Assocodipuglia }\end{array}$ & $\begin{array}{l}\text { precipitation, temperature, solar } \\
\text { radiation, wind speed and direction, } \\
\text { humidity }\end{array}$ & xls \\
\hline $\begin{array}{l}\text { National Agricultural } \\
\text { Census (2010) }\end{array}$ & Istat & $\begin{array}{l}\text { Animal farming and crop (yield, } \\
\text { surface) on a municipal scale }\end{array}$ & xls file \\
\hline
\end{tabular}

\section{Results and Discussion}

Stream water monitoring results

The Canale d'Aiedda stream has a flow regime characterized by typical semi-arid features, with a drought or low flow period (from May to October) with flash floods (De Girolamo et al., 2017a) and a wet season (from November to April). Figure 114 shows the hydrographs measured at the B and $\mathrm{C}$ gauging stations from August 2017 to March 2018, besides the pluviogram recorded at the Grottaglie meteorological station at a sub-hourly time step. 

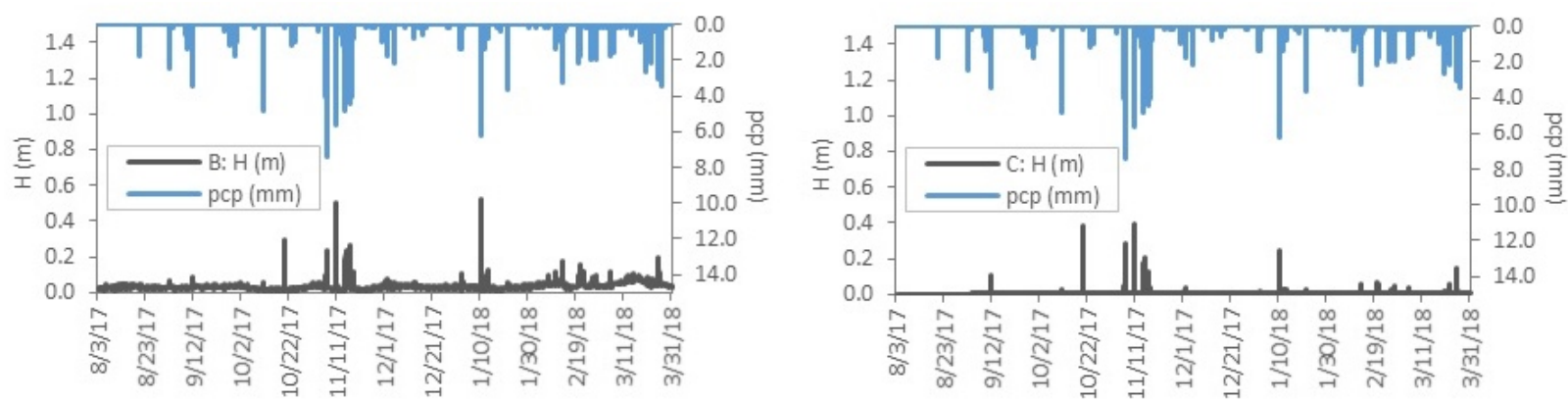

Figure 114. Hydrographs measured at the gauge B (left) and at the gauge C (right). Pluviogram recorded at the Grottaglie meteorological station.

From August 2017 to the end of March 2018 the total amount of precipitation recorded at the Grottaglie gauging station was $394.6 \mathrm{~mm}$, which is about $60 \%$ of annual amount (average annual rainfall from 1951 to 1994 was $645 \mathrm{~mm}$ ). For the gauging station B (Figure 114), only during two events (10 November 2017, 10 January 2018), water levels exceeded $0.3 \mathrm{~m}$, reaching a maximum value of $0.53 \mathrm{~m}$ (10 January 2018). The registered mean velocity was $0.53 \mathrm{~m} \mathrm{~s}^{-1}$. In terms of flow, it varied from 0.001 to $6.3 \mathrm{~m}^{3} \mathrm{~s}^{-1}$, with a mean daily value of $0.08 \mathrm{~m}^{3} \mathrm{~s}^{-}$ 1. The dry condition was never registered. Conversely, for the gauging station $\mathrm{C}$, the completely dry condition was recorded in August 2017 (Figure 114). However, the flow is always so low that the instrument was not able to detect measurements as they are concentrated in the central part of the river section that was slightly eroded. In this situation, the water height was $0.003 \mathrm{~m}$. Only during two events water levels exceeded $0.30 \mathrm{~m}$ (20 October 2017, 10 November 2017). Streamflow varied from 0 to $3.4 \mathrm{~m}^{3} \mathrm{~s}^{-1}$, with a mean daily value of $0.003 \mathrm{~m}^{3} \mathrm{~s}^{-1}$. The mean velocity was $0.50 \mathrm{~m} \mathrm{~s}^{-1}$; it was obtained by means of float measurements.

The river network shows a natural intermittent character due to climate and geological features. The hydrographs recorded in the gauging stations show a very rapid rising stage and a short lag time (time between rainfall peak and discharge peak). Flood duration is typically only a few hours during which streamflow increase and decrease rapidly. The natural flow regime is recorded only in the gauging station $\mathrm{C}$, while altered hydrological conditions are present upstream the gauging stations A and B, where two WWTPs, San Giorgio Jonico and Monteiasi, discharge the sewage. As a result, these river streams are permanent, as a continuous flow is recorded in A and B sections also in the dry season. These results confirm a typical behaviour of the intermittent rivers (De Girolamo et al., 2017c). Concerning the water quality monitoring activities, they are still ongoing and will end at the end of the hydrological year. Currently, data on water quality from the monitoring campaigns carried out from September 2017 are being processed.

Setting inputs for hydrological models

Data and information derived from the territorial analysis were processed in order to obtain current and actual input data for the hydrological models. Table 47 summarizes the procedures adopted for obtaining river network, land use and soil maps, as well as for building soil, point sources, meteorological and agricultural practices database. 
Table 47. Hydrological model input.

\begin{tabular}{|c|c|c|}
\hline Input & Description & Format \\
\hline Digital Terrain Model & The DTM provided by the Puglia Region was used & grid \\
\hline River network & $\begin{array}{l}\text { The stream features provided by the Hydro-geomorphological map and Regional } \\
\text { Technical map were crossed and integrated with information obtained by means of } \\
\text { field inspections. A real river network representation was obtained, with particular } \\
\text { reference to the interaction between the Salina Grande and the Canale d'Aiedda river }\end{array}$ & shp \\
\hline Land use map & $\begin{array}{l}\text { The land use map provided by the Puglia Region (CORINE Land Cover project, } \\
\text { IV level) was reclassified. In particular, arable land was reclassified and divided } \\
\text { between durum wheat, set aside land and herbage. Thus, a detailed land cover map } \\
\text { was obtained, and } 23 \text { soil use classes were identified throughout the catchment }\end{array}$ & grid \\
\hline Soil map & $\begin{array}{l}\text { The } 20 \text { polygonal feature of the soil map provided by the Puglia Region was } \\
\text { further divided, taking into account the differences between the percentage coarse } \\
\text { content provided by JRC- ESDAC. Hence, } 24 \text { soil type were identified throughout } \\
\text { the catchment }\end{array}$ & grid \\
\hline Soil database & $\begin{array}{l}\text { The soil information provided by the Puglia Region for each soil type (i.e., } \\
\text { number of soil layers, soil layer depth, texture, organic carbon content) was integrated } \\
\text { with information provided by JRC-ESDAC (i.e., rock fragment content) and obtained } \\
\text { with the software Soil Water Characteristics }\end{array}$ & $\mathrm{mdb}$ \\
\hline Point sources & $\begin{array}{l}\text { Groundwater monitoring results database (Puglia Region) and Urban wastewater } \\
\text { treatment plant discharges monitoring results database (Arpa Puglia) were used in } \\
\text { order to obtain information on the specific point sources (springs and WWTPs } \\
\text { discharges) present in the study area. A new database was built (Table 48) }\end{array}$ & $\mathrm{mdb}$ \\
\hline Meteorological data & $\begin{array}{l}\text { Daily data (precipitation, temperature, solar radiation, wind speed and direction, } \\
\text { humidity) were acquired from Regione Puglia ( } 13 \text { meteorological stations) and } \\
\text { Assocodipuglia ( } 5 \text { meteorological stations) }\end{array}$ & $\mathrm{mdb}$ \\
\hline Agricultural practices & $\begin{array}{l}\text { A database was implemented including for each crop the agricultural practices } \\
\text { such as tillage operations (type and date), fertilizer and irrigation applications (timing, } \\
\text { amount, type). Grazing was also considered on pasture. At this aim, farmers' and } \\
\text { dealers' interviews were used }\end{array}$ & $\mathrm{mdb}$ \\
\hline
\end{tabular}

Figure 115 shows the reclassified soil use and soil type maps used as model input. Considering the information taken from Zuffianò et al. (2016), areas with outcropping limestone, caves and sinkholes have been identified throughout the catchment. Since in these areas the infiltration is prevalent with respect to the superficial runoff, a map of areas contributing to surface runoff was delineated to support hydrological modeling. The basin $\left(408 \mathrm{~km}^{2}\right)$ was appropriately clipped and the area contributing to surface runoff resulted $309 \mathrm{~km}^{2}$ (Figure 116).

Moreover, the basin was divided into sub-basins on the basis of the dominant hydrological processes in the area. At this aim, it was decided to divide the drainage area of the Salina Grande (Watershed S, $86.9 \mathrm{~km}^{2}$ ) from the remaining part of the catchment (Watershed $\mathrm{N}, 222.1 \mathrm{~km}^{2}$ ), due to the peculiarities of this depressed area (Figure 116). Indeed, this area is often subject to flooding and presents a different hydraulic behaviour than that of the northern part of the basin (Watershed N). For this reason, two different hydrological simulations are carried out for these two watersheds. 

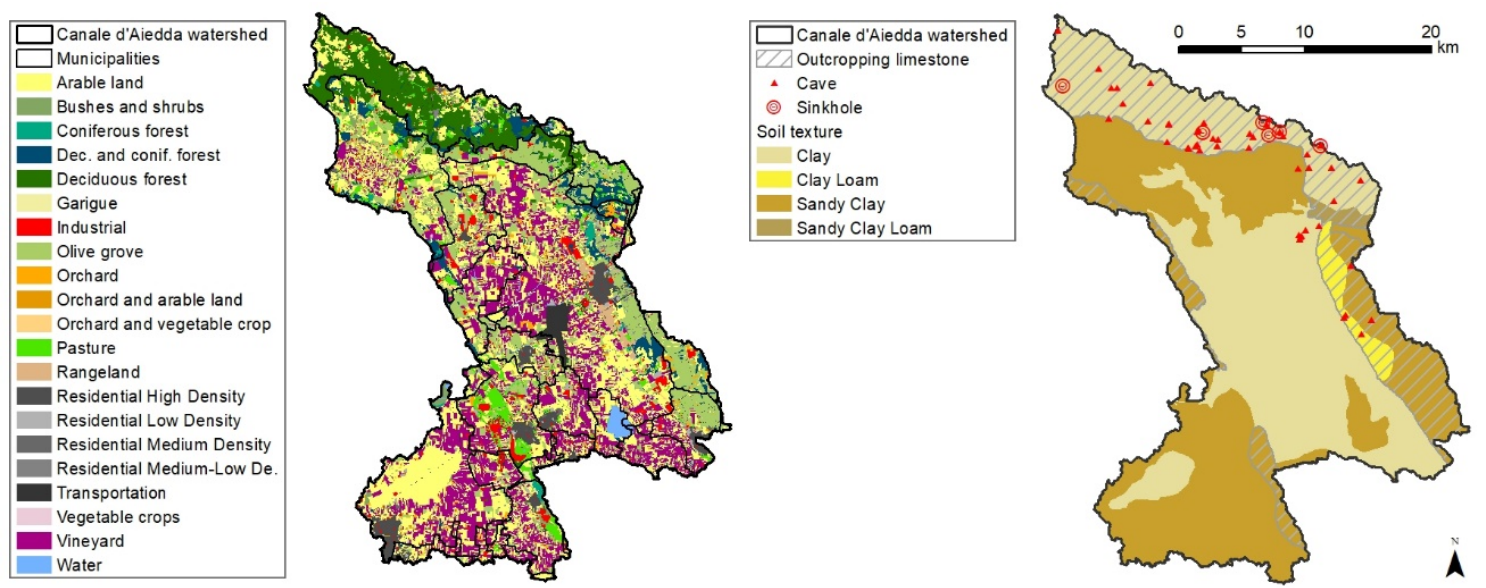

Figure 115. Reclassified land use map (left). Soil texture and main karst units map (right).

Figure 116 shows the boundaries of the Watershed $\mathrm{N}$ and Watershed $\mathrm{S}$, as well as the river network, the point sources and the meteorological stations for the hydrological modelling. The model will be calibrated and validated considering the water quality (TSS and nutrient) and quantity (flow) measurements in the stations A, B and C.

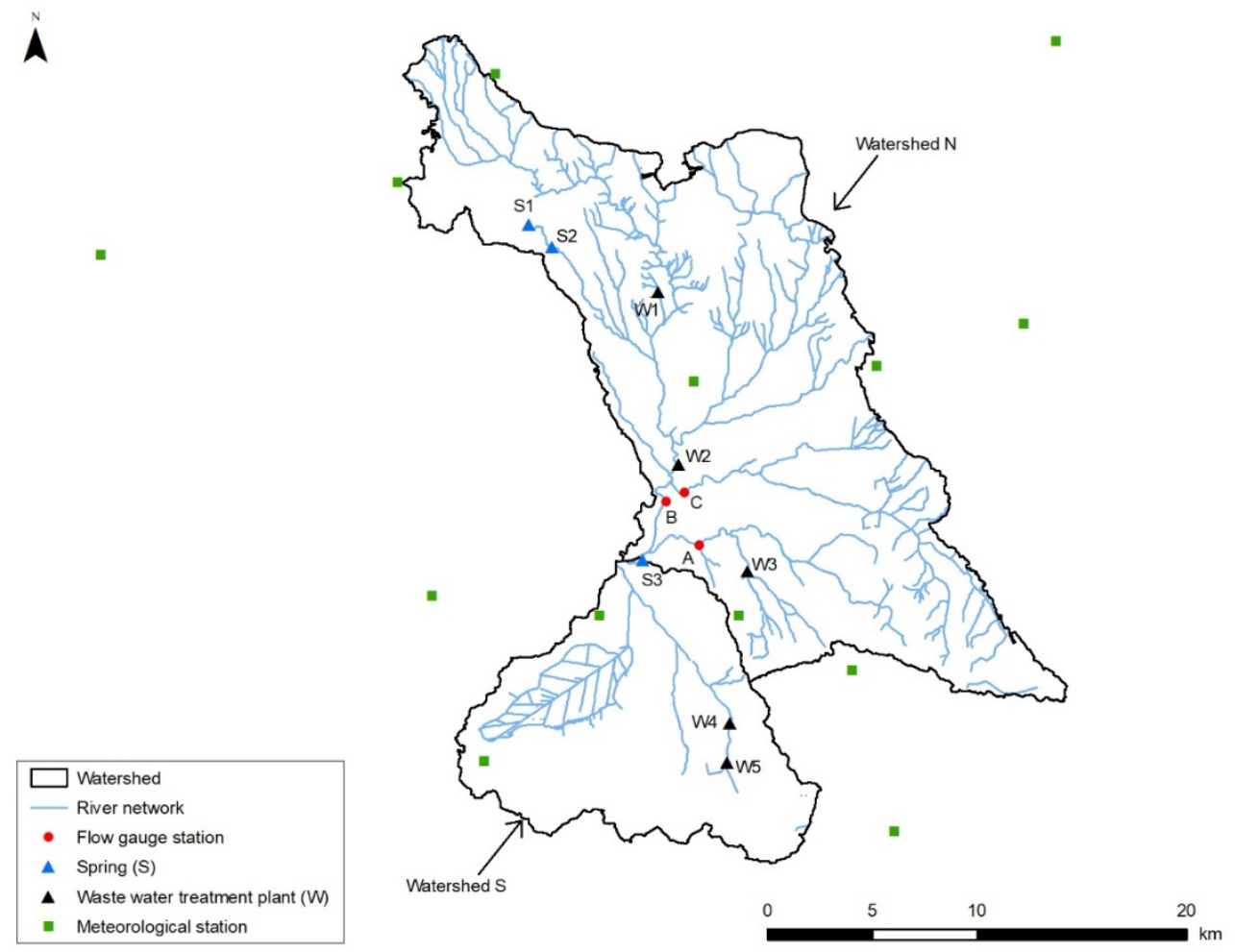

Figure 116. Watershed, sub-basins, point sources (springs and WWTPs), flow gauge and meteorological stations.

Table 48 summarizes the mean daily volume of water discharged, as well as mean daily loads of nutrients (NNO3, N-NH4, P-PO4) and sediment (TSS) associated with the point sources. It is necessary to highlight that the water quality parameters and discharges of the WWTPs are strongly variable over time depending on the treated wastewater characteristics. Here an average value was estimated in order to set up the model. However, when analysing the model results on a daily time scale it is necessary to take into account that input data from WWTPs are affected by an uncertainty. 
Table 48. Point sources characterization derived from territorial analysis to be used as model input.

\begin{tabular}{cccccccc}
\hline Code & Name & Description & $\begin{array}{c}\mathrm{V} \\
\left(\mathrm{m}^{3} \mathrm{~d}^{-1}\right)\end{array}$ & $\begin{array}{c}\mathrm{TSS} \\
\left(\mathrm{kg} \mathrm{d}^{-1}\right)\end{array}$ & $\begin{array}{c}\mathrm{N}-\mathrm{NO} 3 \\
\left(\mathrm{~kg} \mathrm{~d}^{-1}\right)\end{array}$ & $\begin{array}{c}\mathrm{N}-\mathrm{NH} 4 \\
\left(\mathrm{~kg} \mathrm{~d}^{-1}\right)\end{array}$ & $\begin{array}{c}\mathrm{P}-\mathrm{PO} 4 \\
\left(\mathrm{~kg} \mathrm{~d}^{-1}\right)\end{array}$ \\
\hline S1 & Chianca & Spring & 172.8 & - & 2.5 & 0.9 & 0 \\
S2 & Tre Fontane & Spring & 86.4 & - & 0.8 & 0.0 & - \\
S3 & Riso & Spring & 8218.7 & - & 50.5 & 0.9 & 0.2 \\
W1 & Montemesola & WWTP discharge & 743.7 & 0.4 & 4.9 & 2.6 & 0.5 \\
W2 & Monteiasi & WWTP discharge & 6449.9 & 35.8 & 55.6 & 29.4 & 5.2 \\
W3 & S. Giorgio Jonico & WWTP discharge & 5666.0 & 18.3 & 52.0 & 27.5 & 2.5 \\
W4 & Faggiano & WWTP discharge & 516.5 & 2.5 & 4.0 & 2.6 & 0.5 \\
W5 & Pulsano - Leporano & WWTP discharge & 2016.0 & 0.7 & 21.6 & 11.3 & 0.7 \\
\hline \multicolumn{7}{r}{} \\
\hline
\end{tabular}

Concerning the agricultural practices, vineyard, olive grove and durum wheat are the main crops cultivated in the study area. According to the interviewed farmers, durum wheat is mainly fertilized in February and December, meanwhile the vineyard in February and November. Fertilizers operations on olive groves take place in April and August. Manure produced by animals that are free to roam is directly applied to pasture. Conversely, manure produced by indoor farming is spread on set aside land and herbage, after a 90-day storage period, aimed at reducing bacterial load. Concerning the irrigation supply, it is provided only during the dry season (i.e., between May and September) to vineyard, olive grove and orchard. Drip irrigation systems are supplied by groundwater pumping wells.

Despite the fact that the current study gathered high-quality data, due to the collaboration of local dealers and farmers in providing the required agronomic data, it was not possible to assign the actual agricultural practices to each field because of the high fragmentation of land use and of large differences in adopted management practices within the study area. Therefore, the same averaged values will be assigned to specific crops, neglecting sitespecific information.

\section{Conclusions}

Hydrological and water quality models constitute useful tools for river basin management and for identifying source areas of pollutants, as well as to test environmental measures to reduce pollution or to mitigate climate change impacts on water resources. The model applicability may be limited if the data required for its set-up, and measurements for the model calibration and validation are not available and/or are not uniform in terms of time scale and spatial resolution.

The current study presents a territorial analysis at supporting hydrological and water quality models in the Canale d'Aiedda basin. Available data were collected, checked, crossed and integrated with field campaigns, farmers' and citizens' interviews. The results of the data analysis carried out in this study show several inconsistency and missing information in the available datasets. The results also show that collecting, checking and elaborating data is a fundamental task for applying hydrological models. In addition, the territorial analysis developed in the GIS environment is a valid support to watershed management as well as the database created merging previous studies, field surveys and new studies. Moreover, this study shows that a monitoring network is needed to calibrate models and to estimate water resources status. The integration between territorial analysis and monitoring activities could represent a good example of data acquisition and data preparation for the hydrological model set up.

\section{Acknowledgements}

The study was carried out with the financial and technical support of Dr. Vera Corbelli, Commissario Straordinario for Urgent Interventions of Environmental Requalification of Taranto, and her staff, coordinated by Dr. Gennaro Capasso and Eng. Massimo Morea. We also thank Prof. Angelo Tursi for coordinating the project on behalf of University of Bari Aldo Moro. 


\section{References}

Abdelwahab, O.M.M., R.L. Bingner, F. Milillo, F. Gentile, 2016. Evaluation of alternative management practices with the AnnAGNPS model in the Carapelle watershed. Soil Science. 181 (7), $293-305$. doi:10.1097/SS.0000000000000162

Abdelwahab, O.M.M., T. Bisantino, F. Milillo, F. Gentile, 2013. Runoff and sediment yield modeling in a medium-size Mediterranean watershed. Journal of Agricultural Engineering. 44, 31-40. doi:10.4081/jae.2013.s2.e7

Abdelwahab, O.M.M., R.L. Bingner, F. Milillo, F. Gentile, 2014. Effectiveness of alternative management scenarios on the sediment load in a Mediterranean agricultural watershed. Journal of Agricultural Engineering. 45 (3), 125-136. doi:10.4081/jae.2014.430

Abouabdillah, A., M. White, J.G. Arnold, A.M. De Girolamo, O. Oueslati, A. Maataoui, A. Lo Porto, 2014. Evaluation of soil and water conservation measures in a semi-arid river basin in Tunisia using SWAT. Soil Use Manage. 30 (4), 539-549. http://dx.doi.org/10.1111/sum.12146

Arnold, J.G., R. Srinivasan, R.S. Muttiah, J.R. Williams, 1998. Large area hydrologic modeling and assessment - Part 1: Model development. Journal of the American Water Resources Association. 34 (1), $73-89$. https://doi.org/10.1111/j.1752-1688.1998.tb05961.x

Bisantino, T., R. Bingner, W. Chouaib, F. Gentile, G. Trisorio Liuzzi, 2015. Estimation of runoff, peak discharge and sediment load at the event scale in a medium-size Mediterranean watershed using the AnnAGNPS model. Land Degradation \& Development. 26 (4), 340-355. https://doi.org/10.1002/ldr.2213

De Girolamo, A.M., A. Lo Porto, 2012. Land use scenario development as a tool for watershed management within the Rio Mannu Basin. Land Use Policy. 29 (3), 691-701. https://doi.org/10.1016/i.landusepol.2011.11.005

De Girolamo, A.M., A. Lo Porto, G. Pappagallo, O. Tzoraki, F. Gallart, 2015. The Hydrological Status Concept: Application at a Temporary River (Candelaro, Italy). River Research and Applications. 31 (7), 892-903. https://doi.org/10.1002/rra.2786

De Girolamo, A.M., E. Barca, G. Pappagallo, A. Lo Porto, 2017a. Simulating ecologically relevant hydrological indicators in a temporary river system. Agricultural Water Management. 180, 194-204. https://doi.org/10.1016/j.agwat.2016.05.034

De Girolamo, A.M., F. Bouroui, A. Buffagni, G. Pappagallo, A. Lo Porto, 2017b. Hydrology under climate change in a temporary river system: Potential impact on water balance and flow regime. River Research and Applications. 33 (7), 1219-1232. https://doi.org/10.1002/rra.3165

De Girolamo, A.M., R. Balestrini, E. D’Ambrosio, G. Pappagallo, E. Soana, A. Lo Porto, 2017c. Antropogenic input of nitrogen and riverine export from a Mediterranean catchment. The Celone, a temporary river case study. Agricultural Water Management. 187, 190-199. https://doi.org/10.1016/j.agwat.2017.03.025

Fulhage, C.D., D.L. Pfost, D.L. Schuster, 2002. Fertilizer nutrients in livestock and poultry manure. University of Missouri Cooperative Extension Bulletin EQ 351.

Gentile, F., T. Bisantino, R. Corbino, F. Milillo, G. Romano, G. Trisorio Liuzzi, 2010. Monitoring and analysis of suspended sediment transport dynamics in the Carapelle torrent (Southern Italy). Catena. 80 (1), 1-8. https://doi.org/10.1016/j.catena.2009.08.004

Lavarra, P., P. Angelini, R. Augello, P.M. Bianco, R. Capogrossi, R. Gennaio, V. La Ghezza, M. Marrese, 2014. Il sistema Carta della Natura della regione Puglia. ISPRA, Serie Rapporti, 204/2014

Oueslati, O., A.M. De Girolamo, A. Abouabdillah, T.R. Kjeldsen, A. Lo Porto, 2015. Classifying the flow regimes of Mediterranean streams using multi-variate analysis: Classifying the Flow Regimes in Mediterranean Streams. Hydrological Processes. 29 (22), 4666-4682. https://doi.org/10.1002/hyp.10530

Perelli, M., F. Pimpini, 2003. Il nuovo manuale di concimazione. Arvan. Veenezia. 446 p.

Ricci, G.F., A.M. De Girolamo, O.M. Abdelwahab, F. Gentile, 2018. Identifying sediment source areas in a Mediterranean watershed using the SWAT model. Land Degradation \& Development. 29 (4), 1233-1248. https://doi.org/10.1002/ldr.2889

Spanò, M., F. Gentile, C. Davies, R. Lafortezza, 2017. The DPSIR framework in support of green infrastructure planning: a case study in Southern Italy. Land Use Policy. 61, 242-250. https://doi.org/10.1016/j.landusepol.2016.10.051

Theurer, F.D., and R.G. Cronshey, 1998. AnnAGNPS-Reach Routing Processes. In Proceedings First Federal Interagency Hydrologic Modeling Conference. Las Vegas, NV. 19-23 April.

Turnipseed, D.P., and V.B. Sauer, 2010. Discharge measurements at gaging stations. In U.S. USGS, U.S. Geological Survey Techniques and Methods, Book 3, Chapter A8. 87 p.

Zuffianò, L.E., A. Basso, D. Casarano, V. Dragone, P.P. Limoni, A. Romanazzi, F. Santaloia, M. Polemio, 2016. Coastal hydrogeological system of Mar Piccolo (Taranto, Italy). Environmental Science and Pollution Research. 23 (13), 12502-12514. https://doi.org/10.1007/s11356-015-4932-6 


\title{
Landscape Fragmentation in the European Context: Outcomes from a Comparative Approach
}

\author{
Andrea De Montis ${ }^{\text {a,* }}$, Vittorio Serra ${ }^{\mathrm{b}}$, Antonio Ledda ${ }^{\mathrm{a}}$ \\ ${ }^{a}$ Department of Agricultural Sciences, University of Sassari, Sassari, 07100, Italy \\ ${ }^{\mathrm{b}}$ Department of Civil and Environmental Engineering and Architecture, University of Cagliari, Cagliari, 09123 \\ * Corresponding author. Email: E-mail: andreadm@uniss.it
}

\begin{abstract}
In Europe, some policies have focused on landscape and environment quality. Landscape fragmentation (LF) can be considered as one of the main causes that negatively affect landscape quality, because it triggers habitat loss processes, decline of fauna and flora species, and loss of connectivity. Transport and mobility infrastructures (TMIs) and urban settlement have been acknowledged as key factors in increasing LF processes. Scientific literature describes several metrics able to quantify LF, but methods useful to quantify LF caused by TMIs are poorly described. In this study, we measure LF in four landscape units (LUs) - two LUs in Wales (the UK) and two in Sardinia (Italy) - to identify areas where defragmentation measures could contribute in reconnecting isolated patches, allowing wildlife movement. We use the Barrier Fragmentation Index (BFI) to quantify the LF due to TMIs and the Urban Fragmentation Index (UFI) for the one due to urbanized areas. The BFI can measure LF taking into account how specific target species perceive TMIs. We choose the hedgehog (Erinaceus europaeus) as target species, a quite common species in both the countries. Finally, we apply two additional metrics, namely the effective mesh size $\left(\mathrm{m}_{\mathrm{eff}}\right)$ and the connectivity of natural areas (CIx) and verify the correlation between the four metrics. Preliminary results show (i) high values of BFI and UFI in Wales and low values of both indices in Sardinia, and (ii) likely correlation between BFI, UFI and meff. Overall, the coastal areas appear as the most fragmented, and measures may be planned to reconnect patches and allow the target species' movement. Furthermore, soil loss in rural and agrarian areas could be avoided by using urban eco-friendly planning measures.
\end{abstract}

\section{Introduction}

Landscape fragmentation (LF) can be defined as a dynamic process, where larger landscape fragments, or patches, tend to become smaller and more insulated than in their original condition (EEA, 2011). The LF phenomenon is mainly due to the increase in human needs and has led to dramatically higher consumption of the planet's resources (Harrisson et al., 2012), with impacts on land use change and significant loss of habitats and biodiversity (see, for example, Gibson et al., 2013, Foley et al., 2011; Foley et al., 2005). This process can be caused by linear and mobility infrastructures (TMI) such as railways and roads and by urbanised surfaces, which can reduce the movement of animal species and landscape connectivity (Bissonette, Adair, 2008). LF is measurable through indices such as the Infrastructural Fragmentation Index (BFI) that measures LF caused by TMIs considering also the barrier effect, and the UFI that quantifies LF caused by urbanized areas. The effective mesh-size index demonstrates how much the fragmentation value is proportional to the probability that two randomly selected points in an area are connected to each other, i.e. they are not separated by barriers fragments (roads, railways, and urban areas) (Jaeger,2000). The connectivity index (CIx) is the last index applied. This index provides us with information on the connectivity of wilderness areas in cities and is a component of the City Biodiversity Index (CBI) -also known as Singapore Index- which is a combination of 23 indicators (Deslauriers et al 2017).

This essay unfolds as follows. In the next section, we describe the selected methods and the case studies. In the third and fourth section, we respectively present and discuss the results, while in the fifth section we report on the concluding remarks.

\section{Materials and Methods}

Scientific literature is rich in indices able to measure LF, but few indices measure LF caused by TMIs (Bruschi et al., 2015). In this work, we apply the Barrier Fragmentation Index (BFI), which allows us to assess the fragmentation caused by TMIs considering also the so-called barrier effect (De Montis et al., 2017). The barrier effect is measurable by using a parameter ' $\mathrm{F}$ ', which considers how animal species perceive roads 'B' (Scolozzi, Geneletti, 2012). We propose as target species the European Hedgehog and the values of the F(B) are shown in Table 1. 
Table 49. Values of F(B) according the type of TMIs. Source: Scolozzi and Geneletti (2012).

\begin{tabular}{lc}
\hline (B) Type of infrastructure & $\mathrm{F}(\mathrm{B})$ \\
\hline Fast-moving (two-lane) national roads and railways & 0.95 \\
Asphalted secondary (one or two-lane) roads with moderate traffic & 0.50 \\
Rural and forest paved roads & 0.05 \\
\hline
\end{tabular}

The BFI obeys to Equation 1:

$$
B F I=\frac{\left(\sum_{i=1}^{i=n} L_{i} \cdot F(B)_{i}\right) \cdot N \cdot P}{A}
$$

where $L_{i}$ stands for length of TMIs traits, excluding discontinuities (bridges, viaducts, and tunnels), $N$ for the number of fragments, $P$ and $A$ for respectively perimeter and area of the LU.

Urbanization induces effects on ecological networks (De Montiset al., 2016) and causes fragmentation and negative effects on habitats (Astiaso Garcia et al., 2013). LF due to urban areas is measurable through the UFI (Astiaso Garcia et al., 2013; Battisti and Romano, 2007; Romano and Zullo, 2013). According to Romano and Zullo (2013) and Battisti and Romano (2007), the UFI obeys to the following Equation 2:

$$
U F I=\frac{\sum_{i=1}^{i=n} A u r b_{i}}{A t} \cdot \frac{\sum_{i=1}^{i=n} p_{i}}{2 \sqrt{\pi \sum_{i=1}^{i=n} A u r b_{i}}}
$$

where $A u r b_{i}$ stands for the extension in squared meters of the urban area, $p_{i}$ for the perimeter in meters of the urban area, and $A t$ for the extension in squared meters of the LU area. The first term of equation 2 quantifies the incidence of urbanized areas on the LU surface; the second term is the ratio between the perimeter of the urban area and the circumference of the equivalent circle (Romano and Zullo 2013). UFI ranges between zero (for absence of urban areas) and the value of the second term of equation 2 (Battisti et al. 2013).

The Effective Mesh size ( $\mathrm{m}_{\text {eff }}$ ) (Jaeger, 2000) requires an appropriate selection of landscape elements, and it should be measured at federal state, rural district or ecoregions level (Gulinck and Wagendorp, 2002. The more barriers a region faces, the less likely it is that two individuals will meet (EEA, 2011).

The $\mathrm{m}_{\mathrm{eff}}$ can be easily obtained through Equation 3:

$$
m_{e f f}=A_{t} * C=\frac{1}{A_{t}} * \sum_{i=1}^{n} A_{i}^{2}
$$

where $n$ indicates the number of parcels, $A_{i}$ indicates the patches area and $A_{t}$ indicates the total area of the landscape examined. The first part of the formula gives you the probability that two randomly selected points belong to the same patch. The second part (multiplication by the size of the region) converts this probability into a measure. This surface is the "mesh size" of a regular grid with an equal degree of fragmentation. The lower the effective mesh size, the more fragmented the landscape is (EEA, 2011).

$C$ stands for the probability that two randomly chosen points are connectable, without barriers such as roads or urban areas (Equation 4)

$$
C=\sum_{i=1}^{n}\left(\frac{A i}{A t}\right)^{2}
$$

where $n$ is the number of fragments, $A i$ is their surface, while $A t$ is the total area of the landscape unit.

The CBI was revised in 2012 to take into account inter and intra-patches connectivity (Chan et al., 2014). Both connectivity need to be considered as many species depend on the ability to move between and within patches of habitats for their survival (LaPoint et al., 2015; Rudd et al., 2002). The formula is given below (Equation 5): 


$$
C I x=\frac{1}{A_{t o t}}\left(A_{G 1}^{2}+A_{G 2}^{2}+\cdots+A_{G n}^{2}\right)
$$

where $A_{G n}$ is the area of the connected natural patches, $\mathrm{A}_{\text {tot }}$ is the extent of natural areas.

The CBI Manual (Chan et al. 2010) recommends a 100 meters threshold distance for designation of related patches; however, this distance may be changed to meet research requirements, for example, for species specific studies, where dispersion distances are known (Deslauriers, M.R. et al., 2017). We apply the metrics in four Landscape Units (LUs) of approximately the same extent (about $1000 \mathrm{~km}^{2}$ ), in Sardinia (Italy) and Wales (the UK) (Figure 1).

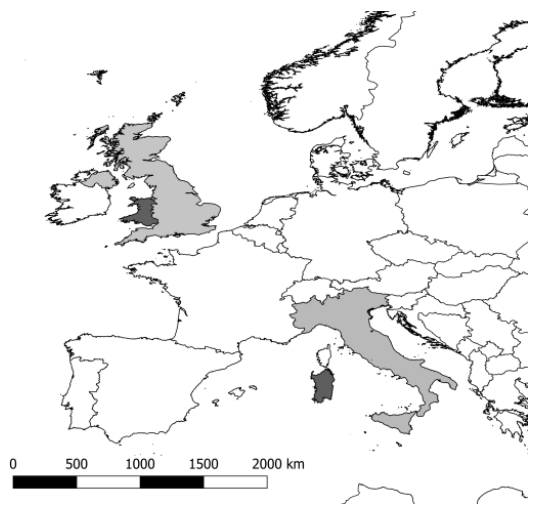

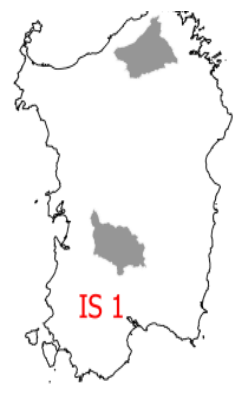

n

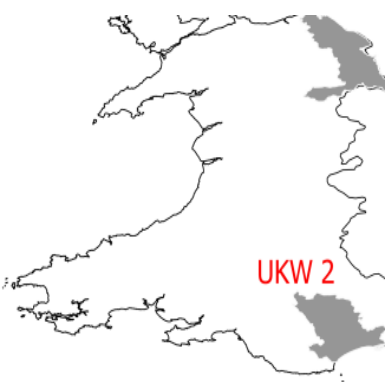

$4 \cap n \mathrm{~km}$

Figure 117. On the left: in dark gray, Sardinia and Wales. On the right: in gray: Regione delle Giare basaltiche (IS1), Limbara (IS2), North-East (UKW1), and South-East (UKW2). Source: our elaboration of data provided by Global Administrative Areas and Regions Sardinia and Wales.

We use the GIS to perform our study, because it has been proved useful in spatial analysis and in measuring landscape (habitat) fragmentation (De Montis et al., 2018; De Montis et al, 2017). We use data freely available online (RAS, 2008; Wales, 2017).

In order to apply the BFI we implement a GIS and use data in shapefile format. Tunnels and bridges were excluded from TMIs traits. Then, we obtained and counted the number of landscape fragments. Finally, the values of the F(B) were associated with each type of TMIs. As for the UFI, we use data in shapefile format relating to urbanized areas, including, inter alia, urban and suburban industrial areas, rural buildings and so on. The $\mathrm{m}_{\mathrm{eff}}$ has been measured by using the same set of shapefile data used for BFI and UFI. Microsoft Excel 2010 has supported the GIS analysis.

Finally, the CIx has been applied by considering the natural areas, where it is easiest to find the hedgehogs (for Sardinia): agroforestry areas, mixed coniferous and broadleaf forests, coniferous forests, broadleaf forests, shrubs and shrubs, orchards and minor fruits, Mediterranean scrub, simple arable crops and open field horticultural crops, cork oak. The Welsh land use map is not rooted into the Corine Land Cover classification. Thus, for Wales, we consider: arable and horticulture, broadleaf woodland, coniferous woodland, improved grassland, suburban. Fragmentation geometries were considered to be urbanized surfaces and infrastructures applying the same buffer as the $m_{\text {eff. }}$ For more details about the calculation of CIx see Deslauriers et al. (2017).

\section{Results and discussion}

Tables 2 and 3 report on the results and show that the most fragmented LUs are those from Wales. We obtained higher values for both BFI (299733.70) and UFI (7.06) in the Welsh LUs. In Wales, we measured the lowest value of $m_{\text {eff }}(9.61)$, the lowest average surface area of non-fragmented patches. Limbara shows the lowest values for both BFI 1441.57 and UFI 0.24, and the highest $\mathrm{m}_{\mathrm{eff}}$ (892.78). 
Table 2. The assessment of LF according to BFI, UFI and $\mathrm{m}_{\text {eff. }}$

\begin{tabular}{lclrrr}
\hline State, region & Code & LUs & \multicolumn{1}{c}{ BFI } & \multicolumn{1}{l}{ UFI } & \multicolumn{1}{c}{ meff $\left(\mathrm{km}^{2}\right)$} \\
\hline Italy, Sardinia & IS1 & Regione delle Giare basaltiche & 13568.89 & 0.43 & 412.45 \\
& IS2 & Limbara & 1441.57 & 0.24 & 892.78 \\
\multirow{2}{*}{ UK, Wales } & UKW1 & North East Wales & 299733.70 & 3.40 & 9.61 \\
& UKW2 & South East Wales & 296164.12 & 7.06 & 10.38 \\
\hline
\end{tabular}

Table 3. The assessment of LF according to the CIx.

\begin{tabular}{lcrr}
\hline LUs & $\begin{array}{c}\text { Intra-patch } \\
\text { (ha) }\end{array}$ & $\begin{array}{c}\text { Inter-patch } \\
\text { (ha) }\end{array}$ & \multicolumn{1}{c}{$\begin{array}{c}\text { Total } \\
\text { (ha) }\end{array}$} \\
\hline Regione delle Giare basaltiche & 2227.71 & 476.52 & 2074.23 \\
Limbara & 50.06 & 18858.15 & 18908.21 \\
North East Wales & 273.20 & 17.66 & 290.87 \\
South East Wales & 256.19 & 274.34 & 530.58 \\
\hline
\end{tabular}

Table 4. Analysis of correlation through Pearson Test.

\begin{tabular}{ccccc}
\hline & BFI & UFI & $\mathrm{m}_{\text {eff }}$ & $\mathrm{Clx}$ \\
\hline $\mathrm{BFI}$ & - & & & \\
$\mathrm{UFI}$ & 0.8800 & - & & \\
$\mathrm{m}_{\mathrm{eff}}$ & -0.8975 & -0.7923 & - & - \\
$\mathrm{Clx}$ & -0.6935 & -0.6066 & 0.9400 & - \\
\hline
\end{tabular}

Table 3 shows the highest Intra-Patch connectivity in Regione delle Giare basaltiche (CIx 2227.71 ha) and the lowest in Limbara (50.06 ha). The Inter-Patch connectivity has the highest value in Regione delle Giare basaltiche (18858.15 ha) and the lowest value in North East Wales (17.66 ha). Regione delle Giare basaltiche has the highest total connectivity (18908.21 ha), while North East Wales has the lowest value (290.87 ha).

According to the Pearson test, the four indices seem to be strongly correlated (Table 4).

The Welsh LUs are the most fragmented LUs, due to high urban pressure and high road density. Limbara appears as the least fragmented LU, with the lowest values of BFI and UFI and highest value of meff. Limbara is a mountainous area with low density of urbanized areas and TMIs. In general, the Welsh coastal areas appear as the most fragmented according to BFI and UFI and show low values of meff. That means the Welsh LUs are characterized by high level of human pressure consisting of infrastructures and settlements, which contribute in splitting up the landscape in several and smaller fragments than the Sardinian landscapes considered in this study. This is also confirmed by the CIx, where its values are greater in the Sardinian case studies than in Welsh one.

The low values of BFI suggest a more favorable habitat for the hedgehog in the Sardinian case studies. On the whole, we stress three main findings. Firstly, we demonstrate that a set of indices can be applied in different geographical contexts according to a comparative approach. Secondly, the BFI, an index scarcely used in scientific literature, has proved consistent with well-known indexes that have been tested in previous international studies. Finally, this study can contribute in stressing fragmented landscape areas where the hedgehog has its habitat. Thus, defragmentation measures could be planned at strategic level in order to reconnect habitat patches, enabling the hedgehog in crossing TMIs or urbanized areas.

\section{Conclusions}

In this work we have developed a comparative approach for assessing LF in two regions of Italy and the UK. We applied four indicators, BFI, UFI, $m_{\text {eff }}$ and CIx. We (1) found that the highest level of landscape fragmentation characterize the coastal and (2) most populated areas and measured how specific target species -European hedgehog - perceive roads as barriers. We have also verified the correlation between the LF metrics, which appear to be related each other, according to the values of the Pearson Test..

This study allows us to identify areas where defragmentation measures can be planned in order to reconnect habitat patches. 


\section{References}

Astiaso Garcia, D., Bruschi, D., Cinquepalmi, F., Cumo, F., 2013. An estimation ofurban fragmentation of natural habitats: case studies of the 24 Italian nationalparks. Chem. Eng. Trans. 32, 49-54.

EEA (2011). Landscape fragmentation in Europe, Joint EEA-FOEN report. Copenhagen: European Environment Agency.

Battisti C., 2004 "Frammentazione ambientale, connettività, reti ecologiche: un contributo teorico e metodologico con particolare riferimento alla fauna selvatica". Provincia di Roma, Assessorato alle politiche agricole, ambientali e protezione civile, $246 \mathrm{pp}$.

Battisti, C., Romano, B., 2007. Frammentazione e connettività. Dall'analisi ecologica alla pianificazione ambientale. CittàStudi, Torino.

Bissonette, J.A., Adair, W. 2008. Restoring habitat permeability to roaded landscapes with isometricallyscale wildlife crossings. Biol. Conserv., 141(2): 482-488.

Chan, L., Hillel, O., Elmqvist, T., Werner, P., Holman, N., Mader, A., Calcaterra, E., 2010. User's Manual on the Singapore Index on Cities' Biodiversity (also Knownas the City Biodiversity Index). National Parks Board, Singapore, Singapore.

Chan, L., Hillel, O., Elmqvist, T., Werner, P., Holman, N., Mader, A., Calcaterra, E., 2014. User's Manual on the Singapore Index on Cities' Biodiversity (also Knownas the City Biodiversity Index). National Parks Board, Singapore, Singapore.

De Montis, A., Caschili, S., Mulas, M., Modica, G., Ganciu, A., Bardi, A., Ledda, A.,Dessena, L., Laudari, L., Fichera, C.R., 2016. Urban-rural ecological networks forlandscape planning. Land Use Policy 50, 312-327, http://dx.doi.org/10.1016/j.landusepol.2015.10.004.

De Montis, A., Martin, B., Ortega, E., Ledda, A., Serra, V. 2017. Landscape fragmentation in Mediterranean Europe: A comparative approach. Land Use Policy, 64: 83-94.

De Montis, A., Ledda, A., Ortega, E., Martín, B., Serra, V., 2018. Landscape planning and defragmentation measures: an assessment of costs and critical issues. Land Use Policy, 72, pp. 313-324.

Deslauriers, M.R., et al. 2017. Implementing the connectivity of natural areas in cities as an indicator in the City Biodiversity Index (CBI). Ecol. Indicat. http://dx.doi.org/10.1016/j.ecolind.2017.02.028.

LaPoint, S., Balkenhol, N., Hale, J., Sadler, J., van der Ree, R., 2015. Ecological connectivity research in urban areas. Funct. Ecol. 29, 868-878, http://dx.doi.org/10.1111/1365-2435.12489

Jaeger, J.A.G., 2000. Landscape division, splitting index, and effective mesh size:new measures of landscape fragmentation. Landsc. Ecol. 15 (2), 115-130.

Foley, J.A., DeFries, R., Asner, G.P., Barford, C., Bonan, G., Carpenter, S.R., Chapin, F.S.,Coe, M.T., Daily, G.C., Gibbs, H.K., Helkowski, J.H., Holloway, T., Howard, E.A.,Kucharik, C.J., Monfreda, C., Patz, J.A., Prentice, I.C., Ramankutty, N., Snyder,P.K., 2005. Global consequences of land use. Science 309 (5734), 570574.

Foley, J.A., Ramankutty, N., Brauman, K.A., Cassidy, E.S., Gerber, J.S., Johnston, M.,Mueller, N.D., O’Connell, C., Ray, D.K., West, P.C., Balzer, C., Bennett, E.M.,Carpenter, S.R., Hill, J., Monfreda, C., Polasky, S., Rockström, J., Sheehan, J.,Siebert, S., Tilman, D., Zaks, D.P.M., 2011. Solutions for a cultivated planet.Nature 478 (7369), 337-342.

Gulinck, H. Wagendorp, T. (2002). References for fragmentation analysis of the rural matrix in cultural landscapes. Landscape Urban Plann 58:137-146

RAS, 2008. Carta dell'uso del suolo [Regional Land Cover Map -RLCM]. http://www.sardegnageoportale.it/argomenti/cartedelsuolo.html.

Romano, B., Zullo, F., 2013. Valutazione della pressione insediativa: indicatori e sperimentazione di soglie. In: Battisti, C., Conigliaro, M., Poeta, G., Teofili, C.(Eds.), Biodiversità, disturbi, minacce. Editrice Universitaria Udinese, Udine,pp. 170-177.

Scolozzi, R., Geneletti, D., 2012. Assessing habitat connectivity for land-use planning: a method integrating landscape graphs and Delphi survey. J. Environ.Plan Manag. 55 (6), 813-830.

Wales, 2017. Land use mao. digimap.edina.ac.uk. https://digimap.edina.ac.uk/?utm_campaign=2118466_EDINA\%20DfS:\%20June \%20Service\%20Update\%20(S tudents)\&utm medium=email\&utm source=dotmailer\&dm i=27JU,19EMA,7O4LSK,41F3R, 1.

Accessed Nov 22017. 


\title{
Effect of Irrigation Systems and Water Quantities on Corn Production under Partial Rootzone Drying Conditions
}

\author{
Abdellateif Abdelwahab Samak ${ }^{\text {a,* }}$, Mohamed Nabih Omar a, Mahmoud Hassan Keshek ${ }^{\text {a }}$ \\ a Agricultural engineering department, Faculty of Agriculture, Menoufia University, 32511 Shebin EL-Kom, Egypt. \\ * Corresponding author. abdellatief.samak@agr.menofia.edu.eg
}

\begin{abstract}
Worldwide, Agriculture is consider the main user of water. In Egypt, Agriculture consumes more than $80 \%$ of the available water. In irrigated agriculture, the right way to save water is to increase irrigation water use efficiency (IWUE) through better irrigation management like Partial Rootzone Drying (PRD). Partial Rootzone Drying is an irrigation method, which increase the irrigation water use efficiency without yield reductions in many different crops. Corn crop is one of the most important crops for human or for animal consumption and planted for grain yield or for forage (FAO, 2018). Corn yield productions under surface and sub-surface drip irrigation systems and their responses to different irrigation water quantities was evaluated in 2017 summer growing seasons. A field experiment was conducted using corn crop (Zea Mays) grown in northern Egypt at Shebin ELKom, Menoufia Governorate. A randomized split-plot design was used with irrigation system as main plots and different irrigation water quantities randomly distributed within either conventional or partial rootzone drying irrigation regimes. The irrigation water quantity was a fraction of crop evapotranspiration (ET) as $0.6,0.8,1.0$, and 1.2 of ET. The experimental treatments were replicated three times. The experimental results showed that, the soil water content significantly affected by irrigation regime and irrigation water quantities. In addition, Corn grain yield was significantly affected by irrigation system and both irrigation regime and irrigation water quantity. Also, corn 100-grain weight were affected by irrigation system, irrigation water quantity and irrigation regime, respectively. Significant differences for the interaction between irrigation regimes and irrigation systems were found only for Corn yield production and 100-grain weight. Finally, the results from this small experiment should extrapolate to a large field to find out the optimal irrigation scheduling under non-uniform of irrigation applications.
\end{abstract}

Keywords: Corn Crop, Irrigation Systems, Water Quantities, Partial Rootzone Drying Regime, Water Use Efficiency

\section{Introduction}

Water supplies are limited worldwide (Fereres and Soriano 2007; Savić et al. 2008), and the Agriculture is consider the main user of water. Water use are growing at more than twice the rate of population increase, and the numbers of regions that are chronically short of water are increasing. Therefore, the problems of water scarcity are mostly acute (FAO 2018). By 2025, the population growth and economic development will lead to more than 1.8 billion people living in countries and regions with absolute water scarcity, and two-thirds of the world population could be under water stress conditions. According to FAO (2018), the situation will be exacerbated. Worldwide, Irrigation of agricultural lands accounts for over $85 \%$ of water usage (van Schilfgaarde 1994). Even any small reduction in irrigation water could substantially increase the water available for other purposes. Therefore, there is an urgent need to discover a better and effective irrigation management strategies under the condition of water scarcity (Fereres and Soriano 2007; Savić et al. 2008).

Partial rootzone drying irrigation (PRDI) is an irrigation method where one-half of the root system is irrigated while leaving the other half in a dry state. After a certain period of time, depending on soil and weather conditions (Kriedemann and Goodwin, 2003), the irrigation is shifted so that the wet part of the root system is allowed to dry out and the dry part is irrigated (Stoll et al. 2000). Partial rootzone drying is one of the deficit irrigation strategies designed to keep half of the root system in a drying state, while the other half of the root area is irrigated. Then, the treatment is reversed, allowing the previously well-irrigated side of the root system to dry down addition to fully irrigating the previously dry side. The principle behind PRDI is to alternately let one part of the root area be exposed to soil drying while another part is irrigated, in order to keep the leaves hydrated. Partial rootzone drying irrigation (PRDI) is a potential water-saving irrigation method where, at each irrigation period, only a part of the rootzone is wetted with the other compartment left to dry to a pre-determined level. PRDI could save water by up to $50 \%$ and yet maintain yield as shown for some crops; e.g. for sugar cane (Shani-Dashtgol et al. 2006), maize (Kang et al. 2000), winter wheat (Sepaskhah and Hosseini 2008), cotton (Du et al., 2008), potato (Jovanovic et al. 2010; and Amer et al., 2016), apple (Zegbe et al., 2008) and tomato (Samak 2012). The PRDI method has either been used as a fixed partial rootzone drying (FPRDI) or as an alternate partial rootzone drying irrigation (APRDI). The FPRDI is a partial rootzone drying irrigation where the irrigation water are applied to a fixed root side during the growing season while keeping the other side in a dry state. On the other hand, the APRDI is a partial rootzone drying irrigation (PRDI), where the irrigated side is changed from one side to the other side; normally PRDI is 
applied as APRDI. Deficit irrigation (DI) strategy is an optimization method in which irrigation water is added during drought sensitive growth stages of a crop according to Geerts and Raes, 2009. English and Raja (1996) reported that, DI consists of the deliberate and systematic under irrigation of crops. In other words, the amount of irrigation water applied is less than that needed to full satisfy the crop's water requirements. Regulated deficit irrigation (RDI) is a special irrigation strategy based on applying only a fraction of the plant's water requirements during certain periods of plant development (Ruiz-Sanchez et al. 2010). Mainly, the Regulated deficit irrigation (RDI) is designed to limit water when the sensitivity of plant to water stress is minimal. The RDI strategies can also be applied when the available water is not enough to optimize maximum yields. According to Farre and Faci, 2009 , deficit irrigation (DI) is an advocated strategy to improve irrigation water use in surface irrigated corn production. DI is the intentional under irrigation of crops below full crop water requirements (Fereres and Soriano, 2007). However, this technique is limited by the fact that water deficits may induce crop water stress, which can reduced the crop growth and development. Soil water stress directly affects plants ability to capture resources needed for photosynthesis and the efficiency with which they convert these physical resources into biological materials, i.e., biomass and grain yield (Yi et al., 2010). For instance, water deficits have been shown to decrease corn dry biomass (Igbadun et al., 2008), plant height (Ḉakir, 2004), leaf area index (Mansouri-Far et al., 2010), and grain yield (Djaman et al., 2013).

Corn (Zea Mays) is one of the most important cereals either for human or for animal consumption and grown for grain and forage. Amer, (2010) studied the responses of corn crop to water deficit as affrication of crop evapotranspiration. Amer (2010) reported that corn is very sensitive to water deficit during vegetative. However, great grain yield reduction was observed by water deficit during flowering period. The production of corn plays a major role in the economic and socio-economic development of many countries owing to the diversification of its uses: food commodity, feed commodity and a significant bioethanol source. However, its optimum production have high irrigation demands (Karam et al., 2003) as corn is among the largest plant user of water (WMO, 2012). In comparing corn to other crops, Sorensen et al. (2004) studied cotton yield response to water applied using subsurface drip irrigation (SSDI). They found that, the SSDI irrigation with a 50\% irrigation treatment had a lower lint yield response when compared with $100 \%$ and $75 \%$ irrigation in both 1999 and 2000 growing seasons. Total rainfall during 1999 and 2000 growing was 329 and $220 \mathrm{~mm}$, respectively. Total irrigation amount for $100 \%$ water applied was 208 and $300 \mathrm{~mm}$, respectively. Lint yield significantly responded with SSDI compared to no-irrigation treatments. In 1999 and 2000 growing seasons, average lint yield for $100 \%$ and $75 \%$ irrigation was $1.482 \mathrm{Mgha}^{-1}$ while $50 \%$ irrigation averaged $1.354 \mathrm{Mgha}^{-1}$, almost a $10 \%$ increase in lint yield attributed to the water applied. Lint yield from the dry treatment (no irrigation) was $1.025 \mathrm{Mgha}^{-1}$. When growing corn, Wang et al. (2008) found out that a maximum biomass accumulation was founded under well-watered conditions, and severe water deficit led to about $50 \%$ reduction of biomass when compared to the conventional irrigation (CI) regime. In their experiments, they tested three irrigation methods: conventional irrigation (CI), alternate partial rootzone drying irrigation (APRDI), and fixed partial rootzone drying irrigation (FPRDI) under three different watering levels: (1) well-watered, (2) mild water deficit and (3) severe water deficit. The irrigation practices needed to give the most efficient use of water may vary widely within soils and locations. Soil properties of planted area are the responsible indicators to calculate the amount of water should be storage into crop rootzone per irrigation, thus, irrigation interval. Therefore, water demands varies based on soils from sandy to clay soils based on the amount of water per irrigation that the soil can be hold it. It varies based on weather condition that crops growing in tropical area or in dry seasons need more water than in semi-tropical and humid regions or wet seasons. Even though, nonuniformity of water application under irrigation system as well as irrigation water salinity has an effect on crop water requirement (Amer, 2010). However, due to the current and expected limited water supplies, interest in subsurface drip irrigation (SSDI) systems to irrigate row crops in Nebraska is growing. Although studies with SSDI-irrigated corn have been conducted in other states (Lamm and Trooien, 2003), local information on the response of corn growth, yield and other crop water dynamics with SSDI is very limited. The agronomic response of the crop to irrigation with SSDI is needed to be able to evaluate the economic and technical feasibility of using SSDI under local conditions and provide scientifically based practical information to the users on best management practices for SSDI-irrigated corn. Under water scarcity conditions, appropriate water management comprises any strategy that achieves adequate yield with significant water savings (García-Tejero et al., 2011). Thus, the implementation of water-saving irrigation management strategies that improve tree growth and/or yield per unit of water used, i.e. irrigation water use efficiency (IWUE), is a key factor for the sustain-ability of agroecosystems.

The main objective of the research was to study and evaluate the corn response to conventional and partial rootzone drying irrigation regimes under surface and subsurface drip irrigation systems and their responses to different irrigation water quantities. One goal of the current study was to assess optimal irrigation schedule for applying the conventional and partial rootzone drying irrigation strategy. 


\section{Materials and Methods}

Field experimental work

Field study was carried out at agriculture faculty farm, Menoufia University, Egypt (Shebin EL-Kom area, $17.9 \mathrm{~m}$ above sea level, $30^{\circ} 32 / \mathrm{N}$, and $31^{\circ} 03 / \mathrm{E}$ ) during the 2017 summer growing seasons to investigate the response of corn crop to different drip irrigation system, irrigation management strategies under different levels of crop evapotranspiration (ET). The crops were planted on beds spaced $90 \mathrm{~cm}$ apart. Soil of the study area was classified as clay loam with $1.27 \mathrm{~g} \mathrm{~cm}^{-3}$ bulk density. The physical and chemical properties of the experimental soil are shown in Tables ( 1 and 2). The Corn crop (Zea Mays) was planted at about 8 seeds per $\mathrm{m}^{2}$ on first of May 2017 in the experimental field, and terminated on 10 of September. The experimental treatments were arranged in a randomized split-plot design with irrigation system as main plots and different irrigation water quantities randomly distributed within either conventional or partial rootzone drying irrigation regimes. The irrigation water quantity was a fraction of crop evapotranspiration (ET) as $0.6,0.8,1.0$, and 1.2 of ET. The experimental treatments were replicated three times. All experimental treatments were separated as surrounded by $1.0 \mathrm{~m}$ no irrigated area as shown in Figure 1. Plants were watered in the first irrigation with the same amount of irrigation water. The irrigating of treatments were initiated with the second irrigating.

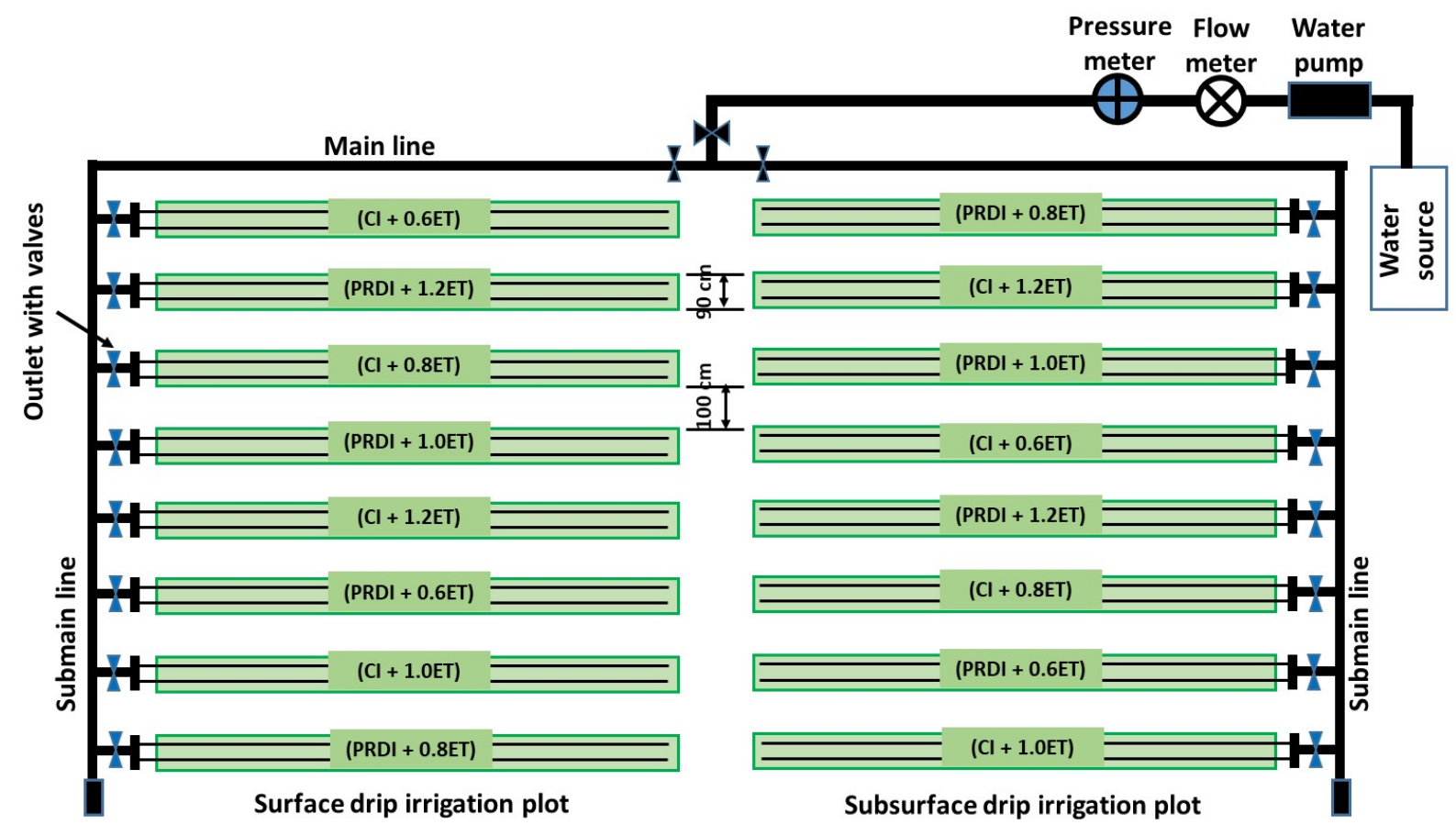

Figure 118. Schematic diagram of the experimental treatments setup and layout.

Table 50. Soil physical properties of the experimental field soil.

\begin{tabular}{ccccccc}
\hline $\begin{array}{c}\text { Soil depth } \\
{[\mathrm{cm}]}\end{array}$ & $\begin{array}{c}\text { Sand } \\
\text { content }\end{array}$ & $\begin{array}{c}\text { Silt } \\
\text { content }\end{array}$ & $\begin{array}{c}\text { Clay } \\
\text { content }\end{array}$ & $\begin{array}{c}\text { Bulk } \\
\text { density } \\
{\left[\mathrm{g} \mathrm{cm}^{-3}\right]}\end{array}$ & $\begin{array}{c}\text { Field } \\
\text { capacity } \\
{\left[\mathrm{g} \mathrm{g}^{-1}\right]}\end{array}$ & $\begin{array}{c}\text { permanent } \\
\text { wilting point } \\
{\left[\mathrm{g} \mathrm{g}^{-1}\right]}\end{array}$ \\
\hline $0-30$ & 23.00 & 31.20 & 45.80 & 1.24 & 0.34 & 0.17 \\
$30-60$ & 17.30 & 26.75 & 55.95 & 1.30 & 0.38 & 0.19 \\
Average & 20.15 & 28.98 & 50.88 & 1.27 & 0.36 & 0.18 \\
\hline
\end{tabular}


Table 51. Some soil chemical properties of the experimental field soil.

\begin{tabular}{|c|c|c|c|c|c|c|c|c|c|}
\hline \multirow{2}{*}{$\begin{array}{l}\text { Soil depth } \\
{[\mathrm{cm}]}\end{array}$} & \multirow{2}{*}{$\mathrm{pH}$} & \multirow{2}{*}{$\begin{array}{c}\text { EC } \\
\text { ds } m^{-1}\end{array}$} & \multicolumn{4}{|c|}{ Soluble cations, meq. $\mathrm{l}^{-1}$} & \multicolumn{3}{|c|}{ Soluble anions, meq. $\mathrm{l}^{-1}$} \\
\hline & & & $\mathrm{Na}^{+}$ & $\mathrm{K}^{+}$ & $\mathrm{Ca}^{+2}$ & $\mathrm{Mg}^{+2}$ & $\mathrm{Cl}^{-1}$ & $\mathrm{HCO}_{3}{ }^{-2}$ & $\mathrm{SO}_{4}^{-2}$ \\
\hline $0-30$ & 8.78 & 0.34 & 3.44 & 0.07 & 0.28 & 0.15 & 1.14 & 1.65 & 1.17 \\
\hline $30-60$ & 8.98 & 0.32 & 3.32 & 0.07 & 0.22 & 0.18 & 1.14 & 1.75 & 1.19 \\
\hline Average & 8.88 & 0.33 & 3.38 & 0.07 & 0.25 & 0.17 & 1.14 & 1.70 & 1.18 \\
\hline
\end{tabular}

Irrigation schedule and management

Irrigation schedule was to refill the top $0.6 \mathrm{~m}$ depth of rootzone. Irrigation scheduling for $0.6 \mathrm{ET}, 0.8 \mathrm{ET}, 1.0 \mathrm{ET}$, and 1.2ET treatments were fractioned from 1.0ET under each of surface and subsurface drip irrigation using conventional and partial rootzone drying irrigation regimes. In the experimental site, there was no rainfall or ground water to avoid the side effecting, where the water table was deeper than $2.6 \mathrm{~m}$, during the study period.

During the irrigation period, water was applied every three days based on crop evapotranspiration calculations. The crop coefficient function for corn was adapted according to FAO 56 (Allen et al., 1998) and ranges from 0.35 to 1.20 as a function of crop age. The fertilizations were applied to the experiment treatments as the recommended rate for corn production in this area, $200 \mathrm{~kg} \mathrm{ha}^{-1} \mathrm{~N}, 60 \mathrm{~kg} \mathrm{ha}^{-1} \mathrm{P}$ and $70 \mathrm{~kg} \mathrm{ha}^{-1} \mathrm{~K}$ (Amer 2010). The calculated fertilizers were applied with irrigation water as recommended by crop science productions. To accurately control of applied irrigation water, two surface drip irrigation (SDI) lines with an emitter spacing of $25 \mathrm{~cm}$ and emitter flow rate of $4 \mathrm{~L} \mathrm{~h}^{-1}$ were fixed for each plant row at a distance approximately $20 \mathrm{~cm}$ away from the plant row on the soil beds surface. In addition, two subsurface drip irrigation (SSDI) with the same specifications were placed for every plant row at a depth approximately $25 \mathrm{~cm}$ below the soil beds surface, as shown in the Figure 2.

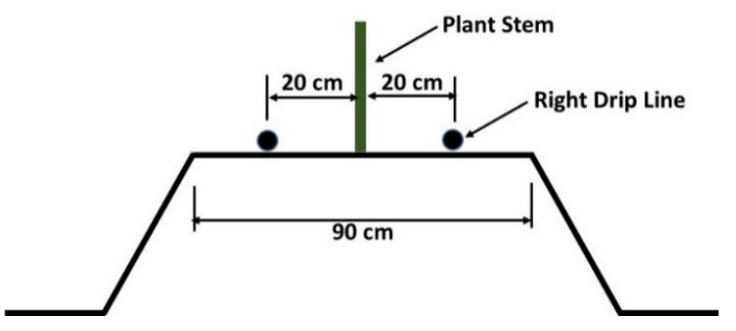

a- $\quad$ Surface Irrigation setup

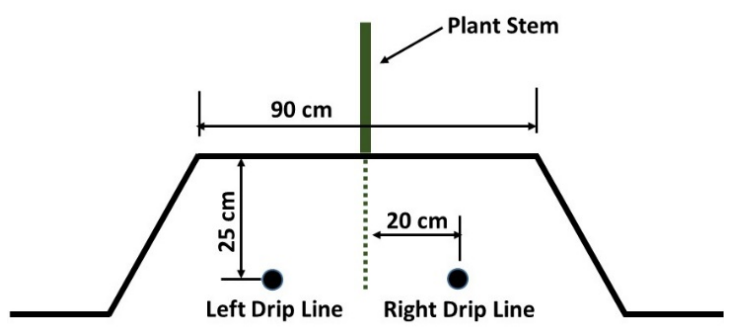

b- Subsurface Irrigation setup

Figure 119. Irrigation systems setup and management: (a) surface drip irrigation and, (b) subsurface drip irrigation.

A water valve was considered to control the irrigation at each side. The irrigation water was measured and controlled by a flow meter and water pump in order to control the amount of applied water. To manage irrigation treatments, the calculated irrigating water were added to both sides of CI treatments. The same amount of water was then added to one side of the PRDI treatment. In the PRDI treatment, two weeks of irrigation time was chosen for shifting the irrigation from wet side to dry side, as shown in Figure 3.

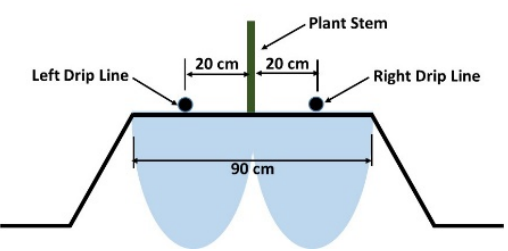

a- Conventional Irrigation

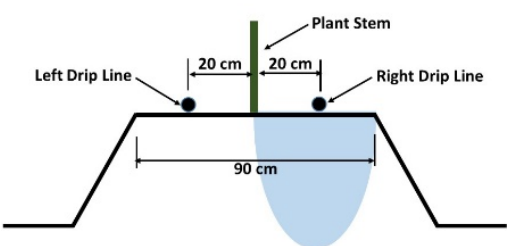

b- Partial rootzone drying irrigation with irrigated right side

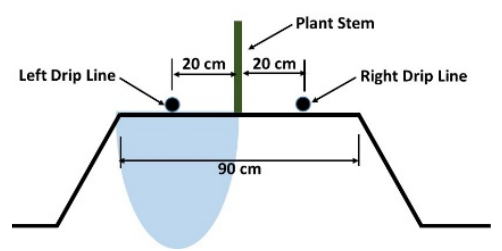

c- Partial rootzone drying irrigation with irrigated left side

Figure 120. Irrigation regime management: (a) both sides of CI treatment were irrigated, (b) only the right side of PRDI treatment was irrigated, and (c) only the left side of PRDI treatment was irrigated. 
Soil water content and irrigation water use efficiency

The soil water content in each side of the experimental treatment were measured at different places during the completely growing season for the top $30 \mathrm{~cm}$ of the soil profile. Soil tube was used to collect the soil samples from each side of the experimental treatments to measure the soil water content using the gravimetric method. However, irrigation water use efficiency (IWUE) of the experimental treatments was calculated using the following equation according to Kirda et al. (2004):

$$
I W U E=\frac{Y}{I}
$$

Where: IWUE: irrigation water use efficiency $\left[\mathrm{Mg} \mathrm{m}^{-3}\right]$; Y: yield per area $\left[\mathrm{Mg} \mathrm{ha}^{-1}\right]$; and I: irrigation water applied per the same area $\left[\mathrm{m}^{3} \mathrm{ha}^{-1}\right]$. Statistical evaluations of the experimental treatments were done using the SPSS-Statistical Package (SPSS 18). A split plot model at a $5 \%$ significance level was used to analyse the results.

\section{Results and Discussion}

Corn crop yields

Table (3) presents the results of grain yield, green forage yield, and number of rows per ear, number of grains per row and 100-grain weight of corn irrigation treatments. Generally, there were significant affected to different applied irrigation water. The results showed that, the average of corn grain yield increased by increasing applied irrigation water. There were no significant affected to irrigation regimes (CI and PRDI) in the treatments with no water stress (1.0ET and 1.2ET), but there were significant affecting in the treatments with water stress (0.6ET and 0.8ET). These results are in agreement with (Vories et al., 2009). The results showed that also, there were significant difference between two irrigation systems (Subsurface and surface drip irrigation systems). The same results were observed for the green forage yield. In addition, the results showed that, 100-grain weight, and number of grains per row were affected by irrigation treatments and there were significant differences between treatments.

The obtained results demonstrated that, both 100-grain weight, and number of grains per row increased by increasing the applied irrigation water; and there were no significant affected to irrigation regimes (CI and PRDI) under no water stress treatments, but there were significant affecting in the treatments with water stress. These results are in agreement with (Vories et al., 2009). Otherwise, a small difference between PRDI and CI could be found concerning the number of rows per ear and there were no significant affect to number of rows per ear for applied irrigation water, irrigation regimes, and irrigations systems. Stamp (2003) reported that, as roots are the first part of the plant to be exposed to changes in soil moisture, it is to be expected that their structure may be affected by the PRDI regime. It has been observed that withholding water from one side of the root system causes roots to grow deeper into the ground soil profile (Dry et al. 2000). From the summary of the effect of PRDI on crop yield, it can be derived that the yield is not affected by the PRDI. No effects were reported between the irrigation methods except from investigations of De la Hera et al. (2007), and Samak (2012) who had clear positive effects using the same amount of water for both irrigation treatments. In the present study, a positive effect could be found for the PRDI regime under treatments with deficit water situations. 
Table 52. Grain and green forage yield, number of rows per ear, number of grains per row and 100-grain weight of corn irrigation treatments.

\begin{tabular}{|c|c|c|c|c|c|c|c|c|}
\hline & Irrigation tre & hent & $\begin{array}{c}\text { Applied } \\
\text { Water }\end{array}$ & $\begin{array}{l}\text { Grain } \\
\text { yield }\end{array}$ & $\begin{array}{c}\text { Green } \\
\text { forage yield }\end{array}$ & $\begin{array}{l}\text { Number } \\
\text { of rows }\end{array}$ & $\begin{array}{l}\text { Number } \\
\text { of grains }\end{array}$ & $\begin{array}{l}100- \\
\text { grain }\end{array}$ \\
\hline & & $0.6 \mathrm{ET}$ & 513 & 12.55 & 66.65 & 11.66 & 41.98 & 36.17 \\
\hline & $\stackrel{\Xi}{0}$. & $0.8 \mathrm{ET}$ & 683 & 14.33 & 72.15 & 11.66 & 46.66 & 38.77 \\
\hline Dे & & $1.0 \mathrm{ET}$ & 854 & 17.35 & 81.78 & 12.00 & 48.88 & 39.69 \\
\hline 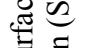 & & $1.2 \mathrm{ET}$ & 1025 & 17.55 & 82.35 & 12.00 & 49.88 & 40.27 \\
\hline ప్రి & 8 & $0.6 \mathrm{ET}$ & 513 & 14.55 & 71.15 & 12.33 & 43.00 & 36.39 \\
\hline $\bar{\omega} \cdot \Xi$ & 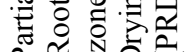 & $0.8 \mathrm{ET}$ & 683 & 16.66 & 76.17 & 12.00 & 48.33 & 38.88 \\
\hline & & $1.0 \mathrm{ET}$ & 854 & 17.00 & 82.45 & 12.66 & 48.50 & 40.78 \\
\hline & & $1.2 \mathrm{ET}$ & 1025 & 17.15 & 82.89 & 12.66 & 50.15 & 41.88 \\
\hline & & $0.6 \mathrm{ET}$ & 513 & 12.35 & 65.50 & 11.66 & 41.66 & 35.18 \\
\hline . 윯 & 芯氙 & $0.8 \mathrm{ET}$ & 683 & 14.55 & 70.75 & 12.00 & 46.00 & 38.15 \\
\hline$\Xi$ & & $1.0 \mathrm{ET}$ & 854 & 16.87 & 80.60 & 12.00 & 48.00 & 39.66 \\
\hline धै & & $1.2 \mathrm{ET}$ & 1025 & 16.95 & 82.10 & 12.33 & 49.50 & 39.98 \\
\hline$\ddot{\delta}$ & & $0.6 \mathrm{ET}$ & 513 & 15.15 & 70.70 & 12.00 & 42.66 & 36.11 \\
\hline$\stackrel{\pi}{\Xi}$ & 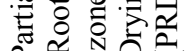 & $0.8 \mathrm{ET}$ & 683 & 16.55 & 75.80 & 12.00 & 47.50 & 38.33 \\
\hline & & $1.0 \mathrm{ET}$ & 854 & 16.35 & 81.00 & 12.66 & 48.00 & 40.15 \\
\hline & & $1.2 \mathrm{ET}$ & 1025 & 16.40 & 81.15 & 12.66 & 49.66 & 41.18 \\
\hline
\end{tabular}

Irrigation Water Use Efficiency (IWUE)

Figure (4) presents the results of irrigation water use efficiency (IWUE) by $\mathrm{kg} \mathrm{m}^{-3}$ based on the corn grain yield. The obtained results showed that in general, the IWUE increased by decreasing the applied irrigation water and there were significant differences to the applied water. However, the results demonstrated that, the IWUE of PRDI treatments were higher than the $\mathrm{CI}$ treatments, and there were significant affecting to irrigation regime. Considering the drip irrigation systems, a small difference between SDI and SSDI could be found concerning the IWUE and the results observed that there were no significant affecting. As mentioned, corn is one of the most important cereals both for human and animal consumption. Therefore, The IWUE was calculated once again based on yields of grain and forage; and the similar results were obtained as shown in Figure 5. 


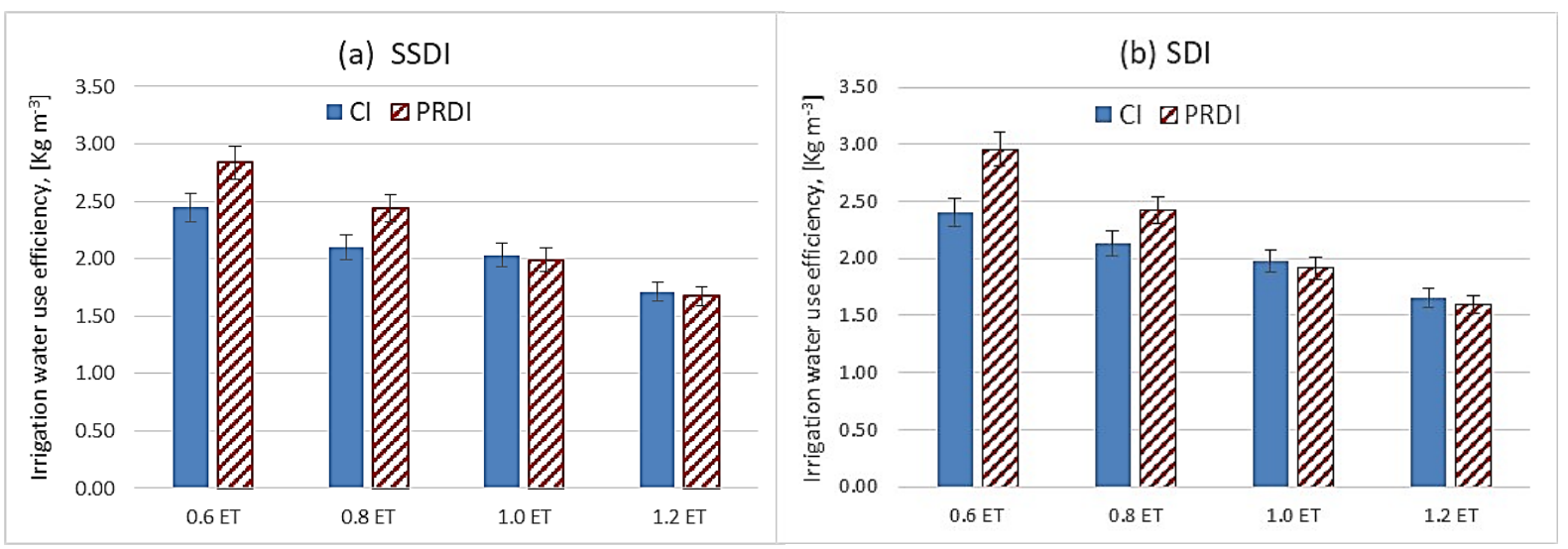

Figure 121. Irrigation water use efficiency for corn irrigation study based on grain yields for: (a) Subsurface drip irrigation treatment (SSDI), and (b) Surface drip irrigation treatment (SDI).

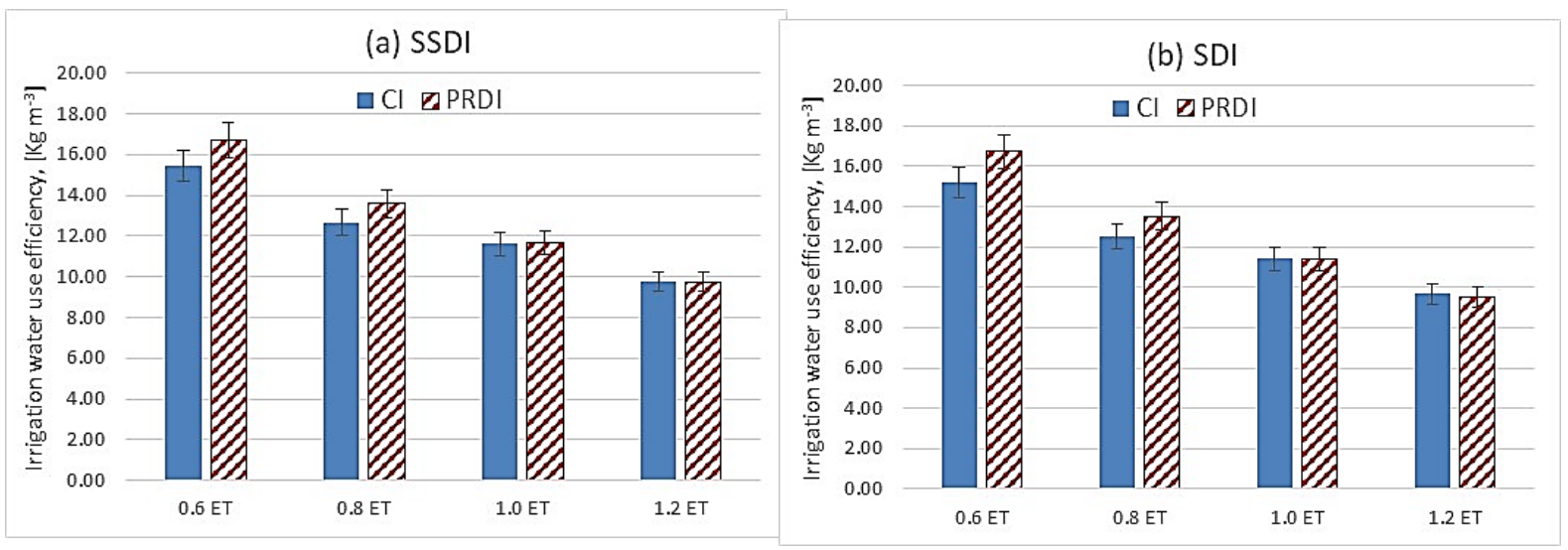

Figure 122. Irrigation water use efficiency for corn irrigation study based on grain and green forage yields for: (a) Subsurface drip irrigation treatment (SSDI), and (b) Surface drip irrigation treatment (SDI).

The IWUE values from this study were the similar with the results reported by (e.g., Caldwell et al., 1994). However, those studies were conducted in more arid and semi-arid locations. Moreover, the obtained results were higher than of the values reported in the literature (e.g., Howell et al. (1989; and Vories, 2009) they observed a value of IWUE for fully irrigated corn (about $1.4 \mathrm{~kg} \mathrm{~m}^{-3}$ ), but most studies reported higher values for the more efficient treatments. Stamp (2003) reported that, the irrigation water use efficiency (IWUE) for commercial application of the PRDI compared to a CI method was on average increased by $75 \%$. The amount of water was reduced by $47 \%$ whilst the yield was lower by $7 \%$ on average (Stamp 2003). This further supports the results of the current study and earlier observations by Loveys et al. (1998). The results of the present study showed that the PRDI method increased the IWUE compared to the CI method when applying it under water stress. The possibility for the improved IWUE under PRDI came from the analysis of the soil water distribution. The soil water content and the depth of the soil profile is almost positive in the PRDI method because the irrigation water moved to deeper soil layers, and there was an upward soil water content gradient in their rootzone (Kang et al. 2002). Therefore, the PRDI method offered a very useful technique to produce a good commercial qualities; Stikic et al. (2003), and Samak (2012) reported similar results.

\section{Soil moisture content distribution}

Figures $(6,7$, and 8) show the distribution of the volumetric soil moisture content for the PRDI and CI treatments of the corn crop experiment at the top $30 \mathrm{~cm}$ of the soil profile. As described in the materials and methods, the PRDI and CI treatments were irrigated with the same amount of irrigation water, but the irrigation water was added only to one side of the PRDI method. Meanwhile, the irrigation water was added to both sides of conventional irrigation method as recommended. The results show that, the soil water content for each side of the PRDI treatment depended on whether the side of the PRDI was irrigated or not. It could be seen that, the irrigation was shifted from side to the other side multi times during the growing season. In comparison to the two sides of PRDI treatment, the difference of the soil water between the dry and wet side of the PRDI were very high as shown in the figures. These obtained results demonstrated that most of applied water in the PRDI method 
moved to the bottom layers or to the other side of the soil profile. Water movement only worked well in the top layers of the soil, but it was influenced by climatic situations, plant, and soil interactions.

The irrigation of the PRDI treatment increased the soil water content of the irrigated side. This is logical because half of the plant area was irrigated with double amount of water compared to the CI method. The soil water content for each side of the PRDI treatment depended on whether the side of the PRDI was irrigated or not. These results are depended on the irrigation management and cropping system. Similar results were also reported for drip irrigated tomato in open-soil conditions inside a greenhouse by Kaman et al. (2006), in big pots with splitting roots by Savić et al. (2008), for furrow irrigated cotton under open-field conditions by Kaman et al. (2006), and for tomato in open field inside greenhouse by Samak, (2012). When comparing CI and PRDI, the soil water content in the irrigated side of the PRDI treatment was lower than either side of the CI treatment; similar results were reported by Samak (2012) for experiments with tomato crop. Otherwise, Kaman et al. (2006), Zegbe et al. (2004), Leib et al. (2004), and Savić et al. (2008) reported for different crops and different irrigation systems that the soil water content in the irrigated side of the PRDI regime was lower than either side of the CI treatment.
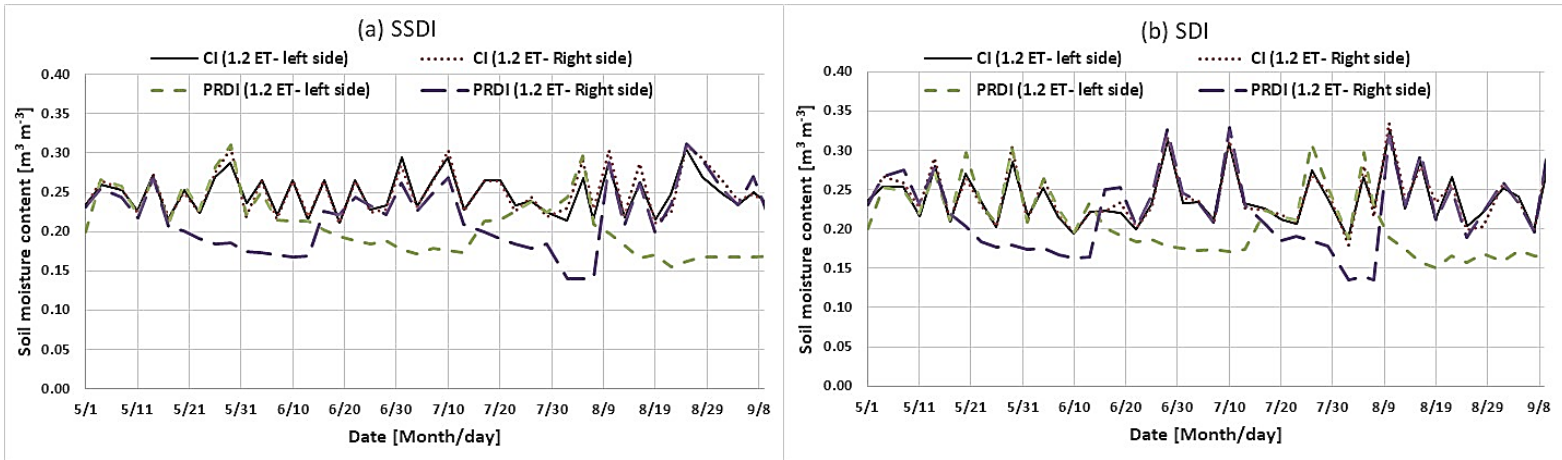

Figure 123. Variation of the soil moisture content in both sides of the different irrigation regimes during the corn crop experiment under 1.2ET for: (a) Subsurface drip irrigation SSDI, and (b) Surface drip irrigation SDI systems.
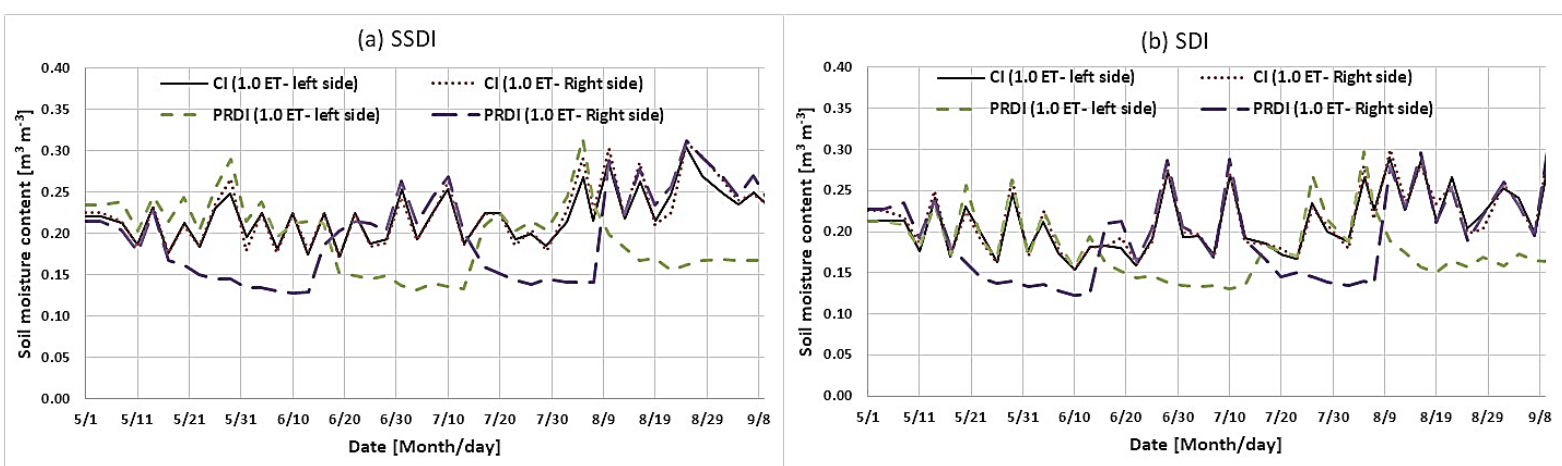

Figure 124. Variation of the soil moisture content in both sides of the different irrigation regimes during the corn crop experiment under 1.0ET for: (a) Subsurface drip irrigation SSDI, and (b) Surface drip irrigation SDI systems.
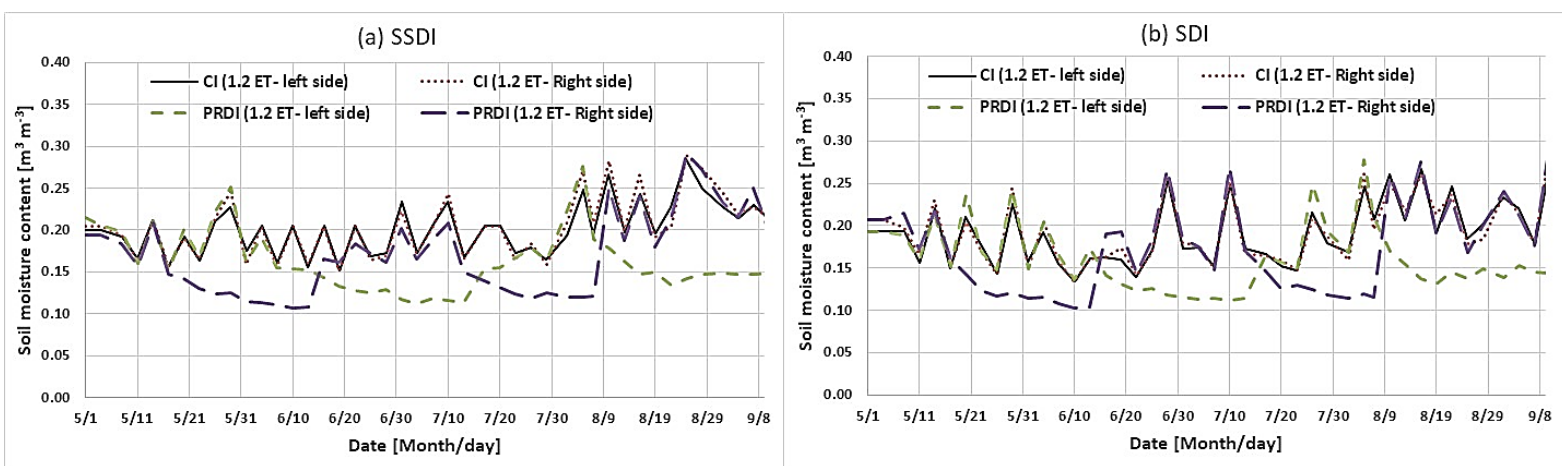

Figure 125. Variation of the soil moisture content in both sides of the different irrigation regimes during the corn crop experiment under 0.8ET for: (a) Subsurface drip irrigation SSDI, and (b) Surface drip irrigation SDI systems. 


\section{Conclusions}

Worldwide, Agriculture is consider the main user of water. In Egypt, Agriculture consumes more than $80 \%$ of the available water. In irrigated agriculture, the right way to save water is to increase irrigation water use efficiency through better irrigation management like Partial Rootzone Drying. Partial Rootzone Drying (PRD) is an irrigation regime, which increase water use efficiency without any yield reductions in many different crops. The results of the current study demonstrated that, the average of corn grain yield increased by increasing applied irrigation water and there were no significant affected to irrigation regimes in the treatments with no water stress (1.0ET and 1.2ET), while there were significant affecting in the treatments with water stress $(0.6 \mathrm{ET}$ and $0.8 \mathrm{ET})$. The results showed that also, there were significant difference between two irrigation systems (Subsurface and surface drip irrigation). In addition, the similar results were observed for the green forage yields. Otherwise, a small difference between PRDI and CI could be found concerning the number of rows per ear and there were no significant affect to number of rows per ear to applied irrigation water, irrigation regimes, and irrigations systems. The obtained results of IWUE showed that, the IWUE increased by decreasing the applied irrigation water and there were significant differences to the applied water. However, the results demonstrated that, the IWUE of PRDI treatments were higher than the CI treatments, and there were significant affecting to irrigation regime. Considering the drip irrigation systems, a small difference between SDI and SSDI could be found and the results observed that there were no significant affecting. The results of soil water distribution show that, the soil water content for each side of the PRDI regime depended on whether the side of the PRDI was irrigated or not, and the difference of the soil water between the dry and wet side of the PRDI were very high. The obtained results also demonstrated that, the irrigating of the PRDI treatment increased the soil moisture content of the irrigated side. Moreover, the results showed that, the soil water content significantly affected by irrigation regime and irrigation water quantities. Finally, the results from this small experiment should extrapolate to a large field to find out the optimal irrigation scheduling under non-uniform of irrigation applications.

\section{References}

Allen, R.G., Pereira, L.S., Raes, D., Smith, M., 1998. Crop evapotranspiration—guidelines for computing crop water requirements (Irrigation and Drainage Paper No. 56). Food and Agriculture Organization of the United Nations (FAO), Rome, Italy.

Amer, K.H., 2010. Corn crop response under managing different irrigation and salinity levels. Agricultural Water Management 97 (2010) 1553-1563.

Amer, K.H., Samak, A.A. and Hatfield, J.L. 2016. Effect of Irrigation Method and Non-Uniformity of Irrigation on Potato Performance and Quality. Journal of Water Resource and Protection, 8, 277-292.

http://dx.doi.org/10.4236/jwarp.2016.83024

Çakir, R., 2004. Effect of water stress at different development stages on vegetative and reproductive growth of corn. Field Crop. Res. 89, 1-16.

Caldwell, D.S., Spurgeon, W.E., Manges, H.L., 1994. Frequency of irrigation for subsurface drip-irrigated corn. Trans. ASAE 31 (4), 1099-1103.

De la Hera, M. L., Romero, P., Gómez-Plaza, E., and Martinez, A., 2007. Is partial root-zone drying an effective irrigation technique to improve water use efficiency and fruit quality in field-grown wine grapes under semiarid conditions? Agricultural water management (87): 261- 274.

Djaman, K., Irmak, S., Rathje, W.R., Martin, D.L., Eisenhauer, D.E., 2013. Maize evapotranspiration, yield production functions, biomass, grain yield, harvest index, and yield response factors under full and limited irrigation. Am. Soc. Agric. Biol. Eng. 56, 273-293.

Dry, P. R., Loveys, B. R., 2000. Partial drying of the rootzone of grape: I. Transient changes in shoot growth and gas exchange. Vitis (39): 3-7.

Du, T., Kang, S., Zhang, J., Li, F., Yan, B., 2008. Water use efficiency and fruit quality of table grape under alternate partial rootzone drip irrigation. Agri. Water Management, (95): 659-668.

English, M., and Raja, S. N., 1996. Perspectives on deficit irrigation. Agr. Water Manag. (32): 1-14.

Farré, I., Faci, J.-M., 2009. Deficit irrigation in maize for reducing agricultural water use in a Mediterranean environment. Agric. Water Manag. 96, 383-394.

Fereres, E., Soriano, M. A., 2007. Deficit irrigation for reducing agricultural water use. Journal of Experimental Botany (58): 147-159.

Food and Agricultural Organization, FAO, 2018. Hot issues: water scarcity water \& poverty, an issue of life \& livelihoods. http://www.fao.org/nr/water/issues/scarcity.html (June, 2018) 
García-Tejero, I., Durán-Zuazo, V.H., Muriel-Fernández, J.L., Martínez-García, G.,Jiménez-Bocanegra, J.A., 2011. Benefits of low-frequency irrigation in citrusorchards. Agron. Sust. Dev. 31, 779-791, http://dx.doi.org/10.1007/s13593-011-0025-1.

Geerts, S., and Raes, D., 2009. Deficit irrigation as an on-farm strategy to maximize crop water productivity in dry areas. Agricultural Water Management 96 (2009) 1275-1284

Howell, T.A., Yazar, A., Schneider, A.D., Dusek, D.A., Copeland, K.S., 1995. Yield and water use efficiency of corn in response to LEPA irrigation. Trans. ASAE 38, 1737-1747.

Igbadun, H.E., Salim, B.A., Tarimo, A.K.P.R., Mahoo, H.F., 2008. Effects of deficit irrigation scheduling on yields and soil water balance of irrigated maize. Irrig. Sci. 27, 11-23.

Jovanovic, Z., Stikic, R., Vucelic-Radovic, B., Paukovic, M., Brocic, Z., Matovic, G., Rovcanin, S., Mojevic, M., 2010. Partial root-zone drying increases WUE, N and antioxidant content in field potatoes. European Jour. of Agro. (33): 124-131.

Kaman, H., Kirda, C., Cetin, M., and Topcu, S., 2006. Salt accumulation in the root zones of tomato and cotton irrigated with partial root-drying technique. Irrig. and Drain. (55): 533- 544.

Kang, S. Z., Shi, P., Pan, Y. H., Liang, Z. S., Hu, X. T., Zhang, J., 2000. Soil water distribution, uniformity and water-use efficiency under alternate furrow irrigation in arid areas. Irrig. Science, (19): 181-190.

Karam, F., Breidy, J., Stephan, C., Rouphael, J., 2003. Evapotranspiration, yield and water use efficiency of drip-irrigated corn in the Bekaa Valley of Lebanon. Agric. Water Manag. 63, 125-137.

Kriedemann, P. E., and Goodwin, I., 2003. Regulated deficit irrigation and partial root-zone drying. An overview of principles and applications. Land \& Water Australia. Product No. PR020382, ISBN 0642760896.

Lamm, F.R., Trooien, T.P., 2003. Subsurface drip irrigation for corn production: a review of 10 years of research in Kansas. Irrig. Sci. 22, 195-200.

Leib, B. G., Caspari, H. W., Andrews, P. K., Redulla, C. A., Jabro, J. D., and Strausz, D., 2004. Deficit Irrigation and Partial Rootzone Drying Compared in Fuji Apples: Fruit Yield, Fruit Quality and Soil Moisture Trends. Written for presentation at the 2004 ASAE/CSAE Annual International Meeting Sponsored by ASAE/CSAE Fairmont Chateau Laurier, The Westin, Government Centre Ottawa, Ontario, Canada, 1 - 4 August 2004, Paper Number: 042284

Loveys, B.R., Stoll, M., Dry, P.R., McCarthy, M., 1998. Partial rootzone drying stimulates stress responses in grapevine to improve water use efficiency while maintaining crop yield and quality. Australian Grape grower and Winemaker, Technical issue (414):108-114.

Mansouri-Far, C., Sanavy, S.A.M.M., Saberali, S.F., 2010. Maize yield response to deficit irrigation during low-sensitive growth stages and nitrogen rate under semi-arid climatic conditions. Agric. Water Manag. 97, 12 22, http://dx.doi.org/10.1016/j.agwat.2009.08.003.

Ruiz-Sanchez, M.C., Domingo, R., and Castel, J. R., 2010. Review. Deficit irrigation in fruit trees and vines in Spain. Spanish Journal of Agricultural Research 8(S2): 5-20.

Samak, A.A. 2012. Soil Moisture, Crop Yield and Soil Salinity Relocation under Partial Rootzone Drying Irrigation. Faculty of Natural Science, university of Hannover, Germany.

Savić, S., Stikić, R., Radović, B. V., Bogičević, B., Jovanović, Z., and Šukalović, V. H., 2008. Comparative effects of regulated deficit irrigation (RDI) and partial root-zone drying (PRD) on growth and cell wall peroxidase activity in tomato fruits. Scientia Horticulturae 117 (2008) 15-20

Sepaskhah, A. R., Hosseini, S. N., 2008. Effects of alternate furrow irrigation and nitrogen application rates on winter wheat (Triticum aestivum L.) yield, water- and nitrogen-use efficiencies. Plant Production Science, (11): 250-259.

Shani-Dashtgol, A., Jaafari, S., Abbasi, N., and Malaki, A., 2006. Effect of alternate furrow irrigation (PRD) on yield quantity and quality of sugarcane in southern farm in Ahvaz. Proceeding of national conference on Irrigation and Drainage Networks Management. Shahid Chamran University of Ahvaz. 2-4 May, Pp: 565-572. [In Farsi].

Sorensen, R.B., Bader, M.J., Wilson, E.H., 2004. Cotton yield and response to nitrogen applied daily through a subsurface drip irrigation system. Appl. Eng. Agric. ASAE 20 (1), 13-16.

Stamp, J., (2003).Partial Rootzone Drying. http://www.winebusiness.com/wbm/?go=getArticle\&dataID=21662 
Stikic, R., Popovic, S., Srdic, M., Savic, D., Jovanovic, Z., Prokic, Lj., and Zdravkovic, J., 2003. Partial root drying (PRD): A new technique for growing plants that saves water and improves the quality of fruit. BULG. J. Plant Physiol., Special ISSUE 2003, 164- 171.

Stoll, M., Loveys, B., Dry, P., 2000. Improving water use efficiency of irrigated horticultural crops. J. Exp. Bot. (51): 1627- 1634.

Van Schilfgaarde, J., 1994. Irrigation a blessing or a curse. Agric. Water Manage. (25): 203- 219.

Vories, E.D.; P.L. Tacker; S.W. Lancaster, and R.E. Glover 2009. Subsurface drip irrigation of corn in the United States Mid-South. Agricultural Water Management 96 (2009) 912-916

Wang, J., Kang, S., Li, F., Zhang, F., Li, Z., Zhang, J., 2008. Effects of alternate partial root-zone irrigation on soil microorganism and maize growth. Plant Soil (302):45-52.

WMO, 2012. Agrometeorlogy of some selected crops. In: Guide to Agricultural Meteorological Practices (GAMP). Chair Publications Board, Geneva, Switzerland, pp. 1-128.

Yi, L., Shenjiao, Y., Shiqing, L., Xinping, C., Fang, C., 2010. Growth and development of maize (Zea mays L.) in response to different field water management practices: resource capture and use efficiency. Agric. For. Meteorol. 150, 606-613, http://dx.doi.org/10.1016/j.agrformet.2010.02.003.

Zegbe, J. A., Behboudian, M. H., and Clothier, B. E., 2004. Partial rootzone drying is a feasible option for irrigating processing tomatoes. Agricultural Water Management, (68): 195- 206.

Zegbe, J.A., Behboudian, M.H., Clothier, B.E., Lang, A., 2008. Postharvest performance of 'Pacific Rose TM' apple grown under partial rootzone drying. Hort. Science 43, 952-954. 


\title{
$\mathrm{NH}_{3}$ Emissions from Treated Buffalo Manure Application in Mediterranean Climate and Comparison to ALFAM Model
}

\author{
Ester Scotto di Perta, Nunzio Fiorentino, Salvatore Faugno, Elena Cervelli, \\ Stefania Pindozzi" \\ Department of Agricultural Sciences, University of Naples Federico II, Via Università 100, Portici (NA), Italy \\ * Corresponding author. Email: stefania.pindozzi@unina.it
}

\begin{abstract}
Ammonia volatilization is widely recognized as one of the major environmental European problems, due to the increase in livestock farming activities. As a consequence, accurate ammonia assessment is needed in order to control ammonia emissions and to update national emission inventories. Besides some uncertainties still related to the measurement methods, another important issue is the necessity of investigating a different kind of fertilizers. In the last few years, considerable attention has been paid to many manure treatments prior to field application. This study aims to assess ammonia emissions from the field application of separated buffalo manure digestate in the Mediterranean climate, in order to improve the emission inventory for this animal species, reared mostly in South Italy. Two measuring methods were used: wind tunnel (WT) and Integrated Horizontal flux (IHF). Moreover, ammonia emission measured were compared to those obtained running the statistical regression model ALFAM. This model based on Michaelis-Menten type equation is often used to predict cumulative ammonia loss and since it is based on a significant dataset is useful to discuss the effectiveness of the emission measured. The total ammonia losses measured in 7 days were 26.39 and $49.24 \mathrm{~kg} \mathrm{~N} \mathrm{ha}^{-1}$, for WT and IHF, respectively. Although the predicted total emissions were 40.99 and $36.56 \mathrm{~kg} \mathrm{~N} \mathrm{ha}^{-1}$, for IHF and WT, respectively, it is possible to observe the good accordance of the ALFAM model with the temporal pattern of both methods.
\end{abstract}

Keywords: Statistical model, open field monitoring, anaerobic digestion, manure application, wind tunnel, Integrated Horizontal flux

\section{Introduction}

Manure treatments have been gaining importance in recent years. In particular, they have been utilized on dairy farms in order to improve the manure management, thanks to economic incentives, increasing the number of animals bred and development of new technologies (Evans et al., 2018). As a consequence, some studies started to investigate the influence of the manure treatment (slurry separation, anaerobic digestion, slurry aeration and straw cover) on greenhouse and $\mathrm{NH}_{3}$ emission after field application (Amon et al., 2006), in order to limit those emissions according to next National Emission Ceilings EU Directive adoption.

Therefore, accurate ammonia assessment is needed in order to control ammonia emissions and to update national emission inventories, but it is necessary to overcome some uncertainties still related to the measurement methods. This study aims to assess ammonia emissions from the field application of separated buffalo manure digestate in the Mediterranean climate, in order to improve the emission inventory for this animal species, reared mostly in South Italy (Infascelli et al., 2010; Pindozzi et al. 2013). Ammonia volatilization measurements were provided using two methods simultaneously: wind tunnel (WT) and Integrated Horizontal flux (IHF). Additionally, ammonia emissions measured were compared to those obtained running the statistical regression model ALFAM (Søgaard, et al. 2002). This is often used to compare ammonia losses estimated with those obtained with experimental trials, in order to discuss the effectiveness of the results (Minoli et al., 2015).

\section{Materials and Methods}

On July 2017 a field trial was carried out in Acerra $\left(40^{\circ} 57^{\prime} 57.5^{\prime \prime} \mathrm{N}, 14^{\circ} 25^{\prime} 34.9^{\prime \prime} \mathrm{E}\right)$, on agricultural soil, in order to measure the ammonia emission, that occurs after the fertilizer application. Climate is Mediterranean according to Emberger's index, while soil is sandy loam texture according to the USDA Textural Classification System. The main chemical soil characteristics are summarized in Table 1. 
Table 53. Main soil characteristics.

\begin{tabular}{ccccccccc}
\hline $\begin{array}{c}\mathrm{pH} \\
(-)\end{array}$ & $\begin{array}{c}\text { Organic C } \\
(\%)\end{array}$ & $\begin{array}{c}\mathrm{TKN} \\
(\%)\end{array}$ & $\begin{array}{c}\mathrm{OM} \\
(\%)\end{array}$ & $\begin{array}{c}\mathrm{CEC} \\
(\mathrm{mS} \mathrm{cm}-1)\end{array}$ & $\begin{array}{c}\mathrm{NO}_{3}-\mathrm{N} \\
(\mathrm{ppm})\end{array}$ & $\begin{array}{c}\mathrm{NH}_{4}-\mathrm{N} \\
(\mathrm{ppm})\end{array}$ & $\begin{array}{c}\mathrm{P}_{2} \mathrm{O}_{5} \\
(\mathrm{ppm})\end{array}$ & $\begin{array}{c}\mathrm{K}_{2} \mathrm{O} \\
(\mathrm{ppm})\end{array}$ \\
\hline 7.55 & 1.61 & 0.19 & 2.77 & 0.23 & 34.21 & 2.92 & 127.13 & 1950.52 \\
\hline
\end{tabular}

A regional weather station $200 \mathrm{~m}$ far from the plot monitored the micrometeorological data during the test. Separated buffalo manure digestate was applied on bare soil using splash plate spreader for the IHF circular plot and by hand on the WT covered surface. The experimental set-up is reported in Table 2 .

Table 54. Experimental set-up.

\begin{tabular}{|c|c|c|c|c|c|c|c|}
\hline \multirow[t]{2}{*}{ Method } & \multirow[t]{2}{*}{ Duration } & \multicolumn{3}{|c|}{ Application } & \multicolumn{3}{|c|}{ Manure properties } \\
\hline & & Technique & $\begin{array}{c}\text { Rate } \\
\left(\mathrm{kg} \text { tot } \mathrm{N} \mathrm{ha}^{-1}\right)\end{array}$ & $\begin{array}{l}\text { Area } \\
\left(\mathrm{m}^{2}\right)\end{array}$ & $\begin{array}{c}\text { TAN } \\
\left(\mathrm{mg} \mathrm{l}^{-1}\right)\end{array}$ & $\begin{array}{l}\mathrm{DM} \\
(\%)\end{array}$ & $\begin{array}{c}\text { TKN } \\
\left(\mathrm{mg} \mathrm{l}^{-1}\right)\end{array}$ \\
\hline IHF & 7 days & Splash plate spreader & 176 & 1256 & 737 & 6.657 & 2214 \\
\hline WT & & Manual & & 0.32 & & & \\
\hline
\end{tabular}

Ammonia losses assessment

Ammonia emission measurements were carried out using a micrometeorological method and a chamber method: Integrated Horizontal Flux method (IHF) by Wood et al. (2000) and Wind tunnel (Scotto di Perta et al., 2016).

Specifically, in the case of Integrated Horizontal Flux method (IHF) by Wood et al. (2000), glass tubes (Schjoerring et al., 1992), previously treated with a solution of oxalic acid and acetone, were employed. These samplers were positioned in a pair at 4 and 3 heights of rotating masts, to catch the main wind direction, placed in the centre of fertilized plot and $80 \mathrm{~m}$ far at the background, respectively. The main principle of the method is to calculate the vertical $\mathrm{NH}_{3}$ flux from the emitting area, equating to horizontal flux measuring ammonia concentration in the air at different heights above the surface.

Wind tunnel (Scotto di Perta et al., 2016), consisting of a chamber placed on a rectangular fertilized surface, that allows to simulate the wind action on the surface, by means of a fan. WT is equipped of two sampling points at the inlet and at the outlet sections. Acid traps were used to trap $\mathrm{NH}_{3}$ volatilized. They were furnished with sulphuric acid solution, flow meter and suction pump. Acid solutions were replaced every 2 hours for the first two days and every three to four hours for the remaining two days. The concentration of $\mathrm{NH}_{3}$ trapped both in oxalic and sulphuric solutions were measured spectrometrically using the FIAstar 5000 system (FOSS, Denmark) in the laboratory.

The ALFAM model

Measured ammonia emissions were compared to the predicted emissions by the ALFAM model (Søgaard, et al. 2002). The model is the result of a statistical analysis of $\mathrm{NH}_{3}$ emissions data, measured in seven European countries (Denmark, Italy, the Netherlands, Norway, Sweden, Switzerland and UK) over the years. At the base of the model there is the kinetic of Michaelis-Menten-type:

$$
\mathrm{N}(\mathrm{t})=\mathrm{N}_{\max } \frac{t}{t+K_{m}}
$$

Where $\mathrm{N}(\mathrm{t})$ is the cumulative $\mathrm{NH}_{3}$ volatilization over time $(\mathrm{t})$; $\mathrm{N}_{\max }$ is the total $\mathrm{NH}_{3}$ loss when time approaches infinity and $\mathrm{K}_{\mathrm{m}}$ is time interval when $\mathrm{N}(\mathrm{t})$ reaches $0.5 \mathrm{~N}_{\max }$.

The cumulative $\mathrm{NH}_{3}$ emission is estimated according to a set of parameters affecting $\mathrm{NH}_{3}$ volatilization, reported in Table 3.

Table 3. Input of ALFAM model.

\begin{tabular}{ccccccc}
\hline Method & $\begin{array}{c}\text { Soil } \\
\text { moisture }\end{array}$ & $\begin{array}{c}\text { Temperature } \\
\left({ }^{\circ} \mathrm{C}\right)\end{array}$ & $\begin{array}{c}\text { Wind } \\
\text { speed }\left(\mathrm{m} \mathrm{s}^{-1}\right)\end{array}$ & $\begin{array}{c}\text { DM } \\
(\%)\end{array}$ & $\begin{array}{c}\text { TKN } \\
\left(\mathrm{g} \mathrm{kg}^{-1}\right)\end{array}$ & $\begin{array}{c}\text { Application Rate } \\
\left(\mathrm{t} \mathrm{ha}^{-1}\right)\end{array}$ \\
\hline IHF & dry & 26.13 & $\begin{array}{c}1.23 \\
0.3\end{array}$ & 6.657 & 0.737 & 79.62 \\
WT & 26 & 0.37 & \\
\hline
\end{tabular}




\section{Statistics}

Ammonia emission rate were evaluated for each method and compared. Linear regression was performed to assess the relationship between the $\mathrm{NH}_{3}$ measured and predict fluxes measured with IHF and WT, respectively. Moreover, RMSE (Fox, 1981), EF (Nash and Sutcliffe, 1970), CRM (Loague and Green, 1991) were also calculated to further investigate the accuracy of linear regression. Specifically, EF contributes to know the efficiency of the model: negative values prove that the mean value of observations is a better predictor than the model; positive values instead show that there is a good agreement between the model and the observed data. The agreement is total if EF is equal to 1. Lastly, CRM indicates the inclination of a method to overestimate (if $<0$ ) or underestimate (if $>0$ ) the $\mathrm{NH}_{3}$ fluxes.

\section{Results and Discussion}

Cumulative ammonia emission curves obtained after 7 days of measurements are shown in Figure 1a. The total ammonia losses were 26.39 and $49.24 \mathrm{~kg} \mathrm{~N} \mathrm{ha}^{-1}$, for WT and IHF method, respectively. The highest ammonia emission rates occurred in the first hours after fertilization and decreased substantially after $24 \mathrm{~h}$. This was due to manure hydrolysis process occurring before application (Ferrara, 2010), usually on the floors of animal house within $24 \mathrm{~h}$ of deposition (Sommer et al., 2013) and for this reason the ammonia emission process proved to be faster.

As may be seen in Figure 1a, ALFAM model curves are in good accordance with the temporal pattern of cumulative ammonia emission curves related to both methods. Nevertheless, modelled losses by ALFAM model were 40.99 and $36.56 \mathrm{~kg} \mathrm{~N} \mathrm{ha}^{-1}$, for IHF and WT, respectively.

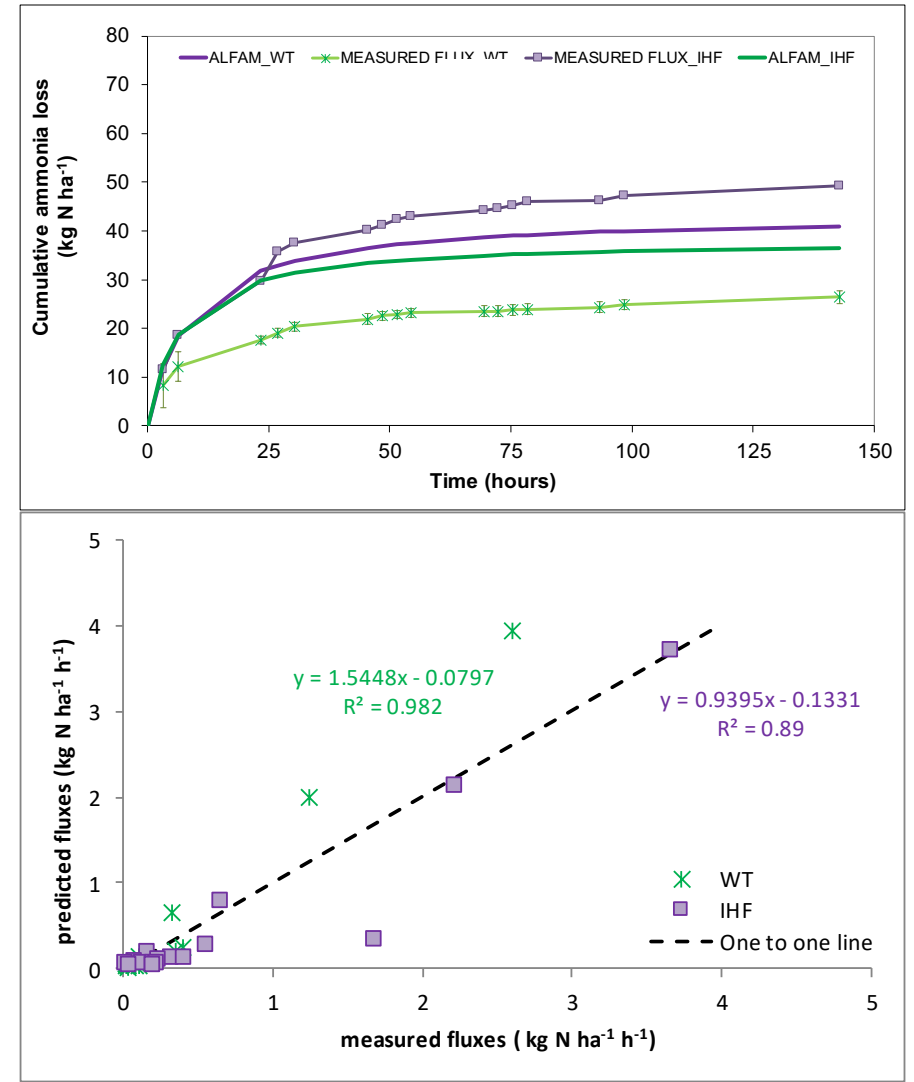

Figure 126. Comparison between $\mathrm{NH}_{3}$ emissions: (a) cumulative measured and predicted cumulative $\mathrm{NH}_{3}$ emissions with IHF and WT; (b) Linear regression between measured and predicted fluxes for each method.

Linear regression was provided between measured and predicted fluxes for each method (Figure 1b), considered more robust than the cumulative emissions, since there is a serial correlation between the successive cumulative emission values (Sogaard et al., 2002; Misselbrook et al., 2005). As it is possible to notice the correlation between measured and predicted values are good, especially for the WT $\left(\mathrm{R}^{2}=0.982\right)$. The prediction of ALFAM fitted well the measured IHF fluxes in the first $24 \mathrm{~h}$, after this time the model seems to underestimate the process, as it is also confirmed by the CRM $=0.275$ (Table 4). In the same time, ALFAM model seemed to over predict the ammonia loss related to the wind tunnel (Figure 1b). similar result is provided by CRM $=-0.324$ (Table 4). 
Table 4. Statistical indexes: coefficient of determination (R2), relative root mean square error (RRMSE), model efficiency (E), coefficient of residual mass (CRM).

\begin{tabular}{lcccc}
\hline & RMSE & RRMSE & EF & CRM \\
\hline ALFAM_WT vs measured_WT & 0.395741 & 109.743 & 0.627 & -0.324 \\
ALFAM_IHF vs measured_IHF & 0.370380 & 54.664 & 0.855 & 0.257 \\
\hline
\end{tabular}

These differences are probably explained with the characteristics of fertilizer used, that is a liquid fraction of digested buffalo manure. On the contrary, ALFAM model derived from the statistical analysis of measured fluxes related to cattle and pig slurry. Generally, ALFAM model proved to be a good tool for discussing the effectiveness of the results, but it may need additional testing to better simulate the emissions from treated manure.

\section{Conclusions}

The total ammonia losses measured in 7 days were 26.39 and $49.24 \mathrm{~kg} \mathrm{~N} \mathrm{ha}^{-1}$, for WT and IHF, respectively. Although the predicted total emissions were 40.99 and $36.56 \mathrm{~kg} \mathrm{~N} \mathrm{ha}^{-1}$, for IHF and WT, respectively, it is possible to observe the good accordance of the ALFAM model with the temporal pattern of both methods, nevertheless more studies will be needed to adapt ALFAM to treated manure.

\section{Acknowledgements}

This research was realized under the Project "Riduzione delle emissioni di Ammoniaca e Gestione delle Risorse Idriche nella piana del Sele (RiAGRI-Sele)" funded by Rural Development Program for 2014 - 2020 of Campania Region. The authors would like to thank Dr. Sabrina Nocerino, Filomena Carannante and Agraria Student Association for their help with data collection during the experiments and Dr. Roberto Maiello for his technical support in laboratory analyses.

\section{References}

Amon, B., V. Kryvoruchko, T. Amon and S. Zechmeister-Boltenstern, 2006. Methane, nitrous oxide and ammonia emissions during storage and after application of dairy cattle slurry and influence of slurry treatment. Agriculture, ecosystems \& environment, 112(2-3), 153-162.

Evans, L., A. C. VanderZaag V. Sokolov, H. Baldé, D. MacDonald, C. Wagner-Riddle, R. Gordon, 2018. Ammonia emissions from the field application of liquid dairy manure after anaerobic digestion or mechanical separation in Ontario, Canada. Agricultural and Forest Meteorology. In press (https://doi.org/10.1016/i.agrformet.2018.02.017)

Ferrara, R.M., 2010. Temporal dynamics of ammonia volatilization from agricultural land: on micrometeorological measurements of slurry and urea. Italian Journal of Agrometeorology, 15(2), 15-24.

Fox, D.G., 1981. Judging air quality model performance. Bulletin of the American Meteorological Society, 62(5), 599-609.

Infascelli, R., S. Faugno, S. Pindozzi, R. Pelorosso, L. Boccia, 2010. The environmental impact of buffalo manure in areas specialized in mozzarella production, southern Italy. Geospatial health, 5(1), 131-137.

Loague, K., R.E. Green, 1991. Statistical and graphical methods for evaluating solute transport models: overview and application. Journal of contaminant hydrology, 7(1-2), 51-73.

Minoli, S., M. Acutis, M. Carozzi, 2015. NH3 emissions from land application of manures and N-fertilisers: a review of the Italian literature. ITALIAN JOURNAL OF AGROMETEOROLOGY - RIVISTA ITALIANA DI AGROMETEOROLOGIA, 20(3), 5-24.

Misselbrook, T.H., F.A. Nicholson, B.J. Chambers, 2005. Predicting ammonia losses following the application of livestock manure to land. Bioresource Technology, 96(2), 159-168.

Nash, J.E., J.V. Sutcliffe, 1970. River flow forecasting through conceptual models part I-A discussion of principles. Journal of hydrology, 10(3), 282-290.

Pindozzi, S., S. Faugno, C. Okello L. Boccia, 2013. Measurement and prediction of buffalo manure evaporation in the farmyard to improve farm management. Biosystems engineering, 115(2), 117-124.

Schjoerring, J.K., S.G., Sommer, M. Ferm, 1992. A simple passive sampler for measuring ammonia emission in the field. Water, air, and soil Pollution, 62(1-2), 13-24.

Scotto di Perta, E., M. A. Agizza, G. Sorrentino, L. Boccia, S. Pindozzi, 2016. Study of aerodynamic performances of different wind tunnel configurations and air inlet velocities, using computational fluid dynamics (CFD). Computers and Electronics in Agriculture, 125(C), 137-148. 
Søgaard, H.T., S.G. Sommer, N.J Hutchings, J.F.M. Huijsmans, D.W. Bussink, F. Nicholson, 2002. Ammonia volatilization from field-applied animal slurry-the ALFAM model. Atmospheric Environment, 36(20), 33093319.

Sommer, S. G., M. L. Christensen, T. Schmidt, L. S. Jensen, 2013. Animal manure recycling: Treatment and management. John Wiley \& Sons. 364 p.

Wood, C.W., S.B. Marshall, M.L. Cabrera, 2000. Improved method for field-scale measurement of ammonia volatilization. Communications in Soil Science \& Plant Analysis, 31(5-6), 581-590. 


\title{
Novel In Situ System for Monitoring Soil Organic Carbon by Using Mobile Vis-NIR Spectroscopy and Machine Learning Techniques
}

\author{
Nikolaos Tziolas ${ }^{a, *}$, Nikolaos Tsakiridis ${ }^{\text {b }}$, Eyal Ben-Dor ${ }^{c}$, Eleni Kalopesa ${ }^{d}$, George Galanis ${ }^{\text {, }}$, George \\ Zalidis ${ }^{\text {a, }}$, \\ ${ }^{a}$ Laboratory of Remote Sensing, Spectroscopy and GIS, Faculty of Agriculture, Forestry, and Natural Environment, \\ Aristotle University of Thessaloniki, 54123, Greece \\ ${ }^{\mathrm{b}}$ Department of Electrical and Computer Engineering, Faculty of Engineering, Aristotle University of Thessaloniki, \\ 54123, Greece \\ ${ }^{c}$ The Remote Sensing and GIS laboratory Department of Geography, School of Earth Science, Tel-Aviv University, \\ 39040, Israel \\ d Interbalkan Environment Center, 18 Loutron Str., Lagadas, 51200, Greece \\ * Corresponding author. Email: ntziolas@agro.auth.gr
}

\begin{abstract}
Recognizing the importance of Soil Organic Carbon (SOC) for effectively reorienting and transforming agricultural systems towards the perspective of climate smart agriculture, it is essential to perform in situ measurements in a novel, efficient, and cost effective way. The objective of the present study is to introduce a Mobile Proximal Soil Sensing System (MPS 3 ) allowing in situ vis-NIR measurements independent of illumination and soil roughness restrictions. The $\mathrm{MPS}_{3}$ towed by a tractor provides some advantages over conventional backpacker and soil penetrator systems such as the fast and high power data acquisition in a near-real time domain. The current study focused on integrating in situ measurements with machine learning algorithms towards delivering quantitative and spatially distributed estimates of SOC to support the implementation of informed decision making in the agro-environmental sector. Specifically, a set of linear and non-linear machine learning algorithms namely the partial least squares regression (PLSR), Cubist, and Support Vector Machines (SVM) are utilized with leave one-out cross-validation in order to establish spectroscopic calibration models of SOC. A detailed in-situ dataset (100 soil samples coupled with in situ spectral and chemical laboratory measurements) was acquired in an experimental layout in Greece to test the performance of the MPS 3 under open field conditions. The results reached coefficients of determination values $\left(\mathrm{R}^{2}\right)$ of 0.68 and a ratio of performance to inter-quartile range (RPIQ) of 1.97. In this context, this study proves that the $\mathrm{MPS}_{3}$ is a rapid and cost effective tool for precision agriculture applications and effectively monitoring of SOC stocks for accounting purposes. Further research should consider the improvement of signal processing, reconstruction techniques to address the effects of soil moisture, and the synergetic use of field acquired spectra with existing soil spectral libraries by employing spiking techniques.
\end{abstract}

Keywords: Mobile Proximal Sensing, vis-NIR Spectroscopy, Machine Learning, In Situ Mapping, Support Vector Machine

\section{Introduction}

Agricultural soils carry out a large potential for additional carbon sequestration, which is essential to increase resilience against climate change and also to improve agricultural productivity. Targeting interventions in agricultural management practices that would not only preserve but potentially enhance soil organic carbon (SOC) requires reliable, accurate, and timely key soil information.

Visible and near-infrared (vis-NIR) diffuse reflectance spectroscopy has emerged in the past decades as an efficient method for the estimation of physical and chemical soil properties (Viscarra Rossel et al., 2016). Among the most studied and well predicted soil properties is SOC. One of the advantages of this technique is that it allows for in situ non-mobile and mobile measurements with significant soil sampling resolution, enabling the deduction of highly detailed soil maps. Thus, mobile proximal soil sensing (PSS) integrated with data mining techniques allows us to perform soil surveys in a far more efficient and cost effective manner compared to conventional laboratory analysis and backpacker systems. In this line, several mobile PSS systems have been developed using diffuse reflectance spectroscopy at 350-2500 wavelength range (Viscarra Rossel et al., 2011). Despite the progress achieved in the development of platforms and multivariate data mining techniques the collection of highresolution information about key soil properties enabling precision agriculture and other environmental applications remains a challenging task. Whereas in laboratory spectral measurements are done under controlled conditions using precise protocols (Ben Dor et al., 2015), mobile spectral acquisitions are affected by a number of factors like the ambient light conditions, the soil moisture and structure, presence of residue and litter, etc. To address these challenges, it is important to establish a precise measuring protocol using configurations that overcome them, exploit effective spectral pre-treatments, and use efficient data mining algorithms. What is more, recent advances in manufacturing and miniaturization (Roy et al., 2015) constitute this technology as a 
complement to vehicle mounted sensor systems for surveying soil.

Our objectives here are to describe the development process of the Mobile Proximal Soil Sensing System (MPS 3 ), its configuration (including sensors, lighting equipment, and other components), and the spectra data analyses and modelling used to perform on-the-go vis-NIR measurements and predict SOC in outdoor terrain conditions.

\section{Materials and Methods}

The MPS 3 semi-automatically estimates biochemical and physical properties of soil surface under real field condition. In this section, we describe the platform, its sensors, and the analyses and modelling of the spectroscopic data to derive predictions of SOC.

Mobile soil sensing platform

The core of the MPS 3 consists of a mobile data acquisition system ("dark box"). The dark box is a closed chamber that keeps out natural sunlight enabling vis-NIR spectral data acquisition under stable ambient light conditions, as indicated in the recent studies of Ben Dor et al. (2015) and Rodionov et al. (2015). The testing and validation of the quantity of incident light, geometrical positioning of the spectral head and fibre optic, and potential limitations due to surface properties and dust have been carried out in a series of iterative test pilots, providing feedback towards their refinement and optimisation. In this context, a complementary set of technological components have been utilized in order to optimize the spectra acquisition. The dark box unit includes three halogen lamps (12V and 20W) to ensure the illumination of the soil surface at constant intensity. The lamps are adjusted at a height of $40 \mathrm{~cm}$ above the targeted soil surface from the nadir position. The reflected light from the soil surface is recorded via a field portable spectroradiometer (Spectral Evolution PSR +3500 ) operating at the range of $350-2500 \mathrm{~nm}$ with an enhanced spectral resolution of $3 \mathrm{~nm}, 8 \mathrm{~nm}$ and $6 \mathrm{~nm}$ at around 700 $\mathrm{nm}, 1500 \mathrm{~nm}$ and $2100 \mathrm{~nm}$, respectively. The spectroradiometer is equipped with a customized cooling system as well as a long duration battery ( $7200 \mathrm{mAH})$ to ensure constant operation under open field conditions. A spectral head is utilized to measure from a $45^{\circ}$ incidence angle, covering a field of view (FOV) of $10 \mathrm{~cm}$ diameter of the sample surface, while it is connected with the spectroradiometer with a fibre optic cable (standard length of $2 \mathrm{~m}$ ). During operation an air compressor makes one spraying every 1 minute in order to account for the effects of dust on mobile proximally sensed spectra. The air nozzle is adjusted to a $90^{\circ}$ incidence angle and at a distance of $5 \mathrm{~cm}$ of the fibre optic. Supply of a constant current for the illumination and air pump is ensured by a standard generator $(12 \mathrm{~V}, 90 \mathrm{~A})$, powered by a standard tractor battery $(12 \mathrm{~V}, 55 \mathrm{~A} \mathrm{~h})$. Moreover, a triangular shape soil flattening unit is placed in front of the platform in order to eliminate the soil roughness, set aside plant residues and other litter, and enable soil surface measurements at $0-2 \mathrm{~cm}$. Finally, the recorded data are transferred to an internal storage system (PS336 Getac equipped with a GPS receiver) with the appropriate metadata for each spectrum (coordinates, date, time). The MPS 3 as finally utilized for the field tests in the current research is illustrated in Figure 127.
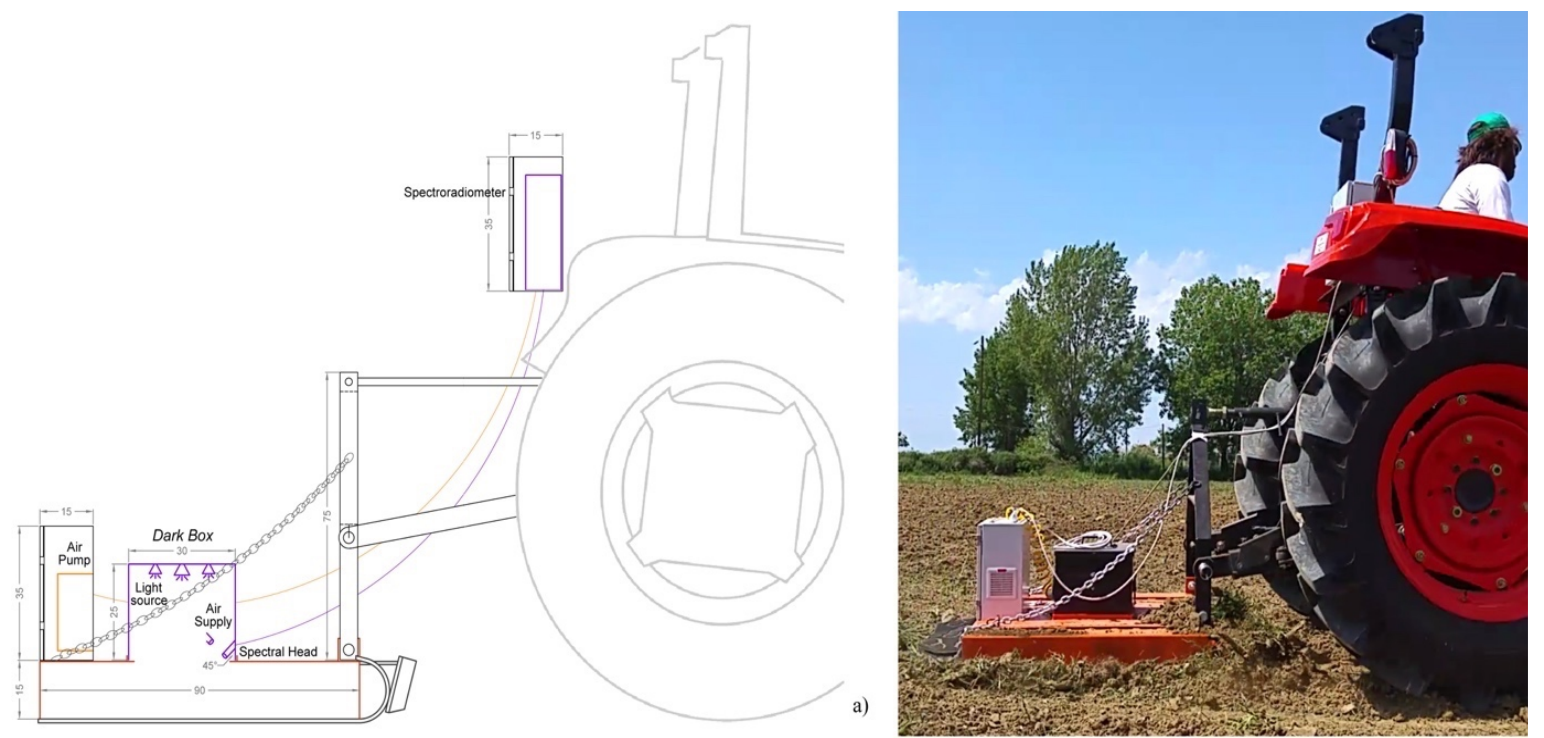

Figure 127. a) Schematic of the $\mathrm{MPS}_{3}$ in lateral view (dimensions in $\mathrm{cm}$ ) and b) the MPS 3 at a field site during soil scanning operation. 


\section{Description of the experimental site}

The capabilities of the MPS 3 were tested and improved on an experimental site ( $0,1 \mathrm{ha})$ in northern Greece, and more concretely in the Farm of Aristotle University of Thessaloniki, School of Agriculture (Thessaloniki, $40^{\circ} 53^{\prime} 58.00^{\prime} \mathrm{N}$ and $22^{\circ} 98^{\prime} 71.20^{\prime}$ ). Soil moisture content was relatively low at the time when the spectral measurements were acquired, and was not significantly varied within the experimental site.

We aimed to build a detailed and variable (with respect to the in-field variability) dataset comprised of surface soil spectral signatures and the corresponding SOC measurement for each recording. In this line, the experimental field was divided into 16 blocks $(5$ by $5 \mathrm{~m}$ ) in which variable rates of organic manure (class A: no application, class B: $0-15 \mathrm{~kg}$ and class $\mathrm{C}: 15-30 \mathrm{~kg}$ ) and chemical fertilizers (class $\mathrm{d}: 2.5 \mathrm{~kg}$ ) were applied.

After the $\mathrm{MPS}_{3}$ had navigated through the extent of the experimental field, we extracted the exact coordinates of the soil spectral signatures captured during the operation. Then, 100 physical soil samples were collected in sealable plastic bags and the Walkley-Black (Heanes, 1984) method was followed for the determination of soil organic carbon $(\% \mathrm{SOC})$.

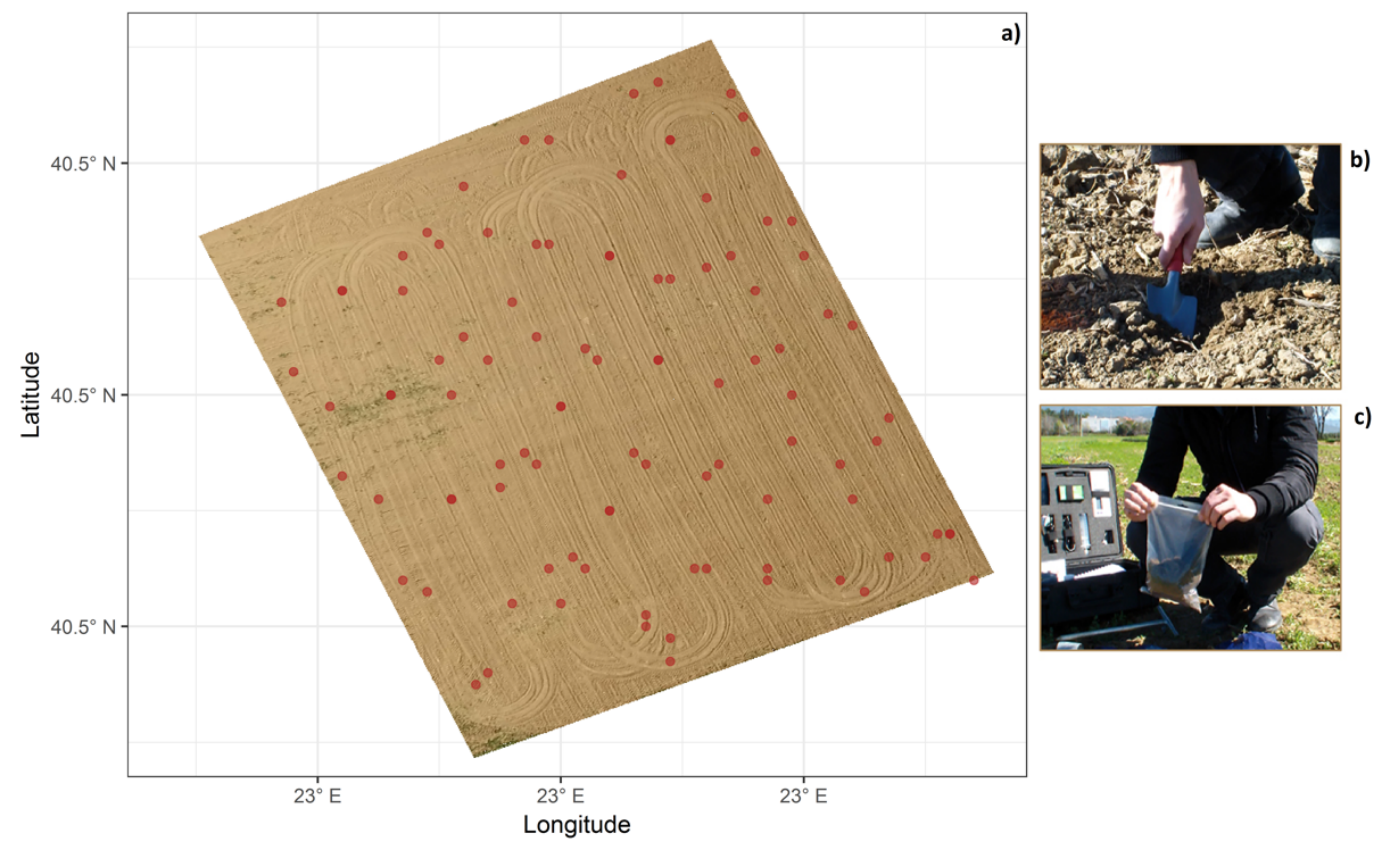

Figure 128. a) The experimental site in Thessaloniki. Red dots in the map indicate the exact position of the surface soil spectral measurements with $\mathrm{MPS}_{3}$. Development of soil spectra dataset: soil samples b) were collected around the point scanned by MPS 3 , and c) were sent to the chemically laboratory for SOC assessment.

\section{Data analysis and modelling}

Advanced data handling and processing techniques are required to fully exploit raw data and generate tailored products and services. The literature on machine learning algorithms revealed several methods of regression analysis and of pre-processing techniques (Nocita et al., 2014; Ramirez-Lopez et al., 2013; Tsakiridis et al., 2017a). The pre-processing techniques are exploited to eliminate effects such as increased scattering due to the presence of quartz, and to enhance the absorption bands so that the machine learning algorithms can more accurately identify them. On the other hand, depending on the task at hand some machine learning algorithms can perform better than others by e.g. addressing non-linearities or performing more efficient feature selection. Prior the analysis, the spectral signatures were limited to $400-2450 \mathrm{~nm}$ in order to eliminate large noise on both edges.

With the goal of comparing the results of state of the art algorithms we selected the most common machine learning algorithms in the literature applied in soil spectroscopy, namely partial least square regression (PLSR), support vector machines for regression (SVM), and regression trees (Cubist). PLSR establishes a linear regression model on a projected space, created through the use of a number of orthogonal factors (latent variables) which maximize the covariance between predictor and response variables. SVM for regression employs a kernel function to map the features into a higher dimensional space and solves an optimization problem subject to some special conditions which reduce the complexity of the training data to a significant subset thereof termed as support vectors. Cubist establishes a decision tree where each node can be reduced to a single rule which has a consequent a linear regression model; Cubist is also able to enhance the prediction through the use of committees (where additional trees using boosting are created), and by employing a correction mechanism on its predictions. The 
algorithms were implemented in the R software (R Development Core Team, 2013) utilizing the caret (Kuhn, 2008) package.

Concerning the pre-processing techniques, the following methods were considered in the present study: (i) the pseudo-absorbance transformation, (ii) smoothing the absorbance spectra using a Savitzky-Golay filter (SG0), (iii) the continuum removal, (iv) de-trending, (v) first-derivative transformation, (vi) the standard normal variate (SNV), (vii) smoothing and SNV (SG0 + SNV), (viii) first-derivative and SNV (Rinnan et al., 2009).

Granted that the number of samples was relatively low (100), the use of leave-one-out cross-validation was preferred (Martens and Dardenne, 1998). For each of these folds an internal 5 -fold cross-validation procedure was used for the hyper-parameter estimation of the respective models.

Model accuracy

The performance of the models was evaluated using the following metrics: the coefficient of determination $\left(R^{2}\right)$, root mean squared error $(R M S E)$, ratio of performance to inter-quartile range ( $R P I Q$, Bellon-Maurel et al., 2010), and bias (Equations (1)-(4)):

$$
\begin{aligned}
& R^{2}=1-\frac{\sum_{i=1}^{n}\left(y_{i}-y_{i}\right)^{2}}{\sum_{i=1}^{n}\left(y_{i}-y_{i}\right)^{2}} \\
& R M S E=\sqrt{\frac{1}{n} \sum_{i=1}^{n}\left(y_{i}-y_{i}\right)^{2}} \\
& \text { bias }=\sum_{i=1}^{n} \frac{\left(y_{i}-y_{i}\right)}{n} \\
& R P I Q=\frac{I Q}{R M S E}
\end{aligned}
$$

where: $y_{i}$ and $y_{i}$ are the measured and predicted values of sample $\mathrm{i}$, respectively, and $\mathrm{n}$ is the number of samples, and $I Q$ is the interquartile range $(I Q=\mathrm{Q} 3-\mathrm{Q} 1)$ of the observed values.

Spatially explicit indicator preparation

Hengl et al. (2018) indicated in a recent study that machine learning models, such as random forest, can be utilized to efficiently map soil properties. A similar approach is adopted in the framework of the current study instead of the common Inverse Distance Weighing method as proposed by other studies (Kodaira and Shibusawa, 2013).

\section{Results and Discussion}

Comparison of spectra under different configurations and operations

To perform a comparison of the acquired spectra under different configurations and operations we performed a series of measurements, taking care to measure at the exact same spots each time so that the acquired spectra are from roughly the same sampling point. The spectra were then visualized graphically as curves as well as in the Principal Component (PC) space using the first 2 PCs which capture the largest variance of the underlying data.

The effect of the flattening unit placed in front of the platform is depicted in Figure 129. It can be seen that the spectra acquired with the use of the flattening unit are less noisy and distorted than the ones without. The plot on the PC space further reveals that the spectra from these two configurations are quite dissimilar. This is attributed to the fact that the flattening unit has a dual purpose: (i) to account for the rough soil surface that is usually present in situ and thus provide a flattened soil surface mitigating the effects of increased scattering due to the mixed distance between the sensor and the surface, and (ii) to set aside litter which can taint the spectral signature of the bare soil. It should be noted that the flattened unit as utilized in the current study significantly eliminate (around 5 times lower) the contact pressure during the scanning operation comparing to drum rollers $\left(25 \mathrm{kN} \mathrm{m}^{-2}\right)$ as indicated in a recent study (Rodionov et al. 2015). 

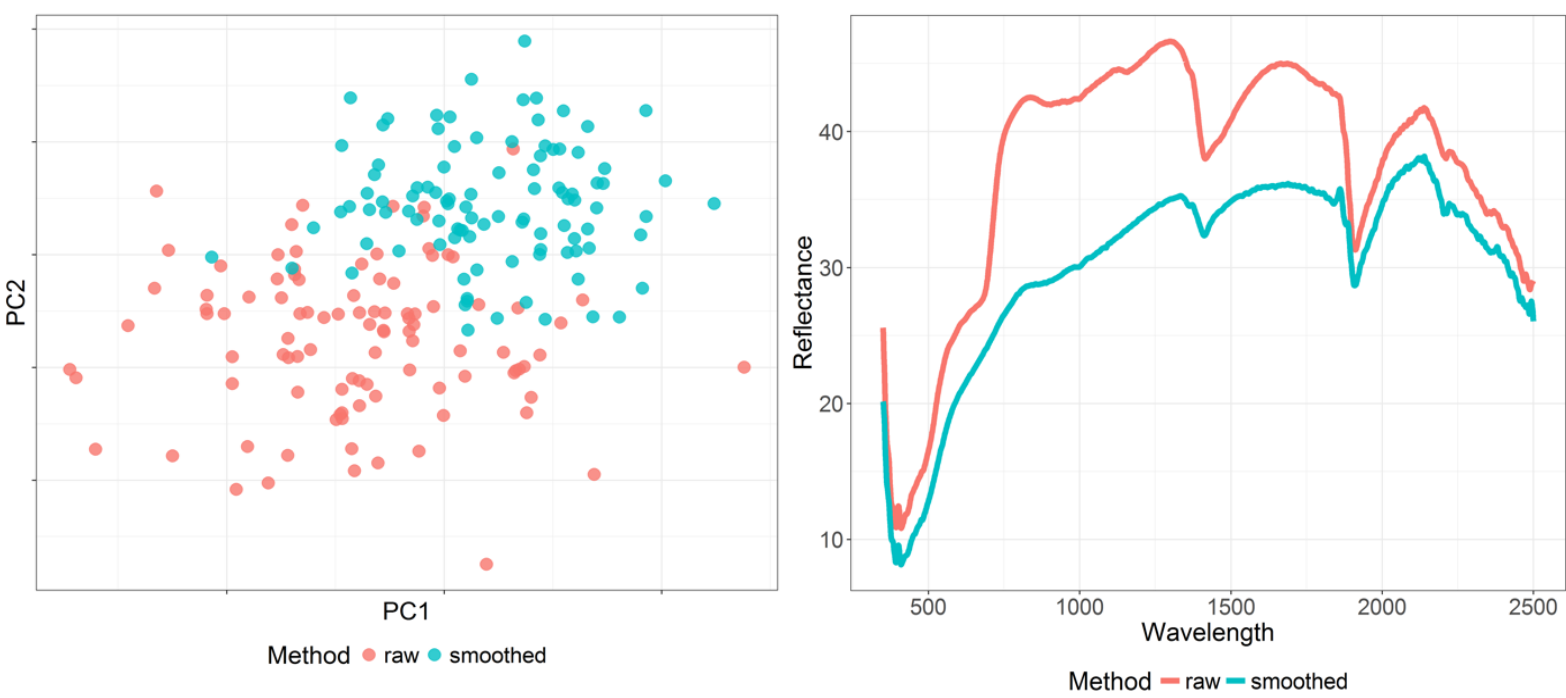

Figure 129. Comparison of samples and acquired spectra of the same point without (raw) and with (smoothed) the use of the soil flattening unit.

Having established that the use of the flattening unit is preferred, the analysis continued with the potential adverse effect the acquisition at a continuous movement might have. To this end, we compared the spectra acquired with the chamber being stationary, i.e. with the tractor stopping at each measurement point (termed intermittent), and with the tractor acquiring the spectra continuously (i.e. without stopping, with the tractor moving at about $4 \mathrm{~km} \mathrm{~h}^{-1}$ ). With regards to measurement speed, the second approach is evidently more favorable and can cover approximately twice the area at the same speed (roughly 12 measurements per minute versus 6 measurements per minute). If the acquired spectra are similar, then the second approach would be more advantageous. The results of the analysis of the spectra are given in Figure 13, and indicate that the acquired spectra are similar. In the PC space, the spectral signatures are close and the sampling points can even be identified. The spectra using the intermittent approach tend to have a slightly larger reflectance, which can be attributed to the more stable lighting conditions in this approach, and the presence of dust which accrues during the movement of the chamber in the continuous approach which diffuses the light.
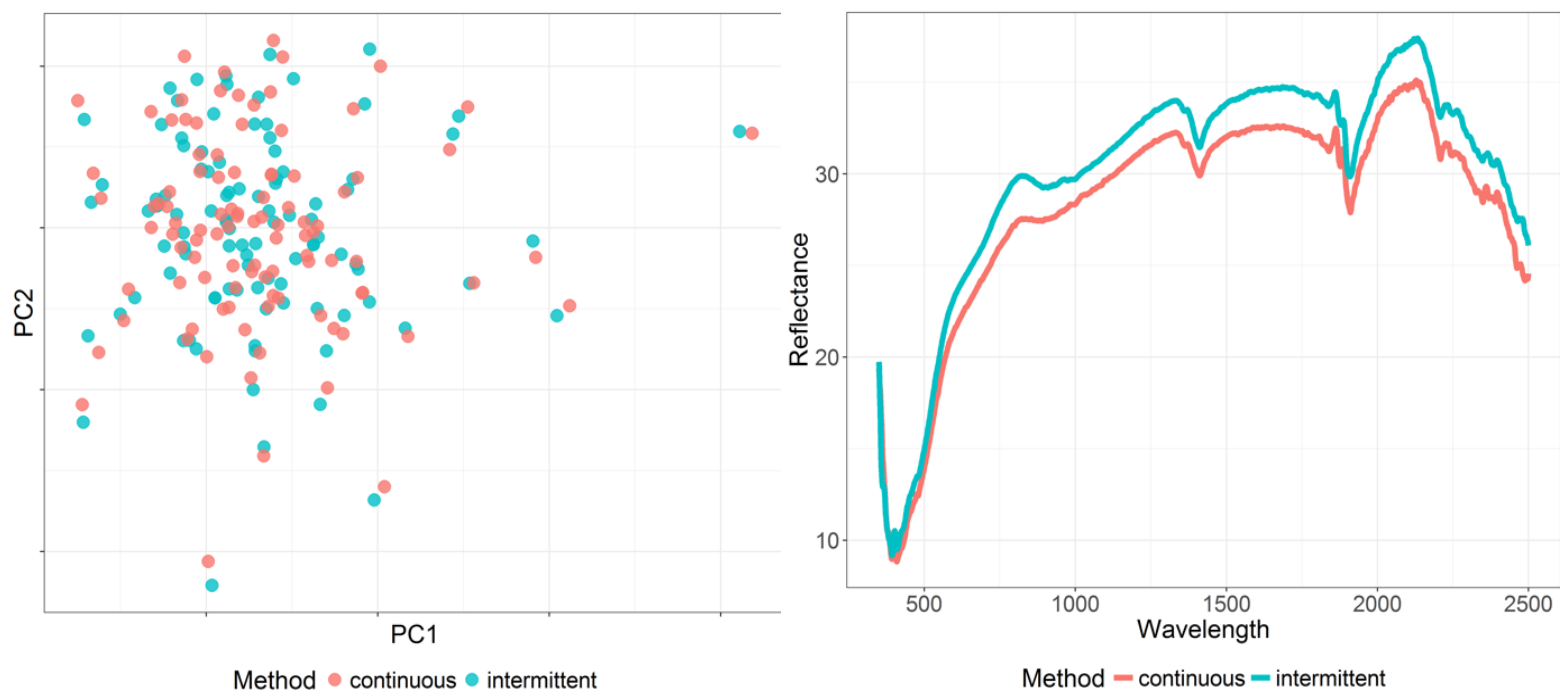

Figure 130. Comparison of acquired spectra between continuous and intermittent approach (a) on the PC space on all sampling points, (b) from one single sampling point.

Results of SOC chemical analysis

In the experimental field where MPS 3 was tested, the Walkley-Black analysis of the collected samples revealed that the variable treatment of organic manure and fertilizers applied to it resulted in good within field variability (Table 55). The availability of a reference and variable analytical dataset from the experimental site allows us the further development of multivariate calibration models. 
Table 55. Statistical summary of the SOC data used in spectroscopic modelling.

\begin{tabular}{ccccccc}
\hline & Min & Max & Mean & Median & SD & skew \\
\hline SOC $(\%)$ & 0.42 & 1.77 & 0.90 & 0.91 & 0.27 & 0.67 \\
\hline
\end{tabular}

Results of the machine learning algorithms

In this section, we present the assessment statistic values obtained by the machine learning algorithms for both intermittent and continuous modes of operation. For brevity, we present only the best models from each of the machine learning algorithms examined (Table 56).

Table 56. Results of the best models per machine learning algorithm and for the two modes of operation considered.

\begin{tabular}{|c|c|c|c|c|c|c|}
\hline & \multicolumn{3}{|c|}{ Intermittent } & \multicolumn{3}{|c|}{ Continuous } \\
\hline & Cubist & PLSR & SVM & Cubist & PLSR & SVM \\
\hline Best source & SG0 & SG0 & SG0 + SNV & SG0 & SG0 & SG0 + SNV \\
\hline RMSE & 0.16 & 0.15 & 0.16 & 0.16 & 0.16 & 0.16 \\
\hline $\mathrm{R}^{2}$ & 0.66 & 0.68 & 0.66 & 0.64 & 0.65 & 0.64 \\
\hline RPIQ & 1.94 & 1.97 & 1.93 & 1.86 & 1.89 & 1.87 \\
\hline Bias & 0.03 & 0.01 & -0.01 & 0.02 & 0.00 & -0.01 \\
\hline
\end{tabular}

According to the assessment statistics, PLS using the absorbances and simple smoothing using a zero-ordered Savitzky-Golay filter attained the best results for both approaches. More elaborate pre-processing techniques such as the first-derivative transformation, which commonly produces good results on large SSLs achieved lower performances. This can be explained by the fact that this local dataset is comprised of spectra from the same soil type, and therefore scatter correction techniques which normally account for the presence of quartz which increases scattering are not so relevant in a local dataset (from one agricultural field).

The two modes of operation not only produce similar spectra as evidenced by Figure 13, but the best models on both modes have attained similar performances. In an effort to present how similar these predictions are, Figure 131 illustrates in a scatter plot the correlation among them. From both the accuracy measures (low Bias, good RPIQ values) and the scatter plot we can conclude that the continuous mode of operation is comparable to the intermittent mode.

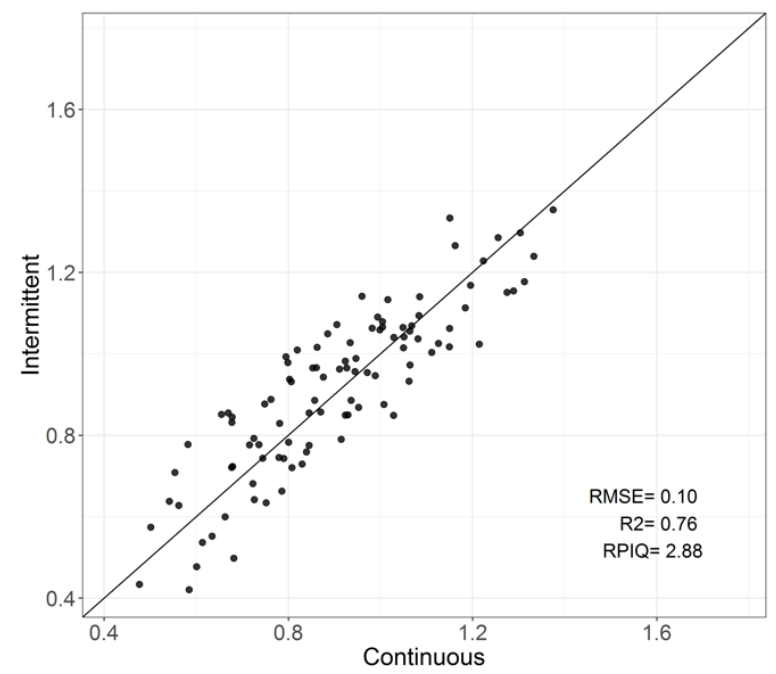

Figure 131. Comparison of predictions of the continuous vs the intermittent mode of operation from the best models.

\section{Soil property mapping}

High-resolution spatially explicit indicators of SOC for the experimental layout are illustrated in Figure 132. The spatial distribution of SOC measured in the laboratory and predicted by the best multivariate calibration model indicated partial similarity at most areas of the experimental field. Furthrmore, the similarity that we observed is valid not only in terms of spatial variability but also at the range of the predicted values, altohufh the estimated range was little smaller $(<5 \%)$. 

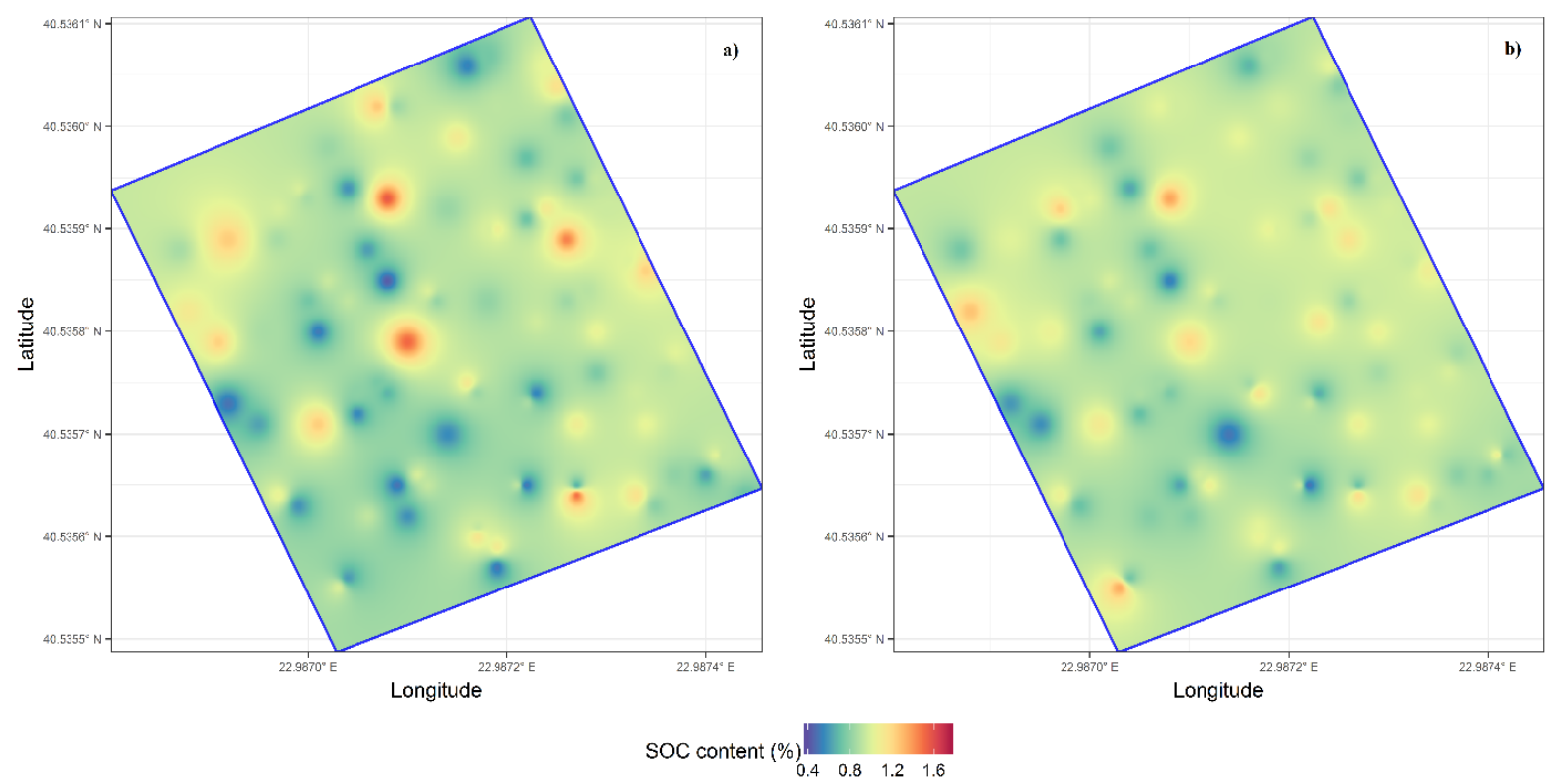

Figure 132. High-resolution spatial explicit indicators of SOC; (a) The measured maps were generated using the data from soil chemical analyses and (b) the predicted maps were drawn using spectral data as captured during the intermittent mode of operation and applied the best model.

Overview and future considerations

With increasingly limited land available for agricultural activities, more intensive food production is resulting in a heightened environment impact. In this line, the MPS 3 could be a valuable tool for several agro-environmental applications, such as determination of soil constraints, adoption of best agronomic practices, assessment of soil degradation (loss of SOC) as well as the development of SOC trading methodologies (England and Rossel, 2018) and site specific soil management strategies. The incorporation of data driven systems, such as MPS3, into agricultural production, are becoming the go-to solution to push towards precision agriculture, since they can provide significant benefits in farmer's working conditions across a series of works on the farm (e.g. specific variable rate fertilization, less labor intensive seeding etc.). It should be mentioned that these systems are hesitantly finding their way to a market for the provision of agricultural information services (Wetterlind J. et al., 2015). Last but not least, forward thinking policy makers are already transitioning their operations to the employment of soil sensors in order to transform many aspects of decision making such as soil carbon accounting (Australian Government, 2018) or monitoring and reporting progress towards SDG target 15.3.1 "Proportion of land that is degraded over total land area".

In the light of the above, it is clearly illustrated that the inherited spatial and temporal variation of soil properties, is critical for determining evidence driven strategies. The conventional backpacker scanning methods of soil analyses are time consuming and allow only limited sampling resolution which is not sufficient to explore within-field variation in soil properties. Proximal soil sensing systems, such as MPS 3 and/or other similar (Rodionov et al., 2015) allow us to undertake soil surveys in a far more efficient manner. Moreover, the continuous approach presented in the current study is proved to be a more favourable method as it is twice as fast and produces similar results, with the intermittent approach or soil penetrator systems (Kodaira and Shibusawa, 2013; Mouazen et al., 2007). Overall, the MPS 3 paves the way to offset costs in human labour, maintenance and upkeep, and its significant performance using the continuous approach reduces the energy requirements.

In previous studies we have shown that Soil Spectra Libraries (SSLs) can be utilized for both qualitative and quantitative analyses, via the development of robust spectroscopic models (Tsakiridis et al., 2017b). This allowed us to investigate the synergistic use of in situ data collection systems and large SSLs to produce comparative quantitative assessments and corresponding mapping over large geographical zones. Future work should deal with gaps and hindrances not covered in this study. In this line, spiking methods could be utilized as a method to improve models' applicability (Kühnel and Bogner, 2017) or the research may focus on techniques for removal of unwanted background effect such as soil moisture (Franceschini et al., 2018).

\section{Conclusions}

Mapping SOC at a high spatial resolution allows for the realisation of a mobile soil spectroscopic sensor derived service that can be easily integrated into daily farming practice, to underpin SOC accounting methodologies or be in line with cross compliance rules as applied in a series of EU strategies. The results showed 
that state of the art machine learning models can be used to develop multivariate calibration models able to fairly estimate SOC and produce relevant maps. The timeliness of the system's measurements were improved by introducing a soil flattening tool that enables the data capturing two times faster than the intermittent approach.

\section{Acknowledgements}

This study is based on the work that has been conducted in the framework of the GEO-CRADLE project, which has received funding from the European Union's Horizon 2020 research and innovation programme under grant agreement No 690133

\section{References}

Australian Government: Carbon Credits (Carbon Farming Initiative - Measurement of Soil Carbon Sequestration in Agricultural Systems) Methodology Determination 2018, https://www. legislation.gov.au/Details/F2018L00089 (last access: 14 June 2018), 2018.

Bellon-Maurel, V., Fernandez-Ahumada, E., Palagos, B., Roger, J.-M., McBratney, A., 2010. Critical review of chemometric indicators commonly used for assessing the quality of the prediction of soil attributes by NIR spectroscopy. TrAC Trends Anal. Chem. 29, 1073-1081. doi:https://doi.org/10.1016/j.trac.2010.05.006

Ben Dor, E., Ong, C., Lau, I.C., 2015. Reflectance measurements of soils in the laboratory: Standards and protocols. Geoderma 245-246, 112-124. doi:10.1016/j.geoderma.2015.01.002

England, J.R., Rossel, R.A. V, 2018. Proximal sensing for soil carbon accounting. SOIL 4, 101-122. doi:10.5194/soil-4-101-2018

Franceschini, M.H.D., Demattê, J.A.M., Kooistra, L., Bartholomeus, H., Rizzo, R., Fongaro, C.T., Molin, J.P., 2018. Effects of external factors on soil reflectance measured on-the-go and assessment of potential spectral correction through orthogonalisation and standardisation procedures. Soil Tillage Res. 177, 19-36. doi:10.1016/j.still.2017.10.004

Heanes, D.L., 1984. Determination of total organic-C in soils by an improved chromic acid digestion and spectrophotometric procedure. Commun. Soil Sci. Plant Anal. 15, 1191-1213. doi:10.1080/00103628409367551

Hengl, T., Nussbaum, M., Wright, M., Heuvelink, G., Graeler, B., 2018. Random Forest as a generic framework for predictive modeling of spatial and spatio-temporal variables. doi:10.7287/peerj.preprints.26693v2

Kodaira, M., Shibusawa, S., 2013. Using a mobile real-time soil visible-near infrared sensor for high resolution soil property mapping. Geoderma 199, 64-79. doi:https://doi.org/10.1016/j.geoderma.2012.09.007

Kuhn, M., 2008. Building Predictive Models in R Using the caret Package. J. Stat. Software, Artic. 28, 1-26. doi: $10.18637 /$ jss.v028.i05

Kühnel, A., Bogner, C., 2017. In-situ prediction of soil organic carbon by vis-NIR spectroscopy: An efficient use of limited field data, European Journal of Soil Science. doi:10.1111/ejss. 12448

Martens, H.A., Dardenne, P., 1998. Validation and verification of regression in small data sets. Chemom. Intell. Lab. Syst. 44, 99-121. doi:10.1016/S0169-7439(98)00167-1

Mouazen, A.M., Maleki, M.R., De Baerdemaeker, J., Ramon, H., 2007. On-line measurement of some selected soil properties using a VIS-NIR sensor. Soil Tillage Res. 93, 13-27. doi:https://doi.org/10.1016/j.still.2006.03.009

Nocita, M., Stevens, A., Toth, G., Panagos, P., van Wesemael, B., Montanarella, L., 2014. Prediction of soil organic carbon content by diffuse reflectance spectroscopy using a local partial least square regression approach. Soil Biol. Biochem. 68, 337-347. doi:10.1016/j.soilbio.2013.10.022

R Development Core Team, 2013. R: A language and environment for statistical computing. R Found. Stat. Comput. Vienna, Austria.

Ramirez-Lopez, L., Behrens, T., Schmidt, K., Stevens, A., Demattê, J.A.M., Scholten, T., 2013. The spectrumbased learner: A new local approach for modeling soil vis-NIR spectra of complex datasets. Geoderma 195-196, 268-279. doi:10.1016/j.geoderma.2012.12.014

Rinnan, Å., Berg, F. van den, Engelsen, S.B., 2009. Review of the most common pre-processing techniques for near-infrared spectra. TrAC Trends Anal. Chem. 28, 1201-1222. doi:10.1016/j.trac.2009.07.007

Rodionov, A., Welp, G., Damerow, L., Berg, T., Amelung, W., Pätzold, S., 2015. Towards on-the-go field assessment of soil organic carbon using Vis-NIR diffuse reflectance spectroscopy: Developing and testing a novel tractor-driven measuring chamber. Soil Tillage Res. 145, 93-102. doi:10.1016/j.still.2014.08.007

Roy, S.C., Kundu, T., Rao, V.R., 2015. Polymer-Based MEMS Photodetector With Spectral Response in UVVis-NIR and Mid-IR Region. J. Light. Technol. 33, 3345-3350. doi:10.1109/JLT.2015.2430618

Tsakiridis, N.L., Theocharis, J.B., Zalidis, G.C., 2017a. DECO3RUM: A Differential Evolution learning approach for generating compact Mamdani fuzzy rule-based models. Expert Syst. Appl. 83, 257-272. doi:10.1016/j.eswa.2017.04.026

Tsakiridis, N.L., Tziolas, N., DImitrakos, A., Galanis, G., Ntonou, E., Tsirika, A., Terzopoulou, E., Kalopesa, E., Zalidis, G.C., 2017b. Predicting soil properties for sustainable agriculture using vis-NIR spectroscopy - A case 
study in northern Greece, in: Proceedings of SPIE - The International Society for Optical Engineering. doi: $10.1117 / 12.2277905$

Viscarra Rossel, R.A., Adamchuk, V.I., Sudduth, K.A., McKenzie, N.J., Lobsey, C., 2011. Proximal Soil Sensing. An Effective Approach for Soil Measurements in Space and Time. Adv. Agron. doi:10.1016/B978-0-12386473-4.00010-5

Viscarra Rossel, R.A., Behrens, T., Ben-Dor, E., Brown, D.J., Demattê, J.A.M., Shepherd, K.D., Shi, Z., Stenberg, B., Stevens, A., Adamchuk, V., Aïchi, H., Barthès, B.G., Bartholomeus, H.M., Bayer, A.D., Bernoux, M., Böttcher, K., Brodský, L., Du, C.W., Chappell, A., Fouad, Y., Genot, V., Gomez, C., Grunwald, S., Gubler, A., Guerrero, C., Hedley, C.B., Knadel, M., Morrás, H.J.M., Nocita, M., Ramirez-Lopez, L., Roudier, P., Campos, E.M.R., Sanborn, P., Sellitto, V.M., Sudduth, K.A., Rawlins, B.G., Walter, C., Winowiecki, L.A., Hong, S.Y., Ji, W., 2016. A global spectral library to characterize the world's soil. Earth-Science Rev. 155, 198-230. doi:https://doi.org/10.1016/j.earscirev.2016.01.012

Wetterlind J., W., K., P., B., S., M., S., 2015. Exploring the predictability of soil texture and organic matter content with a commercial integrated soil profiling tool. Eur. J. Soil Sci. 66, 631-638. doi:10.1111/ejss.12228 


\section{Topic 7: Animal production technologies (AP)}

Research and application of technology and management of systems to control indoor air quality and thermal comfort in barns, improve welfare and health of animals, reduce emissions and their impacts. Examples include precision livestock farming, CFD and ventilation systems, emissions of particulate matter and gases (e.g. ammonia, greenhouse gases, odour), and control of pest and diseases.

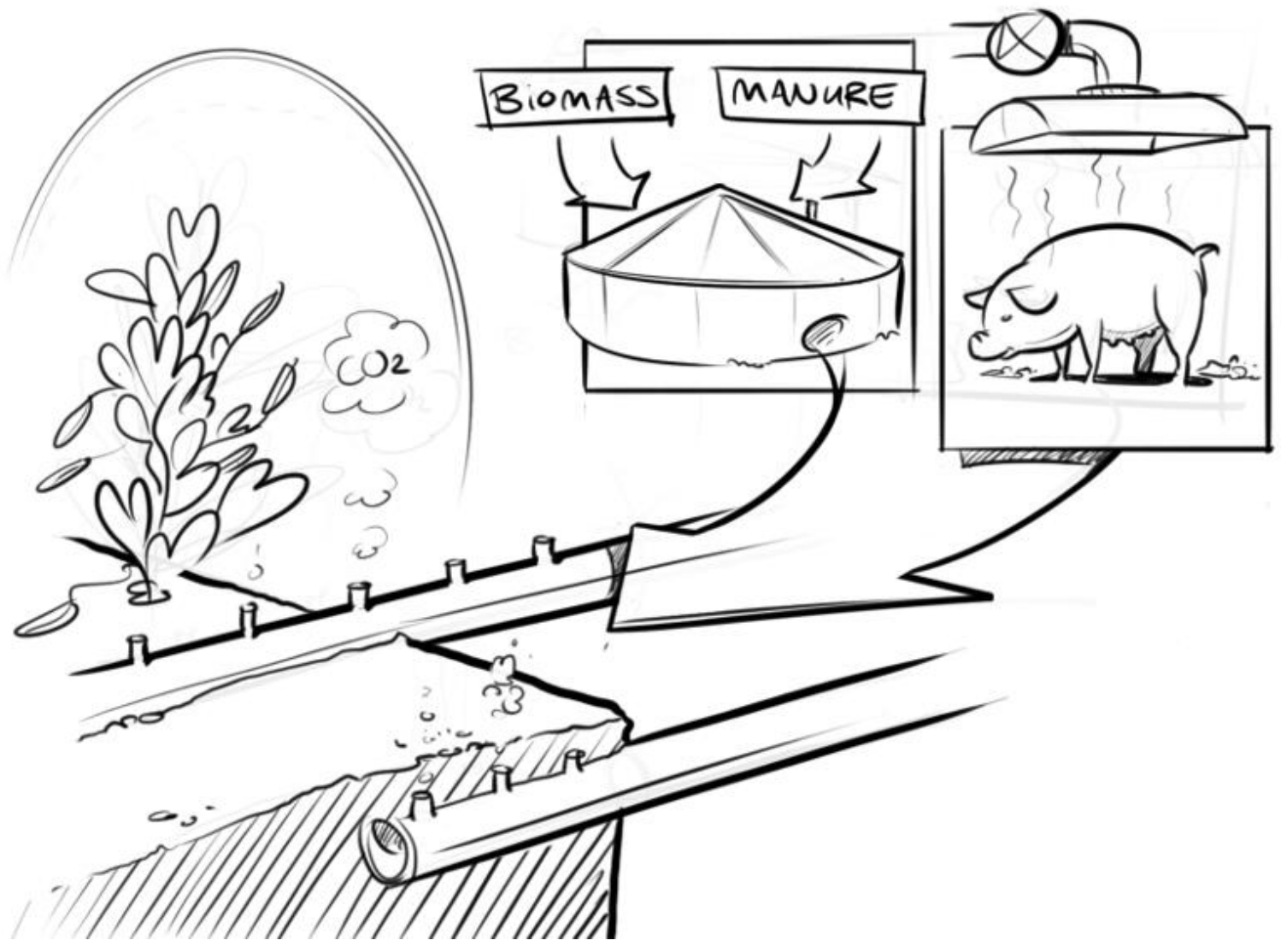




\title{
Evaluation of Novel Farrowing Systems with the Possibility of Temporary Crating of the Sow - Effects on Working Time Requirement
}

\author{
Emil Blumauer a, Franz Handler a, ${ }^{\text {a, }}$, Birgit Heidinger ${ }^{\text {b }}$ \\ ${ }^{a}$ HBLFA Francisco Josephinum, 3250 Wieselburg, Austria \\ ${ }^{\mathrm{b}}$ HBLFA Raumberg-Gumpenstein, 8952 Irdning-Donnersbachtal, Austria \\ *Email: franz.handler@josephinum.at
}

\begin{abstract}
The amendment of the 1. Animal Husbandry Regulation in 2012 prohibited the standard farrowing crates as of 1 January 2033. The aim of was to evaluate five alternative loose pen systems in terms of the working time requirement compared to conventional pens. All investigated loose lactation pen systems led to an increase in working time requirement in the farrowing accommodation in relation to the conventional pens. The main causes for this development were the worse visibility into the pens, more freedom of movement of the sow, the worse accessibility to the pen and the larger surface of the pen.
\end{abstract}

Keywords: Working time requirement, loose pen systems, farrowing accommodation

\section{Introduction}

Conventional piglet producers with a three week production rhythm and a suckling period of 28 days spend without considering the piglet rearing - almost half of their working time in the farrowing accommodation, $40 \%$ in the service section und around $10 \%$ in the gestation unit (KTBL, 2016).

So far it has been standard in Austria to keep breeding sows in pens with standard farrowing crates. The amendment of the 1. Animal Husbandry Regulation in 2012 (1. THVO) prohibits permanent crating in the traditional farrowing crates as of 1 January 2033. The amendment requires new pen dimensions and the possibility of free movement for the sows after the critical phase of life of suckling piglets.

The Chambers of Agriculture, HBLFA Raumberg-Gumpenstein and farmers developed three new farrowing pens with the participation of manufacturers of stable equipment. HBLFA Raumberg-Gumpenstein, the Chamber of Agriculture, the University of Veterinary Medicine Vienna, the Chamber of Agriculture of Styria, HBLFA Francisco Josephinum Wieselburg, Austrian Agency for Health and Food Safety Graz and the University of Natural Resources and Life Sciences in Vienna evaluated the new pens in a research project (Heidinger et al., 2017).

The aim of the project from a work economics perspective was the evaluation of the examined pen types with regard to the individual working time requirement compared to conventional pens. The reasons for more or less working time required were identified in different sub-operations.

\section{Materials and Methods}

All pen types investigated in this project offered possibilities for crating the sow temporarily during the critical phase of life of the suckling piglets. The tested pens are illustrated in Figure 1 to Figure 5. Three farrowing pens were developed within the project (Figure 1-3) and two systems came from abroad. In comparison to conventional pens with standard farrowing crates and a minimum size of $4.0 \mathrm{~m}^{2}$ the three new developed pens had to provide at least $5.5 \mathrm{~m}^{2}$ according to the Animal Husbandry Regulation. With regard to the flooring system emphasis was laid on a comparable composition of plastic-, cast iron- and concrete floor. The concrete elements were situated in the front lying area of the sow when the crate was closed. The cast iron slats were usually installed at the rear of the pen.

Beside the three farrowing pens produced by Austrian manufacturers also two foreign pen types were included in the survey. The first foreign pen (F1) provided an area of $6.0 \mathrm{~m}^{2}$. The floor system included a closed concrete floor with a size of $3.5 \mathrm{~m}^{2}$ situated in the frontal area of the pen (directed towards the stable corridor) and a cast iron slats at the rear (Figure 4). The second foreign pen (F2) had a total surface area of $7.4 \mathrm{~m}^{2}$ (see Figure 5). The solid floor has a surface of $2.3 \mathrm{~m}^{2}$. The solid and the slatted floors were made of plastic. Both pens had closable creep areas. F1 and F2 pens had fixed feeding troughs whereas the three new developed pens were equipped with tiltable troughs.

A survey using questionnaires was carried out to get an overview of the common work processes on Austrian farms keeping breeding sows. Finally forty-five feedbacks could be evaluated.

The working time measurements were performed on the research farms Pig Centre Gießhübl GmbH, LFS Hatzendorf and on the pig farm Medau of the University of Veterinary Medicine Vienna. As a first step semistructured interviews were conducted with the farm managers to identify sub-operations which differ with regard to the working time required between conventional and new farrowing pens. The next step was to measure the 
working time requirement of those sub-operations that differed or were new. For this purpose time measurements were made on site. Also videos for observing the animals were used.
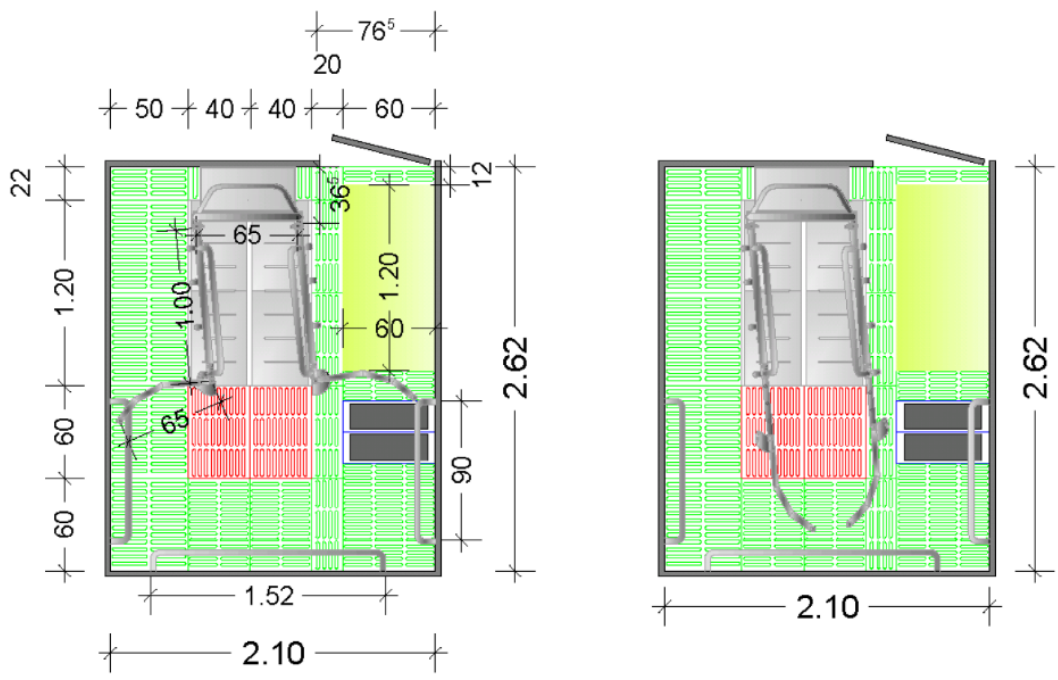

Figure 1. Design of the Wing pen - open on the left / closed on the right (Heidinger et al., 2017).

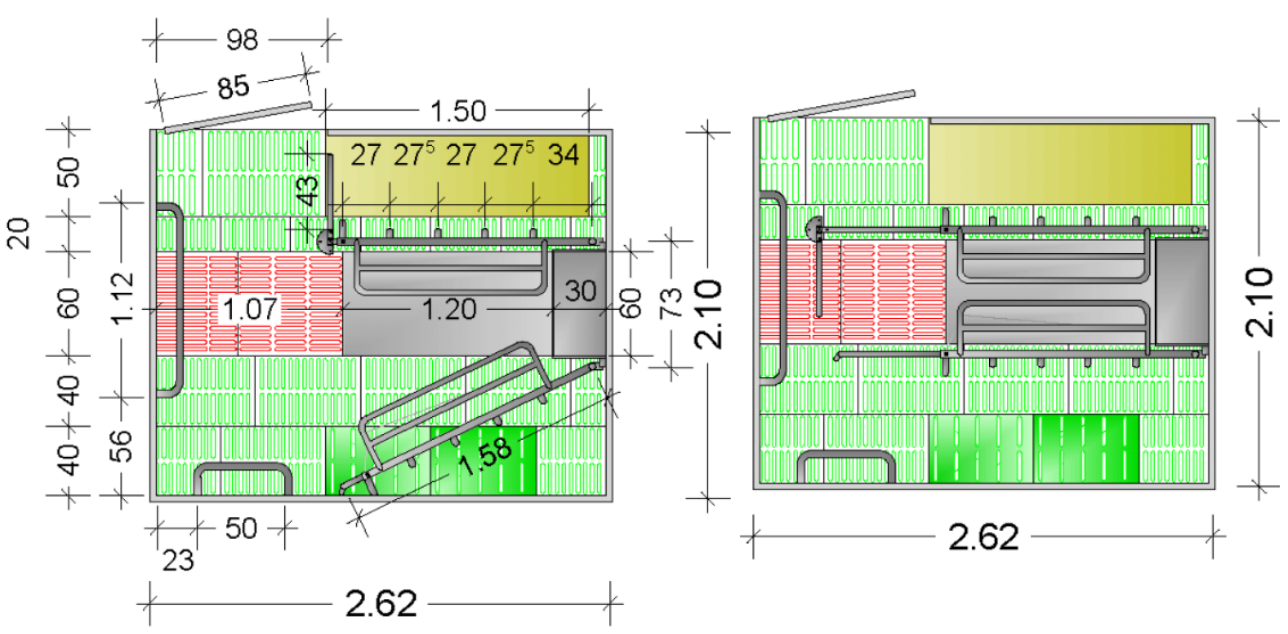

Figure 2. Design of the Kink pen - open on the left / closed on the right (Heidinger et al., 2017).

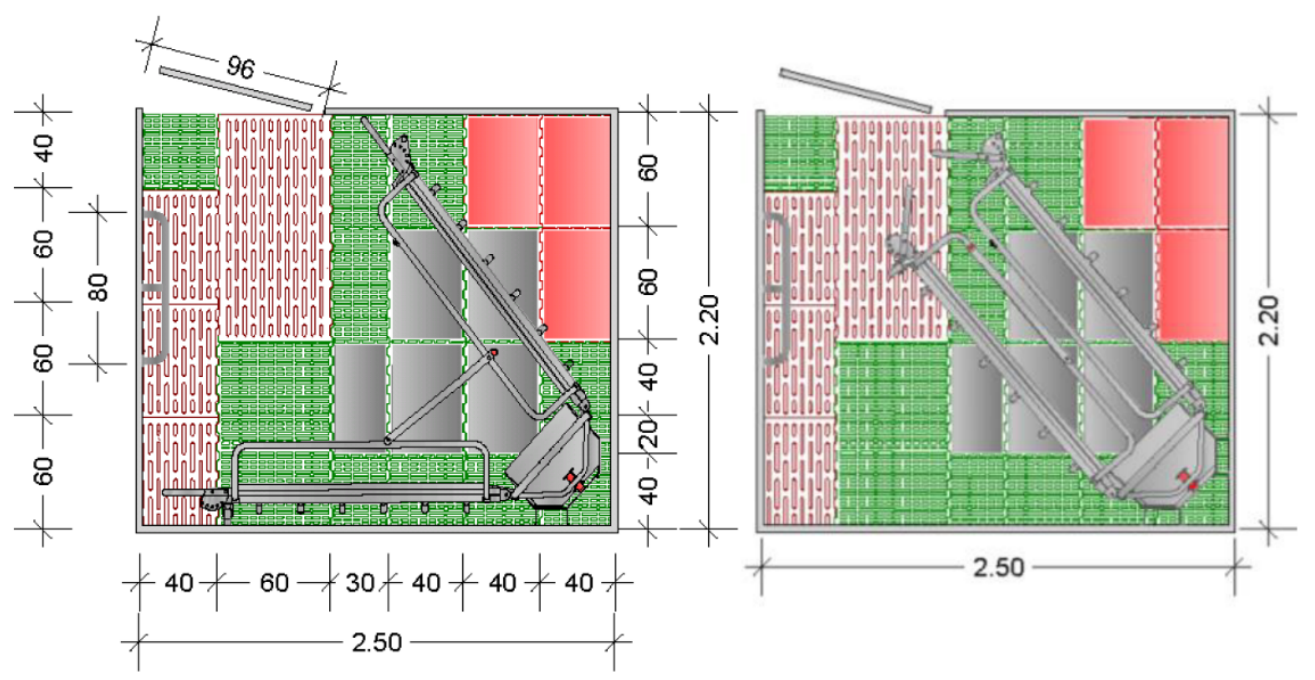

Figure 3. Design of the Trapezium pen - open on the left / closed on the right (Heidinger et al., 2017). 
Time-distance diagrams basing on building plans for model farrowing accommodations with different pen types were created to discuss the impact of differences in the length of the paths. For determining the difference in working time per year it was necessary to survey the number of repeats of operations and sub-operations during a breeding cycle.
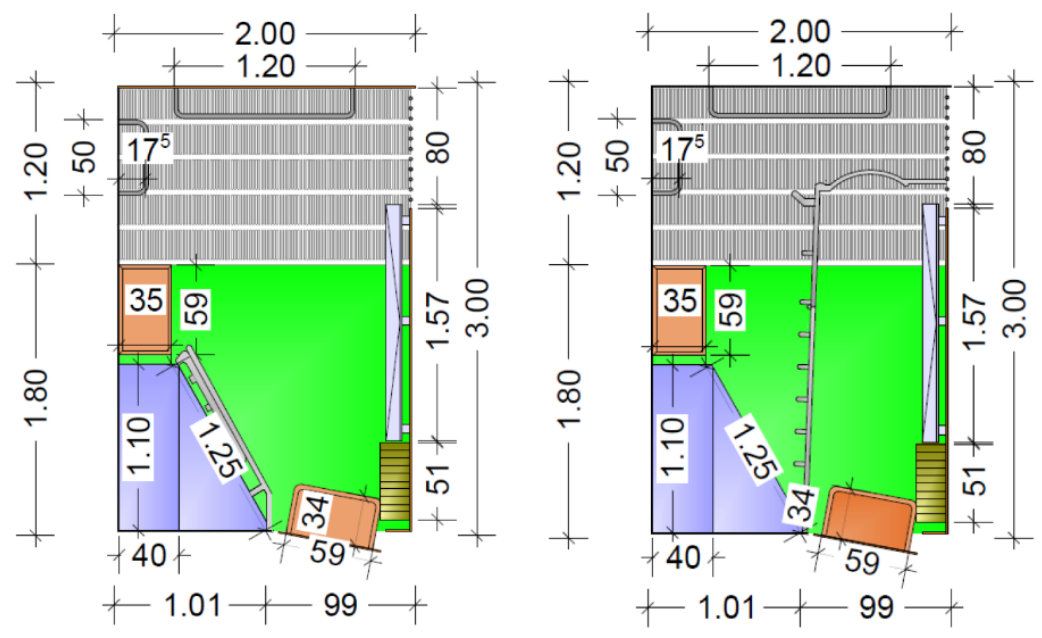

Figure 4. Design of the F1 pen - open on the left / closed on the right (Heidinger et al., 2017).
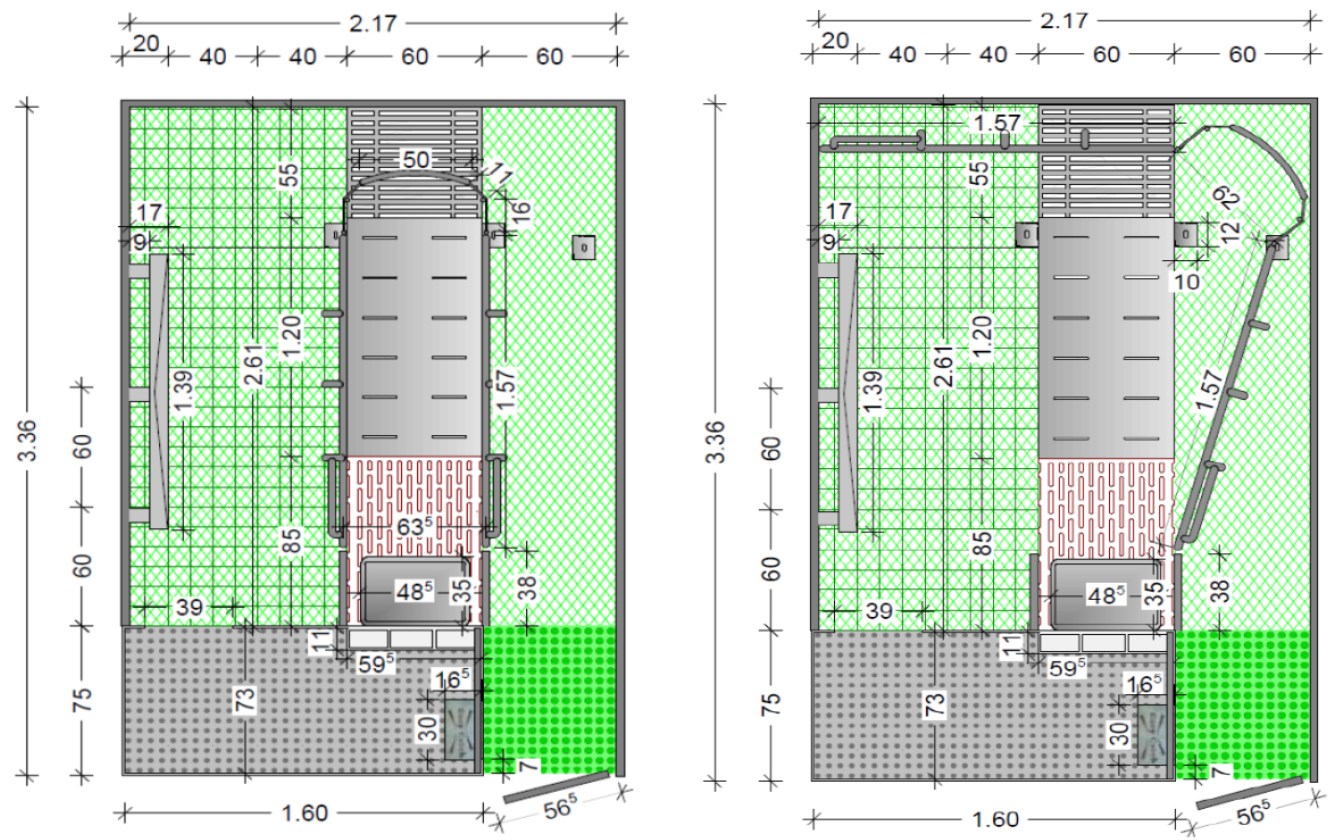

Figure 5. Design of the F2 pen - closed on the left / open on the right (Heidinger et al., 2017).

The statistical evaluation of data from the surveys was made with Statgraphics Centurion XVII and SPSS 19. The working time requirement in conventional farrowing pens refer also to data from literature (Haidn, 1992; Martetschläger, 2007; Kamphues, 2004; ART Tänikon, 2013; Baumgartner et al., 2007).

Basing on the crucial operating and performance data from a work economics perspective, the results from own surveys and literature data, the differences in annual working time requirement regarding the different pen types were calculated in model calculations using Excel spreadsheets.

Four confinement periods (CP) were defined by the Scientific Advisory Board (Heidinger et al., 2017) regarding the critical phase of life of suckling piglets. In CP 0 sows were not confined in the farrowing crate. In CP 3 sows were crated from the end of birth to day 4 of piglets' life. In CP 4 and CP 6 the sows were confined from the day before the calculated farrowing date till day 4 (CP 4) respectively day 6 (CP 6) after the birth of the piglets. 


\section{Results and Discussion}

Working time requirement of the individual operations

The total working time required in the farrowing accommodation has been divided into the following operations: Transfer of the sows from the gestation to the farrowing unit, Adjustment of the feed doser, Offering enrichment / nest building material, Closing the farrowing crate, Birth monitoring, Obstetric aid, Removing afterbirth and dead piglets, Cross-foster, Checking the hygiene in the trough, Cleaning the trough, Health control, Opening of the farrowing crate, Mucking out the pen, Catching piglets 1, Catching piglets 2, Catching piglets 3 , Catching piglets 4, Treatment of piglet, Treatment of sow, Feeding piglets, Moving piglets and sow out of farrowing accommodation, Washing the farrowing accommodation, and Disinfection of the farrowing accommodation. Some working operations will be discussed in more detail below to illustrate that some pen types have a big impact on the working time requirement.

\section{Transfer of the sows from the gestation to the farrowing unit}

In this operation the following activities are included: driving the sows from the entrance of the farrowing accommodation via the corridor to the opened farrowing pen, pushing them into the pen, closing the farrowing crate, closing the pen and going back to leave the farrowing accommodation. The pregnant sows were driven by two workers. Occasionally some pregnant sows wanted to enter the wrong pen although the door was closed, especially in case of pens with a low wall height. For driving the sows paddles and boards were used as an aid. The transfer of the sows from the gestation to the farrowing unit has been divided into the following four suboperations:

Driving the pregnant sows from the entrance of the farrowing accommodation via the corridor to the opened farrowing pen

For the evaluation regarding this sub-operation 95 work observations were available. A statistically verified correlation between the number of driven breeding sows per sub-operation and the average speed of driving could not be established. For this reason all transfers to the farrowing pens were collected and analysed. The median value for the average transfer speed on concrete floor was $1.8 \mathrm{~km} / \mathrm{h}$. Basing on the transfer speed and the distances that had been determined on the basis of pen models provided by the working group for stable construction, the transfer time for five sows was calculated in the model calculations in Chapter 3.2.

Transfer to the farrowing pen

This sub-operation included the work elements opening and closing the door of the farrowing pen, pushing the sow into the pen and - if necessary - to position the farrowing crate so that the sow can no longer leave the pen. The latter was necessary for the F2 and the Wing pen. In the conventional pen also the farrowing crate was closed during this work element. The working time requirement for transferring the sows to the conventional pen or to the Trapezium pen was equal with $44 \mathrm{cmin}$. Closing the two-part door of the Trapezium pen required special skills of the driving person. The Kink pen and the F1 pen had the widest entrances with high pen doors which made the transfer easier. In this case $25 \mathrm{cmin}$ per sow were enough. In case of the F2 pen and the Wing pen the actual transfer to the farrowing pen took the longest time $(96 \mathrm{cmin})$ due to small pen entrances and the required manipulation of the farrowing crate.

Table 1. Standard time for the transfer to the farrowing pen and closing the farrowing crate (homogenous subgroups basing on the median).

\begin{tabular}{ccc}
\hline \multirow{2}{*}{ Pen Type } & \multicolumn{2}{c}{ Working time requirement - Median [cmin] } \\
& Transfer to the farrowing pen & Closing farrowing crate \\
\hline Conventional pen & 44 & - \\
Kink pen & 25 & 74 \\
Wing pen & 96 & 60 \\
Trapezium pen & 44 & 65 \\
F2 pen & 96 & 90 \\
F1 pen & 25 & 146 \\
\hline
\end{tabular}

\section{Changing the pen}

The sub-operation changing the pen included the time for moving on or driving the next sow to the subsequent pen after the previous one had already been transferred to the pen. No differences were found between these pen types. The median value of all measurements was $26 \mathrm{cmin}$.

Leaving the farrowing accommodation (going around in the farrowing accommodation):

This term relates to the sub-operation leaving the farrowing accommodation after all sows of a group (mostly five) had been transferred to their farrowing pens. Basing on 27 measurements the required working time for going around in the farrowing accommodation was calculated by linear regression depending on the distance walked. The regression equation with a coefficient of determination of $74 \%$ was: 


$$
t=7.38+1.73 d
$$

where $t$ is the working time requirement per worker, centiminutes ( $\mathrm{cmin}) ; d$ is distance walked, metre.

Closing the farrowing crate

The operation closing the farrowing crate included the following sub-operations: entering the pen, the actual closing of the farrowing crate, leaving the pen as well as going from pen to pen. The farrowing crate of the conventional pen was closed during the transfer of the sow and stayed closed until the breeding sow was leaving the pen. For this reason no value exists for this pen type in Table 1. In general it is recommended to close the farrowing crate during feeding the sows or luring the sows to the feed trough. The decisive factor is that the farrowing crate can be quickly adapted to the body size of the sow. Telescopic or tiltable elements of the farrowing crate must be adjustable quickly and easily. That was not always the case and was additionally complicated by the behaviour of some sows. In case of Kink pens, Wing pens and Trapezium pens the farrowing crate could be usually closed without any direct sow contact. This was evident from the roughly equal standard times in Table 1. The time required in the F2 pen and in the F1 pen was significantly higher. Both pen types were basically designed for loose farrowing. The built-in farrowing crate therefore represented a compromise with room for further improvement. This became especially evident through increased animal contact when closing the crate and, on the other hand, was noticeable by a higher working time requirement. In order to close the farrowing crate of the F2 pen at least two workers were necessary whereas in the case of the F1 pen strong men managed to close the crate on their own, women only in pairs.

\section{Catching piglets 1}

The operation Catching piglets 1 was carried out by two workers on day 1 of life of the piglets and comprised the following sub-operations catching the piglets, piglet processing, returning the piglets to the pen and going from pen to pen.

\section{Catching the piglets}

The F2 pen was equipped with a self-catching mechanism where the creep area was closed on one side with a slider. Consequently the piglets could only enter the creep area on the other creep side, but not leave it. Usually the creep area was not voluntarily visited by all piglets when the self-catching mechanism was activated. So some of the piglets had to be driven into the creep area, or they were caught in the pen and then lifted into the creep area. This sub-operation required $358 \mathrm{cmin}$ (median) regardless of the litter size. Also the F1 pen had a closable creep area. Piglets that were not in the creep area had to be caught in the pen and were lifted into the treatment trolley. The latter also applied to the piglets caught in the creep area. In the other types of pens the piglets were caught in the pen and lifted into the treatment trolley. The time required to catch the piglets was the same for the conventional pen, the Kink pen, the Wing pen and the F1 pen (see Table 2). In the Trapezium pen, where piglets found shelter under the feeding trough, the catching time increased significantly. When the suckling piglets were on the pen wall opposite the creep area in the $\mathrm{F} 1$ pen, there was an increased time requirement $(19 \mathrm{cmin})$ because the piglets were difficult to reach due to the closed farrowing crate. This special case was not taken into account in the model calculation.

\section{Piglet processing}

To lift the piglets out of the treatment trolley, clip the teeth and dock the tail a median of $44 \mathrm{cmin}$ per piglet (1 person clips teeth, 1 person docks tail) was determined over all measurements. In the F2 pen, an additional requirement of $2 \mathrm{cmin}$ was determined to lift the piglets out of the creep area, as no treatment trolley was used.

\section{Returning the piglets to the pen:}

Each treated piglet was individually returned to the farrowing pen after treatment. The time differences were caused by the different heights of the pen walls and the door (see Table 2).

\section{Going from pen to pen:}

The working time required for going from pen to pen was determined by Eq (1).

Table 2. Standard times for catching the piglets and returning the piglets to the pen (homogenous subgroups basing on the median test).

\begin{tabular}{cccc}
\hline & \multicolumn{3}{c}{ Working time requirement per piglet - Median [cmin] } \\
\cline { 2 - 4 } Pen Type & \multicolumn{2}{c}{ Catching piglets 1 } & Catching piglets 3 \\
& Catching & Returning & piglets \\
piglets & 13 & 5 & Catching piglets \\
\hline Conventional pen & 13 & 10 & 18 \\
Kink pen & 13 & 8 & 18 \\
Wing pen & 19 & 8 & 18 \\
Trapezium pen & - & 8 & - \\
F2 pen & 13 & 10 & 24 \\
F1 pen & & & \\
\hline
\end{tabular}


Catching piglets 2

The operation Catching piglets 2 was done on day 2 of life of the piglets. The work process corresponded to Catching piglets 1 . The main difference was that due to piglet losses fewer piglets had to be caught and treated. The time required to catch the piglets, return the piglets and go from pen to pen corresponded to the values described in the previous chapter. The processing of the piglets included the iron injection and the administration of the suspension directly into the mouth using a pump doser. The median amounted to $16 \mathrm{cmin}$ per piglet.

\section{Catching piglets 3}

The work process corresponded to Catching piglets 1 and was done on day 5 or 6 of life of the piglets. The number of piglets corresponded to that in Catching piglets 2.

\section{Catching the piglets}

To drive the piglets into the creep area, a median working time of $128 \mathrm{cmin}$ was required for the F2 pen, irrespective of the number of piglets. One possible explanation for the less working time requirement compared to Catching piglets 1 could be the escape behaviour of the piglets into the creep area. The conventional pen, the Kink pen, the Wing pen and the Trapezium pen had the same catching time with 18 cmin per piglet (Table 2). With the exception of the Trapezium pen, the average catching time per piglet increased. This is probably due to the fact that, in contrast to the small piglets, the larger piglets no longer found room under the feeding trough. Besides the trough area was better accessible due to the crate being opened. In the F1 pen the time requirement was highest at $24 \mathrm{cmin}$ per piglet due to the location of the creep area and the height of the pen door.

\section{Piglet processing}

It was assumed that $50 \%$ of the piglets were male and had to be castrated. For this work element $200 \mathrm{cmin}$ per castrated piglet were assumed (Haidn, 1992). A median of $16 \mathrm{cmin}$ per piglet was obtained for the mycoplasma vaccination and putting in the earmarks. In case of the F2 pen an extra time of $2 \mathrm{cmin}$ for lifting the piglets out of the creep area was determined.

\section{Returning the piglets to the pen}

The determined working time for returning the piglets to the pen was the same for all pen types. The median was $8 \mathrm{cmin}$ per piglet.

Going from pen to pen

The working time required for going from pen to pen was determined by Eq (1).

Catching piglets 4

The work process corresponded to Catching piglets 3 and took place on the $21^{\text {st }}$ day of the piglets' lives. The time required to catch the piglets, return the piglets into the pen and go from pen to pen corresponded to the values described in the previous chapter. The treatment of the piglets included the second mycoplasma and circovirus vaccination. The working time requirement (median) was $41 \mathrm{cmin}$ per piglet based on own surveys.

Washing the farrowing accommodation

This operation consists of the sub-operations washing the pen, cleaning the corridor and the associated preparation and follow-up work.

\section{Washing the pen:}

This sub-operation consisted of removing the manure, the pre-cleaning, the possible application of an alkaline foam cleaner and the final cleaning. Not all research farms applied an alkaline foam cleaner or allowed it to soak in accordingly. During the application time (approx. half an hour), the worker was engaged in another activity. Since the intensity of the pre-cleaning was different and therefore had an influence on the final cleaning time, only the total time for washing each pen type was shown. This was directly related to the pen size and was lowest for the conventional pen with 4.19 min and highest for the F2 pen with 15.83 min per pen (Table 3 ).

Table 3. Determined standard times for washing the pen (homogenous subgroups basing on the median test).

\begin{tabular}{cc}
\hline Pen Type & Working time requirement - Median [min] \\
\hline Conventional pen & 4.19 \\
Kink pen & 7.42 \\
Wing pen & 7.42 \\
Trapezium pen & 7.42 \\
F2 pen & 15.83 \\
F1 pen & 9.05 \\
\hline
\end{tabular}




\section{Cleaning the corridor}

The cleaning of the corridor was carried out when washing the pen. Five pens and the proportionate corridor area were combined into one washing section.

Basing on the data collected, the following regression equation with a coefficient of determination of $67 \%$ was determined for the working time requirement:

$$
t=1.52+0.14 a
$$

where $t$ is the working time requirement per washing section, minutes (min); $a$ is corridor area per washing section, square metre.

For the model calculation the corridor area was taken from the building plans of the model farrowing accommodations (Heidinger et al., 2017).

Preparation and follow-up work

According to Haidn (1992), the preparation and follow-up work for washing the farrowing accommodation was set at $67 \mathrm{cmin}$ per pen and breeding cycle.

\section{Comparison of total annual working time requirement}

Basing on the operations and data described in Chapter 3.1 and the piglet losses determined in the project (Heidinger et al., 2017), an annual working time requirement per breeding sow in the farrowing accommodation was calculated for the individual pen types in a model calculation. For all pen types, the Scientific Advisory Board (Heidinger et al., 2017) of the project defined the following uniform operational framework conditions:

Stock size: 140 sows (40 farrowing pens)

- Piglets born alive per litter: 13

- Breeding cycles per year: 2.39

- Production system: three week production rhythm

The model calculation for conventional pens with 11.5 weaned piglets per litter showed a working time requirement per breeding sow and year of 4.39 MPh (manpower hours). This was above the KTBL (2016) values of 3.97 to $4.22 \mathrm{MPh}$ for considerably larger herds (252 and 336 sows respectively).

The results of the model calculation for the new pen systems were summarized in Table 4. The confinement periods did not differ significantly from each other in terms of the mean additional working time requirement compared to the conventional pen with permanent crating. The additional working time requirement tended to be less in case of CP 0 than in the other variants of confinement, since fewer piglets had to be caught and treated (Catching piglets 2,3,4) and closing and or opening of the farrowing crate was not necessary. This effect was compensated by the fact that the F2 pen required more working time for mucking out.

In case of the new pen systems prescribed in the future the farrowing crate may only remain closed during the critical phase of the piglet's life. As a result, additional work will be required in connection with opening and closing the farrowing crate outside this specific period to protect the worker during various activities in the farrowing pen (especially animal treatments). With regard to health control and birth monitoring the poorer visibility conditions within the pens became evident. Due to the sow's greater freedom of movement and the sometimes difficult accessibility of the pen, additional working time was required for mucking out the farrowing pen. The operation catching the piglets was negatively affected by the size of the pen, the necessary process of confining the sow and partly the difficult access into the pen. Also the bigger pen area had an effect when washing the farrowing accommodation.

The additional working time required was lowest in case of the Wing pen. When adjusting the feed doser and cleaning the trough, it performed even better than the conventional pen. When using the Trapezium pen, the poor visibility of the feeding trough (checking the hygiene in the trough) and the possibility for the small piglets to hide under the feeding trough in sub-operation catching piglets increased the working time requirement. In case of the Kink pen, the difficult accessibility also had a negative effect on the adjustment of the feed dosers, the feeding of the piglets and the cleaning of the trough. The transfer to the farrowing pen could easily be executed within time. The F1 pen performed worse, especially when mucking out the pen due to the difficult accessibility and to the arrangement of the farrowing crate when catching the piglets. The transfer to the farrowing pen could easily be executed within time.

When working in the F2 pen the time required was by far the highest. The relatively narrow pen door and the slippery plastic floor in the entrance area made it difficult to move the piglets and the sow in and out of the farrowing pen. The construction of the farrowing crate caused additional work load when mucking out the pen both when opened and closed. Opening and closing of the crate usually had to be carried out by two workers. The construction of the trough made cleaning considerably more difficult. The size of the pen also had a negative 
effect on the working time required when washing the pen. With the appropriate body size of the staff, the feed doser could be adjusted quickly. The self-catching device in the creep area had a positive effect when catching the larger piglets.

Table 4. Weaned piglets per litter, annual working time requirement (WTR) per breeding sow and annual additional working time requirement compared to conventional pens.

\begin{tabular}{ccccccc}
\hline $\begin{array}{c}\text { Confine- } \\
\text { ment period }\end{array}$ & Key figure & Kink pen & Wing pen & $\begin{array}{c}\text { Trapezium } \\
\text { pen }\end{array}$ & F2 pen & F1 pen \\
\hline \multirow{3}{*}{ CP 0 } & Weaned piglets/litter & 10.9 & 11.0 & 10.5 & 10.3 & 10.2 \\
& Annual WTR per sow [MPh] & 5.29 & 4.50 & 5.20 & 8.40 & 4.81 \\
& Annual additional WTR [MPh] & 126 & 15 & 113 & 560 & 59 \\
CP 3 & Weaned piglets /litter & 11.4 & 11.4 & 11.1 & 10.9 & 10.8 \\
& Annual WTR per sow [MPh] & 5.51 & 4.59 & 5.28 & 8.17 & 5.04 \\
& Annual additional WTR [MPh] & 156 & 27 & 125 & 528 & 90 \\
CP 4 & Weaned piglets/litter & 11.7 & 11.7 & 11.4 & 11.3 & 11.2 \\
& Annual WTR per sow [MPh] & 5.54 & 4.61 & 5.32 & 8.09 & 5.07 \\
& Annual additional WTR [MPh] & 160 & 30 & 130 & 518 & 94 \\
CP 6 & Weaned piglets/litter & 11.5 & 11.5 & 11.2 & 11.0 & 10.9 \\
& Annual WTR per sow [MPh] & 5.52 & 4.59 & 5.30 & 7.87 & 5.03 \\
& Annual additional WTR [MPh] & 158 & 28 & 126 & 487 & 89 \\
\hline
\end{tabular}

In addition to the increased working time requirement for the new pen systems, work peaks were determined during the transfer of the sows from the gestation to the farrowing unit, birth monitoring, catching the piglets, moving the piglets and the sow out of the farrowing accommodation and washing the farrowing accommodation.

With regard to the design and handling of the farrowing pens examined, there is still potential for improvement, which will be worked on in cooperation with the manufacturing companies.

\section{References}

1. THVO (1. Tierhaltungsverordnung), 2012. In BGBl. II Nr. 61/2012 vom 9.3.2012, Vienna.

ART Tänikon, 2013. Software ART-Arbeitsvoranschlag (Version 1.1.17 / 26.09.2013). Forschungsanstalt Agroscope Reckenholz-Tänikon, CH-8356 Ettenhausen.

Baumgartner, J., C. Winckler, E. Quendler, E. Ofner, E. Zentner, M. Dolezal, F. Schmoll, C. Schwarz, M. Koller, U. Winkler, S. Laister, M. Fröhlich, C. Podiwinsky, R. Martetschläger, W. Schleicher, A. Ladinig, B. Rudorfer, G. Huber, I. Mösenbacher, und J. Troxler, 2008. Beurteilung von serienmäßig hergestellten Abferkelbuchten in Bezug auf Verhalten, Gesundheit und biologische Leistung der Tiere sowie in Hinblick auf Arbeitszeitbedarf und Rechtskonformität. Schlussbericht zum Forschungsprojekt Nr. 1437 BMGFJ \& BMLFUW, GZ. LE.1.3.2/0003-II/1/2005.

Haidn, B., 1992. Arbeitswirtschaftliche Untersuchungen und Modellkalkulationen in der Zuchtsauenhaltung. Dissertation, Technische Universität München.

Heidinger, B., J. Stinglmayr, K. Maschat, M. Oberer, E. Blumauer, S. Kuchling, C. Leeb, E. Hatzmann, E. Zentner, L. Hochfellner, C. Laubichler, M. Dolezal, L. Schwarz, I. Mösenbacher-Molterer, D. Vockenhuber, J. Baumgartner, 2017. Evaluierung von neuen Abferkelbuchten mit Bewegungsmöglichkeit für die Sau. Abschlussbericht zu den Forschungsprojekten Nr. 100964, 100986, 101062 und BMLFUW-LE.1.3.2/0086II/1/2013, HBLFA Raumberg-Gumpenstein, Wien, Juli 2017.

Kamphues, B., 2004. Vergleich von Haltungsvarianten für die Einzelhaltung von säugenden Sauen unter besonderer Berücksichtigung der Auswirkungen auf das Tierverhalten und der Wirtschaftlichkeit. Dissertation, Georg-August-Universität Göttingen.

KTBL, 2016. Betriebsplanung Landwirtschaft 2016/17. Kuratorium für Technik und Bauwesen in der Landwirtschaft e.V. (KTBL), 25. Auflage, Darmstadt.

Martetschläger, R., 2007. Arbeitszeitvergleich von Abferkelbuchttypen mit und ohne Fixierung der Sau. Diplomarbeit, Universität für Bodenkultur Wien. 


\title{
Long term investigations to compare different renewable energy sources for heating and cooling pig houses
}

\author{
Wolfgang Büscher a," ${ }^{\text {, }}$ Manuel S. Krommweh a, Hannah Licharz ${ }^{\text {a }}$, Christian Maack ${ }^{\text {a }}$ \\ ${ }^{a}$ Institute of Agricultural Engineering, Livestock Technology, University of Bonn, Nußallee 5, 53115 Bonn, Germany \\ * Corresponding author. Email: buescher@uni-bonn.de
}

\begin{abstract}
The energy efficiency of three different heating/cooling systems for pig barns using renewable energy sources should be compared under long term conditions in practice. To reach that goal a geothermal heat exchanger in a fattening barn, a ground water heat pump system in a farrowing barn and an air-to-air heat exchanger in a rearing barn were tested with the same measuring equipment and the same method to calculate the "coefficient of performance' after a whole winter and summer season. COP values were used to describe the gain of thermal energy per consumed electrical energy. Typical for case studies the results are not completely comparable because each system was implemented in a specific barn situation and the boundary conditions were different. The observed efficiency values were more different than expected. The investigated geothermal heat exchanger offered a high potential for heating and also for cooling. The heat pump system generated a very constant energy output confined on warm water for supplying floor heating areas in a rearing barn. The air-to-air heat exchanger transferred only one third of the outlet-air energy to the inlet-air system. The value was depending strongly on the dust situation, so a water based cleaning system was added. It can be concluded that all systems had their special benefits and disadvantages. The calculated COP-values $(197.9,2.7,15.8)$ offered a good comparability based on the consumed electrical energy input. The economic parameters (invest and running costs) were not considered in this research.
\end{abstract}

\section{Introduction}

Against the backdrop of global reductions in fossil fuels reserves and rising energy prices, there is increasing interest in the use of renewable energy sources in farm animal production. In recent years, the Institute of Agricultural Engineering of the University of Bonn has researched various technologies for heating and cooling pig houses in practical scale. In this conference contribution, three systems that use renewable energies in different ways will be compared and energetically evaluated:

- Modular housing system with integrated heat exchanger area,

- Groundwater heat pump,

- Air-to-air heat exchanger.

All three systems were examined in long-term studies. The investigations were carried out under winter and summer conditions in Germany. The pig houses used were thermally insulated and mechanically ventilated houses. During the experimental periods all measurement parameters relevant for evaluation the systems were recorded.

Different sustainability criteria can be used to compare the systems. For evaluation of the energy efficiency the coefficient of performance (COP) is the most important criterion. It describes the gain of thermal energy in relation to the consumed electrical energy. It was not the goal of the project to evaluate economic parameters (invest and running costs).

\section{Materials and Methods}

Modular housing system with integrated geothermal heat exchanger

The research work on the modular housing with integrated geothermal heat exchanger presented here are already published by Krommweh et al. (2014).

\section{Description of the modular housing}

In general, the modular housing concept is a housing system with an integrated geothermal heat exchanger in which the building is constructed in modular form on strip foundations. Fresh air is led through a space between slurry pit and soil below the building to condition supply air. Then it is channeled through a vertical shaft into the roof area from where it reaches the animal sections (cf. Büscher et al., 2007). 


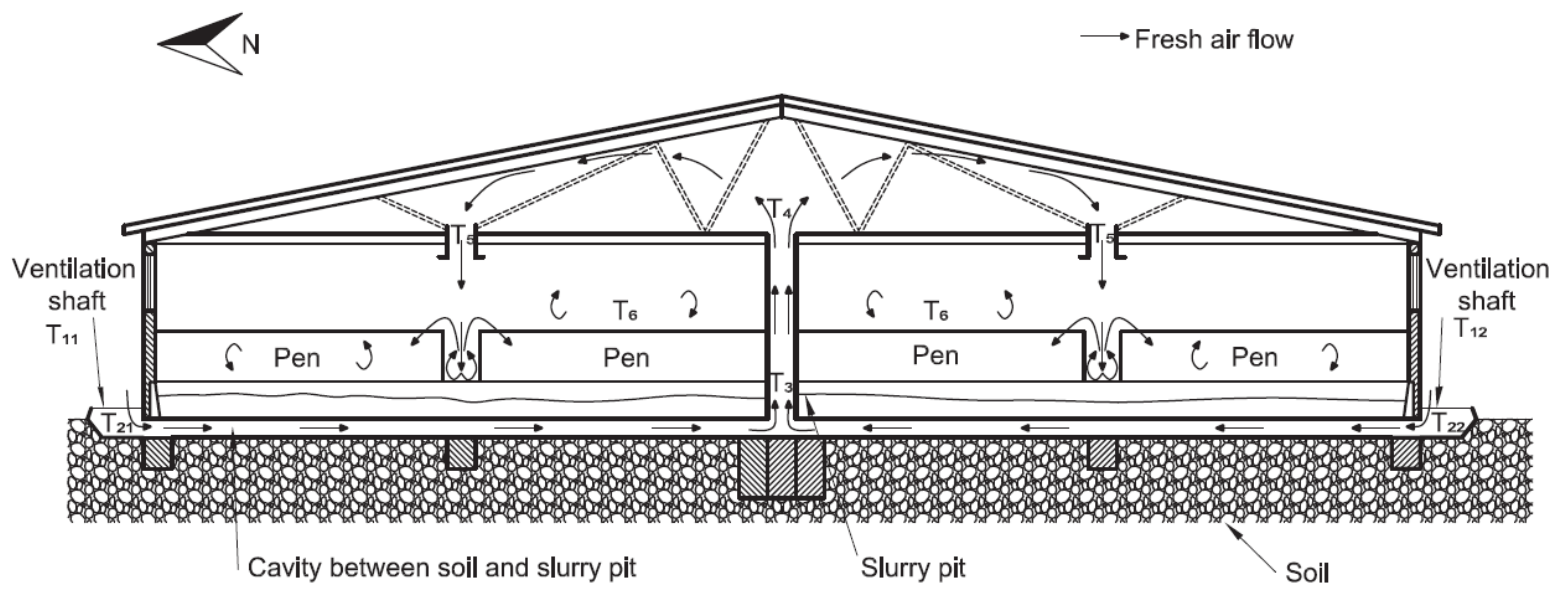

Figure 0.1. Functional principle of the modular housing system with integrated geothermal heat exchanger area below the barn (Krommweh et al., 2014).

The modular housing investigated (Figure 0.1) is located in western Germany. It is a thermally insulated and mechanically ventilated livestock building and designed for 1,280 fattening pigs (floor area $49.8 \mathrm{~m}$ x $26.9 \mathrm{~m}$; large group management on full slatted floor; four compartments with eight pens each; liquid sensor feeding). The supply air is conducted using the under-pressure principle. From all four sides of the modular housing, fresh air flows through 68 ventilation shafts at ground level into the horizontal space between the soil and the slurry pit ( $0.2 \mathrm{~m}$ height). This cavity is the centerpiece of the geothermal heat exchanger system. Through this cavity, the supply air is led to a vertical shaft, which is located in the middle of the livestock building. It extends above the total length of the housing and leads the air into the under-roof area. From there, the air reaches the animal sections through slotted channels, which are installed above the supply corridor.

The modular housing is usually not heated. At the beginning of the fattening period in winter, the animals' thermal output can be too low. In this case, a gas radiator ( $30 \mathrm{~kW}$ rated heating performance) is used to warm up each section for about two days. The aim of the study was to quantify the heating and cooling performance of this system under practical conditions over an extended period.

Experimental period and measured parameters

The modular housing system was investigated for the first time over a one-year experimental period. During this examination time from July $29^{\text {th }}, 2010$ to July $28^{\text {th }}, 2011$, the following parameters were continuously measured and recorded: temperature of outdoor air, fresh air, supply air and section air; air flow rate by use of calibrated measuring fans; electricity consumption of the five exhaust air fans by means of customary electricity meters; gas consumption of the gas radiators by use of a gas meter.

\section{Groundwater heat pump}

Three heat pumps (each $40 \mathrm{~kW}$ electric performance) operating on groundwater have been the subject of further research on a pig farm in North Rhine-Westphalia, Western Germany.

\section{Description of the experimental setup}

A case study from December $8^{\text {th }}, 2011$ to February $15^{\text {th }}, 2012$ was conducted to examine the studied heat pumps. The field experiments were performed in two mechanically ventilated pig houses. These livestock buildings were thermally insulated $\left(\varnothing 0.3 \mathrm{~W} \mathrm{~m}^{-2} \mathrm{~K}^{-1}\right)$. According to the seasonal effects, three identical heat pumps (Fighter 1330, NIBE Systemtechnik GmbH, Celle, Germany) were investigated. The first heat pump was installed in a farrowing barn, while the other two pumps were installed in a piglet-rearing barn. Each heat pump consisted of two modules (A and B).

The farrowing barn consisted of 172 places divided into four compartments. Each compartment had 38 farrowing pens in addition to an extra compartment with 20 farrowing pens. Each pen was equipped with a $0.65 \mathrm{~m}^{2}$ warm-water piglet heater supplied by the heat pump. In this barn, modules A and B fed different reservoirs, and module A was responsible for controlling the temperature of the buffer reservoir (500 1) at approximately $40^{\circ} \mathrm{C}$ (Figure 0.2). This serves as a heat source for the warm-water piglet heater. Module B of the heat pump was a heat source for the water reservoir $(3001)$ and ensured that the water temperature in the reservoir was maintained at approximately $50^{\circ} \mathrm{C}$. 


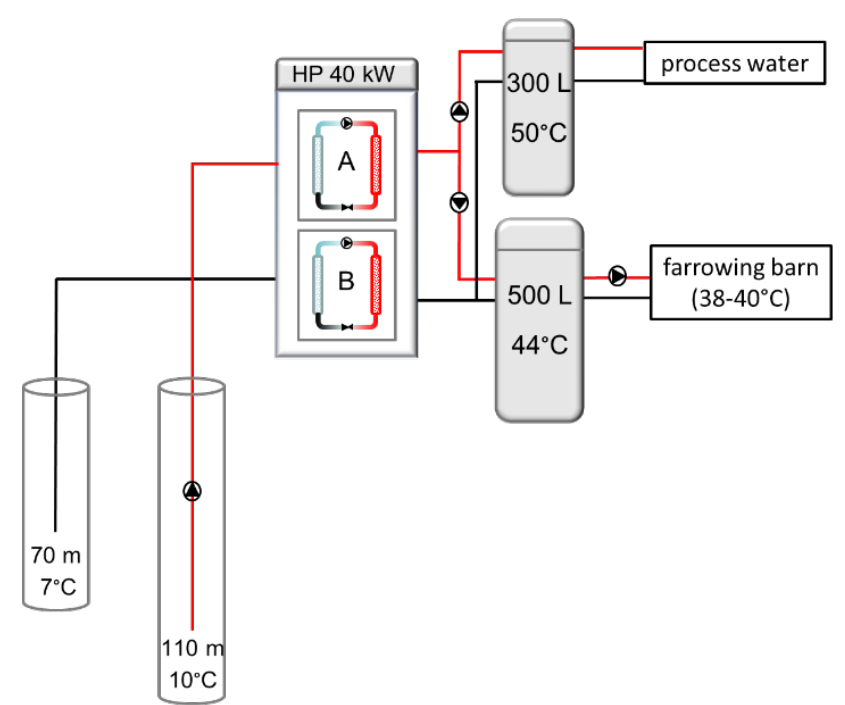

Figure 0.2. Functional principle of the HP-process during the experimental period in the farrowing barn.

The piglet-rearing barn was divided into eight equal compartments, each with 500 breeding places. The compartments were heated by four parallel steel tubes with a diameter of $63 \mathrm{~mm}$. These tubes were mounted on the wall one above the other, and the warm water from the heat pump flowed through them. The lying areas of the compartments were provided with heat-protection covers. The compartment temperature depends on the animals' ages and ranged between $32^{\circ} \mathrm{C}$ and $24^{\circ} \mathrm{C}$. Two heat pumps were regularly used to heat the compartments. These pumps were installed in master-slave combination modules; accordingly, the master heat pump operates separately with low heating capacity requirements. The slave heat pump is controlled by the master heat pump and operates only in conditions requiring high heating. All four modules of the two heat pumps were used to supply a 1,500-1 buffer reservoir with a target temperature of $50^{\circ} \mathrm{C}$. Before restocking the compartments with new animals or to dry the compartments after a cleaning process, $30 \mathrm{~kW}$ heat air blowers heat the compartments.

A groundwater temperature of approximately $10^{\circ} \mathrm{C}$ served as a heat source for both heating circuits of the farrowing and piglet-rearing barns. A frequency-controlled groundwater pump $(7.5 \mathrm{~kW}$, conveying capacity of $\max .40 \mathrm{~m}^{3} \mathrm{~h}^{-1}$ ) lifted the groundwater from common extraction wells with a depth of $110 \mathrm{~m}$ (Figure 0.2 ). The groundwater circulates with an operating pressure of 1.3 bar through the heat pump and transfers its heat energy to the working equipment. Once the water passes through the heat pump, it will be pumped again into the absorption well, which is $70 \mathrm{~m}$ deep and located $20 \mathrm{~m}$ away from the extraction wells. The heat pumps of the farrowing and piglet-weaning barns operated independently. Consequently, these two systems can be directly compared.

\section{Measured parameters}

Four calibrated ALLMESS ultrasonic heat metres (CF Echo II, ALLMESS GmbH, Oldenburg, Germany) were used to measure the heat generated from the groundwater in $\mathrm{kWh}$. To estimate the total amount of water passed through the system, three calibrated flowmeters were used to observe both, the groundwater flow for the heat pump systems and the drinking water supply network. Recording both the total amount of water and the water supply of the investigated stables was necessary because both were driven to the same groundwater-feeding pump. In parallel, the energy requirements of the groundwater-feeding pump were recorded using a three phase-meter, to which each groundwater consumer (farrowing or piglet-weaning barn) could be subsequently quantitatively assigned. To achieve energetic comparability of the heat pump systems, an additional electric energy requirement for both pumps was separately detected using threephase metres. The LPG consumption of the piglet-rearing barn $\left(\right.$ in $^{3}$ ) was determined using a calibrated liquid counter (RS/2001AL, Samgas, Vernate Milano, Italy). The outside and inside temperatures of both stables were recorded using temperature data loggers (Testo T175 T1 AG, Lenzkirchen, Germany) with integrated negative temperature coefficient thermistors as sensors. The range of measurement of the sensors was from $-35^{\circ} \mathrm{C}$ to $+70^{\circ} \mathrm{C}$ within $\pm 0.5^{\circ} \mathrm{C}$ accuracy. A special heat pump software program (NIBE, Systemtechnik) was used to record several operating parameters continuously, including the working hours for each heat pump and the input and output groundwater temperatures of the heat pump in degrees Celsius $\left({ }^{\circ} \mathrm{C}\right)$.

\section{Air-to-air heat exchanger}

The research work on the heat exchanger presented here has already been published by Rösmann (2012) as well as Rösmann and Büscher (2010). 


\section{Description of the experimental setup}

In forced ventilated heated livestock housing between $70-90 \%$ of heat loss in winter takes place through the ventilation (Van Caenegem, 2008; Lindley and Whitaker, 1996; Spengler and Stombaugh, 1983). As early as the 1980s air-to-air heat exchangers were installed in pig housing to minimize such losses. In practice, however, these failed to become established because their design meant they required a high maintenance input. They were also financially inefficient (high investment costs, low primary energy prices) (Van Caenegem and Wechsler, 2000). Increasing energy prices as well as improvements in technological development regarding air-to-air heat exchangers have in recent times made heat recovery from exhaust air in livestock housing interesting once again. The farmer's decision to purchase an air-to-air heat exchanger is hereby first of all intrinsically linked with the estimated advantage of this investment compared with alternative technology. In turn, this depends substantially on energy price development (Klement, 2001).

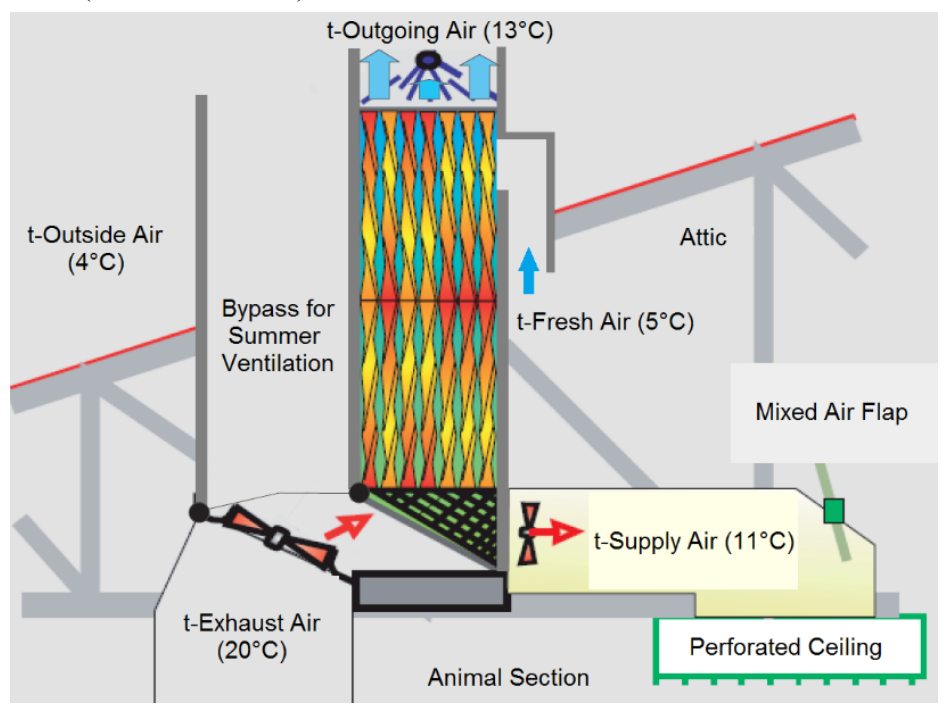

Figure 0.3. Functional principle of the investigated air-to-air heat exchanger with average air temperature registered from March $12^{\text {th }}-18^{\text {th }}, 2010$ (based on Rösmann and Büscher, 2010).

Intensive investigations with a WVTL 480 Schönhammer recuperative heat exchanger were carried out in a piglet-rearing barn in Bavaria, South Germany, with regard to its energy performance potential. The tested heat exchanger (Figure 0.3) is fitted near the front in the roof space of a 2,000-place piglet-rearing barn, the piglets being housed in nine compartments directly post-weaning, remaining until they reach 30-kg body weight. Exhaust air is channeled through a central underfloor suction system. This collects under the central passage the warm exhaust air from the piglet compartments and, during winter, channels it over the heat exchanger and out of the barn. During summer ventilation, directional flaps help channel the exhaust air past the heat exchanger and directly out of the barn. Fresh intake air is drawn into the roof space via eave inlets. From there it is channeled over the heat exchanger with subsequent introduction into the rearing compartments through perforated ceilings.

The air-to-air heat exchanger used is a recuperative counter-current model. The recuperative models transfer heat convectively from the warmer air current through a separation layer to the colder air current. With the WVTL 480 the fresh air is channeled on the one side from above and through the heat exchanger through smooth, spiral exchange surfaces made of plastic. The warm exhaust air is channeled in the opposite direction, i.e. upwards, from the central exhaust air collection canal. The cooled outgoing exhaust air then leaves the barn. The spiral surface within the heat exchanger increases exchange or contact area between intake and exhaust air. Additionally, the air turbulence thus caused is aimed at increasing the heat conduction. If required, the exchange area can be cleaned of dust from the barn through a cleaning system permanently fitted on the exhaust air side.

\section{Experimental period and measured parameters}

The air-to-air heat exchanger was investigated over a one-year experimental period (cf. Rösmann, 2012). During this examination time from October $1^{\text {st }}, 2009$ to September $30^{\text {th }}, 2010$, the following parameters were continuously measured and recorded every ten minutes: airflow volume of intake and outgoing exhaust air, relative air moisture content and temperatures of the fresh, intake, exhaust and outgoing exhaust air as well as the outside temperature and the electricity consumption.

\section{Calculation of the coefficient of performance}

In order to be able to compare the different heating and cooling technologies, for each of the above stated systems, the coefficient of performance was calculated according to VDI 3803-5 (2013), by relating thermal 
energy output in heating and cooling to electricity input for running the system:

$$
\mathrm{COP}=\left(\dot{\mathrm{Q}}_{\mathrm{HR}} / \mathrm{Pel}_{\mathrm{el}}\right)
$$

where COP is the coefficient of performance, non-dimensional; $\dot{\mathrm{Q}}_{\text {HR }}$ is the thermal energy output delivered by heat recovery, $\mathrm{kWh} ; \mathrm{Pel}$ is the electrical power required for running the system, $\mathrm{kWh}$.

\section{Results and Discussion}

Modular housing system with integrated geothermal heat exchanger

The investigation clearly showed that the fresh air supply of the modular housing has a positive effect on the barn climate. Strong fluctuations in the outside air temperature were intercepted (amplitude damping). During winter, supply air was heated on average by $7.9 \mathrm{~K}$. The neutral temperature ${ }^{4}$ of the modular housing system was between $20^{\circ} \mathrm{C}$ and $24^{\circ} \mathrm{C}$. At temperatures above $24^{\circ} \mathrm{C}$, incoming supply air was cooled. The maximum heating effect was $16 \mathrm{~K}$ at an outside temperature of $9^{\circ} \mathrm{C}$, the highest cooling effect was $7 \mathrm{~K}$ at an outside temperature of $31^{\circ} \mathrm{C}$.

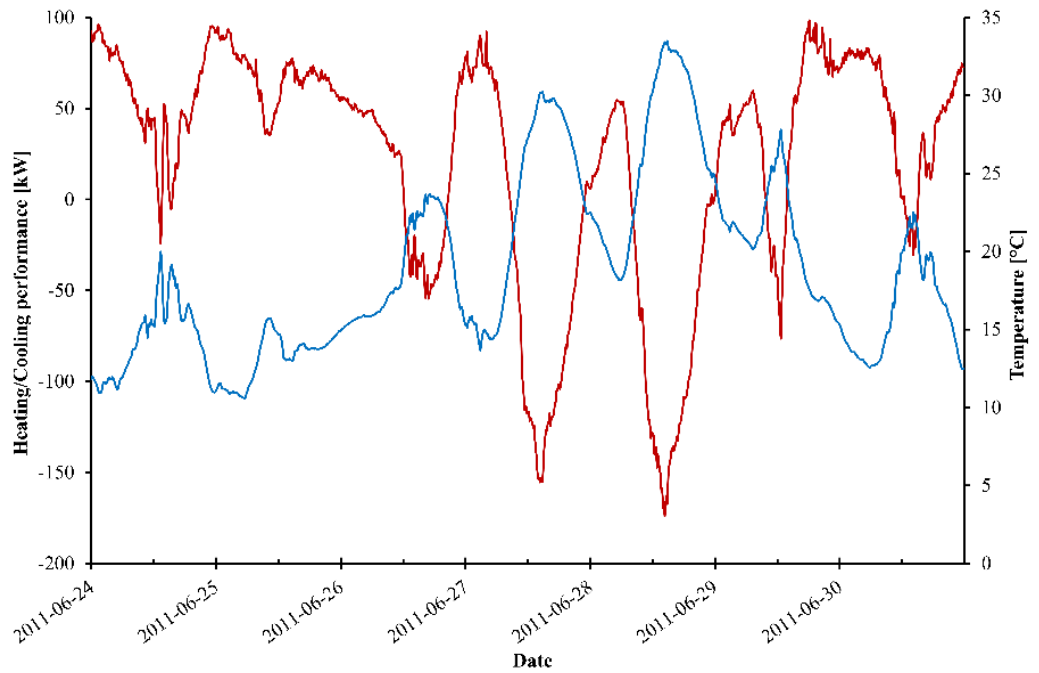

Figure 0.4. Heating and cooling performance of the modular housing system with integrated geothermal heat exchanger area, depending on the fresh air temperature in summer (June, $\left.24^{\text {th }}-30^{\text {th }}, 2011\right)$. Red line: heating and cooling performance; blue line: outside air temperature (based on Krommweh et al., 2014).

Depending on outside air temperature, supply air was warmed or cooled through the GHE and the supply air ducting of the modular housing (Figure 0.4). The relationship between heating performance and outdoor air temperature is described as follows: the higher the outdoor air temperature, the lower the heating power and the higher the cooling capacity. At low outside temperatures, the situation is exactly the other way round.

The continuous acquisition of the measurement parameters described above enabled for the first time a more reliable calculation of the performance of the modular housing system. The modular housing system in this location provided on annual basis an energy amount of $508,275 \mathrm{kWh} .96 \%$ of it heating energy and only $4 \%$ cooling energy (Table 57). The modular housing provided effective heating totalling $489,820 \mathrm{kWh}$, which corresponds to 74,554 litres of liquid gas and a $\mathrm{CO}_{2}$ emission avoidance of about $114 \mathrm{t}$. On the other hand, the electricity consumption for running the exhaust air fans was $10,270 \mathrm{kWh}$. Given the specific supply air ducting of the modular housing, there are higher pressure resistances than in comparable houses with conventional supply air ducting. To account for this pressure resistance, $25 \%$ of electricity expenditure $(2,568 \mathrm{kWh})$ is recognized as an additional expenditure compared to conventional systems. The result is a COP of 1:197.9.

Under German legislation, a minimum indoor temperature of $14{ }^{\circ} \mathrm{C}$ to $22{ }^{\circ} \mathrm{C}$ must be maintained for fattening pigs, depending on their live weight (DIN 18910:2017-08). The average indoor temperature of $26^{\circ} \mathrm{C}$ in this modular housing over the course of the year and the relatively high neutral temperature reveal that more heat is clearly provided (especially in winter) than is needed to fatten pigs. From an economic perspective, it is questionable whether the modular housing for fattening pigs can be justified. The conclusion is drawn that it would be more profitable to use this modular housing in heat-intensive piglet production and rearing.

The power consumption by the ventilation system is around $8 \mathrm{kWh} \mathrm{fp}^{-15} \mathrm{a}^{-1} 46 \%$ lower than in comparable conventional stables. The gas consumption is negligible with about $0.9 \mathrm{kWh} \mathrm{fp}^{-1} \mathrm{a}^{-1}$. The investment costs of the

\footnotetext{
${ }^{4}$ The neutral temperature is known as the average fresh air temperature at which no effective heating or cooling performance is provided (Krommweh et al., 2014).

$5 \mathrm{fp}$ : fattening place
} 
module house are $480 € \mathrm{fp}^{-1}$, around $70 € \mathrm{fp}^{-1}$ higher than comparable conventional barns.

A detailed presentation of all results to the modular housing system with integrated geothermal heat exchanger is given by Krommweh et al. (2014).

\section{Heat pump}

The study illustrates the possibility to use a groundwater heat pump in agricultural breeding stables. The operating hours of the separate pumps detail information about their utilization, ranging in the farrowing from $51.7 \%$ to $61.5 \%$. In piglets, the total utilization was $37.3 \%$ on average. The low utilization rates are due to a doubling of the stable complex, considered at a later date.

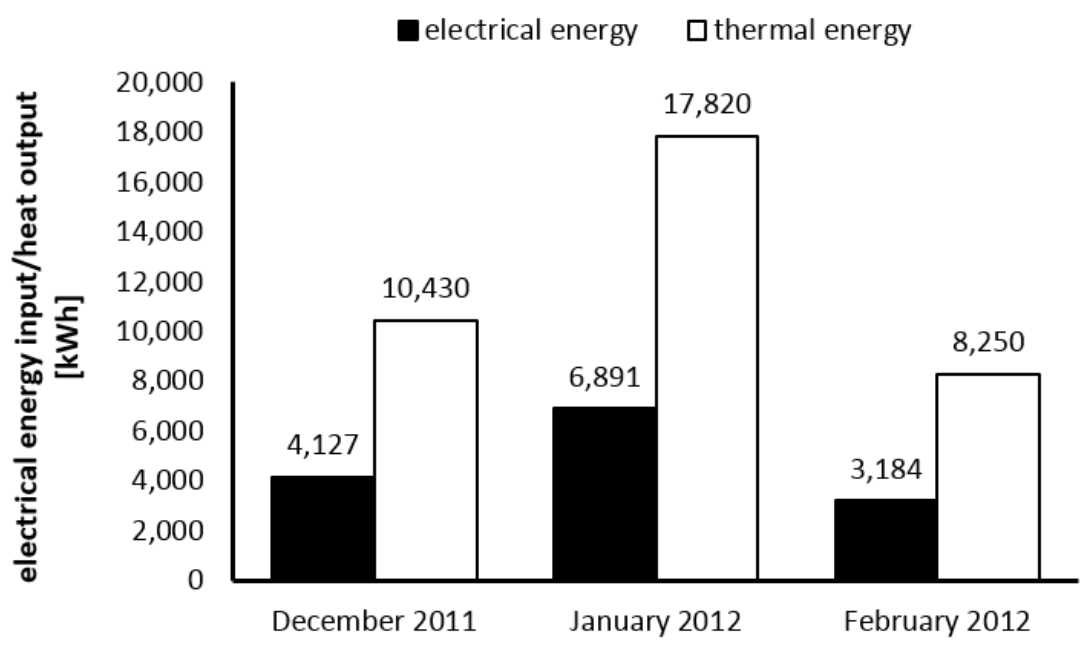

Figure 0.5. Electrical energy input and heat output in HP-process during the experimental period in the farrowing barn.

During the experimental period, electrical energy consumption of the heat pump in the farrowing ranged from $3,184 \mathrm{kWh}$ to $6,891 \mathrm{kWh}$, as shown in Figure 0.5. During the same period the heat pump produced between $8,250 \mathrm{kWh}$ and $17,820 \mathrm{kWh}$ of available thermal energy for heating purposes. The COP of the heat pump between December 2011 and February 2012 ranged from 2.5 to 2.6.

In the piglet rearing, the electrical energy consumption of the heat pumps was between $4,000 \mathrm{kWh}$ and $8,736 \mathrm{kWh}$. The heat pump produced available thermal energy between $8,430 \mathrm{kWh}$ and $23,030 \mathrm{kWh}$ for heating purposes at the same time. In this case, the COP of the heat pump between December 2011 and February 2012 ranged from 2.6 to 2.9 .

\section{Air-to-air heat exchanger}

The results presented in this subchapter refer to March $12^{\text {th }}-18^{\text {th }}, 2010$ (cf. Figure 0.3, Rösmann and Büscher, 2010). In this week, the average outside temperature was $4.1^{\circ} \mathrm{C}$. Through induction of fresh air from the roof space its temperature could already be increased by $1.5 \mathrm{~K}$. The intake air entering the piglet rearing barn achieved an average temperature of $11.0^{\circ} \mathrm{C}$ after passing through the heat exchanger and, with that, lay $6.9 \mathrm{~K} \mathrm{higher} \mathrm{than}$ the outside temperature. The exhaust air temperature before entering the heat exchanger was at $19.8^{\circ} \mathrm{C}$ and was cooled down to $13.0^{\circ} \mathrm{C}$ in the heat exchanger. On the intake air side the fresh air temperature could be increased by an average $5.4 \mathrm{~K}$ after passing through the heat exchanger. On the exhaust air side a $6.8 \mathrm{~K}$ cooling of the air took place. Through the fan installed in the air intake, the air volume flow of the intake and outgoing exhaust air during the investigation could be held almost constant. This represented on average $11,465 \mathrm{~m}^{3} \mathrm{~h}^{-1}$ or $11,488 \mathrm{~m}^{3} \mathrm{~h}^{-}$ ${ }^{1}$. Cleaning of the exhaust air side of the exchanger surface need not be carried out in the winter months because condensate production is sufficient to keep the surface clean during this period.

In this study week, the heating performance of the heat exchanger was $21.6 \mathrm{~kW}$. From this there resulted a heat recovery of $3,100 \mathrm{kWh}$. This represents a heating oil equivalent of around 3101 and an avoidance of $820 \mathrm{~kg}$ $\mathrm{CO}_{2}$ emissions. The energy consumption of the air-to-air heat exchanger is not included in this calculation. The temperature efficiency $\eta_{\mathrm{t}}$ describes the relationship of the intake air heating to total temperature difference between fresh and exhaust air. According to VDI 3803-5 (2013) the temperature efficiency $\eta_{t}$ is calculated as follows:

$$
\eta_{\mathrm{t}}=\left(\mathrm{t}_{22}-\mathrm{t}_{21}\right) /\left(\mathrm{t}_{11}-\mathrm{t}_{21}\right)
$$


Where $\eta_{t}$ is the temperature efficiency, non-dimensional; $t_{11}$ is the exhaust air temperature in $K$; $t_{21}$ the outside air temperature in $\mathrm{K}$ and $\mathrm{t}_{22}$ the supply air temperature in $\mathrm{K}$.

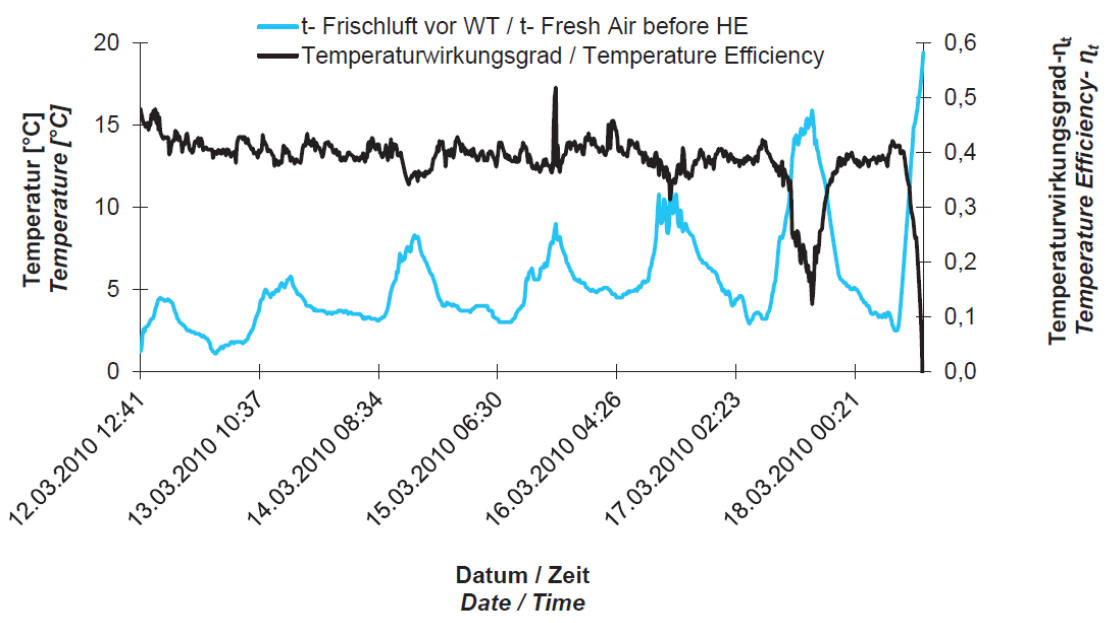

Figure 0.6. Temperature efficiency subjected to fresh air temperature (Rösmann and Büscher, 2010).

The progression of $\eta_{\mathrm{t}}$ is shown in Figure 0.6. With a temperature difference between fresh and exhaust air averaging $14.2 \mathrm{~K}$ a $\eta_{\mathrm{t}}$ of 0.39 is achieved. Increasing fresh air temperatures correspond with decreasing $\eta_{\mathrm{t}}$. The reason for this is the decreasing $\Delta \mathrm{T}$ by stable exhaust air temperature.

Comparison of the different heating and cooling technologies

To compare the three heating and cooling systems investigated in respect to their energy efficiency, Table 57 shows the specific electricity consumption of each system as well as the thermal energy output. The COP is calculated for each system by using equation (1). The data presented for each system in Table 57 relate to the entire data set of the total study period.

Table 57. Performance parameter of modular housing system with integrated geothermal heat exchanger, heat pump and air-to-air heat exchanger.

\begin{tabular}{|c|c|c|c|}
\hline & $\begin{array}{c}\text { Modular } \\
\text { housing system }\end{array}$ & $\begin{array}{c}\text { Heat pump } \\
\text { (piglet rearing / } \\
\text { farrowing barn) }\end{array}$ & $\begin{array}{l}\text { Air-to-air } \\
\text { heat exchanger }\end{array}$ \\
\hline & $\begin{array}{l}\text { Krommweh et } \\
\text { al. (2014) }\end{array}$ & Rösmann (2012) & $\begin{array}{l}\text { Rösmann } \\
\text { (2012) }\end{array}$ \\
\hline Specific electricity consumption (kWh) & 2,568 & $17,636^{* 1} / 14,202^{* 1}$ & 8,958 \\
\hline Thermal energy output (kWh) & 508,275 & $47,160 / 36,500$ & 141,923 \\
\hline of that heating energy & 489,820 & $47,160 / 36,500$ & 141,923 \\
\hline of that cooling energy & 18,455 & $-/-$ & - \\
\hline COP & 197.9 & $2.7^{* 2} / 2.6^{* 2}$ & 15.8 \\
\hline
\end{tabular}

\section{Conclusions}

The results show that each system has specific benefits and disadvantages. Geothermal heat exchangers are able to provide a high amount of heating and cooling energy by comparative very low electrical energy input. They can be recommended for new pig houses if there is a cooling demand in the summer season. The heat pump system examined consumed to much electrical energy for the deep groundwater supply. Also the warm water distribution within the livestock building itself is very energy-intensive. It only can be recommended for 'low temperature warm-water heating systems'. The efficiency of a conventional air-to-air heat exchanger depends, among other things, on the dust loads of the exhaust air. In consequence, own research work shows that an "exchange scrubber" should be used, if exhaust air treatment is demanded for a pig house by environmental legislation. The "exchange scrubber" is a combination of a recuperative air-to-air heat exchanger and a biological trickle-bed reactor for exhaust air cleaning where the heat exchanger surface is sprinkled with scrubbing water on the exhaust air side and thus cleaned (Krommweh, 2018; Krommweh and Büscher, 2017). This fact has a positive 
effect on the COP of the heat exchanger. Under summer conditions it could be observed for the first time that fresh air was cooled down by passing the heat exchanger sprinkled (Krommweh and Büscher, 2017).

\section{References}

Büscher, W., C. Nannen, T. Schneider, 2007. Thermotechnical characteristics of a modular stable. Landtechnik, 62 (5), 340-341

DIN 18910:2017-08. Thermal insulation for closed livestock buildings - Thermal insulation and ventilation Principles for planning and design for closed ventilated livestock buildings. Ed. DIN Deutsches Institut für Normung e.V., Berlin, Germany. Beuth Verlag, Berlin, Germany.

DIN 8900, Teil $61987 . \quad$ Heat pumps; heat pump units equipment with electrically driven compressors, methods of measurement for installed water/water, air/water and brine/water heat pumps. Deutsches Institut für Normung e.V. Beuth Verlag GmbH 10772 Berlin

Klement, S., 2001. Möglichkeiten und Grenzen des Einsatzes rekuperativer Wärmetauscher bei der Klimatisierung von Schweineställen am Beispiel der Mastanlage "Burkersdorfer Schweineproduktion". Doctoral thesis, University of Gießen, Germany.

Krommweh, M. S., 2018. Energy efficiency of different ventilation systems for pig housing. Doctoral thesis, Institute of Agricultural Engineering, University of Bonn, Germany.

Krommweh, M. S., W. Büscher, 2017. Practical use of an exchange scrubber for heat recovery and exhaust air cleaning in pig farming. In $13^{\text {th }}$ Conference Construction, Engineering and Environment in Livestock Farming. Stuttgart-Hohenheim, Germany. Ed. Association for Technology and Structures in Agriculture (KTBL). 439-444

Krommweh, M. S., P. Rösmann, W. Büscher, 2014. Investigation of heating and cooling potential of a modular housing system for fattening pigs with integrated geothermal heat exchanger. Biosystems Engineering, 121, 118129. doi:10.1016/j.biosystemseng.2014.02.008

Lindley, J. A., J. H. Whitaker, 1996. Agricultural buildings and structures. ASAE, The Society for Engineering in Agricultural, Food, and Biological Systems, 2950 Niles Road, St. Joseph, MI 49085-9659, USA.

Rösmann, P., 2012. Einsatz von regenerativen Energiequellen zum Heizen und Kühlen von zwangsbelüfteten Tierställen. Doctoral thesis, Institute of Agricultural Engineering, University of Bonn, Germany.

Rösmann, P., W. Büscher, 2010. Rating of an air-to-air heat exchanger in practice. Landtechnik, 65 (6), 418420

Spengler, R. W., D. P. Stombaugh, 1983. Optimizations of earth-tube heat exchanger for winter ventilation of swine housing. Transaction of the ASAE, 26(4), 1186-1193

Van Caenegem, L., 2008. Energieeffizienz in Abferkelställen durch Erdwärmenutzung. In Energieeffiziente Landwirtschaft. Ed. Association for Technology and Structures in Agriculture (KTBL). 162-172

Van Caenegem, L., B. Wechsler, 2000. Stallklimawerte und ihre Berechnung. Ed. Eidgenössische Forschungsanstalt für Agrarwissenschaft und Landtechnik (FAT). 64-66

VDI 3803-5, 2013. Air-conditioning, system requirements. Part 5: Heat recovery systems (VDI Ventilation Code of Practice). Ed. Verein Deutscher Ingenieure e. V., Düsseldorf, Germany. Beuth Verlag, Berlin, Germany. VDI guidelines, 3803-5. 


\title{
Recent Advances of Modeling and Experimental Verification of Ammonia and Greenhouse Gas Emissions from Dairy Housing
}

\author{
Christopher Y. Choi \\ Department of Biological Systems Engineering, University of Wisconsin, Madison, WI, USA \\ Email: cchoi22@wisc.edu
}

\begin{abstract}
The present study summarizes our recent efforts to design permanent chambers capable of simulating production conditions inside dairy houses so that reliable data pertaining to gas emissions could be collected. We considered replicate chambers, constructed at a selected research facility, in conjunction with an instrumentation system capable of measuring the mass flux of gasses through such chambers. To determine the best locations in which to place sensors and thereby identify best practices intended to reduce the amount of gasses emitted by dairy cows (and also to test mitigation strategies), we developed a computational fluid dynamics model capable of assessing the mixing patterns that gas species would form within the chambers. This work was followed by the creation of a comprehensive computational model of a commercial dairy holding area, a model capable of predicting the biological generation of heat and gas species. The model also considered gas and thermal energy production and was evaluated with experimental data pertaining to air speed and velocity as well as ammonia and methane concentrations. To evaluate the effects of design changes and optimization, the velocity, temperature, and gas concentrations within the animal occupied zone (AOZ) were computationally investigated, and the outcomes derived from modeling cases were compared to the experimental data. The final iterations of the model could be used to improve dairy housing design and provide accurate assessments of GHGs and the volumes of ammonia and methane emitted from a dairy house and, potentially, the related mitigation strategies.
\end{abstract}

\section{Introduction}

Demand for milk and other dairy products has increased dramatically over the last several decades. The corresponding environmental impacts of dairy-related production have increased as producers scale up production to meet the demand. Greenhouse gas emissions from dairy production have been estimated to account for four percent of anthropogenic greenhouse gas emissions (FAO, 2010). These emissions and their byproducts can negatively impact human health (Pope et al, 2009). However, there are many strategies that can be implemented to decrease the environmental impact of milk production while meeting the demand. The United States dairy industry is working to reduce the greenhouse gas emissions from producing fluid milk 25 percent by 2020 (Knapp et al., 2011).

There are opportunities shaping the United States dairy industry by introducing new technologies to dairy producers. From 1940 to 2000 the number of United States dairy operations decreased from 4.6 million to 105,000 along with the percent of dairies with more than 50 head increasing from 0.2 percent to 49.6 percent (Blayney, 2002). Clearly, the concentration of dairy cows into larger farms presents the opportunity to implement strategies to reduced emissions and more efficiently treat the larger volume of the waste streams.

This study sought to use computational modeling and experimental verification to improve the design of dairy housing by reducing gaseous emissions and optimizing ventilation. In addition, the model could be used to identify spatial and temporal patterns in the barn to improve the implementation of mitigation strategies such as air treatment. We expect this model to be a valuable tool in identifying and evaluating methods to reduce greenhouse gas emissions and to optimize ventilation to ensure worker and animal health.

\section{Materials and Methods}

In the present paper, we first outline the procedure to develop and calibrate an emission chamber for the use of measuring gaseous emissions from housed dairy cows. Accurate measurement of gas emissions from dairy cows is essential to assess the effect of cow diets and other management practices on both the composition and rate of gas emissions. Methodologies are described to instrument, calibrate, and assess the uncertainty of gas emissions by cows housed in chambers that simulate production settings. In addition, ideal operating conditions and time to steady state within the chamber were defined.

Then, we document the development of a computational fluid dynamics model of mass, momentum, and heat transport capable of predicting greenhouse gas emissions from dairy housing. The model was validated with experimental data collected in the emission chambers developed. Furthermore, our study sought to identify appropriate modeling assumptions such as turbulence model, animal geometry, and buoyancy effects. While the consideration of buoyancy forces does not significantly affect the overall generation rate of gases, it does affect their spatial distribution within the emission chambers. 
Finally, we describe the development of a computational fluid dynamics (CFD) model of a holding area for a commercial dairy. The results for the previous studies were used to implement models of methane and ammonia generation within the domain. The model was validated with experimental data of velocity and gas concentration. The model results were used to assess the uniformity of velocity, temperature, and gas concentrations within the animal occupied zone. Results of the CFD model can be used to optimize design and operation parameters of commercial dairy holding areas. It should be noted that the present paper is primarily summarized a series of recent research publications supervised by Choi (Mondaca and Choi, 2016; Drewry et al. 2017; Drewry et al., 2018a, Drewry et al. 2018b).

\section{Design and Calibration - Chambers to Measure Diary Cow Gases Emissions}

We developed a computational fluid dynamics model to evaluate air-mixing patterns under steady state conditions. The model was validated with experimental data of air velocity within each chamber. These procedures are intended to facilitate accurate measurement of gas emissions from housed dairy cows and provide a laboratory to test various gas mitigation treatments.

Key design features include: four replicate chambers, isolation of the cow from the external environment, and an instrumentation system capable of measuring the mass flux of gases through the chambers. We proposed calibration procedures for the chambers including the estimation of measurement uncertainty. The CFD model capable of assessing mixing patterns within the chambers were developed to guide experimental sensor placement and appropriate data collection periods. These chambers are designed to identify practices to reduce gaseous emissions from dairy cows as well as to test mitigation strategies.

To provide a suitable field laboratory, four 3-cow emission chambers and a control room were designed and constructed in an existing facility at the USDA-ARS Dairy Forage Research Center in Prairie du Sac, Wisconsin, USA. The chambers were designed to accommodate research while retaining as commercial production setting features as possible. Cows are housed in the chamber, except when taken to the milking parlor, three times per day.

As shown in figure 1, each chamber was built to approximately $4.7 \mathrm{~m}$ deep by $5.4 \mathrm{~m}$ wide by $2.9 \mathrm{~m}$ high; all dimensions can be seen in figures 1, 2, and 3 . Chamber walls were constructed of concrete masonry units and coated with 65 -mil polyurea spray coating to reduce sorption of ammonia to the walls and allow for ease of cleaning between experiments. Industrial grade, insulated garage doors were installed to allow for ease of access to the cows for feeding and milking while minimizing air infiltration to the chambers when closed. Large garage doors (one per chamber) were located behind the gutter to allow access to the cows while small garage doors (two per chamber) were located on both sides of the feed alley to allow access for feeding. The tie-stalls were bedded with rubber mats and sloped to facilitate drainage into the common manure gutter. The

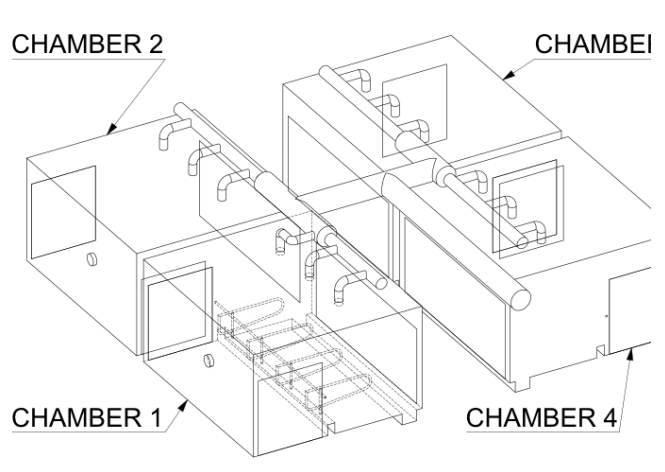

Figure 1. Isometric view of chambers and ventilation assembly. gutter was sealed from the exterior using a block of plastic-wrapped foam that conforms to the gutter shape.
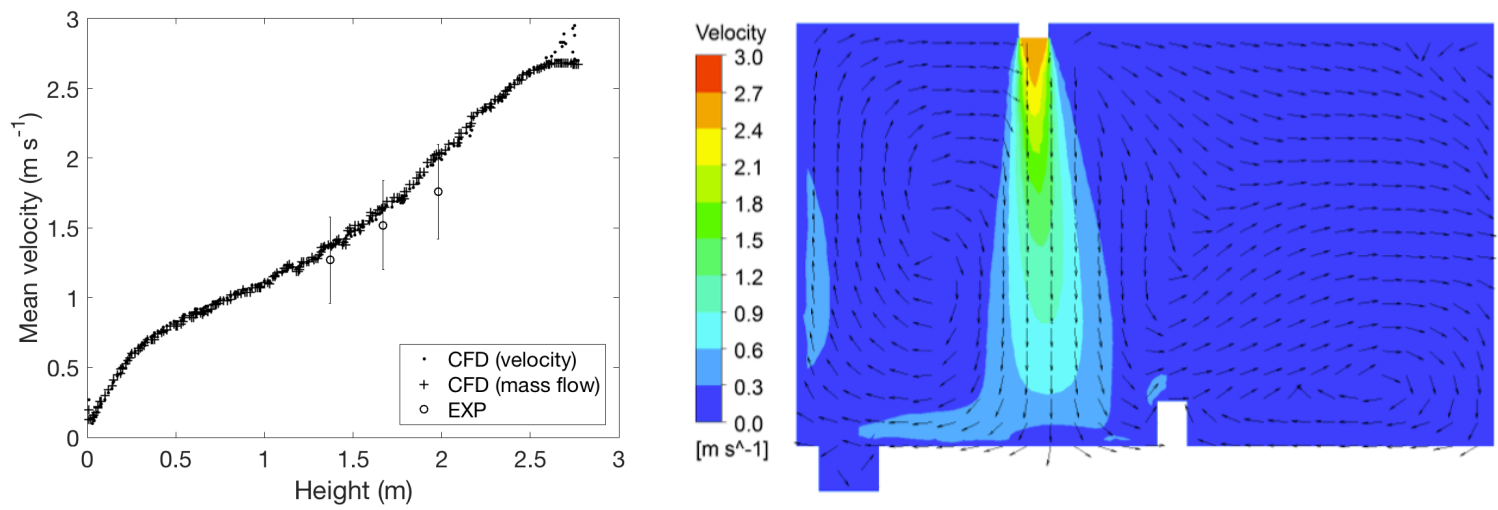

Figure 2. (a, left) Jet velocity, along the jet centerline, as a function of height for both CFD results and experimental data for supply duct 2. (b, right) Contours of velocity overlaid with normalized tangential velocity vectors along the centerline of supply duct 2. 
The velocity profiles generated by the three-dimensional CFD model were benchmarked against data collected using an ultrasonic anemometer within a chamber. Simulated velocity along the centerline of each jet was plotted along with the experimental data of the velocity magnitude along the centerline of the jet at three heights: 1.4, 1.7, and $1.2 \mathrm{~m}$ (figure $2 \mathrm{a}$, featuring supply duct 2 as an example). The CFD model was then used to generate velocity vectors and profiles within the chamber to gain insight into mixing patterns within the chamber on planes intersecting a supply duct and an exhaust duct (figure $2 b$ ). The potential for dead zones to form within the chamber support the use of six air exchange rates as a criterion for steady state, as used in chamber calibration procedures.

The emission chambers designed in this study provide a means to study the effect of diet and management practices on greenhouse gas production from housed dairy cows in a controlled setting. Experiments to determine gas recovery and leakage rates further confirm the expected operation of the chambers. The pressure difference between the chambers and the alley should be monitored to minimize leakage rates and reduce experimental error. Nevertheless, the measurement uncertainty of gas generation in the chambers was found to be within $1.5 \%$ to $4.5 \%$ of the gas generation rate and strongly dependent on the uncertainty in measurements of gas concentration and mass flow rate. Also, the measurement uncertainty, as a percent of the emission rate, was found to decrease with increasing generation rates, especially if mass flow rate is increased as well. The computational fluid dynamics simulations were capable of replicating the experimentally measured velocity within the chamber. Data generated by the CFD model elucidates the mixing patterns within the barn to better understand mixing patterns and optimal sensor placement. In addition, optimized mixing can reduce the time to steady state in the chamber, thereby increasing the amount of data that can be collected from the chambers. Future work should focus on expanding the CFD model to include species generation and transport within the chamber such that the model can be used to develop and evaluate mitigation strategies.

\section{CFD Model and Experimental Validation Of Methane And Ammonia Emission From The Test Chambers}

The subsequent study was to develop a CFD model capable of predicting methane and ammonia emissions from cows housed in the aforementioned emission chambers. Models of methane and ammonia emission were derived from experimental parameters related to cow diet, fecal nitrogen content, and physiological parameters. The study finding should also facilitate the establishment of best modeling practices for future models. To that end, models of ventilation, biological heat production, ammonia production, and methane production were incorporated into a single comprehensive model to evaluate the effects of cow diet and environmental conditions. In addition the effect of buoyancy on thermal and gas species transport was examined. The model was validated with experimental data of gas concentration within an emission chamber. The model is expected to be a powerful tool in identifying and evaluating methods to mitigate greenhouse gas emissions as the dairy industry continues to grow to keep up with the ever-increasing demand for milk and other products while reducing their environmental impact.

The cow was represented as a combination of cylinders to resolve differences in heat transfer at different parts of the cow body while maintaining computational efficiency (Mondaca et al., 2015), as shown in figures 5 and 6 . A six-cylinder geometry was used to represent a standing cow and a two-cylinder geometry to represent a lying cow. Both the six- and two-cylinder cows were of the same volume. Ammonia transport from manure into the air was modeled based on the assumption of equilibrium between nitrogen and ammonia in the manure and of equilibrium between ammonia in the manure and the air. A comprehensive, mechanistic model of cow metabolism was implemented to predict methane production based on cow characteristics and diet. Model inputs were based on the experimental data taken from the cow housed in the emission chamber during spatial gas measurements: a $644 \mathrm{~kg}$ cow consuming $17 \mathrm{~kg}$ of a standard diet per day and producing $41 \mathrm{~kg}$ milk per day, resulting in a yearly production of $196 \mathrm{~kg}$ methane. Results from the model were incorporated as a source term on a single cow surface into the CFD model. This allowed the effect of diet to be considered as an additional parameter used for optimization in the model.

Gas concentration within a chamber was sampled at six locations, twice per day, over a period of four days under standard operating conditions. Prior to the experiment, the cow was housed in the chamber, with the doors open, for four days. The experiment was conducted between the hours of 7:00-10:00 (morning) and 13:00-17:00 (afternoon). The morning measurements were taken after the first milking and before the cow received its daily ration and the afternoon measurements were taken after the second milking and delivery of its daily feed ration. In addition the position of the cow was recorded at the start of each sampling period during the experiment.CFD results of ammonia and methane concentration as a function of height compared with the experimental data in figure 3. For the cases with a lying-cow geometry, buoyancy forces has less of an effect on the results due to the effect of the inlet jet remaining intact for a larger portion of the domain until it dissipated after contacting the cow. Overall, the CFD model predicted higher outlet concentration of ammonia, which could be due to errors in the experimental data due to chamber leakage. The gradients predicted inside the chamber for methane and ammonia were generally well predicted by the model. 
CFD Model of Biological Heat and Gaseous Generation in a Dairy Holding Area

We further developed and evaluated a comprehensive computational model of a commercial dairy holding area capable of predicting biological generation of heat and gas species based on the aforementioned chamber studies. The animal occupied zone (AOZ) within the holding area was modeled as porous media and gas and
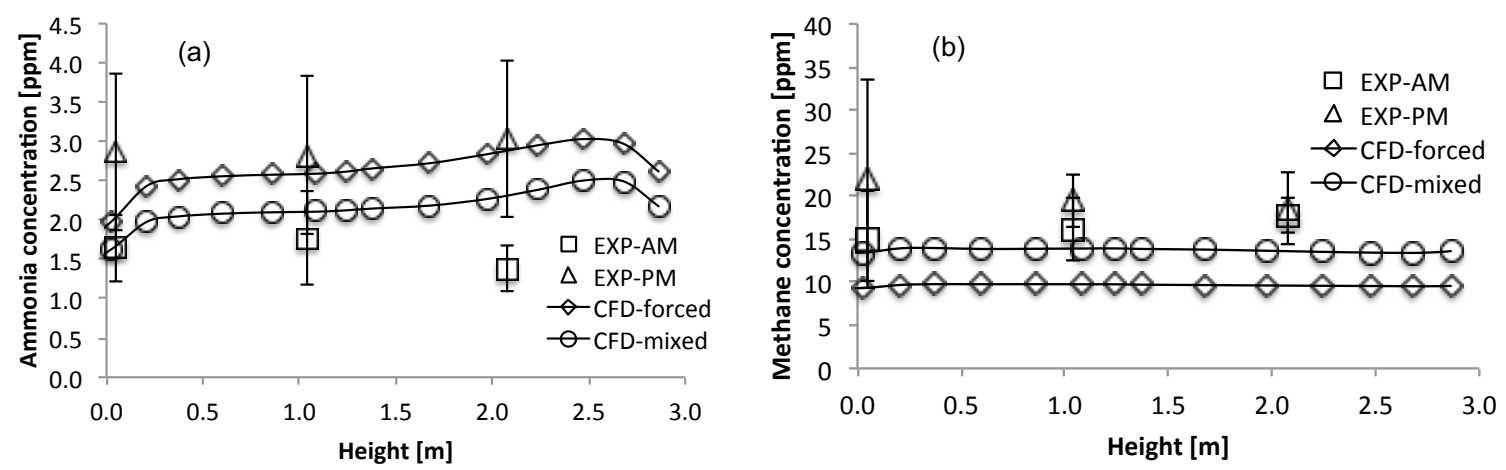

Figure 3. Comparison of experimental (EXP) and computational fluid dynamics (CFD) results for the lying cow: (a, left) ammonia concentration behind cow and $(b$, right) methane concentration behind cow.

thermal energy production was implemented through user-defined functions, largely based on our previous modeling efforts (Mondaca and Choi, 2016). The model was evaluated with experimental data of air speed and velocity and gas concentration. In addition, the effect of design modifications on conditions within the animal occupied zone was explored. The model can be used to improve holding area design to provide more uniform ventilation, increased cooling, and improved air quality.

A commercial tunnel ventilated holding area was selected for modeling due to its small size, compared to a barn, its high but localized animal density, and the use of mechanical ventilation. Experiments were designed to conform to operating procedures while minimizing the effect that cows may have on measurements. All experimental measurements were collected when the side curtains were in the fully-open positions and all ventilation fans were operational. Gas sampling was conducted at a select number of locations to reduce the exposure of the equipment to water (to improve data quality) while keeping the sampling line as short as possible (to ensure proper internal pump performance). A location indoors, that would minimize the effect of the both the sprinklers and the inlet to the holding area from the barn. Gas was sampled at the chosen location at heights of 0.3 $\mathrm{m}$ (location 5), $1.5 \mathrm{~m}$, (location 6) and $5 \mathrm{~m}$ (location7) from the floor using a $15 \mathrm{~m}$ sample line. Gas concentration was also measured outside at a height of $1.5 \mathrm{~m}$ near the east and west curtain openings, at the air intake for the parlor, and at the fan outlets of the holding areas using a $1 \mathrm{~m}$ sample line. Air measurements were taken during morning $(8: 00 \mathrm{~h}-11: 00 \mathrm{~h})$ and afternoon $(13: 00-16: 00 \mathrm{~h})$ periods separated by a cleaning of the parlor and holding area (typically at 11:00 h). The seven locations were randomized and sampled for 15-20 minutes within the two sampling periods.

PTFE tubing (4 mm i.d.) along with a filter (Model 6723-5000, Whatman, Pittsburgh, PA) to reduce dust and moisture were used to collect gas samples. Gas concentration was measured continuously at 20 second intervals using a portable Fourier Transform Infrared (FTIR) gas analyzer (Model DX-4030, Gasmet, Finland). Zero calibration with ultra-pure nitrogen gas was conducted onsite each morning and again in the afternoon if the temperature rose more than $10^{\circ} \mathrm{C}$. Gas concentration was sampled for $20 \mathrm{~min}$ at inside locations; the first $5 \mathrm{~min}$ of data collection was excluded from analysis to account for clearing of the sample line between samples. Gas concentration was sampled for $15 \mathrm{~min}$ at the outside locations, with the first 2 min excluded from analysis, due to a shorter tubing length. An analysis of variance (ANOVA) was applied to the data after assessing the normality and variance (SAS, 2013). Measurement time and location were considered to be factors, while the subsample of gas measurement within morning and afternoon measurement periods was included as a random factor. Type III estimable functions were used due to the unbalanced data set.

As an example, measured gas concentration for each location was summarized in box plots for the morning and afternoon sampling periods in figure 4. Values of methane concentration followed similar patterns over both sampling periods. Ammonia concentrations were below the detection limit of $0.5 \mathrm{ppm}$ during most measurement periods; thus, their results were excluded. The significance of the sampling time and location for all gases could not be determined due to the interaction of the subsamples. Measured values were at the low end of the range measured in other studies carried out in naturally ventilated barns. However, the previous studies have a large variation based on time of day, year, and barn design and location. It would also be expected that gas concentrations in this mechanically ventilated holding area would be lower than a naturally ventilated barn due to its high ventilation rate ( 3 exchanges per min). 

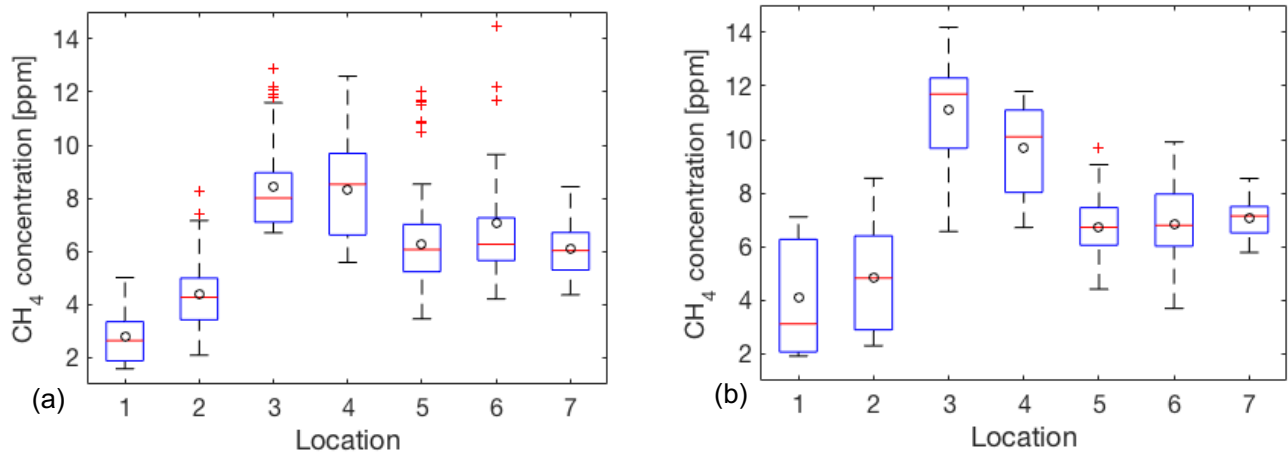

Figure 4. Methane concentration at the seven experimental locations during (a) morning measurements and (b) afternoon measurement. Whiskers were 1.5 times the interquartile above the 75 th and below the $25^{\text {th }}$ percentile. Outliers were represented by red crosses $(+)$ and the mean by black circles (o). Six outliers were removed from location six during the morning measurements for ease of visualization.
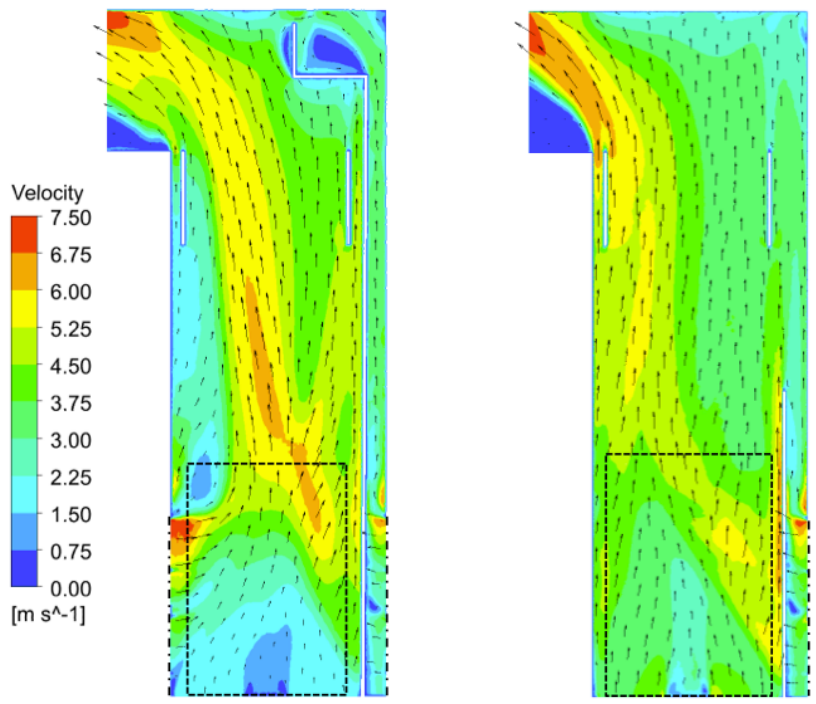

Figure 5. Velocity vectors overlaid on velocity contours $\left(\mathrm{m} \mathrm{s}^{-1}\right)$ at a height of $1 \mathrm{~m}$ from the floor with a 100 cow porous model and the wall separating the walkway (a, left) present design, and (b, right) modified design, reduced in size and the west inlet closed. The dashed line denotes the boundaries of the animal occupied zone (AOZ) and the dashed dotted line denotes the boundaries of the curtain opening.

The 100-cow porous model was used to examine the effect of the design and operation on heat and gas species transport within the holding area, as shown in figure 5. The first design modification was the removal of the concrete wall separating the personnel lane from the holding area. The second design modification was removal of the west curtain opening in favor of allowing more airflow through the parlor entrance/exit. This modification would reduce the volume of air drawn in from the barn outlet thereby improving air quality. The final design modification was to reduce the length of the concrete wall separating the personnel lane from the rest of the holding area. The CFD outcomes clearly demonstrate that we can improve the design and evenly distribute incoming fresh air where 100-cows are standing (figure 5b) in comparison with the current design (figure 5a).

\section{Conclusions}

The three studies presented in this dissertation describe the development and validation of a computational fluid dynamics (CFD) model capable of predicting biological generation of heat and gas species from housed dairy cows. The design and calibration of emission chambers used for the generation of validation data was also presented. A benchmark CFD case was developed to identify the best mathematical relationships for predicting thermal energy generation and methane and ammonia generation as well as best modeling practices. The model was then applied to a commercial dairy holding area. 


\section{Acknowledgements}

This manuscript is based in part on work supported by the USDA National Institute of Food and Agriculture under Grant No. WIS01898.

\section{References}

Blayney, D.P. 2002. The changing landscape of US milk production. Economic Research Service-USDA. Statistical Bulletin No. 978.

Drewry, J.L., M. Mondaca, B.D. Luck, C.Y. Choi, 2018. A Computational Fluid Dynamics Model of Biological Heat and Gas Generation in a Dairy Holding Area. Transactions of ASABE, 61:2, 449-460.

Drewry, J.L., C.Y. Choi, J.M. Powell, BD Luck, 2018. Computational model of methane and ammonia emissions from dairy barns: development and validation, Computers and Electronics in Agriculture, 149, 80-89.

Drewry, J.L., C.Y. Choi, J.M. Powell, 2017. Design and calibration of chambers for the measurement of housed dairy cow gaseous emissions. Transactions of ASABE, 60:4, 1291-1300.

Food and Agriculture Organization of the United Nations 2010. Greenhouse Gas Emissions from the Dairy Sector: A Life Cycle Assessment. Rome, Italy.

Knapp, J.R., G.L. Laur, P.A. Vadas, W.P. Weiss, J.M. Tricarico, 2014. Invited review: enteric methane in dairy cattle production: quantifying the opportunities and impact of reducing emissions. J. Dairy Sci. 97(6): 3231-3261.

Mondaca, M., C.Y. Choi, 2016. An evaluation of simplifying assumptions in dairy cow computational fluid dynamics models, Transactions of the ASABE, 59:6, 1575-1584.

Pope III, C. A., M. Ezzati, D. W. Dockery, 2009. Fine-particulate air pollution and life expectancy in the United States. New England Journal of Medicine, 360(4), 376-386. 


\title{
Climate Conditions - a Status Quo at Dairy Farms in North-East Germany
}

\author{
Jennifer Löbel a,"*, Sandra Rose-Meierhöfer a, Paul Gütschow ${ }^{\text {b }}$, Clemens Fuchs ${ }^{\text {b }}$ \\ ${ }^{\text {a }}$ Department of agricultural machinery, Hochschule Neubrandenburg, University of Applied Science, 17033 \\ Neubrandenburg, Germany \\ ${ }^{\mathrm{b}}$ Department of agricultural economics, Hochschule Neubrandenburg, University of Applied Science, 17033 \\ Neubrandenburg, Germany \\ * Corresponding author. Email: loebel@hs-nb.de
}

\begin{abstract}
The issue of animal welfare is in the focus of politics and customers. This poses enormous challenges for the future viability of modern animal husbandry. The most deficits are the technical design in dairy barns. The energyefficient ventilation and air-conditioning systems could improve the welfare and efficiency of dairy cows and are used in the barn, the living area and the milking parlour. In a research project were collected 202 parameters in each barn of 34 dairy farms. The measurements to evaluate the climate in different barns were implemented with the thermo-hygrometer (temperature and humidity) and the anemometer (air velocity). The data collection occurred three hours after feeding at three different places: at the feeding area, at the cubicles and at the cubicle alley. Every barn has a control element for climate conditions, like eave ridge ventilation, vertical transverse flow system with curtains and jalousie and fans. Only the use of ventilation systems which result of the barn construction are in over $80 \%$ of the analysed farms not sufficiently. 29 of the analysed farms have old GDR barns. Especially in this type, a technical assistance system like ventilators are essential, but only $47.06 \%$ of them have ventilators installed. The temperature values are on average $20,21{ }^{\circ} \mathrm{C}$ in the barn and $18,47{ }^{\circ} \mathrm{C}$ at an outside temperature on average of $22,5^{\circ} \mathrm{C}$. The humidity is on average $77.16 \%$ and except of three barns, no barn has a continuous air circulation. The most air circulation (on average $0.56 \mathrm{~m} / \mathrm{s}$ ) was at the feeding table. The same deficits show the 19 new built barns either. The farmers don't know how to handle it and are often wrong advice.

Keywords: climate conditions, animal welfare, ventilator, ventilation systems, technical design
\end{abstract}

\section{Introduction}

Thermal comfort is one of four components of a satisfactory environment for farm livestock (Anderson, 2018).

A cow needs ventilation to replace her exhaled air, discharge her rumen gases and cool herself down. Fresh air from outside the barn is needed to refresh the air inside (Hulsen, 2014). Cooling is mainly achieved by the flow rate of the air and evaporation. A good ventilation stimulates the health and milk production because the cows feel good (Calegari et al., 2015). Besides that, a good ventilated barn is dry and infection pressure decrease (Hulsen, 2010). The most important factors for the climate conditions in a barn are the air quality, air temperature, air humidity, air rates and air velocity. These factors can be affecting through ventilations systems. It will be decided between the natural and mechanical ventilation. The natural ventilation can be done by cross ventilation over the entire width of the barn and the ridge ventilation. The width of ridge opening is $2 \%$ of the barn width and at the sides are also open (Hulsen, 2010). This system is significant if outside other buildings or high plants are or if the barn is closed by wind and frost. Warm housing barns ventilated with tunnel ventilation systems. Cross tunnel work better than longitudinal ventilation caused of air flows on the side of the cows and not cross over them if they are standing at the lying and feeding area.

Beside the barn constructions should be observed the limit values of the different climate factors to prevent the heat stress of the cows. From the cow physiological point of view is the optimum temperature range between +4 and $+16{ }^{\circ} \mathrm{C}$. Over $21{ }^{\circ} \mathrm{C}$ get started heat stress for the cow and the assistance ventilation should be starting (DLG, 2016, Ofner-Schröck, 2017). The optimum range of humidity in the barn is between $60 \%$ and $80 \%$. The predominant humidity in German barns is $70 \%$ and with the high temperature of over $20^{\circ} \mathrm{C}$ begins the heat stress and high loss of performances (DLG, 2016). If decrease the air velocity under $1 \mathrm{~m} / \mathrm{s}$ is no sufficient air exchange in high barns with open sides available and an assistance ventilation like fans and sprinklers is necessary (DLG, 2016).

In almost all cow houses, fans are needed to keep the cows cool on hot days and sometimes for ventilation as well. The first place where should install a fan is the collecting yard. The second place is the barn especially over the resting and feeding area. The different fan types can be installed horizontal and vertical. The vertical fans can be installed at each place and area and they are very noisy. This type used in low level barns with bad natural ventilation but them use high energy, a large number of fans. The horizontal fans are very quiet and energy efficient and they are only useful in high barns (Hulsen, 2010). Sprinklers used in combination with fans and they are installed over the feeding alley. Sprinklers should soak the cows in a short time until the water is running off their abdomen but their udders remain dry. Then the cows will need to be kept cool with fans (Hulsen, 2014). The sprinklers start automatically controlled by a temperature and humidity sensor like the fans. 


\section{Materials and Methods}

Study design and data collection

This study presents the results of one part from the project: "Animal welfare and economic efficiency in the future- oriented dairy farming - evaluation of various actions and their economic impact". The study took place on 34 dairy farms in North- East Germany, especially in the three federal states: Mecklenburg-Vorpommern, Brandenburg and Schleswig-Holstein. There were collected 202 parameters in 54 barns of 34 dairy farms. The farms are assorted to the number of milking cows in four groups; group 1: up to 300 milking cows, group 2: 301 to 599 milking cows, group 3: 600 to 900 milking cows, group 4: over 900 milking cows. All farms, except one in Schleswig-Holstein with Angler breed and one in Mecklenburg-Vorpommern with Jersey breed, have HolsteinFriesian breed. All farms analysed in the period of April to November 2017. The data collection occurred three hours after feeding. This study focused the climate conditions in the barn and milking parlour. The temperature and humidity would measure with a thermo-hygrometer and the air velocity with an anemometer. The temperature, air humidity and air velocity collected at four different places at the farm. In the barn were measured the light intensity at the feeding area, at the cubicles and the walking area between lying rows (Figure 1). In the milking parlour was the measuring point near the milking pit (Figure 2).

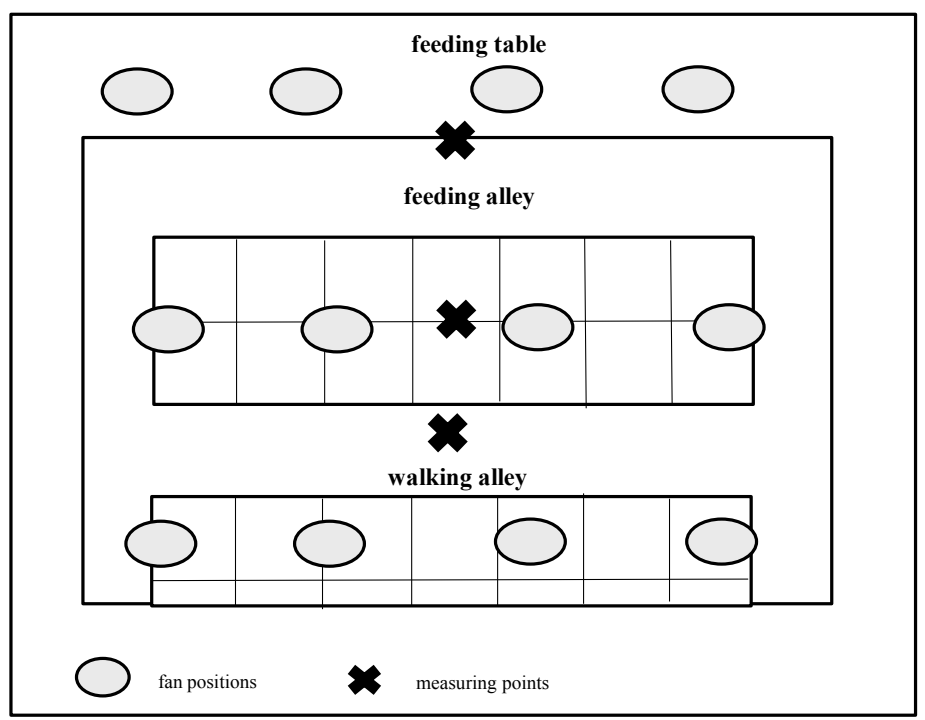

Figure 1 . The measuring points of the study in the barn.

Statistical analysis

In each of the 54 barns were collected three measuring values. Collectively are 162 measuring values of all 34 farms only for the measurement of climate conditions at the farms. Besides that, the presenting ventilation type and the additional assistance ventilation at the farms in each barn would be recorded.

Data were analysed by using the IBM SPSS Statistic 25 software package (IBM Deutschland GmbH., Ehningen, Germany). The descriptive statistics was analysed using the explorative data analysis, especially the Boxplot procedure. The graphics (Figure 2 and 5 to 8) were created by Excel 2016.

\section{Results and Discussion}

Climate conditions in the barn

Figure 2 shows the climate conditions in the fields: air humidity, air temperature and air velocity in the entire barn. Noticeable are the distances to the optimum level and the frequency of the insufficient barns which show still occurring problems in the barn ventilation (Figure 2, Table 1).

The optimum level of air temperature in the entire barn is in a range between $4-16{ }^{\circ} \mathrm{C}$, over $21^{\circ} \mathrm{C}$ get the cows heat stress (Deutscher Tierschutzbund, 2016, LAVES, 2007). Table 1 shows the descriptive statistics of the air temperature, air velocity and air humidity in the entire barn. The graphic (Figure 2) depict that $92.59 \%$ of the analysed barns in the survey period of April until November 2017 the temperature values are mostly under the limit of $21^{\circ} \mathrm{C}$

Figure 3 shows the range of the barn temperature. The median located by $\pm 15^{\circ} \mathrm{C}$. The temperature shows a greatest value of $\pm 25^{\circ} \mathrm{C}$. In sum $75 \%$ of the barns have a temperature under $20^{\circ} \mathrm{C}$. As a result, the temperatures only, do not present the climate problem. Nevertheless, the climate was not good.

Figure 2 and 3 shows the range of the humidity in the barns. $64.81 \%$ of the 54 barns have a very high (over 
$80 \%$ ) or low (fewer than $60 \%$ ) air humidity. The median of humidity is by $\pm 82 \%$. The humidity values show a high variation. The range is by $50 \%$ (lowest value of humidity) to $95 \%$ (greatest value of humidity). The humidity and the temperature are first depending on the climate condition outside, the building construction and the shed occupancy (Polsky et al., 2017). The boxplot of humidity (Figure 3) shows one outrageous value (Nr. 41). This barn is a 3 years old building, very light but all sides were closed on this data collection day and no air circulation could be done. This value in relation to the temperature makes the insufficient climate conditions and the feeling of stuffy air in the barn. Using the temperature - humidity index (THI) that represents the combined effects of air temperature and humidity associated with the level of thermal stress. Villa-Mancera et al. (2010) validated this in his study and came to the same result. The optimum THI value is about 60 . In this current study the THI values are in average by 65 , means no heat stress but regarding to single barns the THI is not in the optimum level. The values moving in the field of low (69-71) and moderately (72-79) heat stress. All barns with a humidity over 60 $\%$ and a temperature over 20 have heat stressed cows.

Normally the air circulation with assistance of a fan, has to start in this moment. TierSchNutztV (2006) says that the cows should be kept in barns with an optimum circulation, dust content, temperature, air humidity and gas concentration of the air. This paragraph can mostly satisfy in the single factors but not included is the air velocity. This value is in each barn not constantly optimal. By taking the air velocity is noticeable that only 22.22 $\%$ (Table 1) of the analysed barns have a constantly air circulation. The most air circulation was at the feeding area. A number of studies (Frazzi et al., 2002, Calegari et al., 2015) have demonstrated that the cooling of the feeding area alone does not totally eliminate the effects of stress. Figure 4 shows the frightening situation of air velocity in the 54 barns. The median is by $0.25 \mathrm{~m} / \mathrm{s}$ caused of the much more than $50 \%$ with none air velocity. The air velocity shows a high difference between the barns caused of the barn construction, additionally ventilation and the function of fans. This graphic show six outrageous values. A reason for these values are the natural cross ventilation and the air velocity outside because some days are very windy outside and the perfect function of the fans. Preferable to the situation of the present construction process would be a value over $70 \%$. It demonstrated, the non-conformity between the ventilation type with the modern construction of barns.

Table 1. Descriptive statistics of the air temperature, air velocity and air humidity in the barn regarding to the optimum level of the different factors $(\mathrm{N}=54)$.

\begin{tabular}{|l|c|c|}
\hline & optimum level & achieved level \\
\hline air temperature in ${ }^{\circ} \mathrm{C}$ & until $21^{\circ} \mathrm{C}$ & $92.59 \%$ \\
\hline air velocity in $\mathrm{m} / \mathrm{s}$ & over $1 \mathrm{~m} / \mathrm{s}$ & $22.22 \%$ \\
\hline air humidity in $\%$ & between $60-80 \%$ & $35.19 \%$ \\
\hline
\end{tabular}

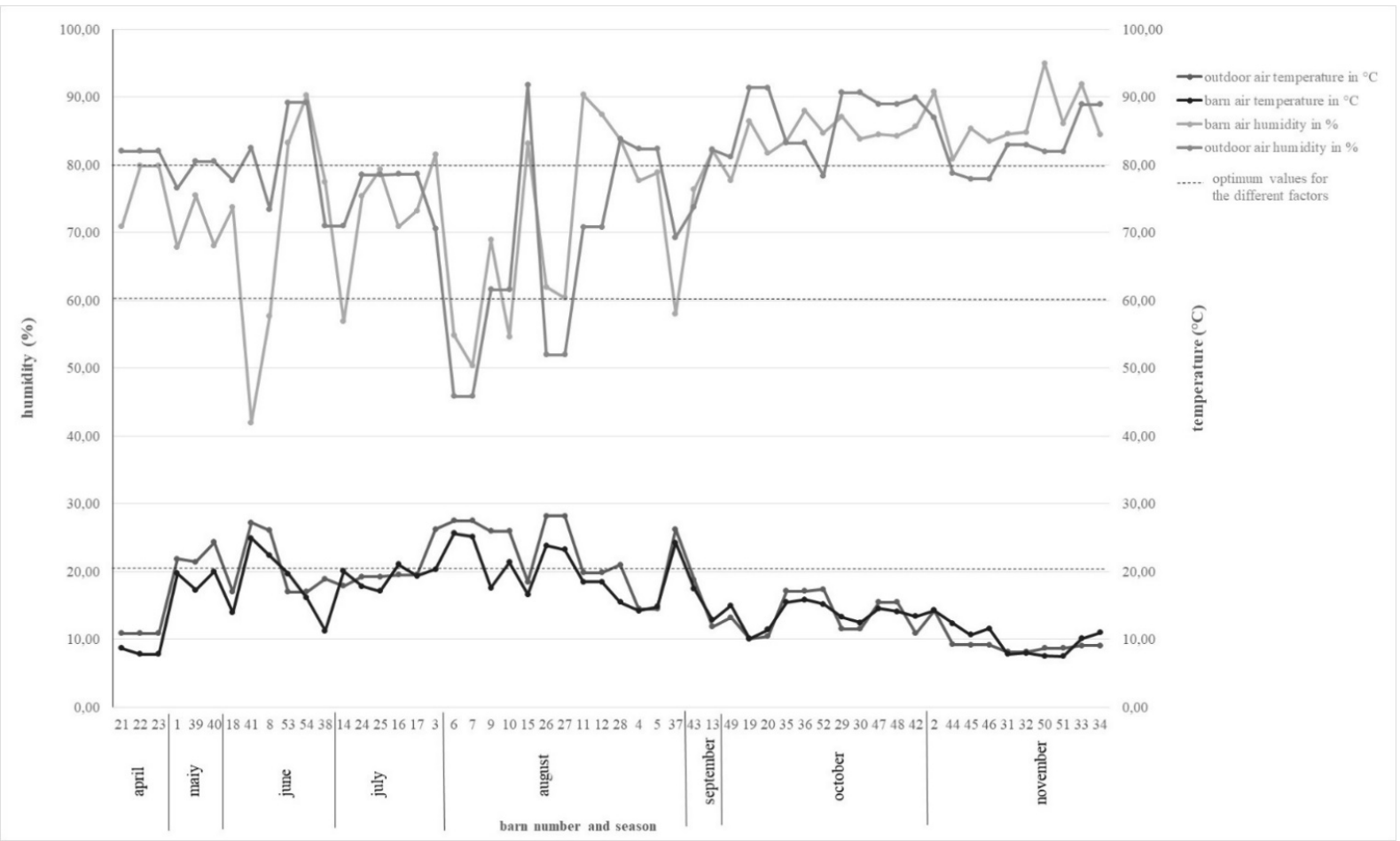

Figure 2. The status quo of the climate conditions at the 54 barns in the survey period April to November. 


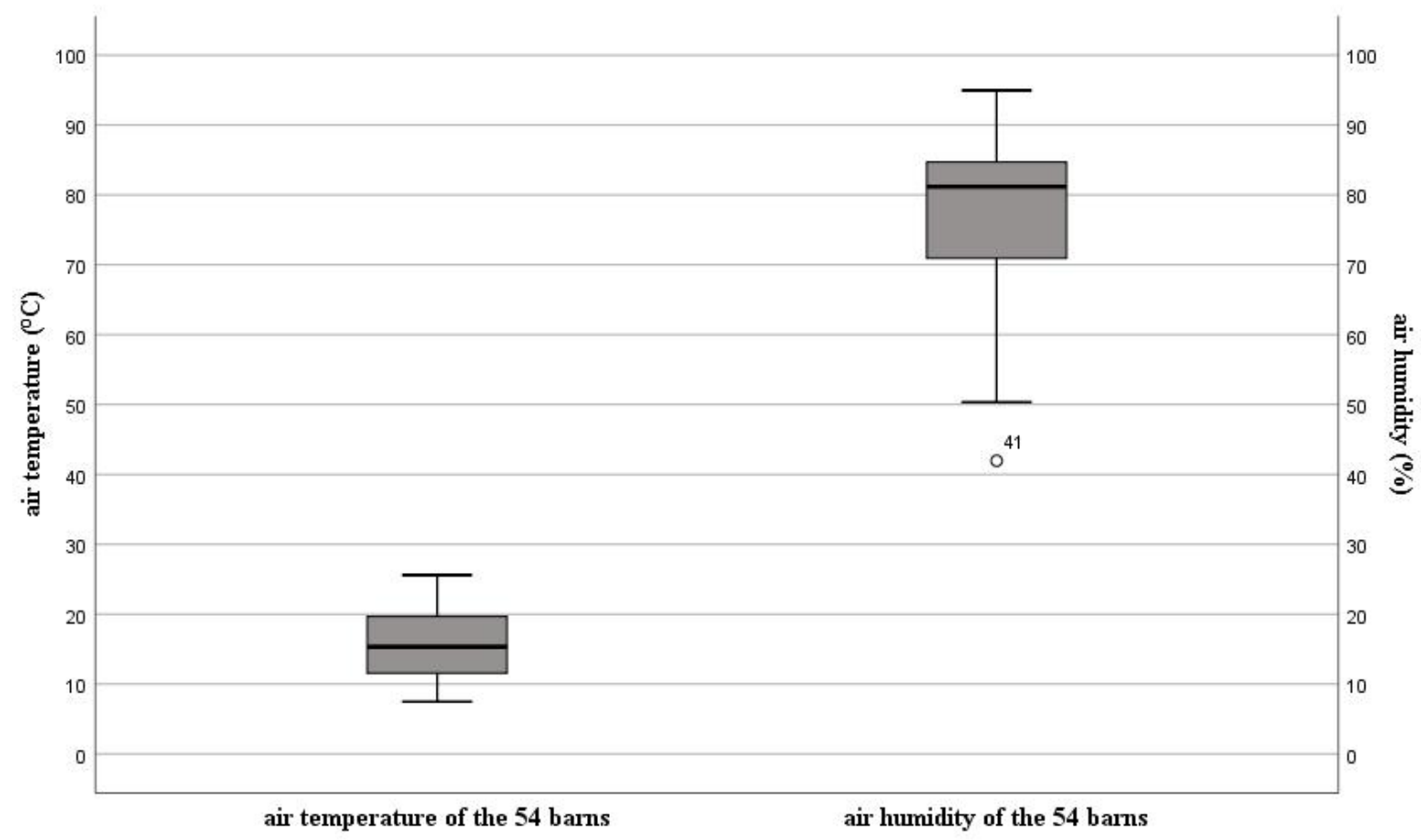

Figure 3. The spread of air temperature and humidity in the 54 barns.

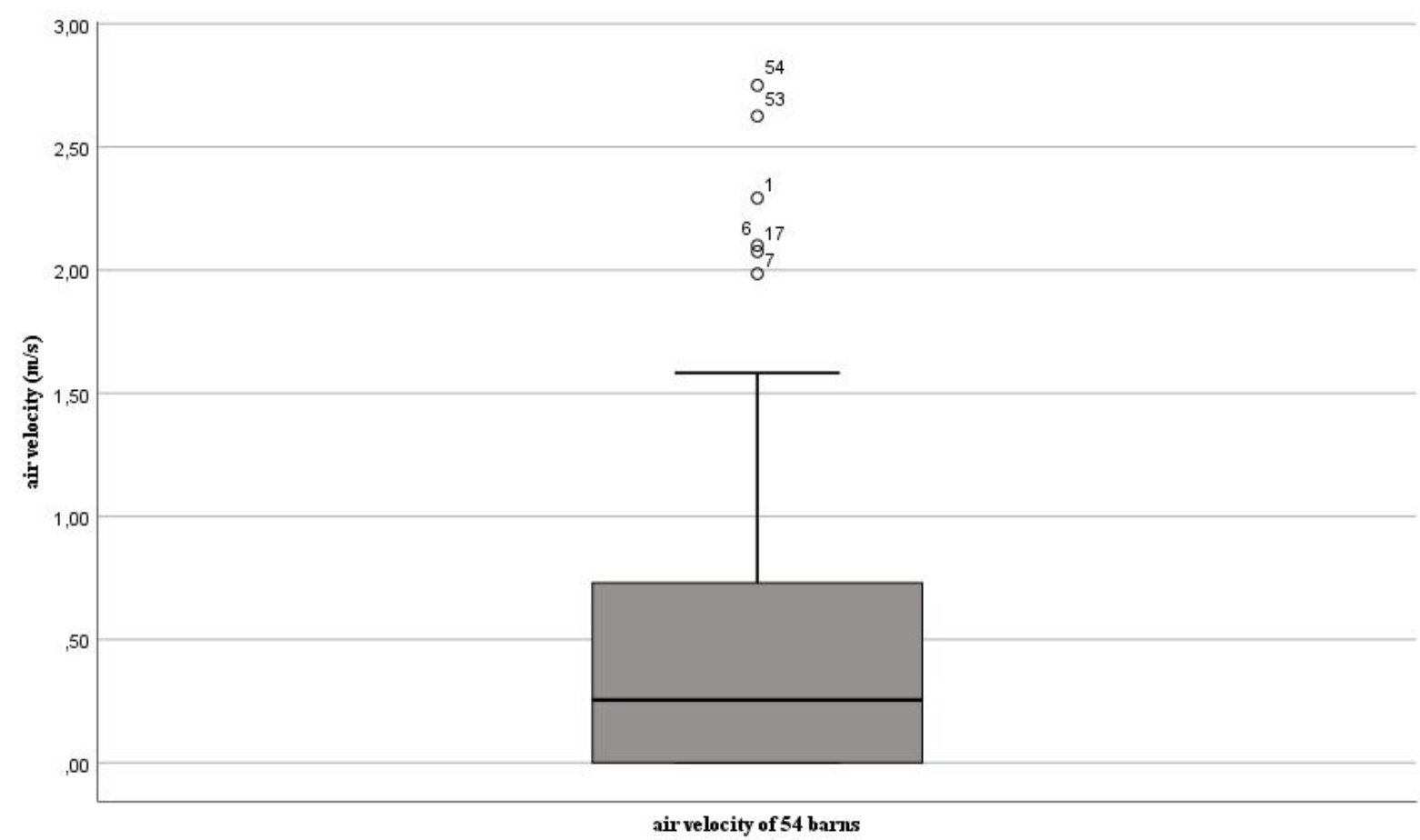

Figure 4. The spread of air velocity in the 54 barns.

Ventilation options and fan types

Figure 5 shows the reason for the insufficient air circulation in the investigated barns. Only $36 \%$ (28 barns) of the analysed farms show ridge ventilation. Calegari et al. (2011) emphasize that this ventilation option in relation with open side walls is the best type for air circulation and the using of encourage cooling like sprinklers and fans. 27 barns have this best type, but only one has sprinklers. $64 \%$ (49 barns) have natural cross ventilation; means they have open side walls, curtains and jalousie and 22 barns of them have additionally fans installed, as a result 27 barns with this ventilation option have no assistance ventilations. 23 of the barns use fans, especially the vertical fans. 

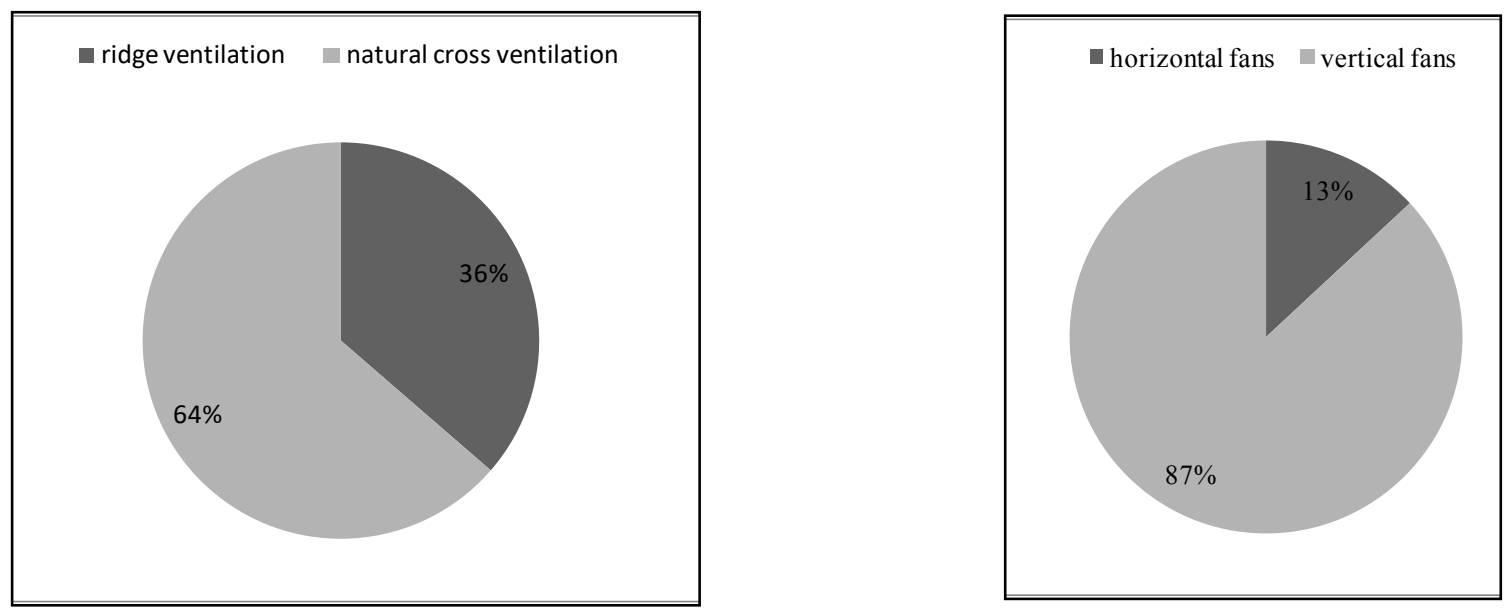

Figure 5. The notation of use of the two different ventilation options. Figure 6 . Notation of the two fan types of the 54 barns.

Noticeable is the influence of fans with the natural ventilation (Figure 6). The fans are positioned on the roof. The vertical fans hang with ropes on the roof and horizontal fans installed directly at the roof. In most barns we have a higher humidity rate (over $80 \%$ ). But in addition to the temperature do not result heat stress. The air velocity is not satisfactory that is a part of wrong installation or underutilized fans that the air circulations only insert near the fan.

Figure 6 shows the climate conditions in none ventilated barns. The temperature is not in the optimum range and surrounded at the limit value of $21^{\circ} \mathrm{C}$. In relation to high humidity brings problems for breath and cow health.

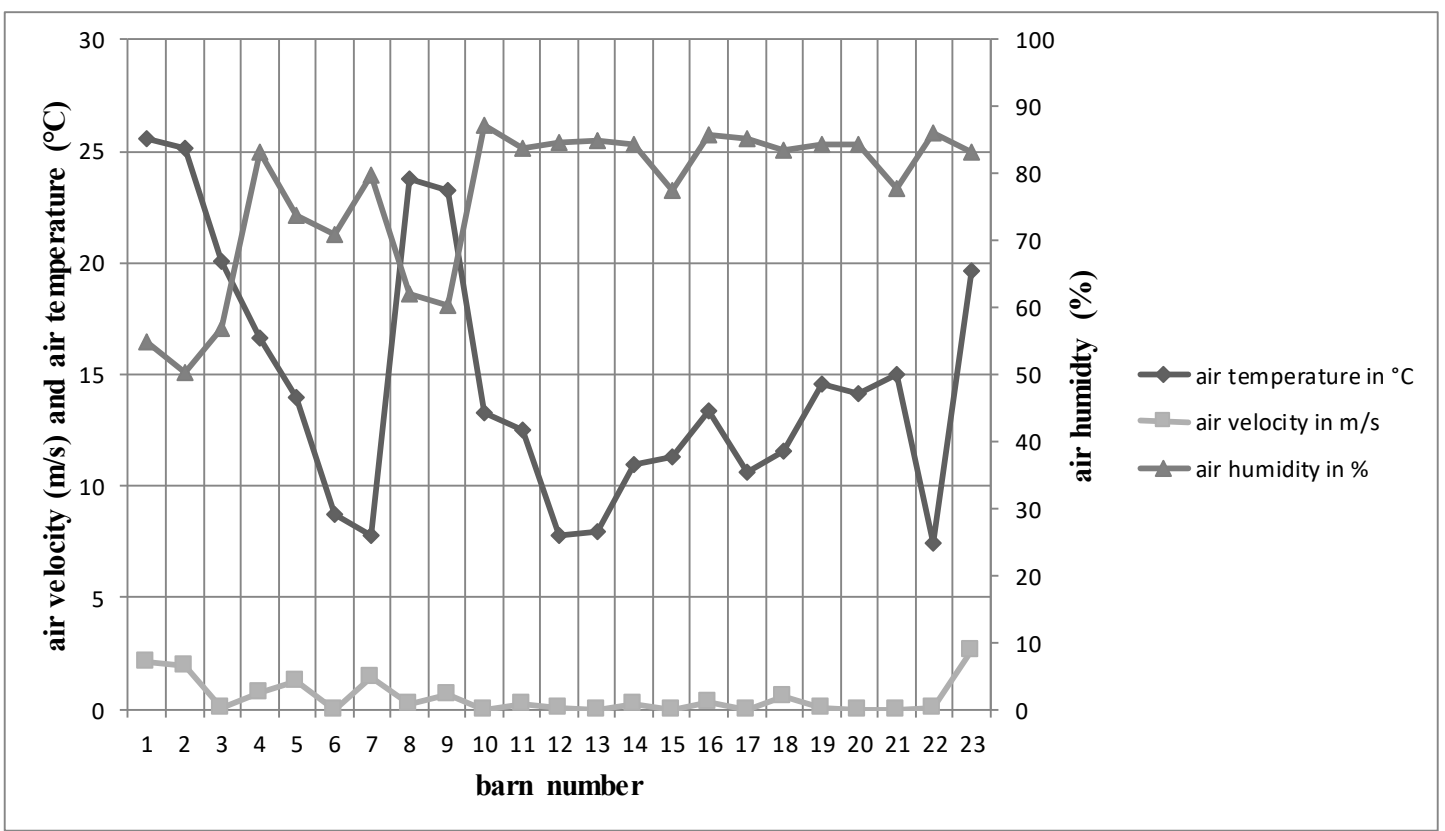

Figure 7. Climate conditions in natural ventilated barns with fan $(\mathrm{N}=23)$. 


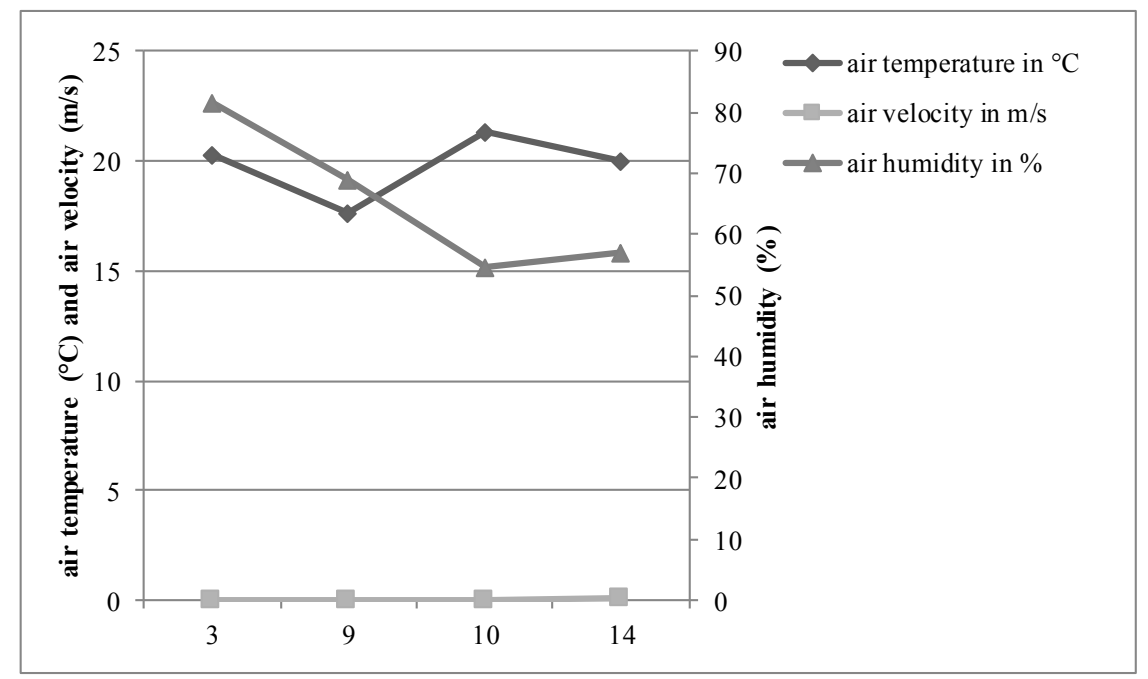

Figure 8. Climate conditions in none ventilated barns $(\mathrm{N}=4)$.

\section{Conclusions}

The special is the climate change which induce sometimes extreme weather conditions. This situation should be given the housing climate more importance. It can be concluded that right ventilation in a barn, irrelevant if new or old building, is also a high challenge for German farmers like the optimum light illumination. Bad ventilation has high influence on the welfare and health of the cows. Optimum climate conditions are one of the requirements for a high status of animal welfare. Positive developments in the construction of barns, in addition to the use of modern climate equipment, have contributed the improvements of the housing conditions.

Finally, can be concluded, the right installation of the fans and open side walls have to be checked to increase animal welfare.

\section{Acknowledgements}

The authors would like to thank all farmers and their employees for their trusted confidence and support. A thank as well the federal ministry of education and research for the financial support. Furthermore, a special thank to the four farms: H. Wilhelm Schaumann GmbH, Gut Hohen Luckow, Agrarhof Brüel eG, LVAT e.V. Ruhlsdorf/Groß Kreutz and Gut Wamckow for the financial and academic support. The university in Hohenheim, Humboldt- Universität zu Berlin, Leibniz - Institut for agricultural engineering and bioeconomy for the scientific support.

\section{References}

Anderson, NG, 2018. Cold housing and open housing - effects on health, management and production of dairy cattle. Journal of Animal Science. 96: 1531 - 1539. Doi: 10.1093/jas/skx065

Calegari, F., Calamari, L., Frazzi, E., 2011. Misting and fan cooling of the rest area in a dairy barn. International Journal of Biometeorology. 56 (2) 287 - 295. https://doi.org/10.1007/s00484-011-0432-7

Calegari, F., Calamari, L., Frazzi, E., 2015. Cooling systems of the resting area in free stall dairy barn. International Journal of Biometeorology. 60 (4) 605 - 614. https://doi.org/10.1007/s00484-015-1056-0

Deutscher Tierschutzbund e.V., 2016. Richtlinie für die Haltung von Milchkühen im Rahmen des Tierschutzlabels „FÜR MEHR TIERSCHUTZ“. 13.

DLG e.V., Heidenreich, T., Büscher, W., Cielejewski, H., 2016. DLG-Merkblatt 336. Vermeidung von Wärmebealstungen bei Milchkühen. Frakfurt am Main, Germany.

Frazzi, E., Calamari, L., Calegari, F., Stefanini, L., 2000. Behavior of dairy cows in response to different barn cooling systems. Transactions of the ASAE. 43 (2) 387. https://doi.org/10.13031/2013.2716

Hulsen, J., 2010. Bauen für die Kuh. Zutphen: RoodBont Verlag.

Hulsen, J., 2014. Cow Signals Checkbook-Working on health, production and welfare. Zutphen: RoodBont Verlag.

LAVES, 2007. Tierschutzleitlinie für die Milchkuhhaltung. Niedersächsisches Ministerium für den ländlichen Raum, Ernährung, Landwirtschaft und Verbraucherschutz, Ref. 204. Hannover, Germany. 57-58.

Ofner-Schröck, E., Breininger, W., Lenz, V., 2017. Stallbau für die Rinderhaltung. Stuttgart: Leopold Stocker Verlag. 
Polsky, L., von Keyserlingk, M. A.G., 2017. Invited review: Effects of heat stress on dairy cattle welfare. Journal of Dairy Science. 100 (11) 8645 - 8657. https://doi.org/10.3168/jds.2017-12651

TierSchNutztV, 2006. Tierschutznutztierhaltungsverordnung. Germany. §3 (3) Satz 2.

Villa-Mancera, A., Méndez-Mendoza, M., Huerta-Crispín, R.,Vázquez-Flores, F., Córdova-Izquierdo, A., 2010. Effect of climate factors on conception rate of lactating dairy cows in Mexico. Tropical Animal Health and Production. 43 (3) 597 - 601. https://doi.org/10.1007/s11250-010-9737-5 


\author{
Measuring the Teat Skin Elasticity with the Help of the Suction Method \\ Susanne Demba ${ }^{\text {a, }}$, Christian Ammon, a , Marie Neumerkel ${ }^{\text {b }}$, Reiner Brunsch a, Sandra Rose- \\ Meierhöfer $^{c}$ \\ ${ }^{a}$ Leibniz Institute for Agricultural Engineering and Bioeconomy e.V. (ATB), Department of Engineering for Livestock \\ Management, 14469 Potsdam, Germany \\ ${ }^{\mathrm{b}}$ Faculty of Life Science, Humboldt-Universität zu Berlin, 10099 Berlin, Germany \\ ${ }^{\mathrm{c}}$ Department of agricultural machinery, Hochschule Neubrandenburg, University of Applied Science, 17033 \\ Neubrandenburg, Germany \\ * Corresponding author. Email: sdemba@atb-potsdam.de
}

\begin{abstract}
The teat of a dairy cow is exposed to a large load during milking because it is the interface between the udder and the liner in the milking process. This results in a fatigue of the teat tissue, especially the teat skin, and can lead to teat damages. The aim of this study was to test the usability of the suction method for teat skin elasticity measurements to get a better understanding of the teat liner interface. The usability of the different measuring parameters was analysed as well. During the present investigation, the Cutometer ${ }^{\mathbb{B}}$ dual MPA 580 was used to investigate the teat skin elasticity of the right rear teats from 101 cows. Measurements were carried out in a sideby-side milking parlour before and after milking using the measuring time-strain mode (suction period about 1 second followed by a 1-second suction-off relaxation period; 450 mbar of suction) of the Cutometer ${ }^{\circledR}$ dual MPA 580. The penetration depth of the skin and the gross elasticity were analysed. Descriptive statistics of both traits were calculated. The penetration depth of the skin before and after milking ranged between $0.50 \mathrm{~mm}$ and 2.86 $\mathrm{mm}$ and $0.50 \mathrm{~mm}$ and $2.82 \mathrm{~mm}$, respectively. The gross elasticity before and after milking was around $26 \%$, respectively and ranges between $0.4 \%$ and $90.6 \%$ before milking and between $20 \%$ and $84 \%$ after milking. Based on the results of our study we concluded that the Cutometer ${ }^{\circledR}$ dual MPA 580 is generally usable to measure teat skin elasticity. The penetration depth of the skin seems to be an important parameter. In conclusion, a combination of several parameters could be most suitable to evaluate teat skin elasticity. Further studies are needed to validate the method. The influence of anatomical, physiological, and technical factors on teat skin elasticity should be investigated as well.
\end{abstract}

Keywords: skin elasticity, teat damages, tissue, dairy cow, machine milking

\title{
1. Introduction
}

The teat skin is the outermost layer of the teat wall. It has a thickness of 1-2 mm, is hairless and usually slightly wrinkled. In addition, it contains a high ratio of elastic fibres, which allow a strong strain of the teat up to $30 \%$ during milking (Hospes and Seeh, 1999). While the udder skin is composed of the epidermis, the dermis and the hypodermis, the teat skin has no hypodermis (Ferdowsi et al., 2013). Thus, a detaching of the skin from their base is impossible. The innervation of the teat is, compared with the udder, pronounced. In the teat skin, especially at the teat tip, many nerve fibres are located (Hospes and Seeh, 1999).

The property of the skin to change and recover shape when it is stretched or deformed is named skin elasticity (Clancy et al., 2010). Skin viscoelasticity combines the elastic properties of the skin with the principle of viscosity, the resistance to flow when a force is applied to a fluid (Everett and Sommers, 2013). The mechanical properties of the skin can be influenced by age (Cua et al., 1990; Ryu et al., 2008; Krueger et al., 2011; Luebberding et al., 2014), gender (Fiedler et al., 2012; Luebberding et al., 2014) and body region (Ryu et al., 2008; Krueger et al., 2011). Teat skin elasticity can be influenced by milking as well (Kuchler, 2011; Krzyś et al., 2011).

The Cutometer $^{\circledR}$ dual MPA 580 (Courage and Khazaka electronic GmbH, Cologne, Germany) is a tool to detect the elasticity of the skin and several skin layers. It consists of a main unit and a measuring probe (Dobrev, 2014) and measures skin elasticity using suction and elongation (Woo et al., 2014). The main unit contains a vacuum pump with pressure sensor and microelectronics to generate a consistent vacuum between 20 and 500 mbar during the measuring procedure. The measuring probe contains the suction head and an optical measuring system (Dobrev, 2014). This optical measuring system includes a light transmitter, a light recipient and two glass prisms (O'goshi, 2006). During the measurement the skin is drawn into the probe and its vertical deformation is measured (Ryu et al., 2008). Depending on the depth of penetration in the probe the incoming light intensity differs. Thus, the ability of the skin to resist the suction (firmness) and to regress to its original condition after removal of the negative pressure is detected (Courage and Khazaka, 2012). There are four measuring modes available: measurement with constant negative pressure (mode 1), measurement with linear increase and decrease in negative pressure (mode 2), measurement with first constant and then linear decrease in negative pressure (mode 3), and measurement with a linear increase in negative pressure and then a sudden elimination of the negative pressure (mode 4). These modes differ in the combination and the meaning of their measurement 
parameters (Dobrev, 2014). The measurement mode 1 is most frequently used in the literature (Cua et al., 1990; Hashmi and Malone-Lee, 2007; Ryu et al., 2008; Krueger et al., 2011; Ohshima et al., 2013; Bonaparte and Chung, 2014; Luebberding et al., 2014; Dykes, 2015;). To evaluate the elastic properties of the skin an output of various parameters is possible. These can be divided into three groups: the R-, F-, and Q-parameters and they are dependent on the mode. While R-parameters are frequently used in skin research (Cua et al., 1990; Dobrev, 2002; Hashmi and Malone-Lee, 2007; Fiedler et al., 2012; Dykes, 2015), F- and Q-parameters are rarely used.

The aim of this study was to test the usability of the suction method for teat skin elasticity measurements, because measuring of teat skin elasticity may help to give information about the fatigue of teat skin during milking and can be a method to evaluate objectively the teat load during milking.

\section{Materials and Methods}

Animals and study design

The study took place on a commercial dairy farm in Germany. The teat skin elasticity of 101 randomly selected German Holstein cows was measured on three consecutive days. The lactating cows were between their first and sixth parity, and the days in milk (DIM) were between 10 and 596 (median = $90 \mathrm{~d}$ ). At the time of investigation, the herd had an average milk yield of $29.8 \mathrm{~kg}$ per cow per day, with a mean fat content of $3.85 \%$ and a protein content of $3.38 \%$. The cows were milked three times a day in a 2 x 24 Side-by-Side milking parlour equipped with conventional milking clusters, and the machine vacuum was adjusted to $42 \mathrm{kPa}$. Alternate pulsation was used with a pulsation rate of $60 \mathrm{~min}^{-1}$ and a pulsation ratio of $60: 40$. The temperature and humidity for test day 1,2 and 3 were $19.3^{\circ} \mathrm{C}$ and $59.5 \%, 24.8^{\circ} \mathrm{C}$ and $60.6 \%$ and $21.5^{\circ} \mathrm{C}$ and $77.4 \%$, respectively.

Data collection

Teat skin elasticity was determined during milking using the Cutometer ${ }^{\circledR}$ dual MPA 580 by Courage and Khazaka electronics GmbH (Cologne, Germany). The opening of the measuring probe was $6 \mathrm{~mm}$ and the timestrain mode (mode 1) was used. For each measurement, a suction period about 1 second followed by a 1-second suction-off relaxation period was used with a setting of 450 mbar of suction. This measuring cycle was repeated ten times, so the measuring time was 20 seconds per measuring. Teat skin elasticity was taken before and after milking on the right rear teat (Figure 1). Before milking indicates after udder cleaning and after milking means directly after cluster removal and before dipping.

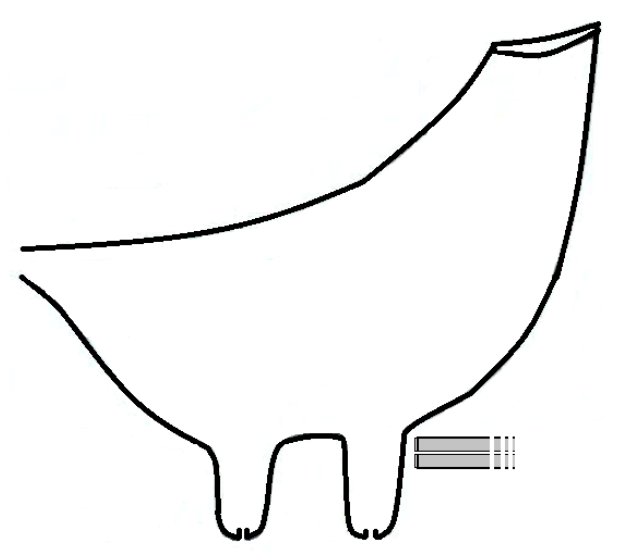

Figure 1. The measuring point on the right rear teat for teat skin elasticity detection.

Statistical analysis

All statistical analyses were performed using the SAS 9.4 software package (SAS Institute Inc., Cary, NC, USA). The penetration depth of the skin (R0 in $\mathrm{mm}$ ) and the gross elasticity (R2 in \%) were used to evaluate the usability of the suction method to measure teat skin elasticity. The descriptive statistics was analysed using the UNIVARIATE procedure. T-tests of the differences were performed to determine differences in R0 and R2 before and after milking, respectively. 


\section{Results and Discussion}

Penetration depth of the skin (R0)

R0 before and after milking ranged between $0.50 \mathrm{~mm}$ and $2.86 \mathrm{~mm}(1.87 \mathrm{~mm} \pm 0.83)$ and $0.50 \mathrm{~mm}$ and 2.82 $\mathrm{mm}(2.10 \mathrm{~mm} \pm 0.76)$, respectively. Table 1 shows the descriptive statistics of R0 before and after milking.

Table 1. Descriptive statistics of the penetration depth of the skin $(\mathrm{mm})$ before and after milking $(\mathrm{N}=105)$.

\begin{tabular}{lcc} 
& Before milking & After milking \\
\cline { 2 - 3 } Mean & 1.87 & 2.10 \\
Standard deviation & 0.83 & 0.76 \\
Variance & 0.68 & 0.57 \\
Range & 2.36 & 2.32 \\
Interquartile range & 1.59 & 1.42 \\
Coefficient of variation & 44.2 & 36.0 \\
\hline
\end{tabular}

The values of R0 show a high variation and mean R0 was significant higher after milking than before $(\mathrm{P}=$ 0.0003). Figure 2 shows the related values of R0 before and after milking. The data points on the right side of the diagonal line illustrate that in some cases the values of $\mathrm{R} 0$ were lower after milking than before.

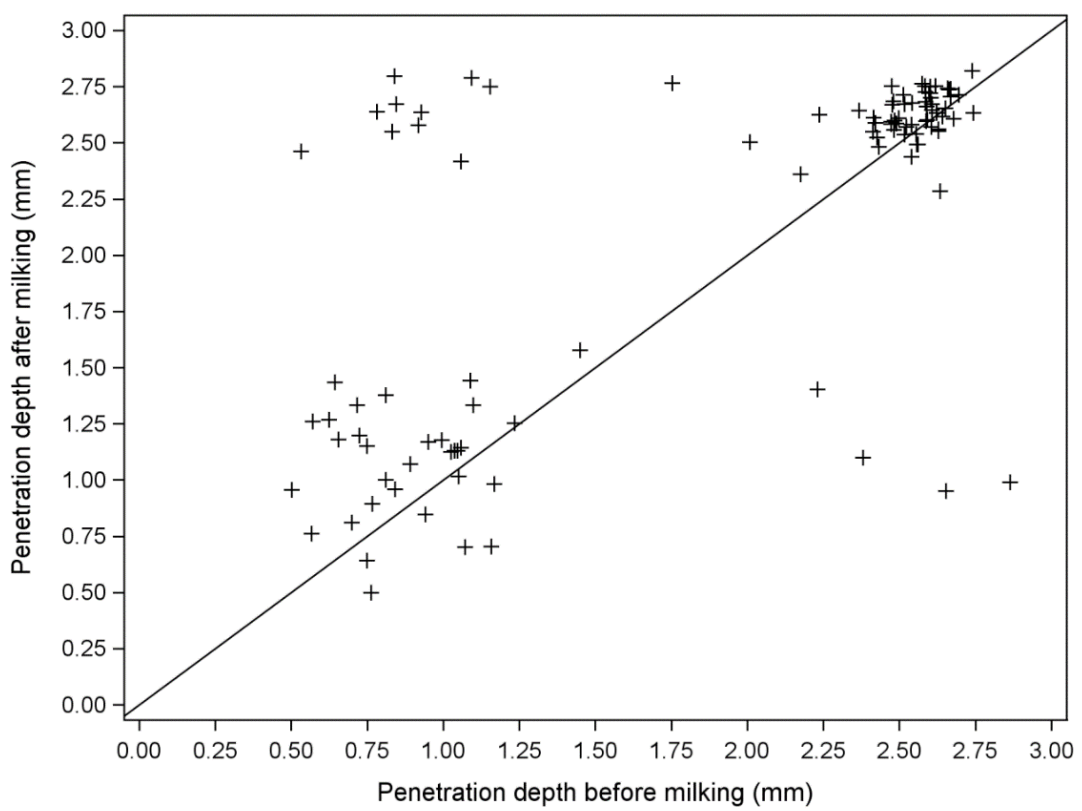

Figure 2. The related values of the penetration depth of the skin $(\mathrm{mm})$ before and after milking with higher values after milking on the left side and higher values before milking on the right side of the diagonal line.

Gross elasticity (R2)

$\mathrm{R} 2$ before and after milking was around $26 \%( \pm 24)$, respectively and ranges between $0.4 \%$ and $90.6 \%$ before milking and between $20 \%$ and $84 \%$ after milking. Table 1 shows the descriptive statistics of R2 before and after milking.

Table 2. Descriptive statistics of the gross elasticity of the skin (\%) before and after milking $(\mathrm{N}=105)$

\begin{tabular}{lcc}
\hline & Before milking & After milking \\
\cline { 2 - 3 } Mean & 26.3 & 26.1 \\
Standard deviation & 23.1 & 23.9 \\
Variance & 5.3 & 5.7 \\
Range & 90.2 & 82.0 \\
Interquartile range & 36.5 & 32.9 \\
Coefficient of variation & 87.8 & 91.5 \\
\hline
\end{tabular}

The values of R2 show a very high variation and no differences between mean R2 before and after milking could be found. Figure 3 shows the related values of R2 before and after milking. This figure illustrates that the values of R2 were higher after milking than before and the other way around. 


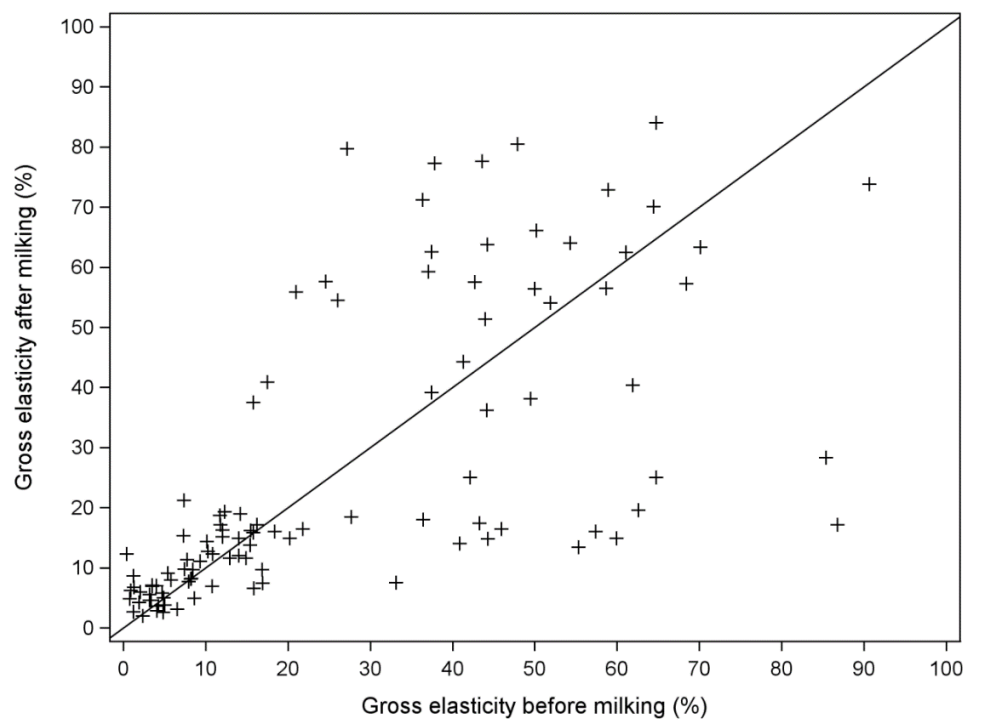

Figure 3. The related values of the gross elasticity of the skin (\%) before and after milking with higher values after milking on the left side and higher values before milking on the right side of the diagonal line.

In this investigation the mean penetration depth of the skin was $1.87 \mathrm{~mm}$ before and $2.10 \mathrm{~mm}$ after milking. For the penetration depth of human skin 0.03-0.08 mm (Krueger et al., 2011), 0.09-0.13 mm (Luebberding et al., 2014), 0.24-0.34 mm (Woo et al., 2014) and 0.61-0.94 mm (Bonaparte and Ellis, 2014) were recorded. The measurements of teat skin were higher, so the teat skin penetrated deeper into the probe during measurements. The gross elasticity for teat skin found in this investigation was before and after milking $0.4-90.6 \%$ and $20-84 \%$, respectively. The mean gross elasticity was $26 \%$ for both times of measurement. For human skin the gross elasticity is stated with 40.98-76.67\% (Krueger et al., 2011), 79.9-87\% (Bonaparte and Chung, 2014), 57.1157.33\% (Luebberding et al., 2014), 67-77\% (Bonaparte and Ellis, 2014), 76-88\% (Woo et al., 2014) and 79-83\% (Nam et al., 2015). The differences in the anatomic structure between human skin and teat skin could be a possible reason for the lower firmness and elasticity of teat skin. Generally human skin is similar to teat skin, but in detail some differences can be seen. Teat skin shows more wrinkles than human skin and has a very high proportion of elastic fibres (Hospes and Seeh, 1999). This could be the reason for the higher R0 values. In contrast, Hibbitt et al. (1992) describe the teat skin as a firm surface which is able to tolerate mechanical shear forces. This should result in a lower R0 of the teat skin. R2 of teat skin was lower than that of human skin. This disagrees with the anatomic differences regarding the higher proportion of elastic fibres (Hospes and Seeh, 1999). Another reason for the lower firmness and elasticity of teat skin could be the differences in skin thickness between teat and human skin, because the mechanical properties of skin are influenced of skin layer thickness (Krueger et al., 2011). With 1-2 mm teat skin is thicker than human skin. Liang and Boppart (2010) found a thickness of 0.09-0.20 mm for human epidermis and stratum corneum. The measured values of teat skin have a high variation for all traits. In our investigation this variation could be explained with the experimental setup. The chosen measuring time of 20 $\mathrm{s}$ was very long and not all of the cows stood still for the whole time. Thus, air got into the probe and could have distorted the measurements. The use of different device settings regarding measuring mode, length of suction and relaxation and number of repetitions could reduce the variability. In some investigations the variation for human skin elasticity was high as well (Luebberding et al., 2014; Bonaparte and Ellis, 2014). R0 seems to be an important trait to evaluate teat skin elasticity, because several parameters were calculated with the help of it. Ohshima et al. (2013) showed in their study that the parameter F3 is appropriate to evaluate cheek skin elasticity, because it is less influenced by environmental factors compared with the R-parameters. Perhaps the area parameters are suitable to detect teat skin elasticity as well. According to the manuals these parameters have no limiting values, which represent good or bad elasticity. This would be a problem to analyse the elastic properties of teat skin with the help of the F-parameters. The most important problem seems to be that there is no gold standard for measuring skin elasticity. Thus, it is not clear which parameter and experimental setup represents the real elastic properties of skin (Bonaparte and Chung, 2014). This discussion can be transferred to teat skin elasticity as well.

\section{Conclusions}

Based on the results of the present study, the suction method is generally usable to measure the elasticity of teat skin. To reduce the high variability of the measured values, other device settings might be helpful. This should be investigated in further studies. A validation of the suction method to measure teat skin elasticity is necessary. 
It cannot be concluded that one of the tested traits is superior to the other regarding the detection of teat skin elasticity. Because several parameters were calculated with the help of the penetration depth of the skin, it seems to be an important parameter. A combination of several parameters is most suitable to evaluate teat skin elasticity.

\section{Acknowledgements}

The authors would like to thank the management and the staff of the Milchhof Rodenwalde KG for their great support during this investigation.

\section{References}

Bonaparte, J.P. and Chung, J. 2014. The effect of probe placement on inter-trial variability when using the Cutometer MPA 580. Journal of Medical Engineering and Technology. 38 (2) 85-89. http://dx.doi.org/10.3109/03091902.2013.876111.

Bonaparte, J.P. and Ellis, D. 2014. Skin Biomechanical Changes after Injection of Onabotulinum Toxin A: Prospective Assessment of Elasticity and Pliability. Otolaryngology-Head and Neck Surgery. 150 (6) 949-955. http://dx.doi.org/10.1177/0194599814526558.

Clancy, N.T., Nilsson, G.E., Anderson, C.D., and Leahy, M.J. 2010. A new device for assessing changes in skin viscoelasticity using indentation and optical measurement. Skin research and Technology. 16 (2) 210-228. http://dx.doi.org/10.1111/j.1600-0846.2010.00433.x.

Courage and Khazaka 2012. User manual of the The Cutometer ${ }^{\circledR}$ dual MPA 580 Cologne, Germany: Courage+Khazaka electronic GmbH.

Cua, A.B., Wilhelm, K.P., and Maibach, H.I. 1990. Elastic properties of human skin - relation to age, sex and anatomical region. Clinical Research. 38 (2) A681-A681. https://doi.org/10.1007/BF00375720.

Dobrev, H. 2014. Cutometer. In Non Invasive Diagnostic Techniques in Clinical Dermatology, Vol. 1, Berardesca, E., Maibach, H. and Wilhelm, K.-P. Eds., Springer, 315-338.

Dobrev, H.P. 2002. A study of human skin mechanical properties by means of Cutometer. Folia medica. 44 (3) 5-10.

Dykes, P. 2015. Increase in skin surface elasticity in normal volunteer subjects following the use of copper oxide impregnated socks. Skin Research and Technology. 21 (3) 272-277. http://dx.doi.org/10.1111/srt.12187.

Everett, J.S. and Sommers, M.S. 2013. Skin Viscoelasticity: Physiologic Mechanisms, Measurement Issues, and Application to Nursing Science. Biological Research for Nursing. 15 (3) 338-346. http://dx.doi.org/10.1177/1099800411434151.

Ferdowsi, H., Moradi, M.A., and Asadi, M. 2013. Histolocical Study on the Sensory Receptors of the Teat Skin in Sarabi Cattle. International Journal of Basic Sciences and Applied Research. 2 (2) 168-171.

Fiedler, M., Gerhardt, L.C., Derler, S., Bischofberger, G., Hurny, C., and Munzer, T. 2012. Assessment of Biophysical Skin Properties at Different Body Sites in Hospitalized Old Patients: Results of a Pilot Study. Gerontology. 58 (6) 513-517. http://dx.doi.org/10.1159/000336623.

Hashmi, F.and Malone-Lee, J. 2007. Measurement of skin elasticity on the foot. Skin Research and Technology. 13 (3) 252-258. http://dx.doi.org/10.1111/j.1600-0846.2007.00216.x.

Hibbitt, K.G., Craven, N., and Batten, E.H. 1992. Anatomy, Physiology and Immunology of the Udder. In Bovine Medicine. Diseases and Husbandry of Cattle, $2^{\text {nd }}$ ed., Andrews, A.H., Blowey, R.W., Boyd, H., and Eddy, R.G. Eds., Blackwell Scientific Publications. Oxford, UK, 311-325.

Hospes, R. and Seeh, C. 1999. Sonographie und Endoskopie an der Zitze des Rindes.(Sonography and endoscopy of the bovine teat) Stuttgart: Schattauer Verlagsgesellschaft $\mathrm{mbH}$.

Krueger, N., Luebberding, S., Oltmer, M., Streker, M., and Kerscher, M. 2011. Age-related changes in skin mechanical properties: a quantitative evaluation of 120 female subjects. Skin Research and Technology. 17 (2) 141-148.

http://dx.doi.org/10.1111/j.1600-0846.2010.00486.x.

Krzyś, A., Luberański, A., Szlachta, J., and Wiercioch, M. 2011. Dependencies of elasticity changes and geometric parameters on tension force of liners used for milking. Agricultural Engineering. 7 (132) 45-55.

Kuchler, K. 2011. Der Einfluss des Melkens auf Durchblutung und Morphologie der Rinderzitze untersucht mittels Color Angiographie und B-Mode Sonographie. (The influence of milking on blood flow and morpholohy of bovine teats using color angiography and B-mode sonography). Dissertation. Ludwig-Maximilian-Universität München, München, Germany.

Liang, X. and Boppart, S.A. 2010. Biomechanical properties of in vivo human skin from dynamic optical coherence elastography. IEEE Transactions on Biomedical Engineering. 57 (4) 953-959. http://dx.doi.org/10.1109/tbme.2009.2033464.

Luebberding, S., Krueger, N., and Kerscher, M. 2014. Mechanical properties of human skin in vivo: a comparative evaluation in 300 men and women. Skin Research and Technology. 20 (2) 127-135. http://dx.doi.org/10.1111/srt.12094. 
Nam, G.W., Baek, J.H., Koh, J.S., and Hwang, J.K. 2015. The seasonal variation in skin hydration, sebum, scaliness, brightness and elasticity in Korean females. Skin Research and Technology. 21 (1) 1-8. http://dx.doi.org/10.1111/srt.12145.

O'goshi, K.I. 2006. Suction Chamber Method for Measurement of Skin Mechanics: The Cutometer ${ }^{\mathbb{R}}$. In Handbook of Non-Invasive Methods and the Skin, $2^{\text {nd }}$ ed., Serup,J., Jemec, G.B. and Grove, G.L., Eds., CRC Press, $579-582$.

Ohshima, H., Kinoshita, S., Oyobikawa, M., Futagawa, M., Takiwaki, H., Ishiko, A., and Kanto, H. 2013. Use of Cutometer area parameters in evaluating age-related changes in the skin elasticity of the cheek. Skin Research and Technology. 19 (1) E238-E242. http://dx.doi.org/10.1111/j.1600-0846.2012.00634.x.

Ryu, H.S., Joo, Y.H., Kim, S.O., Park, K.P., and Youn, S.W. 2008. Influence of age and regional differences on skin elasticity as measured by the Cutometer ${ }^{\mathbb{B}}$. Skin Research and Technology. 14 (3) 354-358. http://dx.doi.org/10.1111/j.1600-0846.2008.00302.x.

Woo, M.S., Moon, K.J., Jung, H.Y., Park, S.R., Moon, T.K., Kim, N.S., and Lee, B.C. 2014. Comparison of skin elasticity test results from the Ballistometer ${ }^{\mathbb{B}}$ and Cutometer $^{\circledR}$. Skin Research and Technology. 20 (4) 422428. http://dx.doi.org/10.1111/srt.12134. 


\title{
Greenhouse Gas Emissions from Dairy Open Lots in China
}

\author{
Luyu Ding ${ }^{\mathrm{a}}$, Chaoyuan Wang ${ }^{\mathrm{b,c}}$, Guoqiang Zhang ${ }^{\mathrm{d}}$, Zhengxiang Shi ${ }^{\mathrm{b}, \mathrm{c}}$, Baoming Li ${ }^{\mathrm{b}, \mathrm{c}}$, Weichao \\ Zheng, ${ }^{\mathrm{b}, \mathrm{c}}$, Ligen Yua, Qifeng $\mathbf{L i}^{\mathrm{a}}$ \\ a National Engineering Research Center for Information Technology in Agriculture (NERCITA), Beijing 100097, P.R. \\ China \\ ${ }^{\mathrm{b}}$ Department of Agricultural Structure and Bioenvironmental Engineering, College of Water Resources and Civil \\ Engineering, China Agricultural University, Beijing 100083, P.R. China \\ ${ }^{\mathrm{c}}$ Key Laboratory of Agricultural Engineering in Structure and Environment, Ministry of Agriculture, Beijing100083, \\ P.R. China \\ ${ }^{\mathrm{d}}$ Department of Engineering, Aarhus University, Tjele 8830, Denmark \\ * Corresponding author. Email: gotowchy@cau.edu.cn
}

\begin{abstract}
Nowadays, open lots are still popular facilities in dairy housing system to provide free ranging space for cows in China. Due to the deposition of feces and urine, a large amount of greenhouse gases (GHG) and ammonia $\left(\mathrm{NH}_{3}\right)$ emit from the open lots, contributing to global warming and causing nitrogen losses. Both field measurements and lab scale experiments were conducted to evaluate gaseous emissions from dairy open lots in this study. In field measurements, GHG emissions $\left(\mathrm{CO}_{2}, \mathrm{CH}_{4}\right.$ and $\left.\mathrm{N}_{2} \mathrm{O}\right)$ from the ground level of a typical open lot in the suburb of Beijing was surveyed by close chambers in four different seasons. Totally 420 sampling events were conducted and the overall estimated $\mathrm{CO}_{2}, \mathrm{CH}_{4}$ and $\mathrm{N}_{2} \mathrm{O}$ emissions were $4.9-341.0 \mathrm{~kg} \cdot \mathrm{hd}^{-1} \cdot \mathrm{yr}^{-1}, 28.8-1306.7$ $\mathrm{g} \cdot \mathrm{hd}^{-1} \cdot \mathrm{yr}^{-1}, 32.9-331.4 \mathrm{~g} \cdot \mathrm{hd}^{-1} \cdot \mathrm{yr}^{-1}$ during the field measurement, respectively. In the simulation experiment, a scale model of dairy open lots was used to investigate gaseous emissions at varied air temperatures $\left(15-35^{\circ} \mathrm{C}\right)$, surface velocities $\left(0.4-1.2 \mathrm{~m} \cdot \mathrm{s}^{-1}\right)$ and floor types (unpaved soil floor or brick-paved floor) in controlled laboratory conditions by wind tunnels. $\mathrm{CO}_{2}, \mathrm{CH}_{4}, \mathrm{~N}_{2} \mathrm{O}$ and $\mathrm{NH}_{3}$ volatilizations were measured in a 24-hour duration after feces and urine deposition. Significantly affected by air temperature and surface air velocity, $\mathrm{CO}_{2}, \mathrm{CH}_{4}, \mathrm{~N}_{2} \mathrm{O}$ and $\mathrm{NH}_{3}$ emissions ranged from 34.4 to $202.6 \mathrm{mg} \cdot \mathrm{kg}^{-1} \cdot \mathrm{h}^{-1}$, from 0.3 to $3.2 \mathrm{mg} \cdot \mathrm{kg}^{-1} \cdot \mathrm{h}^{-1}$, from 0.05 to $0.60 \mathrm{mg} \cdot \mathrm{kg}^{-1} \cdot \mathrm{h}^{-}$ ${ }^{1}$ and from 2.33 to $13.7 \mathrm{mg} \cdot \mathrm{kg}^{-1} \cdot \mathrm{h}^{-1}$ in the latter 12 hours which had a relatively stable emission rate. Compared with unpaved soil floor, higher $\mathrm{CO}_{2}$ and $\mathrm{NH}_{3}$ emitted from the brick-paved floor. Via $\mathrm{N}_{2} \mathrm{O}$ and $\mathrm{NH}_{3}$ volatilization, $1.6 \%-8.6 \%$ of total nitrogen in the manure lost in the latter 12 hours of experimental duration.
\end{abstract}

Key words: field measurements, scale model, dairy manure, nitrogen loss, LivAGE

\section{Introduction}

Dairy operation in China and some other part of the world involve outdoor open lots for free ranging to increase the welfare of dairy cows. Cows spent a lot of time on the open lots and manure deposited on the lots remained from days to months, causing large amount of gas emissions. As an important ground-level area source, the open lots may contribute to $51 \%$ of carbon dioxide $\left(\mathrm{CO}_{2}\right), 72 \%$ of nitrous oxide $\left(\mathrm{N}_{2} \mathrm{O}\right)$, and $63-95 \%$ of ammonia $\left(\mathrm{NH}_{3}\right)$ emissions at ground level in dairy farms (Mukhtar et al., 2008; Borhanet al., 2011a,b). Intensive animal farms in China are subjected to pay their green tax bill based on emission levels since the January $1^{\text {st }}$ of 2018 . However, there are few field and simulation studies on the emissions from the lot because of its open characterization, relative low gas concentration and the potential impact on the daily management of the farms, for China in particular. As a major emission source in housing system, it's essential to investigate the gas emissions from open lots aiding to assess the emission levels of the whole farm.

Manure and floor surface are the major emission sources from the ground level of open lots. Air temperature, wind turbulence, floor type, manure characters and the way of manure collection or temporary storage greatly affect the gaseous emissions from manure or floor surfaces (Gonzalez-Avalos et al., 2001; Saha et al., 2010; Pereira et al., 2011, 2012; Owen and Silver, 2014). In the outdoor open lot condition, these factors are quite different from those in indoor condition. For instance, it is concrete floor inside the dairy barn while unpaved soil floor and brick-paved floor are normally used for outdoor open lots. For unpaved soil floor or brick-paved floor, a part of soluble content in manure is kept in soil and brick layer due to infiltration. This may results to an indirect gas emission and affect the total emissions measured from the ground level. The most common studied material in indoor condition mainly was well mixed slurry or liquid manure which has higher water content than that in open lots and the velocity normally was $0.1-0.5 \mathrm{~m} \cdot \mathrm{s}^{-1}$ which much lower than outdoor condition. Data collected from field measurements are affected by multi factors. It's difficult to clarify the effect of each single factor. For these reasons, a lab scale experiment is needed to simplify the impact factors and it will be very helpful in estimating and characterizing gaseous emissions from the open lots. In this study, both field measurements and laboratory experiments were conducted to investigate gas emissions from the ground level of open lots and evaluate the impacts of air temperature, velocity and floor types on the emissions. 


\section{Material and method}

Field measurements

The field measurements were conducted in four different seasons at a commercial dairy farm in Yanqing district of Beijing. The surveyed open lot is $40 \mathrm{~m}$ long and $27 \mathrm{~m}$ wide with 45 lactation cows on it. GHG emissions $\left(\mathrm{CO}_{2}, \mathrm{CH}_{4}\right.$ and $\left.\mathrm{N}_{2} \mathrm{O}\right)$ from the ground level of the open lot were measured by closed chambers, whose accuracy had been evaluated prior to the measurements (Ding et al., 2015). The closed cylindrical chambers were 0.5 $\mathrm{m} \times 0.3 \mathrm{~m}$ (diameter $\times$ height) and sealed by sand.

Due to the variation of the manure accumulated on the floor surface, the open lot was divided into a cubicle zone (a $1.5 \mathrm{~m}$ width area surrounding the cubicles) and an aisle zone for a better clarification of the emission sources. Gases were sampled at only one location in each zone, due to the disturbance of dairy cow, the high labor intensity of manually gas sampling, and relatively small area of the surveyed open lot. Manure scraped out of the surveyed open lot daily at about 13:00. Gas sampling were conducted before and after manure removal. Totally 420 gas samples were taken in the field measurements. Gas samples were analyzed immediately after finishing all the gas sampling events by gas chromatography method.

Gas fluxes at sampling locations were calculated by:

$$
F=\frac{V}{A} \cdot \frac{d c}{d t}
$$

where $F$ is the gaseous flux of the lot $\left(\mathrm{mg} \cdot \mathrm{m}^{-2} \cdot \mathrm{min}^{-1}\right) ; V$ is the volume of the chamber headspace $\left(\mathrm{m}^{3}\right)$; and $A$ is the emitting surface area covered by the chamber $\left(\mathrm{m}^{2}\right)$; and $d c / d t$ is the gradients of gas concentrations changing over time derived from linear regression $\left(\mathrm{mg} \cdot \mathrm{m}^{-2} \cdot \mathrm{min}^{-1}\right)$.

Greenhouse gas emissions from the whole surveyed open lot $\left(\mathrm{Eol}, \mathrm{kg} \cdot \mathrm{hd}^{-1} \cdot \mathrm{yr}^{-1}\right)$ were estimated based on its area, stocking density of dairy cows, and the fluxes before and after manure removal from different measurement locations in each season by:

$$
E_{o l}=0.131 \cdot D \cdot \frac{A_{1} \sum\left(F_{a 1, i}+F_{b 1, i}\right)+A_{2} \sum\left(F_{a 2, i}+F_{b 2, i}\right)}{2 A_{0}}
$$

where $A_{1}$ and $A_{2}$ are the area of aisle zone and cubicle zone $\left(\mathrm{m}^{2}\right) ; A_{0}$ is the area of open lot $\left(\mathrm{m}^{2}\right) ; \mathrm{F}_{\mathrm{a}}$ and $\mathrm{F}_{\mathrm{b}}$ are the gaseous fluxes of aisle zone right after manure removal and before manure removal $\left(\mathrm{mg} \cdot \mathrm{m}^{-2} \cdot \mathrm{min}^{-1}\right) ; \mathrm{F}_{\mathrm{a} 2}$ and $\mathrm{F}_{\mathrm{b} 2}$ are the gaseous fluxes of cubicle zone right after manure removal and before manure removal $\left(\mathrm{mg} \cdot \mathrm{m}^{-}\right.$ $\left.{ }^{2} \cdot \min ^{-1}\right)$.

Lab scale experiment

In the laboratory experiments, dairy open lot scale models were built to evaluate the impact of air temperature ( $\mathrm{T}, 15$ to $35^{\circ} \mathrm{C}$ ), surface velocity (V, 0.4 to $1.2 \mathrm{~m} \cdot \mathrm{s}^{-1}$ ) and floor type (unpaved soil floor or brick-paved floor) on $\mathrm{CO}_{2}, \mathrm{CH}_{4}, \mathrm{~N}_{2} \mathrm{O}$ and $\mathrm{NH}_{3}$ emissions by a wind tunnel.

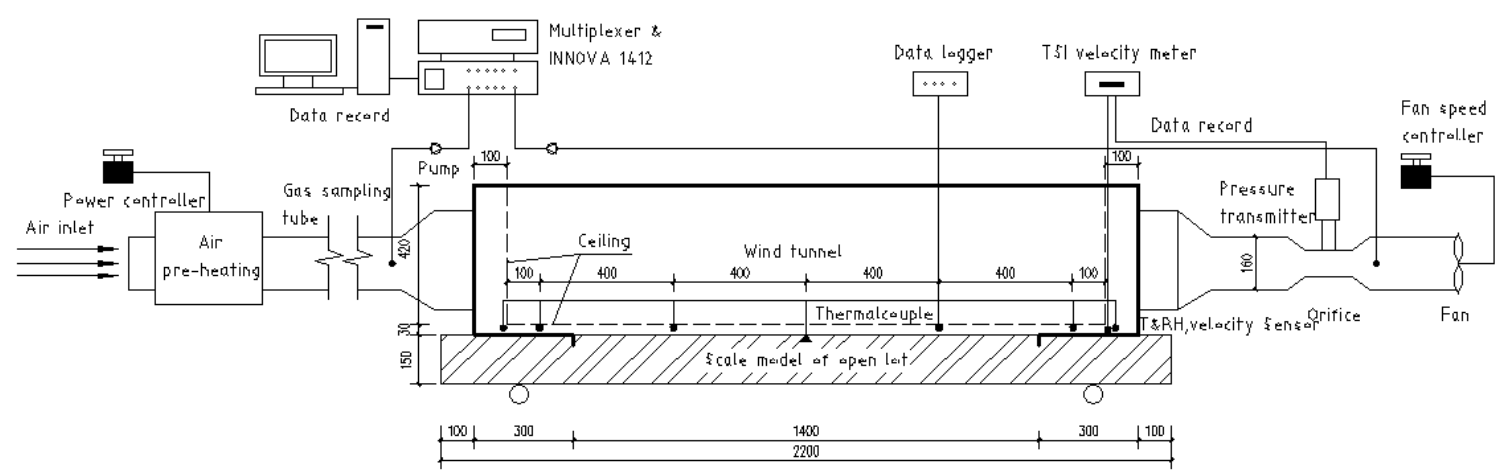

Figure 1. Schematic diagram of experimental setup for simulated open lot (dimensions are in $\mathrm{mm}$ ).

\section{Setup and measurement}

Figure 1 shows the experiment setup which consists of an air pre-heating system, a wind tunnel, a suction fan, a scale model of dairy open lot and a measurement system to monitor thermal environment and gas concentrations. The preheating system and suction fan were to warm the incoming air to a designed temperature and to provide expected air velocity over the emitting surface. The wind tunnel was $2 \mathrm{~m}$ long, $0.5 \mathrm{~m}$ wide and $0.45 \mathrm{~m}$ high, with a working area of $0.7 \mathrm{~m}^{2}(1.4 \times 0.5 \mathrm{~m})$. To get the expected surface velocities at working area and a better concentration difference between inlet and outlet, a ceiling was built inside the wind tunnel with a height of 0.03 $\mathrm{m}$ (figure 1). 
Scale models were built to simulate the open lots with either unpaved soil or brick-paved floors. The unpaved soil open lot scale model was simulated by using a wooden box, $2.2 \times 0.72 \times 0.15 \mathrm{~m}$ (long $\times$ wide $\times$ deep), filled with soil. Soil in wooden box was compacted and its bulk density was $2.1 \mathrm{~g} \mathrm{~cm}^{-3}$. For the brick-paved open lot scale model, commercial red bricks for building were put on the top of soil filled box. Before experiment, floor surfaces of the open lot scale models were fouled with slurry two months to encourage the development of urease or microbs activities. For each experiment, $15 \mathrm{~kg}$ feces and $1.5 \mathrm{~L}$ urine (10:1) were put on the floor surface, leaving an emitting layer about $3 \mathrm{~cm}$ thickness for measurements. Characteristics of the urine and feces in the experiments are shown in table 1.

Table 1. Feces and urine characters collected from lactating cow $(\mathrm{N}=6)$.

\begin{tabular}{cccccc}
\hline & $\mathrm{pH}$ & $\mathrm{DM}, \%$ & $\mathrm{VS}, \% \mathrm{DM}$ & \multicolumn{1}{c}{ Total N, $\mathrm{g}^{\mathrm{kg}} \mathrm{kg}^{-1}$} & $\mathrm{TAN}, \mathrm{g}^{\mathrm{kg}} \mathrm{kg}^{-1}$ \\
\hline Urine & $6.23 \pm 0.13$ & $13.7 \pm 0.9$ & $87.3 \pm 1.2$ & $5.02 \pm 0.45$ & $1.11 \pm 0.17$ \\
Feces & $8.38 \pm 0.11$ & $6.5 \pm 0.3$ & $55.5 \pm 2.8$ & $11.16 \pm 0.71$ & $1.00 \pm 0.21$ \\
\hline
\end{tabular}

The measurement system includes a photoacoustic multi-gas monitor (INNOVA 1412i, LumaSense Technologies A/S, Denmark) and multiplexer (type 1309, LumaSense Technologies A/S, Denmark), an orifice plates (DIRU 160, Lindab, Denmark), a TSI velocity meter which integrated with a differential pressure transmitter, a hot wire anemometer and an air temperature and relative humidity sensor (Model 9565, TSI, USA), and thermal couples. $\mathrm{NH}_{3}, \mathrm{H}_{2} \mathrm{O}, \mathrm{CO}_{2}, \mathrm{CH}_{4}$ and $\mathrm{N}_{2} \mathrm{O}$ concentrations at air inlet and outlet (figure 1) was measured continuously by the multi-gas monitor and multiplexer every 5 minutes with 5 measurements. The last 3 measurements were used to estimate gaseous emission rates. The orifice plate was used to estimate airflow rates through the wind tunnel by measuring the pressure differences between upstream and downstream side with the TSI instrument. Surface velocities, air temperature and humidity during experiments were measured with the same TSI instrument right after the ceiling close to wind tunnel outlet (figure 1) at $0.5 \mathrm{~cm}$ high. Air temperature at working area was measured by thermocouples at five different positions (figure 1) at $3 \mathrm{~cm}$ high and logged every 5 minutes. Manure temperature was measured by a embedded thermocouple inside the manure and logged every 5 minutes.

\section{Experiment design}

Impacts of different surface air velocities and floor types on $\mathrm{CO}_{2}, \mathrm{CH}_{4}, \mathrm{~N}_{2} \mathrm{O}$ and $\mathrm{NH}_{3}$ emissions from the scale model of the open lots were examined at different air temperatures. The examined air temperatures $(15,25$ and $35^{\circ} \mathrm{C}$ ), surface velocities $\left(0.4,0.7,1.0\right.$ and $\left.1.2 \mathrm{~m} \cdot \mathrm{s}^{-1}\right)$ and floor types (soil or brick floors) are summarized in Table 2. Ammonia is included in the study as it can be converted into $\mathrm{N}_{2} \mathrm{O}$ in a certain condition.

Table 2. Examined floor types, air temperatures $\left(\mathrm{T}_{\text {air }}\right)$ and surface air velocities in the study.

\begin{tabular}{lccccc}
\hline \multirow{2}{*}{ Floor Type } & \multirow{2}{*}{$\mathrm{T}_{\text {air }}$} & \multicolumn{4}{c}{ Surface Air Velocity } \\
\cline { 3 - 5 } & $15^{\circ} \mathrm{C}$ & $0.4 \mathrm{~m} \cdot \mathrm{s}^{-1}$ & $0.7 \mathrm{~m} \cdot \mathrm{s}^{-1}$ & $1.0 \mathrm{~m} \cdot \mathrm{s}^{-1}$ & $1.2 \mathrm{~m} \cdot \mathrm{s}^{-1}$ \\
\hline \multirow{3}{*}{ Soil Floor } & $25^{\circ} \mathrm{C}$ & Run 1 & Run 1 & Run 2 & Run 2 \\
& $35^{\circ} \mathrm{C}$ & Run 3 & Run 3 & Run 4 & Run 4 \\
& $15^{\circ} \mathrm{C}$ & Run 7 & Run 5 & Run 6 & Run 6 \\
\multirow{2}{*}{ Brick Floor } & $25^{\circ} \mathrm{C}$ & Run 9 & Run 7 & Run 8 & Run 8 \\
& $35^{\circ} \mathrm{C}$ & Run 11 & Run 11 & Run 10 & Run 10 \\
& & & & Run 12 & Run 12 \\
\hline
\end{tabular}

Twelve 24-hr runs were performed and each test had three replicates during the experiment. Each experimental run was conducted at one air temperature and two surface velocities. Each 24-h measurement was conducted at one temperature and two different surface velocities. The first 12 hours was to get a relatively stable gaseous emission and last 12 hours was used for gas emission tests at two varied surface velocities.

\section{Results and discussion}

GHG emissions from the open lots

Estimated $\mathrm{CO}_{2}, \mathrm{CH}_{4}$ and $\mathrm{N}_{2} \mathrm{O}$ emission rates from ground level of the investigated open lot were $5.7 \pm 2.7 \mathrm{~kg} \cdot \mathrm{m}^{-}$ ${ }^{2} \cdot \mathrm{yr}^{-1}, 18.8 \pm 8.7 \mathrm{~g} \cdot \mathrm{m}^{-2} \cdot \mathrm{yr}^{-1}$ and $5.4 \pm 3.4 \mathrm{~g} \cdot \mathrm{m}^{-2} \cdot \mathrm{yr}^{-1}$, respectively, which were equivalent to $137.5 \pm 64.7 \mathrm{~kg} \cdot \mathrm{hd}^{-1} \cdot \mathrm{yr}^{-1}$, $452.7 \pm 210.6 \mathrm{~g} \cdot \mathrm{hd}^{-1} \cdot \mathrm{yr}^{-1}$ and $129.7 \pm 82.4 \mathrm{~g} \cdot \mathrm{hd}^{-1} \cdot \mathrm{yr}^{-1}$ based on the amount of manure deposited on the lot. Table 3 shows the estimated GHG emissions at different seasons. 
Table 3. Estimated $\mathrm{CO}_{2}, \mathrm{CH}_{4}, \mathrm{~N}_{2} \mathrm{O}$ emissions and global warming potential (GWP) from dairy open lot(N=5).

\begin{tabular}{lllll}
\hline & \multicolumn{1}{c}{$\begin{array}{c}\mathrm{CO}_{2} \\
\mathrm{~kg} \mathrm{~m}^{-2} \mathrm{yr}^{-1}\end{array}$} & \multicolumn{1}{c}{$\begin{array}{c}\mathrm{CH}_{4} \\
\mathrm{~g} \mathrm{~m}^{-2} \mathrm{yr}^{-1}\end{array}$} & \multicolumn{1}{c}{$\begin{array}{c}\mathrm{N}_{2} \mathrm{O} \\
\mathrm{g} \mathrm{m}^{-2} \mathrm{yr}^{-1}\end{array}$} & $\begin{array}{c}\mathrm{GWP}^{*} \\
\mathrm{~kg} \mathrm{CO}_{2} \mathrm{eq}^{-2} \mathrm{~m}^{-2} \mathrm{yr}^{-1}\end{array}$ \\
\hline Spring & $3.4 \pm 1.6$ & $8.6 \pm 4.6$ & $1.4 \pm 1.2$ & $0.6 \pm 0.5$ \\
Summer & $14.2 \pm 6.8$ & $54.4 \pm 23.8$ & $13.8 \pm 9.4$ & $5.5 \pm 3.4$ \\
Autumn & $5.1 \pm 2.3$ & $11.2 \pm 6.1$ & $4.1 \pm 2.8$ & $1.5 \pm 1.0$ \\
Winter & $0.2 \pm 0.1$ & $1.2 \pm 6.5$ & $2.4 \pm 0.3$ & $0.7 \pm 0.1$ \\
Average & $5.7 \pm 2.7$ & $18.9 \pm 8.8$ & $5.4 \pm 3.4$ & $2.1 \pm 1.2$ \\
\hline
\end{tabular}

${ }^{*} \mathrm{CO}_{2}$ was excluded in calculating global warming potential.

Measurement locations and manure removal practice had significant impact on GHG emission rates from the open lot $(P<0.05)$. Compared to the aisle zone, $\mathrm{CO}_{2}$ and $\mathrm{CH}_{4}$ emissions from cubicle zone were higher $(P<0.05)$, which was consistent to the amount of manure enclosed at different locations. Compared with emissions before manure removal, $\mathrm{CH}_{4}$ emissions after manure removal were much lower $(P<0.05)$, while $\mathrm{CO}_{2}$ emissions were in the same level. For $\mathrm{N}_{2} \mathrm{O}$, it was greatly increased both at the aisle and cubicle zones after manure removal $(P<0.05)$. After the accumulated manure being removed, a new surface of the excretion and/or the floor was exposed to ambient air. The change of the microenvironment of the manure surface, oxygen status in particular, as well as the increased surface temperature after manure removal, might be responsible for the higher emission rate during the measurements.
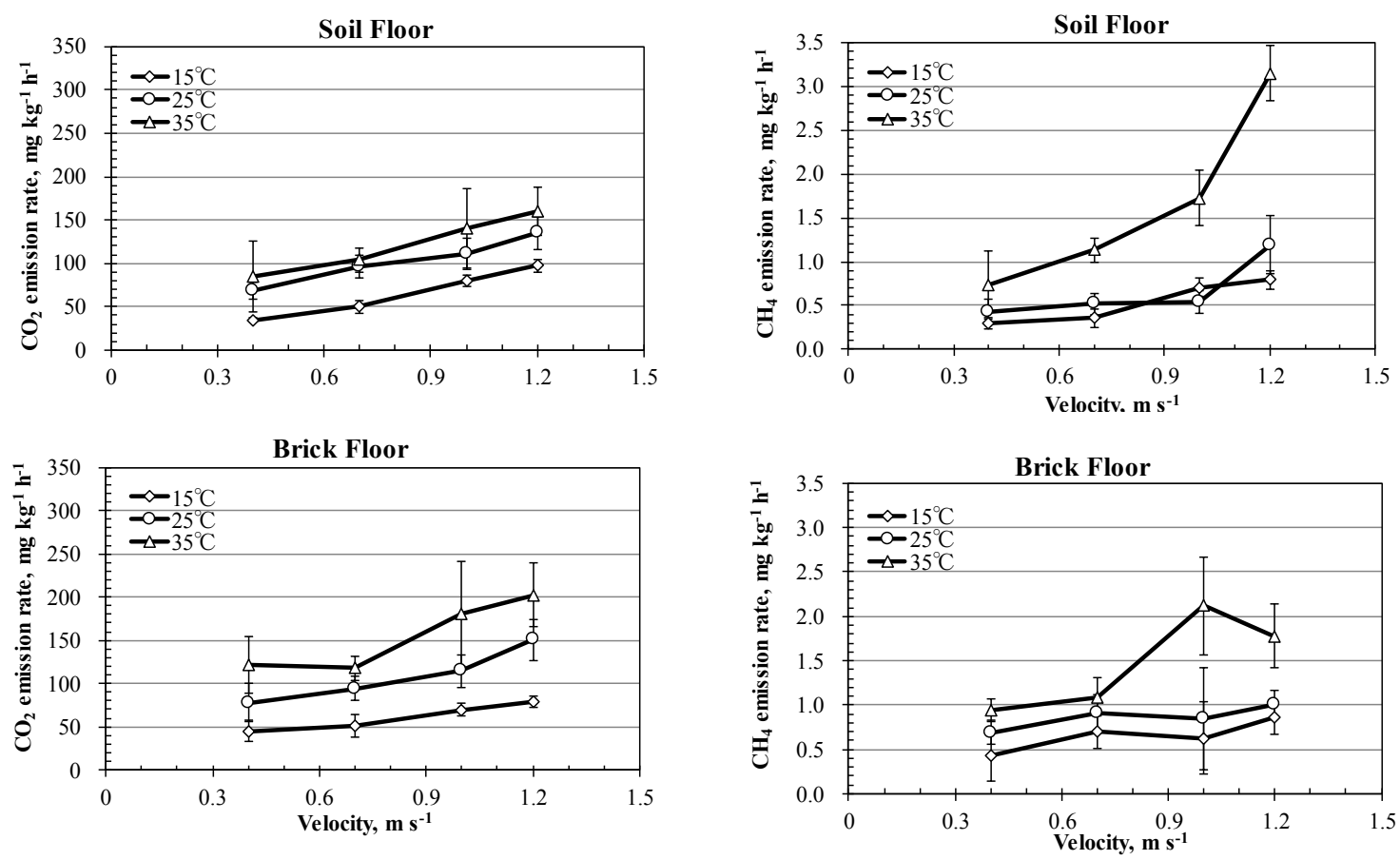

Figure 2. Carbon emissions from the scale model of open lots at different temperatures, velocities and floor types $(\mathrm{N}=5)$. 

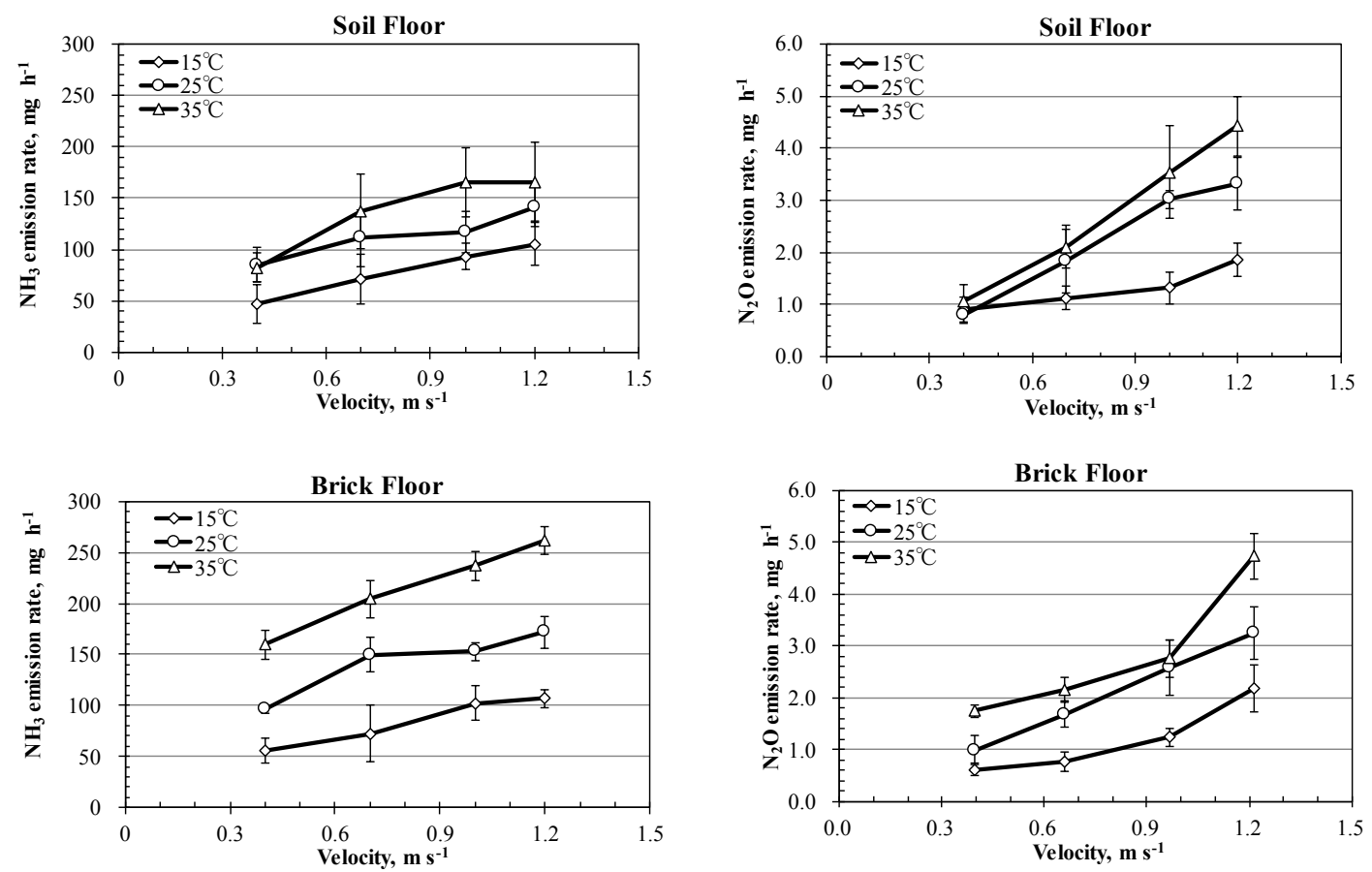

Figure 3. Nitrogen emissions from the scale model of open lots at different temperatures, velocities and floor types $(\mathrm{N}=5)$.

Impacts of air temperature on gas emissions from open lots

As shown in figure 2 and $3, \mathrm{CO}_{2}, \mathrm{CH}_{4}, \mathrm{~N}_{2} \mathrm{O}$ and $\mathrm{NH}_{3}$ emissions is positively related to the air temperature. $\mathrm{Q}_{10}$, which was defined as the ratio of gas emissions measured at air temperatures of $(\mathrm{x}+10){ }^{\circ} \mathrm{C}$ and $\mathrm{x}{ }^{\circ} \mathrm{C}$, to evaluate the temperature change effect. Table 4 shows the $\mathrm{Q}_{10}$ of the surveyed gases at different floor types and air velocities. For $\mathrm{CO}_{2}, \mathrm{~N}_{2} \mathrm{O}$ and $\mathrm{NH}_{3}, \mathrm{Q}_{10}$ calculated by $\left(25 / 15^{\circ} \mathrm{C}\right)$ ranged from 1.9 to 2.2 , while that by $\left(35 / 25^{\circ} \mathrm{C}\right)$ ranged from 1.5 to 1.9. This suggests that the emissions of $\mathrm{CO}_{2}, \mathrm{~N}_{2} \mathrm{O}$ and $\mathrm{NH}_{3}$ were more sensible to the lower temperature gradients. Differing from that, $\mathrm{CH}_{4}$ emissions were more sensible to the higher temperature gradients. $\mathrm{Q}_{10}$ for $\mathrm{CH}_{4}$ were $2.1-3.0$ and $1.6-1.8$ at $\left(35 / 25^{\circ} \mathrm{C}\right)$ and $\left(25 / 15^{\circ} \mathrm{C}\right)$, respectively.

Table 4. Comparison of the $\mathrm{Q}_{10}$ for $\mathrm{CO}_{2}, \mathrm{CH}_{4}, \mathrm{~N}_{2} \mathrm{O}$ and $\mathrm{NH}_{3}$ emitted from brick floor and soil floor at $15-35^{\circ} \mathrm{C}$.

\begin{tabular}{cccccc}
\hline Floor types & $\mathrm{Q}_{10}$ & $\mathrm{CO}_{2}$ & $\mathrm{CH}_{4}$ & $\mathrm{~N}_{2} \mathrm{O}$ & $\mathrm{NH}_{3}$ \\
\hline Soil floor & $25 / 15^{\circ} \mathrm{C}$ & 2.1 & 1.6 & 2.0 & 1.9 \\
& $35 / 25^{\circ} \mathrm{C}$ & 1.5 & 3.0 & 1.6 & 1.5 \\
& $\mathrm{Mean}$ & 1.8 & 2.4 & 1.8 & 1.7 \\
Brick floor & $25 / 15^{\circ} \mathrm{C}$ & 2.2 & 1.8 & 2.2 & 2.1 \\
& $35 / 25^{\circ} \mathrm{C}$ & 1.8 & 2.1 & 1.8 & 1.9 \\
& Mean & 2.0 & 1.9 & 2.0 & 2.0 \\
\hline
\end{tabular}

Impacts of air velocity on gas emissions from open lots

Surface air velocity had a significant impact on gas emissions from the scale model of open lots and it interacted with air temperature $(\mathrm{P}<0.05)$. In general, $\mathrm{CO}_{2}, \mathrm{CH}_{4}, \mathrm{~N}_{2} \mathrm{O}$ and $\mathrm{NH}_{3}$ emissions increased with the increasing of surface air velocity. Variations of gas emissions at the change of every $0.1 \mathrm{~m} \cdot \mathrm{s}^{-1}$ were calculated to evaluate the effect of surface velocity (table 5). 
Table 5. Variations of $\mathrm{CO}_{2}, \mathrm{CH}_{4}, \mathrm{~N}_{2} \mathrm{O}$ and $\mathrm{NH}_{3}$ emissions at the change of every $0.1 \mathrm{~m} \cdot \mathrm{s}^{-1}$ air velocity.

\begin{tabular}{cccccc}
\hline Floor types & Interval & $\mathrm{CO}_{2}$ & $\mathrm{CH}_{4}$ & $\mathrm{~N}_{2} \mathrm{O}$ & $\mathrm{NH}_{3}$ \\
\hline Soil floor & $0.4 \sim 0.7 \mathrm{~m} \cdot \mathrm{s}^{-1}$ & $12 \%$ & $10 \%$ & $28 \%$ & $16 \%$ \\
& $0.7 \sim 1.0 \mathrm{~m} \cdot \mathrm{s}^{-1}$ & $12 \%$ & $17 \%$ & $17 \%$ & $6 \%$ \\
& $1.0 \sim 1.2 \mathrm{~m} \cdot \mathrm{s}^{-1}$ & $10 \%$ & $36 \%$ & $12 \%$ & $6 \%$ \\
Brick floor & Mean & $11 \%$ & $21 \%$ & $19 \%$ & $9 \%$ \\
& $0.4 \sim 0.7 \mathrm{~m} \cdot \mathrm{s}^{-1}$ & $4 \%$ & $13 \%$ & $13 \%$ & $13 \%$ \\
& $0.7 \sim 1.0 \mathrm{~m} \cdot \mathrm{s}^{-1}$ & $12 \%$ & $8 \%$ & $16 \%$ & $7 \%$ \\
& $1.0 \sim 1.2 \mathrm{~m} \cdot \mathrm{s}^{-1}$ & $9 \%$ & $7 \%$ & $29 \%$ & $5 \%$ \\
\hline
\end{tabular}

The variations of $\mathrm{CO}_{2}, \mathrm{CH}_{4}, \mathrm{~N}_{2} \mathrm{O}$ and $\mathrm{NH}_{3}$ emissions were $4-12 \%, 7-2 \%, 12-29 \%$ and $5-16 \%$ for every $0.1 \mathrm{~m} \cdot \mathrm{s}^{-}$ ${ }^{1}$ change of surface velocity in the range of $0.4-1.2 \mathrm{~m} \cdot \mathrm{s}^{-1}$. On both soil floor and brick floor, $\mathrm{NH}_{3}$ emissions were more sensible to the changes at a lower velocity $\left(0.4-0.7 \mathrm{~m} \cdot \mathrm{s}^{-1}\right)$. Differing from ammonia, $\mathrm{CO}_{2}, \mathrm{CH}_{4}$ and $\mathrm{N}_{2} \mathrm{O}$ did not follow the same variation pattern on different floor types. $\mathrm{N}_{2} \mathrm{O}$ was more sensible to the changes at a lower velocity on soil floor but at a higher velocity $\left(1.0-1.2 \mathrm{~m} \cdot \mathrm{s}^{-1}\right)$ on brick floor. To the opposite, $\mathrm{CH}_{4}$ was more sensible to the changes at a higher velocity on soil floor but at a lower velocity.

Impacts of floor types on gas emissions from open lots

\section{$\mathrm{CO}_{2}$ and $\mathrm{CH}_{4}$}

On both soil floor and brick floor, $\mathrm{CO}_{2}$ emission rate peak at $35^{\circ} \mathrm{C}$ and $1.2 \mathrm{~m} \cdot \mathrm{s}^{-1}$ and minimized at $15^{\circ} \mathrm{C}$ and $0.4 \mathrm{~m} \cdot \mathrm{s}^{-1}$. At 15 and $25^{\circ} \mathrm{C}$, there was no significant difference in the $\mathrm{CO}_{2}$ emissions between soil floor and brick $(\mathrm{P}>0.05)$. However, the brick floor had a higher emission than soil floor at $35^{\circ} \mathrm{C}(\mathrm{P}<0.05) \cdot \mathrm{CO}_{2}$ is generated in the process of urea hydrolysis and organic matter degradation of animal manure (Ni et al., 1999; Møller et al., 2004). Compared with the brick-paved floor, it was observed that more liquid leached into the soil floor, which retained more soluble nutrient inside and may retard the total $\mathrm{CO}_{2}$ production and emitting, particularly under higher air temperatures. It might partially explain why the $\mathrm{CO}_{2}$ emission from the brick floor was significantly higher at 35 ${ }^{\circ} \mathrm{C}$.

For $\mathrm{CH}_{4}$, statistical analysis did not show a significant difference in $\mathrm{CH}_{4}$ emissions between the soil and brick floors $(\mathrm{P}>0.05)$. However, the $\mathrm{CH}_{4}$ emission sources on the soil and brick floors were somewhat different. Even after the manure was scraped away from the floor surface, some mixture of urine, feces, and clay still remained, especially for the soil floor. The soil underneath could be either an emission source or a sink of $\mathrm{CH}_{4}$ (Topp and Pattey, 1997; Le Mer and Roger, 2001). This makes the $\mathrm{CH}_{4}$ emission from the open lot with a soil floor to be much more complex. The soil floor may have the potential to emit more $\mathrm{CH}_{4}$ emission when it is flooded, i.e., after a long-term use or with precipitation.

\section{$\mathrm{N}_{2} \mathrm{O}$ and $\mathrm{NH}_{3}$}

Similar to $\mathrm{CO}_{2}$, the highest emissions of $\mathrm{N}_{2} \mathrm{O}$ and $\mathrm{NH}_{3}$ were measured at $35^{\circ} \mathrm{C}$ and $1.2 \mathrm{~m} \cdot \mathrm{s}^{-1}$ on both soil floor and brick floor, and the lowest emissions occurred at $15^{\circ} \mathrm{C}$ and $0.4 \mathrm{~m} \cdot \mathrm{s}^{-1}$. Statistical analysis showed that brick floor had higher $\mathrm{NH}_{3}$ emissions than the soil floor $(\mathrm{P}<0.05)$ while there were no significant differences between different floors in $\mathrm{N}_{2} \mathrm{O}$ emissions. The production of ammonia is highly related to ammonium in the emission source. In this study, manure on the floor surface was the major emission source of gases. Table 7 shows the characteristics of the soil (top $5 \mathrm{~cm}$ ) packed inside the wooden box of the scale model in the beginning and ending of the experiments. Due to the infiltration, a part of ammonium in the manure was remained in the soil and thereby reducing the available ammonium in the manure which reduced the $\mathrm{NH}_{3}$ emissions on soil floor afterwards. As mentioned before, $\mathrm{N}_{2} \mathrm{O}$ production is positively related to inorganic nitrogen in soil which will theoretically promote the production of $\mathrm{N}_{2} \mathrm{O}$ in soil floor. The utilization period of the open lot scale model is relatively short in this study and this may only result to a very small difference in emissions which is hardly to be captured by the experimental setup in this study. The long-term effects of floor types need to be considered in further study.

Table 6. Characteristics of the soil packed inside the wooden box of the scale model in the beginning and ending of the experiments (top $5 \mathrm{~cm}$ soil).

\begin{tabular}{ccccccccc}
\hline & $\begin{array}{c}\mathrm{pH}, \\
-\end{array}$ & $\begin{array}{c}\text { Moisture, } \\
\%\end{array}$ & $\begin{array}{c}\mathrm{TS}, \\
\%\end{array}$ & $\begin{array}{c}\mathrm{VS}, \\
\% \mathrm{TS}\end{array}$ & $\begin{array}{c}\text { Total N, } \\
\mathrm{kg} \cdot \mathrm{Mg}^{-1}\end{array}$ & $\begin{array}{c}\mathrm{NH}_{4}^{+}-\mathrm{N}, \\
\mathrm{mg} \cdot \mathrm{kg}^{-1}\end{array}$ & $\begin{array}{c}\mathrm{NO}_{3}-\mathrm{N}, \\
\mathrm{mg} \cdot \mathrm{kg}^{-1}\end{array}$ & $\begin{array}{c}\text { Mineral-N, } \\
\mathrm{mg} \cdot \mathrm{m}^{-2}\end{array}$ \\
\hline Beginning & 7.53 & 12.7 & 87.3 & 4.1 & 1.22 & 144.75 & 6.12 & 1629.4 \\
Ending & 8.29 & 22.6 & 77.4 & 7.1 & 2.31 & 427.69 & 0.11 & 4620.2 \\
\hline
\end{tabular}

Nitrogen loss via gas emission

$\mathrm{NH}_{3}$ and $\mathrm{N}_{2} \mathrm{O}$ volatilization from manure results to nitrogen loss and affects its fertilizer efficiency after land application. Results showed that $\mathrm{NH}_{3}$ was the major way of gaseous nitrogen loss and the emissions peaked in 200-400 min after manure deposition. Differ from ammonia, $\mathrm{N}_{2} \mathrm{O}$ had a relatively stable emission in each measurement duration. $1.6 \%-8.6 \%$ of total nitrogen in the manure lost via gas emissions at varied operation 
conditions in the latter 12 hours. The brick floor had a higher nitrogen loss comparing with soil floor. Surface velocity had a greater influence than air temperature on nitrogen loss via gaseous volatilization.

Table 7. Nitrogen loss of manure via $\mathrm{NH}_{3}$ and $\mathrm{N}_{2} \mathrm{O}$ emission in the latter 12-hr of experiment at varied conditions.

\begin{tabular}{rcccccccc}
\hline & \multicolumn{3}{c}{ Soil floor } & \multicolumn{3}{c}{ Brick floor } \\
\cline { 2 - 9 } & $0.4 \mathrm{~m} \cdot \mathrm{s}^{-1}$ & $0.7 \mathrm{~m} \cdot \mathrm{s}^{-1}$ & $1 \mathrm{~m} \cdot \mathrm{s}^{-1}$ & $1.2 \mathrm{~m} \cdot \mathrm{s}^{-1}$ & $0.4 \mathrm{~m} \cdot \mathrm{s}^{-1}$ & $0.7 \mathrm{~m} \cdot \mathrm{s}^{-1}$ & $1 \mathrm{~m} \cdot \mathrm{s}^{-1}$ & $1.2 \mathrm{~m} \cdot \mathrm{s}^{-1}$ \\
\hline $15^{\circ} \mathrm{C}$ & $1.6 \%$ & $2.3 \%$ & $3.1 \%$ & $3.5 \%$ & $1.8 \%$ & $2.4 \%$ & $3.4 \%$ & $3.5 \%$ \\
$25^{\circ} \mathrm{C}$ & $2.8 \%$ & $3.7 \%$ & $3.9 \%$ & $4.7 \%$ & $3.2 \%$ & $4.9 \%$ & $5.0 \%$ & $5.7 \%$ \\
$35^{\circ} \mathrm{C}$ & $2.7 \%$ & $4.5 \%$ & $5.5 \%$ & $5.5 \%$ & $5.2 \%$ & $6.7 \%$ & $7.8 \%$ & $8.6 \%$ \\
\hline
\end{tabular}

\section{Conclusion}

Field measurements and laboratory experiments were conducted to illustrate gaseous emissions from dairy open lots and the effects of major influence factors on it in this study. Affected by seasons, sampling locations and manure removal, estimated $\mathrm{CO}_{2}, \mathrm{CH}_{4}$ and $\mathrm{N}_{2} \mathrm{O}$ emission from the ground level of brick-paved dairy open lots were $0.2-14.2 \mathrm{~kg} \cdot \mathrm{m}^{-2} \cdot \mathrm{yr}^{-1}, 4.9-341.0 \mathrm{~g} \cdot \mathrm{m}^{-2} \cdot \mathrm{yr}^{-1}$ and $1.4-13.8 \mathrm{~g} \cdot \mathrm{m}^{-2} \cdot \mathrm{yr}^{-1}$, respectively, by closed chambers in field measurements. Using a scale model of open lots, impacts of air temperature $\left(15-35^{\circ} \mathrm{C}\right)$, velocity $\left(0.4-1.2 \mathrm{~m} \cdot \mathrm{s}^{-1}\right)$ and floor types (unpaved soil floor and brick-paved floor) on $\mathrm{CO}_{2}, \mathrm{CH}_{4}, \mathrm{NH}_{3}$ and $\mathrm{N}_{2} \mathrm{O}$ emission were studied by a wind tunnel in controlled conditions. Generally, the emissions of surveyed gases increased with the increasing of air temperature and velocity. The emission of $\mathrm{CO}_{2}$ and $\mathrm{N}_{2} \mathrm{O}$ were more sensible to temperature changes at the lower interval while $\mathrm{CH}_{4}$ was more sensible to the higher interval conversely. $\mathrm{NH}_{3}$ emissions were more sensible to the lower temperature gradient and the lower velocity range. Brick floor had higher $\mathrm{NH}_{3}$ emission and nitrogen losses than soil floor.

\section{Acknowledgements}

This study was funded by National Nature Science Foundation of China (Grant No. 31472132, and 31172244).

\section{Reference}

Borhan, M.S., S.C. Capareda, S. Mukhtar, W.B. Faulkner, R. Mcgee, C.B. Parnell, 2011. Greenhouse gas emissions from ground level area sources in dairy and cattle feedyard operations. Atmosphere. 2(3), 303-329.

Borhan, M.S., C. Sergio, M. Saqib, W.B. Faulkner, M.G. Russell, C.B. Parnell, 2011. Determining seasonal greenhouse gas emissions from ground-level area sources in a dairy operation in central Texas. Journal of the Air \& Waste Management Association. 61(7), 786-795.

Ding, L., L. Qi, C. Wang, Z. Shi, W. Cao, B. Li, 2015. Effect of configuration and headspace mixing on the accuracy of closed chambers for dairy farm gas emission measurement. Applied Engineering in Agriculture. 31(1), 153-162.

González-Avalos, E., L.G. Ruiz-Suárez, 2001. Methane emission factors from cattle manure in Mexico. Bioresource Technology. 80(1), 63-71.

Le Mer, J., P. Roger, 2001. Production, oxidation, emission and consumption of methane by soils: a review. European Journal of Soil Biology. 37(1), 25-50.

Møller, H.B., S.G. Sommer, B.K. Ahring, 2004. Biological degradation and greenhouse gas emissions during pre-storage of liquid animal manure. Journal of Environmental Quality. 33(1), 27-36.

Mukhtar, S., A. Mutlu, S.C. Capareda, C.B. Parnell, 2008. Seasonal and spatial variations of ammonia emissions from an open-lot dairy operation. Journal of the Air \& Waste Management Association. 58(3), 369-376.

Ni, J.Q., C. Vinckier, J. Hendriks, J. Coenegrachts, 1999. Production of carbon dioxide in a fattening pig house under field conditions. ii. release from the manure. Atmospheric Environment. 33(22), 3697-3703.

Owen, J.J., W.L. Silver, 2014. Greenhouse gas emissions from dairy manure management: a review of fieldbased studies. Global Change Biology. 21(2), 550-565.

Pereira J., T.H. Misselbrook, D.R. Chadwick, J. Coutinho, H. Trindade, 2012. Effects of temperature and dairy cattle excreta characteristics on potential ammonia and greenhouse gas emissions from housing: a laboratory study. Biosystems Engineering. 112(2), 138-150.

Pereira, J., D. Fangueiro, T.H. Misselbrook, D.R. Chadwick, J. Coutinho, H. Trindade, 2011. Ammonia and greenhouse gas emissions from slatted and solid floors in dairy cattle houses: a scale model study. Biosystems Engineering. 109(2), 148-157.

Saha C.K., G. Zhang, J. Ni, 2010. Airflow and concentration characterization and ammonia mass transfer modelling in wind tunnel studies. Biosystems Engineering. 107(4), 328-340.

Topp E., E. Pattey, 1997. Soils as sources and sinks for atmospheric methane. Canadian Journal of Soil Science. 77(2), 167-177. 


\title{
Test of UHF Ear Tags for Growing-Finishing Pigs on an Optimized Static Test Bench
}

\author{
Adrian Förschner*, Felix Adrion, Eva Gallmann \\ Livestock Systems Engineering, University of Hohenheim, 70599 Stuttgart, Germany \\ * Corresponding author. Email: a.foerschner@uni-hohenheim.de
}

\begin{abstract}
Low frequency radio frequency identification (LF-RFID) systems have been established as a standard for animal identification. Ultra-high frequency (UHF-) RFID systems could provide advanced applications such as automated activity measurement and real-time localization of animals. However, strong influence of reflections and absorption lead to signal attenuation, which poses major challenges for UHF systems. Especially ear tissue causes strong absorption of the electromagnetic waves, changes in the resonant frequency of UHF transponders and thus impairs reading performance. In a current research project UHF ear tags, readers and antennas for pig farming are being developed further. The objective of this study is to test a series of small UHF ear tags, which were designed especially for use in growing-finishing pigs on an optimized static test bench. The most promising types of transponder will be scaled down for the application in piglets. An automated test bench was developed to test UHF ear tags, readers and antennas in a standardized manner within a short time and in a measuring field of $3.5 \times 3.5 \mathrm{~m}$. The parameters measured were the received signal strength indicator (RSSI), the read range and the detection area of different types of transponders. In order to minimize the influence of reflections and to lower the measurement bias, the test bench was partially equipped with electromagnetic (EM) absorbers. Five different types of UHF transponder ear tags for growing-finishing pigs were tested on the test bench with and without real pig ears. These ear tags were optimized in terms of directional characteristics, resonance frequency and bandwidth to lower the influence of ear tissue. The results revealed that one of the types of transponder had a good performance also with pig ears and is suited to be scaled down to the size of piglet ear tag.
\end{abstract}

Keywords: Ultra high frequency RFID, ear tags, transponder, read range, pig ears, test bench.

\section{Introduction}

Ultra-high frequency radio frequency identification (UHF-RFID, operating frequency 860 - $960 \mathrm{MHz}$ ) systems are widely used in industry and logistics. This technology offers a high read range up to $10 \mathrm{~m}$ for passive transponders and the capability of bulk detection. These features also provide benefits for many applications in electronic animal identification. The adaptation of UHF RFID in pigs has already been successfully demonstrated in several publications (Hogewerf et al., 2013; Swedberg, 2012). In comparison with the standard technology for electronic animal identification, low-frequency RFID (LF-RFID, operating frequency $134.2 \mathrm{kHz}$ ), UHF-RFID facilitates simultaneous identification of groups of animals (Adrion et al., 2017a). Due to the high read range, it is possible to monitor barn areas in group housing or perform a sector localisation (cell-of-origin) with UHF-RFID (Adrion et al., 2016).

Using UHF-RFID, it has to be considered that many materials absorb and electrically conductive materials reflect UHF radiation. Therefore, materials in the wider application environment, as well as close to UHF transponders, interfere with UHF systems. In the industry or logistics sector, where UHF transponders are usually operated under optimal conditions (free transmission link), they are not subject to interfering influences, contrary to the application in animal husbandry (Kern, 2007). In particular, water and metal are all over in a barn. Absorption or reflection of the electric field can lead to unintentional readings and reading gaps. Materials with a relative permittivity $\varepsilon_{\mathrm{r}}>1$ can affect the impedance of the transponder antenna and may induce a shift of the transponder's resonant frequency. Conversely, the resonance frequency of a transponder can be changed as an adaptation to these adverse effects (Finkenzeller, 2015; Fujimoto and Morishita, 2013). This process is part of the preparation of a transponder for the integration into an ear tag and the attachment to an ear. Other parameters, e.g. the general antenna design, the size of the antenna mass area and the bandwidth of the antenna influence the sensitivity of the transponders to ear tissue and can be utilised during the development.

The successful adaption of transponders to the targeted application can be reviewed e.g. by standardized measurements in anechoic chambers, which are designed for reliable measurements and reproducible results (Stasa et al., 2017). A disadvantage of this approach is the high technical effort for the investigation of relevant parameters (European EPC Compentence Center, 2011). As an alternative to laboratory measurements for new transponders, an application test with animals can be done. Such evaluations in practical tests are not fully generalizable as they always represent a specific application and environment (Hammer et al., 2016). Furthermore, these experiments are elaborate, have to be carried out over a long period of time and possible changes in environmental conditions during the test compromise the reliability of the results. Another possibility would be to test transponders, for example, at a feeder (Maselyne et al., 2014) without animals, but then the informative value of the results would be limited to this special application. 
Thus, with the aim of carrying out reproducible measurements and at the same time remaining applicationoriented, a static test bench was developed (Adrion et al., 2015). This test bench offers the possibility to test the functionality of new UHF ear tags before testing them on animals. Previous studies at this test bench investigated and described first insight into the effects of pig ears and ear tissue phantom on the readability of UHF ear tags (Adrion et al., 2017b). In these experiments, reflections in the test bench environment indicated a relevant bias on the measurements, which is why the test bench is now partially and cost-effectively supplemented with electromagnetic (EM) absorber mats.

In a second innovation project, UHF transponder ear tags for piglets, growing-finishing pigs and sows, as well as stationary readers, antennas and an UHF handheld are currently being engineered. Priority is given to the transponders, because the reduction of size of the UHF transponder antennas is a challenge. The performance of the transponders decreases significantly with the size reduction. At first, new antenna designs for fattening pigs are incrementally scaled down to get an insight into the readability of smaller transponders. In a second step, the most promising transponders will be sized down to fit in piglet ear tags.

The objective of this article is to describe the first measurements of five newly developed types of UHF transponder ear tags on a static test bench. The measurements were performed with a focus on the effects of pig ear tissue on the different UHF transponders. In addition, the improvement of the test environment by EM absorbers is to be discussed briefly.

\section{Materials and Methods}

The experiment was carried out on a static test bench. It was designed to test the read range and signal strength (RSSI) of UHF transponders in a flexibly definable grid of $3.5 \times 3.5 \mathrm{~m}$ in front of a UHF reader. Within this field coordinates for measuring could be programmed. During execution of the program the ear tag would follow the predetermined coordinates. The transponder ear tags and the reader were mounted at $1.65 \mathrm{~m}$ height above the ground. The reader was a TSU 200 model with an integrated antenna (deister electronic GmbH, Barsinghausen, Germany). The operating frequency of the reader was $865.7 \mathrm{MHz}$ and the transmission power was $1 \mathrm{~W}(30 \mathrm{dBm})$ with circular polarization. A tag could be read every $200 \mathrm{~ms}$. The static test bench was equipped with electromagnetic absorbers $(\mathrm{H}+\mathrm{H} \mathrm{GmbH}$, Iserlohn and Albatross Projects $\mathrm{GmbH}$, Nattheim, Germany). This type of absorber is optimized for the frequency of $868 \mathrm{MHz}$. The Pyramid absorbers $(50 \mathrm{x} 50 \mathrm{~cm}$, height $30 \mathrm{~cm}$, reduction of reflection at $800 \mathrm{MHz}$ approx. $-30 \mathrm{~dB}$ ) were mounted on three sides of the test bench. The Advanced broadband truncated pyramidal absorbers $(61 \times 61 \mathrm{~cm}$, height $12.7 \mathrm{~cm}$, reduction of reflection at $800 \mathrm{MHz}$ approx. $-17 \mathrm{~dB}$ ) were laid out on the floor (Figure 1).
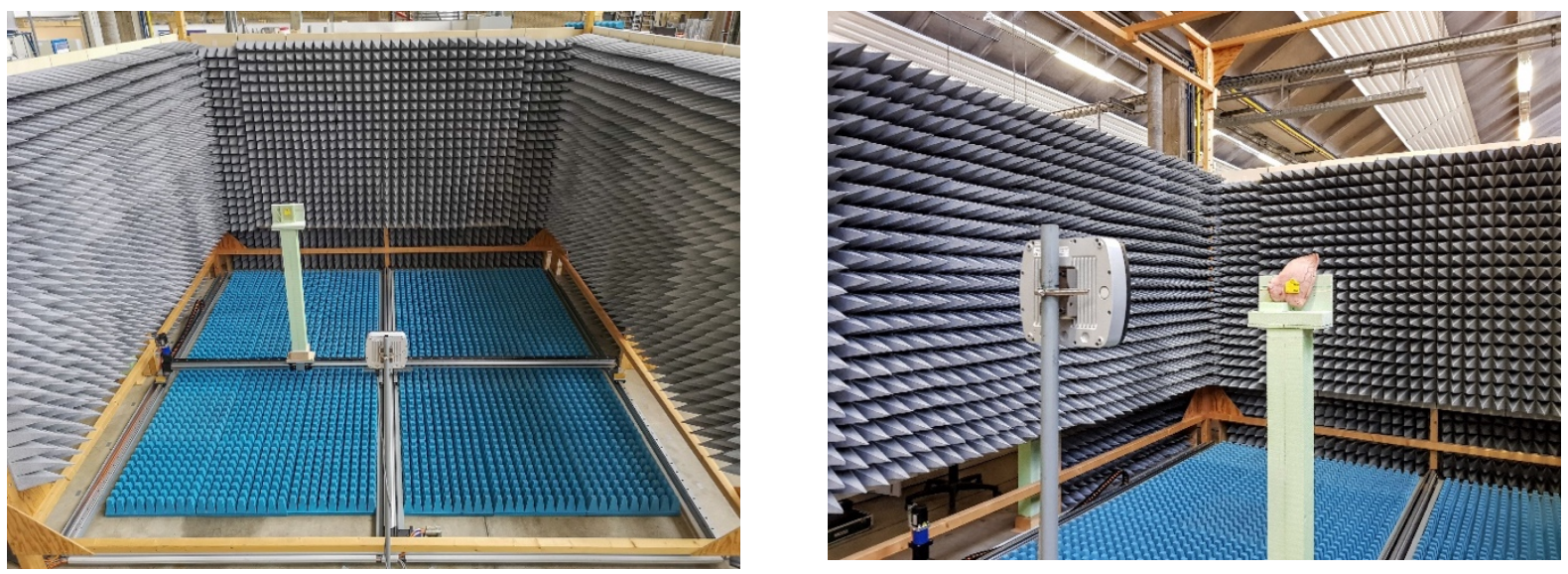

Figure 7. Pictures of the static test bench, (left) top-view, (right) close-up shot from a measurement of an ear tag attached to a pig ear.

The measurements were carried out with five different UHF transponder types. Transponder type B440 was a transponder optimized for use in breeding sows and growing-finishing pigs, which had been developed in a previous research project. The transponder types B510, B520, B530 and B540 were further developments of the previous research project. The transponder designs varied in antenna length and mass area so that various resonant frequencies and impedances could be tested. The aim of all transponder designs was to utilise the pig ear as part of the antenna mass area to maximise the technical flexibility in antenna design. The transponder type B510 was designed as a dipol $(29 \times 31 \mathrm{~mm}$, height $\times$ length). B520 is a monopole Antenna (31 x $31 \mathrm{~mm})$, but the design is kind of PIFA (Planar Inverted F- Shaped Antenna, Fujimoto and Morishita, 2013) with a reduced antenna mass area whereas the antenna length was longer compared to type B440. Type B520 had a monopole antenna (31 x $31 \mathrm{~mm}$ ). With the largest antenna mass area and an antenna emitter routed in parallel to the mass area, type B530 
had the largest antenna $(35 \times 30 \mathrm{~mm})$. Based on the B440 transponder antenna, type B540 (30 x $31 \mathrm{~mm}) \mathrm{had}$ a slightly reduced antenna mass area compared to type B440 and a correspondingly adjusted emitter length. Further details cannot be explained here for reasons of patent. All transponder types were moulded into a standard ear tag for breeding sows and growing-finishing pigs (Primaflex ${ }^{\circledR}$, Caisley International GmbH, Bocholt, Germany). The ear tags had a size of about $45 \times 46 \mathrm{~mm}$ and largest possible transponder size was $33 \times 44 \mathrm{~mm}$. All transponders were located in the male part of the ear tags. The resonance frequency of all transponder antennas were increased in order to compensate for their subsequent lowering by the plastic of the ear tag and the ear tissue.

For this experiment a total of 30 fresh scalded pig ears were used. The ears were taken from the abattoir directly after slaughtering and were chilled down to $1^{\circ} \mathrm{C}$ for the experiment. The male parts of the ear tags were attached on the outside of the ears as they would be on the animals. This is expected to reduce the influence of the concave shape of the ear on the performance of the ear tags. The holes for the ear tags were consistently positioned centrally between the horizontal cartilage strips and $7.5 \mathrm{~cm}$ outwards from the centre of the ears. Measurements were performed both with and without ears. For both variants, the ears were fixed on a polystyrene foam holder using wooden sticks (Figure 2).
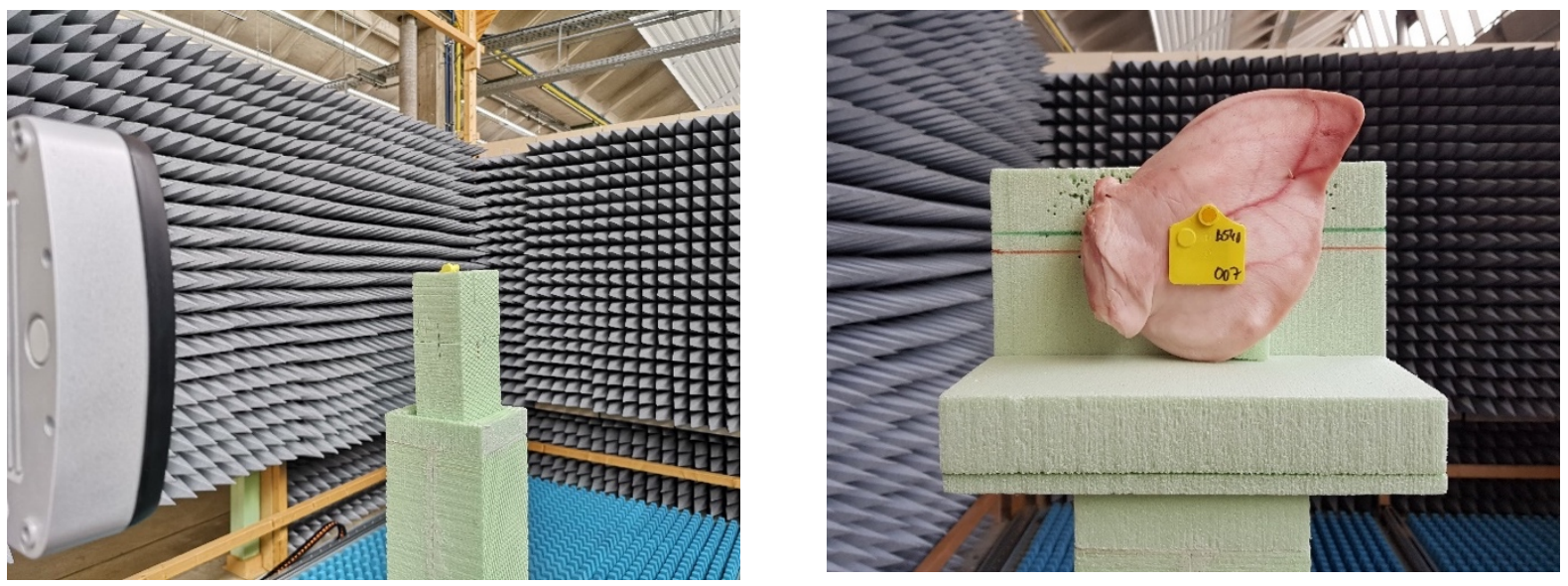

Figure 2. Picture of an ear tag placed in a polystyrene holder, free air variant (left), and close-up view of an ear tag attached to a pig ear on the polystyrene holder (right).

The experimental design contained three treatment factors: type of transponder (five different types), presence of pig ears (with and without) and use of electromagnetic absorbers (with and without). The design consisted of three replicates with two runs (main plots) each. The order of the runs was randomised for each replicate. One of the two runs per replicate was performed with electromagnetic absorbers installed at the test bench and one without absorbers. In each run, one sample of each type of transponder was tested with 10 pig ears and three replications of a reference variant in free air. Hence, in each run 13 measurements were made with each of the five transponder samples. This resulted in 65 measurements per run (completely randomized within run) and in a total of $6 \times 65=$ 390 measurements for the entire experiment. For each replicate 10 new ears and one new sample of each type of transponder were randomly chosen. Thus, in total over the three replicates, 30 ears, nine replications of the reference variant and three transponder samples were tested for each transponder type. On each ear, each of the three transponder types was tested once, meaning that each ear formed a complete block with respect to the transponder types. Due to a wrong assignment, type B530 was only tested two times instead of three times without ears in both runs of replicate 1, whereas type B540 was tested four times without ears in these runs. Each measurement contained a series of (attempted) readings at coordinates on a line directly in front of the reader. The coordinate distance was $0.15 \mathrm{~m}$. The first coordinate was at a distance of $0.40 \mathrm{~m}$, the last coordinate at $3.85 \mathrm{~m}$ distance to the reader ( 24 coordinates). In every measurement, at each coordinate the readings of the transponder tested were recorded and the associated RSSI was measured. Up to five readings and RSSI values could be registered per coordinate (reading time $1 \mathrm{~s}$ ).

Both RSSI and read range were considered for data analysis in all tests. The data for the read range did not need any processing. Regarding the RSSI, at first, an average per coordinate was calculated from the single measurements at each coordinate, which could be up to five RSSI values. These values were used for graphical evaluation. In a second step, these values were averaged again, to get a mean RSSI for each measurement over 24 coordinates. It has to be noted that considering the RSSI as a relevant parameter should be done carefully. There is no standard to calculate the RSSI and as a result, the values from different readers are not reliable for comparison. The measurement data of the first replicate (12 ${ }^{\text {th }}$ December 2017) and the first run (with absorbers) was prepared graphically and evaluated with descriptive statistics for this article. 


\section{Results and Discussion}

First, the effects of the electromagnetic absorber mats will be presented and discussed briefly. In an initial calculation with the statistic software SAS and the function proc mixed, it was found that from the entirety of the collected data the average read range was $126 \mathrm{~cm}$ with absorbers attached. Without absorbers, the read range was $138 \mathrm{~cm}$. This difference was statistically significant $(\mathrm{P}=0.0072)$.

The higher average read range without absorber mats at the test bench was presumably caused by positive interference in the back part of the measuring field due to reflections in the environment of the test bench (Adrion et al., 2015). Initial graphical analyses confirmed, that due to the installation of the electromagnetic absorber mats these reflections were reduced, resulting in less outliers with unexpected high read ranges and in less variance of the RSSI measurements (results not shown). Without absorbers, transponders with a good readability gained read range due to the reflections. For example, during some measurements transponder type B530 was detected again at a greater distance from the reader after missing some coordinates. In previous experiments, reflections in the environment caused significant bias on the measurements (Adrion et al., 2017b). In conclusion, the influence of the pig ears on the transponders should be measurable better with the absorber mats. Hence, the absorber mats should be applied constantly in future experiments. These first results only present the improvement over all transponder types. More details regarding the influence on the different types with and without ears will be analysed in the next step.

The results from the transponder experiment show that the new transponder types B530 and B540 could match the performance of the predecessor B440. Without ears the average read range of these transponders (B440, B530 and B540) was from $100 \mathrm{~cm}$ (B530) to $107.5 \mathrm{~cm}$ (B440) (Table 1). The standard deviation was similar for these types from $39.9 \mathrm{~cm}$ (B530) to $43.8 \mathrm{~cm}$ (B440). Looking at the RSSI, type B440 showed the highest mean with $61.9 \mathrm{dBm}$. The transponder types with reduced antenna mass area, B510 and B520, revealed lower performance and readability. Type B510 was readable only at a distance of $40 \mathrm{~cm}$ to the reader and the B520 at $62.5 \mathrm{~cm}$ distance.

Table 58. RSSI and read range of the transponder types B440, B510, B520, B530 and B540, results of replicate 1 (run1, with absorbers); tested variants: free air (without ears, reference) and with ears (mean \pm standard deviation).

\begin{tabular}{ccccc}
\hline \multirow{2}{*}{ Type } & \multicolumn{2}{c}{ Without ears } & \multicolumn{2}{c}{ With ears } \\
& RSSI $(\mathrm{dBm})$ & Read range $(\mathrm{cm})$ & RSSI $(\mathrm{dBm})$ & Read range $(\mathrm{cm})$ \\
\hline B440 & $-61.9 \pm 4.9$ & $107.5 \pm 43.8$ & $-61.9 \pm 3.8$ & $64.7 \pm 21.8$ \\
B510 & $-66.8 \pm 0.0$ & $40.0 \pm 0.0$ & $-65.4 \pm 1.3$ & $40.0 \pm 0.0$ \\
B520 & $-63.9 \pm 3.5$ & $62.5 \pm 17.5$ & $-67.3 \pm 1.4$ & $50.0 \pm 12.2$ \\
B530 & $-64.8 \pm 4.8$ & $100.0 \pm 39.9$ & $-61.6 \pm 5.7$ & $95.9 \pm 44.3$ \\
B540 & $-66.2 \pm 4.9$ & $103.9 \pm 41.8$ & $-63.3 \pm 3.6$ & $65.7 \pm 22.3$ \\
\hline
\end{tabular}

Table 59. Total number of measurements of the transponder types B440, B510, B520, B530 and B540 conducted with and without ears in replicate 1 (run 1, with absorbers) compared to the number of measurements with at least one successful reading at one of the coordinates.

\begin{tabular}{ccccc}
\hline \multirow{2}{*}{ Type } & \multicolumn{2}{c}{ Without ears } & Target & With ears \\
& Target & Actual & 10 & Actual \\
\hline B440 & 3 & 3 & 10 & 5 \\
B510 & 3 & 1 & 10 & 3 \\
B520 & 3 & 3 & 10 & 5 \\
B530 & 2 & 2 & 10 & 9 \\
B540 & 4 & 4 & & 10 \\
\hline
\end{tabular}

The measurements with ears showed completely different results. The read range of B440 and B540 was reduced in average by approximately $40 \mathrm{~cm}$ to $64.7 \mathrm{~cm}$ for B440 and to $65.7 \mathrm{~cm}$ for type B540. Transponder type B530 almost maintained the average read range of $95.9 \mathrm{~cm}$ with an increase of the average RSSI to $-61.6 \mathrm{dBm}$. Regarding B510, the read range stayed at $40 \mathrm{~cm}$. The B520 reached $50 \mathrm{~cm}$ attached to ears.

The graphical evaluation of the transponders B440 and B530 confirm the results presented in Table 1. The measurements of type B440 without ears displayed a uniform curve without a huge scattering (Figure 3). When type B440 was attached to ears, some individual measurements showed a scattering and reduction of the read range. Only in one measurement an ear tag could be read further. Only in 5 out of 10 measurements in run 1 (Table 2), that were done with type B440 attached to ears, at least one reading was received. 
Transponder type B530 achieved an even higher read range in three of the measurements with ears, compared to the measurements in free air (Figure 4). It should be noted that there were also four measurements, which had a visibly reduced read range.
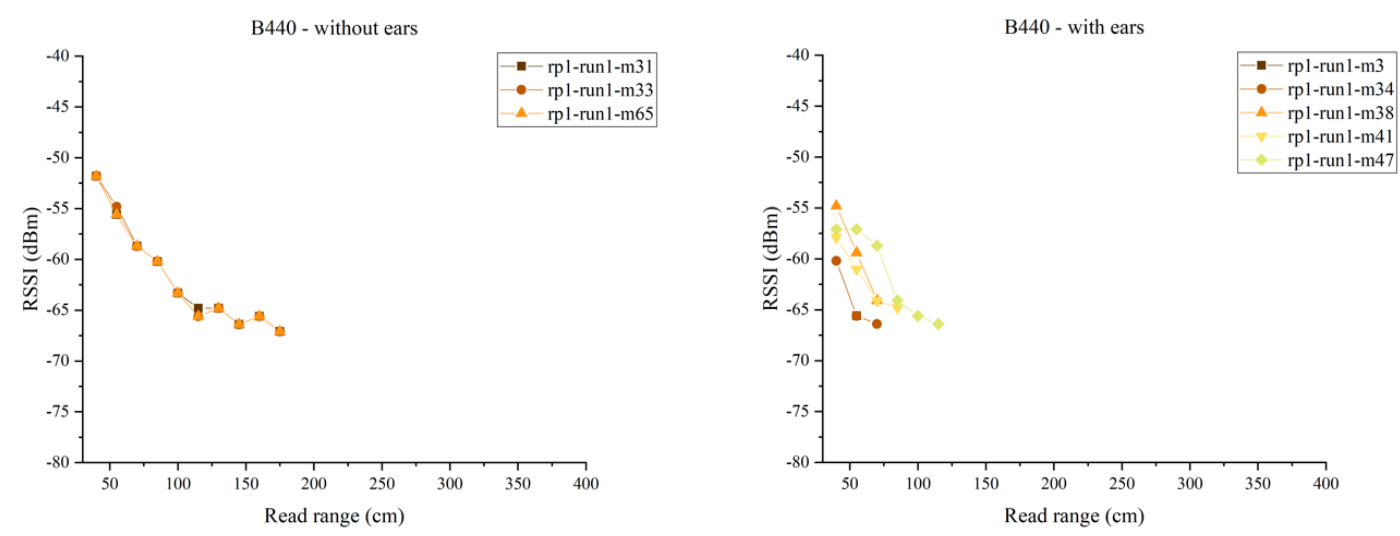

Figure 3. RSSI of the transponder B440 in relation to the distance to the reader, results of replicate 1, run 1 (rp1-run1-m31= replicate 1, run 1, measurement 31). Tested variants: free air (without ears, reference) and with pig ears. All measurements shown were performed with electromagnetic absorber mats at the test bench.
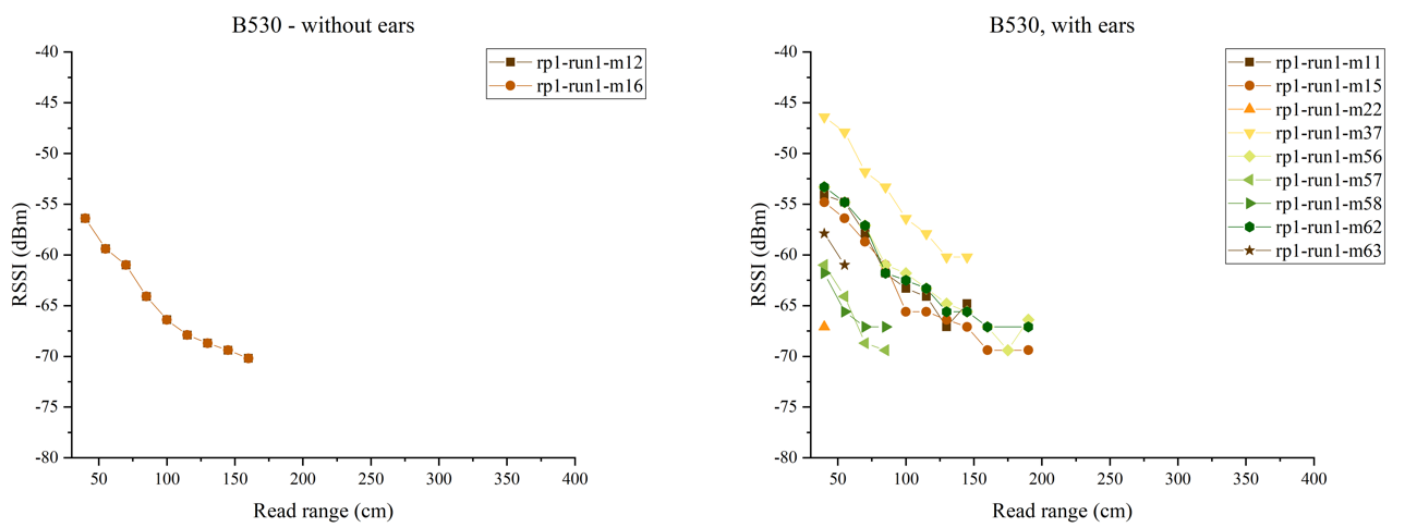

Figure 4. RSSI of the transponder B440 in relation to the distance to the reader, results of replicate 1, run 1 (rp1-run1-m31= replicate 1, run 1, measurement 31). Tested variants: free air (without ears, reference) and with pig ears. All measurements shown were performed with electromagnetic absorber mats at the test bench.

In general, it was observed, that when the transponders were applied to ears the readings dropped to greater or lesser extent (Table 2). Both transponder types B530 and B540 could be read in 9 and 10 measurements out of 10 in replicate 1, respectively. In contrast, only 5 successful measurements could be registered for type B440. It should be noted for the transponder B510, that out of 10 measurements with ears only in 3 at least one reading was received. Mostly all transponders gained readings in the measurements without ears, only the transponder B510 did not have any successful readings during 1 of the 3 measurements without ears in replicate 1.

The results illustrate that the pig ears in close vicinity to the transponders caused a strong scattering of the measurements. A possible explanation for this is that the different shapes of the ears and their varying size resulted in a strong variation of absorption or reflection of the radiation from the reader and transponder. This caused a varying influence on the all antenna designs. Since previous experiments showed similar bias in such measurements (Adrion et al., 2017b), all conclusions were included in the new transponder designs. In general, is important to design different transponder antenna layouts during the development in which also the resonant frequency and impedance are varied incrementally to find a suitable variant with good functional stability on the animal. Not all tissue variations can be covered with a single transponder design. Therefore, the focus must be placed on the types of transponder with the best overall performance and stability to further reduce the size of the antenna.

Close attention was paid to a uniform attachment of the ear tags during the experiments, because the ears should serve as mass area for the transponder antennas. However, a certain degree of variation was inevitable, 
because of the different shapes of the ears. This could be seen as an advantage, because this represents the transfer to the application in animal husbandry, since there is also variation in the position of the ear tag in practice. In addition, the ear tags are often attached into growing ears and should not sit too tightly in the ear. For this reason, ear tags have a small space to move, which leads to fluctuating read ranges of UHF transponders on the animal, too. This fact always has to be taken into account when applying UHF RFID in animal husbandry.

It is of interest, which of the effects (shape of the ear vs. tissue properties) improved or limited the performance more. In previous experiments, the same effects have already been described. Adrion et al. (2017b) stated, that the choice of the outside of the ear as transponder position should reduce the effects of the ears (Adrion et al. $2017 \mathrm{~b}$ ). This indicates, that the shape of the ear should not be neglected. Nevertheless, it is likely that the tissue itself still influenced the transponders a lot, as the scattered RSSI measurements in Figure 3 and 4 show.

The adaptation of different types of transponders to pig ears with varying degree of success could be shown in this series of experiments. The objective was to find a transponder that works well under the influence of ears and presents a good prospect for further reduction of antenna size for application in piglets. As discussed above the types B510 and B520 did not give satisfactory results with a limited reading range of approx. 40 to $50 \mathrm{~cm}$ with ears. Even without ears these types did not accomplish a mean read range of $60 \mathrm{~cm}$. On the contrary, the transponder types B530 and B540 performed very well. In conclusion, a reduced antenna geometry can maintain a good functionality by targeted adaptation of the resonance frequency under the negative influence of ears, but a strong reduction of the antenna mass area (B510 and B520) seems to be contradictive. This shows that the pig ears cannot replace the antenna mass area completely.

Type B540 almost had a similar read range compared to B440 (approx. $65 \mathrm{~cm}$ with ears and approx. $105 \mathrm{~cm}$ without ears), despite of the reduced antenna size in comparison to the B440. Type B530 even had measurements with ears, in which the read range was increased about $30 \mathrm{~cm}$. Thus, it can be stated that type B530 is the most promising antenna design in this experiment.

\section{Conclusions}

The aim of this experiment was to test and evaluate a new set of UHF transponder types. In order to depict the later application, the transponders were attached to ears and tested on an optimized static test bench. The most promising transponder type should be considered for the reduction to piglet size.

In summary, the influence of pig ears on all transponder types was recognizable. A successful development in terms of reducing the antenna size and simultaneously maintaining the performance in terms of read range could be shown. The next step is the analysis of all three replicates of the experiment with focus on the ear and absorber effects using a mixed model and the test of the promising types of transponder with finishing pigs. Based on this data, new types of transponder will be developed to further decrease the size of the antenna.

\section{Acknowledgements}

The project is supported by funds of the german government's special purpose fund held at Landwirtschaftliche Rentenbank. The authors would like to thank deister electronic GmbH, Caisley International $\mathrm{GmbH}$, Agrident GmbH and Prof. Dr.-Ing. W. Knop (University of Applied Sciences and Arts, Hannover) for their support and technical advice during the experiment. Thanks also go to Prof. Dr. H.P. Piepho (University of Hohenheim) for his advice on statistical design and analysis.

\section{References}

Adrion, F., Hammer, N., Rößler, B., Jezierny, D., Kapun, A., Gallmann, E., 2015. Aufbau, Funktion und Test eines statischen Prüfstandes für UHF-RFID-Ohrmarken. 46-66 Seiten / LANDTECHNIK, Bd. 70, Nr. 3 (2015). 10.15150/LT.2015.2660.

Adrion, F., Kapun, A., Eckert, F., Gallmann, E., Jungbluth, T., 2017a. Vergleich von LF- und UHF-RFID ber der Erkennung von Mastschweinen an einem Beschäftigungsgerät, in: 13. Tagung Bau, Technik und Umwelt in der Landwirtschaftlichen Nutztierhaltung. 18.-20. September 2017 in Stuttgart-Hohenheim $=13$ th Conference Construction, Engineering and Environment in Livestock Farming; September 18th-20th, 2017 in Stuttgart-Hohenheim, Germany. Kuratorium für Technik und Bauwesen in der Landwirtschaft, Darmstadt, pp. 276-281.

Adrion, F., Kapun, A., Holland, E.-M., Staiger, M., Löb, P., Gallmann, E., 2017b. Novel approach to determine the influence of pig and cattle ears on the performance of passive UHF-RFID ear tags. Computers and Electronics in Agriculture 140, 168-179. 10.1016/j.compag.2017.06.004.

Adrion, F., Reger, M., Eckert, F., Kapun, A., Staiger, M., Holland, E.-M., Hammer, N., Jungbluth, T., Gallmann, E., 2016. Sektorlokalisation von Mastschweinen mit UHF-RFID. Informatik in der Land-, Forst- und Ernährungswirtschaft 2016, 17-20. 
European EPC Compentence Center, 2011. The UHF Tag Performance Survey. European EPC Compentence Center, Neuss, Germany.

Finkenzeller, K., 2015. RFID-Handbuch: Grundlagen und praktische Anwendungen von Transpondern, kontaktlosen Chipkarten und NFC, 7th ed. Hanser, München, 755 pp.

Fujimoto, K., Morishita, H., 2013. Modern small antennas. Cambridge University Press, Cambridge, 473 pp.

Hammer, N., Adrion, F., Staiger, M., Holland, E., Gallmann, E., Jungbluth, T., 2016. Comparison of different ultra-high-frequency transponder ear tags for simultaneous detection of cattle and pigs. Livestock Science 187, 125-137. 10.1016/j.livsci.2016.03.007.

Hogewerf, P., Dirx, N., Verheijen, R., Ipema, B., 2013. The use of Ultra High Frequency (UHF) tags for fattening pig identification, in: Berckmans, D., Vandermeulen, J. (Eds.), Precision Livestock Farming '13. 6th European Conference on Precision Livestock Farming, Leuven, Belgium, 1012 September, 2013, pp. 440-448.

Kern, C., 2007. Anwendung von RFID-Systemen, 2nd ed. Springer-Verlag Berlin Heidelberg, Berlin, Heidelberg.

Maselyne, J., van Nuffel, A., Ketelaere, B. de, Vangeyte, J., Hessel, E.F., Sonck, B., Saeys, W., 2014. Range measurements of a High Frequency Radio Frequency Identification (HF RFID) system for registering feeding patterns of growing-finishing pigs. Computers and Electronics in Agriculture 108, 209-220. 10.1016/j.compag.2014.08.006.

Stasa, P., Svub, J., Benes, F., 2017. Development and Evaluation of Automated System for RFID Tag Performance Measurements. AEEE 15 (4). 10.15598/aeee.v15i4.2365.

Swedberg, C., 2012. PigTracker Project Finds UHF Tags Effective for Swine: The Danish Agriculture \& Food Council conducted a three-year pilot at five farms to learn how well EPC passive UHF tags performed for tracking pigs. http://www.rfidjournal.com/articles/view?9129. 


\title{
Differences in Light Concepts of Animal Welfare at Dairy Farms in North-East Germany
}

\author{
Jennifer Löbel ${ }^{\text {a,** }}$, Sandra Rose-Meierhöfer a ${ }^{\text {, Paul Gütschow }}{ }^{\text {b }}$, Clemens Fuchs ${ }^{\text {b }}$ \\ ${ }^{\text {a }}$ Department of agricultural machinery, Hochschule Neubrandenburg, University of Applied Science, 17033 \\ Neubrandenburg, Germany \\ ${ }^{\mathrm{b}}$ Department of agricultural economics, Hochschule Neubrandenburg, University of Applied Science, 17033 \\ Neubrandenburg, Germany \\ * Corresponding author. Email: loebel@hs-nb.de
}

\begin{abstract}
Cow comfort is the basic requirement for the health and animal welfare of dairy cows. Farmers underestimate many factors, especially the installation of extra equipment becomes neglected. In a research project was this claim analysed and it were collected 202 parameters in each barn of 34 dairy farms. The farms were assorted to the number of milking cows (group 1: up to 300 milking cows, group 2: 301 to 599 milking cows, group 3: 600 to 900 milking cows, group 4: over 900 milking cows). For this study we analysed especially the light types and light conditions in the barns and milking parlours. The light conditions were measured with lux meters. The data collection occurred three hours after feeding at four different places in the barn: the feeding table, at the cubicles and in the milking parlour at the milker pit and the cow area. Each measurement was carried out with light turned on and off. As a result, were on the feeding table the light conditions in average $327 \mathrm{~lx}$ (light turned off), so it was more than the theoretical optimum value of $200 \mathrm{~lx}$. In the cow area, the light conditions were bad in each barn: on average $95 \mathrm{~lx}$ (light turned off) and $120 \mathrm{~lx}$ (light turned on). The same results were registered in the milking parlours. The broadest grievance is the choice and the intensity of the lamp types. Only one barn has LED lamps. The other 33 farms have light pipes in their barns and 5 of them have also metal halide lamps. These types show that the light intensity cannot be well because light pipes have no big illuminance. It can be concluded that for increasing welfare in the stable the investigation in new light concept can be one activity.
\end{abstract}

Keywords: animal welfare, light conditions, light concepts, climate conditions, technical features

\section{Introduction}

Proper lighting in housing areas and in milking parlours will add to a high quality working environment to improve worker efficiency, safety and comfort especially for increasing milk production and animal welfare (Dahl et al., 2003). Photoperiod is defined as the duration of light exposure within a 24-hour period. That the cows and worker need light to get a better orientation is obviously. But it causes much more in a cow body. Light reception occurs in the retina and both the intensity and length of light exposure will control the hormone response in the pineal gland. When light is received by the eyes, a signal is sent to the hypothalamus, which sends a signal to the pineal gland, inhibiting the production of hormones. The pineal gland secretes melatonin. In lactating cow, melatonin will cause a reduction in appetite and reproduction levels therefore less melatonin will lead to an increase in feed intakes and associated milk production (Tucker, 1981).

A distinction is made between two photoperiods. A long-day photoperiod (LDPP) means exposure to 16 to 18 hours of continuous light followed by 6 to 8 hours of darkness, which means an illumination level by $50 \mathrm{~lx}$. (Buyserie et al., 2001). This light program is typical for lactating cows. The opposite is the short-day photoperiod (SDPP). It is defined as continuous exposure to 8 hours of light followed by 16 hour darkness. In practice, shortday photoperiod is anything less than 12 to 13 hours of light (Buyserie et al., 2001). The illumination level should not be over $50 \mathrm{~lx}$ in this time. This system is traceable by dry cows. The short-day photoperiod during the dry period resets the cows sensitivity to longer photoperiods in the subsequent lactation. Lactating cows exposed to long-day photoperiod consistently show an 8 to 10 percent increase in milk production. The long-day photoperiod should begin after calving and the increased milk production started 3 to 4 weeks later (Buyserie et al., 2001). The photoperiod does not significantly influence protein, lactose, fat or other ingredients in milk. The light intensity, as well, is very important in all parts of dairy barn. For each working area special illumination levels are recommended. For dairy cows are areas like the parlour, the feeding and lying area and the calving pens of crucial meaning. The general illumination level in the parlour, which also important for the cows, should be minimum $200 \mathrm{~lx}$ and in the zone of the udder level, the working area of the milking person, should be 400 to $500 \mathrm{~lx}$. The illumination level in the barn, especially at the lying and feeding area is comparable with the parlour and should be between 200 and $300 \mathrm{~lx}$ (Winter et al.). It is the most important area for cows because they get their energy of the feed and ruminate at the lying position and as a result produce milk. All influencing factors should be in the tolerance range to get a good foundation for milk production.

At least a farmer has to decide which and how much lighting he and the animals need. We decide between incandescent lights, halogen lamps, metal halide, high pressure sodium lights and light emitting diode (LED) 
(Harner, 2014). The incandescent lights are traditional light bulbs, they are cheap, easy to replace and instantaneous. But they are also having low energy efficiency. They are useful for situations that only require illumination for short periods at a time. The halogen or spot lamps are useful where high intensity light needs to be directed for a specific purpose for example in the treatment crate. They are inexpensive and often equipped with roof mountings. This light type can cause sharper shadows and increased glare. The third type, the metal halide, has high outputs making them suitable for general lightning in larger areas with high roofs. They throw a very white light and are about twice as energy efficient but they are very expensive and have a shorter life $(12.000$ hours) than the others. High pressure sodium lights are extremely energy efficient and have a long life (25.000 hours). They have a yellow lighting and are used extensively in very cold climates or in areas requiring continuous lighting (Paschotta, 2010; Delaval 2007). The last type, light emitting diode (LED), is a new generation of barn lighting (Bommel, 2016). This type is very efficient and has a very long life with over 50.000 hours. Main disadvantage is the price. It is the most expensive lamp in purchase but in variable costs cheaper than the other. The LED-Retrofit products should be use with caution. At this LED lamp type, conventional bulbs changed by LED bulbs. The biggest disadvantage of the upgrade by conventional lights is the temperature development. The temperature of the LED bulb should not be higher than the conventional bulb (DLG, 2016; SchwenzfeierHellkamp, 2013). Most farms don't stick to it, result from it are burning lamps. The conventional lamps are not reserved for use of the new LED bulbs. The whole light should be change.

The following formula should be used to calculate how many lights a farmer needs:

$$
\text { Number of lights }=\underline{\text { square footage of barn } * \text { foot candles* } \mathrm{K}} \quad \text { lumen output per lamp }
$$

where foot candles (FC) measure the light intensity, one FC is equal to 10,8 lux (lx), a minimum illumination level of $200 \mathrm{~lx}$ in the barn means $19 \mathrm{FC}$; $\mathrm{K}$ is a constant that accounts for light reflected in and escaping from the barn, $\mathrm{K}=2$ in enclosed barns, $\mathrm{K}=3$ in open-sided cubicle buildings (Buyserie et al., 2001).

\section{Materials and Methods}

Study design and data collection

This study presents the results of one part from the project: "Animal welfare and economic efficiency in the futureoriented dairy farming - evaluation of various actions and their economic impact". The study took place on 34 dairy farms in North- East Germany, especially in the three federal states: Mecklenburg-Vorpommern, Brandenburg and Schleswig-Holstein. There were collected 202 parameters in 54 barns of 34 dairy farms. The farms are assorted to the number of milking cows in four groups; group 1: up to 300 milking cows, group 2: 301 to 599 milking cows, group 3: 600 to 900 milking cows, group 4: over 900 milking cows. All farms, except one in Schleswig-Holstein with Angler breed and one in Mecklenburg-Vorpommern with Jersey breed, have HolsteinFriesian breed. All farms analysed in the period of April to November 2017. The data collection occurred three hours after feeding. This study focused the light conditions in the barn and milking parlour. The illumination levels collected with a lux meter at three different places at the farm. In the barn were measured the light intensity at the feeding table and at the cubicles (Figure 1). In the milking parlour were the measuring points the milking pit and the cow areas (Figure 2).

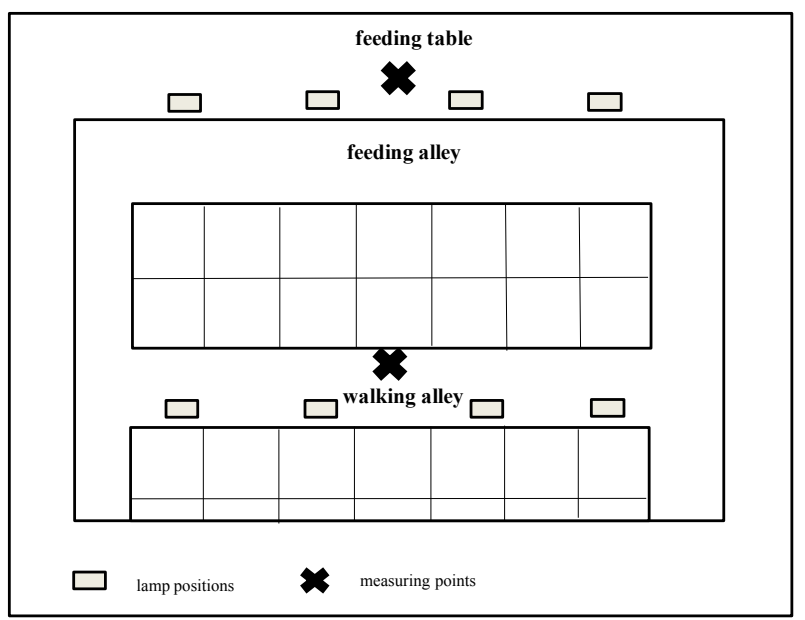

Figure 1. The measuring points of the study in the barn.

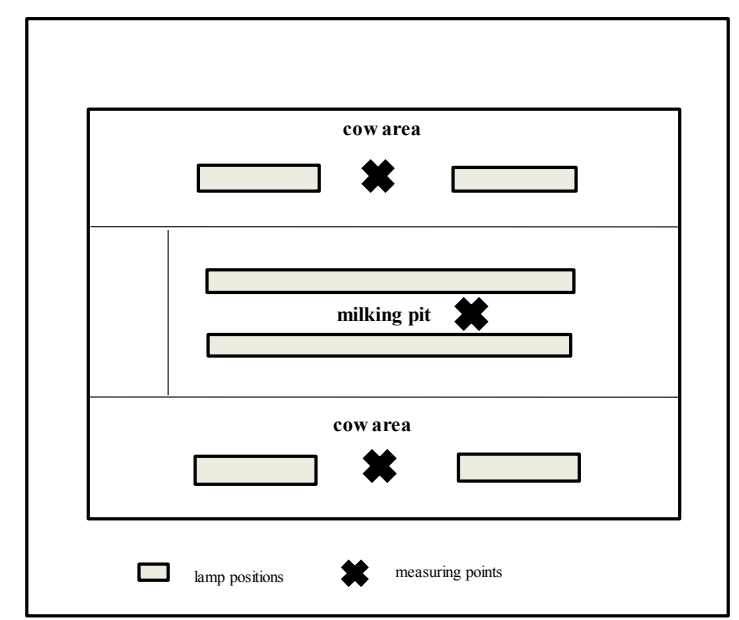

Figure 2. The measuring points of the study in the parlour. 
Statistical analysis

In each of the 54 barns, including the milking area, were collected 6 measuring values. Collectively are 324 measuring values of all 34 farms only for the detection of light intensity. Besides that, the presenting light types at the farms in each barn have been collected.

Data were analysed by using the IBM SPSS Statistic 25 software package (IBM Deutschland GmbH., Ehningen, Germany). The descriptive statistics was analysed using the explorative data analysis, especially the Boxplot procedure. The graphics (Figure 3,4,5 and 6) were created by Excel 2010.

\section{Results and Discussion}

Illumination levels

Figure 3 and 4 show the light intensity at the feeding and lying area and makes attentive to the light problems in the barns. The optimum level in the entire barn is 200 lx (DLG,2016; Hulsen, 2010). Table 1 shows the descriptive statistics of the light intensity of the entire barn. The graphics (Figure 3) depict that $51.85 \%$ of the analysed barns do not reach this value at the feeding area with light off. This outcome changes by $11.11 \%$, when the light turned on $40.74 \%$ are under $200 \mathrm{~lx}$. This modification demonstrates a positive effect that is important to have lighting in the barns and use them efficiently. But it is still a problem that shows the value of $40.74 \%$. Figure 4 indicate the problem if the feeding area too dark. It was the same like in the cubicle area. $74.07 \%$ of the barns at the cubicle area, light turned off; show an unsatisfactory illuminated lying area. Noticeable is the effect by light turned on; the same value result (59.26\%) like the barns over $200 \mathrm{~lx}$ at the feeding area when light turned on. In both figures are barns with very high light intensities ( $\mathrm{Nr} 40,41)$. These barns are very new and would built with free sides without a shadow effect by other buildings around and due to the ridge opening is the feeding table also very bright.

Table 1. Descriptive statistics of the light intensity in the barn regarding to the optimum level of $200 \mathrm{~lx}(\mathrm{~N}=54)$.

\begin{tabular}{|l|c|c|}
\hline & under $200 \mathrm{~lx}$ & over $200 \mathrm{~lx}$ \\
\hline feeding area_light off & $51.85 \%$ & $48.15 \%$ \\
\hline feeding area_light on & $40.74 \%$ & $59.26 \%$ \\
\hline lying cubicles_light off & $74.07 \%$ & $25.93 \%$ \\
\hline lying cubicles_light on & $59.26 \%$ & $40.74 \%$ \\
\hline
\end{tabular}

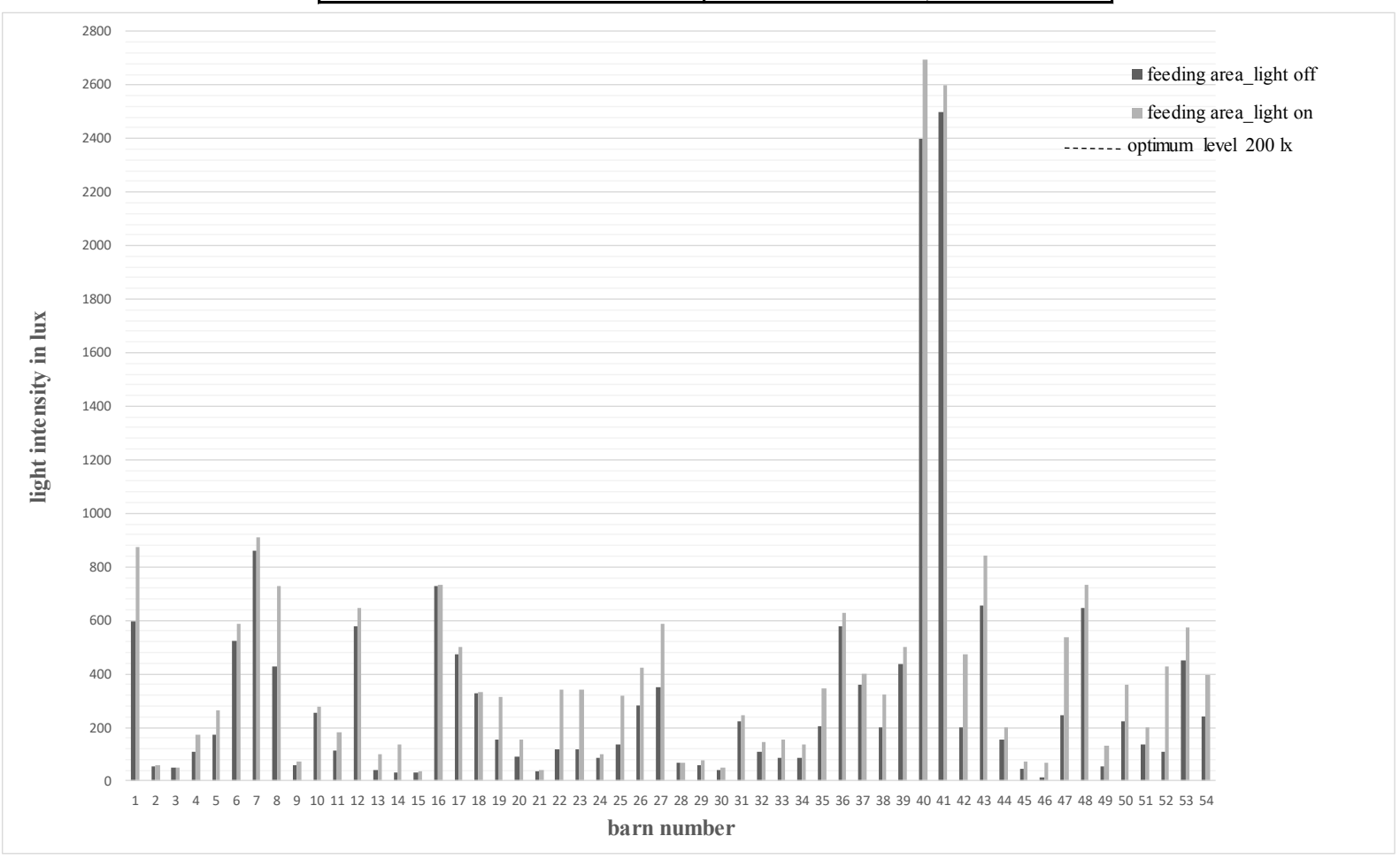

Figure 3. The differences in the measured light intensity at the feeding area with light on and off. 


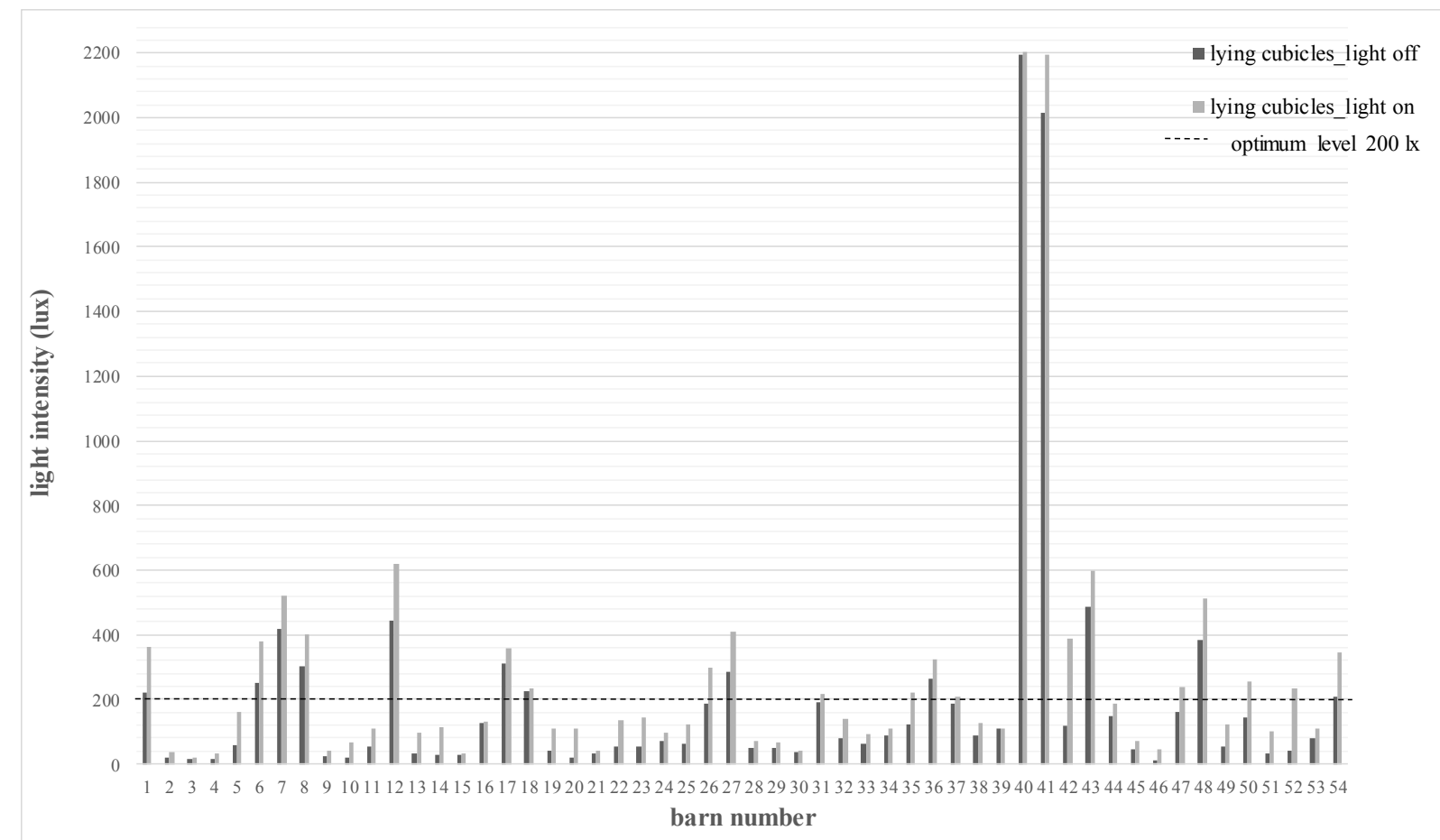

Figure 4. The differences in the measured light intensity at the lying area with light on and off.

In the parlour, the light conditions are mostly better for the worker efficiency, specifically in the milking pit achieved $45.16 \%$ barns the optimum value range of 400 to 500 lx (Table 2). But synoptically more than $50 \%$ of the farms do not reach the optimum range.

Table 2. Descriptive statistics of the light intensity in the barn regarding to the optimum level of $200 \mathrm{~lx}(\mathrm{~N}=31)$.

\begin{tabular}{|l|c|c|}
\hline & under 200 lx & over 400 lx \\
\hline milking pit & & $45.16 \%$ \\
\hline general lighting & $41.94 \%$ & \\
\hline
\end{tabular}

Noticeable is the general lighting in the parlour also the light condition for the cows. General it is a kind of darkness in the parlour, comparative with the illuminate rate in the lying area. The farmers do not pay attention to the area around the milking pit. This area is also important for the animal welfare of the cows. For the cows is an abruptly and continuously change between lightness and darkness very stressful because the orientation time is too short. Figure 5 shows a correlation between illumination values in the milking pit and cow area. Is the light level in the milking pit well, the general lighting is also at the minimum range. This is mostly a result of built translucently. Because the most milking parlour have only lamps at the milking pit and especially by rotary milking parlour are additionally lamps over the parlour. 


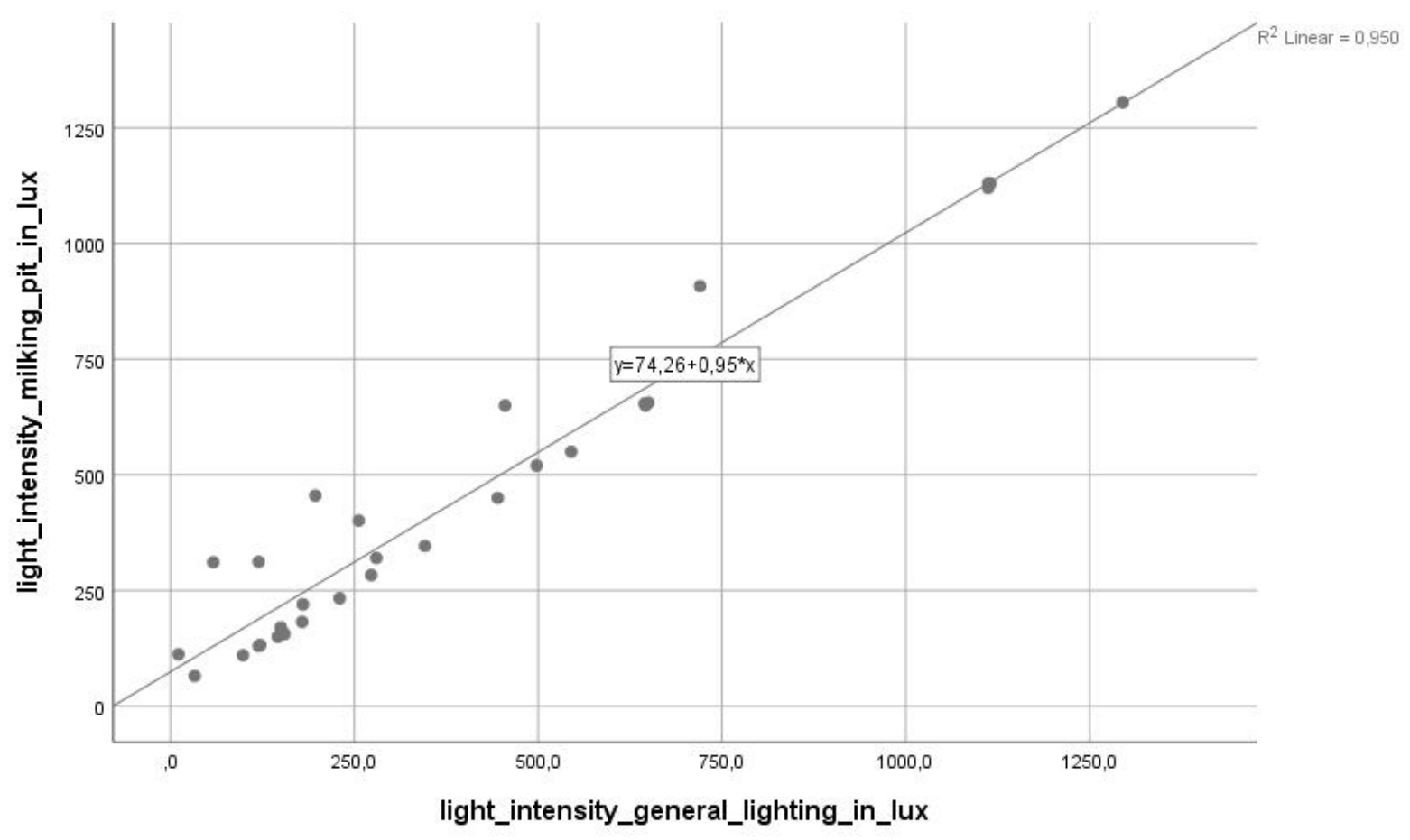

Figure 5. The correlation between the measuring values of light intensity at the milking pit and the general lighting in the milking parlour.

Lighting types

The analysis of the graphics below shows a big problem of light intensity in all areas even if the light turned on. This let's assume that the light type can be cause for it. The notations of the lighting types confirm it (Figure 6). Most of the 34 farmers use in their barns (total of 54) the incandescent light $(66 \%)$ that is the reason for the inadequate light intensity as well, if the light turned on. The second widespread type is with $24 \%$ the metal halide. On the basis of the high output in larger areas with high roofs, these are used. But nobody respected where they are mount the lamps and how many. That is an another reason for the inadequate illuminate rate on the whole farm. Only one farm has the LED light in their barn.

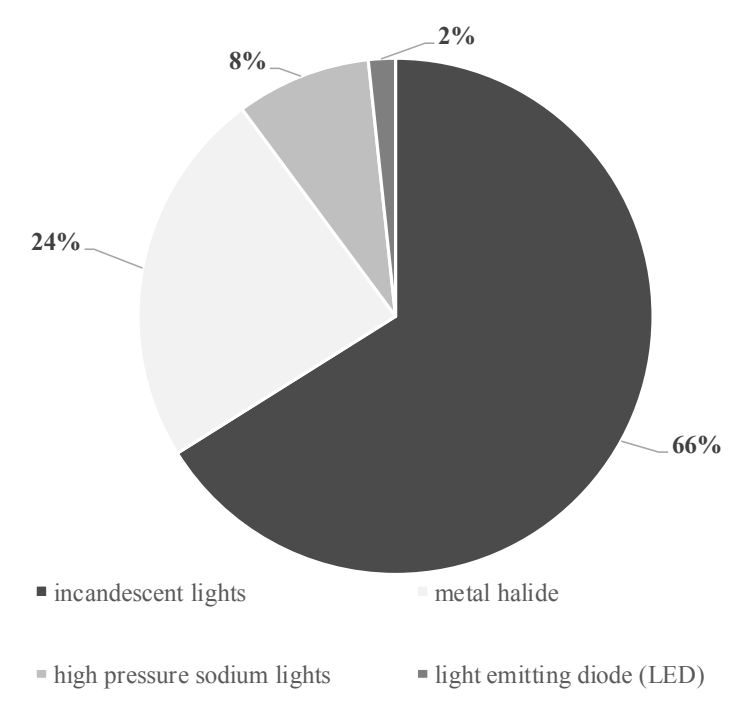

Figure 6. The notation of frequency of use of the different light types. 


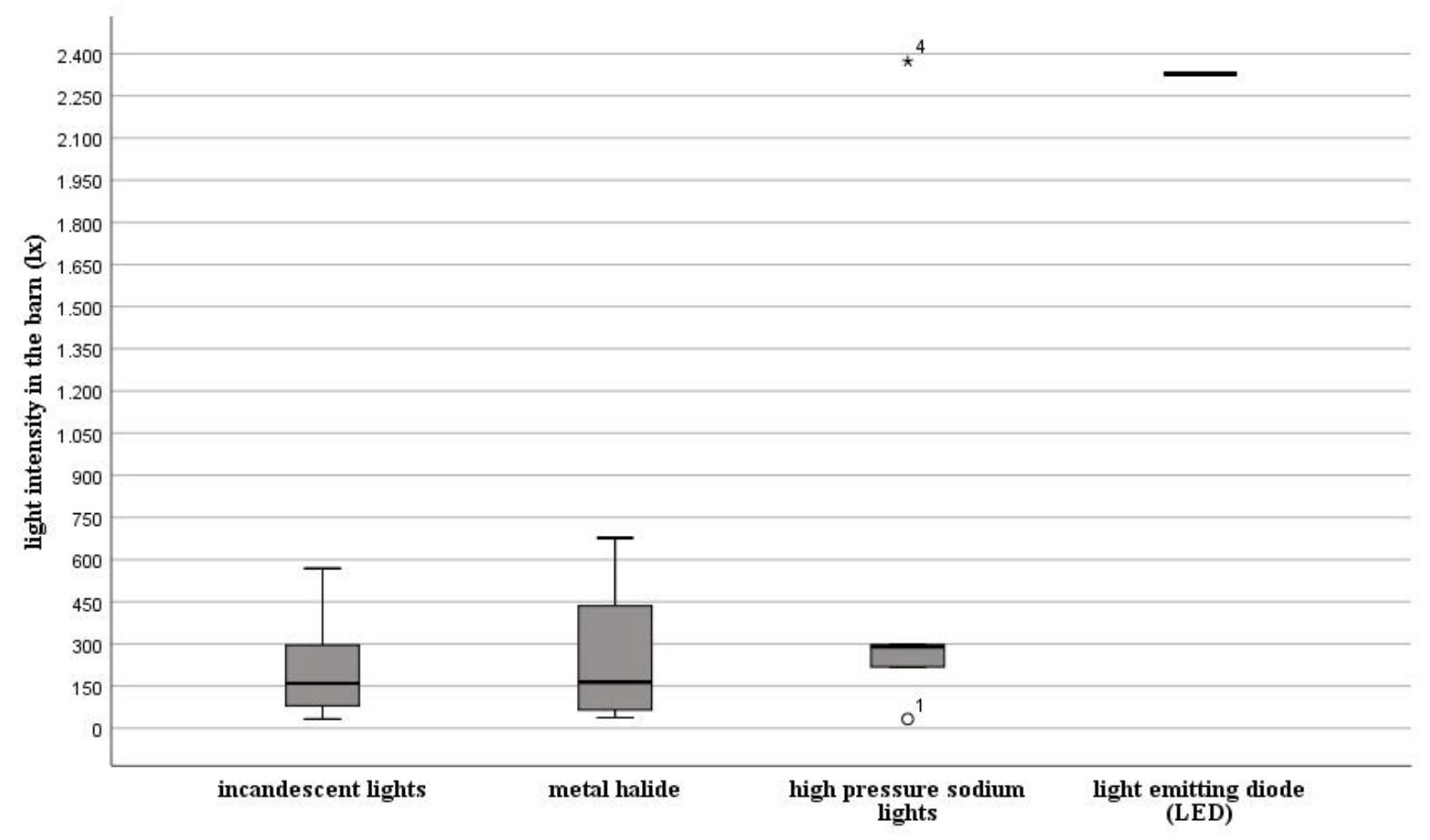

Figure 7. Light intensity of the different light types in the whole barn by light on (average of light intensity at the cubicles (light on) and the feeding area (light on) for each barn).

Figure 7 shows the different light types in the 54 barns. Some barns have more than one type built in. The allocation of the single types agrees with their theoretical description. The median of the incandescent lights located by $\pm 150 \mathrm{~lx}$ and the metal halide by $200 \mathrm{~lx}$. The incandescent light shows a greatest value of $\pm 550 \mathrm{~lx}$. In connection with the light intensity outside and the light incident in the barn evolved these values. But the graphic shows that are only single values and not the entirety. The boxplot of the metal halide shows that $75 \%$ of the barns have a light intensity under $450 \mathrm{~lx}$, the incandescent lights only under $300 \mathrm{~lx}$. This result confirms with the theoretical description of the implementation of Paschotta (2010) and Delaval (2007).

These values have a high delimitation of these from high pressure sodium lights and LED lamps. Paschotta (2010) says that the high pressure sodium lights and LED lamps are extremely energy efficient and have a high output. The median of $\pm 300 \mathrm{~lx}$ by high pressure sodium lights confirm it. The value is much better as the one of incandescent lights and metal halide but you have to consider the environmental influences like rain and different outside temperatures.

So a median of $300 \mathrm{~lx}$ is a good value and over the minimum value of $200 \mathrm{~lx}$ but the measuring was only on one day and not constantly the 16 hours where should be a light intensity of $2001 x$. When it is cloudy outside the light intensity goes automatically down, so it is very hard to get constantly minimum 200 lx for 16 hours in the barn. The farmer has two different choices, he can turn the light on 16 hours for example after milking to get approximately $200 \mathrm{~lx}$ in the barn or he install an automatically system which measured constantly the light intensity in the barn and decide when the light turn on automatically (Dehoff, 2016).

The boxplot of the high pressure sodium lights shows one outrageous and on extreme value. The extreme value with the number 4 is the barn number 40 (Figure 3 and 4). This value is not typical and representative in comparison to the other barns. The value resulting of the barn construction which is very friendly and the sides and roof are open and thus the high light intensity which could come in. As well the outrageous value which is for this lamp type very untypical. This barn was very dark because all sides were closed and there were not enough lamps which can also a factor for inadequate light intensity in the barn. The LED lamp type has only one barn. It is a very new building and so the choice came up to get LED lamps. For this lamp type could not show a range of light intensity because it is only one barn. We only see the high light intensity as well of the barn construction. 


\section{Conclusions}

It can be concluded that the type of the lighting in each area, besides the barn construction are of high importance. A lot of farmers shy away LED's because of the higher price but they don't know the benefit over a long period. Their knowledge about lamp systems is not much pronounced that they use the wrong type for their barns. The farmers should measure the illumination level in each area, compare with the minimum value and react then. Every farmer should get information about the different light types and the right installation of them. In this part is a big catching up. It can be concluded that for increasing welfare in the stable the investigation in new light concepts can be one activity.

\section{Acknowledgements}

The authors would like to thank all farmers and their employees for their trusted confidence and support. A thank as well the federal ministry of education and research for the financial support. Furthermore, a special thank to the four farms: H. Wilhelm Schaumann GmbH, Gut Hohen Luckow, Agrarhof Brüel eG, LVAT e.V. Ruhlsdorf/Groß Kreutz and Gut Wamckow for the financial and academic support. The university in Hohenheim, Humboldt- Universität zu Berlin, Leibniz - Institut for agricultural engineering and bioeconomy for the scientific support.

\section{References}

Bommel, W., 2016. Lighting Controls. In Encyclopedia of Color Science and Technology. 850-861. https://doi.org/10.1007/978-1-4419-8071-7 134

Buyserie, A., Gamroth,M., Dahl, G., 2001.Managing Light in Dairy Barns for Increased Milk Production. Oregon State University, USA. September.

Dahl, G.E., Petitclerc, D., 2003. Management of photoperiod in the dairy herd for improved production and health. Journal of Animal Science. (81) 11-17. https://doi.org/10.2527/2003.81suppl_311x

Dahl G.E., Buchanan B.A., Tucker H.A.,2000. Photoperiodic Effects on Dairy Cattle. A Review. Journal of Dairy Science. (83) 885-893. https://doi.org/10.3168/jds.S0022-0302(00)74952-6

Dehoff, P., 2016. Lighting Controls. In Encyclopedia of Color Science and Technology. 864-871. https://doi.org/10.1007/978-1-4419-8071-7 134

Delaval, 2007. Dairy lighting system for free stall barns and milking centers. University of Minnesota, U.S. Department of Agriculture, and Minnesota Counties Cooperating. http://www.milkproduction.com/Library/Scientific-articles/Housing/Dairy-lighting-system-for-free. Accessed May 3, 2018.

DLG e.V., Werner, D., Schneider, M., Schwenzfeier-Hellkamp., E., Müller, M., Reiter, K., Pelzer, A., 2016. DLG-Merkblatt 415. Beleuchtung und Beleuchtungstechnik im Rinderstall.Frakfurt am Main, Germany.Oktober.

Harner, J. P., Zulovich, J.M., 2014. Application of Light Emitting Diodes in Dairy Facilities. In High Plains

Dairy Conference. Kansas State University and University of Missouri. http://highplainsdairy.org/2014/Harner.pdf

Hulsen, J., 2010. Bauen für die Kuh. Zutphen: RoodBont Verlag.

Paschotta, R., 2010. RP-Energie-Lexikon. https://www.st-energieberatung.de/cms/upload/pdf/LichtLexikon.pdf. Accessed May15, 2018.

Schwenzfeier-Hellkamp, E., 2013. Projekt: „Intelligentes optisches LED Leitsystem“. University of Applied Sciences Bielefeld.

Tucker, H.A.,1981. Photoperiodic control of hormones, growth and lactation in cattle. In Environmental Factors in Mammal Reproduction. 54-73. https://doi.org/10.1007/978-1-349-03964-7_4

Winter, A., Wagner, T. 2002. Lighting of cowhouse and milking area. Hanover Camber of Agriculture. Hanover, Germany. 


\title{
Evaluating Pig Ear Skin Temperature: Intercontinental Transport vs Housing Conditions
}

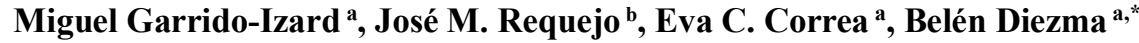 \\ ${ }^{a}$ Laboratorio de Propiedades Físicas y Técnicas Avanzadas en Agroalimentación. ETSIAAB, Universidad Politécnica de \\ Madrid, Madrid, 28040, Spain \\ ${ }^{\text {b } H e n d r i x-G e n e t i c s ~ C o m p a n y . ~ H y p o r ~ B . V . V i l l a ~ ' d e ~ K o ̈ r v e r ', ~ S p o o r s t r a a t ~ 69,5831 ~ C K ~ B o x m e e r, ~ N e t h e r l a n d s ~}$ \\ * Corresponding author. Email: belen.diezma@upm.es
}

\begin{abstract}
Variations in the skin temperature are often used as indicators of stress in animals. With advances in technology, it is now feasible to record high frequency livestock temperatures continuously for extended periods. In this study the objective was to analyse the response of pigs to different environmental conditions. The ear skin temperature of two batches of animals in intercontinental transport and housing was registered and characterized by the use of phase space diagram methodology. Eleven pigs were monitored during transport from a farm located in Reston (Canada), until their arrival at a farm in Montiel (Spain) during May 2016. After the itinerary by airplane and trucks separated with rest housing, pigs arrived to the farm after 94 hours. Fourteen finishers were monitored in one pen on a breeding farm in Villatobas (Spain) during one week in June 2017. To measure ear skin pig temperature, one logger with temperature sensor was glued to the RFID tag of each pig and placed on the inside half of the left ear. The areas and centroids of the phase space diagrams of ear skin temperatures were used to quantify the variability of the time temperature series. It was considered analysis of full times series and time series by periods (transport modality, transport transfers and day and night). The time series pattern for each individual was analysed independently in order to identify differences between animals of the same batch. The thermal variability between animals of the same batch was higher during transport than those identified in housed animals. The maximum and minimum areas for an animal during transport were $60-77 \%$ higher than the areas for housed animals, indicating more exposure to thermal stress during transport management.
\end{abstract}

Keywords: animal welfare, phase space diagram methodology, temperature logger, animal monitoring.

\section{Introduction}

The skin temperature of mammals can be an indicator of health status since stressed or diseased states will tend to cause hyperthermia (Loughmiller et al., 2001). Activity level, eating and psychological stress can cause hyperthermia, changing the thermoregulatory set-point under normal, non-disease conditions (Grant, 1950). In recent years, advances in technology have produced a growing number of electronic devices that can provide more detailed data about diurnal variation in body temperature for farm animals (Langer \& Fietz, 2014), including deep body implants, subcutaneous chips and infra-red (IR) thermographers (Soerensen and Pedersen 2015). Specifically for pigs, Andersen et al. (2008) demonstrated that continuous monitoring of ear skin temperature using small data loggers can give an idea of thermoregulatory effort. In usual conditions, the thermal equilibrium in the body of pigs is achieved through a balance between the metabolic heat production and heat loss from the body, trying to maintain a preferred skin temperature. Therefore, to keep the body temperature relatively constant, variations in heat loss may be expected.

The transport of cattle is a part of the livestock activity of transcendental economic importance around the world, and it is a crucial aspect regarding health, well-being and product quality. The current legislative framework in the European Union defines procedures to protect animals during transport; specifically limits in the range of temperatures where animals are located. The environmental changes suffered during transport, which can involve significant thermal variations in short periods, can lead to a change in the thermoregulatory response of the animal, as well as to the appearance of stress. Genetics has proven to be a factor of great importance in relation to the appearance of thermal stress and difficulties to adapt the thermoregulatory response. Assuming this premise, not all animals will respond in the same way to environmental conditions changes, being of special interest the possibility of characterizing this reaction for each of the animals belonging to a batch by non-invasive methods.

Feed efficiency is one of the most important determinants of animal productivity. Animals with a higher deep body temperature will use more energy to produce metabolic heat, at the expense of productivity (Herd and Arthur 2009; Britt et al. 2003). The lower physical activity associated with reduced energy expenditure improves efficiency in pigs (Meunier-Salaün et al., 2014). Labussière et al. (2013) found that partitioning heat production in growing pigs can help to calculate efficiency, where heat production includes physical activity, the thermal effect of feed and basal metabolism.

With advances in technology, it is now feasible to record high frequency livestock temperatures continuously for extended periods in different locations (transport, housing, etc.). The phase space diagram methodology for analysing temporal series has shown potential to display the behaviour of dynamic systems of temperature. 
The objective of this study is to register and characterise the ear skin temperature of two batches of animals in intercontinental transport and housing conditions by the use of phase space diagram methodology in order to identify differences between animals regarding thermoregulatory response.

\section{Materials and Methods}

Animals

\section{Housing monitoring}

Between June 19th and 26th (2017), 14 Landrace finishers (all male) were monitored in one pen (15 $\left.\mathrm{m}^{2}\right)$ on a breeding farm in Villatobas, Castilla-La Mancha, Spain belonging to Hendrix Genetics. The initial animal density was $56.6 \mathrm{~kg} / \mathrm{m}^{2}$. Environmental temperature was controlled automatically (set point temperature at $30^{\circ} \mathrm{C}$ ) and the light schedule was 14L:8D. All animals were weighed individually at the beginning and end of the trial, and the total feed consumed by each was monitored. The feed efficiency was calculated as the ratio between the increase of weight of each animal and the total feed consumed during the test.

Intercontinental Transport monitoring

The transport of 13 Duroc pigs from specific pathogen-free (SPF) facilities in Reston (Manitoba, Canada) to a farm in Montiel, Ciudad Real, Spain was monitored between May 19th and 22nd (2016). The trip included trucks and plane displacements. Table 60 summarizes the complete itinerary.

Table 60. Summary of the itinerary followed by monitored Duroc pigs.

\begin{tabular}{ccccc}
\hline Departure Date & Departure Location & $\begin{array}{c}\text { Transport and } \\
\text { Duration (hours) }\end{array}$ & Arrival Date & Arrival Location \\
\hline May 19, 20:00 & SPF:Reston (Canada) & Truck $(5 \mathrm{~h})$ & May 20, 01:00 & Airport Toronto \\
May 20, 01:00 & Airport Toronto & Plane $(12 \mathrm{~h})$ & May 20, 13:00 & Luxembourg \\
May 20, 13:00 & Luxembourg & Truck $(13 \mathrm{~h})$ & May 21, 02:00 & Bordeaux \\
May 21, 02:00 & Bordeaux & Temporary rest $(22 \mathrm{~h})$ & May 22, 00:00 & Bordeaux \\
May 22, 00:00 & Bordeaux & Truck $(9 \mathrm{~h})$ & May 22, 09:00 & Lérida \\
May 22, 09:00 & Lérida & Truck $(9 \mathrm{~h})$ & May 22, 18:00 & Farm: Montiel \\
\hline
\end{tabular}

Temperature measurements

To measure ear skin pig temperature, one iButton (DS1923, Dallas Semiconductor, USA) was glued to the RFID tag of each pig and placed on the inside half of the left ear (Figure 13). The iButtons can measure and register temperature $(\mathrm{T})$ and relative humidity $(\mathrm{RH})$, with a resolution of $0.0625^{\circ} \mathrm{C}$ and $0.04 \% \mathrm{RH}$, temperature range -20 to $+85^{\circ} \mathrm{C}$ and relative humidity range from 0 to $100 \%$. Skin temperature was measured every $180 \mathrm{~s}$, due to limits of data storage ( $n=1680$ readings for $T$ and $\mathrm{RH}$ and $\mathrm{n}=3361$ readings for skin temperature). In the housing experiment, four more identical iButtons (numbered E1 to E4) were placed around the pen at pig height to measure $\mathrm{T}$ and $\mathrm{RH}$ and programmed to read every $360 \mathrm{~s}$.

(a)

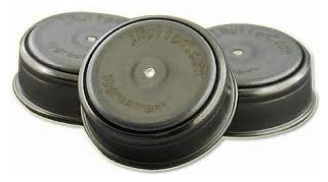

(b)

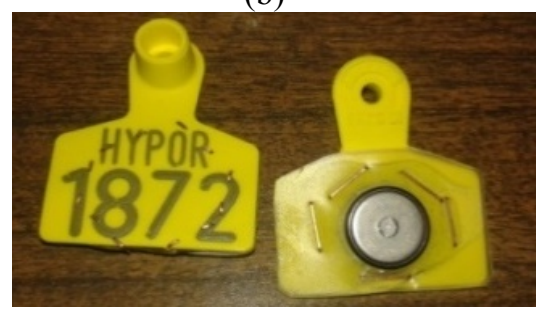

(c)

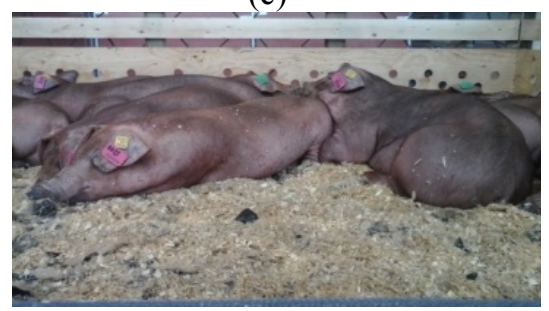

Figure 8. (a) iButton DS1923 devices; (b) device placed in the RFID tag; (c) RFID tag with iButton placed inside the ear of monitored pigs.

Data Analysis

We calculated the descriptive statistics of environmental temperature and relative humidity in housing experiment ( $\mathrm{n}=1680$ data points from 4 sensors) and skin temperature of the pigs in both experiments (14 and 11 sensors respectively. Subsequently a phase space was constructed for each pig based on skin temperature data using time delays, following Eckmann and Ruelle (1985). A dynamic system can tend to move towards a nucleus or attractor which can be seen clearly in a phase space representation. Each phase space was reconstructed in a phase graph using a time series versus itself delayed in time. The appropriate value for the time delays $t_{d}$ and system dimension $\mathrm{N}$ was obtained by heuristics. In this work, the two-dimensional $(N=2)$ phase space representations were obtained by plotting the measured temperature $T(k)$ at each time $t(k)$ versus the temperature $T(k+\Delta)$ at time $t(k+\Delta)$, with an optimum step $\Delta$ established by heuristics. The data acquisition interval (i.e., the fixed time step of the time series) was $\tau$ min ( 3 min in this experiment), making the corresponding time delay $t_{d}$ 
$=\Delta x \tau$ min. To obtain the optimum time delay, different values of $\Delta$ were tested systematically, looking for the maximum expansion of the trajectory loops or attractors in the phase space. The area of the trajectory loops corresponding to one sensor or group of sensors was computed using the Matlab function (MATLAB R2015b, MathWorks, Inc., Natick, MA, USA) convhull and polyarea. This function allows to select a set of points from the trajectory loop in the phase space plot and returns the smallest convex envelope containing the set of points and the corresponding area. The convhulln function is based on the Quickhull (Qhull) algorithm for convex hulls (Barber et al., 1996). The polyarea function returns the area of vertices defined by convhull (Figure 9).

From these phase space diagrams we can extract two variables of interest: 1) the centroid or centre of gravity $\left({ }^{\circ} \mathrm{C}\right)$ of the cloud of points that represents the arithmetic mean of all points of temperature registered, weighted by the local density of points or specific weight, 2$)$ the areas $\left({ }^{\circ} \mathrm{C}^{2}\right)$ that include all the points in the phase space as a measure of the total variability in temperature during the complete periods (total area, as well as during the day and night in housing experiment and stage of the trip in transport experiment.

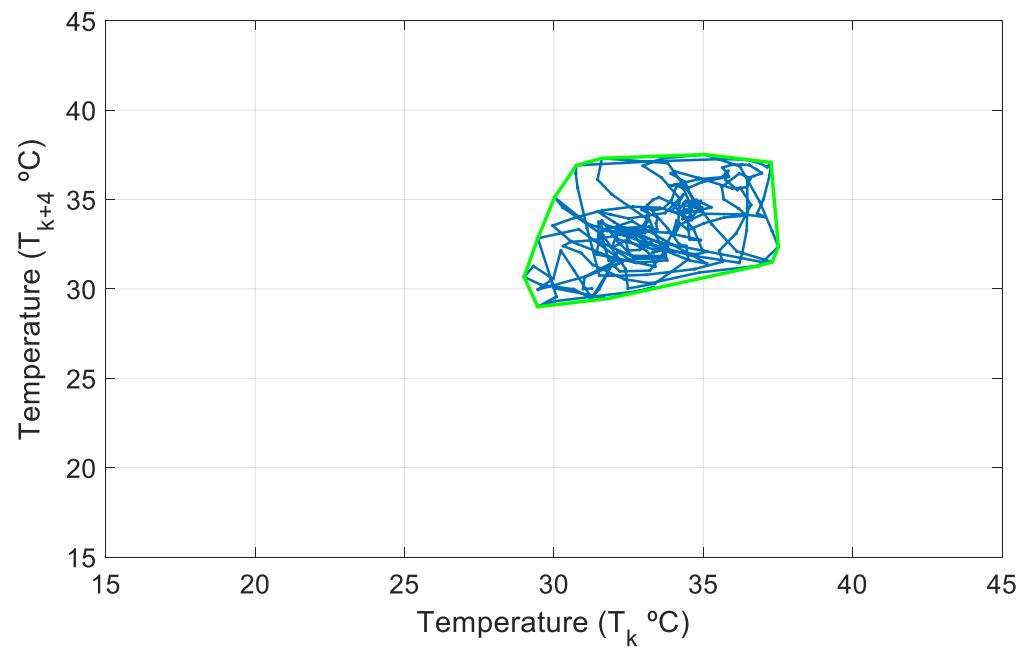

Figure 9. Areas of the phase spaces of one of the pigs. Points included are represented in blue, while border limit is represented by a green line.

\section{Results and Discussion}

Housing monitoring

The average temperature in the pen was $27 \pm 1.82{ }^{\circ} \mathrm{C}$ (range $21.16-30.74{ }^{\circ} \mathrm{C}$ ). The average skin temperature was $34.70{ }^{\circ} \mathrm{C}$ (range $=32.82+/-2.29$ to $35.89+/-1.50{ }^{\circ} \mathrm{C}$ ). The average standard deviation $(\mathrm{n}=3361)$ of the temperatures among animals was $1.75^{\circ} \mathrm{C}$. The average of the differences between the maximum and minimum temperatures in each instant was $6^{\circ} \mathrm{C}$. The time series of the skin temperatures is shown in Figure 10

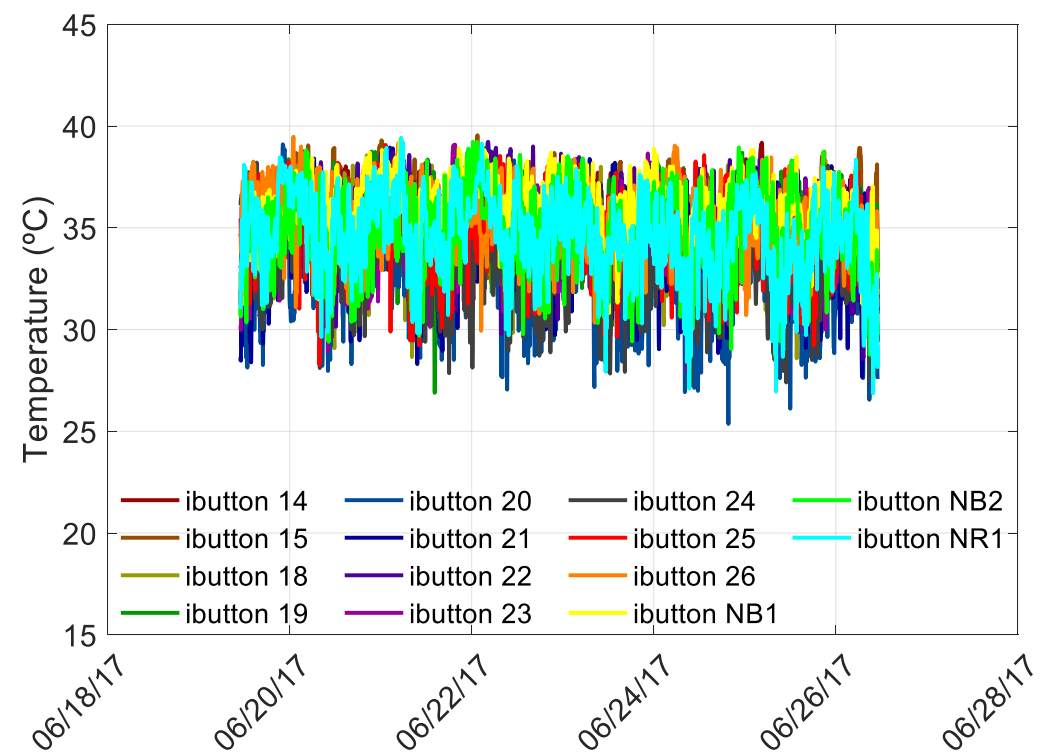

Figure 10. Time series of ear skin temperatures during the housing test of the 14 monitored pigs. 
The phase space in skin temperature was computed with a time delay of $12 \mathrm{~min}$. Areas and centroids were calculated for each animal and period. Analyses of variances were computed for centroids and areas respect to day or L and night or D periods. There were significant differences for both variables, with increase of more than $1{ }^{\circ} \mathrm{C}$ in the centroids during night, and increases of $50 \%$ in the value of the phase spaces area during day, denoting a higher variability of the ear skin temperatures (Figure 11). So, diurnal rhythm in the ear skin temperature could be observed, being highest at night and lowest in the afternoon. Similar results were obtained by Andersen et al. (2008), who states that higher skin temperature is prefer for several species when they are at rest.

(a)

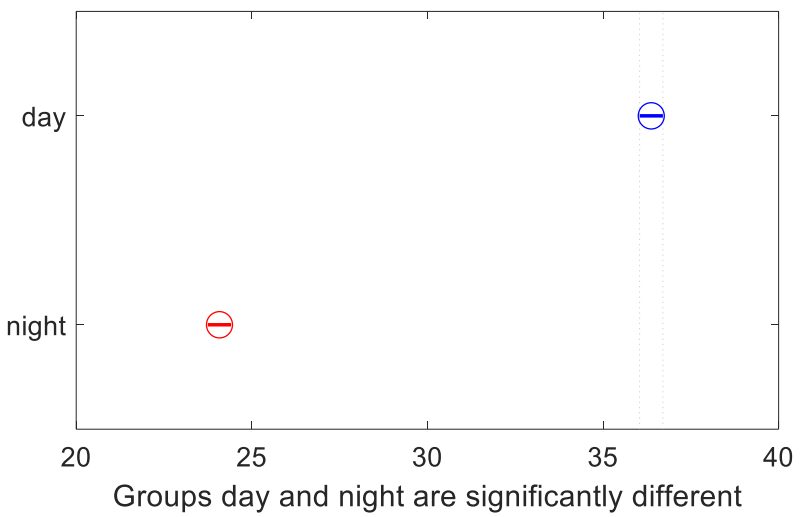

(b)

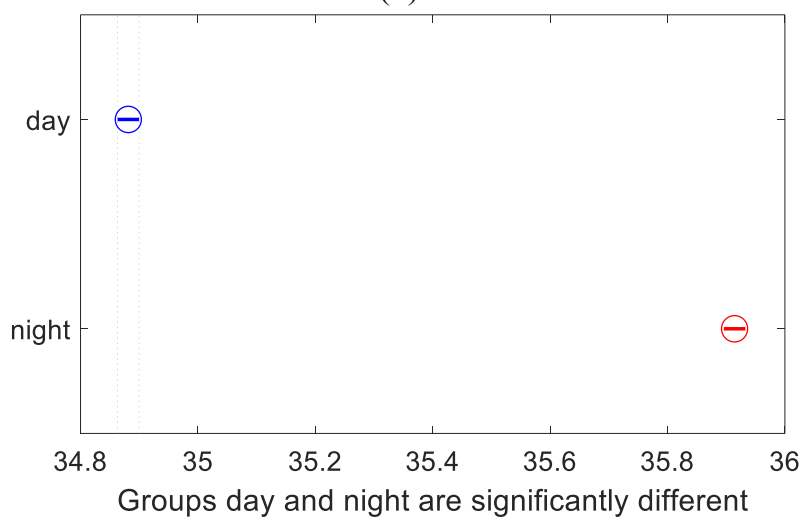

Figure 11. (a) Significant differences in the area of the phase space diagram respect to day and night and; (b) significant differences in the centroids of phase space diagram respect to day and night.

Regarding the animals, there were significant differences between the centroids of the phase space diagram computed for periods of 24 hours in state of lighting conditions (Figure 12). IButtons 20 and NB1 can be highlighted as the extremes values of temperature in terms of area and centroids. Figure 13 presents centroids and areas for each iButton distinguishing day and night periods. It can be observed that the iButtons that presented the higher values of centroids and lower values of areas corresponds to the animals with the animals with lower feed efficiency (

Table 61), as in the case of iButton NB1 for low feed efficiency, and in opposite situation the iButton 20 (low centroid, high area and high feed efficiency). The correlation coefficient between areas and centroids of phase space diagrams was of -0.98 , considering separately periods of day and night.

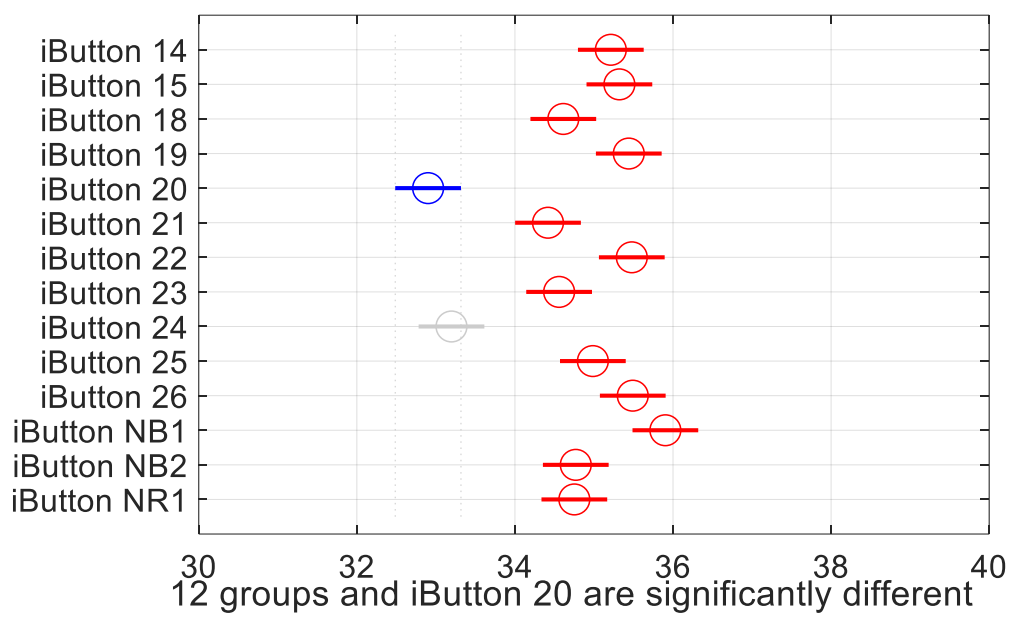

Figure 12. Significant differences in the centroids of phase space diagram respect to the iButton of each animal. 


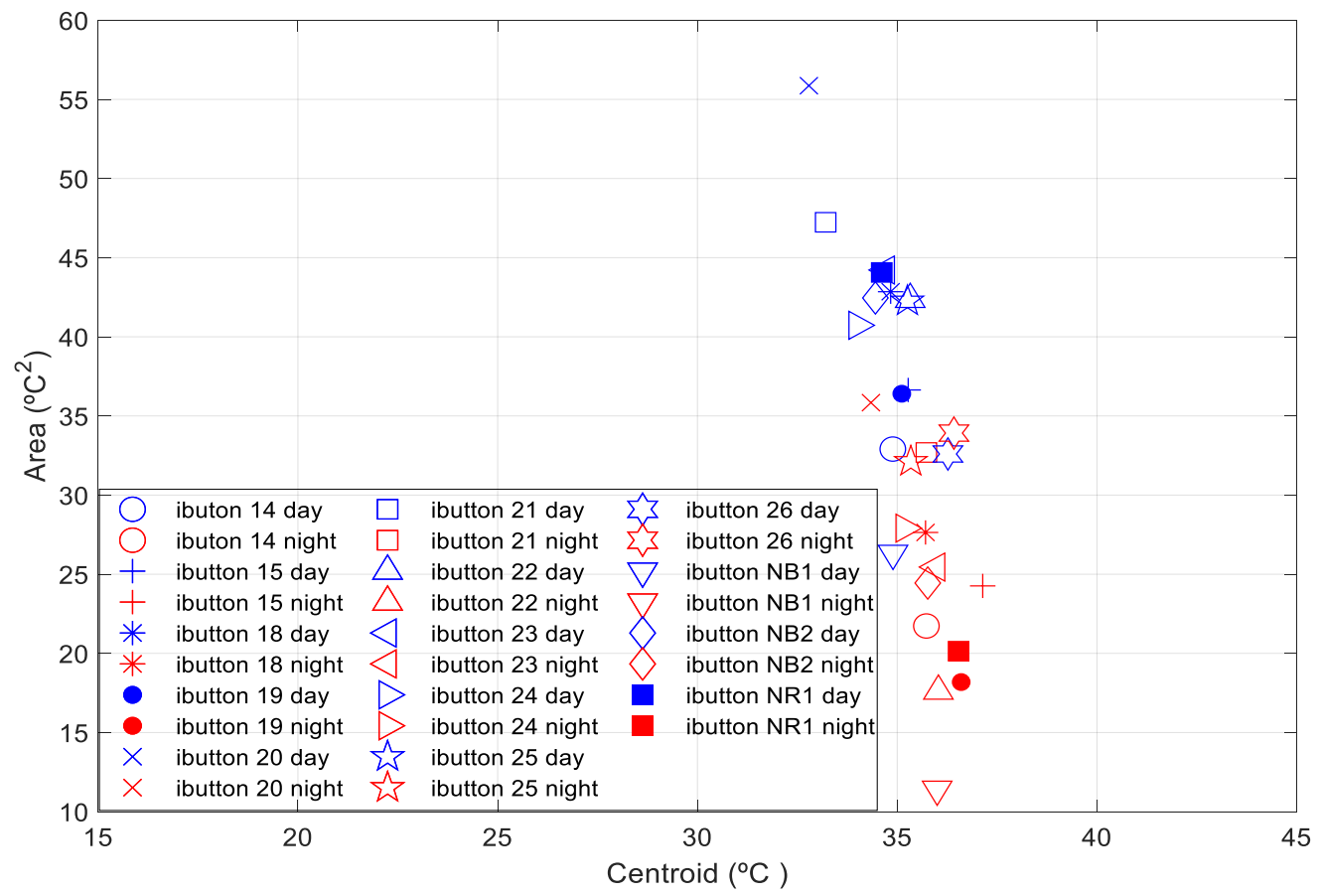

Figure 13. Centroids and areas of the phase space diagram for each iButton for day and night periods.

Table 61. Productivity measures of the pigs under study ( $\mathrm{n}=14)$, including initial weight, final weight, weight gain, cumulative feed intake and feed efficiency ( $\mathrm{kg}$ gained $/ \mathrm{kg}$ ingested).

\begin{tabular}{|c|c|c|c|c|c|c|}
\hline \multicolumn{3}{|c|}{ Weight $(\mathrm{kg})$} & \multirow{2}{*}{$\begin{array}{l}\text { Cumulative } \\
\text { intake }(\mathrm{kg})\end{array}$} & \multirow{2}{*}{$\begin{array}{c}\text { Feed } \\
\text { efficiency }(\mathrm{kg} / \mathrm{kg})\end{array}$} & \multirow{2}{*}{$\begin{array}{l}\text { Group (low, } \\
\text { medium or high } \\
\text { FE) }\end{array}$} & \multirow{2}{*}{ Sensor } \\
\hline Initial & Final & Gain & & & & \\
\hline 65.6 & 71.0 & 5.4 & - & - & & iButton 14 \\
\hline 64.0 & 65.0 & 1 & 12.2 & 0.08 & L1 & iButton 19 \\
\hline 62.0 & 64.0 & 2 & 13.5 & 0.15 & L2 & iButton 22 \\
\hline 61.2 & 64.0 & 2.8 & 16.2 & 0.17 & $\mathbf{L 3}$ & iButtonNB1 \\
\hline 56.4 & 59.0 & 2.6 & 14.6 & 0.18 & L4 & iButton 23 \\
\hline 56.3 & 58.9 & 2.6 & 12.7 & 0.20 & M1 & iButton15 \\
\hline 62.6 & 66.0 & 3.4 & 15.7 & 0.22 & M2 & iButton 24 \\
\hline 64.3 & 68.0 & 3.7 & 16.6 & 0.22 & M3 & iButton 25 \\
\hline 53.8 & 57.0 & 3.2 & 12.8 & 0.25 & M4 & iButton NR1 \\
\hline 56.7 & 61.0 & 4.3 & 15.0 & 0.29 & M5 & iButton NB2 \\
\hline 60.7 & 65.0 & 4.3 & 14.9 & 0.29 & M6 & iButton 26 \\
\hline 61.5 & 66.0 & 4.5 & 15.1 & 0.30 & M7 & iButton 21 \\
\hline 62.2 & 68.0 & 5.8 & 14.3 & 0.40 & H1 & iButton 20 \\
\hline 61.6 & 68.0 & 6.4 & 14.9 & 0.43 & $\mathrm{H} 2$ & iButton 18 \\
\hline
\end{tabular}

The phase space diagrams of the extremes iButtons (20 and NB1) is showed in Figure 14. This representation, in a confined space, allows to the user visualize more easily than in the representation of time series, the differences between iButtons and periods. 
(a)

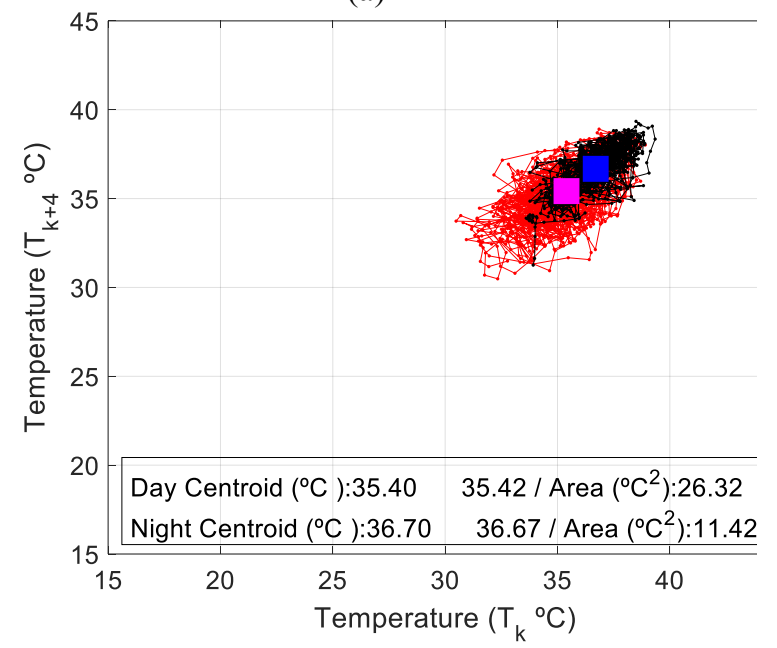

(b)

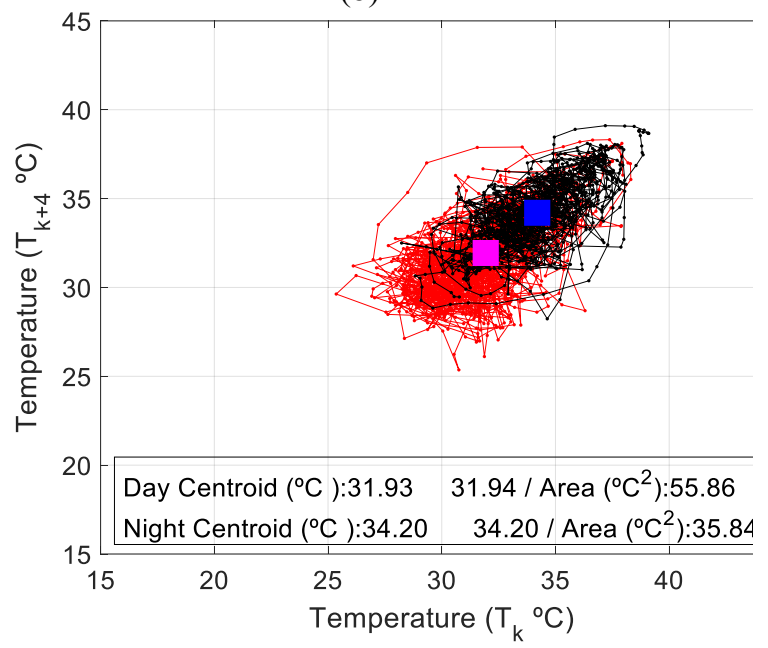

Figure 14. Phase space representation separated by day (in red) and night (in black) of (a) one representative low and (b) high feed efficiency pig.

Intercontinental Transport monitoring

Figure 15 shows the time series of skin temperature during transport. The average skin temperature was 34.11 ${ }^{\circ} \mathrm{C}$ (range $=32.73+/-3.29$ to $35.05+/-2.63{ }^{\circ} \mathrm{C}$ ). The average standard deviation $(\mathrm{n}=1400)$ of the temperatures among animals was $2.03{ }^{\circ} \mathrm{C}$. The average of the differences between the maximum and minimum temperatures in each instant was $6.71{ }^{\circ} \mathrm{C}$. Regarding the different stages of the trip, it is during the period corresponding to the rest in Bordeaux when the maximum average temperature is reached $\left(35.3^{\circ} \mathrm{C}\right)$ with significant differences respect some of the other stages of the trip.

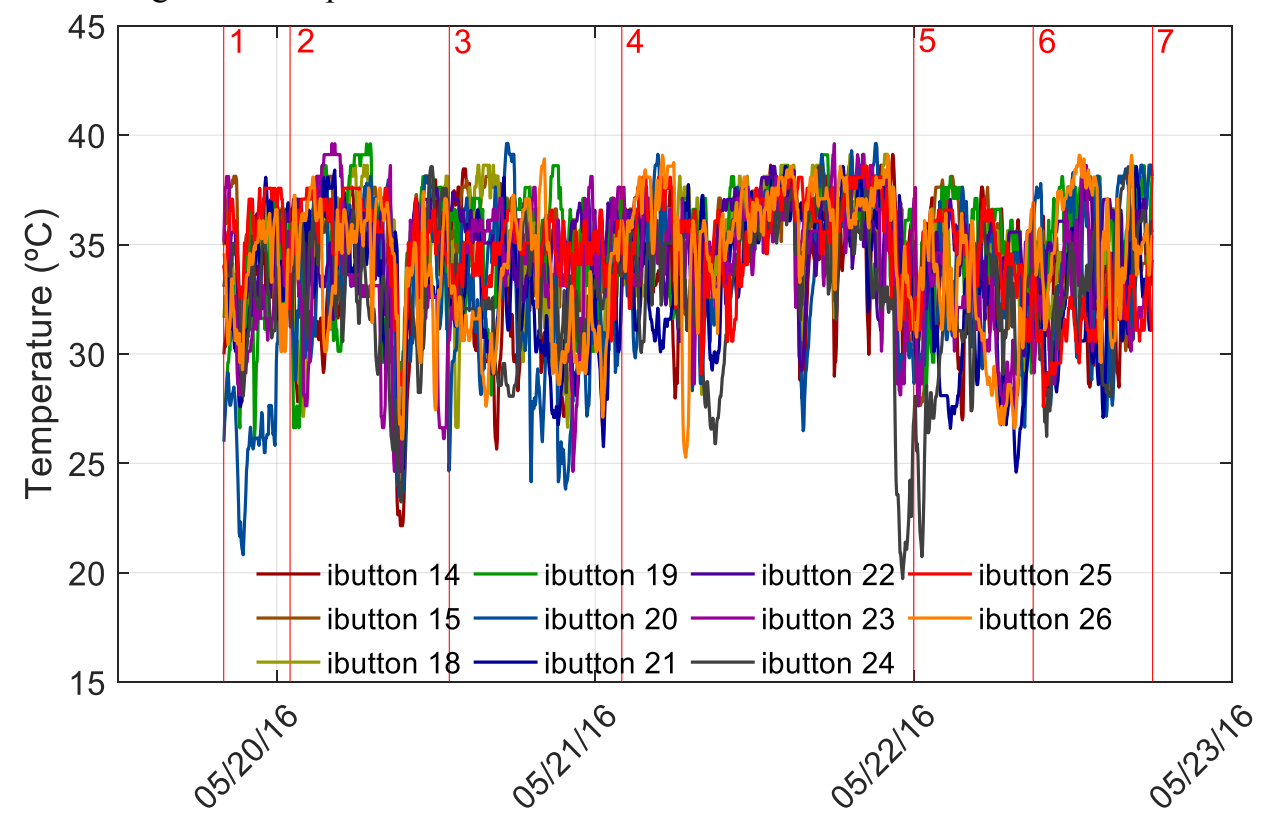

Figure 15. Time series of ear skin temperatures during the transport test of the 11 monitored pigs: 1 SPF departure; 2 Airport Toronto departure; 3 Luxembourg arrival; 4 Bordeaux arrival; 5 Bordeaux departure; 6 Lérida arrival and; 7 Montiel arrival.

There are not significant differences between the values of centroids of phase space diagrams for the stages corresponding to displacements (Figure 16a). Regarding comparison of animals, only iButton 20 and iButton 25 were significantly different (Figure 16b). 
(a)

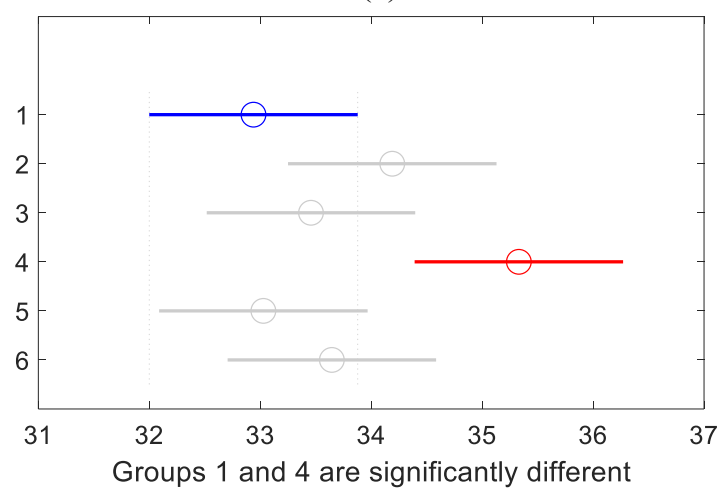

(b)

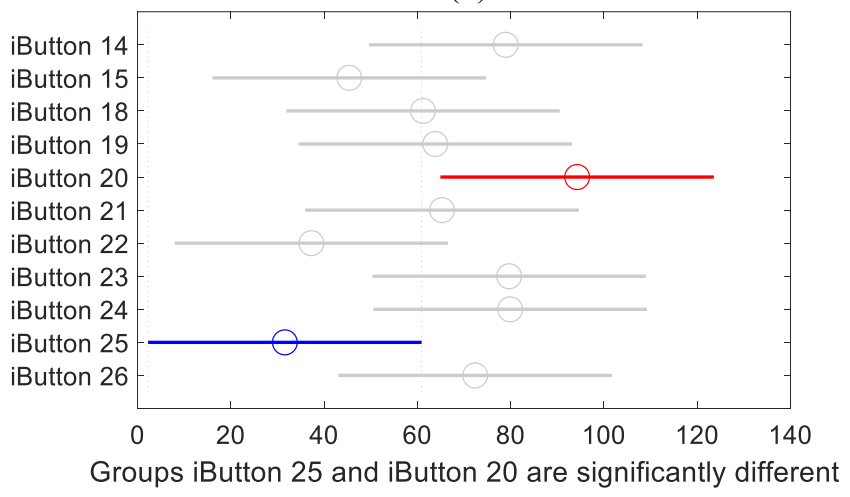

Figure 16. (a) Significant differences in the centroids of the phase space diagrams respect the different stages of the trip: 1 SPF - Airport Toronto; 2 Airport Toronto - Luxembourg; 3 Luxembourg - Bordeaux; 4 Bordeaux (Rest); 5 Bordeaux Lérida and; 6 Lérida - Montiel; (b) significant differences in the areas of the phase space diagrams respect the different sensor iButtons during the trip.

These differences can be observed in the corresponding phase space diagram (Figure 17). Centroid values were 33.10 and 34.83 for iButton 20 and 25 respectively, and areas values were 187.80 and 59.48. In transport test, also has been observed the negative relation between centroids and areas of the phase space diagrams, whit a correlation coefficient of -0.76 (Figure 18).

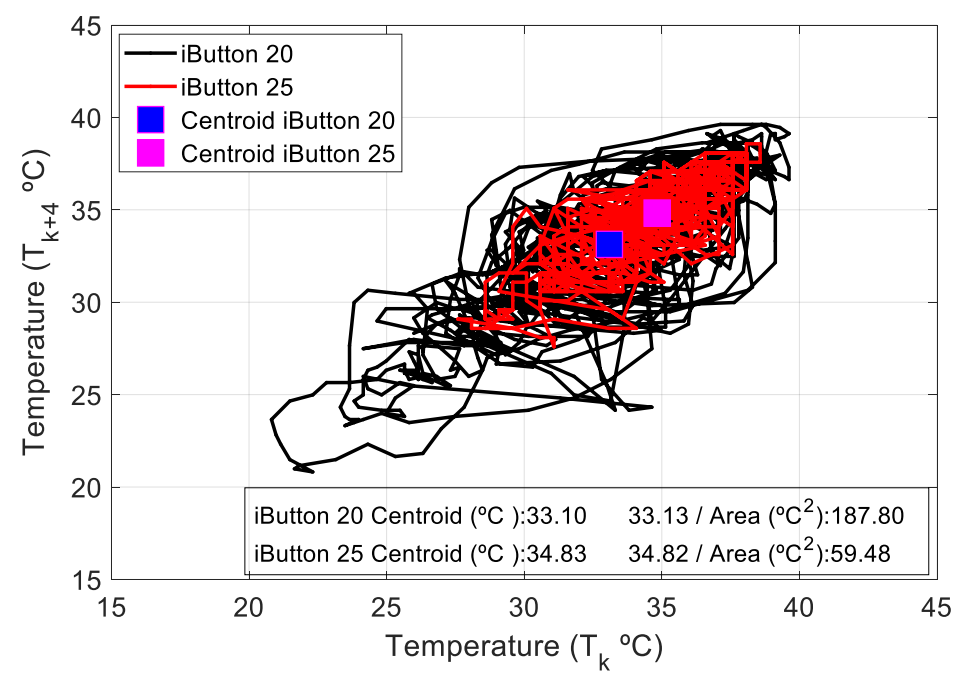

Figure 17. Phase space representation for complete trip of iButton 20 (black line) and iButton 25 (red line).

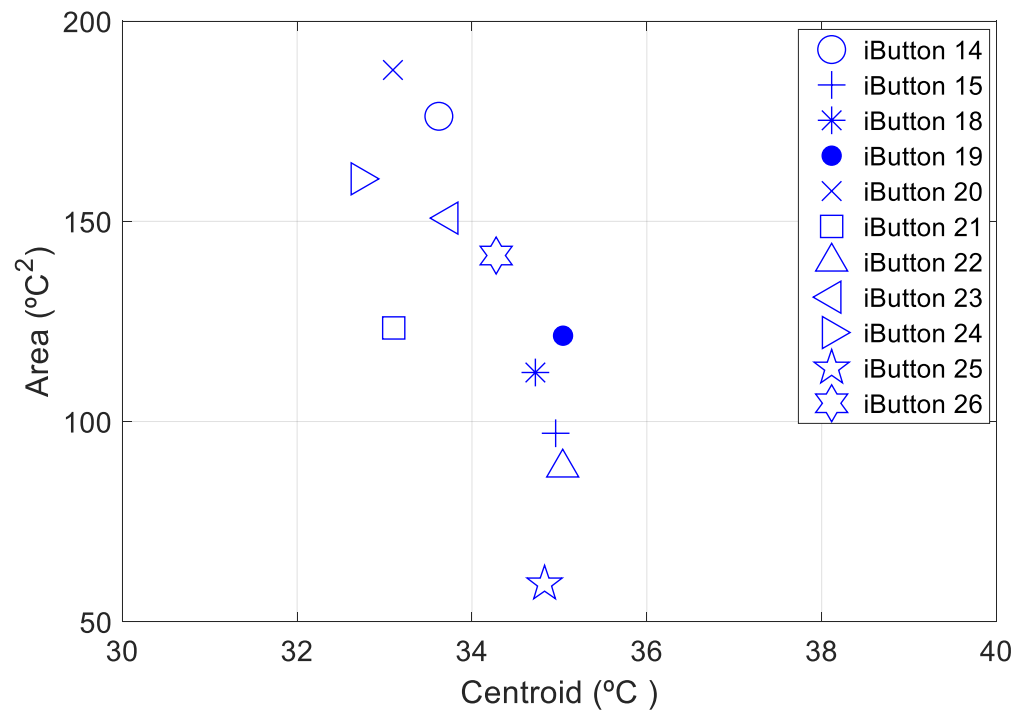

Figure 18. Centroids and areas of the phase space diagram for each iButton during the complete trip transport test. 
Intercontinental Transport vs Housing Conditions

Regarding the centroids similar values have been observed during housing and transport conditions (average values of $34.70+/-0.86{ }^{\circ} \mathrm{C}$ and $34.10+/-0.88^{\circ} \mathrm{C}$ respectively). However, considering the phase space areas, it can be stated that the thermal variability between animals of the same batch was higher during transport than those identified in housed animals (average values of $78.50+/-16.56^{\circ} \mathrm{C}$ and $129.0+/-38.97{ }^{\circ} \mathrm{C}$ ). The maximum and minimum areas for an animal during transport $\left(187.8^{\circ} \mathrm{C}^{2}\right.$ and $\left.59.48{ }^{\circ} \mathrm{C}^{2}\right)$ were higher than the areas for housed animals $\left(112.3^{\circ} \mathrm{C}^{2}\right.$ and $\left.45.8{ }^{\circ} \mathrm{C}^{2}\right)$, in spite of being the duration of the housed experiment twice than the transport experiment. This data highlight a more exposure to thermal stress during transport management which have been state for other authors Villarroel et. al (2011).

\section{Conclusions}

It has been possible to do an individualized non-invasive monitoring along a commercial transport as well as a housed period of the ear skin temperature of each animal, by placing an autonomous recording temperature sensor in the RFID tag of the left ear at each animal.

There was a wide diversity in skin temperature (average and standard deviation of skin temperatures). All of this data could be used as a starting point to differentiate between animal by thermoregulatory pattern. Applying the phase space methodology to the time series of ear skin temperature highlighted the variability among animals, periods of day and night or transport stages.

It was demonstrated that the environmental changes suffered during transport cause higher variability in the ear skin temperature than the variability observed during housing (higher areas in the phase space diagram), which can be related with thermoregulatory changes in the pigs and at worst in the appearance of stress.

\section{Acknowledgements}

The funding of this work has been covered by Comunidad de Madrid and European Union through S2013/ABI-2747 (TAVS-CM) and FEDER Innterconecta EXP 00082820 / ITC-20151192 projects.

\section{References}

Britt, J. S., Thomas, R. C., Speer, N. C., \& Hall, M. B. (2003). Efficiency of converting nutrient dry matter to milk in Holstein herds. Journal of Dairy Science, 86(11), 3796-3801.

Eckmann, J. P., \& Ruelle, D. (1985). Ergodic theory of chaos and strange attractors. Reviews of modern physics, 57(3), 617.

Grant, R. (1950). Emotional hypothermia in rabbits. American Journal of Physiology--Legacy Content, 160(2), 285-290.

Herd, R. M., \& Arthur, P. F. (2009). Physiological basis for residual feed intake. Journal of animal science, 87(14_suppl), E64-E71.

Labussière, E., Dubois, S., van Milgen, J., \& Noblet, J. (2013). Partitioning of heat production in growing pigs as a tool to improve the determination of efficiency of energy utilization. Frontiers in physiology, 4.

Langer, F., \& Fietz, J. (2014). Ways to measure body temperature in the field. Journal of thermal biology, 42, 46-51.

Loughmiller, J. A., Spire, M. F., Dritz, S. S., Fenwick, B. W., Hosni, M. H., \& Hogge, S. B. (2001). Relationship between mean body surface temperature measured by use of infrared thermography and ambient temperature in clinically normal pigs and pigs inoculated with Actinobacillus pleuropneumoniae. American journal of veterinary research, 62(5), 676-681.

Meunier-Salaün, M. C., Guerin, C., Billon, Y., Sellier, P., Noblet, J., \& Gilbert, H. (2014). Divergent selection for residual feed intake in group-housed growing pigs: characteristics of physical and behavioural activity according to line and sex. animal, 8(11), 1898-1906.

Soerensen, D. D., \& Pedersen, L. J. (2015). Infrared skin temperature measurements for monitoring health in pigs: a review. Acta veterinaria scandinavica, 57(1), 5.

Villarroel,M., Barreiro, P., Kettlewell, P., Farish,M., \& Mitchell,M. (2011). Time derivatives in air temperature and enthalpy as non-invasive welfare indicators during long distance animal transport. Biosystems Engineering, 110, 253-260. doi:10.1016/j.biosystemseng.2011.07.011. 


\title{
The Effect of Liner Type on the Teat Load Caused by a Collapsing Liner Using a Pressure-Indicating Film
}

\author{
Susanne Demba a ${ }^{a},{ }^{,}$Viktoria Paul ${ }^{\text {b }}$, Christian Ammon ${ }^{\text {a }}$, Sandra Rose-Meierhöfer ${ }^{\mathrm{c}}$ \\ ${ }^{a}$ Leibniz Institute for Agricultural Engineering and Bioeconomy e.V. (ATB), Department of Engineering for Livestock \\ Management, 14469 Potsdam, Germany \\ ${ }^{\mathrm{b}}$ Faculty of Life Science, Humboldt-Universität zu Berlin, 10099 Berlin, Germany \\ ${ }^{\mathrm{c}}$ Department of agricultural machinery, Hochschule Neubrandenburg, University of Applied Science, 17033 \\ Neubrandenburg, Germany \\ * Corresponding author. Email: sdemba@atb-potsdam.de
}

\begin{abstract}
During milking, the teat cup liner is the interface between the teat and the milking system, so it should be very well adapted. The most commonly used method to detect the impact of liner type is to visually evaluate teat condition based on teat colour, swelling, ring formation at the teat base, and teat-end hyperkeratosis. In addition, sensor-based determination of the influence of liner type on the teat load caused by liner collapse can be performed with several measuring devices, such as ultrasonography, infrared thermography, and pressure-sensitive sensors. As the methods commonly used to detect the effect of liner type on the bovine teat are very subjective and because the tested sensor-based methods are very complex to use or have shown limited usability, the aim of this study was to determine the effect of liner type on the directly measured teat load caused by a collapsing liner with a pressure-indicating film. The Extreme Low pressure-indicating film was used to detect the effect of six different liners on teat load at artificial teats. For each liner, six positions in the teat cup were specified, and six repetitions were performed for each position with a new film piece each time. Analysis of variance was performed to detect differences between the six liners and the measuring areas. The pressure applied to the teat by a liner depends on the technical characteristics of the liner, especially the barrel shape. A square and a concave liner applied the highest teat load at the teat base, while a round silicone liner and a round rubber liner applied the highest load at the teat end. For all tested liners, a higher teat load was found at the teat end compared with the other measuring areas.
\end{abstract}

Keywords: teat load, pressure sensor, liner collapse, machine milking

\section{Introduction}

During machine milking, the teat cup liner is the interface between the teat of a dairy cow and the milking system, so it transfers the force created by the pressure difference between the pulsation chamber and the interior of the liner directly to the teat tissue (Hubal et al., 2010). While the teat of a dairy cow is robustly constructed and well adapted to shear stress (Hibbit et al., 1992), machine milking can worsen the condition of the teat and teat tissue (Mein et al., 2001; Gleeson et al., 2002; Mir et al., 2015). Therefore, it is important that the liner is very well adapted to the teat.

The most commonly used method to detect the impact of liner type is to visually evaluate teat condition based on teat color, swelling, ring formation at the teat base, and teat-end hyperkeratosis (Rasmussen et al., 1998; Capuco et al., 2000; Mein et al., 2001; Gleeson et al., 2005; Zucali et al., 2008; Haeussermann et al., 2016). In addition to visually observing teat condition, sensor-based determination of the influence of liner type on the teat load caused by liner collapse can be performed with several measuring devices. Paulrud et al. (2005) used infrared thermography as well as ultrasonography to monitor the influence of liner type on teat temperature and teat traits such as the teat cistern wall, teat cistern diameter, and teat canal length. The ultrasonography was also used by Gleeson et al. $(2004,2005)$ to investigate differences in teat traits caused by different liners. Several studies have attempted to detect the influence of liner type on the teat load with the help of different pressure-sensitive sensors. Davis et al. (2001) measured the compressive load applied to the teat by the closed liner using an artificial teat equipped with a miniature load cell and found that the compressive load of a liner is proportional to the thickness of the liner wall; the authors determined a curvilinear relationship between the insertion depth and the compressive teat load as well. Tol et al. (2010) investigated the teat-liner interface using a flexible pressure-sensitive layer and found that conventional round liners concentrated the load over two sides of the end of the teat. In contrast, liners with softer material, reduced tension, a smaller barrel, and reduced mouthpiece depth distributed the pressure over a larger area of the teat, but the maximum pressure was always exerted at the teat end. Leonardi et al. (2015) used an artificial teat sensor adapted from Davis et al. (2001) to estimate liner compression, and the liner compression was positively correlated $\left(\mathrm{R}^{2}=0.97-0.91\right)$ with the pressure difference through the liner wall. According to these authors, this sensor is only useful for round liners.

The Prescale film by Fujifilm (KAGER Industrieprodukte GmbH, Dietzenbach, Germany) is a mono- or twosheet pressure-indicating film which is used to measure pressure, pressure distribution and pressure balance. It 
contains colour developing material which indicate the applied pressure. As a result of an applied pressure to the pressure-indicating film, the microcapsules are broken, the colour-forming material reacts with the colourdeveloping material and red areas appear on the film. The different pressure levels were pointed out with the intensity of the red colour. According to Cuffaro (2013) the Prescale film has a pressure range between 0.05 and $300 \mathrm{MPa}$ and it does not support shear stress (Rodriguez-Martinez et al., 2012). The measurements of the film are not influenced by bending or negative pressure (Demba et al., 2016). After measuring the films could be analysed with the associated software which recorded six parameters: the effective rate (ER) in percent, the pressed area (PA) in square millimetres, the average pressure (AP) in megapascals, the maximum pressure (MP) in megapascals, the load (L) in newton, and the measured area (MA) in square millimetres.

As the methods commonly used to detect the effect of liner type on the bovine teat are very subjective and because the tested sensor-based methods are very complex to use or have shown limited usability, the aim of this study was to determine the effect of liner type on the directly measured teat load caused by a collapsing liner with a pressure-indicating film.

\section{Materials and Methods}

Study design and data collection

During this investigation the Extreme Low film was used to determine the influence of different liners on the measured teat load caused by a collapsing liner. This film is a two-sheet type with an A-film and a C-film. The components of the A-film are a polyester base and a microencapsulated colour-forming layer. In contrast, the Cfilm composed of a polyester base and a colour-developing layer. The used film measures pressure between 0.05$0.2 \mathrm{MPa}$.

An artificial teat made of silicone was used to investigate the influence of liner type on the teat load caused by a collapsing liner. The teat had a length and a mean diameter of $56 \mathrm{~mm}$ and $21 \mathrm{~mm}$, respectively, and it was hollow with a teat wall thickness of $4.5 \mathrm{~mm}$. According to the manufacturer, the silicone rubber had a Shore A hardness of 25 .

The teat load on the artificial teat caused by liner collapse was measured for six different liners (Table 1): a round silicone liner (SilRou), a round rubber liner with head ventilation (RubRouHV), a triangular rubber liner (RubTri), a concave rubber liner (RubCon), a round rubber liner (RubRou), and a square rubber liner (RubSqu).

Table 1. Overview of the tested teat cup liners and their different characteristics.

\begin{tabular}{|c|c|c|c|c|c|c|}
\hline Liner & 1 & 2 & 3 & 4 & 5 & 6 \\
\hline $\begin{array}{c}\text { Abbreviation } \\
\text { Material }\end{array}$ & $\begin{array}{l}\text { SilRou } \\
\text { silicone }\end{array}$ & $\begin{array}{c}\text { RubRouHV } \\
\text { rubber }\end{array}$ & $\begin{array}{l}\text { RubTri } \\
\text { rubber }\end{array}$ & $\begin{array}{c}\text { RubCon } \\
\text { rubber }\end{array}$ & $\begin{array}{l}\text { RubRou } \\
\text { rubber }\end{array}$ & $\begin{array}{c}\text { RubSqu } \\
\text { rubber }\end{array}$ \\
\hline $\begin{array}{l}\text { Mouthpiece bore } \\
\text { diameter }(\mathrm{mm})\end{array}$ & 23 & 23 & 23 & 20 & 23 & 23 \\
\hline Barrel shape & round & round & triangular & concave & round & square \\
\hline Touchpoint $(\mathrm{kPa})$ & 18.4 & 15.1 & - & - & 12.9 & - \\
\hline $\begin{array}{l}\text { Mouthpiece } \\
\text { ventilation }\end{array}$ & no & yes & yes & no & no & no \\
\hline
\end{tabular}

The experiments were performed with a conventional milking cluster (GEA Group AG, Düsseldorf, Germany) in the laboratory milking parlour of the Leibniz-Institute for Agricultural Engineering and Bioeconomy e.V. (ATB). The machine vacuum was adjusted to $40 \mathrm{kPa}$. Alternate pulsation was used at a rate of $60 \mathrm{~min}^{-1}$ and a 60:40 pulsation ratio.

The pressure-indicating film was cut into pieces $(15 \mathrm{~mm} \times 55 \mathrm{~mm})$, all of which were attached to the teat with tape. Due to the small size of the sensor, the teat-sensor-combination was rotated five times in intervals of $30^{\circ}$ to record the teat load at each position (Figure 1), and six repetitions were performed for each position. The artificial teat was inserted in the teat cup, and the liner was opened and closed for $30 \mathrm{~s}$. After each session, the artificial teat was turned $30^{\circ}$, and the next measurement was recorded. Thus, the pressure-indicating film measured the pressure at each point of the liner surface at and between the chosen positions. 

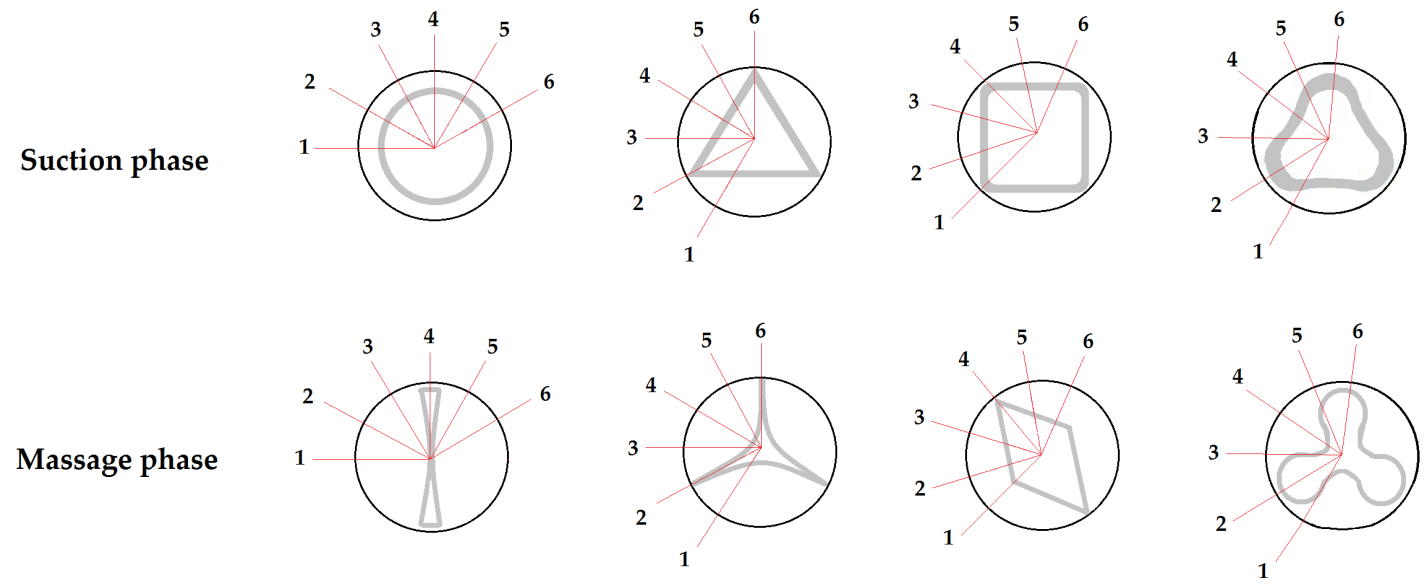

Round barrel shape Triangular barrel shape Square barrel shape Concave barrel shape

Figure 1. The measurement positions in $30^{\circ}$ intervals on the teat cup of the artificial teat for each barrel shape during the suction and massage phases of the milking process.

The positions where the liners compressed the teat (COMP) and the position where the liners did not compress the teat $(\mathrm{CORN})$ were compared between the liners to determine differences between the tested liners. COMP included position 1 of SilRou, RubRouHV, RubRou, and RubSqu, and position 4 of RubTri and RubCon; CORN included the position 4 of SilRou, RubRouHV, RubRou, and RubSqu, and position 6 of RubTri and RubCon.

After measuring, the films were analysed with the FDP-8010E software by Fujifilm (Prescale by Fujifilm; KAGER Industrieprodukte GmbH, Dietzenbach, Germany). L and MP were used to analyse the influence of the different liners. Both traits were calculated for the film at the three measuring areas. Therefore, the scanned film was divided into the teat base area (BASE), the middle teat area (MIDDLE), and the teat end area (END).

\section{Statistical analysis}

Data were analysed using the SAS 9.4 software package (SAS Institute Inc., Cary, NC, USA), and analysis of variance (ANOVA) was used to estimate the differences in $\mathrm{L}$ among the liners, the position of the artificial teat in the teat cup, and the three measuring areas using the MIXED procedure. The null hypothesis was that there were no differences between the liners, the positions, and the measuring areas. The following model was used to calculate the influence of the different liner types on L:

$$
\mathrm{y}_{\mathrm{ijkl}}=\mu+\mathrm{L}_{\mathrm{i}}+\mathrm{P}_{\mathrm{j}}+\mathrm{A}_{\mathrm{k}}+(\mathrm{LP})_{\mathrm{ij}}+(\mathrm{LA})_{\mathrm{ik}}+(\mathrm{PA})_{\mathrm{jk}}+(\mathrm{LPA})_{\mathrm{ijk}}+\varepsilon_{\mathrm{ijk}}
$$

where $y_{\mathrm{ijk}}$ is the observed value of the $\mathrm{i}$-th liner $(\mathrm{i}=1, \ldots, 6)$, the $\mathrm{j}$-th position of the artificial teat in the teat cup $(j=1, \ldots, 6)$, the $k$-th measuring area $(\mathrm{k}=$ BASE, MIDDLE, END), and the 1 -th repetition $(1=1, \ldots, 6) ; \mu$ is the overall mean; $\mathrm{L}_{\mathrm{i}}$ is the fixed effect of the liner type; $\mathrm{P}_{\mathrm{j}}$ is the fixed effect of the position in the teat cup; $A_{k}$ is the fixed effect of the measuring area; (LP) ${ }_{\mathrm{ij}}$ is the interaction between the liner and the teat position; (PA) $)_{\mathrm{k}}$ is the interaction between the position and the measuring area; (LPA) ijk $_{\mathrm{j}}$ is the interaction between the liner, the position and the measuring area; and $\varepsilon_{\mathrm{ijkl}}$ is the residual.

The GLIMMIX procedure was used to examine the differences in MP between the liners, the position of the artificial teat in the teat cup, and the three measuring areas, and the null hypothesis for MP was that there were no differences between the liners, the positions, and the measuring areas. A binomial distribution was assumed for MP, and the following model was used:

$$
\mathrm{P}\left(\mathrm{y}_{\mathrm{ijl}}\right)=\frac{\mathrm{e}^{\eta}}{1+\mathrm{e}^{\eta}}
$$


The linear predictor $\eta$ is calculated as follows:

$$
\eta_{\mathrm{ijl}}=\mu+\mathrm{L}_{\mathrm{i}}+\mathrm{P}_{\mathrm{j}}+\varepsilon_{\mathrm{ijl}}
$$

where $\mu$ is the overall mean; $L_{i}$ is the fixed effect of the $\mathrm{i}$-th liner type $(i=1, \ldots, 6)$; $\mathrm{P}_{\mathrm{j}}$ is the fixed effect of the $j$-th position of the artificial teat in the teat $\operatorname{cup}(j=1, \ldots, 6) ; A_{k}$ is the fixed effect of the $k$-th measuring area $(\mathrm{k}=\mathrm{BASE}, \mathrm{MIDDLE}, \mathrm{END})$; and $\varepsilon_{\mathrm{ijkl}}$ is the residual. All tests were carried out at a significance level of 0.05 .

\section{Results}

Load

The analysis of variance showed a significant influence of the liner $(p<0.0001)$, the position of the teat in the liner $(\mathrm{p}=0.0007)$, and the measuring area $(\mathrm{p}<0.0001)$ on $\mathrm{L}$. The interactions between the position and the liner $(p<0.0001)$, the liner and the measuring area $(p<0.0001)$, and the position and the measuring area $(p=0.0012)$ as well as the triple interaction between the position, the liner, and the measuring area $(\mathrm{p}<0.0001)$ significantly influence $\mathrm{L}$ as well. Within all liners, the highest $\mathrm{L}$ was measured at the END compared to the BASE and MIDDLE. Figure 3 shows the differences in $\mathrm{L}$ between the tested liners depending on the measuring area.

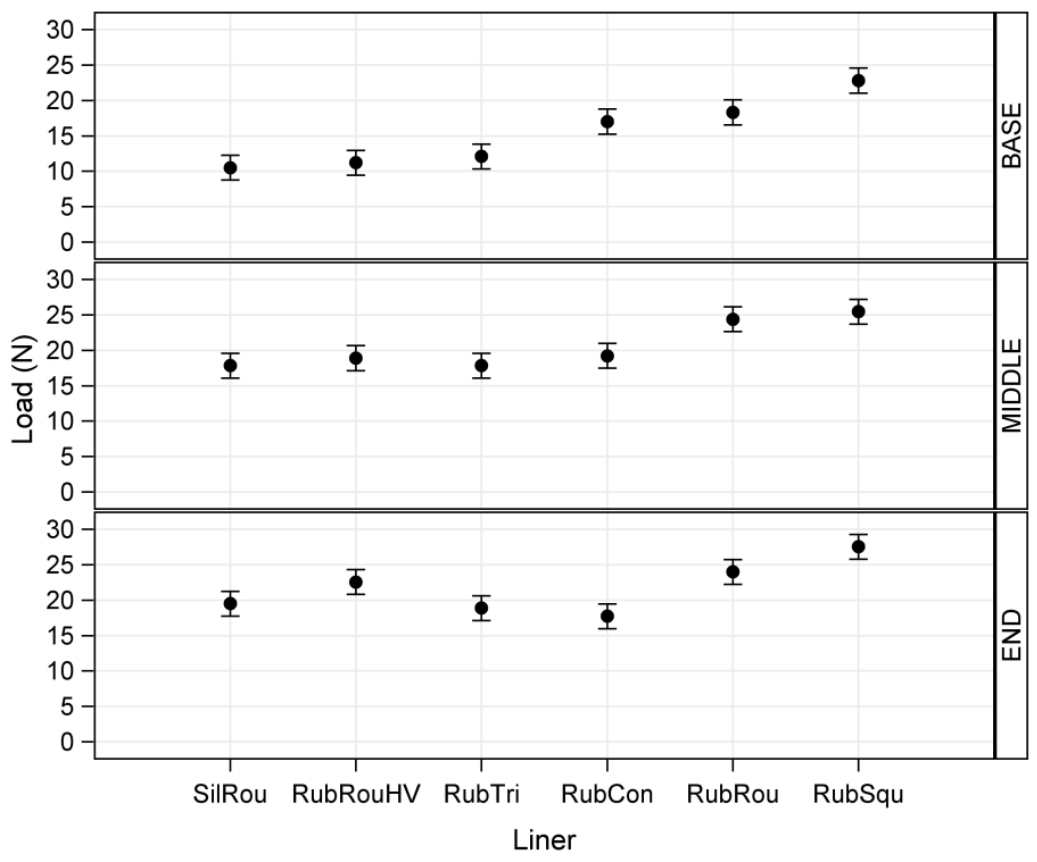

Figure 3. The differences in the measured Load between a round silicone liner (SilRou), a round rubber liner with head ventilation (RubRouH), a triangular rubber liner (RubTri), a concave rubber liner (RubCon), a round rubber liner (RubRou) and a square rubber liner (RubSqu) depending on the measuring area with BASE = the teat base measuring area, MIDDLE $=$ the middle teat measuring area and $\mathrm{END}=$ the teat end measuring area

The comparison of COMP at the BASE area showed that the applied L by RubSqu was $11.00 \mathrm{~N}, 9.67 \mathrm{~N}$, and $9.83 \mathrm{~N}$ higher than this of RubRouHV, RubTri and RubRou, respectively. The L values of RubCon were $10.67 \mathrm{~N}$ and $9.33 \mathrm{~N}$ higher than these of RubRouHV and RubTri, respectively. The L values of SilRou were $9.83 \mathrm{~N}, 13.33$ $\mathrm{N}, 11.17 \mathrm{~N}$, and $19.50 \mathrm{~N}$ higher at the END than these of RubTri, RubCon, RubRou, and RubSqu, respectively. The applied L of RubRouHV was $11.83 \mathrm{~N}$ higher than this of RubSqu. No differences between the liners were found at the MIDDLE.

The comparison of CORN at the BASE resulted in a $9.67 \mathrm{~N}$ and $16.33 \mathrm{~N}$ lower $\mathrm{L}$ for SilRou compared to RubRou, and RubSqu, respectively. L of RubRouHV was $9.67 \mathrm{~N}, 8.83 \mathrm{~N}$, and $15.50 \mathrm{~N}$ lower than of RubCon, RubRou, and RubSqu. The comparison of the three angular liners resulted in a $10.67 \mathrm{~N}$ higher load of RubSqu compared to RubTri and RubCon. At the MIDDLE L of RubRou and RubSqu was $10.00 \mathrm{~N}$ and $10.33 \mathrm{~N}$ higher than this of SilRou, respectively. The L values of RubSqu were 25.00 N, 16.83 N, 9.17 N, 9.17 N, and $16.33 \mathrm{~N}$ higher at the END compared to SilRou, RubRouHV, RubTri, RubCon, and RubRou, respectively.

The standard error for all estimated means was $3.10 \mathrm{~N}$. 


\section{Maximum pressure}

The analysis of variance showed a significant influence of the liner $(p<0.0001)$ and the measuring area $(p=$ 0.0406) on MP, but no significant effect of the position of the teat in the teat cup on MP could be found. The MP values were higher for RubRou and RubSqu compared to the other tested liners; the MP of RubRou was 0.04$0.05 \mathrm{MPa}$ higher than that of SilRou, RubRouHV, RubTri, and RubCon. RubSqu showed the highest MP values; the applied pressure was 0.05-0.06 MPa higher than that of SilRou, RubRouHV, RubTri, and RubCon. The MP values were $0.02 \mathrm{MPa}$ higher at the END compared to the BASE, but no significant differences in MP could be found among the other measuring areas.

\section{Discussion and conclusion}

The comparison of the different liner types resulted in the highest teat load for SilRou at COMP and END, which disagrees with Tol et al. (2010). They found the highest pressure values for a round-square and a triangular liner. Furthermore, the angular liners had a higher teat load at CORN compared with the round liners, which disagrees with the results of Tol et al. (2010) as well. They found no load in the corners of a triangular liner and observed pressure all around the teat with a square liner. In contrast, Zucali et al. (2009) found higher incidences of teat-end hyperkeratosis on farms milking with triangular liners. The use of triangular rubber liners, compared to round rubber liners, did not reduce the traumatization of teats due to milking at different machine vacuum levels (Kunc et al., 1999). Schukken et al. (2006) found a lower frequency of teat ends with crack and teat-end hyperkeratosis in teats milked with square liners. At COMP, the teat load caused by SilRou was higher than that by RubRou, which led to the assumption that a softer material resulted in a higher teat load. Paulrud et al. (2005) found that milking with a liner made of softer material resulted in colder teats after milking, but Tol et al. (2010) found similar pressure values for liners made of silicone and rubber. The observations regarding liner material in the present study could be explained by the higher SilRou Touch Point values. The results of Davis et al. (2001) that the compressive load of a liner is proportional to the thickness of the liner wall can neither be confirmed nor refuted because the majority of the liners tested in the present study had a wall thickness of $2.0 \mathrm{~mm}$.

For SilRou, L was highest at the position where the liner pressed the teat and lowest where the liner bends at the edges (Position 4); these results were confirmed by Tol et al. (2010) who found similar effects. In contrast, the results for RubRou in the present study did not agree with those of Tol et al. (2010). For RubTri, L was higher at the corner than at the position where the liner pressed the teat, which is inconsistent with the results of Tol et al. (2010) who found only three pressure spots (the three sides where the liner touched the teat) within a triangular liner. However, their artificial teat was $20 \mathrm{~mm}$ longer, had a tapered shape, and a $2.5 \mathrm{~mm}$-thinner teat wall compared to the artificial teat used in the present investigation, so this could explain the different results.

Teat load increased from the BASE through the MIDDLE to the END during liner collapse. This result partially agrees with Tol et al. (2010) who found a similar pressure distribution in round liners. In the present study, the highest teat load was found at the END compared to the other measuring areas. Muthukumarappan et al. (1994) confirmed these results, finding that the maximum pressure was applied within 1 or $2 \mathrm{~mm}$ of the teat end, and Tol et al (2010) also found that the maximum pressure was always applied to the teat end.

The measurements with the pressure-indicating film were not influenced by machine vacuum and sensor bending. According to Demba et al. (2016) neither negative pressure nor sensor bending influenced the measurements by pressure-indicating film.

It can be concluded that the type of a teat cup liner influence the teat load caused by a collapsing liner. The pressure applied to the teat depends on the technical characteristics of the teat cup liner, especially the barrel shape. However, there is still a lack of information about the load applied by a collapsing liner, which is necessary to massage the teat, so further studies are needed. Furthermore, it is important to determine the dimensions of the teats in a dairy herd to select the best adapted liner. The artificial teat used in the present investigation was flexible, but in further studies, a teat that is more similar to a natural teat will be used.

\section{Acknowledgements}

The authors would like to thank Eckhard Schütte of Milkrite, who provided some of the examined teat cup liners.

\section{References}

Capuco, A.V., Wood, D.L., Quast, J.W., 2000. Effects of teat cup liner tension on teat canal keratin and teat condition in cows. Journal of Dairy Research. 67 (3), 319-327. http://dx.doi.org/10.1017/S0022029900004295.

Cuffaro, V., 2013. Prediction Method for the Surface Damage in Splined Couplings, Dissertation, Politecnico di Torino, Italy. 
Davis, M.A., Reinemann, D.J., Mein, G.A. 2001. Development and testing of a device to measure compressive teat load applied to a bovine teat by the closed teatcup liner. In Proceedings of the American Society of Agricultural and Biological Engineers Annual Meeting, Sacramento, CA, USA, 29 July-1 August.

Demba, S., Elsholz, S., Ammon, C., Rose-Meierhöfer, S., 2016. The usability of a pressure-indicating film to measure the teat load caused by a collapsing liner. Sensors. 16, 1597. http://dx.doi.org/10.3390/s16101597.

Gleeson, D.E.; O'Callaghan, E.J.; Meaney, W.J.; Rath, M.V., 2005. Effect of two milking systems on the milking characteristics, teat tissue changes and new infection rate of dairy cows. Animal Research. 54 (4), 259267. http://dx.doi.org/10.1051/animres:2005025.

Gleeson, D.E., O’Callaghan, E.J., Rath, M.V., 2004. Effect of liner design, pulsator setting, and vacuum level on bovine teat tissue changes and milking characteristics as measured by ultrasonography. Irish Veterinary Journal. 57 (5), 289-296. http://dx.doi.org/10.1186/2046-0481-57-5-289.

Gleeson, D.E., O’Callaghan, E.J., Rath, M.V., 2002. Effect of milking on bovine teat tissue as measured by ultrasonography. Irish Veterinary Journal. 55 (12), 628-632. https://doi.org/10.1186/2046-0481-57-5-289.

Haeussermann, A., Britten, J., Britten, A., Pahl, C., Älveby, N., Hartung, E., 2016. Effect of a multi-sided concave liner barrel design on thickness and roughness of teat-end hyperkeratosis. Journal of Dairy Research. 83 (2), 188-195. http://dx.doi.org/10.1017/s0022029916000200.

Hibbitt, K.G., Craven, N., Batten, E.H., 1992. Anatomy, physiology and immunology of the udder. In Bovine Medicine. Diseases and Husbandry of Cattle, $2^{\text {nd }}$ ed., Andrews, A.H., Blowey, R.W., Boyd, H., Eddy, R.G., Eds., Blackwell Scientific Publications. Oxford, UK, 311-325.

Hubal, M., 2010. Milking Technique Parameters and the Bovine Teat Condition. Dissertation, Georg-AugustUniversity, Göttingen, Germany.

Kunc, P., Knizkova, I., Koubkova, M., 1999. The influence of milking with different vacuum and different design of liner on the change of teat surface temperature. Czech Journal of Animal Science. 44 (3), 131-134.

Leonardi, S., Penry, J.F., Tangorra, F.M., Thompson, P.D., Reinemann, D.J., 2015. Methods of estimating liner compression. Journal of Dairy Science. 98 (10), 6905-6912. http://dx.doi.org/10.3168/jds.2015-9380.

Mein, G.A., Neijenhuis, F., Morgan, W.F., Reinemann, D.J., Hillerton, J.E., Baines, J.R., Ohnstad, I., Rasmussen, M.D., Timms, L., Britt, J.S., Farnsworth, R., Cook, N., Hemling, T., 2001. Evaluation of bovine teat condition in commercial dairy herds: 1. Non-infectious factors. In Proceedings of the International Symposium on Mastitis and Milk Quality, Lelystad, The Netherlands, 13-15 September.

Mir, A.Q., Bansal, B.K., Gupta, D.K., 2015. Short term changes in teats following machine milking with respect to quarter health status in cows. Journal of Animal Research. 5 (3), 467-471. http://dx.doi.org/10.5958/2277-940X.2015.00080.7.

Muthukumarappan, K., Reinemann, D.J., Mein, G.A., 1994. Compressive load applied to the bovine teat by the teatcup liner. In Proceedings of the American Society of Agricultural and Biological Engineers International Winter Meeting, Atlanta, GA, USA, 13-16 December.

Paulrud, C.O., Clausen, S., Andersen, P.E., Rasmussen, M.D., 2005. Infrared thermography and ultrasonography to indirectly monitor the influence of liner type and overmilking on teat tissue recovery. Acta Veterinaria Scandinavica. 46 (3), 137-147. http://dx.doi.org/10.1186/1751-0147-46-137.

Rasmussen, M.D., Frimer, E.S., Kaartinen, L., Jensen, N.E., 1998. Milking performance and udder health of cows milked with two different liners. Journal of Dairy Research. 65 (3), 353-363. http://dx.doi.org/10.1017/S0022029998002994.

Rodriguez-Martinez, R., Urriolagoitia-Sosa, G., Torres-San Miguel, C.R., L.H., H.-G., G., U.-C., CarbajalRomero, M.F., 2012. Development of an experimental apparatus for testing a total knee prostheses focused on Mexican phenotype. International Journal of Physical Science. 7 (43), 5779-5786. https://doi.org/10.5897/IJPS12.129.

Schukken, Y.H., Petersson, L.G., Rauch, B.J., 2006. Liner and teat end health. In Proceedings of the 45th National Mastitis Council Annual Meeting, Tampa, FL, USA, 22-25 January. 183-196.

Tol, P.P.J.vd, Schrader,W., Aernouts, B., 2010. Pressure distribution at the teat-liner and teat-calf interfaces. Journal of Dairy Science. 93 (1), 45-52. https://doi.org/10.3168/jds.2008-1864.

Zucali, M., Bava, L., Penati, C., Tamburini, A., 2009. Effect of liner characteristics on teat apex condition and milk flow traits. Italian Journal of Animal Science. 8, 447-449. https://doi.org/10.4081/ijas.2009.s2.447

Zucali, M., Reinemann, D.J., Tamburini, A., Bade, R.D., 2008. Effects of liner compression on teat-end hyperkeratosis. In Proceedings of the American Society of Agricultural and Biological Engineers, Providence, RI, USA, 29 June-2 July. 


\title{
Effects of the Accumulation of Heat Load Duration on the Activity Behaviour of Lactating Dairy Cows
}

\author{
Julia Heinicke ${ }^{\mathrm{a}, *}$, Stephanie Ibscher ${ }^{\mathrm{b}}$, Vitaly Belik ${ }^{\mathrm{b}}$, Thomas Amon ${ }^{\mathrm{a}, \mathrm{c}}$ \\ ${ }^{\text {a }}$ Leibniz Institute for Agricultural Engineering and Bioeconomy e.V., Department of Engineering for Livestock \\ Management, 14469 Potsdam, Germany \\ ${ }^{\mathrm{b}}$ Institute of Veterinary Epidemiology and Biostatistcs, Department of Veterinary Medicine, Freie Universität Berlin, \\ 14163 Berlin, Germany \\ ${ }^{c}$ Institute of Animal Hygiene and Environmental Health, Department of Veterinary Medicine, Freie Universität Berlin, \\ 14163 Berlin, Germany \\ * Corresponding author. Email: jheinicke@atb-potsdam.de
}

\begin{abstract}
In the course of the predicted climate change, the problem of welfare and heat load (HL) of dairy cows has become increasingly important even under moderate climate conditions. The objective of the present study was to analyse the effects of the daily duration of different heat load levels (HLL) on the activity of lactating dairy cows. Additionally, the adaptation effects of the cows regarding the accumulation of heat load duration (HLD) during the three days preceding the activity measurement day was investigated. The study was conducted from June 2015 to May 2017 in a naturally ventilated dairy barn. The barn climate was measured at high temporal and spatial resolution, and the average temperature-humidity index (THI) was calculated every $10 \mathrm{~min}(\mathrm{~m}=842,112)$. The THI was used to define the HL the cows were exposed to. HLL were determined by defined THI thresholds. The activity of the dairy cows $(n=177)$ was measured using IceTag3 $3 \mathrm{D}^{\mathrm{TM}}$ pedometers and described with several activity traits per cow and day. The analysis models included autocorrelations in time series as well as effects of individual cows. The results showed significant activity adaptations $(\mathrm{P}<0.01)$ regarding the increasing HLD within each HLL on the measurement day. There was a decrease in lying time, number of lying bouts, average lying bout duration and number of standing bouts per day. The average standing bout duration and the number of steps per day increased with increasing HLD on the measurement day. The accumulation of HLD during the three days preceding the measurement day led to reverse activity adaptations on the measurement day $(\mathrm{P}<0.01)$. This indicates that with persistent $\mathrm{HL}$, signs of tiring occur, leading to a reversal of the initial activity adaptation of cows to HL.
\end{abstract}

Keywords: temperature-humidity index, heat load level, heat load duration, activity adaptation

\section{Introduction}

In recent years, heat load (HL) of dairy cows has become one of the most important challenges facing the dairy industry. Besides of physiological thermoregulatory responses to HL (Ferrazza et al., 2017; Toušová et al., 2017), the cows change their activity behaviour (Brzozowska et al., 2014; Endres and Barberg, 2007) in order to sustain their normal body temperature. Numerous studies in different climatic zones have indicated that the activity behaviour of cows is a sensitive indicator for HL. The main findings were that the total lying time (LT) decreased significantly with increasing HL, and consequently, the standing time increased (Cincović et al., 2011; Cook et al., 2007; De Palo et al., 2005). The average duration of each lying bout decreased as the HL increased (De Palo et al., 2005; Endres and Barberg, 2007).

To reduce the adverse effects of HL, it is important for farmers to know when the cows suffer from HL and to correspondingly cool cows in order to help them effectively off-loading heat. Since the use of pedometers to monitor the activity behaviour is already widely used, using activity data for the early detection of anomalies in the activity behaviour that are associated with $\mathrm{HL}$ would be feasible in practice and could be easily implemented on farms.

Additionally to the currently available knowledge, our study analysed the effects of increasing heat load duration (HLD) of different heat load levels (HLL) on the resting and locomotion behaviour of lactating dairy cows. In addition to the contemporaneous effects of $\mathrm{HL}$ on the measurement day, it is further investigated, how the accumulation of ongoing HL during the three days preceding the measurement day and the associated expected reductions in LT influences the activity adaptation on the measurement day. Cow-individual effects, such as days in milk and lactation number, have been taken into account because they influence the activity behaviour of individual cows (Bewley et al., 2010; Maselyne et al., 2017).

It is hypothesised that increasing HLD leads to a reduction in LT with shorter lying bouts and longer standing bouts as well as an increasing number of steps (NS). However, when the HLD spreads during several days preceding the measurement day, they might lie more on the measurement day even if they are still exposed to HL, because they get exhausted and compensate for the reduced LT during the preceding days. 


\section{Materials and Methods}

Barn designs, animals

The measurements were carried out in a naturally ventilated dairy barn with a loose housing system, located in Groß Kreutz, Germany. The barn was $38.88 \mathrm{~m}$ long, $17.65 \mathrm{~m}$ wide and equipped with 51 lying cubicles (mixture of straw and lime as bedding material), an automatic milking system (Lely Astronaut A4, Maassluis, Netherlands) and three cross ventilators above the lying cubicles and the feeding area. The herd consisted of 51 HolsteinFriesian cows (first to eighth lactation), which had an average daily milk yield of $40.7 \pm 6.8 \mathrm{~kg}$ per cow. During the experimental period there was a permanent fluctuation within the herd, whereby activity data were collected at a total of 177 different cows.

Barn climate and activity behaviour measurements

The climate and activity data were recorded from June 2015 to May 2017. The ambient temperature and relative humidity within the barn were measured automatically every 10 min using EasyLog USB 2+ sensors (Lascar Electronics Inc., USA). The sensors were positioned at eight locations inside the barn $3.4 \mathrm{~m}$ above the floor. The THI based on the formula of NRC (1971) was applied:

$$
T H I=(1.8 \times T+32)-(0.55-0.0055 \times R H) \times(1.8 \times T-26)
$$

where $\mathrm{T}$ is the dry bulb temperature in ${ }^{\circ} \mathrm{C}$ and $\mathrm{RH}$ is the relative humidity in $\%$. The THI calculations of all measurement points were averaged for each time point (every $10 \mathrm{~min}$ ) afterwards $(\mathrm{m}=842,112)$. Thus there were the considered variables: mean THI on the measurement day $\left(\mathrm{THI}_{\mathrm{t}}\right)$, mean THI one/two/three day(s) before the measurement day $\left(\mathrm{THI}_{\mathrm{t}-1}, \mathrm{THI}_{\mathrm{t}-2}, \mathrm{THI}_{\mathrm{t}-3}\right)$. In addition, the $\mathrm{THI}$ for each time point was classified according to $\mathrm{THI}$ thresholds found in the literature (Armstrong, 1994; Zimbelman and Collier, 2011). Correspondingly, the THI intensities for each time point were categorized as $68 \leq \mathrm{THI}<72$ (mild HL), $72 \leq \mathrm{THI}<80$ (moderate HL), and $80 \leq \mathrm{THI}$ (severe HL). The defined THI intensities were used within the variables of HLD (Table 1).

Table 1. Variables of heat load duration.

\begin{tabular}{|c|c|}
\hline \multicolumn{2}{|c|}{ Mean heat load duration (HLD) on the measurement day (t) } \\
\hline $\mathrm{HLD}_{\mathrm{t}}^{\mathrm{THI} \epsilon[68.72]}$ & $\begin{array}{l}\text { Number of time points with } 68 \leq \mathrm{THI}<72 \text { on } \mathrm{t} \text { (gives the number of minutes the temperature- } \\
\text { humidity index (THI) was between } 68 \text { and }<72 \text {, if multiplied by } 10 \text {, because measurements } \\
\text { were taken every } 10 \mathrm{~min} \text { ) }\end{array}$ \\
\hline $\mathrm{HLD}_{\mathrm{t}}^{\mathrm{THI} \epsilon[72,80]}$ & $\begin{array}{l}\text { Number of time points with } 72 \leq \mathrm{THI}<80 \text { on } \mathrm{t} \text { (gives the number of minutes the THI was } \\
\text { between } 72 \text { and }<80 \text {, if multiplied by } 10 \text {, because measurements were taken every } 10 \text { min) }\end{array}$ \\
\hline $\mathrm{HLD}_{\mathrm{t}}^{\mathrm{THI}>80}$ & $\begin{array}{l}\text { Number of time points with THI } \geq 80 \text { on } \mathrm{t} \text { (gives the number of minutes the THI was } 80 \text { or } \\
\text { higher, if multiplied by } 10 \text {, because measurements were taken every } 10 \mathrm{~min} \text { ) }\end{array}$ \\
\hline \multicolumn{2}{|c|}{ Mean HLD during the three days preceding $\mathrm{t}$} \\
\hline $\mathrm{HLD}_{\mathrm{t}-1, \mathrm{t}-2, \mathrm{t}-3}^{\mathrm{THI}[68.72]}$ & $\begin{array}{l}\text { Mean HLD during the preceding three days, given that } 68 \leq \mathrm{THI}<72 \text { on at least one } \\
\text { measurement time on each of the three days }(\mathrm{t}-1, \mathrm{t}-2, \mathrm{t}-3) \text { preceding } \mathrm{t}\end{array}$ \\
\hline $\mathrm{HLD}_{\mathrm{t}-1, \mathrm{t}-2, \mathrm{t}-3}^{\mathrm{THI} \in[72.80]}$ & $\begin{array}{l}\text { Mean HLD during the preceding three days, given that } 72 \leq \text { THI }<80 \text { on at least one } \\
\text { measurement time on each of the three days }(\mathrm{t}-1, \mathrm{t}-2, \mathrm{t}-3) \text { preceding } \mathrm{t}\end{array}$ \\
\hline$H L D_{t-1, t-2, t-3}^{\mathrm{THI}}$ & $\begin{array}{l}\text { Mean HLD during the preceding three days, given that THI } \geq 80 \text { on at least one measurement } \\
\text { time on each of the three days }(\mathrm{t}-1, \mathrm{t}-2, \mathrm{t}-3) \text { preceding } \mathrm{t}\end{array}$ \\
\hline
\end{tabular}

The activity of the dairy cows $(n=177)$ was measured with pedometers. Each cow of the herd was equipped with one IceTag3 $\mathrm{D}^{\mathrm{TM}}$ activity sensor (IceRobotics, Edinburgh, UK) on one hind leg. The IceTag3 $\mathrm{D}^{\mathrm{TM}}$ is a noninvasive electronic sensor that measures animal activity with three-dimensional acceleration technology per second. We calculated and analysed the following activity traits per cow and day: total lying time (LT) in s, number of lying bouts (LB) in times, average lying bout duration (LBD) in $\mathrm{s}$ in the functional group "resting behaviour" as well as number of standing bouts (SB) in times, average standing bout duration (SBD) in $\mathrm{s}$ and number of steps (NS) in steps in the functional group "locomotion behaviour".

\section{Statistical data analysis}

For each of the activity traits, a (generalized) linear mixed model was used to estimate the effects of HL exposure on the measurement day and during the three days preceding the measurement day as well as how these effects depend on cow-individual factors. Cow-individual factors were the milk production levels $\left(\right.$ Milk $_{\mathrm{t}}^{\text {low }}$, Milk $_{t}^{\text {normal }}$, Milk $k_{t}^{\text {high }}$ ), the categorised days in milk $\left(\operatorname{DIM}_{\mathrm{t}}^{1-60}, \operatorname{DIM}_{\mathrm{t}}^{61-150}, \operatorname{DIM}_{\mathrm{t}}^{<150}\right)$, the lactation number $\left(\mathrm{L}_{\mathrm{t}}^{1}, \mathrm{~L}_{\mathrm{t}}^{2,3}\right.$, $\left.\mathrm{L}_{\mathrm{t}}^{\geq 4}\right)$ and the gestation status $\left(\mathrm{G}_{\mathrm{t}}^{0}, \mathrm{G}_{\mathrm{t}}^{1-90}, \mathrm{G}_{\mathrm{t}}^{91-180}, \mathrm{G}_{\mathrm{t}}^{>180}\right)$. The models incorporate random effects of the individual cows on the activity traits as well as the within-cow correlation structure, because the observations of the same cow are temporally correlated. The temporal correlation is modelled by autoregressive-moving average (ARMA)processes. The significance level for the (generalized) linear mixed model was 0.05 . 


\section{Results}

Activity adaptation to heat load "resting behaviour"

The significant effects to results of LT, LB and LBD are presented in table 2. Based on the model equations, it was possible to make predictions of the activity adaptation of dairy cows under different HL conditions. Assuming that there was no HL on the measurement day and the days before (mean THI was on average 46.01 on days without HL), LT was approximately $10.8 \mathrm{~h}^{6}$ (38996.18 s) for cows in the reference group (non-pregnant, $\mathrm{L}_{\mathrm{t}}^{1}, \mathrm{DIM}_{\mathrm{t}}^{1-60}, \mathrm{Milk}_{\mathrm{t}}^{\text {normal }}$ ) and not in oestrus. In general, mean THI and the three HLD effects on the measurement day were negatively correlated with LT. An increase in THI by one unit resulted in a reduction in LT of about $3.21 \mathrm{~min}(192.75 \mathrm{~s})$. Furthermore, the longer HLD on the measurement day, the shorter the cows are expected to lie down in addition to the effect of THI. The effect of HLD depended on HLL. Each additional 10 min of exposure to mild HL on the measurement day was expected to reduce LT by $23.83 \mathrm{~s}$. LT was further reduced by $46.80 \mathrm{~s}$ or $94.08 \mathrm{~s}$ when the exposure to moderate or several HL lasted $10 \mathrm{~min}$ longer, respectively. Therefore, there was a reduced LT of $7.7 \mathrm{~h}^{7}(27862.18 \mathrm{~s})$ for cows in the reference group with HL on the measurement day (exemplary THI and HLD values were highlighted with underscores in the formulas) and without HL the days before. In contrast to the contemporaneous effects, the lagged effects were positively related to LT. The result was that cows' LT increased again to $9 \mathrm{~h}^{8}$ (32270.36 s) when there was HL on the measurement day and additionally during the three days preceding the measurement day (exemplary THI and HLD values are highlighted with underscores in the formulas). Independent of the climatic conditions, the cow-individual variables had significant effects on the expected LT. Low-producing cows were found to lie down more than cows with a normal or high level of milk production. Cows with DIM $_{t}^{>150}$ lay down around $17.90 \mathrm{~min}(1074.28 \mathrm{~s})$ more than cows with less than 150 DIM. This was also reflected in the increasing LT within the gestation status. LT in $\mathrm{L}_{\mathrm{t}}^{\geq 4}$ increased by $47.56 \mathrm{~min}$ (2853.64 s) compared with LT of cows in earlier lactations. Cows in oestrus lay down significantly less than cows that were not in oestrus.

The model for LB was estimated using the log-link function. THI had no significant effect on LB. However, LB was negatively affected by HLD on the measurement day. Each additional 10 min of mild HL reduced LB by $0.03 \%$ and every additional $10 \mathrm{~min}$ of moderate HL further reduced LB by $0.05 \%$. There were lagged effects of THI and HLD on LB. When HLD during the three days preceding the measurement day increased, LB decreased. But, all significant effects of the model influenced the LB only to a small extent. Without the influence of HL, the days in milk and the lactation number had significant effects on the expected LB. Cows with DIM $\mathrm{t}_{\mathrm{t}}^{61-150}$ and $\mathrm{DIM}_{\mathrm{t}}^{>150}$ lay down around $6.63 \%$ and $7.89 \%$ more often, respectively, than cows with $\mathrm{DIM}_{\mathrm{t}}^{1-60}$. In $\mathrm{L}_{\mathrm{t}}^{2,3}$ and $\mathrm{L}_{\mathrm{t}}^{\geq 4}$, LB decreased by $29.17 \%$ and $16.11 \%$, respectively, compared with LB of cows in $\mathrm{L}_{t}^{1}$. No significant differences in LB between cows with different milk production levels or in different gestation status were found. The cows in oestrus lay down $6.17 \%$ less often than cows that were not in oestrus. Thus, the cow-individual effects led to stronger percentage changes in LB compared with HL effects.

The third model in table 2 shows the results of the linear mixed model of the logarithmized LBD. The estimated LBD of cows in the reference group without of HL on the measurement day and the days before was $47.68 \min ^{9}$ (2860.82 s). In general, $\mathrm{THI}_{t}$ and $\mathrm{THI}_{\mathrm{t}-2}$ were negatively correlated with LBD. However, increasing $\mathrm{THI}_{\mathrm{t}-1}$ led to an increase in LBD by $0.24 \%$ per unit. Furthermore, HLD significantly affected LBD. Longer exposure to mild, moderate and several HL on the measurement day resulted in a shorter LBD. The reference cows under HL on the measurement day and without HL the days before had an estimated LBD of $37.73 \mathrm{~min}^{10}$ (2263.89 s). LBD under HL was therefore much shorter than LBD without HL. Concerning the HLD during the three days preceding the measurement day, LBD was significantly affected only by $\operatorname{HLD}_{\mathrm{t}-1, \mathrm{t}-2, \mathrm{t}-3}^{\mathrm{THI} \epsilon \mathrm{C}}$. Ten more min of $\operatorname{HLD}_{\mathrm{t}-1, \mathrm{t}-2, \mathrm{t}-3}^{\mathrm{THI}-3}$ increased the expected LBD by $0.03 \%$. In consideration of all significant effects of the model, LBD under HL on the measurement day and during the three days before increased once again slightly to approximately $38.53 \mathrm{~min}^{11}$ (2311.59 s). Independent of HL, the cow-individual variables had significant effects on the expected LBD. LBD in $\mathrm{DIM}_{\mathrm{t}}^{61-150}$ and $\mathrm{DIM}_{\mathrm{t}}^{>150}$ was about $6.66 \%$ and $10.57 \%$ longer, respectively, than in $\mathrm{DIM}_{\mathrm{t}}^{1-60}$. Cows in $\mathrm{L}_{\mathrm{t}}^{2,3}$ and $\mathrm{L}_{\mathrm{t}}^{\geq 4}$ had longer LBD by $24.80 \%$ and $22.71 \%$, respectively, compared with cows in $\mathrm{L}_{\mathrm{t}}^{1}$. The gestation status also affected LBD. During oestrus days, LBD was around $8.04 \%$ shorter than when the cows were not in oestrus.

\footnotetext{
${ }^{6} 38996.18=41723.2535-192.7514 \times 46.01+111.3501 \times 46.01+22.1299 \times 46.01$

${ }^{7} 27862.18=41723.2535-192.7514 \times \underline{73.49}+111.3501 \times \underline{46.01}+22.1299 \times \underline{46.01}-23.8339 \times \underline{26}-46.7991 \times \underline{56.87}-94.0757 \times \underline{27.17}$

${ }^{8} 32270.36=41723.2535-192.7514 \times \underline{73.49}+111.3501 \times \underline{70.21}+22.1299 \times \underline{69.89}-23.8339 \times \underline{26}-46.7991 \times \underline{56.87}-94.0757 \times \underline{27.17}$ $+17.0419 \times \underline{43.04}+30.1041 \times \underline{15}$

${ }^{9} 2860.82=\exp (8.1291-0.0041 \times 46.01+0.0024 \times 46.01-0.0020 \times 46.01)$

${ }^{10} 2263.89=\exp (8.1291-0.0041 \times \underline{73.49}+0.0024 \times \underline{46.01}-0.0020 \times \underline{46.01}-0.0004 \times \underline{26}-0.0009 \times \underline{56.87}-0.0022 \times \underline{27.17})$

${ }^{11} 2311.59=\exp (8.1291-0.0041 \times \underline{73.49}+0.0024 \times \underline{70.21}-0.0020 \times \underline{71.08}-0.0004 \times \underline{26}-0.0009 \times \underline{56.87}-$ $0.0022 \times \underline{27.17}+0.0003 \times \underline{43.04})$
} 
Table 2. Mean lying time, number of lying bouts and average lying bout duration per day depending on mean temperaturehumidity index (THI), daily heat load duration per heat load level (HLD), milk production level (Milk), days in milk (DIM), lactation number $(\mathrm{L})$, gestation status $(\mathrm{G})$ and indicator days $(\mathrm{I})$ with $\mathrm{t}=$ measurement day and meas $=$ measurement.

\begin{tabular}{|c|c|c|c|}
\hline Variables & Lying time & $\begin{array}{l}\text { Number of } \\
\text { lying bouts }\end{array}$ & $\begin{array}{l}\text { Average lying } \\
\text { bout duration }\end{array}$ \\
\hline $\mathrm{THI}_{\mathrm{t}}$ & $\begin{array}{l}-192.7514^{* * *} \\
(13.6192)\end{array}$ & \multirow{4}{*}{$\begin{array}{l}0.0022^{* * *} \\
(0.0004)\end{array}$} & \multirow{4}{*}{$\begin{array}{l}-0.0041^{* * *} \\
(0.0005) \\
0.0024^{* * *} \\
(0.0006) \\
-0.0020^{* * *} \\
(0.0005)\end{array}$} \\
\hline $\mathrm{THI}_{\mathrm{t}-1}$ & $\begin{array}{l}111.3501^{* * *} \\
(13.3279)\end{array}$ & & \\
\hline $\mathrm{THI}_{\mathrm{t}-2}$ & & & \\
\hline $\mathrm{THI}_{\mathrm{t}-3}$ & $\begin{array}{l}22.1299^{* *} \\
(9.5413)\end{array}$ & & \\
\hline $\mathrm{HLD}_{\mathrm{t}}^{\mathrm{THI} \epsilon[68.72]}$ & $\begin{array}{l}-23.8339^{* * *} \\
(3.7114)\end{array}$ & $\begin{array}{l}-0.0003^{* *} \\
(0.0001)\end{array}$ & $\begin{array}{l}-0.0004^{* * *} \\
(0.0001)\end{array}$ \\
\hline $\begin{array}{c}\mathrm{HLD}_{\mathrm{t}}^{\mathrm{THI} \epsilon[72,80]} \\
\operatorname{HLD}_{\mathrm{t}}^{\mathrm{THI} \geq 80}\end{array}$ & $\begin{array}{l}-46.7991^{* * *} \\
(3.1147) \\
-94.0757^{* * *} \\
(7.1975) \\
\end{array}$ & $\begin{array}{l}-0.0005^{* * *} \\
(0.0001)\end{array}$ & $\begin{array}{l}-0.0009^{* * *} \\
(0.0001)^{*} \\
-0.0022^{* * *} \\
(0.0003)\end{array}$ \\
\hline $\mathrm{HLD}_{\mathrm{t}-1, \mathrm{t}-2, \mathrm{t}-3}^{\mathrm{THI}}$ & & $\begin{array}{l}0.0004^{* *} \\
(0.0002)\end{array}$ & \multirow{3}{*}{$\begin{array}{l}0.0003^{* *} \\
(0.0001)\end{array}$} \\
\hline $\mathrm{HLD}_{\mathrm{t}-1, \mathrm{t}-2, \mathrm{t}-3}^{\mathrm{THI} \epsilon[72.80]}$ & $\begin{array}{l}17.0419^{* * *} \\
(3.5910)\end{array}$ & \multirow[b]{2}{*}{$\begin{array}{l}0.0019^{* * *} \\
(0.0004)\end{array}$} & \\
\hline $\mathrm{HLD}_{\mathrm{t}-1, \mathrm{t}-2, \mathrm{t}-3}^{\mathrm{THI}}$ & $\begin{array}{l}30.1041^{* *} \\
(12.5319)\end{array}$ & & \\
\hline Milk $_{\mathrm{t}}^{\text {low }}$ & $\begin{array}{l}1154.3881^{* *} \\
(540.2129)\end{array}$ & \multirow{8}{*}{$\begin{array}{l}-0.0663^{* * *} \\
(0.0124) \\
-0.0789^{* * *} \\
(0.0183)^{* * *} \\
-0.2917^{* * *} \\
(0.0437) \\
-0.1611^{* * *} \\
(0.0530)\end{array}$} & \multirow{9}{*}{$\begin{array}{l}0.0666^{* * *} \\
(0.0110) \\
0.1057^{* * *} \\
(0.0161) \\
0.2480^{* * *} \\
(0.0347) \\
0.2271^{* * *} \\
(0.0425) \\
0.0502^{* * *} \\
(0.0130) \\
0.0577^{* * *} \\
(0.0210)\end{array}$} \\
\hline Milk $_{\mathrm{t}}^{\text {high }}$ & & & \\
\hline $\mathrm{DIM}_{\mathrm{t}}^{61-150}$ & & & \\
\hline $\operatorname{DIM}_{\mathrm{t}}^{>150}$ & $\begin{array}{l}1074.2758^{* *} \\
(432.1500)\end{array}$ & & \\
\hline $\mathrm{L}_{\mathrm{t}}^{2,3}$ & & & \\
\hline $\mathrm{L}_{\mathrm{t}}^{\geq 4}$ & $\begin{array}{l}2853.6397^{* * *} \\
(1099.4544)\end{array}$ & & \\
\hline $\mathrm{G}_{\mathrm{t}}^{1-90}$ & $\begin{array}{l}1424.5861^{* * *} \\
(342.6211)\end{array}$ & & \\
\hline$G_{t}^{91-180}$ & $\begin{array}{l}1086.6468^{* *} \\
(553.9747)\end{array}$ & & \\
\hline$G_{t}^{>180}$ & & & \\
\hline $\mathrm{I}_{\mathrm{t}, \mathrm{t}-1}^{\text {oestrus }}$ & $\begin{array}{l}-5499.1927^{* * *} \\
(282.9488)\end{array}$ & $\begin{array}{l}-0.0617^{* * *} \\
(0.0106) \\
\end{array}$ & $\begin{array}{l}-0.0804^{* * *} \\
(0.0102)\end{array}$ \\
\hline $\mathrm{I}_{\mathrm{t}}^{\text {meas }}$ & $\begin{array}{l}-2183.4099^{* * *} \\
(98.3097)\end{array}$ & $\begin{array}{l}-0.0349^{* * *} \\
(0.0035)\end{array}$ & $\begin{array}{l}-0.0205^{* * *} \\
(0.0035)\end{array}$ \\
\hline Intercept & $\begin{array}{l}41723.2535^{* * *} \\
(1009.7931)\end{array}$ & $\begin{array}{l}2.5624^{* * *} \\
(0.0406)\end{array}$ & $\begin{array}{l}8.1291^{* * *} \\
(0.0370)\end{array}$ \\
\hline
\end{tabular}

$$
* * * \mathrm{p}<0.01 \text { and } * * \mathrm{p}<0.05
$$

Activity adaptation to heat load "locomotion behaviour"

The significant effects to results of SB, SBD and NS are presented in table 3. The model for SB was estimated using the log-link function. $\mathrm{THI}_{t}$ had no significant effect on SB. However, SB was affected by HLD on the measurement day. The longer the cows were exposed to mild or moderate HL, the smaller was SB on the measurement day. The effect of HLD depended on HLL. The duration of severe HL on the measurement day did not led to further adaptations in $\mathrm{SB}$. $\mathrm{THI}_{\mathrm{t}-2}$ was associated with a significant increase in $\mathrm{SB}$ by approximately $0.21 \%$ per increased unit. Additionally, there was a lagged effect of HLD on SB. When HLD during the three days preceding the measurement day increased, SB increased as well. Similar to LB, all significant HL effects of the model influenced the SB only to a small extent. Independent of the climatic conditions, some cow-individual variables had significant effects on the expected SB. Cows in $\mathrm{DIM}_{\mathrm{t}}^{61-150}$ or $\mathrm{DIM}_{\mathrm{t}}^{>150}$ were expected to stand approximately $6.92 \%$ or $8.54 \%$ less often, respectively, than cows at the beginning of lactation. The lactation number also affected SB of the cows. Compared to primiparous cows, the expected SB of cows in $\mathrm{L}_{\mathrm{t}}^{2,3}$ was $28.61 \%$ 
smaller and cows in $\mathrm{L}_{\mathrm{t}}^{\geq 4}$ were expected to stand $15.52 \%$ less often than cows in $\mathrm{L}_{\mathrm{t}}^{1}$. $\mathrm{SB}$ reduced by about approximately $5.8 \%$, when the cow was in oestrus.

The second model in table 3 shows the results of the linear mixed model of the logarithmized SBD. The cows in the reference group without HL on the measurement day and during the days before had an estimated SBD of $57.86 \mathrm{~min}^{12}(3471.33 \mathrm{~s})$. The effects of increasing THI and HLD on the measurement day led to an increase of SBD. When THI increased by one unit the SBD increased by $0.47 \%$. The effects of HLD on the measurement day were a little less pronounced and depended on HLL. As a result, SBD increased to $79.26 \mathrm{~min}^{13}(4755.74 \mathrm{~s})$ during days with HL (without HL the days before). This pronounced change was lower when there was additionally HL during the three days preceding the measurement day. In this case, SBD was approximately 65.49 $\min ^{14}(3929.48 \mathrm{~s})$. The reason was that SBD became shorter with increasing $\mathrm{THI}_{\mathrm{t}-1}$ and $\mathrm{THI}_{\mathrm{t}-3}$ as well as increasing HLD during the three days preceding the measurement day. An increase in THIt-1 or THIt-3 by one unit was expected to result in a reduction in SBD of about $0.36 \%$ or $0.17 \%$, respectively. An increase in $\operatorname{HLD}_{\mathrm{t}-1, \mathrm{t}-2, \mathrm{t}-3 \text {, }}^{\mathrm{THI}}$, $\mathrm{HLD}_{\mathrm{t}-1, \mathrm{t}-2, \mathrm{t}-3}^{\mathrm{THI}}\left[\mathrm{72.80}\right.$ or $\mathrm{HLD}_{\mathrm{t}-1, \mathrm{t}-2, \mathrm{t}-3}^{\mathrm{THI} \geq 80}$ by $10 \mathrm{~min}$ led to a decrease in SBD of $0.005 \%, 0.007 \%$ or $0.18 \%$, respectively. Compared to cows in the reference group, cows with DIM $_{t}^{61-150}$ had approximately $4.29 \%$ longer SBD and cows within $\mathrm{L}_{\mathrm{t}}^{2,3}$ had $26.26 \%$ longer SBD, independent of the climate conditions. No differences in SBD of cows with different milk production level or pregnancy status were found. In general, cows in oestrus stood $21.1 \%$ longer per standing bout than cows which were not in oestrus.

The last model shows the results of the linear mixed model of the logarithmized NS. NS for cows in the reference group was approximately 2000 steps $^{15}$ under climate conditions without of $\mathrm{Hl}$ on the measurement day and the days before. In general, increasing THI and HLD on the measurement day led to an increase of NS. Keeping all other variables constant, an increase of $\mathrm{THI}_{\mathrm{t}}$ by one unit led to an increase of NS by $0.1 \%$. Additionally, an increase by 10 min of $\operatorname{HLD}_{t}^{\mathrm{THI} \epsilon[68.72]}, \mathrm{HLD}_{\mathrm{t}}^{\mathrm{THI} \epsilon[72,80]}$ or $\operatorname{HLD}_{\mathrm{t}}^{\mathrm{THI} \geq 80}$ led to $0.08 \%, 0.12 \%$ or $0.1 \%$ more steps per day. As a result of these effects, NS increased to 2309 steps $^{16}$ when the reference cows were exposed to HL on the measurement day (without HL the days before). The lagged effects of increasing THI and HLD during the three days preceding the measurement day decreased NS again. However, THI -2 and $\mathrm{THI}_{\mathrm{t}-3}$ did not influence NS. Consequently, the estimated NS for the reference cows was 2123 steps $^{17}$ under HL on the measurement day and additional HL during the three days preceding the measurement day. Independent of the climatic conditions, the cow-individual variables had significant effects on the expected NS. Compared to cows of the reference group, cows with $\mathrm{DIM}_{\mathrm{t}}^{61-150}$ or $\mathrm{DIM}_{\mathrm{t}}^{>150}$ had $4.39 \%$ or $6.13 \%$ less NS, respectively. For the cows in $\mathrm{L}_{\mathrm{t}}^{2,3}$ or $\mathrm{L}_{\mathrm{t}}^{\geq 4}, 12.07 \%$ or $39.37 \%$ less NS were predicted, respectively. Concerning the gestation status, in $\mathrm{G}_{\mathrm{t}}^{1-90}$ and $G_{t}^{91-180}$ NS was significantly lower than in $G_{t}^{>180}$ and non-pregnant cows.

$23471.33=\exp (8.1799+0.0047 \times 46.01-0.0036 \times 46.01-0.0017 \times 46.01)$

$134755.74=\exp (8.1799+0.0047 \times \underline{73.49}-0.0036 \times \underline{46.01}-0.0017 \times \underline{46.01}+0.0008 \times \underline{26}+0.0018 \times \underline{56.67}+0.0023 \times \underline{27.17})$

${ }^{14} 3929.48=\exp (8.1799+0.0047 \times \underline{73.49}-0.0036 \times \underline{70.21}-0.0017 \times \underline{69.89}+0.0008 \times \underline{26}+0.0018 \times \underline{56.67}+0.0023 \times \underline{27.17}-$ $0.0005 \times 12-0.0007 \times 43.04-0.0018 \times 15)$

${ }^{15} 2000=\exp (7.6010+0.0010 \times 46.01-0.0010 \times \underline{46.01})$

${ }^{16} 2309=\exp (7.6010+0.0010 \times \underline{73.49}-0.0010 \times \underline{46.01}+0.0008 \times \underline{26}+0.0012 \times \underline{56.67}+0.0010 \times \underline{27.17})$

${ }^{17} 2123=\exp (7.6010+0.0010 \times \underline{73.49}-0.0010 \times \underline{70.21}+0.0008 \times \underline{26}+0.0012 \times \underline{56.67}+0.0010 \times \underline{27.17}-0.0009 \times \underline{43.04}-0.0014 \times \underline{15})$ 
Table 3. Number of standing bouts, average standing bout duration and number of steps per day depending on mean temperature-humidity index (THI), daily heat load duration per heat load level (HLD), milk production level (Milk), days in milk (DIM), lactation number $(\mathrm{L})$, gestation status $(\mathrm{G})$ and indicator days $(\mathrm{I})$ with $\mathrm{t}=$ measurement day and meas $=$ measurement.

\begin{tabular}{|c|c|c|c|}
\hline Variables & $\begin{array}{l}\text { Number of } \\
\text { standing bouts }\end{array}$ & $\begin{array}{l}\text { Average standing } \\
\text { bout duration }\end{array}$ & $\begin{array}{l}\text { Number of } \\
\text { steps }\end{array}$ \\
\hline $\mathrm{THI}_{\mathrm{t}}$ & & $\begin{array}{l}0.0047^{* * *} \\
(0.0006)\end{array}$ & $\begin{array}{l}0.0010^{* *} \\
(0.0006)\end{array}$ \\
\hline $\mathrm{THI}_{\mathrm{t}-1}$ & & $\begin{array}{l}-0.0036^{* * *} \\
(0.0006)\end{array}$ & $\begin{array}{l}-0.0010^{* * *} \\
(0.0005)\end{array}$ \\
\hline $\mathrm{THI}_{\mathrm{t}-2}$ & $\begin{array}{l}0.0021^{* * *} \\
(0.0004)\end{array}$ & & \\
\hline $\mathrm{THI}_{\mathrm{t}-3}$ & & $\begin{array}{l}-0.0017^{* * *} \\
(0.0005)\end{array}$ & \\
\hline $\mathrm{HLD}_{\mathrm{t}}^{\mathrm{THI} \epsilon[68.72]}$ & $\begin{array}{l}-0.0003^{* *} \\
(0.0001)\end{array}$ & $\begin{array}{l}0.0008^{* * *} \\
(0.0002)\end{array}$ & $\begin{array}{l}0.0008^{* * *} \\
(0.0002)\end{array}$ \\
\hline $\begin{array}{c}\mathrm{HLD}_{\mathrm{t}}^{\mathrm{THI} \epsilon[72,80]} \\
\operatorname{HLD}_{\mathrm{t}}^{\mathrm{THI} \geq 80}\end{array}$ & $\begin{array}{l}-0.0004^{* * *} \\
(0.0001)\end{array}$ & $\begin{array}{l}0.0018^{* * *} \\
(0.0001) \\
0.0023^{* * *} \\
(0.0003)\end{array}$ & $\begin{array}{l}0.0012^{* * *} \\
(0.0001) \\
0.0010^{* * *} \\
(0.0003)\end{array}$ \\
\hline $\mathrm{HLD}_{\mathrm{t}-1, \mathrm{t}-2, \mathrm{t}-3}^{\mathrm{THI} \epsilon[68.72]}$ & $\begin{array}{l}0.0004^{* *} \\
(0.0002)\end{array}$ & $\begin{array}{l}-0.0005^{* *} \\
(0.0002)\end{array}$ & \\
\hline $\mathrm{HLD}_{\mathrm{t}-1, \mathrm{t}-2, \mathrm{t}-3}^{\mathrm{THI}}$ & & $\begin{array}{l}-0.0007^{* * *} \\
(0.0002)\end{array}$ & $\begin{array}{l}-0.0009^{* * *} \\
(0.0001)\end{array}$ \\
\hline $\mathrm{HLD}_{\mathrm{t}-1, \mathrm{t}-2, \mathrm{t}-3}^{\mathrm{THI}}$ & $\begin{array}{l}0.0021^{* * *} \\
(0.0004)\end{array}$ & $\begin{array}{l}-0.0018^{* * *} \\
(0.0006)\end{array}$ & $\begin{array}{l}-0.0014^{* * *} \\
(0.0005)\end{array}$ \\
\hline \multicolumn{4}{|l|}{ Milk $_{\mathrm{t}}^{\text {low }}$} \\
\hline \multicolumn{4}{|l|}{ Milk $_{\mathrm{t}}^{\text {high }}$} \\
\hline $\mathrm{DIM}_{\mathrm{t}}^{61-150}$ & $\begin{array}{l}-0.0692^{* * *} \\
(0.0123)\end{array}$ & $\begin{array}{l}0.0429^{* * *} \\
(0.0152)\end{array}$ & $\begin{array}{l}-0.0439^{* * *} \\
(0.0112)\end{array}$ \\
\hline $\operatorname{DIM}_{t}^{>150}$ & $\begin{array}{l}-0.0854^{* * *} \\
(0.0181)\end{array}$ & & $\begin{array}{l}-0.0613^{* * *} \\
(0.0167)\end{array}$ \\
\hline $\mathrm{L}_{\mathrm{t}}^{2,3}$ & $\begin{array}{l}-0.02861^{* * *} \\
(0.0428)\end{array}$ & $\begin{array}{l}0.2626^{* * *} \\
(0.0557)\end{array}$ & $\begin{array}{l}-0.1207^{* * *} \\
(0.0365)\end{array}$ \\
\hline $\mathrm{L}_{\mathrm{t}}^{\geq 4}$ & $\begin{array}{l}-0.1552^{* * *} \\
(0.0519)\end{array}$ & & $\begin{array}{l}-0.3937^{* * *} \\
(0.0447)\end{array}$ \\
\hline $\mathrm{G}_{\mathrm{t}}^{1-90}$ & & & $\begin{array}{l}-0.0502^{* *} \\
(0.0133)\end{array}$ \\
\hline $\mathrm{G}_{\mathrm{t}}^{91-180}$ & & & $\begin{array}{l}-0.0520^{* *} \\
(0.0218)\end{array}$ \\
\hline $\mathrm{G}_{\mathrm{t}}^{>180}$ & & & \\
\hline $\mathrm{I}_{\mathrm{t}, \mathrm{t}-1}^{\text {oestrus }}$ & $\begin{array}{l}-0.0580^{* * *} \\
(0.0105)\end{array}$ & $\begin{array}{l}0.2110^{* * *} \\
(0.0126)\end{array}$ & $\begin{array}{l}0.5203^{* * *} \\
(0.0118)\end{array}$ \\
\hline $\mathrm{I}_{\mathrm{t}}^{\text {meas }}$ & $\begin{array}{l}-0.0354^{* * *} \\
(0.0035)\end{array}$ & & $\begin{array}{l}-0.0422^{* * *} \\
(0.0040)\end{array}$ \\
\hline Intercept & $\begin{array}{l}2.5687^{* * *} \\
(0.0399)\end{array}$ & $\begin{array}{l}8.1799^{* * *} \\
(0.0578)\end{array}$ & $\begin{array}{l}7.6010^{* * *} \\
(0.0381)\end{array}$ \\
\hline
\end{tabular}

$* * * \mathrm{p}<0.01$ and $* * \mathrm{p}<0.05$ 


\section{Discussion and conclusion}

The present study investigated the activity behaviour of dairy cows as influenced by the THI as it has already been shown in the literature and added novel investigations on effects of HLD and intensity. The effect of the mean THI indicated similar adaptations in the lying and locomotion behaviour of the cows as described in previous studies. It is well known that there is a negative correlation between THI and time spent lying (Herbut and Angrecka, 2018; Zähner et al., 2004). According to Brzozowska et al. (2014) and Steensels et al. (2012), who recorded the activity of cows depending on the season of the year, the time a cow spent lying down per day was significantly higher in winter compared with other seasons. A positive correlation between THI and time spent standing has been recorded by Allen et al. (2015) and Provolo and Riva (2009). Furthermore, several studies found that the length of lying bouts decreased as the THI increased (De Palo et al., 2005; Endres and Barberg, 2007). Additionally, the length of standing bouts increased with increasing THI (Allen et al., 2015). In previous studies, the frequency of lying bouts was not significantly influenced by THI (Endres and Barberg, 2007; Zähner et al., 2004). According to Brzozowska et al. (2014) and Steensels et al. (2012), LB was not associated with the season. Endres and Barberg (2007) compared the steps per hour when the THI was $\geq 72$ or $<72$ and found average values of 71.6 and 120.8, respectively. Therefore, there was an increase of steps per hour with increasing HL intensity. Cows' number of steps was significantly higher in summer than in winter, according to Brzozowska et al. (2014) and Steensels et al. (2012). All these findings are similar to our results regarding the mean THI during the measurement day.

The effect of the mean THI one, two or three days preceding the measurement day was not analysed in the literature until now with regard to the activity behaviour. West et al. (2003) found that during a hot period, the THI value and air temperature two and three days earlier have a greater impact on milk yield and dry matter intake than actual values. For this reason, we examined the lagged THI effects on activity and could identify significant influences on some activity traits. Reverse effects concerning the behavioural adaptation were found. This indicates that the previous climate conditions as well as contemporaneous conditions significantly affect the activity behaviour of the cows.

The scientific novelty of the present study was the analysis of HLD for different HLL and the assessment of its effects on the behavioural adaptations in the functional groups "resting behaviour" and "locomotion behaviour". The main advantage over mean THI values is that HLD provided information on how long the cows had been exposed to HL per day and the HLL included additional information on the intensity of HL. In a similar way, this innovative procedure was used previously only by Herbut and Angrecka (2018). They divided the obtained THI values into the periods neutral (maximum $3 \mathrm{~h}$ with THI $=68$ ), warm (time of THI $>68$ occurrence lasted less than $12 \mathrm{~h}$ ), and hot (time of THI $>68$ occurrence lasted longer than $12 \mathrm{~h}$ ), which were characterized by different durations of THI throughout the entire day. In the present study, the intensity of HL was classified in more detail and the effect of HLD was analysed with a temporal resolution of $10 \mathrm{~min}$. The activity traits influenced by different HLD and HLL during the measurement day showed the same correlations as with the effect of the mean THI during the measurement day. However, it is possible to make more precise predictions concerning the activity on a HL day when there is information to the HLD for different HLL additionally to the mean THI. On days without exposure to HL, the information of the mean THI is sufficient.

An important finding of the study was that HLD during the three days preceding the measurement day showed reversed effects to the activity adaptation compared to HLD on the measurement day. We assumed that when the cows were exposed to HL during all three days preceding the measurement day, they possibly could not further increase their adaptation beyond some limit and reacted weakened to the HL exposure on the measurement day. Consequently, it led to increased LT, LB, LBD and SB. SBD and NS during the measurement day decreased with increasing HLD during the three days preceding the measurement day. Previous studies, which analysed the reactions of cows with lying deprivation, illustrated the need for cows to lie down and the compensatory reactions (Cooper et al., 2007; Cooper et al., 2008). They recognized that the cows recovered their lost lying time by rescheduling feeding and standing time. The reduced resting times and increased standing times on the days preceding the measurement day cause exhaustion, so that the cows' strong need for rest predominates the discomfort of lying down.

Another important effect to evaluate the activity behaviour of dairy cows is the cow-individuality. The results of Bewley et al. (2010) and Maselyne et al. (2017) demonstrated the importance of including information about days in milk when interpreting data on lying and locomotion behaviour. Compared with our results, they also found that LT increased as days in milk increased. Maselyne et al. (2017) recognized additional a significant drop in LT appears during the first weeks after calving. Our results showed on the basis of further significantly cowindividual effects how important it is to estimate the activity of each individual cow.

The HLD of different HLL during the measurement day significantly influenced the activity behaviour of lactating dairy cows in the functional groups "resting behaviour" and "locomotion behaviour". The largest activity changes were found in LT, LBD, SBD and NS. In consideration of the accumulation of HLD during the three 
days preceding the measurement day, the activity adaptation of the cows did not further increase. On the contrary, the cows reversed to a limited extent their activity adaptations during the measurement day, which could indicate that the cows reaction weakened. The studied activity traits included many significant cow-individual effects, that might also affect the sensitivity to HL, indicating that HL activity in the future should be considered in interaction with cow-individual effects. In order to predict the activity behaviour under HL, previous climate conditions as well as cow-individual effects must be additionally taken into account.

\section{References}

Allen, J., Hall, L., Collier, R.J., Smith, J., 2015. Effect of core body temperature, time of day, and climate conditions on behavioral patterns of lactating dairy cows experiencing mild to moderate heat stress. Journal of Dairy Science 98, 118-127.

Armstrong, D.V., 1994. Heat Stress Interaction with Shade and Cooling. Journal of Dairy Science 77, 20442050.

Bewley, J.M., Boyce, R.E., Hockin, J., Munksgaard, L., Eicher, S.D., Einstein, M.E., Schutz, M.M., 2010. Influence of milk yield, stage of lactation, and body condition on dairy cattle lying behaviour measured using an automated activity monitoring sensor. Journal of Dairy Research 77, 1-6.

Brzozowska, A., Łukaszewicz, M., Sender, G., Kolasińska, D., Oprządek, J., 2014. Locomotor activity of dairy cows in relation to season and lactation. Applied Animal Behaviour Science 156, 6-11.

Cincović, M., Belić, B., Toholj, B., Stevančević, M., 2011. On-farm measurement of heat stress load in dairy cows. Veterinarska Stanica 42, 285-289.

Cook, N.B., Mentink, R.L., Bennett, T.B., Burgi, K., 2007. The Effect of Heat Stress and Lameness on Time Budgets of Lactating Dairy Cows. Journal of Dairy Science 90, 1674-1682.

Cooper, M., Arney, D., Phillips, C., 2007. Two-or four-hour lying deprivation on the behavior of lactating dairy cows. Journal of Dairy Science 90, 1149-1158.

Cooper, M., Arney, D., Phillips, C.J., 2008. The effect of temporary deprivation of lying and feeding on the behaviour and production of lactating dairy cows. Animal 2, 275-283.

de Andrade Ferrazza, R., Garcia, H.D.M., Aristizábal, V.H.V., de Souza Nogueira, C., Veríssimo, C.J., Sartori, J.R., Sartori, R., Ferreira, J.C.P., 2017. Thermoregulatory responses of Holstein cows exposed to experimentally induced heat stress. Journal of Thermal Biology 66, 68-80.

De Palo, P., Tateo, A., Padalino, B., Zezza, F., Centoducati, P., 2005. Influence of temperature-humidity index on the preference of primiparous Holstein Friesians for different kinds of cubicle flooring. Italian Journal of Animal Science 4, 194-196.

Endres, M.I., Barberg, A.E., 2007. Behavior of dairy cows in an alternative bedded-pack housing system. Journal of Dairy Science 90, 4192-4200.

Herbut, P., Angrecka, S., 2018. Relationship between THI level and dairy cows' behaviour during summer period. Italian Journal of Animal Science 17, 226-233.

Maselyne, J., Pastell, M., Thomsen, P.T., Thorup, V.M., Hänninen, L., Vangeyte, J., Van Nuffel, A., Munksgaard, L., 2017. Daily lying time, motion index and step frequency in dairy cows change throughout lactation. Research Veterinary Science 110, 1-3.

National Research Council NRC, 1971. A Guide to Environmental Research on Animals. National Academy of Sciences, Washington, DC.

Provolo, G., Riva, E., 2009. One year study of lying and standing behaviour of dairy cows in a frestall barn in Italy. Journal of Agricultural Engineering 40, 27-34.

Steensels, M., Bahr, C., Berckmans, D., Halachmi, I., Antler, A., Maltz, E., 2012. Lying patterns of high producing healthy dairy cows after calving in commercial herds as affected by age, environmental conditions and production. Applied Animal Behaviour Science 136, 88-95.

Toušová, R., Ducháček, J., Stádník, L., Ptáček, M., Pokorná, S., 2017. Influence of Temperature-Humidity Relations During Years on Milk Production and Quality. Acta universitatis agriculturae et silviculturae mendelianae brunensis 65, 211-218.

West, J., Mullinix, B., Bernard, J., 2003. Effects of hot, humid weather on milk temperature, dry matter intake, and milk yield of lactating dairy cows. Journal of Dairy Science 86, 232-242.

Zähner, M., Schrader, L., Hauser, R., Keck, M., Langhans, W., Wechsler, B., 2004. The influence of climatic conditions on physiological and behavioural parameters in dairy cows kept in open stables. Animal Science 78, 139-147.

Zimbelman, R., Collier, R., 2011. Feeding strategies for high-producing dairy cows during periods of elevated heat and humidity, Proceedings of the 20th Annual Tri-State Dairy Nutrition Conference, Grand Wayne Center, Fort Wayne, Indiana, USA, 19-20 April, 2011. Ohio State University, pp. 111-126. 


\title{
Animal Welfare Indicators in Growing and Fattening Pigs With Different Environmental Conditions
}

\author{
Vasco Fitas da Cruz ${ }^{1}$, Rui Charneca ${ }^{2}$, Teresa Morgado ${ }^{3}$, Catarina Martins ${ }^{4}$ \\ ${ }^{1}$ ICAAM, Department of Rural Engineering, University of Évora. vfc@uevora.pt \\ ${ }^{2}$ ICAAM, Department of Veterinary Medicine, University of Évora, rmcc@uevora.pt \\ ${ }^{3}$ ICAAM, Department of Rural Engineering, University of Évora. tsmorgado@uevora.pt \\ ${ }^{4}$ Department of Rural Engineering, University of Évora. m37287@alunos.uevora.pt
}

\begin{abstract}
The application of different technological innovations in the intensive systems of pig production has generated some problems related to health and animal welfare in modern facilities. To measure animal welfare is necessary to use a set of indicators (behavioural, physiological, productive and sanitary) capable of expressing the animals' adaptability to the environment. Temperature is one of the main components of the environment, since it influences the physiology, behaviour and productivity of the pigs. The aim of this study is to verify the adaptive evolution to different environmental conditions (winter, thermoneutrality and summer) in growing and fattening pigs through physiological, behavioural and productive indicators. Seven females with initial weight of $45 \mathrm{~kg}$ were analysed throughout this test. The animals were housed in a room equipped with an environmental control system. The area per animal was $1.5 \mathrm{~m}^{2}$. The environmental data collected were temperature, relative humidity and wind speed. The physiological parameters measured were body surface temperature, rectal temperature and salivary $\alpha$ amylase. The feed intake was monitored through an individual feed machine equipped with animal weight scale. In order to monitor the behaviour of animals, video cameras and microphones were installed. The final weight of the animals was about $95 \mathrm{~kg}$. The daily food intake and mean daily gain of live weight were $2.70 \mathrm{~kg} \mathrm{day}^{-1}$ and $0.611 \mathrm{~kg} \mathrm{~kg}^{-1} ; 2.51 \mathrm{~kg} \mathrm{day}^{-1}$ and $0.947 \mathrm{~kg} \mathrm{~kg}^{-1}$ and $2.17 \mathrm{~kg} \mathrm{day}^{-1}$ and $0.526 \mathrm{~kg} \mathrm{~kg}^{-1}$, respectively in winter, thermoneutrality and summer. The corresponding values of body surface temperature were $25.4{ }^{\circ} \mathrm{C} ; 29.1{ }^{\circ} \mathrm{C}$ and $34.0{ }^{\circ} \mathrm{C}$ and the concentration of salivary $\alpha$-amylase were, on average, $0.33 \mathrm{U} / \mathrm{ml} ; 0.13 \mathrm{U} / \mathrm{ml}$ and $0.25 \mathrm{U} / \mathrm{ml}$, respectively in winter, thermoneutrality and summer.
\end{abstract}

Key words: Smart-Farming, Animal Welfare, Real Time, Environmental Control, Pigs.

\section{Introduction}

The intensification of production has generated some problems related to animal health and welfare in modern facilities. Pig farms, being a highly specialized animal husbandry, have some of these problems. On the other hand, with the population increase, a greater production of food is required, at increasingly reduced costs, where consumers demand the quality of the products. Alongside with product quality, consumers are increasingly concerned about animal welfare issues.

Due to the high demand for more products and higher quality, it becomes obvious that the verification and maintenance of animal welfare can not only be done by the employees of the farms. Early detection and real-time monitoring of normal behaviours (feed intake behaviour) and abnormal behaviour (aggressions) reduces animal production costs, limits the incidence of diseases and is capable of reducing mortality (Nasirahmadi et al., 2017a).

Pigs in intensive systems have to cope with long-term and intense short-term stressful stimuli that affect their welfare. High levels of stress and poor welfare have negatives effects in pig performance (Smulders et al., 2006). Throughout the stages of growth and maturity, one of the most important factors affecting welfare is the environment which animals are maintained. The environmental temperature has direct effects on pig behaviour (Nasirahmadi et al., 2015) and has a negative effect in their productivity (Banhazi et al., 2009; Vieira et al., 2010). When environmental temperature changes pigs adapt their behaviours to maintain body temperature (Nasirahmadi et al., 2017b). Feed intake and gain weight are also affect by the temperature (Vieira et al., 2010).

In order to measured animal welfare it is necessary to use a set of indicators (behavioural, physiological, productive and sanitary) capable of expressing the animals' adaptability to the environment provided. There is no a "gold standard" procedure to determine with accuracy the degree of animal welfare and the level of stress of an animal. Methodologies frequently used to quantify stress in animals include the direct behavioural observations or automated behaviour recognition video analysis (Ott et al., 2014; Nilsson et al., 2015) and biomarkers that can reflect the pathophysiological responses to stress (Ayala et al., 2012 and Escribano et al., 2015 cited by MartinezMiró, 2016). Avoiding a threat, facing up to it or hiding from it can be described as normal behaviour, whereas stereotypes are considered as abnormal behaviours that can appear after a stress (Squires et al., 2003 cited by Martinez-Miro, 2016).

In addition we can measure the animal stress with $\alpha$-amylase present in the saliva (Fuentes et al., 2011). Salivary alpha-amylase levels respond to physiological stressors. This it is measured in saliva and its activity is correlated with plasma catecholamine concentrations, being a marker of the sympathetic-adrenal-medullary axis 
activation (DeCaro, 2008; Nater et al., 2009). The use of saliva as sample for stress evaluation can be obtained by non-invasive procedures that do not produce additional stress or harm to the animals. In addition, saliva sampling procedures are very simple to obtain and can be taken by personnel with minimum training (Gutierrez et al., 2009 cited by Fuentes et al., 2011).

Another way to evaluate the animals' responses to the thermal environment is by observing the temperatures, such as body temperature (Vieira et al., 2010) and surface temperature. This measurement guides the determination of the balance between gain and heat loss of the body, which is often used as an adaptability index (Mota, 1997 cited by Vieira et al., 2010).

The aim of this study is to verify the adaptive evolution to different environmental conditions (winter, thermoneutrality and summer) in growing and fattening pigs through physiological, behavioural and productive indicators.

\section{Materials and Methods}

Animals, Housing and Equipment

Seven crossbred growing gilts were used for the present study. All animals were randomly selected from SIAS commercial farm in Santiago do Cacém and transfer to the experimental farm of Mitra in Évora. The gilts had a average body weight of $45 \pm 0.5 \mathrm{~kg}$, they were housed in a room equipped with an environmental control system with area per animal of $1,5 \mathrm{~m}^{2}$.

At the entrance, all gilts were identified with an RFID ear tag. The animals were adapted for a period of 15 days with ad libitum food with a standard commercial diet with and have free access to water. During this period, the gilts were handle by our staff and trained to be accustomed to saliva sampling. During these days the environmental control system was set to thermoneutrality, the temperature was $18 \pm 2^{\circ} \mathrm{C}$ and relative humidity was $60 \%$.

The room was equipped with sensors of temperature $(\mathrm{T})$, relative humidity $(\mathrm{RH})$ and wind speed (Ws). Cameras and microphones were also installed. The animals were fed with an individual automatic feed machine (schauer compident MLP II) equipped with a scale for the food and other for the animals. The machine was provided with an electronic identification system that was activated by the RFID ear tag. The feeding machine was connected to a computer to record and save the data (feed consumption and animal weight).

\section{Experimental Design}

The environmental control system was capable of simulate three different environmental conditions: winter (W) - cold stress, thermoneutrality (TN) and summer (S) - heat stress. The control system also allows to make a variation of the temperature and the humidity. We set up the control system for winter with $\mathrm{T}: 10 \pm 2{ }^{\circ} \mathrm{C}, \mathrm{HR}: 80 \%$; thermoneutrality with $\mathrm{T}: 17 \pm 2^{\circ} \mathrm{C}$, HR: $60 \%$ and in summer conditions we define $\mathrm{T}: 30 \pm 2^{\circ} \mathrm{C}, \mathrm{HR}: 50 \%$. The experimental period of each condition was $13 \mathrm{~d}$.

\section{Samples}

We measure surface temperature, body temperature and collect saliva. All the samples were collected 2 times by condition at $9 \mathrm{am}$. The first data collection was made $4 \mathrm{~d}$ after the change of the conditions; the other one was made before the change for the next condition. Surface temperature (sT) was measure with an IV thermometer in the neck, this measure was taken very quickly, approximately $5 \mathrm{~s}$ per animal. Body temperature (bT) was measure in rectal area with a digital thermometer, each measure take $1 \mathrm{~min}$. Saliva was collected using commercial salivette tubes with cotton. Each gilt was allowed to chew 2 a 3mints in the cotton. After that the samples were refrigerate. The tubes were centrifuged at $5000 \mathrm{rpm}$ for $5 \mathrm{~min}$. The analyses were made following the protocol by Salimetrics salivary a-amylase kinetic enzyme assay kit.

\section{Results and Discussion}

As expected, depending on the environmental condition, we observed a change in feed intake (FI) and in average daily gain (ADG) (Table 1).

Table 1. Mean values of feed intake and average daily gain (ADG), for the three environmental conditions.

\begin{tabular}{ccc}
\hline Environmental condition & Feed Intake $\left(\mathrm{kg}^{-1} \mathrm{~d}^{-1}\right.$ & ADG $\left(\mathrm{kg} . \mathrm{d}^{-1}\right)$ \\
\hline W & 2.70 & 0.611 \\
TN & 2.51 & 0.947 \\
$\mathrm{~S}$ & 2.17 & 0.526 \\
\hline
\end{tabular}


In summer conditions (heat stress) feed intake decreases (Pearce et al. 2013) and also the mean of average daily gain, these results are also reported (Collin et al., 2001; Banhazi et al., 2009; Kiefer et al., 2009). In W conditions we observed and increase in feed intake, as reported by Quiniou et al. (2000), although there was a decrease in average daily gain. These results are consistent with the reported by Li and Patience (2017) since the animals to increase the heat production consumed more food. Regarding body temperature, rectal temperature and surface temperature were measured, and the results obtained are shown in table 2.

Table 2. Mean temperatures of animals, body temperature and surface temperature, for the 3 environmental conditions.

\begin{tabular}{ccc}
\hline Environmental condition & $\mathrm{bT}\left({ }^{\circ} \mathrm{C}\right)$ & $\mathrm{sT}\left({ }^{\circ} \mathrm{C}\right)$ \\
\hline $\mathrm{W}$ & 38.4 & 25.4 \\
$\mathrm{TN}$ & 38.3 & 29.1 \\
$\mathrm{~S}$ & 39.4 & 34.0 \\
\hline
\end{tabular}

Surface temperature measurement is a quick and practical method of verifying that animals are outside the comfort zone (Mostaço, 2014). This indicator is more variable and more influenced by the environment (Manno et al., 2009), as is observable in the results obtained in this test, where the sT have greater variation between the different simulated environmental conditions. Huynh et al. (2005); Manno et al. (2006) and Kiefer et al. (2009) observed that when the ambient temperature increases the surface temperature also increases.

Regarding the rectal temperature in pigs is, on average, $38.8^{\circ} \mathrm{C}$ (Cunningham, 1993). The values obtained in this study fall within this temperature, however, there is a slight increase during the summer simulation. This increase was observed with increasing ambient temperature, this increase was also reported by Huynh et al. (2005) and Kiefer et al. (2009). The increase in body temperature, as a rule, means that the animal is storing heat, due to the failure of thermoregulation mechanisms (Ferreira, 2002, cited by Rodrigues, 2010). That is, when the ambient temperature rises above the capacity of physiological readjustment, the retained body heat is capable of altering the state of homeothermia, being an increase in the rectal temperature, which becomes more intense with the degree of deviation of the temperature of thermal comfort.

When the ambient temperature does not correspond to comfort levels, pigs present specific behavioral characteristics and are able to change their behavior to adapt to the environment that surrounds them (Quiniou et al., 2000). Indeed, temperature is the main parameter affecting pigs lying behavior (Nasirahmadi et al., 2015). These were the results observed by our team, with the animals gathering when the ambient temperature was below the thermoneutral temperature (winter situation), and the spacing when the ambient temperature was above the thermoneutral temperature (summer situation), which is in accordance with the bibliography consulted (Nasirahmadi et al., 2017a, b).
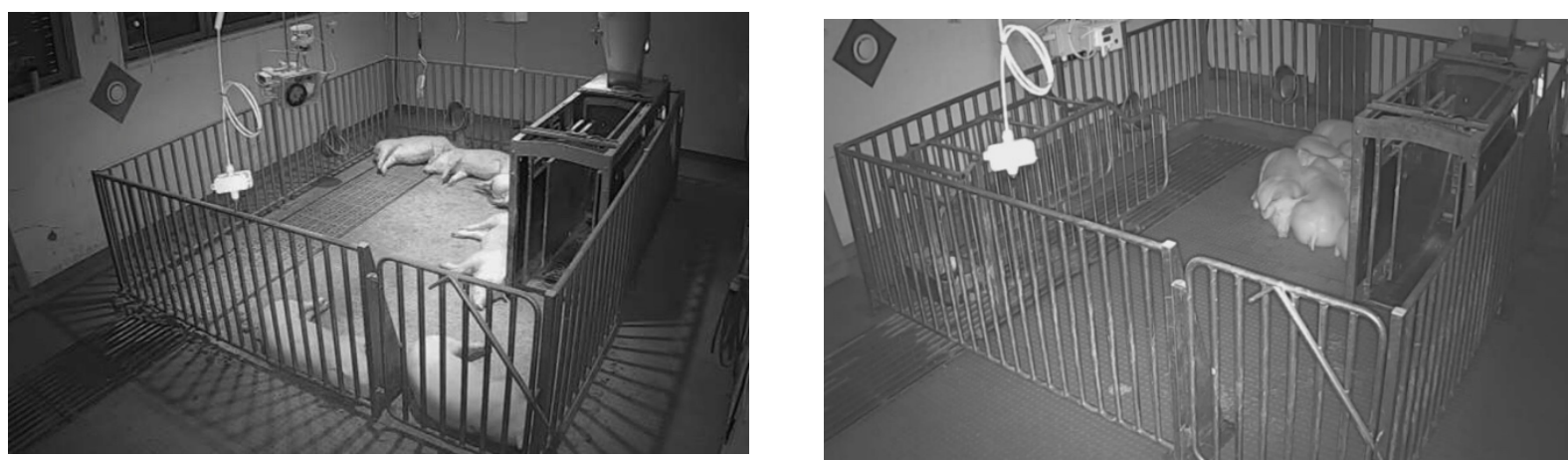

Figure 2. In S conditions (left) the gilts prefer to rest apart, without contact between them. In W conditions (right) the gilts choose to huddle.

Concerning to the $\alpha$-amylase samples (table 3), we found that the values for $\alpha$-amylase are greater in the $\mathrm{W}$ and in the $\mathrm{S}$.

Table 3. Mean values for $\alpha$-amylase activity.

\begin{tabular}{cc}
\hline Environmental condition & $\alpha$-amylase (U/ml) \\
\hline W & 0.3252 \\
TN & 0.1382 \\
S & 0.2544 \\
\hline
\end{tabular}


We didn't find any bibliography that relate thermal stress with $\alpha$-amylase activity, however we found some studies that relate $\alpha$-amylase activity with psychological stress and physical stress. These studies report that $\alpha$ amylase increased with thermal stress (DeCaro, 2008; Nater et al., 2009; Fuentes et al., 2011) and these results agree with the results in our study.

\section{Conclusions}

With this study we can conclude that these welfare indicators can give us the information about the animal welfare. Although we collect some of the data manually, there is technologies that can gather and analyze the data, for example thermo cameras that gave the temperatures. Feed intake, ADG and sT changes with the environmental conditions. In bT changes only occurred in summer conditions, this may suggest that the animal had more difficulties in adapting to the hot temperatures. In relation to alpha amylase, the values increase with thermal stress, however further studies should be done in order to clarify $\alpha$-amylase activity caused by thermal stress.

\section{References}

Banhazi, T. M., Aarnink, A., Thuy, H., Pedersen, S., Hartung, J., Mullan, B., Payne, H., and Berckmans, D. (2009). Review of the Consequences and Control of High Air Temperatures in Intensive Livestock Buildings. Australian Journal of Multi-Disciplinary Engineering, 7(1), 63-78.

Collin, A., van Milgen, J., Dubois, S., and Noblet, J. (2001). Effect of high temperature on feeding behaviour and heat production in group-housed young pigs. British Journal of Nutrition, 86, 63.

Cunningham, J. G. (1993). Tratado de Fisiologia Veterinária. Guanabara Koogan S.A.

DeCaro, J. A. (2008). Methodological considerations in the use of salivary alpha-amylase as a stress marker in field research. American Journal of Human Biology, 20, 617-619.

Deen, J. (2010). Pigs : Behavior and Welfare Assessment, 731-739.

Fuentes, M., Tecles, F., Gutiérrez, A., Otal, J., Martínez-Subiela, S., and Cerón, J. (2011). Validation of an automated method for salivary alpha-amylase measurements in pigs (Sus scrofa domesticus ) and its application as a stress biomarker, 287, 282-287.

Huynh, T. T. T., Aarnink, J., Verstegen, M. W., Gerrits, W. J. J., Heetkamp, M. J. W., Kemp, B., and Canh, T. T. (2005). Effects of increasing temperatures on physiological changes in pigs at different relative humidities. Journal of Animal Science, (83), 1385-1396.

Kiefer, C., Meignen, B. C. G., Sanches, J. F., and Carrijo, A. S. (2009). Resposta de suínos em crescimento mantidos em diferentes temperaturas. Archivos de Zootecnia, 58(221), 55-64.

Li, Q., and Patience, J. F. (2017). Factors involved in the regulation of feed and energy intake of pigs. Animal Feed Science and Technology, 233, 22-33.

Manno, M. C., Oliveira, R. F. M. De, Donzele, J. L., Oliveira, W. P. De, Vaz, R. G. M. V., Silva, B. A. N., Saraiva, E. P. and Lima, K. R. de S. (2006). Efeitos da temperatura ambiente sobre o desempenho de suínos dos 30 ao $60 \mathrm{~kg}$. Revista Brasileira de Zootecnia, 35(2), 471-477.

Martínez-Miró, S., Tecles, F., Ramón, M., Escribano, D., Hernández, F., Madrid, J., Orengo, J., MartínezSubiela, S., Manteca, X. and Cerón, J.J. (2016). Causes, consequences and biomarkers of stress in swine: an update. BMC Veterinary Research. 12, 171.

Mostaço, G.M. (2014). Determinação da temperatura retal e frequência respiratória de suínos em fase de creche por meio da temperatura da superfície corporal em câmara climática. MS dissertation in Engenharia de Sistemas Agrícolas. Escola Superior de Agricultura "Luiz de Queiroz", University of São Paulo, Piracicaba, Brazil.

Nasirahmadi, A., Richter, U., Hensel, O., Edwards, S. and Sturm, B. (2015). Using machine vision for investigation of changes in pig group lying patterns. Computers and Electronics in Agriculture 119, 184-190.

Nasirahmadi, A., Edwards, S.A. and Sturm, B. (2017a). Implementation of machine vision for detecting behaviour of cattle and pigs. Livestock Science, 202, 25-38.

Nasirahmadi, A., Hensel, O., Edwards S.A. and Sturm, B. (2017b). A new approach for categorizing pig lying behaviour based on a Delaunay triangulation method. Animal, 11, 131-139

Nater, U. M., and Rohleder, N. (2009). Salivary alpha-amylase as a non-invasive biomarker for the sympathetic nervous system : Current state of research. Psychoneuroendocrinology, 34, 486-496.

Nilsson, M., Herlin, A. H., Ardö, H., Guzhva, O., Aström, K., and Bergsten, C. (2015). Development of automatic surveillance of animal behaviour and welfare using image analysis and machine learned segmentation technique. Animal, 1-7.

Ott, S., Moons, C. P. H., Kashiha, M. A., Bahr, C., Tuyttens, F. A. M., Berckmans, D., and Niewold, T. A. (2014). Automated video analysis of pig activity at pen level highly correlates to human observations of behavioural activities. Livestock Science, 160, 132-137. 
Pearce, S., Gabler, N., Ross, J. W., Escobar, J., Patience, J. F., Rhoads, R. P., and Baumgard, L. H. (2013). The effects of heat stress and plane of nutrition on metabolism in growing pigs. Journal of Animal Science, 91, 2108-2118.

Quiniou, N., Dubois, S., and Noblet, J. (2000). Voluntary feed intake and feeding behaviour of group-housed growing pigs are affected by ambient temperature and body. Livestock Production Science, 63, 245-253.

Rodrigues, N. E. B., Zangeronimo, M. G., and Fialho, E. T. (2010). Adaptações fisiológicas de suínos sob stress térmico. Revista Eletrónica Nutritime, 7(110), 1197-1211.

Smulders, D., Verbeke, G., Mormède, P., and Geers, R. (2006). Validation of a behavioral observation tool to assess pig welfare. Physiology and Behavior, 89, 438-447.

Vieira, R. de F. N., Silva, K. O., Mello, S. P., and Martins, J. R. (2010). Índices de conforto na avaliação do bem estar animal de matrizes suínas em diferentes sistemas de criação. Nucleus Animalium, 2(1), 1-8. 


\title{
Development of a Test Stand for the Testing of Alternative Manure Removal Methods in Slurry Channels
}

\author{
Bastian Kolb a *, Eva Gallmann ${ }^{\text {a }}$ \\ a Institute of Agricultural Engineering, University of Hohenheim, 70599 Stuttgart, Germany \\ * Corresponding author. Email: bastian.kolb@uni-hohenheim.de
}

\begin{abstract}
Animal welfare is becoming increasingly important in animal husbandry which leads to an increased use of organic material to counteract tail-biting. However liquid manure systems are not designed for increased dry matter and fibre contents. As a result, there are dense, thick and sticky floating layers concentrated under mangers and feeders. These conditions require labour intensive stirring, rinsing and shovelling to remove the remains of solid matter. To remedy these problems, a test stand is being constructed at the University of Hohenheim. Made of transparent acrylic glass and polyvinyl chloride, the test stand allows the simulation of the complete manure removal process and gives an insight into possible bottlenecks, blockage points and residue deposits. A stainless steel frame allows the secure attachment of various newly developed and retrofitted stirring, pulling, chopping, scraping and rinsing modules. Together with variants of basin designs, lock compartments, flushing nozzle positions and flow rates, a large number of combinations can be tested. To ensure the comparability of the test runs despite the heterogeneity of pig manure a substitute fluid will be developed to simulate the rheological properties of a fibrous pseudo plastic suspension. Organic fibre material, roughage pellets, sand and water mixed with natural and synthetic thickening agents serve as the basis for the test medium. Preliminary tests will be conducted to explore the limitations of the system and the substitute fluid in terms of the dry matter contents, fibre lengths and viscosity. Purging intervals and different mixing ratios will be validated to obtain a baseline for the start of the main test series of mechanical manure removal solutions.
\end{abstract}

Keywords: Pig housing, floating layers, rheology, model fluid, animal welfare, organic enrichment material

\section{Introduction}

Animal welfare is becoming increasingly important in the public mind. Together with a general ban on tail docking in pig farming there is an increasing pressure on farmers to limit unwanted behaviours such as tail and ear biting. Changes in animal welfare and behaviour management are required. A more animal-friendly environment can be achieved through increased space, comfort lying areas, different climatic zones, access to open air, wallows or an offering of different materials to manipulate. Destructible and edible organic materials such as straw, hay and pellets in mangers or for bedding is desired - or demanded - by consumers and animal welfare labels alike. However, manure removal is still based on a liquid manure system without any offerings of organic materials in $95 \%$ of all pigsties for fattening pigs over a size of 2,000 animals in Germany (Jungbluth et al., 2017). These liquid manure channels are not designed for the large amounts of organic fibre material. Dense and thick floating layers may clog the outlets or remain in the channels during the manure removal process at the end of a fattening period. Over several fattening periods heavy sinking layers develop which must be removed manually. Additional flushing helps, but it cannot dissolve floating layers. Even with auxiliary stirring, the removal process is tedious and characterized by regular interruptions and the need for constant attention by the operator.

The purpose of this project is therefore to develop systems that completely and reliably remove mineral and organic residues from liquid manure systems in pig farms. A test stand is designed and constructed for this purpose at the University of Hohenheim. This allows the testing of various manure removal techniques in a controlled environment. Furthermore, a model fluid is being developed for the use in the test stand. Solutions which improve the manure removal process will be implemented on a pilot scale under practical conditions in long-term test trials.

\section{Materials and Methods}

The development of the test stand was divided into three phases: Sampling and analysis of pig slurry from manure channels and preliminary tanks for the determination of their physical properties, development of a model fluid based on the findings gained and the design and construction of the test stand. Sampling took place at the federal pig research and training centre "Bildungs- und Wissenszentrum LSZ Boxberg" (LSZ Boxberg) and the rheological analysis of the slurry partly at the University of Hohenheim and the laboratories of Anton Paar in Ostfildern-Scharnhausen, Germany. The test stand was completely designed and put into operation in the workshops at the Institute of Agricultural Engineering in Hohenheim. 


\section{Conception of the Test Stand}

The test stand was created according to the dimensions of the manure channels of the LSZ Boxberg. It is a classic liquid manure channel system. The manure is discharged solely by gravity through a pipe system into a central preliminary tank (Figure 1).

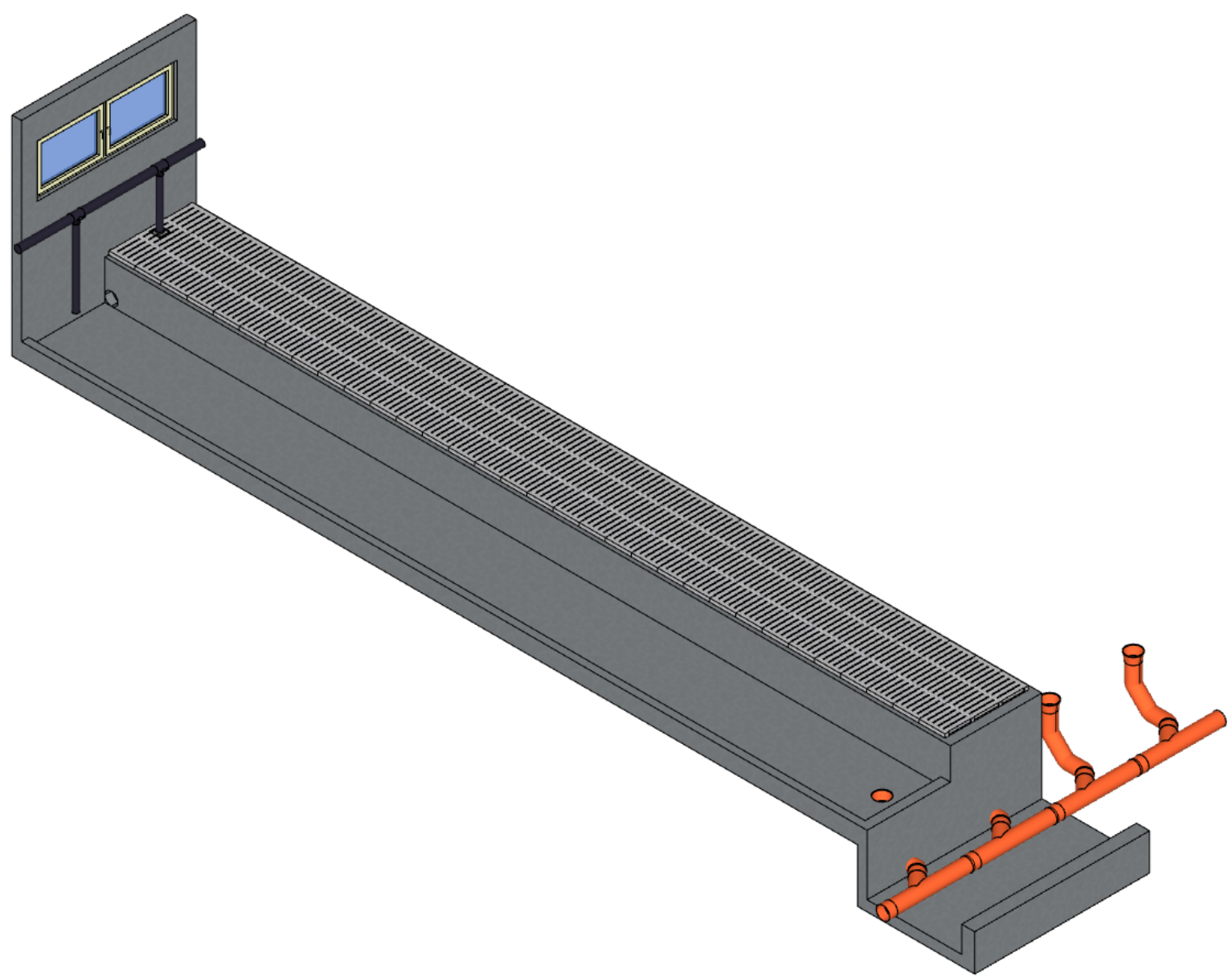

Figure 19. Model of the slurry channels at the Landesanstalt für Schweinezucht Boxberg. Consisting of two combined channels, flushing pipes (top left), discharge outlet and manifold (bottom right). Made in SketchUp (Trimble Navigation Ltd.).

The removal process is supported by a rotary pump for flushing (Eisele DK 22V - 1040, Franz Eisele u. Söhne GmbH und Co. KG, Hauptstraße 4, 72488 Sigmaringen-Laiz, Germany). It allows the use of homogenised slurry from the preliminary tank to flush the channels. The slurry channel used as a template for the test stand has a length of $18.84 \mathrm{~m}$, a width of $1.2 \mathrm{~m}$ and a height of $0.57 \mathrm{~m}$. The pipe diameters are $250 \mathrm{~mm}$ (DN 250) for the outlet and $110 \mathrm{~mm}$ (DN 110) for the flushing pipes. There are two designs of flushing pipes implemented in the pigsty; underground piping with an inlet entering from below through the concrete floors of the channels or an above ground distribution through a central piping system with an inlet through the slatted concrete floorplates (this version is seen in Figure 1). All parallel channels share a manifold for the slurry, converging them into a main pipe leading into the preliminary tank. The height of fall between channel bottom and manifold is $1 \mathrm{~m}$. After two $45^{\circ}$ bends the outlet merges into the manifold through a $45^{\circ} \mathrm{y}$-fitting. The manure removal process takes place in series. Two channels connected in pairs are emptied one after the other. The flushing is constant until the manure removal process is finished. Channels are emptied at the end of a fattening period. Intermediate flushing is not intended.

The priority for the conception of the test stand was the specification of a minimal necessary design. All components and systems had to be assessed for their influencing factor during manure removal procedures. The main points were the number of simulated channels, the design of the piping system, the scale of the test stand and the required pumping power. Since this is a pure gravity discharge, the pipe outlet must be above the upper edge of the collection container used. As the center of gravity of the test stand is high compared to its width and due to the moving liquid in the channel, a structurally stable substructure has to be constructed. Further relevant properties should be: water resistance, mobility, transparent basins and piping for optical evaluation of manure 
removal efficiency, possibilities for the attachment of mechanical manure removal modules at the edge of the basin and a separation of organic material and model fluid from the collection tank. The constructed final test stand should meet a majority of these factors.

\section{Development of the Model Fluid}

Pig manure at rest forms four different layers: A sinking layer - mostly sand and other heavier mineral substances -, floating layers - a mixture of organic fibrous material, excrements and feed -, as well as the slurry, consisting of faeces and urine. Due to the low fill level of manure in the channels (about $50 \mathrm{~cm}$ at the end of the fattening period), no distinction is made between thick and thin slurry layers.

For the simulation of the sinking layer, pure sand will be used in the first generation of model fluids. It is necessary to check which particle size and roughness is closest to the sand in the manure channels. The composition of the floating layer varies based on the enrichment and bedding material used. Accurate assessments of the proportions of eaten, processed (chewed, plucked) and unimpaired organic material are not available. For the simulation of the floating layer, the original organic material from the pigsties at the LSZ Boxberg can be used. However, it is important to consider whether fibrous material such as straw and hay may need to be chopped shorter to avoid clogging the smaller diameter pipes on the test stand too early. Furthermore, a method to simulate the sticky consistency of faeces and food residues in the floating layers needs to be developed.

Pig manure is a non-newtonian pseudoplastic fluid. Compared to newtonian fluids such as water or oil, the viscosity does not increase linearly at a higher shear rate. Slurry becomes thinner with increased flow or agitation. For this reason, water alone cannot be used as a substitute for pig manure. For a better replication, natural or synthetic thickeners should be added. The thickeners tested were locust bean gum powder (E410), guar gum powder (E412), acacia gum powder (E414), xanthan (E415) and carboxymethyl cellulose (CMC, E466). Samples were analysed on their rheological properties via a rotational viscometer (Physica RHEOLAB MC1, Anton Paar $\mathrm{GmbH}$, Anton-Paar-Straße 20, A-8054 Graz, Austria). Slurry samples were taken at the LSZ Boxberg from the preliminary tank, the channels under the lying area and the channels under the faeces area. The experiments were carried out with cooled samples (temperature $10^{\circ} \mathrm{C}$ or $283.15 \mathrm{~K}$ ). Additional trials with different temperature ranges are planned. The limits of the shear rate were set to be between 0 and $1001 / \mathrm{s} .10$ experiments with 3 repetitions per thickener were performed, ranging from 1 to $10 \mathrm{~g} / \mathrm{l}$ in $1 \mathrm{~g}$ sections. Slurry was tested with up to 5 repetitions due to potential issues of homogeneity.

For an additional classification of the analysed pig slurry, a drying and incineration test has been performed. All samples were dried at $105^{\circ} \mathrm{C}$ to constant mass and then incinerated at $550{ }^{\circ} \mathrm{C}$ to constant mass with 2 repetitions. The dry matter (DM) content, organic dry matter (ODM) content and ash content was calculated from the weighed masses.

\section{Results and Discussion}

\section{Test Stand}

\section{Basin and Piping System}

The dimensions of the test stand are based on the actual dimensions of the LSZ Boxberg channels. Since it is planned to use a substitute fluid for pig manure with different amounts of fibrous organic materials, the piping diameter is the limiting factor in the scaling. A reduced pipe diameter massively influences the flow characteristics due to wall friction. Increasing DM content of manure and the chopping length of straw and hay are especially substantial parameters. These factors cannot be avoided when working with models. As a compromise between a practical size of the test stand and adequate flow properties, a scale of 2:1 (real scale to model scale) was chosen for the experimental model. In addition, piping and fittings are available in the standard dimensions of $125 \mathrm{~mm}$ (half of DN 250). The dimensions of the basin would thus be reduced to a length of about $9 \mathrm{~m}$, a width of $0.6 \mathrm{~m}$ and a height of $0.3 \mathrm{~m}$. Since different fill levels of slurry and floating layers are to be tested, the wall height was increased to $0.4 \mathrm{~m}$. The length was reduced by an additional third to $6 \mathrm{~m}$. Observations during the manure removal operations at the LSZ Boxberg have shown, that the floating layers are not contiguous. They form thick and dense mattresses under the lying surfaces and feeding places with very thin floating layers in between. It was deliberately decided to focus on a single floating layer and a shorter channel. As long as the floating layer can be frayed and successfully flushed without a reduction in floating speed, all subsequent layers should undergo a similar sequence without blockage. Since the slurry flows faster than the floating layers move, various arrangements of floating layers can be simulated by changing the fill level of the model fluid and the flow rate of the flushing pump in subsequent experiments.

As a material for the slurry channel and the pipes, transparent acrylic glass and polyvinyl chloride (PVC) were chosen. This allows a complete view of the whole system during the manure removal process. The material thickness of the basin is $15 \mathrm{~mm}$. Since acrylic glass sheets are limited to a length of $3 \mathrm{~m}$, the basin consists of two glued parts. To relieve the stress on the adhesive area and to allow a stable and secure attachment to the 
substructure, the entire basin is harnessed in a stainless steel frame, fixated by felt-covered clamping screws. This makes it possible to compensate for temperature related expansions of the basin and a potential uneven footprint. In addition, various mechanical manure removal modules for the dissolution of floating layers can be attached to the frame without damaging the basin.

As shown in Figure 2, the manure pipe was rotated $90^{\circ}$ compared to the real piping system at the LSZ Boxberg and runs parallel under the basin. This arrangement should only have a minimal impact on the manure removal process but significantly reduces the footprint of the test stand. Plugging sleeves on both ends of the pipe allow direct access into the system should a complete blockage occur.
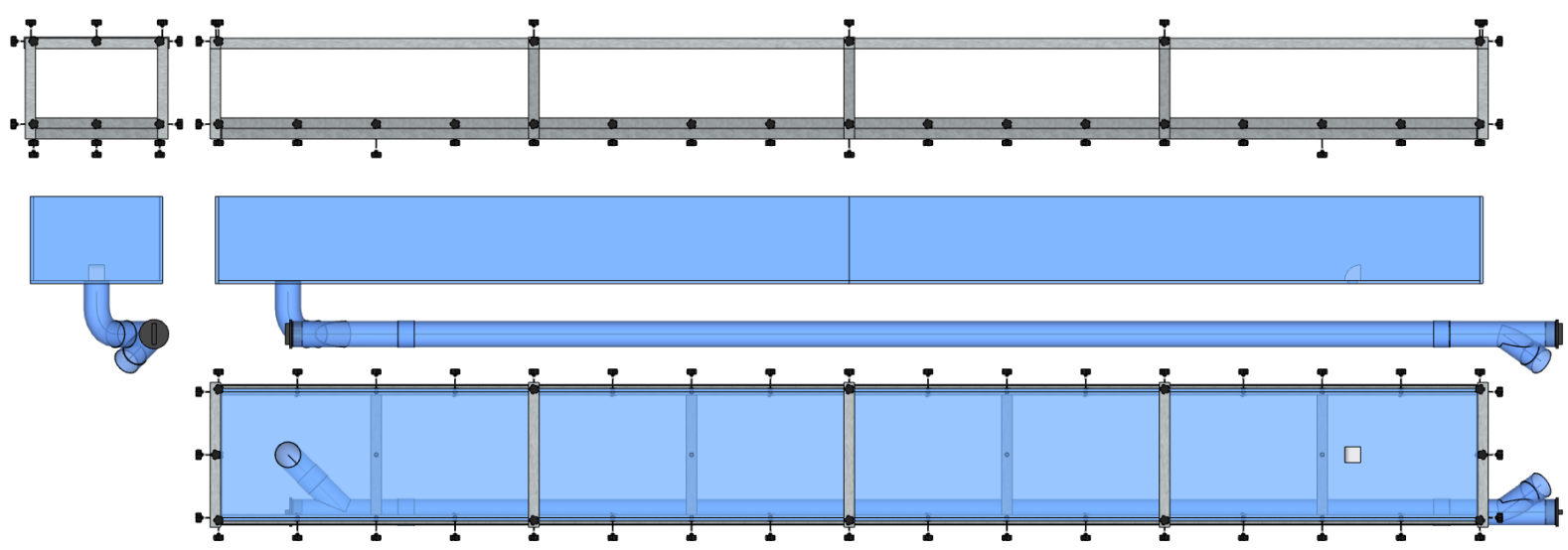

Figure 20. Basin made of transparent acrylic glass, piping made of polyvinyl chloride and the harnessing frame made of stainless steel. Basin is fixated by felt-covered clamping screws. Made in SketchUp (Trimble Navigation Ltd.).

\section{Substructure}

Like the basin frame, the entire load-bearing base is made of stainless steel. The height of the test stand must allow for manure removal by gravity. The top edge of the model fluid tank must be below the outlet fitting of the piping system. The high centre of gravity requires a wide stand. The basin is supported at three points. The substructure is modularly constructed. It consists of welded stainless steel square tubes with an edge length of 50 $\mathrm{mm}$ and a thickness of $3 \mathrm{~mm}$. The crossed bracing of stainless steel rope is connected to the supports at weld eyelets and allows stabilization without leeway. A stiffening against torsional forces is achieved by the connection to the basin frame from above and the horizontal bars at the bottom. The test stand has six heavy-duty castors for mobility. For better stability - and to compensate for the additional weight due to the model fluid - the tests stand will be jacked up on nine rubberized wooden blocks during the experiments. For this purpose, the test stand can be lifted with a fork-lift truck in the middle below the basin frame as well as with two pallet trucks from both ends below. The substructure also holds up the piping by means of pipe clamps. The construction in Figure 3 shows the final variant of the substructure. 

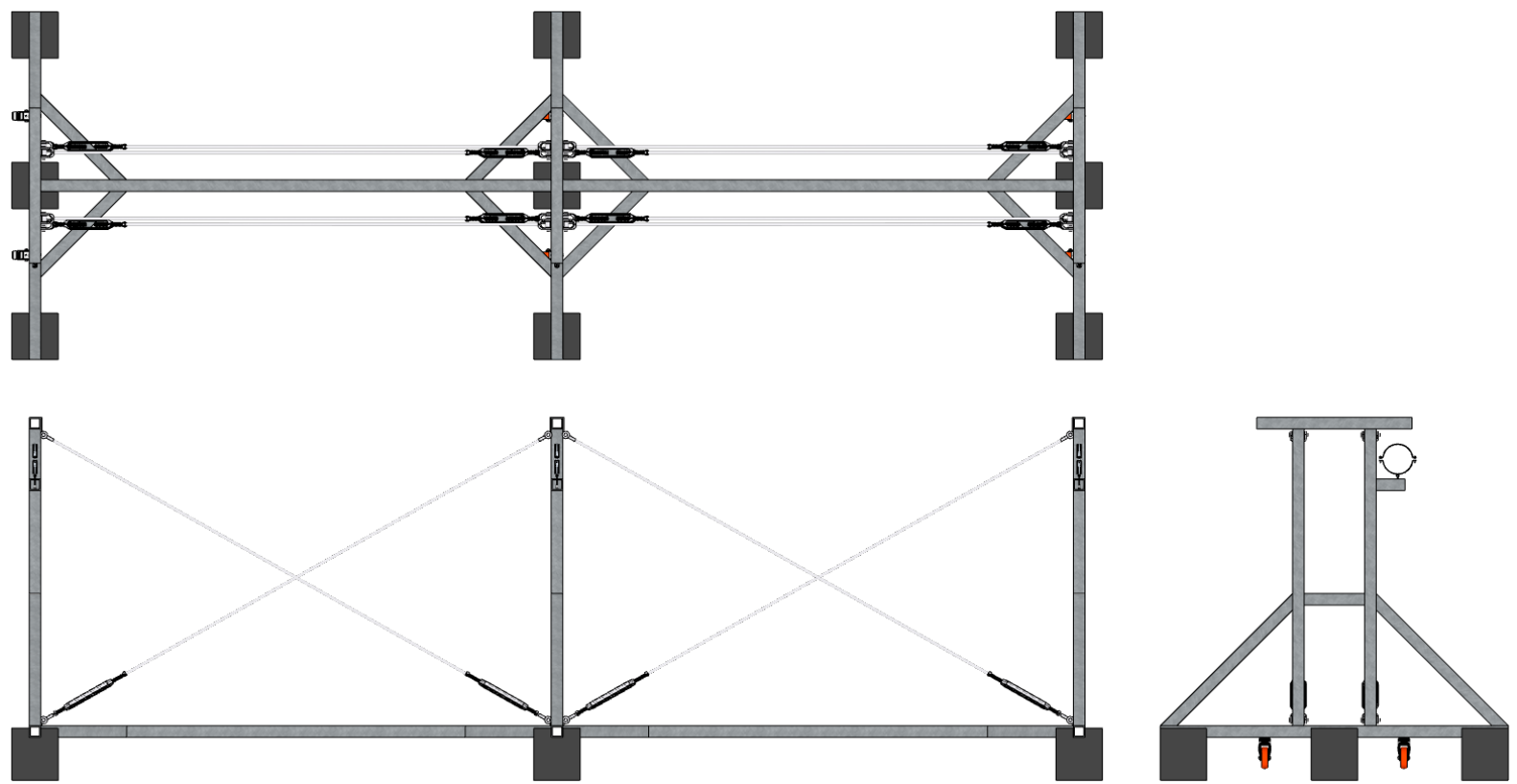

Figure 21. Substructure made of stainless steel square tubes. Fixated by crossed tension cables. Jacked up on nine rubberised wooden blocks while the test stand is in use. Made in SketchUp (Trimble Navigation Ltd.).

Tank, Pump and the complete Test Stand

As a tank for the test stand and the storage of the model fluid, a 10001 Intermediate Bulk Container (IBC) was selected. From a pig stock of 30 , an area of $33.15 \mathrm{~m}^{2}$, a fattening period of $120 \mathrm{~d}$ and an estimated manure volume of $0.5 \mathrm{~m}^{3}$ per fattening pig and fattening period results in a liquid fill level in the slurry channel of about $45 \mathrm{~cm}$. Converted to the 2:1 model scale it results in a maximum volume of about 9001 of model fluid in the basin at a fill level of $25 \mathrm{~cm}$. The remaining space in the IBC allows for a submersible pump to be built directly into the tank

(Figure

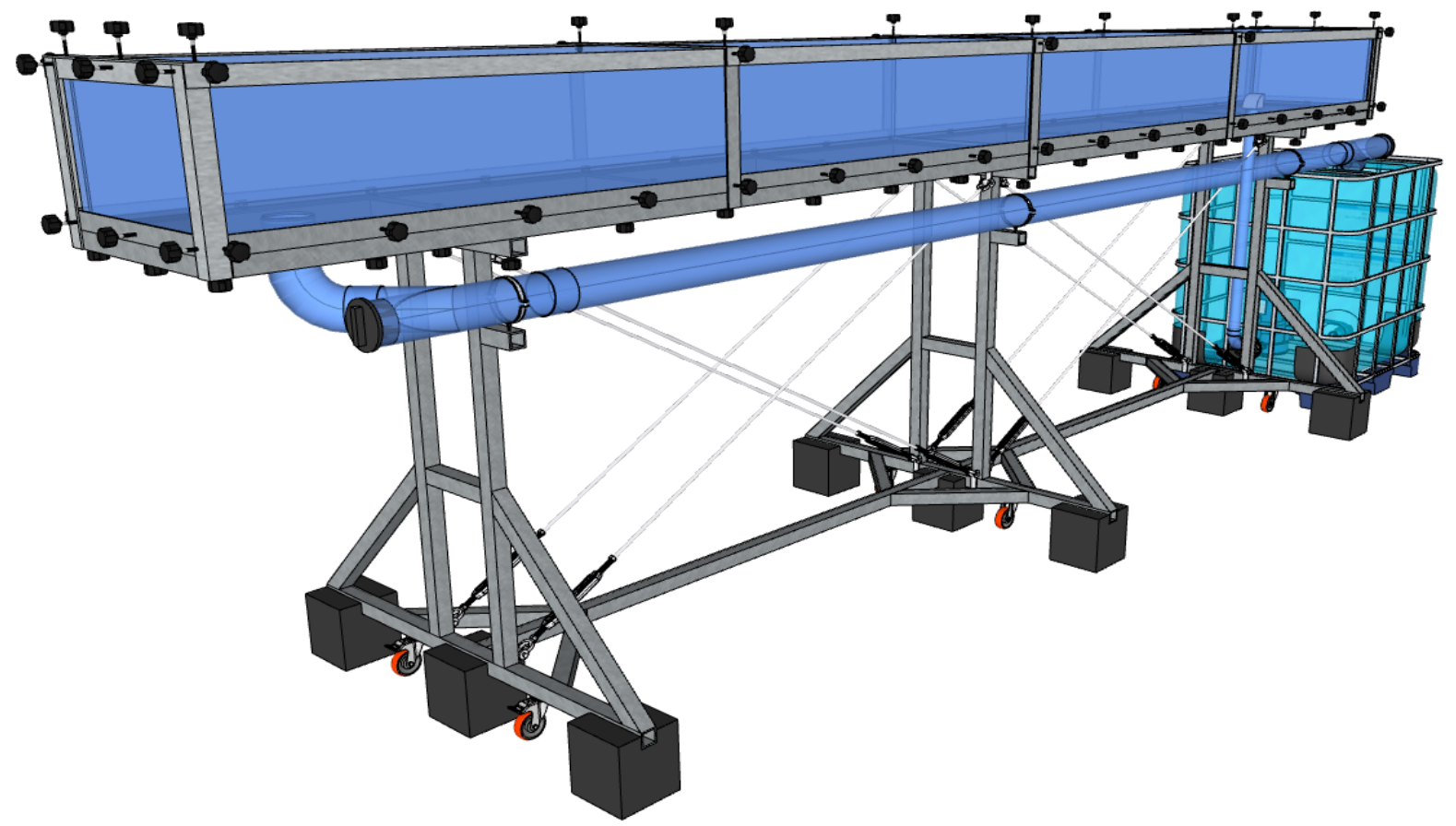

Figure 22. Finalised test stand with tank, pump and flushing pipe. Made in SketchUp (Trimble Navigation Ltd.).

The IBC was prepared and equipped with a sieve to allow the manual removal of organic materials from the system immediately after discharge. Since only minimal straw and hay are pumped back into the basin, a cheaper mud or pond pump can be used instead of a slurry pump. The pump capacity is limited to about 8 to $10 \mathrm{~m}^{3} / \mathrm{h}$, based on the slurry pump capacity of $66 \mathrm{~m}^{3} / \mathrm{h}$ at the LSZ Boxberg and an additional capacity for increased flushing 
volume. This corresponds to an 8 -fold reduction in flow rate compared to the slurry pump, and allows a theoretical replacement rate of the entire basin contents of 11 per hour. This number may be reduced with increasing DM and fibre content.

\section{Model Fluid}

In the drying and incineration experiments, a wide distribution of DM contents was found, depending on the location of the sampling. As can be seen in Table 1, the DM content varies between $0.95 \%$ in the preliminary tank and up to $20.38 \%$ below the faeces area. Samples were deliberately taken without homogenisation of the slurry to show the range of DM-contents before agitation or flushing in different locations.

Table 62. Dry matter, organic dry matter and ash content of slurry samples taken during the fattening period. Samples I to IV were taken under the lying area. Samples V to VIII were taken under the faeces area.

\begin{tabular}{lcccc}
\hline $\begin{array}{c}\text { Sampling } \\
\text { Location }\end{array}$ & $\begin{array}{c}\text { DM-Content } \\
{[\%]}\end{array}$ & $\begin{array}{c}\text { ODM-Content } \\
{[\%]}\end{array}$ & $\begin{array}{c}\text { Ash-Content } \\
{[\%]}\end{array}$ & $\begin{array}{c}\text { ODM per DM } \\
{[\%]}\end{array}$ \\
\hline Preliminary Tank & $00.95 \pm 0.04$ & $00.52 \pm 0.03$ & $00.43 \pm 0.02$ & 54.74 \\
Channel I & $03.50 \pm 0.00$ & $02.55 \pm 0.00$ & $00.95 \pm 0.00$ & 72.86 \\
Channel II & $04.95 \pm 0.16$ & $03.60 \pm 0.14$ & $01.36 \pm 0.02$ & 72.72 \\
Channel III & $05.50 \pm 0.05$ & $04.16 \pm 0.03$ & $01.35 \pm 0.02$ & 75.64 \\
Channel IV & $05.69 \pm 0.09$ & $03.84 \pm 0.10$ & $01.85 \pm 0.01$ & 67.49 \\
Channel V & $11.72 \pm 0.11$ & $08.96 \pm 0.10$ & $02.76 \pm 0.02$ & 76.45 \\
Channel VI & $17.08 \pm 0.00$ & $13.63 \pm 0.01$ & $03.45 \pm 0.00$ & 79.80 \\
Channel VII & $18.78 \pm 0.79$ & $08.13 \pm 2.53$ & $10.65 \pm 1.74$ & 43.29 \\
Channel VIII & $20.38 \pm 0.30$ & $08.40 \pm 0.19$ & $11.98 \pm 0.11$ & 41.22 \\
\hline
\end{tabular}

Such a broad spectrum cannot be replicated with a single model fluid. These differences can also be seen in the viscosity measurements (Figure 5). The diagram shows the results of the rheological analysis of a sample taken under the lying area (channel III) in comparison to a sample taken under the faeces area (channel V). The sample III with a DM-content of $5.5 \%$ shows a spread between replicates of up to 244.16 Pas - between 0.77 and $244.93 \mathrm{Pas}-$ at a shear rate of $33 \mathrm{1} / \mathrm{s}$. This shows a clear influence of particles, fibres and air bubbles on the measuring system when using low viscous slurry samples. While the slurry sample V showed the highest consistency of the first preliminary tests it is not representative for pig slurry in general. Samples taken at the LSZ Boxberg indicate an average of 3 to $7 \%$ in DM-content after homogenisation. The analysis of these samples is problematic however, as the high fibre content prevents the measurement in rotary viscometers. The use of a ball viscometer was deemed a failure due to long fibres enveloping the dragging ball in the slurry sample, changing the flow properties of the measuring system and rendering the experiment results invalid. Getting accurate data on the rheological properties is the biggest hurdle in the classification of pig slurry.

Ultimately these viscosity curves have to be substituted by means of a model fluid. Figure 6 shows the viscosity of the $\mathrm{CMC}$ thickener which came closest to the slurry sample $\mathrm{V}$ in rheological properties in these first trials. A mixture of $10 \mathrm{~g} / 1$ of CMC dissolved in water could be used as a substitute for a high viscous slurry. The ratio could probably be increased to 11 or $12 \mathrm{~g} / \mathrm{l}$ for a better correlation at lower shear rates. Choosing a matching model fluid concentration for the lower viscous slurry (sample III) is not possible based on the available data alone. More trials on low viscous slurry are needed. 


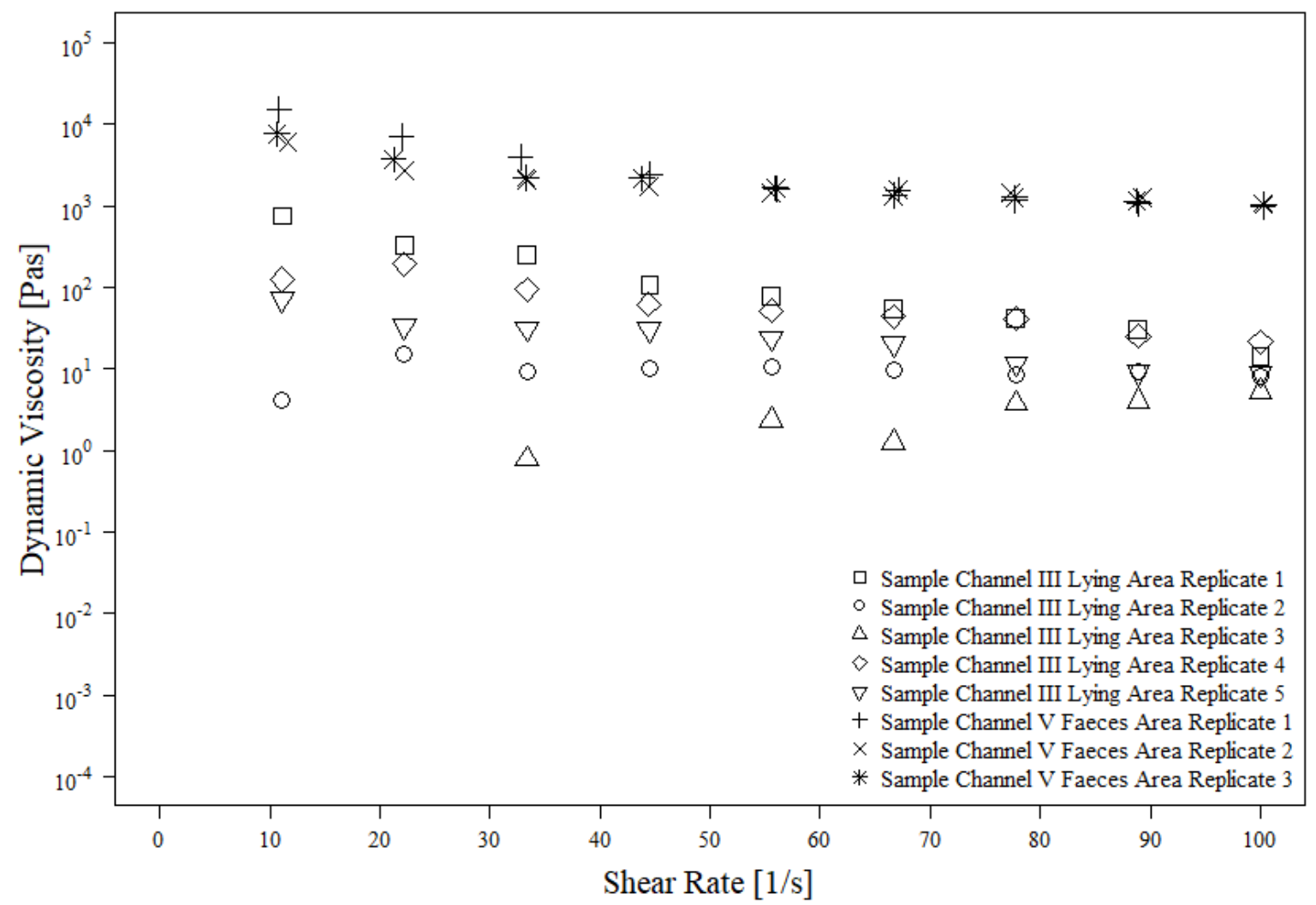

Figure 23. Dynamic viscosity of slurry samples taken under the lying area (sample III, dry matter content $5.5 \%$ ) and the faeces area (sample V, dry matter content $11.72 \%$ )

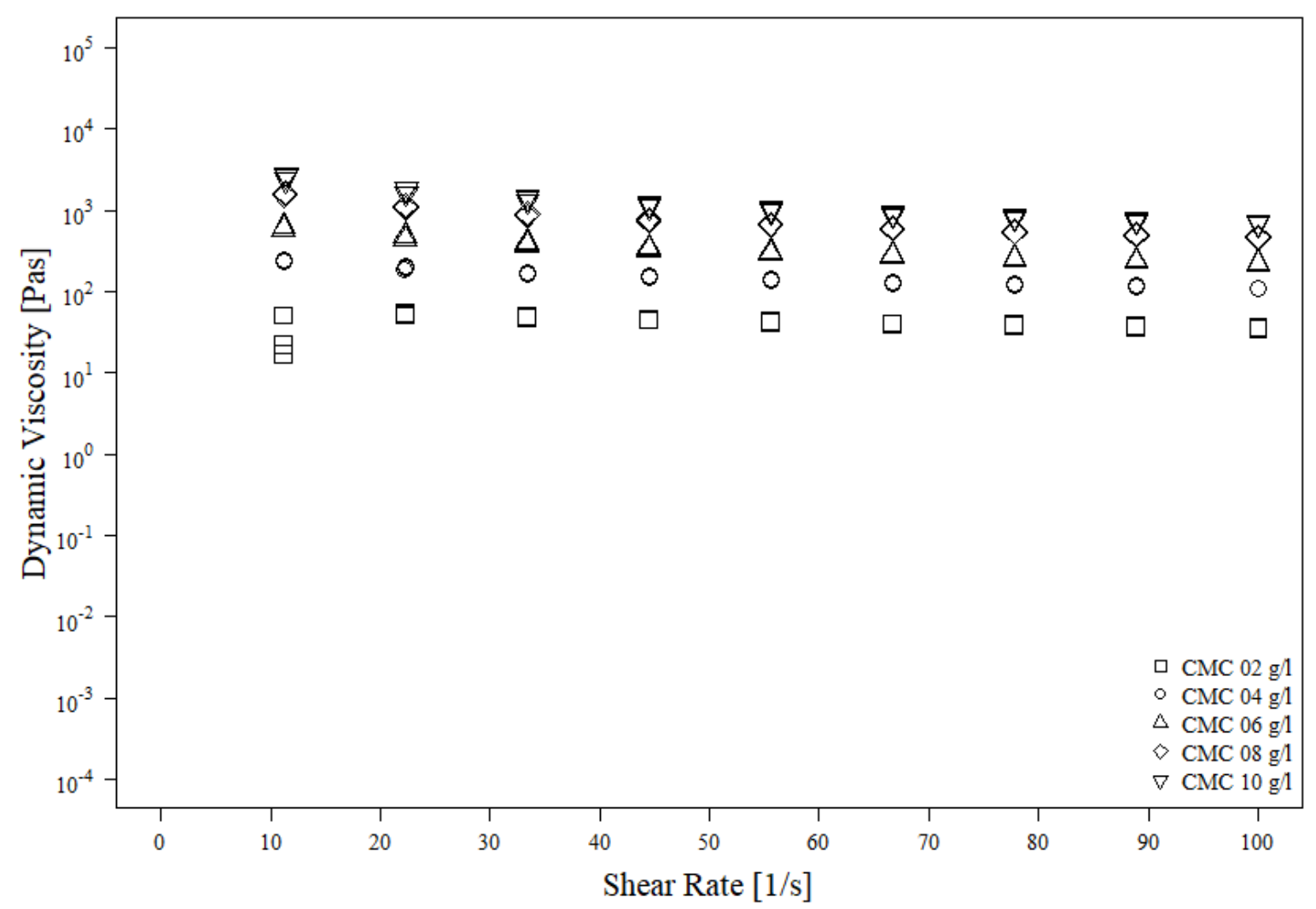

Figure 24. Dynamic viscosity of the thickener carboxymethyl cellulose dissolved in water at a ratio of 2 to $10 \mathrm{~g} / \mathrm{l}$. 


\section{Conclusions and Outlook}

This data serves as the basis for a very early test run of manure removal on the test stand. Due to the reduced scale, it may be necessary to include a conversion factor for the thickening concentration to compensate for the rheological parameters of pipe friction and flow rate. Initial validation trials of the test rig will include a series of tests with increasing amounts of thickeners and organic material. The goal is the recording of changes in the results of manure removal up to the complete obstruction of the pipe system. These conclusions will be compared to empirical values in practice. The validation of the entire model fluid - including organic material and sand and its comparison to pig slurry with a similar solid composition will follow. This also includes the simulation of thicker manure areas in the manure channels und faecal sections. It is not known to what extent these thicker blocks play a role in manure removal. Finding methods for recreating such viscous blockages in the test stand are part of the continuous development of the test stand and the model fluids used.

\section{Acknowledgements}

The project was supported by funds of the Federal Ministry of Food and Agriculture (BMEL) based on a decision of the Parliament of the Federal Republic of Germany via the Federal Office for Agriculture and Food (BLE) under the innovation support programme. FKZ 2819200515.

This contribution is part of the research project "Label-Fit - 'Making pig husbandry fit for animal welfare labels' - Integrated development of housing systems and process technology for the transformation of conventional housings". I want to thank our partners, the German Animal Welfare Federation, the Institute of Animal Welfare and Animal Husbandry at the Friedrich-Loeffler-Institute in Celle, the federal pig research and training centre "Bildungs- und Wissenszentrum LSZ Boxberg" and the Vion food group.

\section{References}

Jungbluth, T., Büscher, W., Krause, M., 2017. Technik Tierhaltung. $2^{\text {nd }}$ Edition. Eugen Ulmer Verlag Stuttgart. ISBN 978-3-8252-4243-5. 322 pp. 


\author{
Ammonia Emission Assessment After Buffalo Manure And Digestate Application \\ Ester Scotto di Perta ${ }^{a}$, Yann Collas ${ }^{\text {b }}$, Nunzio Fiorentino a, Elena Cervelli ${ }^{\text {a }}$, Salvatore Faugno ${ }^{\text {a }}$, \\ Stefania Pindozzi ${ }^{\text {a,* }}$ \\ ${ }^{a}$ Department of Agricultural Sciences, University of Naples Federico II, Via Università 100, Portici (NA), Italy \\ ${ }^{\mathrm{b}}$ General engineering school CESI ,Saint-Nazaire Center, boulevard de l'université, 44600 Saint-Nazaire, France \\ * Corresponding author. Email: stefania.pindozzi@unina.it
}

\begin{abstract}
Quite recently, considerable attention has been paid to the effect of anaerobic digestion on ammonia emissions from digestate spreading in the field, due to the growing interest in $\mathrm{NH}_{3}$ emission monitoring. Unfortunately, there are still some different studies finding about the effect of anaerobic digestion on ammonia emissions. Thus, more research into this topic is still necessary before obtaining a definitive answer to the increment or not in emission. For this purpose, this paper proposes comparison study between ammonia emissions from buffalo raw (Farmyard) manure and digestate on bare soil under Mediterranean climate, using the wind tunnel equipped with acid traps, to assess the ammonia emission fluxes. The sampling campaign, in three replicates, lasted each time, for 6 days to ensure that most of the ammonia has been emitted before the end of each campaign. The results obtained indicate that a diurnal correlation between emission and external temperature occurs, especially during the first days. Specifically, for both fertilizers, ammonia volatilization increased with air temperature raising. Overall, the total digestate cumulative $\mathrm{NH}_{3}$ emission is $54 \%$ higher than raw manure emission. This is certainly due to the Total $\mathrm{NH}_{4}{ }^{+}-\mathrm{N}$ rate, which was $55,8 \mathrm{~kg} \mathrm{ha}^{-1}$ for the raw manure and $107 \mathrm{~kg} \mathrm{ha}^{-1}$ for the digestate, around 1.9 times higher for the digestate TAN content. Finding suggests the need for adjusting digestate application rate based on TAN content, in order to reduce the impact on the environment.
\end{abstract}

Keywords: Ammonia volatilization, Farmyard manure, organic fertilizers comparison, anaerobic digestion, open field monitoring, wind tunnel.

\title{
1. Introduction
}

The intensive livestock breeding leads to the production of high quantities of manure which contribute to ammonia emission especially following manure application to the land, in regions where livestock production increased (Sommer et al., 2013). Ammonia emissions decrease the nutrient availability of organic fertilizers and they are responsible for human and environmental problems (Pedersen et al., 2018).

In the last years, according to the incoming Directive NEC (National Emission Ceiling), there has been a growing interest in $\mathrm{NH}_{3}$ emissions monitoring and reduction. A considerable attention has been paid to field manure application, because of its major contribution to $\mathrm{NH}_{3}$ volatilisation from livestock activities (Carozzi et al., 2013). The intensity of the process is mainly related to the field conditions and manure characteristics, such as $\mathrm{pH}$, dry matter (DM) and total ammoniacal nitrogen (TAN). Specifically, manure characteristics can vary depending on manure pre-treatment (Evans et al., 2018). Therefore, current studies have been focused on some manure pre-treatments such as anaerobic digestion and soil-liquid separation. In this sense, anaerobic digestion (AD), is the only with the advantage of reducing odour and methane emissions along with production of biogas fuel (Neerackal et al., 2015). On the other hand, $\mathrm{AD}$ may potentially increase the $\mathrm{NH}_{3}$ emissions following the digestate field application, due to the increase in $\mathrm{pH}$ and TAN concentration (Sun et al., 2014) because of the mineralization of organic nitrogen to ammonia. The assessment in the field of these possible side-effects is necessary to guarantee the environmental suitability of digestage land application in terms of gaseous $\mathrm{N}$ losses (Chantigny et al., 2009).

Unfortunately, there are still some different finding in literature on the effect of anaerobic digestion on ammonia emissions, that underline the importance of planning new experiments about this topic (Holly et al., 2017; Möller, 2015)

Based on the background presented, the purpose of this study was to assess the effect of $\mathrm{AD}$ on $\mathrm{NH}_{3}$ volatilization on bare soil under Mediterranean climate, comparing ammonia emissions of buffalo digested manure and buffalo farmyard manure (FYM). For this purpose, three field trials were carried out using wind tunnel technique, that is a favoured tool in comparison studies for ammonia emissions evaluation (Sommer and Misselbrook, 2016). 


\section{Materials and Methods}

Trials were carried out in the experimental field of University of Naples Federico II, Portici (Campania region, Southern Italy, $\left.40^{\circ} 48^{\prime} 49.9^{\prime \prime} \mathrm{N} 14^{\circ} 20^{\prime} 48.3^{\prime \prime} \mathrm{E}\right)$. The site is located in a typical Mediterranean environment, characterized by a temperate Mediterranean climate, according to Emberger's index (Leone, A., 2011). A weather station (PCE-FWS 20) has been placed next to the field to measure wind velocity and direction, temperature, rainfall, humidity and relative pressure for all the duration of trials and recorded by a data logger with a resolution of 10 minutes.

Three field trials were carried out in consecutive weeks during the month of June 2017, to keep similar weather conditions, using fertilizers from buffalo species, reared mainly in Southern Italy (Pindozzi et al., 2013). Buffalo raw manure and buffalo digested manure were applied by hand at a rate of $200 \mathrm{~kg} \mathrm{~N} \mathrm{ha}^{-1}$, spreading the same total nitrogen content (TKN), in order to assess $\mathrm{NH}_{3}$ volatilization within a period six consecutive days. Experimental trials and fertilization information are summarized in Table 1.

Table 63. Experimental plan design.

\begin{tabular}{cccccc}
\hline Trial & Starting date & Ending date & $\mathrm{N}$ rate $\left(\mathrm{N} \mathrm{ha}^{-1}\right)$ & Manure $(\mathrm{kg})$ & Digestate $(\mathrm{l})$ \\
\hline 1 & $06 / 06 / 2017$ & $12 / 06 / 2017$ & & & 1.9 \\
2 & $13 / 06 / 2017$ & $19 / 06 / 2017$ & 200 & 1.2 & \\
3 & $20 / 06 / 2017$ & $26 / 06 / 2017$ & & & \\
\hline
\end{tabular}

Before spreading the organic fertilizers, representative samples were collected, to analyse dry matter (DM) content, ammonium-N (TAN) and total-N (TKN). Table 2 gives the main characteristics of fertilisers.

Table 64. Chemical characteristics of fertilizers used in the field trials.

\begin{tabular}{ccccc}
\hline Fertilizers & $\mathrm{pH}$ & $\mathrm{TAN}\left(\mathrm{mg} \mathrm{l}^{-1}\right)$ & $\mathrm{DM}(\%)$ & $\mathrm{TKN}\left(\mathrm{mg} \mathrm{l}^{-1}\right)$ \\
\hline Digestate & 7.73 & 1804.5 & 7.892 & 3374 \\
Manure & 8.13 & 1710 & 18.131 & 6119 \\
\hline
\end{tabular}

After fertilization, ammonia emission rate was measured using two wind tunnels (Scotto di Perta et al., 2016), consisting of an open chamber placed on a rectangular area $\left(0.32 \mathrm{~m}^{2}\right)$, which simulate the wind action on a fertilized surface by means of a fan (Figure 1).

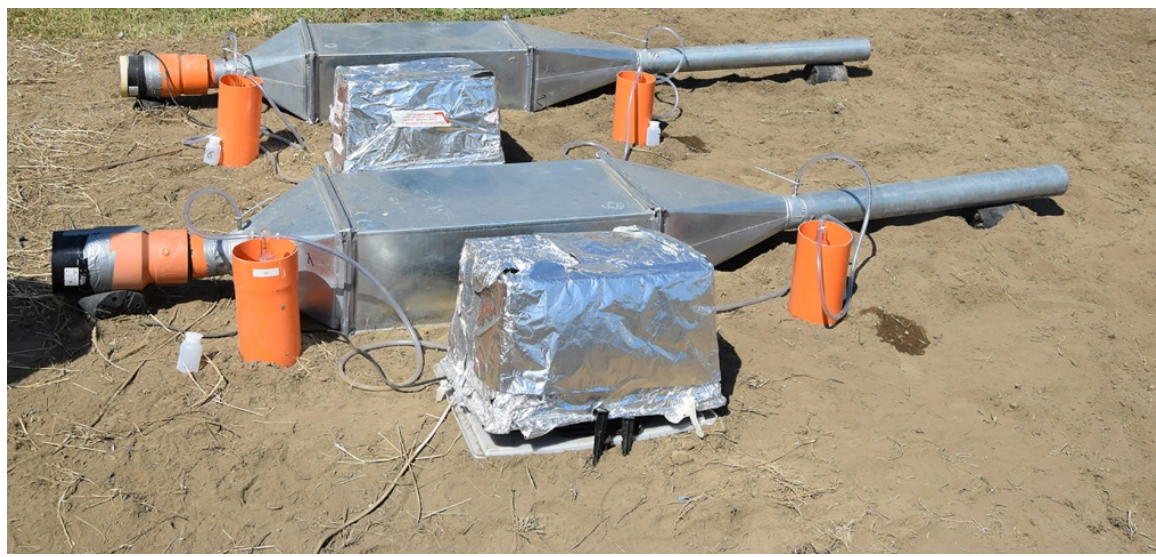

Figure 25. Wind tunnels measuring ammonia emission from bare soil.

Wind tunnel was characterized by two air sampling points, at the inlet and the outlet. $\mathrm{NH}_{3}$ in sampled air was trapped using acid traps system made up of: a bottle contained $1 \%$ sulphuric acid solution, a flow meter stabilizing the rate to $41 \mathrm{~min}^{-1}$ and a suction pump. The sampling lasts for 6 days to ensure that most of the ammonia has been emitted before the end of each campaign. Acid solutions were replaced every 3 hours during the daytime. The concentration of ammonia trapped in acid solutions was measured in lab spectrometrically using a continuous flow analyzer (FIAstar 5000, Foss, Denmark). The $\mathrm{NH}_{3}$ flux can be computed by following equation (1):

$$
J=\frac{Q\left(C_{\text {out }}-C_{\text {in }}\right)}{A}
$$

where $\mathrm{C}_{\text {out }}$ and $\mathrm{C}_{\text {in }}\left(\mu \mathrm{g} \mathrm{m}^{-3}\right)$ are the $\mathrm{NH}_{3}$ concentration in the outlet and inlet air, respectively, while $\mathrm{Q}\left(\mathrm{m}^{3} \mathrm{~s}^{-1}\right)$ is the inlet flow rate and $\mathrm{A}\left(\mathrm{m}^{2}\right)$ is the fertilized area. 


\section{Results and Discussion}

Figure 2 shows selected $\mathrm{NH}_{3}$ emission measured along with air temperature variations. As shown, a diurnal correlation between $\mathrm{NH}_{3}$ emission and air temperature occurred, especially during the first days. Specifically, for both fertilizers, ammonia volatilization increased with air temperature raising. Furthermore, the highest emission rates occurred for both fertilizers during the first hours, because of the high TAN concentration at the application time (Sommer and Misselbrook, 2016).

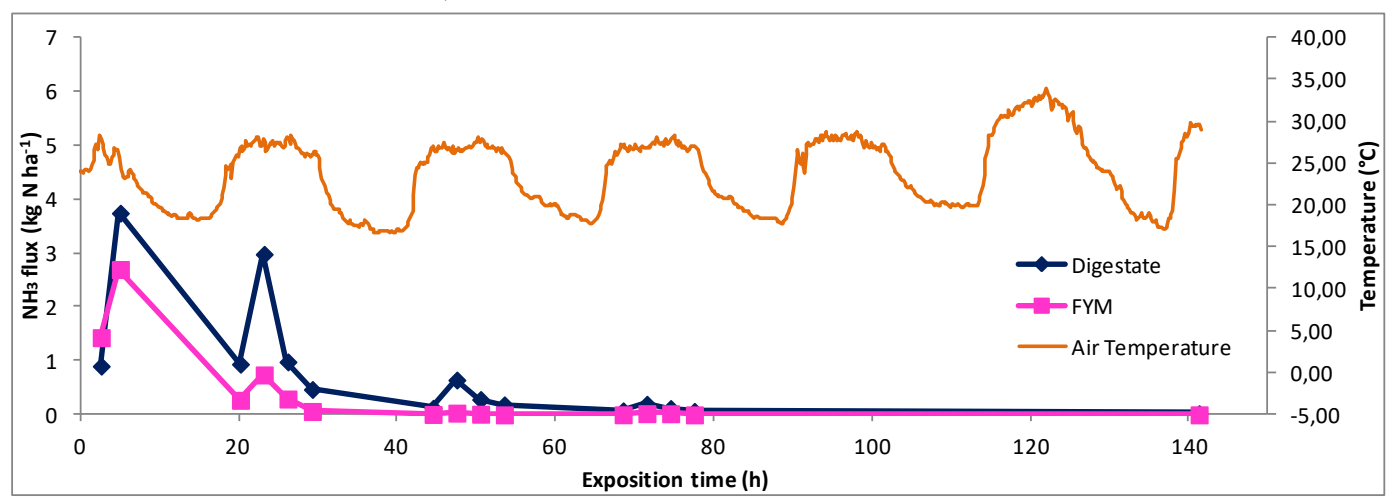

Figure 26. $\mathrm{NH}_{3}$ emissions rate and air temperature variations during trial 1.

The cumulative ammonia emission curves, obtained in the three trials, are illustrated in Figure 3. As can be seen, $\mathrm{NH}_{3}$ emission decreased after the first $24 \mathrm{~h}$ for both treatments. The cumulative ammonia emissions after $141 \mathrm{~h}$ after fertilization were $31.77 \mathrm{~kg} \mathrm{~N} \mathrm{ha}^{-1}$ from the buffalo digested manure and $14.77 \mathrm{~kg} \mathrm{~N}^{-1}$ from the buffalo raw manure, respectively. Thus, it has been found that the digestate emitted around $54 \%$ of total $\mathrm{NH}_{3}$ amount more than raw manure. The higher ammonia emission may be due to the TAN content digestate is twice that of manure, indeed generally the anaerobic process increases the TAN content because of organic nitrogen mineralization (Holly et al., 2017).

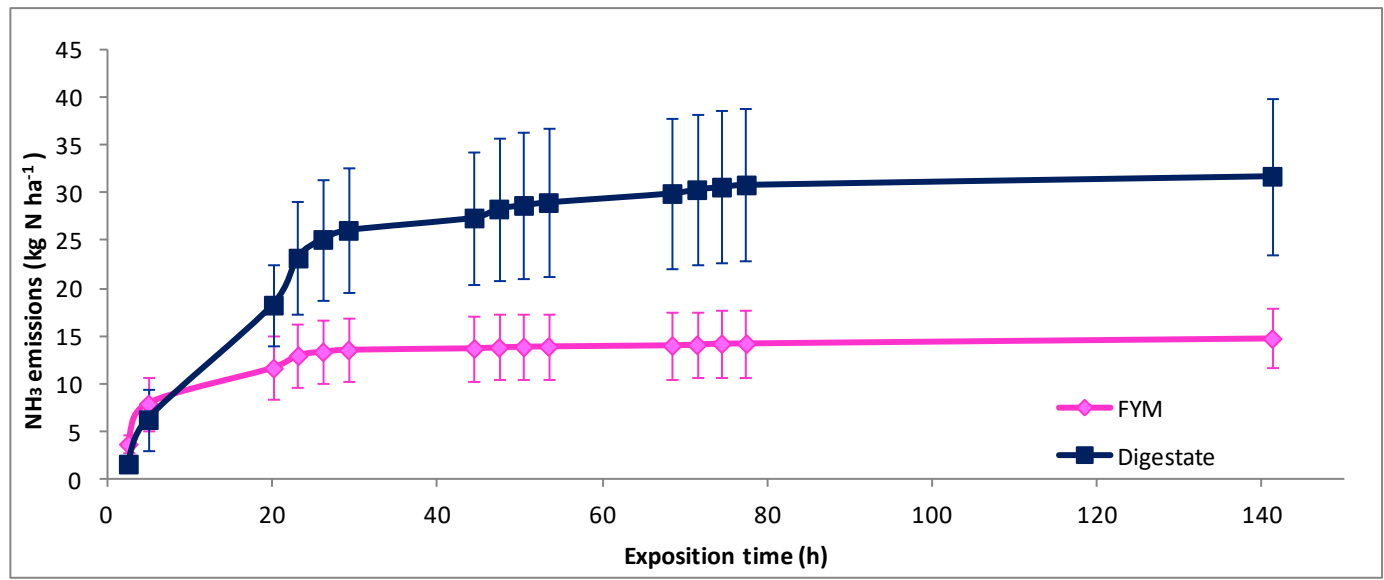

Figure 27. cumulative $\mathrm{NH}_{3}$ emissions. Vertical bars represent standard error of means $(n=3)$.

FYM emission decreased substantially after the first 26 hours, probably due to water evaporation and the crust formation, as reported also by Neerackal et al. (2015) in a previous study, contrary to the digestate that emitted for a longer period.

Normalized emissions by TAN and $\mathrm{N}$ applied for the first $24 \mathrm{~h}$ and for the last day have been considered (Table 3), to improve treatments comparison. 
Table 3. Comparison of cumulative $\mathrm{NH}_{3}$ emission normalized by applied $\mathrm{N}$ and TAN

\begin{tabular}{ccccccc}
\hline \multirow{2}{*}{ Fertilizers } & \multicolumn{2}{c}{$\mathrm{kg} \mathrm{N} \mathrm{ha}^{-1}$} & \multicolumn{2}{c}{ \% of TAN applied } & \multicolumn{2}{c}{ \% of N applied } \\
& $24 \mathrm{~h}$ & Last day & $24 \mathrm{~h}$ & Last day & $24 \mathrm{~h}$ & Last day \\
\hline Digestate & 24.56 & 31.77 & 22.95 & 29.69 & 12.28 & 15.89 \\
FYM & 13.25 & 14.77 & 23.75 & 26.46 & 6.63 & 7.38 \\
\% difference & 46.05 & 53.51 & -3.48 & 10.88 & 46.01 & 53.56 \\
\hline
\end{tabular}

As may be seen from the comparison, when cumulative $\mathrm{NH}_{3}$ emission is normalized by applied TAN, the difference between fertilizers decreases substantially. This could confirm the hypothesis that the difference of 46 and $53 \%$ between the cumulative $\mathrm{NH}_{3}$ emission of digested manure and FYM is mainly due to the greater TAN content of digestate compared to FYM (Evans et al., 2018).

Studies on digestate effect on $\mathrm{NH}_{3}$ emissions after field digestate spreading are still lacking, moreover, sometimes results are different to each other. The most likely explanation is that experiments are carried out in different climate conditions, sometimes introducing also other manure treatments, such as soil/liquid separation or storage, reducing the opportunity of a clear conclusion (Möller et al. 2015). According to relevant literature reports in this field, $\mathrm{NH}_{3}$ losses are greater in case of digestate spreading than manure when the application rate is based on equivalent Total N or volume (Amon et al., 2006; Möller and Stinner, 2009; Rubæk et al., 1996), due to the application of a higher total $\mathrm{NH}_{4}{ }^{+}-\mathrm{N}$ concentration, as in the case of this study. As a matter of fact, when the application rate is based on equivalent total $\mathrm{NH}_{4}{ }^{+}-\mathrm{N}$, literature results showed that anaerobic digestion could reduce $\mathrm{NH}_{3}$ emissions from field-applied digestate (Neerackal et al., 2015; Rubæk et al., 1996).

\section{Conclusions}

The cumulative ammonia emissions of the digestate is 2.15 times higher than the total manure ammonia emission, most likely because of Total $\mathrm{NH}_{4}^{+}-\mathrm{N}$ rate, which was $55.8 \mathrm{~kg} \mathrm{ha}^{-1}$ for the manure and $107 \mathrm{~kg} \mathrm{ha}^{-1}$ for the digestate, around 1.9 times higher for the digestate. Results confirm the need for adjusting digestate application rate based on TAN content, in order to reduce the impact of the spreading activities.

\section{Acknowledgements}

This research was realized under the " Riduzione delle emissioni di Ammoniaca e Gestione delle Risorse Idriche nella piana del Sele (RiAGRI-Sele)" Project funded by Rural Development Program for 2014 - 2020 of Campania Region. The authors would like to thank Dr. Sabrina Nocerino for her help with data collection during the experiments and Dr. Roberto Maiello for his technical support in laboratory analyses.

\section{References}

Amon, B., V. Kryvoruchko, T. Amon, S. Zechmeister-Boltenstern, 2006. Methane, nitrous oxide and ammonia emissions during storage and after application of dairy cattle slurry and influence of slurry treatment. Agriculture, ecosystems \& environment, 112(2), 153-162.

Carozzi, M., R. M. Ferrara, G. Rana, M. Acutis, 2013. Evaluation of mitigation strategies to reduce ammonia losses from slurry fertilisation on arable lands. Science of the Total Environment, 449, 126-133.

Chantigny, M.H., J. D. MacDonald, C. Beaupré, P. Rochette, D. A. Angers, D. Massé, L. E. Parent, 2009. Ammonia volatilization following surface application of raw and treated liquid swine manure. Nutrient cycling in agroecosystems, 85(3), 275-286.

Evans, L., A. C. VanderZaag V. Sokolov, H. Baldé, D. MacDonald, C. Wagner-Riddle, R. Gordon, 2018. Ammonia emissions from the field application of liquid dairy manure after anaerobic digestion or mechanical separation in Ontario, Canada. Agricultural and Forest Meteorology. In press (https://doi.org/10.1016/j.agrformet.2018.02.017)

Holly, M. A., R. A Larson, J. M. Powell, M. D. Ruark, H. Aguirre-Villegas, 2017. Greenhouse gas and ammonia emissions from digested and separated dairy manure during storage and after land application. Agriculture, Ecosystems \& Environment, 239, 410-419.

Leone, A., 2011. Ambiente e pianificazione: analisi, processi, sostenibilità. F. Angeli.

Minoli, S., M. Acutis, M. Carozzi, 2015. NH3 emissions from land application of manures and N-fertilisers: a review of the Italian literature. ITALIAN JOURNAL OF AGROMETEOROLOGY - RIVISTA ITALIANA DI AGROMETEOROLOGIA, 20(3), 5-24.

Möller, K., 2015. Effects of anaerobic digestion on soil carbon and nitrogen turnover, $\mathrm{N}$ emissions, and soil biological activity. A review. Agronomy for sustainable development, 35(3), 1021-1041.

Möller, K., W. Stinner, 2009. Effects of different manuring systems with and without biogas digestion on soil mineral nitrogen content and on gaseous nitrogen losses (ammonia, nitrous oxides). European Journal of Agronomy, 30(1), 1-16. 
Neerackal, G. M., P. M. Ndegwa, H. S. Joo, X. Wang, J. H. Harrison, A. J. Heber, J.Q: Ni, C. Frear, 2015. Effects of anaerobic digestion and solids separation on ammonia emissions from stored and land applied dairy manure. Water, Air, \& Soil Pollution, 226(9), 301.

Pedersen, S. V., E. Scotto di Perta, S. D. Hafner, A. S. Pacholski, S. G. Sommer, 2017. Evaluation of A Simple, Small-Plot Meteorological Technique for Measurement of Ammonia Emission-Feasibility, Costs and Recommendations. Transactions of the ASABE. 61(1): 103-115. (doi: 10.13031/trans.12445)@2018

Pindozzi, S., S. Faugno, C. Okello L. Boccia, 2013. Measurement and prediction of buffalo manure evaporation in the farmyard to improve farm management. Biosystems engineering, 115(2), 117-124.

Rubæk, G. H., K. Henriksen, J. Petersen, B. Rasmussen, S. G. Sommer, 1996. Effects of application technique and anaerobic digestion on gaseous nitrogen loss from animal slurry applied to ryegrass (Lolium perenne). The Journal of Agricultural Science, 126(4), 481-492.

Scotto di Perta, E., M. A. Agizza, G. Sorrentino, L. Boccia, S. Pindozzi, 2016. Study of aerodynamic performances of different wind tunnel configurations and air inlet velocities, using computational fluid dynamics (CFD). Computers and Electronics in Agriculture, 125(C), 137-148.

Sommer, S. G., T. H. Misselbrook, 2016. A review of ammonia emission measured using wind tunnels compared with micrometeorological techniques. Soil Use and Management, 32(S1), 101-108.

Sommer, S. G., M. L. Christensen, T. Schmidt, L. S. Jensen, 2013. Animal manure recycling: Treatment and management. John Wiley \& Sons. $364 \mathrm{p}$.

Sun, F., J. H. Harrison, P. M Ndegwa, K. Johnson, 2014. Effect of manure treatment on ammonia and greenhouse gases emissions following surface application. Water, Air, \& Soil Pollution, 225(4), 1923. 


\title{
Influence of Increased Light Intensity on the Lying and Excretory Behaviour of Fattening Pigs
}

\author{
Svenja Opderbeck ${ }^{\text {a, }}$, Barbara Kessler $^{\text {b }}$, Eva Gallmann ${ }^{\text {a }}$ \\ ${ }^{\text {a }}$ University of Hohenheim, 70599 Stuttgart, Germany \\ ${ }^{\mathrm{b}}$ Bildungs- und Wissenszentrum Boxberg, 97944 Boxberg-Windischbuch, Germany \\ * Corresponding author. Email: svenja.opderbeck@uni-hohenheim.de
}

\begin{abstract}
Due to increasing demands on animal welfare in German pig farming, more and more animal welfare labels have been introduced. The label "The German Animal Welfare Association - For more Animal Welfare" (Deutscher Tierschutzbund - Für mehr Tierwohl) sets besides others, requirements for the design of the area for fattening pigs and weaning piglets. Each fattening pig should have $0.6 \mathrm{~m}^{2}$ of solid lying area with a maximum of $3 \%$ perforation. The project "LabelFit" will investigate how lying and excretory areas can be designed the most attractive, especially in conventional housing systems. The aim is to design a lying area that is used as such throughout the entire year. This paper shows the effect of a strong increased light intensity on the lying and excretory behaviour. This was examined in 4 pens with 18 pigs each over 2 fattening periods (total of $n=108$ pigs). The light intensity above the slatted floor was increased by 2 spotlights hanging above the slatted area in 2 pens. For comparison, 2 pens were rebuilt identically without changing the light intensity. With 2 luxmeters in each pen, the light intensity was measured above the lying and the excretory area. Weekly scores were used to monitor the fouling of the pen $(n=70)$. Using video analysis, the lying behaviour was monitored 3 times a day on 3 days a week $(n=597)$. The results show that about $12 \%$ more animals are lying on the lying area compared to the pens without increased light intensity $(\mathrm{p}<0,0001)$ above the slatted area. The fouling of the lying area seems to be lower in the pens with an increased light intensity. In conclusion, a strong increased light intensity in the slatted area lead to a higher percentage of pigs lying in the lying area.
\end{abstract}

Keywords: pig, lying and excretory behaviour, light intensity

\section{Introduction}

The requirements on animal welfare in pig farming are increasing in Germany. Therefore, more and more animal welfare labels have been introduced. The label of "The German Animal Welfare Association - For more Animal Welfare" sets beside others, requirements for the design of the lying area for fattening pigs and weaning piglets. The maximal perforation of the lying area should be $3 \%$. The main problem is the high risk of fouling of the mainly solid lying area, as known from practical experience with partly slatted floor systems and described in several studies. The consequences are poor animal welfare since they lay in their excrements, poor indoor climate due to more gas emission potential of dirty surfaces and increased working load for manual cleaning.

To address these issues a three years' joint research project called "LabelFit" started in April 2017. One aim is to design and test several constructible and management options for the lying area to ensure that it is used as such throughout the entire year. In two fattening rooms, the influence of different pen structures (arrangement of the slatted floors and feeding), different floor structures (slatted floor, triangular grid and slatted floor with clips) and an increase light intensity will be examined. The influence of a floor cooling or heating will be tested in the two other fattening rooms.

The lying behaviour is influenced by different factors as e.g.: Pigs prefer solid floor to slatted floor in the lying area (Börgemann et al. 2007). Low air speed (Randall et al. 1983) and a low light intensity (Christison 1995; Taylor et al. 2006) are causing a better acceptance of the lying area. For the excretory behaviour pigs prefer wet, cool areas with low activity (Hacker et al. 1994, Randall et al. 1983). Moreover, open partitions promote the excretory behavior in those areas (Hacker et al. 1994). The results of Taylor et al. (2006) show that growing pigs prefer low light intensities for lying and sleeping and areas with higher light intensities for elimination.

The objective of this present paper is to show the results up to date of the investigations of the influence of a strong increased light intensity in the slatted area on the lying and excretory behaviour.

\section{Materials and Methods}

For this purpose, four compartments for fatteners have been rebuilt according to the requirements of the label of "The German Animal Welfare Association - For more Animal Welfare" at the federal pig research and training centre "Bildungs- und Wissenszentrum LSZ Boxberg". The main requirements of the label are: $1.1 \mathrm{~m}^{2}$ per pig $\left(0.6 \mathrm{~m}^{2}\right.$ solid lying area), straw or comparative material for employment, maximum 3:1 animal:feeding place ratio, maximum 12:1 animal:drinking place ratio and no tail docking.

Considering these requirements, four fattening rooms were rebuilt. In two fattening rooms, the influence of 
different pen structures (arrangement of the slatted floors and feeding), different floor structures (slatted floor, triangular grid and slatted floor with clips) and an increase light intensity will be examined. The influence of a floor cooling or heating will be tested in the two other fattening rooms.

In the following only the approach for the investigation of the influence of light intensity will be described in more detail.

Animals

A total of 108 crossbred long-tailed pigs, 54 females and 54 castrated males (German Hybrid x German Piétrain) were used for this study. These pigs were allocated to four pens in two rooms over two fattening periods (Tab. 1). Eighteen pigs were placed in one pen. The average live weight at the beginning and the end of the experiment and the daily gain is shown in Table 1.

Table 1. Dates for each fattening period. Observations took place only in the first twelve weeks of fattening.

\begin{tabular}{clcccccc}
\hline Period & $\begin{array}{c}\text { Date of whole Room } \\
\text { period }\end{array}$ & Pen & \multicolumn{2}{c}{$\begin{array}{c}\text { Days of } \\
\text { Observation }\end{array}$} & \multicolumn{2}{c}{ Average live weight in kg } & Average daily gain in g \\
\hline 1 & $25.09 .17-$ & C1 & 102 & 80 & $29 \pm 3.4$ & $115.9 \pm 4.1$ & $696.7 \pm 80.7$ \\
& 15.01 .18 & & 105 & 80 & $28 \pm 3$ & $119.4 \pm 4,6$ & $712.1 \pm 23.6$ \\
2 & $24.01 .18-$ & C1 & 102 & 85 & $32.4 \pm 5.8$ & $120.6 \pm 4.2$ & $1007.6 \pm 110$ \\
& 22.05 .18 & & 105 & 85 & $30.5 \pm 3.5$ & $118.8 \pm 8$ & $927.6 \pm 79.2$ \\
2 & $03.01 .18-$ & C2 & 202 & 85 & $32 \pm 3.4$ & $119.2 \pm 4.4$ & $939.7 \pm 85.3$ \\
& 30.04 .18 & & 205 & 85 & $33 \pm 4$ & $117.9 \pm 5.8$ & $930.4 \pm 126.3$ \\
\hline
\end{tabular}

Housing and experimental pen design

There were six pens within each fattening room. The rooms are temperature controlled forced ventilated with air supply from outside through the attic and porous ceiling. Each pen had a size of $4.25 \mathrm{~m}$ x $5.2 \mathrm{~m}$ including a lying area of $4.25 \times 3 \mathrm{~m}$ (Fig. 1). The floor in the lying area was solid with a maximum perforation of $3 \%$. The rest of the pen floor was slatted with $17 \%$ perforation. The lights in the fattening room were switched on from 7 am to $6 \mathrm{pm}$. The experimental animals were fed ad libitum at a round tube mash automat type "Optimat" (Schauer Agrotonic GmbH, Prambachkirchen, Austria). This feeder was located in the middle of the lying area and with an animal:feeding place ratio 3:1. The pigs had free access to water from two drinking bowls "Suevia 95S" (Suevia Haiges $\mathrm{GmbH}$, Kirchheim/Neckar, Germany) which were located on the slatted floor. For environmental enrichment in each pen the pigs had free access to hay in a hanging cage, one wooden beam and two hamper ropes. Twice a day (11 am, $3 \mathrm{pm}$ ) approximately $30 \mathrm{~g} / \mathrm{pig}$ chopped straw automatically spread on the lying area by the automatic straw distribution System "Spotmix Welfare” (Schauer Agrotonic GmbH, Prambachkirchen, Austria).

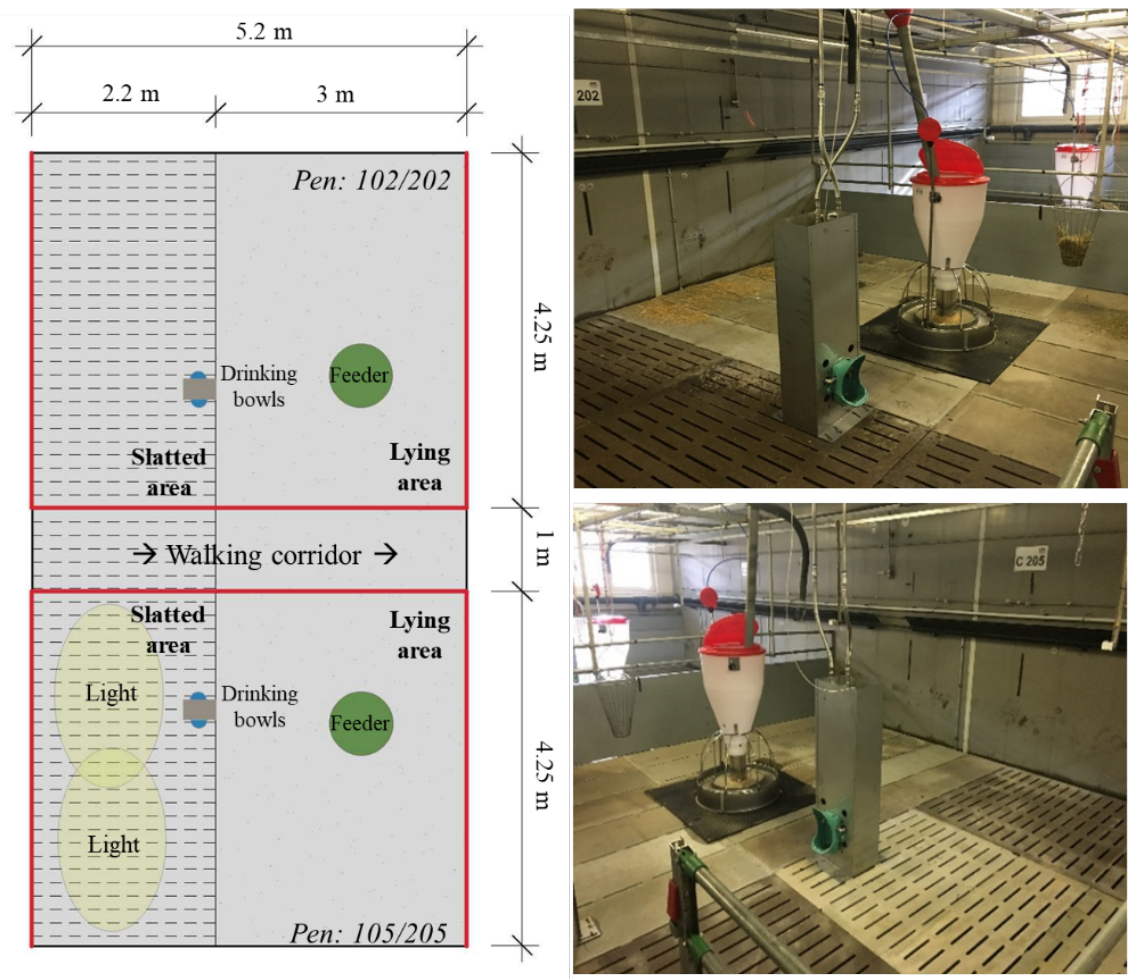

Figure 1. The experimental pens with the lying and slatted area. 
Four pens in two rooms ( $\mathrm{C} 1$ and $\mathrm{C} 2)$ were used to investigate the effect of a strong increase of light intensity over the slatted area on the lying and excretory behaviour. In each room, two identical pens (102/105 and 202/205) were examined. One pen remained unchanged and the other got changing light intensity above the slatted (designated excretory) area. For this purpose, two spotlights were set up over the slatted area (Fig. 2). These spotlights were switched on from 7 am to $6 \mathrm{pm}$ in addition to the general lights in the rooms. The spotlights (LED Fluter $30 \mathrm{~W}, 2400 \mathrm{~lm}$ floodlight) were hung up $1.4 \mathrm{~m}$ above the slatted area and the light was limited by a metal sheet to ensure that only the slatted area was lit up (Fig. 2). After the first fattening period the spotlights were exchanged between the two pens in each room to counteract an effect of the location of the pen within the room.
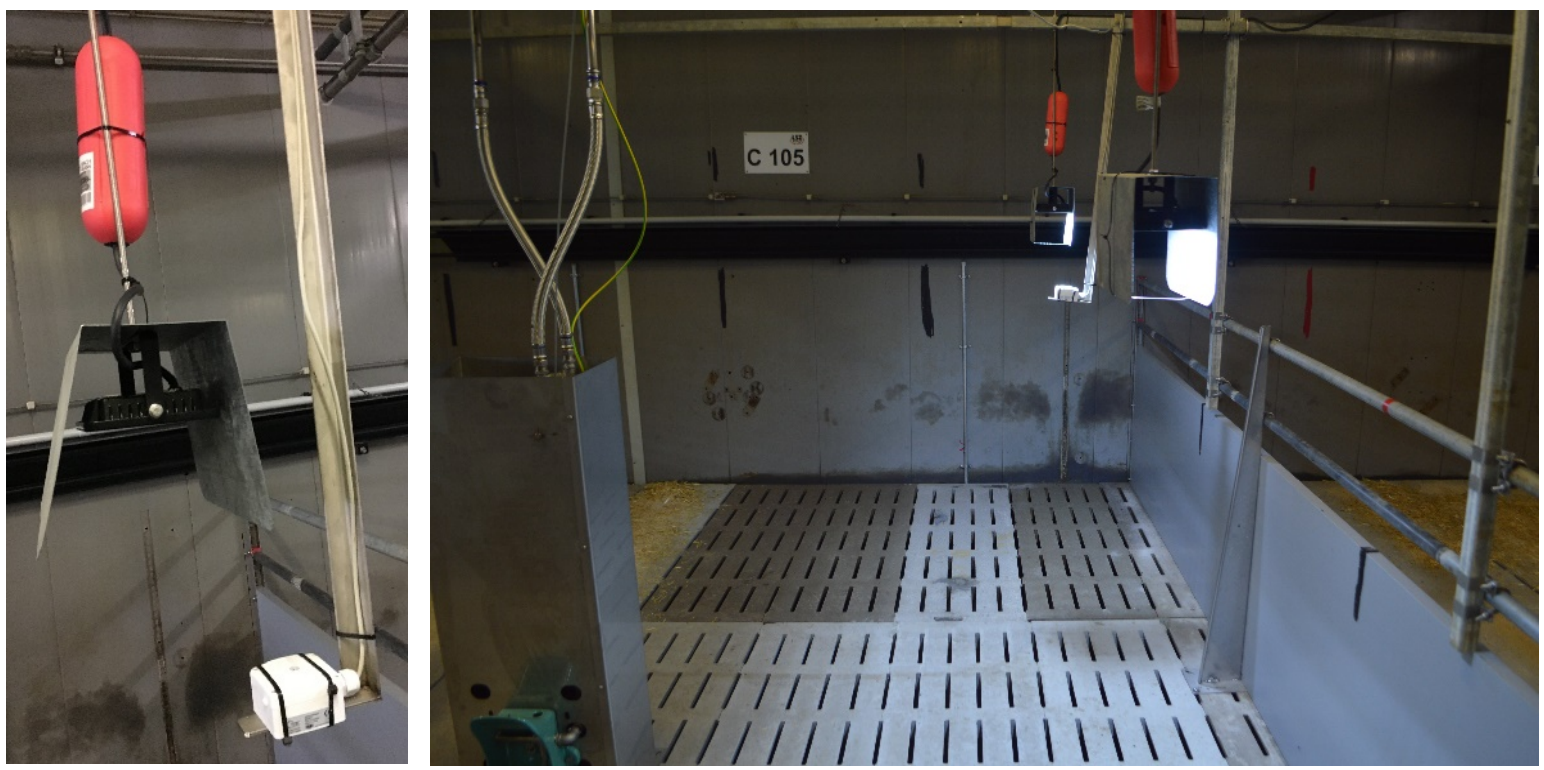

Figure 2. Left: The spotlight above the luxmeter; Right: The lit up slatted floor.

Data collection

During the fattening periods the temperature inside the rooms was recorded. Moreover, the light intensity was measured continuously every second at the lying area as well as at the slatted area. For the analysis an average value over one hour was used. For this purpose, two luxmeters (Apilux, measuring range $0-100 \mathrm{kLUX}$, accuracy $\pm 10 \%$ ) were installed horizontally $1.2 \mathrm{~m}$ above the floor with the detector facing upwards. In the lit up pen one luxmeter was located directly below the spotlight. Measurements with a mobile luxmeter were performed to assess the light intensity on pig eye-height more exactly. For this light intensity was measured with the detector facing upwards and downwards in a high of $50 \mathrm{~cm}$ above the floor directly below the fixed installed luxmeter.

During the first twelve weeks of the fattening period the lying and excretory behaviour of all pigs a video recording was installed (video cameras, HIKVision DS-2CD2125FWD-1) for $24 \mathrm{~h}$ per day during this time. Based on these video records, the lying behaviour was analysed three times a day (5-6 am (morning), 10.30-11.30 am (noon) and 8-9 pm (evening)) on three days a week using scan sampling as observation method. Screenshots of the first moment when all pigs were lying were used for each of the sampling times. The screenshot of the pen was segmented in twelve parts, six parts on the lying area (each 2,13 $\mathrm{m}^{2}$ ) and six parts on the slated area (each $1,56 \mathrm{~m}^{2}$ ). The number of pigs lying in each part was recorded.

For the recording of the excretory behaviour weekly scores were used to monitor the fouling of the pen. Therefore, the pen was segmented in twelve parts. The scoring for the fouling was: grade $0(0 \%-10 \%$ fouling $), 1$ (10\%-25\% fouling), 2 (25\%-50\% fouling), 3 (50\%-75\% fouling) and 4 (75\%-100\% fouling). Only fouling caused by faeces and urine was considered and wet or dry fouling was not distinguished.

\section{Experimental design and data analysis}

In the experimental design the two rooms and the four pens represented spatial and the fattening periods temporal repetitions of the experimental lighting treatment. Thus the measurements on pen level were considered statistically independent. The repeated video observations within one pen and one fattening period (three measurements per pen and day of observation) were considered as measurement repetitions. The pigs were allocated to the pens randomly, but with consideration of an equal distribution of sex and weight over all pens. The experiment ran over two fattening periods in each room. For the statistical analysis of the lying behaviour the percentage of pigs lying on the solid or slatted floor was calculated for each pen and time of sampling. The data 
of room C2 (pen 202 and 205) of the first fattening period was not considered for the data analysis because of severe technical problems causing large gaps in the video data and varying operation times of the LED spotlights.

The lying behaviour data was analysed with a mixed model (procedure MIXED, SAS 9.4®). The fixed effects in the model were light (with and without) and time of day (morning, noon and evening) and their two-way interaction. The design effects room (C1 and C2), pen (102, 105, 202 and 205), fattening period (1 and 2) and day of observation (1-100) were considered as random effects. Their two-way interactions room* day of observation, pen* day of observation and fattening period* day of observation were also included as random effects. All other interactions of the design effects could not be implemented because in room $\mathrm{C} 2$ only data of one period was available. This would have caused over-parametrisation of the model. No fixed or design effect was removed from the model. Repeated measurements were taken into account by setting pen as subject and day of observation*time of day as repetition within the "repeated" statement of proc mixed. The normal distribution of the studentised residuals and their homoscedasticity were confirmed graphically using QQ-plots and plots of residuals versus predicted values. Comparisons of means were conducted using the Edwards-Berry procedure for controlling the family-wise type I error rate at a level of $\alpha=5 \%$ (Edwards and Berry, 1987).

For the results of the excretory behaviour, no statistical analysis was conducted. This data was analysed descriptively. On the basis of this data, an average score for the lying and slatted area was calculated. For considering the different sizes of the segments, the grade of the smaller segments in the slatted area were multiplied with 0.72 . This factor resulted from the surface ratio of the segments.

\section{Results and Discussion}

Temperature and Light intensity

The mean room temperature in $\mathrm{C} 1$ during the first fattening period was $21.4^{\circ} \mathrm{C}\left( \pm 1.4{ }^{\circ} \mathrm{C}\right)$ with a maximum of $26.3^{\circ} \mathrm{C}$, a minimum of $18.9^{\circ} \mathrm{C}$. During the second fattening period $21.2^{\circ} \mathrm{C}\left( \pm 1.6^{\circ} \mathrm{C}\right)$ with a maximum of 29 ${ }^{\circ} \mathrm{C}$, a minimum of $17^{\circ} \mathrm{C}$. The mean room temperature in $\mathrm{C} 2$ during the second fatting period was $20.5^{\circ} \mathrm{C}( \pm 1,9$ ${ }^{\circ} \mathrm{C}$ ) with a maximum of $28.1^{\circ} \mathrm{C}$, a minimum of $15.2{ }^{\circ} \mathrm{C}$. These measurements agree with the set temperature of the temperature curve. The temperature curve decrease from $25^{\circ} \mathrm{C}$ at the beginning to $18^{\circ} \mathrm{C}$ at the end of the fattening period. The ventilation rate was between $10 \%$ and $100 \%$.

The mean light intensities in the pens during the fattening periods are presented in Table 2. Because the room light and spotlights were switched on from 7 am to 6 pm only measurements in this timescale were considered.

The average light intensity measured by the fixed installed luxmeters for the not lit up areas was about 59.7 lux ( \pm 20.9 lux). The light intensity directly below the spotlights was about 29000 lux. The average light intensity on pig eye-height for the lit up area were $860.8 \operatorname{lux}( \pm 295.4 \operatorname{lux} ; \mathrm{n}=16)$ and for the other areas $61.5 \operatorname{lux}( \pm 10.2$ lux; $n=48$ ). To measure the light intensity reflected from the ground these measurements were also done with the detector facing downwards. The results for these measurements were for the lit up area 30.3 lux ( \pm 15 lux; $n$ $=16)$ and for the other areas 8.3 lux $( \pm 2.1$ lux; $n=48)$.

Table 2. Daily average light intensities (in lux) from 7 am to $6 \mathrm{pm}$ in room $\mathrm{C} 1$ and $\mathrm{C} 2$ during the fattening periods. Measured by the luxmeters installed horizontally $1.2 \mathrm{~m}$ above the floor with the detector facing upwards.

\begin{tabular}{|c|c|c|c|c|c|c|c|c|c|c|}
\hline Period & Room & Pen & Light & Sensor position & $\mathrm{n}$ & Average & $\mathrm{SD}$ & Minimum & Maximum & Median \\
\hline \multirow[t]{4}{*}{1} & \multirow[t]{4}{*}{$\mathrm{C} 1$} & \multirow[t]{2}{*}{102} & \multirow[t]{2}{*}{ off } & Lying area & 957 & 72.6 & 13.2 & 26.2 & 98.1 & 75.2 \\
\hline & & & & Slatted area & 957 & 77.1 & 10.5 & 42.2 & 92.5 & 80.3 \\
\hline & & \multirow[t]{2}{*}{105} & \multirow[t]{2}{*}{ on } & Lying area & 925 & 68.3 & 11.9 & 29.6 & 92.2 & 70.3 \\
\hline & & & & Slatted area & $\mathrm{N} / \mathrm{A}$ & $\mathrm{N} / \mathrm{A}$ & $\mathrm{N} / \mathrm{A}$ & $\mathrm{N} / \mathrm{A}$ & $\mathrm{N} / \mathrm{A}$ & $\mathrm{N} / \mathrm{A}$ \\
\hline \multirow[t]{4}{*}{2} & \multirow[t]{4}{*}{$\mathrm{C} 1$} & \multirow[t]{2}{*}{102} & \multirow[t]{2}{*}{ on } & Lying area & 932 & 64.0 & 17.9 & 23.9 & 96.7 & 64.3 \\
\hline & & & & Slatted area & $\mathrm{N} / \mathrm{A}$ & $\mathrm{N} / \mathrm{A}$ & $\mathrm{N} / \mathrm{A}$ & $\mathrm{N} / \mathrm{A}$ & $\mathrm{N} / \mathrm{A}$ & $\mathrm{N} / \mathrm{A}$ \\
\hline & & \multirow[t]{2}{*}{105} & \multirow[t]{2}{*}{ off } & Lying area & 932 & 55.7 & 15.7 & 19.4 & 89.6 & 56.35 \\
\hline & & & & Slatted area & 932 & 74.5 & 18.7 & 23.9 & 102.8 & 79.8 \\
\hline \multirow[t]{4}{*}{2} & \multirow[t]{4}{*}{$\mathrm{C} 2$} & \multirow[t]{2}{*}{202} & \multirow[t]{2}{*}{ on } & Lying area & 921 & 35.9 & 11.1 & 8.3 & 54.8 & 39.2 \\
\hline & & & & Slatted area & $\mathrm{N} / \mathrm{A}$ & N/A & N/A & $\mathrm{N} / \mathrm{A}$ & N/A & $\mathrm{N} / \mathrm{A}$ \\
\hline & & \multirow[t]{2}{*}{205} & \multirow[t]{2}{*}{ off } & Lying area & 921 & 55.0 & 15.6 & 13.6 & 79.7 & 59.4 \\
\hline & & & & Slatted area & 921 & 33.3 & 12.2 & 4.1 & 54.4 & 35.1 \\
\hline
\end{tabular}

To find a possible effect extreme large difference between lit up and other areas was chosen. In future studies the effect of a less increased light intensity should be tested. Taylor et al. (2006) showed that already a light intensity of 400 lux at pig eye-height led to more excretory and less lying behaviour.

Lying behaviour

Summarizing for all observation times and observation days the percentage of pigs lying on the solid lying area in the lit up pens was about $76 \%( \pm 15 \%)$ with a maximum of $100 \%$, a minimum of $33 \%$. In the pens without spotlights, the percentage amount was $69 \%( \pm 17 \%)$ with a maximum of $100 \%$, a minimum of $22 \%$. In the pens 
with increased light intensity $12 \%$ more pig lie on the lying area compared to the pens without spotlights $(\mathrm{p}<0.0001)$ (Fig. 3). Depending on the time of day the results show that more pigs lie on the lying area in the morning then in the noon $(-0,7 \%, \mathrm{p}<0.0001)$ or in the evening $(-0,5 \%, \mathrm{p}<0.0001)$ (Fig. 4$)$.

Considering the results separately for the individual fattening periods and rooms, several differences can be recognized. The most obvious differences are shown during the first fattening period in $\mathrm{C} 1$ (Tab. 3). Compared to this, it seems there are no differences between the pen with and without spotlights in room C2 (Tab. 3).

Table 3. Effect of the light above the slatted area on percentage of pigs (relative of total number of lying pigs) lying on the solid lying area for each pen and fattening period. On each day of observation (three days a week), the lying behaviour observed two or three times (n).

\begin{tabular}{ccccccccccc}
\hline Period & Room & Pen & Light & Days of observation & $\mathrm{n}$ & Average & SD & Minimum & Maximum & Median \\
\hline \multirow{2}{*}{1} & \multirow{2}{*}{$\mathrm{C} 1$} & 102 & 0 & 29 & 85 & 61.6 & 15.8 & 22.2 & 100 & 61.1 \\
\cline { 3 - 11 } & & 105 & 1 & 29 & 85 & 67.3 & 12.7 & 46.7 & 94.4 & 68.8 \\
\hline \multirow{2}{*}{2} & \multirow{2}{*}{$\mathrm{C} 1$} & 102 & 1 & 35 & 107 & 75.2 & 15.7 & 33.3 & 100 & 77.8 \\
\cline { 3 - 11 } & & 105 & 0 & 35 & 106 & 59.4 & 12.3 & 26.7 & 88.9 & 60 \\
\hline \multirow{2}{*}{2} & \multirow{2}{*}{$\mathrm{C} 2$} & 202 & 1 & 36 & 107 & 83.6 & 11.5 & 50 & 100 & 83.3 \\
\cline { 3 - 10 } & & 205 & 0 & 36 & 107 & 84.3 & 10.6 & 53.3 & 100 & 84.6 \\
\hline
\end{tabular}

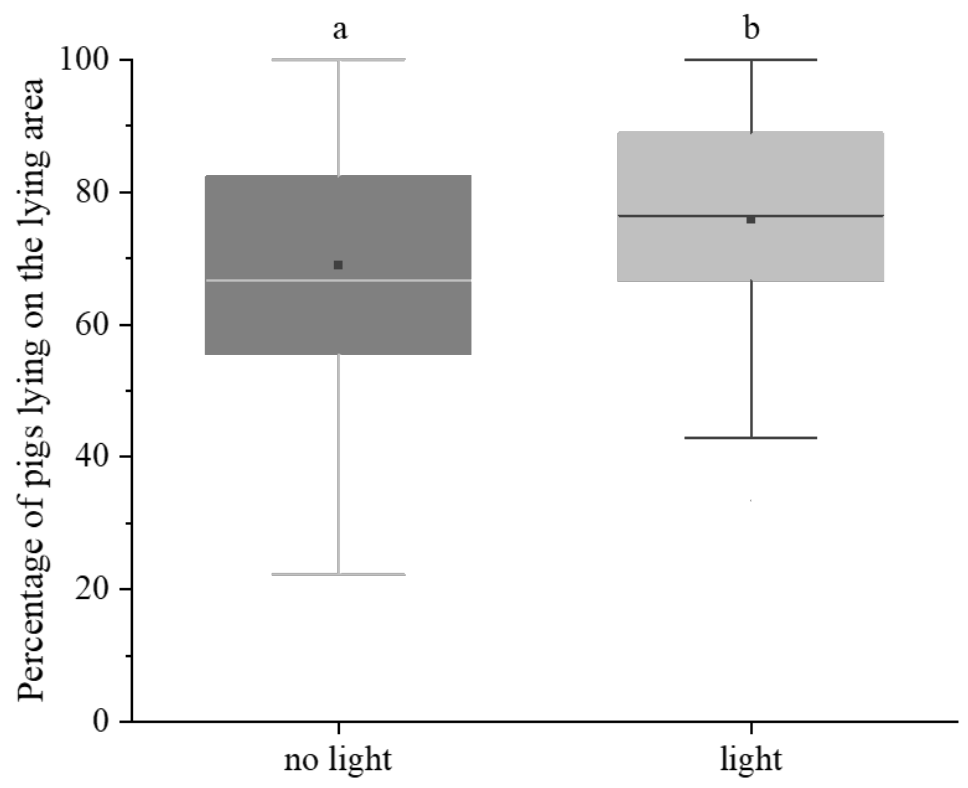

Figure 3. Effect of the light on percentage of pigs (relative of total number of lying pigs) lying on the solid lying area. Dark boxes represent pens without spotlights above the slatted area; light boxes represent the pens with spotlights above the slatted area; $a, b$ : different letters indicate significant differences $(\mathrm{p}<0.05)$.

These results lead to the conclusion that pigs avoid areas with high light intensity for lying and use them mainly for excretory. Other studies show a higher level of resting in darker areas and more activity and excretory behaviour in lighter areas (Christison 1995, 5 lux vs. 100 lux; Taylor et al. 2006 2,4 lux, 4 lux, 40 lux and 400 lux). Therefore, a decreased light intensity in the lying area could be another way to increase the number of pigs lying in the lying area.

Reasons for the missing effect in room $\mathrm{C} 2$ are not clear. A possible reason could be the high fouling of the lying area in the neighboured pen directly at the pen partition. Due to that the faeces and urine ran under the pen partition into the other pen. This could lead to fouling of the lying area in the experimental pen and increased excretory behaviour in this area (Yu et al. 2016). Beside the light intensity among others temperature of room and floor and air speed influence the lying behaviour and should be examined (Randall et al. 1983).

Differences in the results of the two rooms will be examined in further periods. Moreover, the impact of air humidity, room and surface temperature will be investigated. 


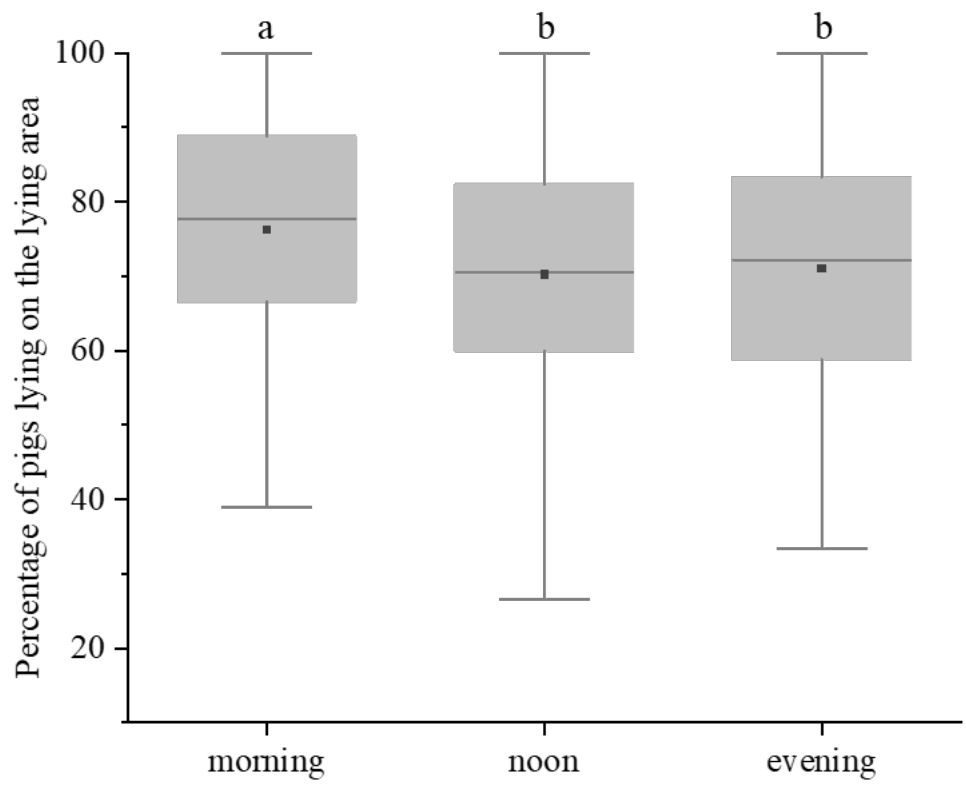

Figure 4. Effect of time of day on percentage of pigs (relative of total number of lying pigs) lying on the solid lying area. Plots that do not share the same letters denote statistical significance $(\mathrm{p}<0.05)$.

Excretory behaviour

Considering the results of the pen fouling they indicate similarities to the lying behaviour. The most obvious differences between the lit up pen and the unchanged pen are in room $\mathrm{C} 1$ during the second fattening period. In $\mathrm{C} 2$ the average grades are nearly the same (Tab. 4). The results of the fouling on the slatted area shows no clear differences between the lit up pens and the others (Tab. 5).

Table 4. Effect of the light above slatted area on fouling on solid lying area. Average grade of weekly scores. $(0(0-10 \%$ fouling), 1 (10-25\% fouling), 2 (25-50\% fouling), 3 (50-75\% fouling) and 4 (75-100\% fouling).

\begin{tabular}{|c|c|c|c|c|c|c|c|c|c|}
\hline Period & Room & Pen & Light & $\mathrm{n}$ & Average & SD & Minimum & Maximum & Median \\
\hline \multirow{2}{*}{1} & \multirow{2}{*}{$\mathrm{C} 1$} & 102 & 0 & 13 & 1.23 & 0.88 & 0 & 2.50 & 1.50 \\
\hline & & 105 & 1 & 13 & 0.73 & 0.44 & 0.33 & 1.83 & 0.67 \\
\hline \multirow{2}{*}{2} & \multirow{2}{*}{$\mathrm{C} 1$} & 102 & 1 & 11 & 0.80 & 0.56 & 0 & 1.67 & 0.67 \\
\hline & & 105 & 0 & 11 & 2.10 & 0.48 & 1.50 & 2.83 & 2.17 \\
\hline \multirow{2}{*}{2} & \multirow{2}{*}{$\mathrm{C} 2$} & 202 & 1 & 11 & 0.82 & 0.80 & 0.17 & 2.33 & 0.50 \\
\hline & & 205 & 0 & 11 & 0.95 & 0.58 & 0.17 & 2.17 & 0.83 \\
\hline
\end{tabular}

Table 5. Effect of the light above slatted area on fouling on slatted area. Average grade of weekly scores. $(0(0-10 \%$ fouling), 1 (10-25\% fouling), 2 (25-50\% fouling), 3 (50-75\% fouling) and 4 (75-100\% fouling).

\begin{tabular}{|c|c|c|c|c|c|c|c|c|c|}
\hline Period & Room & Pen & Light & $\mathrm{n}$ & Average & SD & Minimum & Maximum & Median \\
\hline \multirow{2}{*}{1} & \multirow{2}{*}{$\mathrm{C} 1$} & 102 & 0 & 13 & 0.79 & 0.26 & 0.36 & 1.25 & 0.77 \\
\hline & & 105 & 1 & 13 & 0.73 & 0.44 & 0.33 & 1.83 & 0.67 \\
\hline \multirow{2}{*}{2} & \multirow{2}{*}{$\mathrm{C} 1$} & 102 & 1 & 11 & 1.60 & 0.58 & 0.60 & 2.66 & 1.70 \\
\hline & & 105 & 0 & 11 & 1.40 & 0.39 & 0.96 & 2.16 & 1.32 \\
\hline \multirow{2}{*}{2} & \multirow{2}{*}{$\mathrm{C} 2$} & 202 & 1 & 11 & 1.94 & 0.69 & 0.89 & 2.78 & 2.78 \\
\hline & & 205 & 0 & 11 & 1.72 & 0.66 & 0.72 & 2.64 & 1.92 \\
\hline
\end{tabular}

The highly increased light intensity seems to lead to a lower excretory behaviour in the lying are. However, the light intensity seems to have no effect of the fouling in the slatted area. Tylor et al. (2006) has shown that an increased light intensity lead to higher excretory behaviour.

The reason for the missing effect in room $\mathrm{C} 2$ could be the same that have been discussed in point 3.2. 


\section{Conclusion}

The results show that strong increased light intensity in the slatted area lead to higher percentage of pigs lying in the lying area. Also fouling of the lying area seems to decrease while fouling of the slatted area was not affected. In the morning, more pigs are lying in the solid lying area than in the noon or evening. For this study a strong increase of light intensity was tested. In future studies it should be tested whether a lower increase can also lead to a better acceptance of the excretory areas. Moreover, it should be tested weather a decrease of the light intensity in the lying areas supports the pigs in classifying the functional areas. Influences of floor and room temperature should be examined in detail. In conclusion, a differentiated lighting strategy above different functional areas seems to be a promising tool for further improvement of lying and excretory areas or behaviour.

\section{Acknowledgement}

The project is supported by funds of the Federal Ministry of Food and Agriculture (BMEL) based on a decision of the Parliament of the Federal Republic of Germany via the Federal Office for Agriculture and Food (BLE) under the innovation support programme. We thank our project partners Bildungs- und Wissenszentrum LSZ (Boxberg-Windischbuch, Germany), Deutscher Tierschutzbund (Bonn, Germany), Vion Food International (Bad Bramstedt, Germany) and the Friedrich-Loeffler-Institut (Celle, Germany).

\section{References}

Börgermann, B., Rus, M., Kaufmann O., 2007, Sensorgestützte Überprüfung des Wahlverhaltens von Mastschweinen - Welche Fußböden und Beschäftigungsangebote werden bevorzugt? LandTechnik Agricultural Engineering 62 (4), 228-229. http://dx.doi.org/10.15150/1t.2007.991

Christison, G. I., 1996, Dim light does not reduce fighting or wounding of newly mixed pigs at weaning. Canadian Journal of Animal Science 76(1), 141-143. https://doi.org/10.4141/cjas96-019

Edwards, D., Berry, J.J., 1987, The efficiency of simulation-based multiple comparisons. Biometrics 43 (4), 913-928. http://dx.doi.org/10.2307/2531545

Hacker, R. R., Ogilvie, J. R., Morrison, W. D., Kains, F., 1994, Factors affecting excretory behavior of pigs. Journal of animal sience, 72 (6), 1455-1460. http://jas.fass.org/content/72/6/1455

Randall, J.M., Armsby, A.W., Sharp, J.R., 1983, Cooling gradients across pens in a finishing piggery: II. Effects on excretory behaviour. Journal of Agricultural Engineering Research 28 (3), 247-259. https://doi.org/10.1016/0021-8634(83)90073-2

Taylor, N., Prescott, N., Perry, G., Potter, M., Le Sueur. C., Wathes C., 2006, Preference of growing pigs for illuminance. Applied Animal Behaviour Science 96, 19-31. https://doi.org/10.1016/j.applanim.2005.04.016

Yu, W., Dapeng, L., Xiong, S., Zhengxiang S., 2016, Effects of inductive methods on dunging behavior of weaning pigs in slatted floor pens. International Journal of Agricultural and Biological Engineering 9(4), 192198. https://doi.org/10.3965/j.ijabe.20160904.2560 


\title{
Influence of Wind Direction and Sampling Strategy on the Estimation of Ammonia Emissions in Naturally Ventilated Barns
}

\author{
David Janke a,", Dilya Willink ${ }^{\text {a }}$, Sabrina Hempel a, Christian Ammon ${ }^{\text {a }}$, Barbara Amon a , Thomas \\ Amon ${ }^{\text {a,b }}$ \\ ${ }^{a}$ Leibniz Institute for Agricultural Engineering and Bioeconomy (ATB), Potsdam, Germany \\ ${ }^{\mathrm{b}}$ Department of Veterinary Medicine, Freie Universität Berlin, Berlin, Germany \\ * Corresponding author. Email: djanke@atb-potsdam.de
}

\begin{abstract}
Ammonia and greenhouse gases have a negative impact on the environment. Cattle housing systems are a major contributor to agricultural gaseous emissions, where dairy cattle housing systems are mainly naturally ventilated. Estimating emissions for naturally ventilated barns (NVB) is challenging due to a large number of influencing factors. Especially the direct coupling of the inside flow regime with the ambient, turbulent weather conditions makes it hard to measure the air exchange rate and the emissions. Often, the measurement setup is designed to cover only the main wind direction. In case of deviating wind directions, the measured values are neglected, which extends the required duration of the measurement campaign in order to have a sufficiently large dataset. To overcome this limitation, we equipped an NVB in Northern Germany with an extensive setup, making it measureable for every wind direction. More than $900 \mathrm{~m}$ sampling lines in- and outside the barn with critical orifices every $5-10 \mathrm{~m}$ were installed. Gases like ammonia $\left(\mathrm{NH}_{3}\right)$, methane $\left(\mathrm{CH}_{4}\right)$ and carbon dioxide $\left(\mathrm{CO}_{2}\right)$ were measured in parallel with two Fourier-Transformations-Infrared-Spectrometer (FTIR) devices. Hourly emission factors for $\mathrm{NH}_{3}$ over a period of more than 200 days were derived by using the $\mathrm{CO}_{2}$ balance method and two different sampling methods for inside-outside concentrations. The results show a gain in usable data up to $190 \%$ when also taking into account wind conditions that deviate from the main wind direction. The derived emission factors strongly depend on the choice of the sampling line according to the wind direction. A first analysis shows differences in the $\mathrm{NH}_{3}$ emission factors for different wind directions between $-2.23 \mathrm{~g} / \mathrm{h}$ and $+0.23 \mathrm{~g} / \mathrm{h}$ per livestock unit. Further investigation needs to be done to identify the correlation of wind direction, ambient temperature and emission factors.
\end{abstract}

Keywords: Dairy barn, air exchange, $\mathrm{CO}_{2}$ balance, long term measurements

\section{Introduction}

The first goal of this study was to increase the amount of usable data. This means that, independent of pollutant sources like neighbouring barns or manure stores, the air exchange rates (AER) and emission factors for $\mathrm{NH}_{3}(\mathrm{EF})$ should be measurable for any approaching flow direction. In this way, more measurement values can be taken into account for the estimation of AER and EF. Hence, either the duration of a measurement campaign can be shortened while keeping the required number of samplings for statistical accuracy or the number of samplings can be increased for a constant duration of the campaign.

The second goal was the quantification of the influence of the sampling strategy on the estimation of AER and EF. Since their estimation relies on indirect balancing methods, the difference of measured outside and inside concentration of $\mathrm{CO}_{2}$ (for the $\mathrm{AER}$ ) and $\mathrm{NH}_{3}$ (for the $\mathrm{EF}$ ) is a major influence. The difference is directly dependent on the choice of the sampling location for the outside and inside concentration measurements. With many sampling lines in- and outside the barn, many combinations of in- and outside samplings are possible to determine the concentration differences.

In this study, two exemplifying strategies for the choice of outside and inside sampling will be investigated according to their influence on AER and EF estimation.

\section{Materials and Methods}

The investigated barn is a naturally ventilated dairy barn (NVB) near Rostock in Northern Germany. It has a length of $96.15 \mathrm{~m}$, a width of $34.2 \mathrm{~m}$, and the roof height varies from $4.2 \mathrm{~m}$ at the side walls up to $10.7 \mathrm{~m}$ at the gable top. The side walls are completely open; the gable walls are partially open as depicted in Figure 28 . The barn encloses an overall volume of $25,000 \mathrm{~m}^{3}$ and houses on average 375 dairy cows. It is equipped with a floor scraping system to remove the manure from the two walking alleys with a concrete floor. Four ceiling fans are mounted along the centreline of the barn $5.6 \mathrm{~m}$ above the feeding alley with a diameter of $7.3 \mathrm{~m}$ and are operated in the warmer periods. Further detailed information about the barn, the management or the animals can be found e.g. in (Hempel, 2016) or (Fiedler, 2014). 

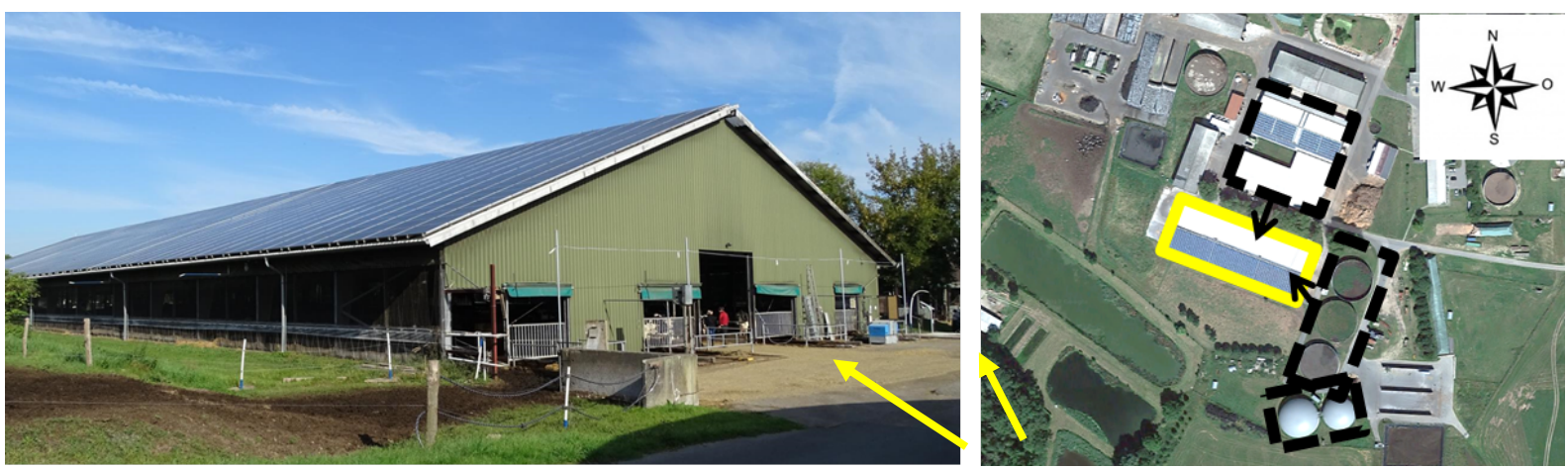

Figure 28. Left: side view of the investigated barn. Arrows mark the underground slurry tank, where the slurry is dumped by the scrapers. Right: top view. The solid yellow box marks the barn, the dashed black boxes mark external sources of $\mathrm{CO}_{2}$ or $\mathrm{NH}_{3}$ that possibly enter the barn and have to be taken into account when using balancing methods.

\section{Measurement setup}

Gaseous concentrations were measured using two high resolution Fourier-Transform-Infrared-Spectrometer (FTIR) measurement devices (Gasmet CX4000). Sample air was sucked through PTFE tubes with an inner diameter of $6 \mathrm{~mm}$. Every $10 \mathrm{~m}$, the tubes had an orifice with a capillary trap, which ensured uniform volumetric flow at every orifice. At the roof of the barn, an ultra-sonic anemometer (USA, Windmaster Pro ultrasonic anemometer, Gill Instruments Limited, Lymington, Hampshire, UK) was installed to measure the wind velocity and direction.

Inside the barn, six sample lines were installed, so that each side or opening was equipped and two lines were placed in the middle. All lines were positioned at a height of $3 \mathrm{~m}$ except the second middle line, which was at a height of $5 \mathrm{~m}$. Outside the barn, six sampling lines were installed, one on each side or opening of the barn and two for additional measurements of potential hot spots. Figure 2 gives an overview of the installations. The duration of measurement per line was 10 minutes and each FTIR was connected to six lines, so that one cycle per hour was measured, providing data for hourly AER and EF estimation.

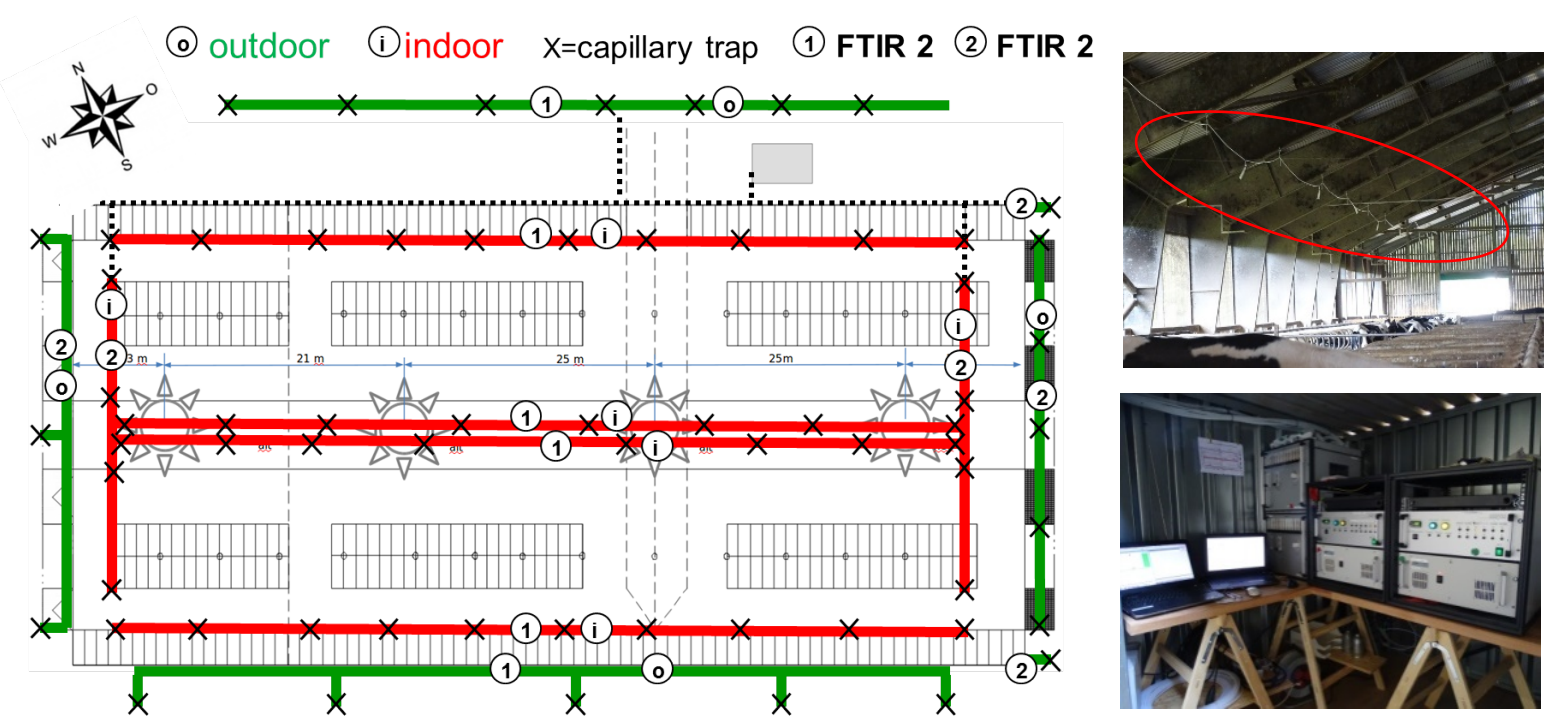

Figure 161. Sketch of the installed sampling lines and pictures of the installation inside the barn and the FTIR devices.

\section{Measurement data and processing}

Measurements with this setup were carried out from November 2016 until September 2017. Due to other experiments in the barn during this period (21/02-16/03/..2017, 23/..05-15/..06/..2017 and 08/..08/..$31 / . .08 / . .2017)$, a number of 1728 hours could not be taken into account for this study. A number of 199 further hours could not be used for analysis due to maintenance of the devices or other technical issues. Overall, this resulted in a total number of 133 days usable measurement data, providing 3379 hourly values.

For every hour, the total volume flow $\mathrm{Q}\left[\mathrm{m}^{3} \mathrm{~h}^{-1}\right]$ was estimated by the $\mathrm{CO}_{2}$ gas balancing method according to (CIGR 2002). The air exchange rate was calculated as the ratio of $\mathrm{Q}$ and $\mathrm{V}$, where $\mathrm{V}$ is the total volume of the 
barn. The EF for $\mathrm{NH}_{3}$ was then calculated as the product of volume flow and measured difference of $\mathrm{CO}_{2}$ concentrations in- and outside the barn, based on one livestock unit LU [500 kg].

For the computation of the AER, no filter (like e.g. a cut-off for differences $\Delta \mathrm{CO} 2=\mathrm{CO} 2_{\text {inside }}-\mathrm{CO} 2_{\text {outside }}<$ $20 \mathrm{ppm}$ ) was used. Only values that resulted in a negative AER or EF, were skipped out (17 values for method 1 and 3 values for method 2 ).

\section{Sampling strategy}

In this study, two different sampling strategies were tested. The first method (method 1) takes into account the hourly wind direction and is sketched in Figure 162. For the outside concentration of $\mathrm{CO}_{2}$ and $\mathrm{NH}_{3}$, the windward sampling line outside the barn is taken, and for the inside concentrations, the sampling line on the leeward side inside the barn is taken.

The second method (method 2), does not take into account the information about the wind direction. The mean value from the inside sampling lines is taken for the inside concentration and the minimum value from the four outside sampling lines is taken as outside concentration.

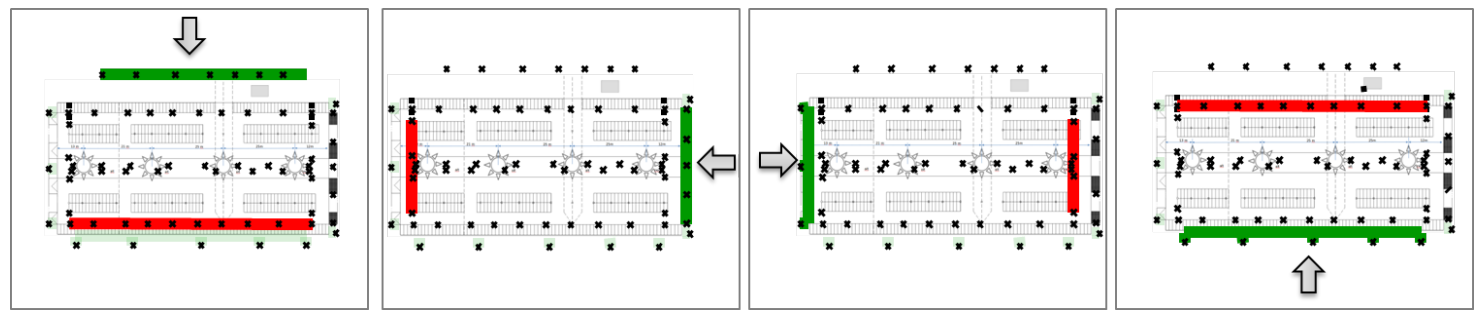

Figure 162. Sampling strategy for method 1 . The arrow marks the approaching flow direction, the green line is the chosen sampling line for outside gas concentrations, the red line is the sampling line for inside concentrations.

\section{Results and Discussion}

Gain in usable data

The approaching flow was divided into four sectors, each sector with an angle of $90^{\circ}$. The angles were adjusted to the orientation of the barn ( $+17^{\circ}$ spin to the north-south axis), so that each sector's symmetry line was perpendicular either to the longitudinal or the lateral openings. In the following, flow entering the barn at the longitudinal side openings will be called north or south, and flow entering at the lateral openings at the gable walls will be called west or east.

A total number of 3379 usable hourly values was measured. They were distributed as follows: 377 events from North, 1158 events from south, 882 events from east and 962 events from west. If the system had been designed only to measure values for the main wind direction (south), the actual setup would result in a gain in usable values of around 190\%. If the system had been designed to measure both cross wind directions approaching the barn (north and south) the actual setup would result in a gain of around $120 \%$.

\section{Air exchange rates}

In Figure 163, the estimated AERs for each method and wind directions are plotted against the approaching flow direction, and a regression model was applied. For all methods and wind directions, an exponential regression model fitted best. Least variations can be seen for method 1 with wind from south $\left(\mathrm{R}^{2}=0.47\right)$ and west $\left(\mathrm{R}^{2}=0.49\right)$, and for method 2 with wind from south $\left(\mathrm{R}^{2}=0.51\right)$. For both methods, the AERs for northern and eastern wind direction show a higher variance in the wind speed range $<1 \mathrm{~m} \mathrm{~s}^{-1}$. A reason for this could be the buildings around the barn. For wind directions north and east, the flow is disturbed and decelerated by these buildings, see Figure 28. Since the wind speed is measured above the roof, the actual speed approaching the barn openings might be lower. Low wind speeds or no wind speed at all can result in a strong overprediction of AERs estimated by $\mathrm{CO}_{2}$ balancing methods, because the measured $\mathrm{CO}_{2}$ concentration at the supposed inlet is actually the out-pouring gas from inside the barn and results in very low $\mathrm{CO}_{2}$ differences, which themselves result in very high incorrectly estimated AERs.

To overcome this, a possible way could be the application of a cut-off filter, which sorts out all the values where either the concentration difference of $\mathrm{CO}_{2}$ inside and $\mathrm{CO}_{2}$ outside the barn is less than a certain value (e.g. $20 \mathrm{ppm}$ ), or a physically reasonable threshold for the estimated volume flow at the actual wind speed is applied (e.g. $1 \mathrm{~m} \mathrm{~s}^{-1}$ on an inlet with $100 \mathrm{~m}^{2}$ cannot result in more than $100 \mathrm{~m}^{3} \mathrm{~s}^{-1}$ ). 

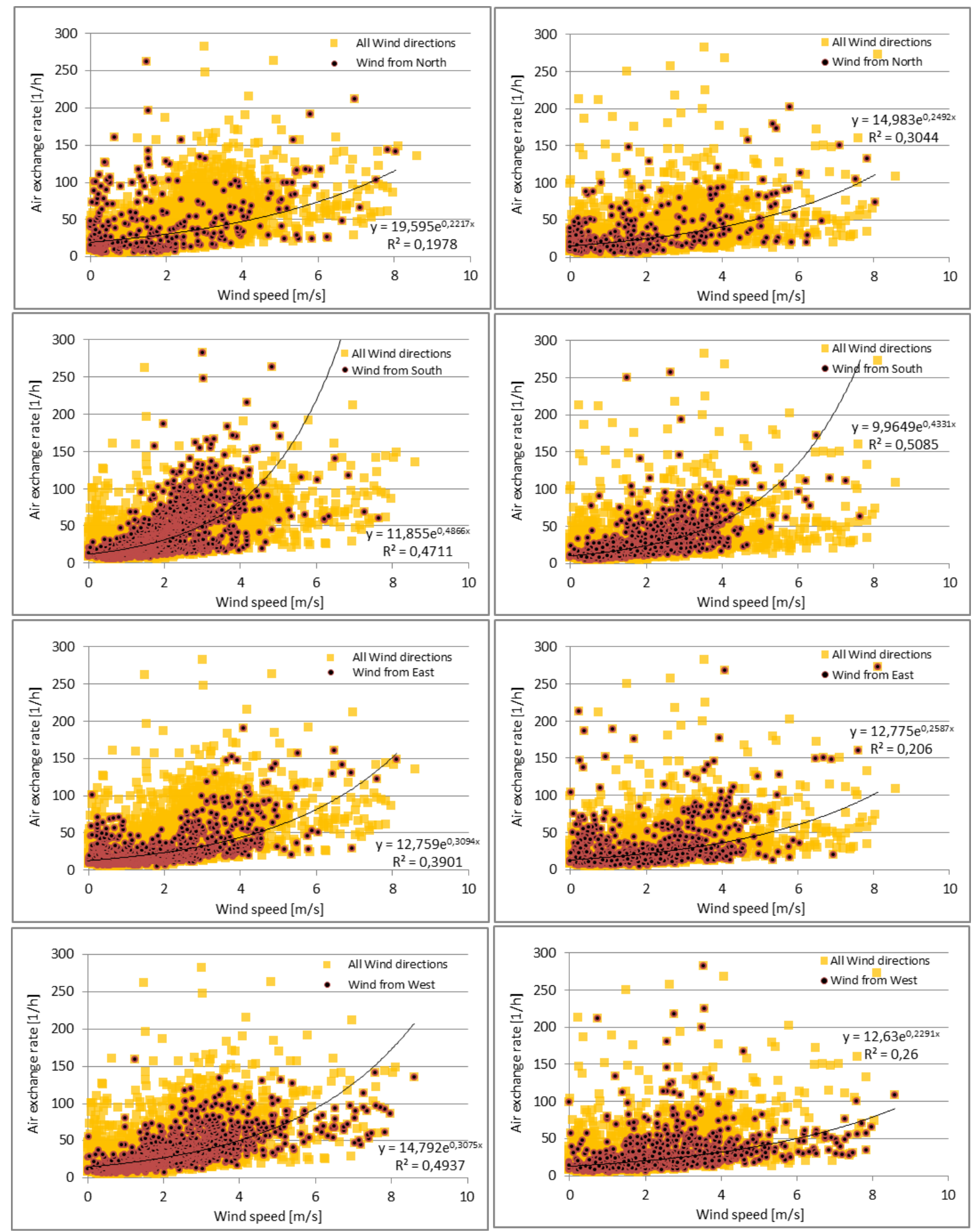

Figure 163. Calculated air exchange rates and measured wind speeds. The yellow rectangles show all AER calculated for a method, the black dotsshow the AER for their respective wind directions. Left: For method 1, the AERs were estimated by choosing the windward sample line outside the barn and the leeward sample line inside the barn for computing the $\mathrm{CO}_{2}$ differences. Right: For method 2, the AERs were estimated by choosing the minimum value of the outside sample lines and the mean value of the inside sampling lines. From top to down: approaching wind from north, south, east and west. 
Emission Factors

The estimated EFs for each wind direction for method 1 and 2 are shown as boxplots in Figure 32. Differences between the EFs for the respective wind directions of the two methods (e.g. AER(method $1_{\text {north }}$ ) AER(method 2 north $)$ ) were tested for statistical significance using a F-test. For all wind directions, method 1 and 2 differ significantly from each other. The mean values of EFs for method 1 and method 2 are compared in table 1.

Table 65. Comparison of method 1 and 2. Shown are emission factors of $\mathrm{NH}_{3}$ for wind from north, east, south and west.

\begin{tabular}{lcccc}
\hline & North & East & South & West \\
\hline Method 1 $\mathrm{EF} \mathrm{NH}_{3}\left[\mathrm{~g} \mathrm{~h}^{-1} \mathrm{LU}^{-1}\right]$ & 1.41 & 1.20 & 1.32 & 3.43 \\
Method 2 $\mathrm{EF} \mathrm{NH}_{3}\left[\mathrm{~g} \mathrm{~h}^{-1} \mathrm{LU}^{-1}\right]$ & 1.13 & 0.90 & 1.14 & 1.20 \\
relative change [\%] & -19.97 & -25.03 & -14.03 & -65.06 \\
\hline
\end{tabular}

For all directions, method 2 estimates lower EFs than method 1. For directions north, east and south, the difference is in the range of $-20 \%$ to $-25 \%$. For method 1 , the EF estimation for western wind directions obviously differs from all other values, both in mean value and variance. The EF for $\mathrm{NH}_{3}$ is calculated as the product of volume flow and the concentration difference of $\mathrm{NH}_{3}$ in- and outside the barn. Since the volume flow for method 1, western wind direction, does not show such obviously higher variances (see Figure 163), the reason for the strong deviation of the EF calculated by method 1 is likely to be found in the values taken for the $\mathrm{NH}_{3}$ difference inside-outside. For western winds, the outside sample line 2-o on the western gable wall was chosen for outside concentrations, and sample line 2-i (east side of the barn) was taken for inside concentrations, see Figure 162. If the EF is high and highly fluctuating for western winds, the most likely reason for that is the concentration measurement inside the barn, because no ambient sources west of the barn are expected to import high levels of external $\mathrm{NH}_{3}$ into the barn. Sample line 2-i was located next to the open underground slurry storage, where the scrapers dump the slurry, see Figure 28 left picture. We suppose the release of $\mathrm{NH}_{3}$ from the surface of the slurry tank to be the source for the higher EFs, by wrongly attributing these $\mathrm{NH}_{3}$ concentrations as inside concentrations that are leaving the barn. A further study, comparing concentration measurements with sample line 2-i eastside inside the barn and line 2-o eastside outside the barn will be done to check this assumption. Within each method, the differences of EF estimations depending on the wind direction were tested for significance shown in Table 2.

Table 66. Differences between $\mathrm{NH}_{3}$-EF for the wind directions within each method. Colored cells show differences with western directions for method 1 . The symbol "-“ means no significant difference could be found.

\begin{tabular}{ccccccc}
\hline & $\mathrm{N}-\mathrm{E}$ & $\mathrm{N}-\mathrm{S}$ & $\mathrm{N}-\mathrm{W}$ & $\mathrm{E}-\mathrm{S}$ & $\mathrm{E}-\mathrm{W}$ & $\mathrm{S}-\mathrm{W}$ \\
\hline Method 1 Difference $\mathrm{NH}_{3}\left[\mathrm{~g} \mathrm{~h}^{-1} \mathrm{LU}^{-1}\right]$ & 0.21 & - & -2.02 & -0.12 & -2.23 & -2.11 \\
Method 2 Difference $\mathrm{NH}_{3}\left[\mathrm{~g} \mathrm{~h}^{-1} \mathrm{LU}^{-1}\right]$ & 0.23 & - & - & -0.24 & -0.3 & - \\
\hline
\end{tabular}

If the emission of $\mathrm{NH}_{3}$ is considered to be independent of the flow direction, then the differences between the estimated EFs for the different wind directions can be used as a an assessment criterion: The smaller the difference of estimated EF among the wind directions, the better the method. In this case, method 2 would be the preferred one, since the differences are lower than those for method 1. 


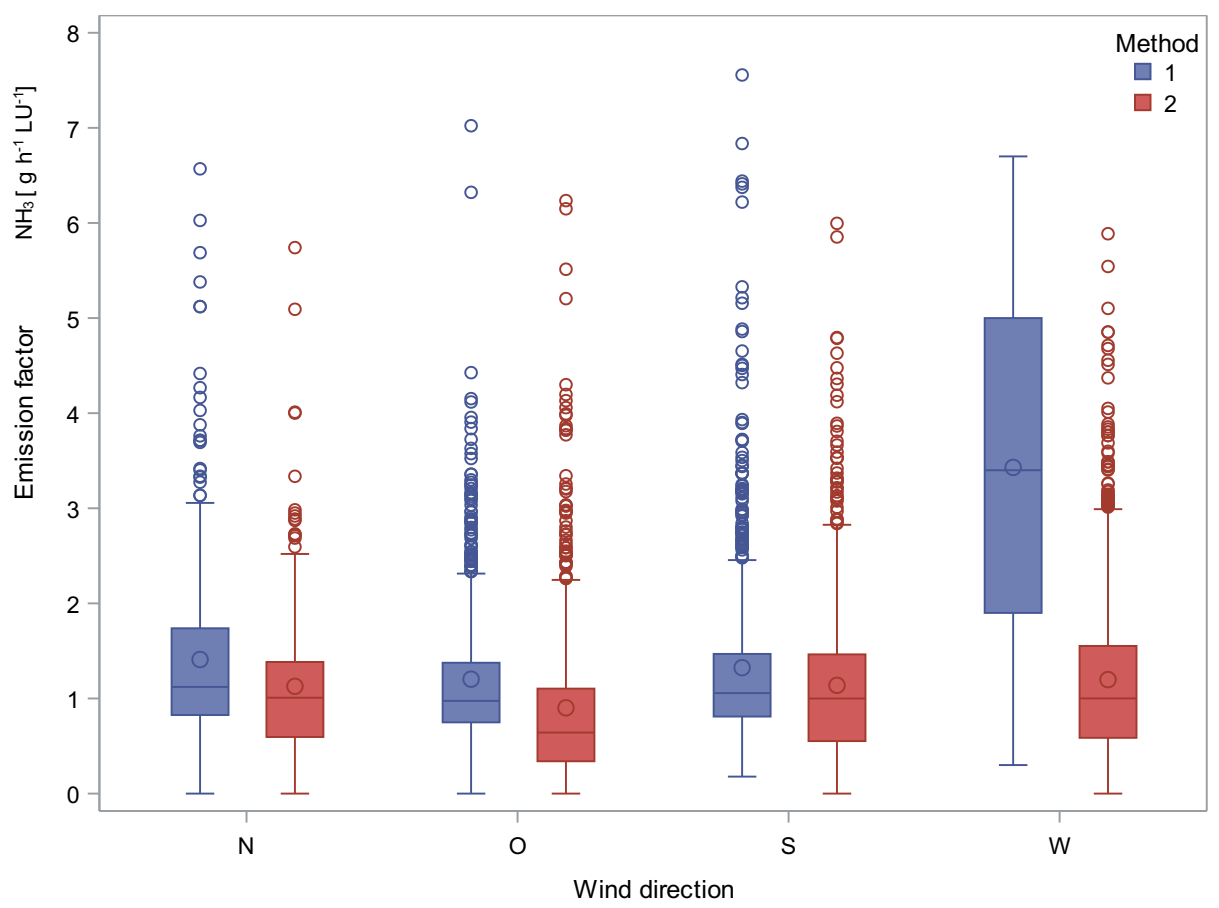

Figure 32. Comparison of the estimated emission factors for $\mathrm{NH}_{3}$ in $\mathrm{g} \mathrm{h}^{-1} \mathrm{LU}^{-1}$. The values are presented as boxplots with the boxes including values from the lower quartile until the upper quartile. The solid line inside the box represents the median, the dot represents the mean value.

\section{Conclusions}

Air exchange rates and emission factors for $\mathrm{NH}_{3}$ were measured in a $\mathrm{NVB}$ with the indirect $\mathrm{CO}_{2}$ balancing method. The measurement setup was designed to be applicable for every approaching flow direction. Two different sampling strategies to derive the in- and outside concentration differences of $\mathrm{CO}_{2}$ and $\mathrm{NH}_{3}$ were applied and their results were compared.

For the measurement period, a gain in usable data of $120 \%$ (compared to a setup designed for the main wind direction and the opposite direction) or 190\% (compared to a setup designed for the main wind direction) could be observed.

Every method differed in the estimation of $\mathrm{NH}_{3}-\mathrm{EFs}$ for the four wind directions. Method 2 is considered to be more reliable under consideration of a wind direction independent emission source of $\mathrm{NH}_{3}$. Further investigations must be done to quantify the influence of the ambient temperature.

The investigation of the influence of wind direction and sampling strategy on AER and EF was done for four $90^{\circ}$-wide wind direction sectors. This results in several values, which cannot clearly be assigned to one wind direction (e.g. the transition from sector south to sector west). Further investigations with four narrower angled sectors will be done to verify if this can lead to results with less variation for the AER and EF estimation..

\section{Acknowledgements}

We like to acknowledge Uli Stollberg and Andreas Reinhard, technicians at ATB, for the technical support during the measurements.

\section{References}

Hempel, S., Saha, C. K., Fiedler, M., Berg, W., Hansen, C., Amon, B., \& Amon, T. (2016). Non-linear temperature dependency of ammonia and methane emissions from a naturally ventilated dairy barn. Biosystems Engineering, 145, 10-21. https://doi.org/10.1016/j.biosystemseng.2016.02.006

Fiedler, M., Fischer, J., Hempel, S., Saha, C. K., Loebsin, C., Berg, W., \& Amon, T. (2014). Flow fields within a dairy barn-Measurements, physical modeling and numerical simulation. In Proceedings of the International Conference of Agricultural Engineering, Zurich (pp. 1-8).

CIGR, 2002. 4th report from working group on climatization of animal houses. In: Pedersen, S., Sällvik, K. (Eds.), Research Centre Bygholm. DIAS, Horsens, Denmark. 45 pp. www.agrsci.dk/jbt/spe/CIGRreport 


\title{
Low Emission Slat Design for Swine Buildings
}

\author{
Stéphane Godbout ${ }^{\text {**, Francy Vanegas }}{ }^{\text {b }}$, Joahnn H. Palacios a, Matthieu Girard a , Ariane Lévesque a , \\ Alain Rousseau ${ }^{c}$ \\ a Research and Development Institute for the Agri-Environment (IRDA), 2700 Einstein, Quebec City, G1P 3W8, \\ Quebec, Canada \\ ${ }^{b}$ Department of Civil and Agricultural Engineering, National University of Colombia, Carrera 45 № 26-85, Bogota, \\ Colombia \\ c Institut National de la Recherche Scientifique (INRS - Eau, Terre et Environnement), Université du Québec, 490 \\ Couronne, Quebec City, G1K 9A9, Quebec, Canada \\ * Corresponding author. Email: stephane.godbout@irda.qc.ca
}

\begin{abstract}
In 2015, agriculture accounted for more than 90\% (455 kt) of all ammonia $\left(\mathrm{NH}_{3}\right)$ emissions in Canada. Livestock production was the largest contributor, emitting around $66 \%(300 \mathrm{kt})$ of the agricultural emissions. $\mathrm{NH}_{3}$ is mainly produced by the degradation of the urea in urine. The contact between air and excreta plays a key role in $\mathrm{NH}_{3}$ emissions from livestock buildings. Consequently, the emission rate is closely related to the soiled floor surface. The objective of this study was to evaluate the impact of a new slat profile design on $\mathrm{NH}_{3}$ emissions in swine buildings. The new profile has a notch along the side of the slat so that the manure will drop directly into the pit without sliding down the side therefore reducing the total soiled area. Two control and two treatment slat sections were placed in two rooms where 16 pigs in each room were bred for 8 weeks. The four soiled sections were then removed from the pig rooms, washed with pressurized water and put in four individual desorption chambers made of galvanized steel where $\mathrm{NH}_{3}$ emissions were measured individually. Additionally, emissions from an empty chamber were measured to determine background $\mathrm{NH}_{3}$ levels. Airflow, $\mathrm{NH}_{3}$ concentration and temperature from each chamber were monitored on a continuous basis for seven days. Average reductions due to the notched profile were $78 \%$ and $43 \%$ for each experimental room respectively, in comparison with a control trapezoidal slat without a notch.
\end{abstract}

Keywords: Air quality, $\mathrm{NH}_{3}$, livestock building, swine

\section{Introduction}

Nitrogen-based emissions such as ammonia $\left(\mathrm{NH}_{3}\right)$ contribute significantly to air pollution, soil and water acidification, eutrophication and loss of biodiversity (IPCC 2013, Steinfeld et al, 2006). Pig farming causes the most important ammonia emissions which can affect the health of workers in swine buildings and the general population (Wojewódzki, 2011).

According to many authors (EEA 2017, IPCC 2013, Steinfeld et al, 2006), agriculture is responsible for 94\% $(455 \mathrm{kt}$ ) of ammonia emissions, with $68 \%$ from animal productions. The main cause of high ammonia emissions in animal production is related to manure management. Moreover, in the building, the presence of slurry on floors significantly influences ammonia emissions (Hamelin et al., 2010, Aarnink et al., 1996).

Most pig buildings have concrete slatted floors because this material has many advantages such as longer life, lower costs and better foot traction (Timmerman et al., 2003). However, in swine buildings, the slatted floor is the main emitting surface due to the contact between the urine and the urease enzyme (present in the faeces) (Mobley \& Hausinger, 1989; Hamelin et al., 2010). Van der Peet-Schwering et al. (1999) proposed that one solution to limit ammonia emissions in swine building would be to reduce emitting surfaces (Misselbrook et al., 1998).

According to the literature, ammonia emissions are proportional to the soiled area in the buildings (Aarnink et al., 1996, Cai et al._2015). Aarnink et al. (1997) tested different slatted systems. Trapezoidal slat profiles with sharp edges produced $4 \%$ less ammonia emission than slat profiles without sharp edges. Ye et al. (2007) compared two trapezoidal slat profiles with and without sharp edges. The latter slat design accumulated more excreta (91.4 $-99.6 \%)$ than a modified slat profile $(25.6-83.7 \%)$. Therefore, it can be assumed that ammonia emissions could be lower for slats with sharp edges.

Moreover, Hamelin et al. (2010) also demonstrated the same phenomenon. This study compared the ammonia emissions of six slat profiles: trapezoidal, T-shaped, and curved, with or without notches. Laboratory tests demonstrated an emission reduction of $23 \%, 42 \%$ and $26 \%$ for notched slats in the trapezoidal, T-shaped, and curved form, respectively $\left(2.0,1.5\right.$ and $1.9 \mathrm{mgNH}_{3} \mathrm{~m}^{-2} \mathrm{~h}^{-1}$, respectively), compared to profiles without notch (2.6, 2.5 and $2.8 \mathrm{mgNH}_{\mathrm{NH}} \mathrm{m}^{-2} \mathrm{~h}^{-1}$, respectively). However, no real scale testing was done to validate the reduction measured in the laboratory by Hamelin et al. (2010).

The objective of this study was to evaluate the impact of a new slat profile design on ammonia emissions in a pre-commercial scale swine building. 


\section{Materials and Methods}

Slat floors and profiles

The slatted floors were made of concrete, manufactured by the company Patio Drummond (Quebec, Canada) with dimensions of $1.52 \mathrm{~m}$ (long), $0.61 \mathrm{~m}$ (wide) and $0.10 \mathrm{~m}$ (thick). The two different slat profiles tested are as follows: control trapezoidal profile without notch (S0) and trapezoidal profile with notches (S1) (Figure 33). The configurations of testing floors were:

S0R1: Slatted floor without notch (S0) from room 1 (R1)

S1R1: Slatted floor with notches (S1) from room 1 (R1)

S0R2: Slatted floor without notch (S0) from room 2 (R2)

S1R2: Slatted floor with notches (S1) from room 2 (R2)

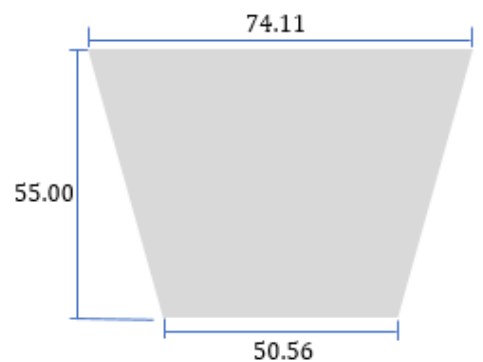

S0: Control trapezoidal profile without notch

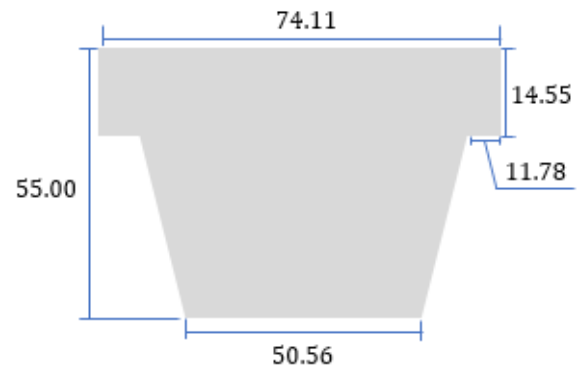

S1: Trapezoidal profile with notch

Figure 33. Slat profiles tested (dimensions in millimetres).

Pig rooms

Two independent experimental rooms with dimensions of $5.18 \mathrm{~m}$ (long) and $4.67 \mathrm{~m}$ (wide) were used for this study. Each room was equipped with individual ventilation and control systems and housed 16 pigs for 8 weeks with a weight ranging from approximately 25 to $80 \mathrm{~kg}$. As mentioned above, one control slat and one test slat were placed in each room for the duration of the 8 weeks. After this, the control and the modified slat sections were removed from each room, washed and introduced in individual desorption chambers for seven days to measure ammonia emissions.

\section{Ammonia emission measurement}

\section{Desorption chamber}

Ammonia emissions were measured using a desorption chamber. The chamber design was based on the setup presented by Pelletier et al. (2005). Five chambers were used to test the four concrete slat sections. One for each floor sample and the fifth desorption chamber was empty in order to measure background ammonia emissions. The covers of the chambers were made in galvanized steel whose length, width and height were $1.78 \mathrm{~m}, 0.76 \mathrm{~m}$ and $0.30 \mathrm{~m}$, respectively. The bases were made in plywood covered with a polyethylene film to prevent ammonia absorption on the wood. To avoid air exchange between the chambers and the laboratory room, two layers of ultra-soft vinyl foam strips were placed between the cover and the base as sealing joints and the covers were screwed to the plywood. Air was introduced into the chambers through an air distributor made of a perforated PVC tube (1-inch diameter) and by a vacuum pump (MOA-P101-AA, Gast) located on the opposite side of the chamber.

\section{Emission measurement}

The ammonia concentration (ppmv) was measured with a gas analyser (DX4040, Gasmet) connected to a rotary valve (Valco, VICI) with PTFE tubing. The rotary valve had 16 positions, the first ten were connected to the air inlet and outlet of each chamber. The rotary valve remained in the same position during 15 min. With this routine, air in each chamber was sampled every $3 \mathrm{~h}$. The temperature of each chamber was measured with a thermocouple (Thermocouple type T, Omega). A Campbell Scientific CR1000 datalogger was used to control the rotary valve and to record data from the thermocouples (figure 2). 


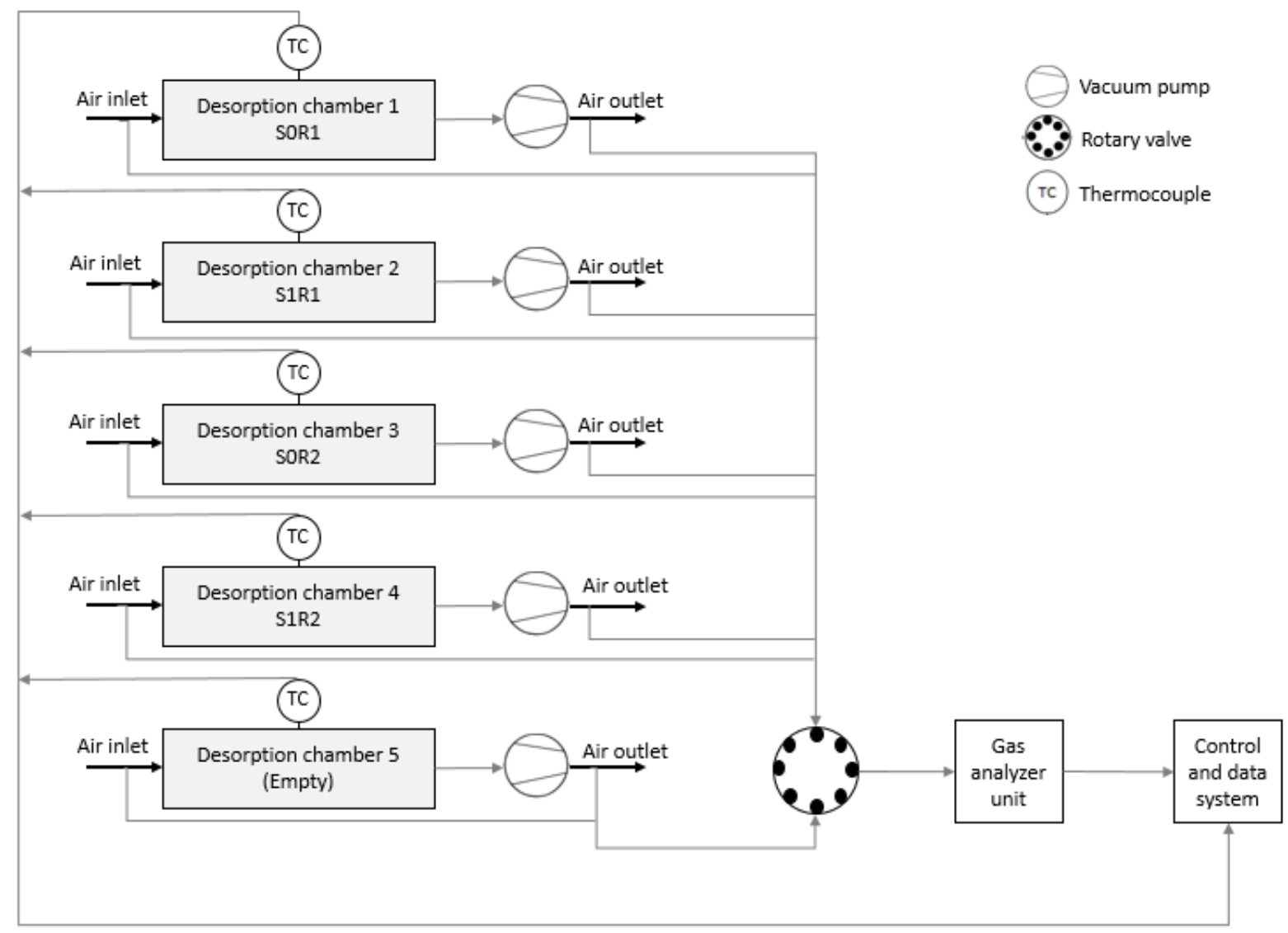

Figure 166. Schema of the ammonia emission measurement setup.

The ammonia emissions from the slat sections were calculated using the following formula:

$$
E_{s}=E_{s+c}-E_{c}
$$

where $E_{s}$ is the $\mathrm{NH}_{3}$ emission rate from slats, $\mathrm{mg} \mathrm{h}^{-1} ; \mathrm{E}_{\mathrm{s}+\mathrm{c}}$ is the $\mathrm{NH}_{3}$ emission rate from the slat sections and desorption chamber, $\mathrm{mg} \mathrm{h}^{-1} ; \mathrm{E}_{\mathrm{c}}$ is the $\mathrm{NH}_{3}$ emission rate from the empty desorption chamber, $\mathrm{mg} \mathrm{h}^{-1}$.

$\mathrm{NH}_{3}$ emissions from chambers were calculated from:

$$
E=\left(C_{o}-C_{i}\right) \times \frac{P_{\text {atm }}-P_{v}}{R_{\text {sair }} \times(T+273)} \times \frac{m_{N H 3}}{m_{\text {air }}} \times Q \times 6
$$

where $\mathrm{E}$ is $\mathrm{NH}_{3}$ emissions rate, $\mathrm{mg} \mathrm{h}^{-1} ; C_{\mathrm{o}}$ and $C_{\mathrm{i}}$ are the airborne $\mathrm{NH}_{3}$ concentrations at the inlet and outlet of the chamber, ppmv; $\mathrm{P}_{\mathrm{atm}}$ is atmospheric pressure, mbar; $\mathrm{P}_{\mathrm{v}}$ is vapor pressure, mbar; $\mathrm{R}_{\mathrm{s}}$ air is air constant, $287 \mathrm{~L} \mathrm{kPa}$ $\mathrm{kg}_{\text {air }}{ }^{-1} \mathrm{~K}^{-1}$; T is desorption chamber temperature, ${ }^{\circ} \mathrm{C}$; $\mathrm{m}_{\mathrm{NH} 3}$ is ammonia molar mass, $17.03 \mathrm{~g} \mathrm{~mol}^{-1}$; mair is air molar mass, $28.964 \mathrm{~g} \mathrm{~mol}^{-1}$; $\mathrm{Q}$ is air exchange rate, $1 \mathrm{pm} ; 6$ is a conversion factor.

Moreover, the $\mathrm{NH}_{3}$ emissions per area were calculated with the following formula:

$$
E_{\text {spa }}=\frac{E_{s}}{A_{s}}
$$

where $E_{\text {spa }}$ is the $\mathrm{NH}_{3}$ emission per area, $\mathrm{mg} \mathrm{h}^{-1} \mathrm{~m}^{-2} ; \mathrm{E}_{\mathrm{s}}$ is the $\mathrm{NH}_{3}$ emission rate from slats, $\mathrm{mg} \mathrm{h}^{-1} ; \mathrm{A}_{\mathrm{s}}$ is slatted floor area, $0.929 \mathrm{~m}^{2}$.

\section{Washing process}

The slats were humidified with a pressure washer (Karcher K3) for two minutes at low pressure, then for two minutes at high pressure. After, the manure excess on the surface was removed with a metallic scraper in order to clear and to standardise the amount of manure on the floor. Finally, concrete slat sections were washed at high pressure for five minutes with the pressure washer, then the slats were placed into the desorption chambers. 


\section{Statistical analysis}

An analysis of variance (ANOVA) and Duncan's multiple-range tests were done for the data recorded. Ammonia emissions were considered as the dependent variable and the desorption chamber or configuration testing as the independent variable, with a $95 \%$ confidence level and a significance level of 0.01 . Statistical analysis was carried out with the software R-Projet version 3.4.4.

\section{Results and Discussion}

The mean $\mathrm{NH}_{3}$ emission rates in the two slat sections in each room are presented in Table 67. Ammonia emissions from room 1 were $1.21 \mathrm{mg} \mathrm{h}^{-1} \mathrm{~m}^{-2}$ for S0 and $0.37 \mathrm{mg} \mathrm{h}^{-1} \mathrm{~m}^{-2}$ for S1. For the concrete slat sections S0 and S1 from room 2, ammonia emissions were 3.42 and $1.96 \mathrm{mg} \mathrm{h}^{-1} \mathrm{~m}^{-2}$, respectively. In the study of Hamelin et al. (2010), $\mathrm{NH}_{3}$ emissions were $2.6 \mathrm{mg} \mathrm{h}^{-1} \mathrm{~m}^{-2}$ from the control trapezoidal slat and $2.0 \mathrm{mg} \mathrm{h}^{-1} \mathrm{~m}^{-2}$ for a notched specimen. As related in the literature, it seems to exist a relationship between emitting surface area and ammonia emissions (Elzing \& Monteny, 1997; Aarnink et al., 1996; Hamelin et al., 2010). The emission rates of this study are similar order of magnitude as values presented by Hamelin et al. (2010) $\left(1.5-2.8 \mathrm{mg} \mathrm{h}^{-1} \mathrm{~m}^{-2}\right)$.

Table 67. Mean $\mathrm{NH}_{3}$ emissions for slatted floors in function of sampling time*.

\begin{tabular}{cccccc}
\hline $\begin{array}{c}\mathrm{t} \\
(\mathrm{h})\end{array}$ & $\begin{array}{c}\text { Empty } \\
\left(\mathrm{mg} \mathrm{h}^{-1} \mathrm{~m}^{-2}\right)\end{array}$ & $\begin{array}{c}\mathrm{S} 1 \mathrm{R} 1 \\
\left(\mathrm{mg} \mathrm{h}^{-1} \mathrm{~m}^{-2}\right)\end{array}$ & $\begin{array}{c}\mathrm{S} 0 \mathrm{R} 1 \\
\left(\mathrm{mg} \mathrm{h}^{-1} \mathrm{~m}^{-2}\right)\end{array}$ & $\begin{array}{c}\mathrm{S} 1 \mathrm{R} 2 \\
\left(\mathrm{mg} \mathrm{h}^{-1} \mathrm{~m}^{-2}\right)\end{array}$ & $\begin{array}{c}\mathrm{S} 0 \mathrm{R} 2 \\
\left(\mathrm{mg} \mathrm{h}^{-1} \mathrm{~m}^{-2}\right)\end{array}$ \\
\hline 0 & $0.033(0.02)$ & $0.9(0.03)$ & $1.56(0.05)$ & $5.71(0.04)$ & $7.6(0.09)$ \\
6 & $0.033(0.022)$ & $1.27(0.02)$ & $2.87(0.05)$ & $7.76(0.04)$ & $12.25(0.08)$ \\
12 & $0.039(0.035)$ & $1.28(0.04)$ & $4.28(0.06)$ & $9.19(0.05)$ & $16.35(0.05)$ \\
18 & $0.031(0.036)$ & $0.76(0.04)$ & $4.28(0.06)$ & $7.76(0.05)$ & $13.44(0.05)$ \\
24 & $0.036(0.031)$ & $0.59(0.04)$ & $3.55(0.05)$ & $5.45(0.05)$ & $8.99(0.05)$ \\
48 & $0.003(0.018)$ & $0.44(0.02)$ & $1.58(0.03)$ & $1.77(0.03)$ & $3.24(0.04)$ \\
72 & $0.002(0.011)$ & $0.35(0.02)$ & $0.93(0.02)$ & $0.94(0.02)$ & $1.82(0.02)$ \\
96 & $0.002(0.007)$ & $0.23(0.01)$ & $0.65(0.02)$ & $0.65(0.02)$ & $1.27(0.02)$ \\
120 & $0.011(0.008)$ & $0.18(0.01)$ & $0.55(0.01)$ & $0.53(0.01)$ & $1.04(0.02)$ \\
144 & $0.015(0.007)$ & $0.17(0.01)$ & $0.52(0.01)$ & $0.48(0.02)$ & $0.96(0.02)$ \\
168 & $0.021(0.007)$ & $0.14(0.01)$ & $0.49(0.01)$ & $0.45(0.01)$ & $0.88(0.02)$ \\
Mean & 0.014 & $0.37 \mathrm{a}$ & $1.30 \mathrm{~b}$ & $1.96 \mathrm{~b}$ & $3.42 \mathrm{c}$ \\
\hline
\end{tabular}

*Standard deviation in parentheses. Mean values with different letters significates that $\mathrm{NH}_{3}$ emissions are significantly different according to Duncan test.

The ANOVA procedure indicated that there was a significant difference between the slats tested $(\mathrm{P}<0.01)$. Meanwhile, the Duncan's test found a significant difference between the slats sections with nothes (S1R1 and S1R2) and without notches (S0R1 and S0R2). The emissions for the slat section S0R1 was not significantly different from those of S1R2.

Figure 35 illustrates the ammonia emissions as a function of time. For all four slat sections, the profile of $\mathrm{NH}_{3}$ emissions is similar with an important increase from $0 \mathrm{~h}$ to $12 \mathrm{~h}$, then ammonia production decreased quickly up to $60 \mathrm{~h}$ before becoming constant. This effect is due to contact between urea and water after washing, which increases the urease activity and activates ammonia production on the slat sections (Aarnink et al., 1997; Mobley and Hausinger, 1989). 


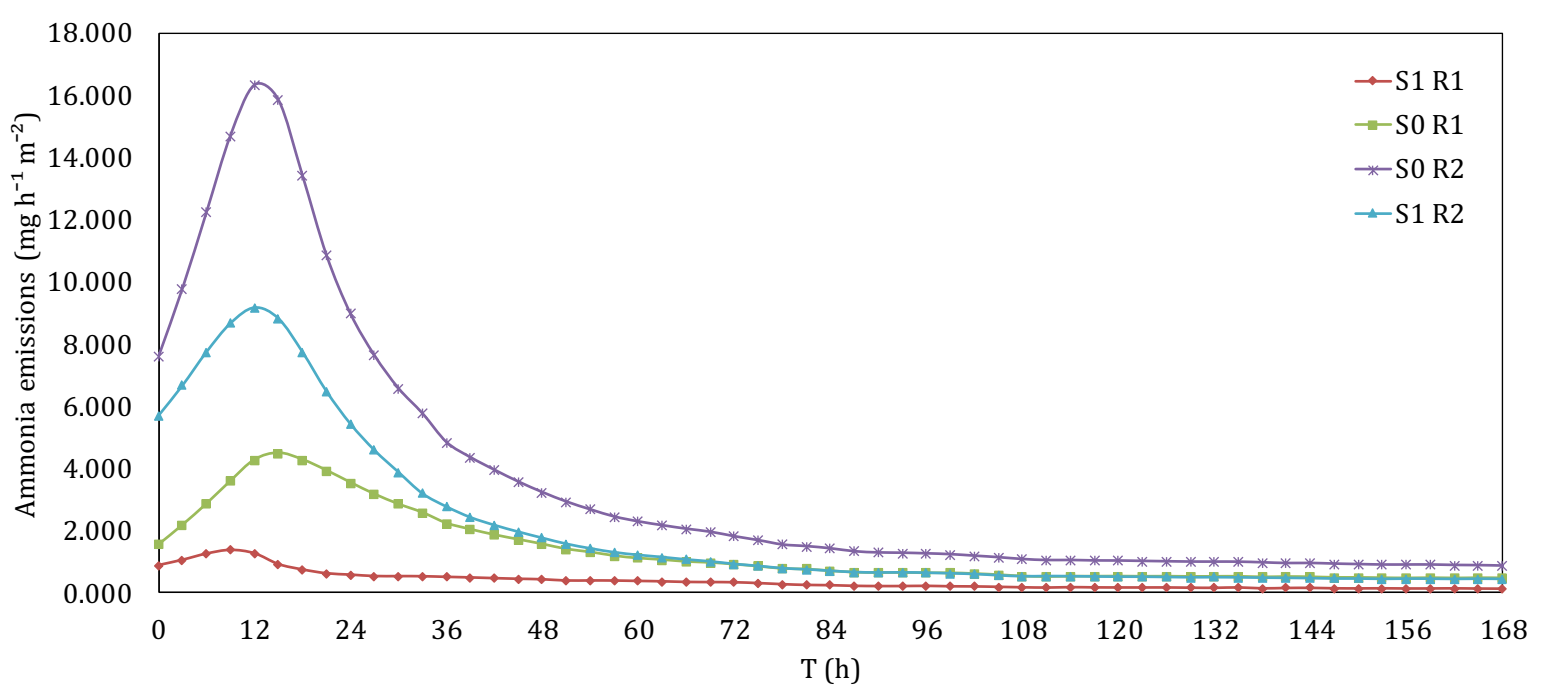

Figure 35. $\mathrm{NH}_{3}$ emissions for slatted floors in each room.

Ammonia emission reductions for notched slats as function of time are presented in Table 68. Average reduction due to the slat sections with notches is $78 \%$ for room 1 and $43 \%$ for room 2 . In general, each room showed important reductions, of 42 to $83 \%$ for room 1 and of 25 to $50 \%$ for room 2 .

Table 68. $\mathrm{NH}_{3}$ emissions reduction (\%) for notched slats compared to control slats.

\begin{tabular}{ccc}
\hline $\mathrm{t}(\mathrm{h})$ & Room 1 & Room 2 \\
\hline 0 & 42 & 25 \\
6 & 56 & 37 \\
12 & 70 & 44 \\
18 & 82 & 42 \\
24 & 83 & 39 \\
48 & 72 & 45 \\
72 & 63 & 49 \\
96 & 65 & 48 \\
120 & 67 & 49 \\
144 & 68 & 50 \\
168 & 71 & 48 \\
Mean & 78 & 43 \\
\hline
\end{tabular}

\section{Conclusion}

The literature has shown that the floor plays a key role in the ammonia emissions process. Few authors mentioned the positive impact of the slat edges on ammonia emissions. The present study aimed to validate this impact at the barn level. Results show a reduction of ammonia emission rates from concrete slat sections with a notch. Average reductions of $78 \%$ and $43 \%$ were measured in comparison with a control trapezoidal slat without a notch. Therefore, slat notches could be seen as a potential solution to reduce ammonia emissions from livestock buildings.

\section{Acknowledgements}

Authors thank the economic support from Agrivita Canada Inc. and IRSST (Institut de recherche Robert Sauvé en santé et sécurité au travail) for funding this project and from the Emerging Leaders in the Americas Program (ELAP) for the abroad student internship. Authors also thank the entire IRDA work team for all the accomplished work and the INRS for welcoming the foreign student.

\section{References}

Aarnink, A., Swierstra, D., van den Berg, A., \& Speelman, L. (1997). Effect of Type of Slatted Floor and Degree of Fouling of Solid Floor on Ammonia Emission Rates from Fattening Piggeries. Journal Of Agricultural Engineering Research, 66(2), 93-102. http://dx.doi.org/10.1006/jaer.1996.0121

Cai, L., Yu, J., Zhang, J., \& Qi, D. (2015). The effects of slatted floors and manure scraper systems on the concentrations and emission rates of ammonia, methane and carbon dioxide in goat buildings. Small Ruminant Research, 132, 103-110. http://dx.doi.org/10.1016/j.smallrumres.2015.10.015 
EEA (2017). European Union emission inventory report 1990-2015 under the UNECE Convention on Longrange Transboundary Air Pollution (LRTAP). European Environment Agency (EEA). DOI:10.2800/478321

Elzing A. \& Monteny, G. (1997). Ammonia emission in a scale model of dairy-cow house. Transaction of ASAE, 40(3), 713-720. doi: 10.13031/2013.21301

Hamelin, L., Godbout, S., Thériault, R., \& Lemay, S. (2010). Evaluating ammonia emission potential from concrete slat designs for pig housing. Biosystems Engineering, 105(4), 455-465. http://dx.doi.org/10.1016/j.biosystemseng.2010.01.004

IPCC (2013). Summary for Policymakers. In: Climate Change 2013: The Physical Science Basis. Contribution of Working Group I to the Fifth Assessment Report of the Intergovernmental Panel on Climate Change. Cambridge University Press, Cambridge, United Kingdom and New York, NY, USA.

Misselbrook, T. H., Pain, B. F., \& Headon, D. M. (1998). Estimates of ammonia emission from dairy cow collecting yards. Journal of Agricultural Engineering Research, 71, 127-135. https://doi.org/10.1006/jaer.1998.0319

Mobley, H. L. T. \& Hausinger, R. P. (1989). Microbial ureases: significance, regulation and molecular characterization. Microbiological Reviews, 53(1), 85-108. PMID: 2651866

Pelletier, F.; Marquis, A.; Godbout, S. ; Joncas, R. ; Larouche, J. P. ; Massé, D. \& Bégué, P. (2005). Gas and odor emissions from swine building materials. Transactions of the ASAE, 48(2), 721-728. DOI: 10.13031/2013.9381

Steinfeld, H., Gerber, P., Wassenarr, T., Caster, V., Rosales, M., Hann, C (2006). Livestock's long shadow environmental issues and options. FAO. Rome, Italy

Timmerman, M., Hoofs, A. I. J., \& Van Wagenberg, A. V. (2003). Ammonia emission from four systems for group-housed sows. Swine Housings II Proceedings of the 12-15 October 2003 Conference. North Carolina. USA. 122-128 pp. doi:10.13031/2013.15477

Van der Peet-Schwering, C. M. C., Aarnink, A. J. A., Rom, H. B., \& Dourmand, J. Y. (1999). Ammonia emissions from pig houses in The Netherlands, Denmark and France. Livestock Production Science, 58, $265-269$. https://doi.org/10.1016/S0301-6226(99)00017-2

Ye, Z., Li, B., Cheng, B., Chen, G., Zhang, G., \& Shi, Z. et al. (2007). A concrete slatted floor system for separation of faeces and urine in pig houses. Biosystems Engineering, 98(2), 206-214. http://dx.doi.org/10.1016/j.biosystemseng.2007.07.007

Wojewódzki, P. (2011). Ammonia emission rates from buildings with pigs intensively reared on slats. Polish Journal of Environmental Studies, 20(5), pp.1343-1352. 


\title{
Effect of Sidewall Openings on Flow Pattern and Gas Emissions of a Naturally Ventilated Dairy Barn - A Wind Tunnel Study
}

\author{
Qianying Yi ${ }^{a}$, Guoqiang Zhang a,", Marcel König b , David Janke ${ }^{\text {b }}$, Sabrina Hempel ${ }^{\text {b }}$, Barbara Amon ${ }^{\text {c, }}$ \\ Thomas Amon b, d \\ a Department of Engineering, Aarhus University, Blichers Allé 20, P.O. Box 50, 8830 Tjele, Denmark \\ ${ }^{b}$ Department of Engineering for Livestock Management, Leibniz Institute for Agricultural Engineering and Bioeconomy \\ (ATB), Max-Eyth-Allee 100, 14469 Potsdam, Germany \\ ${ }^{c}$ Department of Technology Assessment and Substance Cycles, Leibniz Institute for Agricultural Engineering and \\ Bioeconomy (ATB), Max-Eyth-Allee 100, 14469 Potsdam, Germany \\ ${ }^{\mathrm{d}}$ Institut of Animal Hygiene and Environmental Health, Department of Veterinary Medicine, Freie Universität, Berlin, \\ Robert-von-Ostertag-Str. 7-13 Berlin, Germany \\ * Corresponding author. Email: guoqiang.zhang@eng.au.dk
}

\begin{abstract}
To provide good indoor air quality for livestock, and quantify emission of ammonia and greenhouse gases, it is essential to understand indoor air characteristics with respect to ventilation openings in naturally ventilated livestock buildings. The objectives of this study were to investigate the influence of the sidewall opening size and location on indoor airflow patterns and airflow characteristics within the animal occupied zone (AOZ), above the floor and the effects on gas emission and dispersion properties. This study was carried out in a large boundary layer wind tunnel and with a scaled dairy barn model. Eight cases with different opening ratios and opening locations were designed and tested under cross-ventilation conditions. Air velocities inside the scaled model were measured with a 2D Laser Doppler Anemometer. The results showed that when the openings were positioned at the top and the opening ratio was no greater than $62.7 \%$, a 'ceiling-attaching' airflow pattern was observed. The incoming air went across the $\mathrm{AOZ}$ without circulating with the surrounding air when the sidewall heights were below the AOZ height. This airflow pattern was better for removing gaseous emissions from the AOZ but might increase total ammonia release rate and thus give negative effects on gas removal efficiency when applying a partial pit air exhaust. Besides, it was found that the concentration of the air contaminant behind the building was affected by the opening size and unaffected by the opening location, and the plume width was not influenced by the sidewall openings.
\end{abstract}

Keywords: LivAGE, Naturally ventilated buildings, Sidewall opening, Airflow patterns, Gas emission and dispersion.

\section{Introduction}

Naturally ventilated dairy buildings (NVDBs) are a large source of greenhouse gas (GHG) emissions including methane $\left(\mathrm{CH}_{4}\right)$, nitrous oxide $\left(\mathrm{N}_{2} \mathrm{O}\right)$ and carbon dioxide $\left(\mathrm{CO}_{2}\right)$ as well as gaseous contaminant such as ammonia $\left(\mathrm{NH}_{3}\right)$ (König et al., 2017). The GHG emissions, produced from enteric fermentation of ruminants and degradation of excreted manure (Lesschen et al., 2011), contribute a lot to global warming and climate change (Zong et al., 2015). The $\mathrm{NH}_{3}$ emissions, released from stored manure, can not only lead to poor indoor air quality and therefore cause health problems for both animals and workers, but also result in the deterioration of surrounding environment such as soil acidification and eutrophication of aquatic ecosystem (Wang et al., 2006; Zong et al., 2015). Mitigation of gaseous emissions from livestock buildings has become a hot research topic.

The air contaminant levels released from the manure and emitted from livestock buildings are influenced by the airflow patterns (Fiedler et al., 2013). The airflow pattern is largely affected by the configurations of the ventilation system. Therefore, it is essential to understand the airflow characteristics with respect to different ventilation openings.

Some laboratory experiments (Choiniere et al., 1988; Morsing et al., 2002; De Paepe et al., 2012) have been performed to study the impact of ventilation openings on airflow patterns in naturally ventilated livestock buildings, but the detailed airflow fields information inside and around the building are not available.

Therefore, the objectives of this research were to investigate the effects of sidewall opening size and opening location on indoor airflow patterns as well as on the emission and dispersion features of gaseous contaminants for a wind-driven cross-ventilated dairy building. The investigations were implemented with a scaled dairy barn model tested in a large boundary layer wind tunnel. The airflow patterns and the gas emission and dispersion levels were determined by measuring air velocities in and around the scaled dairy building. 


\section{Materials and Methods}

Experimental setup and air velocity measurements

The laboratory experiments were conducted in the boundary layer wind tunnel (BLWT) at the Leibniz Institute for Agricultural Engineering and Bioeconomy (ATB), Germany. The BLWT was $28.5 \mathrm{~m}$ long including the axial fan, the test section and the inlet nozzle, $3 \mathrm{~m}$ wide, and $2 \mathrm{~m}$ high. To generate the atmospheric boundary layer flow, a $9.8 \mathrm{~m}$ length of roughness elements were placed between the inlet and the working section of the wind tunnel. The model building used was 1:40 scale of height and width and 1:80 scale of length of a full-scale naturally ventilated dairy barn. The external dimension of the scaled model was $0.801 \mathrm{~m}$ (length) $\times 0.855 \mathrm{~m}$ (width) $\times 0.279 \mathrm{~m}$ (ridge height). Two sidewall openings with changeable height and one ridge opening with fixed height were installed in the building. The scaled model was placed at the centreline of the BLWT in an orientation of the sidewall openings being perpendicular to the wind. More detailed descriptions about the BLWT and the scaled model have been published elsewhere (Yi, et al., 2017; Yi, et al., 2018).

To investigate the effects of the sidewall opening size and position on the airflow fields as well as on the gas emission and dispersion properties, eight cases with different opening ratios $(18.6 \%-81.4 \%)$ and opening locations (near the roof - denoted as 'upper', near the floor - denoted as 'lower') were performed within an atmospheric boundary layer flow. The vertical profile of the mean stream-wise air velocity obeyed a power law with the exponent of $0.16+/-0.01$.

The stream-wise (U) and vertical (W) air velocities inside and around the scaled model were measured with a 2D Laser Doppler Anemometer (Dantec Dynamics, Skovlunde, Denmark). The measurements were conducted along seven vertical lines in a vertical plane. The sampling time for each measurement position was $60 \mathrm{~s}$. The mean and instantaneous air velocities for $\mathrm{U}$ and $\mathrm{W}$ air velocity components were recorded. Figure 1 shows a photograph of the air velocity measurements inside the BLWT.

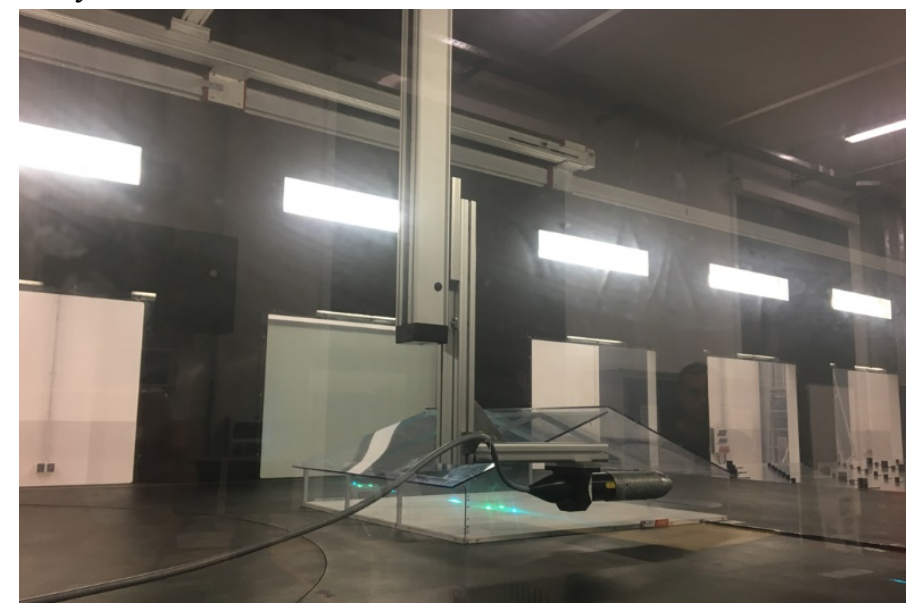

Figure 36. A photograph of the air velocity measurements in the BLWT.

Evaluation parameters of contaminant dispersion

The air velocities in the vertical line behind the building (1.1R away from the building, $\mathrm{R}$ is the reference height equals to $0.29 \mathrm{~m}$ ) were used to analyse the dispersion features of the gaseous contaminants. This study applied the evaluation parameters $\left(P_{U}\right.$ and $\left.P_{W}\right)$ proposed by Ikeguchi and Okushima (2001) to describe the degree of air contaminant dispersion. $P_{U}$, expressed by Eq. (1), was the integrated non-dimensional mean air velocity in stream-wise direction from the ground level to the reference height which was used to reflect the degree of dispersion. $P_{W}$, expressed by Eq. (2), was the integrated turbulence intensity in the vertical direction from the ground to the reference height that was proposed to express the plume width. A higher $P_{U}$ value indicates a larger air momentum and therefore a lower contaminant concentration; a larger $P_{W}$ value indicates a wider plume.

$$
\begin{aligned}
& P_{U}=\int_{G}^{R} U_{D} d z \\
& P_{W}=\int_{G}^{R}\left|I_{W}\right| d z
\end{aligned}
$$

where $G$ and $R$ represent the ground level of the wind tunnel $(0 \mathrm{~m})$ and the reference height $(0.29 \mathrm{~m})$ respectively, $\mathrm{m}$; $d z$ is a small section normalized by the reference height in the vertical direction, dimensionless; $U_{D}$ and $I_{W}$ are the non-dimensional mean air velocity in stream-wise direction and turbulence intensity in the vertical direction respectively expressed by the following equations: 


$$
\begin{gathered}
U_{D}=\frac{U}{U_{R}} \\
I_{W}=\frac{\sigma_{W}}{U}
\end{gathered}
$$

where $U$ is the mean air velocity in the stream-wise direction at each measurement position, $\mathrm{m} \mathrm{s}^{-1} ; U_{R}$ is the reference air velocity measured at the reference height, $\mathrm{m} \mathrm{s}^{-1}$; and $\sigma_{W}$ is the standard deviation of the instantaneous air velocity in the vertical direction, $\mathrm{m} \mathrm{s}^{-1}$.

\section{Results and Discussion}

Internal airflow patterns

When applying the 'upper' sidewall opening location and the opening ratio was below $62.7 \%$ (Cases $1-6)$, a 'ceiling-attaching' airflow pattern in the mainstream was found inside the building (Table 1). In this airflow pattern, the mainstream of the air flowed into the building from windward opening and then attached to the ceiling, and it moved further and exited via leeward opening. Besides, a secondary airflow with clock-wise recirculation appeared at the windward side of the scaled model near the floor.

Table 69. Experimental settings and indoor airflow information of the test cases.

\begin{tabular}{ccccc}
\hline Test Case & $\begin{array}{c}\text { Opening } \\
\text { Location }\end{array}$ & $\begin{array}{c}\text { Opening Ratio } \\
(\%)\end{array}$ & $\begin{array}{c}\text { Airflow Pattern } \\
\begin{array}{c}\text { Average Air Speed } \\
\text { in the AOZ }\left(\mathrm{m} \mathrm{s}^{-1}\right)\end{array}\end{array}$ & $\begin{array}{c}0.26 \\
\text { ceiling-attaching }\end{array}$ \\
\hline 1 & upper & 18.6 & ceiling-attaching & 0.31 \\
3 & upper & 25.4 & ceiling-attaching & 0.39 \\
4 & upper & 37.3 & ceiling-attaching & 0.43 \\
5 & upper & 44.1 & ceiling-attaching & 0.63 \\
6 & upper & 55.9 & ceiling-attaching & 0.97 \\
7 & upper & 62.7 & without circulation & 3.39 \\
8 & upper & 81.4 & floor-attaching & 3.09 \\
\hline
\end{tabular}

When the sidewalls were in fully open (Case 7), a cross-ventilated airflow pattern without air recirculation was obtained. This is inconsistent with the unstable airflow pattern within a similar building model identified by Morsing et al (2002). This is probably due to different flow regimes formed in the two studies since the freestream wind speed in this study $\left(8 \mathrm{~m} \mathrm{~s}^{-1}\right)$ was much higher than that in their experiment $\left(3.2 \mathrm{~m} \mathrm{~s}^{-1}\right)$.

The airflows changed to a 'floor-attaching' flow pattern when putting sidewall openings near the floor for the case with half-opened sidewalls (Case 8). This airflow pattern had the primary airflows attaching to the model floor and flowing across the AOZ until leaving the house. Meanwhile, a secondary airflow was observed near the ceiling and formed an anticlockwise recirculating eddy.

Gas emission

In Cases 1 to 6, the average air speed in the AOZ went up gradually as the opening ratio increased. An obviously higher average air speed in the AOZ was obtained in Cases 7 and 8 . This is attributable to the fact that the sidewalls were lower than the AOZ height and the mainstream air passed through the AOZ directly. Because of this, these opening configurations were better suitable for removing gaseous emissions from the AOZ since the primary airflow went across the AOZ. However, they might increase total ammonia emissions due to higher air speeds occurring near the floor that reduced the boundary layer thickness of the ammonia concentration above the manure surface and thus increased the mass transfer coefficient and enhanced the ammonia release rate. Many studies have proved that the air velocity can lead to increasing gaseous emissions (Zhang, et al., 1994; Arogo, et al., 1999; Ye, et al., 2008). To reduce ammonia and odours cost-effectively, the partial pit ventilation system with an air purification unit has been studied (Wu, et al., 2012) and applied in many livestock buildings (Saha, et al., 2010; Zong, et al., 2014; Zong, et al., 2015). The increased ammonia release rate caused by the 'cross-ventilation without circulation' and 'floor-attaching' airflow patterns will give negative effects on gas removal efficiency when applying a partial pit air exhaust.

\section{Air contaminant dispersion}

The values of $P_{U}$ and $P_{W}$ at 1.1R away from the leeward of the building for Cases 1, 4, 7, and 8 are illustrated in Figure 2. The results show that $P_{U}$ increased with increasing opening size but was not changed with altered opening location. This indicates that larger sidewall openings led to higher air momentum and lower air 
contaminant concentration at this vertical line. The concentration of the contaminated air at this location might be the same for the case with the same opening ratio but different opening location. No obvious differences in $P_{W}$ values among these four cases were seen which means that the plume width in vertical direction was hardly affected by the size and position of the sidewall openings.

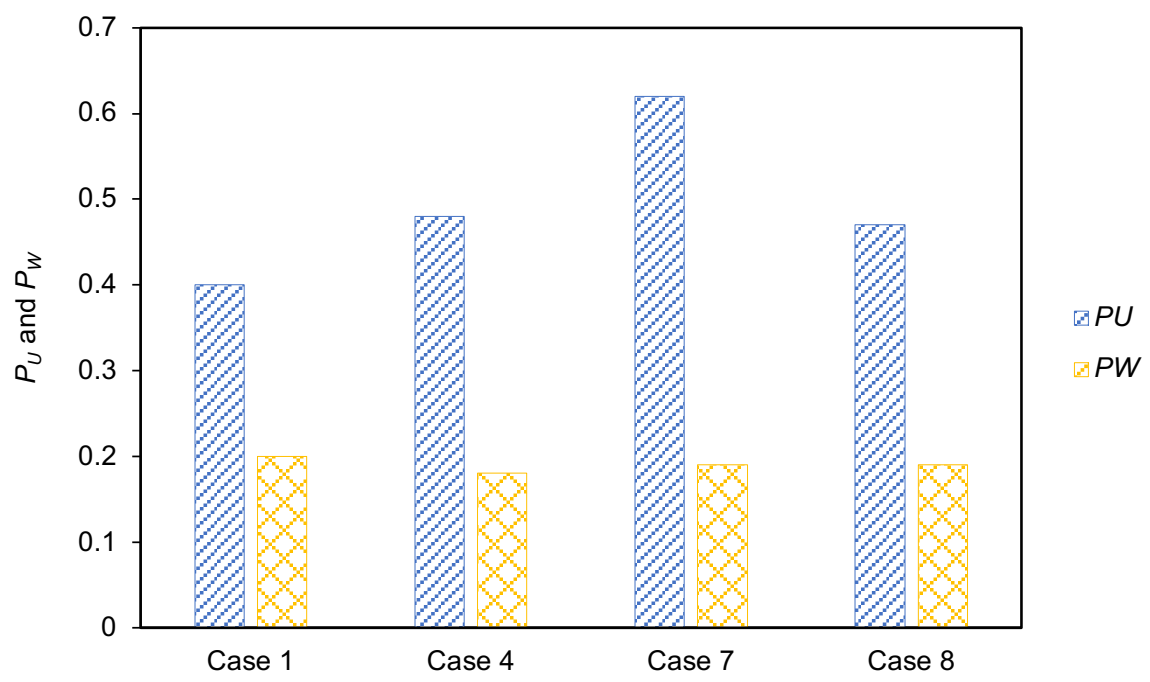

Figure 2. $P_{U}$ and $P_{W}$ values at 1.1R distance behind the building for Cases $1,4,7$, and 8 .

\section{Conclusions}

The influence of the sidewall opening size and opening location on indoor airflow fields and the emission and dispersion properties of the air contaminants were investigated with the method of large boundary layer wind tunnel and scaled model tests. The indoor airflow patterns and the average air speed in the AOZ were identified, and their effects on gaseous emissions were discussed. In addition, the dispersion of the air contaminant in a vertical line behind the building was predicted by two evaluation parameters. This research indicated a 'ceilingattaching' airflow pattern with opening ratio smaller than $62.7 \%$ and 'lower' opening positions. Higher average air speed in the AOZ was obtained when the sidewall heights were below the AOZ height, and this situation was beneficial for removing gaseous emissions from the AOZ but might increase total ammonia release rate. The contaminant concentration behind the building was affected by the opening size and almost unaffected by the opening position. The sidewall openings had no considerable influence on the plume width.

\section{Acknowledgements}

The authors acknowledge the assistance given by technicians Knut Schröter and Andreas Reinhardt at ATB. We also gratefully appreciate the financial support from the research project "Optimized animal-specific barn climatization facing temperature rise and increased climate variability (OptiBarn)" EU-FACCE-JPI project consortium, DK-Grant No. 4215-00004B, Innovation Foundation Denmark and Federal Office for Agriculture and Food (BLE), DE-Grant No. 2814ERA02C, Germany for this study. The fund was also provided by the project COST Action CA16106 "LivAGE: Ammonia and Greenhouse Gases Emissions from Animal Production Buildings", European Cooperation in Science and Technology (COST). China Scholarship Council (Grant number: 201506240160) and the S.C. Van Fonden, Denmark (Grant number: 1674) supported the first author's study in Denmark.

\section{References}

Arogo, J., R.H. Zhang, G.L. Riskowski, L.L. Christianson, D.L. Day, 1999. Mass transfer coefficient of ammonia in liquid swine manure and aqueous solutions. Journal of Agricultural Engineering Research. 73 (1), 77-86.

Choiniere, Y., F. Blais, J.A. Munroe, 1988. A wind tunnel study of airflow patterns in a naturally ventilated building. Canadian Agricultural Engineering. 30 (2), 293-297.

De Paepe, M., J.G. Pieters, W.M. Cornelis, D. Gabriels, B. Merci, P. Demeyer, 2012. Airflow measurements in and around scale model cattle barns in a wind tunnel: Effect of ventilation opening height. Biosystems Engineering. 113 (1), 22-32.

Fiedler, M., W. Berg, C. Ammon, C. Loebsin, P. Sanftleben, M. Samer, K. von Bobrutzki, A. Kiwan, C.K. Saha, 2013. Air velocity measurements using ultrasonic anemometers in the animal zone of a naturally ventilated 
dairy barn. Biosystems Engineering. 116 (3), 276-285.

Ikeguchi, A. and L. Okushima, 2001. Airflow patterns related to polluted air dispersion in open free-stall dairy houses with different roof shapes. Transactions of the ASAE. 44 (6), 1797-1805.

König, M., K. Bonkoß, T. Amon, 2017. Wind tunnel measurements on reduction of near surface concentrations through naturally barrier on emissions from naturally ventilated barn. In International Workshop on Physical Modelling of Flow and Dispersion Phenomena, Dynamics of Urban and Coastal Atmosphere. France, August 2325.

Lesschen, J.P., M. van den Berg, H.J. Westhoek, H.P. Witzke, O. Oenema, 2011. Greenhouse gas emission profiles of European livestock sectors. Animal Feed Science and Technology. 166-167, 16-28.

Morsing, S., A. Ikeguchi, J.C. Bennetsen, J.S. Strøm, P. Ravn, L. Okushima, 2002. Wind induced isothermal airflow patterns in a scale model of a naturally ventilated swine barn with cathedral ceiling. Applied Engineering in Agriculture. 18 (1), 97-101.

Saha, C. K., G. Zhang, P. Kai, B. Bjerg, 2010. Effects of a partial pit ventilation system on indoor air quality and ammonia emission from a fattening pig room. Biosystems Engineering. 105 (3), 279-287.

Wang, C., B. Li, G. Zhang, H.B. Rom, J.S. Strøm, 2006. Model estimation and measurement of ammonia emission from naturally ventilated dairy cattle buildings with slatted floor designs. Journal of the Air \& Waste Management Association. 56 (9), 1252-1259.

Wu, W., P. Kai, G. Zhang, 2012. An assessment of a partial pit ventilation system to reduce emission under slatted floor - Part 1: Scale model study. Computers and Electronics in Agriculture. 83: 127-133.

Ye, Z., G. Zhang, B. Li, J.S. Strøm, P.J. Dahl, 2008. Ammonia emissions affected by airflow in a model pig house: effects of ventilation rate, floor slat opening, and headspace height in a manure storage pit. Transactions of the ASABE. 51 (6), 2113-2122.

Yi, Q., G. Zhang, M. König, D. Janke, T. Amon, S. Hempel, 2017. Determining discharge coefficient of large openings of wind-driven cross ventilation barns by wind tunnel tests. In 2017 ASABE Annual International Meeting, Spokane, America, July 16-19. American Society of Agricultural and Biological Engineers. Manuscript. 1700779.

Yi, Q., G. Zhang, M. König, D. Janke, S. Hempel, T. Amon, 2018. Investigation of discharge coefficient for wind-driven naturally ventilated dairy barns. Energy and Buildings. 165, 132-140.

Zhang, R.H., D.L. Day, L.L. Christianson, W.P. Lepson, 1994. A computer model for predicting ammonia release rates from swine manure pits. Journal of Agricultural Engineering Research. 58 (4), 223-229.

Zong, C., Y. Feng, G. Zhang, M.J. Hansen, 2014. Effects of different air inlets on indoor air quality and ammonia emission from two experimental fattening pig rooms with partial pit ventilation system - Summer condition. Biosystems Engineering. 122, 163-173.

Zong, C., H. Li, G. Zhang, 2015. Ammonia and greenhouse gas emissions from fattening pig house with two types of partial pit ventilation systems. Agriculture, Ecosystems \& Environment. 208, 94-105. 


\title{
Simulation of Nitrous Oxide Emission from Dairy Manure Stockpile Using Dynamic Chamber Technique
}

\author{
Bin Hua , Chaoyuan Wang ${ }^{\text {a,b,c, }}$, Yejian Wang ${ }^{\mathrm{a}}$, Haoxiang Zhao ${ }^{\mathrm{a}}$, Zhengxiang Shi ${ }^{\mathrm{a}, \mathrm{b}, \mathrm{c}}$, Baoming $\mathrm{Li}^{\mathrm{a}, \mathrm{b}, \mathrm{c}}$, \\ Weichao Zheng ${ }^{a, b, c}$, Guanghui Teng ${ }^{a, b, c}$ \\ ${ }^{a}$ Department of Agricultural Structure and Bioenvironmental Engineering, College of Water Resources and Civil \\ Engineering, China Agricultural University, Beijing100083, P.R. China \\ ${ }^{b}$ Key Laboratory of Agricultural Engineering in Structure and Environment, Ministry of Agriculture, Beijing100083, \\ P.R. China \\ 'Beijing Engineering Research Center on Animal Healthy Environment, Beijing 10083, P.R.China \\ * Corresponding author. Email: gotowchy@cau.edu.cn
}

\begin{abstract}
Solid dairy manure is typically stored within the farm from weeks to months before land application, for small and middle sized farms in particular, making the dairy stockpiles an important emission source of greenhouse gases (GHGs) in China, including nitrous oxide $\left(\mathrm{N}_{2} \mathrm{O}\right)$. While little information on the emission is available under the management system. Using dynamic chamber technique, three experiments were conducted to simulate the $\mathrm{N}_{2} \mathrm{O}$ emission from dairy manure stockpile in different seasons, and to explore the influence of temperature, surface wind speed and simulated rainfall. Results show that $\mathrm{N}_{2} \mathrm{O}$ emissions from the simulated dairy manure stockpiles were $5.86 \mathrm{mg} \mathrm{kg}^{-1}, 9.43 \mathrm{mg} \mathrm{kg}^{-1}$ and $0.81 \mathrm{mg} \mathrm{kg}^{-1}$ in spring, summer and autumn, and the overall daily $\mathrm{N}_{2} \mathrm{O}$ emission significantly differed from the temperature. Surface wind speeds had different influences on $\mathrm{N}_{2} \mathrm{O}$, meanwhile the simulated rainfall resulted in a sharp increasing of $\mathrm{N}_{2} \mathrm{O}$ emission and then the emission decreased to previous level after 6-10 hours. According to the results of simulated experiments and field investigation of dairy 26 farms in Yanqing district of Beijing, its total $\mathrm{N}_{2} \mathrm{O}$ emission from manure management system in 2016 was estimated at $736.6 \mathrm{~kg}$, which was $195.2 \mathrm{t} \mathrm{CO}_{2}$ equivalent. .
\end{abstract}

Keywords: LivAGE, dairy manure, stockpile, $\mathrm{N}_{2} \mathrm{O}$, emission

\section{Introduction}

Greenhouse gases emissions of agricultural activities, including nitrous oxide $\left(\mathrm{N}_{2} \mathrm{O}\right)$ generated from manure storage inside the dairy farms, is an important contribution to global warming. For $\mathrm{N}_{2} \mathrm{O}$, its global warming potentials (GWPs) is 265 times as carbon dioxide with its atmospheric lifetimes of 121 years, which is a big concern of public, producers and professionals.

In a typical Chinese dairy farming system, naturally ventilated barns with fully open lots are commonly applied to house dairy cows, which is still a dominating operation, especially for middle and small scaled farms. In such a farming system, solid dairy manure collected from the barn and open lots is typically stored as static stockpile somewhere on the farm for weeks to months before land application, making it is a big emission source of $\mathrm{N}_{2} \mathrm{O}$. As an uncovered storage, air temperature, wind and rainfall are the most potential key factors affecting the $\mathrm{N}_{2} \mathrm{O}$ emission from the stockpile during deposition, and studies have revealed the impacts of air temperature (Miyatake et al., 2006; Arikan et., 2016). Meanwhile, field measurement to evaluate such effects is still challenging because of the difficulties on eliminating the disturbance from climate changing. And due to its relative low concentration in particular, few studies on $\mathrm{N}_{2} \mathrm{O}$ emission from solid manure storage have been documented.

In this paper, three experiments were conducted, and the objectives of were to: (1) simulate $\mathrm{N}_{2} \mathrm{O}$ emissions and obtain the fundamental data from the dairy manure stockpiles using dynamic chamber technique in the laboratory; (2) investigate the impacts of rainfalls and surface wind speeds on the emission; and (3) estimate annual $\mathrm{N}_{2} \mathrm{O}$ emission from dairy manure management in Yanqing, Beijing, based on the results of the simulation.

\section{Material and method}

Experiment design

With the purpose to simulate $\mathrm{N}_{2} \mathrm{O}$ emissions from dairy manure stockpile and assess the impact of rainfall and surface wind speeds, three experiments (Table 1) were conducted in the laboratory using dynamic chambers to simulate the real state of manure temporary storage inside the dairy farm. Fresh dairy manure for the experiments was collected from the open lot of a commercial dairy farm, located in Yanqing, Beijing, China, which had approximately 460 lactating dairy cows of Holstein. The mass of manure was weighted before positioning in the containers.

For the rainfall experiment (experiment II), three containers (reactors) were set and a total of $9.9 \mathrm{~mm}$ of water was spread over the manure storage surface in 20 minutes to simulate the natural rainfall. During the experiment, the manure was treated by two simulated rainfalls with same precipitation $(9.9 \mathrm{~mm}$ of rainfall). When providing 
simulated rainfall, one of the containers was randomly selected as the control group without any precipitation. The air flow of each model was kept constant at $16 \mathrm{~L} \cdot \mathrm{min}^{-1}$.

Based on a field measurement of the surface wind over a dairy stockpile in Beijing, the wind speeds of 0.5 , $0.8,1.2$ and $1.6 \mathrm{~m} \cdot \mathrm{s}^{-1}$ were selected in the experiment (experiment III). Besides, a basic wind speed of $0.2 \mathrm{~m} \cdot \mathrm{s}^{-1}$ for each container was provided to detect gas concentration changes. During the experiments, manure stored in each container was ventilated with the wind speed of the treatments for 3-hour each day, whereas the rest of the day (21-hour) was provided with basic wind speed of $0.2 \mathrm{~m} \cdot \mathrm{s}^{-1}$.

\section{Experimental setup}

Experimental setups were shown in Figure 1.

For experiments I and II with the objectives to simulate $\mathrm{N}_{2} \mathrm{O}$ emission from solid dairy manure storage and the impact of rainfall, the setup was consisted of three brick-containers and air tight Plexiglas box covers, and a gas concentration measuring system (Figure 1 (A) and (B)). During the measurement, fresh air was pumped to the headspace over the manure surface, and gas concentrations were monitored at both the inlet and outlet of each container. For experiment II, sprinklers were installed on the sidewalls of the covered box to uniformly spread water to the manure surface as a simulated precipitation in the nature (Figure 1(B)).

In experiment III, four containers were built to test the effect of various wind speeds on $\mathrm{N}_{2} \mathrm{O}$ emission (Figure $1(\mathrm{C})$ ). For each container, a wind tunnel $(4.5 \mathrm{~m}$ in length) to provide designed wind speeds over the manure underneath, and a measuring system to detect the gas concentrations of the air inlet and outlet. As shown in Figure $1(\mathrm{C})$, two adjustable fans was installed in one end of the wind tunnel to control wind speeds, and three $5 \mathrm{~mm}$ aperture metal screens were set in the other side of the wind tunnel to get the relatively uniform and steady air flow. In order to provide multiple wind speeds over the manure surface, a manual control panel was designed for controlling the fans' operation with relays and adjustable resistances.

Table 1. Basic information of the experiments.

\begin{tabular}{lcc}
\hline Experiment $\#$ & Number of chambers & Chamber description \\
\hline Experiment I: $\mathrm{N}_{2} \mathrm{O}$ emission simulation & Three & A size of $0.9 \mathrm{~m} \times 0.8 \mathrm{~m} \times 0.8 \mathrm{~m}$, made by \\
Experiment II: Rainfall impact & Three & brick with Plexiglas cover boxes \\
Experiment III: Wind speeds impact & Four & \\
\hline
\end{tabular}

Measurement of $\mathrm{N}_{2} \mathrm{O}$ concentration, temperature, relative humidity and wind speed

The gaseous concentration emitted from the container was measured via a photoacoustic multi-gas monitor (INNOVA 1412i, LumaSense Technologies, Denmark) and a self-developed multiplexer. $\mathrm{N}_{2} \mathrm{O}$ concentrations of the inlet (ambient air) and outlet gas were tested at an interval of 10 minutes with five continuous samplings by the INNOVA 1412i. Every sampling lasted for 40 seconds, which was followed by a 20 seconds of air flushing of the testing container. With the multiplexer, the measurement of gas concentrations can switched between inlets and outlets of different containers. Detection range of the gas analyzer was $0.01 \mathrm{ppm}$ for $\mathrm{N}_{2} \mathrm{O}$.

Ambient temperature and relative humidity were automatically monitored by sensors (Testo $175 \mathrm{H} 1$, Testo, Germany). Measurement range and accuracy of the sensor were $-35{ }^{\circ} \mathrm{C}-55{ }^{\circ} \mathrm{C}$ for temperature, and $0 \%-100 \%$ for $\mathrm{RH}$, respectively. The manure core temperature of manure was measured by thermocouples at three different height layers. Air velocities of the wind tunnel were measured by using a handled anemometer (Model KA41L, Kanomax, Japan). The range and accuracy of the anemometer were $0.1 \mathrm{~m} \cdot \mathrm{s}^{-1}-30 \mathrm{~m} \cdot \mathrm{s}^{-1}$ and $\pm 3 \%$, respectively. 


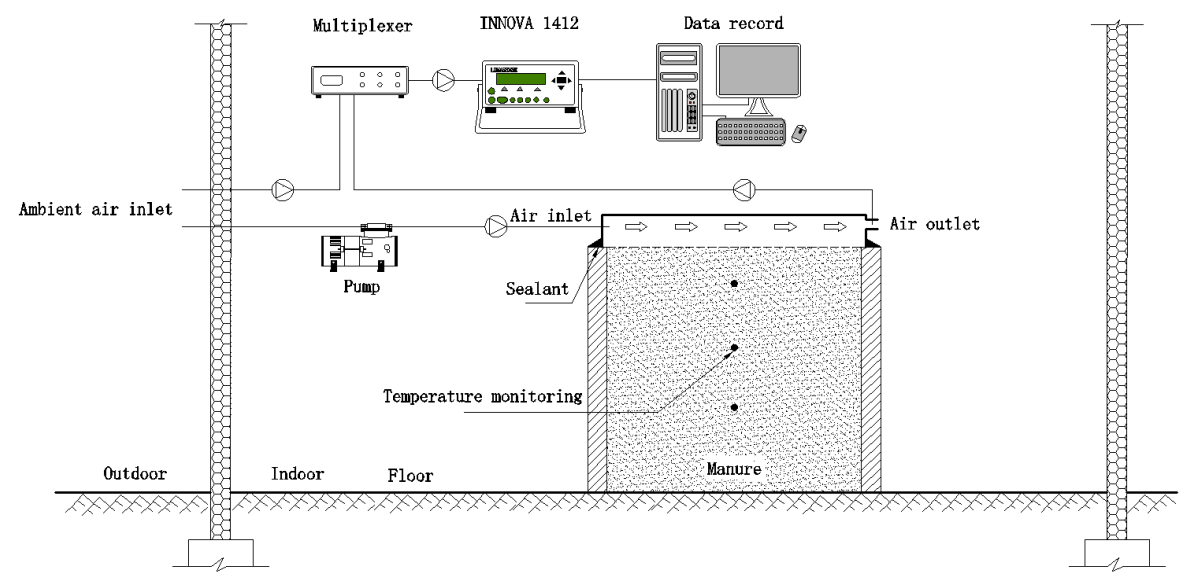

(A)

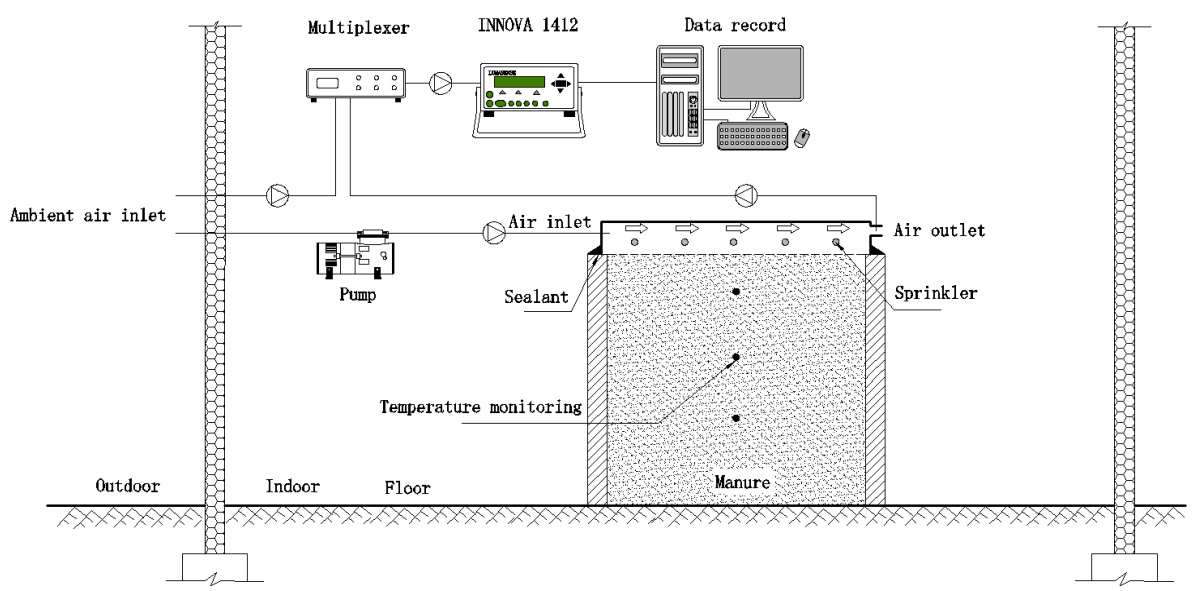

(B)

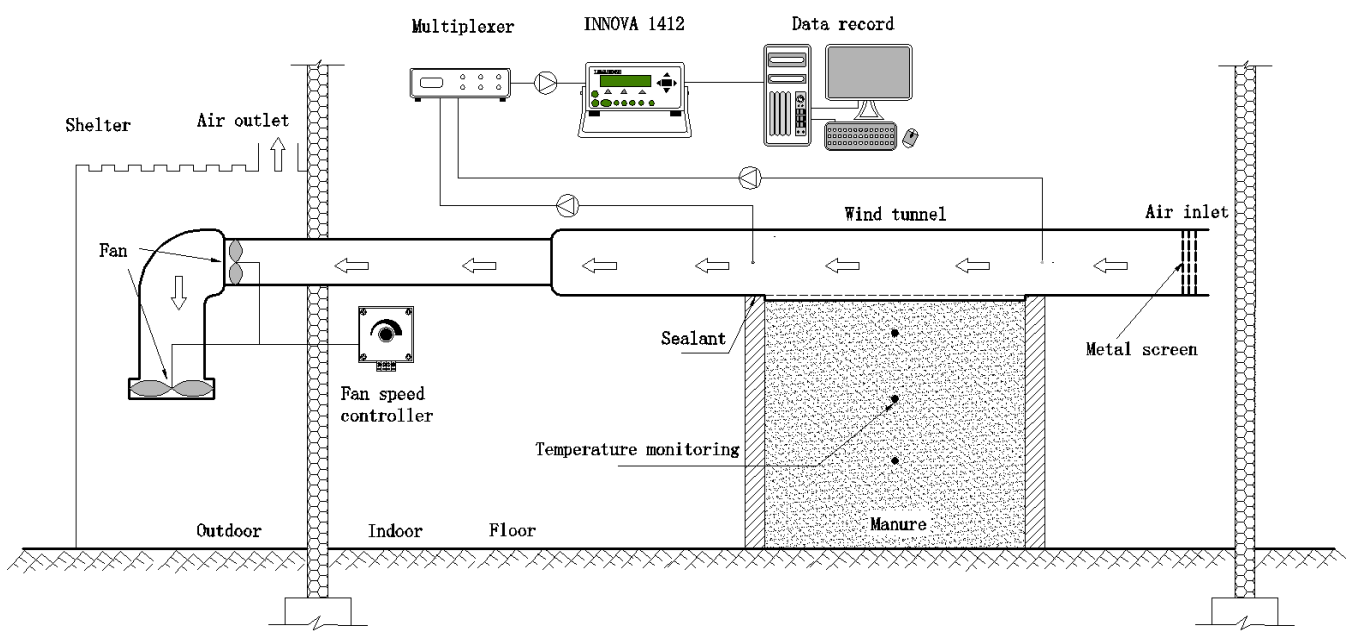

(C)

Figure 1. Schematic diagram of the setups on $\mathrm{N}_{2} \mathrm{O}$ emissions from dairy manure stockpile in different seasons: (A) simulation of $\mathrm{N}_{2} \mathrm{O}$ emissions (experiment I); (B) rainfall impact test (experiment II); and (C) wind speed impact test (experiment III).

Methods for estimating $\mathrm{N}_{2}$ Oemission rate

The $\mathrm{N}_{2} \mathrm{O}$ emissions from the simulated experiments were calculated bythe equation:

$$
E_{N_{2} O}=\frac{\left(C_{O}-C_{I}\right) \times 10^{-3} \times F_{\text {air }} \times 60}{W_{\text {manure }}}
$$

where:

$E_{\mathrm{N}_{2} \mathrm{O}}$ is the $\mathrm{N}_{2} \mathrm{O}$ emission rate $\left(\mathrm{mg} \cdot \mathrm{kg}^{-1} \cdot \mathrm{h}^{-1}\right)$; 
$C_{O}$ is the $\mathrm{N}_{2}$ Oconcentration of air outlet $\left(\mathrm{mg} \cdot \mathrm{m}^{-3}\right)$;

$C_{I}$ is the $\mathrm{N}_{2}$ Oconcentration of air inlet $\left(\mathrm{mg} \cdot \mathrm{m}^{-3}\right)$;

$F_{\text {air }}$ is air flow rate $\left(\mathrm{L} \cdot \mathrm{min}^{-1}\right)$; and

$W_{\text {manure }}$ is the weight of fresh manure $(\mathrm{kg})$.

Estimation of the total $\mathrm{N}_{2} \mathrm{O}$ emission in Yanqing of Beijing

In order to estimatethe total $\mathrm{N}_{2} \mathrm{O}$ emission of the region, basic information on manure management system and manure production of dairy farms in Yanqing district of Beijing was investigated, and a field survey on a total of 26 dairy farms was carried out in 2016.

The average ambient temperature during the trails in experiments I to III was consistent with the temperature of summer, spring and autumn in Beijing, respectively. Thus, the simulated results were used to estimate the total $\mathrm{N}_{2} \mathrm{O}$ emission from dairy manure storage in the three seasons. The $\mathrm{N}_{2} \mathrm{O}$ emission in winter was calculated using the findings of previous studies. The annual $\mathrm{N}_{2} \mathrm{O}$ emission in Yanqing was calculated by the equation:

$$
E_{N_{2} O}=\Sigma\left(Q_{N_{2} O, j} \times W_{\text {manure }, j}\right) \times 10^{-6}
$$

where

$E_{\mathrm{N}_{2} \mathrm{O}}$ is the annual $\mathrm{N}_{2} \mathrm{O}$ emissionfrom dairy manure storage in Yanqing district $(\mathrm{kg})$;

$Q_{N_{2} O, j}$ is the $\mathrm{N}_{2} \mathrm{O}$ emission factor from simulated experiments for different seasons $\left(\mathrm{mg} \cdot \mathrm{kg}^{-1}\right)$ with $j$ presenting different seasons; and

$W_{\text {manure, }}$ is the manure weight of each investigateddairy farm $(\mathrm{kg})$.

Because of the lack of winter experimental data, the $\mathrm{N}_{2} \mathrm{O}$ emission factor in winter of $0.61 \mathrm{mg} \cdot \mathrm{kg}^{-1}$ (for a month duration) was used in the calculation based on the study of $\mathrm{Lu}$, et al. (2007).

\section{Results and discussion}

Effect of temperature for $\mathrm{N}_{2} \mathrm{O}$ emissions from simulated dairy manure storage

Using dynamic chamber technique, experiments were conducted to simulate the $\mathrm{N}_{2} \mathrm{O}$ emission from dairy manure stockpile in different seasons. The average ambient temperature in experiments I, II and III was $25.2^{\circ} \mathrm{C}$, $21.0^{\circ} \mathrm{C}$ and $8.4^{\circ} \mathrm{C}$ during the trials, and the core temperature ( $\mathrm{T}_{\text {core }}$ ) of dairy manure was $25.0^{\circ} \mathrm{C}, 25.0^{\circ} \mathrm{C}$, and $9.1^{\circ} \mathrm{C}$, respectively. Results show that $\mathrm{N}_{2} \mathrm{O}$ emission from dairy manure storage was $9.43 \mathrm{mg} \cdot \mathrm{kg}^{-1}, 5.86 \mathrm{mg} \cdot \mathrm{kg}^{-1}$, and $0.81 \mathrm{mg} \cdot \mathrm{kg}^{-1}$ in the three experiments, respectively; and $\mathrm{N}_{2} \mathrm{O}$ emission was consistent with temperature. Studies have shown that temperature is an important environmental factor affecting gas emissions during the stacking. The activity of microorganisms changes with air temperature, which may affect the rate of related biochemical reactions and ultimately alter the production and release of $\mathrm{N}_{2} \mathrm{O}$ (Sommer S G et al., 2000; Mazzetto A M et al., 2014; ).

Effect of simulated rainfall on $\mathrm{N}_{2} \mathrm{O}$ emission from dairy manure storage

For experiment II, although manure temperature varied in different treatments, an initially increasing tendency was found within each container, and the temperature of the upper layer changed from $18.9-31.5^{\circ} \mathrm{C}, 15.9-22.4^{\circ} \mathrm{C}$ and $17.2-27.3^{\circ} \mathrm{C}$ for the three containers, respectively. Then manure temperature stayed around a constant state during experiment. Compared with the other layers and ambient temperature during all the three experiments, average higher temperature of the upper layer was found, which was considered as an indicator of manure decomposition, and a key factor driving the emission of $\mathrm{N}_{2} \mathrm{O}$ from manure. This phenomenon could be partially explained by the activity of microbes in the upper surfaces, which leaded to heat production during the process of metabolism under the proper environmental temperature. Compared with the wind speed experiment, the change of the interior manure temperature was not closely correlating to ambient temperature, which demonstrated that a steady-state formation appeared in the manure. Furthermore, ambient temperature showed less influence to manure temperature in this experiment by entering a mild weather condition $\left(>10^{\circ} \mathrm{C}\right)$ 。

Although the initial emission rates of $\mathrm{N}_{2} \mathrm{O}$ in varied container were different, the trends were very similar. The difference were originated from different decomposition rate of the manure during the experiment, which might be because of the different initial moisture content of fresh manure positioned in the three groups $(55.7 \pm 1.0 \%$, $61.4 \pm 0.8 \%$ and $56.5 \pm 0.4 \%$, respectively).

The emission of $\mathrm{N}_{2} \mathrm{O}$ initially increased and then decreased rapidly for all the three groups (Figure 2). The rainfall resulted in a sharp increasing to a $\mathrm{N}_{2} \mathrm{O}$ emission rate peak within dozens of minutes, and the increasing typically lased for 10 hours before it returned close to the previous emission level. It was assumed that the increase of $\mathrm{N}_{2} \mathrm{O}$ emission was closely related to the evaporation of moisture after rainfall, which was directly monitored during the experiment, but not to microbial activity. While a clear explanation should be verified with future experimental designs. The result showed that the simulated rainfall greatly increased $\mathrm{N}_{2} \mathrm{O}$ emission in a relatively short time. 


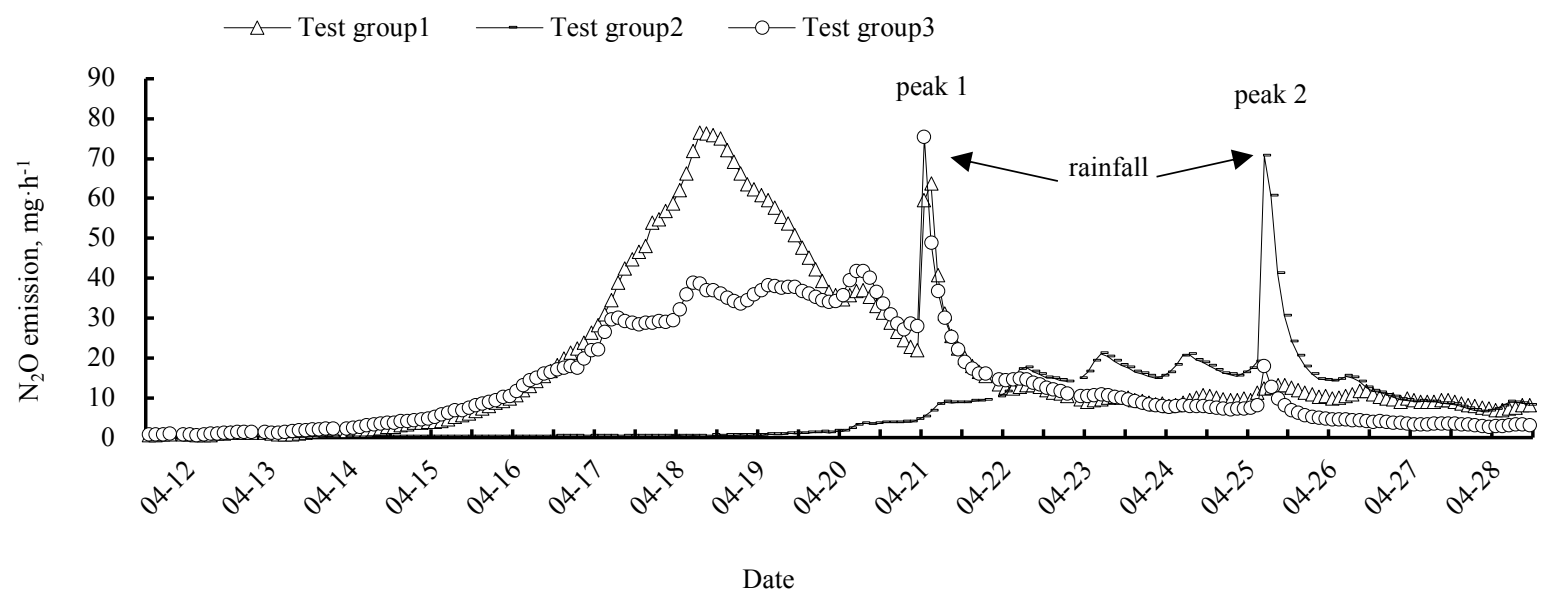

Figure 2. The $\mathrm{N}_{2} \mathrm{O}$ emission flux during experiment II.

Effect of wind speeds for $\mathrm{N}_{2} \mathrm{O}$ emissions from simulated dairy manure storage

During the measurement, manure temperature under different wind speeds treatments slightly varied within a difference of $3^{\circ} \mathrm{C}$, while average manure temperature reached $8.0-10.6^{\circ} \mathrm{C}$, which was overall higher than the mean ambient temperature $\left(7.6^{\circ} \mathrm{C}\right)$ during the trails. In the beginning, manure temperature kept increasing averagely from $9.1^{\circ} \mathrm{C}$ to $10.1-12.6^{\circ} \mathrm{C}$, while the ambient temperature stayed constantly around $10.5^{\circ} \mathrm{C}$, which indicated the microbial activity and decomposition of manure. By some a week later, more influence of air temperature on manure temperature change was observed.

Weekly accumulated $\mathrm{N}_{2} \mathrm{O}$ emission rates from the models under different wind speeds were shown in Figure 3. Change of $\mathrm{N}_{2} \mathrm{O}$ emission rate was complicated under different wind speeds, while the overall emission arose at 1-2 week, and slightly decreased in the 3rd week, and the rate then greatly increased in the end of experiment (fifth week). Due to lack of relevant research on the effect of wind speed, it was possible that the wind speed affected the evaporation of manure moisture and then the activity of microorganism, which need further studies to confirm.

$\mathrm{N}_{2} \mathrm{O}$ emissions from manure under different wind speeds were $0.20 \mathrm{mg} \cdot \mathrm{kg}^{-1}, 0.60 \mathrm{mg} \cdot \mathrm{kg}^{-1}, 1.5 \mathrm{mg} \cdot \mathrm{kg}^{-1}$ and $0.9 \mathrm{mg} \cdot \mathrm{kg}^{-1}$ for wind speeds of $0.5 \mathrm{~m} \cdot \mathrm{s}^{-1}, 0.8 \mathrm{~m} \cdot \mathrm{s}^{-1}, 1.2 \mathrm{~m} \cdot \mathrm{s}^{-1}$ and $1.6 \mathrm{~m} \cdot \mathrm{s}^{-1}$, respectively. As discussed, a clear effect of wind speed on the emission was found. For $\mathrm{N}_{2} \mathrm{O}$, the highest emission rates were at $1.2 \mathrm{~m} \cdot \mathrm{s}^{-1}$, rather than the maximum speed of $1.6 \mathrm{~m} \cdot \mathrm{s}^{-1}$, significant differences on emission rates between varied wind speeds were statically demonstrated with Paired T test.

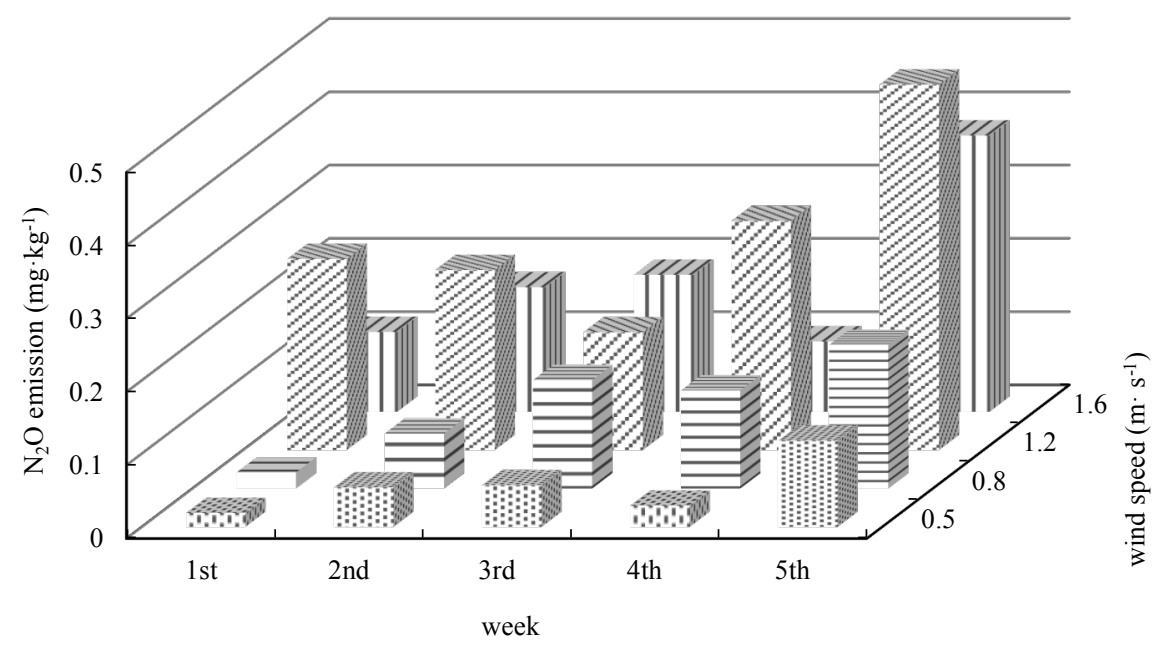

Figure 3. Accumulated $\mathrm{N}_{2} \mathrm{O}$ emissions in every week.

Estimation of the total $\mathrm{N}_{2} \mathrm{O}$ emission in Yanqing of Beijing

The $\mathrm{N}_{2} \mathrm{O}$ emission of dairy manure within the farm highly depends on the daily manure excretion and management strategy, and may vary in countries and regions. Field investigation results showed that there were 
8895 dairy cows in Yanqing in 2016, and the farm sizes were all under 1000 dairy cows. Naturally ventilated barns and open lots were typically applied in those farms, and the cows spent most of the time on the lot even in winter. Manure on the lot was mechanically or artificially removed twice a day to once a week, and was stacked somewhere inside the farm directly in the open air without treatment for less than 4 weeks land application or making composting.

Based on the results of the simulated experiments in this paper and the finding of previous studies for different seasons as well as field survey, the total emission of Yanqing was estimated. Results showed that the annual $\mathrm{N}_{2} \mathrm{O}$ emission from solid dairy manure storage of Yanqing district, Beijing, in 2016 was $736.6 \mathrm{~kg}(195.2 \mathrm{t} \mathrm{CO} 2$ equivalent), which was averaged at $0.08 \mathrm{~kg} \cdot$ head $\cdot \mathrm{year}^{-1}$. The result was within the ranges of the previous findings of 0.10-0.40 kg $\cdot$ head $\cdot y_{e a r}{ }^{-1}$ (Amon B et al., 2001; Sneath R W et al., 2006; Borhan M S et al., 2011).

\section{Conclusion}

$\mathrm{N}_{2} \mathrm{O}$ emission from dairy manure storage and the effects of wind speed and rainfall was simulated by utilizing scaled models in the laboratory, and the annual $\mathrm{N}_{2} \mathrm{O}$ emissions from dairy manure management system in Yanqing, Beijing, was estimated. Results show that $\mathrm{N}_{2} \mathrm{O}$ emissions from the simulated dairy manure stockpiles were $5.86 \mathrm{mg} \mathrm{kg}^{-1}, 9.43 \mathrm{mg} \mathrm{kg}^{-1}$ and $0.81 \mathrm{mg} \mathrm{kg}^{-1}$ in spring, summer and autumn, respectively. $\mathrm{N}_{2} \mathrm{O}$ emission was affected by wind speed over $1.2 \mathrm{~m} \cdot \mathrm{s}^{-1}$, meanwhile the simulated rainfall resulted in a sharp increasing of $\mathrm{N}_{2} \mathrm{O}$ emission and then the emission decreased to previous level for 6-10 hours. Total $\mathrm{N}_{2} \mathrm{O}$ emission from manure management system of 26 dairy farms in Yanqing, Beijing, in 2016 was estimated at 195.2 t $\mathrm{CO}_{2}$ equivalent.

\section{Acknowledgements}

This study was funded by National Nature Science Foundation of China (Grant No. 31472132, and 31172244).

\section{Reference}

Amon B., T. Amon, J. Boxberger, C. Alt, 2001. Emissions of NH3, N2O and CH4 from dairy cows housed in a farmyard manure tying stall (housing, manure storage, manure spreading) [J]. Nutrient Cycling in Agroecosystems. 60(1-3), 103-113.

Arikan, O.A., W. Mulbry, S. Lansing, 2015. Effect of temperature on methane production from field-scale anaerobic digesters treating dairy manure. Waste Management. 43, 108-113.

Borhan, M.S., S.C. Capareda, S. Mukhtar, W. B. Faulkner, R. Mcgee, C. B. Parnell, 2011. Greenhouse gas emissions from ground level area sources in dairy and cattle feedyard operations. Atmosphere. 2(3), 303-329.

Lu, R., Y. Li, Y. Wan, Y. Liu, L. Jin, 2007. Emission of greenhouse gases from stored dairy manure and influence factors. Transactions of the Chinese Society of Agricultural Engineering. 23(8), 198-204.

Mazzetto, A.M., A.S. Barneze, B.J. Feigl, J.W.V. Groenigen, O. Oenema, C.C. Cerri, 2014. Temperature and moisture affect methane and nitrous oxide emission from bovine manure patches in tropical conditions [J]. Soil Biology \& Biochemistry. 76(76), 242-248.

Miyatake, F., K. Iwabuchi, 2006. Effect of compost temperature on oxygen uptake rate, specific growth rate and enzymatic activity of microorganisms in dairy cattle manure. Bioresource Technology. 97(7), 961-965.

Sneath, R.W., F. Beline, M.A. Hilhorst, P. Peu, 2006. Monitoring GHG from manure stores on organic and conventional dairy farms [J]. Agriculture Ecosystems \& Environment. 112(2),122-128.

Sommer, S.G., H.B. Moller, 2000. Emission of greenhouse gases during composting, of deep litter from pig production - effect of straw content [J]. Journal of Agricultural Science. 134(134), 327-335. 


\title{
Optimal Ambient Temperature with Regard to Feed Efficiency and Daily Gain of Finisher pigs
}

\author{
Rikke Koch Hansena, Bjarne Bjerg a,* \\ ${ }^{a}$ Department of Veterinary and Animal Sciences, University of Copenhagen, Groennegaardsvej 2, 1870 Frederiksberg \\ C, Denmark \\ * Corresponding author. Email: $\underline{\text { bsb@sund.ku.dk }}$
}

\begin{abstract}
Optimal thermal conditions for farm animals are crucial preconditions for development of improved climate control methods. Both low and high temperatures are known to comprise both daily gain and feed efficiency. The aim of this work was to utilized published studies to determine how the ambient temperature affects the daily gain and the feed efficiency for finisher pigs. Literature assessing daily gain and feed efficiency at different ambient temperatures was gathered using $\mathrm{Cab}$ Abstract and Google Scholar. The results were transformed to relative values with $100 \%$ being the temperature at which the highest productivity, in that particular study, was found. The slope corresponding to each degree Celsius was calculated within the temperature range investigated in the in the particular study. The mean slope values for all studies were calculated and the average response was illustrated in a graph. Results showed that the two productivity parameters did not peak at same ambient temperature. The daily gain peaked at $15^{\circ} \mathrm{C}$ and was above $95 \%$ of its maximum between 10 and $21^{\circ} \mathrm{C}$. Feed efficiency peaked at $23^{\circ} \mathrm{C}$ and was above $95 \%$ of its maximum between 17 and $29^{\circ} \mathrm{C}$. The optimal ambient temperature for finisher pigs was found in the range between $15^{\circ} \mathrm{C}$ and $23^{\circ} \mathrm{C}$. For the individual farmer, the economic optimal temperature is dependent on feed costs, energy costs, and on whether additional pigs are available to utilize the housing capacity gained by increased grow rate. In the latter case, the optimal temperature will be lower than if the faster growth causes a reduced utilization of the housing system.
\end{abstract}

Keywords: Daily gain, feed efficiency, finisher pigs, ambient temperature

\section{Introduction}

Because pigs are endotherm beings, they spend increased energy on body temperature maintenance when their ambient temperature is too low (Collin et al. 2001, Quiniou et al. 2001). Conversely, they will slow down their metabolic processes to restrict their energy production by reducing their feed intake, when their ambient temperature is too high (Collin et al. 2001, Quiniou et al. 2001). Both situations lead to reduced feed efficiency and weight gain (Morrison \& Heitman 1983, Nienaber et al. 1987, Massabie et al. 1996, Renaudeau et al. 2008). From a farmer's perspective, this is an undesirable outcome, as reduced feed efficiency and weight gain mean additional costs for feed and housing capacity.

Literature disagrees on at which temperature the production parameters begin to decrease and on how much they are impaired at a certain deviation from the temperature with the best performance.

The aim of this work was to combine the published studies to estimate a likely common relationship on how the ambient temperature affects the daily gain and the feed efficiency for finisher pigs.

\section{Materials and methods}

Literature was identified by use of Cab Abstract and Google Scholar. Applied key words were "temperature", "pig*", "swine", "heat stress", "feed efficiency" and "feed conversion". Further, studies cited in the found literature was assessed in order to be included in the review. Three requirements was set as criterion for including a study in the development of the aimed relationship:

The study should report original results for finisher pigs above $30 \mathrm{~kg}$.

The study should report results at at least 3 different ambient temperatures. (Results from two ambient temperatures is insufficient to predict at which temperature the pigs performs best).

The study should be conducted after 1975 (To ensure that the results reflect modern animals and production methods).

The reported feed efficiency or daily gain in each of the included studies was transferred to relative values, where $100 \%$ was defined as the observation were the animal performed best. These relative values were model into a second order relationship and the resulting slope at each one-degree temperature step was averaged for the included studies. To estimate the common relationship, the average value for the slope at each degree Celsius was added to a reference value that ensure that the relationship peaked at $100 \%$. 


\section{Results}

From the literature review, 11 studies were identified that reported data indicating the influence of temperature on daily gain and feed efficiency in pigs (see Table 1). Four of these 11 studies fulfilled the requirements set and were, therefore, used in the development of the aimed common relationships.

For each of the four included studies the agreement with second order relationships between the ambient temperature and the relatively values for daily gain appears from Figure 1, and similar agreements for feed efficiency appears from Figure 2. It can be seen that that the four studies covers different temperature ranges and that the temperature range in the study by Renaudeau et al. $(2008)\left(24-36^{\circ} \mathrm{C}\right)$ is above the temperature where the highest daily gain can be expected.

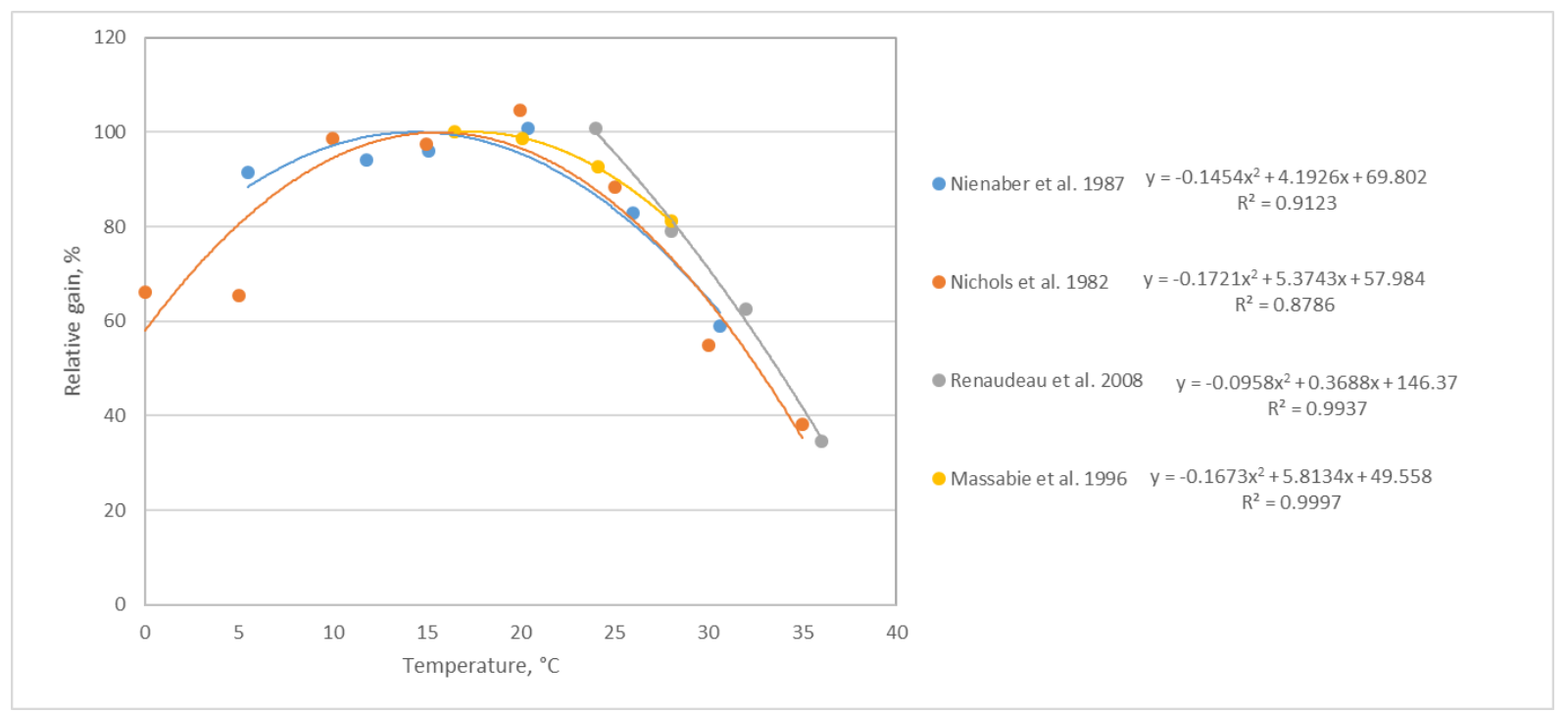

Figure 37 . Relative daily gain at increased ambient temperature for four studies.

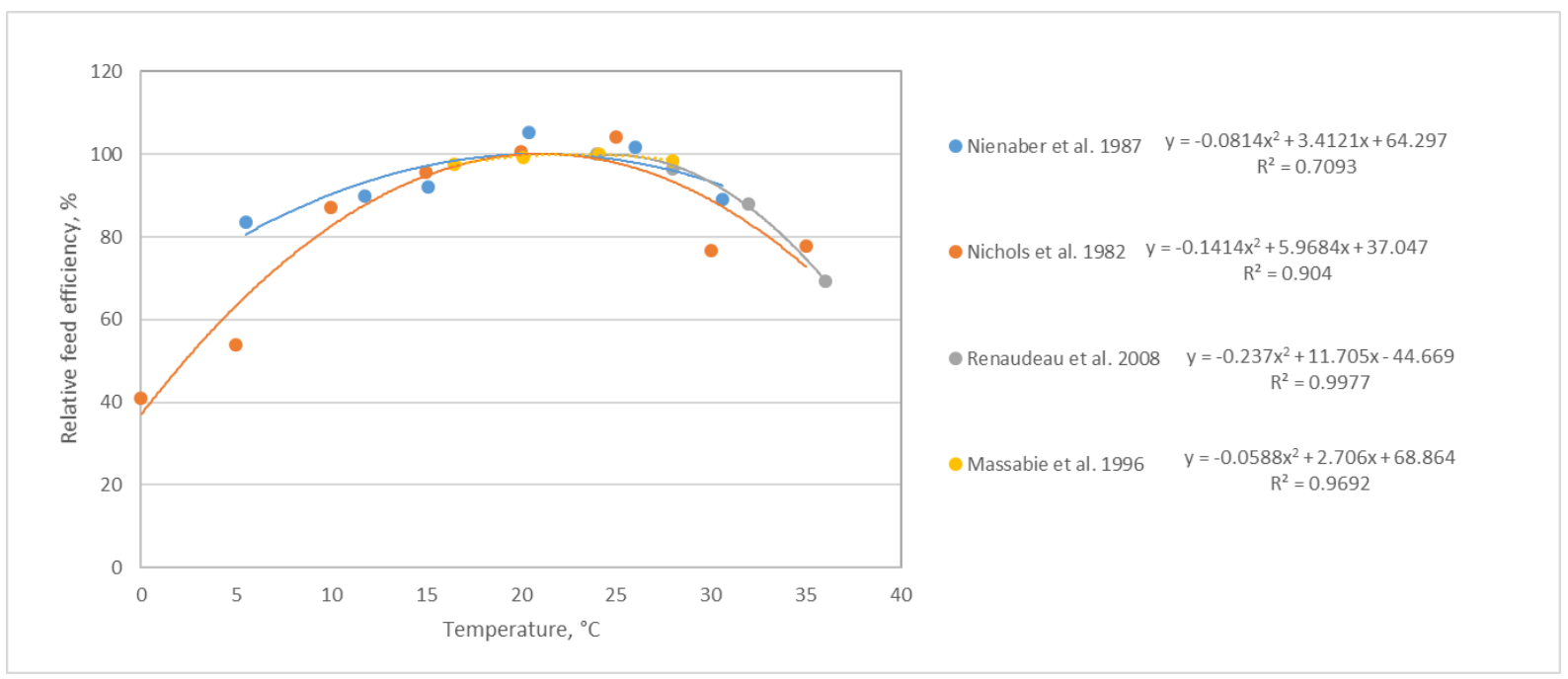

Figure 38. Relative feed efficiency at increased ambient temperature for four studies. 
Table 70 .Overview of former researches and eventual reason for disqualification for use in this study. N.I. $=$ Not informed.

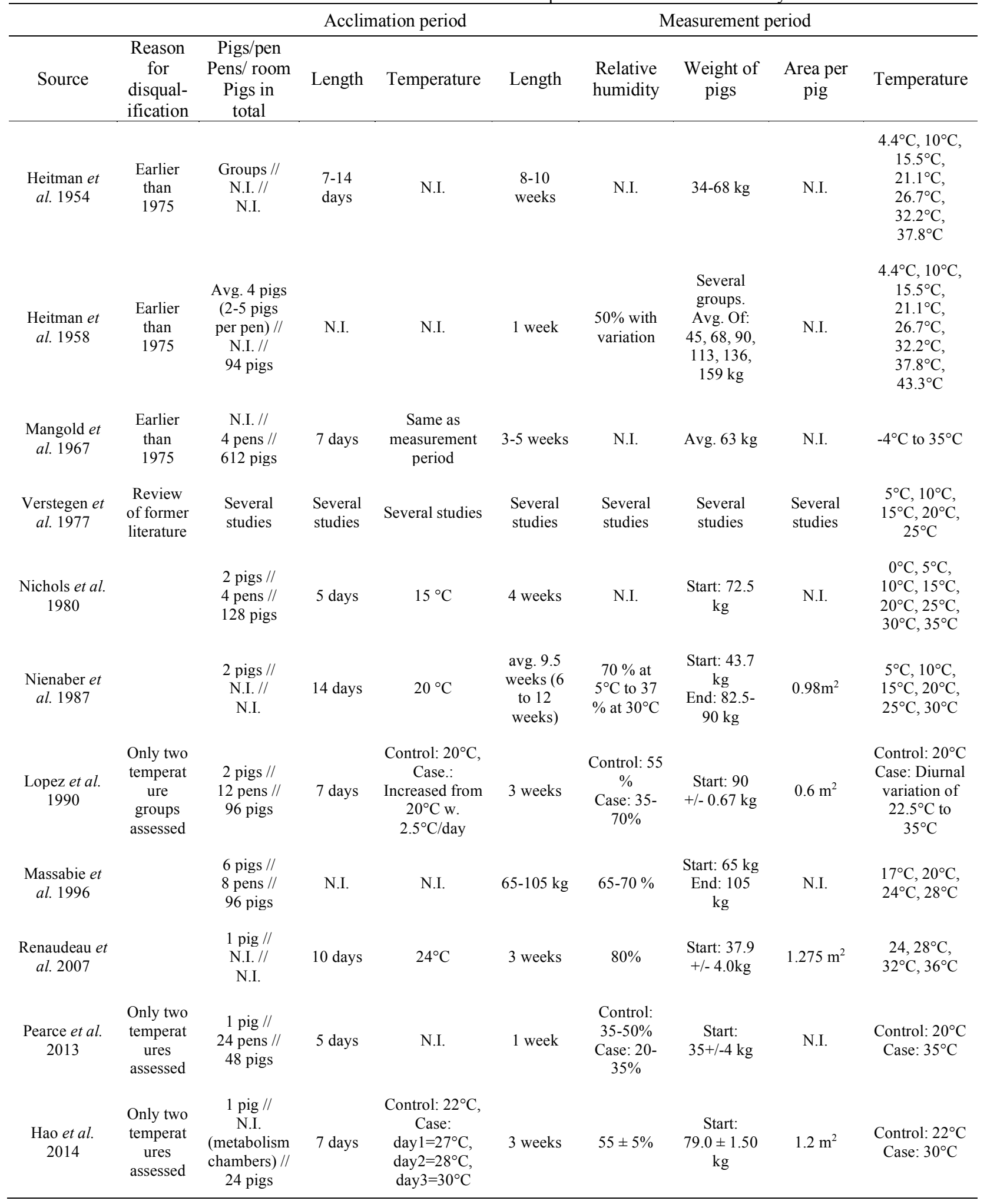


The estimated common relationship between temperature and relatively daily gain, and between temperature and feed efficiency are illustrated in Fig. 3, and it appears that relative daily gain peaks around $15^{\circ} \mathrm{C}$ and the feed efficiency around $23^{\circ} \mathrm{C}$. It also appears that the daily gain is within $95 \%$ of its maximum for all temperatures between 10 and $21^{\circ} \mathrm{C}$ and that the feed efficiency is within $95 \%$ of its maximum when the ambient temperature is between 18 and $29^{\circ} \mathrm{C}$.

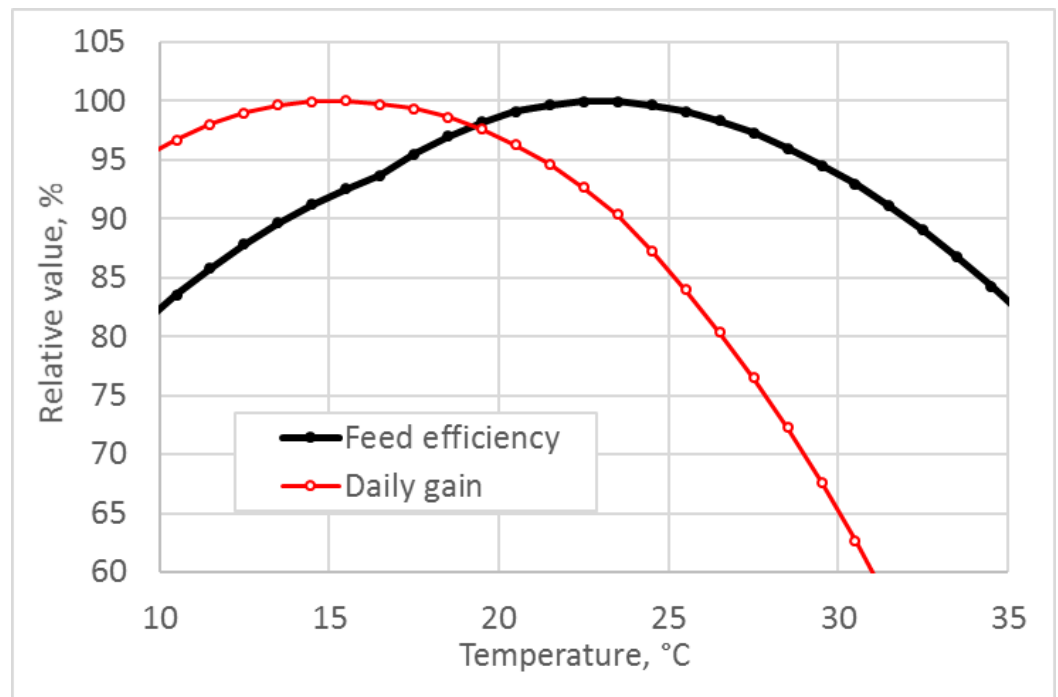

Figure 39. Average response in daily gain and feed efficiency at different ambient temperatures.

\section{Discussion}

Combining results from different studies enabled recommendations that embrace different evaluation methods, different housing conditions and different animals. Thereby a broader basis for the response in production parameter was covered. By doing so, different ambient temperatures were found to be preferable with respect to feed efficiency and weight gain. From this work, it was seen that daily gain peaked at $15{ }^{\circ} \mathrm{C}$, whereas feed efficiency peaked at $23^{\circ} \mathrm{C}$. According to Nienaber et al. (1987) both daily gain and feed efficiency peaked at $20^{\circ} \mathrm{C}$. For daily gain, this was in accordance with the results found by Nichols et al. (1982). This is despite differences in the size of pigs entering the study. In the work carried out by Nichols et al. (1982), only late growth finisher pigs of a weight of $72.5 \mathrm{~kg}$ and four weeks ahead were considered. Nienaber et al. (1987) included a larger range of finisher pigs in their assessment, starting at an average weight of $43.7 \mathrm{~kg}$ and six to 12 weeks ahead. A study by Heitman et al. (1958) confirmed that the daily gain peaked between 18 and $23^{\circ} \mathrm{C}$, depending on the pigs' weight, with a decreasing optimal temperature as the pigs gained weight. It was therefore expected to see a maximised daily gain at a higher temperature in the work by Nienaber et al.(1987) compared to Nichols et al.(1982). No information on indoor velocity or type of ventilation system to cause air movements were given, which could have explained this result. Only one study showed a lower value $\left(17^{\circ} \mathrm{C}\right)$ as the temperature resulting in the highest daily gain among the finisher pigs (Massabie et al. 1996). Their work also concerned finisher pigs in the last part of their growth period $(65-105 \mathrm{~kg})$, which may be a part of the reason for a peak at a lower temperature, at least compared to results by Nienaber et al. (1987). That the highest daily gain was found at this temperature may be because $17^{\circ} \mathrm{C}$ was the lowest assessed temperature. A tendency that applies for several assessed studies is that the maximum daily gain was found at the lowest assessed temperature (Massabie et al. 1996, Pearce et al. 2013, Hao et al. 2014, Lopez et al. 1990, Renaudeau et al. 2008). This could also influence the results found in this work, as data from both Renaudeau et al. (2008) and Massabie et al. (1996) entered the comparison. If mentioned studies had found higher daily gains at lower temperatures, in case lower temperatures were investigated, it would have caused the curve to move left, causing a peak in daily gain at a lower temperature. This was in particular relevant in the study of Renaudeau et al. (2008), as their lowest assessed temperature was $24^{\circ} \mathrm{C}$. However, this was also the study concerning the smallest finisher pigs with an average starting weight of $37.9 \mathrm{~kg}$ and a three-week measurement period, which was expected to result in the highest daily gain at a higher temperature compared to the remaining studies. Both Nichols et al. (1982) and Nienaber et al. (1987) found the highest daily gain at a higher temperature than what was predicted when all observations were considered (using a second order model). It indicates that when the data was fitted to a second order curve, it softened out the response around the peak, resulting in the highest daily gain at a lower temperature than actual experienced. Research assessing more temperature levels, have found a larger decrease in daily gain and feed efficiency as the 
temperature exceeded the optimal level, whereas a smaller response was found at temperatures below (Nichols $e t$ al. 1982, Nienaber et al. 1987).

There was seen a larger discrepancy among the literature when the temperature influence on feed efficiency was assessed. As mentioned, Nienaber et al. (1987) found the highest feed efficiency when the ambient temperature was $20^{\circ} \mathrm{C}$. Higher temperatures were found to cause the highest feed efficiencies in the study by Massabie et al. (1996) and Nichols et al. (1982), who found the highest feed efficiency at $24 \cdot 1^{\circ} \mathrm{C}$ and $25^{\circ} \mathrm{C}$, respectively. Other studies found no effect of temperature on feed efficiency (Lopez et al. 1990, Hao et al. 2014). From the comparison in this work, there was seen a response in feed efficiency as the ambient temperature changed. Compared to the response in daily gain, there was seen a better agreement between the feed efficiency response found in literature and the response, when the results were fitted to one joint curve in this work. A possible explanation is that the researches were conducted within a broader range of high ambient temperatures, which was the range where the feed efficiency was its highest. This means that both temperatures above and below the optimal temperature, with respect to feed efficiency, were assessed.

Studies included in this work all assessed the response in production parameters at constant ambient temperatures. This does not represent a real situation, where the pigs' ambient temperatures variate throughout time. By increasing the ambient temperature from $24^{\circ} \mathrm{C}$ to $27^{\circ} \mathrm{C}$ to $30^{\circ} \mathrm{C}$ with one day intervals, Campos et al. (2014) found relatively large responses in the feed intake, increasing, going from $24^{\circ} \mathrm{C}$ to $27^{\circ} \mathrm{C}$ and decreasing going from $27^{\circ} \mathrm{C}$ to $30^{\circ} \mathrm{C}$. In the subsequent period where pigs were housed at a constant temperature of $30^{\circ} \mathrm{C}$, the feed intake increased slightly, from were it was more stable with a small decrease (Campos et al. 2014). Pearce et al. (2015) measured the body weight gain at day 1, 3 and 7 of the experiment where one group was kept at a constant temperature of $20^{\circ} \mathrm{C}$ and the other at a constant temperature of $35^{\circ} \mathrm{C}$. This showed a stable increase in daily weight gain in the $20^{\circ} \mathrm{C}$-group, whereas the $35^{\circ} \mathrm{C}$ kept pigs showed a large decrease in body weight at the first day, which was equalised at day 3 and a smaller increase in body weight was seen at day 7 . Results from these two studies indicates that a the average response in daily gain, for pigs kept at a constant temperature for a longer time period, could not represent the immediate response in pigs being exposed to a short period with exceeding temperatures. This issue was investigated by Brown-Brandl et al. (1998), who conducted an experiment with pigs housed at $18^{\circ} \mathrm{C}$ prior to a 22-hour exposure to either $18^{\circ} \mathrm{C}, 24^{\circ} \mathrm{C}, 28^{\circ} \mathrm{C}$ or $32^{\circ} \mathrm{C}$. From that it was seen that the feed intake was significantly lower for pigs exposed to $28^{\circ} \mathrm{C}$ and $32^{\circ} \mathrm{C}$ compared to the other groups. This was seen as lower weight of pigs in the $18^{\circ} \mathrm{C}, 28^{\circ} \mathrm{C}$ and $32^{\circ} \mathrm{C}$ group compared to the $24^{\circ} \mathrm{C}$ group. As pigs in the work by Brown-Brandl et al. (1998) did not have time to adjust to the heat, this work was better at describing the reaction expected in a professional pig production.

The discrepancy between the temperatures causing reduction in daily gain and feed efficiency, respectively, shows that the economical optimal temperature for the individual farmer, depends on feed costs, energy costs, and the production cycle with respect to availability of utilizing housing capacity gained by increased growth rate or delay, due to reduced growth rate. It is likely that the desired ambient temperature is equivalent to the temperature that entails the largest monetary gain for the individual farmer, but one temperature does not cause the highest result in both production parameters.

\section{Conclusions}

In conclusion, both daily gain and feed efficiency were affected by the ambient temperature, but the temperatures at which they were affected, were different. The highest daily gain was achieved at lower temperatures $\left(15^{\circ} \mathrm{C}\right)$ than the highest feed efficiency $\left(23^{\circ} \mathrm{C}\right)$. Both productivity parameters showed an exponential response with decreasing values on each side of the peak. In finisher pig facilities, an ambient temperature between 15 and $23^{\circ} \mathrm{C}$ should be aimed.

\section{References}

Brown-Brandl, T. M., J. A. Nienaber, L. W. Turner, 1998. Acute heat stress effects on heat production and respiration rate in swine. Transactions of the ASAE. 41(3):789-793.

Campos, P. H. R. F., J. Noblet, Y. Jaguelin-Peyraud, H. Gilbert, P. Mormede, R. F. M. de Oliveira Donzele, J. L. Donzele \& D. Renaudeau, 2014. Thermoregulatory responses during thermal acclimation in pigs divergently selected for residual feed intake. International Journal of Biometeorology. 58:1545-1557.

Collin, A., J. van Milgen, S. Dubois \& J. Noblet, 2001. Effect of high temperature on feeding behaviour and heat prodution in group-housed young pigs. British Journal of Nutrition. 86, 63-70.

Hao, Y., Y. Feng, P. Yang, J. Feng, H. Lin. \& X. Gu, 2014. Nutritional and physiological responses of finishing pigs exposed to a permanent heat exposure during three weeks. Archives of Animal Nutrition. 68, 296-308.

Heitman, H. J., T. E. Bond \& C. F. Kelly, 1954. Effect of temperature on swine - influence of environment on feed utilization studied for improvement in efficiency of farm structures. California Agriculture. June, 8-9 
Heitman, H. J., C. F. Kelly, \& T. E. Bond, 1958. Ambient air temperature and weight gain in swine.

Lopez, J., G. W. Jesse, B. A. Becker \& M. R. Ellersieck, 1990. Effects of temperature on the performance of finishing swine: I. Effects of a hot, diurnal temperature on average daily gain, feed intake and feed efficiency. Journal of Animal Science. 69, 1843-1849.

Mangold, D. W., T. E. Hazen \& V. W. Hays, 1967. Effect of air temperature on performance of growing finishing swine. Transactions of the ASAE. 370-377.

Massabie, P., R. Granier \& J. L. Dividich, 1996. Influence de la temperature ambiante sur les performances zootechniques du porc a l'engrais alimenté ad libitum. Journées Rech. Porcine en France. 28, 189-194.

Morrison, S.R. \& J. H. Heitman, 1983. Performance of swine following periods of heat stress. Transaction of the ASAE. 522-524.

Nichols, D. A., D. R. Ames \& R. H. Hines, 1980. Effect of temperature on performance of finishing swine. Effect of temperature on performance of finishing swine," Kansas Agricultural Experiment Station Research Reports: Vol. 0: Iss. 10.

Nienaber, J. A., G. L. Hahn \& J. T. Yen, 1987. Thermal environment effects on growing-finishing swine part I - Growth, feed intake and heat production. Transaction of the ASAE. 30(6), 1772-1775.

Pearce, S.C., N. K. Gabler, J. W. Ross, J. Escobar, J. F. Patience,R. P. Rhoads\& L. H. Baumgard, 2013. The effect of heat stress and plane nutrition on metabolism in growing pigs. Journal of Animal Science. 91, 21082118.

Renaudeau, D., M. Kerdoncuff, C. Anaïs \& J. L. Gourdine, 2007. Effect of temperature level on thermal acclimation in large white growing pigs. Animal. 2(11), 1619-1626.

St-Pierre, N. R., B. Cobanov \& G. Schnitkey, 2003. Economic losses from heat stress by US livestock industries. Journal of Dairy Science. 86, E52-E77.

Quiniou, N., J. Noblet, J. van Milgen, \& S. Dubois, 2001. Modelling heat production and energy balance in group-housed growing pigs exposed to low or high ambient temperatures. British Journal of Nutrition. 85, 97106.

Verstegen, M. W. A., E. W. Brascamp \& W. Vanderhel, 1977. Growing and fattening of pigs in relation to temperature of housing and feeding level. Journal of Animal Science. 58:1-13. 


\title{
Influence of evaporative cooling on respiration rate of lactating cows under hot climate conditions
}

\author{
Severino Pinto ${ }^{\text {a,b,c }}$, Harel Levit ${ }^{\text {d,e }}$, Theresa Müschner-Siemens ${ }^{\text {a }}$, Gundula Hoffmann ${ }^{a}$, Christian \\ Ammon $^{\text {a }}$, Ilan Halachmid,e, Wolfgang Heuwieser ${ }^{\mathrm{c}}$ and Thomas Amon ${ }^{\mathrm{a}, \mathrm{b}}$ \\ ${ }^{a}$ Leibniz Institute for Agricultural Engineering and Bioeconomy (ATB), Department of Engineering for Livestock \\ Management, 100 Max-Eyth-Allee, 14469 Potsdam, Germany \\ ${ }^{b}$ Freie Universität Berlin, Department of Veterinary Medicine, Institut of Animal Hygiene and Environmental Health, 7- \\ 13 Robert-von-Ostertag-Str., 14163 Berlin, Germany \\ ${ }^{c}$ Freie Universität Berlin, Department of Veterinary Medicine, Animal Reproduction Clinic, 65 Königsweg, 14163 \\ Berlin, Germany \\ ${ }^{\mathrm{d}}$ Agricultural Research Organization - The Volcani Center (A.R.O.), Institute of Agricultural Engineering, Precision \\ Livestock Farming lab (PLF), 68 HaMaccabim Road, 7505101 Rishon LeZion, Israel \\ e'Department of Animal Sciences, The Robert H. Smith Faculty of Agriculture, Food, and Environment, The Hebrew \\ University of Jerusalem, P.O. Box 12, Rehovot 76100, Israel \\ Corresponding author: severinop@gmail.com
}

\begin{abstract}
The present study aimed to investigate the influence of cooling on the respiration rate (RR) of lactating dairy cows at two different cooling frequencies per day, considering cow-related factors (standing and lying posture) and coat color. RR was hourly measured by visually counting of flank movements in a total of 20 Israeli Holstein dairy cows ( $2^{\text {nd }}$ to $7^{\text {th }}$ lactation), housed in a naturally ventilated barn in Rishon Letsiyon, Israel. The cows were randomly divided in two groups among cooling frequencies: with three times (control) and eight times (experimental) per day. Each cooling phase took place with side fans and sprinklers and lasted 45 minutes. Measurements were done between July and August 2017 on 25 days. The experiment was divided in three different day times (morning, afternoon and night). Data were analyzed for differences between factor levels with a repeated measurements linear mixed model at an overall significance level of 0.05 . During the experimental period, the temperature-humidity index (THI) inside the barn was recorded and was $79.5 \pm 3.16$ (mean $\pm \mathrm{SD}$ ). RR of cows differed between groups $(P<0.001)$. The average RR of the control group was 15 breaths per minute $(\mathrm{BPM})$ higher than in the experimental group $(\mathrm{P}<0.001)$. During the cooling, BPM of standing cows in the control group and in the experimental group decreased by 26 and 16 BPM, respectively, in comparison to the uncooled barn $(P<0.001)$. Standing cows $(73$ and $60 \mathrm{BPM})$ inside the barn showed higher RR than lying cows $(66$ and 52 $\mathrm{BPM})$ in the control and experimental group, respectively $(P<0.001)$. The results showed evaporative cooling helps to relieve heat stress in dairy cows in hot climate zones. An increase of evaporative cooling frequency per day reduced RR and improved heat abatement in lactating dairy cows regardless of cow body posture.
\end{abstract}

Key words: Dairy cows, evaporative cooling, respiration rate, body posture, coat color

\section{Introduction}

With climate change, dairy production requires more specialization to improve the thermal comfort in high yielding dairy cows. Hot weather conditions are regarded as a central problem of dairy production, and impair animal welfare (Kendall et al., 2007). Heat stress influences dairy production negatively due to the cows being specialized in milk production, but sensitive to heat stress conditions, as the optimal temperature range is between -0.5 and $20^{\circ} \mathrm{C}$ (Hahn, 1999) or based on a temperature-humidity index less than 68 (Zimbelman and Collier, 2011).

In response to heat stress, dairy cows increase respiration rate (RR) to initiate a heat stress relief before a significant increase in body temperature (Berman, 2005, Polsky and von Keyserlingk, 2017). RR as a good physiological measure being a non-invasive heat stress indicator assessed only by visually counting (Gaughan et al., 2000).

New strategies are recently required to improve heat stress relief in dairy cows and to reduce production losses. A combination between fans and sprinklers is appropriate to decrease body temperature and RR of dairy cows while improving well-being under hot conditions (Valtorta and Gallardo, 2004). A number of scientific studies have presented positive cooling effects on the respiration rate of cows with different cooling systems. Evaporative cooling provides effective and immediate heat stress relief in dairy cows. This reduction may reach around $60 \%$ in breath per minute (BPM) in comparison with no cooled cows (Kendall et al., 2007).

According Gaughan et al. (2000), the respiratory rate is a suitable heat stress indicator, but its validity may be imparied by environmental and animal individual factors. The respiration effectivesness may be different in cows in a lying down posture (Santos and Overton, 2001), as well as, increase the possibility of heat stress at lower temperaures (Berman, 2005). Differences on heat stress assessment may be also associate with coat color of the 
cows (Tucker et al., 2008). However, most studies of heat stress in dairy cattle did not consider factors, specifically related to the individual cow.

The present study aimed to assess the effectiveness of different evaporative cooling frequencies on respiration rate of lactating dairy cows considering the factor cow body posture and coat color.

\section{Material and Methods}

Experimental design and management of cows

The study was conducted on the research dairy farm of the Agricultural Research Organization Volcani Center in Rishon Letsiyon, Israel $\left(31^{\circ} 59^{\prime} 34.3^{\prime \prime} \mathrm{N} 34^{\circ} 48^{\prime} 59.1^{\prime \prime} \mathrm{E}\right)$. The climate of this region is predominantly semiarid. The experiment was carried out on a total of 25 measurement days during July and August 2017. The cows were housed in a naturally ventilated compost barn aligned in a NW-SE orientation. A total of 20 lactating Israeli Holstein Friesian dairy cows from second to seventh lactation were included in the trial during the experimental period and were fed with a total mixed ration. The cows were milked three times per day in a fishbone milking parlor with capacity of 24 cows milked at the same time. The average daily milk yield of the group was $44.23 \pm$ $7.70 \mathrm{~kg}($ Mean $\pm \mathrm{SD}$ ) per cow. The cows were assigned randomly to 1 of 2 treatments. One of the treatments represented by "control" ( $\mathrm{n}=10$ ) had three times cooling per day (Cooling $\mathrm{C} 1$ from 4:15 to 5:00 am; $\mathrm{C} 2$ from 12:15 to 1:00 pm and $\mathrm{C} 3$ from 7:15 to 8:00 pm), which was carried out before each milking time. In contrast, in the "experimental" group $(\mathrm{n}=10)$ eight times cooling per day were carried out, following the times: first cooling (E1) 1:00 to 1:45 am; second (before first milking; E2) 4:15 to 5:00 am; third (E3) 9:30 to 10:15 am, fourth (before second milking; E4) 12:15 to 1:00 pm; fifth (E5) 02:45 to 03:30 pm; sixth (E6) 5:00 to 5:45 pm; seventh (before third milking; E7) 7:15 to 8:00 pm; and eighth (E8) 10:00 to 10:45 pm. In each group, the coat color of the cows was determined from digital photographs using analysis software (Image J version 1.51, Wayne Rasband NIH, Bethesda, MY, USA). The coat color was categorized in three different groups, black ( $85 \pm 7.6 \%$ black hair, mean $\pm \mathrm{SD})$, mixed $(59 \pm 8 \%$ black hair) or light percentage of black hair $(19 \pm 4.8 \%)$.

The cooling yard had a concrete floor and an area of $3 \times 7 \mathrm{~m}$ for each group, with approximately $1.4 \mathrm{~m}^{2} /$ cow. Three side ventilators and sprinklers with 4 minutes ventilation interval and 40 seconds shower with the sprinklers were installed. Each cooling had duration of $45 \mathrm{~min}$. The sprinklers were fixed $2.8 \mathrm{~m}$ above the ground (approximately $1.4 \mathrm{~m}$ above the cows), with a water flow approximately $75 \mathrm{~mm}$ of water/h.

Hourly the respiration rate was visually assessed by counting right thoracoabdominal movements for thirty seconds and multiplying the value by two (i.e., breaths per minute, BPM). A period of 15 minutes was awaited due to the cows' adaptation on the specific place. During the RR measures, the body posture of the cows (standing and lying) was observed.

\section{Environmental data}

Ambient temperature (AT) and relative humidity (RH) of the air in the barn (seven units) and the cooling yard (two units) were recorded every 5 min over a 24 hour measurement with nine data loggers (EasyLog USB 2+, Lascar Eletronics Inc., Whiteparish England) positioned inside the buildings (barn and cooling pen) at 3 meters above the floor. The temperature-humidity index (THI) was calculated according to NRC (1971) as follows:

$$
\mathrm{THI}=(1.8 \times \mathrm{Tdb}+32)-(0.55-0.0055 \times \mathrm{RH}) \times(1.8 \times \mathrm{Tdb}-26)
$$

where $\mathrm{Tdb}$ is dry bulb temperature (in ${ }^{\circ} \mathrm{C}$ ) and $\mathrm{RH}$ is relative humidity (in \%).

\section{Statistical analysis}

The measures were conducted using two treatments (control and experimental). The environmental condition was recorded during the entire measurement period with a total of 109,793 records. We proceeded the comparisons of respiration rate between treatments over the day and within cooling numbers at the same time cooling of both groups, control: cooling number 1, 2 and 3 and experimental: cooling 2, 4 and 7 respectively. The analyses were conducted with a linear mixed model with repeated measurements per cow to test the influence of the cow-related factors (body posture and coat color group) and places (barn and cooling). The analysis of RR based on the following mixed linear model:

The fixed factors in the models were group (experimental vs. control), body posture (standing vs. lying), color group (dark, mixed and light coat color) and places (barn vs. cooling). A variance component covariance structure was used for random effects and repeated measurements. Factor influences were tested at a significance level of 0.05 . The differences between the factor levels of the significant factors were post hoc tested by t-tests in multiple pairwise comparisons. The $\mathrm{P}$-values of those multiple comparisons were adjusted by a simulation of the true $95 \%$ quantile of the contrasts, maintaining a global significance level of 0.05 . Model viability was checked by a visual examination of the residuals (homogeneity of variance and normality). All analyses were performed using SAS 9.4 (SAS Institute Inc., Cary, NC, USA). 


\section{Results and Discussion}

Table 1. Temperature-humidity index (THI) in the barn and cooling yard during day and night of $25 \mathrm{~d}$ of the experiment

\begin{tabular}{ccccccc}
\multicolumn{7}{c}{ period. } \\
\hline Place & Day and Night Class & Mean & \pm & SD & Min & Max \\
\hline \multirow{2}{*}{ Barn } & Day 0700-1800h & 80.8 & \pm & 2.23 & 69.4 & 86.7 \\
& Night 1900-0600h & 75.9 & \pm & 1.96 & 67.9 & 84.5 \\
\multirow{2}{*}{ Cooling } & Day 0700-1800h & 80.6 & \pm & 2.52 & 68.9 & 85.8 \\
& Night $1900-0600 \mathrm{~h}$ & 75.8 & \pm & 2.06 & 67.7 & 81.9 \\
\hline
\end{tabular}

According to Zimbelman and Collier (2011), the comfort zone for dairy cattle of THI is less than 68. Therefore, we considered that the cows in the present study were constantly under heat stress conditions with THI $78 \pm 3.26$ (mean $\pm \mathrm{SD}$ ) during the measures and did not include the THI in the analyses of effects on RR of the cows. Table 1 summarizes a total of 109,793 records in descriptive analysis of THI inside the barn and the cooling yard during day and night.

The analysis included a total of 4686 RR observations in 20 cows. Figure 1 shows that the RR of cows differed between control and experimental group $(P<0.001)$, correlating with different places (barn and cooling yard) and cow body posture (standing and lying). During the cooling time, the cows remained in standing posture. The cooling lead to a reduction of RR of cows under heat stress conditions in comparison with cows in a standing posture inside the barn $(P<0.001)$. No significant difference of RR was observed between control $(47 \pm 11.14$ $\mathrm{BPM}$; mean $\pm \mathrm{SD})$ and experimental cows $(44 \pm 9.75 \mathrm{BPM})$ during the cooling periods. However, the control group showed a high RR decrease $(25 \%)$ in the cooling yard compared with the experimental group. This finding agrees with Valtorta and Gallardo (2004), who showed that after the cooling cows were able to decrease 17 BPM of RR. We presumed that the control cows decreased the RR more strongly during the cooling period due to the higher accumulation temperature along the day inside the barn.

Long periods of standing posture in cows are associated with an efficient heat release (Berman, 2005). In both treatments, a significant difference of RR on standing and lying cows inside the barn was observed, and the control group showed higher respiration rates in both body postures (standing: $73 \pm 21.5$ BPM and lying: $66 \pm 19.7 \mathrm{BPM}$; mean $\pm \mathrm{SD}$ ) compared with the experimental group (standing: $60 \pm 18.4$ and lying: $52 \pm 17.8 \mathrm{BPM} ; P<0.001$ ). The wind convection improves the body temperature dissipation on the cows (Frazzi et al., 2000). In some not yet published studies, we observed a statistically significant influence of body posture on RR in lactating cows under moderate heat stress conditions ( $\mathrm{THI} \leq 80)$, however, cows tended not differ the RR between body postures in severe heat stress conditions. With cows under severe stress, as in the present study, no difference between body postures was observed. Some authors suggest that the body contours of cows change while lying down by the rumen compression on the diaphragm reducing the lung capacity (Santos and Overton, 2001, Tucker et al., 2008), and the cows increase the respiration frequency to compensate it, which was not observed in the present study.

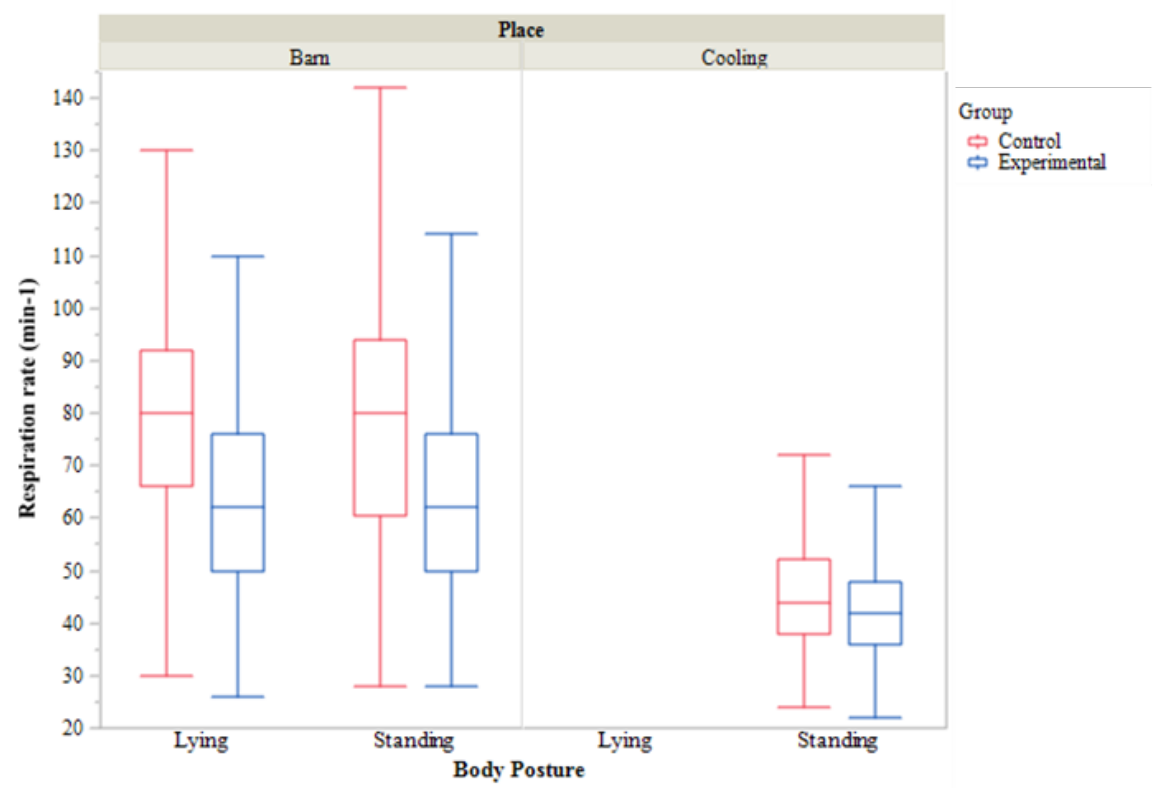

Figure 1. Respiration rate of lactating cows on experimental (blue box) and control (red box) group, inside the barn or cooling yard in different body postures (Lying and Standing). 
There were no statistically significant differences in the average RR associated with coat color in response to the treatments $(P=0.6213)$. Black cows showed a RR of $66 \pm 2.74$ BPM (mean \pm SEM), black and white $65 \pm$ 2.38 and white coat color $64 \pm 3.38$ BPM, respectively. We can postulate that this result is associated with no exposing of cows to direct solar radiation during the experimental period, which would contribute to heat load in animals with a dark coat (Mader et al., 2006). In addition, in a study by Kendall et al. (2007) no differences in average RR associated with coat color were observed.

\section{Conclusions}

The present study provides evidence that the evaporative cooling in hot climate zones is appropriate to relieve heat stress in housed dairy cows. An increase of evaporative cooling frequency per day reduces RR and improves heat abatement in lactating dairy cows. There were no evidences that the cow-related factors as body posture and coat color influence the RR in lactating cows under heat stress conditions.

\section{Acknowledgements}

The authors gratefully acknowledge the staff of the Department at Engineering for Livestock Management at ATB Institute, in particular Julia Heinicke, Annemarie Englisch, Sabrina Hempel and the staff of the dairy farm at the Agricultural Research Organization, Volcani Center in Rishon Letsiyon, Israel, where the experiments were carried out. This project was supported by "Optimized animal-specific barn climatization facing temperature rise and increased climate variability - OptiBarn" project of the FACCE ERA-NET Plus Initiative Climate Smart Agriculture and with scholarship cooperation from the Coordination for Improvement of Higher Education Personnel - CAPES (Brazil).

\section{References}

Berman, A. 2005. Estimates of heat stress relief needs for Holstein dairy cows. Journal of Animal Science 83 (6):1377-1384.

Frazzi, E., L. Calamari, F. Calegari, and L. Stefanini. 2000. Behavior of dairy cows in response to different barn cooling systems. Transactions of the ASAE 43 (2), 387-394.

Gaughan, J. B., S. M. Holt, G. L. Hahn, T. L. Mader, and R. Eigenberg. 2000. Respiration rate - Is it a good measure of heat stress in cattle? Asian-Australasian Journal of Animal Sciences 13, 329-332.

Hahn, G. L. 1999. Dynamic responses of cattle to thermal heat loads. Journal of Animal Science 77 (2), 1020.

Kendall, P. E., G. A. Verkerk, J. R. Webster, and C. B. Tucker. 2007. Sprinklers and shade cool cows and reduce insect-avoidance behavior in pasture-based dairy systems. Journal of Dairy Science 90 (8), 3671-3680.

Mader, T. L., M. S. Davis, and T. Brown-Brandl. 2006. Environmental factors influencing heat stress in feedlot cattle. Journal of Animal Science 84 (3), 712-719.

Polsky, L. and M. A. G. von Keyserlingk. 2017. Invited review: Effects of heat stress on dairy cattle welfare. Journal of Dairy Science 100 (11), 8645-8657.

Santos, J. E. P. and M. W. Overton. 2001. Diet, feeding practices and housing can reduce lameness in dairy cattle. Pages 145-161 in Proc. Intermountain Nutrition Conference, Utah, USA.

Tucker, C. B., A. R. Rogers, and K. E. Schutz. 2008. Effect of solar radiation on dairy cattle behaviour, use of shade and body temperature in a pasture-based system. Applied Animal Behaviour Science 109 (2-4), 141-154.

Valtorta, S. E. and M. R. Gallardo. 2004. Evaporative cooling for Holstein dairy cows under grazing conditions. International Journal of Biometeorology 48 (4), 213-217.

Zimbelman, R. B. and R. J. Collier. 2011. Feeding Strategies for High-Producing Dairy Cows During Periods of Elevated Heat and Humidity. Pages 111-125 in Proc. Tri-State Dairy Nutrition Conference, Fort Wayne, ID. 


\title{
Modelling Environmental Time Series in Livestock Facilities using a Hybrid Model based on ARIMA and Wavelet Transform
}

\author{
Roberto Besteiro $^{\mathrm{a},{ }^{*}, \text { Tamara Arango }}{ }^{\text {, Dolores Rodríguez }}{ }^{\mathrm{a}}$, Manuel Rodríguez ${ }^{\mathrm{a}}$ \\ a Department of Agroforestry Engineering, Campus Terra, Universidade de Santiago de Compostela, Lugo 27002, Spain \\ * Corresponding author. Email: roberto.besteiro@rai.usc.es
}

\begin{abstract}
Environmental control in livestock buildings is a key element to ensure optimal animal welfare and to reduce the environmental impact of animal activity. Although environmental control has been performed mainly by establishing a temperature setpoint, the development of Big Data and smart farming have brought to light new methodologies - such as those based on predictive control - that allow for the incorporation of different environmental variables. The statistical modelling of such variables allows for the use of predictive control algorithms that are more precise than traditional algorithms. Wavelet transform is a recent technique for modelling time series that allows for multi-resolution analysis of the series, which helps improve the performance of the generated models. This paper compares the performance of hybrid models combining a Discrete Wavelet Transform (DWT) and an ARIMA model. For the purposes of comparison, we modelled a relative humidity series from a weaned piglet building in northwest Spain using an ARIMA model and a hybrid Wavelet-ARIMA model, and implemented on-line and off-line predictions of the hybrid model. With the off-line hybrid model, prefiltering the time series considerably reduced prediction errors as compared to conventional ARIMA modelling (Mean Absolute Relative Error, MARE: $0.4 \%$ vs. $1.5 \%$ ), which provided a more precise modelling. The prediction errors for real-time modelling using the hybrid model (MARE: $1.5 \%$ ) were very similar to those obtained with the conventional model and therefore greater than the errors of the off-line hybrid model. Such a decrease in the efficiency of the hybrid model was caused by the influence of the border distortion on the wavelet transform technique. In conclusion, statistical DWT-based hybrid models significantly improve the performance of traditional models. Yet, such an improvent was not verified for on-line predictions. Consequently, our research must continue on the search of the optimal design for these models.
\end{abstract}

Keywords: Wavelet, prediction, hybrid model, livestock facilities, piglets

\section{Introduction}

Many control methods have been developed or proposed for environmental control systems (ECS). However, because of their simplicity, on/off and PID control are still used in many ECS, which results in inconsistent performance among such systems. With advances in data storage, computing, and communication devices, it is now feasible to adopt and implement a proper control approach to overcome the inherent issues in environmental control (Afram and Janabi-Sharifi, 2014). Traditionally, industry has been reluctant to adopt complicated methods in ECS modelling and control. For this reason, most ECS still use very simple on/off or proportional-integralderivative (PID) controllers instead of more modern controllers such as model predictive control (MPC) (Afram et al., 2017). An essential component of modern controllers is the design of reliable prediction models that are capable of understanding the operation of the whole system. In this sense, data-driven models are easy to implement because they do not require a deep definition of the underlying physics of the system.

At the same time, there is a growing interest in the MPC of the systems (Prívara et al., 2013). Among these are the systems based on data-driven models. Similarly, there is a growing interestd in modelling techniques that improve the performance of traditional models, such as the wavelet transform (Nourani et al., 2014). Wavelet analysis appears to be more effective for preprocessing input data due to its ability to reveal both spectral and temporal information simultaneously, within one signal. The wavelet transform decomposes the main time series into subcomponents such that the decomposed data improve the performance of prediction models (Nury et al., 2017). Hybrid models combining traditional models and the wavelet transform improve air temperature forecasts in the range $8-23 \%$, according to the wavelet-neuro-fuzzy model developed by Karimi et al., (2015). In the field of hydrology, the wavelet-artificial neural network (ANN) method proved more successful in anomaly detection for surface water quality managements than the ANN prediction method (Shi et al., 2018). Likewise, Joo and Kim (2015) found that models using wavelet filtering outperformed ARIMA models in most of the analysed cases.

Nevertheless, the wavelet transform must be used carefully, insofar as it shows a number of drawbacks, mainly due to the fact that it uses the future values of the series to perform the decomposition (Du et al., 2017), and also because of the border distortion that can affect model performance (Rana and Koprinska, 2013). For this reason, our paper has two goals:

To compare the effect of wavelet filtering on the performance of a conventional ARIMA model. 
To compare the performance of a hybrid wavelet-ARIMA model for real-time predictions (On-line model) with the performance of a hybrid wavelet-ARIMA model for non-real-time predictions (Off-line model).

\section{Materials and Methods}

Building structure and data collection

Measurements were performed in a weaner room of a pig farm located in Northwest Spain (Figure 1). The room consisted of sixteen $10 \mathrm{~m}^{2}$ pens, each of which housed 50 piglets. The piglets entered the room at 21 days of age, with a body weight of $6.62 \mathrm{~kg}$, and exited the room on the 19th of July, 2016 with a body weight of 19.61 $\mathrm{kg}$.

The room was ventilated through a chimney exhaust fan. The pens, composed of PVC divider panels $0.70 \mathrm{~m}$ in height, had fully slatted floors, and a $3.2 \times 0.5 \mathrm{~m}$ heating plate under a PVC roof placed at a height of $0.70 \mathrm{~m}$. Piglets were fed ad-libitum with three different feeds according to their age (lacto-initiatior, prestarter, starter). Feed and water were provided in a double dry-humid hopper.

The relative humidity of the building in the animal zone was measured using a Temperature/HR smart sensor (ONSET ${ }^{\circledR}$ S-THB-M002) with $\pm 2.5 \%$ accuracy for humidity. The sensor was placed inside a cage at $0.2 \mathrm{~m}$ above the slatted floor so that the animals could not damage it. These variables were sampled every second and recorded at 10 -min intervals by a $\mathrm{HOBO}{ }^{\mathrm{N}}$ data logger.

The measurements performed during the first 5 days were neglected in order to avoid the first measurements, which were affected by the conditions of the room before the entrance of the piglets. Thus, we obtained a time series that consisted of 5031 data of relative humidity measured for 35 days.

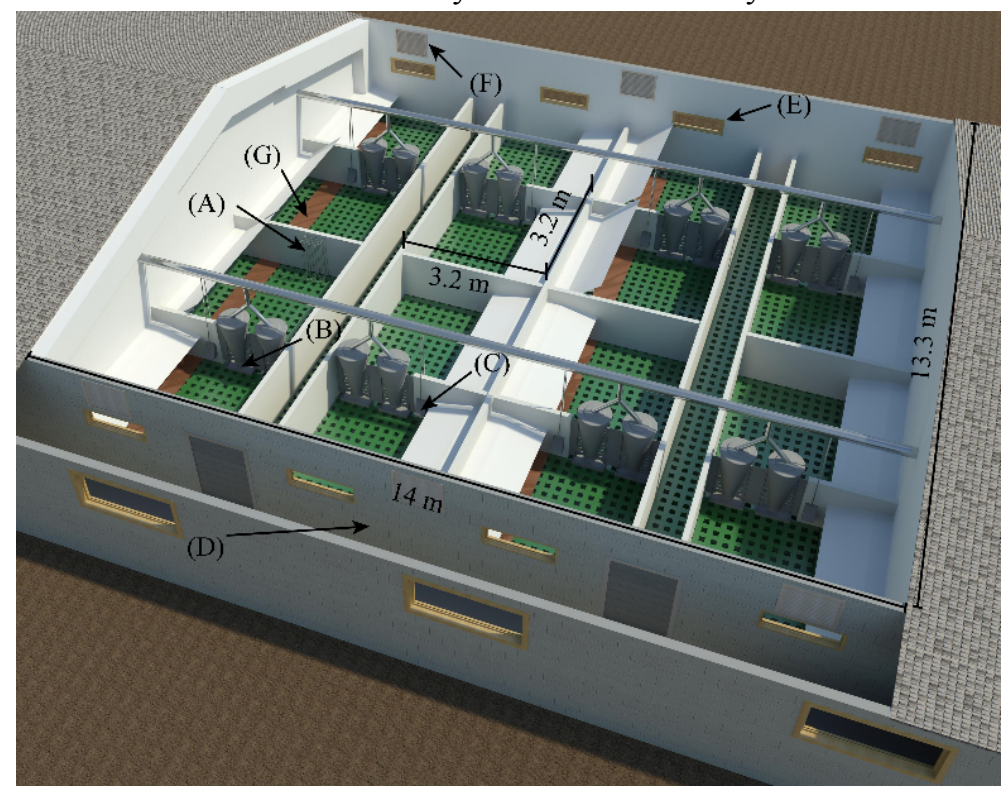

Figure 3. View of the room in which measurements were taken. (A) Location of sensors in the animal zone. (B) Feeder. (C) Drinker. (D) Exterior corridor. (E) Windows. (F) Air inlet. (G) Heating plate.

Model design

To analyse the benefits of wavelet filtering for time series prediction, we compared three statistical models for the humidity signal recorded in the weaner room. To this end, we divided the time series into two periods, a training period $(75 \%)$ and a testing period $(25 \%)$. All the processes described here were performed using the $\mathrm{R}$ statistical language and Rstudio environment (R Core Team, 2017; RStudio Team, 2016). The three designed models are:

ARIMA

The order of the ARIMA model was automatically chosen based on the procedure used by the "forecast v8.2" R package (Hyndman and Khandakar, 2008) for the training period. After that, we performed a one-step prediction with the model selected for the testing period.

Off-line WARIMA

A hybrid model combining the Discrete Wavelet Transform (DWT) and traditional ARIMA models was 
developed. In this case, DWT was used as the filtering method for the original series.

The DWT decomposes the original signal into approximation and detail coefficients using a high-pass filter and low-pass scaling filter. These filters generate an approximation $\left(A_{n}\right)$ that is a high-scale, low-frequency coefficient of the signal and details $\left(\mathrm{D}_{\mathrm{i}}\right)$ that are the low-scale, high-frequency coefficients of the signal. Then, the process is repeated for the low-frequency components of the signal until the desired level of decomposition is reached. A 3-level decomposition with the Daubechies $\mathrm{L}=8(\mathrm{db} 8)$ wavelet filter and a non-decimated DWT were used.

The denoising of the training series was performed by replacing the highest-frequency coefficients with their mean value and applying an Inverse Discrete Wavelet Transform (IDWT). After that, the order and value of the model coefficients were determined by performing an ARIMA modelling of the filtered series. The resulting DWT-filtered model was used for the predictions during the testing period.

\section{On-line WARIMA}

With a view to a potential real-time implementation of the prediction models, we introduced some changes on the hybrid Off-line model to adapt it for real-time predictions.

In this case, the training phase was identical to the one described in section 2.2.2, and included the pre-filtering of the whole dataset and the selection of the appropriate ARIMA model. During the testing phase, a slide window was incorporated such that only the most recent values were filtered and predicted because the slide window gradually moved as the process progressed. To be clear, if $\mathrm{N}$ is the total length of our time series and $\mathrm{M}$ is the size of the slide window, and we want to predict value $\mathrm{K}(\mathrm{K} \leq \mathrm{N})$, we use the $\mathrm{M}$ values preceding $\mathrm{K}$ to apply the DWT and the ARIMA model. Thus, the hybrid model yields predictions that can be considered as real-time predictions, insofar as future information is not available when the new values are predicted (Du et al., 2017). The value of M was set at 512 values to ensure a good DWT signal decomposition.

\section{Model evaluation}

To assess the performance of the generated models, we have used three statistics for measuring the prediction errors: Root Mean Square Error (RMSE), Pearson's correlation coefficient (R) and Mean Absolute Relative Error (MARE). RMSE is used to measure the difference between predicted and observed values. RMSE values are expressed in units, while MARE values suggest the size of the error in percentage terms. The correlation coefficient measures the strength and direction of a linear relationship between the predicted and observed values.

$$
\begin{aligned}
& R M S E=\sqrt{\frac{\sum\left(y-y^{\prime}\right)^{2}}{n}} \\
& R=\frac{\sum(y-\bar{y})\left(y^{\prime}-\bar{y}^{\prime}\right)}{\sqrt{\sum(y-\bar{y})^{2} \sqrt{\sum\left(y^{\prime}-\bar{y}^{\prime}\right)^{2}}}} \\
& M A R E=\frac{1}{n} \sum\left|\frac{y^{\prime}-y}{y}\right|
\end{aligned}
$$

where $y$ is the observed value, $y^{\prime}$ is the predicted value, $\bar{y}$ and $\bar{y}^{\prime}$ are the mean values of $y$ and $y^{\prime}$, respectively, and $n$ is the number of values.

\section{Results and Discussion}

The On-line and Off-line hybrid models proposed here show the same structure because both models were trained using the same data series. Both models have one differentiation and up to 5 autoregressive values. The structure of the WARIMA models differs considerably from the structure of the ARIMA model, which consists of only one autoregressive value and one moving average value.

Table 2. Prediction errors for the three models during testing.

\begin{tabular}{ccccc}
\hline & $\begin{array}{c}\text { ARIMA } \\
(p, d, q)\end{array}$ & RMSE & $R$ & MARE \\
\hline ARIMA & $(1,1,1)$ & 1.156 & 0.984 & 0.015 \\
On-line WARIMA & $(5,1,0)$ & 1.181 & 0.987 & 0.015 \\
Off-line WARIMA & $(5,1,0)$ & 0.344 & 0.999 & 0.004 \\
\hline
\end{tabular}


Overall, the results of the three prediction models were satisfactory, with correlation coefficients over 0.98 and MARE values below $1.5 \%$. As shown by the analysis of the errors for the three models, the Off-line WARIMA model obtained the best results for all the statistics, particularly for RMSE, with a value of 0.344, which demonstrates that the Off-line WARIMA outperforms the other two models (Figure 2). The On-line WARIMA model did not produce predictions for the first 512 values because that was the size assigned to the slide window used in this model. Therefore, on-line WARIMA predictions started at value 513.

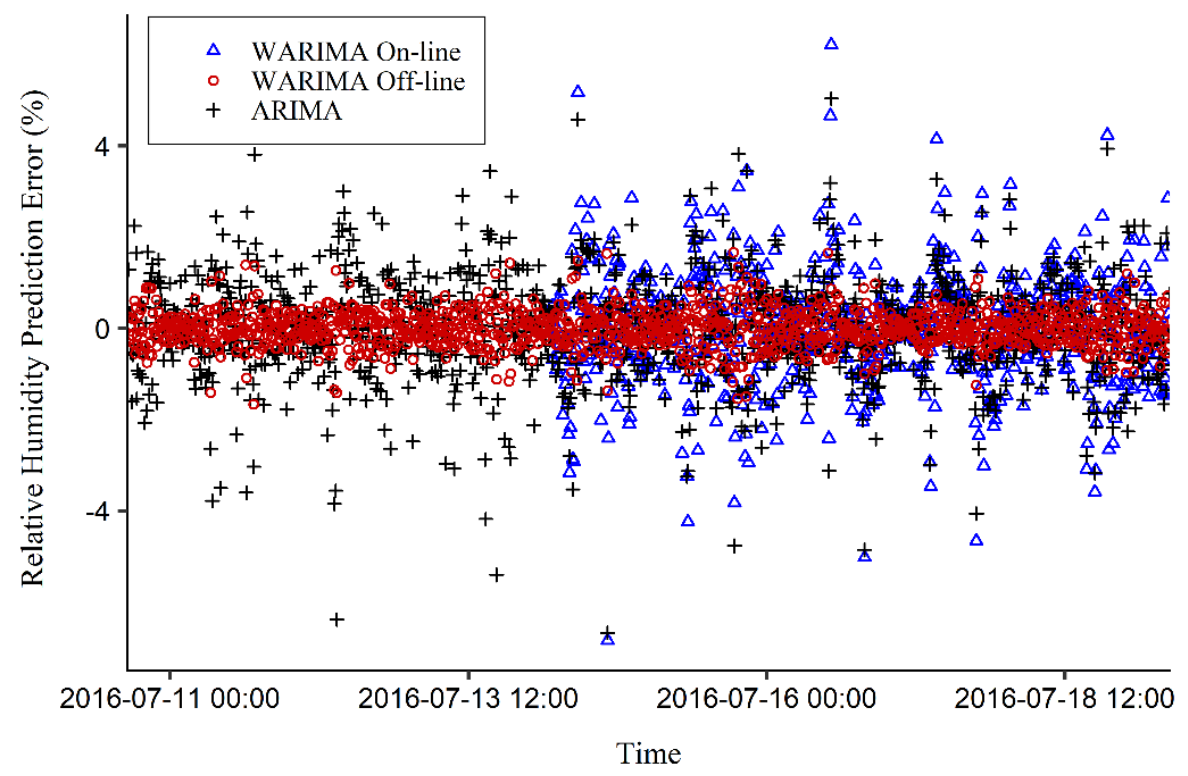

Figure 2. Prediction errors for the models assessed during the complete testing phase.

The improved performance of the Off-line WARIMA is due to the effect of DWT filtering on the original series used for predictions (Figure 3). DWT filtering removes the highest-frequency subseries, which can be associated to noise in the series and hence lead to difficulties in the prediction. Yet, these results must be interpreted with caution because, as suggested by Du et al. (2017), the DWT adopts the future values of the time series. Consequently, the process and aim of the proposed Off-line model must be considered, because it might not be suitable for certain predictions, such as real-time predictions. Only a few authors have considered this issue, which can lead to an overestimation of the predictive capabilities of hybrid models with a structure similar to the structure of the model described here.

In order to use the wavelet method for real-time predictions, a slide window must be created, such that only the most recent values are taken into consideration, thus producing the so-called On-line model. Only slight differences have been found between the statistics of the On-line hybrid model and those of the conventional ARIMA model, which has obtained slightly better results. Such differences between the performance of the Offline and the On-line models are due to the border distortion caused on the left and right borders of the wavelet transform signal. Therefore, when handling signals with finite length, the border should be treated differently from the other parts of the signal (Bastys, 2003). The distortion on the left border does not affect the predicted values, but the distortion on the right border does affect the predictions. For this reason, in this research, we used a boundary condition called 'reflection' based on which the vector beyond its boundaries is assumed to be a symmetric reflection of itself. 


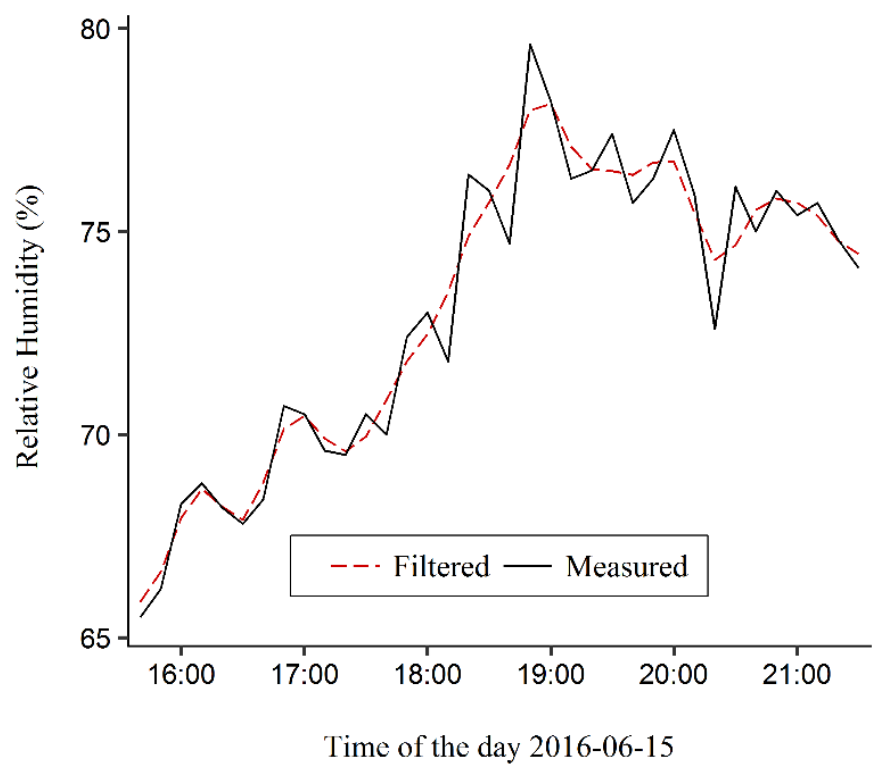

Figure 3. Effect of filtering on the humidity series.

However, this method did not effectively offset the distortion. As shown in Figure 4, the last boundary values of the approximation coefficients for the on-line wavelet transform differ from the coefficients predicted by the off-line DWT, which has been taken as a reference. This effect is due to the discontinuity introduced by the reflection at the signal boundaries, which may lead to incorrect estimation of the wavelet coefficients (Rana and Koprinska, 2013).

Because of the loss of reliability caused by the border distortion for the last values of the series and because of the structure of the ARIMA model $(5,1,0)$, which used the 5 most recent values in the series for the prediction, the performance values of the On-line hybrid model were lower than those of the Off-line model in terms of accuracy.

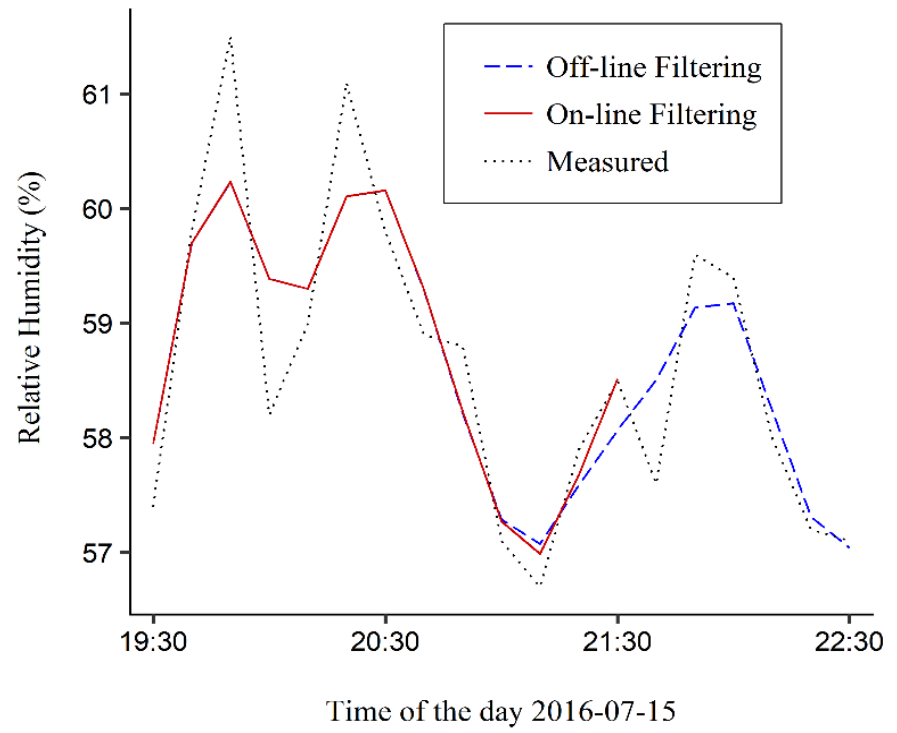

Figure 4. Border distortion caused by the wavelet filtering for the prediction of relative humidity at 21:40 hours.

Given that the Off-line hybrid model cannot be used for real-time predictions despite its great performance, and that the On-line model does not improve the results of the ARIMA model, the structure of the hybrid model must be improved. The following improvements are suggested: the ARIMA model could be trained periodically with recent values; predictions could be performed using the subseries obtained after DWT filtering; and, most importantly, alternative techniques should be studied to offset the effects of the border distortion in this type of signal (Pacola et al., 2016). 


\section{Conclusions}

The best performances were obtained using the off-line hybrid model, which combined DWT and ARIMA modelling, and obtained a MARE value of $0.4 \%$ and an RMSE value of $0.344 \%$. Accordingly, the proposed hybrid model can be a useful method for the analysis of humidity and of other series of environmental data in livestock buildings, and also for the analysis of the relationships between the study variables. Yet, this model could be unsuitable for real-time predictions because it uses the future values of the series during DWT filtering.

The On-line hybrid model, which was the alternative model developed to solve this problem, did not improve the performance of the conventional ARIMA model (MARE: 1.5\% vs. 1.5\%), probably because of the border distortion that affects the values used by the ARIMA model for the predictions. Finally, special attention must be paid to real-time predictions with the wavelet transform because the effect of the border distortion can considerably limit the capabilities of the model.

\section{References}

Afram, A., F. Janabi-Sharifi, 2014. Theory and Applications of HVAC Control Systems - A Review of Model Predictive Control (MPC). Building and Environment 72, 343-55. https://doi.org/10.1016/j.buildenv.2013.11.016.

Afram, A., F. Janabi-Sharifi, A.S. Fung, K. Raahemifar, 2017. Artificial Neural Network (ANN) based Model Predictive Control (MPC) and optimization of HVAC Systems: A state of the art review and case study of a residential HVAC system. Energy and Buildings 141, 96-113. https://doi.org/10.1016/j.enbuild.2017.02.012.

Bastys, A, 2003. The Gibbs phenomenon bounds in wavelet approximations. Proceedings 2003 International Conference on Image Processing (Cat. No.03CH37429) 1, 1017-20. https://doi.org/10.1109/ICIP.2003.1247138.

Du, K., Y. Zhao, J. Lei, 2017. The incorrect usage of singular spectral analysis and discrete wavelet transform in hybrid models to predict hydrological time series. Journal of Hydrology 552, 44-51. https://doi.org/10.1016/j.jhydrol.2017.06.019.

Hyndman, R.J., Khandakar, Y., 2008. Automatic time series forecasting: The forecast package for R. Journal of Statistical Software, 26(3).

Joo, T.W., S.B. Kim, 2015. Time series forecasting based on wavelet filtering. Expert Systems with Applications 42 (8), 3868-74. https://doi.org/10.1016/j.eswa.2015.01.026.

S. Karimi, O. Kisi, J. Shiri1, O. Makarynskyy, 2015. A wavelet and neuro-fuzzy conjunction model to forecast air temperature variations at coastal sites. International Journal of Ocean and Climate System 6(4), 159-72. https://doi.org/10.1260/1759-3131.6.4.159

Nourani V., A.H. Baghanam, J. Adamowski, O. Kisi, 2014. Applications of hybrid wavelet-artificial intelligence models in hydrology: A review. Journal of Hydrology 514, 358-77. https://doi.org/10.1016/j.jhydrol.2014.03.057.

Nury, A. H., K. Hasan, Md.J.B. Alam, 2017. Comparative study of wavelet-arima and wavelet-ann models for temperature time series data in northeastern Bangladesh. Journal of King Saud University-Science 29(1), 47-61. https://doi.org/10.1016/j.jksus.2015.12.002.

Pacola, E.R., V.I. Quandt, P.B.N. Liberalesso, S.F. Pichorim, H.R. Gamba, M.A. Sovierzoski, 2016. Influences of the signal border extension in the discrete wavelet transform in EEG spike detection. Revista Brasileira de Engenharia Biomedica 32(3), 253-62. https://doi.org/10.1590/2446-4740.01815.

Prívara, S., J.Cigler, Z. Ván̆a, F. Oldewurtel, C. Sagerschnig, E. Žáčeková, 2013. Building modeling as a crucial part for building predictive control. Energy and Buildings 56, 8-22. https://doi.org/10.1016/j.enbuild.2012.10.024.

R Core Team, 2017. R: A language and environment for statistical computing. R Foundation for Statistical Computing, Vienna, Austria. URL https://www.R-project.org/.

Rana, M., I. Koprinska, 2013. Wavelet neural networks for electricity load forecasting - Dealing with border distortion and shift invariance. Lecture Notes in Computer Science (Including Subseries Lecture Notes in Artificial Intelligence and Lecture Notes in Bioinformatics) 8131 LNCS: 571-78. https://doi.org/10.1007/978-3642-40728-4 71.

RStudio Team, 2016. RStudio: Integrated Development for R. RStudio, Inc., Boston, MA URL http://www.rstudio.com/.

Shi, B., P.Wang, J. Jiang, R. Liu, 2018. Applying high-frequency surrogate measurements and a Wavelet-ANN model to provide early warnings of rapid surface water quality anomalies. Science of the Total Environment 610 611, 1390-99. https://doi.org/10.1016/j.scitotenv.2017.08.232. 


\title{
Analysis Of Factors Affecting Ammonia And Methane Emissions From Pig Slurries: Slurry Composition And Dietary Factors

\author{
Walter Antezana $^{a, b}$, Alba Cerisuelo ${ }^{c}$, Fernando Estellés ${ }^{b}$, Salvador Calvet ${ }^{b^{*}}$ \\ ${ }^{1}$ Universidad Nacional de San Antonio Abad del Cusco, Facultad de Agronomía y Zootecnia. Av. De la Cultura. Cusco, \\ Perú \\ ${ }^{2}$ Universitat Politècnica de València, Institute of Animal Science and Technique. Camino de Vera s.n. 46022 Valencia, \\ Spain \\ ${ }^{3}$ Centro de Investigación y Tecnología Animal, Instituto Valenciano de Investigaciones Agrarias, Pol. La Esperanza \\ 100, 12400 Segorbe,Castellón, Spain \\ * Corresponding author. Email: salcalsa@upvnet.upv.es
}

\begin{abstract}
Reducing crude protein is a recommended technique to reduce nitrogen excretion and ammonia emissions. Greenhouse gas emissions from slurry are also affected by nutrient composition. However, there are interactions among nutrients in feeds still not clarified. The objective of this study was to evaluate the impacts of different diets on nitrogen $(\mathrm{N})$ and energy balances. A total of 13 diets were evaluated in digestibility trials using 78 animals. Diets were formulated to fulfil commercial standards, although differing in ingredient composition. Nutrient intake, excretion and potential $\mathrm{NH}_{3}$ and $\mathrm{CH}_{4}$ (Biochemical methane potential) emissions from slurry were measured. Animal weight was also monitored. Correlations between emissions and nutrient balance components were done. An analysis of variance was conducted to assess differences in nutrient balance of low, medium and high emitting animals, expressing animals per $\mathrm{kg}$ of live weight increased. For the $\mathrm{N}$ balance, a two-fold range in faeces to urine $\mathrm{N}$ excretion ratio was found throughout the experiments, even considering the low crude protein variations (from 15 to $16 \%$ ). This was related to the ammonia emissions from slurry $(\mathrm{r}=-0.60, \mathrm{p}<0.001$ ). In fact, the amount of crude protein ingested to increase $1 \mathrm{~kg}$ of metabolic weight was positively correlated with the associated emissions $(\mathrm{r}=0.58, \mathrm{p}<0.001)$. However, this was more related to the consumption by the animal than to the crude protein, which was relatively stable among diets. The difference between animals associated with high or low NH3 emissions per weight gain was therefore related to urine losses due to excess $\mathrm{N}$ intake. The energy balance shows that methane potential from slurry was mainly related to the excretion of indigested feed components, mainly the fibrous fraction (particularly the soluble fibre). It was clear that animals emitting high amounts of methane were those with higher dry matter and energy ingestion. The results of this study demonstrate relevant effects on $\mathrm{N}$ and energy balances at diets formulated according to commercial standards.
\end{abstract}

Keywords: Feeding strategies, $\mathrm{NH}_{3}, \mathrm{CH}_{4}$, Slurry, Nutrition balance.

\section{Introduction}

Animal nutrition plays an essential role in the sustainability of livestock production sector. Apart from being a major cost for most intensive livestock producers, it consumes a relevant share of agricultural crops. Therefore, the supply of feed ingredients is a major issue to be solved in the next decades (Lassaletta et al., 2018). Among others, focus is given to the use of more efficient feeds and to recycled feed ingredients such as agro-industrial by-products.

Using by-products contributes to a circularised economy and lowers the dependency on crops. However, the use of by-products for animal feeding poses several challenges. Crucial aspects to be considered are their availability, homogeneity and nutritional value, which are not always fulfilled by the different feedstocks. Composition of by-products is essential and fibrous by products have lower nutritive value for monogastrics compared to ruminants. Additionally, their productive and environmental implications must be evaluated in detail.

Modifying pig slurry characteristics through diet management is a valuable tool to mitigate gaseous emissions (Aarnink and Verstegen, 2007). Several studies have reported the effects of changing diets (particularly protein) on slurry composition (Rigolot et al., 2010, Jarret et al., 2011; Jarret et al., 2012). The relationship between slurry composition and ammonia $\left(\mathrm{NH}_{3}\right)$ and greenhouse gases $(\mathrm{GHG})$ emissions is also evident from these studies.

It has long been demonstrated that crude protein content is positively related to urinary nitrogen excretion and consequently $\mathrm{NH}_{3}$ emissions. However, it has been observed that for similar crude protein content the excretory pattern may also be altered because of other nutritional effects (e.g. different amount or type of fibre). Therefore, further efforts are needed to quantify the complex relationships among feed constituents, animal performance and emissions.

To this aim, we collected information from several studies evaluating nutrient balance and emissions from diets with similar nutritional content. The objective of this work was to analyse the effect of different diets including by-products on the nutritional (nitrogen and energy) balance at animal level. Effects on the related excretory patterns and gaseous $\left(\mathrm{NH}_{3}\right.$ and $\left.\mathrm{CH}_{4}\right)$ emissions were also examined. 


\section{Materials and Methods}

Description of diets

A meta-analysis was conducted using data from the assays described by Antezana et al. (2015); Beccaccia et al. (2015a, b), which analysed the effect of different sources of protein, fibre and fat on nutrition traits, slurry composition and gaseous emissions. All diets tested in these assays (13 diets; Table 1) were formulated according to commercial standards (FEDNA, 2006).

Table 71. Nutritional content (on fresh matter basis) of the experimental diets tested. The number of repetitions (animals) for each diet was $n=6$.

\begin{tabular}{lccccccccccccc}
\hline & \multicolumn{1}{c}{ Beccaccia et al., $2015 \mathrm{a}$} & \multicolumn{8}{c}{ Beccaccia et al., 2015b } & \multicolumn{2}{c}{ Antezana et al., 2015 } \\
\hline & $\# 1$ & $\# 2$ & $\# 3$ & $\# 4$ & $\# 5$ & $\# 6$ & $\# 7$ & $\# 8$ & $\# 9$ & $\# 10$ & $\# 11$ & $\# 12$ & $\# 13$ \\
\hline Dry matter & 912 & 902 & 903 & 895 & 899 & 884 & 896 & 891 & 905 & 905 & 909 & 899 & 889 \\
Ash & 51.8 & 53 & 54.3 & 52.1 & 51 & 57.9 & 52.7 & 50.7 & 45 & 46.4 & 47.8 & 49.4 & 52.1 \\
Crude protein & 158 & 156 & 154 & 153 & 157 & 153 & 164 & 159 & 146 & 145 & 147 & 146 & 144 \\
NDICP* & 21.4 & 26.7 & 21.2 & 23.8 & 20.8 & 9.66 & 12 & 17.4 & 16.1 & 15.8 & 21 & 20.9 & 26.3 \\
Ether extract & 43 & 53.3 & 62.9 & 59.2 & 72.1 & 60.1 & 51.3 & 47.5 & 31.2 & 51.5 & 83.6 & 54.6 & 75.5 \\
Soluble fibre & 61.2 & 75.9 & 97.3 & 60.1 & 71.4 & 62.3 & 17.1 & 25 & 28.4 & 44 & 37.7 & 95.6 & 106 \\
NDF* & 154 & 165 & 158 & 164 & 161 & 148 & 144 & 132 & 167 & 157 & 163 & 169 & 166 \\
ADF* & 45.6 & 52.3 & 56.3 & 61 & 75.4 & 73.6 & 49.4 & 31.5 & 50.8 & 44.7 & 48 & 60.3 & 59.7 \\
Lignin & 8 & 9 & 10.7 & 18.9 & 33.9 & 4.33 & 13.6 & 8.73 & 11 & 8.2 & 9.4 & 7.9 & 8.1 \\
GE (MJ/kg)* & 16.8 & 16.9 & 17 & 16.9 & 17.4 & 16.7 & 16.9 & 16.9 & 16.3 & 16.8 & 17.7 & 16.7 & 17.2 \\
NE (MJ/kg)* & 9.83 & 9.83 & 9.83 & 9.83 & 9.83 & 9.91 & 9.91 & 9.91 & 9.2 & 9.75 & 10.3 & 9.41 & 9.91 \\
\hline
\end{tabular}

*NDICP: Neutral detergent indigestible crude protein; NDF: Neutral detergent fibre; ADF: Acid detergent fibre; GE: Gross energy; NE: Net energy.

Experimental procedure

The experimental period consisted in a 14-day adaptation period to diets followed by a period of 7 consecutive days in which faeces and urine were collected individually (21 days in total). After an initial 9-day adaptation period, animals were individually housed in metabolism pens $(1.2 \times 2 \mathrm{~m} 2)$ until the end of the study. These pens allowed the measurement of individual feed intake and total and separate collection of faeces and urine. The collection period was divided in two parts to facilitate collections for energy and nutrient balance (Days 1-4) and gaseous emission study (Days 5-7). Pigs were individually weighed at the beginning of the adaptation period, at allocation in metabolism pens (Day 9 of experiment) and at the end of the experiment. Feed consumption was measured individually in the metabolism pens.

During the energy and nutrient balance (4 days), total urine and faeces excreted per animal were collected daily in separate buckets, weighed and stored in a chamber at $4{ }^{\circ} \mathrm{C}$ until the end of the collection period. To avoid nitrogen losses due to $\mathrm{NH}_{3}$ volatilization, urine was collected under sulphuric acid $(120 \mathrm{~mL}$ of $\mathrm{H} 2 \mathrm{SO} 4$ at $10 \%$ per bucket and day). Upon final collection the faeces and urine were pooled per pig, mixed, subsampled and stored at $-20^{\circ} \mathrm{C}$ until laboratory analyses were performed. During the next 3 days, urine and faeces were collected in a similar way, but without any addition of sulphuric acid to urine. At the end of the collection period slurries were reconstituted by mixing urine and faeces from each animal in the same proportion as excreted. A part of these slurries was used in fresh for $\mathrm{pH}$ and $\mathrm{NH}_{3}$ emission measurements and another one was subsampled and frozen ($20{ }^{\circ} \mathrm{C}$ ) for biochemical methane potential (BMP) and slurry characteristics determination.

Feeds and faeces from the nutrient balance period were analyzed for dry matter (DM), ash, neutral detergent fibre, acid detergent fibre and lignin, ether extract, gross energy $(\mathrm{GE})$, nitrogen $(\mathrm{N})$ and neutral detergent insoluble crude protein (NDICP) concentration as described in Beccaccia et al. (2015a). The TAN and TKN were determined by steam distillation (APHA, 2005) using an automatic analyser (2300 Kjeltec, Foss Analytical, Hilleroed, Denmark). To avoid N volatilization, the subsample used for TAN analyses was acidified with $\mathrm{HCl}$ immediately after reconstitution. Volatile fatty acids concentration was determined by gas chromatography equipped with a flame ionization detector (HP 68050 series Hewlet Packard, USA) following the method described by Jouany (1982) with the addition of an internal standard (4-metil valeric).

Ammonia emission was measured from fresh samples of reconstituted slurry over 11 days using an ammonia trap system. Slurry samples of $0.5 \mathrm{~kg}$ from each animal $+50 \mathrm{~mL}$ of distilled water to prevent surface crust formation, were placed in a $1 \mathrm{~L}$ closed container and maintained at $25^{\circ} \mathrm{C}$ in a thermostatic water bath (Selecta, Spain). Containers were connected to an air pump which extracted air at a constant airflow rate of $1.2 \mathrm{~L} / \mathrm{min}$. 
During 11 consecutive days, the air was forced to pass through two absorption flasks (impingers) in serial containing $100 \mathrm{~mL}$ of sulphuric acid $0.1 \mathrm{~N}$. The acid solution was replaced daily during the first 5 days, and every $48 \mathrm{~h}$ until the end of the assay (Day 11). The $\mathrm{NH}_{3}$ trapped in the impingers was analyzed following $4500 \mathrm{NH} 3-\mathrm{D}$ procedure (APHA, 2005) using a detection electrode (Orion High Performance $\mathrm{NH}_{3}$ Electrode, model 9512HPBNWP, Thermo Scientific, USA). The cumulative $\mathrm{NH}_{3}$ emission for each sample was calculated by adding the amount retained daily in the flasks during the experimental test.

Biochemical $\mathrm{CH}_{4}$ potential from slurry was measured as the cumulative $\mathrm{CH}_{4}$ production per gram of $\mathrm{OM}$ in a batch assay, using $120 \mathrm{~mL}$ glass bottles incubated at a mesophilic range $\left(35 \pm 1{ }^{\circ} \mathrm{C}\right)$ for 100 days, following the methodology described by Angelidaki et al. (2009). Anaerobic inoculum was pre-incubated during 15 days at $35^{\circ} \mathrm{C}$ in order to deplete the residual biodegradable organic material (degasification). A mixture of pooled slurry and inoculum was made to obtain an inoculum to substrate ratio of 1 on OM basis. Slurry samples from each animal were tested by triplicate. Additionally, three blank bottles containing only anaerobic inoculum were also used in order to determine its endogenous $\mathrm{CH}_{4}$ production. This was subtracted from the $\mathrm{CH}_{4}$ produced by the slurry on each biogas sampling day. After filling, each bottle was sealed with butyl rubber stoppers and aluminium crimps and the headspace was flushed with pure $\mathrm{N}_{2}$ for $2 \mathrm{~min}$. During incubation, biogas volume in each bottle was regularly monitored (from 1 to 10 days depending on biogas production) by pressure measurement of the headspace using a manometer (Delta Ohm, HD 9220, Italy). Methane concentration in the biogas was further analyzed using a Focus Gas Chromatograph (Thermo, Milan, Italy) equipped with a split/splitless injector and a flame ionization detector.

\section{Statistical analysis}

A balance of dry matter (DM), nitrogen (N) and gross energy (GE) was calculated at animal level based on live weight increase. As a result, we obtained values for intake, digestion (intake - faeces excretion), retention (intake - urine and faeces excretion), and total nutrient excretion. The amount of $\mathrm{NH}_{3}$ and $\mathrm{CH}_{4}$ emitted to increase $1 \mathrm{~kg}$ of live weight. The individual data of the pigs used in the three studies were sorted according to the $\mathrm{NH}_{3}$ and $\mathrm{CH}_{4}$ emissions. The range of emissions was divided into three categories according to the rank position (higher third, middle third and lower third of $\mathrm{NH} 3$ and $\mathrm{CH} 4$ emissions emitted per $\mathrm{kg}$ LW increase).

An analysis of variance showed that there were no statistical differences among studies for the above mentioned variables. Therefore, all data from the three studies were analysed together. Correlations were obtained between the nutrients ingested, digested, retained, excreted in faeces and urine and the $\mathrm{NH}_{3}$ and $\mathrm{CH}_{4}$ emissions using PROC CORR of SAS. For the variables correlated to the emissions, an analysis of variance (PROC GLM of SAS) was used to compare nutrient balance components of ranked emissions (higher, medium and lower) of $\mathrm{NH}_{3}$ and $\mathrm{CH}_{4}$.

\section{Results and Discussion}

A summary of average animal performance parameters and nutrient balance for the different diets is provided in Table 2. It can be observed that animal weight and balance components were different for diets \#1 to \#5 (Beccaccia et al., 2015a) compared to diets \#6 to \#13 (Beccaccia et al., 2015b and Antezana et al., 2015). The different animal live weight and average daily gain affected the absolute values of the nutrient balances. On average, nutrient retention was $80 \%, 55 \%$ and $81 \%$ for dry matter, nitrogen and gross energy, respectively. The percentage of $\mathrm{N}$ retained by the animals ranged from $51 \%$ (diet $\# 5$ ) to $64 \%$ (diet \#11). The percentage of dry matter and energy retained ranged from $77 \%$ (diet \#13) to $83 \%$ (diet \#1). 
Table 72. Summary of average animal performance, nutrient balance (dry matter, DM; nitrogen, N; gross energy, GE) and emissions for the different diets. The number of replicates was $n=6$ for each diet.

\begin{tabular}{lcccccccccccccc}
\hline Diet & $\# 1$ & $\# 2$ & $\# 3$ & $\# 4$ & $\# 5$ & $\# 6$ & $\# 7$ & $\# 8$ & $\# 9$ & $\# 10$ & $\# 11$ & $\# 12$ & $\# 13$ \\
\hline $\mathrm{LW}(\mathrm{kg})$ & 107,1 & 107,7 & 105,7 & 104,7 & 109,2 & 55,8 & 56,9 & 56,8 & 62,5 & 61,5 & 65,1 & 61,4 & 62,8 \\
$\Delta \mathrm{LW}(\mathrm{kg} / \mathrm{d})$ & 1,16 & 1,02 & 1,00 & 1,05 & 1,22 & 0,91 & 0,95 & 1,02 & 0,92 & 0,75 & 0,83 & 0,67 & 0,74 \\
DM intake (g/d) & 2437 & 2369 & 2272 & 2282 & 2597 & 1827 & 1703 & 1777 & 1655 & 1509 & 1726 & 1503 & 1560 \\
DM excreted as & 331 & 355 & 311 & 389 & 479 & 291 & 256 & 281 & 276 & 232 & 313 & 235 & 279 \\
faeces (g/d) & & & & & & & & & & & & & \\
DM excreted as & 89,9 & 103,3 & 90,1 & 94,1 & 114,2 & 80,4 & 73,7 & 66,1 & 79,4 & 68,0 & 60,7 & 77,8 & 84,7 \\
urine (g/d) & & & & & & & & & & & & & \\
N intake (g/d) & 67,5 & 65,5 & 62,0 & 62,4 & 72,6 & 52,2 & 49,9 & 49,2 & 42,7 & 38,7 & 44,7 & 39,1 & 40,4 \\
N faeces (g/d) & 9,57 & 11,39 & 11,44 & 12,23 & 15,68 & 10,38 & 7,50 & 11,22 & 8,79 & 6,38 & 8,80 & 8,82 & 9,79 \\
N urine (g/d) & 18,8 & 18,3 & 14,0 & 18,4 & 19,6 & 12,5 & 12,8 & 7,7 & 10,4 & 8,8 & 7,2 & 8,8 & 8,6 \\
GE intake (MJ/d) & 44,9 & 44,4 & 42,8 & 43,1 & 50,3 & 34,7 & 32,1 & 33,6 & 29,8 & 28,0 & 33,6 & 27,9 & 30,2 \\
GE faeces (MJ/d) & 6,05 & 6,58 & 5,89 & 7,30 & 9,19 & 5,46 & 4,67 & 5,22 & 5,14 & 4,54 & 6,70 & 4,84 & 6,05 \\
GE urine (MJ/d) & 1,01 & 1,10 & 1,14 & 0,93 & 1,17 & 0,74 & 0,77 & 0,58 & 0,80 & 0,67 & 0,59 & 0,84 & 0,95 \\
$\mathrm{CH}_{4}(\mathrm{~L} / \mathrm{animal} / \mathrm{d})$ & 116 & 126 & 110 & 123 & 139 & 74 & 66 & 83 & 75 & 65 & 106 & 70 & 94 \\
$\mathrm{NH}_{3}$ (g/animal/d) & 840 & 812 & 597 & 783 & 839 & 488 & 570 & 250 & 412 & 399 & 331 & 373 & 341 \\
\hline
\end{tabular}

From Table 2 it can be observed that animal weight was different among studies and was particularly higher in diets \#1 to \#5 described in Beccaccia et al. (2015a) compared to diets \#6 to \#13. Consequently, components of the nutrient balance and emissions expressed per animal were also different among studies. For this reason, in order to evaluate the real influence of feed composition, balance (DM, energy and protein) components and emissions were both expressed per kg live weight (LW) increase. Each experimental data was classified according to the ranked $\mathrm{NH}_{3}$ and $\mathrm{CH}_{4}$ emissions, resulting in the distribution per rank and diet shown in Table 3. In general terms, all experiments contributed to all ranks of emissions (low, medium and high).

Table 73. Number of experimental data per diet corresponding to lower, medium and higher $\mathrm{NH}_{3}$ and $\mathrm{CH}_{4}$ emissions.

\begin{tabular}{lccccccccccccc}
\hline & \multicolumn{1}{c}{ Beccaccia et al., 2015a } & \multicolumn{1}{c}{ Beccaccia et al., 2015b } & Antezana et al., 2015 & a \\
\hline Diet & $\# 1$ & $\# 2$ & $\# 3$ & $\# 4$ & $\# 5$ & $\# 6$ & $\# 7$ & $\# 8$ & $\# 9$ & $\# 10$ & $\# 11$ & $\# 12$ & $\# 13$ \\
\hline $\mathrm{NH}_{3}$ Low & & & 1 & 1 & & 4 & 2 & 4 & 3 & 2 & 4 & 2 & 3 \\
$\mathrm{NH}_{3}$ Medium & 2 & 3 & 3 & 1 & 2 & & 1 & 2 & 3 & 2 & 2 & 3 & 2 \\
$\mathrm{NH}_{3}$ High & 4 & 3 & 2 & 4 & 4 & 2 & 3 & & & 2 & & 1 & 1 \\
\hline $\mathrm{CH}_{4}$ Low & 3 & & & & 2 & 4 & 5 & 3 & 4 & 3 & & 2 \\
$\mathrm{CH}_{4}$ Medium & 2 & 2 & 4 & 4 & 1 & 1 & & 3 & 2 & 3 & 1 & 2 & 1 \\
$\mathrm{CH}_{4}$ High & 1 & 4 & 2 & 2 & 3 & 1 & 1 & & & & 5 & 2 & 5 \\
\hline
\end{tabular}

An analysis of correlations was conducted among gas emissions, animal performance and diet composition (Table 3). It was observed that $\mathrm{CH}_{4}$ and $\mathrm{NH}_{3}$ were positively correlated $(\mathrm{r}=0.31, \mathrm{p}<0.01)$. The emission of $\mathrm{CH}_{4}$ per $\mathrm{kg}$ of LW increase was positively correlated with LW $(\mathrm{r}=0.36, \mathrm{p}<0.01)$, but inversely correlated with the increment of live weight $(-0.37, \mathrm{p}<0.001)$. On the contrary, $\mathrm{NH}_{3}$ emissions per $\mathrm{kg}$ of $\mathrm{LW}$ increase was not correlated with LW increase, but strongly correlated with live weight $(r=0.52, p<0.001)$.

Methane production per kg LW increased was positively correlated with diet fat and energy contents, as well as with fermentable fibre. Crude protein was positively correlated with crude and indigestible protein, but was not correlated with dry matter, fat or energy contents of the feed. 
Table 74. Coefficients of correlation among gas emissions (expressed per kg live weight increased, $\Delta \mathrm{LW}$ ) animal performance and diet composition. The number of measurements was $n=78$. Statistical significance is also indicated (ns: not significant; $\mathrm{p}<0.05 * ; \mathrm{p}<0.01 * *, \mathrm{p}<0.001 * * *$ ).

\begin{tabular}{lll}
\hline Diet components $(\mathrm{g} / \mathrm{kg}$ fresh matter) & $\mathrm{CH}_{4}(\mathrm{~L} / \mathrm{kg} \mathrm{LW}$ increase $)$ & $\mathrm{NH}_{3}(\mathrm{~g} / \mathrm{kg}$ LW increase $)$ \\
\hline Dry matter & $0.12 \mathrm{~ns}$ & $0.20 \mathrm{~ns}$ \\
Crude protein & $-0.17 \mathrm{~ns}$ & $0.27^{*}$ \\
Indigestible CP & $0.55^{* * *}$ & $0.34^{* *}$ \\
Ether extract & $0.43^{* * *}$ & $-0.10 \mathrm{~ns}$ \\
$\mathrm{NDF}$ & $0.40^{* * *}$ & $0.08 \mathrm{~ns}$ \\
$\mathrm{ADF}$ & $0.19 \mathrm{~ns}$ & $-0.07 \mathrm{~ns}$ \\
Lignin & $0.16 \mathrm{~ns}$ & $0.25^{*}$ \\
Gross energy & $0.41^{* * *}$ & $0.02 \mathrm{~ns}$ \\
\hline
\end{tabular}

Despite the relatively similar nutrient content of diets shown in Table 1, there was a large variation of emissions expressed per kg LW increase for $\mathrm{CH}_{4}$ (Table 4) and $\mathrm{NH}_{3}$ (Table 5). For $\mathrm{CH}_{4}$, emissions in the higher range were on average almost two-fold compared to the lower range. Components of the nutrient balance corresponding to a $1 \mathrm{~kg} \mathrm{LW}$ increase were strongly correlated with $\mathrm{CH}_{4}$ emissions associated with that $\mathrm{kg} \mathrm{LW}$ increase (Table 5). The DM, N and GE intake needed to increase $1 \mathrm{~kg}$ of animal LW were higher in animals associated with higher $\mathrm{CH}_{4}$ emission. Only the ratio of $\mathrm{N}$ between faeces and urine was not affected by the emission rank. Remarkably, the retention of DM, N and GE to increase $1 \mathrm{~kg}$ LW also differed from animals with lower, medium and higher $\mathrm{CH}_{4}$ emissions.

Table 75. Nutrient balance components expressed per kg live weight (LW) increase, as a function of ranked (low, medium or high) $\mathrm{CH}_{4}$ emissions expressed as $\mathrm{L}$ per $\mathrm{kg}$ of live weight increase. The correlation coefficients (r) among balance

\begin{tabular}{|c|c|c|c|c|c|c|c|}
\hline & & $\mathrm{r}$ & $\begin{array}{l}\text { Low } \\
\mathrm{CH}_{4}\end{array}$ & $\begin{array}{c}\text { Medium } \\
\mathrm{CH}_{4}\end{array}$ & $\begin{array}{l}\text { High } \\
\mathrm{CH}_{4}\end{array}$ & SEM & $\mathrm{p}$-Value \\
\hline & $\mathrm{n}$ & & 26 & 26 & 26 & & \\
\hline & $\begin{array}{c}\mathrm{CH}_{4}(\mathrm{~L} / \mathrm{kg} \mathrm{LW} \\
\text { increase })\end{array}$ & & $74.6^{\mathrm{a}}$ & $99.0^{\mathrm{b}}$ & $141.1^{\mathrm{c}}$ & 2.90 & $<0.001$ \\
\hline \multirow{4}{*}{$\begin{array}{l}\text { DM balance } \\
\text { (g/kg LW } \\
\text { increase) }\end{array}$} & DM intake & $0.77 * * *$ & $1792^{\mathrm{a}}$ & $2113^{b}$ & $2436^{\mathrm{c}}$ & 63.5 & $<0.001$ \\
\hline & DM faeces & $0.84 * * *$ & $270^{\mathrm{a}}$ & $329^{\mathrm{b}}$ & $417^{\mathrm{c}}$ & 10.3 & $<0.001$ \\
\hline & DM urine & $0.43 * * *$ & $76.6^{\mathrm{a}}$ & $93.6^{\mathrm{b}}$ & $105.1^{\mathrm{b}}$ & 5.25 & 0.001 \\
\hline & DM retained & $0.71 * * *$ & $1445^{\mathrm{a}}$ & $1691^{\mathrm{b}}$ & $1914^{\mathrm{c}}$ & 54.9 & $<0.001$ \\
\hline \multirow{5}{*}{$\begin{array}{l}\mathrm{N} \text { balance } \\
(\mathrm{g} / \mathrm{kg} \mathrm{LW} \\
\text { increase) }\end{array}$} & $\mathrm{N}$ intake & $0.72 * * *$ & $49.2^{\mathrm{a}}$ & $57.0^{\mathrm{b}}$ & $65.8^{\mathrm{c}}$ & 1.86 & $<0.001$ \\
\hline & $\mathrm{N}$ faeces & $0.75^{* * *}$ & $8.71^{\mathrm{a}}$ & $10.81^{\mathrm{b}}$ & $13.85^{\mathrm{c}}$ & 0.46 & $<0.001$ \\
\hline & $\mathrm{N}$ urine & $0.43 * * *$ & $11.7^{\mathrm{a}}$ & $13.1^{\mathrm{a}}$ & $16.2^{\mathrm{b}}$ & 0.99 & 0.008 \\
\hline & $\mathrm{N}$ retained & $0.53 * * *$ & $28.8^{\mathrm{a}}$ & $33.1^{\mathrm{b}}$ & $35.8^{\mathrm{c}}$ & 1.25 & $<0.001$ \\
\hline & $\begin{array}{l}\text { Ratio } \mathrm{N} \\
\text { faeces:urine }\end{array}$ & $0.14 \mathrm{~ns}$ & 0.83 & 0.91 & 1.01 & 0.078 & 0.25 \\
\hline \multirow{4}{*}{$\begin{array}{l}\text { GE balance } \\
(\mathrm{mJ} / \mathrm{kg} \mathrm{LW} \\
\text { increase) }\end{array}$} & GE intake & $0.80 * * *$ & $33.5^{\mathrm{a}}$ & $39.7^{\mathrm{b}}$ & $46.4^{\mathrm{c}}$ & 1.16 & $<0.001$ \\
\hline & GE faeces & $0.85^{* * *}$ & $5.11^{\mathrm{a}}$ & $6.30^{\mathrm{b}}$ & $8.30^{c}$ & 0.20 & $<0.001$ \\
\hline & GE urine & $0.45^{* * *}$ & $0.75^{\mathrm{a}}$ & $0.99^{\mathrm{b}}$ & $1.15^{\mathrm{b}}$ & 0.065 & $<0.001$ \\
\hline & GE retained & $0.72 * * *$ & $27.6^{\mathrm{a}}$ & $32.4^{\mathrm{b}}$ & $37.0^{\mathrm{c}}$ & 1.04 & $<0.001$ \\
\hline
\end{tabular}

There was a variation of more than two-fold for $\mathrm{NH}_{3}$ emissions expressed per $\mathrm{kg} \mathrm{LW}$ increase when comparing the higher emission range with the lower range (Table 5). Similar to the ranked $\mathrm{CH}_{4}$ emissions presented in Table 4, the components of the nutrient (DM, N and GE) balances changed with the $\mathrm{NH}_{3}$ emissions per $\mathrm{kg} \mathrm{LW}$ increased. Emissions were higher for animals which needed more nutrients to produce a kg of live weight. On the contrary, lower emissions were critically related with urinary losses, and therefore, negatively correlated with the ratio $\mathrm{N}$ urine : $\mathrm{N}$ faeces. These findings are coincident with the idea that animals using resources more efficiently reduce their emissions. 
Table 76. Nutrient balance components expressed per kg live weight (LW) increase, as a function of ranked (low, medium or high) $\mathrm{NH}_{3}$ emissions expressed as $\mathrm{mg} / \mathrm{d}$ per $\mathrm{kg}$ of live weight increase. The correlation coefficients ( $\mathrm{r}$ ) among balance

\begin{tabular}{|c|c|c|c|c|c|c|c|}
\hline & Variable & $\mathrm{r}$ & $\begin{array}{l}\text { Low } \\
\mathrm{NH}_{3}\end{array}$ & $\begin{array}{c}\text { Medium } \\
\mathrm{NH}_{3} \\
\end{array}$ & $\begin{array}{l}\text { High } \\
\mathrm{NH}_{3}\end{array}$ & SEM & p-Value \\
\hline & $\mathrm{n}$ & & 26 & 26 & 26 & & \\
\hline & $\begin{array}{r}\mathrm{NH}_{3}(\mathrm{mg} / \mathrm{d} / \mathrm{kg} \\
\mathrm{LW} \text { increase })\end{array}$ & & $337^{\mathrm{a}}$ & $530^{\mathrm{b}}$ & $860^{c}$ & 27.2 & $<0.001$ \\
\hline \multirow{4}{*}{$\begin{array}{l}\text { DM balance } \\
\text { (g/kg LW increase) }\end{array}$} & DM intake & $0.52 * * *$ & $1840^{\mathrm{a}}$ & $2180^{\mathrm{b}}$ & $2322^{b}$ & 72 & $<0.001$ \\
\hline & DM faeces & $0.33^{* *}$ & $304^{\mathrm{a}}$ & $348^{\mathrm{b}}$ & $363^{\mathrm{b}}$ & 15.0 & 0.018 \\
\hline & DM urine & $0.48^{* * *}$ & $75.1^{\mathrm{a}}$ & $93.6^{\mathrm{b}}$ & $106.7^{\mathrm{b}}$ & 5.13 & $<0.001$ \\
\hline & DM retained & $0.52 * * *$ & $1460^{\mathrm{a}}$ & $1737^{\mathrm{b}}$ & $1851^{\mathrm{b}}$ & 58.3 & $<0.001$ \\
\hline \multirow{5}{*}{$\begin{array}{l}\mathrm{N} \text { balance } \\
\text { (g/kg LW increase) }\end{array}$} & $\mathrm{N}$ intake & $0.55^{* * *}$ & $49.5^{\mathrm{a}}$ & $58.4^{\mathrm{b}}$ & $64.1^{\mathrm{c}}$ & 1.96 & $<0.001$ \\
\hline & $\mathrm{N}$ faeces & $0.15 \mathrm{~ns}$ & 10.3 & 11.7 & 11.4 & 0.61 & 0.21 \\
\hline & $\mathrm{N}$ urine & $0.78^{* * *}$ & $10.1^{\mathrm{a}}$ & $12.4^{\mathrm{b}}$ & $18.6^{\mathrm{c}}$ & 0.78 & $<0.001$ \\
\hline & $\mathrm{N}$ retained & $0.26^{*}$ & $29.2^{\mathrm{a}}$ & $34.3^{\mathrm{b}}$ & $34.2^{\mathrm{b}}$ & 1.29 & 0.008 \\
\hline & $\begin{array}{l}\text { Ratio N } \\
\text { faeces:urine }\end{array}$ & $-0.60 * * *$ & $1.14^{\mathrm{a}}$ & $0.97^{\mathrm{a}}$ & $0.62^{\mathrm{b}}$ & 0.067 & $<0.001$ \\
\hline \multirow{4}{*}{$\begin{array}{l}\text { GE balance } \\
\text { (MJ/kg LW increase) }\end{array}$} & GE intake & $0.51 * * *$ & $34.8^{\mathrm{a}}$ & $40.9^{b}$ & $43.8^{b}$ & 1.38 & $<0.001$ \\
\hline & GE faeces & $0.24 *$ & 6.03 & 6.80 & 6.88 & 0.32 & 0.12 \\
\hline & GE urine & $0.47 * * *$ & $0.754^{\mathrm{a}}$ & $0.961^{\mathrm{b}}$ & $1.17^{\mathrm{c}}$ & 0.064 & $<0.001$ \\
\hline & GE retained & $0.53^{* * *}$ & $28.0^{\mathrm{a}}$ & $33.2^{\mathrm{b}}$ & $35.7^{\mathrm{b}}$ & 1.12 & $<0.001$ \\
\hline
\end{tabular}

Remarkably, there was a significant difference in energy and protein balances when comparing low, medium and high $\mathrm{CH}_{4}$ and $\mathrm{NH}_{3}$ emissions. Apart from potential measurement uncertainties, results suggest that there might nutritional changes affecting the deposition of dry matter and energy per kg of live weight increase. This would involve relevant productive implications, not only considering animal productivity but also in terms of meat quality.

Finally, it must be stressed that animals within each diet were distributed among the different ranked emissions. This suggests an important individual source of variability (even considering that animals belonged to the same genetics). Feed and microbiome relationships may arise as a relevant way to assess this variability and improve results at practical level, as suggested for poultry (Torok et al., 2011) and cattle (Myer et al., 2015).

\section{Conclusions}

This study evidences a high effect on $\mathrm{NH} 3$ and $\mathrm{CH} 4$ emissions for diets formulated with commercial standards, with a low range in crude protein and energy contents. The differences in emissions expressed per $\mathrm{kg}$ of live weight increase were related with nutrient consumption patterns and balances. Efforts can be addressed to investigate the interactions between diet and individual effects.

\section{Acknowledgements}

This project was funded by the Spanish Ministry of Science and Innovation (AGL2014-56653).

\section{References}

Aarnink, A.J.A., Verstegen, M.W.A., 2007. Nutrition, key factor to reduce environmental load from pig production. Livest. Sci. 109, 194-203. DOI: 10.1016/j.livsci.2007.01.112.

Angelidaki, I., Alves, M., Bolzonella, D., Borzacconi, L., Campos, J.L., Guwy, a. J., Kalyuzhnyi, S., Jenicek, P., van Lier, J.B., 2009. Defining the biomethane potential (BMP) of solid organic wastes and energy crops: a proposed protocol for batch assays. Water Sci. Technol. 59, 927. DOI: 10.2166/wst.2009.040.

Antezana, W., Calvet, S., Beccaccia, A., Ferrer, P., De Blas, C., García-Rebollar, P., Cerisuelo, A., 2015. Effects of nutrition on digestion efficiency and gaseous emissions from slurry in growing pigs: III. Influence of varying the dietary level of calcium soap of palm fatty acids distillate with or without orange pulp supplementation. Anim. Feed Sci. Technol. 209, 128-136. DOI: 10.1016/j.anifeedsci.2015.07.022. 
Beccaccia, A., Calvet, S., Cerisuelo, A., Ferrer, P., García-Rebollar, P., De Blas, C., 2015a. Effects of nutrition on digestion efficiency and gaseous emissions from slurry in growing-finishing pigs. I. Influence of the inclusion of two levels of orange pulp and carob meal in isofibrous diets. Anim. Feed Sci. Technol. 208, 158-169. DOI: 10.1016/j.anifeedsci.2015.07.008.

Beccaccia, A., Cerisuelo, A., Calvet, S., Ferrer, P., Estellés, F., De Blas, C., García-Rebollar, P., 2015b. Effects of nutrition on digestion efficiency and gaseous emissions from slurry in growing pigs: II. Effect of protein source in practical diets. Anim. Feed Sci. Technol. 209, 137-144. DOI: 10.1016/j.anifeedsci.2015.07.021.

FEDNA, 2006. In: De Blas, C., Gasa, J., Mateos, G.G. (Eds.), Necesidades nutricionales para ganado porcino: normas FEDNA. Fundación Española para el Desarrollo de la Nutrición Animal, Madrid, Spain, 55 pp.

Jarret, G., Cozannet, P., Martinez, J., Dourmad, J.Y., 2011. Effect of different quality wheat dried distiller's grain solubles (DDGS) in pig diets on composition of excreta and methane production from faeces and slurry. Livestock Science 140, 275-282. DOI: 10.1016/j.livsci.2011.04.006.

Lassaletta, L., Estellés, F., Beusen, A., Bouwman, L., Calvet, S., van Grinsven, H., Doelman, J., Stehfest, E., Uwizeye, A., Westhoek, H. 2018. A Model to assess Environmental Efficiency of Pig Production Systems. 2018 AgEng Conference. Wageningen, The Netherlands.

Myer, P.R., Smith, T.P., Wells, J.E., Kuehn, L.A. and Freetly, H.C., 2015. Rumen microbiome from steers differing in feed efficiency. PloS one, 10(6), p.e0129174. DOI: 10.1371/journal.pone.0129174.

Ndegwa, P.M., Vaddella, V.K., Hristov, a N., Joo, H.S., 2009. Measuring concentrations of ammonia in ambient air or exhaust air stream using acid traps. J. Environ. Qual. 38, 647-653. DOI: 10.2134/jeq2008.0211.

Rigolot, C., Espagnol, S., Pomar, C. and Dourmad, J.Y. 2010. Modelling of manure production by pigs and $\mathrm{NH} 3, \mathrm{~N} 2 \mathrm{O}$ and $\mathrm{CH} 4$ emissions. Part I: animal excretion and enteric $\mathrm{CH} 4$, effect of feeding and performance. Animal 4 (8): 1401-1412. DOI: 10.1017/S1751731110000492

Torok, V.A., Hughes, R.J., Mikkelsen, L.L., Perez-Maldonado, R., Balding, K., MacAlpine, R., Percy, N.J. and Ophel-Keller, K., 2011. Identification and characterization of potential performance-related gut microbiotas in broiler chickens across various feeding trials. Applied and Environmental Microbiology, 77(17), pp.5868-5878. DOI: 10.1128/AEM.00165-11 


\section{Topic 8: Plant production technologies (PP)}

Technology and management systems to control weeds, diseases and pest in crops, and equipment for seeding, maintenance and harvest. Contributions may include automation and autonomous systems, precision farming / agriculture, integrated pest management, precision spraying, geoinformation systems, and related applications of drones.

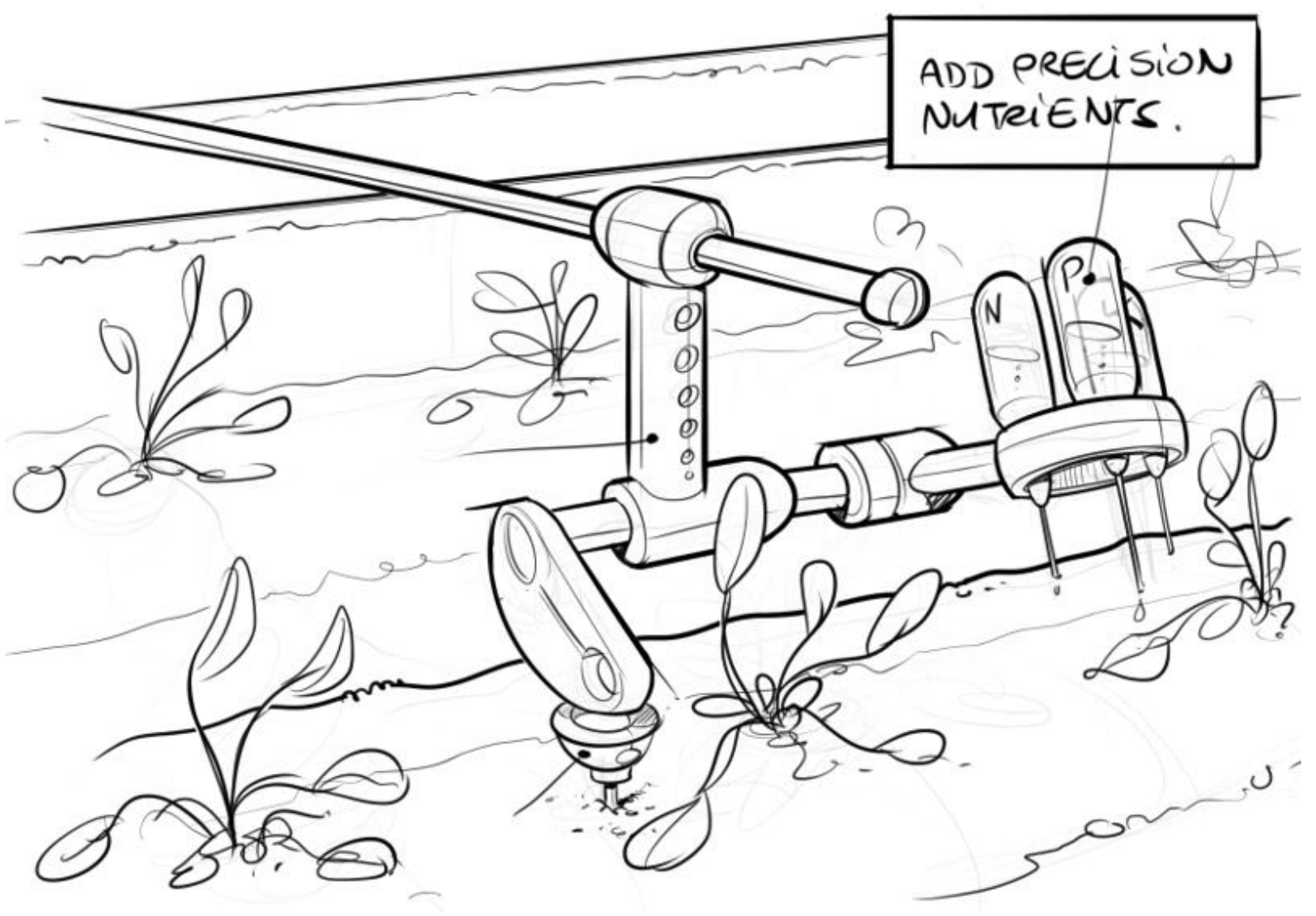




\author{
Mechanical Pruning of Lemon Trees \\ Bernardo Martin-Gorriz ${ }^{\text {a, }}$, Antonio Torregrosa ${ }^{\text {b }}$ Carlos Martinez Barba ${ }^{\text {a }}$ \\ ${ }^{a}$ Universidad Politécnica de Cartagena. Área de Ingeniería Agroforestal. Paseo Alfonso XIII n48, 30203, Cartagena, \\ Spain \\ ${ }^{\mathrm{b}}$ Universitat Politècnica de València. Dpto Ingeniería Rural y Agroalimentaria. Camino de Vera s/n, 46022, Valencia, \\ Spain \\ * Corresponding author. Email: b.martin@upct.es
}

\begin{abstract}
Manual pruning of lemons in the Southeast of Spain entails approximately $39 \%$ of the total labour costs of the crop. Mechanical pruning can be integrated into a management strategy to reduce pruning costs. However, this technique is not widely accepted by farmers for many reasons, such as a lack of rigorous studies with varieties and farm management justifying its potential benefits. This research evaluates mechanical pruning combined with manual pruning in 'Fino 95' lemons over four years. Five pruning treatments have been carried out with different intensities of mechanisation: hand pruning (Mcontrol) and four types of mechanical pruning: FTDR/FTIR (mechanical skirting, topping and hedging of one face of the orchard plus manual pruning of the opposite face to the one hedged), FTR (mechanical skirting and topping plus manual pruning of all the tree), FTDI (mechanical skirting, topping and both faces hedging). Moreover, each year different types of pruning have been applied to each tree. In this work the results of the two first years of experiments are shown, which include the following combinations (year 1+year 2): FTDR+FTIR, Mcontrol+Mcontrol, FTR+FTR, FTDI+hand, FTD+FTI. Yield, fruit size, fruit quality, pruned biomass and pruning cost have been analysed. The accumulated production was significantly higher in the treatment of mechanical pruning with alternate faces hedging during the two years and without manual intervention (FTD+FTI). On the other hand, the least productive treatment was the one where trees were pruned mechanically with a subsequent hand pruning. The treatment with the highest yield was the one with the lowest biomass cut. Additionally, fruit size was inversely proportional to yield.
\end{abstract}

Keywords: Citrus, Crop management, Mechanisation, Lemon, Fino 95.

\title{
1. Introduction
}

Spain is the second largest producer of lemons in the world, after Argentina, maintaining the world leader position of lemon exports for fresh consumption (USDA, 2016). The main use of Spanish lemons is for the fresh market. Eighty percent of the lemon production is concentrated in the Southeast of Spain (Murcia and Alicante) (MAGRAMA, 2014). 'Fino 95' is the leading lemon variety grown in Spain (Porras, 2014). However, despite lemon cultivation playing a major role in Southeast Spain, mechanical pruning has yet to become a widespread practice.

Pruning practices in citriculture are important to support plant health and reduce stress in order to reach an acceptable balance between vegetative and reproductive activities, a key factor in many stages of citrus grove development (Intrigliolo and Roccuzzo, 2011).

Pruning is, after harvesting, the second most expensive operation in the cultivation of citrus fruits. Currently, hand pruning in lemon orchards in Spain requires on average $100 \mathrm{~h} / \mathrm{ha}$ and entails approximately $39 \%$ of the total labour costs of the whole cultivation cycle (Martin-Gorriz et al, 2017), it is therefore quite interesting to mechanise this task and thus reduce crop costs.

Mechanical pruning allows the management of tree dimensions, which makes other tasks like spraying and harvesting easier (Boswell et al., 1977). Nowadays, mechanical pruning, either alone or combined with hand pruning, is used by some Spanish farmers. It is not, however, a technique that is widely accepted by growers, among other reasons due to a lack of experience.

In Spain, the first trials were carried out in the 1970s-1980s (Ortiz-Cañavate, 1979; Zaragoza and Alonso, 1980, 1981). Zaragoza and Alonso (1980, 1981) compared non-pruning, hand pruning, mechanical pruning, and mechanical pruning followed up by hand pruning. After one year of pruning, all the trees remained without pruning the following year, and the experiment was conducted over four years and on two orange varieties, 'Washington Navel' and 'Salustiana'. They noticed that in the year of pruning, the yield in the pruned trees decreased with respect to the unpruned trees, but that in the following year, when all the trees remained unpruned, yields were similar in all the treatments. On average for the two biennia, the yield in all pruning treatments of 'Washington Navel' oranges was lower than in non-pruning treatments (14\%). In 'Salustiana' oranges, however, there were no differences between unpruned or hand-pruned trees, but there was a reduction of $17 \%$ in the yield of those that were pruned mechanically with respect to the unpruned ones. There were no differences between trees pruned mechanically and those that were pruned first mechanically and then followed up by hand. Fruit size was inversely proportional to yield, but no appreciable differences were observed among pruning treatments in 
terms of the soluble solid content, acidity or maturity index. Since then, very few experiences on mechanical pruning have been published in Spain. The first example is that of Velazquez and Fernández (2010) who carried out an experiment with several pre-pruning hedging and topping cutting planes combined or not with handpruning, but the experiment comprised only one year of experiences and over a limited number of trees $(5$ trees/treatment). The main conclusions of the work were related to biomass waste and pruning costs, since conclusions regarding yield require longer period of experiences. On the other hand, Martin-Gorriz el al. (2014) recently compared, over a three-year period, mechanical pruning followed up by hand pruning versus manual pruning in the case of 'Fortune' mandarins. The results showed that there were no significant differences in yield when mechanical pruning was alternated with hand pruning over the years. However, when only mechanical pruning was used for the three years, a $22 \%$ reduction in yield was observed with respect to the treatment involving hand pruning alone. From the economic point of view, mechanical pruning shortened the time needed to complete the follow-up hand pruning by $13 \%$ with respect to only hand pruning, but this reduction in labour costs did not offset the cost of the mechanical equipment.

In other Mediterranean countries such as Italy or Turkey, pruning tests have reported similar results (Raciti et al., 1982; Spina et al., 1984; Giametta and Zimbalatti, 1992; Yildirim et al., 2010; Intrigliolo and Roccuzzo, 2011). It is a well-proven fact that yield decreases in the year in which mechanical pruning is performed, but if the effect of mechanical pruning is analysed over several years, this adverse effect disappears because the tree compensates for the previous reduction in the non-pruning years, probably as a consequence of the accumulation of reserves and better lighting (Zaragoza and Alonso, 1980; Fallahi and Kilby, 1997; Kallsen, 2005; Rouse et al., 2006; Mendonça et al., 2008; Sauls, 2008; Yildirim et al., 2010).

Pruning citrus trees must be a general canopy-management strategy based on the understanding of specific pruning and regrowth management practices that must be combined with cost-effective methods adapted to each orchard period: growth, full production and old trees decline due to age and/or shading (Krajewski and Krajewski, 2011). Mechanical pruning can be integrated into this general strategy.

The aim of the present research was to obtain detailed information on the response of 'Fino 95' lemon trees to mechanical pruning with different levels of severity and how it should be integrated with manual pruning into a management strategy to reduce pruning costs. Such information can aid the farmers in making pruning decisions for the production of fresh market fruit.

\section{Materials and Methods}

Experimental site

Trials were performed in an orchard of 'Fino 95' (Citrus limon L. Burm F.) lemons grafted on 'Cleopatra' (Citrus $\times$ reshni) mandarins, around 20 years old, planted within a frame with an in-row spacing of $6 \mathrm{~m}$ and 7.5 $\mathrm{m}$ between rows (222 trees/ha), and trees reached heights of $4 \mathrm{~m}$. The field, with a total area of 300 ha, was located in 'El Cañarico', Alhama de Murcia (Spain). Five rows with 15 trees per row were used for the pruning trials. The 75 trees selected were uniform in size and vigour. Pruning of the first year of trial was carried out on March 10th and 18th, 2016, and the second year from March 15th to 17th, 2017.

\section{Equipment used}

Manual pruning was conducted with secateurs and hand saws. This labour was carried out by a team of 10 specialised workers who perform this work annually on the farm.

Mechanical pruning was performed with a pruner (Industrias David, Yecla, Murcia, Spain) consisting of a linear arm equipped with five shearing discs. Each disc was driven by a hydraulic motor. The pruner was hitched onto the front of a narrow tractor (Kubota M8540, $63.7 \mathrm{~kW}$ ). The skirts were pruned with a manual hedge trimmer STIHL HS-82 R $60 \mathrm{~cm}$.

\section{Experimental design of trials}

A Latin square ( 5 treatment $x 5$ rows) with three trees per plot was used as the experimental design of the trials (Fig. 1). The pruning trials are to be carried out over a 4-year period.

Table 1 shows the pruning schedule of each treatment for the four years. Each pruning treatment is composed of one or more of the tasks described below:

- Hand pruning of the tree (Mcontrol). This is the control treatment performed by the same team of workers for the two years of trials.

- Manual pruning in treatments 3 and 4 was performed after the mechanical pruning and consisted of: i) manual follow-up and vegetative shoot removal between top and skirt of the tree in treatment 3 , and ii) manual pruning of one side of the tree; the other side had already been mechanically pruned in treatment 4 . 
- Mechanical hedging and topping was carried out with a pruning machine hitched onto the front of a narrow tractor. Double slope topping and two side hedging (treat. 2), and double slope topping and one side hedging (treat. 4 and 5) were performed. Topping and hedging was done at approximately $15^{\circ}$ and $10^{\circ}$ inclination on both sides, respectively.

- Skirt pruning was carried out with a manual hedge trimmer. This task was conducted in all the treatments in which topping was done.

\begin{tabular}{|c|l|l|l|l|l|}
\hline & Row & & & & \\
\hline Tree & \multicolumn{1}{|c|}{1} & \multicolumn{1}{|c|}{2} & \multicolumn{1}{c|}{3} & \multicolumn{1}{|c|}{4} & \multicolumn{1}{|c|}{5} \\
\hline 1 & Mcontrol & FTD & FTDR & FTR & FTDI \\
\hline 2 & Mcontrol & FTD & FTDR & FTR & FTDI \\
\hline 3 & Mcontrol & FTD & FTDR & FTR & FTDI \\
\hline 4 & FTDI & Mcontrol & FTD & FTDR & FTR \\
\hline 5 & FTDI & Mcontrol & FTD & FTDR & FTR \\
\hline 6 & FTDI & Mcontrol & FTD & FTDR & FTR \\
\hline 7 & FTR & FTDI & Mcontrol & FTD & FTDR \\
\hline 8 & FTR & FTDI & Mcontrol & FTD & FTDR \\
\hline 9 & FTR & FTDI & Mcontrol & FTD & FTDR \\
\hline 10 & FTDR & FTR & FTDI & Mcontrol & FTD \\
\hline 11 & FTDR & FTR & FTDI & Mcontrol & FTD \\
\hline 12 & FTDR & FTR & FTDI & Mcontrol & FTD \\
\hline 13 & FTD & FTDR & FTR & FTDI & Mcontrol \\
\hline 14 & FTD & FTDR & FTR & FTDI & Mcontrol \\
\hline 15 & FTD & FTDR & FTR & FTDI & Mcontrol \\
\hline
\end{tabular}

Figure 1. Latin square design for field trials in the first year.

Table 1. Pruning schedule of each treatment for the four years.

\begin{tabular}{|c|c|c|c|c|c|}
\hline $\begin{array}{l}\text { Treat- } \\
\text { ment }\end{array}$ & Year 1 & Year 2 & Year 3 & Year 4 & Comments \\
\hline 1 & Mcontrol & Mcontrol & Mcontrol & Mcontrol & Manual pruning (control). \\
\hline 2 & FTDI & M & FTDI & M & $\begin{array}{l}\text { Mechanical pruning (skirt, top and two sides) in odd } \\
\text { years and manual pruning in even years. }\end{array}$ \\
\hline 3 & FTR & FTR & FTR & FTR & $\begin{array}{l}\text { Mechanical pruning (skirt and top) with manual follow- } \\
\text { up. }\end{array}$ \\
\hline 4 & FTDR & FTIR & FTDR & FTIR & $\begin{array}{l}\text { Mechanical pruning (skirt, top and one side) plus } \\
\text { manual pruning of the other side of the tree. }\end{array}$ \\
\hline 5 & FTD & FTI & FTD & FTI & $\begin{array}{l}\text { Mechanical pruning (skirt, top and one side). North side } \\
\text { (D) is pruned in odd years and South side (I) in even } \\
\text { years. }\end{array}$ \\
\hline
\end{tabular}

Legend: Mcontrol = Manual pruning (control); $\mathrm{M}=$ Manual pruning; $\mathrm{F}=$ Mechanical skirt pruning; $\mathrm{T}=$ Mechanical top pruning; $\mathrm{D}=$ Mechanical pruning of South side hedge; $\mathrm{I}=$ Mechanical pruning of North side hedge; $\mathrm{R}=$ Manual finishing pruning after mechanical pruning.

During the pruning time the following variables were analysed:

- Pruned biomass characterisation. Pruning disposals were weighed and the diameters and length of the cut branches were measured before they were shredded on the ground. Three trees were measured per treatment.

- Productivity (h/ha) of hand and mechanical pruning. In the case of hand pruning, the time taken by a team of ten workers was measured. Productivity of mechanical pruning was calculated according to the number of passes that the machine performed per row; the tractor advance speed $(\mathrm{km} / \mathrm{h})$ and considering $15 \%$ of the time used in manoeuvres to change row.

During harvesting time the following variables were analysed:

- Yield per tree $(\mathrm{kg} / \mathrm{tree})$. The production of twenty-five trees was weighed, i.e. one tree for each treatment and row.

- Fruit equatorial diameter. At harvest, a random sample of 100 fruits per tree were measured with a calliper.

Statistical analyses were performed using a commercially available statistics package (Statgraphics Plus, version 5.1, STSC Inc., Rockville, MD, USA). 


\section{Results}

Total working time

Table 2 shows the total working time (h/ha) by pruning treatment in 2016 and 2017, the total time in two years and the percentage of productivity in relation to the hand-pruned control treatment. Contrary to what one might think, using mechanical pruning equipment combined with manual pruning does not always reduce the working time. The working time in treatment 3 was $9 \%$ higher than the control treatment. However, in treatment 4 the work load was reduced by $12 \%$. This disparity between the results may be due to the fact that the time the workers spent in the task of manual pruning was different each year, despite being the same task. A good option is to alternate mechanical pruning and manual pruning annually as in treatment 2 , where the pruning time was reduced by $29 \%$. Treatment 5 , where only mechanical pruning was performed, obtained the greatest reduction in pruning time $(94 \%)$.

Table 2. Total working time per pruning treatment in 2016 and 2017.

\begin{tabular}{|c|c|c|c|c|c|c|}
\hline Treatment & $\begin{array}{l}\text { Trials } \\
2016 \\
\end{array}$ & $\begin{array}{c}\text { Total pruning time } \\
2016(\mathrm{~h} / \mathrm{ha})\end{array}$ & $\begin{array}{r}\text { Trials } \\
2017 \\
\end{array}$ & $\begin{array}{c}\text { Total pruning } \\
\text { time } 2017(\mathrm{~h} / \mathrm{ha})\end{array}$ & $\begin{array}{c}\text { Total pruning time } \\
\text { two years }(\mathrm{h} / \mathrm{ha})\end{array}$ & $\begin{array}{c}\text { Percentage } \\
(\%)\end{array}$ \\
\hline 1 & Mcontrol & 107.30 & Mcontrol & 55.50 & 162.80 & 100 \\
\hline 2 & FTDI & 6.97 & M & 108.53 & 115.51 & 71 \\
\hline 3 & FTR & 99.69 & FTR & 77.49 & 177.17 & 109 \\
\hline 4 & FTDR & 64.43 & FTIR & 79.23 & 143.66 & 88 \\
\hline 5 & FTD & 5.23 & FTI & 5.23 & 10.46 & 6 \\
\hline
\end{tabular}

Legend: Mcontrol = Manual pruning (control); $\mathrm{M}=$ Manual pruning; $\mathrm{F}=$ Mechanical skirt pruning; $\mathrm{T}=$ Mechanical top pruning; $\mathrm{D}=$ Mechanical pruning of South side hedge; $\mathrm{I}=$ Mechanical pruning of North side hedge; $\mathrm{R}=$ Manual finishing pruning after mechanical pruning.

Fig. 2 shows the annual and accumulated cost of pruning treatments. This has been calculated according to the information provided by farmers, i.e. $8 € / \mathrm{h}$ for manual pruning and $35 € / \mathrm{h}$ for mechanical pruning. The cost of pruning was higher in the treatment with manual and mechanical pruning in the same year than in the control treatment (23\% in treat. 3 and $8 \%$ in treat. 4). Consequently, from the economic point of view it is not advisable to perform mechanical and manual pruning in the same year. However, treatment 5 , where only mechanical pruning was performed, obtained the greatest reduction in pruning cost (92\%). Perhaps the most attractive option for the farmers could be treatment 2, because pruning mechanically or manually in alternate years reduced the pruning cost by $15 \%$. This could be interesting on large farms where the owners have difficulties in finding workers.

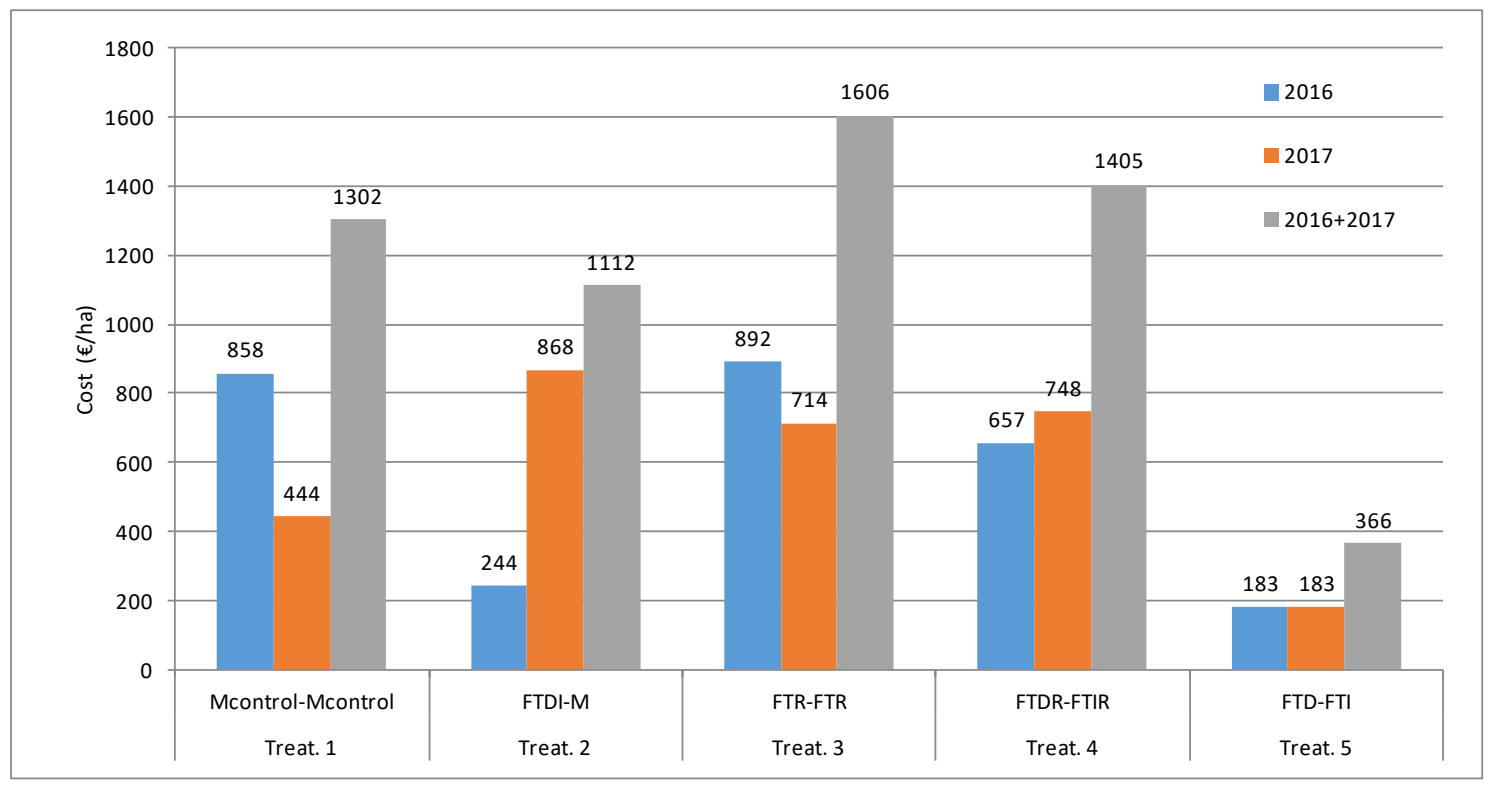

Figure 2. Annual and two years' accumulated cost per pruning treatment (€/ha). 


\section{Pruned biomass}

Table 3 shows the average values of biomass removed per treatment in each year and the total biomass removed in two years of trials. In both years the results show significant differences among the treatments. In the first year, manual pruning (Mcontrol) was the most severe treatment $(65.3 \mathrm{~kg} / \mathrm{tree})$, followed by the treatments involving manual and mechanical pruning (treat. 3 and 4), and the lightest were the treatments with only pruning treatments using mechanical pruning (treat. 2 and 5). In the second year, the hand-pruned treatment (M) was the most severe, although in 2016 it had been a light pruning (FTDI in treat. 2). Then came the treatments which carried out manual and mechanical pruning and the control (treat. 3, 4 and 1), and the least severe was the treatment with only the pruning machine (FTI in treat. 5), even though it was double the amount pruned in 2016 in the same treatment but on the South side of the row (FTD in treat. 5). On the other hand, the analysis of the total amount of biomass pruned in two years shows that treatments 1 and 2 were the most severe, whilst the least severe was the treatment using only mechanical pruning (treat. 5). It should be noted that in the control treatment of manual pruning, in 2017 the workers removed around fifty percent less wood than in 2016. This is in agreement with the time spent for the task (Table 2).

Table 3. Annual and accumulated biomass removed in two years per pruning treatment.

\begin{tabular}{cccccc}
\hline Treatment & Year 2016 & $\begin{array}{c}\text { Biomass 2016 } \\
(\mathrm{kg} / \text { tree })\end{array}$ & Year 2017 & $\begin{array}{c}\text { Biomass 2017 } \\
(\mathrm{kg} / \text { tree })\end{array}$ & $\begin{array}{c}\text { Total biomass } \\
\text { two years }(\mathrm{kg} / \text { tree })\end{array}$ \\
\hline 1 & Mcontrol & $65.3 \mathrm{c}$ & Mcontrol & $35.8 \mathrm{~b}$ & $101.1 \mathrm{c}$ \\
2 & FTDI & $9.0 \mathrm{a}$ & M & $85.2 \mathrm{c}$ & $94.2 \mathrm{c}$ \\
3 & FTR & $29.5 \mathrm{~b}$ & FTR & $36.3 \mathrm{~b}$ & $65.8 \mathrm{~b}$ \\
4 & FTDR & $31.5 \mathrm{~b}$ & FTIR & $39.7 \mathrm{~b}$ & $71.2 \mathrm{~b}$ \\
5 & FTD & $7.9 \mathrm{a}$ & FTI & $15.3 \mathrm{a}$ & $23.1 \mathrm{a}$ \\
\hline
\end{tabular}

Legend: Mcontrol = Manual pruning (control); $\mathrm{M}=$ Manual pruning; $\mathrm{F}=$ Mechanical skirt pruning; $\mathrm{T}=\mathrm{Mechanical}$ top pruning; $\mathrm{D}=$ Mechanical pruning of South side hedge; $\mathrm{I}=$ Mechanical pruning of North side hedge; $\mathrm{R}=$ Manual finishing pruning after mechanical pruning. Treatments with different letters had significant differences according to Duncan's multiple range test at $95.0 \%$.

\section{Yield and diameter of fruits}

Table 4 shows the yield per treatment in each year and the total yield in two years of trials. In the first year, it is clear that in the treatments involving hand pruning (treat. 1, 3 and 4) the yield was significantly lower than in those in which only mechanical pruning was performed (treat. 2 and 5). In the second year, treatments 2 and 4 had the least yield. The other three treatments (Mcontrol, FTI and FTR) showed no significant differences with each other.

Furthermore, it is interesting to compare the accumulated yield of the two years. Treatment 5 (FTD+FTI, with $683 \mathrm{~kg} /$ tree) was the most productive, and treatment 4 (FTDR+FTIR, with $496 \mathrm{~kg} /$ tree) was the least productive. It should be highlighted that the largest accumulated production was obtained in the treatment involving only mechanical pruning, in contrast to the least production which was obtained in the treatment with mechanical and manual pruning for two years. Treatments 1 to 3 showed no significant differences to each other, it is, however, important to point out that treatment 1, pruned manually for two years, was the second least productive (552 $\mathrm{kg} /$ tree); and that treatment 2, mechanical pruning one year and manual pruning in the other year, showed a middle yield $(616 \mathrm{~kg} /$ tree $)$.

Table 4. Annual and two years' accumulated yield by pruning treatments.

\begin{tabular}{cccccc}
\hline Treatment & Year 2016 & \multicolumn{3}{c}{ Year 2017 } & Years 2016 and 2017 \\
\cline { 2 - 6 } & Task & $\begin{array}{c}\text { Yield } \\
(\mathrm{kg} / \text { tree })\end{array}$ & Task & $\begin{array}{c}\text { Yield } \\
(\mathrm{kg} / \text { tree })\end{array}$ & $\begin{array}{c}\text { Total yield two years } \\
(\mathrm{kg} / \text { tree })\end{array}$ \\
\hline 1 & Mcontrol & $221 \mathrm{a}$ & Mcontrol & $331 \mathrm{~b}$ & $552 \mathrm{ab}$ \\
2 & FTDI & $350 \mathrm{~b}$ & M & $266 \mathrm{a}$ & $616 \mathrm{ab}$ \\
3 & FTR & $259 \mathrm{a}$ & FTR & $328 \mathrm{~b}$ & $587 \mathrm{ab}$ \\
4 & FTDR & $250 \mathrm{a}$ & FTIR & $246 \mathrm{a}$ & $496 \mathrm{a}$ \\
5 & FTD & $341 \mathrm{~b}$ & FTI & $342 \mathrm{~b}$ & $683 \mathrm{~b}$ \\
\hline
\end{tabular}

Legend: Mcontrol = Manual pruning (control); $\mathrm{M}=$ Manual pruning; $\mathrm{F}=$ Mechanical skirt pruning; $\mathrm{T}=$ Mechanical top pruning; $\mathrm{D}=$ Mechanical pruning of South side hedge; $\mathrm{I}=$ Mechanical pruning of North side hedge; $\mathrm{R}=$ Manual finishing pruning after mechanical pruning. Treatments with different letters had significant differences according to Duncan's multiple range test at $95.0 \%$.

The average diameter and percentage of lemons with a diameter greater than $60 \mathrm{~mm}$ per pruning treatment in the two years of trials are shown in Table 5. In general, the fruits harvested in 2017 had a lower diameter than those harvested in 2016 (61.78 $\mathrm{mm}$ vs $64.95 \mathrm{~mm})$.

In relation to average diameter, the results show significant differences among the treatments. In the first year, the fruits of treatments 2 and 5 had lower average diameters than those from treatments 1,3 and 4 . However, in 
the second year the distribution of diameters changed, in general less-productive treatments have a higher average diameter of fruits. This is in agreement with other authors (Agustí, 2003; Martin-Gorriz et al., 2014).

Similarly, the percentage of fruits with diameters greater than $60 \mathrm{~mm}$ (commercial size) also decreased in 2017 compared with 2016 in all pruning treatments. Treatment 2 showed the least reduction in the percentage of commercial size fruits $(16 \%)$, probably as a result of the change in pruning strategy. This treatment in the first year involved pruning mechanically (FTDI) and in the second year pruning manually (M) so the amount of pruned biomass increased sharply in 2017 compared to $2016(85.2 \mathrm{~kg} /$ tree vs $9.0 \mathrm{~kg} / \mathrm{tree})$ which produced a decline in yield ( $266 \mathrm{~kg} /$ tree in $2017 \mathrm{vs} 350 \mathrm{~kg} /$ tree in 2016); as a result the reduction in the diameter of the fruit was slight.

On the contrary, the greatest reduction in the percentage of commercial size fruits between 2016 and 2017 was noticed in treatment $3(55 \%)$. The results of this treatment are not clear because in 2017 the trees were pruned slightly more than in $2016(36.3 \mathrm{~kg} /$ tree in $2017 \mathrm{vs} 29.5 \mathrm{~kg} /$ tree in 2016) and yet the yield increased in 2017 , which caused a sharp decline in the diameter of the fruits. It should be noted that the pruning strategy was the same for the two years (FTR-FTR).

Table 5. Average diameter and percentage of lemons with diameter greater than $60 \mathrm{~mm}$ per pruning treatment in 2016 and 2017.

\begin{tabular}{ccccccc}
\hline Treatment & Year 2016 & \multicolumn{5}{c}{ Year 2017 } \\
\cline { 2 - 7 } & Task & $\begin{array}{c}\text { Average } \\
\text { diameter }(\mathrm{mm})\end{array}$ & $\begin{array}{c}\text { Diameter } \\
>60 \mathrm{~mm}(\%)\end{array}$ & Task & $\begin{array}{c}\text { Average } \\
\text { diameter }(\mathrm{mm})\end{array}$ & $\begin{array}{c}\text { Diameter } \\
>60 \mathrm{~mm}(\%)\end{array}$ \\
\hline 1 & Mcontrol & $65.95 \mathrm{~b}$ & 92 & Mcontrol & $62.16 \mathrm{c}$ & 65 \\
2 & FTDI & $64.22 \mathrm{a}$ & 80 & M & $64.19 \mathrm{~d}$ & 75 \\
3 & FTR & $65.46 \mathrm{~b}$ & 88 & FTR & $59.84 \mathrm{a}$ & 48 \\
4 & FTDR & $65.36 \mathrm{~b}$ & 89 & FTIR & $62.01 \mathrm{c}$ & 63 \\
5 & FTD & $63.65 \mathrm{a}$ & 79 & FTI & $60.73 \mathrm{~b}$ & 56 \\
\hline
\end{tabular}

Legend: Mcontrol = Manual pruning (control); $\mathrm{M}=$ Manual pruning; $\mathrm{F}=$ Mechanical skirt pruning; $\mathrm{T}=$ Mechanical top pruning; $\mathrm{D}=$ Mechanical pruning of South side hedge; $\mathrm{I}=$ Mechanical pruning of North side hedge; $\mathrm{R}=$ Manual finishing pruning after mechanical pruning. Treatments with different letters had significant differences according to Duncan's multiple range test at $95.0 \%$.

Fig. 3 shows the average fruit diameter and yield by pruning treatment in the two years. The results show that in treatments with a high production the fruit diameter was lower. In general, fruit diameter was inversely proportional to yield, which is in agreement with other authors (Agustí, 2003; Martin-Gorriz et al., 2014), However, in our study (data not shown) this ratio was not too high $\left(\mathrm{R}^{2}=0.11\right)$ due to the high dispersion of the data.

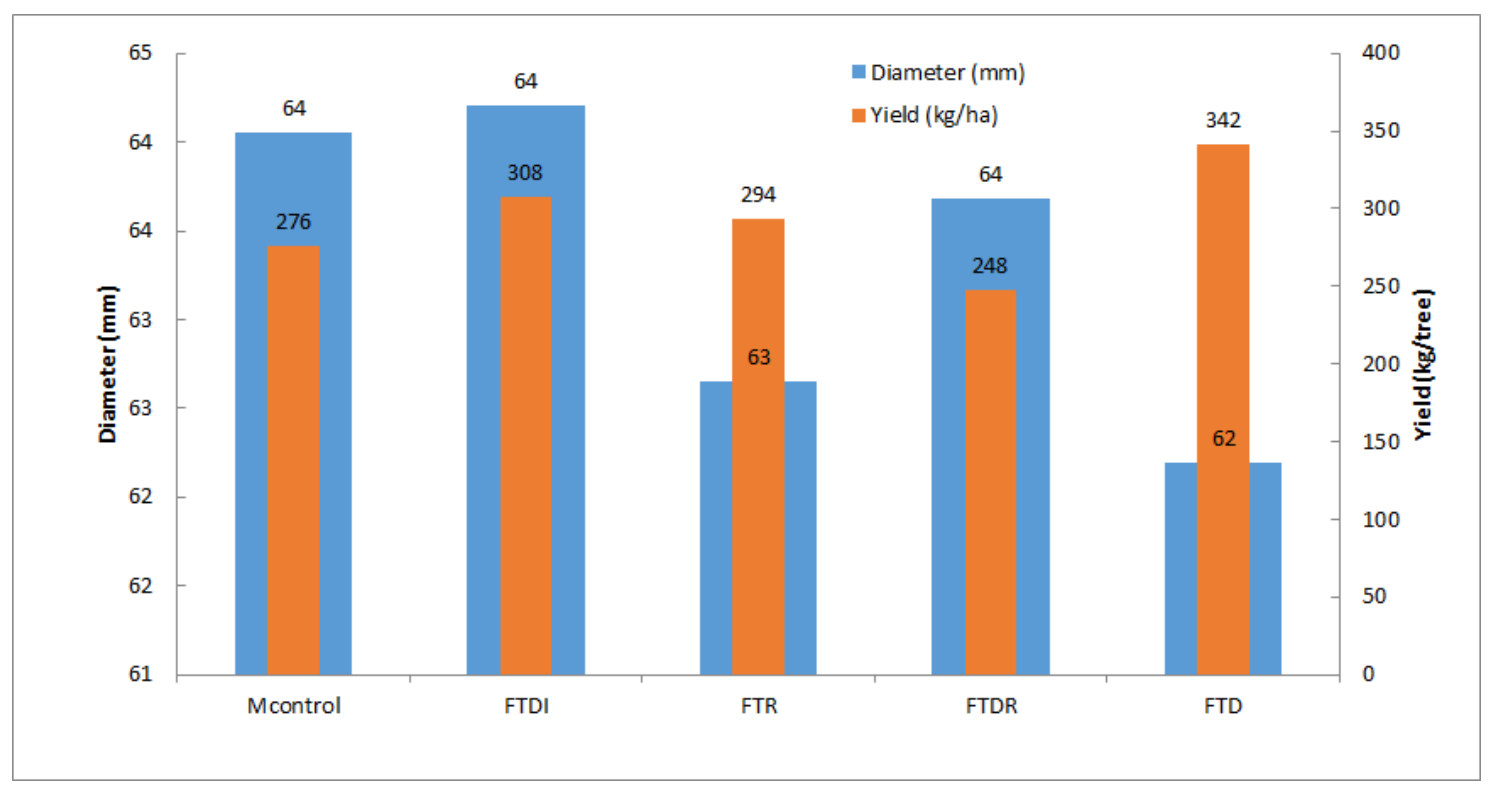

Figure 3. Average fruit diameter and yield by pruning treatment.

Economic assessment

Fig. 4 shows the annual and two years' accumulated net economic value of production by treatment. Net economic value has been calculated as the value of commercial production, i.e. fruits with diameter greater than $60 \mathrm{~mm}$, less the pruning cost. Data from Fig. 2, Table 4 and Table 5 has been used. The sale prices in origin (fresh 
market) of lemons were those officially published by the Government of the Region of Murcia (CARM, 2017) for the harvesting week $(0.29 € / \mathrm{kg}$ in 2016 and $0.36 € / \mathrm{kg}$ in 2017). The net economic value of the production was higher in 2016 than in 2017 in all pruning treatments with the exception of the control treatment (treat. 1). Treatment 2 (FTDI) was the most profitable in 2016, however in 2017 it was treatment 1 (Mcontrol). Over the two years of this study, treatment 2 was the most profitable treatment (FTDI-M) and treatment 4 (FTDR-FTIR) the least profitable, with a difference between them of $7508 € /$ ha.

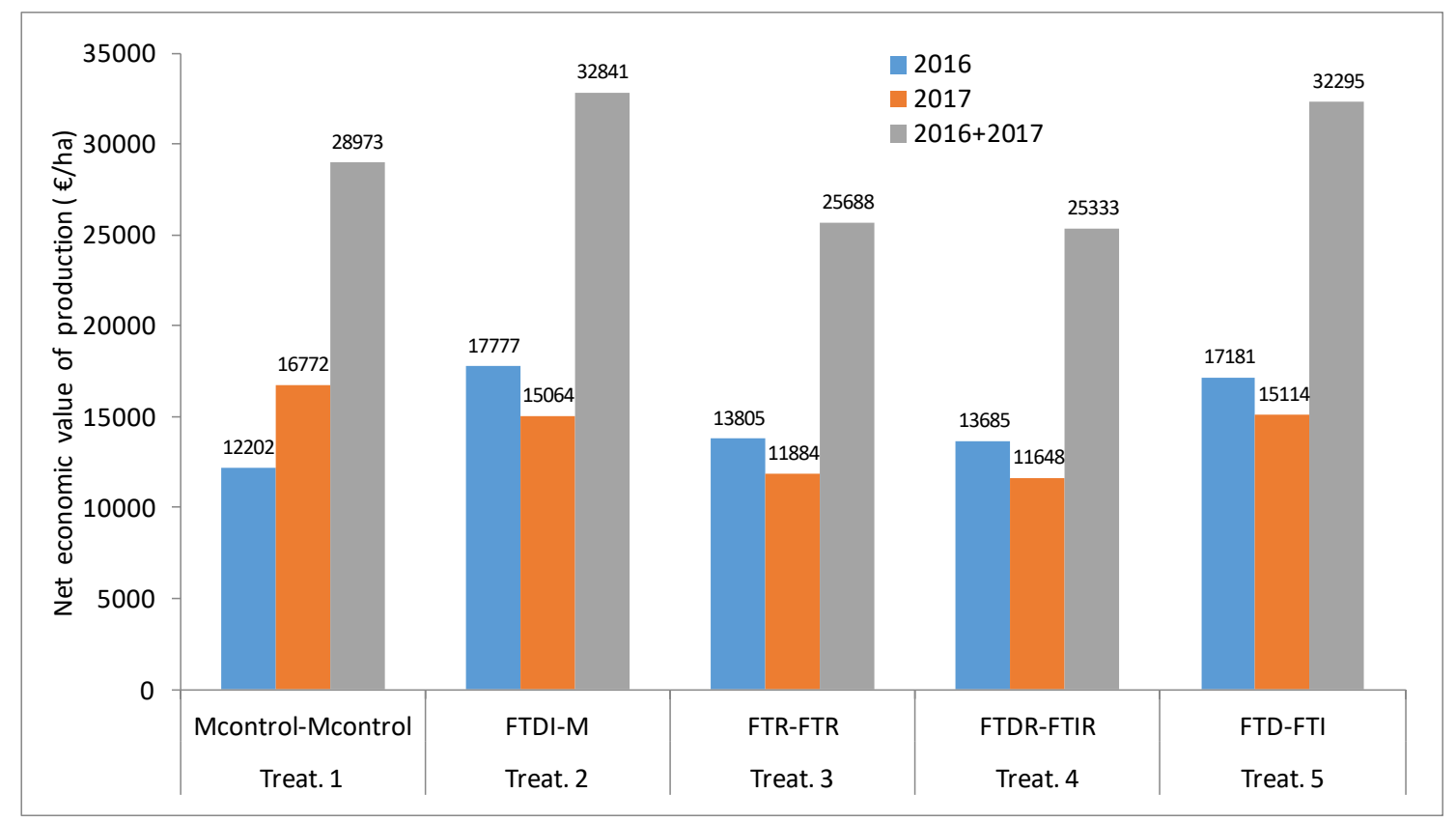

Figure 4. Annual and two years' accumulated net economic value of production by treatment.

\section{Discussion and conclusion}

Fig. 5 shows the relationship between biomass pruned and yield accumulated in two years per treatment. In general, the data show a negative correlation between pruned biomass and yield. The treatment with the most accumulated production was the one that had less biomass eliminated (treat. 5) and treatments 3 and 4 followed the same trend. Bevington (1980) obtained similar results in Valencia orange trees.

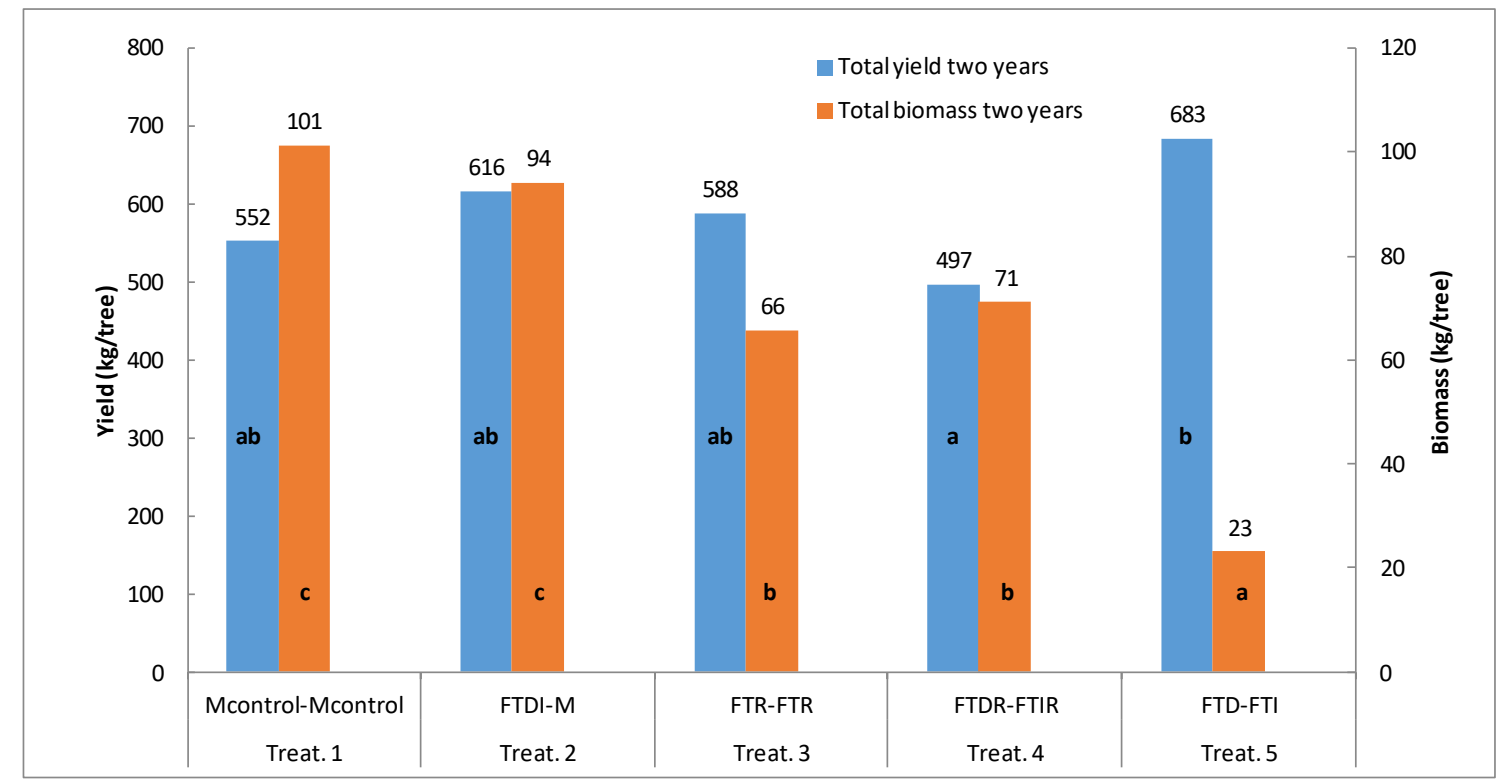

Figure 5. Two years' accumulated yield and biomass pruned per treatment. Treatments with different letters had significant differences according to Duncan's multiple range test at 95.0\%. 
In treatments 1 and 2 the relationship between pruned biomass and yield is best understood analysing annual data, since in these two treatments the amount of biomass pruned one year compared to the following had wide variations ( $89 \%$ in treatment 1 and $-83 \%$ in treatment 2$)$ :

- In treatment 1 the amount of pruned biomass was sharply reduced in 2017 compared to $2016(65.3 \mathrm{~kg} /$ tree in 2016 and $35.8 \mathrm{~kg}$ /tree in 2017; Table 3). According to Bevington (1980) it is possible that the slight pruning stimulated the growth of fruitful shoots, increasing yield from $221 \mathrm{~kg} /$ tree in 2016 to $331 \mathrm{~kg} / \mathrm{tree}$ in 2017 (Table 4). This may explain how its accumulated yield has been maintained despite having been the treatment with the greatest amount of pruned biomass accumulated in two years.

- In treatment 2, as pointed out above, in the first year the amount of pruned biomass increased sharply in 2017 compared to $2016(85.2 \mathrm{~kg} /$ tree vs $9.0 \mathrm{~kg} /$ tree $)$ which produced a decline in yield (266 kg/tree in $2017 \mathrm{vs} 350 \mathrm{~kg} /$ tree in 2016); but the accumulated yield for two years has been maintained because in 2016 it was the treatment with the highest production $(350 \mathrm{~kg} / \mathrm{tree})$.

There is widespread agreement that the correct pruning of citrus trees results in many benefits. However, it is important not to overlook the fact that pruning generally reduces yields in direct proportion to pruning severity (Bevington, 1980; Krajewski and Krajewski, 2011; Zaragoza and Alonso, 1980; 1981). According to Bevington (1980) pruning should always be as light as possible. In this sense the results obtained in our trials over two years suggest that the use of machines to prune may allow a better control of the task, and consequently it is possible to introduce mechanical pruning systems in order to reduce the time and costs involved in the task.

The aim of this research was to obtain detailed information on the response of 'Fino 95' lemon trees to mechanical pruning with different levels of severity and how it should be integrated with manual pruning into a management strategy to reduce pruning costs. This would aid farmers in making pruning decisions for the production of fresh market fruit. The main conclusions drawn from this work are summarised below:

- Mechanical pruning over the two years (treat. 5) and alternating mechanical pruning and manual pruning annually (treat. 2) are presented as the best options to reduce working time.

- The yield was significantly lower in treatments in which manual pruning was involved in the same year (treat. 1, 3 and 4).

- Economic assessment shows that the most profitable treatment over the two years of study was treatment 2, which alternated mechanical pruning and manual pruning annually.

Overall, the data obtained in two years of trials show that it is possible to introduce mechanical pruning systems into a pruning management strategy to reduce costs without decreasing the yield. However, it should be noted that more years of trials will be needed to achieve more conclusive results.

\section{Acknowledgements}

The authors acknowledge the financial support of Instituto Nacional de Investigaciones Agrarias (INIA) and FEDER (Project no. RTA2014-00025-C05-02). The authors are grateful to Juan José Peña and Diego Guerra for their assistance with this research as well as to Fruca S.A. for providing orchards and farm tasks.

\section{References}

Agustí, M., 2003. Citricultura. Madrid. Mundi-Prensa. 416 p.

Bevington, K.B., 1980. Response of Valencia orange trees in Australia to hedging and topping. Proc. Fla. State Hort. Soc. 93, 65-66.

Boswell, S.B., L.L. Ede, C.D. McCarty, 1977. Effect of training young lemon trees for limb-shaker mechanical harvest on yield and tree trunk growth. HortScience. 12 (4), 352-353.

CARM, 2017. Precios agrarios en origen. Retrieved from https://www.carm.es/web/pagina?IDCONTENIDO=1210\&IDTIPO=100\&RASTRO=c212\$m 1230 .

Fallahi, E., M. Kilby, 1997. Rootstock and pruning influence on yield and fruit quality of 'Lisbon' lemon. Fruit Varieties Journal. 51(4), 242-246.

Giametta, G., G. Zimbalatti, 1992. Three-years experiments of citrus fruit mechanical pruning. Proc. Int. Soc. Citriculture. 2, 693-696.

Intrigliolo, F., G. Roccuzzo, 2011. Modern trends of citrus pruning in Italy. Adv Hort Sci. 25(3), 187-192.

Kallsen, C.E., 2005. Topping and manual pruning effects on the production of commercially valuable fruit in a midseason Navel Orange variety. HorTecnology. 15 (2), 335-341.

Krajewski, A.J., S.A. Krajewski, 2011. Canopy management of sweet orange, grapefruit, lemon, lime and mandarin trees in the tropics: Principles, practices and commercial experiences. Acta Hortic. 894, 65-76.

MAGRAMA, 2014. Anuario de estadística. Ministerio de Agricultura, alimentación y medioambiente. Retrieved from http://www.magrama.gob.es/es/estadistica/temas/ publicaciones/ anuario-de-estadistica/ 
Martin-Gorriz, B., I. Porras Castillo, A. Torregrosa, 2014. Effect of mechanical pruning on the yield and quality of 'Fortune' mandarins. Spanish Journal of Agricultural Research. 12(4), 952-959.

Martin-Gorriz, B., C. Martinez Barba, A. Torregrosa, 2017. Influencia de la poda mecánica en la producción y calibre de los frutos de limoneros 'Fino 95'. In IX Congreso Ibérico de AgroIngeniería, AgroIng2017, Braganza, Portugal, September 4-6. Braganza. Eds., J.C. Barbosa. ME O115.

Mendonça, V., J.D. Ramos, S.E.A. Neto, J.C.M. Rufini, 2008. Production of 'Poncan' tangerine tree after pruning recovery. Ciencia e Agrotecnologia. 32 (1), 103-109.

Ortiz-Cañavate, J. 1979. Mechanical pruning of citrus. Anales del Instituto Nacional de Investigaciones Agrarias. Tecnología Agraria. 5, 155-167.

Porras, I. 2014. Limonero, pomelo y lima. In La Fruticultura del siglo XXI en España. Cajamar Caja Rural. Eds., J.J. Hueso Martín; J. Cuevas González. 10, 301-325.

Raciti, G., P. Spina, A. Scuderi, F. Intrigliolo, 1982. Tre anni di potatura meccanica degli agrumi in Italia. Frutticultura. 44, 35-46.

Rouse, B., L. Parsons, T.A. Wheaton, 2006. Hedging, topping and skirting trees in the citrus canker era. Citrus Industry. 12, 1-2.

Sauls, J.W., 2008. Citrus pruning. Texas Citrus and Subtropical fruits. Retrieved from http://aggiehorticulture.tamu.edu/citrus/pruning/L2308.htm

Spina, P., A. Giuffrida, E. Melita, 1984. Comparative trials of citrus mechanical and aided pruning. Proc. Int. Soc. Citriculture. 1, 106-109.

USDA, 2016. Spain's Citrus Report Spain - USDA GAIN reports. Retrieved from https://gain.fas.usda.gov/Recent\%20GAIN\%20Publications/Spain's\%20Citrus\%20Report\%20_Madrid_Spain_1 2-15-2016.pdf

Velázquez, B., E. Fernández, 2010. The influence of mechanical pruning in cost reduction, production of fruit, and biomass waste in citrus orchards. Appl Eng Agric. 26(4), 531-540.

Yildirim, B., T. Yesiloglu, M. Incesu, M. Kamiloglu, F. Ozguven, O. Tuzcu, Y. Aka, 2010. The effects of mechanical pruning on fruit yield and quality in 'Star Ruby' grapefruit. Journal of Food, Agriculture \& Environment. 8 (2), 834-838.

Zaragoza, S., E. Alonso, 1980. La poda mecanizada de los agrios en España. Anales del Instituto Nacional de Investigaciones Agrarias. Serie: Producción Vegetal. 12, 157-180.

Zaragoza, S., E. Alonso, 1981. Citrus pruning in Spain. Proc. Int. Soc. Citriculture. 1, 172-175. 


\title{
Management of Chemical Residual Volume Relative to Use of Stationary Spraying System in Moroccan Greenhouse Crop Production
}

\author{
El Aissaoui Abdellah ${ }^{1 *}$, Khnizrou Larbi ${ }^{2}$, Houmy Karim $^{2}$, Bouzrari Benaissa ${ }^{2}$ \\ ${ }^{1}$ Agricultural Engineering Laboratory, INRA, PO box 586, Settat, Morocco \\ ${ }^{2}$ Agricultural Engineering Department, IAV Hassan II, PO Box 6202, Rabat, Morocco \\ * Corresponding author. Email: ab_elaissaoui@yahoo.fr
}

\begin{abstract}
This study aims to solve problem of residual volume of a chemical applied in a greenhouse using a stationary spraying system with lance. For this purpose, a direct injection method is proposed to avoid chemical mixing as a potential source of residual volume. The work is based on a survey to estimate effective residual chemical volume per square meter. Furthermore, an experiment was done for testing possibility of using direct injection system as an alternative for a clean chemical spraying. a low cost technology based on a venturi injector system using standardized orifices to optimally setting and controlling applied chemical injection rates according to a large range of active ingredient rates of plant protection products used in greenhouse vegetal crop productions. The performance of this injection system was tested in laboratory using fluorescing tracer to simulate injected chemical. Results showed that is possible to design an affordable (less than $100 €$ per unit) venturi metering system to overcome problem of the residual chemical volume estimated at 80 liter per hectare per spraying application.

Keywords: Greenhouse, residual chemical volume, stationary spraying system, direct injection method
\end{abstract}

\section{Introduction}

Several studies focused on performance of spraying plant protection products in greenhouse cropping systems (Sanchez-Hermosilla et al , 2012; Sanchez-Hermosilla et al., 2013; Rincon et al.,2017;). Sanchez-Hermosilla et al. (2012) evaluated spray deposition and soil leaching in a greenhouse tomato crop due to use of manually pulled trolley equipped with two vertical spray booms compared to a manual spray gun. They found that chemical deposits with the spray trolley were significantly the greatest. The trolley resulted in an overall deposition $40.6 \%$ and $33.9 \%$ greater than with a gun using standard flat fan nozzles and air-induction flat fan nozzles, respectively. The spray boom reduced soil leaching by roughly $54 \%$ compared to a spray gun. According to Sanchez-Hermosilla et al. (2013), plant protection products are usually applied in greenhouses by hand-held high-pressure spray guns or lances at high volume rates. This modest spraying technology cannot uniformly deposit chemical mixture on plant canopy; consequently runoff and drift affect environment and operators. These authors showed that high spray pressure can improve deposition and reduce losses on the ground using a hand-held spray lance in a greenhouse tomato crop at 10,15 and 20 bars. In recent work, Rincon et al. (2017) stated that growers believe that a high spray application rate and a high pressure are needed to achieve good pest and disease control in greenhouse cropping system. They evaluated the effects of three application volumes of $100 \%, 75 \%$ and $50 \%$ and found similarity between these applied volume rates of reference of $100 \%$ and reduced volume of $75 \%$.

Moroccan greenhouse production of Tomato turns around 7500 hectares, concentrated mainly at the south in Souss Massa valley. Greenhouse farming systems, equipped with stationary spraying systems for applying plant protection products, are about 2000 units. Those units are surveyed for evaluating conditions of applying plant protection products. Plant protection products application is mainly based on use of stationary spraying equipments and hand operated lances. The technology of stationary sprayers is widely adopted by farmers without paying attention to problem of management and treatment of residual volume kept as dead volume in spraying hydraulic circuit. Volumes of chemical mixtures kept in the circuit of the stationary spraying system after each spraying operation constitutes important point source of pollution. As farmers are actually lacking of recycling measures to protect environment from residual volumes, it is of importance to propose a direct injection spraying technology to be coupled with use of stationary spraying systems. The proposition could totally solve problem of residual volume as only proper carrier water is transported in the hydraulic circuit source of dead volume.

Adaptation of direct injection spraying method to apply plant protection products in greenhouse production system consists of implementing a portable spraying system based on lance or vertical boom coupled to a venturi injector to meter chemical online proportionally to carrier water disserved by the spraying system ( El Aissaoui, 2016). Venturi injector is an affordable and simple technology to inject and meter low chemical flow rate by using only pressure energy of carrier water. The venturi is widely used as injector of fertilizer in drip irrigation systems (Mechael, 2015; Colter , 2011). But its use as direct injector for agricultural sprayer is limited. In fact, venturi use requires implementation of robust design and control for processing low chemical volume rate to ensure stable suction potential and steady chemical injection state. Venturi injector presents a non linear hydraulic behavior and sensibility to cavitation when potential suction is subjected to vary at boundaries pressure limits. For a robust aspiration control, it is of importance to maintain a steady suction level to avoid fluctuation of injection flow rate and ensure precise concentration processing at the lance or boom level. Use of standardized orifice in the suction 
side of a venturi was proposed to manage low volume injection and to stabilize aspiration potential for precise chemical metering.

This work aims to adapt a direct injection system through use of a venturi as a means of plant protection products injection in carrier water to be sprayed by mean of portable lance or boom. Design and evaluation of a venturi injector was achieved by a simulation of a chemical injection of a fluorescent dye tracer

\section{Materials and Methods}

\section{Survey study}

A survey was done to investigate pesticide application practices and management of chemical residual volumes in greenhouse farms using stationary spraying systems. A sample of twelve green house farming systems that produce tomatoes $(84 \%)$ and peppers $(16 \%)$ was achieved. The stationary spraying systems are installed around the greenhouses structures to transfer chemical mixture through hydraulic circuit mounted on the house frame. The chemical mixture is applied by operators using hand lances to be quickly connected at different points situated along hydraulic transfer lines. Data collected concerned prospection of stationary spraying installations and application practices to estimate residual volume per greenhouse area. Extrapolation was done to evaluate the impact on the regional level according to total area used for greenhouse production. The mean residual volume was estimated on the basis of the mean dead volume kept at the bottom of stationary spraying equipment and at the hydraulic circuit used to transfer PPP mixture around the greenhouse frame of each farming production system.
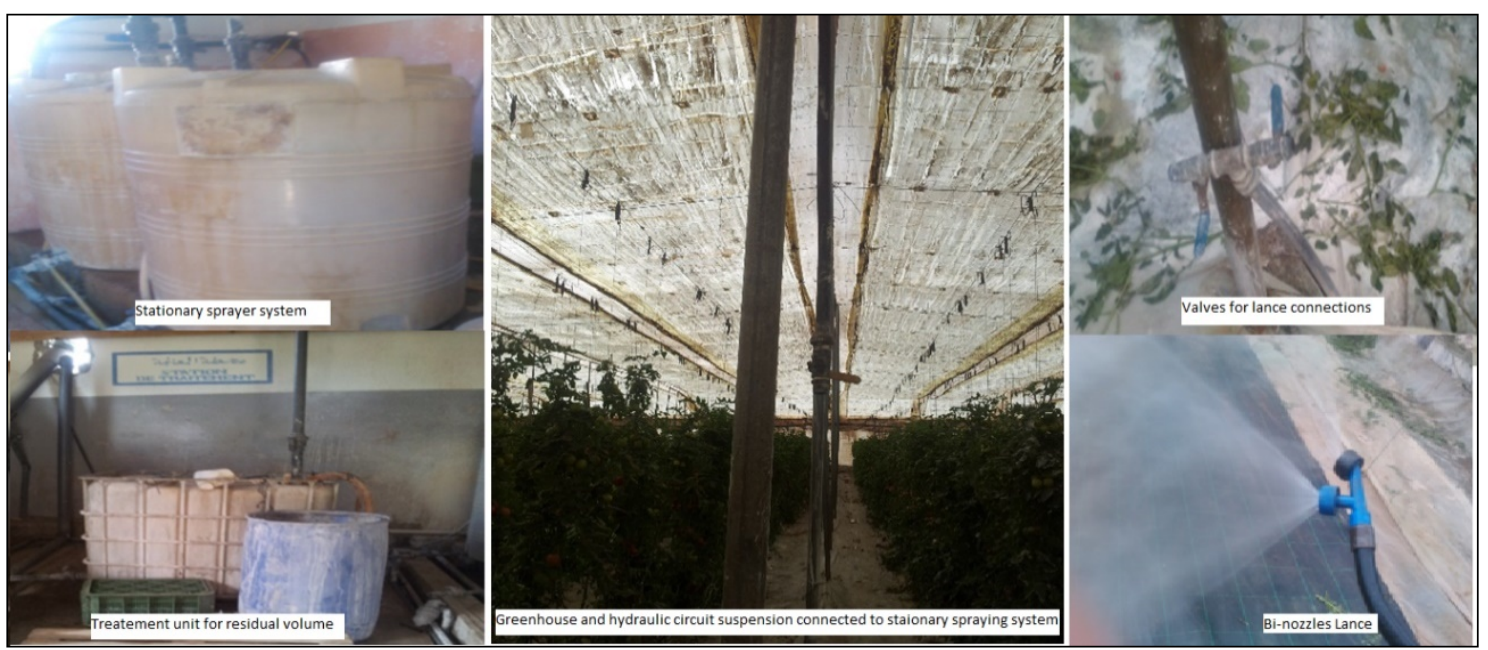

Figure 40. Context of using stationary spraying system in greenhouse system production at Souss Massa valley in Morocco.

Prototype of direct injection spraying system for greenhouse production use

Evaluation of the residual volume at field scale showed the importance to propose a direct injection spraying system as alternative to solve the problem. In fact, a feasibility study was done to implement a direct injection spraying system (DISS) for use with lances to avoid mixing of chemical at the tank level of the stationary spraying system.

A laboratory test bench (Fig. 2) was established to throttle and monitor injection flow rate of two miniature venturi injectors (VAD and MV200, Festo ${ }^{\mathrm{TM}}$ ) having orifice diameters of 1 and $2 \mathrm{~mm}$, respectively. Experimental test was set to manage variable impedance of both venturi injectors at the injection side according to scalar of suction potential and flow rate set with reference to use of five standardized orifices.The prototype (Fig. 2) was based on two venturi injectors mounted in a serial scheme for robust operating conditions. 


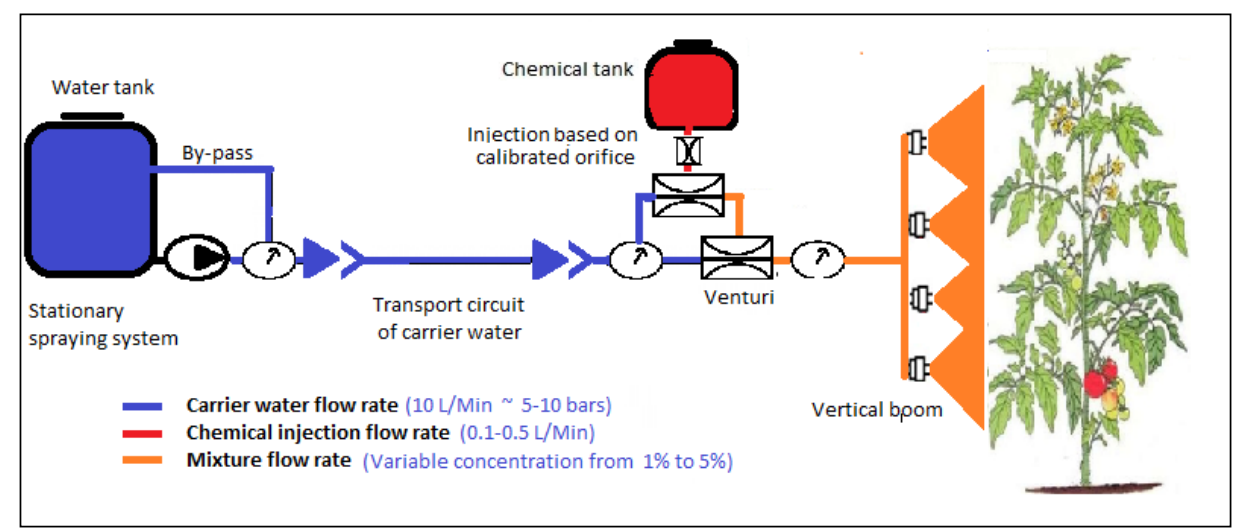

Figure 41. Layout of direct injection spraying system for use in green house cropping system.

The first venturi injector (VAD, $\mathrm{d}=1 \mathrm{~mm}$ ) was used to inject a chemical in an air/liquid stream then the injection of mixture was injected in the second venturi (MV200, $\mathrm{d}=2 \mathrm{~mm}$ ) carrying out the main carrier flow rate (around $10 \mathrm{~L} / \mathrm{min}$ ) feeding a vertical spraying boom of four nozzles (ISO-05 or 06). The suction potential in both venturi injectors was controlled manually using hydraulic valves and manometers.

Controls of chemical injection flow rate and carrier flow rate

Control of injected flow rate was performed according to choice between different standardized orifices (ISO01, ISO-015, ISO-02, ISO-025, ISO-03) mounted at the aspiration side of the venturi VAD. Control of carrier flow rate was set manually using hydraulic valve in upstream side of the venturi MV200.

The standardized orifice plays a main function of regulating injection flow rate and controlling it according to the induced vacuum pressure. The flowing rate trough a standardized orifice is controlled with reference to its area and differential pressure between upstream and downstream sides. Hydraulic orifice model adopted in this study was based on the standard nozzle model (White 1994) as follow:

$$
\mathrm{Q}(\mathrm{L} / \mathrm{min})=\mathrm{K}^{*} \operatorname{sqrt}(\mathrm{P}(\mathrm{cbar}))
$$

The discharge coefficient $\mathrm{K}$ was evaluated experimentally by correlating between flow rate and pressure. To control chemical injection flow rate in a large concentration range 1 to $5 \%$, five nozzle orifices (ISO-01, ISO015, ISO-02, ISO-025, ISO-03) were adapted to process chemical concentrations of different plant protection products formulations. In fact, agricultural pesticides are formulated to be applied at different recommended technical rate. By setting concentration ratio (injected flow rate over carrier flow rate) to be from $1 \%$ to $5 \%$, most of PPP homologated for tomatoes crop production can be processed within the range of [1 to $5 \mathrm{~L} / \mathrm{hL}]$. However, PPP homologated to be applied at low concentrations (less than 1\%) can be processed using pre-dilution method. By adding water to any initial formulated PPP, it is possible to pre-dilute it at any low concentrated technical application rate, then the dilution volume can be processed to be injected at $1 \%$ (instead of 0.1 or $0.01 \%$ ) as requirement for design of the venturi injector coupled to a standardized orifice ISO-01.

Evaluation of injected concentration using fluorescing sensor

The injection system performance was tested in laboratory using fluorescing tracer as injected chemical and sensor that convert the processed tracer concentration to voltage. Calibration of the sensors was based on preparing a solution of fluorescent dye tracer and water $(1 \mathrm{mg} / \mathrm{L})$ to simulate injected chemical at the suction side of the venturi carrier pump (El Aissaoui, 2016).

\section{Results and Discussion}

Characterization of the proposed direct injection spraying system showed the possibility of setting the main venturi injector (MV200) for processing adequate carrier flow rate to be within the range of 5-10 L/min as output for a vertical spraying boom having four nozzles (reference ISO-05 or 06). Furthermore, the chemical injector was designed to process adequately variable concentration within the flow rate range of 0.1 to $0.5 \mathrm{~L} / \mathrm{min}$ according to the engaged reference from the five standardized orifices (ISO-01, ISO-015, ISO-02, ISO-025 and ISO-03).

The operating conditions of the venturi MV200 were evaluated for variable upstream pressure to show the effect on carrier flow rate and on the aspiration potential (Fig. 3). Results showed that flow rate vary according to differential pressure between upstream and downstream sides of the venturi MV200 (Fig. 4). The set of upstream pressure at 12 bars induce a differential pressure of 6 bars and a flow rate of $10 \mathrm{~L} / \mathrm{min}$ as reference condition of carrier flow rate to process chemical injection. 


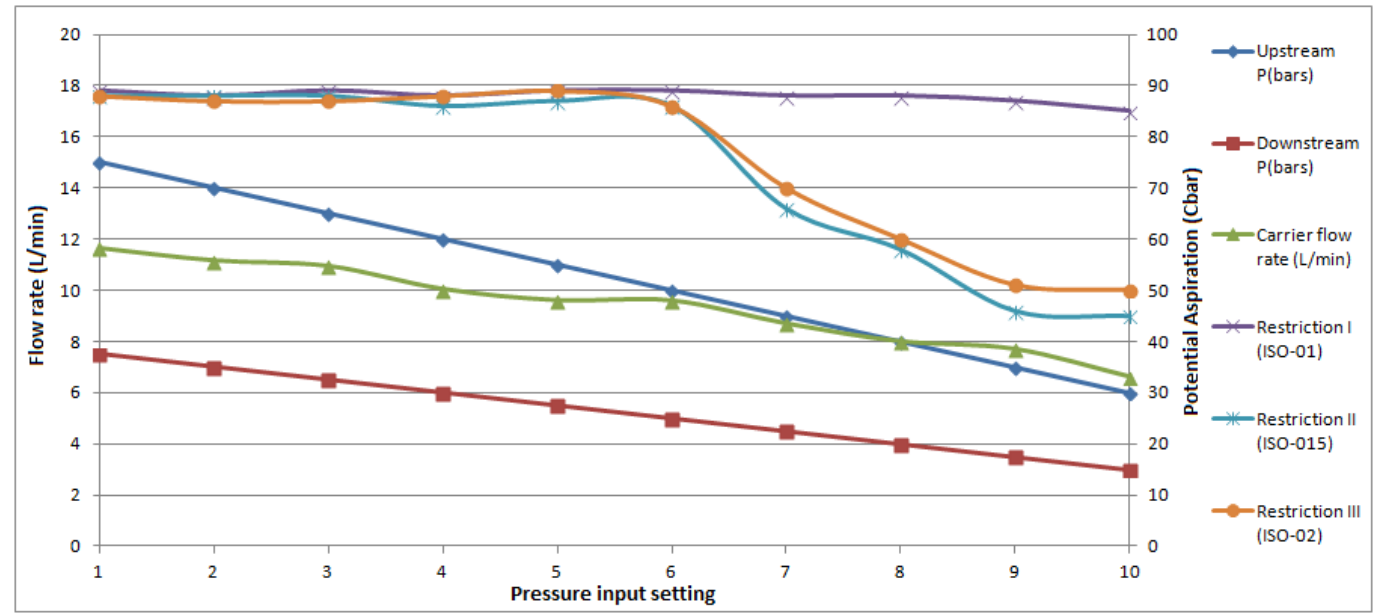

Figure 42. Operating conditions of the venturi MV200: Pressure, carrier flow rate and aspiration potential Induced by three orifice restrictions.

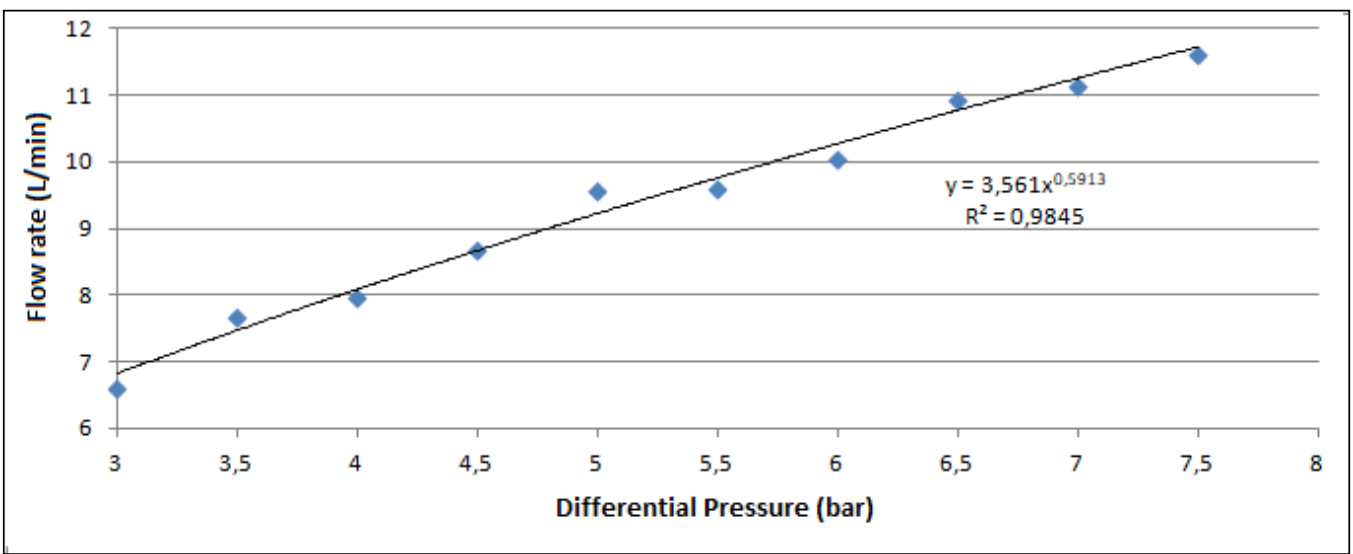

Figure 43. Hydraulic test of venturi MV200: Carrier flow rate versus differential pressure.

The injection models relative to the five standardized orifices were evaluated experimentally. The models (Fig. 4) adequately fit requirement of setting injection flow rate within the range of [0.1 to $0.5 \mathrm{~L} / \mathrm{min}$ ] to apply large brand of PPP formulations at concentration varying from 1 to $5 \%$.

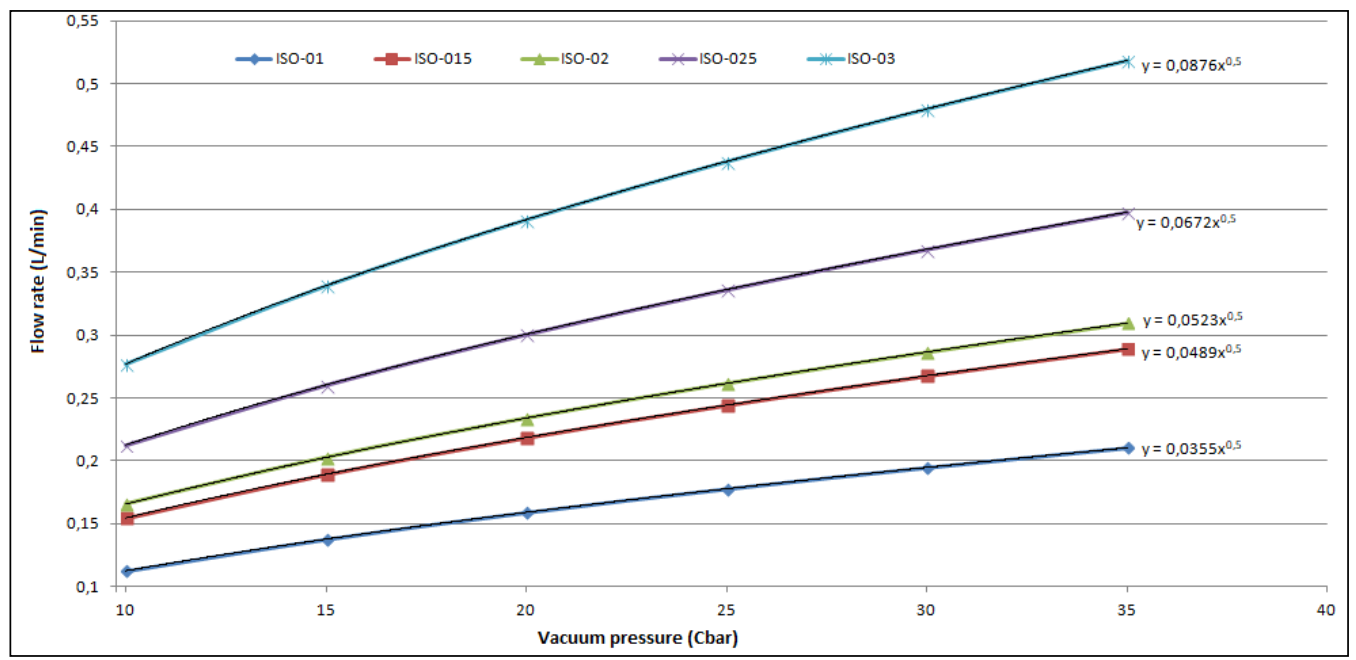

Figure 44. Injection model of five standardized orifices: Flow rate versus Vacuum pressure.

Figure 5 showed that is possible to inject variable flow rate from 0.1 to $0.5 \mathrm{~L} / \mathrm{min}$ by engaging an adequate orifice from the four calibers (ISO-01, 015, 02, 025, 03). The injected flow rate were performed to be processed with a reference carrier flow rate of $10 \mathrm{~L} / \mathrm{min}$ in order to carry out a technical application rate varying from 1 to 
5 liter of PPP per 100 liter of carrier water. The PPP formulated to be applied at low technical application rate (less than 1\%) can be pre-diluted to be processed within de concentration ranging between 1 and $5 \%$.

The test of the direct injection system using fluorescent dye tracer showed that the processed concentration by the system (ratio between the injected flow rate of tracer and the carrier flow rate) proportionally changed by increasing the injected flow rate and keeping the carrier flow rate constant at $10 \mathrm{~L} / \mathrm{min}$. The injection flow rate was switched between the five steps of 100, 200, 300, 400 and 500 with reference to the five standardized orifices of ISO-01, ISO-015, ISO-02, ISO-025 and ISO-03, respectively. The five concentrations of 1, 2, 3, 4 and 5\% were adequately performed by the proposed direct injection system.

\section{Conclusions}

A direct injection spraying system based on two venturi injectors was evaluated. Results showed the possibility of designing a robust hydraulic model for the control of carrier flow rate and injection flow rate in order to satisfy a wide concentration range from 1 to $5 \%$. This later is of importance for processing chemical injection of several PPP formulations used in vegetable cropping system. Furthermore, a method of a prior dilution was proposed to cover chemical injection of low concentration (Less than 1\%) by the design. Altough, venturi is widely adopted for metering flow rate in hydraulic systems; its use for direct injection in spraying systems is still limited. This limitation is due to absence of research for adapting the aspiration potential of the venturi for metering accurately chemical with ability to monitor flow rate and adjust its impedance with reference to the venturi model. The venturi model will served for predicting the concentration change occurrence according to the carrier flow rate and the injected flow rate in order to implement a process controller for an automatic direct injection spraying system and propose it for use in greenhouse cropping system.

\section{References}

Sanchez-Hermosilla J., Rincon V.J., Paez F., and Fernandez M., 2012. Comparative spray deposits by manually pulled trolley sprayer and a spray gun in greenhouse tomato crops. Crop Protection, 31 (1). 119-124. https://doi.org/10.1016/j.cropro.2011.10.007

Sanchez-Hermosilla J., Paez F., Rincon V.J. and Carvajal F., 2013, Evaluation of the effect of spray pressure in hand-held sprayers in a greenhouse tomato crop. Crop Protection, 54 (12) 121-125.

https://doi.org/10.1016/j.cropro.2013.08.006

Rincon V.J., Sanchez-Hermosilla J., Paez F., Alonso J. P. and Callejon A. J., 2017. Assessments of the influence of working pressure and application rate on pesticide spray application with a hand-held spray gun on greenhouse pepper crops. Crop Protection 96 (6) 7-13.https://doi.org/10.1016/j.cropro.2017.01.006

Mechael Reader-Haris, 2015. Orifice plates and venture tubes, Book, Springer Edt, 406p.

El Aissaoui A., 2016. Modelling and design of a direct injection process controller for a small scale farming. PhD thesis, 175p. ULg University.

Colter L. Hollingshead, 2011. Discharge coefficient performance of venturi, standard concenteric orfice plate, V-cone, And wedge flow meters at small reynolds numbers, Master thesis, 94p, Utah State University. 


\title{
Effect of Nozzle Type, Pressure and Height on Spray Distribution Pattern and Droplet Characteristic
}

\author{
Arun Kumar a,*, Bhabani S Dash ${ }^{\text {b }}$ \\ a b Department of Farm Machinery\& Power Engineering, G B Pant University of Ag \& Tech, Pantnagar, Uttarakhand \\ 263145, India \\ * Corresponding author. Email: arunfmp@yahoo.com
}

\begin{abstract}
Various plant protection measures are followed throughout India, among which chemical method is most widely used. The uniformity of spray and droplet size are important parameters for effective disease control. Therefore, selection of nozzles for agricultural sprayers is very important. The nozzles were tested in the laboratory using patternator to find volumetric distribution, swath and spray angle at operating pressures of $1.5(147.1 \mathrm{kPa})$, $2(196.13 \mathrm{kPa}), 2.5(245.16 \mathrm{kPa}), 3(294.2 \mathrm{kPa})$ and $3.5 \mathrm{~kg} / \mathrm{cm}^{2}(343.23 \mathrm{kPa})$ and nozzle heights 200, 300, 400, 500, 545 and $600 \mathrm{~mm}$. The droplet size determination tests were conducted at operating pressure of 1.5, 2, 2.5, 3 and $3.5 \mathrm{~kg} / \mathrm{cm}^{2}$ and fixed nozzle height of $450 \mathrm{~mm}$. The droplet size was measured using Spraytec laser diffraction technique and the Number Median Diameter (NMD) and Volume Median Diameter (VMD) of the droplets were measured. The NMDs and VMDs of the hollow cone nozzle varied between 34 to 23 and 200 to $114 \mu$, respectively while for flat fan nozzle it was 108 to 23 and 199 to $129 \mu$, respectively in pressure range from 1.5 to $3.5 \mathrm{~kg} / \mathrm{cm}^{2}$. The flat fan nozzle gave most uniform volumetric distribution at pressure of $2.5 \mathrm{~kg} / \mathrm{cm}^{2}$ and $300 \mathrm{~mm}$ nozzle height while for the hollow cone nozzle it was observed at pressure of $2 \mathrm{~kg} / \mathrm{cm}^{2}$ and $400 \mathrm{~mm}$ nozzle height. The swath and angle of spray of the nozzles were determined and it was found that with increase in pressure and height the swath increased while the spray angle increased with pressure. The hollow cone nozzle produced droplets ranging from extremely fine to extremely coarse droplets, while the flat fan produced droplets in the range of extremely fine to very coarse for the pressures between $1.5 \mathrm{~kg} / \mathrm{cm}^{2}$ pressure to $3.5 \mathrm{~kg} / \mathrm{cm}^{2}$.
\end{abstract}

Keywords: spray pattern, droplet size, NMD,VMD, nozzle

\section{Introduction}

Mechanization in agriculture holds the key for sustainable development in the terms of increasing the production by timely farm operations, reducing losses, reducing the cost of operations by ensuring better management of costly inputs and enhancing the productivity of natural resources besides it helps in reducing drudgery in farm operations. Vegetables and pulses are important constituents of Indian agriculture and nutritional security due to their short duration, high yield, nutritional richness, economic viability and ability to generate onfarm and off-farm employment. Our country is blessed with diverse agro-climates with distinct seasons, making it possible to grow wide array of vegetables. India is the second largest producer of fruits and vegetables in the world. It is estimated that, diseases, insects, and weeds together annually interfere with the production of, or destroy, between 31 to $42 \%$ of all crops produced worldwide. It has been estimated that of the $36.5 \%$ average total losses, $14.1 \%$ are caused by diseases, $10.2 \%$ by insects and $12.2 \%$ by weeds. Annual losses by nematode are estimated to be $\$ 100$ billion worldwide and in India it is around $\$ 40$ million per year (Agrios, 2005). The impact of pesticides on the environment is alarming. India is placed tenth in the world. Plant protection chemicals currently cover about $30 \%$ of the total cultivated area in India, of which insecticides account for $65 \%$ followed by herbicides $(16 \%)$, fungicides $(15 \%)$ and others $(4 \%)$. Keeping in view these aspects, plant protection becomes a vital option to be dealt with. The uniformity of spray and droplet size are important parameters for effective disease control. Therefore, selection of nozzles for agricultural sprayers is very important. The nozzles were tested in the laboratory to find its volumetric distribution, swath and spray angle at different pressure and height combinations.

\section{Materials and Methods}

Spraying equipments play an important role in effective application of pesticide for pest control. Most of the sprayers spray liquid from the top of the canopy and neglect the underside and the inside of the canopy. This becomes a hiding place for insects and pathogens as chemicals don't reach the target. A self-propelled boom sprayer was developed basically to cover larger area in short period and to perform intra canopy and under leaf spraying. Nozzle selection is one of the most important decisions to be made related to pesticide applications. The type of nozzle affects not only the amount of spray applied to a particular area, but also the uniformity of the applied spray, the coverage obtained on the sprayed surfaces, and the amount of drift that can occur. Each nozzle type has specific characteristics and capabilities and is designed for use under certain application conditions. The types which are commonly used for ground application of agricultural chemicals are flat-fan, even flat-fan, and cone nozzle. Regular flat-fan nozzles are used for most broadcast spraying of herbicides and for certain insecticides when foliar penetration and coverage are not required. 
Experimental variables for testing of nozzles

The nozzles were tested in the laboratory using patternator (Dash 2016) and the details of the experimental variables are given in Table 1. The experimental plan for measuring droplet size is given in Table 2.

Table 1. Experimental plan for patternator testing of the nozzles.

\begin{tabular}{lll}
\hline Independent parameters & \multicolumn{1}{c}{ Levels } & Dependent parameters \\
\hline Nozzle type & Two types: Hollow cone, Flat fan & Discharge rate, ml $/ \mathrm{min}$ \\
Fluid pressure & Five levels: $1.5 \mathrm{~kg} / \mathrm{cm}^{2}(147.1 \mathrm{kPa})$, & Swath width, $\mathrm{mm}$ \\
& $2 \mathrm{~kg} / \mathrm{cm}^{2}(196.13 \mathrm{kPa}), 2.5 \mathrm{~kg} / \mathrm{cm}^{2}(245.16 \mathrm{kPa})$ & Spray angle, degrees \\
& $3 \mathrm{~kg} / \mathrm{cm}^{2}(294.2 \mathrm{kPa}), 3.5 \mathrm{~kg} / \mathrm{cm}^{2}(343.23 \mathrm{kPa})$ & Uniformity of distribution, C.V. \\
& Six levels: $200 \mathrm{~mm}, 300 \mathrm{~mm}, 400 \mathrm{~mm}, 500 \mathrm{~mm}$ & \\
Nozzle height & $545 \mathrm{~mm}, 600 \mathrm{~mm}$ & \\
& & \\
\hline
\end{tabular}

Table 2. Experimental plan for measuring droplet size using Spraytec.

\begin{tabular}{lll}
\hline Independent parameters & Levels & Dependent parameters \\
\hline Nozzle type & Two types: Hollow cone, Flat fan \\
Fluid pressure & Five levels: $1.5 \mathrm{~kg} / \mathrm{cm}^{2}(147.1 \mathrm{kPa}), 2 \mathrm{~kg} / \mathrm{cm}^{2}$ & Volume median diameter, \\
& $(196.13 \mathrm{kPa}), 2.5 \mathrm{~kg} / \mathrm{cm}^{2}(245.16 \mathrm{kPa}), 3 \mathrm{~kg} / \mathrm{cm}^{2}$ & $\begin{array}{l}\text { microns } \\
\text { Number median diameter, } \\
\end{array}$ \\
& & $\begin{array}{l}\text { microns } \\
\text { Uniformity of coefficient, U.C. }\end{array}$ \\
Nozzle height & One level: $450 \mathrm{~mm}), 3.5 \mathrm{~kg} / \mathrm{cm}^{2}(343.23 \mathrm{kPa})$ &
\end{tabular}

Experimental procedure

A $2000 \times 2000 \mathrm{~mm}$ patternator made of acrylic sheet having inner dimensions of equal channels as $2000 \times 25 \times 100 \mathrm{~mm}$ was used for testing. Graduated tubes having $24 \mathrm{~mm}$ inner diameter were used to collect the sprayed liquid. The inclination of the spray channel section has been made adjustable. The nozzles were mounted at the centre of a metallic frame and its axis was kept perpendicular to the horizontal. The height of the nozzle assembly was also adjustable. The nozzles were connected to a constant water supply through a piston pump and a pressure gauge was mounted to check the pressure. The nozzles were run at different pressures $(1.5,2,2.5,3$ and $\left.3.5 \mathrm{~kg} / \mathrm{cm}^{2}\right)$ and heights $(200,300,400,500,545$ and $600 \mathrm{~mm})$ to check nozzle's uniformity of distribution at different heights, since for different crop heights boom height also changes. The collected spray liquid from the channels was poured to test tubes and the readings were taken. There was provision to test more than one nozzle to obtain overlapping of the spray pattern. The test was run for one minute each and replicated three times and the average data was calculated.

\section{Methods of measurement}

Spray angle and swath width

According to Indian Standard, IS: 8548 - 1977, test code for power- operated hydraulic sprayer, the working width (the distance between the outer edges of the outermost channels which at the working pressure received $50 \%$ or more of the largest quantity of liquid collected from any one channel) in millimetres was measured for each of the nozzle height.

The spray angle for each nozzle was calculated on the basis of working width and nozzle height, and was stated in whole degrees. It is also described as the angle subtended at the final orifice by the edges of the spray pattern.

\section{Discharge rate of the nozzles}

The discharge of the two nozzles was collected in a measuring jar for one minute. The readings were taken for all the pressures mentioned above and repeated three times. The average data was calculated and the relationship between pressure and discharge was studied.

\section{Droplet size measurement}

Nozzles produce droplets of various sizes ranging from very fine $(<60 \mu)$ to ultra-coarse $(>665 \mu)$. So it becomes essential to choose the right nozzle to reduce spray drift and get maximum coverage simultaneously. Droplet sizes are usually measured in microns (micrometres). A nozzle with a coarse or very coarse droplet is 
usually selected to minimize off-target spray drift, while a nozzle with a fine droplet is required to obtain maximum surface coverage of the target plant.

- Parameters to measure droplet size

Volume Median Diameter (VMD or DV0.5) refers to the midpoint droplet size (mean), where half of the volume of spray is in droplets smaller, and half of the volume is in droplets larger than the mean. A VMD (DV0.5) of 400 , for example, indicates that half of the volume is in droplet sizes smaller than 400 microns, and half the volume is in droplet sizes larger than 400 microns.

Drift potential depends not only on the VMD, but on the total spectrum of droplet sizes. For more information droplets are also put in to these categories. A DV0.9 value indicates that $90 \%$ of the volume of spray is in droplets smaller (or 10\% larger) than this value. A DV0.1 value indicates that $10 \%$ of the volume of spray is in droplets smaller than this value.

The Number Median Diameter (NMD) is the droplet diameter where the number of droplets above the NMD is equal to the number of droplets below the NMD. The NMD is usually smaller than the VMD because most pesticide sprays usually contain a large number of very small droplets.

Uniformity coefficient is the ratio of VMD and NMD. The closer the ratio of VMD and NMD approaches to one the uniform the size of droplets becomes.

- Measuring the droplet size

The sizes of droplets produced by the nozzles were measured using Malvern Instrument's Spraytec laser diffraction system. The size of droplets produced from both the nozzles were studied for different pressures and compared to the droplet size classification by ASABE standard. While studying the droplet sizes from the nozzles, the maximum $(>60 \%)$ percentage of the droplets produced were taken into account.

\section{Spraytec instrument}

Malvern Instrument's Spraytec laser diffraction system allows measurement of spray particle and spray droplet size distributions in real-time for more efficient product development of sprays and aerosols. It does this by measuring the intensity of light scattered as a laser beam passes through a spray. This data is then analysed to calculate the size of the droplets that created the scattering pattern. It has been specifically designed to address the unique requirements for spray characterization and deliver robust, reproducible droplet size data. It measures across a wide size range $(0.1-2000$ microns $)$ of particle size without requiring constant optics changes. It also resolves rapid changes in droplet size over time, by measuring up to 10,000 measurements a second. It can deliver accurate, concentration-independent results using a patented multiple scattering analysis.

\section{Results and Discussion}

Effect of operating pressure on spray angle of nozzles

The spray angle was calculated at $400 \mathrm{~mm}$ nozzle height for both hollow cone and flat fan nozzles The effect operating pressure on spray angle of the nozzles was described through bar diagrams in the Figs. 3.1 and 3.2.

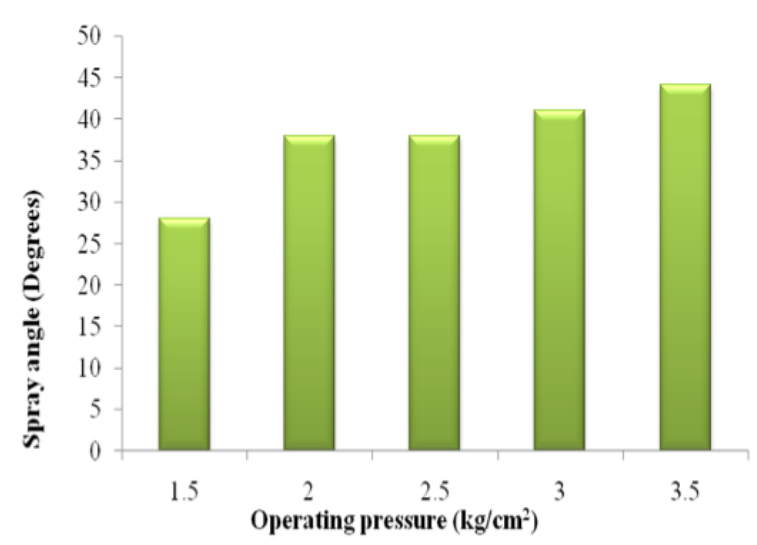

Figure 3.1. Effect of pressure on spray angle of hollow cone nozzle.

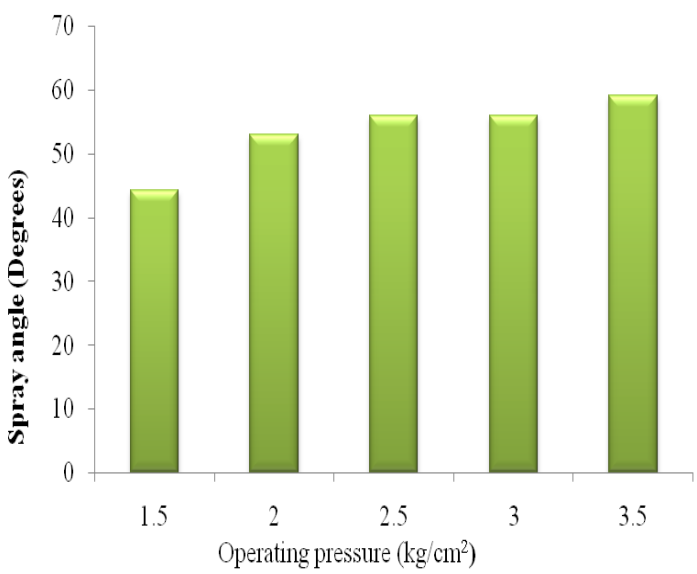

Figure 3.2. Effect of pressure on spray angle of flat fan nozzle. 
Fig. 3.1 shows that as the operating pressure was increased from 1.5 to $3.5 \mathrm{~kg} / \mathrm{cm}^{2}(147.1$ to $343.23 \mathrm{kPa})$, the spray angle for hollow cone nozzle increased from 28 to $44.22^{0}$. It also shows that from $1.5 \mathrm{~kg} / \mathrm{cm}^{2}$ to $2 \mathrm{~kg} / \mathrm{cm}^{2}$ the increase $\left(10^{\circ}\right)$ is sharp and thereafter, the rate of increase is less. The less increase in angle may be due to the fact that, the increase in pressure is gradual and hollow cone nozzles work at higher pressures. The regression analysis for effect of operating pressure on spray angle of hollow cone nozzle shows positive correlation between the factors with $\mathrm{R}$ value of $92.3 \%$. The $\mathrm{R}^{2}$ value $(80.3 \%)$ shows that, spray angle is highly dependent on pressure. The p-value $(<0.05)$ shows that, pressure has significant effect on spray angle.

Fig. 3.2 show that, with increase in operating pressure from 1.5 to $3.5 \mathrm{~kg} / \mathrm{cm}^{2}$ (147.1 to $\left.343.23 \mathrm{kPa}\right)$, the spray angle for flat fan nozzle increased from 44.22 to $59^{\circ}$. Though the increase in value of angle was less, still spray angle varied according to operating pressures. This less increase may be due to gradual increase $\left(0.5 \mathrm{~kg} / \mathrm{cm}^{2}\right)$ in pressure. The regression analysis e for effect of operating pressure on spray angle of flat fan nozzle depicts high positive correlation between pressure and angle. The $\mathrm{R}^{2}$ value of $76.2 \%$ shows the dependency of both the variables. The $\mathrm{p}$-value $(<0.05)$ indicates that, effect of pressure is statistically significant for spray angle.

Effect of operating pressure and height on swath width of nozzles.

Effects of pressure and height on swath of both the nozzles are shown in Figs. 3.3 and 3.4. The maximum swath of $350 \mathrm{~mm}$ for hollow cone nozzle was obtained at $3.5 \mathrm{~kg} / \mathrm{cm}^{2}$ pressure and $600 \mathrm{~mm}$ nozzle height.

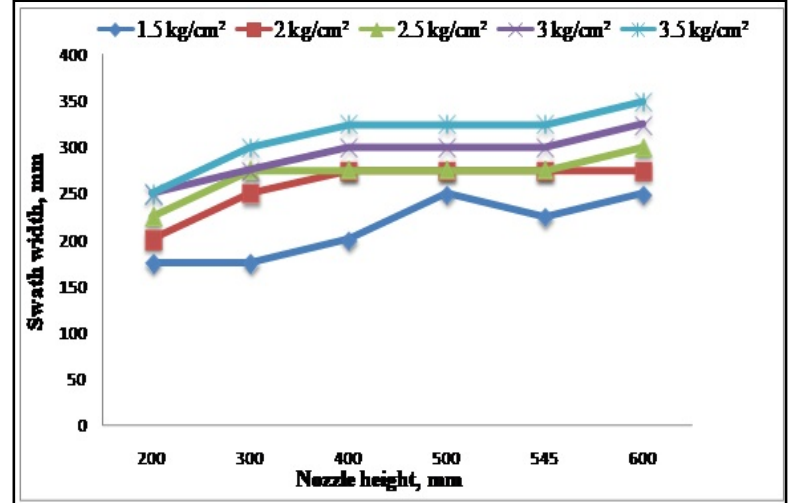

Figure 3.3. Effect of pressure and height on swath of hollow cone nozzle.

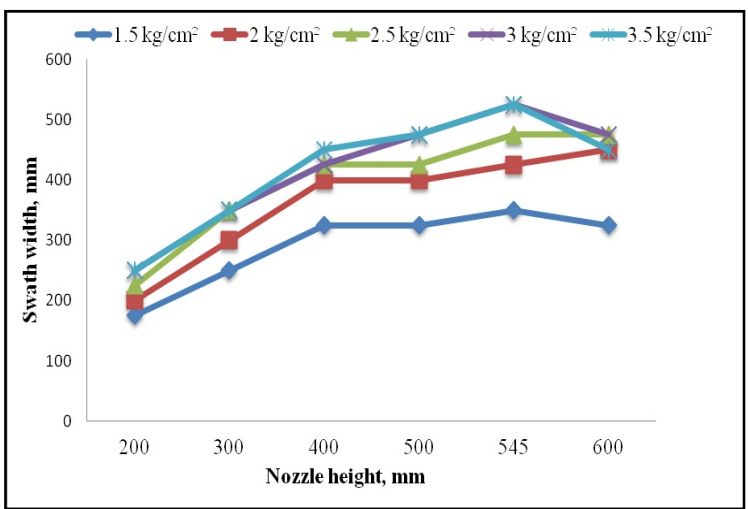

Figure 3.4. Effect of pressure and height on swath of flat fan nozzle.

Fig. 3.4 shows the effect of nozzle height and operating pressure on swath of flat fan nozzles. With increase in pressure the swath increases for all pressures. For operating pressure of $1.5 \mathrm{~kg} / \mathrm{cm}^{2}$ the swath increased from 175 to $325 \mathrm{~mm}$ with increase in height from 200 to $400 \mathrm{~mm}$, and then remained constant for $500 \mathrm{~mm}$ height and again increased to $350 \mathrm{~mm}$ at $545 \mathrm{~mm}$ nozzle height. For 2 and $2.5 \mathrm{~kg} / \mathrm{cm}^{2}$ trend shows an increase of swath with nozzle height then remains same before increasing again. For 3 and $3.5 \mathrm{~kg} / \mathrm{cm}^{2}$ the swath increased from 225 to $525 \mathrm{~mm}$ with increase in nozzle height from 200 $\mathrm{mm}$ to $545 \mathrm{~mm}$ and then decreased to 475 and $450 \mathrm{~mm}$ for both pressures respectively. The maximum swath calculated was $525 \mathrm{~mm}$ for 3 and $3.5 \mathrm{~kg} / \mathrm{cm}^{2}$ at $545 \mathrm{~mm}$ nozzle height. It shows for higher pressures the swath increases with nozzle height up to a certain value and then decreases.

\section{Droplet Size Analysis of Nozzles Using Spraytec Instrument}

Fig. 3.5 depicts the effect of operating pressure on the NMD values of the droplets produced by flat fan nozzle. The number median diameter (NMD) obtained from the flat fan nozzle were 107.6, 25.41, 23.32, 23.21 and 22.8 $\mu$ at operating pressures of $1.5,2,2.5,3$ and $3.5 \mathrm{~kg} / \mathrm{cm}^{2}$, respectively. The NMDs of the flat fan nozzle decreased from 107.6 to 22.8 microns as the pressure was increased from 1.5 to $3.5 \mathrm{~kg} / \mathrm{cm}^{2}$ but after $2 \mathrm{~kg} / \mathrm{cm}^{2}$ the variation in NMD was very less. This shows that after $2 \mathrm{~kg} / \mathrm{cm}^{2}$ pressure there is not much reduction in the NMD value of the droplets produced. The reason might be that after $2 \mathrm{~kg} / \mathrm{cm}^{2}$, more number of smaller droplets were produced from the nozzle resulting in smaller NMD value and after that there was less reduction in the droplet sizes in terms of number with increase of pressure.

There was variation of VMD of the droplets produced from flat fan nozzle with increase of pressure (Fig. 3.6). The VMDs obtained for different operating pressures of $1.5,2,2.5,3$ and $3.5 \mathrm{~kg} / \mathrm{cm}^{2}$ were $199.3,160,140.4,133.9$ and 129.2 $\mu$, respectively. As the operating pressure was increased from 1.5 to $3.5 \mathrm{~kg} / \mathrm{cm}^{2}$ the volume median diameter (VMD) decreased from 199.3 to 129.2 microns. The decrease in value is more pronounced from 1.5 to $2.5 \mathrm{~kg} / \mathrm{cm}^{2}$. After $2.5 \mathrm{~kg} / \mathrm{cm}^{2}$ there is less variation in VMD of the droplets. This shows that after $2.5 \mathrm{~kg} / \mathrm{cm}^{2}$, there was less reduction in the size of droplets produced in terms of volume. Thus, with increase of operating pressure, the VMD of the droplets produced from the nozzles decreases up to a certain size after which the effect of pressure diminished. 


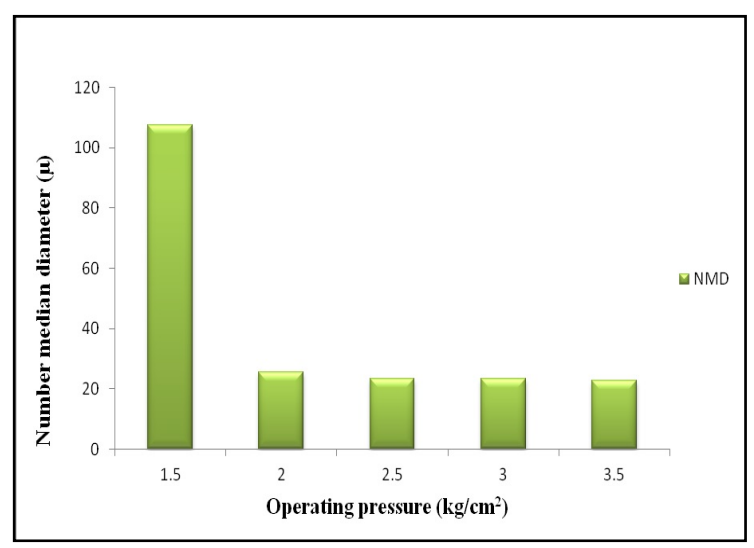

Figure 3.5. Effect of pressure on NMD of flat fan nozzle.

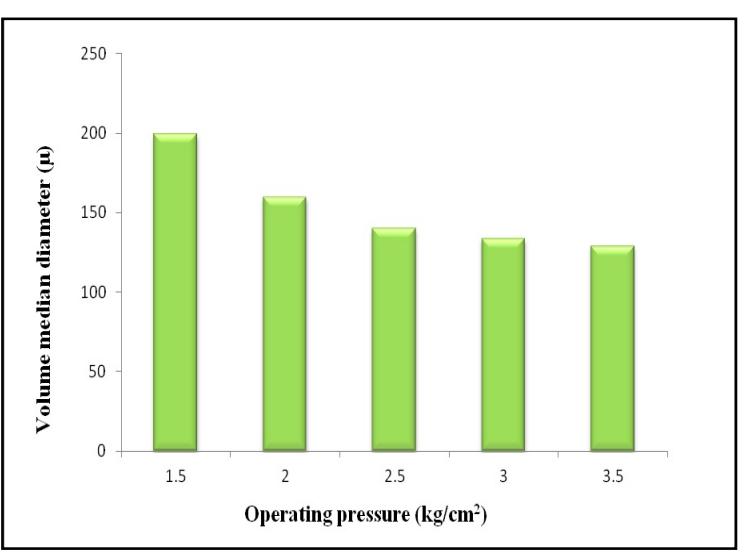

Figure 3.6. Effect of pressure on VMD of flat fan nozzle.

It was observed from Fig. 3.7 that NMD of the hollow cone nozzle decreased from 34.11 to 23.04 microns as the pressure was increased from 1.5 to $3.5 \mathrm{~kg} / \mathrm{cm}^{2}$. It is clearly seen from the figure that, even at $1.5 \mathrm{~kg} / \mathrm{cm}^{2}$ the NMD value of the droplets is quite less and there is less change in the NMD value after $2 \mathrm{~kg} / \mathrm{cm}^{2}$. This can be attributed to the fact that, hollow cone nozzles produce smaller droplets as compared to flat fan nozzles causing lower NMD value. The effect of pressure on NMD also diminished after $2 \mathrm{~kg} / \mathrm{cm}^{2}$ and little variation in the droplet sizes were observed.

Fig. 3.8 depicts the effect of operating pressure on volume median diameter (VMD) of the droplets produced from the hollow cone nozzle at different operating pressures. The VMD value of the hollow cone nozzle decreased from 200 to 113.8 microns as the pressure was increased from 1.5 to $3.5 \mathrm{~kg} / \mathrm{cm}^{2}$. The decrease is much certain from 1.5 to $3 \mathrm{~kg} / \mathrm{cm}^{2}(200$ to $119 \mu)$, and after that there is little variation in the VMD. This shows that as the pressure is increased, the volume of the droplets produced from the nozzles decreases up to a certain value and after which the effect of pressure reduces and there is very little variation in the droplet size.

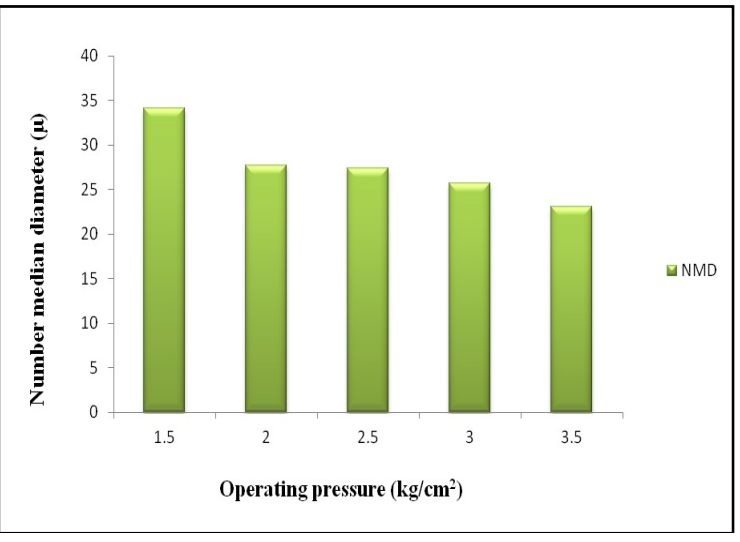

Figure 3.7. Effect of pressure on NMD of hollow cone nozzle.

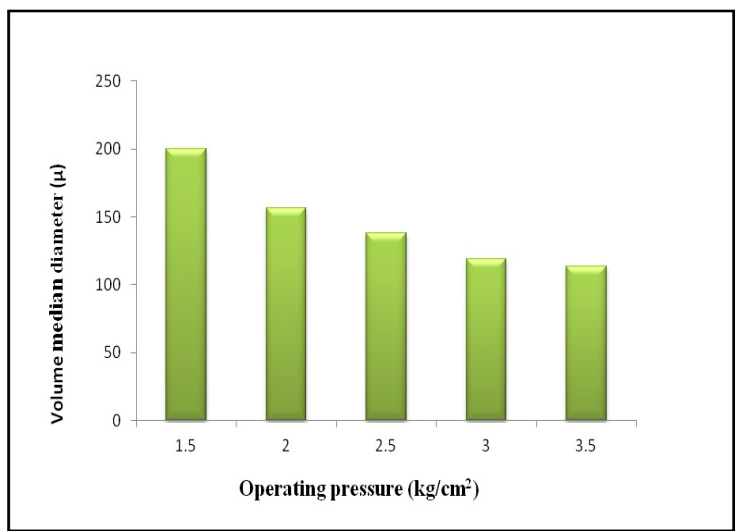

Figure 3.8. Effect of pressure on VMD of hollow cone nozzle. 
The hollow cone nozzle produced droplets ranging from extremely fine to extremely course droplets between 1.5 $\mathrm{kg} / \mathrm{cm}^{2}(147.1 \mathrm{kPa})$, pressure to $3.5 \mathrm{~kg} / \mathrm{cm}^{2}(343.23 \mathrm{kPa})$, while the flat fan produced droplets in the range of extremely fine to very coarse for the above pressures. The range of droplets produced by both hollow cone and flat fan nozzle at different pressures were studied. The hollow cone nozzle generated most of the droplets $(>60 \%)$ in the range of 61 to 340 microns for the pressures ( 1.5 to $3.5 \mathrm{~kg} / \mathrm{cm}^{2}$ ) studied. The data of flat fan nozzle also shows that, most of the droplets produced by the nozzle varied between fine to medium size $(61$ to $340 \mu)$ in-between the operating pressures studied. The droplet size less than $200 \mu$ are considered prone to drift. The nozzle produced some droplets prone to drift. To prevent this low pressure and low boom height should be preferred. The range of droplets produced at various pressures for both the nozzles were classified in Table 3.

Table 3. Classification of droplets according to ASABE standards produced by the nozzles at different operating pressures.

\begin{tabular}{lll}
\hline Operating pressure $\left(\mathrm{kg} / \mathrm{cm}^{2}\right)$ & Hollow cone nozzle & Flat fan nozzle \\
\hline 1.5 & $\mathrm{XF}-\mathrm{XC}$ & $\mathrm{VF}-\mathrm{VC}$ \\
2 & $\mathrm{XF}-\mathrm{C}$ & $\mathrm{XF}-\mathrm{C}$ \\
2.5 & $\mathrm{XF}-\mathrm{C}$ & $\mathrm{XF}-\mathrm{C}$ \\
3 & $\mathrm{XF}-\mathrm{M}$ & $\mathrm{XF}-\mathrm{C}$ \\
3.5 & $\mathrm{XF}-\mathrm{M}$ & $\mathrm{XF}-\mathrm{M}$ \\
\hline
\end{tabular}

\section{Conclusion}

The hollow cone nozzle produced droplets ranging from extremely fine to extremely coarse droplets, while the flat fan produced droplets in the range of extremely fine to very coarse for the pressures between $1.5 \mathrm{~kg} / \mathrm{cm}^{2}$ pressure to $3.5 \mathrm{~kg} / \mathrm{cm}^{2}$. Accordingly, nozzles were selected for development of self propelled sprayer for intra canopy spraying in vegetable row crops.

\section{References} 29.

Agrios, G. N., 2005. Plant Pathology- losses caused by plant diseases, $5^{\text {th }}$ ed. Academic Press, NewYork, p-

Dash, B. S., 2016. Development and performance evaluation of self-propelled boom sprayer for intra canopy spraying in row crops. Unpublished M Tech Thesis. G B Pant University of Agriculture and Technology, Pantnagar, India.

Hand Book on Horticulture Statistics. 2014. Ministry of Agriculture, Department of Agriculture and Cooperation, New Delhi, India. 7 p.

IS 8548 (P 1) 1997 Test Code for Power-Operated Hydraulic Sprayer, New Delhi 


\author{
Hyperspectral Remote Sensing Applications In Raspberry Plantation \\ Boglarka Keller ${ }^{\text {a, }}$, Laszlo Kovacs a, Renata Rak ${ }^{a}$, Andras Jung ${ }^{\text {b }}$, Fanni Sillinger ${ }^{\text {b }}$, Nora Peterfalvi ${ }^{\text {a }}$, \\ Kornel Szalay ${ }^{a}$ \\ a NARIC Institute of Agricultural Engineering, Gödöllő 2100, Hungary \\ b Szent István University, Faculty of Horticulture Science, Technical Department, Budapest 1118, Hungary \\ * Corresponding author. Email: keller.boglarka@mgi.naik.hu
}

\begin{abstract}
In Hungary one of the biggest challenge of raspberry production is the reduction of sunburn related damages (slowing plant growth, yield loss, heat stress, reducing leaf area). Breeding of resilient species is a long period, it can take several years, while the direct physical intervention can be a quick and effective solution. In our experimental area two types of shading nets were applied, in which cases the differences between phenology, morphology and spectral features of plants were investigated by portable hyperspectral remote sensing instruments as snapshot imaging spectroscopy (Cubert Camera) and as non-imaging spectroscopy (ASD FieldSpec Max3). Based on the reflectance curves different vegetation indexes were calculated. Additionally, the chemical features of the leaves were analysed. Preliminary results show significant differences between covered and uncovered plantations where the different species react differently. The first results show close correlation between the cover materials and raspberry yields. Basically more and healthier raspberry yield were occurred in case of covered plantations. During the research the hyperspectral remote sensing proved to be a simple and reliable tool to detect differences between each plantations and species generated by various exposures to sunshine. In the same time further measurements and analysis are necessary to identify the best cover material and production practice.
\end{abstract}

Keywords: raspberry, climate change, shading net, snapshot imaging, hyperspectral remote sensing.

\title{
1. Introduction
}

There has been more and more indication of climate change related stress factors in raspberry plantations all around Hungary in the last couple of years. Farmers regularly experience reduction in plant growth, leaf area, yield and fruit quality. Visual signs of heat stress, sunburn are often registered during the summer periods induced by excessive heat and direct radiation causing decreasing photosynthetic activity of plants and yield losses (Maugan and Black, 2015). Dedicated plant breeding programs has been started to mitigate the effects of climate change (Dénes, 2016) but these programs need long time. Fighting alone by using biological ways is not enough. An immediate action is required to save the raspberry production. A physical protection against excessive direct radiation can be considered as the only way to restore the stability and quality of production on short term. Nevertheless, returning the site of raspberry production to the forests (where the species is originated from) or agroforestry systems (Nagy, 2017) can be also considered as a solution on middle and long term. Combining solar panels with agriculture (Hanley, 2017, Hajdú, 2018) in this particular place can offer an even more reasonable way to solve the question of excessive radiation. An accurately adjusted portion of radiation would be transferred to electricity while the rest can be used by the protected plantation below. In this case the shading system would produce energy which would possibly offer a more sustainable way of fighting against the effects of climate change and energy scarcity (Maughan et al., 2017; Gindaba és Wand, 2007; Kalcsits et al., 2017; Tinyane et al., 2018; Dayioglu és Hepaksoy, 2016). Beside the reduction of direct radiation various shading solutions and applied materials are expected to change the spectral characteristic of incident light and so the light utilisation of plants.

In addition to the different soil analyses (Fenyvesi, 2008, Csorba et al., 2012, Tolner, 2011) spectroscopy allows the examination of plant characteristics. Chlorophyll absorption characteristics, which indicate the changes of phenolic and stress-induced chlorophyll activity (Blackburn and Ferwerda, 2008). Accordingly, the effects of some biochemical components (eg cellulose and lignin) (Houborg and Boegh, 2008) and plant stresses (Thenkabail et al., 2004) affect the short-wave infrared reflectance, which is both quantifiable (Wessman, 1992) with imaging spectrometry (Milics et al., 2008) and not imaging equipment (Virág and Szőke, 2011).

In present study, our aim was to study the beside the traditionally measurable properties the spectral characteristics of plants through hyperspectral remote sensing devices and to isolate the species-specific differences between the covered stocks and within the different stocks.

\section{Materials and Methods}

Experimental area

In order to find a reasonable solution to protect the plants and increase the stability of the production a raspberry plantation with different varieties was established. A sun protective shade tunnel system was erected to 
create a test site at NARIC - Fruitculture Research Institute (FRI), Fruit Culture Research and Development Institute of Fertőd, Hungary. It provides opportunity to measure and evaluate relevant biological and physical parameters playing an important role in berry production (Keller et al., 2018). Experimental plantation (Figure 1) of three different raspberry varieties was set in two repetitions: covered and uncovered versions. Each cover has characteristic interaction with light which is expected to generate different environmental conditions and also differences in plant growth and fruit quality. (Figure 2 and Figure 3).

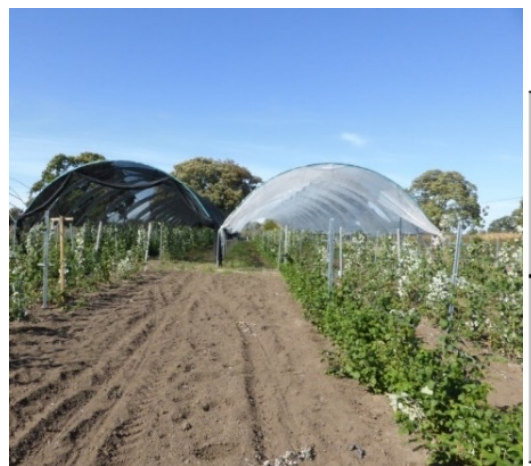

\begin{tabular}{|c|c|c|c|c|c|c|c|c|c|c|}
\hline & \multicolumn{4}{|c|}{ Covered } & & \multicolumn{4}{|c|}{ Uncoverred } \\
\hline & & 1 & \begin{tabular}{|l|l|l}
2 & 3 & 2 \\
\end{tabular} & \begin{tabular}{l|l|l}
4 & 5 & 6 \\
\end{tabular} & \begin{tabular}{l|l}
7 & 8 \\
\end{tabular} & & $1:$ & \begin{tabular}{l|l|l}
2 & 3 & 4 \\
\end{tabular} & \begin{tabular}{|l|l|}
5 & 6 \\
\end{tabular} & \begin{tabular}{|l|l}
7 & 8 \\
\end{tabular} \\
\hline ธ్ & 1. row & \multicolumn{4}{|c|}{ Julcsi } & 1. row & \multicolumn{4}{|c|}{ Julcsi } \\
\hline 莺 & 2. row & \multirow{2}{*}{\multicolumn{4}{|c|}{$\begin{array}{c}\text { Fertödi zamatos } \\
\text { Eszterházai } \\
\text { kétszertermő }\end{array}$}} & 2. row & \multirow{2}{*}{\multicolumn{4}{|c|}{$\begin{array}{c}\text { Fertödi zamatos } \\
\text { Eszterházai } \\
\text { kétszertermő }\end{array}$}} \\
\hline$\stackrel{\frac{\pi}{2}}{=}$ & 3. & & & & & 3. row & & & & \\
\hline 모 & 1. row & \multicolumn{4}{|c|}{ Fertődi zamatos } & 1. row & \multicolumn{4}{|c|}{ Fertödi zamatos } \\
\hline 吾 & 2. row & \multicolumn{4}{|c|}{$\begin{array}{c}\text { Eszterházai } \\
\text { kétszertermő }\end{array}$} & 2. row & \multicolumn{4}{|c|}{$\begin{array}{l}\text { Eszterházai } \\
\text { kétszertermő }\end{array}$} \\
\hline N & 3. row & \multicolumn{4}{|c|}{ Julcsi } & 3. row & \multicolumn{4}{|c|}{ Julcsi } \\
\hline
\end{tabular}

Figure 2 and 3. Illustration of covered and uncovered plantations with the concerned species.

Portable spectral equipments

To measure the spectral conditions (Judd et al., 1964) under the shade nets and the spectral response and features of plants with proximal sensing a portable spectroradimeter and a snapshot hyperspectral camera were used (Figure 4). Portable spectroradiometers can widely be used both in field and laboratory. It is adequate to carry out independent, fast and precise evaluations in an economic way. The VNIR device extends the range of the detectable visible light (Lágymányosi and Szabó, 2009) to NIR (near infrared) and the SWIR (shortwave infrared) region and covers the spectral range of 350 to 2500 [nm] (Csorba et. al, 2014). These spectrometers have successfully mastered several applications; however scanning (Fenyvesi 2008, Fekete et al., 2016) is facing some limitations when the test object or/and camera are randomly or rapidly moving in time and space. To eliminate all these limitations snapshot hyperspectral imaging or spectral frame camera technique (Jung et. al., 2017a) can be successfully applied. In principle, it is capturing the entire hyperspectral image during a single integration time (one shot takes about $1 \mathrm{~ms}$ ). In agricultural field and close-field applications weight and speed have high importance (Jung et al. 2017b).

Portable spectral field measurements were carried out in the control area and under two different types of tunnels. Data acquisition was made by using ASD FieldSpec 3 MAX portable spectroradiometer (350-2500 [nm]) with Plant Probe sensor and Cubert snapshot spectral camera (400-1000 [nm]) on randomly selected leafs (Figure 4). The same reference panel was used as a standard surface reflecting $95 \%$ of all incident radiation for both acquisition methods.
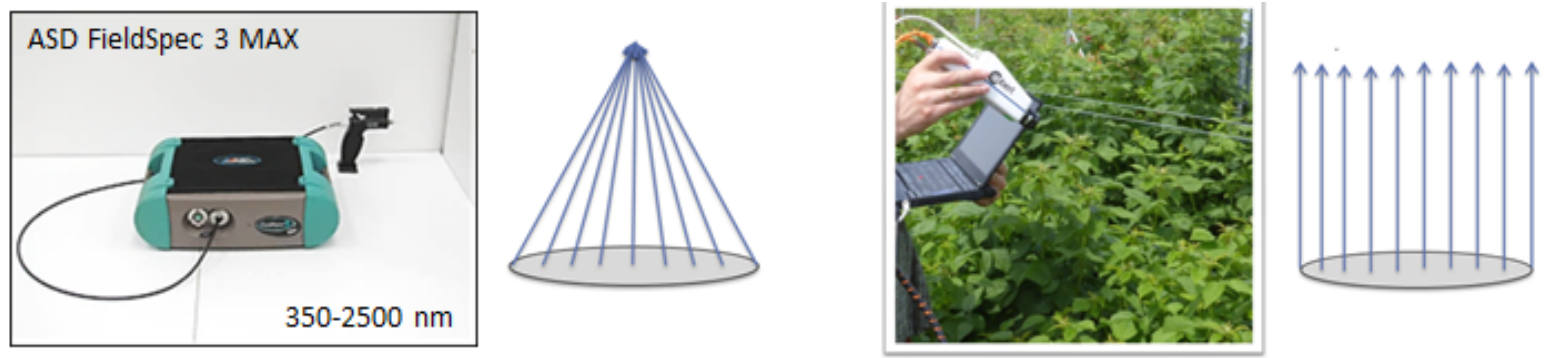

Figure 4. ASD FieldSpec 3 MAX with PlanProbe sensor head and a Cubert snapshot camera.

\section{Equations}

In order to compare the light utilization efficiency, the water and nitrogen management of plants under various shade nets contact measurements was used to calculate vegetation indices such as photochemical reflectance index - PRI (Gamon et al., 1992), water index - WBI (Penuelas et al., 1995), normalized nitrogen index - NDNI ( Serrano et al., 2002) and normalized difference vegetation index - NDVI (Rouse et al., 1973) with the following equations (Figure 5): 


$$
P R I=\frac{\rho_{531}-\rho_{570}}{\rho_{531}+\rho_{570}} \quad W B I=\frac{\rho_{970}}{\rho_{900}} \quad N D N I=\frac{\log \left(\frac{1}{\rho_{1510}}\right)-\log \left(\frac{1}{\rho_{1680}}\right)}{\log \left(\frac{1}{\rho_{1510}}\right)+\log \left(\frac{1}{\rho_{1680}}\right)} \text { NDVI }=\frac{\text { (NIR }- \text { Red })}{(\mathrm{NIR}+\mathrm{Red})}
$$

Figure 5. The applied vegetation indices.

\section{Results and Discussion}

Phenological and chemical properties

Studying the phenological features of strawberry plantation we can stated that the occurring differences are species-specific therefore the effect of shaded nets weren't or just party observable. Well distinguishable discrepancy between the plantation could be observed during the blooming and ripening period. Within the covered plantation the blooming was started and finished earlier, while the ripening process indicated the opposite in case of some species (Eszterházai and Julcsi). In case of Fertödi zamatos the blooming and ripening begun later compared to other species. The blooming and ripening period of covered plantation shifted later. In terms of produce, higher yields were reached in case of covered plantations under white raschel net. The highest yield was measured in case of Eszterházai, which was 16-36\% higher than in outdoor fields. In the case of Julcsi, the yield growing was also occurred under cover with $13-18 \%$ rate. The Fertódi zamatos served weaker results as in outdoor, uncovered plantations. In case of average berries, the volume was different. The largest average berries were found at Julcsi and Eszterházai varieties. The volume of production was doubled compared to 2016, as a result of the growth of the plantation. The species-specific features are visible (Table 1).

Table 1. The mass of whole amount and the average berries during the las two years under different cover system.

\begin{tabular}{lrrrr}
\hline & \multicolumn{2}{c}{2016} & \multicolumn{2}{c}{2017} \\
\cline { 2 - 6 } & $\begin{array}{c}\text { Whole amount } \\
(\mathrm{g})\end{array}$ & $\begin{array}{c}\text { Average berries } \\
(\mathrm{g})\end{array}$ & $\begin{array}{c}\text { Whole amount } \\
(\mathrm{g})\end{array}$ & $\begin{array}{c}\text { Average berries } \\
(\mathrm{g})\end{array}$ \\
\hline White & 156266 & 7,22 & 247707 & 6,74 \\
Black & 89874 & 6,83 & 199904 & 6,52 \\
Outdoor & 85499 & 7,17 & 206167 & 6,31 \\
\hline
\end{tabular}

Spectral characteristic

The processed contact spectral measurement dataset revealed a tendency of differences in case of photochemical reflectance index (PRI). Based on the dataset the light utilisation efficiency of all varieties under shade nets were higher than in the control plantation. Water index (WBI), normalized nitrogen index (NDNI) and normalized vegetation index (NDVI) did not show differences between treatments (Table 2).

Table 2. Different vegetation indices in case of different treatments.

\begin{tabular}{lccr}
\hline & \multicolumn{2}{c}{2017.06 .21} \\
& White & Black & \multicolumn{1}{c}{ Control } \\
\hline PRI & 0,019 & 0,047 & 0,016 \\
WBI & 0,984 & 0,985 & 0,989 \\
NDNI & 0,142 & 0,127 & 0,142 \\
NDVI & 0,822 & 0,830 & 0,822 \\
\hline \multicolumn{4}{c}{2017.08 .02} \\
& White & Black & Control \\
\hline PRI & 0,039 & 0,031 & 0,021 \\
WBI & 1,001 & 0,982 & 0,990 \\
NDNI & 0,138 & 0,139 & 0,150 \\
NDVI & 0,840 & 0,829 & 0,830 \\
\hline
\end{tabular}

It means the soil preparation; nutrient supply and irrigation assure the favourable homogeneity of the plantation and the only variable between treatments really is the difference in illumination. The images acquired with snapshot camera were used to evaluate the shade nets and open sky from below to describe the spectral distribution of the incident light. On the other hand, vegetation survey was performed to visualize reflective features of the vegetation and also the heterogeneity arising within plants. Differences between ratios of significant ranges playing important role in vegetation monitoring to indicate alteration of plant condition (Figure 6-7). 

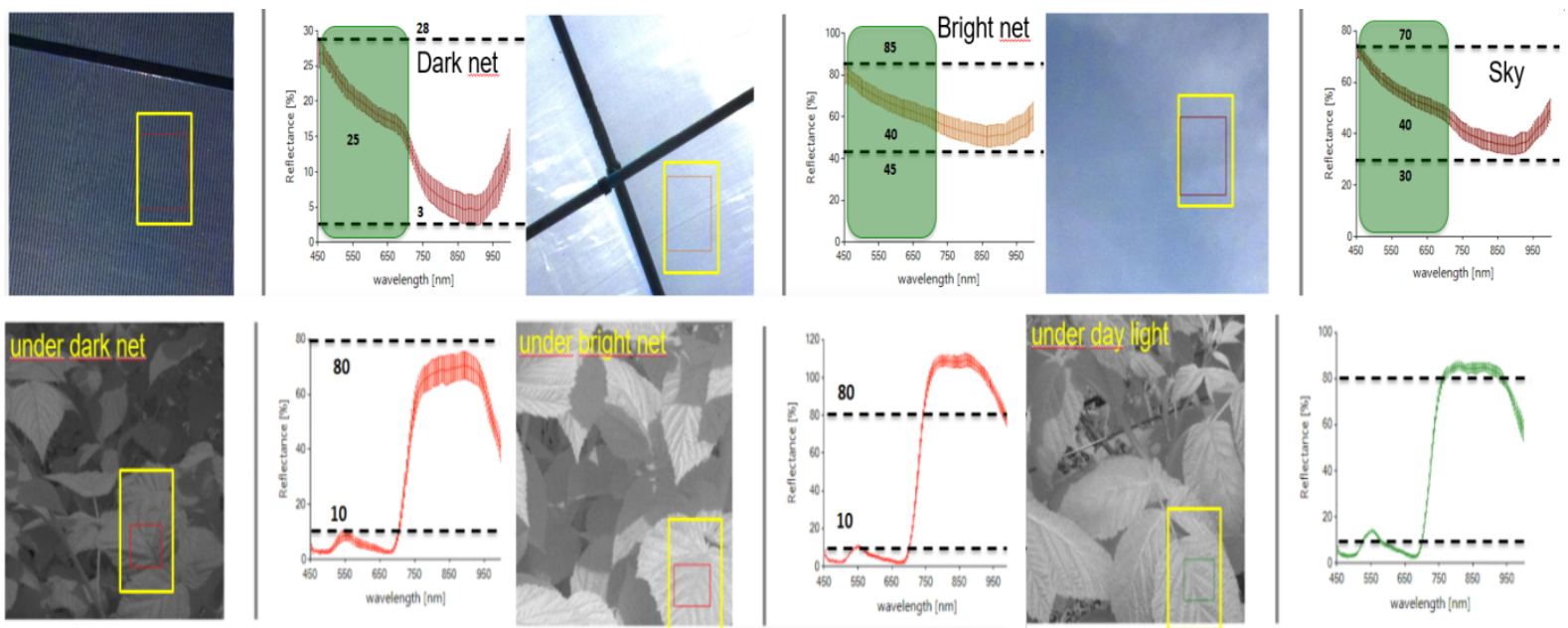

Figure 6 and 7. Hyperspectral images describing the incident and reflected radiation in the VIS and NIR region.

Generally true that the main indicators of different efficiency of production technologies are yield and fruit quality. Within the frame of the research correlations between shade nets and fruit size was observed. Julcsi reacted positively to the white coloured shade net in two consecutive years with extra 72 [\%] (2016) and 24 [\%] of yield. Eszterházai kétszertermö reacted with extra 140 [\%] (2016) and 104 [\%] of yield moreover with extra fruit size 4,6 [\%] (2016) and 36,69 [\%] (2017). Fertödi zamatos did not show such a significant reaction to the treatment.

\section{Conclusions}

A portable spectroradiometer and a snapshot hyperspectral camera with more than 100 spectral channels were used to spectrally map a raspberry plantation under different type of shade nets. It was also a purpose to test and document the usability, flexibility of such techniques in collecting spectro-phenological parameters in a handheld way. An in-situ ambient monitoring system was also set up to collect temperature, humidity and radiation data. Spectral techniques provided opportunity to reveal such differences in natural light conditions that are usually not detected by traditional weather stations and make possible to study the correlation between light condition and plant growth in a more complex way.

The results show significant differences between plantations with or without shade net cover. Measured ambient parameters and spectral analysis of the vegetation revealed differences in plant condition and indicated the effect of shade nets and also the differences between the two experimental shade net materials. Shade nets can increase the yield and also increase the average berry size but the reaction to shade nets seems to be varietyspecific. Yield reacted very positively to white shade nets in case of Eszterházai kétszertermö and Julcsi varieties, in two consecutive years. Fruit size reacted significantly positively in 2017. In case of Fertödi zamatos variety significant positive effect of shade materials was not confirmed. Variety-specific shade net-based production technology can offer a solution to improve yield, quality and production stability of raspberry. The tunnel system can be quickly deployed so it offers an immediate action to mitigate the effects of climate changes. It can also serve as an intermediate step towards agroforestry systems or remain a complementary technique with options to be used as spectral filters adjusted to plant needs or physical barriers for pests or to create a microclimate. Although the first synthesises already show useful correlations for the practice further crop years and additional measurements, analyses are necessary to identify the best production practice.

\section{Acknowledgements}

Authors would like to express their acknowledgements to the Hungarian Ministry of Agriculture to fund this research project and to colleagues at the NARIC - Fruitculture Research Institute (FRI), Fruit Culture Research and Development Institute of Fertőd, Hungary to measure and evaluate relevant biological parameters and for the everyday hard work to maintain and preserve the experimental site. Our special thanks go to Cubert $\mathrm{GmbH}$, Germany, which made it possible to use and test the hyperspectral frame camera. Thank goes to the János Bolyai Research Scholarship of the Hungarian Academy of Sciences to support András Jung's participation in the research project. 


\section{References}

Blackburn, G. A. - Ferwerda, J. G. (2008). Retrieval of chlorophyll concentration from leaf reflectance spectra using wavelet analysis. Remote Sensing of Environment, 112, 1614-1632

Csorba Á. - Láng V. - Fenyvesi L. - Michéli E. (2012): Reflektancia spektroszkópia alkalmazása talajok szervesszén- és CaCO3-tartalmának becslésében. Agrokémia és Talajtan. 6/2, 277-290. o.

Csorba A., Lang V., Fenyvesi L., Micheli E. (2014) Identification of WRB Soil Classification Units from VisNir Spectral Signatures. In: Pil Joo Kim, Hang-Won Kang (Eds.) 20th World Congress of Soil Science In Commemoration of the 90th Anniversary of the IUSS: Soils Embrace Life and Universe. Jeju, South-Korea 06.082014.06.13. Jeju: International Union of Soil Sciences (IUSS), Paper P4-539

Dayioglu, A. and Hepaksoy, S. (2016): Effects of shading nets on sunburn and quality of 'Granny Smith' apple fruits. Acta Hort. (ISHS) 1139:523-528

Dénes F. (2016) Szamócatechnológia és fajtakísérletek Fertődön. Kertészet és Szőlészet 2016.08.31. 65. évf. 35. szám, 18-20.

Fekete Gy., Issa I., Tolner L., Czinkota I., Tolner I. T. (2016) Investigation on the indirect correlation and synergistic effects of soil pH and moisture content detected by remote sensing. Növénytermelés (Suppl) 203-206 pp. 5th Alps-Adria Scientific Workshop. Mali Lošinj, Croatia

Fenyvesi L. (2008): Characterization of the soil - plant condition with hyperspectral analysis of the leaf and land surface, Cereal Res. Com., (Supp 5) 659-663 pp.

Gindaba J. - Wand S.J.E. (2007): Do fruit sunburn control measures affect leaf photosynthetic rate and stomatal conductance in 'Royal Gala' apple?, In Environmental and Experimental Botany, Volume 59, Issue 2, 2007, 160-165. pp

Hajdú J. (2018) Agro-Fotovoltaik berendezések a mezőgazdaságban. Mezőgazdasági Technika LIX. 2018.04. pp. $18-19$

Hanley S. (2017) Combining solar panels with agriculture makes land more productive. CleanTechnica, November 24th, https://cleantechnica.com/2017/11/24/combining-solar-panels-agriculture-makes-landproductive/

Houborg, R. - Boegh, E. (2008). Mapping leaf chlorophyll and leaf area index using inverse and forward canopy reflectance modelling and SPOT reflectance data. Remote Sensing of Environment, 112, 186-202

Judd D. B., MacAdam D. L., Wyszecld G., "Spectral distribution of typical daylight as a function of correlated color temperature,” J. Opt. Soc. Am., 54: 1031-1040 (1964).

Jung A., Dénes F., Kovács L., Szalay K. (2017b) Snapshot Imaging Spectroscopy to Characterize Radiation Scenarios in an Experimental Raspberry Plantation.10th Earsel SIG Imaging Spectroscopy Workshop.

Jung A., Dénes F., Nagy G., Kovács L., Rák R., Szabó B., Nagy J., Deákvári J., Rowlands A., Szalay K. (2017a) Snapshot Hyperspectral Imaging: Hand-held Image Acquisition for Ground Inspection.

Kalcsits L. - Musacchi S. - Layne D.R. - Schmidt T. - Mupambi G. - Serra S. - Mendoza M. - Asteggiano L. - Jarolmasjed S. - Sankaran S. - Khot L.R. - Espinoza C.Z. (2017): Above and below-ground environmental changes associated with the use of photoselective protective netting to reduce sunburn in apple, In Agricultural and Forest Meteorology, Volumes 237-238, 2017, 9-17 pp.

Keller B.; Jung A., Nagy G. M., Dénes F., Péterfalvi N., Szalay K. D. (2018): Hiperspektrális távérzékelés alkalmazási lehetőségeinek bemutatása egy málna ültetvény példáján keresztül. NAIK - Gödöllő. 63-72. pp. ISBN 978-615-5748-09-7

Kollányi G. - Szalay K. (2016) Fizikai paraméterek műszeres mérésének előzetes eredményei. Szamóca és málna fedett termesztése - tanácskozás és technológiai bemutató, Sarród 2016.

Lágymányosi A. and Szabó I., (2009) Calibration procedure for digital imaging, Synergy and Technical Development, Gödöllő, Hungary, 30. Synergy2009 CD-ROM Proceedings, 2009.

Maughan, T. - Black, B.:(2015): Raspberry Management for Utah. Horticulture/Fruit/2015-03pr. https://digitalcommons.usu.edu/extension curall/700/

Maughan T. - Drost D. - Black B. - Day S. (2017): Using Shade for Fruit and Vegetable Production. https://extension.usu.edu/productionhort/files-ou/UsingShadeforFruitandVegetableProduction.pdf

Milics G. - Burai P. - Lénárt Cs. (2008): Pre-Harvest Prediction of spring barley nitrogen content using hyperspectral imaging. Cereal Research Communications, Akadémiai Kiadó, Volume 36, $1863-1866$ p. Proceedings of the VII. Alps-Adria Scientific Workshop. Szlovákia, Stara Lesna. 2008. április 28. - május 2.

Nagy G.M. (2017) Agroforestry - A new start in mixed-use plantations, XVII. International Conference of Forests of Eurasia, Kazan, Oroszország, 2017. 10. 02-07..

Thenkabail, P.S. - Enclona, E. A. - Ashton, M. S. - Van Der Meer, V. (2004). Accuracy assessments of hyperspectral waveband performance for vegetation analysis applications. Remote Sensing of Environment, 91, 354-376

Tinyane P.P - Soundy P. - Sivakumar D. (2018): Growing 'Hass' avocado fruit under different coloured shade netting improves the marketable yield and affects fruit ripening, In Scientia Horticulturae, Volume 230, 43-49. pp. 
Virág I. - Szőke Cs. (2011): Filed and laboratory examinations of corn plants by means of hyperspectral imaging. 10 th Alps-Adria Scientific Workshop. Növénytermelés. Opatija, Croatia 69-72 pp

Wessman, C. A., 1992: Imaging spectrometry for remote sensing of ecosystem processes. Advances in Space Research 12(7): 361-368 


\title{
Analysis of Different Mechanical Pruning Strategies on the Production of 'Clemenules' Mandarin and its Costs
}

\author{
Guillermo Mateua, Antonio Torregrosa ${ }^{\text {b }}$, Patricia Chueca ${ }^{a, *}$ \\ ${ }^{a}$ Centro de Agroingeniería. Instituto Valenciano de Investigaciones Agrarias. Ctra. CV- 315, km. 10. 46113 \\ Moncada (Valencia). España \\ ${ }^{\mathrm{b}}$ Universitat Politècnica de València-DIRA. Camino de Vera s/n 46022 Valencia. España \\ * Corresponding author. Email: chueca_pat@gva.es
}

\begin{abstract}
Spain is the sixth citrus producer in the world and the first exporter for fresh consumption. However, the profitability of citriculture is going down due to the increasing production costs and the low prices perceived by farmers. Between farming operations, pruning is done manually, which represent around $12.5 \%$ of the total production costs. The objective of this study was to analyse the effect of different mechanical pruning strategies on the production of mandarins variety 'Clemenules' comparing them with manual pruning, and to determine their costs. A trial with a random blocks experimental design with 5 repetitions was carried out in a commercial Clemenules orchard located in Chiva (Valencia). During season 2017, eight pruning strategies were assessed: C (control, no pruning); M (manual pruning, with hand saw and pruning shears); and six mechanical pruning with disc pruner: TEW (Topping, Hedging both sides, East and West), TR (Topping, Manual follow-up), TER (Topping, Hedging Est side, Manual follow-up), TE (Topping, Hedging Est side), EW (Hedging both sides) and $\mathrm{E}$ (Hedging Est side). The results of trials showed differences in production and costs between the pruning strategies. C was significantly the most productive with $157 \mathrm{~kg} /$ tree; the following more productive were M, EW and $\mathrm{E}$ ranging between 128 and $132 \mathrm{~kg} /$ tree with no differences between them; and in a third level were all treatments where Topping was carried out (TEW, TE, TER and TR) that oscillate between 72 and $85 \mathrm{~kg} / \mathrm{tree}$ without significant differences between them. Costs were higher for treatments with manual pruning. Cost for $\mathrm{M}$ was $441 € /$ ha and for the treatments with manual follow-up pruning they oscillated between 459 and $293 € /$ ha. However, the cost for mechanical pruning ranged between 25 to $110 € /$ ha depending on the number of machine passes. To get more consistent conclusions, more seasons comparing pruning strategies must be performed.
\end{abstract}

Keywords: disc pruner, mechanization, work capacity, citrus

\section{Introduction}

Spain is the sixth producer of citrus fruits worldwide and the first exporter for fresh consumption, to which it allocates around 50\% of its production (MAGRAMA, 2015). Therefore, citrus fruits are a key product in the Spanish agrarian economy. Despite the social and economic importance of the cultivation of citrus fruits in the Valencian Community, serious problems of profitability are manifest; for example, the abandonment of farm explotations. In fact, the useful agricultural area (UAA) has decreased from 2003 to 2013 by $9 \%$. The lack of profitability is mainly due to excessive production costs. In Spain, production costs are very high and much higher than those of competing countries in foreign markets, such as the USA (Florida or California), or even countries that compete directly in the European market such as Morocco, Egypt or Israel (Juste et al., 2000). The main cause is the excess parcelling of agricultural land present in the Valencian Community, where approximately $28.5 \%$ of farms have sizes of $0.1-1$ ha and $50 \%$ of 1-5 ha.. In this context, reduction of production costs is the only tool available to the farmer to increase their profitability. That reduction could be achieved via the mechanization of the crop.

Pruning is a fundamental task for proper physiological development of citrus fruits. It consists of eliminating less productive or damaged branches, which leads to a better distribution of nutrients in the plant that reduces the amount of fruit produced, but increases its size and, therefore, its quality. In addition, the amount of light and aeration in the tree's crown is increased.

In Spain, this task has traditionally been done manually, gathering together a significant number of workers, which means an increase in production costs. The cost of pruning can amount to $21.6 \%$ of total production costs, not considering the harvest (Mateu., et al 2018).

In the USA, investigation into mechanical pruning began in the 1950s. According to Moore (1958) mechanical pruning supplemented with manual pruning could reduce costs by $30-50 \%$ without affecting the production or quality of lemons. In Spain, Zaragoza et al $(1980,1981)$ conducted the first experiments with mechanical pruning. They observed that, in general, yields from trees pruned both manually and mechanically decreased compared to the controls (without pruning), but the following year, when trees were not pruned, on average the yields were equal for all the treatments for the two biennia the trials lasted. Production in all the pruning treatments on 'Washington Navel' orange trees was lower than the unpruned treatments by $14 \%$. No differences were observed 
between the trees pruned by hand and those that were pruned mechanically. However, there was no difference between unpruned trees and trees pruned by hand for 'Salustiana' oranges, but there was a 17\% reduction in the yield of those that were mechanically pruned compared to those that were not pruned. It is demonstrated that in the year in which mechanical pruning is practiced, production is reduced, but if the effect of mechanical pruning is analysed over several years, this effect is diluted because the tree compensates, in the years that it is not pruned, for the production lost in the years when it was mechanically pruned (Zaragoza and Alonso, 1980, Fallahi and Kilby, 1997, Kallsen, 2005, Rouse et al., 2006, Sauls, 2008, Mendoça et al., 2008, Yildirim et al., 2010; MartínGórriz et al., 2014). However, few studies have been conducted on the influence of mechanized pruning in Spain and its effect on the different citrus varieties cultivated in the Valencian Community is unknown.

The objective of this study is to study the effect of different strategies of mechanical pruning versus manual pruning on the production and quality of 'Clemenules' variety mandarin oranges, the most important mandarin, and to determine their costs.

\section{Materials and methods}

The trial was carried out in a commercial plot of 'Clemenules' variety mandarin oranges of 3.4 ha located in Chiva (Valencia) 39²6'30"N, $0^{\circ} 32^{\prime} 60^{\prime \prime} \mathrm{W}$. The trees were planted with $6 \mathrm{~m}$ by $3 \mathrm{~m}$ row and tree spacing and over raised beds. Prior to the trial, all the treatments had received a pruning of the tree skirts.

The experimental design consisted of a random block design with five repetitions. Eight different treatments or pruning strategies were carried out: C (Control, no pruning), M (manual pruning, with hand saw and pruning shears), TR (Topping, Manual follow-up), TER (Topping, Hedging Est side, Manual follow-up), TE (Topping, Hedging Est side), TEW (Topping, Hedging both sides, East and West), EW (Hedging both sides) and E (Hedging Est side). A minimum of 20 trees was used per treatment and repetition, except the control, which was 5 trees. Two trees were selected at random in each treatment and repetition as an experimental unit for evaluation of production. A pruner with five cutting discs (Model Jumar FH666, Jumar agrícola S.L, La Rioja, Spain) and a tractor Landini 90 REX 90 CV (Landini, Fabbrico, Italy) were used to perform mechanical pruning. Topping was done with two horizontal cuts, one for each side of the crown, to cover the entire crown, and hedging consisted of one cut on the side corresponding to the treatment.

In the case of mechanized pruning, the times used to make the different types of cuts were quantified during the pruning treatments and were used to determine the costs of the pruning operation for each strategy. The times for treatment were attained by totalling the times used in making the various cuts. In the case of manual pruning, the time it took for an operator to perform pruning per tree was quantified.

After pruning the trees, the characterization of the biomass pruned was carried out by measuring the following variables:

- $\quad$ Length of the branches cut according to the type of cut $(\mathrm{cm})$. One hundred branches per type of cut were measured.

- Diameter of the cut branches according to the type of cut ( $\mathrm{mm})$. One hundred branches were measured per type of cut.

- Biomass attained depending on the treatment $(\mathrm{kg} /$ tree $)$. The cut branches were weighed according to the type of cut made with a digital dynamometer (Advanced Force Gauge 500 N, Mecmesin, England,

U.K.) and the weights obtained in the different types of cuts were totalled to obtain the total biomass per strategy.

To study the effect of pruning strategies on production, absolute production ( $\mathrm{kg} / \mathrm{tree})$ was estimated in the two trees of the sample unit by treatment and repetition. To do so, the fruit harvested was weighed with the same digital dynamometer used for the biomass. In addition, the outer diameter calibre of a total of 50 fruits per treatment and repetition $(\mathrm{mm})$ was measured with a digital calliper.

A simple variance analysis (ANOVA) was performed to study the effect of different pruning strategies on the response variables of absolute production and calibre. In all the analyses, it was verified that the data complied with the hypothesis of homoscedasticity by means of the Levene Test. The normality of the data was checked by analysing residuals on normal probability paper. The Least Square Differences (LSD) test was used to compare the means of the treatments. In all analyses, the confidence level was considered as $95 \%$. A descriptive statistical analysis was carried out to study the biomass variable.

To obtain the theoretical work capacities, the time ( $\mathrm{min}$ ) used to prune a tree or to carry out the manual followup of half a tree or a complete tree was used in cases of manual pruning, and in the case of mechanized pruning, the time used in pruning at height or laterally at a known distance and knowing the number of trees in that distance was measured. The time used to make a pruning cut on a tree was calculated. Once the time spent per tree was attained, the number of trees pruned per hour (tree/hour) was calculated. To calculate costs, the cost of the pruner was considered at $7.88 € / \mathrm{h}$, the cost of the tractor with the pruner and tractor operator at $€ 39 / \mathrm{h}$ and a total of 556 trees/ha. 
The following formula was used to obtain costs:

$$
\text { Costs }\left(\frac{€}{\text { ha }}\right)=\frac{1}{\text { TWC }} \times \mathrm{C} \times \mathrm{N}
$$

Where: TWC is the theoretical working capacity (tree/h); $\mathrm{C}$ is the cost in $€ / \mathrm{h}$ of the machinery, plus the tractor operator or the pruners and $\mathrm{N}$ the number of trees/ha. The total costs were calculated by totalling the costs of manual and mechanical pruning.

\section{Results and discussion}

Characterization of biomass pruned

In the manual pruning treatments, the diameter of cut of the branches for $75 \%$ of the values are between 10 $20 \mathrm{~cm}$; maximum values were between 30 and $40 \mathrm{~cm}$, whereas for the cuts with a disc pruner, the thickness of $75 \%$ of the cut branches oscillated between $6-10 \mathrm{~cm}$ and the maximum was between $16-18 \mathrm{~cm}$. The values of diameter of cut braches for lateral and topping pruning were similar (Figure 1).

Similar to the cut diameter, the lengths of the cut branches are much greater for manual pruning, where $75 \%$ of the values are between 80 and $140 \mathrm{~cm}$ whereas $75 \%$ of the lengths of the branches cut with the pruner varies between 40-60 cm with similar values for hedging and topping (Figure 2). This is because the pruner accesses the interior parts of the tree and makes the cuts from the inside on main branches of greater thickness and length, while the disc pruner has no access to the interior and only cuts the twigs that are on the outside.

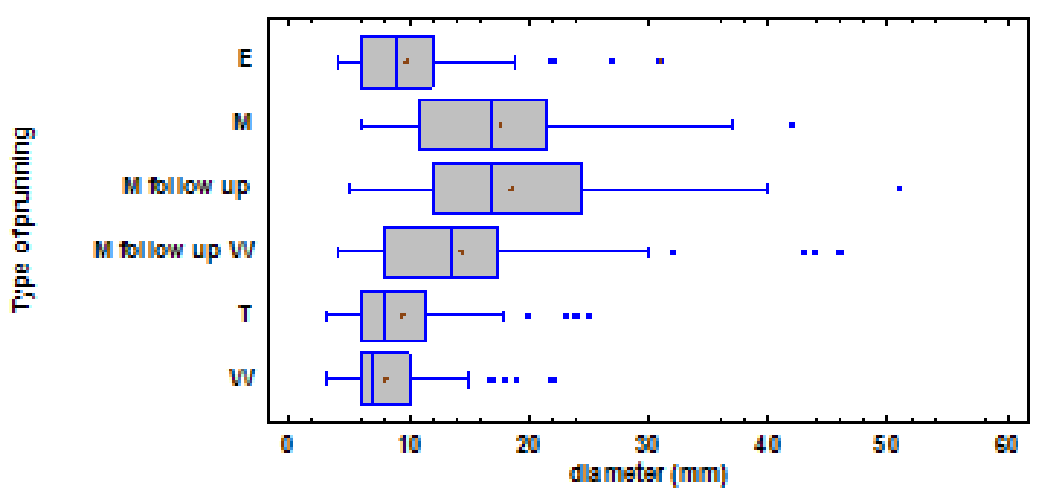

Figure 45. Diagram of boxes and whiskers of the cut diameter of the branches for each type of cut. C: Control; M: Manual prunning; TR: Topping, Manual Follow-up; TER: Topping, Hedging Est Side, Manual follow-up; TEW: Topping, Hedging both sides, East and West; TE: Topping, Hedging Est side; EW: Hedging both sides; E: Hedging Est side.

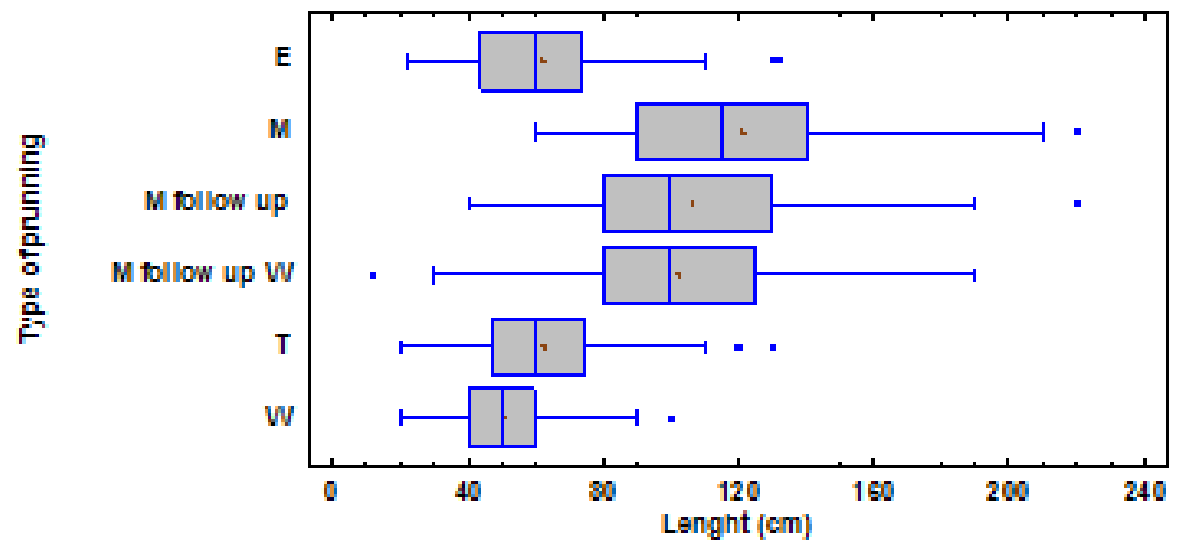

Figure 46. Diagram of boxes and whiskers of the cut length of the branches for each type of cut. C: Control; M: Manual prunning; TR: Topping, Manual Follow-up; TER: Topping, Hedging Est Side, Manual follow-up; TEW: Topping, Hedging both sides, East and West; TE: Topping, Hedging Est side; EW: Hedging both sides; E: Hedging Est side.

Table 77 shows the biomass obtained from the different treatments carried out. As expected, in the treatments that received manual pruning, whether manual pruning or a revision of manual pruning, the biomass is greater than in the treatments carried out with the disc pruner. In general, the biomass value for each pass of the mechanical prunner is around $4 \mathrm{~kg} /$ tree, whereas the biomass in the manual pruning of the total tree is 5 times higher. 
Table 77. Mean biomass per tree depending on treatment. C: Control; M: Manual prunning; TR: Topping, Manual Followup; TER: Topping, Hedging Est Side, Manual follow-up; TEW: Topping, Hedging both sides, East and West; TE: Topping, Hedging Est side; EW: Hedging both sides; E: Hedging Est side.

\begin{tabular}{cc}
\hline Treatment & Mean biomass $(\mathrm{kg} /$ tree $)$ \\
\hline C & 0 \\
M & 25.17 \\
TR & 22.09 \\
TER & 18.34 \\
TEW & 12.44 \\
TE & 8.014 \\
EW & 5.51 \\
E & 4.30 \\
\hline
\end{tabular}

Pruning time, theoretical work capacity and costs

The working speed of the tractor was $2.1 \mathrm{~km} / \mathrm{h}$ regardless of whether it was topping or hedging; hence, each pruning cut, for the given tree spacing, was $0.07 \mathrm{~min} /$ tree and it will take a total 0.31 minutes/tree to prune a tree mechanically on both sides plus topping (TWE). The time used to perform the manual pruning was $6.06 \mathrm{~min} /$ tree whereas the time used to perform the manual follow-up of the entire tree was $5.59 \mathrm{~min} /$ tree and the manual followup of half a tree was $2.93 \mathrm{~min} /$ tree. Table 78 shows the times used for each of the treatments, as well as the theoretical work capacities and costs. It can be observed how the TR treatment presents a higher cost than the M treatment, making evident that manual follow-up after mechanized pruning is not a viable option, since the time used by the pruner is practically the same as in the $\mathrm{M}$ treatment. When manual follow-up is done to only half a tree (TER) the cost is reduced by $34 \%$ compared to the $75 \%$ reduction in the TEW treatment. On the other hand, the reduction of the cost between mechanical pruning ( $€ 25$ to $110 /$ ha depending on the number of the pruner's cuts) and manual pruning ( $€ 441 / \mathrm{ha})$ is very high being on the order of $75 \%$ in the case of making three cuts, and $80 \%$ if only one side and topping are pruned, and $94 \%$ if a single cut is made.

Table 78. Pruning time, theoretical work capacity and costs based on treatment type. C: Control; M: Manual prunning; TR: Topping, Manual Follow-up; TER: Topping, Hedging Est Side, Manual follow-up; TEW: Topping, Hedging both sides, East and West; TE: Topping, Hedging Est side; EW: Hedging both sides; E: Hedging Est side.

\begin{tabular}{|c|c|c|c|c|}
\hline Treatment & Mean time & T.W.C & T.W.C mecanizada & Costs (€/ha) \\
\hline $\mathrm{C}$ & 0 & 0 & 0 & 0 \\
\hline M & 6.06 & 9.9 & 0 & 441 \\
\hline TR & 5.75 & 10.9 & 361.4 & 459 \\
\hline TER & 3.16 & 21.1 & 254.3 & 293 \\
\hline TEW & 0.31 & 0 & 196.1 & 110 \\
\hline TE & 0.24 & 0 & 254.3 & 85 \\
\hline EW & 0.14 & 0 & 428.6 & 50 \\
\hline $\mathrm{E}$ & 0.07 & 0 & 857.1 & 25 \\
\hline
\end{tabular}

Fruit calibre and production

Figure 3 shows the mean calibres of the fruit in the different treatments; although there were significant differences between the different treatments, they are all included in the same commercial category. Therefore, there would be no depreciation in the price.

Regarding the production of all the treatments that received pruning, they reduced production compared to the control treatment. On the other hand, in treatments where pruning at topping was carried out, a decrease in production was observed in comparison to treatments that did not receive this type of pruning, whether it is manual, mechanized pruning or treatment C (Figure 48). No significant differences were observed between the treatments without topping. 


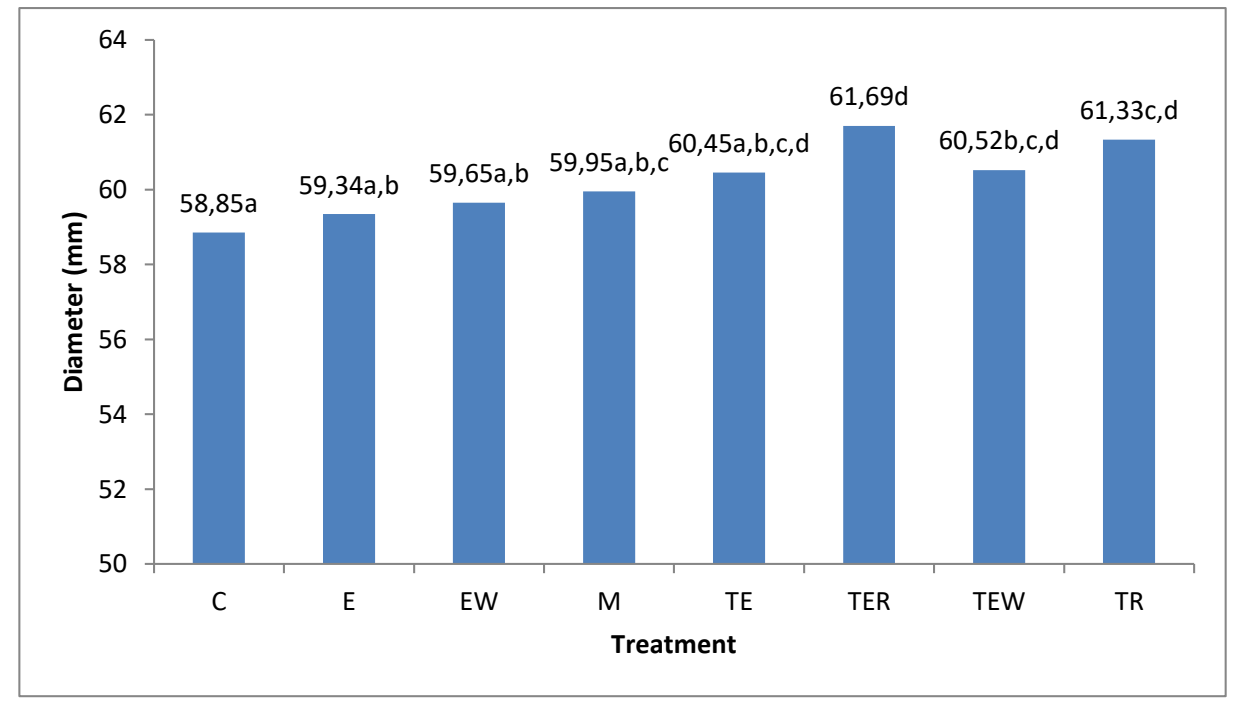

Figure 179. Mean calibres of the fruit $(\mathrm{mm})$ depending on the pruning treatment. Different letters show significant differences between treatments (LSD, $\mathrm{p}<0.05$ ). C: Control; M: Manual prunning; TR: Topping, Manual Follow-up; TER: Topping, Hedging Est Side, Manual follow-up; TEW: Topping, Hedging both sides, East and West; TE: Topping, Hedging Est side; EW: Hedging both sides; E: Hedging Est side.

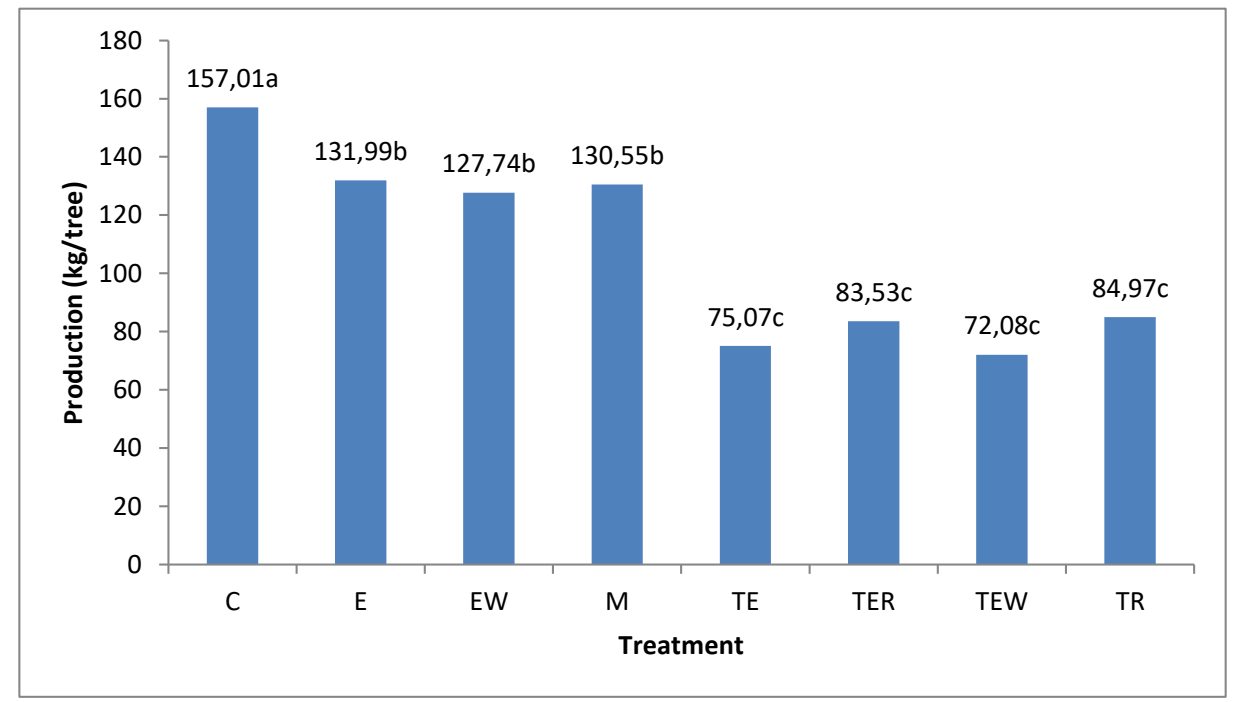

Figure 48. Mean absolute production per tree (kg/tree) based on treatment. Different letters show significant differences between treatment (LSD, p<0.05). C: Control; M: Manual prunning; TR: Topping, Manual Follow-up; TER: Topping, Hedging Est Side, Manual follow-up; TEW: Topping, Hedging both sides, East and West; TE: Topping, Hedging Est side; EW: Hedging both sides; E: Hedging Est side. 


\section{Conclusions}

The highest yields were obtained in treatment $\mathrm{C}$ followed by those obtained in treatments $\mathrm{E}$ and $\mathrm{M}$. If we observe the calibres, the highest is in the TER treatment. Although there is variation in the size of the fruit, the calibres obtained are all in the same commercial category, which means there is no depreciation of the commercial value of the fruit. The totally mechanized pruning implies a $75 \%$ reduction in cost compared to manual pruning.

As has been observed for the 'Clemenules' variety, all treatments in which mechanical pruning was performed at topping had lower production. For that reason, the possibility of combining manual pruning and mechanized lateral pruning in different campaigns could be considered.

These data were attained in a single year; to be able to draw conclusions, it is necessary to repeat this test over several years to observe the performance of this variety with mechanized pruning and its influence on fruit production and quality.

\section{Acknowledgements:}

This study has been conducted within the project "Application of new technologies for a comprehensive strategy of mechanized citrus harvesting (CITRUSREC)" funded by the Spanish National Institute for Agriculture and Food Research and Technology (INIA) and the Ministry of Economy, Industry and Competitiveness of Spain (project RTA2014-00025-C05-00) and co-funded by the European Regional Development Fund (ERDF). We would like to thank La Reva Citrus/Agrimarba group for ceding the experimental plot and their collaboration. Guillermo Mateu is a beneficiary of a scholarship for training and specialization of the European Social Fund (ESF).

\section{References}

Fallahi, E., Kilby, M. 1997. Rootstock and pruning influence on yield and fruit quality of 'Lisbon' lemon. Fruit Varieties Journal 51: 242-246.

Juste, F., Martín, B., Fabado, F., Moltó, E. 2000. Estudio sobre la reducción de los costes de producción de cítricos mediante la mecanización de las prácticas de cultivo. Comunidad valenciana agraria 12: 23-29.

Kallsen, C.E. 2005. Topping and manual pruning effects on the production of commercially valuable fruit in a midseason Navel Orange variety. HorTecnology 15: 335-341.

Martin-Gorriz, B., Castillo, I. P., Torregrosa, A. 2014. Effect of mechanical pruning on the yield and quality of 'Fortune'mandarins. Spanish Journal of Agricultural Research, 12(4): 952-959.

MAGRAMA (Ministerio de Agricultura, Alimentación y Medio Ambiente), 2015. http://www.magrama.gob.es/es/agricultura/temas/producciones-agricolas/frutas-y-hortalizas/). Accessed September, 2017

Mateu, G., Caballero, P., Torregrosa, A., Segura, B., Juste, F., Chueca, P. 2018 Análisis de la influencia de las operaciones de cultivo sobre los costes de producción en la citricultura de la Comunidad Valenciana. Levante Agrícola: Revista internacional de cítricos, 440: 60-64

Mendoça, V., Ramos, J. D., Neto, S. E. A., Rufini, J. C. M. 2008. Production of 'Poncan' tangerine tree after pruning recovery. Ciencia e Agrotecnologia 32: 103-109.

Moore, P.W. 1958. Mechanical pruning for citrus. California Agriculture 11: 7-13.

Rouse, B., Parsons, L., Wheaton, T.A. 2006. Hedging, topping and skirting trees in the citrus canker era. Citrus Industry 12: $2 \mathrm{pp}$.

Sauls, J. W. 2008. Citrus pruning. Texas Citrus and Subtropical fruits. http://aggiehorticulture.tamu.edu/citrus/pruning/L2308.htm. Accessed 14/3/2008.

Yildirim, B., Yesiloglu, T., Incesu, M., Kamiloglu, M., Ozguven, F., Tuzcu, O., Aka, Y. 2010. The effects of mechanical pruning on fruit yield and quality in 'Star Ruby' grapefruit. Journal of Food, Agriculture \& Environment 8: 834-838.

Zaragoza, S., Alonso, E. 1980. La poda mecanizada de los agrios en España. Anales del Instituto Nacional de Investigaciones Agrarias. Serie: Producción Vegetal 12: 157-180.

Zaragoza, S., Alonso, E. 1981. Citrus pruning in Spain. Proc. Int. Soc. Citric. 1: 172-175. 


\title{
Field Performance Evaluation Method for a Machine Vision Based Intra Row Hoeing Machine
}

\author{
Jochen Hemming ${ }^{a, *}$, Hermen de Jong ${ }^{b}$, Lauwrens Struik ${ }^{b}$, Jasper van Meer ${ }^{b}$, Eldert J. van Henten ${ }^{\text {c }}$ \\ a Wageningen University \& Research, Greenhouse Horticulture, P.O. Box 644, 6700 AP, Wageningen, The Netherlands \\ ${ }^{\mathrm{b}}$ Machinefabriek Steketee BV, P.O. Box 6, 3243 ZG Stad aan 't Haringvliet, The Netherlands \\ ${ }^{c}$ Wageningen University \& Research, Farm Technology Group, P.O. Box 16, 6700 AA, Wageningen, The Netherlands \\ * Corresponding author. Email: jochen.hemming@wur.nl
}

\begin{abstract}
Wageningen University and Research developed a machine vision based intra row hoeing machine in collaboration with Steketee, a manufacturer of agricultural machinery. Although the machine is used in agricultural practice for several years already there is still a lack of an experimental method to evaluate the field performance of such a device. Reliable, quantitative information about the performance of the machine in the field is therefore not known and the effect of variations in field conditions and machine settings on the performance were not previously researched. The relative number of crop plants damaged by the machine and the relative amount of soil around the crop plants not hoed were used as criteria to assess the field performance. The performance was measured both manually on the field and with the help of sensors by logging the actuation response of the hoeing elements. In the field trial four treatments were combined: 1) the relative number of lettuce plants, 2) the weed density on the field, 3) the driving speed during hoeing, and 4) the moment of hoeing. Each possible combination of treatments was repeated three times. It was concluded that the developed method was suitable to determine statistically significant differences in the hoeing results for the applied treatments. No difference in the machine performance was found for different numbers of missing crop plants in a plant row. The machine performance decreased in terms of the relative number of damaged crop plants in case of high weed density treatments and decreased in terms of the relative number of not hoed intra row spaces in case of high speed treatments.
\end{abstract}

\section{Introduction}

Weeds and crops are competitors in sharing the availability of resources such as light, water and nutrients. The presence of weeds influences the yield of the crop (Hall et al., 1992; Kropff et al., 1993). The relationship between weed density and yield loss has been described by many researchers (Cousens, 1985; Spitters et al., 1983; Zimdahl, 2004). Growers want to control weeds, to prevent a decrease of their crop yield. The use of synthetic herbicides to control weeds in crop production systems is under discussion, due to negative impact on the environment and human health in case of incorrect use. In North West Europe the use of synthetic herbicides to control weeds is restricted by legislations in conventional production systems and is not allowed in organic crop production systems. At the same time the availability of labour in agriculture is decreasing and the costs of labour are increasing. Therefore, mechanical weed control has become interesting again. Solutions for mechanical weed control between crop rows are available in the form of all kind of inter row hoeing machines. Moreover, since the use of electronics and automation became widely accepted in agriculture, the possibility for automated weed control between single plants in a crop row arose (Hofstee et al., 2014).

Wageningen University and Research developed a machine vision based intra row hoeing machine in collaboration with Steketee, a Dutch manufacturer of agricultural machinery. The machine, the Steketee IC hoeing machine, is carried behind a tractor. It's components and working principles are described in more detail in section 2.1. During the development several prototypes of the machine were tested. In field trials in 2008 it was proven that it was possible to detect the crop rows and single plant positions for the crops lettuce (Lactuca sativa) and celeriac (Apium graveolens var. rapaceum). On average less than $1 \%$ of the crop plants were not detected and less than $1 \%$ of the weeds were classified as crop. However, the number of weeds was small in the beginning of the field experiments and more experiments are needed to define the robustness of the system in crops with high and very high weed intensity (Hemming et al., 2011). During field trials in 2011 it became clear that the due to differences in field conditions variable distance between the cameras and the crop needs to be taken into account to achieve a higher hoeing precision. Ultrasonic vertical distance sensors were added to measure the distance to the crop. Then the machine was tested in sugar beets (Beta vulgaris subsp. vulgaris), endive (Cichorium endivia), cabbage (Brassica oleracea var. capitata), Brussels sprouts (Brassica oleracea var. gemmifera), celeriac (Apium graveolens var. rapaceum) and sweet corn (Zea mays). However, there was no quantitative information collected about the performance in the field (Nieuwenhuizen et al., 2011).

Next to the Steketee IC weeder, more intra row hoeing machines are currently commercially available, e.g. the Garford Robocrop InRow Weeder (Garford, 2018), the Robovator (Poulsen, 2018) or precision spraying equipment from Blue River Technology/John Deere (Blue River Technology, 2018). Only very little information 
is published on evaluation methods for such machines and also about the performance of the machines. One example of an evaluation of intelligent cultivator for use in lettuce production was described by Smith (2015). In that research the Robovator and the Steketee IC were compared. Before and after hoeing, the lettuce plants and weeds were counted. However also in that case, detailed information of the performance per machine was not described.

Objective

There is still a lack of an experimental method to evaluate the field performance of intra row hoeing machines. Reliable, quantitative information about the performance in the field is therefore not known and the effect of variations in field conditions and machine settings on the performance were not previously researched. The objective of the research described here is to develop and to validate a method to obtain reliable, scientifically proven, quantitative information about both the performance of the Steketee IC intra row hoeing machine in the field and the effect of variations in field conditions or machine settings on the performance.

\section{Materials and Methods}

Intra row hoeing machine

The intra row hoeing machine used in this research is the Steketee IC. It is an intelligent hoeing machine that is carried behind a tractor, as shown in Figure 181. The machine is equipped with one set of arced shaped hoeing knives per plant row (Figure 182) that closes in the plant row at the so called intra row spaces to remove unwanted plants and then opens around the desirable crop plants. The machine contains multiple colour cameras that are mounted beside each other on the machine, facing straight downwards, under a cover that blocks natural light. Artificial white LED lighting is used to create a constant level of light under the hood. One of the wheels of the machine is equipped with an encoder, which generates pulses when the wheel is turning. These pulses are used to derive the speed and travelled distance of the machine and to trigger the cameras. During hoeing, the colour cameras take pictures every $0.20 \mathrm{~m}$ forwards. The machine uses an ultrasonic sensor to measure the operating height at the left and right side of the machine and the machine is able to automatically adapt the operating height at both sides of the machine separately, by changing the height of the support wheels with hydraulic cylinders. The images acquired by the machine during operation are analysed using computer vision methods. One image analysis task is to determine the crop row positions. For this task a template fitting algorithm was developed. A side shift mechanism is used to move the machine to the left or right, to guide the hoeing knives onto the exact row centres. The second task of the image analysis is to locate every single crop plant in the row. The method developed for this task is based on a Fast Fourier Transformation (FFT) as described by Bontsema et al. (1991). More details on the computer vision methods are described in Hemming et al. (2011). The system is designed to work in all precision sown and transplanted crops, such as for example lettuce, sugar beets and cabbages, and is able to work in different crop stages.
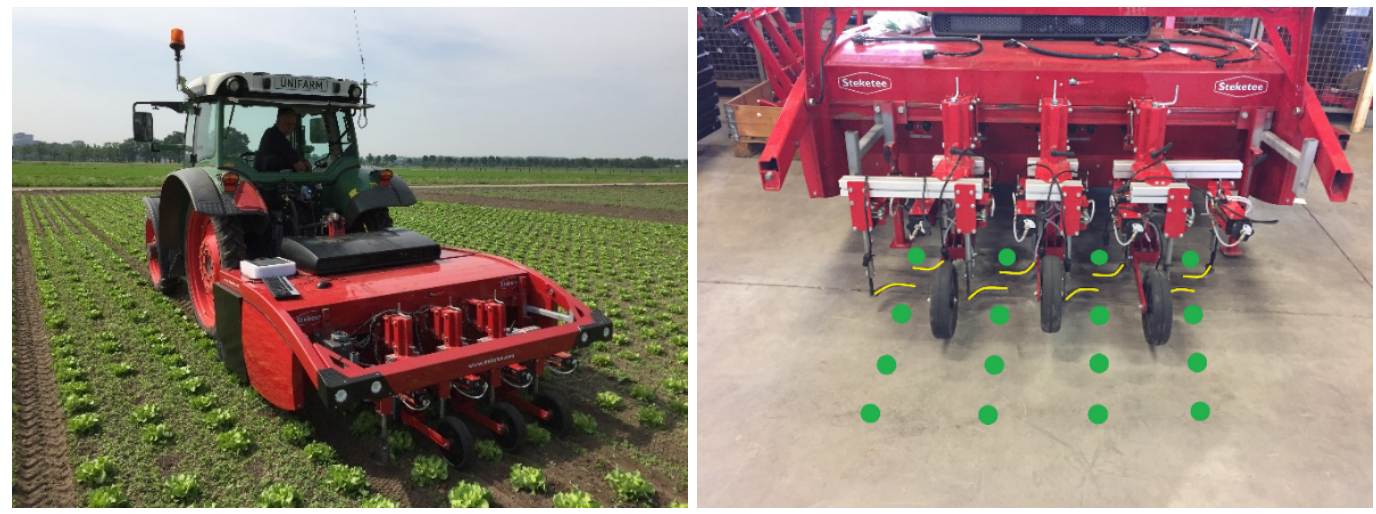

Figure 181. The Steketee IC hoeing machine mounted behind a tractor (left photo) and visualization of the hoeing knives (marked by yellow lines) between the crop plants in the row (marked by green dots) (right photo). 


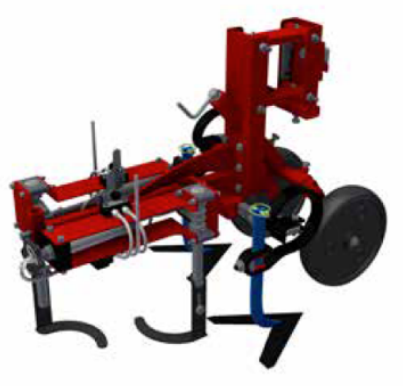

Figure 182. Hoeing actuator of the Steketee IC hoeing machine. The arc shaped knives can rotate to hoe between single crop plants in the row. The $\mathrm{V}$ shaped hoeing element is for hoeing between the crop rows.

Machine settings that were expected to have a high impact on the machine performance were:

Driving speed: The time needed to pneumatically open or close the hoeing knives is fixed for all circumstances, assuming a constant air pressure. Thus, the distance that a hoeing knife covers in the driving direction during opening or closing is depending on the driving speed, even if the algorithm applies a so called speed dependent correction to the actuating signal. Therefore, both a higher number of damaged crop plants and a higher amount of not hoed soil around the crop plants were expected for hoeing with a high driving speed compared to hoeing with a low driving speed.

Virtual safety buffers around crop plant: The machine offers the possibility to set virtual safety buffers around the detected crop plant. These buffers protect the plants for being damaged by the hoeing knives. The larger the safety buffer, the earlier a hoeing knife opens before and the later it closes after a detected plant. Thus, a higher number of damaged crop plants was expected for hoeing with a small safety buffer, compared to hoeing with a large safety buffer. However, a higher amount of not hoed soil around the crop plants was expected for hoeing with a high safety buffer, compared to hoeing with a low safety buffer.

Operating height of the machine: As described above the machine is using two ultrasonic vertical distance sensors to measure and to keep the machine horizontally levelled and to maintain a certain operating height. This operating height has a big influence on the distance between the cameras and the soil or crop plants. Problems in the image processing were expected in case of an incorrect height control. Consecutive images taken at a wrong operating height were expected to result in poorly stitched images, leading to missing or double information. Both a higher number of damaged crop plants and a higher amount of not hoed soil around the crop plants were expected for hoeing with a wrong operating height compared to hoeing with a correct operating height.

\section{Experimental field and crop}

A field at the experimental farm of Wageningen University and Research was used (GPS coordinates: $51^{\circ} 59^{\prime} 42.4^{\prime \prime} 5^{\circ} 39^{\prime} 20.6^{\prime \prime E}$ ). Lettuce (Lactuca sativa var. capitata) was chosen as crop, since this is the crop in which the Steketee IC hoeing machine is most used. The experimental field had 16 beds with a width of $1.50 \mathrm{~m}$ and length of $50 \mathrm{~m}$. Each bed had four crop rows besides each other, the inter row distance (the distance between two crop rows) was $0.375 \mathrm{~m}$. The intra row distance (the distance between two crop plants in one row) was 0.30 $\mathrm{m}$. One bed with a length of $50 \mathrm{~m}$ consist of three field plots of ten metre, taking into account a five metre buffer at the beginning and at the end of the bed and a five metre buffer between each field plot. Each field plot was used only once, to exclude any influence of a previous weeding action.

In the first half of the experimental field the soil was cultivated already two weeks before planting to allow weeds to germinate in order to create a high weed density compared to the second half of the experimental field, were the soil was cultivated just before planting. For both weed densities, half of the field plots were kept fully planted, the other half of the field plots the crop plants were manually thinned out, $25 \%$ of the plants was randomly removed 12 days after planting (see also Figure 187 and Figure 188).

Field variables that were expected to have a high impact on the machine performance were:

Number of missing crop plants: The core of the used computer vision algorithm is based on a FFT and relies on a repetitive plant pattern. Missing crop plants will disturb that pattern, leading to a weaker detection of the row and plant positions. Therefore, both a higher number of damaged crop plants and a higher amount of not hoed soil around the crop plants were expected for hoeing in fields with a high number of missing crop plants, compared to fields with a low number of missing crop plants. 
Weed density: The algorithm distinguishes crop plants from weeds by the specific contribution of the crop plants to a certain frequency band, whereas the weeds are expected to contribute randomly to all frequencies. In case of a high weed density, weed plants could contribute as much to the plant signal as crop plants, causing a decreased distinction between crop plants and weeds. So, both a higher number of damaged crop plants and a higher amount of not hoed soil around the crop plants were expected for hoeing in fields with a high weed density, compared to fields with a low weed density.

Moment in the growing season: A good distinction between crops and weeds was expected early in the growing season when weeds are small and individual crop plants are not touching or overlapping each other, resulting in a clear signal. Both the number of damaged crop plants and a higher amount of not hoed soil around the crop plants were expected for hoeing late in the growing season, compared to hoeing early in the season.

\section{Performance evaluation method}

In order to assess the field performance two criteria were used. The first criterion was that an average relative number of damaged crop plants of at most $1 \%$ was considered to be acceptable. The second criterion was that an average relative amount of soil around the crop plants not hoed by the machine of at most $10 \%$ was considered to be acceptable. Both criteria were based on discussions with several organic growers.

Due to space and time limitations only the following variables were applied to evaluate their effect on the performance of the machine:

1. Two relative numbers of missing crop plants: $0 \%$ and $25 \%$ missing crop plants

2. Two weed densities: a normal and a high weed density

3. Two hoeing moments in the growing season: 19 and 23 days after planting

4. Two driving speeds: 0.6 kilometre per hour and 1.8 kilometre per hour

All variations were applied in each possible combination, which means that two relative numbers of missing crop plants times two weed densities times two moments in the growing season times two driving resulted in 16 treatments. Each treatment was repeated three times which resulted in 48 field plots.

\section{Manual measurements}

The weed density was manually counted before and after hoeing using a counting quadrant with a size of one square metre (Figure 183). Since weed counting is very time consuming, it was decided to count three randomly selected samples for the normal weed density and three randomly selected samples for the high weed density, before and after hoeing, both moments in the growing season. The sample locations were randomly selected, under the condition of a minimal distance of 10 metres between each sample.

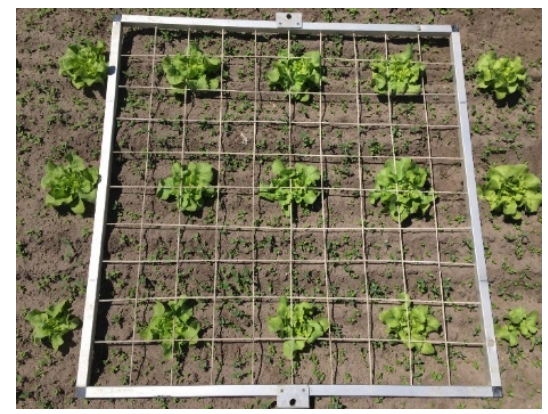

Figure 183. Example of weed counting using a counting quadrant before hoeing 19 days after planting

The relative amount of damaged crop plants was manually determined by counting the damaged crop plants. Counting was done one day after hoeing since at this moment damaged plants were more easily to observe as they had become dried out or wilted. The relative amount of not hoed soil around the crop plants was manually determined after hoeing by counting the number of not hoed intra row spaces (the space between crop plants in one crop row) and the number of not hoed spaces of missing crop plants. The inter row spaces (the space between two crop rows) were assumed to be always hoed, since the machine has fixed hoeing knives between the different crop rows. An intra row space was considered as not hoed when all the weeds between two crop plants were clearly as untouched as before hoeing, an example is shown in Figure 184. A comparison between hoed and not hoed spaces is shown in Figure 185. 


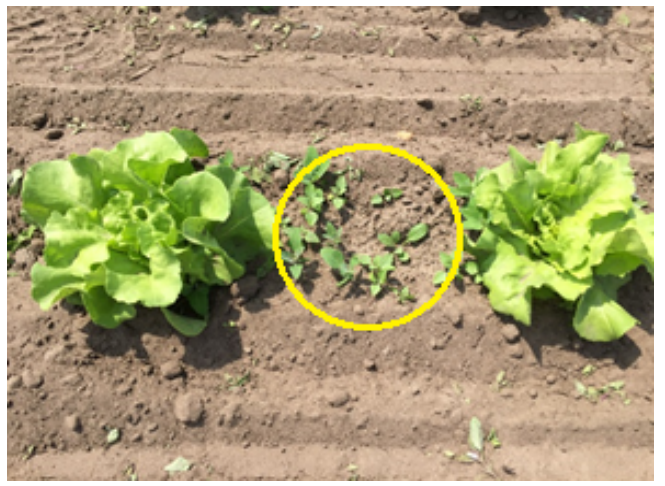

Figure 184. Example of a not hoed intra row space, recognizable by clearly untouched weeds, marked by a yellow circle.

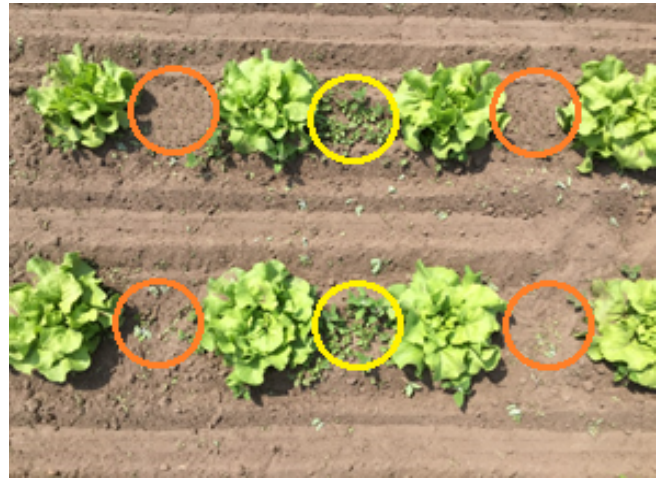

Figure 185. Comparison between hoed intra row spaces, marked by orange circles, and not hoed intra row spaces, marked by yellow circles.

\section{Sensor-based measurements}

The motion of the hoeing knives is actually depending as well on the correctness of the mechanical actuation of the hoeing knives calculated by the vision algorithm as on the correctness of the response of the hoeing knives. This means that the performance of the machine was possibly negatively influenced by an inaccurate or incorrect calculated actuation signal to the hoeing knives or an inaccurate or incorrect response of the hoeing knives, or both. Therefore, two entities were continuously measured during hoeing and analysed afterwards: the actuation of the hoeing knives and the response of the hoeing knives.

The actuation signal to the hoeing knives was logged by simply doubling each signal cable to a hoeing knife. Per hoeing knife, one of the signal cables was plugged into the hoeing knife, whereas the other one was plugged into an Epec 2024 control unit (Seinäjoki, Finland). The control unit was used to receive the actuation signals from the cables and send those together with the encoder position every 3 milliseconds to the computer of the machine via CAN bus. Those actuation signal were logged on the computer. The response of the hoeing knives was also logged with sensors. Each hoeing knife of the hoeing machine is activated by an pneumatic cylinder. If the cylinder is retracted, the hoeing knife is open, if the cylinder is extended, the hoeing knife is closed, Vesta VNPE 3 sensors (Rovigo, Italy) were mounted at the begin and end of each pneumatic cylinder to measure the presence of the magnetic piston. The values of theses sensors were logged with the same Epec 2024 control unit described above.

The images taken by the cameras of the machine were also logged, an example image shown in Figure 186. Both the visualized actuation and the visualised response of the hoeing knives were analysed for the relative number of damaged crop plants and the relative amount of not hoed soil around the crop plants. Figure 189 in the result section shows an example of such a visualisation, overlaid on the camera images.

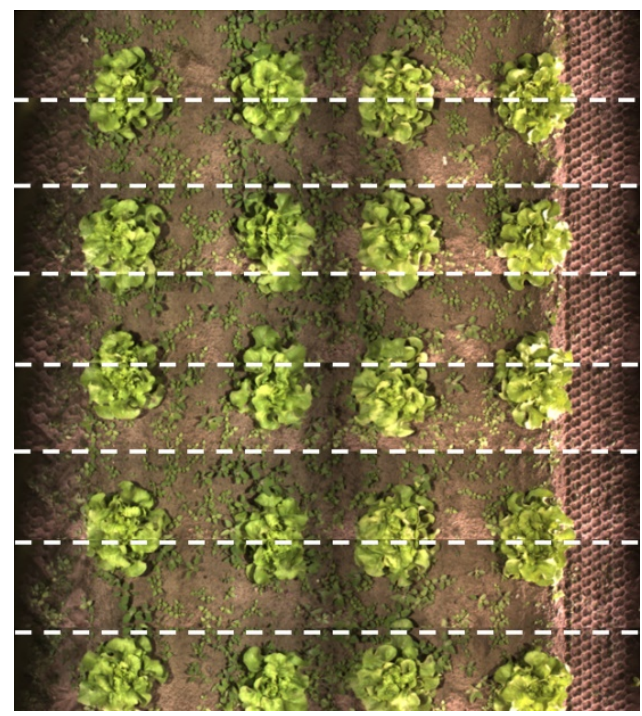

Figure 186. Example of images acquired by the camera system of the machine. Single images are stitched together. The dashed lines indicate the edges of the single images. 


\section{Results}

Validation of applied field conditions

The relative number of missing crop plants after manually thinning for the field plots with a target of $25 \%$ varied roughly between $20 \%$ and $30 \%$. The relative number of missing crop plants for the field plots with a target of $0 \%$ was also slightly different with values roughly between $0 \%$ and $5 \%$. This was caused by a few plants that had died after planting. A Welch's one-way analysis of variance (ANOVA) was used. This test showed that the difference between the mean numbers of crop plants at the field plots with $0-5 \%$ missing crop plants $(96.5 \pm 1.6)$ and the mean numbers of crop plants at the field plots with $20-30 \%$ missing crop plants $(74.8 \pm 3.0)$ was statistically significant, $\mathrm{F}(1.34)=972.6$ and $\mathrm{p}=0.000$. Figure 187 shows a plot without missing plants, Figure 188 shows a plot with $25 \%$ missing crop plants.
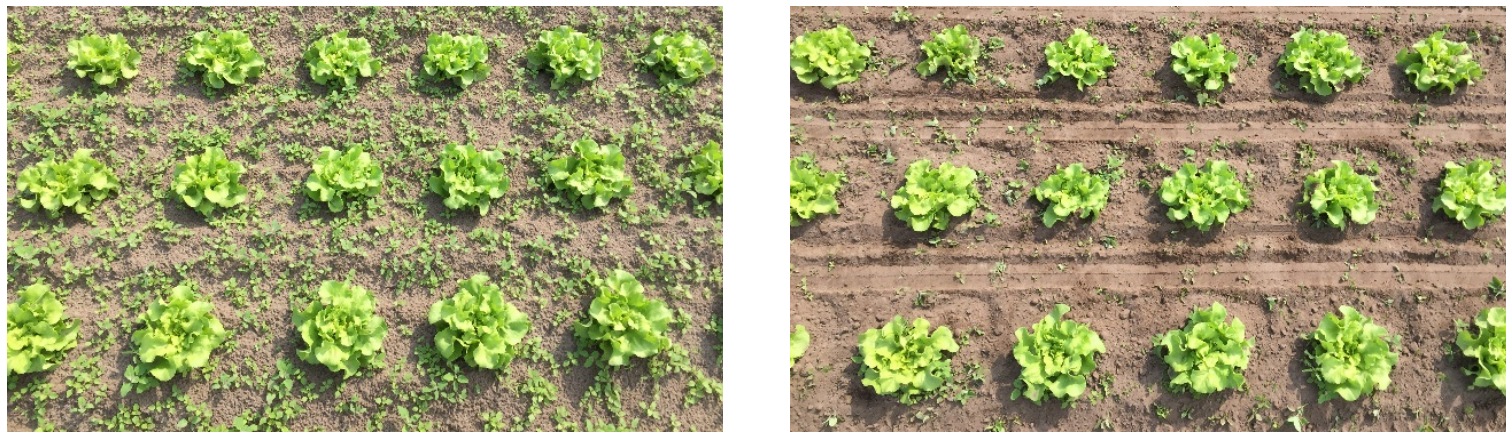

Figure 187. Example of a part of a field plot with no missing crop plants and a normal weed density before hoeing (left) and after hoeing (right) 19 days after planting.
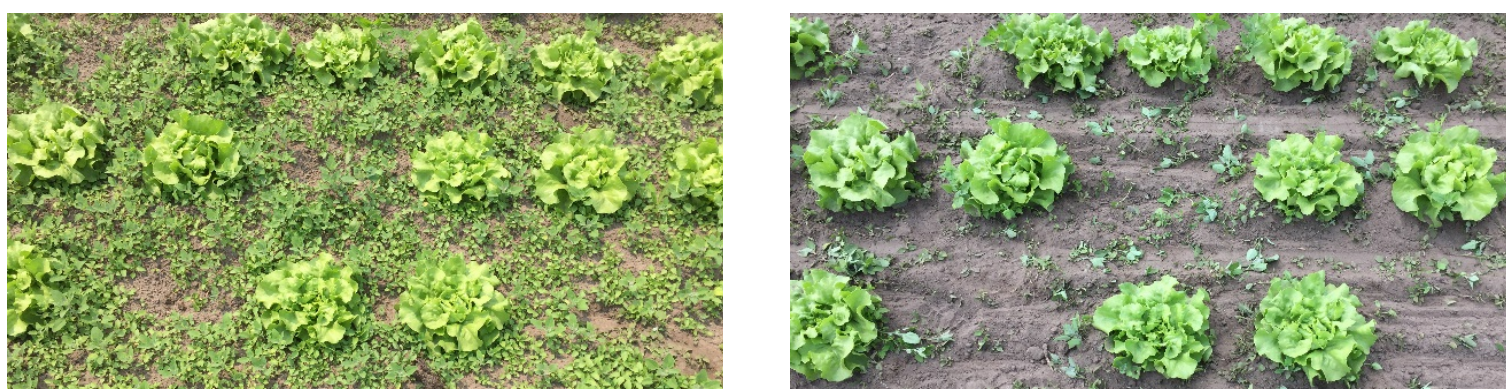

Figure 188. Example of a part of a field plot with $25 \%$ missing crop plants and a normal weed density before hoeing (left) and after hoeing (right) 23 days after planting.

The overall weed density in the experimental field was very high, an impression is given in Figure 187 and Figure 188, which is not common on the fields in practice, since growers used to hoe multiple times between the moment of planting and the moment of harvesting the crop. The weeds that were counted were mostly Orache (Atriplex) and Redshank (Persicaria maculosa) and to a lower extent Stinging nettle (Utica dioica) and Hedge bindweed (Convolvulus sepium). The two different weed densities, that were tried to establish by different moments of soil cultivation as described above were visible by the human eye a view days after planting although, the difference was ambiguous during the weed counting. However, the weeds growing at the part of the field with a normal weed density treatment were smaller than the weeds growing at the part of the field with a high weed density treatment. 
Table 79 shows the manually counted number of weeds divided into categories of weeds bigger and smaller than $5 \mathrm{~cm}$ in size, for the two moments of hoeing, for the different weed densities and before and after hoeing. 
Table 79. Average number of weeds per $\mathrm{m}^{2}$ before and after hoeing.

\begin{tabular}{cccccc}
\hline & & \multicolumn{2}{c}{ Before hoeing } & After hoeing \\
\cline { 3 - 6 } $\begin{array}{c}\text { Days } \\
\text { after } \\
\text { planting }\end{array}$ & $\begin{array}{c}\text { Weed } \\
\text { density } \\
\text { treatment }\end{array}$ & $\begin{array}{c}\text { Number of weeds } \\
\text { per } \mathrm{m}^{2}<5 \mathrm{~cm}\end{array}$ & $\begin{array}{c}\text { Number of weeds } \\
\text { per } \mathrm{m}^{2}>5 \mathrm{~cm}\end{array}$ & $\begin{array}{c}\text { Number of } \\
\text { weeds per } \mathrm{m}^{2}<5 \mathrm{~cm}\end{array}$ & $\begin{array}{c}\text { Number of weeds } \\
\text { per } \mathrm{m}^{2}>5 \mathrm{~cm}^{2}\end{array}$ \\
\hline 19 & Normal & 1358 & 0 & 200 & 7 \\
19 & High & 517 & 10 & 142 & 8 \\
23 & Normal & 1533 & 68 & 208 & 18 \\
23 & High & 867 & 63 & 183 & 17 \\
\hline
\end{tabular}

Machine performance

Weeding result

Based on all the counting samples, the total number of weeds was decreased by $82 \%$ after hoeing.

\section{Number of damaged crop plants}

The difference between the relative number of crop plants that was damaged by hoeing at the field plots with $0-5 \%$ missing crop plants $(1.1 \% \pm 2.3 \%)$ and number that was damaged by hoeing at the field plots with $20-30 \%$ missing crop plants $(2.2 \% \pm 4.7 \%)$ was not statistically significant, $F(1.46)=1.007$ and $p=0.321$ (ANOVA test). Also, the difference between the relative number of crop plants that was damaged by hoeing at the field plots 19 days after planting $(2.5 \% \pm 4.9 \%)$ and the number that was damaged at the field plots hoed 23 days after planting $(0.8 \% \pm 1.5 \%)$ was not statistically significant, $\mathrm{F}(1.27)=2.707$ and $\mathrm{p}=0.111$. Also no significant difference in the relative number of crop plants that was damaged was found for the different driving speeds.

The difference between the relative number of crop plants that was damaged by hoeing at field plots with a normal weed density $(0.4 \% \pm 0.7 \%)$ and the number of crop plants that was damaged by hoeing at the field plots with a high weed density $(2.9 \% \pm 4.9 \%)$ was statistically significant, $\mathrm{F}(1.24)=6.298$ and $\mathrm{p}=0.019$.

In twelve out of the sixteen treatments (75\%) less than $1 \%$ of the crop plants was damaged. As described in section 3.3 this was considered to be acceptable.

\section{Amount of soil around the plant not hoed}

No statistically significance was found for the difference between the relative number of not hoed intra row spaces at the field plots with $0-5 \%$ missing crop plants $(8.2 \% \pm 7.9 \%)$ and at the field plots with $20-30 \%$ missing crop plants $(6.7 \% \pm 4.9 \%), F(1.39)=0.604$ and $\mathrm{p}=0.442$. Also no statistically difference was found for the plots with different weed densities.

There was a significant difference between the relative number of not hoed intra row spaces at the field plots hoed 19 days after planting $(10.0 \% \pm 8.3 \%)$ and field plots hoed 23 days after planting $(4.9 \% \pm 2.4 \%), F(1.27)=$ 8.383 and $\mathrm{p}=0.007$. Also the difference between the relative number of not hoed intra row spaces at field plots hoed with a speed of $0.6 \mathrm{~km} / \mathrm{h}(4.8 \% \pm 2.3 \%)$ and field plots hoed with a speed of $1.8 \mathrm{~km} / \mathrm{h}(10.6 \% \pm 7.9 \%)$ was statistically significant, $\mathrm{F}(1.27)=13.51$ and $\mathrm{p}=0.001$.

The results were assessed using the criterion that an average relative number of not hoed intra row spaces of at most $10 \%$ was considered to be acceptable (see section 3.3). Averaged over all 48 field plots, the relative number of not hoed intra row spaces was $7.6 \%$. In four out of sixteen treatments more than $10 \%$ of the crop plants was damaged. All these four treatments were high speed treatments and it was proven that these treatments led to statistically significant worse results, as previous described. All these four treatments were also hoed 19 days after planting.

\section{Sensor-based measurements}

The measured actuation and response of the hoeing knives could be visualized on the images acquired by the machine vision camera. Figure 189 shows an example. The actuation signals of the hoeing knives are visualized by white lines and the response of the hoeing knives by coloured lines. The time delay between actuation signal and the real movement of the actuator knifes (response) can be observed. 


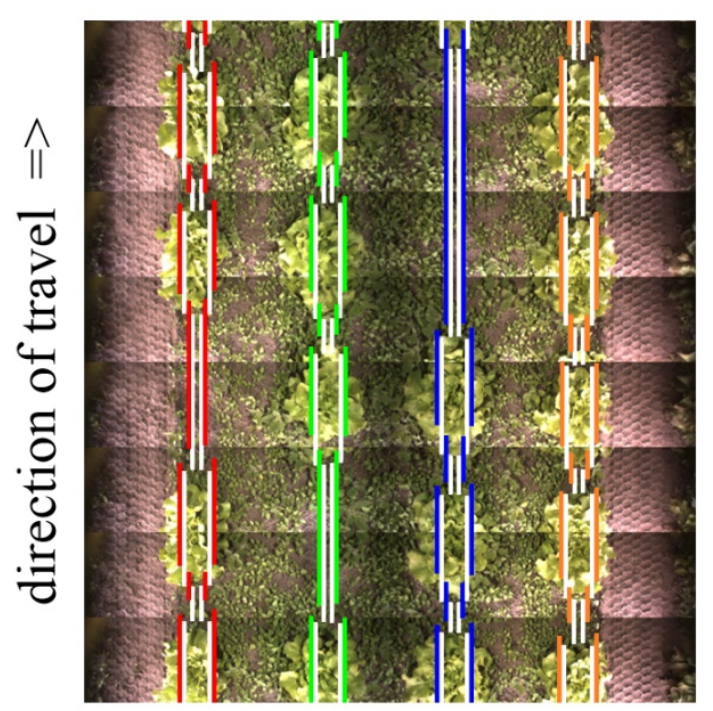

Figure 189. Example of the visualized actuation and response of the hoeing knives on a merged image. The actuation of the hoeing knives is visualized by white lines and the response of the hoeing knives by coloured lines. The difference between closed and opened hoeing knives was visualized by a fixed shift of the lines from the middle of a row to the left and right of that row. This shift does not represent the real shifted distance to the left and right.

The visualisations were analysed by checking if the lines were open at the location of the crop plants and if they were closed at the intra row spaces and spaces of missing crop plants. It turned out that the results based on the manual measurements and the results based on the response signal were worse than the results based on the actuation signal. It is likely that deterioration of the results are caused by mechanics of the machine. Another explanation might be that some of the sensors that measured the knife positions were not properly mounted, resulting in an incomplete signal. The weed density was much higher than usual in practice and sometimes there was so much weed heaping around the hoeing knives that this could have negatively influenced the opening and closing behaviour of the hoeing knives.

\section{Discussion and Conclusions}

To determine the performance of the hoeing machine and the effect of variations in field conditions or machine settings on the performance, an experiment was developed. Multiple variations in the relative number of crop plants on the field, the weed density on the field, the driving speed during hoeing and the moment of hoeing were applied. The differences in the conditions that were provided during the experiment were validated. The differences in the number of crop plants per field plot and the size of the crop plants per hoeing moment were statistically significant. The difference in the weed density was less clear, since the one part of the field contained more weeds and the other part of the field contained bigger weeds. Moreover, the weed density at both parts of the field was much higher than usual in practice. Therefore, conclusions about the field performance of the Steketee IC hoeing machine based on this research are only valid for hoeing fields with a high weed density in general.

The hoeing result was measured both manually on the field and with the help of sensors by logging the actuation and response of the hoeing elements. The actuation signal, calculated by the algorithm, was almost perfect but differed slightly from the results based on the manual in field observations. Studying video recordings revealed that in some rare instances the working depth of the hoeing knives was such shallow that the knives did not even touched the soil. This may explain some of the differences in sensor measurements and manual measurements. The manual assessed relative number of not hoed intra row spaces was statistically significant worse for the field plots that were hoed 19 days after planting compared to the field plots that were hoed 23 days after planting. This result was actually against the expectations, but could be possibly explained by the selection of different machine settings during this hoeing moment. No difference in the machine performance was found for different numbers of missing crop plants in a plant row. The machine performance decreased in terms of the relative number of damaged crop plants in case of high weed density treatments and decreased in terms of the relative number of not hoed intra row spaces in case of high speed treatments.

It can be concluded that the method described is a suitable method to determine statistical significant differences in hoeing results for the applied variations in field conditions and machine settings. 


\section{References}

Blue River Technology, 2018. Blue River Technology Hompage. http://www.bluerivertechnology.com/ Accessed June 8, 2018.

Bontsema, J., T. Grift, K. Pleijsier, 1991. Mechanical weed control in sugar beet growing: the detection of a plant in a row. In Proceedings of the IFAC/ISHS Workshop on mathematics and control applications in agriculture and horticulture, Matsuyama, Japan.

Cousens, R., 1985. An empirical model relating crop yield to weed and crop density and a statistical comparison with other models. The Journal of Agricultural Science, 105 (3), 513-521. https://doi.org/10.1017/S0021859600059396.

Garford, 2018. Robocrop InRow Weeder. http://www.garford.com/products robocropinrow.html Accessed June 8, 2018.

Hall, M. R., C. T. Swanton, G. W. Anderson, 1992. The critical period of weed control in grain corn (Zea mays). Weed Science, 40 (3), 441-447.

Hemming, J., A. T. Nieuwenhuizen, L. E. Struik, 2011. Image analysis system to determine crop row and plant positions for an intra-row weeding machine. In Proceedings of the CIGR International Symposium on Sustainable Bioproduction, Tokyo, Japan, 19-23 September 2011.

Hofstee, J. W., \& A. T. Nieuwenhuizen, 2014. Field Applications of Automated Weed Control: Northwest Europe In: Automation: The future of weed control in cropping systems. Eds., S. L. Young, F. J. Pierce. 171-187. https://doi.org/10.1007/978-94-007-7512-1

Kropff, M. J., H. H. van Laar, 1993. Modelling crop-weed interactions. CAB International, in association with the International Rice Research Institute.

Poulsen, 2018. Robovator vision based hoeing machine for controlling weed in row crops. http://www.visionweeding.com/robovator/ Accessed June 8, 2018.

Smith, 2015. Evaluation of intelligent cultivators for use in lettuce production. UC Weed Science. http://ucanr.edu/blogs/blogcore/postdetail.cfm?postnum=19841. Accessed June 1, 2018.

Spitters, C. J. T., R. Aerts, 1983. Simulation of competition for light and water in crop-weed associations. Aspects of Applied Biology, 4, 1-18. https://doi.org/urn:nbn:nl:ui:32-76628.

Nieuwenhuizen, A. T., J. Hemming, L. E. Struik, 2011. Automatisch onkruid bestrijden in de rij. Fase 4: Technische realisatie prototype meerrijer. Plant Research International, report 425. Wageningen.

Zimdahl, R. L. 2004. Weed- crop competition. Crop Science, 44(6), 2284-a. 


\title{
'Clemenrubi' Mandarin Size Development after Manual and Mechanical Thinning
}

\author{
Antonio Torregrosa ${ }^{a, *}$, Enrique Ortía ${ }^{\text {Emilio Soria }}{ }^{\mathrm{b}}$, Sebastià Balasch ${ }^{\mathrm{c}}$, Coral Ortiz ${ }^{\mathrm{a}}$ \\ ${ }^{a}$ Departamento de Ingeniería Rural y Agroalimentaria, Universitat Politècnica de València, Valencia, Spain \\ ${ }^{\mathrm{b}}$ Departamento de Ingeniería Electrónica, Universitat de València, Valencia, Spain \\ ${ }^{\mathrm{c}}$ Departamento de Estadística i Investigació Operativa Aplicades i Qualitat, Universitat Politècnica de València, \\ Valencia, Spain \\ * Corresponding author. Email: torregro@dmta.upv.es
}

\begin{abstract}
Fruit thinning is the operation of removing fruits on the tree in order to increase final fruit size. Citrus fruit thinning is usually done by hand, selecting the adequate fruits to remove. Mechanical thinning has been tested to reduce costs. In this research work, fruit growth from trees with different thinning treatments (intensities and dates of manual and mechanical thinning) were tested in order to asses fruit size increase with a non-selective mechanical thinning compared to the selective manual thinning. Early severe thinning treatments had fruits with higher diameter close to harvesting time. Mechanical thinning treatments needed more than 30 days to have fruits with higher diameter values than the fruits from the non-thinned trees. However, when the thinning treatment is done close to the harvesting date, the fruits left on the tree do not have enough time to increase fruit size.
\end{abstract}

Keywords: Citrus, shaker, mechanical thinning, growth model

\section{Introduction}

Thinning is the removal of some flowers or fruits in order to increase final fruit size and then achieve greater prices (Agustí, 2003).

In the case of citrus fruits, this technique is important for some orange varieties and particularly for mandarin varieties 'Orri', 'Nadorcott', 'Clemenrubí', and others in which the smallest fruits do not compensate harvest and transport costs.

At present, thinning operation is done by hand, in order to select the adequate fruits to remove. In most of the cases, fruits under a certain diameter are removed, this diameter depends on the time of the year. The operation is also used to remove damaged fruits (Agustí y Almela, 1984).

In order to reduce costs, chemical and mechanical thinning have been tested (Davis et al., 2004, Ouma G., 2012). Chemical thinning has proven to be effective, but their effect is strongly influenced by climatic and environmental factors. In addition, agricultural products with chemicals are recently being rejected by consumers, this fact, in some years, could cause legal problems with some chemical compounds that are already working in citrus thinning (Agustí y Almela, 1991; Guardiola y García Luis, 2000; Agustí, 2003; Mesejo et al, 2003 y 2012 ; Stover, 2002).

For this reason, in this research work different options of mechanical thinning have been tested to reduce costs. Branch shakers have been tested as mechanical devices, associated with their flexibility of use, being the operator responsible of selecting the adequate branch and vibration time according to the fruits that are being removed. Obviously, mechanical thinning does not allow to select the fruits to remove, a part of the fruits on the tree are detached from all the sizes (Ortiz et al, 2016 y 2017; Torregrosa et al, 2017).

Previous research studies as Agustí et al. (1998) showed that the best results for manual thinning of 'Clausellina' mandarin are achieved when an early operation is done but always after flowering, otherwise the number of flowers would increase and the task would be useless. Besides, in order to obtain clear results more than $50 \%$ of the fruitlets should be removed after the 'porga' or physiological drop of the fruit. However, this intense thinning operation causes a crop production reduction.

Citrus producers are disoriented related to thinning date and intensity, since for each variety and development stage the best option is different. All this, in addition to the fact that the most important unknown factor is the market demand at harvest time, years with high demand all the fruits sizes are accepted, on the contrary, years with low demand almost no size is accepted.

In any case, having certain information about tree response to thinning obtained from rigorous tests will be useful for citrus producers in order to make more reasoned decisions.

In this research work fruit growth from trees with different thinning treatments were tested, from different intensities and dates of manual and mechanical thinning operations. The possibility of increasing fruit size with a non-selective mechanical thinning compared to the selective manual thinning was analyzed. In addition, fruit equatorial diameters were recorded from thinning to harvest in order to assess diameter development. 


\section{Materials and Methods}

Trials were performed in a mandarin orchard located in Sagunto (Valencia, Spain, Google maps coordinates: $39.645122,-0.303941)$. Two rows over a two-year period were tested. A total number of 54 trees and 6 treatments were tested (Table 1 and Figure 1).

Table 1. Thinning treatments carried out.

\begin{tabular}{ccc}
\hline Treatments & Date of the main thinning & Type of thinning \\
\hline M & JN 29, 2017 & manual $\mathrm{D}<28 \mathrm{~mm}$ \\
M1 & JN 28, 2017 & manual $\mathrm{D}<30 \mathrm{~mm}$ \\
V & JN 29, 2017 & mechanical \\
V1 & JL 13, 2017 & mechanical \\
M2 & JL 25, 2017 & manual $\mathrm{D}<37 \mathrm{~mm}$ \\
N & & no thinnig \\
\hline
\end{tabular}

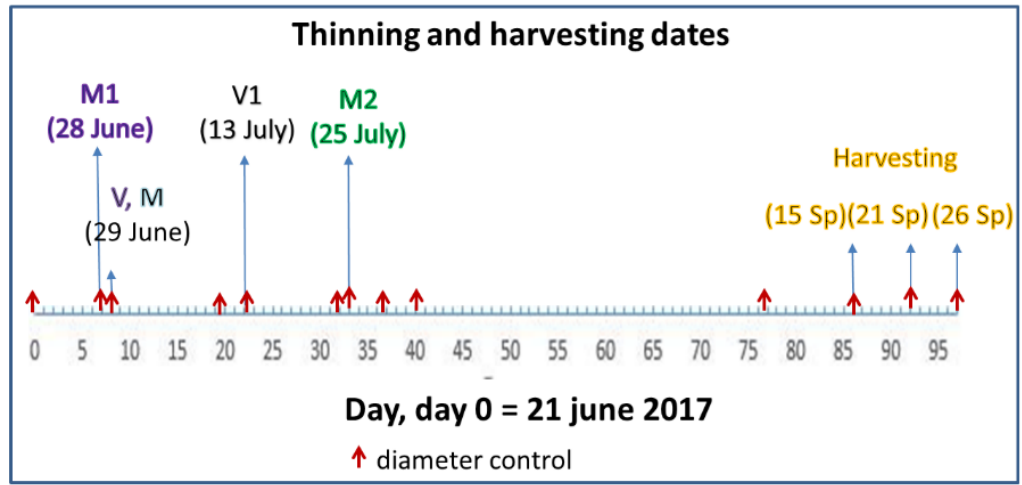

Figure 1. Dates of the thinning and harvesting operations.

The trees were planted in a grid with an in-row spacing of $2 \mathrm{~m}$ and $5.1 \mathrm{~m}$ between rows. Rows were on trapezoidal-shaped ridges, $0.5 \mathrm{~m}$ in height and $1.5 \mathrm{~m}$ wide at the top. Trees were 'Clemenrubi' grafted onto 'Citrange' with an intermediate of 'Salustiano' orange. They were 8-9 years old. Trunks were $0.45 \mathrm{~m}$ in height and $0.11 \mathrm{~m}$ in diameter. Trees had three or four main branches, and the canopies were $2.2 \mathrm{~m}$ above the top of the ridge with the canopy skirt $0.9 \mathrm{~m}$ above the base of the ridge. The canopy diameter was $2.5 \mathrm{~m}$ at $90^{\circ}$ to the axis of the rows and $2.2 \mathrm{~m}$ along the axis of the row. The equivalent canopy volume was $6.8 \mathrm{~m}^{3}$. A manual petrol shaker was used (Vibroli, www.nta.es) $(2.1 \mathrm{~kW}, 60 \mathrm{~mm}$ stroke, $11 \mathrm{~kg}$ weight) along with a $2 \mathrm{~m}$ pole with a terminal, $40 \mathrm{~mm}$ wide hook. Canvases were located under the trees to collect detached fruits (Figure 2).

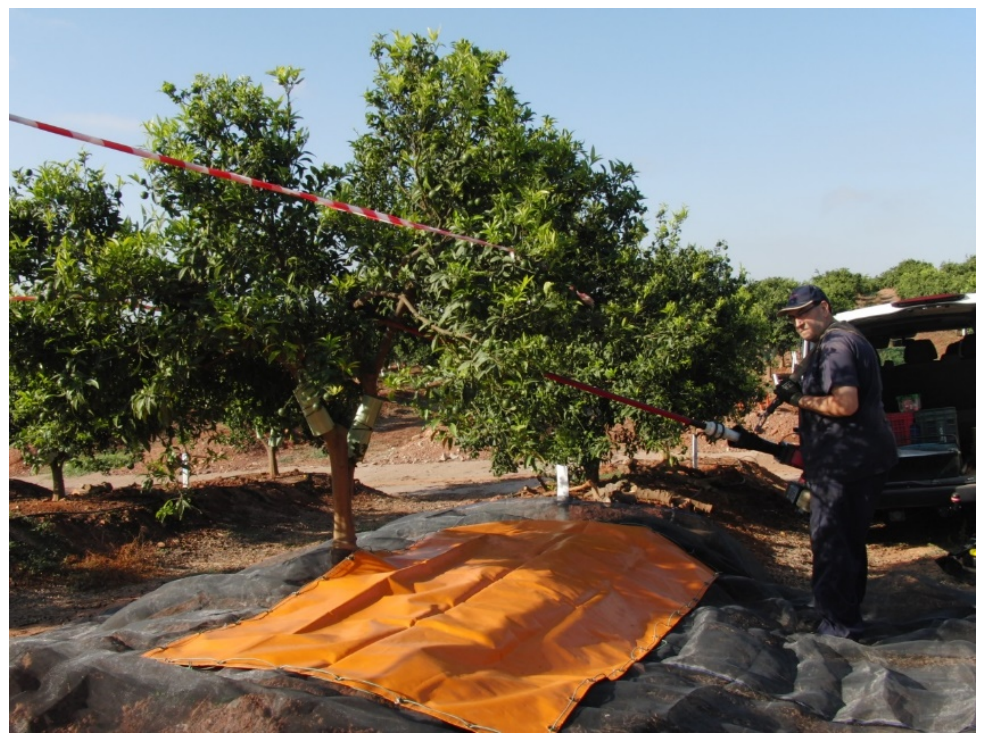

Figure 2. Equipment used for mechanical thinning.

A total of 3 to 5 branches on each tree were shaken, using a very short vibration cycle (less than 1 second). When only a few fruits were detached, a second very short vibration was applied. High-speed video recordings (300 frames/s) were taken, and a triaxial accelerometer (X200-4, www.gcdataconcepts.com) was located on the 
branch closest to the gripping point in order to determine the vibration frequency. Recorded frequencies were between $14 \mathrm{~Hz}$ and $23 \mathrm{~Hz}$, the peak-to-peak displacement of the branch at the gripping point varied from 35 to 39 $\mathrm{mm}$, and the vibration time ranged from 0.5 and $1 \mathrm{~s}$. The peak acceleration registered at the gripping point was between 500 and $700 \mathrm{~ms}^{-2}$ (Figure 3).

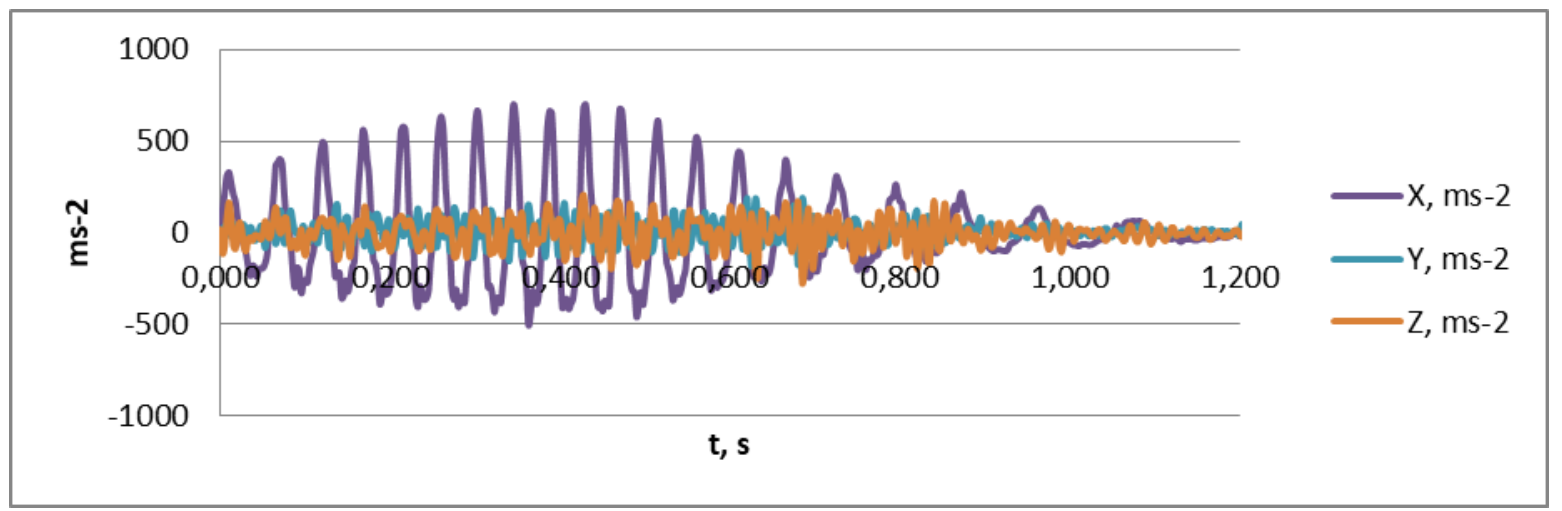

Figure 3. Branch accelerations at the gripping point in a standard vibration.

All the detached fruits, manually and mechanically, were weighted and calibrated. The fruits remaining on the trees were calibrated during their development according to the dates showed in Figure 1.

The harvesting operation was done in three steps, the first one was done on September $15^{\text {th }}$ and all the fruits from the trees and treatments were harvested. Due to some confusion, during the second and third harvests (September $21^{\text {st }}$ and $26^{\text {th }}$ ), part of the information was lost.

Data were analysed using one factor variance analysis in order to assess the effect of the thinning method on the crop production. Tukey LSD intervals were used to compare the mean values of the different thinning methods. In addition, the differences between the detached fruits and the remaining fruits on every vibrated tree were studied using Student's t-test of means for independent samples. Finally, linear regression models were developed in order to relate tree production and fruit number and increase in diameter and time.

\section{Results and Discussion}

Removed fruits

If the population of removed fruit in each thinning treatment is analysed (Table 2) is possible to notice that: a) $\mathrm{V} 1$ treatment was carried out 14 days later than $\mathrm{V}$ treatment and less fruits were removed per tree, 168 compared to 223 respectively; however, V1 removed fruits were larger than those removed by V treatment, $35.7 \mathrm{~mm}$ compared to $31.1 \mathrm{~mm}$ respectively; and b) despite being done very late (12 days later than V1 treatment), M2 treatment removed intermediate size fruits between $\mathrm{V}$ and $\mathrm{V} 1,33.7 \mathrm{~mm}$ average diameter.

Table 2. Equatorial diameters of the removed fruits and number of removed fruits in the thinning treatments. Different letters are significantly different diameter at $\mathrm{P}<0.05$ according to Turkey's multiple range test.

\begin{tabular}{cccccc}
\hline Treatment & Count & Mean & Num. trees & Fruit/tree & Day \\
\hline M & 753 & $27,5^{\text {a }}$ & 5 & 151 & 7 \\
M1 & 1332 & $28,2^{\mathrm{b}}$ & 4 & 333 & 8 \\
V & 1760 & $31,1^{\mathrm{c}}$ & 9 & 223 & 8 \\
M2 & 801 & $33,7^{\mathrm{d}}$ & 8 & 100 & 34 \\
V1 & 1141 & $35,7^{\mathrm{e}}$ & 8 & 168 & 22 \\
\hline
\end{tabular}

Considering tree and treatment, related to the vibrated tree, small differences between the average fruit diameters of each tree were found. In this way, in V treatment average diameter varied between 30.1 and 32.1 $\mathrm{mm}$, and in V1 treatment, apart from tree number 14 with smaller fruits $(33.2 \mathrm{~mm})$, average diameter difference was less than $2 \mathrm{~mm}$.

Comparison between removed fruits and fruits on the tree prior to thinning

\section{Mechanical thinning}

As recorded in 2016 (Torregrosa et al., 2017), it was confirmed that the branch shaker removed fruits from all sizes, without any tendency to remove the largest or smallest fruits (Figure 4). Moreover, the mechanically detached fruits were slightly smaller than the fruits just prior to thinning (Table 3). 


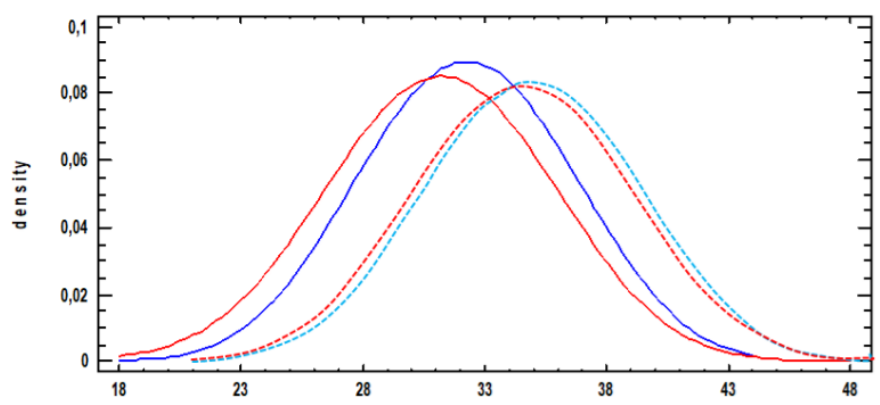

Figure 4. Density functions of the fruit diameters before shaking (red) and detached by the mechanical thinning (blue) in V (continuous) and V1 (dot) treatments.

Table 3. Equatorial diameters $(\mathrm{mm})$ of the fruit on the tree prior to thinning $(\mathrm{A})$ and the fruits removed with the vibration $(\mathrm{V})$.

\begin{tabular}{|c|c|c|c|c|}
\hline \multirow[t]{2}{*}{ Treatment } & \multicolumn{2}{|c|}{$\mathrm{V}$} & \multicolumn{2}{|c|}{ V1 } \\
\hline & A & $\mathrm{V}$ & $\mathrm{A}$ & $\mathrm{V}$ \\
\hline Count & 1000 & 1760 & 800 & 1141 \\
\hline Average (mm) & 32.23 & 31.13 & 35.99 & 35.66 \\
\hline Standard deviation (mm) & 2.84 & 3.30 & 3.48 & 3.62 \\
\hline Coeff. of variation $(\%)$ & 8.8 & 10.6 & 9.7 & 10.1 \\
\hline
\end{tabular}

\section{Manual thinning}

Table 4 shows the average diameters of the fruits on the tree just prior to thinning and the fruits removed by each treatment. Removed fruits were 4-5 mm smaller than the previous fruits on the trees. Meaning that the smallest fruits were removed, as it was expected, and it is clear that the results follow the guidelines given to the workers, removed fruits with $\mathrm{D}<28 \mathrm{~mm}$ in $\mathrm{M}$ treatment, $\mathrm{D}<30 \mathrm{~mm}$ in $\mathrm{M} 1$ and $\mathrm{D}<37 \mathrm{~mm}$ in $\mathrm{M} 2$.

Table 4. Ecuatorial diameters (mm) of fruits prior to thinning (A) and the ones of fruits manually removed (DM).

\begin{tabular}{ccccccc}
\hline Treatment & \multicolumn{2}{c}{ M } & & M1 & \multicolumn{3}{c}{ M2 } \\
\hline & A & DM & A & DM & A & DM \\
Count & 1000 & 753 & 1000 & 1332 & 800 & 801 \\
Average (mm) & 32.2 & 27.5 & 32.2 & 28.2 & 41.5 & 33.7 \\
Standard deviation (mm) & 2.84 & 2.33 & 2.84 & 2.21 & 3.35 & 2.78 \\
Coeff. of variation (\%) & 8.82 & 8.49 & 8.82 & 7.84 & 8.07 & 8.23 \\
\hline
\end{tabular}

\section{Diameters evolution}

Along the season, fruit diameters were recorded on different dates, as explained hereafter.

Fruit diameter development of the $\mathrm{N}$ treatment

From thinning date (June-July) to harvesting (September) fruits are in the linear growth II phase (Agustí et al., 1998), in which the fruits are increasing weight due to the locul development. Although bibliography defines this phase as linear, in fact it is a non-linear phase, depending on the variety and the agricultural practices.

In this study, in order to explain the different treatments, the fruit diameter development during the time period studied is analysed and the treatment without thinning $(\mathrm{N})$ is used as reference.

Figure 5 shows the non-linear development of fruit diameter, with a faster growth at the beginning and a slower growth, nearly linear, at the end. The proposed adjusted equation is:

$$
\mathrm{mm}=29.46+0.426 \mathrm{~d}-0.002317 \mathrm{~d}^{2}
$$

with R2 of $78 \%$, where mm correspond to the fruit equatorial diameter and $\mathrm{d}$ to the days passed from June 21th, 2017. 


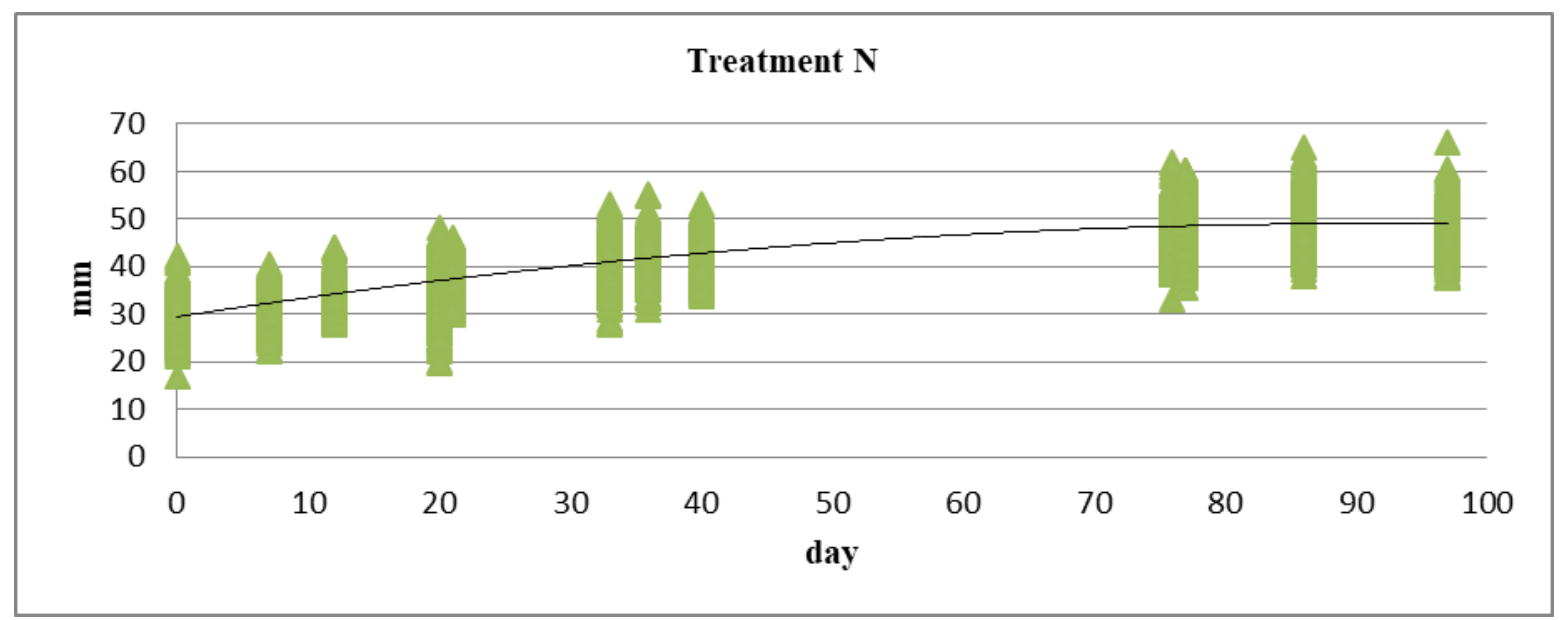

Figure 5. Growth model from N treatment fruits. Day $(d)=0=$ June $21^{\text {th }}, 2017$.

During the first 40 days fruit was growing at a rate of $0.34 \mathrm{~mm} /$ day, but in September, at the harvesting time (days 77-92) fruit growing stop.

\section{Fruit diameter development of all the treatments}

Fruit growth from all the treatments could be adjusted to second-degree polynomial equations (Table 5). The no thinning treatment $(\mathrm{N})$ and the early thinning treatments $(\mathrm{V}, \mathrm{M}$ and $\mathrm{M} 1)$ curves have two parts, a first part with a fast growth rate and a second part with a slow growth rate, nearly linear. In the case of the late thinning treatments (V1 and M2), only data from the last part of the equation was available.

Table 5. Second-degree polynomial equations from all the treatments and their coefficients, $\mathrm{mm}$ is the equatorial diameter and d passed days.

\begin{tabular}{ccc}
\hline Trat & Regression curve & $\mathrm{R}^{2}$ \\
\hline $\mathrm{N}$ & $\mathrm{mm}=29.46+0.426 \mathrm{~d}-0.0023167 \mathrm{~d}^{2}$ & 0.78 \\
V & $\mathrm{mm}=28.71+0.432 \mathrm{~d}-0.0015994 \mathrm{~d}^{2}$ & 0.85 \\
$\mathrm{M}$ & $\mathrm{mm}=30.14+0.360 \mathrm{~d}-0.0014489 \mathrm{~d}^{2}$ & 0.73 \\
M1 & $\mathrm{mm}=29.49+0.455 \mathrm{~d}-0.0021427 \mathrm{~d}^{2}$ & 0.85 \\
V1 & $\mathrm{mm}=27.44+0.477 \mathrm{~d}-0.0024089 \mathrm{~d}^{2}$ & 0.59 \\
M2 & $\mathrm{mm}=29.90+0.398 \mathrm{~d}-0.00185236 \mathrm{~d}^{2}$ & 0.51 \\
\hline
\end{tabular}

In order to better understand the phenomenon, the curves were analysed separately, early treatments (June) and late treatments (July).

Early treatments fruit diameter development

Light manual thinning (M) had similar development to the no thinning treatment $(\mathrm{N})$, as can be noticed in figure 6.

The most severe manual thinning treatment (M1) had a different development than the control treatment (N). However, the first 20-25 days the evolution was similar.

Mechanical treatment (V) started being different from the control treatment (N) after 15-20 days, but its growth rate was slightly higher than M1, reaching and leading M1 fruits diameter at the end of the first part. This fact could be due to the average larger size of the fruits left on the tree after the selective thinning in M1 treatment, and the fruits started the slow rate growth reaching the full ripe stage. However, in $\mathrm{V}$ treatment, no selective thinning was carried out and the fruits had different sizes, larger and smaller, and the smaller ones had a delayed development and they will continue growing with a higher rate at harvest. 


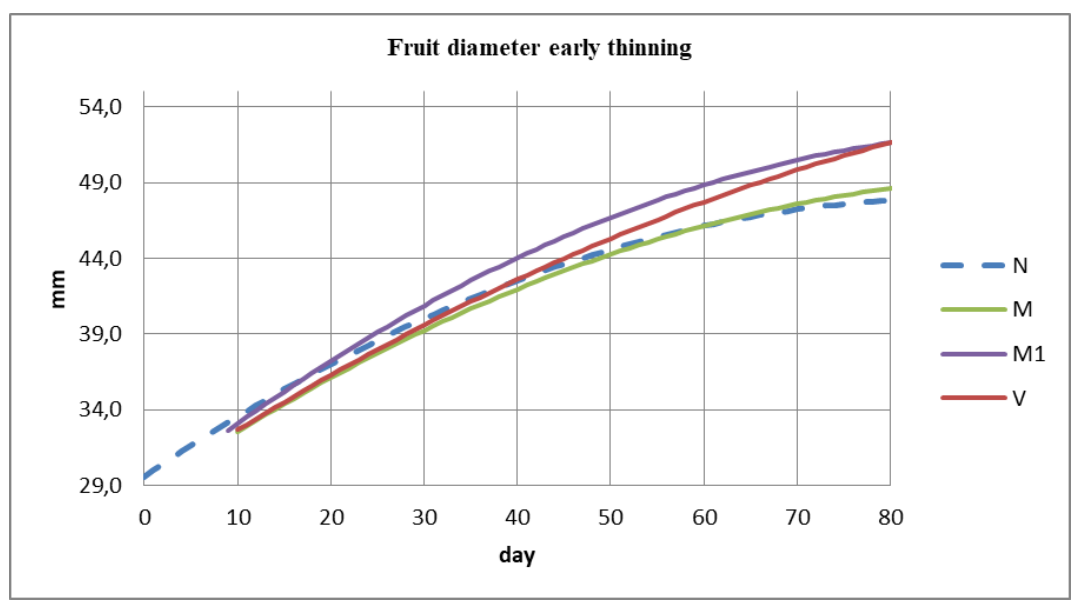

Figure 6. Average fruit diameter development, second-degree polynomial models from the early treatments (M, M1 and V).

Late treatments fruit diameter development

The manual treatment (M2) started being different from the control treatment (N) just after the thinning operation while the mechanical treatment (V1) needed nearly 40 days to have a diameter larger than the control. In the same way than in the mechanical early treatment, early in September fruits from V1 treatment had a higher growing rate than the manual treatment. Due to the short time from thinning to harvesting, differences related to the control treatment were very narrow. These results agree with those found by Agustí et al. (1998), they considered that late thinning (July and August) done by the citrus producers are only related to an aesthetic objective, removing the smaller fruits but without increasing the size of the fruits left on the tree. However, citrus producers also carry out this operation in order to remove the damaged fruits and improve the crop appearance and price.

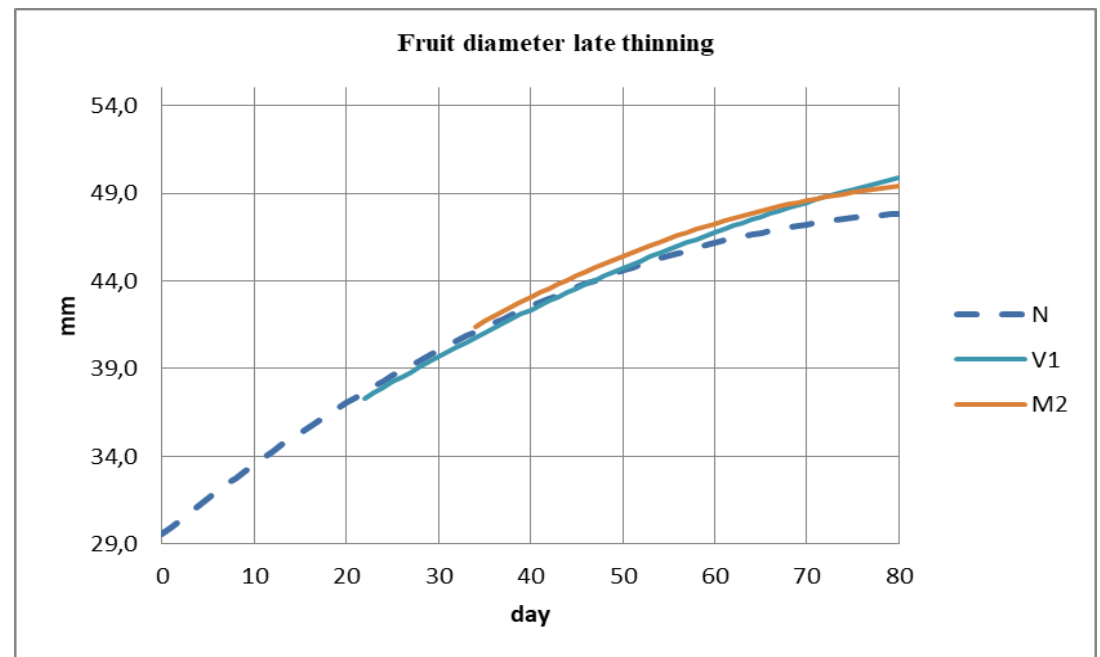

Figure 7. Average fruit diameter development, second-degree polynomial models from the late treatments (V1 y M2).

Fruit diameter just prior to harvesting

One week before harvesting (September 5-6th, 2017) fruit size from all the treatments was measured. Non thinned fruits from the control treatment $(\mathrm{N})$ had the smallest size $(48.25 \mathrm{~mm})$, followed by M treatment (an light and early manual thinning) and M2 (light and late manual thinning), without significant differences between the last two treatments and 0.7-1.0 mm diameter difference to the control. Right after it was V1 (light and late mechanical thinning) without significant differences from M2. Fruits from the early severe treatments (M1, manual) and (V, mechanical) had significantly larger fruits, with diameters 3.0 to $3.5 \mathrm{~mm}$ larger than the control $(\mathrm{N})$, Table 6.

It is remarkable that $\mathrm{V}$ treatment removing less fruits than $\mathrm{M} 1$ and being non-selective had the same final average fruit diameter as the selective M1 treatment. This fact agrees with the results found by other authors (Agustí el al., 2003), producers and technicians, confirming that when an early thinning is carried out the smaller fruits have enough time to grow and reach an adequate final diameter. 
Table 6. Fruit diameter (mm) on September 5-6 $6^{\text {th }}, 2017$. Different letters are significantly different diameter at $\mathrm{P}<0.05$ according to Turkey's multiple range test.

\begin{tabular}{cccc}
\hline Treatment & Count & Diameter $(\mathrm{mm})$ & coefficient of variation \% \\
\hline N & 1700 & $48.25^{\mathrm{a}}$ & 8.33 \\
M & 500 & $48.91^{\mathrm{b}}$ & 9.05 \\
M2 & 800 & $49.28^{\mathrm{bc}}$ & 7.15 \\
V1 & 800 & $49.82^{\mathrm{c}}$ & 8.03 \\
M1 & 400 & $51.28^{\mathrm{d}}$ & 7.18 \\
V & 900 & $51.73^{\mathrm{d}}$ & 7.11 \\
\hline
\end{tabular}

\section{Conclusions}

Fruit diameter from the non-thinned trees $(\mathrm{N})$ had a growing rate of $0.34 \mathrm{~mm} /$ day during June and July, decreasing the growing rate to $0.14 \mathrm{~mm} /$ day during August.

Fruits from the trees with early severe thinning treatments (V and M1) reached higher diameter values close to harvesting time.

Although fruits from the trees with late thinning treatments increased diameter values, the difference with the non-thinned treatment was very narrow.

Considering the mechanical thinning treatments, more than 30 days were needed to have fruits on the thinned trees with higher diameter values than the control treatment $(\mathrm{N})$. Therefore, when the thinning treatment is carried out close to the harvesting date, the fruits left on the tree do not have enough time to increase fruit size at the expenses of the reserve energy destined to the removed fruits. This fact is more clearly observed in the case of mechanical thinned trees.

\section{Acknowledgements}

This work was was supoported by the Instituto Nacional de Investigaciones Agrarias (INIA) and european FEDER founds (Projects RTA2014-00025-C05-02 and RTA2014-00025-C05-05).

To Fontestat by providing experimental fields, and specially to the technicians Federico Izquierdo and Joan Borrás.

To UPV technicians Montano Pérez, Juan José Peña and Diego Guerra.

\section{References}

Agustí, M. y Almela, V., 1984. Mejora de la calidad del fruto de la mandarina Satsuma. Bco. de Santander, Madrid, España. ISBN: 84-398-1798-3.

Agustí, M. y Almela, V., 1991. Aplicación de fitorreguladores en citricultura. ED. AEDOS, Barcelona, España

Agustí, M; Martínez-Fuentes, A; Mesejo, C; Juan, M; Almela, M., 2003. Cuajado y desarrollo de los frutos cítricos. Generalitat de Valencia, Consellería de Agricultura, Pesca y Alimentació.

Agustí, M., 2003. Citricultura. Ed. Mundi-Prensa. Madrid, España.

Davis, K., Stover E. and Wirth, F. 2004. Economics of Fruit Thinning: A Review Focusing on Apple and Citrus. HortTechnology 14(2): 282-289.

Guardiola, J.L. and García-Luis, A. 2000. Increasing fruit size in Citrus. Thinning and stimulation of fruit growth. Plant Growth Regulation 31: 121-132.

Ortiz, C., Martín-Gorriz, B., Ortí, E. and Torregrosa, A. 2016. An approach to mechanical thinning of mandarins. In ASABE Annual International Meeting.1-7. 17-20 July. Orlando, Florida.

Ortiz, C; Orti, E; Balasch, S; Martín-Gorriz, B; Torregrosa, A., 2017. Análisis del aclareo mecánico de mandarinas a través de los vídeos de desprendimiento en laboratorio. IX Congreso Ibérico de Agroingeniería. Braganza (Portugal).

Stover, E., Ciliento, S., Ritenour, M. and Counter, C. 2002. NAA thinning of 'Murcott': comparison of small plot and commercial harvest data. Proceedings of Florida State Horticultural Society 115: 287-291.

Torregrosa, A; Ortí, E; Balasch, S; Ortiz, C., 2017. Aclareo mecánico de mandarinas 'Clemenrubí' con un vibrador de ramas. IX Congresso ibérico de Agroengenharia 2017. 


\title{
Managing Weeds in Wheat and Pulses by Optimising Plant Spatial Arrangement and Sowing Yime
}

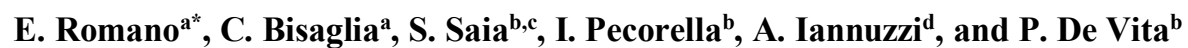 \\ ${ }^{a}$ CREA Centro di ricerca Ingegneria e Trasformazioni Agroalimentari, via Milano 43, 24047 Treviglio (BG), Italy \\ ${ }^{\mathrm{b}}$ CREA Centro di ricerca Cerealicoltura e Colture Industriali, S.S. 16 km 675, 71122 Foggia, Italy \\ ${ }^{c}$ CREA Centro di ricerca Cerealicoltura e Colture Industriali, S.S. 11 per Torino, Km 2.5, 13100 Vercelli, Italy \\ ${ }^{\mathrm{d}}$ Analist Group, via Aldo Pini 10, 83100 Avellino, Italy \\ * Corresponding author. Email: elio.romano@crea.gov.it
}

\begin{abstract}
Weed competition is a major constrain in both organic and non-organic cropping systems. Previous reports on wheat showed that the crop competitive ability against weeds can strongly be improved by a proper management of the plant spatial arrangement. In grain legumes, weed management is frequently achieved by delaying sowing time, which however reduces the length of crop cycle and thus legume ability to derive $\mathrm{N}$ from the atmosphere and transfer it to the grain, with a net effect on yield that does depends on the legume species and water availability during late season. Aim of this work was to evaluate the effects of sowing time and spatial arrangement of chickpea, lentil and durum wheat on weed biomass and yield using an unmanned aerial vehicle (UAV) remote sensing platform for precision agriculture applications. Durum wheat and pulses were sown in two contrasting spatial arrangements (5-cm and $17-\mathrm{cm}$ rows) with constant seed rate per species. Legumes were sown both at the end of autumn (December) and in the middle of winter (February), in a Vertisol located in a semi-arid Mediterranean area in Italy. Preliminary results showed a significant strong decline of weed biomass at delaying sowing time. Reduction of row distance reduced weed biomass when legumes were sown early but not late. The percentage of soil cover during early phase correlated with NDVI computed with UAV taken aerial pictures. These results suggest that avoiding late sowing to allow legumes to outcompete weed can be achieved by a proper management of the sowing pattern.
\end{abstract}

Keywords: UAV, NDVI, wheat, pulses, weeds, soil cover

\section{Introduction}

Mediterranean cropping systems pivoting on cereals, especially wheat, are often characterised by soils with low organic carbon content (Schillaci et al., 2017), which dramatically limit the $\mathrm{N}$ availability for crops. In such systems, legumes have thus an important agro-ecological role, both for increasing the number of species in rotations and $\mathrm{N}$ availability for crops via $\mathrm{N}$ sparing, water saving and $\mathrm{N}$ input after biological $\mathrm{N}$ fixation (Cernay et al., 2016; Siddique et al., 2012; Watson et al., 2017). Nonetheless, area cropped as legumes in Mediterranean strongly declined due to a number of yield limitations of these species (Magrini et al., 2016; Stagnari et al., 2017). Such limitation include the low amount of genotypes adapted to the various environments and especially the scarce ability of these species to contrast weeds, along with low availability of 'ad hoc' active ingredients for weed control (Frenda et al., 2013).

A number of management strategies other than chemical weed control can allow modulating the scarce competitive ability of legumes against weeds. These strategies, which are important in organic and integrated weed managements, include soil tillage, choice of position in the rotation, inoculation with specific rhizobia, time of sowing (Avola et al., 2008; Giambalvo et al., 2012; Kessel et al., 2000; Rubiales and Fernández-Aparicio, 2011; Ruisi et al., 2012), all of which were indicated as important traits in the prediction of legume yield (Boote et al., 1998; Siddique et al., 2012). In particular, delaying time of sowing in the semi-arid areas can allow the avoidance of winter growing weeds, but reduces the length of crops cycle and total biomass, which is related to the crop ability to derive N from the atmosphere (Evans et al., 1989; Ruisi et al., 2012; Saia et al., 2016). Such trade-offs results in unclear effects on the yield that also depend on the plant genotype and other management issues (Horn et al., 1996; Young et al., 1994).

However, the interaction of sowing patterns and time at varying the species and time of sowing is very scarce. Aim of this work was thus testing the growth of two important food legumes (chickpea and lentil) during the early phase and their ability (compared to wheat) to suppress weeds when sown under contrasting spatial arrangements (5- $\mathrm{cm}$ and $17-\mathrm{cm}$ rows with constant seed rate per species) and time of sowing. Wheat was sown at the end of autumn (December), legumes were sown both at the end of autumn (December) and in the middle of winter (February). Soil cover and weed biomass were measured both directly and estimated using multispectral (NDVI) images captured throughout the growing season. 


\section{Materials and Methods}

Experimental site

The study was performed at the Research Centre for Cereal and Industrial crops (CREA-CI, Foggia, Italy; $41^{\circ}$ $28^{\prime} \mathrm{N}, 15^{\circ} 32^{\prime} \mathrm{E}$; $75 \mathrm{~m}$ a.s.l.) in the 2017-18 growing season. The soil of the experiment is clay-loamy and classifies as Typic Chromoxerert (USDA). Texture of the fine fraction is $36 \%$ clay and $47 \%$ sand; $\mathrm{pH}(1: 2.5)$ is 7.8 . The 0 $40 \mathrm{~cm}$ depth soil layer has $17.3 \mathrm{~g} \mathrm{~kg}^{-1}$ total organic $\mathrm{C} ; 1.5 \mathrm{~g} \mathrm{~kg}^{-1}$ total organic N. Mean long-term rainfall of the experimental site is $479 \mathrm{~mm}$, and the mean air temperatures are $12.2^{\circ} \mathrm{C}$ in autumn, $8.2{ }^{\circ} \mathrm{C}$ in winter, and $17.6{ }^{\circ} \mathrm{C}$ in spring. The mean minimum and maximum annual temperatures are $9.9^{\circ} \mathrm{C}$ and $21.0^{\circ} \mathrm{C}$, respectively.

\section{Experimental design and crop management}

The experimental design was a split-plot (3 replicates) with the following treatments: main plots were time of sowing (either early or late) and plant spatial arrangement $(5 \mathrm{~cm}$ and $17 \mathrm{~cm}$ rows with constant seed rate per species). The split-plot was species: durum wheat, chickpea or lentil. Durum wheat was sown only in the early sowing time.

The size of each plot was $40 \mathrm{~m} \times 10 \mathrm{~m}$. The preceding crop was durum wheat. Before sowing, the soil was ploughed in late August and harrowed twice in September and October, to prepare a suitable seedbed and to control the summer weeds. The pre-sowing fertilisation consisted of $36.0 \mathrm{~kg} \mathrm{~N} \mathrm{ha}^{-1}$ and $40.1 \mathrm{~kg} \mathrm{P}^{-1}$ (as diammonium phosphate), top-dressing fertilisation consisted of $54 \mathrm{~kg} \mathrm{~N}^{-1}$ (as ammonium nitrate). The topdressing fertilisation was applied at wheat growth stage 31 .

Early sowing date was on $22^{\text {nd }}$ of December 2017; late sowing date was on $12^{\text {th }}$ of February 2018. Sowing at $5 \mathrm{~cm}$ was performed using a seed drill prototype (SEMINBIO project, RM2013A000332 and/or 202015000006429 ) that was equipped with a series of delivery elements spaced at $5 \mathrm{~cm}$. The sowing of the $17 \mathrm{~cm}$ inter-row distance was performed with a Vicon Accord $4.5 \mathrm{~m}$ seed drill.

Sowing density was 400 viable seeds $\mathrm{m}^{-2}$ in wheat (Cultivar Natal). Sowing density for legumes varied by row distance and was 65 and 40 viable seeds $\mathrm{m}^{-2}$ in chickpea at $17 \mathrm{~cm}$ and $5 \mathrm{~cm}$ row, respectively, and 250 and 150 viable seeds $\mathrm{m}^{-2}$ in lentil at $17 \mathrm{~cm}$ and $5 \mathrm{~cm}$ row, respectively.

\section{Weeds and crop analysis}

Date of complete crop emergence and number of plants per unit area were recorded. NDVI was measured using a UAV-based platform (Parrot Sequoia, Paris, France) equipped with a multi-spectral camera on the $28^{\text {th }}$ of March 2018 (in early sown crops, only, since in late sown plant and weed biomass was near zero) and on $10^{\text {th }}$ of April 2018 and $18^{\text {th }}$ of May 2018. In a sub-plot $\left(3 \mathrm{~m}^{2}\right)$, weed biomass was collected and dry biomass of weeds recorded on the $29^{\text {th }}$ of March 2018 and $28^{\text {th }}$ of May 2018.

\section{Calculations and statistical analysis}

Data were subjected to analysis of variance by a general linear mixed model (GLIMMIX procedure in SAS/STAT 9.2) with sowing time nested in the crop. Least squares means were computed and when each factor or interaction showed a $\mathrm{p}>\mathrm{F}$ lower than 0.05 , leas squares means differences were computed and grouped with Tukey test at 0.05 corrected for Tukey-Kramer Grouping. Correlation among traits was computed.

\section{Results and Discussion}

The meteorological conditions during the growing season were dramatically different than the long term means, with a relatively dry and strongly cold winter and spring, which likely impaired both crop and weed emergence and growth. Plant density at the time of crop emergence strongly varied by the treatments applied, as expected (Table 1). In particular, plant density was on average $15 \% \pm 1.2 \%$ (mean \pm standard error) lower than sowing rate, with few differences among the treatments applied (data not shown). Similarly, time to complete emergence scarcely varied among sowing times and row distance and was, on average 25.7 days and 11.0 days for the legumes and wheat, respectively. Plant density highly correlated with NDVI taken on the $28^{\text {th }}$ of March $2018(\mathrm{r}=0.90)$ but not with NDVI in the $10^{\text {th }}$ of April $(\mathrm{r}=0.49)$ and $18^{\text {th }}$ of May $(\mathrm{r}=0.27)$. This suggests that NDVI taken with an unmanned aerial vehicle (UAV) can be a valuable option to measure plant density, which is related to crop competition against weeds (Weiner et al., 2001) and yield potential in legumes (Sankaran et al., 2016). Similar results were found by (Flowers et al., 2001).

On the $28^{\text {th }}$ of March, crop and weed biomass in late sown stands were negligible and no NDVI could be computed. Differences among treatments for NDVI values mostly occurred in the first sampling: 28/03/18 for early sown and 10/04/18 for late sown) and minimised at increasing crop growth (Figure 1). In particular, on the $10^{\text {th }} / 04$, NDVI differed by the crop and sowing time but not row distance, with, what higher than lentil and 
chickpea and early sown crops showing higher NDVI than late sown crops. On the $18^{\text {th }}$ of May, almost no differences were found between row distances within species and sowing date, except that in late sown chickpea, in which the crop sown at $17-\mathrm{cm}$ rows showed NDVI values $20.3 \%$ higher than the crop sown at 5-cm rows.

Weed biomass in the first sampling of weed biomass strongly varied by row distance, with $17-\mathrm{cm}$ rowed crops showing on average 3.7 fold more biomass than $5-\mathrm{cm}$ rowed crops (Table 1 and Figure 2, sampling of the $29^{\text {th }}$ of March). In the first sampling of weed biomass, only late sowing reduced weeds in lentil but not in chickpea (Figure 2 ). In the second sampling of weed biomass (Figure 2, sampling of the $28^{\text {th }}$ of May), weed amount varied at varying both the row distance and sowing time per species. In particular, reducing row distance reduced weed biomass in the early-sown crops, only. These data confirm those found by De Vita et al. (2017) in durum wheat, where row distance can be important to reduce weed biomass and increase yield. Also, in De Vita et al. (2017) we also found that variation of the sowing patter decreases weed presence the more the weed in the crops. Thus, it is likely that since late sown legumes showed fewer weeds than early sown crops, in such conditions, ability of the reduced row system to hamper weed growth was minimised. Notably, this experiment was performed in a growing season characterised by low weed germination, which scarcity could have reduced the need of favouring the legume competitive ability against weeds and could have increased the amount of resources for the legume stands.

Weed biomass in the first sampling of weed biomass strongly varied by row distance, with 17-cm rowed crops showing on average 3.7 fold more biomass than $5-\mathrm{cm}$ rowed crops (Table 1 and Figure 2, sampling of the $29^{\text {th }}$ of March). In the first sampling of weed biomass, only late sowing reduced weeds in lentil but not in chickpea (Figure 2 ). In the second sampling of weed biomass (Figure 2, sampling of the $28^{\text {th }}$ of May), weed amount varied at varying both the row distance and sowing time per species. In particular, reducing row distance reduced weed biomass in the early-sown crops, only.

These data confirm those found by De Vita et al. (2017) in durum wheat, where row distance can be important to reduce weed biomass and increase yield. Also, in De Vita et al. (2017) we also found that variation of the sowing patter decreases weed presence the more the weed in the crops. Thus, it is likely that since late sown legumes showed fewer weeds than early sown crops, in such conditions, ability of the reduced row system to hamper weed growth was minimised.

Notably, this experiment was performed in a growing season characterised by low weed germination, which scarcity could have reduced the need of favouring the legume competitive ability against weeds and could have increased the amount of resources for the legume stands.

Table 1. Results of the general linear mixed model for row distance (RD), sowing time (ST) and Crop (Cr) with ST nested in

\begin{tabular}{|c|c|c|c|c|c|c|c|c|c|c|c|c|c|c|}
\hline & \multirow{3}{*}{$\begin{array}{c}\text { DF } \\
\text { num }\end{array}$} & \multirow{3}{*}{$\begin{array}{l}\text { DF } \\
\text { den }\end{array}$} & \multirow{2}{*}{\multicolumn{2}{|c|}{ Plant density }} & \multicolumn{6}{|c|}{ NDVI } & \multicolumn{4}{|c|}{ Weed Biomass } \\
\hline & & & & & \multicolumn{2}{|c|}{$28 / 03 / 2018$} & \multicolumn{2}{|c|}{$10 / 04 / 2018$} & \multicolumn{2}{|c|}{$18 / 05 / 2018$} & \multicolumn{2}{|c|}{$29 / 03 / 2018$} & \multicolumn{2}{|c|}{$18 / 05 / 2018$} \\
\hline & & & $\mathrm{F}$ & $\mathrm{p}>\mathrm{F}$ & $\mathrm{F}$ & $\mathrm{p}>\mathrm{F}$ & $\mathrm{F}$ & $\mathrm{p}>\mathrm{F}$ & $\mathrm{F}$ & $\mathrm{p}>\mathrm{F}$ & $\mathrm{F}$ & $\mathrm{p}>\mathrm{F}$ & $\mathrm{F}$ & $\mathrm{p}>\mathrm{F}$ \\
\hline $\mathrm{D}$ & 1 & 6 & 204.4 & $<.001$ & 3.98 & 0.093 & 1.0 & 0.357 & 16.1 & 0.007 & 33.2 & 0.001 & 58.6 & $<.001$ \\
\hline $\mathrm{ST}(\mathrm{Cr})$ & 4 & 8 & 973.9 & $<.001$ & n.a & n.a & 213.8 & $<.001$ & 14.0 & 0.001 & 8.3 & 0.006 & 17.5 & 0.001 \\
\hline $\mathrm{RD} \times \mathrm{ST}(\mathrm{Cr})$ & 4 & 8 & 87.0 & $<.001$ & n.a & n.a & 2.7 & 0.105 & 31.6 & $<.001$ & 3.0 & 0.085 & 6.1 & 0.015 \\
\hline
\end{tabular}




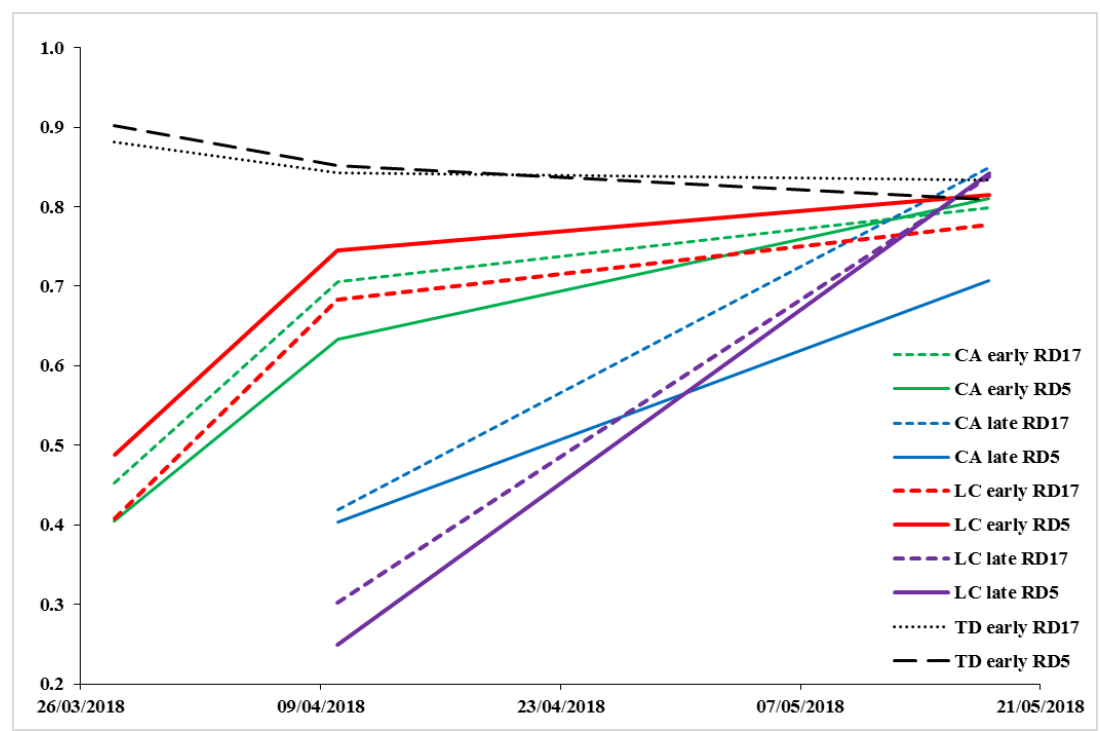

Figure 1. NDVI at varying the crop (Chickpea, CA; lentil, LC; and durum wheat, TD), sowing time (early or late) and row distance (5-cm rows, $\mathrm{RD} 5$; and 17-cm rows, $\mathrm{RD} 17$ ).

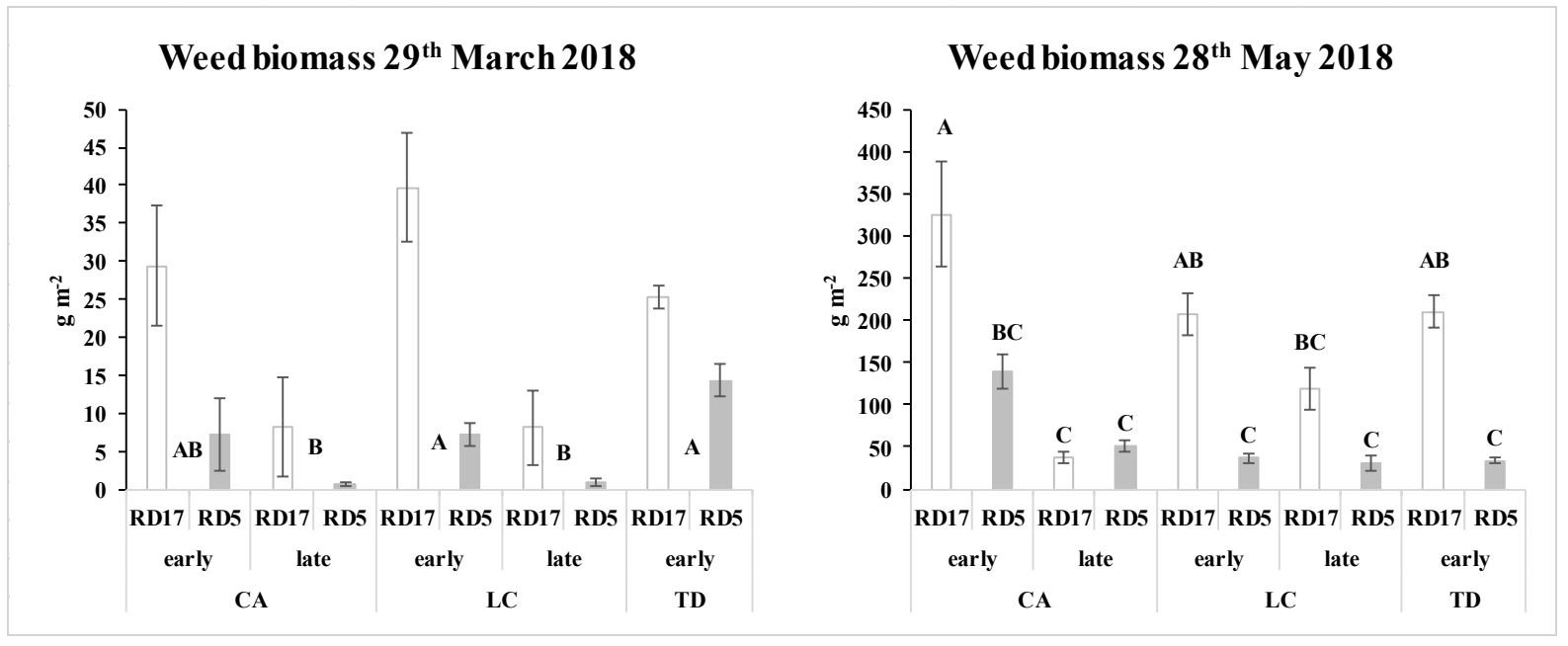

Figure 2. Weed biomass $\left(\mathrm{g} \mathrm{m}^{-2}\right)$ at varying the crop (Chickpea, CA; lentil, LC; and durum wheat, TD), sowing time (early or late) and row distance (5-cm rows, RD5; and 17-cm rows, RD17). Bars are means \pm S.E. For weed biomass at the $28^{\text {th }}$ of May, early sown differed by the late sown chickpea, whereas no differences were found among lentil and wheat. Sowing time (species) treatments with a letter in common are not different according to the Tukey-Kramer test at 5\%. In the left panel, sowing time (species) difference among means were indicated with letters shared between row distances and row

distance was significant (not indicated). In the right panel, letters apply to the row distance $\times$ sowing time (species) interaction.

\section{Conclusions}

In this work we showed that optimization of the sowing pattern by reducing row distance can increase legume competitive ability against weed and that such competitiveness is related to ability of the crop to cover early the soil. Nonetheless, we've seen that such effects mostly occurred in early sown crops, where higher weed biomass were found compared to late-sown crops.

When sowing chickpea in late winter, weed biomass declined compared to sowing in late fall, and optimization of the sowing patter was less efficient in reducing further weed biomass. Nonetheless, such reduction can still have implication for legume yield due to a sparing of water from the soil reserve. Such effect was not seen in lentil and this could be due to both the lower ability of lentil to scavenge soil reserve and its lower plant height compared to chickpea, and thus competitive ability against weeds, as seen in both legumes and cereals (Ruisi et al., 2015, 2012; Saia et al., 2012).

These results also confirm that proximal sensed, automatable information collection, such as the NDVI from aerial pictures collected after the use of a UAV, is a valuable option to measure crop cover in the early phase, which is related to crop ability to outcompete weeds. 
Delaying sowing is a strategy to reduce legume problems related to early weed emergence and especially pathogenic and broomrape attacks (Gan et al., 2006; Rubiales et al., 2003). Nonetheless, in semi-arid areas, the reduction of the crop cycle by late sowing can consist in both a reduction of total biomass and exposure of the plants to warmer temperatures during pod filing, which can reduce yield potential (Thomson et al., 1997). Here we showed that optimization of the sowing pattern can allow the plant to establish and cover the soil earlier and can allow a competition.

Ongoing experiments (Romano et al. 2018; ID 388 in this congress) are also being conducted to identify an effective method to be included in an application for the smartphone or other proximal sensing devices useful for the defining the presence of weeds.

\section{Acknowledgements}

This work was supported by the Italian Ministry of Agriculture (MiPAAF) under the BIODURUM project (D.D. n. 5424, 17 may 2017) and Rete Rurale Nazionale 2014-2020 "Sviluppo di attività di supporto, animazione e collegamento delle politiche per l'innovazione e la conoscenza (PEI, Piano strategico per l'innovazione e la ricerca, politiche regionali"

\section{References}

Avola, G., Tuttobene, R., Gresta, F., Abbate, V., 2008. Weed control strategies for grain legumes. Agron. Sustain. Dev. 28, 389-395. doi:10.1051/agro:2008019

Boote, K.J., Jones, J.W., Hoogenboom, G., Pickering, N.B., 1998. The CROPGRO model for grain legumes, in: Tsuji, G.Y., Hoogenboom, G., Thornton, P.K. (Eds.), Understanding Options for Agricultural Production. Springer, Dordrecht, pp. 99-128. doi:10.1007/978-94-017-3624-4_6

Cernay, C., Pelzer, E., Makowski, D., 2016. A global experimental dataset for assessing grain legume production. Sci. Data 3, 160084. doi:10.1038/sdata.2016.84

De Vita, P., Colecchia, S.A., Pecorella, I., Saia, S., 2017. Reduced inter-row distance improves yield and competition against weeds in a semi-dwarf durum wheat variety. Eur. J. Agron. 85, 69-77. doi:10.1016/j.eja.2017.02.003

Evans, J., O’Connor, G., Turner, G., Coventry, D., Fettell, N., Mahoney, J., Armstrong, E., Walsgott, D., 1989. N 2 fixation and its value to soil N increase in lupin, field pea and other legumes in south-eastern Australia. Aust. J. Agric. Res. 40, 791. doi:10.1071/AR9890791

Flowers, M., Weisz, R., Heiniger, R., 2001. Remote Sensing of Winter Wheat Tiller Density for Early Nitrogen Application Decisions. Agron. J. 93, 783. doi:10.2134/agronj2001.934783x

Frenda, A.S., Ruisi, P., Saia, S., Frangipane, B., Di Miceli, G., Amato, G., Giambalvo, D., 2013. The Critical Period of Weed Control in Faba Bean and Chickpea in Mediterranean Areas. Weed Sci. 61, 452-459. doi:10.1614/WS-D-12-00137.1

Gan, Y.T., Siddique, K.H.M., MacLeod, W.J., Jayakumar, P., 2006. Management options for minimizing the damage by ascochyta blight (Ascochyta rabiei) in chickpea (Cicer arietinum L.). F. Crop. Res. 97, 121-134. doi:10.1016/j.fcr.2005.10.002

Giambalvo, D., Ruisi, P., Saia, S., Di Miceli, G., Frenda, A.S., Amato, G., 2012. Faba bean grain yield, N2fixation, and weed infestation in a long-term tillage experiment under rainfed Mediterranean conditions. Plant Soil 360, 215-227. doi:10.1007/s11104-012-1224-5

Horn, C., Birch, C., Dalal, R., Doughton, J., 1996. Sowing time and tillage practice affect chickpea yield and nitrogen fixation. 1. Dry matter accumulation and grain yield. Aust. J. Exp. Agric. $36,695$. doi:10.1071/EA9960695

Kessel, C. Van, Hartley, C., Van Kessel, C., Hartley, C., 2000. Agricultural management of grain legumes: has it led to an increase in nitrogen fixation? F. Crop. Res. 65, 165-181. doi:10.1016/S0378-4290(99)00085-4

Magrini, M.-B., Anton, M., Cholez, C., Corre-Hellou, G., Duc, G., Jeuffroy, M.-H., Meynard, J.-M., Pelzer, E., Voisin, A.-S., Walrand, S., 2016. Why are grain-legumes rarely present in cropping systems despite their environmental and nutritional benefits? Analyzing lock-in in the French agrifood system. Ecol. Econ. 126, 152162. doi:10.1016/j.ecolecon.2016.03.024

Rubiales, D., Alcántara, C., Alejandro Pérez-de-luque, Gil, J., Sillero, J.C., 2003. Infection of chickpea (Cicer arietinum) by crenate broomrape (Orobanche crenata) as influenced by sowing date and weather conditions. Agronomie 23, 359-362. doi:10.1051/agro:2003016

Rubiales, D., Fernández-Aparicio, M., 2011. Innovations in parasitic weeds management in legume crops. A review. Agron. Sustain. Dev. 32, 433-449. doi:10.1007/s13593-011-0045-x

Ruisi, P., Frangipane, B., Amato, G., Frenda, A.S., Plaia, A., Giambalvo, D., Saia, S., 2015. Nitrogen uptake and nitrogen fertilizer recovery in old and modern wheat genotypes grown in the presence or absence of interspecific competition. Front. Plant Sci. 6, 185. doi:10.3389/fpls.2015.00185

Ruisi, P., Giambalvo, D., Di Miceli, G., Frenda, A.S., Saia, S., Amato, G., 2012. Tillage Effects on Yield and 
Nitrogen Fixation of Legumes in Mediterranean Conditions. Agron. J. 104, 1459. doi:10.2134/agronj2012.0070

Saia, S., Ruisi, P., Amato, G., Di Miceli, G., Frenda, A.S., Giambalvo, D., P, R., G, A., G, D.M., AS, F., D, G., 2012. Effects of soil inoculation with arbuscular mycorrhizal fungi on plant growth and nutrient uptake of some Mediterranean species grown under rainfed field conditions, in: 17th International Nitrogen Workshop. Richards, K; Fenton, O; Watson, CJ, Wexford, pp. 90-91.

Saia, S., Urso, V., Amato, G., Frenda, A.S., Giambalvo, D., Ruisi, P., Di Miceli, G., 2016. Mediterranean forage legumes grown alone or in mixture with annual ryegrass: biomass production, N2 fixation, and indices of intercrop efficiency. Plant Soil 402, 395-407. doi:10.1007/s11104-016-2837-x

Sankaran, S., Khot, L.R., Quirós, J., Vandemark, G.J., McGee, R.J., 2016. UAV-based high-throughput phenotyping in legume crops, in: Valasek, J., Thomasson, J.A. (Eds.), . p. 98660G. doi:10.1117/12.2228550

Schillaci, C., Acutis, M., Lombardo, L., Lipani, A., Fantappiè, M., Märker, M., Saia, S., 2017. Spatio-temporal topsoil organic carbon mapping of a semi-arid Mediterranean region: The role of land use, soil texture, topographic indices and the influence of remote sensing data to modelling. Sci. Total Environ. 601-602, 821-832. doi:10.1016/j.scitotenv.2017.05.239

Siddique, K.H.M., Johansen, C., Turner, N.C., Jeuffroy, M.-H., Hashem, A., Sakar, D., Gan, Y., Alghamdi, S.S., 2012. Innovations in agronomy for food legumes. A review. Agron. Sustain. Dev. 32, $45-64$. doi:10.1007/s13593-011-0021-5

Stagnari, F., Maggio, A., Galieni, A., Pisante, M., 2017. Multiple benefits of legumes for agriculture sustainability: an overview. Chem. Biol. Technol. Agric. 4, 2. doi:10.1186/s40538-016-0085-1

Thomson, B.D., Siddique, K.H.M., Barr, M.D., Wilson, J.M., 1997. Grain legume species in low rainfall mediterranean-type environments I. Phenology and seed yield. F. Crop. Res. 54, 173-187. doi:10.1016/S03784290(97)00047-6

Watson, C.A., Reckling, M., Preissel, S., Bachinger, J., Bergkvist, G., Kuhlman, T., Lindström, K., Nemecek, T., Topp, C.F.E., Vanhatalo, A., Zander, P., Murphy-Bokern, D., Stoddard, F.L., 2017. Grain Legume Production and Use in European Agricultural Systems. pp. 235-303. doi:10.1016/bs.agron.2017.03.003

Weiner, J., Griepentrog, H.W., Kristensen, L., 2001. Suppression of weeds by spring wheat Triticum aestivum increases with crop density and spatial uniformity. J. Appl. Ecol. 38, 784-790. doi:10.1046/j.13652664.2001.00634.x

Young, R., Morthorpe, K., Nicol, H., Croft, P., 1994. Effects of sowing time and grazing on the dry matter yield, phenology, seed yield, and hardseed levels of annual pasture legumes in western New South Wales. Aust. J. Exp. Agric. 34, 189. doi:10.1071/EA9940189 


\title{
The design and development of machinery for planting daffodil bulbs under agricultural upland grassland and harvesting the above ground biomass.

\author{
*D R White-1, I J Loynes', S E Cooper ${ }^{1}$, K Stephens², S Head², M D Fraser ${ }^{3}$, H Vallin³ and J R T \\ Davies ${ }^{3}$.
} \\ ${ }^{1}$ Harper Adams University, Newport, Shropshire, TF10 8NB, UK. \\ ${ }^{2}$ Agroceutical Products Ltd, Fedwlydan, Glasbury-on-Wye, Powys HR3 5ND. \\ ${ }^{3}$ Pwllpeiran Upland Research Centre, Cwmystwyth, Aberystwyth, Ceredigion, SY23 4AD. \\ *Corresponding author: drwhite@harper-adams.ac.uk
}

\begin{abstract}
The number of people suffering from dementia is considerable and growing at a significant rate. Alzheimer's disease accounts for between 50 and $75 \%$ of these cases. Galantamine is a pharmaceutical compound that has been an approved treatment for Alzheimer's disease since 1998. Galantamine can be synthesised chemically but it is a difficult and expensive process. Producing galantamine from the alkeloid galanthamine extracted from daffodils is more cost effective, but supplies are limited. Research has suggested that the environmental challenges associated with upland areas trigger a higher concentration of galanthamine in daffodils compared to daffodils grown under lowland conditions. A 4.5 year UK Agri-Tech Catalyst Industrial Research project is investigating daffodil-derived galanthamine production by integrating daffodil growing into permanent upland sheep pasture. The aim is to increase the economic sustainability of hill farming by providing farmers with a high value supplementary daffodil crop while maintaining a traditional farming system. Machinery is readily available for lowland daffodil production for the cut flower market and for the production of bulbs. Soils are typically deep, fertile and free draining. However, the UK uplands are characterised by low temperatures; exposure to wind; high rainfall; winter snow and frosts; thin impoverished stony soils; a shortage of major nutrients and steep slopes. As part of the research project Harper Adams University agricultural engineers have developed machines for planting daffodil bulbs and harvesting the above ground daffodil biomass in these arduous upland grassland pastures. The planter uses belts to meter and deliver bulbs from the storage hopper to two drop chutes positioned above the purpose built ground opening winged tines. The harvester removes and collects the above ground biomass which is then transferred to sealed containers before being processed.
\end{abstract}

Keywords: Galanthamine, Galantamine, Daffodil, Upland Pasture, Planting, Harvesting.

\section{Introduction}

The number of people suffering from dementia is considerable and growing at a significant rate. In 2017, there were estimated to be 50 million dementia sufferers worldwide (Alzeimers Disease International, 2018) and 4.6 million new cases are diagnosed each year. Alzeimers Disease (AD) accounts for between 50 and $75 \%$ of these cases. The U.S. Food and Drug Administration, the UK Medicines and Healthcare Products Regulatory Agency and The European Medicines Agency has approved galantamine as an AD treatment since 1998. Galantamine can be synthesised chemically but it is a difficult and expensive process (Trost and Toste, 2000; Marco-Contelles et al, 2006). Producing galantamine from galanthamine extracted from plants, for example, daffodils (Narcissus sp) (Torras-Claveria et al, 2013) is more cost effective, but supplies are limited. The annual global consumption of

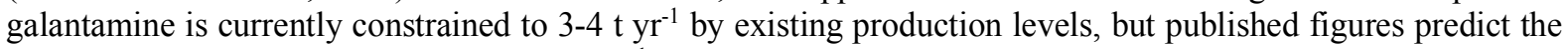
potential global market could be nearer $40 \mathrm{t} \mathrm{yr}^{-1}$. Independent reports project the competitive active pharmaceutical ingredient price for galantamine drugs will remain between $£ 15,000-£ 18,000 \mathrm{~kg}^{-1}$ in the medium term.

Previous research has suggested that the environmental challenges associated with upland areas (low temperatures; exposure to wind; high rainfall; winter snow and frosts; thin impoverished stony soils; a shortage of major nutrients and steep slopes) trigger a higher yield of galanthamine in daffodils (bulbs and above ground bio-mass) that are grown there when compared to those grown in normal lowland conditions (Department for Environment Food \& Ruarl Affairs, 2006). Daffodils grown for galanthamine production, therefore, could offer a novel, high-value crop for upland farmers that will provide an important new income stream, increasing their economic resilience.

Currently, daffodil-derived galanthamine production requires annual mouldboard ploughing of the soil (with the associated release of greenhouse gases and soil erosion) and strict herbicide regimes to control weeds (with associated impacts on biodiversity). A 4.5 year UK Agri-Tech Catalyst Industrial Research project (commenced March 2015) is investigating daffodil-derived galanthamine production by integrating daffodil growing into permanent upland sheep pasture. The aim is to increase both the production of galanthamine and the economic sustainability of hill farming by providing farmers with a high value supplementary daffodil crop while maintaining a traditional farming system. This paper describes the equipment that has been developed for planting daffodil bulbs and harvesting the above ground daffodil biomass in these arduous upland grassland environments. 


\section{Materials and Methods}

Prime mover

A range of vehicles, including compact agricultural tractors, all-terrain vehicles (ATVs) and off road utility vehicles, as well as self-propelled tracked vehicles, were evaluated as potential prime movers for both the planting and harvesting operations. Because the production of the daffodils is designed to integrate with existing pastureland and the required tractive forces needed to pull the planter, a $55 \mathrm{~kW}$ tractor was chosen to pull both the planter and harvester.

Planter

The aim of the planter was to plant commercially available daffodil bulbs (sizes 10/12, 12/14, 14/16 and $16+$ ) under sloping permanent pasture at rates of 4 to $10 \mathrm{t} \mathrm{ha}^{-1}$. Upland soils are typically shallow and stony with the subsoil containing large stones / rocks (Soil Survey of England and Wales, 1984a; Soil Survey of England and Wales, 1984b). Ideally, daffodil bulbs should be planted $150 \mathrm{~mm}$ deep and, in cold climates, the bulbs should be coved by $75 \mathrm{~mm}$ of soil to protect them from frost.

\section{Row spacing}

As it is not known how long daffodils will remain productive under these harsh conditions when they are harvested each year with limited opportunity to regenerate the below ground biomass, it was decided to plant the bulbs in twin rows $800 \mathrm{~mm}$ apart. This will allow, if necessary, daffodils to be planted in between existing rows by moving the tractor / planter combination over by "half a wheel-track width".

\section{Metering mechanism.}

Typically, metering mechanisms on bulb / tuber planters consist of either flat belts or cup mechanisms (Bell, 2016). For simplicity, a belt mechanism was chosen but, unlike machines used on flat / slightly undulating ground, a cleated belt (Figure 1) was used to provide traction for the bulbs and provide consistent feed rates when going up and down hill. To give a controllable, wide range of planting densities, a hydraulic motor was used to drive the metering mechanism and provide planting rates of up to $10 \mathrm{tha}^{-1}$.
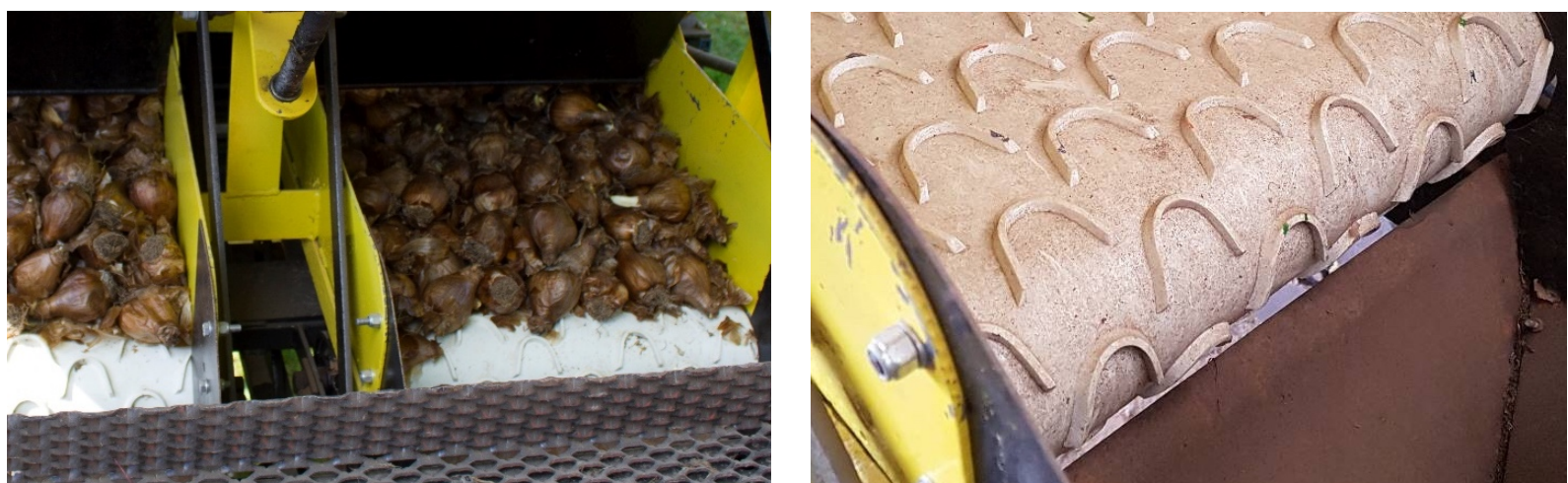

Figure 1. Planter cleated belt.

Depth control

Depth control was via wheels at both the front and rear of the planter. The rear wheels also acted as press wheels to consolidate the loosened sward to give good soil / bulb contact.

Ground opening device

Upland soils are generally shallow (75 to $150 \mathrm{~mm}$ ) and have low shear strengths. To minimise the damage to the existing grass sward, a heavy duty plain disc (Figure 2) was used to make a vertical cut through the sward. To minimise draft forces (Godwin and Spoor, 1974) and to allow the sward to remain intact whilst being lifted and then carefully replaced in its original position once the bulbs had been planted, an opener with a low rake angle was required (Payne and Tanner, 1959). Experiments by Cooper (2015) evaluated a range of commercially available tines. A specially designed furrow opener (Figure 3) was manufactured with the aim of peeling back the sward cut by the leading disc, gently lifting it intact whilst the bulbs were planted before allowing the sward to fall back into place so covering the bulbs. 


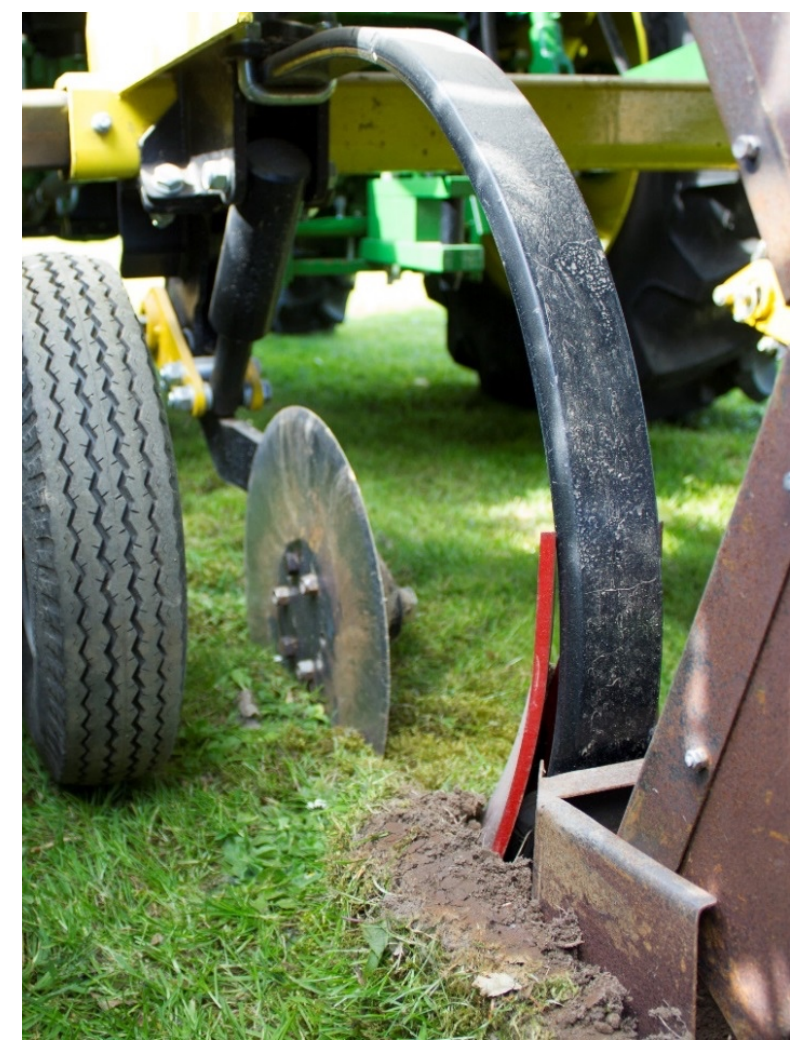

Figure 2. Planter tine and disc unit.

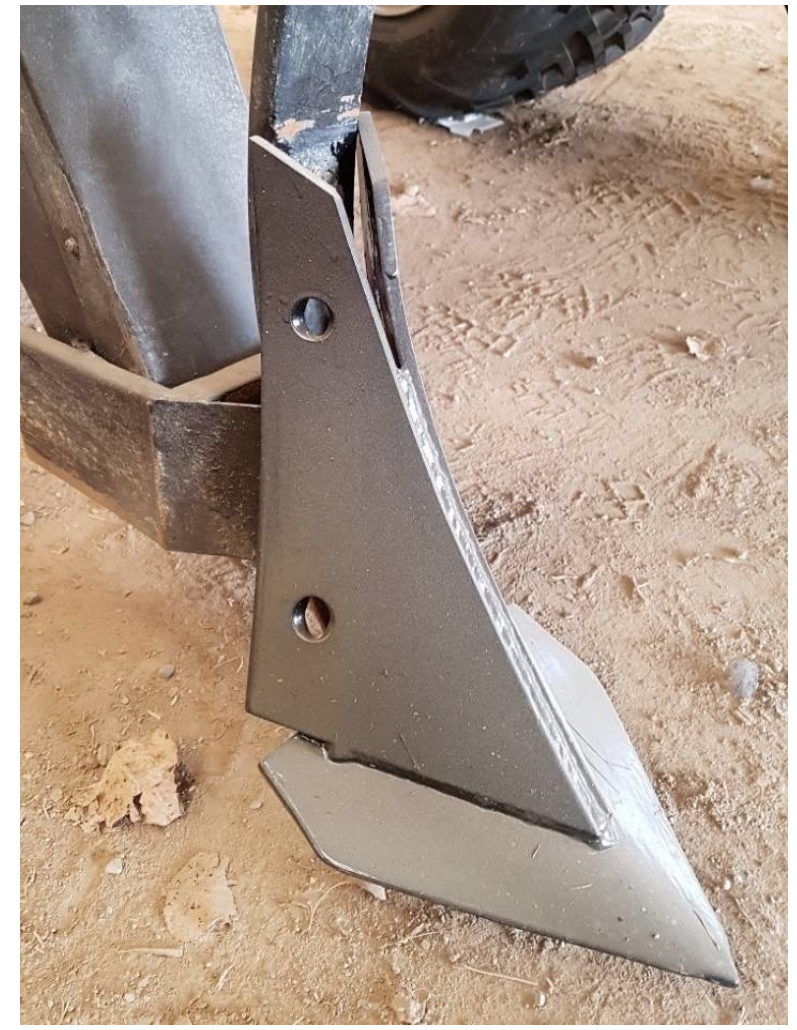

Figure 3. Soil opener.

\section{Frame}

Because of the undulating ground profile, local depressions and mounds, and rocks close to the soil surface, an articulating frame was manufactured to enable independent movement of the front and rear halves of the planter so improving the depth control of the two-row planter.

\section{Harvesting machinery}

The aim of the harvester was to harvest the above ground daffodil bio-mass and grass and deliver the product into water tight boxes sealed with a lid to prevent external contamination from vermin, birds and weather. These boxes are then transported off site where the material is processed and the galantamine extracted.

Typically, above ground bio-mass of agricultural or horticultural products is harvested either by cutting or flailing. A range of commercially available equipment, for example, baby leaf salad harvesters and amenity flail grass harvesters were considered as base units. Both of these techniques were evaluated on daffodils in 2016. In order to eliminate the need for additional processing of the harvested bio-mass at the galantamine extraction phase and reduce transportation costs, flailing was chosen as the preferred method. In 2017, a commercially available flail harvester with a working width of $1.3 \mathrm{~m}$ was purchased. This enabled two rows of daffodils to be harvested at once. Modifications in 2017 and 2018 included changes to the cutting height mechanism (range 0 to $350 \mathrm{~mm}$ ), modifying the rear castor wheels and the addition of a hopper discharge mechanism.

\section{Results and Discussion}

The planter and harvester have been used to establish and harvest a total of 15 ha of experimental plots at the Aberystwyth University Upland Research Centre, Pwllpeiran, Wales $\left(52.3553^{\circ} \mathrm{N}, 3.8006^{\circ} \mathrm{W}\right)$. The project plots are in permanent pasture that ranges from 250 to $430 \mathrm{~m}$ above sea level with slopes of up to 27 degrees. The soil is a clay loam with an average cohesion of $22.5 \mathrm{kN} \mathrm{m}^{-2}$ and angle of internal shearing resistance of $9.5^{\circ}$ and an average stone content of $45 \%$ (Cooper, 2015).

Planting

The first-year planting (2015) was completed using a single row version of the final machine (Figure 4) using a ground wheel drive and a V-belt driven metering mechanism. A total of 6 ha of plots were planted with size 10/12 and 12/14 bulbs at a rate of $4 \mathrm{t} \mathrm{ha}^{-1}$ and rows spaced at $800 \mathrm{~mm}$ apart. For the second year, planting 
rates of up to $10 \mathrm{tha}^{-1}$ were required with bulbs up to $14 / 16$ and the ground wheel V-belt drive system was replaced with a hydraulic motor. An additional 10 ha of project trial plots were planted in the second year using the tworow planter as shown in Figure 4.

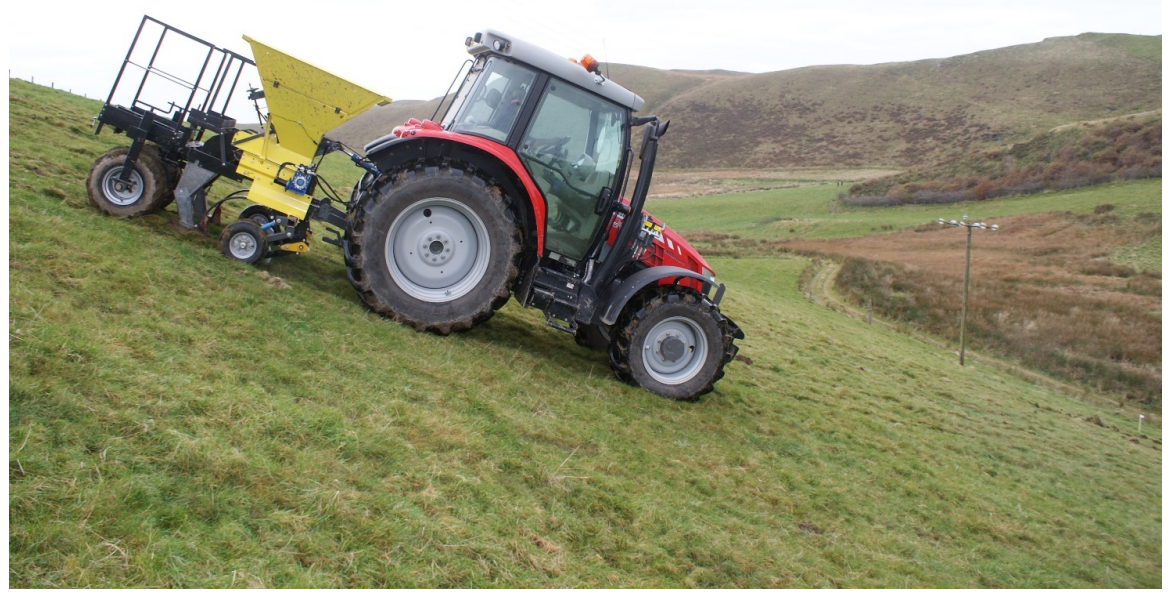

Figure 4. Planter at Aberystwyth University Upland Research Centre.

The articulated frame performed well and enabled good planting depth control over the undulating ground profile. The metering mechanism resulted in the bulbs being randomly orientated between "apex up" and "apex down". Additional plots and small scale plot experiments in 2016 and 2017 showed that bulbs planted "apex down" emerged up to 2 weeks later than those planted "apex up" leading to inconsistencies in growth stage at harvest. This suggests it may be worthwhile designing a metering mechanism that will plant all the bulbs "apex up" but the effects on plant growth in subsequent years is comparatively minimal.

\section{Harvesting}

To maximise the amount of daffodil bio-mass whilst minimising the amount of grass and soil contamination, the flail height was set to give an average cut height of $100 \mathrm{~mm}$. Cutting daffodils just as they are about / have started to flower (Figure 5) is contrary to normal growing advice which is to leave the foliage for at least six weeks after flowering before removing it (Royal Horticultural Society, 2018). It is not known at this stage if flailing the biomass, rather than cutting it with a reciprocating knife, has any effect on making the plants susceptible to viral and bacterial diseases.

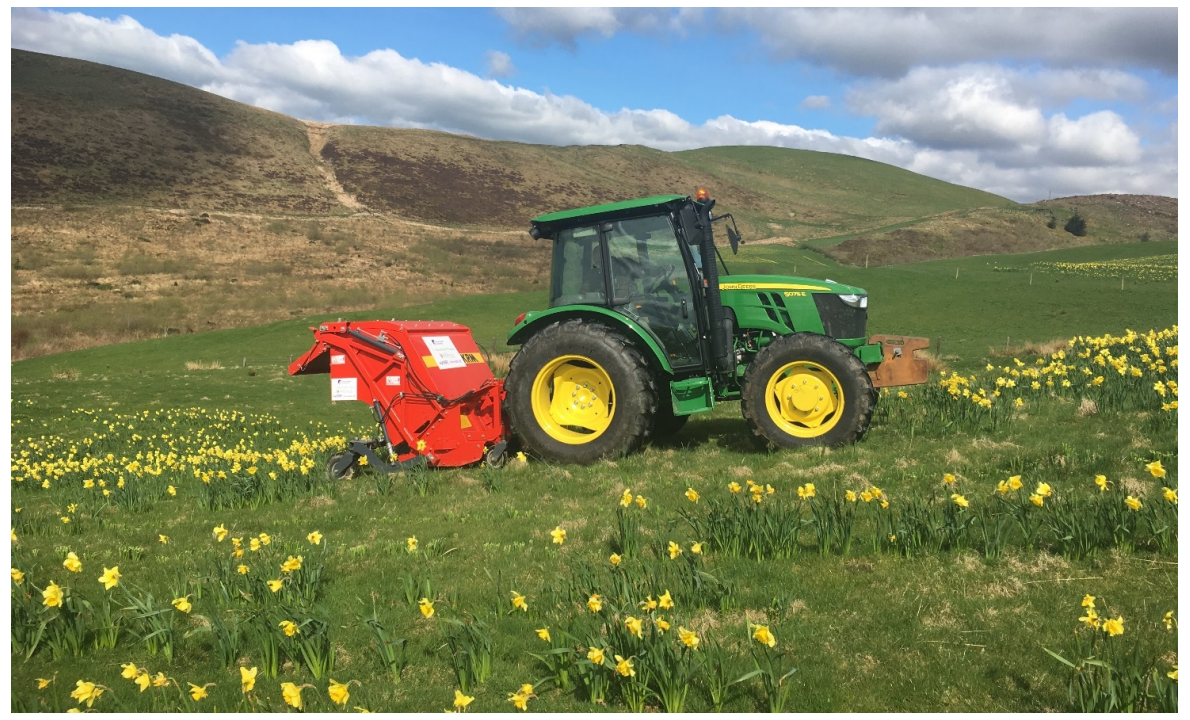

Figure 5. 2018 harvest at AU Upland Research Centre. 


\section{Conclusions}

A two-row planter and flail harvester have been developed to establish and harvest daffodils planted in $800 \mathrm{~mm}$ rows in upland pasture. Future work may include modifying the planter to plant the bulbs "apex" up. Additionally, the harvester may require further modifications to adjust the cut height automatically depending on the ground profile and provide a system for lifting lodged crop that has fallen outside the standard cut width.

\section{Acknowledgements}

The authors would like to express their thanks to the Biotechnology and Biological Sciences Research Council, Innovate UK, Keith Rennie Machinery Ltd, UK and John Deere Ltd, UK.

\section{References}

Journal articles:

Godwin, R. J. and Spoor, G. Soil Failure with Narrow Tines. Journal of Agricultural Engineering Research, Vol 22, No 3, p 213-228, 1977.

Marco-Contelles J., Carreiras, M.D., Rodríguez, C., Villarroya, M., and Garcia, A.G. Synthesis and pharmacology of galantamine. Chemical Reviews 106, 116-133, 2006.

Payne, P.C. and Tanner, D.W. The relationship between rake angle and the performance of simple cultivation implements. J. Agric. Eng. Res. 4(4), 312-325, 1959.

Torras-Claveria, L.,Berkov, S.,Codina, C.,Viladomat, F., and Bastida, J. Daffodils as potential crops of galanthamine. Assessment of more than 100 ornamental varieties for their alkaloid content and acetylcholinesterase inhibitory activity. Industrial Crops and Products. (44). 237-244. 2013.

Trost, B.M. and Toste, F.D. Enantioselective total synthesis of (-)-galanthamine. Journal of the American Chemical Society 122, 1262-1263, 2000.

Books:

Bell, B., 2016. Farm Machinery, 6th edition. Sheffield: Old Pond.

Soil Survey of England and Wales, 1984a. Soils and their use in Northern England. Harpenden: Soil Survey of England and Wales.

Soil Survey of England and Wales, 1984b. Soils and their use in Wales. Harpenden: Soil Survey of England and Wales.

Technical reports:

Department for Environment Food \& Ruarl Affairs, 2006. Sustainable production of the natural product galanthamine. Defra project code NF0612. Institute of Grassland and Environmenatl Research, Plas Gogerddan, Aberstwyth SY23 3EB

Theses and dissertations:

Cooper, M., 2015. An investigation into the terramechanics of a prototype daffodil bulb planter. CREST Report (unpublished). Harper Adams University, Newport, Shropshire, UK.

Internet resources:

Alzeimers Disease International, 2018. Dementia Statistics. https://www.alz.co.uk/research/statistics Accessed May 30, 2018

Royal Horticultural Society, 2018. Daffodils. https://www.rhs.org.uk/advice/profile?PID=91 Accessed June 1,2018 


\title{
Prognosis of Leaf Chlorosis in Catherina Peaches Based on the Mineral Composition of Flowers
}

\author{
Victoria Lafuente $^{1}$; Pilar Barreiro ${ }^{2}$; Lourdes Lleó ${ }^{2} ;$ Jesús Val $^{1}$ \\ 1 Departamento de Nutrición Vegetal, Estación experimental Aula Dei (CSIC), Apartado 727, 50080. Zaragoza Spain \\ 2 LPF_TAGRALIA Departamento de Ingeniería Agroforestal, UPM CEI Moncloa, Madrid, UP4, España
}

\begin{abstract}
The peach tree is one of the fruit species with the highest production and area cultivated in Spain, which rootstock show a marked influence on the mineral content of leaves and consequently on chlorosis. The nutritional diagnosis of the peach tree, based on the mineral analysis of leaves, is often late for in-season amendments, while leaf diagnosis based either on photosynthetic activity (non-destructive) or on iron chlorosis is always late and in many cases imprecise. Previous work from Aula Dei (CSIC) has established the possibility and efficiency of making a mineral prognosis of leafs based on the mineral composition of flowers not only in peaches but in pear and cherry trees. This current work aims to go further in non-destructive prognosis by selecting key features from RGB images and validating them in a set of 300 photographs. The test was carried out in a plot located in the Experimental Station of Aula Dei (CSIC), the soil of the plot being heavy and calcareous. Six plumxpeach trees have been used determining the foliar and floral mineral concentration on flower at full blossom (FB), and corresponding leaf samples at 120 days after full bloom (aFB). The ions in flowers and leaves were determined by plasma-mass spectroscopy, while the measurement of the photosynthetic activity of the leaves and their mineral composition was carried out 120 days aFB using a SPAD meter. The SPAD used as a reference for the identification of chlorosis in peach leaf appears correlated with several leaf minerals: $\mathrm{Ca}, \mathrm{S}, \mathrm{Fe}, \mathrm{Cu}$ and $\mathrm{Sr}$, restricted to $\mathrm{Ca}$ and $\mathrm{Sr}$ when compared with the mineral composition in flowers. The mineral composition of the flowers allows anticipating extreme cases of chlorosis based on $\mathrm{Sr}$ and $\mathrm{Ca}$ levels, while the methodology developed based on RGB images shows promising results and is still ongoing.
\end{abstract}

Keywords: Crop status, Physiological stress, fruit quality, variable rate fertilisation.

\section{Introduction}

Peach [Prunus pérsica (L.) Batsch] is the most important temperate and decidous fruit tree grown in Spain. The abailability of rootstocks for peach is large due to the various species and/or interspecific hybrids that can be used (Iglesias et al, 2001).

Rootstocks are an essential component in modern fruit production because of their capability of adapting scion cultivars to diverse environmental conditions and cultural practices. Peach varieties are significantly influenced by the pattern on which they are grafted. Previous research has demonstrated the rootstock effects on fruit quality (Caruso et al., 1996) and foliar levels of the mineral nutrients (Knowles et al., 1984; Boyhan et al., 1995; Facteau et al., 1996; Moreno et al., 1996; Rosati et al., 1997).

Leaf mineral analysis is a useful tool for the assessment of the nutritional status of crops (Johnson \& Uriu, 1989; Montañés et al., 1993; Guo-yi et al., 2015), and the use of tolerant rootstocks would prevent nutritional disorders that cause high economic losses for the fruit growers (Jiménez et al., 2007; 2008).

Iron chlorosis is the most common nutritional disorder in fruit tree crops growing in calcareous soils (Abadía et al, 2004). The prognosis of leaf iron chlorosis and other deficiencies by foliar analysis or SPAD reading is usually performed too late in the growing season to efficiently apply correcting measures before harvesting. Therefore, methods alternative to leaf analysis have been proposed to prognose nutrient deficiency. For instance, the mineral composition of flower has been used with this purpose in peach (Belkhodja et al, 1998: Zarrouk et al, 2005) and pear (El-Jendoubi et al, 2012).

These methods are accurate but they require destructive sampling, specialist equipment, and are expensive, laborious and time consuming. Therefore, digital image is being developed like an alternative method which is non-destructive, more rapid and straightforward for estimating chlorosis in flower state.

Using RGB images, the researchers successfully calculated total fruit yield in apple (Stajnko et al. 2009) and in mango (Payne et al. 2013). Similarly geometrical attributes of different crops were predicted using RGB based image processing technique (Hahn and Sanchez 2000; Koc 2007; Khojastehnazhand et al. 2009; Wang and Li 2014; Nambi et al. 2015b). Garrido Novell et al. (2012) reported for apple and Donis-González et al. (2013) reported for chest nut for its quality prediction using RGB color image.

The objective was thus to evaluate the applicability of a fast and non-destructive field method for prognosis of leaf chlorosis in Catherina peaches, using RGB image analysis in flower peach. 


\section{Materials and Methods}

Plant material and crop characterization

The trial was carried out at The Experimental Station of Aula Dei (CSIC) on a calcareous soil, with 29-30\% total calcium carbonate, 7.4-7.6\% active lime, $\mathrm{pH}$ in water 7.7 and a clay-loam texture.

Six hexaploid plum rootstocks including three Pollizo plums (P. insititia): Adesoto, P. Soto $67 \mathrm{AD}$ and PM 105 AD; a St. Julien plum (P. insititia): GF 655/2, a common local plum (P. domestica): Constantí 1 were selected as polyvalent clonal rootstocks for different Prunus species, but especially for peaches to avoid waterlogging and iron chlorosis in heavy and calcareous soils. The six rootstocks were budded with 'Catherina' peach.

\section{Leaf chlorophyll assesssment}

The chlorophyll concentration per unit leaf area was estimated in the field, using a SPAD 502 meter (Minolta Co., Osaka, Japan). Thirty leaves per tree, selected from the middle of bearing shoots located all around the crown, were measured to obtain an average concentration representative of the leaves belonging to the outer part of the tree canopy. Measurements were carried out 120 days after full bloom (DAFB) in 2014 and 2016.

Mineral analysis of plant material

Leaf and flower mineral concentrations were determined in 2016. Flower sampling was done at full bloom at the middle of March. Leaf sampling was done at 120 DAFB.

\section{Digital imaging}

The proposed digital image analysis technique was applied on the same flowers used for mineral analysis. A Nikon SLR Nikon D5200 con 24.1MP digital camera mounted on a tripod was used for recording digital images of the flowers. All pictures were taken from the same distance and under identical illuminative conditions.

\section{Results and discussion}

SPAD as a chlorosis reference

Figure 1 gives an overview of SPAD values for the rootstocks analysed in 2016. There appears to be a very large dispersion among SPAD values for the same rootstock (especially 1, 3, 4 and 5) which points the existence of isolated individuals with leave chlorosis, while all trees from rootstock 7 seem to have developed this pathology.
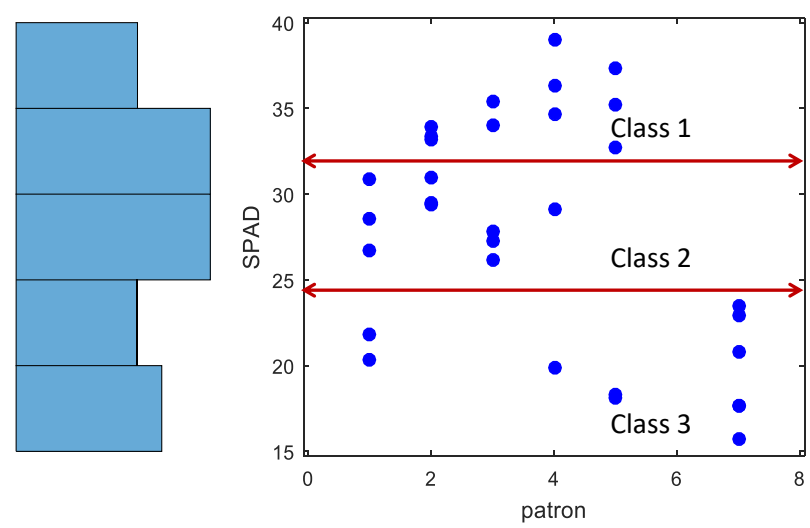

Figure 1. SPAD values for the rootstocks analysed in 2016. Patron 1: Adesoto 101; Patron 2: PM 105; Patron 3: Soto 67; Patron 4: Constatí 1; Patron 5: Montizo; Patron 7: GF 655/2.

According to the visual identification of experts $\mathrm{SPAD}>=32$ was set to sound trees (class 1 ), $\mathrm{SPAD}<24$ was label as trees severely affected by chlorosis (class 3), and intermediate chlorosis level in-between (class 2).

Macro-nutrients at flower and leave level

Table 1 shows macro nutrients (\%) at flower and leave level. Leaves show higher average values than flowers for $\mathrm{K}, \mathrm{Ca}$ and $\mathrm{Mg}$ while being lower for $\mathrm{P}$, and $\mathrm{S}$. Leaves generally show greater $\mathrm{CV}$ than flowers, with the largest variability corresponding to $\mathrm{Ca}$ in both flowers $(\mathrm{CV}=11.7 \%)$ and leaves $(\mathrm{CV}=21.90 \%)$. 
Table 1. \% macro nutrients at flower and leave level.

\begin{tabular}{lcccccccc}
\hline \multirow{5}{*}{ Flower } & & $\%$ & $\%$ & $\%$ & $\%$ & $\%$ & $\%$ & $\%$ \\
& Average & 1.83 & 0.48 & 0.38 & 0.18 & 0.14 & & \\
& STD & 0.14 & 0.06 & 0.03 & 0.01 & 0.01 & & \\
& $\% \mathrm{CV}$ & 7.65 & 11.76 & 9.00 & 6.35 & 8.19 & & \\
Leaves & Average & 2.57 & 1.42 & 0.17 & 0.33 & 0.12 & 42.32 & 2.88 \\
& STD & 0.29 & 0.31 & 0.02 & 0.04 & 0.01 & 0.68 & 0.25 \\
& \%CV & 11.14 & 21.90 & 13.00 & 11.42 & 10.16 & 1.61 & 8.56 \\
\hline
\end{tabular}

Micro-nutrients at flower and leave level

Table 2 shows the eight most relevant micro nutrients. The amount of micro nutrients in the flowers is higher than that of leaves (leave to flower ratio below 1) for $\mathrm{Na}, \mathrm{Al} \mathrm{Fe}$ and $\mathrm{Cu}$, while being lower for $\mathrm{Sr}, \mathrm{Mn}$ and $\mathrm{B}$. A very large difference in average value is found for $\mathrm{Cu}, \mathrm{Na}$ and $\mathrm{Fe}$ between flowers and leaves: $77.1 \mathrm{vs} 7.38 \mathrm{mg} / \mathrm{kg}$ for $\mathrm{Cu}, 176.9$ vs. $46 \mathrm{mg} / \mathrm{kg}$ for $\mathrm{Na}$, and 146.7 vs. $71.60 \mathrm{mg} / \mathrm{kg}$ for Fe. The highest variability in micro nutrients for leaves corresponds also to $\mathrm{Na}, \mathrm{Al}$ and $\mathrm{Fe}(\mathrm{cv}=31.8 \% 24.4 \%$ and $18.9 \%$ ) compared to flowers $(\mathrm{cv}=19.7 \%$, $19.1 \%$ and $13.8 \%$ ). We may speculate that some limitation for $\mathrm{Na}, \mathrm{Al}$ and $\mathrm{Fe}$ absorbance at leave level has occurred, leading to lower average and higher variability in these micro nutrients.

Table 2. Micro nutrient $\left(\mathrm{mg} \mathrm{kg}^{-1}\right)$ at flower and leave level

\begin{tabular}{cccccccccc}
\hline & & $\mathrm{Na}$ & $\mathrm{Al}$ & $\mathrm{Fe}$ & $\mathrm{Cu}$ & $\mathrm{Zn}$ & $\mathrm{Sr}$ & $\mathrm{Mn}$ & $\mathrm{B}$ \\
\hline \multirow{5}{*}{ Flower } & Average & 176.9 & 152.4 & 146.7 & 77.7 & 45.8 & 34.9 & 28.0 & 19.0 \\
& $\mathrm{STD}$ & 34.8 & 29.1 & 20.2 & 15.8 & 4.9 & 4.5 & 2.9 & 3.1 \\
& $\mathrm{CV}(\%)$ & 19.7 & 19.1 & 13.8 & 20.3 & 10.6 & 13.0 & 10.2 & 16.4 \\
Leaves & Average & 46.0 & 71.60 & 46.39 & 7.38 & 24.63 & 149.91 & 40.71 & 26.35 \\
& $\mathrm{STD}$ & 14.6 & 17.35 & 8.76 & 0.99 & 4.67 & 30.73 & 5.23 & 3.45 \\
& $\mathrm{CV}(\%)$ & 31.8 & 24.24 & 18.89 & 13.47 & 18.98 & 20.50 & 12.85 & 13.11 \\
\hline
\end{tabular}

Relationship between SPAD and macro and micro mineral composition of flowers and leaves

Table 3 shows the linear correlation coefficients between macro elements and SPAD at flower and leave level. The correlation coefficients between macro nutrients and SPAD are significant at leave level for Ca, S and $\mathrm{K}$, while at flower level only Ca seems related to SPAD.

\begin{tabular}{cccccc}
\multicolumn{6}{c}{ Table 3. Linear correlation coefficients between macro-nutrients and SPAD at flower and leave level. } \\
\hline & $\mathrm{K}$ & $\mathrm{Ca}$ & $\mathrm{P}$ & $\mathrm{Mg}$ & $\mathrm{S}$ \\
\hline FLOWER & $\mathrm{ns}$ & $0.4455^{* *}$ & $\mathrm{~ns}$ & $\mathrm{~ns}$ & $\mathrm{~ns}$ \\
LEAVE & $-0.3091^{* *}$ & $0.4736^{* *}$ & $\mathrm{~ns}$ & $-0.1487^{*}$ & $0.4336^{* *}$ \\
\hline
\end{tabular}

Table 4 shows the linear correlation coefficients between micro elements and SPAD at flower and leave level. As before, higher correlation coefficients are found at leave as at flower level. For leaves, $\mathrm{Ca}, \mathrm{S}, \mathrm{Fe}, \mathrm{Cu}$ and $\mathrm{Sr}$ seem relevant, while at flower level only $\mathrm{Ca}$ and $\mathrm{Sr}$ show significant correlation.

Table 4. Linear correlation coefficients between micro-nutrients and SPAD at flower and leave level.

\begin{tabular}{ccccccccc}
\hline & $\mathrm{Na}$ & $\mathrm{Al}$ & $\mathrm{Fe}$ & $\mathrm{Cu}$ & $\mathrm{Zn}$ & $\mathrm{Sr}$ & $\mathrm{Mn}$ & $\mathrm{B}$ \\
\hline FLOWER & $\mathrm{ns}$ & $0.1517^{*}$ & $0.1460^{*}$ & $0.1854^{*}$ & $\mathrm{~ns}$ & $0.4096^{* *}$ & $0.1200^{*}$ & $0.2099^{*}$ \\
& & & & & & & \\
LEAVE & $\mathrm{ns}$ & $\mathrm{ns}$ & $0.3345^{* *}$ & $0.3092^{* *}$ & $0.1049^{*}$ & $0.4565^{* *}$ & $0.1448^{*}$ & $0.3710^{* *}$ \\
\hline
\end{tabular}

Significant higher amount of $\mathrm{Ca}$ and $\mathrm{Sr}$ are found at flower stage for class 1 (sound) compared to slight and strong affection of chlorosis $(\mathrm{F}=4.16, \mathrm{p}<0.05$ and $\mathrm{F}=3.48, \mathrm{p}<0.05)$. Therefore $\mathrm{Ca}$ and $\mathrm{Sr}$ are candidates for the early diagnosis of chlorosis at flower level. Figure 3 corroborates this fact; prognosis at flower level can only be done for extreme $\mathrm{Ca}-\mathrm{Sr}$ values. These values refer to much higher deficit that data reported in 1998 (Igartúa et al. 2000). 
Table 5. SPAD values for the years 2012, 2014 and 2016

\begin{tabular}{lccc}
\hline Rootstock & 2012 & 2014 & 2016 \\
\hline Adesoto (1) & 42.6 & 34.3 & 25.7 \\
Monpol (6) & 48.1 & 31.2 & \\
Montizo (5) & 42.0 & 35.5 & 28.3 \\
P. Soto 67 AD (3) & 42.1 & 34.2 & 30.1 \\
PM 105 AD (2) & 44.9 & 37.6 & 31.7 \\
GF 655/2 (7) & 37.7 & 34.3 & 19.7 \\
Constatí 1 (4) & 53.9 & 39.7 & 31.8 \\
\hline
\end{tabular}

SPAD evolution among seasons

Table 5 shows the SPAD values for the years 2012, 2014 and 2016. The ANOVA analysis between campaigns shows an $\mathrm{F}$ value of $\mathrm{F}=20.48$ (significant at $1 \%$ ), which indicates that the trees have deteriorated during the period of analysis. Following the SPAD cut-off values in relation to the development of chlorosis, we observe that only in 2016 extreme levels of chlorosis have occurred, especially in the GF 655/2 rootstock.

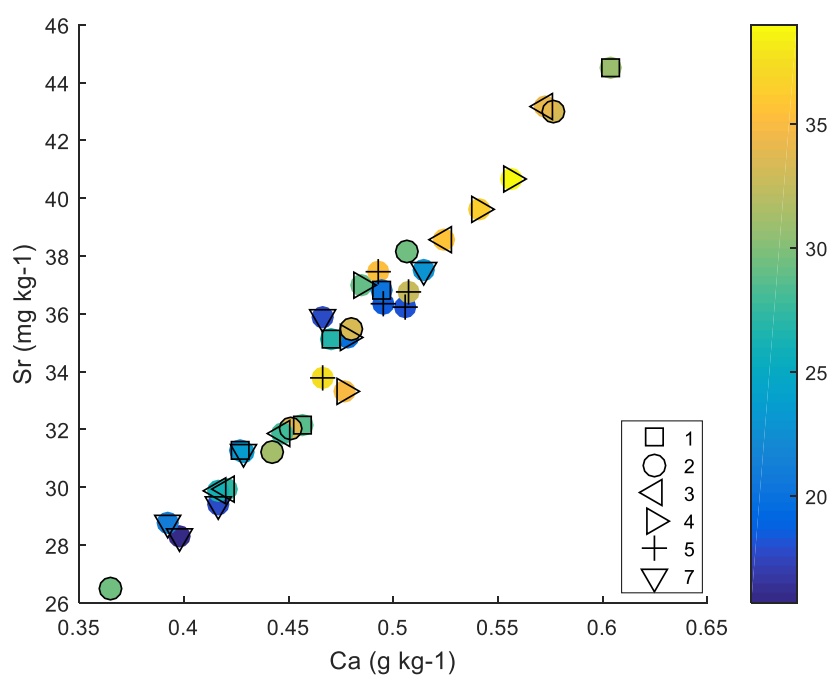

Figure 2. SPAD values for the rootstocks analysed in 2016.

Figure 2 shows the SPAD values in 2016 as compared to Sr content, where marker shape refers to rootstock number as listed in Figure 1 while the color of the marker quantifies the SPAD values; note that SPAD values above 32 corresponds to sound trees while SPAD below 24 points to intense leaf chlorosis.

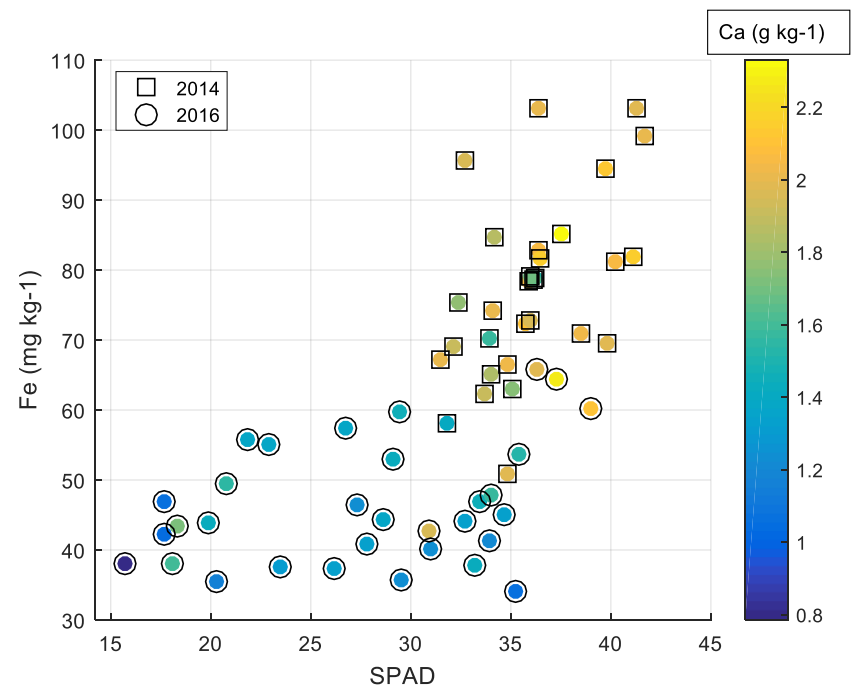

Figure 3. Representation of SPAD values versus Fe content segregated by seasons; the color of the marker quantifies Ca composition in terms of $\mathrm{g}$ per $\mathrm{kg}$ dry weight. 
Figure 3 presents the SPAD values as compared to Fe content segregated by seasons; the color of the marker quantifies Ca composition in terms of $\mathrm{g}$ per kg dry weight. It can be observed a large decrease both in SPAD and $\mathrm{Fe}$ in 2016 compared to 2014, which is also consistent with a systematic decrease in $\mathrm{Ca}$. Therefore, $\mathrm{Ca}$ and $\mathrm{Fe}$ show significant relationship at leaf level already stated in Tables 1 and 2.

Evolution of mineral composition among seasons

The analysis of the mineral evolution between campaigns has only been carried out for 2014 and 2016 in peach leaves, the only complete records available. The composition of the leaves also registered a gradual deterioration, a significant effect at $1 \%$ in both $\mathrm{Ca}$ and $\mathrm{Fe}(\mathrm{F}=59.9$ and 122.9 respectively), only significant at $5 \%$ in $\mathrm{Zn}(\mathrm{F}=$ 4.0).

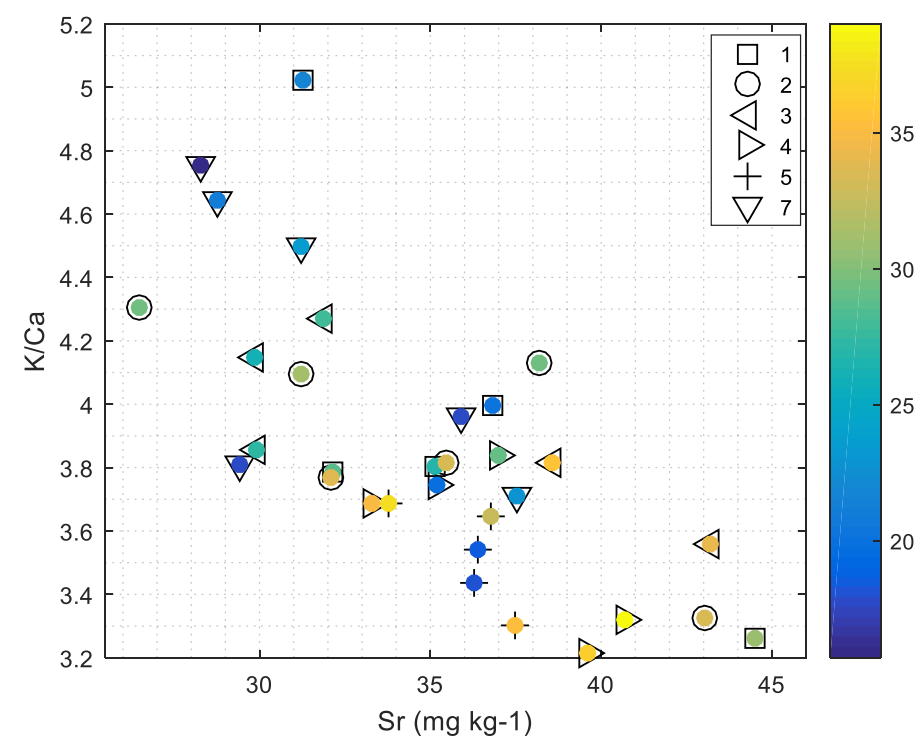

Figure 4. Sr values as compared to K/Ca ratio for all the rootstocks analysed in 2016 (see Table 5).

Figure 3 shows for both campaigns that SPAD values lower than 30 imply leaf values lower than $60 \mathrm{mg} \mathrm{kg-1}$ of Fe and less than $1.6 \mathrm{~g} \mathrm{~kg}-1$ of $\mathrm{Ca}$. This threshold of SPAD corresponds in flower to Sr levels lower than 38.2 $\mathrm{mg} \mathrm{kg-1}$ and Ca values below $0.514 \mathrm{~g} \mathrm{~kg}-1$. These features do not seem rootstock dependent according to Figure 4.

Figure 4 also points the value of the $\mathrm{K} / \mathrm{Ca}$ ratio in flowers as an interesting key performance identifier regarding the prognosis of leave chlorosis with four months of anticipation (120 days aFB). Belkhodja, Ramzi, et al. in 1998, studying BabyGold peaches in Huesca, a nearby region of Zaragoza (Spain), found in a two-year field experiment (1994-1995) that values above three in the K/Ca ratio indicated the future development of leaf chlorosis. These facts indicate the value of flowers in the anticipation of tree fertilization needs, or even for addressing variable rate technologies based on historical values. Only the destructive and expensive nature of mass spectroscopy remains a burden for daily farm management, which could be overcome whenever mass spectroscopy could be substituted by other non-destructive technologies applied to flowering such as vis-NIR spectroscopy or hyperspectral imaging.

\section{Conclusions}

The SPAD used as a reference for the identification of chlorosis in peach leaf appears correlated with several leaf minerals: $\mathrm{Ca}, \mathrm{S}, \mathrm{Fe}, \mathrm{Cu}$ and $\mathrm{Sr}$, restricted to $\mathrm{Ca}$ and $\mathrm{Sr}$ when SPAD is compared with the mineral composition in flower.

Data from several campaigns indicate that a cut-off value in SPAD of 30 is a reflection in visible chlorosis, associated with leaf levels lower than $60 \mathrm{mg} \mathrm{kg}-1 \mathrm{Fe}$ and $1.6 \mathrm{~g} \mathrm{~kg}-1 \mathrm{Ca}$.

The mineral composition of the flowers allows to anticipate extreme cases of chlorosis $(\mathrm{SPAD}<30)$ in Caterina peach based on $\mathrm{Sr}$ and Ca levels. Threshold values of $38 \mathrm{mg} \mathrm{kg}-1$ of $\mathrm{Sr}$ and $0.5 \mathrm{~g} \mathrm{~kg}-1$ in Ca could be useful for the identification of the need for additional fertilization four months in advance of leaf and fruit deficiencies. The $\mathrm{k} / \mathrm{Ca}$ ratio in flower is confirmed as a good predictor of the later development of leaf chlorosis. 


\section{Acknowledgements}

The authors would like to thank the research group Alimentos de Origen Vegetal from Estación experimental Aula Dei (CSIC) from Zaragoza, Spain for the availability of peach plots and their previous experience concerning peach quality as related to soil properties.

\section{References}

J. Abadía, A. Álvarez-Fernández, A. Rombolà, M. Sanz, M. Tagliavini, A. Abadía.2004.Technologies for the diagnosis and remediation of Fe deficiency. Soil Sci Plant Nutr 50:965-971

Belkhodja, Ramzi, et al. "Iron deficiency in peach trees: effects on leaf chlorophyll and nutrient concentrations in flowers and leaves." Plant and Soil 203.2 (1998): 257-268.

Ben Khelil, M., et al. "Inflorescence analysis as alternative to leaf analysis to evaluate the nutritional status of olive trees." VI International Symposium on Mineral Nutrition of Fruit Crops 868. 2008.

T. Caruso, D. Giovanni, A. Liverani, 1996. Rootstock influences the fruit mineral, sugar and organic acid content of a very early ripening peach cultivar. J. Horticult. Sci. 71, 931-937.

Custódio, L., et al. "Floral analysis and seasonal dynamics of mineral levels in carob tree leaves." Journal of plant nutrition30.5 (2007): 739-753.

G.E. Boyhan, J.D. Norton, J.A. Pitts. 1995. Establishment, growth, and foliar nutrient content of plum trees on various rootstocks. HortScience 30, 219-221.

T. Caruso, D. Giovanni, A. Liverani, 1996. Rootstock influences the fruit mineral, sugar and organic acid content of a very early ripening peach cultivar. J. Horticult. Sci. 71, 931-937.

I.R. Donis-González, D.E. Guyer, G.A. Leiva-Valenzuela, J. Burns. 2013. Assessment of chestnut (Castanea.spp.) slice quality using color images. J. Food Eng. 115, 407-414.

I. Iglesias, R. Dalmau, R. Monserrat, J. Carbó, J. Bonany, Guanter, G., 2001. Comportamiento agronómico de 23 patrones de melocotonero con la variedad 'Elegant Lady' (Merdame) en Lleida y Girona. Acta Hort. 29,787795.

El-Jendoubi, Hamdi, et al. "Prognosis of iron chlorosis in pear (Pyrus communis L.) and peach (Prunus persica L. Batsch) trees using bud, flower and leaf mineral concentrations." Plant and soil 354.1-2 (2012): 121-139.

Jiménez, S., et al. "Influence of different vigour cherry rootstocks on leaves and shoots mineral composition." Scientia Horticulturae 112.1 (2007): 73-79.

T. J. Facteau, N.E. Chesnut, K.E. Rowe. 1996. Tree fruit size and yield of 'Bing' sweet cherry as influenced by rootstock, replant area, and training system. Sci. Horticult. 67, 13-26.

C. Garrido-Novell, D. Pérez-Marín, J.M. Amigo, J. Fernández-Novales, J.E. Guerrero, A. Garrido-Varo. 2012. Grading and color evolution of apples using RGB and hyperspectral imaging vision cameras. J. Food Eng. 113, 281-288.

W. Guo-yi, Z. Xin-zhong, Xu. Xue-feng, H. Zhen-hai H. 2015. Key minerals influencing apple quality in Chinese orchard identified by nutrional diagnosis of leaf and soil analysis. J Intreg Agr 14(5): 864-874. https://doi.org/10.1016/S2095- 3119(14)60877-7

F. Hahn, S. Sanchez. 2000. Carrot volume evaluation using imaging algorithms. J. Agric. Eng. Res. 75, 243249.

Igartua, Ernesto, et al. "Prognosis of iron chlorosis from the mineral composition of owers in peach." The Journal of Horticultural Science and Biotechnology 75.1 (2000): 111-118.

I. Iglesias, R. Dalmau, R. Monserrat, J. Carbó, J. Bonany, Guanter, G., 2001. Comportamiento agronómico de 23 patrones de melocotonero con la variedad 'Elegant Lady' (Merdame) en Lleida y Girona. Acta Hort. 29,787795.

S. Jiménez, J. Pinochet, A. Abadía, M.A. Moreno, Y. Gogorcena. 2008. Tolerance response to iron chlorosis of Prunus selections as rootstocks. HortScience 43(2): 304-309.

R.S. Johnson, K. Uriu. 1989. In: Larue, J., Johnson, R.S. (Eds.), Mineral Nutrition. Peach, Plum and Nectarine: Growing and Handling for Fresh Market. University of California: Division of Agriculture Resource, Oakland, CA, pp. 68-81.

Karagiannidis, N., et al. "Prognosis and correction of iron chlorosis in peach trees and relationship between iron concentration and Brown Rot." Scientia horticulturae 118.3 (2008): 212-217.

M. Khojasthnazhand, M. Omid. A. Tabatabaeefar. 2009. Determination of orange volume and surface area using image processing technique. Int. Agrophys. 23, 237-242.

J. W. Knowles, W. A. Dozier, Jr., C.E. Evans, C.C. Carlton, J.M. McGuire. 1984. Peach rootstock influence on foliar and dormant stem nutrient content. J. Am. Horticult. Sci. 109, 440-444.

A.B. Koc. 2007. Determination of watermelon volume using ellipsoid approximation and image processing. Postharv. Biol. Technol. 45, 366-371.

Martinez, Herminia EP, et al. "Coffee-Tree Floral Analysis as a Mean of Nutritional Diagnosis." Journal of plant nutrition 26.7 (2003): 1467-1482. 
Mestre, Lucía, et al. "Influence of plum rootstocks on agronomic performance, leaf mineral nutrition and fruit quality of "Catherina'peach cultivar in heavy-calcareous soil conditions." Spanish Journal of Agricultural Research 15.1 (2017): 0901.

Montañés L, Heras L, Abadía J, Sanz M, 1993. Plant analysis interpretation based on a new index: deviation from optimum percentage (DOP). J Plant Nutr 16: 1289-1308. https://doi.org/10.1080/01904169309364613

M.A. Moreno, L. Montañés, M.C. Tabuenca, R. Cambra. 1996. The performance of Adara as a cherry rootstock. Sci. Horticult. 65, 58-91.

V.E. Nambi. 2015. Development of online grading system based on internal and external qualities of mango using machine vision technology. In Department of Food and Process Engineering and Technology, Vol PhD, Tamil Nadu Agricultural University, Coimbatore.

A.B Payne, K.B. Walsh, P. Subedi, D. Jarvis. 2013. Estimation of mango crop yield using image analysisSegmentation method. Comput. Electron. Agric. 91, 57-64.

Pestana, Maribela, et al. "Floral analysis as a tool to diagnose iron chlorosis in orange trees." Plant and Soil 259.1 (2004): 287-295.

Pestana, Maribela, et al. "Relationships between nutrient composition of flowers and fruit quality in orange trees grown in calcareous soil." Tree physiology 25.6 (2005): 761-767.

A. Rosati, T.M. DeJong, S.M. Southwick. 1997. Comparison of leaf mineral content, carbon assimilation and stem water potential of two apricot (Prunus armeniaca) cultivars grafted on 'Citation' and 'Mariana 2624' rootstocks. Acta Hort. 451, 263-267.

D. Stajnko, J. Rakun, M. Blanke. 2009. Modelling apple fruit yield using image analysis for fruit color, shape and texture. Eur. J. Horticult. Sci. 74, 260.

W. Wang, C. Li. 2014. Size estimation of sweet onions using consumer-grade RGB-depth sensor. J. Food Eng. $142,153-162$.

Zarrouk, O., et al. "Influence of almond $\times$ peach hybrids rootstocks on flower and leaf mineral concentration, yield and vigour of two peach cultivars." Scientia Horticulturae 106.4 (2005): 502-514. 


\section{Topic 9: Greenhouse production technologies (GP)}

Technology and management systems for greenhouse production systems. Contributions may include greenhouse structures, covering materials, climate and energy systems, design of ventilation and CFD applications, automation and decision support systems for greenhouse climate, crop, water and nutrient management, as well as pest and disease detection and control.

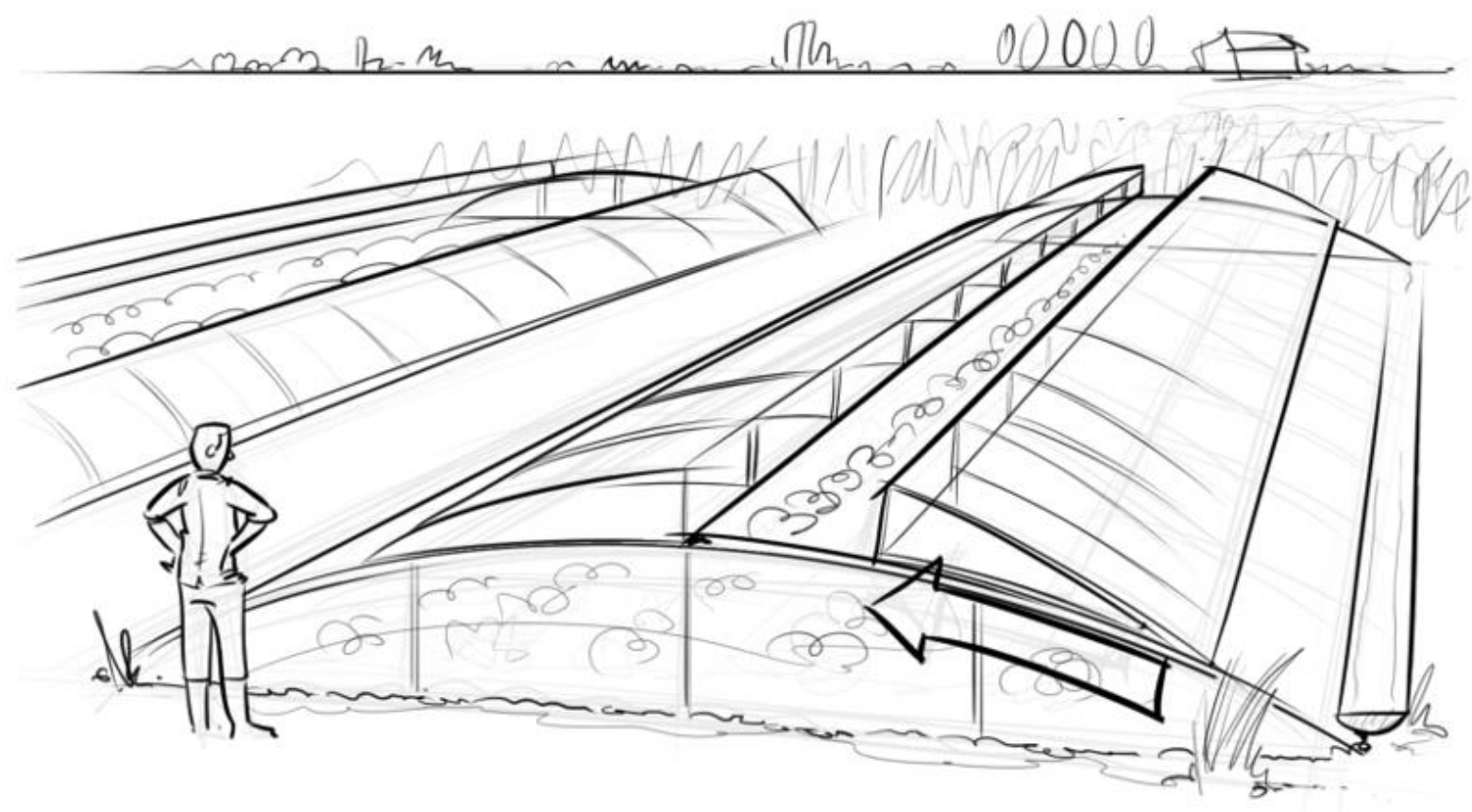

'ARENA' - ROOF. 


\title{
Effect of Bench Heating on Growing Medium Temperature and Heat Loss From a Greenhouse in Wintertime
}

\author{
Massimo Brambilla a,", Elio Romano a, Maurizio Cutini ${ }^{\text {a }}$, Marco Fedrizzi ${ }^{\text {b }}$, Mauro Pagano b , Gianluca \\ Burchi $^{c}$, Sonia Cacini ${ }^{c}$, Daniele Massa ${ }^{c}$, Chiara Terrosi ${ }^{c}$ and Carlo Bisaglia ${ }^{a}$ \\ ${ }^{a}$ CREA Research Centre for Engineering and Agro-Food Processing,. Via Milano, 43 - 24047 Treviglio (BG), Italy \\ ${ }^{\mathrm{b}}$ CREA Research Centre for Engineering and Agro-Food Processing, Via della Pascolare 16 - 00015 - Monterotondo \\ (Roma), Italy \\ ${ }^{c}$ CREA Research Centre for Vegetable and Ornamental Crops, Via dei Fiori, 8 - 51017 - Pescia (PT), Italy \\ * Corresponding author. Email: massimo.brambilla@crea.gov.it
}

\begin{abstract}
The dynamic behaviour of greenhouse microclimate is a combination of physical processes involving energy transfer (solar radiation and heat transfer) and mass balance (water vapour and $\mathrm{CO}_{2}$ fluxes) at varying of outside environmental conditions. In Italy, $52.3 \%$ of greenhouse production sites are placed in the North and in the Centre of the Country and exposed to a variety of climate conditions: greenhouse microclimate control plays a key role in the management of such production systems, in particular when automated. In this work, we present a preliminary study aimed to assess the energy losses occurring in a greenhouse while providing bench heating filled up with a growing medium (peat:perlite 50:50 V/V). In wintertime, inside a greenhouse covered with polycarbonate, cultivation benches were heated by circulating warm water $\left(37.3 \pm 0.06^{\circ} \mathrm{C}\right)$ in polyethylene pipes placed at three different depths (on the top, in the middle and at the bottom). The temperatures of the substrate, as well as the microclimatic parameters inside and outside the greenhouse, were collected. The heat loss (kW) from the structure was assessed by the ASAE EP406.4 Standard net of the heat occurring from solar radiation. Data processing by General Linear Model (using the position of the pipe as a factor and the temperature and the solar radiation as covariates) pointed out that pipe position significantly affected the temperature of the medium and the heat loss. Pipes placed inside the substrate (in the middle) provided the highest temperature of the growth medium $\left(16.3 \pm 0.02{ }^{\circ} \mathrm{C}\right)$ and resulted in the lowest greenhouse heat loss $(1.34 \pm 0.05 \mathrm{kWh})$ on hourly basis that is $34.7 \%$ lower than that related to the heating pipes placed on the top of the growing substrate.
\end{abstract}

Keywords: Energy saving, Protected crops, Environment, Sustainability.

\section{Introduction}

Greenhouses represent a trend in agricultural development that indicates the level of agricultural modernization of a region. In Italy, $52.3 \%$ of greenhouse production sites are placed in the North and in the Centre of the Country and exposed to a variety of climate conditions. Greenhouse microclimate control is a key point in the management of such production systems, in particular when automated.

To achieve high production yields running the required equipment (light-supplementation, dehumidification, heating, cooling and actuators) could account for more than $90 \%$ of the total energy consumption of the greenhouse (Hemming et al., 2015). To reduce this consumption many issues have been tackled placing efforts in optimizing the daily average temperature and humidity as well as solar radiation and $\mathrm{CO}_{2}$ concentration (Shen et al., 2018; Dieleman et al., 2006; Elings et al., 2005).

Heating is required during the cold season of the year because, as well known, it enhances plant growth by maintaining adequate temperature and humidity levels. . Nevertheless, providing heat to the greenhouse unavoidably results in heat loss through conduction, convection and radiation phenomena A variety of heaters is used to provide heat to cultivated plants. Among these there are gas, hot air heaters (mounted on the wall or free standing), hot water heaters and boilers (either gas or electrically fired, they provide radiant, floor and bench heat), electric heaters, wood or pellet stoves, and heat pumps (Bailey, 1983; Kozai, 1986; Garcia, 1998; Aye et al., 2010).

Most greenhouse operations can be ascribed to two main crop production systems both providing excellent results, the floor system and the bench production system that utilizes either fixed or movable benches. Floor production systems utilize floor/soil heating (hot water heating pipes embedded in the floor/soil), but supplementary overhead heating can be required during colder seasons. Bench systems can operate like floor heating systems: hot water heating pipes are installed under the benches to increase rooting media or pot temperatures. An alternative system is to place small diameter rubber pipes on top of the bench underneath the plant containers. The advantage of the bench system is that it elevates plants above the cooler soil providing at the same time greenhouse workers with a more comfortable working height. Conversely, benches represent a cost and bedding plant operators usually place plants directly on the floor of polyethylene film greenhouses even though this growing system could undergo serious problems occurring from low soil temperatures in winter months. 
Neither warm air nor overhead hot water heating systems are able to maintain warm root zone temperatures for many crops, without operating on the greenhouse temperature. Using floor and bench heating systems, the temperature of the microclimate surrounding the plant canopy can generally be maintained at an optimum level. Traditional bench heating systems use steel or specially extruded aluminium finned piping under the bench to deliver heat to the rooting media and to the crop. These bench heating systems result in excellent plant growth and in some cases in reduced energy usage because of the lower ambient air temperatures set they allow. (Both et al., 2008). Thus, floor or bench heating can significantly reduce heating costs, with actual savings depending on weather conditions and temperature crop requirements.

In this work, we present a preliminary study aimed to assess the energy losses occurring in wintertime from a greenhouse while providing bench heating to a growth substrate (peat : perlite 50:50 V/V) using an innovative piping system and comparing this with an unheated bench.

\section{Materials and Methods}

The trial took place in a greenhouse of the experimental facility of CREA Research Centre for Horticulture and Floriculture, located in Pescia, Italy (43'53'00.59' ' N, $10^{\circ} 40^{\prime} 59,86^{\prime \prime}$ E) and lasted 11 days (from 08/12 to 19/12) providing bench heating using an innovative distribution system of thermal vector consisting of a coaxial pipe fuelled by a heat pump.

The trial greenhouse consists of a galvanized steel structure with a polycarbonate roof and double polyethene side surface and it has equipped with a fully automated and motorized opening system and air heating system to maintain air temperature above $5{ }^{\circ} \mathrm{C}$. The air heating system was switched off during the trial; only the basal heating system in the bench was kept running.

The basal heating system was placed in a concrete bench $(0.70 \mathrm{~m}$ width $\times 28.00 \mathrm{~m}$ length $\times 0.30 \mathrm{~m}$ height $)$ placed on cement blocks raising it $0.35 \mathrm{~m}$ above the soil level. The bench interior surfaces were insulated with $3 \mathrm{~cm}$ thickness polystyrene panels.

The innovative heating system consists of a coaxial polyethylene pipe: hot water coming from heat pump flows in the inner pipe (external diameter $16 \mathrm{~mm}$ ), returning water to heat pump flows in the outer pipe (external diameter $32 \mathrm{~mm}$ ). The inner tube was inserted manually for the whole length in the outer one that was closed at the end by a cap.

The water passed from the inner pipe to the outer one due to the presence of this closure. The contact between the coaxial pipes results in a continuous heat exchange between the fluid in the inner pipe and the fluid in the outer pipe. The characteristics of coaxial pipeline are shown in the Table 1. Using coaxial pipes is widespread for general purpose (Carnavos, 1985; Horton et al., 1975), in heat exchange systems or equipment such as air conditioning and refrigeration circuits (Edwards, 1981), in carrying toxic or hazardous gases (Spiegelman, 1992), in pneumatic servo and control systems (Wolf and Reichert, 1995), in dangerous conditions such as in the possibility of air contamination (Wolf, 1996), in thermally insulating systems (Ziemek and Schatz, 1972).

Table 1. Main characteristics of coaxial pipeline.

\begin{tabular}{ccc}
\hline Characteristics & Return line (outer tube) & Delivery line (inner tube) \\
\hline External diameter $(\mathrm{mm})$ & 16.0 & 32.0 \\
Inner diameter $(\mathrm{mm})$ & 12.5 & 23.2 \\
Tube section area $\left(\mathrm{mm}^{2}\right)$ & 122.7 & 422.7 \\
Area of the interstice between the pipes $\left(\mathrm{mm}^{2}\right)$ & 122.7 & 221.7 \\
\hline
\end{tabular}

The coaxial pipe was fuelled by a heat pump Aermec HSI120 having 12kW maximum nominal thermal power. The growth substrate temperature in the bench was measured using specific 2-channels data loggers, model Testo 175-T2 (Testo AG, Germany) with internal and external sensors. These instruments were synchronized to the solar time and were programmed to acquire data every 5 minutes. The growth substrate was irrigated using five polyethylene driplines (flow rate $2 \mathrm{~L} \mathrm{~h}^{-1}$, emitters $30 \mathrm{~cm}$ spaced). The irrigation system was placed on the surface of the substrate and seven a day irrigation interventions lasting for one minute were programmed.

\section{Data acquisitions}

Probes were placed in the greenhouse to record air temperature and the relative humidity of the air. Fourteen temperature probes were placed in rows inside the growing bench starting $5 \mathrm{~cm}$ from its bottom (Figure 1). 

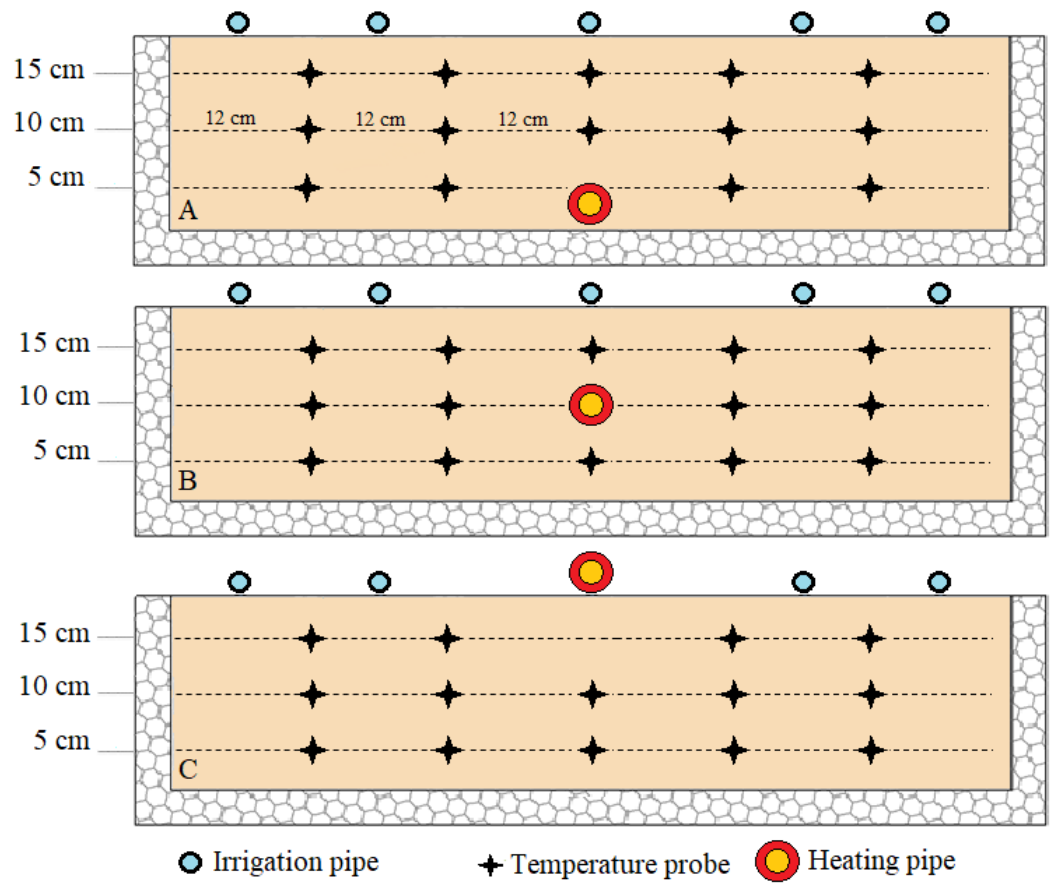

Figure 190. Representation of the cross section of the heated growing bench. Trials were carried out placing the innovative heating pipe (supply pipe - yellow- internal to the return one - red) at the bottom (A), in the middle (B) and in the top of the bench (at the base of the plants $-\mathrm{C}$ ). High density polystyrene insulated the bench at the bottom and at the sides.

During the experiment the temperature of the water in the heating system (supply and return pipes) as well as the temperature of the water provided by the irrigation pipes placed on the top of the benches were also monitored together with the solar irradiance $\left(\mathrm{W} \mathrm{m}^{-2}\right)$. All the data had an acquisition rate of $300 \mathrm{~s}$.

Energy flux

The heat loss $(\mathrm{kW})$ from the structure was assessed by the ASAE EP406.4 standard (ASABE 2008) net of the heat occurring from solar radiation (Eq. 1).

$$
\mathrm{Q}=\mathrm{UA}_{\mathrm{c}}\left(\mathrm{t}_{\mathrm{i}}-\mathrm{t}_{\mathrm{o}}\right)+\rho_{\mathrm{i}} \mathrm{NV}\left[\mathrm{C}_{\mathrm{pi}}\left(\mathrm{t}_{\mathrm{i}}-\mathrm{t}_{\mathrm{o}}\right)+\mathrm{h}_{\mathrm{fg}}\left(\mathrm{W}_{\mathrm{i}}-\mathrm{W}_{\mathrm{o}}\right)\right]-\mathrm{I \tau A}_{\mathrm{f}}(1-\mathrm{E})
$$

where $Q$ is the overall heat loss, $U$ is the overall heat transfer coefficient, $5.7 \mathrm{~W} \mathrm{~m}^{-2}{ }^{\circ} \mathrm{C}^{-1} ; A_{c}$ is the surface of the cover, $\mathrm{m}^{2} ; t_{i}$ is the greenhouse air temperature, ${ }^{\circ} \mathrm{C} ; t_{o}$ is the outside air temperature, ${ }^{\circ} \mathrm{C}, \rho_{i}$ is the greenhouse air density, $\mathrm{kg} \mathrm{m}^{-3} ; N$ is the infiltration rate, $\mathrm{s}^{-1} ; V$ is the greenhouse volume, $\mathrm{m}^{3}, C_{p i}$ is the specific heat of greenhouse air, $\mathrm{J} \mathrm{kg}^{-1}{ }^{\circ} \mathrm{C}^{-1} ; h_{f g}$ is the latent heat of vaporization of water at $t_{i}, \mathrm{~J} \mathrm{~kg}^{-1} ; W_{i}$ is the humidity ratio of greenhouse air, dimensionless; $W_{o}$ is the humidity ratio of the outside air, dimensionless; $A_{f}$ is the floor area, $\mathrm{m}^{2} ; I$ is the solar radiation, $\mathrm{W} \mathrm{m}{ }^{-2} ; \tau$ is the solar PAR transmittance, dimensionless; $E$ is the evapotraspiration coefficient, dimensionless.

\section{Data processing}

Statistical processing was performed using MINITAB 17 (2010): data underwent contour plot graphic representation and analysis of variance by means of General Linear Model $(G L M)$ procedure $(p<0.05)$ using the position of the heating pipe as a factor and the air temperature and the solar radiation as covariates.

\section{Results and Discussion}

Preliminary data processing showed that the days during which trials were performed had different meteorological conditions. The temperature of water used for heating was stable in all the tests (Table 2). Nevertheless, the intensity of the solar radiation varied significantly: the average overall irradiation ranged from $2.76 \mathrm{~kW} \mathrm{day}^{-1}$ when the heating system was at the bottom of the bench, to $3.19 \mathrm{~kW}$ day $^{-1}$ when the heating system was in the middle of it reaching

$6.06 \mathrm{~kW} \mathrm{day}^{-1}$ in case of heating provided with the pipes on the top of the growing substrate. Such a variability of the solar radiation resulted in analogous variations in the greenhouse temperature (Table 3 ). 
Table 2. Average temperatures $\left({ }^{\circ} \mathrm{C}\right)$ of the water flowing within the heating system (supply and return pipes), of the external surface of the heating system and of the growing substrate filling the bench.

\begin{tabular}{ccccc}
\hline $\begin{array}{c}\text { Positioning of the } \\
\text { heating system }\end{array}$ & Supply pipe & Return pipe & External surface & $\begin{array}{c}\text { Growing } \\
\text { substrate }\end{array}$ \\
\hline Bottom & $37.4 \pm 2.82$ & $33.6 \pm 0.97$ & $32.1 \pm 1.03$ & $12.6 \pm 0.06$ \\
Middle & $37.5 \pm 2.75$ & $33.9 \pm 1.01$ & $32.4 \pm 0.82$ & $16.3 \pm 0.02$ \\
Top & $37.0 \pm 3.20$ & $33.1 \pm 1.02$ & $29.7 \pm 1.65$ & $10.5 \pm 0.05$ \\
\hline
\end{tabular}

Table 3. Average temperatures $\left({ }^{\circ} \mathrm{C}\right)$ of the air inside the greenhouse.

\begin{tabular}{ccc}
\hline Positioning of the heating system & Daily temperature & Amplitude $\left(\mathrm{t}_{\max }-\mathrm{t}_{\min }\right)$. \\
\hline Bottom & $9.4 \pm 1.9$ & $13.7 \pm 8.1$ \\
Middle & $13.2 \pm 0.2$ & $23.6 \pm 3.7$ \\
Top & $9.8 \pm 0.7$ & $12.4 \pm 4.7$ \\
\hline
\end{tabular}

Figure 2 represents the trend of the temperatures recorded in heated and unheated growing benches on hourly basis. It shows that, at varying of the environmental conditions, the heated bench is, on average, always warmer than the unheated.

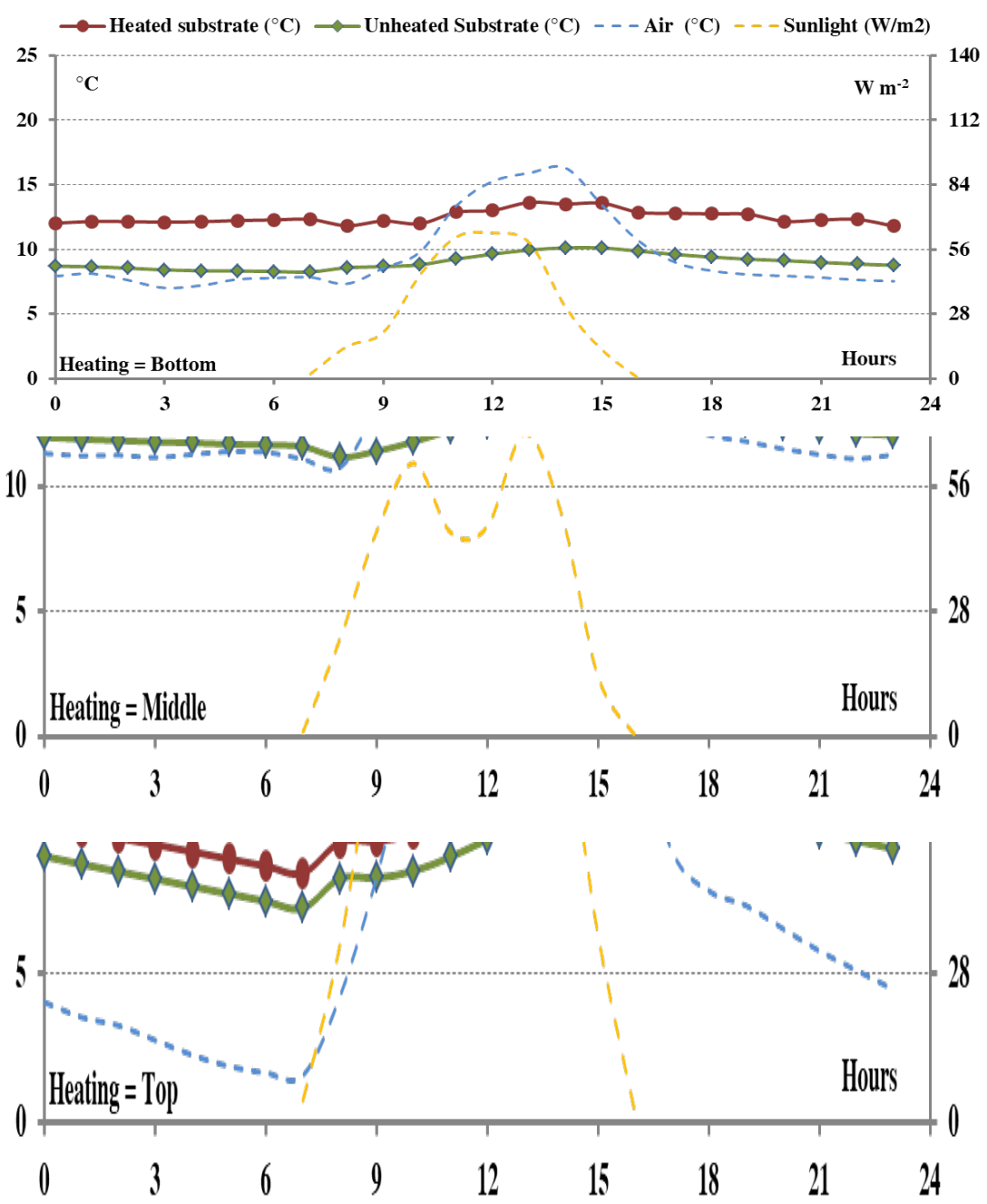

Figure 191. Average hourly temperatures of the peat-perlite substrates, of the air inside the greenhouse during the different tests (Bottom, Middle and Top) and the hourly solar radiation $\left(\mathrm{W} \mathrm{m}^{-2}\right)$. 
The results of the GLM (followed by Tukey pairwise comparisons) pointed out that, the positioning of the heating system significantly affected the average temperature of the growth medium. Following the post hoc comparisons it was possible to point out the following trend:

$$
\mathrm{t}_{\text {Middle }}>\mathrm{t}_{\text {Bottom }}>\mathrm{t}_{\text {Top }} \quad\left({ }^{\circ} \mathrm{C}\right)
$$

In particular, considering the difference of bench temperature in the different trials, it was possible to point out that when the heating system is placed in the middle of the bench, the $\Delta \mathrm{t}$ between heated and unheated benches is significantly higher (around $4^{\circ} \mathrm{C}$ on hourly basis) while in case of heating pipe placed on the top of the bench, the resulting average difference of temperature with the unheated bench is significantly lower with the range 1.1$1.9^{\circ} \mathrm{C}$ following the heat exchange by convection that the pipe has with the air. To better understand the dynamic behaviour of the temperature in the growing medium,, the median values of the temperatures the datalogger recorded (placed as indicated in Figure 1) were plotted in contour plots where the $\mathrm{x}$ - and y-axes report the distances of the dataloggers the origin of the system $(0,0)$ placed in the centre of the section (Figure 3 ). Such visual representation pointed out that, when the heating system is placed in the middle of the bench, the temperatures around it tend to be more uniform across the section than they do in the other cases (Figure 3). In particular, it points out that the heating effect occurring when the heating system is placed on the top of the bench is more localized in the upper central part of the bench while in case of heating system placed at the bottom, the rise of the temperature is able to reach almost half of the height of the bench and it expands throughout the base, but the median temperatures in the upper part tend to remain low. This also results in lower energy loss from the greenhouse, assessed with Eq (1) that, when pipes were at the bottom of the bench, was $1.34 \pm 0.05 \mathrm{kWh}$ that is lower than that occurring when pipes were placed on the top $(2.10 \pm 0.07 \mathrm{kWh})$.
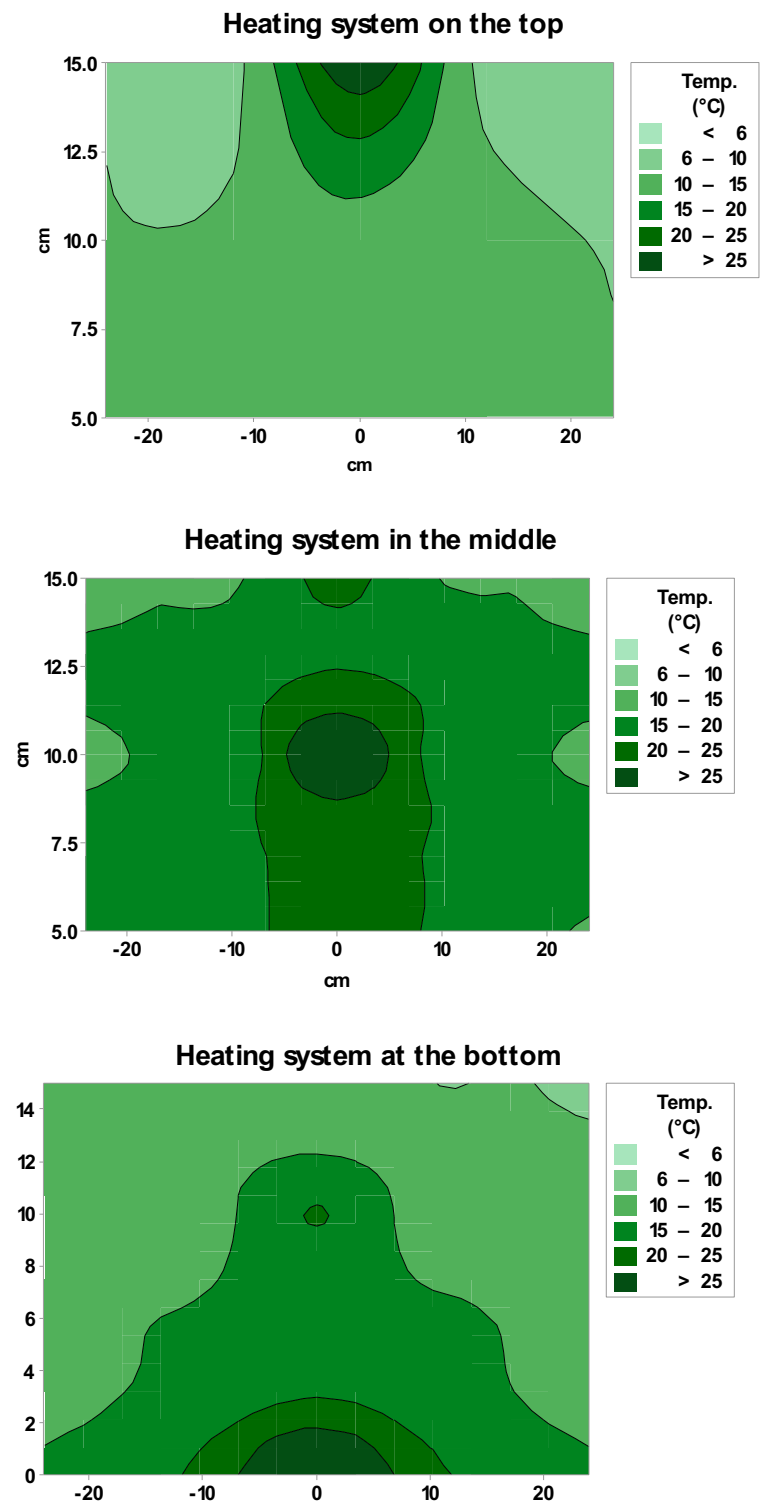

Figure 192. Contour plots representing the median values of the temperatures $\left({ }^{\circ} \mathrm{C}\right)$ recorded by the dataloggers. 
The problem of providing heat to the roots heating the benches is an issue: traditional bench heating systems use steel or specially extruded aluminium finned piping under the bench to deliver heat to the rooting media and to the crop. Maintaining the proper water temperature is critical for the efficient operation of floor heating systems. Generally, as reported by Both (2008), operating temperatures are maintained between 32 and $49^{\circ} \mathrm{C}$ (higher temperatures may inadvertently affect plant growth), and the loops are designed to result in a low temperature drop along the entire length of the loop (e.g., $15^{\circ} \mathrm{C}$ ) resulting in a uniform floor surface temperature. When higher floor temperatures are needed, for example in germination areas, the tubing is generally spaced closer (e.g., 9 inches apart) and the temperature of the water in the tubing is maintained close to $49^{\circ} \mathrm{C}$. This closer tubing spacing generally increases the uniformity of heat distribution. In the presented case, water temperature was lower than the specified range (see Table 2), nevertheless the heating system managed to provide heat to the growth medium successfully: the particular design of the pipes allowed to maintain the drop of temperature around $4{ }^{\circ} \mathrm{C}$ which is lower than the temperature drop described above.

\section{Conclusions}

In our work the effect of the position (on the top, in the middle and at the bottom) of an innovative pipeline to provide bench heating in greenhouse production is introduced. Pipe position significantly affected the temperature of the growth medium and the heat loss. Pipes placed inside the substrate (in the middle) provided the highest temperature of the growth medium $\left(16.3 \pm 0.02{ }^{\circ} \mathrm{C}\right)$ and resulted in the lowest heat loss from the greenhouse $(1.34$ $\pm 0.05 \mathrm{kWh}$ ) on hourly basis that is $36 \%$ lower than that related to the heating pipes placed on the top of the growing substrate.

\section{Acknowledgements}

This work was supported by the Italian Ministry of Agriculture (MiPAAF) under the AGROENER project (D.D. n. 26329, 1 april 2016) - http://agroener.crea.gov.it/

The authors acknowledge the work of Mr. Alex Filisetti, Elia Premoli and Alessio Fedrizzi for the valuable help provided.

\section{References}

ASABE- American Society of Agricultural and Biological Engineers. 2008. ANSI/ASAE EP406.4 Standard: Heating, Ventilating and Cooling Greenhouses. Revision approved February 20034; reaffirmed February 2008 as an American Standard.

Aye, L., Fuller, R.J. Canal, A. 2010, Evaluation of a heat pump system for greenhouse heating. International journal of thermal sciences, vol. 49, pp. 202-208.[DOI]

Bailey, B.J. 1983. Limiting the relative humidity in insulated greenhouses at night, Acta horticulturae, Energy in Protected Cultivation, 148, 1-11. http://dx.doi.org/10.17660/ActaHortic.1984.148.52

Both A.J., Manning T.O, Mears D.R., Reiss E. 2008. Root Zone Heating for Commercial Greenhouses. Technical Report. Center for Controlled Environment Agriculture, Rutgers University. USA. http://ceac.arizona.edu/sites/default/files/aj_both__root_zone heating.pdf. Acessed April 2018.

Carnavos, T.C., 1985. Coaxial finned tube heat exchanger, Noranda Metal Industries, Inc. (Newton, CT) United States Patent $n^{\circ} 4554969$

Dieleman, J. A., Marcelis, L. F. M., Elings, A., Dueck, T. A. \& Meinen, E. 2006. Energy saving in greenhouses: Optimal use of climate conditions and crop management. Acta Horticulturae 718, 203-210.

Edwards, R.C. 1981. Coaxial tube in tube heat exchanger with inner tube support, Edwards Engineering Corporation (Pompton Plains, NJ) United States Patent $n^{\circ} 4286653$

Elings, A., Kempkes, F. L. K., Kaarsemaker, R. C., Ruijs, M. N. A., van de Braak, N. J. \& Dueck, T. A. 2005. The energy balance and energy-saving measures in greenhouse tomato cultivation. Acta Horticulturae 691, 67-74. http://dx.doi.org/10.17660/ActaHortic.2005.691.5

Garcia, J.L., De la Plaza, S., Navas, L.M., Benavente, R.M. and Luna, L. 1998. Evaluation of the feasibility of alternative energy sources for greenhouse heating, Journal of Agricultural Engineering Research 69, 107-114. https://doi.org/10.1006/jaer.1997.0228

Hemming, S.; Balendonck, J.; Dieleman, J.A.; de Gelder, A.; Kempkes, F.L.K.; Swinkels, G.L.A.M.; de Visser, P.H.B.; de Zwart, H.F. 2015. Innovations in greenhouse systems-Energy conservation by system design, sensors and decision support systems. In Proceedings of the ISHS Acta Horticulturae 1170: International Symposium on New Technologies and Management for Greenhouses-GreenSys 2015, Evora, Portugal, 19-23 July 2015

Horton, L.R., Jackson, C.A., Holcomb, W.P., and Horton, C. 1975. Coaxial Tube Coupler Assembly, United States Patent $n^{\circ} 3860269$

Kozai, T. 1986. Thermal performance of an oil engine driven heat pump for greenhouse heating, Journal of Agricultural Engineering Research, 35, 25-37. https://doi.org/10.1016/0021-8634(86)90027-2 
Minitab 17 Statistical Software. 2010. [Computer software]. State College, PA (USA): Minitab, Inc. (www.minitab.com)

Shen, Y., Wei, R., Xu, L. 2018. Energy Consumption Prediction of a Greenhouse and Optimization of Daily Average Temperature. Energies, 11, 65- http://dx.doi.org/10.3390/en11010065

Spiegelman, J.J. 1992. Coupling for interconnection of coaxial tubing, Tylan General, Inc. (San Diego, CA) United States Patent $n^{\circ} 5088774$

Wolf, F.J. and Reichert, U. 1995. Fluid containing coaxial tube for control systems, WOCO Franz-Josef Wolf $\&$ Co. (Bad Soden-Salmunster, DE) United States Patent $\mathrm{n}^{\circ} 5433252$

Wolf, L.W., 1996. Vented bending sleeves for coaxial tubing systems, United States Patent n ${ }^{\circ} 5497809$

Ziemek, G., and Schatz, F. 1972. Spacing in coaxial pipes system, Kabel-und Metallwerke Gutehoffnungshutte Aktiengesellschaft (Hannover, DT) United States Patent n ${ }^{\circ} 3670772$ 


\title{
Sensitivity Analysis of a Modified Equation for Greenhouse Energy Demand Assessment
}

\author{
Massimo Brambilla a,", Elio Romano a, Maurizio Cutini a , Marco Fedrizzi ${ }^{\text {b }}$, Mauro Pagano ${ }^{\text {b }}$, Gianluca \\ Burchi $^{c}$, Sonia Cacini ${ }^{c}$, Daniele Massa ${ }^{c}$, Chiara Terrosi ${ }^{c}$ and Carlo Bisaglia ${ }^{a}$ \\ ${ }^{\text {a }}$ CREA Research Centre for Engineering and Agro-Food Processing, Via Milano, 43 - 24047 Treviglio (BG), Italy \\ ${ }^{\mathrm{b}}$ CREA Research Centre for Engineering and Agro-Food Processing, Via della Pascolare 16 - 00015 - Monterotondo \\ (Roma), Italy \\ ${ }^{c}$ CREA Research Centre for Vegetable and Ornamental Crops, Via dei Fiori, 8 - 51017 - Pescia (PT), Italy \\ * Corresponding author. Email: massimo.brambilla@crea.gov.it
}

\begin{abstract}
Within the framework provided by the Italian Ministry of Agriculture with AGROENER project (http://agroener.crea.gov.it/), a modified equation for the assessment of greenhouse energy demand was developed. As a matter of fact, in Italy, almost 30.000 heated greenhouse production sites undergo variety of climate conditions. With particular reference to the Northern part of the Country (Po and Adige valleys) and to the Central one (low inland) humid subtropical climate can result in moderately cold wintertime. Starting from the recommendations of ASAE EP406.4/08 [B1] standard, the overall heat loss from the greenhouse (Qt) has been assessed as function of the heat losses by radiation, conduction and convection (Qre), by infiltration (Qi) and the heat occurring from solar radiation (Qs). Qt $=$ Qrc+Qi-Qs [W]. All the accessory variables needed for heat loss assessment were assessed in accordance with ASAE D271.2/99 and ASAE EP 460/01. To understand the relationships between information flowing in and out of the model, a sensitivity analysis was carried out by means of Monte Carlo analysis. Multiple evaluations with randomly selected model input were performed to determine both uncertainties in equation predictions and evaluating the importance of each individual input variable with reference to the uncertainty of the output by means of standardized coefficients: 22900 runs were performed. The expected mean value for Qt resulted to be $3.62 \mathrm{~kW}$ corresponding to $11.4 \mathrm{~W} \mathrm{~m}^{-3}$. A preliminary investigation of variable importance using the Pearson correlation coefficient ( $\mathrm{r}$ ) pointed out that the overall heat transfer coefficient (U-value, $\left.\mathrm{W} \mathrm{m}^{-2}{ }^{\circ} \mathrm{C}^{-1}\right)$, the area of the cover $\left(\mathrm{Ac}, \mathrm{m}^{2}\right)$ and the temperatures $\left({ }^{\circ} \mathrm{C}\right)$ inside and outside the greenhouses were the variables whose variations were mostly influencing the equation output. The standardized regression coefficients resulting from linear regression proved the temperatures are the most important variables followed by U-value and the area of the cover.
\end{abstract}

Keywords: Agriculture, Protected Crops, Process Optimization.

\section{Introduction}

Greenhouses provide a suitable environment for plants. The energy from the sun provides sunlight and heat: greenhouse design allows to maintain solar radiation, temperature, and humidity in the greenhouse environment. The materials of the greenhouse covers transmit the useful wavelengths of the light spectrum for photosynthetic activity and create a favourable indoor micro-environment. Providing greenhouses with a proper heating system (e.g. heaters, fans, heating pipes) fosters their protection of plants in cold weathers so that they relieve the discrepancy between supply and demand of off-season vegetables (Kendirli et al., 2006; Ozgener and Hepbasli, 2005; Jain et al., 2002). Of course, selecting the proper site becomes fundamental for profitable and sustainable greenhouse production: the climatic conditions guide the choice of the structural elements (covers, structure and internal equipment for climate control) as they drive the investment costs as well as greenhouse performance to obtain high-quality products at competitive level (Castilla and Baeza, 2013).

Air temperature, solar radiation and relative humidity play a key role in greenhouse climate control. They influence not only crop development and yield but also the energy requirements of the structure. Plant growers can improve greenhouse climate control and energy demand choosing among a range of technologies. Nevertheless, some economic and technologic issues need to be taken into account: as a matter of fact, technologies developed in North European countries hardly ever can be transferred directly to Mediterranean Countries following the investment capacity they have and the different technological know-how. However, greenhouse heating becomes essential even in temperate climate Countries, like those of the Mediterranean region, as it boosts production quality and quantity. The heating system is fundamental to maintain the day and night temperatures crops need: the greenhouses itself is not an efficient solar heat collector and therefore taking into account the heat loss from the structure becomes very important. The ideal heating system must provide heat to the greenhouse at the same rate at which it is lost: inefficiencies may result in loss of profitability and increase of noxious emissions into the atmosphere (Kittas et al., 2013).

The rate of nocturnal heat loss from a heated plastic greenhouse is made up of many heat transfer processes, 
each of which can be described by a mathematical expression. Under a given set of climatic conditions, it is possible to develop a series of equations, input factors, parameters and variables mimicking or approximating processes of different nature. Solving the relevant equations assess the magnitude of each of the involved transfer processes and enable the calculation of the total rate of heat loss (Garzoli et al., 1981).

Nevertheless, the input of such equations is subjected to a number of uncertainty sources (e.g. measurement errors, missing information, imperfect or partial understanding of the involved processes) resulting in a limitation of model output reliability and confidence. Sensitivity analysis (SA) helps is assessing such uncertainty: it focuses the variations the model output undergoes apportioning them to the different inputs (quantitatively or qualitatively) to gain insight into which assumptions are critical and on how the model behaviour depends upon the information fed into it (Saltelli, 2000). The methodologies and the strategies used to perform SA are basically three (Campolongo et al., 2000):

- factor screening: aimed at the identification of the influential factors in a system with many factors acting on it;

- local $S A$ : focused on the single impact (point) factors have on the model;

- global SA: designed to highlight the apportioning the output uncertainty to that of the input factors. Any SA activity is considered global when all parameters vary simultaneously and the sensitivity is measured over the full range of each input parameter.

Monte Carlo (MC) analysis is a global SA strategy based on the performance of multiple simulations with randomly selected model input. The outcome of each simulation is afterwards used to determine the uncertainty of model predictions and their apportioning to the contribution of the input factors. This process foresees various ways of changing model inputs to see the effect on the output value. The random variables or inputs are modelled on the basis of probability distributions (e.g. normal, log normal, uniform, and triangular): running different iterations or simulations generates paths that undergo suitable numerical computations. MC analysis is a reliable method in case of models having uncertain parameters or in case dynamic complex systems need to be explored: it is a probabilistic method that describes the expected model output and its probability of occurrence (Briggs et al., 2001; Qin et al., 2016).

The aim of this study is to present the results of the sensitivity analysis of a modified equation for the assessment of the heat loss from the structure that takes into account the contribution of solar radiation to the overall energetic balance in wintertime.

\section{Materials and Methods}

The used equation

The used model was developed starting from the recommendations of ASAE EP406.4/08 standard (ASABE, 2008), assessing the overall heat loss from the greenhouse $\left(Q_{t}\right)$ as function (Eq. 1) of the heat losses by radiation, conduction and convection $\left(Q_{r c}\right)$, by infiltration $\left(Q_{i}\right)$ net of the heat occurring from solar radiation $\left(Q_{s}\right)$.

$$
\mathrm{Q}_{\mathrm{t}}=\mathrm{Q}_{\mathrm{rc}}+\mathrm{Q}_{\mathrm{i}}-\mathrm{Q}_{\mathrm{s}}
$$

More analytically, each component of the equation is hereafter defined. The standard defines $Q_{r c}(\mathrm{~W})$ the heat loss by long wave radiation, conduction and convection with Eq. (2):

$$
\mathrm{Q}_{\mathrm{rc}}=\mathrm{UA}_{\mathrm{c}}\left(\mathrm{t}_{\mathrm{i}}-\mathrm{t}_{\mathrm{o}}\right)
$$

where $\mathrm{U}$ is the overall heat transfer coefficient, $\mathrm{W} \mathrm{m}{ }^{-2}{ }^{\circ} \mathrm{C}^{-1}$; Ac is the surface of the cover, $\mathrm{m}^{2} ; t_{i}$ and $t_{o}$ are the temperatures inside and outside the greenhouse, ${ }^{\circ} \mathrm{C}$.

The heat loss occurring from infiltration $\left(Q_{i}\right)$ has been estimated according to Eq. (3).

$$
\mathrm{Q}_{\mathrm{i}}=\rho_{\mathrm{i}} \mathrm{NV}\left[\mathrm{C}_{\mathrm{pi}}\left(\mathrm{t}_{\mathrm{i}}-\mathrm{t}_{\mathrm{o}}\right)+\mathrm{h}_{\mathrm{fg}}\left(\mathrm{W}_{\mathrm{i}}-\mathrm{W}_{\mathrm{o}}\right)\right]
$$

where $\rho_{i}$ is the greenhouse air density, $\mathrm{kg} \mathrm{m}^{-3} ; N$ is the infiltration rate, $\mathrm{s}^{-1} ; V$ is the volume of the greenhouse, $\mathrm{m}^{3} ; C_{p i}$ is the specific heat of greenhouse air, $\mathrm{J} \mathrm{kg}^{-1}{ }^{\circ} \mathrm{C}^{-1} ; h_{f g}$ is the latent heat of vaporization of water at saturation, $\mathrm{J} \mathrm{kg}^{-1} ; W_{i}$ and $W_{o}$ are the humidity ratios $\left(\mathrm{kg}_{\text {water }} \mathrm{kgair}^{-1}\right)$ of the air inside and outside the structure, dimensionless.

The contribution of the solar energy $\left(Q_{s}\right)$ was assessed following Eq. (4).

$$
\mathrm{Q}_{\mathrm{s}}=\mathrm{I} \tau A_{f}(1-E)
$$

where $I$ is the incoming solar radiation, $\mathrm{W} \mathrm{m} \mathrm{m}^{-2} ; A_{f}$ is the floor area of the greenhouse, $\mathrm{m}^{2} ; \tau$ is the photosynthetically active radiation (PAR) transmittance of the cover, dimensionless; $E$ is an evapotranspiration coefficient that takes account the presence of the crop, dimensionless. All the accessory variables needed to run the model were assessed in accordance with ASAE EP 460/01 standard (ASAE, 2001) and calculated as recommended by ASAE D271.2/99 standard (ASAE, 1999) and ASHRAE (2012). 
The scenario

Not all the possible climatic conditions were considered to perform this analysis. The considered scenario is that of a gable trussed roof greenhouse (Figure 1) whose heights of the lateral walls (h) and of the apex (ha) were kept constant at $2.2 \mathrm{~m}$ and $3.7 \mathrm{~m}$. On the contrary the greenhouse width and length were considered varying from 3 to $10 \mathrm{~m}$ (the former) and from 5 to $20 \mathrm{~m}$ the latter. The model took into account such variations and the relations existing between them were taken into account.

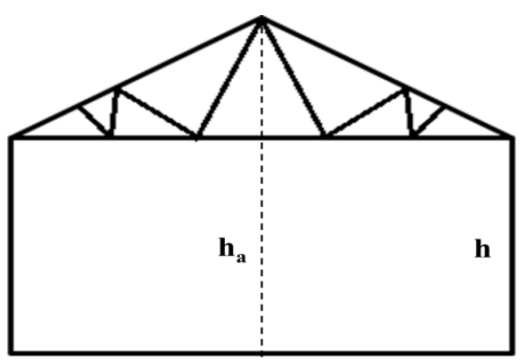

Figure 193. Schematic diagram of the considered greenhouse (modified from ASAE, 2004).

Concerning climatic conditions, attention was focused on the most energy requiring period of the year. Because of this the micrometeorological parameters occurred during the month of December 2017 at the CREA Research Centre for Vegetable and Ornamental Crops experimental site (Pescia, Italy) were considered. The studied dataset contained the records of air temperature $\left({ }^{\circ} \mathrm{C}\right)$, air relative humidity $(\%)$ and solar irradiation $(\mathrm{W}$ $\mathrm{m}^{-2}$ ) recorded with the acquisition rate of $300 \mathrm{~s}^{-1}$.

Concerning the other operative parameters, the used values ranged between the maximum and the minimum contained in the ASAE EP 460/01 standard (ASAE, 2001)

\section{Model evaluation}

The variables feeding the model were varied randomly between the minimum and maximum value that Table 1 reports. To account for a wider range of model outputs their random distribution between the established the uniform and 100,000 runs were performed.

Table 80. Minimum and maximum values used to run the model.

\begin{tabular}{cccc}
\hline Variable Name & Symbol & Min & Max \\
\hline Overall transfer coefficient & $\mathrm{U}$ & 0.60 & 6.50 \\
Surface of the cover & $\mathrm{Ac}$ & 15.8 & 212 \\
Inner temperature & $\mathrm{t}_{\text {in }}$ & 12 & 20 \\
External temperature & $\mathrm{t}_{\mathrm{out}}$ & -3.0 & 18 \\
Greenhouse air density & $\rho_{\mathrm{in}}$ & 1.16 & 1.29 \\
Infiltration rate & $\mathrm{s}^{-1}$ & $2.1010^{-4}$ & $4.1010^{-4}$ \\
Volume of the greenhouse & $\mathrm{m}^{3}$ & 44 & 590 \\
Specific heat of the inner air & $\mathrm{C}_{\mathrm{pi}}$ & 1.059 & 1.061 \\
Inner humidity ratio & $\mathrm{h}_{\mathrm{fg}}$ & $2.4310^{6}$ & $2.510^{6}$ \\
External humidity ratio & $\mathrm{W}_{\mathrm{i}}$ & $3.1010^{-3}$ & $9.4110^{-3}$ \\
Incoming solar radiation & $\mathrm{W}_{\mathrm{o}}$ & $1.8610^{-3}$ & $9.3510^{-3}$ \\
PAR Transmittance & $\mathrm{I}$ & 0 & 213 \\
Evapotranspiration coefficient & $\tau$ & 0.79 & 0.88 \\
Floor area of the greenhouse & $\mathrm{E}$ & 0 & 1 \\
\hline
\end{tabular}

Uncertainty analysis

In this phase the evaluation of the equation was done for each of the sample elements: given that each element enters the model the output. This first evaluation was done considering the distribution the output undergoes.

\section{Sensitivity analysis}

To assess the effect that individual components of the variables have on the outcome of the model the scatterplots representing the relation between model output and each considered variable were considered and, with these, the Pearson correlation coefficients $(r)$ between any input value and the model output.

Following this, further investigation foresaw the adoption of stepwise linear regression analysis (LRA) with 
backward elimination carried out on standardized data. The approach of this more formal analysis is based on the linear regression model Eq. (5)

$$
y_{i}=b_{0}+\sum_{j=1} b_{j} x_{i j}+\varepsilon_{i}
$$

where $b_{j}$ are the regression coefficients and $\varepsilon_{i}$ is the error (residual of the approximation). The computing of the coefficients allows to use them as way to indicate the importance of each variable with respect to the uncertainty of the output $y$. In accordance with Campolongo et al. (2000), the standardized regression coefficients (SRC), calculated according to Eq. (6) were used:

$$
\mathrm{SRC}=\frac{b_{j} \sqrt{\frac{\left(x_{i j}-\bar{x}_{j}\right)^{2}}{N-1}}}{\sqrt{\frac{\left(y_{i-\bar{y}}\right)^{2}}{N-1}}}
$$

These coefficients have the advantage of quantifying the effect of varying each input variable away from its mean by a fixed fraction of its variance while all the other variables remain at their expected value.

\section{Data processing}

Statistical processing was performed using the MINITAB $17.0^{\text {TM }}$ software (Minitab, 2010). Model output underwent graphical summarization with histogram of data with an overlaid normal curve, boxplot, $95 \%$ confidence intervals for both average and median values. Linear Regression Analysis (LRA) allowed to obtain the regression coefficients $(\mathrm{p}<0.05)$ whose standardization to obtain the various SRCs was carried out with Excel spreadsheet.

\section{Results and Discussion}

Figure 2 reports the graphical summary of the model output $(Q t)$ : this first representation shows that model output distribution is not statistically normal (following the established variable ranges and distributions) and how much uncertainty is connected to the output of the equation even when the micrometeorological data are referred only to a small albeit significant period of the year. The average heat loss calculated by the model is $3,264 \mathrm{~W}$ (the median value is $2,339 \mathrm{~W}$ ) that, based on greenhouse volume, correspond to an average dispersion of $11.4 \pm 10.9$ $\mathrm{W} \mathrm{m}{ }^{-3}$. This means that the uncertainty associated to this output is quite high, nevertheless from the picture it can be noticed that the amount of heat exiting the greenhouse (from zero onwards) is higher than that entering it following sun irradiation that, following model construction, have negative values.

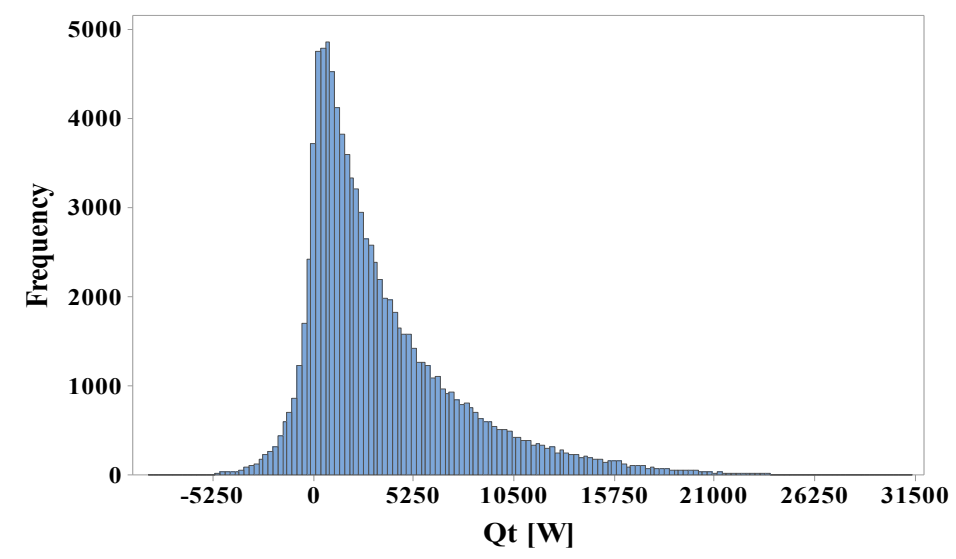

Figure 194. Graphical summary (frequency histogram) of the distribution of the output of the 100,000 model runs.

To overcome such uncertainty and gain insight into the role that the different variables play on model behaviour scatterplots representing the relation they have with Qt were generated (Figure 3). Despite the model seems to have quite a complex behaviour, each single scatterplot results to be simply to interpret, in particular in case they are represented next to each other. According to Helton and Davis (2000) it may happen that scatterplots alone can completely reveal the relationships between model input (i.e. element of $x$ ) and model predictions (i.e. $y$ values) but this is true when only few inputs affectively dominate the model behaviour. In this case it appears evident that (referring to the considered scenario) the variables having the wider effect on model output appear to be the overall transfer coefficient $(\mathrm{U})$, the surface of the cover $(\mathrm{Ac})$, the external temperature $\left(\mathrm{t}_{\text {out }}\right)$, the volume of the structure (V) as well as its floor surface (Af) given the high variation Qt undergoes at their varying.

How well these variables are related to the output they give rise is represented by the correlation coefficient 
they have with Qt (Table 2). Among them those that were assessed affecting model output the most (and significantly) are the overall transfer coefficient, the surface of the cover, the external temperature and the floor area of the greenhouse. Beyond these there are other model inputs that show significant correlation coefficient, nevertheless the extent of their relation can be considered too weak in compared with the former. This starts to be a representation of the dynamics behind the considered equation, nevertheless a more and supported explanation is still required.

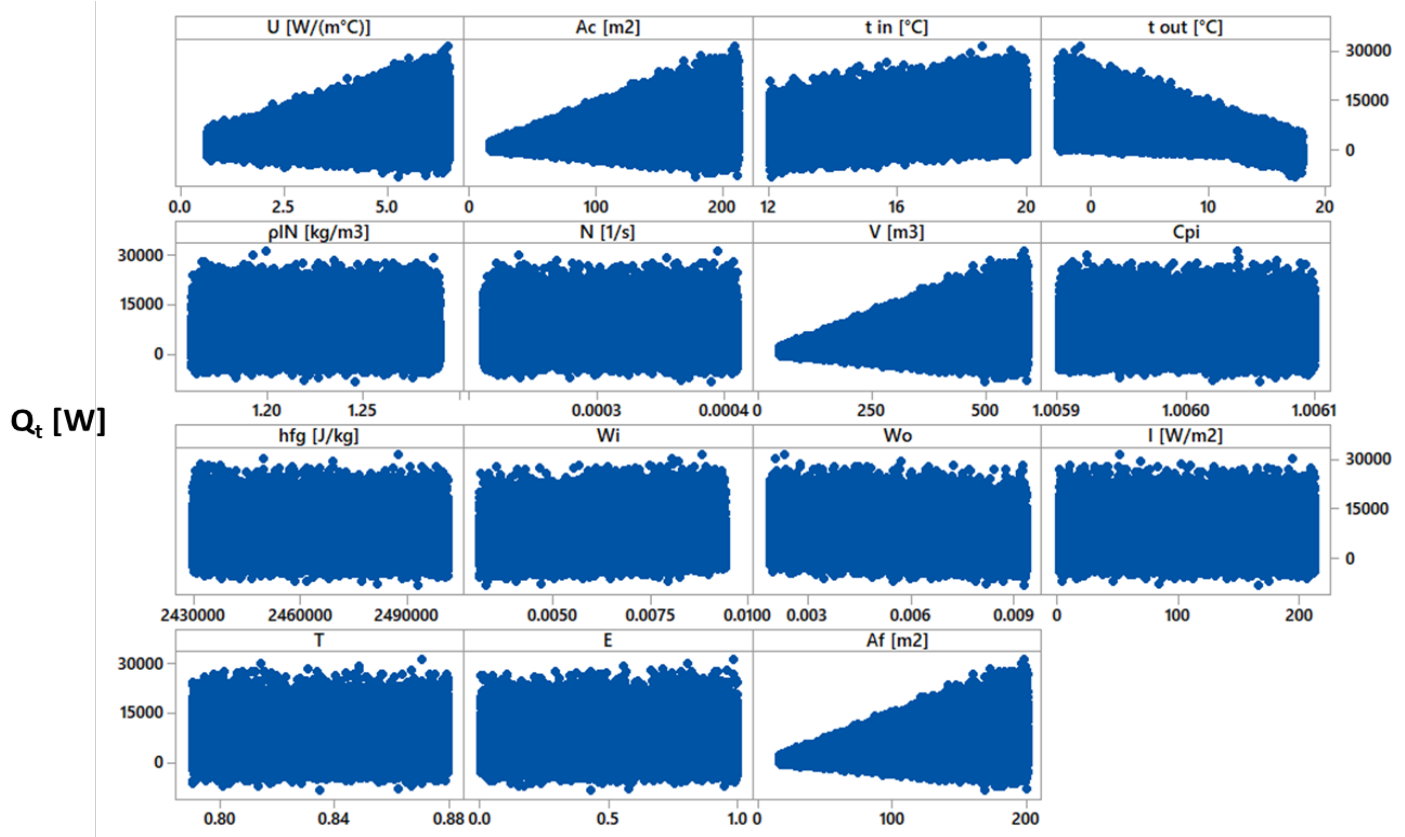

Figure 195. The scatterplot representing the model output (Qt) vs. any considered variable.

The LRA provided a more formal investigation of the mapping of variable's importance to lower the uncertainty attained to the model. The stepwise backward elimination method pointed out a partial albeit important confirmation of what appeared in scatterplot and correlation coefficient analysis. The resulting equation, whose $\mathrm{R}^{2}$ is 0.75 , is:

$$
\begin{aligned}
\mathrm{Qt}=-7342.3+ & 970.23 \mathrm{U}+31.726 \mathrm{~A}_{\mathrm{c}}+406.5 \mathrm{t}_{\text {in }}-404.4 \mathrm{t}_{\text {out }}+667109 \mathrm{~N}+298625 \mathrm{~W}_{\mathrm{i}}-299100 \mathrm{~W}_{0} \\
& +50.6 \mathrm{E}
\end{aligned}
$$

nevertheless the coefficient describing the role need to be taken with extreme attention as they are not standardized and therefore they suffer from the effects of their distribution assumption (in this case, starting from standardized values, the effect connected to the different units of measure is not present). Therefore a better interpretation of their role can't help using the SRCs hereafter reported in Table 2

Table 81. Pearson correlation coefficients of model input with model output together with the standardized regression coefficients resulting from LRA. * significant at $\mathrm{p}<0.05$.

\begin{tabular}{ccc}
\hline Variable & $r$ & SRC \\
\hline $\mathrm{U}$ & $0.38^{*}$ & 0.66 \\
$\mathrm{Ac}$ & $0.42^{*}$ & 24.0 \\
$\mathrm{t}_{\text {in }}$ & $0.22^{*}$ & 0.51 \\
$\mathrm{t}_{\text {out }}$ & $-0.57^{*}$ & -3.49 \\
$\rho_{\text {in }}$ & 0.001 & \\
$\mathrm{~N}$ & $0.014^{*}$ & $5.2510^{-7}$ \\
$\mathrm{~V}$ & $0.42^{*}$ & \\
$\mathrm{C}_{\mathrm{pi}}$ & 0.02 & \\
$\mathrm{~h}_{\mathrm{fg}}$ & -0.001 & \\
$\mathrm{~W}_{\mathrm{i}}$ & $0.128^{*}$ & $2.3510^{-4}$ \\
$\mathrm{~W}_{\mathrm{o}}$ & $-0.152^{*}$ & $-3.3010^{-4}$ \\
$\mathrm{I}$ & $0.001^{*}$ & \\
$\tau$ & -0.002 & $9.9110^{-4}$ \\
$\mathrm{E}$ & -0.001 & \\
$\mathrm{~A}_{\mathrm{f}}$ & $0.42^{*}$ & \\
\hline
\end{tabular}


According to Table 2 the variables most affecting the heat loss of the greenhouse are three (i.e. the overall transfer coefficient, the surface of the cover and the external temperature). Other variables were selected by the stepwise regression, nevertheless their contribution to the final output can be considered negligible on the base of their SRC.

In particular, the area of the cover is the factor contributing the most to the heat loss occurring in winter time and this turns out to be important in view of greenhouse design as it can undergo specific modification (e.g. placing of specific insulation). Of course, the materials greenhouse are made of can contribute to reduce the heat loss occurring from the structure, but it's evident that the extent of their action is quite limited. The inner temperature setting is another interesting variable: its role in affecting the foreseen heat loss is comparable to that of the material of the covering but different runs pointed out that reducing its range results in a frequency histogram of the model output that moves towards statistical normality (data not shown).

\section{Conclusions}

To deep the knowledge related to the structural elements and the micrometeorological variables affecting the output of a modified equation to calculate the heat loss from a greenhouse, a sensitivity analysis based on Monte Carlo strategy was carried out. Model variables underwent uniform distribution between literature and measured minimum and maximum values and 100,000 simulations were performed using the outcome of each simulation to determine the uncertainty of model predictions and their apportioning to the contribution of the input factors.

The standardized regression coefficients showed that in the considered scenario the variables acting the most on heat loss from the greenhouse are the overall transfer coefficient, the surface of the cover and the external temperature suggesting that in the design phase, specific thinking of the covering and of the material it can be made plays a key role. Further work on these aspects is anyway required to better focus model testing in order to meet farmer's needs to increase the sustainability of the production chain.

\section{Acknowledgements}

This work was supported by the Italian Ministry of Agriculture (MiPAAF) under the AGROENER project (D.D. n. 26329, 1 april 2016) - http://agroener.crea.gov.it/

The authors acknowledge the work of Mr. Alex Filisetti and Elia Premoli for the valuable help provided.

\section{References}

ASABE - American Society of Agricultural and Biological Engineers. 2008. ASABE Standard (ANSI/ASAE EP406.4), Heating, ventilating and cooling greenhouses.

ASAE - American Society of Agricultural Engineers. 1999. ASAE standard ASAE D271.2/99. Psychrometric Data.

ASAE - American Society of Agricultural Engineers. 2001. ASAE standard ASAE 460/01. Commercial Greenhouse Design and Layout.

Berna K. 206. Structural analysis of greenhouses: a case study in Turkey. Building and Environment41 (7), 864-871. https://doi.org/10.1016/j.buildenv.2005.04.013

Briggs A, Goeree R, Blackhouse G, O'Brien B. 2001. Probabilistic Analysis of Cost-effectiveness Models: Choosing Between Treatment Strategies for Gastro-Esophogeal Reflux Disease, McMaster University Centre for Health Economics and Policy Analysis Research Working Paper 01-01, February,2001.

Campolongo F., Saltelli A., Sørensen T., Tarantola S. 2000. Hitchhiker's guide to Sensitivity Analysis. In Sensitivity Analysis. Eds. A. Saltelli, K. Chan, E.M. Scott. John Wiley \& Sons Ltd. (UK). 16-45.

Castilla, N. and Baeza E. 2013. Greenhouse site selection. In Good Agricultural Practices for greenhouse vegetable crops. Principles for Mediterranean climate areas. FAO - Food and Agriculture Organization of the United Nations. Eds., W. Baudoin, R. Nono-Womdim, N. Lutaladio, A. Hodder, N. Castilla, C. Leonardi, S. De Pascale, M. Qaryouti. 21-34.

Cooper P.I., Fuller R.J. 1983. A transient model of the interaction between crop, environment and greenhouse structure for predicting crop yield and energy consumption, Journal of Agricultural Engineering Research. 28(5): 401-417. https://doi.org/10.1016/0021-8634(83)90133-6

Halton J. C., Davis F. J. 2000. Sampling -Based Methods. In Sensitivity Analysis. Eds. A. Saltelli, K. Chan, E.M. Scott. John Wiley \& Sons Ltd. (UK). 101-152.

Jain D., Tiwari G.N. 2002. Modeling and optimal design of evaporative cooling system in controlled environment greenhouse, Energy Conversion and Management. 43(16), 2235-2250. https://doi.org/10.1016/S0196-8904(01)00151-0

Kittas C., Katsoulas N., Bartzanas T., Bakker S. 2013. Driving Forces for Greenhouse Climate Control and Sustainable Energy Use in Mediterranean Greenhouses. In Good Agricultural Practices for greenhouse vegetable 
crops. Principles for Mediterranean climate areas. FAO - Food and Agriculture Organization of the United Nations. Eds., W. Baudoin, R. Nono-Womdim, N. Lutaladio, A. Hodder, N. Castilla, C. Leonardi, S. De Pascale, M. Qaryouti. 63-95.

Minitab 17 Statistical Software (2010). State College, Pa.: Minitab, Inc. Retrieved from www.minitab.com.

Qin F., Zhao Y., Shi X., Xu S., Yu D. 2016. Sensitivity and uncertainty analysis for theDeNitrificationDeComposition model, a case study of modeling soil organic carbon dynamics at a long-term observation site with a rice-bean rotation. Computers and Electronics in Agriculture. 124, 263-272. http://dx.doi.org/10.1016/j.compag.2016.04.017

Saltelli A. 2000. What is Sensitivity Analysis? In Sensitivity Analysis. Eds. A. Saltelli, K. Chan, E.M. Scott. John Wiley \& Sons Ltd. (UK). 3-13. 


\title{
Wind Pressures on Arched and Gothic Roof Greenhouses
}

\author{
Anastasios Giannoulis a, Demetres Briassoulis a, Antonis Mistriotis a, \\ ${ }^{a}$ Laboratory of Agricultural Structures, Department of Natural Resources Management and Agricultural Engineering, \\ Agricultural University of Athens, Athens, 11854, Greece \\ * Corresponding author. Email: amistr@aua.gr
}

\begin{abstract}
The scope of the current research work was to investigate the interaction of the wind with two typical Mediterranean greenhouse structures, namely the arched roof and the gothic type, and determine the wind loading distribution on their walls and roof. The greenhouses were considered to be without openings, so as only external pressures were estimated. The analysis has been carried out through two dimensional (2D) numerical simulations. The numerical model was validated against the results of a recent wind tunnel research work regarding loading measurements on different greenhouse types. The relevant provisions of Design Codes and Standards regarding arched roof greenhouses appear to be incomplete as some important geometric configurations are missing. The recommended guidelines may be misleading if applied to geometric configurations different from those considered by the Codes. Specifically, no provisions exist for gothic type greenhouses. The geometric characteristics of the computational models were selected to demonstrate such discrepancies and contribute to the definition of the crucial design parameters for gothic type greenhouses. The numerical model was shown in agreement with wind tunnel results and proved that the computational analysis, if applied correctly, is capable of representing realistically the airflow around these types of greenhouses. Roof pressure distribution comparisons between the arched roof and the gothic type greenhouse showed that the flow separation occurs at the pointy ridge of the gothic type greenhouse while the smoother configuration of the arched roof allows the flow to separate at a point at its leeward side. Consequently, the recirculation created by the separation is differently formed and located. The pressure distribution along the roof shows major differences between the two tested greenhouse types. Analysis of the numerical results and the codes' provisions further reveals the need for more targeted design information. Suggestions are offered for improvements in the relevant design provisions.
\end{abstract}

Keywords: greenhouse, arched roof, gothic type, wind pressure, numerical model, design codes

\section{Introduction}

Greenhouses are light low-rise structures used for protecting cultivation against environmental hazards. Classification of greenhouses is based on their geometric characteristics, namely the height of the side walls and the ridge and the design of the roof. Their supporting frame is mainly made of steel, while the cladding material is usually plastic film such as low-density polyethylene (LDPE). Lately, permeable materials like agricultural nets became popular as covering materials for net-houses (Teitel and Tanny, 1999). Two types widely used in Greece and in the Mediterranean region in general, are the arched roof and the gothic type greenhouses.

Studies regarding greenhouses mostly concern the indoor microclimatic conditions which are directly related to the quantity and the quality of the crop yield (Kittas and Bartzanas, 2007). The interaction of the wind with greenhouses has also been studied in the case of duo-pitch structures, such as the Venlo type (Mistriotis et al., 1997) and for tunnels (Matthews and Meyer, 1988). There are only few published research works concerning wind loads on the arched roof greenhouses (Qiu et al., 2014; Kwon et al., 2016; Kim et al., 2017).

Design Codes and Standards such as the EN1991-1-4 and the EN 13031-1-2001 define the procedures for determining the design wind loads for different greenhouse types depending on their geometric characteristics, duration of life, wind climatic data and include several types of actions and combinations of actions. Strong winds are a frequent cause of structural failures and should be taken under serious consideration. Lately, research studies on arched roof greenhouses revealed several discrepancies indicating that the existing data regarding wind loading provisions in Design Codes are insufficient (Blackmore and Tsokri, 2006). Additionally, provisions for the gothic type greenhouses do not exist and their design is currently based on the guidelines concerning duo-pitch and arched roof greenhouses. Therefore, there is a serious gap in the available provisions and a strong need for updating the current versions of the Greenhouse Design Standard EN 13031-1-2001 and the Eurocode EN19911-4 with new research data.

The present research work concerns a numerical simulation comparative analysis of the wind loading on an arched roof and a gothic type greenhouse without openings. Two dimensional (2D) numerical models were created. A recently published wind tunnel and numerical research work was used for validating the numerical model. The numerical results were shown to realistically represent the wind-structure interaction. Testing the arched roof and the gothic type numerical greenhouse models and comparing their results to each other proved that wind loading distribution especially at the roof of the greenhouse can be highly sensitive to changes with respect to its geometric parameters such as height/span ratio, roof height/span ratio and the curvature of the roof. 
Especially, shifting from a "smooth" (arched roof) to a "pointy" (gothic type) ridge could be a critical factor for the wind pressures distribution along the roof. This study indicates that design guidelines included in design codes and standards should be further enriched. Therefore, the present work is expected to support the revision and updating of the relevant provisions of EN 13031-1-2001, which are currently in progress.

\section{Materials and Methods}

Numerical setup of the greenhouse models

Figure 1 shows the arched roof and the gothic type greenhouse models. The basic geometric features of the greenhouses are also presented. The arrow depicts the wind direction. Only transverse winds were considered in this study.

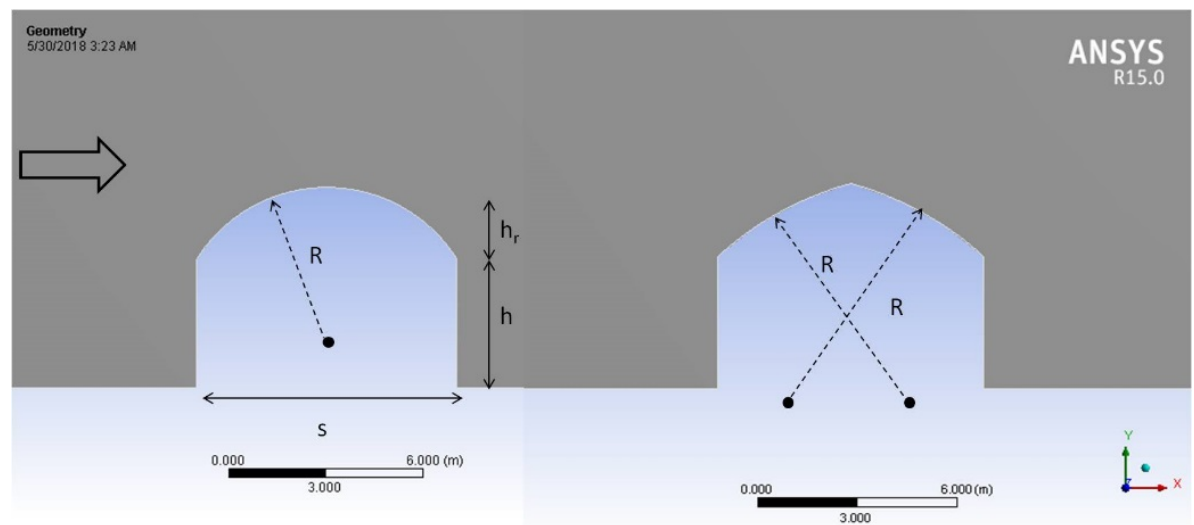

Figure 196. The arched roof and the gothic type greenhouse models.

In Figure $1, h$ is the height from the ground to the gutter, $h_{r}$ is the height from the gutter to the ridge (also called rise height), $s$ is the span length and $R$ is the curvature radius of the roof. For this analysis the arched roof was a circular arc with radius equal to $R$, while for the gothic type roof, each half of the roof (wall to ridge) was a circular arc of radius $(R)$, as it is shown in Figure 1. As mentioned in the previous section, the pressure distribution is directly related to the rise height to span $(h r / s)$ and the gutter height to span $(h / s)$ ratio. Design codes and standards also examine different cases and combinations of these two parameters in order to provide design guidelines.

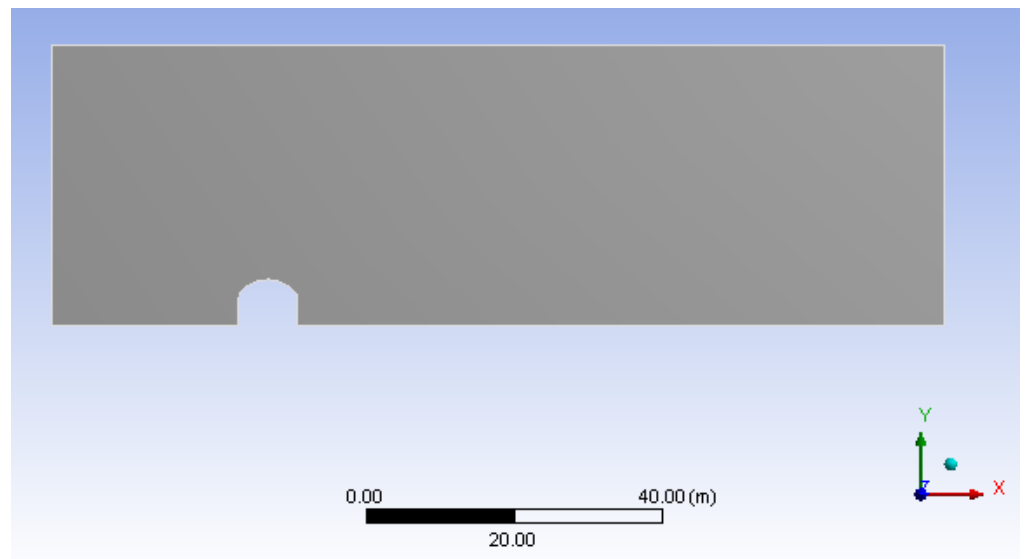

Figure 197. The rectangular tunnel. 
Three two-dimensional (2D) numerical simulations have been carried out. The software package used for the estimation of the wind pressures on the greenhouse models was ANSYS CFX 15.0. The simulation domain was a rectangular area (tunnel) of $120 \mathrm{~m}$ length and $40 \mathrm{~m}$ height (Figure 2). A recently published wind-tunnel study (Kwon et al., 2016) was used for validating the numerical model. Wind pressures on a greenhouse with the same geometric characteristics, named Arched-roof-1, were numerically calculated and compared to the experimental data. In this way, the quality of the numerical results was confirmed. In contrast to the other two modelled greenhouses (described below), where the side walls were vertical, the Arched-roof-1 greenhouse had slightly inclined walls (2-3 from the vertical direction). Arched-roof-1 greenhouse's dimensions were: $h=1.6 \mathrm{~m}, h_{r}=1.9 \mathrm{~m}$, $s=7 \mathrm{~m}$ and a curvature radius $R=4 \mathrm{~m}$. Two additional numerical simulations followed regarding another arched roof (Arched-roof-2), and a gothic type greenhouse (Gothic) model. Their geometric characteristics were: $h=4 \mathrm{~m}$, $h_{r}=2.2, s=8 \mathrm{~m}$. The curvature radius of the Arched-roof- 2 greenhouse was $R=4.7 \mathrm{~m}$ and for each half of the gothic type roof was $R=9.7 \mathrm{~m}$. Table 1 shows all design features and the corresponding aspect ratios of the numerical models.

Table 82. Geometric characteristics of the numerical models.

\begin{tabular}{ccccccc}
\hline $\begin{array}{c}\text { Greenhouse } \\
\text { Type }\end{array}$ & $\begin{array}{c}\text { Gutter height } \\
h(\mathrm{~m})\end{array}$ & $\begin{array}{c}\text { Rise height } \\
h_{r}(m)\end{array}$ & $\begin{array}{c}\text { Span length } \\
S(m)\end{array}$ & $\begin{array}{c}\text { Curvature } \\
\text { radius } R(m)\end{array}$ & $h / s$ & $h_{r} / s$ \\
\hline Arched-roof-1 & 1.6 & 1.9 & 7 & 4.0 & 0.23 & 0.27 \\
Arched-roof-2 & 4.0 & 2.2 & 8 & 4.7 & 0.50 & 0.28 \\
Gothic & 4.0 & 2.2 & 8 & 9.7 & 0.50 & 0.28 \\
\hline
\end{tabular}

Simulation mesh and boundary conditions

Figure 3 shows the mesh of the numerical simulations close to the greenhouses.
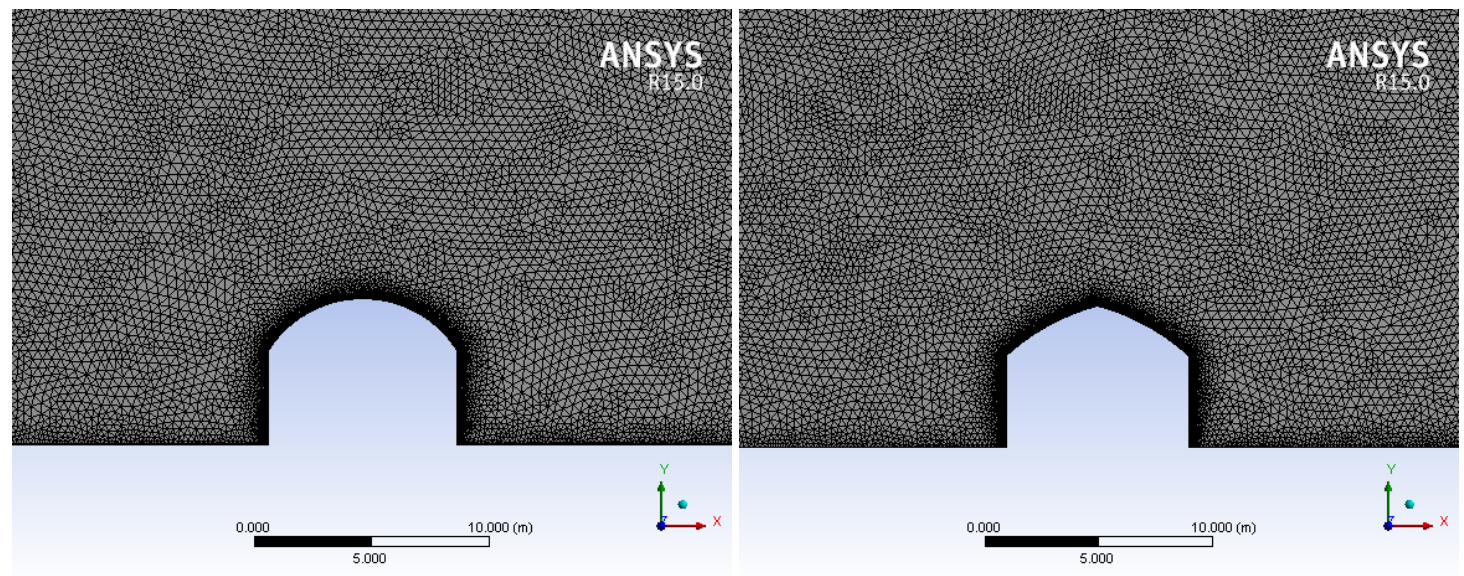

Figure 198. The mesh close to the arched roof and the gothic type greenhouse models.

The mesh consisted of quadrilateral elements in all tested cases. The total number of elements was approximately 1.5 million, depending on the case. The mesh sensitivity was also tested by increasing the total number of elements of the numerical models. A significant factor which was analysed in detail for both greenhouse types is the location of the flow separation at the roof, since it influences the formulation of a leeward recirculation and affects the wind pressure distribution. Such flow separation and recirculation is related to the near wall behaviour of the flow. For this reason, a non-dimensional parameter $y^{+}$that describes the mesh quality (coarse or fine) near the wall has to be adjusted. As suggested by the numerical software (ANSYS CFX 15.0) and also confirmed in literature (Kim et al., 2017) using the shear stress transport $k$ - $\omega$ model (SST) combined with a nearwall first element that satisfies the criterion of $y^{+}$smaller than 5 can be a very efficient choice for the accurate reproduction of the flow separation at the roof. The $k-\omega$ SST turbulence model was applied to all numerical simulation cases in this study and the $y^{+}$value was set equal to 1 .

A log-law profile (Eq. (1)) was used at the inlet with wind velocity at $10 \mathrm{~m}$ high equal to $5 \mathrm{~m} \mathrm{~s}^{-1}$. The roughness length was set equal to $z_{0}=0.05 \mathrm{~m}$ representing an area of low vegetation and the absence of other obstructions in the vicinity. The inlet boundary for the turbulent kinetic energy $k\left(\mathrm{~m}^{2} \mathrm{~s}^{-2}\right)$ and the turbulence frequency $\omega\left(\mathrm{s}^{-1}\right)$ were calculated based on the Eq. (2), (3) and (4). 


$$
\begin{aligned}
& u(z)=\frac{u^{*}}{K} \ln \left(\frac{z+z_{0}}{z_{0}}\right) \\
& k=\frac{u^{* 2}}{\sqrt{C_{\mu}}} \\
& \varepsilon=\frac{u^{* 3}}{K\left(z+z_{0}\right)} \\
& \omega=\frac{\varepsilon}{C_{\mu} k}
\end{aligned}
$$

Where $u(z)$ is the average wind velocity $\left(m s^{-1}\right), u^{*}$ is the wall friction velocity $\left(m s^{-1}\right), z$ is the distance from the wall $(m), z_{0}$ is aerodynamic roughness length $(m), \varepsilon$ is the turbulent energy dissipation $\left(\mathrm{m}^{2} \mathrm{~s}^{-3}\right), C_{\mu}$ is a model fitting parameter equal to 0.09 and $K$ is the von Karman constant (approximately equal to 0.41) (Richards and Hoxey, 1993).

In all walls (tunnel, greenhouse walls and roof) the no-slip wall condition was applied (all velocity components are equal to zero) except at the top wall of the tunnel where a symmetry condition was applied, namely the free slip condition (only the parallel to the wall velocity component is non-zero). At the outlet where the flow moves out of the tunnel and expands to a much larger area the pressure was assumed to be equal to zero. The convergence criterion was set to $10^{-5}$. Testing more strict convergence criteria of up to $10^{-7}$ did not affect the simulation results.

\section{Results and Discussion}

Validation of the numerical model

The numerical model was validated by comparing the Arched-roof-1 model with an arched roof model of the same geometric characteristics tested in a wind tunnel by Kwon et al 2016. Pressure coefficients were given for the exposed areas of the greenhouse, namely the walls and the roof of the structure. Pressure coefficients on the walls and the roof estimated in the present computational study were compared to the wind tunnel results.

Table 83. Comparison of the current study (Arched-roof-1) average walls and roof pressure coefficients to the wind tunnel results of Kwon et al (2016).

\section{Roof angle $\theta\left(^{\circ}\right)$ range}

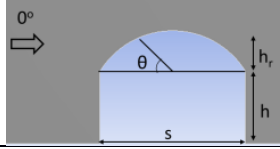

\begin{tabular}{lcccccccccc}
$\begin{array}{c}\text { Location }- \text { Roof } \\
\text { angle } \theta(0) \text { range }\end{array}$ & $\begin{array}{c}\text { Windward } \\
\text { Wall }\end{array}$ & $0-20$ & $20-40$ & $40-65$ & $65-90$ & $90-115$ & $115-140$ & $140-160$ & $160-180$ & $\begin{array}{c}\text { Leeward } \\
\text { Wall }\end{array}$ \\
\hline $\begin{array}{l}\text { Present numerical } \\
\text { study } C_{p}\end{array}$ & 0.45 & -0.18 & -1.10 & -1.78 & -2.07 & -1.86 & -1.10 & -0.60 & -0.60 & -0.58 \\
$\begin{array}{c}\text { Wind tunnel } \\
\text { average } C_{p}\end{array}$ & 0.50 & -0.09 & -1.09 & -1.73 & -1.90 & -1.76 & -1.20 & -0.61 & -0.50 & -0.50 \\
\hline
\end{tabular}

Table 2 presents average values of pressure coefficients at the windward and the leeward wall and at different locations at the roof. The angle $\theta$ ranges presented in Table 2 are those used in the publication of Kwon et al. (2016). Numerical results appear to be in good agreement with the experimental data.

The current numerical results concerning the Arched-roof-1 greenhouse compare well also to the numerical results presented in Kim et al (2017) concerning a similar greenhouse design (Figure 4). A good qualitative and quantitative agreement with respect to the pressure distribution on the greenhouse roof was observed between the two numerical simulations. Small deviations that can be seen in both Table 2 and Figure 3 are related to the fact that the current study results were derived by a $2 \mathrm{D}$ simulation while both the wind tunnel and the numerical simulation research works were 3D. However, discrepancies, which may also be associated with the wind tunnel measurements accuracy, are small and the numerical model can be considered to properly simulate the interaction of the airflow with the specific greenhouse. 


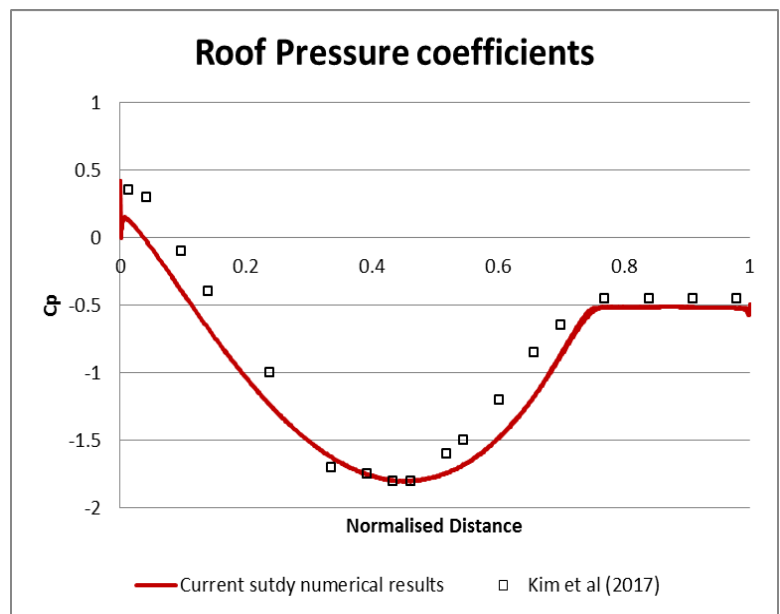

Figure 199. Comparison of the Arched-roof-1 roof pressure coefficients to the numerical results of Kim et al. (2017).

Pressure coefficients on the gothic type greenhouse-Comparisons with the arched roof

The validation of the numerical model allowed for further use of the computational simulation for the calculation of wind pressures on greenhouses of different geometric characteristics. Gothic type greenhouses are widely used, but the interaction of such a structure with the wind has been poorly studied so far. A gothic type greenhouse of the dimensions presented in Table 1 was simulated and was compared to an arched roof greenhouse of similar dimensions but different roof curvature radius.

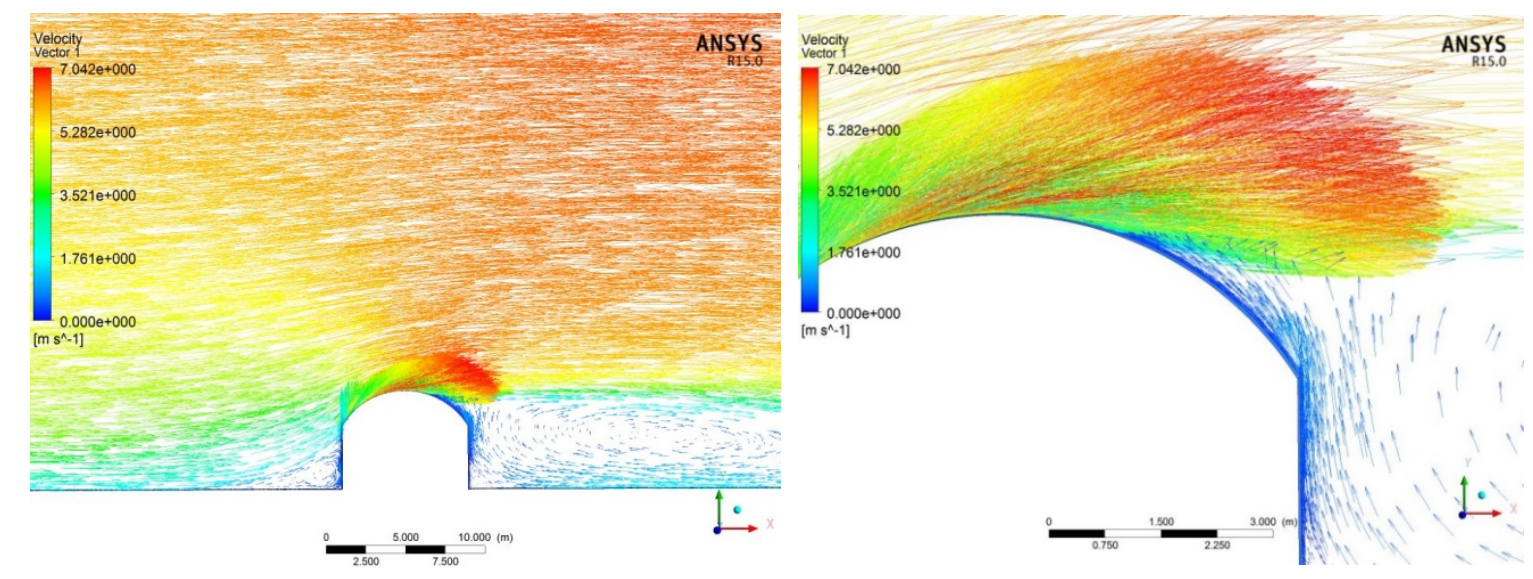

Figure 200. Wind velocity vectors pattern around the arched roof greenhouse and flow separation location.

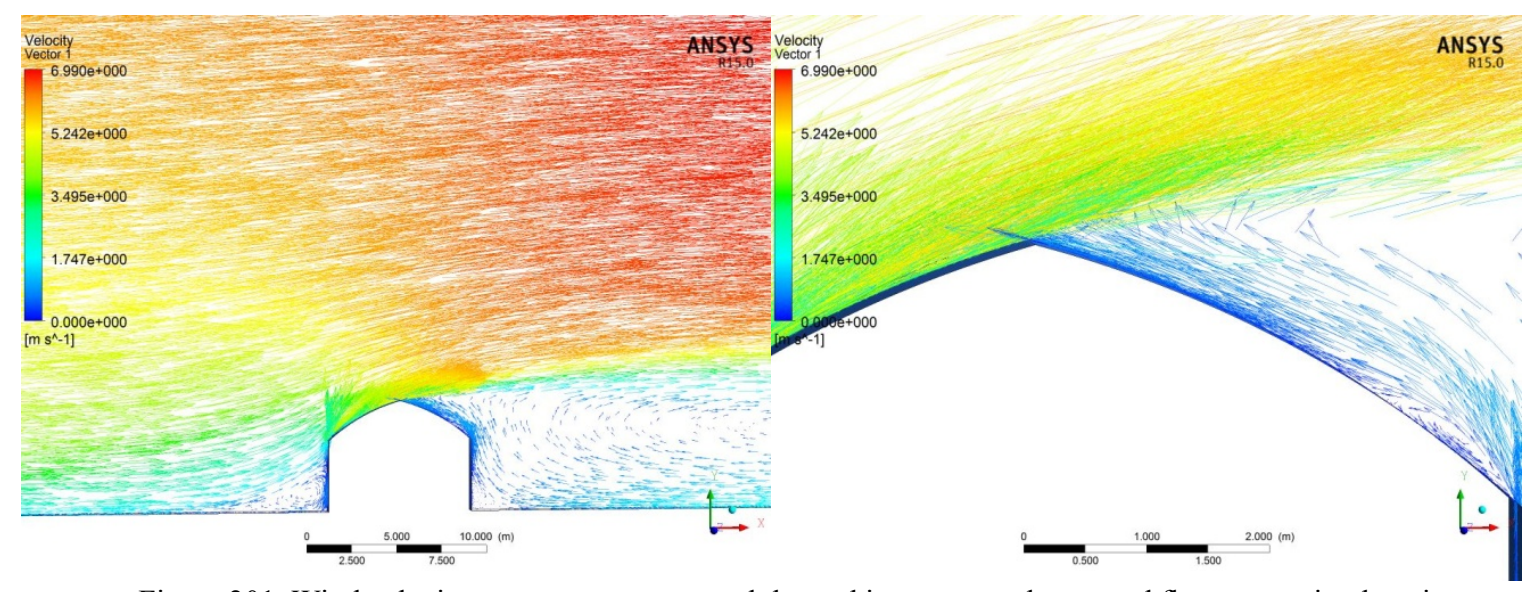

Figure 201. Wind velocity vectors pattern around the gothic type greenhouse and flow separation location. 
Figure 5 and Figure 6 present the airflow around the Arched-roof-2 and the Gothic greenhouses respectively. A close-up of the airflow near the separation point is also shown in these figures. In both cases a small vortex is evident at the windward wall and an extensive recirculation eddy at the leeward one. The different roof geometric configurations, smooth for the arched roof and "pointy" for the gothic type greenhouse, are crucial for the flow separation location. As shown in Figures 5 and 6, the separation occurs exactly at the ridge of the gothic type greenhouse, while for the arched roof separation shifts to the leeward part of the greenhouse roof. This difference directly affects the airflow at the leeward part (roof and wall) of the gothic type greenhouse and hence the pressure at the roof of the two greenhouse models is expected to be differently distributed.

Figure 7 quantifies the observations made regarding the airflow pattern around the tested greenhouses, with respect to the flow separation location and its impact to the wall and roof pressures. Results regarding the Archedroof-1 greenhouse are also included in Figure 7. Near the ridge of the gothic type greenhouse, the roof bears significantly increased suctions due to the discontinuity of the slope. However, the large negative pressure appears as a local discontinuity of the wind pressures distribution exerted on a very small surface and so its effect on the estimated design loads of the roof and structure is not considered significant. It may however affect the local covering or windows supporting systems. In all cases pressure coefficients are negative along the entire length of the roof except for a very small windward location of the Arched-roof-1 greenhouse. Along the windward pitch of the gothic type greenhouse, negative pressure coefficients are lower than these of the arched roof type and increase almost linearly with the distance from the windward gutter. On the other hand, suction increases faster and non-linearly along the arched roof (Figure 7). For the gothic type greenhouse, maximum pressure coefficients appear in the form of a discontinuity near the ridge. The maximum pressure coefficient at the location of the ridge is approximately equal to -3.0. In the arched-roof type greenhouses, maximum suction appears in front of the ridge, at a distance equal to $0.4 \mathrm{~s}$ from the windward gutter. Maximum pressure coefficients were determined equal to -1.8 and -2.0 for the Arched-roof-1 and Arched-roof-2 greenhouses respectively. The distribution of the pressure coefficients along the roof of arched roof and gothic greenhouses appears sensitive to the geometric characteristics of the structure, so extensive investigation of relevant typical configurations is required.

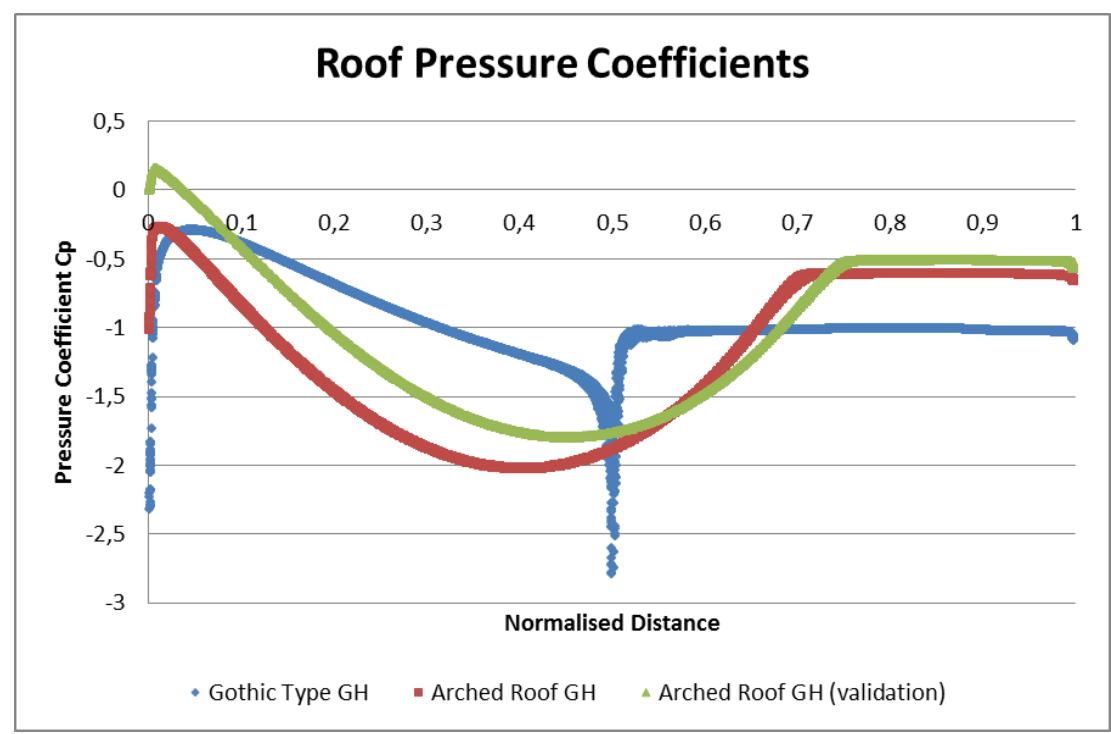

Figure 202. Pressure coefficient distribution at the roof of the arched roof and the gothic type greenhouse.

Figure 7 also shows the difference regarding the flow-separation location in each greenhouse type. It is confirmed that for the gothic type greenhouse flow-separation begins exactly at the ridge location $(0.5 s)$ while for the arched roof greenhouses separation occurs close to a distance equal to $0.7 \mathrm{~s}$. The existence of the recirculation formed directly after the flow separation provides a stable suction at the roof with a constant negative pressure coefficient value, -0.5 for the Arched-roof-1 model, -0.6 for the Arched-roof-2, and -1.0 for the gothic type greenhouse.

Table 84. Greenhouse walls average pressure coefficients $C_{p}$.

\begin{tabular}{|c|c|c|}
\hline Greenhouse Type & Windward wall $C_{p}$ & Leeward wall $C_{p}$ \\
\hline Arched-roof-1 & 0.39 & -0.50 \\
\hline Arched-roof-2 & 0.47 & -0.60 \\
\hline Gothic & 0.78 & -0.97 \\
\hline
\end{tabular}


For the greenhouse walls the average pressure coefficients are given in Table 3. As expected windward values are positive while leeward values are negative due to the presence of the vortex. For both the arched roof models the geometry differences did not significantly affect the walls' pressure coefficients. On the other hand, the pressure coefficients on the walls of the gothic greenhouse are almost double compared to those corresponding to the Arched-roof-2 model, although the sidewalls have the same height. This leads to the conclusion that the geometric configuration of the roof is a critical factor, which directly affects the wind pressures on the greenhouse sidewalls.

A comparison of the pressure coefficients results for the two arched roof greenhouses reveals differences only with respect to the windward side of the roof. Even though the two structures have a similar ratio $h_{r} / s(0.27$ for the Arched-roof- 1 and 0.28 for the Arched-roof-2) the difference regarding the $h / s$ ratio $(0.23$ for the Arched-roof1 and 0.5 for the Arched-roof-2) influences the windward roof pressure distribution. For the leeward side of the roof though, the separation point and the eddy that is formed appear to be the dominant factors that determine the pressure coefficients.

Average Pressure coefficients on the arched roof models - Comparisons with Design Codes

Design Codes and Standards provide guidelines for the optimum design of the arched roof greenhouses. Average pressure coefficients are given for the front, rear and sidewalls as well as for different roof locations for different geometric characteristics of the structure. A literature survey showed that there are currently only a small number of publications related to the wind pressures on arched roof greenhouses. This justifies also the fact that the Design Codes and Standards provisions appear in many cases insufficient and incomplete (Blackmore and Tsokri, 2006). Provisions of EN1991-1-4 and EN 13031-1-2001 regarding arched roof structure are presented in this section.

Table 4 presents comparisons of the two arched roof greenhouse cases simulated with the EN1991-1-4. Table 5 presents similar comparisons with the EN 13031-1-2001. It is important to notice that the greenhouse standard and the Eurocode use different reference heights. The Eurocode EN1991-1-4 defines the top of the structure's roof (ridge) as the reference height, while the Standard EN 13031-1-2001 uses the mid-height between the gutter and the ridge of the structure as reference. EN 13031-1-2001 also clarifies that if this height is lower than 0.75 of the total greenhouse height then the reference height should equal to the 0.75 of the total height. They both divide the roof in three areas (windward, close to the ridge and leeward) and provide average values of pressure coefficients for each one. In the present numerical study the reference height was defined in both ways for comparison purposes.

Table 85. Comparison of the numerical analysis average pressure coefficients to EN1991-1-4.

\begin{tabular}{lccccccc}
\hline & $h / s$ & $h_{r} / s$ & $\begin{array}{c}\text { Windward } \\
\text { wall } C_{p}\end{array}$ & $\begin{array}{c}\text { Leeward } \\
\text { wall } C_{p}\end{array}$ & $\begin{array}{c}\text { Roof } C_{p} \\
\text { (Windward) }\end{array}$ & $\begin{array}{c}\text { Roof (Ridge } \\
\text { Area) } C_{p}\end{array}$ & $\begin{array}{c}\text { Roof } C_{p} \\
\text { (Leeward) }\end{array}$ \\
\hline EN1991-1-4 & 0.23 & 0.27 & 0.70 & -0.30 & 0.25 & -1.00 & -0.40 \\
Arched-roof-1 & & & 0.39 & -0.50 & -0.41 & -1.38 & -0.51 \\
EN1991-1-4 & \multirow{2}{*}{0.5} & \multirow{2}{*}{0.28} & 0.75 & -0.35 & 0.20 or -0.6 & -1.00 & -0.40 \\
Arched-roof-2 & & & 0.47 & -0.60 & -0.82 & -1.50 & -0.65 \\
\hline
\end{tabular}

Table 86. Comparison of the numerical analysis average pressure coefficients to EN 13031-1-2001.

\begin{tabular}{lccccccc}
\hline & $h / s$ & $h_{r} / s$ & $\begin{array}{c}\text { Windward } \\
\text { wall } C_{p}\end{array}$ & $\begin{array}{c}\text { Leeward } \\
\text { wall } C_{p}\end{array}$ & $\begin{array}{c}\text { Roof } C_{p} \\
\text { (Windward) }\end{array}$ & $\begin{array}{c}\text { Roof (Ridge } \\
\text { Area) } C_{p}\end{array}$ & $\begin{array}{c}\text { Roof } C_{p} \\
\text { (Leeward) }\end{array}$ \\
\hline $\begin{array}{l}\text { EN 13031-1- } \\
\quad 0.23\end{array}$ & 0.27 & 0.60 & -0.30 & 0.30 & -1.00 & -0.40 \\
$\begin{array}{l}\text { Arched-roof-1 } \\
\text { EN 13031-1- }\end{array}$ & 0.5 & 0.28 & 0.60 & -0.45 & 0.30 & -1.61 & -0.60 \\
$\quad \begin{array}{l}\text { 2001 } \\
\text { Arched-roof-2 }\end{array}$ & & & 0.51 & -0.66 & -0.89 & -1.63 & -0.40 \\
\hline
\end{tabular}

For the EN1991-1-4 an overestimation exists for the windward wall $C_{p}$. On the other hand, the leeward wall $C_{p}$ given by this Design Code is slightly lower than the numerically estimated value. EN1991-1-4 does not examine the wall pressure coefficients separately for the arched roof structures but redirects the reader to general rules for "walls of rectangular plan buildings". These values can be considered questionable. Regarding the roof pressure coefficients, the EN1991-1-4 proposes different values based on the $\mathrm{h}_{\mathrm{r}} / \mathrm{s}$ value and takes into account variations of the ratio $\mathrm{h} / \mathrm{s}$ only for the windward roof area. Especially for $0.2 \leq h_{r} / s \leq 0.3$ and $h / s \geq 0.5$ two values are proposed, namely a positive and a negative one. However, changes in the ratio $\mathrm{h} / \mathrm{s}$-as previously shown in 
Figure 7- do not only affect the pressure distribution at the windward roof area but at the ridge area of the roof as well. EN 13031-1-2001 provides pressure coefficient values for the windward and the leeward wall of the arched roof greenhouse for two $h / s$ cases, namely for $h / s \leq 0.4$ and $h / s \geq 0$. 6 . Results of the windward wall $C_{p}$ values from the numerical simulations agree with the standard proposed ones. However, leeward wall pressure coefficients calculated by the numerical models appear to be somewhat higher. For the roof pressure distribution EN 130311-2001 examines only the case where $\mathrm{h} / \mathrm{s} \geq 0.2$ and provides a single average pressure coefficient for the three different roof locations (as given in Tables 4 and 5). Also, for the windward roof area only positive values are proposed which contradict the experimental and numerical results of the current and previous research works (Kim et al., 2016; Kwon et al., 2017). Numerical results and EN 13031-1-2001 provisions appear to totally disagree on the roof pressure coefficients. This is attributed to the fact that the standard deals with the arched roof structures by taking under consideration a very small number of variations for the geometric parameters such as the ratios $h_{r} / s$ and $h / s$.

\section{Conclusions}

Two-dimensional numerical simulations have been carried out on three different geometric types of greenhouse models; two arched roof and a gothic type greenhouses. One of the arched roof models was initially used to validate the numerical method. A comparison of the numerical pressure coefficient results against previously published wind tunnel work and numerical simulations on a similar arched roof greenhouse showed very good agreement.

Computational simulations on the gothic type and the arched roof greenhouses showed that the average pressure coefficient value on the windward and leeward walls was affected by the greenhouse roof type. Small differences regarding the average pressure coefficients at the sidewalls were found in the two studied cases of arched roof greenhouses. However, for the gothic type greenhouse the average pressure coefficients for the walls were almost double the ones estimated for the arched roof cases.

Numerical results also showed that for the gothic type greenhouse the wind flow separates at the top of its roof (ridge). This is a result of the geometric configuration of the gothic type roof and its discontinuity at the top ("pointy" ridge). However, for the arched roof greenhouse the airflow separation point occurs at the leeward side of the roof due to its "smooth" geometry. It has also been shown that for the pressure distribution of the windward half of the roof its geometric configuration is highly significant. For the leeward half though, the separation point and the recirculation eddy formed are probably the most important factors and the ones to define the wind pressures at this location of the roof.

Comparisons of the numerical simulation results on the arched roof greenhouses have been made against the relevant provisions of the Design Codes and Standards as well. It was confirmed that the two Design Codes examined, namely the EN1991-1-4 and the EN 13031-1-2001, do not take into account important variations regarding critical geometric parameters such as the ratio $h_{r} / s$ and $h / s$. Similar criticism appeared lately in other recent publications (Blackmore and Tsokri, 2006). Further pre-normative research work is needed for the improvement of the relevant provisions of the Design Codes and Standards.

\section{References}

Blackmore, P.A., Tsokri, E., 2006. Wind loads on curved roofs. Journal of Wind Engineering and Industrial Aerodynamics, 94 (11), 833-844. https://doi.org/10.1016/j.jweia.2006.06.006

EN 1991-1-4:2005 - Eurocode 1: Basis of design and actions on structures-Part 1-1-4: General actions - Wind actions 1-1-4. European Committee for Standardization, Brussels.

EN-13031-1-2001: Greenhouses: Design and construction Part 1: Commercial production greenhouses. European Committee for Standardization, Brussels.

Kittas, K., Bartzanas, T., 2007. Greenhouse microclimate and dehumidification effectiveness under different ventilator configurations. Building and Environement. 42 (10), 3774-3784. https://doi.org/10.1016/j.buildenv.2006.06.020

Kwon, K., Kim, D., Kim, R., Ha, T., \& Lee, I. 2016. Evaluation of wind pressure coefficients of single-span greenhouses built on reclaimed coastal land using a large-sized wind tunnel. Biosystems Engineering, 141, 58-81. https://doi.org/10.1016/j.biosystemseng.2015.11.007

Mathews, E., \& Meyer, J., 1988. Computation of wind loads on a semicircular greenhouse. Journal of Wind Engineering and Industrial Aerodynamics, 29(1), 225-233. https://doi.org/10.1016/0167-6105(88)90160-2

Mistriotis, A., De Jong, T., Wagemans, M., Bot, G., 1997. The analysis of ventilation and indoor microclimate in agricultural buildings by Computational Fluid Dynamics (CFD). IFAC Proceedings Volumes, 30 (5), 289-295 https://doi.org/10.1016/S1474-6670(17)44447-8 
Qiu, Y., Sun, Y., Wu, Y., Tamura, Y., 2014. Modelling the mean wind loads on cylindrical roofs with consideration of the Reynolds number effect in uniform flow with low turbulence. Journal of Wind Engineering and Industrial Aerodynamics, 129, 11-21. https://doi.org/10.1016/j.jweia.2014.02.011

Richards, P., \& Hoxey, R., 1993. Appropriate boundary conditions for computational wind engineering models using the k- $\varepsilon$ turbulence model. Journal of Wind Engineering and Industrial Aerodynamics, 46-47, 145-153. https://doi.org/10.1016/0167-6105(93)90124-7

Teitel, M., Tanny, J., 1999. Natural ventilation on greenhouses: experiments and model. Agricultural and Forest Meteorology, 96 (1-3), 59-70. https://doi.org/10.1016/S0168-1923(99)00041-6 


\title{
Effects of Infrared Radiation on Growth Parameters and Physiological Characteristics of Eggplant Cultivation
}

\author{
Angeliki Kavga ${ }^{a *}$, Fotini Evangelopoulou ${ }^{a}$, Georgios Trypanagnostopoulos ${ }^{b}$, Vassilios Kappatos ${ }^{c}$

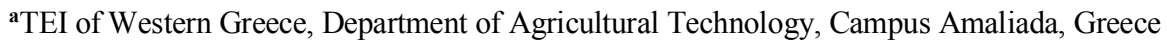 \\ ${ }^{b}$ Department of Mechanical Engineering \& Aeronautics, University of Patras, Patra, Greece \\ ${ }^{c}$ University of Southern Denmark (SDU), Department of Technology and Innovation, Odense M, Denmark \\ \akavga@teiwest.gr
}

\begin{abstract}
The advantages of Infrared (IR) heating is verified and demonstrated in an experimental greenhouse with eggplant cultivation. IR radiation heating systems possess the advantage of high directional control and focused compensation of energy losses, appropriate for creating local temperature conditions in open or thermally unprotected spaces, resulting in an overall reduction of heat losses and consequently heating energy needs up to $50 \%$. IR heating systems can efficiently maintain favourable environmental conditions at the plant canopy. The objective of this research is to investigate the effect of IR radiation on growth parameters and physiological characteristics of eggplant. Extensive experimental results are presented from a full cultivation period inside two identical, small scale experimental greenhouses, with IR and forced air heating system, correspondingly. Eggplant (Solanum melongena L.) is used as the test crop for a three months period. The produced data include solar radiation, relative humidity, greenhouse indoor air temperature and plant growing. Results are compared to conventional cultivation. In parallel with plant growing parameters' evaluation, the antioxidant and metabolic profile of eggplant from the experimental greenhouse was evaluated in comparison to conventional cultivation.
\end{abstract}

\section{Introduction}

Greenhouses are intensive cultivation units for agricultural products, which require optimum combination of allocated particulars and proceeding, as lighting, heating, cooling and ventilation, creating a controlled environment that has a significant impact on the cultivation time, quality, and quantity of the products, resulting in overall higher productivity (Kaushik and Chel, 2011). In Mediterranean countries, where solar radiation is usually sufficient to meet the photosynthetic needs of most greenhouse crops and seedlings and no artificial lighting is mandatory, greenhouse heating is one of the most energy-consuming operational requirements during winter periods, having a significant impact on production cost (De Pascale and Maggio, 2004). Conventional greenhouse heating systems are based either on circulation of hot water through a piping system or on direct use of forced air heaters (Teitel et al., 1999; Perdigones et al., 2006). The above methods, in order to achieve the required plant temperature, have to heat the internal greenhouse air to the same or even to a slightly higher temperature than the value targeted for the plants. This practice results in increased heat losses due to convection and radiation through the cover, and also due to the leakages through openings, caused by unavoidable construction defects.

An alternative method of heating the plants in a greenhouse is the use of low intensity infrared (IR) radiation. The main potential advantage of IR heating is the direct delivery of thermal energy from the power source to the canopy, thus eliminating the need to increase the inside air temperature to deliver the necessary heat by convection. As a result, the cover and inside air may remain at significantly lower temperatures than the target value for the plants, with a concomitant reduction of energy losses. IR heating systems can efficiently maintain favorable environmental conditions at the plant canopy (microclimate), which promote the uniform, quantitative, and qualitative growth of plants and suppress plant pest and diseases. Moreover, when the canopy is retained at a higher temperature than the rest of the greenhouse, the possibility of moisture condensation on plants' surface is drastically reduced. Implementation of IR heating in experimental and production scale greenhouse has indicated energy savings in the range $40-50 \%$ and has contributed to improved product quality (Kavga et al, 2009, 2012, 2015). IR heating systems have only to compensate the heat losses of the canopy, with minimum requirements for supporting and auxiliary systems (boilers, transmissions, lines, fans, etc.) and therefore can respond very quickly to outside temperature changes. Although energy efficiency provides the main motivation of the IR heating, another favorable side-effect is worth noting. IR heating systems can efficiently maintain favorable environmental conditions at the plant canopy. When the canopy is retained at a higher temperature than the rest of the greenhouse, the possibility of moisture condensation on plants' surface is drastically reduced (Teitel et al., 2000).

The objective of the present work is to verify and demonstrate the benefits of IR heating on plant grown. Parallel operation of the two greenhouses allows for a comparison of the two heating systems under identical weather conditions and their effect to plant growing with plant growing parameters' evaluation. 


\section{Materials and methods}

Two identical small-scale experimental greenhouses were used, each one as the test bed of the two heating options that were considered, namely, forced hot air heating (conventional heating) and IR heating. Two alternative heating systems were available: (1) a forced-air unit with two power levels (1 and $2 \mathrm{~kW})$ and a small fan that promoted air mixing, and (2) an IR heating system consisting of four lamps with blown bulb reflectors (1 $\mathrm{kW}$ total power, $50^{\circ}$ beam angle) placed at the greenhouse corners and an elevation of $1 \mathrm{~m}$ above the plants (fig. 1). Both greenhouses are constructed of aluminum framework, with $3 \mathrm{~mm}$ thick glass panels. They have the same orientation and were located in the same field with a sufficient distance between them to avoid mutual interference. Their dimensions are width $2.13 \mathrm{~m}$, length $2.00 \mathrm{~m}$, eaves height $1.00 \mathrm{~m}$ and total height up to the top $1.50 \mathrm{~m}$. The base area of each greenhouse Ap is equal to $4.26 \mathrm{~m}^{2}$, the area of the greenhouse cover is $\mathrm{Ac}=14.05 \mathrm{~m}^{2}$ and the volume of the greenhouse is $\mathrm{V}=5.33 \mathrm{~m}^{3}$.

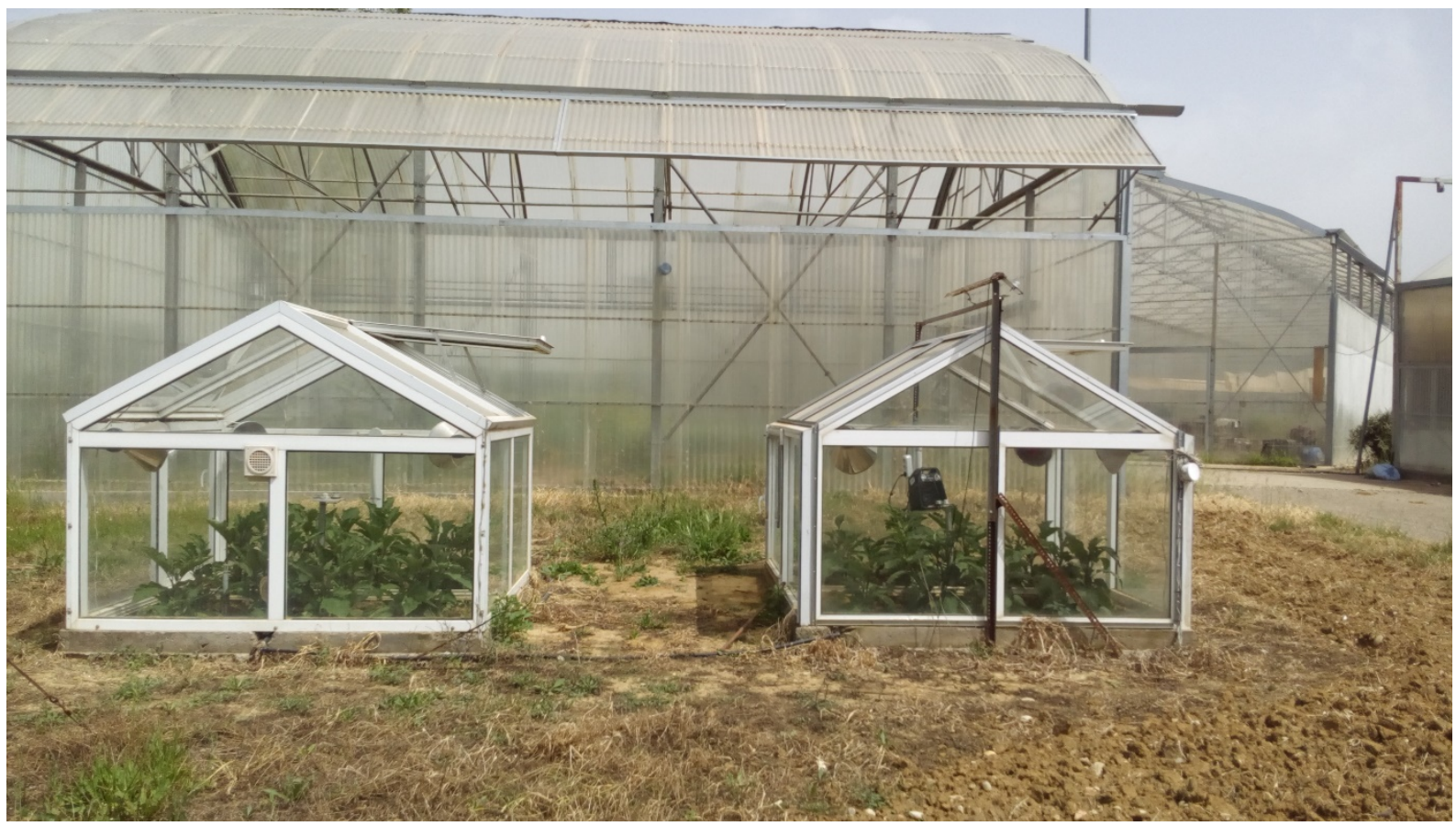

Figure 1. The experimental setup with the cultivation.

Interior microclimatic parameters monitored in both greenhouses are the temperature at several locations at the canopy, inside the greenhouses and at the inside and outside surface of the greenhouse glazing cover, as well as the relative humidity and radiation fluxes (incoming solar radiation and photosynthetically active radiation). The outdoor environmental conditions including temperature, wind speed, relative humidity, sky temperature and rain are monitored at a height of $2.50 \mathrm{~m}$ above the ground level, on a meteorological mast close to the greenhouses.

For a three (3) months operation period (February to April) for both configurations, all data were recorded every minute, and $10 \mathrm{~min}$ averages and were stored in a data logger with a relay analogue multiplexer. Overall day-time and night-time mean values were also computed from the respective time series, based on the interval between the steep radiation and temperature changes at sunrise and sunset. The Analyzer 4.5 Datalogger Software was used for the processing and statistical analysis of the experimental data.

Eggplant was used as the test crop. The plants are cultivated on the soil. In each greenhouse 16 young eggplants were planted, forming four rows of four plants each and planting distances were $36 \mathrm{~cm} \mathrm{x} 24 \mathrm{~cm}$. (plant by plant $\mathrm{x}$ line by line). A drip irrigation system was applied. The supply of each drip emitter was 2 lit/h. The irrigation dose during the experiment was 3.6 lit per plant. Fertilizing of the plants was done with water-soluble fertilizers. During the cultivation, 120 gr N, 280 gr P, 160 gr K, 12 gr Mg and 18 gr Ca were administered on each plant. For the fertilization program, the results of soil analysis prior to the installation of the plants were considered. The full weight and the dry weight of the fruits were measured as well as the perimeter and the length.

Based on corresponding temperature recommendations a night-time temperature of $16^{\circ} \mathrm{C}$ was chosen for the timely growth of eggplant and the heating systems were set to turn on when Tp dropped below $15^{\circ} \mathrm{C}$ and to turn off when $\mathrm{Tp}$ exceeded $17^{\circ} \mathrm{C}$. 


\section{Results and Discussion}

Investigation concerning the variation of the nightly ambient, inside air and plant temperatures to greenhouses, as well as the cultivation results has been performed.

Temperature results

In Figure 2 the variation of the nightly temperatures during the three months period is shown. The presented graphs are for the ambient temperature Ta, the inside temperature and the plants temperature of the IR greenhouse. The diagram shows that the air temperature inside the greenhouse is lower than the plants temperature, creating a local environment on plant canopy in accordance with IR radiation operation principles.

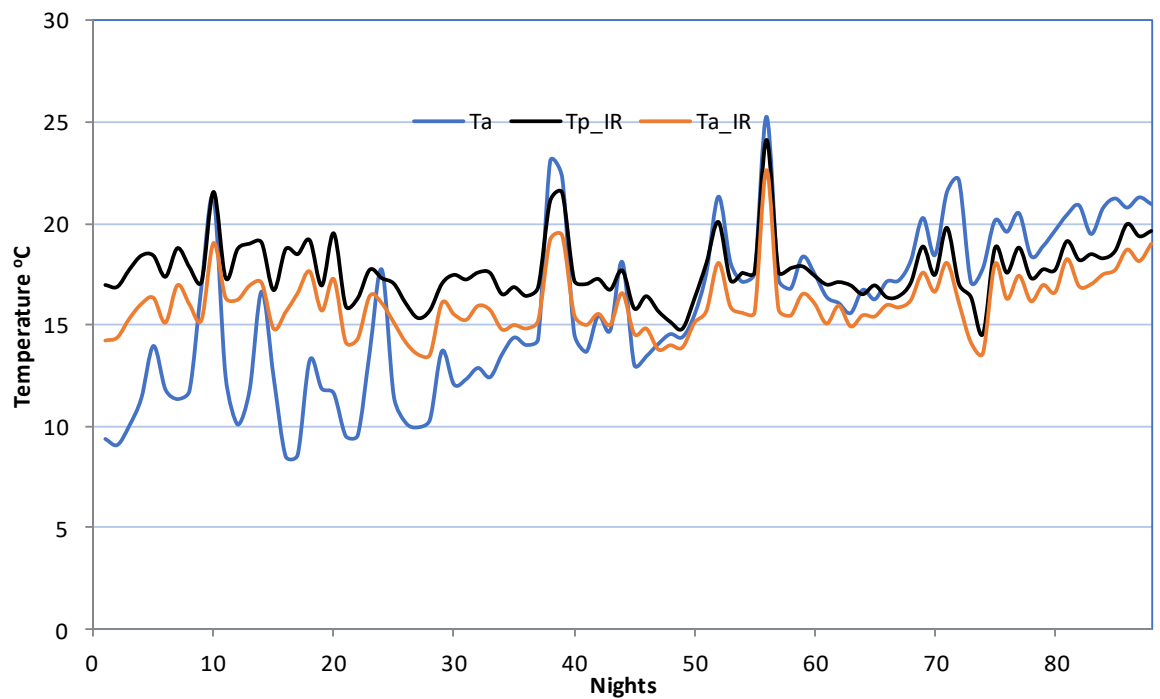

Figure 2. Variation of nightly ambient and inside greenhouses temperatures in IR heated greenhouse.

In Figure 3 the variation of the nightly temperatures during the three months period is shown. The presented graph is for the ambient temperature Ta, the inside temperature and the plants temperature of the conventionally heated greenhouse. The diagram shows that the air temperature inside the greenhouse is the same or a little higher than the value targeted for the plants, creating an isothermal environment throughout the greenhouse interior (whole climate).

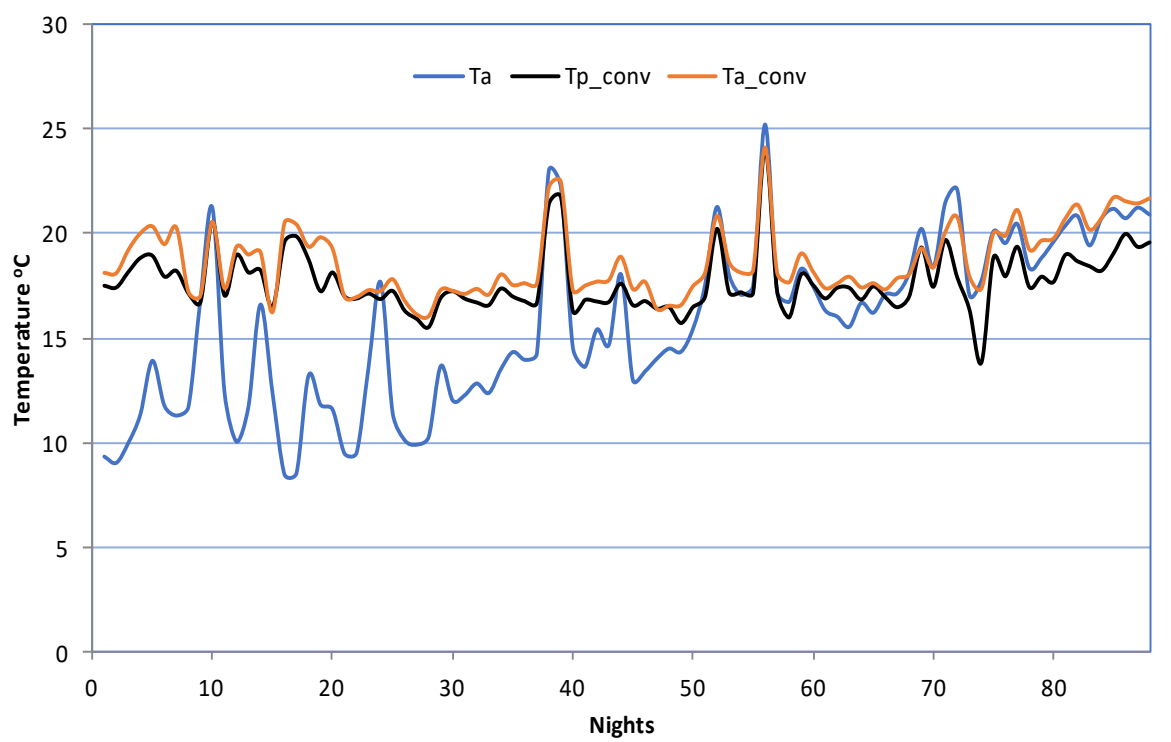

Figure 3. Variation of nightly ambient and inside greenhouses temperatures in conventionally heated greenhouse.

\section{Cultivation results}

In Figure 4 the results for the quantity of fruits, the full and dry weight are presented. Especially, the results give a figure of increased fruit production in the greenhouse with IR heating against the greenhouse with the forced-air unit. In these quantity results, the greenhouse with IR heating produced a larger number of fruits and 
as a natural consequence the weight of the fruits obtained from this greenhouse was higher than the greenhouse with conventional heating.

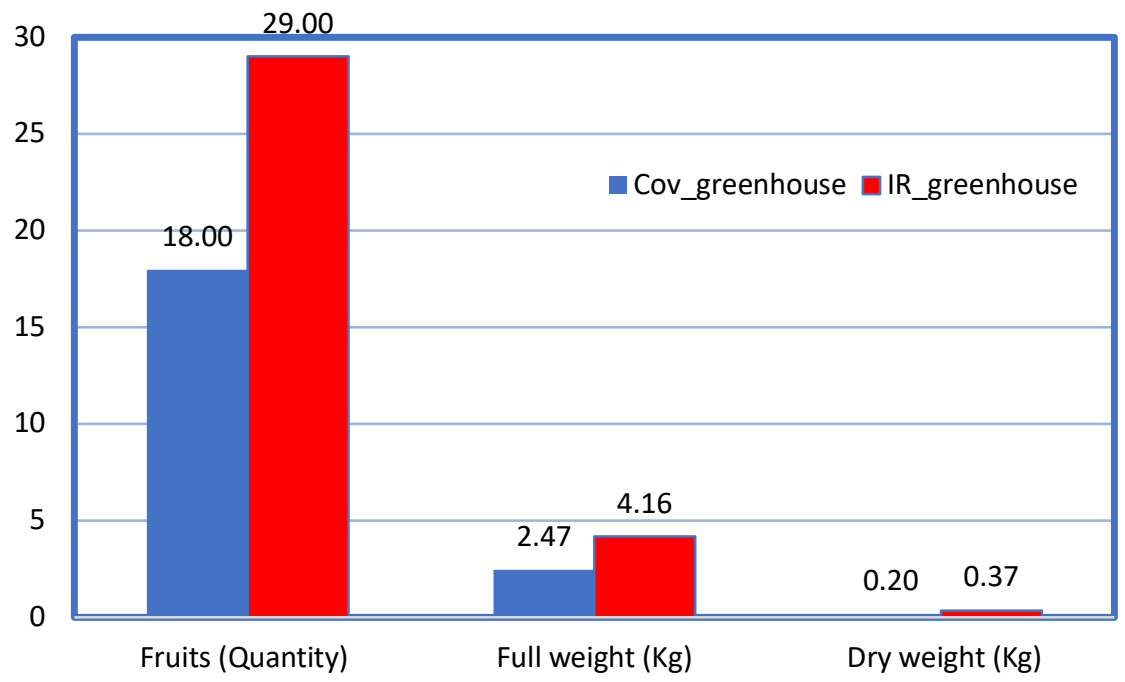

Figure 4. Total values of basic fruits grow indicators I.

In Figure 5 the results regarding the average of full and dry weight and the average of perimeter and height. From these results it is noticed the almost same values of the fruits of the greenhouse with conventional heating compared to those of the fruits of the greenhouse with IR heating. Observing the quality characteristics of the fruits of the two greenhouses, namely their average weight, height and perimeter, we see that there are virtually no differences and that uniform fruits were produced.

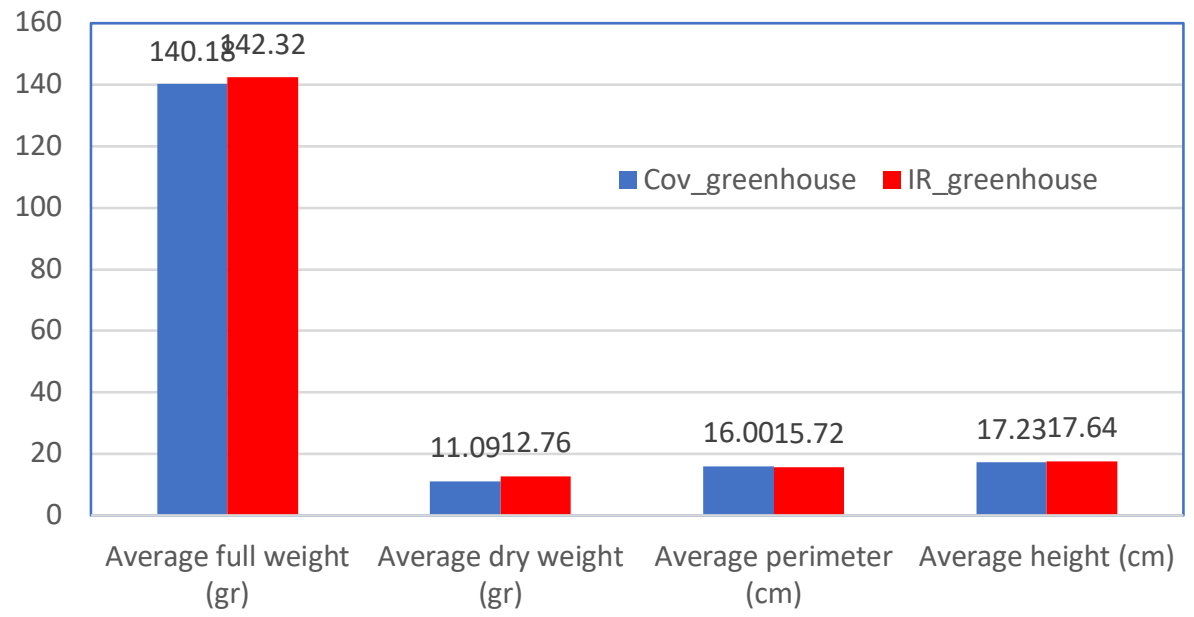

Figure 5. Average values of fruits grow indicators II.

\section{Conclusions and Future Work}

Two identical small-scale experimental greenhouse units were tested for eggplants production. The first greenhouse used IR heating system and the second one used conventional heating system. The implementation of an IR heating system in a greenhouse for eggplants production had satisfactory results, establishing a smooth distribution of the temperature at the canopy, while inside greenhouse air was maintained at lower values than in the conventionally heated greenhouse. Quantitative results on plant growth and yield have been collected, and the favorable conditions resulted in better plant growth than in the conventionally heated greenhouse. Good temperature uniformity was achieved for better distribution over the plants. Plant growth and development was excellent with efficient biomass deposition. Better uniformity also achieved in the early stages of eggplant development, as the microenvironment around them was kept at favorable temperatures.

It would be worthwhile to carry out further experimental investigation in full-scale productive greenhouse. However, full-scale productive greenhouse experiments are quite costly and time consuming. In order to further investigate the effect of IR radiation on growth parameters and physiological characteristics of eggplant, the authors are planning to develop computational models. 


\section{References}

De Pascale, S., \& Maggio, A. 2004. Sustainable protected cultivation at Mediterranean climate, perspectives and challenges. Acta Horticulturae, 691, 29-42.

Kavga, A., Panidis, Th., Bontozoglou, V. and Pantelakis, S. 2009. Infra-Red Heating of Greenhouses Revisited: An Experimental and Modeling Study. Transactions of the ASABE, 52(6):2055-2065

Kavga A., Alexopoulos G. , Bontozoglou B., Pantelakis S. , Panidis Th, 2012. Experimental Investigation of the Energy Needs for a Conventionally and an Infrared-Heated Greenhouse. Advances in Mechanical Engineering, vol. 2012, Article ID 789515, 16 pages. doi:10.1155/2012/789515

Kavga A., and Panidis Th., 2015. Implementation Results of Infrared Heating (IR) in a Production Greenhouse. Applied Engineering in Agriculture, 31(1), 143-151

Kaushik, G., \& Chel, A. 2011. Renewable energy for sustainable agriculture. Agron. Sustainabe Developement, 31(1), 91-118. doi:http://dx.doi.org/10.1051/agro/2010029.

Perdigones, A., García, J. L., Pastor, M., Benavente, R. M., Luna, L., Chaya, C., \& de la Plaza, S. 2006. Effect of heating control strategies on greenhouse energy efficiency: experimental results and modelling. Transactions of the ASABE, 49(1), 143-155. http://dx.doi.org/10.13031/2013.20232.

Teitel, M., Segal, L., Shklyar, A., \& Barak, M. 1999. A comparison between pipe and air heating methods for greenhouses. J. Agric. Eng. Res., 72(3), 259-273.

Teitel, M., Shklyar, A., Elad, Y., Dikhtyar, V., \& Jerby, E. 2000. Development of a microwave system for greenhouse heating. Acta Horticulturae, 534, 189-195. 


\author{
Effect of Dimethyl Ether (DME) Combustion on Lettuce and Chinese Cabbage \\ Growth in Greenhouse \\ Waqas Qasima , Jayanta Kumar Basakª, Frank Gyan Okyere ${ }^{a}$, Fawad Khana ${ }^{\text {, }}$, Yong Jin Lee ${ }^{\text {, }}$ \\ Hyeon Tae Kim ${ }^{a^{*}}$ \\ ${ }^{a}$ Dept. of Bio-systems Engineering, Gyeongsang National University (Institute of Agriculture \& Life Science), Jinju \\ 52828 , Korea. \\ *Corresponding Author-Tel: +82-55-772-1896; Fax: +82-55-772-1899 \\ *E-mail address: bioani@hanmail.net
}

\begin{abstract}
The problems of agriculture in recent years are seriously increased, especially in greenhouse farms where large operating systems for energy are very costly. Therefore, the purpose of this study was to determine whether the thermal energy and carbon dioxide produced by the combustion of DME (dimethyl ether) can be beneficial to the greenhouse plants. DME is a colorless gas and form of natural gas, it is non-toxic and biodegradable synthetic fuel and known as clean fuel. In this study, we investigated the effect of DME combustion on the growth of lettuce and Chinese cabbage in three experimental greenhouses (width $3 \mathrm{~m}$, length $4 \mathrm{~m}$, height $2.5 \mathrm{~m}$ ) at Gyeongsang National University. Three tests were performed to compare and analyze the growth of lettuce and Chinese cabbage, chlorophyll content, $\mathrm{CO}_{2}$ and temperature in greenhouses at different flow quantity of DME. DME supply time was $0.5 \mathrm{hr} / \mathrm{day}, 1 \mathrm{hr} /$ day, $1: 30 \mathrm{hrs} /$ day and $2 \mathrm{hrs} / \mathrm{day}$ on week 1, 2, 3 and 4 respectively. The average DME flow quantity in duct was $17.4 \mathrm{~m} 3 / \mathrm{min}$ and $10.2 \mathrm{~m} 3 / \mathrm{min}$ to Greenhouse 1 and Greenhouse 2 and no DME gas was supplied to Greenhouse 3 which was left as control. As a result, DME combustion significantly affect growth of lettuce and Chinese cabbage in two greenhouses. At end of experiment, the highest fresh weight and dry weight of lettuce and Chinese cabbage were measured in Greenhouse 1 and followed by greenhouse 2. Similarly chlorophyll content of Greenhouse 1 were $15 \%$ higher than greenhouse 3. $\mathrm{CO}_{2}$ were significantly increased at the time of DME supply to greenhouses. In conclusion, DME gas can be used for rapid growth of lettuce and Chinese cabbage in common greenhouses.
\end{abstract}

Keywords: Chinese cabbage, Chlorophyll, CO2, Dimethyl ether, Greenhouse, Lettuce

\title{
1. Introduction
}

As the world population continues to grow, the production of energy from fossil fuels is also expected to increase. However, the amount of fossil fuels that can be used is also diminishing, making it extremely difficult for mankind to meet energy demand. We must look towards renewable energy resources, like geothermal power or solar power, in order to overcome a scenario in which we run out of fossil fuels. Development of energy sources that can replace fossil fuels, and its importance is ever growing and increasing the market size (Bilen et al., 2008). Thus the world, including the major developed countries has put a lot of effort and resources to dominate the fastgrowing renewable energy market. The less developed countries like China, India, Brazil and some other developing countries are also in competition to make new and renewable energy technologies. South Korea also put a lot of resources in the renewable energy sector to the national vision of low-carbon green growth below the world market occupancy and domestic energy for troubleshooting (Choi et al., 2013).

The energy sources for a country includes solar energy, wind energy, biomass energy, nuclear energy, fossil fuels etc. in which energy from agriculture side is consider to be the most dependent on imports. It is expected to conduct more actively research in a variety of ways.

In particular, the energy being consumed to maintain the sustainable environment a lot of flora and fauna is required in the production process of energy. To this end, research is also being conducted continuously on the renewable energy usage, such as in geothermal and solar energy, apart from this the research for more fundamental measures of energy is also needed.

With growing concerns of environmental pollution by using fossils fuel, the Dimethyl Ether (DME) gas is emerged as one alternative (Semelsberger et al., 2005). DME can be produced from coal, biomass and natural gas. The physical properties of DME and LPG are almost similar due to this DME has been specifically identified as substitute fuel for LPG (Arya et al., 2016). DME gas is known as clean fuel by burning it in agriculture facility it will not only provide heating but also provide sufficient $\mathrm{CO} 2$ without any high concentration of toxic gases.

In South Korea growing vegetables inside greenhouse has been increased due to extreme temperature and improvement in automatic control systems of agriculture facilities. The vegetables which are most often used as salad is lettuce and chines cabbage also their consumption has been increased in past few years. This experiment was conducted by growing lettuce and Chinese cabbage in greenhouse by providing appropriate environment for growth. In this study we focused on whether the thermal energy and carbon dioxide produced by the combustion of DME (dimethyl ether) can be beneficial to the greenhouse plants. The objective was to determine the effect of DME combustion gas on lettuce and Chinese cabbage growth in greenhouse. 


\section{Materials and Methods}

Experimental Design

This experiment was performed in three identical greenhouses (width $3 \mathrm{~m}$, length $4 \mathrm{~m}$, height $2.5 \mathrm{~m}$ ) of Gyeongsang National University, South Korea (Figure 1). In all 3 greenhouses there were 2 beds for planting vegetables. In one bed the lettuce were grown and in another bed Chinese cabbage were grown.

Three tests were performed to compare and analyze the growth of lettuce and Chinese cabbage, chlorophyll content, $\mathrm{CO}_{2}$ and temperature in greenhouses at different flow quantity of DME. DME supply time was $0.5 \mathrm{hr} /$ day, $1 \mathrm{hr} /$ day, $1: 30 \mathrm{hrs} /$ day and $2 \mathrm{hrs} /$ day on week $1,2,3$ and 4 respectively. The average DME flow quantity in duct was $17 \mathrm{~m}^{3} / \mathrm{min}$ and $10 \mathrm{~m}^{3} / \mathrm{min}$ to Greenhouse 1 and Greenhouse 2 and no DME gas was supplied to Greenhouse 3 which was left as control.

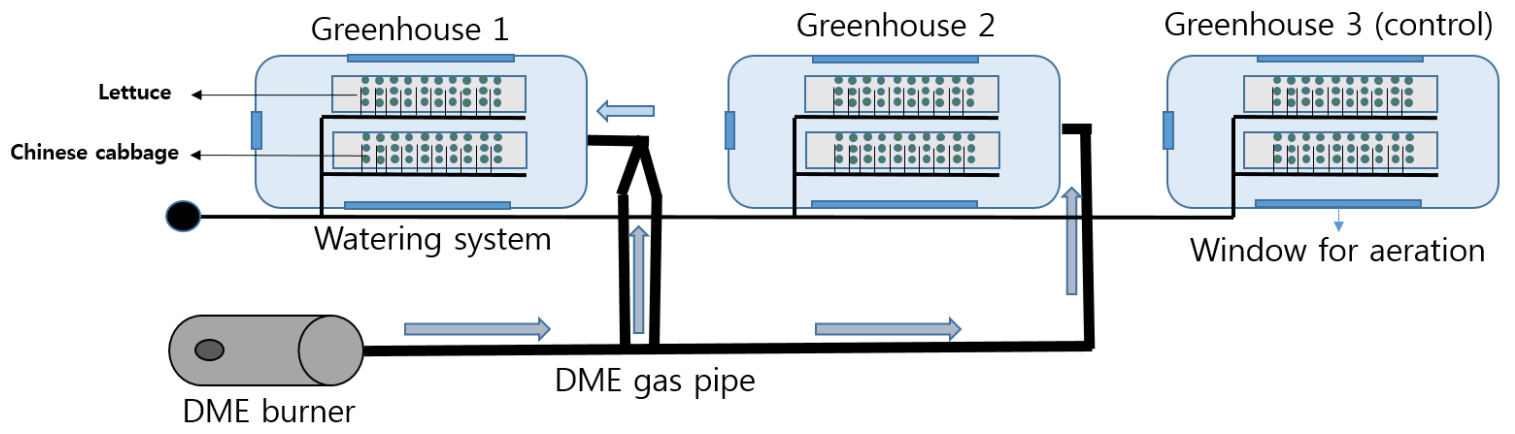

Figure 1. Experimental design of DME supply to greenhouses.

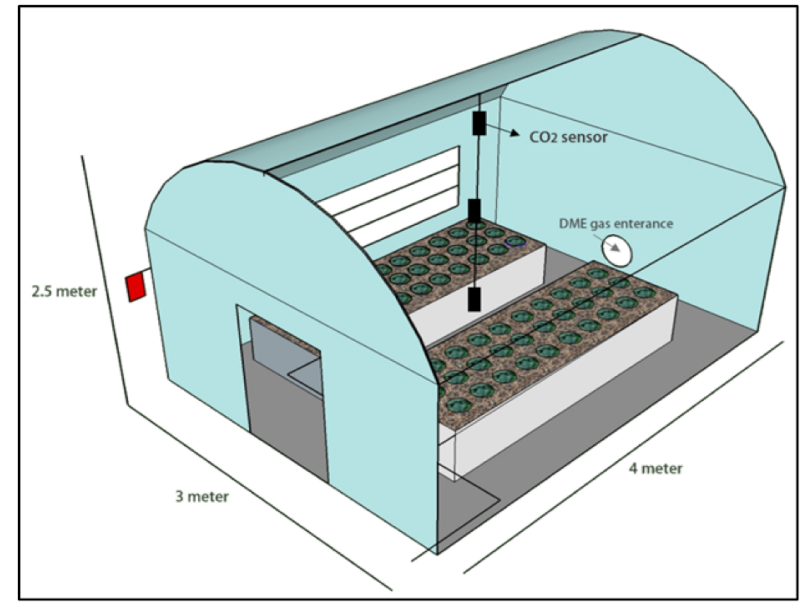

Figure 2. Schematic diagram of Greenhouse for the experiment.
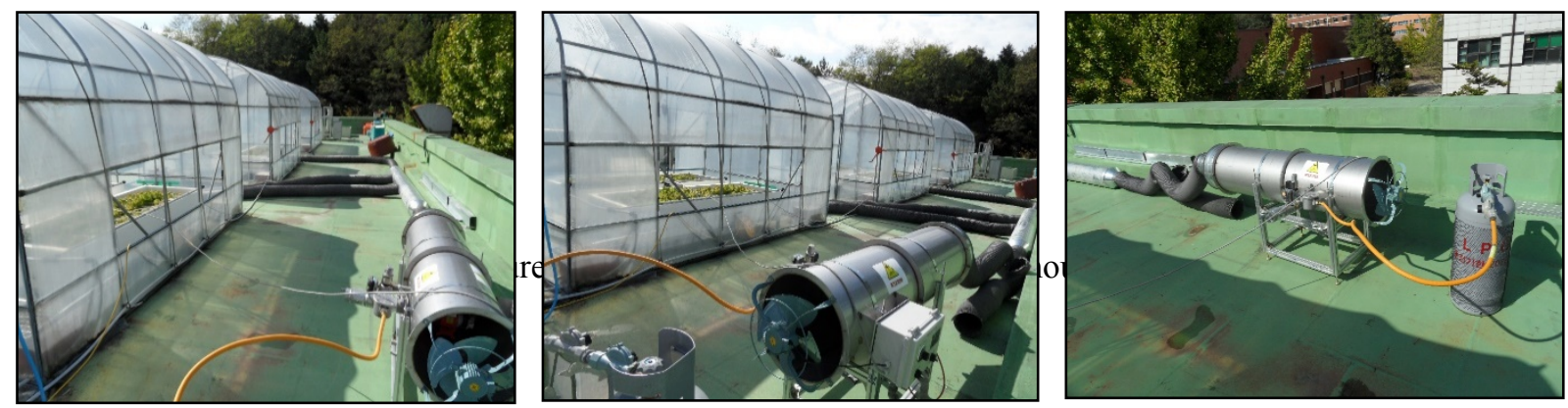

Figure 3. Images of DME supply to greenhouses. 


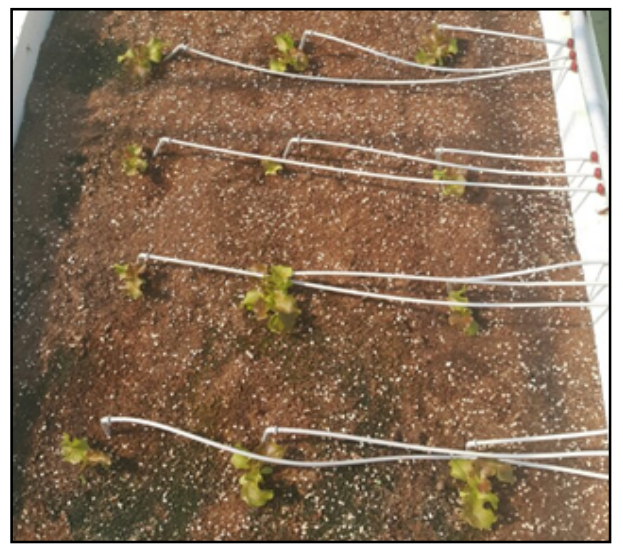

Figure 4. Watering system in greenhouse.

The watering systems were uniform in all three greenhouses. Water were provided by needles to each separate plant as shown in figure 4 . The watering time was $2 \mathrm{~min}$ /day in all greenhouses. For aeration side windows were opened at 9:00 am and closed at 6:00pm to keep the temperature in range.

Physical properties of DME

Dimethyl ether is the simplest ether, with chemical formula of $\mathrm{CH}_{3} \mathrm{OCH}_{3}$. DME is a colorless gas and form of natural gas, it is non-toxic and biodegradable synthetic fuel and known as clean fuel. Its physical properties are similar to propane and butane. DME burns with visible blue flame and is non-peroxide forming in the pure state (Semelsberger et al., 2005). Table 1 shows the physical properties of Dimethyl ether. DME Burner were used for combustion, the specification of burner are described in table 2 .

Table 1. Physical properties of Dimethyl ether (DME).

\begin{tabular}{lc} 
& Table 1. Physical properties of Dimethyl ether (DME). \\
\hline Formula & $\mathrm{CH}_{3} \mathrm{OCH}_{3}$ \\
Molecular weight $\left(\mathrm{g} \mathrm{mol}^{-1}\right)$ & 46.07 \\
Density $\left(\mathrm{g} \mathrm{cm}^{-1}\right)$ & 0.661 \\
Normal boiling point $\left({ }^{\circ} \mathrm{C}\right)$ & -24.9 \\
Carbon Content $(\mathrm{wt} \%)$ & 52.2 \\
Hydrogen content $(\mathrm{wt} \%)$ & 13 \\
Oxygen content $(\mathrm{wt} \%)$ & 34.8 \\
\hline *Density at $\mathrm{P}=1$ atm and $\mathrm{T}=-25^{\circ} \mathrm{C}$ & \\
& Table 2. The specification of the DME gas burner. \\
\hline Items & Specification \\
\hline Quantity of energy $(\mathrm{kcal} / \mathrm{h})$ & 40000 \\
Rotational frequency of motor (rpm) & 1495 \\
Output of motor $(\mathrm{W})$ & 200 \\
Quantity of flow $\left(\mathrm{m}{ }^{3} / \mathrm{min}, \mathrm{CFM}\right)$ & 1060 \\
Diameter of outlet $(\mathrm{mm})$ & 400 \\
Length of burner $(\mathrm{mm})$ & 1360 \\
\hline
\end{tabular}

For checking the concentration of gases the samples were collected in air plastic bags of 201 during operation of DME combustion. Sample were taken to Korean Testing laboratory on same day and ISO 6974-6 standard method were used for determination of gases concentration. The testing environment were $20.0 \pm 5^{\circ} \mathrm{C}$ temperature and $50 \pm 20 \%$ relative humidity. Table 3 shows the concentration of gases during combustion of DME.

Table 3. Concentration of gases during combustion of DME. *samples were collected in front of DME burner.

\begin{tabular}{ll}
\hline Gases (unit) & Amount \\
\hline $\mathrm{CO}_{2} \mu \mathrm{mol} / \mathrm{mol}(\mathrm{ppm})$ & 3316.1 \\
$\mathrm{H}_{2} \mu \mathrm{mol} / \mathrm{mol}(\mathrm{ppm})$ & 0.7 \\
$\mathrm{CO} \mu \mathrm{mol} / \mathrm{mol}(\mathrm{ppm})$ & 11.6 \\
$\mathrm{CH}_{4} \mu \mathrm{mol} / \mathrm{mol}(\mathrm{ppm})$ & 1.7 \\
$\mathrm{O}_{2} \mathrm{cmol} / \mathrm{mol}(\%)$ & 21.3 \\
$\mathrm{~N}_{2} \mathrm{cmol} / \mathrm{mol}(\%)$ & 77.8 \\
\hline
\end{tabular}


Lettuce and Chinese cabbage plantation

Lettuce and Chinese cabbage seeds were purchased from market and seeds trays were used for seedling. After 3 weeks when seeds were grown up 30 lettuce plants and 30 Chinese cabbage plants were transplanted to each two beds of greenhouses as shown in figure 5 .

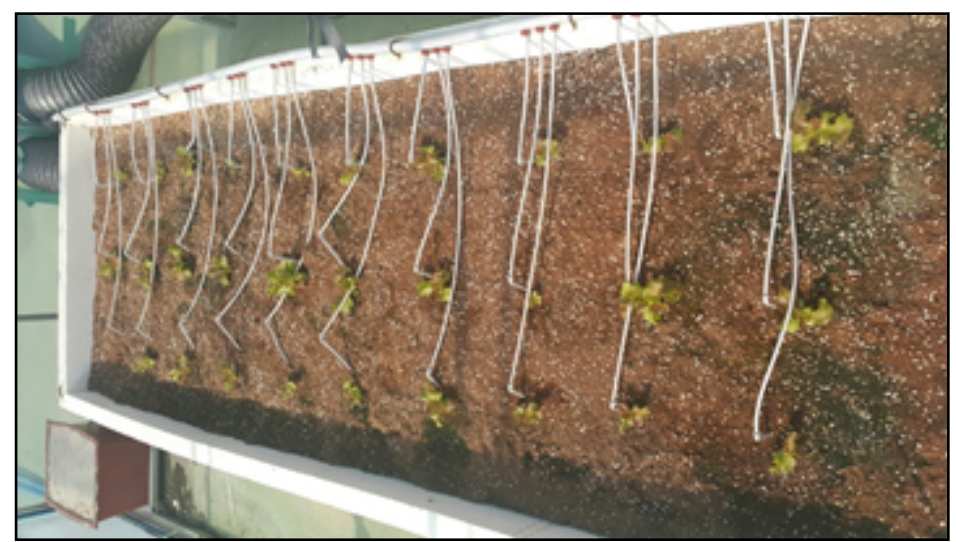

Figure 5. The photo after seedling of lettuce in the greenhouse.

Collection and Analysis of data

For carbon dioxide, Temperature and humidity determination "Lutron MCH-383SD" electrochemical sensors were set at three different height of greenhouses as shown in figure 2. Lutron MCH-383SD is equipped to record the date, time interval and has a memory card to store these data (figure 6). Data were checked at 10-min intervals and recorded data were averaged for further analysis and interpretation (Qasim et al., 2017). The lower sensor were equipped at height of $0.3 \mathrm{~m}$ from floor which was equal with height of plants in bed.

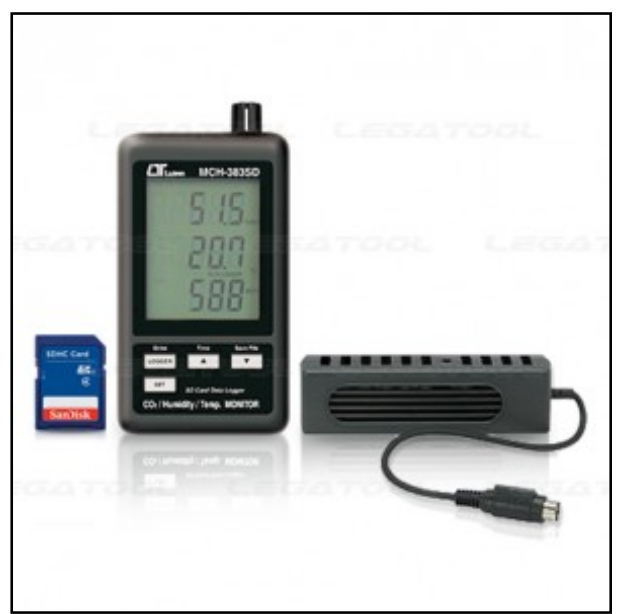

Figure 6. Photo of "Lutron MCH-383SD" electrochemical sensor.

Chlorophyll were measured by using by SPAD 502 chlorophyll meter as shown in figure 7 . Averaged data of each plants were taken for analysis. Data were collected on weekly basis in all three greenhouses. 


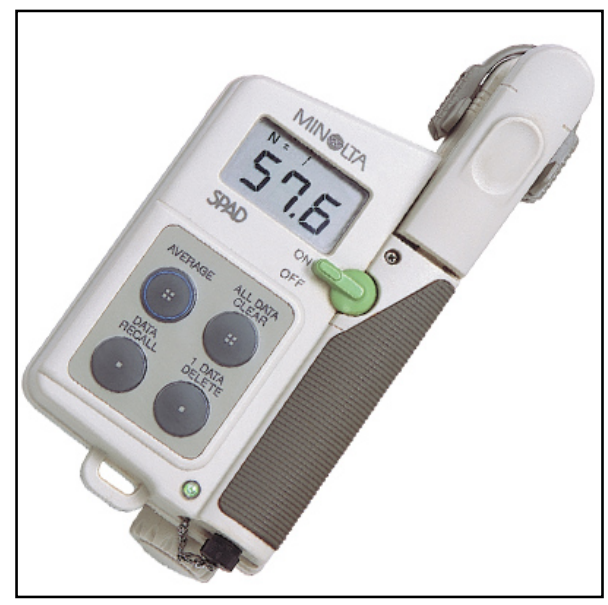

Figure 7. Photo of SPAD 502 chlorophyll meter.

Fresh weight and dry weight of leaves and roots of each plants in greenhouse 1,2 and 3 were compared at end of the experiment. Fresh weight were measured as whole plant but due to high number of leaves and size of Lettuce and Chinese cabbage 5 big leaves from each plants were taken for dry weight analysis. Roots were cleaned from soil and separated from whole plants for analysis. Fresh and dry weight of leaves and roots were measured on electronic balance before and after drying at $80^{\circ} \mathrm{C}$ for $24 \mathrm{hrs}$. in an oven. Leaves number were also compared at end of the study.

Statistical analysis was performed using the Prism 5 Graph Pad program. Data were presented as means (SEM) and analyzed by one-way ANOVA followed by Bonferroni's Multiple Comparison Test with a significance level of $P<0.05$.

\section{Results and Discussion}

Carbon dioxide evaluation

1 day test were performed to evaluate the carbon dioxide pattern in side Greenhouses. Figure 8 shows carbon dioxide pattern in three greenhouse with $17 \mathrm{~m}^{3} / \mathrm{min}, 10 \mathrm{~m}^{3} / \mathrm{min}$ and no DME were supplied. The carbon oxide sharply increases as DME burner were operated from 6:00 am to 6:30am in Greenhouse 1 and 2. With $17 \mathrm{~m}^{3} / \mathrm{min}$ and $10 \mathrm{~m}^{3} / \mathrm{min}$ DME supply the max $\mathrm{CO}_{2}$ were recorded $1380 \mathrm{ppm}$ and $940 \mathrm{ppm}$ respectively. From these results its clearly observed that with DME operation CO2 inside greenhouse significantly increases.

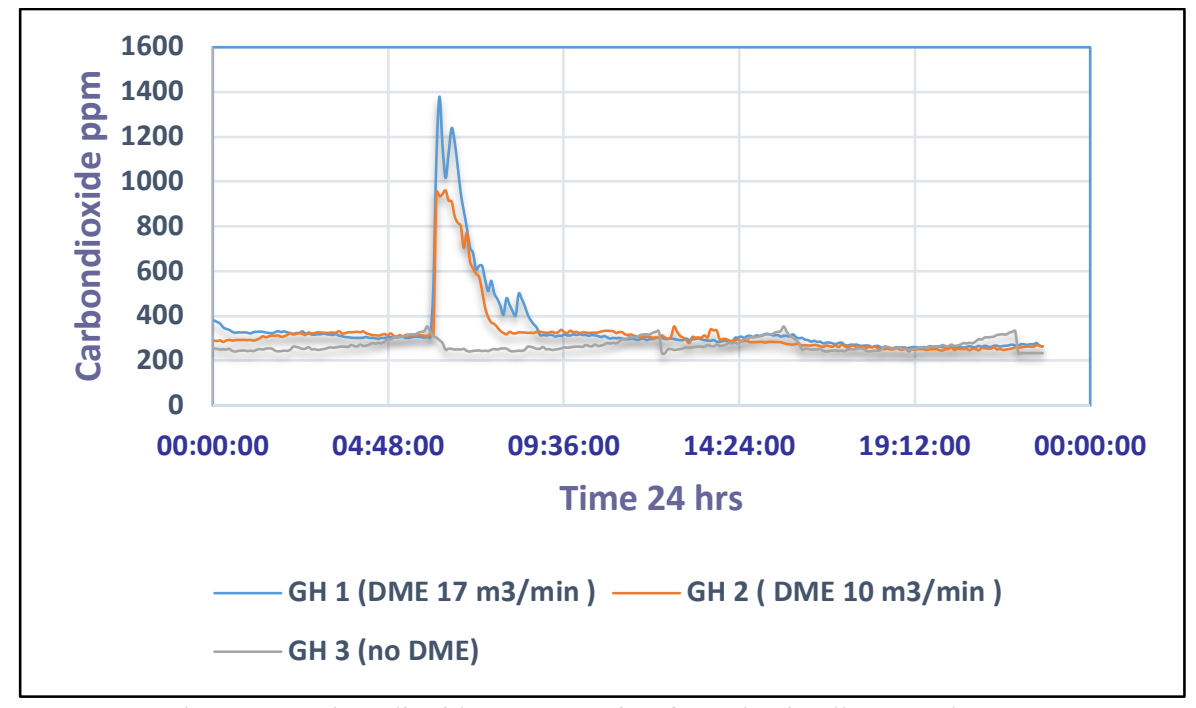

Figure 8. Carbon dioxide concentration for 1 day in all 3 greenhouses. 
Fresh and Dry weight of Lettuce and Chinese cabbage

At end of study we collected all plants and measured the weight of lettuce and chinise cabbage of each plants. Interesting results were observed between Greenhouse 1 and Greenhouse 3 although there were not much difference observed in the results of Greenhouse 1 and 2. Figure $9 a \& 9 b$ shows average fresh and dry weight of lettuce and Chinese cabbage at end of study. The highest fresh weight were measured in Greenhouse 1 which has DME supply $17 \mathrm{~m}^{3} / \mathrm{min}$, followed by Greenhouse 2 with DME supply $10 \mathrm{~m}^{3} / \mathrm{min}$ and Greenhouse 3 with no DME supply which was left as control treatment. By supplying DME gas to greenhouse facility significantly increase the yield.

Total fresh and dry weight of Lettuce

Big leaves fresh and dry weight of Chinese cabbage
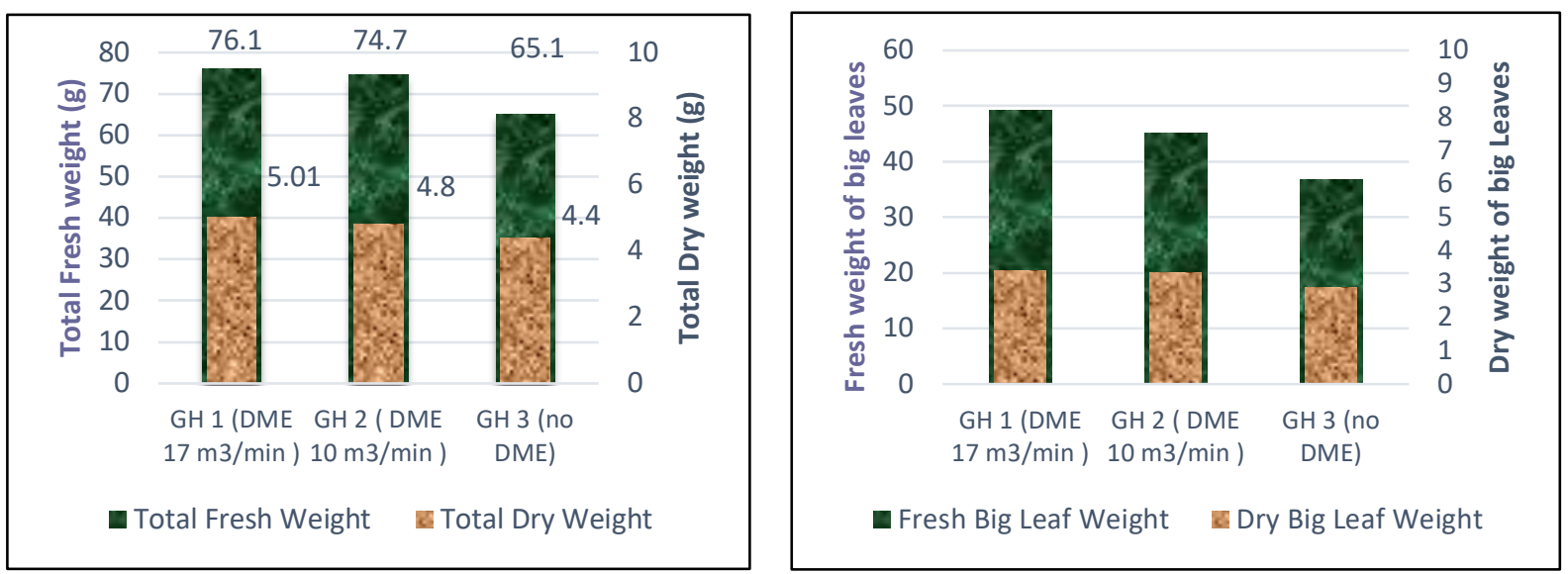

Figure $9 \mathrm{a} \& \mathrm{~b}$. Average fresh and dry weight of lettuce and Chinese cabbage in 3 Greenhouses.

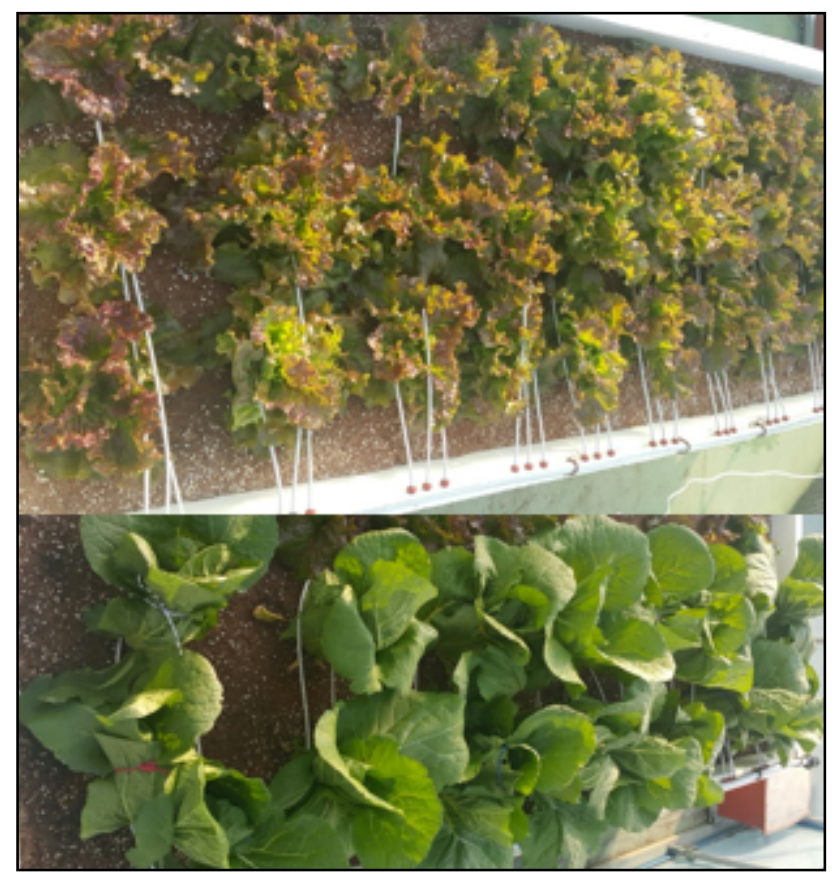

Figure 11. Picture of lettuce and Chinese cabbage at end of study.

Chlorophyll content in Lettuce and Chinese cabbage

The chlorophyll results are presented as an average of the measurement of each line of the bed in all 3 greenhouses. Chlorophyll concentration was measured and compared at end of the study. The highest concentration was measured in Greenhouse 1 as expected because previous studies shows that $\mathrm{CO}_{2}$ increases the chlorophyll content of plants as shown in figure 12. Kim et al., 2017 reported that $\mathrm{CO} 2$ fertilization in greenhouse increases the chlorophyll content. Greenhouse 1 had approximately $15 \%$ more chlorophyll than Greenhouse- 3 . So from these results we are suggesting that DME supply to greenhouse can be effective for growth of plants. 


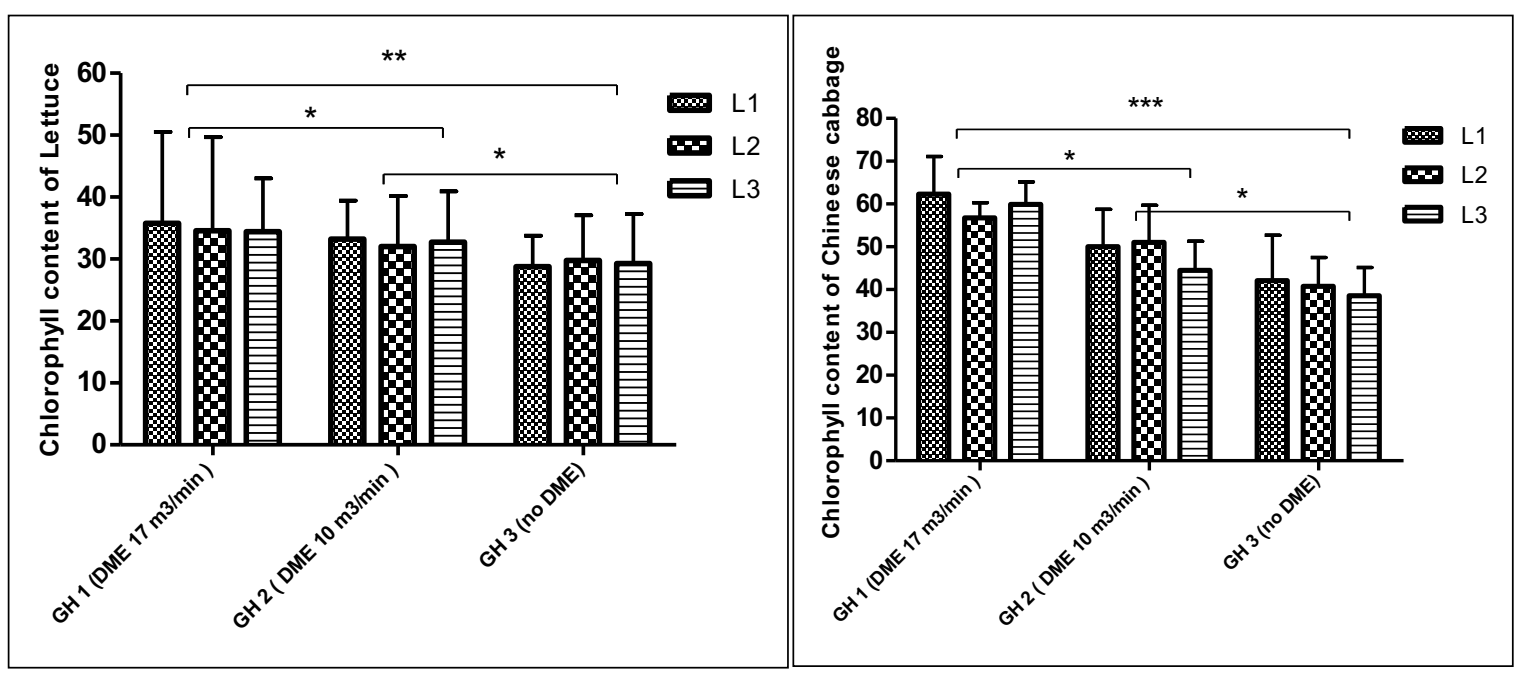

Figure 12a (left) \& b (right). Chlorophyll content of lettuce and Chinese cabbage in all 3 greenhouses.

\section{Conclusion}

From this study we concluded that DME supply not only increase the $\mathrm{CO}_{2}$ but also plants yield. DME supply with $17 \mathrm{~m}^{3} / \mathrm{min}$ was useful for lettuce and Chinese cabbage growth in greenhouse with size (width $3 \mathrm{~m}$, length $4 \mathrm{~m}$, height $2.5 \mathrm{~m}$ ). Although more experiment is required for the max quantity and duration of time for supply in specific volume of greenhouse.

\section{Acknowledgment}

This work was supported by Korea Institute of Planning and Evaluation for Technology in Food, Agriculture and Forestry(IPET) through Agriculture, Food and Rural Affairs Research Center Support Program, funded by Ministry of Agriculture, Food and Rural Affairs(MAFRA)(Grant number : 116113-02)

\section{References}

Bilen, K., Ozyurt, O., Bakırc1, K., Karslı, S., Erdogan, S., Yılmaz, M., \& Comakl1, O. (2008). Energy production, consumption, and environmental pollution for sustainable development: A case study in Turkey. Renewable and Sustainable Energy Reviews, 12(6), 1529-1561.

Choi, M. K., Yun, S. W., \& Yoon, Y. C. (2013). Analysis of surplus solar energy in wide span type greenhouse-case study based on Jinju area. J. Agric. \& Life Sci, 47, 197-207.

Korea Pork Producers Association. 2009. Standard pig house design guide. http://www.koreapork.or.kr/sub3 8.html.

Cho, JK, JH Sung, and MH Hong. 2008. The Mechanical System Integrative Design Strategies for Developing the Sustainable Housing Model. Journal of Architectural Institute of Korea. 24 (8): 269-276.

Semelsberger, T. A., Borup, R. L., \& Greene, H. L. (2006). Dimethyl ether (DME) as an alternative fuel. Journal of Power Sources, 156(2), 497-511.

Arya, P. K., Tupkari, S., Satish, K., Thakre, G. D., \& Shukla, B. M. (2016). DME blended LPG as a cooking fuel option for Indian household: A review. Renewable and Sustainable Energy Reviews, 53, 1591-1601.

Qasim, W., Moon, B. E., Phonsuwan, M., Jo, J. S., Lee, M. H., Nafees, M., \& Kim, H. T. (2017). Effects of an Aluminum Sulfate and Ferric Chloride Blend on Poultry Litter Characteristics in Vitro. The Journal of Applied Poultry Research

Tae, K. H., Jung, H. J., Moon, B. E., Lee, C. S., Ryou, Y. S., \& Kim, H. T. (2017). Purification of CO2 and CH4 From Livestock Biogas and Greenhouse Utilization of the Purified CO2. Journal of Residuals Science \& Technology, 14(2). 


\section{Topic 10: Post-harvest technologies (PH)}

Technology and management systems to monitor and control the quality of products, and for sorting, food processing, packaging and storage. Contributions can address nutrition, flavour and shelf life, and include various types of sensors, actuators, packaging materials and controlled-atmosphere techniques.

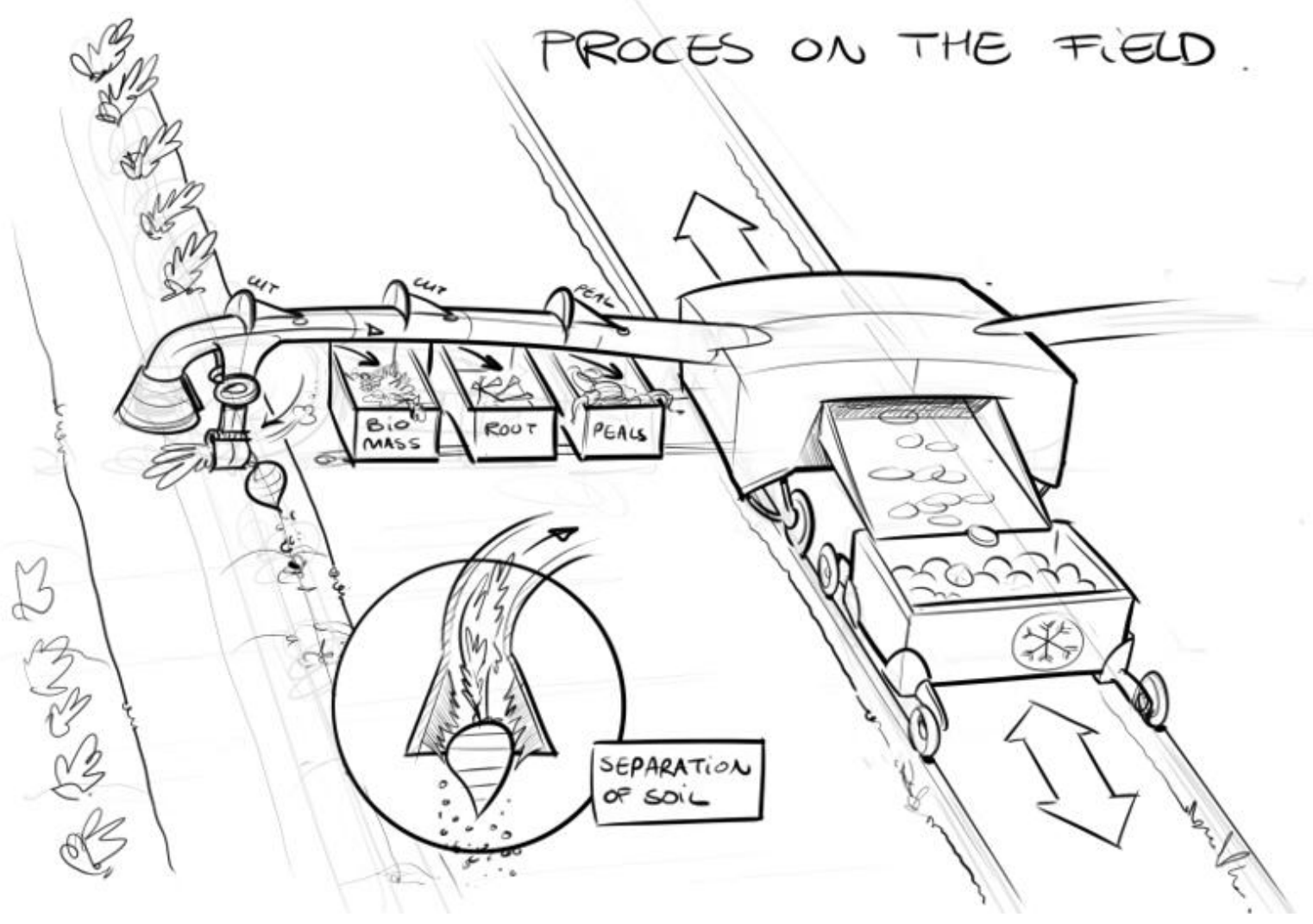




\author{
Wall Pressures on Oblique Hoppers of Steel Silos \\ Eutiquio Gallego $^{\text {a,*, Adam Sadowski }}{ }^{\text {b }}$ Jose María Fuentes $^{\text {a }}$, Francisco Ayuga ${ }^{\text {a }}$ \\ ${ }^{\text {a }}$ BIPREE Research Group, Universidad Politécnica de Madrid, Madrid, Madrid 28040, Spain \\ ${ }^{\mathrm{b}}$ Department of Civil and Environmental Engineering, Imperial College of London, London SW7 2AZ, United Kingdom \\ * Corresponding author. Email: eutiquio.gallego@upm.es
}

\begin{abstract}
Silos are special structures used to store different kind of products that exhibit a complex behaviour because of the existence of specific phenomena such as the "silo effect". EN 1991-4 is a European standard, which is the reference code, employed worldwide to calculate the actions on silo walls produced by the stored material. Bin hopper geometries are one of the main silo typologies existing, especially for steel silos in farm facilities and food industries, and they sometimes have an eccentric outlet in order to improve the flow of material during discharge. The current version of EN 1991-4 considers Walker theory in order to calculate pressures on concentric hoppers, but oblique hoppers are not covered by it. This paper presents a Finite Element Model developed to predict the wall normal pressures expected in oblique hoppers. It has been considered the effect of several parameters, e.g. the ratio of eccentricity, the ratio of radius to the hopper height or the circumferential location, on normal pressures. Results of this research are expected to be of interest in the future updating of EN 1991-4.
\end{abstract}

Keywords: Steel silos, Oblique hoppers, wall pressures

\title{
1. Introduction
}

Silos are special structures used to store different kind of products that exhibit a complex behaviour because of the existence of specific phenomena such as the "silo effect", which are the frictional forces appeared in the contact of the bulk solid with the silo wall. There are many different commercial silo typologies, but one of the most used for steel silos is the named "bin-hopper", which consists of a vertical cylindrical body and a troncoconical hopper.

The distribution of normal pressures exerted by a static bulk granular solid on the walls of a concentric conical hopper is well known in the field of silo design and can be found in many design standards, e.g. European EN 1991-4 (2006). The derivation of this distribution is thought to have been first done by Dąbrowski (1957), though it is commonly attributed to Walker (1966).

The reference concentric system is presented in Figure 1. The mean normal pressure $\mathrm{p}_{\mathrm{n}}$ on the inclined wall is assumed to be related to the mean vertical stress $p_{\mathrm{v}}$ through a hopper pressure ratio $\mathrm{F}$ (Eq. 1), and the mean frictional shear $p_{t}$ is related to the mean normal pressure $p_{n}$ by the fully-developed wall friction coefficient for the hopper $\mu_{\mathrm{h}}$ (Eq. 2). The mean values acknowledge the fact that even pressures under careful concentric filling are never really axisymmetric (Ooi et al., 1990). This approximate description is nonetheless widely applied.

$$
\begin{aligned}
& p_{n}=F \cdot p_{v} \\
& p_{t}=\mu_{h} \cdot p_{n}
\end{aligned}
$$
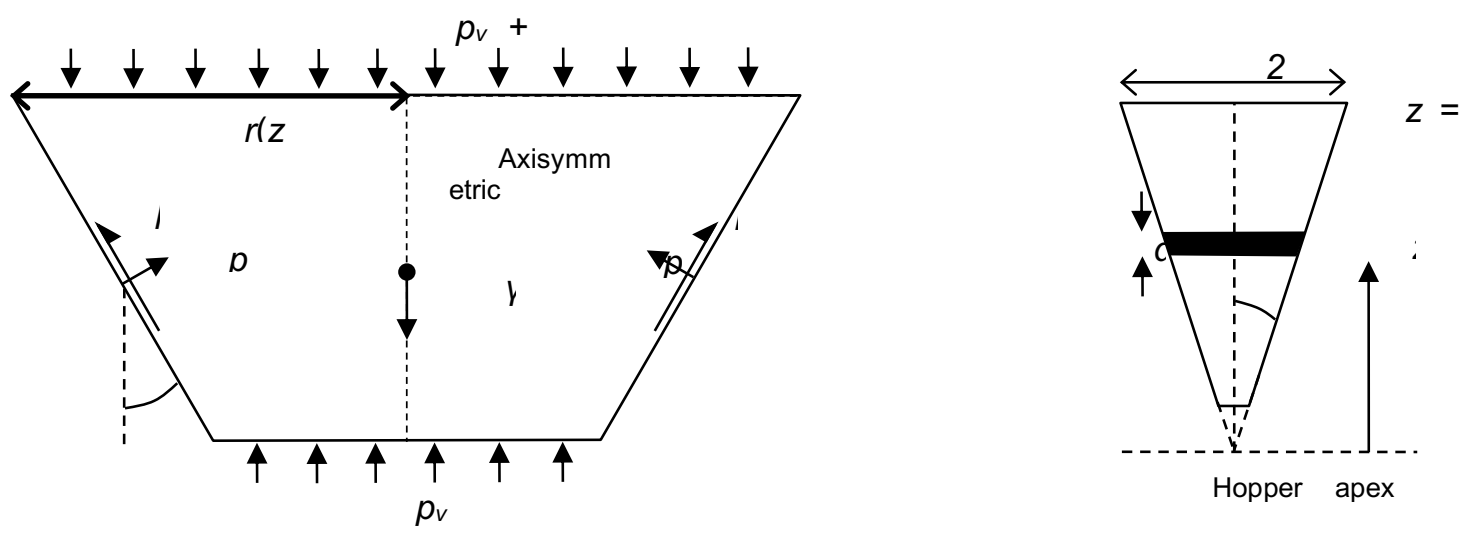

Figure 1. Derivation diagram for concentric hopper pressures, after Rotter (2007).

A differential horizontal slice is selected, where the force balance is set by considering the areas existing at top $A_{\text {top }}$ (Eq. 3) and bottom $A_{\text {bot }}$ (Eq. 4) of the horizontal slice, as well as relevant parameters such as the bulk solid weight, $\gamma$, internal perimeter, $\mathrm{U}$, of the plan cross-section of the hopper walled segment (Eq. 5), or the 
volume, V, of the horizontal slice (Eq. 6).

$$
\begin{aligned}
& A_{\text {top }}=\pi(z+d z)^{2} \tan ^{2} \beta \\
& A_{\text {bot }}=\pi z^{2} \tan ^{2} \beta \\
& U=2 \pi z \tan \beta \sec \beta \\
& V=\pi z^{2} \tan ^{2} \beta d z
\end{aligned}
$$

where $\mathrm{z}$ is the vertical coordinate measured from the hopper apex; $\beta$ is the hopper apex half angle; and dz represents the increment in the vertical coordinate. The force balance applied to the horizontal slice of grain existing within the hopper leads to Equation 7. Assuming the top boundary condition expressed in Equation 8 , where $p_{v f t}$ is the vertical stress in the solid at the bottom of the overlying silo ( $=0$ if absent), then the widespread equation included in standards (EN 1991-4, 2006) to obtain the vertical pressures existing over silo hoppers can be derived (Eq. 9).

$$
\begin{aligned}
& z \frac{d p_{v}}{d z}-2 p_{v}\left[F\left(1+\mu_{h} \cot \beta\right)-1\right]=-\gamma z \\
& \operatorname{pv}(\mathrm{hh})=\operatorname{pvft} \\
& p_{v}(z)=p_{v f t}\left(\frac{z}{h_{h}}\right)^{n}+\frac{\gamma h_{h}}{n-1}\left\{\left(\frac{z}{h_{h}}\right)-\left(\frac{z}{h_{h}}\right)^{n}\right\} \\
& n=2\left[F\left(1+\mu_{h} \cot \beta\right)-1\right]
\end{aligned}
$$

Eurocode EN 1991-4 (2006) sets that the loads on the walls of silo hoppers should be evaluated according to the steepness of the hopper, by applying Equation 11.

$$
\operatorname{tg} \beta<\frac{1-K}{2 \cdot \mu_{h}}
$$

where $\mathrm{K}$ is the lateral pressure ratio; $\beta$ is hopper apex half angle; and $\mu_{\mathrm{h}}$ is the value of wall friction coefficient in the hopper. If condition defined in Equation is met, then the hopper is classified as "steep". Otherwise, the hopper is classified as "shallow". The main difference between both hopper types is that the wall friction is not fully mobilized for shallow hoppers. So, it is required to calculate the mobilized or effective wall friction coefficient, whose value would be used in equations instead of the total wall friction coefficient.

Commercial silos (mainly farm and food industry silos) sometimes are provided with an oblique hopper in order to facilitate the flow of the bulk solid stored during the discharge process. The existing standards, e.g. EN 1991-4 (2006), do not provide guidance or equations to calculate the pressures exerted by the bulk solid over the walls in oblique hoppers. Because of this, some authors (Ayuga et al, 2001; Guaita et al., 2003) developed FE models to analyse this problem, where they found a decrease in normal pressure at the side of the hopper adjacent to the eccentric outlet and a rise in the opposite wall, with relation to the concentric hopper case.

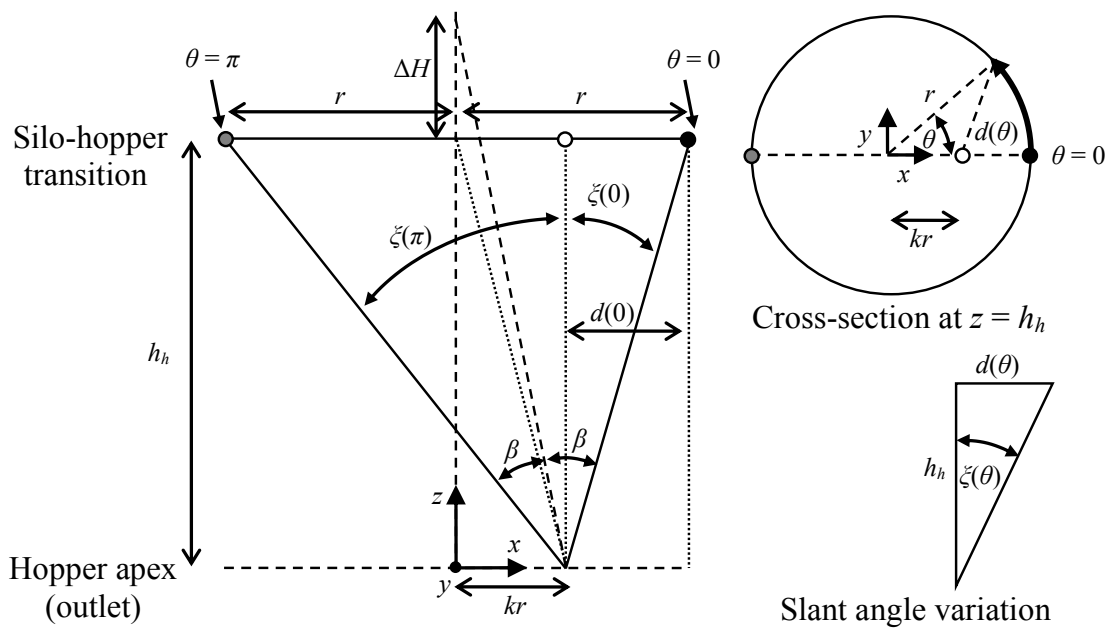

Figure 2. Geometry of the oblique hopper.

In addition, some other authors (Michalowski, 1983; Matchett et al., 2009) derived analytical equations to calculate pressures on oblique hoppers by setting the force equilibrium equation in a horizontal differential slice for any 2D plane. However, these theories are only applicable to wedged hoppers, where any cross section will 
provide always the same left and right wall inclinations. These proposals cannot be applied to oblique circular hoppers (Fig. 2), where the hopper wall inclination varies circumferentially from the highest slope corresponding to the position closest to outlet $(\theta=0)$, to the lowest slope existing on the wall opposite to the outlet $(\theta=\pi)$.

\section{Materials and Methods}

Geometry of the oblique hopper

The geometry of the oblique hopper has been constructed by using the following variables (Fig. 3) and variable $h_{h}$ (Fig. 2):
$\mathrm{R}:$
Oblique hopper radius $(\mathrm{m})$
RT:
Outlet Radius (m)
T: $\quad$ Height of oblique hopper $(\mathrm{m})$
$\mathrm{h}_{\mathrm{h}}: \quad$ vertical height between the hopper apex and the hopper top (m)
EXCT: $\quad$ Outlet eccentricity of the oblique hopper (m)

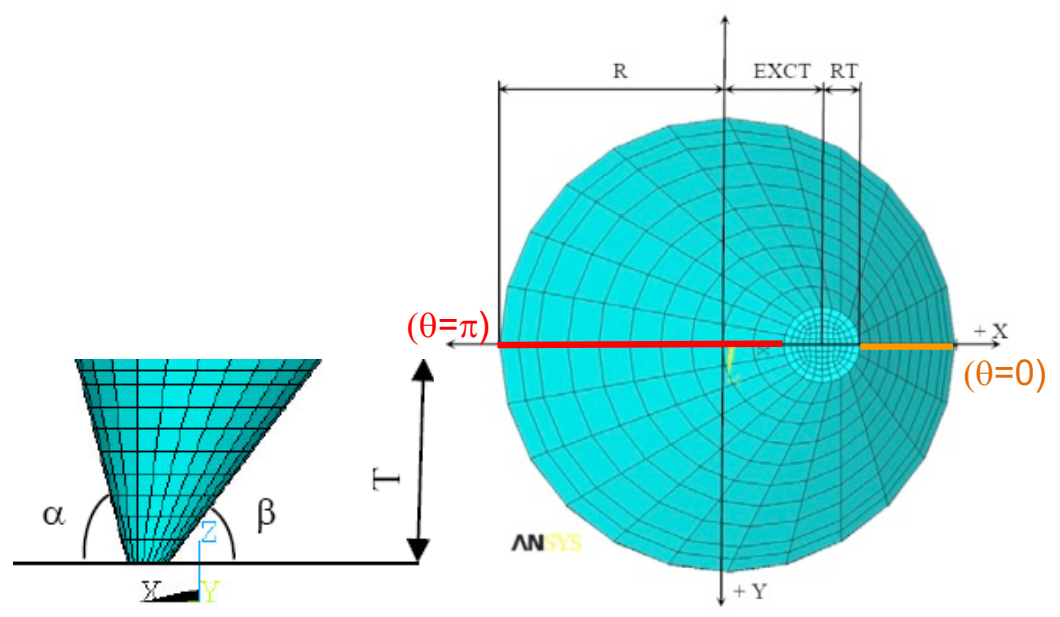

Figure 3. Variables used to develop the Finite Element geometry of the model.

Some of the previous variables were fixed for all the cases studied ( $\mathrm{R}=3 \mathrm{~m} ; \mathrm{RT}=0.5 \mathrm{~m})$, while the rest of them varied. One of the sensitivity analyses performed tried to check the influence of the aspect ratio $\left(\mathrm{R} / \mathrm{h}_{\mathrm{h}}\right)$ on hopper wall pressures at several locations, thus different geometries were tested (Table 1). In addition, 5 different outlet eccentricities were considered for each case: $0 \%$ (concentric hopper), $25 \%, 50 \%, 75 \%$ and $100 \%$. It is important to note that almost all of the silo manufacturers usually design hoppers whose inclination with respect to the horizontal axis is $45^{\circ}$ or $60^{\circ}$, thus leading to corresponding $\mathrm{R} / \mathrm{h}_{\mathrm{h}}$ values of 1.00 and 0.58 , respectively.

Table 87 . Values for the geometry variables of the oblique hopper.

\begin{tabular}{ccc}
\hline $\mathrm{R} / \mathrm{h}_{\mathrm{h}}$ & $\mathrm{h}_{\mathrm{h}}(\mathrm{m})$ & $\mathrm{T}(\mathrm{m})$ \\
\hline 0.40 & 7.50 & 6.25 \\
0.58 & 5.17 & 4.33 \\
0.80 & 3.75 & 3.12 \\
1.00 & 3.00 & 2.50 \\
\hline
\end{tabular}

Numerical model

A 3D Finite Element Model was developed for the study of oblique hoppers by using ANSYS (2012) and assuming the existence of a rigid wall. The bulk solid considered was wheat, and it was simulated by using an elastoplastic model. The classic linear and isotropic hypothesis was considered to represent the elastic region of the model. Thus, only two mechanical parameters are needed to define completely this part: the Poisson's ratio, $v$, and the Modulus of Elasticity, E.

The Drucker and Prager (1952) perfect plasticity law was used to define the plastic yield criterion. The mechanical parameters of the bulk solid required by this criterion are the cohesion, $\mathrm{c}$, the angle of internal friction, $\phi$, and the angle of dilatancy, $\psi$. The value for the Poisson's ratio was obtained according to the lateral pressure ratio, K, defined in EN 1991-4 (2006) for wheat (Eq. 12) in order to compare the normal pressures deduced from Eurocode with the numerical results. 


$$
v=\frac{K}{1+K}
$$

The friction coefficient between wheat and the silo wall is the last mechanical parameter required to properly define the finite element models. The interaction of the bulk solid with the hopper wall was simulated by using the Coulomb frictional law (Eq. 13), where $\tau$ represents the tangential friction stress; $p$, is the normal pressure; and $\mu$ is the friction coefficient between the bulk solid and the hopper wall. It was assumed that there was not sliding cohesion resistance, c, between both materials. The "surface-to-surface" numerical procedure was used in ANSYS to reproduce the interaction between the bulk solid and the hopper wall.

$$
\tau=\mathrm{c}+\mu \cdot \mathrm{p}
$$

The values for the specific weight, the lateral pressure ratio, the Poisson's ratio and the friction coefficient between the bulk solid and the hopper wall (Table 2) were adopted from EN 1991-4 (2006) in order to allow the comparison of pressures between predictions made by European standard and those numerically obtained. The rest of the mechanical parameters needed to simulate the bulk solid were adopted from literature (Couto et al., 2013, Moya et al. 2002).

Table 2. Mechanical parameters considered for wheat.

\begin{tabular}{lc}
\hline \multicolumn{1}{c}{ Parameter } & Value \\
\hline Specific weight $\left(\gamma\right.$, in $\left.\mathrm{kN} / \mathrm{m}^{3}\right)$ & 9.0 \\
Modulus of elasticity $(\mathrm{E}$, in $\mathrm{kPa})$ & 10674 \\
Lateral pressure ratio $(\mathrm{K})$ & 0.54 \\
Poisson's ratio $(v)$ & 0.35 \\
Angle of internal friction $(\phi)$ & 30 \\
Cohesion $(\mathrm{c}$, in $\mathrm{kPa})$ & 2 \\
Dilatancy angle $(\psi)$ & 10 \\
Grain-to-wall friction coefficient $(\mu)$ & 0.38 \\
\hline
\end{tabular}

Commercial silos do not usually have independent hoppers, so cylindrical bins are placed above them. However, the numerical model developed only took into account the hopper geometry. Because of that, a vertical pressure was applied at top of hopper in order to simulate the effects produced by the material stored above the hopper. The value of vertical pressure adopted was $p_{\mathrm{vf}, \mathrm{t}}=65.8 \mathrm{kPa}$, which is the vertical stress in the stored solid at the transition after filling and for an infinite height of the silo bin, so that the results obtained for the hopper would not be affected by the bin height.

\section{Results and Discussion}

Influence of outlet eccentricity

Figure 4 shows the normal pressures obtained for one of the hopper geometries analysed as an example, where a ratio $\mathrm{R} / \mathrm{h}_{\mathrm{h}}=0.58$ was considered and for two different outlet eccentricities: $0 \%$ (corresponding to the concentric hopper) and 50\%. In addition, normal pressures calculated according to provisions of EN 1991-4 (2006) for the corresponding concentric hopper case were also plotted. It is interesting to note that predictions of FE model agreed quite well with formulation of Eurocode, as it can be checked at left image of Figure 4. The numerical model predicts lower normal pressures at top of the hopper, while slightly greater normal pressures are predicted at mid height of hopper. Values of normal pressures are quite similar in the wall hopper close to outlet. 


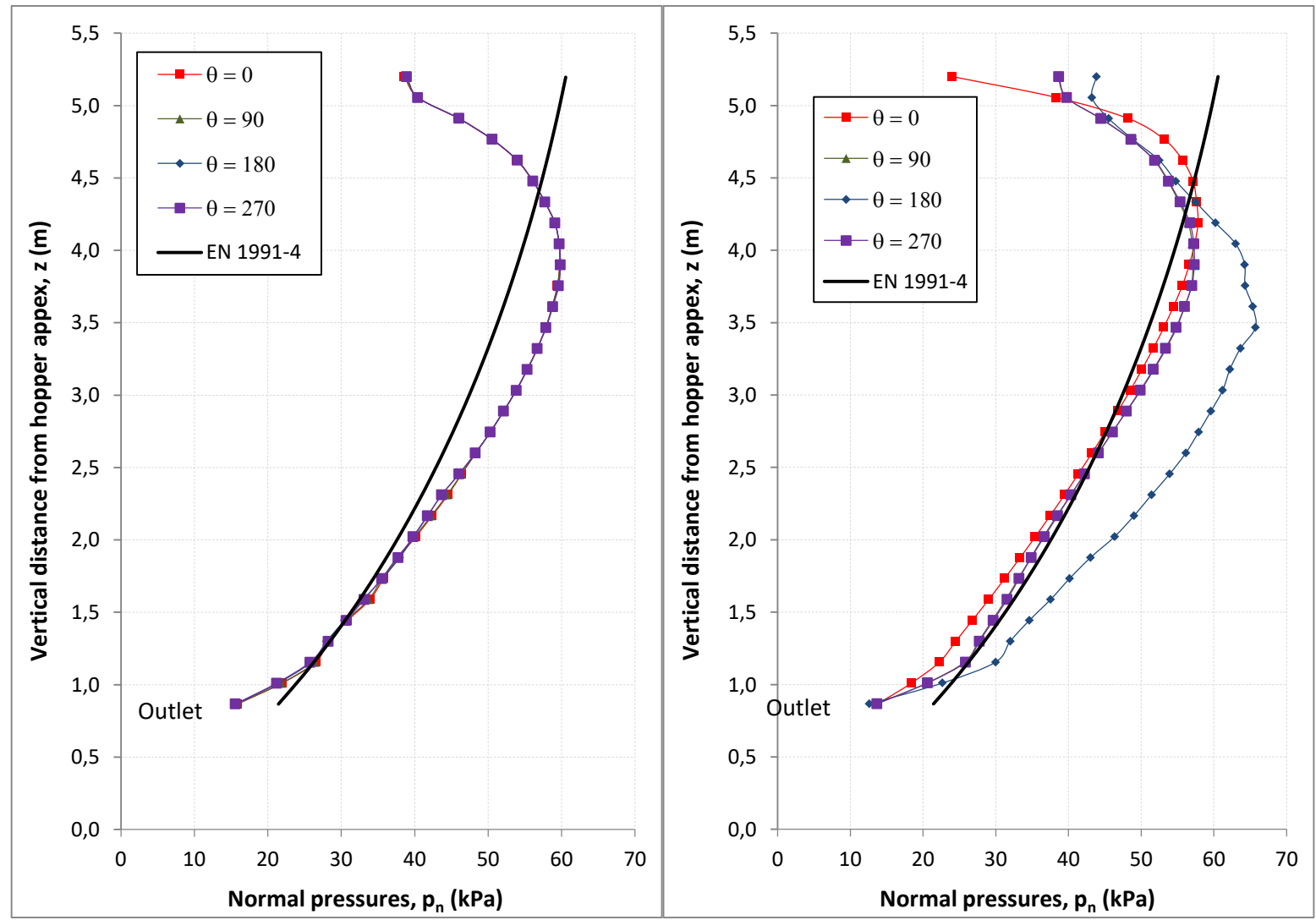

Figure 4. Wall normal pressures obtained for a hopper with $\mathrm{R} / \mathrm{h}_{\mathrm{h}}=0.58$ at different circumferential positions, $\theta$, and for outlet eccentricities of $0 \%$ (left image) and $50 \%$ (right image).

If the numerical results corresponding to the $50 \%$ outlet eccentricity are compared with the concentric case ( $0 \%$ of outlet eccentricity), then it can be checked a decrease of normal pressures for the wall closer to the outlet $\left(\theta=0^{\circ}\right)$, while an increase in pressures is obtained for the wall opposite to the outlet $\left(\theta=180^{\circ}\right)$. This tendency is reported for other eccentricities and agrees with the observations reported by Couto et al. (2003). In addition, it can be checked that normal pressures tend to be similar to the concentric hopper case for circumferential positions located at mid distance $\left(\theta=90^{\circ}\right.$ and $\left.\theta=270^{\circ}\right)$ from these extreme values. Figure 5 shows a 3D representation of normal pressures obtained for one of the hoppers studied, where it can be clearly seen that overpressures tend to concentrate close to the wall opposite to outlet and at mid height of hopper.

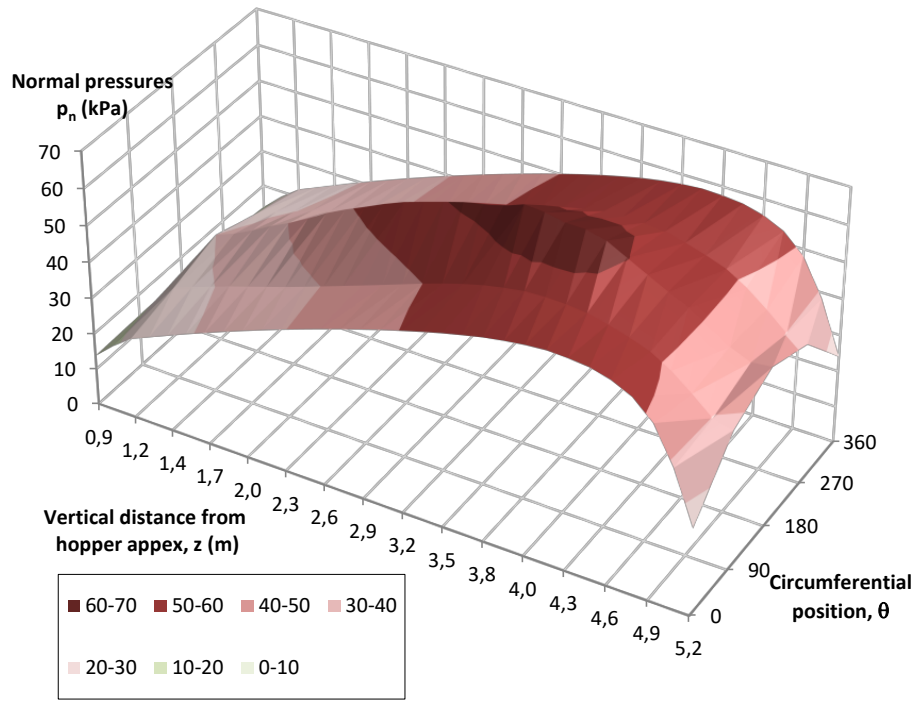

Figure 5. Wall normal pressures obtained for a hopper with $\mathrm{R} / \mathrm{h}_{\mathrm{h}}=0.58$, an outlet eccentricity of $50 \%$ and as a function of circumferential position, $\theta$. 
Influence of aspect ratio $\mathrm{R} / \mathrm{h}_{\mathrm{h}}$

One of the most relevant factors that can modify the normal pressures exerted by the bulk solid on the oblique hopper is the aspect ratio, which can be represented by ratio $\left(\mathrm{R} / \mathrm{h}_{h}\right)$, as defined in the introduction section. Several values of $(\mathrm{R} / \mathrm{h} h)$ were considered, thus different hopper heights would appear for each case (Table 1). Therefore, a dimensionless height, Z, was used (Eq. 14) to facilitate the comparison of pressures between oblique hoppers with different heights.

$$
\bar{Z}=\frac{z}{h_{h}}
$$

For the same reason, it was also calculated a dimensionless pressure (Eq. 15) for each analysed model by dividing the numerical normal pressure obtained by the value calculated according to EN 1991-4 (2006) for the concentric case of the hopper geometry studied.

$$
\bar{p}=\frac{p_{n}}{p_{n, E N}}
$$

Figure 6 shows the influence of aspect ratio $\left(R / h_{h}\right)$ at the wall opposite to the outlet $(\theta=180)$ and by comparing two different eccentricities $(0 \%$ and $50 \%)$. For the concentric hopper, it can be observed that dimensionless pressure is slightly greater than 1.0 for the hopper dimensionless height interval $0.4-0.8$ approximately. The dimensionless height interval with dimensionless pressures greater than 1.0 is larger for hoppers with $50 \%$ outlet eccentricity $(0.25-0.85$ approximately).

The peak dimensionless pressure is around 1.1 for the concentric hopper $(\mathrm{k}=0)$, and close to 1.30 for an outlet eccentricity of $50 \%(\mathrm{k}=0.5)$. For both cases, the maximum dimensionless pressures tend to correspond to the hopper aspect ratio $\mathrm{R} / \mathrm{h}_{\mathrm{h}}=0.58$, and for most of the hopper dimensionless heights. It is interesting to note that there is not an evident relationship between the hopper aspect ratio and its effect on the dimensionless pressures.

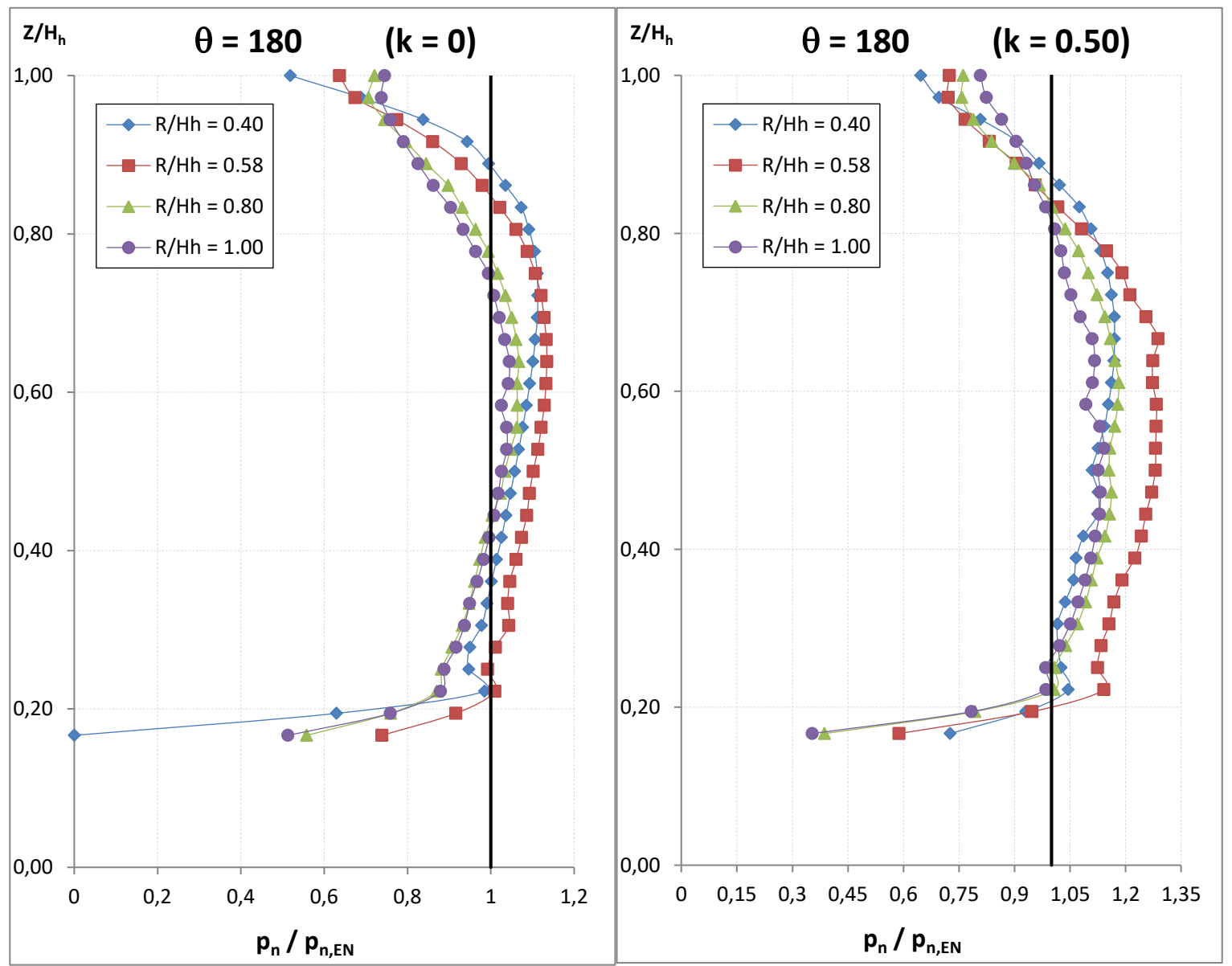

Figure 6. Dimensionless wall normal pressures obtained at circumferential line $\left(\theta=180^{\circ}\right)$ for different hopper aspect ratios $\left(\mathrm{R} / \mathrm{h}_{\mathrm{h}}\right)$, and for outlet eccentricities of $0 \%$ (left image) and $50 \%$ (right image). 
Influence of coefficient of friction $\mu_{\mathrm{h}}$

It was also studied the possible effect of wall friction coefficient on hopper pressures (Fig. 7). FE analyses were made by considering three different wall friction coefficients: $0.25,0.38$ and 0.57 , that correspond to roughness wall types D1, D2 and D3 for wheat, as specified in Annex E of EN 1991-4 (2006). A similar tendency is observed for this analysis that the one observed previously. No clear relationship can be found between the value of the wall friction coefficient and the dimensionless normal pressure. The peak dimensionless normal pressures are again around 1.1 and 1.3 for hopper eccentricities of $0 \%$ and $50 \%$ respectively.

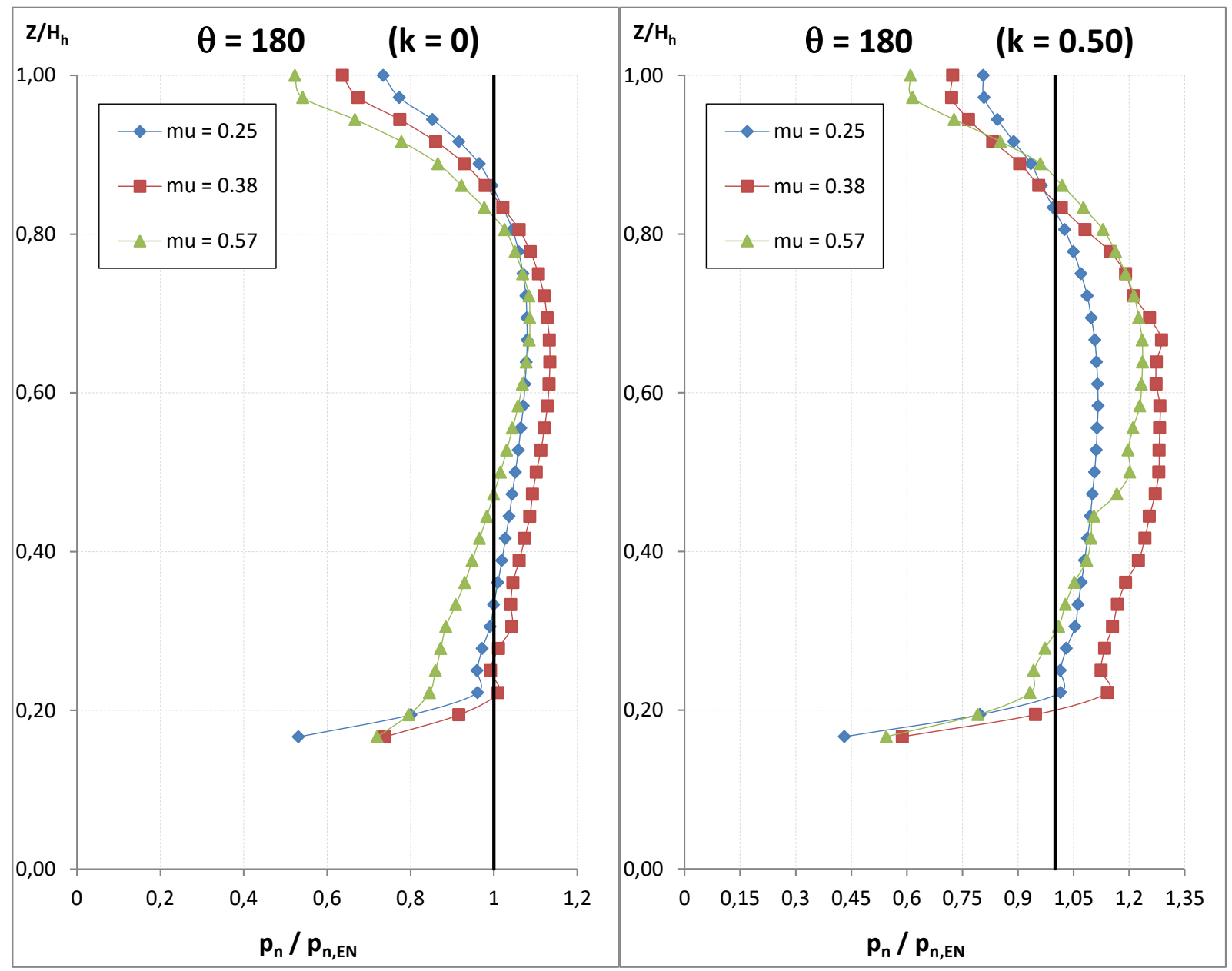

Figure 7. Dimensionless wall normal pressures obtained at circumferential line $\left(\theta=180^{\circ}\right)$ for different wall friction coefficients $\left(\mu_{\mathrm{h}}\right)$, and for outlet eccentricities of $0 \%$ (left image) and $50 \%$ (right image).

\section{Conclusions}

The results obtained with the FE model developed provide the following main conclusions:

- The existence of outlet eccentricity produces in oblique hoppers a decrease of normal pressures over the wall closer to the outlet, and an increase of pressures on the opposite wall.

- If the outlet eccentricity increases, the overpressure over the hopper wall opposite to the outlet is greater.

- No clear effects of hopper aspect ratio $\left(\mathrm{R} / \mathrm{h}_{\mathrm{h}}\right)$ and wall friction coefficient $\left(\mu_{\mathrm{h}}\right)$ have been found over dimensionless normal pressures.

- In all studied cases the pressure values do not exceed more than 1.3 times the values proposed by the Eurocode for a symmetrical hooper. 


\section{References}

ANSYS, 2012. ANSYS User's Manual. Version 13.0. Houston: Swanson Analysis Systems Inc.

Ayuga, F., M. Guaita, P.J. Aguado, A. Couto, 2001. Discharge and the eccentricity of the hopper influence on the silo wall pressures. ASCE Journal of Engineering Mechanics. 127(10), 1067-1074. https://doi.org/10.1061/(ASCE)0733-9399(2001)127:10(1067).

Couto, A., A. Ruiz, P. J. Aguado, 2013. Experimental study of the pressures exerted by wheat stored in slender cylindrical silos, varying the flow rate of material during discharge. Comparison with Eurocode 1. Part4. Powder Technology 237, 450-467. https://doi.org/10.1016/j.powtec.2012.12.030

Dąbrowski, A., 1957. Parcie Materiałów Sypkich w Leju. (In Polish: Pressures in bulk solids in hoppers). Archiwum Inżynierii Lądowej, Z.3, 325-328.

Drucker, D.C., W. Prager, 1952. Soil Mechanics and Plastic Analysis or Limit Design. Quart. Appl. Math. 10, 2, 157-165.

EN 1991-4, 2006. Eurocode 1 - Actions on structures. Part 4: Silos and tanks. European Committee for Standardization, Brussels.

Guaita, M., A. Couto, F. Ayuga, 2003. Numerical Simulation of Wall Pressure during Discharge of Granular Material from Cylindrical Silos with Eccentric Hoppers. Biosystems Engineering. 85 (1), 101-109. doi:10.1016/S1537-5110(03)00037-0.

Matchett, A.J., J. O'Neill, A.P. Shaw, 2009. Stresses in bulk solids in wedge hoppers: A flexible formulation of the co-ordinate specific, Lame-Maxwell equations for circular arc, principal stress systems. Powder Technology. 194, 166-180. https://doi.org/10.1016/j.powtec.2009.04.0.

Michalowski, R. L., 1983. Approximate Theory of Loads in Plane Asymmetrical Converging Hoppers. Powder Technology. 36, 5 - 11. https://doi.org/10.1016/0032-5910(83)80002-3

Moya, M.; Ayuga, F.; Guaita, M.; Aguado, P. (2002). Mechanical Properties of Granular Agricultural Materials. Transactions of the ASAE. 45 (5), 1569-1577. https://doi.org/10.13031/2013.11062

Ooi, J.Y., L. Pham, J.M. Rotter, 1990. Systematic and random features of measured pressures on full-scale silo walls. Engineering Structures. 12, 74-87. https://doi.org/10.1016/0141-0296(90)90012-H

Rotter, J.M., 2007. Silo and hopper design for strength. (Chapter 4 in Bulk Solids Handling Equipment Selection and Operation). Blackwell, Oxford, UK: D. McGlinchey.

Walker, D.M., 1966. An Approximate Theory for Pressure and Arching in Hoppers. Chemical Engineering Science. 21, 957-997. https://doi.org/10.1016/0009-2509(66)85095-9 


\title{
Hyperspectral Imaging for Foal Meat Classification Based on Ageing Time
}

\author{
A. López-Maestresalas ${ }^{a}$, K. Insausti ${ }^{b}$, C. López-Molina ${ }^{c}$, C. Pérez-Roncal ${ }^{a}$, V. Sarriés ${ }^{b}$, C. Jaren ${ }^{a}$ and \\ S. Arazuria \\ ${ }^{\text {a }}$ Department of Engineering, Public University of Navarre, Pamplona, 31006, Spain \\ ${ }^{b}$ Department of Agricultural Engineering, Biotechnology and Food, Public University of Navarre, Pamplona, 31006 , \\ Spain \\ ${ }^{\mathrm{c}}$ Department of Statistics, Computer Science and Mathematics, Public University of Navarre, Pamplona, 31006, Spain \\ * Corresponding author. Email: ainara.lopez@unavarra.es
}

\begin{abstract}
Assessment of meat quality parameters is a major concern in the food industry due to the continuously demand for superior quality of meat. Therefore, it is crucial to assess meat quality parameters correctly and also, do it using sustainable and efficient methods. In this respect, hyperspectral imaging (HSI) technique has been regarded as a smart and promising tool for analyses conducted in research and industries. It integrates both spectroscopy and imaging to directly identify different components and their spatial distribution in the tested product. Thus, a quality evaluation based on HSI would be beneficial to the meat industry. In this study we evaluated the usefulness of HSI to classify foal meat steaks based on ageing time, an aspect that greatly influences meat tenderness. The dataset used comprised 88 foal steaks from twenty-four hours post-mortem Triceps Brachii muscles. Samples were aged $0,4,8$ and 12 days at $4 \pm 1^{\circ} \mathrm{C}$ and then frozen at $-18 \pm 2^{\circ} \mathrm{C}$ until analyses. Steaks were scanned using a hyperspectral camera (Xenics, Xeva-1.7-320) with a 320x256 pixel resolution and a spectrograph (ImSpector N17E, Specim) in the 900-1700 nm spectral range. A Partial Least Squares-Discriminant Analysis (PLS-DA) method was used to classify steaks in 3 classes according to their ageing time (high, medium and low quality). High class was composed of steaks aged 0 and 4 days, medium class comprised steaks aged 8 days and, low class included the rest (steaks aged 12 days). Good classification results were obtained for high and low quality classes (63.6\% and $75 \%$, respectively) in the prediction sets and a low percentage of correctly classified samples was achieved for the medium quality class. Such misclassification for the latter was expected as this was a mixed quality class in which some steaks still maintained an acceptable quality for consumption while others may had lost it.
\end{abstract}

Keywords: HSI, discriminant analysis, chemometrics, quality, Triceps Brachii.

\section{Introduction}

During the last decades, a number of methods have been developed to objectively measure meat quality attributes (Elmasry et al., 2012). Hyperspectral imaging (HSI) technique has been regarded as a smart and promising analytical tool for analyses conducted in research and industries. The main inducement for developing this imaging system is to integrate both spectroscopy and imaging techniques in one method to make direct identification of different components and their spatial distribution in the tested product. By combining spatial and spectral details together, hyperspectral imaging has proved to be a promising technology for objective meat quality evaluation. As reported by those authors, in addition to its ability for effectively quantifying and characterizing quality attributes of some important visual features of meat such as colour, quality grade, marbling, maturity, and texture, it is able to measure multiple chemical constituents simultaneously without monotonous sample preparation. Although this technology has not yet been sufficiently exploited in meat process and quality assessment, its potential is promising. Developing a quality evaluation system based on HSI technology to assess the meat quality parameters and to ensure its authentication would bring economic benefits to the meat industry by increasing consumer confidence in the quality of meat products.

Assessment of meat quality parameters has always been a major concern in all processes of the food industry due to the continuously demand for superior quality of meat and meat products by consumers. In order to meet consumers' requirements, it has become crucial for the meat industry to correctly assess meat quality parameters by improving current techniques for meat and meat products control (Herrero, 2008).

One of the main challenges for the meat industry is the great variability in raw meat and thus, the difficulty to provide the market with tender meat, a parameter directly related to ageing, among other factors. For all these reasons, the main purpose of this preliminary research was to evaluate the usefulness of HSI to classify foal meat based on ageing time. This technology has been previously applied to other types of meats but it has never been applied to foal meat to the best of our knowledge. 


\section{Materials and Methods}

Eleven foals obtained by crossing Galician Mountain mares with a Burguete stallion, were used in this study. The animals were reared under extensive conditions and slaughtered at 13 months. Prior to slaughter, all the foals were supplemented on pasture with a linseed-rich concentrate (5\%) (2 kg per foal and day) for a fattening period of about 104 days.

The foals were then transported $50 \mathrm{~km}$ to the abattoir the day before slaughter in compliance with the current European Community laws of Animal Welfare in transport. They were stunned with a captive bolt, slaughtered and dressed according to the specifications outlined in the European legislation (Council Directive 93/119/EC, 1993).

Twenty-four hours post-mortem, Triceps Brachii muscles were removed from the left half-carcasses. They were sliced into $20 \mathrm{~mm}( \pm 0.2)$ thick steaks. Meat samples were aged $0,4,8$ and 12 days at $4 \pm 1^{\circ} \mathrm{C}$ and then frozen at $-18^{\circ} \mathrm{C}( \pm 2)$ until analyses. Before analysis, samples were thawed at $4^{\circ} \mathrm{C}$ for 24 hours. All measurements were carried out in duplicate. Therefore, 88 foal steaks from Triceps Brachii were scanned using a setup for hyperspectral imaging consisting of: a transportation plate to move the sample, an illumination unit, a hyperspectral camera and a computer equipped with the software Xeneth 2.5. The NIR hyperspectral camera used is an Indium gallium arsenide (InGaAs) CCD camera with $320 \times 256$ pixels (Xenics, Model Xeva-1.7-320, Leuven, Belgium) coupled to a spectrograph (ImSpector N17E, Specim, Spectral Imaging Ltd., Oulu, Finland) both sensitive from 900 to $1700 \mathrm{~nm}$, and to a lens (Xenics, Model OPT-000107, SWIR $16 \mathrm{~mm} \mathrm{f/1.4,} \mathrm{Leuven,}$ Belgium) with a focal length of $16 \mathrm{~mm}$.

This image acquisition system has a line scanning or pushbroom configuration which acquires a whole line of an image each time requiring either to move the camera along the sample or to move the latter under the field of view of the camera. This system works in reflectance mode acquiring a 2-dimensional image at each line in which one dimension represents the spectral axis $(\lambda)$ and the other characterises one spatial dimension $(\mathrm{x})$ of the line scanned composed of 320 pixels. By moving the sample under the scanning area, the third spatial dimension is achieved (y) and after that, all 2-dimensional images are stacked up to obtain a hypercube of the sample $(y, x, \lambda)$ (Fig 1).

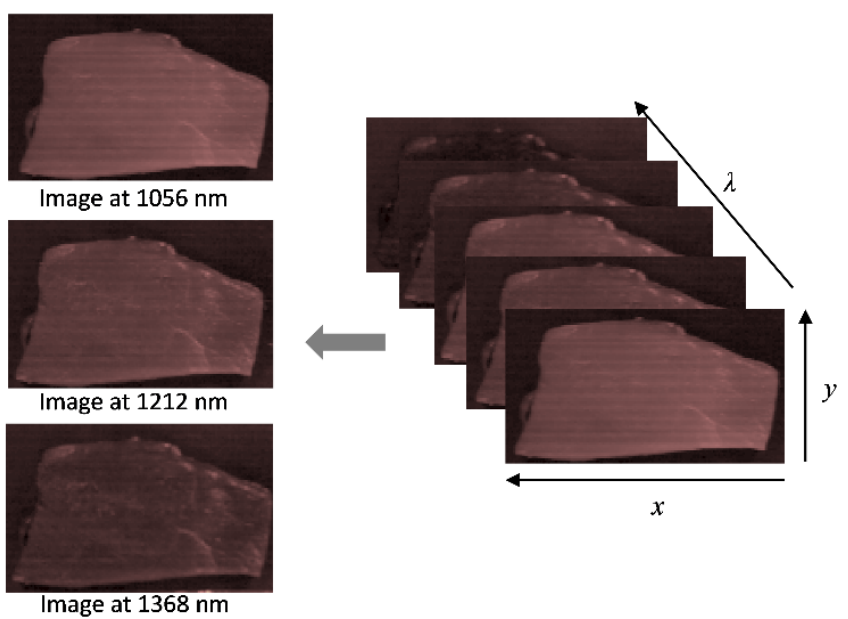

Figure 1. Hypercube of a foal steak. On the left examples of images at different wavelengths.

Image segmentation to eliminate the background and fat areas was performed following the algorithm presented in (Lopez-Molina et al., 2017) which code is available as part of the Kermit ToolKit (Kitt, 2017) Once images were segmented, a region of interest (ROI) consisting of the whole steak was selectedand the mean spectrum was used for further analysis.

Image processing and chemometric analysis of data were performed in MATLAB R2014a (The MathWorks, Natick, MA) and in the PLS_Toolbox (Eigenvector Research Inc., Wenatchee, WA), respectively. A Partial Least Squares-Discriminant Analysis (PLS-DA) method was used to classify steaks in 3 quality classes according to their ageing time (high, medium and low quality). High class was composed of steaks aged 0 and 4 days, medium class comprised steaks aged 8 days and, low class included the rest (steaks aged 12 days). PLS-DA is a supervised classification method that links the spectral information with the sample ageing time. In this study, $70 \%$ of samples $($ ncal $=62)$ randomly of each Class were used for Calibration $(\mathrm{Cal})$ and $30 \%$ (npred $=26)$ for ageing Prediction (Pred). Venetian Blinds cross-validation (CV) method with 10 data splits was applied.

PLS-DA model was evaluated in terms of the sensitivity and specificity and the percentage of Correctly Classified (\%CC) samples of the $\mathrm{CV}$ and Pred set of samples. 


\section{Results and Discussion}

The mean spectrum of each class is shown in Figure 2. Reflectance data from 900 to $1000 \mathrm{~nm}$ were deleted from the study due to noise. Mean spectrum of the high quality class showed the lowest reflectance up to 1200 $\mathrm{nm}$ while the mean spectrum of medium quality class showed the highest reflectance along the spectral range measured.

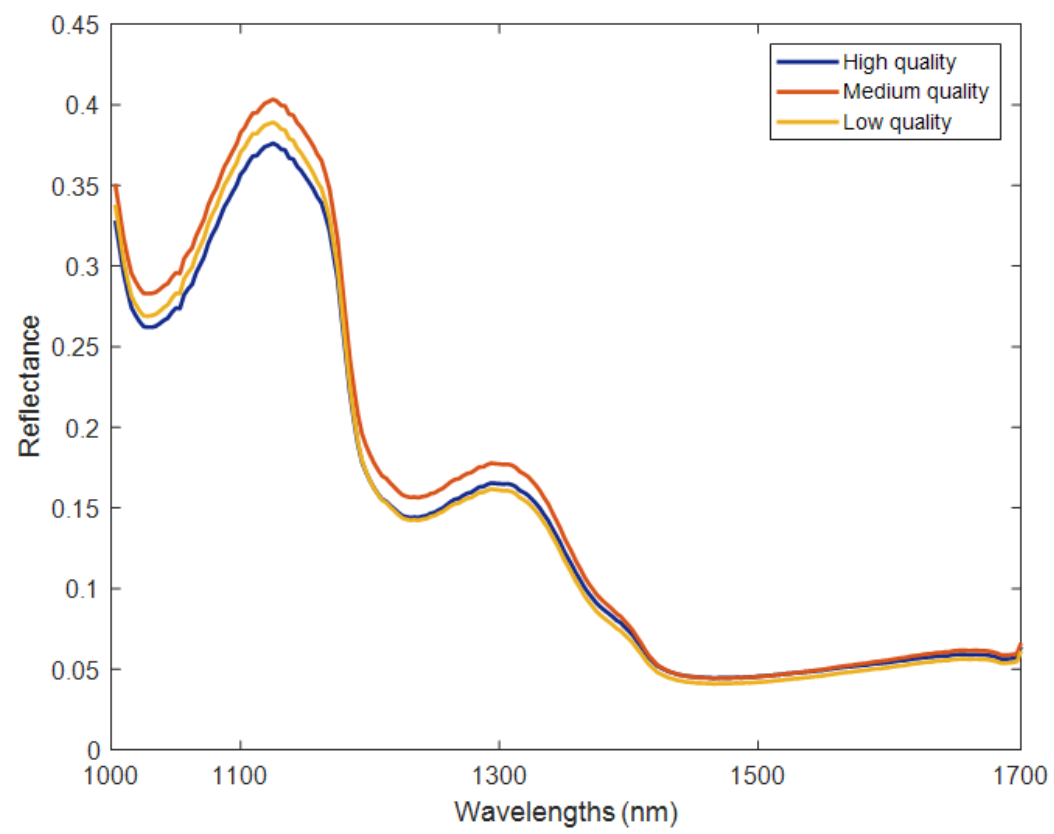

Figure 2. Mean spectrum of each quality class.

Table 1 shows the results of the PLS-DA carried out in this study. A total of 5 Latent Variables (LVs) were used to build the classification model explaining $98.90 \%$ of the spectral variance. Different pre-processing methods were accomplished and the best result was obtained when applying SNV followed by first derivative calculated by Savitzky-Golay method using a second order polynomial and 15 window points and followed by mean center. Three samples belonging to the 0 days Class were identified as outliers and removed from the model.

The $\% \mathrm{CC}$ samples ranged from 60 to $75 \%$ in prediction sets. Best classification results were obtained for the high and low quality Classes in terms of higher \%CC. In overall, good classification results were obtained for the three Classes which indicate the potential of HSI technique to classify foal steaks based on ageing time.

Table 1. PLS-DA model results for the cross-validation and prediction set of samples. Values in bold correspond to the best classification results.

\begin{tabular}{|c|c|c|c|c|c|c|c|c|c|c|c|}
\hline & \multirow{2}{*}{ Class } & \multirow{2}{*}{ Pre-process } & \multirow{2}{*}{$\mathrm{LV}^{-}$} & \multicolumn{4}{|c|}{ Cross-Validation } & \multicolumn{4}{|c|}{ Prediction } \\
\hline & & & & $n$ & sens & spec & $\% \mathrm{CC}$ & $\mathrm{n}$ & sens & spec & $\% \mathrm{CC}$ \\
\hline \multirow{3}{*}{ ஸ் } & High & $\mathrm{SNV}+1^{\text {st }}$ & & & 0.607 & 0.897 & 67.86 & & 0.603 & 0.889 & 63.64 \\
\hline & Medium & Der + Mean & 6 & 62 & 0.600 & 0.857 & 60 & 26 & 0.600 & 0.857 & 60 \\
\hline & Low & center & & & 0.929 & 0.721 & 85.71 & & 0.750 & 0.600 & 75 \\
\hline
\end{tabular}

Although no literature was found regarding foal quality estimation using HSI techniques, different attempts on using such technique as a non-destructive method for beef quality control have been investigated by several authors. Recent studies regarding the assessment of beef tenderness using hyperspectral imaging have shown encouraging results. For instance, in a collaborative study, Naganathan et al. (2008) and Grimes et al. $(2007,2008)$ developed a pushbroom hyperspectral imaging system in the visible and near infrared range of 400-1000 nm with a diffuse-flood lighting system to classify samples based on tenderness of 14-day post-mortem cooked beef from hyperspectral images of fresh beef-ribeye steaks (M. longissimus dorsi) between the 12th and 13th ribs $(\mathrm{n}=111)$. After image acquisition, samples were cooked and SSF values were obtained as tenderness reference. Three beef tenderness categories were established: tender, intermediate and tough and by using a canonical discriminant model a classification accuracy of $96.4 \%$ was achieved.

Other authors have focused on identification and authentication of meat by HSI. Thus, in a study developed by Kamruzzaman et al. (2011) the potential of a NIR HSI system in the 900-1700 nm range was evaluated for 
discriminating three types of lamb muscles: Semitendinosus, Longissimus Dorsi and Psoas Major of Charollais breed. A linear discriminant analysis was carried out for muscles classification reporting an overall accuracy of $100 \%$.

\section{Conclusions}

Promising results were obtained in this study for the discrimination of foal steaks based on their ageing time, using HSI techniques coupled with PLS-DA classification methods. More accurate results were obtained for the high and low quality Classes with correct classification rates above $63 \%$ in prediction sets.

Keeping in mind this is a preliminary study which should be further validated on a larger set of samples and using different chemometric approaches, it seems that NIR hyperspectral imaging systems have potential for quality control of foal meat.

\section{References}

Elmasry, G., Barbin, D. F., Sun, D.-W., \& Allen, P. (2012). Meat Quality Evaluation by Hyperspectral Imaging Technique: An Overview. Critical Reviews in Food Science and Nutrition, 52(August 2012), 689-711. https://doi.org/10.1080/10408398.2010.507908

Grimes, L. M., Naganathan, G. K., Subbiah, J., \& Calkins, C. R. (2007). Hyperspectral imaging: A noninvasive technique to predict beef tenderness. Nebraska Beef Cattle Reports, 97-99.

Grimes, L. M., Naganathan, G. K., Subbiah, J., \& Calkins, C. R. (2008). Predicting aged beef tenderness with a hyperspectral imaging system. Nebraska Beef Cattle Reports, 138-139.

Herrero, A. M. (2008). Raman spectroscopy a promising technique for quality assessment of meat and fish : A review. Food Chemistry, 107, 1642-1651. https://doi.org/10.1016/j.foodchem.2007.10.014

Kamruzzaman, M., Elmasry, G., Sun, D. W., \& Allen, P. (2011). Application of NIR hyperspectral imaging for discrimination of lamb muscles. Journal of Food Engineering, 104(3), 332-340. https://doi.org/10.1016/j.jfoodeng.2010.12.024

Lopez-Molina, C., Ayala-Martini, D., Lopez-Maestresalas, A., \& Bustince, H. (2017). Baddeley's Delta metric for local contrast computation in hyperspectral imagery. Progress in Artificial Intelligence, 6(2), 121-132. https://doi.org/10.1007/s13748-017-0111-y

Naganathan, G. K., Grimes, L. M., Subbiah, J., Calkins, C. R., Samal, A., \& Meyer, G. E. (2008). Visible/nearinfrared hyperspectral imaging for beef tenderness prediction. Computers and Electronics in Agriculture, 64(2), $225-233$.

University, G. (2017). Kermit Image Toolkit (Kitt). 


\title{
Mandarin Mold Inactivation of Storage at Ambient Temperature by Non-thermal Plasma Treatment
}

\author{
Youngwook Seo a, ${ }^{\text {, }}$, Hoeman Park ${ }^{\text {a }}$ Jong Ryul Park ${ }^{\text {a }}$ \\ ${ }^{a}$ Department of Agricultural Engineering, National Institute of Agricultural Sciences, RDA \\ (54875) 310 Nongsaengmyeong-ro, Wansan-gu, Jeonju, Korea \\ *Corresponding author. Email: yws25@korea.kr
}

\begin{abstract}
Inactivation efficiency of mandarin mold was evaluated at room temperature storage using cold plasma based on dielectric barrier discharge (DBD) technology. Tangerine variety (Citrus unshiu) samples were purchased from an orchard in Jeju, Korea. Samples were divided from treatment $(\mathrm{N}=240)$ and control $(\mathrm{N}=240)$ group. The plasma treatment was carried out by the non-thermal plasma actuator with time control condition as $15 \mathrm{sec} \mathrm{ON}, 15 \mathrm{~min}$ OFF and measured inactivation gas concentration with ozone $(\mathrm{O} 3)$ and nitrogen dioxide $(\mathrm{NO} 2)$ detector. In this study, two non-destructive and destructive practices were derived. Samples were stored for 5 weeks and physical properties such as weight, colour, hardness and sugar contents were measured with 10 samples in each group. Non-destructive inspection (weight and colour) was carried out with 60 samples. As a result, weight and hardness of fruit decreased with time in both groups, but colour and sugar contents change was insufficient to evaluate.
\end{abstract}

Keywords: Mandarin mold, Inactivation, Non-thermal plasma, Room temperature storage

\section{Introduction}

Mandarin (Mandarin reticulata) is one of the representative fruits and a major variety of Jeju island, Korea. Total consumption of fruits in one year is $66.7 \mathrm{~kg}$ per person, and mandarin is top with $13.2 \mathrm{~kg}$ per person as $19.7 \%$ (2016, Agricultural Statistics). Harvesting period is from late November to early December, but in winter it is able to breed in greenhouse. Harvested mandarin is processed and packaged in an agricultural processing centre (APC) according to washing, waxing, sorting and boxing processes. In the storage, unexpected conditions such as molds, rots, and quality degradation may occur. In this study, cold plasma was evaluated as a dry sterilization technique to prolong mandarin's quality during storage at room temperature. Usually, farmers harvest mandarins during November to December and store them at ambient temperature for $1-5$ weeks. In this study, cold plasma based on dielectric barrier discharge (DBD) method was used as a plasma actuator and evaluated to inhibit molds on mandarin peels during storage at room temperature and cold storage for comparison. Physical changes such as weight, colour, hardness, and sugar contents were measured for 5 weeks. Control (untreated) group were compared in physical properties with plasma treatment group.

\section{Materials and Methods}

Samples and storage circumstances

Mandarin samples were purchased on the market. As soon as unbox, dehydrated samples at ambient temperature. Degraded samples such as spoilage, scratches and injuries were removed. Samples were divided into 2 groups as treatment and control. Thirty samples from each group were randomly selected and weight and color were measured over 5 weeks. Ten samples from each group by every week which were measured physical properties. Samples of treatment $(\mathrm{N}=240)$ and control $(\mathrm{N}=240)$ were stacked on each plastic carrier $(725 \times 470 \mathrm{x}$ $330 \mathrm{~mm}$ ). Cold plasma actuator based on DBD technique controlled $15 \mathrm{sec} \mathrm{ON}$ and $15 \mathrm{~min}$ OFF for ozone range of $0.1-0.3 \mathrm{ppm}$. Gases from cold plasma actuator were measured using gas monitors for ozone (106-L, 2B Technologies, USA) and nitrogen dioxide (S 500, Aeroqual Ltd., NZ). Temperature and humidity was measured at the ambient temperature and humidity of both storage circumstances using temp \& humidity logger (TR-72nw, T\&D Co., Japan). Figure 1 shows a schematic diagram of DBD cold plasma sterilization with sensors and a diaphragm pump and outlook of DBD generator. 


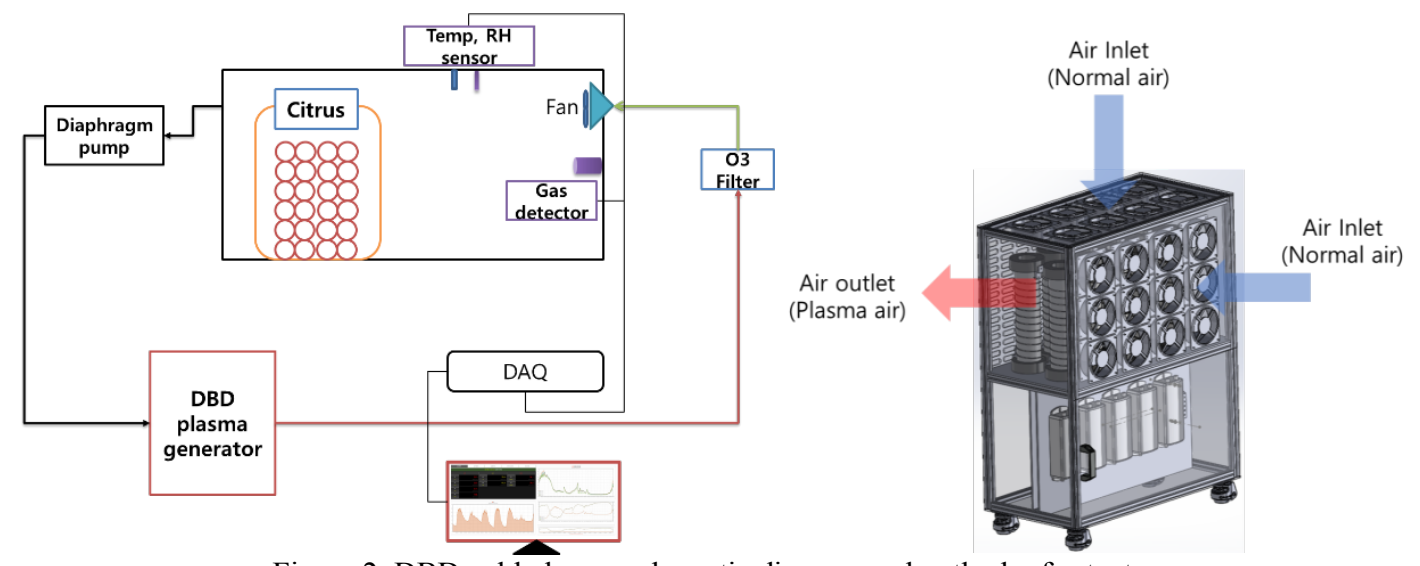

Figure 2. DBD cold plasma schematic diagram and outlook of actuator.

Data acquisition of physical properties

Weight measured by electronic balance (CUW-H, CAS Ltd., Korea) every sample by every week. L*a*b* of selected samples was measured by colorimeter (CM-400, Konica Minolta Ltd., Japan). White calibration was carried out using white plate before measurement. Hardness of flesh was measured 3 spots of the peeled samples using a universal testing machine (AG-X plus, Shimadzu, Japan) with $3 \mathrm{~mm}$ compress rod. Sugar contents were measured using automatic refractometer (Smart-1, Atago Co., LTD., Japan) and averaged of 3 spots per sample. Data visualization and analysis was carried out using R (Ver. 3.3.2, R core team, Austria). We evaluated cold plasma sterilization with inactivation of mold using PDA inoculation for 5 days and more. Mold number was counted with OpenCFU (Geissmann, 2013).

\section{Results and Discussion}

Figure 2 is a box plot of initial weight of total samples $(\mathrm{N}=480)$. Samples for treatment $(\mathrm{N}=240)$ is $89.4 \pm 8.9 \mathrm{~g}$ and samples for control $(\mathrm{N}=240)$ is $86.8 \pm 8.3 \mathrm{~g}$. In order to observe the changes of physical properties during storage, the weight and colour of 30 samples from each group were measured for 5 weeks.

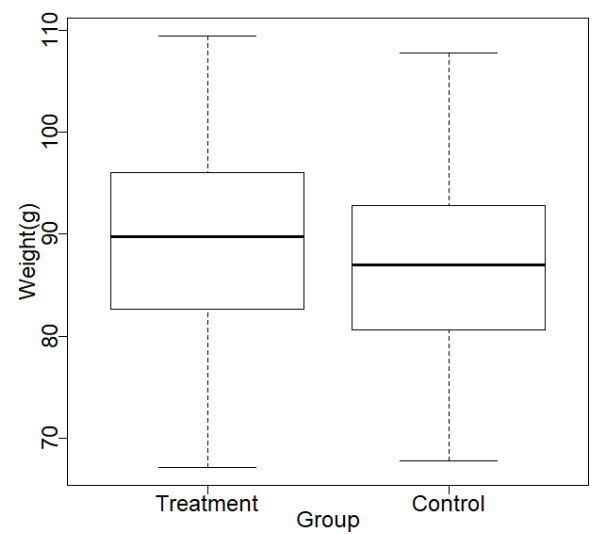

Figure 3. Initial weight of both groups. 


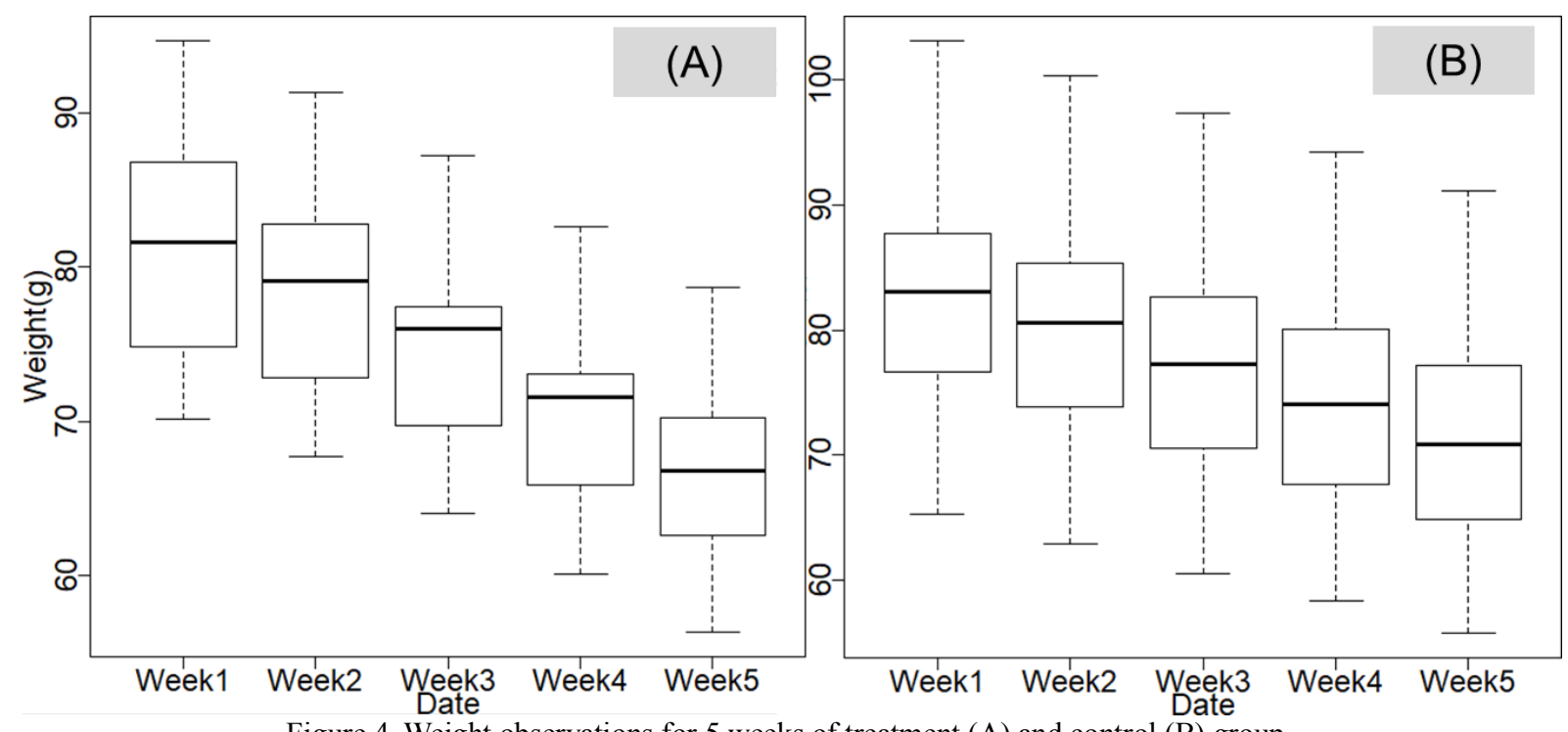

Figure 4. Weight observations for 5 weeks of treatment (A) and control (B) group.

Figure 3 shows that the time series weight changes of 30 samples in treatment and control group for 5 weeks. In order to measure the effect of cold plasma treatment for weight change, we analyzed one factor repeated measures using analysis of variance (ANOVA) and its p-value was 0.3032 (Kuznetsova et al., 2015). That means the treatment is not a cause of weight loss during storage. Color changes also measured and analyzed. $\mathrm{L}^{*}$ has $\mathrm{p}$ value $=0.2138$, $\mathrm{a}^{*}$ has 0.3854 and $\mathrm{b}^{*}$ has 0.2060 . This result obtain that cold plasma treatment for 5 weeks is not a color change cause. In order to evaluate the effect of the treatment to mandarin quality, we measured sugar contents (soluble solid contents, SSC) and flesh hardness.
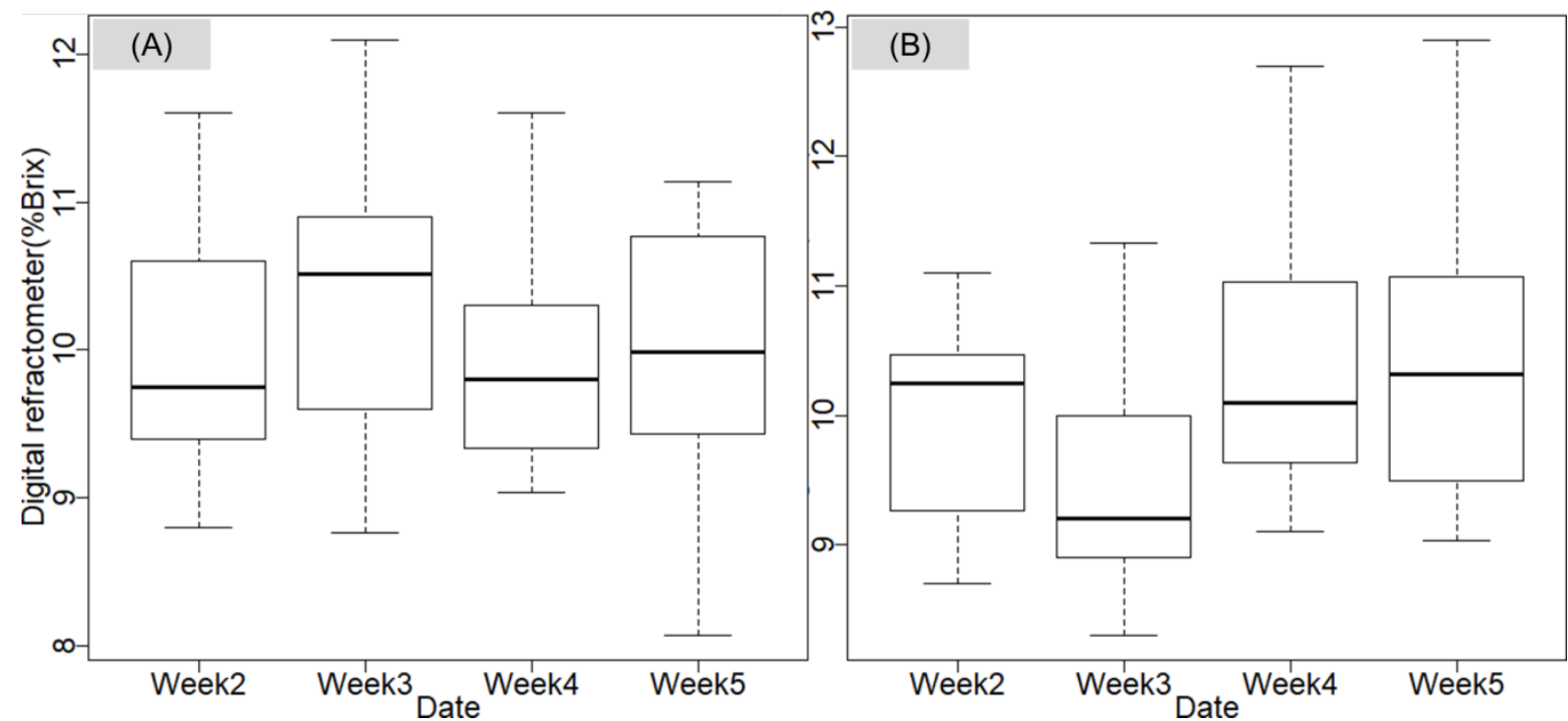

Figure 5. Soluble solid contents using digital refractometer for 5 weeks of treatment (A) and control (B) group. 


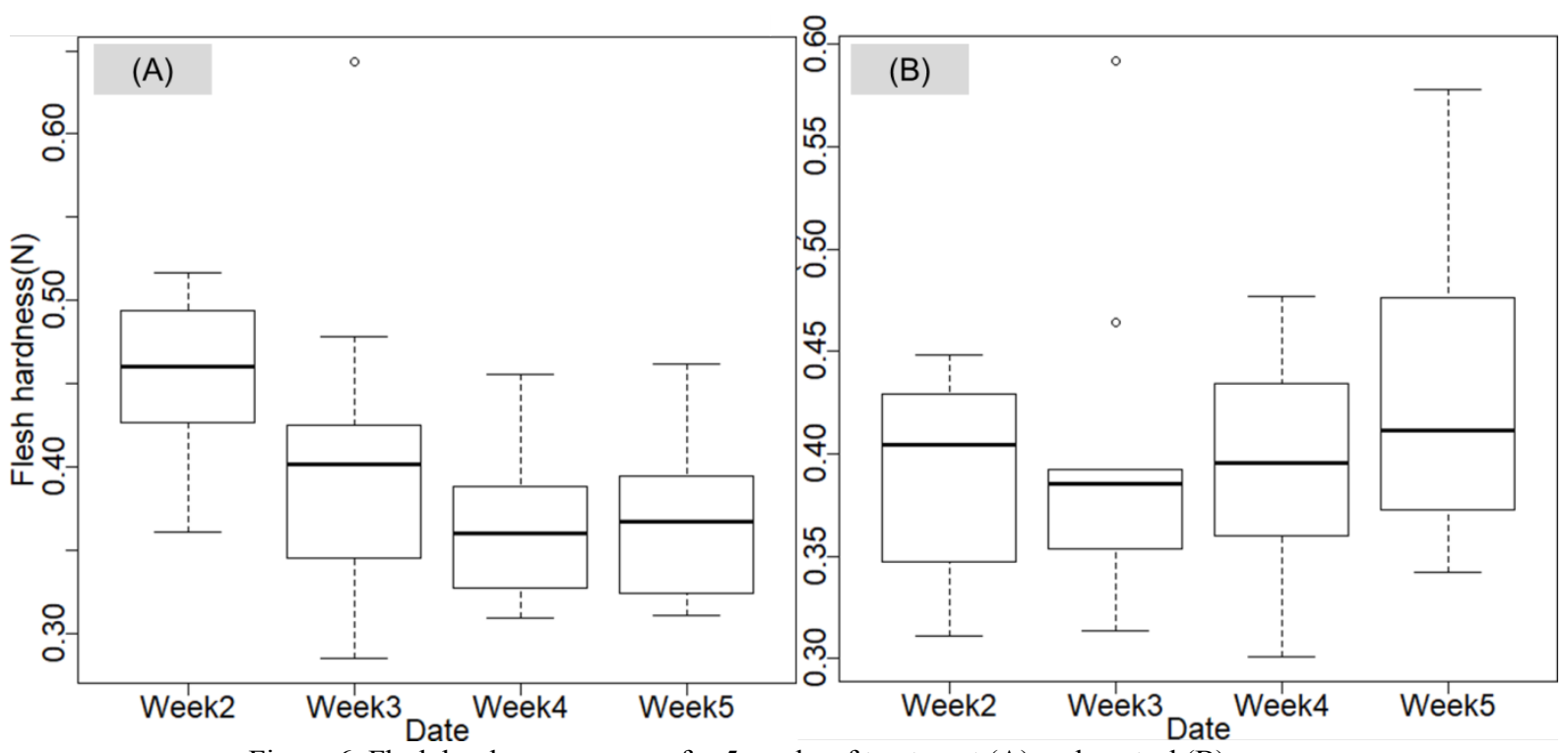

Figure 6. Flesh hardness measures for 5 weeks of treatment (A) and control (B) group.

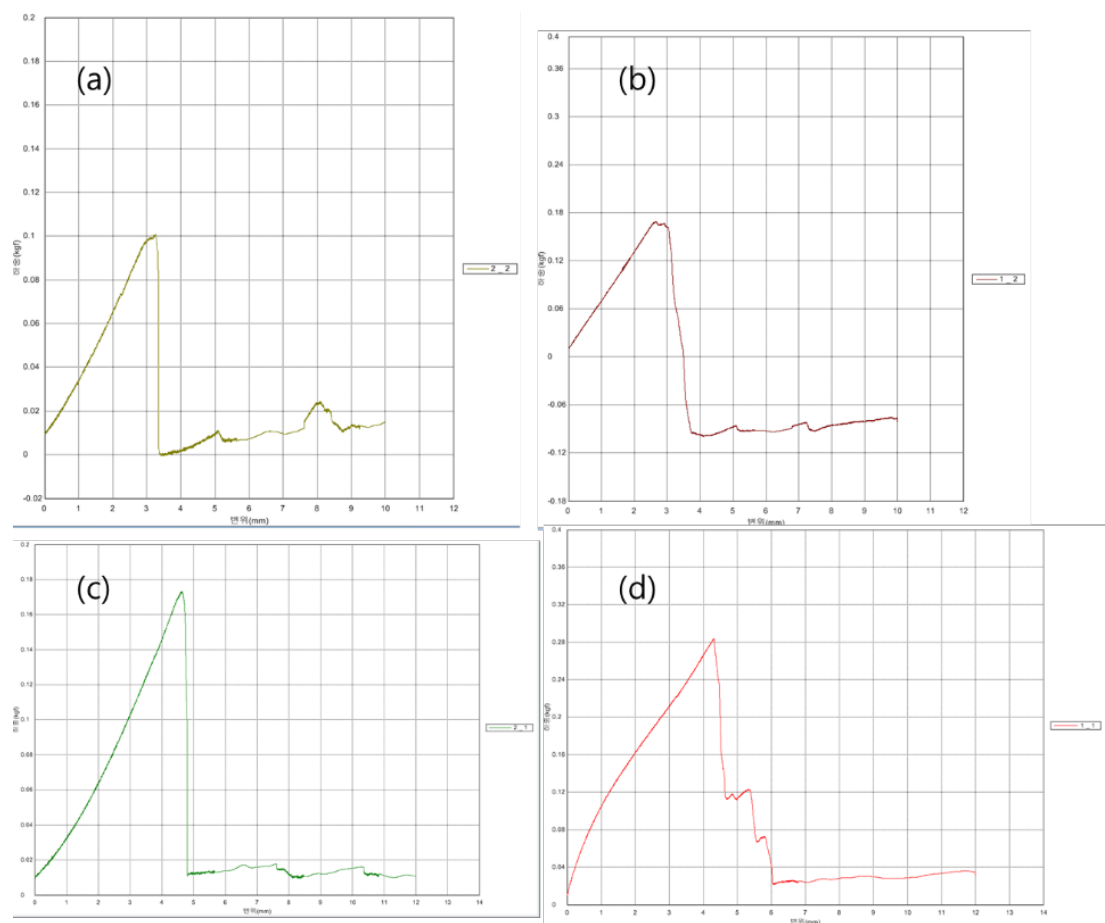

Figure 7. Hardness of flesh and peel of samples. (a) and (b) show initial condition of sample and (c) and (d) show that stored at storage with plasma treatment.

Figures 4 and 5 show the results of SSC and flesh hardness. During 4 weeks, SSC of the treatment group has average $10.03 \pm 0.21 \%$ Brix, the control group has $10.09 \pm 0.46 \%$ Brix. Flesh hardness (Fig. 5 (A)) of treatment samples gradually decreased by the time whereas control group (Fig. 5 (B)) showed a disordered flesh hardness for 5 weeks. Fig 6 shows that a result of hardness of samples. In the figure, (a) and (c) show a hardness of flesh, (b) and (d) show a hardness of peel. Fig $6(a, b)$ is an initial condition of a sample and (c, d) is treated condition with cold storage and plasma. In order to deal detail properties Table 1 summarizes more detail measures. Flesh hardness has similar mean and standard deviation (Std) with treatment versus control, and SSC also shows similar mean value, treatment $(0.21 \%$ Brix $)$ has less half than control $(0.46 \%$ Brix $)$. 
Table 1. SSC and flesh hardness both treatment and control group of 10 samples.

\begin{tabular}{ccccc}
\hline Date & $\begin{array}{c}\text { Treatment } \\
\text { SSC(\%Brix) }\end{array}$ & Hardness(N) & $\begin{array}{c}\text { Control } \\
\text { SSC(\%Brix) }\end{array}$ & Hardness(N) \\
\hline Week2 & 9.93 & 0.458 & 9.97 & 0.390 \\
Week3 & 10.35 & 0.407 & 9.49 & 0.397 \\
Week4 & 9.93 & 0.366 & 10.40 & 0.398 \\
Week5 & 9.92 & 0.372 & 10.51 & 0.431 \\
\hline Mean & 10.03 & 0.401 & 10.09 & 0.404 \\
Std & 0.21 & 0.042 & 0.46 & 0.018 \\
\hline
\end{tabular}

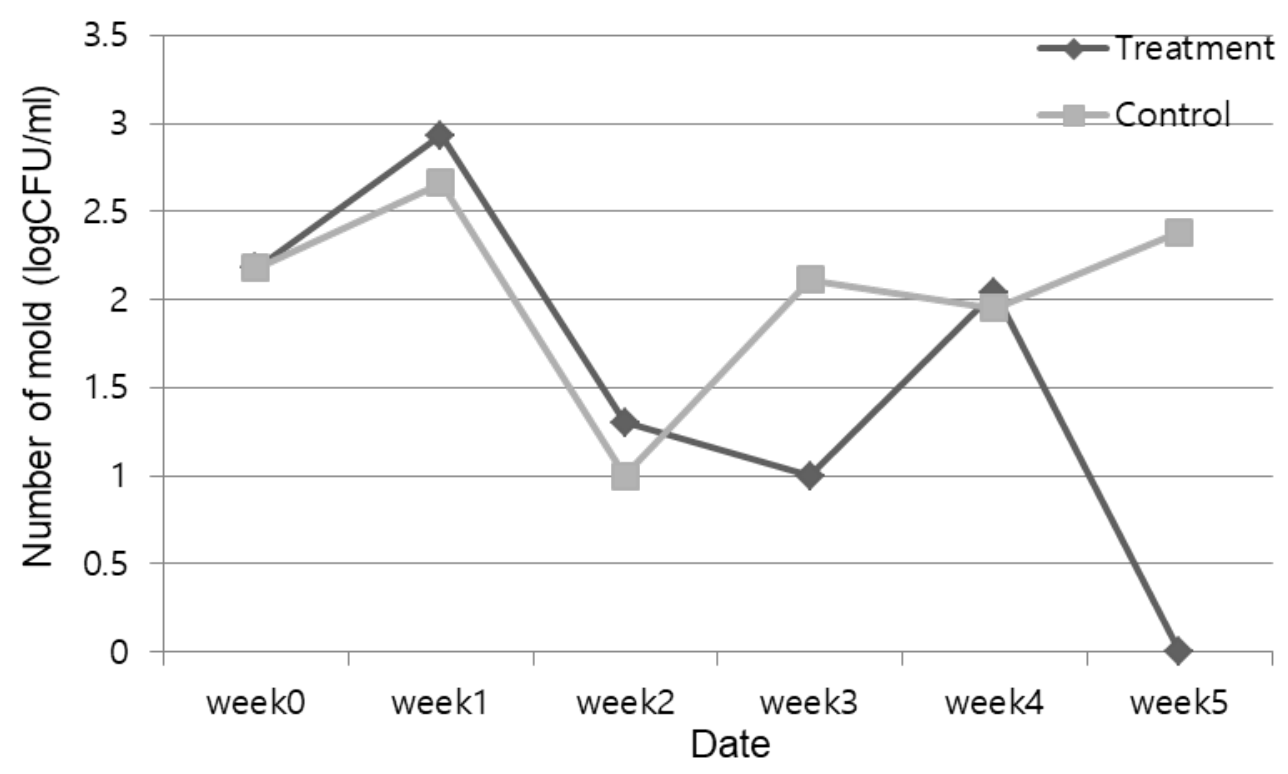

Figure 8. Number of mold for 5 weeks.

\section{Conclusions}

Using cold plasma actuator based on DBD, we evaluated mandarin mold inhibition mandarin storage at room temperature. After 5 weeks, plasma treatment inhibited mold on mandarin. However, physical properties were not able to distinguish quality changes after storage and treatment. In order to compare physical properties of storage we need longer storage period. Further study will obtain both cold storage and ambient storage comparison with plasma treatment.

\section{Acknowledgements}

This study was carried out with the support of "Research Program for Agricultural Science \& Technology Development (Project No. PJ011937)”, National Institute of Agricultural Sciences, RDA, Korea

\section{References}

R Core Team 2016. R: A language and environment for statistical computing. R Foundation for Statistical Computing, Vienna, Austria.

Agricultural Statistics of Korea. 2016. http://www.mafra.go.kr/bbs/mafra/131/189991 accessed June 8th 2018

Geissmann, Q. 2013. OpenCFU, a New Free and Open-Source Software to Count Cell Colonies and Other Circular Objects. PLoS ONE 8(2): e54072 https://doi.org/10.1371/journal.pone.0054072

Kuznetsova, A., Christensen, R.H.B, Bavay, C., Brockhoff, P.B. 2015. Automated mixed ANOVA modeling of sensory and consumer data. Food Quality and Prederence 40, 31-38. https://doi.org/10.1016/j.foodqual.2014.08.004 


\title{
Detection of Astringent and Deastringent Persimmon Fruits using Hyperspectral Imaging Technology
}

\author{
Sandra Munera ${ }^{\mathrm{a}}$, Nuria Aleixos ${ }^{\mathrm{b}}$, Juan Gómez-Sanchís $^{\mathrm{c}}$, Cristina Besada ${ }^{\mathrm{d}}$, Sergio Cubero ${ }^{\mathrm{a}}$, Pau \\ Talens $^{\mathrm{e}}$, Alejandra Salvador ${ }^{\mathrm{d}}$, José Blasco ${ }^{\mathrm{a}}$ \\ ${ }^{a}$ Centro de Agroingeniería. Instituto Valenciano de Investigaciones Agrarias (IVIA). Ctra. CV-315, km 10.7, 46113, \\ Moncada, Valencia (Spain) \\ b Departamento de Ingeniería Gráfica. Universitat Politècnica de València. Camino de Vera, s/n, 46022 Valencia (Spain) \\ ${ }^{\text {c }}$ Departamento de Ingeniería Electrónica. Universitat de València. Av. Universitat, s/n, 46100 Burjassot, Valencia \\ (Spain) \\ d Centro de Poscosecha. Instituto Valenciano de Investigaciones Agrarias (IVIA). Ctra. CV-315, km 10.7, 46113, \\ Moncada, Valencia (Spain) \\ e Departamento de Tecnología de Alimentos. Universitat Politècnica de València. Camino de Vera, s/n, 46022 Valencia \\ (Spain) \\ * Corresponding author. Email: blasco_josiva@gva.es
}

\begin{abstract}
Persimmon fruit cv. 'Rojo Brillante' is an astringent cultivar due to its content of soluble tannins. Traditionally, the consumption of this cultivar has been only possible when the astringency has been naturally removed before harvest, when fruit is overripe and the manipulation is very delicate. In recent years, new postharvest treatments, which allow astringency removal while preserving high flesh firmness, have been developed. Among them, the most widely used in commercial settings is based on exposing fruits to high $\mathrm{CO}_{2}$ concentrations for $24 \mathrm{~h}-36 \mathrm{~h}$. This method promotes anaerobic respiration in the fruit, giving rise to an accumulation of acetaldehyde and insolubilizing tannins at the end of the treatment. The effectiveness of this treatment is controlled by means of methods that are destructive, time-consuming and only a few samples per batch can be analysed. For this reason, the objective of this work is to study the application of the hyperspectral imaging technology in the detection of astringent and deastringent fruits non-destructively. A total of 300 fruits were used and exposed to $\mathrm{CO}_{2}$ during different times in order to obtain fruit with different content of soluble tannins. The hyperspectral images of the fruits were acquired using a VIS-NIR hyperspectral system, which covers the spectral range 450-1040 nm. A reference analysis of soluble tannins was performed in order to find out if the fruits were astringent or deastringent. The spectral information of the two thirds of the fruits was used to build the classification models by means of partial least squares (PLS) and support vector machine (SVM) discriminant analysis methods. The remaining third was used to validate the models as test set. As result, $92.6 \%$ astringent and $84.4 \%$ deastringent fruits were classified correctly using the SVM method. This shows the great potential of hyperspectral imaging technology to detect astringent and deastringent fruits in industrial setups.
\end{abstract}

\section{Introduction}

Currently, Spain is the first producer of persimmon fruit in Europe and the third producer in the world, after China and South Korea (FAOSTAT, 2016). During the last ten years, the harvested area has increased from 2,000 to 14,000 ha, located mainly in the Mediterranean region, and the production has been augmented from 33 to 310 thousand tons (FAOSTAT, 2016). The main variety cultivated is 'Rojo Brillante', which is astringent until the fruit is over-ripe and the texture is too soft. The main reason for this increase in its production is the application of new postharvest techniques that allow obtaining fruit with a crispy texture like an apple and sweet taste without astringency. One of the most effective astringency removal methods is to apply high concentrations of $\mathrm{CO}_{2}(95-$ $98 \%$ ), holding the fruit in airtight chambers for $24 \mathrm{~h}$ at $20^{\circ} \mathrm{C}$ and $90 \%$ relative humidity (Salvador et al., 2008). As the consequence of these anaerobic conditions, soluble tannins (ST) which are responsible of astringency are polymerised by acetaldehyde to form an insoluble compound that is not astringent. This is the current astringency removal method adopted by the industry to commercialise 'Rojo Brillante' persimmon fruit with crispy texture.

The control of the effectiveness of this method is usually assessed by measuring the content of ST that remains in the flesh using analytical techniques, considering the fruit is totally non-astringent when the content of soluble tannins is less than $0.04 \%$ (Tessmer et al., 2016). However, this is a slow and destructive analytical method and not the whole batch is either controlled. The alternative method also employed is based on the reaction of ST with $\mathrm{FeCl}_{3}$, leading to a blue staining; the intensity of the staining observed after a slice of the flesh gets in contact with foils soaked in $\mathrm{FeCl}_{3}$ solution depends on its content level of ST. Although this method is faster and easier than the analytical determination of ST, it is also destructive and subjective and therefore it is necessary to search for new rapid, reliable and non-destructive techniques.

Hyperspectral imaging is a promising optical technique for quality inspection of agricultural and food products integrating the main advantages of spectroscopy and imaging. Hyperspectral imaging can simultaneously acquire 
spectral and spatial information both and detect spectral features in regions of the electromagnetic spectrum such us the ultraviolet, NIR or infrared regions (Lorente et al., 2012). Their use has been widely studied to control the quality of fruit and vegetables during postharvest. Regarding to persimmon fruit and astringency, previous studies have been conducted to predict the content of ST or assess the astringency in different varieties using spectroscopy (Zhang et al., 2013; Altieri et al., 2017; Cortés et al., 2017) and hyperspectral imaging (Munera et al., 2017b \& 2017c). Most of the studies report useful prediction models but they are not precise for ST prediction in fruits with low content because their limit of detection is above $0.10 \% \mathrm{ST}$ and this value does not guarantee a nonastringency sensory value.

In this context, the objective of this work was to study the application of hyperspectral imaging to classify correctly the fruits as astringent (A) and de-astringent (DA) using the value $0.04 \%$ ST content as threshold.

\section{Materials and Methods}

Fruit samples and experimental design

In this work, 100 persimmon fruits (Diospyros kaki cv. 'Rojo Brillante') were harvested in L'Alcudia (Valencia, Spain) at commercial maturity during three consecutive weeks. A total of 300 fruits with apparently similar size and colour and without any defects or bruises were analysed. In order to obtain different levels of soluble tannins content, 90 fruits where not treated, being considered as control. The rest of the fruits were introduced in a container and exposed to air containing $95 \% \mathrm{CO}_{2}$ at $20{ }^{\circ} \mathrm{C}$ and $90 \%$ relative humidity during 12 h (90 fruits) and $24 \mathrm{~h}$ (120 fruits).

\section{Hyperspectral imaging acquisition}

The hyperspectral imaging system consisted of an industrial camera (CoolSNAP ES, Photometrics, AZ, USA), coupled to two liquid-crystal tuneable filters (Varispec VIS-07 and NIR-07, Cambridge Research \& Instrumentation, Inc., MA, USA). The camera was configured to acquire images with a size of 1392x1040 pixels and a spatial resolution of $0.14 \mathrm{~mm} / \mathrm{pixel}$ at 60 different wavelengths every $10 \mathrm{~nm}$, in the working spectral range of $450-1040 \mathrm{~nm}$. The scene was illuminated by indirect light from twelve halogen spotlights (37 W) (Eurostar IR Halogen MR16. Ushio America, Inc., CA, USA) evenly distributed in a circle inside a hemispherical aluminium diffuser. The inner surface of the aluminium diffuser was painted white with a rough texture to maximise its reflectivity and to minimise directional reflections. The fruits were introduced manually into a fruit holder, with the stem-calyx axis lying horizontal. The images were acquired using customised software developed at IVIA, obtaining a total of 300 images.

\section{Reference analyses}

The ST content in each fruit was analysed by spectrophotometry by the Folin-Denis method (Taira, 1995), as described by Arnal \& De Rio (2004), expressing the results as \% of fresh weight. The analysis of variance (ANOVA), followed by Tukey's Honestly Significant Difference (HSD) test, was conducted using the software Statgraphics (Manugistics Corp., Rockville, USA) in order to determine significant differences in ST content in fruits.

\section{Image processing and data analysis}

All images were imported into MATLAB R2017a (The MathWorks, Inc. MA, USA) to be pre-processed using the customised toolbox HYPER-Tools (Mobaraki, N. \& Amigo, J.M., 2018). The image processing started with the correction of the relative reflectance by using white and black references. Then, the corrected images were clipped and compressed to reduce the computation time. A binary mask was applied to remove properly the background and leaves of calyx. The average reflectance spectrum was determined by averaging the relative reflectance spectra of the whole area of each fruit. Finally, the spectra were randomly partitioned into two sets: two thirds of the spectra were used to calibrate the models (training set) while the remaining third was used for independent validation (test set). Second derivative (2D) using Savitzky-Golay smoothing (3 points smoothing window, second order polynomial) was applied to remove physical phenomena in the spectra as additive and multiplicative effects and to improve the subsequent classification models (Rinnan et al., 2009). Then, each pretreated spectrum was normalised using mean centre.

Partial Least Squares (PLS) and Support Vector Machine (SVM) discriminant analysis models were built to classify the fruits as astringent or deastringent. PLS discriminant analysis is a variant of PLS regression in which the independent variable is categorical, expressing the class membership of the samples. It is performed in order to sharpen the separation between groups of observations by maximising the covariance between the spectra and the independent variable so that a maximum separation among classes is obtained (Lorente et al., 2012). SVM is a machine learning technique based on kernels proposed by Cortes and Vapnik (1995). The SVM are linear 
classifiers, in which data are separated by a hyperplane defined by a number of support vectors. These support vectors are part of the training set and are used to define the boundaries of the two classes. The models were calibrated using the pre-processed spectra of the training set and validated using the samples of the test set.

\section{Results and Discussion}

\section{Reference analysis}

The application of the $\mathrm{CO}_{2}$ treatment during different time resulted in fruit with a wide range of values of soluble tannins between $0.91 \%$ in untreated fruits and $0.02 \%$ in fruits treated during $24 \mathrm{~h}$ (Table 1). In the three harvests it was observed the reduction of ST related to the duration of the treatment being the results statistically different.

The value of ST in which 'Rojo Brillante' persimmon fruit is considered non-astringent was evaluated by Tesmeer et al. (2016) using a sensory panel. This cultivar was qualified as non-astringent when the value of ST was $0.04 \%$. However, the fruits were in the over-ripened stage and with a soft texture. Since no studies have been performed in fruits treated with $\mathrm{CO}_{2}$, the value obtained by Tessmer et al. (2016) was considered the threshold value to classify the fruit in this work. Thus, all untreated fruits were considered as astringent, 59 fruits treated during 12 hours were considered as astringent and 31 as deastringent. As the ST content in fruits treated during $24 \mathrm{~h}$ was no higher than $0.03 \%$ these fruits were directly considered as deastringent.

Table 1. Soluble tannins content at each treatment duration and harvest, and number of astringent and de-astringent fruit. Different letters in the same column indicates significant differences between treatment duration ( $\mathrm{p}$-value $<0.05$ ), according to Tukey's (HSD) test.

\begin{tabular}{ccccccccc}
\hline \multirow{2}{*}{ Treatment } & \multicolumn{4}{c}{ Soluble tannins } & \multicolumn{2}{c}{ Harvest 3 } & \multicolumn{2}{c}{ Class } \\
\cline { 2 - 10 } & $\begin{array}{c}\text { Mean } \\
(\%)\end{array}$ & SD & $\begin{array}{c}\text { Mean } \\
(\%)\end{array}$ & SD & $\begin{array}{c}\text { Mean } \\
(\%)\end{array}$ & SD & \#A & \#DA \\
\hline Untreated & $0.69^{\mathrm{a}}$ & 0.16 & $0.61^{\mathrm{a}}$ & 0.08 & $0.91^{\mathrm{a}}$ & 0.13 & 90 & 0 \\
$12 \mathrm{~h}$ & $0.09^{\mathrm{b}}$ & 0.08 & $0.11^{\mathrm{b}}$ & 0.09 & $0.37^{\mathrm{b}}$ & 0.18 & 59 & 31 \\
$24 \mathrm{~h}$ & $0.02^{\mathrm{c}}$ & 0.01 & $0.03^{\mathrm{c}}$ & 0.02 & $0.03^{\mathrm{c}}$ & 0.00 & 0 & 120 \\
\hline
\end{tabular}

\section{Spectral analysis}

The mean spectrum of astringent and deastringent fruits followed a very similar pattern (see Fig. 1). The use of second derivative transformation allowed separating overlapped peaks and therefore more differences could be visualised, especially in the NIR region, between $700 \mathrm{~nm}$ and $800 \mathrm{~nm}$, and between $900 \mathrm{~nm}$ and $1040 \mathrm{~nm}$. These differences are mainly related to the water absorption peak, located at $970 \mathrm{~nm}$ (Lu and Peng, 2006), carbohydrates at $710 \mathrm{~nm}$ and $920 \mathrm{~nm}$ (Siedliska et al., 2018) and phenolic compounds, located at $790 \mathrm{~nm}$ and between $940 \mathrm{~nm}$ and $1000 \mathrm{~nm}$ (Siedliska et al., 2018; Noypitak el al., 2015). All these slight differences are associated to the effect of deastringency treatment. As Salvador et al (2007) pointed out, after treatment with $\mathrm{CO}_{2}$, soluble solids levels drop significantly. This reduction is related to the loss of astringency, since the measurement of soluble solids includes the soluble tannins that cause astringency and, following $\mathrm{CO}_{2}$ treatment, these tannins become insoluble (Arnal and Del Río, 2003). Furthermore, although deastringent treatment does not affect the firmness of the fruit in early stages of ripeness, the softening of flesh is possible in following stages.

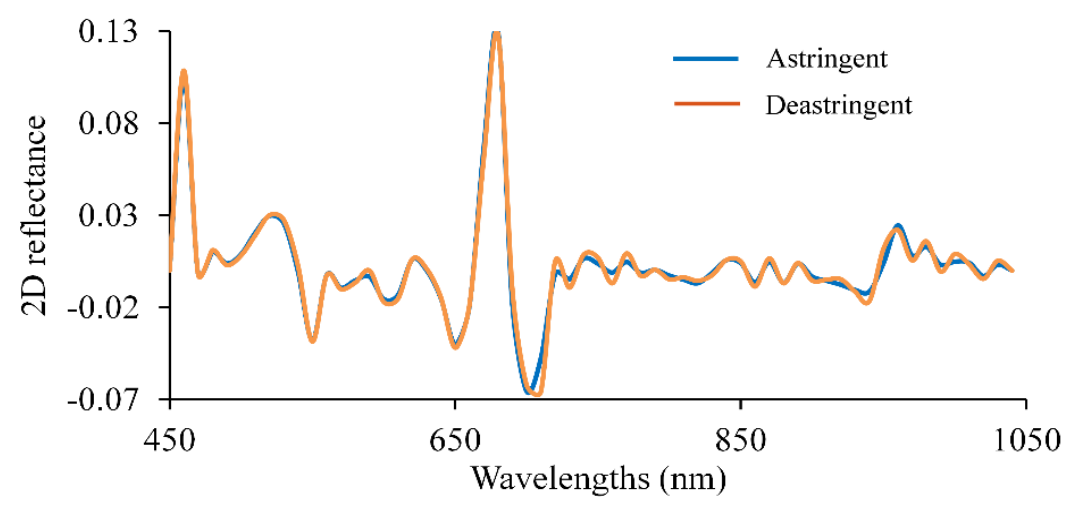

Figure 1. Mean spectra of astringent and deastringent fruits. 
Detection of astringent and deastringent fruits

Due to the importance of detecting as many astringent fruits as possible, the priority of the classification in this work was to try to maximise the correct classification of these fruits by the model. The PLS model was calibrated using 14 latent variables, and the internal validation of the model using a single 10-fold cross validation classified correctly $85.0 \%$ of the samples. The SVM model was calibrated using 89 support vectors, and $89.0 \%$ of the samples were correctly classified. In this case the model using SVM was the more accurate in classifying astringent fruits and also deastringent fruits.

The external validation or classification of the test set is shown in Figure 2. The class for each fruit was predicted by introducing the mean spectrum measured into the previously built models. The result was visualised in the fruit surface showing the fruit coloured in blue if the mean value was assigned by the model to astringent or orange if it was assigned to deastringent class. SVM was the more accurate model to classify astringent fruits and also deastringent fruits of the test set, classifying $92.2 \%$ of astringent and $84.4 \%$ of deastringent fruits correctly.

Table 2. Results of calibration of classification models.

\begin{tabular}{cccccccc}
\hline & & \multicolumn{4}{c}{ Correct classification (\%) } \\
\cline { 3 - 8 } Model & \multirow{2}{*}{ \#LV/\#SV } & \multicolumn{3}{c}{ Calibration } & \multicolumn{3}{c}{ Cross Validation } \\
\cline { 3 - 7 } & & A & DA & Total & A & DA & Total \\
\cline { 3 - 7 } & 14 & 90.7 & 88.3 & 89.5 & 86.0 & 84.0 & 85.0 \\
SVS & 89 & 98.1 & 95.8 & 97.0 & 90.7 & 87.2 & 89.0 \\
\hline
\end{tabular}

$\# \mathrm{LV}=$ number of latent variables; $\# \mathrm{SV}=$ number of support vectors; $\mathrm{A}=$ astringent; $\mathrm{DA}=$ deastringent

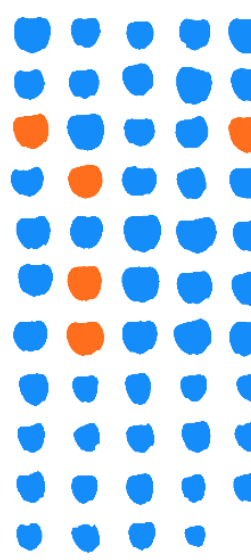

$90.7 \%$
DA

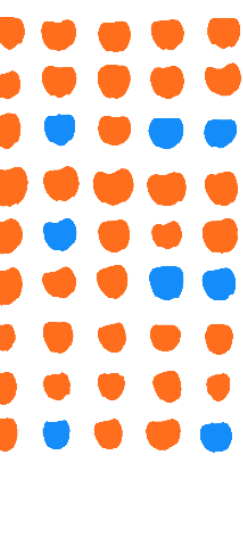

$82.2 \%$
A

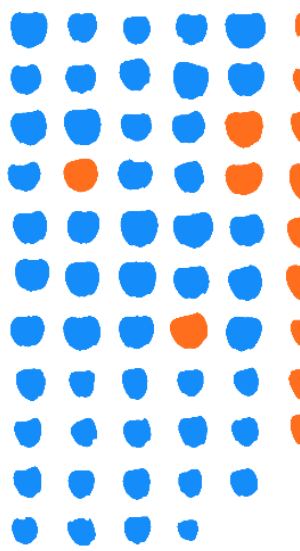

$92.2 \%$
DA

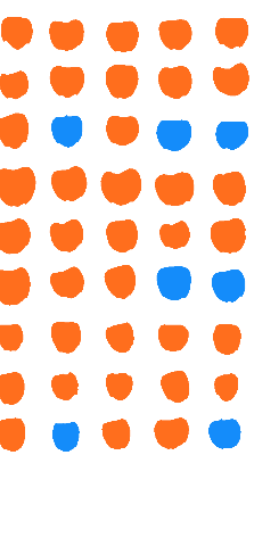

$84.4 \%$

$86.5 \%$

$88.5 \%$

PLS

SVM

Figure 2. Classification of astringent and deastringent fruits of test set using PLS and SVM models.

\section{Conclusions}

This work demonstrates the capability of hyperspectral imaging detecting astringent and deastringent 'Rojo Brillante' persimmon fruits. The more accurate classification was performed using the SVM model obtaining a correct classification of $92.6 \%$ astringent and $84.4 \%$ deastringent fruits. Thus, this technique may have potential as a tool for rapid and non-destructive control of effectiveness of the astringency removal treatment applied to 'Rojo Brillante' persimmon fruit, allowing the detection of fruit that still is astringent before it is marketed.

Nevertheless, the results of this study should be confirmed on a larger sample set of fruits grown in different areas and harvested at different stages of ripeness before they can be implemented in an in-line system. Furthermore, in order to establish a practical tool in industry, an optimal reduction of the spectral information is necessary to speed up this classification. 


\section{Acknowledgements}

This work was partially funded by INIA and FEDER funds through project RTA2015-00078-00-00. Sandra Munera thanks INIA for the FPI-INIA grant num. 43 (CPR2014-0082), partially supported by European Union FSE funds.

\section{References}

Altieri, G., Genovese, F., Tauriello, A. \& Di Renzo, G.C. (2017). Models to improve the non-destructive analysis of persimmon fruit properties by VIS/NIR spectrometry. Journal of the Science of Food and Agriculture 97, 5302-5310.

Arnal, L., \& del Río, M. A. (2004). Quality of persimmon fruit cv. 'Rojo Brillante' during storage at different temperatures. Spanish Journal of Agricultural Research 2, 243-247.

Cortés, V., Rodríguez, A. Blasco, J., Rey, B., Besada, C., Cubero, S., Salvador, A., Talens, P. \& Aleixos, N. (2017). Prediction of the level of astringency in persimmon using visible and near-infrared spectroscopy. Journal of Food Engineering 204, 27-37.

Cortes, C. \& Vapnik, V. (1995). Support-vector networks. Machine Learning 20, 273-297

FAOSTAT. http://www.fao.org/faostat/en/\#data/QC Accessed 02.04.18

Lorente, D., Aleixos, N., Gómez-Sanchis, J., Cubero, S., García-Navarrete, O. L. \& Blasco, J. (2012). Recent advances and applications of hyperspectral imaging for fruit and vegetable quality assessment. Food Bioprocess Technology 5, 1121-1142.

Lu, R. \& Peng, Y. (2006). Hyperspectral scattering for assessing peach fruit firmness. Biosystems Engineering 93, 161-171.

Mobaraki, N. \& Amigo, J.M. (2018). HYPER-Tools. A graphical user-friendly interface for hyperspectral image analysis. Chemometrics and Intelligent Laboratory Systems 172, 174-187.

Munera, S., Besada, C., Blasco, J., Cubero, S., Salvador, A., Talens, P. \& Aleixos, N. (2017b). Astringency assessment of persimmon by hyperspectral imaging. Postharvest Biology and Technology 125, 35-41

Munera, S., Besada, C., Aleixos, N., Talens, P., Salvador, A., Sun, D.-W., et al. (2017c). Non-destructive assessment of the internal quality of intact persimmon using colour and VIS/NIR hyperspectral imaging. $L W T$ Food Science and Technology 77, 241-248.

Noypitak, S., Terdwongworakul, A., Krisanapook, K., Kasemsumran, S., 2015. Evaluation of astringency and tannin content in 'Xichu' persimmons using near infrared spectroscopy. Int. J. Food Prop. 18, 1014-1028.

Rinnan, Å., van den Berg, F., \& Engelsen, S. B. (2009). Review of the most common pre-processing techniques for near-infrared spectra. Trends in Analytical Chemistry 28, 1201-1222.

Salvador, A., Arnal, L., Besada, C., Larrea, V., Quiles, A., Pérez-Munuera, I., 2008. Reduced effectiveness of the treatment for removing astringency in persimmon fruit when stored at $15^{\circ} \mathrm{C}$. Physiological microstructural study. Postharvest Biology and Technology 49, 340-347.

Salvador, A., Arnal, L., Besada, C., Larrea, V., Quiles, A. \& Pérez-Munuera, I., 2007. Physiological and structural changes during ripening and deastringency treatment of persimmon cv. 'Rojo Brillante'. Postharvest Biology and Techonology 46, 181-188.

Siedliska, A., Baranowski, P., Zubik, M., Mazurek, W. \& Sosnowska, B. (2018). Detection of fungal infections in strawberry fruit by VNIR/SWIR hyperspectral imaging. Postharvest Biology and Technology 139, 115-126.

Taira, S. (1995). Astringency in persimmon. In: Fruit analysis. Linskens, H.F., Jackson, J.F. (Eds.). Springer, Hannover (Germany), p.p.: 97-110.

Tessmer, M.A., Besada, C., Hernando, I., Appezzato-da-Glória, B., Quiles, A. \& Salvador, A. (2016). Microstructural changes while persimmon fruits mature and ripen. Comparison between astringent and nonastringent cultivars. Postharvest Biology and Technology 120, 52-60.

Zhang, P., Xue, Y., Li, J., Feng, X. \& Wang, B. (2013). Research on non-destructive measurement of firmness and soluble tannin content of 'mopanshi' persimmon using Vis/NIR diffuse reflection spectroscopy. Acta Horticulturae 996, 447-452. 


\title{
Monitoring Watercress Shelf-life with VNIR Hyperspectral Image through Packaging Film
}

\author{
Miguel A. Lara ${ }^{a}$, Lourdes Lleó ${ }^{b}$, Jean M. Roger ${ }^{c}$, Belén Diezma ${ }^{\text {b, * }}$ \\ a i+dea. Pol. Ind. Los Llanos de San Pedro, Ctra. Ávila 40400 El Espinar, Segovia \\ ${ }^{\mathrm{b}}$ Laboratorio de Propiedades Físicas y Técnicas Avanzadas en Agroalimentación. ETSIAAB, Universidad Politécnica de \\ Madrid, Madrid, 28040, Spain \\ c IRSTEA, 361 Rue Jean-François Breton BP 5095, Montpellier 34196, France \\ * Corresponding author. Email: belen.diezma@upm.es
}

\begin{abstract}
The aim of the present work is to monitor the shelf-life of watercress (Nasturtium officinale) by means of hyperspectral images through packaging films. Fifteen watercress leaves were randomly selected from a commercial bag, put inside Petri dishes covered by plastic film and stored at $4^{\circ} \mathrm{C}$ for 16 days. Hyperspectral images (VNIR 400-1000 nm) were taken during this storage, getting five acquisitions for each leaf. The average spectrum of each image was computed; several models were obtained from these average spectra. A radiometric correction was applied in order to avoid the variation in transmittance of the plastic film along storage in the hyperspectral images. Afterwards, three spectral pre-processing procedures (no pre-process, Savitsky-Golay and Standard Normal Variate, combined with Principal Component Analysis) were applied to obtain different models. These specific models for watercress along with other models previously developed for spinach were applied and compared. The artificial images of scores obtained, applying each model to the original and complete images, were compared by means of Analysis of Variance and Wilks- $\lambda$. Radiometric correction allowed the supervision of shelf-life in leafy vegetables through commercial transparent films. All models applied were able to monitor the aging of the leaves. However, the models developed specifically for watercress spectra were more suitable for monitoring the shelf-life of this vegetable leaf along the storage. There were some differences between the models applied for spinach and for watercress leaves. Despite it, it seems to be necessary to study the spectra of each leafy vegetable independently for developing prediction models more able to monitor the aging of the leaves. Hyperspectral imaging could be used for monitoring the freshness of watercress and/or mix of salad leaves during their shelf-life. Multispectral indexes based on results of PCA and spectral patterns, could contribute to the transference of the technique to the industry.
\end{abstract}

Keywords: Image analysis Non-destructive assessment Ready to use leafy vegetables Transparent plastic films.

\section{Introduction}

The success of ready-to-eat fresh vegetables has caused in the food industry the need to search more varieties in the vegetables offered to the consumers. The development of new types of ready-to-eat salads, using new vegetables, is increasing the sales of these products. Vegetables such as salad rocket (Erucula sativa), alfalfa sprouts (Medicago sativa), baby leaves or watercress (Nasturtium officinale) are being processed and commercialized in recent years although they were not commonly used some years ago. Modified atmosphere packaging (MAP) is an essential technology for the success of fresh-cut produce. Polymeric films are a key element in MAP. The most commonly used packaging films are a few plastic polymers micro perforated, to allow the breath of the products.

Watercress is an aquatic perennial vegetable, from the Brassicaceae family, with wide culinary applications. The raw watercress leaves can be used as salad or steamed. They have a great amount of vitamin $\mathrm{C}$ and provitamin A, folic acid, iodine, iron, proteins and, especially, calcium and sulphur compounds, causing its characteristic smell and its nutritional benefits (Gonçalves et al., 2009).

Understanding the evolution and deterioration processes of watercress leaves during their shelf-life is a key factor for commercializing this vegetable as minimal processed fresh product. To establish the optimal shelf-life of watercress leaves allows the industry to offer high quality products to the consumer, with optimal sensory conditions.

Hyperspectral imaging technique is regarded as an analytical tool for analyses conducted for quality evaluation of food products in research, control, and industries. The hyperspectral imaging system allows integrating spectroscopic and imaging techniques to enable direct identification of different components, or quality characteristics and their spatial distribution in the tested sample. However, few applications of hyperspectral vision system have been focused on ready to use leafy vegetables until now. As an example, there is a research concerning rapid detection of Escherichia coli contamination in packaged fresh spinach using hyperspectral imaging (Siripatrawan et al., 2011). Other recent works focused on freshness of spinach and salinity of lettuce has been carried out by the authors of the present research (Diezma et al. 2013, Lara et al. 2013, Lara et al. 2016). 
Specially related with the present paper, Lara et al. (2013) developed a procedure to avoid the effect of the variation of the transmittance of the plastic applying radiometric correction to the hyperspectral images.

The objective of this research is to propose and compare prediction models based on hyperspectral imaging to monitor the evolution of watercress along cold storage through plastic films. Some of these models specifically obtained from images of watercress, and others previously developed for spinaches.

\section{Materials and Methods}

Experimental set up

Fifteen leaves randomly selected from a commercial bag of ready-to-eat watercress leaves were put individually into Petri dishes, with a reference (grey plastic) and covered with a plastic film (polypropylene 35 microns width). The Petri dishes were stored at $4^{\circ} \mathrm{C}$ during 16 days (

Figure 203).

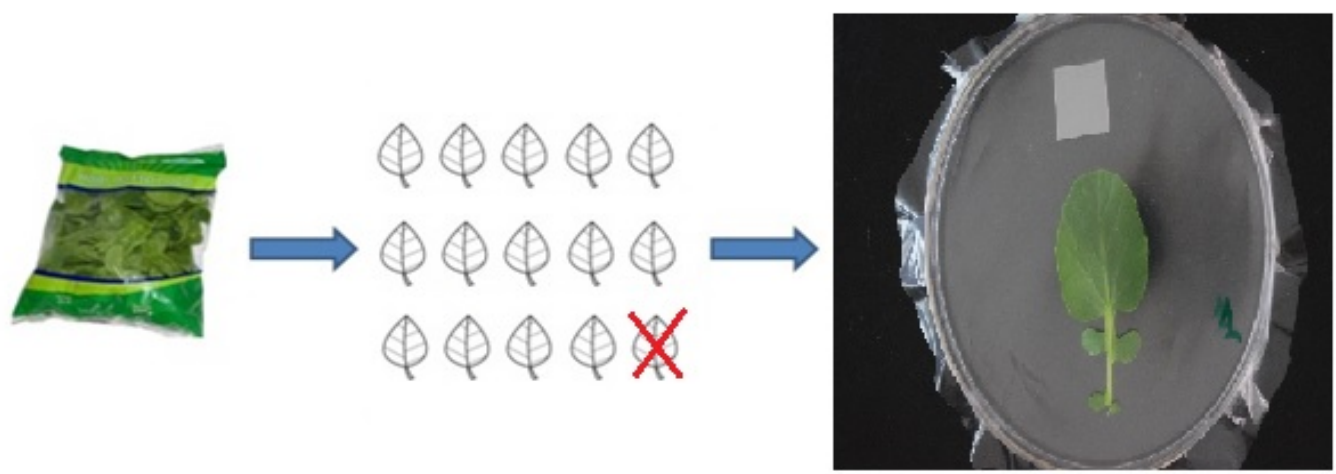

Figure 203. Leaves selection from a watercress commercial bag and placement of the leaves inside Petri dishes for their conservation and image acquisition.

Along this period, hyperspectral images of leaves surface were acquired, through the plastic film, two times per week, with 5 acquisitions till the leaves became deteriorated. One of the 15 leaves was removed due to its fast evolution. For that, the spectral analysis was carried out finally over 14 leaves.

The hyperspectral vision system consists of a push-broom CCD camera (Andor Luca) equipped with a spectrograph Headwall Photonics HyperspecTM VNIR (spectral range: 400 to $1000 \mathrm{~nm}$ ). The spectral binning was configured to obtain 189 wavelengths (spectral resolution $3.17 \mathrm{~nm}$ ). The acquisition and the storage of the images were made through specific software (Headwall HyperespecTM). The illumination was provided by two halogens lamps with regulated and variable intensity. Each Petri disc, with one single leaf, was placed on a platform that moved under the camera (Micos-MOCO motor). The sample was scanned line by line according to the movement (push-broom system). The spatial resolution was $260 \mu \mathrm{m}$. Once the raw images were acquired, the corresponding relative reflectance hypercube was computed, containing the relative reflectance spectrum of each pixel of the image with respect to a reference (mean spectrum of a barium sulphate white reference).

\section{Data analysis}

The average spectrum of each image was computed $(n=2624$ to $n=500213$ spectra extracted from each leaf, depending on its size); then 70 average spectra (14 leaves x 5 days) were considered for pre-processing and model computation. The average spectrum of the reference material (grey plastic) for each image was also calculated for the radiometric correction of the images. This radiometric correction (RC) (Lara et al, 2013) was applied to avoid the variation in transmittance of the plastic film along storage in the hyperspectral images. Afterwards, three spectral pre-processing procedures (no pre-process, Savitsky-Golay SG and Standard Normal Variate SNV), combined with Principal Component Analysis (PCA) were applied to obtain different models. These specific models for watercress along with other models previously developed for spinach were applied and compared. The artificial images of scores obtained applying each model to the original and complete images, were compared by means of Analysis of Variance and Wilks- $\lambda$. The model that showed the greatest values of both parameters was selected as the most suitable to monitor the evolution of the leaves.

\section{Results and Discussion}

Spectral features

Figure 204 shows the mean spectra of the watercress leaves analysed $(n=14)$ on each of the five tested days. Three main features can be observed 
- A reflectance peak in the visible range, between 500 and $660 \mathrm{~nm}$.

- $\quad$ The "red edge", located between 680 and $740 \mathrm{~nm}$.

- High relative reflectance in the infrared region.

In Figure 204, it can be observed an increment of global reflectance in the spectra along the experiment, which can be due to structural changes in the leaves, which cause a decrease in the water content of the cells and an increase of air in the interstitial spaces. This fact induces an increase of scattering in the reflected light and, for that, an increase in the global reflectance of the spectra (Jacquemoud and Baret, 1990; Asner, 1998)
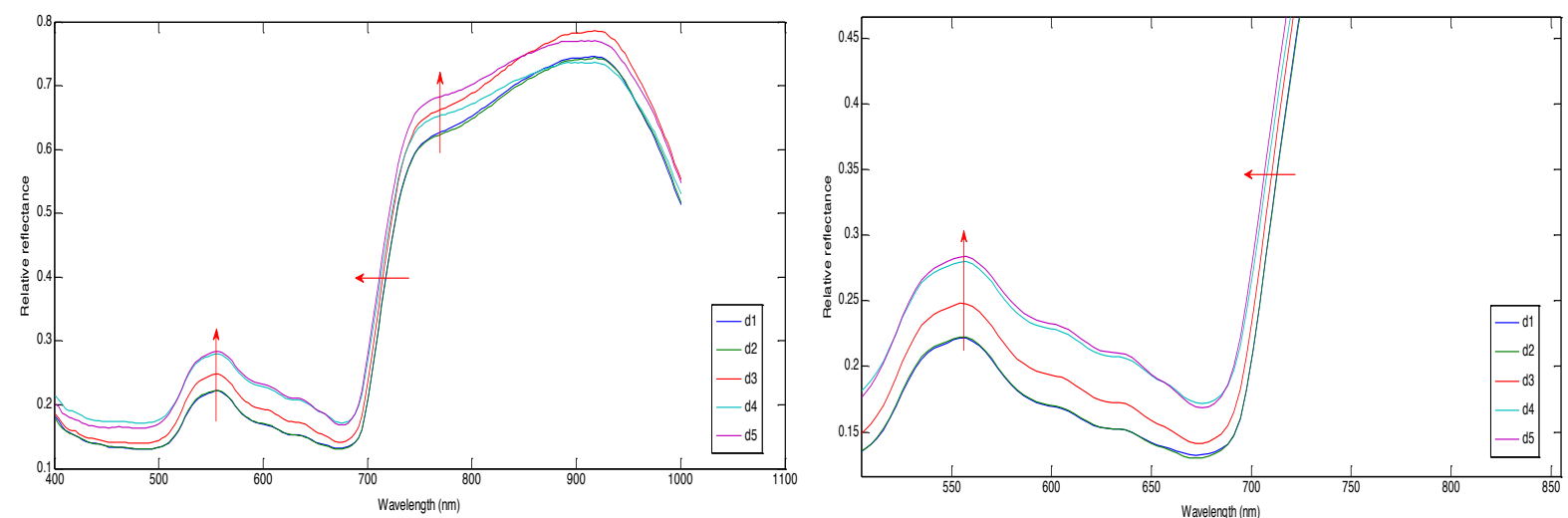

Figure 204. Left: mean spectra, corrected with RC, of the watercress leaves on each tested day. Right: detailed range of spectra on red-edge region. Red arrows mark the evolution of each region along the days $\mathrm{d} 1$ to $\mathrm{d} 5$.

Moreover, a displacement to the left of the red edge position can be clearly observed when the leaves aged. Several authors noticed that, due to the aging of the leaves and chlorophyll degradation, the red edge suffers a displacement to shorter wavelengths (Mutanga and Skidmore, 2007; Van der Meer and Jong, 2002).

Data analysis. PCA computation and score images comparison

The following principal components were selected as the most related with the evolution of the watercress during time, considering the values of the scores along time corresponding to the average spectrum of each leaf:

- $\quad$ PC1 and PC3 from PCA applied on RC + SG corrected spectra.

- $\quad$ PC1 and PC2 from PCA applied on RC + SG + SNV corrected spectra (Figure 205).

Some models obtained for monitoring spinaches (Lara et al., 2013) were also included into this comparison, to study the feasibility of their application onto watercress leaves.

- $\quad$ PC3 from PCA applied on RC corrected spectra.

- $\quad$ PC1 from PCA applied on RC + SG corrected spectra.

- $\quad$ PC1 from PCA applied on RC + SG + SNV corrected spectra.

As an example, Figure 205 (upper) shows the average spectra per days computed on the 14 leaf spectra, subjected to RC, SG and SNV algorithm (very similar in shape to the RC and SG spectra). In the second derivative of the reflectance spectra, the absorbances appear as positive peaks. The patterns of these spectra could be characterized by three main features (indicated by red arrows): 1) a high positive peak around $680 \mathrm{~nm}$ followed by a high negative peak around $740 \mathrm{~nm}$, approximately. Both peaks are placed close to the red edge region of the spectra and a shift to the left can be observed on the spectra along the days of storage. The great positive peak could be due to the main absorbance peak of chlorophyll; 2) a succession of positive and negative peaks between 500 and $585 \mathrm{~nm}$, approximately. These peaks are placed in the green and yellow regions of the visible spectrum and could be due to carotenoids (Gitelson et al., 2006) and chlorophyll (Kleima et al., 2000). A shift to the left can be also observed in the spectra of the last days in this region that could be due to a decrease in chlorophyll content that causes this "green edge" displacement (Gitelson et al., 2003); 3) a slight negative peak around 940 $\mathrm{nm}$ that decreases along the storage. This peak would be attributed to the water content of the leaves. There were two principal components that seem to be related to the evolution of the leaves during time: PC1 and PC2. In the scores of both principal components, there is a clear and progressive rising trend along the period of evolution (Figure 205, second and third rows).

The hyperspectral images of the watercress leaves were projected onto those seven loadings, with the aim of obtaining virtual images of scores able to monitor the evolution of the leaves. The virtual images obtained were compared, leaf by leaf, by means of analysis of variance (ANOVA) and Wilks- $\lambda$.

Scores images corresponding to each loading were considered. ANOVAs were carried out comparing the pixels of each leaf, in the first and in the last tested day. The F-Fisher values obtained are shown in Table 88, considering seven of the 14 leaves, those with more surface available. The Wilks- $\lambda$ values were computed 
similarly, and the results are shown in Table 89 .

According to the F-Fisher values obtained for each model applied on each leaf, there are significant differences between the leaves in the first and in the last tested day in all cases. All models detect the evolution of the leaves along the time. However, the model based on loading 2 from the spectral data corrected with RC $+\mathrm{SG}+\mathrm{SNV}$, is the most sensitive detecting the evolution of the leaves. There are only two leaves in which other models show greater F-Fisher values and, in those cases, the difference is small. Results for PC1 RC+SG of spinach and watercress are very similar. The same occurs with results for PC1 $\mathrm{RC}+\mathrm{SG}+\mathrm{SNV}$ in both leafy vegetables. The loadings of these PCs were almost identical so (data not shown).

Wilks- $\lambda$ values are in most of the cases lower than one so, this indicates that the internal variance of the scores in the leaves is very high in comparison to the variance due to evolution. However, loading $2 \mathrm{RC}+\mathrm{SG}+\mathrm{SNV}$ of watercress leaves shows values higher than one, so that PC could be able to detect the evolution of the leaves.
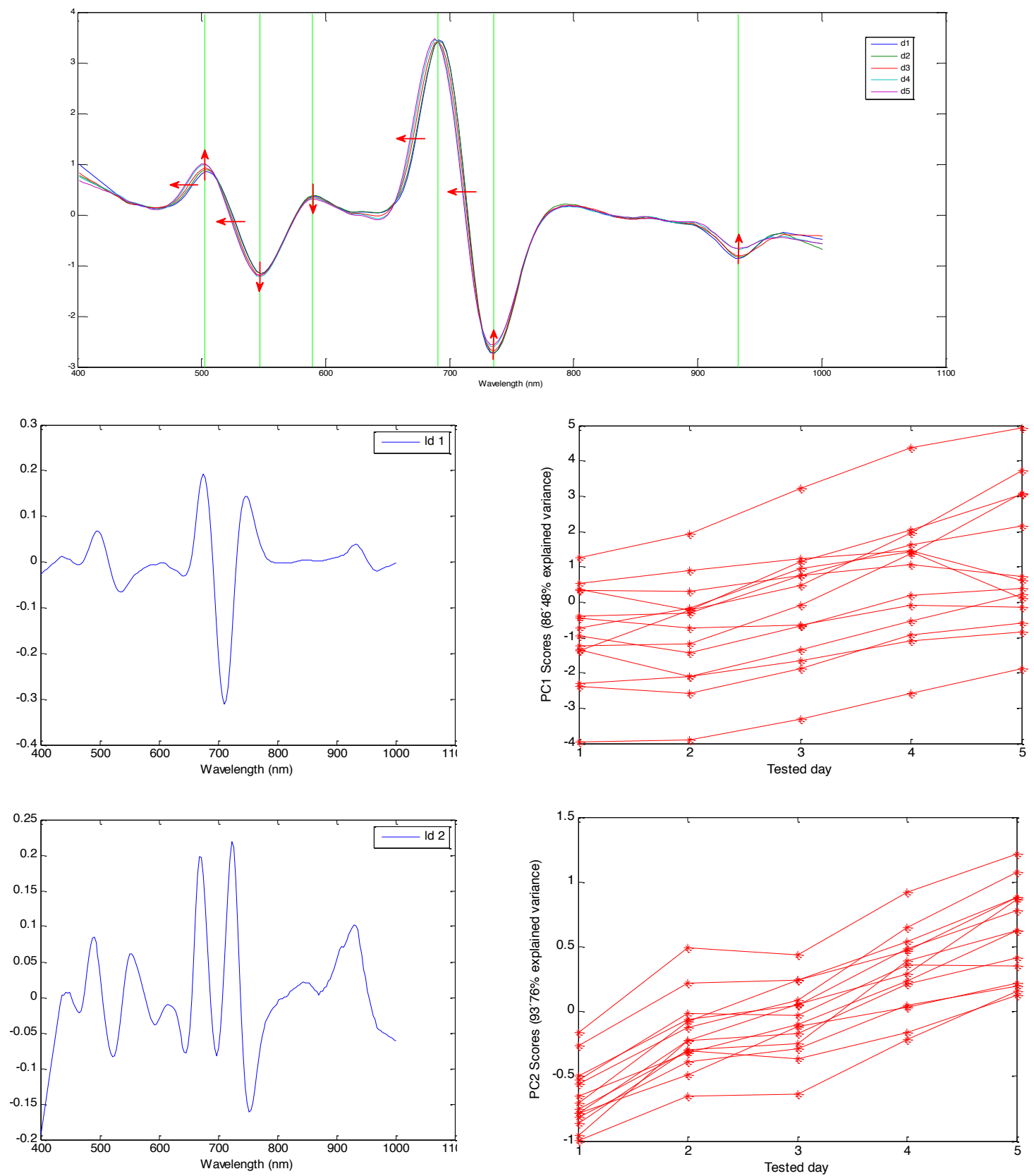

Figure 205. Up: mean RC+SG+SNV spectra of watercress samples measured each day; d1 to d5. Middle left: PC1 loading from PCA applied on $\mathrm{RC}+\mathrm{SG}+\mathrm{SNV}$ corrected spectra. Middle right: scores of $\mathrm{PC} 1$ on mean spectra of the leaves along testing time. Down left: PC2 loading from PCA applied on RC+SG+SNV corrected spectra. Down right: scores of PC2 on mean spectra of the leaves along testing time. 
Table 88. F-Fisher values obtained in each ANOVA computed on the scores images corresponding to each loading used. Pixels $(n=38000)$ from seven of the 14 watercress leaves are compared, each leaf independently, between the first and the last tested day. The greatest F-Fisher value obtained for each leaf is marked in bold.

\begin{tabular}{|c|c|c|c|c|c|c|c|c|}
\hline $\begin{array}{c}\text { Number } \\
\text { of leaf }\end{array}$ & & $\begin{array}{c}\text { Ld 3 } \\
\text { Spinach } \\
\text { RC }\end{array}$ & $\begin{array}{c}\text { Ld 1 } \\
\text { Spinach } \\
\text { RC+SG }\end{array}$ & $\begin{array}{c}\text { Ld 1 } \\
\text { Spinach } \\
\mathrm{RC}+\mathrm{SG}+\mathrm{SNV}\end{array}$ & $\begin{array}{c}\text { Ld 1 } \\
\text { Watercress } \\
\text { RC+SG } \\
\end{array}$ & $\begin{array}{c}\text { Ld 3 } \\
\text { Watercress } \\
\text { RC+SG } \\
\end{array}$ & $\begin{array}{c}\text { Ld 1 } \\
\text { Watercress } \\
\mathrm{RC}+\mathrm{SG}+\mathrm{SNV} \\
\end{array}$ & $\begin{array}{c}\text { Ld } 2 \\
\text { Watercress } \\
\text { RC+SG+SNV }\end{array}$ \\
\hline \multirow{2}{*}{1} & $\mathrm{~F}$ & 136 & 12861 & 6554 & 9845 & 232397 & 7492 & 314562 \\
\hline & $\mathrm{p}$ & $2 \cdot 10^{-31}$ & 0 & 0 & 0 & 0 & 0 & 0 \\
\hline \multirow{2}{*}{2} & $\mathrm{~F}$ & 66 & 729 & 77 & 398 & 253351 & 11 & 276726 \\
\hline & $\mathrm{p}$ & $4 \cdot 10^{-16}$ & 0 & $2 \cdot 10^{-18}$ & 0 & 0 & $4 \cdot 10^{-26}$ & 0 \\
\hline \multirow{2}{*}{4} & $\mathrm{~F}$ & 214 & 5357 & 3384 & 4735 & 133695 & 3842 & 172918 \\
\hline & $\mathrm{p}$ & 0 & 0 & 0 & 0 & 0 & 0 & 0 \\
\hline \multirow{2}{*}{5} & $\mathrm{~F}$ & 35650 & 67358 & 65182 & 65886 & 50081 & 69294 & 66801 \\
\hline & $\mathrm{p}$ & 0 & 0 & 0 & 0 & 0 & 0 & 0 \\
\hline \multirow{2}{*}{6} & $\mathrm{~F}$ & 1323 & 13671 & 10175 & 12222 & 223604 & 11366 & 291248 \\
\hline & $\mathrm{p}$ & 0 & 0 & 0 & 0 & 0 & 0 & 0 \\
\hline \multirow{2}{*}{9} & $\mathrm{~F}$ & 8048 & 31170 & 22675 & 28089 & 128528 & 24514 & 91948 \\
\hline & $\mathrm{p}$ & 0 & 0 & 0 & 0 & 0 & 0 & 0 \\
\hline \multirow{2}{*}{12} & $\mathrm{~F}$ & 27638 & 60146 & 55996 & 61952 & 73731 & 60729 & 91005 \\
\hline & $\mathrm{p}$ & 0 & 0 & 0 & 0 & 0 & 0 & 0 \\
\hline
\end{tabular}

Table 89 . Wilks- $\lambda$ values obtained for each image of scores corresponding to each loading used. Pixels $(n=38000)$ from 7 of the 14 watercress leaves are compared, each leaf independently, between the first and the last tested day. The greatest Wilks- $\lambda$ value obtained for each leaf is marked in bold.

\begin{tabular}{cccccccc}
\hline $\begin{array}{c}\text { Number } \\
\text { of leaf }\end{array}$ & $\begin{array}{c}\text { Ld 3 } \\
\text { Spinach } \\
\text { RC }\end{array}$ & $\begin{array}{c}\text { Ld 1 } \\
\text { Spinach } \\
\text { RC+SG }\end{array}$ & $\begin{array}{c}\text { Ld 1 } \\
\text { Spinach } \\
\text { RC+SG+SNV }\end{array}$ & $\begin{array}{c}\text { Ld 1 } \\
\text { Watercress } \\
\text { RC+SG }\end{array}$ & $\begin{array}{c}\text { Ld 3 } \\
\text { Watercress } \\
\text { RC+SG }\end{array}$ & $\begin{array}{c}\text { Ld 1 } \\
\text { Watercress } \\
\text { RC+SG+SNV }\end{array}$ & $\begin{array}{c}\text { Ld 2 } \\
\text { Watercress } \\
\text { RC+SG+SNV }\end{array}$ \\
\hline 1 & 0.002 & 0.169 & 0.086 & 0.129 & 3.058 & 0.099 & $\mathbf{4 . 1 3 9}$ \\
2 & 0.001 & 0.010 & 0.001 & 0.001 & 3.337 & 0.001 & $\mathbf{3 . 6 4 1}$ \\
4 & 0.003 & 0.070 & 0.045 & 0.062 & 1.759 & 0.051 & $\mathbf{2 . 2 7 5}$ \\
5 & 0.469 & 0.886 & 0.858 & 0.867 & 0.659 & $\mathbf{0 . 8 9 1}$ & 0.879 \\
6 & 0.017 & 0.180 & 0.134 & 0.161 & 2.941 & 0.150 & $\mathbf{3 . 8 3 2}$ \\
9 & 0.106 & 0.410 & 0.298 & 0.370 & $\mathbf{1 . 6 9 1}$ & 0.323 & 1.210 \\
12 & 0.364 & 0.791 & 0.737 & 0.815 & 0.971 & 0.799 & $\mathbf{1 . 1 9 7}$ \\
\hline
\end{tabular}

When the spinach loadings are used directly on the watercress leaves, the results obtained are modest. There are significant differences between first and last tested days in those cases, but not as strong as using some of the own loadings obtained from the spectra of watercress leaves. The main differences between spinach and watercress loadings appear in NIR region, could justify why spinach loadings does not work as good as PC2 loadings of watercress. Watercress leaves suffered a more pronounced dehydration process than spinach leaves along the storage and that effect is not so reflected in spinach loadings. PCs of watercress were also tested in spinach spectra with poor results (not shown). Lee et al. (2014) tried to develop a common model for detecting bovine faeces on both Romaine lettuce and baby spinach leaves. Correlation analysis was used to select the most significant waveband pairs for two-band ratio and difference methods in distinguishing contaminated and uncontaminated leaf areas. For that investigation, two-band ratios using bands at $665.6 \mathrm{~nm}$ and $680.0 \mathrm{~nm}$ (F665.6/F680.0) for lettuce and at $660.8 \mathrm{~nm}$ and $680.0 \mathrm{~nm}$ (F660.8/F680.0) for spinach effectively differentiated all contamination spots applied to the lettuce and spinach leaves, respectively. Both ratios used almost the same spectral bands, with slight but relevant differences.

Figure 206 shows the virtual images of scores obtained by projection of the hyperspectral images of the watercress leaves (correspondingly corrected) onto the best principal component able to detect the evolution of leaves along the storage: PC2 from the spectra of watercress leaves corrected with $\mathrm{RC}+\mathrm{SG}+\mathrm{SNV}$. The fourteen leaves corresponding to the first and the last days of the test are shown. All the leaves evolve during time but with different rates. Some of the leaves suffered changes in shape during storage, becoming smaller and with curved edges, reducing the available surface for acquiring the spectra. 


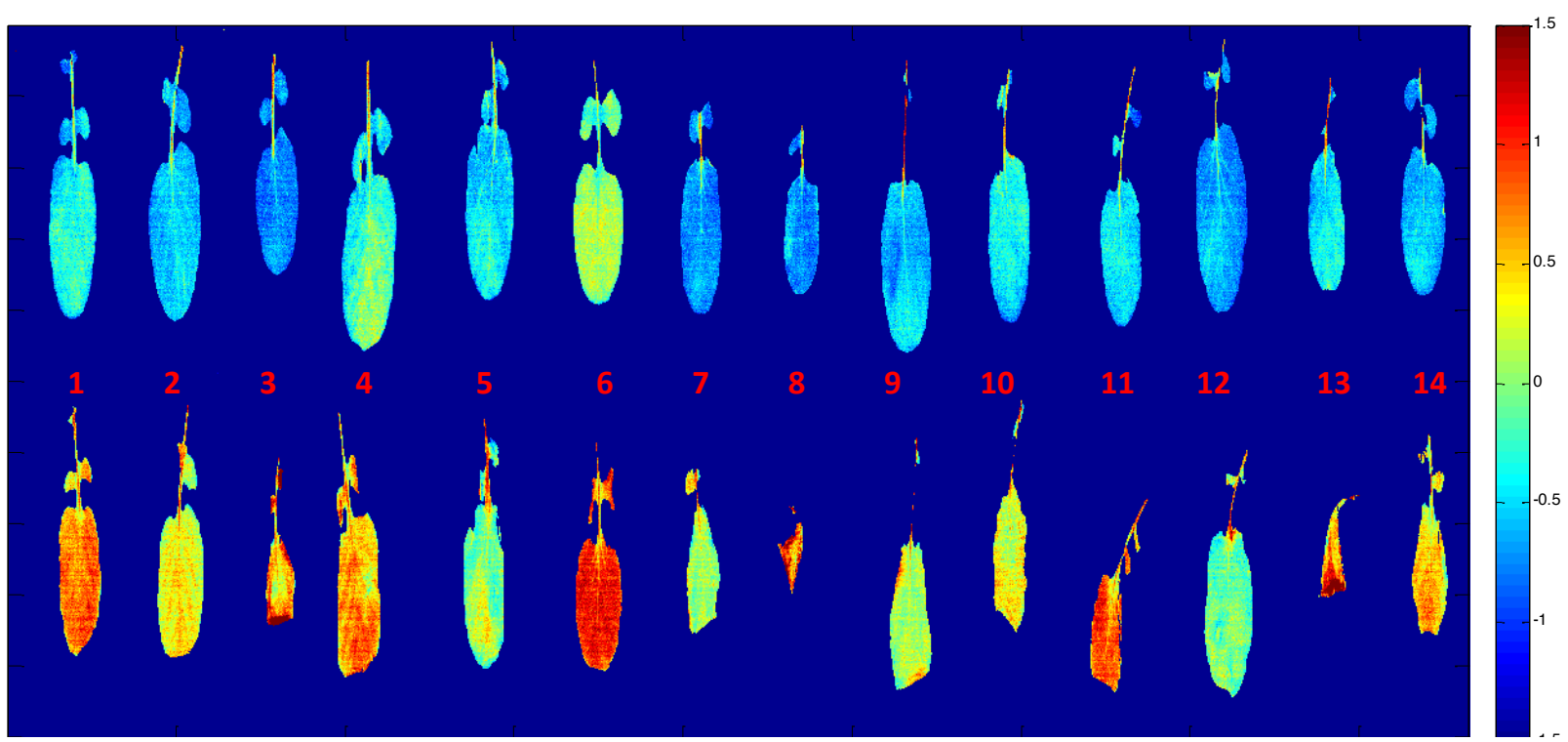

Figure 206. Scores images obtained by projection of the hyperspectral images of the leaves, correspondingly corrected, on PC2 from the spectra of watercress leaves corrected with $\mathrm{RC}+\mathrm{SG}+\mathrm{SNV}$. It is shown the 14 watercress leaves studied, the number of each leaf is marked in red. Upper row: first date. Lower row: last date.

As shown in Table 88 and Table 89, the F-Fisher and Wilks- $\lambda$ values obtained for each leaf are very variable. There are leaves that evolve faster than others, also depending on the initial stage of freshness (Figure 207).

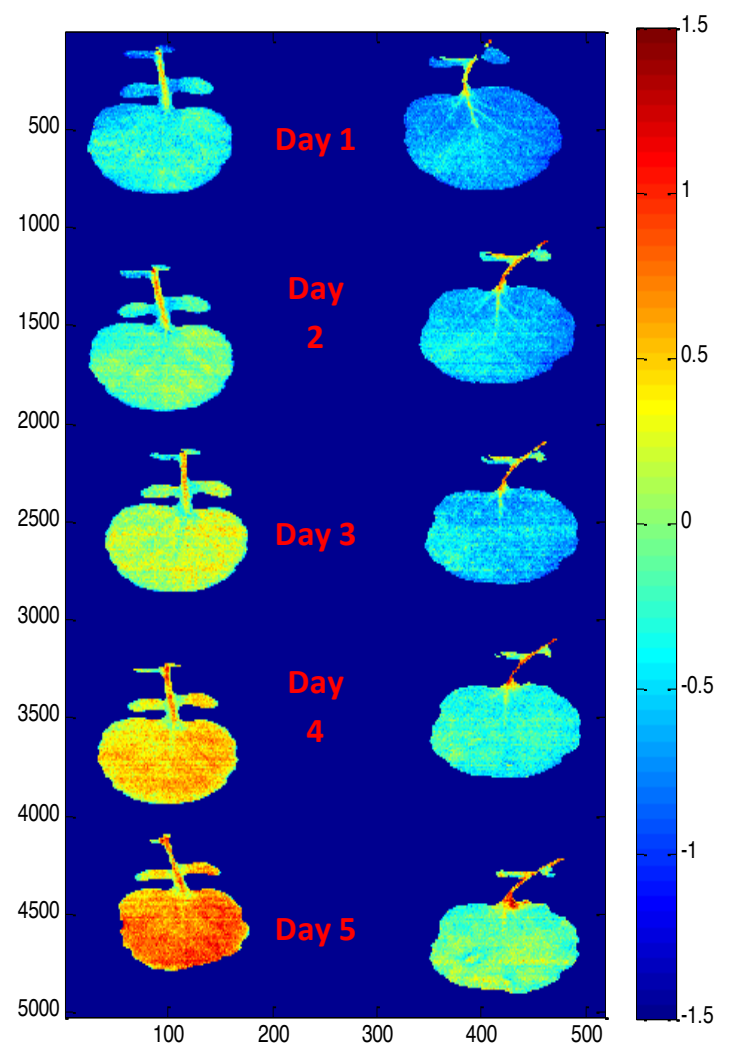

Figure 207. Scores images obtained by projection of the hyperspectral images of the leaves, correspondingly corrected, on PC2 from the spectra of watercress leaves corrected with $\mathrm{RC}+\mathrm{SG}+\mathrm{SNV}$. It is shown two different watercress leaves along the five testing days. 


\section{Conclusions}

The radiometric correction procedure allows removing the variation of the plastic film transmittance along the period storage.

The PCs calculated in the case of spinach leaves can be used in watercress leaves for detecting their evolution, showing significant differences between the leaves at the first and the last day. However, the results are modest. Applying the PCs calculated using the spectra of watercress leaves, the results obtained are better, showing greater differences between the pixels of the leaves in the different stages of evolution.

The loadings retained as the most related to the evolution of the watercress leaves, and those retained in the case of spinach, show the main information in the same spectral bands, with sharpened peaks in the range of 500$550 \mathrm{~nm}$, due to carotenoids, anthocyanins and chlorophylls; in the band of $680 \mathrm{~nm}$, due to chlorophyll; and in the range between 700 and $760 \mathrm{~nm}$, due to the red edge of the leaves. However, in the case of watercress, the most interesting loading shows also information in the NIR region, between 940 and $970 \mathrm{~nm}$. This fact can be the reason why, in this case, other PCs different to those retained in the case of spinach offer better results.

Thus, it seems to be necessary to analyse the spectra of each leafy vegetable species independently, to obtain the most satisfying results for monitoring its shelf-life using hyperspectral imaging. Although each leafy vegetable, spinach and watercress, seems to have different behaviour during shelf-life, there are some common changes that allow to obtain prediction models very similar, able to monitor the evolution of the leaves in both cases. This fact can be relevant for monitoring the shelf-life of mixed salads and ready-to-eat leafy vegetables prepared with different leaves together.

\section{Acknowledgements}

The funding of this work has been covered by Comunidad de Madrid, with the project S2013/ABI-2747 (TAVS-CM) and by the EU.

\section{References}

Asner, G.P., 1998. Biophysical and biochemical sources of variability in canopy reflectance. Remote Sensing of Environment 64 (3), 234-253.

Diezma, B., Lleó, L., Roger, J.M., Herrero-Langreo, A., Lunadei, L., Ruiz-Altisent, M., 2013. Examination of the quality of spinach leaves using hyperspectral imaging. Postharvest Biology and Technology. 85(0), pp. 8-17.

Gitelson, A.A., Keydan, G.P., Merzlyak, M.N. 2006. Three-Band Model for Non-invasive Estimation of Chlorophyll Carotenoids and Anthocyanin Contents in Higher Plant Leaves. Papers in Natural Resources. Paper 258.

Gitelson, A., Viña, A., Arkebauer, T.J., Rundquist, D.C., Keydan, G. And Leavitt, B. 2003. Remote estimation of leaf area index and green leaf biomass in maize canopies. Geophysical Research Letters. 30, 1248.

Gonçalves, E.M., Cruz, R.M.S., Abreu, M., Brandao, T.R.S., Silva, C.L.M. 2009. Biochemical and colour changes of watercress (Nasturtium officinale R.Br.) during freezing and frozen storage. Journal of Food Engineering. 93:32-39.

Jacquemoud, S., Baret, F. 1990. Prospect - a model of leaf optical-properties spectra. Remote Sensing of Environment. 34 (2), 75-91.

Kleima, F.J., Wendling, M., Hofmma, E., Peterman, E.J.G., Van Grondelle, R., Van Amerongen, H. 2000. Peridinin chlorophyll a protein: relating structure and steady-state spectroscopy. Biochemistry 39, 5184-5195.

Lara, M.A., Diezma-Iglesias, B., Lleó, L., Roger, J.M., Ruiz-Altisent, M. 2013. Monitoring spinach shelf-life with hyperspectral image through packaging films. Journal of Food Engineering. 119, 353-361.

Lara, M.A., Lleó, L., Diezma-Iglesias, B., Roger J.M., Garrido Y., Gil M.I,m Ruiz-Altisent, M 2016. Hyperspectral Imaging to Evaluate the Effect of IrrigationWater Salinity in Lettuce. MDPI Applied Sciences. 6, 412, 2-18.

Lee, H., Everard, C.D., Kang, S., Cho, B., Chao, K., Chan, D.E., Kim, M.S. 2014. Multispectral fluorescence imaging for detection of bovine faeces on Romaine lettuce and baby spinach leaves. Biosystems Engineering. 127(0), pp. 125-134.

Mutanga, O., Skidmore, A.K. 2007. Red edge shift and biochemical content in grass canopies. ISPRS Journal of Photogrammetry and Remote Sensing. 62 (1), 34-42.

Siripatrawan, U., Makino, Y., Kawagoe, Y., Oshita, S. 2011. Rapid detection of Escherichia coli contamination in packaged fresh spinach using hyperspectral imaging. Talanta 85 (1), 276-281.

Van Der Meer, F.D., Jong, S.M. 2002. Imaging spectrometry: basic principles and prospective applications. Remote Sensing and Digital Image Processing. 4, 113- 135 (Springer. Chapter 5). 


\author{
Design of an Innovative Plant for Fast Freezing of Potato Dumplings \\ Alessandro Biglia ${ }^{a, *}$, Lorenzo Comba ${ }^{\text {b }}$, Paolo Barge ${ }^{\text {a }}$, Enrico Fabrizio ${ }^{\text {b }}$, Davide Ricauda Aimonino a , \\ Cristina Tortia ${ }^{\text {a }}$, Paolo Gay ${ }^{\text {a }}$ \\ ${ }^{a}$ DiSAFA, Università degli Studi di Torino, Largo Paolo Braccini 2, 10095 Grugliasco (TO), Italy \\ b DENERG, Politecnico di Torino, Corso Duca degli Abruzzi 24, 10129 Torino, Italy \\ * Corresponding author. Email: alessandro.biglia@unito.it
}

\begin{abstract}
The design of an innovative plant for fast freezing of potato dumplings at temperatures much lower than traditional freezing plants is presented in this study. The designed plant is constituted by two separate loops: the first loop, where the cooling energy is obtained at low temperature, is based on a reversed Brayton cycle while the second loop, where the dumplings are frozen, is a forced-air freezer room. Optimal plant parameters, such as temperature and velocity of the freezing medium (air), to obtain the required fast freezing time of dumplings are evaluated. Depending on the requested freezing time and on the mass per hour of dumplings to be processed, the suitable plant solution can be identified and finely designed. The proposed solution overcomes limits of traditional freezing plants, such as ones operated by vapour compression cycles, which cannot reach such low temperatures without exceeding in complexity and number of stages. For this reason, the plant is proved to be a viable, costeffective alternative to traditional freezing plants used in food industries.
\end{abstract}

\title{
1. Introduction
}

Fresh food when processed and/or harvested continues to undergo chemical changes that involve spoilage and deterioration of the product. This food deterioration is due to enzymes and bacteria that cause the loss of texture and nutrients, the colour change, and the taste change. Freezing for short or long term period is a valuable technique of food conservation to extent food shelf life and to reduce food waste. The recommended temperature to storage frozen foods is $-18^{\circ} \mathrm{C}$ (Biglia et al., 2018) as this is the temperature at which enzymes and bacteria stop growing.

Water represents a high percent of weight of most foods, even over $90 \%$ in fruits and vegetables. Part of this water is bound with chemical substances while the remain one is unbound and held within the cell walls. When the temperature of the food drops below freezing point, the unbound fraction of water freezes and expands. The ice crystals cause the cell walls rupture and, consequently, nutrients will be released during food thawing and food texture will be also much softer than it was before freezing. The cell wall damage can be controlled by freezing the food as quickly as possible since quick freezing process allows small ice crystals to be formed. Indeed, slow freezing produces only large ice crystals.

Freezing time can be defined as the time taken for the temperature of the food core to be reduced to $-18^{\circ} \mathrm{C}$. The time of the freezing process depends on: (1) physical and chemical properties of the food; (2) velocity of the medium used to freeze the food, typically purified air, and (3) temperature of the medium (Biglia et al., 2016; Pham, 2014; ASHRAE, 2010). Freezing plants adopted in food industry are usually operated by vapour compressor thermodynamic cycles. These plants allow the medium (air) temperature to be reduced at [-40, -20] ${ }^{\circ} \mathrm{C}$. The low temperature air is usually circulated in closed freezer rooms with a velocity between $[1,5] \mathrm{m} \cdot \mathrm{s}^{-1}$. These freezers are named air blast freezers (Dempsey and Bansal, 2012). Specific air blast freezers have been developed for small and/or thin foods (e.g. slices of fruit, fish fillets, etc.) where air is injected directly on the external surface of the food at very high velocity, more than $20 \mathrm{~m} \cdot \mathrm{s}^{-1}$. This technology, known as impingement (Salvadori and Mascheroni, 2002), significantly reduces the freezing time as the heat transfer coefficient between medium and food is high $\left(>50 \mathrm{~W} \cdot \mathrm{m}^{-2} \mathrm{~K}^{-1}\right)$. Alternatively, a liquid medium (e.g. $\mathrm{CaCl}_{2}$ solutions, $\mathrm{CO}_{2}, \mathrm{~N}_{2}$, etc.) may be used in food freezing (Galetto et al., 2010). An advantage of liquid medium is the enhanced heat transfer coefficient that is 10 to 20 times higher than the one obtained with gaseous medium used in air blast freezers.

Low temperatures for food freezing can also be achieved by means of a reversed Brayton cycle (Biglia et al., 2017; Foster et al., 2011). In this work, an innovative plant configuration for potato dumplings freezing at very low temperatures $\left(<-70^{\circ} \mathrm{C}\right)$ is presented. The designed plant is constituted by two separate loops: the first loop, where the cooling energy is obtained at low temperature, is based on a reversed Brayton cycle while the second loop where, the dumplings are frozen, is a forced-air freezer room. Optimal plant parameters, such as temperature and velocity of the freezing medium (air), to obtain the required fast freezing time of the dumplings are evaluated. Depending on the requested freezing time and on the mass per hour of dumplings to be processed, the suitable plant solution can be identified and finely designed. The proposed solution overcomes limits of traditional freezing plants, such as ones operated by vapour compression cycles, which cannot reach such low temperatures without exceeding in complexity and number of stages. For this reason, the plant is proved to be a viable, cost-effective alternative to traditional freezing plants used in food industries. 
Food freezing plant

The proposed food freezing plant can be outlined into two sub-systems: (1) a freezer room where food is frozen by means of refrigerated air, and (2) a reversed Brayton cycle, which produces cooling energy at very low temperature. A heat exchanger, installed in the freezer room, connects the two systems.

\section{Freezer room}

The final quality of frozen foods is related to the resulting freezing time $\tau$, which, for a specific food product, can be obtained by finding the proper trade-off between temperature $T_{\mathrm{m}}$ and velocity $v_{\mathrm{m}}$ of the medium. Therefore, the design of a freezing room is not a trivial task.

Given the mass of fresh food $G$ that has to be frozen per unit of time, the cooling capacity $Q$ of the freezing room can be easily computed as

$$
\mathrm{Q}=\mathrm{G} \cdot \Delta \mathrm{E}
$$

where $\Delta \mathrm{E}$ is the energy that has to be removed from the food during freezing process (Biglia et a., 2016; ASHRAE 2010). The parameter $\Delta \mathrm{E}$ depends on several food product data: 1) temperature at the beginning and at the end of the freezing process, 2) chemical composition and 3) shape. The higher the $\Delta \mathrm{E}$ is the higher the freezing time $\tau$ will be.

The freezing time $\tau$ can be evaluated as reported by authors Biglia et al. (2016); according to the type of food, the size of the batch lot $\mathrm{M}[\mathrm{kg}]$ can be defined as

$$
\mathrm{M}=\mathrm{G} \cdot \tau
$$

Considering the case of batch processes, an important parameter resides in the mass $\mathrm{M}$ of the batch that can be frozen in the insulated room as a function of medium temperature $T_{\mathrm{m}}$ and velocity $v_{\mathrm{m}}$ and of the plant cooling capacity Q. With this aim, a chart of the batch lot size has been defined for potato dumplings, in which iso-mass curves has been calculated as a function of food production rate G, of freezing time $\tau$ and of cooling capacity Q. Therefore, the proper size of the lot to be frozen by batch can be obtained. In potato dumpling, moisture and ash fractions content have been evaluated experimentally as they deeply affects the freezing time $\tau$.

\section{Reversed Brayton cycle}

The main components of the reversed Brayton cycle are turbo-machineries and heat exchangers. The scheme of the cycle, showing components links, is reported in Figure 1.

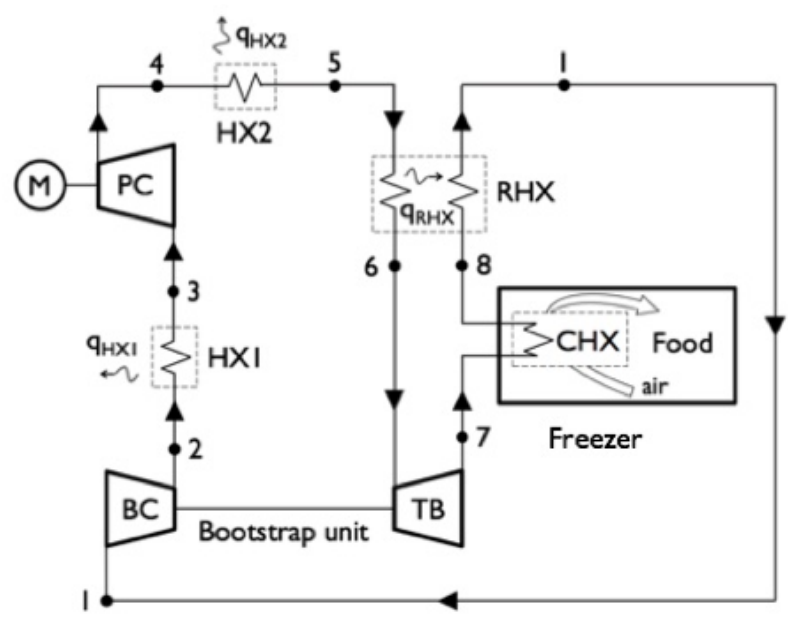

Figure 208. Schematic of the reversed Brayton cycle for fast freezing.

The refrigerant gas enters the bootstrap compressor $(\mathrm{BC})$ at state 1 , where the pressure is minimum, and is compressed to state 2 . The gas is then cooled to state 3 in the heat exchanger $(\mathrm{HX} 1)$ and next, the gas is compressed in the motor-compressor (PC) to the maximum pressure and temperature of the cycle (state 4). Once compressed and before entering the turbine, the gas is sequentially cooled in HX2 (state 5) and in a regenerative heat exchanger (RHX), state 6. In the subsequent expansion through the turbine, the gas achieves the lowest temperature of the cycle at state 7 . The mechanical work produced by the turbine through the expansion is supplied to the bootstrap compressor by installing the two components on the same shaft. The gas exiting the turbine, before re-entering the compressor $\mathrm{BC}$, passes through the cold heat exchanger $(\mathrm{CHX})$ installed in the freezer room, state 8 , and through the RHX, state 1. In the CHX, the gas cools the air in the batch freezer by absorbing heat released by the food during the freezing process. The refrigerant gas always remains in the gaseous state at each state of the cycle. Dry air has been selected as refrigerant gas of the cycle. 
The coefficient of performance $(\mathrm{CoP})$ of the reversed Brayton cycle can be defined as

$$
\operatorname{CoP}=\frac{h_{8}\left(T_{8}, p_{8}\right)-h_{7}\left(T_{7}, p_{7}\right)}{\left(h_{4}\left(T_{4}, p_{4}\right)-h_{3}\left(T_{3}, p_{3}\right)\right) \eta^{-1}}
$$

which is the ratio of the gas enthalpy difference in the $\mathrm{CHX}$ and in the PC, taking into account the electric motor efficiency $\eta$.

The heat released by the refrigerant gas in HX1 and HX2 can be recovered to enhance the performance of the reversed Brayton cycle. Indeed, thermal processes are typically required in the food industry for cooking, heating (Biglia et al., 2015; Comba et al., 2011, 2010), blanching, debacterisation (Biglia et al., 2017), etc.

A numerical model of the proposed reversed Brayton cycle has been developed to evaluate its energy performance. The steady state form of the energy rate balance was used to develop the thermodynamic model of the cycle's components. More in detail, steady-state conditions were considered and heat losses across the cycle's components were neglected. The model was implemented by using Matlab ${ }^{\circledR}$ environment and the thermodynamic properties of the dried air were evaluated by using REFPROP database.

\section{Results and Discussion}

Freezer room

Moisture fraction content and ash in potato dumplings have been measured experimentally. Potato dumplings, after being characterised in terms of shape and weight, have been put into the oven at $105{ }^{\circ} \mathrm{C}$ for 24 hours to remove humidity (Figure 2). Then, dried potato dumplings have been put into the muffle at $450{ }^{\circ} \mathrm{C}$ for 6 hours to obtain ash content.
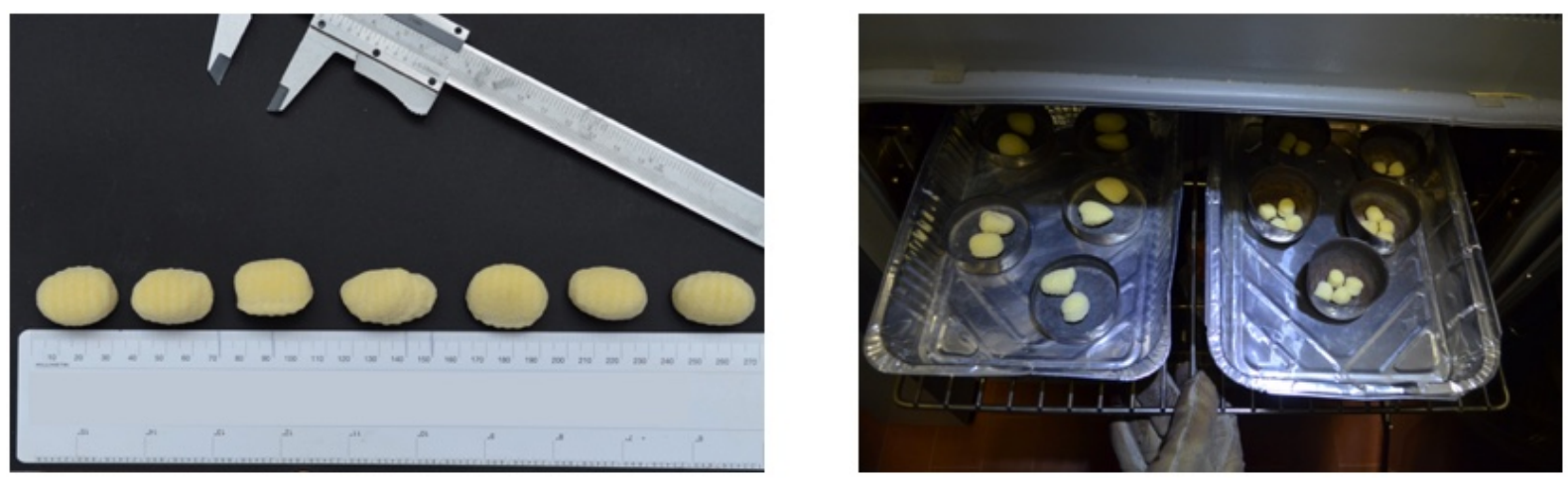

Figure 2. Potato dumplings experimental tests.

Results of potato dumplings characterisation have been reported in Table 1 together with data obtained from the label attached on the food packaging label. Latent heat of potato dumplings has been found on literature (ASHRAE, 2010). 
Table 90. Composition of potato dumpling and geometrical data.

\begin{tabular}{lc}
\hline \multicolumn{1}{c}{ Potato dumplings } \\
\hline Moisture fraction content [\%] & 0.495 \\
Protein fraction content [\%] & 0.137 \\
Fat fraction content [\%] & 0.067 \\
Carbohydrate fraction content [\%] & 0.278 \\
Fiber fraction content $[\%]$ & 0.054 \\
Ash fraction content $[\%]$ & 0.023 \\
Initial food temperature $\left[{ }^{\circ} \mathrm{C}\right]$ & 15.0 \\
Initial freezing temperature $\left[{ }^{\circ} \mathrm{C}\right]$ & -1.0 \\
Final food temperature $\left[{ }^{\circ} \mathrm{C}\right]$ & -25.0 \\
Latent heat $[\mathrm{kJ} / \mathrm{kg}]$ & 228 \\
Ellipsoid shape* & \\
a [cm] & 2.9 \\
$\mathrm{~b}$ [cm] & 2.0 \\
$\mathrm{c}[\mathrm{cm}]$ & 1.6 \\
\hline & *Potato dumplings shape has been modelled as an ellipsoid
\end{tabular}

Figure 3 shows two charts that have been developed to properly size the cooling capacity Q of the freezer room. In particular, the chart in Figure 3 a reports the freezing time as a function of the air temperature and of the air velocity that flows in the freezer room. The chart in Figure $3 \mathrm{~b}$ reports the cooling capacity Q of the freezer room as a function of the freezing time and of the food production rate $\mathrm{G}$.

a)

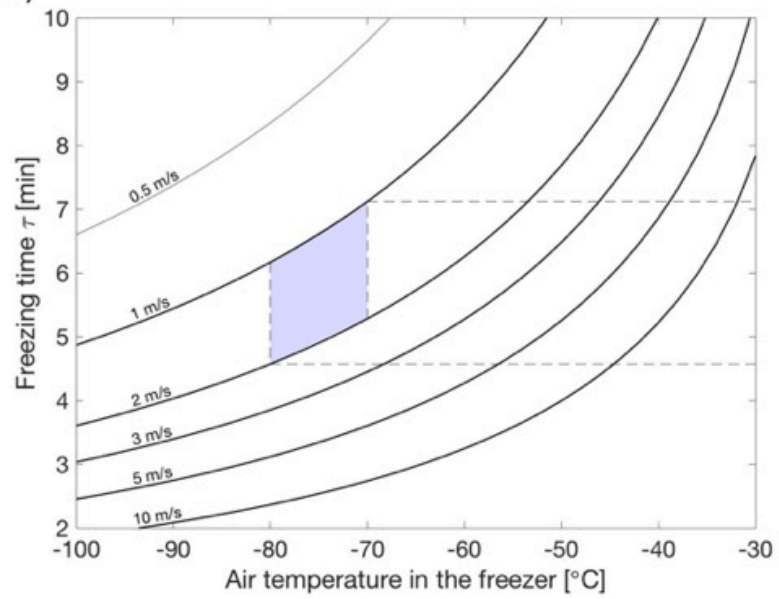

b)

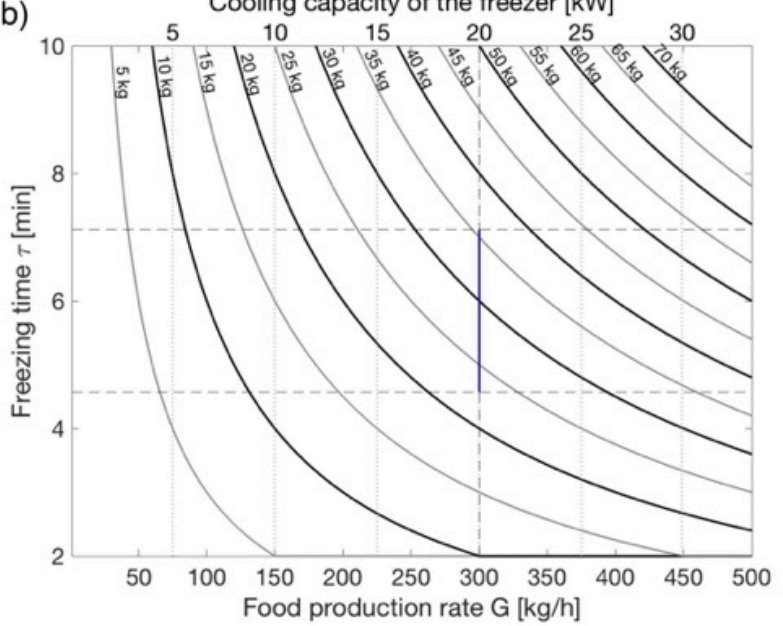

Figure 3. Potato dumplings freezing time $\tau$ as a function of medium temperature $T_{\mathrm{m}}$ and velocity $v_{\mathrm{m}}$ (a), and as a function of food production rate $\mathrm{G}$ and freezer cooling capacity $\mathrm{Q}(\mathrm{b})$.

For example, the blue area indicated in Figure $3 \mathrm{a}$ is representative of some combinations of design parameters (air temperature and velocity) that assure a certain freezing time of the food potato dumplings. The freezing time is linked to the cooling capacity and food production rate in second chart (Figure 3b).

In particular, the time $\tau$ required to process the potato dumplings from an initial temperature of $15^{\circ} \mathrm{C}$ to a final temperature of $-25^{\circ} \mathrm{C}$, was evaluated with an air temperature $T_{\mathrm{m}}$ and velocity $v_{\mathrm{m}}$ and varying within the ranges [$80,-70]^{\circ} \mathrm{C}$ and $[1,2] \mathrm{m} / \mathrm{s}$ respectively. The resulting freezing time for potato dumplings, with the operative ranges reported above, results to be in the range $[4,7]$ minutes. It should be noticed that, for low air velocity values, a reduction in the air temperature involves a significative decrease in the freezing time.

The second chart can be used to size the freezing room in terms of capacity and batch lot. With the aim of freezing $300 \mathrm{~kg} / \mathrm{h}$ of potato dumpling (blue line in Figure $3 \mathrm{~b}$ ), ensuring the freezing time reported in Figure 3a, the suitable cooling capacity of the room resulted to be equal to $20 \mathrm{~kW}$. Once the range of freezing time $\tau$ (highlighted by horizontal dashed lines in Figure 3a and 3b), obtained by the proper trade-off between values of $T_{\mathrm{m}}$ and $v_{\mathrm{m}}$, has been determined, the most adequate batch lot size, in term of mass of potato dumplings, can be obtained (iso-mass curves in Figure 3b). Processing the potato dumplings in the freezing room with air temperature in the range $[-80,-70]^{\circ} \mathrm{C}$ and air velocity in the range $[1,2] \mathrm{m} / \mathrm{s}$, the size of the batch lot $\mathrm{M}$ resulted to be in the range $[22,35] \mathrm{kg}$. According to the results of Figure 3, trolley and freezer room to host potato dumplings were selected and designed respectively (Figure 4). 

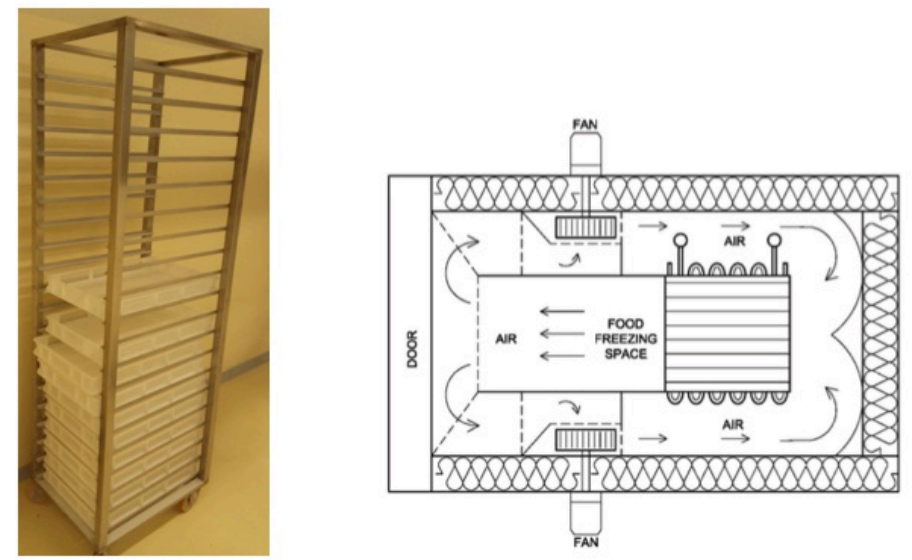

Figure 4. Trolley to host potato dumplings (a) and section of the designed freezer room (b).

\section{Reversed Brayton cycle}

The energy performance of the reversed Brayton cycle has been studied by means of a performance analysis. The results of the performance analysis, considering the main thermodynamic parameters influencing the cycle performance, are shown in Figure 5. Given a fixed cooling capacity of the freezer room, $20 \mathrm{~kW}$ as shown in Section 3.1, the temperature difference of the dry air in the CHX (Figure 5a) affects the mass flow rate of the cycle and, consequently, the size of the cycle's components. The maximum pressure of the cycle (Figure 5a), the TB isentropic efficiency and RHX effectiveness (Figure 5b), given a fixed temperature at the inlet of the freezer room, affect the TB expansion ratio and, consequently, the mechanical work produced in the bootstrap unit and the required mechanical work (electric motor) to drive the primary compressor.

a)

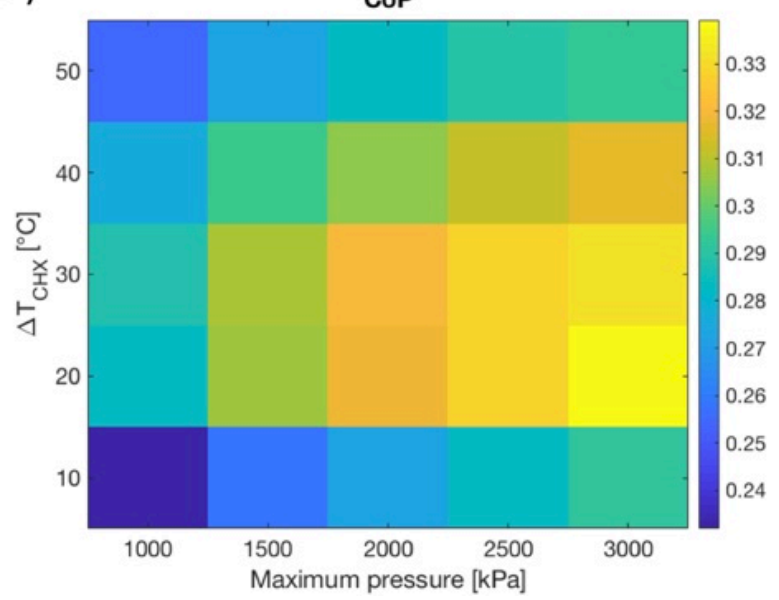

b)

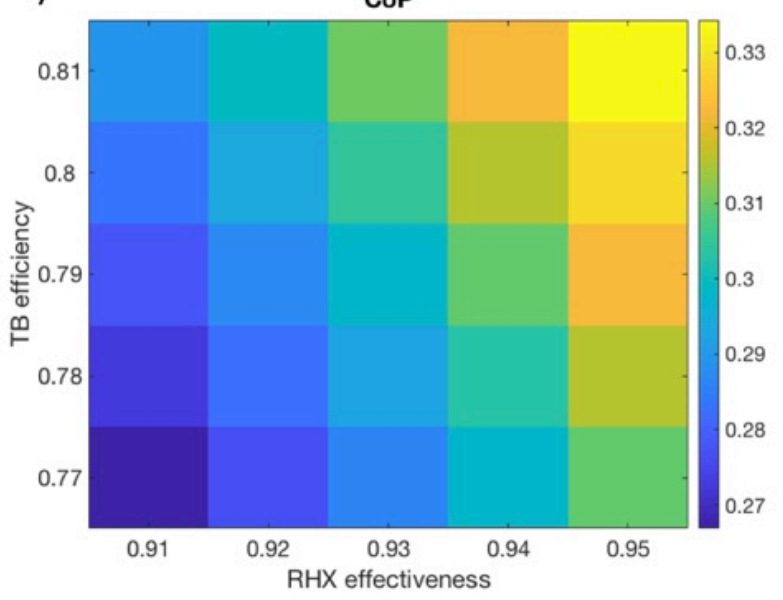

Figure 5. Reversed Brayton cycle performance: Effect of the maximum pressure and temperature difference in CHX (a) and effect of the RHX effectiveness and TB isentropic efficiency (b).

According to the performance analysis results and to real datasheet of the reversed Brayton cycle components, the thermodynamic states (temperature and pressure) of each cycle node of the best operating conditions of the reversed Brayton cycle is reported in Figure 6. 


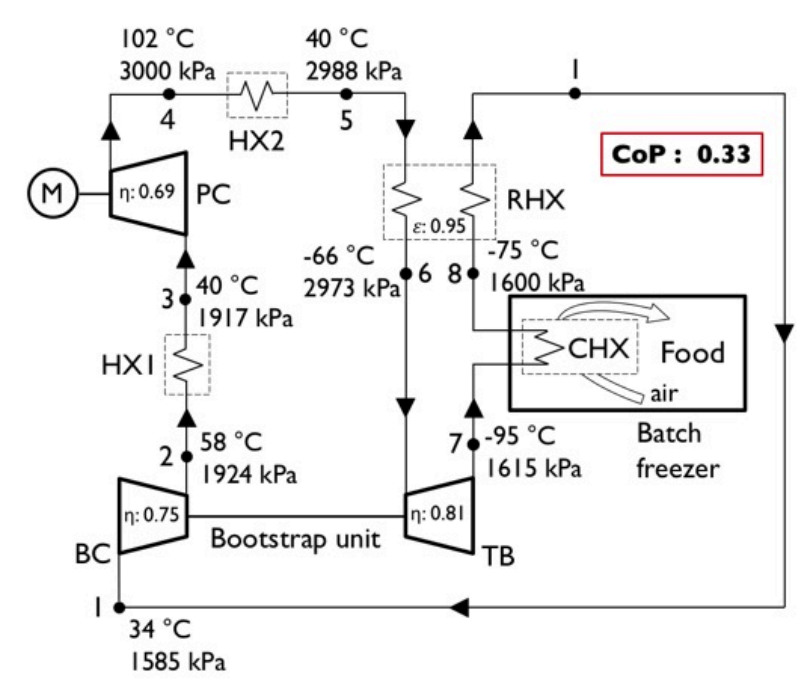

Figure 6. Results of the performance analysis of the reversed Brayton cycle.

The resulting values of the dry air mass flow rate, mechanical power of the turbo machineries and of the heat exchangers, and coefficient of performance are reported in Table 2.

Table 2. Specifications of the reversed Brayton cycle for food freezing.

\begin{tabular}{lc}
\hline \multicolumn{1}{c}{ Parameter } \\
\hline Dry air mass flow rate $[\mathrm{kg} / \mathrm{h}]$ & 3176 \\
Turbine mechanical power $[\mathrm{kW}]$ & 23 \\
Bootstrap compressor mechanical power $[\mathrm{kW}]$ & 21 \\
Primary compressor mechanical power $[\mathrm{kW}]$ & 60 \\
Cooling capacity in the freezer room $[\mathrm{kW}]$ & 20 \\
Capacity of heat exchanger, HX1 $[\mathrm{kW}]$ & 16 \\
Capacity of heat exchanger, HX2 $[\mathrm{kW}]$ & 59 \\
Coefficient of performance & 0.33 \\
Global coefficient of performance & 1.58 \\
\hline
\end{tabular}

The maximum and minimum temperature of the cycle account for $102{ }^{\circ} \mathrm{C}$ and $-95{ }^{\circ} \mathrm{C}$ respectively. It should be noted that, $16 \mathrm{~kW}$ of heat $(\mathrm{HX} 1)$ can be recovered from the cycle at low temperature $\left(<60^{\circ} \mathrm{C}\right)$ while $59 \mathrm{~kW}$ of heat $(\mathrm{HX} 2)$ can be recovered at medium-low temperature $\left(<100{ }^{\circ} \mathrm{C}\right)$. This amount of heat could be used for space heating and/or food processing, allowing a global coefficient of performance equal to 1.58 to be achieved. The mechanical power of the turbine is larger than the mechanical power of the bootstrap compressor to balance mechanical losses of the bootstrap unit. Mechanical power of the primary compressor takes into account the electric motor efficiency. The coefficient of performance, without heat recovery, was found to be 0.33 .

Considering an electricity cost of $18 \mathrm{c} € / \mathrm{kWh}$, a running cost of $55 \mathrm{c} €$ per $\mathrm{kWh}$ of cooling energy has been estimated for the proposed innovative freezing plant.

\section{Conclusions}

The design of an innovative food freezing plant has been presented. The innovative freezing plant is based on a reversed Brayton cycle where the cooling energy is produced and a freezing room where the potato dumplings are frozen. The proposed freezing plant is able to run at very low temperatures $\left(<-70{ }^{\circ} \mathrm{C}\right)$ which guarantee fast food freezing into the freezing room. In addition to the $20 \mathrm{~kW}$ of cooling capacity at low temperatures in the freezer room, the reversed Brayton cycle allows $75 \mathrm{~kW}$ of heat to be recovered at medium-low temperatures in the heat exchangers HX1 and HX2.

The CoP of the reversed Brayton cycle, without heat recovery, resulted to be 0.33 with a running cost of 55 $\mathrm{c} €$ per kWh of cooling energy. The global CoP, including heat recovery, was found to be 1.58 . The freezer room allows a production of $300 \mathrm{~kg} / \mathrm{h}$ of frozen potato dumpling with a batch lot of 25/35 kg. 


\section{Acknowledgements}

This research was partially funded by projects "SACS” and "CRYOFOOD” (POR FESR 2007-2013 and 20142020, Polo Innovazione AgriFood). The authors would like to acknowledge Criotec Impianti, Dyria Sistemi, La Monfortina and Zoppi project partners for their collaboration.

\section{References}

ASHRAE, 2010. Refrigeration ASHRAE Handbook. ISBN 9781933742823.

Biglia, A., E., Fabrizio, M., Ferrara, P., Gay, D., Ricauda Aimonino, 2015. Performance assessment of a multienergy system for a food industry. Energy Procedia. 82, 540-545.

Biglia, A., L., Comba, E., Fabrizio, P., Gay, D., Ricauda Aimonino, 2016. Case studies in food freezing at very low temperature. Energy Procedia. 101, 305-312.

Biglia, A., L., Comba, E., Fabrizio, P., Gay, D., Ricauda Aimonino, 2017. Steam batch thermal processes in unsteady state conditions: Modelling and application to a case study in the food industry. Appl Therm Eng. 118, 638-651.

Biglia, A., A.J., Gemmell, H.J., Foster, J.A., Evans, 2018. Temperature and energy performance of domestic cold appliances in households in England. Int J Refrig. 87, 172-184.

Comba, L., P., Gay, P., Piccarolo, D., Ricauda Aimonino, 2010. Thermal processes in the candy process of chestnut. Acta Horticulturae. 866, 587-594.

Comba, L., G., Belforte, P., Gay, 2011. Modelling techniques for the control of thermal exchanges in mixed continuous-discontinuous flow food plants. J Food Eng. 106, 177-187.

Dempsey, P., P., Bansal, 2012. The art of air blast freezing: Design and efficiency considerations. Appl Therm Eng. 47, 71-83.

Foster, A.M., T., Brown, A.J., Gigiel, A., Alford, J.A., Evans, 2011. Air cycle combined heating and cooling for the food industry. Int J Refrig. 34, 1296-1304.

Galetto, C.D., R.A., Verdini, S.E., Zorrilla, A.C., Rubiolo, 2010. Freezing of strawberries by immersion in $\mathrm{CaCl}_{2}$ solutions. Food Chem. 123, 243-248.

Pham, Q.T., 2014. Freezing time formulas for foods with low moisture content, low freezing point and for cryogenic freezing. J Food Eng. 127, 85-92.

Salvadori, V.O., R.H., Mascheroni, 2002. Analysis of impingement freezers performance. J Food Eng. 54, $133-140$ 


\title{
Simultaneous Production of Functional Lipids and Natural Antioxidants from Passion Fruits Seeds
}

\author{
Carolina Cruzeiro Reis ${ }^{\mathrm{a}}$; Alexandra Mara Goulart Nunes Mamede ${ }^{\mathrm{b}}$; Nina Katia da Silva James ${ }^{\mathrm{a}}$; \\ Antonio Gomes Soares '; Suely Pereira Freitasa,"* \\ ${ }^{\text {a }}$ Escola de Química - Universidade Federal do Rio de Janeiro - UFRJ. \\ ${ }^{b}$ Instituto Federal de Educação, Ciência e Tecnologia da Bahia - IFBA - Campus Barreiras, BA, Brazil \\ ${ }^{\mathrm{c}}$ Embrapa Agroindústria de Alimentos - Rio de Janeiro - RJ, Brazil \\ * Corresponding author. Email: freitasp@eq.ufrj.br
}

\begin{abstract}
The wild passion fruit species, Passiflora setacea, P. alata, and P. tenuifila are native to the Brazilian biomes, particularly Atlantic Forest, Cerrado, and Caatinga. The seeds waste generated after passion fruits juice extraction contains functional polyunsaturated fatty acids and phenolic compounds with high anti-oxidant capacity. The main objectives of this study were to apply sustainable technology to obtain functional lipids and natural antioxidants from passion fruits seeds. Passion seeds oils were obtained using a lab-scale continuous press and their oxidative stability was evaluated using the Rancimat ${ }^{\mathbb{R}}$ method. Regarding the phenolic compounds extracted from pressed cake, a statistical factorial design was applied to select the best solvent polarity and temperature of extraction. The antioxidant capacity (TEAC) of all extracts were the main response to select the best operational conditions. The higher antioxidant capacity from the extracts was found using ethanol-water solution $(70: 30)$ at $45^{\circ} \mathrm{C}$. In these cases, the total phenolic content expressed as gallic acid equivalent from P. setacea, P. alata, and P. tenuifila cakes were approximately 1800,600 and $900 \mathrm{mg} 100 . \mathrm{g}^{-1}$ of extract. Using these extracts as natural antioxidant in their respective oils, the induction periods for P. setacea, P. alata, and P. tenuifila seeds oils increased from 7.30 to 9.60 h, 3.52 to $11.7 \mathrm{~h}$, and 6.87 to $10.27 \mathrm{~h}$, respectively. Therefore, the passion fruit seeds from these selected species can contribute to increase the supply of vegetable polyunsaturated oils with higer oxidative stability.
\end{abstract}

Keywords: Passiflora alata, Passiflora setácea, Passiflora tenuifila, phenolic compounds, natural antioxidants, sustainable technology.

\section{Introduction}

Passion fruits are native of America and grow in tropical and subtropical regions of the world. In Middle West and Northeast regions of Brazil there are near 150 native species, of which about 60 are known to be edible (Dhawan el al. 2004; Bernacci et al. 2003; Cunha et al, 2002). Unfortunately, currently only two are commercially important, Passiflora edulis Sims f. flavicarpa Deg. (yellow passion fruit) and P. edulis f. edulis Sims (purple passion fruit) (Narain et al., 2010). However other species hold promising sensory and functional characteristics and could be explored commercially, such as $P$. setacea, $P$. alata, and $P$. tenuifila, native fruits of Brazilian biomes, particularly Atlantic Forest, Cerrado, and Caatinga.

Brazil is the largest producer and consumer of passion fruit juice in the world. In 2016, 703 thousand tons of yellow passion fruit were produced (IBGE, 2016). At least $40 \%$ of the production goes for juice or pulp industry. Therefore, the passion fruit juice industry discards enormous amounts of pomace, composed particularly of peels and seeds which may still contain high quantity of bioactive compounds. Currently, these natural compounds have been used as functional ingredients for foods, contributing to sustainable development of food and biomass production and processing.

Agro-industry pomace such as peels and seeds have industrial interest due to their composition with high content of lipids, protein, fiber, sugars, vitamins, minerals and bioactive compounds, such as phenolic compounds, phytosterols and carotenoids. In this case, these agro-industrial wastes become to raw materials of interest to the chemical industry, particularly to the pharmaceutical, food and cosmetics sectors (Silva and Jorge, 2014). Besides that, the waste disposal is restricted by legal norms. Thus, the processing of these materials has been shown to be technically and economically feasible in several productive chains (Lowe and Buckmaster, 1995).

Reported data in the literature indicate that the passion fruit pomace contain phenolic compounds of great industrial interest (Mirabella et al., 2014). According to Reis et al. (2016) the phenolic compounds have antiallergic, anti-inflammatory and antimicrobial effects, preventing, in some cases, cardiovascular diseases and tumors.

Passion fruit processing in food industries discards up to $40 \%$ of the fruit, of which the seeds correspond to 615\% (Manica, 2005). The passion fruit seeds oils are rich in mono and polyunsaturated fatty acids, mostly the linoleic acid (C18:2 n-6). According to Paula (2015) the lipids from species $P$. setacea BRS Pérola do Cerrado and P. alata BRS Doce Mel contains between 57 and 59\% of this fatty acid. The fatty acid composition depends, among other factors, on the fruit species and climatic characteristics of the producing region. 
The passion fruit seed present a large amount of piceatannol (3,4,3',5'-tetrahydroxytrans-stilbene) in its phenolic composition. Piceatannol is a hydroxylate of resveratrol and have strong antioxidant activity (UchidaMaruki et al., 2015; Matsui et al., 2010). According to Matsui et al (2010) the high level of piceatannol in passion fruit seeds exert positive effects on cultured dermal cells in inhibition of melanogenesis and synthesis of collagen.

Due to the phytotherapeutic properties of pulp and leaves of native passion fruit species, Embrapa has been developing new passion fruit genotypes for classic genetic enhancement, where the focus is to increase the consumption of fruit by improving the pleasant sensorial characteristics, increasing the resistance in the field and enhanced the functional properties. Thus, due to the properties of passion fruit seeds above mentioned, the main objectives of this study were to obtain functional lipids and natural antioxidants from passion fruits $(P$. setacea, $P$. alata, and $P$ tenuifila) seeds, applying sustainable technology.

\section{Materials and Methods}

Material

Seeds of three species of passion fruit (Passiflora alata, Passiflora setacea and Passiflora tenuifila) were grown in the experimental fields of Embrapa Cerrados (Brasília, Distrito Federal). After fruits were pulped, the seeds were stored at $-20^{\circ} \mathrm{C}$ until their use. After defrosting, the seeds were washed in running water to remove residues of pulp and mucilage still adhered to seeds, then the seeds were autoclaved at $120{ }^{\circ} \mathrm{C}$ for 20 minutes to reduce the microbial load.

Kinetics of seed drying

The passion fruit seeds were dried in a convective dryer at $50{ }^{\circ} \mathrm{C}$ and the samples were weighed at 30 minutes intervals until the mass of the samples remained unchanged over three successive weightings. The drying temperature was optimized in previous experiments for $P$. alata and $P$. setacea seeds drying (Paula, 2015). The physical properties of the air were monitored by dew point of the Cole-Parmer Traceable ${ }^{\circledR}$ Hygrometer thermometer. Drying was performed in triplicate.

Mass diffusion coefficient

For estimation of effective water diffusivity $\left(D_{\text {eff }}\right)$ during seeds drying, the dimensionless moisture $\left(M_{R}\right)$ and time $(\mathrm{t})$ data was fitted using Fick's second law of diffusion model (Eq. 1) in spherical coordinates, considering up to three terms of the infinite series solution using Statistica software (v.13.0).

$$
M r=\frac{M-M s}{M o-M s}=\frac{9}{\pi^{2}} \sum_{n=0}^{10} \frac{1}{(2 n+1)^{2}} \exp \left(-\frac{(2 n+1)^{2} \pi^{2} D_{e f f} t}{r^{2}}\right)
$$

Deff - diffusion coefficient, $\mathrm{m}^{2} \cdot \mathrm{s}^{-1}$

$\mathrm{Ms}_{\mathrm{S}}$ - equilibrium moisture content, in dry basis

$\mathrm{M}_{\mathrm{R}}$ - moisture ratio
$\mathrm{M}$ - moisture content at time $\mathrm{t}$, in dry basis

$\mathrm{M}_{0}$ - initial moisture content, in dry basis

$\mathrm{r}$ - average seeds radius

The average seeds radius was related to a sphere of equivalent volume

Grinding and seeds oil extraction

After drying, the seeds of $P$. alata and $P$. setacea were ground in a knife mill (Macron MA-048). In case of the $P$. tenuifila seeds, this step was not necessary because it has a reduced average diameter $\left(\mathrm{d}_{\mathrm{p}}<3 \mathrm{~mm}\right)$. Crushed passion fruit seeds were processed in a continuous press (Oekotec - Germany, CA59G model) in order to obtain a crude oil, which was decanted for $24 \mathrm{~h}$ in a dark cabinet. The clarified fraction was stored at $-18{ }^{\circ} \mathrm{C}$ until the moment of use and the pressed cake was reserved for phenolic compounds extraction.

Phenolic compounds extraction from pressed cake.

For extraction of phenolic compounds, the pressed cake was triturated in a knife mill (Macron MA-048) coupled to a $1 \mathrm{~mm}$ diameter circular mesh sieve. The crushed sample, with particle diameter smaler than $2 \mathrm{~mm}$, was macerated with ethanol in the 1:5 ratio (pressed cake:ethanolic solution).

For selected the best extraction parameters, the experimental design using three different aqueous-etanolic solutions selected from the preliminary trial, were carried out at 30:70, 50:50 and 70:30 ethanol:water. The suspension was maintained in heating bath at $45^{\circ} \mathrm{C}$ for 30 minutes, with agitation rate of $600 \mathrm{rpm}$. After extraction, the solids and soluble compounds were separated by vacuum filtration. The liquid phase, which contains the bioactive compounds, was stored at $-18.0 \pm 0.5^{\circ} \mathrm{C}$ until analysis. 
Analytical methods

- The oxidative stability of passion seeds oils were measured using Rancimat ${ }^{\circledR} 743$ equipment (Metrohm, 743 model) according to EN 14112 accelerated test, under air flow of $10 \mathrm{~L}^{-h^{-1}}$ at $110{ }^{\circ} \mathrm{C}$ using $3 \mathrm{~g}$ of each oil sample, in duplicates. This test data is expressed as induction period that represents a time interval until the sample reaches a high oxidation level (Metrohm, 2011).

- The antioxidant capacity of passion seeds oils was determined according to modified methodology of Embrapa Tropical Agroindustry for fruits analysis (Rufino, 2007), which uses the DPPH (2,2-diphenyl-1-picrylhidrazil) radical method, adapted for vegetable oils. The DPPH reagent was added to five different dilutions of the original oil in isopropyl alcohol, which remained one hour in the dark before the spectrophotometer analysis at $515 \mathrm{~nm}$. With the absorbance readings, the Inhibitory Concentration ( $\mathrm{IC}_{50}-$ mass needed to reduce in $50 \%$ the initial concentration of DPPH radical) was determined.

- The total phenolic content (TP) of the pressed cake extract was determined according to the spectrophotometric method, proposed by Singleton \& Rossi (1965) using Folin-Ciocalteu reagent. This method is based on the reduction of acids to oxides, and the compound formed is blue. For analysis $0.5 \mathrm{~mL}$ of sample was added to $2.5 \mathrm{~mL}$ of Folin-Ciocalteu reagent $10 \%$ and vortexed for $2 \mathrm{~min}$. After homogenization, $2 \mathrm{~mL}$ of saturated sodium carbonate solution (7.5\%) was added. The solution is incubated for $15 \mathrm{~min}$ at $50{ }^{\circ} \mathrm{C}$ in water bath, and immediately goes to cooling in ice bath for 30 seconds. The absorbance of the mixture was measured at $760 \mathrm{~nm}$ using spectrophotometer (BelFotonix SP 1105). Phenolic compounds quantification was performed using a calibration curve of gallic acid. The results were expressed in mg equivalent of gallic acid by $100 \mathrm{~g}$ of sample $\left(\right.$ GAE $\left.100 . g^{-1}\right)$.

- Antioxidant capacity of the pressed cake was evaluated by the ABTS method expressed in Trolox equivalents (TEAC) according to Re et al., (1999) and Rufino et al. (2007). The ABTS ${ }^{+}$radical was chemically obtained by oxidation of ABTS by potassium persulfate. Aliquots of the aqueous solutions of ABTS $7 \mathrm{mM}$ was mixed to potassium persulfate $140 \mathrm{mM}$ in an amber bottle at stoichiometric ratio of 2:1 (mol:mol). For the complete reaction and stabilization of the radical, the flask remained at room temperature for $14 \mathrm{~h}$, this is the radical solution concentrated. The concentrated $\mathrm{ABTS}^{+}$solution was diluted in $95 \%$ ethanol until an absorbance of $0.700 \pm 0.020$ at $734 \mathrm{~nm}$ to form the test reagent.

Trolox $^{\circledR}$ solutions (300-2000 $\mu \mathrm{M}$ in $95 \%$ ethanol) and the extracts were transferred to test tubes where they reacted for 6 minutes with the diluted $\mathrm{ABTS}^{+}$solution in the proportion of 1:10, at room temperature, and the absorbance at $734 \mathrm{~nm}$ was immediately measured in spectrophotometer (BelFotonix SP 1105). The decolorization of mixed solution indicates that antioxidant compounds in the extract quenched ABTS radical cations. There is a quantitative relationship between the reduction of absorbance at $734 \mathrm{~nm}$ and the concentration of antioxidants present in the sample. The obtained results were expressed in $\mu \mathrm{mol}$ Trolox. $\mathrm{g}^{-1}$ extract

Addition of phenolic extract to enhance the oxidative stability of passion fruit oils

The extracts obtained with the 70:30 ethanol-water solutions from passion fruit seeds of $P$. alata, $P$. setacea and $P$. tenuifila were selected to increase the oxidative stability of the respective oils. To maintain the bioactive properties, the solvent was removed from extract by forced convection with air temperature at $21^{\circ} \mathrm{C}$. The dried extracts were mixed with the oil in a ratio of $10 \%$ mass/mass to the passion fruit seeds oils. To evaluate the oxidative stability of the blend, $3 \mathrm{~g}$ of each samples were then transferred to a Rancimat reaction tube, and analysis were performed in duplicate under air flow of $10 \mathrm{~L} \mathrm{~h}^{-1}$ at $110^{\circ} \mathrm{C}$.

Statistical analysis

All analytical determinations were carried out at least in triplicate, except the RANCIMAT trials. Analysis of variance (ANOVA) followed by Fisher's LSD test was performed using Statistica ${ }^{\circledR}$ v.13.0.

\section{Results and Discussion}

Kinetics of seed drying

The estimated parameters to drying rate and the correspondent R-squared are presented in Table 1. Additionally, kinetic curves, at $50^{\circ} \mathrm{C}$, fitting experimental data to second laws Fick's solution of drying data from passion seeds are illustrated in Figure 1. 
Table 1. Effective diffusion coefficient during mass transport in the drying process of passion seeds fruit.

\begin{tabular}{lccc}
\hline & $\begin{array}{c}\text { Average radius } \\
(\mathrm{mm})\end{array}$ & $\begin{array}{c}\mathrm{D}_{\text {eff }} \\
\left(\mathrm{m}^{2} . \mathrm{s}^{-1}\right) \times 10^{10}\end{array}$ & $\begin{array}{c}\mathrm{R}^{2} \\
(-)\end{array}$ \\
\hline P. alata & 1.9 & 1.14 & 0.98 \\
P. setacea & 1.3 & 0.82 & 0.98 \\
P. tenuifila & 1.3 & 0.83 & 0.98 \\
\hline
\end{tabular}

The estimated diffusion coefficients ranged from $0.82 \times 10^{-10} \mathrm{~m}^{2} \cdot \mathrm{s}^{-1}$ to $1.14 \times 10^{-10} \mathrm{~m}^{2} \cdot \mathrm{s}^{-1}$. The $P$. alata presented higher diffusion coefficients than the other two species studied in this research. From the fitted equation it is possible to estimate the drying time of the passion seeds in order to reach the best moisture to passion seeds pressing, between $10 \%$ and $15 \%$.

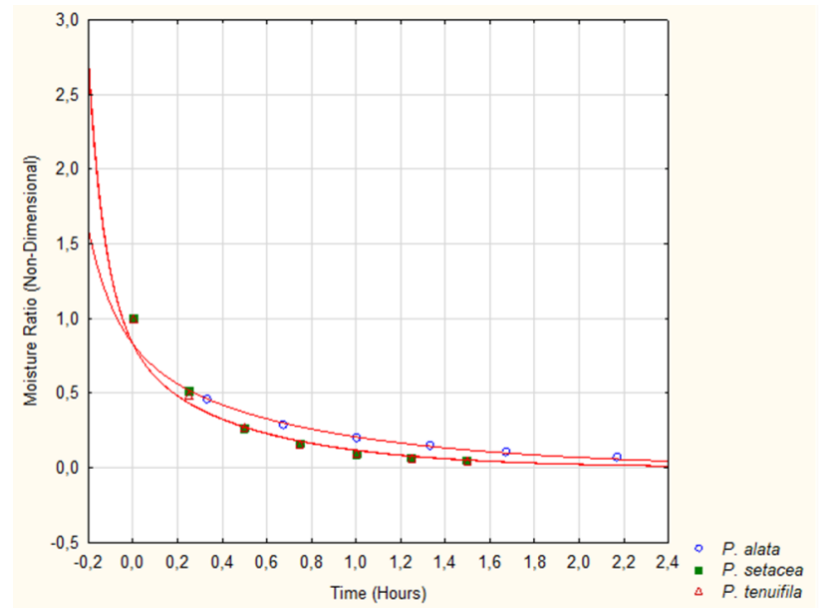

Figure 1. Drying curves of the three species of passion fruit seeds

Oil extraction

The oil content in dry passion fruits seeds was $19 \%, 16 \%$ and $23 \%$ for P. alata, P. setacea and P. tenuifila, respectively (Table 2). Paula (2015), found a lipid content of $22.5 \%$ and $32.2 \%$ for $P$. alata and $P$. setacea, respectively, this difference may be due to seasonality. The efficiency of the continuous oil extraction process, calculated by the ratio between the mass of oil obtained and total oil in the seeds, was from $57 \%$ to $67 \%$, as shown in Table 2. This result is typical for continuous oil extraction process from seeds rich in lignin (up to $40 \%$ in dry matter) and a lipidic content less than 30\%. In addition, the sample with moisture content in the range of $15 \%(P$. alata) presented better extraction efficiency. The extraction yield for $P$. setacea was lower indicating possibly higher lignin content in this sample, which leads to a more resistant cell wall. To improve the extraction yield, the pressed cake can be recycled and processed under heating at $50{ }^{\circ} \mathrm{C}$.

Table 2. Moisture, oil content and extraction yield of passion fruit seeds.

\begin{tabular}{lccc}
\hline Sample & Moisture after drying (\%) & Oil content (\%) & Extraction yield (\%) \\
\hline P. alata & 15 & 19 & 67 \\
P. setacea & 11 & 16 & 57 \\
P. tenuifila & 10 & 23 & 63 \\
\hline
\end{tabular}

Oxidative stability and antioxidant capacity

As shown in Table 3, the induction periods (IP) for $P$. setacea and $P$. tenuifila seed oils at $110^{\circ} \mathrm{C}$ were $7.32 \mathrm{~h}$ and $6.87 \mathrm{~h}$, respectively, which is typical IP for passion fruit seed oils (Paula at al., 2015). However, the P. alata species presented an IP 50\% lower (3.53 h). This result can be explained by the lower proportion of oleic acid and higher linoleic acid in P. alata seed oil (Santana et al., 2015). Due to the two double bonds in linoleic acid it is 40 times more unstable than oleic acid which contains only one double bond.

The $\mathrm{IC}_{50}$, defined as the amount of antioxidant needed to reduce DPPH concentrations by $50 \%$, is considered a good measure of antioxidant capacity (inversely proportional). Regarding the DPPH results, it can be observed that the oil from $P$. setacea seeds presented the higher antioxidant capacity, followed by $P$. alata and $P$. tenuifila oil seeds. These values are consistent with literature data reported by Santana et al. (2015) and can be explained due to the higher vitamin E content in $P$. setacea seed oil $\left(215.3 \mathrm{mg} .100 \mathrm{~g}^{-1}\right)$ and $P$. alata seed oil $\left(114.8 \mathrm{mg}^{1} 100 \mathrm{~g}^{-}\right.$ $\left.{ }^{1}\right)$ as compared with $P$. tenuifila seed oil $\left(80 \mathrm{mg} .100 \mathrm{~g}^{-1}\right)$. 
Table 3. Oxidative stability and antioxidant capacity by DPPH inibition of Passiflora seed oils.

\begin{tabular}{lccc}
\hline Samples & Induction period (h) & DPPH inhibition (\%) & $\mathrm{IC}_{50} \mathrm{mg}_{\text {oil.mg DPPH }}{ }^{-1}$ \\
\hline P. alata & 3.52 & 43 & 1.09 \\
P. setacea & 7.32 & 53 & 1.09 \\
P. tenuifila & 6.87 & 24 & 2.60 \\
\hline
\end{tabular}

Total phenolic content (TP) and antioxidant capacity (TEAC) from pressed cake

The more efficient solid-liquid extraction of bioactive compounds from the pressed cake was reached using ethanol-water solutions $70: 30$ at $45^{\circ} \mathrm{C}$. This occurs probably due to the more solubility of polar compounds from the passion seeds cake in this ethanolic-aqueous solution. The higher value for total TP content and TEAC for pressed cake extracts are shows in Table 3 . In the selected experimental conditions, the ethanolic extract of $P$. setace cake presented higher TP contents and TEAC as compared to the other species. As can be observed, the total phenols from $P$. setacea, $P$. alata, and $P$. tenuifila cakes were approximately 1800,600 and 900 mg GAE.100 $\mathrm{g}^{-1}$ of extract. The TP of the extract obtained in the ratio 70:30 from $P$. setacea cake was 3 and 2 fold greater than the extract obtained from $P$. alata and $P$. tenuifila, respectively (Table 3 ). This result showed a strong positive Pearson correlation $(\mathrm{R}=0.97)$ with antioxidant activity, since the results for the antioxidant capacity in the same experimental conditions of the $P$. setacea cake were 2.3 and 1.8 times higher than $P$ alata and $P$. tenuifila, respectively. According to Vasco et al. (2008) the ethanolic extracts obtained in the present work from $P$. alata and $P$. tenuifila pressed cake can be treated as medium phenolic contents, while for $P$. setacea pressed cake the extract obtained in the ratios 50:50 and 70:30 ethanol:water are treated as high phenolic contents.

Table 3. Total phenolic contents (TP) and antioxidant capacity (TEAC) of the ethanolic extract from pressed cake.

\begin{tabular}{|c|c|c|c|}
\hline Sample & $\begin{array}{c}\text { ethanol:water } \\
\text { ratio }\end{array}$ & $\begin{array}{c}\text { Total phenolic } \\
\left(\mathrm{mg} \mathrm{GAE} .100 \mathrm{~g}^{-1}\right)\end{array}$ & $\begin{array}{c}\text { TEAC } \\
\left(\mu \mathrm{mol} \text { Trolox. } \mathrm{g}^{-1}\right)\end{array}$ \\
\hline \multirow{3}{*}{ P. alata } & $30: 70$ & $293.5^{f}$ & $242.96^{\mathrm{g}}$ \\
\hline & $50: 50$ & $477.2^{\mathrm{e}}$ & $568.24^{\mathrm{ef}}$ \\
\hline & $70: 30$ & $596.9^{d}$ & $670.19^{\text {de }}$ \\
\hline \multirow{3}{*}{$\begin{array}{c}P . \\
\text { setacea }\end{array}$} & $30: 70$ & $220.5^{g}$ & $200.03^{\mathrm{g}}$ \\
\hline & $50: 50$ & $1159.9^{\mathrm{b}}$ & $1032.64^{b}$ \\
\hline & $70: 30$ & $1814.4^{\mathrm{a}}$ & $1569.14^{\mathrm{a}}$ \\
\hline \multirow{3}{*}{$\begin{array}{c}P . \\
\text { tenuifila }\end{array}$} & $30: 70$ & $445.9^{\mathrm{e}}$ & $451.27^{\mathrm{f}}$ \\
\hline & $50: 50$ & $886.7^{\mathrm{c}}$ & $739.88^{d}$ \\
\hline & $70: 30$ & $909.0^{\mathrm{c}}$ & $878.04^{\mathrm{c}}$ \\
\hline
\end{tabular}

*Different letters in the same column indicates significant differences $(\mathrm{p}<0.05)$ according Fisher LSD test.

GAE: $m g$ of gallic acid equivalent

Effect of pressed cake ethanolic extract on oxidative stability of passion fruit seeds oils

The TP and the TEAC values of all extracts were the main response to select the best operational conditions. Thus, the addition of 10\% mass/mass of dried phenolic extracted from the pressed cakes at 70:30 ethanol-water solution in the oils favored their oxidative stability, with a significant increase $(\mathrm{p}<0.05)$. The induction periods (IP) for P. setacea, P. alata, and P. tenuifila seeds oils increase from 7.30 to $9.60 \mathrm{~h}, 3.52$ to $11.7 \mathrm{~h}$, and 6.87 to $10.3 \mathrm{~h}$, respectively. Thus, the use of these phenolic extracts as natural antioxidant in their respective oils increased the IP in 3.4, 1.3 and 1.5 times from $P$. alata, $P$. setacea and $P$. tenuifila, respectively. These results proven that the cake extract to be efficient as a natural antioxidant for crude pressed oils and can be applied as substitute of synthetic antioxidants, harmful to health, traditionally used in the refined commercial oils.

\section{Conclusions}

The passion seeds oils from $P$. setacea, $P$. alata, and $P$ tenuifila, obtained by pressing, presented high quality index probably due to the high phenolic contents in the seeds. Furthermore, it was possible to enhance the oxidative stability for all samples by adding the ethanolic pressed-cake extract, rich in phenolic compounds.

\section{Acknowledgements}

The authors would like to thank the Federal University of Rio de Janeiro (UFRJ/EQ), Embrapa Cerrados, 
Embrapa Food Technology and Brazilian National Research Council (CNPq) for supporting this study.

\section{References}

Bernacci, L.C., L.M.M. Meletti, M.D. Soares-Scott, 2003. Maracujá-doce: o autor, a obra e a data da publicação de Passiflora alata (Passifloraceae). Revista Brasileira de Fruticultura, 25(2), 355-356. https://doi.org/10.1590/S0100-29452003000200046.

Cunha, M.A.P., L.V. Barbosa, N.T.V. Junqueira, 2002. Espécies de maracujazeiro. In: LIMA, A. A. (Ed.). Maracujá Produção: Aspectos Técnicos. Brasília: Embrapa Informação Tecnológica, 104p. (Frutas do Brasil; 15).

Deng, J., W. Cheng, G. Yang, 2011. A novel antioxidant activity index (AAU) for natural products using the DPPH assay. Food Chemistry, 125(4), 1430-1435. https://doi.org/10.1016/j.foodchem.2010.10.031.

Dhawan, K., S. Dhawan, A. Sharma, 2004. Passiflora: A review update. Journal of Ethnopharmacology, 94(1), 1-23. https://doi.org/10.1016/j.jep.2004.02.023.

IBGE, 2016. Produção Agrícola Municipal: Culturas temporárias e permanentes. Retrieved January 5, 2018, from https://www.ibge.gov.br/estatisticas-novoportal/economicas/agricultura-e-pecuaria/9117-producaoagricola-municipal-culturas-temporarias-e-permanentes.html?\&t=resultados. Accessed January 5, 2018.

Lowe, E.D., D.R. Buckmaster, 1995. Dewatering makes big difference in compost strategies, Biocycle, v. 36, p. 78-82.

Nigam N., M.S. Galvão, 2010. "Passion Fruit," in Handbook of Fruit and Vegetable Flavors, Y. H. Hui, Ed. Hoboken, NJ, USA: John Wiley \& Sons, Inc., pp. 345-390.

Manica, I., 2005. Maracujá-doce: tecnologia de produção, pós-colheita, mercado. Porto Alegre: Cinco Continentes. $198 \mathrm{p}$.

Matsui, Y., K. Sugiyama, M. Kamei, T. Takahashi, T. Suzuki, Y. Katagata, T. Ito, 2010. Extract of passion fruit (Passiflora edulis) seed containing high amounts of piceatannol inhibits melanogenesis and promotes collagen synthesis. Journal of Agricultural and Food Chemistry, 58(20), 11112-11118. https://doi.org/10.1021/jf102650d.

Mirabella, N., V. Castellani, S. Sala, 2014. Current options for the valorization of food manufacturing waste: A review. Journal of Cleaner Production, 65, 28-41. https://doi.org/10.1016/j.jclepro.2013.10.051.

Paula, R.C.M. de, A.G. Soares, S.P. Freitas, 2015. Volatile coumponds in passion fruit seed oil (Passiflora setacea BRS Pérola do Cerrado and Passiflora alata BRS Doce Mel). Chemical Engineering Transactions, 44(April 2015), 103-108. https://doi.org/10.3303/CET1544018.

Paula, R.C.M. de, 2015. Óleo de semente de maracujá (Passiflora setacea BRS Pérola do Cerrado e Passiflora alata BRS Doce Mel): Propriedades químicas e efeito do processamento no perfil decompostos voláteis. DS Thesis, Escola de Química, Universidade Federal do Rio de Janeiro, Brasil.

Re, R., N. Pellegrini, A. Proteggente, A. Pannala, M. Yang, C. Rice-Evans, 1999. Antioxidant Activity Applying an Improved Abts Radical Cation Decolorization Assay, 26(98), 1231-1237. https://doi.org/10.1016/S0891-5849(98)00315-3Reis, G. M., Faccin, H., Viana, C., Rosa, M. B. da, \& de Carvalho, L. M. (2016). Vitis vinifera L. cv Pinot noir pomace and lees as potential sources of bioactive compounds. International Journal of Food Sciences and Nutrition, 67(7), 789-796. https://doi.org/10.1080/09637486.2016.1204595.

Rufino, M.do S.M., R.E. Alves, E.S. de Brito, S.M. de Morais, C.deG. Sampaio, J. Pérez-Jiménez and F.D.S. Calixto, 2007. Metodologia Científica: Determinação da atividade antioxidante total em frutas pela captura do radical livre DPPH - A report to the expert group on antioxidant activity. Occasional publication No. 128. Ministério Da Agricultura, Pecuária e Abastecimento, Fortaleza, Ceará. 23(2), 1-4. https://doi.org/10.1017/CBO9781107415324.004. July.

Santana, F.C., F.B. Shinagawa, E.daS. Araujo, A.M. Costa, J. Mancini-Filho, 2015. Chemical Composition and Antioxidant Capacity of Brazilian Passiflora Seed Oils. Journal of Food Science, 80(12), C2647-C2654. https://doi.org/10.1111/1750-3841.13102.

Silva, A.C., N. Jorge, 2014. Bioactive compounds of the lipid fractions of agro-industrial waste. Food Research International, 66, 493-500. https://doi.org/10.1016/j.foodres.2014.10.025.

Singleton, V.L., J.A. Rossi, 1965. Colorimetry of total phenolics with phosphomolybdic-phosphotungstic acid reagents. Am. J. Enol. Vitic., v.16, p.144-168.

Uchida-Maruki, H., H. Inagaki, R. Ito, I. Kurita, M. Sai, T. Ito, 2015. Piceatannol Lowers the Blood Glucose Level in Diabetic Mice. Biological \& Pharmaceutical Bulletin, 38(4), 629-633. https://doi.org/10.1248/bpb.b1500009.

Vasco, C., J. Ruales, A. Kamal-Eldin, 2008. Total phenolic compounds and antioxidant capacities of major fruits from Ecuador. Food Chemistry, 111(4), 816-823. https://doi.org/10.1016/j.foodchem.2008.04.054. 


\title{
Microencapsulated Functional Lipids by the Association of Pomegranate Seed and Soybean Oils
}

\author{
Nina K. S. James ${ }^{a}{ }^{\text {, }}$, Meire J. A. Ferreira ${ }^{a}$, Regina I. Nogueira ${ }^{b}$, Luzimar S. M. Nascimento ${ }^{b}$, Suely P. \\ Freitas $^{\mathbf{a}}$ \\ a Escola de Química - Universidade Federal do Rio de Janeiro/ UFRJ - Rio de Janeiro - RJ - Brazil \\ ${ }^{\mathrm{b}}$ Embrapa Agroindústria de Alimentos - Rio de Janeiro - RJ, Brazil \\ * Corresponding author. Email: ninakatia@ufrj.br
}

\begin{abstract}
Protection against lipids oxidation is the goal supported by microencapsulation. The main objective of this study was to develop an affordable bioactive food ingredient from a blend of pressed pomegranate seed oil and soybean oil microencapsulated by spray drying. A wide variety of delivery systems is available, each with its specific benefits for different applications. A statistical mixture design was applied to select the best carrier material composition from modified starch, maltodextrin and gum arabic; and the best formulation was used to assess the influence of different drying temperatures on encapsulation efficiency. Furthermore, phenolic and fatty acid composition, antioxidant capacity and microcapsules morphology were assessed. The selected carrier agents were modified starch $50 \%$ added to equal parts of either maltodextrin or gum arabic. In this case, the microencapsulated powder can be stored at room temperature for 90 days with minor loss of oxidative stability. A drying temperature of $130{ }^{\circ} \mathrm{C}$, lower than is commonly used in literature, presented higher encapsulation efficiency (95\%), indicating that it is possible to obtain integral microcapsules with lower energy consumption. Compared to liquid oil blends, the microencapsulated blend presented higher oxidative stability ( $42 \%)$ due to the effective protection of the carrier agents and higher antioxidant capacity due to the antioxidant characteristic of the applied carrier material, proving to be an adequate alternative to associate the pressed pomegranate and soybean oils.
\end{abstract}

Keywords: Punicic acid, phenolic compounds, oil blends, polyunsaturated fatty acids, spray drying, drying parameters.

\section{Introduction}

The fruit juices industry dumps large quantities of bagasse in the fields, containing mostly peels and seeds that may still contain high amounts of bioactive compounds. Currently, these natural compounds, such as antioxidants, have been used as functional ingredients for foods, contributing to sustainability in agribusiness. Recent advances in science have revealed the fundamental role of inflammation in mediating metabolic diseases and tumours. Daily intake of foods rich in antioxidant compounds along with a healthy diet, can aid in preventing these diseases (Sharma et al., 2017).

Pomegranate processing in the food industry discards up to $50 \%$ of raw fruit. In this case, the seeds account for about $15 \%$ of the bagasse and contain up to $30 \%$ lipids. Pomegranate seed oil (PSO) contains over $70 \%$ of the rare punicic acid in its fatty acid composition. Punicic acid is an omega-5 long chain polyunsaturated fatty acid (PUFA) comprising 18 carbons and three double bonds (C18:3), a conjugated of linolenic acid (CLnA) that presents anti-inflammatory properties (Hennessy et al., 2016). Punicic acid has been widely studied due to its therapeutic potential, especially against skin and prostate cancer (Sharma et al. 2017) despite its very high sensibility to oxidation.

Furthermore, PSO contains phenolic compounds that, in addition to punicic acid, may be related to the antioxidant capacity attributed to this oil (Singh et al., 2014). Yuan et al. (2009) observed that punicic acid significantly decreased the levels of rat liver triacylglycerol after diet supplementation of $10 \%$ of CLnA for 6 weeks. Besides, the authors observe that punicic acid was metabolized into cis9, trans11-18:2 (CLA) in mouse liver.

Among phenolic compounds, phenolic acids (PA) are a large group found in raw-vegetables that display antioxidants and anti-microbial properties (Heleno et al., 2015). Phenolic acids are well documented in pomegranate juices (Conidi et al., 2017) but few studies addressing their identification in pomegranate oil are available. Due to its health-promoting properties, it is desirable to incorporate PSO as an active ingredient in high consumption food products. However, pomegranate seed oil presents high sensibility to oxidative rancidity and is expensive, which limits its use. Blending of vegetable oils with different fatty acid profiles is a common practice in many countries to improve physical-chemical characteristics and achieve new applications (HashempourBaltork et al., 2016). Blending PSO with a more stable and affordable oil will create a new product, easier to manipulate and commercialize, expanding its potential uses.

Soybean oil (SO) is the most consumed vegetable oil in Brazil, and the Brazilian soybean production is the second largest in the world (USDA, 2017), being considered an important commodity. Pressed SO is a richer 
food, due to higher content of vitamins E, K, and lecithin compared to refined solvent-based oils (Kania et al., 2004). Its major PUFA is linoleic acid, an essential fatty acid. Traditionally, SO is largely consumed due to its availability, competitive price, subtle flavour and good stability against oxidation, which makes it ideal to use as an ingredient in the food industry. The combination of SO and PSO can increase CLnA consumption by virtue of the well-established soybean supply-chain, that will offer consumers a more affordable final product with bioactive and therapeutic properties.

Microencapsulation by spray drying is a traditional and effective method to incorporate polyunsaturated oils into foods, since it minimizes oxidative damage (Hermida; Gallardo, 2015), however it can be further enhanced by a controlled variation of parameters. The physicochemical characteristics of the product are controlled by inlet temperature, inlet air flow rate, feed flow rate and encapsulating material composition. Inlet temperature is particularly important when processing thermo-sensitive compounds like punicic acid. Lower temperatures can be used in spray drying, seeing that the higher humidity on product can be bypassed with inlet air flow variation. Besides, the selection of the carrier agent for wall material is critical, since it protects the core during heating and increases yield by reducing stickiness, facilitating its flow, although its efficiency depends on interactions with the core material. Discovering the optimum conditions for the process is fundamental in order to simultaneously meet process efficiency and product quality (Shishir; Chen, 2017).

In this context, the aim of the present study was to develop a functional ingredient with high CLnA content using microencapsulation by spray drying. For this purpose, the wall material composition was selected by comparing the oxidative stability of the raw and microencapsulated PSO and SO blend. The best formulation was used to evaluate the effect of temperature $\left(110\right.$ to $\left.150{ }^{\circ} \mathrm{C}\right)$ on encapsulation efficiency and shelf life. Few studies on spray drying have explored the influence of temperatures below $150{ }^{\circ} \mathrm{C}$ on product quality, although higher temperatures promote a very high evaporation rate and can form particles with a porous microstructure (Souza and Thomazini, 2013) leaving the core more vulnerable to oxygen transfer.

In addition, the bulk oils were characterized with regard to their fatty acid composition, antioxidant capacity and oxidative stability. Also the particle size and surface structure of microcapsules were assessed.

\section{Materials and Methods}

Material

Fresh pomegranate (Punica granatum - wonderful variety) fruits, growing in the semiarid Brazilian region, were purchased directly from a farm. Cold-pressed soybean Organic $®$ oil was acquired from a local product store. The oil had no added antioxidants, as declared by the supplier. Modified Starch (Capsul ${ }$, Ingredion ${ }^{\mathrm{TM}}$ ), Maltodextrin DE 5 (MD, Globe ${ }^{\circledR}$ 1805) and Gum Arabic (GA, Vetec) were selected as carrier agents. Traditional carrier materials, easily available, were used to focus the results on parameter modifications.

Physical Methods

After pulp and peel separation, the pomegranate seeds containing 10\% moisture content were triturated in a knife mill. Crushed pomegranate seeds were processed in a continuous hydraulic press (Oekotec - Germany, CA59G model), in order to obtain a crude oil, that was decanted for $24 \mathrm{~h}$ in a dark cabinet and the clarified fraction was stored at $-18{ }^{\circ} \mathrm{C}$ until the moment of use. Pressing is an extraction method that obtains the oil with characteristics more similar to the natural oil, however studies using toxic solvents hazardous to both the environment and manipulator health are still observed in the literature.

- Experimental design A simplex centroid three level mixture design according to Montgomery and Calado (2003) was applied to select the best carrier material composition from modified starch (MS), maltodextrin (MD) and gum arabic (GA). Nine assays were carried out. In this case, vertices represents pure material (MD-1, GA-2, MS-3), middle points on edges represent equal binary blending $(4,5,6)$ and the entire inner portion represents ternary blending, with the orthocentre being the central point of the mixture, with all components present in equal parts $(7,8,9)$. Oxidative stability determined using a Rancimat ${ }^{\circledR}$ equipment and microencapsulation efficiency were the evaluated responses, using the Statistica 12.0 Software.

- Formulation The oils blend (OB) were prepared by adding PSO to SO at a 1:4 ratio (w/w), and mixing in Ultra-Turrax (T25 basic IKA) at $13.500 \mathrm{rpm}$ for one minute. An oil:carrier material ratio of 1:4 was applied since punicic acid is highly reactive and the amount of carrier material should be enough to completely involve the oil. The oil-in-water emulsion was prepared during 10 minutes by adding, continuously, the oil blend to the carrier dispersed in water, reaching $37.5 \%$ solids (7.5\% oil, 30\% carrier mix and $62.5 \%$ water) as recommended by $\mathrm{Li}$ et al. (2015).

- Emulsion stability Emulsion stability was evaluated using $10 \mathrm{~mL}$ aliquots of each sample in $12 \mathrm{~mL}$ graduated cylinders, sealed and stored at room temperature for $24 \mathrm{~h}$. The stability was measured by the percentage of separation and expressed as the ratio between the upper phase and the initial height of the emulsion (Carneiro et al., 2013). 
- Processing The spray-drying process was performed using a Buchi B-290 lab scale spray dryer (Buchi Labortechnik AG). At first, the emulsion was fed at a flow rate of $0.36 \mathrm{~L}^{-1} \mathrm{~h}^{-1}$, with a co-current air flow rate of 25 $\mathrm{kg} . \mathrm{h}^{-1}$ and air inlet temperature of $150^{\circ} \mathrm{C}$, using a $0.7 \mathrm{~mm}$ diameter nozzle. The room temperature was maintained at $28^{\circ} \mathrm{C}$ and relative air humidity at $37 \%$. The powder product was stored into sealed metallic packaging at ${ }^{-1} 18$ ${ }^{\circ} \mathrm{C}$ for posterior analysis.

- Temperature influence The most stable formulation was selected to evaluate temperature influence on encapsulation efficiency. A range from 110 to $150{ }^{\circ} \mathrm{C}$ was applied to preserve the bioactive compounds.

Analytical Methods

- Fatty acid identification total fatty acid esterification followed the Hartmann-Lago (1993) method and the chromatographic analysis was performed on an Agilent Technologies 7890A chromatograph, with a DB-WAX column $(30 \mathrm{~m}$ x $250 \mu \mathrm{m} \times 0.25 \mu \mathrm{m})$ and 5975C VL MSD Agilent Technologies mass detector, model. Temperature was maintained at $100{ }^{\circ} \mathrm{C}$ for $2 \mathrm{~min}$, followed ramped up to $150{ }^{\circ} \mathrm{C}$ at $10{ }^{\circ} \mathrm{C} \cdot \mathrm{min}^{-1}$, ramped up to $180{ }^{\circ} \mathrm{C}$ at 3 ${ }^{\circ} \mathrm{C} . \mathrm{min}^{-1}$, and maintained for $20 \mathrm{~min}$. Injector and detector temperatures were adjusted to $210{ }^{\circ} \mathrm{C}$ and $200{ }^{\circ} \mathrm{C}$, respectively. Samples were diluted to $1 \%$ in heptane and the injected volume was $1 \mu \mathrm{L}$ with Split 1:100. Helium was used as carrier at $3 \mathrm{~mL} \cdot \mathrm{min}^{-1}$.

- Phenolic acid and flavonoid identification this analysis was carried out according to Nascimento et al. (2017). The sample was extracted with $4 \mathrm{~mL}$ of methanol: water $(50: 50 \mathrm{v} / \mathrm{v}, \mathrm{pH} 2)$ followed by mechanical stirring for 1 hour and centrifugation $(5000 \mathrm{G})$ for 10 minutes. The supernatant was collected (extract 1$)$. Then, $4 \mathrm{~mL}$ of acetone: water $(70: 30 ; \mathrm{v} / \mathrm{v})$ was added to the residue and the mechanical stirring and centrifugation steps were repeated. The supernatant was collected (extract 2). $3 \mathrm{~mL}$ of the supernatants (extract 1 and extract 2) were mixed and transferred to a $1.5 \mathrm{~mL}$ vial for the chromatographic injection.

Pomegranate pressed cake (PPC) was previously submitted to alkaline hydrolysis to obtain free (PPC1) and linked (PPC2) phenolic acids. Alkaline hydrolysis was carried on with $5 \mathrm{~mL}$ solution of $\mathrm{NaOH} 2 \mathrm{M}$ containing $1 \%$ of ascorbic acid and EDTA $10 \mathrm{mM}$. This solution was added to the samples which was heated at $62^{\circ} \mathrm{C}$ for 60 min. Then $1.5 \mathrm{~mL}$ of $\mathrm{HCl} 6 \mathrm{M}$ was added for the acid hydrolysis. This solution was vortexed for $10 \mathrm{~s}$, left to cool to room temperature, and then centrifuged $(2700 \mathrm{rpm})$ for 10 minutes. The supernatant was collected and $6.5 \mathrm{~mL}$ of ethyl acetate was added. The organic phase was separated and the extraction with ethyl acetate was repeated. The organic fraction was dried under a nitrogen gas $\left(\mathrm{N}_{2}\right)$ flow and then diluted in methanol for chromatographic analysis.

All extracts were analyzed on an Alliance Waters ${ }^{\circledR}$ 2690/5 HPLC with a Waters ${ }^{\circledR} 2996$ photodiode array detector $(270,310,325$ and $370 \mathrm{~nm})$, employing the Empower ${ }^{\circledR}$ software, using a Thermo Hypersil BDS C18 column $(100 \times 4.6 \mathrm{~mm}, 2.4 \mu \mathrm{m})$, with flow ranging from 1.0 to $1.2 \mathrm{~mL} \cdot \mathrm{min}^{-1}$, injection volume of $10 \mu \mathrm{L}$ and run time of $28 \mathrm{~min}$. Column temperature was set at $30^{\circ} \mathrm{C}$ using as elution mode gradient an aqueous $0.15 \%$ phosphoric acid solution ( $95 \%$, phase A) and acetonitrile (5\%, phase B). The quantification of flavonoids and phenolic acids was performed by external standardization.

- Oxidative stability This parameter was measured on a Rancimat ${ }^{\circledR} 743$ equipment according to the EN 14112 accelerated test, at an air flow of $20 \mathrm{~L} \cdot \mathrm{h}^{-1}$ using $3 \mathrm{~g}$ of each oil sample, in duplicate. The air temperature was reduced from $110^{\circ} \mathrm{C}$ to $80^{\circ} \mathrm{C}$, as recommended by Lutterodt et al. (2011), due to the high sensitivity to oxidation of the samples. This test data is expressed as an induction period (IP) that represents the time interval until the samples reach a high oxidation level.

- Antioxidant capacity: This parameter was determined according to a methodology modified from the Embrapa Tropical Agroindustry for fruit analysis (Rufino et al., 2007), that uses the DPPH (2,2-diphenyl-1-picrylhidrazil) radical method, adapted for vegetable oils. The DPPH reagent was added to five different dilutions of the original oil in isopropyl alcohol. In the case of microcapsules, these were previously crushed in a ball mill for 30 minutes (since the microcapsule walls were resistant to isopropyl alcohol diffusion) and the samples with the solvent were maintained under ultrasound treatment for five min. After filtration in $1.6 \mu \mathrm{m}$ glass fiber membrane filters, the DPPH solution was added to the extracts, which remained for one hour in the dark before the chromatographic analysis at $515 \mathrm{~nm}$. The Inhibitory Concentration $\left(\mathrm{IC}_{50}\right.$ - mass needed to reduce the initial concentration of the DPPH radical in 50\%) was determined using the absorbance readings.

Powder analysis

- Dry matter: the moisture content of the powder was determined according to the Association of Official Analytical Chemists (AOAC, 2005) through method 934.01.

- Bulk Density: this analysis was carried out as reported by Kaderides et al. (2015).

- Surface free oil content and Encapsulation Efficiency: The surface free oil was determined by successive washings of microcapsules in hexane according to Carneiro et al. (2013), and the encapsulation efficiency was calculated as the ratio between the encapsulated oil (total oil minus surface oil) and total oil content.

- Particle morphology: the microphotographs were taken on a TM3030 plus Hitachi scanning electron 
microscopy (SEM), using an accelerating voltage of $15 \mathrm{kV}$ at 500x and/or 7000x magnification. A small amount of each sample was sprayed on adhesive carbon tabs, mounted on SEM tubes and introduced in the microscope.

- Powder shelf-life: The microcapsules were stored in sealed packages in a dark cabinet at room temperature $\left(\mathrm{T}=25 \pm 2{ }^{\circ} \mathrm{C}\right.$ ) for 90 days and oxidative stability was determined at $0,30,60$ and 90 days, according to the EN 14112 accelerated test.

\section{Statistical analysis}

All analytical determinations were carried out at least in duplicate. Analysis of variance (ANOVA) followed by Fisher's LSD test was performed using Statistica v.12.0.

\section{Results and Discussion}

Bulk oils and emulsion

- Fatty acids: Table 1 displays the fatty acid profile for PSO, SO and the blend. As reported in literature, punicic acid (C18:3) was the major fatty acid in PSO, comprising $75.5 \%$ of the total area. About $80 \%$ of fatty acid in pomegranate oil is PUFA, confirming its importance as a source of functional fatty acids. Juhaimi, Ozcan and Ghafoor (2017) reported values ranging from 71.7 to $77.6 \%$ using pomegranates from Turkey. The main fatty acids present in SO were palmitic, oleic and linoleic, with 11.5, 25.8 and 48.9\% of total area, respectively. These results are analogue to the characteristic fatty acid profile available in literature (Ferrari et al., 2005). About the oils blend, $60 \%$ of its composition are PUFA ( $15 \%$ of punicic acid) preserving the high insaturation. Since punicic acid ( $\omega-5)$ is a conjugated isomer of linolenic acid $(\omega-3)$, it may contribute to $\omega 6 / \omega-3$ balance in the microcapsules. Recent studies highlight the importance of balanced $\omega 6 / \omega-3$ ratios in human metabolism. In the present study, the $\omega-6 / \omega-3$ ratio in the PSO:SO blend was of 2:1, and can be contribute to a more healthy diet as recommended by Simopoulos (2016).

- Phenolic acids (PA) and flavonoids: The phenolic acids identified in PSO were ferulic, vanillic, ellagic and siringic acids and traces of p-coumaric and in $\mathrm{SO}$ were ellagic and p-hidroxibenzoic acids. Pomegranate pressed cake extract (PPC) presented higher concentrations of phenolic acids (PA). Ellagic, siringic, ferulic, $p$ coumaric and gallic acids were detected, in addition to vanillic, protocatecuic and p-hidroxibenzoic acids. The higher amounts of PA in PPC are due to the extraction method using polar solvents (hydrated methanol and acetone) that present more electronic affinity with the PA that the oils, that are non-polar. Ambigaipalan et al. (2017) also observed ellagic, p-hydroxybenzoic, vanillic and ferulic acids in pomegranate seeds, reporting that insolublebound phenolics from the seeds contributed up to $27 \%$ to total phenolic scavenging activity, which explains the need of a hydrolysis on the analysis method.

Table 1. Fatty acids identified in pomegranate seed oil (PSO), soybean oil (SO) and PSO: SO (1:4) blend.

\begin{tabular}{lccc}
\hline Fatty Acid & PSO (\%) & SO (\%) & PSO:SO (\%) \\
\hline Palmitic C16:0 & $3.00 \pm 0.15$ & $11.5 \pm 0.1$ & 9.82 \\
Estearic C18:0 & $1.67 \pm 0.10$ & $4.43 \pm 0.06$ & 3.88 \\
Oleic C18:1 & $4.75 \pm 0.07$ & $25.8 \pm 0.6$ & 21.6 \\
Linoleic C18:2 $(\omega-6)$ & $5.33 \pm 0.04$ & $48.9 \pm 1.6$ & 40.2 \\
Linolenic C18:3 $(\omega-3)$ & nd & $5.13 \pm 0.25$ & 4.10 \\
Punicic C18:3 $(\omega-5)$ & $75.5 \pm 2.7$ & nd & 15.1 \\
BehenicC22:0 & $3.23 \pm 0.07$ & nd & 0.65 \\
ErucicC22:1n9 & $5.55 \pm 0.18$ & nd & 1.11 \\
$\omega-6 / \omega-3$ & 0.07 & 9.53 & 2.09 \\
PUFA & 80.83 & 54.00 & 59.37 \\
\hline
\end{tabular}

PSO: pomegranate seed oil; SO: soybean oil; nd- non detectable

Phenolic acids are compounds presenting strong polarity (hydrophilic). As a result, the extraction method promotes great influence on the final PA content. This can be observed by the different PA profiles presented in the pressed oils (PSO and $\mathrm{SO}$ ) and pomegranate pressed cake, whose phenolic were extracted using polar solvents. During pressing, mechanical forces expel the oil, which can drag a certain fraction of polar compounds from seeds, such as phenolic acids. However, most polar compounds can only be extracted with polar solvents.

Although the oils presented low phenolic content, these compounds present in PPC can be extracted and added in the oils as natural additives due to their antioxidant potential, since ellagic, siringic, protocatecuic, ferulic, phidroxibenzoic and p-coumaric acids presented antioxidant capacity against free radicals (Perez-Ternero et al., 2017; Heleno et al., 2015). This antioxidant property may protect lipids against oxidative rancidity and may be able to replace synthetic additives. In human metabolism, they may act in the prevention of oxidative-caused diseases, such as atherosclerosis and cancer.

- Oxidative stability: For pomegranate seed oil and soybean oil at $110^{\circ} \mathrm{C}$, the induction periods (IP) were of $0.81 \mathrm{~h}$ and $4.32 \mathrm{~h}$, respectively. Habibnia et al. (2012) determined IP for different Iranian varieties of pomegranate 
seed oil in the range from 0.73 to $1.02 \mathrm{~h}$ at $110^{\circ} \mathrm{C}$. Similar results were reported, by Melo et al. (2016) and Cordeiro et al. (2013) for PSO (IP $0.73 \mathrm{~h}$ ) and for refined SO (5.2 h) at the same temperature, respectively. The presence of three double bonds in a fatty acid molecule makes it unstable and very reactive to hydrogen or oxygen, and additionally, with the bonds in conjugated position (as in punicic acid), there is an increase in the reactivity to oxidation and polymerization. These characteristics of punicic acid generates its low oxidative stability, but its anti-inflammatory properties and its potential against cancer make it worth, while considering that its oxidative sensitivity is managed.

- Antioxidant capacity: The DPPH assay displays many advantages; however, its results have been presented in many different ways, making it difficult to compare data from different studies. $\mathrm{The}^{\mathrm{IC}} \mathrm{C}_{50}$ is defined as the amount of antioxidant needed to reduce DPPH concentrations by $50 \%$, and is considered a good measure of antioxidant capacity (inversely proportional). Pomegranate seed oil presented an $\mathrm{IC}_{50}$ of $0.120 \mathrm{mg} \cdot \mathrm{g}^{-1} \mathrm{DPPH}$, while soybean oil presented $1.021 \mathrm{~g}^{-\mathrm{g}^{-1}} \mathrm{DPPH}, 10$-fold lower. Such antioxidant activity in PSO is due to both punicic acid and phenolic acid. For the PSO:SO (1:4) blend, it is somewhat expected that the antioxidant capacity be nearly the weighted average of these values, but the result $\left(0.552 \mathrm{~g} \mathrm{~g}^{-1} \mathrm{DPPH}\right)$ was remarkably superior than presumed, indicating a synergic effect. Results are displayed in Table 2 . The carrier material extract results will be discussed in item 'Process and powder characterization'- 'Antioxidant capacity', see below

\begin{tabular}{lc}
\multicolumn{2}{c}{ Table 2. $\mathrm{IC}_{50}$ before and after spray drying. } \\
\hline Sample & $\mathrm{IC}_{50} \mathrm{~g}_{\text {oil }} \mathrm{mg}^{-1} \mathrm{DPPH}$ \\
\hline Pomegranate oil & $0.120 \pm 0.003$ \\
Soybean oil & $1.021 \pm 0.021$ \\
Oils blend & $0.552 \pm 0.017$ \\
Microencapsulated blend & $0.212 \pm 0.007$ \\
Carrier material extract & $0.548 \pm 0.015$ \\
\hline
\end{tabular}

- Emulsion Stability: Most emulsions were kinetically stable. Samples 2, 3, 4 and 5 presented maximum value $(100 \%)$, indicating that the attractive forces between the formulation components in these samples were strong enough to maintain emulsion stability. Sample from assay 6 (GA:MS 1:1) and from central points (7, 8 and 9) presented $89 \%$ and $83 \%$ values for emulsion stability, respectively. However, sample 1, using pure MD as carrier agent, presented very low stability (10\%), somewhat expected, since it is well known that maltodextrin has a low film formation capacity and, thus, should not be used as an exclusive carrier material in microencapsulation assays. Wang et at. (2017) reported an obvious oil layer in a sample using only MD, and recommended that it should be used in combination with other carrier agents, due to its low cost, good solubility and neutral aroma.

\section{Process and powder characterization}

- Microencapsulation: By varying operational input parameters, it is possible to control outlet temperature, product moisture and particle size distribution. The final humidity of the microencapsulated PSO:SO blend ranged from 4.8 to $7.6 \%$ and the outlet air temperature was $76 \pm 1{ }^{\circ} \mathrm{C}$. This temperature is the medium temperature on the particle surface during water evaporation, with an increasing correlation to heat sensitive compounds degradation (Shishir and Chen, 2017).

Figure 1 displays the fitted surface of special cubic model regarding the oxidative stability of the microcapsules as a function of wall composition. At $110^{\circ} \mathrm{C}$, oxidation rates were very high (IP 1.2 to $2.1 \mathrm{~h}$ ) and correlation analyses between the influence of the carrier agent and microcapsule quality could not be performed. For this reason, this analysis was carried out at $80^{\circ} \mathrm{C}$, where the induction period was enough (11 to $16 \mathrm{~h}$ ) for comparative purposes. In accelerated tests the oxidation processes occur under drastic conditions, unlike those at normal storage conditions; and since conjugated fatty acid was shown to be more susceptible to autoxidation than their corresponding non-conjugated ones, the use of accelerated oxidation analysis at high temperatures when CLnA ( $\omega-3$ family) are involved is not recommended (Guiotto et al., 2014). At $80^{\circ} \mathrm{C}$, Dreher et al. (2011) determined IP of $12.2 \mathrm{~h}$ for more stabilized pomegranate oil at $80^{\circ} \mathrm{C}$, superior to the IP for the oil blend (PSO:SO 1:4) found in this study $(11.4 \mathrm{~h})$.

For the microencapsulated samples the highest IP $(16.2 \mathrm{~h})$ was $42 \%$ higher using the GA:MS formulation (assay 6), in contrast to reported data by Polavarapu et al. (2011) who found negative influence of spray dryer on oxidative stability of fish oil microcapsules, attributing it to the higher interfacial area of dispersed oil droplets compared to bulk oils. In relation to carrier agents, the same result can be achieved by replacing GA by MD at 25 to $50 \%$, indicating operational flexibility, enclosed in the darker region of the triangular surface, to obtain high oxidative stability powder. Thus, considering the more competitive price of MD as compared to GA, the proportion MD:MS 1:3 was chosen to be applied in further processing.

For mass and energy balance, data from assay 6 (GA:MS 1:1) were selected. In this case, the calculated heat necessary for spray dryer processing was about $1,500 \mathrm{kcal}^{\mathrm{kg}} \mathrm{g}^{-1}$ of evaporated water, representing a $55 \%$ heat loss. Few studies calculate this energy loss for laboratorial scale spray dryers, but this result was $25 \%$ higher compared to data reported by Baker and McKenzie (2002) for industrial spray dryers (about 1,200 $\mathrm{kcal}^{\mathrm{kg}} \mathrm{kg}^{-1}$ of evaporated 
water). This is due to the higher heat loss by conduction and convection through the lab spray drying chamber to the environment as compared to industrial spray dryer energy loss. Regarding the scale-up of the drying chamber, the ratio surface/volume decreases once the surface varies with the square of diameter and volume, with the cube of the diameter, decreasing heat loss to surroundings.

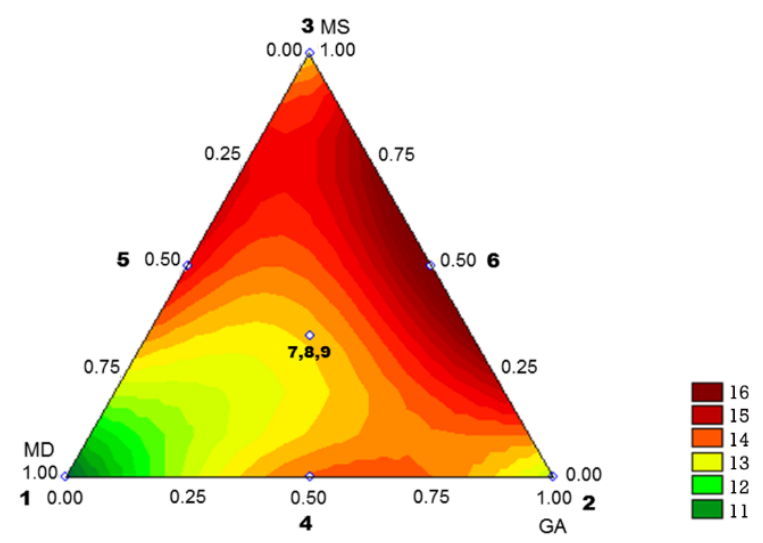

Figure 1. Fitted surface for induction period (h) at $80^{\circ} \mathrm{C}$. 1-MD: maltodextrin; 2-GA: gum Arabic; 3-MS: modified starch; 4, 5,6 : binary mixtures and $7,8,9$ : ternary mixtures.

- Antioxidant capacity: After milling of microcapsules it was possible to enhance solvent diffusion into the core, allowing the oil blend recovery. The microencapsulated oil blend presented an $\mathrm{IC}_{50}$ of $0.212 \mathrm{mg} \cdot \mathrm{g}^{-1} \mathrm{DPPH}$, about three fold higher antioxidant capacity $(\mathrm{p}<0.05)$ than the oil blend itself $\left(0.552 \mathrm{mg} \cdot \mathrm{g}^{-1} \mathrm{DPPH}\right)$, indicating higher antioxidant capacity. These results may be explained due to the carrier formulation containing Capsul ${ }^{\circledR}$ modified starch (MD:MS 1:3), which has an antioxidant capacity $\left(0.545 \mathrm{mg}^{-\mathrm{g}^{-1} \mathrm{DPPH}}\right)$ comparable to the $\mathrm{IC}_{50}$ of the blend (Table 2).

- Encapsulation Efficiency (EE): The air input temperatures displayed a significant influence on EE (from 64.4 to $94.5 \%)$, as observed in Figure 2 . At the lowest temperature $\left(110^{\circ} \mathrm{C}\right)$ a higher standard deviation among the EE values was observed, possibly because particle exposure to air at higher relative moisture leads to the formation of more heterogeneous particles. At $130{ }^{\circ} \mathrm{C}$, EE reached its highest value $(\mathrm{p}<0.05)$, indicating that it is possible to obtain a product with desirable characteristics using milder temperatures then usually found in literature (ranging from 150 to $200^{\circ} \mathrm{C}$ ) by changing other variables, such as feed flow rate and air flow rate. Milder temperatures ensure that thermo-sensitive materials are protected, in addition to consuming less energy for air heating.

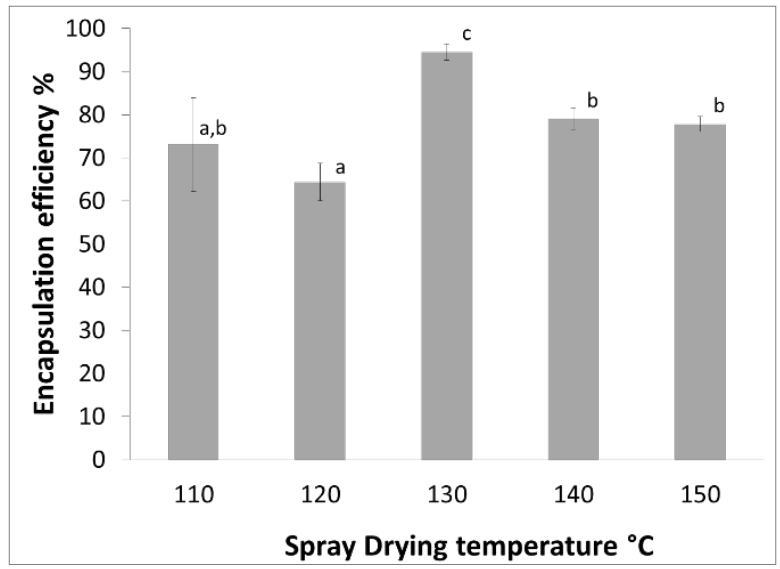

Figure 2. Encapsulation efficiency of spray drying at different temperatures.

Different letters denote significant differences $(\mathrm{p}<0.05)$.

Numerous data in the scientific literature correlate EE with the carrier agents, but correlations between EE and air input temperature is still scarce. Kaderides et al. (2015) investigated the microencapsulation of pomegranate peel extracts and reported values ranging from 69.8 to $99.8 \%$, increasing with increasing drying temperature (from 150 to $\left.190{ }^{\circ} \mathrm{C}\right)$. However, Bustamante et al (2017) observed higher EE $(92.7 \%)$ for pomegranate oil microencapsulation by spray dryer at $150{ }^{\circ} \mathrm{C}$. Furthermore, these authors described that increasing feed solids, speeds up crust formation, diminishing the time the core material is exposed to the surface of the microcapsule. This explanation is valid for increasing temperatures until a certain level, from which the formation of the surface crust may be so fast that is not enough for the interest material to be completely entrapped in the core. 
- Bulk Density: Bulk powder density was distributed from 0.51 to $0.66 \mathrm{~g} \mathrm{~cm}^{-3}$. Assay 6 , encapsulation with equal proportions of GA and MS using a $0.7 \mathrm{~mm}$ nozzle, presented bulk density of $0.51 \pm 0.02 \mathrm{~g} \mathrm{~cm}^{-3}$ and particle size distribution in the range of $1 \mu \mathrm{m}$ to $30 \mu \mathrm{m}$. Carneiro et al. (2013) evaluated microencapsulation of flaxseed oil using different carrier agent combinations at $180^{\circ} \mathrm{C}$ using a $0.5 \mathrm{~mm}$ diameter nozzle. For GA:MD the authors detected $0.40 \mathrm{~g} \mathrm{~cm}^{-3}$, while for MS:MD the value was $0.36 \mathrm{~g} . \mathrm{cm}^{-3}$. Goula and Adamopoulos (2012) observed that particle mean diameter varied from 5.8 to $18.7 \mu \mathrm{m}$ for pomegranate seed oil microencapsulated with skimmed milk powder using a $0.5 \mathrm{~mm}$ nozzle, with temperatures ranging from 150 to $190^{\circ} \mathrm{C}$. Bulk density can be correlated to nozzle diameter, air flow, temperature and final moisture. The higher bulk density observed herein was related to a wider range diameter, allowing for smaller bulk porosity. High density powder occupies smaller storage space, facilitates transport and can contribute to the preservation of the bioavailability of functional compounds, but may reduce the rate of microcapsule solubilization.

- Scanning electron microscopy (SEM): SEM images at a 500x magnification demonstrated the influence of wall material in microcapsule aggregation. The powder aggregation was higher when using MD (assay 1), due to its low film formation capacity, but replacing it by MS (assay 3) led to less aggregation (Figure 3a). Particle aggregation is important, since it has direct influence on microcapsule solubility. Assay 6 (GA:MS 1:1), with higher oxidative stability, exhibited lower aggregation. A range of particle sizes is expected in spray drying, since the pressure nozzle atomizer creates non-homogeneous spray particles, resulting in a wide particle size distribution (Anandharamakrishnan and Padma Ishwarya, 2015).

At 7000x magnification (Figure 3b) it is possible to observe rounded capsules with several cavities. These are characteristic of starchy material (Carneiro, 2013) and are probably formed during the fast shrinking in the spray chamber, where the water evaporates instantly, due to the high contact of particle surfaces with the hot air. Assay 1 (MD) led to cracks on the surface, evidencing that this wall material was too fragile due to the low maltodextrin film formation capacity, while sample 3 (MS), 5 (MD:MS) and 6 (GA:MS) exhibited smooth surfaces. A smooth surface is important, indicating an effective "wall" separating the core from the exterior, ensuring microcapsule integrity. This aspect, along with the oxidative stability results above mentioned, shows that the proportion oil:wall material used in this study was enough to provide a dense, tightly packed matrix around the oil droplets.
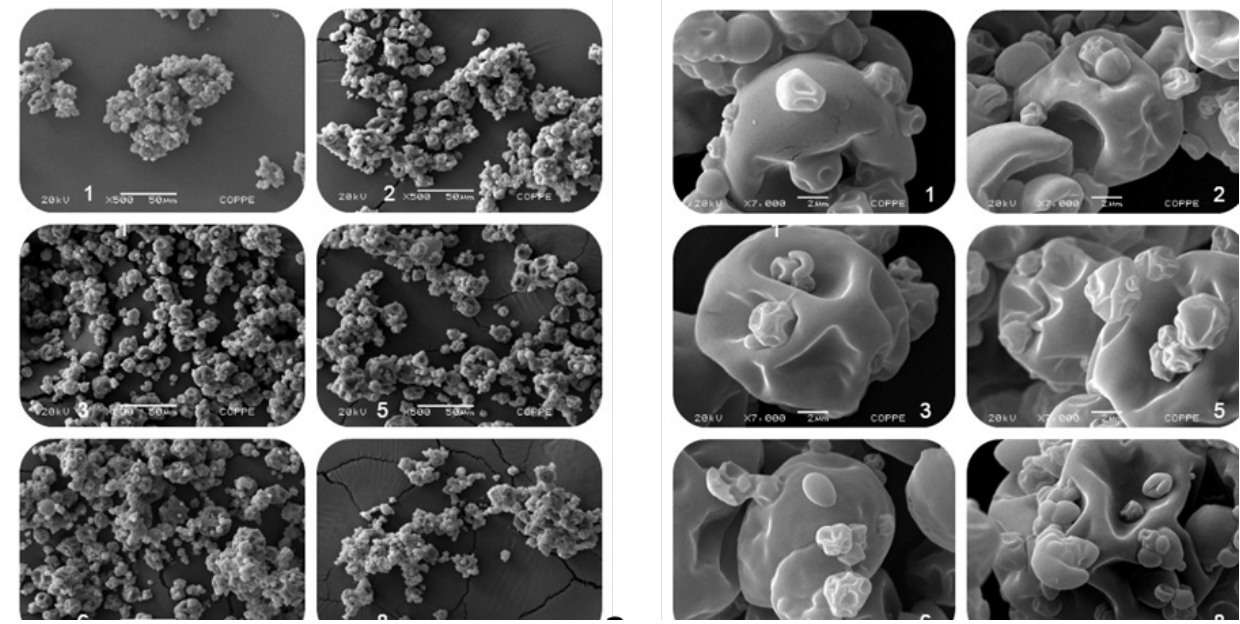

Figure 3. Scanning electron microscopy micrographs of microcapsules: Magnification (a) 500x and (b) 7000x.

- Powder shelf-life: The induction period (IP) may be related to product shelf life and can be used to predict the oxidative stability of foods and oils. The stability of microcapsules produced at $130{ }^{\circ} \mathrm{C}$ slowly decreased in about 30\% until 90 days. In this case, it was not found in literature pomegranate oil blend data for comparison. Guiotto et al. (2014) evaluated the effects of storage conditions on a non-encapsulated blend of chia and sunflower oils $(80: 20)$ at $20^{\circ} \mathrm{C}$ and observed that the IP decreased to almost $50 \%$ during 90 days. Due to the fatty acid profile, the blend chia:sunflower oil is more stable than PSO:SO. This occurred because linolenic acid from chia and oleic acid from sunflower are both more stable than punicic acid (from pomegranate) and linoleic acid (from soybean oil). Thus these results confirms that the selection of temperature range (inferior to the traditionally used in academic studies) very likely made possible a higher oxidative stability of the product and thus promoted a higher shelf life.

\section{Conclusions}

A new technology for pomegranate seed application was developed based on the microencapsulation of PSO:SO blend. The blend presented synergy regarding antioxidant capacity and may be an adequate source of ingredients for food or pharmaceutical industries. Concerning microencapsulation, the best oxidative stability was found in binary blends (1/1) using GA:MS or MD:MS, but for financial reasons, MD:MS should be selected. In 
this condition, oxidative stability was $42 \%$ higher for the microencapsulated powder compared to the nonencapsulated oil blend, although the antioxidant capacity was 2.6-fold higher for the microcapsules, since the carrier material also present antioxidant capacity. The best encapsulation efficiency $(95 \%)$ was reached at $\mathrm{T}=130$ ${ }^{\circ} \mathrm{C}$. The microencapsulated PSO:SO blend was proven to be an interesting way to offer the competitive pomegranate oil ingredient for nutraceutical applications. Considering the high production and low cost of soybean oil in the world, this PSO:SO blend favors the distribution of CLnA in manufactured products, increasing, CLnA intake as recommended by the latest metabolic studies. Pomegranate pressed cake displayed very high phenolic acid content compared with pressed oil, thus, its ethanolic extract can be added to the blend to enhance its bioactive potential.

\section{Acknowledgements}

Coordenação de Aperfeiçoamento de Pessoal de Nível Superior (CAPES), Federal University of Rio de Janeiro (UFRJ/EQ) and Embrapa Food Technology for supporting this study.

\section{References}

Ambigaipalan, P., A. C. de Camargo, F. Shahidi, 2017. Identification of phenolic antioxidants and bioactives of pomegranate seeds following juice extraction using HPLC-DAD-ESI-MSn. Food Chemistry, 221, $1883-1894$. https://doi.org/10.1016/j.foodchem.2016.10.058.

Baker, C. G. J., K. A. Mckenzie, 2002. Energy consumption of industrial spray dryers. In Drying 2002 Proceedings of the 13th International Drying Symposium (IDS' 2002) (pp. 645-652). Beijing, China: Drying Associates Limited.

Bustamante, A., A., Hinojosa, P. Robert, V. Escalona, 2017. Extraction and microencapsulation of bioactive compounds from pomegranate (Punica granatum var. Wonderful) residues. International Journal of Food Science and Technology, 52 (6), 1452-1462. https://doi.org/10.1111/ijfs.13422.

Carneiro, H. C. F., R. V. Tonon, C. R. F. Grosso, M. D. Hubinger, 2013. Encapsulation efficiency and oxidative stability of flaxseed oil microencapsulated by spray drying using different combinations of wall materials. Journal of Food Engineering, 115 (4), 443-451. https://doi.org/10.1016/j.jfoodeng.2012.03.033.

Conidi, C., A. Cassano, F. Caiazzo, E. Drioli, 2017. Separation and purification of phenolic compounds from pomegranate juice by ultrafiltration and nanofiltration membranes. Journal of Food Engineering, 195 , 1-13. https://doi.org/10.1016/j.jfoodeng.2016.09.017.

Cordeiro, A. M. T. M., M. L. Medeiros, M. A. A. D. Silva, I. A. A. Silva, L. E. B. Soledade, A. L. Souza, N. Queiroz, A. G. Souza, 2013. Rancimat and PDSC accelerated techniques for evaluation of oxidative stability of soybean oil with plant extracts. Journal of Thermal Analysis and Calorimetry, 114 (2), 827-832. https://doi.org/10.1007/s10973-013-3036-0.

Dreher, M., V. Lee, J. Costelow, M. Knight, 2011. US9205117 B1. Natural sourcing. USA. Retrieved from https:/www.google.com/patents/US9205117.

Goula, A. M., K. G. Adamopoulos, 2012. A method for pomegranate seed application in food industries: Seed oil encapsulation. Food and Bioproducts Processing, 90 (4), 639-652. https://doi.org/10.1016/j.fbp.2012.06.001

Guiotto, E. N., V. Y. Ixtaina, S. M. Nolasco, M. C. Toma, 2014. Effect of Storage Conditions and Antioxidants on the Oxidative Stability of Sunflower - Chia Oil Blends. Journal of the American Oil Chemists' Society, 91 , 767-776. https://doi.org/10.1007/s11746-014-2410-9

Habibnia, M., M. Ghavami, M., Ansaripourc, S. Vosough, 2012. Chemical evaluation of oils extracted from five different varieties of Iranian pomegranate seeds. Journal of Food Biosciences and Technology, 2 , 35-40. Retrieved from http://www.sid.ir/EN/VEWSSID/J pdf/1023220120206.pdf

Hartman, L., R.C.A. Lago, 1973. Rapid preparation of fatty acid methyl esters from lipids. Lab. Pract. 22, 475476.

Hashempour-Baltork, F., M. Torbati, S. Azadmard-Damirchi, G. P. Savage, 2016. Vegetable oil blending: A review of physicochemical, nutritional and health effects. Trends in Food Science and Technology, 57 , $52-58$. https://doi.org/10.1016/j.tifs.2016.09.007.

Heleno, S. A., A. Martins, M. J. R. P. Queiroz, I. C. F. R. Ferreira, 2015. Bioactivity of phenolic acids: Metabolites versus parent compounds: A review. Food Chemistry, 173 , 501-513. https://doi.org/10.1016/j.foodchem.2014.10.057

Hennessy, A. A., P. R. Ross, G. F. Fitzgerald, C. Stanton, 2016. Sources and Bioactive Properties of Conjugated Dietary Fatty Acids. Lipids, 51 (4), 377-397. https://doi.org/10.1007/s11745-016-4135-Z

Hermida, L. G., G. Gallardo, 2015. Food Applications of Microencapsulated Omega-3 Oils. In Leonard M.C. Sagis (Ed.), Microencapsulation and Microspheres for Food Applications (pp. 271-299). Elsevier. https://doi.org/10.1016/B978-0-12-800350-3.00018-2.

Juhaimi, F. Al, M. M., Özcan, K. Ghafoor, 2017. Characterization of pomegranate (Punica granatum L.) seed and oils. European Journal of Lipid Science and Technology (Vol. 119). https://doi.org/10.1002/ejlt.201700074 
Kaderides, K., A. M. Goula, K. G. Adamopoulos, 2015. A process for turning pomegranate peels into a valuable food ingredient using ultrasound-assisted extraction and encapsulation. Innovative Food Science \& Emerging Technologies, 31, 204-215. https://doi.org/10.1016/j.ifset.2015.08.006.

Kania, M., M. Michalak, M. Gogolewski, 2004. Antioxidative potential of substances contained in cold pressed soybean oil and after each phase of refining process. Acta Sci. Pol., Technol. Aliment., 3 (1), $113-121$. Retrieved from http://www.food.actapol.net/volume3/issue/12_1_2004.pdf.

Li, J., S. Xiong, F. Wang, J. M. Regenstein, R. Liu, 2015. Optimization of Microencapsulation of Fish Oil with Gum Arabic/Casein/Beta-Cyclodextrin Mixtures by Spray Drying. Journal of Food Science, 80 (7), C1445C1452. https://doi.org/10.1111/1750-3841.12928.

Lutterodt, H., M. Slavin, M. Whent, E. Turner, L. Yu, 2011. Fatty acid composition, oxidative stability, antioxidant and antiproliferative properties of selected cold-pressed grape seed oils and flours. Food Chemistry, 128 (2), 391-399. https://doi.org/10.1016/j.foodchem.2011.03.040.

Melo, I. L. P. de, E. B. T. de Carvalho, A. M. de O. E. Silva, L. T. Yoshime, J. A. G. Sattler, R. T. Pavan, J. Mancini-Filho, 2016. Characterization of constituents, quality and stability of pomegranate seed oil (Punica granatum L.). Food Science and Technology (Campinas), 36 (1), 132-139. https://doi.org/10.1590/1678457X.0069.

Montgomery, D.C.; V. Calado, 2003. Planejamento de Experimentos usando Statistica. Rio de Janeiro: EPapers Serviços Editoriais, 260 p.

Nascimento, L., M. Santiago, E. Oliveira, R. Borguini, E. Braga, V. C. Martins, S. Pacheco, M. C. Souza, R. L. O. Gogoy, 2017. Characterization of Bioactive Compounds in Eugenia brasiliensis, Lam. (Grumixama). Nutrition and Food Technology: Open Acess, 3 (3), 1-7. https://doi.org/http://dx.doi. org/10.16966/24706086.146

Perez-Ternero, C., C. M. Werner, A. G. Nickel, M. D. Herrera, M.-J. Motilva, M. Böhm, M. Alvarez de Sotomayor, U. Laufs, 2017. Ferulic acid, a bioactive component of rice bran, improves oxidative stress and mitochondrial biogenesis and dynamics in mice and in human mononuclear cells. The Journal of Nutritional Biochemistry, 48 , 51-61. https://doi.org/10.1016/j.jnutbio.2017.06.011.

Polavarapu, S., C. M. Oliver, S. Ajlouni, M. A. Augustin, 2011. Physicochemical characterisation and oxidative stability of fish oil and fish oil-extra virgin olive oil microencapsulated by sugar beet pectin. Food Chemistry, 127 (4), 1694-1705. https://doi.org/10.1016/j.foodchem.2011.02.044.

Rufino, M., R. E. Alves, E. S. de Brito, S. M. de Morais, C. G. Sampaio, J. Pérez-Jiménez, F. Saura-Calixto, 2007. Metodologia científica: determinação da atividade antioxidante total em frutas pela captura do radical livre DPPH. Comunicado Técnico on line - Embrapa Agroindústria Tropical. Fortaleza, CE. Retrieved from http://scholar.google.com/scholar?hl=en\&btnG=Search\&q=intitle:Metodologia+Cient?fica+:+Determina??o+da + Atividade+Antioxidante+Total+em+Frutas+pela+Captura+do+Radical+Livre\#0.

Sharma, P., S. McClees, F. Afaq, 2017. Pomegranate for Prevention and Treatment of Cancer: An Update. Molecules, 22 (2), 177. https://doi.org/10.3390/molecules22010177

Shishir, M. R. I., W. Chen, 2017. Trends of spray drying: A critical review on drying of fruit and vegetable juices. Trends in Food Science and Technology, 65 , 49-67. https://doi.org/10.1016/j.tifs.2017.05.006

Simopoulos, A. P. 2016. Evolutionary Aspects of the Dietary Omega-6/Omega-3 Fatty Acid Ratio: Medical Implications. In Alexandra Alvergne \& Crispin Jenkinson Charlotte Faurie (Eds.), Evolutionary Thinking in Medicine (1st ed., pp. 119-134). Cham: Springer International Publishing. https://doi.org/10.1007/978-3-31929716-3-9.

Singh, M., A. Jha, A. Kumar, N. Hettiarachchy, A. K. Rai, D. Sharma, 2014. Influence of the solvents on the extraction of major phenolic compounds (Punicalagin, ellagic acid and gallic acid) and their antioxidant activities in pomegranate aril. Journal of Food Science and Technology. https://doi.org/10.1007/s13197-014-1267-0.

Souza, V. de, M. Thomazini, 2013. Effect of spray drying on the physicochemical properties and color stability of the powdered pigment obtained from vinification byproducts of the Bordo grape ( Vitis. Food and Bioproducts Processing, (October), 1-12. https://doi.org/10.1016/j.fbp.2013.11.001.

USDA. 2017. World Agricultural Supply and Demand Estimates. United States Department of Agriculture. https://doi.org/WASDE-525.

Wang, Y., B. Liu, X. Wen, M. Li, K. Wang, Y. Ni, 2017. Quality analysis and microencapsulation of chili seed oil by spray drying with starch sodium octenylsuccinate and maltodextrin. Powder Technology, 312, 294298. https://doi.org/10.1016/j.powtec.2017.02.060.

Yuan, G., H. Sun, A. J. Sinclair, D. Li, 2009. Effects of conjugated linolenic acid and conjugated linoleic acid on lipid metabolism in mice. European Journal of Lipid Science and Technology, 111 (6), 537-545. https://doi.org/10.1002/ejlt.200800200. 


\title{
Development of a Machine for the Production of Liquid Wine Yeast to Induce Grape Juice Fermentations
}

\author{
Lorenzo Guerrini ${ }^{\text {a, }}$, Alessandro Parenti ${ }^{\text {a }}$, Giulia Angeloni ${ }^{\text {a }}$, Piernicola Masella ${ }^{\text {a }}$, Damiano Barbato ${ }^{\text {a }}$, \\ Eleonora Mari ${ }^{a}$, Yuri Romboli ${ }^{a}$, Giacomo Buscioni ${ }^{\text {b }}$, Silvia Mangani ${ }^{\text {b }}$, Simona Guerrini ${ }^{\text {b }}$, Lisa Granchi ${ }^{\text {a }}$ \\ a 1Department of Management of Agricultural, Food and Forestry Systems (GESAAF) University of Florence, Italy \\ ${ }^{\mathrm{b}}$ FoodMicroTeam - University of Florence Academic Spin-off \\ *Corresponding author. Email: lorenzo.guerrini@unifi.it
}

\begin{abstract}
During the winemaking process, the alcoholic fermentation can be carried out either spontaneously or by inoculating commercial dried yeasts. Commercial yeasts ensure effective and quick juice colonization, allowing a better control of the fermentation. Thus, they are usually preferred by winemakers to reduce the fermentation quality-related risks. However, the spontaneous fermentation can produce wines with sensory properties typical of a certain region or even of a specific cellar. This characteristic is particularly appreciated by those winemakers that wish to produce a wine easy to recognize. To keep the advantages of both approaches, a machine able to produce on-demand a liquid yeast biomass from selected local yeasts was studied and developed. In this way, producers can inoculate a quantity of yeasts able to reduce the fermentation risks, with a local selected strain able to give wines typical properties. The machine is composed by two parts: a tank and a centrifuge. The tank is where the yeasts increase in number. It is equipped with to supply systems for water and nutrients. The water supply system includes an UV-lamp for water sterilization and a heat exchanger to set the temperature of the medium water. The feeding system continuously doses the nutrients to keep a low sugar content into the medium, promoting yeasts respiration instead of fermentation. This increases the yeast-growing rate. Furthermore, the fermenter is equipped with a heating/cooling jacket to maintain the set temperature, and with an air supplier to mix the medium and provide the adequate oxygen. At the end of this cycle, the medium is separated by the yeast biomass with a vertical centrifuge. The machine was tested during the 2017 vintage. The produced yeast was used to inoculate must fermentations at industrial scale. The inoculated yeast strains carried out the alcoholic fermentation, as confirmed by the microbial molecular analysis.
\end{abstract}

Keywords: authoctonous yeasts; centrifuge; fermenter; spontaneous fermentations; wine quality; wine typifying,

\section{Introduction}

In oenology fermentation can be either inoculated or spontaneous. The inoculated are usually carried out by commercial yeasts, while the spontaneous by the microorganism that colonize the environment where the fermentations take place. Inoculum of commercial yeasts ensures rapid and reliable fermentations. Furthermore, it reduced the risks of microbial contamination or fermentation stuck (Valero et al., 2007). On the other hand, the spontaneous fermentations are conducted by local yeasts and can give wines sensory properties typical of a specific region or even of a specific cellar (Maqueda et al., 2011). For example, yeasts produce several volatile compounds helpful to give wines recognisable characteristics (Guerrini et al., 2016). Nowadays, winemakers working with local yeasts use the "pied de cuve" practice. Briefly, a certain amount of must is placed in a small tank and the fermentation start is waited. Then, the mass is used for the inoculum of a greater quantity of must. This practice allows to work with local yeasts, but is long and uncertain (Riberau-Gayon et al., 2006). Alternatively, the local yeasts could be isolated, stored, and multiplied before the vintage.

Hence, in order to typify the yeast-related wine properties with a yeast characteristic of one cellar, local yeasts have to be selected and isolated from the other microorganisms present in the winemaking environment. Then, the yeasts have to be inoculated into grape juice in an appropriate amount to be reasonable sure they actually carry out the fermentation.

To reach this goal it is necessary to develop some strategies and machines to multiply the selected yeasts. Thus, we aimed to develop a yeast production plant able to multiply a yeast strain directly in the cellar roughly 37 weeks before the vintage. Producers can inoculate a quantity of yeasts able to reduce the fermentation risks, with a local selected strain able to give wines typical properties. Furthermore, the developed machine was thought to be semi-automatic in order to find a compromise between the labour required to produce the fresh yeasts and the machine production costs.

\section{Materials and Methods}

The test presented in this work aimed to design, size, optimise and test a plant for fresh yeast production. The machine has to multiply the yeasts in a water-sugar solution. Thus, the fermenter was designed to reproduce the 
best conditions for yeasts multiply (i.e. in term of temperature, $\mathrm{pH}$, nutrient concentration, risk of contamination, etc.). Then, the yeasts have to be separated from the culture medium until yeast dough is obtained. For the separation phase five commercial solutions for solid-liquid separation were tested. The yeast dough should contain less than $70 \%$ of water to be easily stored in a fridge for 3-7 weeks.

In order to evaluate the plant performance, to decide the appropriate supplies and to optimise the plant working cycle, several tests were carried out.

2.1. Plant optimization trials

The water sterilization method set up was chosen testing an UV-lamp with water from 4 different aqueducts (namely A, B, C, and D). In order to choose the working flow rate, the lamp treated $250 \mathrm{~L} / \mathrm{h}$ and $350 \mathrm{~L} / \mathrm{h}$ of water from aqueducts $\mathrm{A}$ and $\mathrm{B}$. After the treatments total microorganisms and coliforms were measured into the waters.

The effectiveness of the air supply system was tested measuring the dissolved oxygen into the fermentation medium and comparing the oxygen concentration with and without the air introduction.

The separation systems were tested in the conditions indicated by the system producer. The fresh yeast solid content was measured by oven-drying $15.4 \pm 1.4 \mathrm{ml}$ of yeasts until a constant weight was reached $(24 \mathrm{~h}, 104 \pm 2$ ${ }^{\circ} \mathrm{C}$ ). The yeast losses were measured by sampling the separation device discharge water.

The cleaning cycle was optimized testing total yeasts, total bacteria and coliforms in $2 \mathrm{~L}$ of discharge water. The parameter chosen for optimization was the rinse water volume (30 or $40 \mathrm{~L})$ and the sanitizer (i.e. per-acetic acid) concentration ( $1 \%$ or $2 \%)$.

The produced fresh yeast was used to inoculate $100 \mathrm{hL}$ of Sangiovese red grapes.

2.2. Microbiological measurements

Total yeasts: in order to quantify the yeasts the water samples were collected and immediately transferred to laboratories for analysis. Yeast enumeration was obtained by surface spreading $0.1 \mathrm{~mL}$ of samples of water into plates of WL nutrient agar. Representive colonies developed after incubation of the plates at $28^{\circ} \mathrm{C}$, were counted after $48-72 \mathrm{~h}$.

Total bacteria: The filtered waters were analysed filtering $250 \mathrm{ml}$ of sample on cellulose membranes $(0.20$ $\mu \mathrm{m}$; Pall Corporation). The membranes were plated superficially in Plate Count Agar. Yeast was counted after incubation for $48-72 \mathrm{~h}$ at $30^{\circ} \mathrm{C}$ under aerobic conditions.

Coliforms: water samples were grown at $30^{\circ} \mathrm{C}$ for $24 \mathrm{~h}$ with oxygen, using the specific culture medium VRBA+MUG (Oxoid).

\section{Results and Discussion}

Description of the fermenter

The developed fermenter is shown in Figure 1. A stainless steel tank with nominal volume of $500 \mathrm{~L}$ was chosen as fermenter. The chosen steel was the AISI $316(18 \% \mathrm{Cr}, 10 \% \mathrm{Ni}, 2.5 \% \mathrm{Mo})$. The AISI 316 provides a good resistance to corrosion, required during the cleaning phase when the tank is treated with per-acetic acid (Cubberly et al., 1980). The volume of $500 \mathrm{~L}$ was chosen considering the fresh yeast shelf-life and the volume of grape must to be inoculated. About $1.5 \mathrm{~kg}$ of fresh local yeasts is produced during each cycle (i.e. roughly $24 \mathrm{~h}$ of length). Hence, considering $30 \mathrm{~g} / \mathrm{hL}$ of dough as correct dose $50 \mathrm{hL}$ of must can be inoculated for each cycle.

The tank was equipped with a system for the supply of sterilized water, an automatic feeder, a thermal control system, an air supplier, and an automatic cleaning system. All these parts are electronically handled with a Programmable Logic Controller (PLC).

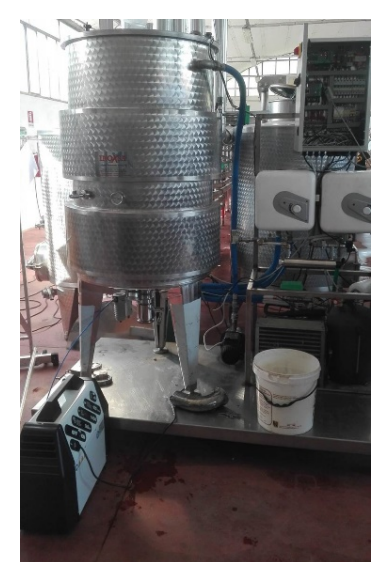

Figure 1. The fermenter developed and tested during the trials. 


\section{Sterilized water supply system}

The system was fed with drinking water, provided by the aqueduct. The system allow to rise, with a dedicated heat exchanger ( $1.2 \mathrm{~kW}$ of nominal power), the water temperature before of its introduction into the fermenter. Then, the water is sterilized with an UV lamp. The need of incoming water sterilization is due to the working conditions of the fermenter, resulting in a high risk of microbial contamination of the yeast culture. A large number of technologies are available for sterilization: boiling, ultra-high temperature, steam, liquid nitrogen, ozone, reverse osmosis, microfiltration, high pressure processing, ultrasound, chlorination, and irradiation. Among them, UV was chosen because it provides high working capacity, low costs and no risk of chemical residues into the water. In fact, the chosen lamp working capacity was $350 \mathrm{l} / \mathrm{h}$. Furthermore, it had a small size $(50 \times 12 \times 4 \mathrm{~cm})$ and a low purchase cost (i.e. $350 €$ ). The results of water contamination after the treatment are reported in Table 1.

Table 91. Total microorganisms and coliforms counts after the water UV treatment. The water was taken from 4 different aqueducts (A, B, C, D) and treated at 2 different flow rates.

\begin{tabular}{cccc}
\hline Sample & Water flow rate $(\mathrm{L} / \mathrm{h})$ & Total microorganisms $(\mathrm{UFC} / \mathrm{ml})$ & $\begin{array}{c}\text { Total coliforms } \\
(\mathrm{UFC} / \mathrm{ml})\end{array}$ \\
\hline Aqueduct A1 & 250 & $<10$ & $<2$ \\
Aqueduct A2 & 350 & $<10$ & $<2$ \\
Aqueduct B1 & 250 & $<10$ & $<2$ \\
Aqueduct B2 & 350 & $<10$ & $<2$ \\
Aqueduct C & 350 & $<10$ & $<2$ \\
Aqueduct D & 350 & $<10$ & $<$ \\
\hline
\end{tabular}

The lamp was tested at the maximum flow rate $(350 \mathrm{~L} / \mathrm{h})$ and at the $70 \%$ of the maximum working capacity $(250 \mathrm{~L} / \mathrm{H})$, with the same results in term of sterilization. Thus, the lamp operative flow rate was the maximum.

\section{Automatic nutrient dosage}

During the plant working cycle yeasts nutrients have to be dosed continually. In this way we wanted to avoid the Crabtree effect. This effect occurs when an excess of glucose is present and lead to a reduction in cell yield (Imura et al., 2018). Hence, the plant was equipped with two continuous doser shown in Figure 2.

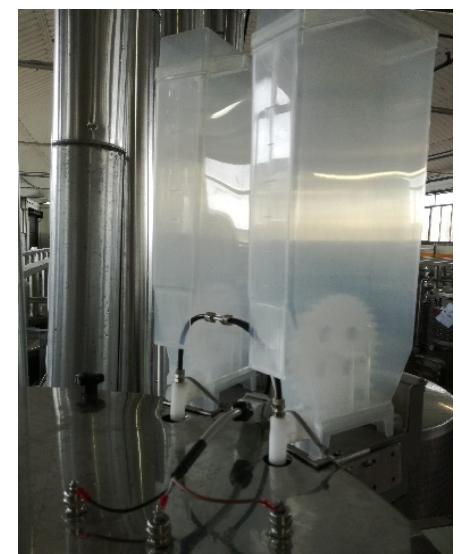

Figure 2. Particular of the plant nutrient dosers

The system is composed by two hoppers, containing granular rectified concentrated must, and two screws for the granules transport, providing a volumetric dosage. In order to ensure the dose of $1 \mathrm{~g} / \mathrm{L}$ at different water levels during the working cycle, the screws velocity was electronically changed.

An inconvenient of this system is the packing of the sugar granules at the end of the screw due to the moisture coming from the fermenter. The problem was solved deriving a small air flux from the air supply system and using the flux to dry the sugar (the black flexible pipes in Figure 2).

\section{Air supply system}

The air supply system is composed by a compressor, a sterilizing air filter ( $0.2 \mu \mathrm{m}$ of nominal porosity) and a sparger. The system have three purposes: it provides microorganisms sufficient oxygen for their metabolism (Chopda et al., 2015), it agitates the medium in order to ensure uniformity in the suspension of yeasts cells, and it improves the cooling efficiency (Guerrini et al., 2017). Furthermore, the air is used to dry the granular nutrients as described above. 


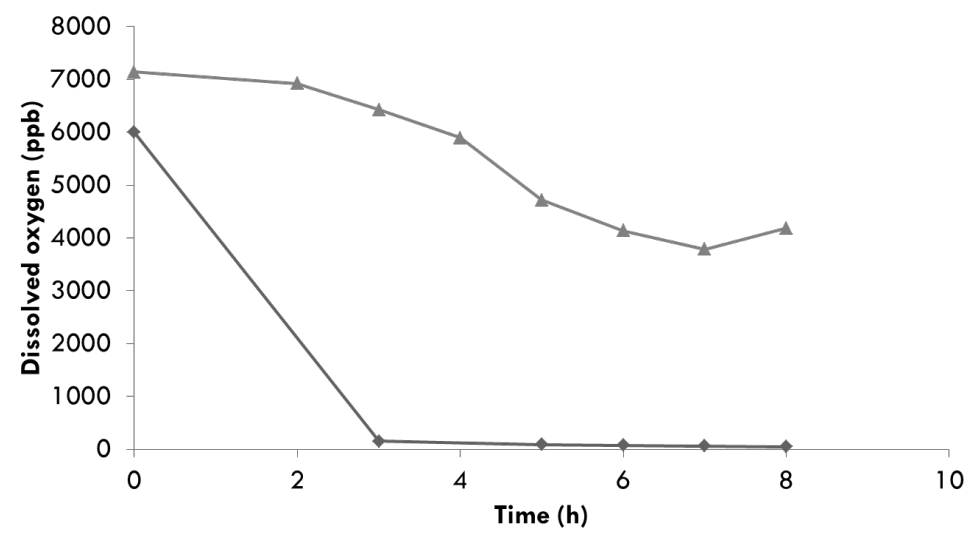

Figure 3. Dissolved oxygen (ppb) during the trials without air (black line) and with air (grey line).

The air was sufficient to homogenize the yeasts cells and nutrients. The yeasts in the trial without air used almost all the oxygen within the $3^{\text {rd }} \mathrm{h}$ since inoculum (Figure 3). On the other hand, small oxygen consumption could be noticed between the $2^{\text {nd }} \mathrm{h}$ and the $7^{\text {th }} \mathrm{h}$ of batch in the trial with air. Furthermore, choosing the appropriate air flow rate, it was possible to remove from the fermenter the mechanical agitation system, making the system easier to clean.

\section{Description of separation system}

The separation system has to fulfil the following requirements. First of all, the obtained yeast dough has to contain live, vital and not damaged cells. Then, the yeast dough has to contain less than the $70 \%$ of water in order to reduce the contamination risks during the products shelf life. Five different technologies were tested. A summary of the results is reported in Table 2 .

Table 2. Resume of the tested methods for yeasts separation.

\begin{tabular}{ccc}
\hline Separation method & Plant type & Comments \\
\hline Centrifugation & Vertical Centrifuge & Ok \\
Centrifugation + Filtration & Bag Centrifuge & The bag was not able to retain the yeasts \\
Centrifugation & Basket Centrifuge & Losses in yeasts higher than $80 \%$ \\
Filtration & Tangential filter & Long separation times \\
Centrifugation & Horizontal centrifuge & Losses in yeasts higher than $80 \%$ \\
\hline
\end{tabular}

Among the tested separation technologies, the best results were achieved with the vertical centrifuge, while the other technologies requires times too long or were not able to separate the yeasts from the medium.

The vertical centrifuge was tested at different feeding rates and the results are reported in Table 3. .

Table 3. Yeasts losses at different centrifuge feeding rates.

\begin{tabular}{ccc}
\hline Feeding rate $(\mathrm{L} / \mathrm{h})$ & Required time $(\mathrm{min})$ & Yeasts losses $(\%)$ \\
\hline 250 & 108 & $<2$ \\
300 & 90 & $<2$ \\
360 & 75 & $<2$ \\
400 & 68 & 18 \\
450 & 60 & 34 \\
\hline
\end{tabular}

Until the $360 \mathrm{~L} / \mathrm{h}$ of feeding rate the losses were not measurable, while at $400 \mathrm{~L} / \mathrm{min}$ and at $450 \mathrm{~L} / \mathrm{min}$ the losses where higher. Hence, the $360 \mathrm{~L} / \mathrm{h}$ was chosen as working flow rate. It is important to point out that at all the feeding rates the yeasts cells were live and vital.

\section{Description of the cleaning cycle}

The performances of the 3 tested cleaning cycles are reported in Table 4 . The $\mathrm{pH}$ sensor was useful to verify the introduction of the per-acetic acid as sanitizer. In fact, the water before the per-acetic acid had $\mathrm{pH}$ of 7.0, while after the sanitizer introduction the $\mathrm{pH}$ falls to 2.5 . At the end of the rinse, when all the acid was washed, the $\mathrm{pH}$ was back to 7.0 . 
Table 4. Yeasts losses at different centrifuge feeding rates.

\begin{tabular}{ccccc}
\hline Rinse water (L) & $\begin{array}{c}\text { Sanitizer } \\
\text { concentration (\%) }\end{array}$ & $\begin{array}{c}\text { Total micro- } \\
\text { organisms (UFC/L) }\end{array}$ & $\begin{array}{c}\text { Total yeasts } \\
(\mathrm{UFC} / \mathrm{ml})\end{array}$ & $\begin{array}{c}\text { Total coliforms } \\
(\mathrm{UFC} / \mathrm{ml})\end{array}$ \\
\hline 30 & 1 & 600 & 19 & 150 \\
30 & 2 & 432 & $<10$ & $<2$ \\
40 & 2 & 250 & $<10$ & $<2$ \\
\hline
\end{tabular}

After the test the chosen rinse time was $30 \mathrm{~min}$ and the $2 \%$ of per-acetic acid was chosen as sanitizer concentration. In fact, the lower rinse water volume and the lower per-acetic acid concentration were discarded for the high contamination allowed.

\section{Description of the working cycle}

The machine working cycle was divided into six steps. The first step is the tank filling. Ninety litres of sterilized water are automatically introduced into the tank. Then, $1.5 \mathrm{~g} / \mathrm{L}$ of nitrogen nutrients are manually added and the $\mathrm{pH}$ is regulated at 5 with orto-phosphoric acid. Ten litres of yeast are finally added to the medium.

During the second step the yeasts multiply. During this step the MCR is automatically dosed ( $1 \mathrm{~g} / \mathrm{Lh})$. The mass is agitated with sterilized air and the temperature is controlled at $28{ }^{\circ} \mathrm{C}$. Temperature, $\mathrm{pH}$ and dissolved oxygen are continuously monitored. This phase lasts $240 \mathrm{~min}$. The third step is the second tank filling. $350 \mathrm{~L}$ of sterilized water are automatically introduced. Then the system alerts the operator for the manual introduction of $1.5 \mathrm{~g} / \mathrm{L}$ of nitrogen nutrients, and orto-phosphoric acid.

The fourth step represents the second yeasts multiply. As in the second step the MCR is automatically dosed $(1 \mathrm{~g} / \mathrm{Lh})$. The mass is agitated with sterilized air and the temperature is controlled at $28^{\circ} \mathrm{C}$. Temperature, $\mathrm{pH}$ and dissolved oxygen are continuously monitored. This phase lasts $900 \mathrm{~min}$.

During the fifth step the yeasts are separated from the culture medium. A pump feeds the centrifuge at $61 / \mathrm{min}$ of flow rate. At the end of the phase the fresh yeasts are manually taken from the centrifuge bowl and stored at 4 ${ }^{\circ} \mathrm{C}$.

The final step is the plant cleaning. Hot sterilized water is introduced for $3 \mathrm{~min}$ in the fermenter. Then the fermenter, the pipes and the centrifuge are cleaned with $40 \mathrm{~L}$ of cold water. Finally, the fermenter is sanitized with a water solution at $2 \%$ of per-acetic acid. After $20 \mathrm{~min}$ the tank is emptied, and the system is rinsed 3 times with hot sterilized water. The centrifuge disks are manually cleaned into a $2 \%$ solution of per-acetic acid in water.

Use of the yeasts in industrial scale fermentation

The produced fresh yeasts were used to inoculate $100 \mathrm{hL}$ of Sangiovese red grapes. A concentration of $4 * 10^{6}$ yeasts $/ \mathrm{ml}$ was inoculated. During the fermentation the maximum concentration of $7.8^{*} 10^{7}$ yeasts $/ \mathrm{ml}$ was measured, and the fermentation length was 8 days. The initial sugar content was $210 \mathrm{~g} / \mathrm{L}$. At the day 8 the concentration of sugar into the wine was $0 \mathrm{~g} / \mathrm{L}$ and the fermentation was considered concluded. The average fermentation temperature was $22{ }^{\circ} \mathrm{C}$, while the maximum was $25^{\circ} \mathrm{C}$. Hence, the yeasts were able to successful conduct the fermentation.

\section{Conclusions}

The work allows to design, size, and optimise a plant for fresh yeast production. This plant in a $24 \mathrm{~h}$ working cycle is able to produce the yeasts dough for the inoculum of $50 \mathrm{hL}$ of grape must. The plant was thought to be semi-automatic and supplied with dissolved oxygen, temperature, $\mathrm{pH}$, and liquid level sensors. The sensors guarantee a continuous plant control. The plant was equipped with an automatic feeding system, to improve the yield in term of yeast biomass. The air was used to agitate the medium, provide the appropriate oxygen to yeasts and to improve the thermal exchange efficiency. Furthermore, it was equipped with an automatic cleaning system. The solid-liquid separation was provided by a vertical centrifuge. In all the tests the produced yeast dough was reliable in term of amount and quality of the produced fresh dough. During the 2017 vintage the obtained yeasts were used to inoculate $100 \mathrm{hL}$ of Sangiovese must, that was successfully fermented into wine.

\section{Acknowledgements}

This work was realized with the FEASR (Piano di Sviluppo Rurale 2014-2020 della Regione Toscana sottomisura 16.2) contribution. Project title "Produzione in cantina di lieviti starter autoctoni - VICAStart". The authors would like to thanks Cantina Sociale Vicas soc. coop., Cantina Tuscania srl and Parsec srl for their technical support during the trials and the machine development. 


\section{References}

Chopda, V. R., Rathore, A. S., \& Gomes, J. (2015). Maximizing biomass concentration in baker's yeast process by using a decoupled geometric controller for substrate and dissolved oxygen. Bioresource technology, 196, 160168.

Cubberly, W. H., P. M. Unterweiser, D. Benjamin, C. W. Kirkpatric, V. Knoll, and K. Nieman, 1980. Stainless steels in corrosion services. In Metals Handbook ( $9^{\text {th }}$ edition).

Imura, M., Iwakiri, R., Bamba, T., \& Fukusaki, E. (2018). Metabolomics approach to reduce the Crabtree effect in continuous culture of Saccharomyces cerevisiae. Journal of bioscience and bioengineering.

Guerrini, L., Masella, P., Spugnoli, P., Spinelli, S., Calamai, L., \& Parenti, A. (2016). A Condenser to Recover Organic Volatile Compounds during Vinification. American Journal of Enology and Viticulture, 67(2), 163-168.

Guerrini, L., Angeloni, G., Baldi, F., \& Parenti, A. (2017). Thermal effects of pump-overs during red wine fermentation. Applied Thermal Engineering, 112, 621-626.

Maqueda, M., Pérez-Nevado, F., Regodón, J. A., Zamora, E., Álvarez, M. L., Rebollo, J. E., \& Ramírez, M. (2011). A low-cost procedure for production of fresh autochthonous wine yeast. Journal of industrial microbiology \& biotechnology, 38(3), 459-469.

Ribâereau-Gayon P, Glories Y, Maujean A. (2006) Handbook of Enology: The Chemistry of Wine: Stabilization and Treatments. John Wiley \& Sons.

Valero, E., Cambon, B., Schuller, D., Casal, M., \& Dequin, S. (2007). Biodiversity of Saccharomyces yeast strains from grape berries of wine-producing areas using starter commercial yeasts. FEMS yeast research, 7, $317-$ 329. 


\title{
Influence of Physical and Chemicals Characteristics from Pumpkin for its Mechanized Processing
}

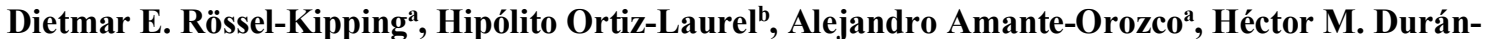 \\ García $^{c}$, L.A. López-Martínez \\ ${ }^{a}$ Colegio de Postgraduados. Iturbide 73, Salinas de Hgo., S.L.P. 78600. México. \\ ${ }^{\mathrm{b}}$ Colegio de Postgraduados. km. 348, Carr. Fed. Córdoba-Veracruz, Congregación Manuel León, Amatlán de los Reyes, \\ Veracruz. 94946. México. \\ 'Universidad Autónoma de San Luis Potosí. Manuel Nava 8, Zona Universitaria. San Luis Potosí. 78290. México. \\ * Corresponding author. Email: edietmar@colpos.mx
}

\begin{abstract}
Dealing with agroindustrial product processing can start from a basic treatment up to a full transformation Thus, it is essential to previously know the physical, chemical and biological characteristics from the raw mass that enters into the process. It is also important to choose processing tools made from suitable materials, as they influence the efficiency of mechanical forces application and its resistance to organic chemical compounds. A study for monitoring the interaction between processing tools with different materials for their blades acting on the raw mass under processing would give data that could orient towards diversification of product transformation, as well as their attributes to generate healthy and well balanced nutritious food products. In this work, a procedure was directed to certain constituents of pumpkin (Cucurbita spp.) in order to obtain bioactive lipids. It started with recording basic data, as dimensions, weight and densities, as well as its physical and chemical attributes from this raw mass before processing. When the mass was processed to obtain flour from pumpkin's seeds with cover and when utilized for cooking, it was recorded a reduction in losses of oil up to $15 \%$. Equally, when its chemical attributes are associated to a proper processing, they contributed to improve the extraction efficiency, as well as the quality and trait for the obtained acid lipids; from 20\% for oleic and 51.9\% for linoleic coming from the same raw mass.
\end{abstract}

Keywords: mechanization, diversification, bioactive compounds, pumpkin seed.

\section{Introduction}

In order to guarantee enough food for the Worldwide population, it is necessary to transform actual food producing and farming systems, to provide economic and social opportunities, as well as to increase resilience against climate change (FAO, 2016). A viable possibility is to whole use the organic matter, reducing losses and to achieve a diversification of final products. Una posibilidad real es usar la materia orgánica producida más ampliamente, reduciendo las perdidas (productos de desecho) y diversificar los productos finales. Esto surge a partir de las limitaciones para aumentar la producción agrícola. La diversificación de la producción agrícola se vería reflejada en una garancia económica, por la elaboración de productos, la rotación agrícola, uso de agroquimicos, contaminacion, más fuentes de empleo, y menos pérdidas en la producción. Un producto suceptible para diversificar es la calabaza, sola o en asociación con otros cultivos.

Pumpkin is a vegetable of great importance in the traditional agricultural systems of the world (Subbaiah, 2016). Farmers have already experience to produce it, however, there is a lack of technology that could aid to increase its productivity and profitability (INIFAP, 2007). Pumpkins have been cultivated through millenniums in the American continent, mainly for obtaining their fruits (contain carbohydrates and vitamins), but also for their seeds; rich in oils and vitamins. They are ease to propagate, grow rapidly on organic wastes and produce high quality food (Figure 1) Different species of pumpkin vary, mainly on the shape, size and color of their fruits (Muschler, 2008).

In Mexico, pumpkin production is around 133334,96 ton; while Zacatecas state leads in the collection of pumpkin seeds of around 6550,32 ton. Pumpkin seeds are flat, ovals, light green color, inside a case or cover, mostly it is used for feeding and medicine. (Applequist, 2006). They have been classified as oily with great amount of protein, essential aminoacids, polyunsaturated lipids, phitoesterols, minerals and vitamins (Ghari, 2011; Martínez, 2012; Habib, 2015). Oil from pumpkin seeds is of great benefit for health. It is highly unsaturated, with a range from $60 \%$ to $90 \%$ between oleic and linoleic acids. Because of that, it is important to evaluate the physical and chemical characteristics of any section from the seed pumpkin var. castilla (Cucúrbita sp), with the purpose of processing and its application on agroindustrial systems. 

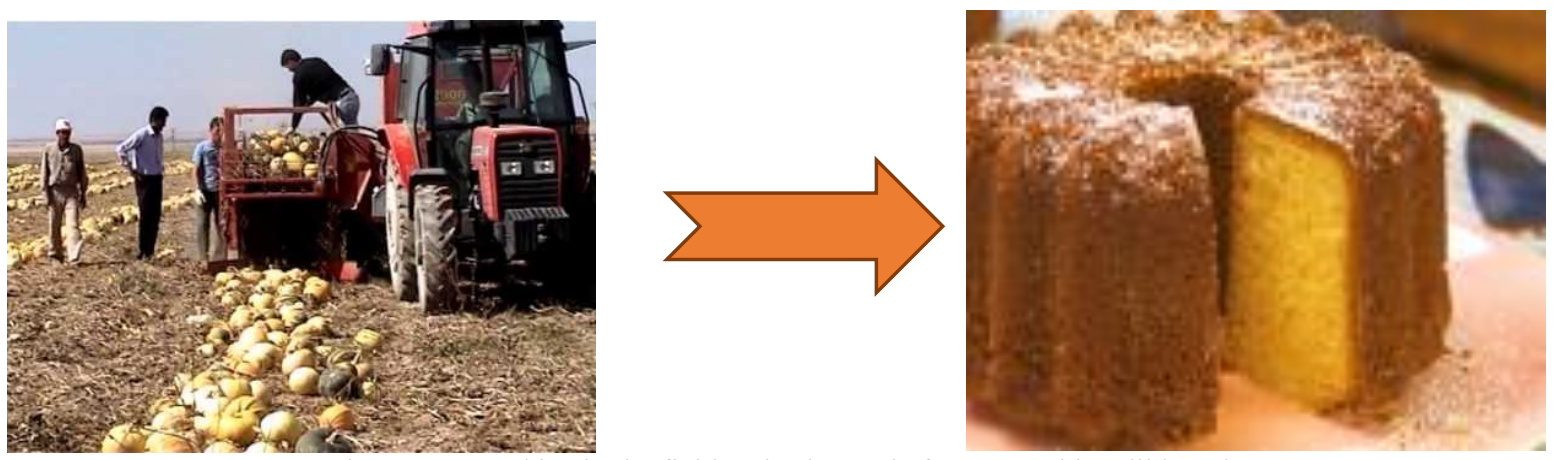

Figure 1. Pumpkins in the field and cake made from pumpkin edible pulp.

\section{Materials and methods}

It was utilized seeds from pumpkin var castilla (Cucúrbita sp) cultivated in Villa de Ramos, San Luis Potosi, Mexico. The climate en this area range from a dry moderate (Bsk) to dry semi-warm (BS h), its average temperature ranges from 16 to $18^{\circ} \mathrm{C}$, an annual mean rainfall from $400 \mathrm{~mm}$ and vegetation is mainly rosetophil and microphil shrubs. The seeds were selected according to traditional procedures, i.e. from the last crop, the best physical condition are separated (size, shape, color) and with a caliper, it was measured its length, wide and thickness. Seeds were carefully prepared to undertake different analysis, described as follows:

Proximal analysis. Moisture content was determined (Nollet, 2004); protein content was registered by using A.O.A.C, Official Methods of Analysis 15, edition.1990, 954.01; lipids were measured with NMX-F-312-1978 and total carbohydrates with NMX-F-089-1978. All measurements were realized three times and they showed their average figures and standard deviations.

Determination of lipid acids profile. Their determinations were realized by using gas chromatography, on pure oil extracted from the seed. The study was realized on the Gas Chromatograph Shimadzu GC-2010, by using a capillary column VARIAN CP 7420. It was used the approved technique AOCS Ce 1f - 96 (Millán et al., 2011).

Milling of pumpkin seed. Before starting, pumpkin seeds with skin and without case were separated milled (Thomas Wiley) inside a four knives mill with a sieve of $2 \mathrm{~mm}$; later, flour obtained was secondly milled inside a two knives mill (KRUPS GX410011). Flour obtained from the seed with case was sieved by using a mechanical strainer device Model (LA-0441).

Technological application. In order to test the technological application for pumpkin seed, crispy tortilla chips were elaborated through several compositions, where flour from pumpkin seed without case (SSC), flour from pumpkin seed with case (SCC) and commercial flour from maize (M) were mixed. Those ratios were mixed according to an at random experimental block design, setting up 10 treatments (Table 1). Ten percent water and $1.5 \%$ salt were added to mix composition, stirring was carried out until it formed a homogenous mass and then the dough was left still for 10 minutes. Later on, dough was placed on a metallic press Brand (Fial) to make a flat cake. Tortilla type chips were cut by a triangle mould $(3 \mathrm{X} 4 \mathrm{~cm})$ and placed inside aluminum trays. Secondly, they were introduced into the $85^{\circ} \mathrm{C}$ oven for around 1 hour. In order to determine acceptance of crispy tortilla chips a sensorial test was carried out by 16 trained testers. The trial was carried out in CARAO-UASLP, where flavor, odor, color, texture, appearance and strength were evaluated through a linear test and three best samples were chosen, considered from better to worst for each sample. The three best samples were evaluated by a proximal analysis employing the same techniques when seeds were evaluated. For the purpose of comparison it was determined the oil content inside the maize's flour.

\section{Results and discussion}

Length, wide and thickness of seeds with case. Table 2 shows average figures obtained through variance analysis which are useful for procedures involve in mixing, dosing, drying, storing, transporting, sieving, milling, etc. Average figures were: length $(20,61 \mathrm{~mm})$; wide $(8,59 \mathrm{~mm})$, thickness $(2,51 \mathrm{~mm})$. Absolute variance was 3,18 $\mathrm{mm}$ for length; 0,50 for wide and 0,14 for thickness, with a standard deviation of \pm de $1,78 \mathrm{~mm}$ (length); $0,71 \mathrm{~mm}$ (wide) and 0,37 $\mathrm{mm}$ (thickness). 
Table 1. Formulae for elaborating crispy tortilla chips.

\begin{tabular}{ccccccccccc}
\hline \multicolumn{10}{c}{ Treatments design } \\
\hline Treatments & T1 & T2 & T3 & T4 & T5 & T6 & T7 & T8 & T9 & T10 \\
SCC & $25 \%$ & & $75 \%$ & & $15 \%$ & & $85 \%$ & & $50 \%$ & \\
SSC & & $25 \%$ & & $75 \%$ & & $15 \%$ & & $85 \%$ & & $50 \%$ \\
M & $75 \%$ & $75 \%$ & $25 \%$ & $25 \%$ & $85 \%$ & $85 \%$ & $15 \%$ & $15 \%$ & $50 \%$ & $50 \%$ \\
\hline
\end{tabular}

$\mathrm{SSC}=$ seed without case, $\mathrm{SCC}=$ seed with case, $\mathrm{M}=$ maize commercial flour.

Table 2. Sizes of pumpkin seed with case.

\begin{tabular}{cccc}
\hline \multicolumn{4}{c}{ Pumpkin seeds with case } \\
\hline Seed & Length $(\mathrm{mm})$ & Wide $(\mathrm{mm})$ & Thickness $(\mathrm{mm})$ \\
Average & 20,61 & 8,59 & 2,51 \\
Absolute variation & 3,16 & 0,504 & 0,143 \\
Standard deviation & 1,784 & 0,710 & 0,378 \\
Maximum figure & 23,90 & 9,60 & 3,00 \\
Minimum figure & 16,30 & 7,00 & 1,60 \\
Range & 7,60 & 2,60 & 1,40 \\
\hline
\end{tabular}

Through a statistical analysis with a Tukey test, it was found significant differences $(\mathrm{P}>0,05)$ for length, wide and thickness, therefore, it was stated that seeds with case develop a largest size. Length, wide and thickness of seeds without case. Table 3 shows average figures. These results are clearly different to those from seeds with case (smaller size), which indicates that the case is an important part. On average, length is $16,01 \mathrm{~mm}$, wide is $6,70 \mathrm{~mm}$ and thickness is $2,13 \mathrm{~mm}$. Absolute variance is; 7,08 $\mathrm{mm}$ for length, $0,48 \mathrm{~mm}$ for wide and $0,17 \mathrm{~mm}$ for thickness. A standard deviation of; \pm de 2,66 $\mathrm{mm}$ for length, 0,69 $\mathrm{mm}$ for wide and 0,41 $\mathrm{mm}$ for thickness. Porosity and density of seeds. Average figures obtained through an air picnometer are showed in Table 5.

Table 3. Sizes of pumpkin seed without case.

\begin{tabular}{cccc}
\hline & Pumpkin seeds without case & \\
\hline Seed & Length $(\mathrm{mm})$ & Wide $(\mathrm{mm})$ & Thickness $(\mathrm{mm})$ \\
Average & 16,01 & 6,70 & 2,13 \\
Absolute variation & 7,081 & 0,483 & 0,170 \\
Standard deviation & 2,661 & 0,695 & 0,412 \\
Maximum figure & 20,50 & 8,40 & 3,00 \\
Minimum figure & 10,70 & 5,30 & 1,00 \\
Range & 9,80 & 3,10 & 2,00 \\
\hline
\end{tabular}

Internal and external friction from the seed. Figures from internal friction ${ }^{d} l^{d}$ (static angle) and external friction ${ }^{e}$ (dynamic angle) obtained are showed in Table 4.

Table 4. Figures for internal and external friction for pumpkin seeds.

\begin{tabular}{lccc}
\hline Seed & Internal friction $(\mu i)$ & \multicolumn{2}{c}{$\begin{array}{c}\text { External friction }(\mu e) \\
\text { Wooden surface }\end{array}$} \\
\hline Seed with case & 0,35 & 0,36 & 0,50 \\
Seed dried on stove & 0,35 & 0,36 & 0,50 \\
Seed without case & 0,54 & 0,59 & 0,58 \\
Case of seed & 0,47 & 0,44 & 0,63 \\
Seed without case (customary 2) & 0,45 & 0,36 & 0,50 \\
Seed without case (customary 3) & 0,50 & 0,42 & 0,50 \\
Seed without case (customary 4) & 0,41 & 0,39 & 0,45 \\
Flour from seed without case & 0,81 & 1,00 & 1,24 \\
Flour from seed with case & 0,56 & 0,59 & 0,81 \\
$\quad$ Seed from stench pumpkin & 0,51 & 0,22 & 1,24 \\
$\quad$ Apodanthera undulata) & 0,42 & 0,30 & 0,41 \\
Nuts & 0,31 & 0,30 & 0,50 \\
Linseed & 0,41 & 0,48 & 0,50 \\
Sunflower & & & Go \\
\hline
\end{tabular}


Table 5. Figures for porosity and density of seed.

\begin{tabular}{lccc}
\hline \multicolumn{1}{c}{ Seed } & $\begin{array}{c}\text { Density of seed } \\
\left(\mathrm{g} / \mathrm{cm}^{3}\right)\end{array}$ & $\begin{array}{c}\text { Density of seed inside a container } \\
\left(\mathrm{g} / \mathrm{cm}^{3}\right)\end{array}$ & $\begin{array}{c}\text { Porosity of seed } \\
\left(\mathrm{cm}^{3}\right)\end{array}$ \\
\hline Seed without case & 0,897 & 0,322 & 80 \\
Seed with case & 0,907 & 0,326 & 80 \\
$\begin{array}{l}\text { Seed with case dried on stove } \\
\quad \text { (up to 4\% moisture) }\end{array}$ & 0,889 & 0,320 & 80 \\
\hline
\end{tabular}

Density from pumpkin oil. Figure 2 shows the behavior of density from pumpkin oil affected by temperature and for figures of $\mathrm{pH} 5,73$ and for an electric conductivity of 6,254 mS. Figure 3 shows degree of viscosity change related to temperature.

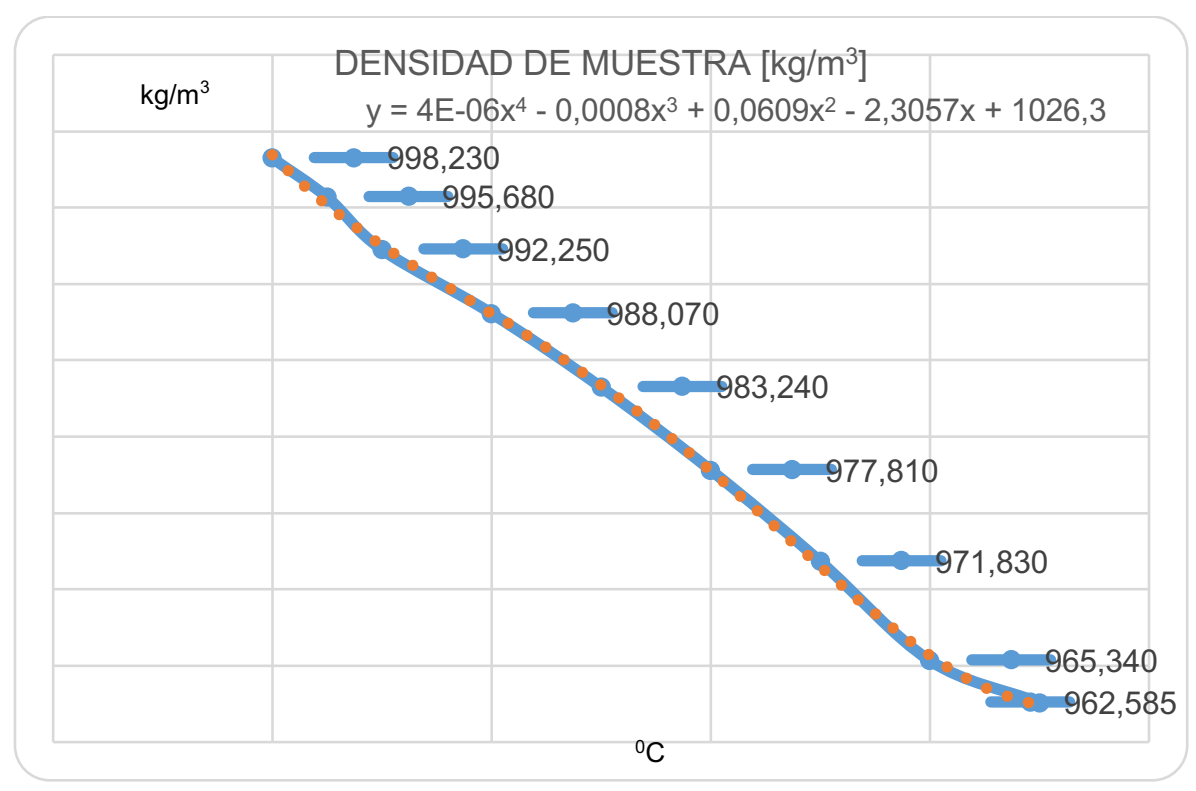

Figure 2. Density from pumpkin seed's oil.

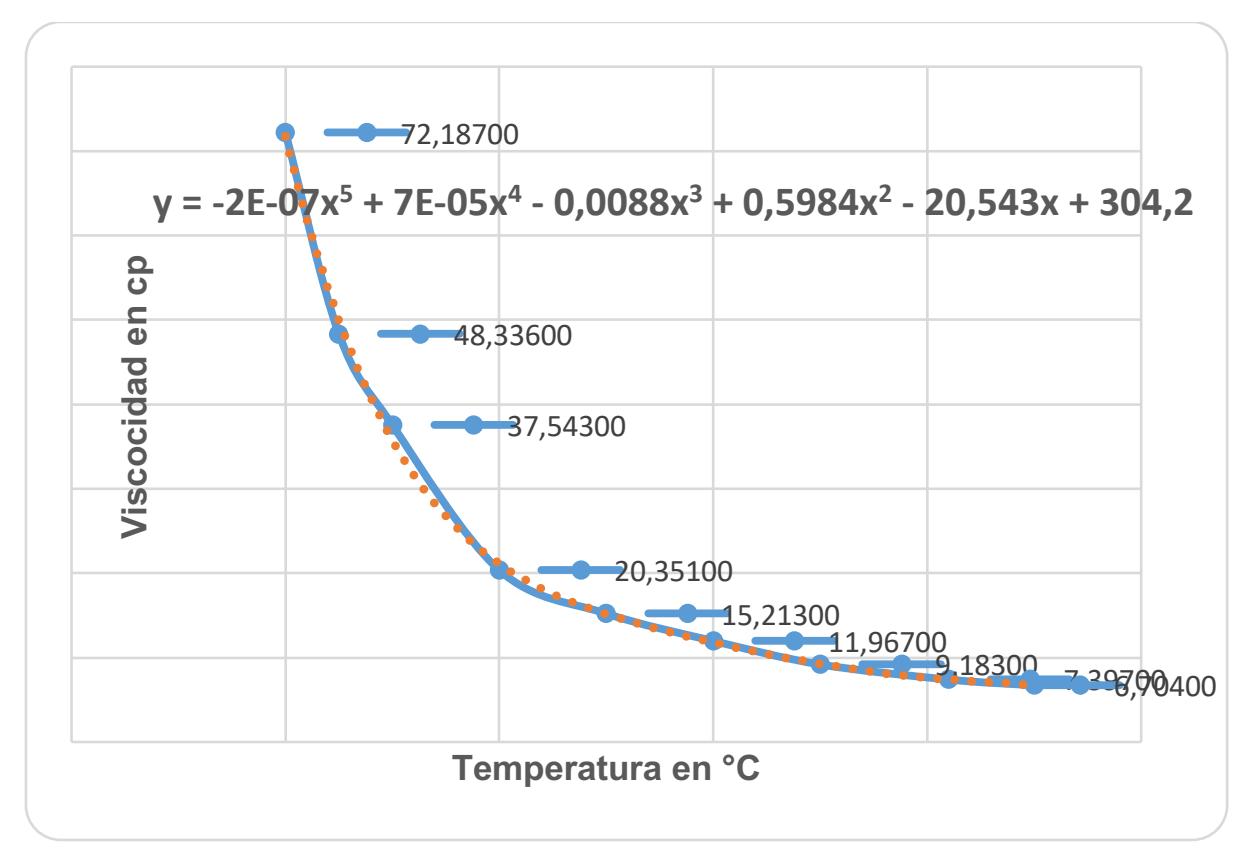

Figure 3. Viscosity profile for pumpkin oil in relation to temperature. 
Table 6. Viscosity from several liquid food products.

\begin{tabular}{ccc}
\hline Material & Temperatura $\left({ }^{\circ} \mathrm{C}\right)$ & Viscosity (cin.) $(\mathrm{mPa})$ \\
\hline Milk & 20 & 2 \\
Buttermilk & 40 & $800 \ldots 1500$ \\
Engine oil SAE 10 & 20 & 50 \\
Olive oil & 40 & 40 \\
Olive oil & 20 & 40 \\
Palm oil & 40 & 45 \\
Paraffin emulsion & 20 & 3000 \\
Flan & 40 & 1000 \\
Oil seed rape & 20 & 160 \\
Cream (30..50\% of fat) & 20 & $15 \ldots 115$ \\
Jatropha oil & 20 & $1000 \ldots 1500$ \\
Cheese cream & 60 & 30000 \\
Pig's lard & 40 & 65 \\
Shampoo & 20 & 3000 \\
Soybean's oil & 20 & 60 \\
Ketchup & 30 & 1000 \\
Oil for transformers & 20 & 30 \\
Oil for transformers & 10 & 75 \\
Oil for turbines & 20 & $200 \ldots 1100$ \\
Vitamins' oil & 10 & 4500 \\
Shark's oil & 29 & 100 \\
\hline
\end{tabular}

Table 6 shows a comparison between oil from pumpkin seeds and several other oil products. The viscosity's path determined in part from the change in temperature shows an acceptable fluid behavior for processing protocols, as well as for making mixtures with other substances. Drying isotherms for pulp of pumpkin at $40^{\circ} \mathrm{C}$ and at one atmosphere pressure as weight against drying time, shows a linear behavior (equation 1), which is adequate as it allows an excellent storage with a minimum of losses. Data for drying pulp from pumpkin to $40^{\circ} \mathrm{C}$ for a pressure of one atmosphere

$$
Y=0,0078 x^{4}-0,1506 x^{3}+0,8613 x^{2}-10,25 x+100,4
$$

Figure 4 shows the drying process for pulp from pumpkin in response to different constant temperature and subjected to a pressure of one atmosphere, which guarantee safeguard to important constituents in the organic mass.

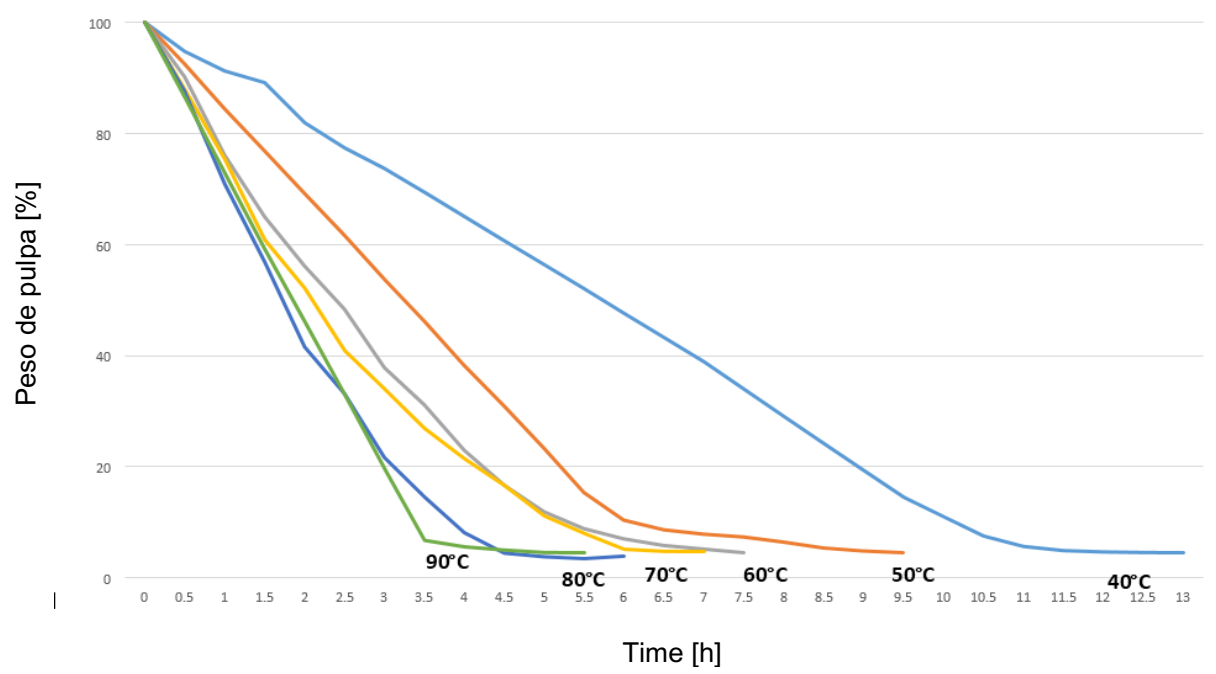

Figure 4. Drying process for pulp of pumpkin tested for different constant temperatures and at one atmosphere pressure. 
In order to demonstrate the importance of the latter, it is showed different equations about isotherms for drying pulp of pumpkin.

Drying isotherm for pulp at $50^{\circ} \mathrm{C}$ and one atmosphere pressure

$$
Y=-0,025 x^{4}+0,5989 x^{3}-3,4484 x^{2}-9,6641 x+98,51
$$

Drying isotherm for pulp at $60^{\circ} \mathrm{C}$ and one atmosphere pressure

$$
Y=0,0125 x^{4}-0,2555 x^{3}-0,2223 x^{2}+23,45 x-0,2527
$$

Drying isotherm for pulp at $70^{\circ} \mathrm{C}$ and one atmosphere pressure

$$
Y=-0,0004 x^{4}-0,0319 x^{3}+2,4755 x^{2}-29,399 x+100,98
$$

Drying isotherm for pulp at $80^{\circ} \mathrm{C}$ and one atmosphere pressure

$$
Y=-0,1076 x^{4}+1,4618 x^{3}-2,9829 x^{2}-27,606 x+100,57
$$

Drying isotherm for pulp at $90^{\circ} \mathrm{C}$ and one atmosphere pressure

$$
Y=-0,1819 x^{5}+2,3171 x^{4}-9,2714 x^{3}+14,187 x^{2}-34,073 x+100,25
$$

Table 7 shows data on calcium, magnesium and lipids inside the pumpkin seeds and compared with other

\begin{tabular}{|c|c|c|c|c|c|c|}
\hline SAMPLE & Case & Pulp & $\begin{array}{c}\text { Pumpkin } \\
\text { Seed } \\
\text { (whole } \\
\text { \& raw) }\end{array}$ & $\begin{array}{l}\text { Pumpkin } \\
\text { pepita } \\
\text { (raw) }\end{array}$ & $\begin{array}{c}\text { Case } \\
\text { from } \\
\text { Pumpkin } \\
\text { seed }\end{array}$ & $\begin{array}{c}\text { Pumpkin } \\
\text { seed } \\
\text { (whole \& } \\
\text { toast) } \\
\end{array}$ \\
\hline Moisture (\%) & 94,986 & 95,595 & 7,229 & 2,558 & 8,806 & 6,158 \\
\hline Adhesion water (\%) & 0,053 & 0,046 & 0,263 & 0,102 & 0,045 & 0,097 \\
\hline Total solids $(\%)$ & 4,961 & 4,359 & 92,508 & 97,340 & 91,149 & 93,745 \\
\hline Ashes $[\mathrm{g} / 100 \mathrm{~g}$ sample $]$ & 0,759 & 0,596 & 0,692 & 0.981 & 0,936 & 0,905 \\
\hline Raw fiber [g/100 g sample] & 3,403 & 3,045 & 4,563 & 2.115 & 75,267 & 4,651 \\
\hline Lipids [g/100 g sample] & 0,196 & 0,273 & 28,324 & 29.659 & 0,146 & 29,571 \\
\hline Total nitrogen $[\mathrm{g} / 100 \mathrm{~g}$ sample $]$ & 1,462 & 1,513 & 1,478 & 1.435 & 1,465 & 1,501 \\
\hline Protein $[\mathrm{g} / 100 \mathrm{~g}$ sample] & 0,364 & 0,711 & 0,129 & 0.282 & 0,008 & 0,275 \\
\hline Carbohydrates [g/100 g sample] & 21,269 & 21,901 & 21,119 & 21.914 & 21,357 & 21,158 \\
\hline Carbon $[\mathrm{g} / 100 \mathrm{~g}$ sample $]$ & 19,79 & 18,41 & 19,534 & 19.478 & 20,913 & 19,852 \\
\hline Ratio $\mathrm{C} / \mathrm{N}$ & $20 / 1$ & $20 / 1$ & $20 / 1$ & $20 / 1$ & $20 / 1$ & $20 / 1$ \\
\hline Calcium [mg/100 g sample] & 30,057 & 30,144 & 29,249 & 28.372 & 28,468 & 29,785 \\
\hline Magnesium [mg/100 g sample] & 10,112 & 11,785 & 12,487 & 10.463 & 12,875 & 11,578 \\
\hline $\mathrm{pH}$ & 5,97 & 5,872 & 5,767 & 5.978 & 5,999 & 5,751 \\
\hline Conductivity $[\mu \mathrm{S}]$ & 323,960 & 330,505 & 320,950 & ------ & ------ & ------ \\
\hline
\end{tabular}
products, and Table 8, shows physical and chemicals figures for different pumpkin's constituents.

Table 7. Calcium, magnesium and lipids content inside the pumpkin seed, and compared to two other products.

\begin{tabular}{lll}
\hline Organic material & Calcium content & Magnesium content \\
\hline Pumpkin (Cucúrbita pepo) & $29 \mathrm{mg}$ in 100g sample & $11 \mathrm{mg}$ in $100 \mathrm{~g}$ sample \\
Col (Brassica oleracea) & $40 \mathrm{mg}$ & $12 \mathrm{mg}$ \\
Purple sweet potato (Ipomea batatas) & $30 \mathrm{mg} \quad$ & - \\
Organic material & & Lipids content \\
Pumpkin seed & $28 \%$ & \\
Sunflower seed & $30 \%$ & \\
Soybean seed & $15-23 \%$ & \\
\hline
\end{tabular}

Table 8. Physical and chemical parameters from different parts of pumpkin.

Table 9 shows useful data that would aid to develop and diversify products from pumpkin within a framework for generating healthy food products.

Table 9. Important components from pumpkin.

\begin{tabular}{cc}
\hline Component & Composition (\%) \\
\hline Raw protein & 36,5 \\
Raw oil & 51,01 \\
Raw fiber & 4,43 \\
Total ash & 3,21 \\
\hline
\end{tabular}


Proximal analysis for pumpkin seed. Quantity of moisture found inside the seed without case was $4,45 \%$ and $5,58 \%$ for pumpkin seed with case (Table 10). This figure is within the range that SAGARPA (2016) indicates for an adequate storage for grain and seeds (less than $9 \%$ moisture). Moisture content is similar to that found by Habib (2015), of around 4,06\% and by Salgin (2011) of around 6,96\%. Similarly, protein content from pumpkin seed without case was $24,36 \%$ and $28,92 \%$ from seed with case (Table 10), which indicates that the case has a protein content of about $4 \%$. Total carbohydrates content was $6,99 \%$ for seeds without case and $5,57 \%$ for seeds with case. Thus, less carbohydrates content inside the pumpkin seeds with case is due that fiber is the main component (Gohari, 2011), so it may reduce the carbohydrates content as well as oil. Oil content in seed with case was $35 \%$, while in seeds without case was $49 \%$. High oil content indicates also, high figures from internal and external friction. In general, nutrimental quality from pumpkin seed was different compared to other pumpkin varieties studied, for instance, protein content for different varieties change from 25 and $40 \%$, while oil was between 31 and 50\% (Rezig, 2012; Gohari, 2011; Petkova, 2015).

\begin{tabular}{lcccc}
\multicolumn{5}{c}{ Table 10. Proximal composition of pumpkin's seed Cucúrbita sp. } \\
\hline & Protein (\%) & Carbohydrates (\%) & Moisture (\%) & Oil (\%) \\
\hline Pumpkin seed without case & $24,36 \pm 0,02$ & $6,99 \pm 0,12$ & $5,58 \pm 0,15$ & $49 \pm 0,52$ \\
Pumpkin seed with case & $28,92 \pm 0,06$ & $5,57 \pm 0,06$ & $4,45 \pm 0,19$ & $35 \pm 0,32$ \\
\hline
\end{tabular}

Due to the high protein and oil content, pumpkin seeds are a potential source for vital technological applications (elaborate new food products, biodiesel). In order to evaluate the most suitable application type, profile from lipid acids was the parameter chosen for analysis through gas chromatography. The study showed that oil from seeds constitutes for $81 \%$ of unsaturated lipid acids and $19 \%$ are saturated lipid acids. Main lipid acids are linoleic acid $(51,87 \%)$ and oleic acid $(29,04 \%)$ (Figure 5), figures similar to those reported by Gohari (2011) and Grisales (2012). Lipid acids omega 6 and omega 9 are related to certain healthy benefits, especially for cardio circulatory preventions disease (Alsina et. al., 2015), reduce the risk for obesity, metabolic syndrome, diabetes type 2 and high pressure arterial (López-Miranda, 2008).

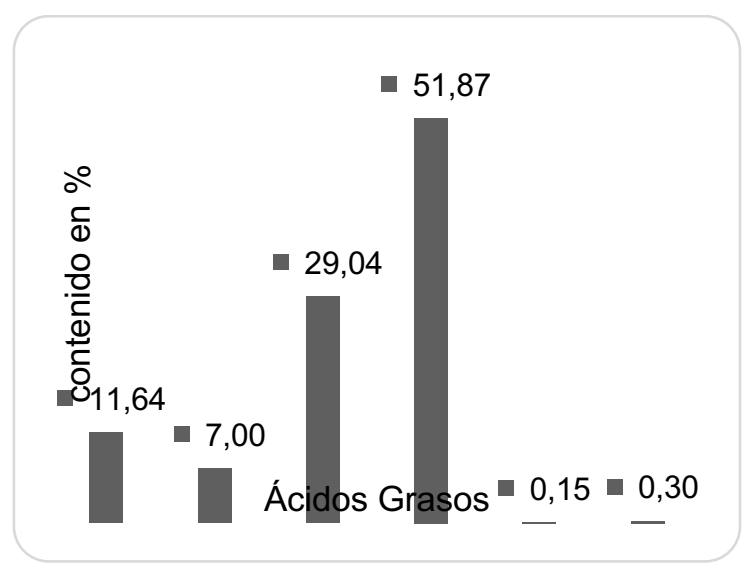

Figure 5. Profile of lipids acids from pumpkin seeds.

Because of the high quality of seed's oil and also, its high protein content, both convert pumpkin seed into a viable option to obtain food products highly healthy (i.e. crispy tortilla chips). In order to prepare a product with optimal characteristics and good appearance, it is important to consider excellent raw matter, the processing and storage. In the latter, it is vital to include; mass separation (sieving), milling, mixing and cooking inside an oven. This study showed that material homogeneity respect to particulate size, milling was easier. Once flours were obtained, sieving was the following process. When milled particulate size from seed were equal or less than 0,39 $\mathrm{mm}$, syneresis is presented, which causes losses higher than $60 \%$ and makes that flour sticks on the sieve. Optimal size of particulate from pumpkin seed with case and without case, where syneresis was not observed and a suitable flour was obtained for preparing crispy tortilla chips was $0,84 \mathrm{~mm}$ for seeds with case and $0,59 \mathrm{~mm}$ for seeds without case. Equally, for mass mixing it was necessary to add water and salt in order to add flavor, except oil as seed is rich on oil. After mass mixing, the product was cooked in the oven (not frying on oil). Cooking was easier since seed had low moisture content and low amount of water was added (10\%). Temperature for cooking was less that $100^{\circ} \mathrm{C}$ all the time, in order to avoid to oxidize oils and to reduce chances of affecting product nutritional value, thus 60 minutes were enough for cooking. Valenzuela et al. (2014) reported that pumpkin seed has a high amount of antioxidants. Product acceptance was trialed by setting up a selecting panel of 16 trained judges, who usually consume crispy tortilla chips once a week. Ten samples from crispy tortilla chips made from mixing 
different flours were evaluated by using a linear order test (from best to worst). The three best samples were selected according to flavor, color, aroma, texture and appearance. In general, judges appreciate crispy tortilla chips, however, best sample has a $85 \%$ SSC and $15 \% \mathrm{M}$ content, in second place was a sample with $85 \%$ SCC and $15 \% \mathrm{M}$. content and third place was a sample with a content of $50 \%$ SCC and $50 \% \mathrm{M}$.

On the other hand, product nutrimental analysis was interesting (Table 11) as protein content of crispy tortilla chips was $6,28 \%$ from a sample made from a content of $85 \%$ SSC and $15 \%$ M; 5,45\% from a sample with $85 \%$ SCC and 15\% M and 3,29\% from a sample with a content of $50 \%$ SCC and 50\% M. It is clear that, protein content of pumpkin seed has an effect (Table 10). Figures for carbohydrates were between 20 and $28 \%$, results considered very different and well far away from commercial crispy tortilla chips (figures for carbohydrates between 60 and 80\%). Here, it is important to stress that higher amount of carbohydrates in the product are effected from maize flour, as pumpkin seed holds a lower amount of carbohydrates. Ash content is related to quantity of minerals already presented in the food, as samples with larger content of flour from pumpkin seeds showed larger amount of minerals.

Table 11. Proximal composition of crispy tortilla chips obtained from a mix of flour from pumpkin seed Cucúrbita ssp and maize flour.

\begin{tabular}{cccccc}
\hline Formulae & Protein ( \%) & $\begin{array}{c}\text { Carbohydrates } \\
(\%)\end{array}$ & Moisture (\%) & Oil (\%) & Ashes (\%) \\
\hline 85\% SSC-15\% M & $6,28 \pm 0,25$ & $27,65 \pm 0,25$ & $1,3 \pm 0,01$ & $31,02 \pm 0,52$ & $5,76 \pm 0,32$ \\
$85 \%$ SCC-15\% M & $5,45 \pm 0,15$ & $19,98 \pm 0,23$ & $3,3 \pm 0,32$ & $21,52 \pm 0,96$ & $5,04 \pm 0,23$ \\
$50 \%$ SCC-50\% M & $3,29 \pm 0,36$ & $28,67 \pm 0,52$ & $3,5 \pm 0,12$ & $19,36 \pm 0,43$ & $3,33 \pm 0,22$ \\
\hline
\end{tabular}

$\mathrm{SSC}=$ pumpkin seeds without case, $\mathrm{SCC}=$ pumpkin seeds with case, $\mathrm{M}=$ maize flour.

Low moisture content inside the product guarantee long self live and presence of microorganisms. Oil content is high (between 20 and 31\%) which is due to flour from pumpkin seed, as maize flour has an oil content of $4,86 \%$. As it was expected, higher oil content was detected inside the product with high percentage of SSC, as pumpkin seed without case has and additional 15\% higher oil content compared to the seed with case. Although, oil content inside the product is high, these kind of oils have mostly lipid acids omega 6 and 9 which have benefits for health. Those products elaborated with seeds without case, synteresis oil was high, it is assumed that the case acts like a barrier that holds oil, as the difference in the amount of oil inside the pumpkin seed with case compared to that without case was $15 \%$. Oil content variation in the crispy tortilla chips was $85 \%$ for SCC and $85 \%$ for SSC (both with $15 \%$ maize seed), was of around $10 \%$. The difference $(5 \%)$ is due to losses during making flour and the crispy tortilla chips. Oil loss was not evaluated during storage, although it can be assumed that it would be higher in those products elaborated from pumpkin seed without case. Both, internal and external friction (a measure of an ideal particulate size that avoids agglutinate while it is sieving), were small on flours elaborated from pumpkin seeds with case.

La fricción tanto interna como externa (medida en los tamaños de partícula que se consideran óptimos para evitar el apelmazamiento durante el cribado), fue menor en las harinas elaboradas a partir de semilla de calabaza con cáscara. This study shows that pumpkin seed has the potential to be use on technological applications, for instance, Gómez (2010), Martínez (2010), Escobar (2012) have made use of it on bakery and pastas.

\section{Conclusions}

Seeds from pumpkin have important nutritive characteristics that aid for improving the nutritious quality of certain food products. The profile of lipid acids from pumpkin seeds showed $29 \%$ of oleic acid and $51.9 \%$ of linoleic acid; both highly related to health benefits. Crispy tortilla chips elaborated from flour obtained from pumpkin seeds with case increase fiber content, being highly accepted, according to judges. Flour from pumpkin seeds with case substitutes flour from pumpkin seeds without case, reducing oil losses by $15 \%$. Oil viscosity from pumpkin seed completes the range of oils for human consumption. Isotherms from pumpkin seeds and pulp achieve an effective control for drying. Physical and Chemicals characteristics are fundamental for technique selection for the process of mixing, dosing, drying, storing, transporting, sieving, milling, etc.

\section{References}

Alsina E, Macri E.V, Zago V, Schreier 1, Friedman S.M. 2015. Aceite de girasol alto oleico: hacia la construcción de una grasa saludable. Actualización en nutrición, 16(4): 115.

Applequist, W. L. 2006. Comparative fatty acid content of seeds of four Cucúrbita species grown in a common (shared) garden. Journal of Food Composition and Analysis, 19(6): 606-611. 
Escobar, G. D. 2012. Estudio de la composición fisicoquímica de harina de semillas de zapallo como ingrediente alimentario. Innotec. 25-30.

FAO. 2016. El Estado Mundial de la Agricultura, y la Alimentación; cambio climatico, agricultura y seguridad alimentaria. http://www.fao.org/3/a-i6030s.pdf. Consultado el 24 de Abril de 2017.

Gohari, A. A. 2011. Chemical composition and physicochemical properties of pumpkin seeds (Cucúrbita pepo Subsp. Pepo Var. Styriaka) grown in Iran. Journal of Agricultural Science and Technology, 13: 1053-1063.

Gómez, B. E. 2010. Evaluación de un produto alimentario con harina de semilla de Cúcurbita moschata. Tesis de grado. Universidad del Zulia. Facultad de Ingenieria., 57.

González, C. F. P. 2016. Caracterización fisicoquímica y microbiologica de calabaza (Cucúrbita moschata 'Bernaut'), utilizada en la industria para la elaboración de cremogenados. Actas de Horticultura, 187-190.

Grisales, S. O. 2012. Fruto y semilla de Cucúrbita moschata fuente de carotenoides y aceite con valor agregado. In Congreso brasileiro de olericultura., Vol 52.

Habib, A. B. 2015. Nutritional and Lipid Composition Analysis of Pumpkin Seed (Cucúrbita maxima linn). Journal Food Science, 5(374): 1-6.

Hernández, M. S. 2009. Multiplicación in vitro vía organogénesis en calabaza. Agronomía mesoamericana, 20(1): 11-22.

INIFAP. 2007. La calabaza pipiana y su manejo integrado. 1-24. http://biblioteca.inifap.gob.mx:8080/jspui/handle/123456789/3057. Consultado el 10 de Abril de 2017.

López-Miranda J. 2008. Olive oil and health: summary of the II international conference on olive oil and health consensus report. España. Nutr. Metab. Cardiovasc. Dis. 2010; 20(4): 284-94.

Martínez, Y. V. 2010. Utilización de la semilla de calabaza (Cucúrbita moschata) en dietas para pollos de ceba. Revista Cubana de Ciencia Agrícola. Instituto de Ciencia Animal la Habana, Cuba., 44(4): 393-398.

Martínez, Y. M. 2012. Factibilidad económica del empleo de la harina de semilla de calabaza en la alimentación de las aves-Economic feasibility the use of pumpkin seed meal in the feed poultry. REDVET, 13(3): $1-9$.

Millán, P. R. 2011. Physicochemical characterization of Macadamia nut (Macadamia integrifolia) oil. CYTAJournal of Food, 9(1): 58-64.

Muschler, R. G. 2008. Escuela de campo para promotores y promotoras de La Selva, Chiapas, México. Módulo 6. Producción ecológica de cultivos anuales básicos: maíz, frijol y calabaza. 30.

NMX-F-312-1978. Determinación nde reductores directos y totales en alimentos. Normas mexicanas. Dirección general de normas. México.

NMX-F-089-1978. Determinación de ácidos grasos. Normas mexicanas. Dirección general de normas.

Nollet, L. 2004. Handbook of food analysis: physical characterization and nutrient analysis. 2a Edición.

Official Methods of Analysis of AOAC INTERNATIONAL. 2006. 20th edition.

Petkova, Z.Y., G.A. Antova. 2015. Changes in the composition of pumpkin seeds (Cucúrbita moschata) during development and maturation. Grasas y aceites. 66(1): 058

Rezig, L. C. 2012. Chemical composition and profile characterization of pumpkin (Cucúrbita maxima) seed oil. Industrial Crops and Products, 37(1): 82-87.

SAGARPA. 2016. Almacenamiento y conservación de granos y semillas. Obtenido de http://www.sagarpa.gob.mx/desarrolloRural/Documents/fichasaapt/Almacenamiento $\% 20 \mathrm{de} \% 20$ semillas.pdf.

Consultado el 28 de Abril de 2017.

Salgin, U. 2011. A green separation process for recovery of healthy oil from pumpkin seed. The Journal of Supercritical Fluids., 58(2), 239-248.

Sánchez Hernández M. V. C. 2000. Variación genética y respuesta a la selección combinada en una variedad criolla de calabaza pipiana (Cucúrbita argyrosperma Huber var. stenosperma). Revista Chapingo Serie Horticultura, 6(2), 221-240.

Subbaiah, M. V. 2016. Adsorption of methyl orange from aqueous solution by aminated pumpkin seed powder: Kinetics, isotherms, and thermodynamic studies. Ecotoxicology and environmental safety, 128, 109-117.

Valenzuela G. M., Cravzov A. L., Soro A. S., Tauguinas A. L., Giménez M. C., Gruszycki M. R. 2014. Relación entre actividad antioxidante y contenido de fenoles y flavonoides totales en semillas de Cucurbita spp. Dominguezia; 30(1): 19-24. 


\title{
A New Method to Calibrate Discrete Element Models of Fibrous Agricultural Materials
}

\author{
Adam Kovacs ${ }^{\text {a,* }}$, Gyorgy Kerenyi ${ }^{\text {a }}$ \\ a Department of Machine and Product Design, Budapest University of Technology and Economics, Budapest, \\ Muegyetem rkp. 3, Hungary \\ *Corresponding author. Email: kovacs.adam@gt3.bme.hu; kovadam19@gmail.com
}

\begin{abstract}
Discrete Element Method (DEM) is widely used to analyse technological processes (e.g. discharging of silos, ploughing) in relation to bulk agricultural materials such as grains and soil but fewer applications can be found in connection with DEM models of crop stems. However, the mechanical and physical behaviours of stems and pile or swath of stems and stalks play an important role in several phases of agricultural production, e.g. during harvesting, mowing, baling, preparation of forage etc. In practice, there is no suitable method to calibrate DEM models of crop stems and stalks, and our study aims to fill this gap by focusing on the development of a new calibration method. The paper first analyses the mechanical structure of fibrous agricultural materials in relation to typical agricultural processes. The steps of the calibration method were defined in a way to provide comparison among the measured and simulated quantitative and qualitative properties. Second, the specific laboratorial tests (transversal compression, three-point bending and dynamic cutting) were conducted on maize (Zea Mays) stalks. Maize was selected for the study as a typical fibrous agricultural material. Third, a suitable discrete element model about an internodal section of a maize stalk was formed and the calibration method was carried out. As a result, a new calibration method for complex DEM models about fibrous agricultural materials was formed and validated on an internodal section of a maize stalk. The results of the validation clearly demonstrate that the new calibration method; that consists of quantitative and qualitative comparison among the observed and simulated values and phenomena of laboratorial tests and simulation results; can be extended to complex DEM models about any fibrous agricultural materials.
\end{abstract}

Keywords: numerical method, DEM, calibration, stalk, stem

\section{Introduction}

Numerical modelling of the mechanical behaviour of agricultural crops and numerical simulations of agricultural processes are becoming more universal in agricultural research and development. Discrete Element Method (DEM) (also called Discrete Element Modelling) is a particle-based simulation technique that is able to predict the interactions among individual particles based on contact laws among them (Cundall and Strack, 1979). Thus, DEM was originally applied to analyse the mechanical behaviour of agricultural granular assemblies such as grains and soil during processes like mixed flow drying (Keppler et al., 2012) or soil cultivation (Tamas et al. 2013). Thanks to the continuous development of DEM, more and more studies now also focus on the analysis of mechanical behaviour of non-granular agricultural materials e.g. stalks and stems (Leblicq et al., 2016a; Leblicq et al., 2016b) during various processes like monitoring of sieve losses in combine harvesters (Liang et al., 2016), overlaid cutting in disc mowers (Kemper et al., 2014) and destemming of grapes (Coetzee and Lombard, 2013). Most of the available studies focus on the numerical modelling of a specific mechanical behaviour or phenomenon (e.g. compression, bending and cutting of stems and stalks), however, real agricultural processes are more complex and are made up of several basic phenomena.

Maize (Zea mays) is one of the most cultivated crop of the world: almost 900 million metric tons of corn were produced in 2016, moreover, corn also plays an important role in agriculture of Hungary: 8.8 million metric tons were harvested in 2016 (Hungarian Statistical Office, KSH, 2017). The stalk, the strongest part of maize plant, is constituted of nodes and internodes, both have the same rind-core (skin-pith) structure. Accordingly, the biological structure of maize is suitable for our study. The general steps of maize production are: soil tillage; sowing; fertilization; control of weeds, diseases and pests; irrigation, harvesting; drying; storing and further processes (Nagy, 2006). Harvesting of shelled maize kernels by a combine harvester is the most wide-spread method, moreover, it is a very complex process, thus, it has been chosen as the object of our study.

To calibrate DEM models on bulk materials two calibration methods can be found: direct measuring (Coetzee, 2017) and bulk calibration approaches (Coetzee, 2017; Keppler et al., 2016). In case of direct measuring parameters are measured directly at the particle or contact level, while in the other case the mechanical behaviour of the assembly of particles is measured. These two methods are also adopted to modelling of stems and stalks because measurements on one stem and on a bulk of stems can be found as well (Leblicq et al., 2016a). In the first case, the interactions among stems and machine parts can be determined while in the other case interactions among individual stems and the bulk can be analysed. During the harvest of shelled maize kernels by a combine harvester 
the interaction among parts of the machine and the individual stalks is more significant, thus, our study focuses on this phenomenon.

To form and calibrate a DEM model related to harvesting of maize mechanical properties, and behaviour of maize is required. As to the mechanical behaviour and properties of maize, three-point bending (Leblicq et al., 2015b; Tongdi et al., 2011; Robertson et al., 2014; Olmedo et al., 2016) and cutting (Chen et al., 2004; Kemper et al., 2012; Igathinathane et al., 2010; Johnson et al., 2012) of maize stalks are the most wide-spread experiments. Less studies can be found related to transversal compression experiments (Leblicq et al., 2016a; Zhang et al., 2017; Robertson et al., 2015; Prasad and Gupta, 1975). These studies on three-point bending, cutting and transversal compression provide several useful information but a complex numerical model cannot be calibrated and validated based on these studies because these experiments were carried out on different maize varieties, in different places of the world, at different times of the year, thus, the relationship among the separately provided data is not justifiable.

Consequently, the objective of this paper to present a new calibration method, that provides comparison among the measured and simulated quantitative and qualitative properties, for complex DEM models about fibrous agricultural materials.

\section{Materials and Methods}

In our study laboratorial experiments were carried out on the $4^{\text {th }}$ internodal section of a maize stalk to define its mechanical behaviour against transversal compression, bending and cutting. Afterwards, the discrete element model (DEM) was formed about the natural structure of the maize stalk. To find an optimal set of the model parameters a calibration loop was determined based on the measured quantitative and observed qualitative characteristics of the investigated samples. All the models about the internodal section of the maize stalk and about the experiments were formed and analysed in EDEM $^{\circledR}$ (DEM Solutions Ltd, Edinburgh, Scotland, UK) discrete element framework.

Mechanical structure of a maize

The main parts of a maize plant are the root system, stalk, leaves, tassel, shank and corn ear. The stalk, the strongest part of maize plant, is constituted of nodes and internodes, both have the same rind-core (skin-pith) structure, see Figure 1(a). Moreover, there is a difference between the orientation of tissues in nodes and internodes as well: the orientation of the tissues is nonuniform while in internodes the tissues are oriented in longitudinal direction of the stalk (Robertson et al., 2015).

The density of the fibre bundles is higher in the skin group than in the core group where the stiffer fibres are surrounded by softer supporting material. Thus, the skin group is proved to be the main supporting material (Huang et al., 2016).

a)

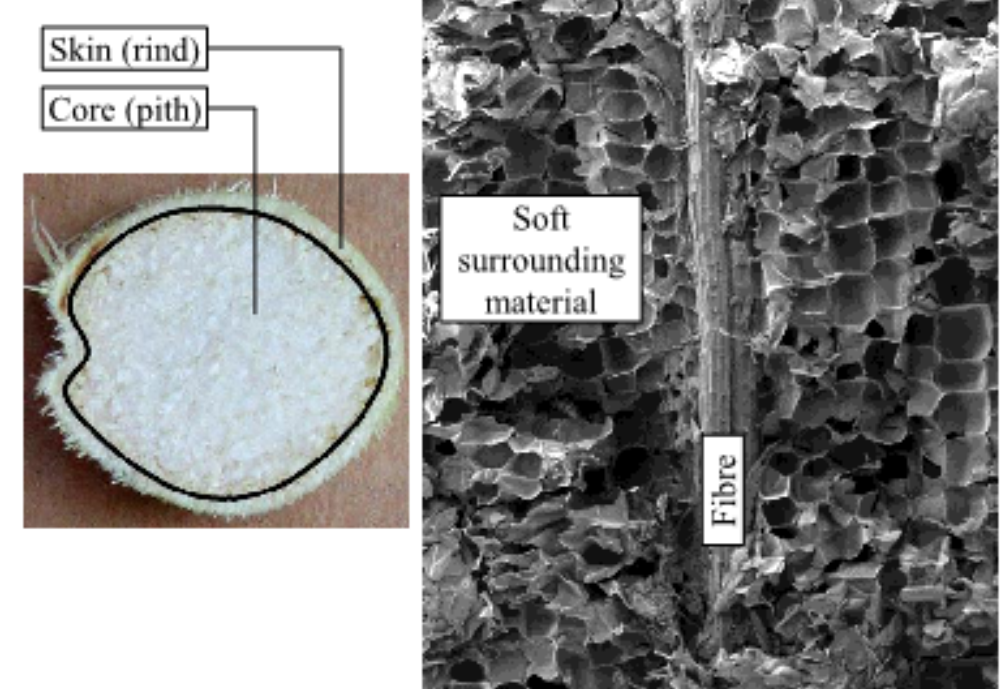

Figure 209. Mechanical structure of a maize stalk: a) zones of the cross-section; b) parts of the micro-structure recorded by scanning electron microscope (SEM). 
Analysis of harvesting

During the harvest by a combine harvester the following major phenomena take place regarding the maize stalk: transversal compression: knives of rotating stalk rollers compress the maize stalk locally in its transversal direction; flexural load by inertia: the stalk is bended thanks to the appearing force of inertia of the corn ear because the whole plant is accelerated by the machine in the direction of movement; oscillation: the stalk oscillates thanks to the interactions among the plants and flexural loads from the eccentric corn ear; longitudinal stretch by inertia: the stalk and shank are stretched by the force of inertia of corn ear because it is accelerated in perpendicular direction to the axes of stalk rollers; longitudinal stretch by gathering: when the corn ear collides on the deck plate a longitudinal force appears in the stalk and shank until separating the corn ear; free cut: knives of the chopping unit cut the stalk that is fixed by the stalk roller.

Based on the consideration of the energy consumption of the previous phenomena and our experiences the longitudinal stretch cases are neglectable, therefore, our study focuses on the compression, cutting and bending of maize stalks.

\section{Laboratorial experiments}

Sectional transversal compression between flat plates (Figure 2(a)) was conducted on the $4^{\text {th }}$ internode of a maize stalk with deformation speed $300 \mathrm{~mm} \mathrm{~min}^{-1}$. Samples of $50 \mathrm{~mm}$ length were cut from the middle section of internodes. The minor axis of the internode was the direction of the load and the compression was carried out until $75 \%$ of compression rate. During the experiments, force-displacement data were collected at $100 \mathrm{~Hz}$ by a universal testing machine (UTM) (Zwick Z020 test frame with a 5000-N load cell) and high-resolution videos were recorded on each sample.

The three-point bending on the $4^{\text {th }}$ internode of a maize stalk was conducted with a deformation speed of 300 $\mathrm{mm} \mathrm{min}{ }^{-1}$, see on Figure 2(b). The span length of $100 \mathrm{~mm}$ has been chosen and the samples were loaded in the middle of the internodal sections. For all the samples the displacement of the bending tool was $30 \mathrm{~mm}$ and the minor axis of the internode was the direction of the load. During the experiment, force-displacement data were collected at $100 \mathrm{~Hz}$ by the UTM (Zwick Z250 test frame with a 5000-N load cell) and high-resolution videos were recorded on each sample.

A pendulum impact machine was modified by replacing the hammer with a cutting blade unit in such a way that the gap between the blade and the supports was approximately $6 \mathrm{~mm}$ on both sides (Figure 2(c)). The cutting test was carried out on the $4^{\text {th }}$ internode of a maize stalk. The pendulum started from the same position each time, thus, the cutting velocity of the blade unit was $3.5 \mathrm{~m} \mathrm{~s}^{-1}$ in each case. The reversal angle was visualized and recorded by the scale of the apparatus.

Based on the recorded data of the transversal compression and the three-point bending tests, typical resistance force curves and the required compression and bending work of the samples were determined. In the case of the dynamic cutting, the required dynamic cutting work was calculated.
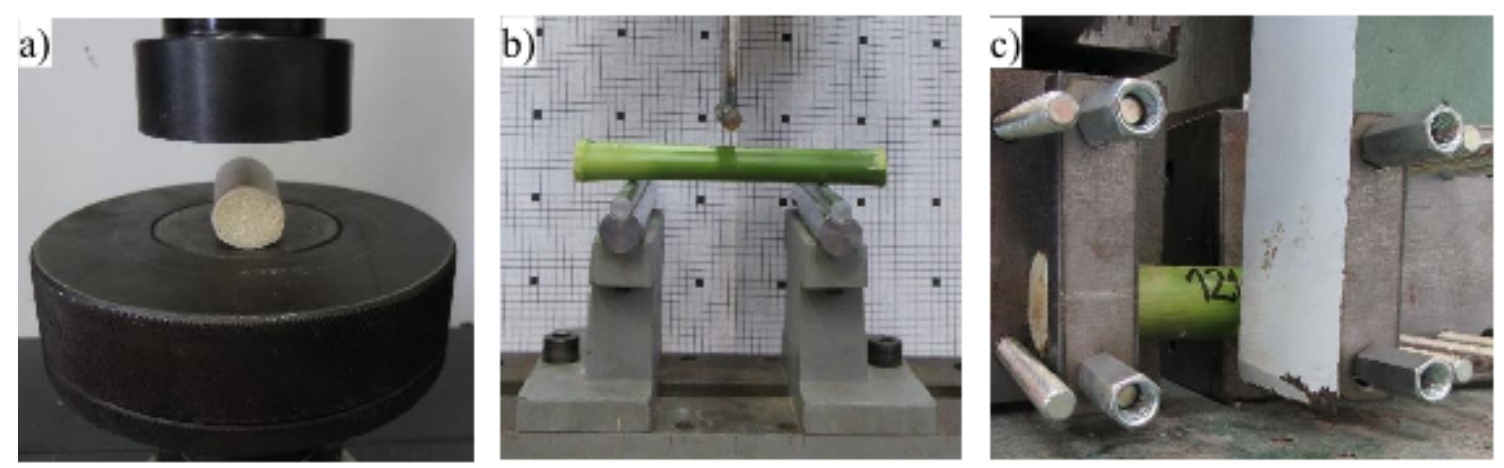

Figure 210. Laboratorial experiments: a) transversal compression; b) three-point bending; c) dynamic cutting.

Numerical model of an internodal section

Figure 3 shows the geometrical model that was inspired by the real mechanical structure (skin-core) of a maize stalk. To reproduce this structure five different particle types were defined: particle P1 and P2 form the skin; particle P4 and P5 form the nodes and particle P3 form the core. In the skin, particle P1 and P2 form a special composition: each cross-section contains 38 particles, 19 P1 particles and 19 P2 particles alternately. Thus, longitudinal fibres can be formed by aligning the cross-sectional layers. In the nodes, a solid cross-section is formed by using 19-19 particle P4 and P5, respectively. Finally, the inner volume is made up by using randomly generated particles type $\mathrm{P} 3$. 
After taking into consideration the existing DEM bonded models the Timoshenko Beam Bond Model $\left(\mathrm{EDEM}^{\circledR}, \mathrm{DEM}\right.$ Solutions Ltd, Edinburgh, UK) was chosen for this study (Brown et al. 2014). In this model a virtual beam element is formed between two spherical particles that can transfer forces and moments between the particles. A bond fails when one of the maximum stresses exceeds the predefined corresponding strength. To define the mechanical behaviour of the bonded model the following bonded properties need to be defined: Young's modulus; Poisson's ratio; mean compressive, tensile and shear strength; coefficients of variation for the compressive, tensile and shear strength and a bond radius multiplier.

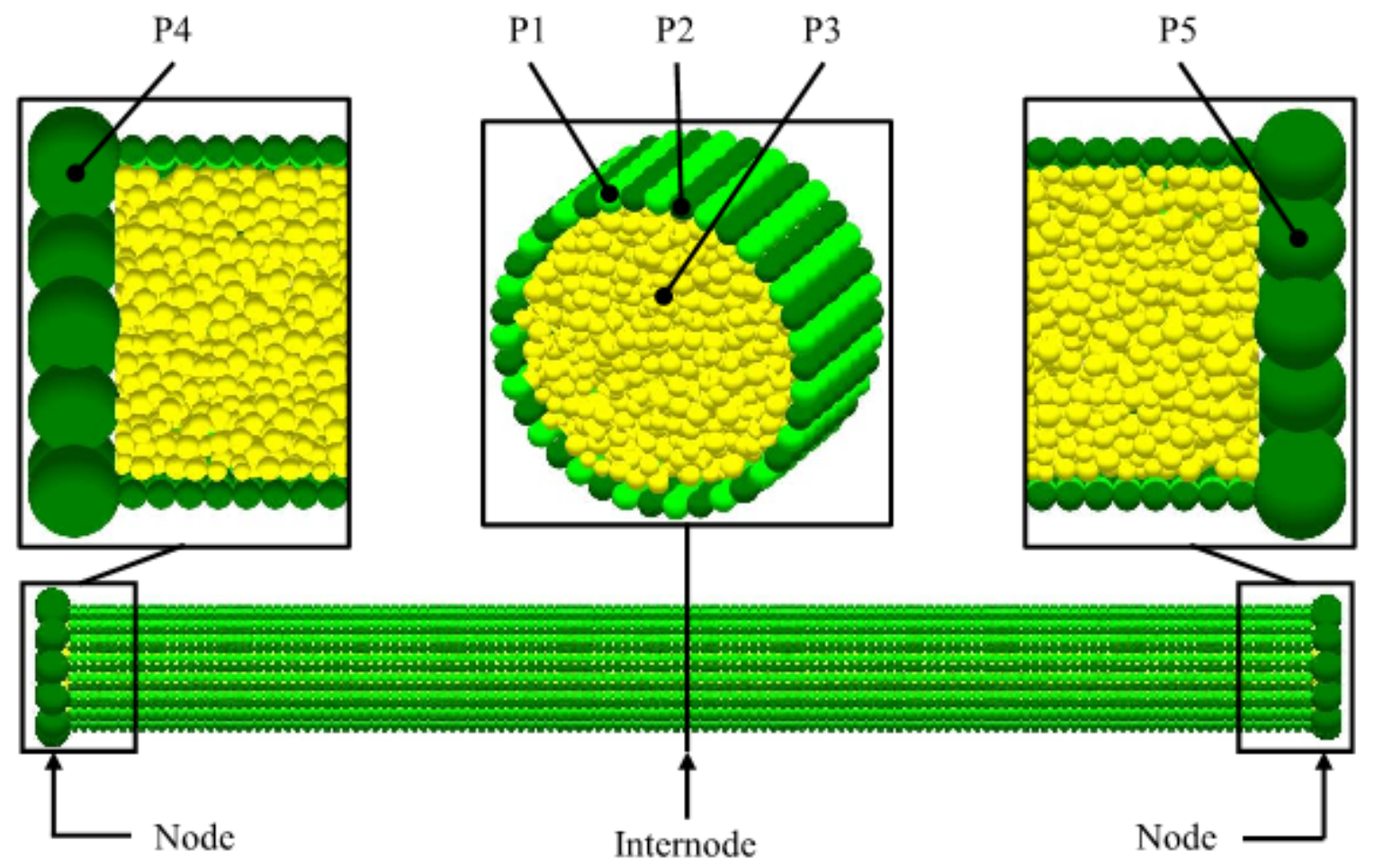

Figure 211. DEM geometrical model about the $4^{\text {th }}$ internodal section of a maize stalk.

\section{Calibration method}

To calibrate the bonded parameters a calibration loop was determined, see on Figure 4. First, the transversal stiffness and strength parameters were calibrated based on the measured force response of the sample and the required compressive work of the sectional transversal compression experiment. Afterwards, the determined parameter set was verified by the breaking phenomena of the internodal section gained during the sectional transversal compression.

Second, the longitudinal stiffness parameters were calibrated based on the measured force response of the sample and the required bending work results of the three-point bending experiment. Afterwards, the determined parameter set was verified by the breaking phenomena of the internodal section gained during the three-point bending experiment.

Third, the longitudinal strength parameters were calibrated based on the measured required cutting work of dynamic cutting experiment. Afterwards, the determined parameter set was verified by the breaking phenomena of the internodal section during dynamic cutting experiment.

Finally, all the simulations were conducted again by using the final parameter set to verify the mechanical behaviour of the model. 


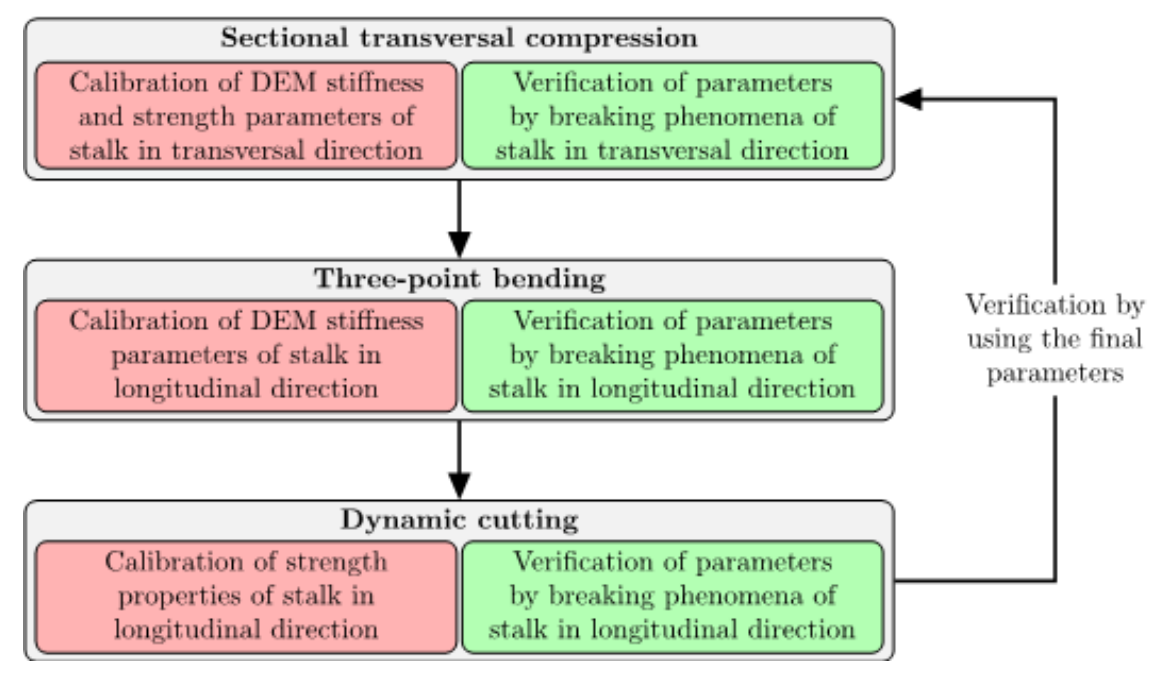

Figure 212. Calibration method for model parameters of an internodal maize stalk section.

\section{Results and Discussion}

Sectional transversal compression

The experimental force response of the real specimen and the DEM predicted force respond of the virtual sample were compared during the quantitative evaluation of sectional transversal compression, see on Figure 5. At the beginning of the compression process the grade of increase of the DEM predicted resistance force is similar to the measured characteristic until reaching the first drop. Between 0.1 and 0.25 compression rate and during the exponential increasing section several drops and linear increasing sections can be observed. Generally, the DEM predicted force response of the virtual specimen aligns well with the experimental result. The DEM predicted required compressive work was $6.4 \mathrm{~J}$, while, the experimentally determined required compression work was 6.3 $\mathrm{J}$, thus, the difference between the results is less than $5 \%$.

Figure 5 (I. - II. - III.) shows the typical breakage of the real and the virtual specimens, that were compared during the qualitative evaluation of sectional transversal compression. Three stages were analysed in detail: I: the ovalized shape after the initial compression; II: the breaks of the skin; III: the final breakage in the end of the process. The results show that the breakage of the virtual and the real specimens nicely line up in each stage, thus, the virtual model can predict the breakage of the real sample with high accuracy.

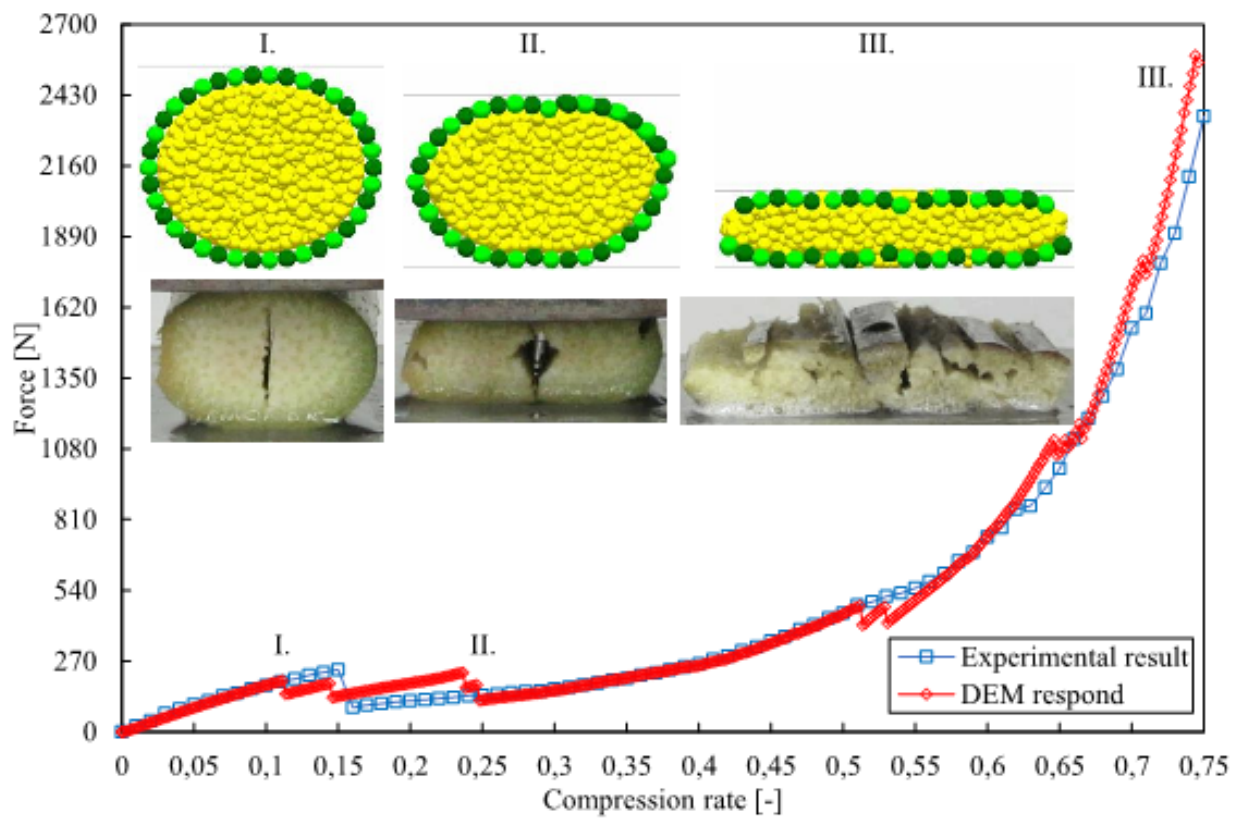

Figure 213. Quantitative and qualitative results of sectional transversal compression.

Three-point bending

The experimental force response of the real specimen and the DEM predicted force response of the virtual sample were compared during the quantitative evaluation of three-point bending, see on Figure 6. The DEM predicted force response shows a good coincidence with the measured curve during the initial section of the 
bending up to $2 \mathrm{~mm}$ of displacement. Before reaching the peak force, the DEM predicted grade of increase drops similarly to the experimental result. After the peak force, the resistance force starts to decrease in both cases, however, this section is governed by two significant breaks in case of the virtual specimen. Afterwards, the DEM predicted resistance force is increasing slightly until the end of the bending process, while the experimental one remains quasi-constant. The DEM predicted required bending work was $3.7 \mathrm{~J}$, while the experimentally determined bending work was $3.1 \mathrm{~J}$, thus, the difference between the results is approximately $20 \%$.

The DEM predicted and the experimental breakage and deformation of the internodal section was compared through an image-based qualitative evaluation between 0-30 $\mathrm{mm}$ displacement of three-point bending, see on Figure 6. Between $0 \mathrm{~mm}$ and $15 \mathrm{~mm}$ displacement the DEM predicted deformation of the internode under the bending tool is similar to the observed deformation of the real specimen. Afterwards, around the $15 \mathrm{~mm}$ displacement the cross-section under the bending tool becomes unstable and a structural collapse takes place. This phenomenon does not occur during the simulation. At $30 \mathrm{~mm}$ displacement the transverse deformation of the cross-section of the real specimen is higher than the transverse deformation of the virtual specimen. Generally, the DEM predicted deformed shape of the entire internodal section is similar to the observations on the real specimen through the entire bending process.

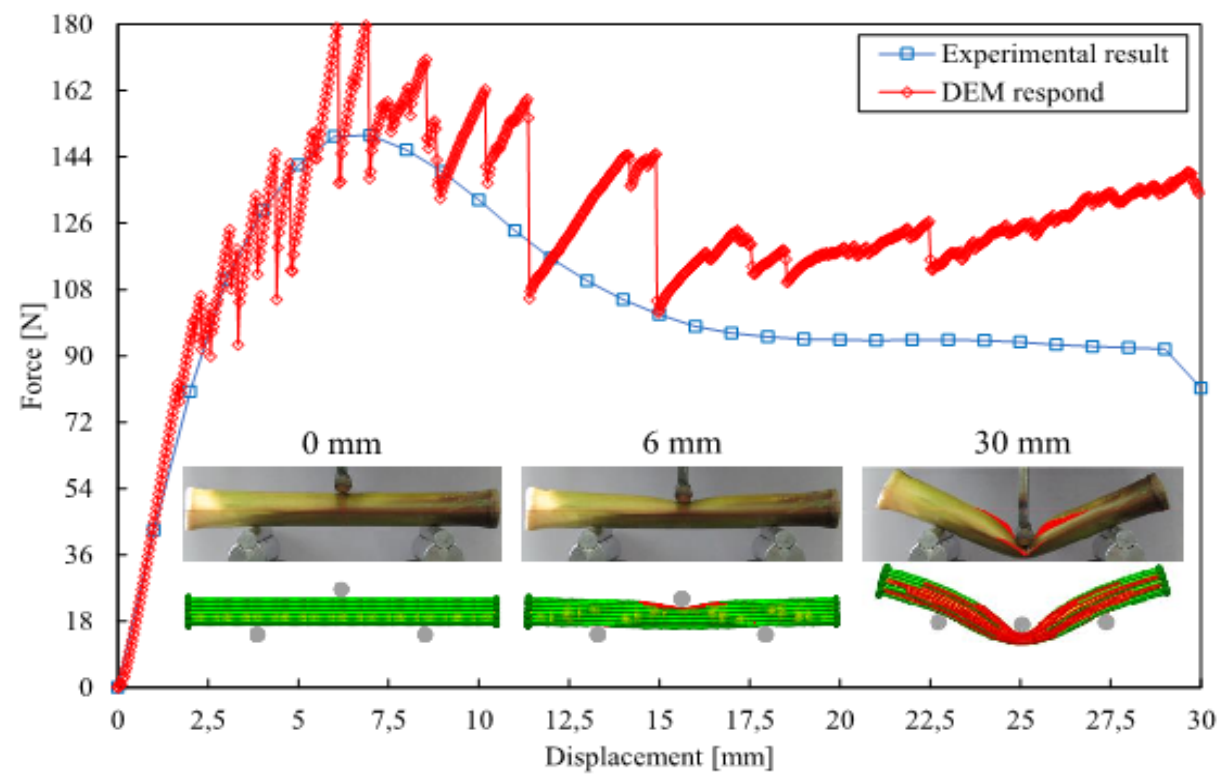

Figure 214. Quantitative and qualitative results of three-point bending.

Dynamic cutting

The DEM predicted force response and the stages of cut are presented on Figure 7. Based on the force response and the breakage of the fibres the cutting process can be divided into five different stages: compression stage between 0 and $12.9 \mathrm{~mm}$ displacement of the cutting blade, when the internodal cross-section is flattening and the resistance force is gradually increasing; cutting stage between $12.9 \mathrm{~mm}$ and $24.7 \mathrm{~mm}$ displacement of the cutting blade, when the skin and the core are cut off while the resistance force reaches its peak; sliding of the cutting blade between $24.7 \mathrm{~mm}$ and $90 \mathrm{~mm}$ displacement, when the cut has been done and the resistance force is decreasing as the cutting blade is passing through the sample. The DEM predicted required cutting work was 16.7 $\mathrm{J}$ while the experimentally determined mean required cutting work was $17.5 \mathrm{~J}$, thus, the difference between the results is less than $5 \%$.

The DEM predicted, and the experimental cutting surface is shown on Figure 7. In case of the real specimens the cutting surface was usually straight, however, a rim of fibres was observable on the side where the cutting blade left the specimen. These properties were also observable in the DEM predicted cutting surface.

Usage of the calibration method

The presented results clearly demonstrate that the new calibration method is usable to determine the DEM model parameters of an internodal maize stalk section to predict the mechanical behaviour of the real specimen. As to the quantitative results, the force response of the virtual specimen aligns nicely with the experimentally determined curves, moreover, the DEM predicted required compression, bending and cutting works are also close to the measured mean values. Only in the case of the three-point bending simulation did the DEM predict a higher difference among the real and experimental results. As to the qualitative results, the DEM predicted breakage of the virtual specimen is similar to the breakage of the real specimen in each case, which verifies the final set of DEM parameters. 


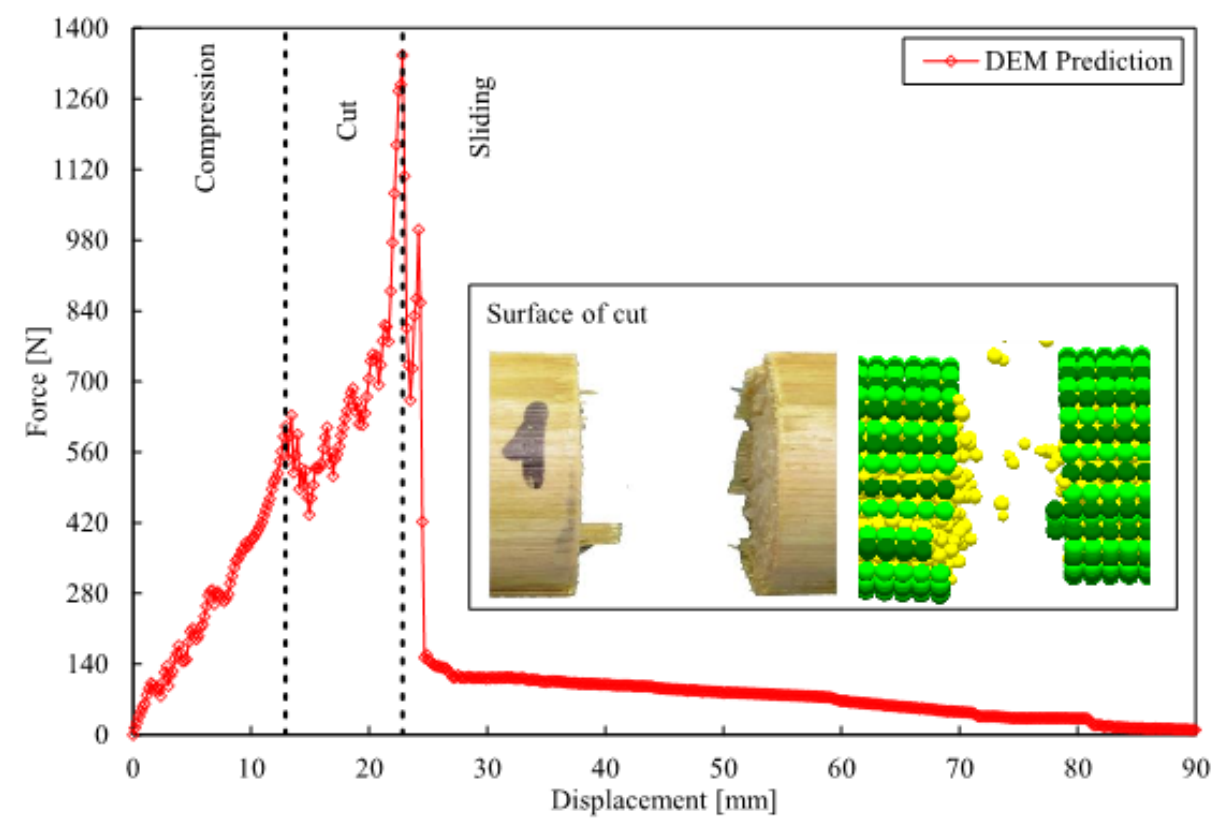

Figure 215. Quantitative and qualitative results of dynamic cutting.

\section{Conclusions}

In this paper the development and verification of a new calibration method for fibrous agricultural materials, especially for an internodal maize stalk section, has been presented.

First, laboratorial compression, bending and cutting experiments were conducted to obtain the necessary input for the calibration method, that consists of quantitative and qualitative comparison among the observed and simulated values and phenomena of laboratorial tests and simulation results. Second, the DEM model about an internodal maize stalk section was formed based on the real mechanical structure of maize. Third, an optimal set of DEM parameters was determined in order to predict the mechanical behaviour and breakage of the real specimen under different loading cases: transversal compression, three-point bending and dynamic cutting.

The presented results clearly demonstrate that the new calibration method can be used to determine the DEM model parameters of an internodal maize stalk section to predict the mechanical behaviour of the real specimen. Moreover, the presented calibration method can be easily extended to complex DEM models about any fibrous agricultural materials.

\section{Acknowledgements}

This research did not receive any specific grant from funding agencies in the public, commercial, or not-forprofit sectors. The authors thank Dr. Gábor Szebényi from the Department of Polymer Engineering, Dr. Imre Orbulov from the Department of Material Science and Engineering and Dr. Tibor Poós from the Department of Building Services and Process Engineering at BUTE for the help they provided in the laboratorial tests. The authors also appreciate the assistance and discussion with the engineers of the EDEM team.

\section{References}

Brown, N. J., J.F. Chen, J.Y. Ooi, 2014. A bond model for DEM simulation of cementitious materials and deformable structures. Granular Matter, 16(3), 299-311. https://doi.org/10.1007/s10035-014-0494-4

Chen, Y., J.L. Gratton, J. Liu, 2004. Power Requirements of Hemp Cutting and Conditioning. Biosystems Engineering, 87(4), 417-424. https://doi.org/10.1016/j.biosystemseng.2003.12.012

Coetzee, C.J., S.G. Lombard, 2013. The destemming of grapes: Experiments and discrete element modelling. Biosystems Engineering, 114(3), 232-248. https://doi.org/10.1016/j.biosystemseng.2012.12.014

Coetzee, C.J., 2017. Review: Calibration of the discrete element method. Powder Technology, 310, $104-142$. https://doi.org/10.1016/j.powtec.2017.01.015

Cundall, P.A., O. D. L. Strack, 1979. A discrete numerical model for granular assemblies. Géotechnique, 29(1), 47-65. https://doi.org/10.1680/geot.1979.29.1.47

Huang, J., W. Liu, F. Zhou, Y. Peng, N. Wang, 2016. Mechanical properties of maize fibre bundles and their contribution to lodging resistance. Biosystems Engineering, 151, 298-307. https://doi.org/10.1016/j.biosystemseng.2016.09.016 
Igathinathane, C., A.R. Womac, S. Sokhansanj, 2010. Corn stalk orientation effect on mechanical cutting. Biosystems Engineering, 107(2), 97-106. https://doi.org/10.1016/j.biosystemseng.2010.07.005

Johnson, P.C., C.L. Clementson, S.K. Mathanker, T.E. Grift, A.C. Hansen, 2012. Cutting energy characteristics of Miscanthus x giganteus stems with varying oblique angle and cutting speed. Biosystems Engineering, 112(1), 42-48. https://doi.org/10.1016/j.biosystemseng.2012.02.003

Kemper S., L. Thorsten, L. Frerichs, 2012. Analysis of the overlaying cut in rotary mowers. Landtechnik, 67, 346-349.

Kemper S., L. Thorsten, L. Frerichs, 2014. The overlaid cut in a disc mower - results from field tests and simulation. Landtechnik, 69(4), 171-175.

Keppler, I., L. Kocsis, I. Oldal, I. Farkas, A. Csatar, 2012. Grain velocity distribution in a mixed flow dryer. Advanced Powder Technology, 23(6), 824-832. https://doi.org/10.1016/j.apt.2011.11.003

Keppler, I., F. Safranyik, I. Oldal, 2016. Shear test as calibration experiment for DEM simulations: a sensitivity study. Engineering Computations, 33(3), 742-758. https://doi.org/10.1108/EC-03-2015-0056

Leblicq, T., B. Smeets, H. Ramon, W. Saeys, 2016a. A discrete element approach for modelling the compression of crop stems. Computers and Electronics in Agriculture, 123, 80-88. https://doi.org/10.1016/j.compag.2016.02.018

Leblicq, T., B. Smeets, S. Vanmaercke, H. Ramon, W. Saeys, 2016b. A discrete element approach for modelling bendable crop stems. Computers and Electronics in Agriculture, 124, 141-149. https://doi.org/10.1016/j.compag.2016.03.022

Liang, Z., Y. Li, L. Xu, Z. Zhao, 2016. Sensor for monitoring rice grain sieve losses in combine harvesters. Biosystems Engineering, 147, 51-66. https://doi.org/10.1016/j.biosystemseng.2016.03.008

Nagy, J., 2006. Maize production. (1st ed.). Budapest: Akadémiai Kiadó.410 p.

Olmedo, I., F. Bourrier, D. Bertrand, F. Berger, A. Limam, 2016. Discrete element model of the dynamic response of fresh wood stems to impact. Engineering Structures, 120, 13-22. https://doi.org/10.1016/i.engstruct.2016.03.025

Prasad, J., C.P. Gupta, 1975. Mechanical properties of maize stalk as related to harvesting. Journal of Agricultural Engineering Research, 20(1), 79-87. https://doi.org/10.1016/0021-8634(75)90098-0

Robertson, D., S. Smith, B. Gardunia, D. Cook, 2014. An Improved Method for Accurate Phenotyping of Corn Stalk Strength. Crop Science, 54(5), 2038. https://doi.org/10.2135/cropsci2013.11.0794

Robertson, D.J., S.L. Smith, D.D. Cook, 2015. On measuring the bending strength of septate grass stems. American Journal of Botany, 102(1), 5-11. https://doi.org/10.3732/ajb.1400183

Tamás, K., I.J. Jóri, A.M. Mouazen, 2013. Modelling soil-sweep interaction with discrete element method. Soil and Tillage Research, 134, 223-231. https://doi.org/10.1016/j.still.2013.09.001

Tongdi Q., L. Yaoming, C. Jin, 2011. Experimental study on flexural mechanical properties of corn stalks. IEEE, 130-134. https://doi.org/10.1109/ICAE.2011.5943766

Zhang, K., Y. He, H. Zhang, H. Li, 2017. Research on mechanical properties of corn stalk. AIP Conference Proceedings 1820, 050007. https://doi.org/10.1063/1.4977311 


\author{
Evaluation of a Citrus Mobile PlatformUusing a Wireless Impact Recording Device \\ Gyomar González $^{a}$, Patricia Chueca ${ }^{a}$, Coral Ortiz ${ }^{\text {* }}$ \\ ${ }^{a}$ Centro de Agroingeniería. Instituto Valenciano de Investigaciones Agrarias (IVIA), Ctra. CV- 315, km. 10. 46113 \\ Moncada (Valencia), Spain \\ ${ }^{\mathrm{b}}$ Dpto. Ingeniería Rural y Agroalimentaria, Universitat Politècnica de València, Camino de Vera s/n, 46022 Valencia, \\ Spain \\ * Corresponding author. Email: cortiz@dmta.upv.es
}

\begin{abstract}
Manual citrus harvesting is an expensive operation that represents between $25-40 \%$ of the total production costs. A mobile platform prototype to assist in the harvesting of citrus fruits designed by the IVIA (Spain) has been tested in order to reduce fruit damages. The mobile platform is fitted with conveyor systems. The multilevel platform can accommodate 4 to 8 workers who pick the fruit and place it on a conveyor belt. The picked fruit is transported to a central conveyor belt on which the fruit is transported to the in-line sorting system that classifies the fruit in two categories using a computer vision system and directs the fruit to one of the two different binfillers. During this process the fruits are subjected to mechanical stress causing physical injuries, including skin punctures, pulp and cell rupture. An impact recording device was used to evaluate damage produced to citrus fruits. The critical points at which damage occurs were determined, and the damage levels assessed. The highest damage level was produced from the bin-fillers to the bins. Seven different shocking absorbing materials were tested in order to reduce fruit damage. Five of the seven tested materials were capable to reduce the recorded impact.
\end{abstract}

Keywords: mechanical harvest, fruit damage, absorbing materials, instrumented spheres

\title{
1. Introduction
}

Spain is the first World's exporter of citrus for fresh market. In current Spanish fresh citrus orchards, fruits are manually harvested, then loaded in boxes and transported to a packing house, where boxes are unloaded, cleaned and fruit are pre-sorted. Manual citrus harvesting represents up to $50 \%$ of current final production costs (Mateu et al., 2018), becoming 10 times higher in Spain than in many competitor countries (Juste et al., 2000). Mechanization of harvest would increase labor productivity and thus result in greater profits for agricultural entrepreneurs.

The system to be used for harvesting the fruit depends largely on what the fruit is going to be used for. Fruit destined to the processing industry could be mechanically harvested because certain types of damage on the peel of the fruit are acceptable. In contrast, fruit destined to be eaten fresh cannot have any kind of damage, whether internal or external.

Between different solutions for citrus mechanical harvesting, platforms seem to be the most adequate for fresh fruit due to its careful fruit management compared with the rest of solutions based on shaker systems. These machines are generally self-propelled and facilitate access to all parts of the tree for the pickers, so that they are only engaged in collecting the fruits and placing them directly in a conveyor belt or a field bag. These machines are being used in other crops like apple and pears grown in trellis systems. The company Argilés Disseny i Fabricació and IVIA have developed a platform machine for harvesting citrus, that is also capable of in-field sorting the harvested fruit into two different classes based on weight, using an electronic weighing system based on load cells, or on colour, using a machine vision system (Gutierrez et al., 2012).

The mechanical damages that occur in the fruits are a consequence of the combination of two main factors: the physical properties of the fruit and the damage inflicted on it during its harvesting and handling (Hernández, 2000). For citrus fruits, these injuries can be due to impact, compression or friction, being the damages by impact and compression undetectable in many occasions through an external observation. There are two alternatives for measuring citrus fruit impact, one is sampling fruit by measuring bruising and physical damage after harvest and the other is using instrumentation (Bollen, 2006). This instrumentation is based on electronic sensors (wireless impact recording device) that capture both the moment and the intensity of the dynamic loads suffered. With them, it is possible to evaluate any process and locate in it the points where the fruits are exposed to impacts, compressions and other situations of mechanical stress (Tennes et al., 1991).

This work objective was to evaluate the mechanical damage to citrus fruits in a part of the process carried out in the mobile platform prototype. After observing where the impacts of greater intensity were produced, seven materials were evaluated in order to assess their shock absorbing capacity. 


\section{Materials and Methods}

Description of the harvest platform prototype

The mobile platform has three levels of fruit discharge: ground level (level 1), a first intermediate height on the platform (level 2) and a second height above the platform (level 3). It has a capacity for 4 to 8 operators positioned on the platform and between 4-6 workers at ground level.

Operators located in level 1 directly drop the fruit to the hopper. Operators of levels 2 and 3 drop the fruit to conveyor belts, where it is directed to the hopper. From this, the fruit is lifted by a conveyor belt with rubber fingers to the top of the platform where the classification system is located. In the upper zone the fruits are singled out into 4 lines or channels (Figure 1) and transported by rollers to the zone of classification by computer vision that classifies the fruit into two categories. In the in-line sorting system, the fruits are classified according to commercial caliber distributing the fruit in different bin-fillers (Figure 2). The internal bin is used for the low quality fruit (or non-commercial fruit) and the external one for the high quality fruit (or commercial fruit).

The prototype includes a trailer where the empty bin-fillers are stored to load them on the platform when needed and also facilitates the unloading of full bin-fillers in the field.

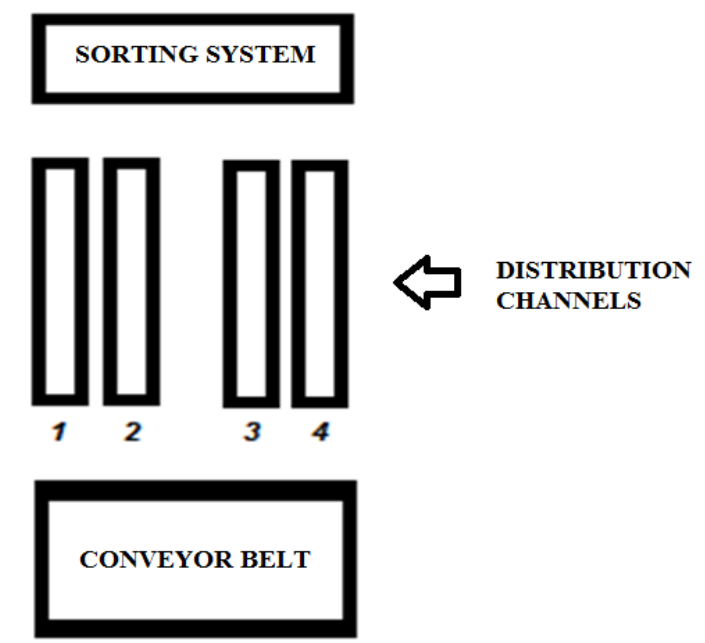

Figure 1. Outline of the top of the mobile platform.

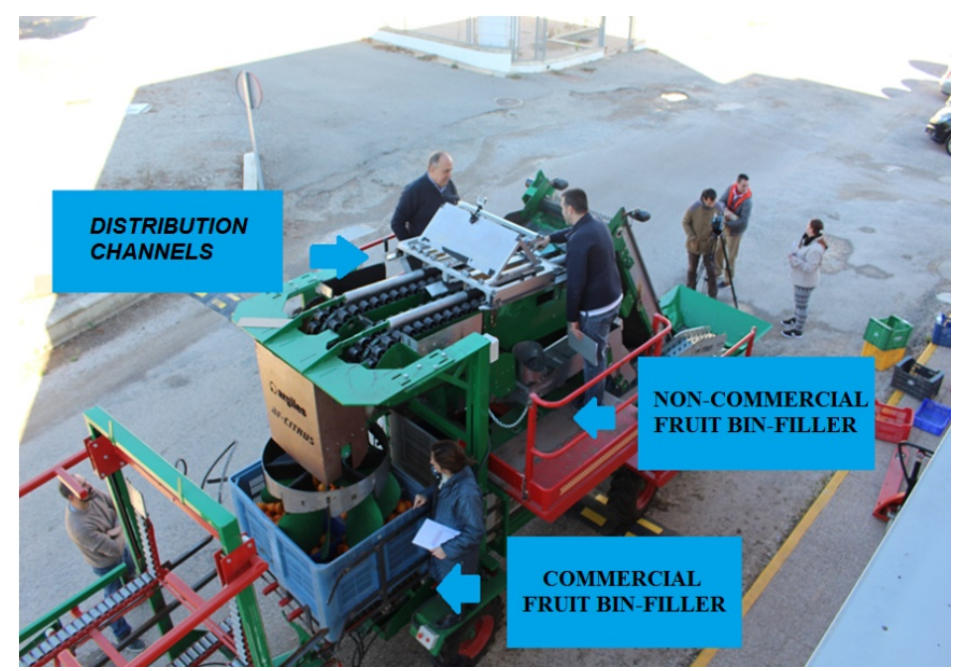

Figure 2. Harvest platform prototype.

Evaluation of impacts in the classification process in the prototype

A trial was carried out to evaluate the critical points during the classification process in the prototype which include the passage of fruit through the 4 conveyor channels of vision system and its destination to one of the two bin-fillers (internal and external). For that, a wireless impact recording device (IRD) (Tuberlog PTR 200, www. martinlishman.com) was used to measure the acceleration impacts. The IRD has an impact range between 30 and 
$500 \mathrm{G}$. The trial consisted in passing the IRD through each of the four channels at uniform advance speed of the conveyor belts established at $0.356 \mathrm{~m} \mathrm{~s}^{-1}$. The caliber was set to establish where the fruit should be deposited forcing the evaluation of the two drop towards the bin-fillers. Three experimental repetitions were carried out per channel (4) and bin-filler (2).

The analysis of data consists of comparing the values of the impact acceleration obtained in the different repetitions for each channel and bin as a function of time. For this comparison, these impacts were displayed in graphs and the time corresponding to the highest impact acceleration value was taken as reference (in all cases this impact corresponds to the drop impact to the bin). This value was set as the origin of coordinates. The points to the right of the y-axis correspond to rebounds after the drop impact while the points to the left of the Y-axis correspond to the impacts originated during the process evaluated in the machine before the drop impact.

\section{Evaluation of impact reducing capacity of different materials}

A trial was carried out to evaluate the impact reducing capacity of seven different shocking absorbing materials (Figure 3) which could be used in the harvest platform to reduce the impacts observed in the previous section. The trial consisted of dropping the IRD described above at uniform advance speed $\left(0.356 \mathrm{~m} \mathrm{~s}^{-1}\right)$ in the conveyor belt located before the fruit hopper and evaluate the impact over the different materials placed in the metallic system, that is between the conveyor belt and the fruit hopper, with a fall of $20 \mathrm{~cm}$ with respect to the conveyor belt (Figure 4). Ten sets of ten repetitions were carried out per material, with a total of 80 tests (70 with materials and 10 without material).

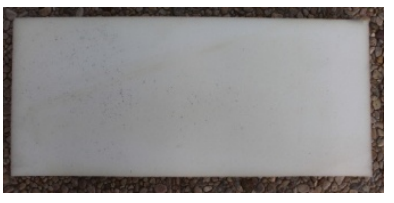

Material 1

Density: $46.36 \mathrm{Kg} \mathrm{m}^{-3}$

Thickness: $41 \mathrm{~mm}$

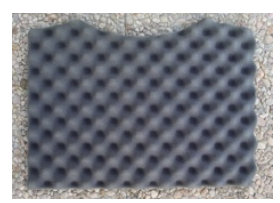

Material 3

Density: $15.56 \mathrm{Kg} \mathrm{m}^{-3}$

Thickness: $23.25 \mathrm{~mm}$

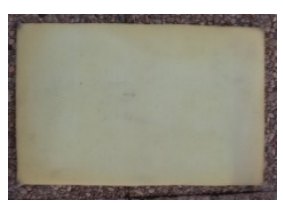

Material 5

Density: $16.44 \mathrm{Kg} \mathrm{m}^{-3}$

Thickness: $9 \mathrm{~mm}$

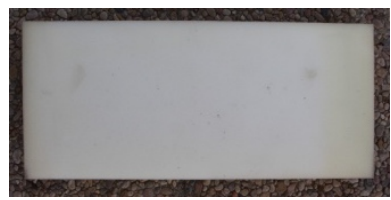

Material 2

Density: $26.74 \mathrm{Kg} \mathrm{m}^{-3}$

Thickness: $43 \mathrm{~mm}$

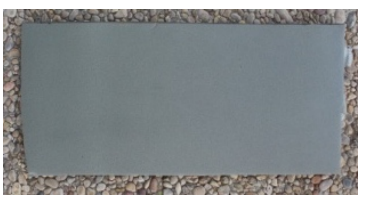

Material 4

Density: $23.37 \mathrm{Kg} \mathrm{m}^{-3}$

Thickness: $10 \mathrm{~mm}$

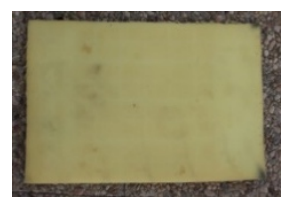

Material 6

Density: $18.26 \mathrm{Kg} \mathrm{m}^{-3}$

Thickness: $18 \mathrm{~mm}$

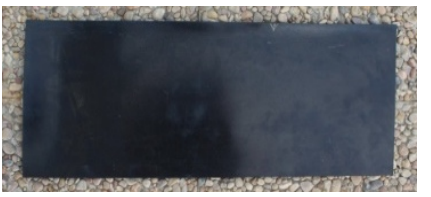

Material 7

Density: $146.87 \mathrm{Kg} \mathrm{m}^{-3}$

Thickness: $3.10 \mathrm{~mm}$

Figure 3. Materials. 


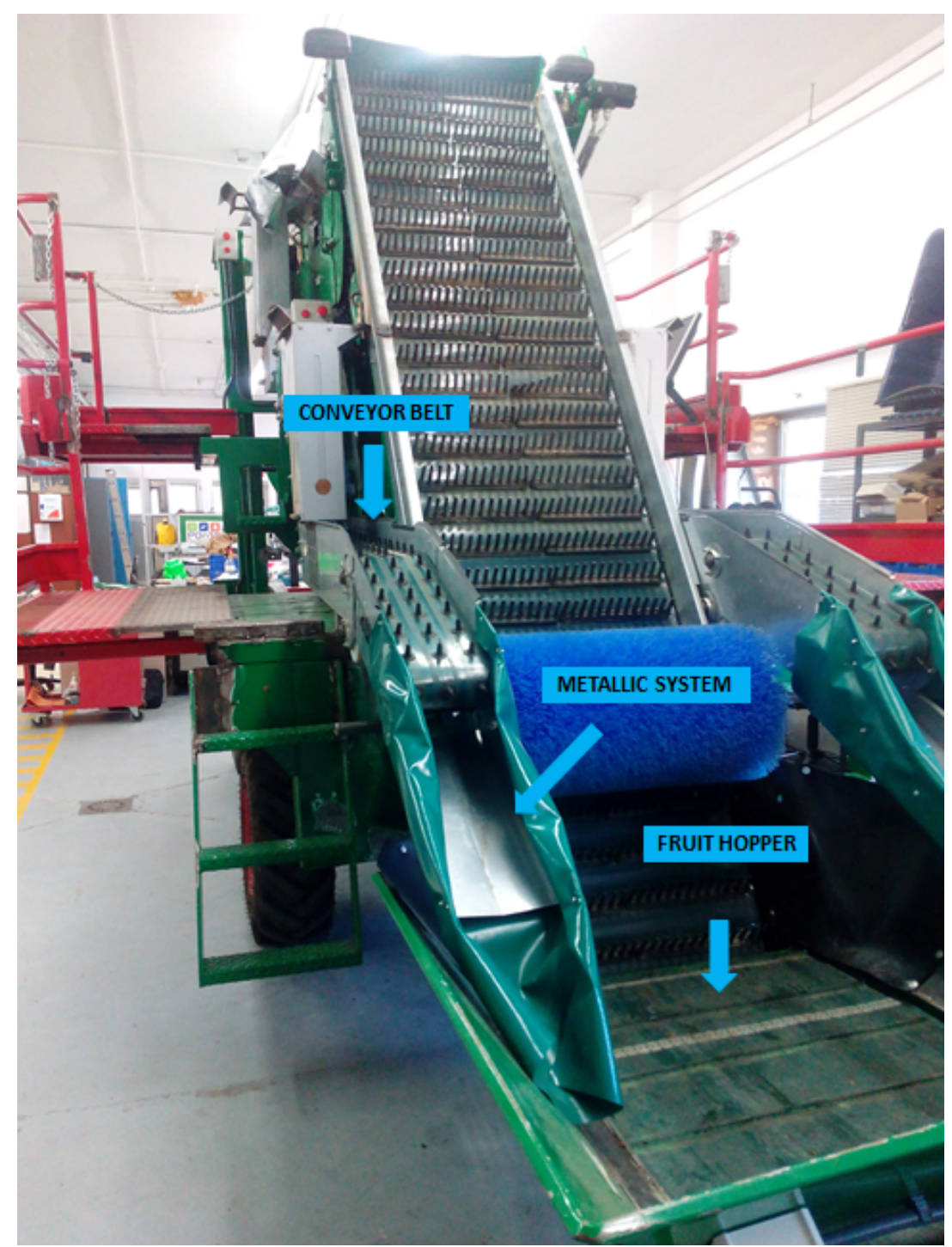

Figure 4. Conveyor belt and metallic system placed before the fruit hopper in the prototype.

Because some impact measures with the IRD were lower than the minimum value range of the sensor (30G), the percentages of impact data lower than $30 \mathrm{G}$ and higher than $30 \mathrm{G}$ for each material were calculated. Later, data (higher than 30G) comparing the drop impact between materials were analyzed using one-way analysis of variance (ANOVA) at $\mathrm{P}<0.05$. The least significant difference (LSD) multiple range test was used for mean separation at $\mathrm{P}<0.05$. Before ANOVA, homocedastiticity and normal distribution of residuals were tested. Although the data were not follow these both hypothesis because of ANOVA results were the same that the obtained with the non-parametric Kruskal-Wallis test, the results of ANOVA were considered valid due to the robustness of the ANOVA (Statgraphics Centurion XVII).

\section{Results and Discussion}

Evaluation of impacts in the classification process in the prototype

Clear differences were found between the impacts registered from the two different bins (Figure 5). 


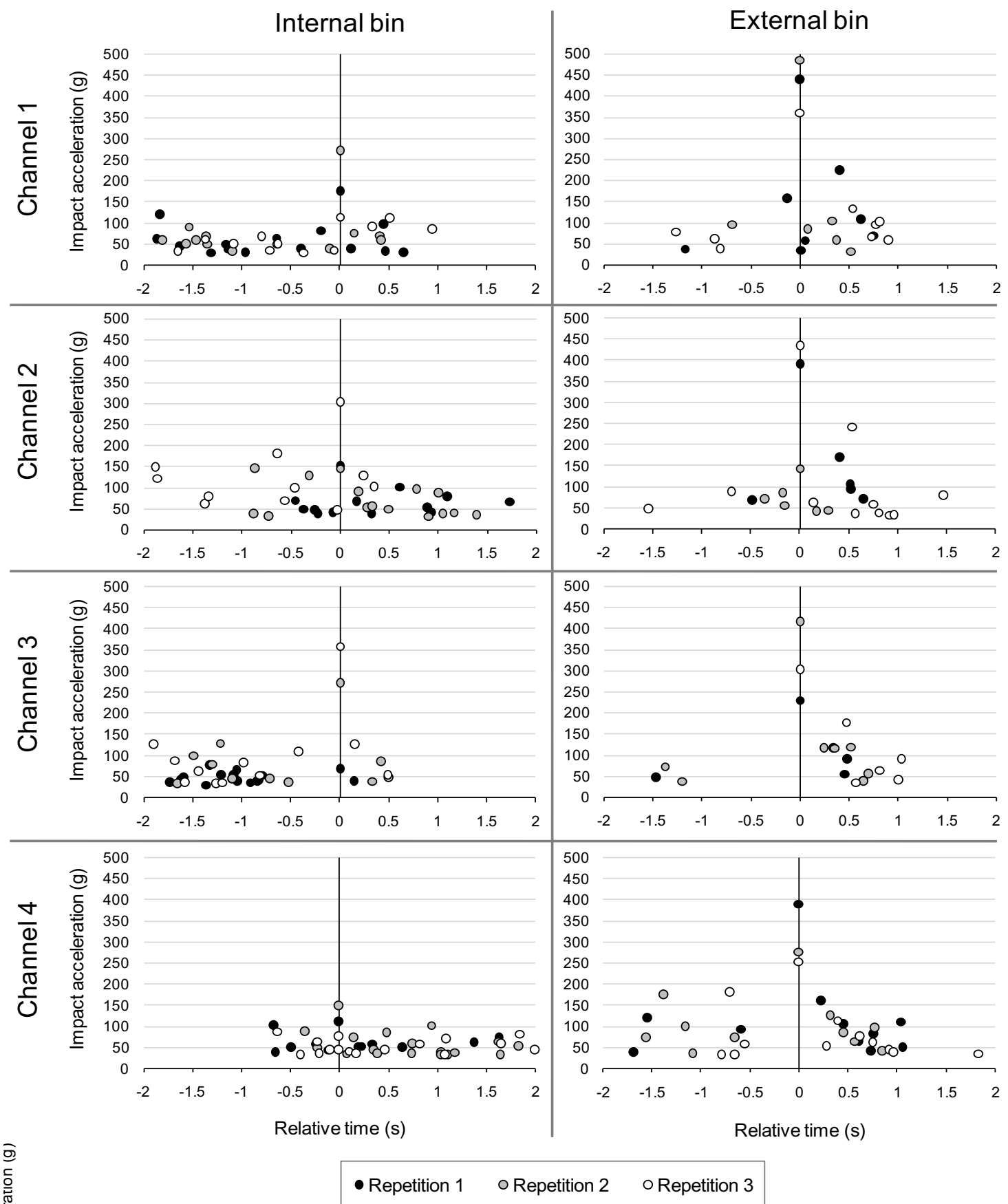

Figure 5. Impacts registered according to the bin and the channel. The highest impact acceleration (drop impact to the bin) value was taken as reference. The points to the right of the y-axis correspond to rebounds after the drop impact while the points to the left of the $\mathrm{Y}$-axis correspond to the impacts before the drop impact.

In the external bin (except the channel 4):

- $\quad$ Few low impacts (from one to three) were registered before the drop impact, with values under 100G.

- A very high drop impact was registered, between 300 and $500 \mathrm{G}$.

- $\quad$ High impact rebounds were produced, in some cases around 200G.

In the internal bin (except channel 4):

- Several medium impacts (between 100 and $200 \mathrm{G}$ ) were found before the drop impact,

- A high drop impact was registered, between 150 and $300 \mathrm{G}$,

- Low impacts rebounds were produced after the dumping. 
No clear differences were found between the impacts recorded in the different channels, except channel 4.

The impacts registered using channel 4 and the external bin before the drop were more numerous and considerably higher (nearly $200 \mathrm{G}$ in some cases) than the ones registered using the other channels. The drop impact registered using the channel 4 was considerably lower (lower than $150 \mathrm{G}$ in all the cases) than the ones registered using the other channels.

Evaluation of impact reducing capacity of different materials

Based on previous studies with potato harvesters using a similar wireless impact recording device, the maximum impact value that does not produce damage is $30 \mathrm{G}$ (Bentini et al., 2006). In citrus, according Miller and Wagner (1991), the value considered is a little bit higher 40G. Anyway, since the lower threshold of IRD is at $30 \mathrm{G}$, and to be in the side of security, this value was considered as the lower threshold for our test.

Table 3. Percentage of impact data lower and higher that $30 \mathrm{G}$ for each material the reference value that not produces

\begin{tabular}{ccc}
\multicolumn{3}{c}{ impact damage. } \\
\hline Material & \multicolumn{2}{c}{$\%$ of data according to impact acceleration value $(\mathrm{g})$} \\
& $<30$ & $>30$ \\
\hline $0^{*}$ & 0 & 100 \\
1 & 100 & 0 \\
2 & 100 & 0 \\
3 & 100 & 0 \\
4 & 0 & 100 \\
5 & 0 & 100 \\
6 & 22.55 & 77.45 \\
7 & 0 & 100 \\
\hline
\end{tabular}

* Material was designated to the metallic system without shocking absorbing material.

Materials 1, 2 and 3 were the most shocking absorbing materials because all the impacts were lower than $30 \mathrm{G}$. The following absorbing material was 6 because $22.55 \%$ of impact data were lower than $30 \mathrm{G}$ while the rest of materials presented all impact values higher that $30 \mathrm{G}($

Table 3). In the statistical analysis of the data comparing the impact higher than $30 \mathrm{G}$ for the five remaining materials was observed that there was a statistically significant difference in the mean of impact acceleration $(\mathrm{g})$ between the different materials $(\mathrm{F}=141.69$; $\mathrm{df}=4,509 ; \mathrm{P}<0)$ (Figure 6).

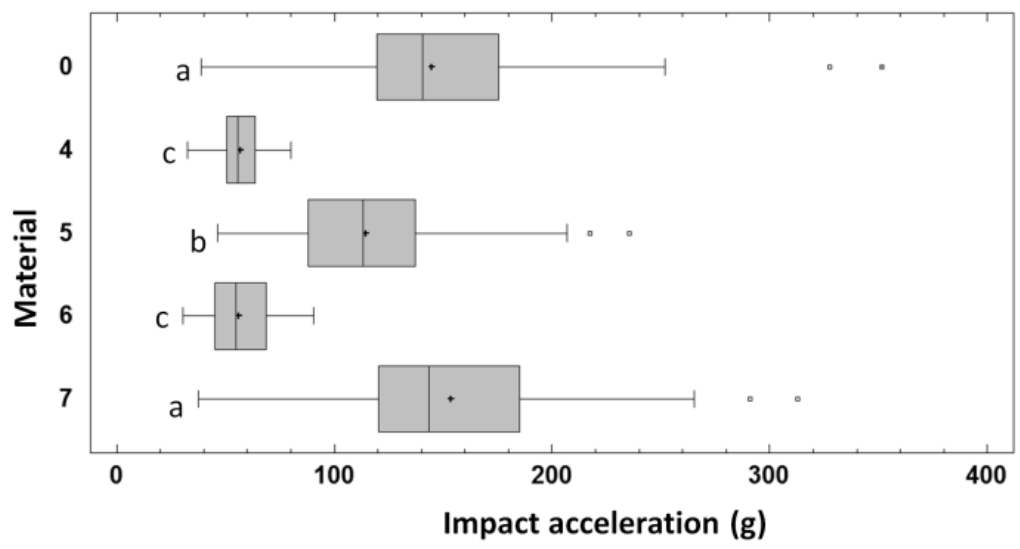

Figure 6. Box and whiskler plot for the variable drop impact higher than $30 \mathrm{G}$ for each material.

Materials 0 and 7, showed the highest variability and the highest mean value of impacts, not having statistically differences between both material 7 and metallic surface. Material 5 was the following less impact absorbing and was statistically different to the rest of the materials. Material 4 and 6 showed the lower value variability and lower values of impact not having statistically differences between both but it has to take into account that for material 6 the $22.55 \%$ of data lower than 30 were not considered, Therefore material 6 is more absorbent material than 4 . With these results the materials can be categorized into 5 distinct groups. A first group composed of materials 1, 2 and 3 which are the most shocking absorbing materials. A second group composed of the material 6. A third group composed of the material 4. A fourth group composed of the material 5 and the last group composed of the material 7 which behavior is the same as if the material had not been put on. In addition to the ability to absorb impacts, other factors like price, durability and impermeability should be taken into account 
to decide the best appropriate material for coating the different areas of the platform. The first three materials and 6 are not waterproof meanwhile material 4 is cover by a plastic surface making it waterproof.

\section{Conclusions}

The highest damage level produced in the citrus mobile platform prototype was registered from the bin-fillers to the bins.

In evaluation of the impacts in the classification process in the prototype, clear differences were found between the impacts from the two different bins. The final drop impact recorded in the external bin was clearly higher. On the other hand, the impacts recorded in the internal bin were more numerous and the final drop impact was lower. The channel 4 of the upper conveyor belt showed differences respect to the other channels.

In evaluation of the impact reducing capacity of different materials, materials 1, 2, 3, 4 and 6 showed a high capacity to absorb impacts being the material 4 the most adequate for its use in outdoor conditions.

The next step will be to analyse the damage on fresh fruit and according to those results to carry out the modifications in the platform prototype to reduce the damage as much as possible with the use, whenever possible, of some of the evaluated materials.

\section{Acknowledgements}

This work has been carried out within the project "Application of new technologies for a comprehensive strategy of mechanized citrus harvesting (CITRUSREC)" funded by the Instituto Nacional de Investigaciones Agrarias (INIA) and the Ministry of Economy, Industry and Competitiveness of Spain (projects RTA2014-00025C05-01 and RTA2014-00025-C05-02) and co-financed by the European Regional Development Fund (ERDF). $\mathrm{M}^{\mathrm{a}}$ Gyomar González is recipient of a postgraduate FPI-INIA scholarship. This work has been possible thanks to the help of the staff of the Agroengineering Center of the IVIA.

\section{References}

Bentini M., Caprara C. and Martelli R., 2006. Harvesting damage to potato tubers by analysis of impacts recorded with an instrumented sphere. Biosystems Engineering (2006). 94 (1), 75-85.

https://doi.org/10.1016/j.biosystemseng.2006.02.007

Bollen A., 2006. Technological innovations in sensors for assessment of postharvest mechanical handling systems. International Journal of Postharvest Technology and Innovation. 1, 16-31. https://doi.org/10.1504/IJPTI.2006.009179

Gutiérrez A., Blasco J., Chueca P., Garcerá C., Alegre S., López S., and Moltó E., 2012. Harvesting and InField Sorting of Citrus with a Self-Propelled Machine. In I International Symposium on Mechanical Harvesting and Handling Systems of Fruits and Nuts. 965, 149-152. https://doi.org/10.17660/actahortic.2012.965.18

Hernández, P., 2000. Caracterización instrumental de "frutos electrónicos" y su utilización en la evaluación de daños en las líneas de confección de cítricos. Trabajo fin de carrera, Escuela Técnica Superior de Ingenieros Agrónomos, Universidad Politécnica de Valencia, Valencia, España.

Juste F., Martín B., Fabado F., and Moltó E., 1999. Estudio sobre la reducción de los costes de producción de cítricos mediante la mecanización de las prácticas de cultivo. Comunitat Valenciana Agraria. 12, 23-29.

Mateu G., Caballero P., Torregrosa A., Segura B., Juste F. and Chueca P., 2018. Análisis de la influencia de las operaciones de cultivo sobre los costes de producción en la citricultura de la Comunidad Valenciana. Levante Agrícola: Revista internacional de cítricos. 440, 60-64.

Miller W.M. and Wagner C., 1991. Florida citrus packing line studies with an instrumented sphere. American Society of Agricultural Engineer. 7(5), 577-581. https://doi.org/10.13031/2013.26272

Tennes B., Zapp H., Armstrong P. and Marshall D., 1991. Combined impact sensing and data acquisition units for studies of bruising during handling of fruits and vegetables. Journal of Agricultural Engineering Research. 49, 189-196. https://doi.org/10.1016/0021-8634(91)80038-G 


\section{Topic 11: Nutrient management and waste treatment (NM)}

Research and application of technology and systems to manage and recycle organic wastes in plant and animal production systems. Contributions may include organic matter and nutrient management on arable farms (e.g. with cover crops) and on animal farms (e.g. processing of animal manure) and include emissions and other nutrient losses.

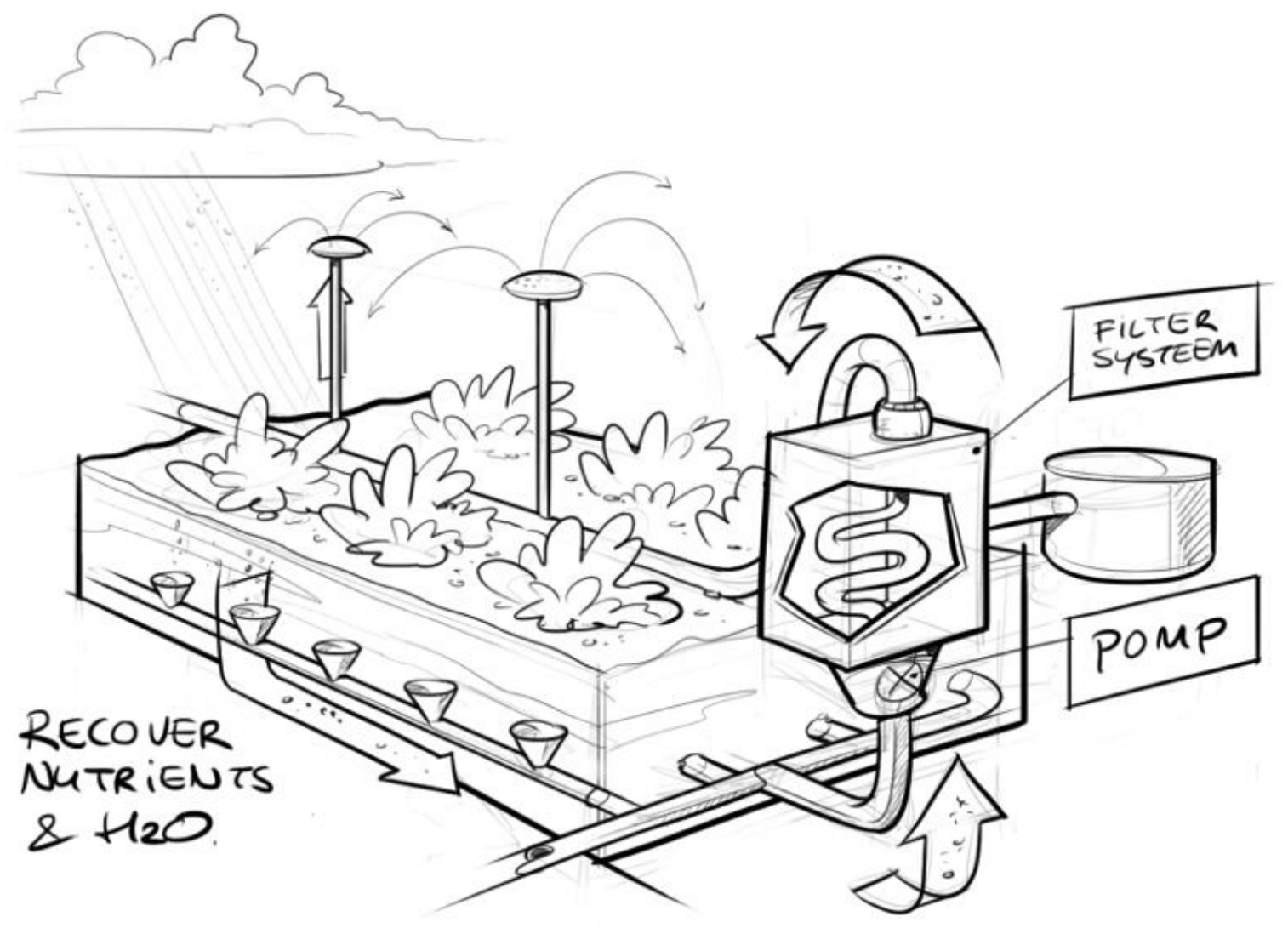




\title{
Recycling Food System Nutrients in a Circular Economy \\ P. Derikx ${ }^{1 *}$, O. Oenema ${ }^{1}$, K. Poppe ${ }^{1}$, T. Vellinga ${ }^{1}$, K. Verloop ${ }^{1}$, J. Weijma ${ }^{1}$, W. Zwanenburg ${ }^{1}$, \\ P. de Wolf ${ }^{1}$, L. de Bie ${ }^{1}$ \\ ${ }^{1}$ Wageningen University and Research, The Netherlands \\ * Corresponding author. Email: piet.derikx@wur.nl
}

\begin{abstract}
Planet Earth already exists for over 5 billion years, while humans have been around for only a million years. The impact of human activity on the natural ecosystem has increased dramatically over the last few hundred years, mainly through agriculture, industry, and urbanisation, resulting in the consumption of natural resources at high rate. Modern agriculture, with the use of fertilizers and agrochemicals, has increased productivity drastically and has loosened the connection between location of food production and location of food consumption. As a result local/regional accumulation of nutrients occurs in terms of waste streams with negative impact on the environment, in combination with regional depletion elsewhere. The circular economy has been generally accepted now by most scientists, policy makers and entrepreneurs, as concept and new paradigm for organizing the food production - consumption cycle. As a consequence any stream of material within that cycle should be considered as an input elsewhere in the cycle. The main question addressed in this paper is "how to organize the recycling of food system nutrients effectively and efficiently'? As socio-economic, environmental and cultural conditions differ from one place to the other on the planet there is not one single solution that fits all food systems for organizing a circular economy. Therefore, a mix of several solutions may occur side by side. This diversity will contribute positively to the robustness of the system towards fluctuations due to impacts generated either by nature or by mankind. An important constraints to modifications to food systems is that the modifications and recycling are acceptable by the stakeholders involved. Therefore, initiatives have to be taken to bring together different stakeholders in order to exchange ideas and to explore common grounds for future cooperation. Position papers are written to stimulate partners to move away from their own comfort zone and think about new types of solutions. As the world changes, new techniques become available and new generations prefer to make different choices. What was good in the past might no longer be good enough for the future. Here the first results from this forward-looking and integrated approach are reported.
\end{abstract}

\section{Introduction}

Planet Earth already exists for over 5 billion years, while humans have been around for only a few million years. The impact of human activity on the natural ecosystem has increased dramatically over the last few hundred years. As an example, the consumption of fossil energy sources has used up a considerable part of all available fossil fuel resources in less than 100 years. The same tendency is observed for many other natural resources.

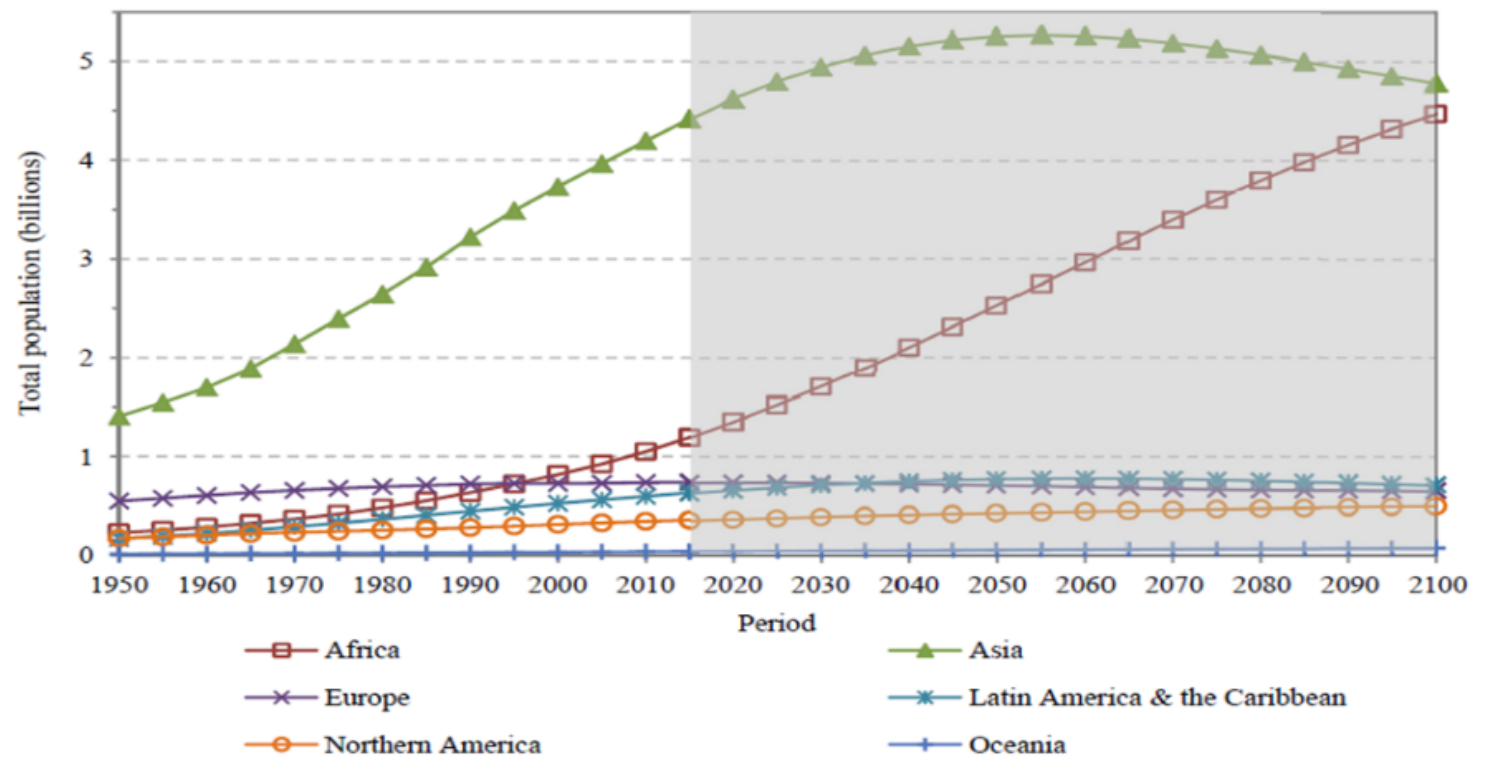

Source: United Nations, Department of Economic and Social Affairs, Population Division (2017). World Population Prospects: The 2017 Revision. New York: United Nations.

Figure 1. Projected increases in the number of people per continent. Source: United Nations World Population Prospects. 
From the early 1970s on people have been worried about this tendency, initiated by a report of the club of Rome (Meadows et al., 1972). Over the years this publication has received a considerable amount of criticism especially on the rather simplified models used to predict future developments. Nevertheless, the awareness among the public for limits to the planet has been increasing. At the beginning of 21th century McDonough and Braungart introduced upcycling way to improve the usability of its constituents by the end of the lifetime of a given product (McDonough and Braungart, 2002). More recently, well accepted models have been published about planetary boundaries which should be respected in order to keep the life on the planet away from major changes and shortages of resources and environmental pollution that might threaten our existence on the long run (Foley et al. 2011, Steffen et al., 2015). For the next decades a steady increase in world population is predicted as shown in figure 1. This increase will result in a population of about 9 billion individuals by 2050. Main increases are foreseen in Asia and Africa.

\section{Agro - food production system}

Pre-industrial communities have coped with the low input principle for thousands of years. Modern agriculture uses fertilizers and agrochemicals more and more as shown in Figure 2.
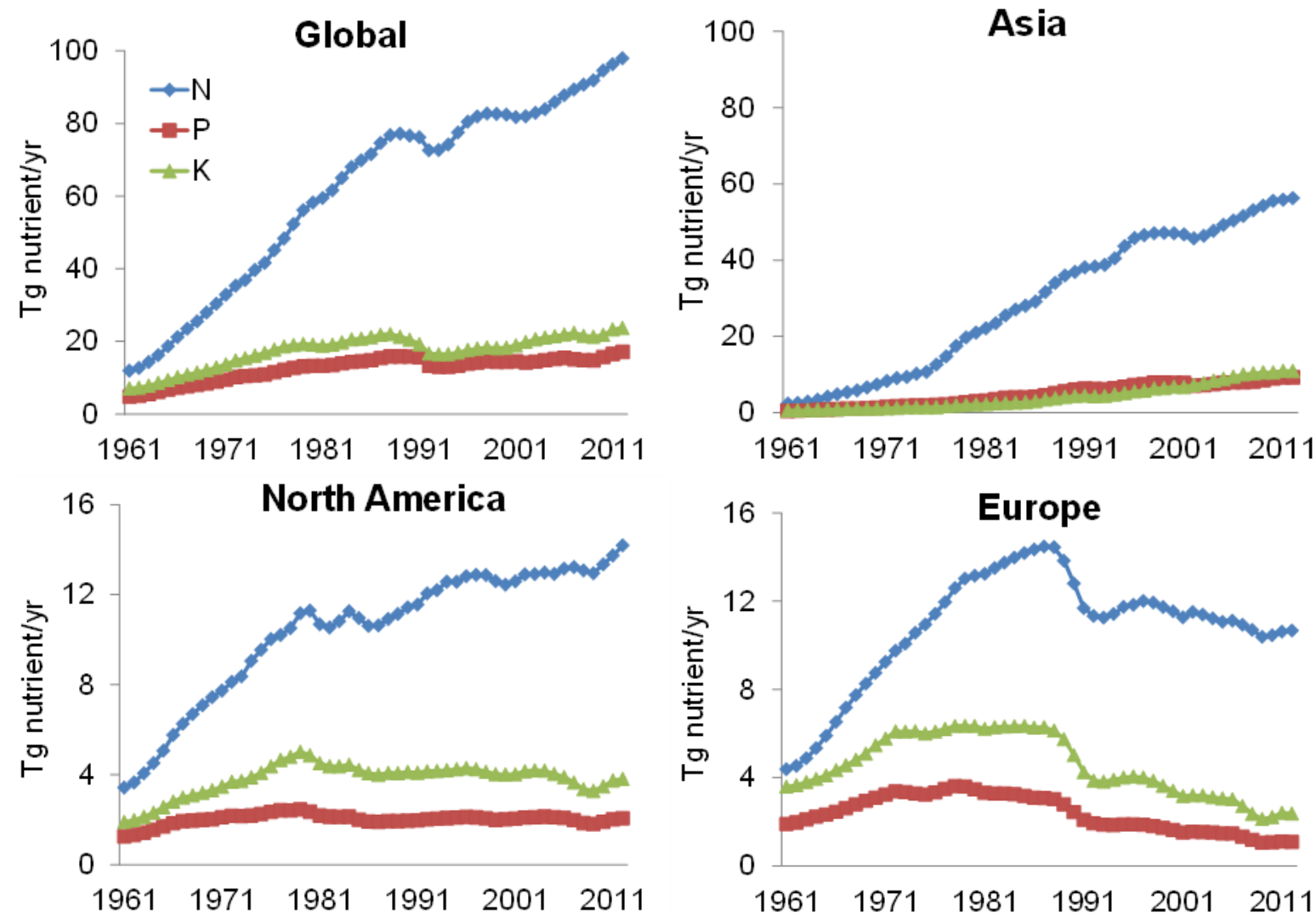

Figure 2. Consumption of synthetic nitrogen $(\mathrm{N})$, phosphorus $(\mathrm{P})$, and potassium $(\mathrm{K})$ fertilizers in the world (upper left panel), Asia, North America and Europe between 1961-2012. Note the differences in Y-axis between the upper two panels and the lower two panels. Data source: FAOSTAT (2015).

About half of the global consumption of fertilizers is situated in Asia. The other half is used in North .America and Europe, with as small additional consumption in Latin America, Oceania and Africa. As a result crop production has increased dramatically in regions with high fertilizer consumption. In addition, and partly as a consequence of the availability of cheap synthetic fertilizers, the connection between the location of food production and the location of food consumption has weakened. The impact of this development is a local/regional accumulation of nutrients in terms of waste streams with negative impact on the environment, in combination with regional depletion elsewhere (Potter et al., 2010). How intensive livestock production will move towards areas where the feed is produced or to regions where the products are consumed is still a topic for many discussions (Poppe et al. 2018). 


\section{Closing nutrient cycles}

The central mission of sustainability is to maintain a liveable planet for the generations to come, and amongst others to keep fulfilling the nutritional needs of the human population. The recycling of all nutrients, maintaining soil quality and fertility and providing clean water on global, regional and local scales are crucial. Making steps on the route towards this situation requires major efforts of all parties involved. Different (economical) interests of the actors generate a complex situation, which requires a new approach to innovate on system level and not only in isolated domains.

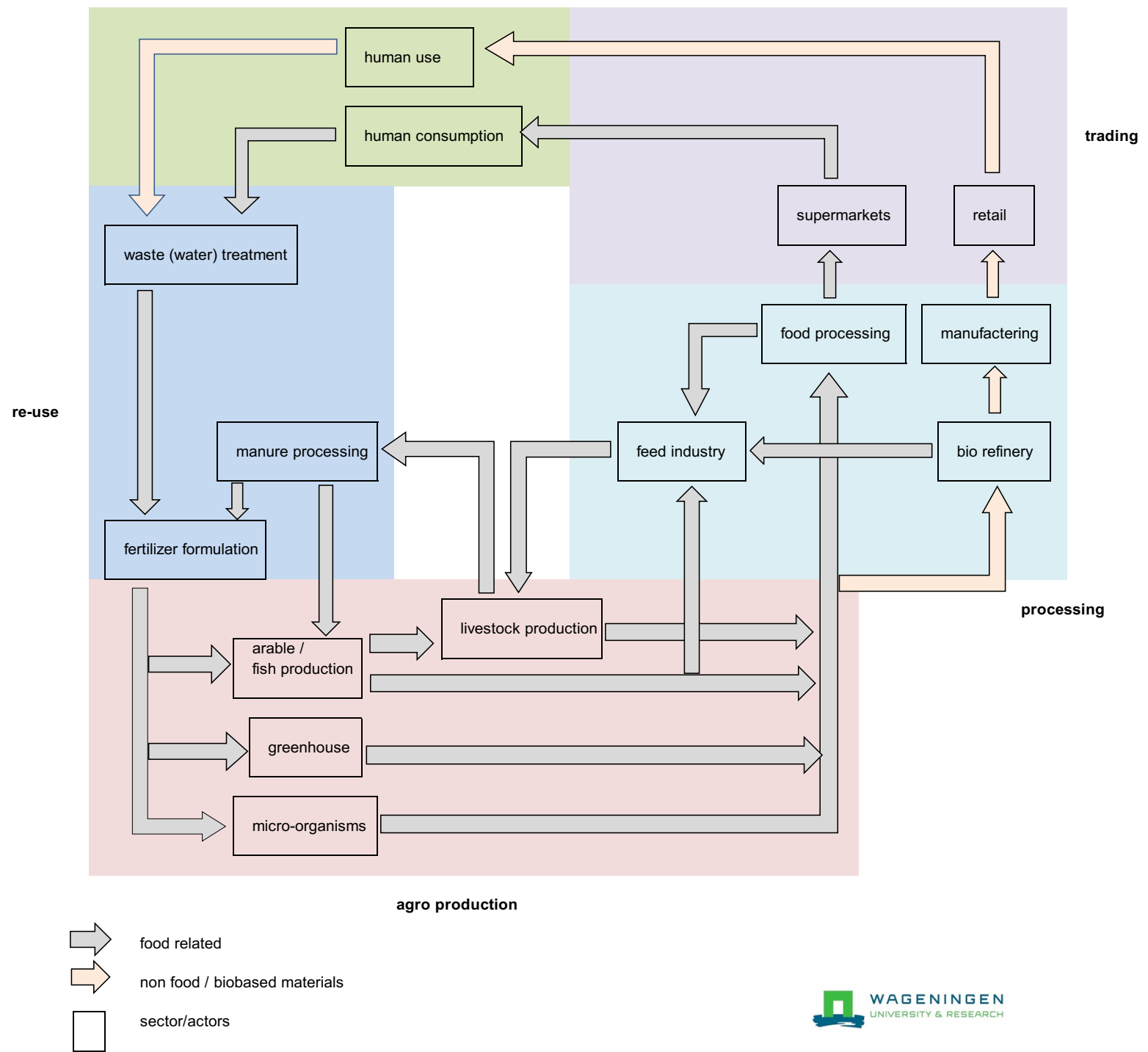

Figure 3. Schematic representation of close nutrient cycles in the Agro-food and Agro-biobased materials production system. Relevant actors and lines of business are shown.

Crucial in the circular economy approach is the awareness that any stream of material considered previously as a waste in the nutrient cycle should be considered as an input for another process in the cycle. In a true circular economy there is no place for the word 'waste'. Organic materials can serve as organic fertilizers and turned into plant nutrients by action of soil micro-organisms. Other side streams, like chemical substances, metals and mineral products can be upcycled to new products or building blocks for new products by the use of clever production processes. Public awareness and political responsible behaviour are currently changing but still have to make additional moves in this aspect. Recent plans to ban single use plastics are promising steps.

As an illustration of how the situation is changing, in The Netherlands 12.000 tons of phosphorus end up in sewage treatment plants originating from household waste water. Slightly over $80 \%$ is recovered into the sewage sludge and incinerated. Ashes are used to stabilize subsoils of high ways rather than being used as a source for plant nutrients Weijma et al, 2018). This implies a loss of nutrients for the agro-food production 
system and will not be affordable on the long run in terms of a circular system. More recently, both public bodies and private enterprises have been working on the recovery of phosphorous from sewage/sewage sludge. This can be seen as a first step. To recover other valuable plant nutrients from sewage/sewage sludge in an economical way, free from risks for environment, animals and humans is a major challenge. Besides technical issues, social and legal aspects needs to be addressed as well.

As circumstances, socio-economic and cultural aspects differ from one place to the other on the planet there is not one single solution that fits all, which is typical for a wicked problem. Therefore, a mix of several solutions may or should arise side by side to move towards closing the nutrient cycles. This diversity will contribute positively to the robustness of the system towards fluctuations due to impacts generated either by nature or by mankind.

The range of solutions may include among others recycling of nutrients from human waste, re allocating of nutrients back to the location of crop production, and the recovery from scarce nutrients from highly diluted sources. For this a range of actors is required involved in creating solutions. As illustrated in scheme of nutrient cycles, several actors will be involved in every step along the cycle. A solution can only contribute significantly when it is embedded in a system approach, with actors working together, each in its own niche and to its own ability, but sensing a shared responsibility for the whole system.

Wageningen University and Research (WUR) has a worldwide reputation in the field of agricultural research, including studies on nutrients recycling (Henckens et al., 2016; Seyhan et al., 2012). WUR has recognized the need for an integrated systems approach for dealing with nutrients in our agri-food system. Therefore, the taskforce "CirculaResource" with a wide range of expertise has started in 2017. Its ambition is to contribute to sustainable solutions by bringing together actors and stimulate cooperation to develop new knowledge and skills that serve a circular nutrient economy.

\section{Outlook}

The taskforce has written a number of position papers (Oenema et al, 2018, Poppe et al, 2018 Weijma et al, 2018 ) and contributions based on these are presented on both national and international level, either scientific or at stakeholders oriented. This is seen as a first step towards a more practical implementation of the lines of thinking described above. Identifying stakeholders and share their concerns for the future with them is a logical next step which is currently in progress. Round table discussions with stakeholders with different backgrounds are organised. For example fertilizer industry is challenged to take the lead in the debate for future pathways of nutrient management (Oenema et al., 2018) Defining common grounds for future developments and visualizing each contribution to it requires courage of all partners involved as they are challenged to come out of their own comfort zone. Most important for future success is the will to pass a liveable planet to our children and grandchildren.

\section{References}

Meadows D.H., D.L. Meadows, J. Randers W.W. Behrens (Club of Rome), 1972. Limits to Growth. 205 p. Universe Books

Foley J.A., N. Ramankutty, K.A. Brauman, E.S. Cassidy, J.S. Gerber, M. Johnston, N.D. Mueller, C.

O’Connell, D.K. Ray, P.C. West, C. Balzer, E.M. Bennett, S.R. Carpenter, J. Hill, C. Monfreda, S. Polasky, J.

Rockström, J. Sheehan, S. Siebert, D. Tilman \& D.P.M. Zaks. (2011) Solutions for a cultivated planet. Nature 478(7369):337-342

Henckens M.L.C.M., E.C. vanIerland, P.P.J. Driessen, E. Worrell. 2016. Mineral resources: Geological scarcity, market price trends, and future generations.. Resources Policy 49, pp 102-111.

McDonough W., and M. Braungart, 2002. Cradle to Cradle: Remaking the Way We Make Things. 193 p. North Point Press.

Oenema, O., M. Guo, P. Derikx, K. Poppe, T. Vellinga, P. de Wolf, J. Weijma. 2018 Trends and developments in the global society and their impacts on plant nutrient and fertilizers needs during next decades. (in preparation)

Poppe, K., P. de Wolf, T. Vellinga, O. Oenema, P. Derikx. J. Weijma. 2018 On the sustainability of manure surpluses in metropoles like the Netherlands (in preparation).

Potter P., N. Ramankutty, E.M. Bennet, and S.D. Donner. 2010. Characterizing the spatial patterns of global fertilizer application and manure production. Earth Interactions, 14 pp 1-22

Seyhan D., H.P. Weikard, E. van Ierland. 2012. An economic model of long-term phosphorus extraction and recycling.. Resources, Conservation and Recycling 61, pp $103-108$.

S Steffen, W.; Richardson, K.; Rockström, J.; Cornell, S.E.; Fetzer, I.; Bennett, E.; Biggs, R.; Vries, W. de. (2015). Planetary boundaries: Guiding human development on a changing planet. Science, 347(6223).. p. 736 - 
United Nations, Department of Economic and Social Affairs, Population Division (2017). World Population Prospects: The 2017 Revision, Key Findings and Advance Tables. Working Paper No. ESA/P/WP/248.

Weijma, J., P. Derikx, O. Oenema, K. Poppe, T. Vellinga, K. Verloop, P. de Wolf. 2018. Sanitation in the Circular Economy. (in preparation) 


\author{
Slurry Application Efficiency Evaluation \\ Christian Toft Madsen ${ }^{\mathrm{a}}$, Nick Skou-Nielsen ${ }^{\mathrm{a}}$, Thorkild Qvist Frandsen ${ }^{\mathrm{b}}$, Claus Aage Grøn Sørensen ${ }^{\mathrm{c}}$, \\ Gareth T.C. Edwards ${ }^{a}$ \& Ole Green ${ }^{\text {a,d }}$ \\ a Agro Intelligence Aps, Agro Food Park 13, Skejby, 8200 Aarhus N, Denmark \\ b Danish Technological Institute, Aarhus, Denmark \\ ${ }^{c}$ Aarhus University, Department of Engineering, Denmark, Aarhus, Denmark \\ d Aarhus University, Department of Agroecology, Denmark, Blichers Allé 20, Tjele, Denmark \\ Corresponding author/presenter: Christian Toft Madsen / CTM@agrointelli.com
}

\begin{abstract}
A continuous key objective for any contractor or farmers in an arable farming context, is to improve the economic viability. The purpose of this paper is to present an efficiency study of slurry application and show how to predict slurry operations time and distance on field scale. A $24 \mathrm{~m}^{3}$ (W24) and a $33 \mathrm{~m}^{3}$ (W33) slurry wagon in the southern part of Jutland, Denmark, were chosen for the study and operated throughout the 2017 slurry season (from 1. February to 1. October). Both used a $30 \mathrm{~m}$ trail hoses boom, and W33 also used a $7 \mathrm{~m}$ injector. Data collection was done with an implemented module that logged the GPS position and recorded outputs from the wagons slurry flow sensor. Based on these data the wagon's operational time was divided into 7 activities: Transporting empty, transporting full, applying slurry, transporting in-between applying slurry (partially loaded), collecting slurry, stopped and parked. During the season the W24 trailer applied $38452 \mathrm{~m}^{3}$ slurry onto 1188.6 hectares and trailer W33 applied $32576 \mathrm{~m}^{3}$ slurry onto 980.6 hectares. W33 had a higher need for maintenance during the season which gave W24 the overall highest time efficiency. However, in-field there was no difference between the time efficiency of the wagons. Statistical modelling of the wagons efficiency showed that the total distance and time usage can be predicted with high accuracy using predetermined factors.
\end{abstract}

\title{
1. Introduction
}

In Denmark there are approximately 38 million tons of animal manure, that each year has to be stored, transported and applied to fields as a nutrition supplement for crops (Nielsen et al 2018). This whole management chain of slurry contains a lot of preparation, timing and specialized equipment. Correct timing of the slurry application is essential to get the best nutritional efficiency of the slurry, which means that the main application occurs within a narrow timeframe in the spring. To apply slurry to the field, equipment like e.g. a self-propelled or tractor pulled slurry wagon, is used in combination with a spreading boom like e.g. an injector or drag hoses. The purchase price and seasonal use of this specialized equipment has caused most Danish farmers to use contractors for applying their slurry. In 2017 an estimated 28 million tons of slurry was transported and applied to fields by contractors (DM\&E 2018). By normal practice a continuous key objective for any contractors in arable farming, is to improve the economic viability, which in case creates a market that generally has a high time efficiency and high professionalism.

However, even though slurry application is a highly professional market, a poorly planned operation can still be costly in the form of wasted diesel and man hours. By overall view, the slurry operation can be separated in two phases; in-field and out-field. Increasing time efficiency from either of these, can be done by achieving higher engine power and/or higher machinery working width and size, but also organizing and coordinating working processes is important (Mederle et al 2015). Currently these processes can be optimized using software solutions, e.g. the introduction of field navigation systems can optimize the combination of tramlines in the field (Couto et al 2016, Edwards et al 2017). Experiments using small fields and/or small machines, may not represent the optimization potential for larger machines working full time. So, to which extent is the contractor capable of improving the time use from farming operations like slurry application? The purpose of this paper is to present knowledge about the current operation of slurry, by recording the activity of two slurry wagons over a whole year season, and present areas that could be optimized to improve the overall operation.

\section{Materials and Methods}

Experimental setup

The experiment was conducted with two slurry wagons in the Southern part of Jutland, Denmark. Throughout the slurry application season (1. February to 1. October) 2017, both wagons had a module implemented, comprising of a datalogging device with a built-in modem and GPS. The module was directly connected to the slurry flow meter on the slurry wagon, and automatically transmitted the slurry flow rate and the GPS position to a server. Data signal from the module was paused $15 \mathrm{~min}$ after the tractor powered down and started again with the ignition. The data collected is based on "business as usual", no interference was made during the season to 
interact with the decision on how slurry where applied.

Slurry wagon number one (W24), was a 3 axels Samson wagon with a $24 \mathrm{~m}^{3}$ volume and a $30 \mathrm{~m}$ trail hose boom. The second wagon (W33) had the same setup, but with a volume of $33 \mathrm{~m}^{3}$, and used a $7 \mathrm{~m}$ black soil injector on approx. $50 \%$ of the applied area.

\section{Data analysis and classification of activities}

Identification of the slurry wagons tasks was done by first GIS mapping the logged GPS positions. The GPS positions were then distinguished as inside a field, outside a field, near a storage tank or near a mobile buffer tank. With these four separations, a drive path from the storage tank (or buffer tank) into the field and back again could be identified. Based on this, the wagon's operational time was divided into 7 activities: (transporting empty, transporting full, applying slurry, transporting in-between applying slurry (partially loaded), collecting slurry, stopped and parked). The activities collecting slurry, parked and stopped, were identified when the logged position was stationary in a fixed time. Stopped is identified as a time frame between 30 to 450 seconds, if it is above 450 seconds it is identified as parked, unless the wagon is near a storage tank, in which case it is always identified as collecting slurry. The total time collection slurry is thus calculated as the time where the wagon is stationary at the storage tank. The 450 second limit was chosen based on the mean refilling time multiplied by a factor of 1.5 . The separation of stopped and parked was necessary to identify times where the tractor was turned-on but inactive.

\section{Statistical Model}

A statistical model predicting the total time and distance for the operation based on parameters that can be calculated before the start of the operation was also created using the recorded data. The model chosen for this work is the multivariate linear model. The reason for choosing this model is to achieve a single equation for both the total distance and time where the relation between the parameters is clear.

To test which activities are significant a t-test was used to reduce the model and decide which activities where relevant. This also gives an indication of how best to approach optimizing the operations by changing the operational parameters.

\section{Results}

Wagons load and distribution

W24 transported 1726 loads of slurry which in total accounted for $38452 \mathrm{~m}^{3}$ with trail hoses. W33 transported 1093 loads of slurry, which in total accounted for $15127 \mathrm{~m}^{3}$ slurry with injection and $17449 \mathrm{~m}^{3}$ with trail hoses. In total, both wagons applied $+/-1.5 \mathrm{~m}^{3}$ from a full load $65 \%$ of the time and $+/-3 \mathrm{~m}^{3}$ from a full tank occurred $83 \%$ of the time. Due to measurements inaccuracies of the slurry flow sensor, recordings of load levels above tank size was expected. $6 \%$ and $7 \%$ of the time for W24 and W33, respectively, a load below $75 \%$ was applied. Ending a slurry application and continuing on a new field occurred 48 and 230 times of accordingly W24 and W33.

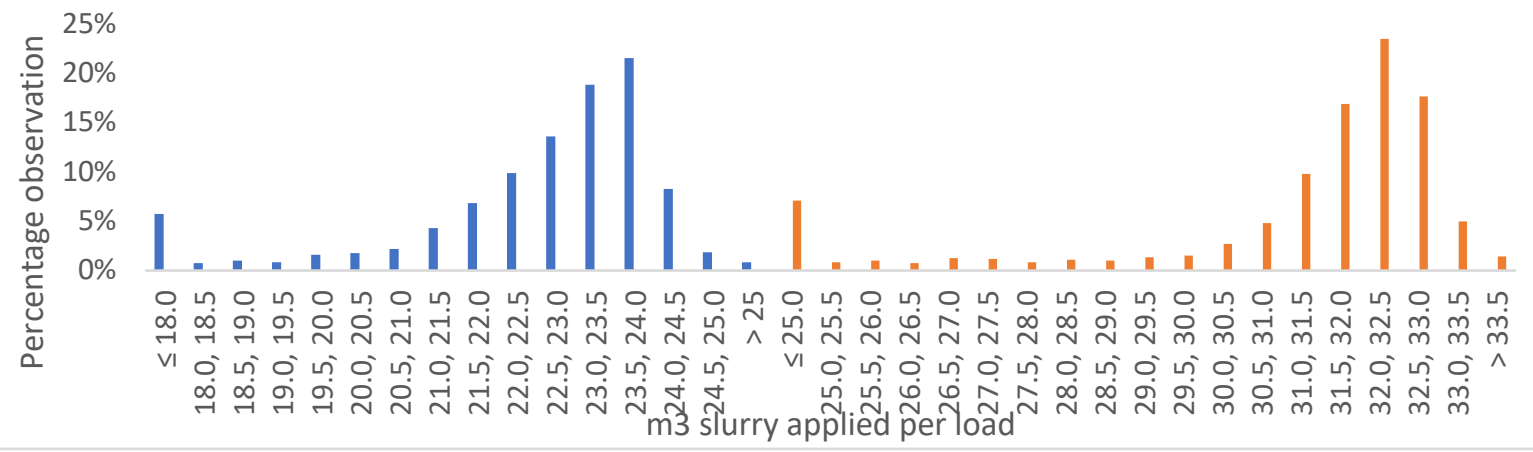

Figure 1. Volume $\left(\mathrm{m}^{3}\right)$ of applied slurry per load, illustrated in percentage observation from $3 / 4$ load to highest recording. The blue bar is the W24 wagon, and the orange is the W33 wagon.

Slurry amount and collection time

The two wagons datasets show that the general approach for applying slurry is to always transport and apply a full wagon load to the field (figure 1). The main exception from this is the first and/or the last application in the field, where a load is often less than $75 \%$. Applications with a load less than full on the first application, is normally due to leftovers from another finished field. This is supported by the wagons refilling time which is 
fairly constant, meaning that the driver often takes a specific amount of time to refill the wagon (figure 2). Time usage between $150-240$ second and $210-300$ seconds cover $80 \%$ and $70 \%$ of the total collecting times for W24 and W33, respectively (figure 2).

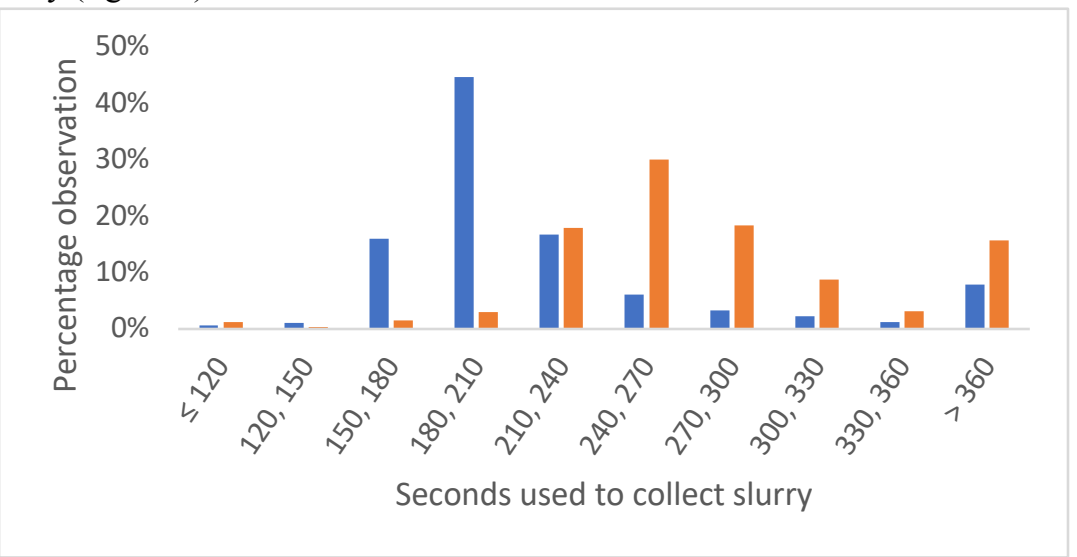

Figure 2. Seconds used to collect slurry per load, illustrated in 30 seconds intervals from 120 to 360 seconds. The blue bar is the W24, and the orange is the W33.

Fields

In the 2017 slurry season (242 days) W24 applied slurry on 71 fields with trail hoses and W33 applied slurry on 68 fields with trail hoses and 49 field with black soil injection. All fields where of varying sizes and shapes, and at no point did the two wagons apply slurry on the same field. W24 applied slurry on more fields above 10 hectares compared to W33 which more frequently applied slurry on fields sizes 5 to 10 hectares. In total, the W24 applied slurry on 1188.6 hectares and W33 applied slurry on 980.6 hectares.

Table 1. Field size and distribution between the wagons.

\begin{tabular}{lllll}
\hline Field size & W24 Trail hoses & W24 Buffer tank & W33 trail hoses & W33 injection \\
\hline$<5$ ha & $18(31 \%)$ & $2(15 \%)$ & $22(32 \%)$ & $18(37 \%)$ \\
5ha-10ha & $12(21 \%)$ & $3(23 \%)$ & $30(44 \%)$ & $20(41 \%)$ \\
10ha-20ha & $10(17 \%)$ & $4(31 \%)$ & $11(16 \%)$ & $9(18 \%)$ \\
$>20$ ha & $18(31 \%)$ & $4(31 \%)$ & $5(7 \%)$ & $2(4 \%)$ \\
Total ha & $959.8 \mathrm{ha}$ & $228.8 \mathrm{ha}$ & $577.4 \mathrm{ha}$ & $403.2 \mathrm{ha}$ \\
\hline
\end{tabular}

Out-field activities

The dataset out-field showed the time use on all of the generated activities, except for applying slurry. The wagons were only active $9 \%$ and $7 \%$ of the season for W24 and W33, respectively, and were thus parked the rest of the time. Stops appeared frequently when transporting. The percentage distribution between the transport and slurry collecting activity out-field are shown in table 2. On average the W33 uses an equal amount of time transporting slurry and collecting slurry, whereas W24, with the exception of when using buffer tanks, used more time on transporting slurry than collecting (table 2). The distance used for transporting slurry empty and full showed a similarity between each field (not shown). However, both wagons had an overall empty transport distance that was higher than when full. This is simply due to the fact that the wagons needed to get to the storage tank from the shelter and transport empty back to the shelter again. Trips like these were in some cases more than $30 \mathrm{~km}$ from the shelter.

W33 had, in general, shorter paths from the storage tank to the field, and when transporting over longer distances the wagon transported through other fields. When W24 had to travel further than $3.5 \mathrm{~km}$ from the storage tank to the field (with the exception of three fields), trucks and buffer tanks where used to transport slurry to the field. 
Table 2. The wagons percentage time distribution separated into out- and in-field activities with standard deviation.

\begin{tabular}{lcccc}
\hline Activity & W24 Trail hoses & W24 Buffer tank & W33 Trail hoses & W33 Injection \\
\hline & $35.0 \% \pm 6.8$ & Out-field activities: & \\
Transport full & $23.7 \% \pm 10.2$ & $21.9 \% \pm 10.4$ & $18.5 \% \pm 12.5$ \\
Transport empty & $31.6 \% \pm 8.7$ & $15.9 \% \pm 10.5$ & $28.2 \% \pm 9.4$ & $26.2 \% \pm 12.2$ \\
Collecting slurry & $33.4 \% \pm 12.1$ & $60.4 \% \pm 17.7$ & $49.8 \% \pm 15.7$ & $55.3 \% \pm 20.5$ \\
& & In-field activities: & & \\
Transport full & $22.2 \% \pm 5.7$ & $24.5 \% \pm 4.8$ & $16.1 \% \pm 5.7$ & $9.3 \% \pm 4.6$ \\
Transport empty & $17.0 \% \pm 7.8$ & $21.8 \% \pm 6.9$ & $15.5 \% \pm 7.1$ & $9.9 \% \pm 5.2$ \\
Transport in-between & $9.6 \% \pm 5.5$ & $6.5 \% \pm 4.8$ & $12.0 \% \pm 6.1$ & $13.9 \% \pm 3.9$ \\
$\quad$ applying slurry & & & \\
Applying slurry & $41.9 \% \pm 7.3$ & $36.6 \% \pm 6.2$ & $47.1 \% \pm 6.5$ & $65.5 \% \pm 7.3$ \\
Stopped & $9.2 \% \pm 5.4$ & $10.6 \% \pm 5.1$ & $9.4 \% \pm 4.8$ & $1.4 \% \pm 1.9$ \\
\hline
\end{tabular}

In-field activities

W33 spent more time applying slurry per load in the field compared to W24. The longest time usage for applying slurry was done by the $7 \mathrm{~m}$ injector, that despite driving faster compared to the $30 \mathrm{~m}$ trail hose boom (table 3), spent on average $65.5 \%$ of the total in-field time applying slurry (table 2). Partially loaded transports also occurred more frequently with the injector, that had nearly one split per load, whereas the W24 overall had one split per every second load. Mean time spent on stops with the injector was more unpredictable than with trail hoses, and some fields had no stops. Stops with trail hoses always occurred before and after applying slurry. Parking in-field occurred as a few times during the season, the mean parking was approx. 15 min but with great inconsistency. Unlike the similarity between the out-field transporting time between empty and full, in-field transport had a great variability between each field.

Mean speed for the wagons seems near constant for each field, thus the variation become larger when comparing all fields (table 3). W33 had a generally lower mean speed than W24, this has possibly multiple reasons, but one is partly because when fully loaded, the W33 is too heavy to legally transport on public roads in Denmark, so there is a lot of transport though neighbouring fields and private dirt roads to the targeted fields.

Table 3. Mean values for the slurry application with standard deviation.

\begin{tabular}{|c|c|c|c|c|c|}
\hline Operation status & Unit & $\begin{array}{c}\text { W24 } \\
\text { Trail hoses } \\
\end{array}$ & $\begin{array}{c}\text { W24 } \\
\text { Buffer tank }\end{array}$ & $\begin{array}{c}\text { W33 } \\
\text { Trail hoses }\end{array}$ & $\begin{array}{c}\text { W33 } \\
\text { Injection }\end{array}$ \\
\hline \multicolumn{6}{|l|}{ Mean transport speed out-field } \\
\hline full & $\mathrm{Km} /$ hour & $23.9 \pm 4.8$ & $24.7 \pm 4.3$ & $17.2 \pm 7.5$ & $15.7 \pm 7.0$ \\
\hline empty & Km/hour & $23.6 \pm 4.9$ & $24.7 \pm 4.1$ & $17.1 \pm 7.3$ & $16.0 \pm 7.0$ \\
\hline \multicolumn{6}{|l|}{ Mean transport speed in-field } \\
\hline full & $\mathrm{Km} /$ hour & $9.9 \pm 3.1$ & $10.7 \pm 2.2$ & $9.1 \pm 2.5$ & $9.6 \pm 2.2$ \\
\hline empty & Km/hour & $14.3 \pm 4.9$ & $15.6 \pm 2.8$ & $12.5 \pm 3.8$ & $12.2 \pm 2.4$ \\
\hline partially & $\mathrm{Km} /$ hour & $6.7 \pm 1.6$ & $6.7 \pm 1.6$ & $7.2 \pm 1.9$ & $7.5 \pm 1.1$ \\
\hline Mean application speed & Km/hour & $6.7 \pm 1.0$ & $6.7 \pm 0.9$ & $8.5 \pm 1.2$ & $8.1 \pm 0.9$ \\
\hline Mean application rate & $\mathrm{m}^{3} / \mathrm{min}$ & $8.1 \pm 0.7$ & $8.1 \pm 1.0$ & $7.4 \pm 0.7$ & $2.7 \pm 0.5$ \\
\hline Mean splits per application & Splits/load & 0.5 & 0.5 & 0.7 & 0.9 \\
\hline Mean stop time in field & $\mathrm{Sec}$ & $67 \pm 39$ & $66 \pm 23$ & $66 \pm 37$ & $104 \pm 77$ \\
\hline Mean parking in field & Sec & $\begin{array}{l}1101 \pm \\
548\end{array}$ & $805 \pm--$ & $3368 \pm 3178$ & $857 \pm 432$ \\
\hline Total time use & Hour & 406 & 100 & 196 & 197 \\
\hline Total distance & $\mathrm{Km}$ & 5434 & 1042 & 1732 & 1692 \\
\hline
\end{tabular}

Statistic calculation between the different parameters

A multivariate linear model has been estimated for both the total time and total distance spent in each recorded field. The field size and the 7 specified activities separated into in-field and out-field was used as input parameters for the model. The combination of the activities, which could predict the best accuracy for distance and time are shown below.

The returned equation from the model, for estimating the total distance for each field ended up, using only the amount of applications, distance for applying slurry and distance to the storage tank as inputs. The R-squared was 0.99 , and there was therefore a good correlation between predicted and measured. And with a mean square error of $5.78 \mathrm{~km}$, the mean total distance for each field could be calculated with an $95 \%$ inaccuracy of $11.33 \mathrm{~km}$. 
Table 4. Results from the multivariate linear model to calculate total distance in the field.

\begin{tabular}{lc}
\hline Total distance for each field & \\
\hline Parameter & Estimate \\
Intercept & -0.2697 \\
Amount of applications & 0.5406 \\
Working distance $[\mathrm{km}]$ & 0.8816 \\
Distance to farm [km] & -0.6806 \\
Amount of applications x Distance to farm & 2.0356 \\
{$[\mathrm{~km}]$} & \\
(Amount of applications) $^{\wedge} 2$ & -0.0030 \\
${\text { (Working distance }[\mathrm{km}])^{\wedge} 2}$ & 0.0054 \\
R-squared & 0.99 \\
Mean Squared Error & 5.7800 \\
\hline
\end{tabular}

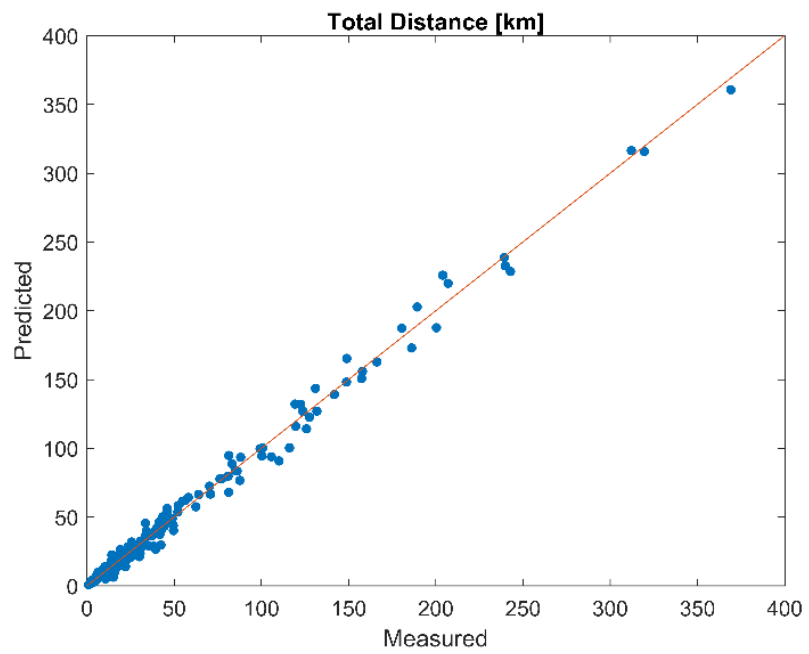

Figure 3. Predicted total distance and measured distance plotted against each other.

The returned equation from the model, for estimating the total time for each field ended up, using 7 parameters as inputs to calculate with the highest prediction. The R-squared was 0.99 , and there was therefore a good correlation between predicted and measured. And with a mean square error of $29.2 \mathrm{~min}$, the mean total time for each field could be calculated with an $95 \%$ inaccuracy of $57.23 \mathrm{~min}$.

Table 5. Results from the multivariate linear model to calculate total time in the field.

\begin{tabular}{lc}
\hline Total time for each field & \\
\hline Parameter & Estimate \\
Intercept & 21.8660 \\
Field size [ha] & -2.8169 \\
Amount of applications & 1.9743 \\
Total amount of slurry [m3] & 0.3920 \\
Total operation time [min] & 0.5705 \\
Distance to farm [km] & -7.6310 \\
Field size x Total amount of slurry [m3] & -0.0096 \\
Amount of applications x Total operation time [min] & 0.0120 \\
Amount of applications x Distance to farm [km] & 3.1661 \\
Total operation time min [min] x Distance to farm [km] & 0.3385 \\
(Field size [ha])^2 & 0.2643 \\
& \\
R-squared & 0.99 \\
Mean Squared Error & 29.2000 \\
\hline
\end{tabular}




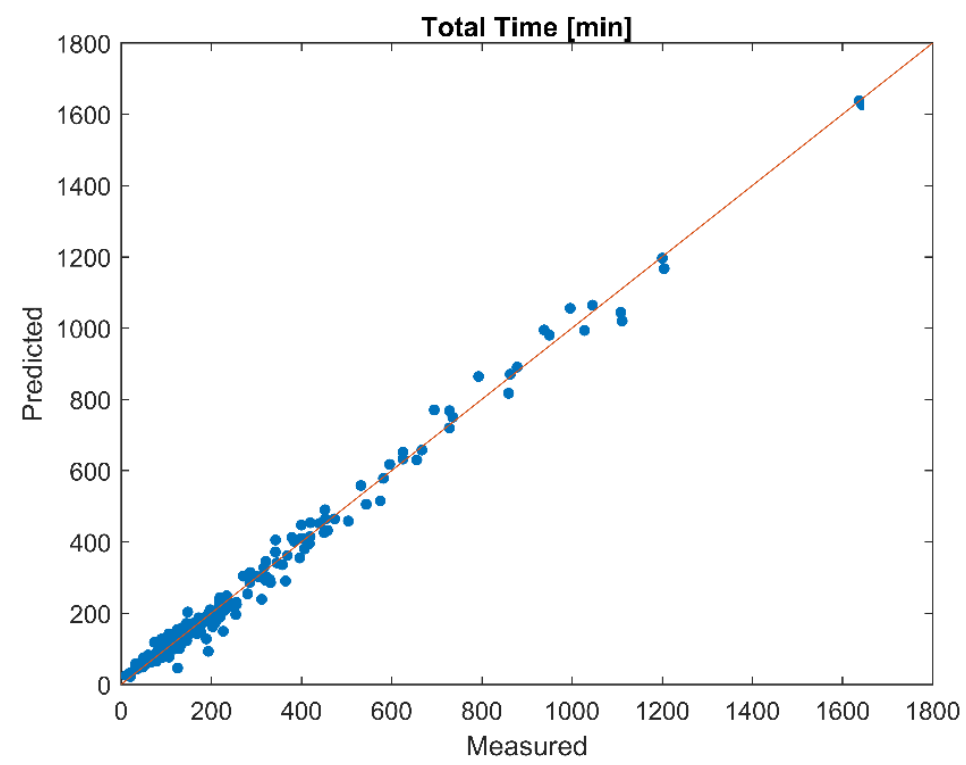

Figure 4. Predicted total time and measured time plotted against each other.

\section{Discussion}

Separating the wagons route into different activities and using these as input for a multivariable model, showed a high predictability of the distance- and time- usage when summarizing on a field level. This is presumably due to some generally predictable distance/time usages during the operation going from the storage tank to the field and back again. To extrapolate on this, assuming that we know the same as a slurry wagon driver when preparing for a job, there are 2 of the 7 activities that are highly predictable whereas 2 are unpredictable and 3 are partly predictable.

\section{Predictable activities}

From table 2 it is seen that applying slurry in the field took almost half of the total in-field time, with only little deviance $\pm 7 \%$. Calculating the time that the wagon needs to apply slurry in the field could therefore give a good indication of the total in-field time usage. The most influential parameters for this calculation is expected to be the wagon size and mean application rate.

A necessary parameter needed for calculating the total time and distance needed is the transportation. The transportation accounts for 35-90\% of the entire distance for each field (data not shown), $20-80 \%$ of the entire time out-field, and $10-40 \%$ of the time in-field (table 2). The lowest percentage is when the field is located close to the storage or a buffer tank is used. With this big variation this is a nonexcludable parameter when calculating the total time and distance of the wagon. Distance needed to get from the storage to the field is easy to estimate, estimating time is however a bit more difficult since it depends on the speed of the wagon. As seen in table 2, speed in-side the field is somewhat predictable, speed outside the field is however a bit more unpredictable. The time uncertainty for out-field transport is small, but not indifferent. An estimation of the uncertainty can be calculated for W24, the time difference between an average $20 \mathrm{~km} \mathrm{hr}^{-1}$. and $30 \mathrm{~km} \mathrm{hr}^{-1}$. over a distance of $1 \mathrm{~km}$ to $3.5 \mathrm{~km}$, gives a time uncertainty of $1 \mathrm{~min}$ to $3.5 \mathrm{~min}$ per trip.

\section{Partly-predictable activities}

Refilling of the two wagons showed a nominal time usage that makes a general pattern. As illustrated in (Figure 1), both wagons always made sure to fill up an entire load and apply as close to an entire load as possible. The general time usage for collecting slurry (figure 2) also supports this. The time that the wagon solely needs to be refilled, can be estimated by using the slurry pump capacity. The pump operates at a maximum speed near 10 $\mathrm{m} 3 / \mathrm{min}$, so the time that the driver needs to completely refill the wagon is approx. 144 and 198 seconds accordingly for W24 and W33. Compared to this the wagons are expected to use approx. 60 seconds on positioning near the storage tank, operating the crane arm and set the wagon in motion again. The collection time is unfortunately not possible to analyse any closer. There is not a clear correlation between the collected slurry and the time that the wagons were stationary at the storage tank. The wagons mean collection time 195 and 255 seconds \pm 45 seconds could predict $80 \%$ and $70 \%$ of the cases for accordingly W24 and W33. Some of the collection times took exceedingly long time, for example, W33 spent 7.7 hours $(8 \%$ of total time spent collecting 
slurry) on 4 loadings (data not shown). The irregular collection time is expected to be caused by the efficiency of the driver and the service requirement for the machine, and that these are significant parameters for the total time spent at the storage tank.

Another partly-predictable activity is the stops in-field with trail hoses. When the wagons use trail hoses, the wagon needs to stop before and after applying slurry to setup the boom. Setting of the $30 \mathrm{~m}$ boom, took approx. 45 seconds, however the amount of settings per load seems irregular, and is assumedly due to repositioning requiring a new setting or other stops not related to the boom.

\section{Un-predictable activities}

Periods above 450 seconds where the wagon was parked in the field occurred seldom and irregularly. The parking is expected to be due to maintenance or getting stuck in the field.

Distance and time spent transporting partially loaded was in all fields below $10 \%$ of total in-field time usage and less than $5 \%$ of total time use no relationship could be found.

\section{Using predictable activities to estimate the total time usage}

The two predictable parameters can be used to roughly calculate the total time needed to finish a field. To give a more accurate prediction the partly-predictable parameters needs to be included as cofactors in the statistical model. The reason for the inclusion of the parameters can't be fully explained for all cases, the reason for them being included is that it may give a slightly better prediction than otherwise. In such a case, there may be hidden parameters that have a correlation with other parameters. E.g. for calculating the total distance, wagon size and working width would be expected to be non-excludable parameters. However, they do not appear in the model equation (table 4) but are most likely hidden in the driven distance in the field and amount of applications.

The multivariate model describes the data, but it also shows that there could be some factors missing from the parameters. This was observed during the validation of the model, but it was decided that multivariate linear model describes the data well enough to be used. This is reasoned from measured vs. the predicted values and the high R-Squared.

The problem of the model is seen as there are more extreme outliers than what you would expect from a normal distribution, which means overall that there is big spread from where the model is highly accurate to where the model is less accurate. To improve the model additional features could be added to the model, explaining some of the extreme behaviours. If the models had been perfectly normal distributed then we would have expected that for the total distance the estimate would have had a precision of plus/minus $11.33 \mathrm{~km}$ in $95 \%$ of the cases and for the time plus/minus $57.23 \mathrm{~min}$ for $95 \%$ of the time.

Since the data for these models are based on observed data, it cannot be determined that parameters for the model does not indicate causation but rather correlation with the true parameters which would mean that the model cannot be used for optimizing individual operation parameters.

\section{Perspectivation}

Further research on slurry application data is needed to get a better model fit. Additionally, adding field boarder and obstacles shape parameters could potentially improve the model fit. One way to approach the problem is investigate the slurry application which deviates the most from the estimated model, by doing this, common factors might be found for further analysis. Also, a better characterization of time usages when collecting slurry, would be useful to separate ineffective time from effective, this would lower the data variation which would lead to stronger model estimation.

Use of precision GPS instead on a standard GPS would give more precise application tracks and could give a better understanding of the slurry distribution accuracy, showing potential overlaps and gaps in the field.

To improve model prediction, further research is needed in the investigation of how govern parameters have cause and correlation. This could be done by having a larger data set and separating it into a training and test set, and then testing the predictive ability of the model.

\section{Conclusions}

During the 2017 season, recording of two slurry wagons activity in totally 188 fields where successfully done. Analysis of the wagons showed a generally predictable activity on a hole field scale.

Statistical modelling of the wagons efficiency showed that the total distance and time usage can be predicted with high accuracy using predetermined factors. 


\section{References}

Journal articles:

Nielsen, O-K., Plejdrup, M.S., Winther, M., Mikkelsen, M.H., Nielsen, M., Gyldenkærne, S., Fauser, P., Albrektsen, R., Hjelgaard, K.H., Bruun, H.G. \& Thomsen, M. 2018. Annual Danish Informative Inventory Report to UNECE. Emission inventories from the base year of the protocols to year 2016. Aarhus University, DCE Danish Centre for Environment and Energy, 495 pp. Scientific Report from DCE - Danish Centre for Environment and Energy No. $267 \mathrm{http}: / / \mathrm{dce} 2 . \mathrm{au} . \mathrm{dk} / \mathrm{pub} / \mathrm{SR} 267 . \mathrm{pdf}$

Mederle, M., Bernhardt, Heinz. 2017. Influences and decision criteria on -infield-logistics in German agriculture farms. Chemical engineering transactions, Vol 58.

Couto L.D., Tran-Jørgensen P.W.V., Edwards G.T.C. (2018) Model-Based Development of a Multi-algorithm Harvest Planning System. In: Obaidat M., Ören T., Merkuryev Y. (eds) Simulation and Modeling Methodologies, Technologies and Applications. SIMULTECH 2016. Advances in Intelligent Systems and Computing, vol 676. Springer, Cham

Gareth T.C. Edwards, Jørgen Hinge, Nick Skou-Nielsen, Andrés Villa-Henriksen, Claus Aage Grøn Sørensen, Ole Green, Route planning evaluation of a prototype optimised infield route planner for neutral material flow agricultural operations, Biosystems Engineering, Volume 153, 2017,

DM\&E 2018. [Personal communication]. 


\title{
Fertigation Equipment and Control Techniques: A Review
}

\author{
Stefan Vidal a,", Tatiana Pinho ${ }^{\text {a }}$, José Boaventura Cunha ${ }^{\text {a,b }}$, Josenalde Oliveira ${ }^{\text {a,c }}$, Paulo Moura \\ Oliveira ${ }^{\mathbf{a}, \mathrm{b}}$ \\ ${ }^{\text {a }}$ INESC TEC -INESC Technology and Science, Campus da FEUP, 4200-465 Porto, Portugal \\ b Department of Engineering, School of Science and Technology, University of Trás-os-Montes and Alto Douro, \\ UTAD, 5001-801 Vila Real, Portugal \\ ${ }^{c}$ Agricultural School of Jundiaí, Federal University of Rio Grande do Norte, UFRN, Brazil \\ * Corresponding author. Email: stefan.c.vidal@inesctec.pt
}

\begin{abstract}
Due to the lack of fertigation control reviews available in literature, this paper presents an overview on these systems used both in scientific research and commercial equipment. The increase in population and consequently on food consumption requires the use of water and nutrients with greater efficiency as well as the reduction of soil and water pollution. The excessive use of fertilizers and plant protection products is a major contributor to excessive standard values concentrations in about one-third of groundwater bodies in Europe. Traditional methods usually involve applying a standard nutrient solution without any concern for environmental aspects. One way to protect the environment is through the irrigation water treatment and/or recirculation. Fertigation techniques have potential to provide optimum nutrients concentrations according to the crops needs, enabling a more efficient use of both water and fertilizers. Plants need several macronutrients and micronutrients, which are not easy to monitor. Typically, the control of Nitrogen, Phosphorus and Potassium (NPK), which are the main nutrients, is recommended. The vast majority of fertigation systems are based on Electrical Conductivity (EC) and $\mathrm{pH}$ sensors to allow nutrient solution dosing. However, despite these variables being related with nutrient concentrations, they do not provide precise measurements of NPK contents. Moreover, current available technologies for NPK measurement have several drawbacks, such as, frequent need of maintenance and calibration, large cost, unfeasible sampling time for feedback control, among others. In this context, the European project Agrinupes comes up with the goals of developing new optical-based NPK sensors to solve these drawbacks. These sensors will be integrated in an easy-to-use, robust and fault-tolerant fertigation controller, and evaluated under several demonstration cases. Besides presenting a fertigation control systems review, this paper identifies current challenges and future trends within this context.
\end{abstract}

Keywords: Fertigation, Control, Nutrients

\section{Introduction}

In the last 30 years the increase in population has forced the expanse of agricultural production, as for example the production of wheat, corn and rice that represent about two-thirds of the human diet. This increment in production is achieved by increasing the yield per unit of time and land [1]. Increased production implies a greater consumption of energy, water and fertilizers, which in turn causes a decrease in biodiversity and in ecosystems. The use of large quantities of fertilizers causes loss of quality of land for long-term production, due to the increase in environmental pollution caused by the chemicals used [2]. To meet the human population and ecosystems immediate needs, it is necessary to have a better management of the resources used to achieve high production without excessively damaging the environment. The concept of precision agriculture arises with the intention of allowing a better management of the resources used based on the specific knowledge of the place where it is being applied (environmental conditions, soil state, etc.). This concept allows to reduce the environmental pollution and to increase the yields by applying only necessary quantities of fertilizers and pesticides and when they are necessary [3].

Fertigation is a technique in which fertilizers are applied to the crop through irrigation water. This technique allows to increase the production and to minimize the environmental pollution through the application of less amount fertilizers at each irrigation performed at a defined frequently. In a fertigation system the irrigation frequency, the quantity and concentration of the applied nutrient solution are usually controlled using measurements provided by EC and $\mathrm{pH}$ sensors [4]. The values are processed by a microprocessor which controls the valves that inject fertilizers into the nutrient solution. There are two ways to apply fertilizers to the irrigation system: the nutrient solution can be prepared in a mixing tank and then injected into the irrigation system; another way is to make the injection of fertilizers directly into the irrigation system using the venturi principle [5]. One way to make the system more sustainable is by recirculating drainage water. This involves the use of another control point where the decision is made if drainage water is good enough to be recirculated or should be discarded.

The nutrient solution must be carefully prepared taking into account the specific needs regarding the plant type (fruit, flower, leafy vegetables) and development stage. Plants require different macronutrients, such as nitrogen 
$(\mathrm{N})$, phosphorus $(\mathrm{P})$, potassium $(\mathrm{K})$, calcium $(\mathrm{Ca})$, magnesium $(\mathrm{Mg})$ and sodium $(\mathrm{Na})$ and different micronutrients such as iron (Fe), manganese $(\mathrm{Mn})$, boron $(\mathrm{B})$, copper $(\mathrm{Cu})$, zinc $(\mathrm{Zn})$ and molybdenum (Mo) [6]. It is extremely complex to control all the nutrients. Therefore, it is necessary to select the most important nutrients for the development of the plant. In several studies, N, P and K (NPK) are considered the main macronutrients $[4,7,8$, $9,10]$. NPK elements usually do not appear in their pure form in fertilizers. $\mathrm{N}$ for instance appears as, urea- $\mathrm{N}$

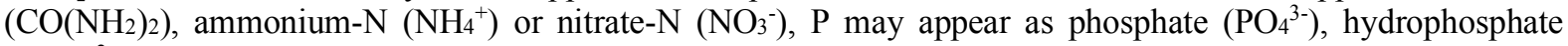
$\left(\mathrm{HPO}_{4}{ }^{2-}\right)$ or dihydrogen phosphate $\left(\mathrm{H}_{2} \mathrm{PO}_{4}^{-}\right)$and $\mathrm{K}$ appears as potash $\left(\mathrm{K}^{+}\right)$[4]. In [11] a study on the efficiency of nutrient management based on $\mathrm{NO}_{3}{ }^{-}$concentration for the production is reported. In [12] different fertigation rates of $\mathrm{P}$ were tested to analyse the influence of the same in the production of pumpkins. In [13] a study is reported about the effects of $\mathrm{K}$ fertigation on tomato productivity and quality. In order to control some of these individual chemical elements, electrochemical sensors are usually used such as: Ion Selective Electrode (ISE), Ion Selective Field Effect Transistor (ISFET) and Chemical Field Effect Transistor (CHEMFET). Electrochemical sensors consist of a sensitive membrane to a particular type of ion and a transducer that converts the reaction into an electrical signal. In [14] an overview is made on electrochemical sensors and the opportunities and challenges for their use discussed. Recently fiber optic sensor was developed and tested to detect NPK nutrients in soil [15]. These sensors are based on the colorimetric principle, where different colors are emitted for the solution and the light is reflected in a greater or lesser quantity depending on the solution coefficient of absorption. The reflective light is absorbed and converted into an electrical signal.

This paper reports a scientific review on fertigation control techniques developed in other projects and used in commercial equipment. The main goal this work is to have a knowledge of the state-of-art in this field and propose possible improvements within the Agrinupes project scope. In the next section, the recent scientific developments in the fertigation control will be addressed and the different control types will be compared. Also, will be presented fertigation control systems available in the market and theire main characteristics are presented. Section 3 addresses a new paradigm and proposal for a new fertigation controller. Finally, section 4 presents the main conclusions and perspectives for the future.

\section{Fertigation Control}

Scientific research efforts in the agriculture area has undergone a significant increase in recent years. When searching in the IEEE Xplore digital library [16] with the keyword Agriculture this returns 15,053 results, among publications in conferences, newspapers, magazines, books and early access papers. Of the presented results, 10,172 are referring to publications between January 2010 and April 2018. Using the same keyword in the same period in ScienceDirect [17] returns 296,948 results and in B-ON [18] 561,041. If the search is done with other keywords in the agriculture area, such as Fertigation and NPK, it is verified that most of the results refer to the last decade, for the keywords given as examples more than half of the results are from that period. This increased interest in the agriculture area in recent years is directly related to the population increase and its needs, which requires an increase in global production and efficiency improvements [19]. In this section are presented the relevant control systems used in fertigation that were developed in research projects and/or that are commercially available.

In a fertigation system a set of components is usually controlled to obtain a greater precision of the amount of nutrients applied in the nutrient solution according to the plants needs. The controller must activate valves that allow the arrival of certain nutrients directly to the irrigation system or to a mixing tank. This must be performed taking into account the reference values of the concentration of each nutrient, $\mathrm{pH}$ and $\mathrm{EC}$ in nutrient solution and drainage water. To control the $\mathrm{pH}$ value, an acid is usually used whose quantity is controlled in the same way as the nutrients. Other components that are normally controlled and monitored are [8]: tanks levels; water temperature; leaching rates; pump controls; irrigation zone valve; line purging; recirculation management. In Figure 1 is showed the layout of a fertigation system with $\mathrm{K}^{+}$concentration feedback used in the article [20]. 


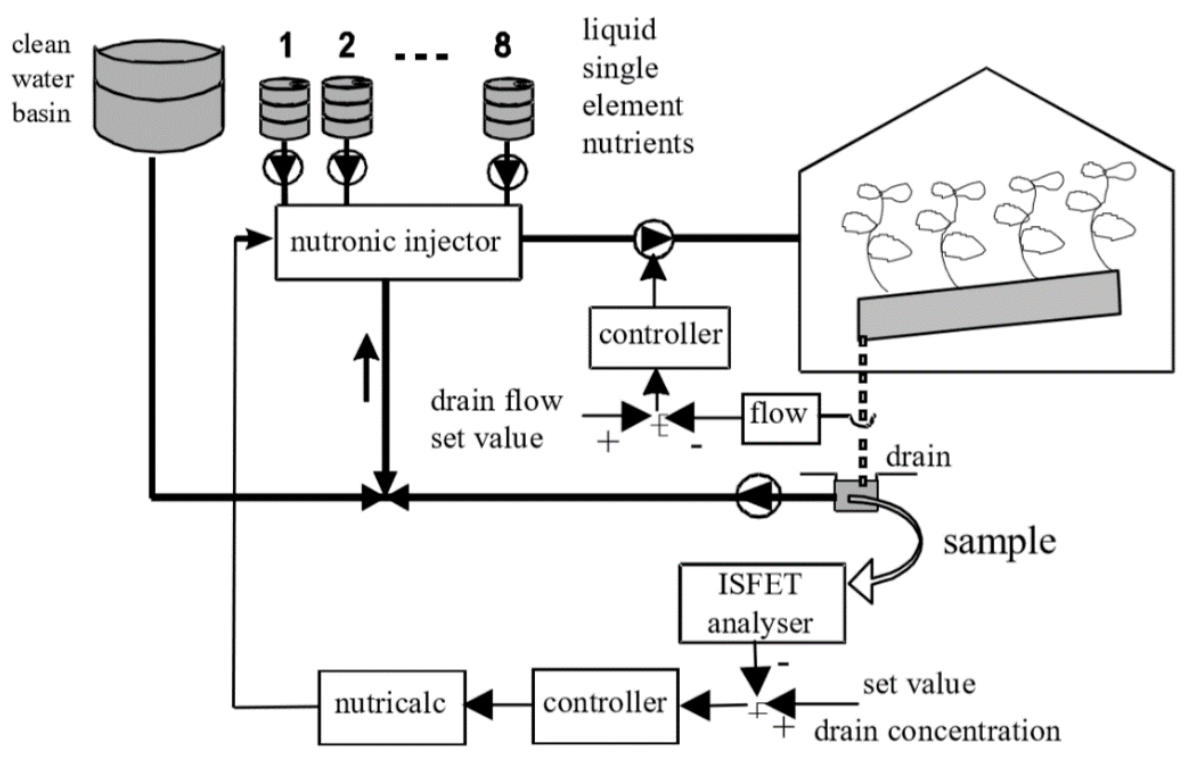

Figure 4. Fertigation system layout [20].

Since the controller is the key to obtain a precise and effective fertigation system from the perspective of plant development, this has motivated the development of different types of control in several projects in the last three decades. In [21] an intelligent hierarchical control system was proposed to improve the growth of long-term hydroponics plants. This system consists of two control techniques, an expert system that is used to determine the reference point for nutrient concentration throughout the plant growth process and a hybrid system that is used to determine the reference point for nutrient concentration only during the initial growth phase (seed), which are based on genetic algorithms and neural networks. In the hybrid system initially, a neural network is used to determine the nutrient concentrations and later the optimum value is obtained through the simulation of the identified model using the genetic algorithm. This control was applied for growing tomatoes and a $10-15 \%$ increase in total leaf length and stem diameter was obtained in the early plant development stage with clever hierarchical control over conventional methods. In [22] an approach is taken on the development of a controller for the supply of water and nutrients, recirculating and treating them. In this case only the injection of potassium $\left(\mathrm{K}^{+}\right)$is controlled for the cultivation of tomatoes that are germinated in rock wool. The controller design was based on robust control theory, which results in a controller with robust performance and stability, which means that it remains stable within a predefined range even with the occurrence of uncertainties (changes in temperature, plant absorption, evaporation, etc.). For the development of the robust controller is used a loop shaping technique, where Bode diagrams allow to define the controller parameters. The performance of the algorithm was tested in a simulation process where it was verified that the controller is capable of suppressing perturbations, keeping the potassium concentration within the defined range. In [23] it is presented a result comparison obtained whith two different controllers over the performance of tomato plants cultivation. In this work a fertigation-control tray method was compared with a new Proportional-Integral-Derivative (PID) controller tuned to maintain the same leaching fraction constant. With the PID controller it was achieved a lower electrical conductivity of drainage and a higher level of nutrient solution absorption by the plants. The amount of production and fruit quality did not improve, however, with the PID controller, larger fruits were obtained. In [24] a control system based on the Penman-Monteith model and the electrical conductivity for the estimation of evapotranspiration is presented. The proposed controller allows the frequency of fertigation cycles adjustment, taking into account the plant need, thus allowing to minimize environmental pollution and reduce the fertilizers and water use. In [25] an ARM microprocessor (Stm32F103ZET6) is used to perform various tasks related to the fertigation control system and a self-adaptive control algorithm is proposed which is combined with the incremental PID control and a methodology presenting a single neuron.

In some projects the control system developed is based on microcontrollers such as in [26] where the BasicStamp 2 microcontroller is used to control the entire electronic component to perform the mixing of the nutrient solution based on the value of the electrical conductivity introduced by the user. In [27] a microcontroller based on PIC16f877A is used to control the solenoid valve that allows to administer the fertilizers in the mixing tank according to the level of $\mathrm{pH}$ and $\mathrm{EC}$, whose values are obtained through two sensors placed in the nutrient solution and on the ground. Due to the lack of a remote control interface it is necessary to enter control data manually.

With the growth of the Internet of Things (IoT) technology in recent years, some projects have emerged [28, $29]$ to monitor and control in real time a fertigation system and enabling the user interfere with the system in 
unexpected situations based on the data acquired. Some articles [30,31] present applications that allow to calculate the best combination of fertilizers and the amount of irrigation to be supplied to different types of crops taking into account the different types of fertilizers and their costs. These applications enable the user to better establish the reference values in the fertigation control system for the nutrient solution.

In order to maximize the yield and to optimize the amount of fertilizers supplied to plants it is not enough to control the concentration in the nutrient solution. Also, it is not enough to control concentration in the nutrient solution, it is necessary to have a strict control of the times and frequency of irrigation and to ensure that the nutrient solution is distributed from one uniform shape. There are several articles $[32,33,34,35]$ where irrigation control systems are proposed with the aim of achieving greater efficiency in irrigation management for cultivation. In $[36,37,38]$ some software's are presented that allow to evaluate and to manage control strategies with the objective of optimizing the irrigation.

\section{Commercial Equipment}

In agriculture, the use of fertigation control systems allows better resource management, which in turn results in an improvement in economic benefits. There are available several commercial equipment for irrigation and fertigation control, with different characteristics and adapted for different application environments. In this section the most relevant characteristics of some available commercial equipment for fertigation control will be addressed (see Table 1).

Table 1. Fertigation Controllers.

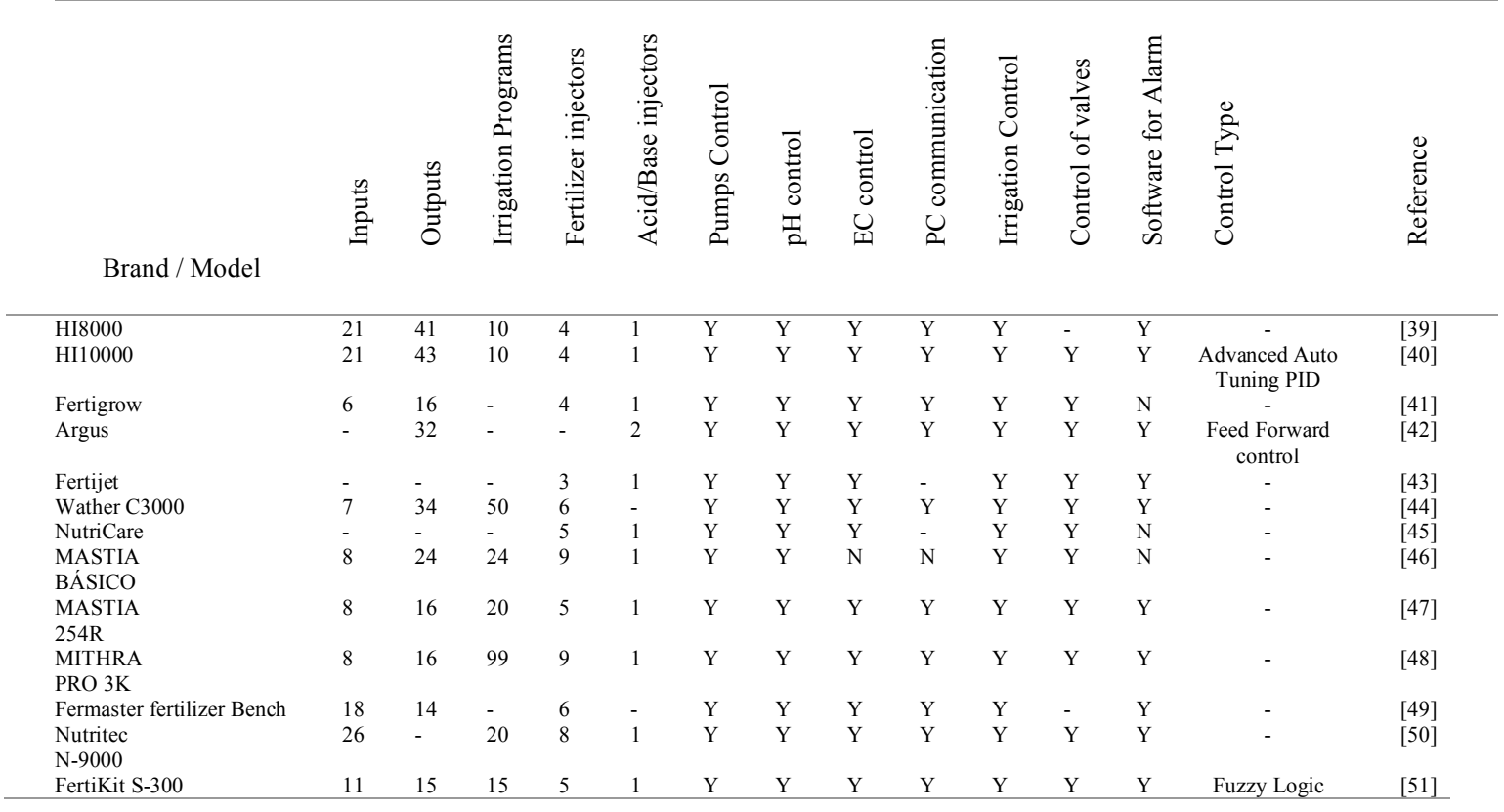

In Table 1 Y represent Yes and N No. Some characteristics for certain equipment are not supplied by the manufacturers, and therefore some cells are filled with (-). Some of these models are from the same manufacturer, HI8000 and HI10000 are both from HANNA Instruments and MASTIA BASIC, MASTIA 254R and MITHRA PRO 3K belong to Nutricontrol. In these cases, the main differences between models are the number of inputs / outputs, the number of possible irrigation programs and the number of fertilizer injectors that can be used. Most commercial fertigation control systems monitor $\mathrm{pH}$ and EC values to control the injectors, pumps, valves and irrigation cycles. Most equipment allow communication with the PC and have an alarm system to notify the user. Despite that the control type used is one of the main characteristics that distinguishes one equipment from another in relation to the quality and precision of the results, this is not always indicated by the manufacturer. However as can be seen in Table 1, in a few cases the type of control used in these devices is presented, highlighting only the Advanced Auto Tuning PID used by the HI10000, the feedforward control used by Argus and the Fuzzy Logic used by FetiKit S-300 [40, 42, 51].

\section{Current Challenges and Trends for Fertigation Control}

Recent studies $[52,53]$ have begun to present as a potential solution the use of fiber optic sensors for the monitoring of nutrients in soils, since they are immune to any type of electromagnetic or radiofrequency interference and have a higher sensitivity and resolution compared to other sensors [54]. Another trend is the use 
of IoT technology $[55,56]$ to have a more centralized control and to be able to notify the user regarding the system state. In most scientific articles reviewed the main focus is the precise macronutrients NPK control, since they are the nutrients considered fundamental for the plant development. Nevertheless, it would be also important to integrate in the fertigation systems the control of micronutrients, such as $\mathrm{Fe}, \mathrm{Mn}, \mathrm{B}, \mathrm{Cu}, \mathrm{Zn}$ and $\mathrm{Mo}$, which are also of extreme importance for the growth and development of plants [57].

This work was developed with the purpose of studying different control systems used in the literature for fertigation, to be able to present improvements in the scope of the European project Agrinupes - Integrated monitoring and control of water, nutrients and plant protection products towards a sustainable agricultural sector. This 3 years project has 8 partners from 5 countries: Portugal, Netherlands, Turkey, Spain and Sweden, and started in 2017. The Agrinupes project has as one of the objectives the integration of NPK sensors data in an innovative Model Predictive Controller (MPC) for precision fertigation, which incorporates robustness and fault-tolerant features. With this project it is expected to contribute to meet both the crop needs and the grower yield/costs expectations in a sustainable way, with proper decision-making options for the grower. The project objectives are: 1) development and evaluation of innovative optical fiber sensors for NPK nutrients monitoring in the supply and drainage water for application in agriculture; 2) development and evaluation of innovative sensors for simple, rapid and sensitive monitoring of Plant Protection Products (PPP); 3) early warning of the water quality, recommending appropriate treatment for maximal recycling of drainage water, or environmentally safe disposal, with the development of strategies to define water quality parameters and thresholds for decision-making; 4) development of participatory policy models for agricultural input optimization and water quality monitoring. Thus, in this project the following impacts are expected: 1) better use and protection of European natural resources; 2) improvement of environmental water quality and protection of human health; 3) reducing operational and energy cost; 4) boosting the European water technology sector; 5) contribution to expand Water RDI.

\section{Conclusions}

In this review, different control systems for fertigation described in the literature are presented and the characteristics of different commercialized equipment for fertigation compared. There are several control techniques available, but all of them are developed with the same objective of optimizing the efficiency of fertigation through the precise control of nutrient application according to the plants needs, which allows a better resource management and a decrease in the environmental pollution. There are several companies that sell specialized equipment for fertigation with identical characteristics. However, the type of control used to optimize the nutrient solution preparation is only presented in few cases. In order to obtain a precise control it is necessary to attend some essential factors in the fertigation systems. Usually to control the injection of nutrients it is also necessary to control the injection of an acid or base to adjust the $\mathrm{pH}$ level in the solution. In these control systems the determination of the amount to be injected of each element is determined by the value read by $\mathrm{pH}$ sensors, EC sensors and in some cases ISE or fiber optic sensors for certain chemical elements. With this review it is possible to verify that in most of the cases the control of the nutrient concentration in general is made through the analysis of the $\mathrm{EC}$ and the $\mathrm{pH}$ and not the individual control of each nutrient. In this way, it becomes simpler to monitor and adjust the solution, and no specific sensors are required for each ion. Although the individual control of each nutrient can make the system more complex, it allows a greater precision in the nutrient solution adjustment and a greater profitability of the resources. Thus, the next challenges for the development of fertigation control systems consist in design the individual control of different nutrients applied, which in turn implies the development of increasingly accurate sensors for each nutrient.

Despite the several technologies presented in this work that provides some relevant contributions to achieve better efficient fertigation systems, it must be referred that these technological solutions and algorithms, incorporating simulation models, plant responses, new sensors, among others, are in most cases not available to the growers. The commercial equipment are limited in the sensors and algorithms employed to guarantee an optimized fertigation. In this way, the Agrinupes project seeks for the development and the transfer of research innovative solutions to the farmers and companies.

\section{Acknowledgements}

The authors would like to thank the EU and FCT for funding in the frame of the collaborative international consortium Agrinupes financed under the ERA-NET Water Works 2015 cofounded call. This ERA-NET is an integral part of the 2016 Joint Activities developed by the Water Challenge for a changing world joint programme initiation (Water JPI).

\section{References}

Cassman, K. G., 1999. Ecological intensification of cereal production systems: Yield potential, soil quality, and precision agriculture. Proc. Natl. Acad. Sci 96(11): 5952-5959. 
Foley, J. A., DeFries, R., Asner, G. P., Barford, C., Bonan, G., Carpenter, S. R., Chapin, F. S., Coe, M. T., Daily, G. C., Gibbs, H. K., Helkowski, J. H., Holloway, T., Howard, E. A., Kucharik, C. J., Monfreda, C., Patz, J. A., Prentice, I. C., Ramankutty, N. and Snyder, P. K., 2005. Global Consequences of Land Use. Science 309(5734): 570-574.

Bongiovanni, R. and Lowenberg-Deboer, J., 2004. Precision Agriculture and Sustainability. Precision Agriculture 5(4): 359-387.

Kafkafi, U. and Tarchitzky, J., 2011. Fertigation A Tool for Efficient Fertilizer and Water Management. International Fertilizer Industry Association, Paris, France.

Carruthers, S., 2014. Fertigation \& Dosing Systems. Practical Hydroponics \& Greenhouses 144:38-47.

Oliveira, J., Boaventura-Cunha, J., Oliveira, P. M., 2017. Automation and Control in Greenhouses: State-ofthe-Art and Future Trends. CONTROLO 2016. Lecture Notes in Electrical Engineering 402.

Boman, B. and Obreza, T., 2015. Fertigation Nutrient Sources and Application Considerations for Citrus. IFAS Extentions, University of Florida.

Oliveira, S., 2005. Adubação com Nitrogênio, Fósforo e Potássio para Experimentos com Cafeeiros em Vasos. Federal University of Larvas. Postgraduate thesis in Agronomy.

Hebbar, S.S., Ramachandrappa, B. K., Nanjappa, H.V. and Prabhakar, M., 2004. Studies on NPK drip fertigation in field grown tomato (Lycopersicon esculentum Mill.). European Journal of Agronomy 21(1): 117127.

Klein, I., Meimon, A. and Skedi, D., 1999. Drip nitrogen, phosphorus, and potassium fertigation of 'spadona' pear. Journal of Plant Nutrition 22(3): 489-499.

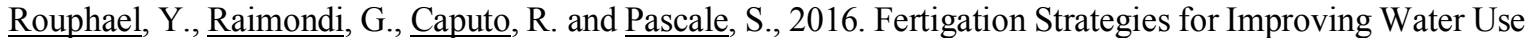
Efficiency and Limiting Nutrient Loss in Soilless Hippeastrum Production. HortScience 51(6): 684-689.

Mohammad, M. J., Ahmed, H. and Elnabi, F. A., 2004. Phosphorus fertigation and preplant conventional soil application of drip irrigated summer squash. Journal of Agronomy 3(3): 162-169.

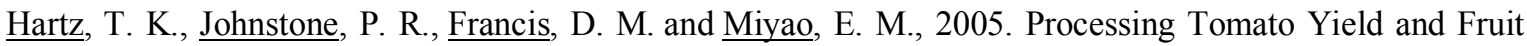
Quality Improved with Potassium Fertigation. HortScience 40(6): 1862-1867.

Lin, J., Wang, M., Zhang, M., Zhang, Y. and Chen, L., 2008. Electrochemical Sensors for Soil Nutrient Detection: Opportunity and Challenge. Computer And Computing Technologies In Agriculture 2: 1349-1353.

Ramane, D. V., Patil, S. S. and Shaligram, A. D., 2015. Detection of NPK nutrients of soil using Fiber Optic Sensor. International Journal of Research in Advent Technology (E-ISSN: 2321-9637) Special Issue National Conference "ACGT 2015”, 13-14 February.

IEEE Xplore digital library. Retrieved from: https://ieeexplore.ieee.org/Xplore/home.jsp, Accessed 9 April 2018.

ScienceDirect. Retrieved from: https://www.sciencedirect.com/, Accessed 9 April 2018.

b-on. Retrieved from: https://www.b-on.pt/, Accessed 9 April 2018.

Charles, H., Godfray, J., Beddington, J. R., Crute, I. R., Haddad, L., Lawrence, D., Muir, J. F., Pretty, J., Robinson, S., Thomas, S. M. and Toulmin, C., 2010. Food Security: The Challenge of Feeding 9 Billion People. Science 327(5967): 812-818.

Gieling, T. H., Corver, F. J. M., Janssen, H. J. J., 2005. Hydrion-line, towards a closed system for water and nutrients: feedback control of water and nutrients in the drain. Acta Horticulture 691: 259-266.

Morimoto, T., Hatou, K. and Hashimoto, Y., 1996. Intelligent Control for a Plant Production System. Control Engineering Practice 4(6): 773-784.

Gieling, T. H., Bontsema, J., Bouwmans, T. W. B. M. and Steeghs, R.H., 1997. Modelling and simulation for control of nutrient application in closed growing systems. Netherlands Journal of Agricultural Science 45(1): $127-$ 142.

Rodríguez, D., Reca, J., Martínez, J., Lao, M. and Urrestarazu, M., 2014. Effect of controlling the leaching fraction on the fertigation and production of a tomato crop under soilless culture. Scientia Horticulturae 179: 153157.

Neto, A., Zolnier, S. and Lopes, D., 2014. Development and evaluation of an automated for fertigation control in soilles tomato production. Computers and Electronics in Agriculture 103: 17-25.

Niu, Y. and Zhang, K., 2015. Development of Fertigation Control System Based on Embedded Platform and Self-Adaptive Control Strategy. 2nd International Conference on Electrical, Computer Engineering and Electronics (ICECEE 2015).

Salih, J. E. M., Adom, A. H. and Shaakaf, A. Y. M., 2012. Solar Powered Automated Fertigation Control System for Cucumis Melo L. Cultivation in Green House. APCBEE Procedia 4: 79-87.

Kaur, B. and Kumar, D., 2013. Development of Automated Nutrients Composition Control Fertigation System. International Journal of Computer Science, Engineering and Applications. 3(3): 67-78. 
Abidin, S. A. H. Z. and Ibrahim, S. N., 2015. Web-based Monitoring of an Automated Fertigation System: An IoT Application. 2015 IEEE 12th Malaysia International Conference on Communications (MICC), 23-25 November, Kuching, Malaysia.

Ruan, J., Liao, P. and Dong, C., 2015. The Design and Research on Intelligent Fertigation System. 2015 7th International Conference on Intelligent Human-Machine Systems and Cybernetics, 26-27 August, Hangzhou, China.

Bueno-Delgado, M. V., Molina-Martínez, J. M., Correoso-Campillo, R. and Pavón-Mariño, P., 2015. Ecofert: An Android application for the optimization of fertilizer cost in fertigation. Computers and Eletronics in Agriculture 121: 32-42.

Pérez-Castro, A., Sánchez-Molina, J. A., Castilla, M., Sánchez-Moreno, J., Moreno-Úbeda, J. C. and Magán, J. J., 2017. cFertigUAL: A fertigation management app for greenhouse vegetable crops. Agricultural Water Management 183: 186-193.

Rodríguez, D., Reca, J., Martínez, J., López-Luque, R. and Urrestarazu, M. 2015. Development of a New Control Algorithm for Automatic Irrigation Scheduling in Soilless Culture. Applied Mathematics \& Information Sciences 9(1): 47-56.

Coates, R. W., Delwiche, M. J. and Brown, P. H., 2006. Control of individual microsprinklers and fault detection strategies. Precision Agric 7(2): 85-99.

Benzekri, A., Meghriche, K. and Refoufi, L., 2007. PC-Based Automation of a Multi-Mode Control for an Irrigation System. 2007 International Symposium on Industrial Embedded Systems, 4-6 July, Lisbon, Portugal.

Kulkarni, A. S., Wakade, T. P., Patki, A. R. and Jamkar, R. G., 2014. Programmable Logic Control (PLC) Based Automation Model for Irrigation and Fertigation Control. International Journal of Advanced Technology in Engineering and Science 2(9): 321-329.

McDaerthy, A. C., Hancock, N. H. and Raine, S. F., 2010. VARIwise: A general-purpose adaptive control simulation framework for spatially and temporally varied irrigation at sub-field scale. Computers and Eletronics in Agriculture 70(1): 117-128.

Barradas, J. M. M., Matula, S. and Dolezal, F., 2012. A Decision Support System-Fertigation Simulator (DSSFS) for design and optimization of sprinkler and drip irrigation systems. Computers and Eletronics in Agriculture 86: 111-119.

Balendonck, J., Pardossi, A., Tuzel, H., Tuzel, Y., Rusan, M. and Karam, F., 2010. FLOW-AID - a Deficit Irrigation Management System using Soil Sensor Activated Control: Case Studies. The Third International Symposium on Soil Water Measurement Using Capacitance, Impedance and TDT, Murcia, Spain.

HANNA instruments. Retrieved from: https://hannainst.com/fertigation-control-system.html, Accessed 9 April 2018.

HANNA instruments. Retrieved from: https://hannainst.com/advanced-fertilizer-injection-system.html, Accessed 9 April 2018.

Spagnol. Retrieved from: http://en.spagnolaut.com/products/irrigation-controllers/fertigationcontroller/fertigrow.php, Accessed 9 April 2018.

Argus Controls. Retrieved from: http://arguscontrols.com, Accessed 9 April 2018.

Galcon. Retrieved from: http://galconc.com/product/fertijet, Accessed 9 April 2018.

Dosing pumps manufacturer. Retrieved from: http://www.itc.es/en/controller/item/185-water-controller3000.html, Accessed 9 April 2018.

Jain Irrigation Systems Ltd. Retrieved from: http://www.jains.com/irrigation/Filter/NutriCare.htm, Accessed 9 April 2018.

Nutricontrol. Retrieved from: http://nutricontrol.com/2015/portfolio item/mastia-configurable/?lang=en, Accessed 9 April 2018.

Nutricontrol. Retrieved from: http://nutricontrol.com/2015/portfolio item/213/?lang=en, Accessed 9 April 2018.

Nutricontrol. Retrieved from: http://nutricontrol.com/2015/portfolio item/mithra-pro/?lang=en, Accessed 9 April 2018.

GOLDTEC Control Systems. Retrieved from: http://goldtecsystems.com.au/product/fertmaster-fertilizerbench, Accessed 9 April 2018.

Ritec. $\quad$ Retrieved from: http:/www.ritec.es/sistemas-riegofertirrigacion/productos/versiones/1/fertirrigacion/1/automatismos/40/nutritec-n-9000.html, Accessed 9 April 2018.

Netafim. Retrieved from: http:/www.netafimuk.com/product/fertikit-s-300, Accessed 9 April 2018. 
Lourembam, D., Laskar, S. and Mukherjee, S., 2017. Framework for an optical sensor system for monitoring of soil nitrogen and tailoring soil pH. Jounal of Optics 46: 1-15.

Gavade, L. C., 2017. Detection of N, P, K Using Fiber Optic Sensor and PIC Controller. Internacional Jornal of Engineering Science and Computing 7(7): 13787-13789.

Batista, J. M. R. T., 2002. Concepção, Analise e Desenvolvimento de Sensores de Fibra Óptica de Intensidade Auto-Referenciados. Faculty of Engineering of the University of Porto. Ph.D. thesis in Electrical and Computer Engineering.

Varma, H., Mulla, Raut R. and Pawar, V. R., 2017. Fertigation \& Irrigation System for Agricultural Application along with Soil Monitoring using IoT. Vishwakarma Journal of Engineering Research 1(2): 241-245.

Mohanraj, I., Gokul, V., Ezhilarasie, R. and Umamakeswari, A., 2017. Intelligent Drip Irrigation and Fertigation Using Wireless Sensor Networks. Technological Innovations in ICT for Agriculture and Rural Development (TIAR), 2017 IEEE, 7-8 April, Chennai, India.

Trivedi, A. and Dhumal, K. N., 2017. Effect of Micronutrients, Growth Regulators and Organic Manures on Yield, Biochemical and Mineral Component of Onion (Allium cepa L.) Grown in Vertisols. International Journal of Current Microbiology and Applied Sciences 6(5): 1759-1771. 


\author{
GO EFLUENTES - A New Approach for Animal Waste Management in Portugal \\ Vasco Fitas da Cruz ${ }^{1}$, Olga Conde Moreira ${ }^{2}$, Elizabeth Duarte ${ }^{3}$, Henrique trindade ${ }^{4}$ \\ ${ }^{1}$ ICAAM, Department of Rural Engineering, University of Évora. vfc@uevora.pt \\ ${ }^{2}$ INIAV, Portugal. olga.moreira@iniav.pt \\ ${ }^{3}$ ISA, University of Lisbon. eduarte@isa.ulisboa.pt \\ ${ }^{4}$ UTAD, Portugal. htrindad@utad.pt
}

\begin{abstract}
This approach aims at valorisation of a resource, focused on different interests that converge in the production and integrated management (social, political, economic, technical and environmental) of the flows generated in the farming systems ensuring the sustainable development. In all steps related to the management of these flows (production, collection, storage, recovery and re-use under local conditions), the aim of recovery will not to eliminate them but to reduce the exploitation of natural resources. Identification, quantification and hierarchization of flows will be fundamental, allowing nutrient balances at farms and consequent sustainable management. The "GO_Efluentes" Operational Group act across the entire production system. Taking into account the different specificities, it seeks to typify the main variables of the flows generated in the animal production systems, testing efficient operational strategies for their sustainable valorisation and management. In the management of livestock effluents from intensive farms of cattle, pigs and poultry, the solutions already developed and of robust and proven use in these productive sectors will be inventoried and a route of applicability will be defined according to the characteristics of the farms and other regional constraints. At the same time, the impact of the introduction of emerging solutions in the treatment of effluents (separation of solids associated with the acidification of liquid fractions or the addition of biochar and solid fraction composting) will be evaluated experimentally, as well as the reduction of emissions pollutants (ammonia and greenhouse gases), as well as the effects on the management of effluents, the economic feasibility of applying these technologies to farms. The development of a Operational Group of this typology allows an approximation between partners in the scope of the agronomic and energy valorisation of the flows generated in the agricultural activity, which until now appears as distant, with evident benefits for all the parties.
\end{abstract}

\title{
1. Introduction
}

Intensive livestock production systems are concentrated in certain regions, some without sufficient area for effluent valorisation by spreading on agricultural land. This sector to be competitive and comply with legal requirements should promote a circular economy, looking for new alternatives for effluent management

This approach aims at valorisation of a resource, focused on different interests that converge in the production and integrated management (social, political, economic, technical and environmental) of the flows generated in the farming systems ensuring the sustainable development. In all steps related to the management of these flows (production, collection, storage, recovery and re-use under local conditions), the aim of recovery will not to eliminate them but to reduce the exploitation of natural resources. Identification, quantification and hierarchization of flows will be fundamental, allowing nutrient balances at farms and consequent sustainable management.

The "GO_Efluentes" Operational Group, a partnership between research institutes, universities, association of farmers and animal producers and private companies, act across the entire production system. Taking into account the different specificities, it seeks to typify the main variables of the flows generated in the animal production systems, testing efficient operational strategies for their sustainable valorisation and management.

The main objectives of this Operational group are:

To valorise the livestock effluents as a resource, focusing on the production and integrated management of the different flows generated

Optimize the use of effluents as secondary raw materials, recovering energy and nutrients, improving nutrient balance at the farm level and promoting sustainable management

\section{Material and methods}

In the management of livestock effluents from intensive farms of cattle, pigs and poultry, the solutions already developed that are robust and proven use in these productive sectors, will be inventoried and a route of applicability will be defined according to the characteristics of the farms and other regional constraints. At the same time, the impact of the introduction of emerging solutions in the treatment of effluents (separation of solids associated with the acidification of liquid fractions or the addition of biochar and solid fraction composting) will be evaluated experimentally, as well as the reduction of emissions pollutants (ammonia and greenhouse gases), 
as well as the effects on the management of effluents, the economic feasibility of applying these technologies to farms.

The work plan is developed in accordance with the following actions lines:

Action 1. Characterization of intensive production systems

Action 2. Measures to mitigate emissions and primary treatment of livestock effluents

Action 3. Valorisation of effluents

Action 4. Socio-economic and multi-criteria impact studies

Action 5. Demonstration / dissemination activities

\section{Results and discussion}

The expected results of this project are:

- A roadmap for effluent management, including technology portfolio, linked to farm characteristics and regional constraints

- Support decision-making on centralized / decentralized solutions

- A contribution to the intensification of sustainable livestock and landscape planning, addressing climate change and scarce resources.

\section{Conclusions}

The development of a Operational group of this typology allows an approximation between partners in the scope of the agronomic and energy valorisation of the flows generated in the agricultural activity, which until now appears as distant, with evident benefits for all the parties since it contributes:

- to improve the efficiency of animal nutrition

- to the mitigation of GHG

- to increase soil fertility

- will create a georeferenced database

- will present innovative solutions

\section{References}

Fangueiro, D., Coutinho, J., Chadwick, D., Moreira, N. and Trindade, H., 2008. Effect of cattle slurry separation on greenhouse gas and ammonia emissions during storage. Journal of Environmental Quality, 37(6), pp.2322-2331.

Hjorth, M., Christensen, K.V., Christensen, M.L. and S.G. Sommer. 2010. Solid-liquid separation of animal slurry in theory and practice. A review. Agronomy for Sustainable Development. 30, 153-180

Soteriades, A., Faverdin, P., March, M. and Stott, A.W. 2015. Improving efficiency assessments using additive data envelopment analysis: an application to contrasting dairy farming systems. Agricultural and Food Science, 24:235-348.

van Huis, A., Van Itterbeeck, J., Klumber, H., Halloran, A., Muir, G., \& Vantomme, P. (2013). Edible insects Future prospects for food and feed security. Rome: FAO. 


\title{
Development of an Empirical Model to Prepare Nutrient Solutions with the Use of Controlled Release Fertilizers
}

\author{
Maria Zografou *, Christos Lykas \\ Department of Agriculture Crop Production and Rural Environment, School of Agricultural Sciences, \\ University of Thessaly, Volos, Magnesia 38446, Greece \\ * Corresponding author. Email: mazografou@uth.gr
}

\begin{abstract}
The use of controlled release fertilizers serves to cover crops needs during the plants growth period. The release of nutrients depends on solution temperature and fertilizers polymer coating, which can control the diffusion of nutrients. However many other parameters may affect the evolution of nutrients elements concentration in aqueous solutions. The aim of this study was to investigate the effect of nutrient solution's $\mathrm{pH}$, $\mathrm{EC}$ and volume as well as the time from its preparation on the evolution of $\mathrm{NO}_{3}{ }^{-}, \mathrm{NH}_{4}{ }^{+}, \mathrm{PO}_{4}{ }^{3-}$ and $\mathrm{K}^{+}$ concentrations and to develop an empirical model to describe the evolution of the above elements concentration during 10 days period. For this reason, $0.5 \mathrm{~g}$ and $265 \mathrm{~g}$ of Multicote fertilizer 14-14-14 dissolved in $100 \mathrm{ml}$ and $2000 \mathrm{ml}$ of deionized water respectively. The solutions remained at $24{ }^{\circ} \mathrm{C}$ and their $\mathrm{pH}$ was adjusted every day at 5.5 during a period of 10 days. The $\mathrm{EC}$ of the nutrient solution was measured every second day, while the volume after sampling and the concentration of the above mentioned elements was measured every two days. A linear empirical mathematical model was developed for the prediction of the above mentioned nutrients concentrations $\left(\mathrm{C}_{\mathrm{X}}\right)$ in relation to the volume removed after sampling (sumVs), the $\mathrm{pH}$ and the $\mathrm{EC}$ of the nutrient solution and the time ( $\mathrm{T}$ ) from its preparation. Values of the above mentioned parameters were measured in six nutrients solutions (three with volume of $100 \mathrm{ml}$ and three with volume $2000 \mathrm{ml}$ ) and were used for the validation of the model. For the evaluation of the model, data from nutrient solutions prepared with the use of 14-14-14 CRF fertilizer with volume of $2000 \mathrm{ml}$ was used. The predicted concentrations of $\mathrm{PO}_{4}{ }^{3-}, \mathrm{NH}_{4}^{+}, \mathrm{K}^{+}$and $\mathrm{NO}_{3}{ }^{-}$compared favourably with the measured one.
\end{abstract}

Keywords: multicote, fertigation, soilless culture, polymer coating, electrical conductivity.

\section{Introduction}

Nowadays one of the most complicated problems concerning crops fertigation, both in soil and soilless cultures, is to match their demands with the appropriate supply of nutrients at the time needed (Shaviv, 2000; Trenkel, 2010). Moreover, there is a continuous need to find ways to reduce fertigation cost and minimize the environmental and ecological impacts deriving from the heavy loss of fertilizers (Yan et al., 2008). These goals could be achieved with the use of appropriate sensors and mechanical equipment or by using mathematical equations to estimate the exact amount of water and fertilizers. CRF fertilizers can achieve this purpose since their granules are covered with a polymer coating that can act as a semi-permeable or impermeable membrane resulting a gradual release of nutrients outside the core of the fertilizer. In other words the polymer coating plays the role of a temperature sensor which can control the diffusion of the nutrients in the solution used from crop irrigation or the medium where plants are grown (Du et al., 2004). The relation between the temperature of the medium where the fertilizer is added and the release rate of nutrients was indicated from many researchers (Fujita and Shoji, 1999; Shaviv, 2005; Shoji and Gandeza, 1992; Liang et al., 2006).

However this relation between temperature and nutrients release rate could lead to elements elimination or toxicity since research revealed that high temperatures during the first month after planting can cause salt problems to the crop due to the high nutrient release of the CRF fertilizer, while low temperatures could cause nutrient deficiency due to the slow nutrient release (Mayer, 2010). Nowadays there is no adequate work that makes possible, through evaluated models, the estimation of nutrient's concentration in aqueous solutions related to temperature or to the other parameters of the nutrient's solution, such as $\mathrm{pH}, \mathrm{EC}$, volume and time from its preparation. This needs a further strengthened especially since CRF fertilizers could be used in crops such as rice (Singh et al., 1995; Carreres et al., 2003) and soilless cultures (Du et al., 2006), where the pH and the EC of the medium where plants are grown and that of the aqueous solution used for their irrigation play a key role to their development. Moreover, less attention of the research so far was paid to the release of nutrients from compound $\mathrm{N}-\mathrm{P}-\mathrm{K}$ coated CRFs. In this type of fertilizers the release of each nutrient/ion is expected to be different from the simple CRF's, due to the interaction of the nutrient elements (Shoji and Gandeza, 1992; Naik et al., 2017). These CRFs are highly recommended to be used for the fertigation of high value crops such as ornamental plants, orchards, vegetables, lawns etc. since they can be applied once in the beginning of the planting, instead of giving an oversupply of common fertilizers which increases the potential for greater nutrient losses (Tian et al., 2016; Kinoshita, 2012).

The above reveal the need a) to control the release rate of nutrients by adjusting other parameters which affect 
the release rate and b) to develop a model that will combine these parameters in order to achieve their adjustment. The aim of this study is to investigate the effect of nutrient solution's $\mathrm{pH}, \mathrm{EC}$ and volume as well as the time from its preparation on the evolution of $\mathrm{NO}_{3}{ }^{-}, \mathrm{NH}_{4}{ }^{+}, \mathrm{PO}_{4}{ }^{3-}$ and $\mathrm{K}^{+}$concentrations and to develop an empirical model to describe the evolution of the above elements concentration during the period that the solution is used for plant irrigation.

\section{Materials and Methods}

Treatments

For the purposes of the experiment, one type of Multicote fertilizer 14-14-14 (100\% coated) was used for the preparation of six aqueous solutions with deionized water. Three of these solution had a volume of $100 \mathrm{ml}$ (each one) and were prepared with the addition of $0.5 \mathrm{~g}$ of 14-14-14 fertilizer. The concentration of the fertilizer in these solutions was $5000 \mathrm{ppm}$. Each one of the other three solutions had a volume of $2000 \mathrm{ml}$ and they were prepared with the addition of $265 \mathrm{~g}$ of 14-14-14 fertilizer. The concentration of the fertilizer in these solutions was 132,500 ppm and can be characterized as very high concentration solutions, similar to stock solutions used in soilless culture. During the experiment period the solutions were kept at a fixed temperature of $24^{\circ} \mathrm{C}$ and their $\mathrm{pH}$ was measured every day, while it was adjusted when necessary with the addition of $\mathrm{NaOH}$ or $\mathrm{HCl}$ at 5.5 during a period of 10 days. The volume that was removed during sampling from the $2000 \mathrm{ml}$ solutions on daily basis was estimated to be proportional to that, which should be removed from a stock solution for the irrigation of a rose soilless culture, grown in $1000 \mathrm{~m}^{2}$ greenhouse area. The EC of the nutrient solutions was measured every second day using the HI991300 Portable $\mathrm{pH} / \mathrm{EC} / \mathrm{TDS}$ meter, while the volume after sampling and the concentration of $\mathrm{NO}_{3}{ }^{-}, \mathrm{NH}_{4}{ }^{+}, \mathrm{PO}_{4}{ }^{3-}$ and $\mathrm{K}^{+}$were measured every two days using a LAMOTTE Smart 2 colorimeter. The type of the 14-14-14 fertilizer was selected due to the same proportion of the nutrients $(\mathrm{N}, \mathrm{P}$ and $\mathrm{K})$ that the fertilizer contain.

\section{Statistical analysis}

Data were statistically analyzed by analysis of variance (ANOVA) using Statgraphics Centurion XVI. Duncan's multiple range test was used at a significance level of 0.05 .

\section{Results and Discussion}

Evolution of $\mathrm{pH}$ in $100 \mathrm{ml}$ and $2000 \mathrm{ml}$ solution

The evolution of $\mathrm{pH}$ in $2000 \mathrm{ml}$ and $100 \mathrm{ml}$ solutions, which are prepared with the use of $265 \mathrm{~g}$ and $0.5 \mathrm{~g}$ of 14-14-14 fertilizer respectively, is shown in Figure 1.

The $\mathrm{pH}$ in $2000 \mathrm{ml}$ solutions, varied slightly from the initial value. It was increased close to 5.6 during the first day after the preparation, but then it was stabilized, close to the initial value until the end of the experiment (ten days after preparation). In contrast $\mathrm{pH}$ of the $100 \mathrm{ml}$ solutions increased continuously during the period of four days after preparation and reached a value close to 6.2. Even with the continuous addition of $\mathrm{HCl}$ the $\mathrm{pH}$ adjustment was not very effective, since in these solutions $\mathrm{pH}$ value reached close to the initial value only during the last day of the experiment. The above results indicate that the $\mathrm{pH}$ alteration was lower and the adjustment more effective in high fertilizer concentration solution compared to low concentration solutions (van Iersel, 1999). This is probably due to a) the ammonium to nitrate $\left(\mathrm{NH}_{4}{ }^{+}: \mathrm{NO}_{3}{ }^{-}\right)$ratio that the fertilizer contains (Fisher et al., 2014) and b) to the volume of the solution by the mean that, the higher the volume of the solution the higher the amount of the diluted fertilizer (or the nutrients release rate) and consequently the higher the $\mathrm{NH}_{4}{ }^{+}$concentration that can decrease the $\mathrm{pH}$ of the solution. 


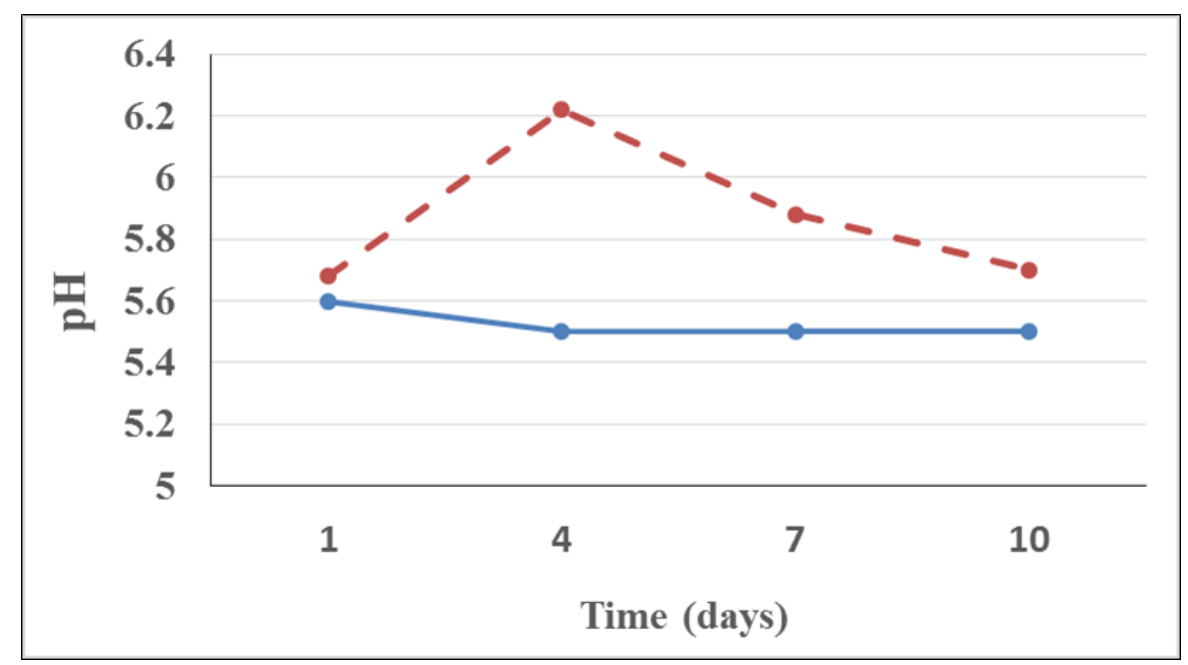

Figure 1. Evolution of pH in solutions with $2000 \mathrm{ml}$ volume (-) and $100 \mathrm{ml}$ volume (- -).

Evolution of nutrient elements concentrations in $100 \mathrm{ml}$ and $2000 \mathrm{ml}$ solution

The results of the measurements concerning the alteration of $\mathrm{NO}_{3}{ }^{-}, \mathrm{NH}_{4}{ }^{+}, \mathrm{PO}_{4}{ }^{3-}$ and $\mathrm{K}^{+}$concentration in 2000 $\mathrm{ml}$ and $100 \mathrm{ml}$ solutions prepared with the addition of 14-14-14 fertilizer, is shown in Figure 3.

Both concentrations of $\mathrm{NO}_{3}{ }^{-}$and $\mathrm{K}^{+}$increased significantly in the solutions during the first four days from the preparation regardless their volume. After this period the concentrations of these two nutrients decreased regarding to the volume of the solution, following a different pattern as far as the time is concerned. In the case of $2000 \mathrm{ml}$ solutions the decrease occurred from the fourth to seventh day, while in the case of $100 \mathrm{ml}$ solutions the decrease of $\mathrm{NO}_{3}{ }^{-}$and $\mathrm{K}^{+}$was observed from seventh day till the tenth. The decrease of these two nutrient elements in the nutrient solutions could be due to $\mathrm{KNO}_{3}$ complexes that may be formatted in the solutions, where the concentrations of $\mathrm{NO}_{3}{ }^{-}$and $\mathrm{K}^{+}$increased significantly (Figure $2 \mathrm{~A}, \mathrm{D}$ ). However, both the $\mathrm{pH}$ alteration as well as the continuous sampling, that resulted to the reduction of the above mentioned nutrients concentration in the nutrient solution, probably caused the redissolving of $\mathrm{KNO}_{3}$ complexes and the increment of $\mathrm{NO}_{3}{ }^{-}$and $\mathrm{K}^{+}$ concentration.

The evolution of $\mathrm{NH}_{4}{ }^{+}$concentration in both $2000 \mathrm{ml}$ and $100 \mathrm{ml}$ solutions followed almost the same pattern (Figure $2 \mathrm{~B}$ ). However the $\mathrm{NH}_{4}{ }^{+}$concentration was significant higher in $2000 \mathrm{ml}$ solutions compared with $100 \mathrm{ml}$ solutions. This is, as expected, in accordance with the $\mathrm{pH}$ evolution in $2000 \mathrm{ml}$ and $100 \mathrm{ml}$ solutions, since the higher concentration of $\mathrm{NH}_{4}{ }^{+}$in $2000 \mathrm{ml}$ solution resulted to the greater reduction of their $\mathrm{pH}$. In addition, differences in $\mathrm{NH}_{4}{ }^{+}$concentration between $2000 \mathrm{ml}$ and $100 \mathrm{ml}$ solutions could be attributed, as mentioned above, to their volume differences that directly affect the amount of fertilizer that could be dissolved in a specific time period.

The concentration of $\mathrm{PO}_{4}{ }^{3-}$ was continuously increased until the seventh day after the $2000 \mathrm{ml}$ solutions preparation, while from the seventh till the tenth day the concentration of $\mathrm{PO}_{4}{ }^{3-}$, tend to stabilize to a certain value (Figure $2 \mathrm{C}$ ). In the case of $100 \mathrm{ml}$ solutions the concentration of $\mathrm{PO}_{4}{ }^{3-}$ increased until the fourth day, while from the fourth till the tenth day a stability is observed to their $\mathrm{PO}_{4}{ }^{3-}$ concentrations. This may be due to $\mathrm{KH}_{2} \mathrm{PO}_{4}$ and $\mathrm{NH}_{4} \mathrm{H}_{2} \mathrm{PO}_{4}$ complexes that could be formed in the solution, because of the high concentrations of $\mathrm{K}^{+}$and $\mathrm{NH}_{4}{ }^{+}$or due to the stabilization of solutions $\mathrm{pH}$ that can significantly affect, in the range of 5.5 to 6.0 , the ionic form of $\mathrm{PO}_{4}{ }^{3-}$ in an aqueous solution. 

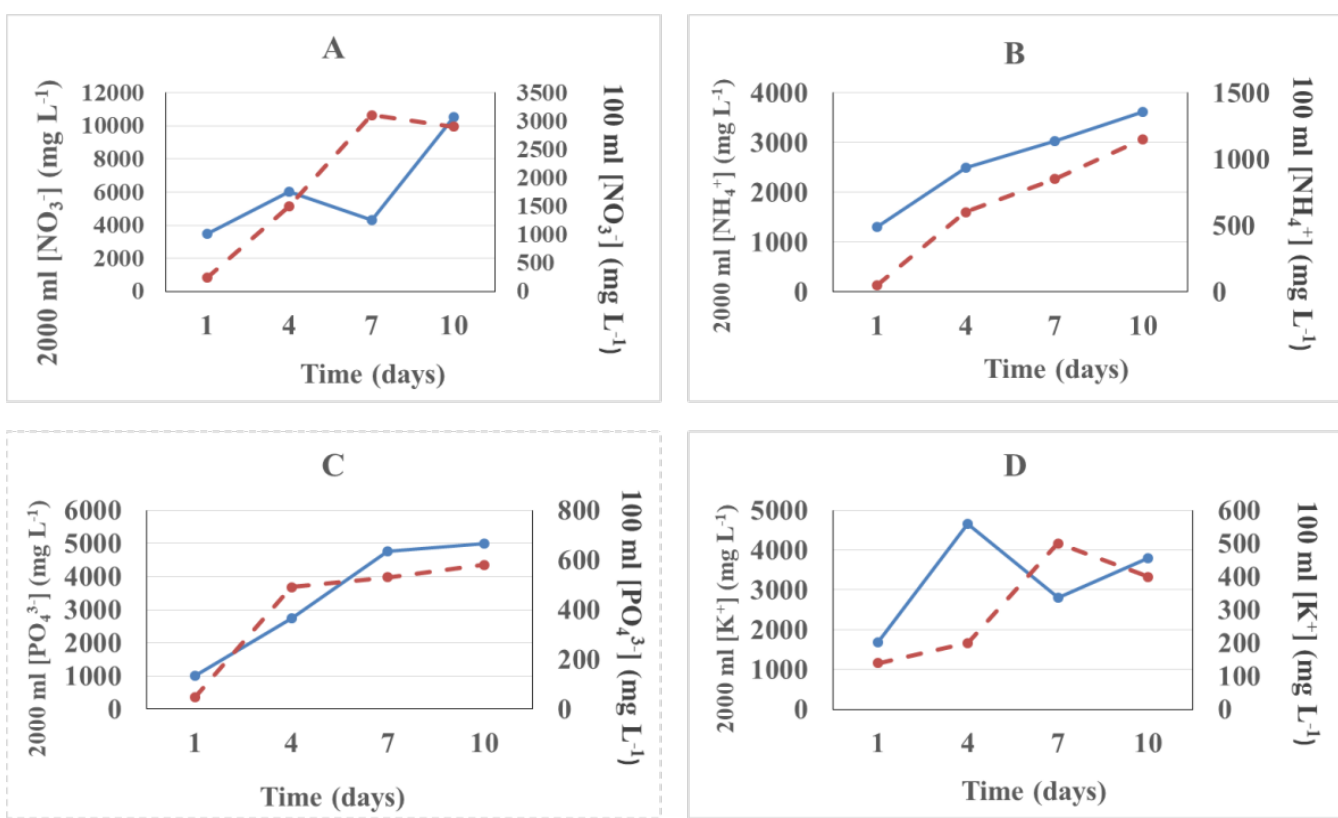

Figure 2. Evolution of $\mathrm{NO}_{3}{ }^{-}(\mathrm{A}), \mathrm{NH}_{4}{ }^{+}(\mathrm{B}), \mathrm{PO}_{4}{ }^{3-}$ (C) and $\mathrm{K}^{+}$(D) concentration in solutions prepared with the use of 14-14-14 with $2000 \mathrm{ml}$ volume (-) and $100 \mathrm{ml}$ volume (- -$)$ where the $\mathrm{pH}$ was adjusted every day.

Evolution of EC in $100 \mathrm{ml}$ solution and $2000 \mathrm{ml}$ solution

The evolution of EC in solutions prepared with the use of 14-14-14 with two different volumes, $2000 \mathrm{ml}$ and $100 \mathrm{ml}$ is shown in Figure 2. The initial $\mathrm{pH}$ of these volumes was 5.5.

According to the diagram the solutions follow the similar evolution of their EC. The initial EC value of 2000 $\mathrm{ml}$ solutions was $10,000 \mathrm{mS} \mathrm{cm}-1$ and reach finally after ten days a value up to $60,000 \mathrm{mS} \mathrm{cm}^{-1}$, while the initial EC value of $100 \mathrm{ml}$ solutions was $500 \mathrm{mS} \mathrm{cm}^{-1}$ and reach during the tenth day up to $2500 \mathrm{mS} \mathrm{cm}^{-1}$. According to the results presented in Figure $2 \mathrm{~A}, \mathrm{D}$, the concentration of $\mathrm{NO}_{3}{ }^{-}$and $\mathrm{K}^{+}$were decreased strongly during the period from fourth to seventh day in $2000 \mathrm{ml}$ solutions, while for the $100 \mathrm{ml}$ solutions a similar decrease occurred during the period from seventh to tenth day. In addition in $100 \mathrm{ml}$ solutions the concentration of $\mathrm{PO}_{4}{ }^{3-}$ almost stabilized after the fourth day while in $2000 \mathrm{ml}$ solution the same phenomenon observed after the seventh day. These alterations in the concentrations affected the EC of the solutions during the same periods. This is indicated by the change of the slope in both curves as it is shown in Figure 3. However because of the continuous increase of the total amount of nutrients in both solutions the EC had a continuous increase until the end of the experiment.

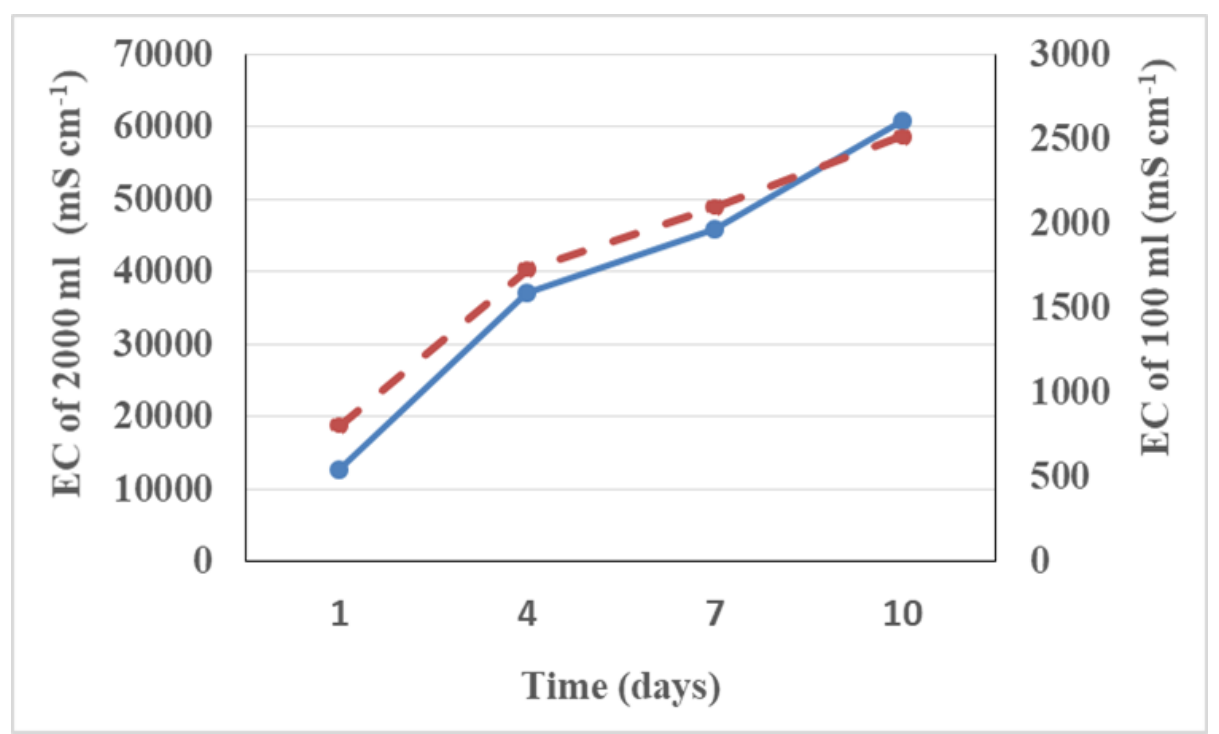

Figure 3. Evolution of EC in solutions with $2000 \mathrm{ml}$ volume (-) and $100 \mathrm{ml}$ volume (- -) where the pH was adjusted every day. 
Model development

From the above results it is concluded that the $\mathrm{pH}$, the volume of the solution and the time from their preparation affect significantly the evolution of $\mathrm{NO}_{3}{ }^{-}, \mathrm{NH}_{4}{ }^{+}, \mathrm{PO}_{4}{ }^{3-}$ and $\mathrm{K}^{+}$concentration as well as their nutrients release rate and consequently the EC. Time is also a parameter that affects the release rate of nutrients since the granules polymer coating material that CRF fertilizers have outside the core permits the income of a specific amount of water over time and therefore the dilution of a specific amount of nutrients. This is the mechanism that CRFs have in order to control the nutrients release in a course of time (Wang et al., 2011).

Taking into account the above mentioned parameters, an empirical model to estimate the $\mathrm{NO}_{3}{ }^{-}, \mathrm{NH}_{4}{ }^{+}, \mathrm{PO}_{4}{ }^{3-}$ and $\mathrm{K}^{+}$concentration in aqueous solutions prepared with 14-14-14 CRF fertilizer was developed. According to this model the concentration of each nutrient element can be calculated by the following equation:

$$
\mathrm{C}_{\mathrm{X}}=\mathrm{a}+\mathrm{b} * \mathrm{pH}+\mathrm{c} * \mathrm{EC}+\mathrm{d} * \operatorname{sumVs}+\mathrm{e} * \mathrm{t}
$$

where $\mathrm{CX}$ is the concentration of $\mathrm{NO}_{3}{ }^{-}, \mathrm{NH}_{4}{ }^{+}, \mathrm{PO}_{4}{ }^{3-}, \mathrm{K}^{+}$at time $\mathrm{t} ; \mathrm{pH}$ and $\mathrm{EC}$ are the $\mathrm{pH}$ and the $\mathrm{EC}$ of the solution at time $t$ respectively; sumVs is the sum of the volume removed by sampling during the time $t$ and $t$ is the time (in hours) from the preparation of the solution.

To calibrate the model described with equation (1), measurements which were performed in 100 and $2000 \mathrm{ml}$ solutions prepared with the use of 14-14-14 CRF, were used. Statgraphics Centurion XVI software was used in order to estimate the values of $\mathbf{a}, \mathbf{b}, \mathbf{c}, \mathbf{d}$ and $\mathbf{e}$ used in equation (1). The values of these parameters are presented in Table 1.

The correlation of the measured and estimated values resulted from the equation (1) is linear, since the equation describing the relation between the measured and calculated values has the form of $y=a_{1} * x+b_{1}$, in which $\mathbf{a}_{1}$ and $\mathbf{b}_{1}$ do not differ statistically from the values 1 and 0 respectively (Gauch et al., 2003).

Table 1. The values of $\mathrm{a}, \mathrm{b}, \mathrm{c}, \mathrm{d}$ and e parameters used in equation (1) for the estimation of $\mathrm{NO}_{3}{ }^{-}, \mathrm{NH}_{4}{ }^{+}, \mathrm{PO}_{4}{ }^{3-}$ and $\mathrm{K}^{+}$, when 14-14-14 fertilizer was used for the preparation of the solutions with $100 \mathrm{ml}$ and $2000 \mathrm{ml}$ volume.

\begin{tabular}{|c|c|c|c|c|c|c|}
\hline \multirow{2}{*}{$\begin{array}{c}\text { Nutrient } \\
\mathrm{C}_{\mathrm{X}}\end{array}$} & \multicolumn{5}{|c|}{ Parameters } & \multirow[b]{2}{*}{$\mathrm{R}^{2}$} \\
\hline & $\mathrm{a}$ & $\mathrm{b}$ & $\mathrm{c}$ & $\mathrm{d}$ & $\mathrm{e}$ & \\
\hline $\mathrm{C}_{\mathrm{NH} 4}{ }^{+}$ & 1796.76 & -183.53 & 0.018 & 3.71 & 0.31 & 0.95 \\
\hline $\mathrm{C}_{\mathrm{PO} 4}{ }^{3-}$ & 1226.44 & -131.76 & 0.009 & 6.46 & 0.32 & 0.95 \\
\hline $\mathrm{C}_{\mathrm{NO} 3}{ }^{-}$ & $-49,850.5$ & 8532.15 & 0.76 & -53.63 & 3.96 & 0.77 \\
\hline $\mathrm{C}_{\mathrm{K}}^{+}$ & $18,555.2$ & -3023.65 & -0.035 & 5.46 & -2.31 & 0.77 \\
\hline
\end{tabular}

\section{Conclusions}

The evolution of $\mathrm{pH}$ seems to be affected strongly from the concentration of the fertilizers in the solution as well as from their volume. In all these solutions parameters such as $\mathrm{pH}, \mathrm{EC}$, the volume of the solution, as well as the time from their preparation affected significantly the rate of nutrients release and the evolution of nutrients concentration. Despite that research indicates that temperature is the most important factor that influence the diffusion and therefore CRFs nutrients release, this work revealed that the above mentioned parameters could affect the concentration of nutrients in the solution released from CRFs.

Based on the above mentioned parameters an empirical mathematical model was developed and calibrated in order to predict the concentration of $\mathrm{NO}_{3}{ }^{-}, \mathrm{NH}_{4}{ }^{+}, \mathrm{PO}_{4}{ }^{3-}$ and $\mathrm{K}^{+}$in aqueous solutions prepared with the use of CRFs. The model output compares favorably with data for the prediction of the concentration of these nutrients. Consequently, this model can be used for the preparation of nutrient solutions with specific characteristics by using CRF fertilizers.

\section{Acknowledgements}

We are thankful $\tau$ o Haifa South East Europe Ltd Company for granting the necessary quantities of Multicote fertilizers to carry out the experiment. 


\section{References}

Carreres, R., Sendra, J., Ballesteros, R., Valiente, E. F., Quesada, A., Carrasco, D., Legane's, F., and de La Cuadra, J. G., 2003. Assessment of slow release fertilizers and nitrification inhibitors in flooded rice. Biology and Fertility of Soils. 39 (2), 80-87. https://doi.org/10.1007/s00374-003-0684-4.

Du, C., Zhou, J., Shaviv, A. Wang, H., 2004. Mathematical Model for Potassium Release from Polymer-coated Fertiliser. Biosystems Engineering. 88 (3), 395-400. https://doi.org/10.1016/j.biosystemseng.2004.03.004.

Du, C., Zhou, J. \& Shaviv, A., 2006. Release Characteristics of Nutrients from Polymer-coated Compound Controlled Release Fertilizers. Journal of Polymers and the Environment. 14 (3), 223-230. https://doi.org/10.1007/s10924-006-0025-4.

Fisher Paul \& Argo, William \& Biernbaum, John, 2014. Validation of a fertilizer potential acidity model to predict the effects of water-soluble fertilizer on substrate $\mathrm{pH}$. HortiScience. 49 (8), 1061-1066.

Fujita T., \& Shoji S., 1999. Kinds and properties of Meister fertilizers. In: Meister controlled release fertilizer - Properties and Utilization. Shoji, S. (ed). Konno Printing Company Ltd. Sendai, Japan. 13-34p.

Gauch Jr, H.G. \& Hwang, J.T.G. \& Fick, Gary, 2003. Model evaluation by comparison of model-based prediction and measured values. Agronomy Journal. 95, 1442-1446. doi:10.2134/agronj2003.144.

Kinoshita, T., 2012. Effects of Different Application Methods of Controlled-release Fertilizers on Capillary Wick Culture of Tomato. Hortscience. 47, 1529-1535.

Liang R, Liu M., Wu L., 2007. Controlled release NPK compound fertilizer with the function of water retention. Reactive and Functional https://doi.org/10.1016/j.reactfunctpolym.2006.12.007.

Mayer Henrique., 2010. Nutrient Release Patterns Of Controlled Release Fertilizers Used In the Ornamental Horticulture Industry Of South Florida. MS Thesis. University Of Florida. USA

Naik, M. Rajeswar, Kumar, B. Kranthi and Manasa, K., 2017. Polymer coated fertilizers as advance technique in nutrient management. Asian J. Soil Sci.. 12 (1), 228-232. doi: 10.15740/HAS/AJSS/12.1/228-232

Singh, U., Cassman, K. G., Ladha, J. K., and Bronson, K. F., 1995. Innovative nitrogen management strategies for lowland rice systems. In Fragile Lives in Fragile Ecosystems. International Rice Research Institute, Manila, Philippines. 229-254.

Shaviv, A. 2001. Advances in controlled-release fertilizers, Advances in Agronomy, Academic Press. 71, 149. https://doi.org/10.1016/S0065-2113(01)71011-5.

Shaviv, A., 2005. Controlled release fertilizers. In IFA International Workshop on Enhanced-Efficiency Fertilizers, Frankfurt, Germany, 28-30 June 2005.

Shoji, S. \& Gandeza A.T., 1992. Controlled release fertilizers with polyolefin resin coating: Development, Properties and Utilization. Konno Printing Co., Sendai, Japan.

Tian, C., Zhou, X., Liu, Q, Peng, J., Wang, W., Zhang, Z., Yang, Y., Song, H., Guan, C., 2016. Effects of a controlled-release fertilizer on yield, nutrient uptake, and fertilizer usage efficiency in early ripening rapeseed (Brassica napus L. Journal of Zhejiang University-SCIENCE B (Biomedicine \& Biotechnology). 17 (10), 775 786. https://doi.org/10.1631/jzus.B1500216.

Trenkel, M.E., 2010. Slow- and Controlled-Release and Stabilized Fertilizers: An Option for Enhancing Nutrient Use Efficiency in Agriculture. International Fertilizer Industry Association (IFA) Paris, France.

Van Iersel, M.W., 1999. Fertilizer concentration affects on growth and nutrient composition of subirrigated pansies. HortScience. 34 (4), 660-663.

Wang, S., Alva, A., Li, Y., Zhang, M., 2011. A Rapid Technique for Prediction of Nutrient Release from Polymer Coated Controlled Release Fertilizers. Open Journal of Soil Science. 1, 40-44. doi: 10.4236/ojss.2011.12005

Yan Xiang, Jin Ji-yun, He Ping, Liang Ming-Zao, 2008. Recent Advances on the Technologies to Increase Fertilizer Use Efficiency. Agricultural Sciences in China. 7 (4), 469-479. https://doi.org/10.1016/S16712927(08)60091-7. 


\title{
Determination of $\mathrm{Ca}^{2+}$ Absorption Rate of Young Rose Plants Grown in Hydroponic System
}

\author{
Christos Lykas*, Apostolos Zantzos, Maria Zografou, \\ Department of Agriculture Crop Production and Rural Environment, School of Agricultural Sciences, \\ University of Thessaly, Volos, Magnesia 38446, Greece \\ * Corresponding author: Email: chlikas@uth.gr
}

\begin{abstract}
During this work, experiments were conducted to determine $\mathrm{Ca}^{2+}$ absorption in relation to transpiration rate of young rose plants growing in closed hydroponic system. For these reason 96 rose plants (Rosa hybrida L. cv. Iceberg) were installed in a closed hydroponic system following the Deep Flow Technique. After a period of twenty days from the planting, the $\mathrm{pH}$ of the nutrient solution, the $\mathrm{Ca}^{2+}$ concentration in the different plant organs (roots, shoots and leaves) and in the nutrient solutions, as well as plants transpiration, were measured every $24 \mathrm{~h}$ for a period of eighteen days. The results of these experiments reviled a correlation between the transpiration rate and leaves $\mathrm{Ca}^{2+}$ content (\% dry mass) as well as $\mathrm{Ca}^{2+}$ increment in the entire plant. The equations describing the above mentioned correlations were included in an empirical model to predict the concentration of $\mathrm{Ca}^{2+}$ in the recycled nutrient solution of the soilless culture $\left(\mathrm{R}^{2}=0.97\right)$. This method can be used to assess the adequacy of calcium in a nutrient solution used for the irrigation of a rose crop established in closed hydroponic system.
\end{abstract}

\section{Introduction}

Calcium plays a significant intra- and extracellular role at all stages of plants development, affecting many processes during their growth (Ferguson and Drøbak, 1988). Normal plants cellular function is highly depended on calcium action, since it regulates intracellular events as a second messenger. Calcium deficiency may alter cell metabolism causing in rose plants visible symptoms like leaves yellowing and loss of cell turgor resulting in wilting (Bush, 1995). In addition it is well documented that adequate calcium concentration in the nutrient solution can reduce the sensitivity of cut rose flowers to diseases (Volpin and Elad, 1991), improve the vase life of rose flower and increase their post-harvest quality (Michalzuk et al., 1989; Starkey and Pedersen, 1997; Torre et al., 1999; Torre et al., 2001; Mortensen et al., 2001).

Calcium absorption by plants is characterized as inactive and their translocation from the root to the shoots, leaves and flowers occurs through the xylem sap with the transpiration stream (Clarkson, 1984; Jeschke and Pate, 1991; Marschner, 1995). Therefore $\mathrm{Ca}^{2+}$ concentrations is generally higher in mature leave that transpire more water than in organs with low transpiration like young leaves, shoots and flowers. For this reason calcium deficiency is most likely in organs with low transpiration rates. However in rose plants $\mathrm{Ca}^{2+}$ concentration along the flower stem, does not follow the general distribution pattern than in other plants, probably because $\mathrm{Ca}^{2+}$ may be translocated through the phloem from mature to younger leaves or because young leaves have higher transpiration rate than the older (Baas et al., 2003) . According to De Kreij et al. (1992) and Oertli, (1966) a sufficient $\mathrm{Ca}^{2+}$ concentration in plants leaves for an adequate growth of roses is at least $10 \mathrm{mg} \mathrm{Ca} \mathrm{g}^{-1}$ of dry matter.

Despite that the effect of greenhouse climate parameters on rose plants transpiration, have been studied (Baille et al., 1994; Dayan et al., 2001; Kim and Lieth, 2003), only limited information concerning air humidity and transpiration on $\mathrm{Ca}^{2+}$ uptake and their translocation in the aerial part of rose plants is available.

The objective of the present study was to investigate the effect of transpiration on $\mathrm{Ca}^{2+}$ absorption by young rose plants grown in hydroponic system. The results of these work can be used for the estimation of $\mathrm{Ca}^{2+}$ concentration in the nutrient solution of closed hydroponic systems.

\section{Materials and Methods}

The experiment was carried out during the winter and spring of 2017 in a $200 \mathrm{~m}^{2}$ glasshouse located at the University of Thessaly in Velestino on the coastal area of Eastern Greece. Rose plants ( $\times$ Rosa hybrida 'Iceberg') were placed with a density of 2 plants per $\mathrm{m}^{-2}$ in, $3 \mathrm{~L}$ plastic containers filled with nutrient solution and grown according to Deep Flow Technique (DFT). The initial mineral composition of a nutrient solution in ppm was as follows: $\mathrm{NO}_{3}^{-} 762, \mathrm{NH}_{4}^{+} 18, \mathrm{PO}_{4}^{3-} 97, \mathrm{SO}_{4}{ }^{2-} 72, \mathrm{~K}^{+} 250, \mathrm{Ca}^{2+} 160, \mathrm{Mg}^{2+} 36, \mathrm{Fe}^{3+} 1.3, \mathrm{~B} 0.28, \mathrm{Cu}^{2+}$ 0.6, Mo 0.027 , $\mathrm{Mn}^{2+} 0.5$ and $\mathrm{Zn}^{2+} 0.23$. Iron was added using Fe EDDHA (6\% in iron). The $\mathrm{pH}$ and EC of the nutrient solution were adjusted daily by the addition of $\mathrm{HNO}_{3}$ and water, in order to remain close to the optimum levels of 5.5 and $1.7 \mathrm{mS} \mathrm{cm}^{1}$, respectively. The nutrient solution used for the irrigation of the plants was replaced with a new one every 10 days. Plants were pruned in order to develop one main flower shoot.

Measurements were performed during April 2017 and started 90 days after planting. The concentration of $\mathrm{Ca}^{2+}$ $\left(\mathrm{C}_{\mathrm{Ca}}\right)$ in the nutrient solution $\left(\mathrm{mg} \mathrm{L}^{-1}\right)$ and in different plant organs $\left(\mathrm{mg} \mathrm{Kg}^{-1}\right)$ as well as the fresh $(\mathrm{FM})$ and dry mass (DM) of leaves, shoots and roots were measured every day, by destructive sampling of 6 plants, for a period 
of 18 days (day 0 was defined the day that the nutrient solution was replaced). Greenhouse air temperature $\left(\mathrm{T}^{\circ} \mathrm{C}\right)$ and relative humidity ( $\mathrm{RH} \%$ ) were measured by means of a ventilated psychrometer (wet and dry bulb) located at the upper level of the flower stems. Solar radiation incident above the rose crop ( $\mathrm{W} \mathrm{m}^{-2}$ ) was measured by means of a solar pyranometer (model CM-6, Kipp and Zonen, Delft, The Netherlands). The climate data were measured every 30 seconds and 10 minute average values were recorded, using a data logger system (model DL3000, Delta-T Devices, Cambridge, UK). Plants transpiration (L/plant) was estimated by measuring the difference of nutrient solution volume used for plants irrigation.

Data were statistically analyzed by analysis of variance (ANOVA) using Statgraphics Centurion XVI. Duncan's multiple range test was used at a significance level of 0.05 .

\section{Results and Discussion}

The evolution of air temperature $\left({ }^{\circ} \mathrm{C}\right)$ and solar radiation $\left(\mathrm{W} \mathrm{m}^{-2}\right)$ inside the greenhouse, as well as the indoor air humidity (\%) and the plants daily transpiration (L/plant) during April 2017, are presented in Figure 1 and Figure 2 respectively. The transpiration, as it was expected, followed the indoor humidity alteration. The average amount of water transpired daily per plant, was estimated at $0.06( \pm 0.018) \mathrm{L}$. According to the results that Baas et al. (2003) presented in their work, the water that was daily transpired from rose plants flowering shoots, ranged from 0.014 to $0.0096 \mathrm{~L}$ during high and low VPD conditions respectively. The above-mentioned values are comparable, considering that during the experiments conducted in this work, the average FM of each plant shoot was 6.5 times lower than those measured in Baas et al. (2003) work. The evolution of $\mathrm{Ca}^{2+}, \mathrm{K}^{+}$and $\mathrm{Mg}^{2+}$ concentration in the nutrient solution used for plants irrigation during the experiment period is presented in Figure 3. The average value of $\mathrm{Ca}^{2+}$ concentration in the nutrient solution during this period was $187.5 \mathrm{ppm}( \pm 55.2)$ whereas the optimum concentration was $160 \mathrm{ppm}$. During the same period $\mathrm{K}^{+}$and $\mathrm{Mg}^{2+}$ concentrations were 101.2 $( \pm 22.7) \mathrm{ppm}$ and $36.3( \pm 2.8) \mathrm{ppm}$ respectively, whereas their optimum concentrations were $250 \mathrm{ppm}$ and $35 \mathrm{ppm}$ respectively. According to these results there was sufficient $\mathrm{Ca}^{2+}$ in the nutrient solution to prevent shortages in plants, whereas the concentrations of $\mathrm{K}^{+}$and $\mathrm{Mg}^{2+}$ were close to the optimum ensuring that no antagonistic relationship among cations will be occurred.

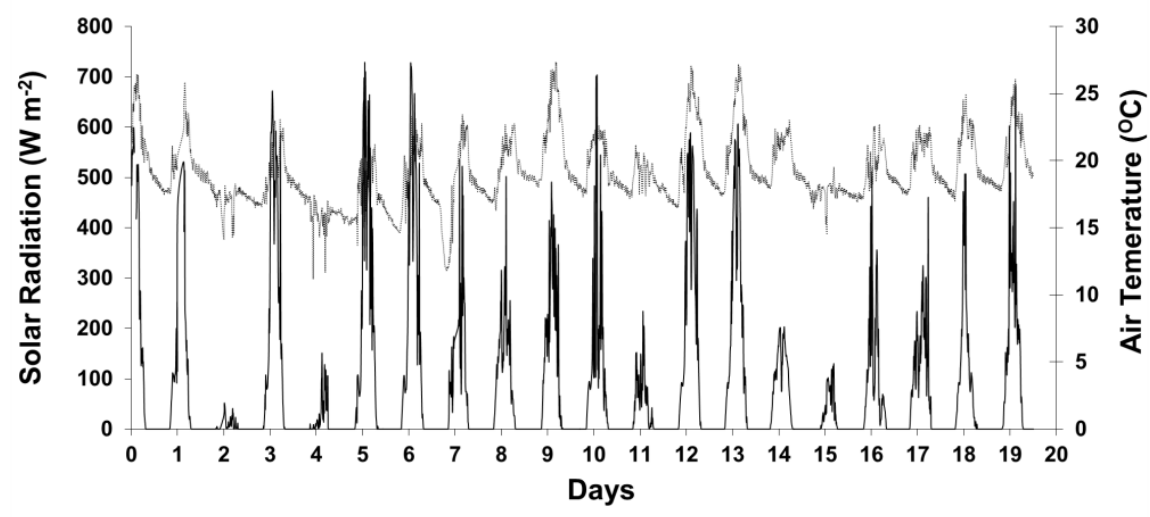

Figure 1. Evolution of air temperature (-) and solar radiation (-) inside the greenhouse during April 2017.

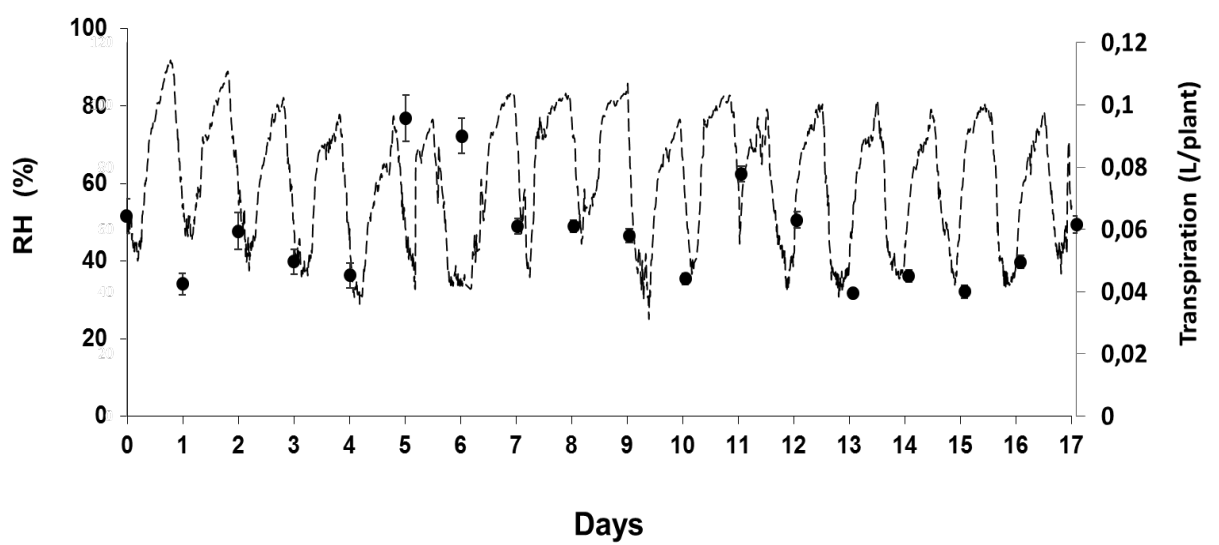

Figure 2. Evolution of air humidity (---) inside the greenhouse as well as the average transpiration $(\bullet)$ of plants during April 2017. 


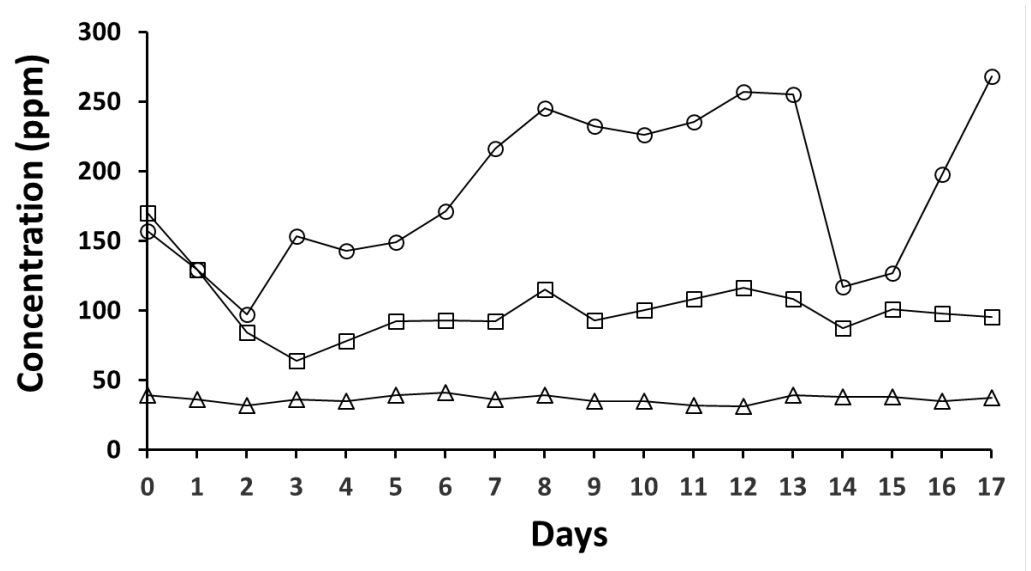

Figure 3. Evolution of $\mathrm{Ca}^{2+}(\circ), \mathrm{K}^{+}(\square)$ and $\mathrm{Mg}^{2+}(\Delta)$ concentration in the nutrient solution during the experiment period.

The results concerning the DM evolution of leaves, shoots and roots (Figure 4) reviled that no significant increment of DM of these organs occurred during the short period of eighteen days that the measurements were performed. This may be attributed to the limited growth of the plants, since the flowering bud was formed on the apex of the shoots. The reduced growth rate of the plants as well as the short period that the measurement conducted, helped the estimation of $\mathrm{Ca}^{2+}$ absorption by plants since leaf and shoots concentrations $\mathrm{Ca}^{2+}$ decreased with increasing age.

The average content $(\% \mathrm{DM})$ of leaves, shoots and roots in $\mathrm{Ca}^{2+}$ was $1.34( \pm 0.14), 0.92( \pm 0.25)$ and 1.26 $( \pm 0.36)$ respectively. Levels of $\mathrm{Ca}^{2+}$ lower than $1 \%$ of $\mathrm{DM}$ in five leaf-let leaves, which are present on the top of the plants, considered deficient for fully plants grown (De Kreij et al., 1992; Ganmore-Neumann and Davidov, 1993). However it may be deduced that Ca deficiency occurs as the $\mathrm{Ca}^{2+}$ content of the upper leaves falls below 0.8 to $1.0 \%$ of DM, in agreement with the recommended critical values in the literature (De Kreij et al., 1992; Baas et al., 2003). In contrast shoots of rose plants have a lower content in Ca ranged according to Tamini et al. (1999) from $0.72 \%$ of DM (upper part) to $0.33 \%$ of DM (basal part). The results presented in this work are in accordance with the above mentioned literature.

Statistical analysis of the results reviled a correlation $\left(\mathrm{R}^{2}=0.73\right)$ between leaves $\mathrm{Ca}^{2+}$ content $(\% \mathrm{DM})$ and the average daily transpiration during the eighteen days period (Figure 5). In contrast, no significant correlation was reviled, as was expected, between plants transpiration and $\mathrm{Ca}^{2+}$ content in shoots and roots, since no transpiration occur in these organs. For this reason the alteration of $\mathrm{Ca}^{2+}$ concentration occurred in the nutrient solution, did not affect $\mathrm{Ca}^{2+}$ content in shoots and roots. Ganmore-Neumann et al. (1992) and Ganmore-Neumann and Davidov (1993) refer that Ca concentration may increase in rose plants leaves, by increasing external Ca levels, but this is not necessarily occurred in low-transpiring organs. It has been suggested that non-transpiring organs like roots, shoots and flowers increase $\mathrm{Ca}^{2+}$ in their tissues by sap delivered to them in response to root pressure during the night (Hanson, 1984). The accumulation of $\mathrm{Ca}^{2+}$ in leaves through the transpiration stream as well as in both shoots and roots, which is attributed to the sap reaches by root pressure, contributed to the increase of $\mathrm{Ca}^{2+}$ content of the whole plant. Since this was generally due to the water absorption, the increased $\mathrm{dC}\left(\mathrm{mg} \mathrm{g}^{-1} \mathrm{DM}\right)$ of Ca $\mathrm{Ca}^{2+}$ (from the initial value at day 0 ), in the whole plant was well correlated $\left(\mathrm{R}^{2}=0.85\right)$ with transpiration as shown in Figure 6. 


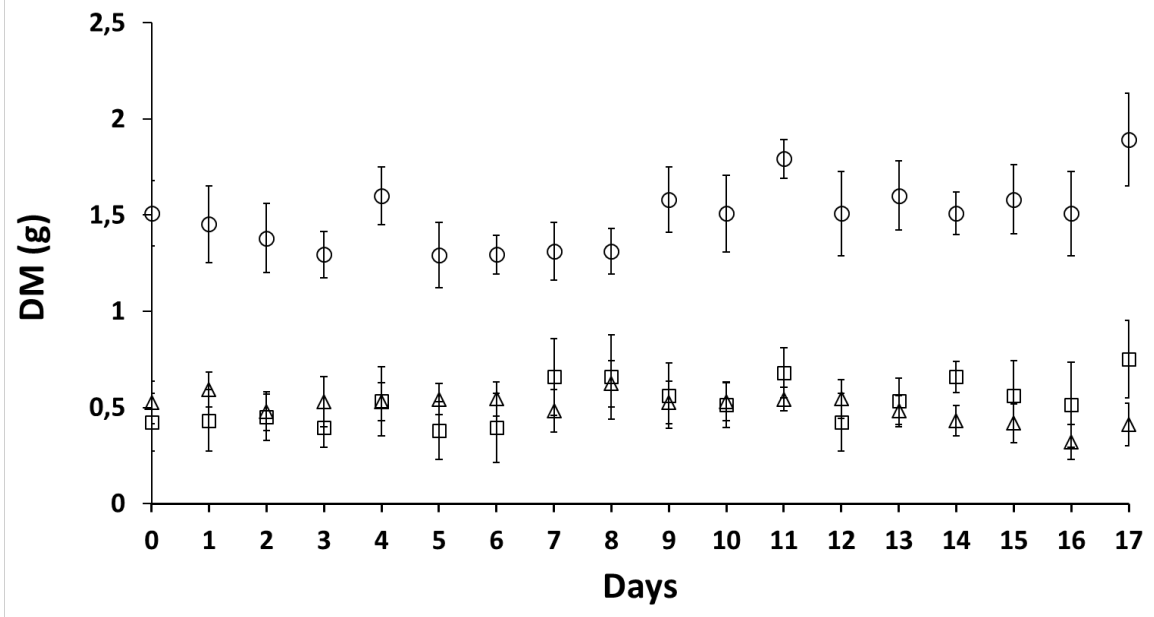

Figure 4. Evolution of DM of plants leaves (०), shoots ( $\square)$ and roots $(\Delta)$.

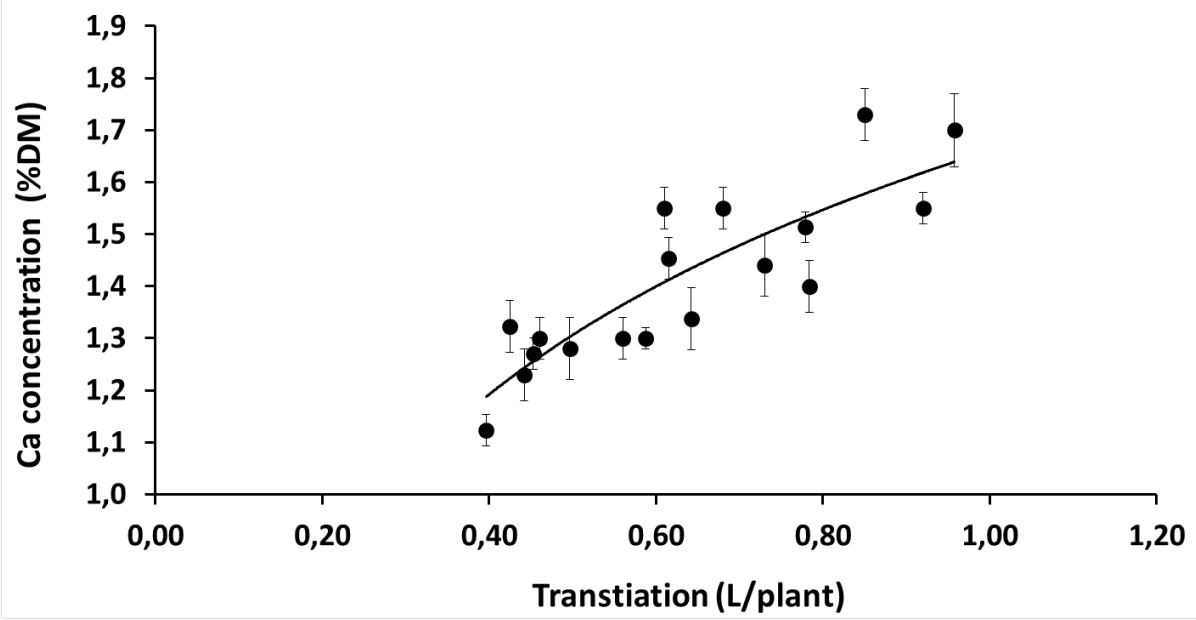

Figure 5. Evolution of leaves $\mathrm{Ca}^{2+}$ content $(\% \mathrm{DM})$ in relation to the average daily transpiration (L/plant) during the eighteen days period when the measurements conducted.

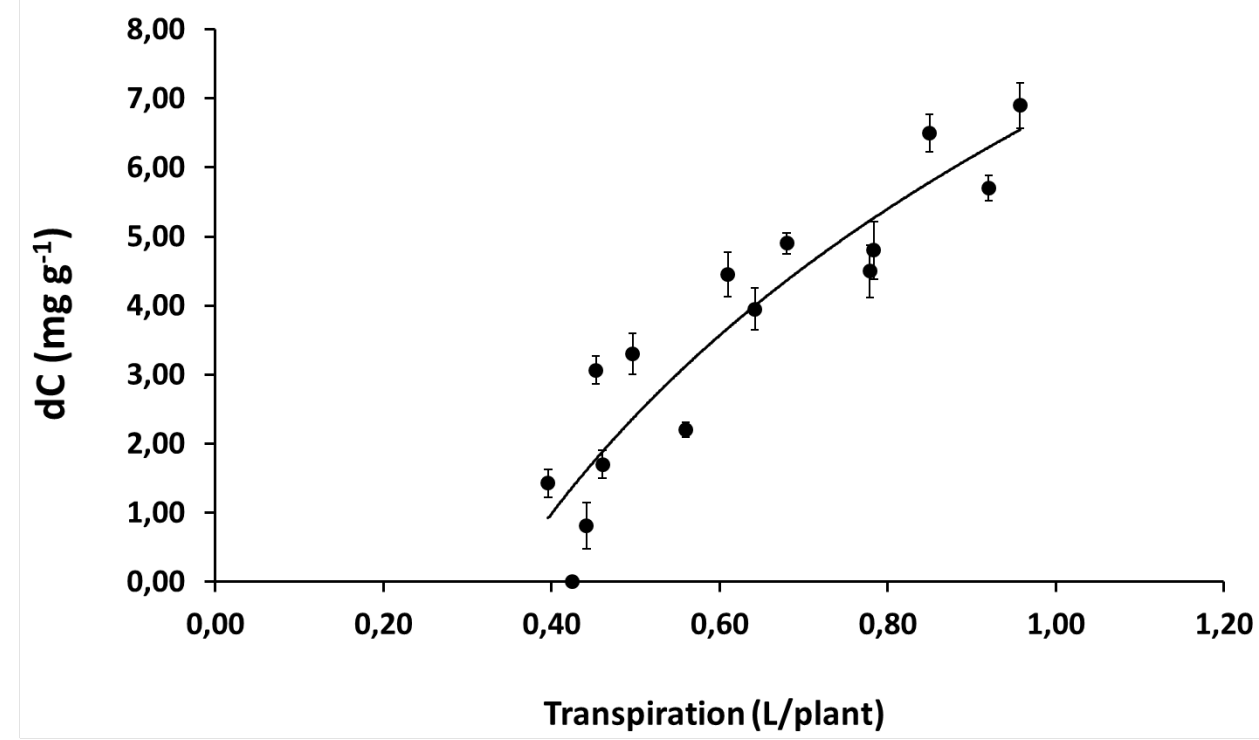

Figure 6. Difference of $\mathrm{Ca}^{2+}$ content in plant ( $\left.\mathrm{mg} \mathrm{g}^{-1} \mathrm{DM}\right)$ from their initial value at day 0 , in relation to the average daily transpiration (L/plant). 
However the content of the whole plant in $\mathrm{Ca}^{2+}(\% \mathrm{DM})$ has a negative correlation with the plants daily transpiration, as shown in Figure 7. This can be attributed mainly to the climate conditions. The low VPD may result to the production of a higher number of flowering stems and increase the transpiration (and consequently $\mathrm{Ca}^{2+}$ absorption), but not necessary in the same proportion (Baas et al., 2003). In this way may cause inbalance between the growth rate and the rate that plants absorb nutrient elements from the nutrient solution. This imbalance is greater in young plants with no adequate root system. In addition, vegetative growth of plants and the proportion of old and new leaves plays an important role in the plant's calcium content. Johansson (1978), Tamini and Matsuyama (1999) and Baas et al. (2003) referred that the concentrations of $\mathrm{Ca}^{2+}$ in young leaves at the top of the stem is nearly twice as great as those in the mature leaves at the bottom. This phenomenon of $\mathrm{Ca}^{2+}$ distribution along the stem is not necessary related to the transpiration, although it was well demonstrated from Baas et al. (2003) that the transpiration of the leaves at the bottom of the flowering shoots was almost the half from that to the top of the shoots.

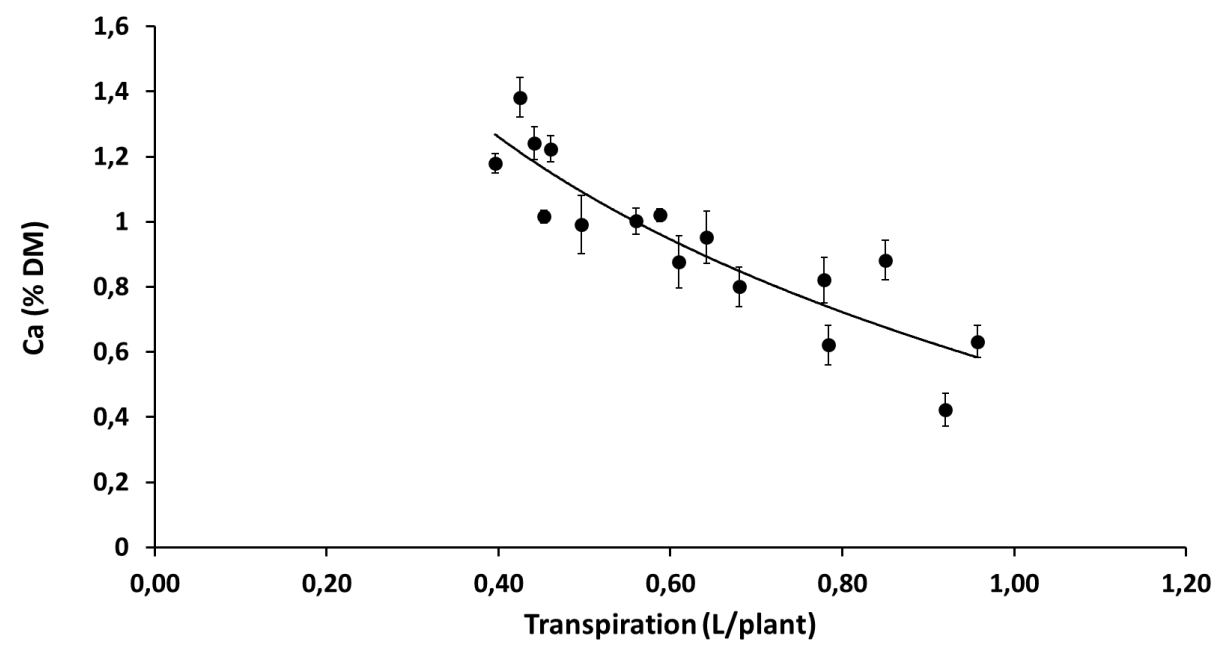

Figure 7. Alteration of the whole plant content in $\mathrm{Ca}^{2+}(\% \mathrm{DM})$ in relation to the plants daily transpiration (L/plant).

\section{Conclusions}

The data presented in this work indicates the correlation between the average daily transpiration and $\mathrm{Ca}^{2+}$ concentration in the leaves of young rose plants, grown in hydroponic system during a short growth period. The increase of $\mathrm{Ca}^{2+}$ content of the whole plant during a short growth period is also related to the transpiration.

Despite that there is no significant correlation between plants transpiration and $\mathrm{Ca}^{2+}$ content in shoots and roots, the content of these organs can be finally calculated from the difference between the $\mathrm{Ca}^{2+}$ content of the whole plant and the content of leaves.

However, the content of the whole plant in $\mathrm{Ca}^{2+}(\% \mathrm{DM})$ has a negative correlation with the plants daily transpiration in young rose plants probably due to the inability of root system to absorb adequate amount of $\mathrm{Ca}^{2+}$. In this way climate conditions that increase the transpiration rate may cause shortages in $\mathrm{Ca}^{2+}$ in young rose plants. Therefore, this can be prevented by increasing $\mathrm{Ca}^{2+}$ concentration in the nutrient solution.

Since most of the data in the literature on mineral content of roses are related to leaf analyses for diagnostic purposes the results of this work may help significantly the management of the nutrient solution in a rose soilless cultivation.

\section{References}

Baas, R., S. Van Oers, A. Silber, N. Bernstein, M. Ioffe, M. Keinan, A. Bar-Tal, 2003. Calcium distribution in cut roses as related to transpiration. Journal of Horticultural Science \& Biotechnology. 78 (1), 1-9.

Baille, M., A. Baille, D. Delmon, 1994. Microclimate and transpiration of greenhouse rose crops. Agricultural and Forest Meteorology. 71, 83-97.

Bush, D.S., 1995. Calcium regulation in plant cells and its role in signaling. Annu Rev Plant Physiol Plant Mol Biol 46, 95-122.

Clarkson, D.T., 1984. Calcium transport between tissues and its distribution in the plant. Plant Cell Environ. 7, 449-456.

Dayan, E., M. Fuchs, Z. Plaut, E. Presnov, A. Grava, E. Matan, A. Solphoy, U. Mugira, N. Pines, 2001. Transpiration of roses in greenhouses. Acta Hortic. 554, 239-250. 
De Kreij C., C. Sonneveld, M.G. Warmenhoven , N.A. Straver, 1992. Guide values for nutrient element contents of vegetables and flowers under glass, PBG brochure No. 15

Ferguson, I.B., B.K. Drøbak, 1988. Calcium and the regulation of plant growth and senescence. HortScience 23, 262-266.

Ganmore-Neumann, R., A. Feigin, S. Davidov, J. Ifrach, A. Hazan, 1992. Optimization of rose nutrition: Calcium, AnnualReport 301-0086-91, A.R.O. (Hebrew).

Ganmore-Neumann, R., S. Davidov, 1993. Uptake and distribution of calcium in rose plantlets as affected by calcium and boron concentration in culture solution. Plant and Soil, 155/156, 151-154.

Hanson J.B., 1984. The function of calcium in plant nutrition, in: Tinker P.B., Lauchli A. (Eds.), Advances in plant nutrition, Vol. 1, Praeger, New York, , pp. 149-208.

Jeschke, W. D., J.S. Pate, 1991. Cation and chloride partitioning through xylem and phloem within the whole plant of Ricinus communis L. under conditions of salt stress. Journal of Experimental Botany. 42, 1105-1116.

Kim, S., J.H. Lieth, 2003. A Coupled Model of Photosynthesis, Stomatal Conductance and Transpiration for a Rose Leaf (Rosa hybrida L.). Annals of Botany. 91(7), 771-781. http://doi.org/10.1093/aob/mcg080.

Marschner, H., 1995. Mineral nutrition of higher plants. Academic Press Ltd., London, UK.

Michalczuk, B., D.M. Goszczynska, R.M. Rudnicki, A.H. Halevy, 1989. Calcium promotes longevity and bud opening in cut rose flowers. Israel Journal of Botany. 38, 209-215.

Mortensen, L. M., C.O. Ottosen, H.R. Gislerod, 2001. Effects of air humidity and K: Ca ratio on growth, morphology, flowering and keeping quality of pot Roses. Scientia Horticulturae. 90, 131-41. doi:10.1016/S03044238(00)00251-X.

Oertli J.J., 1966. Calcium deficiency in rose plants. Flor. Rev. 3.

Starkey, K. R., A.R. Pedersen, 1997. Increased levels of calcium in the nutrient solution improve the post harvest life of potted roses. Journal of the American Society for Horitcultural Science. 122, 863-868.

Tamimi, Y. N., D. T. Matsuyama, K. D. Ison-Takata, R.T. Nakano, 1999. Distribution of Nutrients in Cutflower Roses and the Quantities of Biomass and Nutrients Removed During Harvest. HORTSCIENCE. $34(2), 251-253$.

Torre, S., A. Borochov, A.H. Halevy, 1999. Calcium regulation of senescence in rose petals. Physiologia Plantarum. 107, 214-219.

Torre, S., T. Fjeld, H.R. Gislerod, 2001. Effects of air humidity and K/Ca ratio in the nutrient supply on growth and post harvest characteristics of cut roses. Scientia Horticulturae 90, 291-304.

Volpin, H. Y. Elad, 1991. Influence of Ca nutrition on susceptibility of rose flowers to Botrytis blight. Phytopathology. 81, 1390-1394. 


\title{
Influence of Forced Aeration Rates on Maturation of Composting Poultry Manure AndaWood Shavings in Closed Reactor System \\ Waqas Qasima , Byeong Eun Moon ${ }^{\mathrm{a}}$, Frank Gyan Okyere ${ }^{\mathrm{a}}$, Fawad Khan ${ }^{\mathrm{a}}$ Jayanta Kumar Basak $^{\mathrm{a}}$, Yong Jin Lee ${ }^{a}$, Hyeon Tae Kim ${ }^{\text {a* }}$ \\ ${ }^{a}$ Dept. of Bio-systems Engineering, Gyeongsang National University (Institute of Agriculture \& Life Science), Jinju 52828, Korea. \\ *Corresponding Author-Tel: +82-55-772-1896; Fax: +82-55-772-1899 \\ *E-mail address: bioani@hanmail.net
}

\begin{abstract}
The poultry industry is growing in South Korea, but there are problems associated with the management of poultry manure, and composting is one solution that could be valuable for crops and forage if managed properly. For achieving successful compost, aeration rate must be optimized to provide favorable condition for composting process. We investigate physicochemical properties i.e. Temperature, $\mathrm{pH}, \mathrm{EC}$, organic matter and seed germination index, in the composting of poultry manure with wood shavings under different aeration rates in closed reactor system. Three cylindrical reactors with total volume $60 \mathrm{~L}$ were used in this experiment. The aeration rates in 3 reactors were $0.25,0.50$ and $0.75 \mathrm{~L} / \mathrm{min} \mathrm{kg}$ OM. All parameters were monitored over 30 days of composting. The highest temperature in each treatment was $56.9,55.8$ and $48.1 \mathrm{C}$ for $0.25,0.50$ and $0.75 \mathrm{~L} / \mathrm{min}$ $\mathrm{kg} \mathrm{OM}$, respectively, appearing on the $3^{\text {rd }}$ day at center of compost. Aeration rate of $0.25 \mathrm{~L} / \mathrm{min} \mathrm{kg} \mathrm{OM}$ corresponded to a higher and longer thermophilic phase than other two reactors which had aeration rate of 0.50 and $0.75 \mathrm{~L} / \mathrm{min} \mathrm{kg} \mathrm{OM}$. The maximum organic matter degradation accrued with $0.25 \mathrm{~L} / \mathrm{min} \mathrm{kg} \mathrm{OM}$ of aeration rate which provided adequate oxygen concentration for microorganisms. With $0.75 \mathrm{~L} / \mathrm{min} \mathrm{kg} \mathrm{OM}$ of aeration rate lowest GI were recorded at day 30, suggesting severe phytotoxicity in the substrate. In all 3 reactors, aeration rate of $0.25 \mathrm{~L} / \mathrm{min} \mathrm{kg}$ OM provided most favorable condition for composting poultry manure with wood shavings in closed reactor system.
\end{abstract}

Key words: Poultry manure, Wood shavings, Aeration rate, Temperature, $\mathrm{pH}$, Seed germination

\section{Introduction}

Application of composting to agriculture land to be considered most economical, practical and environmentally beneficial option (Brochier et al. 2012). Proper composting effectively reduces odor emission, nitrogen loss and the organic waste become relatively stable products for use as fertilizer. Composting manure has been recognized as an effective way to solve problem of solid waste (Hoitink, 2000) as this process improves the handling characteristics of the manure by reducing its volume and weight, kills pathogens, and stabilizes the nutrients and organic matter in it (Michel jr et al., 1996, Tiquia et al., 1996, 2000).

Poultry manure is rich in nutrients it is not only beneficial for crop production but it also improve the structural stability of soil. For balance composting C:N ratio must be taken into account properly. Wood shavings are rich with $\mathrm{C}$ content which can be use with poultry manure for better compost. It is most common and easily available from woodworking companies during the wood processing in South Korea.

Composting requires oxygen for aerobic activity, too much aeration can lead to excessive cooling, and preventing the thermophilic conditions required for optimum rates of decomposition however, too little aeration can lead to anaerobic condition. An optimum aeration rate is required which provides sufficient oxygen for aerobic decomposition. The aeration flow rate affects microbial activity, degradation rate and temperature variation in the composting process (Kuter et al., 1985). To achieve a successful composting the factors such as moisture content, $\mathrm{C} / \mathrm{N}$ ratio, aeration rate, temperature, $\mathrm{pH}$, composting materials and method should be appropriately controlled.

Different aeration rates were recommended in some previous studies such as $0.251 \mathrm{~min}^{-1} \mathrm{~kg}^{-1} \mathrm{OM}$ in the composting dairy manure with rice straw (Li et al., 2008), $0.621 \mathrm{~min}^{-1} \mathrm{~kg}^{-1} \mathrm{VS}$ in the composting of vegetables fruit wastes (Arslan et al., 2011), $0.51 \mathrm{~min}^{-1} \mathrm{~kg}^{-1} \mathrm{OM}$ in composting chicken manure and sawdust (Gao et al., 2010). All the aeration rate were different in different materials and condition in composting process. The objective of this work was to investigate physicochemical properties i.e. Temperature, $\mathrm{pH}, \mathrm{EC}$, organic matter and seed germination index, in the composting of poultry manure with wood shavings under different aeration rates in closed reactor system.

\section{Materials and Methods}

Experimental setup

Three cylindrical reactors with total volume $60 \mathrm{~L}$ were used in this experiment as shown in Fig 1 . The reactors were insulated with a layer of polyurethane foam $(2.54 \mathrm{~cm}$ of thickness). A vertical rotating axis with blades were used before taking samples to ensure the complete mixing of the composting mass. The reactors were equipped 
with a valve for dropping the leachate. The airflow rates in 3 reactors were $0.25,0.50$ and $0.75 \mathrm{~L} / \mathrm{min} \mathrm{kg} \mathrm{OM}$. The air was provided at the bottom by air compressor (Air land S45-40-4.5, Korea), the time of the airflow was

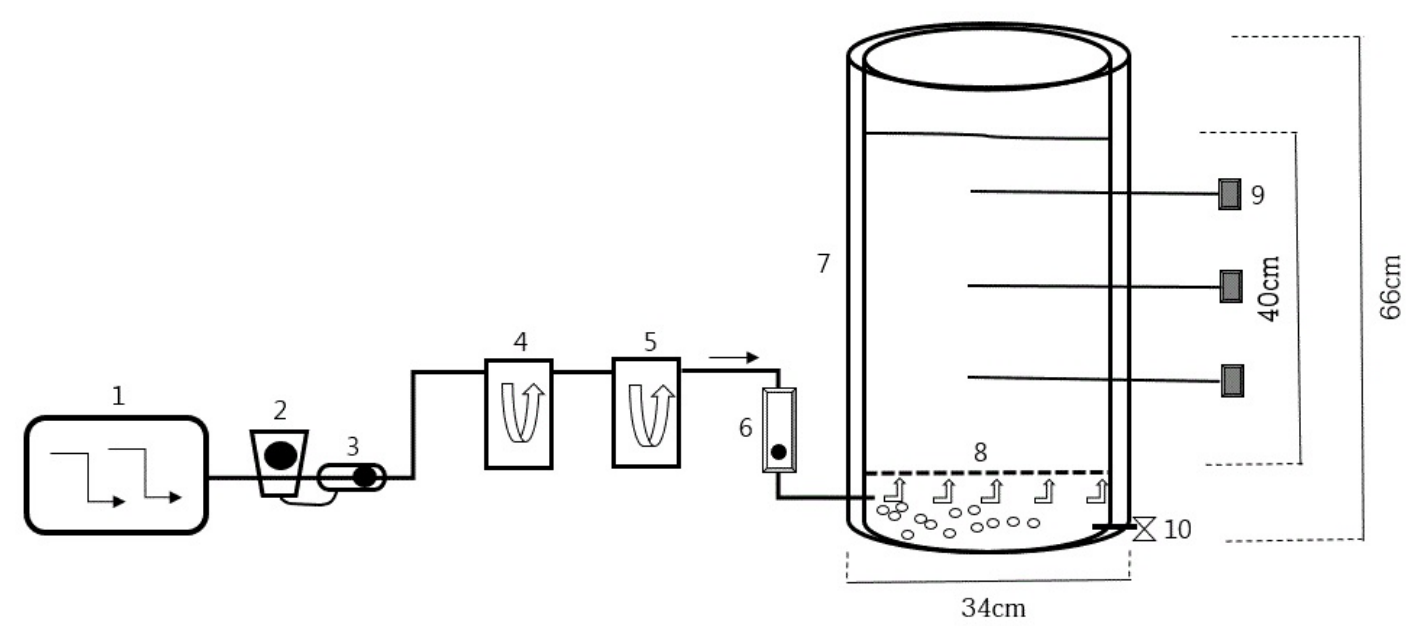

Figure 1. Schematic diagram of reactor with experimental apparatus. (1. Air compressor, 2. Solenoid valve, 3. Timer, 4. Gas washing bottle (Solution of $\mathrm{NaOH}$ ), 5. Gas washing bottle (Distilled water), 6. Air flow meter, 7. Reactor with insulation, 8. Perforated plate, 9. Temperature probe, 10. Leachate outlet.

intermittent with $45 \mathrm{~min}$ of aeration followed by $15 \mathrm{~min}$ without aeration which controlled by timer and solenoid valve (Auto sigma HPW2120), Airflow meter (Valve Acrylic Flowmeter, Cole-Parmer, USA) and pressure regulators were used to control airflow rates. The compost temperature were monitored daily (10 am, 2 pm and 6 $\mathrm{pm}$ ) in 3 different locations of substrate using digital thermometers (Testo $905 \mathrm{~T} 1$ ). The ambient temperature was also recorded daily.

Before inlet to reactors, the air had been introduced into solution of sodium hydroxide in order to remove traces of carbon dioxide. Then air was moisturized through a gas washing bottle i.e. $500 \mathrm{ml}$ distilled water bottle prior to entering the reactor to maintain humidity at reactor.

\section{Composting mixture}

Poultry manure with addition wood shavings were used as experimental materials. Poultry manure was collected from poultry farms while wood shavings were purchased from wood chipping mill. The manure and wood shaving were mixed by concrete mixer to achieve better homogenization of material and initial parameters were determined. The initial moisture content and $\mathrm{C} / \mathrm{N}$ were adjusted to about $65 \%$ and 25 , respectively for filling the 3 reactors (Table1).

\begin{tabular}{ll}
\multicolumn{2}{c}{ Table 1.} \\
\hline Parameters & Values \\
\hline Moisture content, \%ww & $65 \pm 0.7$ \\
Organic matter, \%dw & $85.1 \pm 0.8$ \\
$\mathrm{pH}$ & $7.8 \pm 0.4$ \\
Electrical Conductivity, $\mathrm{mS} / \mathrm{cm}$ & $2.12 \pm 0.6$ \\
$\mathrm{C} / \mathrm{N}$ & $25 \pm 0.2$ \\
\hline
\end{tabular}

\section{Analytical Methods}

The composting material was mixed by vertical rotating axis with blades before taking samples. About $50 \mathrm{~g}$ were taken from 3 different places in the substrate (top, middle, and bottom). The moisture content of the experimental material was analyzed by drying oven method at $105^{\circ} \mathrm{C}$ for $24 \mathrm{~h}$ (APHA, 1995). The carbon and nitrogen content were determined by automated LECO True Mac CNS elemental analyzer. Electrical conductivity and $\mathrm{pH}$ were measured by mechanically shaking the samples with distilled water at a solid to water ratio of $1: 10(\mathrm{w} / \mathrm{v})$ for $1 \mathrm{~h}$. The $\mathrm{pH}$ and electrical conductivity measurements were carried out using digital meters. The organic matter content (volatile solids) of the material was measured by burning oven at $550^{\circ} \mathrm{C}$ for $6 \mathrm{~h}$ (APHA, 1995)

Phytotoxicity assay test

Seed germination and root length test was carried out on water extracts by mechanically shaking the fresh samples for $1 \mathrm{~h}$ at solid: double distilled water ratio of $1: 10$ (w/v, dry weight basis). About $5.0 \mathrm{~mL}$ of each extract 
was pipetted into a sterilized plastic petri dish lined with a Whatman \#2 filter. 12 radish seeds were evenly placed on the filter paper and incubated at $25^{\circ} \mathrm{C}$ in the dark for $72 \mathrm{~h}$. Triplicates were analyzed for samples from each composting mixture. Treatments were evaluated by counting the number of germinated seeds, and measuring the length of roots. The responses were calculated by GI that was determined according to the following Eq. (2) (Zucconi et al., 1981):

$$
G I \%=\frac{\text { Seed germination\% (treatment) *root length (treatment) }}{\text { Seed germination } \% \text { (control)*root length (control) }}
$$

\section{Statistical analysis}

Statistical analysis was performed using the Prism 5 Graph Pad program. Data were presented as means (SD) and analyzed by one-way ANOVA followed by Bonferroni's Multiple Comparison Test with a significance level of $P<0.05$

\section{Results \& discussion}

Temperature profile

The temperature profile can be used to classify the stages of composting. Figure 2 shows the temperature profile of compost with three different aeration rates, the ambient temperature was maintained around $25 \mathrm{C}$. The temperature of compost increased as a result of organic matter degradation by microorganisms in the compost mass. The highest temperature in each treatment was $56.9,55.8$ and $48.1 \mathrm{C}$ for $0.25,0.50$ and $0.75 \mathrm{~L} / \mathrm{min} \mathrm{kg} \mathrm{OM}$, respectively, appearing on the $3^{\text {rd }}$ day at center of compost. The highest temperature last longer time with aeration rate of $0.25 \mathrm{~L} / \mathrm{min} \mathrm{kg} \mathrm{OM}$. The temperature with $0.25 \mathrm{~L} / \mathrm{min} \mathrm{kg} \mathrm{OM}$ was maintained above $55 \mathrm{C}$ for 3 days which should be sufficient to kill pathogens. Also steniford reported that $55 \mathrm{C}$ for 2 days is enough for maximizing sanitation. Throughout experiment with $0.75 \mathrm{~L} / \mathrm{min} \mathrm{kg}$ OM aeration rate the temperature did not reached to $50 \mathrm{C}$ due to poor microbial activity and degradation extent of organic matter was inadequate. The high aeration rate adversely affected temperature rise. Lau also reported that high aeration rates resulted in a cooling effect detrimental to composting process. There were statistically significant difference in temperature regime in all 3 treatments during thermophilic phase $(\mathrm{P}<0.05)$.

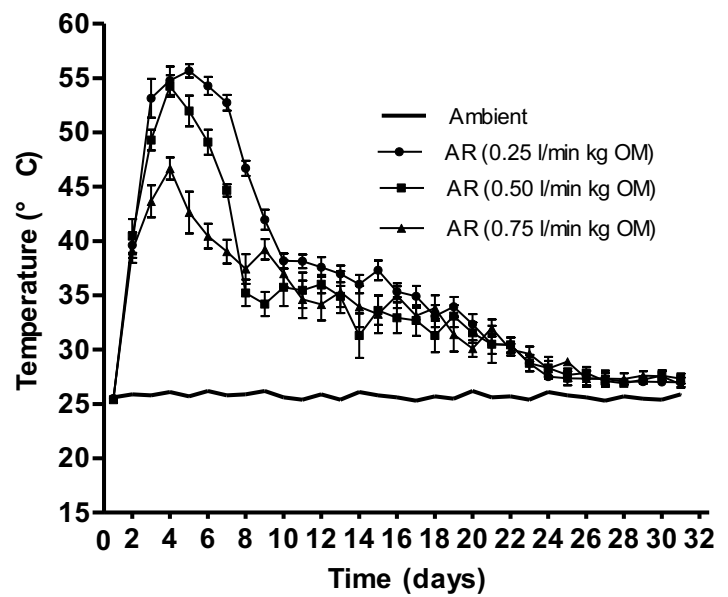

Figure 2. Temperature profiles of compost with three aeration rates.

Evolution of $\mathrm{pH}$ and electrical conductivity

The evaluation of $\mathrm{pH}$ of is presented in Fig 3. During thermophilic stage the $\mathrm{pH}$ increased significantly due to decomposition of organic matter. With $0.25 \mathrm{~L} / \mathrm{min} \mathrm{kg} \mathrm{OM}$ of aeration rate the $\mathrm{pH}$ of compost increased from initial 7.8 to 8.6 on day 4 and then gradually decreases to 6.6 on day 30 . With $0.50 \mathrm{~L} / \mathrm{min} \mathrm{kg} \mathrm{OM}$ of aeration rate the $\mathrm{pH}$ of compost increased from initial 7.8 to 8.5 on day 6 and then gradually decreased to 6.8 on day 30 . With $0.75 \mathrm{~L} / \mathrm{min} \mathrm{kg} \mathrm{OM}$ of aeration rate the $\mathrm{pH}$ of compost increased from initial 7.8 to 8.3 on day 4 and then gradually decreased to 6.9 on day 30 . This increase and decrease is also directly related to ammonia content in the compost, probably due to the decomposition of proteins to ammonium, which also reported by other researchers (Liao et al., 1995 Raviv, 2005). 
EC level indicates the salinity of compost and higher salt levels can be harmful to germination seeds and plants when compost use for growing plants. Excessive salinity cause phytotoxicity depending on plant species (Aslam et al., 2008). The initial value was $2.2 \mathrm{mS} / \mathrm{cm}$ in the reactors. EC values first increased to $4.8,4.14$ and 3.8 with aeration rates of $0.25,0.50$ and $0.75 \mathrm{~L} / \mathrm{min} \mathrm{kg} \mathrm{OM}$, respectively, followed by a gradual decrease till the end of composting process. The initial EC increase may be due to minerals salts such as phosphates and ammonium ion through decomposition of compost substances (Gomez-brandon et al., 2008).
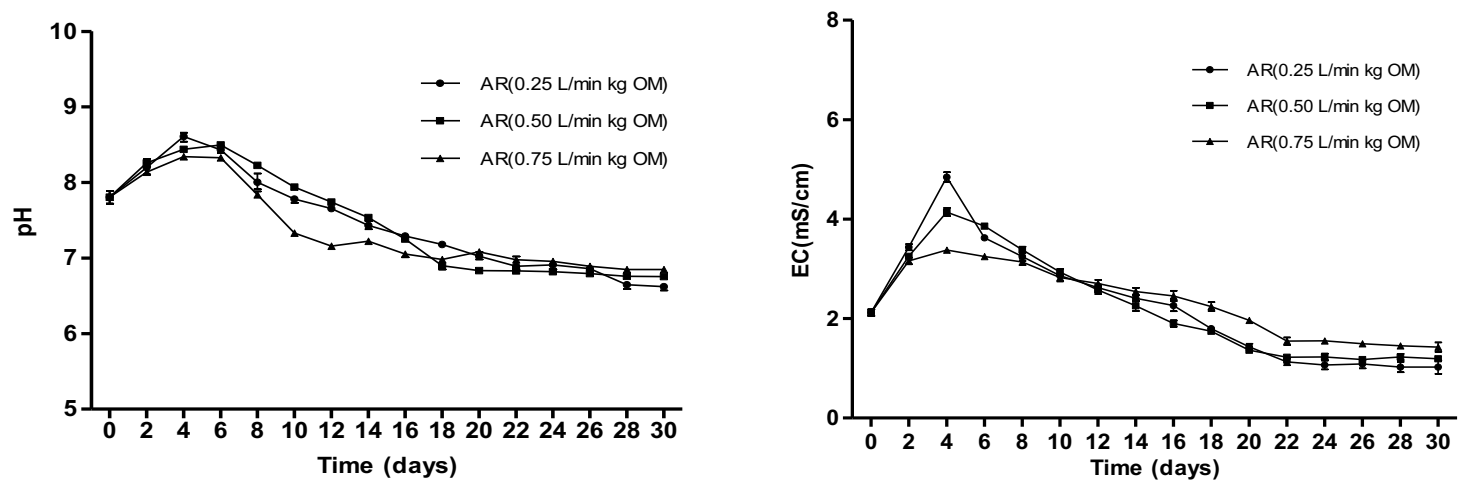

Figure 3. Changes in $\mathrm{pH}$ and $\mathrm{EC}$ in the reactors during composting process.

Table 2. Organic matter degradation (\%) in the reactors during composting process.

\begin{tabular}{cccc}
\hline & $\begin{array}{c}\text { OM } \% \\
\text { Dars } 0.25 \mathrm{~L} / \mathrm{min} \mathrm{kg} \mathrm{OM})\end{array}$ & $\begin{array}{c}\text { OM\% } \\
\mathrm{AR}(0.50 \mathrm{~L} / \mathrm{min} \mathrm{kg} \text { OM})\end{array}$ & $\begin{array}{c}\text { OM\% } \\
\mathrm{AR}(0.75 \mathrm{~L} / \mathrm{min} \mathrm{kg} \mathrm{OM})\end{array}$ \\
\hline 0 & $85.0+0.6$ & $85.0+0.6$ & $85.0+0.6$ \\
2 & $77.4 \pm 0.3$ & $79.5 \pm 0.2$ & $80.3 \pm 0.2$ \\
4 & $74.6 \pm 0.2$ & $76.7 \pm 0.2$ & $79.6 \pm 0.3$ \\
6 & $71.4 \pm 0.3$ & $75.4 \pm 0.2$ & $77.6 \pm 0.2$ \\
8 & $68.6 \pm 0.2$ & $72.1 \pm 0.2$ & $76.6 \pm 0.4$ \\
10 & $65.6 \pm 0.4$ & $70.6 \pm 0.2$ & $73.7 \pm 0.1$ \\
12 & $64.6 \pm 0.3$ & $69.4 \pm 0.2$ & $72.9 \pm 0.2$ \\
14 & $62.6 \pm 0.3$ & $67.5 \pm 0.3$ & $71.4 \pm 0.2$ \\
16 & $62.6 \pm 0.2$ & $65.7 \pm 0.3$ & $70.6 \pm 0.5$ \\
18 & $62.4 \pm 0.4$ & $64.6 \pm 0.3$ & $69.6 \pm 0.5$ \\
20 & $62.0 \pm 0.2$ & $64.6 \pm 0.1$ & $67.5 \pm 0.3$ \\
22 & $61.7 \pm 0.2$ & $64.2 \pm 0.2$ & $67.3 \pm 0.4$ \\
24 & $61.6 \pm 0.3$ & $63.9 \pm 0.2$ & $66.5 \pm 0.3$ \\
26 & $61.5 \pm 0.2$ & $64.3 \pm 0.2$ & $66.6 \pm 0.4$ \\
28 & $61.3 \pm 0.7$ & $64.4 \pm 0.3$ & $65.9 \pm 0.3$ \\
30 & $61.2 \pm 0.4$ & $64.1 \pm 0.2$ & $65.3 \pm 0.3$ \\
\hline
\end{tabular}

Evolution of organic matter

The rate of organic matter is an indicator of overall composting rate (Diaz and Savage, 2007). In this experiment the volume and mass of composting materials were significantly reduce. The evolution of organic matter during composting of chicken manure and mixture of sawdust and wood shavings with different aeration rate are shown in Table 2. The organic matter content decreased in all reactors indicating biodegradation of compost. OM $\%$ of compost decreased gradually from initial $85 \%$ in all reactors to $61 \%, 64 \%$ and $65 \%$ with aeration rate of $0.25 \mathrm{~L} / \mathrm{min} \mathrm{kg} \mathrm{OM}, 0.50 \mathrm{~L} / \mathrm{min} \mathrm{kg} \mathrm{OM}$ and $0.75 \mathrm{~L} / \mathrm{min} \mathrm{kg} O M$ on day 30 . The maximum organic matter degradation accrued in with $0.25 \mathrm{~L} / \mathrm{min} \mathrm{kg} \mathrm{OM}$ of aeration rate which provided adequate oxygen concentration for microorganisms. Too high aeration adversely affect $\mathrm{OM}$ degradation during composting.

Phytotoxicity assay

Germination index shows the maturity and phytotoxicity of compost (Guo et al, 2012). Figure 4 shows the changes in GI with aeration rate of $0.25,0.50$ and $0.75 \mathrm{~L} / \mathrm{min} \mathrm{kg} \mathrm{OM}$ during 30 days composting. The GIs in all reactors decreased in early phase, which shows relatively high salinity at the beginning. After 5 days the GIs of all reactors start to increases gradually due to decomposition of toxic materials, which shows the maturation of compost. The aeration rate had a significant influence on the GI $(\mathrm{P}<0.05)$. At end of compost the GIs were $84.5 \%$, $76.9 \%$ and $67.7 \%$ with aeration rate of $0.25,0.50$ and $0.75 \mathrm{~L} / \mathrm{min} \mathrm{kg}$ OM. A GI more than $80 \%$ indicates phytotoxic-free and mature compost (Guo et al, 2012). With $0.75 \mathrm{~L} / \mathrm{min} \mathrm{kg}$ OM of aeration rate lowest GI were recorded at day 30 , suggesting severe phytotoxicity in the substrate. 


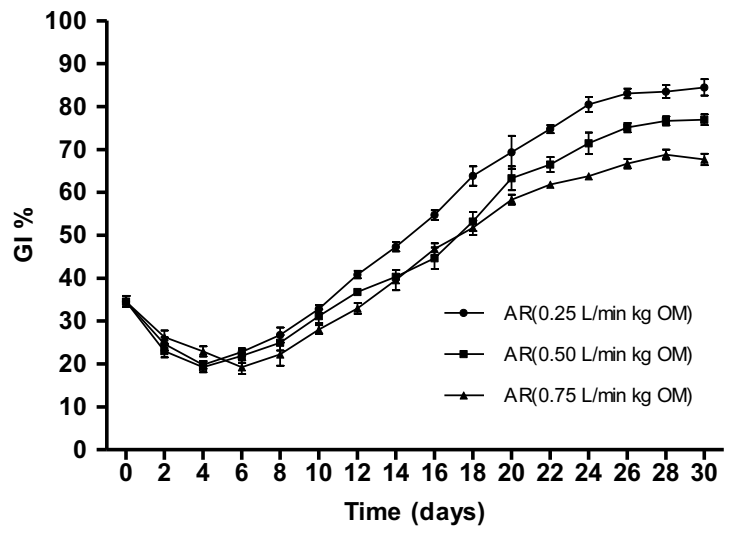

Figure 4. Germination index (\%) with different aeration rate during composting.

Relationship between $\mathrm{pH}$ and germination index

In previous studies $\mathrm{pH}$ values above 8 and below 3 have been reported to inhibit germination for most of the plants (Shoemaker, C. A., \& Carlson, W. H. 1990). pH shows significant negative correlation with germination index of radish seeds as shown in Fig 5. The $\mathrm{R}^{2}$ value were quite higher in all reactors i.e 0.93, 0.92 and 0.72 with aeration rate of $0.25,0.50$ and $0.75 \mathrm{~L} / \mathrm{min} \mathrm{kg}$ OM. At end of compost the GIs were $84.5 \%, 76.9 \%$ and $67.7 \%$ with $\mathrm{pH}$ values $6.64,6.77$ and 6.88 respectively. This study suggested that $\mathrm{pH}$ values 6 to 7 of compost provide better condition for high seed germination.
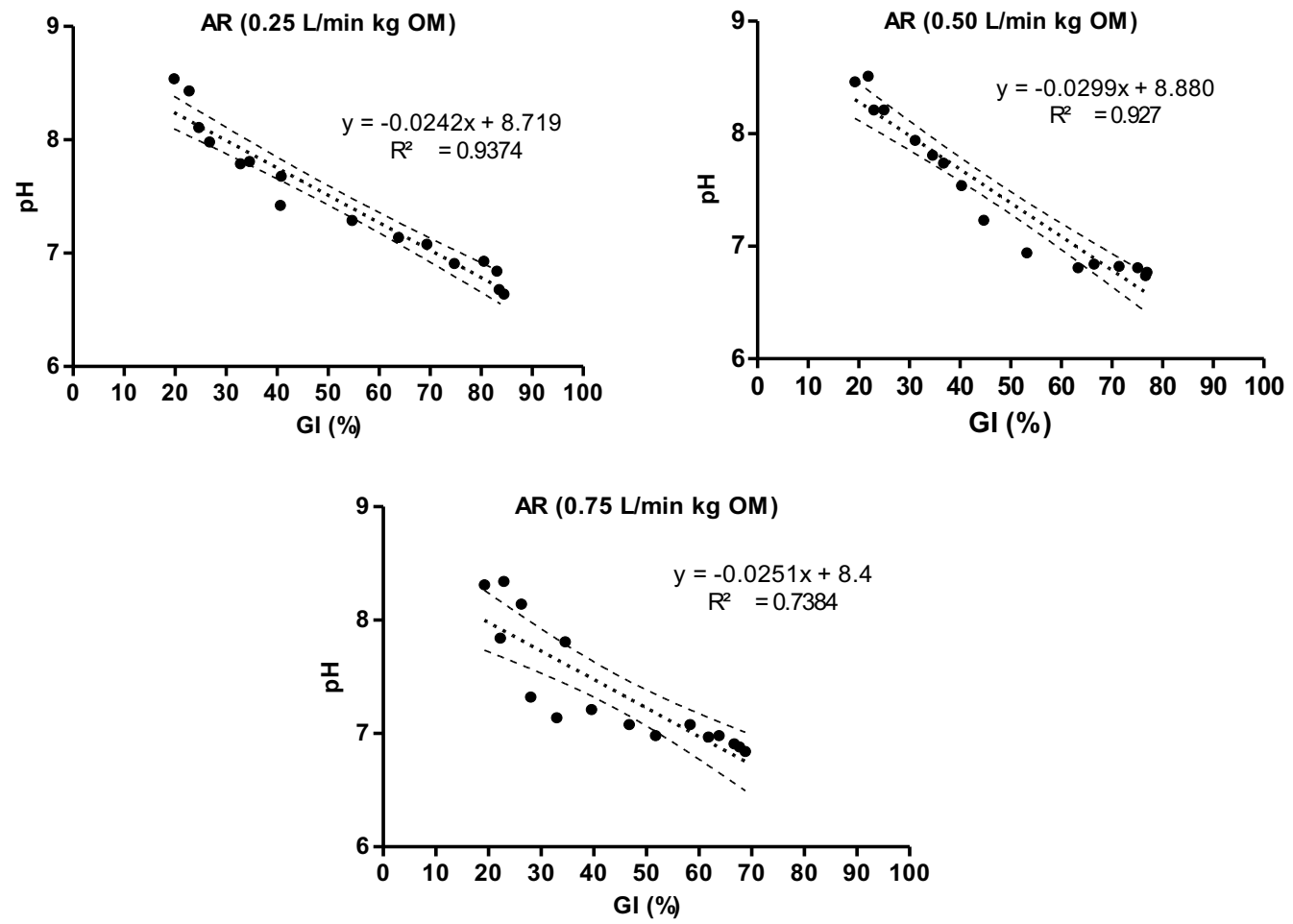

Figure 5. Correlation between $\mathrm{pH}$ and germination index (GI \%).

\section{Conclusions}

The physicochemical properties of composting poultry manure with wood shavings changes with different aeration rates. The highest temperature $56.9 \mathrm{C}$ were recorded at day 3 with aeration rate of $0.25 \mathrm{~L} / \mathrm{min} \mathrm{kg} \mathrm{OM}$, which also last longer time of high temperature than other two reactors with aeration rate of 0.50 and $0.75 \mathrm{~L} / \mathrm{min}$ $\mathrm{kg} \mathrm{OM}$. The maximum organic matter degradation accrued in with $0.25 \mathrm{~L} / \mathrm{min} \mathrm{kg} \mathrm{OM}$ of aeration rate which provided adequate oxygen concentration for microorganisms. With $0.75 \mathrm{~L} / \mathrm{min} \mathrm{kg}$ OM of aeration rate lowest GI were recorded at day 30 , suggesting severe phytotoxicity in the substrate. In all 3 reactors, aeration rate of 0.25 
$\mathrm{L} / \mathrm{min} \mathrm{kg}$ OM provided most favorable condition for composting poultry manure with wood shavings in closed reactor system.

\section{Acknowledgements}

This research was supported by Korea Institute of Planning and Evaluation for Technology (IPET) in food and agriculture, forestry and fisheries, through Research centre support program (Project No. 717001-7), Ministry of Agriculture, Food and rural affairs (MAFRA).

\section{References}

Arslan, E. Iş1l, Ayhan Ünlü, and Murat Topal 2011. Determination of the effect of aeration rate on composting of vegetable-fruit wastes. CLEAN-Soil, Air, Water 39.11: 1014-1021.

Aslam, D. N., Horwath, W., \& VanderGheynst, J. S. 2008. Comparison of several maturity indicators for estimating phytotoxicity in compost-amended soil. Waste Management,28(11), 2070-2076.

Brochier V, Gourland P, Kallassy M, Poitrenaud M, Houot S 2012. Occurrence of pathogens in soils and plants in a long-term field study regularly amended with different composts and manure. Agric Ecosyst Environ 160:91-98.

Diaz, L. F., \& Savage, G. M. 2007. Factors that affect the process. Waste Management Series, 8, 49-65.

F.C. Michel Jr., L.J. Forney, A.J. Huang, S. Drew, M. Czuprenski, J.D. Lindeneg, C.A. Reddy 1996. Effects of turning frequency, leaves to grass ratio and windrow vs pile configuration on composting of yard trimmings Compost Sci. Util., 4 , pp. 26-43

G.A. Kuter, H.A.J. Hoitink, L.A. Rossman 1985. Effects of aeration and temperature on composting of municipal sludge in a full-scale vessel system. J Water Pollut Contr Fed, 57 , pp. 309-315

Gao, Mengchun, et al. 2010. The effect of aeration rate on forced-aeration composting of chicken manure and sawdust." Bioresource Technology 101.6: 1899-1903.

Gómez-Brandón, M., Lazcano, C., \& Domínguez, J. (008. The evaluation of stability and maturity during the composting of cattle manure. Chemosphere, 70(3), 436-444.

Guo, R., Li, G., Jiang, T., Schuchardt, F., Chen, T., Zhao, Y., \& Shen, Y. 2012. Effect of aeration rate, C/N ratio and moisture content on the stability and maturity of compost. Bioresource Technology, 112, 171-178.

Hoitink, H. A. J. 2000. Trends in treatment and utilization of solid waste through composting in the United States. In Proceedings of the International Composting Symposium (ISC 1999) (Vol. 1, pp. 1-13). CBA Press Inc.

Lau, A. K., Lo, K. V., Liao, P. H., \& Yu, J. C. 1992. Aeration experiments for swine waste composting. Bioresource Technology, 41(2), 145-152.

Li, Xiujin, Ruihong Zhang, and Yunzhi Pang. "Characteristics of dairy manure composting with rice straw." Bioresource Technology 99.2 (2008): 359-367.

Liao, P. H., May, A. C., \& Chieng, S. T. 1995. Monitoring process efficiency of a full-scale invessel system for composting fisheries wastes. Bioresource Technology, 54(2), 159-163.

Lin, C. 2008. A negative-pressure aeration system for composting food wastes. Bioresource technology, 99(16), 7651-765.

Pagans, E., Barrena, R., Font, X., \& Sánchez, A. 2006. Ammonia emissions from the composting of different organic wastes. Dependency on process temperature. Chemosphere, 62(9), 1534-1542

Petric, I., Šestan, A., \& Šestan, I. 2009. Influence of wheat straw addition on composting of poultry manure. Process Safety and Environmental Protection, 87(3), 206-212.

Raviv, M. 2005. Production of high-quality composts for horticultural purposes: a minireview. HortTechnology, 15(1), 52-57.

S.M. Tiquia, N.F.Y. Tam, I.J. Hodgkiss 1996. Microbial activities during composting of spent pig-manure sawdust litter at different moisture contents Biores. Technol., 55 , pp. 201-206

S.M. Tiquia, T.L. Richard, M.S. Honeyman 2000. Effects of windrow turning and seasonal temperatures on composting of hog manure from hoop structures Environ Technol., 21 pp. 1037-1046

Shoemaker, C. A., \& Carlson, W. H. 1990. pH affects seed germination of eight bedding plant species. HortScience, 25(7), 762-764.

Stentiford, E. I. 1996. Composting control: principles and practice. In The science of composting (pp. 49-59). Springer Netherlands.

Zucconi, F., Pera, A., Forte, M., \& De Bertoldi, M. A. R. C. 1981. Evaluating toxicity of immature compost. BioCycle (USA). 